\title{
Targeted Investigation at the Former CCC/USDA Grain Storage Facility in York, Nebraska, in 2015-2016
}

\section{Environmental Science Division}




\begin{abstract}
About Argonne National Laboratory
Argonne is a U.S. Department of Energy laboratory managed by UChicago Argonne, LLC under contract DE-AC02-06CH11357. The Laboratory's main facility is outside Chicago, at 9700 South Cass Avenue, Argonne, Illinois 60439. For information about Argonne and its pioneering science and technology programs, see www.anl.gov.
\end{abstract}

\title{
DOCUMENT AVAILABILITY
}

Online Access: U.S. Department of Energy (DOE) reports produced after 1991 and a growing number of pre-1991 documents are available free via DOE's SciTech Connect (http://www.osti.gov/scitech/)

Reports not in digital format may be purchased by the public from the National Technical Information Service (NTIS):

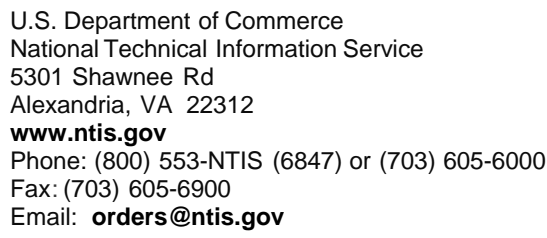

Reports not in digital format are available to DOE and DOE contractors from the Office of Scientific and Technical Information (OSTI):

U.S. Department of Energy

Office of Scientific and Technical Information

P.O. Box 62

Oak Ridge, TN 37831-0062

www.osti.gov

Phone: (865) 576-8401

Fax: (865) 576-5728

Email: reports@osti.gov

\section{Disclaimer}

This report was prepared as an account of work sponsored by an agency of the United States Government. Reference herein to any specific commercial product, process, or service by trade name, trademark, manufacturer, or otherwise, does not necessarily constitute or imply its endorsement, recommendation, or favoring by the United States Government or any agency thereof. The views and opinions of document authors expressed herein do not necessarily state or reflect those of the United States Government or any agency thereof, Argonne National Laboratory, or UChicago Argonne, LLC. 


\section{Targeted Investigation at the Former CCC/USDA Grain Storage Facility in York, Nebraska, in 2015-2016}

by

Applied Geosciences and Environmental Management Section Environmental Science Division, Argonne National Laboratory

February 2017 


\section{Contents}

Notation

1 Introduction

$1-1$

1.1 Technical Objectives and Primary Tasks of the Targeted Investigation $1-3$

1.2 Implementation of the Targeted Investigation Field Program

2 Investigative Methods

2.1 Overview of the Targeted Investigation Field Program

2.1.1 Field Activities in the First Phase of the Targeted Investigation, July 2015 - April 2016

2.1.2 Field Activities in the Second Phase of the Targeted Investigation, May-July 2016.

2.1.3 CCC/USDA Quarterly Sampling of Private and PWS Wells

2.2 Methods to Determine the Characteristics of the Hydrogeologic Sequence in the

Vicinity of the Former CCC/USDA Facility

2.2.1 Characterization of the Lithologic Sequence

2.2.2 Installation of Monitoring Wells and Establishment of a Groundwater Level Monitoring Network

2.3 Methods to Investigate for Possible Carbon Tetrachloride Contamination in Soils.

2.4 Methods to Investigate for Possible Carbon Tetrachloride Contamination in Groundwater

2.4.1 Groundwater Sampling with the Cone Penetrometer $2-14$

2.4.2 Groundwater Sampling from Monitoring Wells $2-15$

2.4.3 Groundwater Sampling from Private and Municipal Supply Wells $2-15$

2.4.4 Analysis of Groundwater Samples

2.5 Methods for the Investigation of Possible Carbon Tetrachloride Vapor Intrusion to Indoor Air

3.1 Lithologic and Physical Property Data for Soils...................................................... 3- 3-1

3.2 Analytical Data for Soil Samples...................................................................... 3-2

3.3 Installation of Permanent Monitoring Wells......................................................... 3-3

3.4 Analytical Data for Water Samples .................................................................... 3-4

3.4.1 Volatile Organic Compounds in Groundwater from the Unconfined Aquifer.

3.4.2 Volatile Organic Compounds in Groundwater from the Upper and Lower Confined Aquifers

3.4.3 Volatile Organic Compounds in Groundwater from Private Wells and PWS Wells

3.4.4 Volatile Organic Compounds in Groundwater from the York Municipal Distribution System 
3.7 Coordinates Survey Data ……....................................................................... 3-9

3.8 Results of Quality Control Activities....................................................................... 3-9

3.9 Waste Characterization, Handling, and Disposal .................................................... 3-11

4 Interpretation of Results................................................................................... 4-1

4.1 Hydrostratigraphy Near the Former CCC/USDA Facility....................................... 4-1

4.1.1 Site-Specific Characteristics of the Hydrostratigraphic Units ....................... 4-2

4.1.2 Preliminary Evaluation of Groundwater Levels ........................................ 4-8

4.2 Evaluation of the Carbon Tetrachloride Distribution in Soils ................................. 4-12

4.3 Evaluation of the Contaminant Distribution in Groundwater................................... 4-14

4.3.1 Contaminant Distribution in the Unconfined Aquifer .................................... 4-15

4.3.2 Contaminant Distribution in the Confined Aquifers..................................... 4-20

4.3.3 Status and Sampling of Private and Municipal Supply Wells ....................... 4-21

4.4 Evaluation of Possible Carbon Tetrachloride Vapor Intrusion to Indoor Air............ 4-28

4.4.1 Analysis Results for Indoor Air at the Hearthstone and Willowbrook Facilities ............................................................................................ 4-29

4.4.2 Analysis Results for Indoor Air at the CVA Corporate Office........................ 4-29

4.4.3 Analysis Results for Indoor Air at the Preslicka Residence ........................... 4-29

4.4.4 Analysis Results for Indoor Air and Sub-Slab Soil Vapor at the Fitness Worx Facility …………….................................................................... 4-30

4.4.5 Analysis Results for Indoor Air and Sub-Slab Soil Vapor at the Garretson/Troester Residence ...................................................................... 4-31

4.4.6 Qualitative Assessment of Potential Carbon Tetrachloride VI at Other Locations.....

5 Conclusions and Recommendations ……………….............................................. 5-1

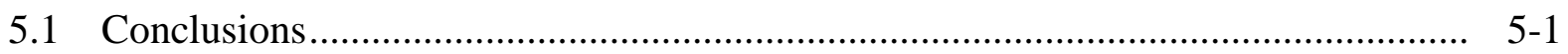

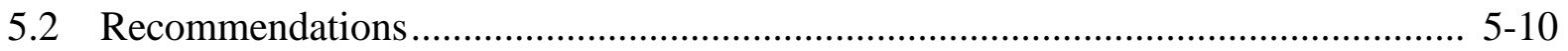

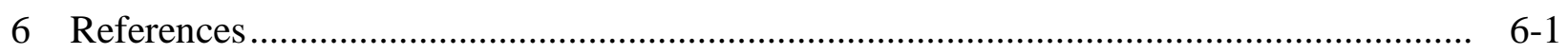

Appendix A: Chronological Summary of Sampling Activities ............................................. A-1

Appendix B: Sub-Slab Soil Vapor Standard Operating Procedures ....................................... B-1

Appendix C: ECPT Logs for Borings TI01-TI28 ……….............................................. C-1

Appendix D: Grain Size Analysis and Lithology Logs for Borings DL1-DL8 ……............... D-1

Appendix E: Analytical Data for Soil Samples ……….................................................... E-1

Appendix F: Monitoring Well Registration Forms................................................................ F-1

Appendix G: Analytical Data for Groundwater Samples ……………............................... G-1 
Supplement 1: Automatically Recorded Groundwater Levels on $\mathrm{CD}$

Supplement 2: Quality Control for Sample Collection, Handling, and Analysis .on $\mathrm{CD}$

Supplement 3: Waste Characterization Data .on $\mathrm{CD}$

\section{Figures}

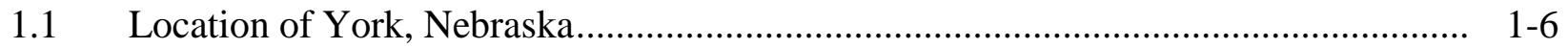

1.2 Locations of contaminated public water supply wells PWS 37-1, PWS 48-1, and PWS 81-1.

1.3 PCE/TCE Northeast Contamination Site, as identified by the EPA, associated with the former Kroy/Alcan facility to the south of the former CCC/USDA facility .......... $1-8$

2.1 Locations of direct-push borings used sample soil for VOCs analyses.....

2.2 Locations of direct-push borings in the unconfined aquifer used to obtain EPCT logs and collect groundwater samples for VOCs analyses

2.3 Locations of private wells investigated during the present study

2.4 Locations of conventionally drilled, continuously cored soil borings .....

2.5 Locations of monitoring wells installed by the CCC/USDA during the current or previous investigations at York, and used to measure groundwater levels and collect groundwater samples for VOCs analyses.

2.6 Locations sampled for indoor air, or sub-slab soil vapor and indoor air, to investigate carbon tetrachloride VI 
2.7 Locations of private wells and public supply wells sampled quarterly for VOCs by the CCC/USDA, at the request of the NDEQ

3.1 Maximum carbon tetrachloride concentrations in groundwater in the unconfined aquifer, determined from samples collected using direct-push techniques

3.2 Carbon tetrachloride concentrations in groundwater in the unconfined aquifer, determined from samples collected from permanent monitoring wells

3.3 Carbon tetrachloride concentrations in groundwater collected from private wells sampled during the targeted investigation, and private and PWS wells sampled quarterly

4.1 Locations of hydrogeologic cross sections D-D', E-E', F-F', and G-G' .....

4.2 Northwest-to-southeast hydrogeologic cross section D-D', showing groundwater levels determined manually on November 3, 2016

4.3 Southwest-to-northeast hydrogeologic cross section E-E', showing groundwater levels determined manually on November 3, 2016

4.4 Southwest-to-northeast hydrogeologic cross section F-F', showing groundwater levels determined manually on November 3, 2016

4.5 Southwest-to-northeast hydrogeologic cross section G-G', showing groundwater levels determined manually on November 3, 2016

4.6 Hydrographs for wells in the upper and lower confined aquifers

4.7 Hydrographs for wells in the unconfined aquifer $4-50$

4.8 Locations of monitoring wells equipped with automatic groundwater level recorders, and the approximate locations of nearby irrigation wells.....

4.9 Hydrographs for monitoring wells DL2-C1 in the unconfined aquifer, and DL2-CU in the upper confined aquifer.

4.10 Hydrographs for monitoring wells DL7-C in the unconfined aquifer, DL7-CU in the upper confined aquifer, and DL7-CL in the lower confined aquifer

4.11 Estimated potentiometric surface for the unconfined aquifer under inferred static conditions, based on groundwater levels measured manually on November 3, 2016. 
4.12 Estimated potentiometric surface for the unconfined aquifer under inferred irrigation pumping conditions, based on groundwater levels measured automatically at 16:00 on August 11, 2016

4.13 Vertical and lateral distribution of carbon tetrachloride concentrations in groundwater along northwest-to-southeast hydrogeologic cross section D-D'

4.14 Vertical and lateral distribution of carbon tetrachloride concentrations in groundwater along southwest-to-northeast hydrogeologic cross section E-E'

4.15 Vertical and lateral distribution of carbon tetrachloride concentrations in groundwater along southwest-to-northeast hydrogeologic cross section F-F'.....

4.16 Vertical and lateral distribution of carbon tetrachloride concentrations in groundwater along southwest-to-northeast hydrogeologic cross section G-G'

4.17 Carbon tetrachloride concentrations in the " $\mathrm{A}$ " sand zone of the unconfined aquifer

4.18 Carbon tetrachloride concentrations in the "B" sand zone of the unconfined aquifer

4.19 Carbon tetrachloride concentrations in the "C-C1" sand zone of the unconfined aquifer

4.20 Carbon tetrachloride concentrations in the "C2" sand zone of the unconfined aquifer

4.21 Estimated potentiometric surface for the unconfined aquifer based on groundwater levels measured manually on November 3, 2016 and the interpreted distribution of carbon tetrachloride contamination in the unconfined aquifer

4.22 Locations of critical groundwater sampling points constraining the interpreted southwestern margin of the carbon tetrachloride distribution in the unconfined aquifer associated with the former CCC/USDA facility....

4.23 Carbon tetrachloride concentrations in groundwater collected from private and municipal wells, and the interpreted distribution of carbon tetrachloride in the unconfined aquifer. Outlying wells omitted for clarity

4.24 Locations sampled for VOCs analyses of indoor air or sub-slab soil vapor and indoor air, York PWS wells, and the interpreted distribution of carbon tetrachloride in the unconfined aquifer 
5.1 Additional locations tentatively proposed for vertical-profile soil sampling for VOCs analyses in the vadose zone

5.2 Locations of monitoring wells proposed for single-well hydraulic testing in the unconfined aquifer

J.1 Approximate locations of the wells and investigative borings that provided lithologic information used to develop local hydrostratigraphic interpretations in the 1993-1995 investigations

J.2 Northwest-to-southeast geologic cross section A-A'

J.3 Southwest-to-northeast geologic cross section B-B'

J.4 Southwest-to-northeast geologic cross section $\mathrm{C}-\mathrm{C}^{\prime}$

J.5 Lateral distribution of carbon tetrachloride in groundwater samples collected in 1993

\section{Tables}

2.1 Summary of field sessions during the York targeted investigation .....

3.1 Summary of carbon tetrachloride and chloroform concentrations in soil samples, as determined by the purge-and-trap method at the AGEM laboratory....

3.2 Monitoring well construction data

3.3 Summary of analytical results for VOCs in groundwater samples collected during the targeted investigation

3.4 Summary of carbon tetrachloride results for groundwater samples collected in quarterly monitoring of selected private and PWS wells

3.5 Hand-measured groundwater levels.

3.6 Summary of results for carbon tetrachloride and chloroform in VI testing samples.... 3-20

4.1 Average grain size distributions for hydrostratigraphic units in the York investigation area

4.2 Construction and groundwater level data for selected irrigation wells. 
4.3a Comparison of carbon tetrachloride concentrations in soils at sampling location TI01-SB01 in 2015 and 1993.

4.3b Comparison of carbon tetrachloride concentrations in soils at sampling location TI02-CPT37 in 2015 and 1993

4.3c Comparison of carbon tetrachloride concentrations in soils at sampling location TI03-SB62 in 2015 and 1993

4.4 Hydrostratigraphic summary of analytical results for VOCs in groundwater samples collected during the targeted investigation

4.5 Historic and current information for selected private wells in the York investigation area.

A.1 Samples collected during the 2015-2016 targeted investigation

D.1 Grain Size Analysis Results

E.1 Analytical results from the AGEM Laboratory for soil samples collected in July 2015 and analyzed by the purge-and-trap method

G.1 Analytical results for volatile organic compounds in groundwater samples G-2

G.2 Field measurements for groundwater samples collected at private and monitoring wells . G-6

H.1 Analytical results from TestAmerica Laboratory for indoor air and sub-slab soil gas samples 


\section{Notation}

AGEM Applied Geosciences and Environmental Management

AMSL above mean sea level

ASTM American Society for Testing and Materials

BGL below ground level

${ }^{\circ} \mathrm{C}$

degree(s) Celsius

CCC Commodity Credit Corporation

CD compact disc

$\mathrm{cm} \quad$ centimeter(s)

COC chain of custody

CPT cone penetrometer

CVA Central Valley Ag

1,1-DCE 1,1-dichloroethylene

1,2-DCA 1,2-dichloroethane

DO dissolved oxygen

ECPT electronic cone penetrometer

EPA U.S. Environmental Protection Agency

$\mathrm{ft} \quad$ foot (feet)

GAC granular activated charcoal

gal gallon(s)

GC-ECD gas chromatograph-electron capture detector

GC-MS gas chromatograph-mass spectrometer

gpm gallon(s) per minute

GPS global positioning system

hr hour(s)

I.D. inner diameter

in. inch(es)

L liter(s)

$\mu \mathrm{g} / \mathrm{kg} \quad$ microgram(s) per kilogram

$\mu \mathrm{g} / \mathrm{L} \quad$ microgram(s) per liter

MCL maximum contaminant level

mi mile(s)

$\min \quad$ minute(s)

$\mathrm{mV} \quad$ millivolt(s)

NDEC Nebraska Department of Environmental Control 
York Targeted Investigation, 2015-2016

Version 01, 02/14/2017

$\begin{array}{ll}\text { NDEQ } & \text { Nebraska Department of Environmental Quality } \\ \text { NDNR } & \text { Nebraska Department of Natural Resources } \\ \text { NDOH } & \text { Nebraska Department of Health } \\ \text { NPL } & \text { National Priorities List } \\ \text { ORP } & \text { oxidation-reduction potential } \\ \text { PCE } & \text { tetrachloroethylene } \\ \text { PVC } & \text { polyvinyl chloride } \\ \text { PWS } & \text { public water supply } \\ \text { QA/QC } & \text { quality assurance/quality control } \\ \text { RPD } & \text { relative percent difference } \\ \text { SDG } & \text { sample delivery group } \\ \text { sec } & \text { second(s) } \\ \text { TCE } & \text { trichloroethylene } \\ \text { TOC } & \text { top of casing } \\ \text { UBB-NRD } & \text { Upper Big Blue Natural Resource District } \\ \text { USDA } & \text { U.S. Department of Agriculture } \\ \text { USGS } & \text { U.S. Geological Survey } \\ \text { VCP } & \text { Voluntary Cleanup Program } \\ \text { VI } & \text { vapor intrusion } \\ \text { VOC } & \text { volatile organic compound } \\ \text { yr } & \text { year(s) }\end{array}$




\section{Targeted Investigation at the Former CCC/USDA Grain Storage Facility in York, Nebraska, in 2015-2016}

\section{Introduction}

From 1950 until 1974, the Commodity Credit Corporation (CCC) of the U.S. Department of Agriculture (USDA) operated a grain storage facility on leased property in the northern portion of York, Nebraska (Figure 1.1). During this time, commercial grain fumigants containing carbon tetrachloride were commonly used by the CCC/USDA and the private grain storage industry to preserve grain in their facilities.

In 1990, routine sampling by the Nebraska Department of Health (NDOH) identified contamination by volatile organic compounds (VOCs) including trichloroethylene (TCE), tetrachloroethylene (PCE), 1,1-dichloroethylene (1,1-DCE), and carbon tetrachloride in several York public water supply (PWS) wells. Levels of TCE significantly above the U.S. Environmental Protection Agency (EPA) maximum contaminant level (MCL) for this compound in drinking water $(5.0 \mu \mathrm{g} / \mathrm{L})$ were detected in well PWS 81-1, and use of the well was discontinued in early 1990. Low concentrations of carbon tetrachloride (less than the MCL of $5.0 \mu \mathrm{g} / \mathrm{L}$ for this compound) were also detected at PWS 81-1 at this time, as well as in two additional municipal wells (PWS 37-1 and PWS 48-1; Figure 1.2). In subsequent studies conducted by the NDOH and the Nebraska Department of Environmental Quality (NDEQ; formerly the Nebraska Department of Environmental Control, NDEC), groundwater contamination by multiple VOCs including carbon tetrachloride was also identified in numerous private wells in the northern portion of the town. In September 1991 the NDEC determined that multiple potential source areas, including the former CCC/USDA grain storage facility, might have contributed to the contamination although no specific sources were conclusively identified (NDEC 1991).

In 1993-1995, the CCC/USDA conducted an investigation of carbon tetrachloride contamination potentially associated with its former York grain storage facility. Carbon tetrachloride was identified in subsurface soils at and immediately to the east of the former CCC/USDA facility (on an adjacent property), as well as in groundwater in the uppermost, unconfined aquifer beneath and to the southeast (downgradient) of the former facility at concentrations that exceeded the current NDEQ regulatory targets for this contaminant ( $39 \mu \mathrm{g} / \mathrm{kg}$ in soils; $5.0 \mu \mathrm{g} / \mathrm{L}$ in groundwater). Low levels of carbon tetrachloride were also detected in association with other VOCs, including TCE and PCE, along an apparent second, parallel trend 
of groundwater contamination south of the former CCC/USDA facility that could not be linked to the CCC/USDA grain storage operations. These findings were interpreted as evidence of one or more additional contaminant source area(s) south of the former facility (Argonne 1993, 1994, 1995a,b).

Further investigations by the EPA and the NDEQ between 1995 and 2014 identified two distinct areas of VOCs contamination (primarily TCE and PCE) unrelated to the former CCC/USDA facility in groundwater in the more southern portions of York (EPA 1996; NDEQ 2010, 2011; SETI 2011; Tetra Tech 2012a,b,c). These areas, now designated the "PCE Southeast Contamination site" and the "PCE/TCE Northeast Contamination site," were placed on the EPA National Priorities List (NPL) in May 2014 (EPA 2013a,b, 2014a,b). Information presently available to the CCC/USDA indicates that the PCE/TCE Northeast Contamination site (associated with the former Kroy/Alcan facility; Figure 1.3) incorporates the region of low-level carbon tetrachloride and other VOCs contamination identified in groundwater south of the former CCC/USDA facility during the 1993-1995 CCC/USDA investigations.

In conjunction with the groundwater contamination issues elsewhere at York, the EPA and NDEQ requested that the CCC/USDA update its earlier studies of carbon tetrachloride contamination associated with its former grain storage operations. Specifically, the CCC/USDA was asked to (1) verify the previous investigation results for carbon tetrachloride in both soil and groundwater, (2) determine the present areal and vertical extent and levels of carbon tetrachloride in soil and groundwater, and (3) identify any risks that the contamination might pose to currently active private or public wells or to public health via other pathways, including potential carbon tetrachloride vapor intrusion.

To address this request, the CCC/USDA has conducted a targeted investigation at the York site in accordance with an Intergovernmental Agreement between the NDEQ and the Farm Service Agency of the USDA. The investigation, which was performed on behalf of the CCC/USDA by the Environmental Science Division of Argonne National Laboratory, was implemented in phases so that information obtained and interpretations developed during each stage of the study could be used to guide subsequent phases, as necessary. 
This report presents the current technical findings of the CCC/USDA targeted investigation. The field activities associated with this effort were conducted in accord with the site-specific Final Work Plan (which incorporates revisions reviewed by the NDEQ; Argonne 2015a, 2016a), and with the standard operating procedures, quality assurance/quality control (QA/QC) measures, and general health and safety policies outlined in the Master Work Plan for operations in Nebraska (Argonne 2002). As the targeted investigation progressed, supplemental recommendations were developed with the approval of the CCC/USDA and the NDEQ project managers to augment the scope of activities outlined in the Final Work Plan, to specifically address (1) the investigation of possible carbon tetrachloride vapor intrusion (VI) to indoor air at selected locations and (2) the installation of permanent monitoring wells, as outlined below and in Section 2 (Argonne 2015b,c; USDA 2016).

\subsection{Technical Objectives and Primary Tasks of the Targeted Investigation}

The York Final Work Plan (Argonne 2016a) summarizes preexisting information for the site and describes the site-specific technical objectives and the intended scope of work for the targeted investigation. The following principal technical objectives for the targeted program were identified in consultation with the CCC/USDA and NDEQ project managers:

- Verify the results of the previous investigations for carbon tetrachloride contamination in both soil and groundwater.

- Update the previous findings to determine the present areal and vertical extent and levels of carbon tetrachloride contamination in soil and groundwater.

- Identify any risks that the carbon tetrachloride contamination might pose to currently active private or public drinking water wells or to public health via other pathways, including potential VI.

To address these objectives, five primary tasks were identified in the Final Work Plan (Argonne 2016a), as follows:

- Verify the present distribution of carbon tetrachloride in the shallow and deeper (vadose zone) soils at and near the former CCC/USDA facility. 
- Determine the potential levels of VOCs contamination in soil vapor at/near the former CCC/USDA facility.

- Determine the current areal and vertical extent of carbon tetrachloride contamination in the unconfined aquifer groundwater downgradient from the former CCC/USDA facility and establish permanent groundwater monitoring points.

- Update the inventory and status of private wells in the targeted investigation area.

- Investigate possible carbon tetrachloride contamination of the deeper (confined) aquifers.

These tasks were developed in response to a request provided by the NDEQ (2014) and were discussed among representatives of the NDEQ, the CCC/USDA, and Argonne in a meeting held on October 1, 2014. The overall goal of the targeted investigation was to address specific data gaps identified by the NDEQ in the current understanding of the carbon tetrachloride distribution in soils and groundwater associated with the former CCC/USDA facility.

As a result of further requests by the NDEQ (2015a), the focus of the second task above (to determine the concentrations of VOCs contamination in soil vapor), was modified to instead consider the possibility of carbon tetrachloride VI to indoor air at selected locations along the anticipated downgradient groundwater (and contaminant) migration pathway from the former CCC/USDA facility. The scope of this modified task was discussed in a conference call among the CCC/USDA and NDEQ project managers and Argonne personnel on June 16, 2015, and summarized in a supplemental VI investigation plan (Argonne 2015b).

\subsection{Implementation of the Targeted Investigation Field Program}

The targeted investigation field program began on July 7, 2015. With the approval of the CCC/USDA and NDEQ project managers, the field activities were temporarily suspended from September 16, 2015 until January 19, 2016, to facilitate access to several investigative locations on agricultural properties. The hiatus was required to accommodate the harvesting of crops and 
the development of reasonably hard/dry ground surface conditions on these properties, prior to use of the required investigation equipment in the fields (see Section 2). Recommendations for continuation of the field program following the hiatus were summarized in a supplemental plan (Argonne 2015c) and were discussed among the CCC/USDA and NDEQ project managers and Argonne personnel in a meeting held on October 7, 2015. Approval for continuation of the direct-push groundwater sampling efforts at three locations identified in the supplemental plan was received from the NDEQ at that time; additional locations for groundwater sampling were also subsequently requested by the NDEQ as the field program progressed (see Section 2.1.1). The installation of permanent monitoring wells and investigation of the deeper, confined aquifers was further considered by these parties in a meeting held on April 18, 2016, and recommendations presented by the CCC/USDA were approved by the NDEQ (USDA 2016; NDEQ 2016a). Data acquisition in conjunction with the primary field program, as documented in this report, was completed on July 21, 2016. Additional sampling and monitoring activities implemented by the CCC/USDA at the request of the NDEQ have continued at selected locations at York since that time (see Sections 2.1.3, 2.2.2, and 2.5).

Section 2 of this report provides a chronological summary of the field program and describes the investigative methods used at York. Section 3 summarizes the resulting field and laboratory data. These data, together with information presented in the Final Work Plan (Argonne 2016a), are interpreted and integrated in Section 4 to (1) develop a preliminary model of the hydrogeologic factors affecting groundwater and contaminant migration in the vicinity of the former CCC/USDA facility, (2) identify the current distribution of carbon tetrachloride in the soils and groundwater associated with the former facility, and (3) serve as a basis for the consideration of contaminant levels and potential exposure pathways. 


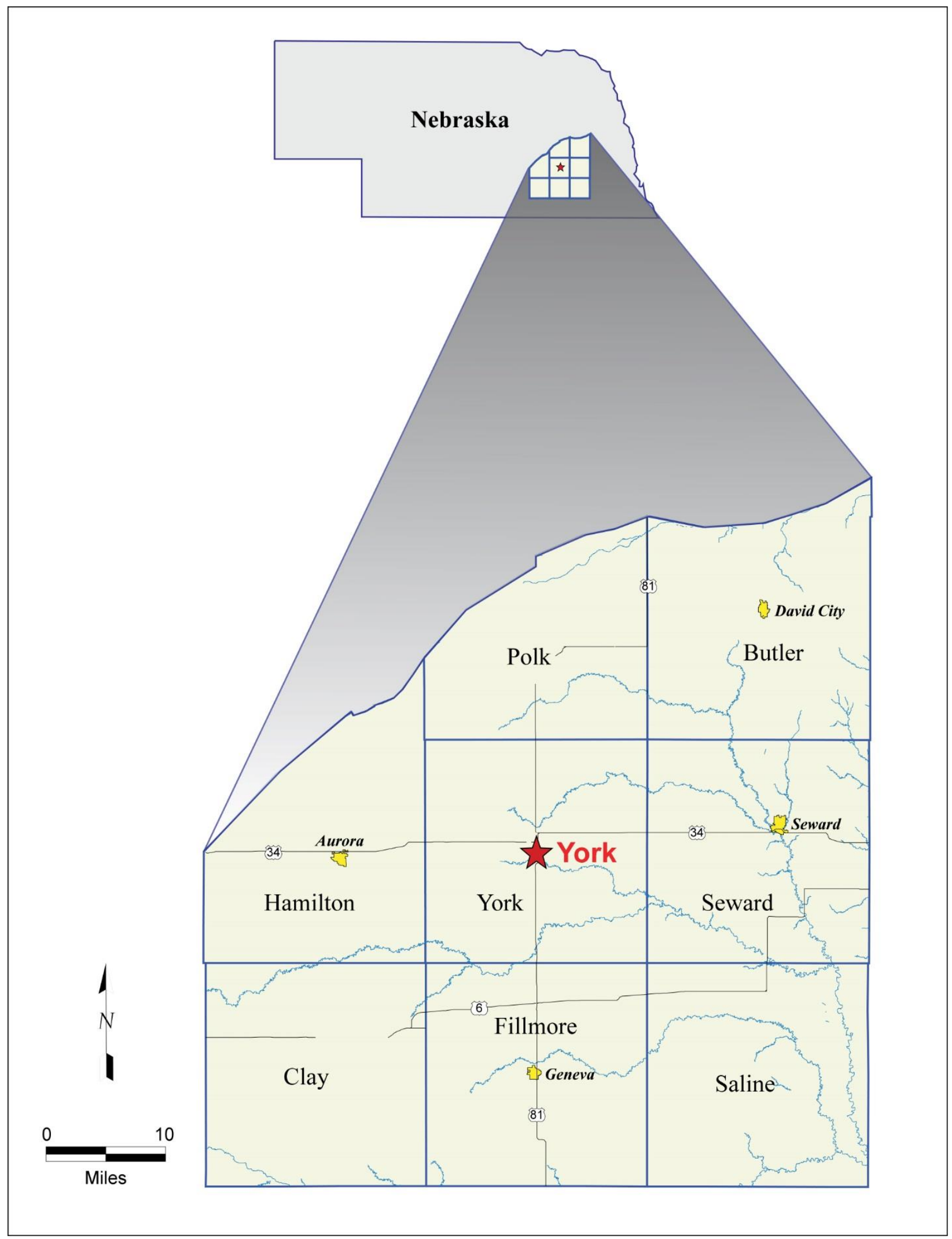

FIGURE 1.1 Location of York, Nebraska. 


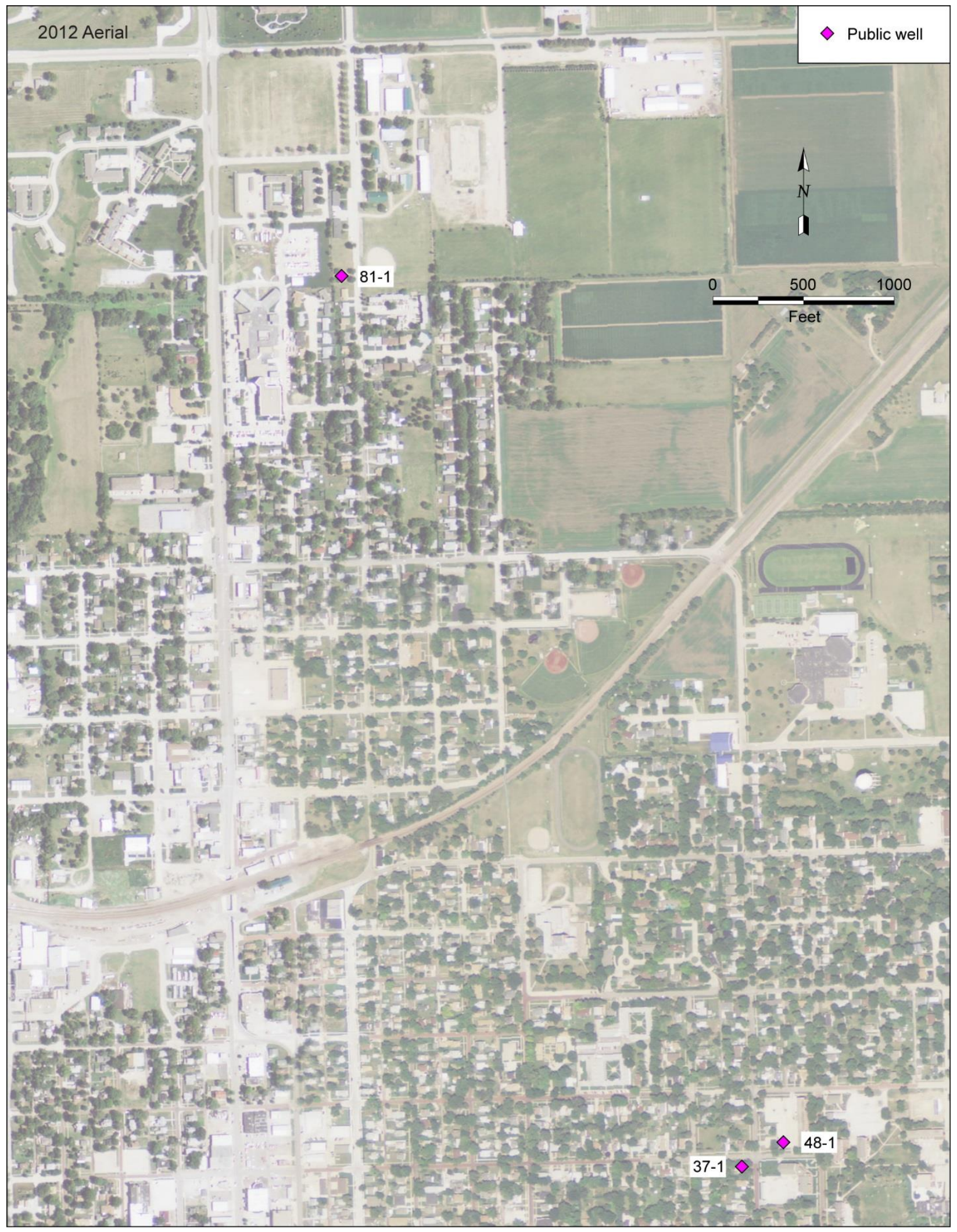

FIGURE 1.2 Locations of contaminated public water supply wells PWS 37-1, PWS 48-1, and PWS 81-1. 


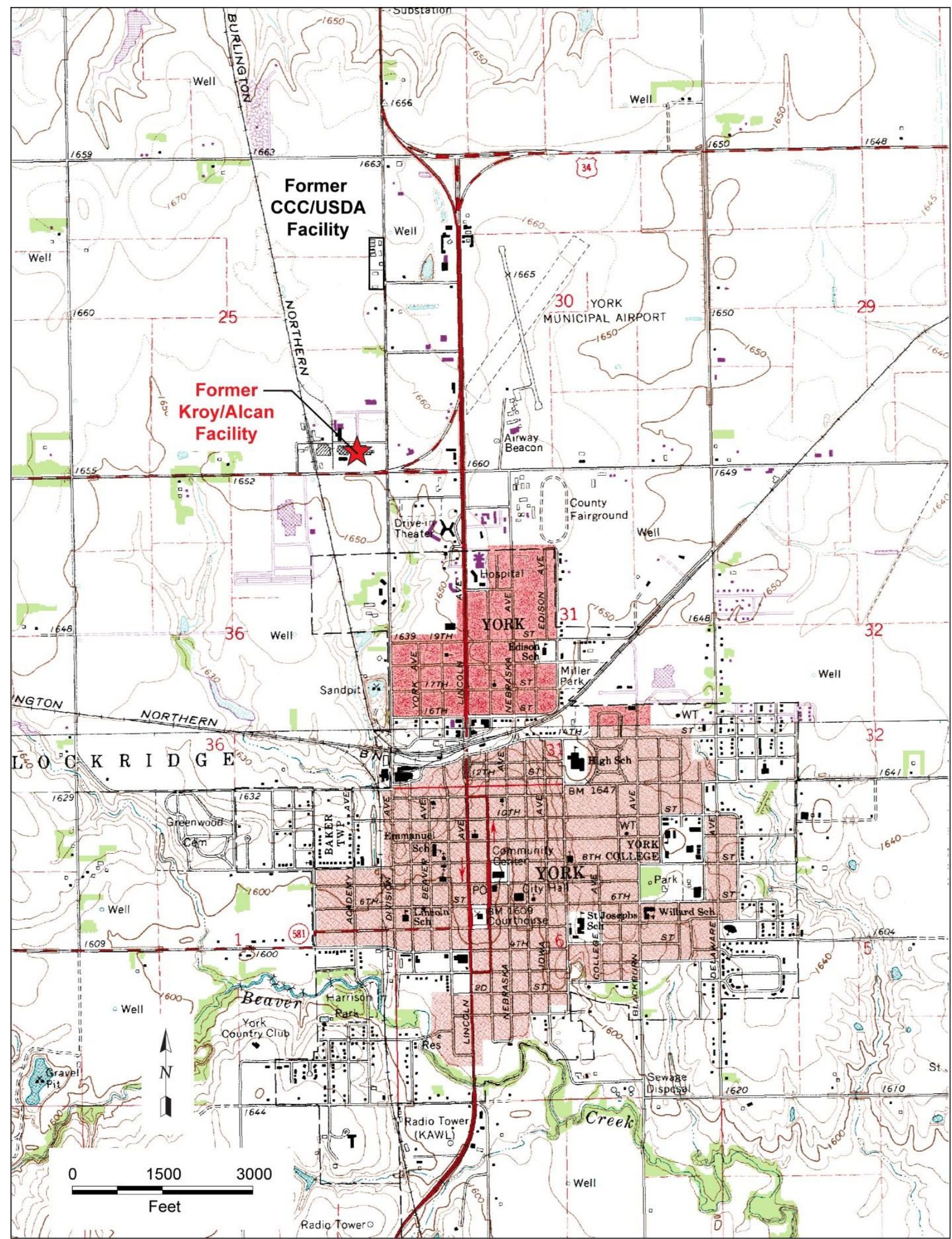

FIGURE 1.3 PCE/TCE Northeast Contamination Site, as identified by the EPA (2014b), associated with the former Kroy/Alcan facility to the south of the former CCC/USDA facility. 


\section{Investigative Methods}

The targeted investigation at York was performed in accord with the site-specific Final Work Plan (Argonne 2016a), with the supplemental plans developed in consultation with the CCC/USDA and NDEQ project managers as the study progressed (Argonne 2015b,c; USDA 2016), and with the standard operating procedures, QA/QC measures, and general health and safety policies in the Master Work Plan (Argonne 2002). As outlined in Section 1, results were reviewed and discussed with the CCC/USDA and NDEQ project managers during the field program to obtain their input and approval for decisions affecting the direction of the work.

Throughout the investigation, a comprehensive QA/QC program was employed to confirm the reliability of all information as it was accumulated (Argonne 2002, 2016a).

The field activities during the targeted investigation included the following:

- Use of direct-push technologies to obtain electronic cone penetrometer (ECPT) logs of the geomechanical properties of the subsurface lithologies.

- Use of direct-push technologies to collect soil and groundwater samples for analyses of VOCs.

- Use of conventional drilling to collect soil samples for lithologic characterization, and for the installation of permanent monitoring wells to facilitate groundwater sampling and the measurement of groundwater levels.

- Sampling of monitoring wells for VOCs analyses.

- Establishment of a preliminary groundwater level monitoring network and the installation of automatic water level recording devices in selected wells.

- Investigation of selected private wells in the study area and sampling of these wells for VOCs analyses (as possible).

- Sampling of indoor air and sub-slab soil vapor for VOCs analyses at selected locations, to test for possible carbon tetrachloride VI. 
The locations investigated during the field program are illustrated as follows:

- Figure 2.1 shows direct-push (CPT) borings advanced for the collection of soil samples for VOCs analyses.

- Figure 2.2 shows direct-push borings advanced for ECPT logging and the collection of groundwater samples for VOCs analyses.

- Figure 2.3 shows previously identified private wells in the vicinity of the former CCC/USDA facility (Argonne 1994, 1995a,b), that were investigated during the current field program.

- Figure 2.4 shows borings advanced by the use of conventional drilling techniques, to obtain continuous soil cores for lithologic evaluation and to facilitate the installation of permanent monitoring wells.

- Figure 2.5 shows monitoring wells installed by the CCC/USDA during the targeted investigation, or in conjunction with previous CCC/USDA investigations at this site (Argonne 1995a,b). These wells were sampled for VOCs analyses and used for the measurement of groundwater levels.

- Figure 2.6 shows the locations sampled for indoor air, or sub-slab soil vapor and indoor air, and for VOCs analyses to investigate possible carbon tetrachloride VI.

\subsection{Overview of the Targeted Investigation Field Program}

The targeted investigation field program was conducted in two main phases that were operationally distinguished by the primary methods employed in each to acquire information on the subsurface characteristics of the study area.

In the first stage of the program, direct-push techniques, implemented using either the Argonne 22-ton track-mounted or 40-ton truck-mounted cone penetrometer (CPT) vehicles, were used to: 
- Acquire preliminary data on the geology and stratigraphy of the vadose zone and unconfined aquifer intervals beneath and in the vicinity of the former CCC/USDA grain storage facility, and

- Investigate the distribution of carbon tetrachloride contamination in these units.

Additional efforts undertaken during the first phase of the targeted study included an evaluation of the status and usage of selected private wells in the vicinity of the former CCC/USDA facility, sampling of these wells as conditions permitted, and the sampling of indoor air and sub-slab soil vapor for VOCs analyses at selected locations to assess the potential for carbon tetrachloride VI.

In consultation with the CCC/USDA and NDEQ project managers, the findings from these efforts were then used to guide the second stage of the field program. In this second phase, conventional (sonic) drilling techniques were employed to:

- Obtain continuous soil cores for confirmation of the local geologic sequence, and the lithologic characteristics of both the unconfined and deeper (confined) aquifers,

- Install permanent monitoring wells penetrating these aquifer units, and

- Establish a preliminary groundwater level monitoring network.

Drilling and monitoring well installation were performed under the direction of Argonne personnel, by Cascade Drilling, L.P., Little Falls, Minnesota, using equipment and staff licensed to operate in Nebraska.

Multiple field sessions were conducted during each phase of the targeted study. The investigative activities performed during each session were implemented, in consultation with the CCC/USDA and NDEQ project managers, to address the principal technical objectives and primary tasks of the targeted investigation (outlined in Section 1.1) in a time- and cost-effective manner. The activities included in each of the field sessions are outlined below, and are summarized in Table 2.1. 


\subsubsection{Field Activities in the First Phase of the Targeted Investigation, July 2015 - April 2016}

The first stage of the targeted investigation began on July 7, 2015, and continued through September 16, 2015. In September 2015, field activities requiring the use of the Argonne CPT vehicles were temporarily suspended to facilitate access to several investigative locations on agricultural properties. Field work with the 40-ton CPT resumed on January 19, 2016, and the activities associated with this phase of the investigation were completed on April 28, 2016. The field activities during the first phase of the targeted investigation were as follows:

- July 7-22, 2015

- Use of the Argonne 22-ton track-mounted CPT to collect continuous soil cores at four locations on the former CCC/USDA facility and the adjacent York (Izaak Walton League) Gun Club property (TI01-TI04; Figure 2.1) from $2 \mathrm{ft}$ below ground level (BGL) to the depth of refusal of the coring apparatus.

- Collection of soil samples for VOCs analyses from the continuous cores, at 4-ft depth intervals, from $4 \mathrm{ft}$ BGL to the depth of CPT refusal.

- Use of the 22-ton CPT vehicle to obtain ECPT logs, to the depth of refusal of the logging probe, at five locations on the former CCC/USDA facility and the adjacent York Gun Club property (TI01-TI05; Figure 2.2) and to collect groundwater samples for VOCs analyses at selected depths at these locations.

- Use of the 40-ton, truck mounted CPT vehicle to obtain ECPT logs, to the depth of CPT refusal, at two locations to the southeast of the former CCC/USDA property (TI06-TI08; Figure 2.2), and to collect groundwater samples for VOCs analyses at selected depths at these locations.

- Interviews were conducted by Argonne personnel with selected property owners to determine the current status and usage of 10 private wells previously identified in the vicinity of the former CCC/USDA facility (Argonne 2016a). At the request of the NDEQ, one additional private well 
was also subsequently investigated during the August 4-8, 2015 field session described below (Figure 2.3).

The soil coring and groundwater sampling activities conducted with the 22ton, track-mounted CPT unit at locations TI01-TI05 demonstrated that it would be difficult for this vehicle to achieve the penetration depths necessary for groundwater sampling at the more downgradient locations proposed in the approved Final Work Plan (Argonne 2016a). The ECPT logging and groundwater sampling performed at locations TI06-TI08 indicated, however, that penetration depths sufficient to complete the investigation could successfully be reached by the Argonne 40-ton, truck-mounted CPT vehicle. This vehicle also provided greater mobility for access to the numerous sampling locations across the study area (Figure 2.2). For these reasons, the remaining direct-push sampling activities outlined below were performed by using the truck-mounted CPT unit.

- August 4-8, 2015

- Collection of groundwater samples for VOCs analyses at two monitoring wells (SB62 and SB77; Figure 2.5) that were previously installed by Argonne on the York Gun Club property (Argonne 1995a,b).

- Collection of indoor and outdoor (background) air samples for VOCs analyses at six locations near, and downgradient of, the former CCC/USDA facility (Figure 2.6).

- An interview was conducted by Argonne personnel with one additional property owner (Volzke; Figure 2.3) to determine the current status and usage of a private well located north of the former CCC/USDA facility, at the specific request of the NDEQ.

- Collection of groundwater samples at five private wells found to still be operable, of the 11 private wells that were investigated during the previous (July 7-22) and current field sessions (Figure 2.3). 
- August 12-28, 2015

- ECPT logging, to the approximate base of the unconfined aquifer, at 10 locations (TI09-TI18; Figure 2.2) along the expected downgradient groundwater and contaminant migration pathway from the former CCC/USDA facility.

- Collection of groundwater samples for VOCs analysis at selected depths at these 10 locations.

- September 9-16, 2015

- ECPT logging, to the approximate base of the unconfined aquifer, at five locations (TI19-TI23; Figure 2.2) in the anticipated direction of downgradient groundwater flow.

- Collection of groundwater samples for VOCs analyses at selected depths at these five locations.

At the end of this field session, investigative activities requiring the use of the CPT vehicle were temporarily suspended to accommodate the harvesting of crops and related agricultural needs at several locations targeted for groundwater sampling in farmed fields.

- January 19-28, 2016

Direct-push operations resumed with the Argonne 40-ton CPT unit on January 19, 2015, at locations that were selected in consultation with the CCC/USDA and NDEQ project managers (Argonne 2015c). The investigation activities that occurred during this field session were as follows:

- ECPT logging, to the approximate base of the unconfined aquifer, at three locations (TI24-TI26; Figure 2.2) along the expected 
downgradient groundwater (and contaminant) migration pathway from the former CCC/USDA facility.

- Collection of groundwater samples for VOCs analyses at selected depths at TI24-TI26.

- Collection of indoor air, sub-slab vapor, and outdoor (background) air samples for VOCs analyses at two locations near the former CCC/USDA facility (Fitness Worx and Garretson/Troester; Figure 2.6).

- February 9, 2016

- At the request of the NDEQ an additional, downgradient location was investigated by using the 40-ton CPT (TI27; Figure 2.2). ECPT logging was performed, to the approximate base of the unconfined aquifer, and groundwater samples for VOCs analyses were collected at selected depths at this location.

- March 2-3, 2016

- At the request of the NDEQ, a second additional location was investigated (TI28; Figure 2.2). ECPT logging was performed, to the approximate base of the unconfined aquifer, and groundwater samples for VOCs analyses were collected at selected depths at this location.

- With the approval of the CCC/USDA and NDEQ project managers, groundwater sampling through the use of direct-push techniques was completed on March 3, 2016.

- $\quad$ April 27-28, 2016

- Indoor air, sub-slab vapor, and outdoor (background) air samples for VOCs analyses were collected at the Fitness Worx facility (Figure 2.6). 


\subsubsection{Field Activities in the Second Phase of the Targeted Investigation, May-July 2016}

The second phase of the targeted investigation at York focused on the use of conventional (sonic) drilling techniques at eight locations selected in consultation with the CCC/USDA and NDEQ project managers (Figure 2.4; Argonne 2015c; USDA 2016; NDEQ 2016a). This method was employed to recover continuous soil cores with depth for lithologic characterization of the unconfined and deeper (confined) aquifer intervals and intervening confining units in the vicinity of the former CCC/USDA grain storage facility. The sonic drilling rig was also use to install a preliminary network of permanent monitoring wells along and adjacent to the identified carbon tetrachloride migration pathway (Figure 2.5).

The field activities during the second phase of the targeted investigation were as follows:

- May 16-25, 2016

- Continuous soil coring and the installation of permanent monitoring wells were completed at drilling locations DL1-DL3 (Figure 2.4 and 2.5).

- The monitoring wells installed at the DL1 drilling location were developed, and groundwater samples were collected from these wells for VOCs analyses.

- May 31-June 13, 2016

- Continuous soil coring and the installation of permanent monitoring wells were completed at drilling locations DL4 and DL6-DL8 (Figures 2.4 and 2.5).

- The monitoring wells installed at the DL2, DL3, and DL6-DL8 drilling locations were developed, and groundwater samples were collected from these wells for VOCs analyses. 
- June 15-16, 2016

- Continuous soil coring and the installation of a permanent monitoring well were completed at the DL5 drilling location (Figures 2.4 and 2.5).

- The monitoring well installed at the DL4 drilling location was developed, and a groundwater sample was collected from the well for VOCs analyses.

- June 21, 2016

- The monitoring well installed at the DL5 drilling location was developed, and a groundwater sample was collected from the well for VOCs analyses.

- July 7-12, 2016

- Manual groundwater level measurements were obtained and automatic groundwater level recording units were installed in monitoring wells DL1-C, DL2-C1, DL2-CU, DL3-C1, DL4-C1, DL5-C1, DL6-C, DL7C, CL7-CU, DL7-CL, and DL8-C1 (Figure 2.5).

- July 21, 2016

- A professional coordinates survey was obtained to provide selfconsistent elevation and location control data for CPT boring locations TI01-TI24, new and existing permanent monitoring wells, and private wells investigated during the field program (Figures 2.2-2.5). Coordinate survey data for additional private wells and PWS wells in the York area that are sampled quarterly by the CCC/USDA, as discussed in Section 2.1.3, were also obtained at this time (Figure 2.7). Coordinates data for CPT borings TI25-TI28 were obtained by Argonne personnel through the use of a hand-held (cell phone) GPS device at the time of installation (in January-March 2016), due to 
access limitations in the agricultural fields at these locations at the time of the professional survey (during the growing season).

- Data acquisition in conjunction with the targeted investigation field program, as documented in this report, was completed on July 21, 2016.

\subsubsection{CCC/USDA Quarterly Sampling of Private and PWS Wells}

In early 2014, the NDEQ and EPA requested that the CCC/USDA conduct groundwater sampling for VOCs analyses at selected active private and York PWS wells generally located to the east and southeast of the former CCC/USDA facility, to supplement private well testing being done by these agencies in the more southern portions of York at that time. An initial sampling event was performed by Argonne on behalf of the CCC/USDA at the identified target wells in April 2014, and additional sampling was conducted in July 2014 (Argonne 2014). Based on the results of these events, an ongoing program of quarterly private and PWS well sampling was implemented by the CCC/USDA at the request of the NDEQ. Routine sampling under this program began in December 2014, and four sampling events were completed during the timeframe of the targeted investigation field activities - in August and November 2015, and February and June 2016 (Argonne 2014; 2015d,e,f;. 2016b,c). Additional sampling events have subsequently occurred.

The findings of the CCC/USDA quarterly well sampling program provide supplemental data that contribute to interpretation of the carbon tetrachloride contamination in the groundwater in the vicinity of the former CCC/USDA grain storage facility. Therefore, the results of the quarterly sampling events are included in this report. The locations of the private and PWS wells addressed under the quarterly monitoring program are shown in Figure 2.7.

\subsection{Methods to Determine the Characteristics of the Hydrogeologic Sequence in the Vicinity of the Former CCC/USDA Facility}

Existing data pertinent to the geologic sequence in the vicinity of the former CCC/USDA grain storage facility at York were summarized in Section 2.2.1 of the Final Work Plan (Argonne 2016a). To obtain a more detailed understanding of the lithologic, hydrologic, and 
stratigraphic characteristics of this sequence, the methods below were employed in the targeted investigation.

\subsubsection{Characterization of the Lithologic Sequence}

At 28 locations across the study area (TI01-TI28; Figure 2.2), the electronicgeomechanical logging capabilities of the ECPT units were used to obtain logs of tip pressure, sleeve friction, and tip-versus-sleeve ratio from the land surface to penetration depths ranging from approximately $131 \mathrm{ft}$ BGL (at TI01) to $164 \mathrm{ft}$ BGL (at TI28), corresponding to or exceeding the interpreted depth to the base of the unconfined aquifer at each location. The resulting logs were employed as a basis for preliminary, field identification and correlation of predominantly coarse-grained versus fine-grained lithologic intervals in the unsaturated (vadose zone) and unconfined aquifer units at each location. Application of the ECPT logging information in this manner proved successful in previous geologic studies conducted by the $\mathrm{CCC} / \mathrm{USDA}$ at this site, based on qualitative calibration of the logs against lithologic data obtained from corresponding soil cores (Argonne 1994, 1995a).

To obtain corroborating lithologic data in the present study, continuous soil cores were recovered at 8 locations (DL1-DL8; Figure 2.4) through the use of sonic drilling techniques. At locations DL1, DL3, DL4, DL6, and DL8, the investigative borings were advanced to the approximate base of the unconfined aquifer, at depths ranging from $123 \mathrm{ft}$ BGL (at DL1) to $165 \mathrm{ft}$ BGL (at DL4). At location DL5, the boring was terminated at a depth of $120 \mathrm{ft}$ BGL, within the unconfined aquifer, to permit the completion of a monitoring well at this target depth.

With the approval of the CCC/USDA and NDEQ project managers, drilling locations DL2 and DL7 (Figure 2.4) were selected for investigation of the deeper, confined aquifers underlying the study area. Boring DL2 was continuously cored to a depth of $245 \mathrm{ft}$ BGL, and is interpreted to have penetrated the local stratigraphic sequence to the top of the confining layer at the base of the upper confined aquifer. Boring DL7 was drilled to a depth of $280 \mathrm{ft} \mathrm{BGL}$, and is interpreted to have penetrated into an uppermost sand interval of the lower confined aquifer (see Section 4.1.1.4).

All cores were characterized lithologically in accord with procedures in the Master Work Plan (Argonne 2002). To supplement visual evaluation and description of the cores, samples of 
selected core intervals from borings DL1-DL8 were also shipped to Alfred Benesch and Company (Lincoln, Nebraska) for laboratory analysis of particle size distribution. All cores are archived at an Argonne storage facility in Lincoln, Nebraska.

The addition of water to the drilled borings was periodically required (1) to advance the outer, override casing used during drilling and (2) in the construction of monitoring wells. Water for these purposes was obtained from the York municipal water supply, samples of which were analyzed and demonstrated to be free of carbon tetrachloride contamination (see Section 3.4.4).

\subsubsection{Installation of Monitoring Wells and Establishment of a Groundwater Level Monitoring Network}

To establish a preliminary network of groundwater monitoring points in the vicinity of the former CCC/USDA grain storage facility, 17 permanent monitoring wells were installed during the present investigation at eight locations approved by the CCC/USDA and NDEQ Project managers (DL1-DL8; Figure 2.4). The designations for the wells are shown in Figure 2.5; each well name includes a suffix (A, B, CL, etc.) reflecting the aquifer interval intercepted by the well, based on the stratigraphic interpretations outlined in Section 4.1.1. The 17 new wells supplement two existing monitoring wells (SB62 and SB77; Figure 2.5) installed by Argonne during the previous studies conducted by the CCC/USDA at this site (Argonne 1994, 1995a,b).

The monitoring wells were constructed in accord with applicable NDEQ requirements, each in a separate borehole. The wells were installed with the sonic drilling rig, by placing a nominal 2.0-in.-I.D., Schedule 40 or Schedule 80, mill-slotted (0.010-in.) polyvinyl chloride (PVC) screen and PVC riser to the selected target depth in each borehole. A sand filter pack was placed around the screen, extending at least $2 \mathrm{ft}$ above the screened interval. A bentonite pellet seal was installed above each filter pack, and the remaining annulus zone was grouted by using a tremie pipe. At the request of the respective property owners, all wells were finished with flushmounted surface completions.

Self-contained, downhole pressure-sensing and data-logging devices (Instrumentation Northwest, Inc. Model PT2X ${ }^{\mathrm{TM}}$ sensor/loggers) were installed in 11 of the monitoring wells in July 2016, to provide continuous information on groundwater relationships. Monitoring units were placed in wells DL1-C, DL2-C1, DL3-C1, DL4-C1, DL5-C1, DL6-C, DL7-C, and DL8-C1 
to obtain water level measurements at comparable stratigraphic positions within the unconfined aquifer. Units were also installed in wells DL2-CU and DL7-CU to monitor groundwater levels in the upper confined aquifer, and in well DL7-CL in the lower confined aquifer (Figure 2.5).

The monitoring units are programmed to measure and record the static water level in each well once every four hours. Automated monitoring is recommended for a minimum of one year, to document possible seasonal or other factors that might influence the local groundwater levels. Water levels are also periodically measured manually, through the use of an electronic water level meter, to obtain information from the full suite of 19 monitoring wells at the site (see Section 3.5).

To assist in the evaluation of possible variations or trends in the groundwater levels at York, daily precipitation data for the weather monitoring station at the York Municipal Airport were obtained from Weather Underground; these data are available online (www.wunderground.com/us/ne/york/zmw:68467.1.99999).

\subsection{Methods to Investigate for Possible Carbon Tetrachloride Contamination in Soils}

In 1993-1995, carbon tetrachloride contamination was identified in soils at several locations on both the former CCC/USDA property and the adjacent York Gun Club property to the east, at levels above the current NDEQ Voluntary Cleanup Program (VCP) target level for this contaminant ( $39 \mu \mathrm{g} / \mathrm{kg}$; for protection of the soil-to-groundwater pathway). To determine the present distribution of residual carbon tetrachloride contamination in the soils, vertical-profile soil sampling for VOCs analyses was conducted at locations TI01-TI04 (Figure 2.1). A chronological summary of the soil sample collection is in Appendix A, Table A.1.

Locations TI01-TI03 were selected, with the approval of the CCC/USDA and NDEQ project managers, to approximately coincide with previous soil boring locations SB01, SB37/CPT37, and SB62, respectively (Figures 2.22 and 2.23 in Argonne 2016a). At the request of the NDEQ (2014), boring TI04 was located on the York Gun Club property directly east of location TI01. At each location, soil cores were obtained with the 22-ton CPT unit by progressively advancing a 4-ft-long core barrel equipped with a 1.25-in.-I.D. disposable liner (Geoprobe dual-tube coring system), from $2 \mathrm{ft}$ BGL to the top of the saturated zone (encountered at approximately $73 \mathrm{ft}$ BGL at the time of this investigation). 
Soil samples for VOCs analyses were retrieved immediately from the cores as they were removed from the core barrels, at 4-ft intervals beginning at $4 \mathrm{ft}$ BGL. The samples were placed immediately in jars, sealed, and preserved on dry ice, then shipped overnight to the Applied Geosciences and Environmental Management (AGEM) Laboratory at Argonne for analysis by the purge-and-trap sample preparation method with analysis by GC-MS (EPA Methods 5030B and 8260B), as described in the Master Work Plan (Argonne 2002).

\subsection{Methods to Investigate for Possible Carbon Tetrachloride Contamination in Groundwater}

To obtain information on the distribution of carbon tetrachloride contamination in groundwater associated with the former CCC/USDA facility, groundwater samples were collected for VOCs analyses from CPT borings TI01-TI28 (Figure 2.2), from the full suite of monitoring wells shown in Figure 2.5, and from five private wells (Figure 2.3). Groundwater samples were also collected during this timeframe in conjunction with the quarterly monitoring program at York (see Section 2.1.3), at the locations shown in Figure 2.7.

Specific information for all of the groundwater samples collected during the targeted investigation is in Appendix A, Table A.1.

\subsubsection{Groundwater Sampling with the Cone Penetrometer}

The cone penetrometer vehicles were used to collect groundwater samples from the unconfined aquifer at the locations shown in Figure 2.2 (TI01-TI28). At each boring, groundwater samples were typically recovered from 2 to $4 \mathrm{ft}$ depth intervals that were selected upon review of the corresponding ECPT log responses for that location. The samples were retrieved by advancing the rod string to the target sample depth, then temporarily inserting a 0.5 in.-I.D. screen and riser into the rods. The rods were then retracted to expose the surrounding formation over the desired depth interval, and a bailer was lowered through the temporary casing to obtain the groundwater sample (without purging). The casing and screen were then removed.

At most locations, sufficient groundwater for sampling accumulated in the temporary casing within several minutes to hours; at a small number of locations, the temporary casing was left in place overnight before sampling, as noted in Appendix A, Table A.1. 


\subsubsection{Groundwater Sampling from Monitoring Wells}

Groundwater samples for VOCs analyses were obtained from the 19 monitoring wells shown in Figure 2.5. Wells installed during the current field program (at locations DL1-DL8; Figure 2.5) were sampled immediately following development by purging through the use of a Waterra inertial foot valve and tubing pump assembly, a Redi-Flow submersible pump, or both depending on the location and physical characteristics of the well and the volume of groundwater to be removed. Purge volumes (ranging from 450 gal to 2,500 gal) were in part determined on the basis of the volume of water reported (by the driller) as used during well installation, as noted in Section 2.2.1, and typically exceeded the casing storage volume by several orders of magnitude (see Appendix A, Table A.1). Groundwater samples for analyses of VOCs were collected from the pump discharge following purging, in accord with the procedures in the Master Work Plan (Argonne 2002).

Monitoring wells SB62 and SB77 (Figure 2.5), installed during the 1993-1995 CCC/USDA investigations at York, were sampled using a low-flow technique. After groundwater levels were measured, a small volume was purged from each well by using a bladder pump in accord with EPA procedure EPA/540/S-95/504 (Puls and Barcelona 1996; Yeskis and Zavala 2002) and the equipment manufacturer's instructions. Field measurements of temperature, $\mathrm{pH}$, conductivity, $\mathrm{DO}$, and ORP were taken during purging until the measurements stabilized, prior to sample collection from the pump outflow.

\subsubsection{Groundwater Sampling from Private and Municipal Supply Wells}

Groundwater samples were collected from five private wells - at the Volzke, Liermann, Preslicka, and Nordlund residences and the York County maintenance garage - in conjunction with the targeted investigation (Figure 2.3). At six additional locations investigated as part of this study (Figure 2.3), private wells that had previously been identified and sampled in 1993-1995 were found to have been abandoned or were otherwise unavailable for sampling (see Section 4.3.1.1). As discussed in Section 2.1.3, groundwater samples were also obtained from 13 private wells and four PWS wells (Figure 2.7) prior to and during the timeframe of the targeted investigation, as part of the quarterly well sampling program implemented by the CCC/USDA at York. 
Direct access to the private and PWS wells for the measurement of well depths and groundwater levels could not be obtained. At each private well location, groundwater was sampled from an indoor or outdoor tap, as close to the well as possible, after running the water for several minutes. PWS wells 77-1, 2009-4, 2009-5, and 2009-6 were sampled from taps in the respective well houses, after running each well for approximately 10 minutes.

\subsubsection{Analysis of Groundwater Samples}

Water samples for the determination of VOCs were preserved and shipped overnight, on ice at $4^{\circ} \mathrm{C}$, to the AGEM Laboratory for purge-and-trap sample preparation and analysis on a GC-MS system (EPA Methods 5030N and 8260B). To ensure reproducibility, aliquots of 30 groundwater samples selected in the field (representing approximately $27 \%$ of the total samples collected) were sent to TestAmerica Laboratories, Inc., South Burlington, Vermont, for verification analysis with the EPA's Contract Laboratory Program methods, in accord with the procedures in the Master Work Plan (Argonne 2002).

\subsection{Methods for the Investigation of Possible Carbon Tetrachloride Vapor Intrusion to Indoor Air}

With the approval of the CCC/USDA and NDEQ project managers, an initial screening of indoor air for contamination by VOCs was performed at six locations in the vicinity of the former CCC/USDA facility, to investigate for possible carbon tetrachloride VI (Argonne 2015b). The selected locations, shown in Figure 2.6, include two long-term health care facilities affiliated with the York General Hospital (Hearthstone and Willow Brook facilities), two commercial buildings (the Central Valley Ag [CVA] Coop and Fitness Worx facilities), and two private residences (Preslicka and Garretson/Troester) located to the southeast, and downgradient, from the former CCC/USDA facility.

A third possible residence originally proposed for the initial air screening (326 Marquis Ave; Figure 1 in Argonne 2015b) was not tested, because the status of the buildings on this property was questionable and access to the property for sampling purposes was not resolved during the targeted investigation field program. 
The indoor air screening event was performed on August 4-5, 2015. At each facility, samples of the indoor air were collected from the breathing zone at locations on the ground floor and the lower floor (or basement), if applicable, over a 24-hr period using certified-clean, 6-L stainless steel canisters equipped with timed-inlet fittings. Background samples of outdoor air were also collected near the Fitness Worx and Hearthstone facilities, at the Garretson/Troester residence, and at the former CCC/USDA facility during this event.

On the basis of findings obtained from the August screening event (see Section 3.5), the NDEQ requested that the CCC/USDA conduct additional sampling of the indoor air, as well as sub-slab vapor, for VOCs analyses at 2 locations - the Fitness Worx facility and the Garretson/Troester home — on a quarterly basis for one year, beginning in January 2016. Sampling of indoor air and sub-slab vapor was therefore performed at these properties on January 27-28, 2016 during the targeted investigation field program. With the approval of the respective property owners, one permanent sub-slab vapor sampling probe was installed in the basement/lower level of each building, by using the standard operating procedures outlined in Appendix B. Sub-slab vapor samples were collected as "grab" samples in certified-clean, 1-L stainless steel canisters. Indoor air and outdoor air (background) samples were collected at the two locations in a manner similar to that described for the August 2015 sampling event.

On April 27-28, 2016, indoor air, sub-slab vapor, and background air samples were collected at the Fitness Worx facility as described above. With the approval of the CCC/USDA and NDEQ project managers, sampling was postponed at the Garretson/Troester residence at this time, pending the installation of a sub-slab vapor mitigation system by the CCC/USDA at this location. Indoor air, sub-slab vapor, and background air samples were again collected at the Fitness Worx facility, and indoor air and background air samples were collected at the Garretson/Troester home (following completion of the vapor mitigation system), on August 910, 2016 and December 13-14, 2016.

All samples of indoor air and sub-slab vapor were shipped overnight to TestAmerica Laboratories, Inc., South Burlington, Vermont, for VOCs analyses by EPA Method TO-15. 
rsion 01, 02/14/2017

TABLE 2.1 Summary of field sessions during the York targeted investigation.

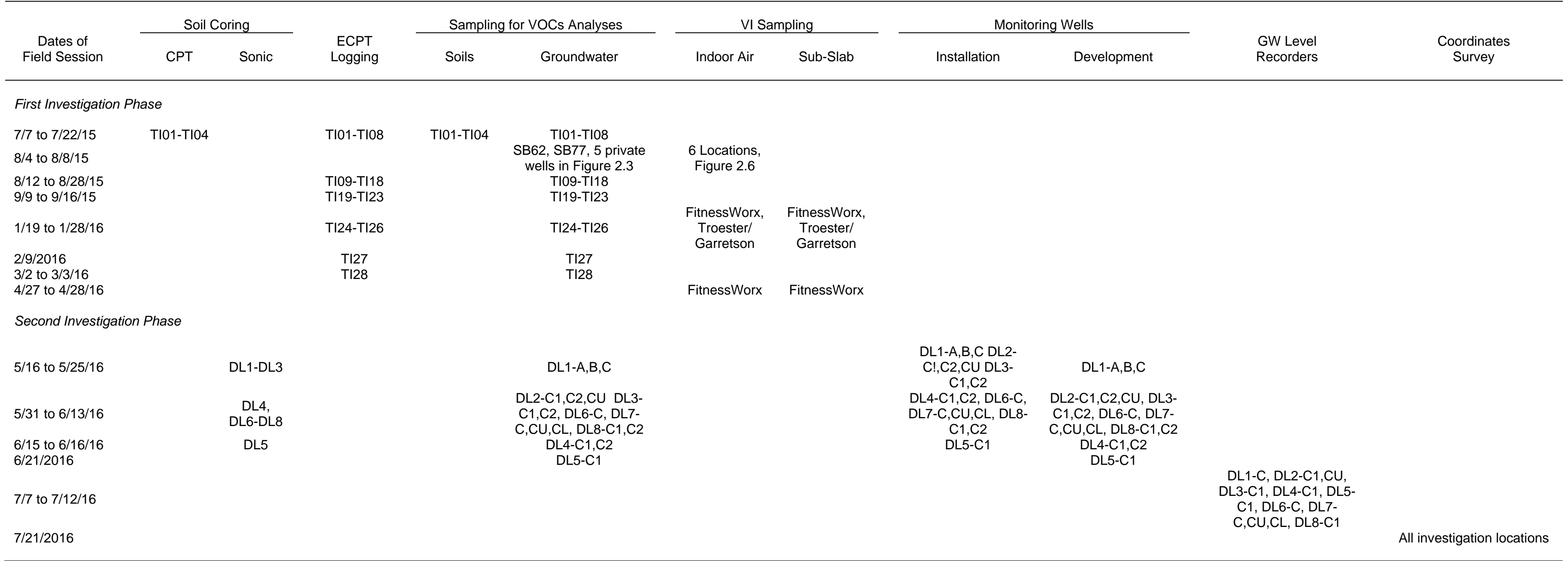




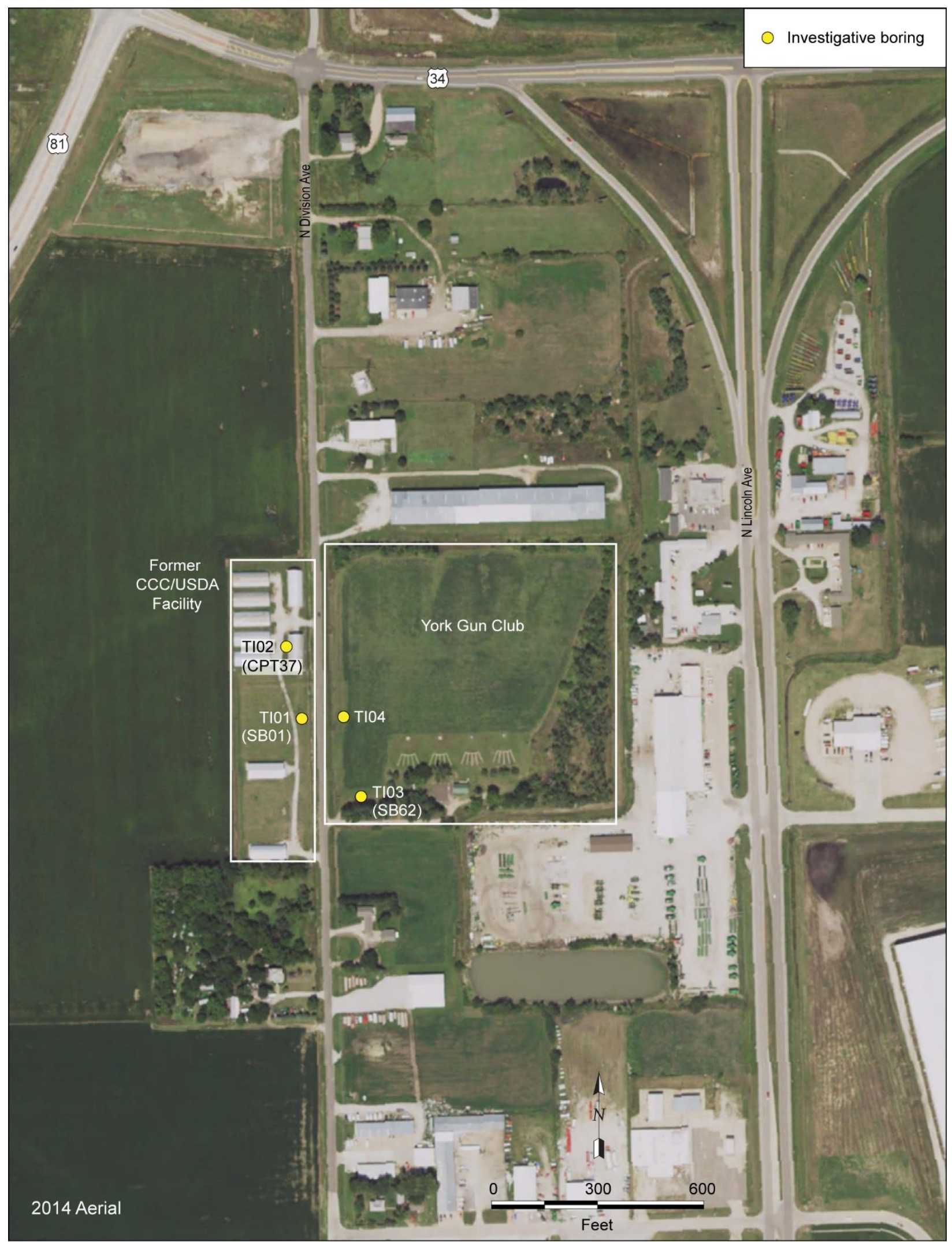

FIGURE 2.1 Locations of direct-push (CPT) borings used to sample soil for VOCs analyses. 


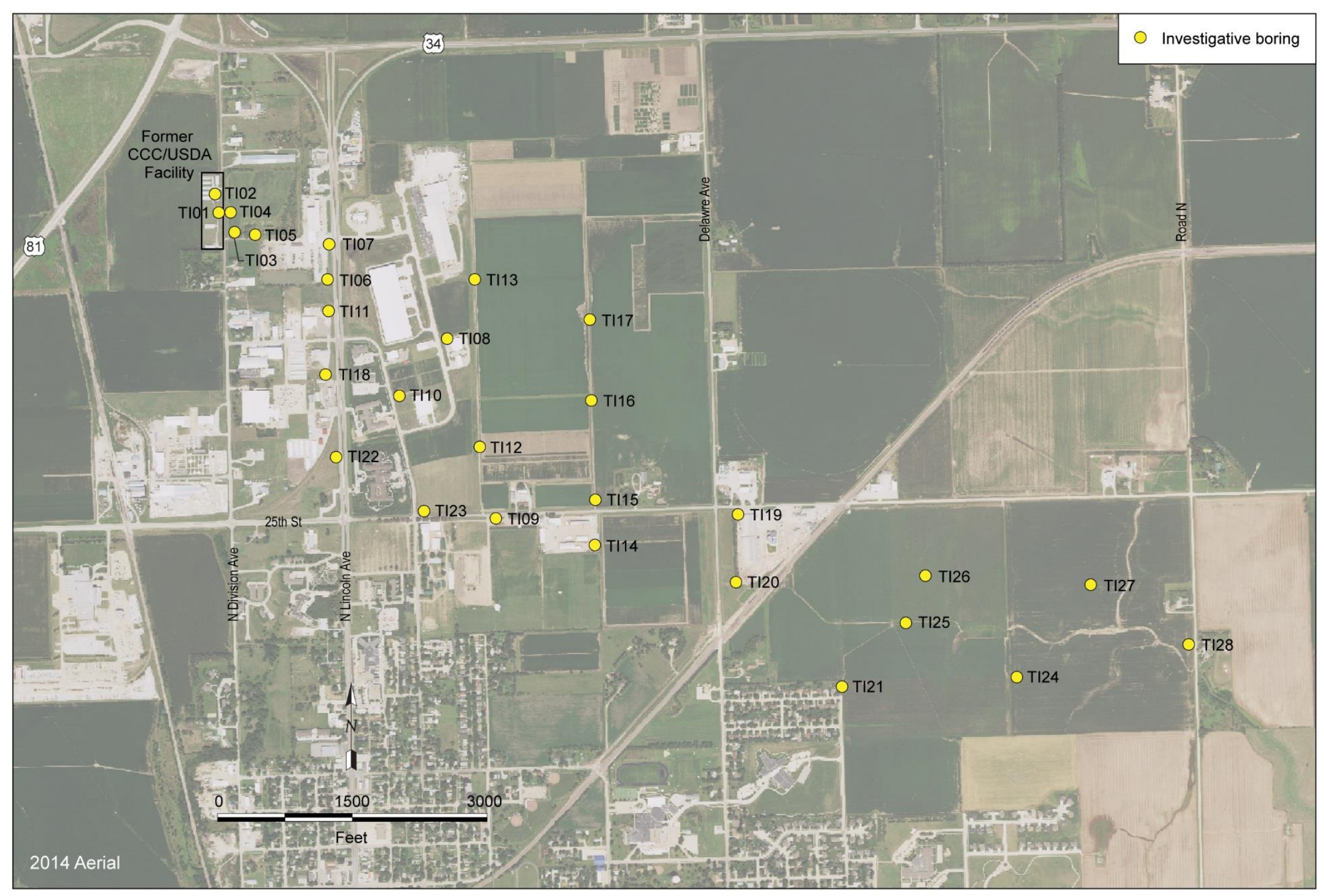

FIGURE 2.2 Locations of direct-push borings in the unconfined aquifer used to obtain EPCT logs and collect groundwater samples for VOCs analyses. 


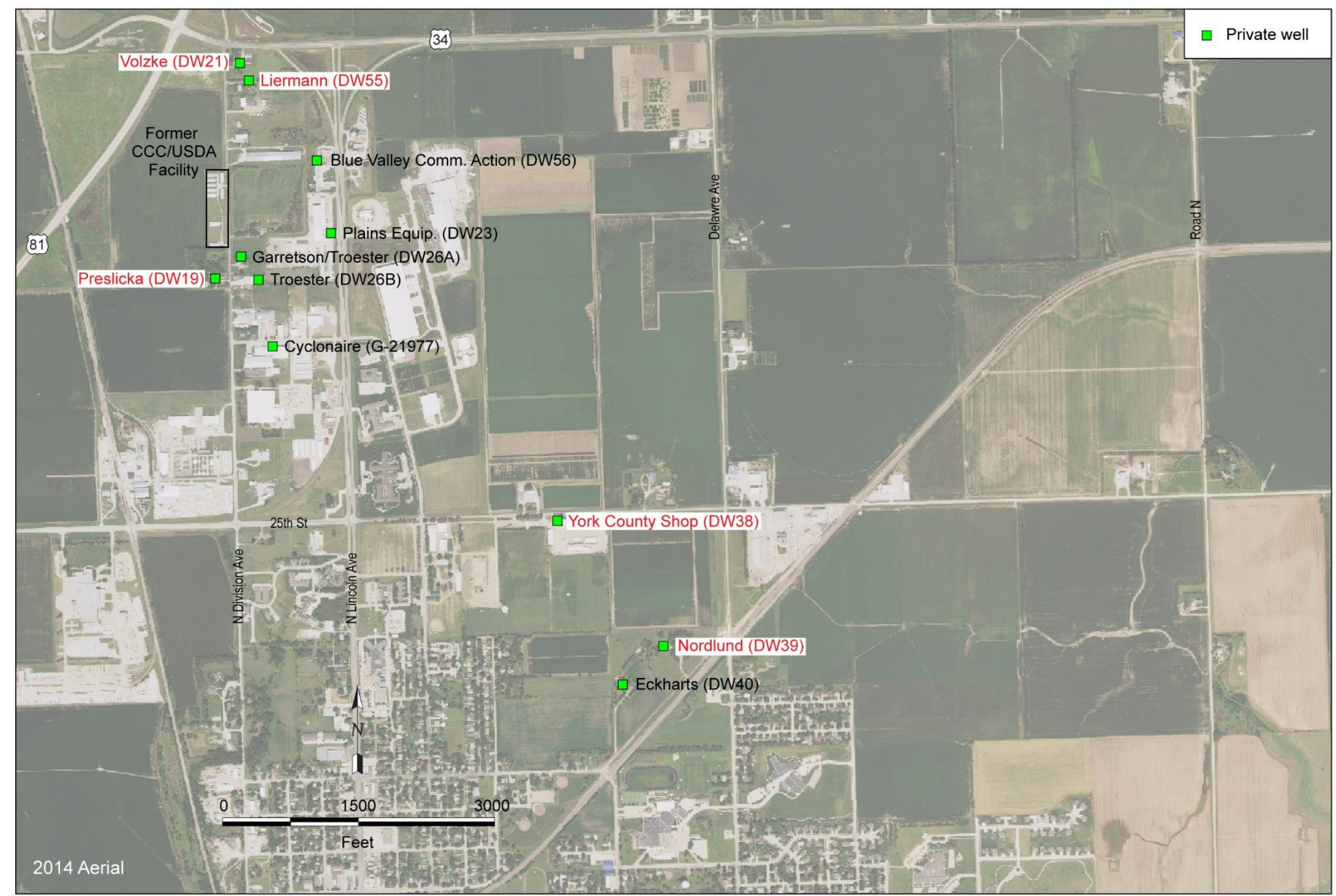

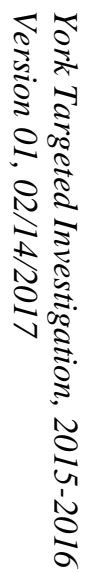

FIGURE 2.3 Locations of private wells investigated during the present study. 


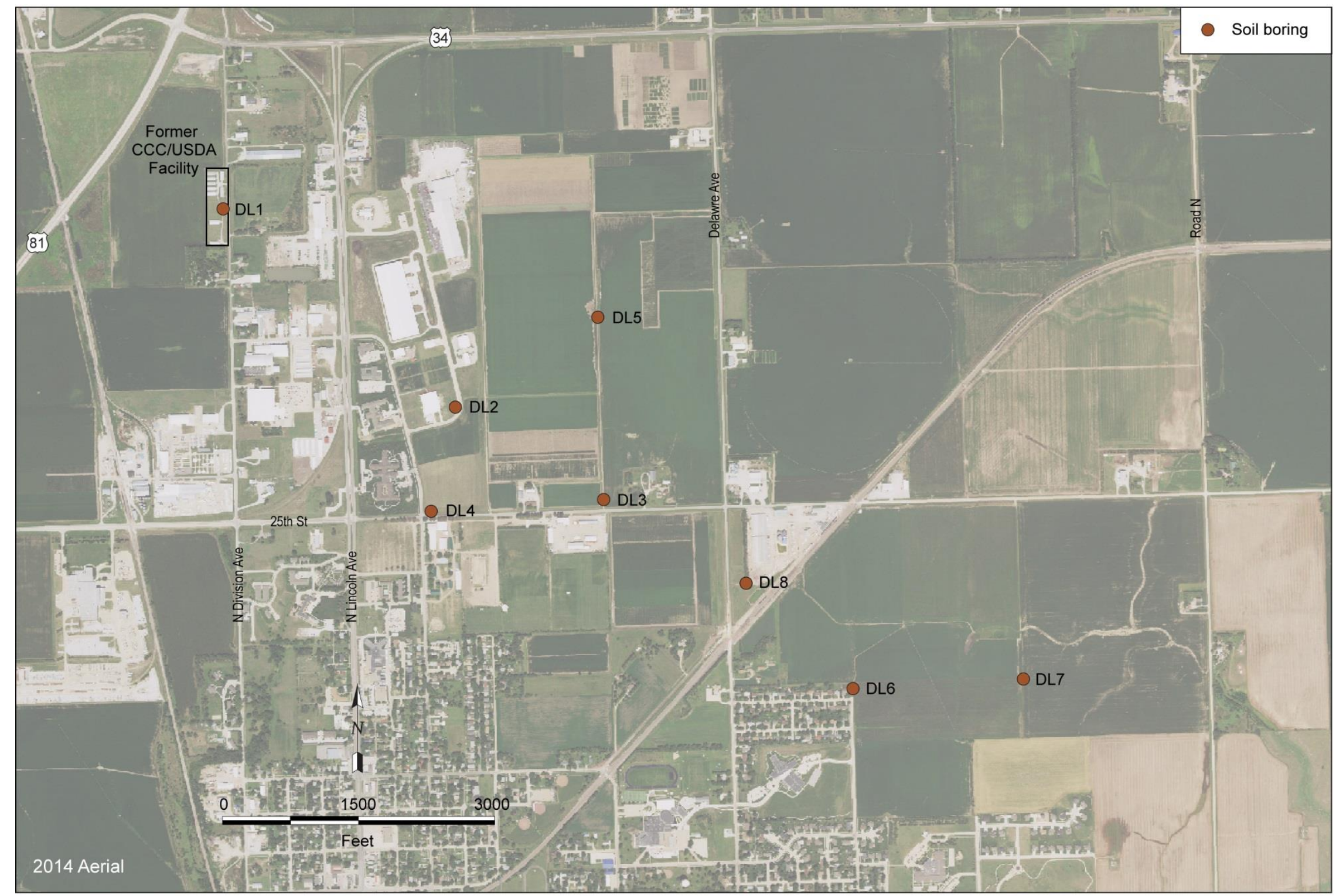

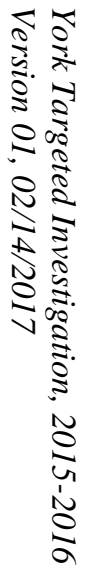

FIGURE 2.4 Locations of conventionally drilled, continuously cored soil borings. 


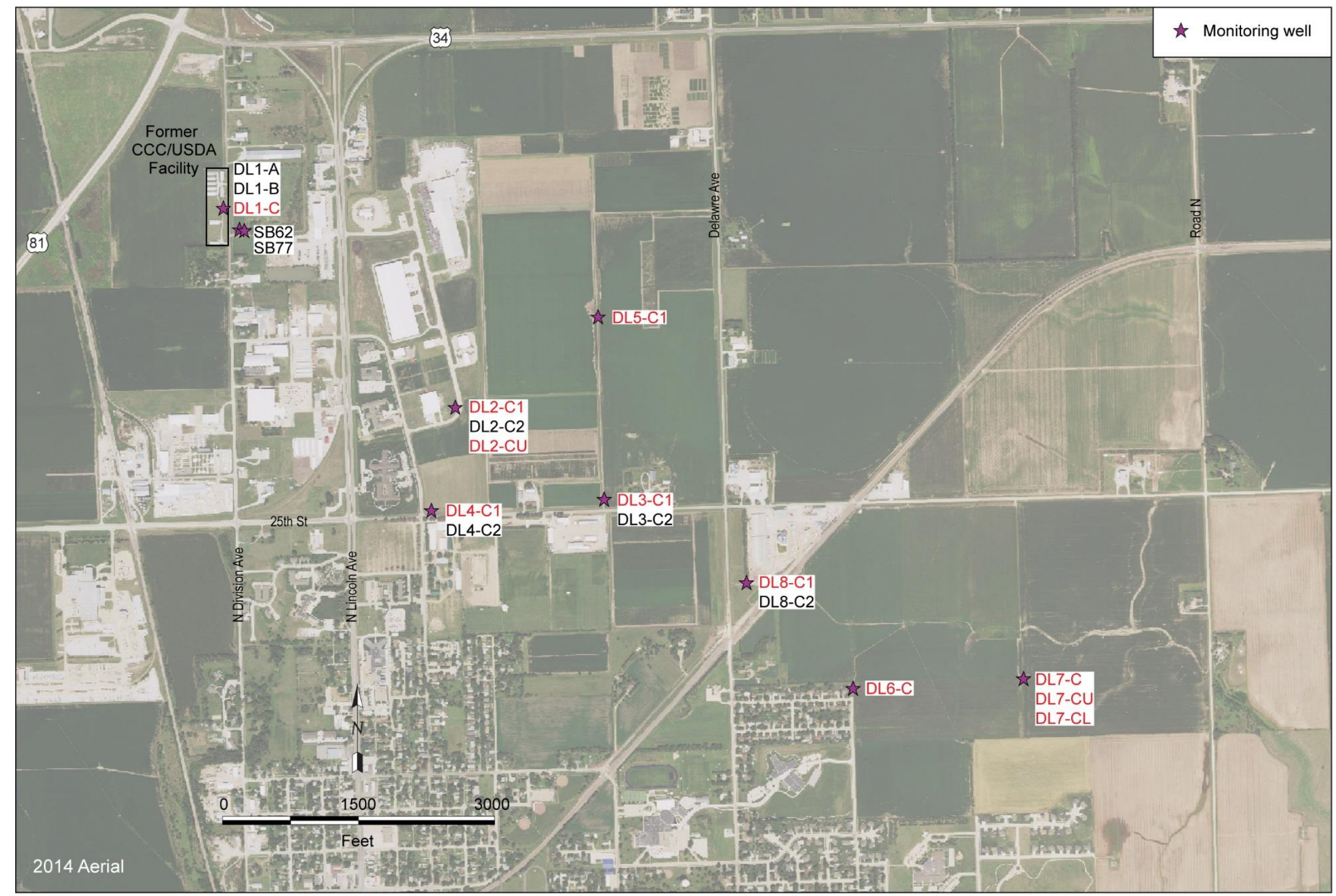

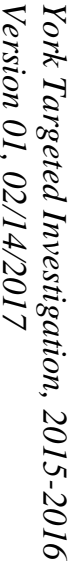

FIGURE 2.5 Locations of monitoring wells installed by the CCC/USDA during the current or previous investigations at York, and used to measure groundwater levels and collect groundwater samples for VOCs analyses. 


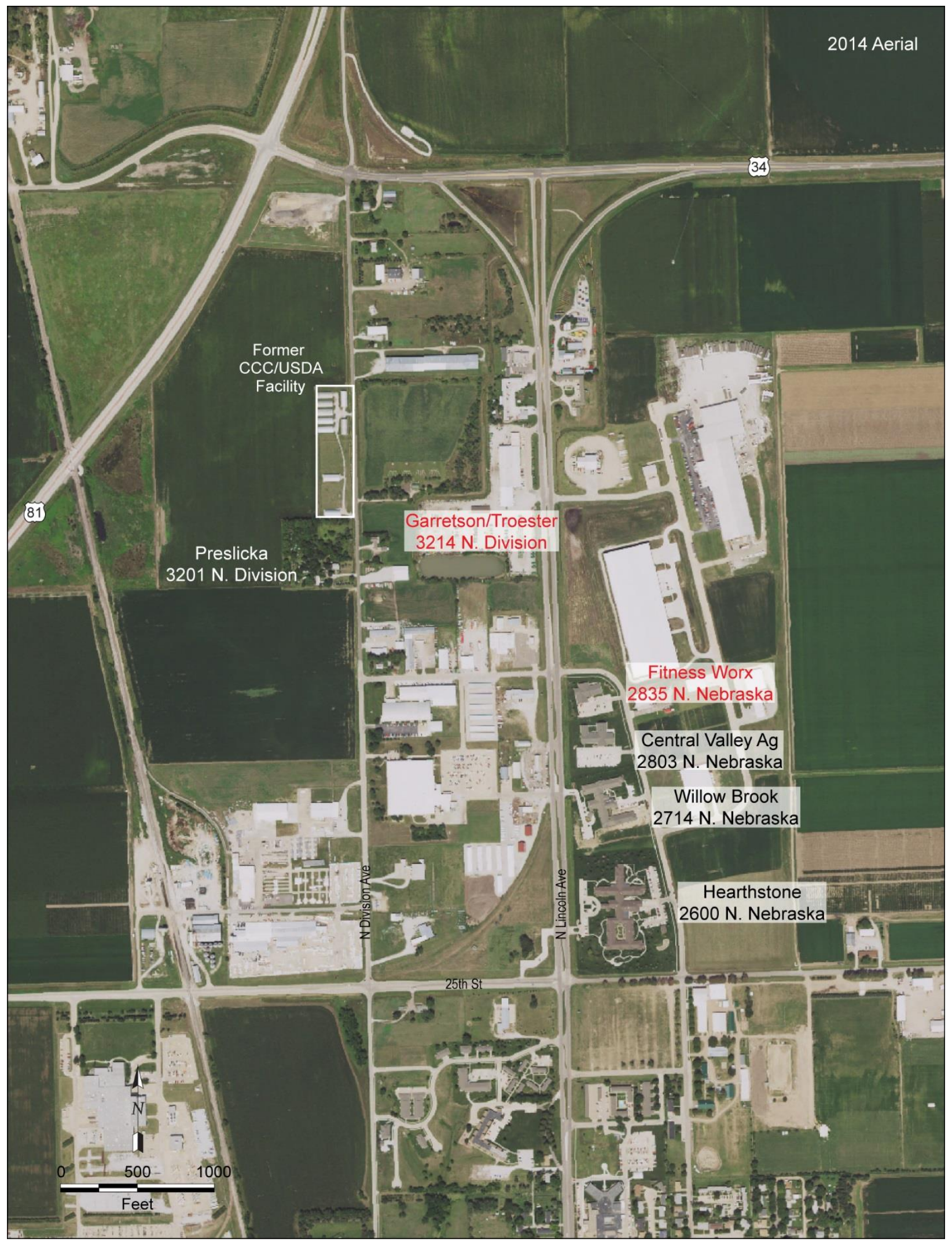

FIGURE 2.6 Locations sampled for indoor air, or sub-slab soil vapor and indoor air, to investigate carbon tetrachloride $\mathrm{VI}$. 


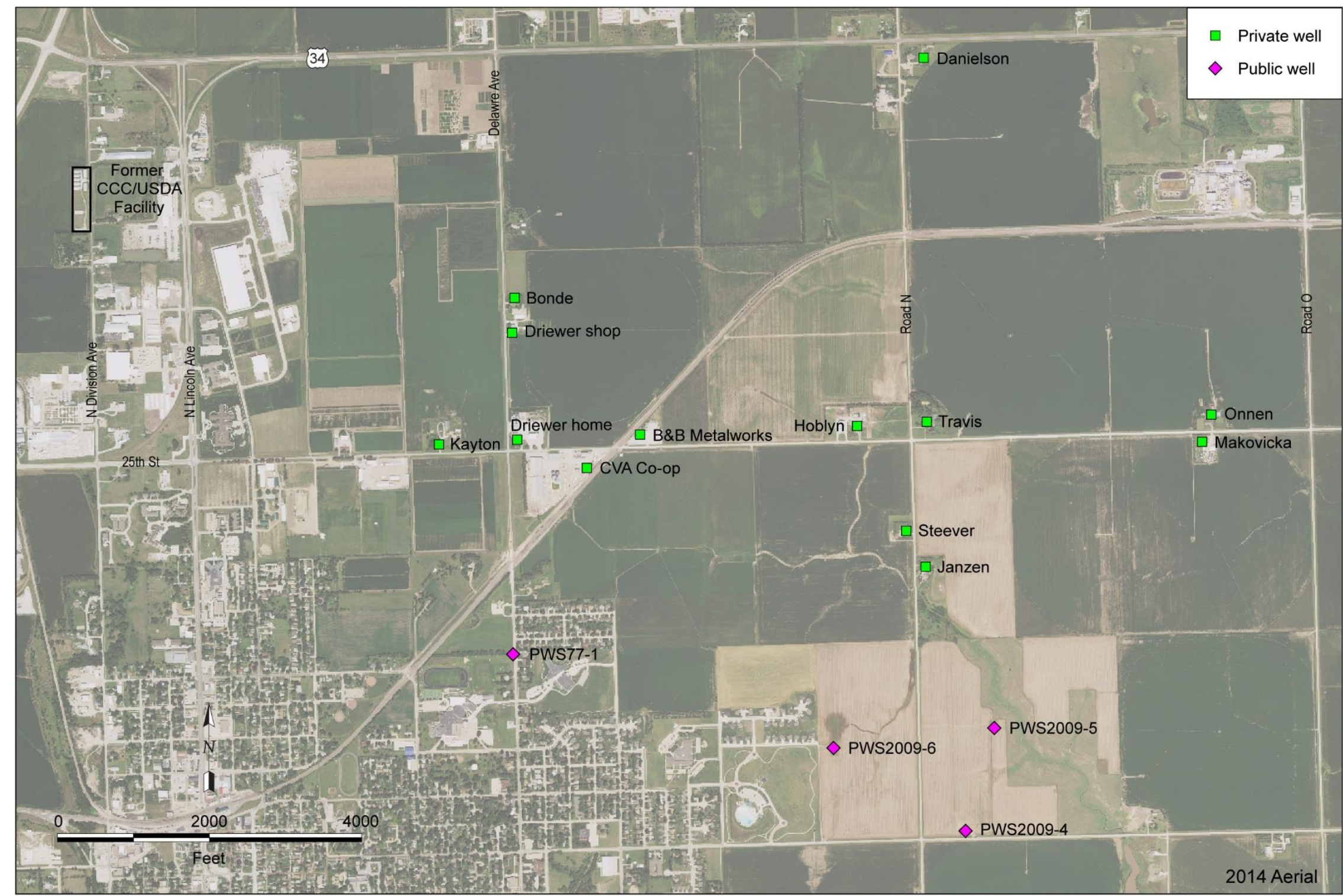

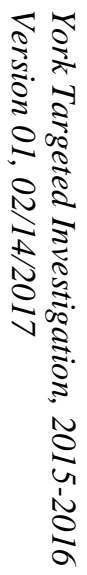

FIGURE 2.7 Locations of private wells and PWS wells sampled quarterly for VOCs by the CCC/USDA, at the request of the NDEQ. 


\section{Field and Laboratory Data}

This section presents the field and laboratory data acquired during the targeted investigation at York. A chronological summary of the sampling activities is in Appendix A, Table A.1. The methods and procedures used in collecting the data are discussed in Section 2, in the Master Work Plan (Argonne 2002), and in the Final Work Plan (Argonne 2016a)

\subsection{Lithologic and Physical Property Data for Soils}

The Argonne ECPT units were used to obtain continuous vertical profiles of tip pressure, sleeve friction, and tip-versus-sleeve ratio through the interpreted vadose zone and unconfined aquifer sequence at the locations shown in Figure 2.2 (TI01-TI28). The resulting geomechanical logs, in Appendix C, were used during the first phase of the targeted investigation field program as a basis for identification and correlation of predominantly coarse-grained versus finer-grained lithologic intervals within the unconfined aquifer, and hence for the selection of depth intervals for groundwater sampling (Section 2.1.1). Qualitative interpretations of the sensor responses developed during this phase of the field program were based on comparisons of the logs to sediment (core) and equivalent log-response data acquired at selected locations during the previous CCC/USDA studies conducted by Argonne at York (Argonne 1994, 1995a.b).

To obtain additional data for evaluation of the local lithologic sequence, continuous soil cores were recovered at conventional drilling locations DL1-DL8 (Figure 2.4). At DL1, DL3DL6, and DL8 the borings were advanced through the vadose zone and unconfined aquifer sediments. At location DL2 coring continued into and through the upper confined aquifer; at DL7 the boring was advanced into the upper portion of the lower confined aquifer (Section 4.1).

Lithologic descriptions for the continuous soil cores recovered at borings DL1-DL8 are in Appendix D. The lithologies identified at DL1-DL8 are compared to the geomechanical log traces for corresponding ECPT investigative locations TI01, TI12, TI15, TI23, TI17, TI21, TI24, and TI20, respectively, in Appendix C (Figures 2.2 and 2.4).

The results of sediment particle-size analyses for selected intervals in borings DL1-DL8 are in Appendix D, Table D.1. The core intervals submitted for laboratory analysis were chosen to reflect the range of predominant sediment types penetrated at the investigation site, as well as subtle variations in the properties of the soils suggested by visual inspection. The lithologic and 
hydrologic characteristics of the sediments identified during the targeted investigation are discussed in Sections 4.1 and 4.2.

\subsection{Analytical Data for Soil Samples}

A total of 78 soil samples were collected for VOCs analysis by the purge-and-trap method at direct-push boring locations TI01-TI04 (Figure 2.1). At each location, the samples were collected at approximate 4-ft intervals with depth, from $4 \mathrm{ft}$ BGL into the top of the saturated zone (identified in samples from 75-80 ft BGL at the time of the sampling event).

The results of the purge-and-trap analyses for the soil samples for carbon tetrachloride and chloroform are summarized in Table 3.1. The full results of these analyses are in Appendix E, Table E.1.

Carbon tetrachloride was identified at concentrations equal to or greater than the method detection limit for the purge-and-trap analysis technique $(10 \mu \mathrm{g} / \mathrm{kg})$ in 28 soil samples, from borings TI01, TI02, and TI04; only trace levels of carbon tetrachloride $(<10 \mu \mathrm{g} / \mathrm{kg})$ were detected at boring TI03. Levels of carbon tetrachloride exceeding the NDEQ VCP target concentration for this contaminant in soils $(39 \mu \mathrm{g} / \mathrm{kg}$; for protection of the soil-to-groundwater pathway) were identified in nine samples from boring TI01 (at depths of 20-44 ft, $52 \mathrm{ft}$, and $80 \mathrm{ft}$ BGL), and five samples from boring TI04 (at $24 \mathrm{ft}$ and 32-44 ft BGL; Table 3.1). Maximum carbon tetrachloride concentrations ranging from $60 \mu \mathrm{g} / \mathrm{kg}$ to $167 \mu \mathrm{g} / \mathrm{kg}$ were identified at boring TI01; the maximum levels observed at boring TI04 are 43-61 $\mu \mathrm{g} / \mathrm{kg}$. No carbon tetrachloride was identified at concentrations greater than the NDEQ VCP target levels for direct-contact exposure under the residential or industrial soil scenarios $(720 \mu \mathrm{g} / \mathrm{kg}$ and 3,600 $\mu \mathrm{g} / \mathrm{kg}$, respectively).

At TI01, TI02, and TI04, carbon tetrachloride was identified in the deepest soil sample(s) analyzed (at depths of 75-80 ft BGL; Table 3.1), beneath an overlying interval of samples showing no detectable contamination. Groundwater levels observed at the time of sampling suggest that the carbon tetrachloride in these deepest soils is likely associated with groundwater present in the saturated, unconfined aquifer zone.

With only five exceptions, chloroform was not detected in the soil samples at levels equal to or greater than the method detection limit for the purge-and-trap analysis $(10 \mu \mathrm{g} / \mathrm{kg})$. 
Chloroform concentrations ranging from $12-17 \mu \mathrm{g} / \mathrm{kg}$ were identified at $32-52 \mathrm{ft}$ BGL in boring TI01, in association with carbon tetrachloride. Trace concentrations of chloroform $(<10 \mu \mathrm{g} / \mathrm{kg})$ were also detected in soil samples at TI01 at depths of $12 \mathrm{ft}$ to $28 \mathrm{ft} \mathrm{BGL}$, and at 16-36 ft and $80 \mathrm{ft}$ BGL at boring TI04. No chloroform was detected at boring TI02 or TI03 (Table 3.1).

No methylene chloride was detected in the soil samples from borings TI01-TI04. The compound 1,2-dichloroethane was identified in two isolated soil samples from boring TI01 $(20 \mu \mathrm{g} / \mathrm{kg}$, at $36 \mathrm{ft} \mathrm{BGL}$; and $19 \mu \mathrm{g} / \mathrm{kg}$, at $44 \mathrm{ft} \mathrm{BGL})$, but was not detected in the remaining samples from this location or borings TI02-TI04. Argonne is unaware of any known usage of this chemical by the CCC/USDA at its former grain storage facilities. The observed detections of 1,2dichloroethane are below the NDEQ VCP target for this contaminant $(28 \mu \mathrm{g} / \mathrm{kg}$; for protection of the soil-to-groundwater pathway). No other VOCs were identified in the soils analyzed from borings TI01-TI04 (Appendix E, Table E.1).

\subsection{Installation of Permanent Monitoring Wells}

A total of 17 permanent monitoring wells were installed in eight locations (DL1-DL8) to facilitate groundwater sampling and the measurement of groundwater levels (Figure 2.5). The wells were constructed by using the conventional (sonic) drilling rig, in accord with the procedures outlined in Section 2.2.2. At each location, the specific zone(s) targeted for well installation were initially selected through evaluation of the ECPT $\log$ responses for the corresponding direct-push investigative borings; final completion depths were adjusted, if warranted, based on the characteristics of the sediment cores retrieved as the drilled borings were advanced.

All wells were installed in individual borings, in accord with NDEQ requirements. A suffix (i.e., A, CU, CL; Table 3.2) is included in the designation for each well signifying the stratigraphic interval penetrated by the well screen, as locally interpreted in the present study area (See Section 4.1 for discussion of the hydrogeologic intervals).

Construction data for the monitoring wells are summarized in Table 3.2. Construction data for two permanent monitoring wells (SB62 and SB77; Figure 2.5) installed during the previous investigations conducted on behalf of the CCC/USDA at this site are included in 
Table 3.2 (Argonne 1994, 1995a,b). Nebraska Department of Natural Resources (NDNR) registration forms for the wells installed during the targeted investigation are in Appendix F.

\subsection{Analytical Data for Water Samples}

A total of 111 water samples were collected in conjunction with the targeted investigation, from 28 direct-push investigative borings (Figure 2.2), five private wells (Figure 2.3), 19 permanent monitoring wells (Figure 2.5), and the York municipal water supply used during the drilling operations.

The water sampling and analyses were performed as described in Section 2.4. The specific conditions under which each sample was collected are in Appendix A, Table A.1. The results for carbon tetrachloride and chloroform identified in the groundwater are summarized in Table 3.3. The complete results of the water analyses for VOCs and groundwater parameters measured in the field for the monitoring well samples are in Appendix G, Tables G.1 and G.2, respectively.

As noted in Sections 2.1.3 and 2.4.3, groundwater samples for VOCs analyses are collected on behalf of the CCC/USDA on a quarterly basis at 13 private wells and 4 PWS wells located to the east and southeast of the former CCC/USDA facility, at the request of the NDEQ (Figure 2.7). Groundwater sampling at these locations was conducted in four events that occurred during the timeframe of the targeted investigation field program - in August and November 2015 and February and June 2016 - as well as in earlier and subsequent episodes. The full results of these events are reported separately (Argonne 2014; 2015e,f; 2016b,c,d,e); for convenience, carbon tetrachloride data from the quarterly groundwater sampling program are summarized in Table 3.4.

\subsubsection{Volatile Organic Compounds in Groundwater from the Unconfined Aquifer}

A total of 85 groundwater samples were collected from the unconfined aquifer by the direct-push method, at the locations shown in Figure 2.2. Groundwater samples from this unit were also obtained from 16 permanent monitoring wells (Table 3.2 and Figure 2.5). The distribution of carbon tetrachloride identified in the unconfined aquifer is summarized in Figures 3.1 and 3.2. 
Carbon tetrachloride was identified in 52 groundwater samples at levels equal to or greater than the method detection limit for the purge-and-trap analysis technique $(1 \mu \mathrm{g} / \mathrm{L})$; trace levels of this contaminant $(<1 \mu \mathrm{g} / \mathrm{L})$ were detected in eight additional samples. Carbon tetrachloride concentrations exceeding the MCL and NDEQ VCP target level for this contaminant in drinking water $(5 \mu \mathrm{g} / \mathrm{L})$ were determined for 35 samples (Table 3.3). Concentrations of carbon tetrachloride ranging from $150 \mu \mathrm{g} / \mathrm{L}$ to $1,875 \mu \mathrm{g} / \mathrm{L}$ were identified in the shallowest groundwater sampled (at depths of approximately 76-82 ft BGL) at direct-push locations TI01-TI05 and monitoring wells DL1-A and SB77, on and adjacent to the former CCC/USDA facility property, and in one sample (from a depth of 112-122 ft BGL) at location TI11 immediately to the southeast of the former facility (Figures 3.1 and 3.2). Figures 3.1 and 3.2 illustrate that the maximum carbon tetrachloride concentrations identified in the groundwater generally decrease, and occur at greater depths within the aquifer, with distance to the southeast from the former CCC/USDA facility. The areal and vertical distribution of carbon tetrachloride within this unit are discussed further in Section 4.3.1.

Chloroform was identified in 27 of the unconfined aquifer groundwater samples at levels greater than the method detection limit for the purge-and-trap analysis technique $(1 \mu \mathrm{g} / \mathrm{L})$; trace concentrations of chloroform $(<1 \mu \mathrm{g} / \mathrm{L})$ were detected in 15 additional samples. With few exceptions, chloroform concentrations were generally low $(<10 \mu \mathrm{g} / \mathrm{L})$. Maximum chloroform levels ranging from $11 \mu \mathrm{g} / \mathrm{L}$ to $164 \mu \mathrm{g} / \mathrm{L}$ were identified in six groundwater samples only, representing the shallowest saturated intervals sampled (at depths of approximately 76-82 ft BGL) at locations TI01-TI05 and monitoring well DL1-A on the former CCC/USDA and adjacent York Gun Club properties (Table 3.3). No methylene chloride was detected in the unconfined aquifer groundwater (Appendix G, Table G.1).

Appendix G, Table G.1 indicates that trace levels of PCE and 1,2-dichloropropane were detected, together with carbon tetrachloride and chloroform, in the shallowest groundwater samples recovered at direct-push location TI01 on the former CCC/USDA property, and a trace of PCE was detected at direct-push location TI04 on the adjacent York Gun Club property. PCE and 1,2-dichloropropane are not associated with fumigants known to have been routinely used by the CCC/USDA to treat grain in storage, and the origin of these chemicals at these locations is unknown. Trace to low levels of PCE, TCE, 1,2 dichloropropane, 1,2-dichloroethane, and 1,1DCE were also detected in isolated samples from several additional locations to the southeast of the former CCC/USDA facility, in the absence of associated carbon tetrachloride Appendix G, Table G.1). The occurrence of these chemicals is discussed further in Section 4.3.1.3. 


\subsubsection{Volatile Organic Compounds in Groundwater from the Upper and Lower Confined Aquifers}

With the approval of the CCC/USDA and NDEQ project managers, samples of groundwater were collected from the upper confined aquifer at two locations (monitoring wells DL2-CU and DL7-CU), and groundwater from the lower confined aquifer was sampled at one location (monitoring well DL7-CL; Table 3.2 and Figure 2.5) during the present study. No carbon tetrachloride, chloroform or other VOCs were detected in these samples (Table 3.3 and Appendix G, Table G.1).

Groundwater samples are also collected on a quarterly basis from York municipal supply wells PWS 77-1, 2009-4, 2009-5, and 2009-6 (Figure 3.3). Well PWS 77-1 is screened in both the upper and lower confined aquifers; wells 2009-4, 2009-5, and 2009-6 are screened exclusively in the lower confined aquifer. No carbon tetrachloride, chloroform, or methylene chloride have been detected at these wells (Table 3.4; see below).

\subsubsection{Volatile Organic Compounds in Groundwater from Private Wells and PWS Wells}

Five private wells - at the Volzke, Liermann, Preslicka, and Nordlund residences and the York County maintenance shop - were sampled in conjunction with the targeted investigation (Figure 2.3). As discussed in Section 2.1.3, groundwater samples were also obtained from 13 private wells and 4 PWS wells (Figure 2.7) prior to and during the timeframe of the targeted field studies, as part of the quarterly well sampling program implemented by the CCC/USDA at York.

Carbon tetrachloride and chloroform analysis data for the samples from the five private wells sampled during the targeted investigation are in Table 3.3; the full results of the analyses for these wells are in Appendix G, Table G.1. Carbon tetrachloride data for the private and PWS wells that were sampled quarterly are summarized in Table 3.4; the full results of the analyses for these wells were previously reported (Argonne 2014; 2015d,e,f; 2016b,c,d, e). The results for carbon tetrachloride for all of the private and PWS wells sampled are illustrated in Figure 3.3.

Carbon tetrachloride was identified at concentrations of $1.3 \mu \mathrm{g} / \mathrm{L}$ and $53 \mu \mathrm{g} / \mathrm{L}$, respectively, in the Preslicka private well and the well at the York County maintenance shop; a trace of chloroform $(<1 \mu \mathrm{g} / \mathrm{L})$ was also detected at the latter well. No carbon tetrachloride or chloroform was detected at the Volzke, Nordlund, or Liermann private wells (Table 3.3). Low 
levels of 1,1-DCE, PCE, and TCE were identified, however, at the Nordlund private well. No methylene chloride was detected in any of these wells (Appendix G, Table G.1).

No carbon tetrachloride has been detected to-date in municipal supply wells PWS 77-1, 2009-4, 2009-5, or 2009-6, or in a majority of the private wells that are sampled quarterly (Table 3.4 and Figure 2.7).

Levels of carbon tetrachloride at or near the MCL and NDEQ VCP target value for this contaminant in drinking water $(5 \mu \mathrm{g} / \mathrm{L})$ have consistently been identified at the CVA Coop and Kayton (former Olson) private wells; however, carbon tetrachloride concentrations of 13-17 $\mu \mathrm{g} / \mathrm{L}$ were identified at the Kayton well in the June and October 2016 sampling events, and $9.2 \mu \mathrm{g} / \mathrm{L}$ at the Coop well in the October 2016 event (Table 3.4). The contaminated well at the CVA Coop is currently used for domestic purposes, without treatment. The Kayton well has historically been used for domestic purposes, but this property is currently vacant, and the CCC/USDA is working with the property owner (as of this report) to have the property connected to the York municipal water supply.

At the request of the NDEQ, quarterly sampling of the private well at the Driewer residence (at 1402 Road 14) was initiated in December 2016; the CCC/USDA had previously been denied access for sampling by the property owner. Carbon tetrachloride was identified at this well at a concentration of $5.1 \mu \mathrm{g} / \mathrm{L}$ in the December 2016 sampling event. The groundwater from this well is treated by reverse osmosis before usage for domestic purposes.

No chloroform or methylene chloride, or other VOCs have been detected to-date in groundwater from the private and PWS wells included in the quarterly well sampling program (Figure 2.7; Argonne 2014; 2015d,e,f; 2016b,c).

\subsubsection{Volatile Organic Compounds in Groundwater from the York Municipal Distribution System}

As noted in Section 2.2.1, water from the York municipal distribution system was used as needed during drilling and monitoring well installation with the sonic drilling rig. Samples of this water were collected for VOCs analyses from (1) a fire hydrant located just east of the York County maintenance shop and (2) the integral water storage tank on the drilling rig support truck, after filling of the tank from the hydrant. These samples are considered equipment blanks. The 
analytical results are in Supplement 2, Table S2.2 (on the compact disc [CD] in the back cover of this report). No carbon tetrachloride, chloroform, or methylene chloride was detected in these samples.

\subsection{Groundwater Level Data}

To obtain information on the levels and possible direction(s) of groundwater flow in the vicinity of the former CCC/USDA facility, groundwater levels were periodically measured manually in the network of 19 monitoring wells (Table 3.2; Figure 2.5). The specific dates on which groundwater levels were measured and the results of the manual measurements are in Table 3.5.

Automatic groundwater level measuring and recording units were installed in 11 monitoring wells in July 2016 (Figure 2.5). To-date, recorded data from the monitoring units were recovered on November 3, 2016. The monitoring devices will remain in the wells for an anticipated period of one year, to investigate possible seasonal or other influences on the local groundwater levels. Additional data will be recovered from the recorders approximately quarterly.

The complete set of water levels recorded by the data loggers is in Supplement 1, Tables S1.1 and S1.2 (on CD). The patterns of groundwater level variations, apparent hydraulic gradients, and flow directions developed from the data logger records and the manual water level measurements are discussed in Section 4.1.2.

Information on precipitation in the York area during the groundwater monitoring period was acquired to assist in the evaluation of observed groundwater level trends. Daily rainfall data for the weather station at the York Municipal airport were obtained from Weather Underground; these data are available online (www.wunderground.com/us/ne/york/zmw:68467.1.99999). The precipitation data are summarized in Supplement 1, Table S1.3 (on CD).

\subsection{Analytical Data for VI Samples}

With the approval of the CCC/USDA and NDEQ project managers, sampling of indoor air was initially conducted at six locations selected in consultation with the NDEQ, to screen for 
possible vapor intrusion by VOCs that might be linked to the past use of grain fumigants at the former CCC/USDA facility (i.e., carbon tetrachloride, chloroform, methylene chloride, and chloromethane). The selected locations included two long-term health care facilities (Hearthstone and Willow Brook facilities), two commercial buildings (the CVA Coop and Fitness Worx facilities), and two private residences (Preslicka and Garretson/Troester) located to the southeast, and downgradient, from the former CCC/USDA facility. The initial VI sampling event was conducted in August 2015. At the request of the NDEQ, the CCC/USDA has subsequently conducted additional sampling of the indoor air and sub-slab vapor at two locations - the Fitness Worx facility and the Garretson/Troester residence - on a quarterly basis (Figure 2.6).

The results of analyses of the indoor air and sub-slab vapor samples for carbon tetrachloride and chloroform are summarized in Table 3.6. The complete results of the analyses for VOCs of these samples are in Appendix H, Table H.1. Because the specific locations sampled, and the series of VI sampling events conducted, at each of these facilities is somewhat unique, the results of the VOCs analyses for each location are discussed individually in Section 4.4.

\subsection{Coordinates Survey Data}

To provide control for use in correlation and interpretation of the lithologic, stratigraphic, analytical, and groundwater level information acquired in this study, the locations and elevations of investigative borings SB01-SB24 and the monitoring wells, private wells, and PWS wells addressed in this report were surveyed by licensed professional surveyors of Catlett Surveying, Bennet, Nebraska. The locations of investigative borings TI25-TI28 were determined by using a hand-held (cell phone) GPS device. The measurements for all of the investigated locations are in Appendix I, Table I.1

\subsection{Results of Quality Control Activities}

The QA/QC procedures for sample collection, handling, and analysis during the 20152016 investigation are described in detail in the Master Work Plan (Argonne 2002). A detailed QA/QC report addressing sample collection, handling, and analysis during the investigation is in Supplement 2 (on CD). 
The results of the QA/QC activities are summarized as follows:

- Sample integrity was maintained successfully during sample collection, shipping, and analysis through documentation of samples as they were collected and the use of custody seals and chain-of-custody (COC) records.

- All samples were received with custody seals intact and at the appropriate preservation conditions, with the exception of the samples shipped with COC number YK29161700, which were received frozen. One vial of each sample with COC YK29161700 remained unbroken and was analyzed. Samples were analyzed within required holding times.

- Carbon tetrachloride and chloroform, the primary contaminants of concern, were not detected in laboratory method blanks at the AGEM Laboratory.

- Quality control samples (equipment blanks, equipment rinsates, and trip blanks) were collected to monitor sample-handling activities. Neither carbon tetrachloride nor chloroform was detected, with the exception of trip blank YKQCTB-W-37869, in which a trace concentration of chloroform was detected. In addition, three trip blanks contained trace concentrations of methylene chloride but were marked with the B qualifier, indicating that the compound was also detected in an associated laboratory blank.

- Soil and groundwater samples were analyzed for VOCs including carbon tetrachloride, chloroform, and methylene chloride at the AGEM Laboratory by using the purge-and-trap method (a GC-MS method) for quantitative determination of contaminant distribution. Indoor air and soil gas samples were analyzed for VOCs at TestAmerica Laboratories, Inc., South Burlington, Vermont (Supplement 4, on CD). Dual analyses were accomplished through either analysis of replicate samples submitted to the laboratory or (for soil and groundwater samples at the AGEM Laboratory) duplicate analysis of samples selected by the laboratory. The average relative percent difference values were $13 \%$ for carbon tetrachloride and $19 \%$ for chloroform in dual analyses by the AGEM Laboratory and TestAmerica with the contaminants present above the respective method detection limits. The quality control range for relative 
percent difference between dual analyses is $\pm 20 \%$. Dual analyses of samples at AGEM Laboratory and TestAmerica therefore demonstrated consistency in the sampling and analytical methodologies.

- The analyses of soil and groundwater samples by the AGEM Laboratory were verified by TestAmerica (Supplement 4, on CD). Agreement in the results from the two laboratories is good over the range of contaminant concentrations detected, with relative percent differences of $16 \%$ and $12 \%$ for carbon tetrachloride and chloroform in verification analyses with the contaminants present above the respective method detection limits. The quality control range for relative percent difference between verification analyses is $\pm 20 \%$. The concentrations detected in soil and groundwater in analyses at the AGEM Laboratory are therefore supported by the verification analyses at TestAmerica.

\subsection{Waste Characterization, Handling, and Disposal}

Waste soil and water generated as potentially contaminated investigation-derived waste were containerized on-site. The accumulated waste soil and water were sampled on June 9, 2016, and June 21, 2016. The waste soil and water samples were analyzed by Pace Analytical Services, Inc., Lenexa, Kansas, on June 14, 2016; June 24, 2016; and July 1, 2016. Waste soil was analyzed by EPA Method 8260 for VOCs and ASTM D2974 for percent moisture. Waste water was analyzed by EPA Method 5030B/8260 for VOCs. No VOCs were detected in the soil; carbon tetrachloride was detected at a concentration of $2.7 \mu \mathrm{g} / \mathrm{L}$ in the water.

The laboratory results are in Supplement 3 (on CD). Per NDEQ approval on July 11, 2016, the soil cuttings were disposed of by Waste Management at the Pheasant Point Landfill facility on August 8, 2016, while the waste water was discharged on-site near the point of generation on November 1, 2016. 
TABLE 3.1 Summary of carbon tetrachloride and chloroform concentrations in soil samples, as determined by the purge-and-trap method at the AGEM Laboratory.

\begin{tabular}{|c|c|c|c|c|c|}
\hline \multirow[b]{2}{*}{ Location } & \multirow[b]{2}{*}{ Sample Date } & \multirow[b]{2}{*}{$\begin{array}{l}\text { Depth } \\
\text { (ft BGL) }\end{array}$} & \multirow[b]{2}{*}{$\begin{array}{c}\text { Number of } \\
\text { Samples }\end{array}$} & \multicolumn{2}{|c|}{ Concentration $(\mu \mathrm{g} / \mathrm{kg})$} \\
\hline & & & & $\begin{array}{c}\text { Carbon } \\
\text { Tetrachloride }\end{array}$ & Chloroform \\
\hline \multirow[t]{16}{*}{ TI01 } & 7/7-10/15 & 4 & 1 & $9.6 \mathrm{Ja}^{\mathrm{a}}$ & $N D^{b}$ \\
\hline & & 8 & 1 & 13 & ND \\
\hline & & 12 & 1 & 18 & $4.8 \mathrm{~J}$ \\
\hline & & 16 & 1 & 29 & $6.0 \mathrm{~J}$ \\
\hline & & 20 & 1 & 84 & $5.0 \mathrm{~J}$ \\
\hline & & 24 & 1 & 139 & $7.9 \mathrm{~J}$ \\
\hline & & 28 & 1 & 110 & $6.2 \mathrm{~J}$ \\
\hline & & 32 & 1 & 121 & 12 \\
\hline & & 36 & 1 & 167 & 12 \\
\hline & & 40 & 1 & 129 & 14 \\
\hline & & 44 & 1 & 60 & $9.3 \mathrm{~J}$ \\
\hline & & 48 & 1 & 29 & 12 \\
\hline & & 52 & 1 & 53 & 17 \\
\hline & & $56-72$ & 5 & ND & ND \\
\hline & & 75 & 1 & 23 & ND \\
\hline & & 80 & 1 & 110 & ND \\
\hline \multirow[t]{10}{*}{ TI02 } & $7 / 7-8 / 15$ & $4-20$ & 5 & ND & ND \\
\hline & & 24 & 1 & $8.4 \mathrm{~J}$ & ND \\
\hline & & 28 & 1 & ND & ND \\
\hline & & 32 & 1 & $4.8 \mathrm{~J}$ & ND \\
\hline & & 36 & 1 & $5.9 \mathrm{~J}$ & ND \\
\hline & & 40 & 1 & 10 & ND \\
\hline & & 44 & 1 & 13 & ND \\
\hline & & 48 & 1 & $7.7 \mathrm{~J}$ & ND \\
\hline & & $52-72$ & 6 & ND & ND \\
\hline & & 75 & 1 & 10 & ND \\
\hline \multirow[t]{5}{*}{ TI03 } & $7 / 9 / 15$ & $4-32$ & 8 & ND & ND \\
\hline & & 36 & 1 & $5.6 \mathrm{~J}$ & ND \\
\hline & & 40 & 1 & $8.7 \mathrm{~J}$ & ND \\
\hline & & 44 & 1 & $6.3 \mathrm{~J}$ & ND \\
\hline & & $48-75$ & 8 & ND & ND \\
\hline \multirow[t]{14}{*}{ TI04 } & $7 / 8 / 15$ & $4-8$ & 2 & ND & ND \\
\hline & & 12 & 1 & $7.2 \mathrm{~J}$ & ND \\
\hline & & 16 & 1 & 19 & $4.4 \mathrm{~J}$ \\
\hline & & 20 & 1 & 27 & $5.1 \mathrm{~J}$ \\
\hline & & 24 & 1 & 49 & $6.3 \mathrm{~J}$ \\
\hline & & 28 & 1 & 35 & ND \\
\hline & & 32 & 1 & 61 & $4.1 \mathrm{~J}$ \\
\hline & & 36 & 1 & 43 & $2.9 \mathrm{~J}$ \\
\hline & & 40 & 1 & 59 & ND \\
\hline & & 44 & 1 & 56 & ND \\
\hline & & 48 & 1 & 11 & ND \\
\hline & & $52-72$ & 6 & ND & ND \\
\hline & & 76 & 1 & 28 & ND \\
\hline & & 80 & 1 & 21 & $3.0 \mathrm{~J}$ \\
\hline
\end{tabular}

a J, compound identified with an estimated concentration between the instrument detection limit and the method detection limit.

b ND, compound analyzed for but not detected at a level greater than or equal to the method detection limit $(<10 \mu \mathrm{g} / \mathrm{kg})$. 
TABLE 3.2 Monitoring well construction data.

\begin{tabular}{|c|c|c|c|c|c|c|c|}
\hline \multirow[b]{2}{*}{$\begin{array}{c}\text { Map } \\
\text { Location }^{\mathrm{a}}\end{array}$} & \multirow[b]{2}{*}{$\begin{array}{l}\text { Corresponding } \\
\text { CPT Location }\end{array}$} & \multirow[b]{2}{*}{ Well } & \multirow[b]{2}{*}{$\begin{array}{c}\text { Casing } \\
\text { Diam. (in.) }\end{array}$} & \multicolumn{3}{|c|}{ Depth (ft BGL) } & \multirow{2}{*}{$\begin{array}{c}\text { Interpreted } \\
\text { Stratigraphic } \\
\text { Unitc }\end{array}$} \\
\hline & & & & Total & $\begin{array}{l}\text { Screen } \\
\text { Interval }\end{array}$ & $\begin{array}{c}\text { Filter Pack } \\
\text { Interval }\end{array}$ & \\
\hline DL1 & TI-01 & $\begin{array}{l}\text { DL1-A } \\
\text { DL1-B } \\
\text { DL1-C }\end{array}$ & $\begin{array}{l}2 \\
2 \\
2\end{array}$ & $\begin{array}{r}85 \\
98 \\
118\end{array}$ & $\begin{array}{c}75-85 \\
88-98 \\
108-118\end{array}$ & $\begin{array}{c}73-85 \\
86-98 \\
106-118\end{array}$ & $\begin{array}{l}\text { Unconf. 'A' } \\
\text { Unconf. 'B' } \\
\text { Unconf. 'C' }\end{array}$ \\
\hline SB62d & $\mathrm{TI}-03$ & SB62 & 2 & 125 & $118-123$ & $111-125$ & Unconf. 'C' \\
\hline SB77 ${ }^{d}$ & TI-03 & SB77 & 4 & 102.75 & $67-102$ & $63-102.75$ & Unconf. 'A'-'B' \\
\hline DL2 & $\mathrm{TI}-12$ & $\begin{array}{l}\text { DL2-C1d } \\
\text { DL2-C2 } \\
\text { DL2-Cud }\end{array}$ & $\begin{array}{l}2 \\
2 \\
2\end{array}$ & $\begin{array}{l}122 \\
146 \\
225\end{array}$ & $\begin{array}{l}112-122 \\
136-146 \\
215-225\end{array}$ & $\begin{array}{l}110-122 \\
134-146 \\
213-225\end{array}$ & $\begin{array}{l}\text { Unconf. 'C1' } \\
\text { Unconf. 'C2' } \\
\text { Upper Conf. }\end{array}$ \\
\hline DL3 & $\mathrm{Tl}-15$ & $\begin{array}{l}\text { DL3-C1 } \\
\text { DL3-C2 }\end{array}$ & $\begin{array}{l}2 \\
2\end{array}$ & $\begin{array}{l}123 \\
143\end{array}$ & $\begin{array}{l}113-123 \\
133-143\end{array}$ & $\begin{array}{l}110-123 \\
130-143\end{array}$ & $\begin{array}{l}\text { Unconf. 'C1' } \\
\text { Unconf. 'C2' }\end{array}$ \\
\hline DL4 & TI-23 & $\begin{array}{l}\text { DL4-C1 } \\
\text { DL4-C2 }\end{array}$ & $\begin{array}{l}2 \\
2\end{array}$ & $\begin{array}{l}130 \\
155\end{array}$ & $\begin{array}{l}120-130 \\
145-155\end{array}$ & $\begin{array}{l}116-130 \\
140-155\end{array}$ & $\begin{array}{l}\text { Unconf. 'C1' } \\
\text { Unconf. 'C2' }\end{array}$ \\
\hline DL5 & $\mathrm{Tl}-17$ & DL5-C $1^{d}$ & 2 & 116 & $106-116$ & $103-116$ & Unconf. 'C1' \\
\hline DL6 & Tl-21 & DL6-C ${ }^{d}$ & 2 & 120 & $110-120$ & $105-120$ & Unconf. 'C' \\
\hline DL7 & TI-24 & $\begin{array}{l}\text { DL7-C } \\
\text { DL7-CU } \\
\text { DL7-CL }\end{array}$ & $\begin{array}{l}2 \\
2 \\
2\end{array}$ & $\begin{array}{l}110 \\
202 \\
275\end{array}$ & $\begin{array}{l}100-110 \\
192-202 \\
265-275\end{array}$ & $\begin{array}{c}95-110 \\
188-202 \\
260-275\end{array}$ & $\begin{array}{l}\text { Unconf. 'C' } \\
\text { Upper Conf. } \\
\text { Lower Conf. }\end{array}$ \\
\hline DL8 & TI-20 & $\begin{array}{l}\text { DL8-C1 } \\
\text { DL8-C2 }\end{array}$ & $\begin{array}{l}2 \\
2\end{array}$ & $\begin{array}{l}114 \\
135\end{array}$ & $\begin{array}{l}104-114 \\
125-135\end{array}$ & $\begin{array}{l}100-114 \\
123-135\end{array}$ & $\begin{array}{l}\text { Unconf. 'C1' } \\
\text { Unconf. 'C2' }\end{array}$ \\
\hline
\end{tabular}

a See Figure 2.5 .

b Location of comparable direct-push boring used to guide the preliminary selection of screen intervals. See Figure 2.2.

c See Section 4.1 for discussion of the local stratigraphy.

d Well equipped with groundwater level measurement and recording unit in July 2016; see Section 3.5.

e Wells previously installed by Argonne in 1993-1995. 
TABLE 3.3 Summary of analytical results for VOCs in groundwater samples collected during the targeted investigation.

\begin{tabular}{|c|c|c|c|c|c|}
\hline \multirow[b]{2}{*}{ Location } & \multirow[b]{2}{*}{ Sample ID } & \multirow[b]{2}{*}{ Sample Date } & \multirow[b]{2}{*}{$\begin{array}{l}\text { Depth } \\
\text { (ft BGL) }\end{array}$} & \multicolumn{2}{|c|}{ Concentration $(\mu \mathrm{g} / \mathrm{L})$} \\
\hline & & & & $\begin{array}{c}\text { Carbon } \\
\text { Tetrachloride }\end{array}$ & Chloroform \\
\hline \multicolumn{6}{|c|}{ Samples collected from direct-push investigative borings } \\
\hline TI01 & YKTI01-W-37844 & $7 / 10 / 15$ & $77.4-82.4$ & 373 & 108 \\
\hline TI01 & YKTI01100-W-37854 & 7/16/15 & $88-98$ & ND & $0.6 \mathrm{~J}$ \\
\hline TI01 & YKTI01120-W-37855 & $7 / 15 / 15$ & $108-118$ & $0.9 \mathrm{~J}$ & 2.0 \\
\hline TI02 & YKTI02-W-37847 & $7 / 10 / 15$ & 77-82 & 200 & 54 \\
\hline TI02 & YKTI02100-W-37849 & 7/13/15 & $100-103.5$ & 1.4 & 7.2 \\
\hline TI02 & YKTI02120-W-37852 & $7 / 14 / 15$ & $108.5-118.5$ & ND & ND \\
\hline TI03 & YKTI03-W-37836 & $7 / 9 / 15$ & $76.8-81.8$ & 150 & 11 \\
\hline TI04 & YKTI04-W-37833 & $7 / 9 / 15$ & $74.6-79.6$ & 159 & 164 \\
\hline TI04 & YKTI04100-W-37861 & 7/16/15 & $89-99$ & ND & $0.8 \mathrm{~J}$ \\
\hline TI04 & YKTI04120-W-37860 & $7 / 16 / 15$ & $108-118$ & ND & 1.1 \\
\hline TI05 & YKTI0580-W-37868 & 7/18/15 & $76-81$ & 254 & 69 \\
\hline TI05 & YKTI05100-W-37865 & 7/17/15 & $88-98$ & 82 & 6.7 \\
\hline TI05 & YKTI05120-W-37864 & $7 / 17 / 15$ & $108-118$ & 2.0 & $0.6 \mathrm{~J}$ \\
\hline TI06 & YKTI0680-W-37872 & $7 / 19 / 15$ & $77-82$ & 7.0 & $0.4 \mathrm{~J}$ \\
\hline TI06 & YKTI06100-W-37873 & 7/19/15 & $90-100$ & $0.8 \mathrm{~J}$ & 2.7 \\
\hline TI06 & YKTI06120-W-37870 & $7 / 19 / 15$ & $110-120$ & ND & ND \\
\hline TI07 & YKTI0780-W-37875 & $7 / 20 / 15$ & $77-82$ & 40 & 2.2 \\
\hline TI07 & YKTI07100-W-37876 & $7 / 20 / 15$ & $90-100$ & ND & ND \\
\hline TI07 & YKTI07120-W-37877 & $7 / 20 / 15$ & $110-120$ & ND & ND \\
\hline T108 & YKT10885-W-37879 & $7 / 21 / 15$ & $80-85$ & ND & ND \\
\hline T108 & YKTI08107-W-37881 & $7 / 21 / 15$ & $97-107$ & 27 & $0.9 \mathrm{~J}$ \\
\hline TI08 & YKTI08125-W-37882 & $7 / 21 / 15$ & $115-125$ & 8.7 & ND \\
\hline TI09 & YKTI09-80-W-37926 & $8 / 13 / 15$ & $80-85$ & $0.7 \mathrm{~J}$ & ND \\
\hline TI09 & YKTI09-95-W-37928 & $8 / 13 / 15$ & $95-105$ & 3.8 & ND \\
\hline TI09 & YKTI09-135-W-37929 & $8 / 13 / 15$ & $135-145$ & 1.8 & ND \\
\hline Tl10 & YKTI10-78-W-37930 & $8 / 14 / 15$ & $78-83$ & 6.6 & $0.9 \mathrm{~J}$ \\
\hline TI10 & YKTI10-95-W-37931 & $8 / 14 / 15$ & $95-105$ & 18 & 1.2 \\
\hline TI10 & YKTI10-117-W-37933 & $8 / 14 / 15$ & $117-127$ & 29 & 1.1 \\
\hline Tl11 & YKTI11-80-W-37935 & $8 / 17 / 15$ & $80-85$ & 36 & 6.4 \\
\hline Tl11 & YKTI11-92-W-37936 & $8 / 18 / 15$ & $92-102$ & ND & ND \\
\hline TI11 & YKTI11-112-W-37937 & $8 / 18 / 15$ & $112-122$ & 202 & 5.1 \\
\hline Tl12 & YKTI12-80-W-37939 & $8 / 20 / 15$ & $80-85$ & 6.2 & 1.2 \\
\hline TI12 & YKTI12-92-W-37940 & $8 / 20 / 15$ & $92-102$ & 45 & 1.5 \\
\hline Tl12 & YKTI12-120-W-37942 & $8 / 20 / 15$ & $110-120$ & 63 & 1.7 \\
\hline TI12 & YKTI12-145-W-37943 & $8 / 20 / 15$ & $135-145$ & 73 & 1.5 \\
\hline Tl13 & YKTI13-82-W-37955 & $8 / 24 / 15$ & $81-86$ & ND & ND \\
\hline TI13 & YKTI13-93-W-37947 & $8 / 22 / 15$ & $93-103$ & ND & ND \\
\hline Tl13 & YKTI13-111-W-37948 & $8 / 22 / 15$ & $111-121$ & ND & ND \\
\hline
\end{tabular}


TABLE 3.3 (Cont.)

\begin{tabular}{|c|c|c|c|c|c|}
\hline \multirow[b]{2}{*}{ Location } & \multirow[b]{2}{*}{ Sample ID } & \multirow[b]{2}{*}{ Sample Date } & \multirow[b]{2}{*}{$\begin{array}{c}\text { Depth } \\
\text { (ft BGL) }\end{array}$} & \multicolumn{2}{|c|}{ Concentration $(\mu \mathrm{g} / \mathrm{L})$} \\
\hline & & & & $\begin{array}{c}\text { Carbon } \\
\text { Tetrachloride }\end{array}$ & Chloroform \\
\hline \multicolumn{6}{|c|}{ Samples collected from direct-push investigative borings (cont.) } \\
\hline TI14 & YKTI14-86-W-37951 & $8 / 23 / 15$ & $86-96$ & 1.9 & ND \\
\hline TI14 & YKTI14-113-W-37952 & $8 / 23 / 15$ & $113-123$ & 36 & 1.4 \\
\hline TI14 & YKTI14-136-W-37954 & $8 / 23 / 15$ & $136-146$ & 59 & $0.8 \mathrm{~J}$ \\
\hline Tl15 & YKTI15-90-W-37956 & $8 / 24 / 15$ & $90-100$ & 1.7 & ND \\
\hline Tl15 & YKTI15-113-W-37957 & $8 / 24 / 15$ & $113-123$ & 22 & $0.7 \mathrm{~J}$ \\
\hline TI15 & YKTI15-134-W-37958 & $8 / 24 / 15$ & $134-144$ & 68 & 1.7 \\
\hline TI16 & YKTI16-86-W-37961 & $8 / 25 / 15$ & $86-96$ & ND & ND \\
\hline TI16 & YKTI16-105-W-37963 & $8 / 25 / 15$ & $105-115$ & 2.9 & ND \\
\hline TI16 & YKTI16-136-W-37964 & $8 / 25 / 15$ & $136-146$ & 35 & $0.7 \mathrm{~J}$ \\
\hline TI17 & YKTI17-84-W-37966 & $8 / 26 / 15$ & $84-94$ & ND & ND \\
\hline TI17 & YKTI17-104-W-37967 & $8 / 26 / 15$ & $104-114$ & ND & ND \\
\hline TI17 & YKTI17-133-W-37968 & $8 / 26 / 15$ & $133-143$ & ND & $0.4 \mathrm{~J}$ \\
\hline Tl18 & YKTI18-81-W-37970 & $8 / 27 / 15$ & $81-86$ & 12 & 9.0 \\
\hline Tl18 & YKTI18-95-W-37971 & $8 / 27 / 15$ & $95-105$ & 11 & $0.9 \mathrm{~J}$ \\
\hline TI18 & YKTI18-114-W-37973 & $8 / 28 / 15$ & $114-124$ & 2.9 & ND \\
\hline Tl19 & YKTI19-84-W-37975 & 9/9/15 & $84-94$ & ND & ND \\
\hline TI19 & YKTI19-107-W-37976 & $9 / 9 / 15$ & $107-117$ & 4.8 & ND \\
\hline TI19 & YKTI19-129-W-37977 & $9 / 9 / 15$ & 129-139 & 22 & $0.8 \mathrm{~J}$ \\
\hline TI20 & YKTI20-81-W-37980 & 9/10/15 & $81-91$ & ND & ND \\
\hline TI20 & YKTI20-105-W-37981 & $9 / 10 / 15$ & $105-115$ & 8.2 & ND \\
\hline TI20 & YKTI20-131-W-37982 & 9/10/15 & $131-141$ & 1.8 & ND \\
\hline TI21 & YKTI21-77-W-37985 & $9 / 11 / 15$ & $77-87$ & $0.8 \mathrm{~J}$ & ND \\
\hline TI21 & YKTI21-99-W-37986 & $9 / 11 / 15$ & $99-109$ & $0.8 \mathrm{~J}$ & ND \\
\hline $\mathrm{TI} 21$ & YKTI21-115-W-37987 & $9 / 11 / 15$ & $115-120$ & ND & ND \\
\hline TI22 & YKTI22-79-W-37990 & $9 / 14 / 15$ & $79-84$ & ND & ND \\
\hline TI22 & YKTI22-98-W-37991 & $9 / 14 / 15$ & $98-108$ & 3.3 & ND \\
\hline TI22 & YKTI22-113-W-37992 & $9 / 14 / 15$ & $113-123$ & ND & ND \\
\hline TI23 & YKTI23-80-W-37995 & $9 / 15 / 15$ & $80-90$ & $0.5 \mathrm{~J}$ & $0.6 \mathrm{~J}$ \\
\hline TI23 & YKTI23-99-W-37996 & $9 / 15 / 15$ & $99-109$ & ND & ND \\
\hline TI23 & YKTI23-120-W-37998 & $9 / 15 / 15$ & $120-130$ & ND & ND \\
\hline TI23 & YKTI23-145-W-37999 & $9 / 16 / 15$ & $145-155$ & ND & ND \\
\hline TI24 & YKTI24-80-W-38017 & $1 / 20 / 16$ & $80-90$ & ND & ND \\
\hline TI24 & YKTI24-90-W-38018 & $1 / 20 / 16$ & $90-100$ & ND & ND \\
\hline TI24 & YKTI24-100-W-38019 & $1 / 20 / 16$ & $100-110$ & ND & ND \\
\hline TI25 & YKTI25-77-W-38023 & $1 / 21 / 16$ & $77-87$ & ND & ND \\
\hline TI25 & YKTI25-98-W-38024 & $1 / 21 / 16$ & $98-108$ & 1.3 & ND \\
\hline TI25 & YKTI25-108-W-38027 & $1 / 21 / 16$ & $113-118$ & ND & ND \\
\hline
\end{tabular}


TABLE 3.3 (Cont.)

\begin{tabular}{|c|c|c|c|c|c|}
\hline \multirow[b]{2}{*}{ Location } & \multirow[b]{2}{*}{ Sample ID } & \multirow[b]{2}{*}{ Sample Date } & \multirow[b]{2}{*}{$\begin{array}{c}\text { Depth } \\
\text { (ft BGL) }\end{array}$} & \multicolumn{2}{|c|}{ Concentration $(\mu \mathrm{g} / \mathrm{L})$} \\
\hline & & & & $\begin{array}{c}\text { Carbon } \\
\text { Tetrachloride }\end{array}$ & Chloroform \\
\hline \multicolumn{6}{|c|}{ Samples collected from direct-push investigative borings (cont.) } \\
\hline TI26 & YKTI26-78-W-38029 & $1 / 25 / 16$ & $78-88$ & ND & ND \\
\hline TI26 & YKTI26-96-W-38030 & $1 / 25 / 16$ & $96-106$ & 2.0 & ND \\
\hline TI26 & YKTI26-113-W-38033 & $1 / 25 / 16$ & $113-123$ & $0.4 \mathrm{~J}$ & ND \\
\hline TI27 & YKTI27-77-W-38050 & $2 / 9 / 16$ & $77-87$ & ND & ND \\
\hline TI27 & YKTI27-77-W-38056 & $2 / 9 / 16$ & $77-87$ & ND & ND \\
\hline TI27 & YKTI27-100-W-38053 & $2 / 9 / 16$ & $100-110$ & $0.4 \mathrm{~J}$ & ND \\
\hline TI27 & YKTI27-100-W-38057 & $2 / 9 / 16$ & $100-110$ & ND & ND \\
\hline TI28 & YKTI28-78-W-38075 & $3 / 2 / 16$ & $78-88$ & ND & ND \\
\hline TI28 & YKTI28-90-W-38076 & $3 / 2 / 16$ & $90-100$ & ND & ND \\
\hline TI28 & YKTI28-104-W-38078 & $3 / 3 / 16$ & 104-109 & ND & ND \\
\hline \multicolumn{6}{|c|}{ Samples collected from monitoring wells } \\
\hline SB62 & YKSB62-W-37924 & $8 / 7 / 15$ & $118-123$ & 23 & 2.2 \\
\hline SB77 & YKSB77-W-37921 & $8 / 7 / 15$ & $75.8-79$ & 220 & 9.8 \\
\hline DL1-A & YKDL1A-75-W-38094 & $5 / 25 / 16$ & $75-85$ & 1875 & 11 \\
\hline DL1-B & YKDL1B-88-W-38093 & $5 / 24 / 16$ & 88-98 & 1.6 & ND \\
\hline DL1-C & YKDL1C-108-W-38091 & $5 / 23 / 16$ & $108-118$ & 1.9 & ND \\
\hline DL2-C1 & YKDL2-C1-112-W-38100 & $6 / 2 / 16$ & $112-122$ & 41 & $0.7 \mathrm{~J}$ \\
\hline DL2-C2 & YKDL2C2-136-W-38096 & $5 / 31 / 16$ & $136-146$ & 42 & 1.0 \\
\hline DL2-CU & YKDL2CU-215-W-38097 & $5 / 31 / 16$ & $215-225$ & ND & ND \\
\hline DL3-C1 & YKDL3-C1-113-W-38103 & $6 / 6 / 16$ & $113-123$ & 39 & $0.7 \mathrm{~J}$ \\
\hline DL3-C2 & YKDL3-C2-134-W-38102 & $6 / 5 / 16$ & $134-144$ & 77 & 1.1 \\
\hline DL4-C1 & YKDL4-C1-120-W-38116 & $6 / 16 / 16$ & $120-130$ & ND & ND \\
\hline DL4-C2 & YKDL4-C2-145-W-38115 & $6 / 15 / 16$ & $145-155$ & ND & ND \\
\hline DL5-C1 & YKDL5-C1-106-W-38118 & $6 / 21 / 16$ & $106-116$ & ND & ND \\
\hline DL6-C & YKDL6-C-110-W-38113 & $6 / 13 / 16$ & $110-120$ & ND & ND \\
\hline DL7-C & YKDL7-C-100-W-38111 & $6 / 12 / 16$ & $100-110$ & ND & ND \\
\hline DL7-CU & YKDL7-CU-192-W-38112 & $6 / 12 / 16$ & $192-202$ & ND & ND \\
\hline DL7-CL & YKDL7-CL-265-W-38108 & $6 / 9 / 16$ & $265-275$ & ND & ND \\
\hline DL8-C1 & YKDL8C1-104-W-38106 & $6 / 7 / 16$ & 104-114 & 16 & ND \\
\hline DL8-C2 & YKDL8-C2-125-W-38104 & $6 / 6 / 16$ & $125-135$ & 2.2 & ND \\
\hline \multicolumn{6}{|c|}{ Samples collected from private wells } \\
\hline Preslicka & YKPRESLICKA-W-37916 & $8 / 5 / 15$ & - & 1.3 & ND \\
\hline Volzke & YKVOLZKE-W-37923 & $8 / 7 / 15$ & - & ND & ND \\
\hline
\end{tabular}


York Targeted Investigation, 2015-2016

Version 01, 02/14/2017

TABLE 3.3 (Cont.)

\begin{tabular}{|c|c|c|c|c|c|}
\hline \multirow[b]{2}{*}{ Location } & \multirow[b]{2}{*}{ Sample ID } & \multirow[b]{2}{*}{ Sample Date } & \multirow[b]{2}{*}{$\begin{array}{l}\text { Depth } \\
\text { (ft BGL) }\end{array}$} & \multicolumn{2}{|c|}{ Concentration $(\mu \mathrm{g} / \mathrm{L})$} \\
\hline & & & & $\begin{array}{c}\text { Carbon } \\
\text { Tetrachloride }\end{array}$ & Chloroform \\
\hline \multicolumn{6}{|c|}{ Samples collected from private wells (cont.) } \\
\hline $\begin{array}{l}\text { York County Shop } \\
\text { Nordlund } \\
\text { Liermann }\end{array}$ & $\begin{array}{l}\text { YKYORKCOUNTYSHOP-W-37917 } \\
\text { YKNORDLUND-W-37918 } \\
\text { YKLIERMANN-W-37920 }\end{array}$ & $\begin{array}{l}8 / 5 / 15 \\
8 / 5 / 15 \\
8 / 5 / 15\end{array}$ & $\begin{array}{l}- \\
- \\
-\end{array}$ & $\begin{array}{l}53 \\
\text { ND } \\
\text { ND }\end{array}$ & $\begin{array}{l}0.8 \mathrm{~J} \\
\mathrm{ND} \\
\mathrm{ND}\end{array}$ \\
\hline
\end{tabular}

a ND, compound analyzed for but not detected at a level greater than or equal to the method detection limit $(<1 \mu \mathrm{g} / \mathrm{L})$.

b $\mathrm{J}$, compound identified with an estimated concentration between the instrument detection limit and the method detection limit. 
TABLE 3.4 Summary of carbon tetrachloride results for groundwater samples collected in quarterly monitoring of selected private and PWS wells

\begin{tabular}{lccccccccc}
\hline & \multicolumn{7}{c}{ Carbon Tetrachloride Concentration ( $\mu \mathrm{g} / \mathrm{L})$} & \\
\cline { 2 - 8 } \multicolumn{1}{c}{ Location } & $\begin{array}{c}\text { April \& July } \\
2014\end{array}$ & $\begin{array}{c}\text { December } \\
2014\end{array}$ & $\begin{array}{c}\text { March } \\
2015\end{array}$ & $\begin{array}{c}\text { August } \\
2015\end{array}$ & $\begin{array}{c}\text { November } \\
2015\end{array}$ & $\begin{array}{c}\text { February } \\
2016\end{array}$ & $\begin{array}{c}\text { June } \\
2016\end{array}$ & $\begin{array}{c}\text { October } \\
2016\end{array}$ & $\begin{array}{c}\text { December } \\
2016\end{array}$ \\
\hline PWS 77-1 & ND & ND & ND & ND & ND & ND & ND & ND & ND \\
PWS 2009-4 & ND & - & ND & ND & ND & ND & - & ND & ND \\
PWS 2009-5 & - & - & - & ND & ND & ND & ND & - & - \\
PWS 2009-6 & - & ND & ND & - & - & - & ND & ND & ND \\
B\&B Metalworks & ND & ND & ND & ND & ND & ND & ND & ND & ND \\
Bonde & ND & ND & ND & ND & ND & ND & ND & ND & ND \\
CVA Coop & 2.3 & 4.9 & 5.1 & 6.6 & 5.9 & 5.7 & 5.7 & 9.2 & 6.7 \\
Danielson & - & ND & ND & ND & ND & ND & ND & ND & ND \\
Driewer Shop & - & - & ND & ND & ND & ND & ND & ND & ND \\
Driewer Home & - & - & - & - & - & - & - & - & 5.1 \\
Hoblyn & ND & ND & ND & ND & ND & ND & ND & ND & ND \\
Janzen & ND & ND & ND & ND & ND & ND & ND & ND & ND \\
Kayton (former Olson) & 2.2 & 7.4 & 2.4 & 6.5 & 4.2 & 5.3 & 17 & 13 & 5.3 \\
Makovicka & ND & ND & ND & ND & ND & ND & ND & ND & ND \\
Onnen & ND & ND & ND & ND & ND & ND & ND & ND & ND \\
Steever & - & ND & ND & ND & ND & ND & ND & ND & ND \\
Travis & ND & ND & ND & ND & ND & ND & ND & ND & ND \\
\hline
\end{tabular}

a ND, compound analyzed for but not detected at a level greater than or equal to the method detection limit $(<1 \mu \mathrm{g} / \mathrm{L})$. 
TABLE 3.5 Hand-measured groundwater levels

\begin{tabular}{|c|c|c|c|c|c|}
\hline \multirow[b]{4}{*}{ Well } & \multirow{2}{*}{\multicolumn{2}{|c|}{ Reference Elevation }} & \multicolumn{3}{|c|}{ November 3, 2016} \\
\hline & & & & & \\
\hline & \multirow[b]{2}{*}{$\begin{array}{l}\text { Ground } \\
\text { (ft AMSL)a }\end{array}$} & \multirow[b]{2}{*}{$\begin{array}{c}\text { TOC }^{b} \\
\text { (ft AMSL) }\end{array}$} & & \multicolumn{2}{|c|}{ Depth (ft) } \\
\hline & & & Time & $\begin{array}{l}\text { from } \\
\text { TOC }\end{array}$ & $\begin{array}{c}\text { Elevation } \\
\text { (AMSL) }\end{array}$ \\
\hline SB62 & 1658.11 & 1659.81 & $11: 30$ & 76.01 & 1583.80 \\
\hline SB77 & 1658.96 & 1658.34 & $11: 25$ & 74.39 & 1583.95 \\
\hline DL1-A & 1658.66 & 1658.45 & $11: 10$ & 73.41 & 1585.04 \\
\hline DL1-B & 1658.77 & 1658.47 & $11: 15$ & 74.44 & 1584.03 \\
\hline DL1-C & 1658.77 & 1658.47 & $11: 20$ & 74.49 & 1583.98 \\
\hline DL2-C1 & 1658.25 & 1657.85 & $11: 21$ & 81.07 & 1576.78 \\
\hline DL2-C2 & 1658.16 & 1657.87 & $11: 40$ & 81.23 & 1576.64 \\
\hline DL2-CU & 1658.16 & 1657.89 & $11: 28$ & 82.43 & 1575.46 \\
\hline DL3-C1 & 1653.53 & 1653.31 & $10: 31$ & 79.31 & 1574.00 \\
\hline DL3-C2 & 1653.53 & 1653.17 & $10: 25$ & 79.20 & 1573.97 \\
\hline DL4-C1 & 1664.23 & 1663.93 & $13: 55$ & 87.95 & 1575.98 \\
\hline DL4-C2 & 1664.37 & 1664.06 & $14: 00$ & 88.08 & 1575.98 \\
\hline DL5-C1 & 1648.99 & 1648.63 & $10: 45$ & 72.92 & 1575.71 \\
\hline DL6-C & 1641.91 & 1641.62 & $13: 30$ & 72.90 & 1568.72 \\
\hline DL7-C & 1637.64 & 1637.29 & $13: 08$ & 71.59 & 1565.70 \\
\hline DL7-CU & 1637.66 & 1637.26 & $13: 13$ & 73.63 & 1563.63 \\
\hline DL7-CL & 1637.66 & 1637.15 & $13: 15$ & 97.89 & 1539.26 \\
\hline DL8-C1 & 1644.10 & 1643.90 & $11: 05$ & 72.57 & 1571.33 \\
\hline DL8-C2 & 1644.02 & 1643.79 & $11: 00$ & 72.53 & 1571.26 \\
\hline
\end{tabular}

a AMSL, above mean sea level.

b TOC, top of casing. 
Concentration $\left(\mu \mathrm{g} / \mathrm{m}^{3}\right)$

\begin{tabular}{|c|c|c|c|c|c|c|c|c|c|c|c|c|c|c|c|c|c|c|}
\hline \multirow[b]{4}{*}{ Location } & \multicolumn{18}{|c|}{ Concentration $\left(\mu \mathrm{g} / \mathrm{m}^{3}\right)$} \\
\hline & \multirow{2}{*}{\multicolumn{2}{|c|}{$\begin{array}{l}\text { August } 2015 \\
\text { Indoor Air }\end{array}$}} & \multicolumn{4}{|c|}{ January 2016} & \multicolumn{4}{|c|}{ April 2016} & \multicolumn{4}{|c|}{ August 2016} & \multicolumn{4}{|c|}{ December 2016} \\
\hline & & & \multicolumn{2}{|c|}{ Indoor Air } & \multicolumn{2}{|c|}{ Subslab } & \multicolumn{2}{|c|}{ Indoor Air } & \multicolumn{2}{|c|}{ Subslab } & \multicolumn{2}{|c|}{ Indoor Air } & \multicolumn{2}{|c|}{ Subslab } & \multicolumn{2}{|c|}{ Indoor Air } & \multicolumn{2}{|c|}{ Subslab } \\
\hline & $\mathrm{CT}^{\mathrm{a}}$ & $\mathrm{CF}^{\mathrm{b}}$ & СT & $\mathrm{CF}$ & Ст & $\mathrm{CF}$ & СT & $\mathrm{CF}$ & CT & $\mathrm{CF}$ & CT & $\mathrm{CF}$ & СT & $\mathrm{CF}$ & CT & $\mathrm{CF}$ & CT & $\mathrm{CF}$ \\
\hline $\begin{array}{l}\text { Hearthstone } \\
\text { Ground floor, } 6 \text { locations }\end{array}$ & $\mathrm{ND}(1.3)$ & $\mathrm{ND}(0.98)$ & - & & - & & - & & - & & - & & - & - & - & - & & \\
\hline $\begin{array}{l}\text { Willowbrook } \\
\text { Ground floor, } 6 \text { locations }\end{array}$ & ND (1.3) & $\mathrm{ND}(0.98)$ & - & & - & & - & & - & & : & & - & - & - & - & - & \\
\hline $\begin{array}{l}\text { Fitness Worx } \\
\text { Ground floor, 2-4 locations } \\
\text { Lower floor, 1-2 locations } \\
\text { Sub-slab, } 1 \text { location }\end{array}$ & $\begin{array}{l}1.5-7.9 \\
6.3 \\
-\end{array}$ & $\begin{array}{l}\text { ND-1.2 } \\
\text { ND (0.98) }\end{array}$ & $\begin{array}{c}4.6 \\
3.5-4.7\end{array}$ & $\begin{array}{l}1.2-1.4 \\
\text { ND-1.0 }\end{array}$ & 50 & $\mathrm{ND}(9.9)$ & $\begin{array}{c}2.4 \\
1.8-2.9\end{array}$ & $\begin{array}{l}4.9-5.2 \\
6.3-7.3\end{array}$ & 51 & ND (9.8) & $\begin{array}{l}7.9-8.2 \\
6.1-7.2\end{array}$ & $\begin{array}{l}7.9-8.5 \\
9.2-9.8\end{array}$ & 20 & ND (9.8) & $\begin{array}{l}3.6-3.8 \\
2.3-3.1\end{array}$ & $\begin{array}{l}4.1-4.4 \\
4.5-5.3\end{array}$ & ND (13) & ND (9.8) \\
\hline $\begin{array}{l}\text { CVA Coop Hdqtrs. } \\
\text { Ground floor, } 3 \text { locations }\end{array}$ & ND (1.3) & $\mathrm{ND}(0.98)$ & - & - & - & - & - & - & - & - & - & - & - & - & - & - & - & - \\
\hline $\begin{array}{l}\text { Preslicka } \\
\text { Ground floor, } 1 \text { location } \\
\text { Basement, } 1 \text { location }\end{array}$ & $\begin{array}{l}\text { ND (1.3) } \\
\text { ND (1.3) }\end{array}$ & $\begin{array}{l}\text { ND (0.98) } \\
\text { ND (.98) }\end{array}$ & - & - & - & - & - & - & - & - & - & - & - & - & - & - & - & - \\
\hline $\begin{array}{l}\text { Garretson/Troester } \\
\text { Ground floor, } 1 \text { location } \\
\text { Lower level, } 1 \text { location } \\
\text { Sub-slab, } 1 \text { location }\end{array}$ & $\begin{array}{c}8 \\
16 \\
-\end{array}$ & $\begin{array}{l}\mathrm{ND}(0.98) \\
\mathrm{ND}(0.98)\end{array}$ & $\begin{array}{l}21 \\
28\end{array}$ & $\begin{array}{l}\mathrm{ND}(0.98) \\
\mathrm{ND}(0.98)\end{array}$ & 6500 & 110 & $\begin{array}{l}- \\
-\end{array}$ & $\begin{array}{l}- \\
-\end{array}$ & $\begin{array}{l}- \\
-\end{array}$ & $\begin{array}{l}- \\
-\end{array}$ & $\begin{array}{l}\mathrm{ND}(1.3)^{\mathrm{c}} \\
\mathrm{ND}(1.3)^{\mathrm{c}}\end{array}$ & $\begin{array}{l}\text { ND }(0.98)^{c} \\
\operatorname{ND}(0.98)^{c}\end{array}$ & _- & - & $\begin{array}{l}\mathrm{ND}(1.3)^{\mathrm{c}} \\
\mathrm{ND}(1.3)^{\mathrm{c}}\end{array}$ & $\begin{array}{l}\mathrm{ND}(0.98)^{\mathrm{c}} \\
\operatorname{ND}(0.98)^{\mathrm{c}}\end{array}$ & - & - \\
\hline
\end{tabular}

\section{a $\mathrm{CT}$, Carbon tetrachloride}

b $\mathrm{CF}$, Chloroform.

c Indoor air sampled after installtion of subslab vapor mitigation system by the CCC/USDA. 


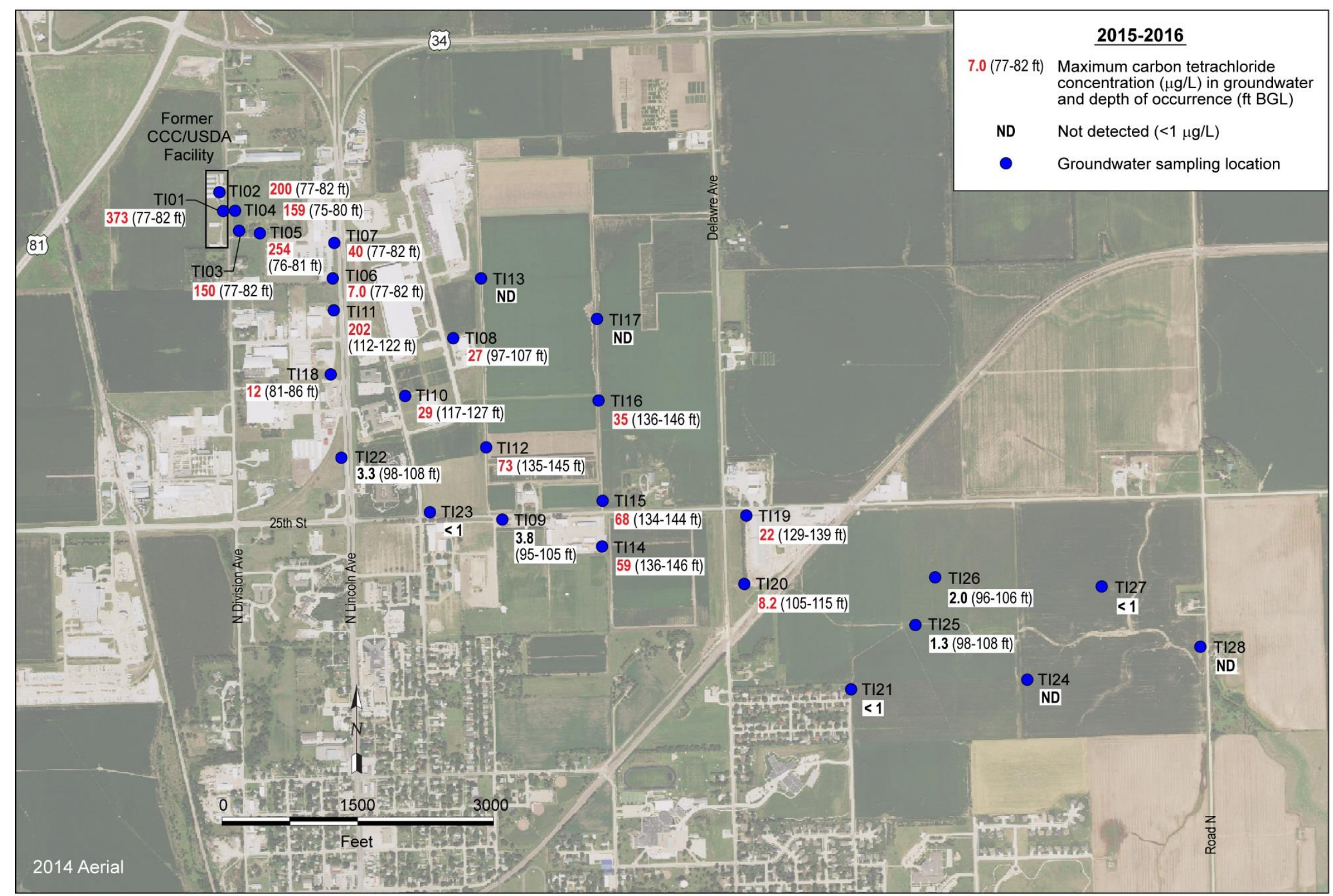

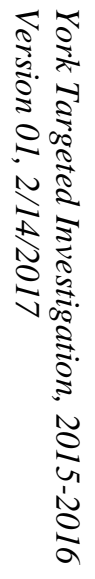

FIGURE 3.1 Maximum carbon tetrachloride concentrations in groundwater in the unconfined aquifer, determined from samples collected using direct-push techniques. 


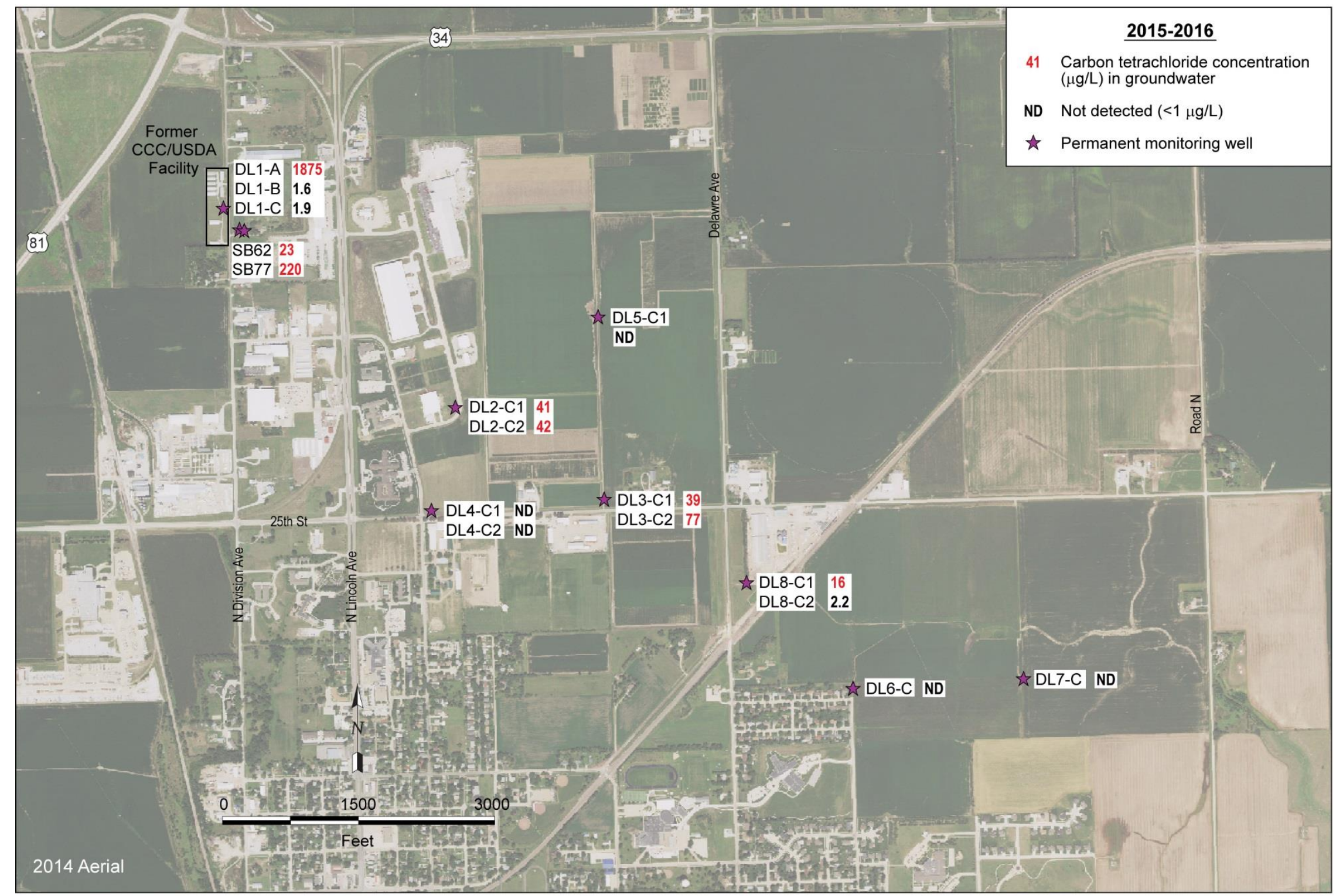

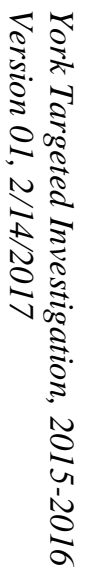

FIGURE 3.2 Carbon tetrachloride concentrations in groundwater in the unconfined aquifer, determined from samples collected from permanent monitoring wells. 


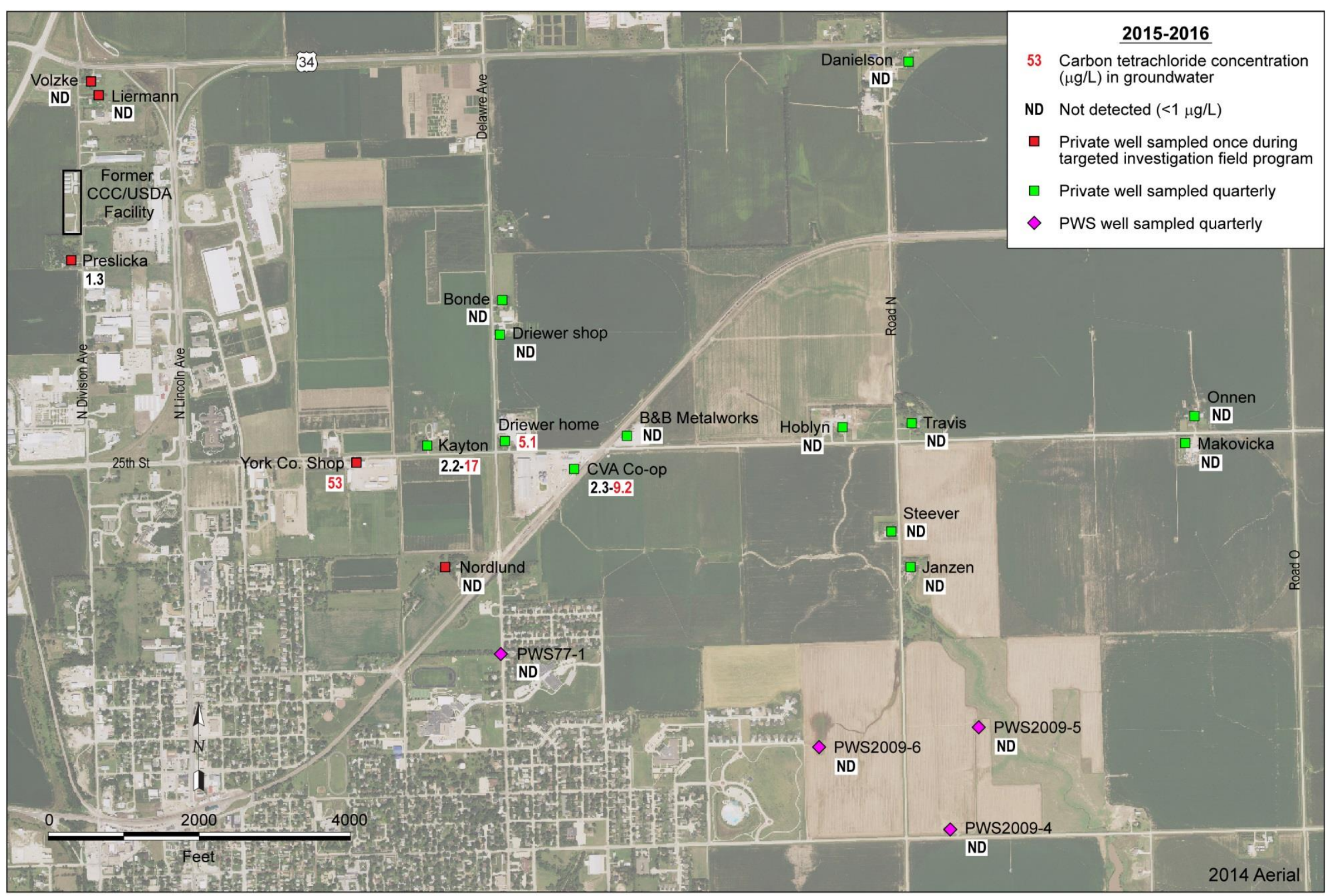

FIGURE 3.3 Carbon tetrachloride concentrations in groundwater collected from private wells sampled during the targeted investigation, and private and PWS wells sampled quarterly. 


\section{Interpretation of Results}

In this section, the investigation results summarized in Section 3 are discussed and interpreted in the context of the primary technical objectives and tasks outlined in Section 1. These results and interpretations, together with regional and local data for the York area summarized in the Final Work Plan (Argonne 2016a), provide an updated working model of the geologic and hydrologic setting, the movement of groundwater, and the distribution of carbon tetrachloride contamination in the vicinity of the former CCC/USDA facility.

\subsection{Hydrostratigraphy Near the Former CCC/USDA Facility}

A relatively consistent hydrogeologic sequence has been regionally identified in the unconsolidated sediments in the vicinity of York. In accord with terminology previously adopted by the U.S. Geological Survey (USGS; Landon and Turco 2007; Clark et al. 2008; Landon et al. 2008), the primary hydrostratigraphic units of this sequence, in order of increasing depth, include (1) an uppermost, predominantly unsaturated interval of silty-clay loess, (2) a laterally continuous unconfined aquifer composed of coarse-grained sediments with lesser interbedded thin layers and lenses of silt and clay, (3) a relatively thick upper confining unit of silty clay and/or silty clay till, and (4) a laterally continuous sandy upper confined aquifer. These units overlie a more heterogeneous zone consisting primarily of clay and silt, with variably interbedded sandy lenses. These heterogeneous deposits generally form (5) a lower confining unit; however, where locally present, the deeper sandy lenses constitute (6) a lower confined aquifer.

More detailed information on the geology and hydrostratigraphy in the immediate vicinity of the former CCC/USDA facility was obtained during the 1993-1995 CCC/USDA studies from an evaluation of available drillers' logs for private and public wells and geologic logs for several state stratigraphic test borings. Other information came from examination of continuous and selectively collected sediment cores, ECPT logs, and downhole geophysical logs obtained during these studies. (Note that all borings advanced during the 1993-1995 CCC/USDA studies were targeted to investigate the unconfined aquifer only.) These sources were used to construct several interpretive hydrostratigraphic cross sections for the York study area that were presented and discussed in the targeted investigation Work Plan (Figures 2.15-2.18 in Argonne 2016a). For convenience, these sections and their locations are shown in the current report in Appendix J, Figures J.1-J.4. 
A relatively complex and locally varying sequence of interbedded sand-gravel and siltclay layers was identified in the unconfined aquifer, although silty-clay intervals appeared in generally greater abundance (1) immediately south and southeast of the former CCC/USDA facility, and (2) in the lower portion of the unconfined aquifer than elsewhere in this unit. Limited groundwater level data acquired in conjunction with the 1993-1995 studies indicated a predominant direction of groundwater flow to the southeast from the former CCC/USDA facility (Argonne 1994, 1995a,b, 2016a).

Additional information for evaluation of the local lithologic sequence was obtained during the current investigation from continuous vertical ECPT log profiles obtained through the vadose zone and unconfined aquifer at 28 locations (TI01-TI28; Figure 2.2), and from continuous soil cores recovered at conventional drilling locations DL1-DL8 (Figure 2.4). At DL1, DL3-DL6, and DL8 the borings were advance through the vadose zone and unconfined aquifer sediments. With the approval of the CCC/USDA and NDEQ project managers, coring continued into and through the upper confined aquifer at location DL2, and at DL7 the boring was advanced into the upper portion of the lower confined aquifer. The resulting ECPT logs and lithologic descriptions for these borings are in Appendices $\mathrm{C}$ and D, respectively. The results of sediment particle-size analyses for selected core intervals in borings DL1-DL8 are also in Appendix D, Table D.1, and are summarized in Table 4.1.

\subsubsection{Site-Specific Characteristics of the Hydrostratigraphic Units}

Figure 4.1 illustrates the locations of four additional hydrogeologic cross sections, constructed primarily from data acquired during the current investigation, that are intended to augment the sections provided in the Work Plan and Appendix J (Argonne 2016a). Sections D-D', E-E', F-F', and G-G' are shown in Figures 4.2-4.5, respectively. The site-specific characteristics of the principal hydrostratigraphic units that were identified as a result of the current studies, as illustrated in these sections, are summarized below. Preliminary groundwater level relationships identified among these units are discussed in Section 4.1.2.

\subsubsection{Surficial Silt-Clay (Loess)}

The uppermost stratigraphic unit throughout the York study area consists of relatively uniform brown to yellowish-brown, reddish-brown, or grayish brown clayey silt to silty clay, 
interpreted as wind-blown loess. Thin intervals of fine sand, sandy silt, sandy clay, or clay were occasionally observed in this unit, as well as occasional iron oxide staining. The identified thickness of these deposits varies from approximately $60 \mathrm{ft}$ at the topographically highest point penetrated (at location TI23; Figures 4.1 and 4.4) to 39-40 ft in the topographically lowest area near locations TI24-TI28 (Figures 2.2 and 4.2), reflecting possible thinning of this unit as a result of local surface erosion. The base of the loess was encountered at a relatively consistent elevation of approximately 1,595-1,605 ft above mean sea level (AMSL) throughout the study area.

Groundwater level measurements obtained at the permanent monitoring wells (Table 3.5, Figure 2.5), together with the physical characteristics of cores recovered from the loess interval, indicated that this unit was unsaturated throughout the study area, and thus represented the upper portion of the vadose zone under the conditions identified at the time of the targeted investigation field activities (July 2015-July 2016).

\subsubsection{Unconfined Aquifer}

In the 1993-1995 investigations conducted at York, Argonne identified a vertical lithologic sequence consisting of three primarily sandy units, separated by intervening finergrained intervals, in the sediments hosting the unconfined aquifer beneath the former CCC/USDA facility (Argonne 1994, 1995a,b). The results of the present investigation demonstrate that while this stratigraphic zonation is not strictly observed throughout the study area, a generally similar vertical sequence of alternating coarse- and fine-grained intervals is suggested across much of the area. For convenience during discussions held among representatives of the CCC/USDA, the NDEQ, and Argonne as the field program progressed, an informal nomenclature was developed to represent these primarily sandy intervals as the "A," "B," and "C" (or "C1-C2") zones, respectively, with increasing depth. The interpreted distribution of these zones is shown in Figures 4.2-4.5.

Detailed visual examination of the sediment cores recovered at borings DL1-DL8 (Figure 2.4), coupled with the results of particle-size analyses performed for representative materials from each of the sandy units penetrated at these locations (Appendix D, Table D.1; Table 4.1), demonstrates that the lithologic characteristics of the unconfined aquifer sands are relatively heterogeneous and can vary both vertically and laterally over short distances, but show no clearly distinct differences among the "A," "B," and "C" intervals (Table 4.1). The coarser- 
grained deposits comprising the unconfined aquifer vary from occasional thin horizons (typically less than $5 \mathrm{ft}$ ) of relatively well sorted, silty to fine sand to thin intervals of coarse sand and gravel; however, the majority of the sediments in this unit consist of thicker intervals of light brown or light gray to brownish gray or grayish brown, poorly sorted mixtures of predominantly fine to medium sand with lesser quantities (individually varying from trace amounts to approximately 25\%) of coarse sand, silt, clay, and dispersed fine gravel.

Occasional thin lenses of clay, silty clay, or silt occur throughout the complex of unconfined aquifer sands. The distribution of these fine-grained units is discussed below, in the context of the associated sandy intervals.

- "A" Sand Zone - An uppermost sandy interval ranging in thickness from approximately $20-25 \mathrm{ft}$ was consistently identified in the study area at elevations ranging from approximately $1,570 \mathrm{ft}$ to $1,610 \mathrm{ft}$ AMSL (Figures 4.2-4.5). The base of this interval is defined by a typically $5-10 \mathrm{ft}$ thick interval of light gray to yellowish brown, brownish gray, or olive gray clayey to sandy silt, that also appears to be laterally persistent across the majority of the study area. At the time of the 2015-2016 field activities, the onset of groundwater saturation was noted in the lowermost several feet of the " $\mathrm{A}$ " sand zone beneath the former CCC/USDA facility and in the north-central portion of the study area; however, the sands of this unit were predominantly unsaturated in the central and southeastern portions of the area.

- "B" Sand Zone - At most of the locations investigated in the present study, a second, approximate 10-20 ft-thick interval of sand, here termed the "B" sand zone, was penetrated at elevations ranging from approximately $1,540 \mathrm{ft}$ to $1,570 \mathrm{ft}$ AMSL. In the northwestern portion of the study area and locally elsewhere, the "B" sand zone is underlain by silty-clay to clayey-silt deposits that range in thickness from approximately 2-10 ft. At other locations, however, the "B" sands appear to be vertically continuous with the underlying "C" sands. Groundwater saturation of the "B" (and deeper) sand zones was identified throughout the study area during the present investigation.

In the 1993-1995 CCC/USDA investigations (Argonne 1994, 1995a,b), an absence of sand at this stratigraphic position was noted south and southeast of 
the former CCC/USDA facility (at former investigative location SB63; Appendix J, Figures J.1 and J.2). This observation was confirmed during the present study, at locations TI06 and TI11 (Figures 4.1 and 4.2). ECPT log traces recovered at adjacent locations directly north, east, and south of the TI06 and TI11 positions (locations TI07, TI08, TI13, and TI18; Figure 2.2) demonstrate that the "B" sand zone is present in these directions; however, lithologic data available for several irrigation wells presented in the sitespecific Work Plan (Argonne 2016a) suggest that the "B" sand may also be absent to the southwest of SB63, TI06 and TI11 (Appendix J, Figures J.1 and J.3).

- "C" Sand Zones - In the northwestern and southeastern portions of the study area (Figures 4.1-4.3 and 4.5), approximately 10-20 ft of sand was identified at the interpreted base of the unconfined aquifer unit, overlying the upper confining layer (See Section 4.1.3). In the more central portion of the study area, however (Figures 4.1, 4.2, and 4.4), this lowermost sandy unit thickens to an average of approximately $40 \mathrm{ft}$, and deepens. This interval has been designated as the " $\mathrm{C}$ " sand zone where it is relatively thinner to the northwest and southeast; in the central portion of the study area, the thicker deposits at this stratigraphic positon have been subdivided into upper ("C1") and lower (“C2") portions (Figures 4.2-4.5).

As noted above, Figures 4.2-4.5 illustrate that the distinction between the interpreted "B" and "C" sand zones is in some cases relatively arbitrary, particularly in the more central portion of the study area where the lowermost ("C1-C2") sands thicken. In contrast to the more continuous silt-clay horizon noted at the base of the "A" sand zone, the fine-grained horizons associated with the "B" and "C-C1-C2" sands are often thinner, more discontinuous, and often cannot be readily correlated among adjacent boring locations. Similar findings for the unconfined aquifer were previously reported (Argonne 2016a; Keech et al. 1967; Clark et al. 2008). The results of aquifer pumping tests conducted by the CCC/USDA in the immediate vicinity of the former CCC/USDA facility further demonstrated that while the interbedded siltclay horizons may locally influence the vertical movement of groundwater, they do not appear to represent hydraulic barriers among the sand zones within the unconfined at a wider scale (Argonne 1995b, 2016a). 
In the present study area, the base of the unconfined aquifer was identified at an elevation of approximately 1,540 $\mathrm{ft}$ AMSL beneath the former CCC/USDA facility and the northwestern portion of the area, and at elevations of 1,500-1,510 $\mathrm{ft}$ AMSL in the central portion of the area beneath the locally thicker basal ("C1-C2") sand deposits of the unit. In the southeastern portion of the study area, the base of the aquifer was encountered at approximately 1,520 $\mathrm{ft}$ AMSL. Together with preliminary groundwater level relationships discussed in Section 4.1.2, these observations suggest ambient saturated thicknesses for the unconfined aquifer ranging from approximately 41-67 ft during the period of Argonne's observations.

\subsubsection{Upper Confining Unit}

Lithologic information obtained in the current investigation (Appendix D) indicates that the upper confining unit is composed of yellowish, reddish, or grayish brown to olive gray silty clay to clayey silt in the study area. The unit is locally soft to very hard, variably iron-oxide stained, and variably calcareous and may contain calcareous grains, small pebbles, or carbonatecemented patches and minor fine sand that are consistent with a possible origin of this unit as till.

Full penetration of the upper confining unit was achieved at two locations only (drilling locations DL2 and DL7; Figures 4.1 and 4.2) in the current study; however, partial penetrations achieved with the CPT at several locations (TI11, TI24-TI28; Appendix C and Figures 2.2, 4.1, and 4.2) suggest a possible minimum thickness for this unit of approximately $27 \mathrm{ft}$ in the northwestern portion of the study area, and 41-46 ft in the southeastern portion of the area. The latter values are consistent with the thickness of this unit identified at drilling location DL7 (46 $\mathrm{ft}$; Figure 4.2)

Significant penetration of the upper confining layer through the use of the CPT proved difficult in the central portion of the study area, because of the increased thickness of the "C1-C2" sands encountered in this region. Core data recovered in this area at drilled boring DL2 (Figures 4.1 and 4.2) indicate a thickness of $23 \mathrm{ft}$ for the upper confining unit at this location, while a drillers' log available for a boring advanced by the Upper Big Blue Natural Resource District (UBB-NRD), adjacent to Argonne drilling location DL4 (also on the UBB-NRD property; Figure 4.1) suggests a thickness of approximately $56 \mathrm{ft}$ for the upper confining layer near the southwest-central margin of the study area (Figures 4.1 and 4.4). 


\subsubsection{Deeper Hydrostratigraphic Units}

With the approval of the CCC/USDA and NDEQ project managers, the upper confined aquifer and lower confining layer were only investigated at conventional drilling locations DL2 and DL7, and an uppermost sand of the lower confined aquifer was penetrated at location DL7 (Figures 4.1 and 4.2); lithologic/hydrostratigraphic characterization of these deeper units across the complete targeted investigation area was beyond the identified scope of the present study (Argonne 2016a). Data on the lithologic properties of these intervals were obtained from examination of the continuous cores recovered at DL2 and DL7 (Appendix D). The characteristics of the deeper hydrostratigraphic units that were identified on this basis are as follows:

- Upper Confined Aquifer - The identified thickness of the upper confined aquifer at drilling location DL2 is $80 \mathrm{ft}$; at DL7, the unit is $76.5 \mathrm{ft}$ thick. The sediments comprising the upper confined aquifer at these locations predominantly consist of generally well sorted, yellowish gray to yellowish brown, grayish brown, or olive gray fine sands, with lesser medium sand (up to approximately $15 \%$ ) and minor to trace quantities of clay and silt (Table 4.1 and Appendix D, Table D.1). Occasional lenses or layers, up to approximately $10 \mathrm{ft}$ thick, of silty sand to sand-silt-and clay were also noted at DL2.

Limited data suggest that the upper confined aquifer may locally thin near the southwestern margin of the CCC/USDA study area, as shown in Figures 4.1 and 4.4. A drillers' log available for the boring advanced by the UBB-NRD, adjacent to Argonne boring DL4 (Figure 4.1) suggests a thickness of approximately $20 \mathrm{ft}$ for the upper confined aquifer at this location. Similar thinning of the upper confined aquifer, and thickening of the overlying upper confining unit, near this location is suggested in the drillers' log for irrigation well, G-15313, located directly east of the UBB-NRD well, as shown in Figures J.1-J.2.

- Lower Confining Unit - At location DL2, drilling of the investigative boring was terminated in the lower confining layer (at $245 \mathrm{ft} \mathrm{BGL}$ ), after penetrating approximately $15 \mathrm{ft}$ into the unit. At boring DL7, the fine-grained unit directly underlying the upper confined aquifer, interpreted here to represent the 
uppermost sediments of the lower confining unit, was $16.5 \mathrm{ft}$ thick, with an additional interval (approximately $5 \mathrm{ft}$ ) of fine-grained sediments having been penetrated at the base of this boring before drilling was terminated at $280 \mathrm{ft}$ BGL. Local thicknesses for the lower confining unit (to the top of the first sand encountered within this unit) of approximately $45-50 \mathrm{ft}$ are indicated at the locations of the UBB-NRD and PWS 77-1 wells, on the basis of the available drillers' logs for these borings.

At borings DL2 and DL7, the sediments representing the lower confining unit consist of dark gray to olive gray, calcareous, silty-clayey fine sand to clayey silt or silty clay.

- Lower Confined Aquifer - Approximately $19 \mathrm{ft}$ of sand, representing the first coarser-grained unit below the identified top of the lower confining layer described above, was penetrated at boring DL7 at an approximate depth of 256-275 ft BGL. As outlined in the approved Work Plan (Argonne 2016a), the sand penetrated at DL7 is generally comparable in depth and stratigraphic position to the shallowest, lower confined aquifer interval screened in nearby PWS well 77-1 (at 255-310 ft BGL; Figures 4.1, 4.4, and 4.5). The lower confined aquifer sand identified at DL7 is lithologically similar to those of the upper confined aquifer, consisting predominantly of well sorted, yellowish gray, fine sand with small to trace amounts of associated medium sand, silt, and clay (Appendix D, Table D.1 and Table 4.1).

\subsubsection{Preliminary Evaluation of Groundwater Levels}

To obtain information on groundwater levels and the potential influences on groundwater flow (and possible contaminant migration) in the vicinity of the former CCC/USDA facility at York, a preliminary network of 19 permanent monitoring wells has been established in the present study area. The locations and distribution of these wells among the aquifer intervals discussed in Section 4.1.1 is shown in Figure 2.5; construction details for the wells are summarized in Table 3.2. Groundwater levels that have been measured manually in the full suite of monitoring wells are in Table 3.5 . 
Automatic groundwater level measuring and recording units were installed in 11 of the permanent monitoring wells on July 8-9, 2016 (Figure 2.5). The measurements recovered from all units to date (through November 2-3, 2016) are summarized as hydrographs in Figures 4.6 and 4.7, together with daily rainfall data for the weather station located at the York Municipal airport. The full suite of recorder data is in Supplement 1 (on CD). Continued automated monitoring of the complete observation well network is recommended for at least one year, to further investigate possible seasonal or other longer-term influences on the local groundwater levels.

\subsubsection{Groundwater Levels in the Confined Aquifers}

Figure 4.6 illustrates significant variations in the groundwater levels at confined aquifer monitoring points DL2-CU, DL7-CU, and DL7-CL (Figure 2.5) during the period of record. Numerous cycles of drop-and-recovery of the groundwater levels, each typically 5 to 7 days in duration, are indicated from the onset of monitoring (in July 2016) through approximately September 3, 2016, followed by an approach to apparently steady levels during the remainder of the observation period. Transient changes in level are the most pronounced for upper confined aquifer wells DL2-CU and DL7-CU, with decline or recovery of up to 23-24 ft noted in conjunction with individual oscillations at well DL2-CU, and 13-17 ft at well DL7-CU. Individual recovery periods in some cases coincide with the occurrence of local rainfall events but this relationship is not consistent throughout the period of observation, and recoveries do not appear proportional to the precipitation received. Although the influences of the individual summer response cycles are much more subdued for lower confined aquifer well DL7-CL, the overall pattern of water level decline and recovery at this well is consistent with those observed for the upper confined aquifer wells. Figure 4.6 suggests, however, that a possible second pattern of subtle decline-and-recovery cycles, occurring approximately every three days throughout the period of record, is evident in the hydrograph for well DL7-CL that is not observed at the upper confined aquifer wells. The groundwater levels at well DL7-CL continued to rise at the end of the current review period; therefore, the relationship of static head levels in the lower and upper confined aquifers could not be determined. The available data indicate that the seasonal water level changes in the lower confined aquifer have been greater in magnitude (approximately $41 \mathrm{ft}$ to date), and are persisting over a longer duration, than those observed in the upper confined aquifer. 
The summer occurrence and cyclic pattern of rapid groundwater level declines and recovery observed at the upper confined aquifer wells strongly suggest that these responses reflect the influence of seasonal groundwater withdrawal for irrigation purposes. Figure 4.8 shows that numerous irrigation wells have been registered (with the NDNR) in the southeastern, predominantly agricultural portion of the study area in the general vicinity of the DL2 and DL7 monitoring locations. Information pertaining to the aquifer zone(s) tapped by these wells is generally unavailable; however, limited data reported online by the NDNR (dnr.nebraska.gov/gwr/groundwaterwelldata), summarized in Table 4.2, indicate that with only two exceptions the wells shown have completion depths, ranging from 232-247 ft BGL, that are roughly consistent with the base of the upper confined aquifer identified at drilling locations DL2 and DL7 (Figure 4.2; see also Appendix J, Figures J.2-J.4). Well G-11922 has a reported total depth of $115 \mathrm{ft}$ BGL, which suggests probable completion in the unconfined aquifer; well G22760, completed at $327 \mathrm{ft}$ BGL, is likely to penetrate both the upper and lower confined aquifers based on the depths of these units identified during the current study (Figures 4.2, 4.5, and Appendix J, Figure J.4).

All of the identified irrigation wells in the vicinity of DL2 and DL7 have large pumping capacities (500-1,600 gpm), and are capable of generating reported groundwater level drawdowns (at the well) ranging from 25-82 ft (Table 4.2). Specific information on the usage of these wells during the current observation period has not been identified; however, the inferred operation of one or more local irrigation wells appears as a probable source for the summer drawdown-and-recovery responses evident in the groundwater level traces for upper confined aquifer wells DL2-CU and DL7-CU and lower confined aquifer well DL7-CL (Figure 4.6). It may also be hypothesized that potential regional groundwater withdrawal from the upper and lower confined aquifers, as a result of irrigation pumping as well as possible seasonal demands on the York PWS well network, might contribute to the more sustained reduction of groundwater levels observed at monitoring wells DL2-CU, DL7-CU, and particularly DL7-CL during the summer months of 2016 (Figure 4.6).

\subsubsection{Groundwater Levels in the Unconfined Aquifer}

Water level monitoring units were installed at observation wells DL1-C, DL2-C1, DL3C1, DL4-C1, DL5-C1, DL6-C, DL7-C, and DL8-C1 to obtain groundwater level measurements at a consistent stratigraphic level within the unconfined aquifer across the current study area (Figure 2.5). The results of these measurements are illustrated in Figure 4.7. 
Figure 4.7 demonstrates that groundwater levels at the unconfined aquifer monitoring points variably show a summer pattern of cyclic drawdown and recovery, followed by an apparent approach to relatively stable values during the fall, that mirrors the trends observed at upper confined aquifer wells DL2-CU and DL7-CU (Figure 4.6). At all locations in the unconfined aquifer, however, the observed responses to individual drawdown events are substantially smaller than the corresponding changes observed in the upper confined aquifer (Figure 4.6), as are the longer-term decreases in groundwater levels noted throughout the summer months. As noted for the deeper aquifers, groundwater level variations in the unconfined aquifer show no direct correlation or immediate response to individual local rainfall events.

While the timing and duration of the summer pumping-recovery cycles in Figure 4.7 appear effectively identical among the individual hydrographs, variations in the magnitude of the groundwater level responses are evident that appear related to the distribution of the monitoring points across the study area. Specifically, the traces for monitoring wells DL1-C and DL7-C near the northwestern and southeastern limits of the study area show little (less than one foot) to no response to the inferred individual pumping events. Progressively larger responses are noted, respectively, at locations DL6-C, DL4-C1, DL2-C1, DL8-C1, and finally DL3-C1 and DL5-C1 with approach toward the north-central portion of the study area (Figures 4.7 and 4.8).

The groundwater level relationships outlined above indicate that inferred irrigation pumping in the vicinity of the former CCC/USDA facility exerts a transient and spatially varying impact on groundwater levels in the unconfined aquifer. Localized vertical "leakage" of groundwater from the unconfined aquifer across the intervening upper confining layer under pumping conditions might in part account for the observed unconfined aquifer responses; limited data suggest, however, that this pathway may not be effective. Figures 4.9 and 4.10 demonstrate that, under the relatively stable groundwater conditions observed during the later (fall) portion of the current review period, relatively little vertical hydraulic head difference (and hence driving force for vertical groundwater migration) is indicated between the unconfined and upper confined aquifers at monitoring locations DL2 and DL7 (in the central and southeastern portions of the study area, respectively). The apparent vertical hydraulic gradients between these units are increased, however, under the transient pumping conditions observed in July-August 2016. As shown, despite evidence of substantial drawdowns in the upper confined aquifer at locations DL2 and DL7, little to no corresponding response is apparent in the directly overlying unconfined aquifer, suggesting that the upper confining layer effectively inhibits vertical hydraulic communication at these locations. 
Figures 4.7 and 4.8 empirically indicate that drawdown responses observed in the unconfined aquifer are most pronounced at monitoring wells (DL8-C1, DL3-C1 and DL5-C1) in the north-central portion of the study area that are effectively surrounded by irrigation wells to the north, east, and south. As noted in Section 4.1.2.1, construction details for the irrigation wells are unknown; however, it may be hypothesized that the presence of continuous gravel packs, and/or multiple screened intervals, in one or more of these wells could provide a plausible mechanism for localized, coincident drawdown influences in both the unconfined and upper confined aquifers,

In Figure 4.11, groundwater levels measured manually on November 3, 2016 (Table 3.5) have been mechanically contoured to generate a representation of the potentiometric surface for the unconfined aquifer under approximate static conditions. The resulting map shows a relatively uniform hydraulic gradient and flow direction to the southeast that is consistent with the flow direction identified in this aquifer in1993-1995 (Argonne 1994, 1995a,b). Under the conditions observed in November 2016, data for monitoring well pairs DL1-B/-C, DL2-C1/C2, DL3-C1/C2, DL4-C1/C2, and DL8-C1/C2 provide evidence of little to no vertical hydraulic gradient among the principal saturated sand units of the unconfined aquifer.

In Figure 4.12, groundwater level measurements obtained from the automated data loggers have been mechanically contoured to estimate the configuration of the potentiometric surface under the conditions of maximum transient drawdown observed in the unconfined aquifer, on August 11, 2016 (Figure 4.7). Evaluation of the hydrograph traces indicates that hydraulic head relationships similar to those identified on this date existed in the study area primarily in late July-early August 2016 during the current review period, over a combined total of less than four weeks. Under this scenario, a shift toward more easterly groundwater flow is suggested in the central portion of the study area, and toward locally north-northeastern flow in the extreme southeastern portion of the study area. Figure 4.12 empirically suggests focusing of the groundwater flow toward a possible, transient groundwater low in the northeastern portion of the area; it must be noted, however, that the hydraulic heads in the latter portion of the diagram are relatively unconstrained.

\subsection{Evaluation of the Carbon Tetrachloride Distribution in Soils}

In 1993-1995, the CCC/USDA conducted sampling of the near-surface and shallow subsurface $(\leq 4 \mathrm{ft}$ BGL) soils at the former CCC/USDA facility, and sampling of vegetation at 
selected locations at the former facility and on the adjacent York Gun Club property, for screening VOCs analyses to prioritize locations for sampling and analysis of the deeper soils (Argonne 1994, 1995a,b). On the basis of these studies, vertical-profile soil sampling was performed at this time at two locations at the former CCC/USDA facility (SB01 and CPT37) and one location on the Gun Club property (SB62; Figure 2.1). Carbon tetrachloride concentrations exceeding the current NDEQ VCP target for this contaminant in soils $(39 \mu \mathrm{g} / \mathrm{kg})$ were detected in the soils at all three of these locations (Tables 4.3a-c).

At SB01, carbon tetrachloride concentrations of $89-111 \mu \mathrm{g} / \mathrm{kg}$ were identified in the nearsurface soils $(<2.5 \mathrm{ft}$ BGL) and 73-772 $\mu \mathrm{g} / \mathrm{kg}$ over the depth interval 12.6-40 ft BGL; concentrations of up to $644 \mu \mathrm{g} / \mathrm{kg}$ were sporadically observed in isolated samples at greater depths. A generally similar vertical distribution of carbon tetrachloride was identified at boring CPT37, at lower overall concentrations $(67-281 \mu \mathrm{g} / \mathrm{kg})$. At boring SB62 on the Gun Club property, carbon tetrachloride concentrations exceeding the NDEQ VCP target were detected in two samples $(46-49 \mu \mathrm{g} / \mathrm{kg}$ ) over the isolated depth range 38-41 ft BGL (Tables 4.3a-c). The vertical distributions identified suggested that carbon tetrachloride was preferentially associated with the unsaturated, silty-clayey (loess) deposits overlying the sands hosting the unconfined aquifer (Section 4.1.1.1).

To evaluate the present distribution and levels of residual carbon tetrachloride contamination in soils at the former CCC/USDA facility and the York Gun Club, vertical-profile soil samples were collected for VOCs analyses at locations TI01-TI04 (Figure 2.1). With the approval of the CCC/USDA and NDEQ project managers, locations TI01-TI03 were chosen to coincide, respectively, with the previous SB01, CPT37, and SB62 soil boring locations. At the request of the NDEQ (2014), boring TI04 was advanced on Gun Club property, directly east of location TI01/SB01 (Figure 2.1). The results of the analyses for these soil samples are summarized in Table 3.1, and are compared (for TI01-TI03) to the corresponding 1993-1995 analysis results for these locations in Tables 4.3a-c. The full results of the recent analyses (for TI01-TI04) are in Appendix E, Table E.1.

Tables 4.3a-c demonstrate that the present (2015) concentrations of carbon tetrachloride and chloroform in soils at and near the former CCC/USDA facility are significantly reduced relative to the previously identified (1993-1995) levels. At both TI03/SB62 (on the Gun Club property) and TI02/CPT37 (at the former site), only very low to trace concentrations (less than the current method detection limit of $10 \mu \mathrm{g} / \mathrm{kg}$ ) of carbon tetrachloride and no chloroform are 
indicated, reflecting an apparent decrease in concentrations of from one to two orders of magnitude for these contaminants. At location TI01/SB01 at the former facility, the recent analyses indicate both a decrease in contaminant levels (by up to an approximate factor of five), and in the apparent vertical extent of the residual carbon tetrachloride distribution, in comparison to the earlier findings.

Historic analysis data are not available for soil sampling location TI04, located directly east of the former CCC/USDA facility on the Gun Club property (Figure 2.1). The results of the current analyses for this boring (Table 3.2) demonstrate a similar vertical distribution of carbon tetrachloride and chloroform contamination to that observed at TI01. At each of these locations, carbon tetrachloride concentrations greater than the NDEQ VCP target level $(39 \mu \mathrm{g} / \mathrm{kg})$ are restricted to an approximate 20-30 ft depth interval within the vadose zone - from 20-52 ft BGL at TI01, and 24-44 $\mathrm{ft}$ BGL at TI04 - but the observed concentrations at equivalent depths are generally two to three times lower at TI04 in comparison to the corresponding levels at TI01. At each location, the maximum levels of residual carbon tetrachloride contamination occur at 32$36 \mathrm{ft}$ BGL $(167 \mu \mathrm{g} / \mathrm{kg}$ at TI01; $61 \mu \mathrm{g} / \mathrm{kg}$ at TI04).

Consideration of the full suite (1993-1995 and 2015) of soil (and vegetation) sampling and analysis data available for the former CCC/USDA facility and York Gun Club properties demonstrates that both the areal and vertical distribution, and maximum residual levels, of carbon tetrachloride contamination in the shallow and deeper soils have decreased over the period of the CCC/USDA studies at this site. The presently available data suggest that residual contaminant levels that may pose a threat of continued contamination to the underlying groundwater (in keeping with the NDEQ VCP target for this contaminant) are generally limited to depths of 20-50 ft BGL within the fine-grained vadose zone soils beneath and near the central portion of the former CCC/USDA facility. Limited additional soil sampling and analyses for VOCs in this area might be warranted, however, to fully constrain the lateral and vertical extent of this contamination.

\subsection{Evaluation of the Contaminant Distribution in Groundwater}

Groundwater samples for VOCs analyses were obtained from the 28 CPT borings illustrated in Figure 2.2, as well as from the permanent monitoring wells (Figure 2.5) and selected private wells that were investigated in conjunction with the current study (Figure 2.3), and from selected private and public supply wells that are sampled quarterly at the request of the 
NDEQ (Figure 2.7). The results for carbon tetrachloride and chloroform in groundwater sampled as part of the targeted study are summarized by investigative location and sample depth in Table 3.3; the complete results of the analyses for these samples are in Appendix G, Table G.1. In Table 4.4, the data for carbon tetrachloride and chloroform are presented on the basis of sampling location and their relationship to the hydrostratigraphic zones outlined in Section 4.1.1. The vertical distribution of groundwater sampling intervals at selected locations and the results of the analyses for carbon tetrachloride are illustrated in Figures 4.13-4.16. Summary data for carbon tetrachloride at the private and York PWS wells that are sampled quarterly are in Table 3.4. No methylene chloride has been identified in any groundwater sample recovered during the targeted investigation and quarterly sampling programs.

\subsubsection{Contaminant Distribution in the Unconfined Aquifer}

\subsubsection{Carbon Tetrachloride}

Groundwater samples for VOCs analyses were recovered from the unconfined aquifer through the use of direct-push (CPT) techniques at 28 locations (Figure 2.2) and from 19 permanent monitoring wells (Figure 2.5). With only one exception (at TI03; Table 3.3), groundwater samples were collected with the CPT from multiple discrete depth intervals at each location, in order to develop an understanding of the 3-dimensional distribution of the contaminant within the aquifer. The areal distribution of carbon tetrachloride among the primary sandy intervals identified in the unconfined aquifer (Section 4.1.1.2) is illustrated in Figures 4.17-4.19. In these diagrams interpretive shading, developed based on consideration of the complete suite of groundwater analyses for the unconfined aquifer sampling points, has been employed to suggest the potential lateral extent of the carbon tetrachloride contamination at each hydrostratigraphic level (Section 4.1.1.2).

Carbon tetrachloride identified in the " $\mathrm{A}$ " sand interval of the unconfined aquifer is illustrated in Figure 4.17. As noted in Section 4.1.1.2, at the time of this investigation the "A" sand zone was predominantly unsaturated; sufficient groundwater for VOCs analyses could typically only be recovered from this zone at locations beneath the former CCC/USDA facility and in the more northwestern portion of the study area, where saturation was generally identified, at most, in the lowermost several feet of the interval. At some sampled locations, extension of the temporarily screened interval into the fine-grained sediments beneath the "A" sand was 
required, in order to obtain a sufficient column of groundwater in the screen and CPT rods for sampling.

The maximum concentrations of carbon tetrachloride identified in the unconfined aquifer were encountered in the "A" sand zone, beneath the former CCC/USDA facility and the western portion of the adjacent York Gun Club property (Figure 4.17). Carbon tetrachloride levels generally ranged from $150 \mu \mathrm{g} / \mathrm{L}$ (at TI03) to $373 \mu \mathrm{g} / \mathrm{L}$ (at TI01) in this area; the single highest carbon tetrachloride concentration $(1,875 \mu \mathrm{g} / \mathrm{L})$ was identified in a groundwater sample recovered from monitoring well DL1-A (Table 4.4), Figure 4.17 illustrates a fairly rapid lateral decrease in carbon tetrachloride concentrations in this zone, to the southeast and downgradient (see Section 4.1.2.2 and below), reaching levels of less than $50 \mu \mathrm{g} / \mathrm{L}$ approximately 1,200 ft from the former CCC/USDA facility, and concentrations near or below the NDEQ and EPA target level for this contaminant in drinking water (MCL; $5 \mu \mathrm{g} / \mathrm{L}$ ) at a distance of approximately $0.5 \mathrm{mi}$.

Carbon tetrachloride concentrations exceeding the MCL were primarily identified in the somewhat more downgradient, central portion of the study area in the " $B$ " sand zone of the unconfined aquifer, as shown in Figure 4.18. Carbon tetrachloride concentrations were very low $(<2 \mu \mathrm{g} / \mathrm{L})$ at locations beneath and to the southeast (downgradient) of the former CCC/USDA and Gun Club properties, (with the exception of the isolated, elevated level [82 $\mu \mathrm{g} / \mathrm{L}]$ identified at CPT boring TI05) and in the southeastern portion of the area (Table 4.4 and Figure 4.18). Carbon tetrachloride concentrations in the central portion of the study area in the " $\mathrm{B}$ " sand zone vary from $11 \mu \mathrm{g} / \mathrm{L}$ (at TI18) to $45 \mu \mathrm{g} / \mathrm{L}$ (at TI12; Table 4.4)

The " $\mathrm{B}$ " sand is absent directly to the southeast of the former facility and the Gun Club, where this stratigraphic zone is represented by a vertically continuous interval of silty-clayey materials separating the "A" and "C" sand zones (at CPT locations TI06, TI07, and TI11; Figures 2.2, 4.12, and 4.13). Groundwater sampling at the latter locations confirms that these fine-grained deposits are effectively free of carbon tetrachloride contamination (Table 4.4).

As described in Section 4.1.1.2, the thickness of the basal sandy unit identified within the unconfined aquifer varies across the study area. In the northwestern and southeastern portions of the area (Figures 4.12, 4.13, 4.14, and 4.16), the basal "C" sand zone typically has a thickness of 10-20 ft. In the central portion of the study area, however, this unit locally thickens (to about 
$40 \mathrm{ft}$ ) and deepens (Figures 4.12, 4.13, and 4.15). In this area, the unit has been informally subdivided into upper ("C1") and lower ("C2") zones for discussion purposes.

In Figure 4.19, the carbon tetrachloride concentrations identified in the "C" and stratigraphically equivalent " $\mathrm{C} 1$ " sand zone(s) are illustrated. The results shown suggest continued, further downgradient extension of the carbon tetrachloride distribution in the groundwater at this hydrostratigraphic level, with the maximum observed contaminant levels indicated toward the central portion of the study area. With one exception, carbon tetrachloride concentrations greater than the MCL in this zone generally range from 8-63 $\mu \mathrm{g} / \mathrm{L}$; a localized, maximum concentration of $202 \mu \mathrm{g} / \mathrm{L}$ was identified at CPT sampling point TI11, in the " $\mathrm{C}$ " sand beneath the thick, vertically continuous deposits of silty-clay materials described above (Table 4.4 and Figure 4.13).

The carbon tetrachloride levels identified in the central, deepest ("C2") portion of the unconfined aquifer are depicted in Figure 4.20. Concentrations greater than the MCL in this hydrostratigraphic zone are generally slightly higher than those in the overlying ("C-C1") zone, ranging from $22 \mu \mathrm{g} / \mathrm{L}$ (at TI19) to $77 \mu \mathrm{g} / \mathrm{L}$ (at monitoring well DL3-C2).

In Figure 4.21, the interpreted areas of carbon tetrachloride contamination at each hydrostratigraphic level have been superimposed to yield a composite "footprint" of the carbon tetrachloride distribution associated with the former CCC/USDA facility in the unconfined aquifer. The resulting distribution is shown in Figure 4.21, together with the potentiometric surface estimated for the aquifer under inferred ambient conditions, on November 3, 2016 (Section 4.1.2.2 and Figure 4.11). Figure 4.21 demonstrates an effectively linear, northwestsoutheast trend for the carbon tetrachloride distribution in the unconfined aquifer that is consistent with the groundwater flow pattern identified in the study area under non-pumping conditions.

Figure 4.21 suggests migration of the carbon tetrachloride contamination associated with the former CCC/USDA facility (at concentrations equal to or greater than the MCL for this contaminant in drinking water; $5 \mu \mathrm{g} / \mathrm{L}$ ) to a distance of approximately $1.6 \mathrm{mi}$ downgradient from the former facility at the time of the current study. In comparison, the results of the 1993 groundwater sampling studies conducted by the CCC/USDA (Argonne 1994, 1995a,b) indicated a carbon tetrachloride distribution extending approximately $0.8 \mathrm{mi}$ downgradient from the former facility (see Figure 2.26 of the targeted investigation Work Plan [Argonne 2016a], included here 
as Appendix J, Figure J.5), suggesting an average linear downgradient migration rate of approximately $200 \mathrm{ft} / \mathrm{yr}$ over 22 years (from 1993 to 2015).

\subsubsection{Chloroform}

Concentrations of chloroform identified in the groundwater of the unconfined aquifer are summarized in Tables 3.3 and 4.4. No methylene chloride was detected in any of the samples from this aquifer.

Tables 3.3 and 4.4 demonstrate that both the absolute concentrations of chloroform, and the relative level of this compound to the associated carbon tetrachloride at each sampling location and hydrostratigraphic interval, were generally very low, providing little to no evidence for possible degradation of carbon tetrachloride via natural (reductive dechlorination) processes in the unconfined aquifer. Notable exceptions to this relationship are observed, however, at direct-push (CPT) sampling points TI01, TI02, TI04, and TI05 in the uppermost, "A" sand zone of the aquifer beneath and immediately to the east of the former CCC/USDA facility (Figure 2.2). At these locations, chloroform concentrations ranging from $54 \mu \mathrm{g} / \mathrm{L}$ to $164 \mu \mathrm{g} / \mathrm{L}$ were identified in association with carbon tetrachloride concentrations ranging from $159 \mu \mathrm{g} / \mathrm{L}$ to 373 $\mu \mathrm{g} / \mathrm{L}$, yielding chloroform-to-carbon tetrachloride ratios ranging from $27 \%$ to $103 \%$. Although these values empirically suggest that reductive dechlorination of carbon tetrachloride might have occurred (or is occurring) to a limited degree, the available data also suggest that the influence of this process may be extremely localized, as similar concentration relationships are not apparent at nearby sampling points TI03, SB77, and DL1-A in this same area.

\subsubsection{Occurrence of Other Volatile Contaminants}

Complete results of the VOCs analyses for groundwater samples from the current investigation are in Appendix G. With only two exceptions, the data in Appendix G demonstrate that no other volatile compounds were identified in association with the carbon tetrachloride distribution in the unconfined aquifer depicted in Figure 4.21. At sampling location TI01 (at the former CCC/USDA facility), trace concentrations $(<1 \mu \mathrm{g} / \mathrm{L})$ of PCE and 1,2 dichloropropane were detected, and a trace of PCE was also detected at location TI04 (on the York Gun Club property; Figure 2.2), in the "A" sand zone. These compounds are unrelated to the grain fumigation chemicals known to have been typically used by the CCC/USDA at their former 
grain storage sites (carbon tetrachloride and carbon disulfide), and the origin of the trace quantities detected at these locations is unknown.

During the 1993-1995 groundwater investigations conducted at this site (Argonne 1994, 1995a,b), the CCC/USDA identified contamination of the unconfined aquifer groundwater by several, non-fumigant related chemicals including PCE, TCE, 1,1-DCE and others, at some locations together with low levels of carbon tetrachloride, along a separate, parallel trend to the south and west of the (exclusively) carbon tetrachloride plume that could be linked to the former CCC/USDA facility (Appendix J, Figure J.5). As outlined in Section 1, this second, parallel trend of PCE-TCE contamination has subsequently been identified by the EPA as the PCE/TCE Northeast Contamination NPL site by the EPA (EPA 2013a,b, 2014a,b).

The results of the present CCC/USDA studies are consistent with these previous observations (Argonne 1994, 1995a,b) in suggesting that the carbon tetrachloride distribution in groundwater associated with the former CCC/USDA facility, as discussed in Section 4.3.1.1 and depicted in Figure 4.21, remains distinct from the PCE/TCE plume identified by the EPA to the south and west. Specific observations which support this interpretation are as follows (Appendix G, Table G.1; Figure 4.22):

- At CPT sampling location TI22, PCE was detected at a low level $(1.0 \mu \mathrm{g} / \mathrm{L})$ in the "A" sand zone, in the absence of carbon tetrachloride, while carbon tetrachloride was exclusively identified at a similarly low level $(3.3 \mu \mathrm{g} / \mathrm{L})$ in the " $\mathrm{B}$ " sand zone, and neither contaminant was detected in the " $\mathrm{C}$ " sand zone.

- At CPT sampling point TI23, a trace of carbon tetrachloride was detected in the "A" sand zone, while the "B" sand zone was free of identified volatile contaminants. Low levels of 1,2-DCE (1.7-4.9 $\mu \mathrm{g} / \mathrm{L})$, and a trace of PCE, were identified in the underlying " $\mathrm{C} 1$ " and " $\mathrm{C} 2$ " zones, however, in the absence of carbon tetrachloride. Subsequent sampling of monitoring wells DL4-C1 and DL4-C2 revealed similar, low levels of 1,1-DCE (approximately $0.9-2.7 \mu \mathrm{g} / \mathrm{L})$ at this location. 
- Low quantities of 1,1-DCE $(2.3 \mu \mathrm{g} / \mathrm{L}), \mathrm{TCE}(6.3 \mu \mathrm{g} / \mathrm{L})$ and PCE $(4.1 \mu \mathrm{g} / \mathrm{L})$ were identified in the Nordlund private well, in the absence of carbon tetrachloride contamination.

- At CPT location TI21 (and related monitoring well DL6-C), low levels of TCE were identified $(4-5 \mu \mathrm{g} / \mathrm{L})$ in the absence of, or associated with a trace quantity $(<1 \mu \mathrm{g} / \mathrm{L})$ of, carbon tetrachloride.

Together, the contaminant relationships identified at these sampling points appear consistent with their possible location at marginal positions along both the carbon tetrachloride distribution delineated in the current study (as shown in Figure 4.22), and the adjacent (to the southwest) mixed-contaminant plume identified by the EPA. At each location trace to low levels of each contaminant have been identified separately, but no clear evidence of mixing among the contaminant distributions is evident.

\subsubsection{Contaminant Distribution in the Confined Aquifers}

With the approval of the CCC/USDA and NDEQ project managers, groundwater was sampled for VOCs analyses at two locations in the upper confined aquifer (DL2-CU and DL7$\mathrm{CU}$ ) and at one location in the lower confined aquifer (DL7-CL; Figures 4.12 and 4.13). The DL2-CU location was selected to facilitate sampling of the upper confined aquifer groundwater along the identified contaminant migration pathway in the overlying unconfined aquifer, at a central point downgradient from the highest concentrations identified in the unconfined aquifer. Monitoring points DL7-CU and DL7-CL were similarly chosen to permit sampling of groundwater from the upper and lower confined aquifers, at a location lying beyond the presently identified, downgradient "toe" of the carbon tetrachloride distribution in the unconfined aquifer (Figures 4.21, 4.12 and 4.13).

The results of the analyses for these wells (Tables 3.3 and 4.4, and Appendix E) indicate that no carbon tetrachloride, chloroform, methylene chloride, or other volatile contaminants were detected in the groundwater from the upper and lower confined aquifers at these locations. The results therefore provide no evidence of potential carbon tetrachloride cross-contamination from the unconfined aquifer to the underlying, upper or lower confined aquifers at monitoring well locations DL2-CU, DL7-CU, and DL7-CL (Figures 4.12 and 4.13). 
As discussed further in Section 4.3.3.2 below, groundwater samples for VOCs analyses are collected quarterly at York PWS wells 77-1, 2009-4, 2009-5, and 2009-6 (Figure 3.3). Well PWS 77-1 is screened in both the upper and lower confined aquifers; wells 2009-4, 2009-5, and 2009-6 are screened exclusively in the lower confined aquifer. No carbon tetrachloride, chloroform, or methylene chloride have been detected at these wells.

\subsubsection{Status and Sampling of Private and Municipal Supply Wells}

Groundwater samples for VOCs analyses were obtained from five private wells that were investigated in conjunction with the current study (Figure 2.3), and from 13 private and four public supply wells that are sampled quarterly at the request of the NDEQ (Figure 2.7). The results of the VOC analyses for these wells are summarized in Tables 3.3 and 3.4, and Figure 3.3. The results for selected wells are shown together with the distribution of carbon tetrachloride contamination identified in the unconfined aquifer in Figure 4.23.

\subsubsection{Private Wells Investigated as Part of the Targeted Study}

In accord with the Final Work Plan (Argonne 2016a) and at the specific request of the NDEQ, interviews were conducted by Argonne personnel (in July and August 2015; Section 2.1.1) with selected property owners to determine the current status and usage of 11 private wells in the vicinity of the former CCC/USDA grain storage facility (Figure 2.3). The wells chosen for investigation were located along, or near, the apparent trend of carbon tetrachloride contamination in groundwater in the unconfined aquifer associated with the former CCC/USDA facility, as identified in the earlier investigations conducted by the CCC/USDA at York (in 1993-1995; Argonne 1994, 1995a,b) and confirmed by the present studies (Appendix J, Figure J.5 and Figures 4.17-4.21).

Information obtained for the targeted private wells during both the early (Argonne 1994, 1995a) and recent CCC/USDA investigations is summarized below and in Table 4.5. Complete analysis results for groundwater samples collected from the accessible private wells during the current study are in Appendix G, Table G.2, and are summarized in Table 3.3 and Figures 3.3 and 4.23; sampling data obtained during the 1993-1995 site investigations were documented previously (Argonne 1994, 2016a). 
DW19-3201 N. Division Ave.

Well DW19 is located at a private residence, owned throughout the period of CCC/USDA's investigations at York (1993-2016) by resident owner M. Preslicka. The well is located in the yard near the southeast corner of the house, and provides groundwater for all domestic purposes.

Levels of carbon tetrachloride below the MCL and NDEQ VCP target concentration of $5 \mu \mathrm{g} / \mathrm{L}$ were identified in samples collected from the well during the 1993-1995 and present studies $(2.0 \mu \mathrm{g} / \mathrm{L}$ and $1.3 \mu \mathrm{g} / \mathrm{L}$, respectively). No other VOCs have been detected in the groundwater at this location.

DW21-3816 N. Division Ave.

DW21, formerly identified (in 1993) as the Krocker well, is located on property now owned by J. Volzke. The residence at this location is currently used as a rental property, and was occupied as of August 2015. The well is located near the southeast corner of the house, and is available for all domestic purposes. Bottled drinking water is being provided to the tenants by the property owner, however, because of water quality concerns (mentioned by the owner) linked to an onsite sceptic system. No carbon tetrachloride has been detected in the groundwater at this location. Low levels $(1 \mu \mathrm{g} / \mathrm{L})$ of TCE, PCE, and TCA were identified in sampling of the well conducted in 1993, but these contaminants were not detected in the current investigation (Table 4.5 and Appendix G, Table G.1).

DW23 - 3221 N. Lincoln Ave.

Well DW23 is located on property formerly occupied (in 1993) by the Welsh Implement Company. The property is now a retail outlet and equipment service center for Plains Equipment Group. A representative for Plains Equipment indicated that, during expansion of the southern (warehouse) portion of the current facility, a cistern-type well was located on the property. The representative indicated that, with permission from the State, the well was filled in and the building foundation was constructed over its former location. Water for all purposes at the facility is obtained from the municipal water supply. 
Carbon tetrachloride was identified in groundwater from the former well at a concentration of $1.0 \mu \mathrm{g} / \mathrm{L}$, during sampling conducted at this location in 1993 (Table 4.5). No other VOCs were detected in the groundwater at that time.

DW26A-3214 N. Division Ave.

The well at this location, formerly identified (in 1993) as the Cotner residence, was previously used for domestic purposes. The property is now owned by the Garretsons, and is connected to the municipal water supply. The well is located in the lawn at the north side of the house, but has been disconnected from the home's plumbing and electrical systems and is presently not operable.

Carbon tetrachloride was identified in groundwater from the well at a concentration of $49 \mu \mathrm{g} / \mathrm{L}$ in 1993; no other VOCs were detected at that time. The well was not sampled during the current study, due to existing obstructions in the well surface completion (pitless adaptor and former electric and other connections) that prevent ready access to the well bore.

DW26B-3132 N. Division Ave.

The property at this location, formerly identified (in 1993) as the Wize residence, is currently owned by D. Troester. The owner reported to Argonne staff that the former home at this location burned down at some point following the earlier CCC/USDA site investigations, and was demolished. At that time, the associated domestic well was reportedly capped and buried. The property is now occupied by an equipment storage building and parking area, constructed by Troester over the location of the former house and well. Water for all uses is obtained at this location from the municipal water supply.

Carbon tetrachloride was identified at a concentration of $16 \mu \mathrm{g} / \mathrm{L}$ in groundwater sampled from this well in 1993 (Table 4.5). No other volatile contaminants were detected at that time. 
DW38 - 722 E. $25^{\text {th }}$ St.

Well DW38 is located near the northwest corner of the York County Highway Department maintenance facility. Information regarding the well was provided to Argonne staff by Highway Superintendent H. Keim. The well is used to supply groundwater for bathrooms and for washing at the facility, and may be connected to one old water fountain in one of the garage buildings that, to his knowledge, is not in use. Bottled water is provided for drinking at the facility.

No carbon tetrachloride was detected in groundwater sampled from this well in 1993; carbon tetrachloride was identified at a concentration of $53 \mu \mathrm{g} / \mathrm{L}$ during the current investigation. No other VOCs were detected at this location during the 1993 or recent sampling events (Table 4.5 and Appendix G, Table G.1).

DW39- 1111 E. $19^{\text {th }}$ St.

The Nordlund private well (DW39; Figure 2.3) is located near the southwestern corner of the residence, and is now used for lawn and garden watering only. The well was previously used for all domestic purposes. The property owner indicated that he had contacted the EPA with concerns regarding the local groundwater quality and had privately installed a water filtration system (type unspecified) approximately 15 years ago. He stated that the EPA had done testing of the well and subsequently upgraded the treatment system, and then connected the property to the York municipal water supply in the spring of 2015.

No carbon tetrachloride was detected in the Nordlund private well during the sampling events conducted by the CCC/USDA in 1993 or 2015 (Table 4.5). No other volatile contaminants were detected in the groundwater during the 1993 CCC/USDA investigation (Argonne 1994, 2016a); however, low levels of TCE $(6.3 \mu \mathrm{g} / \mathrm{L})$, PCE $(4.1 \mu \mathrm{g} / \mathrm{L})$, and 1,1-DCE $(2.3 \mu \mathrm{g} / \mathrm{L})$ were identified during the recent sampling event. Information presently available to the CCC/USDA indicates that the latter compounds represent contamination associated with the PCE/TCE Northeast Contamination NPL site established by the EPA in 2014 (NDEQ 2011; EPA 2013a, 2014a). 
DW40 - 901 E.19 th $^{\text {St. }}$

Well DW40, previously identified (in 1993) as the Euchner well, is located on property adjacent to, and directly southwest of, the Nordlund property (Figure 2.3). The property is now owned and occupied by the Eckharts. The well had previously been used for domestic purposes, and had been equipped with a filtration system. A representative for the Eckharts (C. Banvelos) indicated to Argonne personnel that the well had been located in the yard behind the house, but was abandoned when the property was connected to the York municipal system by the EPA in spring of 2015.

No carbon tetrachloride was detected in the groundwater from DW40 in the sampling conducted by the CCC/USDA in 1993; a high level of TCE $(79 \mu \mathrm{g} / \mathrm{L})$ and low concentrations of PCE $(1 \mu \mathrm{g} / \mathrm{L})$ and 1,1-DCE $(1 \mu \mathrm{g} / \mathrm{L})$ were identified in this event (Table 4.5). Information presently available to the CCC/USDA indicates that the contamination at this location is also associated with the PCE/TCE Northeast Contamination site (NDEQ 2011; EPA 2013a, 2014a).

DW55 - 3720 N. Division Ave.

The Liermann private well (DW55; Figure 2.3) remains in use for all domestic purposes. The well is located near the southern edge of the Liermann property, in the back yard.

No carbon tetrachloride or other volatile contaminants were detected in this well in the 1993 or 2015 sampling events conducted by the CCC/USDA (Table 4.5).

DW56-3401 N. Lincoln Ave.

Well DW56 was formerly located on property owned and occupied by the Blue Valley Community Action Partnership, and used for domestic purposes. A representative for the facility (L. Epp) indicated to Argonne personnel that the property now receives water for all domestic purposes from the municipal water supply. The representative indicated that the onsite facilities were expanded within the last 20 years, with construction occurring in the northwestern portion of the property where the well had previously been located; aerial photographic evidence (Figures 2.11-2.14 in the Final Work Plan; Argonne 2016a) is consistent with these statements. The fate of this well could not be definitively 
determined; however, Argonne staff could find no evidence of the former well, which may now be located under one of the present building foundations.

No carbon tetrachloride or other volatile contaminants were detected in this well in the 1993 sampling conducted by the CCC/USDA (Table 4.5 And Argonne 1994).

G-21977-2922 N. Division Ave.

Well G-21977 was installed in 1962 by the York Cold Storage Company as an irrigation well, on property now owned and occupied by the Cyclonaire Corporation. Information regarding the well was provided to Argonne personnel by Cyclonaire staff member A. Quail. The well was formerly located in the northeastern portion of the Cyclonaire property; the well was reported to have been cut off at the surface and capped, during expansion of the onsite facilities and construction of a parking area in this portion of the property which took place within one year of the (July 2015) interview. Mr. Quail indicated that the well had previously been used to provide groundwater to the facility for undisclosed purposes, but that water is now obtained from the York municipal supply.

Well G-21977 was not sampled by the CCC/USDA during the 1993 or current investigations.

In conjunction with the assessment of these private wells, Argonne conducted a search of available NDNR well registration data for the York investigation area, and informally contacted local residents and business owners for information on any new drinking water wells that might have been installed in the study area subsequent to the 1993-1995 investigations by the CCC/USDA. No new drinking water wells were identified. One new monitoring well, located on property owned by the UBB-NRD was installed in the area in February 2016 (at 319 E. $25^{\text {th }}$ St.). Records indicate that the UBB-NRD well was drilled to a depth of $300 \mathrm{ft}$ BGL then screened over the depth interval 120-160 ft BGL. The UBB-NRD General Manager D. Eigenberg indicated to Argonne personnel that the well is used for the measurement and recording of groundwater levels.

The UBB-NRD well was not sampled during the current investigation, because of its proximity to Argonne drilling location DL4 on the same (UBB-NRD) property (Figure 4.1). 


\subsubsection{Private and Public Wells Sampled Quarterly}

The CCC/USDA conducts quarterly groundwater sampling and analyses for VOCs at 13 additional, more outlying private wells and four York municipal supply wells located generally to the east and southeast of the former CCC/USDA facility, at the request of the NDEQ. The results of these analyses for carbon tetrachloride are summarized in Table 3.4, and are illustrated in Figures 3.3 and 4.23. No chloroform or methylene chloride has been detected in any of the 17 wells that are sampled quarterly.

Three of the private wells are affected by carbon tetrachloride contamination, and appear to lie along the migration pathway of this contaminant identified in the unconfined aquifer (Kayton, CVA Coop, and Driewer [home]; Table 4.5 and Figure 4.23). Information on the depths and construction of these wells is not available.

- CVA Coop - Carbon tetrachloride concentrations of 4.9-9.2 $\mu \mathrm{g} / \mathrm{L}$ have been identified at the CVA Coop well (at 1403 Road 14) since regular sampling began in December 2014 (Table 3.4); this well was not sampled during the 1993-1995 investigations at this site (Table 4.5). Groundwater from the well is currently used at the Coop facility for domestic and other purposes, without treatment.

- Kayton - Carbon tetrachloride levels of 6-8 $\mu \mathrm{g} / \mathrm{L}$ were identified at the Kayton well in 1993 (former Olson, at 1007 E. 25th St.; Table 4.5). Values ranging from $2.4 \mu \mathrm{g} / \mathrm{L}$ to $17 \mu \mathrm{g} / \mathrm{L}$ have been identified to-date during the quarterly monitoring program, but show no clear, net change from the earlier sampling results. The Kayton well has historically been used for domestic purposes, but this property is now used for rental income and is currently (in 2015-2016) vacant, and the property owner is working with the CCC/USDA to have the home connected to the York municipal water supply.

- Driewer (home well) - At the request of the NDEQ, quarterly sampling of the private well at the Driewer residence (at 1402 Road 14) began in December 2016; access to this well for sampling by the CCC/USDA had previously been denied by the property owner. In prior sampling conducted by the EPA, carbon tetrachloride was reported at this well at a concentration of $8.7 \mu \mathrm{g} / \mathrm{L}$; a 
granular activated carbon (GAC) filtration system was subsequently provided for the house by the EPA (NDEQ 2016b). Carbon tetrachloride was identified at a (pretreatment) concentration of $5.1 \mathrm{~g} / \mathrm{L}$ in the December 2016, CCC/USDA sampling event.

The remaining 10 private wells that are sampled quarterly (B\&B Metalworks, Bonde, Danielson, Driewer shop, Hoblyn, Janzen, Makovicka, Onnen, Steever, and Travis; Table 3.4, Figures 3.3 and 4.23 ) have consistently tested free of carbon tetrachloride contamination.

York municipal well PWS 77-1 is screened in both the upper and lower confined aquifers; wells 2009-4, 2009-5, and 2009-6 are screened exclusively in the lower confined aquifer. No carbon tetrachloride has been detected at these wells (Table 3.4; Figures 3.3 and 4.23). Figure 4.23 indicates that well PWS $77-1$ is located approximately $0.25 \mathrm{mi}$ south, and generally cross-gradient, from the identified trend of carbon tetrachloride migration in the unconfined aquifer. PWS wells 2009-4, 2009-5 and 2009-6 appear to lie in the potential direction of continued contaminant migration in the unconfined aquifer, but at locations that are presently 0.75-1.1 mi beyond the interpreted downgradient limit of this contamination.

\subsection{Evaluation of Possible Carbon Tetrachloride Vapor Intrusion to Indoor Air}

An initial screening of indoor air was performed at six locations in the vicinity of the former CCC/USDA facility, to investigate for possible carbon tetrachloride VI (Argonne 2015b). The locations investigated included the Hearthstone and Willowbrook long-term health care facilities (affiliated with York General Hospital), the headquarters building of the CVA Coop, the Fitness Worx (York Physical Therapy) facility, and the Preslicka and Garretson/Troester private residences (Figure 2.6). These locations were chosen, with the approval of the CCC/USDA and NDEQ project managers, as residential and other sites of potentially sensitive populations lying along or near the more upgradient portion of the anticipated migration pathway of carbon tetrachloride in the unconfined aquifer.

The results of the VOCs analyses for carbon tetrachloride and chloroform are summarized in Table 3.6; the complete results of the analyses are in Appendix H, Table H.1. The sampled locations are shown, relative to the identified carbon tetrachloride distribution in the 
unconfined aquifer, in Figure 4.24. The sampling efforts conducted and findings for each location are discussed below.

\subsubsection{Analysis Results for Indoor Air at the Hearthstone and Willowbrook Facilities}

The Hearthstone and Willowbrook facilities are associated with the York General Hospital. Willowbrook is an assisted-living facility; Hearthstone provides skilled nursing and rehabilitation services to extended-stay residents with varying levels of disabilities. In August 2015, samples of indoor air were obtained at each facility from the breathing zone in three large, communal-use areas and in three smaller, staff offices or resident apartments distributed throughout the facility. Building staff and residents of the facilities are variably present in these areas on a 24-hr basis. No carbon tetrachloride or chloroform was detected in the indoor air at either facility, at analytical method detection limits for these compounds of $1.3 \mu \mathrm{g} / \mathrm{m}^{3}$ and 0.98 $\mu \mathrm{g} / \mathrm{m}^{3}$, respectively (Table 3.6). On the basis of these findings, and with the approval of the CCC/USDA and NDEQ project managers, no further VI monitoring was performed at these facilities.

\subsubsection{Analysis Results for Indoor Air at the CVA Corporate Office}

Indoor air samples for VOCs analyses were collected at the headquarters building of the CVA Coop. The CVA facility is regularly occupied during normal business hours (8am-5pm, Monday-Friday). Three samples were collected from the breathing zone in one large conference room, one smaller conference room, and one staff office. No carbon tetrachloride or chloroform was detected in the indoor air at these locations, at the analytical method detection limits noted above (Table 3.6). On the basis of these findings, and with the approval of the CCC/USDA and NDEQ project managers, no further VI monitoring has been conducted at this facility.

\subsubsection{Analysis Results for Indoor Air at the Preslicka Residence}

The Preslicka residence is a private, two-story home occupied by the owner. Indoor air samples for VOCs analyses were collected at one location on the ground floor of the residence, and at one location in an unfinished basement used for storage only. No carbon tetrachloride or chloroform was detected on either of these levels (at the analytical method detection limits noted 
above; Table 3.6), and no additional VI sampling has been undertaken by the CCC/USDA at this location.

\subsubsection{Analysis Results for Indoor Air and Sub-Slab Soil Vapor at the Fitness Worx Facility}

Fitness Worx is a public-use facility providing access to fitness equipment and personal training services (via membership) to the York community. The Fitness Worx facility also houses York Physical Therapy, which provides skilled physical therapy and rehabilitation services. The facility is open to staff and visitors for a maximum of 16 hours per day ( 5 a.m. to 9 p.m., Monday-Friday; 7a.m. to 4p.m., Saturday; and 12 p.m. to 4 p.m., Sunday). Indoor air samples for VOCs analyses were obtained at two locations in a main exercise area and two smaller (physical therapy) rooms on the ground floor, and in a large exercise room located on the lower (basement) level of the building during the August 2015 sampling event.

Carbon tetrachloride levels ranging from $1.5 \mu \mathrm{g} / \mathrm{m}^{3}$ to $7.9 \mu \mathrm{g} / \mathrm{m}^{3}$ for the ground floor air samples, and $6.3 \mu \mathrm{g} / \mathrm{m}^{3}$ for the air sample from the lower level of the building, were identified during this event (Table 3.6). These concentrations exceed the minimum NDEQ VCP target level for this contaminant in residential indoor air (established on the basis of excess cancer risk) of $0.41 \mu \mathrm{g} / \mathrm{m}^{3}$, but are below the corresponding minimum target level for indoor air in an industrial environment (20 $\mu \mathrm{g} / \mathrm{m}^{3}$; NDEQ 2012). Chloroform was also detected in the two small therapy rooms at a level $\left(1.2 \mu \mathrm{g} / \mathrm{m}^{3}\right)$ greater than the NDEQ VCP minimum residential target for this contaminant $\left(0.106 \mu \mathrm{g} / \mathrm{m}^{3}\right)$, but less than the associated industrial target for indoor air $\left(5.33 \mu \mathrm{g} / \mathrm{m}^{3}\right)$. Based on these findings, the NDEQ requested that the CCC/USDA implement sampling of the indoor air, and also sub-slab vapor, at the Fitness Worx facility over four consecutive quarters, beginning in January 2016, as a basis for the consideration of possible mitigation requirements (NDEQ 2015a). For this purpose, a sub-slab vapor sampling point was installed, with the approval of the NDEQ, at the base of a stairwell connecting the two levels of the building. The sampling point was constructed in accord with the procedures in Appendix B.

In compliance with the NDEQ's requests, additional indoor air and sub-slab vapor samples for VOCs analyses were collected in January, April, August, and December 2016 at four locations (two ground floor therapy rooms, the lower level exercise room, and at the base of the stairwell connecting the building levels). The results of these analyses (Table 3.6) demonstrate that carbon tetrachloride concentrations ranging from $2.4 \mu \mathrm{g} / \mathrm{m}^{3}$ to $8.2 \mu \mathrm{g} / \mathrm{m}^{3}$ and from $1.8 \mu \mathrm{g} / \mathrm{m}^{3}$ to $7.2 \mu \mathrm{g} / \mathrm{m}^{3}$, respectively, were consistently identified at the ground floor and lower level 
sampling locations. The identified concentrations remained well below the NDEQ VCP target level for this contaminant in non-residential settings $\left(20 \mu \mathrm{g} / \mathrm{m}^{3}\right)$ throughout the period of monitoring from August 2015 through December 2016. Carbon tetrachloride concentrations ranging from not-detected $\left(<13 \mu \mathrm{g} / \mathrm{m}^{3}\right) 50 \mu \mathrm{g} / \mathrm{m}^{3}$ were identified in corresponding samples of the sub-slab vapor. These levels are also significantly less than the permissible action level for this contaminant in the sub-slab vapor (approximately $666 \mu \mathrm{g} / \mathrm{m}^{3}$ ), based on guidance values for this parameter outlined to the CCC/USDA by the NDEQ (NDEQ 2015b).

Chloroform concentrations ranging from not-detected $\left(<0.98 \mu \mathrm{g} / \mathrm{m}^{3}\right)$ to $9.8 \mu \mathrm{g} / \mathrm{m}^{3}$ were identified in indoor air during the January-December 2016 monitoring events, despite no detectable occurrence of this contaminant (at an analytical method detection limit of 9.8-9.9 $\mu \mathrm{g} / \mathrm{m}^{3}$ for these analyses) in corresponding samples of the sub-slab vapor (Table 3.6). This observation, in conjunction with the high levels of chloroform identified in relation to carbon tetrachloride during the April, August, and December 2016 sampling events, might be indicative of a possible source of chloroform contamination to the indoor air unrelated to the underlying soil vapor.

\subsubsection{Analysis Results for Indoor Air and Sub-Slab Soil Vapor at the Garretson/Troester Residence}

The Garretson/Troester residence is a one-story home with a partially finished lower level (basement) used as living space. In August 2015, at the time of the initial indoor air sampling event, the house was used as an income property by owner D. Troester, and occupied by renters. In September 2015, the house was sold by Troester to the Garrretsons, who have occupied the property since that time.

Indoor air samples were collected during the August 2015 sampling event from one location in the main living area on the ground floor, and from one location in the finished portion of the lower level. Carbon tetrachloride concentrations of $8 \mu \mathrm{g} / \mathrm{m}^{3}$ and $16 \mu \mathrm{g} / \mathrm{m}^{3}$ were identified, respectively, at these locations (Table 3.6). These concentrations exceed the NDEQ VCP minimum target concentration for this contaminant in residential indoor air $\left(0.41 \mu \mathrm{g} / \mathrm{m}^{3}\right)$. No chloroform was detected in the indoor air samples, at an analytical method detection limit of 0.98 $\mu \mathrm{g} / \mathrm{m}^{3}$ for this analysis. On the basis of these findings, the NDEQ requested that the CCC/USDA also implement quarterly sampling of the indoor air, and sub-slab vapor, at this residence for one year, beginning in January 2016. For this purpose, a sub-slab vapor sampling point was installed, 
with the approval of the NDEQ, in an uncarpeted portion of the floor on the lower level of the building. The sampling point was constructed in accord with the procedures in Appendix B.

In compliance with the NDEQ requests, indoor air and sub-slab vapor sampling for VOCs analyses was initiated in January 2016. The results of the January analyses revealed increased concentrations of carbon tetrachloride in the indoor air on both the ground and lower levels of the structure $\left(21 \mu \mathrm{g} / \mathrm{m}^{3}\right.$ and $28 \mu \mathrm{g} / \mathrm{m}^{3}$, respectively) and high concentrations of both carbon tetrachloride $\left(6500 \mu \mathrm{g} / \mathrm{m}^{3}\right)$ and chloroform $\left(110 \mu \mathrm{g} / \mathrm{m}^{3}\right)$ in the sub-slab vapor (Table 3.6). Because of these high levels, the CCC/USDA elected to pursue the installation of a sub-slab vapor mitigation system for the residence. A second quarterly sampling event, originally planned for April 2016, was postponed with the approval of the NDEQ, pending construction of the mitigation system by a local contractor (Thrasher; La Vista, Nebraska) experienced with the installation of these systems in the York area.

Following the completion of the sub-slab vapor mitigation system, sampling of indoor air only was conducted at the Garretson/Troester residence in August and December 2016. No carbon tetrachloride or chloroform was detected in samples from the ground floor or lower level of the home, at analytical method detection limits of $1.3 \mu \mathrm{g} / \mathrm{m}^{3}$ and $0.98 \mu \mathrm{g} / \mathrm{m}^{3}$, respectively, for these analyses (Table 3.6).

\subsubsection{Qualitative Assessment of Potential Carbon Tetrachloride VI at Other Locations}

The Garretson residence and Fitness Worx facility directly overlie the trends of elevated carbon tetrachloride migration identified in the contaminated "A" and "B" sand zones, at approximate depths of 75-100 ft BGL (Figures 4.17 and 4.18). Under the presently observed conditions, concentrations of carbon tetrachloride greater than the NDEQ VCP and MCL level $(5 \mu \mathrm{g} / \mathrm{L})$ for this contaminant in drinking water extend approximately $0.9 \mathrm{mi}$ to the southeast of the former CCC/USDA facility in these zones. At greater distances from the former facility (from $0.9 \mathrm{mi}$ to the current margin of the contaminant distribution, approximately $1.6 \mathrm{mi}$ downgradient), elevated levels of carbon tetrachloride are restricted to the underlying "C-C1" and "C2" sand zones, at depths of approximately 110-160 ft BGL (Figures 4.19 and 4.20). In this downgradient area, sampling has demonstrated that the groundwater in the shallowest saturated interval (the "B" sand zone; Figures 4.13 and 4.18) remains effectively uncontaminated. 
Reconnaissance conducted by Argonne demonstrates that the area along the carbon tetrachloride migration pathway lying downgradient of the (6) locations that have already been screened for VI is relatively sparsely populated, and consists predominantly of agricultural land (Figure 4.24). Occupied properties along the identified migration trend consist primarily of several commercial or warehouse/garage facilities. Only two residential properties fall along the more distal, and deeper, carbon tetrachloride migration pathway that have not been investigated for VI; these are the Kayton (rental property; currently vacant) and Driewer (home) properties (Figure 4.24). Carbon tetrachloride concentrations measured to-date in private wells located at each of these residences (Section 4.3.3.2 and Table 3.4) have been generally low (Kayton property, at 2.2-17 $\mu \mathrm{g} / \mathrm{L}$; and Driewer property, at 5.1 $\mu \mathrm{g} / \mathrm{L})$.

Together, the above observations suggest that the potential risks to human health at downgradient locations in the remainder of the present study area, as a result of possible intrusion of carbon tetrachloride vapors to indoor air, are minimal. Further consideration of these risks may be warranted, however, as the distribution of the carbon tetrachloride contamination in the unconfined aquifer continues to evolve with time. 
TABLE 4.1 Average grain size distributions for hydrostratigraphic units in the York investigation area. ${ }^{a}$

\begin{tabular}{|c|c|c|c|c|c|c|c|c|c|c|c|}
\hline \multirow{3}{*}{$\begin{array}{l}\text { Stratigraphic } \\
\text { Interval }\end{array}$} & \multirow{3}{*}{$\begin{array}{l}\text { Number of } \\
\text { Samples }\end{array}$} & \multicolumn{10}{|c|}{ Composition, \% } \\
\hline & & \multicolumn{3}{|c|}{ Gravel } & \multicolumn{4}{|c|}{ Sand } & \multicolumn{3}{|c|}{ Fines } \\
\hline & & Coarse & Fine & Total & Coarse & Medium & Fine & Total & Silt & Clay & Total \\
\hline \multicolumn{12}{|l|}{ Unconfined Aquifer } \\
\hline "A" Sand & 16 & 0.0 & 2.8 & 2.8 & 7.1 & 33.6 & 44.2 & 85.0 & 8.2 & 4.0 & 12.3 \\
\hline "B" Sand & 27 & 0.0 & 2.9 & 2.9 & 6.1 & 25.3 & 54.6 & 85.9 & 7.2 & 4.0 & 11.2 \\
\hline "C" Sand & 8 & 0.1 & 1.3 & 1.3 & 3.4 & 40.7 & 49.2 & 93.3 & 3.1 & 2.4 & 5.4 \\
\hline "C1" Sand & 13 & 0.0 & 2.1 & 2.1 & 4.0 & 40.1 & 44.9 & 89.0 & 5.6 & 3.3 & 8.9 \\
\hline "C2" Sand & 12 & 0.0 & 1.8 & 1.8 & 3.7 & 25.0 & 60.7 & 89.4 & 5.8 & 3.1 & 8.8 \\
\hline Upper Confining Unit & 2 & 0.0 & 0.6 & 0.6 & 0.8 & 2.8 & 12.2 & 15.7 & 43.9 & 40.0 & 83.8 \\
\hline Upper Confined Aquifer & 18 & 0.0 & 0.0 & 0.0 & 0.0 & 4.4 & 86.4 & 90.8 & 6.0 & 3.2 & 9.0 \\
\hline Lower Confining Unit & 2 & 0.0 & 0.0 & 0.0 & 0.0 & 0.0 & 0.3 & 0.3 & 60.2 & 39.6 & 99.7 \\
\hline Lower Confined Aquifer & 4 & 0.0 & 0.0 & 0.0 & 0.0 & 3.8 & 88.5 & 92.3 & 3.8 & 4.0 & 7.7 \\
\hline
\end{tabular}

a Complete data for all samples are in Appendix D, Table D.1. 
TABLE 4.2 Construction and groundwater level data for selected irrigation wells. ${ }^{\mathrm{a}}$

\begin{tabular}{|c|c|c|c|c|c|c|c|}
\hline \multirow{2}{*}{$\begin{array}{c}\text { Well } \\
\text { Registration } \\
\text { Number }\end{array}$} & \multirow[b]{2}{*}{$\begin{array}{l}\text { Registered } \\
\text { Owner }\end{array}$} & \multirow[b]{2}{*}{$\begin{array}{c}\text { Completion } \\
\text { Date }\end{array}$} & \multirow{2}{*}{$\begin{array}{l}\text { Pumping } \\
\text { Column } \\
\text { Diam. (in.) }\end{array}$} & \multicolumn{3}{|c|}{ Depth (ft BGL) } & \multirow{2}{*}{$\begin{array}{l}\text { Pumping } \\
\text { Capacity } \\
\text { (gpm) }\end{array}$} \\
\hline & & & & Well & $\begin{array}{c}\text { Static } \\
\text { Water Level }\end{array}$ & $\begin{array}{c}\text { Pumping } \\
\text { Water Level }\end{array}$ & \\
\hline G-7037 & W. Driewer & $4 / 29 / 1957$ & 8 & 232 & 73 & 130 & 1200 \\
\hline G-15313 & Olsson Trusts & 2/23/1954 & 9 & 241 & 76 & 101 & 1000 \\
\hline G-49958 & J. \& M. Stahr & $4 / 21 / 1976$ & 8 & 247 & 79 & 118 & 1300 \\
\hline G-8320 & R. Barr & 10/6/1956 & 9 & 238 & 75 & 102 & 1000 \\
\hline G-22760 & Olson Trusts & 2/4/1964 & 9 & 327 & 78 & 114 & 1200 \\
\hline G-19067 & York Pub Sch Dist 12 & $11 / 11 / 1956$ & 9 & 239 & 76 & 106 & 1000 \\
\hline G-38337 & Halstead Trust & $11 / 15 / 1963$ & 7 & 244 & 80 & 127 & 1600 \\
\hline G-11921 & M. Forsberg & $10 / 1 / 1949$ & 6 & 115 & 85 & 110 & 500 \\
\hline G-11922b & M. Forsberg & 4/3/1998 & 9 & 240 & 78 & 160 & 700 \\
\hline
\end{tabular}

a Data as reported in NDNR well registration forms, available online (dnr.nebraska.gov/gwr/groundwaterwelldata).

b Replacement for earlier (1952), 152-ft deep well with this registration number, decommissioned on May 1, 1998. 
York Targeted Investigation, 2015-2016

TABLE 4.3a Comparison of carbon tetrachloride concentrations in soils at sampling location TI01-SB01 in 2015 and 1993.

\begin{tabular}{|c|c|c|c|c|c|}
\hline \multicolumn{3}{|c|}{$2015-$ TIO1 $^{\mathrm{a}}$} & \multirow[b]{2}{*}{$\begin{array}{l}\text { Depth }^{b} \\
\text { (ft BGL) }\end{array}$} & \multicolumn{2}{|c|}{$1993-\mathrm{SB} 01^{\mathrm{C}}$} \\
\hline & $\begin{array}{c}\text { Carbon } \\
\text { Tetrachloride } \\
(\mathrm{mg} / \mathrm{kg})\end{array}$ & $\begin{array}{l}\text { Chloroform } \\
(\mathrm{mg} / \mathrm{kg})\end{array}$ & & $\begin{array}{c}\text { Carbon } \\
\text { Tetrachloride } \\
(\mathrm{mg} / \mathrm{kg})\end{array}$ & $\begin{array}{l}\text { Chloroform } \\
(\mathrm{mg} / \mathrm{kg})\end{array}$ \\
\hline \multicolumn{2}{|r|}{$9.6 \mathrm{~J}$} & ND & 4 & & \\
\hline \multirow{2}{*}{\multicolumn{2}{|c|}{$\begin{array}{l}13 \\
18\end{array}$}} & ND & 8 & Uc & \\
\hline & & $4.8 \mathrm{~J}$ & 12 & 70 & 10 \\
\hline \multirow{2}{*}{\multicolumn{2}{|c|}{29}} & $6.0 \mathrm{~J}$ & $\begin{array}{c}12.6-12.9 \\
16\end{array}$ & 73 & 16 \\
\hline & & & $17.6-17.9$ & 486 & 42 \\
\hline \multirow{2}{*}{\multicolumn{2}{|c|}{84}} & $5.0 \mathrm{~J}$ & 20 & 400 & 32 \\
\hline & & $7.9 \mathrm{~J}$ & & 289 & 37 \\
\hline \multirow{2}{*}{\multicolumn{2}{|c|}{139}} & & $24.8-24.9$ & 772 & 42 \\
\hline & & $6.2 \mathrm{~J}$ & $\begin{array}{c}25.8-26 \\
28\end{array}$ & 522 & 28 \\
\hline \multirow{2}{*}{\multicolumn{2}{|c|}{110}} & & $29.8-30$ & 343 & 15 \\
\hline & & & $30.5-30.7$ & 370 & 25 \\
\hline \multirow{2}{*}{\multicolumn{2}{|c|}{121}} & 12 & 32 & & \\
\hline & & & $34.5-34.7$ & 299 & 16 \\
\hline \multirow{4}{*}{\multicolumn{2}{|c|}{167}} & 12 & 36 & & \\
\hline & & & $37-37.2$ & 563 & 39 \\
\hline & & & $38.8-39$ & 367 & 32 \\
\hline & & & $39.8-40$ & 443 & 19 \\
\hline \multirow{2}{*}{\multicolumn{2}{|c|}{129}} & 14 & 40 & & \\
\hline & & & $41.5-41.7$ & 236 & 26 \\
\hline \multirow{2}{*}{\multicolumn{2}{|c|}{60}} & $9.3 \mathrm{~J}$ & 44 & & \\
\hline & & & $47-47.2$ & 27 & 12 \\
\hline \multirow{5}{*}{\multicolumn{2}{|c|}{29}} & 12 & 48 & & \\
\hline & & & $48-48.2$ & 644 & 55 \\
\hline & & & $49.3-49.6$ & 4 & 14 \\
\hline & & & $50.4-50.6$ & 12 & 25 \\
\hline & & & $51.3-51.5$ & 402 & $55^{*}$ \\
\hline \multirow{2}{*}{\multicolumn{2}{|c|}{53}} & 17 & 52 & & \\
\hline & & & $52.5-52.7$ & 26 & $45^{\star}$ \\
\hline \multirow{2}{*}{\multicolumn{2}{|c|}{ ND }} & ND & 56 & & \\
\hline & & & 57.5 & 12 & $41^{*}$ \\
\hline \multirow{2}{*}{\multicolumn{2}{|c|}{ ND }} & ND & 60 & & \\
\hline & & & $61.7-61.9$ & 24 & $52^{*}$ \\
\hline \multicolumn{2}{|r|}{ ND } & ND & 63.8 & 15 & 17 \\
\hline \multirow{2}{*}{\multicolumn{2}{|c|}{ ND }} & ND & $\begin{array}{c}66.1-66.9 \\
68\end{array}$ & 15 & \\
\hline & & & $71.3-71.4$ & 542 & 23 \\
\hline & ND & ND & 72 & & \\
\hline & 23 & ND & 75 & & \\
\hline & 110 & ND & 80 & & \\
\hline & \multicolumn{5}{|c|}{$\begin{array}{l}\text { Results for samples preserved on dry ice and prepared by the purge-and-trap } \\
\text { method for analysis by GC-MS at the AGEM Laboratory. }\end{array}$} \\
\hline \multirow[t]{4}{*}{ b } & \multicolumn{5}{|c|}{ Color codes for depth intervals are: } \\
\hline & \multicolumn{5}{|c|}{$\begin{array}{c}\text { Color codes for depth intervals are: } \\
\text { Dry silty/clayey }\end{array}$} \\
\hline & & Dry sandy & & & \\
\hline & & Wet sandy & & & \\
\hline c & $\begin{array}{l}\text { Results for } \\
\text { mobile labor }\end{array}$ & les preserve & nethanol a & analyzed by $\mathrm{t}$ & rgonne \\
\hline
\end{tabular}


York Targeted Investigation, 2015-2016

Version 01, 02/14/2017

TABLE 4.3b Comparison of carbon tetrachloride concentrations in soils at sampling location TI02-CPT37 in 2015 and 1993.

\begin{tabular}{|c|c|c|c|c|}
\hline \multicolumn{2}{|c|}{$2015-$ TIO2 $^{\mathrm{a}}$} & \multirow[b]{3}{*}{$\begin{array}{l}\text { Depth } \\
\text { (ft BGL) }\end{array}$} & \multicolumn{2}{|c|}{1993 - СРТЗ7' } \\
\hline Carbon & & & Carbon & \\
\hline $\begin{array}{l}\text { Tetrachloride } \\
(\mathrm{mg} / \mathrm{kg})\end{array}$ & $\begin{array}{l}\text { Chloroform } \\
(\mathrm{mg} / \mathrm{kg})\end{array}$ & & $\begin{array}{l}\text { Tetrachloride } \\
(\mathrm{mg} / \mathrm{kg})\end{array}$ & $\begin{array}{c}\text { Chloroform } \\
(\mathrm{mg} / \mathrm{kg})\end{array}$ \\
\hline \multirow[t]{2}{*}{ ND } & ND & 4 & & \\
\hline & & $4-6$ & 26 & 35 \\
\hline \multirow[t]{3}{*}{ ND } & ND & 7.8 & & \\
\hline & & $7-9$ & 26 & 35 \\
\hline & & $10-12$ & 39 & 42 \\
\hline \multirow[t]{2}{*}{ ND } & ND & 12 & & \\
\hline & & $13-15$ & 25 & 27 \\
\hline \multirow[t]{3}{*}{ ND } & ND & 16 & & \\
\hline & & $16-18$ & 78 & 42 \\
\hline & & $19-21$ & 84 & 45 \\
\hline \multirow[t]{2}{*}{ ND } & ND & 20 & & \\
\hline & & $22-24$ & 201 & 43 \\
\hline \multirow[t]{3}{*}{$8.4 \mathrm{~J}$} & ND & 24 & & \\
\hline & & $24-24.5$ & 281 & 60 \\
\hline & & $26-28$ & 148 & 32 \\
\hline \multirow[t]{2}{*}{ ND } & ND & 28 & & \\
\hline & & $29-31$ & 43 & 23 \\
\hline \multirow[t]{3}{*}{$4.8 \mathrm{~J}$} & ND & 32 & & \\
\hline & & $32-34$ & 70 & 30 \\
\hline & & $35-37$ & 126 & 34 \\
\hline \multirow[t]{2}{*}{$5.9 \mathrm{~J}$} & ND & 36 & & \\
\hline & & $38-40$ & 128 & 43 \\
\hline \multirow[t]{2}{*}{10} & ND & 40 & & \\
\hline & & $41-43$ & 57 & 48 \\
\hline \multirow[t]{4}{*}{13} & ND & 44 & & \\
\hline & & $44-45$ & 10 & 22 \\
\hline & & $45-46$ & 37 & 32 \\
\hline & & $47-48$ & 20 & 34 \\
\hline \multirow[t]{3}{*}{$7.7 \mathrm{~J}$} & ND & 48 & & \\
\hline & & $48-49$ & 67 & 23 \\
\hline & & $50-52$ & ND & 25 \\
\hline \multirow[t]{3}{*}{ ND } & ND & 52 & & \\
\hline & & $53-54$ & ND & 42 \\
\hline & & $54-55$ & ND & 17 \\
\hline \multirow[t]{3}{*}{ ND } & ND & 55.8 & & \\
\hline & & $56.5-57.5$ & ND & 24 \\
\hline & & $59-61$ & ND & 33 \\
\hline \multirow[t]{2}{*}{ ND } & ND & 59.8 & & \\
\hline & & $62-64$ & ND & 17 \\
\hline \multirow[t]{2}{*}{ ND } & ND & 64 & & \\
\hline & & $65-67$ & ND & 17 \\
\hline \multirow[t]{2}{*}{ ND } & ND & 67.8 & & \\
\hline & & $68-70$ & ND & 11 \\
\hline ND & ND & 72 & & \\
\hline 10 & ND & 75 & & \\
\hline
\end{tabular}

a Results for samples preserved on dry ice and prepared by the purge-and-trap method for analysis by GC-MS at the AGEM Laboratory.

b Color codes for depth intervals are:

Dry silty/clayey

Dry sandy

Wet sandy

c Results for samples preserved in methanol and analyzed by the Argonne mobile laboratory. 
York Targeted Investigation, 2015-2016

TABLE 4.3c Comparison of carbon tetrachloride concentrations in soils at sampling location TI03-SB62 in 2015 and 1993.

\begin{tabular}{|c|c|c|c|c|}
\hline \multicolumn{2}{|c|}{$2015-$ TI03 $^{\mathrm{a}}$} & \multirow[b]{2}{*}{$\begin{array}{l}\text { Depth }^{b} \\
\text { (ft BGL) }\end{array}$} & \multicolumn{2}{|c|}{$1993-\mathrm{SB} 62^{\mathrm{C}}$} \\
\hline $\begin{array}{c}\text { Carbon } \\
\text { Tetrachloride } \\
(\mathrm{mg} / \mathrm{kg})\end{array}$ & $\begin{array}{l}\text { Chloroform } \\
(\mathrm{mg} / \mathrm{kg})\end{array}$ & & $\begin{array}{c}\text { Carbon } \\
\text { Tetrachloride } \\
(\mathrm{mg} / \mathrm{kg})\end{array}$ & $\begin{array}{c}\text { Chloroform } \\
(\mathrm{mg} / \mathrm{kg})\end{array}$ \\
\hline \multirow[t]{3}{*}{ ND } & ND & 4 & & \\
\hline & & 4-4.5 & 10 & 34 \\
\hline & ND & $\begin{array}{c}6-6.5 \\
8\end{array}$ & ND & ND \\
\hline \multirow{2}{*}{ ND } & INU & 8-8.5 & 6 & 42 \\
\hline & & $10.3-10.6$ & ND & ND \\
\hline ND & ND & 12 & & \\
\hline ND & ND & 16 & & \\
\hline \multirow[t]{2}{*}{ ND } & ND & 20 & & \\
\hline & & 23-23.5 & 6 & 30 \\
\hline \multirow[t]{2}{*}{ ND } & ND & 24 & & \\
\hline & & $26-26.5$ & 8 & 26 \\
\hline \multirow[t]{3}{*}{ ND } & ND & 28 & & \\
\hline & & $29-29.5$ & 25 & 28 \\
\hline & & $31.7-32$ & 22 & 30 \\
\hline ND & ND & 32 & & \\
\hline $5.6 \mathrm{~J}$ & ND & $\begin{array}{c}36 \\
38-38.6\end{array}$ & 46 & 31 \\
\hline \multirow[t]{2}{*}{$8.7 \mathrm{~J}$} & ND & 40 & 40 & I \\
\hline & & $40.5-41$ & 49 & 33 \\
\hline \multirow[t]{2}{*}{$6.3 \mathrm{~J}$} & ND & 44 & & \\
\hline & & $46.5-47$ & 31 & 8 \\
\hline \multirow[t]{3}{*}{ ND } & ND & 48 & & \\
\hline & & $48-48.5$ & 3 & 6 \\
\hline & & $51.6-52$ & ND & ND \\
\hline ND & ND & 52 & & \\
\hline ND & ND & 55.5 & & \\
\hline ND & ND & 59.4 & & \\
\hline ND & ND & 63.5 & & \\
\hline ND & ND & 67.5 & & \\
\hline ND & ND & 72 & & \\
\hline ND & ND & 75 & & \\
\hline
\end{tabular}

a Results for samples preserved on dry ice and prepared by the purge-and-trap method for analysis by GC-MS at the AGEM Laboratory.

b Color codes for depth intervals are:

Dry silty/clayey

Dry sandy

Wet sandy

c Results for samples preserved in methanol and analyzed by the Argonne mobile laboratory. 
TABLE 4.4 Hydrostratigraphic summary of analytical results for VOCs in groundwater samples collected during the targeted investigation.

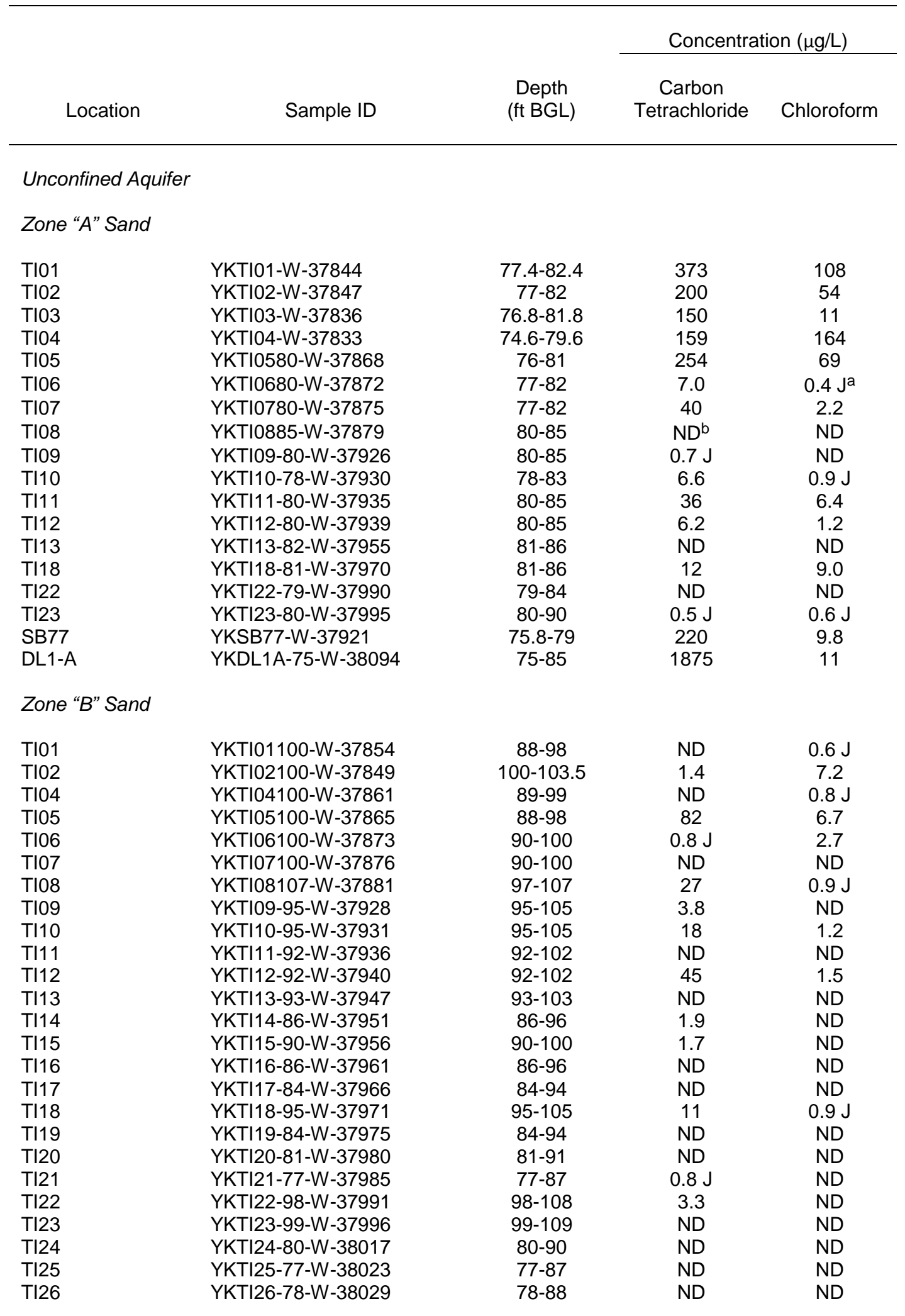


TABLE 4.4 (Cont.)

\begin{tabular}{|c|c|c|c|c|}
\hline \multirow[b]{2}{*}{ Location } & \multirow[b]{2}{*}{ Sample ID } & \multirow[b]{2}{*}{$\begin{array}{l}\text { Depth } \\
\text { (ft BGL) }\end{array}$} & \multicolumn{2}{|c|}{ Concentration $(\mu \mathrm{g} / \mathrm{L})$} \\
\hline & & & $\begin{array}{c}\text { Carbon } \\
\text { Tetrachloride }\end{array}$ & Chloroform \\
\hline \multicolumn{5}{|c|}{ Zone "B" Sand (cont.) } \\
\hline TI27 & YKTI27-77-W-38050 & $77-87$ & ND & ND \\
\hline TI27 & YKTI27-77-W-38056 & $77-87$ & ND & ND \\
\hline TI28 & YKTI28-78-W-38075 & $78-88$ & ND & ND \\
\hline DL1-B & YKDL1B-88-W-38093 & $88-98$ & 1.6 & ND \\
\hline \multicolumn{5}{|c|}{ Zone "C-C1" Sand } \\
\hline TI01 & YKTI01120-W-37855 & 108-118 & $0.9 \mathrm{~J}$ & 2.0 \\
\hline TI02 & YKTI02120-W-37852 & $108.5-118.5$ & ND & ND \\
\hline TI04 & YKTI04120-W-37860 & $108-118$ & ND & 1.1 \\
\hline TI05 & YKTI05120-W-37864 & $108-118$ & 2.0 & $0.6 \mathrm{~J}$ \\
\hline TI06 & YKTI06120-W-37870 & $110-120$ & ND & ND \\
\hline TI07 & YKTI07120-W-37877 & $110-120$ & ND & ND \\
\hline T108 & YKTI08125-W-37882 & $115-125$ & 8.7 & ND \\
\hline TI09 & YKTI09-135-W-37929 & $135-145$ & 1.8 & ND \\
\hline TI10 & YKTI10-117-W-37933 & $117-127$ & 29 & 1.1 \\
\hline TI11 & YKTI11-112-W-37937 & $112-122$ & 202 & 5.1 \\
\hline Tl12 & YKTI12-120-W-37942 & $110-120$ & 63 & 1.7 \\
\hline Tl13 & YKTI13-111-W-37948 & $111-121$ & ND & ND \\
\hline TI14 & YKTI14-113-W-37952 & $113-123$ & 36 & 1.4 \\
\hline TI15 & YKTI15-113-W-37957 & $113-123$ & 22 & $0.7 \mathrm{~J}$ \\
\hline TI16 & YKTI16-105-W-37963 & $105-115$ & 2.9 & ND \\
\hline TI17 & YKTI17-104-W-37967 & $104-114$ & ND & ND \\
\hline TI18 & YKTI18-114-W-37973 & $114-124$ & 2.9 & ND \\
\hline Tl19 & YKTI19-107-W-37976 & $107-117$ & 4.8 & ND \\
\hline TI20 & YKTI20-105-W-37981 & $105-115$ & 8.2 & ND \\
\hline TI21 & YKTI21-99-W-37986 & $99-109$ & $0.8 \mathrm{~J}$ & ND \\
\hline TI21 & YKTI21-99-W-37986 & $99-109$ & $0.8 \mathrm{~J}$ & ND \\
\hline TI21 & YKTI21-115-W-37987 & $115-120$ & ND & ND \\
\hline TI21 & YKTI21-115-W-37987 & $115-120$ & ND & ND \\
\hline TI22 & YKTI22-113-W-37992 & $113-123$ & ND & ND \\
\hline TI23 & YKTI23-120-W-37998 & $120-130$ & ND & ND \\
\hline TI24 & YKTI24-90-W-38018 & $90-100$ & ND & ND \\
\hline TI24 & YKTI24-100-W-38019 & $100-110$ & ND & ND \\
\hline TI25 & YKTI25-98-W-38024 & $98-108$ & 1.3 & ND \\
\hline TI26 & YKTI26-96-W-38030 & $96-106$ & 2.0 & ND \\
\hline TI26 & YKTI26-113-W-38033 & $113-123$ & $0.4 \mathrm{~J}$ & ND \\
\hline TI27 & YKTI27-100-W-38053 & $100-110$ & $0.4 \mathrm{~J}$ & ND \\
\hline TI27 & YKTI27-100-W-38057 & $100-110$ & ND & ND \\
\hline TI28 & YKTI28-90-W-38076 & $90-100$ & ND & ND \\
\hline TI28 & YKTI28-104-W-38078 & 104-109 & ND & ND \\
\hline SB62 & YKSB62-W-37924 & $118-123$ & 23 & 2.2 \\
\hline DL1-C & YKDL1C-108-W-38091 & $108-118$ & 1.9 & ND \\
\hline DL2-C1 & YKDL2-C1-112-W-38100 & $112-122$ & 41 & $0.7 \mathrm{~J}$ \\
\hline DL3-C1 & YKDL3-C1-113-W-38103 & $113-123$ & 39 & $0.7 \mathrm{~J}$ \\
\hline DL4-C1 & YKDL4-C1-120-W-38116 & $120-130$ & ND & ND \\
\hline DL5-C1 & YKDL5-C1-106-W-38118 & $106-116$ & ND & ND \\
\hline DL6-C & YKDL6-C-110-W-38113 & $110-120$ & ND & ND \\
\hline DL7-C & YKDL7-C-100-W-38111 & $100-110$ & ND & ND \\
\hline DL8-C1 & YKDL8C1-104-W-38106 & $104-114$ & 16 & ND \\
\hline
\end{tabular}


York Targeted Investigation, 2015-2016

Version 01, 02/14/2017

TABLE 4.4 (Cont.)

\begin{tabular}{|c|c|c|c|c|}
\hline \multirow[b]{2}{*}{ Location } & \multirow[b]{2}{*}{ Sample ID } & \multirow[b]{2}{*}{$\begin{array}{l}\text { Depth } \\
\text { (ft BGL) }\end{array}$} & \multicolumn{2}{|c|}{ Concentration $(\mu \mathrm{g} / \mathrm{L})$} \\
\hline & & & $\begin{array}{c}\text { Carbon } \\
\text { Tetrachloride }\end{array}$ & Chloroform \\
\hline \multicolumn{5}{|c|}{ Zone "C2" Sand } \\
\hline TI12 & YKTI12-145-W-37943 & $135-145$ & 73 & 1.5 \\
\hline TI14 & YKTI14-136-W-37954 & $136-146$ & 59 & $0.8 \mathrm{~J}$ \\
\hline Tl15 & YKTI15-134-W-37958 & $134-144$ & 68 & 1.7 \\
\hline TI16 & YKTI16-136-W-37964 & $136-146$ & 35 & $0.7 \mathrm{~J}$ \\
\hline TI17 & YKTI17-133-W-37968 & $133-143$ & ND & $0.4 \mathrm{~J}$ \\
\hline TI19 & YKTI19-129-W-37977 & $129-139$ & 22 & $0.8 \mathrm{~J}$ \\
\hline TI20 & YKTI20-131-W-37982 & $131-141$ & 1.8 & ND \\
\hline TI23 & YKTI23-145-W-37999 & $145-155$ & ND & ND \\
\hline TI25 & YKTI25-108-W-38027 & $113-118$ & ND & ND \\
\hline DL2-C2 & YKDL2C2-136-W-38096 & $136-146$ & 42 & 1.0 \\
\hline DL3-C2 & YKDL3-C2-134-W-38102 & $134-144$ & 77 & 1.1 \\
\hline DL4-C2 & YKDL4-C2-145-W-38115 & $145-155$ & ND & ND \\
\hline DL8-C2 & YKDL8-C2-125-W-38104 & $125-135$ & 2.2 & ND \\
\hline \multicolumn{5}{|c|}{ Upper Confined Aquifer } \\
\hline DL2-CU & YKDL2CU-215-W-38097 & $215-225$ & ND & ND \\
\hline DL7-CU & YKDL7-CU-192-W-38112 & $192-202$ & ND & ND \\
\hline \multicolumn{5}{|c|}{ Lower Confined Aquifer } \\
\hline DL7-CL & YKDL7-CL-265-W-38108 & $265-275$ & ND & ND \\
\hline
\end{tabular}

a J, compound identified with an estimated concentration between the instrument detection limit and the method detection limit.

b ND, compound analyzed for but not detected at a level greater than or equal to the method detection limit $(<1 \mu \mathrm{g} / \mathrm{L})$. 
TABLE 4.5 Historic and current information for selected private wells in the York investigation area.

\begin{tabular}{|c|c|c|c|c|c|c|c|}
\hline \multicolumn{5}{|c|}{ Characteristics in 1993} & \multicolumn{3}{|c|}{ Characteristics in July-August 2015} \\
\hline $\begin{array}{c}\text { Well } \\
\text { Designation }\end{array}$ & Address & Property Owner & $\begin{array}{l}\text { Carbon } \\
\text { Tetrachloride } \\
\text { (mg/L) }\end{array}$ & Well Use & Property Owner & $\begin{array}{l}\text { Carbon } \\
\text { Tetrachloride } \\
(\mathrm{mg} / \mathrm{L})\end{array}$ & Well Status \\
\hline DW19 & 3201 N. Division Ave. & Preslicka & 2.0 & domestic & Preslicka & 1.3 & In use for domestic purposes. \\
\hline DW21 & 3816 N. Division Ave. & Krocker & $N D^{a}$ & domestic & Volzke & ND & $\begin{array}{l}\text { Bottled water provided by owner for } \\
\text { drinking; well available for other } \\
\text { domestic uses. }\end{array}$ \\
\hline DW23 & 3221 N. Lincoln Ave. & Welsh Implement & 1.0 & domestic & Plains Equipment & - & $\begin{array}{l}\text { Reported as abandoned in conjunction } \\
\text { with facilities expansion; location of } \\
\text { well now under new building } \\
\text { foundation. }\end{array}$ \\
\hline DW26A & 3214 N. Division Ave. & Cotner & 49 & domestic & Garretson & - & $\begin{array}{l}\text { Well exists but disconnected from piping } \\
\text { and power and not in use. Municipal } \\
\text { supply for domestic uses. }\end{array}$ \\
\hline DW26B & 3132 N. Division Ave. & Wize & 16 & domestic & Troester & - & $\begin{array}{l}\text { Reported as abandoned. Original } \\
\text { residence burned down and was } \\
\text { demolished; location of well now under } \\
\text { new building foundation. }\end{array}$ \\
\hline DW38 & 722 E. 25th St. & York County & ND & domestic & York County & 53 & $\begin{array}{l}\text { Bottled water provided by owner for } \\
\text { drinking; well available for other } \\
\text { domestic uses. }\end{array}$ \\
\hline DW39 & 1111 E. 19th St. & Nordlund & ND & domestic & Nordlund & $N D^{c}$ & $\begin{array}{l}\text { Well is used for lawn watering. } \\
\text { Residence connected to municipal } \\
\text { supply by the EPA. }\end{array}$ \\
\hline DW40 & 901 E. 19th St. & Euchner & $N D^{b}$ & domestic & Eckharts & - & $\begin{array}{l}\text { Abandoned in conjunction with } \\
\text { connection of residence to the } \\
\text { municipal supply by the EPA. }\end{array}$ \\
\hline DW55 & 3720 N. Division Ave. & Liermann & ND & domestic & Liermann & ND & In use for domestic purposes. \\
\hline DW56 & 3401 N. Lincoln Ave. & $\begin{array}{l}\text { Blue Valley } \\
\text { Community Center }\end{array}$ & ND & domestic & $\begin{array}{l}\text { Blue Valley } \\
\text { Community Action } \\
\text { Partnership }\end{array}$ & - & $\begin{array}{l}\text { Reported as abandoned in conjunction } \\
\text { with facilities expansion; location of } \\
\text { well now under new building } \\
\text { foundation. }\end{array}$ \\
\hline
\end{tabular}




\begin{tabular}{|c|c|c|c|c|c|c|c|}
\hline \multicolumn{5}{|c|}{ Characteristics in 1993} & \multicolumn{3}{|c|}{ Characteristics in July-August 2015} \\
\hline $\begin{array}{c}\text { Well } \\
\text { Designation }\end{array}$ & Address & Property Owner & $\begin{array}{l}\text { Carbon } \\
\text { Tetrachloride } \\
(\mathrm{mg} / \mathrm{L})\end{array}$ & Well Use & Property Owner & $\begin{array}{l}\text { Carbon } \\
\text { Tetrachloride } \\
(\mathrm{mg} / \mathrm{L})\end{array}$ & Well Status \\
\hline G-21977 & 2922 N. Division Ave. & Cyclonaire Corp. & - & irrigation & Cyclonaire Corp. & - & $\begin{array}{l}\text { Reported as abandoned in conjunction } \\
\text { with facilities expansion; location of } \\
\text { well now under new paved parking lot. }\end{array}$ \\
\hline DW37 & 1007 E. 25th St. & Olson & $6.0-8.0$ & domestic & Kayton ${ }^{d}$ & 6.5 & $\begin{array}{l}\text { Well available for domestic uses; } \\
\text { residence currently vacant. Property } \\
\text { owner seeking connection to municipal } \\
\text { supply with assistance from } \\
\text { CCC/USDDA. }\end{array}$ \\
\hline- & 1403 Road 14 & not investigated & - & domestic & CVA Coop ${ }^{d}$ & 6.6 & In use for domestic purposes. \\
\hline- & 1402 Road 14 & not investigated & - & domestic & Driewer (home) ${ }^{\mathrm{d}, \mathrm{e}}$ & $5.1^{e}$ & $\begin{array}{l}\text { In use for domestic purposes; treatment } \\
\text { provided by the EPA. }\end{array}$ \\
\hline
\end{tabular}

a TCE (1 mg/L), PCE (1 mg/L), and TCA (1 mg/L) identified in sample.

b TCE $(79 \mathrm{mg} / \mathrm{L})$, DCE (1 mg/L), and PCE $(1 \mathrm{mg} / \mathrm{L})$ identified in sample.

c $\quad \operatorname{TCE}(6.3 \mathrm{mg} / \mathrm{L}), \mathrm{PCE}(4.1 \mathrm{mg} / \mathrm{L})$, and DCE $(2.3 \mathrm{mg} / \mathrm{L})$ identified in sample.

d Sampled quarterly by the CCC/USDA.

e First sampling by the CCC/USDA in December, 2016. Carbon tetrachloride concentration of $8.7 \mathrm{mg} / \mathrm{L}$ previously reported by the EPA. 


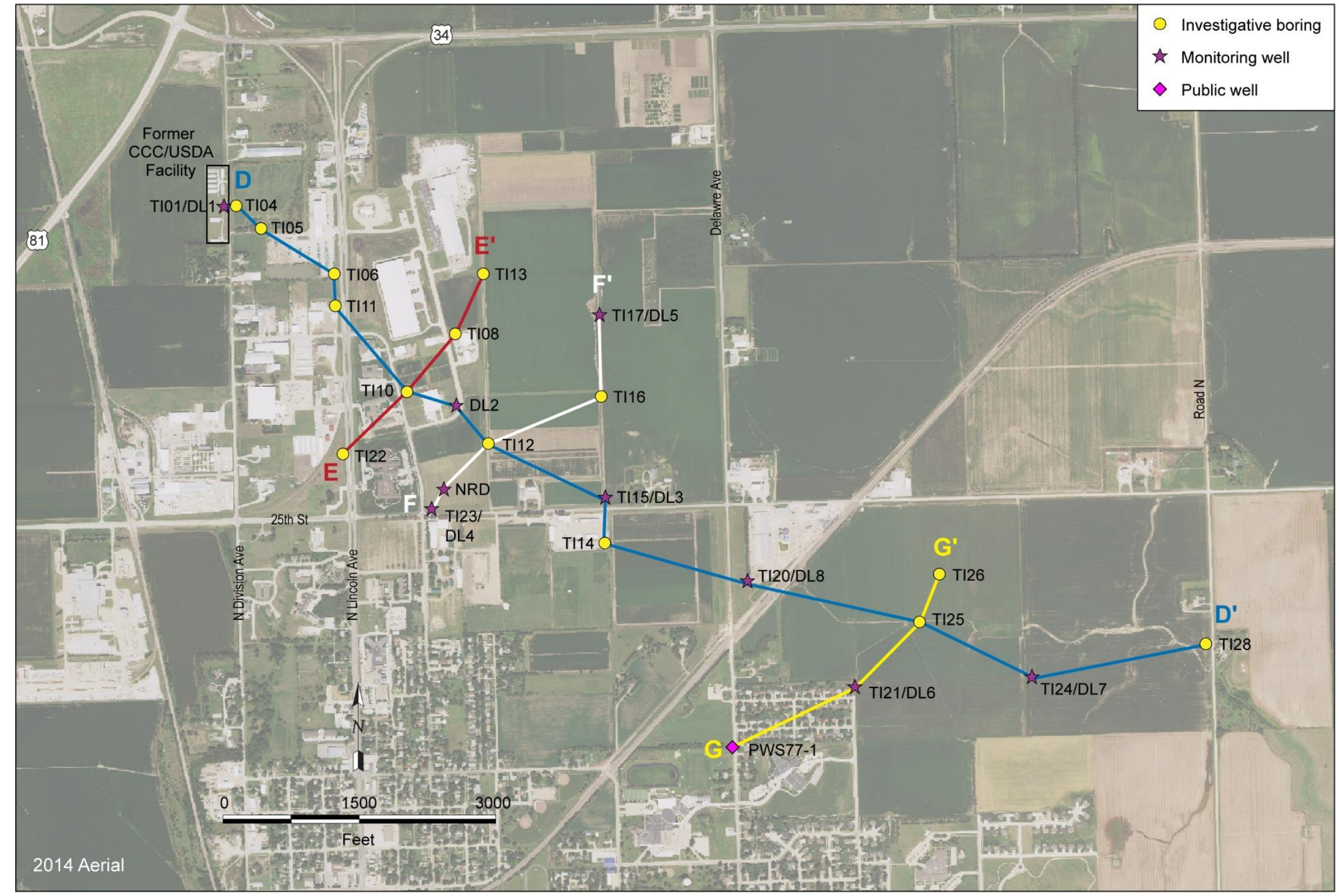

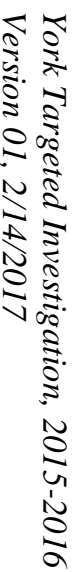

FIGURE 4.1 Locations of hydrogeologic cross sections D-D', E-E', F-F', and G-G'. 
Former CCCUSDA Facility Northwest

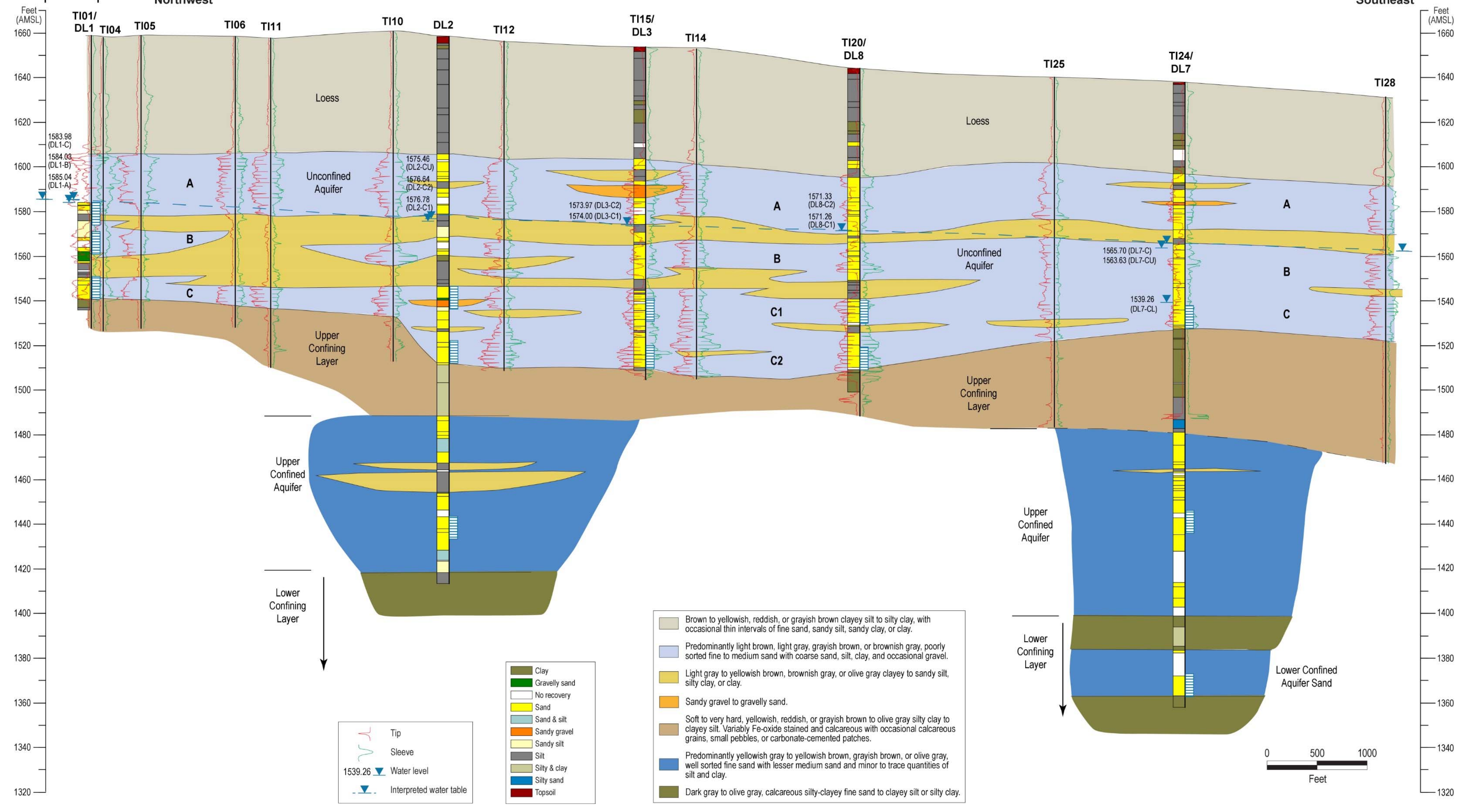

FIGURE 4.2 Northwest-to-southeast hydrogeologic cross section D-D' (vertically exaggerated), showing groundwater levels determined manually on November 3, 2016. 
E

\section{Southwest}

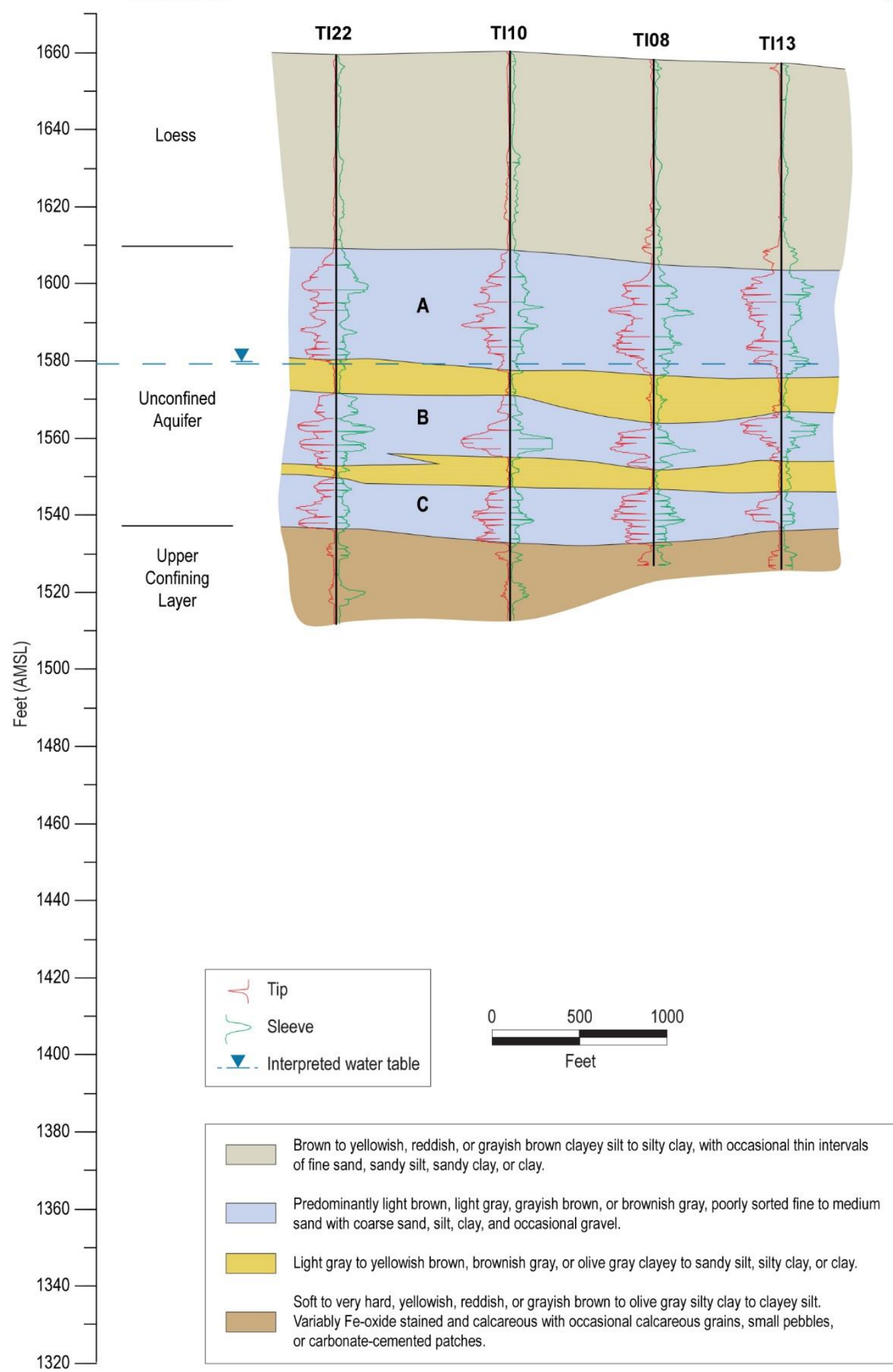

$E^{\prime}$ Northeast

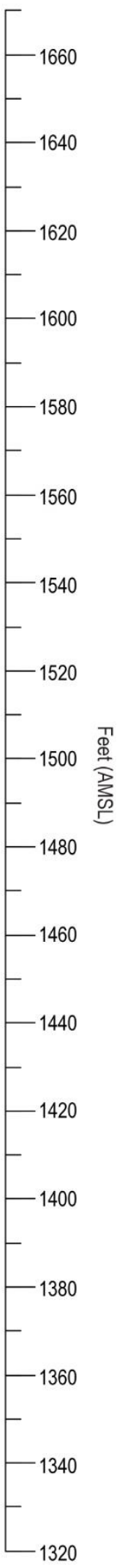

FIGURE 4.3 Southwest-to-northeast hydrogeologic cross section E-E' (vertically exaggerated), showing groundwater levels determined manually on November 3, 2016. 
$F$

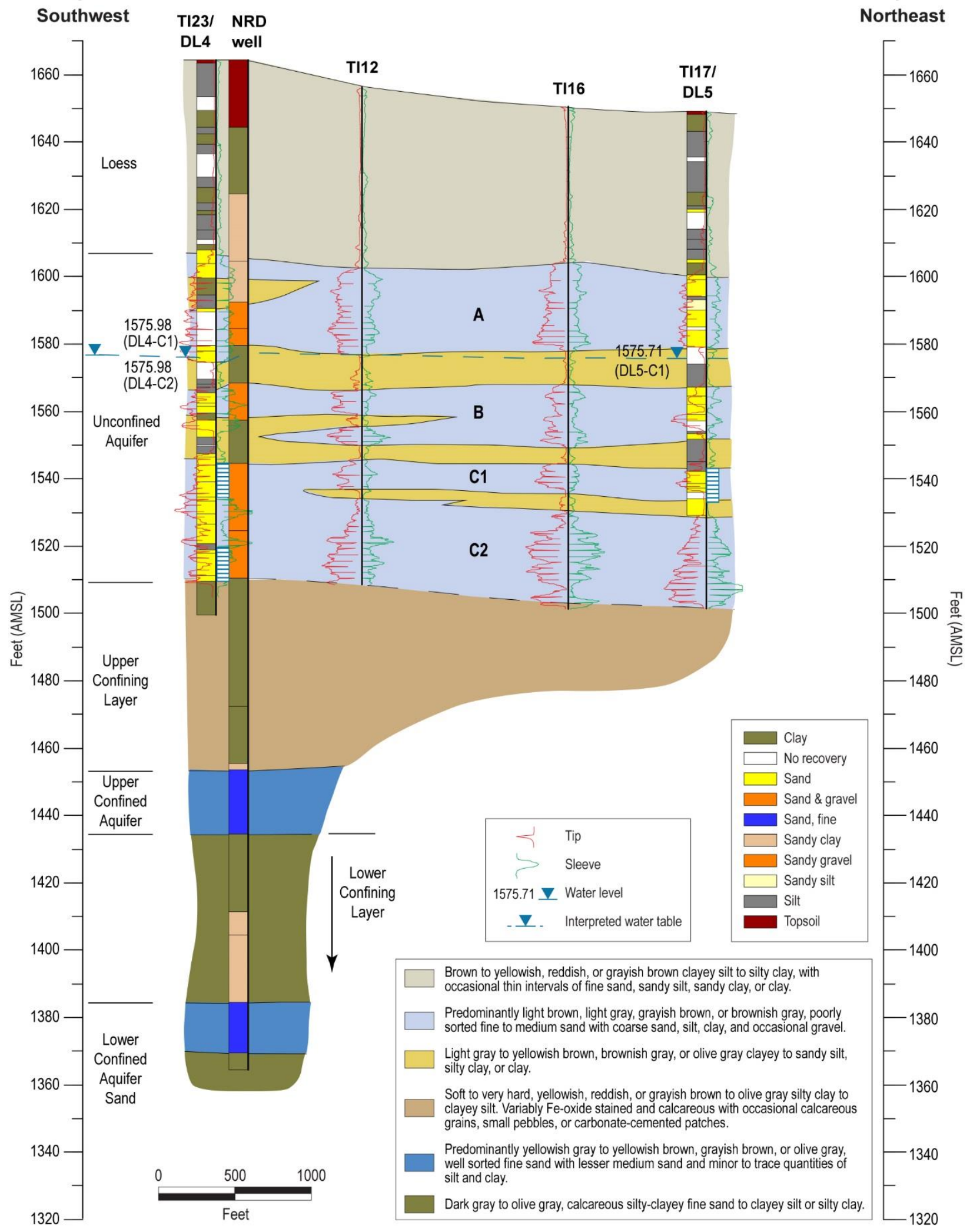

FIGURE 4.4 Southwest-to-northeast hydrogeologic cross section F-F' (vertically exaggerated), showing groundwater levels determined manually on November 3, 2016. 
G

Southwest<smiles>CCCCCCC</smiles>
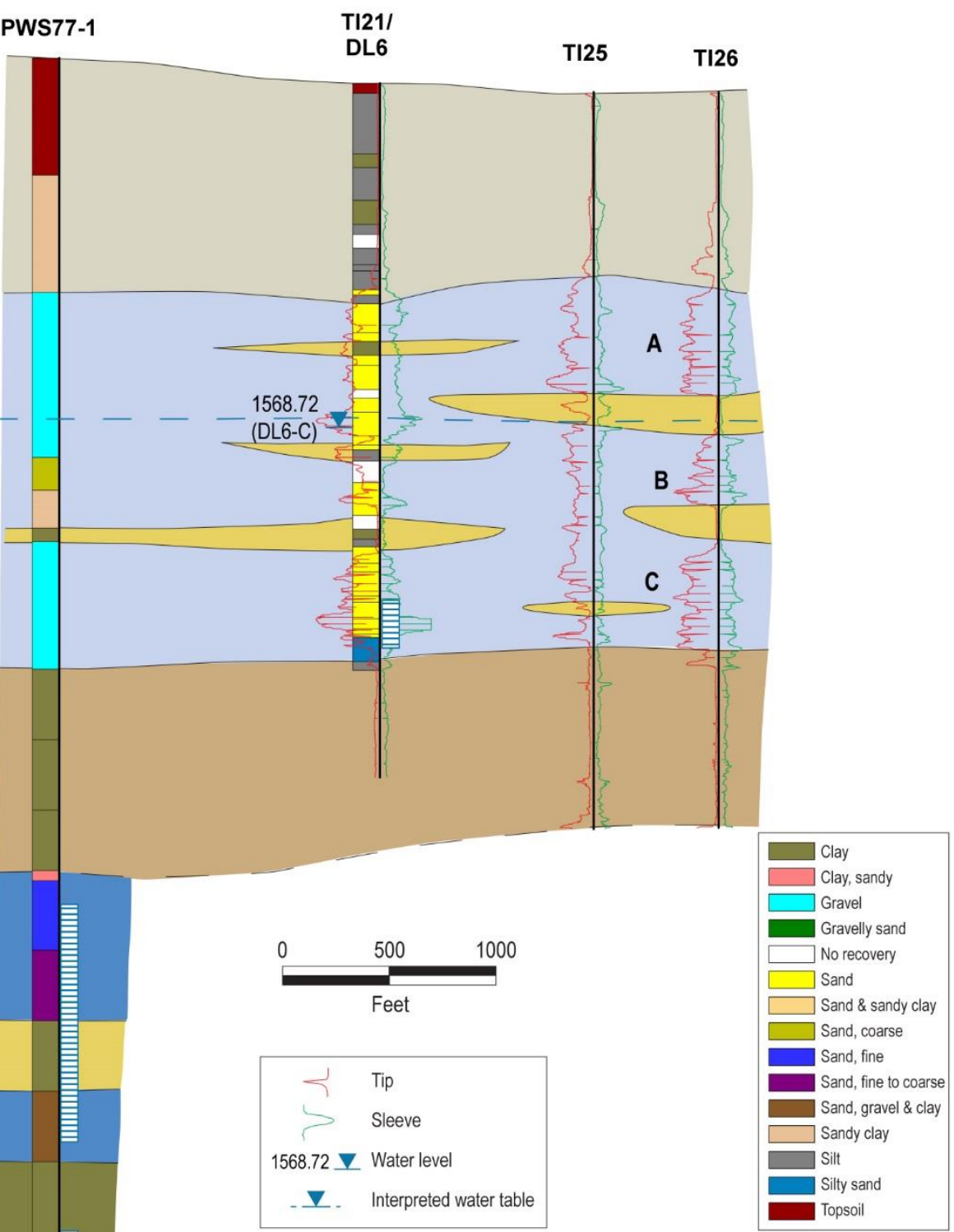

Brown to yellowish, reddish, or grayish brown clayey silt to silty clay, with occasional thin intervals of fine sand, sandy silt, sandy clay, or clay.

Predominantly light brown, light gray, grayish brown, or brownish gray, poorly sorted fine to medium sand with coarse sand, silt, clay, and occasional gravel.

Light gray to yellowish brown, brownish gray, or olive gray clayey to sandy silt, silty clay, or clay.

Soft to very hard, yellowish, reddish, or grayish brown to olive gray silty clay to clayey silt. Variably Fe-oxide stained and calcareous with occasional calcareous grains, small pebbles, or carbonate-cemented patches.

Predominantly yellowish gray to yellowish brown, grayish brown, or olive gray, well sorted fine sand with lesser medium sand and minor to trace quantities of silt and clay.

Dark gray to olive gray, calcareous silty-clayey fine sand to clayey silt or silty clay.
G'

Northeast

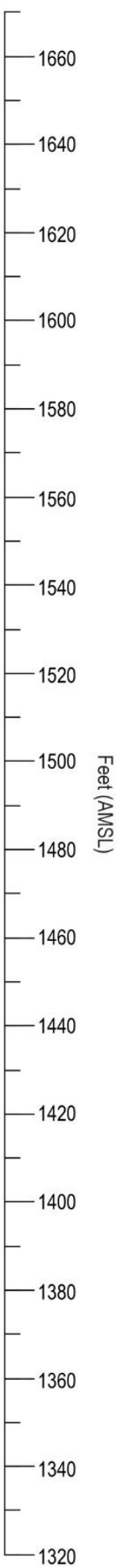

FIGURE 4.5 Southwest-to-northeast hydrogeologic cross section G-G' (vertically exaggerated), showing groundwater levels determined manually on November 3, 2016. 


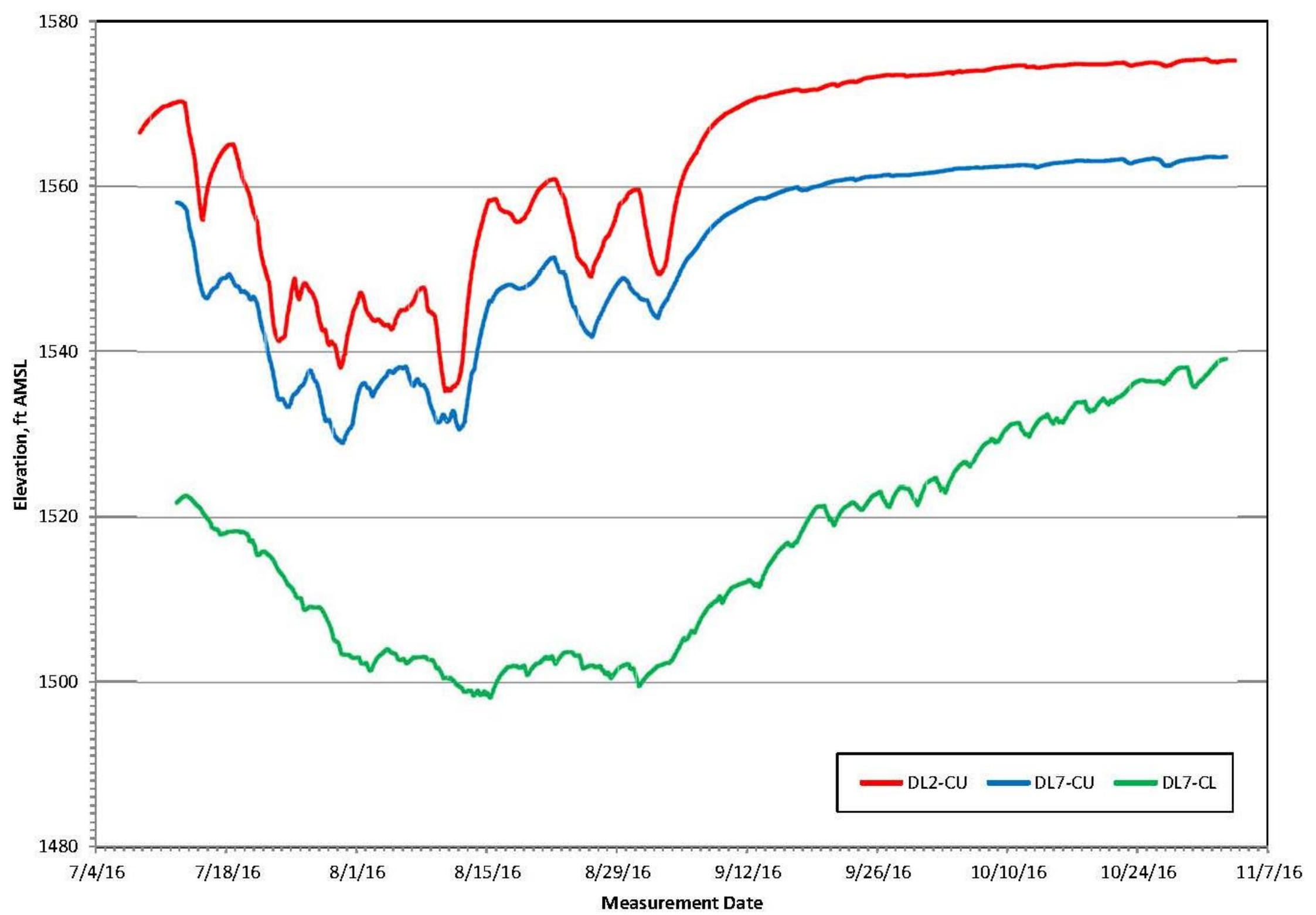

FIGURE 4.6 Hydrographs for wells in the upper and lower confined aquifers. 


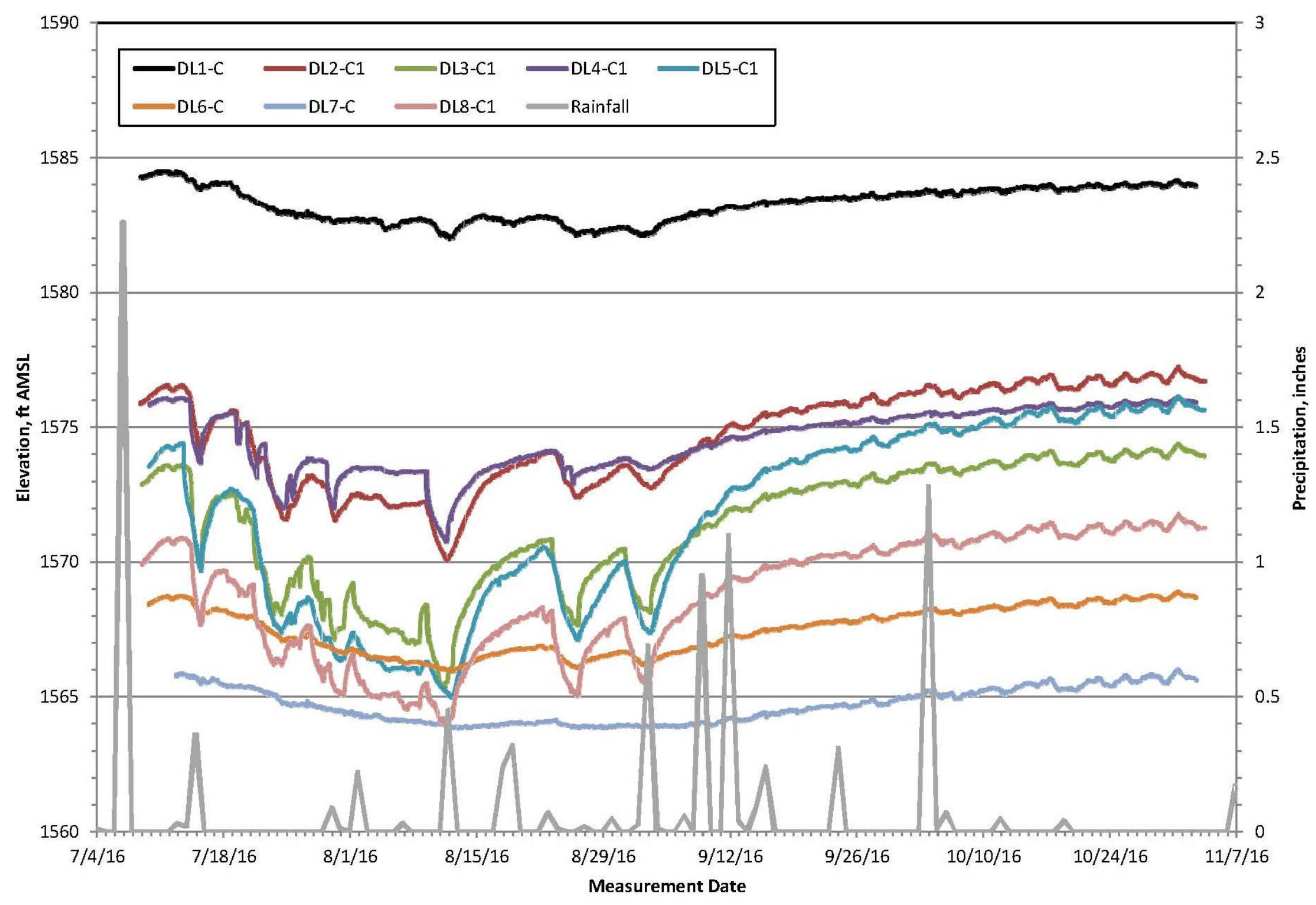

FIGURE 4.7 Hydrographs for wells in the unconfined aquifer. 


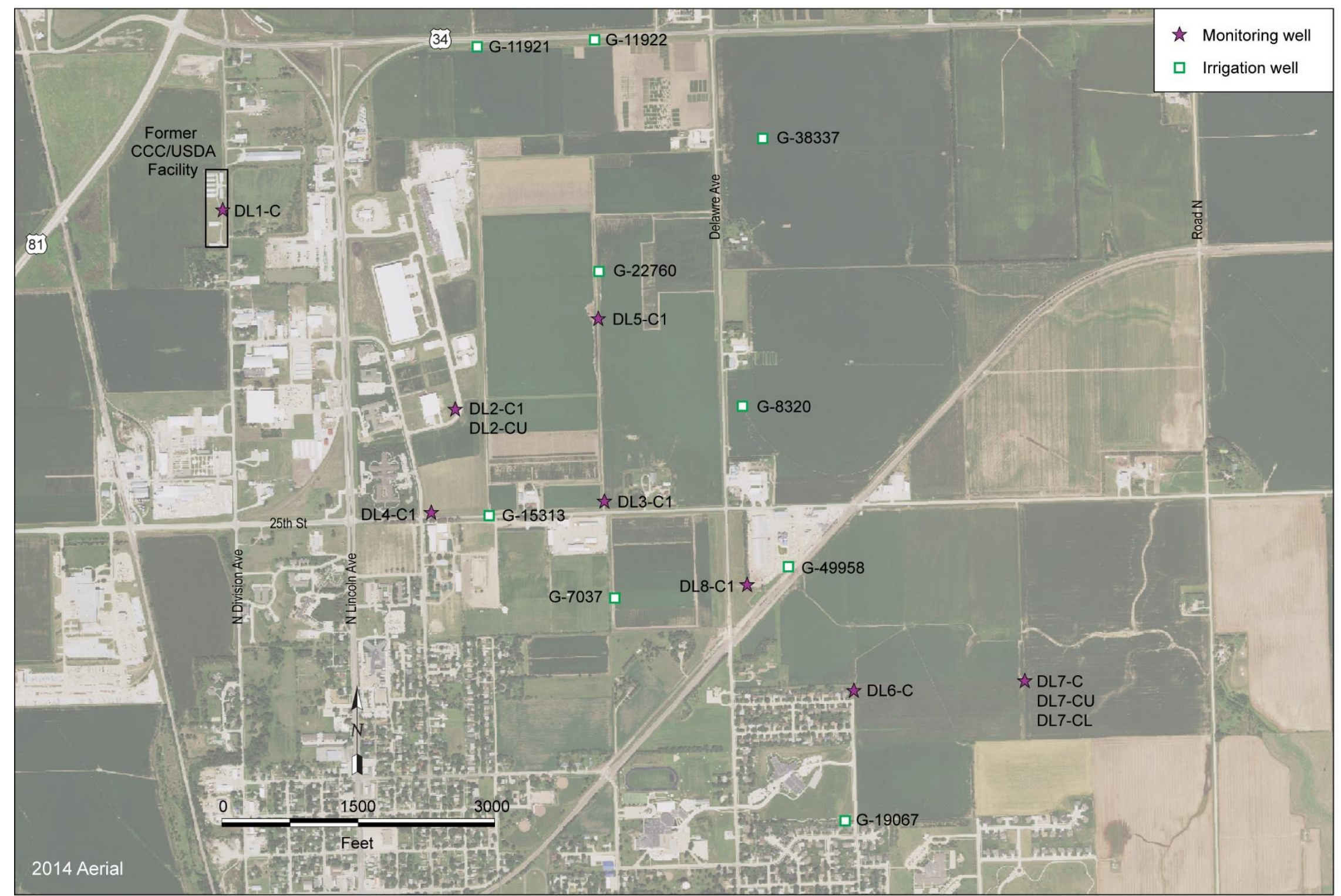

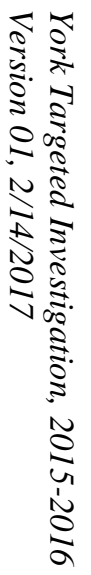

FIGURE 4.8 Locations of monitoring wells equipped with automatic groundwater level recorders, and the approximate locations of nearby irrigation wells. 


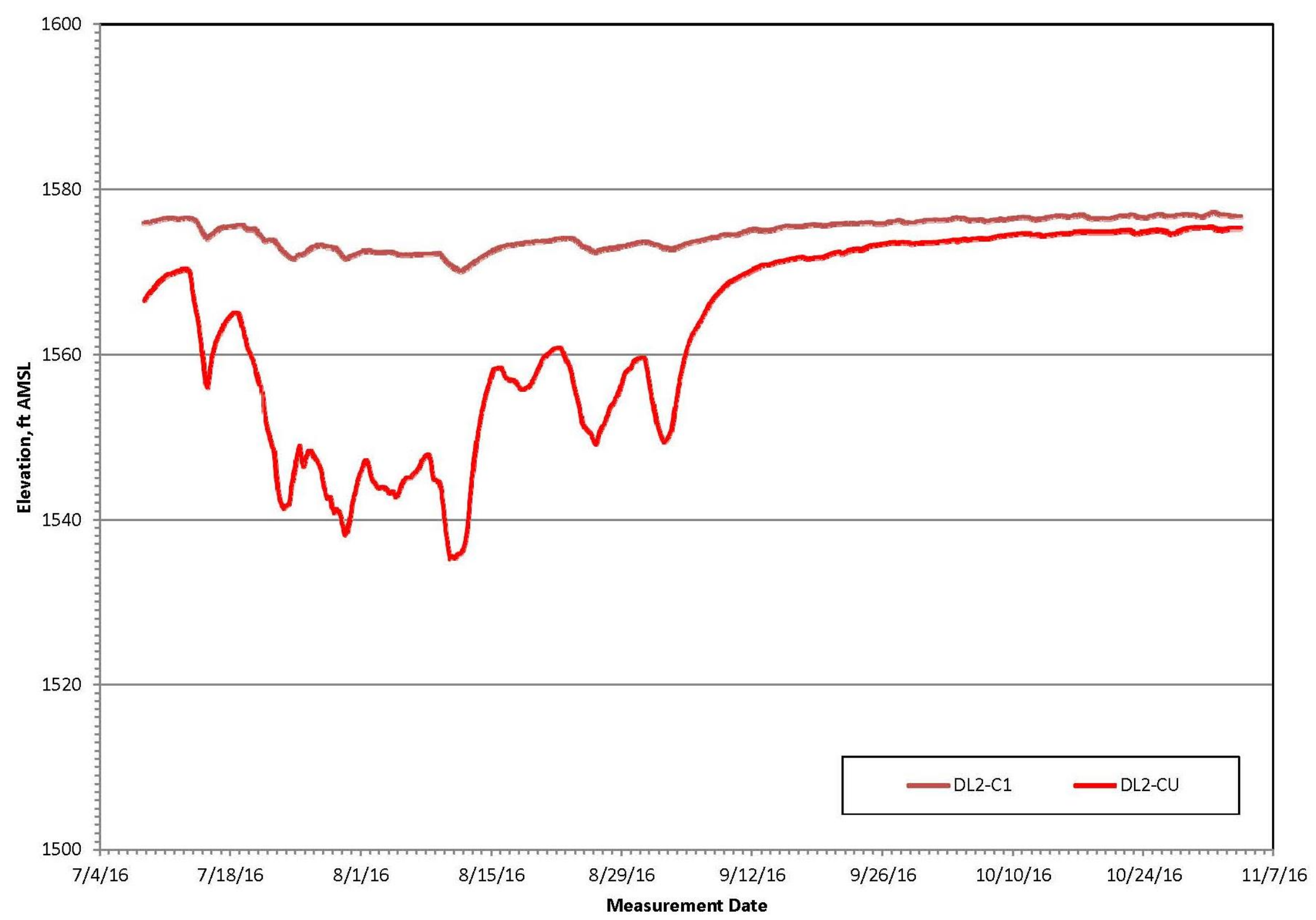

FIGURE 4.9 Hydrographs for monitoring wells DL2-C1 in the unconfined aquifer, and DL2-CU in the upper confined aquifer. 


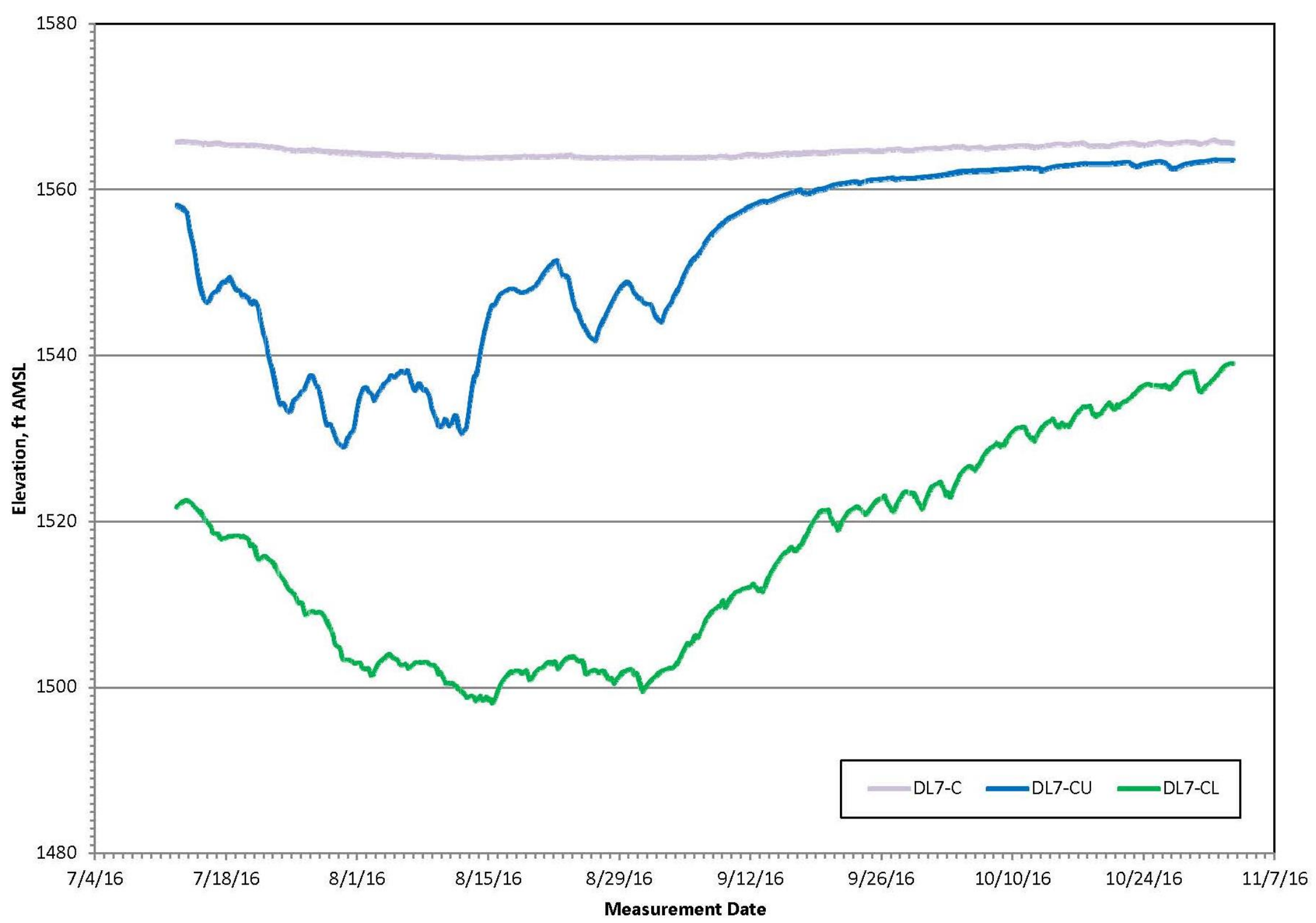

FIGURE 4.10 Hydrographs for monitoring wells DL7-C in the unconfined aquifer, DL7-CU in the upper confined aquifer, and DL7-CL in the lower confined aquifer. 


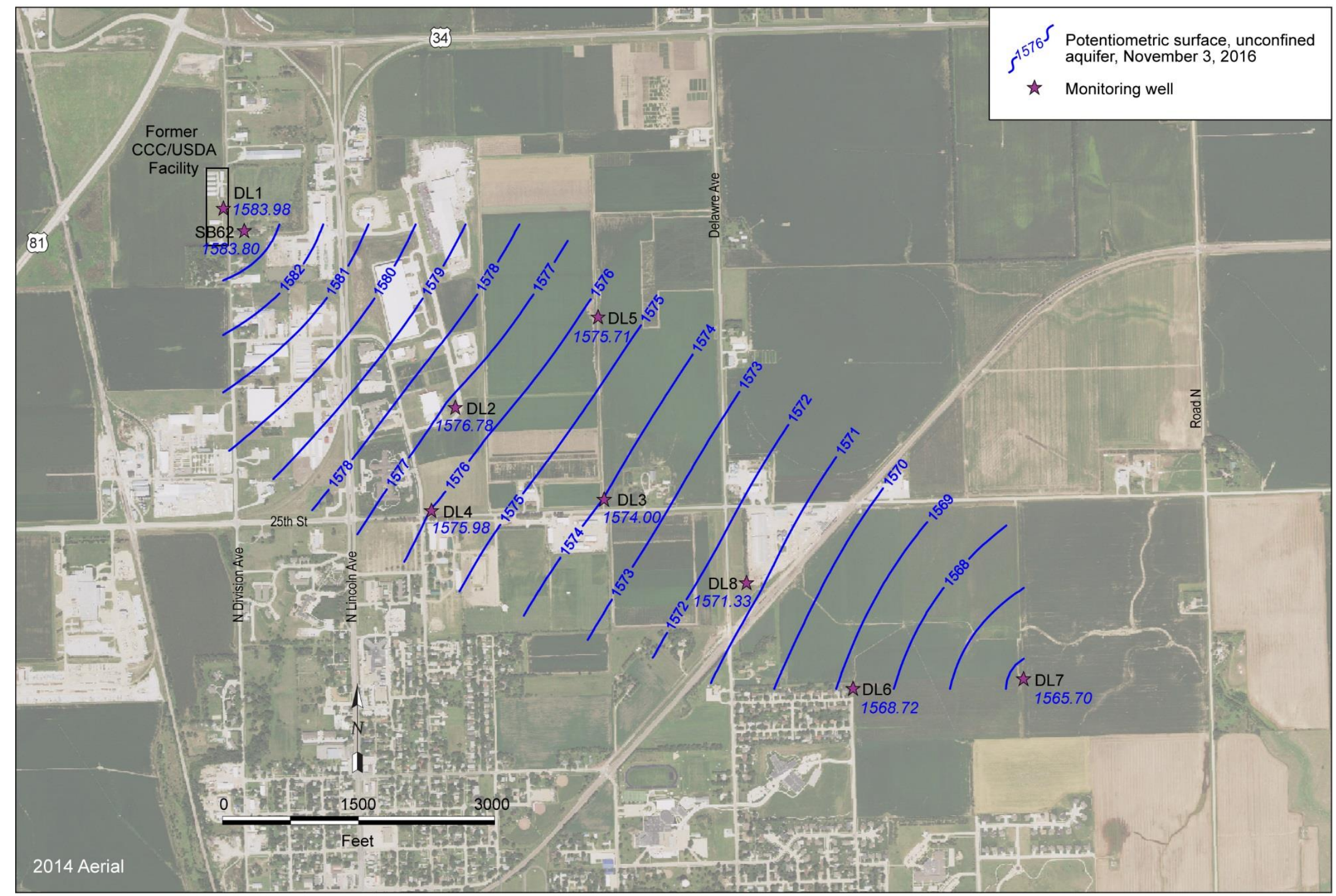

FIGURE 4.11 Estimated potentiometric surface for the unconfined aquifer ("C-C1" zone) under inferred static conditions, based on groundwater levels measured manually on November 3, 2016. 


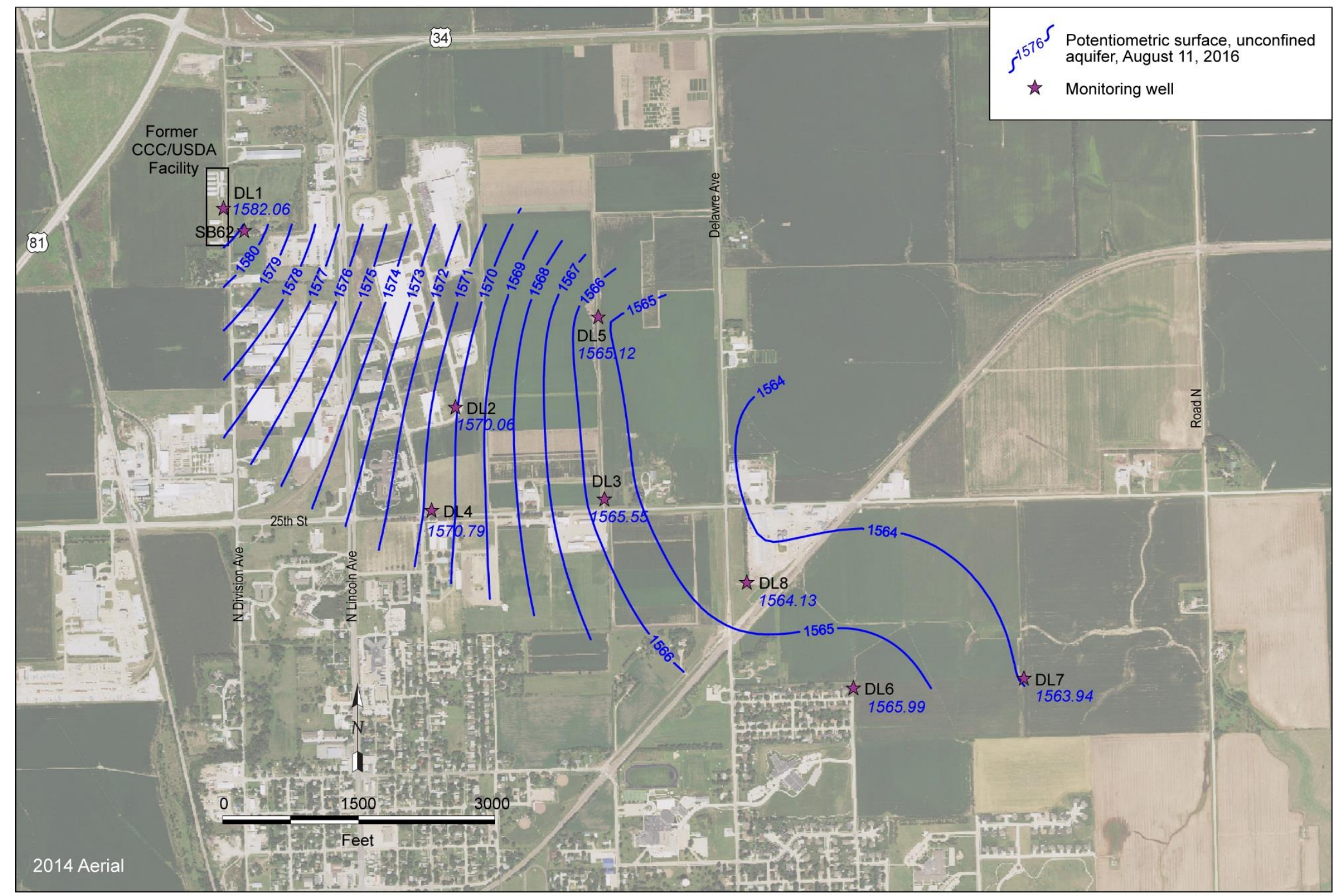

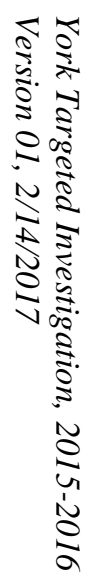

FIGURE 4.12 Estimated potentiometric surface for the unconfined aquifer ("C-C1" zone) under inferred irrigation pumping conditions, based on groundwater levels measured automatically at 16:00 on August 11, 2016. 


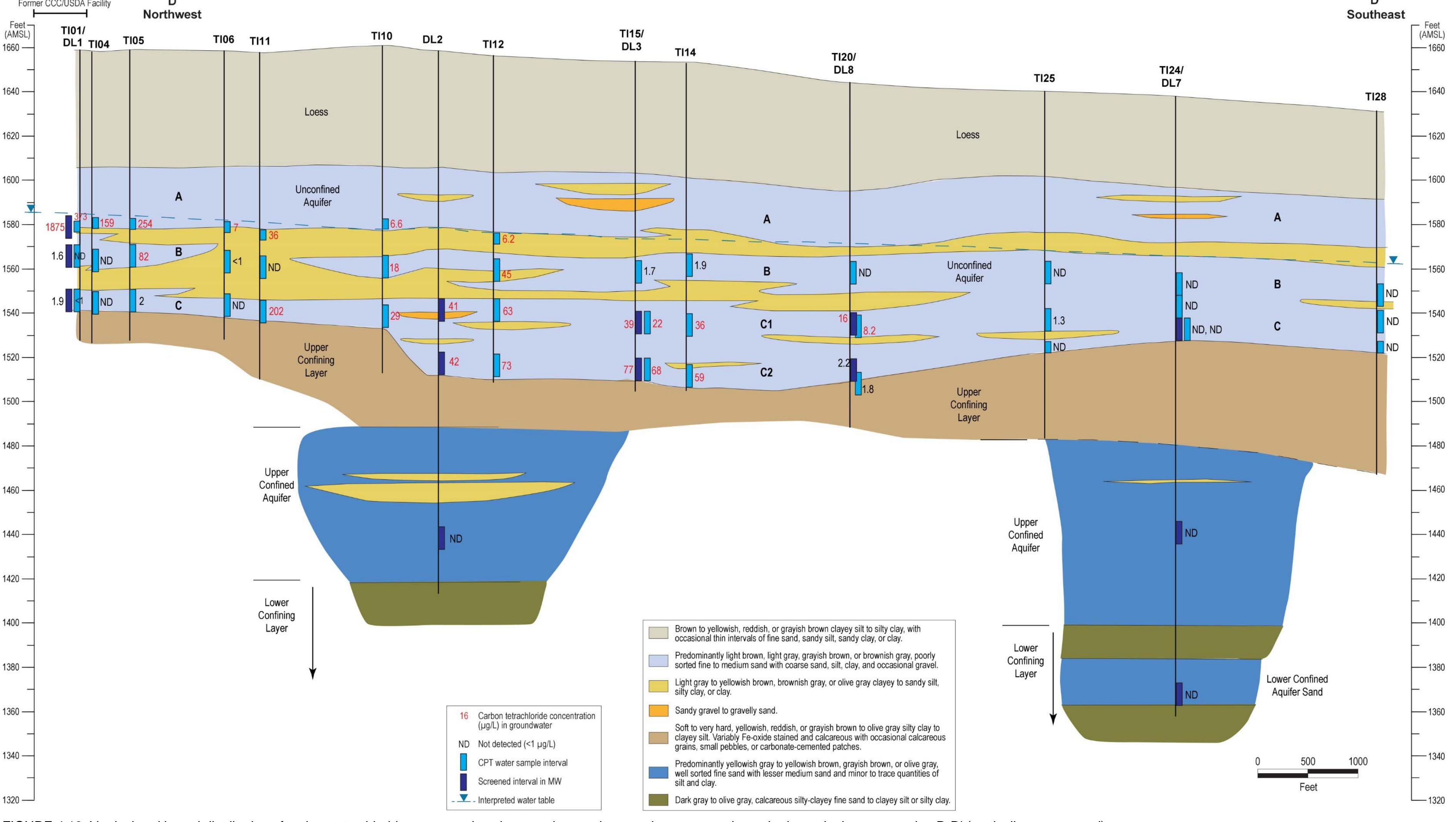

FIGURE 4.13 Vertical and lateral distribution of carbon tetrachloride concentrations in groundwater along northwest-to-southeast hydrogeologic cross section D-D' (vertically exaggerated). 
E

\section{Southwest}

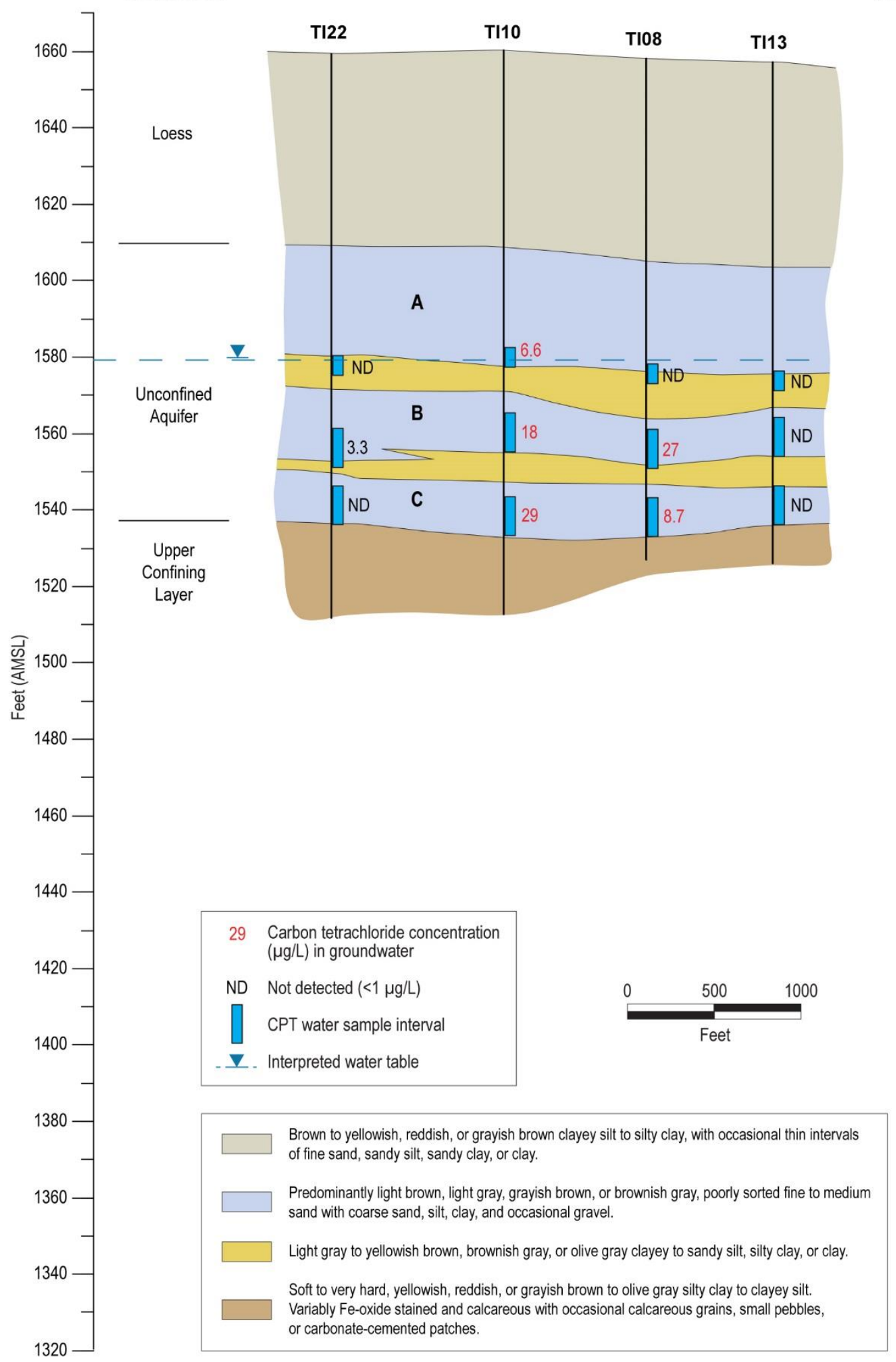

$E^{\prime}$ Northeast

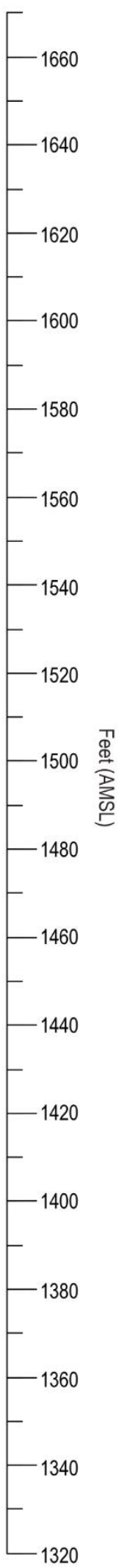

FIGURE 4.14 Vertical and lateral distribution of carbon tetrachloride concentrations in groundwater along southwest-to-northeast hydrogeologic cross section E-E' (vertically exaggerated). 
$F$

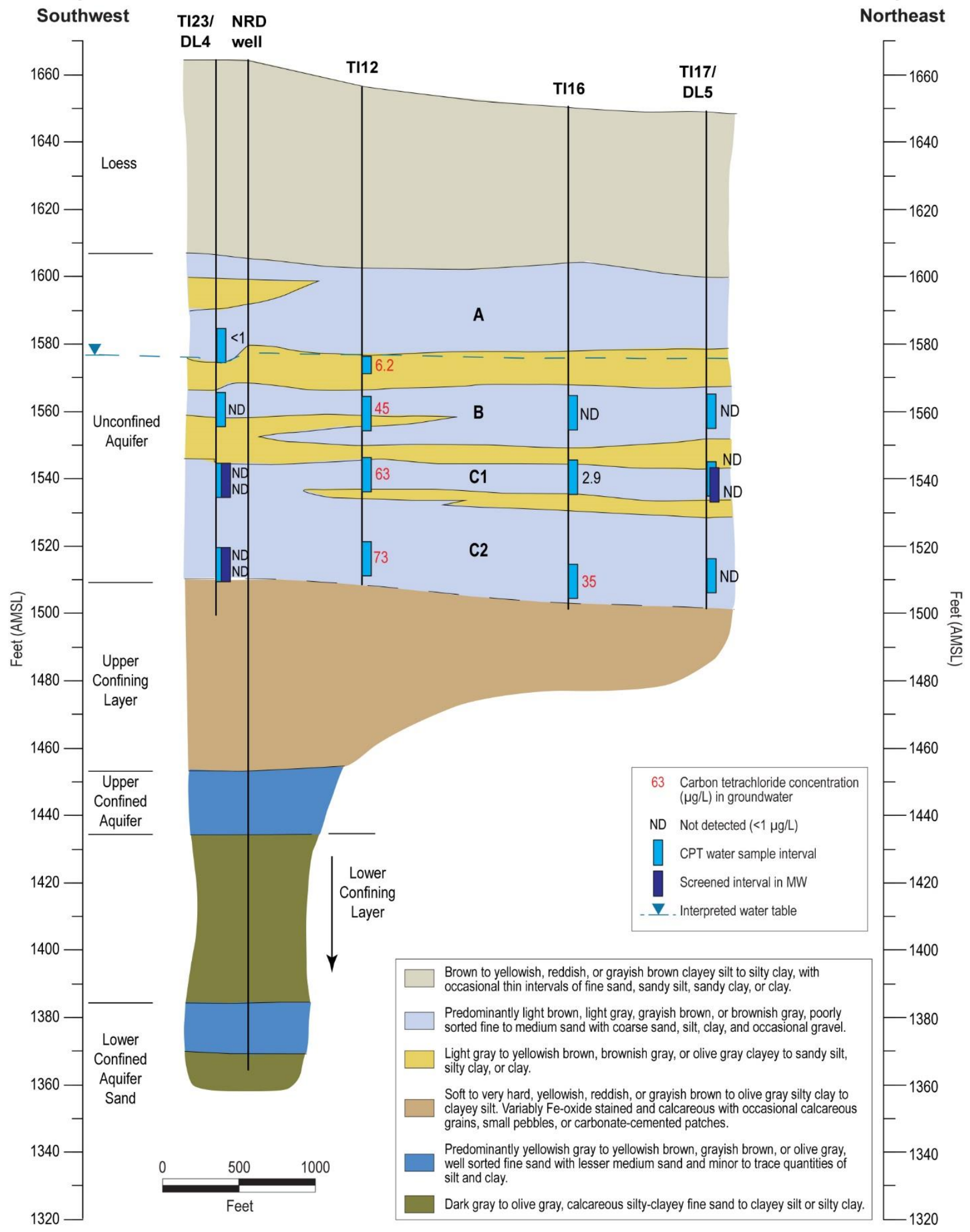

FIGURE 4.15 Vertical and lateral distribution of carbon tetrachloride concentrations in groundwater along southwest-to-northeast hydrogeologic cross section F-F' (vertically exaggerated). 
G

\section{Southwest}

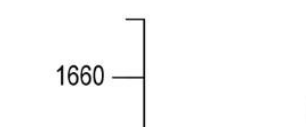

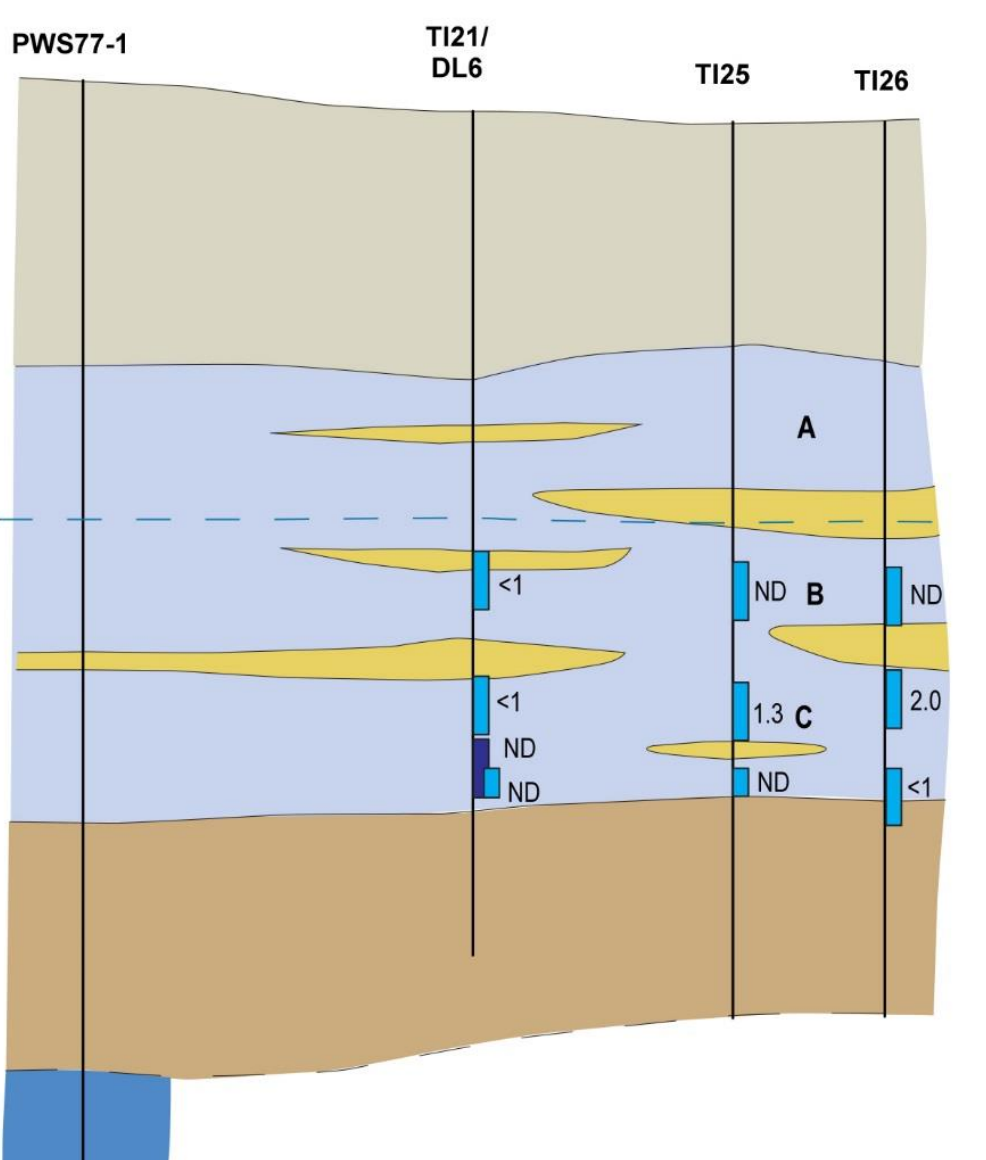
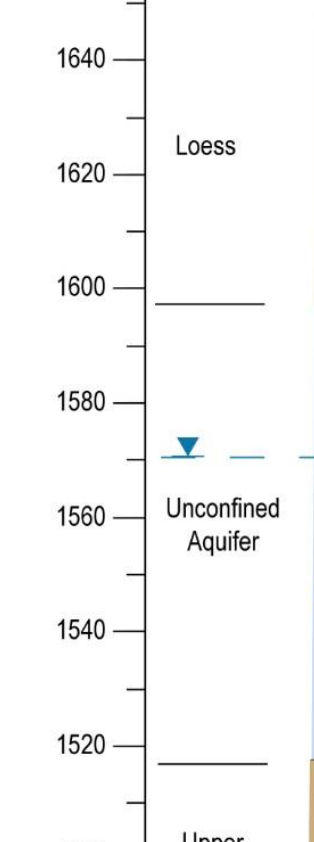

夏 150
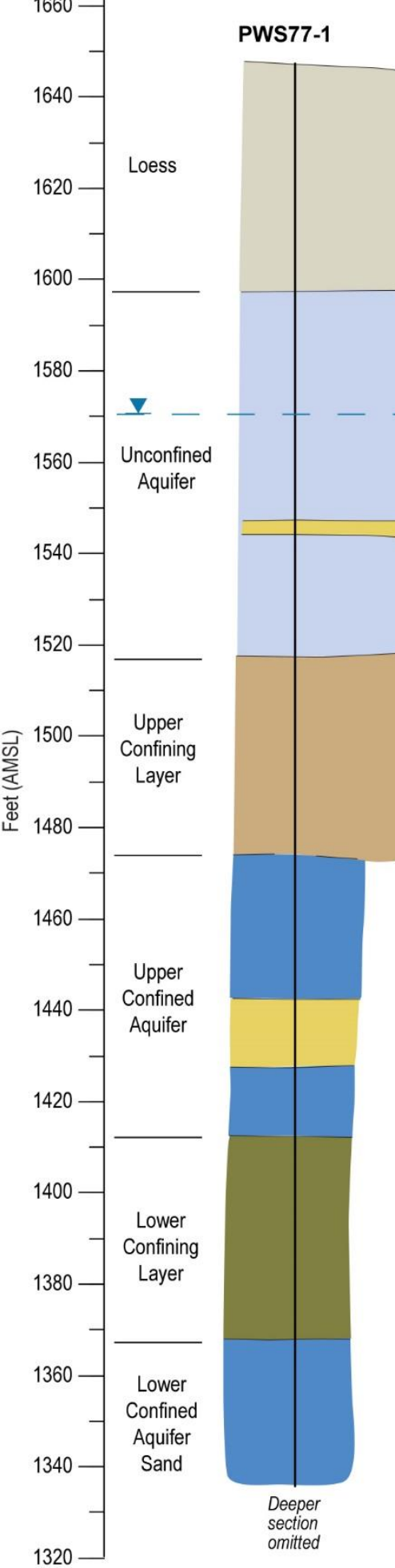

G'

Northeast

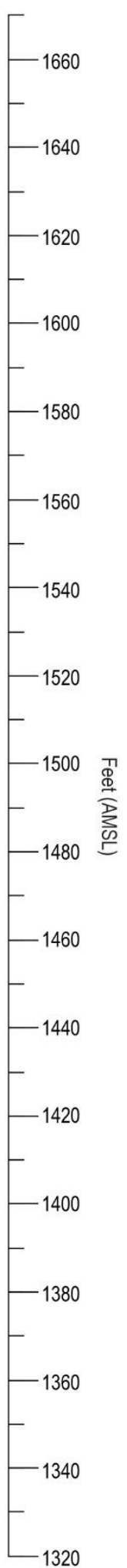

FIGURE 4.16 Vertical and lateral distribution of carbon tetrachloride concentrations in groundwater along southwest-to-northeast hydrogeologic cross section G-G' (vertically exaggerated). 


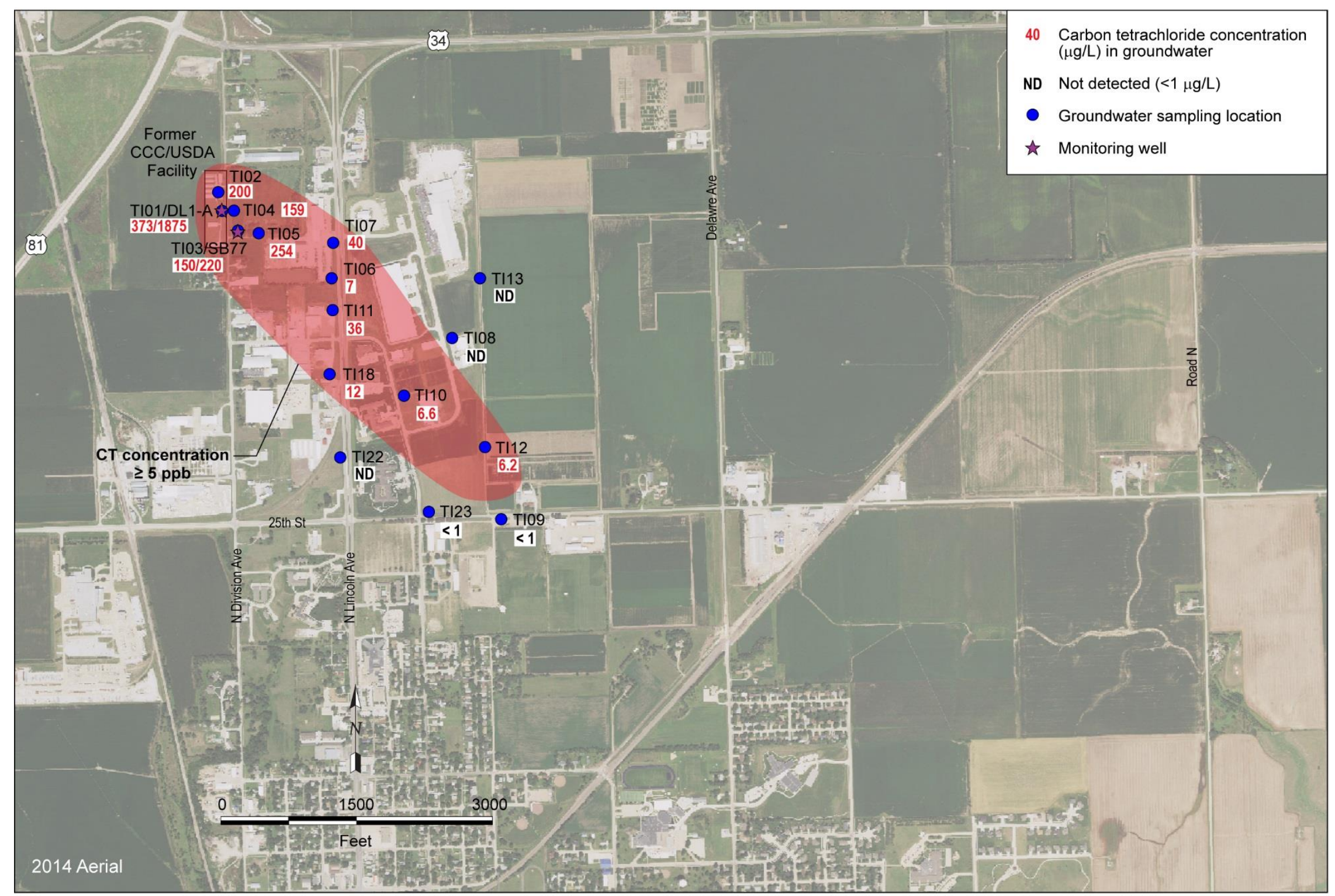

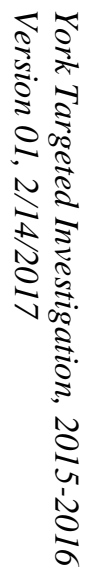

FIGURE 4.17 Carbon tetrachloride concentrations in the "A" sand zone of the unconfined aquifer. 


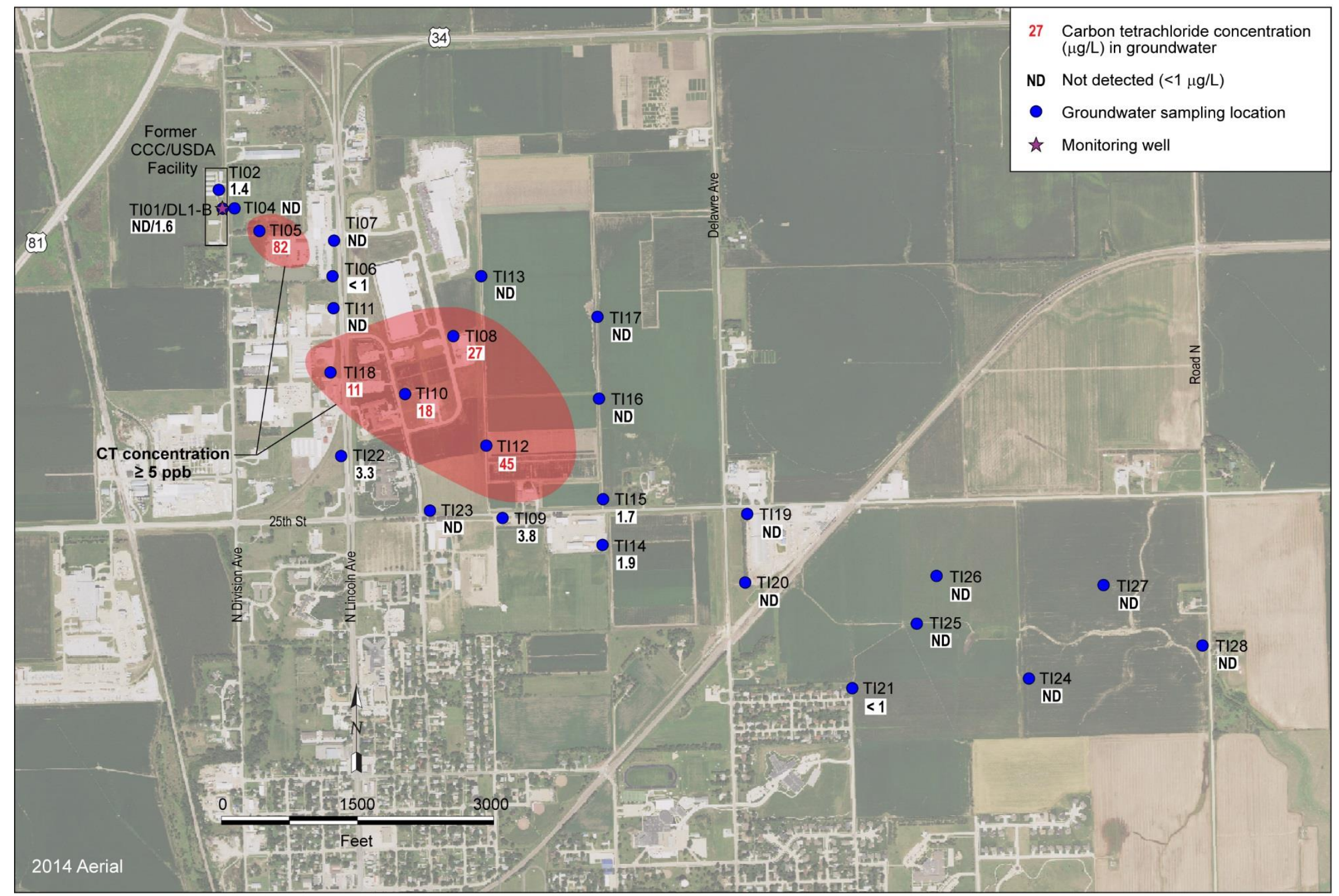




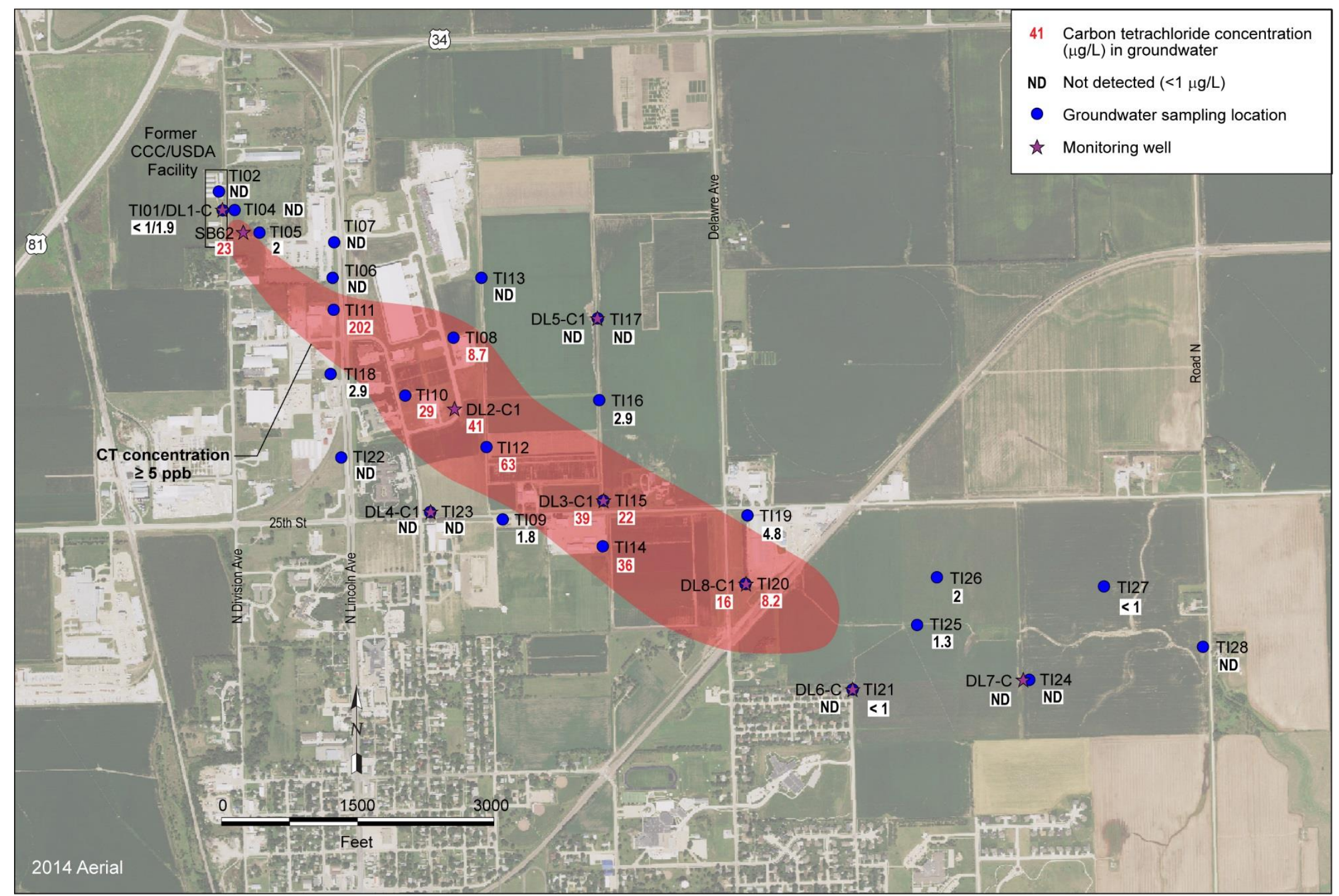

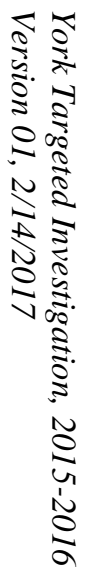

FIGURE 4.19 Carbon tetrachloride concentrations in the "C-C1" sand zone of the unconfined aquifer. 


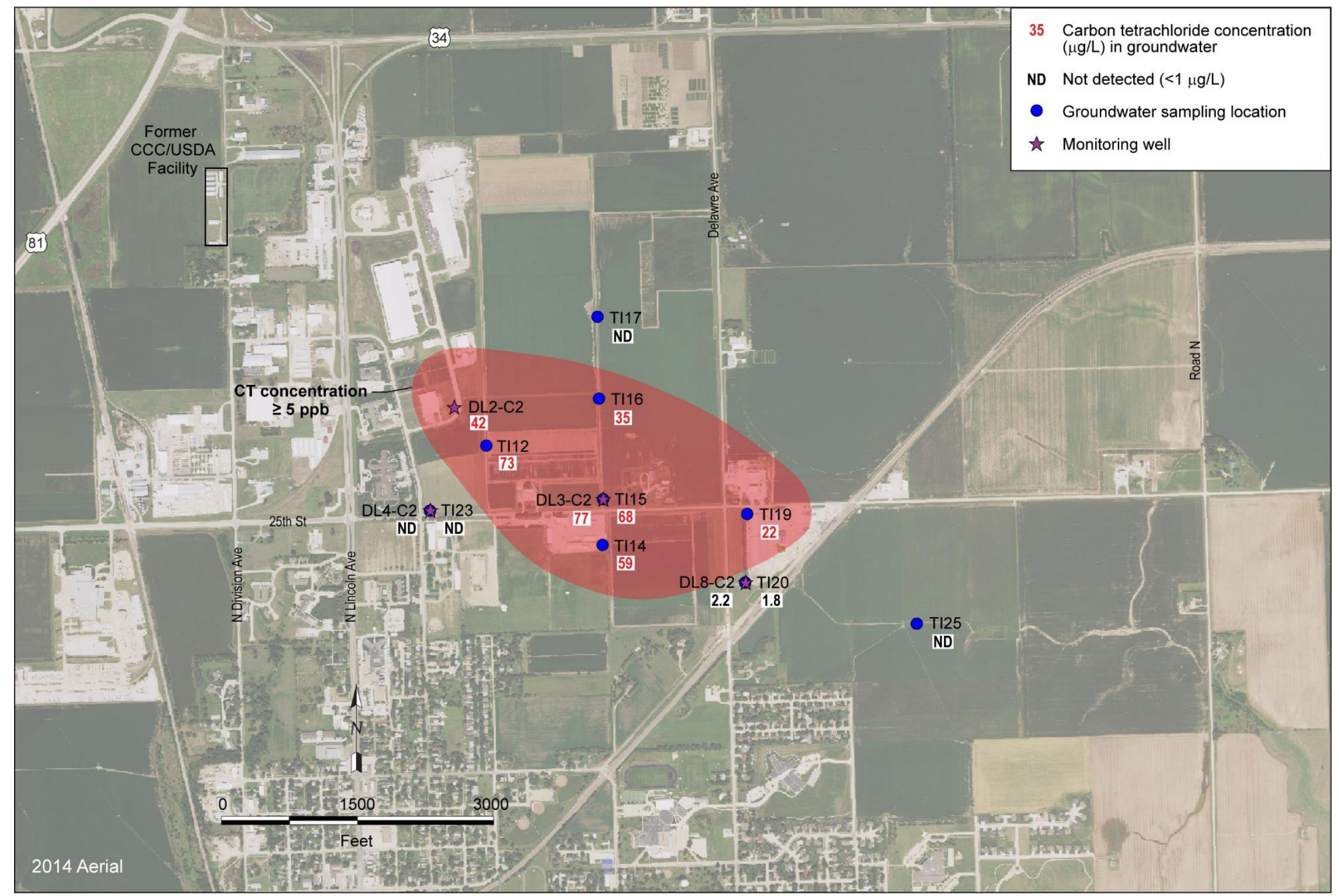

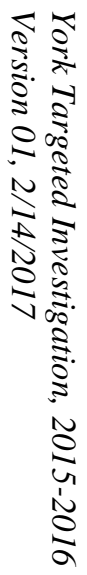

FIGURE 4.20 Carbon tetrachloride concentrations in the "C2" sand zone of the unconfined aquifer. 


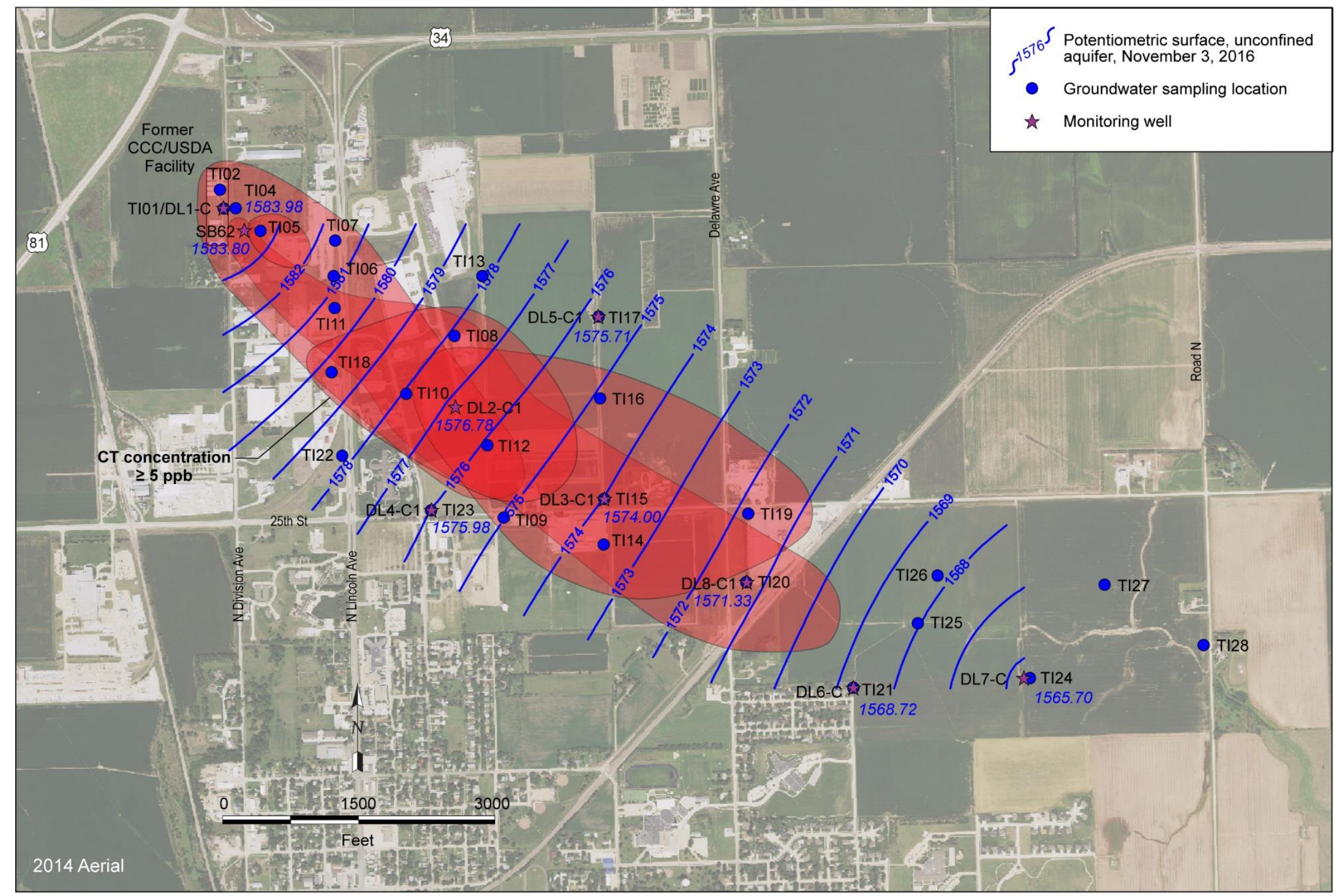

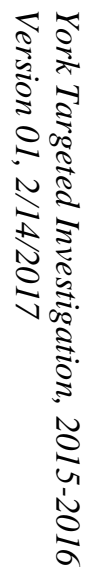

FIGURE 4.21 Estimated potentiometric surface for the unconfined aquifer ("C-C1" zone) based on groundwater levels measured manually on November 3, 2016 and the interpreted distribution of carbon tetrachloride contamination in the unconfined aquifer. 


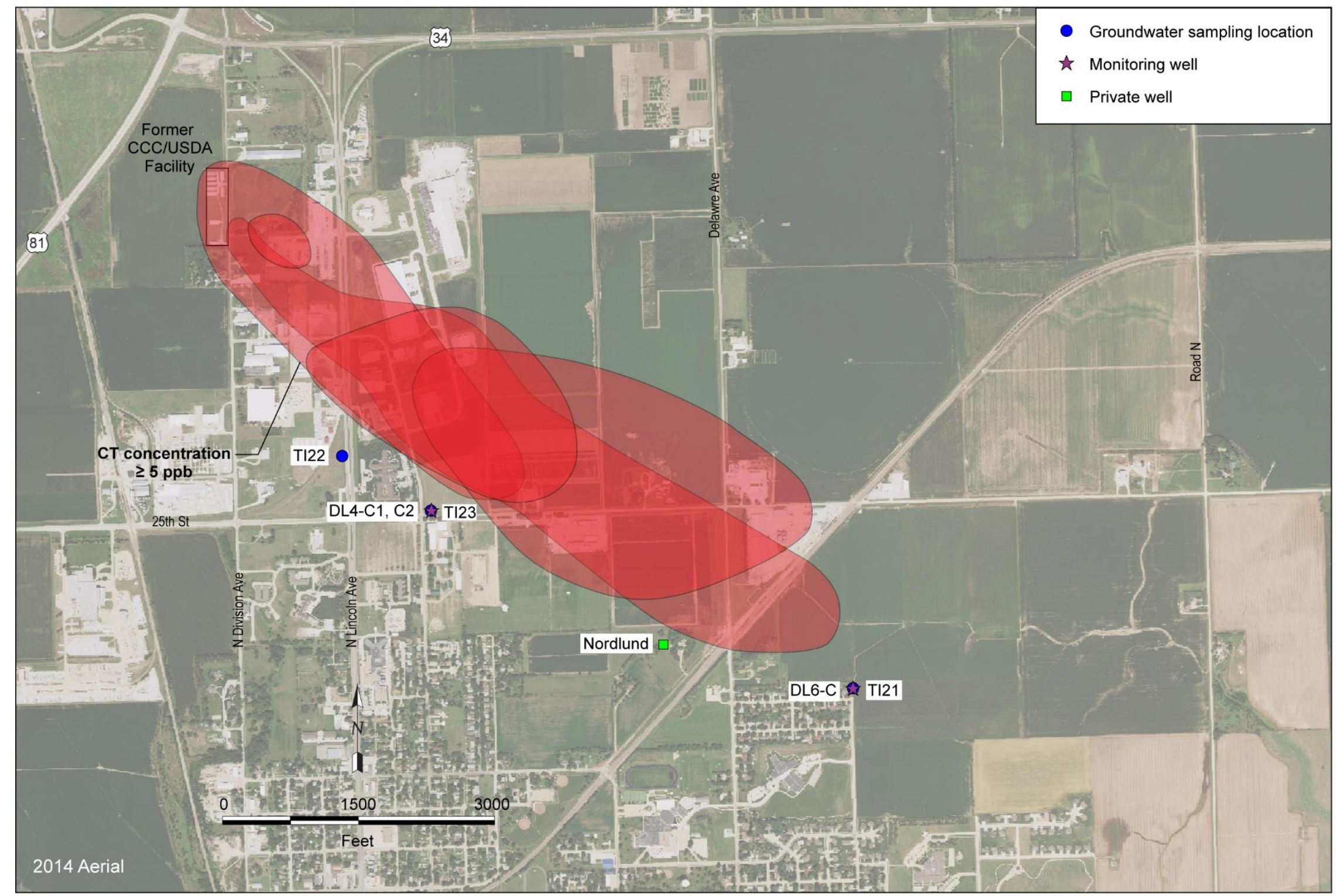

FIGURE 4.22 Locations of critical groundwater sampling points constraining the interpreted southwestern margin of the carbon tetrachloride distribution in the unconfined aquifer associated with the former CCC/USDA facility. 


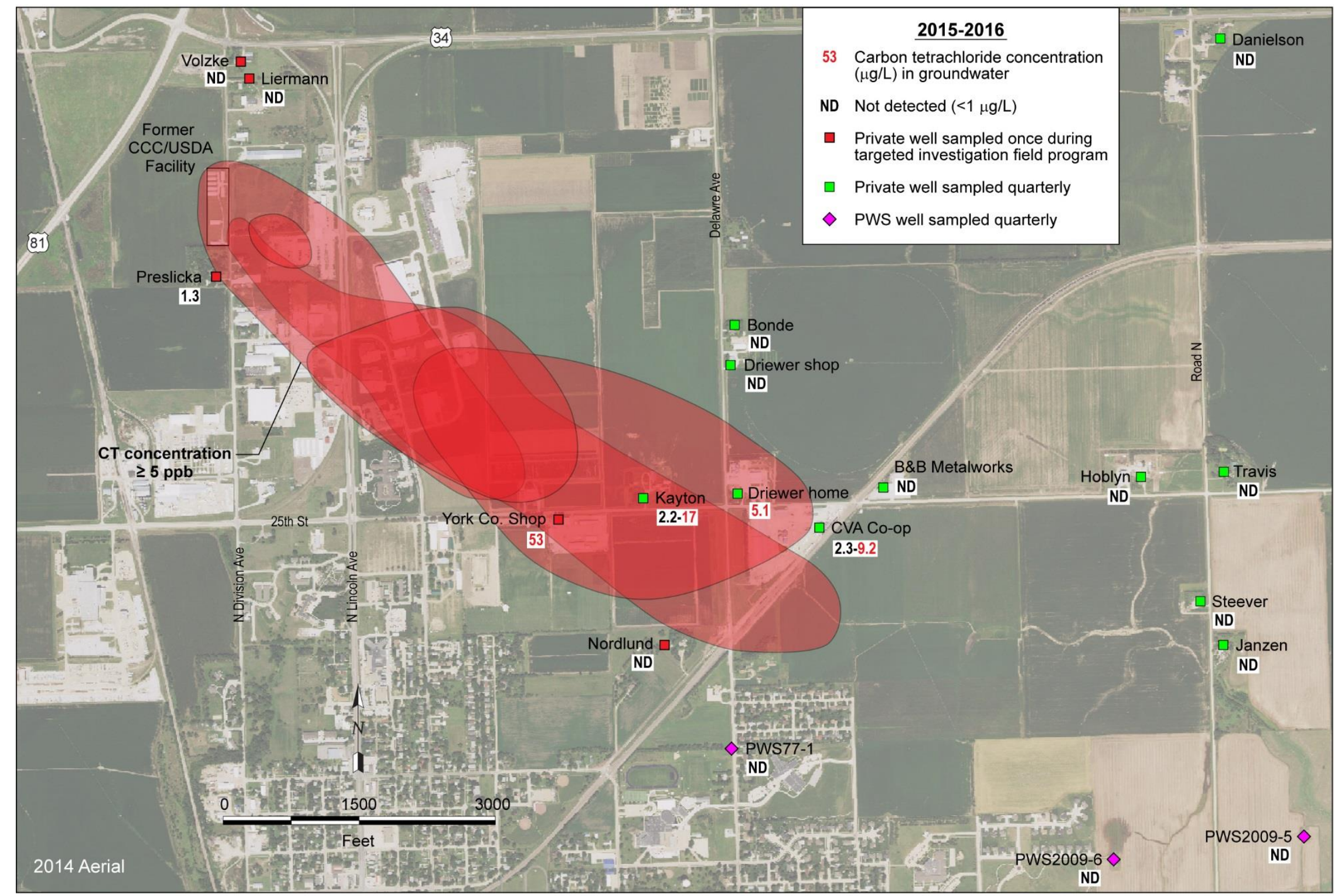

FIGURE 4.23 Carbon tetrachloride concentrations in groundwater collected from private and municipal wells, and the interpreted distribution of carbon tetrachloride in the unconfined aquifer. Outlying wells (Onnen, Makovicka, PWS 2009-4) omitted for clarity. 


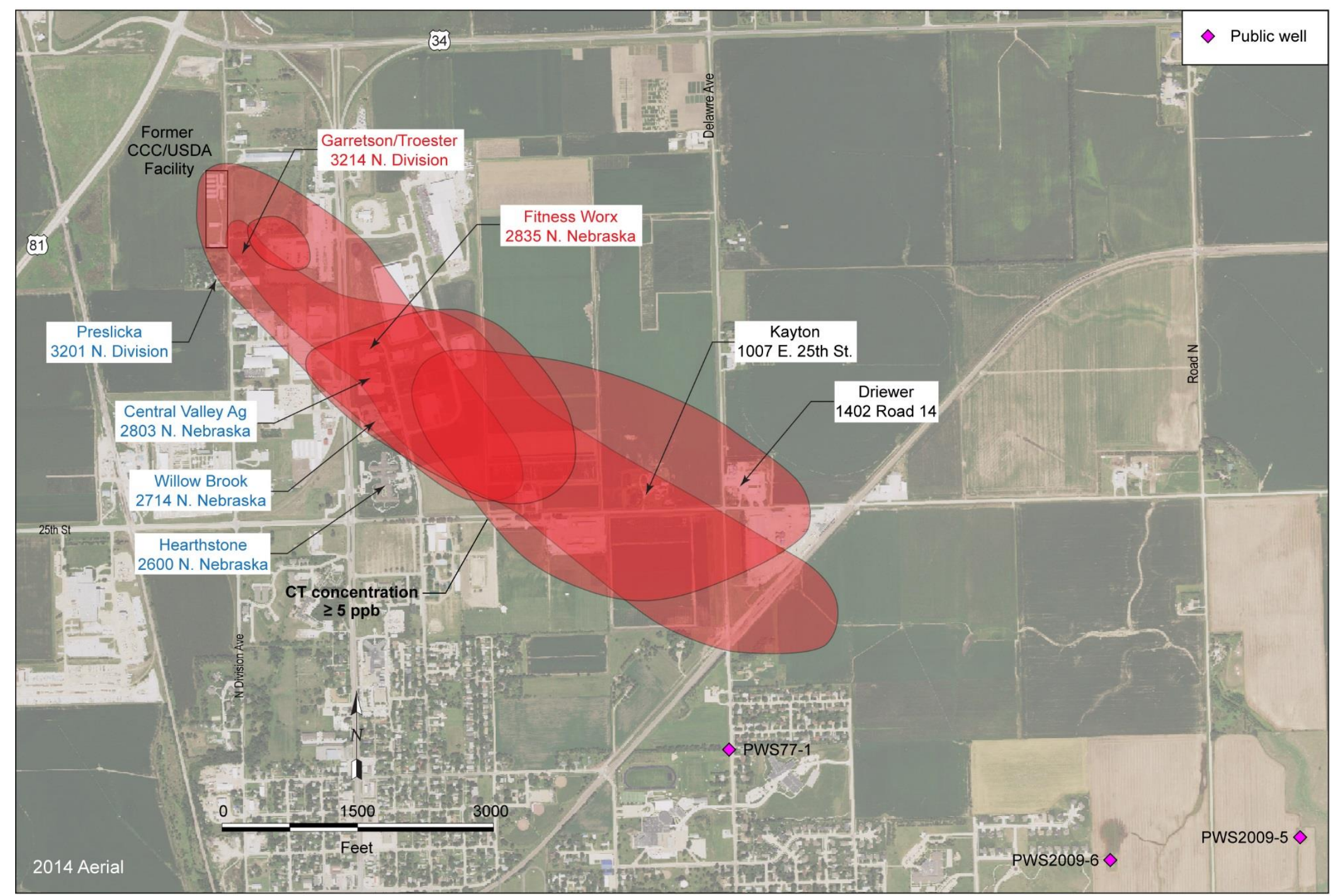

FIGURE 4.24 Locations sampled for VOCs analyses of indoor air (shown in blue) or sub-slab soil vapor and indoor air (shown in red), York PWS wells, and the interpreted distribution of carbon tetrachloride in the unconfined aquifer. Locations shown in black have not been tested for VI. 


\section{Conclusions and Recommendations}

\subsection{Conclusions}

The hydrogeologic, physiographic, and soil and groundwater analysis data evaluated in Section 4 provide an updated understanding of the factors affecting the migration of carbon tetrachloride contamination associated with the former CCC/USDA facility at York, and the present distribution and impacts of this contamination. The key elements of this interpretation identified in the present study are summarized as follows.

- The regional hydrogeologic sequence of unconsolidated deposits that was previously identified in the vicinity of York was confirmed in the local study area, beneath and to the southeast of the former CCC/USDA facility. The primary units of this sequence, in order of increasing depth, include (1) an interval of loess, (2) a sandy unconfined aquifer, (3) a variably thick, siltyclayey upper confining unit, (4) a sandy upper confined aquifer, (5) variably interbedded silty-clayey and sandy deposits, which together comprise a lower confining unit, and (6) a lower confined aquifer.

- The specific characteristics of the units identified in the study area are as follows:

- Loess. The uppermost unit throughout the study area consists of approximately $40-60 \mathrm{ft}$ of relatively uniform clayey silt to silty clay, interpreted as wind-blown silt (loess). Thin intervals of fine sand, sandy silt, sandy clay, or clay were occasionally observed in this unit, as well as occasional iron oxide staining.

- Unconfined Aquifer. The unconfined aquifer consists predominantly of sandy deposits that are interbedded with thin horizons and lenses of clay, silty clay, or silts that vary both vertically and laterally in their abundance and continuity. The coarser-grained materials comprising the unconfined aquifer vary from occasional thin horizons of relatively well sorted, silty to fine sand to thin intervals of coarse sand and gravel; however, the majority of the sediments in this unit consist of thicker intervals of poorly 
sorted mixtures of predominantly fine to medium sand with lesser quantities of coarse sand, silt, clay, and dispersed fine gravel. For the present study, an informal nomenclature was adopted to represent the general vertical sequence of these sandy deposits as the " $\mathrm{A}$," "B," and " $\mathrm{C}$ " (or "C1-C2") sand zones with increasing depth. The aggregate saturated thickness of the unconfined aquifer varied from approximately $43 \mathrm{ft}$ to $67 \mathrm{ft}$ at the time of this study, with the greatest thickness of saturated sandy deposits identified in the central portion of the study area.

Intervals of silt-clay that appear laterally continuous across most of the current study area were identified at the base of the (predominantly unsaturated) "A" sand zone and (saturated) "B" sand zone; however, the vertical distinction among the sand zones can be relatively arbitrary locally, particularly in the more central portion of the area where the lowermost ("C1-C2") sands thicken. The fine-grained zones associated with the "B" and "C-C1-C2" sands are frequently thin and discontinuous, and cannot be correlated with certainty among adjacent boring locations. The results of previous studies conducted by the CCC/USDA in the immediate vicinity of the former CCC/USDA facility have demonstrated that while the interbedded silt-clay horizons may influence the vertical movement of groundwater locally, they do not appear to represent hydraulic barriers among the sandy zones within the unconfined aquifer at a wider scale.

- Upper Confining Unit. Site-specific data for the upper confining unit indicates that this interval consists of locally soft to very hard silty clay to clayey silt that is variably iron-oxide stained, variably calcareous, and may contain calcareous grains, small pebbles, or carbonate-cemented patches and minor fine sand that are consistent with a possible origin of this unit as till. Thicknesses varying from approximately $23 \mathrm{ft}$ to $56 \mathrm{ft}$ were identified for this unit, on the basis of the locations investigated in the current study.

- Upper Confined Aquifer. Where penetrated in the current investigation (at drilling locations DL2 and DL7), the upper confined aquifer consists of approximately $77-80 \mathrm{ft}$ of generally well-sorted fine sands, with lesser 
medium sand and minor to trace quantities of clay and silt. Occasional, interbedded lenses or layers of silty sand to sand-silt-and clay were also noted at DL2. Limited additional data obtained from several driller's logs for nearby wells suggest apparent thinning of the upper confined aquifer, to approximately $20 \mathrm{ft}$, near the south-central margin of the study area.

- Lower Confining Unit. As penetrated in the study area, the sediments representing the uppermost portion of the lower confining unit consist of calcareous silty-clayey fine sand to clayey silt or silty clay, that ranged in (net) thickness from approximately $15 \mathrm{ft}$ to $21.5 \mathrm{ft}$. Thicknesses for this interval ranging from $45 \mathrm{ft}$ to $50 \mathrm{ft}$ are indicated in the drillers' logs available for several wells identified near the southwestern margin of the present study area.

- Lower Confined Aquifer. Approximately $19 \mathrm{ft}$ of sand, representing the first coarser-grained unit beneath the lower confining layer described above, was penetrated at one location (DL7) at an approximate depth of 256-275 ft BGL. The deposits identified were lithologically similar to those of the upper confined aquifer, consisting predominantly of wellsorted fine sand with small to trace amounts of associated medium sand, silt, and clay.

- Groundwater level measurements obtained under ambient conditions in the unconfined aquifer demonstrate a relatively uniform hydraulic gradient and direction of groundwater flow to the southeast in this unit consistent with the flow pattern interpreted for this aquifer in the 1993-1995 CCC/USDA investigations at this site (Figure 4.11). Under these conditions, little to no vertical hydraulic head difference was indicted among the sandy zones within the unconfined aquifer.

- Continuous monitoring of groundwater levels revealed the occurrence of frequent, short-duration episodes of drawdown and subsequent recovery of the groundwater levels in the unconfined aquifer during the summer months of 2016, that were coincident with similar individual (but of a much larger magnitude) events, and more sustained seasonal lowering of the groundwater 
levels, that were also identified in the upper and lower confined aquifers. Together, these observations suggest that the groundwater levels in all three aquifers may be influenced by the pumping of one or more large-capacity irrigation wells that are located predominantly in the southeastern and eastern portions of the study area. Available data suggest possible local, transient shifts in the predominant direction of groundwater flow in the unconfined aquifer, to a more eastward or northeastward direction, in the eastern and southeastern portions of the study area under the inferred pumping conditions (Figure 4.12). Additional monitoring of the levels in these units will be required, however, to further determine the local effects of this, or other, seasonal or longer-term influence(s) on the groundwater levels in the unconfined and confined aquifers.

- Residual carbon tetrachloride contamination was identified, at levels greater than the current NDEQ VCP target for this contaminant in soils ( $39 \mu \mathrm{g} / \mathrm{kg})$, in the vadose zone soils at one location at the former CCC/USDA facility (TI01; 53-167 $\mu \mathrm{g} / \mathrm{kg}$ ), and one location on the York Gun Club property (TI04; 43$61 \mu \mathrm{g} / \mathrm{kg}$ ). Only trace levels of carbon tetrachloride were detected in soils at two additional locations (TI02 and TI03) on these properties (Figure 2.1). Available data suggest that residual contaminant levels that may pose a threat of continued contamination to the underlying groundwater are generally limited to depths of 20-50 ft BGL within the fine-grained vadose zone soils beneath and near the east-central portion of the former CCC/USDA facility. Limited additional soil sampling and analyses for VOCs in this area might be warranted, however, to fully constrain the lateral and vertical extent of this contamination.

- Groundwater sampling for VOCs analyses conducted in vertical profiles at 28 locations in the study area has delineated a relatively complex distribution of carbon tetrachloride contamination in the groundwater of the unconfined aquifer, along an apparent migration pathway extending approximately $1.6 \mathrm{mi}$ to the southeast and downgradient from the former CCC/USDA facility (at levels greater than the NDEQ VCP and EPA-MCL targets for this compound in drinking water; $5 \mu \mathrm{g} / \mathrm{L}$ ). The observed contaminant distribution is 
consistent with the groundwater flow pattern identified under ambient (nonpumping) conditions during the present study (Figure 4.21).

- With two exceptions, the maximum carbon tetrachloride concentrations in the unconfined aquifer, ranging from approximately $150 \mu \mathrm{g} / \mathrm{L}$ to $373 \mu \mathrm{g} / \mathrm{L}$, were identified in the uppermost, " $\mathrm{A}$ " sand zone of the aquifer directly beneath and immediately adjacent to the former facility (Figure 4.17). The single highest carbon tetrachloride concentration $(1,875 \mu \mathrm{g} / \mathrm{L})$ was identified in a groundwater sample recovered from " $\mathrm{A}$ " zone monitoring well DL1-A, at the east-central edge of the former facility. (A carbon tetrachloride concentration of $202 \mu \mathrm{g} / \mathrm{L}$ was also identified in an isolated groundwater sample from the deeper " $\mathrm{C}$ " sand zone, at one location,TI11, in the northwestern portion of the study area; Figure 4.19.)

- The available data indicate a rapid drop in the groundwater concentrations both vertically and laterally beneath and in the vicinity of the former CCC/USDA facility. Carbon tetrachloride concentrations in the " $\mathrm{A}$ " sandy zone decrease to levels of less than $50 \mu \mathrm{g} / \mathrm{L}$ approximately $1,200 \mathrm{ft}$ to the southeast and downgradient of the former facility, and to concentrations near or below the MCL within approximately $0.5 \mathrm{mi}$ downgradient of the former facility (Figure 4.17).

- Carbon tetrachloride concentrations greater than the NDEQ VCP target and MCL ( $5 \mu \mathrm{g} / \mathrm{L})$ in the deeper ("B," "C," and "C1-C2") zones of the unconfined aquifer were primarily identified in the central portion of the study area, at levels ranging from approximately $9 \mu \mathrm{g} / \mathrm{L}$ to $82 \mu \mathrm{g} / \mathrm{L}$ with the higher values in this range primarily occurring in the deepest ("C2") sand zone (Figures 4.184.20).

- Groundwater sampling conducted by the CCC/USDA in 1993 identified carbon tetrachloride contamination in the unconfined aquifer extending approximately $0.8 \mathrm{mi}$ to the southeast and downgradient from the former CCC/USDA facility in the unconfined aquifer. The presently observed distribution outlined above suggests an average linear downgradient migration 
rate of approximately $200 \mathrm{ft} / \mathrm{yr}$ in this aquifer, over the $(22 \mathrm{yr})$ period from 1993 to 2015.

- The absolute concentrations of chloroform, and the relative level of this compound to the associated carbon tetrachloride at each sampling point in the unconfined aquifer, were generally very low, providing little to no evidence for possible degradation of carbon tetrachloride via natural (reductive dechlorination) processes in the unconfined aquifer. Exceptions to this relationship were observed, however, in the groundwater at several locations (TI01, TI02, TI04, and TI05) in the uppermost, "A" sand zone beneath and immediate to the east of the former CCC/USDA facility, suggesting the possibility of a very localized influence of these processes.

- With two trace exceptions, no other volatile contaminants were detected in the unconfined aquifer in association with the carbon tetrachloride distribution delineated in this study. (At sampling location TI01 at the former CCC/USDA facility, trace concentrations $[<1 \mu \mathrm{g} / \mathrm{L}]$ of PCE and 1,2 dichloropropane were detected, and a trace of PCE was also detected at location TI04 on the York Gun Club property.) The results of the present CCC/USDA investigation, together with those of the earlier studies conducted by the CCC/USDA in 1993-1995, demonstrate that the carbon tetrachloride distribution in groundwater associated with the former CCC/USDA facility is distinct from, and trends parallel to, the mixed (predominantly PCE and TCE) contaminant plume that has been identified by the EPA to the south and west of the present study area, and that has been linked to the PCE/TCE Northeast Contamination NPL site.

- Groundwater samples for VOCs analyses were collected at two locations in the upper confined aquifer (DL2-CU and DL7-CU) and at one location in the lower confined aquifer (DL7-CL) in the present study. The DL2-CU location was selected to facilitate sampling of the upper confined aquifer groundwater along the identified contaminant migration pathway in the overlying unconfined aquifer, at a central point downgradient from the highest concentrations identified in the unconfined aquifer. Monitoring points DL7CU and DL7-CL were similarly chosen to permit sampling of groundwater 
from the upper and lower confined aquifers, at a location lying beyond the presently identified, downgradient "toe" of the carbon tetrachloride distribution in the unconfined aquifer. No carbon tetrachloride, chloroform, methylene chloride, or other volatile contaminants were detected in these samples, therefore providing no evidence of potential carbon tetrachloride cross-contamination from the unconfined aquifer to the underlying, upper or lower confined aquifers at these locations.

- Groundwater samples for VOCs analyses are collected quarterly at York well PWS 77-1, which lies generally cross-gradient to the carbon tetrachloride distribution identified in the unconfined aquifer and is screened in the upper and lower confined aquifers, and at wells PWS 2009-4, 2009-5, and 2009-6, which appear to lie downgradient from the contaminant distribution and are screened exclusively in the lower confined aquifer. No carbon tetrachloride, chloroform, or methylene chloride have been detected at these wells (Figure 4.23).

- Three private wells were identified in the present study area that are impacted by carbon tetrachloride contamination and might be associated with the former CCC/USDA facility (although the depths of these wells are not known). These wells are currently used as a source of drinking water, or might be used for this purpose in the future. The identified wells are as follows (Figure 4.23):

- CVA Coop. Carbon tetrachloride concentrations of 4.9-9.2 $\mu \mathrm{g} / \mathrm{L}$ have been identified at the CVA Coop well (at 1403 Road 14) since regular sampling began in December 2014 (Table 3.4). This well was not sampled during the 1993-1995 investigations at this site (Table 4.5). Groundwater from the well is currently used at the Coop facility for domestic and other purposes, without treatment.

- Kayton. Carbon tetrachloride levels of 6-8 $\mu \mathrm{g} / \mathrm{L}$ were identified at the Kayton well in 1993 (former Olson, at 1007 E. 25th St.; Table 4.5). Values ranging from $2.4 \mu \mathrm{g} / \mathrm{L}$ to $17 \mu \mathrm{g} / \mathrm{L}$ have been identified to date during the quarterly monitoring program, but show no clear, net change from the 
earlier sampling results. The Kayton well has historically been used for domestic purposes, but this property is now used for rental income and is currently (in 2015-2016) vacant, and the property owner is working with the CCC/USDA to have the home connected to the York municipal water supply.

- Driewer (home well). At the request of the NDEQ, quarterly sampling of the private well at the Driewer residence (at 1402 Road 14) began in December 2016. Access to this well for sampling by the CCC/USDA had previously been denied by the property owner. In prior sampling conducted by the EPA, carbon tetrachloride was reported at this well at a concentration of $8.7 \mu \mathrm{g} / \mathrm{L}$; a GAC filtration system was subsequently provided for the house by the EPA (NDEQ 2016b). Carbon tetrachloride was identified at a (pretreatment) concentration of $5.1 \mu \mathrm{g} / \mathrm{L}$ in the December 2016, CCC/USDA sampling event.

- Sampling of indoor air was performed at six locations in the vicinity of the former CCC/USDA facility, to investigate for possible carbon tetrachloride VI. The locations were chosen, with the approval of the CCC/USDA and NDEQ project managers, as residential or other sites of potentially sensitive populations lying along or near the more upgradient portion of the identified migration pathway of carbon tetrachloride in the unconfined aquifer. The locations investigated included the Hearthstone and Willowbrook long-term health care facilities, the headquarters building of the CVA Coop, the Fitness Worx (York Physical Therapy) facility, and the Preslicka and Garretson/Troester private residences (Figure 4.24).

- No carbon tetrachloride or chloroform were detected in the indoor air in an initial sampling event conducted at the Willowbrook, Hearthstone and CVA Coop facilities or at the Preslicka residence. With the approval of the CCC/USDA and NDEQ project managers, no further investigations were conducted at these sites.

- Carbon tetrachloride concentrations ranging from $1.5 \mu \mathrm{g} / \mathrm{m}^{3}$ to $7.9 \mu \mathrm{g} / \mathrm{m}^{3}$ were identified during the initial sampling of the indoor air at the Fitness 
Worx facility. At the request of the NDEQ, subsequent sampling conducted by the CCC/USDA at this location over four consecutive quarters consistently identified levels of carbon tetrachloride in the indoor air $\left(1.8-8.2 \mu \mathrm{g} / \mathrm{m}^{3}\right)$ and also in sub-slab soil vapor $\left(20-50 \mu \mathrm{g} / \mathrm{m}^{3}\right)$ at levels well below the respective NDEQ VCP targets for this compound for nonresidential facilities $\left(20 \mu \mathrm{g} / \mathrm{m}^{3}\right.$ and $\left.666 \mu \mathrm{g} / \mathrm{m}^{3}\right)$. Unexpectedly high relative levels of chloroform detected in the indoor air during several of these sampling events strongly suggests a possible origin for this compound unrelated to the underlying soil vapor at this location.

- Carbon tetrachloride was identified in both the indoor air $\left(8-29 \mu \mathrm{g} / \mathrm{m}^{3}\right)$ and in sub-slab vapor $\left(6,500 \mu \mathrm{g} / \mathrm{m}^{3}\right)$ at the Garretson residence, at levels exceeding the respective NDEQ VCP targets for this contaminant in a residential setting $\left(0.41 \mu \mathrm{g} / \mathrm{m}^{3}\right.$ and $13.7 \mu \mathrm{g} / \mathrm{m}^{3}$, respectively). Because of these high levels, the CCC/USDA installed of a sub-slab vapor mitigation system for the residence. Following the completion of this system, sampling of the indoor air was subsequently conducted only at the Garretson/Troester residence in two consecutive quarterly events, and no carbon tetrachloride or chloroform was detected.

- Under the presently observed conditions, concentrations of carbon tetrachloride greater than the NDEQ VCP and MCL level $(5 \mu \mathrm{g} / \mathrm{L})$ for this contaminant in drinking water extend approximately $0.9 \mathrm{mi}$ to the southeast of the former CCC/USDA facility in these zones. At greater distances from the former facility (from $0.9 \mathrm{mi}$ to the current margin of the contaminant distribution, approximately $1.6 \mathrm{mi}$ downgradient), elevated levels of carbon tetrachloride are restricted to the underlying "C-C1" and "C2" sand zones, at depths of approximately 110-160 ft BGL (Figures 4.19 and 4.20). In this downgradient area, sampling has demonstrated that the groundwater in the shallowest saturated interval (the "B" sand zone; Figures 4.13 and 4.18) remains effectively uncontaminated.

Reconnaissance conducted by Argonne demonstrates that the area along the carbon tetrachloride migration pathway lying downgradient from the six locations that have already been screened for VI is relatively sparsely 
populated, and consists predominantly of agricultural land. Occupied properties along the identified migration trend consist primarily of several commercial or warehouse/garage facilities. Only two residential properties fall along the more distal, and deeper, carbon tetrachloride migration pathway that have not been investigated for VI; these are the Kayton (rental property; currently vacant) and Driewer (home) properties (Figure 4.24). Carbon tetrachloride concentrations measured to-date in private wells located at each of these residences have been generally low (Kayton property, at 2.2-17 $\mu \mathrm{g} / \mathrm{L}$; Driewer home, at $5.1 \mu \mathrm{g} / \mathrm{L}$ ). Together, the above observations suggest that the potential risks to human health at downgradient locations in the remainder of the present study area, as a result of possible intrusion of carbon tetrachloride vapors to indoor air, are minimal. Further consideration of these risks may be warranted, however, as the distribution of the carbon tetrachloride contamination in the unconfined aquifer continues to evolve with time.

\subsection{Recommendations}

The results of the targeted investigation demonstrate that carbon tetrachloride is present in vadose zone soils in a limited area at and near the former CCC/USDA facility, and in the groundwater of the unconfined aquifer beneath and downgradient of the former facility, at concentrations greater than the current EPA MCL and NDEQ VCP risk-based targets for this contaminant (39 $\mu \mathrm{g} / \mathrm{kg}$ in soil and $5.0 \mu \mathrm{g} / \mathrm{L}$ in groundwater). Comparison of the current findings to data previously obtained by the CCC/USDA (in 1993-1995) has shown that the concentrations and distribution of carbon tetrachloride in the impacted soils and groundwater has evolved in a manner consistent with earlier CCC/USDA interpretations. The potential for carbon tetrachloride VI to indoor air in association with the identified contamination in groundwater has also been demonstrated, above the more upgradient, generally shallower portion of the carbon tetrachloride distribution in groundwater.

In light of these observations, additional investigation at the site is recommended to (1) further delineate the areal and (vertical) extent of the carbon tetrachloride contamination in vadose zone soils, (2) determine the transient characteristics of groundwater flow in the unconfined aquifer, and (3) obtain additional data on the hydraulic characteristics of the unconfined aquifer. Together with existing data, the results of these additional studies will provide a basis for quantitative estimation of groundwater flow and potential contaminant 
migration rates in the unconfined aquifer. In addition, (4) limited additional screening of indoor air is also proposed at selected locations, to assess the potential for VI above the more downgradient, generally deeper portion of the carbon tetrachloride distribution.

The investigative activities proposed to address these technical objectives are as follows:

- The current study has demonstrated that residual levels of carbon tetrachloride contamination in the vadose zone soils at and near the former CCC/USDA facility have declined since the 1993-1995 CCC/USDA investigations (Argonne 1994, 1995a,b). The recent data suggest that residual contaminant levels that may pose a threat of continued contamination to the underlying groundwater are generally limited to depths of 20-50 ft BGL within the finegrained vadose zone soils beneath and near the east-central portion of the former CCC/USDA facility (Figure 2.1, Table 3.1, and Tables 4.3a-c).

To confirm this interpretation, limited additional vertical-profile sampling of the vadose zone soils for VOCs analyses is recommended. Tentative locations for the collection of soil samples are shown in Figure 5.1; however, the actual number and positions of locations to be investigated will be determined in the field, in consultation with the CCC/USDA and NDEQ project managers, as the additional data are acquired and evaluated. The soil samples will be collected using the direct-push techniques described in Section 2.3.

- Groundwater level measurements suggest that groundwater flow in the unconfined aquifer under ambient conditions is to the southeast, in response to a relatively uniform hydraulic gradient that is consistent with previous CCC/USDA findings (Figure 4.11). Under these conditions, little to no vertical hydraulic head difference was indicted among the sandy zones within the unconfined aquifer. Continuous monitoring data obtained thus far (at 11 of 19 permanent monitoring wells; Table 3.2 and Figure 2.5) has suggested, however, that the groundwater levels in the unconfined, upper confined, and lower confined aquifers may be influenced by the seasonal pumping of one or more large-capacity irrigation wells that are located predominantly in the southeastern and eastern portions of the study area (Figure 4.8). 
Continued, automated monitoring of the groundwater levels in the existing network of permanent monitoring wells is therefore recommended to document transient impacts on groundwater flow in these units over, at minimum, a complete annual cycle. The installation of groundwater level measuring and data logging units in additional existing monitoring wells completed in the "B" and "C2" sand zones (Table 3.2) is also proposed, to provide information on possible seasonal or other influences on vertical groundwater (and contaminant) movement within the unconfined aquifer.

- To obtain quantitative estimates of the hydraulic properties of the sediments composing the unconfined aquifer, single-well slug testing of monitoring wells DL1-A, DL1-B, DL1-C, DL2C1, DL2-C2, DL3-C1, DL3-C2, DL4C-1, DL4-C2. DL5-C1, DL6-C, DL7-C, DL8-C1 and DL8-C2 is recommended (Figure 5.2). The testing will be conducted by using a solid rod or slug of suitable length and diameter to perturb the static water column in each well. The test will be repeated a minimum of three times at each location. The resulting test data will be interpreted (to determine the horizontal hydraulic conductivity) by using established analysis methods that are suitable to the well and aquifer conditions encountered at each testing location (Argonne 2002; Section 6.7).

- The area along the carbon tetrachloride migration pathway lying downgradient of the six locations that have already been screened for carbon tetrachloride VI is relatively sparsely populated, and consists predominantly of agricultural land (Figure 4.24). Occupied properties along the identified migration trend consist primarily of non-residential facilities. Only two residential properties fall along the more distal, and deeper, carbon tetrachloride migration pathway that have not been investigated for VI; these are the Kayton (rental property; currently vacant) and Driewer (home) properties (Figure 4.24). Carbon tetrachloride concentrations measured to-date in private wells located at each of these residences (Section 4.3.3.2 and Table 3.4) have been generally low (Kayton property, at 2.2-17 $\mu \mathrm{g} / \mathrm{L}$; Driewer home, at $5.1 \mu \mathrm{g} / \mathrm{L}$ ). As a precautionary measure, however, one-time sampling of the indoor air at these locations is proposed, to screen for the presence of carbon tetrachloride VI. 
- At each location, samples of the indoor air will be collected from the breathing zone at locations on the ground floor and the lower floor (or basement), if applicable, over a 24-hr period using certified-clean, 6-L stainless steel canisters equipped with timed-inlet fittings. Background samples of outdoor air also will be collected. All samples will be analyzed for a full suite of VOCs, by EPA Method TO-15.

In conjunction with these studies, the following actions are also recommended:

- In October 2015, the NDEQ requested that the CCC/USDA conduct sampling of the indoor air, as well as sub-slab vapor, for VOCs analyses at two locations - the Fitness Worx facility and the Garretson/Troester home - on a quarterly basis for one year, beginning in 2016. The requested sampling was conducted at the Fitness Worx facility in January, April, August, and December 2016. Sampling of the indoor air and sub-slab vapor was performed at the Garretson/Troester home in January 2016, but was postponed in April 2016 to facilitate the installation of a sub-slab vapor mitigation system by the CCC/USDA at this location. Indoor air samples were subsequently collected at the Garretson/Troester home (following completion of the vapor mitigation system) in August and December 2016). The results of these analyses (Table 3.6) demonstrated that (1) concentrations of carbon tetrachloride in indoor air at the Fitness Worx facility remained well below the NDEQ VCP target level for this contaminant in non-residential settings $\left(20 \mu \mathrm{g} / \mathrm{m}^{3}\right)$ throughout the 12-month monitoring period, and (2) no carbon tetrachloride was identified in indoor air at the Garrestson/Troester residence in August and December 2016, confirming that the vapor mitigation system is functioning satisfactorily.

On the basis of these data, the CCC/USDA proposes discontinuation of the quarterly VI sampling at these locations, in keeping with recommendations outlined to the CCC/USDA by the NDEQ (NDEQ 2015b). The CCC/USDA will, however, inspect and test the vapor mitigation system installed at the Garretson/Troester home annually, and perform any maintenance identified as necessary, to ensure the continued protectiveness of this system. 
- The results of the current study demonstrate that the carbon tetrachloride distribution in groundwater in the unconfined aquifer has evolved over many decades in a manner that is consistent with both previous (Argonne 1994, 1995a,b) and present interpretations of the factors controlling groundwater flow and contaminant migration at this site. At the request of the NDEQ, the CCC/USDA has conducted quarterly sampling of groundwater for VOCs analyses at 13 private wells and four PWS wells located in the more distal, eastern and southeastern portions of the current study area since December 2014 (Figure 2.7). The results of these analyses, summarized in Table 3.4 and Figure 4.23, demonstrate that carbon tetrachloride contamination has been detected to-date (at levels at or above the EPA MCL and NDEQ VCP target for this contaminant in drinking water; $5 \mu \mathrm{g} / \mathrm{L}$ ) at only three of these wells Kayton, CVA Coop, and Driewer (home) - that lie along the established path of carbon tetrachloride migration in the unconfined aquifer.

Figure 4.23 illustrates that a majority of the remaining private wells sampled lie cross-gradient to the identified contaminant migration pathway, and/or fall in excess of one mile beyond the existing limits of the carbon tetrachloride distribution. Further, no evidence of contamination of the deeper, upper and lower confined aquifers by carbon tetrachloride has been identified as a result of the CCC/USDA investigations, or quarterly sampling of the York PWS wells summarized in Table 3.4 and Figure 4.23. Documentation provided in Table 2.1 of the targeted investigation Work Plan (Argonne 2016a) indicates that the latter PWS wells (77-1, 2009-4, 2009-5, and 2009-6) are completed exclusively in the confined aquifers.

In light of these findings, CCC/USDA will pursue an alternate source of drinking water (via connection to the York municipal system) for the CVACoop facility and the Kayton residence and, if warranted, the Driewer residence (Figure 4.23). Information presently available to the CCC/USDA indicates that the Driewer residence has already been provided with a pointof-use treatment system for the domestic well at this location. The status of this system has not, however, been determined by the CCC/USDA to-date. 
In this context, the CCC/USDA also proposes discontinuation of groundwater sampling at the Bonde, Danielson, Hoblyn, Travis, Steever, Janzen, Onnen, and Makovicka private wells, and a reduction in frequency to annual groundwater sampling for VOCs analyses at the Kayton, CVA-Coop, Driewer (home), Driewer (shop), and B\&B Metalworks private wells, and at PWS wells 77-1, 2009-4, 2009-5, and 2009-6 (Figure 2.7). 


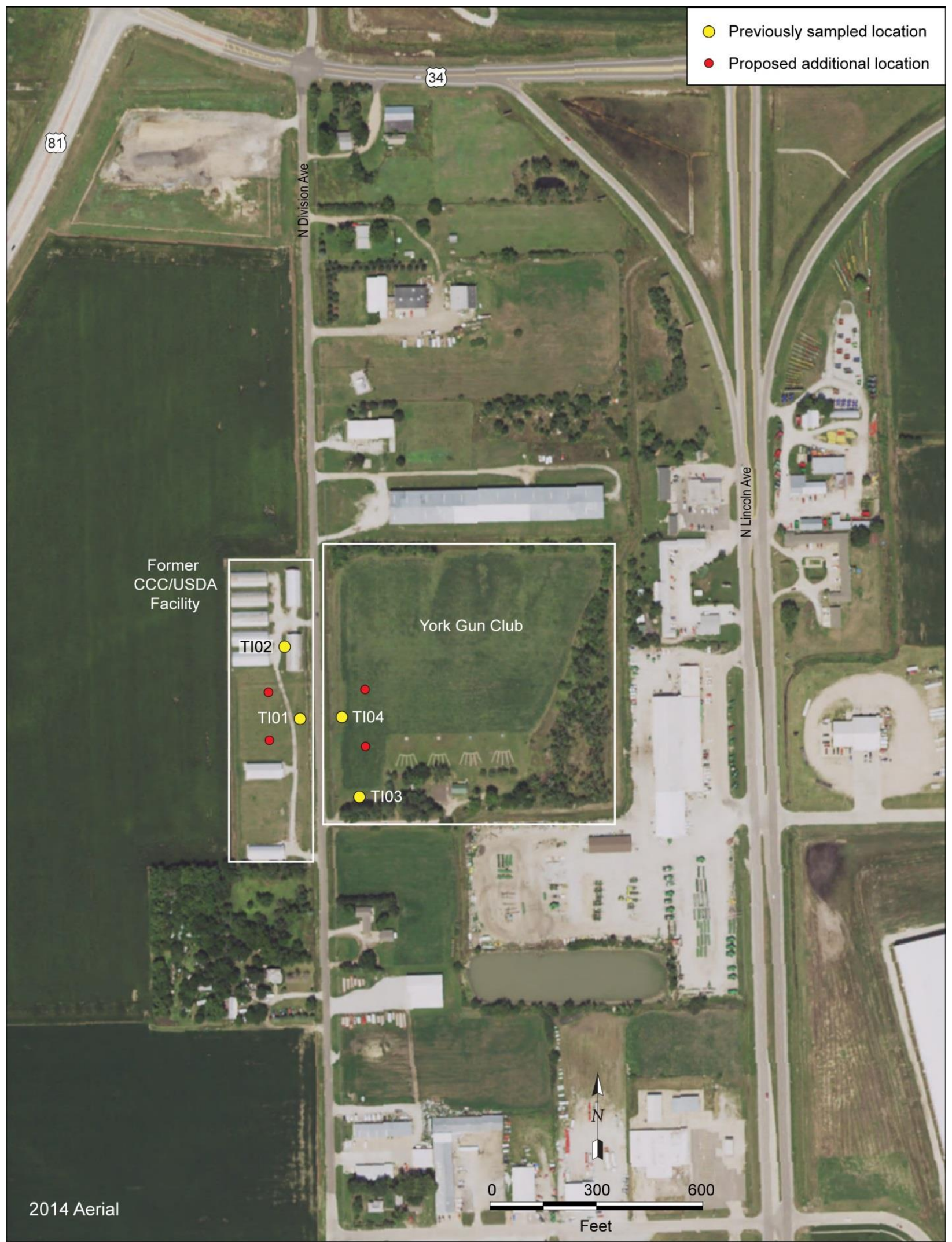

FIGURE 5.1 Additional locations tentatively proposed for vertical-profile soil sampling for VOCs analyses in the vadose zone. 


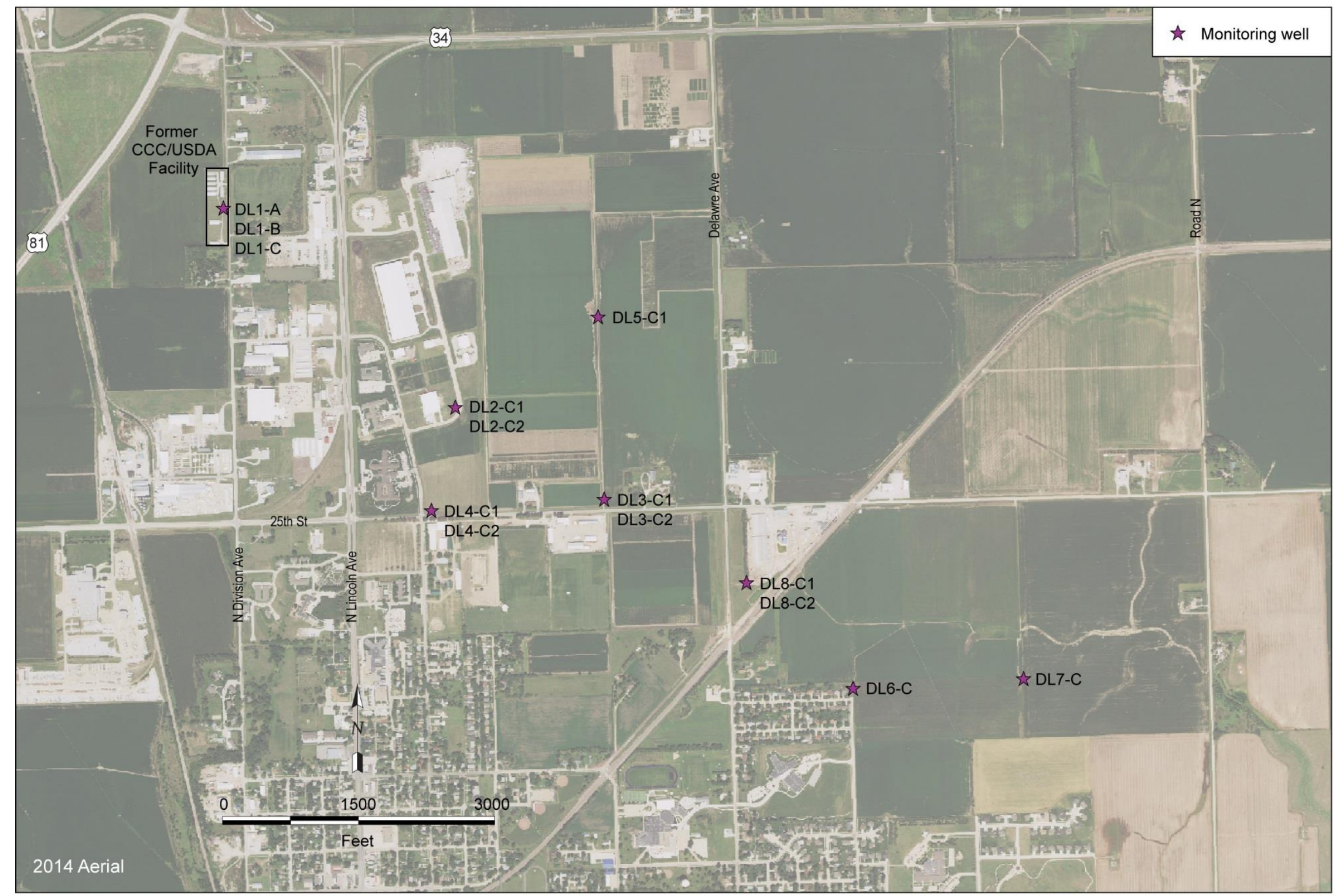

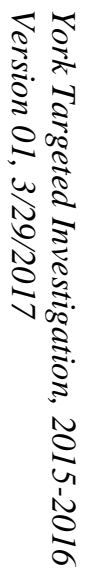

FIGURE 5.2 Locations of monitoring wells proposed for single-well hydraulic (slug) testing in the unconfined aquifer. 


\section{References}

Argonne, 1993, Final Work Plan: Phase I Extended Site Characterization, York, Nebraska, prepared for the Commodity Credit Corporation, U.S. Department of Agriculture, Washington, D.C., by Argonne National Laboratory, Argonne, Illinois, June.

Argonne, 1994, Final Phase I Report and Phase II Work Plan: Expedited Site Characterization, York, Nebraska, prepared for the Commodity Credit Corporation, U.S. Department of Agriculture, Washington, D.C., by Argonne National Laboratory, Argonne, Illinois, February.

Argonne, 1995a, Final Phase II Report: Expedited Site Characterization, York, Nebraska, prepared for the Commodity Credit Corporation, U.S. Department of Agriculture, Washington, D.C., by Argonne National Laboratory, Argonne, Illinois, April.

Argonne, 1995b, Final Feasibility Study for Remedial Action at York, Nebraska, prepared for the Commodity Credit Corporation, U.S. Department of Agriculture, Washington, D.C., by Argonne National Laboratory, Argonne, Illinois, November.

Argonne, 2002, Final Master Work Plan: Environmental Investigations at Former CCC/USDA Facilities in Nebraska, 2002 Revision, ANL/ER/TR-02/003, prepared for the Commodity Credit Corporation, U.S. Department of Agriculture, Washington, D.C., by Argonne National Laboratory, Argonne, Illinois, December.

Argonne, 2014, Argonne National Laboratory, AGEM field program, unpublished information.

Argonne, 2015a, Responses to NDEQ Comments of June 3, 2015 on Draft Work Plan: Targeted Investigation at York, Nebraska, Version 01, 04/30/15, prepared for the Commodity Credit Corporation, U.S. Department of Agriculture, Washington, D.C., by Argonne National Laboratory, Argonne, Illinois, June.

Argonne, 2015b, Recommendations for Initial Screening of Indoor Air at Selected Locations in York, Nebraska, prepared for the Commodity Credit Corporation, U.S. Department of Agriculture, Washington, D.C., by Argonne National Laboratory, Argonne, Illinois, July. 
Argonne, 2015c, Draft Recommendations for Continuation of the CCC/USDA Targeted Groundwater Investigation at York, NE, ANL/EVS/AGEM/CHRON-1922, prepared for the Commodity Credit Corporation, U.S. Department of Agriculture, Washington, D.C., by Argonne National Laboratory, Argonne, Illinois, October.

Argonne, 2015d, Results for Private Well Sampling on March 24, 2015, ANL/EVS/AGEM/CHRON-1827, prepared for the Commodity Credit Corporation, U.S. Department of Agriculture, Washington, D.C., by Argonne National Laboratory, Argonne, Illinois, March.

Argonne, 2015e, Results for Private Well Sampling on August 5-7, 2015, ANL/EVS/AGEM/CHRON-1904, prepared for the Commodity Credit Corporation, U.S. Department of Agriculture, Washington, D.C., by Argonne National Laboratory, Argonne, Illinois, August.

Argonne, 2015f, Results for Private Well Sampling on November 23, 2015, ANL/EVS/AGEM/CHRON-1927, prepared for the Commodity Credit Corporation, U.S. Department of Agriculture, Washington, D.C., by Argonne National Laboratory, Argonne, Illinois, December.

Argonne, 2016a, Final Work Plan: Targeted Investigation at York, Nebraska, ANL/EVS/TR-15-04, prepared for the Commodity Credit Corporation, U.S. Department of Agriculture, Washington, D.C., by Argonne National Laboratory, Argonne, Illinois, August.

Argonne, 2016b, Results for Private Well Sampling on February 23, 2016, ANL/EVS/AGEM/CHRON-1968, prepared for the Commodity Credit Corporation, U.S. Department of Agriculture, Washington, D.C., by Argonne National Laboratory, Argonne, Illinois, March.

Argonne, 2016c, Results for Private Well Sampling on June 21, 2016, ANL/EVS/AGEM/CHRON-2030, prepared for the Commodity Credit Corporation, U.S. Department of Agriculture, Washington, D.C., by Argonne National Laboratory, Argonne, Illinois, June. 
Argonne, 2016d, Results for Private Well Sampling on October 31, 2016, ANL/EVS/AGEM/CHRON-2054 through 2067, prepared for the Commodity Credit Corporation, U.S. Department of Agriculture, Washington, D.C., by Argonne National Laboratory, Argonne, Illinois, November 7.

Argonne, 2016e, Results for Private Well Sampling on December 5, 2016, ANL/EVS/AGEM/CHRON-2088, prepared for the Commodity Credit Corporation, U.S. Department of Agriculture, Washington, D.C., by Argonne National Laboratory, Argonne, Illinois, December.

Clark, B.R., M.K. Landon, L.J. Kauffman, and G.Z. Hornberger, 2008, Simulations of GroundWater Flow, Transport, Age, and Particle Tracking near York, Nebraska, for a Study of Transport of Anthropogenic and Natural Contaminants (TANC) to Public-Supply Wells, Scientific Investigations Report 2007-5068, U.S. Geological Survey, Reston, Virginia (http://pubs.usgs.gov/sir/2007/5068/).

EPA, 1995, Method 524.2: Measurement of Purgeable Organic Compounds in Water by Capillary Column Gas Chromatography/Mass Spectrometry, Revision 4.1, edited by J.W. Munch, National Exposure Research Laboratory, Office of Research and Development, U.S. Environmental Protection Agency, Cincinnati, Ohio.

EPA, 1996, Expanded Site Inspection for the York Public Water Supply Site (Volumes I and II), October.

EPA, 1997, "Definitions and Procedures for the Determination of the Method Detection Limit," pp. 317-319, Appendix B, Part 136, in Guidelines Establishing Test Procedures for the Analysis of Pollutants, U.S. Code of Federal Regulations, Title 40, Revised July 1 (http://www.epa.gov/region9/qa/pdfs/40cfr136_03.pdf).

EPA, 1998, Test Methods for Evaluating Solid Waste: Physical/Chemical Methods, EPA SW 846, 3rd edition, Draft Update IVA, U.S. Environmental Protection Agency, Washington, D.C., January.

EPA, 2008, National Functional Guidelines for Superfund Organic Methods Data Review, OSWER 9240.1-48, USEPA-540-R-08-01, Contract Laboratory Program, Office of Superfund 
Remediation and Technology Innovation, U.S. Environmental Protection Agency, Washington, D.C., June (http://www.epa.gov/superfund/programs/clp/download/somnfg.pdf).

EPA, 2013a, HRS Documentation Record, PCE/TCE Northeast Contamination, U.S. Environmental Protection Agency, Region 7, Lenexa, Kansas, December (http://www.epa.gov/superfund/sites/docrec/pdoc1887.pdf).

EPA, 2013b, HRS Documentation Record, PCE Southeast Contamination, U.S. Environmental Protection Agency, Region 7, Lenexa, Kansas, December (http://www.epa.gov/superfund/sites/docrec/pdoc1886.pdf).

EPA, 2014a, National Priorities List: PCE Southeast Contamination, York, Nebraska, narrative summary, U.S. Environmental Protection Agency, Washington, D.C., May (http://www.epa.gov/superfund/sites/narr/nar1886.pdf).

EPA, 2014b, National Priorities List: PCE/TCE Northeast Contamination, York, Nebraska, narrative summary, U.S. Environmental Protection Agency, Washington, D.C., May (http://www.epa.gov/superfund/sites/narr/nar1887.pdf).

Keech, C.F., V.H. Dreezen, P.A. Emery, 1967, Availability of Ground Water in York County, Nebraska, U.S. Geological Survey Water-Supply Paper 1839-F, U.S. Government Printing Office, Washington, D.C. (http://pubs.usgs.gov/wsp/1839f/report.pdf).

Landon, M.K., and M.J. Turco, 2007, "Hydrogeologic Setting and Ground-Water Flow Simulations of the Eastern High Plains Regional Study Area, Nebraska," Section 8 of Hydrogeologic Settings and Ground-Water Flow Simulations for Regional Studies of the Transport of Anthropogenic and Natural Contaminants to Public-Supply Sells - Studies Begun in 2001, edited by S.S. Paschke, Professional Paper 1737-A, U.S. Geological Survey, Reston, Virginia (http://pubs.usgs.gov/pp/2007/1737a/Section8.pdf).

Landon, M.K., B.R. Clark, P.B. McMahon, V.L. McGuire, and M.J. Turco, 2008, Hydrogeology, Chemical Characteristics, and Transport Processes in the Zone of Contribution of a PublicSupply Well in York, Nebraska, Scientific Investigations Report 2008-5050, U.S. Geological Survey, Reston, Virginia (http://pubs.usgs.gov/sir/2008/5050/). 
NDEC, 1991, Site Investigation: York Ground Water Contamination Site, York, Nebraska, Report prepared for U.S. Environmental Protection Agency, Region VII, by Nebraska Department of Environmental Control, September 10.

NDEQ, 2010, Final Site Inspection, Revision 01, York Northeast Groundwater Site, York, Nebraska, May 17.

NDEQ, 2011, Final Expanded Site Inspection Report, Revision 01, January.

NDEQ, 2012, Nebraska Voluntary Cleanup Program Guidance, September.

NDEQ, 2014, letter from K. Boone (Remediation Section, Waste Management Division, Nebraska Department of Environmental Quality, Lincoln, Nebraska) to S. Gilmore (Commodity Credit Corporation, U.S. Department of Agriculture, Washington, D.C.), regarding the former CCC/USDA grain bin facility at York, January 17.

NDEQ, 2015a, email message from L. Brunner (Remediation Section, Waste Management Division, Nebraska Department of Environmental Quality, Lincoln, Nebraska) to S. Gilmore (Commodity Credit Corporation, U.S. Department of Agriculture, Washington, D.C.) requesting monitoring of indoor air and sub-slab vapor at the FitnessWorx facility and Garretson/Troester residence over four consecutive quarters, December 22.

NDEQ, 2015b, email message from L. Brunner (Remediation Section, Waste Management Division, Nebraska Department of Environmental Quality, Lincoln, Nebraska) to S. Gilmore (Commodity Credit Corporation, U.S. Department of Agriculture, Washington, D.C.) discussing site-specific, risk-based target indoor air and subslab carbon tetrachloride concentrations for the FitnessWorx facility, October 21.

NDEQ, 2016a, email message from L. Brunner (Remediation Section, Waste Management Division, Nebraska Department of Environmental Quality, Lincoln, Nebraska) to S. Gilmore (Commodity Credit Corporation, U.S. Department of Agriculture, Washington, D.C.) providing approval of revised locations proposed by the CCC/USDA for the installation of permanent groundwater monitoring wells in the unconfined and confined aquifers at York, April 26. 
NDEQ, 2016b, email message from L. Brunner (Remediation Section, Waste Management Division, Nebraska Department of Environmental Quality, Lincoln, Nebraska) to S. Gilmore (Commodity Credit Corporation, U.S. Department of Agriculture, Washington, D.C.) discussing sampling of the Driewer house well, July 11.

Puls, R.W., and Barcelona, M.J., 1996, "Low-Flow (Minimal Drawdown) Ground-Water Sampling Procedures," EPA/540/S-95/504, in Ground Water Issue, Superfund Technology Support Center for Ground Water, National Risk Management Research Laboratory, Ada, Oklahoma, April (www.epa.gov/tio/tsp/download/lwflw2a.pdf).

SETI, 2011, Removal Site Evaluation/Site Inspection Report for Southeast York Groundwater Site, York, York County, Nebraska, Seagull Environmental Technologies, Inc., Wheat Ridge, Colorado, February 16.

Tetra Tech, 2012a, Integrated Site Assessment York Tetrachloroethene (PCE) Site, York, Nebraska, prepared for the U.S.EPA, Region 7, February 14.

Tetra Tech, 2012b, Removal Site Evaluation and Site Inspection Southeast York Groundwater Site York County, Nebraska, prepared for the U.S.EPA, Region 7, February 16.

Tetra Tech, 2012c, Trip Report and Data Summary, Removal Action Support, York Tetrachloroethene (PCE) Site, York, Nebraska, prepared for the U.S.EPA, Region 7, December 4.

USDA, 2016, email message from S. Gilmore (Commodity Credit Corporation, U.S. Department of Agriculture, Washington, D.C.) to L. Brunner (Remediation Section, Waste Management Division, Nebraska Department of Environmental Quality, Lincoln, Nebraska) outlining revised locations proposed for the installation of permanent groundwater monitoring wells in the unconfined and confined aquifers at York, April 22.

Yeskis, D., and B. Zavala, 2002, Ground-Water Sampling Guidelines for Superfund and RCRA Project Managers: Ground Water Forum Issue Paper, EPA 542-S-02-001, Technology Innovative Office, Office of Solid Waste and Emergency Response, U.S. Environmental Protection Agency, Washington, May (http://www.epa.gov/tio/tsp/download/gw_sampling_guide.pdf). 
Appendix A:

Chronological Summary of Sampling Activities 
TABLE A.1 Samples collected during the 2015-2016 targeted investigation.

\begin{tabular}{|c|c|c|c|c|c|c|c|c|c|}
\hline \multicolumn{2}{|c|}{$\begin{array}{l}\text { Sample Date } \\
\text { and Time }\end{array}$} & Location & Sample & $\begin{array}{l}\text { Sample } \\
\text { Type }\end{array}$ & $\begin{array}{l}\text { Depth } \\
\text { (ft BGL) }\end{array}$ & Matrix & Chain of Custody & $\begin{array}{l}\text { Shipping } \\
\text { Date }\end{array}$ & Sample Description \\
\hline 7/7/15 & $12: 39$ & TI01 & YKTI01-S-37744 & $\mathrm{N}$ & 4 & SOIL & 7234 & $7 / 7 / 15$ & \\
\hline 7/7/15 & $12: 42$ & TI01 & YKTI01-S-37745 & $\mathrm{N}$ & 8 & SOIL & 7234 & $7 / 7 / 15$ & \\
\hline 7/7/15 & $12: 46$ & TI01 & YKTI01-S-37746 & $\mathrm{N}$ & 12 & SOIL & 7234 & $7 / 7 / 15$ & \\
\hline 7/7/15 & $12: 49$ & TI01 & YKTI01-S-37747 & $\mathrm{N}$ & 16 & SOIL & 7234 & $7 / 7 / 15$ & \\
\hline 7/7/15 & $12: 53$ & TI01 & YKTI01-S-37748 & $\mathrm{N}$ & 20 & SOIL & 7234 & $7 / 7 / 15$ & \\
\hline 7/7/15 & $12: 58$ & TI01 & YKTI01-S-37749 & $\mathrm{N}$ & 24 & SOIL & 7234 & $7 / 7 / 15$ & \\
\hline 7/7/15 & $12: 58$ & TI01 & YKTI01-S-37749DUP & DUP-L & 24 & SOIL & 7234 & $7 / 7 / 15$ & Duplicate laboratory analysis. \\
\hline $7 / 7 / 15$ & $13: 02$ & TI01 & YKTI01-S-37750 & $\mathrm{N}$ & 28 & SOIL & 7234 & $7 / 7 / 15$ & \\
\hline 7/7/15 & $13: 08$ & TI01 & YKTI01-S-37751 & $\mathrm{N}$ & 32 & SOIL & 7234 & $7 / 7 / 15$ & \\
\hline 7/7/15 & $13: 08$ & TI01 & YKTI01-S-37752 & DUP-F & 32 & SOIL & 7234 & $7 / 7 / 15$ & Field replicate. \\
\hline 7/7/15 & $13: 15$ & TI01 & YKTI01-S-37753 & $\mathrm{N}$ & 36 & SOIL & 7234 & $7 / 7 / 15$ & \\
\hline $7 / 7 / 15$ & $13: 22$ & TI01 & YKTI01-S-37754 & $\mathrm{N}$ & 40 & SOIL & 7234 & $7 / 7 / 15$ & \\
\hline 7/7/15 & $13: 29$ & TI01 & YKTI01-S-37755 & $\mathrm{N}$ & 44 & SOIL & 7234 & $7 / 7 / 15$ & \\
\hline 7/7/15 & $13: 45$ & TI01 & YKTI01-S-37756 & $\mathrm{N}$ & 48 & SOIL & 7234 & $7 / 7 / 15$ & \\
\hline $7 / 7 / 15$ & $14: 38$ & QC & YKQCTB-S-37763 & TB & - & SOIL & 7234 & $7 / 7 / 15$ & $\begin{array}{l}\text { Trip blank with soil samples } \\
\text { shipped to the AGEM laboratory. }\end{array}$ \\
\hline $7 / 7 / 15$ & $15: 02$ & TI02 & YKTI02-S-37764VER & VER & 4 & SOIL & 4916 & $7 / 16 / 15$ & $\begin{array}{l}\text { Verification sample to TestAmerica, } \\
\text { Inc. }\end{array}$ \\
\hline 7/7/15 & $15: 02$ & TI02 & YKTI02-S-37764 & $\mathrm{N}$ & 4 & SOIL & 7235 & $7 / 7 / 15$ & \\
\hline $7 / 7 / 15$ & $15: 13$ & QC & YKQCTB-S-37777 & TB & - & SOIL & 7235 & $7 / 7 / 15$ & $\begin{array}{l}\text { Trip blank with soil samples } \\
\text { shipped to the AGEM laboratory. }\end{array}$ \\
\hline 7/7/15 & $15: 18$ & TI02 & YKTI02-S-37765 & $\mathrm{N}$ & 7.8 & SOIL & 7235 & $7 / 7 / 15$ & \\
\hline $7 / 7 / 15$ & $15: 21$ & TI02 & YKTI02-S-37766 & $\mathrm{N}$ & 12 & SOIL & 7235 & $7 / 7 / 15$ & \\
\hline $7 / 7 / 15$ & $15: 24$ & TI02 & YKTI02-S-37767VER & VER & 16 & SOIL & 4916 & $7 / 16 / 15$ & $\begin{array}{l}\text { Verification sample to TestAmerica, } \\
\text { Inc. }\end{array}$ \\
\hline 7/7/15 & $15: 24$ & TI02 & YKTI02-S-37767 & $\mathrm{N}$ & 16 & SOIL & 7235 & $7 / 7 / 15$ & \\
\hline $7 / 7 / 15$ & $15: 28$ & TI02 & YKTI02-S-37768VER & VER & 20 & SOIL & 4916 & $7 / 16 / 15$ & $\begin{array}{l}\text { Verification sample to TestAmerica, } \\
\text { Inc. }\end{array}$ \\
\hline $7 / 7 / 15$ & $15: 28$ & TI02 & YKTI02-S-37768 & $\mathrm{N}$ & 20 & SOIL & 7235 & $7 / 7 / 15$ & \\
\hline $7 / 7 / 15$ & $15: 28$ & TI02 & YKTI02-S-37776 & DUP-F & 20 & SOIL & 7235 & $7 / 7 / 15$ & Field replicate. \\
\hline 7/7/15 & $15: 32$ & TI02 & YKTI02-S-37769 & $\mathrm{N}$ & 24 & SOIL & 7235 & $7 / 7 / 15$ & \\
\hline $7 / 7 / 15$ & $15: 36$ & TI02 & YKTI02-S-37770 & $\mathrm{N}$ & 28 & SOIL & 7235 & $7 / 7 / 15$ & \\
\hline 7/7/15 & $15: 43$ & TI02 & YKTI02-S-37771 & $\mathrm{N}$ & 32 & SOIL & 7235 & $7 / 7 / 15$ & \\
\hline 7/7/15 & $15: 53$ & TI02 & YKTI02-S-37772 & $\mathrm{N}$ & 36 & SOIL & 7235 & $7 / 7 / 15$ & \\
\hline 7/7/15 & $15: 53$ & TI02 & YKTI02-S-37772DUP & DUP-L & 36 & SOIL & 7235 & $7 / 7 / 15$ & Duplicate laboratory analysis. \\
\hline 7/7/15 & $15: 59$ & TI02 & YKTI02-S-37773 & $\mathrm{N}$ & 40 & SOIL & 7235 & $7 / 7 / 15$ & \\
\hline $7 / 7 / 15$ & $16: 05$ & TI02 & YKTI02-S-37774 & $\mathrm{N}$ & 44 & SOIL & 7235 & $7 / 7 / 15$ & \\
\hline 7/7/15 & $16: 12$ & TI02 & YKTI02-S-37775 & $\mathrm{N}$ & 48 & SOIL & 7235 & $7 / 7 / 15$ & \\
\hline 7/7/15 & $16: 20$ & TI02 & YKTI02-S-37778 & $\mathrm{N}$ & 52 & SOIL & 7235 & $7 / 7 / 15$ & \\
\hline 7/7/15 & $16: 32$ & TI02 & YKTI02-S-37779 & $\mathrm{N}$ & 55.8 & SOIL & 7236 & $7 / 7 / 15$ & \\
\hline 7/7/15 & $16: 46$ & TI02 & YKTI02-S-37780 & $\mathrm{N}$ & 59.8 & SOIL & 7236 & $7 / 7 / 15$ & \\
\hline $7 / 7 / 15$ & $17: 05$ & TI02 & YKTI02-S-37781 & $\mathrm{N}$ & 64 & SOIL & 7237 & $7 / 8 / 15$ & \\
\hline
\end{tabular}


TABLE A.1 (Cont.)

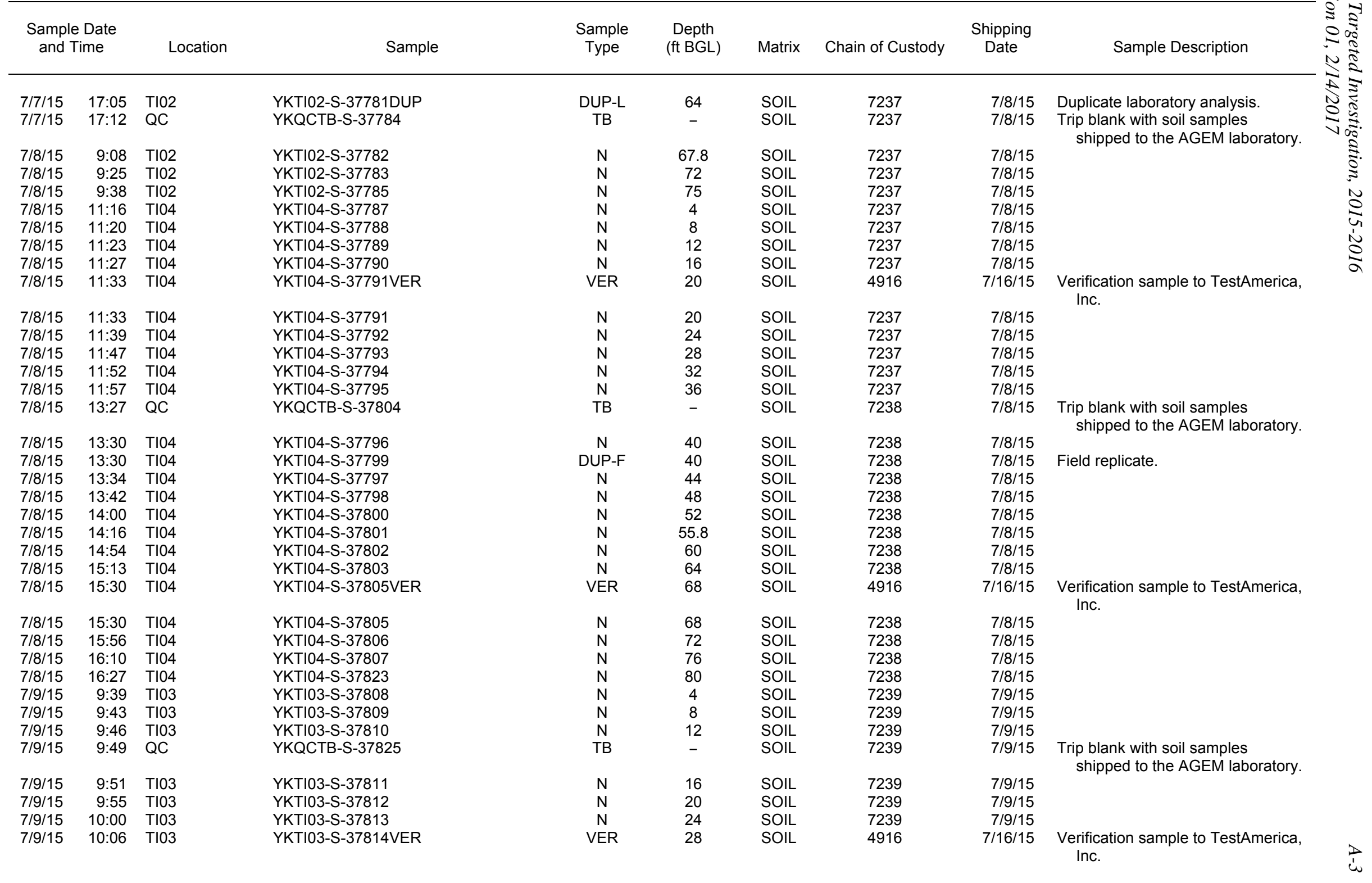


TABLE A.1 (Cont.)

\begin{tabular}{|c|c|c|c|c|c|c|c|c|c|}
\hline \multicolumn{2}{|c|}{$\begin{array}{l}\text { Sample Date } \\
\text { and Time }\end{array}$} & Location & Sample & $\begin{array}{l}\text { Sample } \\
\text { Type }\end{array}$ & $\begin{array}{l}\text { Depth } \\
\text { (ft BGL) }\end{array}$ & Matrix & Chain of Custody & $\begin{array}{l}\text { Shipping } \\
\text { Date }\end{array}$ & Sample Description \\
\hline $7 / 9 / 15$ & $10: 06$ & TI03 & YKTI03-S-37814 & $\mathrm{N}$ & 28 & SOIL & 7239 & $7 / 9 / 15$ & \\
\hline $7 / 9 / 15$ & $10: 12$ & TI03 & YKTI03-S-37815 & $\mathrm{N}$ & 32 & SOIL & 7239 & $7 / 9 / 15$ & \\
\hline $7 / 9 / 15$ & $10: 19$ & TI03 & YKTI03-S-37816 & $\mathrm{N}$ & 36 & SOIL & 7239 & $7 / 9 / 15$ & \\
\hline $7 / 9 / 15$ & $10: 27$ & TI03 & YKTI03-S-37817 & $\mathrm{N}$ & 40 & SOIL & 7239 & $7 / 9 / 15$ & \\
\hline $7 / 9 / 15$ & $10: 35$ & TI03 & YKTI03-S-37818 & $\mathrm{N}$ & 44 & SOIL & 7239 & $7 / 9 / 15$ & \\
\hline $7 / 9 / 15$ & $10: 44$ & TI03 & YKTI03-S-37819 & $\mathrm{N}$ & 48 & SOIL & 7239 & $7 / 9 / 15$ & \\
\hline $7 / 9 / 15$ & $10: 44$ & TI03 & YKTI03-S-37824 & DUP-F & 48 & SOIL & 7239 & $7 / 9 / 15$ & Field replicate. \\
\hline $7 / 9 / 15$ & $10: 54$ & TI03 & YKTI03-S-37820 & $\mathrm{N}$ & 52 & SOIL & 7239 & $7 / 9 / 15$ & \\
\hline $7 / 9 / 15$ & $11: 29$ & TI03 & YKTI03-S-37821 & $\mathrm{N}$ & 55.5 & SOIL & 7240 & $7 / 9 / 15$ & \\
\hline $7 / 9 / 15$ & $11: 48$ & TI03 & YKTI03-S-37822 & $\mathrm{N}$ & 59.4 & SOIL & 7240 & 7/9/15 & \\
\hline $7 / 9 / 15$ & $12: 02$ & TI03 & YKTI03-S-37826VER & VER & 63.5 & SOIL & 4916 & $7 / 16 / 15$ & $\begin{array}{l}\text { Verification sample to TestAmerica, } \\
\text { Inc. }\end{array}$ \\
\hline $7 / 9 / 15$ & $12: 02$ & TI03 & YKTI03-S-37826 & $\mathrm{N}$ & 63.5 & SOIL & 7240 & $7 / 9 / 15$ & \\
\hline $7 / 9 / 15$ & $12: 02$ & TI03 & YKTI03-S-37826DUP & DUP-L & 63.5 & SOIL & 7240 & $7 / 9 / 15$ & Duplicate laboratory analysis. \\
\hline $7 / 9 / 15$ & $12: 15$ & TIO3 & YKTI03-S-37827 & $\mathrm{N}$ & 67.5 & SOIL & 7240 & $7 / 9 / 15$ & \\
\hline $7 / 9 / 15$ & $12: 30$ & TI03 & YKTI03-S-37828 & $\mathrm{N}$ & 72 & SOIL & 7240 & $7 / 9 / 15$ & \\
\hline $7 / 9 / 15$ & $13: 00$ & QC & YKQCTB-W-37835 & TB & - & WQC & 7241 & $7 / 9 / 15$ & $\begin{array}{l}\text { Trip blank with water samples } \\
\text { shipped to the AGEM laboratory. }\end{array}$ \\
\hline $7 / 9 / 15$ & $14: 37$ & QC & YKQCTB-S-37832 & TB & - & SOIL & 7240 & $7 / 9 / 15$ & $\begin{array}{l}\text { Trip blank with soil samples } \\
\text { shipped to the AGEM laboratory. }\end{array}$ \\
\hline $7 / 9 / 15$ & $14: 45$ & TI03 & YKTI03-S-37829 & $\mathrm{N}$ & 75 & SOIL & 7240 & $7 / 9 / 15$ & \\
\hline $7 / 9 / 15$ & $15: 54$ & TI04 & YKTI04-W-37833 & $\mathrm{N}$ & $74.6-79.6$ & WG & 7241 & $7 / 9 / 15$ & $\begin{array}{l}\text { Depth to water }=73.9 \mathrm{ft} \mathrm{BGL} \text {. } \\
\text { Depth of } 0.5 \text { in. well }=79.6 \mathrm{ft} \\
\text { BGL. Soil sampled with crawler } \\
\text { to } 82 \mathrm{ft} \mathrm{BGL} \mathrm{on} 7 / 8 / 15 \text {. Installed } \\
0.5 \text { in. screen and PVC riser } \\
\text { using sand pack+bentonite at } \\
\text { end of day. Water came in } \\
\text { overnight. Sampled using bailer } \\
\text { without purge. }\end{array}$ \\
\hline $7 / 9 / 15$ & $15: 54$ & TI04 & YKTI04-W-37834 & DUP-F & $74.6-79.6$ & WG & 7241 & $7 / 9 / 15$ & Field replicate. \\
\hline $7 / 9 / 15$ & $16: 30$ & TI03 & YKTI03-W-37836 & $\mathrm{N}$ & $76.8-81.8$ & WG & 7241 & $7 / 9 / 15$ & $\begin{array}{l}\text { Depth to water }=74.0 \mathrm{ft} \mathrm{BGL} \text {. } \\
\text { Depth of } 0.5 \text { in. well }=81.8 \mathrm{ft} \\
\text { BGL. Pushed to } 82 \mathrm{ft} \mathrm{BGL} \mathrm{with} \\
\text { crawler for soil sampling on } \\
7 / 9 / 15 . \text { Installed } 0.5 \text { in. Screen } \\
\text { and riser in push hole. Water } \\
\text { came in almost immediately. } \\
\text { Sampled using bailer without } \\
\text { purge. }\end{array}$ \\
\hline 7/9/15 & $16: 30$ & TI03 & YKTI03-W-37837 & DUP-F & $76.8-81.8$ & WG & 7241 & $7 / 9 / 15$ & Field replicate. \\
\hline
\end{tabular}


TABLE A.1 (Cont.)

\begin{tabular}{|c|c|c|c|c|c|c|c|c|}
\hline $\begin{array}{c}\text { Sample Date } \\
\text { and Time }\end{array}$ & Location & Sample & $\begin{array}{c}\text { Sample } \\
\text { Type }\end{array}$ & $\begin{array}{l}\text { Depth } \\
\text { (ft BGL) }\end{array}$ & Matrix & Chain of Custody & $\begin{array}{c}\text { Shipping } \\
\text { Date }\end{array}$ & Sample Description \\
\hline
\end{tabular}

\begin{tabular}{rrll}
\hline $7 / 9 / 15$ & $16: 41$ & QC & YKQCBR-W-37839 \\
& & & \\
$7 / 10 / 15$ & $9: 14$ & TI01 & YKTI01-S-37757 \\
$7 / 10 / 15$ & $9: 14$ & TI01 & YKTI01-S-37757DUP \\
$7 / 10 / 15$ & $9: 22$ & QC & YKQCTB-S-37840 \\
& & & \\
$7 / 10 / 15$ & $9: 24$ & TI01 & YKTI01-S-37758VER \\
& & & \\
$7 / 10 / 15$ & $9: 24$ & TI01 & YKTI01-S-37758 \\
$7 / 10 / 15$ & $9: 35$ & TI01 & YKTI01-S-37759 \\
$7 / 10 / 15$ & $9: 47$ & TI01 & YKTIO1-S-37760 \\
$7 / 10 / 15$ & $10: 05$ & TI01 & YKTI01-S-37761 \\
$7 / 10 / 15$ & $10: 05$ & TI01 & YKTI01-S-37761DUP \\
$7 / 10 / 15$ & $10: 17$ & TI01 & YKTI01-S-37762 \\
$7 / 10 / 15$ & $10: 31$ & TI01 & YKTI01-S-37841 \\
$7 / 10 / 15$ & $10: 44$ & TI01 & YKTIO1-S-37842 \\
$7 / 10 / 15$ & $13: 10$ & TI01 & YKTI01-W-37844
\end{tabular}

$\begin{array}{llll}7 / 10 / 15 & 13: 10 & \text { TI01 } & \text { YKTI01-W-37845 } \\ 7 / 10 / 15 & 13: 30 & \text { QC } & \text { YKQCTB-W-37846 } \\ & & & \\ 7 / 10 / 15 & 14: 44 & \text { TI02 } & \text { YKTI02-W-37847 }\end{array}$

$\begin{array}{cccc}\text { RI } & - & \text { WQC } & 7241 \\ & & & \\ \text { N } & 52 & \text { SOIL } & 7242 \\ \text { DUP-L } & 52 & \text { SOIL } & 7242 \\ \text { TB } & - & \text { SOIL } & 7242 \\ \text { VER } & 56 & \text { SOIL } & 4916 \\ \text { N } & 56 & \text { SOIL } & 7242 \\ N & 60 & \text { SOIL } & 7242 \\ N & 63.8 & \text { SOIL } & 7242 \\ \text { N } & 68 & \text { SOIL } & 7242 \\ \text { DUP-L } & 68 & \text { SOIL } & 7242 \\ N & 72 & \text { SOIL } & 7242 \\ N & 75 & \text { SOIL } & 7242 \\ N & 80 & \text { SOIL } & 7242 \\ \text { N } & 77.4-82.4 & \text { WG } & 7243\end{array}$

7/9/15

7/10/15

$7 / 10 / 15$
$7 / 10 / 15$

$7 / 10 / 15$

$7 / 16 / 15$

$7 / 10 / 15$

$7 / 10 / 15$

$7 / 10 / 15$

$7 / 10 / 15$

$7 / 10 / 15$

$7 / 10 / 15$

$7 / 10 / 15$

$7 / 10 / 15$

$7 / 10 / 15$

$7 / 10 / 15$

5 D

Depth to water $=74.3 \mathrm{ft} \mathrm{BGL}$.

Depth of 0.5 in. well $=82.4 \mathrm{ft}$

BGL. Moved back to TI01

$7 / 10 / 15$ to complete soiL.

Pushed to $82 \mathrm{ft}$ and installed

temporaryorary $0.5 \mathrm{in}$. by $5 \mathrm{ft}$

screen and riser with sand and

bentonite. Water came in

immediately. Sampled using

bailer without purge.

$\begin{array}{cccc}\text { DUP-F } & 77.4-82.4 & \text { WG } & 7243 \\ \text { TB } & - & \text { WQC } & 7243 \\ & & & \end{array}$

$7 / 10 / 15$

Field replicate.

Trip blank with water samples

$\begin{array}{llll}N & 77-82 & \text { WG } & 7243\end{array}$

$7 / 10 / 15$

shipped to the AGEM laboratory.

Depth to water $=74 \mathrm{ft} \mathrm{BGL}$. Depth

of $0.5 \mathrm{in}$. well $=82 \mathrm{ft} \mathrm{BGL}$.

Moved crawler back to TI02

$7 / 10 / 15$. Pushed with solid tip to

$82 \mathrm{ft}$ and installed $0.5 \mathrm{in}$. by $5 \mathrm{ft}$

screen and riser with sand and

bentonite. Water came in

immediately. Sampled using

bailer without purge.

$\begin{array}{rrll}\text { 7/10/15 } & 14: 44 & \text { TIO2 } & \text { YKTI02-W-37848 } \\ 7 / 10 / 15 & 14: 44 & \text { TIO2 } & \text { YKTIO2-W-37848DUP } \\ 7 / 13 / 15 & 9: 18 & \text { QC } & \text { YKQCTB-W-37851 }\end{array}$

$\begin{array}{cccc}\text { DUP-F } & 77-82 & \text { WG } & 7243 \\ \text { DUP-L } & 77-82 & \text { WG } & 7243 \\ \text { TB } & - & \text { WQC } & 7244\end{array}$

7/10/15 Duplicate laboratory analysis.
7/10/15 Field replicate.

7/15/15 Trip blank with water samples

shipped to the AGEM laboratory. 
TABLE A.1 (Cont.)

\begin{tabular}{|c|c|c|c|c|c|c|c|c|c|}
\hline \multicolumn{2}{|c|}{$\begin{array}{l}\text { Sample Date } \\
\text { and Time }\end{array}$} & \multirow{2}{*}{$\begin{array}{r}\text { Location } \\
\text { TI02 }\end{array}$} & \multirow{2}{*}{$\begin{array}{r}\text { Sample } \\
\text { YKTI02100-W-37849 }\end{array}$} & \multirow{2}{*}{$\begin{array}{c}\text { Sample } \\
\text { Type }\end{array}$} & \multirow{2}{*}{$\begin{array}{c}\begin{array}{c}\text { Depth } \\
\text { (ft BGL) }\end{array} \\
100-103.5\end{array}$} & \multirow{2}{*}{$\begin{array}{c}\text { Matrix } \\
\text { WG }\end{array}$} & \multirow{2}{*}{$\begin{array}{c}\text { Chain of Custody } \\
7244\end{array}$} & \multirow{2}{*}{$\begin{array}{l}\begin{array}{c}\text { Shipping } \\
\text { Date }\end{array} \\
7 / 15 / 15\end{array}$} & \multirow{2}{*}{ 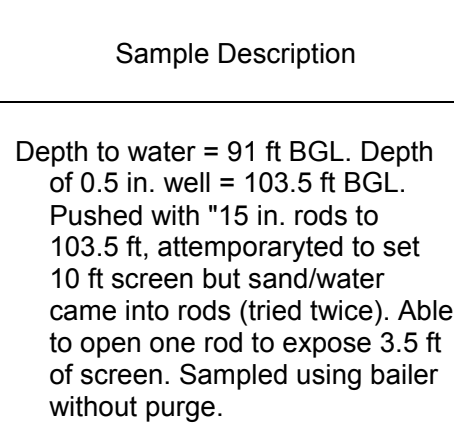 } \\
\hline $7 / 13 / 15$ & $20: 00$ & & & & & & & & \\
\hline $7 / 13 / 15$ & $20: 00$ & TI02 & YKTI02100-W-37850 & DUP-F & $100-103.5$ & WG & 7244 & $7 / 15 / 15$ & Field replicate. \\
\hline $7 / 14 / 15$ & $15: 00$ & TI02 & YKTI02120-W-37852 & $\mathrm{N}$ & $\begin{array}{l}108.5- \\
118.5\end{array}$ & WG & 7244 & $7 / 15 / 15$ & $\begin{array}{l}\text { Depth to water }=99.5 \mathrm{ft} \mathrm{BGL} \text {. } \\
\text { Depth of } 0.5 \text { in. well }=118.5 \mathrm{ft} \\
\text { BGL. Pushed for electronics to } \\
131 \mathrm{ft} \text {. Pushed back in with } 15 \\
\text { rods to target depth and pulled } \\
\text { back rods to expose } 0.5 \text { in. by } \\
10 \mathrm{ft} \text { temporary screen and riser. } \\
\text { Sampled using bailer without } \\
\text { purge. }\end{array}$ \\
\hline $7 / 14 / 15$ & $15: 00$ & TI02 & YKTI02120-W-37852VER & VER & $\begin{array}{l}108.5- \\
118.5\end{array}$ & WG & 7245 & $7 / 15 / 15$ & $\begin{array}{l}\text { Verification sample to TestAmerica, } \\
\text { Inc. }\end{array}$ \\
\hline $7 / 14 / 15$ & $15: 30$ & QC & YKQCTB-W-37853 & TB & - & WQC & 7245 & $7 / 15 / 15$ & $\begin{array}{l}\text { Trip blank with water samples } \\
\text { shipped to TestAmerica } \\
\text { laboratory. }\end{array}$ \\
\hline $7 / 15 / 15$ & $16: 20$ & QC & YKQCIR-W-37857 & $\mathrm{RI}$ & - & WQC & 7244 & $7 / 15 / 15$ & $\begin{array}{l}\text { Rinsate of decontaminated bailer } \\
\text { after sample YKTI01120-W- } \\
37855 \text {. }\end{array}$ \\
\hline $7 / 15 / 15$ & $16: 36$ & TI01 & YKTI01120-W-37855 & $\mathrm{N}$ & $108-118$ & WG & 7244 & $7 / 15 / 15$ & $\begin{array}{l}\text { Depth to water }=98 \mathrm{ft} \text { BGL. Depth } \\
\text { of well }=118 \mathrm{ft} \text { BGL. Pushed to } \\
\text { approximately } 131 \mathrm{ft} \text { for } \\
\text { electronics with } 10 \text { rods. Go } \\
\text { back down with } 15 \text { rods, set } \\
\text { temporary } 0.5 \text { in. by } 10 \mathrm{ft} \text { screen } \\
\text { in riser through rods and pull } \\
\text { back to expose. Sampled using } \\
\text { bailer without purge. }\end{array}$ \\
\hline 7/15/15 & $16: 36$ & TI01 & YKTI01120-W-37855DUP & DUP-L & $108-118$ & WG & 7244 & 7/15/15 & Duplicate laboratory analysis. \\
\hline $7 / 15 / 15$ & $16: 36$ & TI01 & YKTI01120-W-37855VER & VER & $108-118$ & WG & 7245 & $7 / 15 / 15$ & $\begin{array}{l}\text { Verification sample to TestAmerica, } \\
\text { Inc. }\end{array}$ \\
\hline
\end{tabular}


TABLE A.1 (Cont.)

\begin{tabular}{|c|c|c|c|c|c|c|c|c|c|}
\hline $\begin{array}{r}\text { Sample } \\
\text { and } \mathrm{Ti}\end{array}$ & $\begin{array}{l}\text { Date } \\
\text { ime }\end{array}$ & Location & Sample & $\begin{array}{l}\text { Sample } \\
\text { Type }\end{array}$ & $\begin{array}{l}\text { Depth } \\
\text { (ft BGL) }\end{array}$ & Matrix & Chain of Custody & $\begin{array}{l}\text { Shipping } \\
\text { Date }\end{array}$ & Sample Description \\
\hline $7 / 16 / 15$ & $9: 15$ & TI01 & YKTI01100-W-37854 & $\mathrm{N}$ & $88-98$ & WG & 7246 & $7 / 16 / 15$ & $\begin{array}{l}\text { Depth to water }=82 \mathrm{ft} \mathrm{BGL} \text {. Depth } \\
\text { of } 0.5 \mathrm{in} \text {. well }=98 \mathrm{ft} \text { BGL. Push } \\
\text { to } 98 \mathrm{ft} B G L \text { with } 15 \text { rods end of } \\
\text { day on } 7 / 15 / 16 . \text { On } 7 / 16 / 16 \\
\text { (morning) pull back rods to } \\
\text { expose } 0.5 \text { in. screen and riser } \\
88-98 \mathrm{ft} \mathrm{BGL.} \mathrm{Sampled} \mathrm{using} \\
\text { bailer without purge. }\end{array}$ \\
\hline $7 / 16 / 15$ & $9: 15$ & TI01 & YKTI01100-W-37858 & DUP-F & $88-98$ & WG & 7246 & $7 / 16 / 15$ & Field replicate. \\
\hline $7 / 16 / 15$ & $9: 42$ & QC & YKQCTB-W-37859 & TB & - & WQC & 7246 & $7 / 16 / 15$ & $\begin{array}{l}\text { Trip blank with water samples } \\
\text { shipped to the AGEM laboratory. }\end{array}$ \\
\hline 7/16/15 & $9: 50$ & QC & YK-S-MEOHBLANK16JUL2015 & TB & - & $\begin{array}{c}\text { MEO } \\
\mathrm{H}\end{array}$ & 4916 & $7 / 16 / 15$ & $\begin{array}{l}\text { Trip blank with soil samples } \\
\text { shipped to TestAmerica } \\
\text { laboratory. }\end{array}$ \\
\hline $7 / 16 / 15$ & $14: 00$ & QC & YKQCIR-W-37863 & $\mathrm{RI}$ & - & WQC & 7246 & $7 / 16 / 15$ & $\begin{array}{l}\text { Rinsate of decontaminated bailer } \\
\text { after sample YKTI04120-W- } \\
37860 \text {. }\end{array}$ \\
\hline $7 / 16 / 15$ & $14: 20$ & TI04 & YKTI04120-W-37860 & $\mathrm{N}$ & $108-118$ & WG & 7246 & $7 / 16 / 15$ & $\begin{array}{l}\text { Depth to water }=108 \mathrm{ft} \text { BGL. Depth } \\
\text { of } 0.5 \mathrm{in} \text {. well }=98 \mathrm{ft} \text { BGL. } \\
\text { Pushed electronics with } 10 \text { rods } \\
\text { to } 131 \mathrm{ft} \text {. Push back down with } \\
15 \text { rods to target depth and pull } \\
\text { back } 10 \mathrm{ft} \text { to expose } 0.5 \text { in. } \\
\text { screen and riser. Sampled using } \\
\text { bailer without purge. }\end{array}$ \\
\hline $7 / 16 / 15$ & $16: 00$ & TI04 & YKTI04100-W-37861 & $\mathrm{N}$ & $89-99$ & WG & 7246 & $7 / 16 / 15$ & $\begin{array}{l}\text { Depth to water }=86 \mathrm{ft} \text {. Depth of } \\
0.5 \text { in. well }=99 \mathrm{ft} \text { BGL. Pushed } \\
\text { with } 15 \text { rods to target depth and } \\
\text { pull back to expose } 0.5 \text { in. } x 10 \mathrm{ft} \\
\text { screen. Sampled using bailer } \\
\text { without purge. }\end{array}$ \\
\hline $7 / 16 / 15$ & $16: 00$ & TI04 & YKTI04100-W-37862 & DUP-F & $89-99$ & WG & 7246 & $7 / 16 / 15$ & Field replicate. \\
\hline $7 / 16 / 15$ & $16: 00$ & TI04 & YKTI04100-W-37862DUP & DUP-L & $89-99$ & WG & 7246 & $7 / 16 / 15$ & Duplicate laboratory analysis. \\
\hline $7 / 17 / 15$ & $12: 43$ & QC & YKQCTB-W-37866 & TB & - & WQC & 7247 & $7 / 17 / 15$ & $\begin{array}{l}\text { Trip blank with water samples } \\
\text { shipped to the AGEM laboratory. }\end{array}$ \\
\hline 7/17/15 & $12: 52$ & QC & YKQCTB-W-37869 & TB & - & WQC & 7248 & $7 / 20 / 15$ & $\begin{array}{l}\text { Trip blank with water samples } \\
\text { shipped to TestAmerica } \\
\text { laboratory. }\end{array}$ \\
\hline
\end{tabular}


TABLE A.1 (Cont.)

\begin{tabular}{|c|c|c|c|c|c|c|c|c|c|}
\hline $\begin{array}{r}\text { Sample } \\
\text { and } \mathrm{Ti}\end{array}$ & $\begin{array}{l}\text { Date } \\
\text { me }\end{array}$ & Location & Sample & $\begin{array}{l}\text { Sample } \\
\text { Type }\end{array}$ & $\begin{array}{l}\text { Depth } \\
\text { (ft BGL) }\end{array}$ & Matrix & Chain of Custody & $\begin{array}{l}\text { Shipping } \\
\text { Date }\end{array}$ & Sample Description \\
\hline $7 / 17 / 15$ & $13: 20$ & TI05 & YKTI05120-W-37864 & $\mathrm{N}$ & $108-118$ & WG & 7247 & $7 / 17 / 15$ & $\begin{array}{l}\text { Depth to water }=90 \mathrm{ft} \text { BGL. Depth } \\
\text { of } 0.5 \text { in. well }=118 \mathrm{ft} \text { BGL. } \\
\text { Pushed to } 131 \mathrm{ft} \text { for electronics } \\
10 \text { rods. Pushed back down with } \\
15 \text { rods to } 118 \mathrm{ft} \text { and pulled back } \\
\text { to expose } 0.5 \text { in. Screen and } \\
\text { riser } 108-118 \mathrm{ft} \text {. Sampled using } \\
\text { bailer without purge. }\end{array}$ \\
\hline $7 / 17 / 15$ & $15: 35$ & TI05 & YKTI05100-W-37865 & $\mathrm{N}$ & $88-98$ & WG & 7247 & $7 / 17 / 15$ & $\begin{array}{l}\text { Depth to water }=87 \mathrm{ft} \text { BGL. Depth } \\
\text { of } 0.5 \text { in. well }=98 \mathrm{ft} \mathrm{BGL} \text {. } \\
\text { Pushed with } 15 \text { rods to } 98 \mathrm{ft} \text {. } \\
\text { Pulled back to } 10 \mathrm{ft} \text { to expose } \\
0.5 \text { in. screen and riser } 88-98 \mathrm{ft} \\
\text { BGL. Sampled using bailer } \\
\text { without purge. }\end{array}$ \\
\hline 7/17/15 & $15: 35$ & TI05 & YKTI05100-W-37867 & DUP-F & 88-98 & WG & 7247 & $7 / 17 / 15$ & Field replicate. \\
\hline $7 / 17 / 15$ & $15: 35$ & TI05 & YKTI05100-W-37867DUP & DUP-L & $88-98$ & WG & 7247 & $7 / 17 / 15$ & Duplicate laboratory analysis. \\
\hline $7 / 18 / 15$ & $9: 40$ & TI05 & YKTI0580-W-37868VER & VER & $76-81$ & WG & 7248 & $7 / 20 / 15$ & $\begin{array}{l}\text { Verification sample to TestAmerica, } \\
\text { Inc. }\end{array}$ \\
\hline $7 / 18 / 15$ & $9: 40$ & TI05 & YKTI0580-W-37868 & $\mathrm{N}$ & $76-81$ & WG & 7249 & $7 / 20 / 15$ & $\begin{array}{l}\text { Depth to water }=74.32 \mathrm{ft} \mathrm{BGL} \text {. } \\
\text { Depth of } 0.5 \mathrm{in} \text {. well }=81 \mathrm{ft} \text { BGL. } \\
\text { Pushed to } 81 \mathrm{ft} \text { with } 15 \text { rods at } \\
\text { end of day on } 7 / 17 / 16 \text {. Water } \\
\text { started to come in, but slowly. } \\
\text { Left big/rods overnight with } \\
\text { screen exposed at } 76-81 \mathrm{ft} . \\
\text { Sampled using bailer without } \\
\text { purge. }\end{array}$ \\
\hline 7/18/15 & $9: 40$ & TI05 & YKTI0580-W-37868DUP & DUP-L & $76-81$ & WG & 7249 & $7 / 20 / 15$ & Duplicate laboratory analysis. \\
\hline $7 / 18 / 15$ & $10: 31$ & QC & YKQCTB-W-37871 & TB & - & WQC & 7249 & $7 / 20 / 15$ & $\begin{array}{l}\text { Trip blank with water samples } \\
\text { shipped to the AGEM laboratory. }\end{array}$ \\
\hline $7 / 19 / 15$ & $10: 25$ & TI06 & YKTI06120-W-37870 & $\mathrm{N}$ & $110-120$ & WG & 7249 & $7 / 20 / 15$ & $\begin{array}{l}\text { Depth to water }=81.85 \mathrm{ft} \mathrm{BGL} \text {. } \\
\text { Depth of } 0.5 \text { in. well }=120 \mathrm{ft} \\
\text { BGL. Ran electronic with } 40 \text { ton } \\
\text { CPT, } 10 \text { rods, to } 131 \mathrm{ft} \text { on } \\
7 / 18 / 16 \text {. On } 7 / 19 / 16 \text {, pushed to } \\
120 \mathrm{ft} \text { with } 15 \text { rods, pulled back } \\
10 \mathrm{ft} \text { to expose } 0.5 \text { in. screen } \\
\text { and riser. Sampled using bailer } \\
\text { without purge. }\end{array}$ \\
\hline $7 / 19 / 15$ & $15: 50$ & TI06 & YKTI0680-W-37872VER & VER & $77-82$ & WG & 7248 & $7 / 20 / 15$ & $\begin{array}{l}\text { Verification sample to TestAmerica, } \\
\text { Inc. }\end{array}$ \\
\hline
\end{tabular}


TABLE A.1 (Cont.)

\begin{tabular}{|c|c|c|c|c|c|c|c|c|}
\hline $\begin{array}{l}\text { Sample Date } \\
\text { and Time }\end{array}$ & Location & Sample & $\begin{array}{c}\text { Sample } \\
\text { Type }\end{array}$ & $\begin{array}{c}\text { Depth } \\
\text { (ft BGL) }\end{array}$ & Matrix & Chain of Custody & $\begin{array}{l}\text { Shipping } \\
\text { Date }\end{array}$ & Sample Description \\
\hline
\end{tabular}

$7 / 20 / 15$

Depth to water $=75.5 \mathrm{ft} \mathrm{BGL}$

Depth of 0.5 in. well $=82 \mathrm{ft} \mathrm{BGL}$ Pushed to target depth with ARA rod and pulled back $5 \mathrm{ft}$ to expose 0.5 in. screen and riser Water came in immediately. Sampled using bailer without purge.

pth to water $=79.4 \mathrm{ft} \mathrm{BGL}$

Depth of 0.5 in. well $=$

$100 \mathrm{ft}$ BGL. Pushed to depth,

opened rods, exposed screen.

Set temporary screen and riser

sand pack and bentonite. Wate

came in to indicated depth over

several hr. Sampled using bailer without purge.

7/19/15 16:40 TI06

YKTI06100-W-37873DUP

7/20/15 9:35 TIO

YKTI06100-W-37874

YKTI0780-W-37875

$\begin{array}{cccc}\text { DUP-L } & 90-100 & \text { WG } & 7249 \\ \text { DUP-F } & 90-100 & \text { WG } & 7249 \\ \text { N } & 77-82 & \text { WG } & 7249\end{array}$

$7 / 20 / 15$

Duplicate laboratory analysis.

Field replicate.

$7 / 20 / 15$ Depth to water $=75.2 \mathrm{ft} \mathrm{BGL}$. Depth of 0.5 in. well $=82 \mathrm{ft} \mathrm{BGL}$. Pushed to $82 \mathrm{ft}$ and pulled back $10 \mathrm{ft}$ to expose $0.5 \mathrm{in}$. screen and riser $72-82 \mathrm{ft}$. Water almost immediately. Sampled using bailer without purge.

$\begin{array}{llll}7 / 20 / 15 & 12: 25 & \text { TI07 } & \text { YKTI07100-W-37876VER } \\ 7 / 20 / 15 & 12 \cdot 25 & \text { TI07 } & \text { YKTI07100-W-37876 }\end{array}$

$\begin{array}{cccc}\text { VER } & 90-100 & \text { WG } & 7248 \\ \text { N } & 90-100 & \text { WG } & 7249\end{array}$

7/20/15 Verification sample to TestAmerica, Inc.

7/20/15 12:25 TI07 $\quad$ YKTI07100-W-37876

7249

$7 / 20 / 15$ Depth Depth of 0.5 in. well $=100 \mathrm{ft}$ BGL. Pushed to target depth with 40-ton CPT and pulled back rods to expose $0.5 \mathrm{in} . \times 10 \mathrm{ft}$ screen and riser $90 \mathrm{ft}-100 \mathrm{ft}$. Water came in over several hr. Sampled using bailer without purge. 
TABLE A.1 (Cont.)

\begin{tabular}{|c|c|c|c|c|c|c|c|c|c|}
\hline $\begin{array}{r}\text { Sample } \\
\text { and } \mathrm{Ti}\end{array}$ & $\begin{array}{l}\text { Date } \\
\text { ime }\end{array}$ & Location & Sample & $\begin{array}{l}\text { Sample } \\
\text { Type }\end{array}$ & $\begin{array}{l}\text { Depth } \\
\text { (ft BGL) }\end{array}$ & Matrix & Chain of Custody & $\begin{array}{l}\text { Shipping } \\
\text { Date }\end{array}$ & Sample Description \\
\hline $7 / 20 / 15$ & $14: 50$ & TI07 & YKTI07120-W-37877 & $\mathrm{N}$ & $110-120$ & WG & 7249 & $7 / 20 / 15$ & $\begin{array}{l}\text { Depth to water }=94 \mathrm{ft} \mathrm{BGL} \text {. Depth } \\
\text { of } 0.5 \text { in. well }=120 \mathrm{ft} \text { BGL. } \\
\text { Pushed with } 40 \text {-ton to depth and } \\
\text { pulled back to expose } 0.5 \mathrm{in} \text {. by } \\
10 \mathrm{ft} \text { screen and riser } 110-120 \mathrm{ft} \text {. } \\
\text { Water came in almost } \\
\text { immediately. Sampled using } \\
\text { bailer without purge. }\end{array}$ \\
\hline $7 / 20 / 15$ & $14: 50$ & TI07 & YKTI07120-W-37878 & DUP-F & $110-120$ & WG & 7249 & $7 / 20 / 15$ & Field replicate. \\
\hline $7 / 20 / 15$ & $14: 50$ & TI07 & YKTI07120-W-37878DUP & DUP-L & $110-120$ & WG & 7249 & $7 / 20 / 15$ & Duplicate laboratory analysis. \\
\hline $7 / 21 / 15$ & $12: 12$ & QC & YKQCTB-W-37883 & TB & - & WQC & 7250 & $7 / 22 / 15$ & $\begin{array}{l}\text { Trip blank with water samples } \\
\text { shipped to the AGEM laboratory. }\end{array}$ \\
\hline $7 / 21 / 15$ & $12: 12$ & QC & YKQCTB-W-37883VER & VER & - & WQC & 7252 & $7 / 22 / 15$ & $\begin{array}{l}\text { Verification sample to TestAmerica, } \\
\text { Inc. }\end{array}$ \\
\hline $7 / 21 / 15$ & $14: 45$ & TI08 & YKTI0885-W-37879 & $\mathrm{N}$ & $80-85$ & WG & 7250 & $7 / 22 / 15$ & $\begin{array}{l}\text { Depth to water }=76 \mathrm{ft} \mathrm{BGL} \text {. Depth } \\
\text { of } 0.5 \text { in. well }=85 \mathrm{ft} \text { BGL. } \\
\text { Pushed to target depth with } 40- \\
\text { ton and pulled back } 5 \mathrm{ft} \text { to } \\
\text { expose } 0.5 \text { in. screen and riser } \\
\text { at } 80-85 \mathrm{ft} \text { BGL. Sampled using } \\
\text { bailer without purge. }\end{array}$ \\
\hline $7 / 21 / 15$ & $14: 45$ & TI08 & YKTI0885-W-37879DUP & DUP-L & $80-85$ & WG & 7250 & $7 / 22 / 15$ & Duplicate laboratory analysis. \\
\hline $7 / 21 / 15$ & $14: 45$ & TI08 & YKTI0885-W-37879VER & VER & $80-85$ & WG & 7252 & $7 / 22 / 15$ & $\begin{array}{l}\text { Verification sample to TestAmerica, } \\
\text { Inc. }\end{array}$ \\
\hline $7 / 21 / 15$ & $16: 15$ & TI08 & YKTI08107-W-37881 & $\mathrm{N}$ & $97-107$ & WG & 7250 & $7 / 22 / 15$ & $\begin{array}{l}\text { Depth to water }=83.8 \mathrm{ft} \mathrm{BGL} \text {. } \\
\text { Depth of } 0.5 \text { in. well }=107 \mathrm{ft} \text {. } \\
\text { Pushed to target depth with } 40- \\
\text { ton and pulled rods up } 10 \mathrm{ft} \text { to } \\
\text { expose } 0.5 \text { in. screen and riser } \\
97-107 \mathrm{ft} \mathrm{BGL.} \mathrm{Water} \mathrm{in} \\
\text { immediately. Sampled using } \\
\text { bailer without purge. }\end{array}$ \\
\hline $7 / 21 / 15$ & $16: 15$ & TI08 & YKTI08107-W37884 & DUP-F & $97-107$ & WG & 7250 & $7 / 22 / 15$ & Field replicate. \\
\hline 7/21/15 & $17: 40$ & TI08 & YKTI08125-W-37882 & $\mathrm{N}$ & $115-125$ & WG & 7250 & $7 / 22 / 15$ & $\begin{array}{l}\text { Depth to water }=100 \mathrm{ft} \mathrm{BGL} \text {. Depth } \\
\text { of } 0.5 \text { in. well = } 125 \mathrm{ft} \mathrm{BGL} \text {. } \\
\text { Pushed to target depth with } 40 \\
\text { ton. Pulled rods back } 10 \mathrm{ft} \text { to } \\
\text { expose } 0.5 \text { in. screen and riser } \\
\text { at } 115-125 \mathrm{ft} \mathrm{BGL} \text {. Immediate } \\
\text { water. Sampled using bailer } \\
\text { without purge. }\end{array}$ \\
\hline
\end{tabular}


TABLE A.1 (Cont.)

\begin{tabular}{|c|c|c|c|c|c|c|c|c|c|}
\hline \multicolumn{2}{|c|}{$\begin{array}{c}\text { Sample Date } \\
\text { and Time }\end{array}$} & \multirow{2}{*}{$\begin{array}{r}\text { Location } \\
\text { WILLOW }\end{array}$} & \multirow{2}{*}{$\begin{array}{r}\text { Sample } \\
\text { YKWILLOW-G-37887 }\end{array}$} & \multirow{2}{*}{$\begin{array}{c}\text { Sample } \\
\text { Type }\end{array}$} & \multirow{2}{*}{$\begin{array}{c}\text { Depth } \\
\text { (ft BGL) } \\
-\end{array}$} & \multirow{2}{*}{$\begin{array}{c}\text { Matrix } \\
\text { AIR }\end{array}$} & \multirow{2}{*}{$\begin{array}{l}\text { Chain of Custody } \\
\text { TA-06Aug2015-3 }\end{array}$} & \multirow{2}{*}{$\begin{array}{l}\begin{array}{l}\text { Shipping } \\
\text { Date }\end{array} \\
8 / 6 / 15\end{array}$} & \multirow{3}{*}{$\begin{array}{l}\text { Sample Description } \\
\text { Project search room, across } \\
\text { hallway from ice cream parlor; } \\
\text { on center of conference table. } \\
\text { Room remained } \\
\text { closed/unoccupied throughout } \\
\text { collection. Sample collected over } \\
24 \mathrm{hr} \text {. Begin pressure }-27 \text { in. Hg. } \\
\text { End pressure }-10 \mathrm{in} \text {. Hg. } \\
\text { Ice cream parlor, on top of popcorn } \\
\text { maker (not in use). Sample } \\
\text { collected over } 24 \mathrm{hr} \text {. Begin } \\
\text { pressure }-29 \mathrm{in} . \mathrm{Hg} \text {. End } \\
\text { pressure }-6 \text { in. } \mathrm{Hg} \text {. }\end{array}$} \\
\hline $8 / 5 / 15$ & 9:07 & & & & & & & & \\
\hline $8 / 5 / 15$ & 9:09 & WILLOW & YKWILLOW-G-37885 & $\mathrm{N}$ & - & AIR & TA-06Aug2015-3 & $8 / 6 / 15$ & \\
\hline $8 / 5 / 15$ & $9: 13$ & WILLOW & YKWILLOW-G-37886 & $\mathrm{N}$ & - & AIR & TA-06Aug2015-2 & $8 / 6 / 15$ & $\begin{array}{l}\text { Resident lounge, on counter } \\
\text { against walL; area open to } \\
\text { adjacent hallways. Sample } \\
\text { collected over } 24 \mathrm{hr} \text {. Begin } \\
\text { pressure }-30 \mathrm{in} . \mathrm{Hg} \text {. End } \\
\text { pressure }-8 \mathrm{in} . \mathrm{Hg} \text {. }\end{array}$ \\
\hline $8 / 5 / 15$ & $9: 15$ & WILLOW & YKWILLOW-G-37889 & $\mathrm{N}$ & - & AIR & TA-06Aug2015-2 & $8 / 6 / 15$ & $\begin{array}{l}\text { In sitting area, along hallway on top } \\
\text { of end table. Sample collected } \\
\text { over } 24 \mathrm{hr} \text {. Begin } \\
\text { pressure }-30 \text { in. Hg. End } \\
\text { pressure }-6 \text { in. Hg. }\end{array}$ \\
\hline 8/5/15 & $9: 20$ & WILLOW & YKWILLOW-G-37890 & $\mathrm{N}$ & - & AIR & TA-06Aug2015-3 & $8 / 6 / 15$ & $\begin{array}{l}\text { Private office behind activity room; } \\
\text { single entry door, room } \\
\text { remained closed/unoccupied } \\
\text { through collection. Sample } \\
\text { collected over } 24 \mathrm{hr} \text {. Begin } \\
\text { pressure }-29 \mathrm{in} . \mathrm{Hg} \text {. End } \\
\text { pressure }-6 \mathrm{in} . \mathrm{Hg} \text {. }\end{array}$ \\
\hline $8 / 5 / 15$ & $9: 22$ & WILLOW & YKWILLOW-G-37888 & $\mathrm{N}$ & - & AIR & TA-06Aug2015-3 & $8 / 6 / 15$ & $\begin{array}{l}\text { Empty Apt. \#304, on bay window } \\
\text { sill in main room across from } \\
\text { entry door; single entry door, } \\
\text { room remained closed through } \\
\text { collection. Sample collected over } \\
24 \mathrm{hr} \text {. Begin pressure }-27 \text { in. } \mathrm{Hg} \text {. } \\
\text { End pressure }-6 \text { in. } \mathrm{Hg} \text {. }\end{array}$ \\
\hline
\end{tabular}


TABLE A.1 (Cont.)

\begin{tabular}{|c|c|c|c|c|c|c|c|c|}
\hline $\begin{array}{l}\text { Sample Date } \\
\text { and Time }\end{array}$ & Location & Sample & $\begin{array}{c}\text { Sample } \\
\text { Type }\end{array}$ & $\begin{array}{c}\text { Depth } \\
\text { (ft BGL) }\end{array}$ & Matrix & Chain of Custody & $\begin{array}{l}\text { Shipping } \\
\text { Date }\end{array}$ & Sample Description \\
\hline
\end{tabular}

8/5/15 9:57 CVA

YKCVA-G-37910

$8 / 5 / 15$

CVA

YKCVA-G-37912

8/5/15 10:44 PRES

YKPRES-G-37904

8/5/15 10:47 PRES

YKPRES-G-37903
N

AIR

TA-06Aug2015-3

AIR

TA-06Aug2015-1

AIR

TA-06Aug2015-2

N
$8 / 6 / 15$ on table near center-front of room; 2 entry doors, both closed when can set out. One open when checked $8 / 4$; both shut when retrieved $8 / 5$. Sample collected over $24 \mathrm{hr}$. Begin pressure -28 in. $\mathrm{Hg}$. End pressure -6 in. $\mathrm{Hg}$.

8/6/15 Office of Jeff Grawe, on corner of desk near center of room. Door closed throughout collection. Sample collected over $24 \mathrm{hr}$. Begin pressure -30 in. $\mathrm{Hg}$. End pressure -7 in. $\mathrm{Hg}$.

8/6/15 Platte River Room, conference

room across hall from Grawe office on center of conference table. Glass walL on 2 long sides of room, doors closed throughout collection. Sample collected over $24 \mathrm{hr}$. Begin pressure -30 in. $\mathrm{Hg}$. End pressure -6 in. $\mathrm{Hg}$

$8 / 6 / 15$ In living room on top of book case in center of room. Large wood stove in center of room. ALo box with aerosol spray cans. House does not have $A / C$, much clutter. Sample collected over $24 \mathrm{hr}$. Begin pressure $-30+$ in. $\mathrm{Hg}$. End pressure $-10 \mathrm{in}$. $\mathrm{Hg}$

basement on top of sink against wall. Cement floor throughout largely empty room, utilities in separate room. Sample collected over $24 \mathrm{hr}$. Begin

pressure -29 in. $\mathrm{Hg}$. End pressure -6 in. $\mathrm{Hg}$. 
TABLE A.1 (Cont.)

\begin{tabular}{|c|c|c|c|c|c|c|c|c|c|}
\hline \multicolumn{2}{|c|}{$\begin{array}{l}\text { Sample Date } \\
\text { and Time }\end{array}$} & \multirow{2}{*}{$\begin{array}{r}\text { Location } \\
\text { FITWORX }\end{array}$} & \multirow{2}{*}{$\begin{array}{r}\text { Sample } \\
\text { YKFITWORX-G-37898 }\end{array}$} & \multirow{2}{*}{$\begin{array}{c}\text { Sample } \\
\text { Type }\end{array}$} & \multirow{2}{*}{$\begin{array}{c}\text { Depth } \\
\text { (ft BGL) } \\
-\end{array}$} & \multirow{2}{*}{$\begin{array}{c}\text { Matrix } \\
\text { AIR }\end{array}$} & \multirow{2}{*}{$\begin{array}{l}\text { Chain of Custody } \\
\text { TA-06Aug2015-1 }\end{array}$} & \multirow{2}{*}{$\begin{array}{l}\begin{array}{l}\text { Shipping } \\
\text { Date }\end{array} \\
8 / 6 / 15\end{array}$} & Sample Description \\
\hline $8 / 5 / 15$ & 11:05 & & & & & & & & $\begin{array}{l}\text { Main exercise area, in northeast } \\
\text { corner on window sill facing } \\
\text { north. Sample collected over } \\
24 \mathrm{hr} \text {. Begin pressure }-30 \mathrm{in} \text {. } \mathrm{Hg} \text {. } \\
\text { End pressure }-6 \text { in. } \mathrm{Hg} \text {. }\end{array}$ \\
\hline $8 / 5 / 15$ & $11: 09$ & FITWORX & YKFITWORX-G-37897 & $\mathrm{N}$ & - & AIR & TA-06Aug2015-1 & $8 / 6 / 15$ & $\begin{array}{l}\text { Lower level exercise room, on top } \\
\text { of display case to left of entrance } \\
\text { door off stairway. Sample } \\
\text { collected over } 24 \mathrm{hr} \text {. Begin } \\
\text { pressure }-28 \text { in. } \mathrm{Hg} \text {. End } \\
\text { pressure }-5 \mathrm{in} . \mathrm{Hg} \text {. }\end{array}$ \\
\hline
\end{tabular}

$\begin{array}{llll}8 / 5 / 15 & 11: 10 & \text { FITWORX } & \text { YKFITWORX-G-37901 } \\ \text { 8/5/15 } & 11: 16 & \text { FITWORX } & \text { YKFITWORX-G-37900 }\end{array}$

8/5/15 11:16 FITWORX YKFITWORX-G-37900

8/5/15 11:18 FITWORX

YKFITWORX-G-37899

8/5/15 11:20 FITWORX

YKFITWORX-G-37902

8/5/15 11:25 FITWORX
YKBKGND-G-37908
DUP-F

$\mathrm{N}$

AIR

TA-06Aug2015-2

$\mathrm{N}$

$-$

AIR

TA-06Aug2015-2

N

$-$

AIR

TA-06Aug2015-1

N

- $\quad$ AIR TA-06Aug2015-1

pressure $-5 \mathrm{in.} \mathrm{Hg}$.
8/6/15 Field replicate. Sample collected over $24 \mathrm{hr}$. Begin pressure -30 in. $\mathrm{Hg}$. End pressure -6 in. $\mathrm{Hg}$.

8/6/15 Treatment room 3 in physical

therapy area off main exercise room. On top of cabinet against wall. Door to room remained wall. Door to room remained Sample collected over $24 \mathrm{hr}$. Begin pressure -30 in. $\mathrm{Hg}$. End pressure -6 in. $\mathrm{Hg}$.

8/6/15 Office across hall from treatment room 7 on built-in counter across from door. Printer on counter along opposite wall. Laundry room down hall to left leaving office. Sample collected over $24 \mathrm{hr}$. Begin pressure $-30 \mathrm{in}$. $\mathrm{Hg}$. End pressure -7 in. $\mathrm{Hg}$.

8/6/15 Main exercise room, on window sill at northwest corner of room. Sample collected over $24 \mathrm{hr}$. Begin pressure $-30 \mathrm{in}$. Hg. End pressure -7 in. $\mathrm{Hg}$.

8/6/15 In corner against outside walL, to left of main entrance. On top of 5 gal empty bucket. Background 5 sample collected over $24 \mathrm{hr}$. Begin pressure $-30 \mathrm{in}$. Hg. End pressure -6 in. $\mathrm{Hg}$. 
TABLE A.1 (Cont.)

\begin{tabular}{|c|c|c|c|c|c|c|c|c|c|}
\hline \multicolumn{2}{|c|}{$\begin{array}{l}\text { Sample Date } \\
\text { and Time }\end{array}$} & \multirow{2}{*}{$\begin{array}{r}\text { Location } \\
\text { QC }\end{array}$} & \multirow{2}{*}{$\begin{array}{r}\text { Sample } \\
\text { YKQCTB-W-37925 }\end{array}$} & \multirow{2}{*}{$\begin{array}{c}\text { Sample } \\
\text { Type }\end{array}$} & \multirow{2}{*}{$\begin{array}{c}\text { Depth } \\
\text { (ft BGL) } \\
-\end{array}$} & \multirow{2}{*}{$\begin{array}{l}\text { Matrix } \\
\text { WQC }\end{array}$} & \multirow{2}{*}{$\begin{array}{l}\text { Chain of Custody } \\
\text { YK810151705 }\end{array}$} & \multirow{2}{*}{$\begin{array}{l}\begin{array}{l}\text { Shipping } \\
\text { Date }\end{array} \\
8 / 10 / 15\end{array}$} & \multirow{3}{*}{$\begin{array}{l}\text { Sample Description } \\
\text { Trip blank with water samples for } \\
\text { VOC analyses shipped to the } \\
\text { AGEM laboratory. } \\
\text { Verification sample to TestAmerica, } \\
\text { Inc. }\end{array}$} \\
\hline $8 / 5 / 15$ & $12: 00$ & & & & & & & & \\
\hline $8 / 5 / 15$ & $12: 00$ & QC & YKQCTB-W-37925VER & VER & - & WQC & YK810151710 & 8/10/15 & \\
\hline $8 / 5 / 15$ & $12: 37$ & DW19 & YKPRESLICKA-W-37916 & $\mathrm{N}$ & - & WG & YK810151705 & $8 / 10 / 15$ & $\begin{array}{l}\text { Sample collected from tap at } \\
\text { kitchen sink after running for } \\
\sim 10 \text { min. Well is on southwest } \\
\text { side of house in lawn, } 8 \mathrm{ft} \text { from } \\
\text { building. Owner reports well } \\
\text { used for all domestic purposes. }\end{array}$ \\
\hline $8 / 5 / 15$ & $13: 35$ & DW38 & YKYORKCOUNTYSHOP-W-37917 & $\mathrm{N}$ & - & WG & YK810151705 & $8 / 10 / 15$ & $\begin{array}{l}\text { Sample collected from tap in } \\
\text { bathroom of garage building } \\
\text { after running for } \sim 5 \text { min. Well is } \\
\text { in parking area } 5 \mathrm{ft} \text { directly west } \\
\text { of garage building. Hwy } \\
\text { Superintendent reports well used } \\
\text { for bathroom, wash sinks, etc. } \\
\text { Have Culligan service for } \\
\text { drinking water. }\end{array}$ \\
\hline $8 / 5 / 15$ & 13:35 & DW38 & YKYORKCOUNTYSHOP-W-37917VER & VER & - & WG & YK810151710 & $8 / 10 / 15$ & $\begin{array}{l}\text { Verification sample to TestAmerica, } \\
\text { Inc. }\end{array}$ \\
\hline $8 / 5 / 15$ & $13: 54$ & DW39 & YKNORDLUNDDUP-W-37919 & DUP-F & - & WG & YK810151705 & $8 / 10 / 15$ & Field replicate. \\
\hline $8 / 5 / 15$ & $13: 54$ & DW39 & YKNORDLUND-W-37918 & $\mathrm{N}$ & - & WG & YK810151705 & $8 / 10 / 15$ & $\begin{array}{l}\text { Sample collected from hydrant } \\
\text { adjacent to well after running for } \\
\sim 5 \text { min. Well } 50 \text { yards W-SW } \\
\text { from house. Owner reports well } \\
\text { filtered until spring } 2015 \text {; house } \\
\text { connected to city water then by } \\
\text { EPA. Hydrants by well and barn } \\
\text { NW of house still used for yard. }\end{array}$ \\
\hline $8 / 5 / 15$ & $14: 20$ & DW55 & YKLIERMANN-W-37920 & $\mathrm{N}$ & - & WG & YK810151705 & $8 / 10 / 15$ & $\begin{array}{l}\text { Sample collected from yard hydrant } \\
\text { adjacent to well after running for } \\
\sim 5 \text { min. Well in backyard, } \\
25 \text { yards east-northeast from } \\
\text { house. }\end{array}$ \\
\hline
\end{tabular}


TABLE A.1 (Cont.)

\begin{tabular}{|c|c|c|c|c|c|c|c|c|}
\hline $\begin{array}{c}\text { Sample Date } \\
\text { and Time }\end{array}$ & Location & Sample & $\begin{array}{c}\text { Sample } \\
\text { Type }\end{array}$ & $\begin{array}{c}\text { Depth } \\
\text { (ft BGL) }\end{array}$ & Matrix & Chain of Custody & $\begin{array}{l}\text { Shipping } \\
\text { Date }\end{array}$ & Sample Description \\
\hline
\end{tabular}

8/5/15 15:56 HEARTH YKHEARTH-G-37893

$8 / 5 / 15$

YKHEARTH-G-37896

$8 / 5 / 15$

16:01 HEARTH

YKHEARTH-G-37895

8/5/15 16:05 HEARTH

YKHEARTH-G-37891

YKHEARTH-G-37894
$\mathrm{N}$

AIR

TA-06Aug2015-4

N

AIR

TA-06Aug2015-4

$\mathrm{N}$

AIR

TA-06Aug2015-4
8/6/15 Jay Colburn's office off main lobby In use when can placed $8 / 4$ afternoon and $8 / 5$ afternoon retrieval. Closed/unoccupied morning of $8 / 5$ when checked. Sample collected over $24 \mathrm{hr}$. Begin pressure -30 in. $\mathrm{Hg}$. End pressure -10 in. Hg.

8/6/15 Counter top at back of activity room along central hallway from lobby, leading area $\mathrm{B}$ to area $\mathrm{C}$. Sample collected over $24 \mathrm{hr}$.
Begin pressure -26 in. $\mathrm{Hg}$. End pressure -5 in. $\mathrm{Hg}$.

8/6/15 Nurse coordinator's office, wing 500 , adjacent to dining room. Unoccupied when placed $8 / 4$ and checked $8 / 5$; in use $8 / 5$ and checked $8 / 5$; in use $8 / 5$ throughout collection. Sample collected over $24 \mathrm{hr}$. Begin pressure -30 in. $\mathrm{Hg}$. End pressure -10 in. $\mathrm{Hg}$.

8/6/15 At nurse's station connected to dining room, on back counter in 500 wing. Sample collected over $24 \mathrm{hr}$. Begin pressure $-28 \mathrm{in}$. $\mathrm{Hg}$. End pressure -6 in. $\mathrm{Hg}$.

8/6/15 Neighborhood director's office, on bookcase along back wall opposite door. Room unoccupied $8 / 4$ and $8 / 5$ early, in use $8 / 5$ retrieval. Door closed throughout collection. Sample collected over $24 \mathrm{hr}$. Begin pressure -30 in. $\mathrm{Hg}$. End pressure -6 in. Hg.

$\begin{array}{llll}\mathrm{N} & - & \text { AIR } & \text { TA-06Aug2015-4 }\end{array}$

$8 / 6 / 15$

Side counter in dining area, area A special care units. Sample collected over $24 \mathrm{hr}$. Begin pressure $-25.5 \mathrm{in}$. Hg. End pressure -5 in. $\mathrm{Hg}$. 
TABLE A.1 (Cont.)

\begin{tabular}{|c|c|c|c|c|c|c|c|c|}
\hline $\begin{array}{l}\text { Sample Date } \\
\text { and Time }\end{array}$ & Location & Sample & $\begin{array}{l}\text { Sample } \\
\text { Type }\end{array}$ & $\begin{array}{c}\text { Depth } \\
\text { (ft BGL) }\end{array}$ & Matrix & Chain of Custody & $\begin{array}{c}\text { Shipping } \\
\text { Date }\end{array}$ & Sample Description \\
\hline
\end{tabular}

8/5/15 16:56 TROES

YKTROES-G-37913

8/5/15 16:59 TROES

YKTROES-G-37906

8/5/15 17:02 TROES

YKBKGND-G-37914

8/5/15 17:50 MILLER

YKBKGND-G-37915

8/7/15 $12: 00 \quad$ SB77

8/7/15 12:00 SB77

8/7/15 12:00 SB77
YKSB77DUP-W-37922
YKSB77DUP-W-37922DUP
YKSB77-W-37921

N

AIR

TA-06Aug2015-5

N

AIR

TA-06Aug2015-5

$-$

AIR

TA-06Aug2015-5
N

AIR

TA-06Aug2015-5

$\begin{array}{cr}\text { DUP-F } & 75.8-79 \\ \text { DUP-L } & 75.8-79 \\ \text { N } & 75.8-79\end{array}$

$8 / 6 / 15$

alcove on north side of main entrance door. Can placed on empty coleman cooler in corner against wall. Background sample collected over $24 \mathrm{hr}$. Begin pressure -26 in. $\mathrm{Hg}$. End

pressure 0 in. $\mathrm{Hg}$.

8/6/15 Ground floor on counter opposite kitchen sink, south end of living room. Interior recently updated new carpeting/paint. Tenant moved in 2 weeks prior; house largely empty. Sample collected over $24 \mathrm{hr}$. Begin pressure -

26 in. $\mathrm{Hg}$. End

pressure -5 in. $\mathrm{Hg}$.

8/6/15 Lower level family room, in corner opposite bottom of stairs. Lowe level is finished with bedroom, utility room, bathroom. Sample collected over $24 \mathrm{hr}$. Begin pressure -29 in. Hg. End pressure $-5 \mathrm{in}$. $\mathrm{Hg}$.

8/6/15 Under south edge of small open deck/porch at front door of house. Background sample collected over $24 \mathrm{hr}$. Begin pressure $-30 \mathrm{in}$. $\mathrm{Hg}$. End pressure -5 in. $\mathrm{Hg}$.

8/6/15 Canister at north end of gravel drive, directly between 2 northernmost quonsets still on site. Background sample collected over $24 \mathrm{hr}$. Begin pressure $-29 \mathrm{in}$. $\mathrm{Hg}$. End pressure -5 in. $\mathrm{Hg}$.

8/10/15 Field replicate.

8/10/15 Duplicate laboratory analysis.

$8 / 10 / 15$ Depth to water $=75.50 \mathrm{ft} \mathrm{TOC}$.

Depth of 4 in. well $=103.1 \mathrm{ft}$ Depth of 4 in. well $=103.1 \mathrm{ft}$
$B G L$ (obstruction at $79 \mathrm{ft} \mathrm{BGL}$ ). Sample collected using low flow bladder pump after purging $4 \mathrm{~L}$. 
TABLE A.1 (Cont.)

\begin{tabular}{|c|c|c|c|c|c|c|c|c|c|}
\hline $\begin{array}{l}\text { Sample } \\
\text { and Tir }\end{array}$ & $\begin{array}{l}\text { Date } \\
\text { me }\end{array}$ & Location & Sample & $\begin{array}{l}\text { Sample } \\
\text { Type }\end{array}$ & $\begin{array}{l}\text { Depth } \\
\text { (ft BGL) }\end{array}$ & Matrix & Chain of Custody & $\begin{array}{l}\text { Shipping } \\
\text { Date }\end{array}$ & Sample Description \\
\hline $8 / 7 / 15$ & $12: 00$ & SB77 & YKSB77-W-37921DUP & DUP-L & 75.8-79 & WG & YK810151705 & $8 / 10 / 15$ & Duplicate laboratory analysis. \\
\hline $8 / 7 / 15$ & $13: 00$ & DW21 & YKVOLZKE-W-37923 & $\mathrm{N}$ & - & WG & YK810151705 & $8 / 10 / 15$ & $\begin{array}{l}\text { Sample collected from hydrant next } \\
\text { to well after running for about } \\
5 \text { min. Well behind house } \\
\text { opposite back door off drive. } \\
\text { Owner reports well used for all } \\
\text { domestic purposes by tenant } \\
\text { Jody Krause and kids. Currently } \\
\text { for commercial sale; trying to get } \\
\text { city water. }\end{array}$ \\
\hline $8 / 7 / 15$ & $14: 02$ & SB62 & YKSB62-W-37924 & $\mathrm{N}$ & $118-123$ & WG & YK810151705 & 8/10/15 & $\begin{array}{l}\text { Depth to water }=75.80 \mathrm{ft} \mathrm{TOC} \text {. } \\
\text { Depth of } 2 \text { in. well }=122.9 \mathrm{ft} \\
\text { BGL. Sample collected using low } \\
\text { flow bladder pump after purging } \\
5 \mathrm{~L} \text {. }\end{array}$ \\
\hline $8 / 7 / 15$ & $14: 02$ & SB62 & YKSB62-W-37924VER & VER & - & WG & YK810151710 & 8/10/15 & $\begin{array}{l}\text { Verification sample to TestAmerica, } \\
\text { Inc. }\end{array}$ \\
\hline 8/13/15 & $8: 00$ & QC & YKQCTB-W-37934 & TB & - & WQC & YK814151700 & 8/14/15 & $\begin{array}{l}\text { Trip blank with water samples } \\
\text { shipped to the AGEM laboratory. }\end{array}$ \\
\hline 8/13/15 & $8: 00$ & QC & YKQCTB-W-37934VER & VER & - & WQC & YK814151710 & $8 / 14 / 15$ & $\begin{array}{l}\text { Verification sample to TestAmerica, } \\
\text { Inc. }\end{array}$ \\
\hline 8/13/15 & $11: 30$ & TI09 & YKTI09-80DUP-W-37927 & DUP-F & $80-85$ & WG & YK814151700 & $8 / 14 / 15$ & Field replicate. \\
\hline 8/13/15 & $11: 30$ & TI09 & YKTI09-80-W-37926 & $\mathrm{N}$ & $80-85$ & WG & YK814151700 & $8 / 14 / 15$ & $\begin{array}{l}\text { Depth to water }=81 \mathrm{ft} \mathrm{BGL} \text {. Depth } \\
\text { of } 0.5 \text { in. well }=85 \mathrm{ft} \mathrm{BGL} \text {. } \\
\text { Collect water with CPT. Push to } \\
\text { depth and pull back rods to } \\
\text { expose } 0.5 \text { in. PVC screen and } \\
\text { riser. Sampled using bailer } \\
\text { without purge. }\end{array}$ \\
\hline 8/13/15 & $14: 00$ & TI09 & YKTI09-95-W-37928 & $\mathrm{N}$ & $95-105$ & WG & YK814151700 & $8 / 14 / 15$ & $\begin{array}{l}\text { Depth to water }=100 \mathrm{ft} \mathrm{BGL} \text {. Depth } \\
\text { of } 0.5 \text { in. well }=105 \mathrm{ft} \mathrm{BGL} \text {. } \\
\text { Collect water with CPT. Push to } \\
\text { depth and pull back rods to } \\
\text { expose } 0.5 \text { in. PVC screen and } \\
\text { riser. Sampled using bailer } \\
\text { without purge. }\end{array}$ \\
\hline 8/13/15 & $16: 00$ & TI09 & YKTI09-135-W-37929 & $\mathrm{N}$ & $135-145$ & WG & YK814151700 & $8 / 14 / 15$ & $\begin{array}{l}\text { Depth to water }=99 \mathrm{ft} \mathrm{BGL} \text {. Depth } \\
\text { of } 0.5 \text { in. well }=145 \mathrm{ft} \text { BGL. } \\
\text { Collect water with CPT. Push to } \\
\text { depth and pull back rods to } \\
\text { expose } 0.5 \text { in. PVC screen and } \\
\text { riser. Sampled using bailer } \\
\text { without purge. }\end{array}$ \\
\hline
\end{tabular}


TABLE A.1 (Cont.)

\begin{tabular}{|c|c|c|c|c|c|c|c|c|c|}
\hline \multicolumn{2}{|c|}{$\begin{array}{l}\text { Sample Date } \\
\text { and Time }\end{array}$} & \multirow{2}{*}{$\begin{array}{r}\text { Location } \\
\text { TI09 }\end{array}$} & \multirow{2}{*}{$\begin{array}{c}\text { Sample } \\
\text { YKTI09-135-W-37929DUP }\end{array}$} & \multirow{2}{*}{$\begin{array}{l}\text { Sample } \\
\text { Type }\end{array}$} & \multirow{2}{*}{$\begin{array}{c}\begin{array}{c}\text { Depth } \\
\text { (ft BGL) }\end{array} \\
135-145 \\
135-145\end{array}$} & \multirow{3}{*}{$\begin{array}{l}\text { Matrix } \\
\text { WG } \\
\text { WG }\end{array}$} & \multirow{3}{*}{$\begin{array}{l}\text { Chain of Custody } \\
\text { YK814151700 } \\
\text { YK814151710 }\end{array}$} & \multirow{3}{*}{$\begin{array}{l}\begin{array}{c}\text { Shipping } \\
\text { Date }\end{array} \\
8 / 14 / 15 \\
8 / 14 / 15\end{array}$} & \multirow{3}{*}{$\begin{array}{l}\text { Sample Description } \\
\text { Duplicate laboratory analysis. } \\
\text { Verification sample to TestAmerica, } \\
\text { Inc. }\end{array}$} \\
\hline $8 / 13 / 15$ & $16: 00$ & & & & & & & & \\
\hline 8/13/15 & $16: 00$ & TI09 & YKTI09-135-W-37929VER & VER & $135-145$ & & & & \\
\hline 8/14/15 & $12: 00$ & TI10 & YKTI10-78-W-37930 & $\mathrm{N}$ & $78-83$ & WG & YK814151700 & $8 / 14 / 15$ & $\begin{array}{l}\text { Depth to water }=80 \mathrm{ft} \mathrm{BGL} \text {. Depth } \\
\text { of } 0.5 \text { in. well }=83 \mathrm{ft} \mathrm{BGL} \text {. } \\
\text { Collect water with CPT. Push to } \\
\text { depth and pull back rods to } \\
\text { expose } 0.5 \text { in. PVC screen and } \\
\text { riser. Sampled using bailer } \\
\text { without purge. }\end{array}$ \\
\hline $8 / 14 / 15$ & $13: 30$ & TI10 & YKTI10-95DUP-W-37932 & DUP-F & $95-105$ & WG & YK814151700 & $8 / 14 / 15$ & Field replicate. \\
\hline
\end{tabular}
of $0.5 \mathrm{in}$. well $=105 \mathrm{ft} \mathrm{BGL}$. Collect water with CPT. Push to depth and pull back rods to expose 0.5 in. PVC screen and riser. Sampled using bailer without purge.

Depth to water $=90 \mathrm{ft} \mathrm{BGL}$. Depth of $0.5 \mathrm{in}$. well $=127 \mathrm{ft} \mathrm{BGL}$. Collect water with CPT. Push to depth and pull back rods to expose 0.5 in. PVC screen and riser. Sampled using bailer without purge.

$\begin{array}{llll}\text { 8/14/15 } & 15: 30 & \text { TI10 } & \text { YKTI10-117-W-37933DUP } \\ 8 / 14 / 15 & 15: 30 & \text { TI10 } & \text { YKTI10-117-W-37933VER } \\ \text { 8/17/15 } & 17: 30 & \text { QC } & \text { YKQCTB-W-37938 } \\ \text { 8/17/15 } & 17: 30 & \text { QC } & \text { YKQCTB-W-37938VER } \\ \text { 8/17/15 } & 18: 00 & \text { TI11 } & \text { YKTI11-80-W-37935 }\end{array}$

$\begin{array}{cccc}\text { DUP-L } & 117-127 & \text { WG } & \text { YK814151700 } \\ \text { VER } & 117-127 & \text { WG } & \text { YK814151710 } \\ \text { TB } & - & \text { WQC } & \text { YK819151710 } \\ \text { VER } & - & \text { WQC } & \text { YK820151755 } \\ \text { N } & 80-85 & \text { WG } & \text { YK819151710 }\end{array}$

\section{$8 / 14 / 15$}

8/14/15 Duplicate laboratory analysis. Verification sample to TestAmerica, Inc.

8/19/15 Trip blank with water samples shipped to the AGEM laboratory. 8/20/15 Verification sample to TestAmerica, Inc.

$8 / 19 / 15$ of $0.5 \mathrm{in}$. well $=85 \mathrm{ft} \mathrm{BGL}$. Collect water with CPT. Push to depth and pull back rods to expose 0.5 in. PVC screen and riser. Sampled using bailer without purge. 
TABLE A.1 (Cont.)

\begin{tabular}{|c|c|c|c|c|c|c|c|c|c|}
\hline $\begin{array}{r}\text { Sample } \\
\text { and T }\end{array}$ & $\begin{array}{l}\text { Date } \\
\text { me }\end{array}$ & Location & Sample & $\begin{array}{l}\text { Sample } \\
\text { Type }\end{array}$ & $\begin{array}{l}\text { Depth } \\
\text { (ft BGL) }\end{array}$ & Matrix & Chain of Custody & $\begin{array}{l}\text { Shipping } \\
\text { Date }\end{array}$ & Sample Description \\
\hline 8/18/15 & $16: 30$ & TI11 & YKTI11-92-W-37936 & $\mathrm{N}$ & $92-102$ & WG & YK819151710 & $8 / 19 / 15$ & $\begin{array}{l}\text { Depth to water }=88 \mathrm{ft} \mathrm{BGL} \text {. Depth } \\
\text { of } 0.5 \text { in. well }=102 \mathrm{ft} \mathrm{BGL} \text {. } \\
\text { Collect water with CPT. Push to } \\
\text { depth and pull back rods to } \\
\text { expose } 0.5 \text { in. PVC screen and } \\
\text { riser. Sampled using bailer } \\
\text { without purge. }\end{array}$ \\
\hline 8/18/15 & $16: 30$ & TI11 & YKTI11-92-W-37936DUP & DUP-L & $92-102$ & WG & YK819151710 & $8 / 19 / 15$ & Duplicate laboratory analysis. \\
\hline $8 / 18 / 15$ & $16: 30$ & TI11 & YKTI11-92-W-37936VER & VER & $92-102$ & WG & YK820151755 & $8 / 20 / 15$ & $\begin{array}{l}\text { Verification sample to TestAmerica, } \\
\text { Inc. }\end{array}$ \\
\hline 8/18/15 & 18:00 & Tl11 & YKTI11-112-W-37937 & $\mathrm{N}$ & $112-122$ & WG & YK819151710 & $8 / 19 / 15$ & $\begin{array}{l}\text { Depth to water }=102 \mathrm{ft} \mathrm{BGL} \text {. Depth } \\
\text { of } 0.5 \text { in. well }=122 \mathrm{ft} \mathrm{BGL} \text {. } \\
\text { Collect water with CPT. Push to } \\
\text { depth and pull back rods to } \\
\text { expose } 0.5 \text { in. PVC screen and } \\
\text { riser. Sampled using bailer } \\
\text { without purge. }\end{array}$ \\
\hline 8/18/15 & $18: 00$ & TI11 & YKTI11-112-W-37937DUP & DUP-L & $112-122$ & WG & YK819151710 & 8/19/15 & Duplicate laboratory analysis. \\
\hline $8 / 20 / 15$ & 9:00 & QC & YKQCTB-W-37944 & TB & - & WQC & YK820151750 & $8 / 20 / 15$ & $\begin{array}{l}\text { Trip blank with water samples } \\
\text { shipped to the AGEM laboratory. }\end{array}$ \\
\hline $8 / 20 / 15$ & $9: 30$ & TI12 & YKTI12-80-W-37939 & $\mathrm{N}$ & $80-85$ & WG & YK820151750 & $8 / 20 / 15$ & $\begin{array}{l}\text { Depth to water }=83 \mathrm{ft} \mathrm{BGL} \text {. Depth } \\
\text { of } 0.5 \text { in. well }=85 \mathrm{ft} \mathrm{BGL} \text {. } \\
\text { Collect water with CPT. Push to } \\
\text { depth and pull back rods to } \\
\text { expose } 0.5 \text { in. PVC screen and } \\
\text { riser. Sampled using bailer } \\
\text { without purge. }\end{array}$ \\
\hline $8 / 20 / 15$ & $9: 30$ & Tl12 & YKTI12-80-W-37939DUP & DUP-L & $80-85$ & WG & YK820151750 & 8/20/15 & Duplicate laboratory analysis. \\
\hline $8 / 20 / 15$ & $11: 45$ & TI12 & YKTI12-92-W-37940 & $\mathrm{N}$ & $92-102$ & WG & YK820151750 & $8 / 20 / 15$ & $\begin{array}{l}\text { Depth to water }=90 \mathrm{ft} \text { BGL. Depth } \\
\text { of } 0.5 \text { in. well }=102 \mathrm{ft} \text { BGL. } \\
\text { Collect water with CPT. Push to } \\
\text { depth and pull back rods to } \\
\text { expose } 0.5 \text { in. PVC screen and } \\
\text { riser. Sampled using bailer } \\
\text { without purge. }\end{array}$ \\
\hline $8 / 20 / 15$ & $11: 45$ & TI12 & YKTI12-DUP92-W-37941 & DUP-F & $92-102$ & WG & YK820151750 & $8 / 20 / 15$ & Field replicate. \\
\hline $8 / 20 / 15$ & $15: 30$ & TI12 & YKTI12-120-W-37942 & $\mathrm{N}$ & $110-120$ & WG & YK820151750 & $8 / 20 / 15$ & $\begin{array}{l}\text { Depth to water }=102 \mathrm{ft} \mathrm{BGL} \text {. Depth } \\
\text { of } 0.5 \text { in. well }=120 \mathrm{ft} \mathrm{BGL} \text { B } \\
\text { Collect water with CPT. Push to } \\
\text { depth and pull back rods to } \\
\text { expose } 0.5 \text { in. PVC screen and } \\
\text { riser. Sampled using bailer } \\
\text { without purge. }\end{array}$ \\
\hline
\end{tabular}


TABLE A.1 (Cont.)

\begin{tabular}{|c|c|c|c|c|c|c|c|c|c|}
\hline \multicolumn{2}{|c|}{$\begin{array}{l}\text { Sample Date } \\
\text { and Time }\end{array}$} & \multirow[t]{2}{*}{ Location } & \multirow[b]{2}{*}{ YKTI12-145-W-37943 } & \multirow{2}{*}{$\begin{array}{c}\text { Sample } \\
\text { Type } \\
\text { N }\end{array}$} & \multirow{2}{*}{$\begin{array}{c}\text { Depth } \\
\text { (ft BGL) }\end{array}$} & \multirow{2}{*}{$\begin{array}{c}\text { Matrix } \\
\text { WG }\end{array}$} & \multirow{2}{*}{$\begin{array}{l}\text { Chain of Custody } \\
\text { YK820151750 }\end{array}$} & \multirow{2}{*}{$\begin{array}{l}\begin{array}{l}\text { Shipping } \\
\text { Date }\end{array} \\
8 / 20 / 15\end{array}$} & Sample Description \\
\hline $8 / 20 / 15$ & $17: 15$ & & & & & & & & $\begin{array}{l}\text { Depth to water }=119 \mathrm{ft} \text { BGL. Depth } \\
\text { of } 0.5 \text { in. well }=145 \mathrm{ft} \mathrm{BGL} \text {. } \\
\text { Collect water with CPT. Push to } \\
\text { depth and pull back rods to } \\
\text { expose } 0.5 \text { in. PVC screen and } \\
\text { riser. Sampled using bailer } \\
\text { without purge. }\end{array}$ \\
\hline $8 / 20 / 15$ & $17: 15$ & TI12 & YKTI12-145-W-37943VER & VER & $135-145$ & WG & YK820151755 & $8 / 20 / 15$ & $\begin{array}{l}\text { Verification sample to TestAmerica, } \\
\text { Inc. }\end{array}$ \\
\hline $8 / 22 / 15$ & $8: 00$ & QC & YKQCTB-W-37950 & TB & - & WQC & YK8222151150 & $8 / 22 / 15$ & $\begin{array}{l}\text { Trip blank with water samples } \\
\text { shipped to the AGEM laboratory. }\end{array}$ \\
\hline $8 / 22 / 15$ & $8: 30$ & TI13 & YKTI13-93-W-37947 & $\mathrm{N}$ & $93-103$ & WG & YK8222151150 & $8 / 22 / 15$ & Depth to water $=85 \mathrm{ft}$ BGL. Depth \\
\hline
\end{tabular}

$\begin{array}{rrrl}8 / 22 / 15 & 8: 30 & \text { TI13 } & \text { YKTI13-93-W-37947DUP } \\ 8 / 22 / 15 & 10: 30 & \text { TI13 } & \text { YKTI13-111DUP-W-37949 } \\ 8 / 22 / 15 & 10: 30 & \text { TI13 } & \text { YKTI13-111-W-37948 }\end{array}$

8/22/15 $10: 30 \quad$ Tl13

8/23/15 9:00 QC

8/23/15 9:00 QC

8/23/15 10:30 TI14

8/23/15 12:30 TI14
YKTI14-113DUP-W-37953

\section{YKQCTB-W-37960}

YKQCTB-W-37960VER

YKTI14-86-W-37951

$\begin{array}{cccc}\text { TB } & - & \text { WQC } & \text { YK824151700 } \\ \text { VER } & - & \text { WQC } & \text { YK828151310 } \\ \text { N } & 86-96 & \text { WG } & \text { YK824151700 }\end{array}$

DUP-F

$\begin{array}{cccc}\text { DUP-L } & 93-103 & \text { WG } & \text { YK8222151150 } \\ \text { DUP-F } & 111-121 & \text { WG } & \text { YK8222151150 } \\ \text { N } & 111-121 & \text { WG } & \text { YK8222151150 }\end{array}$

$8 / 22 / 15$

$8 / 22 / 15$

$8 / 22 / 15$

$8 / 24 / 15$

$8 / 28 / 15$

$8 / 28 / 15$

$8 / 24 / 15$ shipped to the AGEM laborator of $0.5 \mathrm{in}$. well $=103 \mathrm{ft} \mathrm{BGL}$.
. Collect water with CPT. Push to depth and pull back rods to expose 0.5 in. PVC screen and riser. Sampled using bailer

without purge.
Duplicate laboratory analysis. Field replicate.

Depth to water $=106 \mathrm{ft} \mathrm{BGL}$. Depth of $0.5 \mathrm{in}$. well $=121 \mathrm{ft} \mathrm{BGL}$.

Collect water with CPT. Push to depth and pull back rods to expose 0.5 in. PVC screen and riser. Sampled using bailer without purge.

Trip blank with water samples shipped to the AGEM laboratory. erification sample to TestAmerica, Inc.

Depth to water $=82.5 \mathrm{ft} \mathrm{BGL}$

Depth of 0.5 in. well $=96 \mathrm{ft} \mathrm{BGL}$. Collect water with CPT. Push to depth and pull back rods to expose 0.5 in. PVC screen and riser. Sampled using bailer without purge.

8/24/15 Field replicate. 
TABLE A.1 (Cont.)

\begin{tabular}{|c|c|c|c|c|c|c|c|c|c|}
\hline \multicolumn{2}{|c|}{$\begin{array}{l}\text { Sample Date } \\
\text { and Time }\end{array}$} & \multirow{2}{*}{$\begin{array}{r}\text { Location } \\
\text { TI14 }\end{array}$} & \multirow{2}{*}{$\begin{array}{r}\text { Sample } \\
\text { YKTI14-113-W-37952 }\end{array}$} & \multirow{2}{*}{$\begin{array}{c}\text { Sample } \\
\text { Type }\end{array}$} & \multirow{2}{*}{$\begin{array}{c}\text { Depth } \\
\text { (ft BGL) } \\
113-123\end{array}$} & \multirow{2}{*}{$\begin{array}{c}\text { Matrix } \\
\text { WG }\end{array}$} & \multirow{2}{*}{$\begin{array}{l}\text { Chain of Custody } \\
\text { YK824151700 }\end{array}$} & \multirow{2}{*}{$\begin{array}{l}\text { Shipping } \\
\text { Date }\end{array}$} & \multirow{2}{*}{$\begin{array}{l}\text { Sample Description } \\
\text { Depth to water }=90 \mathrm{ft} \text { BGL. Depth } \\
\text { of } 0.5 \text { in. well }=123 \mathrm{ft} \text { BGL. } \\
\text { Collect water with CPT. Push to } \\
\text { depth and pull back rods to } \\
\text { expose } 0.5 \text { in. PVC screen and } \\
\text { riser. Sampled using bailer } \\
\text { without purge. }\end{array}$} \\
\hline $8 / 23 / 15$ & $12: 30$ & & & & & & & & \\
\hline $8 / 23 / 15$ & $14: 45$ & TI14 & YKTI14-136-W-37954 & $\mathrm{N}$ & $136-146$ & WG & YK824151700 & $8 / 24 / 15$ & $\begin{array}{l}\text { Depth to water }=100 \mathrm{ft} \mathrm{BGL} \text {. Depth } \\
\text { of } 0.5 \mathrm{in} \text {. well }=146 \mathrm{ft} \mathrm{BGL} \text {. } \\
\text { Collect water with CPT. Push to } \\
\text { depth and pull back rods to } \\
\text { expose } 0.5 \text { in. PVC screen and } \\
\text { riser. Sampled using bailer } \\
\text { without purge. }\end{array}$ \\
\hline $8 / 23 / 15$ & $14: 45$ & TI14 & YKTI14-136-W-37954VER & VER & $136-146$ & WG & YK828151310 & $8 / 28 / 15$ & $\begin{array}{l}\text { Verification sample to TestAmerica, } \\
\text { Inc. }\end{array}$ \\
\hline $8 / 24 / 15$ & $8: 30$ & TI13 & YKTI13-82-W-37955 & $\mathrm{N}$ & $81-86$ & WG & YK824151700 & $8 / 24 / 15$ & $\begin{array}{l}\text { Depth to water }=74 \mathrm{ft} \mathrm{BGL} \text {. Depth } \\
\text { of } 0.5 \text { in. well }=86 \mathrm{ft} \mathrm{BGL} \text {. } \\
\text { Collect water with CPT. Push to } \\
\text { depth and pull back rods to } \\
\text { expose } 0.5 \text { in. PVC screen and } \\
\text { riser. Sampled using bailer } \\
\text { without purge. }\end{array}$ \\
\hline $8 / 24 / 15$ & $9: 45$ & TI15 & YKTI15-90-W-37956 & $\mathrm{N}$ & $90-100$ & WG & YK824151700 & $8 / 24 / 15$ & $\begin{array}{l}\text { Depth to water }=89 \mathrm{ft} \mathrm{BGL} \text {. Depth } \\
\text { of } 0.5 \text { in. well }=100 \mathrm{ft} \mathrm{BGL} \text {. } \\
\text { Collect water with CPT. Push to } \\
\text { depth and pull back rods to } \\
\text { expose } 0.5 \text { in. PVC screen and } \\
\text { riser. Sampled using bailer } \\
\text { without purge. }\end{array}$ \\
\hline $8 / 24 / 15$ & $11: 30$ & TI15 & YKTI15-113-W-37957 & $\mathrm{N}$ & $113-123$ & WG & YK824151700 & $8 / 24 / 15$ & $\begin{array}{l}\text { Depth to water }=90 \mathrm{ft} \text { BGL. Depth } \\
\text { of } 0.5 \mathrm{in} \text {. well }=123 \mathrm{ft} \mathrm{BGL} \text {. } \\
\text { Collect water with CPT. Push to } \\
\text { depth and pull back rods to } \\
\text { expose } 0.5 \text { in. PVC screen and } \\
\text { riser. Sampled using bailer } \\
\text { without purge. }\end{array}$ \\
\hline $8 / 24 / 15$ & $11: 30$ & TI15 & YKTI15-113-W-37957DUP & DUP-L & $113-123$ & WG & YK824151700 & $8 / 24 / 15$ & Duplicate laboratory analysis. \\
\hline $8 / 24 / 15$ & $14: 15$ & TI15 & YKTI15-134DUP-W-37959 & DUP-F & $134-144$ & WG & YK824151700 & $8 / 24 / 15$ & Field replicate. \\
\hline
\end{tabular}


TABLE A.1 (Cont.)

\begin{tabular}{|c|c|c|c|c|c|c|c|c|c|}
\hline $\begin{array}{r}\text { Sample } \\
\text { and Ti }\end{array}$ & $\begin{array}{l}\text { Date } \\
\text { me }\end{array}$ & Location & Sample & $\begin{array}{l}\text { Sample } \\
\text { Type }\end{array}$ & $\begin{array}{l}\text { Depth } \\
\text { (ft BGL) }\end{array}$ & Matrix & Chain of Custody & $\begin{array}{l}\text { Shipping } \\
\text { Date }\end{array}$ & Sample Description \\
\hline $8 / 24 / 15$ & $14: 15$ & TI15 & YKTI15-134-W-37958 & $\mathrm{N}$ & $134-144$ & WG & YK824151700 & $8 / 24 / 15$ & $\begin{array}{l}\text { Depth to water }=110 \mathrm{ft} \mathrm{BGL} \text {. Depth } \\
\text { of } 0.5 \text { in. well }=144 \mathrm{ft} \mathrm{BGL} \text {. } \\
\text { Collect water with CPT. Push to } \\
\text { depth and pull back rods to } \\
\text { expose } 0.5 \text { in. PVC screen and } \\
\text { riser. Sampled using bailer } \\
\text { without purge. }\end{array}$ \\
\hline $8 / 25 / 15$ & 9:00 & QC & YKQCTB-W-37965 & TB & - & WQC & YK825151700 & $8 / 25 / 15$ & $\begin{array}{l}\text { Trip blank with water samples } \\
\text { shipped to the AGEM laboratory. }\end{array}$ \\
\hline $8 / 25 / 15$ & $10: 00$ & TI16 & YKTI16-86DUP-W-37962 & DUP-F & $86-96$ & WG & YK825151700 & $8 / 25 / 15$ & Field replicate. \\
\hline $8 / 25 / 15$ & $10: 00$ & TI16 & YKTI16-86-W-37961 & $N$ & $86-96$ & WG & YK825151700 & $8 / 25 / 15$ & $\begin{array}{l}\text { Depth to water }=84 \mathrm{ft} \mathrm{BGL} \text {. Depth } \\
\text { of } 0.5 \text { in. well }=96 \mathrm{ft} \text { BGL. } \\
\text { Collect water with CPT. Push to } \\
\text { depth and pull back rods to } \\
\text { expose } 0.5 \text { in. PVC screen and } \\
\text { riser. Sampled using bailer } \\
\text { without purge. }\end{array}$ \\
\hline $8 / 25 / 15$ & $11: 45$ & TI16 & YKTI16-105-W-37963 & $\mathrm{N}$ & $105-115$ & WG & YK825151700 & $8 / 25 / 15$ & $\begin{array}{l}\text { Depth to water }=85 \mathrm{ft} \mathrm{BGL} \text {. Depth } \\
\text { of } 0.5 \text { in. well }=115 \mathrm{ft} \mathrm{BGL} \text {. } \\
\text { Collect water with CPT. Push to } \\
\text { depth and pull back rods to } \\
\text { expose } 0.5 \text { in. PVC screen and } \\
\text { riser. Sampled using bailer } \\
\text { without purge. }\end{array}$ \\
\hline $8 / 25 / 15$ & $11: 45$ & TI16 & YKTI16-105-W-37963DUP & DUP-L & $105-115$ & WG & YK825151700 & $8 / 25 / 15$ & Duplicate laboratory analysis. \\
\hline 8/25/15 & $14: 50$ & TI16 & YKTI16-136-W-37964 & $\mathrm{N}$ & $136-146$ & WG & YK825151700 & $8 / 25 / 15$ & $\begin{array}{l}\text { Depth to water }=92 \mathrm{ft} \mathrm{BGL} \text {. Depth } \\
\text { of } 0.5 \text { in. well }=146 \mathrm{ft} \text { BGL. } \\
\text { Collect water with CPT. Push to } \\
\text { depth and pull back rods to } \\
\text { expose } 0.5 \text { in. PVC screen and } \\
\text { riser. Sampled using bailer } \\
\text { without purge. }\end{array}$ \\
\hline $8 / 25 / 15$ & $14: 50$ & TI16 & YKTI16-136-W-37964VER & VER & $136-146$ & WG & YK828151310 & $8 / 28 / 15$ & $\begin{array}{l}\text { Verification sample to TestAmerica, } \\
\text { Inc. }\end{array}$ \\
\hline $8 / 26 / 15$ & $9: 00$ & QC & YKQCTB-W-37969 & TB & - & WQC & YK826151700 & $8 / 26 / 15$ & $\begin{array}{l}\text { Trip blank with water samples } \\
\text { shipped to the AGEM laboratory. }\end{array}$ \\
\hline 8/26/15 & $10: 15$ & TI17 & YKTI17-84-W-37966 & $\mathrm{N}$ & $84-94$ & WG & YK826151700 & $8 / 26 / 15$ & $\begin{array}{l}\text { Depth to water }=81 \mathrm{ft} \text { BGL. Depth } \\
\text { of } 0.5 \text { in. well }=94 \mathrm{ft} \text { BGL. } \\
\text { Collect water with CPT. Push to } \\
\text { depth and pull back rods to } \\
\text { expose } 0.5 \text { in. PVC screen and } \\
\text { riser. Sampled using bailer } \\
\text { without purge. }\end{array}$ \\
\hline
\end{tabular}


TABLE A.1 (Cont.)

\begin{tabular}{|c|c|c|c|c|c|c|c|c|c|}
\hline $\begin{array}{l}\text { Sample } \\
\text { and Ti }\end{array}$ & $\begin{array}{l}\text { Date } \\
\text { ime }\end{array}$ & Location & Sample & $\begin{array}{l}\text { Sample } \\
\text { Type }\end{array}$ & $\begin{array}{l}\text { Depth } \\
\text { (ft BGL) }\end{array}$ & Matrix & Chain of Custody & $\begin{array}{l}\text { Shipping } \\
\text { Date }\end{array}$ & Sample Description \\
\hline $8 / 26 / 15$ & $15: 00$ & TI17 & YKTI17-104-W-37967 & $\mathrm{N}$ & $104-114$ & WG & YK826151700 & $8 / 26 / 15$ & $\begin{array}{l}\text { Depth to water }=91 \mathrm{ft} \mathrm{BGL} \text {. Depth } \\
\text { of } 0.5 \text { in. well }=114 \mathrm{ft} \mathrm{BGL} \text {. } \\
\text { Collect water with CPT. Push to } \\
\text { depth and pull back rods to } \\
\text { expose } 0.5 \text { in. PVC screen and } \\
\text { riser. Sampled using bailer } \\
\text { without purge. }\end{array}$ \\
\hline $8 / 26 / 15$ & $16: 35$ & TI17 & YKTI17-133DUP-W-37968 & DUP-F & $133-143$ & WG & YK826151700 & $8 / 26 / 15$ & Field replicate. \\
\hline $8 / 26 / 15$ & $16: 35$ & TI17 & YKTI17-133-W-37968 & $\mathrm{N}$ & $133-143$ & WG & YK826151700 & $8 / 26 / 15$ & $\begin{array}{l}\text { Depth to water }=90 \mathrm{ft} \text { BGL. Depth } \\
\text { of } 0.5 \text { in. well }=143 \mathrm{ft} \mathrm{BGL} \text {. } \\
\text { Collect water with CPT. Push to } \\
\text { depth and pull back rods to } \\
\text { expose } 0.5 \text { in. PVC screen and } \\
\text { riser. Sampled using bailer } \\
\text { without purge. }\end{array}$ \\
\hline 8/26/15 & $16: 35$ & TI17 & YKTI17-133-W-37968DUP & DUP-L & $133-143$ & WG & YK826151700 & $8 / 26 / 15$ & Duplicate laboratory analysis. \\
\hline $8 / 27 / 15$ & $9: 00$ & QC & YKQCTB-W-37974 & TB & - & WQC & YK828151300 & 8/28/15 & $\begin{array}{l}\text { Trip blank with water samples } \\
\text { shipped to the AGEM laboratory. }\end{array}$ \\
\hline $8 / 27 / 15$ & $16: 30$ & TI18 & YKTI18-81-W-37970 & $\mathrm{N}$ & $81-86$ & WG & YK828151300 & 8/28/15 & $\begin{array}{l}\text { Depth to water }=82 \mathrm{ft} \text { BGL. Depth } \\
\text { of } 0.5 \text { in. well }=86 \mathrm{ft} \mathrm{BGL} \text {. } \\
\text { Collect water with CPT. Push to } \\
\text { depth and pull back rods to } \\
\text { expose } 0.5 \text { in. PVC screen and } \\
\text { riser. Sampled using bailer } \\
\text { without purge. }\end{array}$ \\
\hline $8 / 27 / 15$ & $18: 00$ & TI18 & YKTI18-95DUP-W-37972 & DUP-F & $95-105$ & WG & YK828151300 & $8 / 28 / 15$ & Field replicate. \\
\hline $8 / 27 / 15$ & $18: 00$ & TI18 & YKTI18-95-W-37971 & $\mathrm{N}$ & $95-105$ & WG & YK828151300 & $8 / 28 / 15$ & $\begin{array}{l}\text { Depth to water }=90 \mathrm{ft} \text { BGL. Depth } \\
\text { of } 0.5 \text { in. well }=105 \mathrm{ft} \mathrm{BGL} \text {. } \\
\text { Collect water with CPT. Push to } \\
\text { depth and pull back rods to } \\
\text { expose } 0.5 \text { in. PVC screen and } \\
\text { riser. Sampled using bailer } \\
\text { without purge. }\end{array}$ \\
\hline $8 / 28 / 15$ & $11: 00$ & TI18 & YKTI18-114-W-37973 & $\mathrm{N}$ & $114-124$ & WG & YK828151300 & 8/28/15 & $\begin{array}{l}\text { Depth to water }=102 \mathrm{ft} \mathrm{BGL} \text {. Depth } \\
\text { of } 0.5 \text { in. well }=124 \mathrm{ft} \mathrm{BGL} \text {. } \\
\text { Collect water with CPT. Push to } \\
\text { depth and pull back rods to } \\
\text { expose } 0.5 \text { in. PVC screen and } \\
\text { riser. Sampled using bailer } \\
\text { without purge. }\end{array}$ \\
\hline 8/28/15 & $11: 00$ & TI18 & YKTI18-114-W-37973DUP & DUP-L & $114-124$ & WG & YK828151300 & $8 / 28 / 15$ & Duplicate laboratory analysis. \\
\hline $8 / 28 / 15$ & $11: 00$ & TI18 & YKTI18-114-W-37973VER & VER & $114-124$ & WG & YK828151310 & $8 / 28 / 15$ & $\begin{array}{l}\text { Verification sample to TestAmerica, } \\
\text { Inc. }\end{array}$ \\
\hline
\end{tabular}


TABLE A.1 (Cont.)

\begin{tabular}{|c|c|c|c|c|c|c|c|c|c|}
\hline $\begin{array}{l}\text { Sample } \\
\text { and Tir }\end{array}$ & $\begin{array}{l}\text { Date } \\
\text { ime }\end{array}$ & Location & Sample & $\begin{array}{l}\text { Sample } \\
\text { Type }\end{array}$ & $\begin{array}{l}\text { Depth } \\
\text { (ft BGL) }\end{array}$ & Matrix & Chain of Custody & $\begin{array}{l}\text { Shipping } \\
\text { Date }\end{array}$ & Sample Description \\
\hline 9/9/15 & $8: 00$ & QC & YKQCTB-W-37979 & TB & - & WQC & YK99151700 & $9 / 9 / 15$ & $\begin{array}{l}\text { Trip blank with water samples } \\
\text { shipped to the AGEM laboratory. }\end{array}$ \\
\hline 9/9/15 & $9: 30$ & TI19 & YKTI19-84-W-37975 & $\mathrm{N}$ & $84-94$ & WG & YK99151700 & $9 / 9 / 15$ & $\begin{array}{l}\text { Depth to water }=80 \mathrm{ft} \mathrm{BGL} \text {. Depth } \\
\text { of } 0.5 \text { in. well }=94 \mathrm{ft} \mathrm{BGL} \text {. } \\
\text { Collect water with CPT. Push to } \\
\text { depth and pull back rods to } \\
\text { expose } 0.5 \text { in. PVC screen and } \\
\text { riser. Sampled using bailer } \\
\text { without purge. }\end{array}$ \\
\hline 9/9/15 & $11: 30$ & TI19 & YKTI19-107-W-37976 & $\mathrm{N}$ & $107-117$ & WG & YK99151700 & $9 / 9 / 15$ & $\begin{array}{l}\text { Depth to water }=107 \mathrm{ft} \mathrm{BGL} \text {. Depth } \\
\text { of } 0.5 \text { in. well }=117 \mathrm{ft} \mathrm{BGL} \text {. } \\
\text { Collect water with CPT. Push to } \\
\text { depth and pull back rods to } \\
\text { expose } 0.5 \text { in. PVC screen and } \\
\text { riser. Sampled using bailer } \\
\text { without purge. }\end{array}$ \\
\hline 9/9/15 & $11: 30$ & TI19 & YKTI19-107-W-37976DUP & DUP-L & $107-117$ & WG & YK99151700 & $9 / 9 / 15$ & Duplicate laboratory analysis. \\
\hline 9/9/15 & $14: 00$ & TI19 & YKTI19-129DUP-W-37978 & DUP-F & 129-139 & WG & YK99151700 & 9/9/15 & Field replicate. \\
\hline 9/9/15 & $14: 00$ & TI19 & YKTI19-129-W-37977 & $\mathrm{N}$ & $129-139$ & WG & YK99151700 & $9 / 9 / 15$ & $\begin{array}{l}\text { Depth to water }=110 \mathrm{ft} \mathrm{BGL} \text {. Depth } \\
\text { of } 0.5 \text { in. well }=139 \mathrm{ft} \mathrm{BGL} \text {. } \\
\text { Collect water with CPT. Push to } \\
\text { depth and pull back rods to } \\
\text { expose } 0.5 \text { in. PVC screen and } \\
\text { riser. Sampled using bailer } \\
\text { without purge. }\end{array}$ \\
\hline 9/10/15 & $8: 00$ & QC & YKQCTB-W-37984 & TB & - & WQC & YK910151700 & $9 / 10 / 15$ & $\begin{array}{l}\text { Trip blank with water samples } \\
\text { shipped to the AGEM laboratory. }\end{array}$ \\
\hline 9/10/15 & $8: 00$ & QC & YKQCTB-W-37984VER & VER & - & WQC & YK915151755 & $9 / 15 / 15$ & $\begin{array}{l}\text { Verification sample to TestAmerica, } \\
\text { Inc. }\end{array}$ \\
\hline 9/10/15 & $10: 30$ & TI20 & YKTI20-81-W-37980 & $\mathrm{N}$ & $81-91$ & WG & YK910151700 & $9 / 10 / 15$ & $\begin{array}{l}\text { Depth to water }=80 \mathrm{ft} \mathrm{BGL} \text {. Depth } \\
\text { of } 0.5 \text { in. well }=91 \mathrm{ft} \mathrm{BGL} \text {. } \\
\text { Collect water with CPT. Push to } \\
\text { depth and pull back rods to } \\
\text { expose } 0.5 \text { in. PVC screen and } \\
\text { riser. Sampled using bailer } \\
\text { without purge. }\end{array}$ \\
\hline $9 / 10 / 15$ & $12: 00$ & TI20 & YKTI20-105-W-37981 & $\mathrm{N}$ & $105-115$ & WG & YK910151700 & $9 / 10 / 15$ & $\begin{array}{l}\text { Depth to water }=91 \mathrm{ft} \mathrm{BGL} \text {. Depth } \\
\text { of } 0.5 \text { in. well }=115 \mathrm{ft} \mathrm{BGL} \text {. } \\
\text { Collect water with CPT. Push to } \\
\text { depth and pull back rods to } \\
\text { expose } 0.5 \text { in. PVC screen and } \\
\text { riser. Sampled using bailer } \\
\text { without purge. }\end{array}$ \\
\hline
\end{tabular}


TABLE A.1 (Cont.)

\begin{tabular}{|c|c|c|c|c|c|c|c|c|c|}
\hline \multicolumn{2}{|c|}{$\begin{array}{l}\text { Sample Date } \\
\text { and Time }\end{array}$} & Location & Sample & $\begin{array}{l}\text { Sample } \\
\text { Type }\end{array}$ & $\begin{array}{l}\text { Depth } \\
\text { (ft BGL) }\end{array}$ & Matrix & Chain of Custody & $\begin{array}{l}\text { Shipping } \\
\text { Date }\end{array}$ & Sample Description \\
\hline 9/10/15 & $12: 00$ & $\mathrm{~T} 120$ & YKTI20-105-W-37981DUP & DUP-L & $105-115$ & WG & YK910151700 & 9/10/15 & Duplicate laboratory analysis. \\
\hline 9/10/15 & $12: 00$ & $\mathrm{TI} 20$ & YKTI20-105-W-37981VER & VER & $105-115$ & WG & YK915151755 & $9 / 15 / 15$ & $\begin{array}{l}\text { Verification sample to TestAmerica, } \\
\text { Inc. }\end{array}$ \\
\hline 9/10/15 & $15: 00$ & TI20 & YKTI20-131DUP-W-37983 & DUP-F & $131-141$ & WG & YK910151700 & 9/10/15 & Field replicate. \\
\hline 9/10/15 & $15: 00$ & TI20 & YKTI20-131-W-37982 & $\mathrm{N}$ & $131-141$ & WG & YK910151700 & $9 / 10 / 15$ & $\begin{array}{l}\text { Depth to water }=101 \mathrm{ft} \mathrm{BGL} \text {. Depth } \\
\text { of } 0.5 \text { in. well }=141 \mathrm{ft} \text { BGL. } \\
\text { Collect water with CPT. Push to } \\
\text { depth and pull back rods to } \\
\text { expose } 0.5 \text { in. PVC screen and } \\
\text { riser. Sampled using bailer } \\
\text { without purge. }\end{array}$ \\
\hline 9/11/15 & $8: 00$ & QC & YKQCTB-W-37989 & TB & - & WQC & YK911151800 & $9 / 11 / 15$ & $\begin{array}{l}\text { Trip blank with water samples } \\
\text { shipped to the AGEM laboratory. }\end{array}$ \\
\hline 9/11/15 & $14: 00$ & $\mathrm{~T} 121$ & YKTI21-77-W-37985 & $\mathrm{N}$ & $77-87$ & WG & YK911151800 & $9 / 11 / 15$ & $\begin{array}{l}\text { Depth to water }=75.5 \mathrm{ft} \text { BGL. } \\
\text { Depth of } 0.5 \text { in. well }=87 \mathrm{ft} \mathrm{BGL} \text {. } \\
\text { Collect water with CPT. Push to } \\
\text { depth and pull back rods to } \\
\text { expose } 0.5 \text { in. PVC screen and } \\
\text { riser. Sampled using bailer } \\
\text { without purge. }\end{array}$ \\
\hline 9/11/15 & $15: 30$ & $\mathrm{~T} 121$ & YKTI21-99-W-37986 & $\mathrm{N}$ & $99-109$ & WG & YK911151800 & $9 / 11 / 15$ & $\begin{array}{l}\text { Depth to water }=102 \mathrm{ft} \mathrm{BGL} \text {. Depth } \\
\text { of } 0.5 \mathrm{in} \text {. well }=109 \mathrm{ft} \text { BGL. } \\
\text { Collect water with CPT. Push to } \\
\text { depth and pull back rods to } \\
\text { expose } 0.5 \text { in. PVC screen and } \\
\text { riser. Sampled using bailer } \\
\text { without purge. }\end{array}$ \\
\hline 9/11/15 & $15: 30$ & TI21 & YKTI21-99-W-37986DUP & DUP-L & $99-109$ & WG & YK911151800 & $9 / 11 / 15$ & Duplicate laboratory analysis. \\
\hline $9 / 11 / 15$ & $15: 30$ & $\mathrm{~T} 121$ & YKTI21-99-W-37986VER & VER & $99-109$ & WG & YK915151755 & $9 / 15 / 15$ & $\begin{array}{l}\text { Verification sample to TestAmerica, } \\
\text { Inc. }\end{array}$ \\
\hline 9/11/15 & $17: 45$ & TI21 & YKTI21-115DUP-W-37988 & DUP-F & $115-120$ & WG & YK911151800 & $9 / 11 / 15$ & Field replicate. \\
\hline 9/11/15 & $17: 45$ & TI21 & YKTI21-115-W-37987 & $\mathrm{N}$ & $115-120$ & WG & YK911151800 & $9 / 11 / 15$ & $\begin{array}{l}\text { Depth to water }=110 \mathrm{ft} \text { BGL. Depth } \\
\text { of } 0.5 \text { in. well }=120 \mathrm{ft} \mathrm{BGL} \text {. } \\
\text { Collect water with CPT. Push to } \\
\text { depth and pull back rods to } \\
\text { expose } 0.5 \text { in. PVC screen and } \\
\text { riser. Sampled using bailer } \\
\text { without purge. }\end{array}$ \\
\hline 9/14/15 & $8: 00$ & QC & YKQCTB-W-37994 & TB & - & WQC & YK915151800 & $9 / 15 / 15$ & $\begin{array}{l}\text { Trip blank with water samples } \\
\text { shipped to the AGEM laboratory. }\end{array}$ \\
\hline
\end{tabular}

YKQCTB-W-37994 
TABLE A.1 (Cont.)

\begin{tabular}{|c|c|c|c|c|c|c|c|c|c|}
\hline \multicolumn{2}{|c|}{$\begin{array}{l}\text { Sample Date } \\
\text { and Time }\end{array}$} & \multirow{2}{*}{$\begin{array}{r}\text { Location } \\
\mathrm{TI} 22\end{array}$} & \multirow{2}{*}{$\begin{array}{r}\text { Sample } \\
\text { YKTI22-79-W-37990 }\end{array}$} & \multirow{2}{*}{$\begin{array}{c}\text { Sample } \\
\text { Type }\end{array}$} & \multirow{2}{*}{$\begin{array}{c}\text { Depth } \\
\text { (ft BGL) } \\
79-84\end{array}$} & \multirow{2}{*}{$\begin{array}{c}\text { Matrix } \\
\text { WG }\end{array}$} & \multirow{2}{*}{$\begin{array}{l}\text { Chain of Custody } \\
\text { YK915151800 }\end{array}$} & \multirow{2}{*}{$\begin{array}{l}\begin{array}{l}\text { Shipping } \\
\text { Date }\end{array} \\
9 / 15 / 15\end{array}$} & \multirow{2}{*}{$\begin{array}{l}\text { Sample Description } \\
\text { Depth to water }=81 \mathrm{ft} \mathrm{BGL} \text {. Depth } \\
\text { of } 0.5 \text { in. well }=84 \mathrm{ft} \mathrm{BGL} \text {. } \\
\text { Collect water with CPT. Push to } \\
\text { depth and pull back rods to } \\
\text { expose } 0.5 \text { in. PVC screen and } \\
\text { riser. Sampled using bailer } \\
\text { without purge. }\end{array}$} \\
\hline 9/14/15 & $14: 30$ & & & & & & & & \\
\hline 9/14/15 & $16: 30$ & TI22 & YKTI22-98DUP-W-37993 & DUP-F & $98-108$ & WG & YK915151800 & $9 / 15 / 15$ & Field replicate. \\
\hline 9/14/15 & $16: 30$ & $\mathrm{TI} 22$ & YKTI22-98-W-37991 & $\mathrm{N}$ & $98-108$ & WG & YK915151800 & $9 / 15 / 15$ & $\begin{array}{l}\text { Depth to water }=89 \mathrm{ft} \text { BGL. Depth } \\
\text { of } 0.5 \text { in. well }=108 \mathrm{ft} \mathrm{BGL} \text {. } \\
\text { Collect water with CPT. Push to } \\
\text { depth and pull back rods to } \\
\text { expose } 0.5 \text { in. PVC screen and } \\
\text { riser. Sampled using bailer } \\
\text { without purge. }\end{array}$ \\
\hline 9/14/15 & $18: 30$ & $\mathrm{~T} 122$ & YKTI22-113-W-37992 & $\mathrm{N}$ & $113-123$ & WG & YK915151800 & $9 / 15 / 15$ & $\begin{array}{l}\text { Depth to water }=112 \mathrm{ft} \mathrm{BGL} \text {. Depth } \\
\text { of } 0.5 \text { in. well }=123 \mathrm{ft} \mathrm{BGL} \text {. } \\
\text { Collect water with CPT. Push to } \\
\text { depth and pull back rods to } \\
\text { expose } 0.5 \text { in. PVC screen and } \\
\text { riser. Sampled using bailer } \\
\text { without purge. }\end{array}$ \\
\hline 9/15/15 & $13: 40$ & $\mathrm{~T} 123$ & YKTI23-80-W-37995 & $\mathrm{N}$ & $80-90$ & WG & YK915151800 & $9 / 15 / 15$ & $\begin{array}{l}\text { Depth to water }=88.5 \mathrm{ft} \mathrm{BGL} \text {. } \\
\text { Depth of } 0.5 \text { in. well }=90 \mathrm{ft} \text { BGL. } \\
\text { Collect water with CPT. Push to } \\
\text { depth and pull back rods to } \\
\text { expose } 0.5 \text { in. PVC screen and } \\
\text { riser. Sampled using bailer } \\
\text { without purge. }\end{array}$ \\
\hline 9/15/15 & $13: 40$ & $\mathrm{~T} 123$ & YKTI23-80-W-37995DUP & DUP-L & $80-90$ & WG & YK915151800 & $9 / 15 / 15$ & Duplicate laboratory analysis. \\
\hline 9/15/15 & $15: 15$ & TI23 & YKTI23-99DUP-W-37997 & DUP-F & $99-109$ & WG & YK915151800 & $9 / 15 / 15$ & Field replicate. \\
\hline 9/15/15 & $15: 15$ & TI23 & YKTI23-99-W-37996 & $\mathrm{N}$ & $99-109$ & WG & YK915151800 & $9 / 15 / 15$ & $\begin{array}{l}\text { Depth to water }=96 \mathrm{ft} \text { BGL. Depth } \\
\text { of } 0.5 \text { in. well }=109 \mathrm{ft} \text { BGL. } \\
\text { Collect water with CPT. Push to } \\
\text { depth and pull back rods to } \\
\text { expose } 0.5 \text { in. PVC screen and } \\
\text { riser. Sampled using bailer } \\
\text { without purge. }\end{array}$ \\
\hline 9/15/15 & $16: 45$ & $\mathrm{TI} 23$ & YKTI23-120-W-37998VER & VER & $120-130$ & WG & YK915151755 & $9 / 15 / 15$ & $\begin{array}{l}\text { Verification sample to TestAmerica, } \\
\text { Inc. }\end{array}$ \\
\hline
\end{tabular}


TABLE A.1 (Cont.)

\begin{tabular}{|c|c|c|c|c|c|c|c|c|c|}
\hline \multicolumn{2}{|c|}{$\begin{array}{l}\text { Sample Date } \\
\text { and Time }\end{array}$} & \multirow[t]{2}{*}{ Location } & \multirow[b]{2}{*}{ YKTI23-120-W-37998 } & \multirow{2}{*}{$\begin{array}{c}\text { Sample } \\
\text { Type } \\
\mathrm{N}\end{array}$} & \multirow{2}{*}{$\begin{array}{c}\text { Depth } \\
\text { (ft BGL) } \\
120-130\end{array}$} & \multirow{2}{*}{$\begin{array}{c}\text { Matrix } \\
\text { WG }\end{array}$} & \multirow{2}{*}{$\begin{array}{l}\text { Chain of Custody } \\
\text { YK915151800 }\end{array}$} & \multirow{2}{*}{$\begin{array}{l}\begin{array}{l}\text { Shipping } \\
\text { Date }\end{array} \\
9 / 15 / 15\end{array}$} & \multirow{2}{*}{$\begin{array}{l}\text { Sample Description } \\
\text { Depth to water }=111 \mathrm{ft} \mathrm{BGL} \text {. Depth } \\
\text { of } 0.5 \text { in. well }=130 \mathrm{ft} \mathrm{BGL} \text {. } \\
\text { Collect water with CPT. Push to } \\
\text { depth and pull back rods to } \\
\text { expose } 0.5 \text { in. PVC screen and } \\
\text { riser. Sampled using bailer } \\
\text { without purge. }\end{array}$} \\
\hline $9 / 15 / 15$ & $16: 45$ & & & & & & & & \\
\hline $9 / 16 / 15$ & $9: 00$ & QC & YKQCTB-W-38000 & TB & - & WQC & YK916151700 & $9 / 16 / 15$ & $\begin{array}{l}\text { Trip blank with water samples } \\
\text { shipped to the AGEM laboratory. }\end{array}$ \\
\hline $9 / 16 / 15$ & $11: 00$ & $\mathrm{TI} 23$ & YKTI23-145-W-37999 & $N$ & $145-155$ & WG & YK916151700 & $9 / 16 / 15$ & $\begin{array}{l}\text { Depth to water }=115 \mathrm{ft} \text { BGL. Depth } \\
\text { of } 0.5 \text { in. well }=155 \mathrm{ft} \mathrm{BGL} \text {. } \\
\text { Collect water with CPT. Push to } \\
\text { depth and pull back rods to } \\
\text { expose } 0.5 \text { in. PVC screen and } \\
\text { riser. Sampled using bailer } \\
\text { without purge. }\end{array}$ \\
\hline 9/16/15 & $11: 00$ & TI23 & YKTI23-145-W-37999DUP & DUP-L & $145-155$ & WG & YK916151700 & 9/16/15 & Duplicate laboratory analysis. \\
\hline $1 / 20 / 16$ & $9: 00$ & QC & YKQCTB-W-38021 & TB & - & WQC & YK120161700 & $1 / 20 / 16$ & $\begin{array}{l}\text { Trip blank with water samples } \\
\text { shipped to the AGEM laboratory. }\end{array}$ \\
\hline $1 / 20 / 16$ & 9:00 & QC & YKQCTB-W-38021VER & VER & - & WQC & YK121161810 & $1 / 21 / 16$ & $\begin{array}{l}\text { Verification sample to TestAmerica, } \\
\text { Inc. }\end{array}$ \\
\hline $1 / 20 / 16$ & $10: 55$ & $\mathrm{TI} 24$ & YKTI24-90-W-38018 & $\mathrm{N}$ & $90-100$ & WG & YK120161700 & $1 / 20 / 16$ & $\begin{array}{l}\text { Depth to water }=71 \mathrm{ft} \mathrm{BGL} \text {. Depth } \\
\text { of } 0.5 \mathrm{in} \text {. well }=100 \mathrm{ft} \mathrm{BGL} \text {. Push } \\
\text { the } 2.125 \text { in. rods and } 2.125 \text { in. } \\
\text { water tip to depth and expose } \\
\text { the screen. Sampled without } \\
\text { purge. }\end{array}$ \\
\hline $1 / 20 / 16$ & $10: 55$ & $\mathrm{TI} 24$ & YKTI24-90-W-38018DUP & DUP-L & $90-100$ & WG & YK120161700 & 1/20/16 & Duplicate laboratory analysis. \\
\hline $1 / 20 / 16$ & $11: 30$ & QC & YKQCIR-W-38022 & RI & - & WQC & YK120161700 & $1 / 20 / 16$ & $\begin{array}{l}\text { Rinsate of decontaminated bailer } \\
\text { after sample YKTI24-90-W- } \\
38018 \text {. }\end{array}$ \\
\hline $1 / 20 / 16$ & $13: 20$ & $\mathrm{TI} 24$ & YKTI24-100-W-38019 & $\mathrm{N}$ & $100-110$ & WG & YK120161700 & $1 / 20 / 16$ & $\begin{array}{l}\text { Depth to water }=88 \mathrm{ft} \mathrm{BGL} \text {. Depth } \\
\text { of } 0.5 \mathrm{in} \text {. well }=110 \mathrm{ft} \text { BGL. Push } \\
\text { the } 2.125 \text { in. rods and } 2.125 \text { in. } \\
\text { water tip to depth and expose } \\
\text { the screen. Sampled without } \\
\text { purge. }\end{array}$ \\
\hline $1 / 20 / 16$ & $13: 20$ & $\mathrm{TI} 24$ & YKTI24-100-W-38019VER & VER & $100-110$ & WG & YK121161810 & $1 / 21 / 16$ & $\begin{array}{l}\text { Verification sample to TestAmerica, } \\
\text { Inc. }\end{array}$ \\
\hline $1 / 20 / 16$ & $15: 40$ & $\mathrm{TI} 24$ & YKTI24-80DUP-W-38020 & DUP-F & $80-90$ & WG & YK120161700 & $1 / 20 / 16$ & Field replicate. \\
\hline
\end{tabular}


TABLE A.1 (Cont.)

\begin{tabular}{|c|c|c|c|c|c|c|c|c|c|}
\hline $\begin{array}{r}\text { Sample } \\
\text { and } \mathrm{Ti}\end{array}$ & $\begin{array}{l}\text { Date } \\
\text { me }\end{array}$ & Location & Sample & $\begin{array}{l}\text { Sample } \\
\text { Type }\end{array}$ & $\begin{array}{l}\text { Depth } \\
\text { (ft BGL) }\end{array}$ & Matrix & Chain of Custody & $\begin{array}{l}\text { Shipping } \\
\text { Date }\end{array}$ & Sample Description \\
\hline $1 / 20 / 16$ & $15: 40$ & $\mathrm{TI} 24$ & YKTI24-80-W-38017 & $\mathrm{N}$ & $80-90$ & WG & YK120161700 & $1 / 20 / 16$ & $\begin{array}{l}\text { Depth to water }=87 \mathrm{ft} \mathrm{BGL} \text {. Depth } \\
\text { of } 0.5 \mathrm{in} \text {. well }=90 \mathrm{ft} \text { BGL. Push } \\
\text { the } 2.125 \text { in. rods and } 2.125 \text { in. } \\
\text { water tip to depth and expose } \\
\text { the screen. Sampled without } \\
\text { purge. }\end{array}$ \\
\hline $1 / 21 / 16$ & 9:00 & QC & YKQCTB-W-38028 & TB & - & WQC & YK121161800 & $1 / 21 / 16$ & $\begin{array}{l}\text { Trip blank with water samples } \\
\text { shipped to the AGEM laboratory. }\end{array}$ \\
\hline $1 / 21 / 16$ & $14: 00$ & $\mathrm{TI} 25$ & YKTI25-77-W-38023 & $\mathrm{N}$ & $77-87$ & WG & YK121161800 & $1 / 21 / 16$ & $\begin{array}{l}\text { Depth to water }=67 \mathrm{ft} \text { BGL. Depth } \\
\text { of } 0.5 \mathrm{in} \text {. well }=87 \mathrm{ft} \mathrm{BGL} \text {. Push } \\
\text { the } 2.125 \text { in. rods and } 2.125 \text { in. } \\
\text { water tip to depth and expose } \\
\text { the screen. Sampled without } \\
\text { purge. }\end{array}$ \\
\hline $1 / 21 / 16$ & $15: 27$ & $\mathrm{TI} 25$ & YKTI25-98DUP-W-38025 & DUP-F & $98-108$ & WG & YK121161800 & $1 / 21 / 16$ & Field replicate. \\
\hline $1 / 21 / 16$ & $15: 27$ & TI25 & YKTI25-98-W-38024 & $\mathrm{N}$ & $98-108$ & WG & YK121161800 & $1 / 21 / 16$ & $\begin{array}{l}\text { Depth to water }=70 \mathrm{ft} \mathrm{BGL} \text {. Depth } \\
\text { of } 0.5 \mathrm{in} \text {. well }=108 \mathrm{ft} \text { BGL. Push } \\
\text { the } 2.125 \text { in. rods and } 2.125 \text { in. } \\
\text { water tip to depth and expose } \\
\text { the screen. Sampled without } \\
\text { purge. }\end{array}$ \\
\hline $1 / 21 / 16$ & $16: 45$ & QC & YKQCIR-W-38026 & RI & - & WQC & YK121161800 & $1 / 21 / 16$ & $\begin{array}{l}\text { Rinsate of decontaminated bailer } \\
\text { after samples YKTI25-98-W- } \\
38024 \text { and YKTI25-98DUP-W- } \\
38025 \text {. }\end{array}$ \\
\hline $1 / 21 / 16$ & $17: 45$ & $\mathrm{TI} 25$ & YKTI25-108-W-38027 & $\mathrm{N}$ & $113-118$ & WG & YK121161800 & $1 / 21 / 16$ & $\begin{array}{l}\text { Depth to water }=80 \mathrm{ft} \mathrm{BGL} \text {. Depth } \\
\text { of } 0.5 \text { in. well }=118 \mathrm{ft} \text { BGL. Push } \\
\text { the } 2.125 \text { in. rods and } 2.125 \text { in. } \\
\text { water tip to depth and expose } \\
\text { the screen. Sampled without } \\
\text { purge. }\end{array}$ \\
\hline $1 / 21 / 16$ & $17: 45$ & TI25 & YKTI25-108-W-38027DUP & DUP-L & $113-118$ & WG & YK121161800 & $1 / 21 / 16$ & Duplicate laboratory analysis. \\
\hline $1 / 21 / 16$ & $17: 45$ & TI25 & YKTI25-108-W-38027VER & VER & $113-118$ & WG & YK121161810 & $1 / 21 / 16$ & $\begin{array}{l}\text { Verification sample to TestAmerica, } \\
\text { Inc. }\end{array}$ \\
\hline $1 / 25 / 16$ & $8: 00$ & QC & YKQCTB-W-38035 & TB & - & WQC & YK126161700 & $1 / 26 / 16$ & $\begin{array}{l}\text { Trip blank with water samples } \\
\text { shipped to the AGEM laboratory. }\end{array}$ \\
\hline $1 / 25 / 16$ & $13: 20$ & TI26 & YKTI26-78-W-38029 & $\mathrm{N}$ & $78-88$ & WG & YK126161700 & $1 / 26 / 16$ & $\begin{array}{l}\text { Depth to water }=69 \mathrm{ft} \mathrm{BGL} \text {. Depth } \\
\text { of } 0.5 \mathrm{in} \text {. well }=89 \mathrm{ft} \text { BGL. Push } \\
\text { the } 2.125 \text { in. rods and } 2.125 \text { in. } \\
\text { water tip to depth and expose } \\
\text { the screen. Sampled without } \\
\text { purge. }\end{array}$ \\
\hline $1 / 25 / 16$ & $13: 20$ & $\mathrm{TI} 26$ & YKTI26-78-W-38029DUP & DUP-L & $78-88$ & WG & YK126161700 & $1 / 26 / 16$ & Duplicate laboratory analysis. \\
\hline
\end{tabular}


TABLE A.1 (Cont.)

\begin{tabular}{|c|c|c|c|c|c|c|c|c|c|}
\hline $\begin{array}{l}\text { Sample } \\
\text { and Tir }\end{array}$ & $\begin{array}{l}\text { Date } \\
\text { ime }\end{array}$ & Location & Sample & $\begin{array}{c}\text { Sample } \\
\text { Type }\end{array}$ & $\begin{array}{c}\text { Depth } \\
\text { (ft BGL) }\end{array}$ & Matrix & Chain of Custody & $\begin{array}{l}\text { Shipping } \\
\text { Date }\end{array}$ & Sample Description \\
\hline $1 / 25 / 16$ & $15: 15$ & TI26 & YKTI26-96DUP-W-38031 & DUP-F & $96-106$ & WG & YK126161700 & $1 / 26 / 16$ & Field replicate. \\
\hline $1 / 25 / 16$ & $15: 15$ & $\mathrm{TI} 26$ & YKTI26-96-W-38030 & $\mathrm{N}$ & $96-106$ & WG & YK126161700 & $1 / 26 / 16$ & $\begin{array}{l}\text { Depth to water }=81 \mathrm{ft} \mathrm{BGL} \text {. Depth } \\
\text { of } 0.5 \mathrm{in} \text {. well }=106 \mathrm{ft} \text { BGL. Push } \\
\text { the } 2.125 \mathrm{in} \text {. rods and } 2.125 \mathrm{in} \text {. } \\
\text { water tip to depth and expose } \\
\text { the screen. Sampled without } \\
\text { purge. }\end{array}$ \\
\hline $1 / 25 / 16$ & $16: 30$ & QC & YKQCIR-W-38032 & $\mathrm{RI}$ & - & WQC & YK126161700 & $1 / 26 / 16$ & $\begin{array}{l}\text { Rinsate of decontaminated bailer } \\
\text { after samples YKTI26-96-W- } \\
38030 \text { and YKTI26-96DUP-W- } \\
38031 \text {. }\end{array}$ \\
\hline $1 / 25 / 16$ & $17: 55$ & TI26 & YKTI26-113DUP-W-38034 & DUP-F & $113-123$ & WG & YK126161700 & $1 / 26 / 16$ & Field replicate. \\
\hline $1 / 25 / 16$ & $17: 55$ & TI26 & YKTI26-113-W-38033 & $N$ & $113-123$ & WG & YK126161700 & $1 / 26 / 16$ & $\begin{array}{l}\text { Depth to water }=71 \mathrm{ft} \mathrm{BGL} \text {. Depth } \\
\text { of } 0.5 \mathrm{in} \text {. well }=123 \mathrm{ft} \text { BGL. Push } \\
\text { the } 2.125 \text { in. rods and } 2.125 \text { in. } \\
\text { water tip to depth and expose } \\
\text { the screen. Sampled without } \\
\text { purge. }\end{array}$ \\
\hline $1 / 28 / 16$ & $8: 20$ & GARRETSON & YKGARRETSON-G-38036 & $\mathrm{N}$ & - & AIR & YK128161000 & $1 / 28 / 16$ & $\begin{array}{l}\text { Garretson kitchen. Sample } \\
\text { collected over } 24 \mathrm{hr} \text {. Begin } \\
\text { pressure }-28 \text { in. } \mathrm{Hg} \text {. End } \\
\text { pressure }-8 \mathrm{in} . \mathrm{Hg} \text {. }\end{array}$ \\
\hline $1 / 28 / 16$ & $8: 24$ & GARRETSON & YKGARRETSON-G-38037 & $\mathrm{N}$ & - & AIR & YK128161000 & $1 / 28 / 16$ & $\begin{array}{l}\text { Garretson basement. Sample } \\
\text { collected over } 24 \mathrm{hr} \text {. Begin } \\
\text { pressure }-30 \mathrm{in} . \mathrm{Hg} \text {. End } \\
\text { pressure }-7 \mathrm{in} . \mathrm{Hg} .\end{array}$ \\
\hline $1 / 28 / 16$ & $8: 24$ & GARRETSON & YKGARRETSON-G-38038 & DUP-F & - & AIR & YK128161000 & $1 / 28 / 16$ & $\begin{array}{l}\text { Field replicate. Sample collected } \\
\text { over } 24 \mathrm{hr} \text {. Begin } \\
\text { pressure }-26 \text { in. } \mathrm{Hg} \text {. End } \\
\text { pressure }-3 \mathrm{in} . \mathrm{Hg} \text {. }\end{array}$ \\
\hline $1 / 28 / 16$ & $8: 40$ & GARRETSON & YKBKGND-G-38039 & $\mathrm{N}$ & - & AIR & YK128161000 & $1 / 28 / 16$ & $\begin{array}{l}\text { Garretson ambient air. Sample } \\
\text { collected over } 24 \mathrm{hr} \text {. Begin } \\
\text { pressure }-29 \text { in. } \mathrm{Hg} \text {. End } \\
\text { pressure }-5 \mathrm{in} . \mathrm{Hg} \text {. }\end{array}$ \\
\hline $1 / 28 / 16$ & $9: 23$ & FITWORX & YKFITWORX-G-38040 & $\mathrm{N}$ & - & AIR & YK128161000 & $1 / 28 / 16$ & $\begin{array}{l}\text { FitnessWorx basement. Sample } \\
\text { collected over } 24 \mathrm{hr} \text {. Begin } \\
\text { pressure }-32 \mathrm{in.} \mathrm{Hg} \text {. End } \\
\text { pressure }-6 \mathrm{in.} \mathrm{Hg} \text {. }\end{array}$ \\
\hline $1 / 28 / 16$ & $9: 30$ & FITWORX & YKFITWORX-G-38042 & $\mathrm{N}$ & - & AIR & YK128161000 & $1 / 28 / 16$ & $\begin{array}{l}\text { FitnessWorx stairwell. Sample } \\
\text { collected over } 24 \mathrm{hr} \text {. Begin } \\
\text { pressure }-29 \text { in. Hg. End } \\
\text { pressure }-5 \text { in. } \mathrm{Hg} \text {. }\end{array}$ \\
\hline
\end{tabular}


TABLE A.1 (Cont.)

\begin{tabular}{|c|c|c|c|c|c|c|c|c|c|}
\hline $\begin{array}{r}\text { Sample } \\
\text { and } \mathrm{Ti}\end{array}$ & $\begin{array}{l}\text { Date } \\
\text { ime }\end{array}$ & Location & Sample & $\begin{array}{l}\text { Sample } \\
\text { Type }\end{array}$ & $\begin{array}{l}\text { Depth } \\
\text { (ft BGL) }\end{array}$ & Matrix & Chain of Custody & $\begin{array}{l}\text { Shipping } \\
\text { Date }\end{array}$ & Sample Description \\
\hline $1 / 28 / 16$ & $9: 32$ & FITWORX & YKFITWORX-G-38041 & DUP-F & - & AIR & YK128161000 & $1 / 28 / 16$ & $\begin{array}{l}\text { Field replicate. Sample collected } \\
\text { over } 24 \mathrm{hr} \text {. Begin } \\
\text { pressure }-27 \text { in. } \mathrm{Hg} \text {. End } \\
\text { pressure }-4 \text { in. } \mathrm{Hq} \text {. }\end{array}$ \\
\hline $1 / 28 / 16$ & $9: 35$ & FITWORX & YKFITWORX-G-38043 & $\mathrm{N}$ & - & AIR & YK128161000 & $1 / 28 / 16$ & $\begin{array}{l}\text { FitnessWorx Room \#3. Sample } \\
\text { collected over } 24 \mathrm{hr} \text {. Begin } \\
\text { pressure }-28 \text { in. } \mathrm{Hg} \text {. End } \\
\text { pressure }-6 \text { in. } \mathrm{Hg} \text {. }\end{array}$ \\
\hline $1 / 28 / 16$ & $9: 36$ & FITWORX & YKFITWORX-G-38044 & $\mathrm{N}$ & - & AIR & YK128161000 & $1 / 28 / 16$ & $\begin{array}{l}\text { FitnessWorx Room \#7. Sample } \\
\text { collected over } 24 \mathrm{hr} \text {. Begin } \\
\text { pressure }-31 \text { in. Hg. End } \\
\text { pressure }-6 \text { in. } \mathrm{Hg} \text {. }\end{array}$ \\
\hline $1 / 28 / 16$ & $9: 39$ & FITWORX & YKBKGND-G-38045 & $\mathrm{N}$ & - & AIR & YK128161000 & $1 / 28 / 16$ & $\begin{array}{l}\text { FitnessWorx ambient air. Sample } \\
\text { collected over } 24 \mathrm{hr} \text {. Begin } \\
\text { pressure }-30 \mathrm{in} . \mathrm{Hg} \text {. End } \\
\text { pressure }-5 \mathrm{in} . \mathrm{Hg} \text {. }\end{array}$ \\
\hline $1 / 28 / 16$ & $10: 15$ & GARRETSON & YKGARRETSON-G-38046 & $\mathrm{N}$ & - & $\begin{array}{l}\text { SOIL } \\
\text { GAS }\end{array}$ & HP-28Jan2016 & $1 / 28 / 16$ & $\begin{array}{l}\text { Sub-slaboratory soil gas sampling } \\
\text { in the Garretson home. Begin } \\
\text { pressure }-27 \text { in. Hg. End } \\
\text { pressure } 0 \text { in. } \mathrm{Hg} \text {. }\end{array}$ \\
\hline $1 / 28 / 16$ & $10: 47$ & FITWORX & YKFITWORX-G-38048 & $\mathrm{N}$ & - & $\begin{array}{l}\text { SOIL } \\
\text { GAS }\end{array}$ & HP-28Jan2016 & $1 / 28 / 16$ & $\begin{array}{l}\text { Sub-slaboratory soil gas sampling } \\
\text { in FitnessWorx. Begin } \\
\text { pressure }-28 \text { in. Hg. End } \\
\text { pressure }-1 \text { in. Hg. }\end{array}$ \\
\hline $2 / 9 / 16$ & $13: 00$ & QC & YKQCTB-W-38055 & TB & - & WQC & YK29161700 & $2 / 9 / 16$ & $\begin{array}{l}\text { Trip blank with water samples } \\
\text { shipped to the AGEM laboratory. } \\
\text { Sample frozen; one vial was } \\
\text { unbroken and analyzed. }\end{array}$ \\
\hline $2 / 9 / 16$ & $14: 45$ & $\mathrm{TI} 27$ & YKTI27-77-W-38056 & $\mathrm{N}$ & $77-87$ & WG & YK210161200 & $2 / 10 / 16$ & $\begin{array}{l}\text { Back-up TI27 } 77 \mathrm{ft} \text { sample sent to } \\
\text { replace the frozen YKTI27-77- } \\
\text { W-38050 vial sent in shipment } \\
\text { with COC YK29161700. }\end{array}$ \\
\hline $2 / 9 / 16$ & $14: 45$ & $\mathrm{TI} 27$ & YKTI27-77DUP-W-38051 & DUP-F & $77-87$ & WG & YK29161700 & $2 / 9 / 16$ & $\begin{array}{l}\text { Field replicate. Sample frozen; one } \\
\text { vial was unbroken and analyzed. }\end{array}$ \\
\hline $2 / 9 / 16$ & $14: 45$ & $\mathrm{TI} 27$ & YKTI27-77-W-38050 & $\mathrm{N}$ & $77-87$ & WG & YK29161700 & $2 / 9 / 16$ & $\begin{array}{l}\text { Depth to water }=71 \mathrm{ft} \mathrm{BGL} \text {. Depth } \\
\text { of } 0.5 \text { in. well }=87 \mathrm{ft} \text { BGL. Push } \\
2.125 \text { in. rods and } 2.125 \text { in. } \\
\text { water tip to depth and expose } \\
\text { the screen. Sampled without } \\
\text { purge. Sample frozen; one vial } \\
\text { was unbroken and analyzed. } \\
\text { Back-up sample YKTI27-77-W- } \\
38056 \text { shipped } 2 / 10 / 16 \text {. }\end{array}$ \\
\hline
\end{tabular}


TABLE A.1 (Cont.)

\begin{tabular}{|c|c|c|c|c|c|c|c|c|c|}
\hline \multicolumn{2}{|c|}{$\begin{array}{l}\text { Sample Date } \\
\text { and Time }\end{array}$} & \multirow[t]{2}{*}{ Location } & \multirow[b]{2}{*}{ YKQCIR-W-38052 } & \multirow{2}{*}{$\begin{array}{c}\text { Sample } \\
\text { Type } \\
\text { RI }\end{array}$} & \multirow{2}{*}{$\begin{array}{c}\text { Depth } \\
\text { (ft BGL) } \\
-\end{array}$} & \multirow{2}{*}{$\begin{array}{l}\text { Matrix } \\
\text { WQC }\end{array}$} & \multirow{2}{*}{$\begin{array}{l}\text { Chain of Custody } \\
\text { YK29161700 }\end{array}$} & \multirow{2}{*}{$\begin{array}{l}\begin{array}{l}\text { Shipping } \\
\text { Date }\end{array} \\
2 / 9 / 16\end{array}$} & \multirow{2}{*}{$\begin{array}{c}\text { Sample Description } \\
\text { Rinsate of decontaminated bailer } \\
\text { after samples YKTI27-77-W- } \\
38050 \text { and YKTI27-77DUP-W- } \\
\text { 38051. Sample frozen; one vial } \\
\text { was unbroken and analyzed. }\end{array}$} \\
\hline 2/9/16 & $15: 15$ & & & & & & & & \\
\hline 2/9/16 & $16: 30$ & $\mathrm{TI} 27$ & YKTI27-100-W-38057 & $\mathrm{N}$ & $100-110$ & WG & YK210161200 & $2 / 10 / 16$ & $\begin{array}{l}\text { Back-up TI27 } 100 \mathrm{ft} \text { sample sent to } \\
\text { replace the frozen YKTI27-100- } \\
\text { W-38053 vial sent in shipment } \\
\text { with COC YK29161700. }\end{array}$ \\
\hline 2/9/16 & $16: 30$ & TI27 & YKTI27-100-W-38057DUP & DUP-L & $100-110$ & WG & YK210161200 & 2/10/16 & Duplicate laboratory analysis. \\
\hline $2 / 9 / 16$ & $16: 30$ & $\mathrm{TI} 27$ & YKTI27-100DUP-W-38054 & DUP-F & $100-110$ & WG & YK29161700 & $2 / 9 / 16$ & $\begin{array}{l}\text { Field replicate. Sample frozen; one } \\
\text { vial was unbroken and analyzed. }\end{array}$ \\
\hline 2/9/16 & $16: 30$ & $\mathrm{TI} 27$ & YKTI27-100-W-38053 & $\mathrm{N}$ & $100-110$ & WG & YK29161700 & 2/9/16 & $\begin{array}{l}\text { Depth to water }=100 \mathrm{ft} \mathrm{BGL} \text {. Depth } \\
\text { of } 0.5 \text { in. well }=110 \mathrm{ft} \text { BGL. Push } \\
2.125 \text { in. rods and } 2.125 \text { in. } \\
\text { water tip to depth and expose } \\
\text { screen. Sampled without purge. } \\
\text { Sample frozen; one vial was } \\
\text { unbroken and analyzed. Back-up } \\
\text { sample YKTI27-100-W-38057 } \\
\text { shipped } 2 / 10 / 16 \text {. }\end{array}$ \\
\hline 2/9/16 & $16: 30$ & TI27 & YKTI27-100-W-38053DUP & DUP-L & $100-110$ & WG & YK29161700 & 2/9/16 & Duplicate laboratory analysis. \\
\hline $2 / 10 / 16$ & $10: 00$ & QC & YKQCTB-W-38058 & TB & - & WQC & YK210161200 & $2 / 10 / 16$ & $\begin{array}{l}\text { Trip blank with water samples } \\
\text { shipped to the AGEM laboratory. }\end{array}$ \\
\hline 3/2/16 & 13:00 & QC & YKQCTB-W-38080 & TB & - & WQC & YK33161400 & $3 / 3 / 16$ & $\begin{array}{l}\text { Trip blank with water samples } \\
\text { shipped to the AGEM laboratory. }\end{array}$ \\
\hline $3 / 2 / 16$ & 13:00 & QC & YKQCTB-W-38080VER & VER & - & WQC & YK33161410 & $3 / 3 / 16$ & $\begin{array}{l}\text { Verification sample to TestAmerica, } \\
\text { Inc. }\end{array}$ \\
\hline $3 / 2 / 16$ & $17: 00$ & $\mathrm{TI} 28$ & YKTI28-78-W-38075 & $\mathrm{N}$ & $78-88$ & WG & YK33161400 & $3 / 3 / 16$ & $\begin{array}{l}\text { Depth to water }=68 \mathrm{ft} \text { BGL. Depth } \\
\text { of } 0.5 \mathrm{in} \text {. well }=88 \mathrm{ft} \text { BGL. Push } \\
\text { the } 2.125 \mathrm{in} . \text { rods and } 2.125 \mathrm{in} \text {. } \\
\text { water tip to depth and expose } \\
\text { the screen. Sampled without } \\
\text { purge. }\end{array}$ \\
\hline $3 / 2 / 16$ & $17: 00$ & TI28 & YKTI28-78-W-38075DUP & DUP-L & $78-88$ & WG & YK33161400 & $3 / 3 / 16$ & Duplicate laboratory analysis. \\
\hline $3 / 2 / 16$ & 18:00 & QC & YKQCIR-W-38079 & RI & - & WQC & YK33161400 & $3 / 3 / 16$ & $\begin{array}{l}\text { Rinsate of decontaminated bailer } \\
\text { after sample YKTI28-78-W- } \\
38075\end{array}$ \\
\hline $3 / 2 / 16$ & $18: 30$ & TI28 & YKTI28-90-DUP-W-38077 & DUP-F & $90-100$ & WG & YK33161400 & $3 / 3 / 16$ & Field replicate. \\
\hline
\end{tabular}


TABLE A.1 (Cont.)

\begin{tabular}{|c|c|c|c|c|c|c|c|c|c|}
\hline $\begin{array}{r}\text { Sample } \\
\text { and T }\end{array}$ & $\begin{array}{l}\text { Date } \\
\text { ime }\end{array}$ & Location & Sample & $\begin{array}{l}\text { Sample } \\
\text { Type }\end{array}$ & $\begin{array}{l}\text { Depth } \\
\text { (ft BGL) }\end{array}$ & Matrix & Chain of Custody & $\begin{array}{l}\text { Shipping } \\
\text { Date }\end{array}$ & Sample Description \\
\hline $3 / 2 / 16$ & $18: 30$ & $\mathrm{TI} 28$ & YKTI28-90-W-38076 & $\mathrm{N}$ & $90-100$ & WG & YK33161400 & $3 / 3 / 16$ & $\begin{array}{l}\text { Depth to water }=90 \mathrm{ft} \text { BGL. Depth } \\
\text { of } 0.5 \text { in. well }=100 \mathrm{ft} \text { BGL. Push } \\
\text { the } 2.125 \text { in. rods and } 2.125 \text { in. } \\
\text { water tip to depth and expose } \\
\text { the screen. Sampled without } \\
\text { purge. }\end{array}$ \\
\hline $3 / 3 / 16$ & $10: 50$ & $\mathrm{TI} 28$ & YKTI28-104-W-38078 & $\mathrm{N}$ & 104-109 & WG & YK33161400 & $3 / 3 / 16$ & $\begin{array}{l}\text { Depth to water }=100 \mathrm{ft} \mathrm{BGL} \text {. Depth } \\
\text { of } 0.5 \mathrm{in} \text {. well }=109 \mathrm{ft} \text { BGL. Push } \\
\text { the } 2.125 \text { in. rods and } 2.125 \text { in. } \\
\text { water tip to depth and expose } \\
\text { the screen. Sampled without } \\
\text { purge. }\end{array}$ \\
\hline $3 / 3 / 16$ & $10: 50$ & $\mathrm{TI} 28$ & YKTI28-104-W-38078VER & VER & 104-109 & WG & YK33161410 & $3 / 3 / 16$ & $\begin{array}{l}\text { Verification sample to TestAmerica, } \\
\text { Inc. }\end{array}$ \\
\hline $4 / 28 / 16$ & $13: 55$ & FITWORX & YKFITWORX-G-38081 & $\mathrm{N}$ & - & AIR & YK428161700 & $4 / 28 / 16$ & $\begin{array}{l}\text { FitnessWorx basement. Sample } \\
\text { collected over } 24 \mathrm{hr} \text {. Begin } \\
\text { pressure }-30 \mathrm{in.} \mathrm{Hg} \text {. End } \\
\text { pressure }-3 \mathrm{in} . \mathrm{Hg} \text {. }\end{array}$ \\
\hline $4 / 28 / 16$ & $13: 57$ & FITWORX & YKFITWORX-G-38082 & DUP-F & - & AIR & YK428161700 & $4 / 28 / 16$ & $\begin{array}{l}\text { Field replicate. Sample collected } \\
\text { over } 24 \mathrm{hr} \text {. Begin } \\
\text { pressure }-31 \mathrm{in} . \mathrm{Hg} \text {. End } \\
\text { pressure }-5 \mathrm{in} . \mathrm{Hg} \text {. }\end{array}$ \\
\hline $4 / 28 / 16$ & $14: 00$ & FITWORX & YKFITWORX-G-38083 & $\mathrm{N}$ & - & AIR & YK428161700 & $4 / 28 / 16$ & $\begin{array}{l}\text { FitnessWorx stairwell. Sample } \\
\text { collected over } 24 \mathrm{hr} \text {. Begin } \\
\text { pressure }-30 \text { in. } \mathrm{Hg} \text {. End } \\
\text { pressure }-6 \text { in. } \mathrm{Hg} \text {. }\end{array}$ \\
\hline $4 / 28 / 16$ & $14: 05$ & FITWORX & YKFITWORX-G-38084 & $N$ & - & AIR & YK428161700 & $4 / 28 / 16$ & $\begin{array}{l}\text { FitnessWorx room \#3. Sample } \\
\text { collected over } 24 \mathrm{hr} \text {. Begin } \\
\text { pressure }-29 \text { in. } \mathrm{Hg} \text {. End } \\
\text { pressure }-2 \mathrm{in.} \mathrm{Hg} \text {. }\end{array}$ \\
\hline $4 / 28 / 16$ & $14: 08$ & FITWORX & YKFITWORX-G-38085 & $\mathrm{N}$ & - & AIR & YK428161700 & $4 / 28 / 16$ & $\begin{array}{l}\text { FitnessWorx room \#7. Sample } \\
\text { collected over } 24 \mathrm{hr} \text {. Begin } \\
\text { pressure }-27 \text { in. } \mathrm{Hg} \text {. End } \\
\text { pressure }-3 \text { in. } \mathrm{Hg} \text {. }\end{array}$ \\
\hline $4 / 28 / 16$ & $14: 15$ & FITWORX & YKBKGND-G-38086 & $\mathrm{N}$ & - & AIR & YK428161700 & $4 / 28 / 16$ & $\begin{array}{l}\text { FitnessWorx ambient air. Sample } \\
\text { collected over } 24 \mathrm{hr} \text {. Begin } \\
\text { pressure }-29 \text { in. } \mathrm{Hg} \text {. End } \\
\text { pressure }-3 \mathrm{in} . \mathrm{Hg} \text {. }\end{array}$ \\
\hline $4 / 28 / 16$ & $14: 28$ & FITWORX & YKFITWORX-G-38087 & $\mathrm{N}$ & - & $\begin{array}{l}\text { SOIL } \\
\text { GAS }\end{array}$ & YK428161700 & $4 / 28 / 16$ & $\begin{array}{l}\text { Sub-slaboratory soil gas sampling } \\
\text { in FitnessWorx stairwell. Begin } \\
\text { pressure }-26 \text { in. Hg. End } \\
\text { pressure } 1 \mathrm{in.} \mathrm{Hg} \text {. }\end{array}$ \\
\hline
\end{tabular}


TABLE A.1 (Cont.)

\begin{tabular}{|c|c|c|c|c|c|c|c|c|c|}
\hline $\begin{array}{l}\text { Sample } \\
\text { and } \mathrm{Ti}\end{array}$ & $\begin{array}{l}\text { Date } \\
\text { me }\end{array}$ & Location & Sample & $\begin{array}{l}\text { Sample } \\
\text { Type }\end{array}$ & $\begin{array}{l}\text { Depth } \\
\text { (ft BGL) }\end{array}$ & Matrix & Chain of Custody & $\begin{array}{l}\text { Shipping } \\
\text { Date }\end{array}$ & Sample Description \\
\hline $4 / 28 / 16$ & $14: 38$ & FITWORX & YKFITWORX-G-38088 & DUP-F & - & $\begin{array}{l}\text { SOIL } \\
\text { GAS }\end{array}$ & YK428161700 & $4 / 28 / 16$ & $\begin{array}{l}\text { Field replicate. Begin pressure - } \\
28 \mathrm{in} . \mathrm{Hg} \text {. End pressure } 1 \mathrm{in.} \mathrm{Hg} .\end{array}$ \\
\hline $5 / 17 / 16$ & $13: 00$ & QC & YKQCTB-W-38092 & TB & - & WQC & YK523161710 & $5 / 23 / 16$ & $\begin{array}{l}\text { Trip blank with water samples } \\
\text { shipped to the AGEM laboratory. }\end{array}$ \\
\hline $5 / 17 / 16$ & $14: 00$ & QC & YKCITY-W-38089 & EB & - & WG & YK523161710 & $5 / 23 / 16$ & $\begin{array}{l}\text { Sample collected from the city fire } \\
\text { hydrant east of the county shop. }\end{array}$ \\
\hline $5 / 17 / 16$ & $15: 30$ & QC & YKTRUCK-W-38090 & EB & - & WG & YK523161710 & $5 / 23 / 16$ & $\begin{array}{l}\text { Sample collected from the Cascade } \\
\text { Drilling water truck. }\end{array}$ \\
\hline $5 / 23 / 16$ & $17: 00$ & DL1C & YKDL1C-108-W-38091 & $\mathrm{N}$ & $108-118$ & WG & YK523161710 & $5 / 23 / 16$ & $\begin{array}{l}\text { Depth to water }=73.7 \mathrm{ft} \mathrm{BGL} \text {. } \\
\text { Depth of } 2 \text { in. well }=118.8 \mathrm{ft} \\
\text { BGL. Cascade used } 400 \text { gal to } \\
\text { drill and set well. Purged } 50 \text { gal } \\
\text { each from top and bottom halves } \\
\text { of screen with foot valve. Purged } \\
\text { additional } 350 \text { gal with Redi- } \\
\text { Flow pump before collecting a } \\
\text { sample. }\end{array}$ \\
\hline $5 / 23 / 16$ & $17: 00$ & DL1C & YKDL1C-108-W-38091DUP & DUP-L & $108-118$ & WG & YK523161710 & $5 / 23 / 16$ & Duplicate laboratory analysis. \\
\hline $5 / 23 / 16$ & $17: 00$ & DL1C & YKDL1C-108-W-38091VER & VER & $108-118$ & WG & YK531161735 & $5 / 31 / 16$ & $\begin{array}{l}\text { Verification sample to TestAmerica, } \\
\text { Inc. }\end{array}$ \\
\hline $5 / 24 / 16$ & $18: 20$ & DL1B & YKDL1B-88-W-38093 & $\mathrm{N}$ & $88-98$ & WG & YK525161800 & $5 / 25 / 16$ & $\begin{array}{l}\text { Depth to water }=73.6 \mathrm{ft} \text { BGL. } \\
\text { Depth of } 2 \text { in. well }=98.3 \mathrm{ft} \\
\text { BGL. Cascade used } 400 \text { gal to } \\
\text { drill and set well. Purged } 50 \text { gal } \\
\text { each from top and bottom halves } \\
\text { of screen with foot valve. Purged } \\
\text { additional } 350 \text { gal with Redi- } \\
\text { Flow pump before collecting a } \\
\text { sample. }\end{array}$ \\
\hline $5 / 24 / 16$ & $18: 20$ & DL1B & YKDL1B-88-W-38093VER & VER & $88-98$ & WG & YK531161735 & $5 / 31 / 16$ & $\begin{array}{l}\text { Verification sample to TestAmerica, } \\
\text { Inc. }\end{array}$ \\
\hline $5 / 25 / 16$ & $15: 10$ & DL1A & YKDL1A-75-W-38094 & $\mathrm{N}$ & $75-85$ & WG & YK525161800 & $5 / 25 / 16$ & $\begin{array}{l}\text { Depth to water }=73.6 \mathrm{ft} \text { BGL. } \\
\text { Depth of } 2 \text { in. well = } 86 \mathrm{ft} \text { BGL. } \\
\text { Cascade used } 400 \text { gal to drill } \\
\text { and set well. Purged } 50 \text { gal each } \\
\text { from top and bottom halves of } \\
\text { screen with foot valve. Purged } \\
\text { additional } 350 \text { gal with Redi- } \\
\text { Flow pump before collecting a } \\
\text { sample. }\end{array}$ \\
\hline $5 / 25 / 16$ & $15: 30$ & QC & YKQCTB-W-38095 & TB & - & WQC & YK525161800 & $5 / 25 / 16$ & $\begin{array}{l}\text { Trip blank with water samples } \\
\text { shipped to the AGEM laboratory. }\end{array}$ \\
\hline
\end{tabular}


TABLE A.1 (Cont.)

\begin{tabular}{|c|c|c|c|c|c|c|c|c|c|}
\hline \multicolumn{2}{|c|}{$\begin{array}{l}\text { Sample Date } \\
\text { and Time }\end{array}$} & \multirow{2}{*}{$\begin{array}{r}\text { Location } \\
\mathrm{QC}\end{array}$} & \multirow{2}{*}{$\begin{array}{r}\text { Sample } \\
\text { YKQCTB-W-38099VER }\end{array}$} & \multirow{2}{*}{$\begin{array}{c}\text { Sample } \\
\text { Type }\end{array}$} & \multirow{2}{*}{$\begin{array}{c}\text { Depth } \\
\text { (ft BGL) } \\
-\end{array}$} & \multirow{2}{*}{ Matrix } & \multirow{2}{*}{$\begin{array}{l}\text { Chain of Custody } \\
\text { YK531161735 }\end{array}$} & \multirow{2}{*}{$\begin{array}{l}\begin{array}{l}\text { Shipping } \\
\text { Date }\end{array} \\
5 / 31 / 16\end{array}$} & \multirow{2}{*}{$\begin{array}{c}\text { Sample Description } \\
\begin{array}{l}\text { Verification sample to TestAmerica, } \\
\text { Inc. }\end{array}\end{array}$} \\
\hline $5 / 31 / 16$ & $16: 00$ & & & & & & & & \\
\hline $5 / 31 / 16$ & $16: 00$ & QC & YKQCTB-W-38099 & TB & - & WQC & YK531161800 & $5 / 31 / 16$ & $\begin{array}{l}\text { Trip blank with water samples } \\
\text { shipped to the AGEM laboratory. }\end{array}$ \\
\hline $5 / 31 / 16$ & $16: 45$ & DL2C2 & YKDL2C2-136-W-38096VER & VER & $136-146$ & WG & YK531161735 & $5 / 31 / 16$ & $\begin{array}{l}\text { Verification sample to TestAmerica, } \\
\text { Inc. }\end{array}$ \\
\hline $5 / 31 / 16$ & $16: 45$ & DL2C2 & YKDL2C2-136-W-38096 & $\mathrm{N}$ & $136-146$ & WG & YK531161800 & $5 / 31 / 16$ & $\begin{array}{l}\text { Depth to water }=80 \mathrm{ft} \mathrm{BGL} \text {. Depth } \\
\text { of } 2 \text { in. well }=145.7 \mathrm{ft} \mathrm{BGL} \text {. } \\
\text { Cascade used } 400 \text { gal to drill } \\
\text { and set well. Purged } 300 \text { gal } \\
\text { each from top and bottom halves } \\
\text { of screen with foot valve before }\end{array}$ \\
\hline
\end{tabular}

$\begin{array}{llll}5 / 31 / 16 & 16: 45 & \text { DL2C2 } & \text { YKDL2C2-136-W-38096DUP } \\ 5 / 31 / 16 & 17: 00 & \text { DL2CU } & \text { YKDL2CU-215-W-38097VER } \\ & & & \\ 5 / 31 / 16 & 17: 00 & \text { DL2CU } & \text { YKDL2CU-215-DUP-W-38098 } \\ 5 / 31 / 16 & 17: 00 & \text { DL2CU } & \text { YKDL2CU-215-W-38097 }\end{array}$

$\begin{array}{lll}5 / 31 / 16 & 17: 00 & \text { DL2CU } \\ 5 / 31 / 16 & 17: 00 & \text { DL2CU }\end{array}$

$6 / 2 / 16$

9:15 DL2C1

6/2/16 9:15 DL2C1

6/2/16 19:00 QC

6/5/16 9:15 DL3C2
YKDL2-C1-112-W-38100DUP YKQCTB-W-38101

YKDL3-C2-134-W-38102

$\begin{array}{cccc}\text { DUP-L } & 136-146 & \text { WG } & \text { YK531161800 } \\ \text { VER } & 215-225 & \text { WG } & \text { YK531161735 } \\ \text { DUP-F } & 215-225 & \text { WG } & \text { YK531161800 } \\ \text { N } & 215-225 & \text { WG } & \text { YK531161800 }\end{array}$

N

$112-122$

WG

YK62161910

TB

N

\section{$112-122$}

$-$

134-144
WG
WQC

WG
YK62161910 YK62161910

YK66161800
$5 / 31 / 16$ of screen with foot valve before collecting a sample.

Duplicate laboratory analysis.

Verification sample to TestAmerica,

5/31/16 Field replicate

$5 / 31 / 16$ Depth to water $=48.6 \mathrm{ft} \mathrm{BGL}$.

Depth of 2 in. well $=225 \mathrm{ft} \mathrm{BGL}$ Cascade used 2000 gal to drill and set well. Purged 1250 gal each from top and bottom halves of screen with Redi-Flow pump before collecting a sample.

6/2/16 Depth to water $=80.1 \mathrm{ft} \mathrm{BGL}$

Depth of 2 in. well $=122.75 \mathrm{ft}$ BGL. Cascade used 400 gal to drill and set well. Purged 300 gal each from top and bottom halves of screen with foot valve before collecting a sample.

6/2/16 Duplicate laboratory analysis.

6/2/16 Trip blank with water samples shipped to the AGEM laboratory.

6/6/16 Depth to water $=78.1 \mathrm{ft} \mathrm{BGL}$. Depth of 2 in. well $=142.6 \mathrm{ft}$ BGL. Cascade used $400 \mathrm{gal}$ to drill and set well. Purged 300 gal each from top and bottom halves of screen with foot valve before of screen with foot valing a sample. 
TABLE A.1 (Cont.)

\begin{tabular}{|c|c|c|c|c|c|c|c|c|c|}
\hline $\begin{array}{r}\text { Sample } \\
\text { and T }\end{array}$ & $\begin{array}{l}\text { Date } \\
\text { ime }\end{array}$ & Location & Sample & $\begin{array}{l}\text { Sample } \\
\text { Type }\end{array}$ & $\begin{array}{l}\text { Depth } \\
\text { (ft BGL) }\end{array}$ & Matrix & Chain of Custody & $\begin{array}{l}\text { Shipping } \\
\text { Date }\end{array}$ & Sample Description \\
\hline $6 / 6 / 16$ & 8:05 & DL3C1 & YKDL3-C1-113-W-38103 & $\mathrm{N}$ & $113-123$ & WG & YK66161800 & $6 / 6 / 16$ & $\begin{array}{l}\text { Depth to water }=78.1 \mathrm{ft} \mathrm{BGL} \text {. } \\
\text { Depth of } 2 \text { in. well }=122.9 \mathrm{ft} \\
\text { BGL. Cascade used } 400 \text { gal to } \\
\text { drill and set well. Purged } 300 \text { gal } \\
\text { each from top and bottom halves } \\
\text { of screen with foot valve before } \\
\text { collecting a sample. }\end{array}$ \\
\hline $6 / 6 / 16$ & 8:05 & DL3C1 & YKDL3-C1-113-W-38103DUP & DUP-L & $113-123$ & WG & YK66161800 & $6 / 6 / 16$ & Duplicate laboratory analysis. \\
\hline $6 / 6 / 16$ & $10: 20$ & QC & YKQCTB-W-38105 & TB & - & WQC & YK66161800 & $6 / 6 / 16$ & $\begin{array}{l}\text { Trip blank with water samples } \\
\text { shipped to the AGEM laboratory. }\end{array}$ \\
\hline $6 / 6 / 16$ & $17: 30$ & DL8C2 & YKDL8-C2-125-W-38104 & $\mathrm{N}$ & $125-135$ & WG & YK66161800 & $6 / 6 / 16$ & $\begin{array}{l}\text { Depth to water }=71.4 \mathrm{ft} \mathrm{BGL} \text {. } \\
\text { Depth of } 2 \text { in. well }=135 \mathrm{ft} \text { BGL. } \\
\text { Cascade used } 400 \text { gal to drill } \\
\text { and set well. Purged } 300 \text { gal } \\
\text { each from top and bottom halves } \\
\text { of screen with foot valve before } \\
\text { collecting a sample. }\end{array}$ \\
\hline $6 / 7 / 16$ & 8:05 & QC & YKQCTB-W-38107 & TB & - & WQC & YK67161530 & $6 / 7 / 16$ & $\begin{array}{l}\text { Trip blank with water samples } \\
\text { shipped to the AGEM laboratory. }\end{array}$ \\
\hline $6 / 7 / 16$ & $14: 25$ & DL8C1 & YKDL8C1-104-W-38106 & $\mathrm{N}$ & $104-114$ & WG & YK67161530 & $6 / 7 / 16$ & $\begin{array}{l}\text { Depth to water }=71.4 \mathrm{ft} \text { BGL. } \\
\text { Depth of } 2 \text { in. well }=114.7 \mathrm{ft} \\
\text { BGL. Cascade used } 400 \text { gal to } \\
\text { drill and set well. Purged } 300 \text { gal } \\
\text { each from top and bottom halves } \\
\text { of screen with foot valve before } \\
\text { collecting a sample. }\end{array}$ \\
\hline $6 / 7 / 16$ & $14: 25$ & DL8C1 & YKDL8C1-104-W-38106DUP & DUP-L & $104-114$ & WG & YK67161530 & $6 / 7 / 16$ & Duplicate laboratory analysis. \\
\hline $6 / 9 / 16$ & $17: 10$ & DL7CL & YKDL7-CL-265-W-38108 & $N$ & $265-275$ & WG & YK69161730 & $6 / 9 / 16$ & $\begin{array}{l}\text { Depth to water }=91.7 \mathrm{ft} \mathrm{BGL} \text {. } \\
\text { Depth of } 2 \text { in. well }=275 \mathrm{ft} \text { BGL. } \\
\text { Cascade used } 2000 \text { gal to drill } \\
\text { and set well. Purged } 2500 \text { gal } \\
\text { total at } 150 \mathrm{ft} \mathrm{BGL} \mathrm{before} \\
\text { collecting a sample. }\end{array}$ \\
\hline $6 / 9 / 16$ & $17: 10$ & DL7CL & YKDL7-CL-265-W-38108DUP & DUP-L & $265-275$ & WG & YK69161730 & $6 / 9 / 16$ & Duplicate laboratory analysis. \\
\hline $6 / 9 / 16$ & $17: 10$ & DL7CL & YKDL7-CL-265-W-38108VER & VER & $265-275$ & WG & YK69161740 & $6 / 9 / 16$ & $\begin{array}{l}\text { Verification sample to TestAmerica, } \\
\text { Inc. }\end{array}$ \\
\hline $6 / 9 / 16$ & $17: 20$ & QC & YKIDW-W-38109 & WASTE & - & WG & Pace-9Jun2016 & $6 / 9 / 16$ & $\begin{array}{l}\text { Composite sample of the purge } \\
\text { water containerized on-site } \\
\text { during monitoring well } \\
\text { development. }\end{array}$ \\
\hline $6 / 9 / 16$ & $17: 30$ & QC & YKQCTB-W-38110PACE & TB & - & WQC & Pace-9Jun2016 & $6 / 9 / 16$ & $\begin{array}{l}\text { Trip blank sent with water samples } \\
\text { shipped to Pace Analytical for } \\
\text { organic analysis. }\end{array}$ \\
\hline
\end{tabular}


TABLE A.1 (Cont.)

\begin{tabular}{|c|c|c|c|c|c|c|c|c|c|}
\hline \multicolumn{2}{|c|}{$\begin{array}{l}\text { Sample Date } \\
\text { and Time }\end{array}$} & \multirow{2}{*}{$\begin{array}{r}\text { Location } \\
\mathrm{QC}\end{array}$} & \multirow{2}{*}{$\begin{array}{r}\text { Sample } \\
\text { YKQCTB-W-38110 }\end{array}$} & \multirow{2}{*}{$\begin{array}{c}\begin{array}{c}\text { Sample } \\
\text { Type }\end{array} \\
\text { TB }\end{array}$} & \multirow{2}{*}{$\begin{array}{c}\text { Depth } \\
\text { (ft BGL) } \\
-\end{array}$} & \multirow{2}{*}{$\begin{array}{l}\text { Matrix } \\
\text { WQC }\end{array}$} & \multirow{2}{*}{$\begin{array}{l}\text { Chain of Custody } \\
\text { YK69161730 }\end{array}$} & \multirow{2}{*}{$\begin{array}{l}\begin{array}{c}\text { Shipping } \\
\text { Date }\end{array} \\
6 / 9 / 16\end{array}$} & \multirow{2}{*}{$\begin{array}{c}\text { Sample Description } \\
\begin{array}{l}\text { Trip blank with water samples } \\
\text { shipped to the AGEM laboratory. }\end{array}\end{array}$} \\
\hline $6 / 9 / 16$ & $17: 30$ & & & & & & & & \\
\hline $6 / 9 / 16$ & $17: 30$ & QC & YKQCTB-W-38110VER & VER & - & WQC & YK69161740 & $6 / 9 / 16$ & $\begin{array}{l}\text { Verification sample to TestAmerica, } \\
\text { Inc. }\end{array}$ \\
\hline $6 / 12 / 16$ & $11: 50$ & DL7C & YKDL7-C-100-W-38111 & $\mathrm{N}$ & $100-110$ & WG & YK613161500 & $6 / 13 / 16$ & $\begin{array}{l}\text { Depth to water }=69.5 \mathrm{ft} \mathrm{BGL} \text {. } \\
\text { Depth of } 2 \text { in. well }=109.5 \mathrm{ft} \\
\text { BGL. Cascade used } 400 \text { gal to } \\
\text { drill and set well. Purged } 300 \text { gal } \\
\text { each from top and bottom halves } \\
\text { of screen with foot valve before } \\
\text { collecting a sample. }\end{array}$ \\
\hline $6 / 12 / 16$ & $11: 50$ & DL7C & YKDL7-C-100-W-38111DUP & DUP-L & $100-110$ & WG & YK613161500 & $6 / 13 / 16$ & Duplicate laboratory analysis. \\
\hline
\end{tabular}

6/12/16 11:50 DL7C $\quad$ YKDL7-C-100-W-38111DUP

6/12/16 12:30 DL7CU

YKDL7-CU-192-W-38112

6/12/16 12:30 DL7CU

6/13/16 11:00 DL6C

YKDL7-CU-192-W-38112VER

YKDL6-C-110-W-38113

$\begin{array}{cccc}\text { DUP-L } & 100-110 & \text { WG } & \text { YK613161500 } \\ \text { N } & 192-202 & \text { WG } & \text { YK613161500 }\end{array}$

$6 / 13 / 16$ collecting a sample.

Duplicate laboratory analysis. Depth to water $=72 \mathrm{ft} \mathrm{BGL}$. Depth of 2 in. well $=202 \mathrm{ft} \mathrm{BGL}$. Cascade used 1000 gal to drill and set well. Purged $1400 \mathrm{gal}$ total with Redi-Flow at $150 \mathrm{ft}$ BGL before collecting a sample.

VER 192-202 WG YK613161510 6/13/16 $\begin{gathered}\text { Verification sample to TestAmerica, } \\ \text { Inc. }\end{gathered}$
Inc.

6/13/16 Depth to water $=71.9 \mathrm{ft} \mathrm{BGL}$. Depth of 2 in. well $=119.6 \mathrm{ft}$ BGL. Cascade used 400 gal to drill and set well. Purged 300 gal each from top and bottom halves of screen with foot valve before collecting a sample.
6/13/16 11:00 DL6C

6/13/16 14:30 QC

6/13/16 14:30 QC

6/15/16 13:10 DL4C2
YKDL6-C-110-W-38113VER

YKQCTB-W-38114

YKQCTB-W-38114VER
YKDL4-C2-145-W-38115

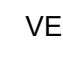

TB

VER

N

145-155

$110-120$

WQC

WQC

WG

YK616161500

K613161500

161500

6/15/16 13:10 DL4C2 
TABLE A.1 (Cont.)

\begin{tabular}{|c|c|c|c|c|c|c|c|c|c|}
\hline \multicolumn{2}{|c|}{$\begin{array}{l}\text { Sample Date } \\
\text { and Time }\end{array}$} & \multirow{2}{*}{$\begin{array}{r}\text { Location } \\
\text { DL4C1 }\end{array}$} & \multirow{2}{*}{$\begin{array}{r}\text { Sample } \\
\text { YKDL4-C1-120-W-38116 }\end{array}$} & \multirow{2}{*}{$\begin{array}{c}\text { Sample } \\
\text { Type }\end{array}$} & \multirow{2}{*}{$\begin{array}{c}\begin{array}{c}\text { Depth } \\
\text { (ft BGL) }\end{array} \\
120-130\end{array}$} & \multirow{2}{*}{$\begin{array}{c}\text { Matrix } \\
\text { WG }\end{array}$} & \multirow{2}{*}{$\begin{array}{l}\text { Chain of Custody } \\
\text { YK616161500 }\end{array}$} & \multirow{2}{*}{$\begin{array}{l}\begin{array}{c}\text { Shipping } \\
\text { Date }\end{array} \\
6 / 16 / 16\end{array}$} & \multirow{2}{*}{$\begin{array}{l}\text { Sample Description } \\
\text { Depth to water }=86.95 \mathrm{ft} \mathrm{BGL} \text {. } \\
\text { Depth of } 2 \text { in. well = } 130 \mathrm{ft} \text { BGL. } \\
\text { Cascade used } 400 \text { gal to drill } \\
\text { and set well. Purged } 300 \text { gal } \\
\text { each from top and bottom halves } \\
\text { of screen with foot valve before } \\
\text { collecting a sample. }\end{array}$} \\
\hline $6 / 16 / 16$ & $14: 30$ & & & & & & & & \\
\hline $6 / 16 / 16$ & $14: 30$ & DL4C1 & YKDL4-C1-120-W-38116VER & VER & $120-130$ & WG & YK616161510 & $6 / 16 / 16$ & $\begin{array}{l}\text { Verification sample to TestAmerica, } \\
\text { Inc. }\end{array}$ \\
\hline $6 / 16 / 16$ & $14: 50$ & QC & YKQCTB-W-38117 & TB & - & WQC & YK616161500 & $6 / 16 / 16$ & $\begin{array}{l}\text { Trip blank with water samples } \\
\text { shipped to the AGEM laboratory. }\end{array}$ \\
\hline $6 / 16 / 16$ & $14: 50$ & QC & YKQCTB-W-38117VER & VER & - & WQC & YK616161510 & $6 / 16 / 16$ & $\begin{array}{l}\text { Verification sample to TestAmerica, } \\
\text { Inc. }\end{array}$ \\
\hline $6 / 21 / 16$ & 9:00 & QC & YKQCTB-W-38120PACE & TB & - & WQC & Pace-21Jun2016 & $6 / 21 / 16$ & $\begin{array}{l}\text { Trip blank sent with water samples } \\
\text { shipped to Pace Analytical for } \\
\text { organic analysis. }\end{array}$ \\
\hline $6 / 21 / 16$ & 9:00 & QC & YKQCTB-W-38120 & TB & - & WQC & YK621161730 & $6 / 21 / 16$ & $\begin{array}{l}\text { Trip blank with water samples } \\
\text { shipped to the AGEM laboratory. }\end{array}$ \\
\hline $6 / 21 / 16$ & $15: 30$ & DL5C1 & YKDL5-C1-106-W-38118 & $\mathrm{N}$ & $106-116$ & WG & YK621161730 & $6 / 21 / 16$ & $\begin{array}{l}\text { Depth to water }=75.7 \mathrm{ft} \mathrm{BGL} \text {. } \\
\text { Depth of } 2 \text { in. well }=116 \mathrm{ft} \text { BGL. } \\
\text { Cascade used } 400 \text { gal to drill } \\
\text { and set well. Purged } 300 \text { gal } \\
\text { each from top and bottom halves } \\
\text { of screen with foot valve before } \\
\text { collecting a sample. }\end{array}$ \\
\hline $6 / 21 / 16$ & $15: 30$ & DL5C1 & YKDL5-C1-106-W-38118DUP & DUP-L & $106-116$ & WG & YK621161730 & $6 / 21 / 16$ & Duplicate laboratory analysis. \\
\hline $6 / 21 / 16$ & $16: 00$ & QC & YKIDW-W-38119 & WASTE & - & WG & Pace-21Jun2016 & $6 / 21 / 16$ & $\begin{array}{l}\text { Composite purge water sample } \\
\text { from poly tanks containing the } \\
\text { development water stored on- } \\
\text { site during monitoring well } \\
\text { development. }\end{array}$ \\
\hline $6 / 21 / 16$ & $16: 55$ & QC & YKROLLFRONT-S-38121 & WASTE & - & SOIL & Pace-21Jun2016 & $6 / 21 / 16$ & $\begin{array}{l}\text { Composite soil sample of the drill } \\
\text { cuttings stored on-site in a } \\
\text { closed top roll off dumpster. }\end{array}$ \\
\hline $6 / 21 / 16$ & $17: 00$ & QC & YKROLLREAR-S-38122 & WASTE & - & SOIL & Pace-21Jun2016 & $6 / 21 / 16$ & $\begin{array}{l}\text { Composite soil sample of the drill } \\
\text { cuttings stored on-site in a } \\
\text { closed top roll off dumpster. }\end{array}$ \\
\hline $8 / 10 / 16$ & $10: 40$ & GARRETSON & YKGARRETSON-G-38140 & $\mathrm{N}$ & - & AIR & YK810161710 & $8 / 10 / 16$ & $\begin{array}{l}\text { Garretson ground floor. Sample } \\
\text { collected over } 24 \mathrm{hr} \text {. Begin } \\
\text { pressure }-26 \text { in. } \mathrm{Hg} \text {. End } \\
\text { pressure }-4 \text { in. } \mathrm{Hg} \text {. }\end{array}$ \\
\hline
\end{tabular}


TABLE A.1 (Cont.)

\begin{tabular}{|c|c|c|c|c|c|c|c|c|c|}
\hline $\begin{array}{r}\text { Sample } \\
\text { and Ti }\end{array}$ & $\begin{array}{l}\text { Date } \\
\text { ime }\end{array}$ & Location & Sample & $\begin{array}{c}\text { Sample } \\
\text { Type }\end{array}$ & $\begin{array}{l}\text { Depth } \\
\text { (ft BGL) }\end{array}$ & Matrix & Chain of Custody & $\begin{array}{l}\text { Shipping } \\
\text { Date }\end{array}$ & Sample Description \\
\hline $8 / 10 / 16$ & $10: 42$ & GARRETSON & YKGARRETSON-G-38138 & $\mathrm{N}$ & - & AIR & YK810161710 & 8/10/16 & $\begin{array}{l}\text { Garretson basement. Sample } \\
\text { collected over } 24 \mathrm{hr} \text {. Begin } \\
\text { pressure }-30 \mathrm{in.} \mathrm{Hg} \text {. End } \\
\text { pressure }-5 \mathrm{in} . \mathrm{Hg} \text {. }\end{array}$ \\
\hline $8 / 10 / 16$ & $10: 43$ & GARRETSON & YKGARRETSON-G-38139 & DUP-F & - & AIR & YK810161710 & 8/10/16 & $\begin{array}{l}\text { Field replicate from Garretson } \\
\text { basement. Sample collected } \\
\text { over } 24 \mathrm{hr} \text {. Begin } \\
\text { pressure }-30 \text { in. Hg. End } \\
\text { pressure }-4 \text { in. Hg. Results not } \\
\text { reported by TestAmerica } \\
\text { because sample was } \\
\text { contaminated with laboratory } \\
\text { standard due to instrument } \\
\text { malfunction. }\end{array}$ \\
\hline $8 / 10 / 16$ & $10: 44$ & GARRETSON & YKBKGND-G-38141 & $\mathrm{N}$ & - & AIR & YK810161710 & 8/10/16 & $\begin{array}{l}\text { Garretson ambient air. Sample } \\
\text { collected over } 24 \mathrm{hr} \text {. Begin } \\
\text { pressure }-24 \mathrm{in} . \mathrm{Hg} \text {. End } \\
\text { pressure } 0 \mathrm{in} . \mathrm{Hg} .\end{array}$ \\
\hline $8 / 10 / 16$ & $11: 02$ & FITWORX & YKFITWORX-G-38148 & $N$ & - & $\begin{array}{l}\text { SOIL } \\
\text { GAS }\end{array}$ & YK810161710 & 8/10/16 & $\begin{array}{l}\text { Sub-slaboratory soil gas sampling } \\
\text { in FitnessWorx stairwell. Begin } \\
\text { pressure }-29 \text { in. Hg. End } \\
\text { pressure } 0 \text { in. } \mathrm{Hg} \text {. }\end{array}$ \\
\hline $8 / 10 / 16$ & $11: 15$ & FITWORX & YKFITWORX-G-38149 & DUP-F & - & $\begin{array}{l}\text { SOIL } \\
\text { GAS }\end{array}$ & YK810161710 & $8 / 10 / 16$ & $\begin{array}{l}\text { Field replicate from FitnessWorx } \\
\text { stairwell. Begin pressure }-30 \text { in. } \\
\mathrm{Hg} \text {. End pressure } 0 \text { in. } \mathrm{Hg} \text {. }\end{array}$ \\
\hline $8 / 10 / 16$ & $11: 30$ & FITWORX & YKFITWORX-G-38142 & $\mathrm{N}$ & - & AIR & YK810161710 & $8 / 10 / 16$ & $\begin{array}{l}\text { FitnessWorx basement. Sample } \\
\text { collected over } 24 \mathrm{hr} \text {. Begin } \\
\text { pressure }-28 \text { in. } \mathrm{Hg} \text {. End } \\
\text { pressure }-4 \mathrm{in} . \mathrm{Hg} \text {. }\end{array}$ \\
\hline $8 / 10 / 16$ & $11: 31$ & FITWORX & YKFITWORX-G-38143 & DUP-F & - & AIR & YK810161710 & $8 / 10 / 16$ & $\begin{array}{l}\text { Field replicate from FitnessWorx } \\
\text { basement. Sample collected } \\
\text { over } 24 \mathrm{hr} \text {. Begin } \\
\text { pressure }-29 \text { in. Hg. End } \\
\text { pressure }-5 \text { in. } \mathrm{Hg} \text {. }\end{array}$ \\
\hline 8/10/16 & $11: 32$ & FITWORX & YKFITWORX-G-38144 & $\mathrm{N}$ & - & AIR & YK810161710 & $8 / 10 / 16$ & $\begin{array}{l}\text { FitnessWorx stairwell. Sample } \\
\text { collected over } 24 \mathrm{hr} \text {. Begin } \\
\text { pressure }-30 \mathrm{in} . \mathrm{Hg} \text {. End } \\
\text { pressure }-5 \mathrm{in} . \mathrm{Hg} \text {. }\end{array}$ \\
\hline 8/10/16 & $11: 34$ & FITWORX & YKFITWORX-G-38145 & $\mathrm{N}$ & - & AIR & YK810161710 & $8 / 10 / 16$ & $\begin{array}{l}\text { FitnessWorx room \#3. Sample } \\
\text { collected over } 24 \mathrm{hr} \text {. Begin } \\
\text { pressure }-26 \text { in. } \mathrm{Hg} \text {. End } \\
\text { pressure }-2 \text { in. } \mathrm{Hg} \text {. }\end{array}$ \\
\hline
\end{tabular}


TABLE A.1 (Cont.)

\begin{tabular}{|c|c|c|c|c|c|c|c|c|}
\hline $\begin{array}{l}\text { Sample Date } \\
\text { and Time }\end{array}$ & Location & Sample & $\begin{array}{l}\text { Sample } \\
\text { Type }\end{array}$ & $\begin{array}{l}\text { Depth } \\
\text { (ft BGL) }\end{array}$ & Matrix & Chain of Custody & $\begin{array}{l}\text { Shipping } \\
\text { Date }\end{array}$ & Sample Description \\
\hline 8/10/16 & FITWORX & YKFITWORX-G-38146 & $\mathrm{N}$ & - & AIR & YK810161710 & $8 / 10 / 16$ & $\begin{array}{l}\text { FitnessWorx room \#7. Sample } \\
\text { collected over } 24 \mathrm{hr} \text {. Begin } \\
\text { pressure }-29 \mathrm{in} . \mathrm{Hg} \text {. End } \\
\text { pressure }-6 \text { in. } \mathrm{Hg} \text {. }\end{array}$ \\
\hline $8 / 10 / 16 \quad 11: 37$ & FITWORX & YKBKGND-G-38147 & $\mathrm{N}$ & - & AIR & YK810161710 & $8 / 10 / 16$ & $\begin{array}{l}\text { FitnessWorx ambient air. Sample } \\
\text { collected over } 24 \mathrm{hr} \text {. Begin } \\
\text { pressure }-29 \mathrm{in} . \mathrm{Hg} \text {. End } \\
\text { pressure }-6 \mathrm{in} . \mathrm{Hg} \text {. }\end{array}$ \\
\hline 12/14/16 10:14 & GARRETSON & YKGARRETSON-G-39533 & $\mathrm{N}$ & - & AIR & YK1214161700 & $12 / 14 / 16$ & $\begin{array}{l}\text { Garretson basement. Sample } \\
\text { collected over } 24 \mathrm{hr} \text {. Begin } \\
\text { pressure }-26 \text { in. Hg. End } \\
\text { pressure }-4 \text { in. } \mathrm{Hg} \text {. }\end{array}$ \\
\hline 12/14/16 10:15 & GARRETSON & YKGARRETSON-G-39535 & $\mathrm{N}$ & - & AIR & YK1214161700 & $12 / 14 / 16$ & $\begin{array}{l}\text { Garretson ground floor. Sample } \\
\text { collected over } 24 \mathrm{hr} \text {. Begin } \\
\text { pressure }-21 \mathrm{in} . \mathrm{Hg} \text {. End } \\
\text { pressure } 0 \text { in. } \mathrm{Hg} \text {. }\end{array}$ \\
\hline 12/14/16 10:18 & GARRETSON & YKGARRETSON-G-39534 & DUP-F & - & AIR & YK1214161700 & $12 / 14 / 16$ & $\begin{array}{l}\text { Field replicate of basement sample. } \\
\text { Sample collected over } 24 \mathrm{hr} \text {. } \\
\text { Begin pressure }-29 \mathrm{in} \text {. } \mathrm{Hg} \text {. End } \\
\text { pressure }-7 \mathrm{in} . \mathrm{Hg} \text {. }\end{array}$ \\
\hline 12/14/16 10:20 & GARRETSON & YKBKGND-G-39536 & $\mathrm{N}$ & - & AIR & YK1214161700 & $12 / 14 / 16$ & $\begin{array}{l}\text { Garretson ambient air. Sample } \\
\text { collected over } 24 \mathrm{hr} \text {. Begin } \\
\text { pressure }-26 \text { in. } \mathrm{Hg} \text {. End } \\
\text { pressure }-1 \mathrm{in} . \mathrm{Hg} \text {. }\end{array}$ \\
\hline 12/14/16 11:08 & FITWORX & YKFITWORX-G-39537 & $\mathrm{N}$ & - & AIR & YK1214161710 & $12 / 14 / 16$ & $\begin{array}{l}\text { FitnessWorx basement. Sample } \\
\text { collected over } 24 \mathrm{hr} \text {. Begin } \\
\text { pressure }-30 \text { in. } \mathrm{Hg} \text {. End } \\
\text { pressure }-5 \text { in. } \mathrm{Hg} \text {. }\end{array}$ \\
\hline 12/14/16 11:08 & FITWORX & YKFITWORX-G-39538 & DUP-F & - & AIR & YK1214161710 & $12 / 14 / 16$ & $\begin{array}{l}\text { Field replicate of basement sample. } \\
\text { Sample collected over } 24 \mathrm{hr} \text {. } \\
\text { Begin pressure }-27 \text { in. } \mathrm{Hg} \text {. End } \\
\text { pressure }-4 \text { in. } \mathrm{Hg} \text {. }\end{array}$ \\
\hline 12/14/16 11:10 & FITWORX & YKFITWORX-G-39539 & $\mathrm{N}$ & - & AIR & YK1214161710 & $12 / 14 / 16$ & $\begin{array}{l}\text { FitnessWorx stairwell. Sample } \\
\text { collected over } 24 \mathrm{hr} \text {. Begin } \\
\text { pressure }-30 \mathrm{in} . \mathrm{Hg} \text {. End } \\
\text { pressure }-8 \mathrm{in.} \mathrm{Hg} \text {. }\end{array}$ \\
\hline 12/14/16 11:12 & FITWORX & YKFITWORX-G-39540 & $\mathrm{N}$ & - & AIR & YK1214161710 & $12 / 14 / 16$ & $\begin{array}{l}\text { FitnessWorx room \#3. Sample } \\
\text { collected over } 24 \mathrm{hr} \text {. Begin } \\
\text { pressure }-31 \text { in. Hg. End } \\
\text { pressure }-5 \text { in. } \mathrm{Hg} \text {. }\end{array}$ \\
\hline
\end{tabular}


TABLE A.1 (Cont.)

\begin{tabular}{|c|c|c|c|c|c|c|c|c|}
\hline $\begin{array}{l}\text { Sample Date } \\
\text { and Time }\end{array}$ & Location & Sample & $\begin{array}{l}\text { Sample } \\
\text { Type }\end{array}$ & $\begin{array}{c}\text { Depth } \\
\text { (ft BGL) }\end{array}$ & Matrix & Chain of Custody & $\begin{array}{l}\text { Shipping } \\
\text { Date }\end{array}$ & Sample Description \\
\hline 12/14/16 11:13 & FITWORX & YKFITWORX-G-39541 & $\mathrm{N}$ & - & AIR & YK1214161710 & $12 / 14 / 16$ & $\begin{array}{l}\text { FitnessWorx room \#7. Sample } \\
\text { collected over } 24 \mathrm{hr} \text {. Begin } \\
\text { pressure }-27 \mathrm{in.} \mathrm{Hg.} \mathrm{End} \\
\text { pressure }-4 \text { in. } \mathrm{Hg} \text {. }\end{array}$ \\
\hline 12/14/16 11:16 & FITWORX & YKBKGND-G-39542 & $\mathrm{N}$ & - & AIR & YK1214161710 & $12 / 14 / 16$ & $\begin{array}{l}\text { FitnessWorx ambient air. Sample } \\
\text { collected over } 24 \mathrm{hr} \text {. Begin } \\
\text { pressure }-29 \text { in. } \mathrm{Hg} \text {. End } \\
\text { pressure }-4 \text { in. } \mathrm{Hg} \text {. }\end{array}$ \\
\hline 12/14/16 11:22 & FITWORX & YKFITWORX-G-39543 & $\mathrm{N}$ & - & $\begin{array}{l}\text { SOIL } \\
\text { GAS }\end{array}$ & YK1214161710 & $12 / 14 / 16$ & $\begin{array}{l}\text { Sub-slaboratory soil gas sampling } \\
\text { in FitnessWorx stairwell. Begin } \\
\text { pressure }-28 \text { in. Hg. End } \\
\text { pressure } 0 \text { in. } \mathrm{Hg} \text {. }\end{array}$ \\
\hline 12/14/16 11:34 & FITWORX & YKFITWORX-G-39544 & DUP-F & - & $\begin{array}{l}\text { SOIL } \\
\text { GAS }\end{array}$ & YK1214161710 & $12 / 14 / 16$ & $\begin{array}{l}\text { Field replicate of soil gas sample. } \\
\text { Begin pressure }-26 \mathrm{in} . \mathrm{Hg} \text {. End } \\
\text { pressure } 0 \mathrm{in.} \mathrm{Hg} \text {. }\end{array}$ \\
\hline
\end{tabular}

Sample type codes: DUP-F, field replicate; DUP-L, laboratory duplicate; FB, field blank; N, primary sample; RI, rinsate; TB, trip blank; VER, verification sample; WASTE, waste sample. Matrix type codes: AIR, indoor air; MEOH, methanol; SOIL, soil; SOIL GAS, sub-slab soil gas; WG, groundwater; WQC, quality control water sample, e.g., trip blank. 


\section{Appendix B:}

\section{Sub-Slab Soil Vapor Standard Operating Procedures (For Vapor Intrusion Applications)}

Reproduced with the written permission of H\&P Mobile Geochemistry, Carlsbad, California 


\section{Sub-Slab Soil Vapor Standard Operating Procedures (For Vapor Intrusion Applications)}

Revised January 2008

Prepared by:

H\&P Mobile Geochemistry

Carlsbad, California

760-804-9678

www.handpmg.com 


\section{Soil Gas Probe/lmplant Construction}

Materials: Stainless steel tubing

Diameter: $1 / 8$ " OD or $1 / 4$ " OD

Length: Cut to desired length within or just below base of slab

Tip: SS or plastic (typically no tip required for sub-slab)

Surface Termination: Swagelok fittings or 2-way valve

Refer to Photos 1 and 2.

Notes:

- Tips (aluminum, ceramic, SS) can be put on the end if desired to give a longer screen interval, but typically are not used for sub-slab samples.

- Various surface terminations are available and the selection often depends on whether the probes are temporary or permanent and whether they need to be installed flush with the surface.

\section{Probe Installation Protocol}

1. Ensure all sub-slab utilities (public and building specific) are marked prior to installation.

2. Drill a $1 / 2$ " to 1 " OD hole through the slab with a drill and spline bit. Do not use water. If dust prevention necessary, cover the location with a towel/cloth and drill through a pre-cut hole in the cloth (Photo 3).

3. Measure slab thickness. Cut tubing to appropriate length to reach base of slab and to give required type of surface termination (flush, recessed, protruding). If a flush or recessed surface termination is required, a larger diameter hole (1") in the upper 1-inch of the slab may be useful to leave enough room for the fitting on the probe tubing (Photo 4).

4. Insert tubing. Add sand to cover tip with about 1 inch of sand.

5. Grout to the surface using bentonite (if temporary installation) or cement (if permanent installation).

6. Wait 15 to 30 minutes prior to sampling for bentonite to congeal. 


\section{Soil Gas Sample Collection}

Since sub-slab sampling is from very shallow depths (typically 2 " to 6" below surface), minimum purge volumes and low volume samples are preferred to minimize potential breakthrough from the surface. Tracer/leak gas is necessary to ensure breakthrough does not occur.

Materials: $1 / 8$ " or $1 / 4$ " OD nylon or Teflon tubing.

Sample Canister: syringe, tedlar bag, SS canister, gas-tight glass.

Plastic 3-way valve.

Vacuum gauge and sampling train as necessary.

Notes: If canisters with flow chokes are used, ensure flow chokes are dedicated to the canister or cleaned before reuse on another canister.

1. If syringe samples only to be collected, connect syringe to probe tubing using a 3-way valve (Photo 5). Purge out $250 \mathrm{cc}$ or 4 deadvolumes of the probe and connecting tubing ( $4 \mathrm{cc} / \mathrm{ft}$ for $1 / 8$ "OD tubing and $20 \mathrm{cc} / \mathrm{ft}$ for $1 / 4$ " OD tubing), whichever is larger. Leave syringe connected to implant tubing.

2. If canister samples are being collected, connect $60 \mathrm{cc}$ syringe to sampling train and purge out $250 \mathrm{cc}$ or 4 dead-volumes of the probe and sampling train, whichever is larger (Photo 6).

3. For canisters with valves (not mini-cans with quick-disconnects), connect canister to sampling train or connecting tubing. Do not connect mini-cans with quick-disconnects until step 6 .

4. For canister samples, check canister vacuum immediately before use. Ensure any flow chokes \& filters have been flushed between samples and all connections/fittings are tight.

5. Place tracer/leak compound, typically difluoroethane or a gas tracer such as helium around the implant at the ground surface and at connections in the sampling system. Liquid tracers are easily emplaced by wetting a paper towel and wrapping around the test locations. Gas tracers require a shroud to hold the gas tracer over the probe and sampling train.

6. Once leak compound in place, open 3-way valve and collect soil gas sample in syringe. For canister samples with valves, open valve. For mini-cans with quick-disconnects, remove purging syringe and connect mini-can to sampling train (Photos $7 \& 8$ ). 
7. If measurements with a portable meter to be made (e.g. oxygen), conduct measurements after collection of the soil gas sample(s) for VOC analysis.

Notes:

- For larger canisters (>1 liter), sample flow rates are not to exceed 500 $\mathrm{ml} / \mathrm{min}$ (200 $\mathrm{ml} / \mathrm{min}$ for CA-EPA) to minimize potential for vacuum extraction of contaminants from the soil phase.

- The presence of the tracer compound in the analysis confirms a leak and another sample is collected until no leak is detected (if on-site analysis exists).

- If large volume canisters used (3 or more liters), a purge volume test may be required to ensure sample dilution from other zones is not occurring. 


\section{Field Records}

The field technician maintains a log sheet summarizing:

- Sample identification

- Probe location

- Date and time of sample collection

- Sampling depth

- Identity of samplers

- Weather conditions

- Sampling methods and devices

- Soil gas purge volumes

- Volume of soil gas extracted

- Vacuum of canisters before and after samples collected.

- Apparent moisture content (dry, moist or saturated etc.) of the sampling zone

Chain of custody protocols and records used to track samples from sampling point to analysis. 


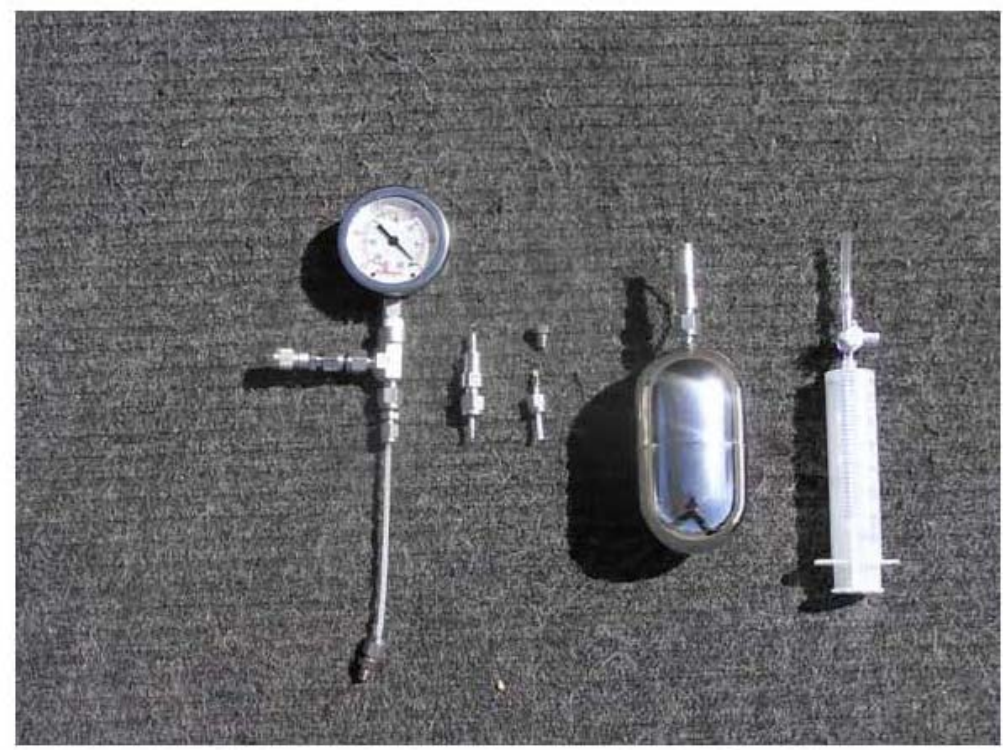

Photo 1 - Soil gas sampling materials: sample collection train, vapor implants, mini-can $(400 \mathrm{cc})$, purging syringe.

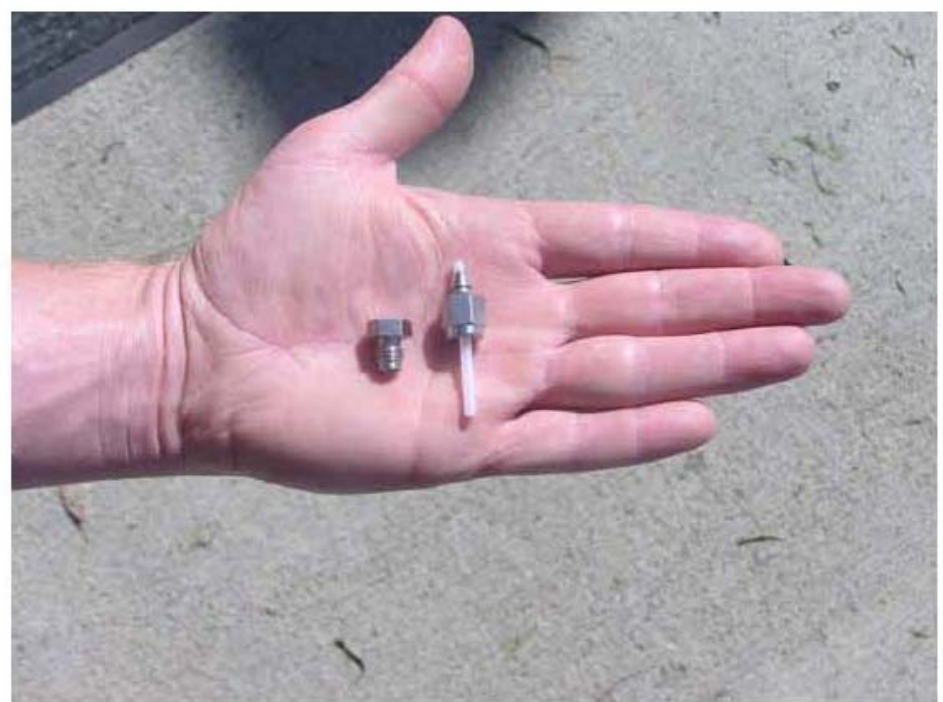

Photo 2 - Sub-slab vapor implant (1/8" OD) and cap. Tubing can be cut to any length (nylon showed). 


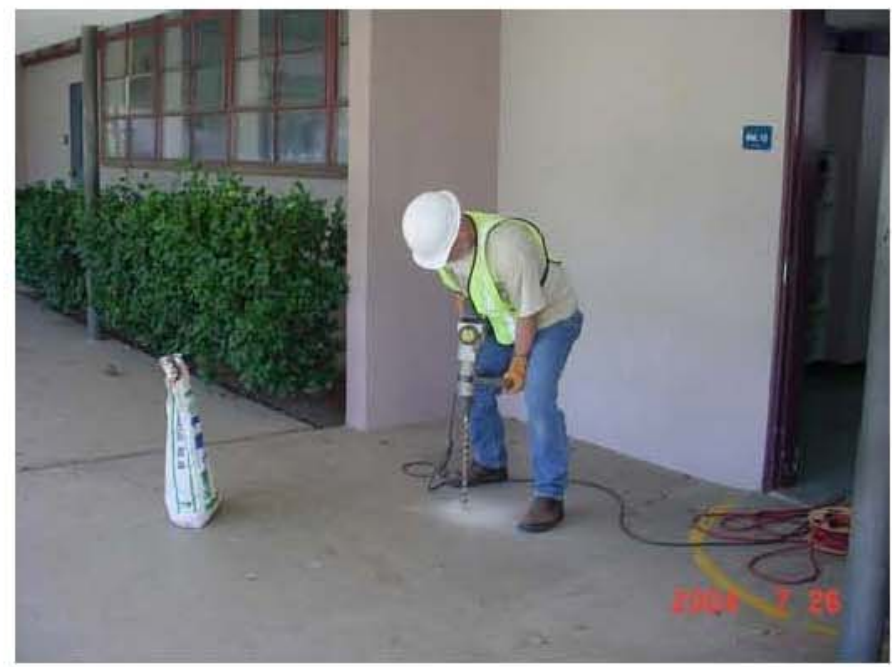

Photo 3 - Drilling hole through slab (1/2" OD).
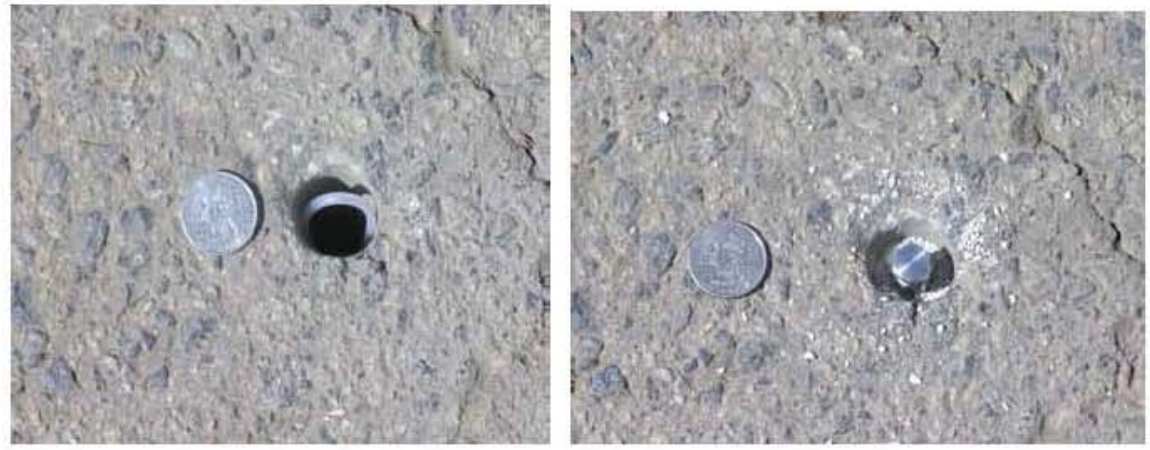

Photo 4 - Surface completions. Left: tubing coiled in hole. Removable plug installed to make flush with surface. Right: cemented flush to surface (hole slightly recessed purposely to enable fitting to be visible in photo). 


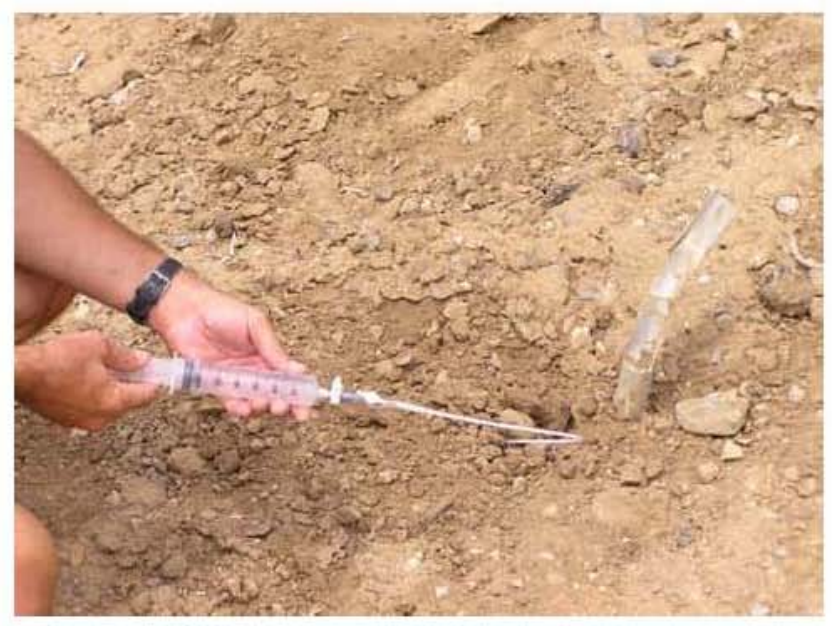

Photo 5 - Collecting sample with $60 \mathrm{cc}$ syringe. Note small dead-volume of tubing connecting syringe to implant.

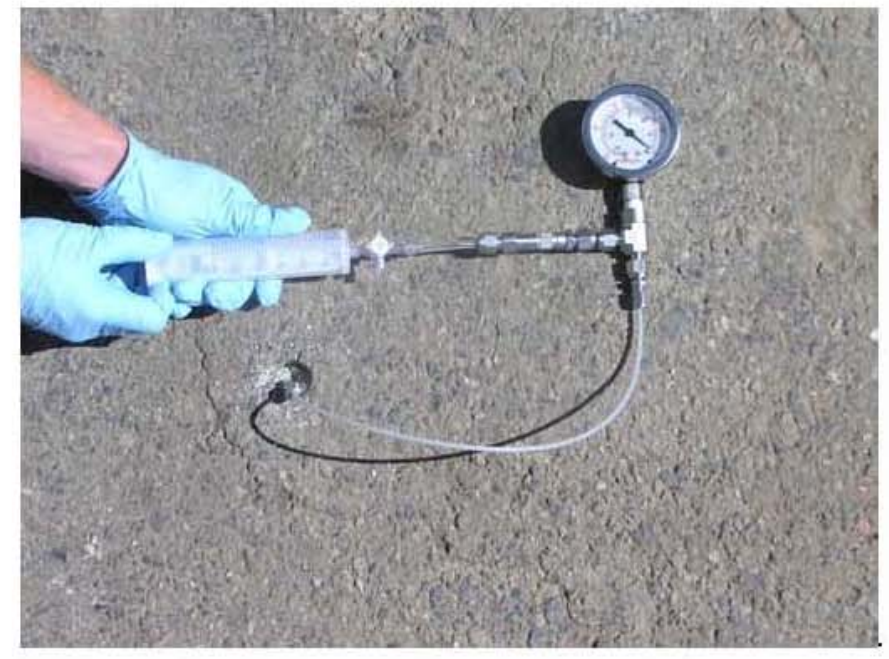

Photo 6 - Purging implant \& sampling train with $60 \mathrm{cc}$ syringe. Note small dead-volume of tubing connecting syringe to implant. 


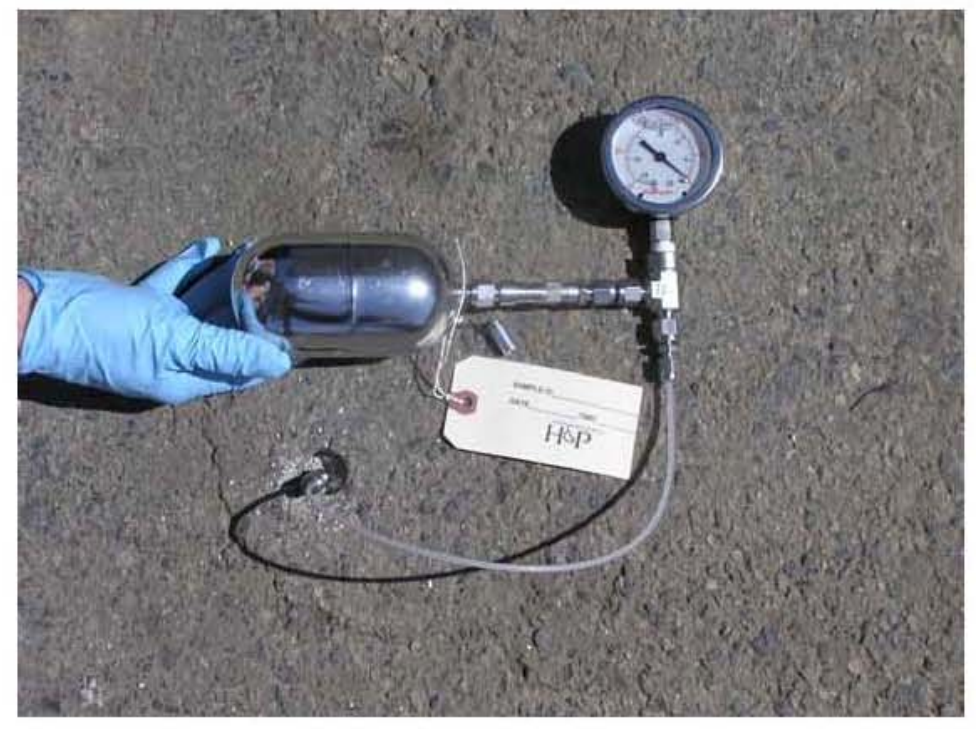

Photo 7 - Collecting soil gas sample in mini-can $(400 \mathrm{cc})$.

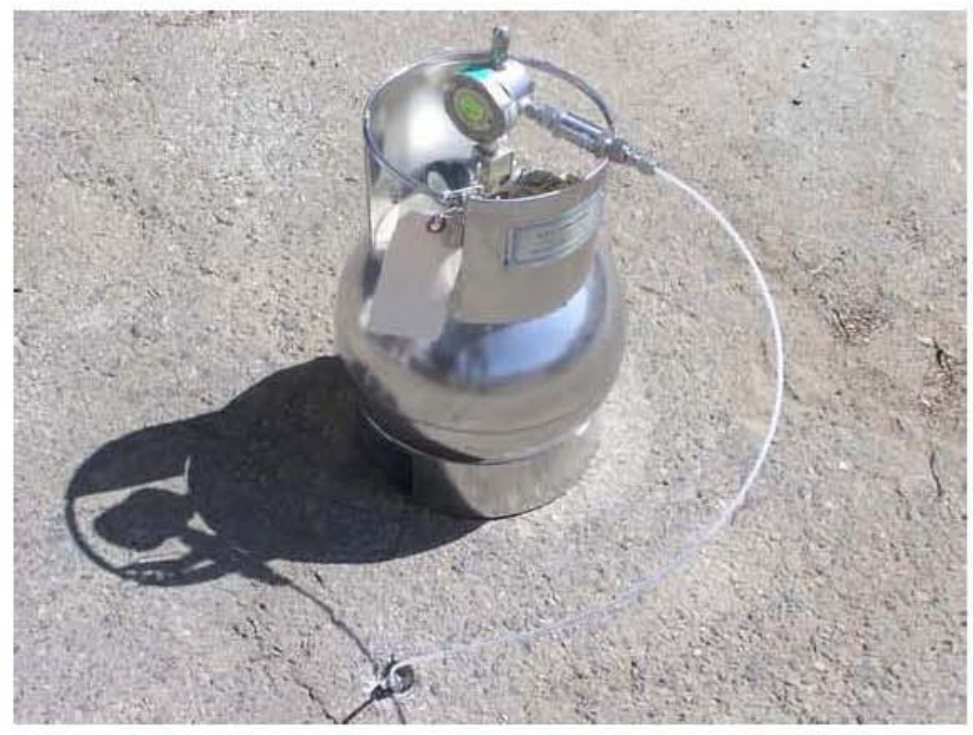

Photo 8 - Collecting sample in larger canister (6 liter). No sampling train is required since the canister is already equipped with valve, vacuum gauge, and flow choke. 


\section{Appendix C:}

ECPT Logs for Borings TI01-TI28 


\section{Argonne National Laboratory}

Project: York, NE

\section{Boring ID: TI01/DL1}

Elevation: $1658.77 \mathrm{ft} \quad$ Log Date: 5/18/2016

Depth: $131.23 \mathrm{ft} \mathrm{BGL}$
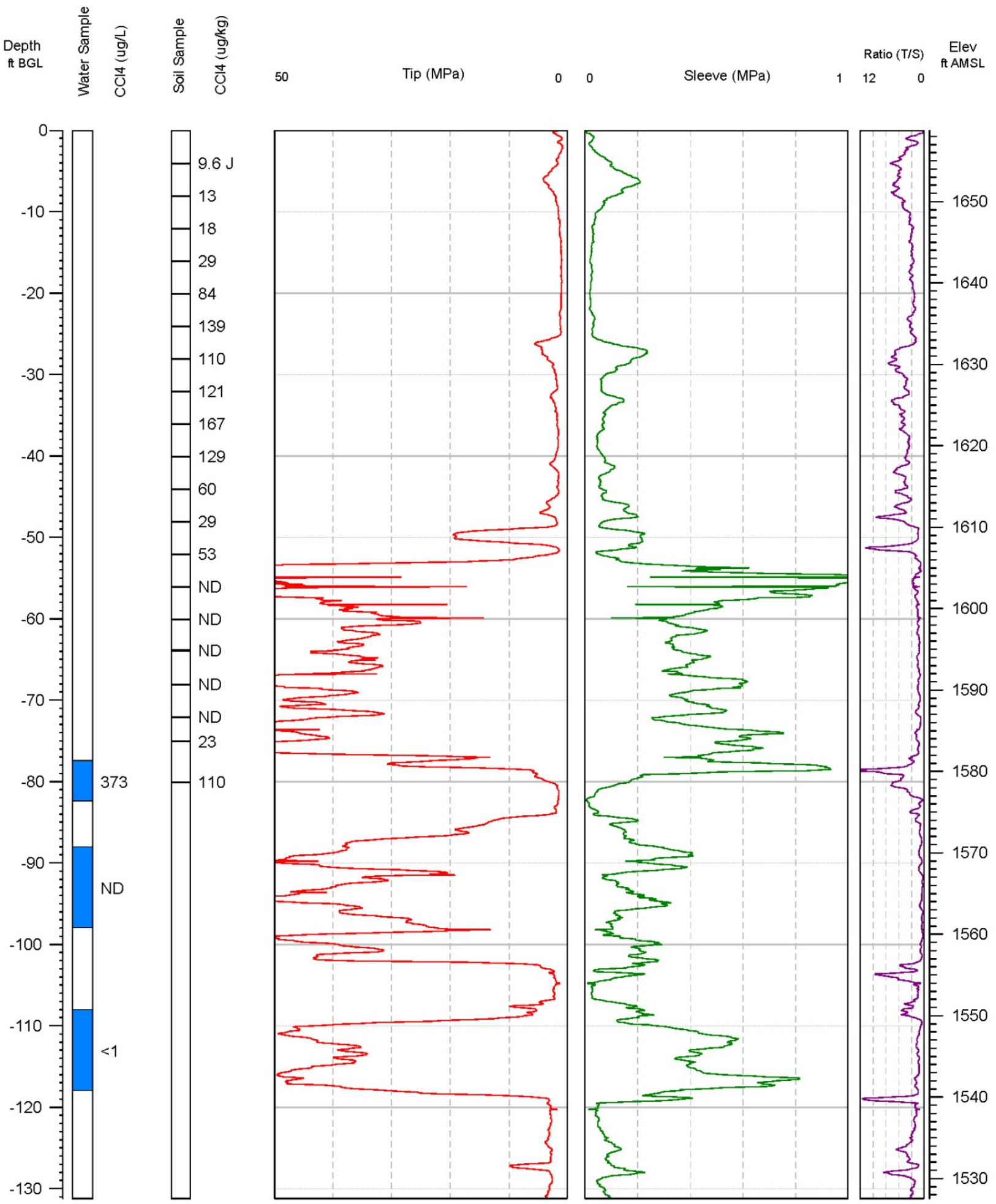
Version 01, 2/14/2017

Argonne National Laboratory

Project: York, NE

\section{Boring ID: T102}

Elevation: $1659.08 \mathrm{ft}$

Log Date: 7/14/2015

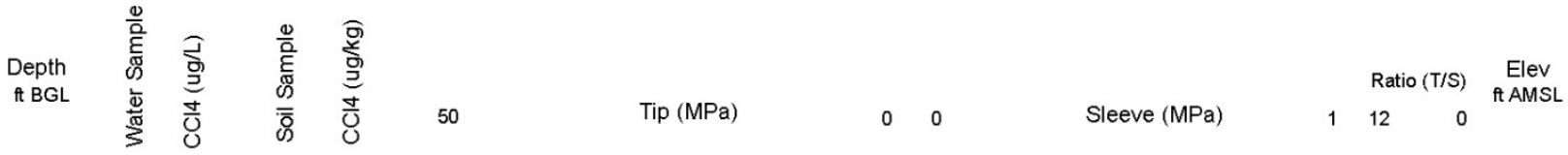
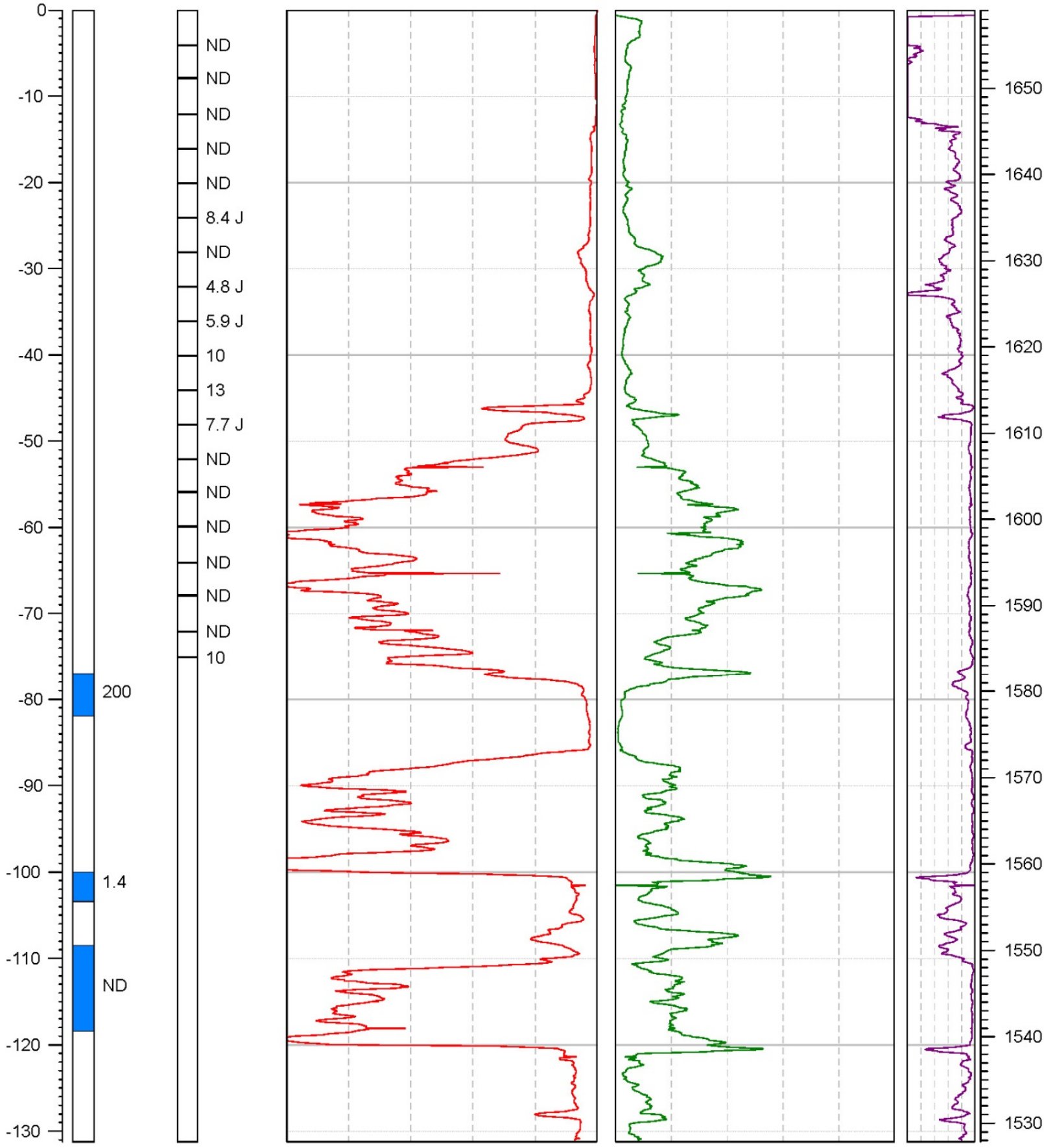


\section{Argonne National Laboratory}

Project: York, NE

\section{Boring ID: TI04}

Elevation: $1657.66 \mathrm{ft}$

Log Date: 7/16/2015

Depth: $131.23 \mathrm{ft} \mathrm{BGL}$

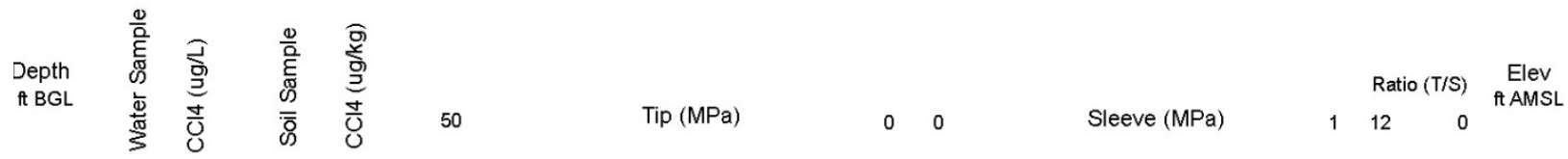
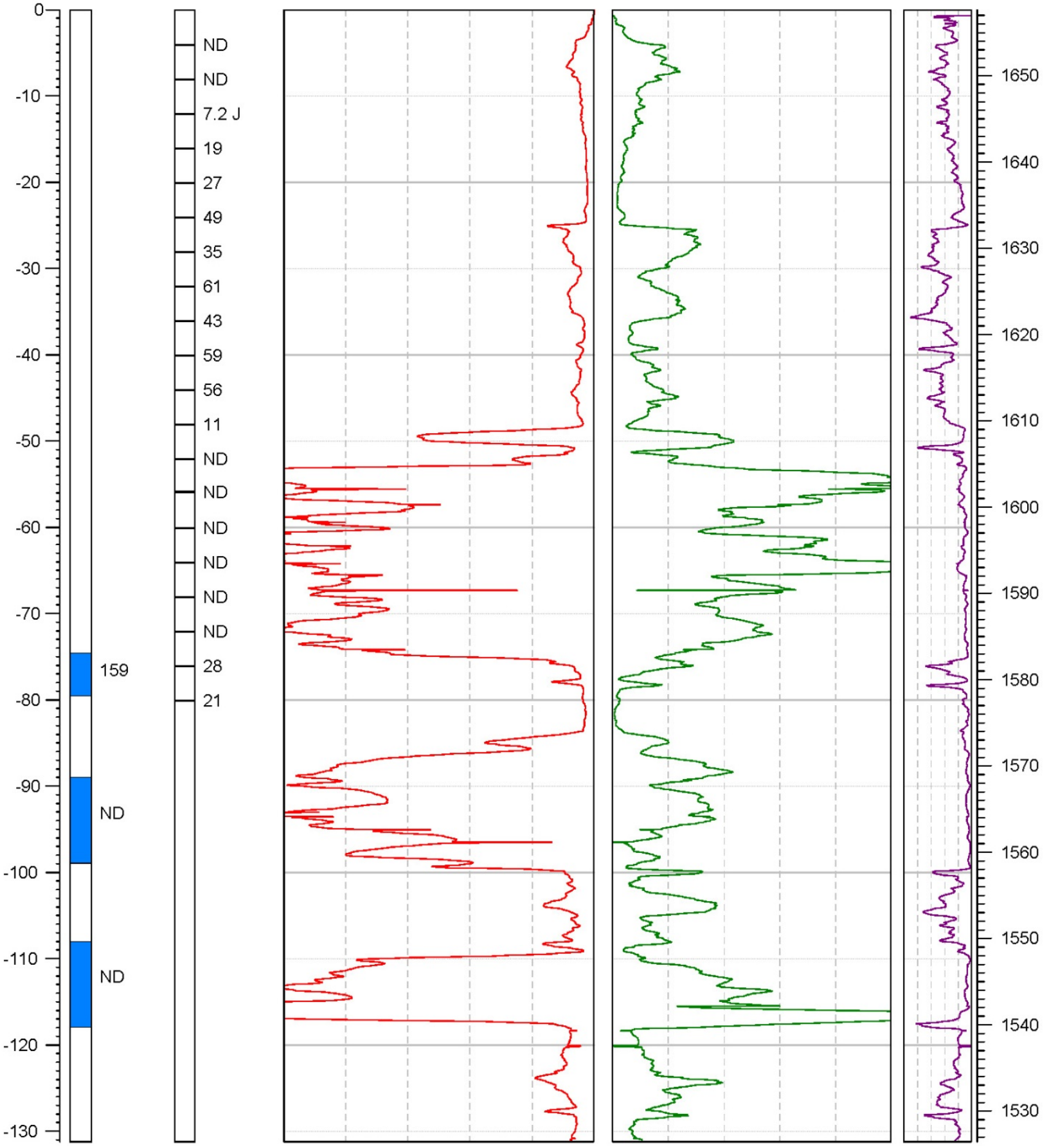


\section{Argonne National Laboratory}

Project: York, NE
Elevation: $1658.65 \mathrm{ft}$

Depth: $131.23 \mathrm{ft} \mathrm{BGL}$

\section{Boring ID: TI05}

Log Date: 7/17/2015

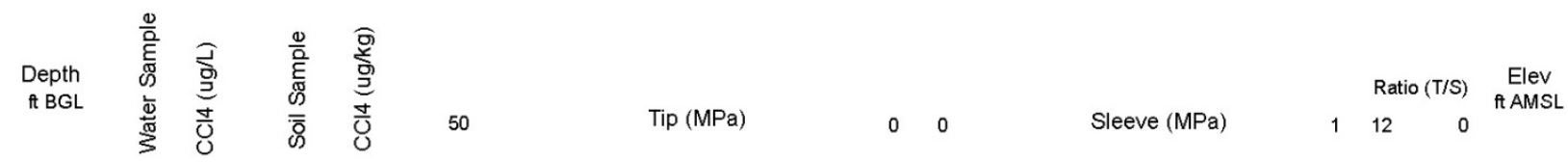

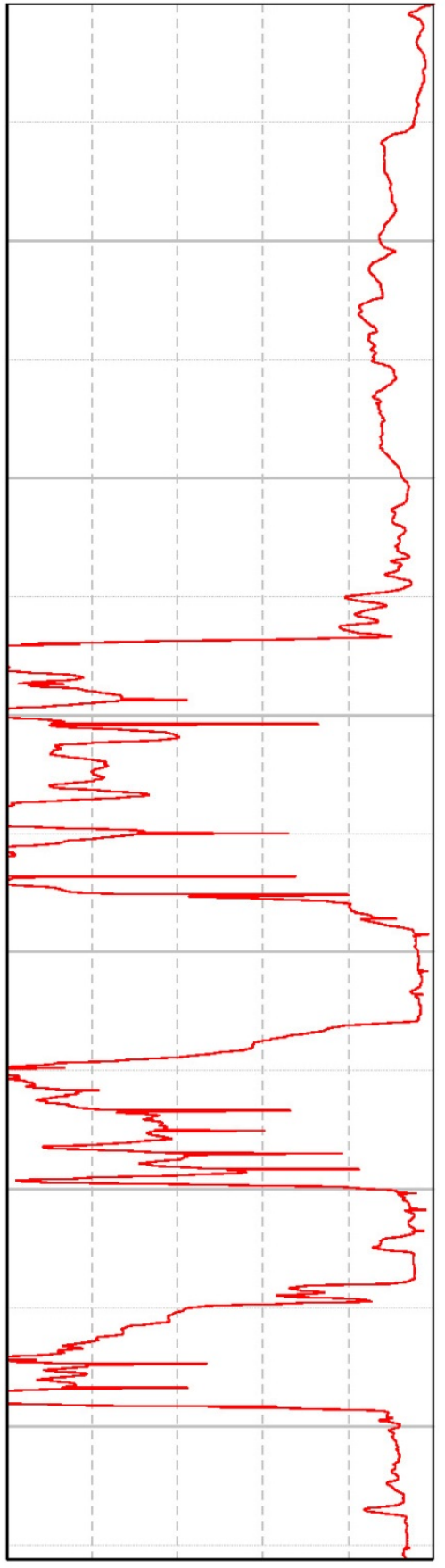
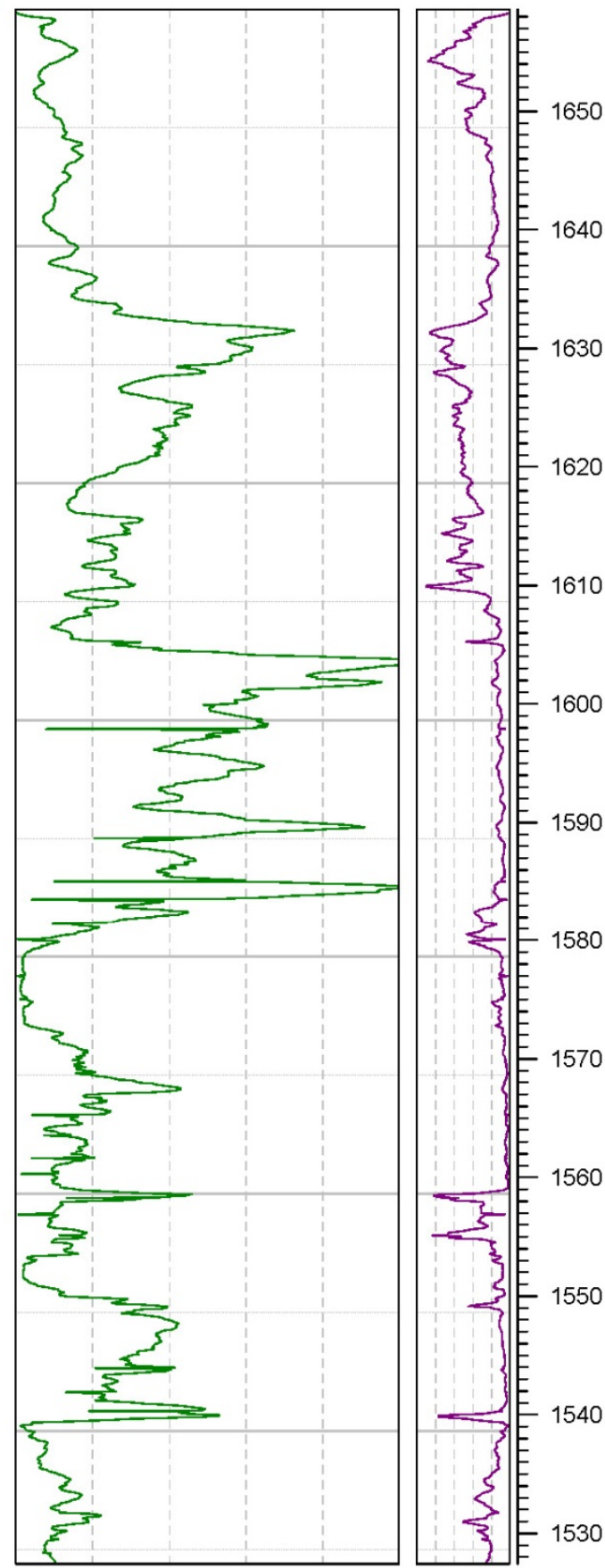
Version 01, 2/14/2017

\section{Argonne National Laboratory}

Project: York, NE
Elevation: $1658.26 \mathrm{ft}$

Depth: $130.31 \mathrm{ft} \mathrm{BGL}$

\section{Boring ID: TI06}

Log Date: 7/18/2015

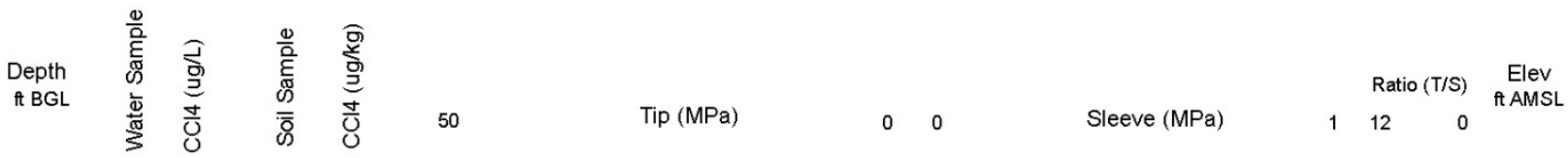
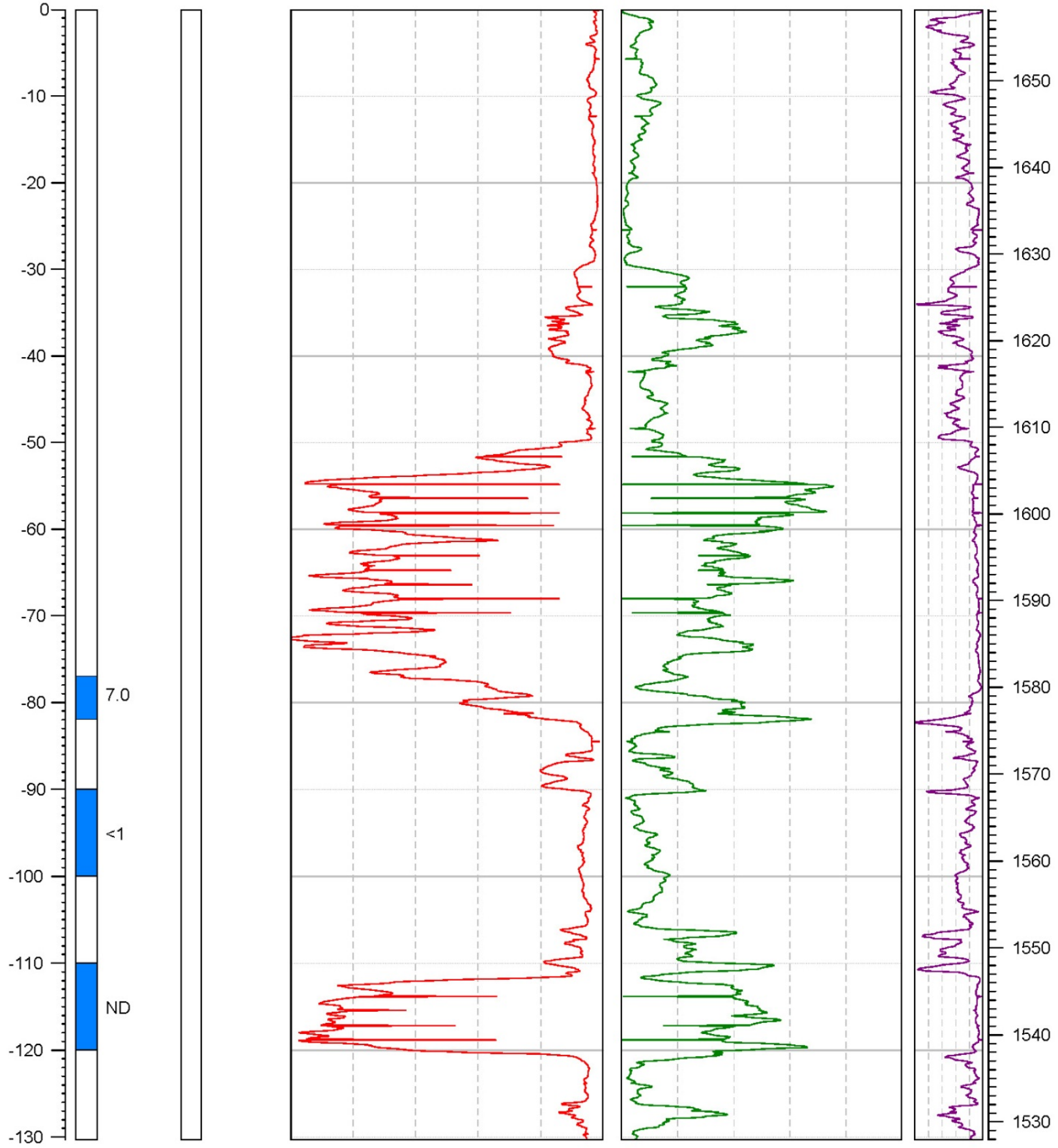
Version 01, 2/14/2017

\section{Argonne National Laboratory}

Project: York, NE
Elevation: $1658.21 \mathrm{ft}$

Depth: $131.23 \mathrm{ft} \mathrm{BGL}$

\section{Boring ID: T108}

Log Date: 7/21/2015

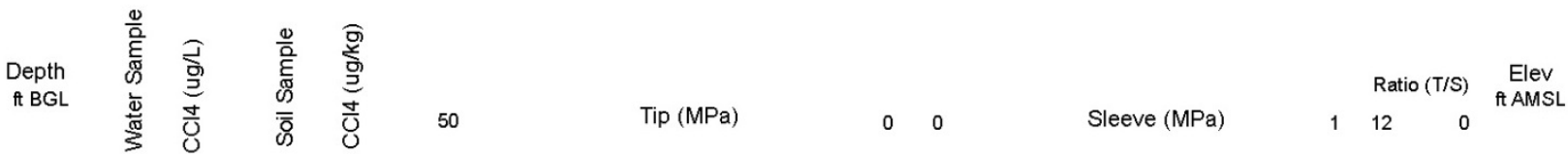
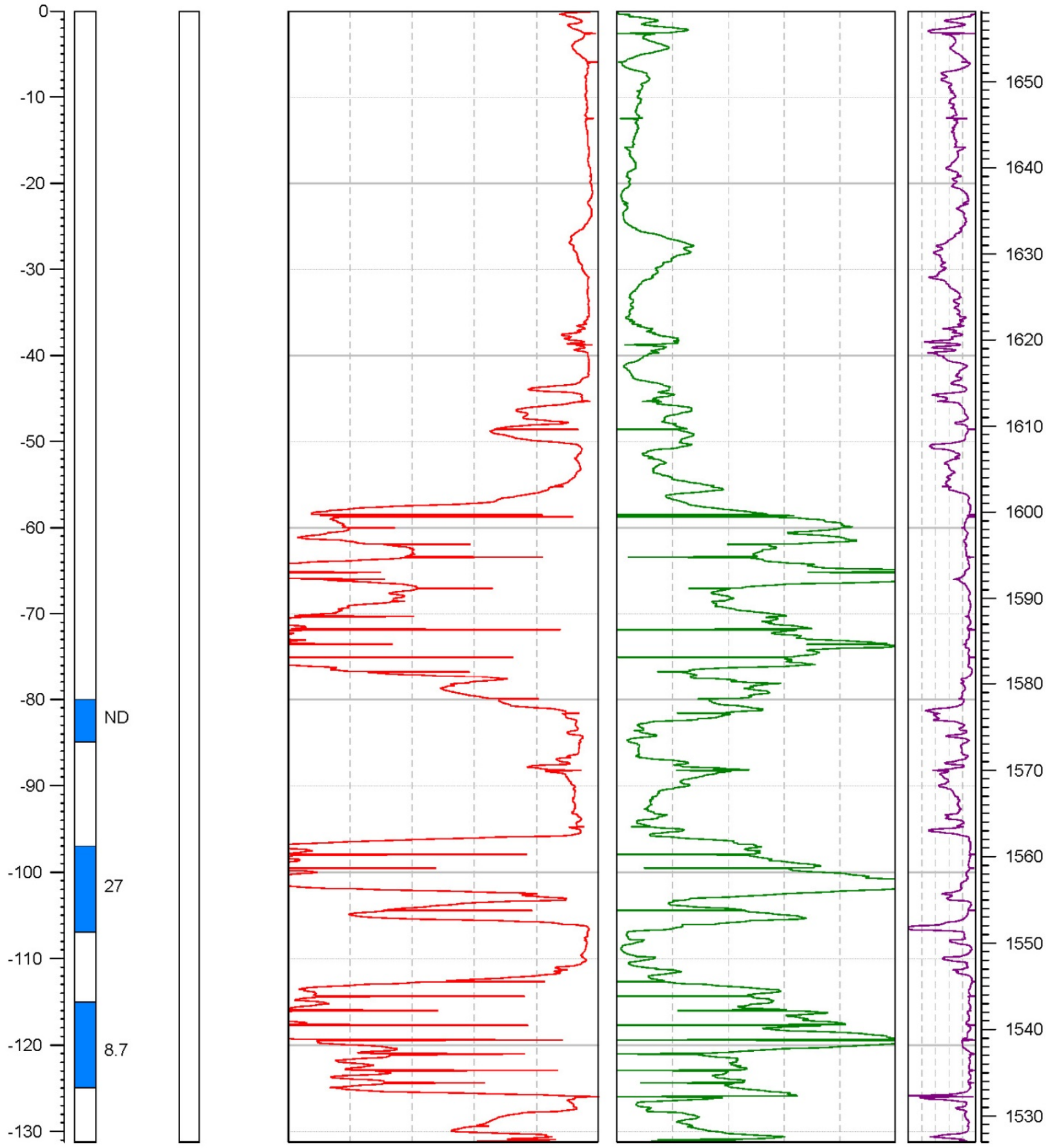


\section{Argonne National Laboratory}

Project: York, NE
Elevation: $1659.39 \mathrm{ft}$

Depth: $147.63 \mathrm{ft} \mathrm{BGL}$

\section{Boring ID: TI09}

Log Date: 8/12/2015
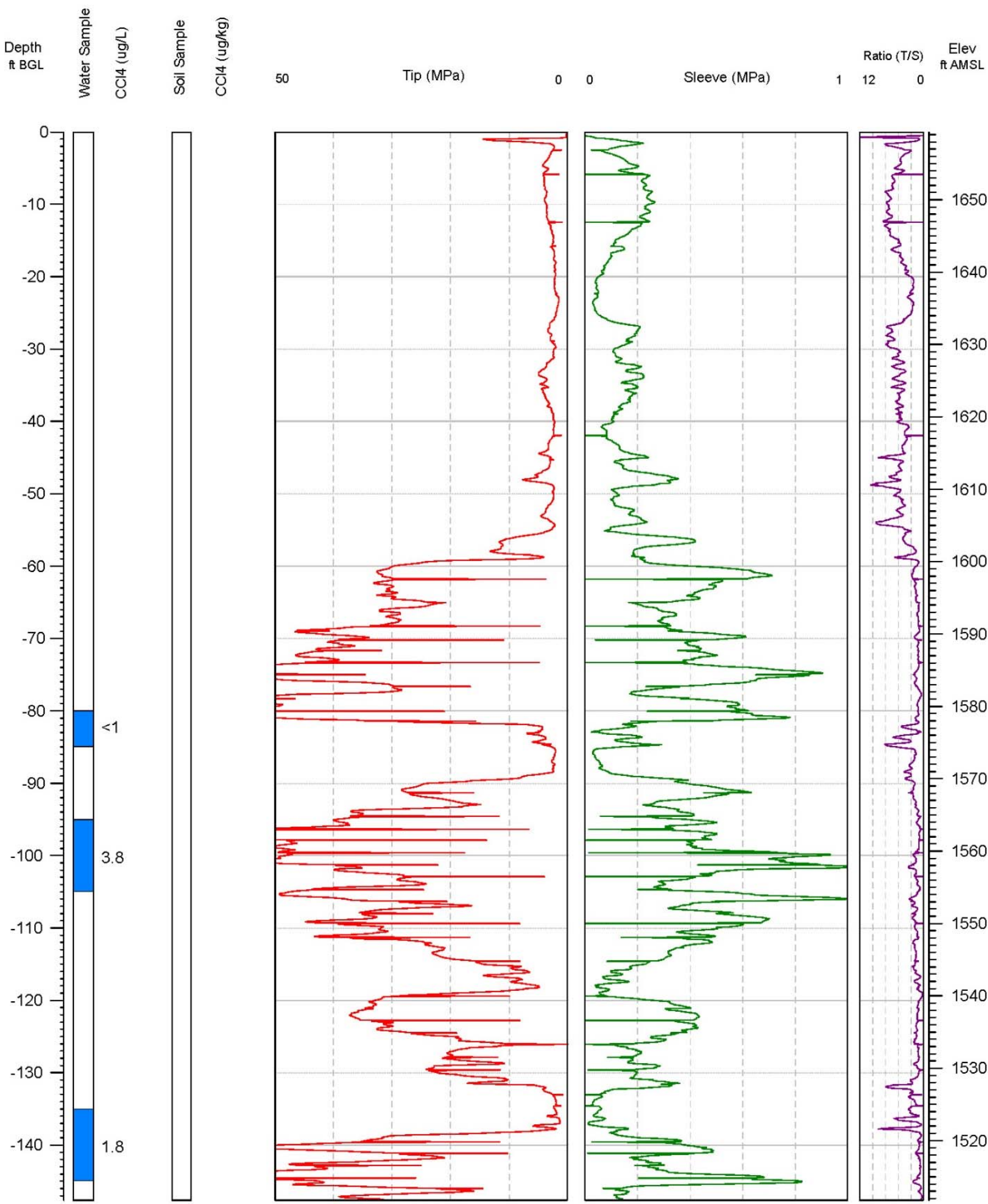
Argonne National Laboratory

Project: York, NE

\section{Boring ID: TI10}

Elevation: $1660.48 \mathrm{ft}$

Depth: $147.63 \mathrm{ft} \mathrm{BGL}$

Log Date: 8/13/2015
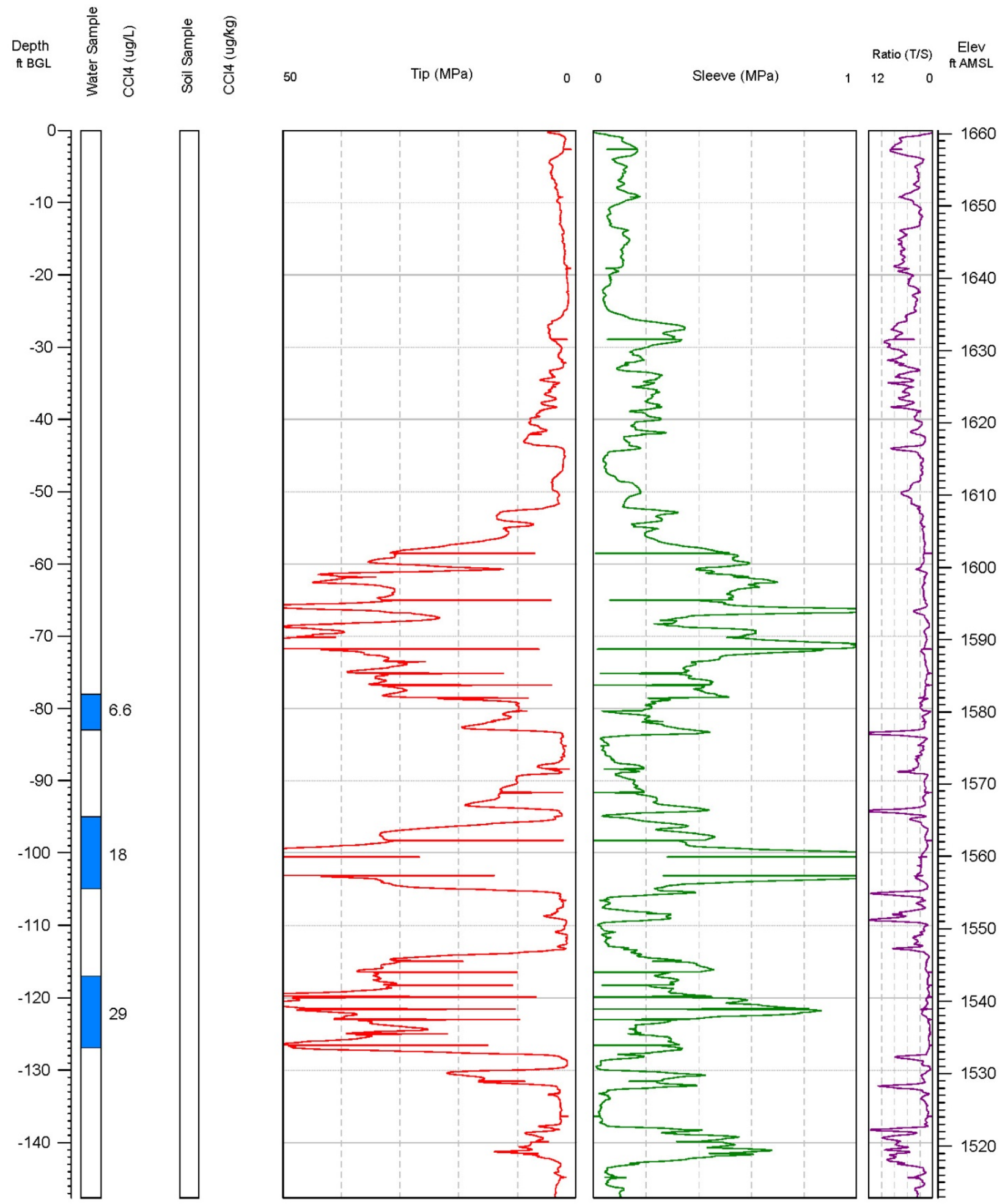
Argonne National Laboratory

Project: York, NE
Elevation: $1657.54 \mathrm{ft}$

Depth: $147.63 \mathrm{ft} \mathrm{BGL}$

\section{Boring ID: TI11}

Log Date: 8/17/15

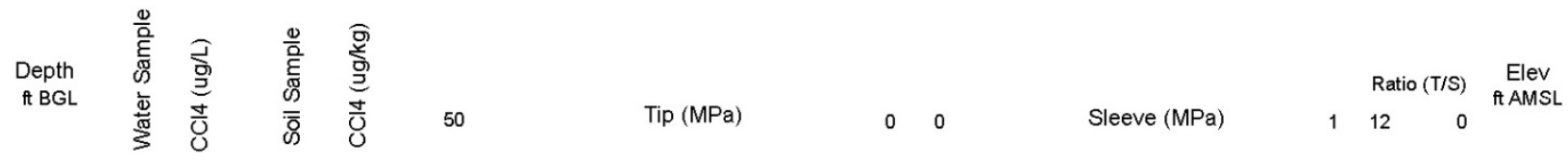
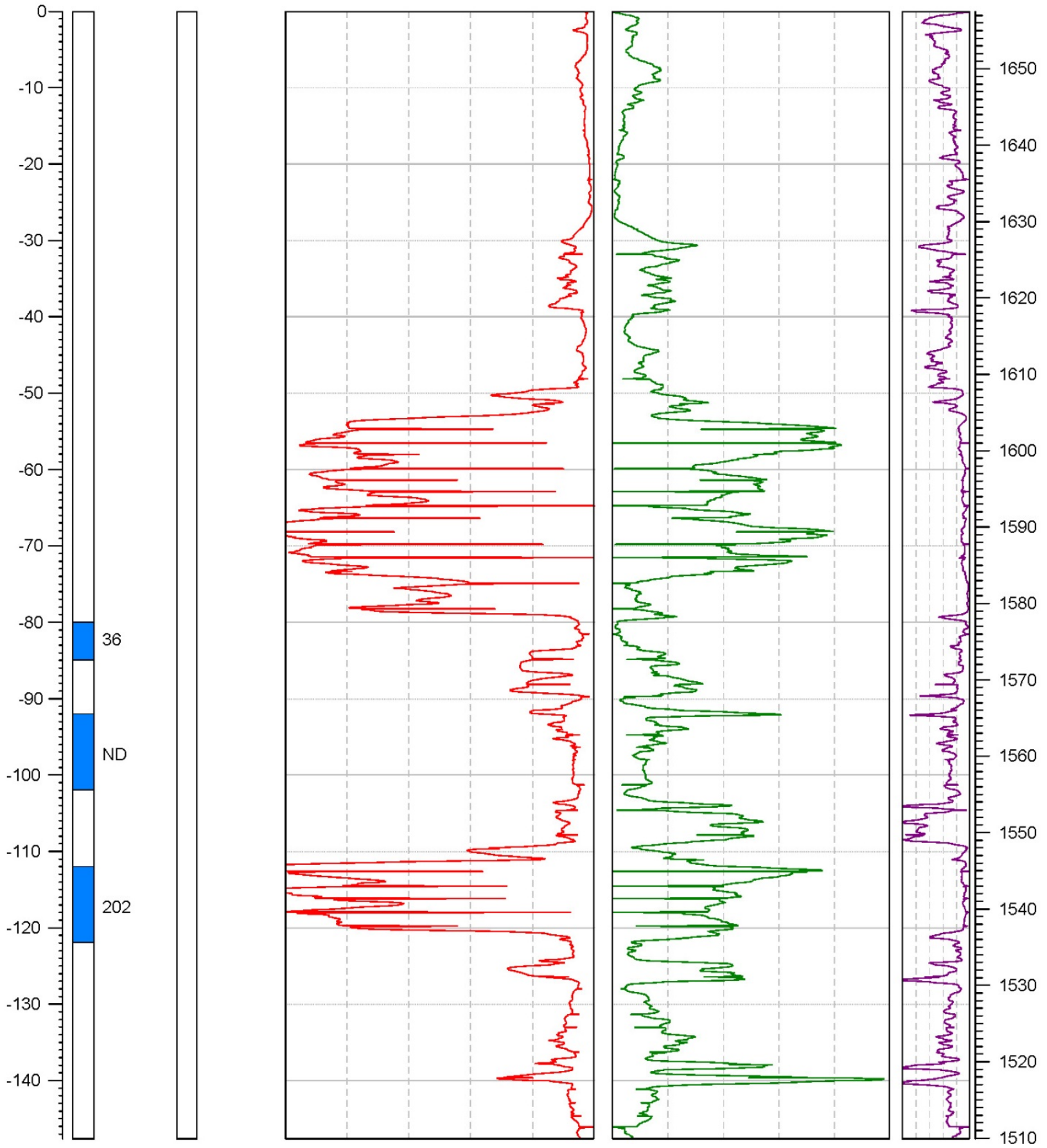


\section{Argonne National Laboratory}

Project: York, NE
Elevation: $1656.11 \mathrm{ft}$

Depth: $147.63 \mathrm{ft} \mathrm{BGL}$
Boring ID: TI12

Log Date: 8/19/2015

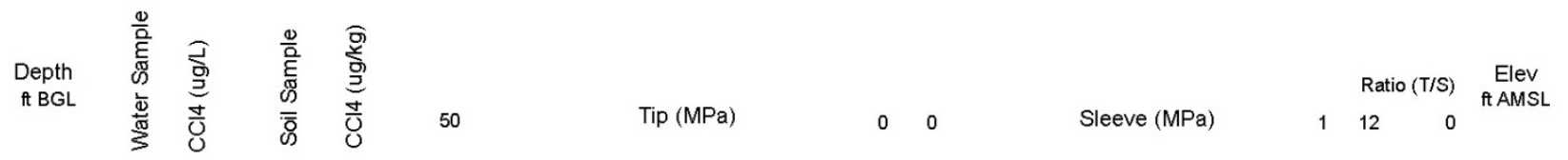
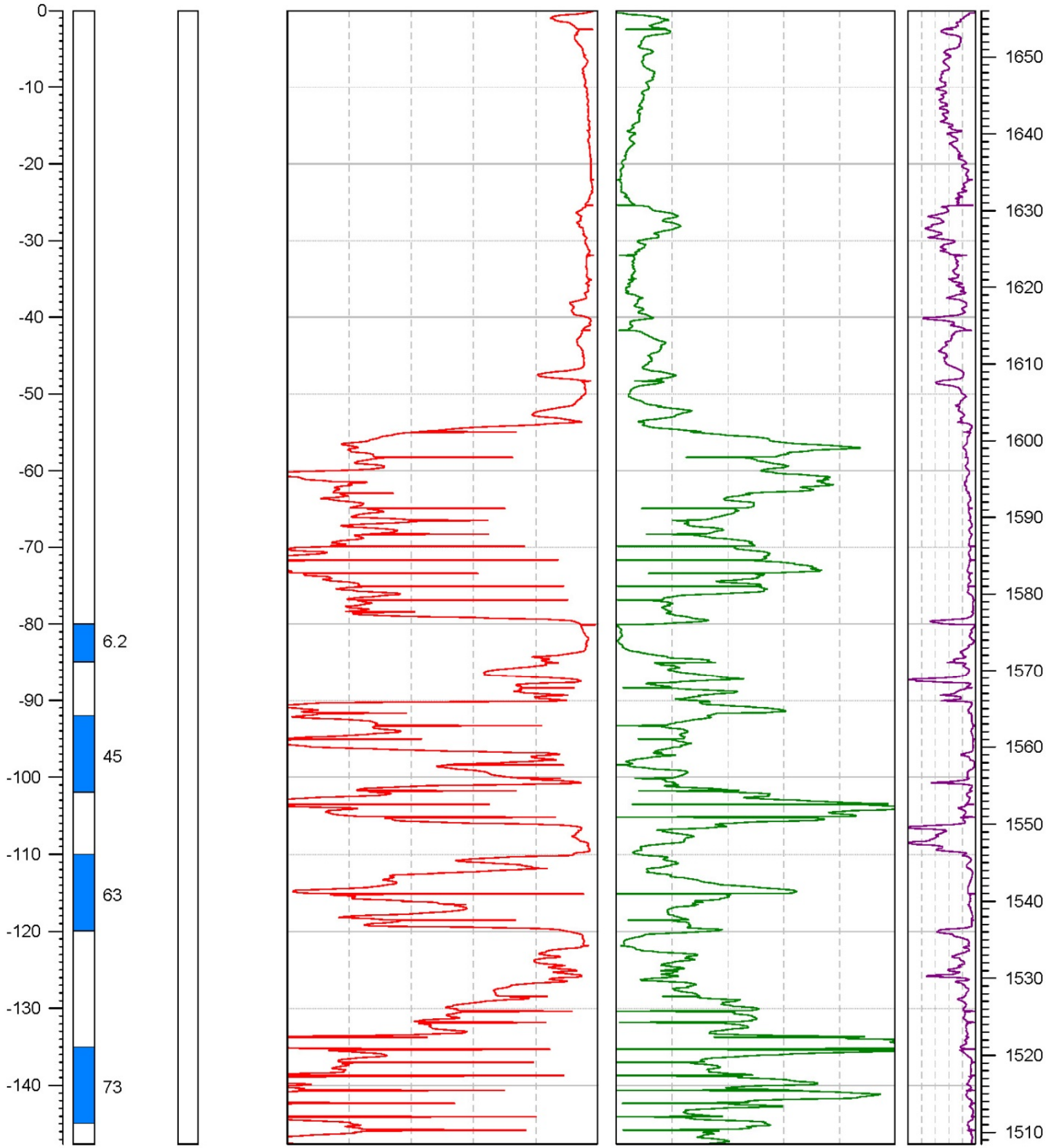
Version 01, 2/14/2017

\section{Argonne National Laboratory}

Project: York, NE

\section{Boring ID: TI13}

Elevation: $1657.26 \mathrm{ft}$

Depth: $131.23 \mathrm{ft} \mathrm{BGL}$
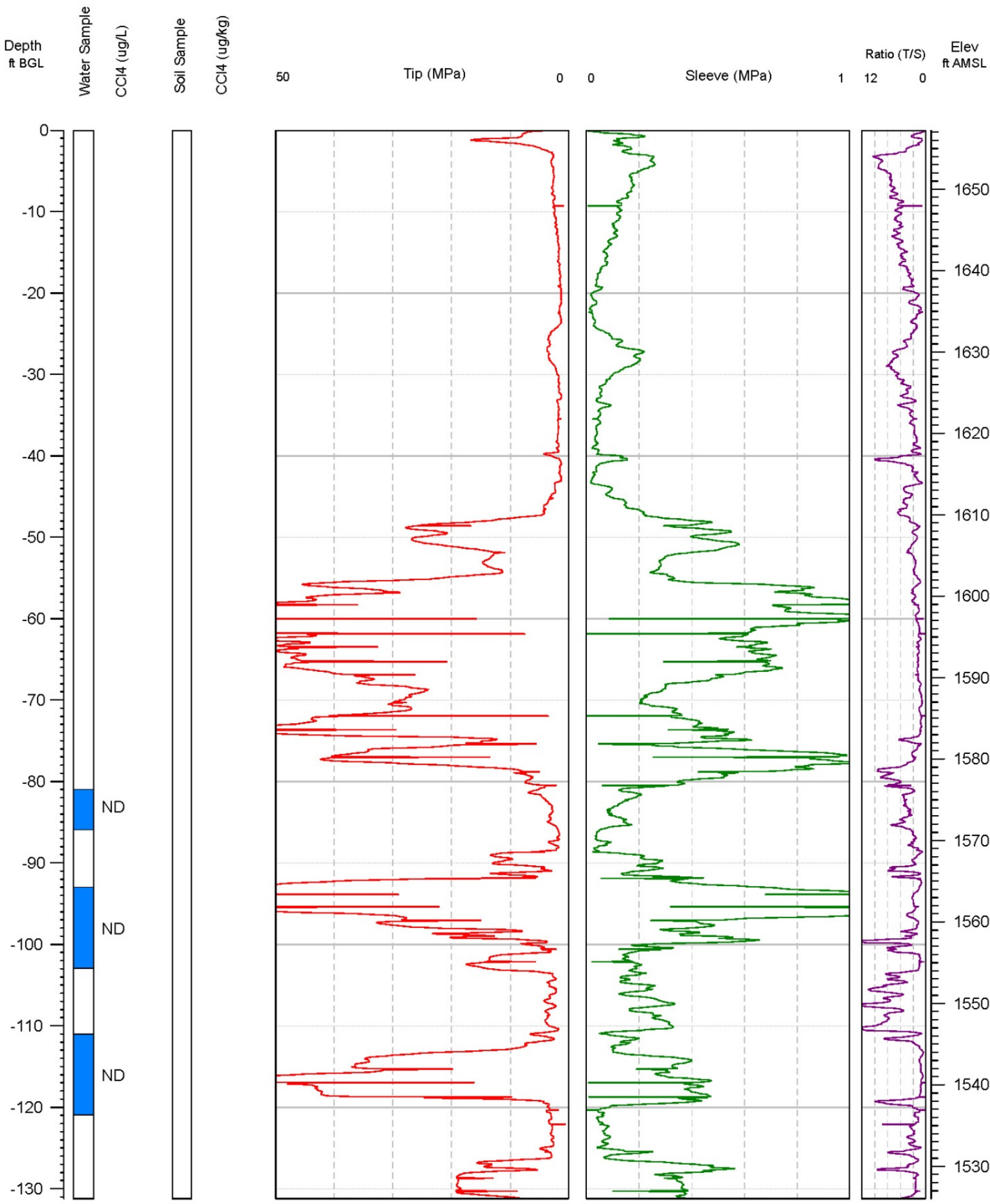
Version 01, 2/14/2017

\section{Argonne National Laboratory}

Project: York, NE
Elevation: $1652.52 \mathrm{ft}$

Depth: $147.63 \mathrm{ft} \mathrm{BGL}$

\section{Boring ID: TI14}

Log Date: $8 / 22 / 2015$

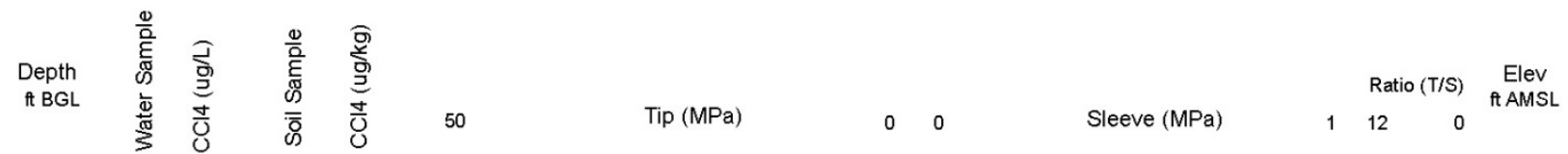
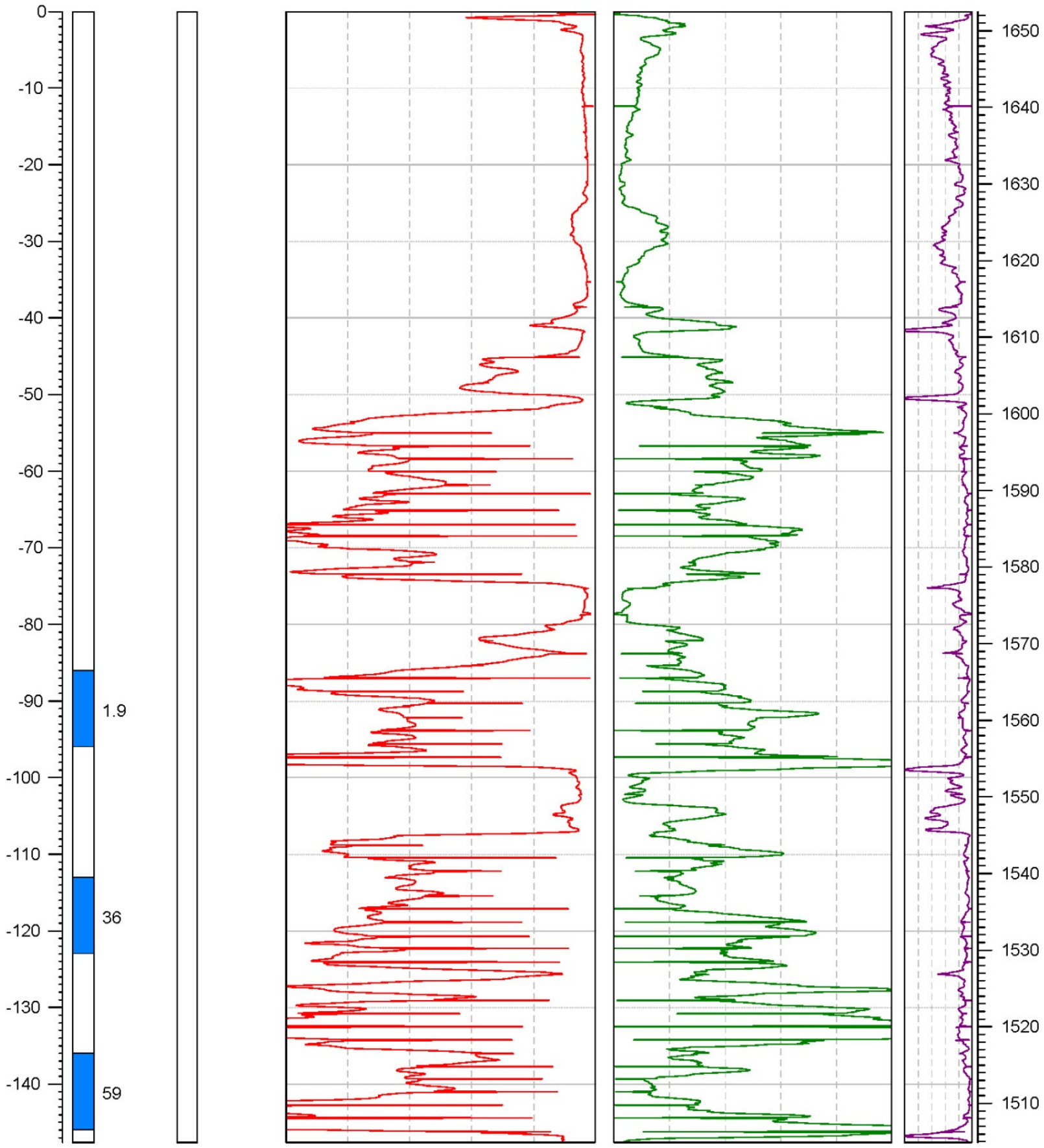


\section{Argonne National Laboratory}

Project: York, NE
Elevation: $1653.52 \mathrm{ft}$

Depth: $149.27 \mathrm{ft} \mathrm{BGL}$

\section{Boring ID: TI15/DL3}

Log Date: 5/24/2016

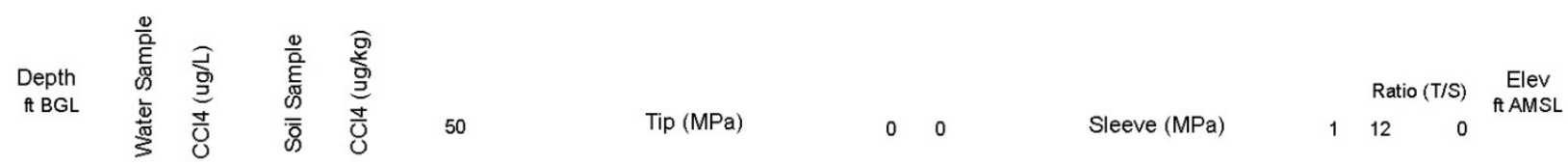
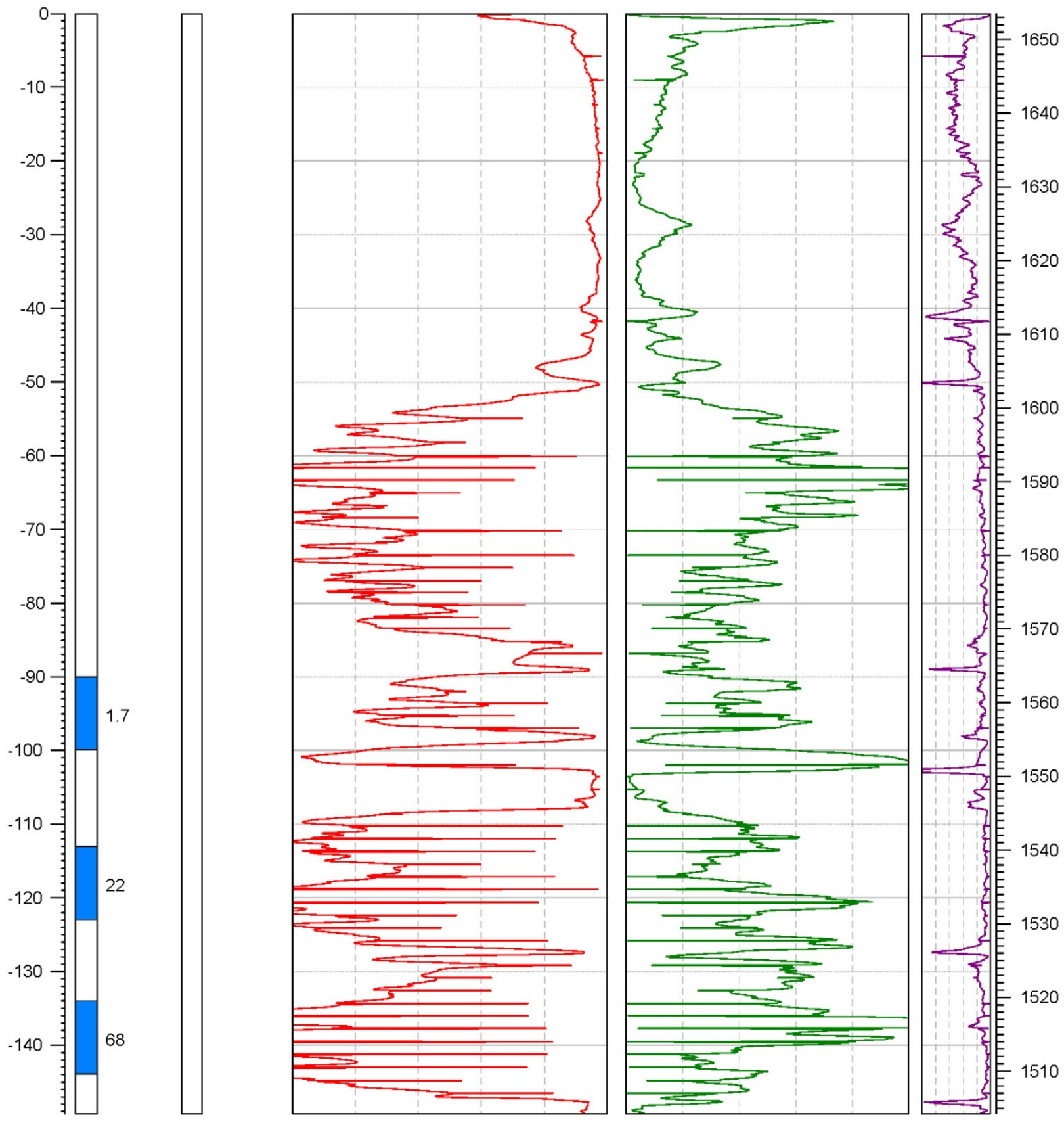


\section{Argonne National Laboratory}

Project: York, NE
Elevation: $1650.49 \mathrm{ft}$

Depth: $149.27 \mathrm{ft} \mathrm{BGL}$

\section{Boring ID: TI16}

Log Date: 8/24/2015

Depth

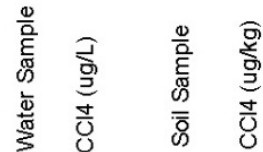

50

Tip (MPa)

00

Sleeve (MPa)

Ratio (T/S) Elev

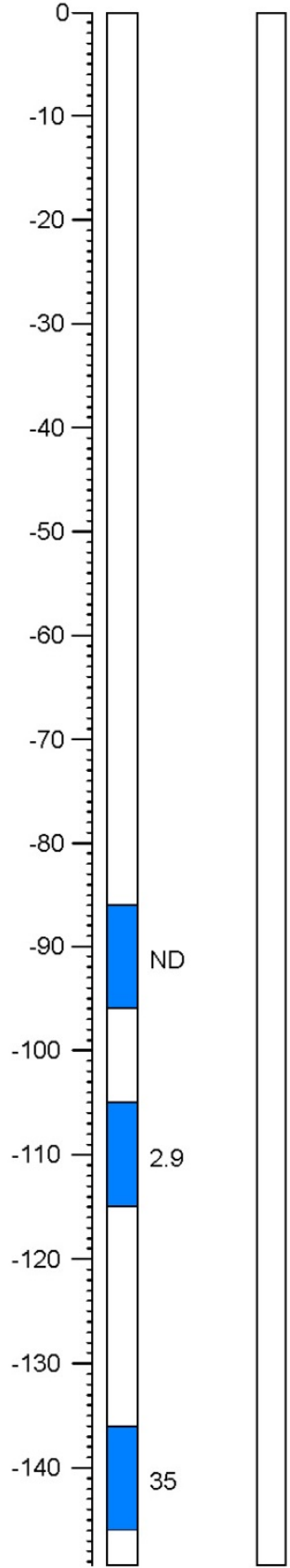

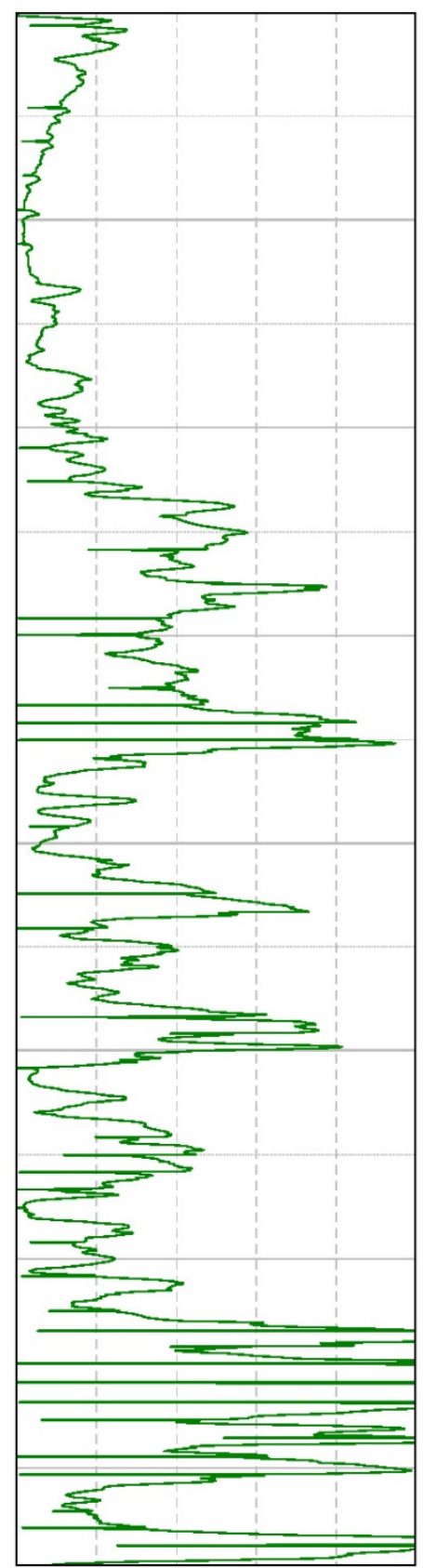

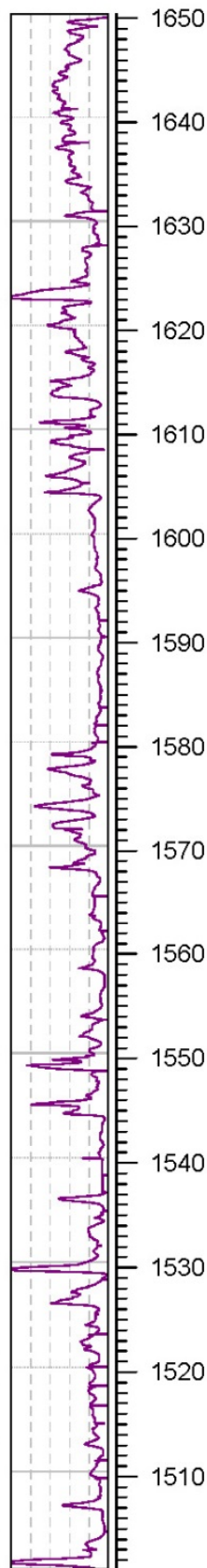




\section{Argonne National Laboratory}

Project: York, NE
Elevation: $1648.99 \mathrm{ft}$

Depth: $147.63 \mathrm{ft} \mathrm{BGL}$

\section{Boring ID: TI17/DL5}

Log Date: 6/16/16

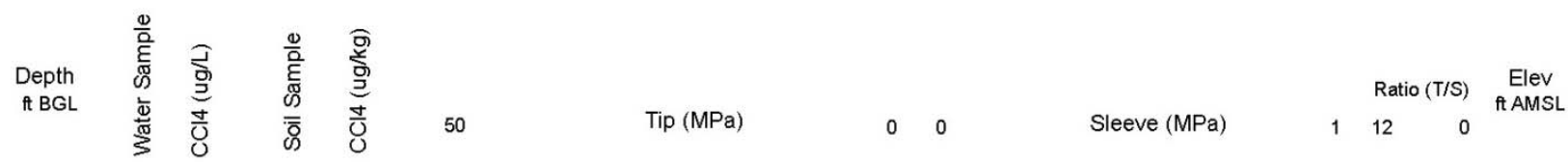

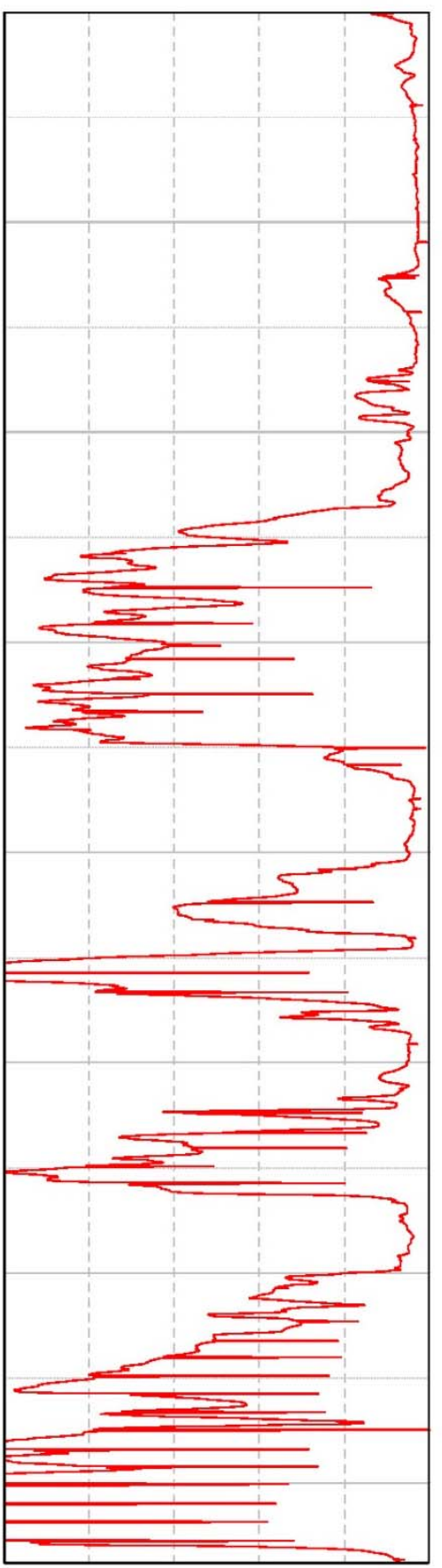

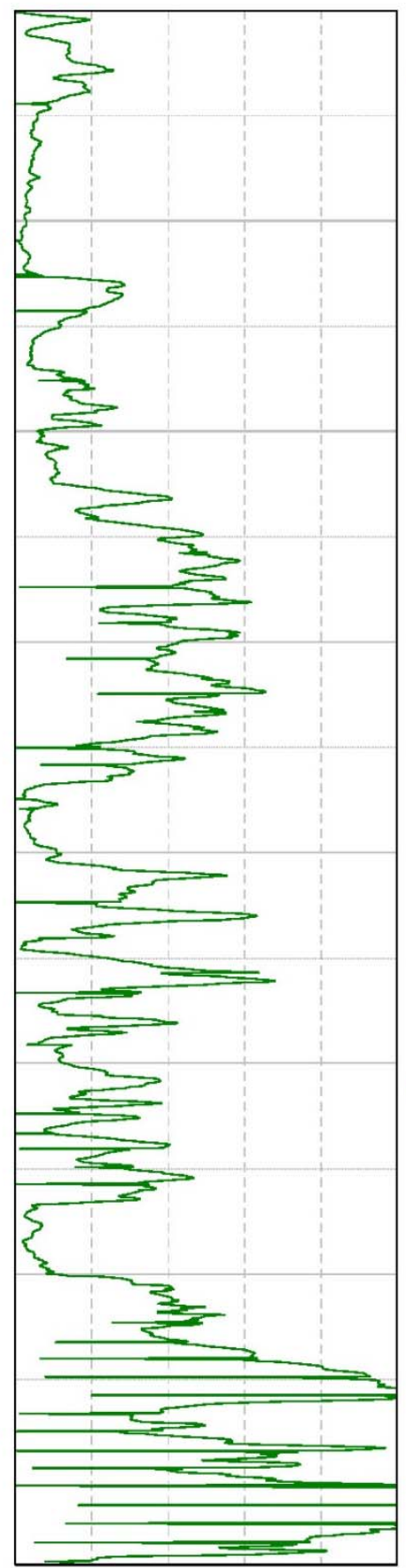

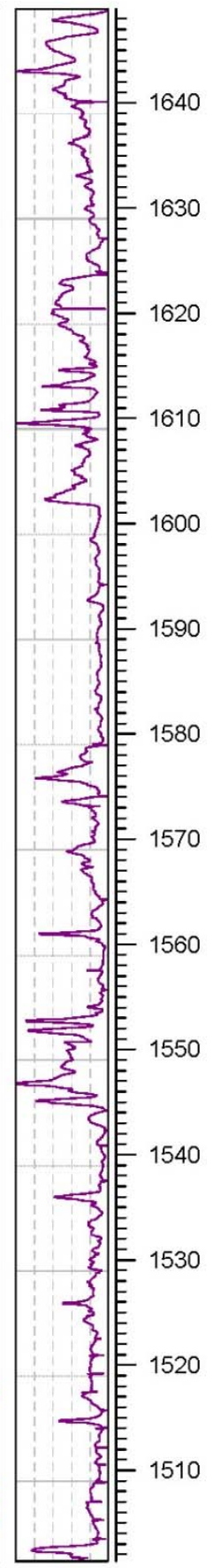


Version 01, 2/14/2017

\section{Argonne National Laboratory}

Project: York, NE
Elevation: $1661.42 \mathrm{ft}$

Depth: $147.83 \mathrm{ft} \mathrm{BGL}$
Boring ID: TI18

Log Date: 8/27/2015

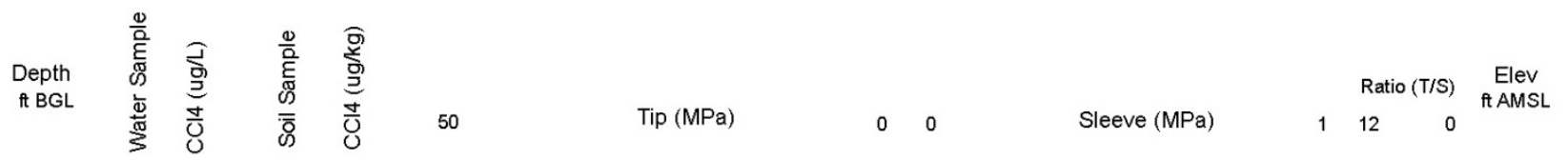
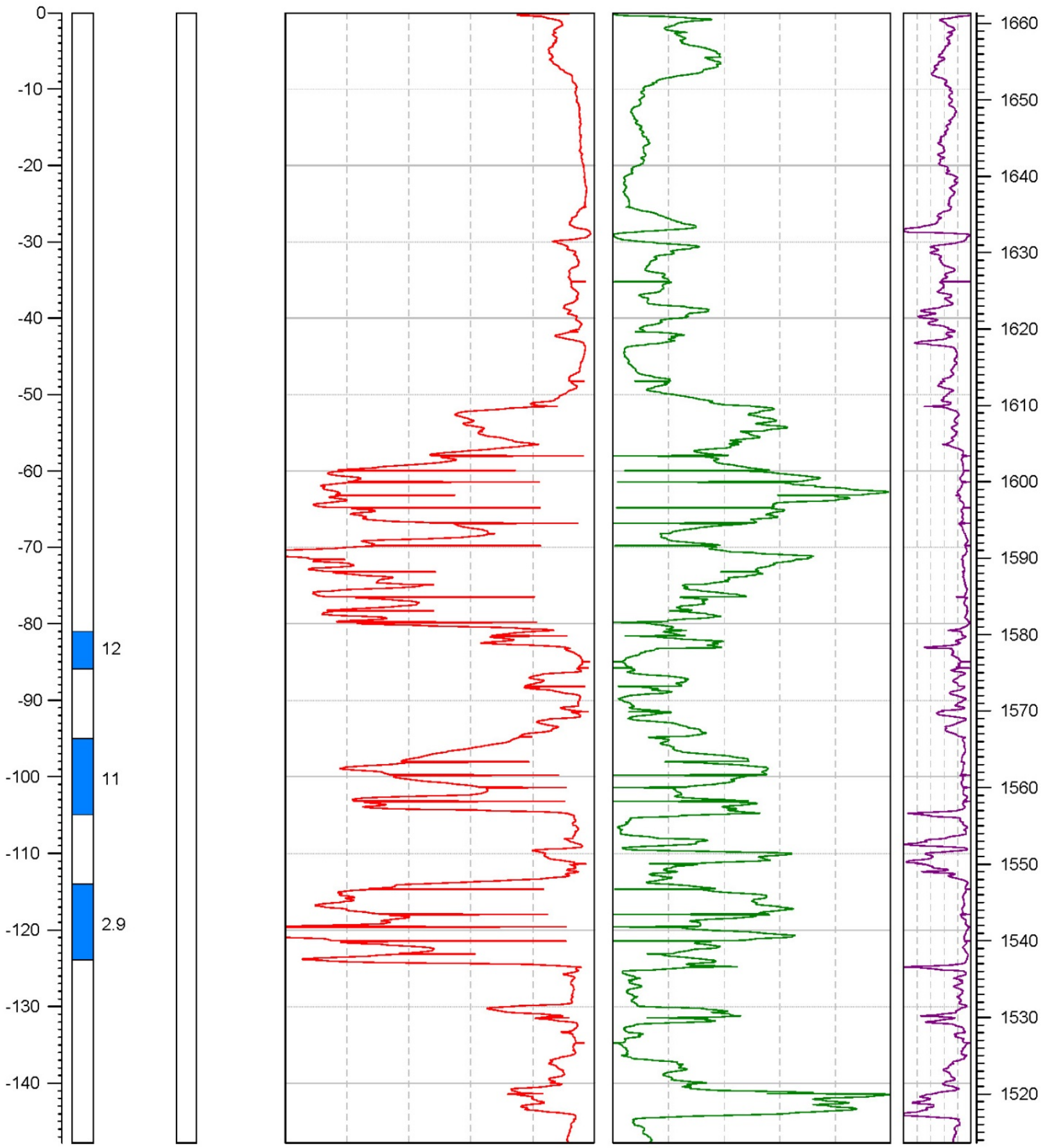
Version 01, 2/14/2017

\section{Argonne National Laboratory}

Project: York, NE
Elevation: $1646.21 \mathrm{ft}$

Depth: $147.63 \mathrm{ft} \mathrm{BGL}$

\section{Boring ID: TI19}

Log Date: 9/8/2015

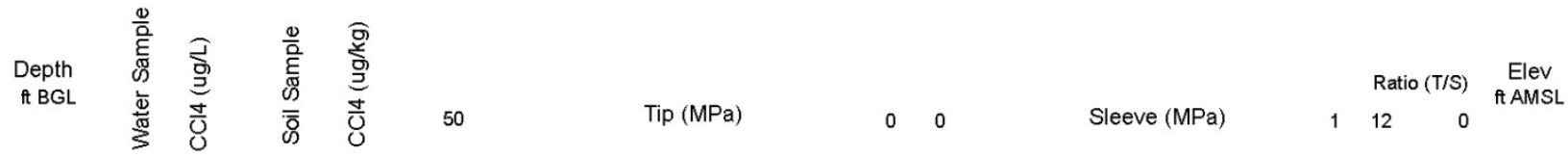
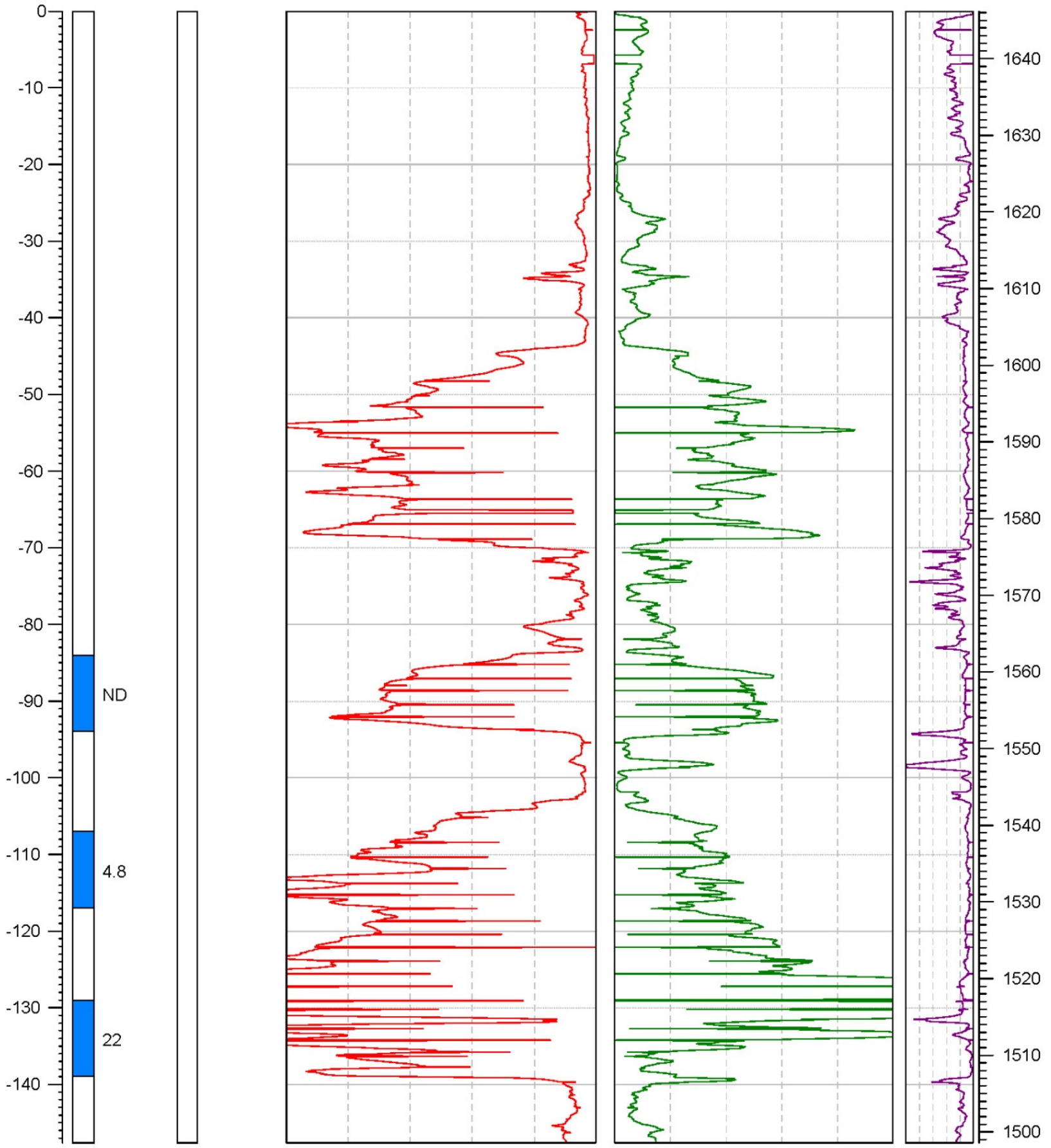


\section{Argonne National Laboratory}

Project: York, NE
Elevation: $1644.01 \mathrm{ft}$

Depth: $155.83 \mathrm{ft} \mathrm{BGL}$

\section{Boring ID: TI20/DL8}

Log Date: 6/1/2016

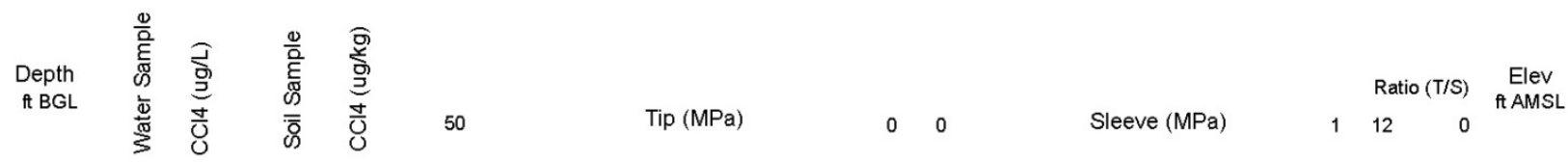
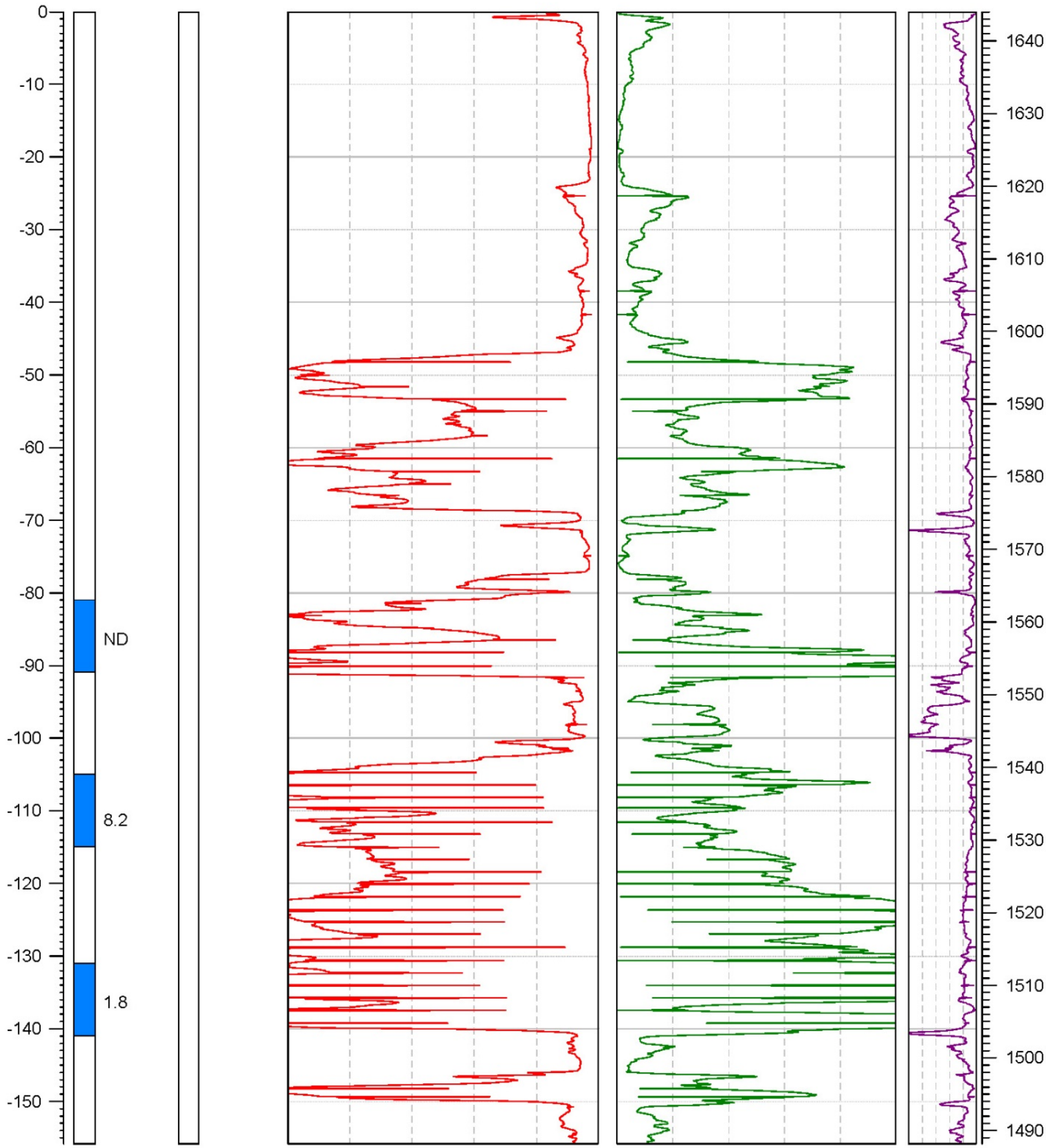


\section{Argonne National Laboratory}

Project: York, NE

\section{Boring ID: TI21/DL6}

Elevation: $1641.91 \mathrm{ft} \quad$ Log Date: 6/7/2016

Depth: $147.63 \mathrm{ft} \mathrm{BGL}$
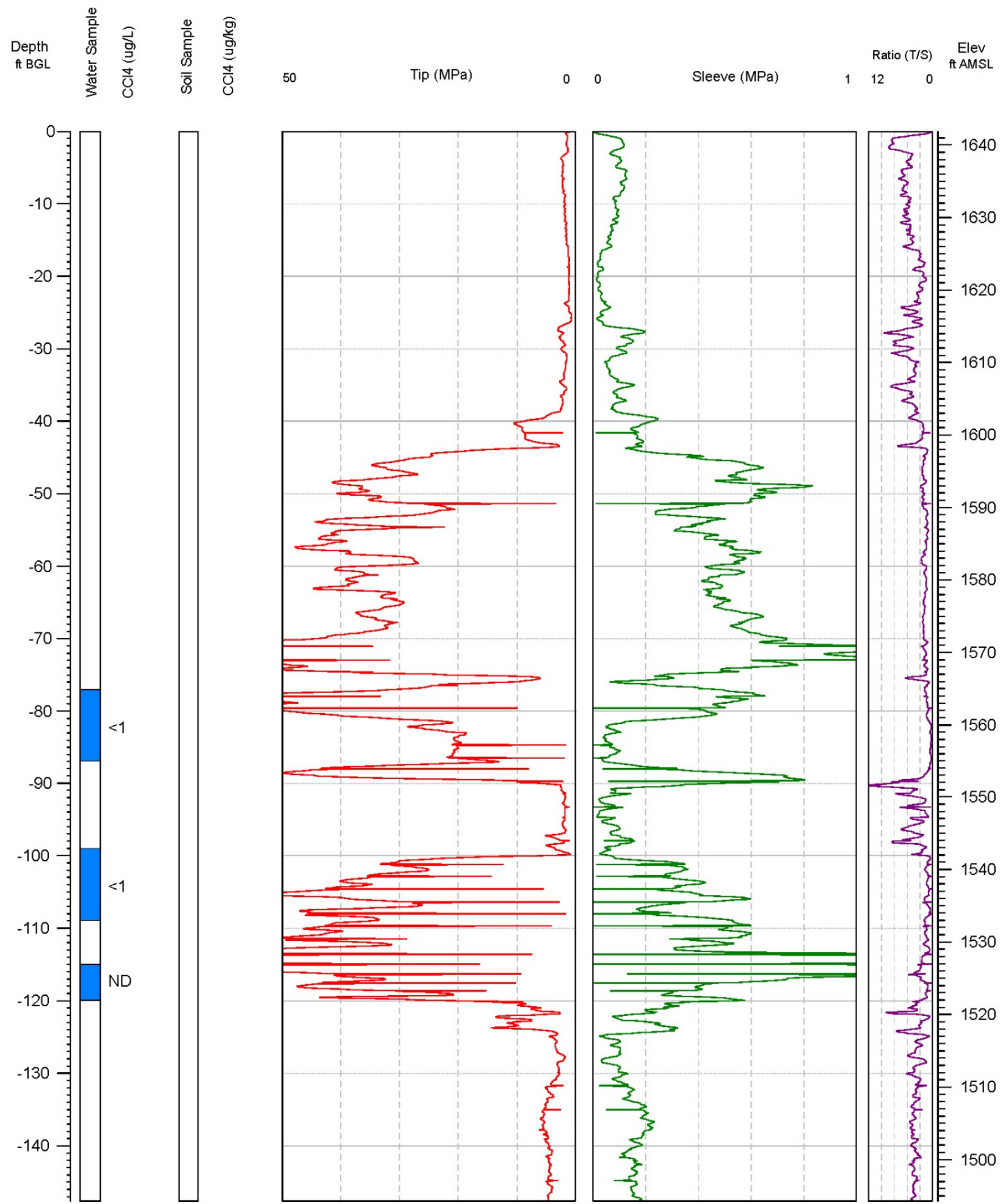


\section{Argonne National Laboratory}

Project: York, NE
Elevation: $1659.38 \mathrm{ft}$

Depth: $147.63 \mathrm{ft} \mathrm{BGL}$

\section{Boring ID: T122}

Log Date: 9/14/2015

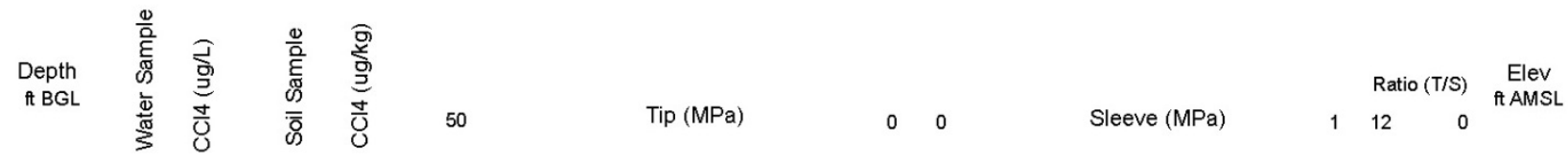
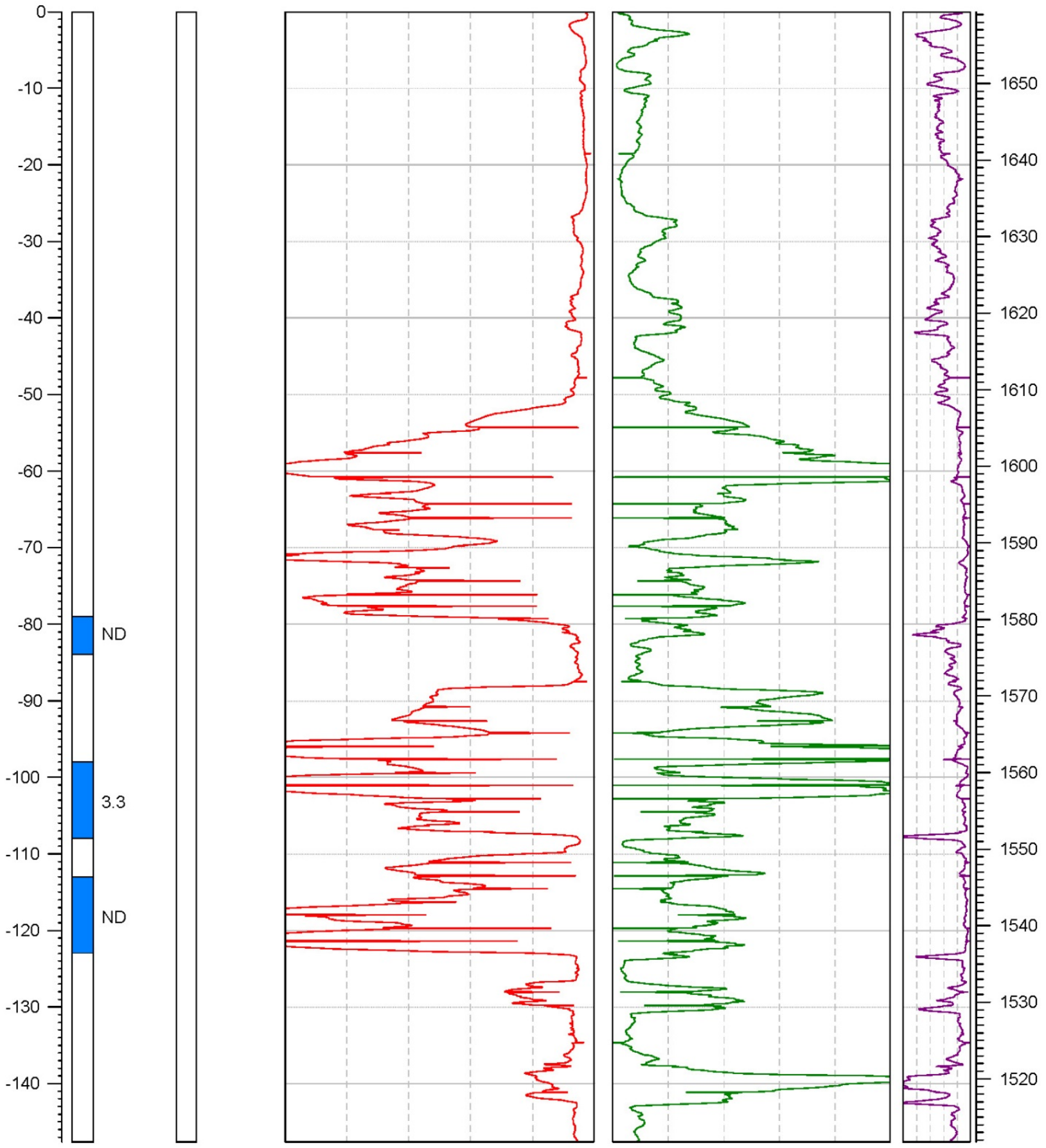


\section{Argonne National Laboratory}

Project: York, NE
Elevation: $1664.37 \mathrm{ft}$

Depth: $159.77 \mathrm{ft} \mathrm{BGL}$

\section{Boring ID: TI23/DL4}

Log Date: 6/8/2016

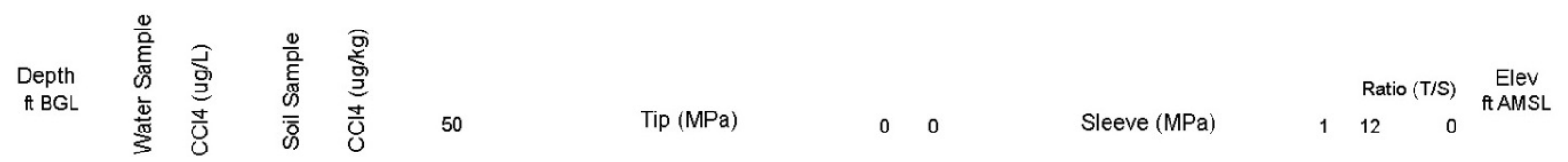
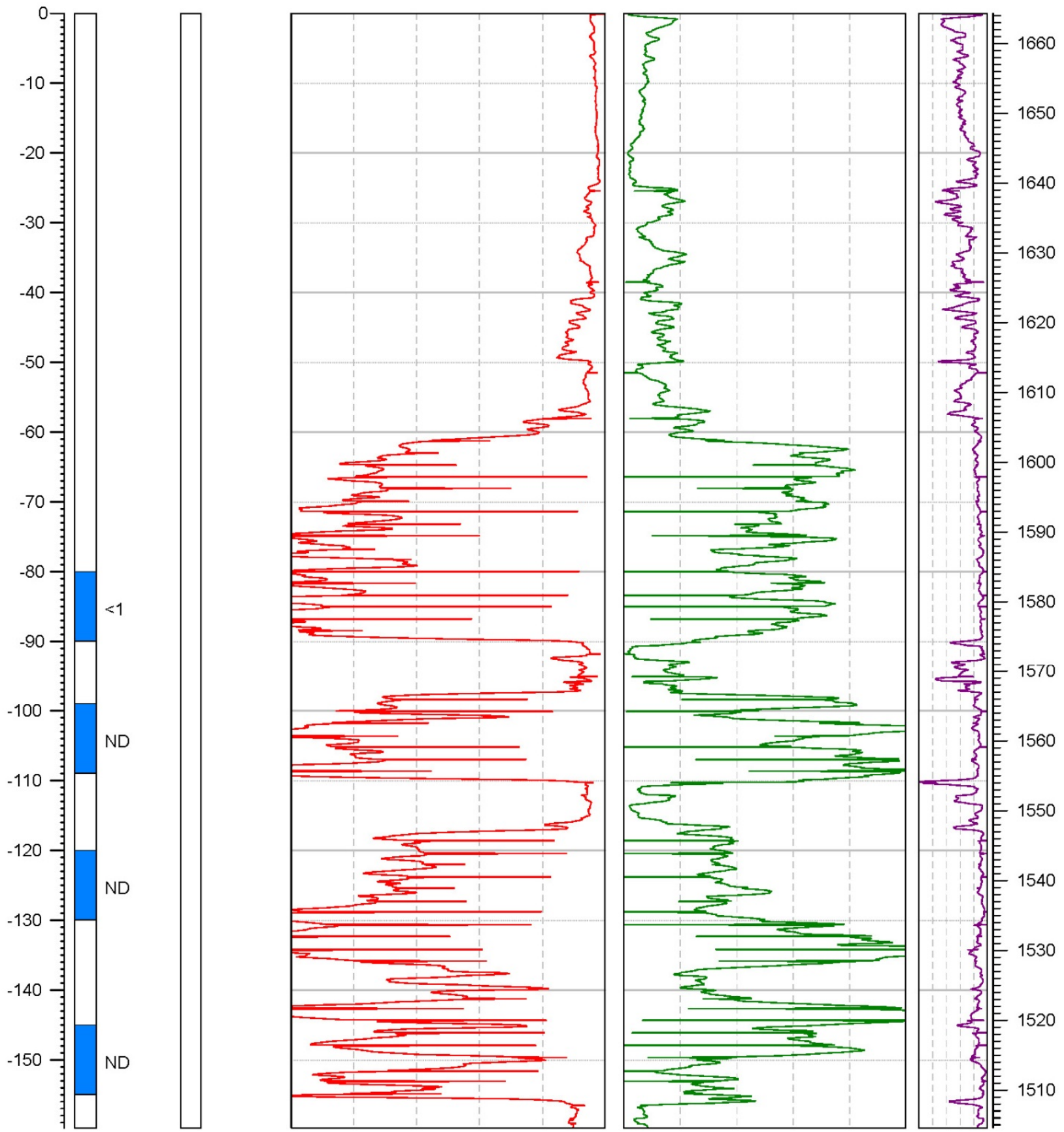


\section{Argonne National Laboratory}

Project: York, NE
Elevation: $1637.66 \mathrm{ft}$

Depth: $151.18 \mathrm{ft} \mathrm{BGL}$

\section{Boring ID: TI24/DL7}

Log Date: 6/2-5/2016

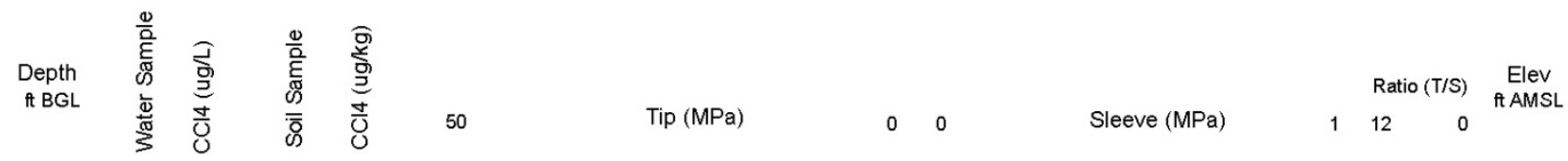
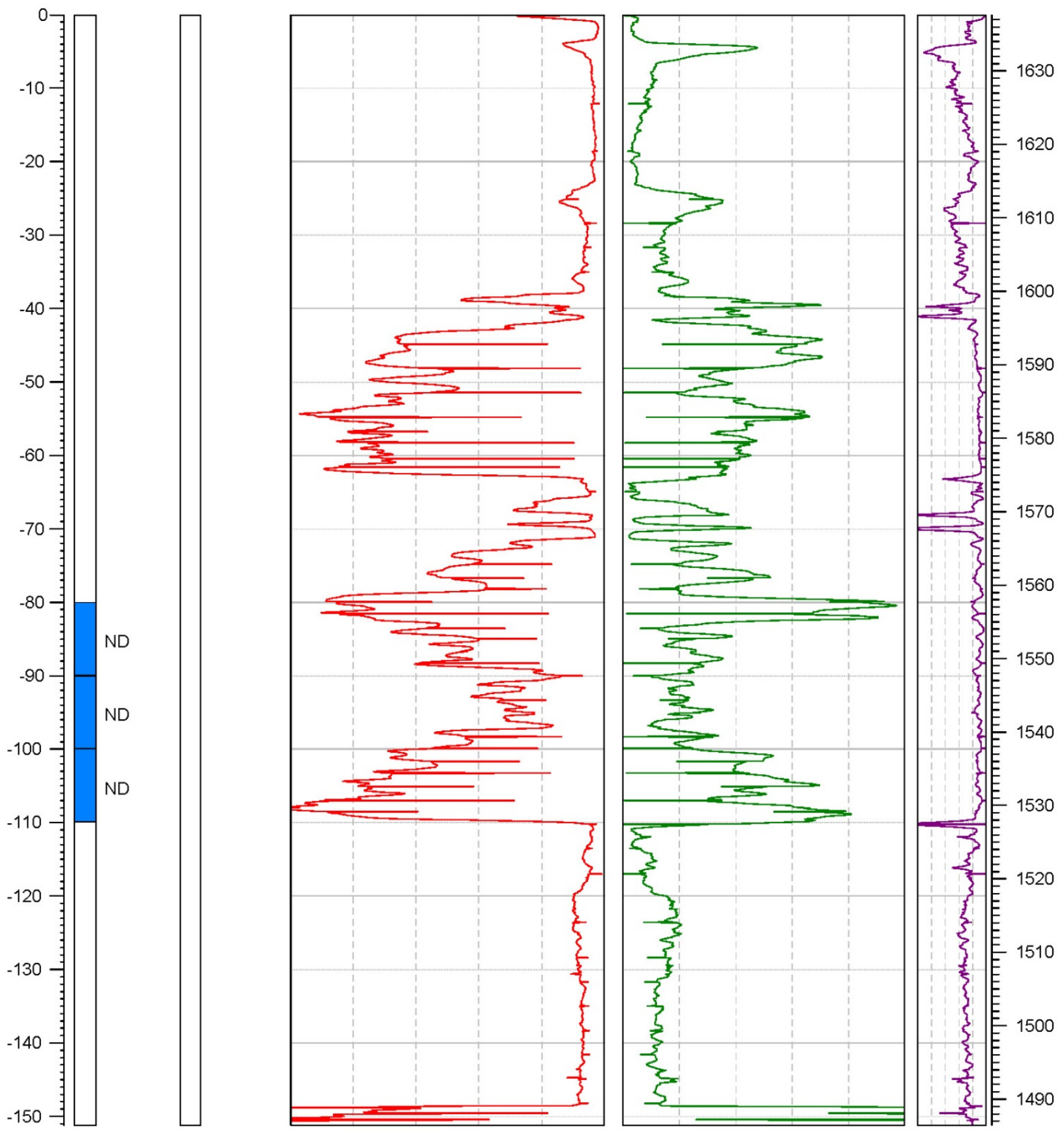
Version 01, 2/14/2017

\section{Argonne National Laboratory}

Project: York, NE

\section{Boring ID: Ti25}

Elevation: $1640 \mathrm{ft}$

Log Date: 1/21/2016

Depth: $156.75 \mathrm{ft} \mathrm{BGL}$

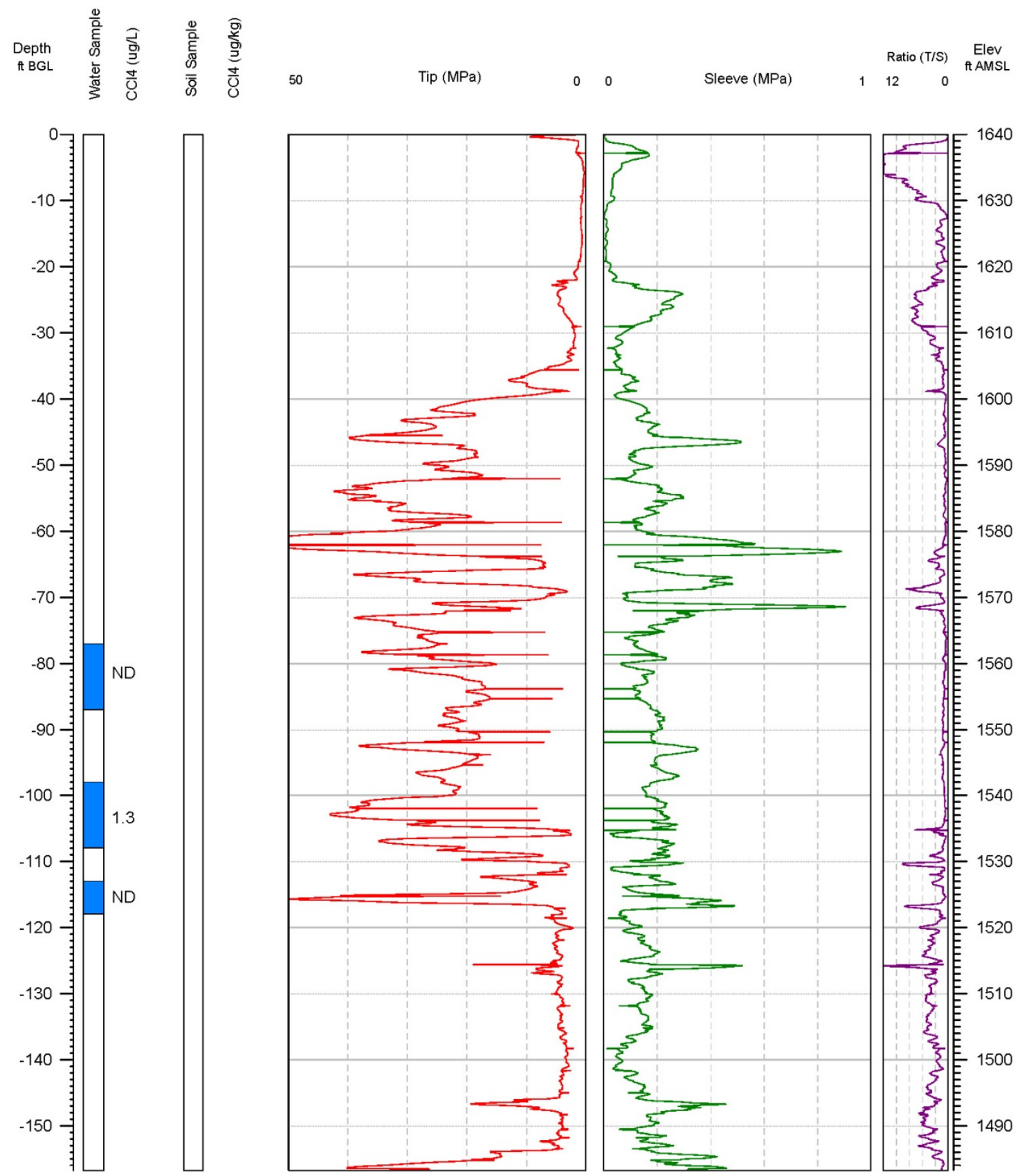


Version 01, 2/14/2017

\section{Argonne National Laboratory}

Project: York, NE
Elevation: $1640 \mathrm{ft}$

Depth: $156.43 \mathrm{ft} \mathrm{BGL}$

\section{Boring ID: TI26}

Log Date: 1/25/2016

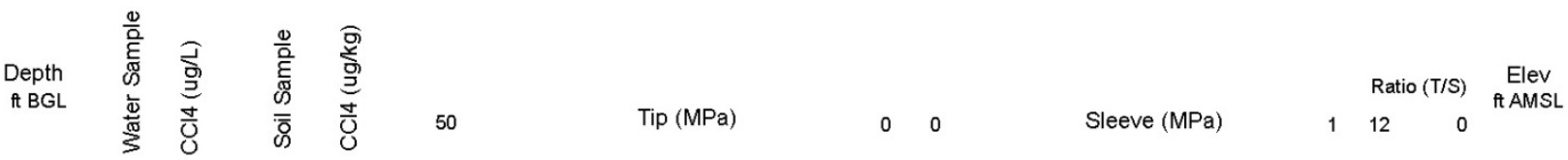
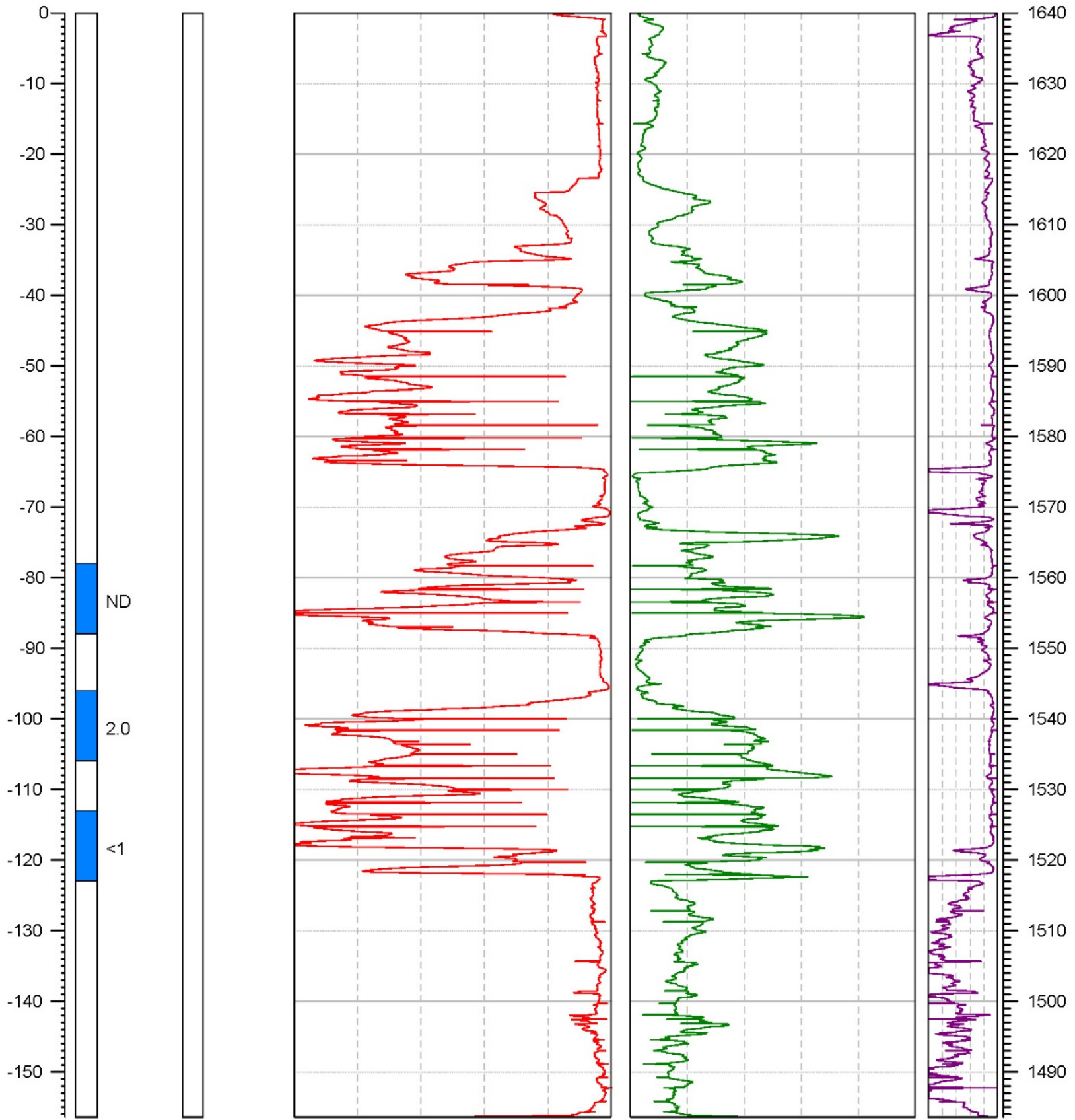


\section{Argonne National Laboratory}

Project: York, NE
Elevation: $1635 \mathrm{ft}$

Depth: $150.59 \mathrm{ft} \mathrm{BGL}$

\section{Boring ID: T127}

Log Date: 2/9/2016

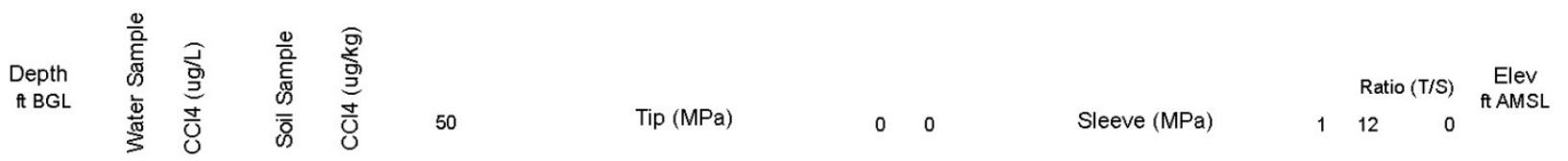
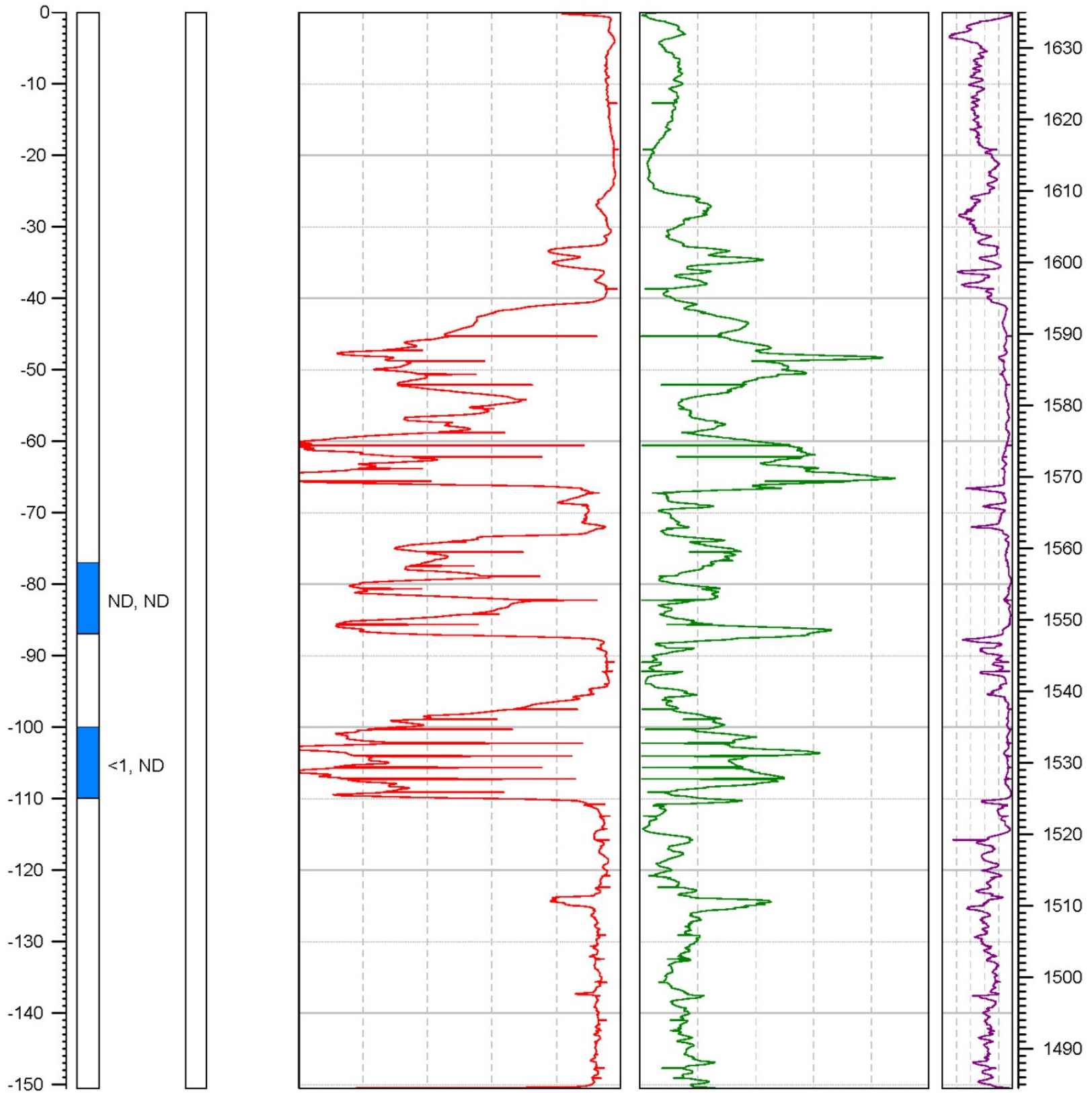
Version 01, 2/14/2017

\section{Argonne National Laboratory}

Project: York NE
Elevation: $1631 \mathrm{ft}$

Depth: $164.04 \mathrm{ft} \mathrm{BGL}$

\section{Boring ID: TI28}

Log Date: 2/3/2016

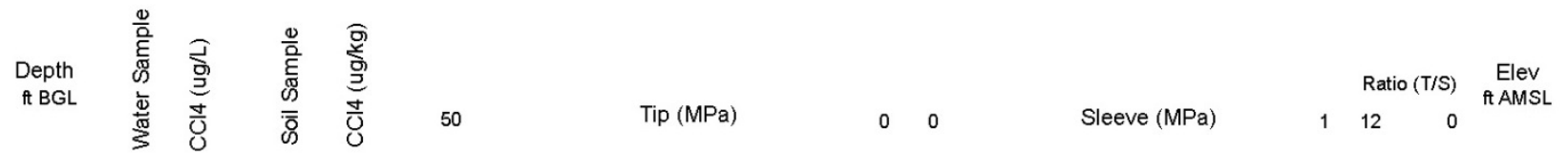
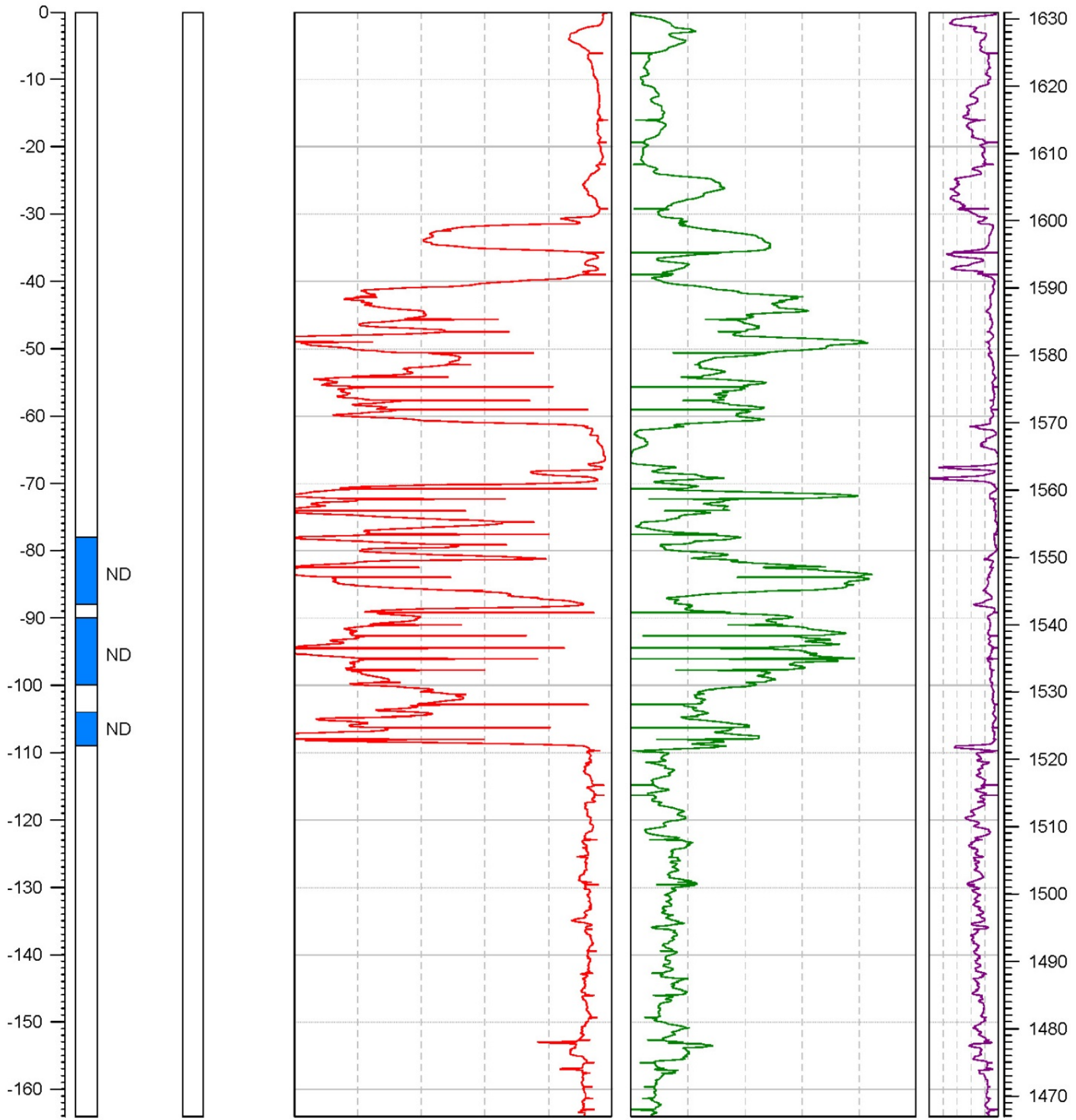


\section{Appendix D:}

\section{Grain Size Analysis and Lithology Logs for Borings DL1-DL8}


TABLE D.1 Grain Size Analysis Results.

\begin{tabular}{|c|c|c|c|c|c|c|c|c|c|c|c|c|c|c|c|c|c|c|c|c|c|c|c|c|c|c|c|c|}
\hline \multirow[b]{3}{*}{ Boring } & \multirow{3}{*}{$\begin{array}{c}\text { Sample } \\
\text { Depth } \\
\text { (tt BGLL) }\end{array}$} & \multirow{3}{*}{$\begin{array}{l}\text { Stratigraphic } \\
\text { Interval| }\end{array}$} & \multirow{2}{*}{\multicolumn{15}{|c|}{ Sieve Analysis - Percent Finer than Indicated Size }} & \multicolumn{11}{|c|}{ Composition \% } \\
\hline & & & & & & & & & & & & & & & & & & \multirow[b]{2}{*}{ Cobbles } & \multicolumn{3}{|c|}{ Gravel } & \multicolumn{4}{|c|}{ Sand } & \multicolumn{3}{|c|}{ Fines } \\
\hline & & & $3 / 4 \mathrm{in.}$ & $1 / 2 \mathrm{in.}$ & $3 / 8$ in. & \#4 & $\# 10$ & $\# 18$ & $\# 35$ & $\# 40$ & $\# 60$ & $\# 100$ & $\# 120$ & $\# 140$ & \#200 & $\# 230$ & \#270 & & Coarse & Fine & Total & Coarse & Medium & Fine & Total & Silt & Clay & Total \\
\hline DL-1 & $\begin{array}{c}75-76 \\
80.0 \\
85.0 \\
88.0 \\
92.0 \\
98-100 \\
105.0 \\
112-113 \\
116-118\end{array}$ & $\begin{array}{c}\text { A } \\
\text { A } \\
\text { B } \\
\text { B } \\
\text { B } \\
\text { B } \\
\text { B-C Silt } \\
\text { C } \\
\text { C }\end{array}$ & 100.0 & $\begin{array}{r}100.0 \\
96.4\end{array}$ & $\begin{array}{r}99.1 \\
100.0 \\
100.0 \\
100.0 \\
98.3 \\
91.4\end{array}$ & $\begin{array}{r}98.5 \\
99.6 \\
99.9 \\
99.9 \\
91.1 \\
80.2 \\
100.0 \\
99.0 \\
100.0\end{array}$ & $\begin{array}{l}96.2 \\
98.9 \\
99.5 \\
99.6 \\
75.8 \\
59.6 \\
99.8 \\
96.2 \\
99.9\end{array}$ & $\begin{array}{l}89.8 \\
97.8 \\
98.2 \\
98.7 \\
59.8 \\
42.9 \\
98.2 \\
91.1 \\
99.5\end{array}$ & $\begin{array}{l}76.9 \\
95.1 \\
94.2 \\
95.5 \\
38.4 \\
27.0 \\
91.1 \\
70.2 \\
91.2\end{array}$ & $\begin{array}{l}73.3 \\
93.9 \\
92.6 \\
94.0 \\
33.4 \\
23.2 \\
88.2 \\
62.9 \\
84.3\end{array}$ & $\begin{array}{l}53.9 \\
85.8 \\
82.5 \\
86.0 \\
17.2 \\
11.8 \\
76.3 \\
37.4 \\
40.4\end{array}$ & $\begin{array}{r}23.6 \\
71.1 \\
68.1 \\
74.1 \\
7.6 \\
66.5 \\
66.9 \\
22.7 \\
19.4\end{array}$ & $\begin{array}{r}17.5 \\
64.0 \\
61.8 \\
68.4 \\
6.1 \\
5.6 \\
64.4 \\
20.5 \\
14.0\end{array}$ & $\begin{array}{r}14.6 \\
58.1 \\
56.9 \\
64.3 \\
5.4 \\
5.1 \\
62.6 \\
19.4 \\
10.8\end{array}$ & $\begin{array}{r}10.6 \\
44.7 \\
47.1 \\
54.6 \\
4.6 \\
4.2 \\
58.6 \\
18.1 \\
7.0\end{array}$ & $\begin{array}{r}9.3 \\
37.9 \\
42.0 \\
50.0 \\
4.3 \\
3.8 \\
54.6 \\
17.6 \\
6.0\end{array}$ & $\begin{array}{r}8.5 \\
34.1 \\
38.8 \\
46.9 \\
4.2 \\
30.6 \\
50.9 \\
17.2 \\
5.7\end{array}$ & $\begin{array}{l}0.0 \\
0.0 \\
0.0 \\
0.0 \\
0.0 \\
0.0 \\
0.0 \\
0.0 \\
0.0\end{array}$ & $\begin{array}{l}0.0 \\
0.0 \\
0.0 \\
0.0 \\
0.0 \\
0.0 \\
0.0 \\
1.0 \\
0.0\end{array}$ & $\begin{array}{r}1.5 \\
0.4 \\
0.1 \\
0.1 \\
8.9 \\
19.8 \\
0.0 \\
1.0 \\
0.0\end{array}$ & $\begin{array}{r}1.5 \\
0.4 \\
0.1 \\
0.1 \\
8.9 \\
19.8 \\
0.0 \\
1.0 \\
0.0\end{array}$ & $\begin{array}{r}2.3 \\
0.7 \\
0.4 \\
0.3 \\
15.3 \\
20.6 \\
0.2 \\
2.8 \\
0.1\end{array}$ & $\begin{array}{r}22.9 \\
5.0 \\
6.9 \\
5.6 \\
42.4 \\
36.4 \\
11.6 \\
33.3 \\
15.6\end{array}$ & $\begin{array}{l}62.7 \\
49.2 \\
45.5 \\
39.4 \\
28.8 \\
19.0 \\
29.6 \\
44.8 \\
77.3\end{array}$ & $\begin{array}{l}87.9 \\
54.9 \\
52.8 \\
45.3 \\
86.5 \\
76.0 \\
41.4 \\
80.9 \\
93.0\end{array}$ & $\begin{array}{c}7.1 \\
32.2 \\
32.6 \\
36.1 \\
1.9 \\
3.1 \\
49.8 \\
12.9 \\
4.3\end{array}$ & $\begin{array}{r}3.5 \\
12.5 \\
14.5 \\
18.5 \\
2.7 \\
1.1 \\
8.8 \\
5.2 \\
2.7\end{array}$ & $\begin{array}{r}10.6 \\
44.7 \\
47.1 \\
54.6 \\
4.6 \\
4.2 \\
58.6 \\
18.1 \\
7.0\end{array}$ \\
\hline DL-2 & $\begin{array}{r}70-72 \\
7-78 \\
90-92 \\
95-96 \\
115-117 \\
12-1124 \\
133-135 \\
137-139 \\
144-146 \\
144-150 \\
164-165 \\
172-173 \\
177-178 \\
184-186 \\
198-189 \\
208-299 \\
216-218 \\
22-224 \\
227-229 \\
231-232 \\
243-244\end{array}$ & $\begin{array}{c}\text { A } \\
\text { A } \\
\text { B } \\
\text { B } \\
\text { C1 } \\
\text { C1-C2 Trans } \\
\text { C2 } \\
\text { C2 } \\
C 2 \\
\text { CL } \\
\text { UCL } \\
\text { UCL } \\
C U \\
C U \\
\text { CU Sand-Silt } \\
\text { CU Silt-Clay } \\
C U \\
C U \\
C U \\
C U \\
C U \\
C U \\
\text { LCL }\end{array}$ & 100.0 & $\begin{array}{r}99.5 \\
100.0\end{array}$ & $\begin{array}{r}100.0 \\
99.7 \\
100.0 \\
99.5\end{array}$ & $\begin{array}{r}96.7 \\
99.3 \\
100.0 \\
96.0 \\
99.1 \\
100.0 \\
100.0 \\
98.1 \\
99.0 \\
99.7 \\
99.2\end{array}$ & $\begin{array}{r}84.6 \\
97.3 \\
99.9 \\
85.8 \\
97.0 \\
99.9 \\
99.7 \\
89.6 \\
94.2 \\
99.3 \\
98.1 \\
100.0\end{array}$ & $\begin{array}{l}63.4 \\
95.6 \\
99.5 \\
71.7 \\
89.7 \\
99.8 \\
98.5 \\
80.2 \\
85.4 \\
98.9 \\
96.7 \\
99.9 \\
100.0\end{array}$ & $\begin{array}{l}32.4 \\
94.4 \\
97.0 \\
50.1 \\
58.4 \\
99.5 \\
93.9 \\
69.5 \\
65.7 \\
98.4 \\
94.4 \\
99.9 \\
99.9\end{array}$ & $\begin{array}{l}25.1 \\
94.0 \\
95.1 \\
43.8 \\
49.7 \\
99.2 \\
91.1 \\
64.6 \\
56.6 \\
98.2 \\
93.7 \\
99.8 \\
99.8 \\
100.0 \\
96.2 \\
98.0 \\
83.7 \\
97.2 \\
96.4 \\
99.8\end{array}$ & $\begin{array}{l}8.3 \\
87.4 \\
76.3 \\
27.8 \\
26.8 \\
96.0 \\
74.5 \\
33.9 \\
22.3 \\
97.1 \\
89.6 \\
76.3 \\
90.4 \\
99.7 \\
91.9 \\
51.8 \\
38.2 \\
47.5 \\
51.9 \\
97.7\end{array}$ & $\begin{array}{r}3.1 \\
61.2 \\
46.7 \\
17.5 \\
10.6 \\
78.7 \\
38.5 \\
14.9 \\
8.2 \\
95.5 \\
85.3 \\
21.2 \\
32.8 \\
95.6 \\
85.1 \\
13.2 \\
7.5 \\
10.0 \\
14.2 \\
91.9 \\
100.0\end{array}$ & $\begin{array}{r}2.3 \\
47.9 \\
36.9 \\
15.2 \\
7.2 \\
64.2 \\
18.7 \\
11.6 \\
6.1 \\
94.6 \\
83.8 \\
17.2 \\
20.3 \\
88.3 \\
81.8 \\
8.2 \\
4.6 \\
7.7 \\
9.8 \\
85.1 \\
99.9\end{array}$ & $\begin{array}{r}1.9 \\
39.1 \\
31.3 \\
13.7 \\
5.4 \\
52.4 \\
11.3 \\
10.0 \\
5.0 \\
53.3 \\
82.6 \\
15.6 \\
15.4 \\
75.0 \\
79.2 \\
6.3 \\
3.5 \\
6.9 \\
8.2 \\
73.8 \\
99.9\end{array}$ & $\begin{array}{r}1.3 \\
26.8 \\
24.3 \\
11.3 \\
3.7 \\
33.0 \\
5.9 \\
7.7 \\
3.8 \\
88.0 \\
79.6 \\
13.3 \\
10.5 \\
55.7 \\
72.9 \\
4.1 \\
2.4 \\
6.1 \\
6.4 \\
42.4 \\
99.8\end{array}$ & $\begin{array}{r}1.2 \\
22.7 \\
21.8 \\
10.2 \\
3.2 \\
27.4 \\
5.0 \\
6.9 \\
3.4 \\
84.4 \\
77.1 \\
11.9 \\
9.0 \\
42.5 \\
68.8 \\
3.4 \\
2.0 \\
5.9 \\
50.7 \\
30.8 \\
99.7\end{array}$ & $\begin{array}{r}1.1 \\
20.8 \\
20.3 \\
9.6 \\
2.9 \\
24.8 \\
4.6 \\
6.4 \\
3.2 \\
82.4 \\
74.7 \\
11.0 \\
8.2 \\
37.4 \\
65.3 \\
2.9 \\
1.8 \\
5.7 \\
55.5 \\
25.6 \\
99.5\end{array}$ & $\begin{array}{l}0.0 \\
0.0 \\
0.0 \\
0.0 \\
0.0 \\
0.0 \\
0.0 \\
0.0 \\
0.0 \\
0.0 \\
0.0 \\
0.0 \\
0.0 \\
0.0 \\
0.0 \\
0.0 \\
0.0 \\
0.0 \\
0.0 \\
0.0 \\
0.0\end{array}$ & $\begin{array}{l}0.0 \\
0.0 \\
0.0 \\
0.0 \\
0.0 \\
0.0 \\
0.0 \\
0.0 \\
0.0 \\
0.0 \\
0.0 \\
0.0 \\
0.0 \\
0.0 \\
0.0 \\
0.0 \\
0.0 \\
0.0 \\
0.0 \\
0.0 \\
0.0\end{array}$ & $\begin{array}{l}3.3 \\
0.7 \\
0.0 \\
4.0 \\
0.9 \\
0.0 \\
0.0 \\
1.9 \\
1.0 \\
0.3 \\
0.8 \\
0.0 \\
0.0 \\
0.0 \\
0.1 \\
0.0 \\
0.0 \\
0.0 \\
0.0 \\
0.0 \\
0.0\end{array}$ & $\begin{array}{l}3.3 \\
0.7 \\
0.0 \\
4.0 \\
0.9 \\
0.0 \\
0.0 \\
1.9 \\
1.0 \\
0.3 \\
0.8 \\
0.0 \\
0.0 \\
0.0 \\
0.1 \\
0.0 \\
0.0 \\
0.0 \\
0.0 \\
0.0 \\
0.0\end{array}$ & $\begin{aligned} 12.1 \\
2.0 \\
0.1 \\
10.2 \\
2.1 \\
0.1 \\
0.1 \\
8.5 \\
4.8 \\
0.4 \\
1.1 \\
0.0 \\
0.0 \\
0.0 \\
0.6 \\
0.0 \\
0.0 \\
0.0 \\
0.0 \\
0.0 \\
0.0\end{aligned}$ & $\begin{array}{r}59.5 \\
3.3 \\
4.8 \\
42.0 \\
47.3 \\
0.7 \\
8.6 \\
25.0 \\
37.6 \\
1.1 \\
4.4 \\
0.2 \\
0.2 \\
0.0 \\
3.1 \\
2.0 \\
16.3 \\
2.8 \\
3.6 \\
0.2 \\
0.0\end{array}$ & $\begin{array}{l}23.8 \\
67.2 \\
70.8 \\
32.5 \\
46.0 \\
66.2 \\
85.2 \\
56.9 \\
52.8 \\
10.2 \\
14.1 \\
86.5 \\
89.3 \\
48.3 \\
23.3 \\
93.9 \\
81.3 \\
91.1 \\
90.0 \\
57.4 \\
0.2\end{array}$ & $\begin{array}{l}95.4 \\
72.5 \\
75.7 \\
84.7 \\
95.4 \\
67.0 \\
94.1 \\
90.4 \\
95.2 \\
11.7 \\
19.6 \\
86.7 \\
89.5 \\
48.3 \\
27.0 \\
95.9 \\
97.6 \\
93.9 \\
93.6 \\
57.6 \\
0.2\end{array}$ & $\begin{array}{c}0.5 \\
16.3 \\
17.3 \\
6.9 \\
1.5 \\
22.9 \\
3.1 \\
5.3 \\
1.5 \\
45.2 \\
42.5 \\
8.8 \\
6.9 \\
41.4 \\
50.9 \\
2.8 \\
1.5 \\
1.6 \\
3.7 \\
36.5 \\
65.7\end{array}$ & $\begin{array}{r}0.8 \\
10.5 \\
7.0 \\
4.4 \\
2.2 \\
10.1 \\
2.8 \\
2.4 \\
2.3 \\
42.8 \\
37.1 \\
4.5 \\
3.6 \\
10.3 \\
22.0 \\
1.3 \\
0.9 \\
4.5 \\
2.7 \\
5.9 \\
34.1\end{array}$ & $\begin{array}{r}1.3 \\
26.8 \\
24.3 \\
11.3 \\
3.7 \\
33.0 \\
5.9 \\
7.7 \\
3.8 \\
8.0 \\
79.6 \\
13.3 \\
10.5 \\
55.7 \\
72.9 \\
4.1 \\
2.4 \\
6.1 \\
6.4 \\
42.4 \\
99.8\end{array}$ \\
\hline DL-3 & $\begin{array}{c}70-72 \\
76-78 \\
93-95 \\
115-117 \\
123-125 \\
133-135 \\
136-138 \\
140-142\end{array}$ & $\begin{array}{l}\text { A } \\
\text { A } \\
\text { B } \\
\text { C1 } \\
\text { C1 } \\
\text { C2 } \\
\text { C2 } \\
\text { C2 }\end{array}$ & & 100.0 & $\begin{array}{r}100.0 \\
100.0 \\
100.0 \\
100.0 \\
99.6\end{array}$ & $\begin{array}{r}100.0 \\
96.6 \\
100.0 \\
93.6 \\
99.2 \\
99.9 \\
99.7 \\
98.4\end{array}$ & $\begin{array}{l}99.9 \\
81.8 \\
99.9 \\
80.4 \\
96.6 \\
99.5 \\
98.9 \\
90.9\end{array}$ & $\begin{array}{l}98.9 \\
58.9 \\
99.8 \\
65.4 \\
85.3 \\
98.5 \\
97.2 \\
71.5\end{array}$ & $\begin{array}{l}89.3 \\
32.4 \\
98.9 \\
38.7 \\
37.9 \\
90.5 \\
90.9 \\
38.3\end{array}$ & $\begin{array}{l}82.0 \\
26.8 \\
97.0 \\
30.3 \\
24.6 \\
86.5 \\
87.4 \\
30.3\end{array}$ & $\begin{array}{r}34.7 \\
13.1 \\
60.9 \\
11.0 \\
8.1 \\
70.4 \\
57.3 \\
13.3\end{array}$ & $\begin{array}{r}10.7 \\
6.5 \\
25.5 \\
4.5 \\
5.7 \\
42.5 \\
22.4 \\
6.6\end{array}$ & $\begin{array}{r}6.5 \\
5.2 \\
15.9 \\
3.5 \\
55.1 \\
25.3 \\
13.7 \\
5.4\end{array}$ & $\begin{array}{r}4.6 \\
4.4 \\
10.4 \\
3.0 \\
4.6 \\
16.8 \\
9.6 \\
4.7\end{array}$ & $\begin{array}{l}2.7 \\
3.4 \\
4.6 \\
2.2 \\
3.5 \\
6.1 \\
5.4 \\
3.9\end{array}$ & $\begin{array}{l}2.2 \\
3.0 \\
3.5 \\
2.0 \\
3.2 \\
3.6 \\
4.5 \\
3.6\end{array}$ & $\begin{array}{l}1.9 \\
2.8 \\
3.0 \\
1.9 \\
3.0 \\
2.8 \\
4.0 \\
3.4\end{array}$ & $\begin{array}{l}0.0 \\
0.0 \\
0.0 \\
0.0 \\
0.0 \\
0.0 \\
0.0 \\
0.0\end{array}$ & $\begin{array}{l}0.0 \\
0.0 \\
0.0 \\
0.0 \\
0.0 \\
0.0 \\
0.0 \\
0.0\end{array}$ & $\begin{array}{l}0.0 \\
3.4 \\
0.0 \\
6.4 \\
0.8 \\
0.1 \\
0.3 \\
1.6\end{array}$ & $\begin{array}{l}0.0 \\
3.4 \\
0.0 \\
6.4 \\
0.8 \\
0.1 \\
0.3 \\
1.6\end{array}$ & $\begin{array}{r}0.1 \\
14.8 \\
0.1 \\
13.2 \\
2.6 \\
0.4 \\
0.8 \\
7.5\end{array}$ & $\begin{array}{r}17.9 \\
55.0 \\
2.9 \\
50.1 \\
72.0 \\
13.0 \\
11.5 \\
60.6\end{array}$ & $\begin{array}{l}79.3 \\
23.4 \\
92.4 \\
28.1 \\
21.1 \\
80.4 \\
82.0 \\
26.4\end{array}$ & $\begin{array}{l}97.3 \\
93.2 \\
95.4 \\
91.4 \\
95.7 \\
93.8 \\
94.3 \\
94.5\end{array}$ & $\begin{array}{l}2.1 \\
2.7 \\
2.9 \\
1.1 \\
1.8 \\
4.4 \\
3.0 \\
2.3\end{array}$ & $\begin{array}{l}0.6 \\
0.7 \\
1.7 \\
1.1 \\
1.7 \\
1.7 \\
2.4 \\
1.6\end{array}$ & $\begin{array}{l}2.7 \\
3.4 \\
4.6 \\
2.2 \\
3.5 \\
6.1 \\
5.4 \\
3.9\end{array}$ \\
\hline DL-4 & $\begin{array}{c}85-86 \\
88-90 \\
100-102 \\
102-103 \mathrm{ft} \\
104-105 \\
108-110 \\
110-112 \\
12-124 \\
126-128 \\
132-134 \\
141-143 \\
152-153 \\
154-155\end{array}$ & $\begin{array}{l}\text { A } \\
\text { A } \\
\text { B } \\
\text { B } \\
\text { B } \\
\text { B } \\
\text { C1 } \\
C 1 \\
C 1 \\
C 2 \\
C 2 \\
C 2\end{array}$ & 100.0 & $\begin{array}{r}100.0 \\
100.0 \\
99.4\end{array}$ & $\begin{array}{r}99.5 \\
99.6 \\
99.4 \\
100.0 \\
100.0 \\
99.7 \\
99.4 \\
98.5 \\
100.0 \\
99.7 \\
99.3 \\
96.0 \\
100.0\end{array}$ & $\begin{array}{l}95.8 \\
95.7 \\
99.8 \\
99.8 \\
99.5 \\
99.5 \\
95.0 \\
95.2 \\
98.6 \\
99.4 \\
98.4 \\
86.7 \\
99.2\end{array}$ & $\begin{array}{l}82.3 \\
84.6 \\
88.9 \\
98.9 \\
94.1 \\
83.6 \\
81.6 \\
99.4 \\
94.9 \\
99.0 \\
95.9 \\
70.5 \\
96.7\end{array}$ & $\begin{array}{l}63.1 \\
71.6 \\
75.2 \\
97.7 \\
87.4 \\
70.6 \\
62.6 \\
82.3 \\
85.9 \\
96.6 \\
87.4 \\
56.5 \\
93.0\end{array}$ & $\begin{array}{l}38.7 \\
47.1 \\
53.5 \\
95.8 \\
73.7 \\
49.9 \\
40.7 \\
51.0 \\
52.7 \\
79.4 \\
43.4 \\
38.0 \\
76.7\end{array}$ & $\begin{array}{l}34.2 \\
40.3 \\
46.0 \\
95.0 \\
66.9 \\
42.8 \\
34.4 \\
42.2 \\
41.6 \\
71.8 \\
30.5 \\
33.3 \\
6.1\end{array}$ & $\begin{array}{l}25.0 \\
21.9 \\
23.8 \\
87.4 \\
29.9 \\
16.4 \\
12.6 \\
22.4 \\
13.1 \\
40.8 \\
10.9 \\
22.7 \\
31.9\end{array}$ & $\begin{array}{r}18.9 \\
11.6 \\
9.6 \\
48.6 \\
10.6 \\
7.0 \\
5.2 \\
12.5 \\
5.0 \\
14.7 \\
6.2 \\
14.3 \\
14.8\end{array}$ & $\begin{array}{r}17.1 \\
9.5 \\
6.9 \\
30.0 \\
8.2 \\
6.2 \\
4.5 \\
10.3 \\
4.2 \\
10.5 \\
5.4 \\
11.5 \\
10.2\end{array}$ & $\begin{array}{r}15.8 \\
9.2 \\
5.5 \\
21.5 \\
6.9 \\
5.8 \\
4.1 \\
9.0 \\
3.8 \\
8.8 \\
4.9 \\
9.8 \\
8.1\end{array}$ & $\begin{array}{r}13.5 \\
8.3 \\
3.8 \\
12.9 \\
5.2 \\
5.3 \\
3.6 \\
7.1 \\
3.3 \\
6.9 \\
4.8 \\
7.5 \\
5.8\end{array}$ & $\begin{array}{r}12.4 \\
6.4 \\
3.3 \\
10.8 \\
4.5 \\
4.9 \\
3.3 \\
6.4 \\
3.1 \\
6.2 \\
4.6 \\
6.8 \\
5.1\end{array}$ & $\begin{array}{r}11.7 \\
5.2 \\
3.1 \\
9.6 \\
4.2 \\
4.6 \\
3.1 \\
6.0 \\
3.0 \\
5.8 \\
4.5 \\
6.4 \\
4.7\end{array}$ & $\begin{array}{l}0.0 \\
0.0 \\
0.0 \\
0.0 \\
0.0 \\
0.0 \\
0.0 \\
0.0 \\
0.0 \\
0.0 \\
0.0 \\
0.0 \\
0.0\end{array}$ & $\begin{array}{l}0.0 \\
0.0 \\
0.0 \\
0.0 \\
0.0 \\
0.0 \\
0.0 \\
0.0 \\
0.0 \\
0.0 \\
0.0 \\
0.0 \\
0.0\end{array}$ & $\begin{array}{r}4.2 \\
4.3 \\
2.2 \\
0.2 \\
1.5 \\
4.0 \\
4.8 \\
3.4 \\
1.3 \\
0.6 \\
1.7 \\
13.3 \\
0.8\end{array}$ & $\begin{array}{r}4.2 \\
4.3 \\
2.2 \\
0.2 \\
1.5 \\
4.0 \\
4.8 \\
3.4 \\
1.3 \\
0.6 \\
1.7 \\
13.3 \\
0.8\end{array}$ & $\begin{array}{r}13.5 \\
11.1 \\
8.9 \\
0.9 \\
4.4 \\
12.4 \\
13.6 \\
4.2 \\
3.8 \\
0.4 \\
2.4 \\
16.2 \\
2.5\end{array}$ & $\begin{array}{l}48.1 \\
44.3 \\
42.9 \\
3.9 \\
27.2 \\
40.8 \\
47.2 \\
50.2 \\
55.3 \\
27.2 \\
65.4 \\
37.2 \\
30.6\end{array}$ & $\begin{array}{l}20.7 \\
32.0 \\
42.2 \\
82.1 \\
61.7 \\
37.5 \\
30.8 \\
35.1 \\
38.3 \\
64.9 \\
25.7 \\
25.8 \\
60.3\end{array}$ & $\begin{array}{l}82.3 \\
87.4 \\
94.0 \\
86.9 \\
93.3 \\
90.7 \\
91.6 \\
89.5 \\
95.4 \\
92.5 \\
93.5 \\
79.2 \\
93.4\end{array}$ & $\begin{array}{l}9.5 \\
6.7 \\
2.4 \\
9.8 \\
3.4 \\
3.1 \\
1.9 \\
4.3 \\
1.7 \\
4.7 \\
2.1 \\
4.2 \\
3.0\end{array}$ & $\begin{array}{l}4.0 \\
1.6 \\
1.4 \\
3.1 \\
1.8 \\
2.2 \\
1.7 \\
2.8 \\
1.6 \\
2.2 \\
2.7 \\
3.3 \\
2.8\end{array}$ & $\begin{array}{r}13.5 \\
8.3 \\
3.8 \\
12.9 \\
5.2 \\
5.3 \\
3.6 \\
7.1 \\
3.3 \\
6.9 \\
4.8 \\
7.5 \\
5.8\end{array}$ \\
\hline
\end{tabular}




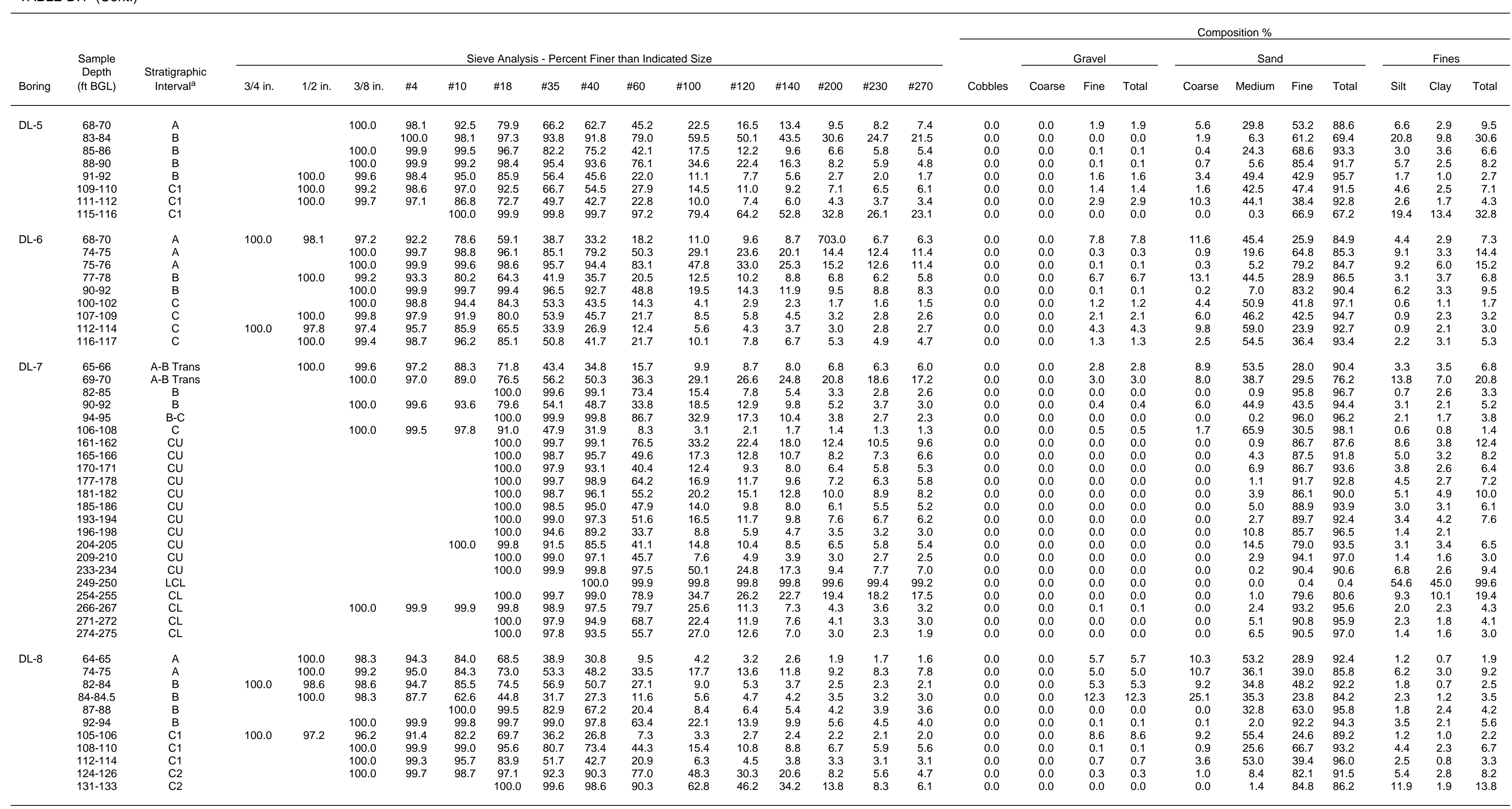

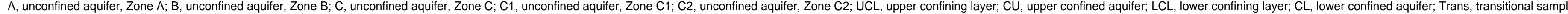
between to units. 


\section{Argonne National Laboratory}

Project: York, NE

Geologist: Eugene Yan

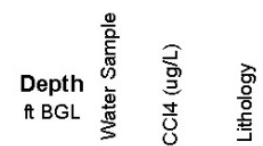

Well ID: TI01/DL1

Elevation: $1658.77 \mathrm{ft}$

Depth: $123 \mathrm{ft} \mathrm{BGL}$
Log Date: 5/18/2016

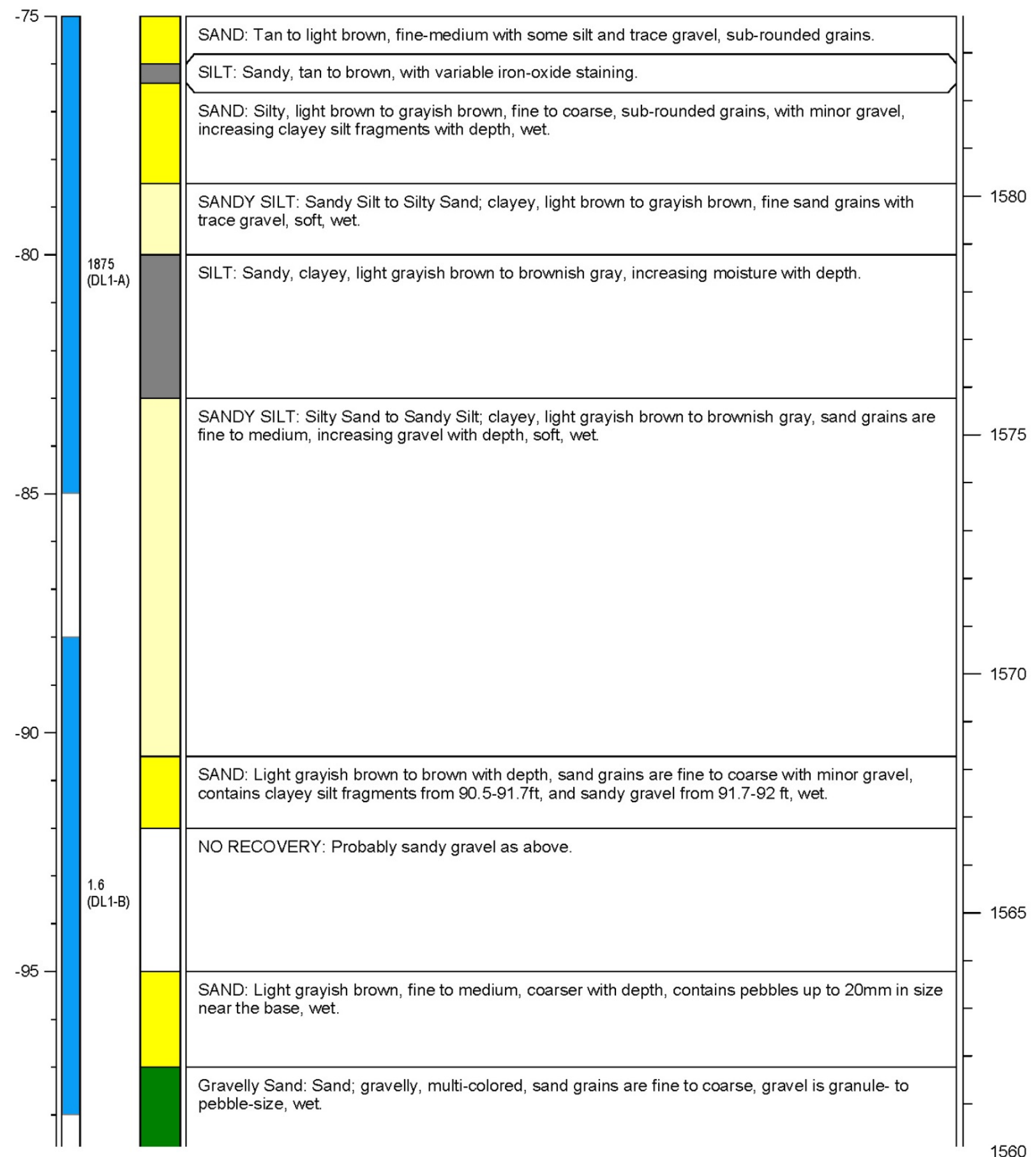




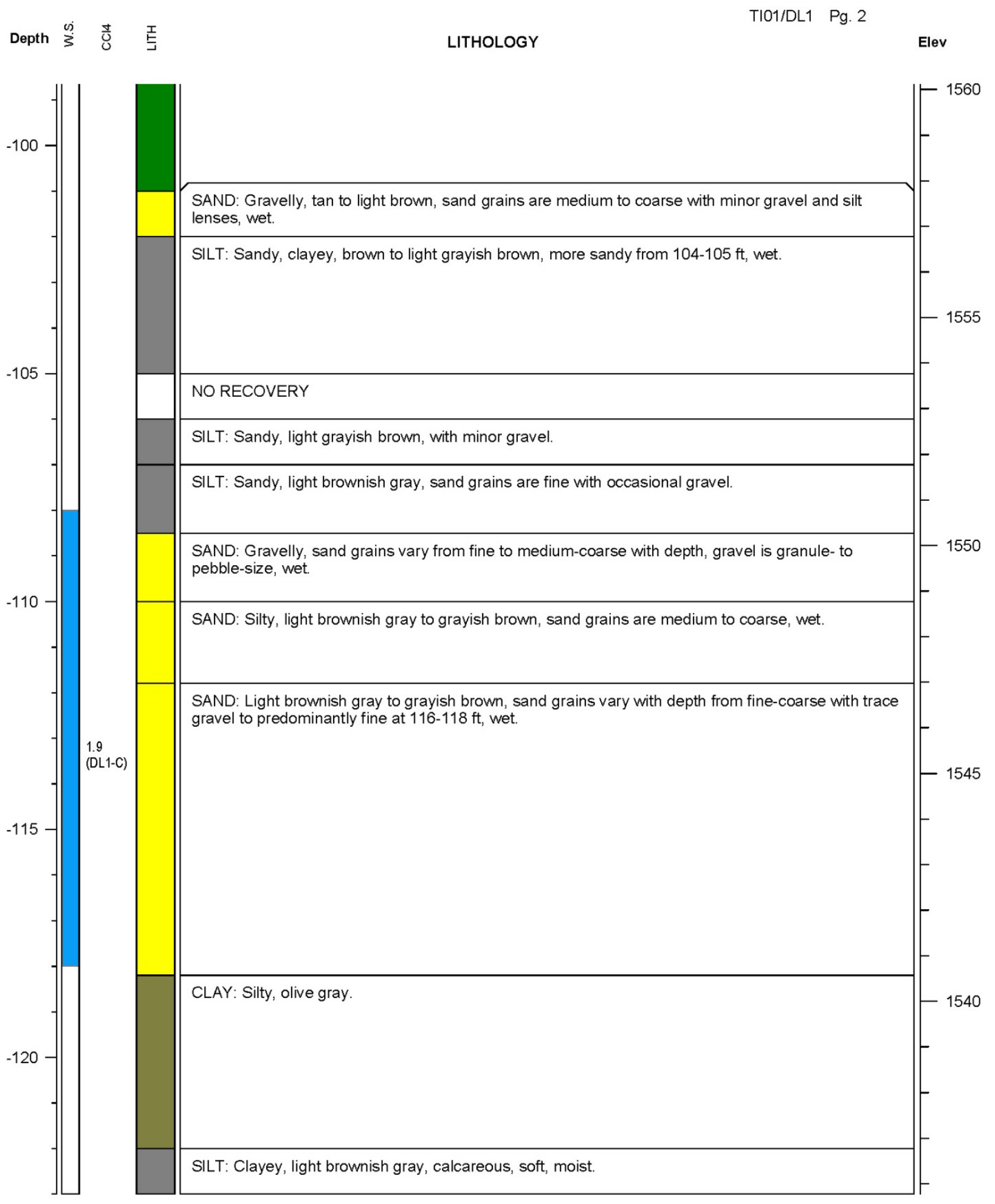




\section{Argonne National Laboratory}

Project: York, NE

Geologist: Eugene Yan

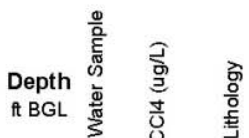

Elevation: $1658.16 \mathrm{ft}$

Depth: $245 \mathrm{ft}$ BGL
Well ID: DL2

Log Date: 5/20-21/2016

\section{LITHOLOGY DESCRIPTION}

Elev

ft AMSL

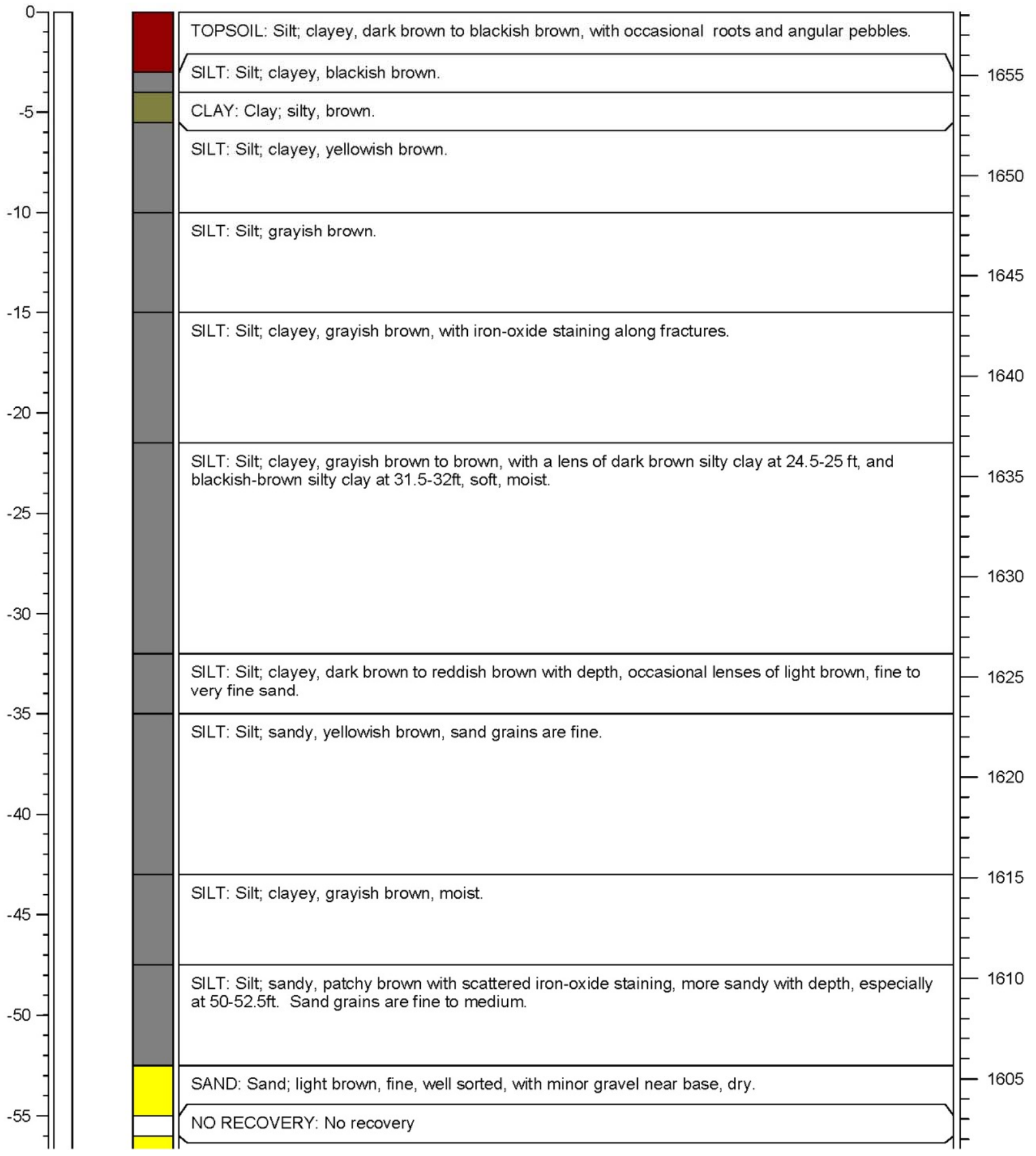




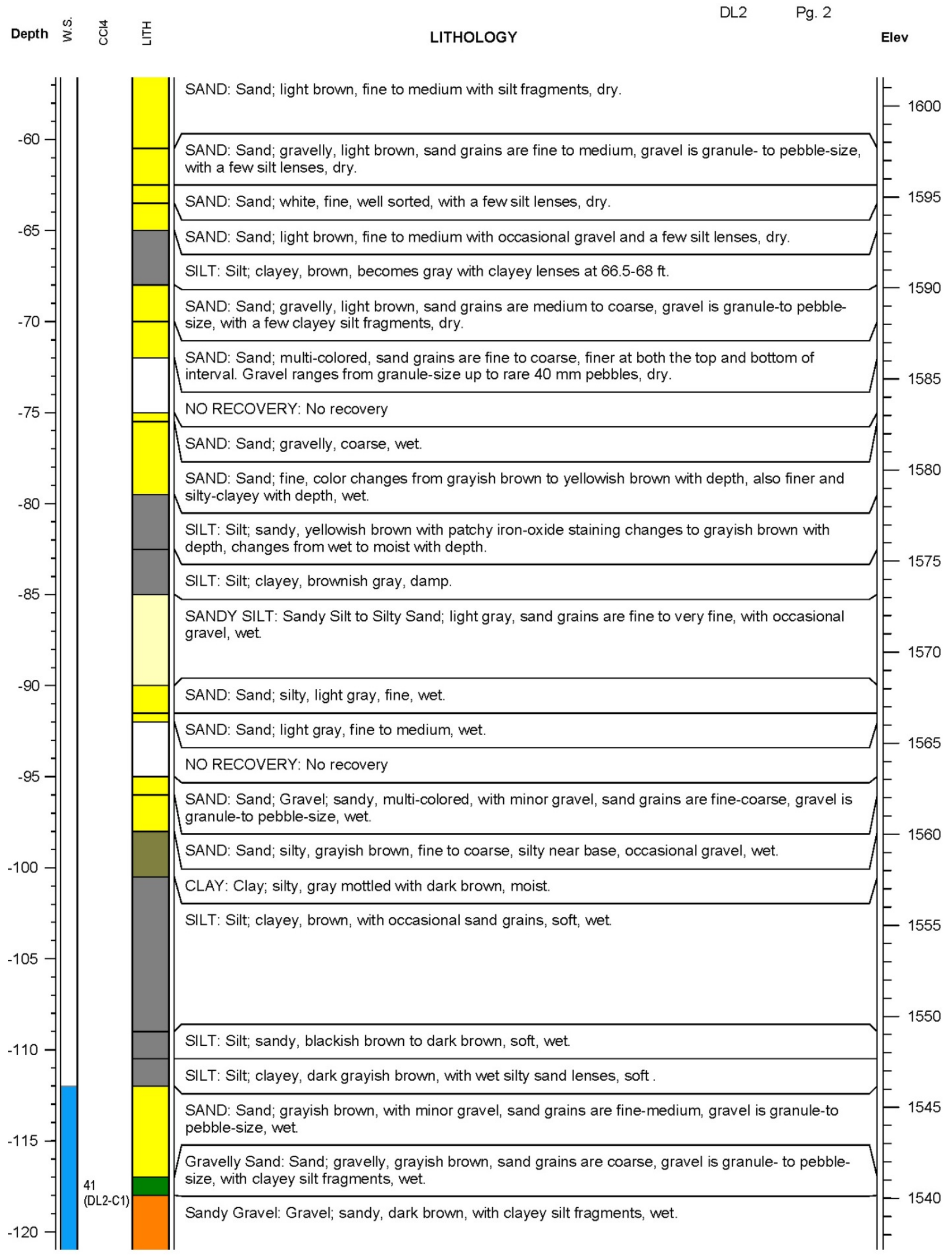




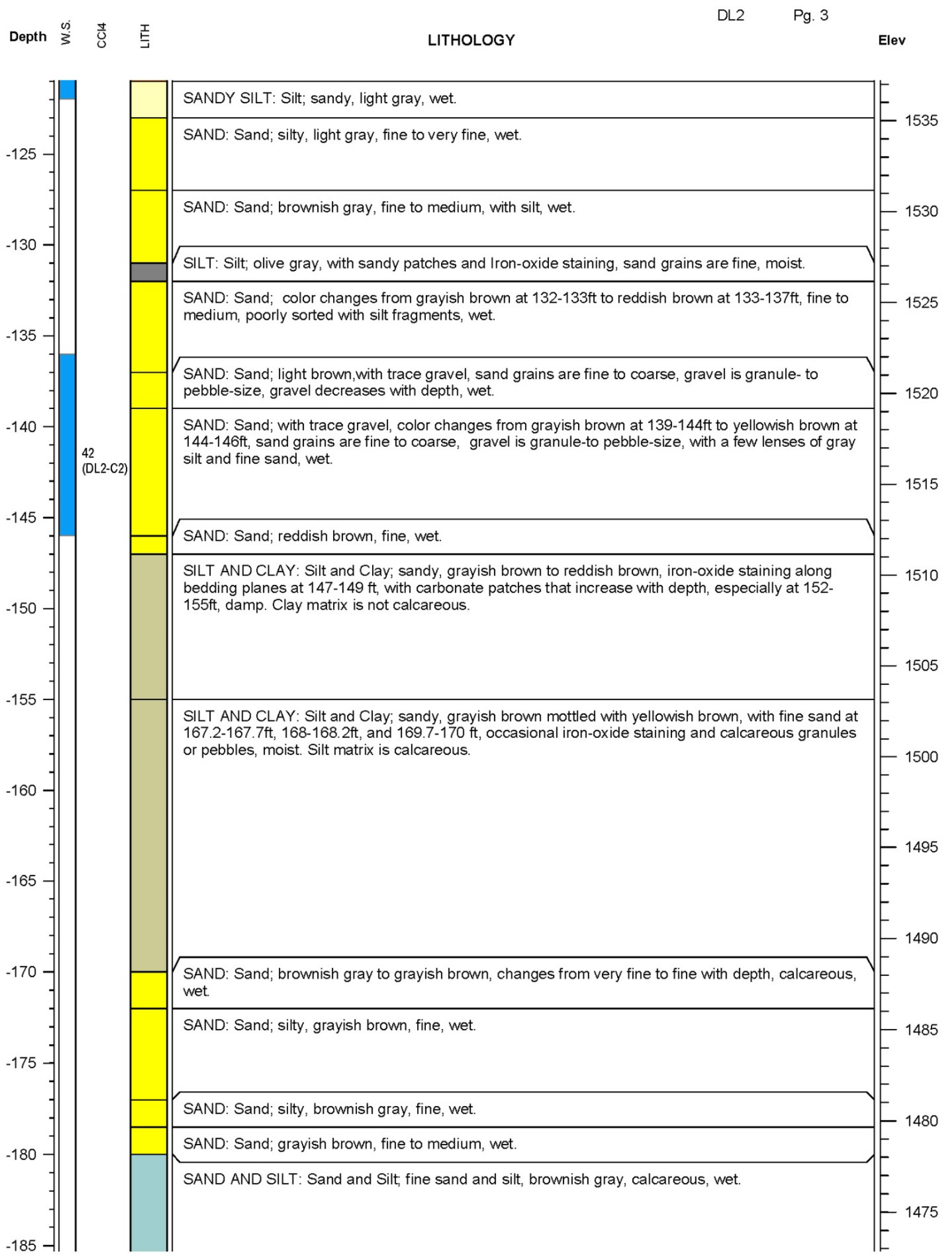




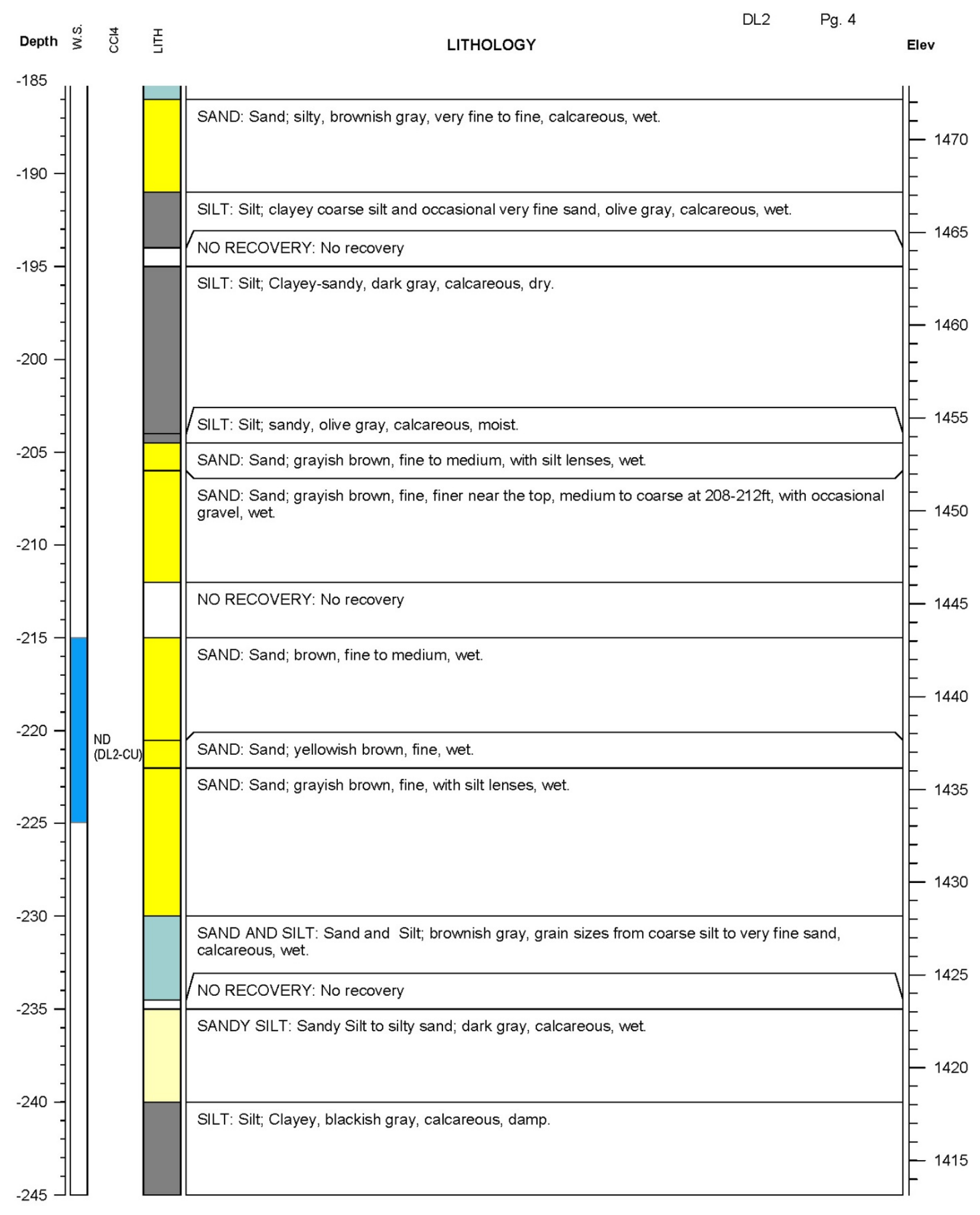




\section{Argonne National Laboratory} Project: York, NE

Geologist: Eugene Yan

Elevation: $1653.52 \mathrm{ft}$

Depth: $145 \mathrm{ft}$ BGL

\section{Well ID: TI15/DL3}

Log Date: 5/24/2016

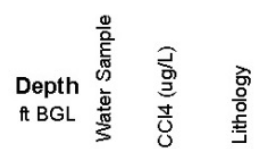

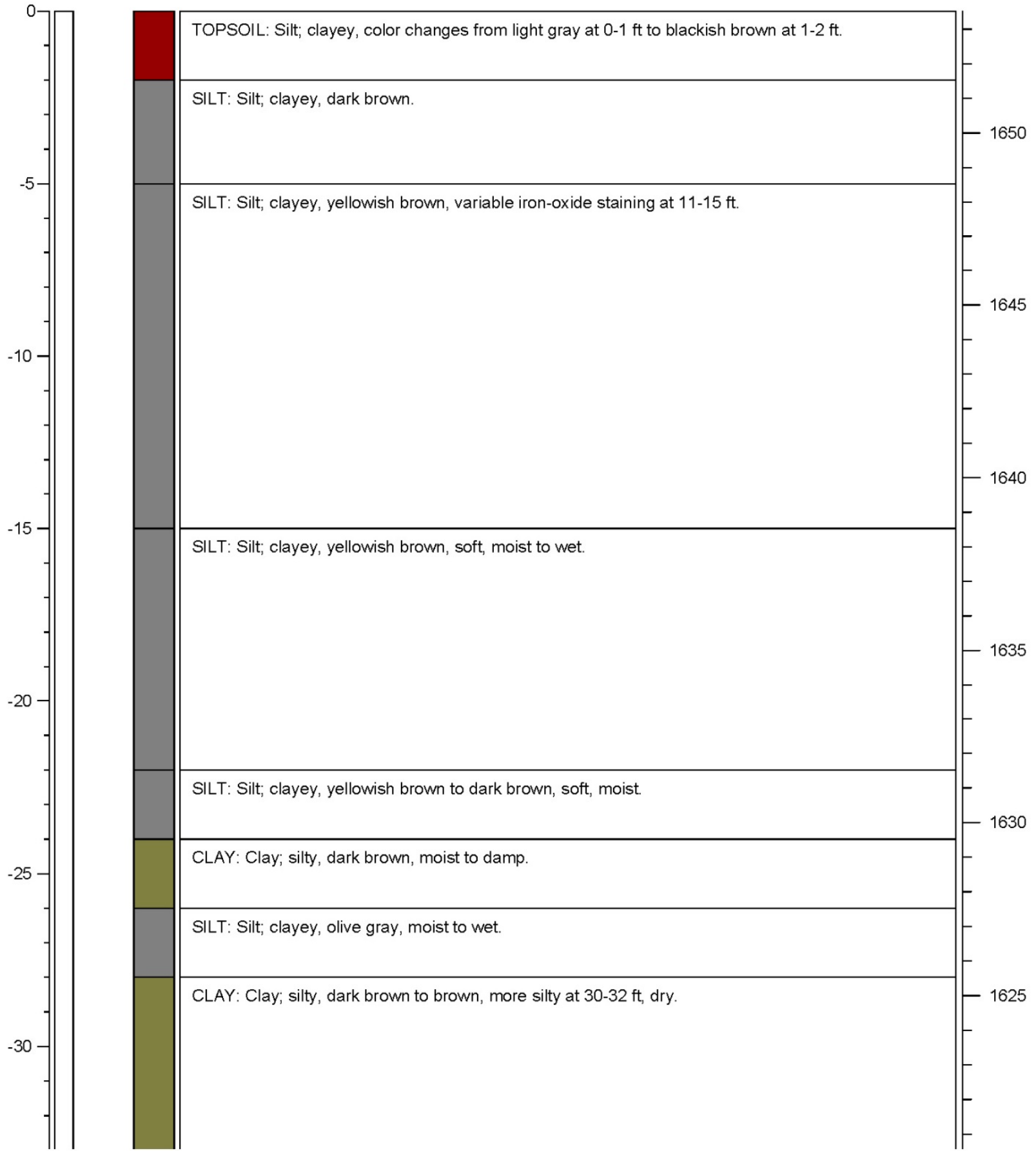




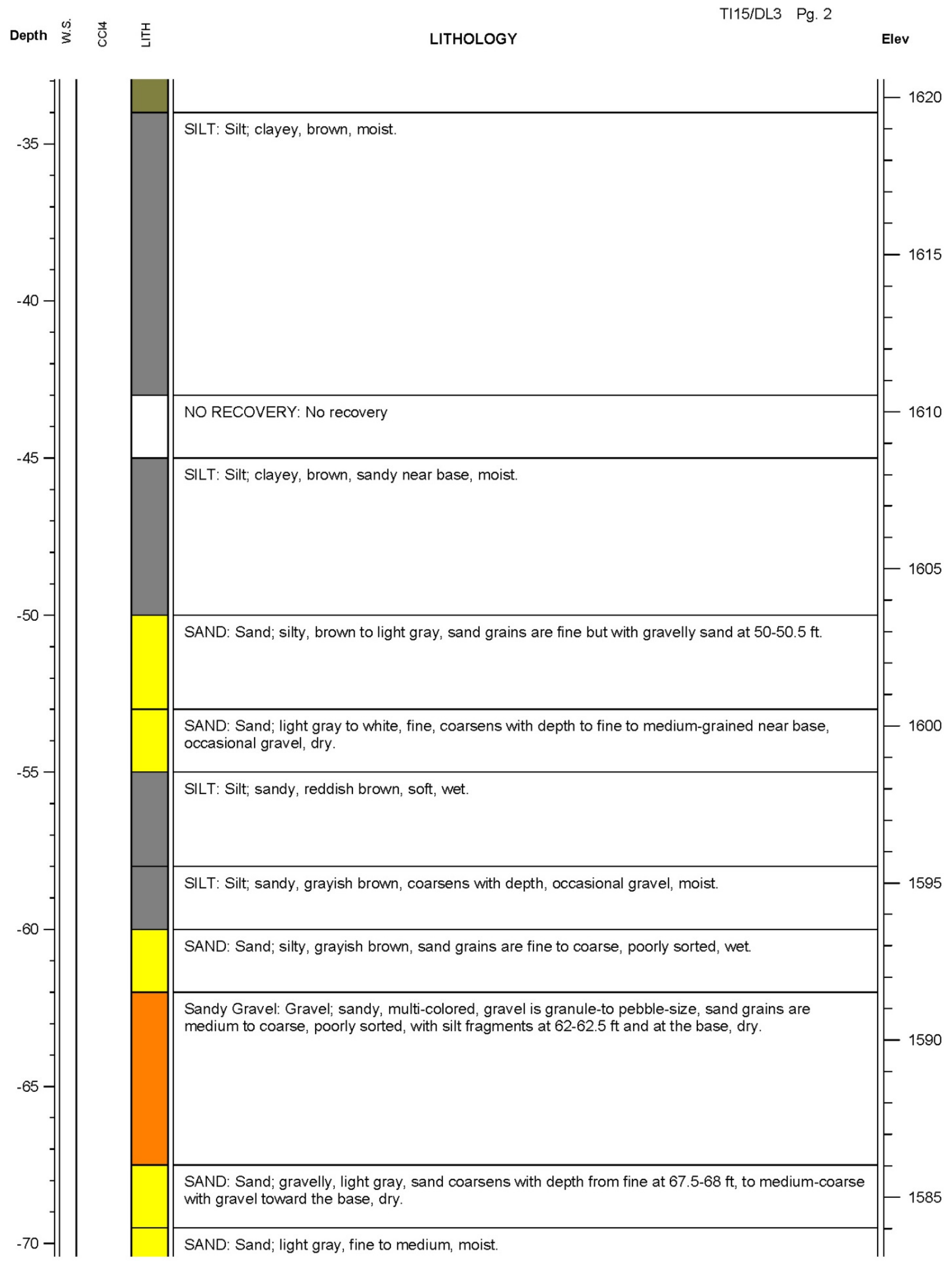




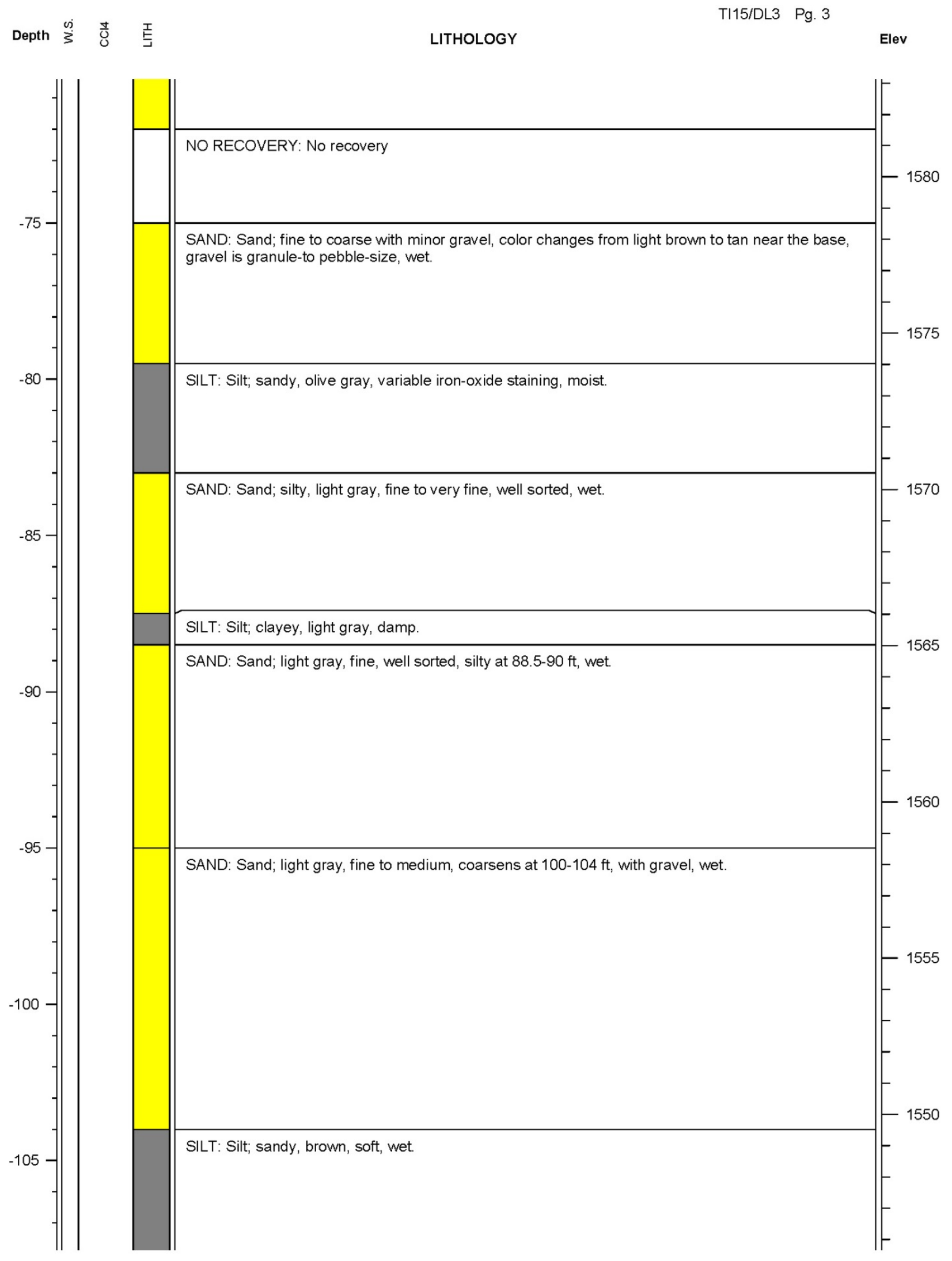




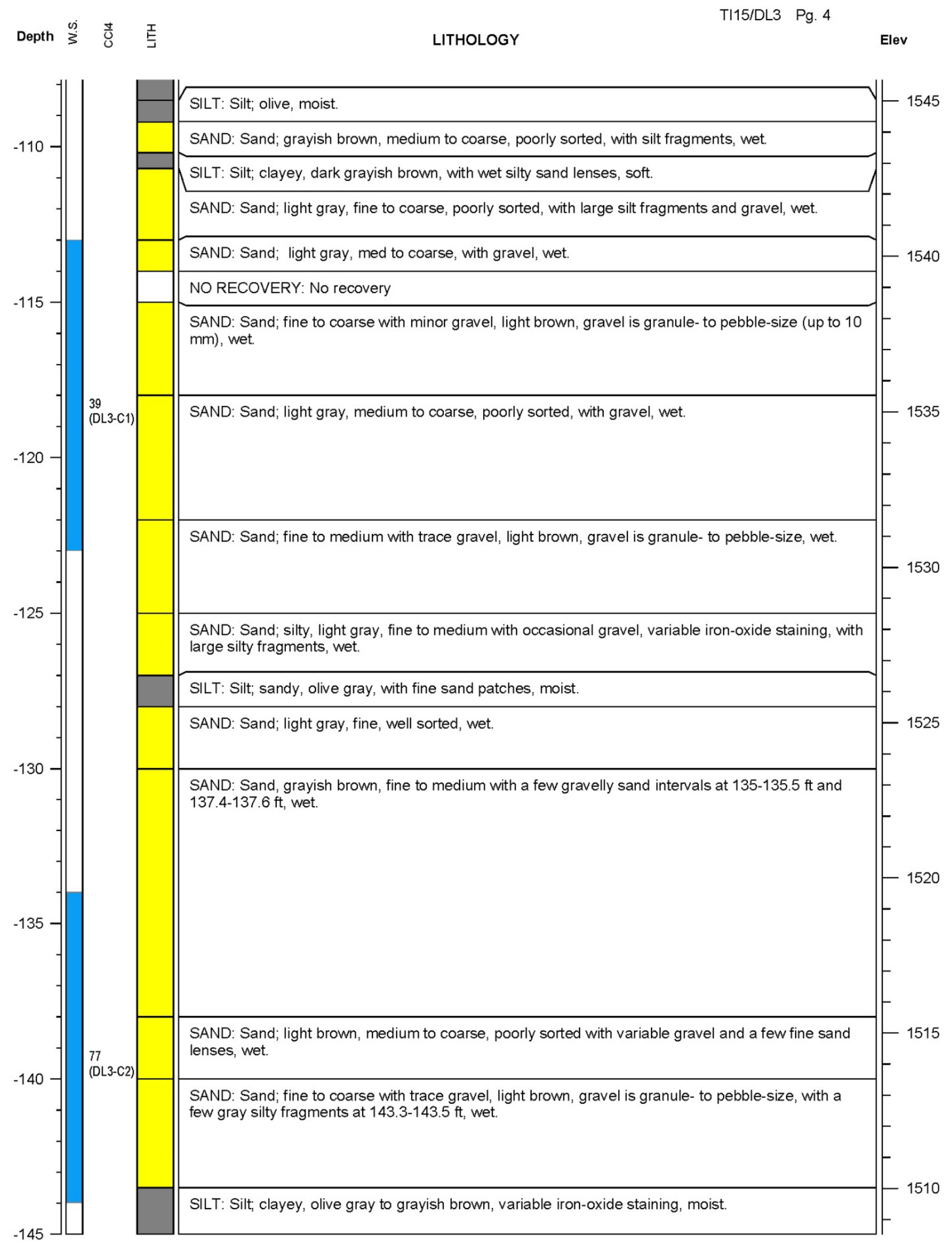




\section{Argonne National Laboratory}

Project: York, NE

Geologist: Eugene Yan

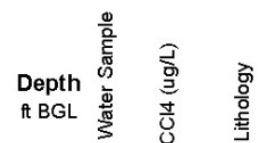

Well ID: TI23/DL4

Elevation: $1664.37 \mathrm{ft}$

Depth: $165 \mathrm{ft} \mathrm{BGL}$

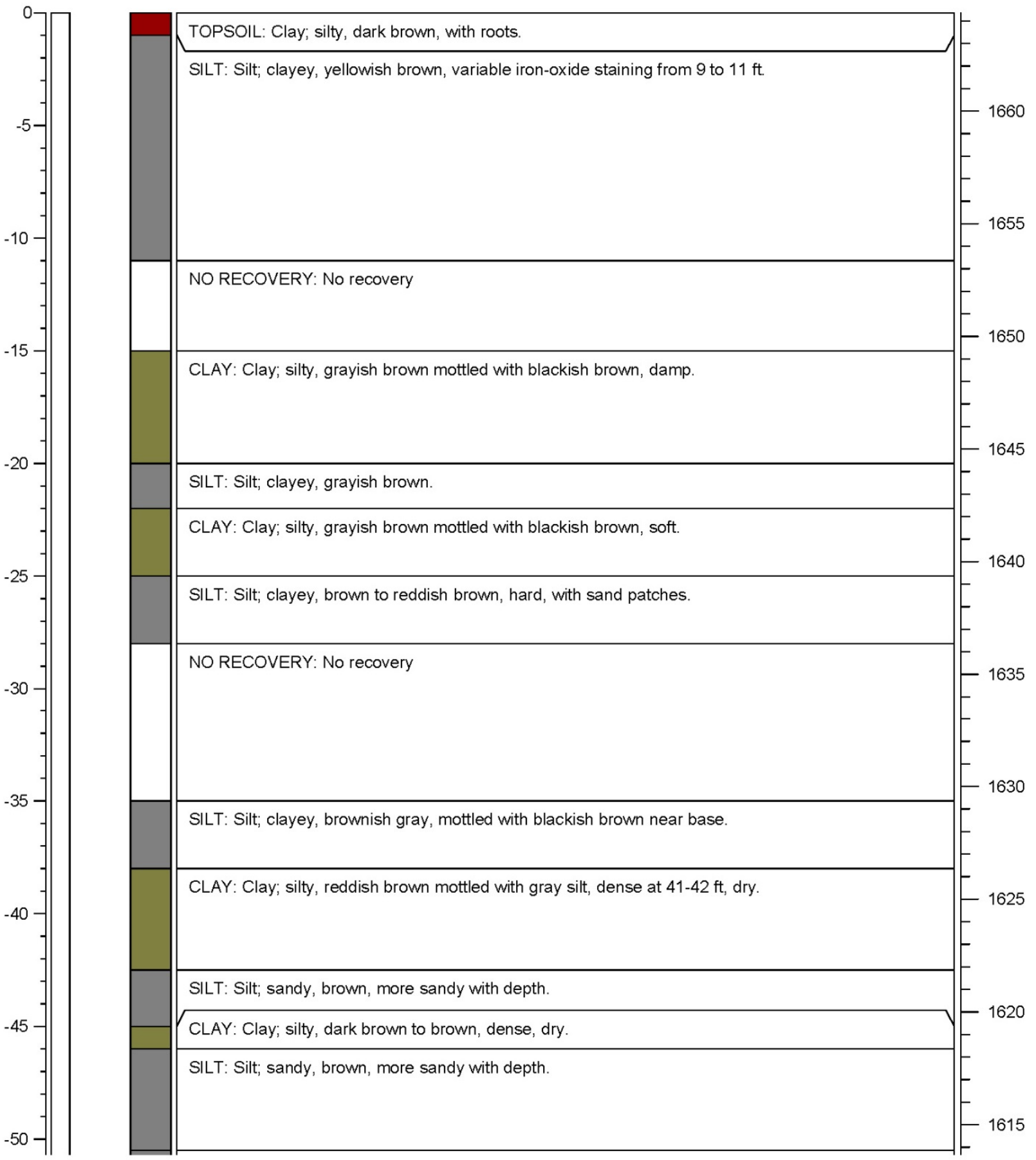




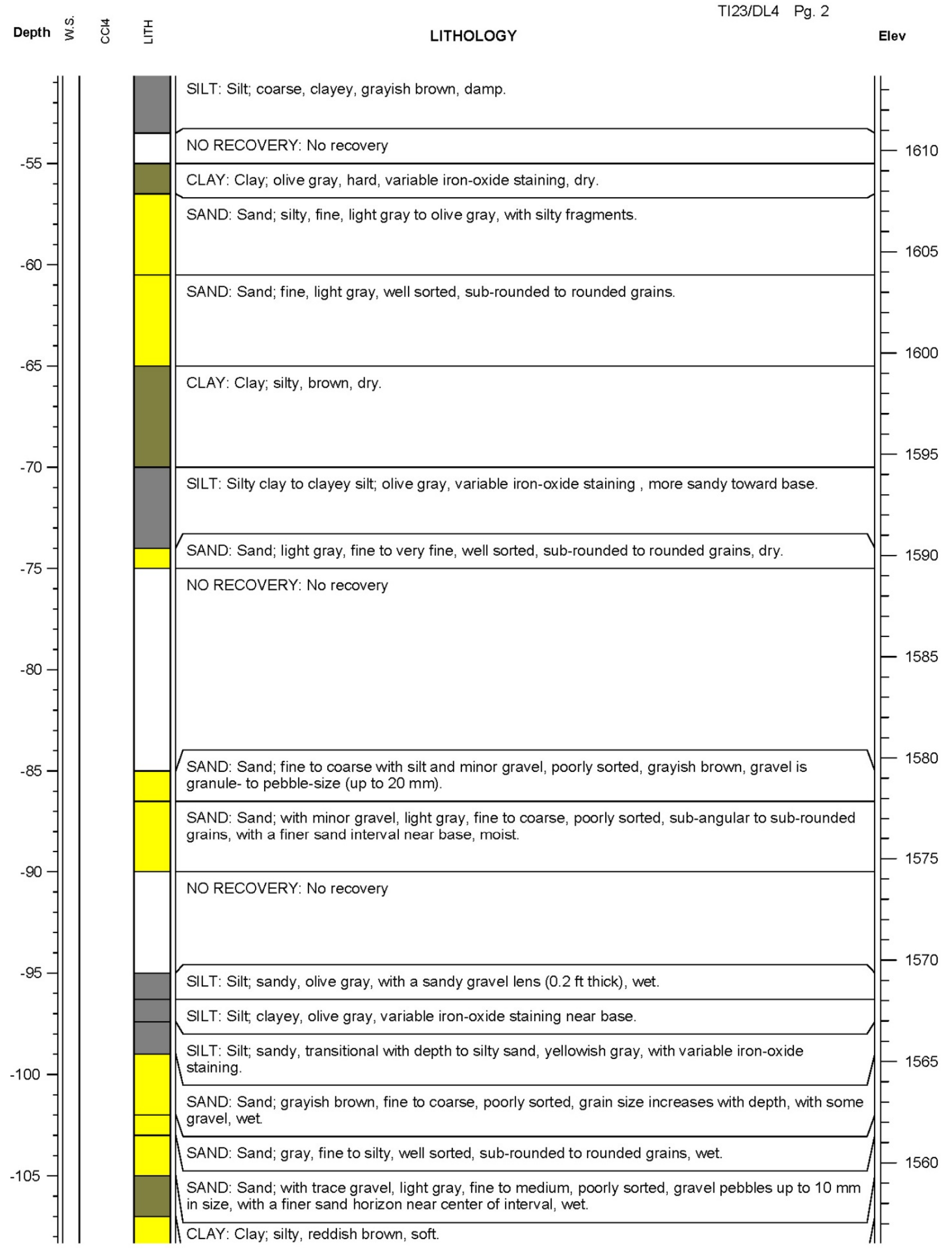




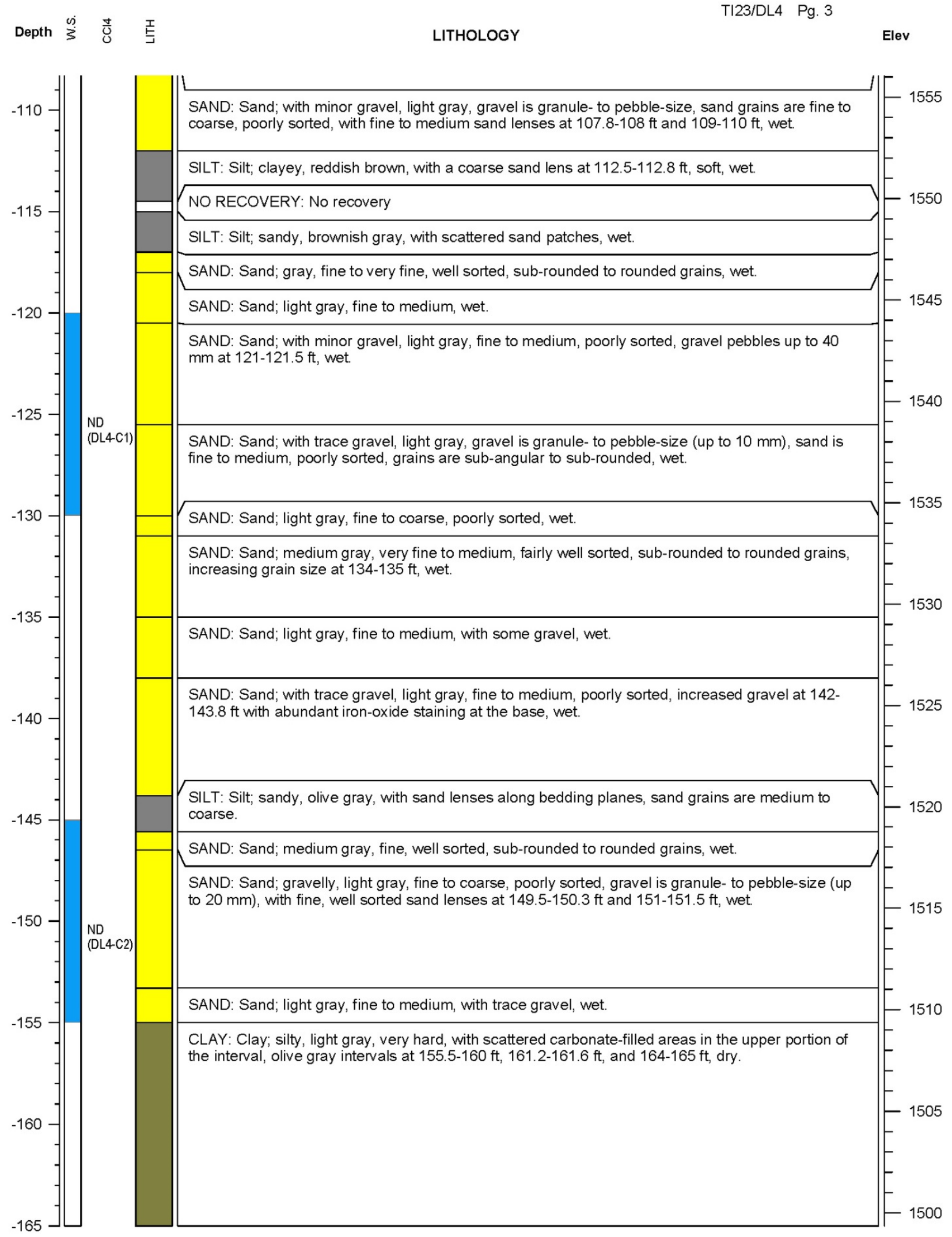




\section{Argonne National Laboratory}

Project: York, NE

Geologist: Eugene Yan

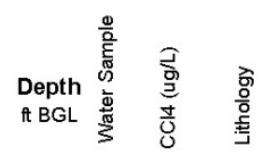

\section{Well ID: TI17/DL5}

Elevation: $1648.99 \mathrm{ft}$

Depth: $120 \mathrm{ft} \mathrm{BGL}$
Log Date: 6/16/16

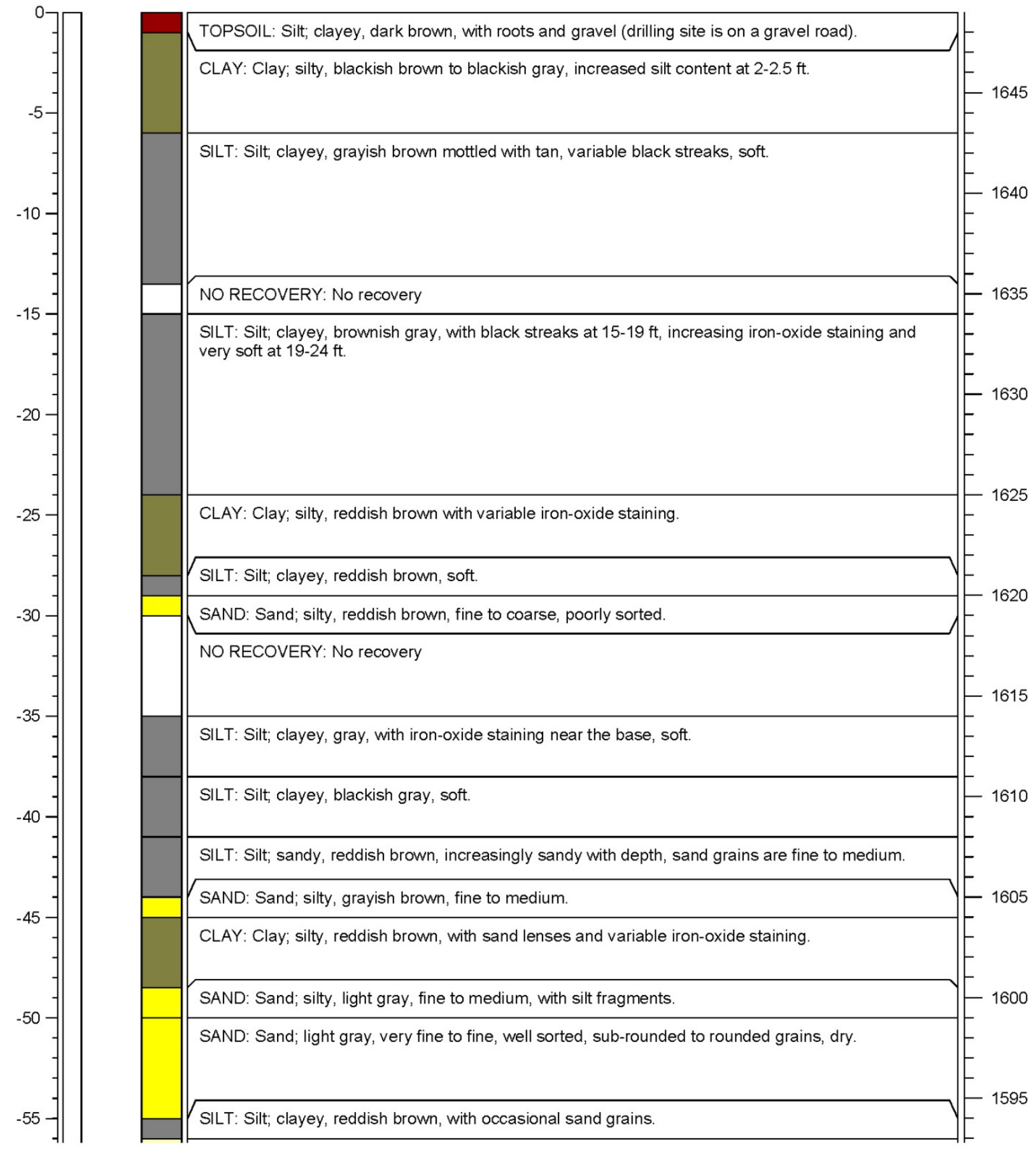




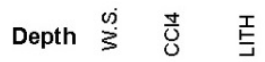

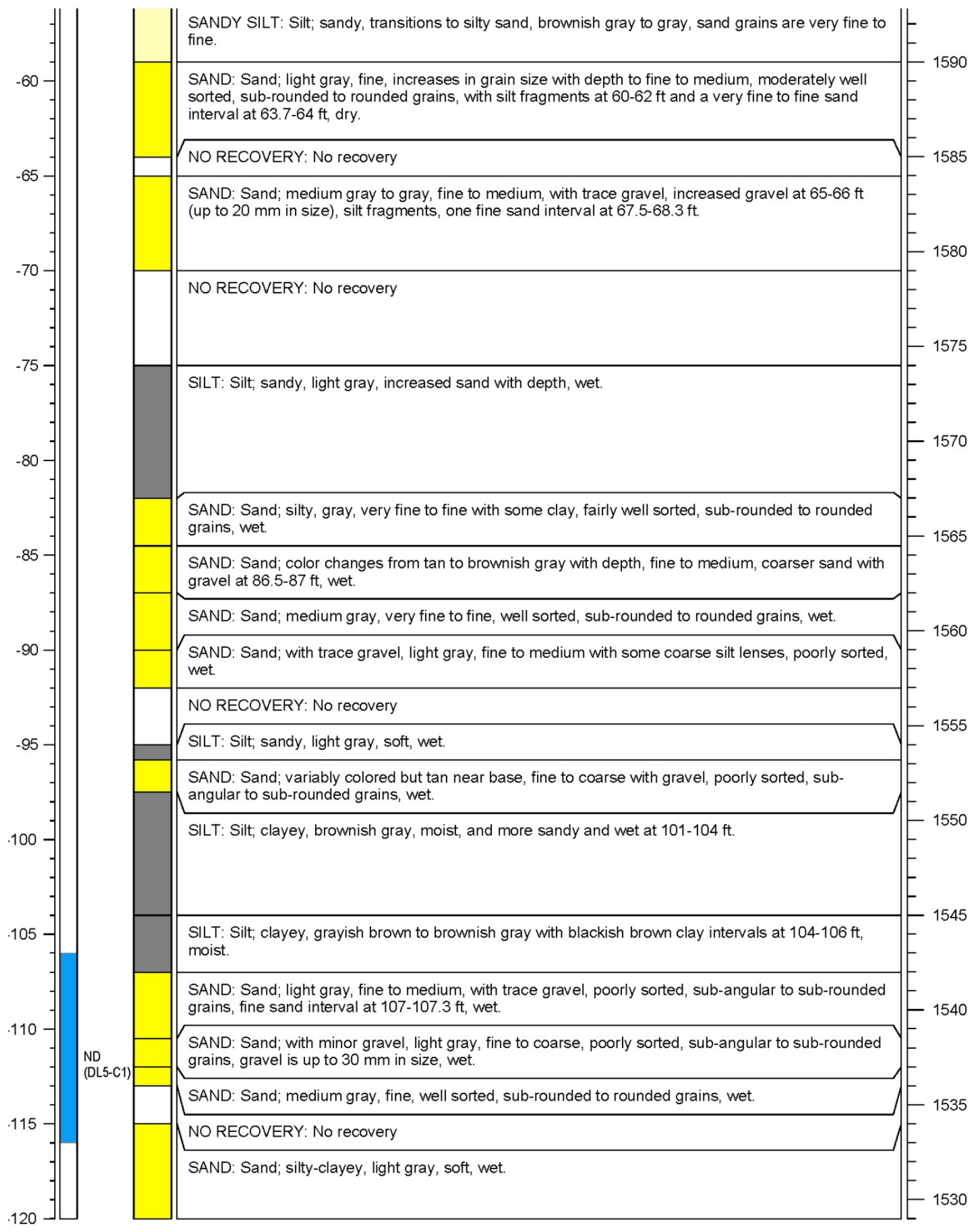




\section{Argonne National Laboratory}

Project: York, NE

Geologist: Eugene Yan

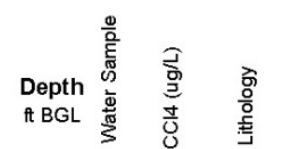

Elevation: $1641.91 \mathrm{ft}$

Depth: $125 \mathrm{ft} \mathrm{BGL}$
Well ID: TI21/DL6

Log Date: 6/7/2016
LITHOLOGY DESCRIPTION

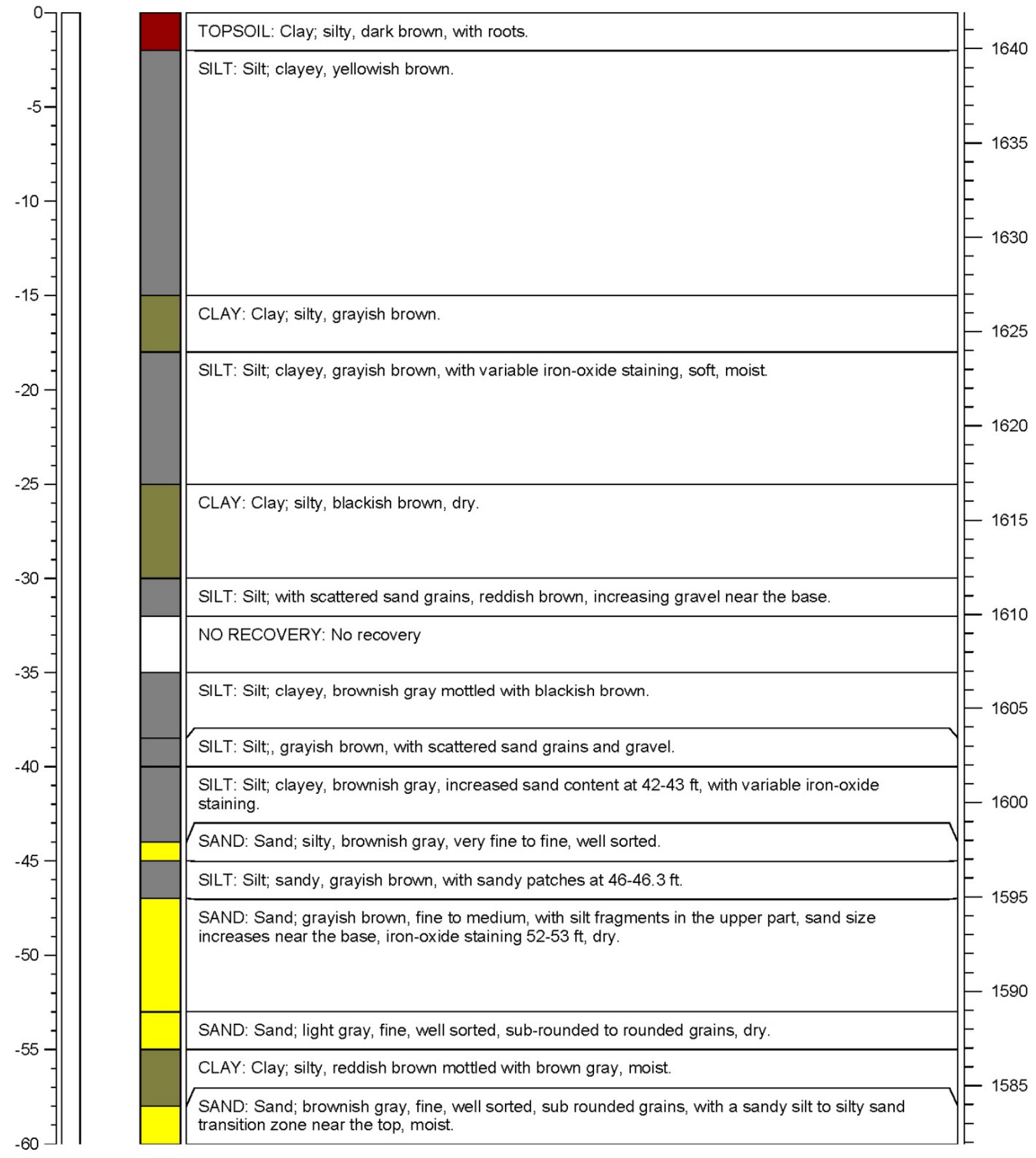




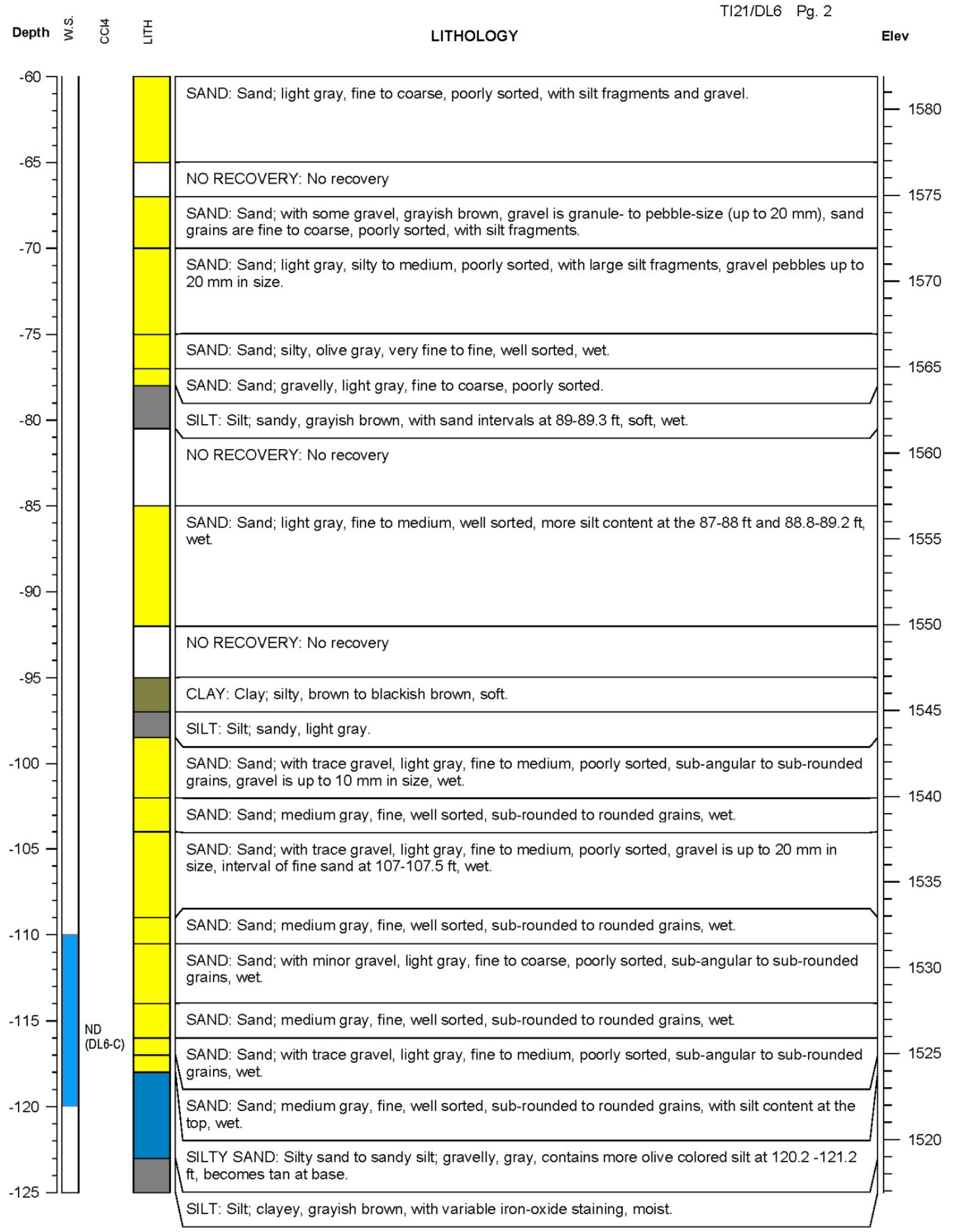




\section{Argonne National Laboratory}

Project: York, NE

Geologist: Eugene Yan

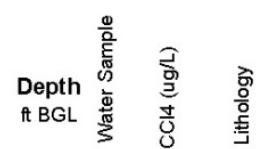

\section{Well ID: TI24/DL7}

Elevation: $1637.66 \mathrm{ft}$

Depth: $280 \mathrm{ft} \mathrm{BGL}$

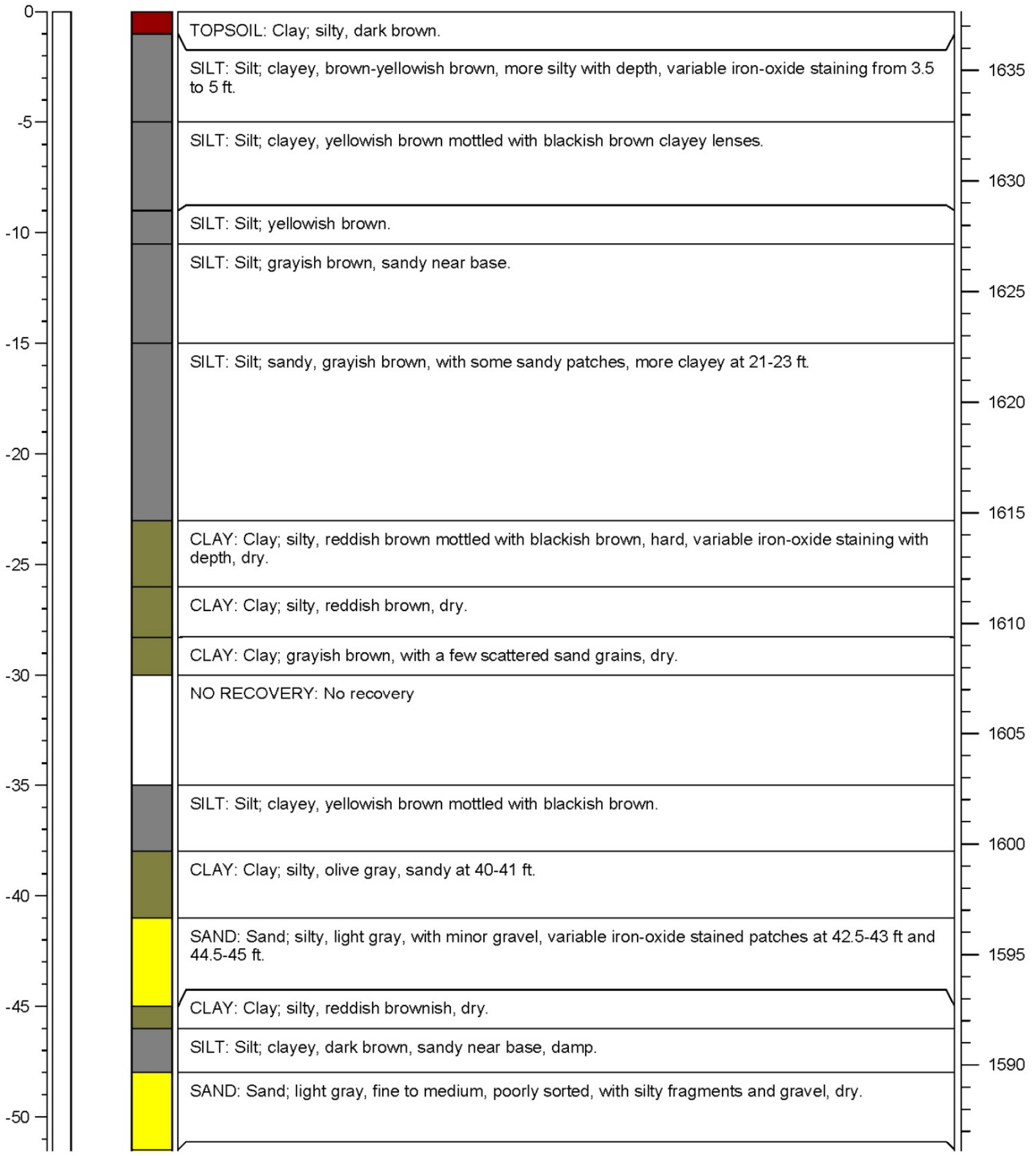




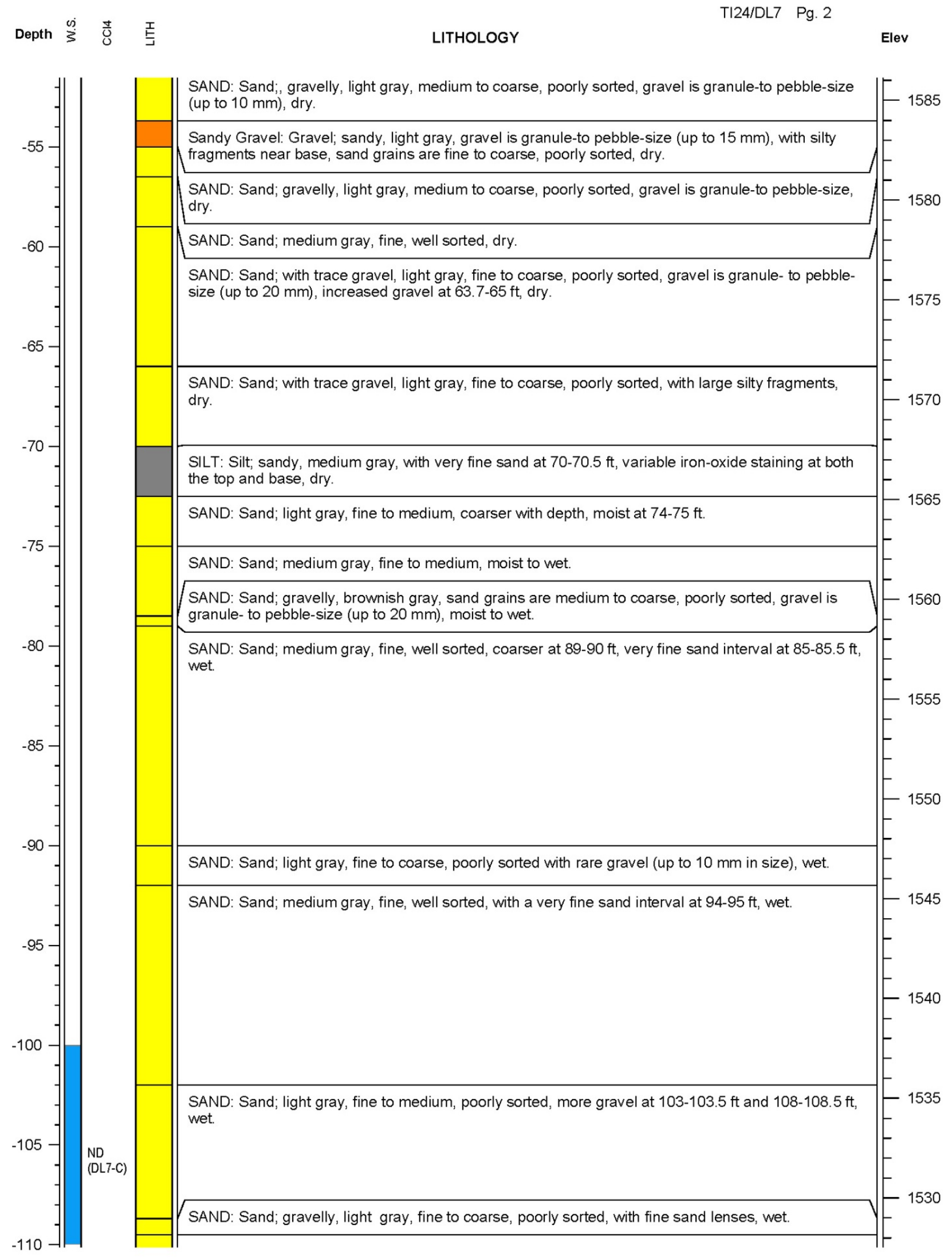




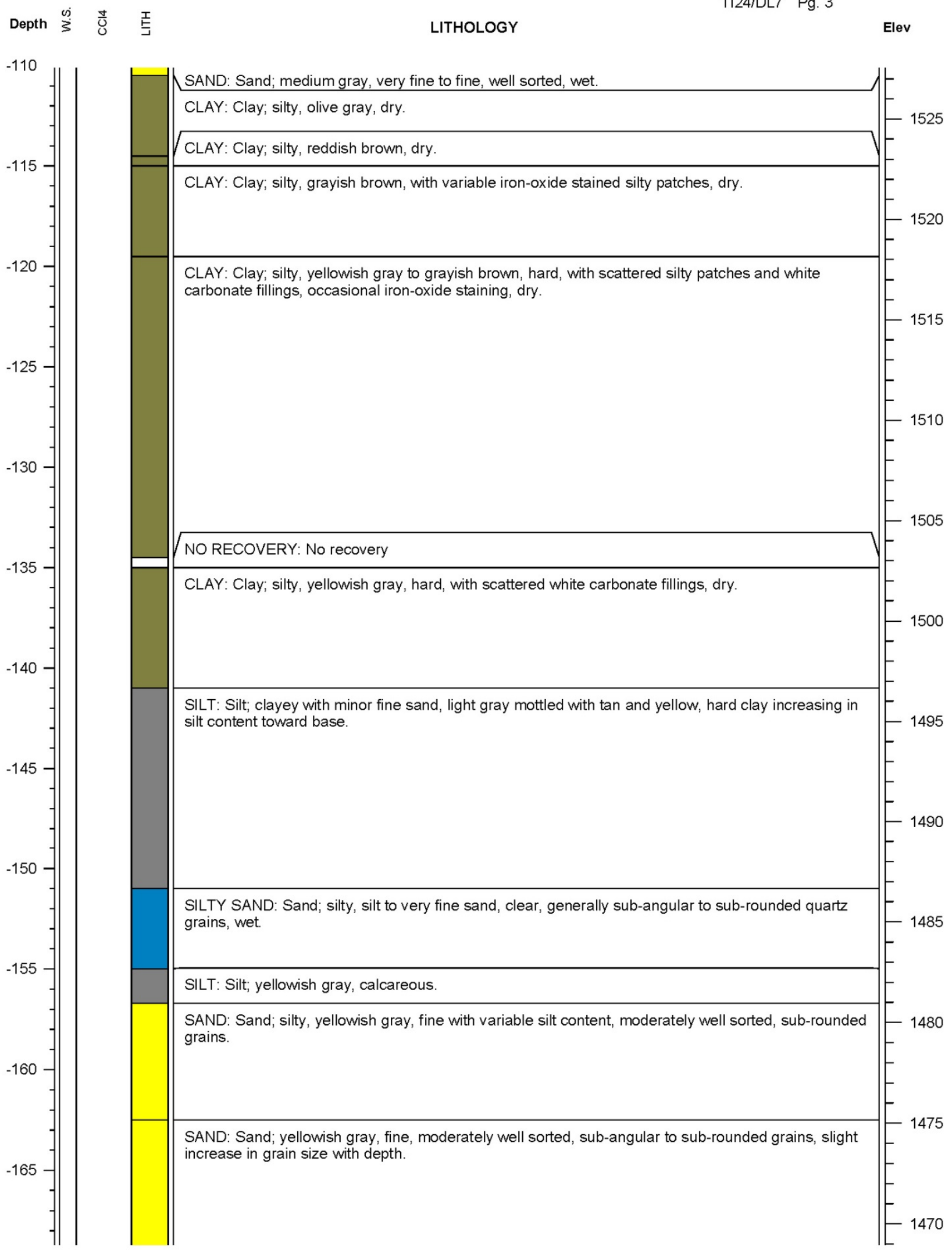




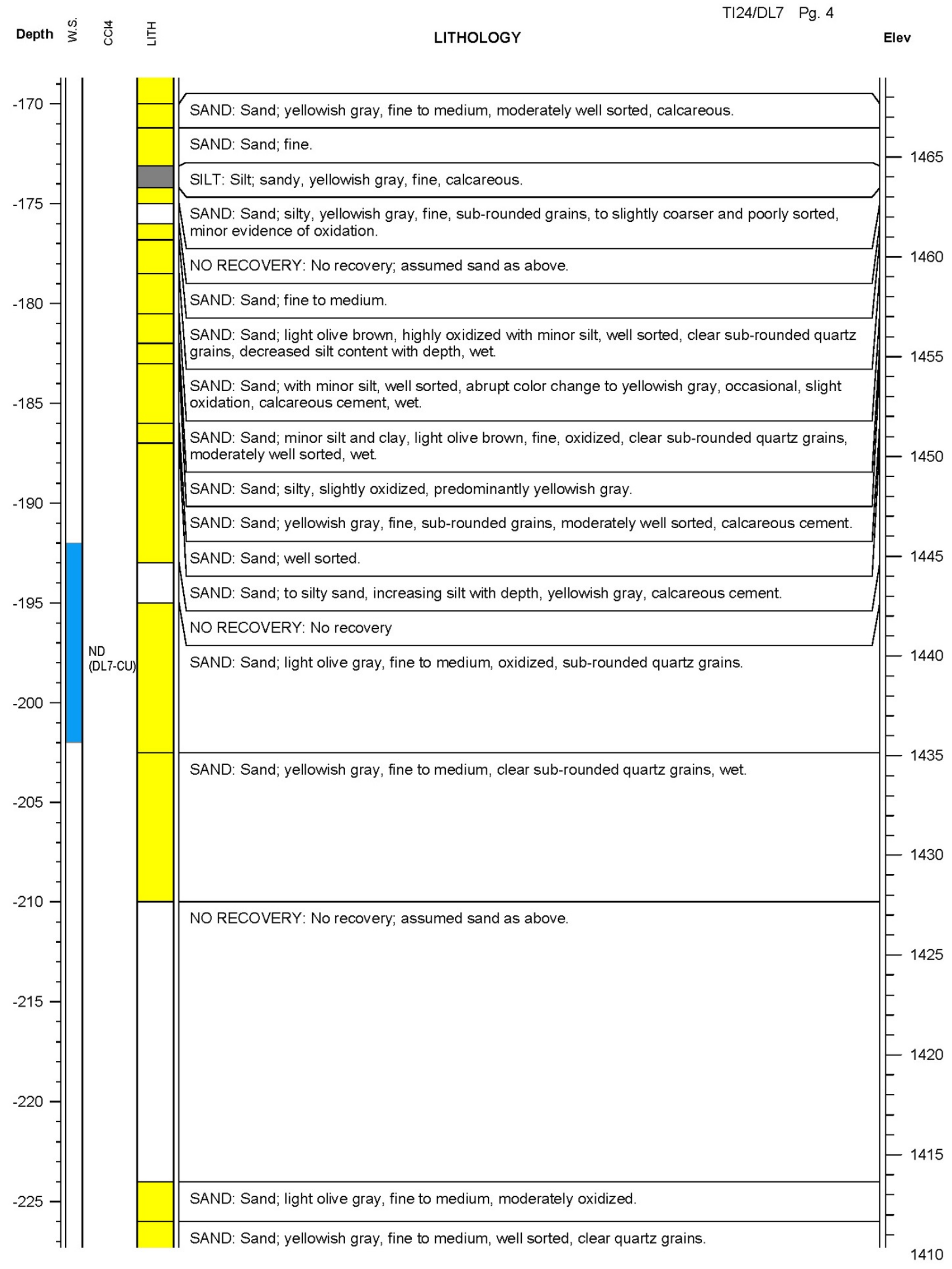




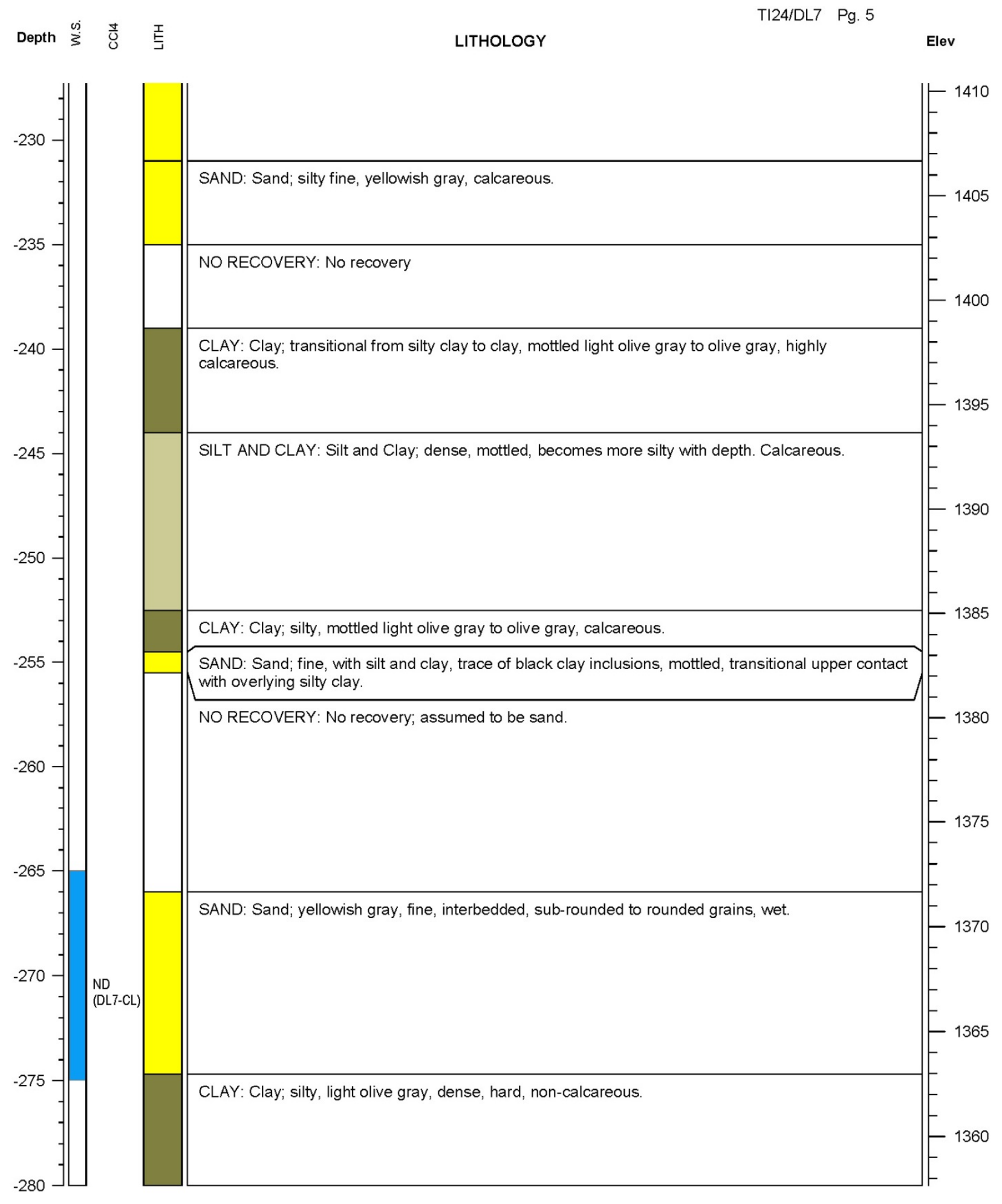




\section{Argonne National Laboratory}

Project: York, NE

Geologist: Eugene Yan

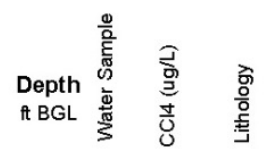

Well ID: TI20/DL8

Elevation: $1644.01 \mathrm{ft}$

Depth: $145 \mathrm{ft} \mathrm{BGL}$

Log Date: 6/1/2016

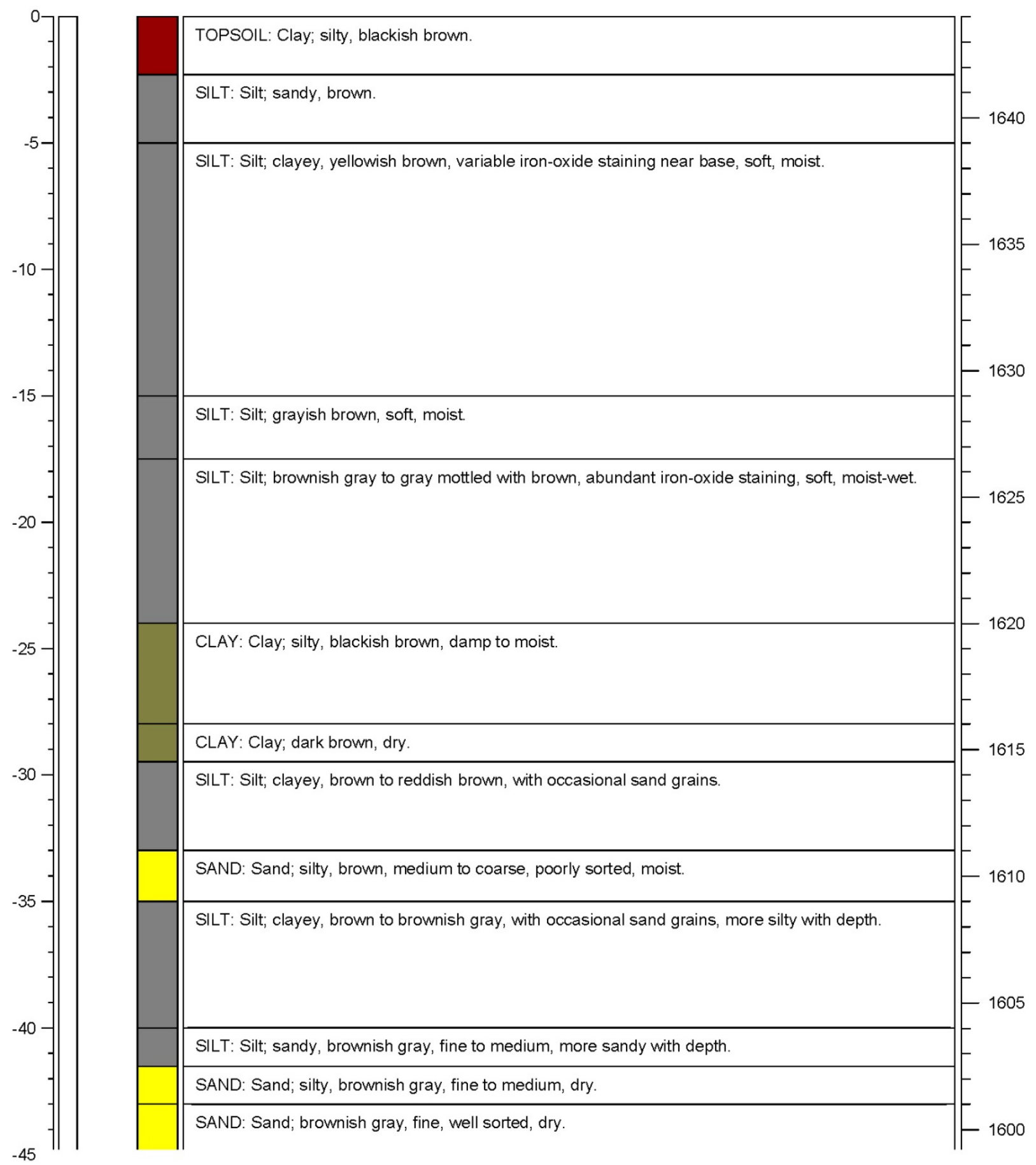




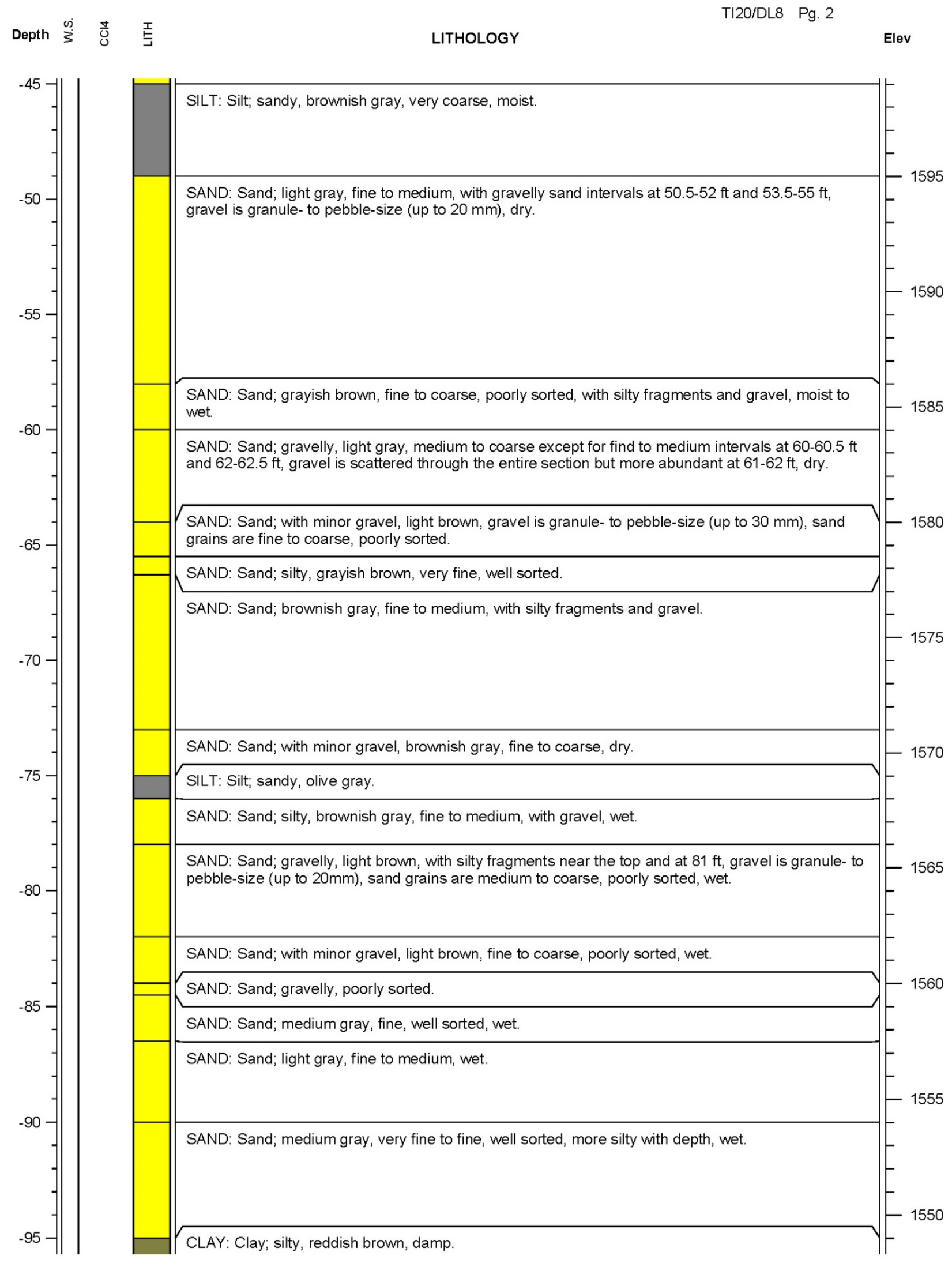




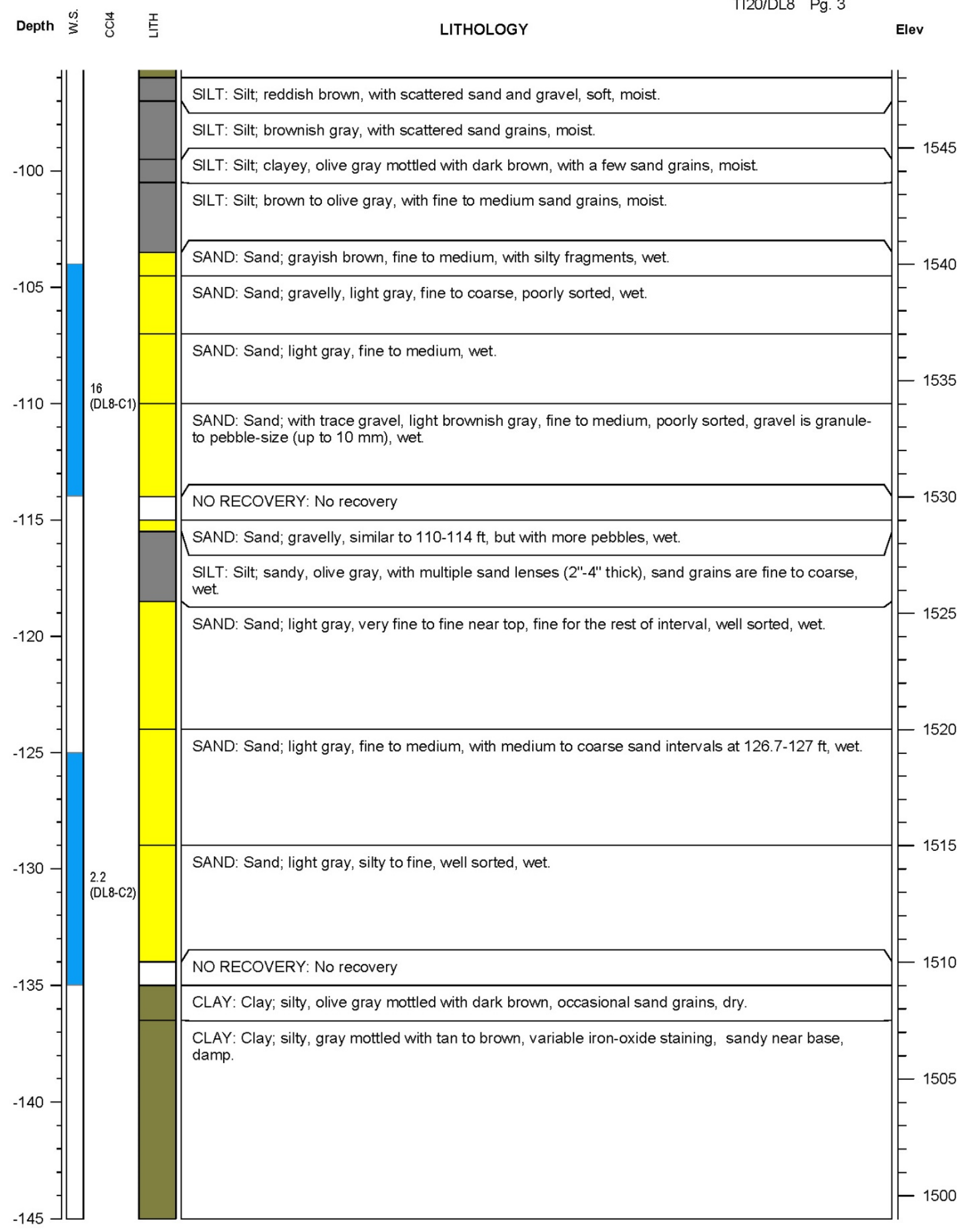




\section{Appendix E:}

\section{Analytical Data for Soil Samples}


TABLE E.1 Analytical results from the AGEM Laboratory for soil samples collected in July 2015 and analyzed by the purge-and-trap method.

\begin{tabular}{|c|c|c|c|c|c|c|c|}
\hline \multirow[b]{2}{*}{ Location } & \multirow[b]{2}{*}{ Sample } & \multirow[b]{2}{*}{$\begin{array}{l}\text { Sample } \\
\text { Date }\end{array}$} & \multirow[b]{2}{*}{$\begin{array}{l}\text { Depth } \\
\text { (ft BGL) }\end{array}$} & \multicolumn{4}{|c|}{ Concentration $(\mu \mathrm{g} / \mathrm{kg})$} \\
\hline & & & & $\begin{array}{c}\text { Carbon } \\
\text { Tetrachloride }\end{array}$ & Chloroform & $\begin{array}{l}\text { Methylene } \\
\text { Chloride }\end{array}$ & $\begin{array}{c}1,2- \\
\text { Dichloroethane }\end{array}$ \\
\hline TI01 & YKTI01-S-37744 & 7/7/15 & 4 & $9.6 \mathrm{Ja}$ & $N D^{b}$ & ND & ND \\
\hline TI01 & YKTI01-S-37745 & 7/7/15 & 8 & 13 & ND & ND & ND \\
\hline TI01 & YKTI01-S-37746 & 7/7/15 & 12 & 18 & $4.8 \mathrm{~J}$ & ND & ND \\
\hline TI01 & YKTI01-S-37747 & 7/7/15 & 16 & 29 & $6.0 \mathrm{~J}$ & ND & ND \\
\hline TI01 & YKTI01-S-37748 & 7/7/15 & 20 & 84 & $5.0 \mathrm{~J}$ & ND & ND \\
\hline TI01 & YKTI01-S-37749 & 7/7/15 & 24 & 139 & $7.9 \mathrm{~J}$ & ND & ND \\
\hline TI01 & YKTI01-S-37750 & 7/7/15 & 28 & 110 & $6.2 \mathrm{~J}$ & ND & ND \\
\hline TI01 & YKTI01-S-37751 & 7/7/15 & 32 & 121 & 12 & ND & ND \\
\hline TI01 & YKTI01-S-37753 & 7/7/15 & 36 & 167 & 12 & ND & 20 \\
\hline TI01 & YKTI01-S-37754 & 7/7/15 & 40 & 129 & 14 & ND & ND \\
\hline TI01 & YKTI01-S-37755 & 7/7/15 & 44 & 60 & $9.3 \mathrm{~J}$ & ND & 19 \\
\hline TI01 & YKTI01-S-37756 & 7/7/15 & 48 & 29 & 12 & ND & ND \\
\hline TI01 & YKTI01-S-37757 & $7 / 10 / 15$ & 52 & 53 & 17 & ND & ND \\
\hline TI01 & YKTI01-S-37758 & $7 / 10 / 15$ & 56 & ND & ND & ND & ND \\
\hline TI01 & YKTI01-S-37759 & $7 / 10 / 15$ & 60 & ND & ND & ND & ND \\
\hline TI01 & YKTI01-S-37760 & 7/10/15 & 63.8 & ND & ND & ND & ND \\
\hline TI01 & YKTI01-S-37761 & $7 / 10 / 15$ & 68 & ND & ND & ND & ND \\
\hline TI01 & YKTI01-S-37762 & $7 / 10 / 15$ & 72 & ND & ND & ND & ND \\
\hline TI01 & YKTI01-S-37841 & 7/10/15 & 75 & 23 & ND & ND & ND \\
\hline TI01 & YKTI01-S-37842 & $7 / 10 / 15$ & 80 & 110 & ND & ND & ND \\
\hline TI02 & YKTI02-S-37764 & $7 / 7 / 15$ & 4 & ND & ND & ND & ND \\
\hline TI02 & YKTI02-S-37765 & 7/7/15 & 7.8 & ND & ND & ND & ND \\
\hline TI02 & YKTI02-S-37766 & 7/7/15 & 12 & ND & ND & ND & ND \\
\hline TI02 & YKTI02-S-37767 & 7/7/15 & 16 & ND & ND & ND & ND \\
\hline TI02 & YKTI02-S-37768 & 7/7/15 & 20 & ND & ND & ND & ND \\
\hline TI02 & YKTI02-S-37769 & 7/7/15 & 24 & $8.4 \mathrm{~J}$ & ND & ND & ND \\
\hline TIO2 & YKTI02-S-37770 & 7/7/15 & 28 & ND & ND & ND & ND \\
\hline TI02 & YKTI02-S-37771 & 7/7/15 & 32 & $4.8 \mathrm{~J}$ & ND & ND & ND \\
\hline TI02 & YKTI02-S-37772 & 7/7/15 & 36 & $5.9 \mathrm{~J}$ & ND & ND & ND \\
\hline TI02 & YKTI02-S-37773 & 7/7/15 & 40 & 10 & ND & ND & ND \\
\hline TI02 & YKTI02-S-37774 & 7/7/15 & 44 & 13 & ND & ND & ND \\
\hline TI02 & YKTI02-S-37775 & 7/7/15 & 48 & $7.7 \mathrm{~J}$ & ND & ND & ND \\
\hline TIO2 & YKTI02-S-37778 & 7/7/15 & 52 & ND & ND & ND & ND \\
\hline TI02 & YKTI02-S-37779 & 7/7/15 & 55.8 & ND & ND & ND & ND \\
\hline TI02 & YKTI02-S-37780 & 7/7/15 & 59.8 & ND & ND & ND & ND \\
\hline TI02 & YKTI02-S-37781 & 7/7/15 & 64 & ND & ND & ND & ND \\
\hline TI02 & YKTIO2-S-37782 & $7 / 8 / 15$ & 67.8 & ND & ND & ND & ND \\
\hline TI02 & YKTI02-S-37783 & $7 / 8 / 15$ & 72 & ND & ND & ND & ND \\
\hline TIO2 & YKTI02-S-37785 & $7 / 8 / 15$ & 75 & 10 & ND & ND & ND \\
\hline TI03 & YKTI03-S-37808 & $7 / 9 / 15$ & 4 & ND & ND & ND & ND \\
\hline TI03 & YKTI03-S-37809 & $7 / 9 / 15$ & 8 & ND & ND & ND & ND \\
\hline TI03 & YKTI03-S-37810 & 7/9/15 & 12 & ND & ND & ND & ND \\
\hline TI03 & YKTI03-S-37811 & 7/9/15 & 16 & ND & ND & ND & ND \\
\hline TI03 & YKTI03-S-37812 & $7 / 9 / 15$ & 20 & ND & ND & ND & ND \\
\hline TI03 & YKTI03-S-37813 & 7/9/15 & 24 & ND & ND & ND & ND \\
\hline TI03 & YKTI03-S-37814 & 7/9/15 & 28 & ND & ND & ND & ND \\
\hline TI03 & YKTI03-S-37815 & $7 / 9 / 15$ & 32 & ND & ND & ND & ND \\
\hline TI03 & YKTI03-S-37816 & 7/9/15 & 36 & $5.6 \mathrm{~J}$ & ND & ND & ND \\
\hline TI03 & YKTI03-S-37817 & 7/9/15 & 40 & $8.7 \mathrm{~J}$ & ND & ND & ND \\
\hline
\end{tabular}


York Targeted Investigation, 2015-2016

Version 01, 2/14/2017

TABLE E.1 (Cont.)

\begin{tabular}{|c|c|c|c|c|c|c|c|}
\hline \multirow[b]{2}{*}{ Location } & \multirow[b]{2}{*}{ Sample } & \multirow[b]{2}{*}{$\begin{array}{l}\text { Sample } \\
\text { Date }\end{array}$} & \multirow[b]{2}{*}{$\begin{array}{l}\text { Depth } \\
\text { (ft BGL) }\end{array}$} & \multicolumn{4}{|c|}{ Concentration $(\mu \mathrm{g} / \mathrm{kg})$} \\
\hline & & & & $\begin{array}{c}\text { Carbon } \\
\text { Tetrachloride }\end{array}$ & Chloroform & $\begin{array}{l}\text { Methylene } \\
\text { Chloride }\end{array}$ & $\begin{array}{c}1,2- \\
\text { Dichloroethane }\end{array}$ \\
\hline TI03 & YKTI03-S-37818 & 7/9/15 & 44 & $6.3 \mathrm{~J}$ & ND & ND & ND \\
\hline TI03 & YKTI03-S-37819 & $7 / 9 / 15$ & 48 & ND & ND & ND & ND \\
\hline TI03 & YKTI03-S-37820 & 7/9/15 & 52 & ND & ND & ND & ND \\
\hline TI03 & YKTI03-S-37821 & $7 / 9 / 15$ & 55.5 & ND & ND & ND & ND \\
\hline TI03 & YKTI03-S-37822 & $7 / 9 / 15$ & 59.4 & ND & ND & ND & ND \\
\hline TI03 & YKTI03-S-37826 & 7/9/15 & 63.5 & ND & ND & ND & ND \\
\hline TI03 & YKTI03-S-37827 & $7 / 9 / 15$ & 67.5 & ND & ND & ND & ND \\
\hline TI03 & YKTI03-S-37828 & $7 / 9 / 15$ & 72 & ND & ND & ND & ND \\
\hline TIO3 & YKTI03-S-37829 & $7 / 9 / 15$ & 75 & ND & ND & ND & ND \\
\hline TI04 & YKTI04-S-37787 & $7 / 8 / 15$ & 4 & ND & ND & ND & ND \\
\hline TI04 & YKTI04-S-37788 & $7 / 8 / 15$ & 8 & ND & ND & ND & ND \\
\hline TI04 & YKTI04-S-37789 & $7 / 8 / 15$ & 12 & $7.2 \mathrm{~J}$ & ND & ND & ND \\
\hline TI04 & YKTI04-S-37790 & $7 / 8 / 15$ & 16 & 19 & $4.4 \mathrm{~J}$ & ND & ND \\
\hline TI04 & YKTI04-S-37791 & $7 / 8 / 15$ & 20 & 27 & $5.1 \mathrm{~J}$ & ND & ND \\
\hline TI04 & YKTI04-S-37792 & $7 / 8 / 15$ & 24 & 49 & $6.3 \mathrm{~J}$ & ND & ND \\
\hline TI04 & YKTI04-S-37793 & $7 / 8 / 15$ & 28 & 35 & ND & ND & ND \\
\hline TI04 & YKTI04-S-37794 & $7 / 8 / 15$ & 32 & 61 & $4.1 \mathrm{~J}$ & ND & ND \\
\hline TI04 & YKTI04-S-37795 & $7 / 8 / 15$ & 36 & 43 & $2.9 \mathrm{~J}$ & ND & ND \\
\hline TI04 & YKTI04-S-37796 & $7 / 8 / 15$ & 40 & 59 & ND & ND & ND \\
\hline TI04 & YKTI04-S-37797 & $7 / 8 / 15$ & 44 & 56 & ND & ND & ND \\
\hline TI04 & YKTI04-S-37798 & $7 / 8 / 15$ & 48 & 11 & ND & ND & ND \\
\hline TI04 & YKTI04-S-37800 & $7 / 8 / 15$ & 52 & ND & ND & ND & ND \\
\hline TI04 & YKTI04-S-37801 & $7 / 8 / 15$ & 55.8 & ND & ND & ND & ND \\
\hline TI04 & YKTI04-S-37802 & $7 / 8 / 15$ & 60 & ND & ND & ND & ND \\
\hline TI04 & YKTI04-S-37803 & $7 / 8 / 15$ & 64 & ND & ND & ND & ND \\
\hline TI04 & YKTI04-S-37805 & 7/8/15 & 68 & ND & ND & ND & ND \\
\hline TIO4 & YKTI04-S-37806 & $7 / 8 / 15$ & 72 & ND & ND & ND & ND \\
\hline TI04 & YKTI04-S-37807 & $7 / 8 / 15$ & 76 & 28 & ND & ND & ND \\
\hline TI04 & YKTI04-S-37823 & 7/8/15 & 80 & 21 & $3.0 \mathrm{~J}$ & ND & ND \\
\hline
\end{tabular}

a $\mathrm{J}$, compound identified with an estimated concentration between the instrument detection limit and the method detection limit.

b ND, compound analyzed for but not detected at a level greater than or equal to the method detection limit $(<10 \mu \mathrm{g} / \mathrm{kg})$. 
Appendix F:

Monitoring Well Registration Forms 
Mail to

Department of Natural Resources PO Box 94676

Lincoln, NE 68509-4676

Phone (402)471-2363

STATE OF NEBRASKA

DEPARTMENT OF NATURAL RESOURCES

WATER WELL REGISTRATION

Please indicate NA for items unknown

\section{FOR DEPARTMENT USE ONLY}

Date Filed Owner Code No. Registration No. G-1801

.244227 Receipt

Well ID

1. a. Well Owner's First Name Last Name

OR Company Name_USDA/CCC

b. Attention Name Steve Gilmore - 202-720-5/04

c. Street Address 1400 Fadependence Ave 40

Address 2/PO Address Stop 05/3 Roon 47/4-5

City Washingtor State $D C \quad$ Zip 20250_-05/3 Telephone

2. a. Contractor's License No_ 39508 Contractor's Name STEVEN D JOHNSON

Contractor's Email Address_SJOHNSON@CASCADEDRILLING.COM

b. Drilling Firm Name CASCADE DRILLING

Address 209 LEMIEUR ST

City LITTLE FALLS State_ MN Zip $\quad 56345$ Telephone $\quad 320 \quad 632 \quad 6552$

Drilling Firm's Email Address_STHALACKER@CASCADEDRILLING.COM

3. a. Well location $\quad N W \quad 1 / 4$ of the $S E^{1 / 4}$ of Section 30 , Township 11 North, Range 2 IWEST

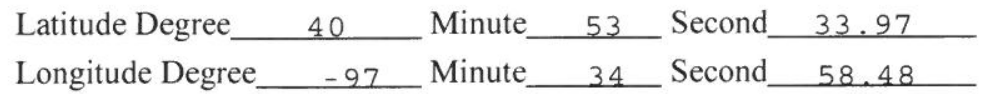

b. Natural Resources District $\quad-97$ Minute_ 34 Second $\frac{58.48}{\text { UPPER BIG BLUE }}$ feet from the (North/ Sogxth) section line and (circle one)

c. The well is: 2185 GPS Required County

d. Street address and subdivision, if applicable

e. Location of water use (give legal descriptions)

1355 (BLOCK: LOT:)

$\mathrm{N} / \mathrm{A}$

f. If for irrigation, the land to be irrigated is 0 acres.

Location of water use is required on all wells

g. Well reference letter(s), if applicable

DL- \#5 HHSS PWSID

4. Permits

Management Area Permit Number

Geothermal Permit Number

Municipal Permit Number

Well Spacing Permit Number

HHSS
Surface Water Permit Number

Industrial Permit Number

Transfer Out-Of-State Permit Number

Conduct Permit Number

Other Permit Number

NDEQ
5. Purpose of well (indicate one) Domestic Livestock Ground Heat Exchanger Monitoring Public Water Supply (without spacing)
Aquaculture

Commercial/Industrial Groundwater Source Heat Pump Observation Recovery
Pit (for irrigation) Other
Location of well for a pit is the location of the pump feet from the (Eqst/West) section line (circle one)
Dewatering (over 90 days) Irrigation
Injection

Public Water Supply (with spacing (46-638))

6. Wells in a Series.
a. Is this well a part of a series?
Yes go to part $b$ of this section
$\mathrm{x} \quad$ No go to part 7 of this application ( $\mathrm{Y} / \mathrm{N}$ required)
b. If one or more of the wells in the series is currently registered, give well registration number
c. How many wells in the series are you registering at this time? d. How many total wells in the series? 
7. Replacement and decommissioned/modified well information.

a. Is this well a replacement well? Yes $\mathrm{x}$ No go to part 8 of this application

b. Registration number of original well

c. Original well last operated $(\mathrm{m}) \_$_ $/$(d) (d) $l^{(y)}$ If not registered, date origin d. Replacement well is feet from original well.

e. Location of water use of original well Please Select One:

f.1. $\square$ Original water well decommissioned on (m)__/(d__/(y)__ OR

2. $\square$ I hereby certify that the original water well will be decommissioned within 180 days after such construction of the replacement water well. OR

3. $\square$ I hereby certify that the original water well will be modified and equipped to pump 50 gallons per minute or less within 180 days after such construction of the replacement water well. It will be used for one of the following: a. $\square$ Livestock

b. $\square$ Monitoring c. $\square$ Observation

d. $\square$ nonconsumptive or de minimus use approved by the applicable natural resources district. State use:

If $3 \mathrm{~d}$ is chosen. NRD signature is required. (Signature can be submitted on NRD Approval form to DNR prior to registration)

NRD signature

Date

OR

4. $\square$ Decommission/Modification Certification form is submitted by landowner. (Must be submitted before registering well)

8. Pump Information.

(Pump information is required if registering a pit)

a. Is pump installed at this time

Is pump installed by well owner in section 1 ?

Is pump installed by contractor in section 2 ?

Yes $\mathrm{X}$ No

Is this a free flowing well ___ Yes(no pump to be installed) $\quad x \quad$ No

Is this a free flowing well ___ Yes(no pump to be installed) $\mathrm{x}$ No

Yes $\mathrm{x}$ No

If pump installed by pump installer, please fill out license number below

b. Pump Installer's License No. Pump Installer's Name

Pump Installer's Email Address

Pump Installer's Firm Name

Pump Installer's Firm Address

City State Zip $\quad-0000$ Telephone

Pump Installer's Firm Email Address

c. Measured Pumping rate gallons per minute d. Pumping water level feet

e. Drop pipe diameter inches

f. Length of drop pipe feet

g. Pumping equipment installed $(\mathrm{m}) \_/\left(\mathrm{d} \_/ /(\mathrm{y}) \ldots\right.$

h. Pump Brand

i. This well is designed and constructed to pump less than $50 \mathrm{gpm} \quad \mathrm{x}$ Yes __ No (8H is required on ALL wells)

9. Well Construction Information.

a. Total well depth

116 feet.

b. Static water level

75 feet. (required)

c. Well Construction Began $(\mathrm{m}) \quad 0 \phi_{(6)}\left(6 / 20 A_{6}\right.$

d.. Well Construction Completed $(\mathrm{m})$ $06 / / 1+6 / 20,76$

Wells drilled prior to stays or NRD signature Date moratoriums require NRD signature

(Signature can be submitted on NRD Approval form to DNR prior to registration)

e. Bore hole diameter in inches Top Bottom 6

f. Casing and Screen Joints are Welded Glued Threaded $\mathrm{x}$ Other

g. Capacity of Well _ 4 gallons per minute (to be used to determine sustainability of aquifer)

h. Pumping water level at this capacity feet 
10. Well Construction (Casing \& Screen)- c, d, e, \& f measurements should be in inches to three decimal places (From not less than zero)

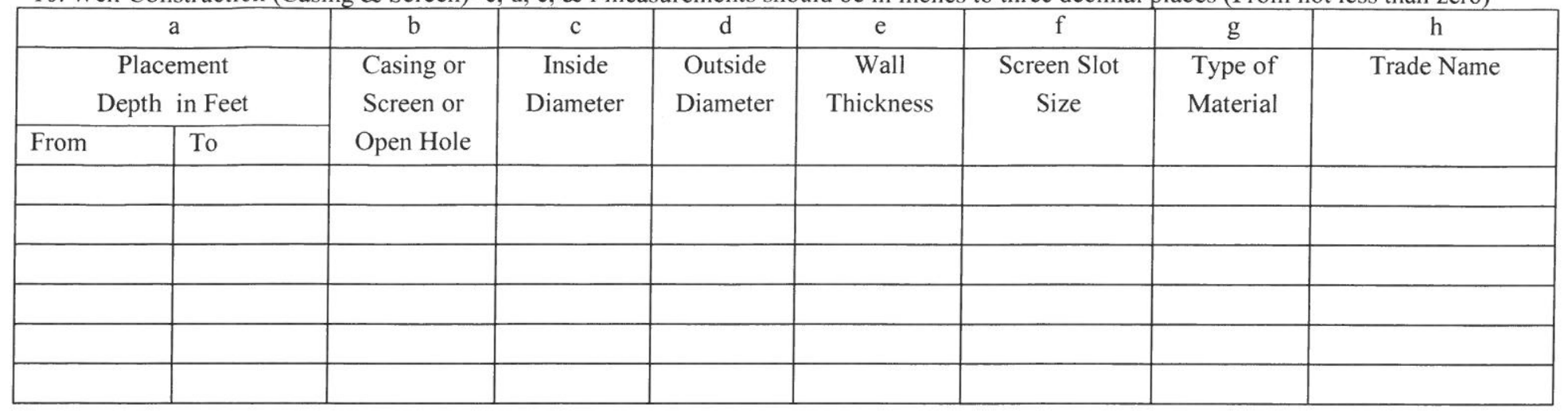

Additional information for Double cased or Nested wells:

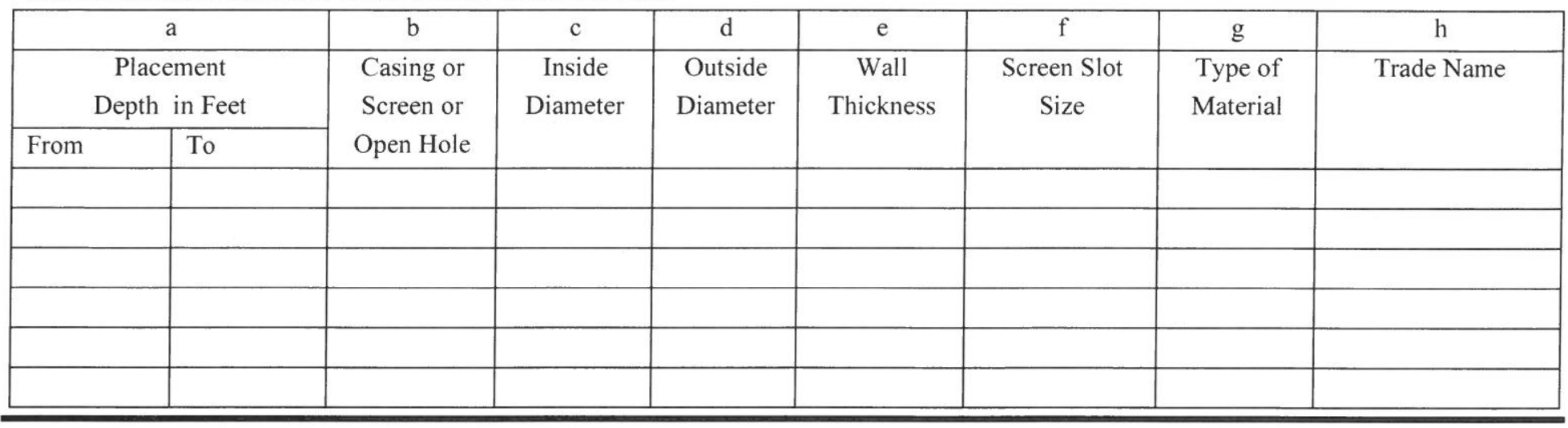

11. Grout and Gravel Pack (must start at zero)

\begin{tabular}{|c|c|c|c|c|c|}
\hline \multicolumn{2}{|c|}{$\begin{array}{c}\text { Placement Depth in } \\
\text { Feet }\end{array}$} & \multirow[t]{2}{*}{$\begin{array}{l}\text { Grout/Gravel/ } \\
\text { Open Hole }\end{array}$} & \multirow[t]{2}{*}{$\begin{array}{l}\text { Material Description } \\
\text { *See Desc for gravel }\end{array}$} & \multirow[t]{2}{*}{$\begin{array}{c}\text { Quantity gravel } \\
\text { *See Desc }\end{array}$} & \multirow[t]{2}{*}{$\begin{array}{c}\text { Volume \& Type Grout } \\
\text { *See Desc }\end{array}$} \\
\hline From & To & & & & \\
\hline & & & & & \\
\hline & & & & & \\
\hline & & & & & \\
\hline & & & & & \\
\hline & & & & & \\
\hline & & & & & \\
\hline & & & & & \\
\hline
\end{tabular}

Desc: Description of gravel pack i.e. engineered gravel pack, or gravel pit description (1/4 down), or brand name (Best Sand), natural formation, drilling cuttings, soil backfill

Quantity \#cubic yards, \#tons, \#sacks, - (for drilling cuttings and soil backfill estimate quantity) Calculation assistance available on web Volume \& Type: \#gallons of a slurry, \#barrels of a slurry, \#sacks used in the slurry, \#bags of non-slurry bentonite (chip-pellet -granular) Additional information for Double cased or Nested wells:

\begin{tabular}{|l|l|l|l|l|l|}
\hline \multicolumn{2}{|c|}{$\begin{array}{c}\text { Placement Depth in } \\
\text { Feet }\end{array}$} & $\begin{array}{c}\text { Grout/Gravel/ } \\
\text { Open Hole }\end{array}$ & $\begin{array}{c}\text { Material Description } \\
\text { *See Desc for gravel }\end{array}$ & $\begin{array}{c}\text { Quantity gravel } \\
\text { *See Desc }\end{array}$ & $\begin{array}{c}\text { Volume \& Type Grout } \\
\text { *See Desc }\end{array}$ \\
\hline From & To & & & & \\
\hline & & & & & \\
\hline & & & & & \\
\hline \\
\hline
\end{tabular}


WELL CONSTRUCTION LOG - 146999119710669

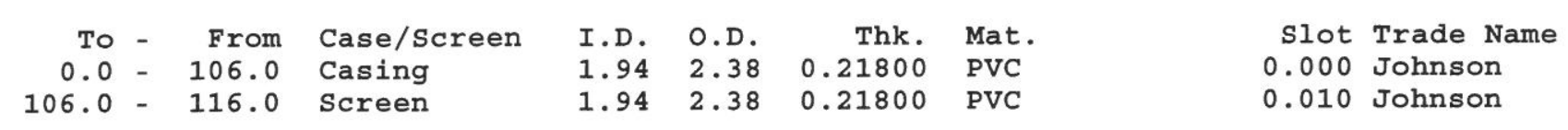

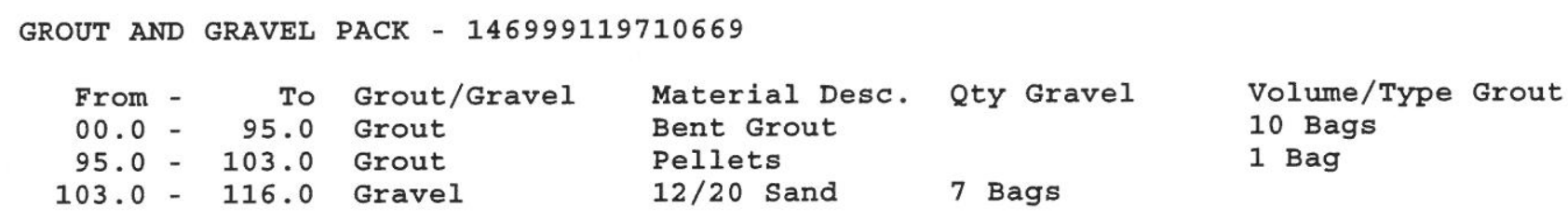


GEOLOGICAL LOGS - 146999119710669

\begin{tabular}{|c|c|c|c|c|c|c|}
\hline From & - & To & Type & Hardness & Color & Other/Drilling \\
\hline 00.0 & - & 15.0 & clay & Soft & Brown & \\
\hline 15.0 & - & 55.0 & Sand with Clay/silt & Hard & Brown & \\
\hline 55.0 & - & 95.0 & silt & Dense/Stiff & Brown & \\
\hline 95.0 & - & 116.0 & Sand med-coarse & Dense/Stiff & Brown & \\
\hline
\end{tabular}


12. Geologic Materials Logged (must start at zero) - Use only options provided below for Type, Hardness and Color

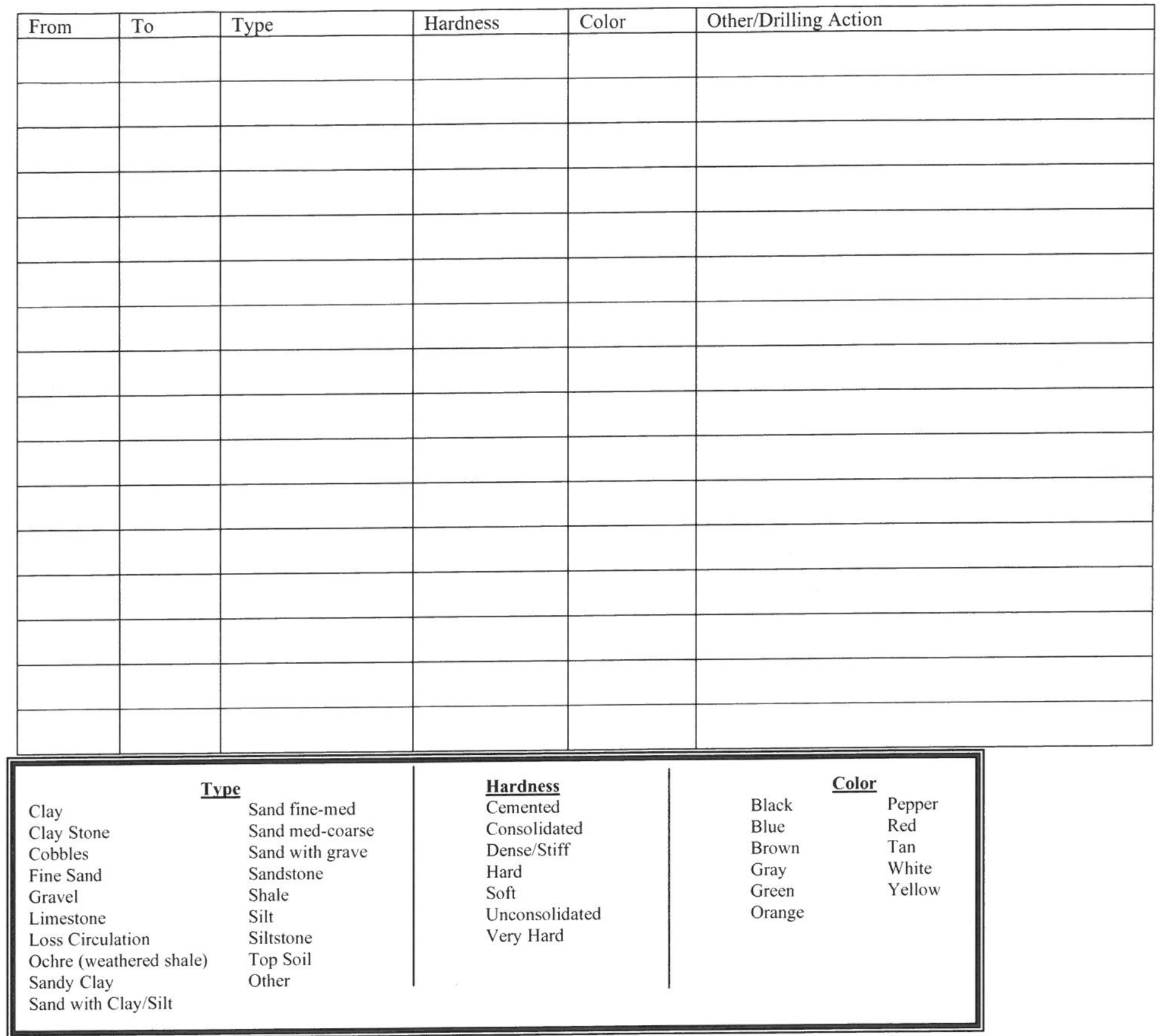

(Additional sheets may be submitted)

13. I hereby certify that the information provided on this registration is true and accurate to the best of my knowledge.
Water Well Contractor's Signature
Date
Well Owner's Signature
(if Contractor is unknown or Deceased or for pits)
(Not required if signed by Contractor)
(not required for pits)

Date

Please note this document contains four pages.

Sections 9F, 9G, 10, $11 \& 12$ are not required if registering a pit.

Owner Registering well drilled prior to 2002: Minimum Required Sections - 1, 3(a,b,c,e,f), 5, 6, 7, 8(a,f,h), 9e 
Mail to

Department of Natural Resources

PO Box 94676

Lincoln, NE 68509-4676

Phone (402)471-2363
STATE OF NEBRASKA

DEPARTMENT OF NATURAL RESOURCES

WATER WELL REGISTRATION Please indicate NA for items unknown

\section{FOR DEPARTMENT USE ONLY}

Date Filed

Owner Code No.
.244566

Well ID
Registration No. $.6-180345$

Receipt

NRD

1. a. Well Owner's First Name

Last Name

OR Company Name_nSDA/CCC

b. Attention Name Steve Giluore

c. Street Address 1400 INDEPENDENCE HVE SW

Address 2/PO Address Stop 0513 Room 4714 - 5

City WAShington State DC Zip 20250=.0513 Telephone 202. $720-5704$

2. a. Contractor's License No_ 39508 Contractor's Name_STEVEN D JOHNSON

Contractor's Email Address_SJOHNSON@CASCADEDRILLING.COM

b. Drilling Firm Name CASCADE DRILLING

Address 209 LEMIEUR ST

City LITTLE FALLS State_MN_Zip_ 56345 Telephone_ $320 \quad 632 \quad 6552$

Drilling Firm's Email Address_STHALACKER@CASCADEDRILLING.COM

3. a. Well location $\mathrm{SE}^{1 / 4}$ of the $\mathrm{NE}^{1 / 4}$ of Section 25 , Township 11 North, Range 3 IWEST

\begin{tabular}{|c|c|c|c|c|c|c|}
\hline Latitude Degree & 40 & Minute & 53 & Second & 47 & GPS \\
\hline Longitude Degree & -97 & Minute & 35 & Second & 51.9 & Required \\
\hline
\end{tabular}

b. Natural Resources District

UPPER BIG BIUE

c. The well is: 1768

feet from the (Noxh/ South) section line and (circle one)

62

(BLOCK: LOT: )

$\mathrm{N} / \mathrm{A}$

d. Street address and subdivision, if applicable

e. Location of water use (give legal descriptions)

YORK County
f. If for irrigation, the land to be irrigated is $0 \quad$ acres.
g. Well reference letter(s), if applicable
$D L-1 A$
HHSS PWSID
Location of water use is required on all wells

4. Permits

Management Area Permit Number

Geothermal Permit Number

Municipal Permit Number

Well Spacing Permit Number

HHSS

5. Purpose of well (indicate one) Ground Heat Exchanger Domestic

Public Water Supply (without spacing)
Livestock $\mathrm{x}$ Monitoring
Observation

Recovery
Surface Water Permit Number

Industrial Permit Number

Transfer Out-Of-State Permit Number

Conduct Permit Number

Other Permit Number

NDEQ

Commercial/Industrial Groundwater Source Heat Pump

Pit (for irrigation) Dewatering (over 90 days) Irrigation

Public Water Supply (with spacing (46-638)) Other (further description of use can be provided under other)

(indicate use)

6. Wells in a Series.
a. Is this well a part of a series?
Yes go to part $\mathrm{b}$ of this section
$\mathrm{x}$ No go to part 7 of this application ( $\mathrm{Y} / \mathrm{N}$ required)
b. If one or more of the wells in the series is currently registered, give well registration number
c. How many wells in the series are you registering at this time?
d. How many total wells in the series? 
7. Replacement and decommissioned/modified well information.

a. Is this well a replacement well? ___ Yes $\mathrm{x}$ No go to part 8 of this application

b. Registration number of original well

c. Original well last operated $(\mathrm{m}) \_$_ (d) (d) If not registered, date original well was constructed ${ }_{(\mathrm{m}) \_} /(\mathrm{d}$

e. Location of water use of original well Please Select One:

f.1. $\square$ Original water well decommissioned on $(\mathrm{m}) \_/\left(\mathrm{d} \_/ / \mathrm{y}\right) \_$OR

2. $\square$ I hereby certify that the original water well will be decommissioned within 180 days after such construction of the replacement water well. OR

3. $\square$ I hereby certify that the original water well will be modified and equipped to pump 50 gallons per minute or less within 180 days after such construction of the replacement water well. It will be used for one of the following: a. $\square$ Livestock b. $\square$ Monitoring c. $\square$ Observation

d. $\square$ nonconsumptive or de minimus use approved by the applicable natural resources district. State use:

If $3 \mathrm{~d}$ is chosen. NRD signature is required. (Signature can be submitted on NRD Approval form to DNR prior to registration)

NRD signature

Date

OR

4. $\square$ Decommission/Modification Certification form is submitted by landowner. (Must be submitted before registering well)

8. Pump Information.

(Pump information is required if registering a pit)

a. Is pump installed at this time ___ Yes $\quad \mathrm{x}$ No
Is pump installed by well owner in section 1 ?
Is pump installed by contractor in section 2 ?
Yes $\mathrm{X}$ No
Yes $\mathrm{X}$ No

Is this a free flowing well ___ Yes(no pump to be installed) $\mathrm{x}$ No

If pump installed by pump installer, please fill out license number below

b. Pump Installer's License No. Pump Installer's Name

Pump Installer's Email Address

Pump Installer's Firm Name

Pump Installer's Firm Address

City State Zip $-0000$ Telephone

Pump Installer's Firm Email Address

c. Measured Pumping rate gallons per minute d. Pumping water level feet

e. Drop pipe diameter inches f. Length of drop pipe feet

g. Pumping equipment installed (in)___ (d___ (y)

h. Pump Brand

i. This well is designed and constructed to pump less than $50 \mathrm{gpm} \quad \mathrm{X}$ Yes No ( $8 \mathrm{H}$ is required on $\mathrm{ALL}$ wells)

9. Well Construction Information.

a. Total well depth 85 feet.

b. Static water level 75 feet. (required)

c. Well Construction Began (m) $07(6) 06 / 2016$ d.. Well Construction Completed $(\mathrm{m})$ $07 / 406 / 20,16$

Wells drilled prior to stays or NRD signature Date moratoriums require NRD signature (Signature can be submitted on NRD Approval form to DNR prior to registration)

e. Bore hole diameter in inches Top $\quad 6 \quad$ Bottom

f. Casing and Screen Joints are Welded Glued Threaded $\mathrm{X} \quad$ Other

g. Capacity of Well _ـ 4 gallons per minute (to be used to determine sustainability of aquifer)

h. Pumping water level at this capacity feet 
10. Well Construction (Casing \& Screen)- c, d, e, \& f measurements should be in inches to three decimal places (From not less than zero)

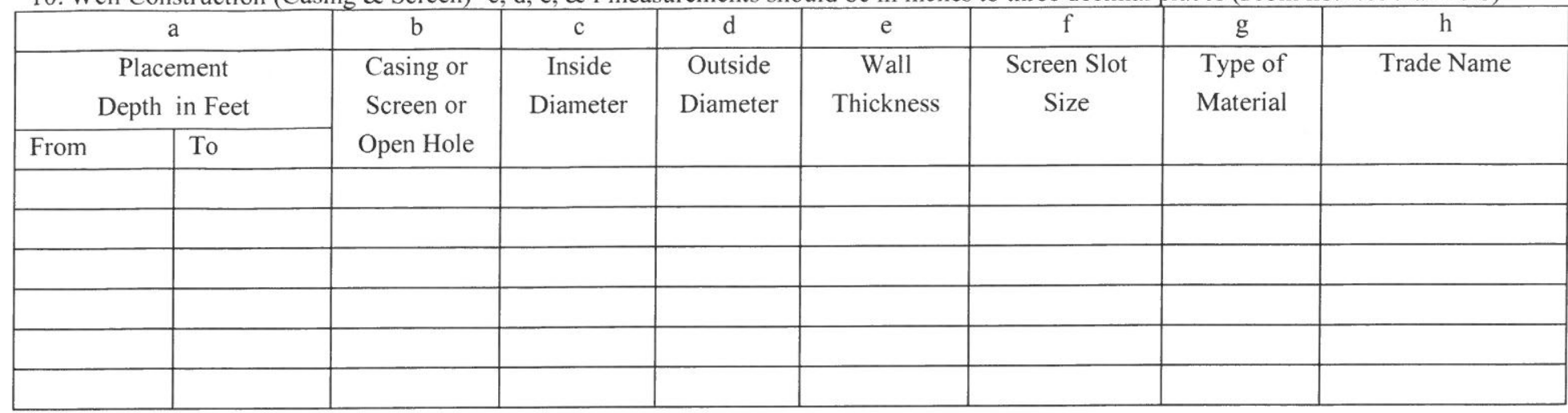

Additional information for Double cased or Nested wells:

\begin{tabular}{|c|c|c|c|c|c|c|c|c|}
\hline \multicolumn{2}{|c|}{$\mathrm{a}$} & $\mathrm{b}$ & c & d & $\mathrm{e}$ & f & g & h \\
\hline \multicolumn{2}{|c|}{$\begin{array}{c}\text { Placement } \\
\text { Depth in Feet }\end{array}$} & $\begin{array}{l}\text { Casing or } \\
\text { Screen or }\end{array}$ & $\begin{array}{c}\text { Inside } \\
\text { Diameter }\end{array}$ & $\begin{array}{l}\text { Outside } \\
\text { Diameter }\end{array}$ & $\begin{array}{c}\text { Wall } \\
\text { Thickness }\end{array}$ & $\begin{array}{c}\text { Screen Slot } \\
\text { Size }\end{array}$ & $\begin{array}{l}\text { Type of } \\
\text { Material }\end{array}$ & Trade Name \\
\hline From & To & Open Hole & & & & & & \\
\hline & & & & & & & & \\
\hline & & & & & & & & \\
\hline & & & & & & & & \\
\hline & & & & & & & & \\
\hline & & & & & & & & \\
\hline & & & & & & & & \\
\hline
\end{tabular}

11. Grout and Gravel Pack (must start at zero)

\begin{tabular}{|c|c|c|c|c|c|}
\hline \multicolumn{2}{|c|}{$\begin{array}{c}\text { Placement Depth in } \\
\text { Feet }\end{array}$} & \multirow[t]{2}{*}{$\begin{array}{l}\text { Grout/Gravel/ } \\
\text { Open Hole }\end{array}$} & \multirow[t]{2}{*}{$\begin{array}{l}\text { Material Description } \\
\text { *See Desc for gravel }\end{array}$} & \multirow[t]{2}{*}{$\begin{array}{l}\text { Quantity gravel } \\
\text { *See Desc }\end{array}$} & \multirow[t]{2}{*}{$\begin{array}{c}\text { Volume \& Type Grout } \\
\text { *See Desc }\end{array}$} \\
\hline From & To & & & & \\
\hline & & & & & \\
\hline & & & & & \\
\hline & & & & & \\
\hline & & & & & \\
\hline & & & & & \\
\hline & & & & & \\
\hline & & & & & \\
\hline
\end{tabular}

Desc: Description of gravel pack i.e. engineered gravel pack, or gravel pit description ( $1 / 4$ down), or brand name (Best Sand), natural formation, drilling cuttings, soil backfill

Quantity \#cubic yards, \#tons, \#sacks, - (for drilling cuttings and soil backfill estimate quantity) Calculation assistance available on web

Volume \& Type: \#gallons of a slurry, \#barrels of a slurry, \#sacks used in the slurry, \#bags of non-slurry bentonite (chip-pellet -granular)

Additional information for Double cased or Nested wells:

\begin{tabular}{|c|c|c|c|c|c|}
\hline \multicolumn{2}{|c|}{$\begin{array}{c}\text { Placement Depth in } \\
\text { Feet }\end{array}$} & \multirow[t]{2}{*}{$\begin{array}{c}\text { Grout/Gravel/ } \\
\text { Open Hole }\end{array}$} & \multirow[t]{2}{*}{$\begin{array}{l}\text { Material Description } \\
\text { *See Desc for gravel }\end{array}$} & \multirow[t]{2}{*}{$\begin{array}{l}\text { Quantity gravel } \\
\text { *See Desc }\end{array}$} & \multirow[t]{2}{*}{$\begin{array}{c}\text { Volume \& Type Grout } \\
\text { *See Desc }\end{array}$} \\
\hline From & To & & & & \\
\hline & & & & & \\
\hline & & & & & \\
\hline & & & & & \\
\hline & & & & & \\
\hline & & & & & \\
\hline & & & & & \\
\hline & & & & & \\
\hline
\end{tabular}


WELL CONSTRUCTION LOG - 147213725830877

\begin{tabular}{|c|c|c|c|c|c|c|c|}
\hline $\begin{array}{l}\text { To } \\
0.0\end{array}$ & $\begin{array}{l}\text { From } \\
75.0\end{array}$ & $\begin{array}{l}\text { Case/Screen } \\
\text { Casing }\end{array}$ & $\begin{array}{l}\text { I.D. } \\
2.07\end{array}$ & $\begin{array}{l}0 . D . \\
2.38\end{array}$ & $\begin{array}{l}\text { Thk. } \\
0.15400\end{array}$ & $\begin{array}{l}\text { Mat. } \\
\text { PVC }\end{array}$ & $\begin{array}{l}\text { Slot Trade Name } \\
0.000 \text { Johnson }\end{array}$ \\
\hline 75.0 & 85.0 & Screen & 2.07 & 2.38 & 0.15400 & PVC & 0.010 Johnson \\
\hline
\end{tabular}

GROUT AND GRAVEL PACK - 147213725830877

\begin{tabular}{|c|c|c|c|c|c|c|}
\hline From & - & To & Grout/Gravel & Material Desc. & Qty Gravel & Volume/Type Grout \\
\hline 00.0 & - & 67.0 & Grout & Bent Grout & & 5 Bags \\
\hline 67.0 & - & 73.0 & Grout & Pellets & & 1/2 Pail \\
\hline 73.0 & - & 85.0 & Gravel & \#40 & 5 Bags & \\
\hline
\end{tabular}


GEOLOGICAL LOGS - 147213725830877

$\begin{array}{lrlll}\text { From - } & \text { To Type } & \text { Hardness } & \text { Color } & \text { Other/Drilling Action } \\ 00.0- & 52.0 & \text { Clay } & \text { Soft } & \text { Brown } \\ 52.0- & 75.0 & \text { Sand fine-med } & \text { Dense/Stiff } & \text { Brown } \\ 75.0- & 85.0 & \text { Sand med-coarse } & \text { Dense/Stiff } & \text { Brown }\end{array}$


12. Geologic Materials Logged (must start at zero) - Use only options provided below for Type, Hardness and Color

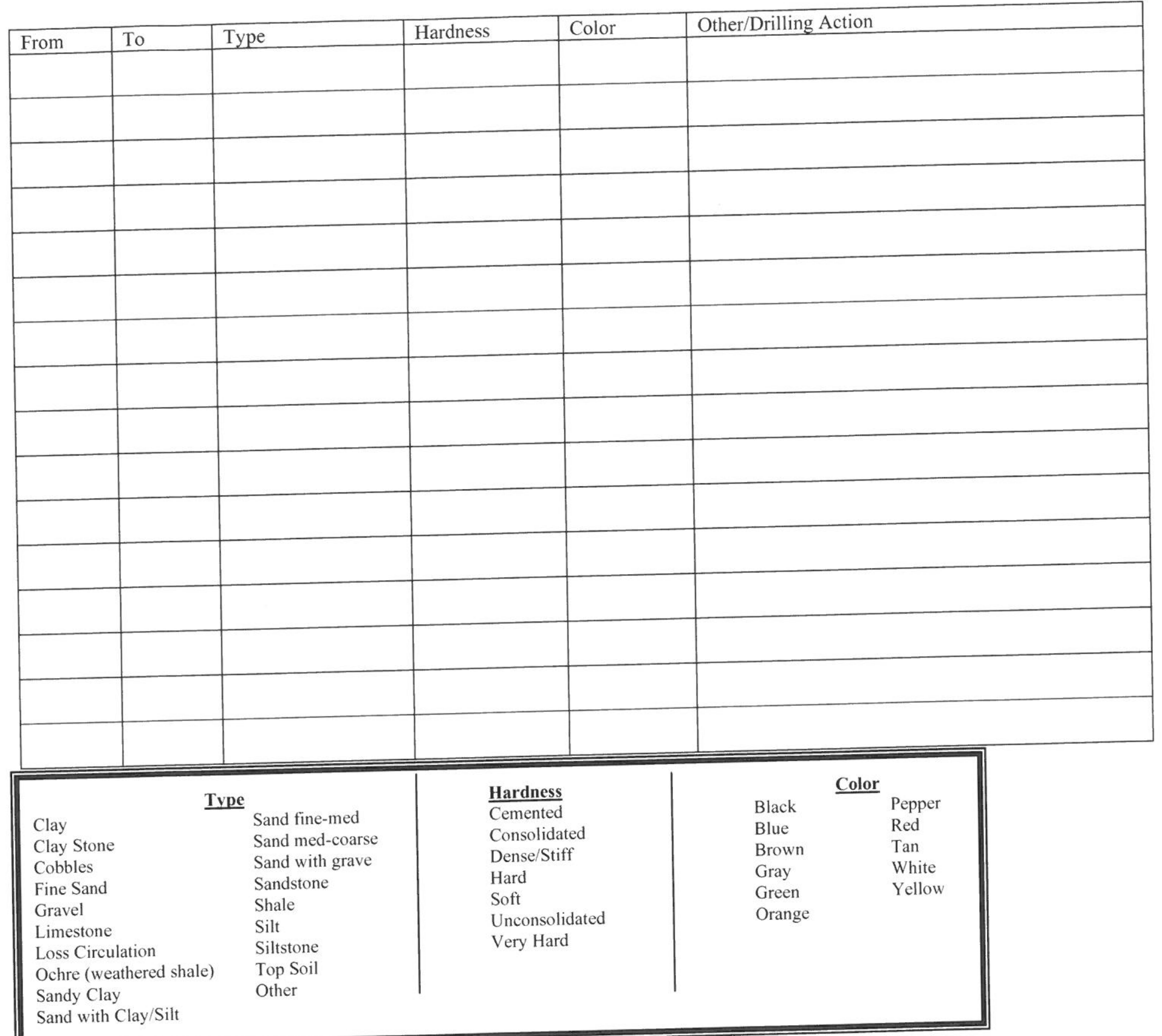

(Additional sheets may be submitted)

13. I hereby certify that the information provided on this registration is true and accurate to the best of my knowledge.
Water Well Contractor's Signature
Date
Well Owner's Signature
(not required for pits)
(if Contractor is unknown or Deceased or for pits)
(Not required if signed by Contractor)

Date

Please note this document contains four pages.

Sections 9F, 9G, 10, $11 \& 12$ are not required if registering a pit.

Owner Registering well drilled prior to 2002: Minimum Required Sections - 1, 3(a,b,c,e,f), 5, 6, 7, 8(a,f,h), 9e 
Mail to

Department of Natural Resources

PO Box 94676

Lincoln, NE 68509-4676

Phone (402)471-2363

STATE OF NEBRASKA

DEPARTMENT OF NATURAL RESOURCES

WATER WELL REGISTRATION

Please indicate NA for items unknown

\section{FOR DEPARTMENT USE ONLY}

Date Filed

Owner Code No.

Registration No.G - 180332

$$
\frac{-244571}{\text { Well ID }}
$$

Receipt

1. a. Well Owner's First Name

Last Name

OR Company Name USDA/CCC

b. Attention Name Steve Gilmore

c. Street Address 1400 IUdependence AUE SW Address 2/PO Address Stop 0513 Room 4714-5 City Washingtow State DC Zip 20250-05\%3 Telephone 202-720-5704

2. a. Contractor's License No_ 39508 Contractor's Name_STEVEN D JOHNSON

Contractor's Email Address_SJOHNSON@CASCADEDRILLING.COM

b. Drilling Firm Name CASCADE DRILLING

Address 209 LEMIEUR ST

City LITTLE FALLS State_MN_Zip_ 56345 Telephone $\quad 320 \quad 632 \quad 6552$

Drilling Firm's Email Address_STHALACKER@CASCADEDRILLING.COM

3. a. Well location SE $1 / 4$ of the $\mathrm{NE}^{1 / 4}$ of Section 25 , Township 11 North, Range 3 IWEST

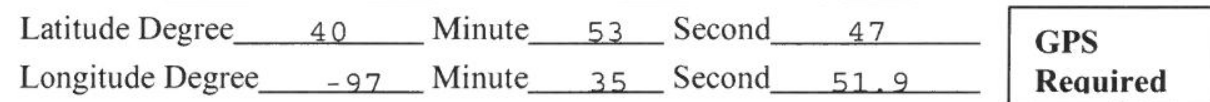

YORK County

b. Natural Resources District UPPER BIG BIUE

c. The well is: 1768 feet from the (Nokh/ South) section line and (circle one)

62 (BLOCK: LOT:)

d. Street address and subdivision, if applicable

e. Location of water use (give legal descriptions)

$\mathrm{N} / \mathrm{A}$

f. If for irrigation, the land to be irrigated is 0 acres.

$D L-1 B \& 1 C$

g. Well reference letter(s), if applicable HHSS PWSID
Location of well for a pit is the location of the pump
4. Permits

Management Area Permit Number

Geothermal Permit Number

Municipal Permit Number

Well Spacing Permit Number HHSS

5. Purpose of well (indicate one) ___ Aquaculture Domestic Ground Heat Exchanger Observation Livestock $\mathrm{x}$ Monitoring Public Water Supply (without spacing) Recovery
Surface Water Permit Number

Industrial Permit Number

Transfer Out-Of-State Permit Number

Conduct Permit Number

Other Permit Number

NDEQ

Commercial/Industrial Groundwater Source Heat Pump Pit (for irrigation) Other
Dewatering (over 90 days) Irrigation Injection

6. Wells in a Series.

a. Is this well a part of a series?___ Yes go to part b of this section

$\mathrm{x}$ No go to part 7 of this application ( $\mathrm{Y} / \mathrm{N}$ required)

b. If one or more of the wells in the series is currently registered, give well registration number

c. How many wells in the series are you registering at this time? d. How many total wells in the series? 
7. Replacement and decommissioned/modified well information.
a. Is this well a replacement well?
Yes $\mathrm{x}$ No go to part 8 of this application

b. Registration number of original well

c. Original well last operated $(\mathrm{m})$ (d) $I_{(y)}$

If not registered, date origin

e. Location of water use of original well Please Select One:

f.1. $\square$ Original water well decommissioned on $(\mathrm{m}) \_/\left(\mathrm{d} \_-(\mathrm{y}) \_ \text {OR }\right.$

2. $\square$ I hereby certify that the original water well will be decommissioned within 180 days after such construction of the replacement water well. OR

3. $\square$ I hereby certify that the original water well will be modified and equipped to pump 50 gallons per minute or less within 180 days after such construction of the replacement water well. It will be used for one of the following: a. $\square$ Livestock

b. $\square$ Monitoring c. $\square$ Observation

d. $\square$ nonconsumptive or de minimus use approved by the applicable natural resources district. State use:

If $3 \mathrm{~d}$ is chosen. NRD signature is required. (Signature can be submitted on NRD Approval form to DNR prior to registration)
NRD signature
Date
OR

4. $\square$ Decommission/Modification Certification form is submitted by landowner. (Must be submitted before registering well)

8. Pump Information.

(Pump information is required if registering a pit)

a. Is pump installed at this time

Yes $\mathrm{x}$ No

Is pump installed by well owner in section 1 ?

Is pump installed by contractor in section 2 ?

Yes $\mathrm{X}$ No

Yes $\mathrm{x}$ No

Is this a free flowing well ___ Yes(no pump to be installed) $\mathrm{x}$ No

If pump installed by pump installer, please fill out license number below

b. Pump Installer's License No.

Pump Installer's Name

Pump Installer's Email Address

Pump Installer's Firm Name

Pump Installer's Firm Address

City State Zip $\quad-0000$ Telephone

Pump Installer's Firm Email Address

c. Measured Pumping rate gallons per minute

d. Pumping water level feet

e. Drop pipe diameter inches

f. Length of drop pipe feet

g. Pumping equipment installed $(\mathrm{m}) / /(\mathrm{d}$

h. Pump Brand

i. This well is designed and constructed to pump less than $50 \mathrm{gpm} \quad \mathbf{x}$ Yes

No (8H is required on ALL wells)

9. Well Construction Information.

a. Total well depth 118 feet.

b. Static water level 75 feet. (required)

c. Well Construction Began $(\mathrm{m}) \quad 07(6) 11 / 204(6)$

d.. Well Construction Completed ${ }_{(\mathrm{m})}$ $07 / / 1+1 / 20,76$

Wells drilled prior to stays or

NRD signature Date moratoriums require NRD signature

(Signature can be submitted on NRD Approval form to DNR prior to registration)

e. Bore hole diameter in inches Top $\quad 10 \quad$ Bottom $\quad 6$

f. Casing and Screen Joints are Welded Glued Threaded $\mathrm{x}$ Other

g. Capacity of Well _ـ 4 gallons per minute (to be used to determine sustainability of aquifer)

h. Pumping water level at this capacity feet 
10. Well Construction (Casing \& Screen)- c, d, e, \& f measurements should be in inches to three decimal places (From not less than zero)

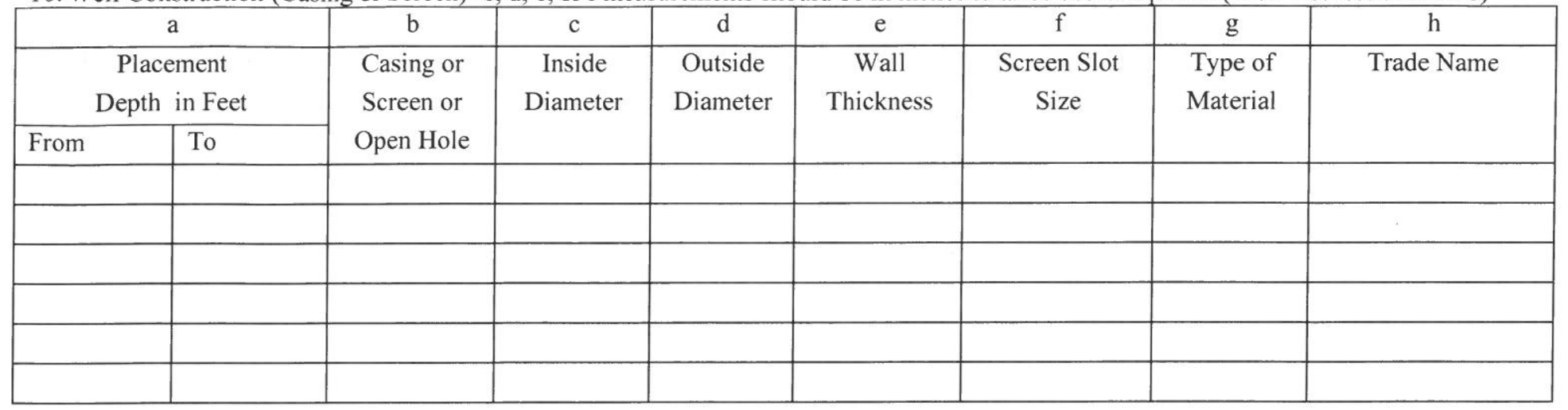

Additional information for Double cased or Nested wells:

\begin{tabular}{|c|c|c|c|c|c|c|c|c|}
\hline \multicolumn{2}{|c|}{$\mathrm{a}$} & $\mathrm{b}$ & $\mathrm{c}$ & $\mathrm{d}$ & $\mathrm{e}$ & $\mathrm{f}$ & $\mathrm{g}$ & $\mathrm{h}$ \\
\hline \multicolumn{2}{|c|}{$\begin{array}{c}\text { Placement } \\
\text { Depth in Feet }\end{array}$} & \multirow{2}{*}{$\begin{array}{l}\text { Casing or } \\
\text { Screen or } \\
\text { Open Hole }\end{array}$} & \multirow[t]{2}{*}{$\begin{array}{c}\text { Inside } \\
\text { Diameter }\end{array}$} & \multirow[t]{2}{*}{$\begin{array}{c}\text { Outside } \\
\text { Diameter }\end{array}$} & \multirow[t]{2}{*}{$\begin{array}{c}\text { Wall } \\
\text { Thickness }\end{array}$} & \multirow[t]{2}{*}{$\begin{array}{c}\text { Screen Slot } \\
\text { Size }\end{array}$} & \multirow[t]{2}{*}{$\begin{array}{l}\text { Type of } \\
\text { Material }\end{array}$} & \multirow[t]{2}{*}{ Trade Name } \\
\hline From & To & & & & & & & \\
\hline & & & & & & & & \\
\hline & & & & & & & & \\
\hline & & & & & & & & \\
\hline & & & & & & & & \\
\hline & & & & & & & & \\
\hline & & & & & & & & \\
\hline
\end{tabular}

11. Grout and Gravel Pack (must start at zero)

\begin{tabular}{|l|l|l|l|l|l|}
\hline \multicolumn{2}{|c|}{$\begin{array}{c}\text { Placement Depth in } \\
\text { Feet }\end{array}$} & $\begin{array}{c}\text { Grout/Gravel/ } \\
\text { Open Hole }\end{array}$ & $\begin{array}{c}\text { Material Description } \\
\text { *See Desc for gravel }\end{array}$ & $\begin{array}{c}\text { Quantity gravel } \\
\text { *See Desc }\end{array}$ & $\begin{array}{c}\text { Volume \& Type Grout } \\
\text { *See Desc }\end{array}$ \\
\hline From & To & & & & \\
\hline & & & & & \\
\hline & & & & & \\
\hline & & & & & \\
\hline
\end{tabular}

Desc: Description of gravel pack i.e. engineered gravel pack, or gravel pit description ( $1 / 4$ down), or brand name (Best Sand), natural formation, drilling cuttings, soil backfill

Quantity \#cubic yards, \#tons, \#sacks, - (for drilling cuttings and soil backfill estimate quantity) Calculation assistance available on web

Volume \& Type: \#gallons of a slurry, \#barrels of a slurry, \#sacks used in the slurry, \#bags of non-slurry bentonite (chip-pellet -granular)

Additional information for Double cased or Nested wells:

\begin{tabular}{|c|c|c|c|c|c|}
\hline \multicolumn{2}{|c|}{$\begin{array}{c}\text { Placement Depth in } \\
\text { Feet }\end{array}$} & \multirow[t]{2}{*}{$\begin{array}{c}\text { Grout/Gravel/ } \\
\text { Open Hole }\end{array}$} & \multirow[t]{2}{*}{$\begin{array}{l}\text { Material Description } \\
\text { *See Desc for gravel }\end{array}$} & \multirow[t]{2}{*}{$\begin{array}{l}\text { Quantity gravel } \\
\text { *See Desc }\end{array}$} & \multirow[t]{2}{*}{$\begin{array}{c}\text { Volume \& Type Grout } \\
\text { *See Desc }\end{array}$} \\
\hline From & To & & & & \\
\hline & & & & & \\
\hline & & & & & \\
\hline & & & & & \\
\hline & & & & & \\
\hline & & & & & \\
\hline & & & & & \\
\hline & & & & & \\
\hline
\end{tabular}


WELL CONSTRUCTION LOG - 14721388711780

\begin{tabular}{|c|c|c|c|c|c|c|c|c|c|}
\hline $\begin{array}{r}\text { To } \\
0.0\end{array}$ & - & $\begin{array}{l}\text { From } \\
88.0\end{array}$ & $\begin{array}{l}\text { Case/Screen } \\
\text { Casing }\end{array}$ & $\begin{array}{l}\text { I.D. } \\
1.94\end{array}$ & $\begin{array}{l}\text { O.D. } \\
2.38\end{array}$ & $\begin{array}{l}\text { Thk. } \\
0.21800\end{array}$ & $\begin{array}{l}\text { Mat. } \\
\text { PVC }\end{array}$ & $\begin{array}{l}\text { Slot } \\
0.000\end{array}$ & $\begin{array}{l}\text { Trade Name } \\
\text { Johnson }\end{array}$ \\
\hline 88.0 & - & 98.0 & Screen & 1.94 & 2.38 & 0.21800 & PVC & 0.010 & Johnson \\
\hline 0.0 & - & 108.0 & Casing & 2.07 & 2.38 & 0.15400 & PVC & 0.000 & Johnson \\
\hline 108.0 & - & 118.0 & Screen & 2.07 & 2.38 & 0.15400 & PVC & 0.010 & Johnson \\
\hline
\end{tabular}

GROUT AND GRAVEL PACK - 14721388711780

\begin{tabular}{|c|c|c|c|c|c|}
\hline From & To & Grout/Gravel & Material Desc. & Qty Gravel & Volume/Type Grout \\
\hline 00.0 & 80.0 & Grout & Bent Grout & & 6 Bags \\
\hline 80.0 & 86.0 & Grout & Pellets & & 1/2 Pail \\
\hline 86.0 & 98.0 & Gravel & $\# 40$ & 6 Bags & \\
\hline 98.0 & 106.0 & Grout & Pellets & & 1/2 Pail \\
\hline 06.0 & 118.0 & Gravel & $\# 40$ & 6Bags & \\
\hline
\end{tabular}


GEOLOGICAL LOGS - 14721388711780

\begin{tabular}{|c|c|c|c|c|c|c|}
\hline From & - & To & Type & Hardness & Color & Other/Drilling \\
\hline 00.0 & - & 52.0 & clay & Soft & Brown & \\
\hline 52.0 & - & 75.0 & Sand fine-med & Dense/Stiff & Brown & \\
\hline 75.0 & - & 80.0 & Sand med-coarse & Dense/stiff & Brown & \\
\hline 80.0 & - & 89.0 & Silt & Dense/stiff & Brown & \\
\hline 89.0 & - & 100.0 & Sand fine-med & Dense/stiff & Brown & \\
\hline 100.0 & - & 107.0 & Shale & Dense/Stiff & Brown & \\
\hline 107.0 & - & 118.0 & Sand med-coarse & Dense/Stiff & Brown & \\
\hline
\end{tabular}


12. Geologic Materials Logged (must start at zero) - Use only options provided below for Type, Hardness and Color

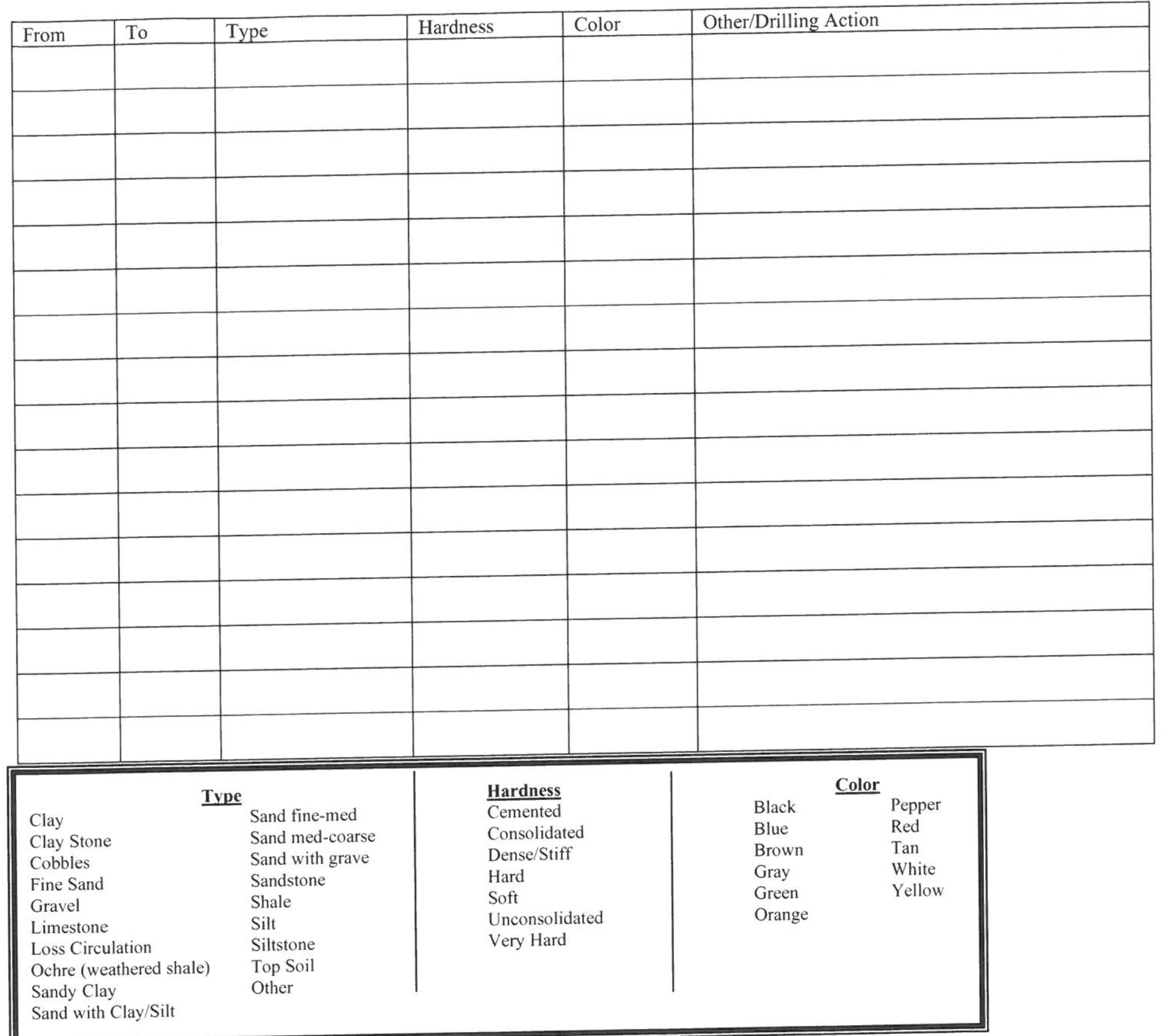

(Additional sheets may be submitted)

13. I hereby certify that the information provided on this registration is true and accurate to the best of my knowledge.
Water Well Contractor's Signature
Date
Well Owner's Signature (not required for pits)
(if Contractor is unknown or Deceased or for pits)
(Not required if signed by Contractor)

Date

Please note this document contains four pages.

Sections 9F, 9G, 10, $11 \& 12$ are not required if registering a pit.

Owner Registering well drilled prior to 2002: Minimum Required Sections - 1, 3(a,b,c,e,f), 5, 6, 7, 8(a,f,h), 9e 
Mail to

Department of Natural Resources

PO Box 94676

Lincoln, NE 68509-4676

Phone (402)471-2363

STATE OF NEBRASKA

\section{DEPARTMENT OF NATURAL RESOURCES}

WATER WELL REGISTRATION Please indicate NA for items unknown

\section{FOR DEPARTMENT USE ONLY}

Date Filed

Owner Code No.

Registration No. $6-180329$

$$
\frac{-244568}{\text { Well ID }}
$$

Receipt

1. a. Well Owner's First Name

Last Name

OR Company Name USDA/ CCC

b. Attention Name Steve GIIMORE

c. Street Address 1400 INDEPENDENCE AVE SW Address 2/PO Address Stop 0513 Room 4714-5

City Washington

State DC

Zip $20250-0513$

Telephone

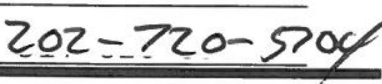

2. a. Contractor's License No_ 39508 Contractor's Name STEVEN D JOHNSON

Contractor's Email Address_SJOHNSON@CASCADEDRILLING.COM

b. Drilling Firm Name CASCADE DRILLING

Address 209 LEMIEUR ST

City LITTLE FALLS

State $\quad$ MN

Zip

56345

Telephone $\quad 320 \quad 632 \quad 6552$

Drilling Firm's Email Address_STHALACKER@CASCADEDRILLING.COM

3. a. Well location $S_{\text {SE }} 1 / 4$ of the $\mathrm{SW}^{1 / 4}$ of Section 30 , Township 11 North, Range 2 IWEST Latitude Degree__ 40 Minute_ 53 Second_ 24.6 Longitude Degree

b. Natural Resources District Minute_ 35 Second_ 19.4 UPPER BIG BLUE

c. The well is: 1230 feet from the (North/ Soxth) section line and

d. Street address and subdivision, if applicable (circle one)

e. Location of water use (give legal descriptions)

GPS Required (BI.OCK: IOT:)

f. If for irrigation, the land to be irrigated is

g. Well reference letter(s), if applicable $0 \quad$ acres $\mathrm{DL}-2 \mathrm{C} 1$ HHSS PWSID
4. Permits

Management Area Permit Number

Geothermal Permit Number

Municipal Permit Number

Well Spacing Permit Number

HHSS

5. Purpose of well (indicate one) ___ Aquaculture Domestic Ground Heat Exchanger Livestock $\mathrm{x}$ Monitoring Public Water Supply (without spacing)
Observation Recovery
Surface Water Permit Number

Industrial Permit Number

Transfer Out-Of-State Permit Number

Conduct Permit Number

Other Permit Number NDEQ
Commercial/Industrial Groundwater Source Heat Pump Other
Pit (for irrigation) $\mathrm{N} / \mathrm{A}$

Location of water use is required on all wells

Location of well for a pit is the location of the pump

feet from the (East/West) section line (circle one)

(further description of use can be provided under other)

Dewatering (over 90 days) Irrigation

Public Water Supply (with spacing (46-638))

6. Wells in a Series.

a. Is this well a part of a series? ___ Yes go to part $\mathrm{b}$ of this section _ $\quad \mathrm{x} \quad$ No go to part 7 of this application (Y/N required)

b. If one or more of the wells in the series is currently registered, give well registration number

c. How many wells in the series are you registering at this time? d. How many total wells in the series? 
7. Replacement and decommissioned/modified well information.

a. Is this well a replacement well?___ Yes $\mathrm{x}$ No go to part 8 of this application

b. Registration number of original well

c. Original well last operated $(\mathrm{m}) \_$_ $/$(d) (d)__ $/(y)$ $I_{(y)}$

If not registered, date or

e. Location of water use of original well Please Select One:

f.1. $\square$ Original water well decommissioned on ${ }_{(\mathrm{m}) \_/\left(\mathrm{d} \_/(\mathrm{y}) \_ \text {OR }\right.}$

2. $\square$ I hereby certify that the original water well will be decommissioned within 180 days after such construction of the replacement water well. OR

3. $\square$ I hereby certify that the original water well will be modified and equipped to pump 50 gallons per minute or less within 180 days after such construction of the replacement water well. It will be used for one of the following: a. $\square$ Livestock b. $\square$ Monitoring c. $\square$ Observation

d. $\square$ nonconsumptive or de minimus use approved by the applicable natural resources district. State use:

If $3 \mathrm{~d}$ is chosen. NRD signature is required. (Signature can be submitted on NRD Approval form to DNR prior to registration)

NRD signature

Date

OR

4. $\square$ Decommission/Modification Certification form is submitted by landowner. (Must be submitted before registering well)

8. Pump Information.

(Pump information is required if registering a pit)

a. Is pump installed at this time _Yes $\quad \mathrm{x}$ No

Is pump installed by well owner in section 1? ___ Yes $\quad \mathrm{x}$ No

Is pump installed by contractor in section 2? Y__ Yes $\mathrm{x} \quad$ No

Is this a free flowing well ___ Yes(no pump to be installed) $\mathrm{x}$ No

If pump installed by pump installer, please fill out license number below

b. Pump Installer's License No. Pump Installer's Name

Pump Installer's Email Address

Pump Installer's Firm Name

Pump Installer's Firm Address

City State Zip $\quad-0000$ Telephone

Pump Installer's Firm Email Address

c. Measured Pumping rate gallons per minute

d. Pumping water level feet

e. Drop pipe diameter inches

f. Length of drop pipe feet

g. Pumping equipment installed $(\mathrm{m}) \_/\left(\mathrm{d} \_/(\mathrm{y}) \ldots\right.$

h. Pump Brand

i. This well is designed and constructed to pump less than $50 \mathrm{gpm} \quad \mathrm{x}$ Yes

No ( $8 \mathrm{H}$ is required on ALL wells)

9. Well Construction Information.

a. Total well depth 122 feet.

b. Static water level feet. (required)

c. Well Construction Began $(\mathrm{m}) \_\quad 07(6) 09 / 20469$ d.. Well Construction Completed $(\mathrm{m})$ $07 / 409 / 20,16$

Wells drilled prior to stays or NRD signature Date moratoriums require NRD signature (Signature can be submitted on NRD Approval form to DNR prior to registration)

e. Bore hole diameter in inches Top 6 Bottom 6

f. Casing and Screen Joints are Welded Glued Threaded $\mathrm{x}$ Other

g. Capacity of Well 4 gallons per minute (to be used to determine sustainability of aquifer)

h. Pumping water level at this capacity feet 
10. Well Construction (Casing \& Screen)- c, d, e, \& f measurements should be in inches to three decimal places (From not less than zero)

\begin{tabular}{|c|c|c|c|c|c|c|c|c|}
\hline \multicolumn{2}{|c|}{ a } & $\mathrm{b}$ & $\mathrm{c}$ & $\mathrm{d}$ & $\mathrm{e}$ & $\mathrm{f}$ & $\mathrm{g}$ & $\mathrm{h}$ \\
\hline \multicolumn{2}{|c|}{$\begin{array}{c}\text { Placement } \\
\text { Depth in Feet }\end{array}$} & $\begin{array}{c}\text { Casing or } \\
\text { Screen or } \\
\text { Open Hole }\end{array}$ & $\begin{array}{c}\text { Inside } \\
\text { Diameter }\end{array}$ & $\begin{array}{c}\text { Outside } \\
\text { Diameter }\end{array}$ & $\begin{array}{c}\text { Wall } \\
\text { Thickness }\end{array}$ & $\begin{array}{c}\text { Screen Slot } \\
\text { Size }\end{array}$ & $\begin{array}{c}\text { Type of } \\
\text { Material }\end{array}$ & Trade Name \\
\hline From & To & & & & & & & \\
\hline & & & & & & & & \\
\hline & & & & & & & & \\
\hline & & & & & & & & \\
\hline
\end{tabular}

Additional information for Double cased or Nested wells:

\begin{tabular}{|c|c|c|c|c|c|c|c|c|}
\hline \multirow{2}{*}{\multicolumn{2}{|c|}{$\begin{array}{c}\mathrm{a} \\
\text { Placement } \\
\text { Depth in Feet }\end{array}$}} & $\mathrm{b}$ & $\mathrm{c}$ & $\mathrm{d}$ & $\mathrm{e}$ & $f$ & $\mathrm{~g}$ & $\mathrm{~h}$ \\
\hline & & \multirow{2}{*}{$\begin{array}{l}\text { Casing or } \\
\text { Screen or } \\
\text { Open Hole }\end{array}$} & \multirow[t]{2}{*}{$\begin{array}{c}\text { Inside } \\
\text { Diameter }\end{array}$} & \multirow[t]{2}{*}{$\begin{array}{c}\text { Outside } \\
\text { Diameter }\end{array}$} & \multirow[t]{2}{*}{$\begin{array}{c}\text { Wall } \\
\text { Thickness }\end{array}$} & \multirow[t]{2}{*}{$\begin{array}{l}\text { Screen Slot } \\
\text { Size }\end{array}$} & \multirow[t]{2}{*}{$\begin{array}{l}\text { Type of } \\
\text { Material }\end{array}$} & \multirow[t]{2}{*}{ Trade Name } \\
\hline From & To & & & & & & & \\
\hline & & & & & & & & \\
\hline & & & & & & & & \\
\hline & & & & & & & & \\
\hline & & & & & & & & \\
\hline & & & & & & & & \\
\hline & & & & & & & & \\
\hline
\end{tabular}

11. Grout and Gravel Pack (must start at zero)

\begin{tabular}{|c|c|c|c|c|c|}
\hline \multicolumn{2}{|c|}{$\begin{array}{c}\text { Placement Depth in } \\
\text { Feet }\end{array}$} & \multirow[t]{2}{*}{$\begin{array}{c}\text { Grout/Gravel/ } \\
\text { Open Hole }\end{array}$} & \multirow[t]{2}{*}{$\begin{array}{l}\text { Material Description } \\
\text { *See Desc for gravel }\end{array}$} & \multirow[t]{2}{*}{$\begin{array}{l}\text { Quantity gravel } \\
* \text { See Desc }\end{array}$} & \multirow[t]{2}{*}{$\begin{array}{c}\text { Volume \& Type Grout } \\
\text { *See Desc }\end{array}$} \\
\hline From & To & & & & \\
\hline & & & & & \\
\hline & & & & & \\
\hline & & & & & \\
\hline & & & & & \\
\hline & & & & & \\
\hline & & & & & \\
\hline & & & & & \\
\hline
\end{tabular}

Desc: Description of gravel pack i.e. engineered gravel pack, or gravel pit description ( $1 / 4$ down), or brand name (Best Sand), natural formation, drilling cuttings, soil backfill

Quantity \#cubic yards, \#tons, \#sacks, - (for drilling cuttings and soil backfill estimate quantity) Calculation assistance available on web

Volume \& Type: \#gallons of a slurry, \#barrels of a slurry, \#sacks used in the slurry, \#bags of non-slurry bentonite (chip-pellet -granular)

Additional information for Double cased or Nested wells:

\begin{tabular}{|c|c|c|c|c|c|}
\hline \multicolumn{2}{|c|}{$\begin{array}{c}\text { Placement Depth in } \\
\text { Feet }\end{array}$} & \multirow[t]{2}{*}{$\begin{array}{l}\text { Grout/Gravel/ } \\
\text { Open Hole }\end{array}$} & \multirow[t]{2}{*}{$\begin{array}{l}\text { Material Description } \\
\text { *See Desc for gravel }\end{array}$} & \multirow[t]{2}{*}{$\begin{array}{l}\text { Quantity gravel } \\
\text { *See Desc }\end{array}$} & \multirow[t]{2}{*}{$\begin{array}{c}\text { Volume \& Type Grout } \\
\text { *See Desc }\end{array}$} \\
\hline From & To & & & & \\
\hline & & & & & \\
\hline & & & & & \\
\hline & & & & & \\
\hline & & & & & \\
\hline & & & & & \\
\hline & & & & & \\
\hline & & & & & \\
\hline
\end{tabular}


WELL CONSTRUCTION LOG - 14721381252724

$\begin{array}{rrlrrrr}\text { To - } & \text { From } & \text { Case/Screen } & \text { I.D. } & \text { O.D. } & \text { Thk. } & \text { Mat. } \\ 0.0- & 112.0 & \text { Casing } & 1.94 & 2.38 & 0.21800 & \text { PVC } \\ 112.0- & 122.0 & \text { Screen } & 1.94 & 2.38 & 0.21800 & \text { PVC }\end{array}$

112.0 - 122.0 Screen

$1.94 \quad 2.38$

0.21800

PVC
Slot Trade Name

0.000 Johnson

0.010 Johnson

GROUT AND GRAVEL PACK - 14721381252724

\begin{tabular}{|c|c|c|c|c|c|}
\hline $\begin{array}{l}\text { From } \\
00.0\end{array}$ & $\begin{array}{lr}\text { - } & \text { To } \\
\text { - } & 104.0\end{array}$ & $\begin{array}{l}\text { Grout/Gravel } \\
\text { Grout }\end{array}$ & $\begin{array}{l}\text { Material Desc. } \\
\text { Bent Grout }\end{array}$ & Qty Gravel & $\begin{array}{l}\text { Volume/Type } \\
7 \text { Bags }\end{array}$ \\
\hline 04.0 & 110.0 & Grout & Pellets & & 1/2 Pail \\
\hline 10.0 & 122.0 & Gravel & \#40 & 5 Bags & \\
\hline
\end{tabular}


GEOLOGICAL LOGS - 14721381252724

$\begin{array}{rrl}\text { From - } & \text { To } & \text { Type } \\ 00.0- & 52.0 & \text { Clay } \\ 52.0- & 75.0 & \text { Sand fine-med } \\ 75.0- & 98.0 & \text { Sand med-coarse } \\ 98.0- & 112.0 & \text { Clay } \\ 112.0- & 122.0 & \text { Sand med-coarse }\end{array}$

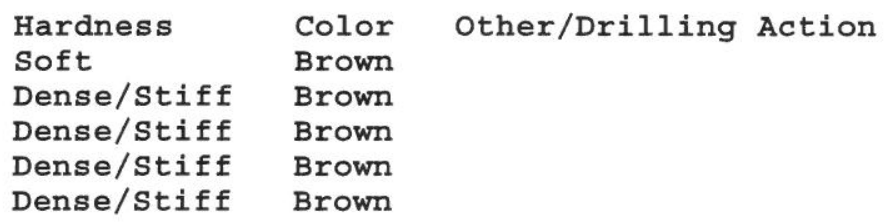


12. Geologic Materials Logged (must start at zero) - Use only options provided below for Type, Hardness and Color

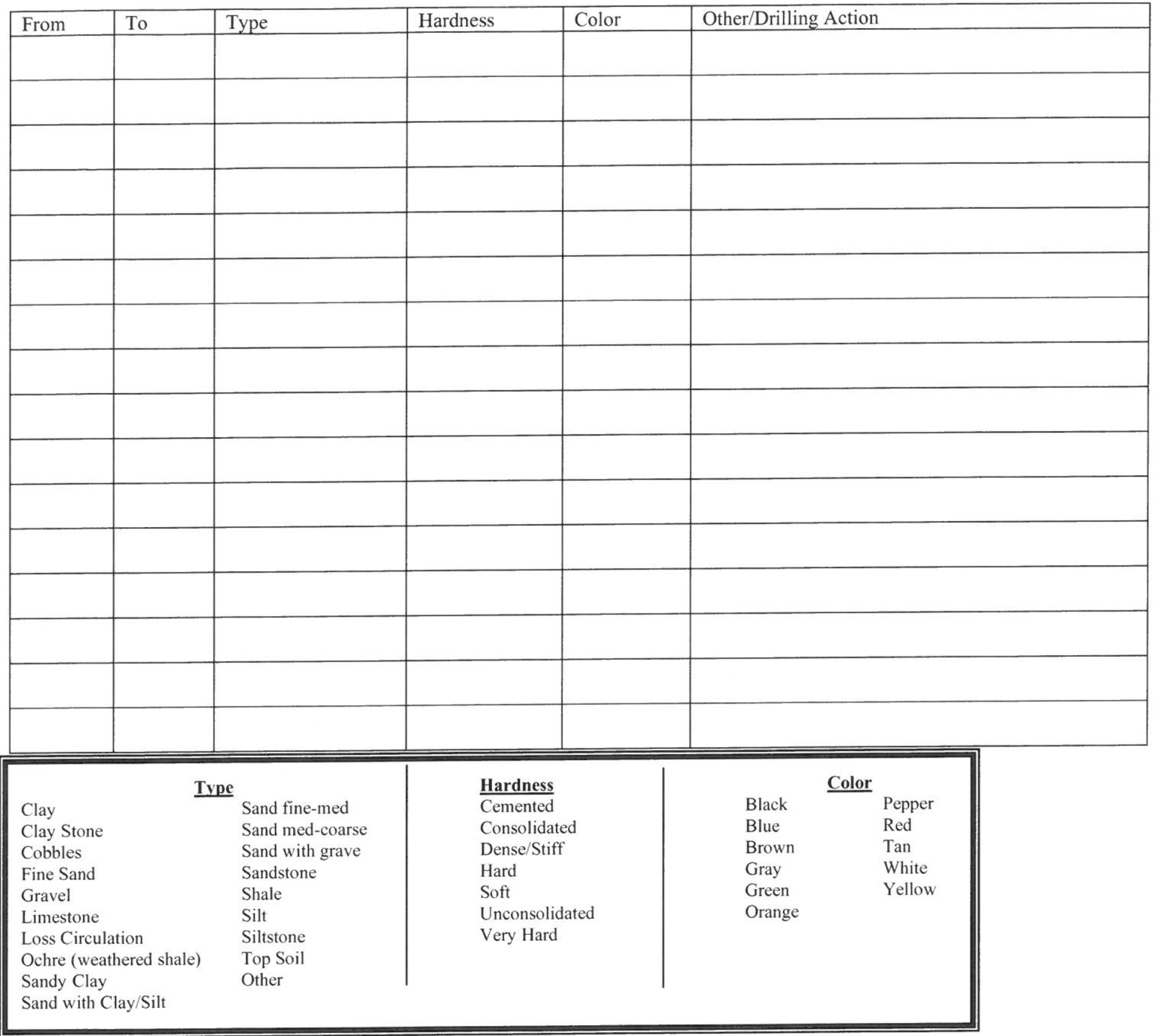

(Additional sheets may be submitted)

13. I hereby certify that the information provided on this registration is true and accurate to the best of my knowledge.

$\begin{array}{ccc}\begin{array}{c}\text { Water Well Contractor's Signature } \\ \text { (not required for pits) }\end{array} & \text { Date } & \begin{array}{c}\text { Well Owner's Signature } \\ \text { (if Contractor is unknown or Deceased or for pits) } \\ \text { (Not required if signed by Contractor) }\end{array}\end{array}$

(Not required if signed by Contractor)

Please note this document contains four pages.

Sections 9F, 9G, 10, $11 \& 12$ are not required if registering a pit.

Owner Registering well drilled prior to 2002: Minimum Required Sections - 1, 3(a,b,c,e,f), 5, 6, 7, 8(a,f,h), 9e 
Mail to

Department of Natural Resources

PO Box 94676

Lincoln, NE 68509-4676

Phone (402)471-2363

STATE OF NEBRASKA

DEPARTMENT OF NATURAL RESOURCES

WATER WELL REGISTRATION

Please indicate NA for items unknown

\section{FOR DEPARTMENT USE ONLY}

Date Filed

Owner Code No.

Registration No. G-180325

$$
\frac{-244563}{\text { Well ID }}=\text { Receipt }
$$

1. a. Well Owner's First Name Last Name

OR Company Name USDA_ CCC

b. Attention Name Steve Gilmure

c. Street Address 1400 IndEPENDENCE AVE SW

Address 2/PO Address Stop 0513 Room 4714-5

City Washing to 0

State $D C$

Zip 20250-0513

Telephone

$202=220=5704$

2. a. Contractor's License No_ 39508 Contractor's Name_STEVEN D JOHNSON

Contractor's Email Address_SJOHNSON@CASCADEDRILLING.COM

b. Drilling Firm Name CASCADE DRILLING

Address 209 LEMIEUR ST

City LITTLE FALLS

State MN

Zip $\quad 56345$

Telephone $\quad 320 \quad 632 \quad 6552$

Drilling Firm's Email Address_STHALACKER@CASCADEDRILLING.COM

3. a. Well location $\mathrm{SE}^{1 / 4}$ of the $\mathrm{SW}^{1 / 4}$ of Section 30 , Township 11 North, Range 2 IWEST

\begin{tabular}{|c|c|c|c|c|c|}
\hline Latitude Degree & 40 & Minute & 53 & Second & \\
\hline Longitude Degree & -97 & Minute & 35 & Second & Required \\
\hline
\end{tabular}

b. Natural Resources District

UPPER BIG BLUE

c. The well is: 1220

feet from the (North/ Soxth) section line and (circle one)

d. Street address and subdivision, if applicable

e. Location of water use (give legal descriptions)

2434

(BIOCK : IOT:)

$\mathrm{N} / \mathrm{A}$

f. If for irrigation, the land to be irrigated is

0 acres.

g. Well reference letter(s), if applicable

$\mathrm{DL}-2 \mathrm{C} 2$

HHSS PWSID

4. Permits

Management Area Permit Number

Geothermal Permit Number

Municipal Permit Number

Well Spacing Permit Number

HHSS

5. Purpose of well (indicate one) Domestic Livestock Ground Heat Exchanger Aquaculture Observation Recovery

Public Water Supply
Surface Water Permit Number

Industrial Permit Number

Transfer Out-Of-State Permit Number

Conduct Permit Number

Other Permit Number NDEQ
Commercial/Industrial Groundwater Source Heat Pump

Other

Pit (for irrigation)
Dewatering (over 90 days) Irrigation

Injection

Public Water Supply (with spacing (46-638))
Location of well for a pit is the location of the pump
(further description

Wells in a Series.

6. Wells in a Series.

a. Is this well a part of a series?___ Yes go to part $\mathrm{b}$ of this section $\quad \mathrm{x} \quad$ No go to part 7 of this application (Y/N required)

b. If one or more of the wells in the series is currently registered, give well registration number

c. How many wells in the series are you registering at this time? d. How many total wells in the series? 
7. Replacement and decommissioned/modified well information.

a. Is this well a replacement well? ___ Yes $\mathrm{x}$ No go to part 8 of this application

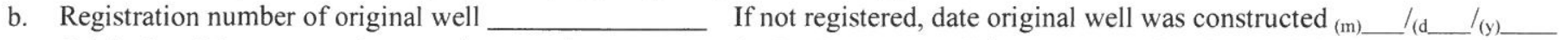

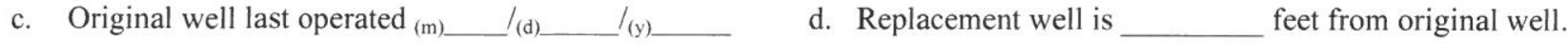

e. Location of water use of original well

Please Select One:

f.1. $\square$ Original water well decommissioned on $(\mathrm{m}) \_/{ }_{\left(\mathrm{d} \_-\right.} /(\mathrm{y}) \_$OR

2. $\square$ I hereby certify that the original water well will be decommissioned within 180 days after such construction of the replacement water well. OR

3. $\square$ I hereby certify that the original water well will be modified and equipped to pump 50 gallons per minute or less within 180 days after such construction of the replacement water well. It will be used for one of the following: a. $\square$ Livestock

b. $\square$ Monitoring c. $\square$ Observation

d. $\square$ nonconsumptive or de minimus use approved by the applicable natural resources district. State use:

If $3 \mathrm{~d}$ is chosen. NRD signature is required. (Signature can be submitted on NRD Approval form to DNR prior to registration)

NRD signature

Date

OR

4. $\square$ Decommission/Modification Certification form is submitted by landowner. (Must be submitted before registering well)

8. Pump Information.

(Pump information is required if registering a pit)

a. Is pump installed at this time __ Yes $\quad \mathrm{X}$ No

Is pump installed by well owner in section 1? ___ Yes $\mathrm{X}$ No

Is pump installed by contractor in section 2? ___ Yes $\mathrm{x} \quad$ No

Is this a free flowing well ___ Yes(no pump to be installed) $\quad \mathrm{x}$ No

If pump installed by pump installer, please fill out license number below

b. Pump Installer's License No. Pump Installer's Name

Pump Installer's Email Address

Pump Installer's Firm Name

Pump Installer's Firm Address

City_ State_Zip__ - 0000 Telephone

Pump Installer's Firm Email Address

c. Measured Pumping rate gallons per minute d. Pumping water level feet

e. Drop pipe diameter inches

f. Length of drop pipe feet

g. Pumping equipment installed $(\mathrm{m}) \_/(\mathrm{d}$

h. Pump Brand

i. This well is designed and constructed to pump less than $50 \mathrm{gpm} \quad \mathrm{x}$ Yes

No ( $8 \mathrm{H}$ is required on ALL wells)

9. Well Construction Information.

a. Total well depth 146 feet. b. Static water level 75 feet. (required)

c. Well Construction Began $(\mathrm{m}) \quad 07, \quad 06202 / 204,6$

d.. Well Construction Completed $(\mathrm{m})$ $07 / / Q 2 / 20,76$

Wells drilled prior to stays or moratoriums require NRD signature
NRD signature Date

e. Bore hole diameter in inches Top Bottom 6

f. Casing and Screen Joints are Welded Glued Threaded $x$ Other

g. Capacity of Well 4 gallons per minute (to be used to determine sustainability of aquifer)

h. Pumping water level at this capacity feet 
10. Well Construction (Casing \& Screen)- c, d, e, \& f measurements should be in inches to three decimal places (From not less than zero)

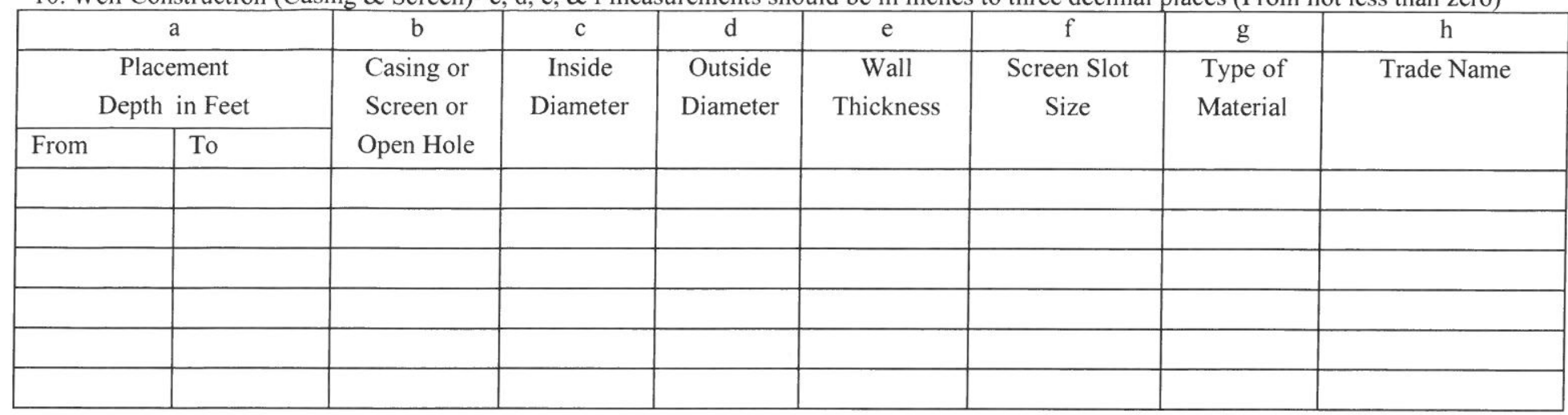

Additional information for Double cased or Nested wells:

\begin{tabular}{|c|c|c|c|c|c|c|c|c|}
\hline & & $\mathrm{b}$ & $\mathrm{c}$ & d & $\mathrm{e}$ & $\mathrm{f}$ & $\mathrm{g}$ & $\mathrm{h}$ \\
\hline & $\begin{array}{l}\text { ment } \\
\text { in Feet }\end{array}$ & $\begin{array}{l}\text { Casing or } \\
\text { Screen or }\end{array}$ & $\begin{array}{c}\text { Inside } \\
\text { Diameter }\end{array}$ & $\begin{array}{l}\text { Outside } \\
\text { Diameter }\end{array}$ & $\begin{array}{c}\text { Wall } \\
\text { Thickness }\end{array}$ & $\begin{array}{c}\text { Screen Slot } \\
\text { Size }\end{array}$ & $\begin{array}{l}\text { Type of } \\
\text { Material }\end{array}$ & Trade Name \\
\hline From & To & Open Hole & & & & & & \\
\hline & & & & & & & & \\
\hline & & & & & & & & \\
\hline & & & & & & & & \\
\hline & & & & & & & & \\
\hline & & & & & & & & \\
\hline & & & & & & & & \\
\hline
\end{tabular}

11. Grout and Gravel Pack (must start at zero)

\begin{tabular}{|l|l|l|l|l|l|}
\hline \multicolumn{2}{|c|}{$\begin{array}{c}\text { Placement Depth in } \\
\text { Feet }\end{array}$} & $\begin{array}{c}\text { Grout/Gravel/ } \\
\text { Open Hole }\end{array}$ & $\begin{array}{c}\text { Material Description } \\
\text { *See Desc for gravel }\end{array}$ & $\begin{array}{c}\text { Quantity gravel } \\
\text { *See Desc }\end{array}$ & $\begin{array}{c}\text { Volume \& Type Grout } \\
\text { *See Desc }\end{array}$ \\
\hline From & To & & & & \\
\hline & & & & & \\
\hline & & & & & \\
\hline & & & & & \\
\hline
\end{tabular}

Desc: Description of gravel pack i.e. engineered gravel pack, or gravel pit description ( $1 / 4$ down), or brand name (Best Sand), natural formation, drilling cuttings, soil backfill

Quantity \#cubic yards, \#tons, \#sacks, - (for drilling cuttings and soil backfill estimate quantity) Calculation assistance available on web

Volume \& Type: \#gallons of a slurry, \#barrels of a slurry, \#sacks used in the slurry, \#bags of non-slurry bentonite (chip-pellet -granular)

Additional information for Double cased or Nested wells:

\begin{tabular}{|c|c|c|c|c|c|}
\hline \multicolumn{2}{|c|}{$\begin{array}{c}\text { Placement Depth in } \\
\text { Feet }\end{array}$} & \multirow[t]{2}{*}{$\begin{array}{l}\text { Grout/Gravel/ } \\
\text { Open Hole }\end{array}$} & \multirow[t]{2}{*}{$\begin{array}{l}\text { Material Description } \\
\text { *See Desc for gravel }\end{array}$} & \multirow[t]{2}{*}{$\begin{array}{l}\text { Quantity gravel } \\
\text { *See Desc }\end{array}$} & \multirow[t]{2}{*}{$\begin{array}{c}\text { Volume \& Type Grout } \\
\text { *See Desc }\end{array}$} \\
\hline From & To & & & & \\
\hline & & & & & \\
\hline & & & & & \\
\hline & & & & & \\
\hline & & & & & \\
\hline & & & & & \\
\hline & & & & & \\
\hline & & & & & \\
\hline
\end{tabular}


WELL CONSTRUCTION LOG - 147213634526245

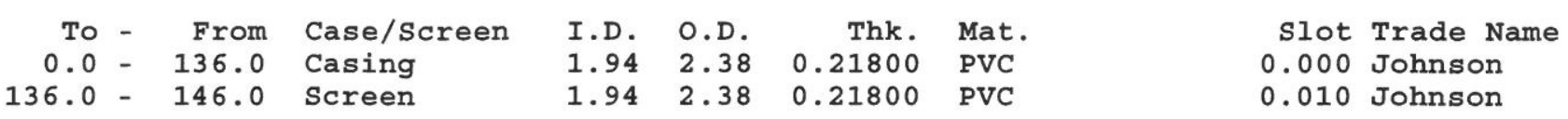

GROUT AND GRAVEL PACK - 147213634526245

\begin{tabular}{|c|c|c|c|c|c|}
\hline $\begin{array}{l}\text { From } \\
00.0\end{array}$ & $\begin{array}{l}-\quad \text { To } \\
-\quad 128.0\end{array}$ & $\begin{array}{l}\text { Grout/Gravel } \\
\text { Grout }\end{array}$ & $\begin{array}{l}\text { Material Desc. } \\
\text { Bent Grout }\end{array}$ & Qty Gravel & $\begin{array}{l}\text { Volume/Type } \\
9 \text { Bags }\end{array}$ \\
\hline 28.0 & 134.0 & Grout & Pellets & & 1/2 Pail \\
\hline 34.0 & 146.0 & Gravel & \#40 & 5 Bags & \\
\hline
\end{tabular}


GEOLOGICAL LOGS - 147213634526245

\begin{tabular}{|c|c|c|c|c|c|}
\hline From & To & Type & Hardness & Color & Other/Drilling \\
\hline 00.0 & 52.0 & clay & Soft & Brown & \\
\hline 52.0 & 75.0 & Sand fine-med & Dense/stiff & Brown & \\
\hline 75.0 & 98.0 & Sand med-coarse & Dense/Stiff & Brown & \\
\hline 98.0 & 112.0 & Clay & Dense/stiff & Brown & \\
\hline 112.0 & 146.0 & Sand med-coarse & Dense/Stiff & Brown & \\
\hline
\end{tabular}




\section{Geologic Materials Logged (must start at zero) - Use only options provided below for Type, Hardness and Color}

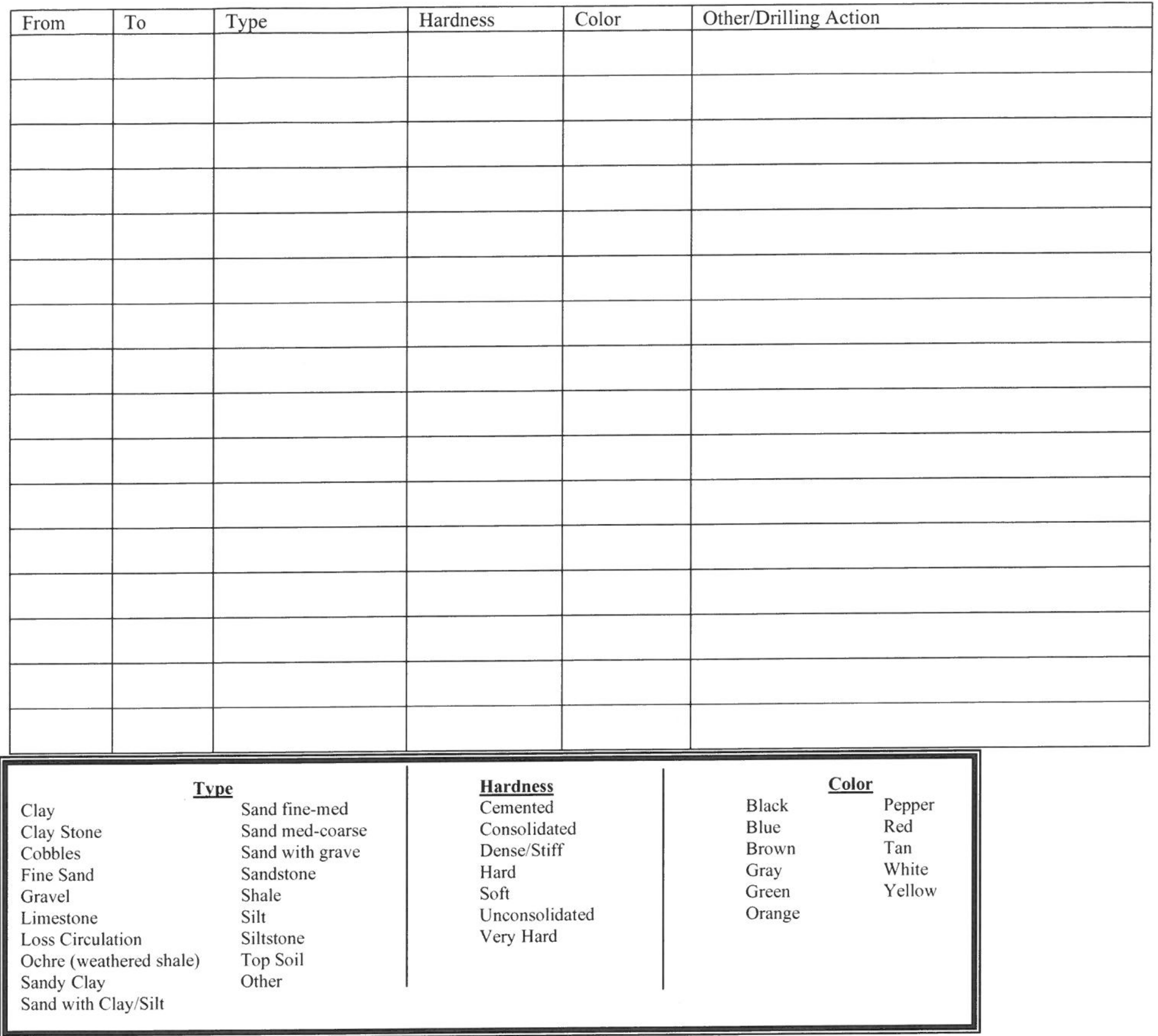

(Additional sheets may be submitted)

13. I hereby certify that the information provided on this registration is true and accurate to the best of my knowledge.
Water Well Contractor's Signature (not required for pits)

\section{Date}

Well Owner's Signature

(if Contractor is unknown or Deceased or for pits)

(Not required if signed by Contractor)

Please note this document contains four pages.

\section{Sections 9F, 9G, 10, $11 \& 12$ are not required if registering a pit.}

Owner Registering well drilled prior to 2002: Minimum Required Sections - 1, 3(a,b,c,e,f), 5, 6, 7, 8(a,f,h), 9e 
Mail to

Department of Natural Resources

PO Box 94676

Lincoln, NE 68509-4676

Phone (402)471-2363
STATE OF NEBRASKA

DEPARTMENT OF NATURAL RESOURCES

WATER WELL REGISTRATION

Please indicate NA for items unknown

\section{FOR DEPARTMENT USE ONLY}

Date Filed Owner Code No.

Registration No. G-180326

$$
-\frac{244564}{\text { Well ID }}-\text { Receipt }
$$

1. a. Well Owner's First Name

Last Name

OR Company Name USDA/CCC

b. Attention Name Steve Gilmore

c. Street Address 1400 IndEPENDENCE AVE SW

Address 2/PO Address Stop 0513 Room $4714-5$

City WAshington State DC Zip 20250-05/3 Telephone 202-720-5704

2. a. Contractor's License No_ 39508 Contractor's Name_STEVEN D JOHNSON

Contractor's Email Address_SJOHNSON@CASCADEDRILLING.COM

b. Drilling Firm Name CASCADE DRILLING

Address 209 LEMIEUR ST

City LITTLE FALLS State_MN_Zip_ 56345

Telephone $\quad 320 \quad 632 \quad 6552$

Drilling Firm's Email Address_STHALACKER@CASCADEDRILLING.COM

3. a. Well location SE $1 / 4$ of the $S_{W} 1 / 4$ of Section 30 , Township 11 North, Range 2 IWEST

\begin{tabular}{lllll|l} 
Latitude Degree & 40 & Minute & 53 & Second & 24.4 \\
Longitude Degree & -97 & Minute & 35 & Second & 19.5
\end{tabular}$\quad \begin{aligned} & \text { GPS } \\
& \text { Required }\end{aligned}$

UPPER BIG BLUE

b. Natural Resources District feet from the (North/ Soxth) section line and feet from the (North/ Soath) section line and
(circle one)

c. The well is: 1211

d. Street address and subdivision, if applicable

e. Location of water use (give legal descriptions)

2434 (BLOCK: IOT:)

$\mathrm{N} / \mathrm{A}$

f. If for irrigation, the land to be irrigated is

g. Well reference letter(s), if applicable acres. HHSS PWSID

4. Permits

Management Area Permit Number

Geothermal Permit Number

Municipal Permit Number

Well Spacing Permit Number

HHSS
Surface Water Permit Number

Industrial Permit Number

Transfer Out-Of-State Permit Number

Conduct Permit Number

Other Permit Number

NDEQ
5. Purpose of well (indicate one) Domestic Livestock

Public Water Supply (without spacing)
Aquaculture

Commercial/Industrial Groundwater Source Heat Pump

Pit (for irrigation)
YORK County

Location of well for a pit is the location of the pump __ Recovery Other

Dewatering (over 90 days) Irrigation

Public Water Supply (with spacing (46-638))
(East/West) section line (circle one) 
7. Replacement and decommissioned/modified well information.

a. Is this well a replacement well? ___ Yes $\quad \mathrm{x} \quad$ No go to part 8 of this application

b. Registration number of original well

c. Original well last operated $(\mathrm{m}) \_$_ $/$(d)

(d)

If not registered, date original well was constructed $(\mathrm{m}) \_/ /(\mathrm{d}$

e. Location of water use of original well

Please Select One:

f.1. $\square$ Original water well decommissioned on $(\mathrm{m}) \_/ /\left(\mathrm{d} \_/ /(\mathrm{y}) \_ \text {OR }\right.$

2. $\square$ I hereby certify that the original water well will be decommissioned within 180 days after such construction of the replacement water well. $\mathbf{O R}$

3. $\square$ I hereby certify that the original water well will be modified and equipped to pump 50 gallons per minute or less within 180 days after such construction of the replacement water well. It will be used for one of the following: a. $\square$ Livestock

b. $\square$ Monitoring c. $\square$ Observation

d. $\square$ nonconsumptive or de minimus use approved by the applicable natural resources district. State use:

If $3 \mathrm{~d}$ is chosen. NRD signature is required. (Signature can be submitted on NRD Approval form to DNR prior to registration)

NRD signature

Date

OR

4. $\square$ Decommission/Modification Certification form is submitted by landowner. (Must be submitted before registering well)

8. Pump Information.

(Pump information is required if registering a pit)

a. Is pump installed at this time ___ Yes $\quad \mathrm{X}$ No
Is pump installed by well owner in section 1 ?
Yes $\mathrm{x}$ No
Is pump installed by contractor in section 2 ?
Yes $\mathrm{X}$ No

Is this a free flowing well ___ Yes(no pump to be installed) $\quad \mathrm{x}$ No

If pump installed by pump installer, please fill out license number below

b. Pump Installer's License No. Pump Installer's Name

Pump Installer's Email Address

Pump Installer's Firm Name

Pump Installer's Firm Address

City State

Zip $-0000$ Telephone

Pump Installer's Firm Email Address

c. Measured Pumping rate gallons per minute d. Pumping water level feet

e. Drop pipe diameter inches

f. Length of drop pipe feet

g. Pumping equipment installed (m)___ $/(\mathrm{d}$

h. Pump Brand

i. This well is designed and constructed to pump less than $50 \mathrm{gpm} \quad \mathrm{X}$ Yes

No (8H is required on ALL wells)

9. Well Construction Information.

a. Total well depth feet.

b. Static water level 75 feet. (required)

c. Well Construction Began $(\mathrm{m}) \quad 07(6) 06 / 20146$ d.. Well Construction Completed $(\mathrm{m}) \_07 / /\left(0,6 / 2 Q_{y}\right) 6$

Wells drilled prior to stays or NRD signature Date moratoriums require NRD signature (Signature can be submitted on NRD Approval form to DNR prior to registration)

e. Bore hole diameter in inches Top_ $6 \quad$ Bottom

f. Casing and Screen Joints are Welded Glued Threaded $\mathrm{x} \quad$ Other

g. Capacity of Well 4 gallons per minute (to be used to determine sustainability of aquifer)

h. Pumping water level at this capacity feet 
10. Well Construction (Casing \& Screen)- c, d, e, \& f measurements should be in inches to three decimal places (From not less than zero)

\begin{tabular}{|c|c|c|c|c|c|c|c|c|}
\hline \multicolumn{2}{|c|}{$\mathrm{a}$} & $\mathrm{b}$ & $\mathrm{c}$ & d & $\mathrm{e}$ & $\mathrm{f}$ & $\mathrm{g}$ & $\mathrm{h}$ \\
\hline \multicolumn{2}{|c|}{$\begin{array}{c}\text { Placement } \\
\text { Depth in Feet }\end{array}$} & \multirow{2}{*}{$\begin{array}{l}\text { Casing or } \\
\text { Screen or } \\
\text { Open Hole }\end{array}$} & \multirow[t]{2}{*}{$\begin{array}{c}\text { Inside } \\
\text { Diameter }\end{array}$} & \multirow[t]{2}{*}{$\begin{array}{c}\text { Outside } \\
\text { Diameter }\end{array}$} & \multirow[t]{2}{*}{$\begin{array}{c}\text { Wall } \\
\text { Thickness }\end{array}$} & \multirow[t]{2}{*}{$\begin{array}{c}\text { Screen Slot } \\
\text { Size }\end{array}$} & \multirow[t]{2}{*}{$\begin{array}{l}\text { Type of } \\
\text { Material }\end{array}$} & \multirow[t]{2}{*}{ Trade Name } \\
\hline From & To & & & & & & & \\
\hline & & & & & & & & \\
\hline & & & & & & & & \\
\hline & & & & & & & & \\
\hline & & & & & & & & \\
\hline & & & & & & & & \\
\hline
\end{tabular}

Additional information for Double cased or Nested wells:

\begin{tabular}{|c|c|c|c|c|c|c|c|c|}
\hline \multicolumn{2}{|c|}{ a } & $\mathrm{b}$ & $\mathrm{c}$ & $\mathrm{d}$ & $\mathrm{e}$ & $\mathrm{f}$ & $g$ & $\mathrm{~h}$ \\
\hline \multicolumn{2}{|c|}{$\begin{array}{c}\text { Placement } \\
\text { Depth in Feet }\end{array}$} & \multirow{2}{*}{$\begin{array}{l}\text { Casing or } \\
\text { Screen or } \\
\text { Open Hole }\end{array}$} & \multirow[t]{2}{*}{$\begin{array}{c}\text { Inside } \\
\text { Diameter }\end{array}$} & \multirow[t]{2}{*}{$\begin{array}{l}\text { Outside } \\
\text { Diameter }\end{array}$} & \multirow[t]{2}{*}{$\begin{array}{c}\text { Wall } \\
\text { Thickness }\end{array}$} & \multirow[t]{2}{*}{$\begin{array}{l}\text { Screen Slot } \\
\text { Size }\end{array}$} & \multirow[t]{2}{*}{$\begin{array}{l}\text { Type of } \\
\text { Material }\end{array}$} & \multirow[t]{2}{*}{ Trade Name } \\
\hline From & To & & & & & & & \\
\hline & & & & & & & & \\
\hline & & & & & & & & \\
\hline & & & & & & & & \\
\hline & & & & & & & & \\
\hline & & & & & & & & \\
\hline & & & & & & & & \\
\hline
\end{tabular}

11. Grout and Gravel Pack (must start at zero)

\begin{tabular}{|c|c|c|c|c|c|}
\hline \multicolumn{2}{|c|}{$\begin{array}{c}\text { Placement Depth in } \\
\text { Feet }\end{array}$} & \multirow[t]{2}{*}{$\begin{array}{l}\text { Grout/Gravel/ } \\
\text { Open Hole }\end{array}$} & \multirow[t]{2}{*}{$\begin{array}{l}\text { Material Description } \\
* \text { See Desc for gravel }\end{array}$} & \multirow[t]{2}{*}{$\begin{array}{l}\text { Quantity gravel } \\
\text { *See Desc }\end{array}$} & \multirow[t]{2}{*}{$\begin{array}{c}\text { Volume \& Type Grou } \\
\text { *See Desc }\end{array}$} \\
\hline From & To & & & & \\
\hline & & & & & \\
\hline & & & & & \\
\hline & & & & & \\
\hline & & & & & \\
\hline & & & & & \\
\hline & & & & & \\
\hline & & & & & \\
\hline
\end{tabular}

Desc: Description of gravel pack i.e. engineered gravel pack, or gravel pit description ( $1 / 4$ down), or brand name (Best Sand), natural formation, drilling cuttings, soil backfill

Quantity \#cubic yards, \#tons, \#sacks, - (for drilling cuttings and soil backfill estimate quantity) Calculation assistance available on web Volume \& Type: \#gallons of a slurry, \#barrels of a slurry, \#sacks used in the slurry, \#bags of non-slurry bentonite (chip-pellet -granular) Additional information for Double cased or Nested wells:

\begin{tabular}{|c|c|c|c|c|c|}
\hline \multicolumn{2}{|c|}{$\begin{array}{c}\text { Placement Depth in } \\
\text { Feet }\end{array}$} & \multirow[t]{2}{*}{$\begin{array}{l}\text { Grout/Gravel/ } \\
\text { Open Hole }\end{array}$} & \multirow[t]{2}{*}{$\begin{array}{l}\text { Material Description } \\
\text { *See Desc for gravel }\end{array}$} & \multirow[t]{2}{*}{$\begin{array}{l}\text { Quantity gravel } \\
\text { *See Desc }\end{array}$} & \multirow[t]{2}{*}{$\begin{array}{c}\text { Volume \& Type Grout } \\
\text { *See Desc }\end{array}$} \\
\hline From & To & & & & \\
\hline & & & & & \\
\hline & & & & & \\
\hline & & & & & \\
\hline & & & & & \\
\hline & & & & & \\
\hline & & & & & \\
\hline & & & & & \\
\hline
\end{tabular}


WELL CONSTRUCTION LOG - 147213668023463

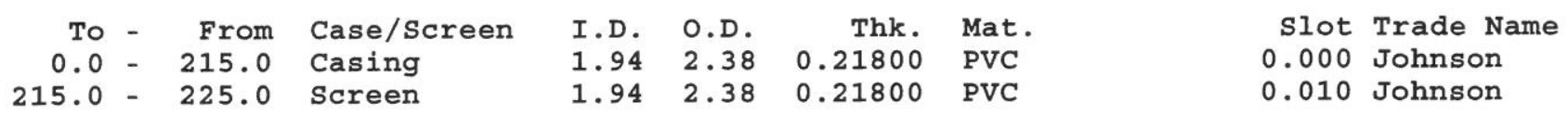

GROUT AND GRAVEL PACK - 147213668023463

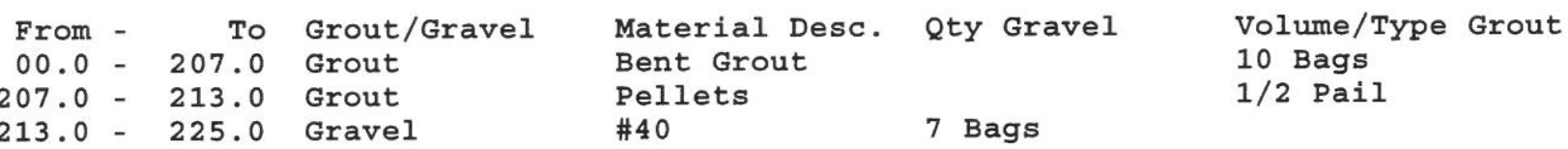


GEOLOGICAL LOGS - 147213668023463

\begin{tabular}{|c|c|c|c|c|c|}
\hline From & To & Type & Hardness & Color & Other/Drilling \\
\hline 00.0 & 52.0 & clay & Soft & Brown & \\
\hline 52.0 & 75.0 & Sand fine-med & Dense/Stiff & Brown & \\
\hline 75.0 & 98.0 & Sand med-coarse & Dense/stiff & Brown & \\
\hline 98.0 & 112.0 & Clay & Dense/stiff & Brown & \\
\hline 112.0 & 147.0 & Sand med-coarse & Dense/stiff & Brown & \\
\hline 147.0 & 170.0 & Clay & Dense/Stiff & Brown & \\
\hline 170.0 & 192.0 & Sand fine-med & Dense/Stiff & Brown & \\
\hline 192.0 & 202.0 & Clay & Dense/Stiff & Brown & \\
\hline 202.0 & $-\quad 225.0$ & Sand med-coarse & Dense/stiff & Brown & \\
\hline
\end{tabular}


12. Geologic Materials Logged (must start at zero) - Use only options provided below for Type, Hardness and Color

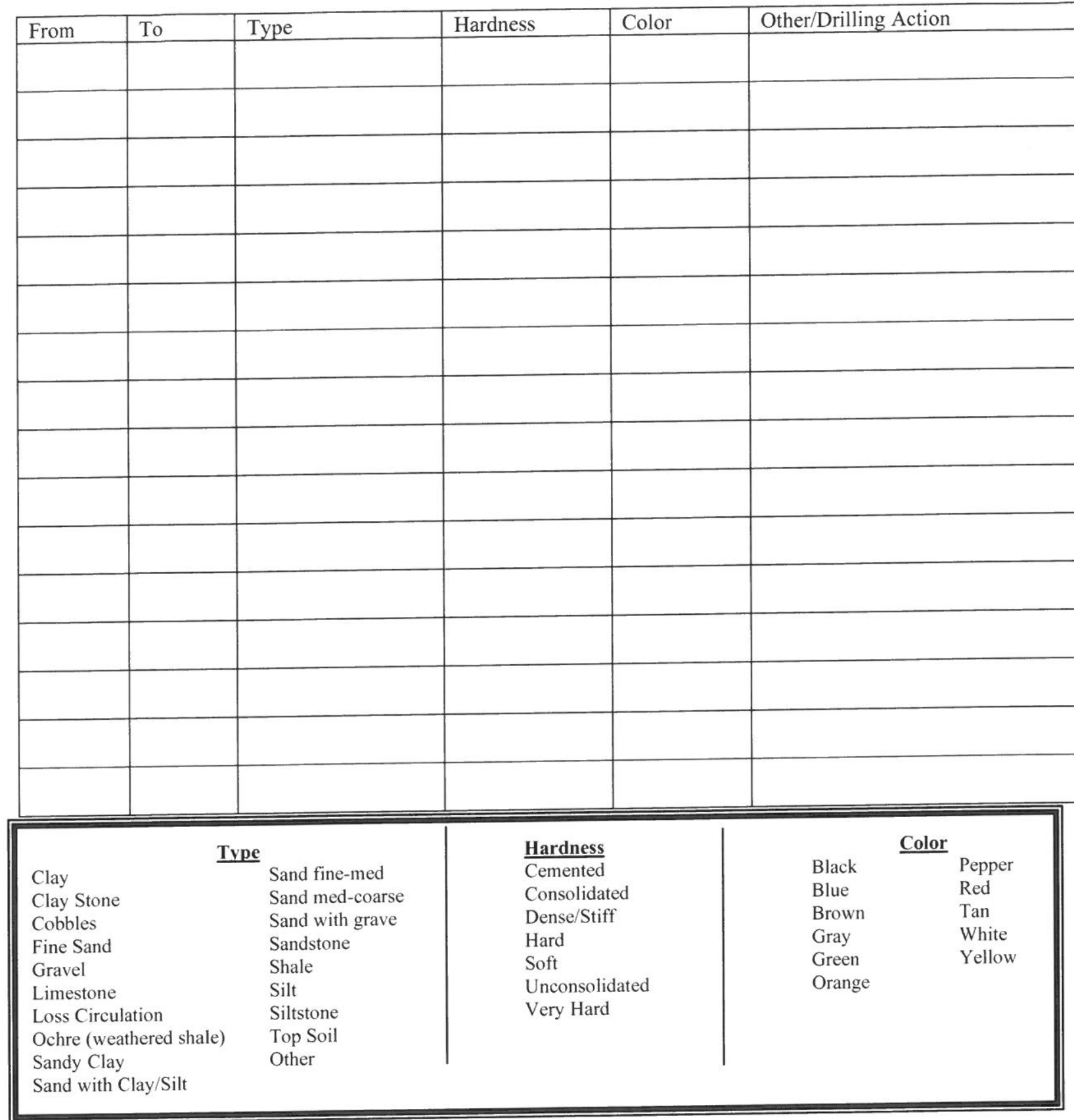

(Additional sheets may be submitted)

13. I hereby certify that the information provided on this registration is true and accurate to the best of my knowledge.

Water Well Contractor's Signature (not required for pits)
Date

ate




Mail to

Department of Natural Resources

PO Box 94676

Lincoln, NE 68509-4676

Phone (402)471-2363
STATE OF NEBRASKA

DEPARTMENT OF NATURAL RESOURCES

WATER WELL REGISTRATION Please indicate NA for items unknown

\section{FOR DEPARTMENT USE ONLY}

Date Filed

Owner Code No.

Registration No. G-180331

\section{$-\frac{244570}{\text { Well ID }}$}

1. a. Well Owner's First Name

Last Name

OR Company Name USDA/CCC

b. Attention Name steve Gilmore -

c. Street Address 1400 INDEPENDENCE AVE $5 W$ Address 2/PO Address Stop 0513 Room 4714-5 City WAshirgtan

2. a. Contractor's License No_39508 Contractor's Name_STEVEN D JOHNSON

Contractor's Email Address_SJOHNSON@CASCADEDRILLING.COM

b. Drilling Firm Name CASCADE DRILLING

Address 209 LEMIEUR ST

City LITTLE FALLS

State MN

Zip $\quad 56345$

Telephone $\quad 320 \quad 632 \quad 6552$

Drilling Firm's Email Address_STHALACKER@CASCADEDRILLING.COM

3. a. Well location SW $1 / 4$ of the $\mathrm{SE}^{1 / 4}$ of Section 30 , Township 11 North, Range 2 IWEST

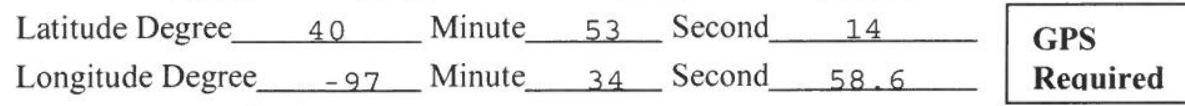

YORK

County

Longitude Degree ___

b. Natural Resources District UPPER BIG BLUE

c. The well is: 164 feet from the (North/ Soath) section line and (circle one)

d. Street address and subdivision, if applicable

e. Location of water use (give legal descriptions)

1368

(BLOCK : TOT: )

$\mathrm{N} / \mathrm{A}$

f. If for irrigation, the land to be irrigated is

0 acres.

g. Well reference letter(s), if applicable $D L-3 C 1$
HHSS PWSID
Location of well for a pit is the location of the pump
4. Permits

Management Area Permit Number

Geothermal Permit Number

Municipal Permit Number

Well Spacing Permit Number

HHSS

5. Purpose of well (indicate one) Aquaculture Ground Heat Exchanger Domestic Monitoring Livestock $\mathrm{x}$ Monitoring

Public Water Supply (without spacing) ___ Recovery __ Other Observation

Public Water Supply (without spacing) ___ Recovery __ Other

Location of water use is required on all wells
Exst/West) section line (circle one)

(further description of use can be provided under other)

Dewatering (over 90 days) Irrigation Injection
Surface Water Permit Number

Industrial Permit Number

Transfer Out-Of-State Permit Number

Conduct Permit Number

Other Permit Number

NDEQ
Commercial/Industrial Groundwater Source Heat Pump Pit (for irrigation)

Public Water Supply (with spacing (46-638))

(indicate use)

6. Wells in a Series.
a. Is this well a part of a series?
Yes go to part $b$ of this section
$\mathrm{x} \quad$ No go to part 7 of this application (Y/N required)
b. If one or more of the wells in the series is currently registered, give well registration number
c. How many wells in the series are you registering at this time? d. How many total wells in the series? 
7. Replacement and decommissioned/modified well information.

a. Is this well a replacement well? ___ Yes $\quad \mathrm{x} \quad$ No go to part 8 of this application

b. Registration number of original well

c. Original well last operated (m)__ / (d)__ / $/(\mathrm{y})$

If not registered, date or

d. Replacement well is feet from original well.

e. Location of water use of original well

Please Select One:

f.1. $\square$ Original water well decommissioned on (m) $\_/\left(\mathrm{d} \_/(\mathrm{y}) \_ \text {OR }\right.$

2. $\square$ I hereby certify that the original water well will be decommissioned within 180 days after such construction of the replacement water well. OR

3. $\square$ I hereby certify that the original water well will be modified and equipped to pump 50 gallons per minute or less within 180 days after such construction of the replacement water well. It will be used for one of the following: a. $\square$ Livestock b. $\square$ Monitoring c. $\square$ Observation

d. $\square$ nonconsumptive or de minimus use approved by the applicable natural resources district. State use:

If $3 \mathrm{~d}$ is chosen. NRD signature is required. (Signature can be submitted on NRD Approval form to DNR prior to registration)

NRD signature

Date

OR

4. $\square$ Decommission/Modification Certification form is submitted by landowner. (Must be submitted before registering well)

8. Pump Information.

(Pump information is required if registering a pit)

a. Is pump installed at this time___ Yes $\mathrm{x}$ No

Is pump installed by well owner in section $1 ? \quad$ Yes $\quad \mathrm{x} \quad$ No

Is pump installed by contractor in section 2?__ Yes $\mathrm{x}$ No

Is this a free flowing well ___ Yes(no pump to be installed) $\quad \mathrm{x}$ No

If pump installed by pump installer, please fill out license number below

b. Pump Installer's License No. Pump Installer's Name

Pump Installer's Email Address

Pump Installer's Firm Name

Pump Installer's Firm Address

City State Zip $\quad-0000$ Telephone

Pump Installer's Firm Email Address

c. Measured Pumping rate gallons per minute

d. Pumping water level feet

e. Drop pipe diameter inches

f. Length of drop pipe feet

g. Pumping equipment installed (m)__ / $/$ d__ $/(y)$

h. Pump Brand

i. This well is designed and constructed to pump less than $50 \mathrm{gpm} \quad \mathrm{x}$ Yes

No (8H is required on ALL wells)

9. Well Construction Information.

a. Total well depth 123 feet.

b. Static water level feet. (required)

c. Well Construction Began $(\mathrm{m}) \quad 07(6) 10 / 204,6$

d.. Well Construction Completed $(\mathrm{m}) \_07 / / \alpha^{2} 0 / 2,0,76$

Wells drilled prior to stays or

NRD signature Date moratoriums require NRD signature (Signature can be submitted on NRD Approval form to DNR prior to registration)

e. Bore hole diameter in inches Top 6 Bottom 6

f. Casing and Screen Joints are Welded Glued Threaded $\mathrm{x}$ Other

g. Capacity of Well __ 4 gallons per minute (to be used to determine sustainability of aquifer)

h. Pumping water level at this capacity feet 
10. Well Construction (Casing \& Screen)- c, d, e, \& f measurements should be in inches to three decimal places (From not less than zero)

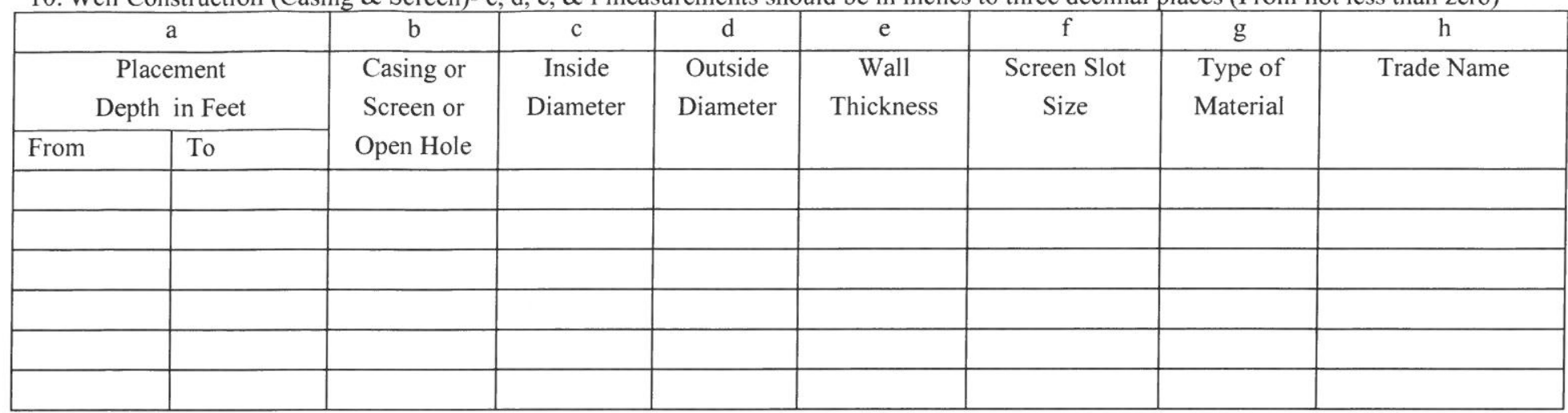

Additional information for Double cased or Nested wells:

\begin{tabular}{|c|c|c|c|c|c|c|c|c|}
\hline \multicolumn{2}{|c|}{ a } & $\mathrm{b}$ & $\mathrm{c}$ & $\mathrm{d}$ & $\mathrm{e}$ & $\mathrm{f}$ & $\mathrm{g}$ & $\mathrm{h}$ \\
\multicolumn{2}{|c|}{$\begin{array}{c}\text { Placement } \\
\text { Depth in Feet }\end{array}$} & $\begin{array}{c}\text { Casing or } \\
\text { Screen or } \\
\text { Open Hole }\end{array}$ & $\begin{array}{c}\text { Inside } \\
\text { Diameter } \\
\text { From }\end{array}$ To & & $\begin{array}{c}\text { Outside } \\
\text { Diameter }\end{array}$ & $\begin{array}{c}\text { Wall } \\
\text { Thickness }\end{array}$ & $\begin{array}{c}\text { Screen Slot } \\
\text { Size }\end{array}$ & $\begin{array}{c}\text { Type of } \\
\text { Material }\end{array}$ \\
\hline \\
\hline
\end{tabular}

11. Grout and Gravel Pack (must start at zero)

\begin{tabular}{|c|c|c|c|c|c|}
\hline \multicolumn{2}{|c|}{$\begin{array}{l}\text { Placement Depth in } \\
\text { Feet }\end{array}$} & \multirow[t]{2}{*}{$\begin{array}{l}\text { Grout/Gravel/ } \\
\text { Open Hole }\end{array}$} & \multirow[t]{2}{*}{$\begin{array}{l}\text { Material Description } \\
\text { *See Desc for gravel }\end{array}$} & \multirow[t]{2}{*}{$\begin{array}{c}\text { Quantity gravel } \\
\text { *See Desc }\end{array}$} & \multirow[t]{2}{*}{$\begin{array}{c}\text { Volume \& Type Grout } \\
\text { *See Desc }\end{array}$} \\
\hline From & To & & & & \\
\hline & & & & & \\
\hline & & & & & \\
\hline & & & & & \\
\hline & & & & & \\
\hline & & & & & \\
\hline & & & & & \\
\hline & & & & & \\
\hline
\end{tabular}

Desc: Description of gravel pack i.e. engineered gravel pack, or gravel pit description ( $1 / 4$ down), or brand name (Best Sand), natural formation, drilling cuttings, soil backfill

Quantity \#cubic yards, \#tons, \#sacks, - (for drilling cuttings and soil backfill estimate quantity) Calculation assistance available on web Volume \& Type: \#gallons of a slurry, \#barrels of a slurry, \#sacks used in the slurry, \#bags of non-slurry bentonite (chip-pellet-granular) Additional information for Double cased or Nested wells:

\begin{tabular}{|c|c|c|c|c|c|}
\hline \multicolumn{2}{|c|}{$\begin{array}{c}\text { Placement Depth in } \\
\text { Feet }\end{array}$} & \multirow[t]{2}{*}{$\begin{array}{c}\text { Grout/Gravel/ } \\
\text { Open Hole }\end{array}$} & \multirow[t]{2}{*}{$\begin{array}{l}\text { Material Description } \\
\text { *See Desc for gravel }\end{array}$} & \multirow[t]{2}{*}{$\begin{array}{l}\text { Quantity gravel } \\
* \text { See Desc }\end{array}$} & \multirow[t]{2}{*}{$\begin{array}{c}\text { Volume \& Type Grout } \\
\text { *See Desc }\end{array}$} \\
\hline From & To & & & & \\
\hline & & & & & \\
\hline & & & & & \\
\hline & & & & & \\
\hline & & & & & \\
\hline & & & & & \\
\hline & & & & & \\
\hline & & & & & \\
\hline
\end{tabular}


WELL CONSTRUCTION LOG - 14721384835714

\begin{tabular}{|c|c|c|c|c|c|c|c|}
\hline $\begin{array}{l}\text { To } \\
0.0\end{array}$ & $\begin{array}{r}\text { From } \\
113.0\end{array}$ & $\begin{array}{l}\text { Case/Screen } \\
\text { Casing }\end{array}$ & $\begin{array}{l}\text { I.D. } \\
1.94\end{array}$ & $\begin{array}{l}0 . D . \\
2.38\end{array}$ & $\begin{array}{r}\text { Thk. } \\
0.21800\end{array}$ & $\begin{array}{l}\text { Mat. } \\
\text { PVC }\end{array}$ & $\begin{array}{l}\text { Slot Trade Name } \\
0.000 \text { Johnson }\end{array}$ \\
\hline 0 & 123.0 & Screen & 1.94 & 2.38 & 0.21800 & PVC & 0.010 Johnson \\
\hline
\end{tabular}

GROUT AND GRAVEL PACK - 14721384835714

\begin{tabular}{|c|c|c|c|c|c|}
\hline $\begin{array}{l}\text { From } \\
00.0\end{array}$ & $\begin{array}{lr}- & \text { To } \\
- & 104.0\end{array}$ & $\begin{array}{l}\text { Grout/Gravel } \\
\text { Grout }\end{array}$ & $\begin{array}{l}\text { Material Desc. } \\
\text { Bent Grout }\end{array}$ & Qty Gravel & $\begin{array}{l}\text { Volume/Type } \\
8 \text { Bags }\end{array}$ \\
\hline 104.0 & 110.0 & Grout & Pellets & & 1/2 Pail \\
\hline 110.0 & 123.0 & Gravel & $\# 40$ & 5 Bags & \\
\hline
\end{tabular}




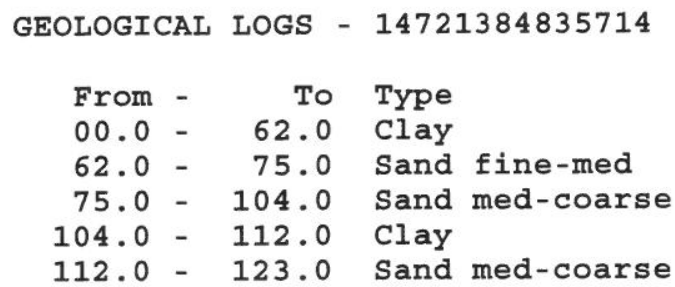

$\begin{array}{lll}\text { Hardness } & \text { Color } & \text { Other/Drilling Action } \\ \text { Soft } & \text { Brown } & \\ \text { Dense/Stiff } & \text { Brown } & \\ \text { Dense/Stiff } & \text { Brown } \\ \text { Dense/Stiff } & \text { Brown } \\ \text { Dense/Stiff } & \text { Brown }\end{array}$


12. Geologic Materials Logged (must start at zero) - Use only options provided below for Type, Hardness and Color

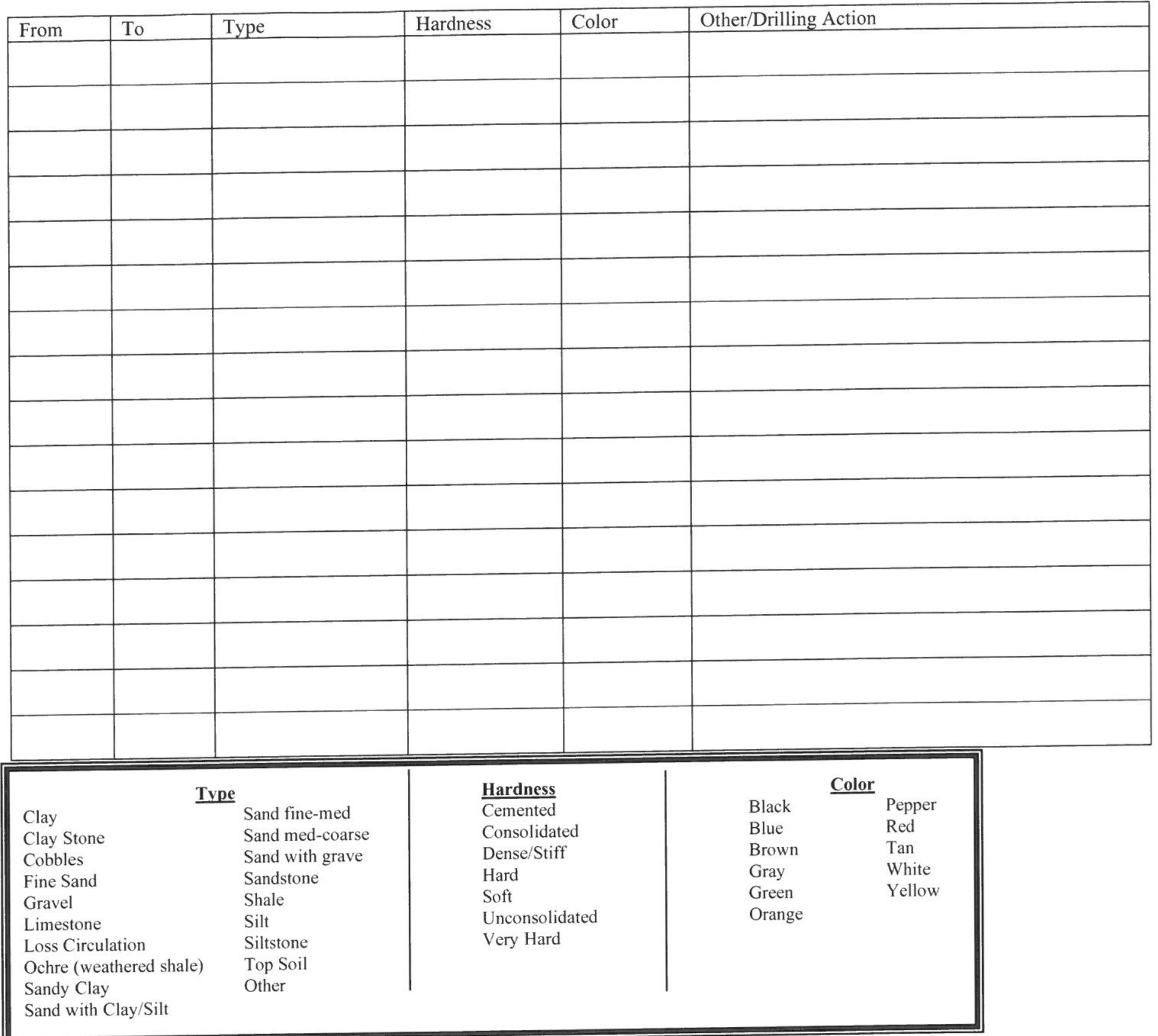

(Additional sheets may be submitted)

13. I hereby certify that the information provided on this registration is true and accurate to the best of my knowledge.
Water Well Contractor's Signature
Date
Well Owner's Signature
(not required for pits)
(if Contractor is unknown or Deceased or for pits)
(Not required if signed by Contractor)

Date

Please note this document contains four pages.

Sections 9F, 9G, 10, $11 \& 12$ are not required if registering a pit.

Owner Registering well drilled prior to 2002: Minimum Required Sections - 1, 3(a,b,c,e,f), 5, 6, 7, 8(a,f,h), 9e 
Mail to

Department of Natural Resources

PO Box 94676

Lincoln, NE 68509-4676

Phone (402)471-2363
STATE OF NEBRASKA

DEPARTMENT OF NATURAL RESOURCES

WATER WELL REGISTRATION

\section{FOR DEPARTMENT USE ONLY}

Registration No. G-180328

Receipt

1. a. Well Owner's First Name Last Name

OR Company Name: USDA / C CC

b. Attention Name Steve Gilmore-

c. Street Address 1400 INDEPENDENCE AUE -SW Address 2/PO Address 5 top 0513 Room $4714-5$ City Washington State DC Zip 20250-0513

2. a. Contractor's License No_ 39508 Contractor's Name STEVEN D JOHNSON Contractor's Email Address_SJOHNSON@CASCADEDRILLING.COM

b. Drilling Firm Name CASCADE DRILLING

Address 209 LEMIEUR ST

City LITTLE FALLS State $\mathrm{MN}$ Zip $\quad 56345$ Telephone $320 \quad 632 \quad 6552$

Drilling Firm's Email Address_STHALACKER@CASCADEDRILLING.COM

3. a. Well location $\mathrm{SW}^{1 / 4}$ of the $\mathrm{SE}^{1 / 4}$ of Section 30 , Township 11 North, Range 2 IWEST

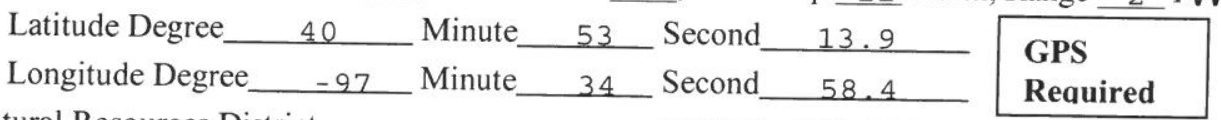

b. Natural Resources District UPPER BIG BLUE

c. The well is: 154 feet from the (North/ Soxth) section line and

d. Street address and subdivision, if applicable (circle one)

e. Location of water use (give legal descriptions) (BIOCK . LOT.) $\mathrm{N} / \mathrm{A}$

f. If for irrigation, the land to be irrigated is

g. Well reference letter(s), if applicable HHSS PWSID Location of water use is required on all wells

4. Permits

Management Area Permit Number

Geothermal Permit Number

Municipal Permit Number

Well Spacing Permit Number HHSS
Surface Water Permit Number

Industrial Permit Number

Transfer Out-Of-State Permit Number

Conduct Permit Number

Other Permit Number

NDEQ
5. Purpose of well (indicate one) __ Aquaculture __ Commercial/Industrial Domestic Ground Heat Exchanger Livestock $\mathrm{X}$ Monitoring Public Water Supply (without spacing)
Observation $\mathrm{G}$ Recovery Other
Pit (for irrigation)

(further description of use can be provided under other) (indicate use)

Dewatering (over 90 days) Irrigation

Public Water Supply (with spacing (46-638))
Location of well for a pit is the location of the pump

6. Wells in a Series.

a. Is this well a part of a series? ___ Yes go to part $\mathrm{b}$ of this section _ $\quad \mathrm{x} \quad$ No go to part 7 of this application (Y/N required)

b. If one or more of the wells in the series is currently registered, give well registration number

c. How many wells in the series are you registering at this time? d. How many total wells in the series? 
7. Replacement and decommissioned/modified well information.
a. Is this well a replacement well?
Yes $\mathrm{x}$ No go to part 8 of this application

b. Registration number of original well

c. Original well last operated $(\mathrm{m}) \_$_ $/$(d) $I_{(y)}$

If not registered, date or

e. Location of water use of original well

Please Select One:

f.1. $\square$ Original water well decommissioned on $(\mathrm{m}) \_/$(d_ $/$(y)

OR

2. $\square$ I hereby certify that the original water well will be decommissioned within 180 days after such construction of the replacement water well. OR

3. $\square$ I hereby certify that the original water well will be modified and equipped to pump 50 gallons per minute or less within 180 days after such construction of the replacement water well. It will be used for one of the following: a. $\square$ Livestock

b. $\square$ Monitoring c. $\square$ Observation

d. $\square$ nonconsumptive or de minimus use approved by the applicable natural resources district. State use:

If $3 \mathrm{~d}$ is chosen. NRD signature is required. (Signature can be submitted on NRD Approval form to DNR prior to registration)

NRD signature

Date

OR

4. $\square$ Decommission/Modification Certification form is submitted by landowner. (Must be submitted before registering well)

8. Pump Information.

(Pump information is required if registering a pit)

a. Is pump installed at this time

Is pump installed by well owner in section 1 ? ___ Yes _ $\mathrm{X} \quad$ No

Is pump installed by contractor in section 2 ? ___ Yes $\quad \mathrm{x} \quad$ No

Is this a free flowing well ___ Yes(no pump to be installed) $\quad \mathrm{x} \quad$ No

If pump installed by pump installer, please fill out license number below

b. Pump Installer's License No.

Pump Installer's Name

Pump Installer's Email Address

Pump Installer's Firm Name

Pump Installer's Firm Address

City State Zip $-0000$ Telephone

Pump Installer's Firm Email Address

c. Measured Pumping rate gallons per minute d. Pumping water level feet

e. Drop pipe diameter inches

f. Length of drop pipe feet

g. Pumping equipment installed $(\mathrm{m}) /_{\left(\mathrm{d} \_\right.} /(\mathrm{y})$

h. Pump Brand

i. This well is designed and constructed to pump less than $50 \mathrm{gpm} \quad \mathrm{x}$ Yes

No ( $8 \mathrm{H}$ is required on $\mathrm{ALL}$ wells)

9. Well Construction Information.

a. Total well depth 143 feet.

b. Static water level 75 feet. (required)

c. Well Construction Began $(\mathrm{m})$ $07(6) 08 / 2046$

d.. Well Construction Completed ${ }_{(\mathrm{m})}$ $07 / /(Q 8) 8 / 20,76$

Wells drilled prior to stays or NRD signature Date moratoriums require NRD signature

(Signature can be submitted on NRD Approval form to DNR prior to registration)

e. Bore hole diameter in inches Top Bottom 6

f. Casing and Screen Joints are Welded Glued Threaded $\mathrm{X}$ Other

g. Capacity of Well ___ gallons per minute (to be used to determine sustainability of aquifer)

h. Pumping water level at this capacity feet 
10. Well Construction (Casing \& Screen)- c, d, e, \& f measurements should be in inches to three decimal places (From not less than zero)

\begin{tabular}{|c|c|c|c|c|c|c|c|c|}
\hline & & b & c & d & $\mathrm{e}$ & f & $\mathrm{g}$ & $\mathrm{h}$ \\
\hline & $\begin{array}{l}\text { ment } \\
\text { in Feet }\end{array}$ & $\begin{array}{l}\text { Casing or } \\
\text { Screen or }\end{array}$ & $\begin{array}{c}\text { Inside } \\
\text { Diameter }\end{array}$ & $\begin{array}{c}\text { Outside } \\
\text { Diameter }\end{array}$ & $\begin{array}{c}\text { Wall } \\
\text { Thickness }\end{array}$ & $\begin{array}{l}\text { Screen Slot } \\
\text { Size }\end{array}$ & $\begin{array}{l}\text { Type of } \\
\text { Material }\end{array}$ & Trade Name \\
\hline From & To & Open Hole & & & & & & \\
\hline & & & & & & & & \\
\hline & & & & & & & & \\
\hline & & & & & & & & \\
\hline & & & & & & & & \\
\hline & & & & & & & & \\
\hline & & & & & & & & \\
\hline
\end{tabular}

Additional information for Double cased or Nested wells:

\begin{tabular}{|c|c|c|c|c|c|c|c|c|}
\hline \multicolumn{2}{|c|}{ a } & $\mathrm{b}$ & $\mathrm{c}$ & $\mathrm{d}$ & $\mathrm{e}$ & $\mathrm{f}$ & $\mathrm{g}$ & $\mathrm{h}$ \\
\hline \multicolumn{2}{|c|}{$\begin{array}{c}\text { Placement } \\
\text { Depth in Feet }\end{array}$} & \multirow{2}{*}{$\begin{array}{l}\text { Casing or } \\
\text { Screen or } \\
\text { Open Hole }\end{array}$} & \multirow[t]{2}{*}{$\begin{array}{c}\text { Inside } \\
\text { Diameter }\end{array}$} & \multirow[t]{2}{*}{$\begin{array}{c}\text { Outside } \\
\text { Diameter }\end{array}$} & \multirow[t]{2}{*}{$\begin{array}{c}\text { Wall } \\
\text { Thickness }\end{array}$} & \multirow[t]{2}{*}{$\begin{array}{c}\text { Screen Slot } \\
\text { Size }\end{array}$} & \multirow[t]{2}{*}{$\begin{array}{l}\text { Type of } \\
\text { Material }\end{array}$} & \multirow[t]{2}{*}{ Trade Name } \\
\hline From & To & & & & & & & \\
\hline & & & & & & & & \\
\hline & & & & & & & & \\
\hline & & & & & & & & \\
\hline & & & & & & & & \\
\hline & & & & & & & & \\
\hline & & & & & & & & \\
\hline
\end{tabular}

11. Grout and Gravel Pack (must start at zero)

\begin{tabular}{|l|l|l|l|l|l|}
\hline \multicolumn{2}{|c|}{$\begin{array}{c}\text { Placement Depth in } \\
\text { Feet }\end{array}$} & $\begin{array}{c}\text { Grout/Gravel/ } \\
\text { Open Hole }\end{array}$ & $\begin{array}{c}\text { Material Description } \\
\text { *See Desc for gravel }\end{array}$ & $\begin{array}{c}\text { Quantity gravel } \\
\text { *See Desc }\end{array}$ & $\begin{array}{c}\text { Volume \& Type Grout } \\
\text { *See Desc }\end{array}$ \\
\hline From & To & & & & \\
\hline & & & & & \\
\hline & & & & & \\
\hline & & & & & \\
\hline
\end{tabular}

Desc: Description of gravel pack i.e. engineered gravel pack, or gravel pit description ( $1 / 4$ down), or brand name (Best Sand), natural formation, drilling cuttings, soil backfill

Quantity \#cubic yards, \#tons, \#sacks, - (for drilling cuttings and soil backfill estimate quantity) Calculation assistance available on web Volume \& Type: \#gallons of a slurry, \#barrels of a slurry, \#sacks used in the slurry, \#bags of non-slurry bentonite (chip-pellet -granular) Additional information for Double cased or Nested wells:

\begin{tabular}{|c|c|c|c|c|c|}
\hline \multicolumn{2}{|c|}{$\begin{array}{c}\text { Placement Depth in } \\
\text { Feet }\end{array}$} & \multirow[t]{2}{*}{$\begin{array}{l}\text { Grout/Gravel/ } \\
\text { Open Hole }\end{array}$} & \multirow[t]{2}{*}{$\begin{array}{l}\text { Material Description } \\
\text { *See Desc for gravel }\end{array}$} & \multirow[t]{2}{*}{$\begin{array}{l}\text { Quantity gravel } \\
{ }^{*} \text { See Desc }\end{array}$} & \multirow[t]{2}{*}{$\begin{array}{c}\text { Volume \& Type Grout } \\
\text { *See Desc }\end{array}$} \\
\hline From & To & & & & \\
\hline & & & & & \\
\hline & & & & & \\
\hline & & & & & \\
\hline & & & & & \\
\hline & & & & & \\
\hline & & & & & \\
\hline & & & & & \\
\hline
\end{tabular}


WELL CONSTRUCTION LOG - 147213777025740

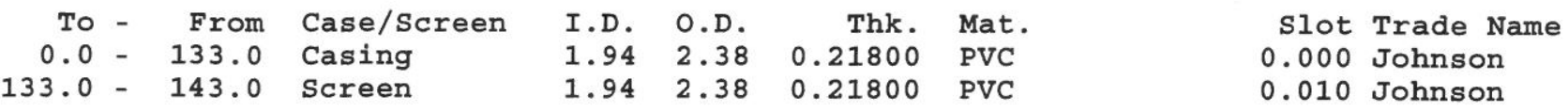

GROUT AND GRAVEL PACK - 147213777025740

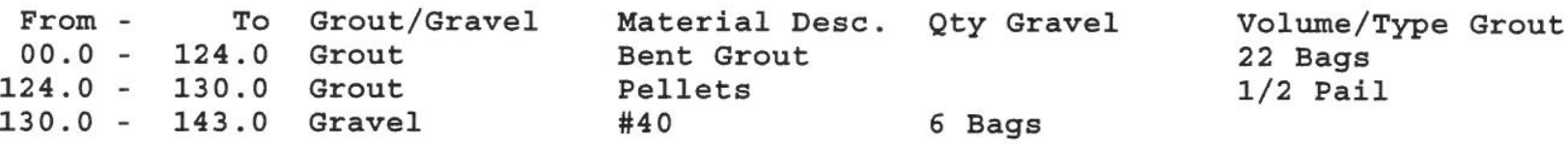


GEOLOGICAL LOGS - 147213777025740

\begin{tabular}{|c|c|c|c|c|c|}
\hline From & To & Type & Hardness & Color & other/Drilling \\
\hline 00.0 & 62.0 & clay & Soft & Brown & \\
\hline 62.0 & 75.0 & Sand fine-med & Dense/stiff & Brown & \\
\hline 75.0 & 104.0 & Sand med-coarse & Dense/stiff & Brown & \\
\hline 104.0 & $-\quad 112.0$ & Clay & Dense/stiff & Brown & \\
\hline 112.0 & 143.0 & Sand med-coarse & Dense/stiff & Brown & \\
\hline
\end{tabular}




\section{Geologic Materials Logged (must start at zero) - Use only options provided below for Type, Hardness and Color}

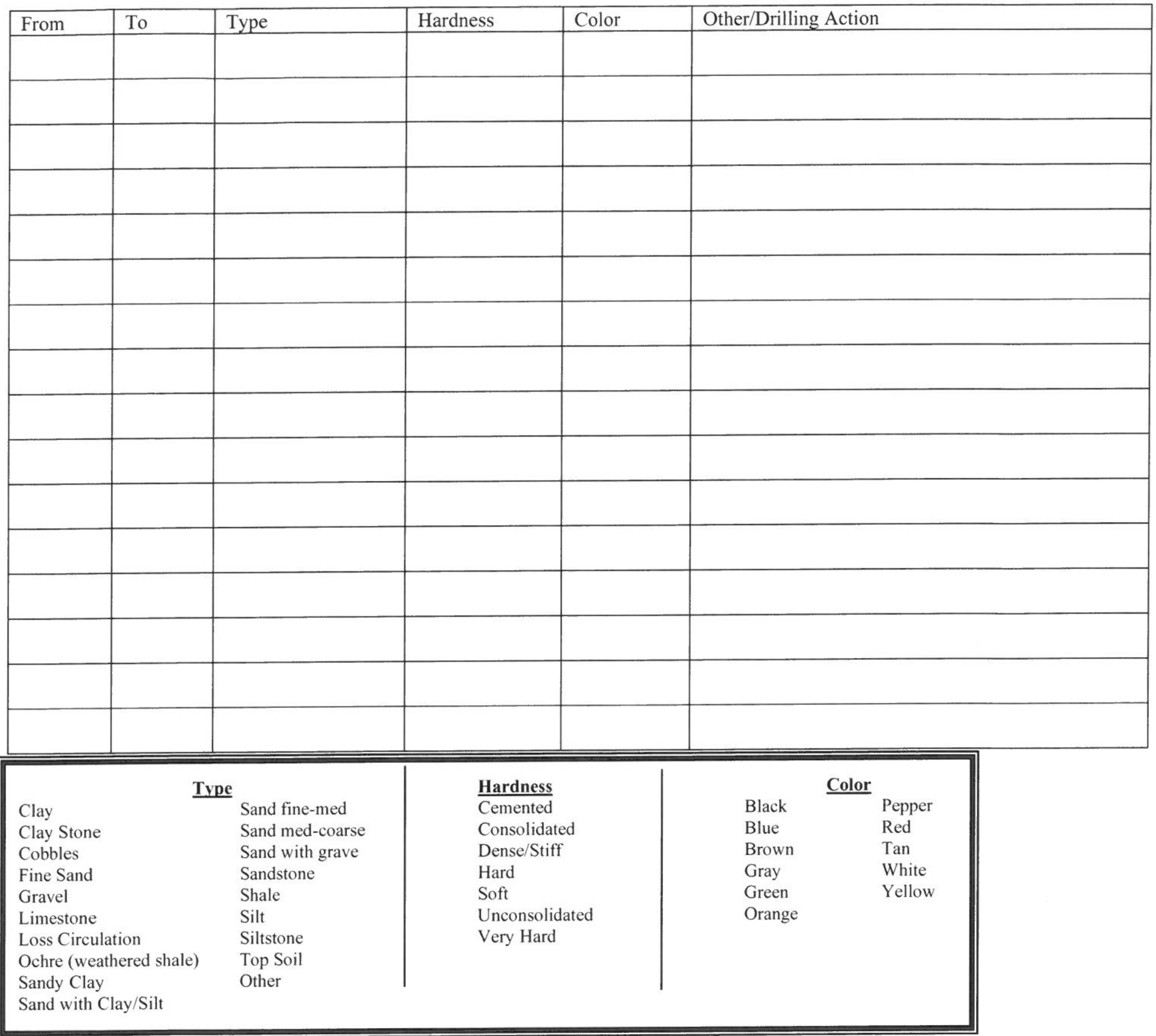

(Additional sheets may be submitted)

13. I hereby certify that the information provided on this registration is true and accurate to the best of my knowledge.
Water Well Contractor's Signature
Date
Well Owner's Signature
(not required for pits)
(if Contractor is unknown or Deceased or for pits)
(Not required if signed by Contractor)

Date

Please note this document contains four pages.

Sections 9F, 9G, 10, $11 \& 12$ are not required if registering a pit.

Owner Registering well drilled prior to 2002: Minimum Required Sections - 1, 3(a,b,c,e,f), 5, 6, 7, 8(a,f,h), 9e 
STATE OF NEBRASKA

\section{DEPARTMENT OF NATURAL RESOURCES}

WATER WELL REGISTRATION

Please indicate NA for items unknown

\section{FOR DEPARTMENT USE ONLY}

Date Filed

Owner Code No.

Registration No.

$6-180323$

$-\frac{244561}{\text { Well ID }}$

Receipt

NRD

1. a. Well Owner's First Name

Last Name

OR Company Name USDA/CCC

b. Attention Name Steve GIMore

c. Street Address 1400 INDEPENDENCE AUE $5 \%$

Address 2/PO Address Stop osß Room 4714 -5

City WASHINGTON

State $P C \quad$ Zip 20250-0513

Telephone_202-720-5104

2. a. Contractor's License No_ 39508 Contractor's Name STEVEN D JOHNSON

Contractor's Email Address_SJOHNSON@CASCADEDRILLING.COM

b. Drilling Firm Name CASCADE DRILLING

Address 209 LEMIEUR ST

City LITTLE FALLS

State_ MN

Zip $\quad 56345$

Telephone $\quad 320 \quad 632 \quad 6552$

Drilling Firm's Email Address_STHALACKER@CASCADEDRILLING.COM

3. a. Well location SE $1 / 4$ of the $S^{1 / 4}$ of Section 30 , Township 11 North, Range 2 IWEST

$\begin{array}{lllll}\text { Latitude Degree } \quad 40 & \text { Minute } & 53 & \text { Second } & 23.29 \\ \text { Longitude Degree } \quad-97 & \text { Minute } \quad 35 & \text { Second } & 23.31\end{array}$

b. Natural Resources District

UPPER BIG BLUE

c. The well is: 1099 feet from the (North/ Soxth) section line and

d. Street address and subdivision, if applicable (circle one)

e. Location of water use (give legal descriptions)

GPS

Required

2142

(BLOCK : TOT:

$\mathrm{N} / \mathrm{A}$

f. If for irrigation, the land to be irrigated is

g. Well reference letter(s), if applicable

$0 \quad$ acres

DL4-C1

HHSS PWSID
4. Permits

Management Area Permit Number

Geothermal Permit Number

Municipal Permit Number

Well Spacing Permit Number

HHSS

5. Purpose of well (indicate one) ___ Aquaculture Domestic Ground Heat Exchanger

Livestock $\mathrm{x}$ Monitoring

Public Water Supply (without spacing)
Surface Water Permit Number

Industrial Permit Number

Transfer Out-Of-State Permit Number

Conduct Permit Number

Other Permit Number NDEQ
Commercial/Industrial Groundwater Source Heat Pump

Observation Recovery
Other
Dewatering (over 90 days) Irrigation

Public Water Supply (with spacing (46-638))
Location of well for a pit is the location of the pump feet from the (East/Wesst) section line (circle one)

6. Wells in a Series.

a. Is this well a part of a series? ___ Yes go to part $\mathrm{b}$ of this section _ $\quad \mathrm{x} \quad$ No go to part 7 of this application (Y/N required)

b. If one or more of the wells in the series is currently registered, give well registration number

c. How many wells in the series are you registering at this time? d. How many total wells in the series? 
7. Replacement and decommissioned/modified well information.

a. Is this well a replacement well? ___ Yes $\mathrm{x}$ No go to part 8 of this application

b. Registration number of original well

c. Original well last operated (m)___ (d)

(d) $\quad$ (y)

$I_{(y)}$

If not registered, date or

e. Location of water use of original well

Please Select One:

f.1. $\square$ Original water well decommissioned on $(\mathrm{m}) \_/ \mathrm{d}_{\text {(___ } /(\mathrm{y}) \_ \text {OR }}$

2. $\square$ I hereby certify that the original water well will be decommissioned within 180 days after such construction of the replacement water well. OR

3. $\square$ I hereby certify that the original water well will be modified and equipped to pump 50 gallons per minute or less within 180 days after such construction of the replacement water well. It will be used for one of the following: a. $\square$ Livestock

b. $\square$ Monitoring c. $\square$ Observation

d. $\square$ nonconsumptive or de minimus use approved by the applicable natural resources district. State use:

If $3 \mathrm{~d}$ is chosen. NRD signature is required. (Signature can be submitted on NRD Approval form to DNR prior to registration)

NRD signature

Date

OR

4. $\square$ Decommission/Modification Certification form is submitted by landowner. (Must be submitted before registering well)

8. Pump Information.

(Pump information is required if registering a pit)

a. Is pump installed at this time __ Yes $\quad \mathrm{x}$ No

Is pump installed by well owner in section 1? ___ Yes $\quad \mathrm{X} \quad$ No

Is pump installed by contractor in section 2? __ Yes $\mathrm{x} \quad$ No

Is this a free flowing well ___ Yes(no pump to be installed) $\quad \mathrm{x}$ No

If pump installed by pump installer, please fill out license number below

b. Pump Installer's License No. Pump Installer's Name

Pump Installer's Email Address

Pump Installer's Firm Name

Pump Installer's Firm Address

City State

Zip $\quad-0000$
Telephone

Pump Installer's Firm Email Address

c. Measured Pumping rate gallons per minute

d. Pumping water level feet

e. Drop pipe diameter inches

f. Length of drop pipe feet

g. Pumping equipment installed $(\mathrm{m}) \_/\left(\mathrm{d} \_/ /(\mathrm{y})\right.$

h. Pump Brand

i. This well is designed and constructed to pump less than $50 \mathrm{gpm} \quad \mathrm{x}$ Yes

No (8H is required on ALL wells)

9. Well Construction Information.

a. Total well depth 130 feet.

b. Static water level 75 feet. (required)

c. Well Construction Began $(\mathrm{m}) \quad 07(6) 02 / 20 \mathbb{A}_{1}, \quad$

d.. Well Construction Completed $(\mathrm{m})$ $071 / 102 / 20,16$

Wells drilled prior to stays or

NRD signature Date moratoriums require NRD signature

(Signature can be submitted on NRD Approval form to DNR prior to registration)

e. Bore hole diameter in inches Top 6 Bottom 6

f. Casing and Screen Joints are Welded Glued Threaded $\mathrm{x}$ Other

g. Capacity of Well 4 gallons per minute (to be used to determine sustainability of aquifer)

h. Pumping water level at this capacity feet 
10. Well Construction (Casing \& Screen)- c, d, e, \& f measurements should be in inches to three decimal places (From not less than zero)

\begin{tabular}{|c|c|c|c|c|c|c|c|c|}
\hline \\
\hline & a & $\mathrm{b}$ & $\mathrm{c}$ & $\mathrm{d}$ & $\mathrm{e}$ & $\mathrm{f}$ & $\mathrm{g}$ & $\mathrm{h}$ \\
\hline \multirow{2}{*}{\multicolumn{2}{|c|}{$\begin{array}{c}\text { Placement } \\
\text { Depth in Feet }\end{array}$}} & \multirow{3}{*}{$\begin{array}{l}\text { Casing or } \\
\text { Screen or } \\
\text { Open Hole }\end{array}$} & \multirow{3}{*}{$\begin{array}{c}\text { Inside } \\
\text { Diameter }\end{array}$} & \multirow{3}{*}{$\begin{array}{c}\text { Outside } \\
\text { Diameter }\end{array}$} & \multirow{3}{*}{$\begin{array}{c}\text { Wall } \\
\text { Thickness }\end{array}$} & \multirow{3}{*}{$\begin{array}{l}\text { Screen Slot } \\
\text { Size }\end{array}$} & \multirow{3}{*}{$\begin{array}{l}\text { Type of } \\
\text { Material }\end{array}$} & \multirow[t]{3}{*}{ Trade Name } \\
\hline & & & & & & & & \\
\hline From & To & & & & & & & \\
\hline & & & & & & & & \\
\hline & & & & & & & & \\
\hline & & & & & & & & \\
\hline & & & & & & & & \\
\hline & & & & & & & & \\
\hline & & & & & & & & \\
\hline
\end{tabular}

Additional information for Double cased or Nested wells:

\begin{tabular}{|c|c|c|c|c|c|c|c|c|}
\hline \multicolumn{2}{|c|}{ a } & $\mathrm{b}$ & $\mathrm{c}$ & d & $\mathrm{e}$ & f & $\mathrm{g}$ & $\mathrm{h}$ \\
\hline \multicolumn{2}{|c|}{$\begin{array}{c}\text { Placement } \\
\text { Depth in Feet }\end{array}$} & $\begin{array}{l}\text { Casing or } \\
\text { Screen or }\end{array}$ & $\begin{array}{c}\text { Inside } \\
\text { Diameter }\end{array}$ & $\begin{array}{c}\text { Outside } \\
\text { Diameter }\end{array}$ & $\begin{array}{c}\text { Wall } \\
\text { Thickness }\end{array}$ & $\begin{array}{c}\text { Screen Slot } \\
\text { Size }\end{array}$ & $\begin{array}{l}\text { Type of } \\
\text { Material }\end{array}$ & Trade Name \\
\hline From & To & Open Hole & & & & & & \\
\hline & & & & & & & & \\
\hline & & & & & & & & \\
\hline & & & & & & & & \\
\hline & & & & & & & & \\
\hline & & & & & & & & \\
\hline & & & & & & & & \\
\hline
\end{tabular}

11. Grout and Gravel Pack (must start at zero)

\begin{tabular}{|l|l|l|l|l|l|}
\hline \multicolumn{2}{|c|}{$\begin{array}{c}\text { Placement Depth in } \\
\text { Feet }\end{array}$} & $\begin{array}{c}\text { Grout/Gravel/ } \\
\text { Open Hole }\end{array}$ & $\begin{array}{c}\text { Material Description } \\
\text { *See Desc for gravel }\end{array}$ & $\begin{array}{c}\text { Quantity gravel } \\
\text { *See Desc }\end{array}$ & $\begin{array}{c}\text { Volume \& Type Grout } \\
\text { *See Desc }\end{array}$ \\
\hline From & To & & & & \\
\hline & & & & & \\
\hline & & & & & \\
\hline & & & & & \\
\hline
\end{tabular}

Desc: Description of gravel pack i.e. engineered gravel pack, or gravel pit description ( $1 / 4$ down), or brand name (Best Sand), natural formation, drilling cuttings, soil backfill

Quantity \#cubic yards, \#tons, \#sacks, - (for drilling cuttings and soil backfill estimate quantity) Calculation assistance available on web Volume \& Type: \#gallons of a slurry, \#barrels of a slurry, \#sacks used in the slurry, \#bags of non-slurry bentonite (chip-pellet -granular) Additional information for Double cased or Nested wells:

\begin{tabular}{|c|c|c|c|c|c|}
\hline \multicolumn{2}{|c|}{$\begin{array}{c}\text { Placement Depth in } \\
\text { Feet }\end{array}$} & \multirow[t]{2}{*}{$\begin{array}{l}\text { Grout/Gravel/ } \\
\text { Open Hole }\end{array}$} & \multirow[t]{2}{*}{$\begin{array}{l}\text { Material Description } \\
\text { *See Desc for gravel }\end{array}$} & \multirow[t]{2}{*}{$\begin{array}{l}\text { Quantity gravel } \\
\text { *See Desc }\end{array}$} & \multirow[t]{2}{*}{$\begin{array}{c}\text { Volume \& Type Grout } \\
\text { *See Desc }\end{array}$} \\
\hline From & To & & & & \\
\hline & & & & & \\
\hline & & & & & \\
\hline & & & & & \\
\hline & & & & & \\
\hline & & & & & \\
\hline & & & & & \\
\hline & & & & & \\
\hline
\end{tabular}


WELL CONSTRUCTION LOG - 147213594319355

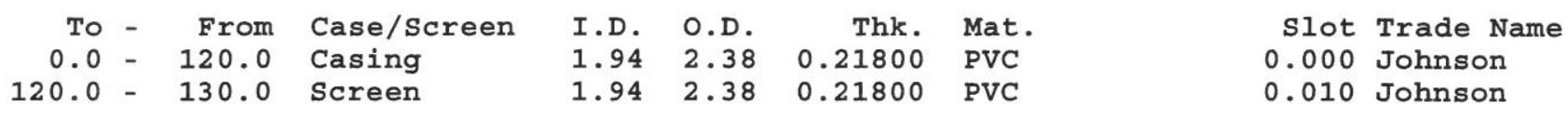

GROUT AND GRAVEL PACK - 147213594319355

\begin{tabular}{|c|c|c|c|c|c|}
\hline From & To & Grout/Gravel & Material Desc. & Qty Gravel & Volume/Type \\
\hline 00.0 & 108.0 & Grout & Bent Grout & & 7 Bags \\
\hline 108.0 & 116.0 & Grout & Pellets & & 1 Pail \\
\hline 116.0 & 130.0 & Gravel & \#40 & 7 Bags & \\
\hline
\end{tabular}


GEOLOGICAL LOGS - 147213594319355

$\begin{array}{lrlll}\text { From - } & \text { To } & \text { Type } & \text { Hardness } & \text { Color Other/Drilling Action } \\ 00.0- & 55.0 & \text { Clay } & \text { Soft } & \text { Brown } \\ 55.0- & 85.0 & \text { Sand fine-med } & \text { Dense/stiff } & \text { Brown } \\ 85.0- & 130.0 & \text { Sand med-coarse } & \text { Dense/stiff } & \text { Brown }\end{array}$


12. Geologic Materials Logged (must start at zero) - Use only options provided below for Type, Hardness and Color

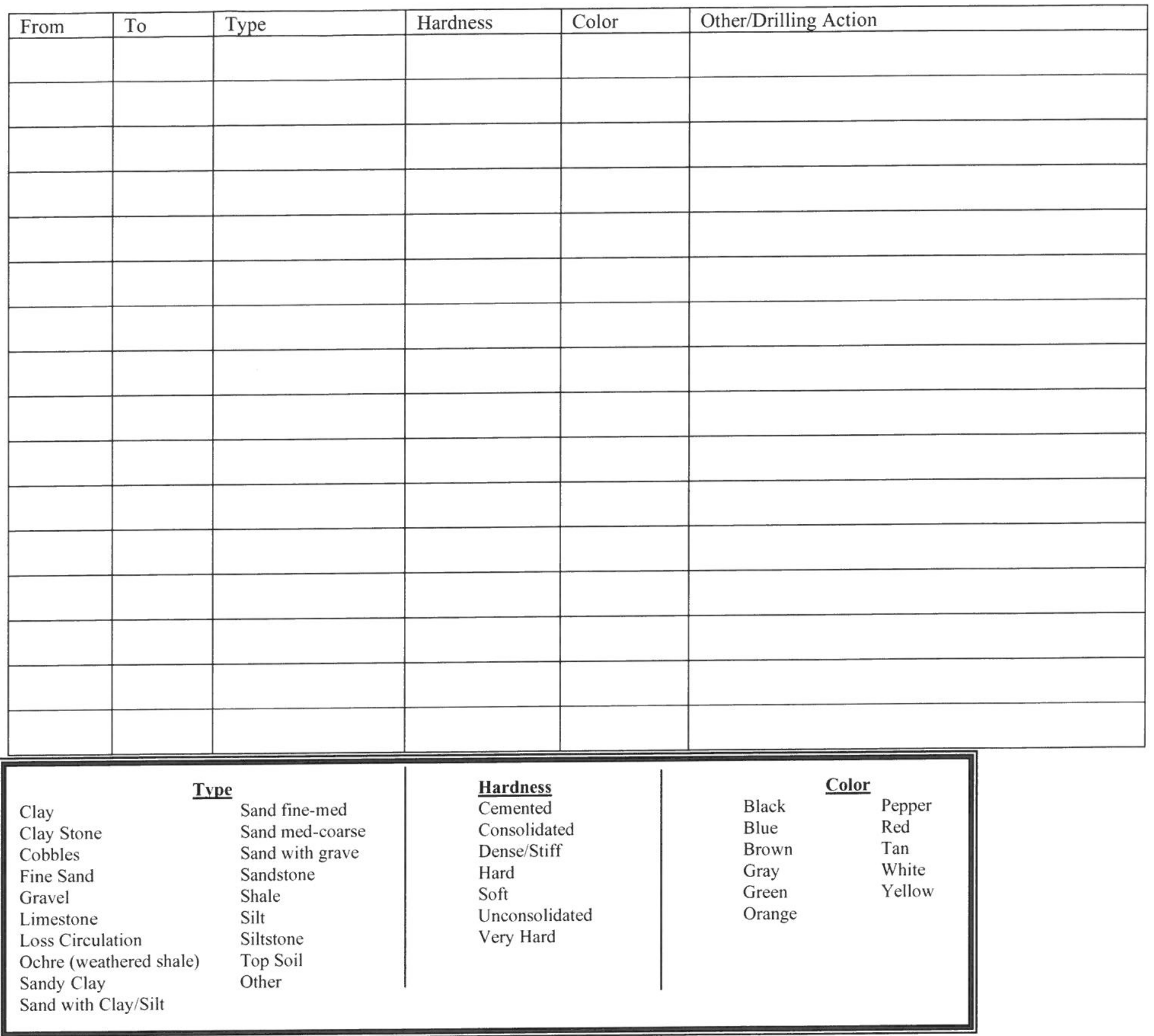

(Additional sheets may be submitted)

13. I hereby certify that the information provided on this registration is true and accurate to the best of my knowledge.
Water Well Contractor's Signature
Date
Well Owner's Signature
(not required for pits)
(if Contractor is unknown or Deceased or for
(Not required if signed by Contractor)

Date

Please note this document contains four pages.

Sections 9F, 9G, 10, $11 \& 12$ are not required if registering a pit.

Owner Registering well drilled prior to 2002: Minimum Required Sections - 1, 3(a,b,c,e,f), 5, 6, 7, 8(a,f,h), 9e 
Mail to

Department of Natural Resources

PO Box 94676

Lincoln, NE 68509-4676

Phone (402)471-2363

STATE OF NEBRASKA

\section{DEPARTMENT OF NATURAL RESOURCES}

WATER WELL REGISTRATION Please indicate NA for items unknown

\section{FOR DEPARTMENT USE ONLY}

Date Filed

Owner Code No.

Registration No. $6-180321$

\section{9 Well ID}

Receipt NRD

1. a. Well Owner's First Name Last Name

OR Company Name USDA $/ \mathrm{CCC}$

b. Attention Name Steve Gluare

c. Street Address 1400 INDOPENDENCE ALE SW

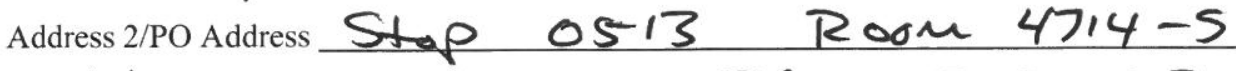
City WASHINGTON

2. a. Contractor's License No 39508 Contractor's Name STEVEN D JOHNSON

Contractor's Email Address_SJOHNSON@CASCADEDRILLING.COM

b. Drilling Firm Name CASCADE DRILLING

Address 209 LEMIEUR ST

City LITTLE FALLS

State MN

Zip $\quad 56345$

Telephone $\quad 320 \quad 632 \quad 6552$

Drilling Firm's Email Address_STHALACKER@CASCADEDRILLING.COM

3. a. Well location $S E E^{1 / 4}$ of the $S_{W}^{1 / 4}$ of Section _ 30, Township 11 North, Range _2 IWEST

\begin{tabular}{|c|c|c|c|c|c|}
\hline Latitude Degree & 40 & Minute & 53 Second & 13.17 & GPS \\
\hline Longitude Degre & -97 & Minute & 35 Second & 23.31 & Required \\
\hline
\end{tabular}

b. Natural Resources District

feet from the (North/ Sogth) section line and (circle one)

d. Street address and subdivision, if applicable

e. Location of water use (give legal descriptions)

f. If for irrigation, the land to be irrigated is

g. Well reference letter(s), if applicable acres.

HHSS PWSID
4. Permits

Management Area Permit Number

Geothermal Permit Number

Municipal Permit Number

Well Spacing Permit Number HHSS

5. Purpose of well (indicate one) Aquaculture Domestic Ground Heat Exchanger Livestock $\mathrm{X}$ Monitoring

Public Water Supply (without spacing) __ Recovery __ Other

Public Water Supply (without spacing) __ Recovery __ Other Observation

0
2146 (BIOCK : TOT. $\mathrm{N} / \mathrm{A}$ feet from the (East/West) section line (circle one)

Location of well for a pit is the location of the pump

Surface Water Permit Number

Industrial Permit Number

Transfer Out-Of-State Permit Number

Conduct Permit Number

Other Permit Number NDEQ
Commercial/Industrial Groundwater Source Heat Pump Pit (for irrigation)
Location of water use is required on all wells
Dewatering (over 90 days) Irrigation Injection

6. Wells in a Series.

a. Is this well a part of a series? ___ Yes go to part $\mathrm{b}$ of this section $\quad \mathrm{x} \quad$ No go to part 7 of this application (Y/N required)

b. If one or more of the wells in the series is currently registered, give well registration number

c. How many wells in the series are you registering at this time? d. How many total wells in the series? 
7. Replacement and decommissioned/modified well information.
a. Is this well a replacement well?
Yes $\mathrm{x}$ No go to part 8 of this application
b. Registration number of original well
If not registered, date ori
c. Original well last operated $(\mathrm{m}) \_$_ $/$(d) $I^{\prime}(y)$
d. Replacement well is was constructed $(\mathrm{m}) /$
feet from original well.
e. Location of water use of original well

Please Select One:

f.1. $\square$ Original water well decommissioned on $(\mathrm{m}) \_/ /_{\text {(d__ } \_/(y) \_}$OR

2. $\square$ I hereby certify that the original water well will be decommissioned within 180 days after such construction of the replacement water well. $\mathbf{O R}$

3. $\square$ I hereby certify that the original water well will be modified and equipped to pump 50 gallons per minute or less within 180 days after such construction of the replacement water well. It will be used for one of the following: a. $\square$ Livestock

b. $\square$ Monitoring c. $\square$ Observation

d. $\square$ nonconsumptive or de minimus use approved by the applicable natural resources district. State use:

If $3 \mathrm{~d}$ is chosen. NRD signature is required. (Signature can be submitted on NRD Approval form to DNR prior to registration)

NRD signature__ Date___ OR

4. $\square$ Decommission/Modification Certification form is submitted by landowner. (Must be submitted before registering well)

8. Pump Information.

(Pump information is required if registering a pit)

a. Is pump installed at this time

Yes $\mathrm{x}$ No

Is pump installed by well owner in section 1? Y_ Yes $\quad \mathrm{X}$ No

Is pump installed by contractor in section 2? __ Yes $\quad \mathrm{x} \quad$ No

Is this a free flowing well ___ Yes(no pump to be installed) $\mathrm{x}$ No

If pump installed by pump installer, please fill out license number below

b. Pump Installer's License No.

Pump Installer's Name

Pump Installer's Email Address

Pump Installer's Firm Name

Pump Installer's Firm Address

City State Zip $\quad-0000$ Telephone

Pump Installer's Firm Email Address

c. Measured Pumping rate gallons per minute

d. Pumping water level feet

e. Drop pipe diameter inches

f. Length of drop pipe feet

g. Pumping equipment installed $(\mathrm{m}) \_/ /_{\left(\mathrm{d} \_\right.} /(\mathrm{y}) \ldots$

h. Pump Brand

i. This well is designed and constructed to pump less than $50 \mathrm{gpm} \quad \mathrm{X}$ Yes

No (8H is required on ALL wells)

9. Well Construction Information.

a. Total well depth 155 feet. $\quad$ b. Static water level 75 feet. (required)

c. Well Construction Began ${ }_{(\mathrm{m})} \_07(6) 01 / 204(6) \_$d.. Well Construction Completed $(\mathrm{m})$

$07 / / Q 2 / 2 Q, 476$

Wells drilled prior to stays or

NRD signature

Date

moratoriums require NRD signature

(Signature can be submitted on NRD Approval form to DNR prior to registration)

e. Bore hole diameter in inches Top

Bottom

6

f. Casing and Screen Joints are Welded

Glued

Threaded Other

g. Capacity of Well _ 4 gallons per minute (to be used to determine sustainability of aquifer)

h. Pumping water level at this capacity feet 
10. Well Construction (Casing \& Screen)- c, d, e, \& f measurements should be in inches to three decimal places (From not less than zero)

\begin{tabular}{|c|c|c|c|c|c|c|c|c|}
\hline & a & $\mathrm{b}$ & c & d & $\mathrm{e}$ & $\mathrm{f}$ & $\mathrm{g}$ & $\mathrm{h}$ \\
\hline & $\begin{array}{l}\text { ment } \\
\text { in Feet }\end{array}$ & $\begin{array}{l}\text { Casing or } \\
\text { Screen or }\end{array}$ & $\begin{array}{c}\text { Inside } \\
\text { Diameter }\end{array}$ & $\begin{array}{l}\text { Outside } \\
\text { Diameter }\end{array}$ & $\begin{array}{c}\text { Wall } \\
\text { Thickness }\end{array}$ & $\begin{array}{c}\text { Screen Slot } \\
\text { Size }\end{array}$ & $\begin{array}{l}\text { Type of } \\
\text { Material }\end{array}$ & Trade Name \\
\hline From & To & Open Hole & & & & & & \\
\hline & & & & & & & & \\
\hline & & & & & & & & \\
\hline & & & & & & & & \\
\hline & & & & & & & & \\
\hline & & & & & & & & \\
\hline
\end{tabular}

Additional information for Double cased or Nested wells:

\begin{tabular}{|c|c|c|c|c|c|c|c|c|}
\hline & & $\mathrm{b}$ & c & $\mathrm{d}$ & $\mathrm{e}$ & $\mathrm{f}$ & $\mathrm{g}$ & $\mathrm{h}$ \\
\hline & $\begin{array}{l}\text { ment } \\
\text { in Feet }\end{array}$ & $\begin{array}{l}\text { Casing or } \\
\text { Screen or }\end{array}$ & $\begin{array}{c}\text { Inside } \\
\text { Diameter }\end{array}$ & $\begin{array}{l}\text { Outside } \\
\text { Diameter }\end{array}$ & $\begin{array}{c}\text { Wall } \\
\text { Thickness }\end{array}$ & $\begin{array}{c}\text { Screen Slot } \\
\text { Size }\end{array}$ & $\begin{array}{l}\text { Type of } \\
\text { Material }\end{array}$ & Trade Name \\
\hline From & To & Open Hole & & & & & & \\
\hline & & & & & & & & \\
\hline & & & & & & & & \\
\hline & & & & & & & & \\
\hline & & & & & & & & \\
\hline & & & & & & & & \\
\hline & & & & & & & & \\
\hline
\end{tabular}

11. Grout and Gravel Pack (must start at zero)

\begin{tabular}{|c|c|c|c|c|c|}
\hline \multicolumn{2}{|c|}{$\begin{array}{c}\text { Placement Depth in } \\
\text { Feet }\end{array}$} & \multirow[t]{2}{*}{$\begin{array}{l}\text { Grout/Gravel/ } \\
\text { Open Hole }\end{array}$} & \multirow[t]{2}{*}{$\begin{array}{l}\text { Material Description } \\
\text { *See Desc for gravel }\end{array}$} & \multirow[t]{2}{*}{$\begin{array}{l}\text { Quantity gravel } \\
* \text { See Desc }\end{array}$} & \multirow[t]{2}{*}{$\begin{array}{c}\text { Volume \& Type Grout } \\
\text { *See Desc }\end{array}$} \\
\hline From & To & & & & \\
\hline & & & & & \\
\hline & & & & & \\
\hline & & & & & \\
\hline & & & & & \\
\hline & & & & & \\
\hline & & & & & \\
\hline & & & & & \\
\hline
\end{tabular}

Desc: Description of gravel pack i.e. engineered gravel pack, or gravel pit description ( $1 / 4$ down), or brand name (Best Sand), natural formation, drilling cuttings, soil backfill

Quantity \#cubic yards, \#tons, \#sacks, - (for drilling cuttings and soil backfill estimate quantity) Calculation assistance available on web Volume \& Type: \#gallons of a slurry, \#barrels of a slurry, \#sacks used in the slurry, \#bags of non-slurry bentonite (chip-pellet-granular) Additional information for Double cased or Nested wells:

\begin{tabular}{|c|c|c|c|c|c|}
\hline \multicolumn{2}{|c|}{$\begin{array}{c}\text { Placement Depth in } \\
\text { Feet }\end{array}$} & \multirow[t]{2}{*}{$\begin{array}{c}\text { Grout/Gravel/ } \\
\text { Open Hole }\end{array}$} & \multirow[t]{2}{*}{$\begin{array}{l}\text { Material Description } \\
\text { * See Desc for gravel }\end{array}$} & \multirow[t]{2}{*}{$\begin{array}{l}\text { Quantity gravel } \\
\text { *See Desc }\end{array}$} & \multirow[t]{2}{*}{$\begin{array}{c}\text { Volume \& Type Grout } \\
\text { *See Desc }\end{array}$} \\
\hline From & To & & & & \\
\hline & & & & & \\
\hline & & & & & \\
\hline & & & & & \\
\hline & & & & & \\
\hline & & & & & \\
\hline & & & 1 & & \\
\hline & & & & & \\
\hline
\end{tabular}


WELL CONSTRUCTION LOG - 147213548922249

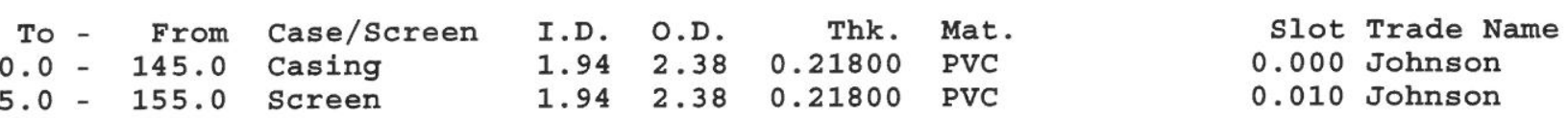

GROUT AND GRAVEL PACK - 147213548922249

\begin{tabular}{|c|c|c|c|c|c|}
\hline From & To & Grout/Gravel & Material Desc. & Qty Gravel & Volume/Type Grout \\
\hline $\begin{array}{l}00.0 \\
35.0\end{array}$ & $\begin{array}{l}135.0 \\
140.0\end{array}$ & $\begin{array}{l}\text { Grout } \\
\text { Grout }\end{array}$ & $\begin{array}{l}\text { Bent Grout } \\
\text { Pellets }\end{array}$ & & $\begin{array}{l}8 \text { Bags } \\
1 / 2 \text { Pail }\end{array}$ \\
\hline 40.0 & 155.0 & Gravel & $\# 40$ & 6 Bags & \\
\hline
\end{tabular}


GEOLOGICAL LOGS - 147213548922249

$\begin{array}{lrlll}\text { From - } & \text { To } & \text { Type } & \text { Hardness } & \text { Color Other/Drilling Action } \\ 00.0- & 55.0 & \text { Clay } & \text { Soft } & \text { Brown } \\ 55.0- & 75.0 & \text { Sand fine-med } & \text { Dense/Stiff } & \text { Brown } \\ 75.0- & 155.0 & \text { Sand med-coarse } & \text { Dense/Stiff } & \text { Brown }\end{array}$


12. Geologic Materials Logged (must start at zero) - Use only options provided below for Type, Hardness and Color

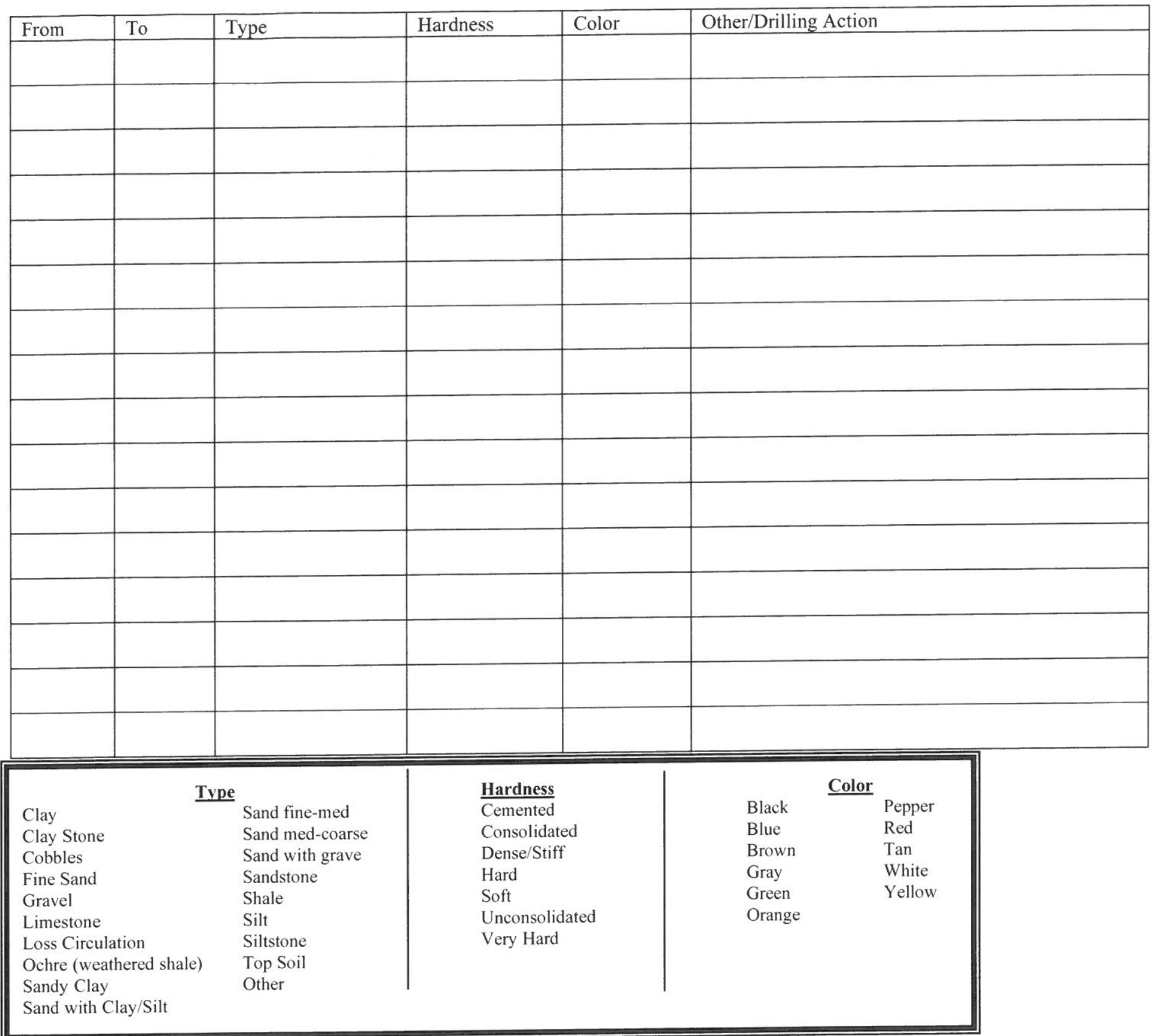

(Additional sheets may be submitted)

13. I hereby certify that the information provided on this registration is true and accurate to the best of my knowledge.
Water Well Contractor's Signature
Date
Well Owner's Signature
(not required for pits)
(if Contractor is unknown or Deceased or for pits)
(Not required if signed by Contractor)

Date

Please note this document contains four pages.

Sections 9F, 9G, 10, $11 \& 12$ are not required if registering a pit.

Owner Registering well drilled prior to 2002: Minimum Required Sections - 1, 3(a,b,c,e,f), 5, 6, 7, 8(a,f,h), 9e 
Mail to

Department of Natural Resources

PO Box 94676

Lincoln, NE 68509-4676

Phone (402)471-2363
STATE OF NEBRASKA

\section{DEPARTMENT OF NATURAL RESOURCES}

WATER WELL REGISTRATION Please indicate NA for items unknown

\section{FOR DEPARTMENT USE ONLY}

Date Filed

Owner Code No.

Registration No. G-180320

Well ID

1. a. Well Owner's First Name Last Name

OR Company Name USDA/CCC

b. Attention Name Steve G'lmoret

c. Street Address 1400 INDEPENDENCE ACE SW Address 2/PO Address Stop 0513 Room $4714-5$ City Washingtor State $D C \quad$ Zip_20250-0513 Telephone

2. a. Contractor's License No_ 39508 Contractor's Name_STEVEN D JOHNSON

Contractor's Email Address_SJOHNSON@CASCADEDRILLING.COM

b. Drilling Firm Name CASCADE DRILLING

Address 209 LEMIEUR ST

City LITTLE FALLS State_MN_Zip_ 56345 Telephone $\quad 320 \quad 632 \quad 6552$

Drilling Firm's Email Address_STHALACKER@CASCADEDRILLING.COM

3. a. Well location $5 E^{1 / 4}$ of the NW $1 / 4$ of Section 32 , Township 11 North, Range _ 2 IWEST

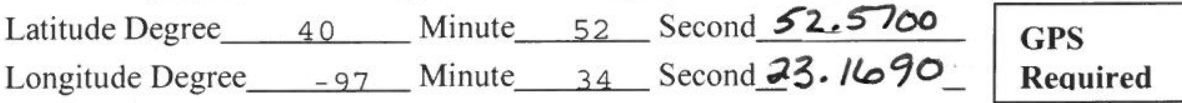

b. Natural Resources District UPPER BIG BLUE

c. The well is: 1352 feet from the (Noth/ South) section line and (circle one) 1362 (BIOCK: LOT:)

d. Street address and subdivision, if applicable

e. Location of water use (give legal descriptions) $\mathrm{N} / \mathrm{A}$

f. If for irrigation, the land to be irrigated is 0 acres.

g. Well reference letter(s), if applicable DL6-C HHSS PWSID
4. Permits

Management Area Permit Number

Geothermal Permit Number

Municipal Permit Number

Well Spacing Permit Number HHSS

5. Purpose of well (indicate one) Aquaculture Ground Heat Exchanger Observation Domestic $\mathrm{x}$ Monitoring Livestock

Public Water Supply (without spacing) Recovery

Surface Water Permit Number

Industrial Permit Number

Transfer Out-Of-State Permit Number

Conduct Permit Number

Other Permit Number

NDEQ

Commercial/Industrial Groundwater Source Heat Pump Pit (for irrigation)
YORK County

Location of well for a pit is the location of the pump feet from the (Exst/West) section line (circle one)
Location of water use is required on all wells
Dewatering (over 90 days) Irrigation Injection Public Water Supply (with spacing (46-638))

6. Wells in a Series.

a. Is this well a part of a series?

b. If one or more of the wells in the series is currently registered, give well registration number

c. How many wells in the series are you registering at this time? d. How many total wells in the series? 
7. Replacement and decommissioned/modified well information.
a. Is this well a replacement well?
Yes $\mathrm{X}$ No go to part 8 of this application
b. Registration number of original well
-
(d) $\quad /(y)$

(y)
If not registered, date o
c. Original well last operated $(\mathrm{m}) \_$_ (d)
e. Location of water use of original well

Please Select One:

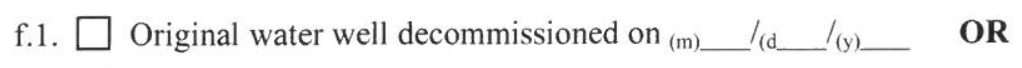

2. $\square$ I hereby certify that the original water well will be decommissioned within 180 days after such construction of the replacement water well. OR

3. $\square$ I hereby certify that the original water well will be modified and equipped to pump 50 gallons per minute or less within 180 days after such construction of the replacement water well. It will be used for one of the following: a. $\square$ Livestock b. $\square$ Monitoring c. $\square$ Observation

d. $\square$ nonconsumptive or de minimus use approved by the applicable natural resources district. State use:

If $3 \mathrm{~d}$ is chosen. NRD signature is required. (Signature can be submitted on NRD Approval form to DNR prior to registration)

NRD signature___ Date_ OR

4. $\square$ Decommission/Modification Certification form is submitted by landowner. (Must be submitted before registering well)
8. Pump Information.
(Pump information is required if registering a pit)

a. Is pump installed at this time __ Yes $\quad \mathrm{x}$ No
Is pump installed by well owner in section 1 ?
Is pump installed by contractor in section 2 ?
Yes $\mathrm{x}$ No
Yes $\mathrm{X}$ No

Is this a free flowing well ___ Yes(no pump to be installed) $\mathrm{x}$ No

If pump installed by pump installer, please fill out license number below

b. Pump Installer's License No. Pump Installer's Name

Pump Installer's Email Address

Pump Installer's Firm Name

Pump Installer's Firm Address

City State Zip $\quad-0000$ Telephone

Pump Installer's Firm Email Address

c. Measured Pumping rate gallons per minute

d. Pumping water level feet

e. Drop pipe diameter inches

f. Length of drop pipe feet

g. Pumping equipment installed $(\mathrm{m}) \_/ /\left(\mathrm{d} \_/(\mathrm{y})\right.$

h. Pump Brand

i. This well is designed and constructed to pump less than $50 \mathrm{gpm} \mathrm{X}$ Yes

No (8H is required on $A L L$ wells)

9. Well Construction Information.

a. Total well depth 120 feet. b. Static water level 75 feet. (required)

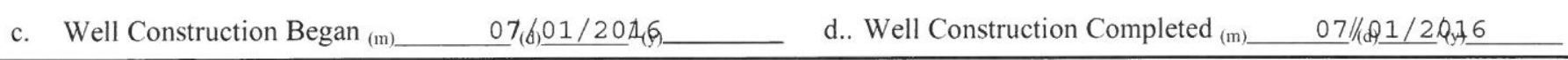

Wells drilled prior to stays or NRD signature Date moratoriums require NRD signature

(Signature can be submitted on NRD Approval form to DNR prior to registration)

e. Bore hole diameter in inches Top $\quad 6 \quad$ Bottom

f. Casing and Screen Joints are Welded Glued Threaded Other

g. Capacity of Well _ـ 4 gallons per minute (to be used to determine sustainability of aquifer)

h. Pumping water level at this capacity feet 
10. Well Construction (Casing \& Screen)- c, d, e, \& f measurements should be in inches to three decimal places (From not less than zero)

\begin{tabular}{|c|c|c|c|c|c|c|c|c|}
\hline & a & $\mathrm{b}$ & $\mathrm{c}$ & d & $\mathrm{e}$ & f & $\mathrm{g}$ & $\mathrm{h}$ \\
\hline & $\begin{array}{l}\text { ment } \\
\text { in Feet }\end{array}$ & $\begin{array}{l}\text { Casing or } \\
\text { Screen or }\end{array}$ & $\begin{array}{c}\text { Inside } \\
\text { Diameter }\end{array}$ & $\begin{array}{l}\text { Outside } \\
\text { Diameter }\end{array}$ & $\begin{array}{c}\text { Wall } \\
\text { Thickness }\end{array}$ & $\begin{array}{c}\text { Screen Slot } \\
\text { Size }\end{array}$ & $\begin{array}{l}\text { Type of } \\
\text { Material }\end{array}$ & Trade Name \\
\hline From & To & Open Hole & & & & & & \\
\hline & & & & & & & & \\
\hline & & & & & & & & \\
\hline & & & & & & & & \\
\hline & & & & & & & & \\
\hline & & & & & & & & \\
\hline & & & & & & & & \\
\hline
\end{tabular}

Additional information for Double cased or Nested wells:

\begin{tabular}{|c|c|c|c|c|c|c|c|c|}
\hline \multicolumn{2}{|c|}{ a } & $\mathrm{b}$ & c & d & $\mathrm{e}$ & $f$ & g & h \\
\hline \multicolumn{2}{|c|}{$\begin{array}{c}\text { Placement } \\
\text { Depth in Feet }\end{array}$} & \multirow{2}{*}{$\begin{array}{l}\text { Casing or } \\
\text { Screen or } \\
\text { Open Hole }\end{array}$} & \multirow[t]{2}{*}{$\begin{array}{c}\text { Inside } \\
\text { Diameter }\end{array}$} & \multirow[t]{2}{*}{$\begin{array}{l}\text { Outside } \\
\text { Diameter }\end{array}$} & \multirow[t]{2}{*}{$\begin{array}{c}\text { Wall } \\
\text { Thickness }\end{array}$} & \multirow[t]{2}{*}{$\begin{array}{c}\text { Screen Slot } \\
\text { Size }\end{array}$} & \multirow[t]{2}{*}{$\begin{array}{l}\text { Type of } \\
\text { Material }\end{array}$} & \multirow[t]{2}{*}{ Trade Name } \\
\hline From & To & & & & & & & \\
\hline & & & & & & & & \\
\hline & & & & & & & & \\
\hline & & & & & & & & \\
\hline & & & & & & & & \\
\hline & & & & & & & & \\
\hline & & & & & & & & \\
\hline
\end{tabular}

11. Grout and Gravel Pack (must start at zero)

\begin{tabular}{|c|c|c|c|c|c|}
\hline \multicolumn{2}{|c|}{$\begin{array}{c}\text { Placement Depth in } \\
\text { Feet }\end{array}$} & \multirow[t]{2}{*}{$\begin{array}{l}\text { Grout/Gravel/ } \\
\text { Open Hole }\end{array}$} & \multirow[t]{2}{*}{$\begin{array}{l}\text { Material Description } \\
\text { *See Desc for gravel }\end{array}$} & \multirow[t]{2}{*}{$\begin{array}{l}\text { Quantity gravel } \\
\text { *See Desc }\end{array}$} & \multirow[t]{2}{*}{$\begin{array}{c}\text { Volume \& Type Grout } \\
\text { *See Desc }\end{array}$} \\
\hline From & To & & & & \\
\hline & & & & & \\
\hline & & & & & \\
\hline & & & & & \\
\hline & & & & & \\
\hline & & & & & \\
\hline & & & & & \\
\hline & & & & & \\
\hline
\end{tabular}

Desc: Description of gravel pack i.e. engineered gravel pack, or gravel pit description ( $1 / 4$ down), or brand name (Best Sand), natural formation, drilling cuttings, soil backfill

Quantity \#cubic yards, \#tons, \#sacks, - (for drilling cuttings and soil backfill estimate quantity) Calculation assistance available on web

Volume \& Type: \#gallons of a slurry, \#barrels of a slurry, \#sacks used in the slurry, \#bags of non-slurry bentonite (chip-pellet -granular) Additional information for Double cased or Nested wells:

\begin{tabular}{|c|c|c|c|c|c|}
\hline \multicolumn{2}{|c|}{$\begin{array}{c}\text { Placement Depth in } \\
\text { Feet }\end{array}$} & \multirow[t]{2}{*}{$\begin{array}{l}\text { Grout/Gravel/ } \\
\text { Open Hole }\end{array}$} & \multirow[t]{2}{*}{$\begin{array}{l}\text { Material Description } \\
\text { *See Desc for gravel }\end{array}$} & \multirow[t]{2}{*}{$\begin{array}{l}\text { Quantity gravel } \\
\text { *See Desc }\end{array}$} & \multirow[t]{2}{*}{$\begin{array}{c}\text { Volume \& Type Grout } \\
\text { *See Desc }\end{array}$} \\
\hline From & To & & & & \\
\hline & & & & & \\
\hline & & & & & \\
\hline & & & & & \\
\hline & & & & & \\
\hline & & & & & \\
\hline & & & & & \\
\hline & & & & & \\
\hline
\end{tabular}


WELL CONSTRUCTION LOG - 147213516416877

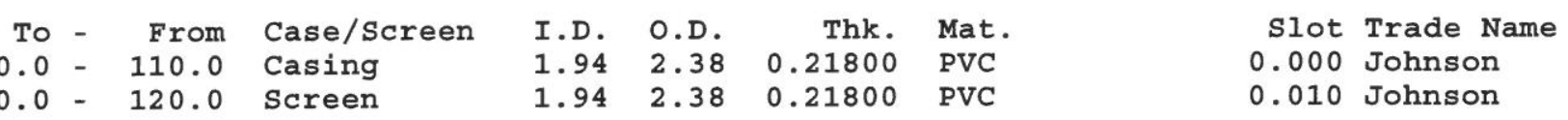

0.000 Johnson

$2.38-0.21800$ PVC

O.010 Johnson

GROUT AND GRAVEL PACK - 147213516416877

\begin{tabular}{|c|c|c|c|c|c|}
\hline $\begin{array}{l}\text { From } \\
00.0\end{array}$ & $\begin{array}{lr}- & \text { To } \\
- & 100.0\end{array}$ & $\begin{array}{l}\text { Grout/Gravel } \\
\text { Grout }\end{array}$ & $\begin{array}{l}\text { Material Desc. } \\
\text { Bent Grout }\end{array}$ & Qty Gravel & $\begin{array}{l}\text { Volume/Type } \\
6 \text { Bags }\end{array}$ \\
\hline 100.0 & 105.0 & Grout & Pellets & & 1/2 Pail \\
\hline 105.0 & 120.0 & Gravel & \#40 & 6 Bags & \\
\hline
\end{tabular}


GEOLOGICAL LOGS - 147213516416877

$\begin{array}{rrlll}\text { From - } & \text { To } & \text { Type } & \text { Hardness } & \text { Color Other/Drilling Action } \\ 00.0- & 65.0 & \text { Clay } & \text { Soft } & \text { Brown } \\ 65.0- & 80.0 & \text { Sand fine-med } & \text { Dense/stiff } & \text { Brown } \\ 80.0- & 120.0 & \text { Sand med-coarse } & \text { Dense/stiff } & \text { Brown }\end{array}$


12. Geologic Materials Logged (must start at zero) - Use only options provided below for Type, Hardness and Color

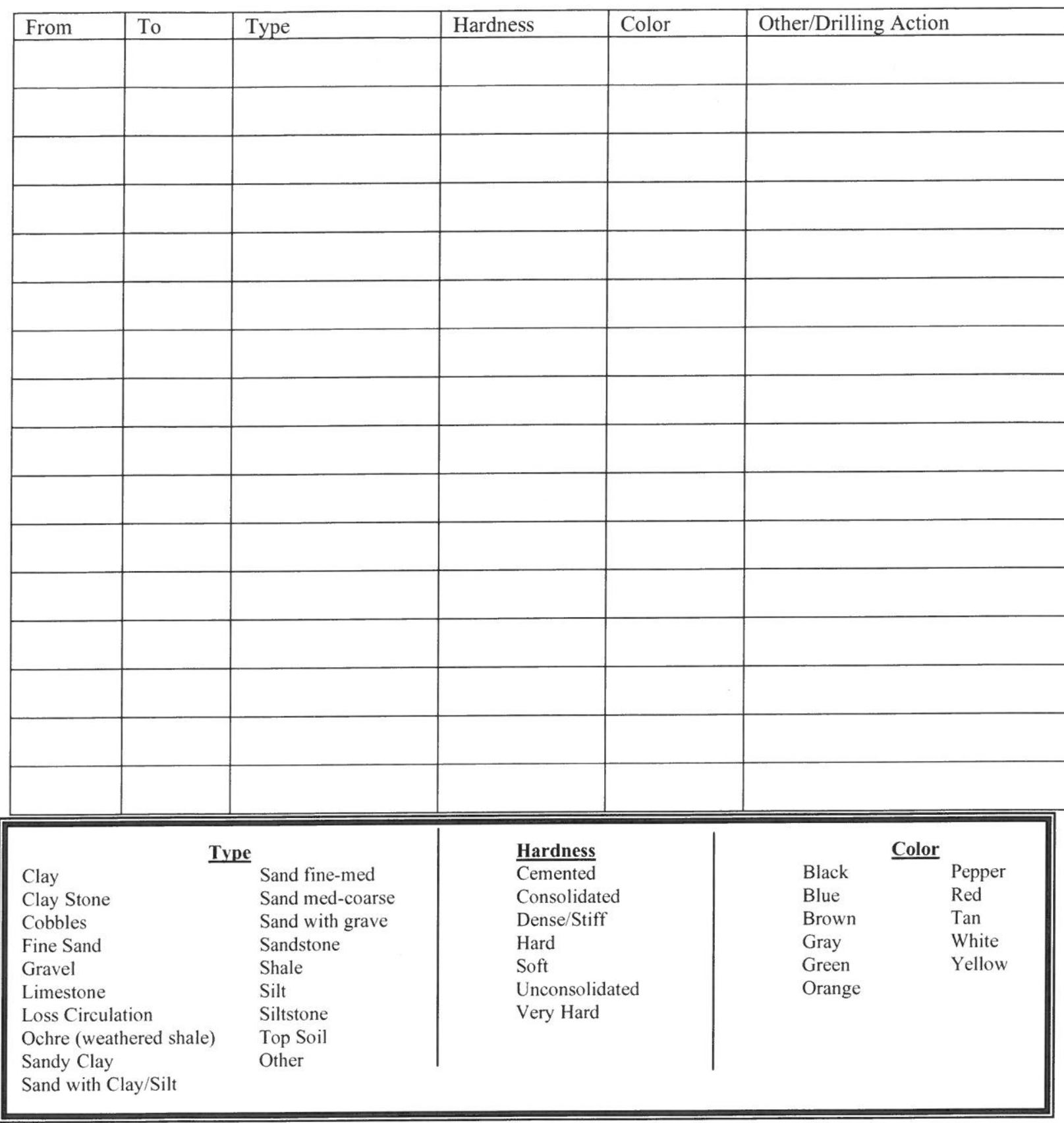

(Additional sheets may be submitted)

13. I hereby certify that the information provided on this registration is true and accurate to the best of my knowledge.

$\begin{array}{ccc}\begin{array}{c}\text { Water Well Contractor's Signature } \\ \text { (not required for pits) }\end{array} & \text { Date } & \begin{array}{c}\text { Well Owner's Signature } \\ \text { (if Contractor is unknown or Deceased or for pits) } \\ \text { (Not required if signed by Contractor) }\end{array}\end{array}$

(Not required if signed by Contractor)

Please note this document contains four pages.

Sections 9F, 9G, 10, $11 \& 12$ are not required if registering a pit.

Owner Registering well drilled prior to 2002: Minimum Required Sections - 1, 3(a,b,c,e,f), 5, 6, 7, 8(a,f,h), 9e 
Mail to

Department of Natural Resources

PO Box 94676

Lincoln, NE 68509-4676

Phone (402)471-2363
14721331819151

STATE OF NEBRASKA

\section{DEPARTMENT OF NATURAL RESOURCES}

WATER WELL REGISTRATION Please indicate NA for items unknown

\section{FOR DEPARTMENT USE ONLY}

January 201

Date Filed Owner Code No. Registration No. $6-180344$

\section{5 Well ID}

Receipt

1. a. Well Owner's First Name Last Name

OR Company Name_ USDA / CCC

b. Attention Name Steve Gilmore

c. Street Address $/ 400$ IUdEPENDENCE AuE $5 \mathrm{~W}$ Address 2/PO Address StoP O5/3 Room $47 / 4-5$ City WAshington State DC Zip 26250-0513 Telephone 202=720=5704

2. a. Contractor's License No 39508 Contractor's Name STEVEN D JOHNSON

Contractor’s Email Address_SJOHNSON@CASCADEDRILLING.COM

b. Drilling Firm Name CASCADE DRILLING

Address 209 LEMIEUR ST

City LITTLE FALLS

State MN

Zip $\quad 56345$

Telephone $\quad 320 \quad 632 \quad 6552$

Drilling Firm's Email Address_STHALACKER@CASCADEDRILLING.COM

3. a. Well location $\mathrm{SW}^{1 / 4}$ of the $\mathrm{NE}^{1 / 4}$ of Section 32 , Township 11 North, Range_ 2 IWEST

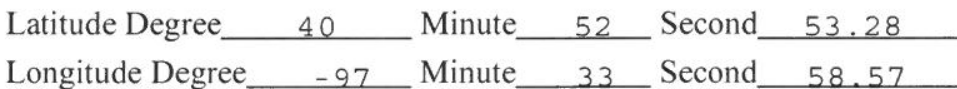

atural Resources District UPPER BIG BLUE

c. The well is: 1949 feet from the (Noxh/ South) section line and (circle one)

d. Street address and subdivision, if applicable

e. Location of water use (give legal descriptions)

GPS Required County

f. If for irrigation, the land to be irrigated is

g. Well reference letter(s), if applicable

\section{)} 2044 (BL.OCK : LOT:) feet from the (Exst/West) section line (circle one)

Location of well for a pit is the location of the pump

\section{Permits}

Management Area Permit Number

Geothermal Permit Number

Municipal Permit Number

Well Spacing Permit Number HHSS

5. Purpose of well (indicate one) ___ Aquaculture Domestic _ Ground Heat Exchanger Livestock $\quad \mathrm{x}$ Monitoring Observation

$\underset{\text { Public Water Supply (withou spacing) }}{\triangle}$ Recovery

Surface Water Permit Number
Industrial Permit Number
Transfer Out-Of-State Permit Number
Conduct Permit Number
Other Permit Number
NDEQ
cres.

__ Commercial/Industrial
Groundwater Source Heat Pump Other Pit (for irrigation)
Dewatering (over 90 days) Irrigation Injection Public Water Supply (with spacing (46-638)) (further description of use can be provided under other)

6. Wells in a Series.
a. Is this well a part of a series?
Yes go to part $b$ of this section
$\mathrm{X} \quad$ No go to part 7 of this application (Y/N required)
b. If one or more of the wells in the series is currently registered, give well registration number
c. How many wells in the series are you registering at this time? d. How many total wells in the series? 
7. Replacement and decommissioned/modified well information.
a. Is this well a replacement well? ___ Yes $\mathrm{x}$ No go to part 8 of this application
b. Registration number of original well
c. Original well last operated $(\mathrm{m}) \_$/(d) (d)__ $\quad /(y)$
If not registered, date ori
d. Replacement well is was constructed
feet from original well.
e. Location of water use of original well

Please Select One:

f.1. $\square$ Original water well decommissioned on $(\mathrm{m}) \_/ / \mathrm{d} \_/$(y)

OR

2. $\square$ I hereby certify that the original water well will be decommissioned within 180 days after such construction of the replacement water well. OR

3. $\square$ I hereby certify that the original water well will be modified and equipped to pump 50 gallons per minute or less within 180 days after such construction of the replacement water well. It will be used for one of the following: a. $\square$ Livestock

b. $\square$ Monitoring c. $\square$ Observation

d. $\square$ nonconsumptive or de minimus use approved by the applicable natural resources district. State use:

If $3 \mathrm{~d}$ is chosen. NRD signature is required. (Signature can be submitted on NRD Approval form to DNR prior to registration)

NRD signature

Date

OR

4. $\square$ Decommission/Modification Certification form is submitted by landowner. (Must be submitted before registering well)

8. Pump Information.

(Pump information is required if registering a pit)

a. Is pump installed at this time ___ Yes $\quad \mathrm{x}$ No

Is pump installed by well owner in section 1 ?

Is pump installed by contractor in section 2 ?

Yes $\mathrm{x}$ No

Yes $\mathrm{X}$ No

Is this a free flowing well ___ Yes(no pump to be installed) $\quad \mathrm{X} \quad$ No

If pump installed by pump installer, please fill out license number below

b. Pump Installer's License No. Pump Installer's Name

Pump Installer's Email Address

Pump Installer's Firm Name

Pump Installer's Firm Address

City State Zip $-0000$ Telephone

Pump Installer's Firm Email Address

c. Measured Pumping rate gallons per minute d. Pumping water level feet

e. Drop pipe diameter inches

f. Length of drop pipe feet

g. Pumping equipment installed $(\mathrm{m}) \_/ /\left(\mathrm{d} \_/(\mathrm{y})\right.$

h. Pump Brand

i. This well is designed and constructed to pump less than $50 \mathrm{gpm} \mathrm{x}$ Yes

No ( $8 \mathrm{H}$ is required on ALL wells)

9. Well Construction Information.

a. Total well depth 110 feet.

b. Static water level 75 feet. (required)

c. Well Construction Began $(\mathrm{m}) \quad 0 \phi_{(6)}(28 / 20146$ d.. Well Construction Completed $(\mathrm{m}) \_06 / 4,8 / 20,16$

Wells drilled prior to stays or NRD signature Date moratoriums require NRD signature

(Signature can be submitted on NRD Approval form to DNR prior to registration)

e. Bore hole diameter in inches Top $6 \quad$ Bottom $\quad 6$

f. Casing and Screen Joints are Welded Glued Threaded $\mathrm{x}$ Other

g. Capacity of Well _ 4 gallons per minute (to be used to determine sustainability of aquifer)

h. Pumping water level at this capacity feet 
10. Well Construction (Casing \& Screen)- c, d, e, \& f measurements should be in inches to three decimal places (From not less than zero)

\begin{tabular}{|c|c|c|c|c|c|c|c|c|}
\hline & & $b$ & $\mathrm{c}$ & d & $\mathrm{e}$ & f & $\mathrm{g}$ & $\mathrm{h}$ \\
\hline & $\begin{array}{l}\text { ment } \\
\text { n Feet }\end{array}$ & $\begin{array}{l}\text { Casing or } \\
\text { Screen or }\end{array}$ & $\begin{array}{c}\text { Inside } \\
\text { Diameter }\end{array}$ & $\begin{array}{c}\text { Outside } \\
\text { Diameter }\end{array}$ & $\begin{array}{c}\text { Wall } \\
\text { Thickness }\end{array}$ & $\begin{array}{c}\text { Screen Slot } \\
\text { Size }\end{array}$ & $\begin{array}{l}\text { Type of } \\
\text { Material }\end{array}$ & Trade Name \\
\hline From & To & Open Hole & & & & & & \\
\hline & & & & & & & & \\
\hline & & & & & & & & \\
\hline & & & & & & & & \\
\hline & & & & & & & & \\
\hline & & & & & & & & \\
\hline & & & & & & & & \\
\hline
\end{tabular}

Additional information for Double cased or Nested wells:

\begin{tabular}{|c|c|c|c|c|c|c|c|c|}
\hline \multicolumn{2}{|c|}{ a } & $\mathrm{b}$ & $\mathrm{c}$ & $\mathrm{d}$ & $\mathrm{e}$ & $\mathrm{f}$ & $\mathrm{g}$ & $\mathrm{h}$ \\
\hline \multicolumn{2}{|c|}{$\begin{array}{c}\text { Placement } \\
\text { Depth in Feet }\end{array}$} & \multirow{2}{*}{$\begin{array}{l}\text { Casing or } \\
\text { Screen or } \\
\text { Open Hole }\end{array}$} & \multirow[t]{2}{*}{$\begin{array}{c}\text { Inside } \\
\text { Diameter }\end{array}$} & \multirow[t]{2}{*}{$\begin{array}{c}\text { Outside } \\
\text { Diameter }\end{array}$} & \multirow[t]{2}{*}{$\begin{array}{c}\text { Wall } \\
\text { Thickness }\end{array}$} & \multirow[t]{2}{*}{$\begin{array}{c}\text { Screen Slot } \\
\text { Size }\end{array}$} & \multirow[t]{2}{*}{$\begin{array}{l}\text { Type of } \\
\text { Material }\end{array}$} & \multirow[t]{2}{*}{ Trade Name } \\
\hline From & To & & & & & & & \\
\hline & & & & & & & & \\
\hline & & & & & & & & \\
\hline & & & & & & & & \\
\hline & & & & & & & & \\
\hline & & & & & & & & \\
\hline & & & & & & & & \\
\hline
\end{tabular}

11. Grout and Gravel Pack (must start at zero)

\begin{tabular}{|c|c|c|c|c|c|}
\hline \multicolumn{2}{|c|}{$\begin{array}{c}\text { Placement Depth in } \\
\text { Feet }\end{array}$} & \multirow[t]{2}{*}{$\begin{array}{l}\text { Grout/Gravel/ } \\
\text { Open Hole }\end{array}$} & \multirow[t]{2}{*}{$\begin{array}{l}\text { Material Description } \\
\text { *See Desc for gravel }\end{array}$} & \multirow[t]{2}{*}{$\begin{array}{l}\text { Quantity gravel } \\
\text { *See Desc }\end{array}$} & \multirow[t]{2}{*}{$\begin{array}{c}\text { Volume \& Type Grout } \\
\text { *See Desc }\end{array}$} \\
\hline From & To & & & & \\
\hline & & & & & \\
\hline & & & & & \\
\hline & & & & & \\
\hline & & & & & \\
\hline & & & & & \\
\hline & & & & & \\
\hline & & & & & \\
\hline
\end{tabular}

Desc: Description of gravel pack i.e. engineered gravel pack, or gravel pit description ( $1 / 4$ down), or brand name (Best Sand), natural formation, drilling cuttings, soil backfill

Quantity \#cubic yards, \#tons, \#sacks, - (for drilling cuttings and soil backfill estimate quantity) Calculation assistance available on web Volume \& Type: \#gallons of a slurry, \#barrels of a slurry, \#sacks used in the slurry, \#bags of non-slurry bentonite (chip-pellet -granular) Additional information for Double cased or Nested wells:

\begin{tabular}{|c|c|c|c|c|c|}
\hline \multicolumn{2}{|c|}{$\begin{array}{c}\text { Placement Depth in } \\
\text { Feet }\end{array}$} & \multirow[t]{2}{*}{$\begin{array}{l}\text { Grout/Gravel/ } \\
\text { Open Hole }\end{array}$} & \multirow[t]{2}{*}{$\begin{array}{l}\text { Material Description } \\
\text { *See Desc for gravel }\end{array}$} & \multirow[t]{2}{*}{$\begin{array}{l}\text { Quantity gravel } \\
\text { *See Desc }\end{array}$} & \multirow[t]{2}{*}{$\begin{array}{c}\text { Volume \& Type Grout } \\
\text { *See Desc }\end{array}$} \\
\hline From & To & & & & \\
\hline & & & & & \\
\hline & & & & & \\
\hline & & & & & \\
\hline & & & & & \\
\hline & & & & & \\
\hline & & & & & \\
\hline & & & & & \\
\hline
\end{tabular}


WELL CONSTRUCTION LOG - 14721331819151

$\begin{array}{rrlrrrr}\text { To - } & \text { From } & \text { Case/Screen } & \text { I.D. } & \text { O.D. } & \text { Thk. } & \text { Mat. } \\ 0.0- & 100.0 & \text { Casing } & 1.94 & 2.38 & 0.21800 & \text { PVC } \\ 100.0- & 110.0 & \text { Screen } & 1.94 & 2.38 & 0.21800 & \text { PVC }\end{array}$

Slot Trade Name 0.000 Johnson 0.010 Johnson

GROUT AND GRAVEL PACK - 14721331819151

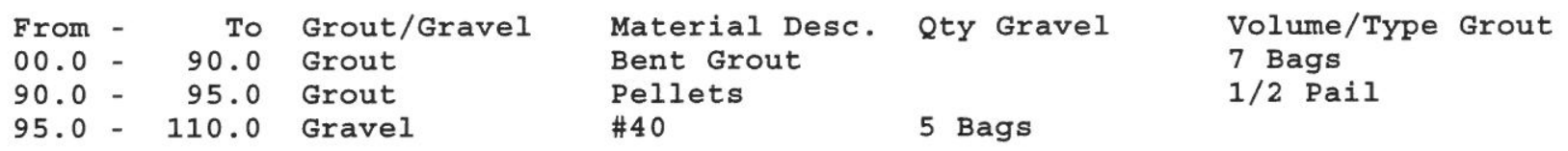


GEOLOGICAL LOGS - 14721331819151

$\begin{array}{rrlll}\text { From - } & \text { To Type } & \text { Hardness } & \text { Color Other/Drilling Action } \\ 00.0- & 65.0 & \text { Clay } & \text { Soft } & \text { Brown } \\ 65.0- & 85.0 & \text { Sand fine-med } & \text { Dense/Stiff } & \text { Brown } \\ 85.0- & 110.0 & \text { Sand med-coarse } & \text { Dense/Stiff } & \text { Brown }\end{array}$


12. Geologic Materials Logged (must start at zero) - Use only options provided below for Type, Hardness and Color

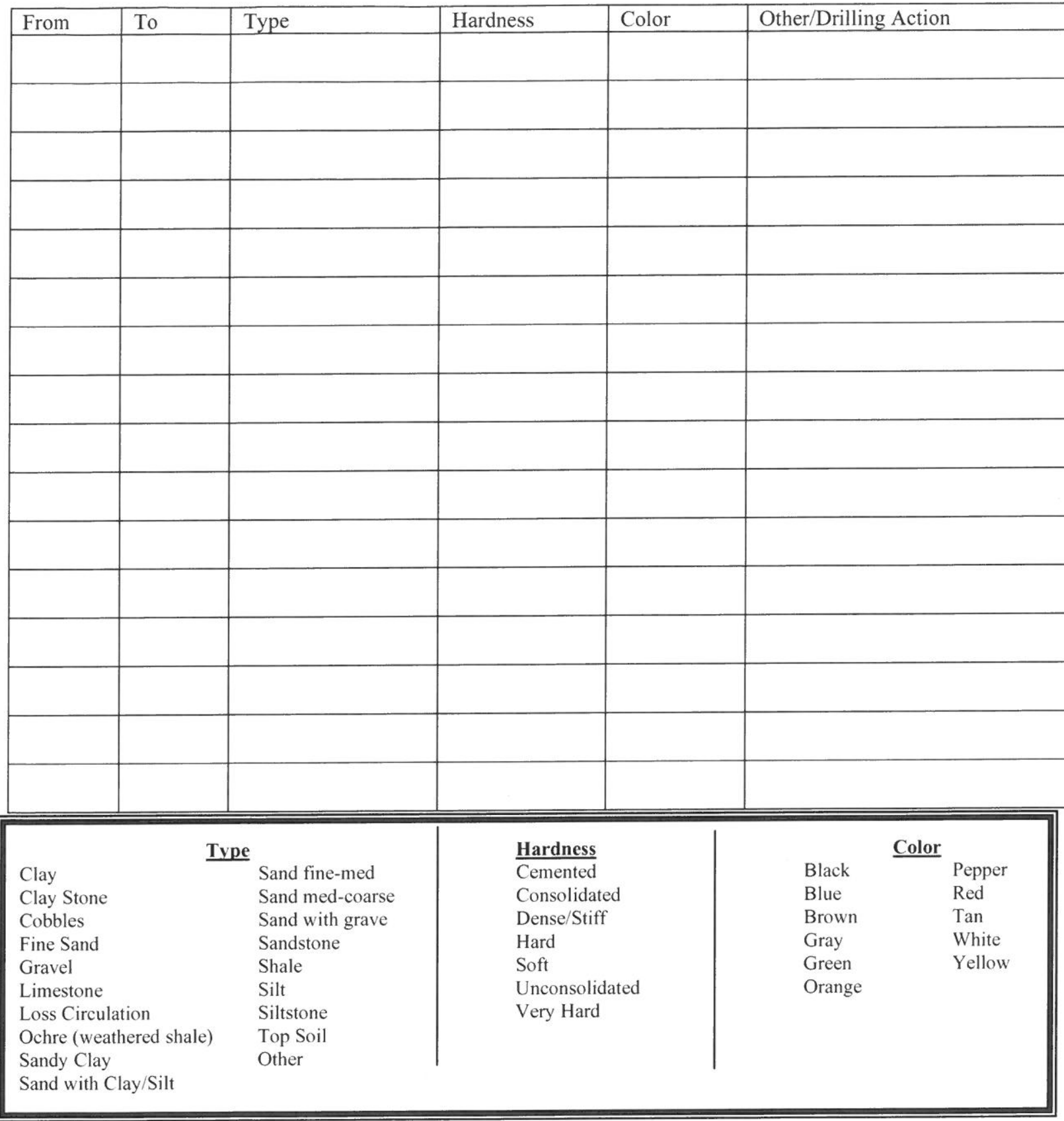

(Additional sheets may be submitted)

13. I hereby certify that the information provided on this registration is true and accurate to the best of my knowledge.

Water Well Contractor's Signature (not required for pits)

\section{Date}

Well Owner's Signature

(if Contractor is unknown or Deceased or for pits)

(Not required if signed by Contractor)

Please note this document contains four pages.

Sections 9F, 9G, 10, $11 \& 12$ are not required if registering a pit.

Owner Registering well drilled prior to 2002: Minimum Required Sections - 1, 3(a,b,c,e,f), 5, 6, 7, 8(a,f,h), 9e 
Mail to

Department of Natural Resources

PO Box 94676

Lincoln, NE 68509-4676

Phone (402)471-2363

STATE OF NEBRASKA

DEPARTMENT OF NATURAL RESOURCES

WATER WELL REGISTRATION

Please indicate NA for items unknown

\section{FOR DEPARTMENT USE ONLY}

Date Filed

Owner Code No.

Registration No. $6-180319$

$-\frac{244557}{\text { Well ID }}$

Receipt

1. a. Well Owner's First Name Last Name

OR Company Name USDA /CCC

b. Attention Name Steve Gilmoret

c. Street Address 1400 INDEPENDENCE AUE su Address 2/PO Address Stop 0513 Room 4714-5 City Washingtan State DC Zip 20250-0513 Telephone Z02-720-5104

2. a. Contractor's License No_ 39508 Contractor's Name_STEVEN D JOHNSON

Contractor's Email Address_SJOHNSON@CASCADEDRILLING.COM

b. Drilling Firm Name CASCADE DRILLING

Address 209 LEMIEUR ST

City LITTLE FALLS State_MN_Zip_ 56345

Telephone $\quad 320 \quad 632 \quad 6552$

Drilling Firm's Email Address_STHALACKER@CASCADEDRILLING.COM

3. a. Well location $\mathrm{SW}^{1 / 4}$ of the $\mathrm{NE}^{1 / 4}$ of Section $\quad 32$, Township 11 North, Range 2 IWEST

\begin{tabular}{|c|c|c|c|c|c|}
\hline Latitude Degree_ & 40 & Minute & Second & 53.19 & GPS \\
\hline Longitude Degree & -97 & Minute & 33 Second & 58.55 & Required \\
\hline
\end{tabular}

YORK County
Natural Resources District
UPPER BIG BLUE

c. The well is: 1959 feet from the (Nokh/ South) section line and (circle one)

2041 (BIOCK: LOT:)

d. Street address and subdivision, if applicable

e. Location of water use (give legal descriptions)

$\mathrm{N} / \mathrm{A}$

f. If for irrigation, the land to be irrigated is $0 \quad$ acres.

DL7-LC

g. Well reference letter(s), if applicable HHSS PWSID
Location of well for a pit is the location of the pump

Location of water use is required on all wells

4. Permits

Management Area Permit Number

Geothermal Permit Number

Municipal Permit Number

Well Spacing Permit Number HHSS

5. Purpose of well (indicate one) ___ Aquaculture ___ Commercial/Industrial Domestic Ground Heat Exchanger Groundwater Source Heat Pump Livestock $\mathrm{X}$ Monitoring Observation Public Water Supply (without spacing) Recovery

Pit (for irrigation)

Surface Water Permit Number

Industrial Permit Number

Transfer Out-Of-State Permit Number

Conduct Permit Number

Other Permit Number

NDEQ

Commercial/Industrial ___ Dewatering (over 90 days)

Public Water Supply (with spacing (46-638)

\section{(circle one)}

(indicate use)

6. Wells in a Series.

a. Is this well a part of a series? ___ Yes go to part b of this section $\quad \mathrm{x} \quad$ No go to part 7 of this application (Y/N required)

b. If one or more of the wells in the series is currently registered, give well registration number

c. How many wells in the series are you registering at this time? d. How many total wells in the series? 
7. Replacement and decommissioned/modified well information.

a. Is this well a replacement well? ___ Yes $\quad \mathrm{x} \quad$ No go to part 8 of this application

b. Registration number of original well

c. Original well last operated $(\mathrm{m}) \_$_ $/$(d) $I_{(y)}$

If not registered, date original well was constructed ${ }_{(m)}$ feet from original well.

e. Location of water use of original well

Please Select One:

f.1. $\square$ Original water well decommissioned on ${ }_{(\mathrm{m}) \_/\left(\mathrm{d} \_/(\mathrm{y}) \_ \text {OR }\right.}$

2. $\square$ I hereby certify that the original water well will be decommissioned within 180 days after such construction of the replacement water well. $\mathbf{O R}$

3. $\square$ I hereby certify that the original water well will be modified and equipped to pump 50 gallons per minute or less within 180 days after such construction of the replacement water well. It will be used for one of the following: a. $\square$ Livestock

b. $\square$ Monitoring c. $\square$ Observation

d. $\square$ nonconsumptive or de minimus use approved by the applicable natural resources district. State use:

If $3 \mathrm{~d}$ is chosen. NRD signature is required. (Signature can be submitted on NRD Approval form to DNR prior to registration)

NRD signature

Date

OR

4. $\square$ Decommission/Modification Certification form is submitted by landowner. (Must be submitted before registering well)

8. Pump Information.

(Pump information is required if registering a pit)

a. Is pump installed at this time ___ Yes $\mathrm{x}$ No

Is pump installed by well owner in section 1? Y__ Yes $\quad \mathrm{X} \quad$ No

Is pump installed by contractor in section 2? ___ Yes $\mathrm{x} \quad$ No

Is this a free flowing well ___ Yes(no pump to be installed) $\quad \mathrm{X} \quad$ No

If pump installed by pump installer, please fill out license number below

b. Pump Installer's License No. Pump Installer's Name

Pump Installer's Email Address

Pump Installer's Firm Name

Pump Installer's Firm Address

City State Zip $\quad-0000$ Telephone

Pump Installer's Firm Email Address

c. Measured Pumping rate gallons per minute

d. Pumping water level feet

e. Drop pipe diameter inches

f. Length of drop pipe feet

g. Pumping equipment installed (m)___ $/(\mathrm{d}$

h. Pump Brand

i. This well is designed and constructed to pump less than $50 \mathrm{gpm} \quad \mathrm{X}$ Yes

No (8H is required on ALL wells)

9. Well Construction Information.

a. Total well depth_275 feet. b. Static water level 75 feet. (required)

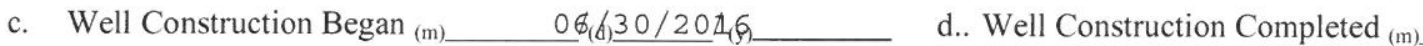

$06 / 1 / 30 / 20,76$

Wells drilled prior to stays or

NRD signature Date moratoriums require NRD signature

(Signature can be submitted on NRD Approval form to DNR prior to registration)

e. Bore hole diameter in inches Top_ 6 Bottom 6

f. Casing and Screen Joints are Welded Glued Threaded $\mathrm{x}$ Other

g. Capacity of Well _ 4 gallons per minute (to be used to determine sustainability of aquifer)

h. Pumping water level at this capacity feet 
10. Well Construction (Casing \& Screen)- c, d, e, \& f measurements should be in inches to three decimal places (From not less than zero)

\begin{tabular}{|c|c|c|c|c|c|c|c|c|}
\hline & & $\mathrm{b}$ & $\mathrm{c}$ & d & $\mathrm{e}$ & $\mathrm{f}$ & $\mathrm{g}$ & $\mathrm{h}$ \\
\hline & $\begin{array}{l}\text { ment } \\
\text { in Feet }\end{array}$ & $\begin{array}{l}\text { Casing or } \\
\text { Screen or }\end{array}$ & $\begin{array}{c}\text { Inside } \\
\text { Diameter }\end{array}$ & $\begin{array}{l}\text { Outside } \\
\text { Diameter }\end{array}$ & $\begin{array}{c}\text { Wall } \\
\text { Thickness }\end{array}$ & $\begin{array}{c}\text { Screen Slot } \\
\text { Size }\end{array}$ & $\begin{array}{l}\text { Type of } \\
\text { Material }\end{array}$ & Trade Name \\
\hline From & To & Open Hole & & & & & & \\
\hline & & & & & & & & \\
\hline & & & & & & & & \\
\hline & & & & & & & & \\
\hline & & & & & & & & \\
\hline & & & & & & & & \\
\hline & & & & & & & & \\
\hline
\end{tabular}

Additional information for Double cased or Nested wells:

\begin{tabular}{|c|c|c|c|c|c|c|c|c|}
\hline \multicolumn{2}{|c|}{$\mathrm{a}$} & $\mathrm{b}$ & $\mathrm{c}$ & $\mathrm{d}$ & $\mathrm{e}$ & $f$ & $\mathrm{~g}$ & $\mathrm{~h}$ \\
\hline \multicolumn{2}{|c|}{$\begin{array}{c}\text { Placement } \\
\text { Depth in Feet }\end{array}$} & \multirow{2}{*}{$\begin{array}{l}\text { Casing or } \\
\text { Screen or } \\
\text { Open Hole }\end{array}$} & \multirow[t]{2}{*}{$\begin{array}{c}\text { Inside } \\
\text { Diameter }\end{array}$} & \multirow[t]{2}{*}{$\begin{array}{c}\text { Outside } \\
\text { Diameter }\end{array}$} & \multirow[t]{2}{*}{$\begin{array}{c}\text { Wall } \\
\text { Thickness }\end{array}$} & \multirow[t]{2}{*}{$\begin{array}{c}\text { Screen Slot } \\
\text { Size }\end{array}$} & \multirow[t]{2}{*}{$\begin{array}{l}\text { Type of } \\
\text { Material }\end{array}$} & \multirow[t]{2}{*}{ Trade Name } \\
\hline From & To & & & & & & & \\
\hline & & & & & & & & \\
\hline & & & & & & & & \\
\hline & & & & & & & & \\
\hline & & & & & & & & \\
\hline & & & & & & & & \\
\hline & & & & & & & & \\
\hline
\end{tabular}

11. Grout and Gravel Pack (must start at zero)

\begin{tabular}{|l|l|l|l|l|l|}
\hline \multicolumn{2}{|c|}{$\begin{array}{c}\text { Placement Depth in } \\
\text { Feet }\end{array}$} & $\begin{array}{c}\text { Grout/Gravel/ } \\
\text { Open Hole }\end{array}$ & $\begin{array}{c}\text { Material Description } \\
\text { *See Desc for gravel }\end{array}$ & $\begin{array}{c}\text { Quantity gravel } \\
\text { *See Desc }\end{array}$ & $\begin{array}{c}\text { Volume \& Type Grout } \\
\text { *See Desc }\end{array}$ \\
\hline From & To & & & & \\
\hline & & & & & \\
\hline & & & & & \\
\hline \\
\hline
\end{tabular}

Desc: Description of gravel pack i.e. engineered gravel pack, or gravel pit description (1/4 down), or brand name (Best Sand), natural formation, drilling cuttings, soil backfill

Quantity \#cubic yards, \#tons, \#sacks, - (for drilling cuttings and soil backfill estimate quantity) Calculation assistance available on web Volume \& Type: \#gallons of a slurry, \#barrels of a slurry, \#sacks used in the slurry, \#bags of non-slurry bentonite (chip-pellet -granular) Additional information for Double cased or Nested wells:

\begin{tabular}{|l|l|l|l|l|l|}
\hline \multicolumn{2}{|c|}{$\begin{array}{c}\text { Placement Depth in } \\
\text { Feet }\end{array}$} & $\begin{array}{c}\text { Grout/Gravel/ } \\
\text { Open Hole }\end{array}$ & $\begin{array}{c}\text { Material Description } \\
\text { *See Desc for gravel }\end{array}$ & $\begin{array}{c}\text { Quantity gravel } \\
\text { *See Desc }\end{array}$ & $\begin{array}{c}\text { Volume \& Type Grout } \\
\text { *See Desc }\end{array}$ \\
\hline From & To & & & & \\
\hline & & & & & \\
\hline & & & & & \\
\hline \\
\hline
\end{tabular}


WELL CONSTRUCTION LOG - 147213454913020

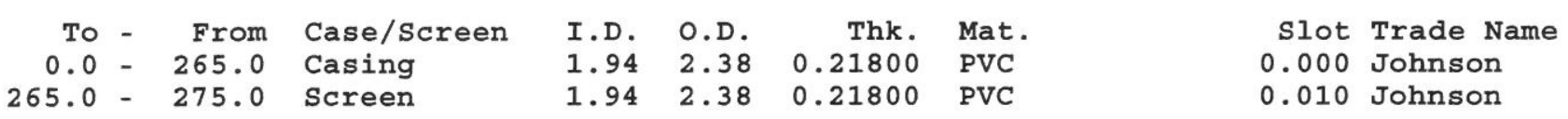

GROUT AND GRAVEL PACK - 147213454913020

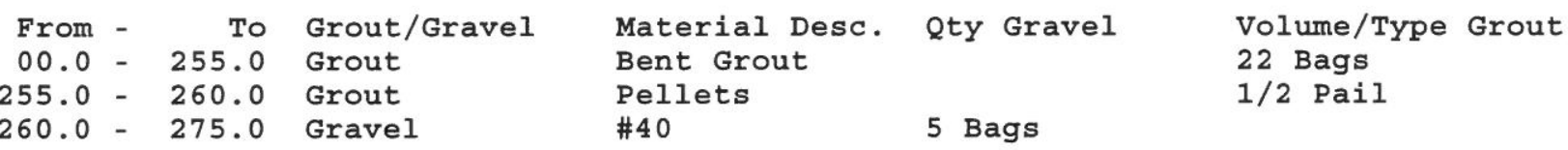


GEOLOGICAL LOGS - 147213454913020

$\begin{array}{rrl}\text { From - } & \text { To } & \text { Type } \\ 00.0- & 65.0 & \text { Clay } \\ 65.0- & 85.0 & \text { Sand fine-med } \\ 85.0- & 155.0 & \text { Sand med-coarse } \\ 155.0- & 190.0 & \text { Clay } \\ 190.0- & 275.0 & \text { Sand med-coarse }\end{array}$

$\begin{array}{lll}\text { Hardness } & \text { Color } & \text { Other/Drilling Action } \\ \text { Soft } & \text { Brown } & \\ \text { Dense/stiff } & \text { Brown } & \\ \text { Dense/Stiff } & \text { Brown } \\ \text { Dense/Stiff } & \text { Brown } \\ \text { Dense/Stiff } & \text { Brown }\end{array}$




\section{Geologic Materials Logged (must start at zero) - Use only options provided below for Type, Hardness and Color}

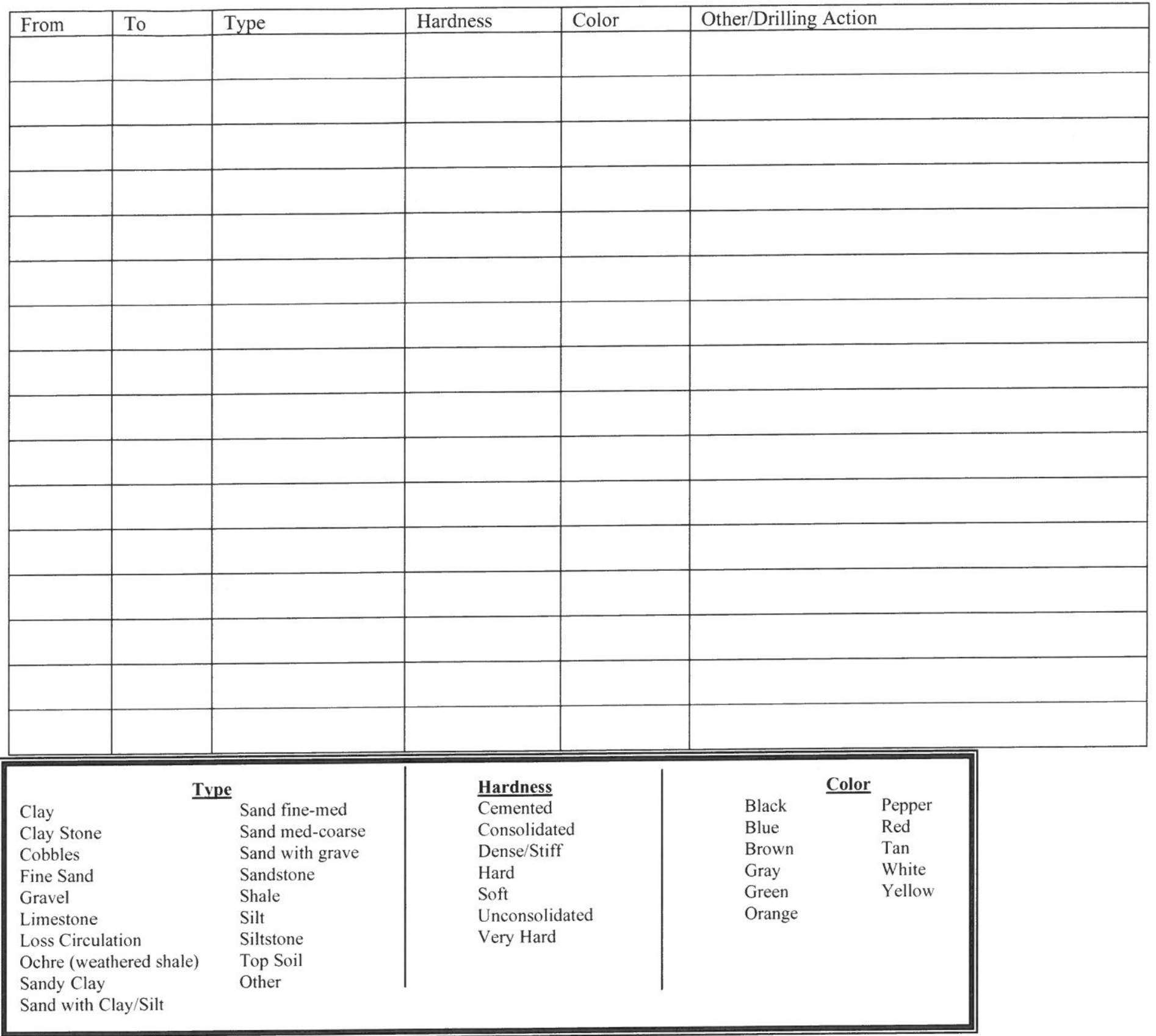

(Additional sheets may be submitted)

13. I hereby certify that the information provided on this registration is true and accurate to the best of my knowledge.
Water Well Contractor's Signature
Date
Well Owner's Signature
(not required for pits)
(if Contractor is unknown or Deceased or for
(Not required if signed by Contractor)

Date

Please note this document contains four pages.

Sections 9F, 9G, 10, 11 \& 12 are not required if registering a pit.

Owner Registering well drilled prior to 2002: Minimum Required Sections - 1, 3(a,b,c,e,f), 5, 6, 7, 8(a,f,h), 9e 
Mail to

Department of Natural Resources

PO Box 94676

Lincoln, NE 68509-4676

Phone (402)471-2363

STATE OF NEBRASKA

DEPARTMENT OF NATURAL RESOURCES

WATER WELL REGISTRATION

Please indicate NA for items unknown

\section{FOR DEPARTMENT USE ONLY}

Date Filed

Owner Code No.

Registration No. $6-180318$

$$
-\frac{244556}{\text { Well ID }}-\text { Receipt }
$$

1. a. Well Owner's First Name

Last Name

OR Company Name USDA/CCC

b. Attention Name Steve Gilmore

c. Street Address 1400 Independence Ave SW Address 2/PO Address Stop 0513 Room 4714-5 City Washing tow State DC Zip 20250-0513 Telephone 202-720-5104

2. a. Contractor's License No_ 39508 Contractor's Name STEVEN D JOHNSON

Contractor's Email Address_SJOHNSON@CASCADEDRILLING.COM

b. Drilling Firm Name CASCADE DRILLING

Address 209 LEMIEUR ST

City LITTLE FALLS

State_ MN

Zip $\quad 56345$

Telephone $\quad 320 \quad 632 \quad 6552$

Drilling Firm's Email Address_STHALACKER@CASCADEDRILLING.COM

3. a. Well location $S_{S} W^{1 / 4}$ of the $\mathrm{NE}^{1 / 4}$ of Section 32 , Township 11 North, Range 2 IWEST

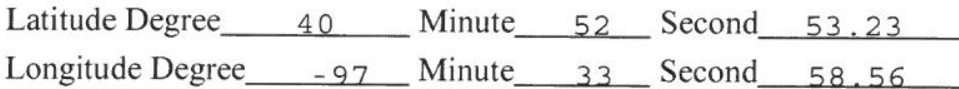

b. Natural Resources District UPPER BIG BLUE

c. The well is: 1955 feet from the (Noxh/ South) section line and

d. Street address and subdivision, if applicable

e. Location of water use (give legal descriptions)

\begin{tabular}{l} 
GPS \\
Required \\
\hline
\end{tabular}

County

Location of well for a pit is the location of the pump

f. If for irrigation, the land to be irrigated is (circle one)

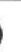

.
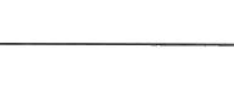

2041

(BLOCK: I.OT:)

$\mathrm{N} / \mathrm{A}$

feet from the (Egst/West) section line (circle one) g. Well reference letter(s), if applicable

4. Permits

Management Area Permit Number

Geothermal Permit Number

Municipal Permit Number

Well Spacing Permit Number

HHSS

5. Purpose of well (indicate one) Domestic Ground Heat Exchanger Aquaculture DL7-UC Livestock $\frac{\mathrm{x} \text { Monitoring }}{n}$ Public Water Supply (without spacing)

acres.

HHSS PWSID
Location of water use is required on all wells

Surface Water Permit Number

Industrial Permit Number

Transfer Out-Of-State Permit Number

Conduct Permit Number

Other Permit Number NDEQ

Commercial/Industrial Groundwater Source Heat Pump Pit (for irrigation) Other
(further description of use can be provided under other)
(indicate use)

Dewatering (over 90 days) Irrigation

Public Water Supply (with spacing (46-638))

6. Wells in a Series.

a. Is this well a part of a series?___ Yes go to part $\mathrm{b}$ of this section _ $\quad \mathrm{x} \quad$ No go to part 7 of this application (Y/N required)

b. If one or more of the wells in the series is currently registered, give well registration number

c. How many wells in the series are you registering at this time? d. How many total wells in the series? 
7. Replacement and decommissioned/modified well information.

a. Is this well a replacement well? ___ Yes $\mathrm{x}$ No go to part 8 of this application

b. Registration number of original well ___ If not registered, date original well was constructed (m)__ / $/$ ___ / $/(\mathrm{y}) \_$

c. Original well last operated $(\mathrm{m}) \_/(\mathrm{d}) \_{ }_{(\mathrm{y})} \_$d. Replacement well is___ feet from original well.

e. Location of water use of original well

Please Select One:

f.1. $\square$ Original water well decommissioned on $(\mathrm{m}) \_/ /_{\left(\mathrm{d} \_\right.} /(\mathrm{y}) \_$OR

2. $\square$ I hereby certify that the original water well will be decommissioned within 180 days after such construction of the replacement water well. $\mathbf{O R}$

3. $\square$ I hereby certify that the original water well will be modified and equipped to pump 50 gallons per minute or less within 180 days after such construction of the replacement water well. It will be used for one of the following: a. $\square$ Livestock b. $\square$ Monitoring c. $\square$ Observation

d. $\square$ nonconsumptive or de minimus use approved by the applicable natural resources district. State use:

If $3 \mathrm{~d}$ is chosen. NRD signature is required. (Signature can be submitted on NRD Approval form to DNR prior to registration)

NRD signature

Date

OR

4. $\square$ Decommission/Modification Certification form is submitted by landowner. (Must be submitted before registering well)

8. Pump Information.

(Pump information is required if registering a pit)

a. Is pump installed at this time __ Yes $\quad \mathrm{x}$ No

Is pump installed by well owner in section 1? __ Yes

Is pump installed by contractor in section 2?__ Yes $\mathrm{X}$ No

Is this a free flowing well ___ Yes(no pump to be installed) $\quad \mathrm{X}$ No

If pump installed by pump installer, please fill out license number below

b. Pump Installer's License No. Pump Installer's Name

Pump Installer's Email Address

Pump Installer's Firm Name

Pump Installer's Firm Address

City State

Zip $\quad-0000$
Telephone

Pump Installer's Firm Email Address

c. Measured Pumping rate gallons per minute

d. Pumping water level feet

e. Drop pipe diameter inches

f. Length of drop pipe feet

g. Pumping equipment installed $(\mathrm{m}) \_/(\mathrm{d}$

h. Pump Brand

i. $\quad$ This well is designed and constructed to pump less than $50 \mathrm{gpm} \quad \mathrm{x}$ Yes

No ( $8 \mathrm{H}$ is required on $\mathrm{ALL}$ wells)

9. Well Construction Information.

a. Total well depth 202 feet. b. Static water level 75 feet. (required)

c. Well Construction Began $(\mathrm{m}) \quad 06(6) 29 / 2046$

d.. Well Construction Completed $(\mathrm{m})$ $06 / 1 / 29 / 20,76$

Wells drilled prior to stays or

NRD signature Date moratoriums require NRD signature

(Signature can be submitted on NRD Approval form to DNR prior to registration)

e. Bore hole diameter in inches Top $\quad 6 \quad$ Bottom $\quad 6$

f. Casing and Screen Joints are Welded Glued Threaded $\mathrm{X}$ Other

g. Capacity of Well _ـ_ gallons per minute (to be used to determine sustainability of aquifer)

h. Pumping water level at this capacity feet 
10. Well Construction (Casing \& Screen)- c, d, e, \& f measurements should be in inches to three decimal places (From not less than zero)

\begin{tabular}{|c|c|c|c|c|c|c|c|c|}
\hline & $a$ & $\mathrm{~b}$ & $\mathrm{c}$ & $\mathrm{d}$ & $\mathrm{e}$ & $f$ & $\mathrm{~g}$ & $\mathrm{~h}$ \\
\hline & $\begin{array}{l}\text { in Feet } \\
\text { int }\end{array}$ & $\begin{array}{l}\text { Casing or } \\
\text { Screen or }\end{array}$ & $\begin{array}{c}\text { Inside } \\
\text { Diameter }\end{array}$ & $\begin{array}{l}\text { Outside } \\
\text { Diameter }\end{array}$ & $\begin{array}{c}\text { Wall } \\
\text { Thickness }\end{array}$ & $\begin{array}{c}\text { Screen Slot } \\
\text { Size }\end{array}$ & $\begin{array}{l}\text { Type of } \\
\text { Material }\end{array}$ & Trade Name \\
\hline From & To & Open Hole & & & & & & \\
\hline & & & & & & & & \\
\hline & & & & & & & & \\
\hline & & & & & & & & \\
\hline & & & & & & & & \\
\hline & & & & & & & & \\
\hline & & & & & & & & \\
\hline
\end{tabular}

Additional information for Double cased or Nested wells:

\begin{tabular}{|c|c|c|c|c|c|c|c|c|}
\hline \multicolumn{2}{|c|}{$a$} & $\mathrm{~b}$ & $\mathrm{c}$ & $\mathrm{d}$ & $\mathrm{e}$ & f & $\mathrm{g}$ & $\mathrm{h}$ \\
\hline \multicolumn{2}{|c|}{$\begin{array}{c}\text { Placement } \\
\text { Depth in Feet }\end{array}$} & $\begin{array}{l}\text { Casing or } \\
\text { Screen or }\end{array}$ & $\begin{array}{c}\text { Inside } \\
\text { Diameter }\end{array}$ & $\begin{array}{l}\text { Outside } \\
\text { Diameter }\end{array}$ & $\begin{array}{c}\text { Wall } \\
\text { Thickness }\end{array}$ & $\begin{array}{c}\text { Screen Slot } \\
\text { Size }\end{array}$ & $\begin{array}{l}\text { Type of } \\
\text { Material }\end{array}$ & Trade Name \\
\hline From & To & Open Hole & & & & & & \\
\hline & & & & & & & & \\
\hline & & & & & & & & \\
\hline & & & & & & & & \\
\hline & & & & & & & & \\
\hline & & & & & & & & \\
\hline & & & & & & & & \\
\hline
\end{tabular}

11. Grout and Gravel Pack (must start at zero)

\begin{tabular}{|c|c|c|c|c|c|}
\hline \multicolumn{2}{|c|}{$\begin{array}{c}\text { Placement Depth in } \\
\text { Feet }\end{array}$} & \multirow[t]{2}{*}{$\begin{array}{c}\text { Grout/Gravel/ } \\
\text { Open Hole }\end{array}$} & \multirow[t]{2}{*}{$\begin{array}{l}\text { Material Description } \\
\text { *See Desc for gravel }\end{array}$} & \multirow[t]{2}{*}{$\begin{array}{l}\text { Quantity gravel } \\
* \text { See Desc }\end{array}$} & \multirow[t]{2}{*}{$\begin{array}{c}\text { Volume \& Type Grout } \\
\text { *See Desc }\end{array}$} \\
\hline From & To & & & & \\
\hline & & & & & \\
\hline & & & & & \\
\hline & & & & & \\
\hline & & & & & \\
\hline & & & & & \\
\hline & & & & & \\
\hline & & & & & \\
\hline
\end{tabular}

Desc: Description of gravel pack i.e. engineered gravel pack, or gravel pit description ( $1 / 4$ down), or brand name (Best Sand), natural formation, drilling cuttings, soil backfill

Quantity \#cubic yards, \#tons, \#sacks, - (for drilling cuttings and soil backfill estimate quantity) Calculation assistance available on web Volume \& Type: \#gallons of a slurry, \#barrels of a slurry, \#sacks used in the slurry, \#bags of non-slurry bentonite (chip-pellet -granular) Additional information for Double cased or Nested wells:

\begin{tabular}{|c|c|c|c|c|c|}
\hline \multicolumn{2}{|c|}{$\begin{array}{c}\text { Placement Depth in } \\
\text { Feet }\end{array}$} & \multirow[t]{2}{*}{$\begin{array}{l}\text { Grout/Gravel/ } \\
\text { Open Hole }\end{array}$} & \multirow[t]{2}{*}{$\begin{array}{l}\text { Material Description } \\
\text { *See Desc for gravel }\end{array}$} & \multirow[t]{2}{*}{$\begin{array}{c}\text { Quantity gravel } \\
* \text { See Desc }\end{array}$} & \multirow[t]{2}{*}{$\begin{array}{c}\text { Volume \& Type Grout } \\
\text { *See Desc }\end{array}$} \\
\hline From & To & & & & \\
\hline & & & & & \\
\hline & & & & & \\
\hline & & & & & \\
\hline & & & & & \\
\hline & & & & & \\
\hline & & & & & \\
\hline & & & & & \\
\hline
\end{tabular}


WELL CONSTRUCTION LOG - 147213389814480

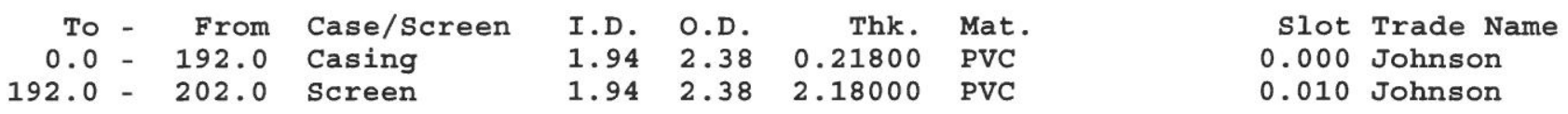

GROUT AND GRAVEL PACK - 147213389814480

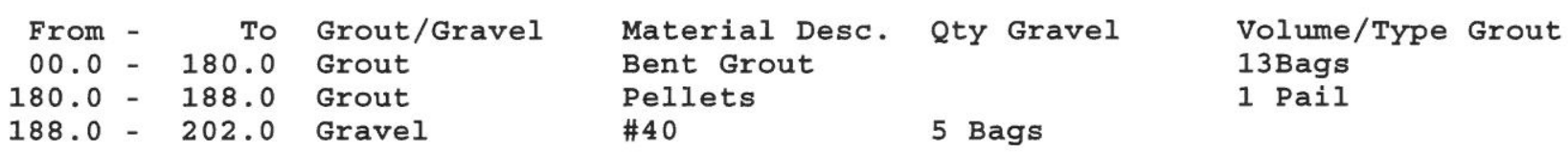




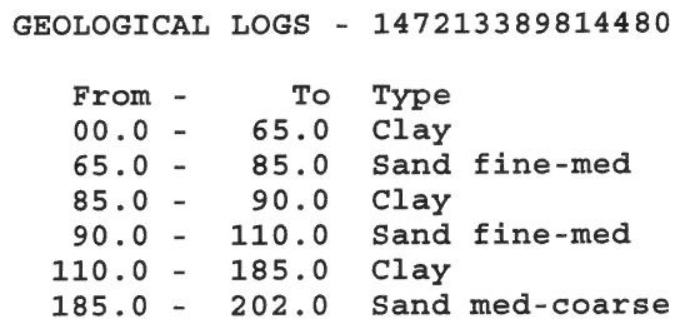

$\begin{array}{ll}\text { Hardness } & \text { Color } \\ \text { Soft } & \text { Brown } \\ \text { Dense/stiff } & \text { Brown } \\ \text { Soft } & \text { Brown } \\ \text { Dense/Stiff } & \text { Brown } \\ \text { Dense/Stiff } & \text { Brown } \\ \text { Dense/Stiff } & \text { Brown }\end{array}$


12. Geologic Materials Logged (must start at zero) - Use only options provided below for Type, Hardness and Color

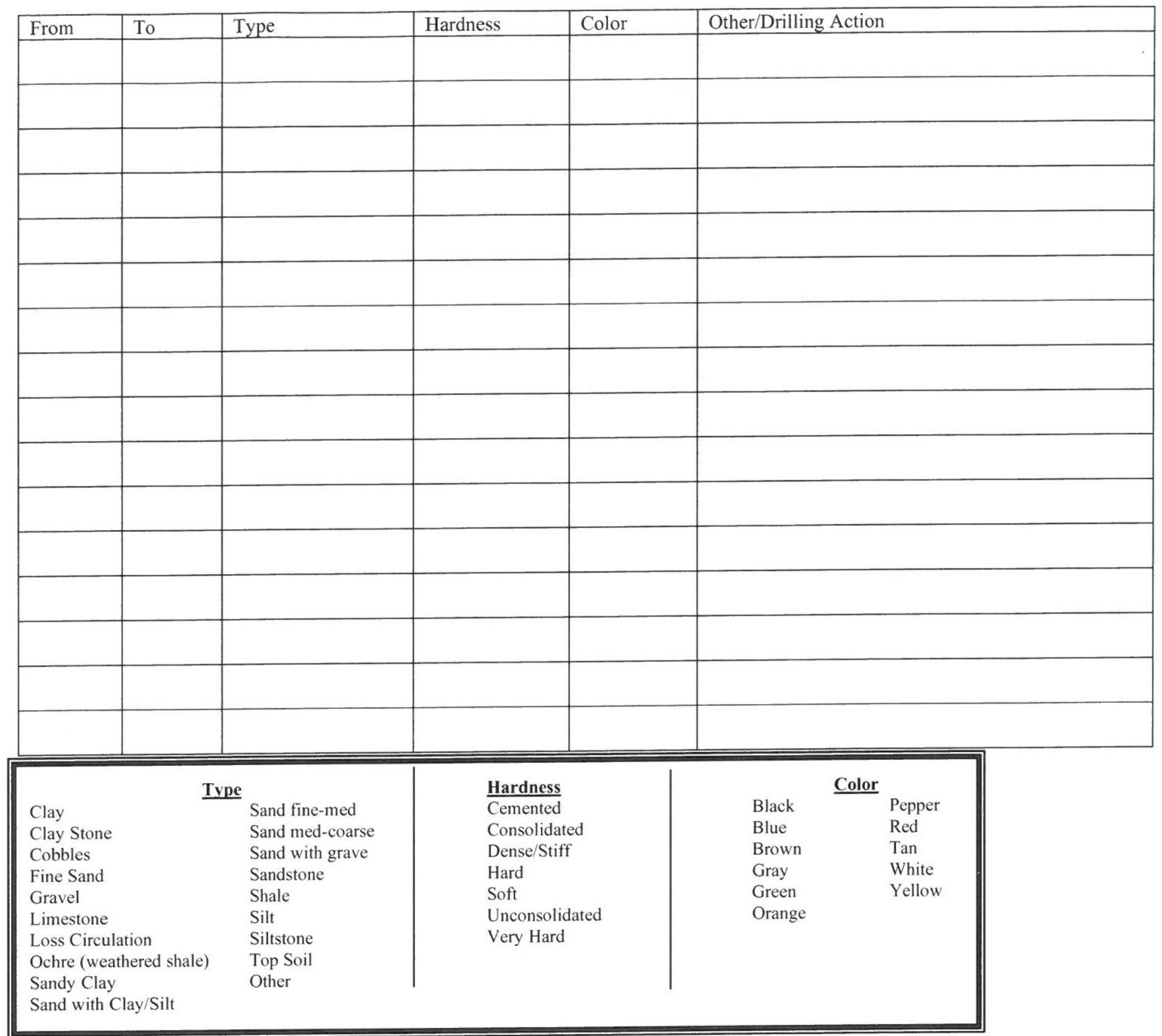

(Additional sheets may be submitted)

13. I hereby certify that the information provided on this registration is true and accurate to the best of my knowledge.
Water Well Contractor's Signature
Date
Well Owner's Signature
(not required for pits)
(if Contractor is unknown or Deceased or for
(Not required if signed by Contractor)

Date

Please note this document contains four pages.

Sections 9F, 9G, 10, $11 \& 12$ are not required if registering a pit.

Owner Registering well drilled prior to 2002: Minimum Required Sections - 1, 3(a,b,c,e,f), 5, 6, 7, 8(a,f,h), 9e 
Mail to

Department of Natural Resources

PO Box 94676

Lincoln, NE 68509-4676

Phone (402)471-2363
STATE OF NEBRASKA

DEPARTMENT OF NATURAL RESOURCES

WATER WELL REGISTRATION Please indicate NA for items unknown

Date Filed Owner Code No.

Registration No. $6-180112$ $-244223$ Receipt NRD Well ID

1. a. Well Owner's First Name Last Name

OR Company Name USDA / CCC

b. Attention Name Steve Gilmoke

c. Street Address 1400 IndEPENDENCE AVE SW Address 2/PO Address Stop 0573 Room 4714-S City WAshing ton State DC Zip20250-0513 elephone 202-720-5104

2. a. Contractor's License No_ 39508 Contractor's Name STEVEN D JOHNSON Contractor's Email Address_SJOHNSON@CASCADEDRILLING.COM

b. Drilling Firm Name CASCADE DRILLING

Address 209 LEMIEUR ST

City LITTLE FALLS State_MN_Zip_ 56345 Telephone $\quad 320 \quad 632 \quad 6552$

Drilling Firm's Email Address_STHALACKER@CASCADEDRILLING.COM

3. a. Well location NW $1 / 4^{1 / 2}$ of the NW $1 / 4$ of Section 32 , Township $11^{\text {North, Range }}$

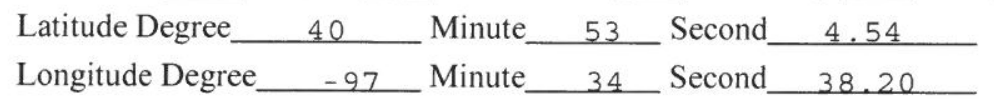

b. Natural Resources District UPPER BIG BLUE

GPS
Required

Require 197 feet from the (Noxh/ South) section line and (circle one)

d. Street address and subdivision, if applicable

e. Location of water use (give legal descriptions) (BIOCK: T.OT:)

$\mathrm{N} / \mathrm{A}$

f. If for irrigation, the land to be irrigated is $0 \quad$ acres.

g. Well reference letter(s), if applicable DL8-C1 HHSS PWSID
4. Permits

Management Area Permit Number

Geothermal Permit Number

Municipal Permit Number

Well Spacing Permit Number HHSS
5. Purpose of well (indicate one) ___ Aquaculture ___ Commercial/Industrial Domestic Ground Heat Exchanger

Livestock $\mathrm{x}$ Monitoring Public Water Supply (without spacing)
Observation Recovery Groundwater Source Heat Pump Pit (for irrigation) Other feet from the (East/West) section line (circle one)

Location of well for a pit is the location of the pump
Dewatering (over 90 days) Irrigation

Public Water Supply (with spacing (46-638))
Surface Water Permit Number

Transfer Out-Of-State Permit Numbe

Conduct Permit Number

Other Permit Number

NDEQ

Wells in a Series.
a. Is this well a part of a series?
Yes go to part $b$ of this section
$\mathrm{X} \quad$ No go to part 7 of this application (Y/N required)
b. If one or more of the wells in the series is currently registered, give well registration number
c. How many wells in the series are you registering at this time? d. How many total wells in the series? 
7. Replacement and decommissioned/modified well information.
a. Is this well a replacement well?___ Yes $\mathrm{x}$ No go to part 8 of this application

b. Registration number of original well

c. Original well last operated $(\mathrm{m})$ /(d) $I_{(y)}$

If not registered, date origir

e. Location of water use of original well Please Select One:

f.1. $\square$ Original water well decommissioned on ${ }_{(\mathrm{m}) \_} /_{\left(\mathrm{d} \_\_\right.} /_{(\mathrm{y})} \_$OR

2. $\square$ I hereby certify that the original water well will be decommissioned within 180 days after such construction of the replacement water well. OR

3. $\square$ I hereby certify that the original water well will be modified and equipped to pump 50 gallons per minute or less within 180 days after such construction of the replacement water well. It will be used for one of the following: a. $\square$ Livestock

b. $\square$ Monitoring c. $\square$ Observation

d. $\square$ nonconsumptive or de minimus use approved by the applicable natural resources district. State use:

If $3 \mathrm{~d}$ is chosen. NRD signature is required. (Signature can be submitted on NRD Approval form to DNR prior to registration)
NRD signature
Date
OR

4. $\square$ Decommission/Modification Certification form is submitted by landowner. (Must be submitted before registering well)

8. Pump Information.

(Pump information is required if registering a pit)

a. Is pump installed at this time Yes $\mathrm{x}$ No

Is pump installed by well owner in section 1 ?

Is pump installed by contractor in section 2 ? Yes $\mathrm{X}$ No

Yes $\mathrm{X}$ No

Is this a free flowing well ___ Yes(no pump to be installed) $\mathrm{x}$ No

If pump installed by pump installer, please fill out license number below

b. Pump Installer's License No. Pump Installer's Name

Pump Installer's Email Address

Pump Installer's Firm Name

Pump Installer's Firm Address

City State Zip $\quad-0000$ Telephone

Pump Installer's Firm Email Address

c. Measured Pumping rate gallons per minute d. Pumping water level feet

e. Drop pipe diameter inches

f. Length of drop pipe feet

g. Pumping equipment installed $(\mathrm{m}) \_/$(d _ _ / $/(\mathrm{y}) \ldots$

h. Pump Brand

i. This well is designed and constructed to pump less than $50 \mathrm{gpm} \quad \mathrm{X}$ Yes

No (8H is required on ALL wells)

9. Well Construction Information.

a. Total well depth 114 feet. b. Static water level 75 feet. (required)

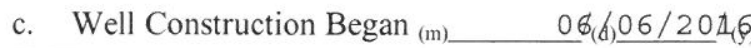

d.. Well Construction Completed ${ }_{(\mathrm{m})}$

$06 / 10,6 / 20,76$

Wells drilled prior to stays or

NRD signature Date moratoriums require NRD signature

(Signature can be submitted on NRD Approval form to DNR prior to registration)

e. Bore hole diameter in inches Top Bottom 6

f. Casing and Screen Joints are Welded Glued Threaded $\mathrm{x}$ Other

g. Capacity of Well 4 gallons per minute (to be used to determine sustainability of aquifer)

h. Pumping water level at this capacity feet 
10. Well Construction (Casing \& Screen)- c, d, e, \& f measurements should be in inches to three decimal places (From not less than zero)

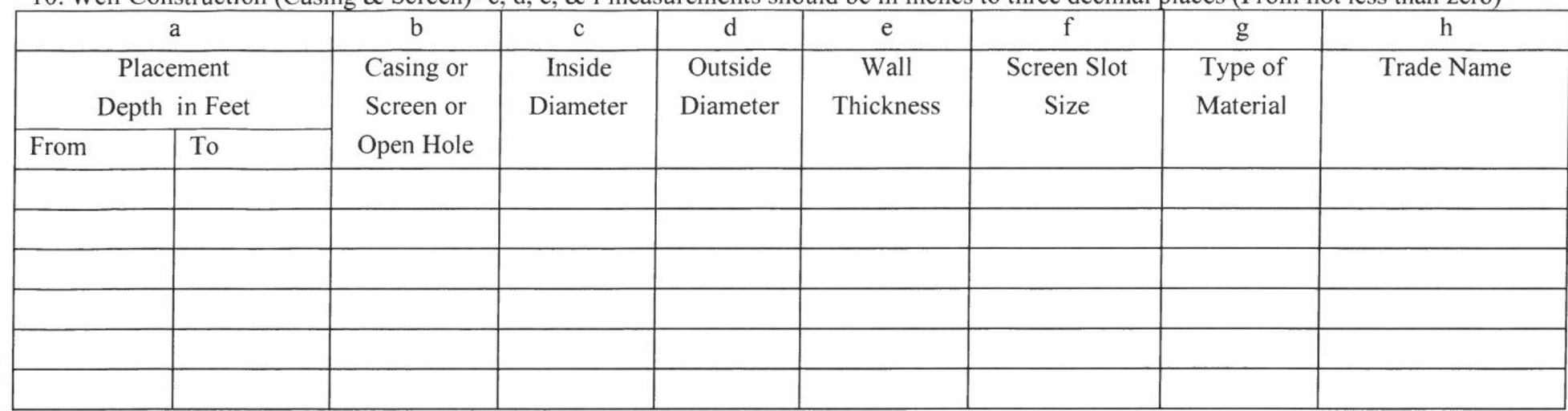

Additional information for Double cased or Nested wells:

\begin{tabular}{|c|c|c|c|c|c|c|c|c|}
\hline \multicolumn{2}{|c|}{$\begin{array}{c}\text { Placement } \\
\text { Depth in Feet }\end{array}$} & $\begin{array}{c}\text { Casing or } \\
\text { Screen or } \\
\text { Open Hole }\end{array}$ & $\begin{array}{c}\text { Inside } \\
\text { Diameter } \\
\text { From }\end{array}$ & To & $\begin{array}{c}\text { Outside } \\
\text { Diameter }\end{array}$ & $\begin{array}{c}\text { Wall } \\
\text { Thickness }\end{array}$ & $\begin{array}{c}\text { Screen Slot } \\
\text { Size }\end{array}$ & $\begin{array}{c}\text { Type of } \\
\text { Material }\end{array}$ \\
\hline \\
\hline
\end{tabular}

11. Grout and Gravel Pack (must start at zero)

\begin{tabular}{|c|c|c|c|c|c|}
\hline \multicolumn{2}{|c|}{$\begin{array}{c}\text { Placement Depth in } \\
\text { Feet }\end{array}$} & \multirow[t]{2}{*}{$\begin{array}{l}\text { Grout/Gravel/ } \\
\text { Open Hole }\end{array}$} & \multirow[t]{2}{*}{$\begin{array}{l}\text { Material Description } \\
\text { *See Desc for gravel }\end{array}$} & \multirow[t]{2}{*}{$\begin{array}{l}\text { Quantity gravel } \\
\text { *See Desc }\end{array}$} & \multirow[t]{2}{*}{$\begin{array}{c}\text { Volume \& Type Grout } \\
\text { *See Desc }\end{array}$} \\
\hline From & To & & & & \\
\hline & & & & & \\
\hline & & & & & \\
\hline & & & & & \\
\hline & & & & & \\
\hline & & & & & \\
\hline & & & & & \\
\hline & & & & & \\
\hline
\end{tabular}

Desc: Description of gravel pack i.e. engineered gravel pack, or gravel pit description ( $1 / 4$ down), or brand name (Best Sand), natural formation, drilling cuttings, soil backfill

Quantity \#cubic yards, \#tons, \#sacks, - (for drilling cuttings and soil backfill estimate quantity) Calculation assistance available on web Volume \& Type: \#gallons of a slurry, \#barrels of a slurry, \#sacks used in the slurry, \#bags of non-slurry bentonite (chip-pellet -granular) Additional information for Double cased or Nested wells:

\begin{tabular}{|l|l|l|l|l|l|}
\hline \multicolumn{2}{|c|}{$\begin{array}{c}\text { Placement Depth in } \\
\text { Feet }\end{array}$} & $\begin{array}{c}\text { Grout/Gravel/ } \\
\text { Open Hole }\end{array}$ & $\begin{array}{c}\text { Material Description } \\
\text { *See Desc for gravel }\end{array}$ & $\begin{array}{c}\text { Quantity gravel } \\
\text { *See Desc }\end{array}$ & $\begin{array}{c}\text { Volume \& Type Grout } \\
\text { *See Desc }\end{array}$ \\
\hline From & To & & & & \\
\hline & & & & & \\
\hline & & & & & \\
\hline \\
\hline
\end{tabular}


WELL CONSTRUCTION LOG - 146999179810378

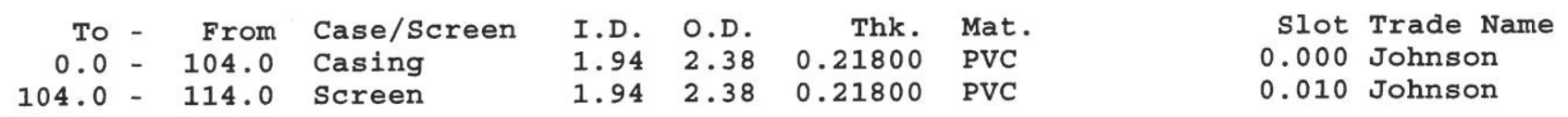

GROUT AND GRAVEL PACK - 146999179810378

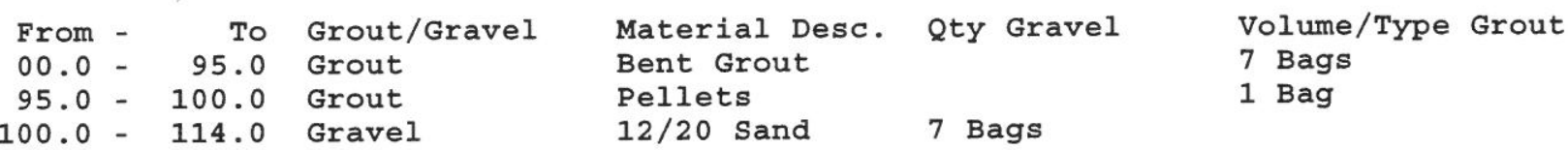


GEOLOGICAL LOGS - 146999179810378

$\begin{array}{lrlll}\text { From - } & \text { To } & \text { Type } & \text { Hardness } & \text { Color Other/Drilling Action } \\ 00.0- & 65.0 & \text { Clay } & \text { Dense/stiff } & \text { Brown } \\ 65.0- & 85.0 & \text { Fine Sand } & \text { Hard } & \text { Brown } \\ 85.0- & 114.0 & \text { Sand med-coarse } & \text { Dense/stiff } & \text { Brown }\end{array}$


12. Geologic Materials Logged (must start at zero) - Use only options provided below for Type, Hardness and Color

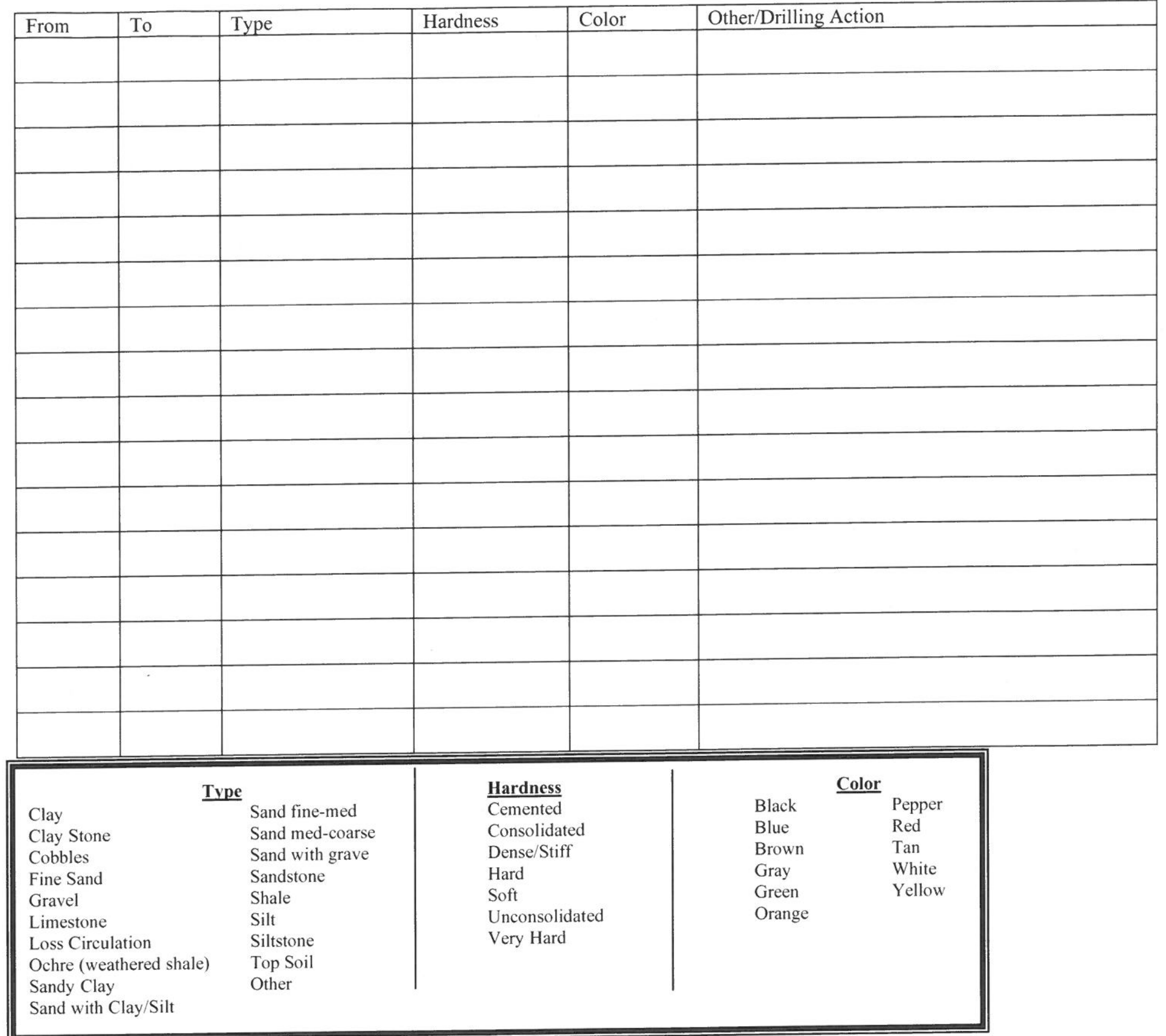

(Additional sheets may be submitted)

13. I hereby certify that the information provided on this registration is true and accurate to the best of my knowledge.
Water Well Contractor's Signature
Date
Well Owner's Signature (not required for pits)
(if Contractor is unknown or Deceased or for pits)
(Not required if signed by Contractor)

Date

Please note this document contains four pages.

Sections 9F, 9G, 10, $11 \& 12$ are not required if registering a pit.

Owner Registering well drilled prior to 2002: Minimum Required Sections - 1, 3(a,b,c,e,f), 5, 6, 7, 8(a,f,h), 9e 
Mail to

Department of Natural Resources

PO Box 94676

Lincoln, NE 68509-4676

Phone (402)471-2363

STATE OF NEBRASKA

DEPARTMENT OF NATURAL RESOURCES

WATER WELL REGISTRATION Please indicate NA for items unknown

FOR DEPARTMENT USE ONLY

Date Filed

Owner Code No.

Registration No. $6-180317$

$$
-\frac{24455 \%}{\text { Well ID }}-\text { Receipt }
$$

1. a. Well Owner's First Name

OR Company Name USPA/CCC

b. Attention Name Steve Gilmore-

c. Street Address 1400 FUDEPENDENCF AUE SW

Address 2/PO Address Stop 0513 Room 4714-S

City WasHINGTON State DC Zip_ 20250-0513

2. a. Contractor's License No_ 39508 Contractor's Name STEVEN D JOHNSON

Contractor's Email Address SJOHNSON@CASCADEDRILLING.COM

b. Drilling Firm Name CASCADE DRILLING

Address 209 LEMIEUR ST

City LITTLE FALLS

State_ MN

Zip

56345

Telephone $\quad 320 \quad 632 \quad 6552$

Drilling Firm's Email Address_STHALACKER@CASCADEDRILLING.COM

3. a. Well location NW $1 / 4$ of the NW $1 / 4$ of Section _ 32, Township 11 North, Range 2 IWEST

$\begin{array}{llll}\text { Latitude Degree__ } 40 & \text { Minute__ } 53 & \text { Second } & 4.54 \\ \text { Longitude Degree__ } \quad-97 & \text { Minute_ } 34 & \text { Second } \quad 38.20\end{array}$

b. Natural Resources District

UPPER BIG BLUE

c. The well is: 787

feet from the (Nokh/ South) section line and

d. Street address and subdivision, if applicable (circle one)

e. Location of water use (give legal descriptions)

GPS

Required feet from the (East/Wesst) section line (circle one)
Location of well for a pit is the location of the pump

f. If for irrigation, the land to be irrigated is

g. Well reference letter(s), if applicable

$0 \quad$ acres.

DL8-C2

HHSS PWSID

(BLOCK: LOT:) $\mathrm{N} / \mathrm{A}$
4. Permits

Management Area Permit Number

Geothermal Permit Number

Municipal Permit Number

Well Spacing Permit Number

HHSS

5. Purpose of well (indicate one) Domestic Livestock Ground Heat Exchanger Aquaculture Observation Recovery

Public Water Supply

$\frac{\mathrm{x}}{\text { Supply }}$ Monitoring
Surface Water Permit Number

Industrial Permit Number

Transfer Out-Of-State Permit Number

Conduct Permit Number

Other Permit Number NDEQ

6. Wells in a Series.
a. Is this well a part of a series?
Yes go to part $\mathrm{b}$ of this section
$\mathrm{x}$ No go to part 7 of this application ( $\mathrm{Y} / \mathrm{N}$ required)
b. If one or more of the wells in the series is currently registered, give well registration number
c. How many wells in the series are you registering at this time?

Commercial/Industrial

Groundwater Source Heat Pump Other

\section{Dewatering (over 90 days)} Irrigation

Public Water Supply (with spacing (46-638)) 
7. Replacement and decommissioned/modified well information.
a. Is this well a replacement well?
Yes $\mathrm{X}$ No go to part 8 of this application
b. Registration number of original well
c. Original well last operated $(\mathrm{m})$
/(d) $I^{\prime}(y)$
If not registered, date ori
d. Replacement well is
riginal well feet from original well.
e. Location of water use of original well Please Select One:

f.1. $\square$ Original water well decommissioned on $(\mathrm{m}) \_/\left(\mathrm{d} \_/(\mathrm{y}) \_ \text {OR }\right.$

2. $\square$ I hereby certify that the original water well will be decommissioned within 180 days after such construction of the replacement water well. OR

3. $\square$ I hereby certify that the original water well will be modified and equipped to pump 50 gallons per minute or less within 180 days after such construction of the replacement water well. It will be used for one of the following: a. $\square$ Livestock

b. $\square$ Monitoring c. $\square$ Observation

d. $\square$ nonconsumptive or de minimus use approved by the applicable natural resources district. State use:

If $3 \mathrm{~d}$ is chosen. NRD signature is required. (Signature can be submitted on NRD Approval form to DNR prior to registration)

NRD signature_ Date___ OR

4. $\square$ Decommission/Modification Certification form is submitted by landowner. (Must be submitted before registering well)

8. Pump Information.

(Pump information is required if registering a pit)

a. Is pump installed at this time Yes $\mathrm{x}$ No

Is pump installed by well owner in section 1? Y_Yes $\quad \mathrm{x}$ No

Is pump installed by contractor in section 2? ___ Yes $\mathrm{x} \quad$ No

Is this a free flowing well ___ Yes(no pump to be installed) $\quad \mathrm{x}$ No

If pump installed by pump installer, please fill out license number below

b. Pump Installer's License No. Pump Installer's Name

Pump Installer's Email Address

Pump Installer's Firm Name

Pump Installer's Firm Address

City State Zip $\quad-0000$ Telephone

Pump Installer's Firm Email Address

c. Measured Pumping rate gallons per minute d. Pumping water level feet

e. Drop pipe diameter inches

f. Length of drop pipe feet

g. Pumping equipment installed $(\mathrm{m}) \_/\left(\mathrm{d} \_/ \text {(y) _ }\right.$

h. Pump Brand

i. This well is designed and constructed to pump less than $50 \mathrm{gpm} \quad \mathrm{x}$ Yes

No (8H is required on ALL wells)

9. Well Construction Information.

a. Total well depth 135 feet. b. Static water level 75 feet. (required)

c. Well Construction Began (m)__ $\quad 06(6) 27 / 2046$

d.. Well Construction Completed $(\mathrm{m}) \quad 06 / 2,87 / 20,736$

Wells drilled prior to stays or

NRD signature Date moratoriums require NRD signature

(Signature can be submitted on NRD Approval form to DNR prior to registration)

e. Bore hole diameter in inches Top 6 Bottom 6

f. Casing and Screen Joints are Welded Glued Threaded $\mathrm{x}$ Other

g. Capacity of Well _ 4 gallons per minute (to be used to determine sustainability of aquifer)

h. Pumping water level at this capacity feet 
10. Well Construction (Casing \& Screen)- c, d, e, \& f measurements should be in inches to three decimal places (From not less than zero)

\begin{tabular}{|c|c|c|c|c|c|c|c|c|}
\hline & 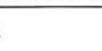 & $\mathrm{b}$ & $\mathrm{c}$ & $\mathrm{d}$ & $\mathrm{e}$ & $f$ & g & $\mathrm{h}$ \\
\hline & $\begin{array}{l}\text { ment } \\
\text { in Feet }\end{array}$ & $\begin{array}{l}\text { Casing or } \\
\text { Screen or }\end{array}$ & $\begin{array}{c}\text { Inside } \\
\text { Diameter }\end{array}$ & $\begin{array}{l}\text { Outside } \\
\text { Diameter }\end{array}$ & $\begin{array}{c}\text { Wall } \\
\text { Thickness }\end{array}$ & $\begin{array}{c}\text { Screen Slot } \\
\text { Size }\end{array}$ & $\begin{array}{l}\text { Type of } \\
\text { Material }\end{array}$ & Trade Name \\
\hline From & To & Open Hole & & & & & & \\
\hline & & & & & & & & \\
\hline & & & & & & & & \\
\hline & & & & & & & & \\
\hline & & & & & & & & \\
\hline & & & & & & & & \\
\hline & & & & & & & & \\
\hline
\end{tabular}

Additional information for Double cased or Nested wells:

\begin{tabular}{|c|c|c|c|c|c|c|c|c|}
\hline \multicolumn{2}{|c|}{$\mathrm{a}$} & $\mathrm{b}$ & $\mathrm{c}$ & $\mathrm{d}$ & $\mathrm{e}$ & $\mathrm{f}$ & $\mathrm{g}$ & $\mathrm{h}$ \\
\hline \multicolumn{2}{|c|}{$\begin{array}{c}\text { Placement } \\
\text { Depth in Feet }\end{array}$} & \multirow{2}{*}{$\begin{array}{l}\text { Casing or } \\
\text { Screen or } \\
\text { Open Hole }\end{array}$} & \multirow[t]{2}{*}{$\begin{array}{c}\text { Inside } \\
\text { Diameter }\end{array}$} & \multirow[t]{2}{*}{$\begin{array}{c}\text { Outside } \\
\text { Diameter }\end{array}$} & \multirow[t]{2}{*}{$\begin{array}{c}\text { Wall } \\
\text { Thickness }\end{array}$} & \multirow[t]{2}{*}{$\begin{array}{c}\text { Screen Slot } \\
\text { Size }\end{array}$} & \multirow[t]{2}{*}{$\begin{array}{l}\text { Type of } \\
\text { Material }\end{array}$} & \multirow[t]{2}{*}{ Trade Name } \\
\hline From & To & & & & & & & \\
\hline & & & & & & & & \\
\hline & & & & & & & & \\
\hline & & & & & & & & \\
\hline & & & & & & & & \\
\hline & & & & & & & & \\
\hline & & & & & & & & \\
\hline
\end{tabular}

11. Grout and Gravel Pack (must start at zero)

\begin{tabular}{|l|l|l|l|l|l|}
\hline \multicolumn{2}{|c|}{$\begin{array}{c}\text { Placement Depth in } \\
\text { Feet }\end{array}$} & $\begin{array}{c}\text { Grout/Gravel/ } \\
\text { Open Hole }\end{array}$ & $\begin{array}{c}\text { Material Description } \\
\text { *See Desc for gravel }\end{array}$ & $\begin{array}{c}\text { Quantity gravel } \\
\text { *See Desc }\end{array}$ & $\begin{array}{c}\text { Volume \& Type Grout } \\
\text { *See Desc }\end{array}$ \\
\hline From & To & & & & \\
\hline & & & & & \\
\hline & & & & & \\
\hline & & & & & \\
\hline & & & & & \\
\hline
\end{tabular}

Desc: Description of gravel pack i.e. engineered gravel pack, or gravel pit description ( $1 / 4$ down), or brand name (Best Sand), natural formation, drilling cuttings, soil backfill

Quantity \#cubic yards, \#tons, \#sacks, - (for drilling cuttings and soil backfill estimate quantity) Calculation assistance available on web Volume \& Type: \#gallons of a slurry, \#barrels of a slurry, \#sacks used in the slurry, \#bags of non-slurry bentonite (chip-pellet -granular) Additional information for Double cased or Nested wells:

\begin{tabular}{|c|c|c|c|c|c|}
\hline \multicolumn{2}{|c|}{$\begin{array}{c}\text { Placement Depth in } \\
\text { Feet }\end{array}$} & \multirow[t]{2}{*}{$\begin{array}{c}\text { Grout/Gravel/ } \\
\text { Open Hole }\end{array}$} & \multirow[t]{2}{*}{$\begin{array}{l}\text { Material Description } \\
\text { *See Desc for gravel }\end{array}$} & \multirow[t]{2}{*}{$\begin{array}{c}\text { Quantity gravel } \\
* \text { See Desc }\end{array}$} & \multirow[t]{2}{*}{$\begin{array}{c}\text { Volume \& Type Grout } \\
\text { *See Desc }\end{array}$} \\
\hline From & To & & & & \\
\hline & & & & & \\
\hline & & & & & \\
\hline & & & & & \\
\hline & & & & & \\
\hline & & & & & \\
\hline & & & & & \\
\hline & & & & & \\
\hline
\end{tabular}


WELL CONSTRUCTION LOG - 14721322886886

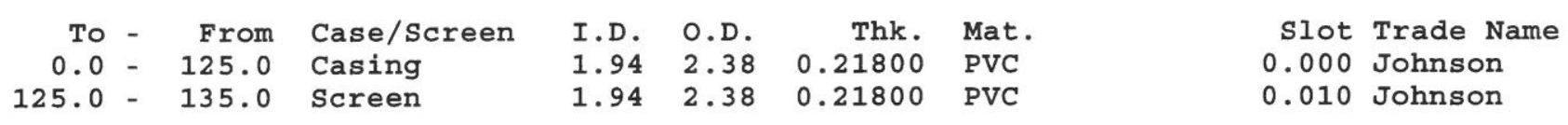

0.000 Johnson

0.010 Johnson

GROUT AND GRAVEL PACK - 14721322886886

\begin{tabular}{|c|c|c|c|c|c|}
\hline From & To & Grout/Gravel & Material Desc. & Qty Gravel & Volume/Type Grout \\
\hline 00.0 & 115.0 & Grout & Bent Grout & & 15 Bags \\
\hline 115.0 & 123.0 & Grout & Pellets & & 1/2 Pail \\
\hline 123.0 & 135.0 & Gravel & $\# 40$ & 5 Bags & \\
\hline
\end{tabular}


GEOLOGICAL LOGS - 14721322886886

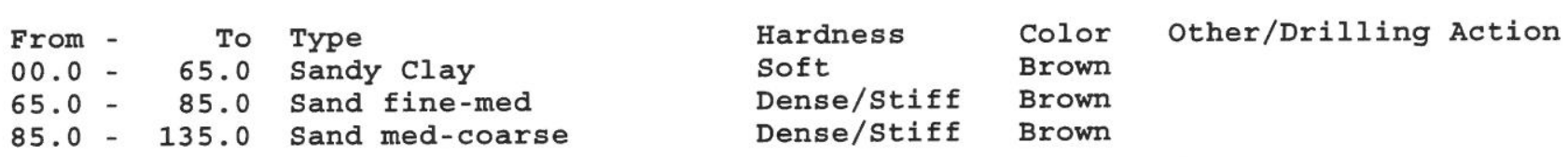


12. Geologic Materials Logged (must start at zero) - Use only options provided below for Type, Hardness and Color

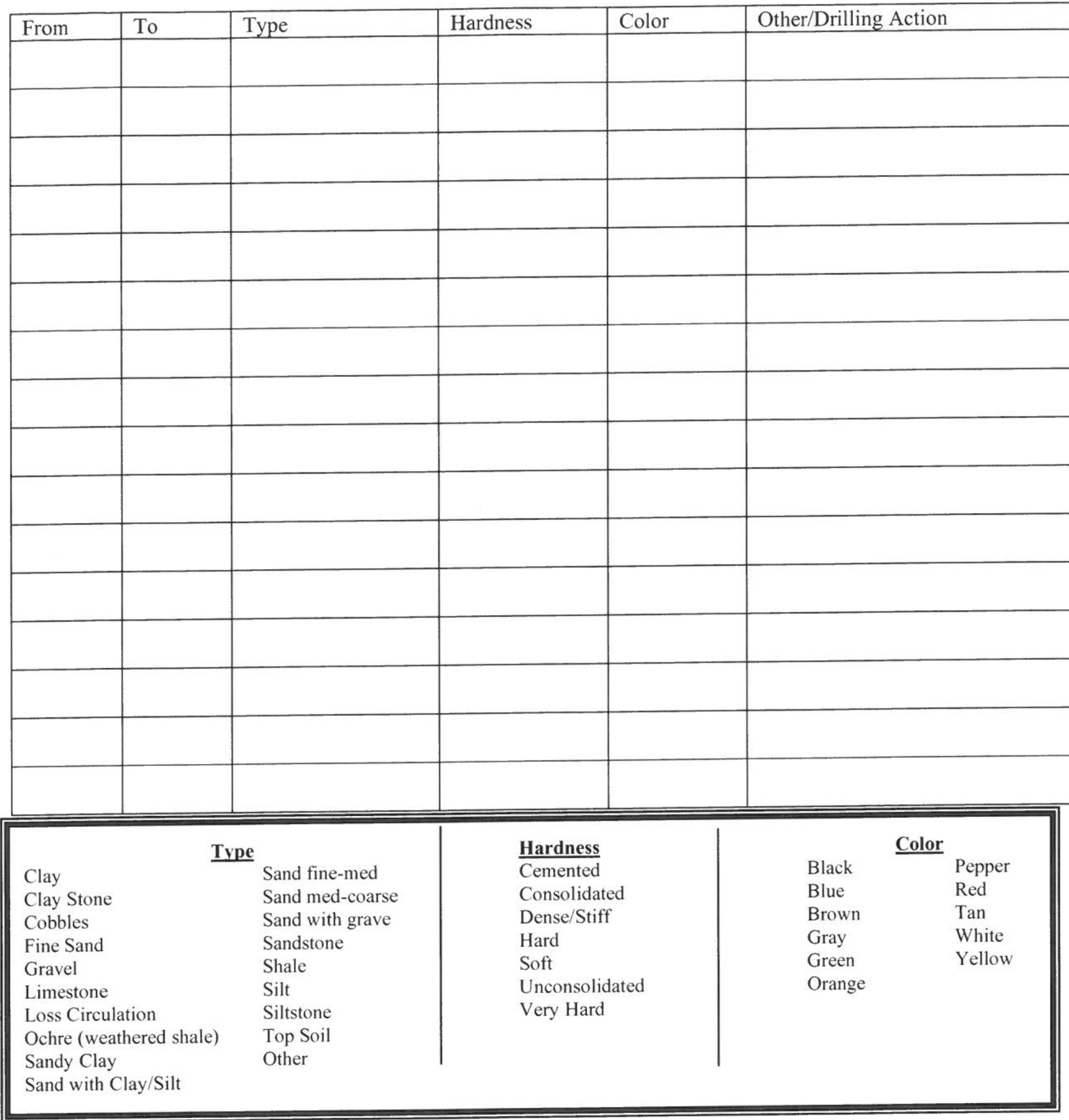

(Additional sheets may be submitted)

13. I hereby certify that the information provided on this registration is true and accurate to the best of my knowledge.
Water Well Contractor's Signature
Date
Well Owner's Signature (not required for pits)
(if Contractor is unknown or Deceased or for pits)
(Not required if signed by Contractor)

Date

Please note this document contains four pages.

Sections 9F, 9G, 10, $11 \& 12$ are not required if registering a pit.

Owner Registering well drilled prior to 2002: Minimum Required Sections - 1, 3(a,b,c,e,f), 5, 6, 7, 8(a,f,h), 9e 


\section{Appendix G:}

\section{Analytical Data for Groundwater Samples}


TABLE G.1 Analytical results for volatile organic compounds in groundwater samples.

\begin{tabular}{|c|c|c|c|c|c|c|c|c|c|c|c|}
\hline \multirow[b]{2}{*}{ Location } & \multirow[b]{2}{*}{ Sample } & \multirow{2}{*}{$\begin{array}{c}\text { Sample } \\
\text { Date }\end{array}$} & \multirow{2}{*}{$\begin{array}{c}\text { Depth } \\
\text { (ft BGL) }\end{array}$} & \multicolumn{8}{|c|}{ Concentration $(\mu \mathrm{g} / \mathrm{L})$} \\
\hline & & & & $\begin{array}{l}\text { Carbon } \\
\text { Tetrachloride }\end{array}$ & Chloroform & $\begin{array}{l}\text { Methylene } \\
\text { Chloride }\end{array}$ & 1,1-DCE & 1,2-DCA & 1,2-Dichloropropane & PCE & TCE \\
\hline \multicolumn{12}{|c|}{ Samples collected from direct-push investigative borings } \\
\hline TI01 & YKTI01-W-37844 & 7/10/15 & $77.4-82.4$ & 373 & 108 & ND & ND & ND & $0.9 \mathrm{~J}$ & $0.6 \mathrm{~J}$ & ND \\
\hline TI01 & YKTI01100-W-37854 & $7 / 16 / 15$ & 88-98 & $N D^{b}$ & $0.6 \mathrm{~J}$ & ND & ND & ND & ND & ND & ND \\
\hline TI01 & YKTI01120-W-37855 & $7 / 15 / 15$ & $108-118$ & $0.9 \mathrm{~J}^{\mathrm{c}}$ & 2.0 & ND & ND & ND & ND & ND & ND \\
\hline T102 & YKTI02-W-37847 & $7 / 10 / 15$ & 77-82 & 200 & 54 & ND & ND & ND & ND & ND & ND \\
\hline TI02 & YKTI02100-W-37849 & $7 / 13 / 15$ & $100-103.5$ & 1.4 & 7.2 & ND & ND & ND & ND & ND & ND \\
\hline TI02 & YKTI02120-W-37852 & $7 / 14 / 15$ & $108.5-118.5$ & ND & ND & ND & ND & ND & ND & ND & ND \\
\hline TI03 & YKTI03-W-37836 & 7/9/15 & $76.8-81.8$ & 150 & 11 & ND & ND & ND & ND & ND & ND \\
\hline TI04 & 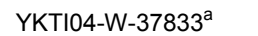 & $7 / 9 / 15$ & $74.6-79.6$ & 159 & 164 & ND & ND & ND & ND & $0.5 \mathrm{~J}$ & ND \\
\hline TI04 & YKTI04100-W-37861 & 7/16/15 & 89-99 & ND & $0.8 \mathrm{~J}$ & ND & ND & ND & ND & ND & ND \\
\hline TI04 & YKTI04120-W-37860 & 7/16/15 & $108-118$ & ND & 1.1 & ND & ND & ND & ND & ND & ND \\
\hline T105 & YKTI0580-W-37868 & $7 / 18 / 15$ & $76-81$ & 254 & 69 & ND & ND & ND & ND & ND & ND \\
\hline T105 & YKTI05100-W-37865 & 7/17/15 & $88-98$ & 82 & 6.7 & ND & ND & ND & ND & ND & ND \\
\hline T105 & YKTI05120-W-37864 & 7/17/15 & $108-118$ & 2.0 & $0.6 \mathrm{~J}$ & ND & ND & ND & ND & ND & ND \\
\hline TI06 & YKTI0680-W-37872 & $7 / 19 / 15$ & $77-82$ & 7.0 & $0.4 \mathrm{~J}$ & ND & ND & ND & ND & ND & ND \\
\hline T106 & YKTI06100-W-37873 & 7/19/15 & $90-100$ & $0.8 \mathrm{~J}$ & 2.7 & ND & ND & ND & ND & ND & ND \\
\hline TI06 & YKTI06120-W-37870 & 7/19/15 & $110-120$ & ND & ND & ND & ND & ND & ND & ND & ND \\
\hline TI07 & YKTI0780-W-37875 & $7 / 20 / 15$ & $77-82$ & 40 & 2.2 & ND & ND & ND & ND & ND & ND \\
\hline TI07 & YKTI07100-W-37876 & 7/20/15 & $90-100$ & ND & ND & ND & ND & ND & ND & ND & ND \\
\hline TI07 & YKT107120-W-37877 & 7/20/15 & $110-120$ & ND & ND & ND & ND & ND & ND & ND & ND \\
\hline TI08 & YKT10885-W-37879 & $7 / 21 / 15$ & $80-85$ & ND & ND & ND & ND & ND & ND & ND & ND \\
\hline TI08 & YKTI08107-W-37881 & $7 / 21 / 15$ & $97-107$ & 27 & $0.9 \mathrm{~J}$ & ND & ND & ND & ND & ND & ND \\
\hline TI08 & YKTI08125-W-37882 & $7 / 21 / 15$ & $115-125$ & 8.7 & ND & ND & ND & ND & ND & ND & ND \\
\hline T109 & YKTI09-80-W-37926 & $8 / 13 / 15$ & $80-85$ & $0.7 \mathrm{~J}$ & ND & ND & ND & ND & ND & ND & ND \\
\hline TI09 & YKTI09-95-W-37928 & $8 / 13 / 15$ & $95-105$ & 3.8 & ND & ND & ND & ND & ND & ND & ND \\
\hline TI09 & YKTI09-135-W-37929 & $8 / 13 / 15$ & $135-145$ & 1.8 & ND & ND & ND & ND & ND & ND & ND \\
\hline TI10 & YKTI10-78-W-37930 & $8 / 14 / 15$ & $78-83$ & 6.6 & $0.9 \mathrm{~J}$ & ND & ND & ND & ND & ND & ND \\
\hline TI10 & YKTI10-95-W-37931 & $8 / 14 / 15$ & $95-105$ & 18 & 1.2 & ND & ND & ND & ND & ND & ND \\
\hline TI10 & YKTI10-117-W-37933 & $8 / 14 / 15$ & $117-127$ & 29 & 1.1 & ND & ND & ND & ND & ND & ND \\
\hline TI11 & YKTI11-80-W-37935 & $8 / 17 / 15$ & $80-85$ & 36 & 6.4 & ND & ND & ND & ND & ND & ND \\
\hline TI11 & YKTI11-92-W-37936 & $8 / 18 / 15$ & $92-102$ & ND & ND & ND & ND & ND & ND & ND & ND \\
\hline TI11 & YKTI11-112-W-37937 & $8 / 18 / 15$ & $112-122$ & 202 & 5.1 & ND & ND & ND & ND & ND & ND \\
\hline Tl12 & YKTI12-80-W-37939 & $8 / 20 / 15$ & $80-85$ & 6.2 & 1.2 & ND & ND & ND & ND & ND & ND \\
\hline TI12 & YKTI12-92-W-37940 & $8 / 20 / 15$ & $92-102$ & 45 & 1.5 & ND & ND & ND & ND & ND & ND \\
\hline TI12 & YKTI12-120-W-37942 & $8 / 20 / 15$ & $110-120$ & 63 & 1.7 & ND & ND & ND & ND & ND & ND \\
\hline TI12 & YKTI12-145-W-37943 & $8 / 20 / 15$ & $135-145$ & 73 & 1.5 & ND & ND & ND & ND & ND & ND \\
\hline
\end{tabular}




\begin{tabular}{|c|c|c|c|c|c|c|c|c|c|c|c|}
\hline \multirow[b]{2}{*}{ Location } & \multirow[b]{2}{*}{ Sample } & \multirow[b]{2}{*}{$\begin{array}{c}\text { Sample } \\
\text { Date }\end{array}$} & \multirow[b]{2}{*}{$\begin{array}{c}\text { Depth } \\
\text { (ft BGL) }\end{array}$} & \multicolumn{8}{|c|}{ Concentration $(\mu \mathrm{g} / \mathrm{L})$} \\
\hline & & & & $\begin{array}{c}\text { Carbon } \\
\text { Tetrachloride }\end{array}$ & Chloroform & $\begin{array}{l}\text { Methylene } \\
\text { Chloride }\end{array}$ & 1,1-DCE & 1,2-DCA & 1,2-Dichloropropane & PCE & $\mathrm{TCH}$ \\
\hline \multicolumn{12}{|c|}{ Samples collected from direct-push investigative borings (cont.) } \\
\hline TI13 & YKT113-82-W-37955 & $8 / 24 / 15$ & $81-86$ & ND & ND & ND & ND & ND & ND & ND & ND \\
\hline TI13 & YKTI13-93-W-37947 & $8 / 22 / 15$ & $93-103$ & ND & ND & ND & ND & ND & ND & ND & ND \\
\hline TI13 & YKTI13-111-W-37948 & $8 / 22 / 15$ & $111-121$ & ND & ND & ND & ND & ND & ND & ND & ND \\
\hline TI14 & YKTI14-86-W-37951 & $8 / 23 / 15$ & $86-96$ & 1.9 & ND & ND & ND & ND & ND & ND & ND \\
\hline TI14 & YKTI14-113-W-37952 & $8 / 23 / 15$ & $113-123$ & 36 & 1.4 & ND & ND & $\mathrm{ND}$ & ND & $\mathrm{ND}$ & $\mathrm{ND}$ \\
\hline TI14 & YKTI14-136-W-37954 & $8 / 23 / 15$ & $136-146$ & 59 & $0.8 \mathrm{~J}$ & ND & ND & ND & ND & ND & ND \\
\hline TI15 & YKTI15-90-W-37956 & $8 / 24 / 15$ & $90-100$ & 1.7 & ND & ND & ND & ND & ND & ND & ND \\
\hline TI15 & YKTI15-113-W-37957 & $8 / 24 / 15$ & $113-123$ & 22 & $0.7 \mathrm{~J}$ & ND & $\mathrm{ND}$ & ND & ND & ND & $\mathrm{ND}$ \\
\hline TI15 & YKTI15-134-W-37958 & $8 / 24 / 15$ & $134-144$ & 68 & 1.7 & ND & ND & ND & ND & ND & ND \\
\hline TI16 & YKTI16-86-W-37961 & $8 / 25 / 15$ & $86-96$ & ND & ND & ND & ND & ND & ND & ND & ND \\
\hline TI16 & YKTI16-105-W-37963 & $8 / 25 / 15$ & $105-115$ & 2.9 & ND & ND & ND & ND & ND & & ND \\
\hline TI16 & YKTI16-136-W-37964 & $8 / 25 / 15$ & $136-146$ & 35 & $0.7 \mathrm{~J}$ & $\mathrm{ND}$ & $\mathrm{ND}$ & $\mathrm{ND}$ & ND & ND & $\mathrm{ND}$ \\
\hline TI17 & YKTI17-84-W-37966 & $8 / 26 / 15$ & $84-94$ & ND & ND & ND & ND & ND & ND & ND & ND \\
\hline T117 & YKTI17-104-W-37967 & $8 / 26 / 15$ & $104-114$ & ND & ND & ND & ND & ND & ND & ND & ND \\
\hline TI17 & YKTI17-133-W-37968 & $8 / 26 / 15$ & $133-143$ & $\mathrm{ND}$ & $0.4 \mathrm{~J}$ & $\mathrm{ND}$ & $\mathrm{ND}$ & $\mathrm{ND}$ & $\mathrm{ND}$ & $\mathrm{ND}$ & $\mathrm{ND}$ \\
\hline T118 & YKTI18-81-W-37970 & $8 / 27 / 15$ & $81-86$ & 12 & 9.0 & ND & ND & ND & ND & ND & ND \\
\hline T118 & YKTI18-95-W-37971 & $8 / 27 / 15$ & $95-105$ & 11 & $0.9 \mathrm{~J}$ & ND & ND & ND & $\mathrm{ND}$ & ND & ND \\
\hline TI18 & YKTI18-114-W-37973 & $8 / 28 / 15$ & $114-124$ & 2.9 & ND & ND & $\mathrm{ND}$ & $\mathrm{ND}$ & ND & ND & $\mathrm{ND}$ \\
\hline T119 & YKTI19-84-W-37975 & 9/9/15 & $84-94$ & ND & ND & ND & ND & ND & ND & ND & ND \\
\hline T119 & YKTI19-107-W-37976 & 9/9/15 & $107-117$ & 4.8 & ND & ND & ND & ND & ND & ND & ND \\
\hline TI19 & YKTI19-129-W-37977 & $9 / 9 / 15$ & $129-139$ & 22 & $0.8 \mathrm{~J}$ & $\mathrm{ND}$ & $\mathrm{ND}$ & $\mathrm{ND}$ & $\mathrm{ND}$ & $\mathrm{ND}$ & $\mathrm{ND}$ \\
\hline TI20 & YKTI20-81-W-37980 & $9 / 10 / 15$ & $81-91$ & ND & ND & ND & ND & ND & ND & ND & ND \\
\hline T120 & YKTI20-105-W-37981 & $9 / 10 / 15$ & $105-115$ & 8.2 & ND & ND & ND & ND & ND & ND & ND \\
\hline $\mathrm{T} 120$ & YKTI20-131-W-37982 & $9 / 10 / 15$ & $131-141$ & 1.8 & $\mathrm{ND}$ & $\mathrm{ND}$ & $\mathrm{ND}$ & $\mathrm{ND}$ & $\mathrm{ND}$ & $\mathrm{ND}$ & $\mathrm{ND}$ \\
\hline TI21 & YKTI21-77-W-37985 & $9 / 11 / 15$ & $77-87$ & $0.8 \mathrm{~J}$ & ND & ND & ND & ND & ND & ND & ND \\
\hline T121 & YKTI21-99-W-37986 & $9 / 11 / 15$ & $99-109$ & $0.8 \mathrm{~J}$ & ND & ND & ND & ND & ND & ND & 4.9 \\
\hline T121 & YKTI21-115-W-37987 & $9 / 11 / 15$ & $115-120$ & ND & ND & ND & ND & ND & ND & ND & ND \\
\hline TI22 & YKTI22-79-W-37990 & $9 / 14 / 15$ & $79-84$ & ND & ND & ND & ND & ND & ND & 1.0 & ND \\
\hline T122 & YKTI22-98-W-37991 & $9 / 14 / 15$ & $98-108$ & 3.3 & ND & ND & ND & ND & ND & ND & ND \\
\hline T122 & YKTI22-113-W-37992 & $9 / 14 / 15$ & $113-123$ & ND & ND & ND & ND & ND & ND & ND & ND \\
\hline T123 & YKTI23-80-W-37995 & $9 / 15 / 15$ & $80-90$ & $0.5 \mathrm{~J}$ & $0.6 \mathrm{~J}$ & ND & ND & ND & ND & ND & ND \\
\hline $\mathrm{T} 123$ & YKTI23-99-W-37996 & $9 / 15 / 15$ & $99-109$ & ND & ND & $\mathrm{ND}$ & $\mathrm{ND}$ & ND & $\mathrm{ND}$ & ND & $\mathrm{ND}$ \\
\hline T123 & YKTI23-120-W-37998 & $9 / 15 / 15$ & $120-130$ & ND & ND & ND & ND & $1.7 \mathrm{E}^{\mathrm{c}}$ & ND & ND & ND \\
\hline T123 & YKT123-145-W-37999 & 9/16/15 & $145-155$ & ND & ND & ND & ND & $4.9 \mathrm{E}$ & ND & $0.6 \mathrm{~J}$ & ND \\
\hline
\end{tabular}


TABLE G.1 (Cont.)

\begin{tabular}{|c|c|c|c|c|c|c|c|c|c|c|c|}
\hline \multirow[b]{2}{*}{ Location } & \multirow[b]{2}{*}{ Sample } & \multirow{2}{*}{$\begin{array}{l}\text { Sample } \\
\text { Date }\end{array}$} & \multirow{2}{*}{$\begin{array}{l}\text { Depth } \\
\text { (ft BGL) }\end{array}$} & \multicolumn{8}{|c|}{ Concentration $(\mu \mathrm{g} / \mathrm{L})$} \\
\hline & & & & Tetrachloride & Chloroform & Chloride & 1,1-DCE & 1,2-DCA & 1,2-Dichloropropane & PCE & TCE \\
\hline \multicolumn{12}{|c|}{ Samples collected from direct-push investigative borings (cont.) } \\
\hline TI24 & YKTI24-80-W-38017 & 1/20/16 & $80-90$ & ND & ND & ND & ND & ND & ND & ND & ND \\
\hline TI24 & YKTI24-90-W-38018 & $1 / 20 / 16$ & $90-100$ & ND & ND & ND & ND & ND & ND & ND & ND \\
\hline TI24 & YKTI24-100-W-38019 & 1/20/16 & $100-110$ & ND & ND & ND & ND & ND & ND & ND & ND \\
\hline TI25 & YKTI25-77-W-38023 & 1/21/16 & $77-87$ & ND & ND & ND & ND & ND & ND & ND & ND \\
\hline TI25 & YKTI25-98-W-38024 & $1 / 21 / 16$ & $98-108$ & 1.3 & ND & ND & ND & ND & ND & ND & ND \\
\hline TI25 & YKTI25-108-W-38027 & $1 / 21 / 16$ & $113-118$ & ND & ND & ND & ND & ND & ND & ND & ND \\
\hline TI26 & YKTI26-78-W-38029 & $1 / 25 / 16$ & $78-88$ & ND & ND & ND & ND & ND & ND & ND & ND \\
\hline TI26 & YKTI26-96-W-38030 & $1 / 25 / 16$ & $96-106$ & 2.0 & ND & ND & ND & ND & ND & ND & ND \\
\hline TI26 & YKTI26-113-W-38033 & $1 / 25 / 16$ & $113-123$ & $0.4 \mathrm{~J}$ & ND & ND & ND & ND & ND & ND & ND \\
\hline TI27 & YKTI27-77-W-38050 & 2/9/16 & $77-87$ & ND & ND & ND & ND & ND & ND & ND & ND \\
\hline TI27 & YKTI27-77-W-38056 & 2/9/16 & $77-87$ & ND & ND & ND & ND & ND & ND & ND & ND \\
\hline TI27 & YKTI27-100-W-38053 & 2/9/16 & $100-110$ & $0.4 \mathrm{~J}$ & ND & ND & ND & ND & ND & ND & ND \\
\hline TI27 & YKTI27-100-W-38057 & 2/9/16 & $100-110$ & ND & ND & ND & ND & ND & ND & ND & ND \\
\hline TI28 & YKTI28-78-W-38075 & $3 / 2 / 16$ & $78-88$ & ND & ND & ND & ND & ND & ND & ND & ND \\
\hline TI28 & YKTI28-90-W-38076 & $3 / 2 / 16$ & $90-100$ & ND & ND & ND & ND & ND & ND & ND & ND \\
\hline TI28 & YKTI28-104-W-38078 & $3 / 3 / 16$ & 104-109 & ND & ND & ND & ND & ND & ND & ND & ND \\
\hline \multicolumn{12}{|c|}{ Samples collected from monitoring wells } \\
\hline SB62 & YKSB62-W-37924 & $8 / 7 / 15$ & $118-123$ & 23 & 2.2 & ND & ND & ND & ND & ND & ND \\
\hline SB77 & YKSB77-W-37921 & $8 / 7 / 15$ & $75.8-79$ & 220 & 9.8 & ND & ND & ND & ND & ND & ND \\
\hline DL1A & YKDL1A-75-W-38094 & $5 / 25 / 16$ & $75-85$ & 1875 & 11 & ND & ND & ND & ND & ND & ND \\
\hline DL1B & YKDL1B-88-W-38093 & $5 / 24 / 16$ & $88-98$ & 1.6 & ND & ND & ND & ND & ND & ND & ND \\
\hline DL1C & YKDL1C-108-W-38091 & $5 / 23 / 16$ & 108-118 & 1.9 & ND & ND & ND & ND & ND & ND & ND \\
\hline DL2C1 & YKDL2-C1-112-W-38100 & $6 / 2 / 16$ & $112-122$ & 41 & $0.7 \mathrm{~J}$ & ND & ND & ND & ND & ND & ND \\
\hline DL2C2 & YKDL2C2-136-W-38096 & $5 / 31 / 16$ & 136-146 & 42 & 1.0 & ND & ND & ND & ND & ND & ND \\
\hline DL2CU & YKDL2CU-215-W-38097 & $5 / 31 / 16$ & $215-225$ & ND & ND & ND & ND & ND & ND & ND & ND \\
\hline DL3C1 & YKDL3-C1-113-W-38103 & $6 / 6 / 16$ & $113-123$ & 39 & $0.7 \mathrm{~J}$ & ND & ND & ND & ND & ND & ND \\
\hline DL3C2 & YKDL3-C2-134-W-38102 & $6 / 5 / 16$ & $134-144$ & 77 & 1.1 & ND & ND & ND & ND & ND & ND \\
\hline DL4C1 & YKDL4-C1-120-W-38116 & $6 / 16 / 16$ & $120-130$ & ND & ND & ND & $0.9 \mathrm{~J}$ & ND & ND & ND & ND \\
\hline DL4C2 & YKDL4-C2-145-W-38115 & $6 / 15 / 16$ & 145-155 & ND & ND & ND & 2.7 & ND & ND & ND & ND \\
\hline DL5C1 & YKDL5-C1-106-W-38118 & $6 / 21 / 16$ & $106-116$ & ND & ND & ND & ND & ND & ND & ND & ND \\
\hline DL6C & YKDL6-C-110-W-38113 & 6/13/16 & $110-120$ & ND & ND & ND & ND & ND & ND & ND & 4.0 \\
\hline
\end{tabular}




\begin{tabular}{|c|c|c|c|c|c|c|c|c|c|c|c|}
\hline \multirow[b]{2}{*}{ Location } & \multirow[b]{2}{*}{ Sample } & \multirow[b]{2}{*}{$\begin{array}{l}\text { Sample } \\
\text { Date }\end{array}$} & \multirow[b]{2}{*}{$\begin{array}{l}\text { Depth } \\
\text { (ft BGL) }\end{array}$} & \multicolumn{8}{|c|}{ Concentration $(\mu \mathrm{g} / \mathrm{L})$} \\
\hline & & & & $\begin{array}{c}\text { Carbon } \\
\text { Tetrachloride }\end{array}$ & Chloroform & $\begin{array}{l}\text { Methylene } \\
\text { Chloride }\end{array}$ & 1,1-DCE & 1,2-DCA & 1,2-Dichloropropane & PCE & TCE \\
\hline \multicolumn{12}{|c|}{ Samples collected from monitoring wells (cont.) } \\
\hline DL7C & YKDL7-C-100-W-38111 & $6 / 12 / 16$ & $100-110$ & ND & ND & ND & ND & ND & ND & ND & ND \\
\hline DL7CU & YKDL7-CU-192-W-38112 & $6 / 12 / 16$ & 192-202 & ND & ND & ND & ND & ND & ND & ND & ND \\
\hline $\mathrm{DL7CL}$ & YKDL7-CL-265-W-38108 & $6 / 9 / 16$ & $265-275$ & ND & ND & ND & ND & ND & ND & ND & ND \\
\hline DL8C1 & YKDL8C1-104-W-38106 & $6 / 7 / 16$ & $104-114$ & 16 & ND & ND & ND & ND & ND & ND & ND \\
\hline DL8C2 & YKDL8-C2-125-W-38104 & $6 / 6 / 16$ & $125-135$ & 2.2 & ND & ND & ND & ND & ND & ND & ND \\
\hline \multicolumn{12}{|c|}{ Samples collected from private wells } \\
\hline Preslicka & YKPRESLICKA-W-37916 & $8 / 5 / 15$ & - & 1.3 & ND & ND & ND & ND & ND & ND & ND \\
\hline Volzke & YKVOLZKE-W-37923 & $8 / 7 / 15$ & - & ND & ND & ND & ND & ND & ND & ND & ND \\
\hline York County Shop & YKYORKCOUNTYSHOP-W-37917 & $8 / 5 / 15$ & - & 53 & $0.8 \mathrm{~J}$ & ND & ND & ND & ND & ND & ND \\
\hline Nordlund & YKNORDLUND-W-37918 & $8 / 5 / 15$ & - & ND & ND & ND & 2.3 & ND & ND & 4.1 & 6.3 \\
\hline Liermann & YKLIERMANN-W-37920 & $8 / 5 / 15$ & - & ND & ND & ND & ND & ND & ND & ND & ND \\
\hline
\end{tabular}

a Xylene was detected in field replicate YKTI04-W-37834 at a trace level that was not quantified by the instrument; it was not detected in the primary sample (YKTI04-W-37833).

b ND, compound analyzed for but not detected at a level greater than or equal to the method detection limit $(<1 \mu \mathrm{g} / \mathrm{L})$.

c J, compound identified with an estimated concentration between the instrument detection limit and the method detection limit.

d E, result exceeds calibration range.

e Back-up TI27, $77 \mathrm{ft}$ sample sent to replace the frozen YKTI27-77-W-38050 vial.

f Back-up T127, $100 \mathrm{ft}$ sample sent to replace the frozen YKTI27-100-W-38053 vial. 
TABLE G.2 Field measurements for groundwater samples collected at private and monitoring wells.

\begin{tabular}{|c|c|c|c|c|c|c|c|}
\hline Location & Sample & $\begin{array}{l}\text { Sample } \\
\text { Date }\end{array}$ & $\begin{array}{c}\text { Temperature } \\
\text { (Deg. C) }\end{array}$ & $\mathrm{pH}$ & $\begin{array}{l}\text { Conductivity } \\
(\mu \mathrm{S} / \mathrm{cm})\end{array}$ & $\begin{array}{c}\text { Dissolved } \\
\text { Oxygen } \\
(\mathrm{mg} / \mathrm{L})\end{array}$ & $\begin{array}{l}\text { Oxidation- } \\
\text { Reduction } \\
\text { Potential } \\
(\mathrm{mV})\end{array}$ \\
\hline DL1A & YKDL1A-75-W-38094 & $5 / 25 / 16$ & 14.9 & 6.26 & 797 & 14.50 & 144.9 \\
\hline DL1B & YKDL1B-88-W-38093 & $5 / 24 / 16$ & 14.7 & 6.86 & 802 & 6.28 & 3.5 \\
\hline DL1C & YKDL1C-108-W-38091 & $5 / 23 / 16$ & 14.7 & 6.86 & 802 & 6.28 & 3.5 \\
\hline DL2C1 & YKDL2-C1-112-W-38100 & $6 / 2 / 16$ & 13.4 & 7.17 & 624 & 4.83 & 156.3 \\
\hline DL2C2 & YKDL2C2-136-W-38096 & $5 / 31 / 16$ & 16.3 & 7.02 & 770 & 7.26 & -47.6 \\
\hline DL2CU & YKDL2CU-215-W-38097 & $5 / 31 / 16$ & 14.6 & 7.29 & 743 & 1.24 & -141.2 \\
\hline DL3C1 & YKDL3-C1-113-W-38103 & $6 / 6 / 16$ & 14.2 & 6.88 & 624 & 9.17 & 99.3 \\
\hline DL3C2 & YKDL3-C2-134-W-38102 & $6 / 5 / 16$ & 14.6 & 7.47 & 613 & 6.71 & 129.5 \\
\hline DL4C1 & YKDL4-C1-120-W-38116 & $6 / 16 / 16$ & 22.4 & 6.55 & 651 & 6.11 & 166.3 \\
\hline DL4C2 & YKDL4-C2-145-W-38115 & $6 / 15 / 16$ & 22.8 & 6.87 & 661 & 5.24 & 146.8 \\
\hline DL5C1 & YKDL5-C1-106-W-38118 & $6 / 21 / 16$ & 15.0 & 6.54 & 780 & 81.70 & 145.5 \\
\hline DL6C & YKDL6-C-110-W-38113 & $6 / 13 / 16$ & 15.1 & 7.03 & 702 & 6.41 & 72.0 \\
\hline DL7C & YKDL7-C-100-W-38111 & $6 / 12 / 16$ & 18.9 & 6.98 & 771 & 6.29 & 129.8 \\
\hline DL7CL & YKDL7-CL-265-W-38108 & $6 / 9 / 16$ & 15.9 & 6.94 & 751 & 15.90 & 73.1 \\
\hline DL7CU & YKDL7-CU-192-W-38112 & $6 / 12 / 16$ & 15.5 & 7.05 & 905 & 0.32 & -70.1 \\
\hline DL8C1 & YKDL8C1-104-W-38106 & $6 / 7 / 16$ & 15.9 & 6.94 & 751 & 15.90 & 73.1 \\
\hline DL8C2 & YKDL8-C2-125-W-38104 & $6 / 6 / 16$ & 15.2 & 7.00 & 610 & 11.67 & 84.6 \\
\hline LIERMANN & YKLIERMANN-W-37920 & $8 / 5 / 15$ & 15.8 & 7.05 & 1101 & 9.39 & 203.8 \\
\hline NORDLUND & YKNORDLUND-W-37918 & $8 / 5 / 15$ & 19.0 & 7.03 & 674 & 8.48 & 200.4 \\
\hline PRESLICKA & YKPRESLICKA-W-37916 & $8 / 5 / 15$ & 15.9 & 6.84 & 688 & 9.89 & 179.9 \\
\hline SB62 & YKSB62-W-37924 & $8 / 7 / 15$ & 18.4 & 7.08 & 753 & 6.49 & 168.0 \\
\hline SB77 & YKSB77-W-37921 & $8 / 7 / 15$ & 19.8 & 6.85 & 688 & 7.69 & 174.2 \\
\hline VOLZKE & YKVOLZKE-W-37923 & $8 / 7 / 15$ & 15.7 & 6.74 & 995 & 7.74 & 196.6 \\
\hline YORKCOUNTYSHOP & YKYORKCOUNTYSHOP-W-37917 & $8 / 5 / 15$ & 15.0 & 7.02 & 590 & 9.13 & 186.8 \\
\hline
\end{tabular}




\section{Appendix H:}

Analytical Data for Indoor Air and Sub-Slab Soil Gas Samples 
Concentration $\left(\mu \mathrm{g} / \mathrm{m}^{3}\right)$

\begin{tabular}{|c|c|c|c|c|c|c|c|c|c|c|c|c|c|c|c|c|}
\hline \multirow[b]{2}{*}{ Location } & \multirow[b]{2}{*}{ Sample } & \multirow[b]{2}{*}{$\begin{array}{l}\text { Sample } \\
\text { Date }\end{array}$} & \multirow[b]{2}{*}{$\begin{array}{l}\text { Sample } \\
\text { Type }^{\mathrm{b}}\end{array}$} & \multirow[b]{2}{*}{$\begin{array}{l}\text { Sample } \\
\text { Matrix }\end{array}$} & \multirow[b]{2}{*}{$\begin{array}{l}\text { Ambient } \\
\text { Sample }^{\mathrm{C}}\end{array}$} & \\
\hline & & & & & & $\begin{array}{c}\text { Carbon } \\
\text { Tetrachloride }\end{array}$ & Chloroform & $\begin{array}{l}\text { Methylene } \\
\text { Chloride }\end{array}$ & $\begin{array}{l}1,1,1,2- \\
\text { Tetrachloroethane }\end{array}$ & $\begin{array}{l}1,1,1- \\
\text { Trichloroethane }\end{array}$ & $\begin{array}{l}1,1,2,2- \\
\text { Tetrachloroethane }\end{array}$ & $\begin{array}{l}1,1,2- \\
\text { Trichloroethane }\end{array}$ & $\begin{array}{l}1,1- \\
\text { Dichloroethane }\end{array}$ & $\begin{array}{c}1,1- \\
\text { Dichloroethylene }\end{array}$ & $\begin{array}{l}1,2,4- \\
\text { Trichlorobenzene }\end{array}$ & $\begin{array}{l}1,2,4- \\
\text { Trimethylbenzen }\end{array}$ \\
\hline WILLOW & YKWILLOW-G-37887 & $8 / 5 / 15$ & $\mathrm{~N}$ & AIR & & $N D^{d}(1.3)$ & $\mathrm{ND}(0.98)$ & ND (1.7) & $\_^{\mathrm{e}}$ & $N D(1.1)$ & ND (1.4) & ND (1.1) & $\mathrm{ND}(0.81)$ & $\mathrm{ND}(0.79)$ & ND (3.7) & $\mathrm{ND}(0.98)$ \\
\hline WILLOW & YKWILLOW-G-37885 & $8 / 5 / 15$ & $\mathrm{~N}$ & AIR & & ND (1.3) & ND (0.98) & ND (1.7) & - & ND (1.1) & ND (1.4) & ND (1.1) & ND $(0.81)$ & ND (0.79) & ND (3.7) & ND (0.98) \\
\hline WILLOW & YKWILLOW-G-37886 & $8 / 5 / 15$ & $\mathrm{~N}$ & AIR & & ND (1.3) & ND $(0.98)$ & ND (1.7) & - & ND (1.1) & ND (1.4) & ND (1.1) & ND $(0.81)$ & ND $(0.79)$ & ND (3.7) & ND $(0.98)$ \\
\hline WILLOW & YKWILLOW-G-37889 & $8 / 5 / 15$ & $\mathrm{~N}$ & AIR & & $\mathrm{ND}(1.3)$ & ND (0.98) & $\mathrm{ND}(1.7)$ & - & ND (1.1) & ND (1.4) & ND (1.1) & ND (0.81) & ND (0.79) & ND (3.7) & $\mathrm{ND}(0.98)$ \\
\hline WILLOW & YKWILLOW-G-37890 & $\begin{array}{l}8 / 5 / 15 \\
8 / 5115\end{array}$ & $N$ & AIR & & ND (1.3) & ND $(0.98)$ & ND (1.7) & - & ND (1.1) & ND (1.4) & $N D(1.1)$ & ND $(0.81)$ & ND $(0.79)$ & ND (3.7) & ND $(0.98)$ \\
\hline $\begin{array}{l}\text { WILLOW } \\
\text { CVA }\end{array}$ & $\begin{array}{l}\text { YKWILLOW-G-307888 } \\
\text { YKCVA-G-37909 }\end{array}$ & $\begin{array}{l}8 / 5 / 15 \\
8 / 5 / 15\end{array}$ & $\stackrel{N}{N}$ & $\begin{array}{l}\text { AlR } \\
\text { AIR }\end{array}$ & & $\begin{array}{l}\text { ND (1.3) } \\
\text { ND (1.3) }\end{array}$ & $\begin{array}{l}\mathrm{ND}(0.98) \\
\mathrm{ND}(0.98)\end{array}$ & $\begin{array}{l}\text { ND (1.7) } \\
\text { ND (1.7) }\end{array}$ & $\begin{array}{lll}- & & \\
-\end{array}$ & $\begin{array}{l}\mathrm{ND}(1.1) \\
\mathrm{ND}(1.1)\end{array}$ & $\begin{array}{l}\text { ND (1.4) } \\
\text { ND (1.4) }\end{array}$ & $\begin{array}{l}\mathrm{ND}(1.1) \\
\mathrm{ND}(1.1)\end{array}$ & $\begin{array}{l}\mathrm{ND}(0.81) \\
\mathrm{ND}(0.81)\end{array}$ & $\begin{array}{l}\mathrm{ND}(0.79) \\
\mathrm{ND}(0.79)\end{array}$ & $\begin{array}{l}\mathrm{ND}(3.7) \\
\mathrm{ND}(3.7)\end{array}$ & $\begin{array}{l}\mathrm{ND}(0.98) \\
\mathrm{ND}(0.98)\end{array}$ \\
\hline CVA & $\begin{array}{l}\text { YKCVA-G-37910 } \\
\text { YKCVA-G }\end{array}$ & $\begin{array}{l}8 / 5 / 15 \\
8 / 515\end{array}$ & $n^{n}$ & AlR & & $\begin{array}{l}\text { ND }(1.3) \\
\text { ND (1.3) }\end{array}$ & $\begin{array}{l}\mathrm{ND}(0.98) \\
\mathrm{ND}(0.98)\end{array}$ & $\begin{array}{l}\text { ND (1.7) } \\
\text { ND (1.7) }\end{array}$ & - & $\begin{array}{l}\mathrm{ND}(1.1) \\
\mathrm{ND}(1.1)\end{array}$ & $\begin{array}{l}\mathrm{ND}(1.4) \\
\mathrm{ND}(1.4)\end{array}$ & (1.1) & ND (8.81) & ND (79) & (3) & $\begin{array}{llll} & \end{array}$ \\
\hline CVA & $\begin{array}{l}\text { YKCVA-G-G37912 } \\
\text { YKCVA }\end{array}$ & $\begin{array}{l}8 / 5 / 15 \\
8 / 5\end{array}$ & 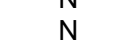 & AlR & & ND (1.3) & $\begin{array}{l}\mathrm{ND}(0.98) \\
\mathrm{N}\end{array}$ & ND (1.7) & - & ND (1.1) & ND (1.4) & ND (1.1) & ND $(.0 .81)$ & ND $(.79)$ & ND (3.7) & $\begin{array}{l}\mathrm{ND}(0.98) \\
\text { ND) }\end{array}$ \\
\hline $\begin{array}{l}\text { PRES } \\
\text { PRA }\end{array}$ & YKPRES-G-37904 & $8 / 5 / 15$ & $\mathrm{~N}$ & AlR & & ND (1.3) & ND (0.98) & ND (1.7) & - & ND (1.1) & ND (1.4) & ND (1.1) & ND $(0.81)$ & ND (0.79) & ND (3.7) & ND (0.98) \\
\hline PRES & YKPRES-G-37903 & $8 / 5 / 15$ & $\mathrm{~N}$ & AlR & & ND (1.3) & ND (0.98) & ND (1.7) & - & ND (1.1) & ND (1.4) & ND (1.1) & ND $(0.81)$ & ND (0.79) & ND (3.7) & ND $(0.98)$ \\
\hline FITWORX & YKFITWORX-G-37897 & $8 / 5 / 15$ & $n$ & AIR & & 6.3 & ND $(0.98)$ & ND (1.7) & - & ND (1.1) & ND (1.4) & ND (1.1) & $\mathrm{ND}(0.81)$ & ND (0.79) & ND (3.7) & 2.1 \\
\hline $\begin{array}{l}\text { FITWORX } \\
\text { FITWORX }\end{array}$ & 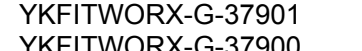 & $\begin{array}{l}8 / 5 / 15 \\
8 / 5115\end{array}$ & DUP-F & $\begin{array}{l}\text { AR } \\
A A R\end{array}$ & & 5.9 & $\mathrm{ND}(0.98)$ & ND (1.7) & - & ND (1.1) & ND (1.4) & ND (1.1) & $\begin{array}{l}\mathrm{ND}(0.81) \\
\mathrm{ND}(0.81)\end{array}$ & ND $(0.79)$ & ND (3.7) & 2.0 \\
\hline $\begin{array}{l}\text { FITWORX } \\
\text { FIVW }\end{array}$ & 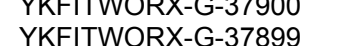 & $\begin{array}{l}8 / 5 / 15 \\
8 / 5 / 15\end{array}$ & ${ }_{N}^{N}$ & $\begin{array}{l}\text { AlR } \\
A A R\end{array}$ & & $\begin{array}{l}6.3 \\
79\end{array}$ & $\begin{array}{l}1.2 \\
1.2\end{array}$ & $\begin{array}{l}\mathrm{ND}(1.7) \\
\mathrm{ND}(1.7)\end{array}$ & $\begin{array}{lll}- \\
-\end{array}$ & $\begin{array}{l}\mathrm{ND}(1.1) \\
\mathrm{ND}(1.1)\end{array}$ & $\begin{array}{l}\text { ND (1.4) } \\
\text { ND (1.4) }\end{array}$ & $\begin{array}{l}\mathrm{ND}(1.1) \\
\mathrm{ND}(1.1)\end{array}$ & $\begin{array}{l}\text { ND }(0.81) \\
\text { ND }(0.81)\end{array}$ & ND (0.79) & $\begin{array}{l}\mathrm{ND}(3 .) \\
\mathrm{ND}(3.7)\end{array}$ & $\begin{array}{l}2.9 \\
2.9\end{array}$ \\
\hline $\begin{array}{l}\text { FITWORX } \\
\text { FITWORX }\end{array}$ & $\begin{array}{l}\text { YKHIWORX-G-37899 } \\
\text { YKFITWORX-G-37902 }\end{array}$ & $\begin{array}{l}8 / 1515 \\
8 / 515\end{array}$ & $N_{N}$ & $\begin{array}{l}A_{A R} \\
A R\end{array}$ & & 15 & ND (. 0.98$)$ & $\begin{array}{l}\text { ND (1.7) } \\
\text { ND (1.7) }\end{array}$ & $=$ & ND (1.1) & ND (1.4) & ND (1.1) & ND (0.81) & ND (79) & 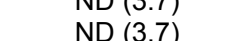 & ND $(0.98)$ \\
\hline $\begin{array}{l}\text { FITWORX } \\
\end{array}$ & YKBKGND-G-37908 & $8 / 5 / 15$ & $\mathrm{~N}$ & AlR & $x$ & ND (1.3) & $\begin{array}{ll}\mathrm{ND}(0.08) \\
\text { (n) }\end{array}$ & ND (1.7) & - & $\mathrm{ND}(1.1)$ & ND (1.4) & ND (1.1) & ND ( 0.81$)$ & ND ( $(.79)$ & ND (3.7) & ND $(0.98)$ \\
\hline HEARTH & YKHEARTH-G-37892 & $8 / 5 / 15$ & $\mathrm{~N}$ & AIR & & ND (1.3) & ND (0.98) & ND (1.7) & - & ND (1.1) & ND (1.4) & ND (1.1) & ND $(0.81)$ & ND (0.79) & ND (3.7) & ND (0.98) \\
\hline HEARTH & YKHEARTH-G-37893 & $8 / 5 / 15$ & $\mathrm{~N}$ & AIR & & ND (1.3) & ND $(0.98)$ & ND (1.7) & - & ND (1.1) & ND (1.4) & ND (1.1) & ND $(0.81)$ & ND $(0.79)$ & ND (3.7) & ND $(0.98)$ \\
\hline HEARTH & YKHEARTH-G-37896 & $8 / 5 / 15$ & $\mathrm{~N}$ & AIR & & ND (1.3) & ND (0.98) & ND (1.7) & - & ND (1.1) & ND (1.4) & ND (1.1) & $\mathrm{ND}(0.81)$ & ND (0.79) & ND (3.7) & ND (0.98) \\
\hline $\begin{array}{l}\text { HEARTH } \\
\text { HEARTH }\end{array}$ & YKHEARTH-G-37895 & $\begin{array}{l}8 / 5 / 15 \\
885115\end{array}$ & $N$ & AIR & & ND (1.3) & ND $(0.98)$ & ND $(1.7)$ & - & ND (1.1) & ND (1.4) & $N D(1.1)$ & ND $(0.81)$ & ND $(0.79)$ & ND (3.7) & ND $(0.98)$ \\
\hline $\begin{array}{l}\text { IEERTH } \\
\text { HEARTH }\end{array}$ & $\begin{array}{l}\text { YKHEARTH-G-38891 } \\
\text { YKHEARTH-G-37894 }\end{array}$ & $\begin{array}{l}885515 \\
8 / 5 / 15\end{array}$ & $\mathrm{~N}$ & $\begin{array}{l}\text { AR } \\
\text { AIR }\end{array}$ & & $\begin{array}{l}\mathrm{ND}(1.3) \\
\mathrm{ND}(1.3)\end{array}$ & $\begin{array}{l}\mathrm{ND}(0.98) \\
\mathrm{ND}(0.98)\end{array}$ & $\begin{array}{l}\text { ND (1.7) } \\
\text { ND (1.7) }\end{array}$ & $\begin{array}{ll}- \\
-\end{array}$ & $\begin{array}{l}\mathrm{ND}(1.1) \\
\mathrm{ND}(1.1)\end{array}$ & $\begin{array}{l}\mathrm{ND}(1.4) \\
\mathrm{ND}(1.4)\end{array}$ & $\begin{array}{l}\mathrm{ND}(1.1) \\
\mathrm{ND}(1.1)\end{array}$ & $\begin{array}{l}\mathrm{ND}(0.81) \\
\mathrm{ND}(0.81)\end{array}$ & $\begin{array}{l}\mathrm{ND}(0.79) \\
\mathrm{ND}(0.79)\end{array}$ & $\begin{array}{l}\mathrm{ND}(3.7) \\
\mathrm{ND}(3.7)\end{array}$ & $\begin{array}{l}\mathrm{ND}(0.98) \\
\mathrm{ND}(0.98)\end{array}$ \\
\hline $\begin{array}{l}\text { HEARRTH } \\
\text { HEARTH }\end{array}$ & $\begin{array}{l}\text { YKHEARTHA-G-378944 } \\
\text { YKBKGND-G-37907 }\end{array}$ & $\begin{array}{l}8 / 5 / 15 \\
8 / 5 / 15\end{array}$ & $N^{n}$ & $\begin{array}{l}\text { AR } \\
\text { AIR }\end{array}$ & $x$ & $\begin{array}{l}\text { ND }(1.3) \\
\text { ND (1.3) }\end{array}$ & $\begin{array}{l}\text { ND (0.98) } \\
\text { ND }(0.98)\end{array}$ & ND (1.7) & $\begin{array}{l}- \\
-\end{array}$ & $\begin{array}{l}\mathrm{ND}(1.1) \\
\mathrm{ND}(1.1)\end{array}$ & $\begin{array}{l}\mathrm{ND}(1.4) \\
\mathrm{ND}(1.4)\end{array}$ & $\begin{array}{l}\mathrm{ND}(1.1) \\
\mathrm{ND}(1.1)\end{array}$ & (N) & (1) & (1) & $\begin{array}{llll} & \end{array}$ \\
\hline TROES & 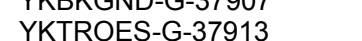 & $\begin{array}{l}81515 \\
8 / 515\end{array}$ & $\begin{array}{lllll}\mathrm{N} \\
\mathrm{N}\end{array}$ & $\begin{array}{l}\text { AR } \\
A \text { AR }\end{array}$ & & 8.0 & $\begin{array}{l}\text { ND }(0.98) \\
\text { No8) }\end{array}$ & $\begin{array}{l}\mathrm{ND}(1.7) \\
\mathrm{ND}(1.7)\end{array}$ & - & ND (1.1) & ND (1.4) & ND (1.1) & 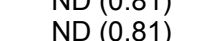 & ND (.79) & ND (3.7) & $\begin{array}{l}\text { ND }(0.98) \\
\text { Nog) }\end{array}$ \\
\hline TROES & YKTROES-G-37906 & $\begin{array}{l}8 / 5 / 15 \\
8 / 515\end{array}$ & $\mathrm{~N}$ & AlR & & 16 & ND $(0.98)$ & ND (1.7) & - & ND (1.1) & ND (1.4) & ND (1.1) & ND $(0.81)$ & ND (0.79) & ND (3.7) & 1.1 \\
\hline TROES & YKBKGND-G-37914 & $8 / 5 / 15$ & $\mathrm{~N}$ & AlR & $x$ & $\mathrm{ND}(1.3)$ & ND (0.98) & ND (1.7) & - & ND (1.1) & ND (1.4) & ND (1.1) & ND ( $(0.81)$ & ND $(0.79)$ & ND (3.7) & ND $(0.98)$ \\
\hline GARRETSON & & $1 / 28 / 16$ & $\frac{n^{2}}{N}$ & AIR & & $\begin{array}{l}28 \\
28\end{array}$ & $\begin{array}{l}\mathrm{ND}(0.98) \\
\mathrm{ND}(0.98)\end{array}$ & & - & ND (1.1) & $\begin{array}{l}N D(1.4) \\
\text { N }\end{array}$ & ND (1.1) & ND $(0.81)$ & ND $(0.79)$ & ND (3.7) & $\mathrm{ND}(0.98)$ \\
\hline $\begin{array}{l}\text { GARRETSON } \\
\text { GARRETSON }\end{array}$ & $\begin{array}{l}\text { YKGARRETSONN-G-38038 } \\
\text { YKBKGND-G-38039 }\end{array}$ & $\begin{array}{l}1 / 28816 \\
1 / 2816\end{array}$ & DUP-F & $\begin{array}{l}\text { AR } \\
A A R\end{array}$ & $x$ & $\begin{array}{l}28 \\
\mathrm{ND}(1.3)\end{array}$ & & ND (1.7) & - & ND (1.1) & ND (1.4) & ND (1.1) & ND $(0.81)$ & ND $(0.79)$ & ND (3.7) & 1.22098 \\
\hline $\begin{array}{l}\text { GARRETSON } \\
\text { FITWORX }\end{array}$ & $\begin{array}{l}\text { YKBKGND-G-38039 } \\
\text { YKFITWORX-G-38040 }\end{array}$ & $\begin{array}{l}1 / 28816 \\
1 / 28 / 16\end{array}$ & $\int_{n}^{N}$ & AR & & $\begin{array}{l}\mathrm{ND}(1.3) \\
47\end{array}$ & $\begin{array}{ll}\mathrm{ND}(0.98) \\
10\end{array}$ & $\begin{array}{l}\mathrm{ND}(1.7) \\
\mathrm{ND}(1.7)\end{array}$ & 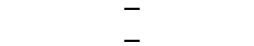 & $\begin{array}{l}\mathrm{ND}(1.1) \\
\mathrm{ND}(1.1)\end{array}$ & $\begin{array}{l}\mathrm{ND}(1.4) \\
\mathrm{ND}(1.4)\end{array}$ & ND (1.1) & ND $(0.81)$ & ND (0.79) & ND (3.7) & $\begin{array}{ll}N D(0.98) \\
N D(0.98)\end{array}$ \\
\hline $\begin{array}{l}\text { FITWORX } \\
\text { FIVWU }\end{array}$ & $\begin{array}{l}\text { YKFITWORX-G-38040 } \\
\text { YKFITWORX }\end{array}$ & $1 / 28 / 16$ & $\mathrm{~N}^{\mathrm{N}}$ & $\begin{array}{l}\text { AR } \\
\text { AIR }\end{array}$ & & $\begin{array}{l}4.1 \\
3.5\end{array}$ & $\mathrm{ND}(0.98)$ & $\mathrm{ND}(1.7)$ & $\begin{array}{l}- \\
-\end{array}$ & ND (1.1) & ND (1.4) & ND (1.1) & ND $(0.81)$ & ND (0.79) & ND (3.7) & 14 \\
\hline FITWORX & YKFITWORX-G-38041 & $1 / 28 / 16$ & DUP-F & AIR & & 5.3 & 1.1 & ND (1.7) & - & ND (1.1) & ND (1.4) & ND (1.1) & ND (0.81) & ND ( 0.79$)$ & ND (3.7) & 1.3 \\
\hline FITWORX & YKFITWORX-G-38043 & $1 / 28 / 16$ & $\mathrm{~N}$ & AIR & & 4.6 & 1.2 & ND (1.7) & - & ND (1.1) & ND (1.4) & ND (1.1) & ND $(0.81)$ & ND $(0.79)$ & ND (3.7) & 1.8 \\
\hline FITWORX & YKFITWORX-G-38044 & 1/28/16 & $\mathrm{N}$ & AIR & & 4.6 & 1.4 & ND (1.7) & - & ND (1.1) & ND (1.4) & ND (1.1) & ND (0.81) & ND (0.79) & ND (3.7) & 1.5 \\
\hline FITWORX & YKBKGND-G-38045 & $1 / 28 / 16$ & $n$ & AIR & $\mathrm{x}$ & ND (1.3) & $\mathrm{ND}(0.98)$ & ND $(1.7)$ & $N^{-}(11)$ & ND (1.1) & ND (1.4) & ND (1.1) & ND $(0.81)$ & ND $(0.79)$ & ND (3.7) & ND $(0.98)$ \\
\hline $\begin{array}{l}\text { GARRETSON } \\
\text { FITWORX }\end{array}$ & $\begin{array}{l}\text { YKGARRETSON-G-33046 } \\
\text { YKEITWORX-G-38084 }\end{array}$ & $\begin{array}{l}1 / 28816 \\
1 / 2816\end{array}$ & $N^{n}$ & $\begin{array}{l}\text { SOLL GAS } \\
\text { SOII GAS }\end{array}$ & & & $\begin{array}{l}110 \\
1109)\end{array}$ & $\begin{array}{l}\text { ND }(7.1) \\
N D(71)\end{array}$ & ND (14) & ND (11) & ND (14) & ND (11) & $\mathrm{ND}(8.2)$ & $\begin{array}{l}\mathrm{ND}(8) \\
\mathrm{ND}(8)\end{array}$ & $\begin{array}{l}\text { ND (75) } \\
N D(75)\end{array}$ & $\begin{array}{l}N D(10) \\
G 7\end{array}$ \\
\hline FITWORX & $\begin{array}{l}\text { YKFIIWURX-G-30048 } \\
\text { YKFITWORX-G-38081 }\end{array}$ & $\begin{array}{l}1728816 \\
4 / 28 / 16\end{array}$ & $\mathrm{~N}^{\mathrm{N}} \mathrm{r}-\mathrm{r}$ & $\begin{array}{l}\text { SOIL GAS } \\
\text { AIR }\end{array}$ & & $\begin{array}{l}50 \\
29\end{array}$ & $\begin{array}{l}\mathrm{ND}(9.9) \\
6.3\end{array}$ & $\begin{array}{l}\text { ND (7.1) } \\
\text { ND (1.7) }\end{array}$ & $\mathrm{ND}(14)$ & $\begin{array}{l}\text { ND (17) } \\
\text { ND (1.1) }\end{array}$ & $\begin{array}{l}\mathrm{ND}(14) \\
\mathrm{ND}(1.4)\end{array}$ & $\begin{array}{l}\text { ND (11) } \\
\text { ND (1.1) }\end{array}$ & $\begin{array}{l}\mathrm{ND}(8.2) \\
\mathrm{ND}(0.81)\end{array}$ & $\begin{array}{l}\mathrm{ND}(8) \\
\mathrm{ND}(0.79)\end{array}$ & $\begin{array}{l}\text { ND ( (5) } \\
\text { ND (3.7) }\end{array}$ & $\begin{array}{l}6.1 \\
14\end{array}$ \\
\hline $\begin{array}{l}\text { FITWORX } \\
\text { FITW }\end{array}$ & $\begin{array}{l}\text { YKIIWORX-G-30081 } \\
\text { YKFITWORX-G-38082 }\end{array}$ & $\begin{array}{l}4 / 28816 \\
4 / 28 / 16\end{array}$ & DUP-F & $\begin{array}{l}\text { AlR } \\
\text { AlR }\end{array}$ & & $\begin{array}{l}2.9 \\
3.3\end{array}$ & $\begin{array}{l}6.3 \\
6.9\end{array}$ & $\begin{array}{l}\mathrm{ND}(1.7) \\
\mathrm{ND}(1.7)\end{array}$ & - & $\begin{array}{l}\mathrm{ND}(1.1) \\
\mathrm{ND}(1.1)\end{array}$ & $\begin{array}{l}\mathrm{ND}(1.4) \\
\mathrm{ND}(1.4)\end{array}$ & ND (1.1) & ND $(0.81)$ & ND $(0.79)$ & $\mathrm{ND}(3.7)$ & $\begin{array}{l}.1 .4 \\
1.1\end{array}$ \\
\hline $\begin{array}{l}\text { FITWORX } \\
\text { FITW }\end{array}$ & YKEITWORX-G-30883 & $42 / 28116$ & $\mathrm{~N}$ & $A \cap$ & & 18 & 73 & ND 01 & - & ND (1.1) & ND (1.4) & ND (1 1) & ND $(0.81)$ & ND (7.79) & ND ( 37 ) & ND $(0.98)$ \\
\hline FITWORX & YKFITWORX-G-38084 & $4 / 28 / 16$ & $\mathrm{~N}$ & AlR & & 2.4 & 5.2 & ND (1.7) & - & $\mathrm{ND}(1.1)$ & ND (1.4) & ND (1.1) & $\begin{array}{l}\text { ND }(0.81) \\
\text { No. }\end{array}$ & ND ( 0.79$)$ & ND (3.7) & $\begin{array}{ll}\mathrm{ND}(0.08) \\
\text { No }\end{array}$ \\
\hline FITWORX & YKFITWORX-G-38085 & $4 / 28 / 16$ & $\mathrm{~N}$ & AIR & & 2.4 & 4.9 & ND (1.7) & - & ND (1.1) & ND (1.4) & ND (1.1) & ND $(0.81)$ & ND $(0.79)$ & ND (3.7) & \\
\hline FITWORX & YKBKGND-G-38086 & $4 / 28 / 16$ & $N$ & AIR & $\mathrm{x}$ & ND (1.3) & ND $(0.98)$ & ND (1.7) & - & ND (1.1) & ND (1.4) & ND (1.1) & $\mathrm{ND}(0.81)$ & $\mathrm{ND}(0.79)$ & ND (3.7) & $\mathrm{ND}(0.98)$ \\
\hline $\begin{array}{l}\text { FITWORX } \\
\text { FITWORX }\end{array}$ & YKFITWORX-G-38087 & $4 / 28 / 16$ & 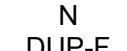 & SOIL GAS & & $\begin{array}{l}51 \\
46\end{array}$ & ND (9.8) & ND (17) & - & ND (11) & ND (14) & ND (11) & ND (8.1) & ND (7.9) & ND (37) & 26 \\
\hline $\begin{array}{l}\text { GARRETSON } \\
\text { GARRETSON }\end{array}$ & $\begin{array}{l}\text { YKFIIWORX-G-38088 } \\
\text { YKGARRETSON-G-38140 }\end{array}$ & $\begin{array}{l}4 / 28016 \\
8 / 10 / 16\end{array}$ & DUP-F & SOIL GAS & & $\begin{array}{l}46 \\
\text { ND }(1.3)\end{array}$ & $\begin{array}{l}\mathrm{ND}(9.8) \\
\mathrm{ND}(0.98)\end{array}$ & $\begin{array}{l}\text { ND (17) } \\
\text { ND (17) }\end{array}$ & - & $\begin{array}{l}\mathrm{ND}(11) \\
\mathrm{ND}(11)\end{array}$ & $\begin{array}{l}\mathrm{ND}(14) \\
\mathrm{ND}(144)\end{array}$ & $\begin{array}{l}\mathrm{ND}(11) \\
\mathrm{ND}(11)\end{array}$ & $\begin{array}{l}\mathrm{ND}(8.1) \\
\mathrm{ND}(0.81)\end{array}$ & $\begin{array}{l}\mathrm{ND}(7.9) \\
\mathrm{ND}(079)\end{array}$ & $\begin{array}{l}\mathrm{ND}(37) \\
\mathrm{ND}(37)\end{array}$ & $\begin{array}{l}32 \\
12\end{array}$ \\
\hline GARRETSON & YKGARRETSON-G-38130 & $\begin{array}{l}8 / 1 / 0016 \\
8 / 10 / 16\end{array}$ & ${ }_{N}^{N}$ & $\begin{array}{l}\text { AlR } \\
\text { AlR }\end{array}$ & & ND (1.3) & $\begin{array}{l}\mathrm{ND}(0.98) \\
\mathrm{ND}(0.98)\end{array}$ & ND (1.7) & $\begin{array}{l}- \\
-\end{array}$ & $\begin{array}{l}\text { ND }(1.1) \\
\text { ND (1.1) }\end{array}$ & $\begin{array}{l}\text { ND (1.4) } \\
\text { ND (1.4) }\end{array}$ & ND (1.1) & ND $(0.81)$ & ND $(0.79)$ & $\mathrm{ND}(3.7)$ & 1.2 \\
\hline GARRETSON & YKBKGND-G-38141 & $8 / 10 / 16$ & $\mathrm{~N}$ & AIR & $x$ & ND (1.3) & ND (0.98) & ND (1.7) & 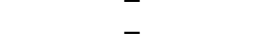 & ND (1.1) & ND (1.4) & ND (1.1) & ND $(0.81)$ & ND (0.79) & ND (3.7) & 2.1 \\
\hline $\begin{array}{l}\text { FITWORX } \\
\text { FI }\end{array}$ & YKFITWORX-G-38148 & $8 / 10 / 16$ & $\mathrm{~N}$ & SOIL GAS & & 20 & ND $(9.8)$ & ND (17) & - & ND (11) & ND (14) & ND (11) & ND (8.1) & ND (7.9) & ND D (37) & ND $(9.8)$ \\
\hline FITWORX & YKFITWORX-G-38149 & $8 / 10 / 16$ & DUP-F & SOIL GAS & & 24 & ND $(9.8)$ & ND (17) & - & ND (11) & ND (14) & ND (11) & ND (8.1) & ND (7.9) & ND (37) & ND (9.8) \\
\hline FITWORX & YKFITWORX-G-38142 & $8 / 10 / 16$ & $\mathrm{~N}$ & AlR & & 7.2 & 9.2 & ND (1.7) & - & ND (1.1) & ND (1.4) & ND (1.1) & $\mathrm{ND}(0.81)$ & $\operatorname{ND}(0.79)$ & ND $(3.7)$ & 2.2 \\
\hline 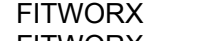 & YKFITWORX-G-38143 & 8/10/16 & DUP-F & AIR & & 6.5 & 9.5 & ND (1.7) & - & ND (1.1) & ND (1.4) & ND (1.1) & ND $(0.81)$ & ND $(0.79)$ & ND (3.7) & 2.5 \\
\hline FITWORX & YKFITWORX-G-38144 & $8 / 10 / 16$ & N & AIR & & 6.1 & 9.8 & ND (1.7) & - & ND (1.1) & $\begin{array}{l}N D(1.4) \\
N\end{array}$ & ND (1.1) & $\begin{array}{lll}N D & (0.81) \\
\end{array}$ & 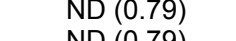 & ND (3.7) & 1.9 \\
\hline $\begin{array}{l}\text { FIITWORX } \\
\text { FITWORX }\end{array}$ & $\begin{array}{l}\text { YKITITWORX-G-3145 } \\
\text { YKFITWORX-G-38146 }\end{array}$ & $\begin{array}{l}\text { 8/110/16 } \\
8 / 10 / 16\end{array}$ & $\mathrm{~N}_{\mathrm{N}}$ & $\begin{array}{l}\text { AlR } \\
\text { AIR }\end{array}$ & & $\begin{array}{l}7.9 \\
8.2\end{array}$ & $\begin{array}{l}8.5 \\
7.9\end{array}$ & $\begin{array}{l}\text { ND (1.7) } \\
\text { ND (1.7) }\end{array}$ & $\overline{-}$ & $\begin{array}{l}\mathrm{ND}(1.1) \\
\mathrm{ND}(1.1)\end{array}$ & $\begin{array}{l}\text { ND (1.4) } \\
\text { ND (1.4) }\end{array}$ & $\begin{array}{l}\mathrm{ND}(1.1) \\
\mathrm{ND}(1.1)\end{array}$ & $\begin{array}{l}\mathrm{ND}(0.81) \\
\mathrm{ND}(0.81)\end{array}$ & $\begin{array}{l}\mathrm{ND}(0 . / 9) \\
\mathrm{ND}(0.79)\end{array}$ & $\begin{array}{l}\mathrm{ND}(3.1) \\
\mathrm{ND}(3.7)\end{array}$ & $\begin{array}{l}2.9 \\
3.0\end{array}$ \\
\hline $\begin{array}{l}\text { ITWORX } \\
\text { ITWVR }\end{array}$ & YKBKGND-G-38147 & $8 / 10 / 16$ & $\mathrm{~N}$ & AIR & $x$ & ND (1.3) & $\operatorname{ND}(0.98)$ & $\mathrm{ND}(1.7)$ & - & ND (1.1) & ND (1.4) & ND (1.1) & ND $(0.81)$ & ND (0.79) & ND (3.7) & ND (0.98) \\
\hline
\end{tabular}


Concentration $\left(\mu \mathrm{g} / \mathrm{m}^{3}\right)$

\begin{tabular}{|c|c|c|c|c|c|c|c|c|c|c|c|c|c|c|c|c|}
\hline \multirow[b]{2}{*}{ Location } & \multirow[b]{2}{*}{ Sample } & \multirow[b]{2}{*}{$\begin{array}{l}\text { Sample } \\
\text { Date }\end{array}$} & \multirow[b]{2}{*}{$\begin{array}{l}\text { Sample } \\
\text { Type }\end{array}$} & \multirow[b]{2}{*}{$\begin{array}{l}\text { Sample } \\
\text { Matrix }\end{array}$} & \multirow[b]{2}{*}{$\begin{array}{l}\text { Ambient } \\
\text { Sample }\end{array}$} & \multicolumn{11}{|c|}{ Concentration $\left(\mu \mathrm{g} / \mathrm{m}^{3}\right)$} \\
\hline & & & & & & $\begin{array}{c}\text { Carbon } \\
\text { Tetrachloride }\end{array}$ & Chloroform & $\begin{array}{l}\text { Methylene } \\
\text { Chloride }\end{array}$ & $\begin{array}{l}1,1,1,2- \\
\text { Tetrachloroethane }\end{array}$ & $\begin{array}{c}1,1,1- \\
\text { Trichloroethane }\end{array}$ & $\begin{array}{l}1,1,2,2- \\
\text { Tetrachloroethane }\end{array}$ & $\begin{array}{c}1,1,2- \\
\text { Trichloroethane }\end{array}$ & $\begin{array}{c}1,1- \\
\text { Dichloroethane }\end{array}$ & $\begin{array}{c}1,1- \\
\text { Dichloroethylene }\end{array}$ & $\begin{array}{c}1,2,4- \\
\text { Trichlorobenzene }\end{array}$ & $\begin{array}{c}1,2,4- \\
\text { Trimethylbenzene }\end{array}$ \\
\hline GARRETSON & YKGARRETSON-G-39533 & $12 / 14 / 16$ & $\mathrm{~N}$ & AIR & & $\mathrm{ND}(1.3)$ & $\mathrm{ND}(0.98)$ & 4.3 & - & ND (1.1) & ND (1.4) & ND (1.1) & $\mathrm{ND}(0.81)$ & $\mathrm{ND}(0.79)$ & ND (3.7) & $\mathrm{ND}(0.98)$ \\
\hline GARRETSON & YKGARRETSON-G-39535 & $12 / 14 / 16$ & $\mathrm{~N}$ & AIR & & ND (1.3) & ND $(0.98)$ & 8.6 & - & ND (1.1) & ND (1.4) & ND (1.1) & ND $(0.81)$ & ND $(0.79)$ & ND (3.7) & 1.1 \\
\hline GARRETSON & YKBKGND-G-39536 & $12 / 14 / 16$ & $N$ & AIR & $\mathrm{x}$ & ND (1.3) & ND $(0.98)$ & ND (1.7) & - & $N D(1.1)$ & ND (1.4) & ND (1.1) & ND $(0.81)$ & ND $(0.79)$ & ND (3.7) & ND (0.98) \\
\hline $\begin{array}{l}\text { FITWORX } \\
\text { FITWORX }\end{array}$ & $\begin{array}{l}\text { YKFFTWORX-G-39537 } \\
\text { YKITWORX-G-39538 }\end{array}$ & $12 / 14416$ & DUP-F & AIR & & $\begin{array}{l}3.1 \\
32\end{array}$ & $\begin{array}{l}4.5 \\
46\end{array}$ & ND (1.7) & - & ND (1.1) & ND (1.4) & ND (1.1) & ND $(0.81)$ & ND $(0.79)$ & ND (3.7) & 1.0 \\
\hline $\begin{array}{l}\text { FITWORX } \\
\text { FITWORX }\end{array}$ & $\begin{array}{l}\text { YKITWORX-G-39558 } \\
\text { YKFITWORX-3939 }\end{array}$ & $\begin{array}{l}12 / 14416 \\
121 / 4116\end{array}$ & DUP-F & $\begin{array}{l}\text { ARR } \\
A A R\end{array}$ & & 3.2 & $\begin{array}{l}4.6 \\
53\end{array}$ & ND (1.7) & - & ND (1.1) & $\begin{array}{l}N D(1.4) \\
N D(1.4)\end{array}$ & ND (1.1) & $\begin{array}{ll}N D(0.81) \\
N D(8.81\end{array}$ & $\begin{array}{ll}N D(0.79) \\
N D(079)\end{array}$ & $\begin{array}{ll}N D(3.7) \\
N D(37)\end{array}$ & 1.3 \\
\hline $\begin{array}{l}\text { FITWORX } \\
\text { FITWOR }\end{array}$ & 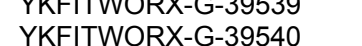 & $\begin{array}{l}12 / 144 / 16 \\
12 / 14 / 16\end{array}$ & $\mathrm{~N}_{\mathrm{N}}^{\mathrm{N}} \mathrm{r}-\mathrm{r}$ & $\begin{array}{l}\text { AlR } \\
\text { AlR }\end{array}$ & & $\begin{array}{l}2.3 \\
3.8\end{array}$ & $\begin{array}{l}5.3 \\
4.4\end{array}$ & $\begin{array}{l}\mathrm{ND}(1 .) \\
\mathrm{ND}(1.7)\end{array}$ & - & $\begin{array}{l}\text { ND (1.) } \\
\text { ND (1.1) }\end{array}$ & $\begin{array}{l}\text { ND (1.4) } \\
\text { ND (1.4) }\end{array}$ & ND (1.1) & ND $(0.81)$ & ND (0.79) & $\mathrm{ND}(3.7)$ & $\mathrm{ND}(0.98)$ \\
\hline $\begin{array}{l}\text { FITWORX } \\
\text { FITW }\end{array}$ & YKFITWORX-G-39541 & $\begin{array}{l}1 / 2 / 14 / 16 \\
12\end{array}$ & n & $\begin{array}{l}\text { ARr } \\
\text { AIR }\end{array}$ & & 3.6 & 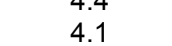 & ND (1.7) & - & ND (1.1) & ND (1.4) & ND (1.1) & ND $(0.81)$ & ND $(0.79)$ & $\mathrm{ND}(3.7)$ & $\mathrm{ND}(0.98)$ \\
\hline FITWORX & YKBKGND-G-39542 & $12 / 14 / 16$ & $\mathrm{~N}$ & $\mathrm{AIR}$ & $\mathrm{x}$ & ND (1.3) & $\mathrm{ND}(0.98)$ & ND (1.7) & - & ND (1.1) & ND (1.4) & ND (1.1) & ND $(0.81)$ & ND (0.79) & ND (3.7) & ND (0.98) \\
\hline FITWORX & YKFITWORX-G-39543 & $12 / 14 / 16$ & $\mathrm{~N}$ & SOIL GAS & & ND (13) & ND $(9.8)$ & ND (17) & - & ND (11) & ND (14) & ND (11) & ND $(8.1)^{\prime}$ & $\operatorname{ND}(7.9)$ & ND (37) & $\mathrm{ND}(9.8)$ \\
\hline
\end{tabular}

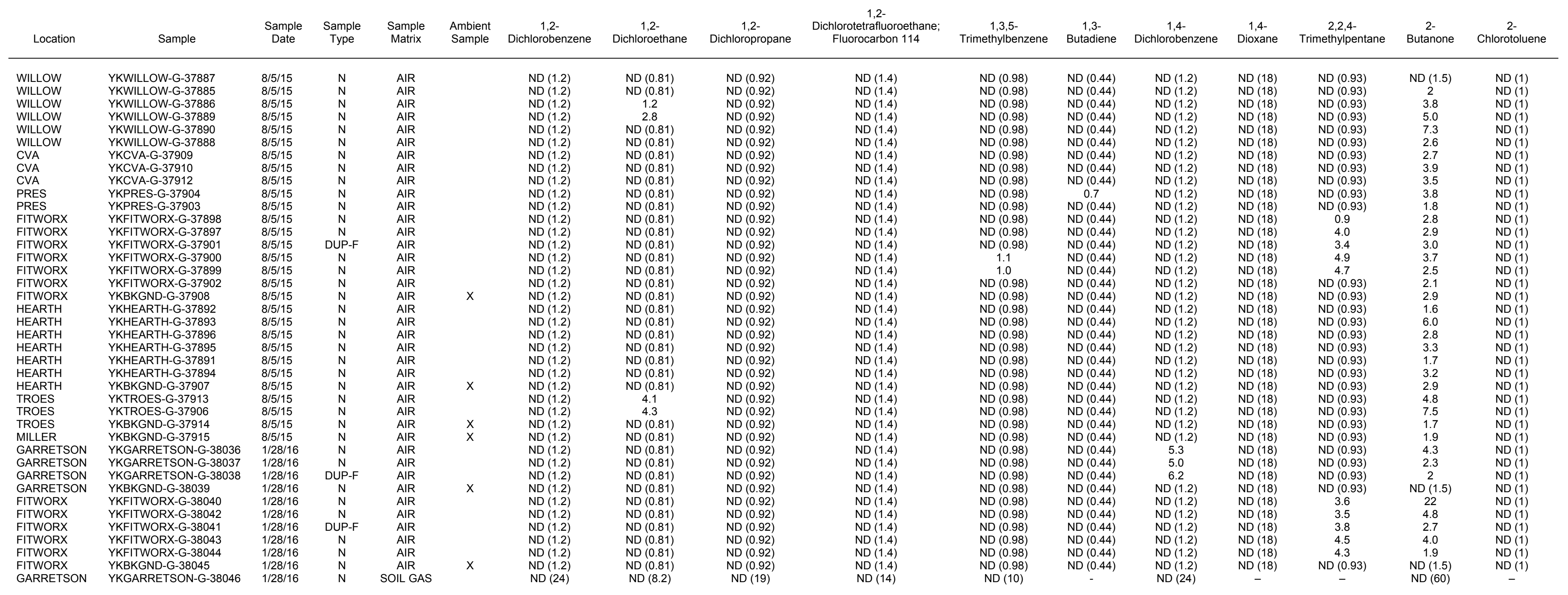


Concentration $\left(\mu \mathrm{g} / \mathrm{m}^{3}\right)$

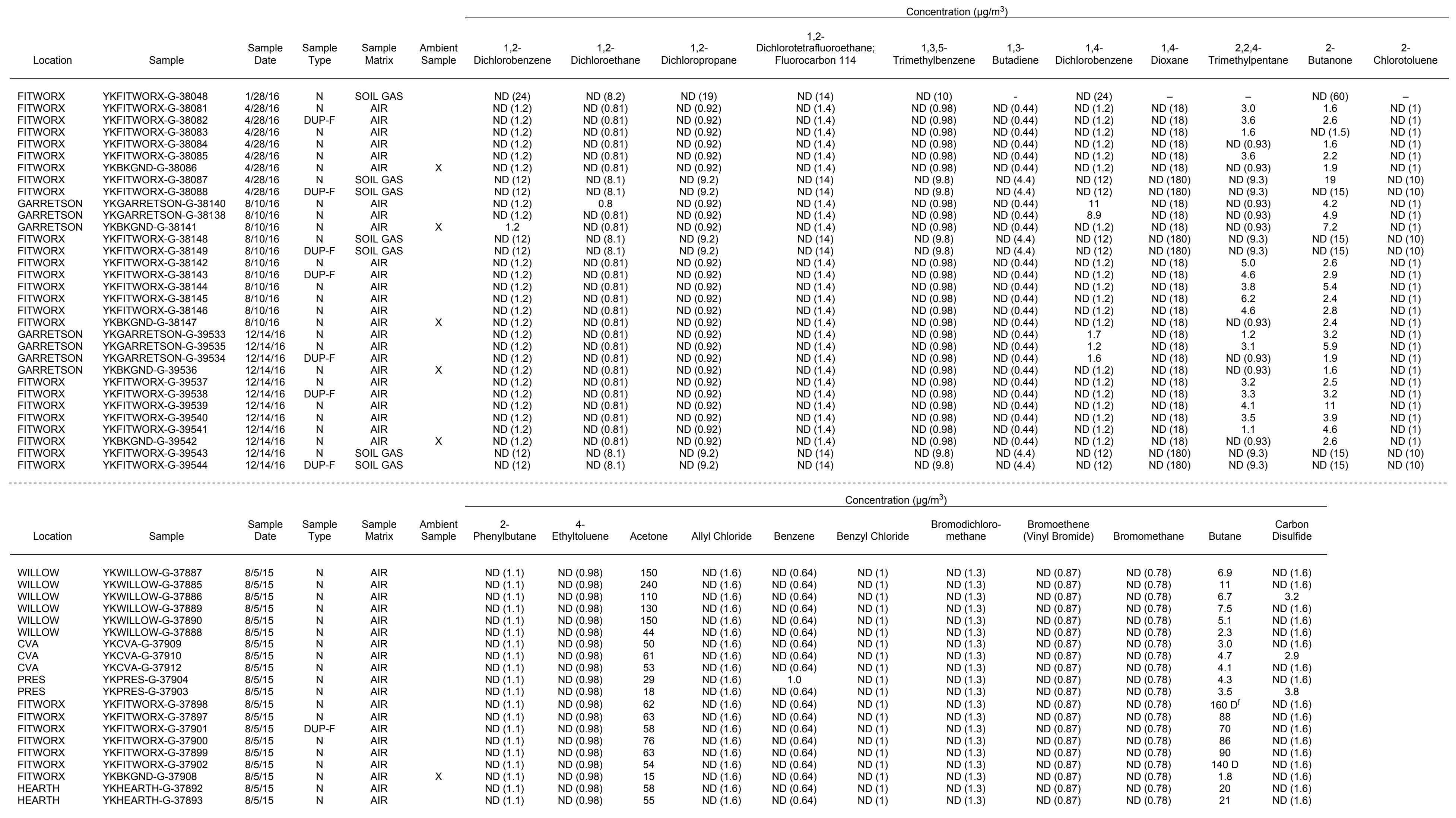


Concentration $\left(\mu \mathrm{g} / \mathrm{m}^{3}\right)$

\begin{tabular}{|c|c|c|c|c|c|c|c|c|c|c|c|c|c|c|c|c|}
\hline \multirow[b]{2}{*}{ Location } & \multirow[b]{2}{*}{ Sample } & \multirow[b]{2}{*}{$\begin{array}{l}\text { Sample } \\
\text { Date }\end{array}$} & \multirow[b]{2}{*}{$\begin{array}{c}\text { Sample } \\
\text { Type }\end{array}$} & \multirow[b]{2}{*}{$\begin{array}{l}\text { Sample } \\
\text { Matrix }\end{array}$} & \multirow[b]{2}{*}{$\begin{array}{l}\text { Ambient } \\
\text { Sample }\end{array}$} & \multicolumn{11}{|c|}{ Concentration $\left(\mu \mathrm{g} / \mathrm{m}^{3}\right)$} \\
\hline & & & & & & $\begin{array}{c}2- \\
\text { Phenylbutane }\end{array}$ & $\begin{array}{l}\text { 4- } \\
\text { Ethyltoluene }\end{array}$ & Acetone & Allyl Chloride & Benzene & Benzyl Chloride & $\begin{array}{l}\text { Bromodichloro- } \\
\text { methane }\end{array}$ & $\begin{array}{l}\text { Bromoethene } \\
\text { (Vinyl Bromide) }\end{array}$ & Bromomethane & Butane & $\begin{array}{l}\text { Carbon } \\
\text { Disulfide }\end{array}$ \\
\hline HEARTH & YKHEARTH-G-37896 & $8 / 5 / 15$ & $\mathrm{~N}$ & AIR & & ND (1.1) & ND $(0.98)$ & & ND (1.6) & $\mathrm{ND}(0.64)$ & ND (1) & ND (1.3) & ND (0.87) & ND (0.78) & 34 & ND (1.6) \\
\hline $\begin{array}{l}\text { HEARTH } \\
\text { HEATH }\end{array}$ & YKHEARTH-G-37895 & $8 / 5 / 15$ & $\mathrm{~N}$ & AIR & & ND (1.1) & ND (0.98) & 100 & ND (1.6) & ND $(0.64)$ & ND (1) & ND (1.3) & ND (0.87) & ND (0.78) & 57 & ND (1.6) \\
\hline HEARTH & YKHEARTH-G-37891 & $8 / 5 / 15$ & $\mathrm{~N}$ & AIR & & ND (1.1) & ND $(0.98)$ & 40 & ND (1.6) & ND $(0.64)$ & ND (1) & ND (1.3) & ND $(0.87)$ & ND $(0.78)$ & 7.9 & ND (1.6) \\
\hline HEARTH & YKHEARTH-G-37894 & $8 / 5 / 15$ & $N$ & AIR & & $\mathrm{ND}(1.1)$ & ND $(0.98)$ & 50 & ND (1.6) & $\mathrm{ND}(0.64)$ & ND (1) & ND (1.3) & ND $(0.87)$ & ND (0.78) & 9.6 & ND (1.6) \\
\hline HEARTH & YKBKGND-G-37907 & $8 / 5 / 15$ & $\mathrm{~N}$ & AIR & $x$ & $\mathrm{ND}(1.1)$ & ND (0.98) & 16 & $\mathrm{ND}(1.6)$ & ND (0.64) & & ND (1.3) & ND $(0.87)$ & ND (0.78) & 1.7 & \\
\hline TROES & YKTROES-G-37913 & $8 / 5 / 15$ & N & AlR & & ND (1.1) & ND (0.98) & 46 & ND (1.6) & 1.2 & ND (1) & $\mathrm{ND}(1.3)$ & ND $(0.87)$ & ND $(0.78)$ & 8.5 & ND (1.6) \\
\hline TROES & & 8/5/15 & $\mathrm{N}$ & AIR & & ND (1.1) & ND (0.98) & & ND (1.6) & 1.2 & ND (1) & ND (1.3) & ND $(0.87)$ & ND $(0.78)$ & 8.7 & ND (1.6) \\
\hline TROES & YKBKGND-G-37914 & $8 / 5 / 15$ & $\mathrm{~N}$ & AIR & $x$ & ND (1.1) & ND $(0.98)$ & ND (12) & ND (1.6) & $\mathrm{ND}(0.64)$ & ND (1) & ND (1.3) & ND $(0.87)$ & ND $(0.78)$ & 1.5 & 3.4 \\
\hline MILLER & YKBKGND-G-37915 & $8 / 5 / 15$ & $\mathrm{~N}$ & AIR & $\mathrm{x}$ & ND (1.1) & ND $(0.98)$ & ND (12) & ND (1.6) & ND $(0.64)$ & ND (1) & $\mathrm{ND}(1.3)$ & ND $(0.87)$ & ND (0.78) & 1.4 & ND (1.6) \\
\hline GARRETSON & YKGARRETSON-G-38036 & $1 / 28 / 16$ & $N$ & AIR & & ND (1.1) & ND $(0.98)$ & 81 & ND (1.6) & 0.7 & ND (1) & $\mathrm{ND}(1.3)$ & ND $(0.87)$ & ND (0.78) & ND (1.2) & 17 \\
\hline & & & & & & & & & & ND (0.64) & & $\mathrm{ND}(1.3)$ & ND (0.87) & ND (0.78) & $\mathrm{ND}(1.2)$ & ND (1.6) \\
\hline GARRETSON & YKGARRETSON-G-38038 & 1/28/16 & DUP-F & AIR & & ND (1.1) & ND (0.98) & 44 & ND (1.6) & 0.6 & ND (1) & ND (1.3) & ND (0.87) & ND (0.78) & ND (1.2) & ND (1.6) \\
\hline GARRETSON & YKBKGND-G-38039 & $1 / 28 / 16$ & $N$ & AIR & $\mathrm{x}$ & $\mathrm{ND}(1.1)$ & ND (0.98) & ND (12) & ND (1.6) & $\mathrm{ND}(0.64)$ & ND (1) & ND (1.3) & $N D(0.87)$ & ND (0.78) & $\mathrm{ND}(1.2)$ & ND (1.6) \\
\hline $\begin{array}{l}\text { FITWORX } \\
\text { FITWORX }\end{array}$ & $\begin{array}{l}\text { YKFITWORX-G-38040 } \\
\text { YKFITWORX-G-38042 }\end{array}$ & $\begin{array}{l}1 / 28 / 16 \\
1 / 2816\end{array}$ & $\begin{array}{l}\mathrm{N} \\
\mathrm{N}\end{array}$ & $\begin{array}{l}{ }_{A}^{A} R \\
\text { AlR }\end{array}$ & & ND (1.1) & $\begin{array}{l}\mathrm{ND}(0.98) \\
N D(0.98)\end{array}$ & - & ND (1.6) & 1.2 & ND (1) & ND (1.3) & ND (0.87) & ND (0.78) & 64 & ND (1.6) \\
\hline $\begin{array}{l}\text { FITWORX } \\
\text { FITWORX }\end{array}$ & $\begin{array}{l}\text { YKFIIWORX-G-30042 } \\
\text { YKFITWORX-G-38041 }\end{array}$ & $\begin{array}{l}1 / 28816 \\
1 / 28 / 16\end{array}$ & $\begin{array}{c}N \\
\text { DUP-F }\end{array}$ & $\begin{array}{l}\text { AIR } \\
\text { AIR }\end{array}$ & & $\begin{array}{l}\mathrm{ND}(1.1) \\
\mathrm{ND}(1.1)\end{array}$ & $\begin{array}{l}\text { NDD (0.98) } \\
\text { ND (0.98) }\end{array}$ & $\begin{array}{l}85 \\
64\end{array}$ & $\begin{array}{l}\mathrm{ND}(1.6) \\
\mathrm{ND}(1.6)\end{array}$ & $\begin{array}{l}1.2 \\
1.3\end{array}$ & $\begin{array}{l}\text { ND (1) } \\
\text { ND (1) }\end{array}$ & $\begin{array}{l}\mathrm{ND}(1.3) \\
\mathrm{ND}(1.3)\end{array}$ & ND (0.87) & $\begin{array}{l}\text { ND (0.78) } \\
\text { ND (0.78) }\end{array}$ & $\begin{array}{l}80 \\
66\end{array}$ & $\begin{array}{ll}\mathrm{ND}(1.6) \\
2.3\end{array}$ \\
\hline $\begin{array}{l}\text { FITWORX } \\
\text { FITX }\end{array}$ & YKFITWORX-G-38043 & 1/28/16 & $\operatorname{noph}^{n}$ & $\begin{array}{l}\text { AR } \\
\text { AlR }\end{array}$ & & $\begin{array}{l}\mathrm{ND}(1.1) \\
\mathrm{ND}(1.1)\end{array}$ & ND (0.98) & $\begin{array}{l}04 \\
57\end{array}$ & ND (1.6) & 1.2 & ND (1) & $\begin{array}{l}\mathrm{ND}(1.3) \\
\mathrm{ND}(1.3)\end{array}$ & ND ( $(.87)$ & ND (0.78) & $\begin{array}{l}66 \\
58\end{array}$ & $\mathrm{ND}(1.6)$ \\
\hline FITWORX & $\begin{array}{l}\text { YKFITWORX-G-38045 } \\
\text { YKOA }\end{array}$ & $\begin{array}{l}1 / 28 / 16 \\
1 / 2816\end{array}$ & $N^{N}$ & AlR & & $\mathrm{ND}(1.1)$ & ND (0.98) & 53 & $\mathrm{ND}(1.6)$ & 1.1 & ND (1) & $\mathrm{ND}(1.3)$ & ND ( 0.87$)$ & ND (0.78) & 55 & ND (1.6) \\
\hline FITWORX & YKBKGND-G-38045 & $1 / 28 / 16$ & $\mathrm{~N}$ & AlR & $\mathrm{x}$ & $\mathrm{ND}(1.1)$ & ND $(0.98)$ & ND (12) & ND (1.6) & ND $(0.64)$ & ND (1) & ND (1.3) & ND $(0.87)$ & ND (0.78) & ND (1.2) & ND (1.6) \\
\hline GARRETSON & YKGARRETSON-G-38046 & $1 / 28 / 16$ & $\mathrm{~N}$ & SOIL GAS & & & $\mathrm{ND}(10)$ & ND (48) & & ND (6.5) & & ND (14) & (1) & ND (32) & & ND (13) \\
\hline FITWORX & YKFITWORX-G-38048 & 1/28/16 & $\mathrm{N}$ & SOIL GAS & & & ND (10) & ND (48) & - & ND (6.5) & - & ND (14) & & ND (32) & 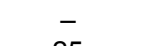 & 6.9 \\
\hline $\begin{array}{l}\text { FITWORX } \\
\text { FTWOPX }\end{array}$ & 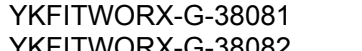 & $\begin{array}{l}4 / 28 / 16 \\
4 / 2816\end{array}$ & $\frac{D^{2}}{n}$ & AIR & & ND (1.1) & $\begin{array}{l}\mathrm{ND}(0.98) \\
N(0.98)\end{array}$ & 25 & $N D(1.6)$ & 0.8 & ND (1) & 1.7 & ND $(0.87)$ & ND (0.78) & 25 & ND (1.6) \\
\hline $\begin{array}{l}\text { FITWORX } \\
\text { FTTWORX }\end{array}$ & $\begin{array}{l}\text { YKFITWORX-G-38082 } \\
\text { YKKFITWORX-G-38083 }\end{array}$ & $\begin{array}{l}4 / 28 / 16 \\
4 / 2816\end{array}$ & DUP-F & $\begin{array}{l}A_{A l R} \\
A \| R\end{array}$ & & $\begin{array}{l}\mathrm{ND}(1.1) \\
\mathrm{ND}(1.1)\end{array}$ & $\begin{array}{l}\mathrm{ND}(0.98) \\
\text { ND (0.98) }\end{array}$ & $\begin{array}{l}34 \\
23\end{array}$ & $\begin{array}{l}\mathrm{ND}(1.6) \\
\mathrm{ND}(1.6)\end{array}$ & $\begin{array}{l}1.0 \\
\text { ND }(0.64)\end{array}$ & $\begin{array}{l}\mathrm{ND}(1) \\
\mathrm{ND}(1)\end{array}$ & $\begin{array}{l}2.1 \\
19\end{array}$ & $\begin{array}{l}N D(0.87) \\
N D(0.87)\end{array}$ & $\begin{array}{l}\mathrm{ND}(0.78) \\
\text { ND (0.78) }\end{array}$ & ${ }_{27}^{28}$ & ND (1.6) \\
\hline $\begin{array}{l}\text { FITWORX } \\
\text { FITWORX }\end{array}$ & $\begin{array}{l}\text { YKFITWWORX-G-33083 } \\
\text { YKFITWORX-G-38084 }\end{array}$ & $\begin{array}{l}4 / 28816 \\
4 / 28 / 16\end{array}$ & $\mathrm{~N}_{\mathrm{N}}^{\mathrm{N}} \mathrm{r}-\mathrm{r}$ & $\begin{array}{l}\text { AIR } \\
\text { AIR }\end{array}$ & & $\begin{array}{l}\mathrm{ND}(1.1) \\
\mathrm{ND}(1.1)\end{array}$ & $\begin{array}{l}\text { NDD (0.98) } \\
\text { ND (0.98) }\end{array}$ & $\begin{array}{l}23 \\
26\end{array}$ & $\begin{array}{l}\mathrm{ND}(1.6) \\
\mathrm{ND}(1.6)\end{array}$ & $\begin{array}{l}\text { ND (0.64) } \\
\text { ND (0.64) }\end{array}$ & $\begin{array}{l}\text { NDD (1) } \\
\text { ND (1) }\end{array}$ & $\begin{array}{l}1.9 \\
\mathrm{ND}(1.3)\end{array}$ & $\begin{array}{l}\text { ND }(0.87) \\
\text { ND (0.87) }\end{array}$ & $\begin{array}{l}\text { ND } 0(0.78) \\
\text { ND (0.78) }\end{array}$ & 24 & $\begin{array}{ll}N D(1.6) \\
N D(16)\end{array}$ \\
\hline $\begin{array}{l}\text { FITWORX } \\
\text { FIVWORX }\end{array}$ & $\begin{array}{l}\text { YKFITWORX-G-38085 } \\
\text { YKFITUO-G085 }\end{array}$ & $\begin{array}{l}4 / 28 / 10 \\
4 / 28 / 16\end{array}$ & $N^{n}$ & $\begin{array}{l}\text { AlR } \\
\text { AlR }\end{array}$ & & ND (1.1) & ND (0.98) & $\begin{array}{l}26 \\
29\end{array}$ & ND (1.6) & $\begin{array}{l}\mathrm{ND}(0.84) \\
0.8\end{array}$ & ND (1) & ND (1.3) & ND $(0.87)$ & ND $(0.78)$ & 22 & ND (1.6) \\
\hline FITWORX & YKBKGND-G-38086 & $4 / 28 / 16$ & N & AlR & $x$ & ND (1.1) & ND (0.98) & 20 & ND (1.6) & ND $(0.64)$ & $\mathrm{ND}(1)$ & ND (1.3) & ND (0.87) & ND (0.78) & $\mathrm{ND}(1.2)$ & ND (1.6) \\
\hline FITWORX & YKFITWORX-G-38087 & $4 / 28 / 16$ & $\mathrm{~N}$ & SOIL GAS & & ND (11) & $\mathrm{ND}(9.8)$ & ND (120) & ND (16) & ND (6.4) & ND (10) & ND (13) & $\mathrm{ND}(8.7)$ & $\mathrm{ND}(7.8)$ & ND (12) & ND (16) \\
\hline FITWORX & YKFITWORX-G-38088 & $4 / 28 / 16$ & DUP-F & SOIL GAS & & ND (11) & $\mathrm{ND}(9.8)$ & ND (120) & ND (16) & $\mathrm{ND}(6.4)$ & ND (10) & ND (13) & $\mathrm{ND}(8.7)$ & $\mathrm{ND}(7.8)$ & ND (12) & ND (16) \\
\hline GARRETSON & YKGARRETSON-G-38140 & $8 / 10 / 16$ & $N$ & AIR & & ND (1.1) & ND (0.98) & 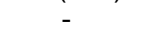 & ND (1.6) & 0.9 & $\mathrm{ND}(1)$ & ND (1.3) & ND $(0.87)$ & ND (0.78) & & ND (1.6) \\
\hline $\begin{array}{l}\text { GARRETSON } \\
\text { GARRFTSON }\end{array}$ & $\begin{array}{l}\text { YKGARRETSON-G-38138 } \\
\text { YKRKGAND--38141 }\end{array}$ & $8 / 10 / 16$ & $N$ & AIR & & $N D(1.1)$ & ND $(0.98)$ & 19 & $\mathrm{ND}(1.6)$ & 0.7 & ND (1) & $N D(1.3)$ & $N D(0.87)$ & ND $(0.78)$ & 20 & ND (1.6) \\
\hline $\begin{array}{l}\text { GARRETSON } \\
\text { FITWORX }\end{array}$ & $\begin{array}{l}\text { YKBKGND-G-38141 } \\
\text { YKFITWOR-G-38148 }\end{array}$ & $\begin{array}{l}8 / 10 / 16 \\
8 / 10 / 16\end{array}$ & $\mathrm{~N}_{\mathrm{N}}^{\mathrm{N}} \mathrm{r}$ & $\begin{array}{l}\text { AlR } \\
\text { SOIL GAS }\end{array}$ & $\mathrm{x}$ & $\begin{array}{l}\mathrm{ND}(1.1) \\
\mathrm{ND}(11)\end{array}$ & $\begin{array}{l}\mathrm{ND}(0.98) \\
\mathrm{ND}(9.8)\end{array}$ & $\begin{array}{l}19 \\
\text { ND (120) }\end{array}$ & $\begin{array}{l}\mathrm{ND}(1.6) \\
\mathrm{ND}(16)\end{array}$ & $\begin{array}{l}\mathrm{ND}(0.64) \\
\mathrm{ND}(6.4)\end{array}$ & $\begin{array}{l}\mathrm{ND}(1) \\
\mathrm{ND}(10)\end{array}$ & $\begin{array}{l}\mathrm{ND}(1.3) \\
\mathrm{ND}(13)\end{array}$ & $\begin{array}{l}\mathrm{ND}(0.87) \\
\mathrm{ND}(87)\end{array}$ & $\begin{array}{l}\mathrm{ND}(0.78) \\
\mathrm{ND}(7.8)\end{array}$ & $\begin{array}{l}2.0 \\
20\end{array}$ & ND (1.6) \\
\hline $\begin{array}{l}\text { FITWORX } \\
\text { FITWOR }\end{array}$ & $\begin{array}{l}\text { YKFITWORX-G-38149 } \\
\text { YKFIIT) }\end{array}$ & $\begin{array}{l}8 / 10 / 16 \\
8 / 16\end{array}$ & DUP-F & $\begin{array}{l}\text { SOIL GAS } \\
\text { SAIL }\end{array}$ & & ND (11) & ND (9.8) & ND (120) & ND (16) & ND (6.4) & ND (10) & ND (13) & ND (8.7) & ND ( 7.8$)$ & ND (12) & $\begin{array}{l}\text { NDD (16) } \\
\text { ND (16) }\end{array}$ \\
\hline FITWORX & $\begin{array}{l}\text { YKFITWORX-G-38142 } \\
\text { YKFIITU }\end{array}$ & 8 8/10/16 & $n^{n-1}$ & AIR & & ND (1.1) & ND (0.98) & 39 & ND (1.6) & 1.1 & $\mathrm{ND}(1)$ & ND (1.3) & ND $(0.87)$ & ND (0.78) & 23 & ND (1.6) \\
\hline FITWORX & YKFITWORX-G-38143 & $8 / 10 / 16$ & DUP-F & AlR & & ND (1.1) & ND (0.98) & 42 & ND (1.6) & 1.0 & ND (1) & $\mathrm{ND}(1.3)$ & $\mathrm{ND}(0.87)$ & ND $(0.78)$ & 24 & ND (1.6) \\
\hline FITWORX & YKFITWORX-G-38144 & $8 / 10 / 16$ & $\mathrm{~N}$ & AlR & & $\mathrm{ND}(1.1)$ & ND $(0.98)$ & 60 & $\mathrm{ND}(1.6)$ & 0.9 & ND (1) & ND (1.3) & ND $(0.87)$ & ND $(0.78)$ & 25 & 2.4 \\
\hline FITWORX & YKFITWORX-G-38145 & $8 / 10 / 16$ & $\mathrm{~N}$ & AIR & & ND (1.1) & ND $(0.98)$ & 44 & ND (1.6) & 1.2 & ND (1) & ND (1.3) & ND $(0.87)$ & ND $(0.78)$ & 22 & ND (1.6) \\
\hline FITWORX & YKFITWORX-G-38146 & $8 / 10 / 16$ & $\mathrm{~N}$ & AIR & & $N D(1.1)$ & ND $(0.98)$ & 44 & $N D(1.6)$ & 1.0 & ND (1) & ND (1.3) & ND $(0.87)$ & ND $(0.78)$ & 21 & ND (1.6) \\
\hline FITWORX & YKBKGND-G-38147 & 8/10/16 & $\mathrm{N}$ & AIR & $\mathrm{x}$ & ND (1.1) & ND (0.98) & 15 & ND (1.6) & $\mathrm{ND}(0.64)$ & ND (1) & ND (1.3) & ND $(0.87)$ & ND (0.78) & 1.6 & ND (1.6) \\
\hline GARRETSON & YKGARRETSON-G-39533 & $12 / 14 / 16$ & $N$ & AIR & & ND (1.1) & ND (0.98) & 64 & ND (1.6) & 1.5 & ND (1) & ND (1.3) & ND $(0.87)$ & ND (0.78) & $180 \mathrm{Eg}^{\mathrm{g}}$ & ND (1.6) \\
\hline $\begin{array}{l}\text { GARRETSON } \\
\text { GARRETSON }\end{array}$ & $\begin{array}{l}\text { YKGARRETSON-G-39535 } \\
\text { YKGARETSON-G-39534 }\end{array}$ & $\begin{array}{l}12 / 14 / 16 \\
12 / 44 / 16\end{array}$ & $\begin{array}{c}\text { DUP-F } \\
n\end{array}$ & $\begin{array}{l}\text { AlR } \\
\text { AlR }\end{array}$ & & $\begin{array}{l}\mathrm{ND}(1.1) \\
\mathrm{ND}(1.1)\end{array}$ & $\begin{array}{l}\text { ND (0.98) } \\
\text { ND (0.98) }\end{array}$ & $\begin{array}{l}93 \\
52\end{array}$ & $\begin{array}{l}\mathrm{ND}(1.6) \\
\mathrm{ND}(1.6)\end{array}$ & 2.7 & $\begin{array}{l}\text { NDD (1) } \\
\text { ND (1) }\end{array}$ & $\begin{array}{l}\mathrm{ND}(1.3) \\
\mathrm{ND}(1.3)\end{array}$ & $\begin{array}{l}\text { ND }(0.87) \\
\text { ND }(0.87)\end{array}$ & $\begin{array}{l}\mathrm{ND}(0.78) \\
\text { ND }(0.78)\end{array}$ & $\begin{array}{l}160 \mathrm{E} \\
170 \mathrm{E}\end{array}$ & $\begin{array}{ll}N D(1.6) \\
N D(16)\end{array}$ \\
\hline $\begin{array}{l}\text { GARRETSON } \\
\text { GARRET ISON }\end{array}$ & YKBKGND-G-39536 & $\begin{array}{l}T 2 / 14 / 16 \\
12 / 14 / 16\end{array}$ & N $-F$ & $\begin{array}{l}\text { AlR } \\
\text { AlR }\end{array}$ & $x$ & ND (1.1) & ND (0.98) & ND (12) & ND (1.6) & $\begin{array}{l}1.0 \\
0.8\end{array}$ & ND (1) & ND (1.3) & ND (0.87) & ND (0.78) & 4.9 & $\begin{array}{ll}N D(1.6) \\
N D(16)\end{array}$ \\
\hline $\begin{array}{l}\text { FITWORX } \\
\text { FITWON }\end{array}$ & YKFITWORX-G-39537 & $\begin{array}{l}12 / 14 / 16 \\
1274 / 10\end{array}$ & $\mathrm{~N}$ & AIR & & ND (1.1) & ND (0.98) & 34 & ND (1.6) & 0.0 & ND (1) & ND (1.3) & ND $(0.87)$ & $\begin{array}{l}\mathrm{ND}(0.78) \\
\mathrm{N}(0.78)\end{array}$ & 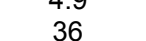 & 17 \\
\hline FITWORX & YKFITWORX-G-39538 & $12 / 14 / 16$ & DUP-F & AlR & & ND (1.1) & ND (0.98) & 42 & ND (1.6) & 1.0 & ND (1) & ND (1.3) & ND $(0.87)$ & ND $(0.78)$ & 33 & ND (1.6) \\
\hline FITWORX & YKFITWORX-G-39539 & $12 / 14 / 16$ & $\mathrm{~N}$ & AIR & & ND (1.1) & ND $(0.98)$ & 45 & ND (1.6) & 1.2 & ND (1) & ND (1.3) & ND $(0.87)$ & ND $(0.78)$ & 49 & 19 \\
\hline FITWORX & YKFITWORX-G-39540 & 12/14/16 & $\mathrm{N}$ & AIR & & $\mathrm{ND}(1.1)$ & ND (0.98) & 40 & $\mathrm{ND}(1.6)$ & 0.9 & ND (1) & ND (1.3) & ND (0.87) & & 32 & ND (1.6) \\
\hline $\begin{array}{l}\text { FITWORX } \\
\text { FITWODPY }\end{array}$ & YKFITWORX-G-39541 & $12 / 14 / 16$ & N & AIR & & $N D(1.1)$ & ND $(0.98)$ & 38 & $N D(1.6)$ & 0.8 & ND (1) & $\mathrm{ND}(1.3)$ & ND $(0.87)$ & ND $(0.78)$ & 29 & ND (1.6) \\
\hline $\begin{array}{l}\text { FITWORX } \\
\text { FTWOAP }\end{array}$ & 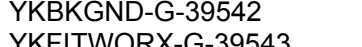 & $12 / 14 / 16$ & $\mathrm{~N}$ & AlR & $\mathrm{x}$ & $\mathrm{ND}(1.1)$ & ND $(0.98)$ & ND (12) & ND (1.6) & ND (0.64) & ND (1) & $N D(1.3)$ & & & 2.5 & \\
\hline $\begin{array}{l}\text { FITWORX } \\
\text { FITWORX }\end{array}$ & $\begin{array}{l}\text { YKFITWORXX-G-39543 } \\
\text { YKFITWORX-G-39544 }\end{array}$ & $\begin{array}{l}12 / 14 / 16 \\
12 / 4 / 16\end{array}$ & $\begin{array}{c}N \\
\text { DUP-F }\end{array}$ & $\begin{array}{l}\text { SOIL GAS } \\
\text { SOll GAS }\end{array}$ & & $\begin{array}{l}\text { ND (11) } \\
\text { ND (11) }\end{array}$ & $\begin{array}{l}\mathrm{ND}(9.8) \\
\mathrm{ND}(9.8)\end{array}$ & $\begin{array}{l}\text { ND (120) } \\
\text { ND (120) }\end{array}$ & $\begin{array}{l}\mathrm{ND}(16) \\
\mathrm{ND}(16)\end{array}$ & $\begin{array}{l}\mathrm{ND}(6.4) \\
\mathrm{ND}(6.4)\end{array}$ & $\begin{array}{ll}\text { ND } & (10) \\
\text { ND (10) }\end{array}$ & $\begin{array}{l}\mathrm{ND}(13) \\
\mathrm{ND}(13)\end{array}$ & $\begin{array}{l}\mathrm{ND}(8.7) \\
\mathrm{ND}(8.7)\end{array}$ & ND (7.8) & $\begin{array}{l}57 \\
58\end{array}$ & ND (16) \\
\hline & & & & & & & & & & & & & $\mathrm{ND}(8.7)$ & & & ND (16) \\
\hline
\end{tabular}


Concentration $\left(\mu \mathrm{g} / \mathrm{m}^{3}\right)$

\begin{tabular}{|c|c|c|c|c|c|c|c|c|c|c|c|c|c|c|c|c|}
\hline \multirow[b]{2}{*}{ Location } & \multirow[b]{2}{*}{ Sample } & \multirow[b]{2}{*}{$\begin{array}{l}\text { Sample } \\
\text { Date }\end{array}$} & \multirow[b]{2}{*}{$\begin{array}{c}\text { Sample } \\
\text { Type }\end{array}$} & \multirow[b]{2}{*}{$\begin{array}{l}\text { Sample } \\
\text { Matrix }\end{array}$} & \multirow[b]{2}{*}{$\begin{array}{l}\text { Ambient } \\
\text { Sample }\end{array}$} & \multicolumn{11}{|c|}{ coniveriluc } \\
\hline & & & & & & CFC-11 & CFC-12 & $\begin{array}{l}\text { Chlorinated } \\
\text { Fluorocarbon } \\
\text { (Freon 113) }\end{array}$ & Chlorobenzene & $\begin{array}{l}\text { Chlorodibromo- } \\
\text { methane }\end{array}$ & $\begin{array}{l}\text { Chlorodifluoro- } \\
\text { methane }\end{array}$ & Chloroethane & Chloromethane & $\begin{array}{l}\text { Cis-1,2- } \\
\text { Dichloroethene }\end{array}$ & $\begin{array}{l}\text { Cis-1,3- } \\
\text { Dichloropropene }\end{array}$ & Cyclohexane \\
\hline WILLOW & YKWILLOW-G-37887 & $8 / 5 / 15$ & $\mathrm{~N}$ & AIR & & 1.2 & ND (2.5) & ND (1.5) & $\mathrm{ND}(0.92)$ & ND (1.7) & 19 & ND (1.3) & 1.1 & ND (0.79) & ND (0.91) & $\mathrm{ND}(0.69)$ \\
\hline WILLOW & YKWILLOW-G-37885 & $8 / 5 / 15$ & $\mathrm{~N}$ & AIR & & 1.2 & ND (2.5) & ND (1.5) & ND (0.92) & ND $(1.7)$ & 13 & $\mathrm{ND}(1.3)$ & 1.2 & ND (0.79) & ND (0.91) & $\mathrm{ND}(0.69)$ \\
\hline WILLOW & YKWILLOW-G-37886 & $8 / 5 / 15$ & $N$ & AIR & & $\mathrm{ND}(1.1)$ & ND (2.5) & ND (1.5) & ND (0.92) & ND (1.7) & 4.7 & ND (1.3) & 1.1 & ND $(0.79)$ & ND (0.91) & $\mathrm{ND}(0.69)$ \\
\hline $\begin{array}{l}\text { WILLOW } \\
\text { WLLOW }\end{array}$ & $\begin{array}{l}\text { YKWLLLOW-G-37889 } \\
\text { YKWLLLOW-G-37890 }\end{array}$ & $\begin{array}{l}8 / 5 / 115 \\
85115\end{array}$ & $N^{n}$ & $\begin{array}{l}A A R \\
A A R\end{array}$ & & 1.2 & $N$ N & $\begin{array}{l}N D(1.5) \\
N D(15)\end{array}$ & ND (0.92) & ND (1.1) & 3.3 & ND (1.3) & 1.1 & 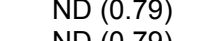 & ND & $\mathrm{ND}(0.69)$ \\
\hline $\begin{array}{l}\text { WILLOW } \\
\text { WILLOW }\end{array}$ & $\begin{array}{l}\text { YKWLLLOW-G-38980 } \\
\text { YKWLLLO-G-37888 }\end{array}$ & $\begin{array}{l}8 / 1 / 15 \\
8 / 5 / 15\end{array}$ & $N^{N}$ & $\begin{array}{l}\text { AR } \\
A \mid R\end{array}$ & & $\begin{array}{l}1.2 \\
1.2\end{array}$ & $\begin{array}{l}\text { ND (2.5) } \\
\text { ND (25) }\end{array}$ & $\begin{array}{l}\mathrm{ND}(1.5) \\
\mathrm{ND}(1.5)\end{array}$ & $\begin{array}{l}\mathrm{ND}(0.92) \\
\mathrm{ND}(0.92)\end{array}$ & ND (1.1) & 72 & $\mathrm{ND}(1.3)$ & 1.2 & $\begin{array}{ll}\mathrm{ND}(0.99) \\
N D(79)\end{array}$ & $\begin{array}{ll}N D(0.91) \\
N D\end{array}$ & ND $(0.69)$ \\
\hline CVA & YKCVA-G-37909 & $\begin{array}{l}8 / 5 / 15 \\
8 / 15\end{array}$ & N & AlR & & 1.2 & ND (2.5) & ND (1.5) & ND (0.92) & ND (1.7) & $\mathrm{ND}(1.8)$ & ND (1.3) & 1.2 & ND (0.79) & ND (0.91) & ND $(0.69)$ \\
\hline CVA & YKCVA-G-37910 & $8 / 5 / 15$ & $\mathrm{~N}$ & AIR & & 1.4 & 2.7 & ND (1.5) & ND (0.92) & ND (1.7) & ND (1.8) & $\mathrm{ND}(1.3)$ & 1.6 & ND $(0.79)$ & ND (0.91) & $\begin{array}{l}\text { ND (0.69) } \\
\text { ND ( } 0.69)\end{array}$ \\
\hline CVA & YKCVA-G-37912 & $8 / 5 / 15$ & $\mathrm{~N}$ & AlR & & 1.2 & ND (2.5) & ND (1.5) & ND (0.92) & ND (1.7) & 2.4 & ND (1.3) & 1.3 & ND $(0.79)$ & ND (0.91) & ND (0.69) \\
\hline PRES & YKPRES-G-37904 & $8 / 5 / 15$ & $\mathrm{~N}$ & AIR & & 1.4 & ND $(2.5)$ & ND (1.5) & ND (0.92) & ND (1.7) & ND (1.8) & ND (1.3) & 2.3 & $\mathrm{ND}(0.79)$ & ND (0.91) & $\mathrm{ND}(0.69)$ \\
\hline PRES & YKPRES-G-37903 & $8 / 5 / 15$ & $\mathrm{~N}$ & AIR & & 1.5 & 4.2 & ND (1.5) & ND (0.92) & ND (1.7) & ND (1.8) & $\mathrm{ND}(1.3)$ & 1.4 & ND (0.79) & ND (0.91) & $\mathrm{ND}(0.69)$ \\
\hline $\begin{array}{l}\text { FITWORX } \\
\text { FITWRD }\end{array}$ & YKFITWORX-G-378988 & 8 & $N$ & AlR & & 1.2 & ND (2.5) & ND (1.5) & ND (0.92) & & 4.8 & ND (1.3) & ND (1) & ND $(0.79)$ & ND $(0.91)$ & ND (0.69) \\
\hline FITWORX & YKFITWORX-G-37897 & $8 / 5 / 15$ & & AlR & & 1.9 & ND (2.5) & ND (1.5) & ND (0.92) & ND (1.7) & 9.1 & ND (1.3) & 1.2 & ND $(0.79)$ & ND (0.91) & ND (0.69) \\
\hline FITWORX & YKFITWORX-G-37901 & $8 / 5 / 15$ & DUP-F & AIR & & 1.7 & ND (2.5) & ND (1.5) & ND (0.92) & ND (1.7) & 8.3 & ND (1.3) & 1.1 & $\mathrm{ND}(0.79)$ & $\mathrm{ND}(0.91)$ & 0.7 \\
\hline FITWORX & YKFITWORX-G-37900 & $8 / 5 / 15$ & $\mathrm{~N}$ & AIR & & 2.1 & 2.6 & ND (1.5) & ND (0.92) & ND (1.7) & 11 & ND (1.3) & 1.7 & ND (0.79) & $\mathrm{ND}(0.91)$ & 1.0 \\
\hline FITWORX & YKFITWORX-G-37899 & $8 / 5 / 15$ & $\mathrm{~N}$ & AIR & & 2.0 & 2.6 & ND (1.5) & ND (0.92) & ND (1.7) & 11 & $\mathrm{ND}(1.3)$ & 1.3 & ND (0.79) & $\mathrm{ND}(0.91)$ & 1.0 \\
\hline $\begin{array}{l}\text { FITWORX } \\
\text { FITWROY }\end{array}$ & & & $\mathrm{N}$ & & & ND (1.1) & & $\mathrm{ND}(1.5)$ & & $\mathrm{ND}(1.7)$ & & ND (1.3) & $N D(1)$ & ND $(0.79)$ & ND (0.91) & ND (0.69) \\
\hline FITWORX & YKBKGND-G-37908 & $8 / 5 / 15$ & $\mathrm{~N}$ & AIR & $\mathrm{x}$ & 1.2 & 2.5 & ND (1.5) & ND (0.92) & $\mathrm{ND}(1.7)$ & ND (1.8) & ND (1.3) & 1.5 & ND $(0.79)$ & ND (0.91) & ND $(0.69)$ \\
\hline HEARTH & YKHEARTH-G-37892 & $8 / 5 / 15$ & $N$ & AIR & & $N D(1.1)$ & ND (2.5) & ND (1.5) & ND $(0.92)$ & $\mathrm{ND}(1.7)$ & 88 & $\mathrm{ND}(1.3)$ & ND (1) & ND $(0.79)$ & $\mathrm{ND}(0.91)$ & $\mathrm{ND}(0.69)$ \\
\hline HEARTH & YKHEARTH-G-37893 & $8 / 5 / 15$ & $\mathrm{~N}$ & AIR & & ND (1.1) & ND (2.5) & ND (1.5) & ND (0.92) & ND (1.7) & $180 \mathrm{D}$ & ND (1.3) & 1.2 & ND (0.79) & $\mathrm{ND}(0.91)$ & $\mathrm{ND}(0.69)$ \\
\hline HEARTH & $\begin{array}{l}\text { YKHEARTH-G-37896 } \\
\text { YKHEARTHG-37895 }\end{array}$ & $8 / 5 / 15$ & N & AlR & & ND (1.1) & ND (2.5) & ND (1.5) & ND (0.92) & ND (1.7) & 48 & ND (1.3) & 1.1 & ND $(0.79)$ & ND (0.91) & $\mathrm{ND}(0.69)$ \\
\hline HEARIH & YKHEARTH-G-37895 & 8) $8 / 1 / 15$ & $\mathrm{~N}$ & AlR & & 1.2 & 2.5 & ND (1.5) & ND (0.92) & ND (1.7) & 36 & $\mathrm{ND}(1.3)$ & 1.4 & ND (0.79) & $\mathrm{ND}(0.91)$ & $\mathrm{ND}(0.69)$ \\
\hline HEARTH & YKHEARTH-G-37891 & $8 / 5 / 15$ & $\mathrm{~N}$ & AIR & & ND (1.1) & ND (2.5) & ND (1.5) & ND (0.92) & ND (1.7) & 11 & ND (1.3) & 1.1 & ND (0.79) & $\mathrm{ND}(0.91)$ & $\mathrm{ND}(0.69)$ \\
\hline $\begin{array}{ll}\text { HEARTH } \\
\text { HEATH }\end{array}$ & $\begin{array}{l}\text { YYHEARTH-G-37894 } \\
\text { Y }\end{array}$ & $8 / 5 / 15$ & N & AIR & & 1.2 & $\begin{array}{l}2.5 \\
(25)\end{array}$ & ND (1.5) & ND (0.92) & ND (1.7) & 6.5 & ND (1.3) & 1.3 & ND $(0.79)$ & ND (0.91) & ND $(0.69)$ \\
\hline $\begin{array}{l}\text { HEARTH } \\
\text { TROES }\end{array}$ & $\begin{array}{l}\text { YKBKGGND-G-37907 } \\
\text { YKTROES-G-37913 }\end{array}$ & 88515 & $N^{n}$ & $\begin{array}{l}A A R \\
A A R\end{array}$ & $x$ & $\begin{array}{l}1.2 \\
9.5\end{array}$ & $\mathrm{ND}(2.5)$ & $\begin{array}{l}\mathrm{ND}(1.5) \\
\mathrm{ND}(1.5)\end{array}$ & ND (0.92) & $\begin{array}{l}\mathrm{ND}(1.7) \\
\mathrm{ND}(1.7)\end{array}$ & $\begin{array}{l}\mathrm{ND}(1.8) \\
\mathrm{ND}(1.8)\end{array}$ & $\begin{array}{l}\mathrm{ND}(1.3) \\
\mathrm{ND}(1.3)\end{array}$ & 1.2 & ND (0.79) & $\begin{array}{l}\mathrm{ND}(0.91) \\
N D(0.91)\end{array}$ & ND $(0.69)$ \\
\hline $\begin{array}{l}\text { IRUES } \\
\text { TROES }\end{array}$ & YKTROES-G-3/9713 & $\begin{array}{l}8 / 5 / 15 \\
8 / 5 / 15\end{array}$ & $\begin{array}{l}n \\
N\end{array}$ & AlR & & $\begin{array}{l}9.5 \\
9.5\end{array}$ & $\begin{array}{l}\text { ND (2.5) } \\
\text { ND (2.5) }\end{array}$ & $\begin{array}{l}\mathrm{ND}(1.5) \\
\mathrm{ND}(1.5)\end{array}$ & $\begin{array}{l}\mathrm{ND}(0.92) \\
\mathrm{ND}(0.92)\end{array}$ & $\begin{array}{l}\mathrm{ND}(1.1) \\
\mathrm{ND}(1.7)\end{array}$ & $\begin{array}{l}\text { ND }(1.8) \\
\text { ND (1.8) }\end{array}$ & ND (1.3) & $\begin{array}{l}1.6 \\
1.8\end{array}$ & ND (0.79) & ND (0.91) & $\begin{array}{l}\mathrm{ND}(0.69) \\
\mathrm{ND}(0.69)\end{array}$ \\
\hline TROES & YKBKGND-G-37906 & $\begin{array}{l}8 / 5 / 15 \\
8 / 5 / 15\end{array}$ & $N^{n}$ & AlR & $x$ & 1.1 & ND (2.5) & ND (1.5) & $\mathrm{ND}(0.92)$ & ND (1.7) & $\mathrm{ND}(1.8)$ & ND (1.3) & 1.2 & ND (0.79) & ND (0.91) & (N) (1.69) \\
\hline MILLER & $\begin{array}{l}\text { YKBKGNDD-G-3/7914 } \\
\text { YKBKGN-G-37915 }\end{array}$ & $\begin{array}{l}8 / 5 / 15 \\
8 / 5 / 15\end{array}$ & $\frac{N}{N}$ & $\begin{array}{l}\text { AlR } \\
\text { AlR }\end{array}$ & $\begin{array}{l}x \\
x\end{array}$ & $\begin{array}{l}1.1 \\
1.2\end{array}$ & $\begin{array}{l}\mathrm{ND}(2.5) \\
\mathrm{ND}(2.5)\end{array}$ & $\begin{array}{l}\mathrm{ND}(1.5) \\
\mathrm{ND}(1.5)\end{array}$ & ND (0.92) & $\begin{array}{l}\mathrm{ND}(1 . /) \\
\mathrm{ND}(1.7)\end{array}$ & $\begin{array}{l}\mathrm{ND}(1.8) \\
\mathrm{ND}(1.8)\end{array}$ & $\begin{array}{l}\mathrm{ND}(1.3) \\
\mathrm{ND}(1.3)\end{array}$ & 1.2 & ND (0.79) & ND (0.91) & ND (0.69) \\
\hline GARRETSON & YKGARRETSON-G-38036 & $1 / 28 / 16$ & $\mathrm{~N}$ & AlR & & 1.8 & ND (2.5) & ND (1.5) & ND (0.92) & ND (1.7) & ND (1.8) & ND (1.3) & 1.6 & ND (0.79) & ND (0.91) & ND (0.69) \\
\hline GARRETSON & YKGARRETSON-G-38037 & $1 / 28 / 16$ & $\mathrm{~N}$ & AIR & & 1.8 & ND $(2.5)$ & $\mathrm{ND}(1.5)$ & $\mathrm{ND}(0.92)$ & ND (1.7) & $\mathrm{ND}(1.8)$ & ND (1.3) & 1.6 & ND $(0.79)$ & $\mathrm{ND}(0.91)$ & $\mathrm{ND}(0.69)$ \\
\hline GARRETSON & YKGARRETSON-G-38038 & $1 / 28 / 16$ & DUP-F & AIR & & 1.8 & ND (2.5) & ND (1.5) & ND $(0.92)$ & ND (1.7) & ND (1.8) & ND (1.3) & 1.6 & $\mathrm{ND}(0.79)$ & $\mathrm{ND}(0.91)$ & $\mathrm{ND}(0.69)$ \\
\hline $\begin{array}{l}\text { GARRETSON } \\
\text { FTTPX }\end{array}$ & $\begin{array}{l}\text { YKBKGNDD-G-38039 } \\
\text { YKETHNOPX-38040 }\end{array}$ & $1 / 28 / 16$ & ${ }_{N}^{N}$ & AIR & $\mathrm{x}$ & 1.2 & 2.5 & ND (1.5) & ND (0.92) & ND (1.7) & $\mathrm{ND}(1.8)$ & ND (1.3) & 1.2 & ND $(0.79)$ & $N D(0.91)$ & ND $(0.69)$ \\
\hline $\begin{array}{l}\text { FIIWWORX } \\
\text { FITWORX }\end{array}$ & $\begin{array}{l}\text { YKHIWORX-G-38040 } \\
\text { YKFITWORX-G-38042 }\end{array}$ & $\begin{array}{l}1 / 128 / 16 \\
1 / 28 / 16\end{array}$ & ${ }_{N}^{N}$ & $\begin{array}{l}\text { AR } \\
A A R\end{array}$ & & $\begin{array}{l}1.6 \\
1.6\end{array}$ & $\begin{array}{l}2.5 \\
2.5\end{array}$ & $\begin{array}{l}\mathrm{ND}(1.5) \\
\mathrm{ND}(1.5)\end{array}$ & $\begin{array}{l}\mathrm{ND}(0.92) \\
\mathrm{ND}(0.92)\end{array}$ & $\begin{array}{l}\mathrm{ND}(1.7) \\
\mathrm{ND}(1.7)\end{array}$ & $\begin{array}{l}9.9 \\
9.6\end{array}$ & $\begin{array}{l}\mathrm{ND}(1.3) \\
\mathrm{ND}(1.3)\end{array}$ & $\begin{array}{l}1.4 \\
1.6\end{array}$ & $\begin{array}{l}\text { ND }(0.79) \\
\text { ND }(079)\end{array}$ & $\begin{array}{l}\text { ND }(0.91) \\
\text { ND ( } 0.911)\end{array}$ & ND $(0.69)$ \\
\hline $\begin{array}{l}\text { FITWORX } \\
\text { FITWORX }\end{array}$ & $\begin{array}{l}\text { YKIIWORX-G-38042 } \\
\text { YKFITWORX-G-38041 }\end{array}$ & $\begin{array}{l}\begin{array}{l}1 / 28 / 166 \\
1 / 28 / 16\end{array} \\
-\end{array}$ & DUP-F & $\begin{array}{l}\text { AR } \\
\text { AIR }\end{array}$ & & $\begin{array}{l}1.6 \\
1.7\end{array}$ & $\begin{array}{l}2.5 \\
2.6\end{array}$ & $\begin{array}{l}\mathrm{ND}(1.5) \\
\mathrm{ND}(1.5)\end{array}$ & $\begin{array}{l}\mathrm{ND}(0.92) \\
\mathrm{ND}(0.92)\end{array}$ & $\mathrm{ND}(1.7)$ & $\begin{array}{l}9.6 \\
10\end{array}$ & ND (1.3) & 1.6 & ND (0.79) & ND (0.91) & $\begin{array}{l}\mathrm{ND}(0.69) \\
0.7\end{array}$ \\
\hline FITWORX & $\begin{array}{l}\text { YKFITWORX-G-38041 } \\
\text { YKOTX }\end{array}$ & $\begin{array}{l}1 / 28 / 16 \\
1 / 28 / 16\end{array}$ & $n$ & AlR & & 1.7 & $\mathrm{ND}(2.5)$ & ND (1.5) & ND (0.92) & $\mathrm{ND}(1.7)$ & 10 & $\mathrm{ND}(1.3)$ & $\begin{array}{l}1.5 \\
1.4\end{array}$ & ND (0.79) & ND (0.91) & 0.18 \\
\hline FITWORX & YKFITWORX-G-38044 & $1 / 28 / 16$ & $\mathrm{~N}$ & AlR & & 1.7 & 2.7 & ND (1.5) & ND (0.92) & ND (1.7) & 11 & ND (1.3) & 1.3 & ND (0.79) & ND (0.91) & 0.8 \\
\hline FITWORX & YKBKGND-G-38045 & $1 / 28 / 16$ & $\mathrm{~N}$ & AIR & $x$ & ND (1.1) & ND $(2.5)$ & $\mathrm{ND}(1.5)$ & $\mathrm{ND}(0.92)$ & ND (1.7) & ND (1.8) & ND (1.3) & 1.3 & ND $(0.79)$ & ND (0.91) & ND (0.69) \\
\hline GARRETSON & YKGARRETSON-G-38046 & $1 / 28 / 16$ & $\mathrm{~N}$ & SOIL GAS & & 160 & ND (10) & ND (15) & $\mathrm{ND}(9.4)$ & ND (17) & & ND (16) & ND (4.1) & $\mathrm{ND}(8)$ & ND (9.2) & \\
\hline FITWORX & YKFITWORX-G-38048 & $1 / 28 / 16$ & $\mathrm{~N}$ & SOIL GAS & & $\mathrm{ND}(11)$ & ND (10) & ND (15) & ND (9.4) & ND (17) & & ND (16) & $\mathrm{ND}(4.1)$ & ND (8) & ND $(9.2)$ & \\
\hline $\begin{array}{l}\text { FITWORX } \\
\text { FITWORX }\end{array}$ & $\begin{array}{l}\text { YKFITWORX-G-38081 } \\
\text { YYKITWO-G }\end{array}$ & $4 / 28 / 16$ & 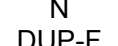 & $\begin{array}{l}\text { AlR } \\
\text { AlR }\end{array}$ & & $\begin{array}{l}1.7 \\
1.9\end{array}$ & 2.5 & ND (1.5) & ND (0.92) & ND (1.7) & $\begin{array}{l}8.6 \\
95\end{array}$ & ND (1.3) & $\begin{array}{l}1.1 \\
1.3\end{array}$ & ND (0.79) & ND (0.91) & $\mathrm{ND}(0.69)$ \\
\hline $\begin{array}{l}\text { FITWORX } \\
\text { FITWORX }\end{array}$ & $\begin{array}{l}\text { YKITWORX-G-38082 } \\
\text { YKFITWORX-G-38083 }\end{array}$ & $\begin{array}{l}4 / 28 / 16 \\
4 / 2816\end{array}$ & $\begin{array}{l}\text { DUP-F } \\
\text { N }\end{array}$ & $\begin{array}{l}\text { AR } \\
A A R\end{array}$ & & $\begin{array}{l}1.9 \\
1.4\end{array}$ & $\begin{array}{l}2.7 \\
\mathrm{ND}(2.5)\end{array}$ & $\begin{array}{l}\mathrm{ND}(1.5) \\
\mathrm{ND}(1.5)\end{array}$ & $\begin{array}{l}\mathrm{ND}(0.92) \\
\mathrm{ND}(0.92)\end{array}$ & $\begin{array}{l}\mathrm{ND}(1.7) \\
\mathrm{ND}(1.7)\end{array}$ & $\begin{array}{l}9.5 \\
7.4\end{array}$ & $\begin{array}{l}\mathrm{ND}(1.3) \\
\mathrm{ND}(1.3)\end{array}$ & $\begin{array}{l}1.3 \\
1.2\end{array}$ & $\begin{array}{l}\text { ND ( }(0.79) \\
\text { ND }(0.79)\end{array}$ & $\begin{array}{ll}N D(0.91) \\
N D\end{array}$ & $\begin{array}{l}0.8 \\
N D(069)\end{array}$ \\
\hline $\begin{array}{l}\text { FITWORX } \\
\text { FIVWORX }\end{array}$ & 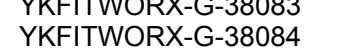 & $\begin{array}{l}4 / 28 / 16 \\
4 / 28 / 16\end{array}$ & ${ }_{N}^{N}$ & $\begin{array}{l}\text { AIR } \\
\text { AlR }\end{array}$ & & $\begin{array}{l}1.4 \\
1.8\end{array}$ & 2.5 & ND (1.5) & ND (0.92) & $\mathrm{ND}(1.7)$ & $\begin{array}{l}.4 \\
9.5\end{array}$ & ND (1.3) & 1.2 & ND (0.79) & $\mathrm{ND}(0.91)$ & $\begin{array}{l}\mathrm{ND}(0.69) \\
\mathrm{ND}(0.69)\end{array}$ \\
\hline $\begin{array}{l}\text { FITWORX } \\
\text { Fith }\end{array}$ & YKFITWORX-G-38085 & $\begin{array}{l}4 / 28 / 16 \\
4 / 28\end{array}$ & n & AlR & & $\begin{array}{l}1.0 \\
1.6\end{array}$ & $\mathrm{ND}(2.5)$ & ND (1.5) & ND (0.92) & $\mathrm{ND}(1.7)$ & $\begin{array}{l}5.5 \\
8.8\end{array}$ & $\mathrm{ND}(1.3)$ & 1.1 & ND (0.79) & ND (0.91) & 0.7 \\
\hline FITWORX & YKBKGND-G-38086 & $4 / 28 / 16$ & $\mathrm{~N}$ & AlR & $x$ & 1.3 & 2.6 & ND (1.5) & ND (0.92) & ND (1.7) & ND (1.8) & ND (1.3) & 1.2 & ND (0.79) & ND (0.91) & ND (0.69) \\
\hline FITWORX & YKFITWORX-G-38087 & $4 / 28 / 16$ & $\mathrm{~N}$ & SOIL GAS & & ND (11) & ND (25) & ND (15) & $\mathrm{ND}(9.2)$ & ND (17) & ND (18) & ND (13) & ND (10) & ND $(7.9)$ & ND $(9.1)$ & ND $(6.9)$ \\
\hline FITWORX & YKFITWORX-G-38088 & $4 / 28 / 16$ & DUP-F & SOIL GAS & & ND (11) & ND (25) & ND (15) & ND (9.2) & ND (17) & ND (18) & ND (13) & ND (10) & ND (7.9) & ND (9.1) & ND (6.9) \\
\hline GARRETSON & YKGARRETSON-G-38140 & $8 / 10 / 16$ & $\mathrm{~N}$ & AlR & & 1.3 & $\mathrm{ND}(2.5)$ & ND (1.5) & ND (0.92) & $\mathrm{ND}(1.7)$ & ND (1.8) & ND (1.3) & 1.8 & ND $(0.79)$ & $\mathrm{ND}(0.91)$ & $\mathrm{ND}(0.69)$ \\
\hline $\begin{array}{l}\text { GARRETSON } \\
\end{array}$ & YKGARRETSON-G-38138 & 8/10/16 & $N$ & AIR & $x$ & 1.441, & 2.8 & ND (1.5) & ND (0.92) & ND (1.7) & ND (1.8) & ND (1.3) & 2.1 & ND $(0.79)$ & ND (0.91) & $\mathrm{ND}(0.69)$ \\
\hline GARRETSON & YKBKGND-G-38141 & $8 / 10 / 16$ & $\mathrm{~N}$ & & $x$ & ND (1.1) & 2.5 & ND (1.5) & ND (0.92) & $\mathrm{ND}(1.7)$ & ND (1.8) & $\mathrm{ND}(1.3)$ & 1.1 & ND (0.79) & ND (0.91) & 1.0 \\
\hline FITWORX & YKFITWORX-G-38148 & $8 / 10 / 16$ & $\mathrm{~N}$ & SOIL GAS & & ND (11) & ND (25) & ND (15) & $\mathrm{ND}(9.2)$ & ND (17) & ND (18) & ND (13) & $\mathrm{ND}(10)$ & ND (7.9) & ND $(9.1)$ & ND (6.9) \\
\hline WORX & YKFITWORX-G-38149 & $8 / 10 / 16$ & DUP-F & SOIL GAS & & $N D(11)$ & ND (25) & ND (15) & ND (9.2) & ND (17) & ND (18) & ND (13) & ND (10) & & & ND (6.9) \\
\hline NORX & YKFITWORX-G-38142 & $8 / 10 / 16$ & $\mathrm{~N}$ & AIR & & 1.7 & 2.8 & ND (1.5) & ND (0.92) & ND (1.7) & 11 & ND (1.3) & ND (1) & ND (0.79) & ND (0.91) & 0.9 \\
\hline FITWORX & YKFITWORX-G-38143 & & DUP-F & AIR & & 1.8 & 2.7 & ND (1.5) & ND (0.92) & ND (1.7) & 11 & ND (1.3) & 1.1 & ND (0.79) & ND (0.91) & 0.8 \\
\hline FITWORX & YKFITWORX-G-38144 & 8/10/16 & $\mathrm{N}$ & AIR & & 2.1 & 2.9 & ND (1.5) & ND (0.92) & $N D(1.7)$ & 11 & ND (1.3) & 1.2 & ND $(0.79)$ & ND (0.91) & 0.8 \\
\hline FITWORX & YKFITWORX-G-38145 & $8 / 10 / 16$ & $\mathrm{~N}$ & AIR & & 2.1 & 2.8 & ND (1.5) & ND (0.92) & ND (1.7) & 12 & ND (1.3) & 1.0 & ND (0.79) & $\mathrm{ND}(0.91)$ & 1.1 \\
\hline $\begin{array}{l}\text { FITWORX } \\
\text { FITWORX }\end{array}$ & $\begin{array}{l}\text { YKITITORX-G-38146 } \\
\text { YKBKGND-G-38147 }\end{array}$ & $\begin{array}{l}8 / 10 / 16 \\
8 / 10 / 16\end{array}$ & $\begin{array}{l}\mathrm{N} \\
\mathrm{N}\end{array}$ & $\begin{array}{l}{ }_{A A R}^{A R} \\
\text { AlR }\end{array}$ & $x$ & $\begin{array}{l}2.1 \\
1.2\end{array}$ & $\begin{array}{l}2.8 \\
2.5\end{array}$ & $\begin{array}{l}\mathrm{ND}(1.5) \\
\mathrm{ND}(1.5)\end{array}$ & $\begin{array}{l}\mathrm{ND}(0.92) \\
\mathrm{ND}(0.92)\end{array}$ & $\begin{array}{l}\text { ND (1.7) } \\
\text { ND (1.7) }\end{array}$ & $\begin{array}{l}11 \\
N D(1.8)\end{array}$ & $\begin{array}{l}\text { ND (1.3) } \\
\text { ND (1.3) }\end{array}$ & $\begin{array}{l}1.1 \\
\mathrm{ND}(1)\end{array}$ & $\begin{array}{l}\text { ND }(0.79) \\
\text { ND }(0.79)\end{array}$ & ND (0.91) & $\begin{array}{c}1.0 \\
1.069)\end{array}$ \\
\hline & & & & & & & & & & & & & & & & \\
\hline
\end{tabular}


Concentration $\left(\mu \mathrm{g} / \mathrm{m}^{3}\right)$

\begin{tabular}{|c|c|c|c|c|c|c|c|c|c|c|c|c|c|c|c|c|}
\hline \multirow[b]{2}{*}{ Location } & \multirow[b]{2}{*}{ Sample } & \multirow[b]{2}{*}{$\begin{array}{c}\text { Sample } \\
\text { Date }\end{array}$} & \multirow[b]{2}{*}{$\begin{array}{c}\text { Sample } \\
\text { Type }\end{array}$} & \multirow[b]{2}{*}{$\begin{array}{l}\text { Sample } \\
\text { Matrix }\end{array}$} & \multirow[b]{2}{*}{$\begin{array}{l}\text { Ambient } \\
\text { Sample }\end{array}$} & \multicolumn{11}{|c|}{ Concentration $\left(\mu \mathrm{g} / \mathrm{m}^{3}\right)$} \\
\hline & & & & & & CFC-11 & CFC-12 & $\begin{array}{l}\text { Chlorinated } \\
\text { Fluorocarbon } \\
\text { (Freon 113) }\end{array}$ & Chlorobenzene & $\begin{array}{l}\text { Chlorodibromo- } \\
\text { methane }\end{array}$ & $\begin{array}{l}\text { Chlorodifluoro- } \\
\text { methane }\end{array}$ & Chloroethane & Chloromethane & $\begin{array}{c}\text { Cis-1,2- } \\
\text { Dichloroethene }\end{array}$ & $\begin{array}{c}\text { Cis-1,3- } \\
\text { Dichloropropene }\end{array}$ & Cyclohexane \\
\hline GARRETSON & YKGARRETSON-G-39533 & $12 / 14 / 16$ & $N$ & AIR & & 1.2 & $\mathrm{ND}(2.5)$ & ND (1.5) & ND (0.92) & $\mathrm{ND}(1.7)$ & $\mathrm{ND}(1.8)$ & ND (1.3) & 2.0 & $\mathrm{ND}(0.79)$ & ND (0.91) & 1.4 \\
\hline GARRETSON & YKGARRETSON-G-39535 & 12/14/16 & $\mathrm{N}$ & AIR & & 1.2 & $\mathrm{ND}(2.5)$ & $\mathrm{ND}(1.5)$ & $\mathrm{ND}(0.92)$ & ND (1.7) & $\mathrm{ND}(1.8)$ & $\mathrm{ND}(1.3)$ & 1.9 & & ND (0.91) & \\
\hline GARRETSON & YKGARRETSON-G-39534 & 12/14/16 & DUP-F & AIR & & 1.2 & $\mathrm{ND}(2.5)$ & $\mathrm{ND}(1.5)$ & ND (0.92) & $\mathrm{ND}(1.7)$ & $\mathrm{ND}(1.8)$ & ND (1.3) & 1.9 & ND (0.79) & ND (0.91) & $N D(0.69)$ \\
\hline GARRETSON & YKBKGND-G-39536 & $12 / 14 / 16$ & $\mathrm{~N}$ & AlR & $x$ & 1.2 & ND $(2.5)$ & ND (1.5) & ND $(0.92)$ & ND (1.7) & ND $(1.8)$ & $\mathrm{ND}(1.3)$ & $N D(1)$ & ND $(0.79)$ & ND $(0.91)$ & $\mathrm{ND}(0.69)$ \\
\hline FITWORX & YKFITWORX-G-39537 & $12 / 14 / 16$ & $\mathrm{~N}$ & AIR & & 1.7 & ND (2.5) & ND (1.5) & ND $(0.92)$ & ND (1.7) & 18 & $\mathrm{ND}(1.3)$ & 1.3 & ND $(0.79)$ & ND $(0.91)$ & 0.8 \\
\hline FITWORX & YKFITWORX-G-39538 & $12 / 14 / 16$ & DUP-F & AIR & & 1.7 & 2.5 & $\mathrm{ND}(1.5)$ & $\mathrm{ND}(0.92)$ & ND (1.7) & 18 & $\mathrm{ND}(1.3)$ & 1.2 & ND (0.79) & ND (0.91) & 0.7 \\
\hline FITWORX & YKFITWORX-G-39539 & 12/14/16 & $\mathrm{N}$ & AIR & & 1.6 & 2.4 & ND (1.5) & ND (0.92) & ND (1.7) & 17 & ND (1.3) & 1.1 & ND (0.79) & ND (0.91) & $\mathrm{ND}(0.69)$ \\
\hline FITWORX & YKFITWORX-G-39540 & $12 / 14 / 16$ & $\mathrm{~N}$ & AIR & & 1.8 & ND (2.5) & $\mathrm{ND}(1.5)$ & $\mathrm{ND}(0.92)$ & ND (1.7) & 20 & ND (1.3) & 1.3 & ND $(0.79)$ & $\mathrm{ND}(0.91)$ & ND (0.69) \\
\hline FITWORX & YKFITWORX-G-39541 & $12 / 14 / 16$ & $\mathrm{~N}$ & AIR & & 1.9 & 2.5 & $\mathrm{ND}(1.5)$ & ND $(0.92)$ & ND (1.7) & 20 & ND (1.3) & 1.4 & ND $(0.79)$ & ND (0.91) & ND $(0.69)$ \\
\hline \multirow{3}{*}{$\begin{array}{l}\text { FITWORX } \\
\text { FITWORX } \\
\text { FITWORX }\end{array}$} & YKBKGND-G-39542 & $12 / 14 / 16$ & $N$ & AlR & $\mathrm{x}$ & 1.2 & ND (2.5) & ND (1.5) & $N D(0.92)$ & ND (1.7) & ND (1.8) & ND (1.3) & ND (1) & $\mathrm{ND}(0.79)$ & ND $(0.91)$ & $\mathrm{ND}(0.69)$ \\
\hline & $\begin{array}{l}\text { YKFITWORX-G-39543 } \\
\text { YKFITWORX-G-39544 }\end{array}$ & $\begin{array}{l}12 / 14 / 16 \\
12 / 14 / 16\end{array}$ & $\begin{array}{l}\mathrm{N} \\
\text { DUP-F }\end{array}$ & $\begin{array}{l}\text { SOIL GAS } \\
\text { SOIL GAS }\end{array}$ & & $\begin{array}{l}\text { ND (11) } \\
\text { ND (11) }\end{array}$ & $\begin{array}{l}\text { ND (25) } \\
\text { ND (25) }\end{array}$ & $\begin{array}{l}\text { ND (15) } \\
\text { ND (15) }\end{array}$ & $\begin{array}{l}\text { ND (9.2) } \\
\text { ND (9.2) }\end{array}$ & $\begin{array}{l}\text { ND (17) } \\
\text { ND (17) }\end{array}$ & $\begin{array}{l}\text { ND (18) } \\
\text { ND (18) }\end{array}$ & $\begin{array}{l}\text { ND (13) } \\
\text { ND (13) }\end{array}$ & $\begin{array}{l}\text { ND (10) } \\
\text { ND (10) }\end{array}$ & $\begin{array}{l}\text { ND }(7.9) \\
\text { ND }(7.9)\end{array}$ & $\begin{array}{l}\mathrm{ND}(9.1) \\
\mathrm{ND}(9.1)\end{array}$ & $\begin{array}{l}\text { ND (6.9) } \\
\text { ND (6.9) }\end{array}$ \\
\hline & & & & & & \multicolumn{11}{|c|}{ Concentration $\left(\mu \mathrm{g} / \mathrm{m}^{3}\right)$} \\
\hline Location & Sample & $\begin{array}{c}\text { Sample } \\
\text { Date }\end{array}$ & $\begin{array}{c}\text { Sample } \\
\text { Type }\end{array}$ & $\begin{array}{l}\text { Sample } \\
\text { Matrix }\end{array}$ & $\begin{array}{l}\text { Ambient } \\
\text { Sample }\end{array}$ & Cymene & Ethylbenzene & $\begin{array}{l}\text { Ethylene } \\
\text { Dibromide }\end{array}$ & $\begin{array}{l}\text { Hexachloro-1,3- } \\
\text { Butadiene }\end{array}$ & Hexane & $\begin{array}{l}\text { Isopropyl } \\
\text { Alcohol }\end{array}$ & Isopropylbenzene & M,P-Xylene & $\begin{array}{c}\text { M- } \\
\text { Dichlorobenzene }\end{array}$ & $\begin{array}{l}\text { Methyl Isobutyl } \\
\text { Ketone }\end{array}$ & $\begin{array}{c}\text { Methyl } \\
\text { Methacrylate }\end{array}$ \\
\hline WILLOW & YKWILLOW-G-37887 & $8 / 5 / 15$ & $N$ & AIR & & ND (11) & $N D(0,87)$ & $N D(1.5)$ & $\mathrm{ND}(21)$ & ND $(07)$ & 89 & & & & & \\
\hline WILLOW & YKWILLOW-G-37885 & $8 / 5 / 15$ & $\mathrm{~N}$ & AlR & & ND (1.1) & ND $(0.87)$ & ND (1.5) & ND (2.1) & ND $(0.7)$ & $\begin{array}{l}89 \\
150\end{array}$ & $\begin{array}{l}\text { ND ( }(0.98) \\
\text { ND ( } 998)\end{array}$ & $\begin{array}{l}\mathrm{ND}(2.2) \\
\text { ND }(2.2)\end{array}$ & $\begin{array}{l}\mathrm{ND}(1.2) \\
\mathrm{ND}(1.2)\end{array}$ & $\begin{array}{l}\text { ND (2) } \\
\text { ND (2) }\end{array}$ & ND (2) \\
\hline WILLOW & YKWILLOW-G-37886 & $8 / 5 / 15$ & $\mathrm{~N}$ & AIR & & ND (1.1) & $N D(0.87)$ & ND (1.5) & $\mathrm{ND}(2.1)$ & ND $(0.7)$ & 58 & ND (1.98) & ND (2) & ND (1, 1 ) & ND (2) & ND (2) \\
\hline WILLOW & YKWILLOW-G-37889 & $8 / 5 / 15$ & $\mathrm{~N}$ & AIR & & ND (1.1) & $N D(0.87)$ & ND (1.5) & ND (2.1) & ND $(0.7)$ & 61 & ND $(0.98)$ & ND (2.2) & $\mathrm{ND}(12)$ & ND (2) & $\mathrm{ND}(2)$ \\
\hline WILLOW & YKWILLOW-G-37890 & $8 / 5 / 15$ & $\mathrm{~N}$ & AIR & & ND (1.1) & ND $(0.87)$ & ND (1.5) & ND (2.1) & ND $(0.7)$ & 78 & ND $(0.98)$ & $\mathrm{ND}(2.2)$ & ND (1.2) & $\mathrm{ND}(2)$ & ND (2) \\
\hline WILLOW & YKWILLOW-G-37888 & $8 / 5 / 15$ & $\mathrm{~N}$ & AIR & & ND (1.1) & ND $(0.87)$ & ND (1.5) & ND (2.1) & ND $(0.7)$ & 22 & ND $(0.98)$ & ND (2.2) & ND (1.2) & $\mathrm{ND}(2)$ & ND (2) \\
\hline CVA & YKCVA-G-37909 & $8 / 5 / 15$ & $\mathrm{~N}$ & AIR & & ND (1.1) & 0.9 & ND (1.5) & ND $(2.1)$ & ND $(0.7)$ & 46 & ND $(0.98)$ & $\mathrm{ND}(2.2)$ & ND (1.2) & $\mathrm{ND}(2)$ & ND (2) \\
\hline CVA & YKCVA-G-37910 & 8/5/15 & $\mathrm{N}$ & AIR & & 2.9 & 1.7 & $\mathrm{ND}(1.5)$ & $\mathrm{ND}(2.1)$ & ND $(0.7)$ & 56 & ND (0.98) & 2.6 & $\mathrm{ND}(1.2)$ & $\mathrm{ND}(2)$ & ND (2) \\
\hline CVA & & $8 / 5 / 15$ & $\mathrm{~N}$ & AIR & & 3.0 & 1.4 & ND (1.5) & ND $(2.1)$ & ND $(0.7)$ & 42 & ND $(0.98)$ & ND (2.2) & ND (1.2) & ND (2) & $\mathrm{ND}(2)$ \\
\hline PRES & YKPRES-G-37904 & $8 / 5 / 15$ & $\mathrm{~N}$ & AIR & & ND (1.1) & ND $(0.87)$ & ND (1.5) & ND (2.1) & ND $(0.7)$ & ND (12) & ND $(0.98)$ & ND (2.2) & ND (1.2) & 4.6 & ND (2) \\
\hline PRES & YKPRES-G-37903 & $8 / 5 / 15$ & $\mathrm{~N}$ & AIR & & ND (1.1) & 1.1 & $\mathrm{ND}(1.5)$ & $\mathrm{ND}(2.1)$ & $\mathrm{ND}(0.7)$ & ND (12) & ND $(0.98)$ & 3.8 & $\mathrm{ND}(1.2)$ & ND (2) & $\mathrm{ND}(2)$ \\
\hline FITWORX & YKFITWORX-G-37898 & $8 / 5 / 15$ & $\mathrm{~N}$ & AIR & & ND (1.1) & 0.9 & ND (1.5) & $\mathrm{ND}(2.1)$ & ND $(0.7)$ & ND (12) & ND $(0.98)$ & 2.9 & ND (1.2) & ND (2) & ND (2) \\
\hline $\begin{array}{l}\text { FITWORX } \\
\text { FITWORX }\end{array}$ & YKFITWORX-G-37897 & $8 / 5 / 15$ & $\frac{D^{2}}{N}$ & AIR & & 1.4 & 1.2 & & $\mathrm{ND}(2.1)$ & $\begin{array}{l}1.3 \\
12\end{array}$ & $\begin{array}{l}22 \\
19\end{array}$ & ND (0.98) & $\begin{array}{l}3.9 \\
37\end{array}$ & ND (1.2) & ND (2) & ND (2) \\
\hline $\begin{array}{l}\text { FITWORX } \\
\text { FITWORX }\end{array}$ & $\begin{array}{l}\text { YKFITWORX-G-37901 } \\
\text { YKFFITWORX-G-37900 }\end{array}$ & $\begin{array}{l}8 / 5 / 15 \\
8 / 5 / 15\end{array}$ & $\begin{array}{l}\text { DUP-F } \\
N\end{array}$ & $\begin{array}{l}\text { AIR } \\
\text { AIR }\end{array}$ & & $\begin{array}{l}1.4 \\
1.4\end{array}$ & $\begin{array}{l}1.2 \\
1.6\end{array}$ & $\begin{array}{l}\text { ND (1.5) } \\
\text { ND (1.5) }\end{array}$ & $\begin{array}{l}\mathrm{ND}(2.1) \\
\mathrm{ND}(2.1)\end{array}$ & $\begin{array}{l}1.2 \\
1.8\end{array}$ & $\begin{array}{l}19 \\
22\end{array}$ & $\begin{array}{l}\text { ND ( (0.98) } \\
\text { ND (0.98) }\end{array}$ & $\begin{array}{l}3.7 \\
5.1\end{array}$ & $\begin{array}{l}\mathrm{ND}(1.2) \\
\mathrm{ND}(1.2)\end{array}$ & $\begin{array}{ll}\mathrm{ND}(2) \\
\mathrm{ND}(2)\end{array}$ & ND (2) \\
\hline $\begin{array}{l}\text { FITWORX } \\
\text { FITX }\end{array}$ & $\begin{array}{l}\text { YKFITWORX-G-37899 } \\
\text { YKOAX }\end{array}$ & $8 / 5 / 15$ & $\mathrm{~N}$ & $\begin{array}{l}\text { AlR } \\
\text { AIR }\end{array}$ & & $\begin{array}{l}1.8 \\
1.8\end{array}$ & $\begin{array}{l}1.6 \\
1.6\end{array}$ & ND (1.5) & $\mathrm{ND}(2.1)$ & $\begin{array}{l}1.8 \\
1.7\end{array}$ & 21 & ND (0.98) & $\begin{array}{l}.1 \\
5.0\end{array}$ & ND (1.2) & $\mathrm{ND}(2)$ & ND (2) \\
\hline FITWORX & YKFITWORX-G-37902 & $8 / 5 / 15$ & $\mathrm{~N}$ & AlR & \multirow{6}{*}{$x$} & $\mathrm{ND}(1.1)$ & ND $(0.87)$ & ND (1.5) & ND (2.1) & ND $(0.7)$ & $\mathrm{ND}(12)$ & ND $(0.98)$ & ND (2.2) & ND (1.2) & $\mathrm{ND}(2)$ & ND (2) \\
\hline FITWORX & YKBKGND-G-37908 & $8 / 5 / 15$ & $\mathrm{~N}$ & AlR & & $\mathrm{ND}(1.1)$ & ND (0.87) & ND (1.5) & ND (2.1) & ND (0.7) & ND (12) & ND (0.98) & ND (2.2) & ND (1.2) & ND (2) & ND (2) \\
\hline HEARTH & YKHEARTH-G-37892 & $8 / 5 / 15$ & $\mathrm{~N}$ & AIR & & ND (1.1) & ND $(0.87)$ & ND (1.5) & ND (2.1) & ND $(0.7)$ & 340 & ND $(0.98)$ & ND (2.2) & ND (1.2) & $\mathrm{ND}(2)$ & ND (2) \\
\hline HEARTH & YKHEARTH-G-37893 & $8 / 5 / 15$ & $\mathrm{~N}$ & AIR & & ND (1.1) & ND $(0.87)$ & $\mathrm{ND}(1.5)$ & $\mathrm{ND}(2.1)$ & ND $(0.7)$ & 230 & ND $(0.98)$ & $\mathrm{ND}(2.2)$ & ND (1.2) & ND (2) & ND (2) \\
\hline $\begin{array}{l}\text { HEARTH } \\
\text { HEARTH }\end{array}$ & YKHEARTH-G-37896 & $8 / 5 / 15$ & $\mathrm{~N}$ & AIR & & ND (1.1) & ND $(0.87)$ & ND (1.5) & ND (2.1) & $\mathrm{ND}(0.7)$ & 1100 & ND (0.98) & ND (2.2) & ND (1.2) & 3.7 & ND (2) \\
\hline $\begin{array}{l}\text { HEARTH } \\
\text { HEARTH }\end{array}$ & $\begin{array}{l}\text { YKHEARTH-G-37895 } \\
\text { YKHHEARTH-G-37891 }\end{array}$ & $\begin{array}{l}8 / 5 / 115 \\
8 / 5 / 15\end{array}$ & $\begin{array}{r}n \\
N\end{array}$ & $\begin{array}{l}\text { AIR } \\
\text { AlR }\end{array}$ & & ND (1.1) & ND $(0.87)$ & $\begin{array}{l}\mathrm{ND}(1.5) \\
\mathrm{ND}(1.5)\end{array}$ & ND (2.1) & ND (0.7) & $\begin{array}{l}1300 \\
370\end{array}$ & $\begin{array}{l}N D(0.98) \\
N D(0.9)\end{array}$ & $\mathrm{ND}(2.2)$ & ND (1.2) & 5.7 & ND (2) \\
\hline $\begin{array}{l}\text { HEARTH } \\
\text { HEARTH }\end{array}$ & $\begin{array}{l}\text { YKEEARTH-G-38781 } \\
\text { YKHEART-G-37894 }\end{array}$ & $\begin{array}{l}8 / 5 / 15 \\
8 / 5 / 15\end{array}$ & $\begin{array}{l}\mathrm{N} \\
\mathrm{N}\end{array}$ & $\begin{array}{l}\text { AlR } \\
\text { AlR }\end{array}$ & & $\begin{array}{l}\text { ND (1.1) } \\
\text { ND (1.1) }\end{array}$ & $\begin{array}{l}\text { ND }(0.87) \\
\text { ND }(0.87)\end{array}$ & $\begin{array}{l}\text { ND (1.5) } \\
\text { ND (1.5) }\end{array}$ & $\begin{array}{l}\mathrm{ND}(2.1) \\
\mathrm{ND}(2.1)\end{array}$ & $\begin{array}{l}\text { ND (0.7) } \\
\text { ND }(0.7)\end{array}$ & $\begin{array}{l}370 \\
570\end{array}$ & $\begin{array}{l}\text { ND (0.98) } \\
\text { ND (0.98) }\end{array}$ & $\begin{array}{l}\mathrm{ND}(2.2) \\
\mathrm{ND}(2.2)\end{array}$ & $\begin{array}{l}\mathrm{ND}(1.2) \\
\mathrm{ND}(1.2)\end{array}$ & $\begin{array}{l}\text { ND (2) } \\
2.7\end{array}$ & $\begin{array}{l}\mathrm{ND}(2) \\
\mathrm{ND}(2)\end{array}$ \\
\hline HEARTH & 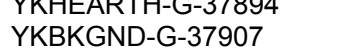 & $\begin{array}{l}8 / 5 / 15 \\
8 / 15\end{array}$ & $\mathrm{~N}$ & AIR & & ND (1.1) & ND $(0.87)$ & ND (1.5) & $\mathrm{ND}(2.1)$ & $\mathrm{ND}(0.7)$ & ND (12) & ND (0.98) & $\mathrm{ND}(2.2)$ & ND (1.2) & $\mathrm{ND}(2)$ & ND (2) \\
\hline TROES & YKTROES-G-37913 & $8 / 5 / 15$ & $\mathrm{~N}$ & AlR & \multirow{2}{*}{$x$} & ND (1.1) & ND $(0.87)$ & ND (1.5) & $\mathrm{ND}(2.1)$ & 2.1 & 65 & ND (0.98) & $\mathrm{ND}(2.2)$ & $\mathrm{ND}(1.2)$ & ND (2) & ND (2) \\
\hline TROES & YKTROES-G-37906 & $8 / 5 / 15$ & $\mathrm{~N}$ & AlR & & 1.1 & ND $(0.87)$ & ND (1.5) & ND (2.1) & 2.2 & 65 & ND (0.98) & ND (2.2) & ND (1.2) & ND (2) & ND (2) \\
\hline TROES & YKBKGND-G-37914 & $8 / 5 / 15$ & $\mathrm{~N}$ & AlR & $x$ & ND (1.1) & ND $(0.87)$ & ND (1.5) & $\mathrm{ND}(2.1)$ & ND $(0.7)$ & ND (12) & ND $(0.98)$ & $\mathrm{ND}(2.2)$ & $\mathrm{ND}(1.2)$ & ND (2) & $\mathrm{ND}(2)$ \\
\hline MILLER & YKBKGND-G-37915 & $8 / 5 / 15$ & $\mathrm{~N}$ & AIR & \multirow[t]{2}{*}{$x$} & ND (1.1) & ND $(0.87)$ & ND $(1$ & $\mathrm{ND}(2.1)$ & ND $(0.7)$ & ND (12) & ND $(0.98)$ & $\mathrm{ND}(2.2)$ & ND (1.2) & ND (2) & ND (2) \\
\hline GARRETSON & YKGARRETSON-G-38036 & $1 / 28 / 16$ & $\mathrm{~N}$ & AIR & & $\begin{array}{l}1.1 \\
11\end{array}$ & 1.2 & ND (1.5) & $\mathrm{ND}(2.1)$ & $N D(0.7)$ & - & ND (0.98) & $\begin{array}{l}4.5 \\
4\end{array}$ & ND (1.2) & ND (2) & ND (2) \\
\hline $\begin{array}{l}\text { GARRETSON } \\
\text { GARRETSON }\end{array}$ & $\begin{array}{l}\text { YKGARREETSON-G-38037 } \\
\text { YKGGARRTSONONG-G830 }\end{array}$ & $\begin{array}{l}1 / 28 / 16 \\
1 / 2816\end{array}$ & $\begin{array}{l}\text { DUP-F } \\
\text { DUP }\end{array}$ & $\begin{array}{l}\text { AIR } \\
\text { AlR }\end{array}$ & \multirow[b]{2}{*}{$x$} & ND (1.1) & ND $(0.87)$ & $\begin{array}{l}\mathrm{ND}(1.5) \\
\mathrm{ND}(1.5)\end{array}$ & $\begin{array}{ll}\mathrm{ND}(2.1) \\
\mathrm{ND}(2.1)\end{array}$ & $\begin{array}{ll}\mathrm{ND}(0.7) \\
\mathrm{ND}(0.7)\end{array}$ & - & $\begin{array}{l}N D(0.98) \\
N D(0.98)\end{array}$ & $\begin{array}{l}\mathrm{ND}(2.2) \\
24\end{array}$ & $\mathrm{ND}(1.2)$ & $\mathrm{ND}(2)$ & ND (2) \\
\hline $\begin{array}{l}\text { GARREISON } \\
\text { GARRETSON }\end{array}$ & YKBKGND-G-3803980 & $\begin{array}{l}1 / 28 / 16 \\
1 / 28 / 16\end{array}$ & & $\begin{array}{l}\text { AlR } \\
\text { AlR }\end{array}$ & & ND (1.1) & ND $(0.87)$ & $\begin{array}{l}\mathrm{ND}(1.5) \\
\mathrm{ND}(1.5)\end{array}$ & $\begin{array}{l}\mathrm{ND}(2.1) \\
\mathrm{ND}(2.1)\end{array}$ & $\begin{array}{l}\mathrm{ND}(0.9) \\
\mathrm{ND}(0.7)\end{array}$ & ND (12) & ND (0.98) & $\begin{array}{l}2.4 \\
\mathrm{ND}(2.2)\end{array}$ & $\begin{array}{l}\mathrm{ND}(1.2) \\
\mathrm{ND}(1.2)\end{array}$ & $\mathrm{ND}(2)$ & $\begin{array}{ll}\text { ND (2) } \\
\text { ND (2) }\end{array}$ \\
\hline $\begin{array}{l}\text { FITWORX } \\
\text { FITW }\end{array}$ & $\begin{array}{l}\text { YKBKGNDU-G-380039 } \\
\text { YKFITWOR-G-38040 }\end{array}$ & $\begin{array}{l}1 / 28 / 16 \\
1 / 28 / 6\end{array}$ & $\mathrm{~N}^{\mathrm{N}} \mathrm{r}-\mathrm{a}$ & AIR & A & 1.2 & 1.2 & ND (1.5) & $\mathrm{ND}(2.1)$ & 1.6 & 80 & ND (0.98) & 3.6 & $\mathrm{ND}(1.2)$ & $\mathrm{ND}(2)$ & 44 \\
\hline FITWORX & YKFITWORX-G-38042 & $1 / 28 / 16$ & $\mathrm{~N}$ & AlR & & ND (1.1) & 1.1 & ND (1.5) & ND (2.1) & 1.8 & 72 & ND $(0.98)$ & 3.7 & ND (1.2) & $\mathrm{ND}(2)$ & ND (2) \\
\hline FITWORX & YKFITWORX-G-38041 & $1 / 28 / 16$ & DUP-F & AlR & & ND (1.1) & 1.3 & ND 11 & ND (2.1) & 1.9 & 91 & ND $(0.98)$ & 4.3 & ND (1.2) & $\mathrm{ND}(2)$ & ND (2) \\
\hline FITWORX & YKFITWORX-G-38043 & $1 / 28 / 16$ & $\mathrm{~N}$ & AIR & & ND (1.1) & 1.3 & ND (1.5) & $\mathrm{ND}(2.1)$ & 2.1 & 78 & $\mathrm{ND}(0.98)$ & 4.5 & $\mathrm{ND}(1.2)$ & ND (2) & $\mathrm{ND}(2)$ \\
\hline FITWORX & YKFITWORX-G-38044 & $1 / 28 / 16$ & $\mathrm{~N}$ & AIR & & ND (1.1) & 1.3 & ND (1.5) & $\mathrm{ND}(2.1)$ & 2.2 & 74 & ND (0.98) & 4.6 & $\mathrm{ND}(1.2)$ & $\mathrm{ND}(2)$ & ND (2) \\
\hline FITWORX & YKBKGND-G-38045 & 1/288/16 & $\mathrm{N}$ & AIR & $x$ & ND (1.1) & ND $(0.87)$ & ND (1.5) & $\mathrm{ND}(2.1)$ & ND $(0.7)$ & ND (12) & ND (0.98) & ID (2.2) & ND (1.2) & & ND (2) \\
\hline GARRETSON & YKGARRETSON-G-38046 & 1/28/16 & & & & & ND (8.8) & ND (16) & ND (110) & & & & & ND (24) & & \\
\hline
\end{tabular}


Concentration $\left(\mu \mathrm{g} / \mathrm{m}^{3}\right)$

\begin{tabular}{|c|c|c|c|c|c|c|c|c|c|c|c|c|c|c|c|c|}
\hline \multirow[b]{2}{*}{ Location } & \multirow[b]{2}{*}{ Sample } & \multirow[b]{2}{*}{$\begin{array}{c}\text { Sample } \\
\text { Date }\end{array}$} & \multirow[b]{2}{*}{$\begin{array}{c}\text { Sample } \\
\text { Type }\end{array}$} & \multirow[b]{2}{*}{$\begin{array}{l}\text { Sample } \\
\text { Matrix }\end{array}$} & \multirow[b]{2}{*}{$\begin{array}{l}\text { Ambient } \\
\text { Sample }\end{array}$} & \multicolumn{11}{|c|}{ Concentration $\left(\mu \mathrm{g} / \mathrm{m}^{3}\right)$} \\
\hline & & & & & & Cymene & Ethylbenzene & $\begin{array}{l}\text { Ethylene } \\
\text { Dibromide }\end{array}$ & $\begin{array}{l}\text { Hexachloro-1,3- } \\
\text { Butadiene }\end{array}$ & Hexane & $\begin{array}{l}\text { Isopropyl } \\
\text { Alcohol }\end{array}$ & Isopropylbenzene & M,P-Xylene & $\begin{array}{c}\text { M- } \\
\text { Dichlorobenzene }\end{array}$ & $\begin{array}{l}\text { Methyl Isobutyl } \\
\text { Ketone }\end{array}$ & $\begin{array}{c}\text { Methyl } \\
\text { Methacrylate }\end{array}$ \\
\hline FITWORX & YKFITWORX-G-38048 & 1/28/16 & $\mathrm{N}$ & SOIL GAS & & & 5.1 & ND (16) & ND (110) & - & - & & 21 & ND (24) & ND (17) & \\
\hline FITWORX & YKFITWORX-G-38081 & $4 / 28 / 16$ & 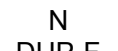 & AIR & & $\mathrm{ND}(1.1)$ & 1.0 & $\mathrm{ND}(1.5)$ & $\mathrm{ND}(2.1)$ & 1.3 & \multirow{2}{*}{$\begin{array}{l}29 \\
38\end{array}$} & $\mathrm{ND}(0.98)$ & 3.8 & ND (1.2) & $\mathrm{ND}(2)$ & ND (2) \\
\hline $\begin{array}{ll}\text { FITWORX } \\
\text { FITWODPY }\end{array}$ & YKFITWORX-G-38082 & $4 / 28 / 16$ & DUP-F & AIR & & ND (1.1) & 1.2 & ND (1.5) & ND (2.1) & 1.7 & & ND (0.98) & 4.3 & $\mathrm{ND}(1.2)$ & ND (2) & $\mathrm{ND}(2)$ \\
\hline $\begin{array}{l}\text { FITWORX } \\
\text { FITWORX }\end{array}$ & $\begin{array}{l}\text { YKITWORX-G-38803 } \\
\text { YKFITWORX-G-3084 }\end{array}$ & $\begin{array}{l}4 / 28 / 16 \\
4 / 28 / 16\end{array}$ & $\stackrel{N}{N}$ & $\begin{array}{l}\text { AIR } \\
\text { AIR }\end{array}$ & & $\begin{array}{l}\mathrm{ND}(1.1) \\
\mathrm{ND}(1.1)\end{array}$ & $\begin{array}{l}\text { ND }(0.87) \\
\text { ND }(0.87)\end{array}$ & $\begin{array}{l}\mathrm{ND}(1.5) \\
\mathrm{ND}(1.5)\end{array}$ & $\begin{array}{l}\mathrm{ND}(2.1) \\
\mathrm{ND}(2.1)\end{array}$ & $\begin{array}{l}0.7 \\
1.2\end{array}$ & 19 & $\begin{array}{l}\mathrm{ND}(0.98) \\
\mathrm{ND}(0.98)\end{array}$ & $\mathrm{ND}(2.2)$ & $\mathrm{ND}(1.2)$ & $\mathrm{ND}(2)$ & $\mathrm{ND}(2)$ \\
\hline $\begin{array}{l}\text { FITWORX } \\
\text { FITW }\end{array}$ & 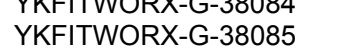 & $\begin{array}{l}4 / 28 / 16 \\
4 / 28 / 6\end{array}$ & $\begin{array}{l}\mathrm{N} \\
\mathrm{N}\end{array}$ & $\begin{array}{l}\text { ARR } \\
\text { AIR }\end{array}$ & & ND (1.1) & 1.2 & ND (1.5) & ND $(2.1)$ & 1.6 & \multirow{2}{*}{$\begin{array}{l}41 \\
39\end{array}$} & ND (0.98) & 4.5 & ND (1.2) & ND (2) & $\mathrm{ND}(2)$ \\
\hline FITWORX & $\begin{array}{l}\text { YKBKGND-G-38086 } \\
\text { YKKGN }\end{array}$ & $4 / 28 / 16$ & $\mathrm{~N}$ & AlR & $\mathrm{x}$ & ND (1.1) & & $\mathrm{ND}(1.5)$ & ND (2.1) & ND $(0.7)$ & & ND (0.98) & $\mathrm{ND}(2.2)$ & $\mathrm{ND}(1.2)$ & ND (2) & ND (2) \\
\hline FITWORX & $\begin{array}{l}\text { YKFITWORX-G-38087 } \\
\text { YKFIUT) }\end{array}$ & $4 / 28 / 16$ & $\mathrm{~N}$ & SOIL GAS & & ND (11) & ND (8.7) & ND (15) & ND (21) & ND ( (7) & $\begin{array}{l}\text { ND (12) } \\
\text { ND (120) }\end{array}$ & ND (9.8) & 27 & ND (12) & ND (20) & ND (20) \\
\hline FITWORX & YKFITWORX-G-38088 & $4 / 28 / 16$ & DUP-F & SOIL GAS & & ND (11) & ND (8.7) & ND (15) & ND (21) & $\mathrm{ND}(7)$ & \multirow{2}{*}{$\begin{array}{c}\text { ND (120) } \\
89\end{array}$} & ND (9.8) & ND (22) & ND (12) & ND (20) & ND (20) \\
\hline GARRETSON & YKGARRETSON-G-38140 & $8 / 10 / 16$ & $\mathrm{~N}$ & AIR & & $\mathrm{ND}(1.1)$ & 1.0 & ND (1.5) & $\mathrm{ND}(2.1)$ & 0.7 & & $\mathrm{ND}(0.98)$ & 3.1 & $\mathrm{ND}(1.2)$ & $\mathrm{ND}(2)$ & ND (2) \\
\hline GARRETSON & YKGARRETSON-G-38138 & $8 / 10 / 16$ & $N$ & AIR & & 1.1 & 1.0 & $\mathrm{ND}(1.5)$ & $\mathrm{ND}(2.1)$ & 0.8 & \multirow{2}{*}{$\mathrm{ND}^{-}(12)$} & $\mathrm{ND}(0.98)$ & 3.0 & ND (1.2) & ND (2) & ND (2) \\
\hline $\begin{array}{l}\text { GARRETSON } \\
\text { FITWRPX }\end{array}$ & $\begin{array}{l}\text { YKBKGND-G-38141 } \\
\text { YKEITOPY-3148 }\end{array}$ & $8 / 10 / 16$ & $\mathrm{~N}_{\mathrm{N}}^{\mathrm{N}} \mathrm{r}-\mathrm{r}$ & AIR & $\mathrm{x}$ & $\mathrm{ND}(1.1)$ & $N D(0.87)$ & $\mathrm{ND}(1.5)$ & $\mathrm{ND}(2.1)$ & 0.9 & & $N D(0.98)$ & $\mathrm{ND}(2.2)$ & $\mathrm{ND}(1.2)$ & ND (2) & ND (2) \\
\hline $\begin{array}{l}\text { FIIWWORX } \\
\text { FITWORX }\end{array}$ & $\begin{array}{l}\text { YKITWORX-G-38148 } \\
\text { YKFITWORX-G-38149 }\end{array}$ & $\begin{array}{l}8 / 10 / 16 \\
8 / 10 / 16\end{array}$ & $\begin{array}{l}N \\
\text { DUP-F }\end{array}$ & $\begin{array}{l}\text { SOIL GAS } \\
\text { SOIL GAS }\end{array}$ & & $\begin{array}{l}\text { ND (11) } \\
\text { ND (11) }\end{array}$ & $\begin{array}{l}\mathrm{ND}(8.7) \\
\mathrm{ND}(8.7)\end{array}$ & $\begin{array}{l}\text { ND (15) } \\
\text { ND (15) }\end{array}$ & $\begin{array}{l}\mathrm{ND}(21) \\
\mathrm{ND}(21)\end{array}$ & $\begin{array}{l}\mathrm{ND}(7) \\
\mathrm{ND}(7)\end{array}$ & $\begin{array}{l}\text { ND ( (120) } \\
\text { ND (120) }\end{array}$ & $\begin{array}{l}\mathrm{ND}(9.8) \\
\mathrm{ND}(9.8)\end{array}$ & $\begin{array}{l}\mathrm{ND}(22) \\
\mathrm{ND}(22)\end{array}$ & $\begin{array}{l}\mathrm{ND}(12) \\
\mathrm{ND}(12)\end{array}$ & $\begin{array}{l}\mathrm{ND}(20) \\
\text { ND }(20)\end{array}$ & $\begin{array}{ll}N D & (20) \\
N D(20)\end{array}$ \\
\hline $\begin{array}{l}\text { FITWORX } \\
\text { FIVR }\end{array}$ & $\begin{array}{l}\text { YKFITWORX-G-38142 } \\
\text { YKFOC-38) }\end{array}$ & $8 / 10 / 16$ & $n^{n-1}$ & AIR & & 1.4 & $\begin{array}{l}1.6 \\
1.17)\end{array}$ & $\mathrm{ND}(1.5)$ & $\mathrm{ND}(2.1)$ & 1.5 & 32 & ND ( $(9.98)$ & 5.6 & ND (1.2) & ND (2) & $\mathrm{ND}(2)$ \\
\hline $\begin{array}{l}\text { FITWORX } \\
\text { FITWOA }\end{array}$ & 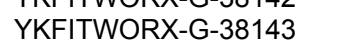 & $8 / 10 / 16$ & DUP-F & AIR & & 1.6 & 1.6 & ND (1.5) & ND (2.1) & 1.5 & 32 & ND ( $(0.98)$ & $\begin{array}{l}6.0 \\
6.1\end{array}$ & ND (1.2) & $\begin{array}{lll}\mathrm{ND}(2) & \\
\end{array}$ & $\mathrm{ND}(2)$ \\
\hline FITWORX & YKFITWORX-G-38144 & $8 / 10 / 16$ & $\mathrm{~N}$ & AlR & & 1.4 & 1.5 & ND (1.5) & ND (2.1) & 1.7 & 39 & ND (0.98) & 5.1 & ND (1.2) & $\mathrm{ND}(2)$ & ND (2) \\
\hline FITWORX & YKFITWORX-G-38145 & $8 / 10 / 16$ & $\mathrm{~N}$ & AlR & & 1.5 & 2.1 & ND (1.5) & ND (2.1) & 1.7 & 33 & $\mathrm{ND}(0.98)$ & 7.3 & ND (1.2) & ND (2) & $\mathrm{ND}(2)$ \\
\hline FITWORX & YKFITWORX-G-38146 & $8 / 10 / 16$ & $\mathrm{~N}$ & AIR & & 1.5 & 2.1 & ND (1.5) & $\mathrm{ND}(2.1)$ & 1.5 & 33 & $\mathrm{ND}(0.98)$ & 7.3 & $\mathrm{ND}(1.2)$ & ND (2) & $\mathrm{ND}(2)$ \\
\hline FITWORX & YKBKGND-G-38147 & $8 / 10 / 16$ & N & $A I R$ & $\mathrm{x}$ & $\mathrm{ND}(1.1)$ & $\mathrm{ND}(0.87)$ & ND (1.5) & $\mathrm{ND}(2.1)$ & $\mathrm{ND}(0.7)$ & ND (12) & ND $(0.98)$ & $\mathrm{ND}(2.2)$ & $\mathrm{ND}(1.2)$ & $\mathrm{ND}(2)$ & ND (2) \\
\hline $\begin{array}{l}\text { GARRETSON } \\
\text { GARRFTSON }\end{array}$ & $\begin{array}{l}\text { YKGARRETSON-G-39533 } \\
\text { YKGARRETSON-G-39535 }\end{array}$ & $\begin{array}{l}12 / 14416 \\
121 / 416\end{array}$ & $N^{N}$ & AIR & & ND (1.1) & $\begin{array}{l}3.6 \\
98\end{array}$ & ND (1.5) & $\mathrm{ND}(2.1)$ & $\begin{array}{l}1.4 \\
25\end{array}$ & $\begin{array}{l}\mathrm{ND}(12) \\
65\end{array}$ & $\begin{array}{l}\mathrm{ND}(0.98) \\
\mathrm{ND}(0.98)\end{array}$ & $\begin{array}{l}13 \\
36\end{array}$ & $\mathrm{ND}(1.2)$ & $\mathrm{ND}(2)$ & ND (2) \\
\hline $\begin{array}{l}\text { GARRETSON } \\
\text { GARRETSON }\end{array}$ & $\begin{array}{l}\text { YKGARRETSON-G-39535 } \\
\text { YKGARRETSON-G-3953 }\end{array}$ & $\begin{array}{l}121 / 14416 \\
12 / 14 / 16\end{array}$ & $\begin{array}{c}N \\
\text { DUP-F }\end{array}$ & $\begin{array}{l}\text { AR } \\
\text { AIR }\end{array}$ & & $\begin{array}{l}\mathrm{ND}(1.1) \\
\mathrm{ND}(1.1)\end{array}$ & $\begin{array}{l}9.8 \\
1.3\end{array}$ & $\begin{array}{l}\mathrm{ND}(1.5) \\
\mathrm{ND}(1.5)\end{array}$ & $\begin{array}{l}\mathrm{ND}(2.1) \\
\mathrm{ND}(2.1)\end{array}$ & $\begin{array}{l}2.5 \\
0.9\end{array}$ & $\begin{array}{c}65 \\
\text { ND (12) }\end{array}$ & $\begin{array}{l}\text { ND ( }(0.98) \\
\text { ND }(0.98)\end{array}$ & $\begin{array}{l}36 \\
4.4\end{array}$ & $\begin{array}{l}\mathrm{ND}(1.2) \\
\mathrm{ND}(1.2)\end{array}$ & $\mathrm{ND}(2)$ & $\mathrm{ND}(2)$ \\
\hline GARRETSON & YKBKGND-G-39536 & $12 / 14 / 16$ & $n$ & $\begin{array}{l}\text { AR } \\
\text { AIR }\end{array}$ & $x$ & $\begin{array}{l}\mathrm{ND}(1.1) \\
\mathrm{ND}(1.1)\end{array}$ & $\begin{array}{l}1.3 \\
1.3\end{array}$ & $\begin{array}{l}\mathrm{ND}(1.5) \\
\mathrm{ND}(1.5)\end{array}$ & ND (2.1) & ND $(0.7)$ & ND (12) & ND (0.98) & $\begin{array}{l}4.4 \\
4.7\end{array}$ & ND (1.2) & ND (2) & $\mathrm{ND}(2)$ \\
\hline $\begin{array}{l}\text { FITWORX } \\
\text { FITOR }\end{array}$ & 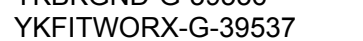 & $12 / 14 / 16$ & $\mathrm{~N}$ & AlR & & ND (1.1) & 1.5 & ND (1.5) & ND (2.1) & 1.4 & 24 & ND (0.98) & 5.1 & ND (1.2) & ND ( 21 & $\mathrm{ND}(2)$ \\
\hline FITWORX & YKFITWORX-G-39538 & $12 / 14 / 16$ & DUP-F & AIR & & ND (1.1) & 1.3 & ND (1.5) & ND (2.1) & 1.4 & 25 & ND (0.98) & 4.3 & ND (1.2) & $\mathrm{ND}(2)$ & ND (2) \\
\hline FITWORX & YKFITWORX-G-39539 & $12 / 14 / 16$ & $\mathrm{~N}$ & AIR & & ND (1.1) & 2.3 & ND (1.5) & $\mathrm{ND}(2.1)$ & 1.7 & 30 & $\mathrm{ND}(0.98)$ & 7.6 & $\mathrm{ND}(1.2)$ & $\mathrm{ND}(2)$ & $\mathrm{ND}(2)$ \\
\hline FITWORX & YKFITWORX-G-39540 & $12 / 14 / 16$ & $\mathrm{~N}$ & AIR & & ND (1.1) & 1.0 & ND (1.5) & $\mathrm{ND}(2.1)$ & 1.4 & 22 & ND $(0.98)$ & 3.4 & $\mathrm{ND}(1.2)$ & $\mathrm{ND}$ (2) & $\mathrm{ND}(2)$ \\
\hline $\begin{array}{l}\text { FITWORX } \\
\text { FITWORDY }\end{array}$ & YKFITWORX-G-39541 & $12 / 14 / 16$ & $\mathrm{~N}$ & AIR & & ND (1.1) & $N D(0.87)$ & ND (1.5) & ND (2.1) & 1.2 & 21 & ND (0.98) & $\mathrm{ND}(2.2)$ & ND (1.2) & $\mathrm{ND}(2)$ & ND (2) \\
\hline $\begin{array}{l}\text { FITWORX } \\
\text { FTWWORX }\end{array}$ & $\begin{array}{l}\text { YKBKGND-G-39542 } \\
\text { YKEITHOPX }\end{array}$ & $12 / 14416$ & $N_{n}^{N}$ & $\begin{array}{l}\text { AIR } \\
\text { SOIL GAS }\end{array}$ & $\mathrm{x}$ & $\mathrm{ND}(1.1)$ & $\mathrm{ND}(0.87)$ & ND (1.5) & $\mathrm{ND}(2.1)$ & ND (0.7) & ND (12) & ND (0.98) & $\mathrm{ND}(2.2)$ & ND (1.2) & ND (2) & $\mathrm{ND}(2)$ \\
\hline $\begin{array}{l}\text { FITWORX } \\
\text { FITS }\end{array}$ & 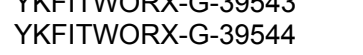 & $\begin{array}{l}12 / 14 / 16 \\
12 / 16\end{array}$ & DUP-F & SOIL GAS & & ND (11) & $\mathrm{ND}(8.7)$ & ND (15) & ND (21) & ND (7) & ND (120) & ND (9.8) & 24 & ND (12) & ND (20) & ND (20) \\
\hline & & & & & & & & & & & Concentra & $\left(\mu \mathrm{g} / \mathrm{m}^{3}\right)$ & & & & \\
\hline & & & Sample & Sample & & & & & & & & & & & & \\
\hline Location & Sample & Date & Type & Matrix & Sample & Butyl Ketone & $\begin{array}{l}\text { Butyl Ether } \\
\text { But }\end{array}$ & Methylbenzene & Naphthalene & N-Butylbenzene & $\mathrm{N}$-Heptane & Propylbenzene & (Monomer) & Alcohol & Tert-Buty|benzene & Tetrachloroethene \\
\hline WILLOW & YKWILLOW-G-37887 & $8 / 5 / 15$ & $\mathrm{~N}$ & AIR & & ND (2) & ND (0.72) & 1.6 & $\mathrm{ND}(2.6)$ & ND (1.1) & $\mathrm{ND}(0.82)$ & ND (0.98) & $\mathrm{ND}(0.85)$ & ND (15) & $\mathrm{ND}(1.1)$ & ND (1.4) \\
\hline WILLOW & YKWILLOW-G-37885 & $8 / 5 / 15$ & $\mathrm{~N}$ & AIR & & ND (2) & $N D(0.72)$ & 2.0 & ND ( & ND (1.1) & $\mathrm{ND}(0.82)$ & & & & & \\
\hline $\begin{array}{l}\text { WLLOW } \\
\text { WHLIOW }\end{array}$ & $\begin{array}{l}\text { YKWLLLOW-G-37886 } \\
\text { YKWWLLOW-G-3788 }\end{array}$ & $\begin{array}{l}8 / 5 / 15 \\
851 / 15\end{array}$ & $\begin{array}{c}n \\
N\end{array}$ & $\begin{array}{l}A A R \\
A A R\end{array}$ & & $\begin{array}{ll}\mathrm{ND}(2) \\
\mathrm{ND}(2)\end{array}$ & $\begin{array}{l}\text { ND }(0.72) \\
N D(072)\end{array}$ & $\begin{array}{l}1.1 \\
10\end{array}$ & $\begin{array}{ll}\mathrm{ND}(2.6) \\
\mathrm{ND}(2.6)\end{array}$ & $\begin{array}{l}\mathrm{ND}(1.1) \\
\mathrm{ND}(1.1)\end{array}$ & $\begin{array}{l}\mathrm{ND}(0.82) \\
\mathrm{ND}(0.82)\end{array}$ & $\mathrm{ND}(0.98)$ & & & & ND (1.4) \\
\hline $\begin{array}{l}\text { WILLOW } \\
\text { WILLOW }\end{array}$ & $\begin{array}{l}\text { YKWILLOW-G-G3889 } \\
\text { YKWILLOW-G-3789- }\end{array}$ & $\begin{array}{l}85 / 15 \\
85 / 15\end{array}$ & $\begin{array}{l}n \\
N\end{array}$ & $\begin{array}{l}\text { AAR } \\
\text { AlR }\end{array}$ & & $\begin{array}{l}\text { ND } \\
\text { ND }\end{array}$ & $\begin{array}{l}\mathrm{ND}(0 . \\
\mathrm{ND}(0 .\end{array}$ & $\begin{array}{l}1.0 \\
1.0\end{array}$ & $\begin{array}{l}\text { ND } 2(2 \\
\text { ND } 2(2\end{array}$ & ND (1.1) & $N 0(0.82)$ & ND $(0.98)$ & $\begin{array}{ll}\mathrm{ND}(0.85) \\
\mathrm{ND}(085)\end{array}$ & ND & $\begin{array}{ll}\mathrm{ND} & 1 \\
\mathrm{ND} & 1\end{array}$ & $\begin{array}{l}\mathrm{ND}(1.4) \\
\mathrm{ND}(1.4)\end{array}$ \\
\hline WILLOW & YKWWILLOW-G-G-37890 & $\begin{array}{l}8 / 5 / 15 \\
8 / 5 / 15\end{array}$ & $n^{n}$ & $\begin{array}{l}\text { AAR } \\
\text { AlR }\end{array}$ & & ND & ND $(0$. & $\begin{array}{l}1.5 \\
0.9\end{array}$ & ND $(2$ & ND (1.1) & ND $(0.82)$ & ND $(0.98)$ & ND $(0.85)$ & & ND (1.1) & $\begin{array}{l}\mathrm{ND}(1.4) \\
\mathrm{ND}(1.4)\end{array}$ \\
\hline CVA & YKCVA-G-37909 & $8 / 5 / 15$ & $\mathrm{~N}$ & AlR & & $\mathrm{ND}(2)$ & $\mathrm{ND}(0.72)$ & 4.6 & ND $(2.6)$ & ND (1.1) & ND (0.82) & ND $(0.98)$ & ND $(0.85)$ & ND & ND (1.1) & ND (1.4) \\
\hline CVA & YKCVA & & $\mathrm{N}$ & AlR & & & ND $(0.7$ & 6.5 & ND $(2.6)$ & ND (1.1) & ND (0.82) & ND (0.98) & 1.2 & & ND (1.1) & ND (1.4) \\
\hline CVA & YKCVA-G-37912 & $8 / 5$ & $\mathrm{~N}$ & AIR & & $\mathrm{ND}(2)$ & $\mathrm{ND}(0.72)$ & 4.9 & $\mathrm{ND}(2.6)$ & ND (1.1) & $\mathrm{ND}(0.82)$ & $\mathrm{ND}(0.98)$ & 1.1 & ND (15) & $\mathrm{ND}(1.1)$ & $\mathrm{ND}(1.4)$ \\
\hline PRES & YKPRES-G-37904 & $8 / 5 /$ & $\mathrm{N}$ & AIR & & ND & ND $(0$ & 5.4 & & ND (1.1) & $\mathrm{ND}(0.82)$ & ND (0.98) & $\mathrm{ND}(0.85)$ & ND & $\mathrm{ND}(1.1)$ & ND (1.4) \\
\hline $\begin{array}{l}\text { PRES } \\
\text { FITWORX }\end{array}$ & $\begin{array}{l}\text { YKPRES-G-37903 } \\
\text { YKRETWORX-G-378 }\end{array}$ & $8 / 5 / 15$ & $N$ & AIR & & $\mathrm{ND}(2)$ & $N D(0.72)$ & $\begin{array}{l}42 \\
17\end{array}$ & ND (2.6) & ND (1.1) & ND $(0.82)$ & ND (0.98) & ND $(0.85)$ & & ND (1.1) & ND (1.4) \\
\hline $\begin{array}{l}\text { FIWWORX } \\
\text { FITWORX }\end{array}$ & $\begin{array}{l}\text { YKFIIWORX-G-3898 } \\
\text { YKEITWORX-G-37897 }\end{array}$ & $\begin{array}{l}8 / 5 / 15 \\
8 / 5 / 15\end{array}$ & $n^{N}$ & $\begin{array}{l}A A R \\
A \| R\end{array}$ & & $\begin{array}{ll}\mathrm{ND}(2) \\
\mathrm{ND}(2)\end{array}$ & $\begin{array}{l}\text { ND }(0 . \\
\text { ND } 0 .\end{array}$ & $\begin{array}{l}1.7 \\
29\end{array}$ & $\begin{array}{l}\mathrm{ND}(2.6) \\
\mathrm{ND}(2.6)\end{array}$ & $\begin{array}{l}\mathrm{ND}(1.1) \\
\mathrm{ND}(1.1)\end{array}$ & $\begin{array}{ll}\mathrm{ND}(0.82) \\
0.9\end{array}$ & $\begin{array}{l}\text { ND }(0.98) \\
\text { ND }(0.98)\end{array}$ & ND $(0.85)$ & $\begin{array}{ll}\mathrm{ND} \\
\mathrm{ND}\end{array}$ & $\begin{array}{l}\mathrm{ND}(1.1) \\
\mathrm{ND}(1.1)\end{array}$ & ND (1.4) \\
\hline $\begin{array}{l}\text { FITWORX } \\
\text { FilW }\end{array}$ & 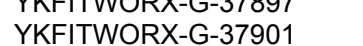 & $\begin{array}{l}8 / 51 \\
8151\end{array}$ & DUP-F & $\begin{array}{l}\text { AIR } \\
\text { AlR }\end{array}$ & & ND & $\begin{array}{l}\text { NDD ( } 0 . \\
\text { ND } 0 .\end{array}$ & $\begin{array}{l}2.9 \\
2.6\end{array}$ & $\mathrm{ND}(2$ & ND (1.1) & $\begin{array}{l}0.9 \\
1.1\end{array}$ & ND (0.98) & ND i 10 & ND & ND (1.1) & ND (1.4) \\
\hline & $\begin{array}{l}\text { RX-G-375 } \\
\text { RX-G-37 }\end{array}$ & $\begin{array}{l}8 / 5 / 15 \\
8 / 5\end{array}$ & $n^{n-1}$ & AlR & & ND & ND 10 & $\begin{array}{l}.26 \\
3.9\end{array}$ & ND & ND (1.1) & $\begin{array}{l}1.1 \\
1.7\end{array}$ & ND $(0.98)$ & ND 1085 & & $\mathrm{ND}(1.1)$ & ND (1.4) \\
\hline FITWORX & YKFITWORX-G-37899 & $8 / 5 / 15$ & $\mathrm{~N}$ & AlR & & ND (2) & ND $(0$ & $\begin{array}{l}.5 \\
3.8\end{array}$ & ND $(2.6)$ & ND (1.1) & 1.4 & ND (0.98) & ND (0.85) & ND (15) & ND (1.1) & ND (1.4) \\
\hline FITWORX & YKFITWORX-G-37902 & $8 / 5 / 15$ & $\mathrm{~N}$ & AlR & & & ND $(0.72)$ & 1.0 & ND (2.6) & ND (1.1) & ND $(0.82)$ & ND $(0.98)$ & ND $(0.85)$ & ND & ND (1.1) & ND (1.4) \\
\hline FITWORX & YKBKGND-G-37908 & $8 / 5$ & $\mathrm{~N}$ & AIR & $x$ & $\mathrm{ND}(2)$ & ND $(0$. & $\mathrm{ND}(0.75)$ & ND i & ND (1.1) & $\mathrm{ND}(0.82)$ & $\mathrm{ND}(0.98)$ & $\mathrm{ND}(\mathrm{C}$ & ND & ND (1.1) & ND (1.4) \\
\hline HEARTH & YKHEARTH-G-37892 & $8 / 5 / 15$ & $\mathrm{~N}$ & $\mathrm{AIR}$ & & ND (2) & $N D(0.72)$ & 0.8 & ND (2.6) & ND (1.1) & ND $(0.82)$ & ND $(0.98)$ & $\mathrm{ND}(0.85)$ & ND (15) & ND (1.1) & ND (1.4) \\
\hline $\begin{array}{l}\text { HEATH } \\
\text { HEARTH }\end{array}$ & $\begin{array}{l}\text { YKHEARTH-G-37893 } \\
\text { YKHEARTH-G-37896 }\end{array}$ & $885 / 15$ & $\begin{array}{l}\mathrm{N} \\
\mathrm{N}\end{array}$ & $\begin{array}{l}{ }_{A}^{A R R} \\
\text { AlR }\end{array}$ & & $\mathrm{ND}(2)$ & $\begin{array}{ll}N D(0.72) \\
N D\end{array}$ & 0.9 & $\mathrm{ND}(2.6)$ & ND (1.1) & $\begin{array}{ll}N D(0.82) \\
N D(0.82)\end{array}$ & $\begin{array}{l}\mathrm{ND}(0.98) \\
\mathrm{ND}(0.98)\end{array}$ & $\begin{array}{ll}\mathrm{ND}(0.85) \\
\mathrm{ND}(0.85)\end{array}$ & $\begin{array}{ll}N D(15) \\
N D(15)\end{array}$ & $\mathrm{ND}(1.1)$ & ND (1.4) \\
\hline HEARTH & & & & & & & & & & & & & & & & $\mathrm{ND}(1.4)$ \\
\hline
\end{tabular}


Concentration $\left(\mu \mathrm{g} / \mathrm{m}^{3}\right)$

\begin{tabular}{|c|c|c|c|c|c|c|c|c|c|c|c|c|c|c|c|c|}
\hline \multirow[b]{2}{*}{ Location } & \multirow[b]{2}{*}{ Sample } & \multirow[b]{2}{*}{$\begin{array}{c}\text { Sample } \\
\text { Date }\end{array}$} & \multirow[b]{2}{*}{$\begin{array}{c}\text { Sample } \\
\text { Type }\end{array}$} & \multirow[b]{2}{*}{$\begin{array}{l}\text { Sample } \\
\text { Matrix }\end{array}$} & \multirow[b]{2}{*}{$\begin{array}{l}\text { Ambient } \\
\text { Sample }\end{array}$} & \multicolumn{11}{|c|}{ Concentration $\left(\mu \mathrm{g} / \mathrm{m}^{3}\right)$} \\
\hline & & & & & & $\begin{array}{l}\text { Methyl N- } \\
\text { Butyl Ketone }\end{array}$ & $\begin{array}{l}\text { Methyl Tert- } \\
\text { Butyl Ether }\end{array}$ & Methylbenzene & Naphthalene & N-Butylbenzene & N-Heptane & $\begin{array}{c}N- \\
\text { Propylbenzene }\end{array}$ & $\begin{array}{l}\text { Styrene } \\
\text { (Monomer) }\end{array}$ & $\begin{array}{l}\text { Tert-Butyl } \\
\text { Alcohol }\end{array}$ & Tert-Butylbenzene & Tetrachloroethene \\
\hline HEARTH & YKHEARTH-G-37895 & $8 / 5 / 15$ & $\mathrm{~N}$ & AIR & & ND (2) & $\mathrm{ND}(0.72)$ & 1.7 & $\mathrm{ND}(2.6)$ & $\mathrm{ND}(1.1)$ & $\mathrm{ND}(0.82)$ & $\mathrm{ND}(0.98)$ & ND (0.85) & ND (15) & $\mathrm{ND}(1.1)$ & 2.6 \\
\hline HEARTH & YKHEARTH-G-37891 & $8 / 5 / 15$ & $\mathrm{~N}$ & AIR & & ND (2) & $\mathrm{ND}(0.72)$ & 0.8 & ND (2.6) & ND (1.1) & $\mathrm{ND}(0.82)$ & ND $(0.98)$ & ND $(0.85)$ & ND (15) & ND (1.1) & ND (1.4) \\
\hline HEARTH & YKHEARTH-G-37894 & $8 / 5 / 15$ & $\mathrm{~N}$ & AIR & & $\mathrm{ND}(2)$ & ND (0.72) & 1.3 & $\mathrm{ND}(2.6)$ & ND (1.1) & $\mathrm{ND}(0.82)$ & ND (0.98) & ND (0.85) & ND (15) & ND (1.1) & ND (1.4) \\
\hline $\begin{array}{l}\text { IEARTH } \\
\text { TROES }\end{array}$ & $\begin{array}{l}\text { YYBKGNDD-G-37907 } \\
\text { YKTROFS-G-37913 }\end{array}$ & $8 / 5 / 15$ & $\begin{array}{r}\mathrm{N} \\
\mathrm{N}\end{array}$ & AIR & $x$ & ND (2) & ND (0.72) & ND (0.75) & $\mathrm{ND}(2.6)$ & ND (1.1) & $\mathrm{ND}(0.82)$ & ND (0.98) & $\mathrm{ND}(0.85)$ & ND (15) & ND (1.1) & $\mathrm{ND}(1.4)$ \\
\hline $\begin{array}{l}\text { TROES } \\
\text { TROES }\end{array}$ & $\begin{array}{l}\text { YKTROES-G-37913 } \\
\text { YKTROES-G-37906 }\end{array}$ & $885 / 15$ & $\begin{array}{l}n \\
N\end{array}$ & $\begin{array}{l}A I R \\
A I R\end{array}$ & & $\begin{array}{l}\mathrm{ND}(2) \\
\mathrm{ND}(2)\end{array}$ & $\begin{array}{l}\mathrm{ND}(0.72) \\
\mathrm{ND}(072)\end{array}$ & $\begin{array}{l}180 \mathrm{D}^{\mathrm{e}} \\
190 \mathrm{D}\end{array}$ & $\mathrm{ND}(2.6)$ & $\mathrm{ND}(1.1)$ & 1.4 & $\mathrm{ND}(0.98)$ & 2.1 & ND (15) & $\mathrm{ND}(1.1)$ & 2.7 \\
\hline $\begin{array}{l}\text { TROES } \\
\text { TROES }\end{array}$ & $\begin{array}{l}\text { YKTROES-G-397906 } \\
\text { YKBKGND-G-37914 }\end{array}$ & $\begin{array}{l}8 / 5 / 15 \\
8 / 5 / 15\end{array}$ & $\begin{array}{l}\mathrm{N} \\
\mathrm{N}\end{array}$ & $\begin{array}{l}\text { AlR } \\
\text { AlR }\end{array}$ & $x$ & $\begin{array}{l}\mathrm{ND}(2) \\
\mathrm{ND}(2)\end{array}$ & $\begin{array}{l}\mathrm{ND}(0.72) \\
\mathrm{ND}(0.72)\end{array}$ & $\begin{array}{l}190 \mathrm{D} \\
\mathrm{ND}(0.75)\end{array}$ & $\begin{array}{l}\mathrm{ND}(2.6) \\
\mathrm{ND}(2.6)\end{array}$ & $\begin{array}{l}\mathrm{ND}(1.1) \\
\mathrm{ND}(1.1)\end{array}$ & $\begin{array}{l}11.8 \\
\mathrm{ND}(0.82)\end{array}$ & $\begin{array}{l}\mathrm{ND}(0.98) \\
\mathrm{ND}(0.98)\end{array}$ & $\begin{array}{l}2.6 \\
\text { ND }(0.85)\end{array}$ & $\begin{array}{l}\text { ND (15) } \\
\text { ND (15) }\end{array}$ & $\begin{array}{l}\mathrm{ND}(1.1) \\
\mathrm{ND}(1.1)\end{array}$ & $\begin{array}{l}3.0 \\
\mathrm{ND}(1.4)\end{array}$ \\
\hline MILLER & YKBKGND-G-37915 & $88 / 15$ & $\mathrm{~N}$ & AlR & $\hat{x}$ & ND (2) & ND (0.72) & ND (0.75) & ND (2.6) & ND (1.1) & ND (0.82) & ND (0.98) & $\mathrm{ND}(0.85)$ & ND (15) & ND (1.1) & $\begin{array}{l}\text { ND (1.4) } \\
\text { ND (1.4) }\end{array}$ \\
\hline GARRETSON & YKGARRETSON-G-38036 & $1 / 28 / 16$ & $\mathrm{~N}$ & AIR & & ND (2) & ND (0.72) & 2.9 & ND (2.6) & ND (1.1) & ND $(0.82)$ & 1.3 & ND $(0.85)$ & ND (15) & ND (1.1) & 2.0 \\
\hline GARRETSON & YKGARRETSON-G-38037 & $1 / 28 / 16$ & $\mathrm{~N}$ & AIR & & ND (2) & ND (0.72) & 3.0 & ND (2.6) & ND (1.1) & $\mathrm{ND}(0.82)$ & ND $(0.98)$ & ND $(0.85)$ & ND (15) & ND (1.1) & 2.3 \\
\hline GARRETSON & YKGARRETSON-G-38038 & $1 / 28 / 16$ & DUP-F & AIR & & ND (2) & $\mathrm{ND}(0.72)$ & 3.2 & $\mathrm{ND}(2.6)$ & $\mathrm{ND}(1.1)$ & ND $(0.82)$ & ND $(0.98)$ & 1.1 & ND (15) & $\mathrm{ND}(1.1)$ & 2.4 \\
\hline GARRETSON & YKBKGND-G-38039 & $1 / 28 / 16$ & $N$ & AIR & $\mathrm{x}$ & ND (2) & ND $(0.72)$ & ND (0.75) & ND (2.6) & ND (1.1) & ND $(0.82)$ & ND $(0.98)$ & $N D(0.85)$ & ND (15) & ND (1.1) & ND (1.4) \\
\hline $\begin{array}{l}\text { FITWORX } \\
\text { FITWORX }\end{array}$ & $\begin{array}{l}\text { YKFITWORX-G-38040 } \\
\text { YKEITWWORX-G-3040 }\end{array}$ & $\begin{array}{l}1 / 28 / 16 \\
1 / 2816\end{array}$ & $\stackrel{N}{N}$ & AlR & & ND (2) & $\mathrm{ND}(0.72)$ & 7.6 & $\mathrm{ND}(2.6)$ & $\mathrm{ND}(1.1)$ & $\mathrm{ND}(0.82)$ & ND (0.98) & $N D(0.85)$ & ND (15) & ND (1.1) & $N D(1.4)$ \\
\hline $\begin{array}{l}\text { FITWOORX } \\
\text { FITWORX }\end{array}$ & 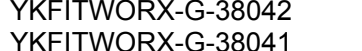 & $\begin{array}{l}1 / 28 / 16 \\
1 / 28 / 16\end{array}$ & $\begin{array}{l}N \\
\text { DUP-F }\end{array}$ & $\begin{array}{l}{ }_{A I R} \\
\text { AIR }\end{array}$ & & $\begin{array}{l}\mathrm{ND}(2) \\
\mathrm{ND}(2)\end{array}$ & $\begin{array}{l}\mathrm{ND}(0.72) \\
\mathrm{ND}(0.72)\end{array}$ & $\begin{array}{l}6.3 \\
8.5\end{array}$ & $\begin{array}{l}\mathrm{ND}(2.6) \\
\mathrm{ND}(2.6)\end{array}$ & $\begin{array}{l}\mathrm{ND}(1.1) \\
\mathrm{ND}(1.1)\end{array}$ & $\begin{array}{l}1.0 \\
1.2\end{array}$ & $\begin{array}{l}\mathrm{ND}(0.98) \\
\mathrm{ND}(0.98)\end{array}$ & $\begin{array}{ll}N D(0.85) \\
N\end{array}$ & ND (15) & $\begin{array}{l}\mathrm{ND}(1.1) \\
\mathrm{ND}(1.1)\end{array}$ & ND (1.4) \\
\hline $\begin{array}{l}\text { FITWORX } \\
\text { FIWWOAX }\end{array}$ & $\begin{array}{l}\text { YKFIIWORX-G-30041 } \\
\text { YKFITWORX-G-38043 }\end{array}$ & $\begin{array}{l}1 / 28 / 16 \\
1 / 28 / 6\end{array}$ & DUP-F & AIR & & $\begin{array}{l}\mathrm{ND}(2) \\
\mathrm{ND}(2)\end{array}$ & $\begin{array}{l}\mathrm{ND}(0.72) \\
\mathrm{ND}(0.72)\end{array}$ & $\begin{array}{l}8.5 \\
7.5\end{array}$ & $\mathrm{ND}(2.6)$ & $\mathrm{ND}(1.1)$ & $\begin{array}{l}1.2 \\
1.3\end{array}$ & $\mathrm{ND}(0.98)$ & ND ( $(0.85)$ & ND (15) & ND (1.1) & $\begin{array}{l}\mathrm{ND}(1.4) \\
\mathrm{ND}(1.4)\end{array}$ \\
\hline $\begin{array}{l}\text { FITWORX } \\
\text { FITW }\end{array}$ & 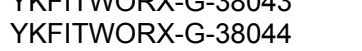 & $\begin{array}{l}1 / 28 / 16 \\
1 / 28 / 6\end{array}$ & $\mathrm{~N}^{\mathrm{N}} \mathrm{r}-\mathrm{a}$ & $\begin{array}{l}\text { AlR } \\
\text { AIR }\end{array}$ & & $\mathrm{ND}(2)$ & $\mathrm{ND}(0.72)$ & 7.7 & $\mathrm{ND}(2.6)$ & ND (1.1) & 1.2 & ND (0.98) & $\mathrm{ND}(0.85)$ & ND (15) & ND (1.1) & ND (1.4) \\
\hline FITWORX & YKBKGND-G-38045 & $1 / 28 / 16$ & N & AIR & $\mathrm{x}$ & ND (2) & ND $(0.72)$ & 0.8 & $\mathrm{ND}(2.6)$ & ND (1.1) & ND $(0.82)$ & $\mathrm{ND}(0.98)$ & ND $(0.85)$ & ND (15) & ND (1.1) & $\mathrm{ND}(1.4)$ \\
\hline & YKGARRETSON-G-38046 & $1 / 28 / 16$ & $\mathrm{~N}$ & SOIL GAS & & ND (17) & $=1$ & 38 & (No) & 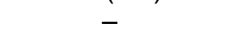 & $-x_{1}$ & $-x_{1}$ & $\mathrm{ND}(8.6)^{\prime}$ & & $=$ - & 30 \\
\hline FITWORX & YKFITWORX-G-38048 & $1 / 28 / 16$ & $\mathrm{~N}$ & SOIL GAS & & ND (17) & - & 30 & - & - & - & $-{ }^{-}$ & $\mathrm{ND}(8.6)$ & - & - & ND (14) \\
\hline $\begin{array}{l}\text { FITWORX } \\
\text { FITWORX }\end{array}$ & $\begin{array}{l}\text { YKFITWORX-G-38081 } \\
\text { YKFITORX-G-308 }\end{array}$ & $\begin{array}{l}4 / 28 / 16 \\
4 / 2816\end{array}$ & $\frac{1}{n}$ & $\begin{array}{l}\text { AIR } \\
\text { AIR }\end{array}$ & & ND (2) & $N D(0.72)$ & $\begin{array}{l}6.4 \\
8.1\end{array}$ & $\mathrm{ND}(2.6)$ & $\mathrm{ND}(1.1)$ & 0.8 & ND (0.98) & $N D(0.85)$ & ND (15) & ND (1.1) & ND (1.4) \\
\hline $\begin{array}{l}\text { FITWWORX } \\
\text { FITWORX }\end{array}$ & $\begin{array}{l}\text { YKFITWORX-G-38082 } \\
\text { YKFITWWORX-G-30083 }\end{array}$ & $\begin{array}{l}4 / 28 / 16 \\
4 / 28 / 16\end{array}$ & $\begin{array}{l}\text { DUP-F } \\
\text { N }\end{array}$ & $\begin{array}{l}\text { AIR } \\
\text { AlR }\end{array}$ & & $\begin{array}{l}\mathrm{ND}(2) \\
\mathrm{ND}(2)\end{array}$ & $\begin{array}{l}\mathrm{ND}(0.72) \\
\mathrm{ND}(0.72)\end{array}$ & $\begin{array}{l}8.1 \\
3.6\end{array}$ & $\begin{array}{l}\mathrm{ND}(2.6) \\
\mathrm{ND}(2.6)\end{array}$ & $\begin{array}{l}\text { ND (1.1) } \\
\text { ND (1.1) }\end{array}$ & $\begin{array}{l}1.1 \\
\mathrm{ND}(0.82)\end{array}$ & $\begin{array}{l}\text { ND ( }(0.98) \\
\text { ND }(0.98)\end{array}$ & $\begin{array}{l}\text { NDD ( }(0.85) \\
\text { ND }(0.85)\end{array}$ & $\begin{array}{l}\text { ND (15) } \\
\text { ND (15) }\end{array}$ & $\begin{array}{l}\text { ND (1.1) } \\
\text { ND (1.1) }\end{array}$ & $\begin{array}{l}\text { ND (1.4) } \\
\text { ND (1.4) }\end{array}$ \\
\hline FITWORX & YKFITWORX-G-38084 & $4 / 28 / 16$ & $\mathrm{~N}$ & AlR & & ND (2) & $\mathrm{ND}(0.72)$ & ND $(0.75)$ & $\mathrm{ND}(2.6)$ & ND (1.1) & ND (0.82) & ND (0.98) & ND ( $(0.85)$ & ND (15) & ND (1.1) & ND (1.4) \\
\hline FITWORX & YKFITWORX-G-38085 & $4 / 28 / 16$ & $\mathrm{~N}$ & AIR & & ND (2) & $\mathrm{ND}(0.72)$ & 7.5 & ND (2.6) & ND (1.1) & 0.9 & ND $(0.98)$ & $\mathrm{ND}(0.85)$ & ND (15) & ND (1.1) & ND (1.4) \\
\hline $\begin{array}{l}\text { FITWORX } \\
\text { FITWORX }\end{array}$ & $\begin{array}{l}\text { YKBKGNDD-G-38086 } \\
\text { YKEITHOPX-38087 }\end{array}$ & $4 / 28 / 16$ & $\mathrm{~N}$ & AIR & $\mathrm{x}$ & $\mathrm{ND}(2)$ & $\mathrm{ND}(0.72)$ & $\mathrm{ND}(0.75)$ & $\mathrm{ND}(2.6)$ & $\mathrm{ND}(1.1)$ & $N D(0.82)$ & ND $(0.98)$ & $N D(0.85)$ & ND (15) & $\mathrm{ND}(1.1)$ & $N D(1.4)$ \\
\hline $\begin{array}{l}\text { FITWORX } \\
\text { FIWUR }\end{array}$ & $\begin{array}{l}\text { YKFIIWWORX-G-G3008/ } \\
\text { YKFITWORX-G-38088 }\end{array}$ & $\begin{array}{l}4 / 28816 \\
4 / 28 / 16\end{array}$ & DUP-F & $\begin{array}{l}\text { SOIL GAS } \\
\text { SOIL GAS }\end{array}$ & & $\begin{array}{l}\text { ND ( } 20) \\
\text { ND (20) }\end{array}$ & $\begin{array}{l}\mathrm{ND}(7.2) \\
\mathrm{ND}(7.2)\end{array}$ & $\mathrm{ND}(7.5)$ & $\begin{array}{l}\mathrm{ND}(26) \\
\mathrm{ND}(26)\end{array}$ & $\begin{array}{l}\text { ND (11) } \\
\text { ND (11) }\end{array}$ & $\begin{array}{l}\mathrm{ND}(8.2) \\
\mathrm{ND}(8.2)\end{array}$ & $\begin{array}{l}\mathrm{ND}(9.8) \\
\mathrm{ND}(9.8)\end{array}$ & $\begin{array}{l}\mathrm{ND}(8.5) \\
\mathrm{ND}(8.5)\end{array}$ & $\begin{array}{l}\mathrm{ND}(150) \\
\mathrm{ND}(150)\end{array}$ & $\begin{array}{l}\text { ND (11) } \\
\text { ND (11) }\end{array}$ & $\begin{array}{l}\text { ND (14) } \\
\text { ND (14) }\end{array}$ \\
\hline GARRETSON & YKGARRETSON-G-38140 & $8 / 10 / 16$ & $\mathrm{~N}$ & AIR & & $\mathrm{ND}(2)$ & ND (0.72) & 5.9 & ND (2.6) & $\mathrm{ND}(1.1)$ & 1.0 & ND (0.98) & 3.0 & ND (15) & ND (1.1) & 4.0 \\
\hline GARRETSON & YKGARRETSON-G-38138 & $8 / 10 / 16$ & $\mathrm{~N}$ & AIR & & $\mathrm{ND}(2)$ & $\mathrm{ND}(0.72)$ & 5.7 & ND (2.6) & ND (1.1) & ND $(0.82)$ & ND (0.98) & 2.7 & ND (15) & $\mathrm{ND}(1.1)$ & 4.2 \\
\hline $\begin{array}{l}\text { GARRETSON } \\
\text { FITWORX }\end{array}$ & $\begin{array}{l}\text { YKBKGND-G-38141 } \\
\text { YKEITWORX-G-38148 }\end{array}$ & $\begin{array}{l}8 / 10 / 16 \\
8 / 10 / 6\end{array}$ & $\begin{array}{c}n \\
N\end{array}$ & $\begin{array}{l}\text { AIR } \\
\text { SOlL GAS }\end{array}$ & $\mathrm{x}$ & ND (2) & $\mathrm{ND}(0.72)$ & $\begin{array}{c}7.9 \\
\text { ND }(7.5)\end{array}$ & $\begin{array}{l}\mathrm{ND}(2.6) \\
\mathrm{ND}(26)\end{array}$ & $\begin{array}{l}\mathrm{ND}(1.1) \\
\mathrm{ND}(11)\end{array}$ & ND $(0.82)$ & $\begin{array}{l}\mathrm{ND}(0.98) \\
\mathrm{ND}(98)\end{array}$ & $\mathrm{ND}(0.85)$ & ND (15) & $\mathrm{ND}(1.1)$ & $\mathrm{ND}(1.4)$ \\
\hline $\begin{array}{l}\text { FITWORX } \\
\text { FITUR }\end{array}$ & 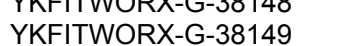 & $\begin{array}{l}8 / 10 / 16 \\
8 / 16\end{array}$ & DUP-F & $\begin{array}{l}\text { SOIL GAS } \\
\text { SAL }\end{array}$ & & ND (20) & $\mathrm{ND}(7.2)$ & $\mathrm{ND}(7.5)$ & ND (26) & ND (11) & ND (8.2) & ND (9.8) & $\mathrm{ND}(8.5)$ & ND (150) & ND (11) & $\begin{array}{l}\text { ND (14) } \\
\text { ND (14) }\end{array}$ \\
\hline $\begin{array}{l}\text { FITWORX } \\
\text { FIN }\end{array}$ & YKFITWORX-G-38142 & $\begin{array}{l}8 / 10 / 16 \\
8 / 16\end{array}$ & n & AIR & & ND (2) & $\mathrm{ND}(0.72)$ & 10 & ND (2.6) & ND (1.1) & 1.6 & ND (0.98) & $\mathrm{ND}(0.85)$ & ND (15) & ND (1.1) & ND (1.4) \\
\hline FITWO & G-38143 & $8 / 10 / 16$ & DUP-F & AIR & & ND (2) & ND (0.72) & 12 & $\mathrm{ND}(2.6)$ & ND (1.1) & 1.5 & ND $(\mathrm{C}$ & ND $(0.85)$ & ND & ND (1.1) & $\mathrm{ND}(1.4)$ \\
\hline FITWORX & RX-G-38144 & $8 / 10 / 16$ & $\mathrm{~N}$ & AIR & & ND (2) & $\mathrm{ND}(0.72)$ & 9.7 & ND (2.6) & ND (1.1) & 1.2 & $\mathrm{ND}(0.98)$ & ND $(0.85)$ & ND (15) & ND (1.1) & ND (1.4) \\
\hline $\begin{array}{l}\text { FITWORX } \\
\text { FITWOA }\end{array}$ & YKFITWORX-G-38145 & $8 / 10 / 16$ & $\mathrm{~N}$ & AIR & & ND (2) & $\mathrm{ND}(0.72)$ & 13 & ND (2.6) & ND (1.1) & 1.8 & $\mathrm{ND}(0.98)$ & ND $(0.85)$ & ND (15) & ND (1.1) & ND (1.4) \\
\hline FITWORX & YKFITWORX-G-38146 & $8 / 10 / 16$ & $\mathrm{~N}$ & AIR & & ND (2) & $\mathrm{ND}(0.72)$ & 13 & ND (2.6) & ND (1.1) & 1.5 & ND ( & ND $(0.85)$ & ND (15) & ND (1.1) & ND (1.4) \\
\hline $\begin{array}{l}\text { FITWORX } \\
\text { GARRFTSON }\end{array}$ & $\begin{array}{l}\text { YKBKGND-G-38147 } \\
\text { YKGABRETSONG-39533 }\end{array}$ & $\begin{array}{l}8 / 10 / 16 \\
12 / 14 / 16\end{array}$ & $N^{n}$ & $\begin{array}{ll}A I R \\
A I R\end{array}$ & $\mathrm{x}$ & ND (2) & ND (0.72) & 0.8 & $\mathrm{ND}(2.6)$ & ND (1.1) & $\mathrm{ND}(0.82)$ & ND (0.98) & ND (0.85) & ND ( & ND (1.1) & ND (1.4) \\
\hline $\begin{array}{l}\text { GARREISON } \\
\text { GARRETSON }\end{array}$ & $\begin{array}{l}\text { YKGARRETSON-G-39533 } \\
\text { YKGARRETSON-G-39535 }\end{array}$ & $\begin{array}{l}12 / 14 / 16 \\
12 / 4 / 16\end{array}$ & $\mathrm{~N}_{\mathrm{N}} \mathrm{r}-\mathrm{r}$ & $\begin{array}{l}\text { AlR } \\
\text { AlR }\end{array}$ & & $\begin{array}{l}\text { ND (2) } \\
\text { ND (2) }\end{array}$ & $\begin{array}{l}\mathrm{ND}(0.72) \\
\mathrm{ND}(0.72)\end{array}$ & $\begin{array}{l}6.7 \\
13\end{array}$ & $\begin{array}{l}\mathrm{ND}(2.6) \\
\mathrm{ND}(2.6)\end{array}$ & $\begin{array}{l}\mathrm{ND}(1.1) \\
\mathrm{ND}(1.1)\end{array}$ & $\begin{array}{l}11 \\
32\end{array}$ & $\begin{array}{l}\mathrm{ND}(0.98) \\
\mathrm{ND}(0.98)\end{array}$ & 0.9 & 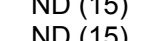 & $\mathrm{ND}(1.1)$ & ${ }_{53}^{2.5}$ \\
\hline $\begin{array}{l}\text { GARREISON } \\
\text { GARRETSON }\end{array}$ & $\begin{array}{l}\text { YKGARREISON-G-39535 } \\
\text { YKGARRETSON-G-39534 }\end{array}$ & $\begin{array}{l}12 / 14 / 16 \\
12 / 14 / 16\end{array}$ & $\begin{array}{l}N \\
\text { DUP-F }\end{array}$ & $\begin{array}{l}\text { AlR } \\
\text { AlR }\end{array}$ & & $\begin{array}{l}\text { ND (2) } \\
\text { ND (2) }\end{array}$ & ND (0.72) & $\begin{array}{l}13 \\
3.7\end{array}$ & $\begin{array}{l}\mathrm{ND}(2.6) \\
\mathrm{ND}(2.6)\end{array}$ & $\begin{array}{l}\mathrm{ND}(1.1) \\
\mathrm{ND}(1.1)\end{array}$ & $\begin{array}{l}32 \\
3.1\end{array}$ & $\begin{array}{l}\mathrm{ND}(0.98) \\
\mathrm{ND}(0.98)\end{array}$ & $\begin{array}{ll}1.1 \\
\text { ND }(0.85)\end{array}$ & $\begin{array}{l}\text { ND (15) } \\
\text { ND (15) }\end{array}$ & $\begin{array}{l}\mathrm{ND}(1.1) \\
\mathrm{ND}(1.1)\end{array}$ & $\begin{array}{l}5.3 \\
1.9\end{array}$ \\
\hline GARRETSON & 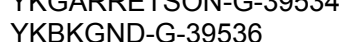 & $12 / 14 / 16$ & n & AIR & $x$ & ND (2) & ND 10 & $\begin{array}{l}3.1 \\
1.7\end{array}$ & ND & $\mathrm{ND}(1.1)$ & 4.1 & ND (0.98) & ND (0.85) & ND & ND (1.1) & ND (1.4) \\
\hline $\begin{array}{l}\text { FITWORX } \\
\text { FIT }\end{array}$ & $\begin{array}{l}\text { YKFITWORX-G-39537 } \\
\text { YKITX }\end{array}$ & $12 / 14 / 16$ & $\mathrm{~N}$ & AlR & & ND (2) & ND $(0.72)$ & 5.3 & ND (2.6) & ND (1.1) & 3.6 & $\mathrm{ND}(0.98)$ & ND $(0.85)$ & ND (15) & ND (1.1) & ND (1.4) \\
\hline FITWOF & YKFITWORX-G-39538 & $12 / 14 / 16$ & DUP-F & AIR & & $\mathrm{ND}(2)$ & $\mathrm{ND}(0.72)$ & 5.1 & ND (2.6) & ND (1.1) & 3.1 & ND (0.98) & $\mathrm{ND}(0.85)$ & ND (15) & $\mathrm{ND}(1.1)$ & ND (1.4) \\
\hline FITWORX & YKFITWORX-G-39539 & $12 / 14 / 16$ & $\mathrm{~N}$ & AIR & & ND (2) & $\mathrm{ND}(0.72)$ & 5.9 & $\mathrm{ND}(2.6)$ & $\mathrm{ND}(1.1)$ & 9.5 & ND $(C$ & ND $(0.85)$ & ND (15) & ND (1.1) & ND (1.4) \\
\hline FITWORX & YKFITWORX-G-39540 & 12/14/16 & $\mathrm{N}$ & AIR & & $\mathrm{ND}(2)$ & $\mathrm{ND}(0.72)$ & 4.6 & $\mathrm{ND}(2.6)$ & $\mathrm{ND}(1.1)$ & 1.7 & ND $(\mathrm{C}$ & ND $(0.85)$ & ND & $\mathrm{ND}(1.1)$ & ND (1.4) \\
\hline $\begin{array}{l}\text { FITWORX } \\
\text { FITWORP }\end{array}$ & YKFITWORX-G-39541 & $12 / 14 / 16$ & $N$ & AIR & $x$ & $\mathrm{ND}(2)$ & $N D(0.72)$ & $\begin{array}{l}1.7 \\
075)\end{array}$ & $\begin{array}{l}\mathrm{ND}(2.6) \\
\mathrm{ND}(26)\end{array}$ & ND (1.1) & 0.8 & ND (0.98) & $N D(0.85)$ & ND (15) & ND (1.1) & ND (1.4) \\
\hline & 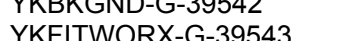 & 12/14/16 & $N_{N}^{N}$ & SOII GAS & $x$ & (2) & ND(7) & ND 17.5$)$ & (2) & (1,1) & ND (82) & (1) & (N) & (150) & (1.1) & $\begin{array}{l}\mathrm{ND}(1.4) \\
\mathrm{ND}(14)\end{array}$ \\
\hline $\begin{array}{l}\text { FITWORX } \\
\text { Fit }\end{array}$ & YKFITWORX-G-39544 & $\begin{array}{l}12 / 14 / 16 \\
12716\end{array}$ & DUP-F & SOIL GAS & & ND (20) & $\mathrm{ND}(7.2)$ & $\mathrm{ND}(7.5)$ & $\begin{array}{l}\mathrm{ND}(26) \\
\mathrm{ND}(26)\end{array}$ & ND (11) & ND (8.2) & ND (9.8) & $\begin{array}{l}\mathrm{ND}(8.5) \\
\mathrm{ND}(8.5)\end{array}$ & ND (150) & ND (11) & ND (14) \\
\hline
\end{tabular}


Concentration $\left(\mu \mathrm{g} / \mathrm{m}^{3}\right)$

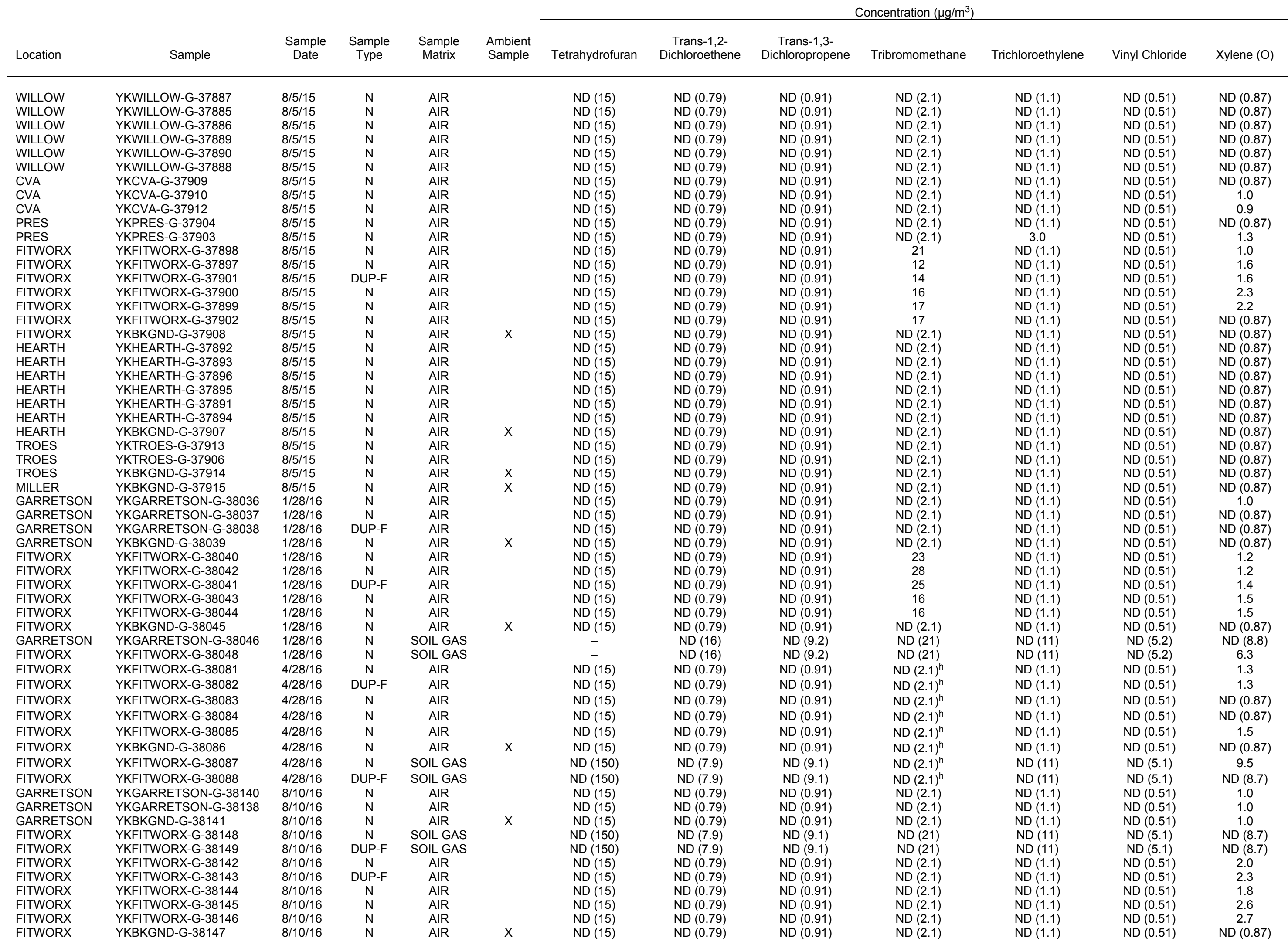


Concentration $\left(\mu \mathrm{g} / \mathrm{m}^{3}\right)$

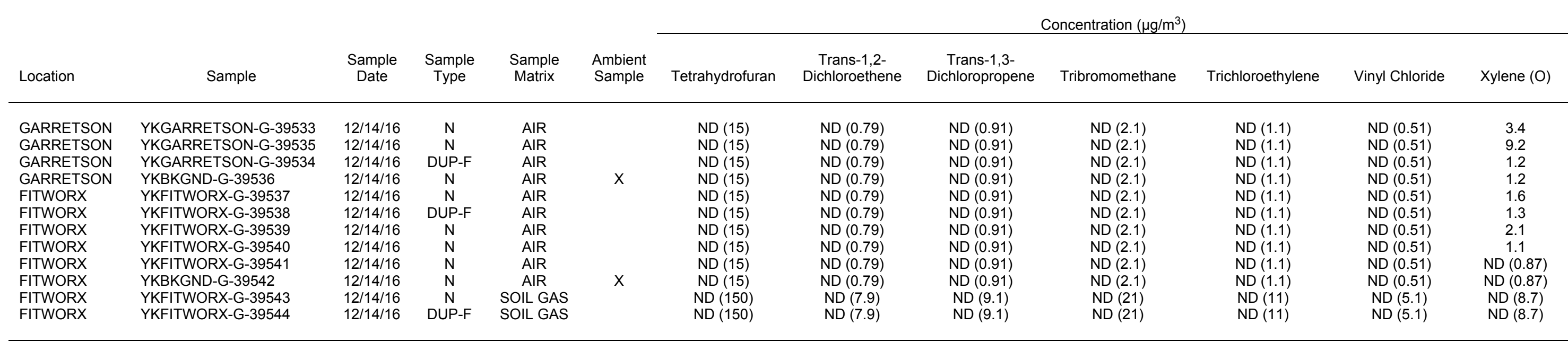

a Results for January 2016 sub-slab soil gas sampling are from H\&P Mobile Geochemistry, Inc.

b Sample type codes: DUP-F, field replicate; $\mathrm{N}$, primary sample.

Ambient air samples are "background" samples of outdoor air collected outside the location specified. Background samples are designated with the sample prefix "YKBKGND.

d ND, compound analyzed for but not detected at a level greater than or equal to the indicated method detection limit.

e Sample not analyzed for specified compound.

f D, sample analyzed at secondary dilution factor.

E, result exceeds calibration range

Laboratory control sample (LCS) or LCS-duplicate is outside QC limits. 
Appendix I:

Coordinates Survey Data 
TABLE I.1 Coordinates survey data.

$2309888.947 \quad 390474.604$ 388150.888

1647.358 1638.820

Monitoring Wells

SB-62

SB-77

DL1-A

DL1-B

DL1-C

DL2-C1

$\mathrm{DL} 2-\mathrm{C} 2$

DL2-CU

DL3-C1

DL3-C2

DL4-C1

DL4-C2

DL5-C1

DL6-C

DL7-C

DL7-CU

DL7-CL

DL8-C1

DL8-C2

Private Wells

Volzke

Liermann

Preslicka

Troester/Garretson

Nordlund

York Co. Shop

Kayton

Bonde

Driewer Shop

B\&B Metalworks

Hoblyn

Travis
2304490.692 2304451.377 2304280.013

2304280.258

2304280.044

2306845.223

2306841.671

2306838.177

2308482.891

2308483.156

2306573.437

2306566.931

2308415.297

2311244.778

2313133.976

2313134.105

2313133.880

2310061.132

2310066.097

396199.330

396210.614

396449.209

396444.056

396443.897

394256.725

394243.718

393234.842

393227.033

393098.816

393098.693

395249.325

391138.781

391243.091

391247.781

391253.725

392319.438

392319.713

2304448.783

2304547.867

2304185.808

2304465.981

2309148.269

2307966.319

308902.282

2309916.343

2309874.065

311557.303

2314417.957

2315337.395
398076.406

397877.108

395691.844

395922.957

391623.226

393006.103

393237.035

395161.077

394704.154

393360.483

393475.726

393529.896
1658.106

1658.958

1658.664

1658.771

1658.771

1658.246

1658.161

1658.164

1653.531

1653.526

1664.226

1664.373

1648.990

1641.911

1637.636

1637.658

1637.660

1644.102

1644.015

1659.814

1658.335

1658.453

1658.468

1658.472

1657.853

1657.874

1657.891

1653.308

1653.167

1663.929

1664.060

1648.628

1641.621

1637.291

1637.256

1637.151

1643.895

1643.794
1660.491

1658.506

1661.055

1655.372

1650.874

1655.897

1651.132

1651.105

1652.166

1644.607

1636.731

1638.882 
a Coordinates are in the Nebraska State Plane, North American Datum (NAD) 83

b Vertical datum is North American Vertical Datum (NAVD) 88.

c Location determined by Argonne, using hand-held (cellular telephone) GPS. Elevation estimated from U.S. Geological Survey topographic data. 


\section{Appendix J:}

Locations of Wells and Borings Used in the 1993-1995 Investigation (with CrossSections and Carbon Tetrachloride Distribution in Groundwater) 


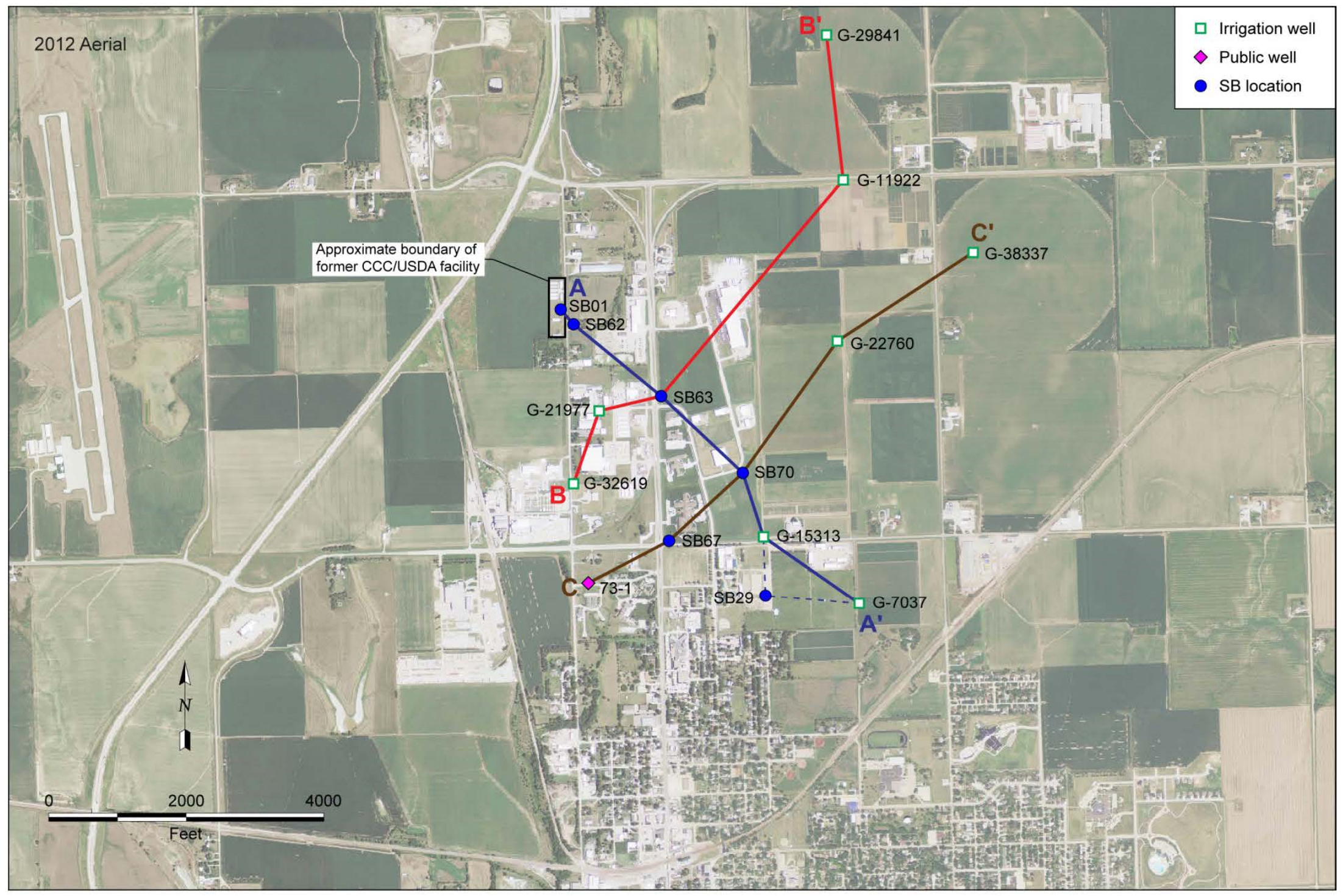

FIGURE J.1 Approximate locations of the wells and investigative borings that provided lithologic information used to develop local hydrostratigraphic interpretations in the 1993-1995 investigations (Argonne 1993, 1994, 1995a,b, with locations of interpretive cross sections A-A', B-B', and C-C'. Source of aerial photograph: USDA (2012). 


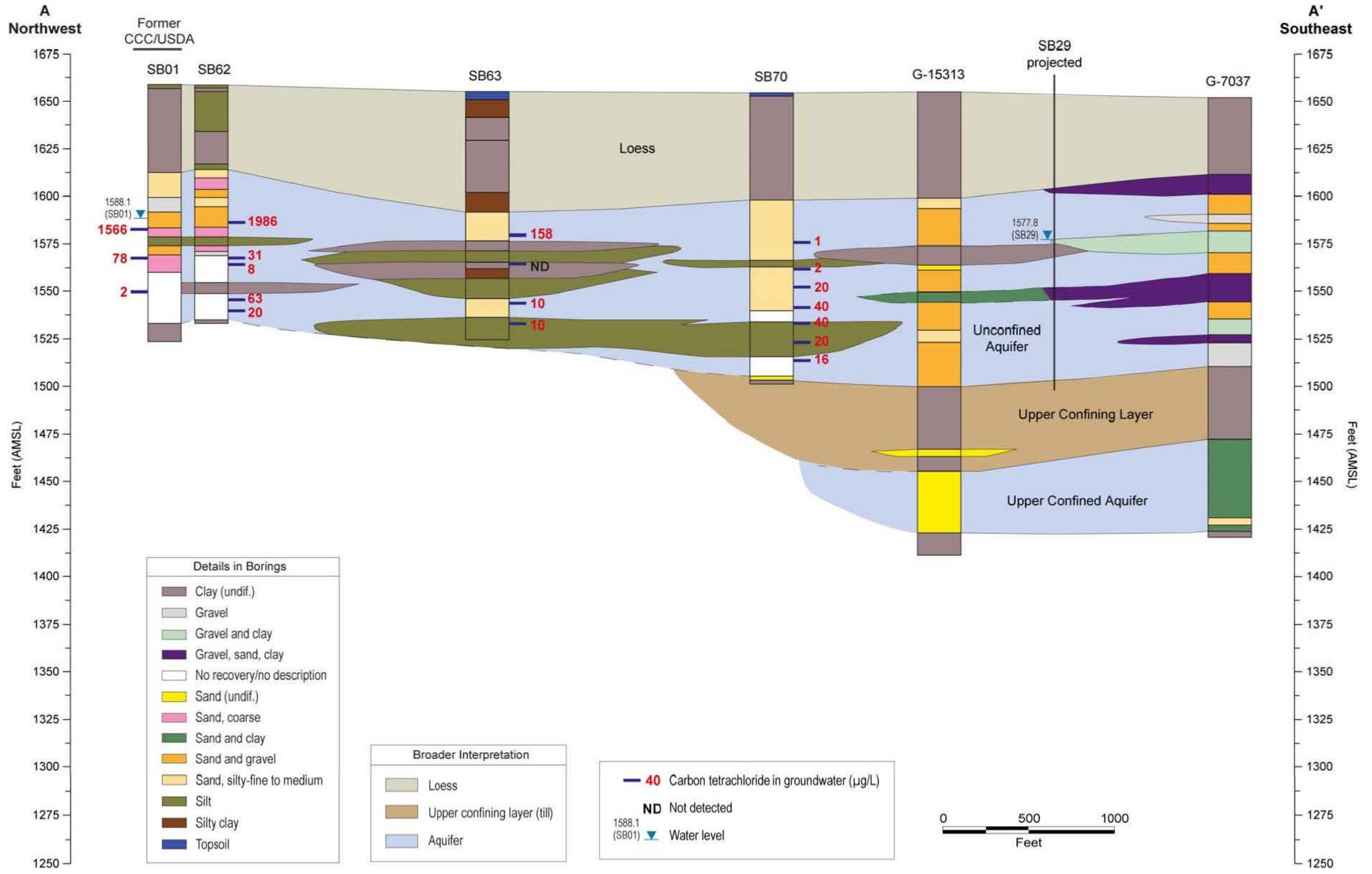




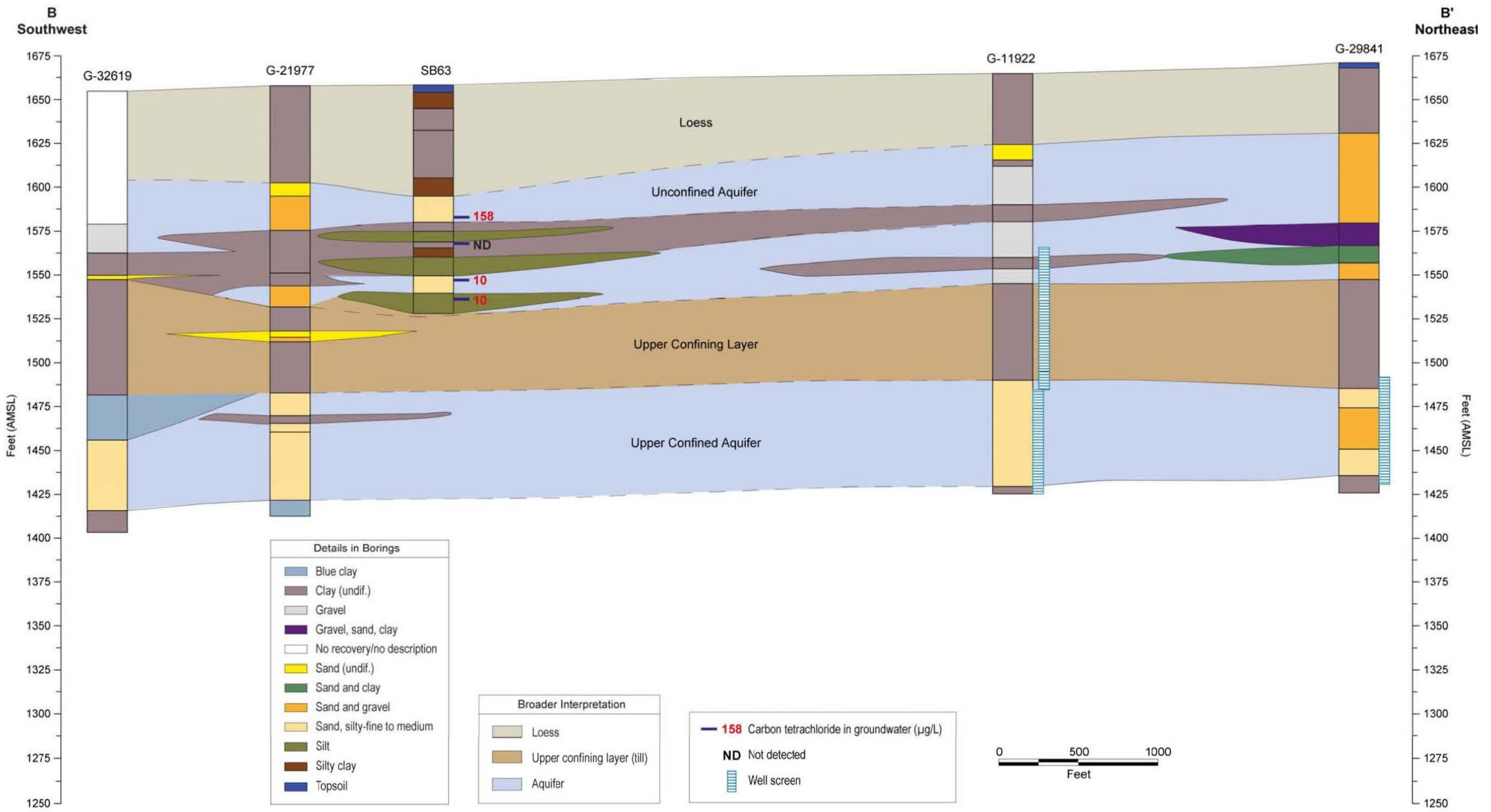

FIGURE J.3 Southwest-to-northeast geologic cross section B-B'. 


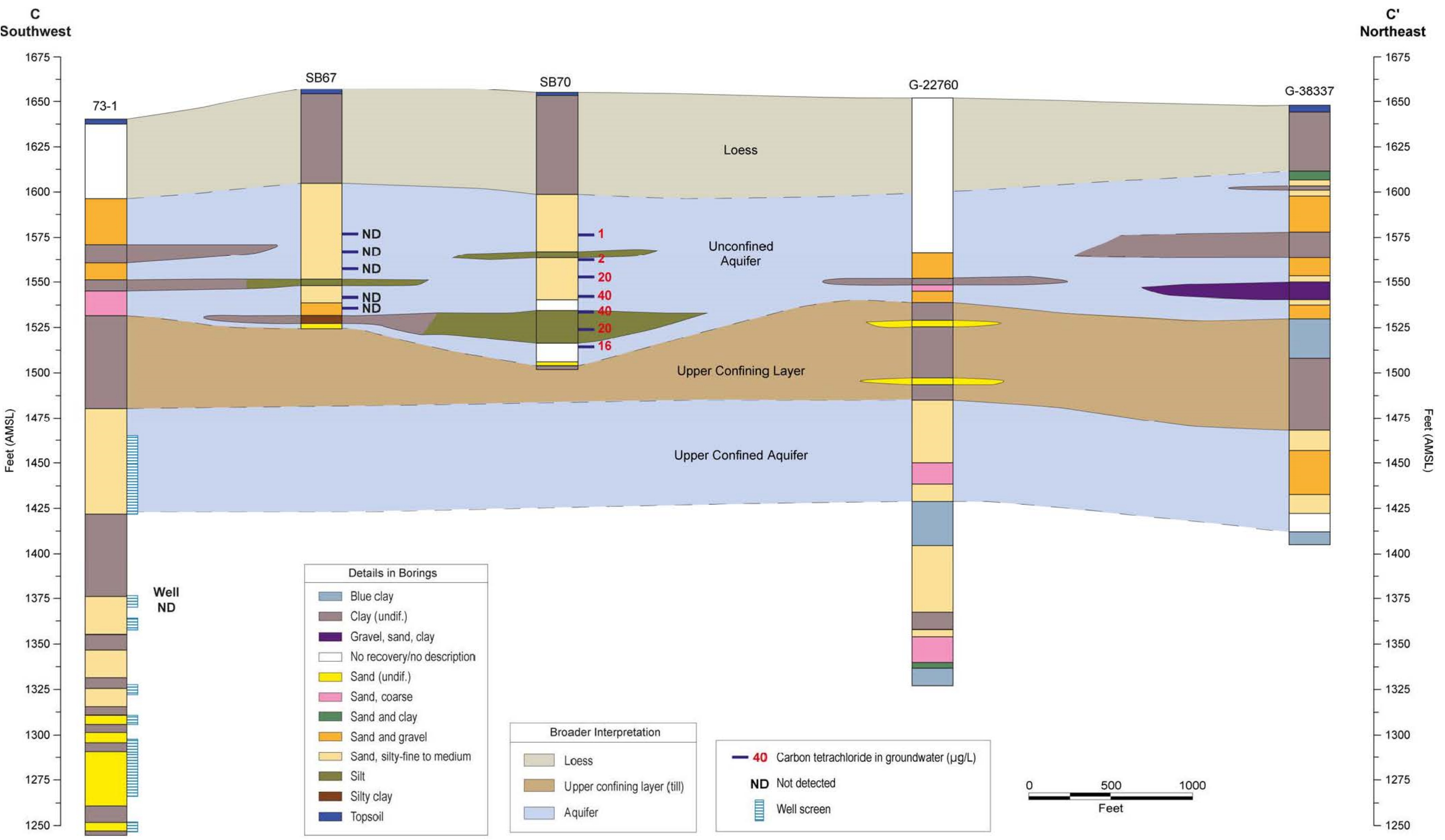

FIGURE J.4 Southwest-to-northeast geologic cross section C-C'. 


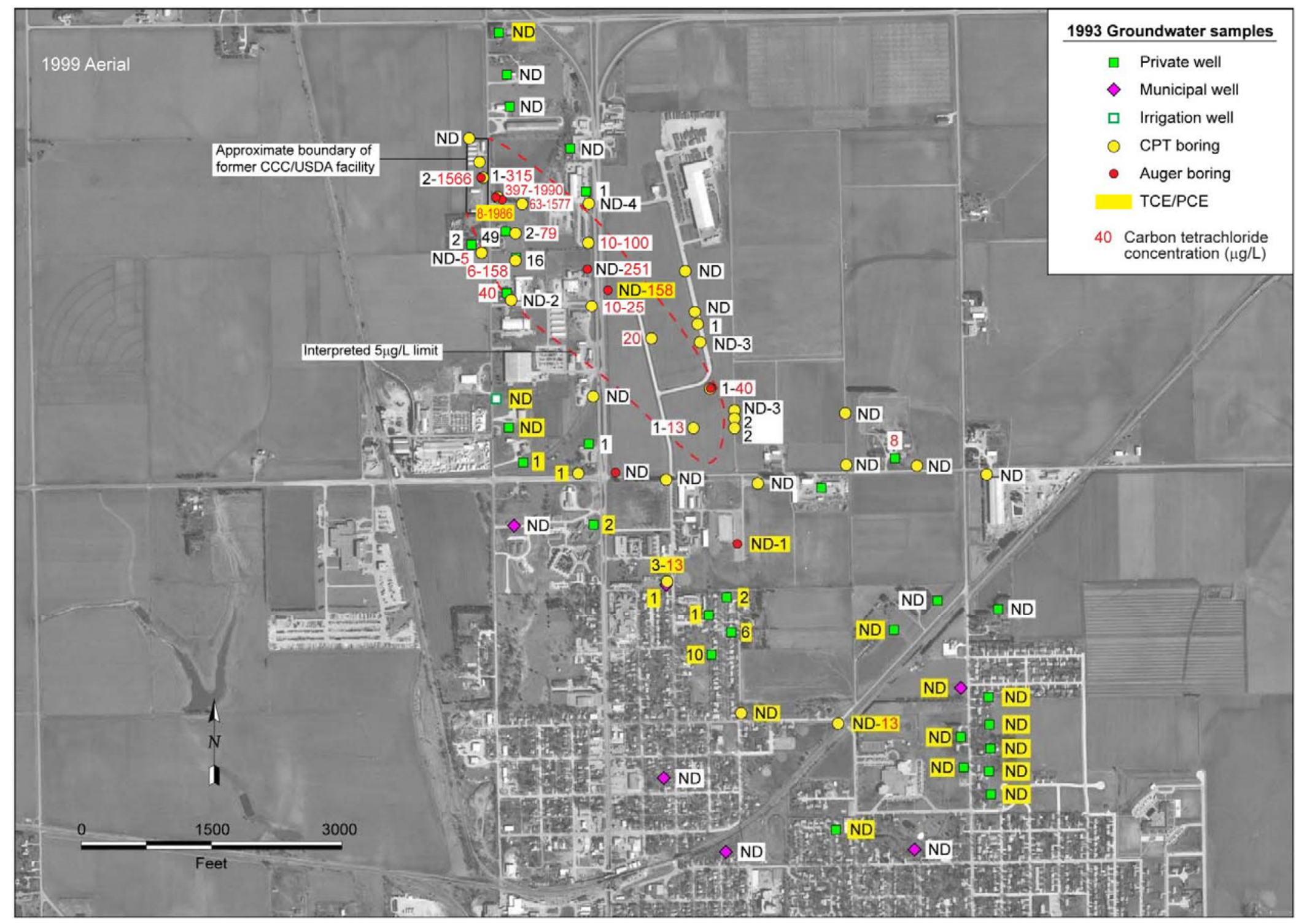

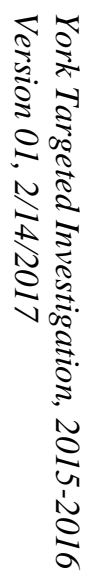

FIGURE J.5 Lateral distribution of carbon tetrachloride in groundwater samples collected in 1993 (maximum at each location). Source of aerial photograph: USDA (1999) 


\section{Supplement 1:}

Automatically Recorded Groundwater Levels 
TABLE S1.1 Automatically recorded groundwater levels in the unconfined aquifer.

\begin{tabular}{|c|c|c|c|c|c|c|c|c|c|c|c|c|c|c|c|c|}
\hline \multirow[b]{2}{*}{ Date and Time } & \multicolumn{8}{|c|}{ Measured Depth to Water (ft TOC ${ }^{\mathrm{a}}$ ) } & \multicolumn{8}{|c|}{ Groundwater Elevation $\left(\mathrm{ft} A M S L^{\mathrm{b}}\right.$ ) } \\
\hline & DL1-C & DL2-C1 & DL3-C1 & DL4-C1 & DL5-C1 & DL6-C & DL7-C & DL8-C1 & DL1-C & DL2-C1 & DL3-C1 & DL4-C1 & DL5-C1 & DL6-C & DL7-C & DL8-C1 \\
\hline 7/8/16 18:00 & & 81.948 & & & & & & & & 1575.905 & & & & & & \\
\hline $7 / 8 / 1619: 00$ & 74.19 & 81.934 & & & & & & & 1584.282 & 1575.919 & & & & & & \\
\hline $7 / 8 / 1620: 00$ & 74.185 & 81.918 & & & & & & & 1584.287 & 1575.935 & & & & & & \\
\hline 7/8/16 21:00 & 74.19 & 81.92 & & & & & & & 1584.282 & 1575.933 & & & & & & \\
\hline $7 / 8 / 1622: 00$ & 74.193 & 81.918 & 80.406 & & & & & 73.947 & 1584.279 & 1575.935 & 1572.902 & & & & & 1569.948 \\
\hline 7/8/16 23:00 & 74.209 & 81.925 & 80.413 & & & & & 73.956 & 1584.263 & 1575.928 & 1572.895 & & & & & 1569.939 \\
\hline $7 / 9 / 160: 00$ & 74.211 & 81.932 & 80.413 & & & & & 73.949 & 1584.261 & 1575.921 & 1572.895 & & & & & 1569.946 \\
\hline $7 / 9 / 161: 00$ & 74.207 & 81.923 & 80.404 & & & & & 73.935 & 1584.265 & 1575.93 & 1572.904 & & & & & 1569.96 \\
\hline $7 / 9 / 162: 00$ & 74.185 & 81.897 & 80.378 & & & & & 73.905 & 1584.287 & 1575.956 & 1572.93 & & & & & 1569.99 \\
\hline 7/9/16 3:00 & 74.176 & 81.888 & 80.364 & & & & & 73.882 & 1584.296 & 1575.965 & 1572.944 & & & & & 1570.013 \\
\hline 7/9/16 4:00 & 74.171 & 81.874 & 80.355 & & & & & 73.868 & 1584.301 & 1575.979 & 1572.953 & & & & & 1570.027 \\
\hline 7/9/16 5:00 & 74.162 & 81.862 & 80.334 & & & & & 73.824 & 1584.31 & 1575.991 & 1572.974 & & & & & 1570.071 \\
\hline $7 / 9 / 166: 00$ & 74.174 & 81.869 & 80.339 & & & & & 73.845 & 1584.298 & 1575.984 & 1572.969 & & & & & 1570.05 \\
\hline $7 / 9 / 167: 00$ & 74.174 & 81.862 & 80.327 & & & & & 73.787 & 1584.298 & 1575.991 & 1572.981 & & & & & 1570.108 \\
\hline $7 / 9 / 168: 00$ & 74.174 & 81.857 & 80.329 & & & & & 73.833 & 1584.298 & 1575.996 & 1572.979 & & & & & 1570.062 \\
\hline 7/9/16 9:00 & 74.162 & 81.846 & 80.315 & & & & & 73.814 & 1584.31 & 1576.007 & 1572.993 & & & & & 1570.081 \\
\hline 7/9/16 10:00 & 74.162 & 81.834 & 80.306 & & & & & 73.8 & 1584.31 & 1576.019 & 1573.002 & & & & & 1570.095 \\
\hline 7/9/16 11:00 & 74.152 & 81.823 & 80.292 & & & & & 73.777 & 1584.32 & 1576.03 & 1573.016 & & & & & 1570.118 \\
\hline $7 / 9 / 1612: 00$ & 74.145 & 81.811 & 80.278 & & & & & 73.759 & 1584.327 & 1576.042 & 1573.03 & & & & & 1570.136 \\
\hline 7/9/16 13:00 & 74.136 & 81.797 & 80.257 & & & & & 73.735 & 1584.336 & 1576.056 & 1573.051 & & & & & 1570.16 \\
\hline 7/9/16 14:00 & 74.127 & 81.776 & 80.238 & & & & & 73.715 & 1584.345 & 1576.077 & 1573.07 & & & & & 1570.18 \\
\hline $7 / 9 / 1615: 00$ & 74.122 & 81.764 & 80.227 & & & & & 73.698 & 1584.35 & 1576.089 & 1573.081 & & & & & 1570.197 \\
\hline 7/9/16 16:00 & 74.112 & 81.746 & 80.206 & & & 73.199 & & 73.67 & 1584.36 & 1576.107 & 1573.102 & & & 1568.422 & & 1570.225 \\
\hline $7 / 9 / 1617: 00$ & 74.105 & 81.723 & 80.185 & 88.057 & 75.077 & 73.181 & & 73.647 & 1584.367 & 1576.13 & 1573.123 & 1575.872 & 1573.551 & 1568.44 & & 1570.248 \\
\hline 7/9/16 18:00 & 74.091 & 81.699 & 80.161 & 88.069 & 75.044 & 73.162 & & 73.585 & 1584.381 & 1576.154 & 1573.147 & 1575.86 & 1573.584 & 1568.459 & & 1570.31 \\
\hline $7 / 9 / 1619: 00$ & 74.087 & 81.681 & 80.143 & 88.078 & 75.021 & 73.155 & & 73.598 & 1584.385 & 1576.172 & 1573.165 & 1575.851 & 1573.607 & 1568.466 & & 1570.297 \\
\hline $7 / 9 / 1620: 00$ & 74.084 & 81.669 & 80.131 & 88.076 & 75.002 & 73.148 & & 73.578 & 1584.388 & 1576.184 & 1573.177 & 1575.853 & 1573.626 & 1568.473 & & 1570.317 \\
\hline $7 / 9 / 1621: 00$ & 74.087 & 81.662 & 80.119 & 88.055 & 74.984 & 73.146 & & 73.564 & 1584.385 & 1576.191 & 1573.189 & 1575.874 & 1573.644 & 1568.475 & & 1570.331 \\
\hline $7 / 9 / 1622: 00$ & 74.094 & 81.664 & 80.119 & 88.043 & 74.974 & 73.151 & & 73.564 & 1584.378 & 1576.189 & 1573.189 & 1575.886 & 1573.654 & 1568.47 & & 1570.331 \\
\hline 7/9/16 23:00 & 74.112 & 81.674 & 80.126 & 88.048 & 74.977 & 73.158 & & 73.566 & 1584.36 & 1576.179 & 1573.182 & 1575.881 & 1573.651 & 1568.463 & & 1570.329 \\
\hline $7 / 10 / 160: 00$ & 74.105 & 81.664 & 80.117 & 88.036 & 74.956 & 73.146 & & 73.552 & 1584.367 & 1576.189 & 1573.191 & 1575.893 & 1573.672 & 1568.475 & & 1570.343 \\
\hline $7 / 10 / 161: 00$ & 74.096 & 81.646 & 80.098 & 88.025 & 74.935 & 73.134 & & 73.533 & 1584.376 & 1576.207 & 1573.21 & 1575.904 & 1573.693 & 1568.487 & & 1570.362 \\
\hline $7 / 10 / 162: 00$ & 74.079 & 81.623 & 80.078 & 88.013 & 74.907 & 73.118 & & 73.501 & 1584.393 & 1576.23 & 1573.23 & 1575.916 & 1573.721 & 1568.503 & & 1570.394 \\
\hline $7 / 10 / 163: 00$ & 74.065 & 81.604 & 80.054 & 87.999 & 74.876 & 73.099 & & 73.475 & 1584.407 & 1576.249 & 1573.254 & 1575.93 & 1573.752 & 1568.522 & & 1570.42 \\
\hline $7 / 10 / 164: 00$ & 74.063 & 81.59 & 80.043 & 87.994 & 74.86 & 73.097 & & 73.462 & 1584.409 & 1576.263 & 1573.265 & 1575.935 & 1573.768 & 1568.524 & & 1570.433 \\
\hline $7 / 10 / 165: 00$ & 74.065 & 81.588 & 80.033 & 87.997 & 74.846 & 73.095 & & 73.45 & 1584.407 & 1576.265 & 1573.275 & 1575.932 & 1573.782 & 1568.526 & & 1570.445 \\
\hline $7 / 10 / 166: 00$ & 74.07 & 81.588 & 80.033 & 87.997 & 74.837 & 73.097 & & 73.443 & 1584.402 & 1576.265 & 1573.275 & 1575.932 & 1573.791 & 1568.524 & & 1570.452 \\
\hline $7 / 10 / 167: 00$ & 74.077 & 81.585 & 80.029 & 87.997 & 74.83 & 73.097 & & 73.441 & 1584.395 & 1576.268 & 1573.279 & 1575.932 & 1573.798 & 1568.524 & & 1570.454 \\
\hline $7 / 10 / 168: 00$ & 74.072 & 81.576 & 80.019 & 87.987 & 74.811 & 73.085 & & 73.427 & 1584.4 & 1576.277 & 1573.289 & 1575.942 & 1573.817 & 1568.536 & & 1570.468 \\
\hline $7 / 10 / 169: 00$ & 74.058 & 81.557 & 80.001 & 87.973 & 74.788 & 73.071 & & 73.413 & 1584.414 & 1576.296 & 1573.307 & 1575.956 & 1573.84 & 1568.55 & & 1570.482 \\
\hline $7 / 10 / 16$ 10:00 & 74.049 & 81.541 & 79.987 & 87.969 & 74.762 & 73.062 & & 73.387 & 1584.423 & 1576.312 & 1573.321 & 1575.96 & 1573.866 & 1568.559 & & 1570.508 \\
\hline 7/10/16 11:00 & 74.037 & 81.518 & 79.959 & 87.955 & 74.736 & 73.051 & & 73.359 & 1584.435 & 1576.335 & 1573.349 & 1575.974 & 1573.892 & 1568.57 & & 1570.536 \\
\hline $7 / 10 / 1612: 00$ & 74.035 & 81.509 & 79.949 & 87.95 & 74.718 & 73.041 & & 73.345 & 1584.437 & 1576.344 & 1573.359 & 1575.979 & 1573.91 & 1568.58 & & 1570.55 \\
\hline 7/10/16 13:00 & 74.037 & 81.509 & 79.942 & 87.955 & 74.704 & 73.041 & & 73.336 & 1584.435 & 1576.344 & 1573.366 & 1575.974 & 1573.924 & 1568.58 & & 1570.559 \\
\hline $7 / 10 / 1614: 00$ & 74.039 & 81.502 & 79.938 & 87.948 & 74.692 & 73.039 & & 73.329 & 1584.433 & 1576.351 & 1573.37 & 1575.981 & 1573.936 & 1568.582 & & 1570.566 \\
\hline 7/10/16 15:00 & 74.025 & 81.476 & 79.912 & 87.934 & 74.664 & 73.018 & & 73.301 & 1584.447 & 1576.377 & 1573.396 & 1575.995 & 1573.964 & 1568.603 & & 1570.594 \\
\hline $7 / 10 / 1616: 00$ & 74.018 & 81.46 & 79.898 & 87.929 & 74.641 & 73.013 & & 73.28 & 1584.454 & 1576.393 & 1573.41 & 1576 & 1573.987 & 1568.608 & & 1570.615 \\
\hline 7/10/16 17:00 & 74.009 & 81.436 & 79.872 & 87.915 & 74.615 & 72.995 & & 73.26 & 1584.463 & 1576.417 & 1573.436 & 1576.014 & 1574.013 & 1568.626 & & 1570.635 \\
\hline $7 / 10 / 16$ 18:00 & 74.004 & 81.423 & 79.861 & 87.913 & 74.592 & 72.99 & & 73.239 & 1584.468 & 1576.43 & 1573.447 & 1576.016 & 1574.036 & 1568.631 & & 1570.656 \\
\hline
\end{tabular}


TABLE S1.1 Automatically recorded groundwater levels in the unconfined aquifer.

\begin{tabular}{|c|c|c|c|c|c|c|c|c|c|c|c|c|c|c|c|c|}
\hline \multirow[b]{2}{*}{ Date and Time } & \multicolumn{8}{|c|}{ Measured Depth to Water (ft TOC ${ }^{\mathrm{a}}$ ) } & \multicolumn{8}{|c|}{ Groundwater Elevation ( $\mathrm{ft} A M \mathrm{AL}^{\mathrm{b}}$ ) } \\
\hline & DL1-C & DL2-C1 & DL3-C1 & DL4-C1 & DL5-C1 & DL6-C & DL7-C & DL8-C1 & DL1-C & DL2-C1 & DL3-C1 & DL4-C1 & DL5-C1 & DL6-C & DL7-C & DL8-C1 \\
\hline 7/10/16 19:00 & 73.995 & 81.404 & 79.837 & 87.898 & 74.564 & 72.971 & & 73.222 & 1584.477 & 1576.449 & 1573.471 & 1576.031 & 1574.064 & 1568.65 & & 1570.673 \\
\hline $7 / 10 / 1620: 00$ & 73.995 & 81.397 & 79.828 & 87.898 & 74.55 & 72.971 & & 73.204 & 1584.477 & 1576.456 & 1573.48 & 1576.031 & 1574.078 & 1568.65 & & 1570.691 \\
\hline 7/10/16 21:00 & 74.006 & 81.402 & 79.826 & 87.905 & 74.545 & 72.976 & & 73.199 & 1584.466 & 1576.451 & 1573.482 & 1576.024 & 1574.083 & 1568.645 & & 1570.696 \\
\hline 7/10/16 22:00 & 74.016 & 81.404 & 79.83 & 87.908 & 74.543 & 72.983 & & 73.204 & 1584.456 & 1576.449 & 1573.478 & 1576.021 & 1574.085 & 1568.638 & & 1570.691 \\
\hline 7/10/16 23:00 & 74.021 & 81.402 & 79.835 & 87.903 & 74.534 & 72.983 & & 73.197 & 1584.451 & 1576.451 & 1573.473 & 1576.026 & 1574.094 & 1568.638 & & 1570.698 \\
\hline $7 / 11 / 160: 00$ & 74.018 & 81.399 & 79.823 & 87.903 & 74.524 & 72.978 & & 73.188 & 1584.454 & 1576.454 & 1573.485 & 1576.026 & 1574.104 & 1568.643 & & 1570.707 \\
\hline $7 / 11 / 161: 00$ & 74.013 & 81.392 & 79.814 & 87.898 & 74.508 & 72.969 & & 73.174 & 1584.459 & 1576.461 & 1573.494 & 1576.031 & 1574.12 & 1568.652 & & 1570.721 \\
\hline $7 / 11 / 162: 00$ & 74.011 & 81.383 & 79.882 & 87.894 & 74.494 & 72.964 & & 73.174 & 1584.461 & 1576.47 & 1573.426 & 1576.035 & 1574.134 & 1568.657 & & 1570.721 \\
\hline $7 / 11 / 163: 00$ & 74.011 & 81.383 & 79.872 & 87.896 & 74.487 & 72.967 & & 73.171 & 1584.461 & 1576.47 & 1573.436 & 1576.033 & 1574.141 & 1568.654 & & 1570.724 \\
\hline $7 / 11 / 164: 00$ & 74.002 & 81.367 & 79.87 & 87.884 & 74.468 & 72.953 & & 73.157 & 1584.47 & 1576.486 & 1573.438 & 1576.045 & 1574.16 & 1568.668 & & 1570.738 \\
\hline $7 / 11 / 165: 00$ & 73.99 & 81.35 & 79.851 & 87.875 & 74.445 & 72.941 & & 73.139 & 1584.482 & 1576.503 & 1573.457 & 1576.054 & 1574.183 & 1568.68 & & 1570.756 \\
\hline $7 / 11 / 166: 00$ & 73.983 & 81.341 & 79.814 & 87.866 & 74.424 & 72.934 & & 73.125 & 1584.489 & 1576.512 & 1573.494 & 1576.063 & 1574.204 & 1568.687 & & 1570.77 \\
\hline $7 / 11 / 167: 00$ & 73.98 & 81.327 & 79.758 & 87.863 & 74.41 & 72.927 & & 73.104 & 1584.492 & 1576.526 & 1573.55 & 1576.066 & 1574.218 & 1568.694 & & 1570.791 \\
\hline 7/11/16 8:00 & 73.978 & 81.32 & 79.744 & 87.861 & 74.396 & 72.922 & & 73.09 & 1584.494 & 1576.533 & 1573.564 & 1576.068 & 1574.232 & 1568.699 & & 1570.805 \\
\hline 7/11/16 9:00 & 73.98 & 81.32 & 79.747 & 87.861 & 74.391 & 72.92 & & 73.097 & 1584.492 & 1576.533 & 1573.561 & 1576.068 & 1574.237 & 1568.701 & & 1570.798 \\
\hline $7 / 11 / 1610: 00$ & 73.98 & 81.318 & 79.735 & 87.856 & 74.375 & 72.918 & & 73.081 & 1584.492 & 1576.535 & 1573.573 & 1576.073 & 1574.253 & 1568.703 & & 1570.814 \\
\hline $7 / 11 / 1611: 00$ & 73.98 & 81.306 & 79.723 & 87.852 & 74.361 & 72.911 & & 73.074 & 1584.492 & 1576.547 & 1573.585 & 1576.077 & 1574.267 & 1568.71 & & 1570.821 \\
\hline $7 / 11 / 1612: 00$ & 73.983 & 81.309 & 79.723 & 87.852 & 74.357 & 72.913 & & 73.083 & 1584.489 & 1576.544 & 1573.585 & 1576.077 & 1574.271 & 1568.708 & & 1570.812 \\
\hline 7/11/16 13:00 & 73.99 & 81.316 & 79.723 & 87.859 & 74.354 & 72.918 & & 73.069 & 1584.482 & 1576.537 & 1573.585 & 1576.07 & 1574.274 & 1568.703 & & 1570.826 \\
\hline $7 / 11 / 1614: 00$ & 73.992 & 81.306 & 79.723 & 87.854 & 74.345 & 72.915 & & 73.083 & 1584.48 & 1576.547 & 1573.585 & 1576.075 & 1574.283 & 1568.706 & & 1570.812 \\
\hline $7 / 11 / 1615: 00$ & 73.988 & 81.297 & 79.712 & 87.849 & 74.331 & 72.906 & & 73.064 & 1584.484 & 1576.556 & 1573.596 & 1576.08 & 1574.297 & 1568.715 & & 1570.831 \\
\hline $7 / 11 / 1616: 00$ & 73.985 & 81.297 & 79.707 & 87.845 & 74.324 & 72.906 & & 73.064 & 1584.487 & 1576.556 & 1573.601 & 1576.084 & 1574.304 & 1568.715 & & 1570.831 \\
\hline $7 / 11 / 1617: 00$ & 73.978 & 81.285 & 79.7 & 87.842 & 74.305 & 72.897 & & 73.048 & 1584.494 & 1576.568 & 1573.608 & 1576.087 & 1574.323 & 1568.724 & & 1570.847 \\
\hline $7 / 11 / 1618: 00$ & 73.985 & 81.288 & 79.695 & 87.868 & 74.307 & 72.899 & & 73.03 & 1584.487 & 1576.565 & 1573.613 & 1576.061 & 1574.321 & 1568.722 & & 1570.865 \\
\hline 7/11/16 19:00 & 73.995 & 81.297 & 79.7 & 87.887 & 74.307 & 72.904 & & 73.023 & 1584.477 & 1576.556 & 1573.608 & 1576.042 & 1574.321 & 1568.717 & & 1570.872 \\
\hline $7 / 11 / 1620: 00$ & 74.006 & 81.309 & 79.707 & 87.898 & 74.317 & 72.913 & & 73.032 & 1584.466 & 1576.544 & 1573.601 & 1576.031 & 1574.311 & 1568.708 & & 1570.863 \\
\hline 7/11/16 21:00 & 74.016 & 81.325 & 79.714 & 87.887 & 74.319 & 72.92 & & 73.006 & 1584.456 & 1576.528 & 1573.594 & 1576.042 & 1574.309 & 1568.701 & & 1570.889 \\
\hline $7 / 11 / 1622: 00$ & 74.032 & 81.348 & 79.73 & 87.887 & 74.338 & 72.934 & & 73.062 & 1584.44 & 1576.505 & 1573.578 & 1576.042 & 1574.29 & 1568.687 & & 1570.833 \\
\hline $7 / 11 / 1623: 00$ & 74.051 & 81.374 & 79.751 & 87.894 & 74.359 & 72.955 & & 73.039 & 1584.421 & 1576.479 & 1573.557 & 1576.035 & 1574.269 & 1568.666 & & 1570.856 \\
\hline $7 / 12 / 160: 00$ & 74.037 & 81.364 & 79.744 & 87.884 & 74.345 & 72.941 & & 73.076 & 1584.435 & 1576.489 & 1573.564 & 1576.045 & 1574.283 & 1568.68 & & 1570.819 \\
\hline $7 / 12 / 161: 00$ & 74.044 & 81.381 & 79.756 & 87.892 & 74.359 & 72.957 & & 73.085 & 1584.428 & 1576.472 & 1573.552 & 1576.037 & 1574.269 & 1568.664 & & 1570.81 \\
\hline $7 / 12 / 162: 00$ & 74.061 & 81.406 & 79.779 & 87.905 & 74.38 & 72.974 & & 73.111 & 1584.411 & 1576.447 & 1573.529 & 1576.024 & 1574.248 & 1568.647 & & 1570.784 \\
\hline $7 / 12 / 163: 00$ & 74.051 & 81.402 & 79.779 & 87.896 & 74.373 & 72.962 & & 73.106 & 1584.421 & 1576.451 & 1573.529 & 1576.033 & 1574.255 & 1568.659 & & 1570.789 \\
\hline $7 / 12 / 164: 00$ & 74.07 & 81.436 & 79.798 & 87.92 & 74.398 & 72.99 & & 73.129 & 1584.402 & 1576.417 & 1573.51 & 1576.009 & 1574.23 & 1568.631 & & 1570.766 \\
\hline $7 / 12 / 165: 00$ & 74.072 & 81.448 & 79.807 & 87.92 & 74.403 & 72.988 & & 73.141 & 1584.4 & 1576.405 & 1573.501 & 1576.009 & 1574.225 & 1568.633 & & 1570.754 \\
\hline $7 / 12 / 166: 00$ & 74.094 & 81.474 & 79.837 & 87.936 & 74.429 & 73.013 & & 73.164 & 1584.378 & 1576.379 & 1573.471 & 1575.993 & 1574.199 & 1568.608 & & 1570.731 \\
\hline $7 / 12 / 167: 00$ & 74.094 & 81.476 & 79.84 & 87.927 & 74.429 & 73.004 & & 73.176 & 1584.378 & 1576.377 & 1573.468 & 1576.002 & 1574.199 & 1568.617 & & 1570.719 \\
\hline $7 / 12 / 168: 00$ & 74.082 & 81.483 & 79.851 & 87.929 & 74.436 & 73.009 & & 73.178 & 1584.39 & 1576.37 & 1573.457 & 1576 & 1574.192 & 1568.612 & & 1570.717 \\
\hline $7 / 12 / 169: 00$ & 74.077 & 81.483 & 79.87 & 87.929 & 74.436 & 73.009 & & 73.183 & 1584.395 & 1576.37 & 1573.438 & 1576 & 1574.192 & 1568.612 & & 1570.712 \\
\hline $7 / 12 / 1610: 00$ & 74.072 & 81.488 & 79.875 & 87.931 & 74.433 & 73.009 & & 73.197 & 1584.4 & 1576.365 & 1573.433 & 1575.998 & 1574.195 & 1568.612 & & 1570.698 \\
\hline $7 / 12 / 1611: 00$ & 74.077 & 81.49 & 79.877 & 87.934 & 74.433 & 73.009 & & 73.194 & 1584.395 & 1576.363 & 1573.431 & 1575.995 & 1574.195 & 1568.612 & & 1570.701 \\
\hline $7 / 12 / 1612: 00$ & 74.065 & 81.478 & 79.868 & 87.92 & 74.422 & 72.995 & & 73.199 & 1584.407 & 1576.375 & 1573.44 & 1576.009 & 1574.206 & 1568.626 & & 1570.696 \\
\hline $7 / 12 / 1613: 00$ & 74.051 & 81.462 & 79.835 & 87.908 & 74.399 & 72.981 & & 73.181 & 1584.421 & 1576.391 & 1573.473 & 1576.021 & 1574.229 & 1568.64 & & 1570.714 \\
\hline $7 / 12 / 1614: 00$ & 74.042 & 81.443 & 79.835 & 87.901 & 74.382 & 72.969 & & 73.162 & 1584.43 & 1576.41 & 1573.473 & 1576.028 & 1574.246 & 1568.652 & & 1570.733 \\
\hline $7 / 12 / 1615: 00$ & 74.03 & 81.423 & 79.819 & 87.887 & 74.359 & 72.955 & & 73.141 & 1584.442 & 1576.43 & 1573.489 & 1576.042 & 1574.269 & 1568.666 & & 1570.754 \\
\hline $7 / 12 / 1616: 00$ & 74.028 & 81.413 & 79.807 & 87.885 & 74.345 & 72.946 & & 73.109 & 1584.444 & 1576.44 & 1573.501 & 1576.044 & 1574.283 & 1568.675 & & 1570.786 \\
\hline $7 / 12 / 1617: 00$ & 74.028 & 81.39 & 79.786 & 87.877 & 74.324 & 72.939 & 71.518 & 73.118 & 1584.444 & 1576.463 & 1573.522 & 1576.052 & 1574.304 & 1568.682 & 1565.773 & 1570.777 \\
\hline $7 / 12 / 1618: 00$ & 74.016 & 81.367 & 79.749 & 87.863 & 74.305 & 72.925 & 71.494 & 73.078 & 1584.456 & 1576.486 & 1573.559 & 1576.066 & 1574.323 & 1568.696 & 1565.797 & 1570.817 \\
\hline $7 / 12 / 16$ 19:00 & 74.011 & 81.353 & 79.735 & 87.859 & 74.293 & 72.915 & 71.482 & 73.067 & 1584.461 & 1576.5 & 1573.573 & 1576.07 & 1574.335 & 1568.706 & 1565.809 & 1570.828 \\
\hline
\end{tabular}


TABLE S1.1 Automatically recorded groundwater levels in the unconfined aquifer.

\begin{tabular}{|c|c|c|c|c|c|c|c|c|c|c|c|c|c|c|c|c|}
\hline \multirow[b]{2}{*}{ Date and Time } & \multicolumn{8}{|c|}{ Measured Depth to Water (ft TOC ${ }^{\mathrm{a}}$ ) } & \multicolumn{8}{|c|}{ Groundwater Elevation (ft AMSL) } \\
\hline & DL1-C & DL2-C1 & DL3-C1 & DL4-C1 & DL5-C1 & DL6-C & DL7-C & DL8-C1 & DL1-C & DL2-C1 & DL3-C1 & DL4-C1 & DL5-C1 & DL6-C & DL7-C & DL8-C1 \\
\hline $7 / 12 / 1620: 00$ & 74.016 & 81.343 & 79.726 & 87.859 & 74.286 & 72.915 & 71.477 & 73.053 & 1584.456 & 1576.51 & 1573.582 & 1576.07 & 1574.342 & 1568.706 & 1565.814 & 1570.842 \\
\hline 7/12/16 21:00 & 74.028 & 81.348 & 79.723 & 87.861 & 74.282 & 72.918 & 71.485 & 73.048 & 1584.444 & 1576.505 & 1573.585 & 1576.068 & 1574.346 & 1568.703 & 1565.806 & 1570.847 \\
\hline $7 / 12 / 1622: 00$ & 74.046 & 81.362 & 79.728 & 87.87 & 74.289 & 72.929 & 71.499 & 73.03 & 1584.426 & 1576.491 & 1573.58 & 1576.059 & 1574.339 & 1568.692 & 1565.792 & 1570.865 \\
\hline $7 / 12 / 1623: 00$ & 74.068 & 81.374 & 79.744 & 87.877 & 74.303 & 72.941 & 71.518 & 73.067 & 1584.404 & 1576.479 & 1573.564 & 1576.052 & 1574.325 & 1568.68 & 1565.773 & 1570.828 \\
\hline $7 / 13 / 160: 00$ & 74.063 & 81.362 & 79.735 & 87.868 & 74.296 & 72.936 & 71.509 & 73.064 & 1584.409 & 1576.491 & 1573.573 & 1576.061 & 1574.332 & 1568.685 & 1565.782 & 1570.831 \\
\hline 7/13/16 1:00 & 74.058 & 81.353 & 79.726 & 87.863 & 74.277 & 72.927 & 71.497 & 73.048 & 1584.414 & 1576.5 & 1573.582 & 1576.066 & 1574.351 & 1568.694 & 1565.794 & 1570.847 \\
\hline $7 / 13 / 162: 00$ & 74.042 & 81.332 & 79.768 & 87.845 & 74.254 & 72.913 & 71.475 & 73.034 & 1584.43 & 1576.521 & 1573.54 & 1576.084 & 1574.374 & 1568.708 & 1565.816 & 1570.861 \\
\hline $7 / 13 / 163: 00$ & 74.03 & 81.311 & 79.761 & 87.833 & 74.233 & 72.897 & 71.454 & 73.016 & 1584.442 & 1576.542 & 1573.547 & 1576.096 & 1574.395 & 1568.724 & 1565.837 & 1570.879 \\
\hline $7 / 13 / 164: 00$ & 74.035 & 81.306 & 79.763 & 87.838 & 74.223 & 72.894 & 71.451 & 73.013 & 1584.437 & 1576.547 & 1573.545 & 1576.091 & 1574.405 & 1568.727 & 1565.84 & 1570.882 \\
\hline $7 / 13 / 165: 00$ & 74.058 & 81.32 & 79.777 & 87.852 & 74.24 & 72.911 & 71.468 & 73.023 & 1584.414 & 1576.533 & 1573.531 & 1576.077 & 1574.388 & 1568.71 & 1565.823 & 1570.872 \\
\hline $7 / 13 / 166: 00$ & 74.046 & 81.302 & 79.735 & 87.835 & 74.221 & 72.897 & 71.449 & 73.009 & 1584.426 & 1576.551 & 1573.573 & 1576.094 & 1574.407 & 1568.724 & 1565.842 & 1570.886 \\
\hline $7 / 13 / 167: 00$ & 74.058 & 81.309 & 79.693 & 87.842 & 74.226 & 72.904 & 71.461 & 73.006 & 1584.414 & 1576.544 & 1573.615 & 1576.087 & 1574.402 & 1568.717 & 1565.83 & 1570.889 \\
\hline $7 / 13 / 168: 00$ & 74.058 & 81.297 & 79.7 & 87.831 & 74.219 & 72.901 & 71.458 & 73.013 & 1584.414 & 1576.556 & 1573.608 & 1576.098 & 1574.409 & 1568.72 & 1565.833 & 1570.882 \\
\hline 7/13/16 9:00 & 74.056 & 81.292 & 79.698 & 87.835 & 74.203 & 72.89 & 71.441 & 72.981 & 1584.416 & 1576.561 & 1573.61 & 1576.094 & 1574.425 & 1568.731 & 1565.85 & 1570.914 \\
\hline $7 / 13 / 1610: 00$ & 74.058 & 81.29 & 79.684 & 87.831 & 74.193 & 72.887 & 71.437 & 72.997 & 1584.414 & 1576.563 & 1573.624 & 1576.098 & 1574.435 & 1568.734 & 1565.854 & 1570.898 \\
\hline $7 / 13 / 1611: 00$ & 74.077 & 81.306 & 79.702 & 87.847 & 74.212 & 72.906 & 71.461 & 73.018 & 1584.395 & 1576.547 & 1573.606 & 1576.082 & 1574.416 & 1568.715 & 1565.83 & 1570.877 \\
\hline $7 / 13 / 1612: 00$ & 74.087 & 81.311 & 79.7 & 87.847 & 74.205 & 72.904 & 71.458 & 73.006 & 1584.385 & 1576.542 & 1573.608 & 1576.082 & 1574.423 & 1568.717 & 1565.833 & 1570.889 \\
\hline $7 / 13 / 1613: 00$ & 74.117 & 81.339 & 79.712 & 87.863 & 74.231 & 72.925 & 71.494 & 73.027 & 1584.355 & 1576.514 & 1573.596 & 1576.066 & 1574.397 & 1568.696 & 1565.797 & 1570.868 \\
\hline 7/13/16 14:00 & 74.129 & 81.353 & 79.742 & 87.863 & 75.126 & 72.929 & 71.499 & 73.037 & 1584.343 & 1576.5 & 1573.566 & 1576.066 & 1573.502 & 1568.692 & 1565.792 & 1570.858 \\
\hline $7 / 13 / 1615: 00$ & 74.117 & 81.353 & 79.728 & 87.859 & 75.46 & 72.913 & 71.482 & 73.02 & 1584.355 & 1576.5 & 1573.58 & 1576.07 & 1573.168 & 1568.708 & 1565.809 & 1570.875 \\
\hline $7 / 13 / 1616: 00$ & 74.119 & 81.35 & 79.723 & 87.856 & 75.602 & 72.913 & 71.475 & 73.016 & 1584.353 & 1576.503 & 1573.585 & 1576.073 & 1573.026 & 1568.708 & 1565.816 & 1570.879 \\
\hline $7 / 13 / 1617: 00$ & 74.143 & 81.364 & 79.728 & 87.859 & 75.723 & 72.911 & 71.475 & 73.018 & 1584.329 & 1576.489 & 1573.58 & 1576.07 & 1572.905 & 1568.71 & 1565.816 & 1570.877 \\
\hline $7 / 13 / 1618: 00$ & 74.169 & 81.378 & 79.737 & 87.887 & 75.835 & 72.913 & 71.475 & 73.018 & 1584.303 & 1576.475 & 1573.571 & 1576.042 & 1572.793 & 1568.708 & 1565.816 & 1570.877 \\
\hline 7/13/16 19:00 & 74.2 & 81.402 & 79.751 & 87.91 & 75.94 & 72.92 & 71.487 & 73.037 & 1584.272 & 1576.451 & 1573.557 & 1576.019 & 1572.688 & 1568.701 & 1565.804 & 1570.858 \\
\hline $7 / 13 / 1620: 00$ & 74.23 & 81.43 & 79.756 & 87.924 & 76.04 & 72.932 & 71.499 & 73.037 & 1584.242 & 1576.423 & 1573.552 & 1576.005 & 1572.588 & 1568.689 & 1565.792 & 1570.858 \\
\hline 7/13/16 21:00 & 74.251 & 81.453 & 79.772 & 87.91 & 76.131 & 72.941 & 71.506 & 73.074 & 1584.221 & 1576.4 & 1573.536 & 1576.019 & 1572.497 & 1568.68 & 1565.785 & 1570.821 \\
\hline $7 / 13 / 1622: 00$ & 74.277 & 81.481 & 79.793 & 87.908 & 76.222 & 72.953 & 71.525 & 73.09 & 1584.195 & 1576.372 & 1573.515 & 1576.021 & 1572.406 & 1568.668 & 1565.766 & 1570.805 \\
\hline 7/13/16 23:00 & 74.294 & 81.506 & 79.809 & 87.913 & 76.301 & 72.962 & 71.53 & 73.106 & 1584.178 & 1576.347 & 1573.499 & 1576.016 & 1572.327 & 1568.659 & 1565.761 & 1570.789 \\
\hline $7 / 14 / 160: 00$ & 74.306 & 81.527 & 79.823 & 87.915 & 76.369 & 72.962 & 71.535 & 73.125 & 1584.166 & 1576.326 & 1573.485 & 1576.014 & 1572.259 & 1568.659 & 1565.756 & 1570.77 \\
\hline $7 / 14 / 161: 00$ & 74.306 & 81.534 & 79.828 & 87.908 & 76.425 & 72.957 & 71.523 & 73.083 & 1584.166 & 1576.319 & 1573.48 & 1576.021 & 1572.203 & 1568.664 & 1565.768 & 1570.812 \\
\hline $7 / 14 / 162: 00$ & 74.306 & 81.541 & 79.828 & 87.898 & 76.469 & 72.95 & 71.509 & 73.125 & 1584.166 & 1576.312 & 1573.48 & 1576.031 & 1572.159 & 1568.671 & 1565.782 & 1570.77 \\
\hline $7 / 14 / 163: 00$ & 74.317 & 81.564 & 79.849 & 87.913 & 76.53 & 72.96 & 71.518 & 73.148 & 1584.155 & 1576.289 & 1573.459 & 1576.016 & 1572.098 & 1568.661 & 1565.773 & 1570.747 \\
\hline $7 / 14 / 164: 00$ & 74.315 & 81.576 & 79.861 & 87.915 & 76.576 & 72.96 & 71.513 & 73.162 & 1584.157 & 1576.277 & 1573.447 & 1576.014 & 1572.052 & 1568.661 & 1565.778 & 1570.733 \\
\hline $7 / 14 / 165: 00$ & 74.303 & 81.588 & 79.87 & 87.917 & 76.614 & 72.962 & 71.513 & 73.171 & 1584.169 & 1576.265 & 1573.438 & 1576.012 & 1572.014 & 1568.659 & 1565.778 & 1570.724 \\
\hline $7 / 14 / 166: 00$ & 74.336 & 81.65 & 79.924 & 87.957 & 76.7 & 73.006 & 71.568 & 73.229 & 1584.136 & 1576.203 & 1573.384 & 1575.972 & 1571.928 & 1568.615 & 1565.723 & 1570.666 \\
\hline $7 / 14 / 167: 00$ & 74.332 & 81.667 & 79.947 & 87.955 & 76.739 & 73.002 & 71.563 & 73.248 & 1584.14 & 1576.186 & 1573.361 & 1575.974 & 1571.889 & 1568.619 & 1565.728 & 1570.647 \\
\hline $7 / 14 / 168: 00$ & 74.315 & 81.704 & 80.073 & 88.375 & 76.77 & 73.002 & 71.549 & 73.255 & 1584.157 & 1576.149 & 1573.235 & 1575.554 & 1571.858 & 1568.619 & 1565.742 & 1570.64 \\
\hline 7/14/16 9:00 & 74.294 & 81.771 & 80.175 & 88.674 & 76.784 & 72.983 & 71.532 & 73.234 & 1584.178 & 1576.082 & 1573.133 & 1575.255 & 1571.844 & 1568.638 & 1565.759 & 1570.661 \\
\hline $7 / 14 / 1610: 00$ & 74.301 & 81.867 & 80.294 & 88.881 & 76.835 & 72.999 & 71.551 & 73.262 & 1584.171 & 1575.986 & 1573.014 & 1575.048 & 1571.793 & 1568.622 & 1565.74 & 1570.633 \\
\hline $7 / 14 / 1611: 00$ & 74.31 & 81.967 & 80.411 & 89.022 & 76.884 & 73.018 & 71.558 & 73.364 & 1584.162 & 1575.886 & 1572.897 & 1574.907 & 1571.744 & 1568.603 & 1565.733 & 1570.531 \\
\hline $7 / 14 / 1612: 00$ & 74.292 & 82.032 & 81.154 & 89.11 & 76.945 & 73.032 & 71.525 & 73.831 & 1584.18 & 1575.821 & 1572.154 & 1574.819 & 1571.683 & 1568.589 & 1565.766 & 1570.064 \\
\hline $7 / 14 / 1613: 00$ & 74.306 & 82.146 & 81.504 & 89.222 & 77.047 & 73.078 & 71.549 & 74.063 & 1584.166 & 1575.707 & 1571.804 & 1574.707 & 1571.581 & 1568.543 & 1565.742 & 1569.832 \\
\hline $7 / 14 / 1614: 00$ & 74.334 & 82.265 & 81.732 & 89.323 & 77.164 & 73.116 & 71.566 & 74.281 & 1584.138 & 1575.588 & 1571.576 & 1574.606 & 1571.464 & 1568.505 & 1565.725 & 1569.614 \\
\hline $7 / 14 / 1615: 00$ & 74.334 & 82.348 & 81.896 & 89.379 & 77.25 & 73.132 & 71.551 & 74.434 & 1584.138 & 1575.505 & 1571.412 & 1574.55 & 1571.378 & 1568.489 & 1565.74 & 1569.461 \\
\hline 7/14/16 16:00 & 74.334 & 82.43 & 82.01 & 89.456 & 77.322 & 73.144 & 71.542 & 74.553 & 1584.138 & 1575.423 & 1571.298 & 1574.473 & 1571.306 & 1568.477 & 1565.749 & 1569.342 \\
\hline $7 / 14 / 1617: 00$ & 74.341 & 82.516 & 82.117 & 89.484 & 77.406 & 73.169 & 71.542 & 74.671 & 1584.131 & 1575.337 & 1571.191 & 1574.445 & 1571.222 & 1568.452 & 1565.749 & 1569.224 \\
\hline $7 / 14 / 1618: 00$ & 74.353 & 82.597 & 82.217 & 89.53 & 77.495 & 73.193 & 71.547 & 74.757 & 1584.119 & 1575.256 & 1571.091 & 1574.399 & 1571.133 & 1568.428 & 1565.744 & 1569.138 \\
\hline 7/14/16 19:00 & 74.379 & 82.676 & 82.317 & 89.572 & 77.582 & 73.214 & 71.544 & 74.885 & 1584.093 & 1575.177 & 1570.991 & 1574.357 & 1571.046 & 1568.407 & 1565.747 & 1569.01 \\
\hline $7 / 14 / 1620: 00$ & 74.414 & 82.755 & 82.411 & 89.622 & 77.673 & 73.239 & 71.549 & 74.989 & 1584.058 & 1575.098 & 1570.897 & 1574.307 & 1570.955 & 1568.382 & 1565.742 & 1568.906 \\
\hline
\end{tabular}


TABLE S1.1 Automatically recorded groundwater levels in the unconfined aquifer.

\begin{tabular}{|c|c|c|c|c|c|c|c|c|c|c|c|c|c|c|c|c|}
\hline \multirow[b]{2}{*}{ Date and Time } & \multicolumn{8}{|c|}{ Measured Depth to Water (ft TOC ${ }^{\mathrm{a}}$ ) } & \multicolumn{8}{|c|}{ Groundwater Elevation (ft AMSL) } \\
\hline & DL1-C & DL2-C1 & DL3-C1 & DL4-C1 & DL5-C1 & DL6-C & DL7-C & DL8-C1 & DL1-C & DL2-C1 & DL3-C1 & DL4-C1 & DL5-C1 & DL6-C & DL7-C & DL8-C1 \\
\hline 7/14/16 21:00 & 74.445 & 82.839 & 82.509 & 89.671 & 77.768 & 73.267 & 71.561 & 75.087 & 1584.027 & 1575.014 & 1570.799 & 1574.258 & 1570.86 & 1568.354 & 1565.73 & 1568.808 \\
\hline $7 / 14 / 1622: 00$ & 74.482 & 82.932 & 82.611 & 89.727 & 77.863 & 73.302 & 71.58 & 75.187 & 1583.99 & 1574.921 & 1570.697 & 1574.202 & 1570.765 & 1568.319 & 1565.711 & 1568.708 \\
\hline 7/14/16 23:00 & 74.511 & 83.009 & 82.707 & 89.773 & 77.957 & 73.33 & 71.592 & 75.277 & 1583.961 & 1574.844 & 1570.601 & 1574.156 & 1570.671 & 1568.291 & 1565.699 & 1568.618 \\
\hline 7/15/16 0:00 & 74.532 & 83.09 & 82.791 & 89.818 & 78.046 & 73.353 & 71.602 & 75.365 & 1583.94 & 1574.763 & 1570.517 & 1574.111 & 1570.582 & 1568.268 & 1565.689 & 1568.53 \\
\hline $7 / 15 / 161: 00$ & 74.546 & 83.16 & 82.87 & 89.864 & 78.127 & 73.372 & 71.609 & 75.449 & 1583.926 & 1574.693 & 1570.438 & 1574.065 & 1570.501 & 1568.249 & 1565.682 & 1568.446 \\
\hline $7 / 15 / 162: 00$ & 74.563 & 83.228 & 83.026 & 89.907 & 78.209 & 73.393 & 71.614 & 75.53 & 1583.909 & 1574.625 & 1570.282 & 1574.022 & 1570.419 & 1568.228 & 1565.677 & 1568.365 \\
\hline 7/15/16 3:00 & 74.574 & 83.307 & 83.103 & 89.953 & 78.295 & 73.421 & 71.623 & 75.625 & 1583.898 & 1574.546 & 1570.205 & 1573.976 & 1570.333 & 1568.2 & 1565.668 & 1568.27 \\
\hline $7 / 15 / 164: 00$ & 74.603 & 83.397 & 83.196 & 90.011 & 78.391 & 73.456 & 71.657 & 75.714 & 1583.869 & 1574.456 & 1570.112 & 1573.918 & 1570.237 & 1568.165 & 1565.634 & 1568.181 \\
\hline $7 / 15 / 165: 00$ & 74.631 & 83.493 & 83.315 & 90.072 & 78.495 & 73.5 & 71.699 & 75.82 & 1583.841 & 1574.36 & 1569.993 & 1573.857 & 1570.133 & 1568.121 & 1565.592 & 1568.075 \\
\hline $7 / 15 / 166: 00$ & 74.633 & 83.569 & 83.38 & 90.112 & 78.582 & 73.53 & 71.716 & 75.909 & 1583.839 & 1574.284 & 1569.928 & 1573.817 & 1570.046 & 1568.091 & 1565.575 & 1567.986 \\
\hline $7 / 15 / 167: 00$ & 74.61 & 83.602 & 83.383 & 90.126 & 78.631 & 73.523 & 71.69 & 75.964 & 1583.862 & 1574.251 & 1569.925 & 1573.803 & 1569.997 & 1568.098 & 1565.601 & 1567.931 \\
\hline $7 / 15 / 168: 00$ & 74.596 & 83.662 & 83.429 & 90.166 & 78.689 & 73.54 & 71.697 & 76.006 & 1583.876 & 1574.191 & 1569.879 & 1573.763 & 1569.939 & 1568.081 & 1565.594 & 1567.889 \\
\hline 7/15/16 9:00 & 74.633 & 83.774 & 83.52 & 90.238 & 78.799 & 73.602 & 71.762 & 76.111 & 1583.839 & 1574.079 & 1569.788 & 1573.691 & 1569.829 & 1568.019 & 1565.529 & 1567.784 \\
\hline $7 / 15 / 1610: 00$ & 74.638 & 83.828 & 83.522 & 90.023 & 78.873 & 73.628 & 71.797 & 76.173 & 1583.834 & 1574.025 & 1569.786 & 1573.906 & 1569.755 & 1567.993 & 1565.494 & 1567.722 \\
\hline $7 / 15 / 1611: 00$ & 74.65 & 83.851 & 83.492 & 89.757 & 78.955 & 73.649 & 71.814 & 76.215 & 1583.822 & 1574.002 & 1569.816 & 1574.172 & 1569.673 & 1567.972 & 1565.477 & 1567.68 \\
\hline $7 / 15 / 1612: 00$ & 74.614 & 83.802 & 83.418 & 89.731 & 78.456 & 73.612 & 71.785 & 76.134 & 1583.858 & 1574.051 & 1569.89 & 1574.198 & 1570.172 & 1568.009 & 1565.506 & 1567.761 \\
\hline $7 / 15 / 1613: 00$ & 74.588 & 83.758 & 83.359 & 89.538 & 78.344 & 73.595 & 71.757 & 76.118 & 1583.884 & 1574.095 & 1569.949 & 1574.391 & 1570.284 & 1568.026 & 1565.534 & 1567.777 \\
\hline $7 / 15 / 1614: 00$ & 74.558 & 83.7 & 82.814 & 89.405 & 78.241 & 73.563 & 71.721 & 75.867 & 1583.914 & 1574.153 & 1570.494 & 1574.524 & 1570.387 & 1568.058 & 1565.57 & 1568.028 \\
\hline 7/15/16 15:00 & 74.537 & 83.63 & 82.441 & 89.318 & 78.148 & 73.549 & 71.704 & 75.637 & 1583.935 & 1574.223 & 1570.867 & 1574.611 & 1570.48 & 1568.072 & 1565.587 & 1568.258 \\
\hline $7 / 15 / 1616: 00$ & 74.494 & 83.539 & 82.21 & 89.194 & 78.006 & 73.498 & 71.642 & 75.444 & 1583.978 & 1574.314 & 1571.098 & 1574.735 & 1570.622 & 1568.123 & 1565.649 & 1568.451 \\
\hline 7/15/16 17:00 & 74.534 & 83.53 & 82.124 & 89.162 & 77.964 & 73.53 & 71.688 & 75.375 & 1583.938 & 1574.323 & 1571.184 & 1574.767 & 1570.664 & 1568.091 & 1565.603 & 1568.52 \\
\hline $7 / 15 / 1618: 00$ & 74.527 & 83.474 & 82.021 & 89.124 & 77.877 & 73.512 & 71.673 & 75.252 & 1583.945 & 1574.379 & 1571.287 & 1574.805 & 1570.751 & 1568.109 & 1565.618 & 1568.643 \\
\hline $7 / 15 / 1619: 00$ & 74.541 & 83.451 & 81.952 & 89.108 & 77.822 & 73.526 & 71.692 & 75.221 & 1583.931 & 1574.402 & 1571.356 & 1574.821 & 1570.806 & 1568.095 & 1565.599 & 1568.674 \\
\hline $7 / 15 / 1620: 00$ & 74.553 & 83.418 & 81.896 & 89.087 & 77.765 & 73.528 & 71.709 & 75.187 & 1583.919 & 1574.435 & 1571.412 & 1574.842 & 1570.863 & 1568.093 & 1565.582 & 1568.708 \\
\hline $7 / 15 / 1621: 00$ & 74.56 & 83.393 & 81.84 & 89.045 & 77.71 & 73.533 & 71.721 & 75.143 & 1583.912 & 1574.46 & 1571.468 & 1574.884 & 1570.918 & 1568.088 & 1565.57 & 1568.752 \\
\hline $7 / 15 / 1622: 00$ & 74.579 & 83.374 & 81.809 & 89.022 & 77.677 & 73.547 & 71.752 & 75.115 & 1583.893 & 1574.479 & 1571.499 & 1574.907 & 1570.951 & 1568.074 & 1565.539 & 1568.78 \\
\hline $7 / 15 / 1623: 00$ & 74.581 & 83.339 & 81.763 & 88.984 & 77.624 & 73.542 & 71.754 & 75.08 & 1583.891 & 1574.514 & 1571.545 & 1574.945 & 1571.004 & 1568.079 & 1565.537 & 1568.815 \\
\hline 7/16/16 0:00 & 74.579 & 83.311 & 81.721 & 88.963 & 77.575 & 73.54 & 71.764 & 74.994 & 1583.893 & 1574.542 & 1571.587 & 1574.966 & 1571.053 & 1568.081 & 1565.527 & 1568.901 \\
\hline $7 / 16 / 161: 00$ & 74.581 & 83.288 & 81.69 & 88.947 & 77.535 & 73.544 & 71.778 & 75.019 & 1583.891 & 1574.565 & 1571.618 & 1574.982 & 1571.093 & 1568.077 & 1565.513 & 1568.876 \\
\hline $7 / 16 / 162: 00$ & 74.563 & 83.232 & 81.637 & 88.907 & 77.465 & 73.521 & 71.757 & 74.978 & 1583.909 & 1574.621 & 1571.671 & 1575.022 & 1571.163 & 1568.1 & 1565.534 & 1568.917 \\
\hline 7/16/16 3:00 & 74.548 & 83.19 & 81.593 & 88.881 & 77.404 & 73.514 & 71.749 & 74.924 & 1583.924 & 1574.663 & 1571.715 & 1575.048 & 1571.224 & 1568.107 & 1565.542 & 1568.971 \\
\hline $7 / 16 / 164: 00$ & 74.541 & 83.151 & 81.558 & 88.865 & 77.357 & 73.505 & 71.75 & 74.903 & 1583.931 & 1574.702 & 1571.75 & 1575.064 & 1571.271 & 1568.116 & 1565.541 & 1568.992 \\
\hline $7 / 16 / 165: 00$ & 74.539 & 83.118 & 81.525 & 88.84 & 77.311 & 73.505 & 71.747 & 74.871 & 1583.933 & 1574.735 & 1571.783 & 1575.089 & 1571.317 & 1568.116 & 1565.544 & 1569.024 \\
\hline $7 / 16 / 166: 00$ & 74.544 & 83.095 & 81.499 & 88.83 & 77.274 & 73.505 & 71.752 & 74.852 & 1583.928 & 1574.758 & 1571.809 & 1575.099 & 1571.354 & 1568.116 & 1565.539 & 1569.043 \\
\hline $7 / 16 / 167: 00$ & 74.546 & 83.065 & 81.474 & 88.819 & 77.234 & 73.505 & 71.759 & 74.827 & 1583.926 & 1574.788 & 1571.834 & 1575.11 & 1571.394 & 1568.116 & 1565.532 & 1569.068 \\
\hline $7 / 16 / 168: 00$ & 74.544 & 83.034 & 81.441 & 88.805 & 77.19 & 73.498 & 71.761 & 74.794 & 1583.928 & 1574.819 & 1571.867 & 1575.124 & 1571.438 & 1568.123 & 1565.53 & 1569.101 \\
\hline $7 / 16 / 169: 00$ & 74.537 & 83.002 & 81.406 & 88.788 & 77.143 & 73.493 & 71.757 & 74.762 & 1583.935 & 1574.851 & 1571.902 & 1575.141 & 1571.485 & 1568.128 & 1565.534 & 1569.133 \\
\hline $7 / 16 / 16$ 10:00 & 74.522 & 82.962 & 81.369 & 88.765 & 77.087 & 73.477 & 71.742 & 74.732 & 1583.95 & 1574.891 & 1571.939 & 1575.164 & 1571.541 & 1568.144 & 1565.549 & 1569.163 \\
\hline $7 / 16 / 1611: 00$ & 74.511 & 82.925 & 81.334 & 88.746 & 77.038 & 73.463 & 71.733 & 74.694 & 1583.961 & 1574.928 & 1571.974 & 1575.183 & 1571.59 & 1568.158 & 1565.558 & 1569.201 \\
\hline 7/16/16 12:00 & 74.501 & 82.89 & 81.294 & 88.73 & 76.987 & 73.453 & 71.726 & 74.664 & 1583.971 & 1574.963 & 1572.014 & 1575.199 & 1571.641 & 1568.168 & 1565.565 & 1569.231 \\
\hline $7 / 16 / 1613: 00$ & 74.485 & 82.851 & 81.257 & 88.709 & 76.933 & 73.442 & 71.711 & 74.622 & 1583.987 & 1575.002 & 1572.051 & 1575.22 & 1571.695 & 1568.179 & 1565.58 & 1569.273 \\
\hline $7 / 16 / 1614: 00$ & 74.466 & 82.804 & 81.215 & 88.685 & 76.877 & 73.423 & 71.688 & 74.578 & 1584.006 & 1575.049 & 1572.093 & 1575.244 & 1571.751 & 1568.198 & 1565.603 & 1569.317 \\
\hline $7 / 16 / 1615: 00$ & 74.445 & 82.755 & 81.171 & 88.662 & 76.819 & 73.404 & 71.666 & 74.497 & 1584.027 & 1575.098 & 1572.137 & 1575.267 & 1571.809 & 1568.217 & 1565.625 & 1569.398 \\
\hline $7 / 16 / 1616: 00$ & 74.431 & 82.716 & 81.131 & 88.641 & 76.761 & 73.393 & 71.652 & 74.497 & 1584.041 & 1575.137 & 1572.177 & 1575.288 & 1571.867 & 1568.228 & 1565.639 & 1569.398 \\
\hline 7/16/16 17:00 & 74.428 & 82.683 & 81.101 & 88.627 & 76.719 & 73.39 & 71.649 & 74.467 & 1584.044 & 1575.17 & 1572.207 & 1575.302 & 1571.909 & 1568.231 & 1565.642 & 1569.428 \\
\hline $7 / 16 / 1618: 00$ & 74.416 & 82.646 & 81.068 & 88.601 & 76.668 & 73.374 & 71.628 & 74.439 & 1584.056 & 1575.207 & 1572.24 & 1575.328 & 1571.96 & 1568.247 & 1565.663 & 1569.456 \\
\hline $7 / 16 / 1619: 00$ & 74.423 & 82.634 & 81.052 & 88.604 & 76.64 & 73.381 & 71.644 & 74.42 & 1584.049 & 1575.219 & 1572.256 & 1575.325 & 1571.988 & 1568.24 & 1565.647 & 1569.475 \\
\hline $7 / 16 / 1620: 00$ & 74.428 & 82.609 & 81.033 & 88.59 & 76.607 & 73.384 & 71.645 & 74.404 & 1584.044 & 1575.244 & 1572.275 & 1575.339 & 1572.021 & 1568.237 & 1565.646 & 1569.491 \\
\hline $7 / 16 / 1621: 00$ & 74.428 & 82.592 & 81.022 & 88.59 & 76.579 & 73.384 & 71.652 & 74.388 & 1584.044 & 1575.261 & 1572.286 & 1575.339 & 1572.049 & 1568.237 & 1565.639 & 1569.507 \\
\hline
\end{tabular}


TABLE S1.1 Automatically recorded groundwater levels in the unconfined aquifer.

\begin{tabular}{|c|c|c|c|c|c|c|c|c|c|c|c|c|c|c|c|c|}
\hline \multirow[b]{2}{*}{ Date and Time } & \multicolumn{8}{|c|}{ Measured Depth to Water (ft TOC ${ }^{\mathrm{a}}$ ) } & \multicolumn{8}{|c|}{ Groundwater Elevation ( $\mathrm{ft} \mathrm{AMSL}^{\mathrm{b}}$ ) } \\
\hline & DL1-C & DL2-C1 & DL3-C1 & DL4-C1 & DL5-C1 & DL6-C & DL7-C & DL8-C1 & DL1-C & DL2-C1 & DL3-C1 & DL4-C1 & DL5-C1 & DL6-C & DL7-C & DL8-C1 \\
\hline $7 / 16 / 1622: 00$ & 74.435 & 82.59 & 81.008 & 88.587 & 76.556 & 73.388 & 71.666 & 74.376 & 1584.037 & 1575.263 & 1572.3 & 1575.342 & 1572.072 & 1568.233 & 1565.625 & 1569.519 \\
\hline $7 / 16 / 1623: 00$ & 74.452 & 82.588 & 81.017 & 88.597 & 76.549 & 73.407 & 71.692 & 74.383 & 1584.02 & 1575.265 & 1572.291 & 1575.332 & 1572.079 & 1568.214 & 1565.599 & 1569.512 \\
\hline $7 / 17 / 160: 00$ & 74.433 & 82.553 & 80.984 & 88.573 & 76.502 & 73.386 & 71.673 & 74.358 & 1584.039 & 1575.3 & 1572.324 & 1575.356 & 1572.126 & 1568.235 & 1565.618 & 1569.537 \\
\hline $7 / 17 / 161: 00$ & 74.423 & 82.537 & 80.966 & 88.564 & 76.467 & 73.381 & 71.673 & 74.332 & 1584.049 & 1575.316 & 1572.342 & 1575.365 & 1572.161 & 1568.24 & 1565.618 & 1569.563 \\
\hline $7 / 17 / 162: 00$ & 74.412 & 82.509 & 80.935 & 88.543 & 76.43 & 73.367 & 71.664 & 74.258 & 1584.06 & 1575.344 & 1572.373 & 1575.386 & 1572.198 & 1568.254 & 1565.627 & 1569.637 \\
\hline $7 / 17 / 163: 00$ & 74.414 & 82.495 & 80.928 & 88.545 & 76.404 & 73.372 & 71.671 & 74.311 & 1584.058 & 1575.358 & 1572.38 & 1575.384 & 1572.224 & 1568.249 & 1565.62 & 1569.584 \\
\hline $7 / 17 / 164: 00$ & 74.402 & 82.472 & 80.907 & 88.529 & 76.371 & 73.363 & 71.661 & 74.283 & 1584.07 & 1575.381 & 1572.401 & 1575.4 & 1572.257 & 1568.258 & 1565.63 & 1569.612 \\
\hline $7 / 17 / 165: 00$ & 74.388 & 82.437 & 80.879 & 88.515 & 76.334 & 73.353 & 71.649 & 74.263 & 1584.084 & 1575.416 & 1572.429 & 1575.414 & 1572.294 & 1568.268 & 1565.642 & 1569.632 \\
\hline $7 / 17 / 166: 00$ & 74.398 & 82.439 & 80.875 & 88.513 & 76.32 & 73.358 & 71.666 & 74.228 & 1584.074 & 1575.414 & 1572.433 & 1575.416 & 1572.308 & 1568.263 & 1565.625 & 1569.667 \\
\hline $7 / 17 / 167: 00$ & 74.431 & 82.467 & 80.898 & 88.531 & 76.331 & 73.393 & 71.714 & 74.281 & 1584.041 & 1575.386 & 1572.41 & 1575.398 & 1572.297 & 1568.228 & 1565.577 & 1569.614 \\
\hline $7 / 17 / 168: 00$ & 74.452 & 82.476 & 80.914 & 88.545 & 76.334 & 73.407 & 71.745 & 74.288 & 1584.02 & 1575.377 & 1572.394 & 1575.384 & 1572.294 & 1568.214 & 1565.546 & 1569.607 \\
\hline $7 / 17 / 169: 00$ & 74.447 & 82.469 & 80.896 & 88.531 & 76.311 & 73.398 & 71.737 & 74.276 & 1584.025 & 1575.384 & 1572.412 & 1575.398 & 1572.317 & 1568.223 & 1565.554 & 1569.619 \\
\hline $7 / 17 / 1610: 00$ & 74.454 & 82.472 & 80.896 & 88.536 & 76.306 & 73.411 & 71.761 & 74.274 & 1584.018 & 1575.381 & 1572.412 & 1575.393 & 1572.322 & 1568.21 & 1565.53 & 1569.621 \\
\hline $7 / 17 / 1611: 00$ & 74.464 & 82.481 & 80.905 & 88.545 & 76.304 & 73.418 & 71.785 & 74.249 & 1584.008 & 1575.372 & 1572.403 & 1575.384 & 1572.324 & 1568.203 & 1565.506 & 1569.646 \\
\hline $7 / 17 / 1612: 00$ & 74.464 & 82.472 & 80.898 & 88.538 & 76.292 & 73.418 & 71.793 & 74.225 & 1584.008 & 1575.381 & 1572.41 & 1575.391 & 1572.336 & 1568.203 & 1565.498 & 1569.67 \\
\hline $7 / 17 / 1613: 00$ & 74.461 & 82.479 & 80.9 & 88.538 & 76.28 & 73.423 & 71.807 & 74.281 & 1584.011 & 1575.374 & 1572.408 & 1575.391 & 1572.348 & 1568.198 & 1565.484 & 1569.614 \\
\hline $7 / 17 / 1614: 00$ & 74.456 & 82.465 & 80.889 & 88.506 & 76.259 & 73.421 & 71.809 & 74.274 & 1584.016 & 1575.388 & 1572.419 & 1575.423 & 1572.369 & 1568.2 & 1565.482 & 1569.621 \\
\hline $7 / 17 / 1615: 00$ & 74.456 & 82.469 & 80.886 & 88.517 & 76.252 & 73.428 & 71.823 & 74.269 & 1584.016 & 1575.384 & 1572.422 & 1575.412 & 1572.376 & 1568.193 & 1565.468 & 1569.626 \\
\hline $7 / 17 / 1616: 00$ & 74.449 & 82.458 & 80.877 & 88.517 & 76.229 & 73.421 & 71.821 & 74.265 & 1584.023 & 1575.395 & 1572.431 & 1575.412 & 1572.399 & 1568.2 & 1565.47 & 1569.63 \\
\hline $7 / 17 / 1617: 00$ & 74.438 & 82.441 & 80.861 & 88.51 & 76.213 & 73.414 & 71.823 & 74.253 & 1584.034 & 1575.412 & 1572.447 & 1575.419 & 1572.415 & 1568.207 & 1565.468 & 1569.642 \\
\hline $7 / 17 / 16$ 18:00 & 74.435 & 82.439 & 80.856 & 88.531 & 76.196 & 73.421 & 71.828 & 74.244 & 1584.037 & 1575.414 & 1572.452 & 1575.398 & 1572.432 & 1568.2 & 1565.463 & 1569.651 \\
\hline $7 / 17 / 16$ 19:00 & 74.431 & 82.423 & 80.847 & 88.534 & 76.173 & 73.416 & 71.826 & 74.237 & 1584.041 & 1575.43 & 1572.461 & 1575.395 & 1572.455 & 1568.205 & 1565.465 & 1569.658 \\
\hline $7 / 17 / 1620: 00$ & 74.428 & 82.418 & 80.837 & 88.531 & 76.159 & 73.418 & 71.831 & 74.23 & 1584.044 & 1575.435 & 1572.471 & 1575.398 & 1572.469 & 1568.203 & 1565.46 & 1569.665 \\
\hline $7 / 17 / 1621: 00$ & 74.438 & 82.42 & 80.837 & 88.508 & 76.152 & 73.423 & 71.845 & 74.235 & 1584.034 & 1575.433 & 1572.471 & 1575.421 & 1572.476 & 1568.198 & 1565.446 & 1569.66 \\
\hline $7 / 17 / 1622: 00$ & 74.44 & 82.413 & 80.835 & 88.496 & 76.143 & 73.425 & 71.852 & 74.186 & 1584.032 & 1575.44 & 1572.473 & 1575.433 & 1572.485 & 1568.196 & 1565.439 & 1569.709 \\
\hline $7 / 17 / 1623: 00$ & 74.445 & 82.416 & 80.835 & 88.494 & 76.136 & 73.432 & 71.866 & 74.23 & 1584.027 & 1575.437 & 1572.473 & 1575.435 & 1572.492 & 1568.189 & 1565.425 & 1569.665 \\
\hline $7 / 18 / 160: 00$ & 74.438 & 82.404 & 80.826 & 88.489 & 76.117 & 73.425 & 71.859 & 74.23 & 1584.034 & 1575.449 & 1572.482 & 1575.44 & 1572.511 & 1568.196 & 1565.432 & 1569.665 \\
\hline $7 / 18 / 161: 00$ & 74.435 & 82.385 & 80.814 & 88.48 & 76.101 & 73.421 & 71.854 & 74.214 & 1584.037 & 1575.468 & 1572.494 & 1575.449 & 1572.527 & 1568.2 & 1565.437 & 1569.681 \\
\hline $7 / 18 / 162: 00$ & 74.426 & 82.369 & 80.875 & 88.468 & 76.075 & 73.411 & 71.848 & 74.209 & 1584.046 & 1575.484 & 1572.433 & 1575.461 & 1572.553 & 1568.21 & 1565.443 & 1569.686 \\
\hline $7 / 18 / 163: 00$ & 74.423 & 82.362 & 80.865 & 88.461 & 76.059 & 73.414 & 71.852 & 74.2 & 1584.049 & 1575.491 & 1572.443 & 1575.468 & 1572.569 & 1568.207 & 1565.439 & 1569.695 \\
\hline $7 / 18 / 164: 00$ & 74.438 & 82.372 & 80.889 & 88.473 & 76.066 & 73.428 & 71.876 & 74.221 & 1584.034 & 1575.481 & 1572.419 & 1575.456 & 1572.562 & 1568.193 & 1565.415 & 1569.674 \\
\hline $7 / 18 / 165: 00$ & 74.431 & 82.36 & 80.886 & 88.466 & 76.054 & 73.421 & 71.869 & 74.223 & 1584.041 & 1575.493 & 1572.422 & 1575.463 & 1572.574 & 1568.2 & 1565.422 & 1569.672 \\
\hline $7 / 18 / 166: 00$ & 74.438 & 82.358 & 80.854 & 88.463 & 76.05 & 73.425 & 71.879 & 74.221 & 1584.034 & 1575.495 & 1572.454 & 1575.466 & 1572.578 & 1568.196 & 1565.412 & 1569.674 \\
\hline $7 / 18 / 167: 00$ & 74.442 & 82.36 & 80.823 & 88.466 & 76.042 & 73.456 & 71.89 & 74.334 & 1584.03 & 1575.493 & 1572.485 & 1575.463 & 1572.586 & 1568.165 & 1565.401 & 1569.561 \\
\hline $7 / 18 / 168: 00$ & 74.447 & 82.362 & 80.823 & 88.468 & 76.04 & 73.481 & 71.905 & 74.4 & 1584.025 & 1575.491 & 1572.485 & 1575.461 & 1572.588 & 1568.14 & 1565.386 & 1569.495 \\
\hline $7 / 18 / 169: 00$ & 74.452 & 82.36 & 80.84 & 88.468 & 76.031 & 73.493 & 71.912 & 74.416 & 1584.02 & 1575.493 & 1572.468 & 1575.461 & 1572.597 & 1568.128 & 1565.379 & 1569.479 \\
\hline $7 / 18 / 1610: 00$ & 74.445 & 82.353 & 80.828 & 88.464 & 76.017 & 73.495 & 71.907 & 74.451 & 1584.027 & 1575.5 & 1572.48 & 1575.465 & 1572.611 & 1568.126 & 1565.384 & 1569.444 \\
\hline $7 / 18 / 1611: 00$ & 74.447 & 82.358 & 80.842 & 88.471 & 76.017 & 73.526 & 71.926 & 74.571 & 1584.025 & 1575.495 & 1572.466 & 1575.458 & 1572.611 & 1568.095 & 1565.365 & 1569.324 \\
\hline $7 / 18 / 1612: 00$ & 74.447 & 82.351 & 80.842 & 88.466 & 76.005 & 73.526 & 71.924 & 74.502 & 1584.025 & 1575.502 & 1572.466 & 1575.463 & 1572.623 & 1568.095 & 1565.367 & 1569.393 \\
\hline $7 / 18 / 1613: 00$ & 74.44 & 82.341 & 80.837 & 88.457 & 75.998 & 73.528 & 71.926 & 74.504 & 1584.032 & 1575.512 & 1572.471 & 1575.472 & 1572.63 & 1568.093 & 1565.365 & 1569.391 \\
\hline $7 / 18 / 1614: 00$ & 74.442 & 82.334 & 80.842 & 88.452 & 75.991 & 73.54 & 71.928 & 74.518 & 1584.03 & 1575.519 & 1572.466 & 1575.477 & 1572.637 & 1568.081 & 1565.363 & 1569.377 \\
\hline $7 / 18 / 1615: 00$ & 74.428 & 82.309 & 80.83 & 88.424 & 75.968 & 73.53 & 71.91 & 74.52 & 1584.044 & 1575.544 & 1572.478 & 1575.505 & 1572.66 & 1568.091 & 1565.381 & 1569.375 \\
\hline $7 / 18 / 1616: 00$ & 74.416 & 82.288 & 80.814 & 88.417 & 75.949 & 73.53 & 71.895 & 74.534 & 1584.056 & 1575.565 & 1572.494 & 1575.512 & 1572.679 & 1568.091 & 1565.396 & 1569.361 \\
\hline $7 / 18 / 1617: 00$ & 74.409 & 82.267 & 80.798 & 88.405 & 75.926 & 73.53 & 71.886 & 74.539 & 1584.063 & 1575.586 & 1572.51 & 1575.524 & 1572.702 & 1568.091 & 1565.405 & 1569.356 \\
\hline $7 / 18 / 16$ 18:00 & 74.407 & 82.244 & 80.784 & 88.396 & 75.907 & 73.535 & 71.876 & 74.55 & 1584.065 & 1575.609 & 1572.524 & 1575.533 & 1572.721 & 1568.086 & 1565.415 & 1569.345 \\
\hline $7 / 18 / 1619: 00$ & 74.421 & 82.227 & 80.772 & 88.389 & 75.898 & 73.537 & 71.866 & 74.546 & 1584.051 & 1575.626 & 1572.536 & 1575.54 & 1572.73 & 1568.084 & 1565.425 & 1569.349 \\
\hline $7 / 18 / 1620: 00$ & 74.447 & 82.218 & 80.765 & 88.384 & 75.898 & 73.542 & 71.862 & 74.546 & 1584.025 & 1575.635 & 1572.543 & 1575.545 & 1572.73 & 1568.079 & 1565.429 & 1569.349 \\
\hline $7 / 18 / 1621: 00$ & 74.482 & 82.216 & 80.767 & 88.391 & 75.91 & 73.554 & 71.876 & 74.555 & 1583.99 & 1575.637 & 1572.541 & 1575.538 & 1572.718 & 1568.067 & 1565.415 & 1569.34 \\
\hline $7 / 18 / 1622: 00$ & 74.511 & 82.216 & 80.77 & 88.393 & 75.921 & 73.563 & 71.886 & 74.569 & 1583.961 & 1575.637 & 1572.538 & 1575.536 & 1572.707 & 1568.058 & 1565.405 & 1569.326 \\
\hline
\end{tabular}


TABLE S1.1 Automatically recorded groundwater levels in the unconfined aquifer.

\begin{tabular}{|c|c|c|c|c|c|c|c|c|c|c|c|c|c|c|c|c|}
\hline \multirow[b]{2}{*}{ Date and Time } & \multicolumn{8}{|c|}{ Measured Depth to Water (ft TOC ${ }^{\mathrm{a}}$ ) } & \multicolumn{8}{|c|}{ Groundwater Elevation (ft AMSL) } \\
\hline & DL1-C & DL2-C1 & DL3-C1 & DL4-C1 & DL5-C1 & DL6-C & DL7-C & DL8-C1 & DL1-C & DL2-C1 & DL3-C1 & DL4-C1 & DL5-C1 & DL6-C & DL7-C & DL8-C1 \\
\hline 7/18/16 23:00 & 74.539 & 82.227 & 80.777 & 88.398 & 75.938 & 73.575 & 71.895 & 74.588 & 1583.933 & 1575.626 & 1572.531 & 1575.531 & 1572.69 & 1568.046 & 1565.396 & 1569.307 \\
\hline $7 / 19 / 160: 00$ & 74.567 & 82.234 & 80.784 & 88.396 & 75.954 & 73.591 & 71.907 & 74.597 & 1583.905 & 1575.619 & 1572.524 & 1575.533 & 1572.674 & 1568.03 & 1565.384 & 1569.298 \\
\hline 7/19/16 1:00 & 74.588 & 82.234 & 80.786 & 88.403 & 75.968 & 73.595 & 71.919 & 74.602 & 1583.884 & 1575.619 & 1572.522 & 1575.526 & 1572.66 & 1568.026 & 1565.372 & 1569.293 \\
\hline $7 / 19 / 162: 00$ & 74.596 & 82.225 & 80.784 & 88.401 & 75.972 & 73.593 & 71.912 & 74.576 & 1583.876 & 1575.628 & 1572.524 & 1575.528 & 1572.656 & 1568.028 & 1565.379 & 1569.319 \\
\hline 7/19/16 3:00 & 74.603 & 82.223 & 80.781 & 88.394 & 75.977 & 73.598 & 71.907 & 74.639 & 1583.869 & 1575.63 & 1572.527 & 1575.535 & 1572.651 & 1568.023 & 1565.384 & 1569.256 \\
\hline 7/19/16 4:00 & 74.631 & 82.241 & 80.802 & 88.41 & 76.008 & 73.616 & 71.933 & 74.66 & 1583.841 & 1575.612 & 1572.506 & 1575.519 & 1572.62 & 1568.005 & 1565.358 & 1569.235 \\
\hline $7 / 19 / 165: 00$ & 74.624 & 82.237 & 80.828 & 88.41 & 76.012 & 73.619 & 71.928 & 74.671 & 1583.848 & 1575.616 & 1572.48 & 1575.519 & 1572.616 & 1568.002 & 1565.363 & 1569.224 \\
\hline $7 / 19 / 166: 00$ & 74.61 & 82.23 & 80.821 & 88.403 & 76.01 & 73.616 & 71.928 & 74.678 & 1583.862 & 1575.623 & 1572.487 & 1575.526 & 1572.618 & 1568.005 & 1565.363 & 1569.217 \\
\hline $7 / 19 / 167: 00$ & 74.614 & 82.234 & 80.837 & 88.429 & 76.017 & 73.63 & 71.933 & 74.701 & 1583.858 & 1575.619 & 1572.471 & 1575.5 & 1572.611 & 1567.991 & 1565.358 & 1569.194 \\
\hline $7 / 19 / 168: 00$ & 74.577 & 82.241 & 80.945 & 88.846 & 75.984 & 73.609 & 71.898 & 74.68 & 1583.895 & 1575.612 & 1572.363 & 1575.083 & 1572.644 & 1568.012 & 1565.393 & 1569.215 \\
\hline 7/19/16 9:00 & 74.572 & 82.306 & 81.033 & 89.122 & 75.987 & 73.616 & 71.9 & 74.694 & 1583.9 & 1575.547 & 1572.275 & 1574.807 & 1572.641 & 1568.005 & 1565.391 & 1569.201 \\
\hline 7/19/16 10:00 & 74.584 & 82.381 & 81.143 & 89.316 & 75.996 & 73.626 & 71.902 & 74.725 & 1583.888 & 1575.472 & 1572.165 & 1574.613 & 1572.632 & 1567.995 & 1565.389 & 1569.17 \\
\hline 7/19/16 11:00 & 74.631 & 82.462 & 81.25 & 89.467 & 76.017 & 73.642 & 71.912 & 74.739 & 1583.841 & 1575.391 & 1572.058 & 1574.462 & 1572.611 & 1567.979 & 1565.379 & 1569.156 \\
\hline 7/19/16 12:00 & 74.678 & 82.534 & 81.304 & 89.5 & 76.043 & 73.654 & 71.914 & 74.827 & 1583.794 & 1575.319 & 1572.004 & 1574.429 & 1572.585 & 1567.967 & 1565.377 & 1569.068 \\
\hline $7 / 19 / 1613: 00$ & 74.73 & 82.572 & 81.231 & 89.166 & 76.066 & 73.661 & 71.924 & 74.806 & 1583.742 & 1575.281 & 1572.077 & 1574.763 & 1572.562 & 1567.96 & 1565.367 & 1569.089 \\
\hline $7 / 19 / 1614: 00$ & 74.77 & 82.595 & 81.336 & 89.106 & 76.094 & 73.668 & 71.926 & 74.862 & 1583.702 & 1575.258 & 1571.972 & 1574.823 & 1572.534 & 1567.953 & 1565.365 & 1569.033 \\
\hline $7 / 19 / 1615: 00$ & 74.796 & 82.602 & 81.415 & 88.959 & 76.113 & 73.67 & 71.919 & 74.913 & 1583.676 & 1575.251 & 1571.893 & 1574.97 & 1572.515 & 1567.951 & 1565.372 & 1568.982 \\
\hline $7 / 19 / 1616: 00$ & 74.803 & 82.616 & 81.565 & 89.283 & 76.12 & 73.672 & 71.9 & 75.136 & 1583.669 & 1575.237 & 1571.743 & 1574.646 & 1572.508 & 1567.949 & 1565.391 & 1568.759 \\
\hline 7/19/16 17:00 & 74.822 & 82.676 & 81.709 & 89.516 & 76.138 & 73.67 & 71.893 & 75.07 & 1583.65 & 1575.177 & 1571.599 & 1574.413 & 1572.49 & 1567.951 & 1565.398 & 1568.825 \\
\hline 7/19/16 18:00 & 74.834 & 82.723 & 81.749 & 89.433 & 76.159 & 73.67 & 71.886 & 75.075 & 1583.638 & 1575.13 & 1571.559 & 1574.496 & 1572.469 & 1567.951 & 1565.405 & 1568.82 \\
\hline 7/19/16 19:00 & 74.843 & 82.723 & 81.707 & 89.199 & 76.171 & 73.64 & 71.874 & 74.961 & 1583.629 & 1575.13 & 1571.601 & 1574.73 & 1572.457 & 1567.981 & 1565.417 & 1568.934 \\
\hline $7 / 19 / 1620: 00$ & 74.852 & 82.723 & 81.681 & 89.075 & 76.187 & 73.633 & 71.876 & 74.938 & 1583.62 & 1575.13 & 1571.627 & 1574.854 & 1572.441 & 1567.988 & 1565.415 & 1568.957 \\
\hline $7 / 19 / 1621: 00$ & 74.855 & 82.72 & 81.667 & 88.986 & 76.196 & 73.628 & 71.878 & 74.896 & 1583.617 & 1575.133 & 1571.641 & 1574.943 & 1572.432 & 1567.993 & 1565.413 & 1568.999 \\
\hline $7 / 19 / 1622: 00$ & 74.859 & 82.723 & 81.662 & 88.921 & 76.21 & 73.633 & 71.888 & 74.887 & 1583.613 & 1575.13 & 1571.646 & 1575.008 & 1572.418 & 1567.988 & 1565.403 & 1569.008 \\
\hline $7 / 19 / 1623: 00$ & 74.871 & 82.732 & 81.676 & 88.9 & 76.231 & 73.642 & 71.912 & 74.95 & 1583.601 & 1575.121 & 1571.632 & 1575.029 & 1572.397 & 1567.979 & 1565.379 & 1568.945 \\
\hline $7 / 20 / 160: 00$ & 74.878 & 82.732 & 81.679 & 88.875 & 76.238 & 73.64 & 71.917 & 74.959 & 1583.594 & 1575.121 & 1571.629 & 1575.054 & 1572.39 & 1567.981 & 1565.374 & 1568.936 \\
\hline $7 / 20 / 161: 00$ & 74.881 & 82.727 & 81.683 & 88.856 & 76.247 & 73.64 & 71.919 & 74.964 & 1583.591 & 1575.126 & 1571.625 & 1575.073 & 1572.381 & 1567.981 & 1565.372 & 1568.931 \\
\hline $7 / 20 / 162: 00$ & 74.885 & 82.723 & 81.763 & 88.842 & 76.257 & 73.635 & 71.929 & 74.973 & 1583.587 & 1575.13 & 1571.545 & 1575.087 & 1572.371 & 1567.986 & 1565.362 & 1568.922 \\
\hline $7 / 20 / 163: 00$ & 74.895 & 82.732 & 81.788 & 88.835 & 76.273 & 73.647 & 71.943 & 74.999 & 1583.577 & 1575.121 & 1571.52 & 1575.094 & 1572.355 & 1567.974 & 1565.348 & 1568.896 \\
\hline $7 / 20 / 164: 00$ & 74.895 & 82.725 & 81.784 & 88.821 & 76.276 & 73.637 & 71.94 & 75.008 & 1583.577 & 1575.128 & 1571.524 & 1575.108 & 1572.352 & 1567.984 & 1565.351 & 1568.887 \\
\hline $7 / 20 / 165: 00$ & 74.895 & 82.711 & 81.812 & 88.812 & 76.282 & 73.633 & 71.936 & 75.01 & 1583.577 & 1575.142 & 1571.496 & 1575.117 & 1572.346 & 1567.988 & 1565.355 & 1568.885 \\
\hline $7 / 20 / 166: 00$ & 74.907 & 82.716 & 81.809 & 88.809 & 76.294 & 73.637 & 71.945 & 75.015 & 1583.565 & 1575.137 & 1571.499 & 1575.12 & 1572.334 & 1567.984 & 1565.346 & 1568.88 \\
\hline $7 / 20 / 16$ 7:00 & 74.916 & 82.709 & 81.756 & 88.807 & 76.294 & 73.656 & 71.95 & 75.129 & 1583.556 & 1575.144 & 1571.552 & 1575.122 & 1572.334 & 1567.965 & 1565.341 & 1568.766 \\
\hline $7 / 20 / 168: 00$ & 74.918 & 82.713 & 81.576 & 88.8 & 76.303 & 73.672 & 71.95 & 75.119 & 1583.554 & 1575.14 & 1571.732 & 1575.129 & 1572.325 & 1567.949 & 1565.341 & 1568.776 \\
\hline $7 / 20 / 169: 00$ & 74.921 & 82.706 & 81.483 & 88.8 & 76.306 & 73.684 & 71.952 & 75.115 & 1583.551 & 1575.147 & 1571.825 & 1575.129 & 1572.322 & 1567.937 & 1565.339 & 1568.78 \\
\hline $7 / 20 / 1610: 00$ & 74.925 & 82.695 & 81.439 & 88.791 & 76.308 & 73.691 & 71.953 & 75.115 & 1583.547 & 1575.158 & 1571.869 & 1575.138 & 1572.32 & 1567.93 & 1565.338 & 1568.78 \\
\hline $7 / 20 / 1611: 00$ & 74.923 & 82.688 & 81.413 & 88.784 & 76.308 & 73.691 & 71.95 & 75.061 & 1583.549 & 1575.165 & 1571.895 & 1575.145 & 1572.32 & 1567.93 & 1565.341 & 1568.834 \\
\hline $7 / 20 / 1612: 00$ & 74.93 & 82.69 & 81.378 & 88.788 & 76.322 & 73.707 & 71.955 & 75.126 & 1583.542 & 1575.163 & 1571.93 & 1575.141 & 1572.306 & 1567.914 & 1565.336 & 1568.769 \\
\hline $7 / 20 / 1613: 00$ & 74.93 & 82.679 & 81.343 & 88.777 & 76.327 & 73.707 & 71.945 & 75.108 & 1583.542 & 1575.174 & 1571.965 & 1575.152 & 1572.301 & 1567.914 & 1565.346 & 1568.787 \\
\hline $7 / 20 / 1614: 00$ & 74.928 & 82.667 & 81.341 & 88.765 & 76.339 & 73.707 & 71.941 & 75.11 & 1583.544 & 1575.186 & 1571.967 & 1575.164 & 1572.289 & 1567.914 & 1565.35 & 1568.785 \\
\hline $7 / 20 / 1615: 00$ & 74.916 & 82.679 & 81.387 & 89.164 & 76.341 & 73.705 & 71.926 & 75.089 & 1583.556 & 1575.174 & 1571.921 & 1574.765 & 1572.287 & 1567.916 & 1565.365 & 1568.806 \\
\hline $7 / 20 / 1616: 00$ & 74.916 & 82.734 & 81.485 & 89.472 & 76.35 & 73.705 & 71.919 & 75.105 & 1583.556 & 1575.119 & 1571.823 & 1574.457 & 1572.278 & 1567.916 & 1565.372 & 1568.79 \\
\hline 7/20/16 17:00 & 74.921 & 82.799 & 81.565 & 89.659 & 76.369 & 73.693 & 71.917 & 75.01 & 1583.551 & 1575.054 & 1571.743 & 1574.27 & 1572.259 & 1567.928 & 1565.374 & 1568.885 \\
\hline $7 / 20 / 1618: 00$ & 74.925 & 82.865 & 81.609 & 89.75 & 76.387 & 73.67 & 71.917 & 74.913 & 1583.547 & 1574.988 & 1571.699 & 1574.179 & 1572.241 & 1567.951 & 1565.374 & 1568.982 \\
\hline 7/20/16 19:00 & 74.928 & 82.918 & 81.632 & 89.808 & 76.406 & 73.651 & 71.907 & 74.85 & 1583.544 & 1574.935 & 1571.676 & 1574.121 & 1572.222 & 1567.97 & 1565.384 & 1569.045 \\
\hline $7 / 20 / 1620: 00$ & 74.942 & 82.988 & 81.655 & 89.876 & 76.427 & 73.649 & 71.909 & 74.783 & 1583.53 & 1574.865 & 1571.653 & 1574.053 & 1572.201 & 1567.972 & 1565.382 & 1569.112 \\
\hline $7 / 20 / 1621: 00$ & 74.951 & 83.041 & 81.688 & 89.949 & 76.451 & 73.642 & 71.912 & 74.783 & 1583.521 & 1574.812 & 1571.62 & 1573.98 & 1572.177 & 1567.979 & 1565.379 & 1569.112 \\
\hline $7 / 20 / 1622: 00$ & 74.973 & 83.12 & 81.723 & 90.021 & 76.488 & 73.649 & 71.931 & 74.773 & 1583.499 & 1574.733 & 1571.585 & 1573.908 & 1572.14 & 1567.972 & 1565.36 & 1569.122 \\
\hline $7 / 20 / 1623: 00$ & 74.998 & 83.202 & 81.767 & 90.084 & 76.532 & 73.656 & 71.952 & 74.773 & 1583.474 & 1574.651 & 1571.541 & 1573.845 & 1572.096 & 1567.965 & 1565.339 & 1569.122 \\
\hline
\end{tabular}


TABLE S1.1 Automatically recorded groundwater levels in the unconfined aquifer.

\begin{tabular}{|c|c|c|c|c|c|c|c|c|c|c|c|c|c|c|c|c|}
\hline \multirow[b]{2}{*}{ Date and Time } & \multicolumn{8}{|c|}{ Measured Depth to Water (ft TOC ${ }^{\mathrm{a}}$ ) } & \multicolumn{8}{|c|}{ Groundwater Elevation ( $\mathrm{ft} \mathrm{AMSL}^{\mathrm{b}}$ ) } \\
\hline & DL1-C & DL2-C1 & DL3-C1 & DL4-C1 & DL5-C1 & DL6-C & DL7-C & DL8-C1 & DL1-C & DL2-C1 & DL3-C1 & DL4-C1 & DL5-C1 & DL6-C & DL7-C & DL8-C1 \\
\hline $7 / 21 / 160: 00$ & 75.01 & 83.265 & 81.795 & 90.126 & 76.556 & 73.651 & 71.957 & 74.771 & 1583.462 & 1574.588 & 1571.513 & 1573.803 & 1572.072 & 1567.97 & 1565.334 & 1569.124 \\
\hline $7 / 21 / 161: 00$ & 75.013 & 83.321 & 81.816 & 90.17 & 76.581 & 73.644 & 71.957 & 74.75 & 1583.459 & 1574.532 & 1571.492 & 1573.759 & 1572.047 & 1567.977 & 1565.334 & 1569.145 \\
\hline $7 / 21 / 162: 00$ & 75.015 & 83.372 & 81.835 & 90.21 & 76.6 & 73.637 & 71.953 & 74.741 & 1583.457 & 1574.481 & 1571.473 & 1573.719 & 1572.028 & 1567.984 & 1565.338 & 1569.154 \\
\hline $7 / 21 / 163: 00$ & 75.022 & 83.425 & 81.858 & 90.252 & 76.626 & 73.635 & 71.955 & 74.739 & 1583.45 & 1574.428 & 1571.45 & 1573.677 & 1572.002 & 1567.986 & 1565.336 & 1569.156 \\
\hline $7 / 21 / 164: 00$ & 75.034 & 83.483 & 81.889 & 90.294 & 76.658 & 73.635 & 71.965 & 74.732 & 1583.438 & 1574.37 & 1571.419 & 1573.635 & 1571.97 & 1567.986 & 1565.326 & 1569.163 \\
\hline $7 / 21 / 165: 00$ & 75.027 & 83.514 & 81.893 & 90.315 & 76.668 & 73.616 & 71.943 & 74.713 & 1583.445 & 1574.339 & 1571.415 & 1573.614 & 1571.96 & 1568.005 & 1565.348 & 1569.182 \\
\hline $7 / 21 / 166: 00$ & 75.041 & 83.567 & 81.917 & 90.357 & 76.7 & 73.616 & 71.95 & 74.706 & 1583.431 & 1574.286 & 1571.391 & 1573.572 & 1571.928 & 1568.005 & 1565.341 & 1569.189 \\
\hline $7 / 21 / 167: 00$ & 75.055 & 83.616 & 82.273 & 90.392 & 76.749 & 73.63 & 71.95 & 74.887 & 1583.417 & 1574.237 & 1571.035 & 1573.537 & 1571.879 & 1567.991 & 1565.341 & 1569.008 \\
\hline $7 / 21 / 168: 00$ & 75.06 & 83.669 & 82.683 & 90.423 & 76.842 & 73.677 & 71.953 & 75.272 & 1583.412 & 1574.184 & 1570.625 & 1573.506 & 1571.786 & 1567.944 & 1565.338 & 1568.623 \\
\hline $7 / 21 / 169: 00$ & 75.086 & 83.737 & 82.877 & 90.465 & 76.959 & 73.714 & 71.971 & 75.495 & 1583.386 & 1574.116 & 1570.431 & 1573.464 & 1571.669 & 1567.907 & 1565.32 & 1568.4 \\
\hline $7 / 21 / 1610: 00$ & 75.093 & 83.807 & 83 & 90.511 & 77.073 & 73.738 & 71.981 & 75.628 & 1583.379 & 1574.046 & 1570.308 & 1573.418 & 1571.555 & 1567.883 & 1565.31 & 1568.267 \\
\hline $7 / 21 / 1611: 00$ & 75.107 & 83.865 & 83.105 & 90.546 & 77.187 & 73.761 & 71.998 & 75.73 & 1583.365 & 1573.988 & 1570.203 & 1573.383 & 1571.441 & 1567.86 & 1565.293 & 1568.165 \\
\hline $7 / 21 / 1612: 00$ & 75.112 & 83.916 & 83.206 & 90.602 & 77.292 & 73.772 & 71.998 & 75.814 & 1583.36 & 1573.937 & 1570.102 & 1573.327 & 1571.336 & 1567.849 & 1565.293 & 1568.081 \\
\hline $7 / 21 / 1613: 00$ & 75.126 & 83.983 & 83.299 & 90.656 & 77.407 & 73.784 & 72.005 & 75.895 & 1583.346 & 1573.87 & 1570.009 & 1573.273 & 1571.221 & 1567.837 & 1565.286 & 1568 \\
\hline $7 / 21 / 1614: 00$ & 75.137 & 84.037 & 83.39 & 90.707 & 77.519 & 73.803 & 72.01 & 75.988 & 1583.335 & 1573.816 & 1569.918 & 1573.222 & 1571.109 & 1567.818 & 1565.281 & 1567.907 \\
\hline $7 / 21 / 1615: 00$ & 75.145 & 84.09 & 83.469 & 90.745 & 77.621 & 73.817 & 72.01 & 76.062 & 1583.327 & 1573.763 & 1569.839 & 1573.184 & 1571.007 & 1567.804 & 1565.281 & 1567.833 \\
\hline $7 / 21 / 1616: 00$ & 75.147 & 84.139 & 83.536 & 90.78 & 77.724 & 73.826 & 72.005 & 76.132 & 1583.325 & 1573.714 & 1569.772 & 1573.149 & 1570.904 & 1567.795 & 1565.286 & 1567.763 \\
\hline $7 / 21 / 1617: 00$ & 75.145 & 84.163 & 83.499 & 90.474 & 77.817 & 73.835 & 72 & 76.185 & 1583.327 & 1573.69 & 1569.809 & 1573.455 & 1570.811 & 1567.786 & 1565.291 & 1567.71 \\
\hline $7 / 21 / 1618: 00$ & 75.149 & 84.151 & 83.45 & 90.229 & 77.908 & 73.849 & 72.003 & 76.243 & 1583.323 & 1573.702 & 1569.858 & 1573.7 & 1570.72 & 1567.772 & 1565.288 & 1567.652 \\
\hline $7 / 21 / 1619: 00$ & 75.156 & 84.135 & 83.418 & 90.079 & 77.996 & 73.866 & 72.005 & 76.299 & 1583.316 & 1573.718 & 1569.89 & 1573.85 & 1570.632 & 1567.755 & 1565.286 & 1567.596 \\
\hline $7 / 21 / 1620: 00$ & 75.161 & 84.118 & 83.401 & 89.979 & 78.085 & 73.88 & 72.012 & 76.345 & 1583.311 & 1573.735 & 1569.907 & 1573.95 & 1570.543 & 1567.741 & 1565.279 & 1567.55 \\
\hline $7 / 21 / 1621: 00$ & 75.166 & 84.102 & 83.38 & 89.879 & 78.172 & 73.868 & 72.019 & 76.271 & 1583.306 & 1573.751 & 1569.928 & 1574.05 & 1570.456 & 1567.753 & 1565.272 & 1567.624 \\
\hline $7 / 21 / 1622: 00$ & 75.185 & 84.1 & 83.394 & 89.815 & 78.269 & 73.877 & 72.043 & 76.283 & 1583.287 & 1573.753 & 1569.914 & 1574.114 & 1570.359 & 1567.744 & 1565.248 & 1567.612 \\
\hline $7 / 21 / 1623: 00$ & 75.194 & 84.086 & 83.404 & 89.764 & 78.358 & 73.884 & 72.053 & 76.303 & 1583.278 & 1573.767 & 1569.904 & 1574.165 & 1570.27 & 1567.737 & 1565.238 & 1567.592 \\
\hline $7 / 22 / 160: 00$ & 75.194 & 84.062 & 83.406 & 89.72 & 78.428 & 73.882 & 72.053 & 76.317 & 1583.278 & 1573.791 & 1569.902 & 1574.209 & 1570.2 & 1567.739 & 1565.238 & 1567.578 \\
\hline $7 / 22 / 161: 00$ & 75.196 & 84.049 & 83.413 & 89.687 & 78.503 & 73.884 & 72.057 & 76.341 & 1583.276 & 1573.804 & 1569.895 & 1574.242 & 1570.125 & 1567.737 & 1565.234 & 1567.554 \\
\hline $7 / 22 / 162: 00$ & 75.199 & 84.03 & 83.492 & 89.654 & 78.573 & 73.889 & 72.057 & 76.368 & 1583.273 & 1573.823 & 1569.816 & 1574.275 & 1570.055 & 1567.732 & 1565.234 & 1567.527 \\
\hline $7 / 22 / 163: 00$ & 75.196 & 84.007 & 83.511 & 89.626 & 78.636 & 73.884 & 72.055 & 76.348 & 1583.276 & 1573.846 & 1569.797 & 1574.303 & 1569.992 & 1567.737 & 1565.236 & 1567.547 \\
\hline $7 / 22 / 164: 00$ & 75.192 & 83.99 & 83.522 & 89.601 & 78.698 & 73.882 & 72.05 & 76.415 & 1583.28 & 1573.863 & 1569.786 & 1574.328 & 1569.93 & 1567.739 & 1565.241 & 1567.48 \\
\hline $7 / 22 / 165: 00$ & 75.206 & 83.979 & 83.546 & 89.589 & 78.771 & 73.896 & 72.06 & 76.443 & 1583.266 & 1573.874 & 1569.762 & 1574.34 & 1569.857 & 1567.725 & 1565.231 & 1567.452 \\
\hline $7 / 22 / 166: 00$ & 75.213 & 83.979 & 83.529 & 89.575 & 78.836 & 73.903 & 72.069 & 76.475 & 1583.259 & 1573.874 & 1569.779 & 1574.354 & 1569.792 & 1567.718 & 1565.222 & 1567.42 \\
\hline $7 / 22 / 167: 00$ & 75.229 & 83.979 & 83.518 & 89.57 & 78.915 & 73.919 & 72.086 & 76.503 & 1583.243 & 1573.874 & 1569.79 & 1574.359 & 1569.713 & 1567.702 & 1565.205 & 1567.392 \\
\hline $7 / 22 / 168: 00$ & 75.234 & 83.976 & 83.532 & 89.556 & 78.976 & 73.938 & 72.098 & 76.559 & 1583.238 & 1573.877 & 1569.776 & 1574.373 & 1569.652 & 1567.683 & 1565.193 & 1567.336 \\
\hline $7 / 22 / 169: 00$ & 75.244 & 83.974 & 83.548 & 89.547 & 79.037 & 73.945 & 72.103 & 76.587 & 1583.228 & 1573.879 & 1569.76 & 1574.382 & 1569.591 & 1567.676 & 1565.188 & 1567.308 \\
\hline $7 / 22 / 1610: 00$ & 75.251 & 83.974 & 83.567 & 89.54 & 79.095 & 73.954 & 72.105 & 76.617 & 1583.221 & 1573.879 & 1569.741 & 1574.389 & 1569.533 & 1567.667 & 1565.186 & 1567.278 \\
\hline $7 / 22 / 1611: 00$ & 75.258 & 83.981 & 83.571 & 89.538 & 79.158 & 73.963 & 72.122 & 76.633 & 1583.214 & 1573.872 & 1569.737 & 1574.391 & 1569.47 & 1567.658 & 1565.169 & 1567.262 \\
\hline $7 / 22 / 1612: 00$ & 75.267 & 83.988 & 83.585 & 89.538 & 79.218 & 73.97 & 72.136 & 76.617 & 1583.205 & 1573.865 & 1569.723 & 1574.391 & 1569.41 & 1567.651 & 1565.155 & 1567.278 \\
\hline $7 / 22 / 1613: 00$ & 75.267 & 83.988 & 83.611 & 89.531 & 79.272 & 73.998 & 72.141 & 76.803 & 1583.205 & 1573.865 & 1569.697 & 1574.398 & 1569.356 & 1567.623 & 1565.15 & 1567.092 \\
\hline $7 / 22 / 1614: 00$ & 75.267 & 83.995 & 83.639 & 89.524 & 79.328 & 74.022 & 72.148 & 76.921 & 1583.205 & 1573.858 & 1569.669 & 1574.405 & 1569.3 & 1567.599 & 1565.143 & 1566.974 \\
\hline $7 / 22 / 1615: 00$ & 75.269 & 83.988 & 83.672 & 89.521 & 79.372 & 74.04 & 72.148 & 77.037 & 1583.203 & 1573.865 & 1569.636 & 1574.408 & 1569.256 & 1567.581 & 1565.143 & 1566.858 \\
\hline $7 / 22 / 1616: 00$ & 75.267 & 84.03 & 83.828 & 89.939 & 79.673 & 74.05 & 72.151 & 76.984 & 1583.205 & 1573.823 & 1569.48 & 1573.99 & 1568.955 & 1567.571 & 1565.14 & 1566.911 \\
\hline $7 / 22 / 1617: 00$ & 75.265 & 84.076 & 83.954 & 90.184 & 79.792 & 74.05 & 72.134 & 77.023 & 1583.207 & 1573.777 & 1569.354 & 1573.745 & 1568.836 & 1567.571 & 1565.157 & 1566.872 \\
\hline $7 / 22 / 1618: 00$ & 75.265 & 84.132 & 84.047 & 90.348 & 79.857 & 74.052 & 72.122 & 77.044 & 1583.207 & 1573.721 & 1569.261 & 1573.581 & 1568.771 & 1567.569 & 1565.169 & 1566.851 \\
\hline $7 / 22 / 1619: 00$ & 75.291 & 84.204 & 84.138 & 90.474 & 79.927 & 74.068 & 72.134 & 77.077 & 1583.181 & 1573.649 & 1569.17 & 1573.455 & 1568.701 & 1567.553 & 1565.157 & 1566.818 \\
\hline $7 / 22 / 1620: 00$ & 75.307 & 84.276 & 84.217 & 90.57 & 79.993 & 74.078 & 72.131 & 77.118 & 1583.165 & 1573.577 & 1569.091 & 1573.359 & 1568.635 & 1567.543 & 1565.16 & 1566.777 \\
\hline $7 / 22 / 1621: 00$ & 75.319 & 84.351 & 84.292 & 90.649 & 80.053 & 74.092 & 72.136 & 77.146 & 1583.153 & 1573.502 & 1569.016 & 1573.28 & 1568.575 & 1567.529 & 1565.155 & 1566.749 \\
\hline $7 / 22 / 1622: 00$ & 75.345 & 84.437 & 84.376 & 90.731 & 80.128 & 74.115 & 72.158 & 77.204 & 1583.127 & 1573.416 & 1568.932 & 1573.198 & 1568.5 & 1567.506 & 1565.133 & 1566.691 \\
\hline $7 / 22 / 1623: 00$ & 75.371 & 84.528 & 84.464 & 90.803 & 80.207 & 74.143 & 72.186 & 77.2 & 1583.101 & 1573.325 & 1568.844 & 1573.126 & 1568.421 & 1567.478 & 1565.105 & 1566.695 \\
\hline $7 / 23 / 160: 00$ & 75.38 & 84.597 & 84.534 & 90.857 & 80.27 & 74.157 & 72.186 & 77.299 & 1583.092 & 1573.256 & 1568.774 & 1573.072 & 1568.358 & 1567.464 & 1565.105 & 1566.596 \\
\hline
\end{tabular}


TABLE S1.1 Automatically recorded groundwater levels in the unconfined aquifer.

\begin{tabular}{|c|c|c|c|c|c|c|c|c|c|c|c|c|c|c|c|c|}
\hline \multirow[b]{2}{*}{ Date and Time } & \multicolumn{8}{|c|}{ Measured Depth to Water (ft TOC ${ }^{\mathrm{a}}$ ) } & \multicolumn{8}{|c|}{ Groundwater Elevation ( $\mathrm{ft} \mathrm{AMSL}^{\mathrm{b}}$ ) } \\
\hline & DL1-C & DL2-C1 & DL3-C1 & DL4-C1 & DL5-C1 & DL6-C & DL7-C & DL8-C1 & DL1-C & DL2-C1 & DL3-C1 & DL4-C1 & DL5-C1 & DL6-C & DL7-C & DL8-C1 \\
\hline $7 / 23 / 161: 00$ & 75.373 & 84.649 & 84.576 & 90.894 & 80.312 & 74.147 & 72.17 & 77.304 & 1583.099 & 1573.204 & 1568.732 & 1573.035 & 1568.316 & 1567.474 & 1565.121 & 1566.591 \\
\hline $7 / 23 / 162: 00$ & 75.38 & 84.714 & 84.632 & 90.943 & 80.366 & 74.159 & 72.175 & 77.334 & 1583.092 & 1573.139 & 1568.676 & 1572.986 & 1568.262 & 1567.462 & 1565.116 & 1566.561 \\
\hline $7 / 23 / 163: 00$ & 75.383 & 84.767 & 84.681 & 90.983 & 80.412 & 74.164 & 72.17 & 77.367 & 1583.089 & 1573.086 & 1568.627 & 1572.946 & 1568.216 & 1567.457 & 1565.121 & 1566.528 \\
\hline $7 / 23 / 164: 00$ & 75.42 & 84.865 & 84.765 & 91.051 & 80.494 & 74.206 & 72.215 & 77.434 & 1583.052 & 1572.988 & 1568.543 & 1572.878 & 1568.134 & 1567.415 & 1565.076 & 1566.461 \\
\hline $7 / 23 / 165: 00$ & 75.406 & 84.914 & 84.839 & 91.079 & 80.538 & 74.203 & 72.208 & 77.467 & 1583.066 & 1572.939 & 1568.469 & 1572.85 & 1568.09 & 1567.418 & 1565.083 & 1566.428 \\
\hline $7 / 23 / 166: 00$ & 75.378 & 84.953 & 84.879 & 91.104 & 80.569 & 74.199 & 72.189 & 77.485 & 1583.094 & 1572.9 & 1568.429 & 1572.825 & 1568.059 & 1567.422 & 1565.102 & 1566.41 \\
\hline $7 / 23 / 167: 00$ & 75.364 & 85.002 & 84.916 & 91.137 & 80.606 & 74.203 & 72.191 & 77.52 & 1583.108 & 1572.851 & 1568.392 & 1572.792 & 1568.022 & 1567.418 & 1565.1 & 1566.375 \\
\hline $7 / 23 / 168: 00$ & 75.364 & 85.058 & 84.963 & 91.172 & 80.643 & 74.215 & 72.196 & 77.546 & 1583.108 & 1572.795 & 1568.345 & 1572.757 & 1567.985 & 1567.406 & 1565.095 & 1566.349 \\
\hline $7 / 23 / 169: 00$ & 75.368 & 85.114 & 85.005 & 91.214 & 80.692 & 74.248 & 72.205 & 77.604 & 1583.104 & 1572.739 & 1568.303 & 1572.715 & 1567.936 & 1567.373 & 1565.086 & 1566.291 \\
\hline $7 / 23 / 1610: 00$ & 75.364 & 85.156 & 85.049 & 91.244 & 80.729 & 74.25 & 72.201 & 77.611 & 1583.108 & 1572.697 & 1568.259 & 1572.685 & 1567.899 & 1567.371 & 1565.09 & 1566.284 \\
\hline $7 / 23 / 1611: 00$ & 75.361 & 85.209 & 85.11 & 91.289 & 80.774 & 74.264 & 72.208 & 77.666 & 1583.111 & 1572.644 & 1568.198 & 1572.64 & 1567.854 & 1567.357 & 1565.083 & 1566.229 \\
\hline $7 / 23 / 1612: 00$ & 75.376 & 85.263 & 85.159 & 91.333 & 80.822 & 74.266 & 72.213 & 77.685 & 1583.096 & 1572.59 & 1568.149 & 1572.596 & 1567.806 & 1567.355 & 1565.078 & 1566.21 \\
\hline $7 / 23 / 1613: 00$ & 75.401 & 85.314 & 85.201 & 91.373 & 80.874 & 74.273 & 72.222 & 77.713 & 1583.071 & 1572.539 & 1568.107 & 1572.556 & 1567.754 & 1567.348 & 1565.069 & 1566.182 \\
\hline $7 / 23 / 1614: 00$ & 75.425 & 85.377 & 85.247 & 91.412 & 80.932 & 74.289 & 72.241 & 77.724 & 1583.047 & 1572.476 & 1568.061 & 1572.517 & 1567.696 & 1567.332 & 1565.05 & 1566.171 \\
\hline $7 / 23 / 1615: 00$ & 75.437 & 85.419 & 85.049 & 91.445 & 80.948 & 74.299 & 72.239 & 77.685 & 1583.035 & 1572.434 & 1568.259 & 1572.484 & 1567.68 & 1567.322 & 1565.052 & 1566.21 \\
\hline $7 / 23 / 1616: 00$ & 75.444 & 85.456 & 84.895 & 91.471 & 80.951 & 74.303 & 72.236 & 77.594 & 1583.028 & 1572.397 & 1568.413 & 1572.458 & 1567.677 & 1567.318 & 1565.055 & 1566.301 \\
\hline $7 / 23 / 1617: 00$ & 75.451 & 85.479 & 84.87 & 91.487 & 80.946 & 74.303 & 72.236 & 77.543 & 1583.021 & 1572.374 & 1568.438 & 1572.442 & 1567.682 & 1567.318 & 1565.055 & 1566.352 \\
\hline $7 / 23 / 1618: 00$ & 75.456 & 85.507 & 84.858 & 91.499 & 80.946 & 74.306 & 72.239 & 77.504 & 1583.016 & 1572.346 & 1568.45 & 1572.43 & 1567.682 & 1567.315 & 1565.052 & 1566.391 \\
\hline $7 / 23 / 1619: 00$ & 75.46 & 85.532 & 84.856 & 91.513 & 80.946 & 74.306 & 72.236 & 77.474 & 1583.012 & 1572.321 & 1568.452 & 1572.416 & 1567.682 & 1567.315 & 1565.055 & 1566.421 \\
\hline $7 / 23 / 1620: 00$ & 75.479 & 85.574 & 84.872 & 91.539 & 80.962 & 74.324 & 72.261 & 77.469 & 1582.993 & 1572.279 & 1568.436 & 1572.39 & 1567.666 & 1567.297 & 1565.03 & 1566.426 \\
\hline $7 / 23 / 1621: 00$ & 75.505 & 85.621 & 84.893 & 91.532 & 80.988 & 74.343 & 72.284 & 77.474 & 1582.967 & 1572.232 & 1568.415 & 1572.397 & 1567.64 & 1567.278 & 1565.007 & 1566.421 \\
\hline $7 / 23 / 1622: 00$ & 75.526 & 85.67 & 84.928 & 91.555 & 81.016 & 74.364 & 72.315 & 77.485 & 1582.946 & 1572.183 & 1568.38 & 1572.374 & 1567.612 & 1567.257 & 1564.976 & 1566.41 \\
\hline $7 / 23 / 1623: 00$ & 75.54 & 85.714 & 84.958 & 91.576 & 81.04 & 74.378 & 72.332 & 77.501 & 1582.932 & 1572.139 & 1568.35 & 1572.353 & 1567.588 & 1567.243 & 1564.959 & 1566.394 \\
\hline $7 / 24 / 160: 00$ & 75.536 & 85.749 & 84.977 & 91.592 & 81.047 & 74.385 & 72.339 & 77.508 & 1582.936 & 1572.104 & 1568.331 & 1572.337 & 1567.581 & 1567.236 & 1564.952 & 1566.387 \\
\hline $7 / 24 / 161: 00$ & 75.54 & 85.793 & 85.01 & 91.627 & 81.07 & 74.403 & 72.365 & 77.532 & 1582.932 & 1572.06 & 1568.298 & 1572.302 & 1567.558 & 1567.218 & 1564.926 & 1566.363 \\
\hline $7 / 24 / 162: 00$ & 75.533 & 85.83 & 85.038 & 91.648 & 81.082 & 74.415 & 72.377 & 77.546 & 1582.939 & 1572.023 & 1568.27 & 1572.281 & 1567.546 & 1567.206 & 1564.914 & 1566.349 \\
\hline $7 / 24 / 163: 00$ & 75.531 & 85.867 & 85.073 & 91.676 & 81.103 & 74.432 & 72.396 & 77.58 & 1582.941 & 1571.986 & 1568.235 & 1572.253 & 1567.525 & 1567.189 & 1564.895 & 1566.315 \\
\hline $7 / 24 / 164: 00$ & 75.526 & 85.9 & 85.1 & 91.697 & 81.121 & 74.441 & 72.408 & 77.599 & 1582.946 & 1571.953 & 1568.208 & 1572.232 & 1567.507 & 1567.18 & 1564.883 & 1566.296 \\
\hline $7 / 24 / 165: 00$ & 75.519 & 85.93 & 85.145 & 91.711 & 81.135 & 74.448 & 72.413 & 77.618 & 1582.953 & 1571.923 & 1568.163 & 1572.218 & 1567.493 & 1567.173 & 1564.878 & 1566.277 \\
\hline $7 / 24 / 166: 00$ & 75.522 & 85.97 & 85.17 & 91.739 & 81.156 & 74.464 & 72.435 & 77.636 & 1582.95 & 1571.883 & 1568.138 & 1572.19 & 1567.472 & 1567.157 & 1564.856 & 1566.259 \\
\hline $7 / 24 / 167: 00$ & 75.529 & 86.012 & 85.203 & 91.763 & 81.184 & 74.48 & 72.456 & 77.659 & 1582.943 & 1571.841 & 1568.105 & 1572.166 & 1567.444 & 1567.141 & 1564.835 & 1566.236 \\
\hline $7 / 24 / 168: 00$ & 75.531 & 86.042 & 85.231 & 91.788 & 81.254 & 74.511 & 72.475 & 77.701 & 1582.941 & 1571.811 & 1568.077 & 1572.141 & 1567.374 & 1567.11 & 1564.816 & 1566.194 \\
\hline $7 / 24 / 169: 00$ & 75.54 & 86.091 & 85.271 & 91.823 & 81.201 & 74.534 & 72.499 & 77.741 & 1582.932 & 1571.762 & 1568.037 & 1572.106 & 1567.427 & 1567.087 & 1564.792 & 1566.154 \\
\hline $7 / 24 / 1610: 00$ & 75.555 & 86.142 & 85.168 & 91.863 & 81.177 & 74.55 & 72.528 & 77.696 & 1582.917 & 1571.711 & 1568.14 & 1572.066 & 1567.451 & 1567.071 & 1564.763 & 1566.199 \\
\hline $7 / 24 / 1611: 00$ & 75.557 & 86.174 & 85.086 & 91.886 & 81.159 & 74.55 & 72.54 & 77.625 & 1582.915 & 1571.679 & 1568.222 & 1572.043 & 1567.469 & 1567.071 & 1564.751 & 1566.27 \\
\hline $7 / 24 / 1612: 00$ & 75.54 & 86.186 & 85.024 & 91.891 & 81.126 & 74.529 & 72.525 & 77.569 & 1582.932 & 1571.667 & 1568.284 & 1572.038 & 1567.502 & 1567.092 & 1564.766 & 1566.326 \\
\hline $7 / 24 / 1613: 00$ & 75.54 & 86.212 & 84.972 & 91.914 & 81.112 & 74.536 & 72.54 & 77.539 & 1582.932 & 1571.641 & 1568.336 & 1572.015 & 1567.516 & 1567.085 & 1564.751 & 1566.356 \\
\hline $7 / 24 / 1614: 00$ & 75.536 & 86.23 & 84.942 & 91.928 & 81.096 & 74.539 & 72.537 & 77.497 & 1582.936 & 1571.623 & 1568.366 & 1572.001 & 1567.532 & 1567.082 & 1564.754 & 1566.398 \\
\hline $7 / 24 / 1615: 00$ & 75.519 & 86.23 & 84.879 & 91.928 & 81.061 & 74.529 & 72.528 & 77.495 & 1582.953 & 1571.623 & 1568.429 & 1572.001 & 1567.567 & 1567.092 & 1564.763 & 1566.4 \\
\hline $7 / 24 / 1616: 00$ & 75.51 & 86.23 & 84.842 & 91.933 & 81.037 & 74.525 & 72.518 & 77.462 & 1582.962 & 1571.623 & 1568.466 & 1571.996 & 1567.591 & 1567.096 & 1564.773 & 1566.433 \\
\hline $7 / 24 / 1617: 00$ & 75.507 & 86.24 & 84.809 & 91.917 & 81.014 & 74.527 & 72.521 & 77.446 & 1582.965 & 1571.613 & 1568.499 & 1572.012 & 1567.614 & 1567.094 & 1564.77 & 1566.449 \\
\hline $7 / 24 / 1618: 00$ & 75.51 & 86.24 & 84.774 & 91.903 & 80.99 & 74.527 & 72.521 & 77.418 & 1582.962 & 1571.613 & 1568.534 & 1572.026 & 1567.638 & 1567.094 & 1564.77 & 1566.477 \\
\hline $7 / 24 / 1619: 00$ & 75.5 & 86.23 & 84.728 & 91.879 & 80.944 & 74.508 & 72.511 & 77.348 & 1582.972 & 1571.623 & 1568.58 & 1572.05 & 1567.684 & 1567.113 & 1564.78 & 1566.547 \\
\hline $7 / 24 / 1620: 00$ & 75.512 & 86.24 & 84.702 & 91.893 & 80.923 & 74.499 & 72.526 & 77.246 & 1582.96 & 1571.613 & 1568.606 & 1572.036 & 1567.705 & 1567.122 & 1564.765 & 1566.649 \\
\hline $7 / 24 / 1621: 00$ & 75.517 & 86.244 & 84.679 & 91.903 & 80.89 & 74.499 & 72.537 & 77.197 & 1582.955 & 1571.609 & 1568.629 & 1572.026 & 1567.738 & 1567.122 & 1564.754 & 1566.698 \\
\hline $7 / 24 / 1622: 00$ & 75.536 & 86.254 & 84.595 & 91.669 & 80.874 & 74.508 & 72.564 & 77.172 & 1582.936 & 1571.599 & 1568.713 & 1572.26 & 1567.754 & 1567.113 & 1564.727 & 1566.723 \\
\hline $7 / 24 / 1623: 00$ & 75.55 & 86.223 & 84.492 & 91.426 & 80.853 & 74.508 & 72.58 & 77.139 & 1582.922 & 1571.63 & 1568.816 & 1572.503 & 1567.775 & 1567.113 & 1564.711 & 1566.756 \\
\hline $7 / 25 / 160: 00$ & 75.552 & 86.179 & 84.394 & 91.263 & 80.82 & 74.499 & 72.583 & 77.097 & 1582.92 & 1571.674 & 1568.914 & 1572.666 & 1567.808 & 1567.122 & 1564.708 & 1566.798 \\
\hline $7 / 25 / 161: 00$ & 75.548 & 86.121 & 84.306 & 91.139 & 80.783 & 74.49 & 72.576 & 77.06 & 1582.924 & 1571.732 & 1569.002 & 1572.79 & 1567.845 & 1567.131 & 1564.715 & 1566.835 \\
\hline
\end{tabular}


TABLE S1.1 Automatically recorded groundwater levels in the unconfined aquifer.

\begin{tabular}{|c|c|c|c|c|c|c|c|c|c|c|c|c|c|c|c|c|}
\hline \multirow[b]{2}{*}{ Date and Time } & \multicolumn{8}{|c|}{ Measured Depth to Water (ft TOC ${ }^{\mathrm{a}}$ ) } & \multicolumn{8}{|c|}{ Groundwater Elevation ( $\mathrm{ft} \mathrm{AMSL}^{\mathrm{b}}$ ) } \\
\hline & DL1-C & DL2-C1 & DL3-C1 & DL4-C1 & DL5-C1 & DL6-C & DL7-C & DL8-C1 & DL1-C & DL2-C1 & DL3-C1 & DL4-C1 & DL5-C1 & DL6-C & DL7-C & DL8-C1 \\
\hline $7 / 25 / 162: 00$ & 75.522 & 86.047 & 84.275 & 91.027 & 80.722 & 74.462 & 72.549 & 77.009 & 1582.95 & 1571.806 & 1569.033 & 1572.902 & 1567.906 & 1567.159 & 1564.742 & 1566.886 \\
\hline $7 / 25 / 163: 00$ & 75.515 & 85.979 & 84.208 & 90.948 & 80.676 & 74.45 & 72.547 & 76.981 & 1582.957 & 1571.874 & 1569.1 & 1572.981 & 1567.952 & 1567.171 & 1564.744 & 1566.914 \\
\hline $7 / 25 / 164: 00$ & 75.524 & 85.93 & 84.159 & 90.892 & 80.648 & 74.462 & 72.561 & 76.956 & 1582.948 & 1571.923 & 1569.149 & 1573.037 & 1567.98 & 1567.159 & 1564.73 & 1566.939 \\
\hline $7 / 25 / 165: 00$ & 75.536 & 85.886 & 84.14 & 90.845 & 80.625 & 74.466 & 72.58 & 76.954 & 1582.936 & 1571.967 & 1569.168 & 1573.084 & 1568.003 & 1567.155 & 1564.711 & 1566.941 \\
\hline $7 / 25 / 166: 00$ & 75.531 & 85.823 & 84.049 & 90.784 & 80.585 & 74.453 & 72.573 & 76.907 & 1582.941 & 1572.03 & 1569.259 & 1573.145 & 1568.043 & 1567.168 & 1564.718 & 1566.988 \\
\hline $7 / 25 / 167: 00$ & 75.545 & 85.795 & 83.975 & 90.754 & 80.562 & 74.462 & 72.597 & 76.895 & 1582.927 & 1572.058 & 1569.333 & 1573.175 & 1568.066 & 1567.159 & 1564.694 & 1567 \\
\hline $7 / 25 / 168: 00$ & 75.564 & 85.76 & 83.963 & 90.731 & 80.545 & 74.483 & 72.621 & 76.847 & 1582.908 & 1572.093 & 1569.345 & 1573.198 & 1568.083 & 1567.138 & 1564.67 & 1567.048 \\
\hline $7 / 25 / 169: 00$ & 75.569 & 85.728 & 83.942 & 90.787 & 80.599 & 74.49 & 72.63 & 76.884 & 1582.903 & 1572.125 & 1569.366 & 1573.142 & 1568.029 & 1567.131 & 1564.661 & 1567.011 \\
\hline $7 / 25 / 1610: 00$ & 75.566 & 85.723 & 84.017 & 91.102 & 80.876 & 74.483 & 72.623 & 76.87 & 1582.906 & 1572.13 & 1569.291 & 1572.827 & 1567.752 & 1567.138 & 1564.668 & 1567.025 \\
\hline $7 / 25 / 1611: 00$ & 75.559 & 85.726 & 84.084 & 91.27 & 80.89 & 74.469 & 72.614 & 76.868 & 1582.913 & 1572.127 & 1569.224 & 1572.659 & 1567.738 & 1567.152 & 1564.677 & 1567.027 \\
\hline $7 / 25 / 1612: 00$ & 75.552 & 85.73 & 84.122 & 91.375 & 80.888 & 74.476 & 72.602 & 76.93 & 1582.92 & 1572.123 & 1569.186 & 1572.554 & 1567.74 & 1567.145 & 1564.689 & 1566.965 \\
\hline $7 / 25 / 1613: 00$ & 75.538 & 85.73 & 84.143 & 91.445 & 80.876 & 74.471 & 72.585 & 76.944 & 1582.934 & 1572.123 & 1569.165 & 1572.484 & 1567.752 & 1567.15 & 1564.706 & 1566.951 \\
\hline $7 / 25 / 1614: 00$ & 75.526 & 85.737 & 84.163 & 91.503 & 80.869 & 74.466 & 72.576 & 76.93 & 1582.946 & 1572.116 & 1569.145 & 1572.426 & 1567.759 & 1567.155 & 1564.715 & 1566.965 \\
\hline $7 / 25 / 1615: 00$ & 75.526 & 85.739 & 84.168 & 91.543 & 80.853 & 74.473 & 72.559 & 77 & 1582.946 & 1572.114 & 1569.14 & 1572.386 & 1567.775 & 1567.148 & 1564.732 & 1566.895 \\
\hline $7 / 25 / 1616: 00$ & 75.54 & 85.749 & 84.177 & 91.574 & 80.851 & 74.462 & 72.552 & 76.947 & 1582.932 & 1572.104 & 1569.131 & 1572.355 & 1567.777 & 1567.159 & 1564.739 & 1566.948 \\
\hline $7 / 25 / 1617: 00$ & 75.543 & 85.744 & 84.182 & 91.576 & 80.837 & 74.45 & 72.53 & 76.921 & 1582.929 & 1572.109 & 1569.126 & 1572.353 & 1567.791 & 1567.171 & 1564.761 & 1566.974 \\
\hline $7 / 25 / 1618: 00$ & 75.557 & 85.719 & 84.063 & 91.268 & 80.829 & 74.45 & 72.525 & 76.916 & 1582.915 & 1572.134 & 1569.245 & 1572.661 & 1567.799 & 1567.171 & 1564.766 & 1566.979 \\
\hline $7 / 25 / 1619: 00$ & 75.581 & 85.693 & 83.986 & 91.095 & 80.832 & 74.457 & 72.53 & 76.909 & 1582.891 & 1572.16 & 1569.322 & 1572.834 & 1567.796 & 1567.164 & 1564.761 & 1566.986 \\
\hline $7 / 25 / 1620: 00$ & 75.606 & 85.67 & 83.935 & 90.985 & 80.862 & 74.473 & 72.547 & 76.921 & 1582.866 & 1572.183 & 1569.373 & 1572.944 & 1567.766 & 1567.148 & 1564.744 & 1566.974 \\
\hline $7 / 25 / 1621: 00$ & 75.63 & 85.639 & 83.881 & 90.878 & 80.662 & 74.48 & 72.561 & 76.916 & 1582.842 & 1572.214 & 1569.427 & 1573.051 & 1567.966 & 1567.141 & 1564.73 & 1566.979 \\
\hline $7 / 25 / 1622: 00$ & 75.661 & 85.628 & 83.856 & 90.81 & 80.606 & 74.501 & 72.592 & 76.926 & 1582.811 & 1572.225 & 1569.452 & 1573.119 & 1568.022 & 1567.12 & 1564.699 & 1566.969 \\
\hline $7 / 25 / 1623: 00$ & 75.679 & 85.6 & 83.828 & 90.754 & 80.566 & 74.513 & 72.604 & 76.935 & 1582.793 & 1572.253 & 1569.48 & 1573.175 & 1568.062 & 1567.108 & 1564.687 & 1566.96 \\
\hline $7 / 26 / 160: 00$ & 75.691 & 85.57 & 83.793 & 90.705 & 80.531 & 74.515 & 72.614 & 76.93 & 1582.781 & 1572.283 & 1569.515 & 1573.224 & 1568.097 & 1567.106 & 1564.677 & 1566.965 \\
\hline $7 / 26 / 161: 00$ & 75.689 & 85.53 & 83.76 & 90.661 & 80.494 & 74.52 & 72.614 & 76.935 & 1582.783 & 1572.323 & 1569.548 & 1573.268 & 1568.134 & 1567.101 & 1564.677 & 1566.96 \\
\hline $7 / 26 / 162: 00$ & 75.665 & 85.481 & 83.721 & 90.614 & 80.436 & 74.513 & 72.599 & 76.919 & 1582.807 & 1572.372 & 1569.587 & 1573.315 & 1568.192 & 1567.108 & 1564.692 & 1566.976 \\
\hline $7 / 26 / 163: 00$ & 75.649 & 85.432 & 83.683 & 90.579 & 80.384 & 74.506 & 72.595 & 76.87 & 1582.823 & 1572.421 & 1569.625 & 1573.35 & 1568.244 & 1567.115 & 1564.696 & 1567.025 \\
\hline $7 / 26 / 164: 00$ & 75.632 & 85.388 & 83.648 & 90.544 & 80.338 & 74.504 & 72.59 & 76.886 & 1582.84 & 1572.465 & 1569.66 & 1573.385 & 1568.29 & 1567.117 & 1564.701 & 1567.009 \\
\hline $7 / 26 / 165: 00$ & 75.63 & 85.356 & 83.644 & 90.521 & 80.305 & 74.506 & 72.597 & 76.849 & 1582.842 & 1572.497 & 1569.664 & 1573.408 & 1568.323 & 1567.115 & 1564.694 & 1567.046 \\
\hline $7 / 26 / 166: 00$ & 75.621 & 85.314 & 83.613 & 90.493 & 80.261 & 74.504 & 72.592 & 76.865 & 1582.851 & 1572.539 & 1569.695 & 1573.436 & 1568.367 & 1567.117 & 1564.699 & 1567.03 \\
\hline $7 / 26 / 167: 00$ & 75.621 & 85.279 & 83.59 & 90.469 & 80.226 & 74.506 & 72.597 & 76.844 & 1582.851 & 1572.574 & 1569.718 & 1573.46 & 1568.402 & 1567.115 & 1564.694 & 1567.051 \\
\hline $7 / 26 / 168: 00$ & 75.614 & 85.251 & 83.569 & 90.451 & 80.186 & 74.497 & 72.599 & 76.77 & 1582.858 & 1572.602 & 1569.739 & 1573.478 & 1568.442 & 1567.124 & 1564.692 & 1567.125 \\
\hline $7 / 26 / 169: 00$ & 75.611 & 85.223 & 84.066 & 90.43 & 80.195 & 74.497 & 72.602 & 76.986 & 1582.861 & 1572.63 & 1569.242 & 1573.499 & 1568.433 & 1567.124 & 1564.689 & 1566.909 \\
\hline $7 / 26 / 1610: 00$ & 75.609 & 85.202 & 84.189 & 90.418 & 80.228 & 74.501 & 72.609 & 77.081 & 1582.863 & 1572.651 & 1569.119 & 1573.511 & 1568.4 & 1567.12 & 1564.682 & 1566.814 \\
\hline $7 / 26 / 1611: 00$ & 75.604 & 85.172 & 83.765 & 90.397 & 80.214 & 74.49 & 72.602 & 76.891 & 1582.868 & 1572.681 & 1569.543 & 1573.532 & 1568.414 & 1567.131 & 1564.689 & 1567.004 \\
\hline $7 / 26 / 1612: 00$ & 75.606 & 85.144 & 83.637 & 90.38 & 80.207 & 74.469 & 72.597 & 76.782 & 1582.866 & 1572.709 & 1569.671 & 1573.549 & 1568.421 & 1567.152 & 1564.694 & 1567.113 \\
\hline $7 / 26 / 1613: 00$ & 75.614 & 85.105 & 83.557 & 90.357 & 80.193 & 74.45 & 72.59 & 76.707 & 1582.858 & 1572.748 & 1569.751 & 1573.572 & 1568.435 & 1567.171 & 1564.701 & 1567.188 \\
\hline $7 / 26 / 1614: 00$ & 75.614 & 85.06 & 83.499 & 90.336 & 80.175 & 74.432 & 72.571 & 76.654 & 1582.858 & 1572.793 & 1569.809 & 1573.593 & 1568.453 & 1567.189 & 1564.72 & 1567.241 \\
\hline $7 / 26 / 1615: 00$ & 75.621 & 85.025 & 83.462 & 90.315 & 80.165 & 74.422 & 72.566 & 76.603 & 1582.851 & 1572.828 & 1569.846 & 1573.614 & 1568.463 & 1567.199 & 1564.725 & 1567.292 \\
\hline $7 / 26 / 1616: 00$ & 75.63 & 84.993 & 83.397 & 90.289 & 80.154 & 74.411 & 72.556 & 76.58 & 1582.842 & 1572.86 & 1569.911 & 1573.64 & 1568.474 & 1567.21 & 1564.735 & 1567.315 \\
\hline $7 / 26 / 1617: 00$ & 75.623 & 84.951 & 83.362 & 90.264 & 80.135 & 74.399 & 72.54 & 76.547 & 1582.849 & 1572.902 & 1569.946 & 1573.665 & 1568.493 & 1567.222 & 1564.751 & 1567.348 \\
\hline $7 / 26 / 1618: 00$ & 75.628 & 84.923 & 83.324 & 90.247 & 80.128 & 74.392 & 72.54 & 76.501 & 1582.844 & 1572.93 & 1569.984 & 1573.682 & 1568.5 & 1567.229 & 1564.751 & 1567.394 \\
\hline $7 / 26 / 16$ 19:00 & 75.639 & 84.9 & 83.296 & 90.236 & 80.121 & 74.383 & 72.54 & 76.473 & 1582.833 & 1572.953 & 1570.012 & 1573.693 & 1568.507 & 1567.238 & 1564.751 & 1567.422 \\
\hline $7 / 26 / 1620: 00$ & 75.646 & 84.881 & 83.282 & 90.229 & 80.121 & 74.387 & 72.552 & 76.445 & 1582.826 & 1572.972 & 1570.026 & 1573.7 & 1568.507 & 1567.234 & 1564.739 & 1567.45 \\
\hline $7 / 26 / 1621: 00$ & 75.642 & 84.863 & 83.264 & 90.217 & 80.114 & 74.385 & 72.556 & 76.431 & 1582.83 & 1572.99 & 1570.044 & 1573.712 & 1568.514 & 1567.236 & 1564.735 & 1567.464 \\
\hline $7 / 26 / 1622: 00$ & 75.644 & 84.86 & 83.257 & 90.215 & 80.116 & 74.39 & 72.573 & 76.422 & 1582.828 & 1572.993 & 1570.051 & 1573.714 & 1568.512 & 1567.231 & 1564.718 & 1567.473 \\
\hline $7 / 26 / 1623: 00$ & 75.654 & 84.863 & 83.257 & 90.217 & 80.119 & 74.401 & 72.597 & 76.426 & 1582.818 & 1572.99 & 1570.051 & 1573.712 & 1568.509 & 1567.22 & 1564.694 & 1567.469 \\
\hline $7 / 27 / 160: 00$ & 75.642 & 84.844 & 83.247 & 90.201 & 80.114 & 74.397 & 72.595 & 76.412 & 1582.83 & 1573.009 & 1570.061 & 1573.728 & 1568.514 & 1567.224 & 1564.696 & 1567.483 \\
\hline $7 / 27 / 161: 00$ & 75.628 & 84.823 & 83.224 & 90.187 & 80.098 & 74.385 & 72.595 & 76.343 & 1582.844 & 1573.03 & 1570.084 & 1573.742 & 1568.53 & 1567.236 & 1564.696 & 1567.552 \\
\hline $7 / 27 / 162: 00$ & 75.628 & 84.821 & 83.292 & 90.187 & 80.093 & 74.39 & 72.607 & 76.389 & 1582.844 & 1573.032 & 1570.016 & 1573.742 & 1568.535 & 1567.231 & 1564.684 & 1567.506 \\
\hline
\end{tabular}


TABLE S1.1 Automatically recorded groundwater levels in the unconfined aquifer.

\begin{tabular}{|c|c|c|c|c|c|c|c|c|c|c|c|c|c|c|c|c|}
\hline \multirow[b]{2}{*}{ Date and Time } & \multicolumn{8}{|c|}{ Measured Depth to Water (ft TOC ${ }^{\mathrm{a}}$ ) } & \multicolumn{8}{|c|}{ Groundwater Elevation ( $\mathrm{ft} \mathrm{AMSL}^{\mathrm{b}}$ ) } \\
\hline & DL1-C & DL2-C1 & DL3-C1 & DL4-C1 & DL5-C1 & DL6-C & DL7-C & DL8-C1 & DL1-C & DL2-C1 & DL3-C1 & DL4-C1 & DL5-C1 & DL6-C & DL7-C & DL8-C1 \\
\hline $7 / 27 / 163: 00$ & 75.668 & 84.853 & 83.338 & 90.212 & 80.14 & 74.432 & 72.659 & 76.433 & 1582.804 & 1573 & 1569.97 & 1573.717 & 1568.488 & 1567.189 & 1564.632 & 1567.462 \\
\hline $7 / 27 / 164: 00$ & 75.609 & 84.777 & 83.287 & 90.147 & 80.072 & 74.366 & 72.58 & 76.385 & 1582.863 & 1573.076 & 1570.021 & 1573.782 & 1568.556 & 1567.255 & 1564.711 & 1567.51 \\
\hline $7 / 27 / 165: 00$ & 75.5 & 84.644 & 83.185 & 90.068 & 79.951 & 74.266 & 72.451 & 76.264 & 1582.972 & 1573.209 & 1570.123 & 1573.861 & 1568.677 & 1567.355 & 1564.84 & 1567.631 \\
\hline $7 / 27 / 166: 00$ & 75.519 & 84.642 & 83.15 & 90.091 & 79.953 & 74.292 & 72.482 & 76.248 & 1582.953 & 1573.211 & 1570.158 & 1573.838 & 1568.675 & 1567.329 & 1564.809 & 1567.647 \\
\hline $7 / 27 / 167: 00$ & 75.557 & 84.66 & 83.115 & 90.112 & 79.971 & 74.327 & 72.53 & 76.255 & 1582.915 & 1573.193 & 1570.193 & 1573.817 & 1568.657 & 1567.294 & 1564.761 & 1567.64 \\
\hline $7 / 27 / 168: 00$ & 75.618 & 84.716 & 83.164 & 90.149 & 80.023 & 74.39 & 72.602 & 76.317 & 1582.854 & 1573.137 & 1570.144 & 1573.78 & 1568.605 & 1567.231 & 1564.689 & 1567.578 \\
\hline $7 / 27 / 169: 00$ & 75.658 & 84.746 & 83.194 & 90.163 & 80.058 & 74.413 & 72.642 & 76.357 & 1582.814 & 1573.107 & 1570.114 & 1573.766 & 1568.57 & 1567.208 & 1564.649 & 1567.538 \\
\hline $7 / 27 / 1610: 00$ & 75.654 & 84.735 & 83.189 & 90.149 & 80.056 & 74.408 & 72.635 & 76.366 & 1582.818 & 1573.118 & 1570.119 & 1573.78 & 1568.572 & 1567.213 & 1564.656 & 1567.529 \\
\hline $7 / 27 / 1611: 00$ & 75.639 & 84.721 & 83.212 & 90.133 & 80.051 & 74.394 & 72.623 & 76.354 & 1582.833 & 1573.132 & 1570.096 & 1573.796 & 1568.577 & 1567.227 & 1564.668 & 1567.541 \\
\hline $7 / 27 / 1612: 00$ & 75.635 & 84.709 & 83.182 & 90.124 & 80.056 & 74.38 & 72.618 & 76.32 & 1582.837 & 1573.144 & 1570.126 & 1573.805 & 1568.572 & 1567.241 & 1564.673 & 1567.575 \\
\hline $7 / 27 / 1613: 00$ & 75.628 & 84.695 & 83.157 & 90.117 & 80.051 & 74.369 & 72.614 & 76.299 & 1582.844 & 1573.158 & 1570.151 & 1573.812 & 1568.577 & 1567.252 & 1564.677 & 1567.596 \\
\hline $7 / 27 / 1614: 00$ & 75.616 & 84.677 & 83.138 & 90.103 & 80.051 & 74.359 & 72.597 & 76.283 & 1582.856 & 1573.176 & 1570.17 & 1573.826 & 1568.577 & 1567.262 & 1564.694 & 1567.612 \\
\hline $7 / 27 / 1615: 00$ & 75.611 & 84.66 & 83.243 & 90.093 & 80.058 & 74.355 & 72.592 & 76.331 & 1582.861 & 1573.193 & 1570.065 & 1573.836 & 1568.57 & 1567.266 & 1564.699 & 1567.564 \\
\hline $7 / 27 / 1616: 00$ & 75.602 & 84.639 & 83.693 & 90.082 & 80.093 & 74.364 & 72.573 & 76.647 & 1582.87 & 1573.214 & 1569.615 & 1573.847 & 1568.535 & 1567.257 & 1564.718 & 1567.248 \\
\hline $7 / 27 / 1617: 00$ & 75.599 & 84.635 & 83.853 & 90.077 & 80.147 & 74.383 & 72.568 & 76.823 & 1582.873 & 1573.218 & 1569.455 & 1573.852 & 1568.481 & 1567.238 & 1564.723 & 1567.072 \\
\hline $7 / 27 / 1618: 00$ & 75.599 & 84.635 & 83.94 & 90.096 & 80.189 & 74.394 & 72.564 & 76.928 & 1582.873 & 1573.218 & 1569.368 & 1573.833 & 1568.439 & 1567.227 & 1564.727 & 1566.967 \\
\hline $7 / 27 / 1619: 00$ & 75.606 & 84.632 & 84.012 & 90.114 & 80.254 & 74.413 & 72.568 & 77.026 & 1582.866 & 1573.221 & 1569.296 & 1573.815 & 1568.374 & 1567.208 & 1564.723 & 1566.869 \\
\hline $7 / 27 / 1620: 00$ & 75.623 & 84.656 & 84.084 & 90.138 & 80.321 & 74.446 & 72.595 & 77.135 & 1582.849 & 1573.197 & 1569.224 & 1573.791 & 1568.307 & 1567.175 & 1564.696 & 1566.76 \\
\hline $7 / 27 / 1621: 00$ & 75.639 & 84.672 & 84.149 & 90.131 & 80.387 & 74.466 & 72.607 & 77.225 & 1582.833 & 1573.181 & 1569.159 & 1573.798 & 1568.241 & 1567.155 & 1564.684 & 1566.67 \\
\hline $7 / 27 / 1622: 00$ & 75.656 & 84.702 & 84.212 & 90.121 & 80.457 & 74.487 & 72.628 & 77.295 & 1582.816 & 1573.151 & 1569.096 & 1573.808 & 1568.171 & 1567.134 & 1564.663 & 1566.6 \\
\hline $7 / 27 / 1623: 00$ & 75.67 & 84.723 & 84.278 & 90.159 & 80.527 & 74.513 & 72.65 & 77.36 & 1582.802 & 1573.13 & 1569.03 & 1573.77 & 1568.101 & 1567.108 & 1564.641 & 1566.535 \\
\hline $7 / 28 / 160: 00$ & 75.663 & 84.721 & 84.315 & 90.159 & 80.571 & 74.515 & 72.638 & 77.427 & 1582.809 & 1573.132 & 1568.993 & 1573.77 & 1568.057 & 1567.106 & 1564.653 & 1566.468 \\
\hline $7 / 28 / 161: 00$ & 75.665 & 84.737 & 84.357 & 90.166 & 80.625 & 74.534 & 72.645 & 77.487 & 1582.807 & 1573.116 & 1568.951 & 1573.763 & 1568.003 & 1567.087 & 1564.646 & 1566.408 \\
\hline $7 / 28 / 162: 00$ & 75.658 & 84.742 & 84.392 & 90.156 & 80.664 & 74.539 & 72.638 & 77.52 & 1582.814 & 1573.111 & 1568.916 & 1573.773 & 1567.964 & 1567.082 & 1564.653 & 1566.375 \\
\hline $7 / 28 / 163: 00$ & 75.661 & 84.751 & 84.427 & 90.159 & 80.711 & 74.553 & 72.647 & 77.576 & 1582.811 & 1573.102 & 1568.881 & 1573.77 & 1567.917 & 1567.068 & 1564.644 & 1566.319 \\
\hline $7 / 28 / 164: 00$ & 75.649 & 84.744 & 84.448 & 90.14 & 80.746 & 74.548 & 72.623 & 77.615 & 1582.823 & 1573.109 & 1568.86 & 1573.789 & 1567.882 & 1567.073 & 1564.668 & 1566.28 \\
\hline $7 / 28 / 165: 00$ & 75.665 & 84.774 & 84.492 & 90.156 & 80.809 & 74.578 & 72.654 & 77.659 & 1582.807 & 1573.079 & 1568.816 & 1573.773 & 1567.819 & 1567.043 & 1564.637 & 1566.236 \\
\hline $7 / 28 / 166: 00$ & 75.677 & 84.788 & 84.553 & 90.142 & 80.867 & 74.597 & 72.661 & 77.724 & 1582.795 & 1573.065 & 1568.755 & 1573.787 & 1567.761 & 1567.024 & 1564.63 & 1566.171 \\
\hline $7 / 28 / 167: 00$ & 75.696 & 84.814 & 84.555 & 90.152 & 80.923 & 74.618 & 72.681 & 77.741 & 1582.776 & 1573.039 & 1568.753 & 1573.777 & 1567.705 & 1567.003 & 1564.61 & 1566.154 \\
\hline $7 / 28 / 168: 00$ & 75.694 & 84.825 & 84.059 & 90.145 & 80.916 & 74.627 & 72.683 & 77.562 & 1582.778 & 1573.028 & 1569.249 & 1573.784 & 1567.712 & 1566.994 & 1564.608 & 1566.333 \\
\hline $7 / 28 / 169: 00$ & 75.701 & 84.835 & 84.399 & 90.149 & 80.937 & 74.643 & 72.692 & 77.703 & 1582.771 & 1573.018 & 1568.909 & 1573.78 & 1567.691 & 1566.978 & 1564.599 & 1566.192 \\
\hline $7 / 28 / 1610: 00$ & 75.698 & 84.846 & 84.56 & 90.149 & 80.967 & 74.66 & 72.692 & 77.815 & 1582.774 & 1573.007 & 1568.748 & 1573.78 & 1567.661 & 1566.961 & 1564.599 & 1566.08 \\
\hline $7 / 28 / 1611: 00$ & 75.689 & 84.849 & 84.613 & 90.138 & 80.991 & 74.664 & 72.683 & 77.889 & 1582.783 & 1573.004 & 1568.695 & 1573.791 & 1567.637 & 1566.957 & 1564.608 & 1566.006 \\
\hline $7 / 28 / 1612: 00$ & 75.684 & 84.853 & 84.653 & 90.133 & 81.018 & 74.66 & 72.683 & 77.878 & 1582.788 & 1573 & 1568.655 & 1573.796 & 1567.61 & 1566.961 & 1564.608 & 1566.017 \\
\hline $7 / 28 / 1613: 00$ & 75.677 & 84.853 & 84.672 & 90.128 & 81.042 & 74.66 & 72.673 & 77.922 & 1582.795 & 1573 & 1568.636 & 1573.801 & 1567.586 & 1566.961 & 1564.618 & 1565.973 \\
\hline $7 / 28 / 1614: 00$ & 75.679 & 84.846 & 84.69 & 90.121 & 81.068 & 74.66 & 72.664 & 77.95 & 1582.793 & 1573.007 & 1568.618 & 1573.808 & 1567.56 & 1566.961 & 1564.627 & 1565.945 \\
\hline $7 / 28 / 1615: 00$ & 75.701 & 84.849 & 84.732 & 90.121 & 81.1 & 74.667 & 72.661 & 77.973 & 1582.771 & 1573.004 & 1568.576 & 1573.808 & 1567.528 & 1566.954 & 1564.63 & 1565.922 \\
\hline $7 / 28 / 1616: 00$ & 75.715 & 84.858 & 84.483 & 90.124 & 81.166 & 74.669 & 72.661 & 77.88 & 1582.757 & 1572.995 & 1568.825 & 1573.805 & 1567.462 & 1566.952 & 1564.63 & 1566.015 \\
\hline $7 / 28 / 1617: 00$ & 75.734 & 84.863 & 84.455 & 90.126 & 81.186 & 74.664 & 72.671 & 77.766 & 1582.738 & 1572.99 & 1568.853 & 1573.803 & 1567.442 & 1566.957 & 1564.62 & 1566.129 \\
\hline $7 / 28 / 1618: 00$ & 75.738 & 84.86 & 84.893 & 90.121 & 81.221 & 74.662 & 72.664 & 77.933 & 1582.734 & 1572.993 & 1568.415 & 1573.808 & 1567.407 & 1566.959 & 1564.627 & 1565.962 \\
\hline $7 / 28 / 1619: 00$ & 75.755 & 84.881 & 85.056 & 90.133 & 81.273 & 74.674 & 72.681 & 78.024 & 1582.717 & 1572.972 & 1568.252 & 1573.796 & 1567.355 & 1566.947 & 1564.61 & 1565.871 \\
\hline $7 / 28 / 1620: 00$ & 75.76 & 84.888 & 85.142 & 90.133 & 81.305 & 74.671 & 72.676 & 78.075 & 1582.712 & 1572.965 & 1568.166 & 1573.796 & 1567.323 & 1566.95 & 1564.615 & 1565.82 \\
\hline $7 / 28 / 1621: 00$ & 75.774 & 84.914 & 85.224 & 90.149 & 81.354 & 74.69 & 72.697 & 78.133 & 1582.698 & 1572.939 & 1568.084 & 1573.78 & 1567.274 & 1566.931 & 1564.594 & 1565.762 \\
\hline $7 / 28 / 1622: 00$ & 75.8 & 84.951 & 85.303 & 90.168 & 81.413 & 74.709 & 72.724 & 78.189 & 1582.672 & 1572.902 & 1568.005 & 1573.761 & 1567.215 & 1566.912 & 1564.567 & 1565.706 \\
\hline $7 / 28 / 1623: 00$ & 75.83 & 84.995 & 85.385 & 90.187 & 81.473 & 74.732 & 72.752 & 78.258 & 1582.642 & 1572.858 & 1567.923 & 1573.742 & 1567.155 & 1566.889 & 1564.539 & 1565.637 \\
\hline $7 / 29 / 160: 00$ & 75.826 & 85.012 & 85.431 & 90.187 & 81.506 & 74.727 & 72.748 & 78.296 & 1582.646 & 1572.841 & 1567.877 & 1573.742 & 1567.122 & 1566.894 & 1564.543 & 1565.599 \\
\hline $7 / 29 / 161: 00$ & 75.819 & 85.025 & 85.471 & 90.189 & 81.536 & 74.732 & 72.747 & 78.328 & 1582.653 & 1572.828 & 1567.837 & 1573.74 & 1567.092 & 1566.889 & 1564.544 & 1565.567 \\
\hline $7 / 29 / 162: 00$ & 75.788 & 85.018 & 85.135 & 90.185 & 81.525 & 74.718 & 72.731 & 78.145 & 1582.684 & 1572.835 & 1568.173 & 1573.744 & 1567.103 & 1566.903 & 1564.56 & 1565.75 \\
\hline $7 / 29 / 163: 00$ & 75.767 & 85.014 & 84.937 & 90.18 & 81.501 & 74.706 & 72.719 & 78.008 & 1582.705 & 1572.839 & 1568.371 & 1573.749 & 1567.127 & 1566.915 & 1564.572 & 1565.887 \\
\hline
\end{tabular}


TABLE S1.1 Automatically recorded groundwater levels in the unconfined aquifer.

\begin{tabular}{|c|c|c|c|c|c|c|c|c|c|c|c|c|c|c|c|c|}
\hline \multirow[b]{2}{*}{ Date and Time } & \multicolumn{8}{|c|}{ Measured Depth to Water (ft TOC ${ }^{\mathrm{a}}$ ) } & \multicolumn{8}{|c|}{ Groundwater Elevation ( $\mathrm{ft} \mathrm{AMSL}^{\mathrm{b}}$ ) } \\
\hline & DL1-C & DL2-C1 & DL3-C1 & DL4-C1 & DL5-C1 & DL6-C & DL7-C & DL8-C1 & DL1-C & DL2-C1 & DL3-C1 & DL4-C1 & DL5-C1 & DL6-C & DL7-C & DL8-C1 \\
\hline $7 / 29 / 164: 00$ & 75.75 & 85.014 & 84.87 & 90.184 & 81.485 & 74.702 & 72.721 & 77.947 & 1582.722 & 1572.839 & 1568.438 & 1573.745 & 1567.143 & 1566.919 & 1564.57 & 1565.948 \\
\hline $7 / 29 / 165: 00$ & 75.731 & 85.004 & 84.823 & 90.177 & 81.464 & 74.688 & 72.707 & 77.871 & 1582.741 & 1572.849 & 1568.485 & 1573.752 & 1567.164 & 1566.933 & 1564.584 & 1566.024 \\
\hline $7 / 29 / 166: 00$ & 75.729 & 85.007 & 84.76 & 90.184 & 81.455 & 74.688 & 72.716 & 77.84 & 1582.743 & 1572.846 & 1568.548 & 1573.745 & 1567.173 & 1566.933 & 1564.575 & 1566.055 \\
\hline $7 / 29 / 167: 00$ & 75.753 & 85.049 & 84.73 & 90.212 & 81.478 & 74.727 & 72.762 & 77.833 & 1582.719 & 1572.804 & 1568.578 & 1573.717 & 1567.15 & 1566.894 & 1564.529 & 1566.062 \\
\hline $7 / 29 / 168: 00$ & 75.774 & 85.072 & 84.735 & 90.222 & 81.492 & 74.739 & 72.783 & 77.845 & 1582.698 & 1572.781 & 1568.573 & 1573.707 & 1567.136 & 1566.882 & 1564.508 & 1566.05 \\
\hline $7 / 29 / 169: 00$ & 75.776 & 85.095 & 84.753 & 90.324 & 81.494 & 74.753 & 72.795 & 77.866 & 1582.696 & 1572.758 & 1568.555 & 1573.605 & 1567.134 & 1566.868 & 1564.496 & 1566.029 \\
\hline $7 / 29 / 1610: 00$ & 75.76 & 85.13 & 84.825 & 90.712 & 81.483 & 74.76 & 72.781 & 77.912 & 1582.712 & 1572.723 & 1568.483 & 1573.217 & 1567.145 & 1566.861 & 1564.51 & 1565.983 \\
\hline $7 / 29 / 1611: 00$ & 75.753 & 85.188 & 84.737 & 90.934 & 81.478 & 74.758 & 72.778 & 77.822 & 1582.719 & 1572.665 & 1568.571 & 1572.995 & 1567.15 & 1566.863 & 1564.513 & 1566.073 \\
\hline $7 / 29 / 1612: 00$ & 75.753 & 85.256 & 84.73 & 91.086 & 81.482 & 74.762 & 72.781 & 77.78 & 1582.719 & 1572.597 & 1568.578 & 1572.843 & 1567.146 & 1566.859 & 1564.51 & 1566.115 \\
\hline $7 / 29 / 1613: 00$ & 75.745 & 85.312 & 84.73 & 91.191 & 81.473 & 74.762 & 72.771 & 77.75 & 1582.727 & 1572.541 & 1568.578 & 1572.738 & 1567.155 & 1566.859 & 1564.52 & 1566.145 \\
\hline $7 / 29 / 1614: 00$ & 75.734 & 85.36 & 84.725 & 91.277 & 81.462 & 74.758 & 72.762 & 77.717 & 1582.738 & 1572.493 & 1568.583 & 1572.652 & 1567.166 & 1566.863 & 1564.529 & 1566.178 \\
\hline $7 / 29 / 1615: 00$ & 75.734 & 85.419 & 84.728 & 91.349 & 81.464 & 74.76 & 72.762 & 77.694 & 1582.738 & 1572.434 & 1568.58 & 1572.58 & 1567.164 & 1566.861 & 1564.529 & 1566.201 \\
\hline $7 / 29 / 1616: 00$ & 75.731 & 85.467 & 84.723 & 91.403 & 81.457 & 74.758 & 72.757 & 77.669 & 1582.741 & 1572.386 & 1568.585 & 1572.526 & 1567.171 & 1566.863 & 1564.534 & 1566.226 \\
\hline $7 / 29 / 1617: 00$ & 75.743 & 85.512 & 84.816 & 91.452 & 81.459 & 74.753 & 72.745 & 77.69 & 1582.729 & 1572.341 & 1568.492 & 1572.477 & 1567.169 & 1566.868 & 1564.546 & 1566.205 \\
\hline $7 / 29 / 1618: 00$ & 75.762 & 85.57 & 85.31 & 91.525 & 81.499 & 74.76 & 72.743 & 77.926 & 1582.71 & 1572.283 & 1567.998 & 1572.404 & 1567.129 & 1566.861 & 1564.548 & 1565.969 \\
\hline $7 / 29 / 1619: 00$ & 75.783 & 85.628 & 85.478 & 91.576 & 81.546 & 74.772 & 72.745 & 78.031 & 1582.689 & 1572.225 & 1567.83 & 1572.353 & 1567.082 & 1566.849 & 1564.546 & 1565.864 \\
\hline $7 / 29 / 1620: 00$ & 75.8 & 85.688 & 85.574 & 91.625 & 81.592 & 74.788 & 72.75 & 78.131 & 1582.672 & 1572.165 & 1567.734 & 1572.304 & 1567.036 & 1566.833 & 1564.541 & 1565.764 \\
\hline $7 / 29 / 1621: 00$ & 75.828 & 85.763 & 85.66 & 91.655 & 81.648 & 74.804 & 72.774 & 78.2 & 1582.644 & 1572.09 & 1567.648 & 1572.274 & 1566.98 & 1566.817 & 1564.517 & 1565.695 \\
\hline $7 / 29 / 1622: 00$ & 75.852 & 85.83 & 85.734 & 91.683 & 81.704 & 74.827 & 72.793 & 78.272 & 1582.62 & 1572.023 & 1567.574 & 1572.246 & 1566.924 & 1566.794 & 1564.498 & 1565.623 \\
\hline $7 / 29 / 1623: 00$ & 75.861 & 85.888 & 85.8 & 91.744 & 81.748 & 74.834 & 72.793 & 78.328 & 1582.611 & 1571.965 & 1567.508 & 1572.185 & 1566.88 & 1566.787 & 1564.498 & 1565.567 \\
\hline $7 / 30 / 160: 00$ & 75.877 & 85.953 & 85.865 & 91.8 & 81.8 & 74.853 & 72.807 & 78.377 & 1582.595 & 1571.9 & 1567.443 & 1572.129 & 1566.828 & 1566.768 & 1564.484 & 1565.518 \\
\hline $7 / 30 / 161: 00$ & 75.882 & 86.007 & 85.928 & 91.835 & 81.839 & 74.858 & 72.805 & 78.419 & 1582.59 & 1571.846 & 1567.38 & 1572.094 & 1566.789 & 1566.763 & 1564.486 & 1565.476 \\
\hline $7 / 30 / 162: 00$ & 75.88 & 86.047 & 85.97 & 91.858 & 81.872 & 74.858 & 72.8 & 78.446 & 1582.592 & 1571.806 & 1567.338 & 1572.071 & 1566.756 & 1566.763 & 1564.491 & 1565.449 \\
\hline $7 / 30 / 163: 00$ & 75.892 & 86.102 & 86.021 & 91.889 & 81.914 & 74.872 & 72.807 & 78.491 & 1582.58 & 1571.751 & 1567.287 & 1572.04 & 1566.714 & 1566.749 & 1564.484 & 1565.404 \\
\hline $7 / 30 / 164: 00$ & 75.894 & 86.142 & 86.058 & 91.912 & 81.951 & 74.872 & 72.805 & 78.465 & 1582.578 & 1571.711 & 1567.25 & 1572.017 & 1566.677 & 1566.749 & 1564.486 & 1565.43 \\
\hline $7 / 30 / 165: 00$ & 75.889 & 86.184 & 86.105 & 91.931 & 81.982 & 74.879 & 72.802 & 78.544 & 1582.583 & 1571.669 & 1567.203 & 1571.998 & 1566.646 & 1566.742 & 1564.489 & 1565.351 \\
\hline $7 / 30 / 166: 00$ & 75.88 & 86.226 & 86.138 & 91.947 & 82.019 & 74.886 & 72.802 & 78.569 & 1582.592 & 1571.627 & 1567.17 & 1571.982 & 1566.609 & 1566.735 & 1564.489 & 1565.326 \\
\hline $7 / 30 / 167: 00$ & 75.88 & 86.277 & 86.175 & 91.935 & 82.054 & 74.907 & 72.821 & 78.6 & 1582.592 & 1571.576 & 1567.133 & 1571.994 & 1566.574 & 1566.714 & 1564.47 & 1565.295 \\
\hline $7 / 30 / 168: 00$ & 75.877 & 86.284 & 86.096 & 91.606 & 82.077 & 74.911 & 72.821 & 78.628 & 1582.595 & 1571.569 & 1567.212 & 1572.323 & 1566.551 & 1566.71 & 1564.47 & 1565.267 \\
\hline $7 / 30 / 169: 00$ & 75.873 & 86.279 & 86.047 & 91.426 & 82.108 & 74.925 & 72.833 & 78.658 & 1582.599 & 1571.574 & 1567.261 & 1572.503 & 1566.52 & 1566.696 & 1564.458 & 1565.237 \\
\hline $7 / 30 / 1610: 00$ & 75.885 & 86.288 & 86.019 & 91.321 & 82.147 & 74.944 & 72.86 & 78.679 & 1582.587 & 1571.565 & 1567.289 & 1572.608 & 1566.481 & 1566.677 & 1564.431 & 1565.216 \\
\hline $7 / 30 / 1611: 00$ & 75.835 & 86.216 & 85.958 & 91.193 & 82.131 & 74.909 & 72.802 & 78.658 & 1582.637 & 1571.637 & 1567.35 & 1572.736 & 1566.497 & 1566.712 & 1564.489 & 1565.237 \\
\hline $7 / 30 / 1612: 00$ & 75.837 & 86.209 & 85.942 & 91.142 & 82.152 & 74.928 & 72.821 & 78.683 & 1582.635 & 1571.644 & 1567.366 & 1572.787 & 1566.476 & 1566.693 & 1564.47 & 1565.212 \\
\hline $7 / 30 / 1613: 00$ & 75.844 & 86.188 & 85.916 & 91.09 & 82.18 & 74.942 & 72.838 & 78.7 & 1582.628 & 1571.665 & 1567.392 & 1572.839 & 1566.448 & 1566.679 & 1564.453 & 1565.195 \\
\hline $7 / 30 / 1614: 00$ & 75.833 & 86.154 & 85.884 & 91.034 & 82.185 & 74.937 & 72.819 & 78.697 & 1582.639 & 1571.699 & 1567.424 & 1572.895 & 1566.443 & 1566.684 & 1564.472 & 1565.198 \\
\hline $7 / 30 / 1615: 00$ & 75.816 & 86.114 & 85.832 & 90.983 & 82.185 & 74.93 & 72.805 & 78.679 & 1582.656 & 1571.739 & 1567.476 & 1572.946 & 1566.443 & 1566.691 & 1564.486 & 1565.216 \\
\hline $7 / 30 / 1616: 00$ & 75.816 & 86.091 & 85.811 & 90.952 & 82.196 & 74.937 & 72.817 & 78.7 & 1582.656 & 1571.762 & 1567.497 & 1572.977 & 1566.432 & 1566.684 & 1564.474 & 1565.195 \\
\hline $7 / 30 / 1617: 00$ & 75.807 & 86.056 & 85.79 & 90.915 & 82.201 & 74.939 & 72.809 & 78.702 & 1582.665 & 1571.797 & 1567.518 & 1573.014 & 1566.427 & 1566.682 & 1564.482 & 1565.193 \\
\hline $7 / 30 / 1618: 00$ & 75.804 & 86.023 & 85.783 & 90.885 & 82.205 & 74.937 & 72.805 & 78.709 & 1582.668 & 1571.83 & 1567.525 & 1573.044 & 1566.423 & 1566.684 & 1564.486 & 1565.186 \\
\hline $7 / 30 / 16$ 19:00 & 75.797 & 85.995 & 85.769 & 90.854 & 82.245 & 74.939 & 72.802 & 78.718 & 1582.675 & 1571.858 & 1567.539 & 1573.075 & 1566.383 & 1566.682 & 1564.489 & 1565.177 \\
\hline $7 / 30 / 1620: 00$ & 75.8 & 85.97 & 85.767 & 90.836 & 82.238 & 74.946 & 72.807 & 78.725 & 1582.672 & 1571.883 & 1567.541 & 1573.093 & 1566.39 & 1566.675 & 1564.484 & 1565.17 \\
\hline $7 / 30 / 1621: 00$ & 75.809 & 85.958 & 85.769 & 90.819 & 82.247 & 74.958 & 72.824 & 78.755 & 1582.663 & 1571.895 & 1567.539 & 1573.11 & 1566.381 & 1566.663 & 1564.467 & 1565.14 \\
\hline $7 / 30 / 1622: 00$ & 75.835 & 85.965 & 85.786 & 90.822 & 82.271 & 74.988 & 72.86 & 78.788 & 1582.637 & 1571.888 & 1567.522 & 1573.107 & 1566.357 & 1566.633 & 1564.431 & 1565.107 \\
\hline $7 / 30 / 1623: 00$ & 75.837 & 85.951 & 85.786 & 90.801 & 82.273 & 74.99 & 72.857 & 78.753 & 1582.635 & 1571.902 & 1567.522 & 1573.128 & 1566.355 & 1566.631 & 1564.434 & 1565.142 \\
\hline $7 / 31 / 160: 00$ & 75.833 & 85.928 & 85.783 & 90.777 & 82.268 & 74.993 & 72.855 & 78.813 & 1582.639 & 1571.925 & 1567.525 & 1573.152 & 1566.36 & 1566.628 & 1564.436 & 1565.082 \\
\hline $7 / 31 / 161: 00$ & 75.828 & 85.914 & 85.776 & 90.763 & 82.259 & 74.995 & 72.857 & 78.813 & 1582.644 & 1571.939 & 1567.532 & 1573.166 & 1566.369 & 1566.626 & 1564.434 & 1565.082 \\
\hline $7 / 31 / 162: 00$ & 75.821 & 85.888 & 85.767 & 90.742 & 82.245 & 74.995 & 72.85 & 78.816 & 1582.651 & 1571.965 & 1567.541 & 1573.187 & 1566.383 & 1566.626 & 1564.441 & 1565.079 \\
\hline $7 / 31 / 163: 00$ & 75.807 & 85.858 & 85.744 & 90.721 & 82.222 & 74.986 & 72.836 & 78.802 & 1582.665 & 1571.995 & 1567.564 & 1573.208 & 1566.406 & 1566.635 & 1564.455 & 1565.093 \\
\hline $7 / 31 / 164: 00$ & 75.804 & 85.84 & 85.739 & 90.71 & 82.215 & 74.99 & 72.841 & 78.804 & 1582.668 & 1572.013 & 1567.569 & 1573.219 & 1566.413 & 1566.631 & 1564.45 & 1565.091 \\
\hline
\end{tabular}


TABLE S1.1 Automatically recorded groundwater levels in the unconfined aquifer.

\begin{tabular}{|c|c|c|c|c|c|c|c|c|c|c|c|c|c|c|c|c|}
\hline \multirow[b]{2}{*}{ Date and Time } & \multicolumn{8}{|c|}{ Measured Depth to Water ( $\mathrm{ft}$ TOC ${ }^{\mathrm{a}}$ ) } & \multicolumn{8}{|c|}{ Groundwater Elevation (ft $\mathrm{AMSL}^{\mathrm{b}}$ ) } \\
\hline & DL1-C & DL2-C1 & DL3-C1 & DL4-C1 & DL5-C1 & DL6-C & DL7-C & DL8-C1 & DL1-C & DL2-C1 & DL3-C1 & DL4-C1 & DL5-C1 & DL6-C & DL7-C & DL8-C1 \\
\hline $7 / 31 / 165: 00$ & 75.807 & 85.826 & 85.753 & 90.698 & 82.208 & 75 & 72.843 & 78.82 & 1582.665 & 1572.027 & 1567.555 & 1573.231 & 1566.42 & 1566.621 & 1564.448 & 1565.075 \\
\hline $7 / 31 / 166: 00$ & 75.804 & 85.8 & 85.732 & 90.684 & 82.194 & 74.995 & 72.836 & 78.813 & 1582.668 & 1572.053 & 1567.576 & 1573.245 & 1566.434 & 1566.626 & 1564.455 & 1565.082 \\
\hline $7 / 31 / 167: 00$ & 75.814 & 85.798 & 85.732 & 90.684 & 82.196 & 75.011 & 72.852 & 78.832 & 1582.658 & 1572.055 & 1567.576 & 1573.245 & 1566.432 & 1566.61 & 1564.439 & 1565.063 \\
\hline $7 / 31 / 168: 00$ & 75.837 & 85.809 & 85.49 & 90.691 & 82.199 & 75.035 & 72.886 & 78.734 & 1582.635 & 1572.044 & 1567.818 & 1573.238 & 1566.429 & 1566.586 & 1564.405 & 1565.161 \\
\hline $7 / 31 / 169: 00$ & 75.863 & 85.828 & 85.161 & 90.7 & 82.192 & 75.056 & 72.926 & 78.57 & 1582.609 & 1572.025 & 1568.147 & 1573.229 & 1566.436 & 1566.565 & 1564.365 & 1565.325 \\
\hline $7 / 31 / 1610: 00$ & 75.807 & 85.744 & 84.965 & 90.637 & 82.084 & 74.993 & 72.85 & 78.402 & 1582.665 & 1572.109 & 1568.343 & 1573.292 & 1566.544 & 1566.628 & 1564.441 & 1565.493 \\
\hline 7/31/16 11:00 & 75.826 & 85.746 & 84.907 & 90.647 & 82.056 & 75.016 & 72.879 & 78.335 & 1582.646 & 1572.107 & 1568.401 & 1573.282 & 1566.572 & 1566.605 & 1564.412 & 1565.56 \\
\hline $7 / 31 / 1612: 00$ & 75.814 & 85.714 & 84.835 & 90.623 & 81.998 & 75.002 & 72.867 & 78.263 & 1582.658 & 1572.139 & 1568.473 & 1573.306 & 1566.63 & 1566.619 & 1564.424 & 1565.632 \\
\hline 7/31/16 13:00 & 75.816 & 85.702 & 84.774 & 90.616 & 81.963 & 75 & 72.879 & 78.166 & 1582.656 & 1572.151 & 1568.534 & 1573.313 & 1566.665 & 1566.621 & 1564.412 & 1565.729 \\
\hline 7/31/16 14:00 & 75.823 & 85.686 & 84.718 & 90.607 & 81.923 & 74.988 & 72.886 & 78.042 & 1582.649 & 1572.167 & 1568.59 & 1573.322 & 1566.705 & 1566.633 & 1564.405 & 1565.853 \\
\hline $7 / 31 / 1615: 00$ & 75.821 & 85.667 & 84.651 & 90.595 & 81.886 & 74.974 & 72.888 & 77.966 & 1582.651 & 1572.186 & 1568.657 & 1573.334 & 1566.742 & 1566.647 & 1564.403 & 1565.929 \\
\hline $7 / 31 / 1616: 00$ & 75.819 & 85.639 & 84.597 & 90.579 & 81.837 & 74.963 & 72.881 & 77.947 & 1582.653 & 1572.214 & 1568.711 & 1573.35 & 1566.791 & 1566.658 & 1564.41 & 1565.948 \\
\hline 7/31/16 17:00 & 75.811 & 85.612 & 84.543 & 90.565 & 81.786 & 74.946 & 72.874 & 77.875 & 1582.661 & 1572.241 & 1568.765 & 1573.364 & 1566.842 & 1566.675 & 1564.417 & 1566.02 \\
\hline $7 / 31 / 1618: 00$ & 75.816 & 85.591 & 84.494 & 90.551 & 81.748 & 74.937 & 72.874 & 77.773 & 1582.656 & 1572.262 & 1568.814 & 1573.378 & 1566.88 & 1566.684 & 1564.417 & 1566.122 \\
\hline 7/31/16 19:00 & 75.819 & 85.57 & 84.453 & 90.556 & 81.707 & 74.932 & 72.879 & 77.764 & 1582.653 & 1572.283 & 1568.855 & 1573.373 & 1566.921 & 1566.689 & 1564.412 & 1566.131 \\
\hline $7 / 31 / 1620: 00$ & 75.816 & 85.546 & 84.408 & 90.556 & 81.667 & 74.921 & 72.881 & 77.706 & 1582.656 & 1572.307 & 1568.9 & 1573.373 & 1566.961 & 1566.7 & 1564.41 & 1566.189 \\
\hline 7/31/16 21:00 & 75.821 & 85.539 & 84.38 & 90.553 & 81.637 & 74.923 & 72.893 & 77.671 & 1582.651 & 1572.314 & 1568.928 & 1573.376 & 1566.991 & 1566.698 & 1564.398 & 1566.224 \\
\hline $7 / 31 / 1622: 00$ & 75.833 & 85.535 & 84.362 & 90.539 & 81.618 & 74.93 & 72.915 & 77.648 & 1582.639 & 1572.318 & 1568.946 & 1573.39 & 1567.01 & 1566.691 & 1564.376 & 1566.247 \\
\hline $7 / 31 / 1623: 00$ & 75.837 & 85.526 & 84.336 & 90.546 & 81.588 & 74.925 & 72.919 & 77.618 & 1582.635 & 1572.327 & 1568.972 & 1573.383 & 1567.04 & 1566.696 & 1564.372 & 1566.277 \\
\hline $8 / 1 / 160: 00$ & 75.828 & 85.505 & 84.299 & 90.542 & 81.546 & 74.909 & 72.91 & 77.573 & 1582.644 & 1572.348 & 1569.009 & 1573.387 & 1567.082 & 1566.712 & 1564.381 & 1566.322 \\
\hline 8/1/16 1:00 & 75.816 & 85.474 & 84.259 & 90.521 & 81.504 & 74.895 & 72.9 & 77.532 & 1582.656 & 1572.379 & 1569.049 & 1573.408 & 1567.124 & 1566.726 & 1564.391 & 1566.363 \\
\hline 8/1/16 2:00 & 75.802 & 85.442 & 84.292 & 90.509 & 81.455 & 74.876 & 72.886 & 77.488 & 1582.67 & 1572.411 & 1569.016 & 1573.42 & 1567.173 & 1566.745 & 1564.405 & 1566.407 \\
\hline 8/1/16 3:00 & 75.793 & 85.414 & 84.25 & 90.49 & 81.41 & 74.865 & 72.876 & 77.45 & 1582.679 & 1572.439 & 1569.058 & 1573.439 & 1567.218 & 1566.756 & 1564.415 & 1566.445 \\
\hline $8 / 1 / 164: 00$ & 75.769 & 85.372 & 84.217 & 90.465 & 81.352 & 74.839 & 72.852 & 77.418 & 1582.703 & 1572.481 & 1569.091 & 1573.464 & 1567.276 & 1566.782 & 1564.439 & 1566.477 \\
\hline $8 / 1 / 16$ 5:00 & 75.762 & 85.346 & 84.205 & 90.451 & 81.303 & 74.834 & 72.848 & 77.374 & 1582.71 & 1572.507 & 1569.103 & 1573.478 & 1567.325 & 1566.787 & 1564.443 & 1566.521 \\
\hline $8 / 1 / 166: 00$ & 75.786 & 85.351 & 84.149 & 90.448 & 81.291 & 74.848 & 72.883 & 77.355 & 1582.686 & 1572.502 & 1569.159 & 1573.481 & 1567.337 & 1566.773 & 1564.408 & 1566.54 \\
\hline 8/1/16 7:00 & 75.816 & 85.356 & 84.124 & 90.446 & 81.289 & 74.874 & 72.912 & 77.371 & 1582.656 & 1572.497 & 1569.184 & 1573.483 & 1567.339 & 1566.747 & 1564.379 & 1566.524 \\
\hline 8/1/16 8:00 & 75.816 & 85.346 & 84.112 & 90.434 & 81.256 & 74.869 & 72.915 & 77.355 & 1582.656 & 1572.507 & 1569.196 & 1573.495 & 1567.372 & 1566.752 & 1564.376 & 1566.54 \\
\hline 8/1/16 9:00 & 75.821 & 85.349 & 84.448 & 90.437 & 81.261 & 74.881 & 72.931 & 77.515 & 1582.651 & 1572.504 & 1568.86 & 1573.492 & 1567.367 & 1566.74 & 1564.36 & 1566.38 \\
\hline $8 / 1 / 1610: 00$ & 75.819 & 85.377 & 84.723 & 90.448 & 81.315 & 74.911 & 72.969 & 77.692 & 1582.653 & 1572.476 & 1568.585 & 1573.481 & 1567.313 & 1566.71 & 1564.322 & 1566.203 \\
\hline 8/1/16 11:00 & 75.797 & 85.381 & 84.835 & 90.439 & 81.347 & 74.902 & 72.967 & 77.759 & 1582.675 & 1572.472 & 1568.473 & 1573.49 & 1567.281 & 1566.719 & 1564.324 & 1566.136 \\
\hline $8 / 1 / 1612: 00$ & 75.781 & 85.388 & 84.902 & 90.437 & 81.387 & 74.911 & 72.979 & 77.81 & 1582.691 & 1572.465 & 1568.406 & 1573.492 & 1567.241 & 1566.71 & 1564.312 & 1566.085 \\
\hline $8 / 1 / 1613: 00$ & 75.769 & 85.386 & 84.951 & 90.434 & 81.424 & 74.914 & 72.984 & 77.873 & 1582.703 & 1572.467 & 1568.357 & 1573.495 & 1567.204 & 1566.707 & 1564.307 & 1566.022 \\
\hline 8/1/16 14:00 & 75.762 & 85.379 & 84.982 & 90.427 & 81.452 & 74.909 & 72.972 & 77.908 & 1582.71 & 1572.474 & 1568.326 & 1573.502 & 1567.176 & 1566.712 & 1564.319 & 1565.987 \\
\hline $8 / 1 / 1615: 00$ & 75.753 & 85.363 & 84.986 & 90.418 & 81.471 & 74.916 & 72.958 & 78.01 & 1582.719 & 1572.49 & 1568.322 & 1573.511 & 1567.157 & 1566.705 & 1564.333 & 1565.885 \\
\hline $8 / 1 / 1616: 00$ & 75.755 & 85.344 & 85.005 & 90.402 & 81.494 & 74.937 & 72.948 & 78.105 & 1582.717 & 1572.509 & 1568.303 & 1573.527 & 1567.134 & 1566.684 & 1564.343 & 1565.79 \\
\hline $8 / 1 / 1617: 00$ & 75.753 & 85.33 & 85.031 & 90.397 & 81.515 & 74.925 & 72.938 & 78.098 & 1582.719 & 1572.523 & 1568.277 & 1573.532 & 1567.113 & 1566.696 & 1564.353 & 1565.797 \\
\hline $8 / 1 / 16$ 18:00 & 75.75 & 85.307 & 85.024 & 90.406 & 81.529 & 74.904 & 72.924 & 78.059 & 1582.722 & 1572.546 & 1568.284 & 1573.523 & 1567.099 & 1566.717 & 1564.367 & 1565.836 \\
\hline $8 / 1 / 16$ 19:00 & 75.757 & 85.302 & 85.04 & 90.425 & 81.557 & 74.914 & 72.926 & 78.107 & 1582.715 & 1572.551 & 1568.268 & 1573.504 & 1567.071 & 1566.707 & 1564.365 & 1565.788 \\
\hline $8 / 1 / 1620: 00$ & 75.783 & 85.319 & 85.073 & 90.448 & 81.604 & 74.946 & 72.953 & 78.177 & 1582.689 & 1572.534 & 1568.235 & 1573.481 & 1567.024 & 1566.675 & 1564.338 & 1565.718 \\
\hline 8/1/16 21:00 & 75.807 & 85.335 & 85.112 & 90.444 & 81.658 & 74.972 & 72.977 & 78.235 & 1582.665 & 1572.518 & 1568.196 & 1573.485 & 1566.97 & 1566.649 & 1564.314 & 1565.66 \\
\hline $8 / 1 / 1622: 00$ & 75.814 & 85.342 & 85.145 & 90.423 & 81.69 & 74.984 & 72.984 & 78.277 & 1582.658 & 1572.511 & 1568.163 & 1573.506 & 1566.938 & 1566.637 & 1564.307 & 1565.618 \\
\hline 8/1/16 23:00 & 75.826 & 85.349 & 85.18 & 90.446 & 81.727 & 74.993 & 72.986 & 78.316 & 1582.646 & 1572.504 & 1568.128 & 1573.483 & 1566.901 & 1566.628 & 1564.305 & 1565.579 \\
\hline $8 / 2 / 160: 00$ & 75.809 & 85.332 & 85.184 & 90.439 & 81.737 & 74.984 & 72.965 & 78.337 & 1582.663 & 1572.521 & 1568.124 & 1573.49 & 1566.891 & 1566.637 & 1564.326 & 1565.558 \\
\hline $8 / 2 / 161: 00$ & 75.781 & 85.307 & 85.184 & 90.432 & 81.732 & 74.972 & 72.938 & 78.337 & 1582.691 & 1572.546 & 1568.124 & 1573.497 & 1566.896 & 1566.649 & 1564.353 & 1565.558 \\
\hline $8 / 2 / 162: 00$ & 75.852 & 85.409 & 85.285 & 90.511 & 81.841 & 75.067 & 73.053 & 78.442 & 1582.62 & 1572.444 & 1568.023 & 1573.418 & 1566.787 & 1566.554 & 1564.238 & 1565.453 \\
\hline 8/2/16 3:00 & 75.816 & 85.377 & 85.294 & 90.458 & 81.832 & 75.021 & 73.012 & 78.409 & 1582.656 & 1572.476 & 1568.014 & 1573.471 & 1566.796 & 1566.6 & 1564.279 & 1565.486 \\
\hline $8 / 2 / 164: 00$ & 75.804 & 85.391 & 85.308 & 90.46 & 81.851 & 75.023 & 73.024 & 78.381 & 1582.668 & 1572.462 & 1568 & 1573.469 & 1566.777 & 1566.598 & 1564.267 & 1565.514 \\
\hline $8 / 2 / 165: 00$ & 75.819 & 85.419 & 85.359 & 90.481 & 81.898 & 75.042 & 73.051 & 78.405 & 1582.653 & 1572.434 & 1567.949 & 1573.448 & 1566.73 & 1566.579 & 1564.24 & 1565.49 \\
\hline
\end{tabular}


TABLE S1.1 Automatically recorded groundwater levels in the unconfined aquifer.

\begin{tabular}{|c|c|c|c|c|c|c|c|c|c|c|c|c|c|c|c|c|}
\hline \multirow[b]{2}{*}{ Date and Time } & \multicolumn{8}{|c|}{ Measured Depth to Water (ft TOC ${ }^{\mathrm{a}}$ ) } & \multicolumn{8}{|c|}{ Groundwater Elevation ( $\mathrm{ft} \mathrm{AMSL}^{\mathrm{b}}$ ) } \\
\hline & DL1-C & DL2-C1 & DL3-C1 & DL4-C1 & DL5-C1 & DL6-C & DL7-C & DL8-C1 & DL1-C & DL2-C1 & DL3-C1 & DL4-C1 & DL5-C1 & DL6-C & DL7-C & DL8-C1 \\
\hline $8 / 2 / 166: 00$ & 75.783 & 85.384 & 85.348 & 90.432 & 81.884 & 75.009 & 73 & 78.386 & 1582.689 & 1572.469 & 1567.96 & 1573.497 & 1566.744 & 1566.612 & 1564.291 & 1565.509 \\
\hline 8/2/16 7:00 & 75.776 & 85.388 & 85.352 & 90.434 & 81.898 & 75.011 & 73.005 & 78.381 & 1582.696 & 1572.465 & 1567.956 & 1573.495 & 1566.73 & 1566.61 & 1564.286 & 1565.514 \\
\hline 8/2/16 8:00 & 75.769 & 85.379 & 85.371 & 90.425 & 81.9 & 75.002 & 72.994 & 78.335 & 1582.703 & 1572.474 & 1567.937 & 1573.504 & 1566.728 & 1566.619 & 1564.297 & 1565.56 \\
\hline 8/2/16 9:00 & 75.774 & 85.395 & 85.376 & 90.434 & 81.923 & 75.011 & 73.008 & 78.388 & 1582.698 & 1572.458 & 1567.932 & 1573.495 & 1566.705 & 1566.61 & 1564.283 & 1565.507 \\
\hline $8 / 2 / 1610: 00$ & 75.786 & 85.407 & 85.401 & 90.444 & 81.944 & 75.035 & 73.017 & 78.479 & 1582.686 & 1572.446 & 1567.907 & 1573.485 & 1566.684 & 1566.586 & 1564.274 & 1565.416 \\
\hline $8 / 2 / 1611: 00$ & 75.807 & 85.437 & 85.438 & 90.462 & 81.984 & 75.044 & 73.048 & 78.439 & 1582.665 & 1572.416 & 1567.87 & 1573.467 & 1566.644 & 1566.577 & 1564.243 & 1565.456 \\
\hline $8 / 2 / 1612: 00$ & 75.814 & 85.446 & 85.459 & 90.462 & 82.005 & 75.07 & 73.048 & 78.549 & 1582.658 & 1572.407 & 1567.849 & 1573.467 & 1566.623 & 1566.551 & 1564.243 & 1565.346 \\
\hline $8 / 2 / 1613: 00$ & 75.826 & 85.449 & 85.466 & 90.458 & 82.026 & 75.077 & 73.041 & 78.593 & 1582.646 & 1572.404 & 1567.842 & 1573.471 & 1566.602 & 1566.544 & 1564.25 & 1565.302 \\
\hline $8 / 2 / 1614: 00$ & 75.84 & 85.446 & 85.476 & 90.455 & 82.052 & 75.084 & 73.041 & 78.604 & 1582.632 & 1572.407 & 1567.832 & 1573.474 & 1566.576 & 1566.537 & 1564.25 & 1565.291 \\
\hline $8 / 2 / 1615: 00$ & 75.847 & 85.449 & 85.457 & 90.451 & 82.073 & 75.091 & 73.036 & 78.648 & 1582.625 & 1572.404 & 1567.851 & 1573.478 & 1566.555 & 1566.53 & 1564.255 & 1565.247 \\
\hline $8 / 2 / 1616: 00$ & 75.847 & 85.442 & 85.441 & 90.439 & 82.084 & 75.086 & 73.02 & 78.653 & 1582.625 & 1572.411 & 1567.867 & 1573.49 & 1566.544 & 1566.535 & 1564.271 & 1565.242 \\
\hline $8 / 2 / 1617: 00$ & 75.847 & 85.432 & 85.459 & 90.432 & 82.096 & 75.088 & 73.015 & 78.66 & 1582.625 & 1572.421 & 1567.849 & 1573.497 & 1566.532 & 1566.533 & 1564.276 & 1565.235 \\
\hline $8 / 2 / 1618: 00$ & 75.847 & 85.425 & 85.441 & 90.425 & 82.107 & 75.088 & 73.005 & 78.667 & 1582.625 & 1572.428 & 1567.867 & 1573.504 & 1566.521 & 1566.533 & 1564.286 & 1565.228 \\
\hline $8 / 2 / 16$ 19:00 & 75.849 & 85.425 & 85.443 & 90.425 & 82.124 & 75.093 & 73 & 78.683 & 1582.623 & 1572.428 & 1567.865 & 1573.504 & 1566.504 & 1566.528 & 1564.291 & 1565.212 \\
\hline $8 / 2 / 1620: 00$ & 75.854 & 85.423 & 85.45 & 90.42 & 82.14 & 75.1 & 73.005 & 78.693 & 1582.618 & 1572.43 & 1567.858 & 1573.509 & 1566.488 & 1566.521 & 1564.286 & 1565.202 \\
\hline 8/2/16 21:00 & 75.866 & 85.444 & 85.48 & 90.432 & 82.161 & 75.119 & 73.027 & 78.716 & 1582.606 & 1572.409 & 1567.828 & 1573.497 & 1566.467 & 1566.502 & 1564.264 & 1565.179 \\
\hline $8 / 2 / 1622: 00$ & 75.88 & 85.467 & 85.525 & 90.448 & 82.201 & 75.151 & 73.058 & 78.769 & 1582.592 & 1572.386 & 1567.783 & 1573.481 & 1566.427 & 1566.47 & 1564.233 & 1565.126 \\
\hline $8 / 2 / 1623: 00$ & 75.866 & 85.467 & 85.541 & 90.441 & 82.21 & 75.151 & 73.051 & 78.79 & 1582.606 & 1572.386 & 1567.767 & 1573.488 & 1566.418 & 1566.47 & 1564.24 & 1565.105 \\
\hline 8/3/16 0:00 & 75.847 & 85.465 & 85.543 & 90.434 & 82.208 & 75.147 & 73.041 & 78.797 & 1582.625 & 1572.388 & 1567.765 & 1573.495 & 1566.42 & 1566.474 & 1564.25 & 1565.098 \\
\hline $8 / 3 / 161: 00$ & 75.826 & 85.458 & 85.532 & 90.432 & 82.208 & 75.144 & 73.032 & 78.797 & 1582.646 & 1572.395 & 1567.776 & 1573.497 & 1566.42 & 1566.477 & 1564.259 & 1565.098 \\
\hline $8 / 3 / 162: 00$ & 75.816 & 85.453 & 85.611 & 90.425 & 82.203 & 75.149 & 73.032 & 78.811 & 1582.656 & 1572.4 & 1567.697 & 1573.504 & 1566.425 & 1566.472 & 1564.259 & 1565.084 \\
\hline $8 / 3 / 163: 00$ & 75.809 & 85.453 & 85.62 & 90.427 & 82.206 & 75.154 & 73.032 & 78.83 & 1582.663 & 1572.4 & 1567.688 & 1573.502 & 1566.422 & 1566.467 & 1564.259 & 1565.065 \\
\hline 8/3/16 4:00 & 75.809 & 85.46 & 85.634 & 90.434 & 82.217 & 75.165 & 73.041 & 78.797 & 1582.663 & 1572.393 & 1567.674 & 1573.495 & 1566.411 & 1566.456 & 1564.25 & 1565.098 \\
\hline $8 / 3 / 165: 00$ & 75.804 & 85.458 & 85.676 & 90.43 & 82.226 & 75.172 & 73.036 & 78.867 & 1582.668 & 1572.395 & 1567.632 & 1573.499 & 1566.402 & 1566.449 & 1564.255 & 1565.028 \\
\hline $8 / 3 / 166: 00$ & 75.797 & 85.453 & 85.644 & 90.425 & 82.229 & 75.16 & 73.034 & 78.867 & 1582.675 & 1572.4 & 1567.664 & 1573.504 & 1566.399 & 1566.461 & 1564.257 & 1565.028 \\
\hline $8 / 3 / 167: 00$ & 75.795 & 85.456 & 85.604 & 90.427 & 82.229 & 75.172 & 73.032 & 78.871 & 1582.677 & 1572.397 & 1567.704 & 1573.502 & 1566.399 & 1566.449 & 1564.259 & 1565.024 \\
\hline 8/3/16 8:00 & 75.788 & 85.449 & 85.611 & 90.427 & 82.226 & 75.17 & 73.029 & 78.874 & 1582.684 & 1572.404 & 1567.697 & 1573.502 & 1566.402 & 1566.451 & 1564.262 & 1565.021 \\
\hline 8/3/16 9:00 & 75.79 & 85.456 & 85.604 & 90.437 & 82.231 & 75.177 & 73.034 & 78.878 & 1582.682 & 1572.397 & 1567.704 & 1573.492 & 1566.397 & 1566.444 & 1564.257 & 1565.017 \\
\hline $8 / 3 / 1610: 00$ & 75.79 & 85.46 & 85.611 & 90.439 & 82.236 & 75.184 & 73.039 & 78.888 & 1582.682 & 1572.393 & 1567.697 & 1573.49 & 1566.392 & 1566.437 & 1564.252 & 1565.007 \\
\hline $8 / 3 / 1611: 00$ & 75.786 & 85.449 & 85.618 & 90.437 & 82.229 & 75.184 & 73.029 & 78.883 & 1582.686 & 1572.404 & 1567.69 & 1573.492 & 1566.399 & 1566.437 & 1564.262 & 1565.012 \\
\hline $8 / 3 / 1612: 00$ & 75.783 & 85.451 & 85.623 & 90.439 & 82.229 & 75.181 & 73.029 & 78.885 & 1582.689 & 1572.402 & 1567.685 & 1573.49 & 1566.399 & 1566.44 & 1564.262 & 1565.01 \\
\hline $8 / 3 / 1613: 00$ & 75.776 & 85.444 & 85.611 & 90.434 & 82.217 & 75.179 & 73.02 & 78.881 & 1582.696 & 1572.409 & 1567.697 & 1573.495 & 1566.411 & 1566.442 & 1564.271 & 1565.014 \\
\hline $8 / 3 / 1614: 00$ & 75.764 & 85.428 & 85.609 & 90.432 & 82.21 & 75.172 & 73.005 & 78.878 & 1582.708 & 1572.425 & 1567.699 & 1573.497 & 1566.418 & 1566.449 & 1564.286 & 1565.017 \\
\hline $8 / 3 / 16$ 15:00 & 75.771 & 85.419 & 85.588 & 90.427 & 82.203 & 75.17 & 73 & 78.871 & 1582.701 & 1572.434 & 1567.72 & 1573.502 & 1566.425 & 1566.451 & 1564.291 & 1565.024 \\
\hline $8 / 3 / 1616: 00$ & 75.778 & 85.412 & 85.583 & 90.416 & 82.206 & 75.165 & 72.991 & 78.876 & 1582.694 & 1572.441 & 1567.725 & 1573.513 & 1566.422 & 1566.456 & 1564.3 & 1565.019 \\
\hline $8 / 3 / 1617: 00$ & 75.79 & 85.395 & 85.571 & 90.411 & 82.203 & 75.158 & 72.981 & 78.862 & 1582.682 & 1572.458 & 1567.737 & 1573.518 & 1566.425 & 1566.463 & 1564.31 & 1565.033 \\
\hline $8 / 3 / 1618: 00$ & 75.802 & 85.384 & 85.557 & 90.43 & 82.206 & 75.156 & 72.972 & 78.853 & 1582.67 & 1572.469 & 1567.751 & 1573.499 & 1566.422 & 1566.465 & 1564.319 & 1565.042 \\
\hline $8 / 3 / 16$ 19:00 & 75.816 & 85.381 & 85.569 & 90.451 & 82.217 & 75.16 & 72.977 & 78.86 & 1582.656 & 1572.472 & 1567.739 & 1573.478 & 1566.411 & 1566.461 & 1564.314 & 1565.035 \\
\hline $8 / 3 / 1620: 00$ & 75.826 & 85.379 & 85.581 & 90.455 & 82.222 & 75.158 & 72.972 & 78.862 & 1582.646 & 1572.474 & 1567.727 & 1573.474 & 1566.406 & 1566.463 & 1564.319 & 1565.033 \\
\hline $8 / 3 / 1621: 00$ & 75.84 & 85.381 & 85.59 & 90.446 & 82.24 & 75.163 & 72.981 & 78.871 & 1582.632 & 1572.472 & 1567.718 & 1573.483 & 1566.388 & 1566.458 & 1564.31 & 1565.024 \\
\hline $8 / 3 / 1622: 00$ & 75.861 & 85.393 & 85.606 & 90.427 & 82.264 & 75.177 & 72.996 & 78.89 & 1582.611 & 1572.46 & 1567.702 & 1573.502 & 1566.364 & 1566.444 & 1564.295 & 1565.005 \\
\hline $8 / 3 / 1623: 00$ & 75.877 & 85.405 & 85.62 & 90.448 & 82.287 & 75.181 & 73.003 & 78.864 & 1582.595 & 1572.448 & 1567.688 & 1573.481 & 1566.341 & 1566.44 & 1564.288 & 1565.031 \\
\hline 8/4/16 0:00 & 75.88 & 85.4 & 85.625 & 90.458 & 82.296 & 75.186 & 73.003 & 78.913 & 1582.592 & 1572.453 & 1567.683 & 1573.471 & 1566.332 & 1566.435 & 1564.288 & 1564.982 \\
\hline $8 / 4 / 161: 00$ & 75.892 & 85.414 & 85.632 & 90.467 & 82.315 & 75.195 & 73.012 & 78.925 & 1582.58 & 1572.439 & 1567.676 & 1573.462 & 1566.313 & 1566.426 & 1564.279 & 1564.97 \\
\hline $8 / 4 / 162: 00$ & 75.892 & 85.416 & 85.623 & 90.458 & 82.32 & 75.193 & 73.012 & 78.925 & 1582.58 & 1572.437 & 1567.685 & 1573.471 & 1566.308 & 1566.428 & 1564.279 & 1564.97 \\
\hline 8/4/16 3:00 & 75.877 & 85.414 & 85.625 & 90.446 & 82.32 & 75.195 & 73.008 & 78.906 & 1582.595 & 1572.439 & 1567.683 & 1573.483 & 1566.308 & 1566.426 & 1564.283 & 1564.989 \\
\hline 8/4/16 4:00 & 75.856 & 85.407 & 85.623 & 90.432 & 82.31 & 75.186 & 72.998 & 78.927 & 1582.616 & 1572.446 & 1567.685 & 1573.497 & 1566.318 & 1566.435 & 1564.293 & 1564.968 \\
\hline $8 / 4 / 165: 00$ & 75.844 & 85.405 & 85.625 & 90.432 & 82.308 & 75.186 & 72.996 & 78.932 & 1582.628 & 1572.448 & 1567.683 & 1573.497 & 1566.32 & 1566.435 & 1564.295 & 1564.963 \\
\hline $8 / 4 / 166: 00$ & 75.844 & 85.412 & 85.639 & 90.423 & 82.315 & 75.2 & 73.01 & 78.934 & 1582.628 & 1572.441 & 1567.669 & 1573.506 & 1566.313 & 1566.421 & 1564.281 & 1564.961 \\
\hline
\end{tabular}


TABLE S1.1 Automatically recorded groundwater levels in the unconfined aquifer.

\begin{tabular}{|c|c|c|c|c|c|c|c|c|c|c|c|c|c|c|c|c|}
\hline \multirow[b]{2}{*}{ Date and Time } & \multicolumn{8}{|c|}{ Measured Depth to Water ( $\mathrm{ft}$ TOC ${ }^{\mathrm{a}}$ ) } & \multicolumn{8}{|c|}{ Groundwater Elevation (ft $\mathrm{AMSL}^{\mathrm{b}}$ ) } \\
\hline & DL1-C & DL2-C1 & DL3-C1 & DL4-C1 & DL5-C1 & DL6-C & DL7-C & DL8-C1 & DL1-C & DL2-C1 & DL3-C1 & DL4-C1 & DL5-C1 & DL6-C & DL7-C & DL8-C1 \\
\hline $8 / 4 / 167: 00$ & 75.856 & 85.432 & 85.66 & 90.434 & 82.336 & 75.214 & 73.032 & 78.96 & 1582.616 & 1572.421 & 1567.648 & 1573.495 & 1566.292 & 1566.407 & 1564.259 & 1564.935 \\
\hline 8/4/16 8:00 & 75.863 & 85.444 & 85.665 & 90.434 & 82.345 & 75.205 & 73.046 & 78.883 & 1582.609 & 1572.409 & 1567.643 & 1573.495 & 1566.283 & 1566.416 & 1564.245 & 1565.012 \\
\hline 8/4/16 9:00 & 75.863 & 85.453 & 85.667 & 90.439 & 82.352 & 75.195 & 73.051 & 78.834 & 1582.609 & 1572.4 & 1567.641 & 1573.49 & 1566.276 & 1566.426 & 1564.24 & 1565.061 \\
\hline $8 / 4 / 1610: 00$ & 75.894 & 85.465 & 85.669 & 90.444 & 82.362 & 75.191 & 73.053 & 78.811 & 1582.578 & 1572.388 & 1567.639 & 1573.485 & 1566.266 & 1566.43 & 1564.238 & 1565.084 \\
\hline $8 / 4 / 1611: 00$ & 75.946 & 85.486 & 85.676 & 90.462 & 82.385 & 75.193 & 73.07 & 78.799 & 1582.526 & 1572.367 & 1567.632 & 1573.467 & 1566.243 & 1566.428 & 1564.221 & 1565.096 \\
\hline $8 / 4 / 1612: 00$ & 75.993 & 85.507 & 85.683 & 90.476 & 82.408 & 75.198 & 73.084 & 78.795 & 1582.479 & 1572.346 & 1567.625 & 1573.453 & 1566.22 & 1566.423 & 1564.207 & 1565.1 \\
\hline 8/4/16 13:00 & 76.031 & 85.526 & 85.686 & 90.483 & 82.429 & 75.195 & 73.084 & 78.783 & 1582.441 & 1572.327 & 1567.622 & 1573.446 & 1566.199 & 1566.426 & 1564.207 & 1565.112 \\
\hline $8 / 4 / 1614: 00$ & 76.061 & 85.542 & 85.697 & 90.497 & 82.453 & 75.2 & 73.091 & 78.818 & 1582.411 & 1572.311 & 1567.611 & 1573.432 & 1566.175 & 1566.421 & 1564.2 & 1565.077 \\
\hline $8 / 4 / 16$ 15:00 & 76.09 & 85.563 & 85.711 & 90.509 & 82.478 & 75.207 & 73.103 & 78.818 & 1582.382 & 1572.29 & 1567.597 & 1573.42 & 1566.15 & 1566.414 & 1564.188 & 1565.077 \\
\hline $8 / 4 / 1616: 00$ & 76.101 & 85.572 & 85.711 & 90.504 & 82.49 & 75.202 & 73.098 & 78.804 & 1582.371 & 1572.281 & 1567.597 & 1573.425 & 1566.138 & 1566.419 & 1564.193 & 1565.091 \\
\hline $8 / 4 / 1617: 00$ & 76.111 & 85.593 & 85.716 & 90.509 & 82.504 & 75.2 & 73.103 & 78.785 & 1582.361 & 1572.26 & 1567.592 & 1573.42 & 1566.124 & 1566.421 & 1564.188 & 1565.11 \\
\hline $8 / 4 / 1618: 00$ & 76.127 & 85.605 & 85.734 & 90.514 & 82.523 & 75.202 & 73.108 & 78.795 & 1582.345 & 1572.248 & 1567.574 & 1573.415 & 1566.105 & 1566.419 & 1564.183 & 1565.1 \\
\hline $8 / 4 / 1619: 00$ & 76.137 & 85.628 & 85.744 & 90.523 & 82.541 & 75.207 & 73.122 & 78.792 & 1582.335 & 1572.225 & 1567.564 & 1573.406 & 1566.087 & 1566.414 & 1564.169 & 1565.103 \\
\hline $8 / 4 / 1620: 00$ & 76.16 & 85.656 & 85.774 & 90.537 & 82.574 & 75.219 & 73.137 & 78.804 & 1582.312 & 1572.197 & 1567.534 & 1573.392 & 1566.054 & 1566.402 & 1564.154 & 1565.091 \\
\hline 8/4/16 21:00 & 76.167 & 85.674 & 85.786 & 90.544 & 82.595 & 75.219 & 73.141 & 78.818 & 1582.305 & 1572.179 & 1567.522 & 1573.385 & 1566.033 & 1566.402 & 1564.15 & 1565.077 \\
\hline $8 / 4 / 1622: 00$ & 76.174 & 85.693 & 85.795 & 90.551 & 82.611 & 75.223 & 73.149 & 78.825 & 1582.298 & 1572.16 & 1567.513 & 1573.378 & 1566.017 & 1566.398 & 1564.142 & 1565.07 \\
\hline $8 / 4 / 1623: 00$ & 76.177 & 85.714 & 85.814 & 90.56 & 82.63 & 75.233 & 73.158 & 78.839 & 1582.295 & 1572.139 & 1567.494 & 1573.369 & 1565.998 & 1566.388 & 1564.133 & 1565.056 \\
\hline $8 / 5 / 160: 00$ & 76.151 & 85.716 & 85.811 & 90.556 & 82.625 & 75.221 & 73.146 & 78.837 & 1582.321 & 1572.137 & 1567.497 & 1573.373 & 1566.003 & 1566.4 & 1564.145 & 1565.058 \\
\hline 8/5/16 1:00 & 76.13 & 85.714 & 85.804 & 90.549 & 82.614 & 75.212 & 73.132 & 78.816 & 1582.342 & 1572.139 & 1567.504 & 1573.38 & 1566.014 & 1566.409 & 1564.159 & 1565.079 \\
\hline $8 / 5 / 162: 00$ & 76.13 & 85.735 & 85.888 & 90.558 & 82.627 & 75.226 & 73.151 & 78.839 & 1582.342 & 1572.118 & 1567.42 & 1573.371 & 1566.001 & 1566.395 & 1564.14 & 1565.056 \\
\hline 8/5/16 3:00 & 76.13 & 85.749 & 85.914 & 90.567 & 82.635 & 75.228 & 73.156 & 78.853 & 1582.342 & 1572.104 & 1567.394 & 1573.362 & 1565.993 & 1566.393 & 1564.135 & 1565.042 \\
\hline $8 / 5 / 164: 00$ & 76.123 & 85.765 & 85.916 & 90.572 & 82.639 & 75.233 & 73.163 & 78.86 & 1582.349 & 1572.088 & 1567.392 & 1573.357 & 1565.989 & 1566.388 & 1564.128 & 1565.035 \\
\hline 8/5/16 5:00 & 76.113 & 85.765 & 85.947 & 90.57 & 82.637 & 75.23 & 73.156 & 78.869 & 1582.359 & 1572.088 & 1567.361 & 1573.359 & 1565.991 & 1566.391 & 1564.135 & 1565.026 \\
\hline $8 / 5 / 166: 00$ & 76.094 & 85.758 & 85.9 & 90.56 & 82.623 & 75.214 & 73.139 & 78.846 & 1582.378 & 1572.095 & 1567.408 & 1573.369 & 1566.005 & 1566.407 & 1564.152 & 1565.049 \\
\hline 8/5/16 7:00 & 76.097 & 85.772 & 85.867 & 90.574 & 82.628 & 75.219 & 73.156 & 78.843 & 1582.375 & 1572.081 & 1567.441 & 1573.355 & 1566 & 1566.402 & 1564.135 & 1565.052 \\
\hline 8/5/16 8:00 & 76.113 & 85.805 & 85.884 & 90.598 & 82.646 & 75.251 & 73.187 & 78.871 & 1582.359 & 1572.048 & 1567.424 & 1573.331 & 1565.982 & 1566.37 & 1564.104 & 1565.024 \\
\hline 8/5/16 9:00 & 76.111 & 85.812 & 85.891 & 90.598 & 82.653 & 75.249 & 73.187 & 78.874 & 1582.361 & 1572.041 & 1567.417 & 1573.331 & 1565.975 & 1566.372 & 1564.104 & 1565.021 \\
\hline $8 / 5 / 1610: 00$ & 76.099 & 85.812 & 85.877 & 90.6 & 82.644 & 75.242 & 73.18 & 78.86 & 1582.373 & 1572.041 & 1567.431 & 1573.329 & 1565.984 & 1566.379 & 1564.111 & 1565.035 \\
\hline $8 / 5 / 1611: 00$ & 76.092 & 85.816 & 85.867 & 90.598 & 82.637 & 75.242 & 73.175 & 78.841 & 1582.38 & 1572.037 & 1567.441 & 1573.331 & 1565.991 & 1566.379 & 1564.116 & 1565.054 \\
\hline $8 / 5 / 1612: 00$ & 76.082 & 85.812 & 85.858 & 90.6 & 82.628 & 75.233 & 73.165 & 78.836 & 1582.39 & 1572.041 & 1567.45 & 1573.329 & 1566 & 1566.388 & 1564.126 & 1565.059 \\
\hline 8/5/16 13:00 & 76.073 & 85.809 & 85.851 & 90.598 & 82.618 & 75.228 & 73.158 & 78.823 & 1582.399 & 1572.044 & 1567.457 & 1573.331 & 1566.01 & 1566.393 & 1564.133 & 1565.072 \\
\hline $8 / 5 / 1614: 00$ & 76.064 & 85.8 & 85.837 & 90.593 & 82.604 & 75.221 & 73.149 & 78.811 & 1582.408 & 1572.053 & 1567.471 & 1573.336 & 1566.024 & 1566.4 & 1564.142 & 1565.084 \\
\hline $8 / 5 / 1615: 00$ & 76.052 & 85.788 & 85.83 & 90.591 & 82.59 & 75.212 & 73.137 & 78.809 & 1582.42 & 1572.065 & 1567.478 & 1573.338 & 1566.038 & 1566.409 & 1564.154 & 1565.086 \\
\hline $8 / 5 / 1616: 00$ & 76.047 & 85.779 & 85.828 & 90.586 & 82.583 & 75.209 & 73.137 & 78.804 & 1582.425 & 1572.074 & 1567.48 & 1573.343 & 1566.045 & 1566.412 & 1564.154 & 1565.091 \\
\hline 8/5/16 17:00 & 76.04 & 85.774 & 85.832 & 90.581 & 82.574 & 75.205 & 73.129 & 78.799 & 1582.432 & 1572.079 & 1567.476 & 1573.348 & 1566.054 & 1566.416 & 1564.162 & 1565.096 \\
\hline $8 / 5 / 1618: 00$ & 76.031 & 85.765 & 85.828 & 90.6 & 82.562 & 75.198 & 73.125 & 78.778 & 1582.441 & 1572.088 & 1567.48 & 1573.329 & 1566.066 & 1566.423 & 1564.166 & 1565.117 \\
\hline $8 / 5 / 1619: 00$ & 76.031 & 85.76 & 85.828 & 90.616 & 82.558 & 75.195 & 73.122 & 78.778 & 1582.441 & 1572.093 & 1567.48 & 1573.313 & 1566.07 & 1566.426 & 1564.169 & 1565.117 \\
\hline $8 / 5 / 1620: 00$ & 76.028 & 85.753 & 85.814 & 90.623 & 82.551 & 75.188 & 73.122 & 78.765 & 1582.444 & 1572.1 & 1567.494 & 1573.306 & 1566.077 & 1566.433 & 1564.169 & 1565.13 \\
\hline 8/5/16 21:00 & 76.035 & 85.76 & 85.811 & 90.616 & 82.56 & 75.198 & 73.134 & 78.767 & 1582.437 & 1572.093 & 1567.497 & 1573.313 & 1566.068 & 1566.423 & 1564.157 & 1565.128 \\
\hline $8 / 5 / 1622: 00$ & 76.054 & 85.781 & 85.83 & 90.614 & 82.574 & 75.221 & 73.163 & 78.778 & 1582.418 & 1572.072 & 1567.478 & 1573.315 & 1566.054 & 1566.4 & 1564.128 & 1565.117 \\
\hline $8 / 5 / 1623: 00$ & 76.061 & 85.786 & 85.837 & 90.637 & 82.588 & 75.219 & 73.165 & 78.778 & 1582.411 & 1572.067 & 1567.471 & 1573.292 & 1566.04 & 1566.402 & 1564.126 & 1565.117 \\
\hline $8 / 6 / 160: 00$ & 76.066 & 85.793 & 85.844 & 90.651 & 82.593 & 75.221 & 73.17 & 78.783 & 1582.406 & 1572.06 & 1567.464 & 1573.278 & 1566.035 & 1566.4 & 1564.121 & 1565.112 \\
\hline 8/6/16 1:00 & 76.061 & 85.791 & 85.839 & 90.651 & 82.588 & 75.216 & 73.163 & 78.778 & 1582.411 & 1572.062 & 1567.469 & 1573.278 & 1566.04 & 1566.405 & 1564.128 & 1565.117 \\
\hline $8 / 6 / 162: 00$ & 76.064 & 85.8 & 85.849 & 90.649 & 82.6 & 75.221 & 73.17 & 78.781 & 1582.408 & 1572.053 & 1567.459 & 1573.28 & 1566.028 & 1566.4 & 1564.121 & 1565.114 \\
\hline 8/6/16 3:00 & 76.057 & 85.793 & 85.846 & 90.637 & 82.595 & 75.216 & 73.163 & 78.788 & 1582.415 & 1572.06 & 1567.462 & 1573.292 & 1566.033 & 1566.405 & 1564.128 & 1565.107 \\
\hline 8/6/16 4:00 & 76.052 & 85.784 & 85.844 & 90.633 & 82.59 & 75.212 & 73.158 & 78.776 & 1582.42 & 1572.069 & 1567.464 & 1573.296 & 1566.038 & 1566.409 & 1564.133 & 1565.119 \\
\hline $8 / 6 / 165: 00$ & 76.038 & 85.77 & 85.849 & 90.621 & 82.576 & 75.198 & 73.141 & 78.765 & 1582.434 & 1572.083 & 1567.459 & 1573.308 & 1566.052 & 1566.423 & 1564.15 & 1565.13 \\
\hline $8 / 6 / 166: 00$ & 76.031 & 85.763 & 85.842 & 90.6 & 82.565 & 75.195 & 73.137 & 78.755 & 1582.441 & 1572.09 & 1567.466 & 1573.329 & 1566.063 & 1566.426 & 1564.154 & 1565.14 \\
\hline 8/6/16 7:00 & 76.045 & 85.779 & 85.877 & 90.607 & 82.581 & 75.228 & 73.158 & 78.862 & 1582.427 & 1572.074 & 1567.431 & 1573.322 & 1566.047 & 1566.393 & 1564.133 & 1565.033 \\
\hline
\end{tabular}


TABLE S1.1 Automatically recorded groundwater levels in the unconfined aquifer.

\begin{tabular}{|c|c|c|c|c|c|c|c|c|c|c|c|c|c|c|c|c|}
\hline \multirow[b]{2}{*}{ Date and Time } & \multicolumn{8}{|c|}{ Measured Depth to Water (ft TOC ${ }^{\mathrm{a}}$ ) } & \multicolumn{8}{|c|}{ Groundwater Elevation ( $\mathrm{ft} \mathrm{AMSL}^{\mathrm{b}}$ ) } \\
\hline & DL1-C & DL2-C1 & DL3-C1 & DL4-C1 & DL5-C1 & DL6-C & DL7-C & DL8-C1 & DL1-C & DL2-C1 & DL3-C1 & DL4-C1 & DL5-C1 & DL6-C & DL7-C & DL8-C1 \\
\hline 8/6/16 8:00 & 76.052 & 85.798 & 85.893 & 90.621 & 82.595 & 75.258 & 73.18 & 78.939 & 1582.42 & 1572.055 & 1567.415 & 1573.308 & 1566.033 & 1566.363 & 1564.111 & 1564.956 \\
\hline 8/6/16 9:00 & 76.024 & 85.791 & 85.914 & 90.612 & 82.595 & 75.27 & 73.175 & 78.985 & 1582.448 & 1572.062 & 1567.394 & 1573.317 & 1566.033 & 1566.351 & 1564.116 & 1564.91 \\
\hline $8 / 6 / 16$ 10:00 & 75.991 & 85.786 & 85.923 & 90.602 & 82.588 & 75.275 & 73.17 & 79.008 & 1582.481 & 1572.067 & 1567.385 & 1573.327 & 1566.04 & 1566.346 & 1564.121 & 1564.887 \\
\hline 8/6/16 11:00 & 75.967 & 85.786 & 85.937 & 90.602 & 82.59 & 75.284 & 73.172 & 79.031 & 1582.505 & 1572.067 & 1567.371 & 1573.327 & 1566.038 & 1566.337 & 1564.119 & 1564.864 \\
\hline 8/6/16 12:00 & 75.946 & 85.779 & 85.954 & 90.595 & 82.583 & 75.286 & 73.17 & 79.05 & 1582.526 & 1572.074 & 1567.354 & 1573.334 & 1566.045 & 1566.335 & 1564.121 & 1564.845 \\
\hline $8 / 6 / 16$ 13:00 & 75.927 & 85.774 & 85.956 & 90.593 & 82.583 & 75.296 & 73.168 & 79.025 & 1582.545 & 1572.079 & 1567.352 & 1573.336 & 1566.045 & 1566.325 & 1564.123 & 1564.87 \\
\hline $8 / 6 / 16$ 14:00 & 75.908 & 85.758 & 85.956 & 90.586 & 82.574 & 75.293 & 73.156 & 79.087 & 1582.564 & 1572.095 & 1567.352 & 1573.343 & 1566.054 & 1566.328 & 1564.135 & 1564.808 \\
\hline $8 / 6 / 16$ 15:00 & 75.887 & 85.737 & 85.926 & 90.574 & 82.56 & 75.291 & 73.141 & 79.055 & 1582.585 & 1572.116 & 1567.382 & 1573.355 & 1566.068 & 1566.33 & 1564.15 & 1564.84 \\
\hline $8 / 6 / 16$ 16:00 & 75.877 & 85.726 & 85.923 & 90.572 & 82.551 & 75.296 & 73.144 & 79.085 & 1582.595 & 1572.127 & 1567.385 & 1573.357 & 1566.077 & 1566.325 & 1564.147 & 1564.81 \\
\hline 8/6/16 17:00 & 75.873 & 85.721 & 85.919 & 90.567 & 82.544 & 75.3 & 73.141 & 79.104 & 1582.599 & 1572.132 & 1567.389 & 1573.362 & 1566.084 & 1566.321 & 1564.15 & 1564.791 \\
\hline $8 / 6 / 1618: 00$ & 75.868 & 85.712 & 85.921 & 90.56 & 82.534 & 75.307 & 73.146 & 79.101 & 1582.604 & 1572.141 & 1567.387 & 1573.369 & 1566.094 & 1566.314 & 1564.145 & 1564.794 \\
\hline $8 / 6 / 16$ 19:00 & 75.856 & 85.695 & 85.97 & 90.556 & 82.52 & 75.307 & 73.137 & 79.115 & 1582.616 & 1572.158 & 1567.338 & 1573.373 & 1566.108 & 1566.314 & 1564.154 & 1564.78 \\
\hline $8 / 6 / 1620: 00$ & 75.859 & 85.7 & 86.072 & 90.558 & 82.532 & 75.324 & 73.151 & 79.182 & 1582.613 & 1572.153 & 1567.236 & 1573.371 & 1566.096 & 1566.297 & 1564.14 & 1564.713 \\
\hline $8 / 6 / 1621: 00$ & 75.863 & 85.702 & 86.114 & 90.563 & 82.541 & 75.337 & 73.163 & 79.224 & 1582.609 & 1572.151 & 1567.194 & 1573.366 & 1566.087 & 1566.284 & 1564.128 & 1564.671 \\
\hline $8 / 6 / 1622: 00$ & 75.875 & 85.716 & 86.149 & 90.572 & 82.565 & 75.354 & 73.18 & 79.266 & 1582.597 & 1572.137 & 1567.159 & 1573.357 & 1566.063 & 1566.267 & 1564.111 & 1564.629 \\
\hline $8 / 6 / 1623: 00$ & 75.877 & 85.721 & 86.175 & 90.57 & 82.576 & 75.368 & 73.187 & 79.296 & 1582.595 & 1572.132 & 1567.133 & 1573.359 & 1566.052 & 1566.253 & 1564.104 & 1564.599 \\
\hline 8/7/16 0:00 & 75.873 & 85.712 & 86.187 & 90.56 & 82.579 & 75.365 & 73.177 & 79.312 & 1582.599 & 1572.141 & 1567.121 & 1573.369 & 1566.049 & 1566.256 & 1564.114 & 1564.583 \\
\hline 8/7/16 1:00 & 75.866 & 85.705 & 86.194 & 90.553 & 82.576 & 75.365 & 73.172 & 79.326 & 1582.606 & 1572.148 & 1567.114 & 1573.376 & 1566.052 & 1566.256 & 1564.119 & 1564.569 \\
\hline 8/7/16 2:00 & 75.849 & 85.691 & 86.191 & 90.544 & 82.567 & 75.361 & 73.16 & 79.331 & 1582.623 & 1572.162 & 1567.117 & 1573.385 & 1566.061 & 1566.26 & 1564.131 & 1564.564 \\
\hline 8/7/16 3:00 & 75.842 & 85.686 & 86.201 & 90.542 & 82.567 & 75.363 & 73.156 & 79.347 & 1582.63 & 1572.167 & 1567.107 & 1573.387 & 1566.061 & 1566.258 & 1564.135 & 1564.548 \\
\hline 8/7/16 4:00 & 75.844 & 85.691 & 86.212 & 90.546 & 82.576 & 75.37 & 73.17 & 79.322 & 1582.628 & 1572.162 & 1567.096 & 1573.383 & 1566.052 & 1566.251 & 1564.121 & 1564.573 \\
\hline $8 / 7 / 165: 00$ & 75.854 & 85.693 & 86.254 & 90.551 & 82.597 & 75.375 & 73.18 & 79.306 & 1582.618 & 1572.16 & 1567.054 & 1573.378 & 1566.031 & 1566.246 & 1564.111 & 1564.589 \\
\hline $8 / 7 / 166: 00$ & 75.859 & 85.698 & 86.264 & 90.553 & 82.602 & 75.37 & 73.187 & 79.299 & 1582.613 & 1572.155 & 1567.044 & 1573.376 & 1566.026 & 1566.251 & 1564.104 & 1564.596 \\
\hline 8/7/16 7:00 & 75.866 & 85.712 & 86.296 & 90.563 & 82.616 & 75.379 & 73.201 & 79.292 & 1582.606 & 1572.141 & 1567.012 & 1573.366 & 1566.012 & 1566.242 & 1564.09 & 1564.603 \\
\hline 8/7/16 8:00 & 75.87 & 85.723 & 86.294 & 90.572 & 82.628 & 75.386 & 73.213 & 79.357 & 1582.602 & 1572.13 & 1567.014 & 1573.357 & 1566 & 1566.235 & 1564.078 & 1564.538 \\
\hline 8/7/16 9:00 & 75.87 & 85.728 & 86.299 & 90.57 & 82.63 & 75.377 & 73.215 & 79.236 & 1582.602 & 1572.125 & 1567.009 & 1573.359 & 1565.998 & 1566.244 & 1564.076 & 1564.659 \\
\hline $8 / 7 / 16$ 10:00 & 75.87 & 85.735 & 86.301 & 90.572 & 82.639 & 75.377 & 73.218 & 79.264 & 1582.602 & 1572.118 & 1567.007 & 1573.357 & 1565.989 & 1566.244 & 1564.073 & 1564.631 \\
\hline 8/7/16 11:00 & 75.868 & 85.739 & 86.308 & 90.572 & 82.639 & 75.375 & 73.22 & 79.261 & 1582.604 & 1572.114 & 1567 & 1573.357 & 1565.989 & 1566.246 & 1564.071 & 1564.634 \\
\hline $8 / 7 / 1612: 00$ & 75.852 & 85.726 & 86.303 & 90.56 & 82.628 & 75.358 & 73.199 & 79.241 & 1582.62 & 1572.127 & 1567.005 & 1573.369 & 1566 & 1566.263 & 1564.092 & 1564.654 \\
\hline $8 / 7 / 16$ 13:00 & 75.835 & 85.712 & 86.282 & 90.556 & 82.614 & 75.344 & 73.189 & 79.175 & 1582.637 & 1572.141 & 1567.026 & 1573.373 & 1566.014 & 1566.277 & 1564.102 & 1564.72 \\
\hline $8 / 7 / 1614: 00$ & 75.833 & 85.712 & 86.28 & 90.558 & 82.609 & 75.347 & 73.191 & 79.208 & 1582.639 & 1572.141 & 1567.028 & 1573.371 & 1566.019 & 1566.274 & 1564.1 & 1564.687 \\
\hline $8 / 7 / 1615: 00$ & 75.83 & 85.709 & 86.259 & 90.556 & 82.607 & 75.342 & 73.191 & 79.203 & 1582.642 & 1572.144 & 1567.049 & 1573.373 & 1566.021 & 1566.279 & 1564.1 & 1564.692 \\
\hline $8 / 7 / 1616: 00$ & 75.826 & 85.709 & 86.254 & 90.556 & 82.602 & 75.342 & 73.191 & 79.196 & 1582.646 & 1572.144 & 1567.054 & 1573.373 & 1566.026 & 1566.279 & 1564.1 & 1564.699 \\
\hline $8 / 7 / 1617: 00$ & 75.816 & 85.695 & 86.247 & 90.546 & 82.595 & 75.335 & 73.187 & 79.192 & 1582.656 & 1572.158 & 1567.061 & 1573.383 & 1566.033 & 1566.286 & 1564.104 & 1564.703 \\
\hline $8 / 7 / 1618: 00$ & 75.809 & 85.688 & 86.238 & 90.565 & 82.583 & 75.331 & 73.182 & 79.173 & 1582.663 & 1572.165 & 1567.07 & 1573.364 & 1566.045 & 1566.29 & 1564.109 & 1564.722 \\
\hline $8 / 7 / 16$ 19:00 & 75.8 & 85.677 & 86.222 & 90.577 & 82.569 & 75.321 & 73.175 & 79.15 & 1582.672 & 1572.176 & 1567.086 & 1573.352 & 1566.059 & 1566.3 & 1564.116 & 1564.745 \\
\hline $8 / 7 / 1620: 00$ & 75.8 & 85.677 & 86.217 & 90.588 & 82.569 & 75.326 & 73.18 & 79.152 & 1582.672 & 1572.176 & 1567.091 & 1573.341 & 1566.059 & 1566.295 & 1564.111 & 1564.743 \\
\hline $8 / 7 / 1621: 00$ & 75.802 & 85.681 & 86.224 & 90.579 & 82.569 & 75.328 & 73.191 & 79.152 & 1582.67 & 1572.172 & 1567.084 & 1573.35 & 1566.059 & 1566.293 & 1564.1 & 1564.743 \\
\hline $8 / 7 / 1622: 00$ & 75.814 & 85.691 & 86.236 & 90.563 & 82.579 & 75.337 & 73.206 & 79.164 & 1582.658 & 1572.162 & 1567.072 & 1573.366 & 1566.049 & 1566.284 & 1564.085 & 1564.731 \\
\hline $8 / 7 / 1623: 00$ & 75.811 & 85.688 & 86.238 & 90.579 & 82.574 & 75.335 & 73.201 & 79.162 & 1582.661 & 1572.165 & 1567.07 & 1573.35 & 1566.054 & 1566.286 & 1564.09 & 1564.733 \\
\hline 8/8/16 0:00 & 75.807 & 85.681 & 86.233 & 90.591 & 82.567 & 75.331 & 73.199 & 79.152 & 1582.665 & 1572.172 & 1567.075 & 1573.338 & 1566.061 & 1566.29 & 1564.092 & 1564.743 \\
\hline $8 / 8 / 161: 00$ & 75.8 & 85.679 & 86.229 & 90.588 & 82.555 & 75.324 & 73.194 & 79.148 & 1582.672 & 1572.174 & 1567.079 & 1573.341 & 1566.073 & 1566.297 & 1564.097 & 1564.747 \\
\hline 8/8/16 2:00 & 75.804 & 85.684 & 86.296 & 90.591 & 82.558 & 75.328 & 73.203 & 79.15 & 1582.668 & 1572.169 & 1567.012 & 1573.338 & 1566.07 & 1566.293 & 1564.088 & 1564.745 \\
\hline 8/8/16 3:00 & 75.819 & 85.7 & 86.315 & 90.593 & 82.572 & 75.344 & 73.223 & 79.178 & 1582.653 & 1572.153 & 1566.993 & 1573.336 & 1566.056 & 1566.277 & 1564.068 & 1564.717 \\
\hline 8/8/16 4:00 & 75.816 & 85.695 & 86.343 & 90.586 & 82.576 & 75.344 & 73.218 & 79.18 & 1582.656 & 1572.158 & 1566.965 & 1573.343 & 1566.052 & 1566.277 & 1564.073 & 1564.715 \\
\hline 8/8/16 5:00 & 75.8 & 85.684 & 86.357 & 90.579 & 82.56 & 75.321 & 73.199 & 79.131 & 1582.672 & 1572.169 & 1566.951 & 1573.35 & 1566.068 & 1566.3 & 1564.092 & 1564.764 \\
\hline $8 / 8 / 166: 00$ & 75.802 & 85.691 & 86.324 & 90.574 & 82.567 & 75.331 & 73.211 & 79.173 & 1582.67 & 1572.162 & 1566.984 & 1573.355 & 1566.061 & 1566.29 & 1564.08 & 1564.722 \\
\hline 8/8/16 7:00 & 75.814 & 85.705 & 86.32 & 90.579 & 82.579 & 75.342 & 73.227 & 79.185 & 1582.658 & 1572.148 & 1566.988 & 1573.35 & 1566.049 & 1566.279 & 1564.064 & 1564.71 \\
\hline 8/8/16 8:00 & 75.802 & 85.702 & 86.303 & 90.572 & 82.572 & 75.337 & 73.215 & 79.173 & 1582.67 & 1572.151 & 1567.005 & 1573.357 & 1566.056 & 1566.284 & 1564.076 & 1564.722 \\
\hline
\end{tabular}


TABLE S1.1 Automatically recorded groundwater levels in the unconfined aquifer.

\begin{tabular}{|c|c|c|c|c|c|c|c|c|c|c|c|c|c|c|c|c|}
\hline \multirow[b]{2}{*}{ Date and Time } & \multicolumn{8}{|c|}{ Measured Depth to Water (ft TOC ${ }^{\mathrm{a}}$ ) } & \multicolumn{8}{|c|}{ Groundwater Elevation ( $\mathrm{ft} \mathrm{AMSL}^{\mathrm{b}}$ ) } \\
\hline & DL1-C & DL2-C1 & DL3-C1 & DL4-C1 & DL5-C1 & DL6-C & DL7-C & DL8-C1 & DL1-C & DL2-C1 & DL3-C1 & DL4-C1 & DL5-C1 & DL6-C & DL7-C & DL8-C1 \\
\hline 8/8/16 9:00 & 75.802 & 85.7 & 86.296 & 90.579 & 82.572 & 75.342 & 73.22 & 79.162 & 1582.67 & 1572.153 & 1567.012 & 1573.35 & 1566.056 & 1566.279 & 1564.071 & 1564.733 \\
\hline $8 / 8 / 16$ 10:00 & 75.788 & 85.693 & 86.289 & 90.567 & 82.691 & 75.326 & 73.203 & 79.162 & 1582.684 & 1572.16 & 1567.019 & 1573.362 & 1565.937 & 1566.295 & 1564.088 & 1564.733 \\
\hline 8/8/16 11:00 & 75.781 & 85.688 & 86.282 & 90.565 & 82.737 & 75.326 & 73.201 & 79.131 & 1582.691 & 1572.165 & 1567.026 & 1573.364 & 1565.891 & 1566.295 & 1564.09 & 1564.764 \\
\hline $8 / 8 / 1612: 00$ & 75.783 & 85.686 & 86.299 & 90.572 & 82.76 & 75.324 & 73.206 & 79.15 & 1582.689 & 1572.167 & 1567.009 & 1573.357 & 1565.868 & 1566.297 & 1564.085 & 1564.745 \\
\hline 8/8/16 13:00 & 75.783 & 85.693 & 86.287 & 90.577 & 82.777 & 75.331 & 73.208 & 79.15 & 1582.689 & 1572.16 & 1567.021 & 1573.352 & 1565.851 & 1566.29 & 1564.083 & 1564.745 \\
\hline $8 / 8 / 1614: 00$ & 75.781 & 85.688 & 86.287 & 90.579 & 82.781 & 75.319 & 73.203 & 79.145 & 1582.691 & 1572.165 & 1567.021 & 1573.35 & 1565.847 & 1566.302 & 1564.088 & 1564.75 \\
\hline $8 / 8 / 1615: 00$ & 75.778 & 85.686 & 86.266 & 90.579 & 82.786 & 75.331 & 73.201 & 79.182 & 1582.694 & 1572.167 & 1567.042 & 1573.35 & 1565.842 & 1566.29 & 1564.09 & 1564.713 \\
\hline $8 / 8 / 1616: 00$ & 75.76 & 85.667 & 86.261 & 90.565 & 82.777 & 75.337 & 73.18 & 79.268 & 1582.712 & 1572.186 & 1567.047 & 1573.364 & 1565.851 & 1566.284 & 1564.111 & 1564.627 \\
\hline $8 / 8 / 1617: 00$ & 75.753 & 85.658 & 86.247 & 90.565 & 82.774 & 75.347 & 73.177 & 79.296 & 1582.719 & 1572.195 & 1567.061 & 1573.364 & 1565.854 & 1566.274 & 1564.114 & 1564.599 \\
\hline $8 / 8 / 16$ 18:00 & 75.753 & 85.649 & 85.658 & 90.563 & 82.737 & 75.351 & 73.18 & 79.041 & 1582.719 & 1572.204 & 1567.65 & 1573.366 & 1565.891 & 1566.27 & 1564.111 & 1564.854 \\
\hline $8 / 8 / 16$ 19:00 & 75.748 & 85.63 & 85.427 & 90.553 & 82.688 & 75.342 & 73.172 & 78.888 & 1582.724 & 1572.223 & 1567.881 & 1573.376 & 1565.94 & 1566.279 & 1564.119 & 1565.007 \\
\hline $8 / 8 / 1620: 00$ & 75.753 & 85.621 & 85.317 & 90.553 & 82.653 & 75.344 & 73.18 & 78.79 & 1582.719 & 1572.232 & 1567.991 & 1573.376 & 1565.975 & 1566.277 & 1564.111 & 1565.105 \\
\hline $8 / 8 / 1621: 00$ & 75.769 & 85.628 & 85.254 & 90.563 & 82.628 & 75.363 & 73.208 & 78.72 & 1582.703 & 1572.225 & 1568.054 & 1573.366 & 1566 & 1566.258 & 1564.083 & 1565.175 \\
\hline $8 / 8 / 1622: 00$ & 75.793 & 85.644 & 85.217 & 90.577 & 82.621 & 75.384 & 73.239 & 78.702 & 1582.679 & 1572.209 & 1568.091 & 1573.352 & 1566.007 & 1566.237 & 1564.052 & 1565.193 \\
\hline $8 / 8 / 1623: 00$ & 75.802 & 85.649 & 85.18 & 90.574 & 82.6 & 75.391 & 73.246 & 78.662 & 1582.67 & 1572.204 & 1568.128 & 1573.355 & 1566.028 & 1566.23 & 1564.045 & 1565.233 \\
\hline $8 / 9 / 160: 00$ & 75.788 & 85.621 & 85.119 & 90.553 & 82.553 & 75.375 & 73.223 & 78.611 & 1582.684 & 1572.232 & 1568.189 & 1573.376 & 1566.075 & 1566.246 & 1564.068 & 1565.284 \\
\hline 8/9/16 1:00 & 75.797 & 85.63 & 85.091 & 90.565 & 82.539 & 75.391 & 73.244 & 78.583 & 1582.675 & 1572.223 & 1568.217 & 1573.364 & 1566.089 & 1566.23 & 1564.047 & 1565.312 \\
\hline 8/9/16 2:00 & 75.802 & 85.628 & 85.061 & 90.565 & 82.516 & 75.396 & 73.249 & 78.565 & 1582.67 & 1572.225 & 1568.247 & 1573.364 & 1566.112 & 1566.225 & 1564.042 & 1565.33 \\
\hline 8/9/16 3:00 & 75.8 & 85.616 & 85.028 & 90.558 & 82.485 & 75.391 & 73.246 & 78.491 & 1582.672 & 1572.237 & 1568.28 & 1573.371 & 1566.143 & 1566.23 & 1564.045 & 1565.404 \\
\hline 8/9/16 4:00 & 75.802 & 85.609 & 85 & 90.553 & 82.46 & 75.396 & 73.246 & 78.518 & 1582.67 & 1572.244 & 1568.308 & 1573.376 & 1566.168 & 1566.225 & 1564.045 & 1565.377 \\
\hline 8/9/16 5:00 & 75.795 & 85.593 & 84.991 & 90.542 & 82.436 & 75.389 & 73.234 & 78.486 & 1582.677 & 1572.26 & 1568.317 & 1573.387 & 1566.192 & 1566.232 & 1564.057 & 1565.409 \\
\hline $8 / 9 / 166: 00$ & 75.802 & 85.595 & 84.97 & 90.549 & 82.42 & 75.4 & 73.249 & 78.47 & 1582.67 & 1572.258 & 1568.338 & 1573.38 & 1566.208 & 1566.221 & 1564.042 & 1565.425 \\
\hline 8/9/16 7:00 & 75.809 & 85.588 & 84.951 & 90.549 & 82.394 & 75.4 & 73.251 & 78.458 & 1582.663 & 1572.265 & 1568.357 & 1573.38 & 1566.234 & 1566.221 & 1564.04 & 1565.437 \\
\hline 8/9/16 8:00 & 75.811 & 85.586 & 84.93 & 90.556 & 82.376 & 75.417 & 73.261 & 78.456 & 1582.661 & 1572.267 & 1568.378 & 1573.373 & 1566.252 & 1566.204 & 1564.03 & 1565.439 \\
\hline 8/9/16 9:00 & 75.826 & 85.591 & 84.916 & 90.556 & 82.362 & 75.431 & 73.27 & 78.451 & 1582.646 & 1572.262 & 1568.392 & 1573.373 & 1566.266 & 1566.19 & 1564.021 & 1565.444 \\
\hline $8 / 9 / 16$ 10:00 & 75.83 & 85.605 & 84.982 & 90.862 & 82.341 & 75.428 & 73.275 & 78.433 & 1582.642 & 1572.248 & 1568.326 & 1573.067 & 1566.287 & 1566.193 & 1564.016 & 1565.462 \\
\hline 8/9/16 11:00 & 75.828 & 85.653 & 85.082 & 91.17 & 82.327 & 75.421 & 73.268 & 78.409 & 1582.644 & 1572.2 & 1568.226 & 1572.759 & 1566.301 & 1566.2 & 1564.023 & 1565.486 \\
\hline 8/9/16 12:00 & 75.835 & 85.716 & 85.504 & 91.357 & 82.334 & 75.421 & 73.273 & 78.53 & 1582.637 & 1572.137 & 1567.804 & 1572.572 & 1566.294 & 1566.2 & 1564.018 & 1565.365 \\
\hline $8 / 9 / 1613: 00$ & 75.837 & 85.777 & 85.846 & 91.49 & 82.357 & 75.428 & 73.268 & 78.706 & 1582.635 & 1572.076 & 1567.462 & 1572.439 & 1566.271 & 1566.193 & 1564.023 & 1565.189 \\
\hline $8 / 9 / 16$ 14:00 & 75.842 & 85.844 & 86 & 91.592 & 82.385 & 75.433 & 73.263 & 78.792 & 1582.63 & 1572.009 & 1567.308 & 1572.337 & 1566.243 & 1566.188 & 1564.028 & 1565.103 \\
\hline $8 / 9 / 16$ 15:00 & 75.844 & 85.909 & 86.091 & 91.681 & 82.411 & 75.44 & 73.263 & 78.869 & 1582.628 & 1571.944 & 1567.217 & 1572.248 & 1566.217 & 1566.181 & 1564.028 & 1565.026 \\
\hline $8 / 9 / 1616: 00$ & 75.847 & 85.97 & 86.175 & 91.751 & 82.432 & 75.442 & 73.263 & 78.918 & 1582.625 & 1571.883 & 1567.133 & 1572.178 & 1566.196 & 1566.179 & 1564.028 & 1564.977 \\
\hline 8/9/16 17:00 & 75.844 & 86.03 & 86.25 & 91.81 & 82.448 & 75.445 & 73.258 & 78.955 & 1582.628 & 1571.823 & 1567.058 & 1572.119 & 1566.18 & 1566.176 & 1564.033 & 1564.94 \\
\hline $8 / 9 / 16$ 18:00 & 75.847 & 86.084 & 86.303 & 91.868 & 82.471 & 75.447 & 73.254 & 78.983 & 1582.625 & 1571.769 & 1567.005 & 1572.061 & 1566.157 & 1566.174 & 1564.037 & 1564.912 \\
\hline 8/9/16 19:00 & 75.852 & 86.135 & 86.354 & 91.924 & 82.495 & 75.452 & 73.256 & 79.011 & 1582.62 & 1571.718 & 1566.954 & 1572.005 & 1566.133 & 1566.169 & 1564.035 & 1564.884 \\
\hline $8 / 9 / 1620: 00$ & 75.866 & 86.2 & 86.415 & 91.982 & 82.525 & 75.461 & 73.265 & 79.052 & 1582.606 & 1571.653 & 1566.893 & 1571.947 & 1566.103 & 1566.16 & 1564.026 & 1564.843 \\
\hline 8/9/16 21:00 & 75.887 & 86.267 & 86.473 & 92.013 & 82.56 & 75.477 & 73.285 & 79.092 & 1582.585 & 1571.586 & 1566.835 & 1571.916 & 1566.068 & 1566.144 & 1564.006 & 1564.803 \\
\hline $8 / 9 / 1622: 00$ & 75.915 & 86.347 & 86.543 & 92.05 & 82.609 & 75.501 & 73.308 & 79.136 & 1582.557 & 1571.506 & 1566.765 & 1571.879 & 1566.019 & 1566.12 & 1563.983 & 1564.759 \\
\hline 8/9/16 23:00 & 75.934 & 86.409 & 86.604 & 92.122 & 82.649 & 75.514 & 73.323 & 79.175 & 1582.538 & 1571.444 & 1566.704 & 1571.807 & 1565.979 & 1566.107 & 1563.968 & 1564.72 \\
\hline $8 / 10 / 160: 00$ & 75.941 & 86.461 & 86.65 & 92.169 & 82.681 & 75.512 & 73.318 & 79.201 & 1582.531 & 1571.392 & 1566.658 & 1571.76 & 1565.947 & 1566.109 & 1563.973 & 1564.694 \\
\hline 8/10/16 1:00 & 75.943 & 86.509 & 86.69 & 92.209 & 82.705 & 75.517 & 73.316 & 79.227 & 1582.529 & 1571.344 & 1566.618 & 1571.72 & 1565.923 & 1566.104 & 1563.975 & 1564.668 \\
\hline $8 / 10 / 162: 00$ & 75.946 & 86.556 & 86.783 & 92.237 & 82.73 & 75.505 & 73.308 & 79.206 & 1582.526 & 1571.297 & 1566.525 & 1571.692 & 1565.898 & 1566.116 & 1563.983 & 1564.689 \\
\hline $8 / 10 / 163: 00$ & 75.958 & 86.605 & 86.825 & 92.265 & 82.76 & 75.496 & 73.311 & 79.178 & 1582.514 & 1571.248 & 1566.483 & 1571.664 & 1565.868 & 1566.125 & 1563.98 & 1564.717 \\
\hline $8 / 10 / 164: 00$ & 75.958 & 86.642 & 86.867 & 92.286 & 82.784 & 75.489 & 73.304 & 79.166 & 1582.514 & 1571.211 & 1566.441 & 1571.643 & 1565.844 & 1566.132 & 1563.987 & 1564.729 \\
\hline $8 / 10 / 165: 00$ & 75.969 & 86.688 & 86.926 & 92.321 & 82.812 & 75.489 & 73.306 & 79.117 & 1582.503 & 1571.165 & 1566.382 & 1571.608 & 1565.816 & 1566.132 & 1563.985 & 1564.778 \\
\hline $8 / 10 / 166: 00$ & 75.981 & 86.737 & 86.926 & 92.337 & 82.849 & 75.484 & 73.311 & 79.162 & 1582.491 & 1571.116 & 1566.382 & 1571.592 & 1565.779 & 1566.137 & 1563.98 & 1564.733 \\
\hline $8 / 10 / 167: 00$ & 75.995 & 86.782 & 86.926 & 92.363 & 82.877 & 75.514 & 73.32 & 79.275 & 1582.477 & 1571.071 & 1566.382 & 1571.566 & 1565.751 & 1566.107 & 1563.971 & 1564.62 \\
\hline $8 / 10 / 168: 00$ & 76.007 & 86.828 & 86.96 & 92.391 & 82.907 & 75.526 & 73.323 & 79.282 & 1582.465 & 1571.025 & 1566.348 & 1571.538 & 1565.721 & 1566.095 & 1563.968 & 1564.613 \\
\hline 8/10/16 9:00 & 76.045 & 86.875 & 86.988 & 92.426 & 82.933 & 75.526 & 73.328 & 79.278 & 1582.427 & 1570.978 & 1566.32 & 1571.503 & 1565.695 & 1566.095 & 1563.963 & 1564.617 \\
\hline
\end{tabular}


TABLE S1.1 Automatically recorded groundwater levels in the unconfined aquifer.

\begin{tabular}{|c|c|c|c|c|c|c|c|c|c|c|c|c|c|c|c|c|}
\hline \multirow[b]{2}{*}{ Date and Time } & \multicolumn{8}{|c|}{ Measured Depth to Water ( $\mathrm{ft}$ TOC ${ }^{\mathrm{a}}$ ) } & \multicolumn{8}{|c|}{ Groundwater Elevation (ft AMSL ${ }^{\mathrm{b}}$ ) } \\
\hline & DL1-C & DL2-C1 & DL3-C1 & DL4-C1 & DL5-C1 & DL6-C & DL7-C & DL8-C1 & DL1-C & DL2-C1 & DL3-C1 & DL4-C1 & DL5-C1 & DL6-C & DL7-C & DL8-C1 \\
\hline 8/10/16 10:00 & 76.071 & 86.907 & 87.016 & 92.449 & 82.952 & 75.533 & 73.318 & 79.352 & 1582.401 & 1570.946 & 1566.292 & 1571.48 & 1565.676 & 1566.088 & 1563.973 & 1564.543 \\
\hline 8/10/16 11:00 & 76.104 & 86.949 & 87.056 & 92.484 & 82.98 & 75.533 & 73.325 & 79.37 & 1582.368 & 1570.904 & 1566.252 & 1571.445 & 1565.648 & 1566.088 & 1563.966 & 1564.525 \\
\hline 8/10/16 12:00 & 76.125 & 86.989 & 87.089 & 92.533 & 83.001 & 75.535 & 73.318 & 79.384 & 1582.347 & 1570.864 & 1566.219 & 1571.396 & 1565.627 & 1566.086 & 1563.973 & 1564.511 \\
\hline 8/10/16 13:00 & 76.151 & 87.021 & 87.103 & 92.573 & 83.061 & 75.538 & 73.316 & 79.398 & 1582.321 & 1570.832 & 1566.205 & 1571.356 & 1565.567 & 1566.083 & 1563.975 & 1564.497 \\
\hline 8/10/16 14:00 & 76.179 & 87.058 & 87.259 & 92.608 & 83.071 & 75.535 & 73.306 & 79.466 & 1582.293 & 1570.795 & 1566.049 & 1571.321 & 1565.557 & 1566.086 & 1563.985 & 1564.429 \\
\hline 8/10/16 15:00 & 76.203 & 87.093 & 87.385 & 92.643 & 83.085 & 75.542 & 73.304 & 79.554 & 1582.269 & 1570.76 & 1565.923 & 1571.286 & 1565.543 & 1566.079 & 1563.987 & 1564.341 \\
\hline 8/10/16 16:00 & 76.219 & 87.119 & 87.464 & 92.664 & 83.094 & 75.54 & 73.289 & 79.568 & 1582.253 & 1570.734 & 1565.844 & 1571.265 & 1565.534 & 1566.081 & 1564.002 & 1564.327 \\
\hline 8/10/16 17:00 & 76.233 & 87.144 & 87.529 & 92.683 & 83.108 & 75.542 & 73.28 & 79.631 & 1582.239 & 1570.709 & 1565.779 & 1571.246 & 1565.52 & 1566.079 & 1564.011 & 1564.264 \\
\hline 8/10/16 18:00 & 76.245 & 87.168 & 87.562 & 92.666 & 83.12 & 75.542 & 73.27 & 79.663 & 1582.227 & 1570.685 & 1565.746 & 1571.263 & 1565.508 & 1566.079 & 1564.021 & 1564.232 \\
\hline 8/10/16 19:00 & 76.264 & 87.193 & 87.604 & 92.678 & 83.141 & 75.549 & 73.273 & 79.691 & 1582.208 & 1570.66 & 1565.704 & 1571.251 & 1565.487 & 1566.072 & 1564.018 & 1564.204 \\
\hline 8/10/16 20:00 & 76.29 & 87.233 & 87.65 & 92.708 & 83.169 & 75.561 & 73.289 & 79.726 & 1582.182 & 1570.62 & 1565.658 & 1571.221 & 1565.459 & 1566.06 & 1564.002 & 1564.169 \\
\hline 8/10/16 21:00 & 76.313 & 87.275 & 87.695 & 92.741 & 83.192 & 75.573 & 73.306 & 79.716 & 1582.159 & 1570.578 & 1565.613 & 1571.188 & 1565.436 & 1566.048 & 1563.985 & 1564.179 \\
\hline 8/10/16 22:00 & 76.335 & 87.319 & 87.744 & 92.776 & 83.227 & 75.587 & 73.316 & 79.788 & 1582.137 & 1570.534 & 1565.564 & 1571.153 & 1565.401 & 1566.034 & 1563.975 & 1564.107 \\
\hline 8/10/16 23:00 & 76.346 & 87.347 & 87.769 & 92.795 & 83.243 & 75.591 & 73.318 & 79.809 & 1582.126 & 1570.506 & 1565.539 & 1571.134 & 1565.385 & 1566.03 & 1563.973 & 1564.086 \\
\hline $8 / 11 / 160: 00$ & 76.346 & 87.384 & 87.8 & 92.813 & 83.262 & 75.601 & 73.32 & 79.821 & 1582.126 & 1570.469 & 1565.508 & 1571.116 & 1565.366 & 1566.02 & 1563.971 & 1564.074 \\
\hline 8/11/16 1:00 & 76.337 & 87.407 & 87.83 & 92.841 & 83.271 & 75.598 & 73.318 & 79.83 & 1582.135 & 1570.446 & 1565.478 & 1571.088 & 1565.357 & 1566.023 & 1563.973 & 1564.065 \\
\hline $8 / 11 / 162: 00$ & 76.33 & 87.435 & 87.856 & 92.865 & 83.287 & 75.584 & 73.318 & 79.781 & 1582.142 & 1570.418 & 1565.452 & 1571.064 & 1565.341 & 1566.037 & 1563.973 & 1564.114 \\
\hline $8 / 11 / 163: 00$ & 76.316 & 87.451 & 87.863 & 92.883 & 83.29 & 75.57 & 73.308 & 79.74 & 1582.156 & 1570.402 & 1565.445 & 1571.046 & 1565.338 & 1566.051 & 1563.983 & 1564.155 \\
\hline 8/11/16 4:00 & 76.311 & 87.475 & 87.879 & 92.904 & 83.299 & 75.568 & 73.306 & 79.73 & 1582.161 & 1570.378 & 1565.429 & 1571.025 & 1565.329 & 1566.053 & 1563.985 & 1564.165 \\
\hline $8 / 11 / 165: 00$ & 76.311 & 87.503 & 87.914 & 92.928 & 83.318 & 75.563 & 73.308 & 79.686 & 1582.161 & 1570.35 & 1565.394 & 1571.001 & 1565.31 & 1566.058 & 1563.983 & 1564.209 \\
\hline $8 / 11 / 166: 00$ & 76.316 & 87.528 & 87.939 & 92.951 & 83.334 & 75.566 & 73.318 & 79.737 & 1582.156 & 1570.325 & 1565.369 & 1570.978 & 1565.294 & 1566.055 & 1563.973 & 1564.158 \\
\hline 8/11/16 7:00 & 76.318 & 87.556 & 87.963 & 92.965 & 83.35 & 75.573 & 73.316 & 79.77 & 1582.154 & 1570.297 & 1565.345 & 1570.964 & 1565.278 & 1566.048 & 1563.975 & 1564.125 \\
\hline 8/11/16 8:00 & 76.328 & 87.586 & 88.002 & 92.986 & 83.369 & 75.605 & 73.328 & 79.874 & 1582.144 & 1570.267 & 1565.306 & 1570.943 & 1565.259 & 1566.016 & 1563.963 & 1564.021 \\
\hline 8/11/16 9:00 & 76.337 & 87.619 & 88.005 & 93.007 & 83.385 & 75.622 & 73.335 & 79.909 & 1582.135 & 1570.234 & 1565.303 & 1570.922 & 1565.243 & 1565.999 & 1563.956 & 1563.986 \\
\hline 8/11/16 10:00 & 76.342 & 87.649 & 87.972 & 93.052 & 83.409 & 75.624 & 73.344 & 79.918 & 1582.13 & 1570.204 & 1565.336 & 1570.877 & 1565.219 & 1565.997 & 1563.947 & 1563.977 \\
\hline 8/11/16 11:00 & 76.344 & 87.677 & 87.863 & 93.087 & 83.42 & 75.615 & 73.344 & 79.833 & 1582.128 & 1570.176 & 1565.445 & 1570.842 & 1565.208 & 1566.006 & 1563.947 & 1564.062 \\
\hline 8/11/16 12:00 & 76.351 & 87.705 & 87.825 & 93.11 & 83.441 & 75.622 & 73.347 & 79.805 & 1582.121 & 1570.148 & 1565.483 & 1570.819 & 1565.187 & 1565.999 & 1563.944 & 1564.09 \\
\hline 8/11/16 13:00 & 76.365 & 87.724 & 87.795 & 93.126 & 83.451 & 75.621 & 73.342 & 79.784 & 1582.107 & 1570.129 & 1565.513 & 1570.803 & 1565.177 & 1566 & 1563.949 & 1564.111 \\
\hline 8/11/16 14:00 & 76.379 & 87.742 & 87.776 & 93.145 & 83.469 & 75.622 & 73.337 & 79.77 & 1582.093 & 1570.111 & 1565.532 & 1570.784 & 1565.159 & 1565.999 & 1563.954 & 1564.125 \\
\hline $8 / 11 / 16$ 15:00 & 76.398 & 87.768 & 87.774 & 93.171 & 83.488 & 75.626 & 73.344 & 79.777 & 1582.074 & 1570.085 & 1565.534 & 1570.758 & 1565.14 & 1565.995 & 1563.947 & 1564.118 \\
\hline 8/11/16 16:00 & 76.415 & 87.793 & 87.758 & 93.14 & 83.504 & 75.636 & 73.347 & 79.765 & 1582.057 & 1570.06 & 1565.55 & 1570.789 & 1565.124 & 1565.985 & 1563.944 & 1564.13 \\
\hline $8 / 11 / 16$ 17:00 & 76.431 & 87.784 & 87.639 & 92.816 & 83.525 & 75.64 & 73.354 & 79.779 & 1582.041 & 1570.069 & 1565.669 & 1571.113 & 1565.103 & 1565.981 & 1563.937 & 1564.116 \\
\hline 8/11/16 18:00 & 76.427 & 87.74 & 87.541 & 92.648 & 83.516 & 75.626 & 73.328 & 79.758 & 1582.045 & 1570.113 & 1565.767 & 1571.281 & 1565.112 & 1565.995 & 1563.963 & 1564.137 \\
\hline 8/11/16 19:00 & 76.424 & 87.703 & 87.455 & 92.54 & 83.514 & 75.624 & 73.316 & 79.74 & 1582.048 & 1570.15 & 1565.853 & 1571.389 & 1565.114 & 1565.997 & 1563.975 & 1564.155 \\
\hline $8 / 11 / 1620: 00$ & 76.438 & 87.686 & 87.399 & 92.461 & 83.525 & 75.636 & 73.342 & 79.721 & 1582.034 & 1570.167 & 1565.909 & 1571.468 & 1565.103 & 1565.985 & 1563.949 & 1564.174 \\
\hline 8/11/16 21:00 & 76.488 & 87.71 & 87.415 & 92.4 & 83.588 & 75.689 & 73.406 & 79.779 & 1581.984 & 1570.143 & 1565.893 & 1571.529 & 1565.04 & 1565.932 & 1563.885 & 1564.116 \\
\hline $8 / 11 / 1622: 00$ & 76.474 & 87.675 & 87.375 & 92.314 & 83.588 & 75.682 & 73.394 & 79.777 & 1581.998 & 1570.178 & 1565.933 & 1571.615 & 1565.04 & 1565.939 & 1563.897 & 1564.118 \\
\hline 8/11/16 23:00 & 76.457 & 87.633 & 87.331 & 92.272 & 83.588 & 75.678 & 73.382 & 79.772 & 1582.015 & 1570.22 & 1565.977 & 1571.657 & 1565.04 & 1565.943 & 1563.909 & 1564.123 \\
\hline $8 / 12 / 160: 00$ & 76.45 & 87.61 & 87.296 & 92.239 & 83.593 & 75.68 & 73.392 & 79.723 & 1582.022 & 1570.243 & 1566.012 & 1571.69 & 1565.035 & 1565.941 & 1563.899 & 1564.172 \\
\hline 8/12/16 1:00 & 76.45 & 87.589 & 87.273 & 92.211 & 83.605 & 75.68 & 73.406 & 79.705 & 1582.022 & 1570.264 & 1566.035 & 1571.718 & 1565.023 & 1565.941 & 1563.885 & 1564.19 \\
\hline $8 / 12 / 162: 00$ & 76.445 & 87.561 & 87.312 & 92.167 & 83.612 & 75.673 & 73.402 & 79.672 & 1582.027 & 1570.292 & 1565.996 & 1571.762 & 1565.016 & 1565.948 & 1563.889 & 1564.223 \\
\hline $8 / 12 / 163: 00$ & 76.452 & 87.551 & 87.303 & 92.141 & 83.626 & 75.682 & 73.423 & 79.67 & 1582.02 & 1570.302 & 1566.005 & 1571.788 & 1565.002 & 1565.939 & 1563.868 & 1564.225 \\
\hline $8 / 12 / 164: 00$ & 76.448 & 87.528 & 87.289 & 92.314 & 83.635 & 75.682 & 73.447 & 79.64 & 1582.024 & 1570.325 & 1566.019 & 1571.615 & 1564.993 & 1565.939 & 1563.844 & 1564.255 \\
\hline $8 / 12 / 165: 00$ & 76.431 & 87.484 & 87.28 & 92.101 & 83.633 & 75.668 & 73.418 & 79.642 & 1582.041 & 1570.369 & 1566.028 & 1571.828 & 1564.995 & 1565.953 & 1563.873 & 1564.253 \\
\hline $8 / 12 / 166: 00$ & 76.429 & 87.454 & 87.215 & 92.034 & 83.635 & 75.671 & 73.423 & 79.633 & 1582.043 & 1570.399 & 1566.093 & 1571.895 & 1564.993 & 1565.95 & 1563.868 & 1564.262 \\
\hline $8 / 12 / 167: 00$ & 76.426 & 87.435 & 87.147 & 91.987 & 83.63 & 75.663 & 73.411 & 79.614 & 1582.046 & 1570.418 & 1566.161 & 1571.942 & 1564.998 & 1565.958 & 1563.88 & 1564.281 \\
\hline 8/12/16 8:00 & 76.452 & 87.442 & 86.748 & 91.992 & 83.635 & 75.691 & 73.456 & 79.433 & 1582.02 & 1570.411 & 1566.56 & 1571.937 & 1564.993 & 1565.93 & 1563.835 & 1564.462 \\
\hline 8/12/16 9:00 & 76.426 & 87.405 & 86.494 & 91.959 & 83.595 & 75.673 & 73.447 & 79.268 & 1582.046 & 1570.448 & 1566.814 & 1571.97 & 1565.033 & 1565.948 & 1563.844 & 1564.627 \\
\hline $8 / 12 / 1610: 00$ & 76.394 & 87.37 & 86.38 & 91.929 & 83.549 & 75.689 & 73.449 & 79.403 & 1582.078 & 1570.483 & 1566.928 & 1572 & 1565.079 & 1565.932 & 1563.842 & 1564.492 \\
\hline
\end{tabular}


TABLE S1.1 Automatically recorded groundwater levels in the unconfined aquifer.

\begin{tabular}{|c|c|c|c|c|c|c|c|c|c|c|c|c|c|c|c|c|}
\hline \multirow[b]{2}{*}{ Date and Time } & \multicolumn{8}{|c|}{ Measured Depth to Water ( $\mathrm{ft}$ TOC ${ }^{\mathrm{a}}$ ) } & \multicolumn{8}{|c|}{ Groundwater Elevation (ft $\mathrm{AMSL}^{\mathrm{b}}$ ) } \\
\hline & DL1-C & DL2-C1 & DL3-C1 & DL4-C1 & DL5-C1 & DL6-C & DL7-C & DL8-C1 & DL1-C & DL2-C1 & DL3-C1 & DL4-C1 & DL5-C1 & DL6-C & DL7-C & DL8-C1 \\
\hline 8/12/16 11:00 & 76.37 & 87.34 & 86.289 & 91.901 & 83.504 & 75.673 & 73.452 & 79.185 & 1582.102 & 1570.513 & 1567.019 & 1572.028 & 1565.124 & 1565.948 & 1563.839 & 1564.71 \\
\hline 8/12/16 12:00 & 76.346 & 87.307 & 86.217 & 91.866 & 83.453 & 75.664 & 73.449 & 79.052 & 1582.126 & 1570.546 & 1567.091 & 1572.063 & 1565.175 & 1565.957 & 1563.842 & 1564.843 \\
\hline 8/12/16 13:00 & 76.313 & 87.258 & 86.135 & 91.833 & 83.392 & 75.64 & 73.435 & 78.957 & 1582.159 & 1570.595 & 1567.173 & 1572.096 & 1565.236 & 1565.981 & 1563.856 & 1564.938 \\
\hline 8/12/16 14:00 & 76.283 & 87.221 & 86.072 & 91.8 & 83.332 & 75.638 & 73.428 & 78.943 & 1582.189 & 1570.632 & 1567.236 & 1572.129 & 1565.296 & 1565.983 & 1563.863 & 1564.952 \\
\hline $8 / 12 / 1615: 00$ & 76.264 & 87.179 & 86.01 & 91.77 & 83.273 & 75.624 & 73.43 & 78.922 & 1582.208 & 1570.674 & 1567.298 & 1572.159 & 1565.355 & 1565.997 & 1563.861 & 1564.973 \\
\hline $8 / 12 / 1616: 00$ & 76.243 & 87.14 & 85.958 & 91.739 & 83.222 & 75.617 & 73.425 & 78.82 & 1582.229 & 1570.713 & 1567.35 & 1572.19 & 1565.406 & 1566.004 & 1563.866 & 1565.075 \\
\hline 8/12/16 17:00 & 76.217 & 87.093 & 85.898 & 91.707 & 83.157 & 75.601 & 73.416 & 78.753 & 1582.255 & 1570.76 & 1567.41 & 1572.222 & 1565.471 & 1566.02 & 1563.875 & 1565.142 \\
\hline $8 / 12 / 16$ 18:00 & 76.196 & 87.042 & 85.839 & 91.674 & 83.094 & 75.591 & 73.409 & 78.695 & 1582.276 & 1570.811 & 1567.469 & 1572.255 & 1565.534 & 1566.03 & 1563.882 & 1565.2 \\
\hline $8 / 12 / 16$ 19:00 & 76.177 & 87 & 85.783 & 91.646 & 83.036 & 75.58 & 73.409 & 78.621 & 1582.295 & 1570.853 & 1567.525 & 1572.283 & 1565.592 & 1566.041 & 1563.882 & 1565.274 \\
\hline $8 / 12 / 1620: 00$ & 76.167 & 86.97 & 85.741 & 91.623 & 82.987 & 75.577 & 73.421 & 78.579 & 1582.305 & 1570.883 & 1567.567 & 1572.306 & 1565.641 & 1566.044 & 1563.87 & 1565.316 \\
\hline $8 / 12 / 1621: 00$ & 76.163 & 86.942 & 85.706 & 91.588 & 82.945 & 75.58 & 73.435 & 78.544 & 1582.309 & 1570.911 & 1567.602 & 1572.341 & 1565.683 & 1566.041 & 1563.856 & 1565.351 \\
\hline $8 / 12 / 1622: 00$ & 76.165 & 86.928 & 85.683 & 91.578 & 82.914 & 75.587 & 73.456 & 78.521 & 1582.307 & 1570.925 & 1567.625 & 1572.351 & 1565.714 & 1566.034 & 1563.835 & 1565.374 \\
\hline $8 / 12 / 1623: 00$ & 76.16 & 86.905 & 85.655 & 91.569 & 82.87 & 75.587 & 73.468 & 78.497 & 1582.312 & 1570.948 & 1567.653 & 1572.36 & 1565.758 & 1566.034 & 1563.823 & 1565.398 \\
\hline $8 / 13 / 160: 00$ & 76.141 & 86.865 & 85.609 & 91.541 & 82.816 & 75.573 & 73.459 & 78.456 & 1582.331 & 1570.988 & 1567.699 & 1572.388 & 1565.812 & 1566.048 & 1563.832 & 1565.439 \\
\hline 8/13/16 1:00 & 76.125 & 86.835 & 85.574 & 91.525 & 82.767 & 75.566 & 73.461 & 78.426 & 1582.347 & 1571.018 & 1567.734 & 1572.404 & 1565.861 & 1566.055 & 1563.83 & 1565.469 \\
\hline $8 / 13 / 162: 00$ & 76.108 & 86.791 & 85.529 & 91.501 & 82.709 & 75.554 & 73.447 & 78.374 & 1582.364 & 1571.062 & 1567.779 & 1572.428 & 1565.919 & 1566.067 & 1563.844 & 1565.521 \\
\hline $8 / 13 / 163: 00$ & 76.082 & 86.74 & 85.483 & 91.469 & 82.646 & 75.538 & 73.433 & 78.337 & 1582.39 & 1571.113 & 1567.825 & 1572.46 & 1565.982 & 1566.083 & 1563.858 & 1565.558 \\
\hline $8 / 13 / 164: 00$ & 76.061 & 86.695 & 85.436 & 91.445 & 82.583 & 75.524 & 73.425 & 78.289 & 1582.411 & 1571.158 & 1567.872 & 1572.484 & 1566.045 & 1566.097 & 1563.866 & 1565.606 \\
\hline $8 / 13 / 165: 00$ & 76.052 & 86.663 & 85.422 & 91.42 & 82.534 & 75.514 & 73.43 & 78.224 & 1582.42 & 1571.19 & 1567.886 & 1572.509 & 1566.094 & 1566.107 & 1563.861 & 1565.671 \\
\hline $8 / 13 / 166: 00$ & 76.038 & 86.621 & 85.39 & 91.406 & 82.483 & 75.51 & 73.428 & 78.228 & 1582.434 & 1571.232 & 1567.918 & 1572.523 & 1566.145 & 1566.111 & 1563.863 & 1565.667 \\
\hline $8 / 13 / 167: 00$ & 76.038 & 86.6 & 85.362 & 91.389 & 82.441 & 75.512 & 73.445 & 78.184 & 1582.434 & 1571.253 & 1567.946 & 1572.54 & 1566.187 & 1566.109 & 1563.846 & 1565.711 \\
\hline $8 / 13 / 168: 00$ & 76.04 & 86.582 & 85.345 & 91.382 & 82.404 & 75.517 & 73.459 & 78.189 & 1582.432 & 1571.271 & 1567.963 & 1572.547 & 1566.224 & 1566.104 & 1563.832 & 1565.706 \\
\hline 8/13/16 9:00 & 76.033 & 86.554 & 85.32 & 91.366 & 82.357 & 75.519 & 73.459 & 78.307 & 1582.439 & 1571.299 & 1567.988 & 1572.563 & 1566.271 & 1566.102 & 1563.832 & 1565.588 \\
\hline $8 / 13 / 1610: 00$ & 76.026 & 86.526 & 85.294 & 91.347 & 82.313 & 75.514 & 73.464 & 78.179 & 1582.446 & 1571.327 & 1568.014 & 1572.582 & 1566.315 & 1566.107 & 1563.827 & 1565.716 \\
\hline 8/13/16 11:00 & 76.005 & 86.486 & 85.264 & 91.322 & 82.257 & 75.503 & 73.449 & 78.163 & 1582.467 & 1571.367 & 1568.044 & 1572.607 & 1566.371 & 1566.118 & 1563.842 & 1565.732 \\
\hline 8/13/16 12:00 & 75.991 & 86.451 & 85.236 & 91.303 & 82.206 & 75.491 & 73.447 & 78.096 & 1582.481 & 1571.402 & 1568.072 & 1572.626 & 1566.422 & 1566.13 & 1563.844 & 1565.799 \\
\hline 8/13/16 13:00 & 75.974 & 86.416 & 85.196 & 91.282 & 82.15 & 75.487 & 73.442 & 78.128 & 1582.498 & 1571.437 & 1568.112 & 1572.647 & 1566.478 & 1566.134 & 1563.849 & 1565.767 \\
\hline $8 / 13 / 1614: 00$ & 75.955 & 86.37 & 85.154 & 91.256 & 82.091 & 75.47 & 73.428 & 78.038 & 1582.517 & 1571.483 & 1568.154 & 1572.673 & 1566.537 & 1566.151 & 1563.863 & 1565.857 \\
\hline 8/13/16 15:00 & 75.934 & 86.323 & 85.091 & 91.233 & 82.028 & 75.452 & 73.416 & 77.994 & 1582.538 & 1571.53 & 1568.217 & 1572.696 & 1566.6 & 1566.169 & 1563.875 & 1565.901 \\
\hline $8 / 13 / 1616: 00$ & 75.915 & 86.279 & 85.049 & 91.202 & 81.97 & 75.442 & 73.404 & 78.04 & 1582.557 & 1571.574 & 1568.259 & 1572.727 & 1566.658 & 1566.179 & 1563.887 & 1565.855 \\
\hline $8 / 13 / 1617: 00$ & 75.901 & 86.235 & 85.017 & 91.179 & 81.912 & 75.44 & 73.399 & 77.994 & 1582.571 & 1571.618 & 1568.291 & 1572.75 & 1566.716 & 1566.181 & 1563.892 & 1565.901 \\
\hline $8 / 13 / 16$ 18:00 & 75.889 & 86.193 & 84.972 & 91.177 & 81.853 & 75.424 & 73.392 & 77.905 & 1582.583 & 1571.66 & 1568.336 & 1572.752 & 1566.775 & 1566.197 & 1563.899 & 1565.99 \\
\hline $8 / 13 / 1619: 00$ & 75.877 & 86.151 & 84.937 & 91.167 & 81.795 & 75.41 & 73.394 & 77.82 & 1582.595 & 1571.702 & 1568.371 & 1572.762 & 1566.833 & 1566.211 & 1563.897 & 1566.075 \\
\hline 8/13/16 20:00 & 75.882 & 86.13 & 84.912 & 91.163 & 81.758 & 75.414 & 73.409 & 77.845 & 1582.59 & 1571.723 & 1568.396 & 1572.766 & 1566.87 & 1566.207 & 1563.882 & 1566.05 \\
\hline $8 / 13 / 1621: 00$ & 75.889 & 86.114 & 84.898 & 91.135 & 81.725 & 75.421 & 73.43 & 77.826 & 1582.583 & 1571.739 & 1568.41 & 1572.794 & 1566.903 & 1566.2 & 1563.861 & 1566.069 \\
\hline $8 / 13 / 1622: 00$ & 75.908 & 86.114 & 84.898 & 91.125 & 81.707 & 75.438 & 73.461 & 77.827 & 1582.564 & 1571.739 & 1568.41 & 1572.804 & 1566.921 & 1566.183 & 1563.83 & 1566.068 \\
\hline 8/13/16 23:00 & 75.908 & 86.091 & 84.877 & 91.135 & 81.672 & 75.433 & 73.461 & 77.815 & 1582.564 & 1571.762 & 1568.431 & 1572.794 & 1566.956 & 1566.188 & 1563.83 & 1566.08 \\
\hline $8 / 14 / 160: 00$ & 75.899 & 86.063 & 84.851 & 91.128 & 81.623 & 75.421 & 73.454 & 77.787 & 1582.573 & 1571.79 & 1568.457 & 1572.801 & 1567.005 & 1566.2 & 1563.837 & 1566.108 \\
\hline $8 / 14 / 161: 00$ & 75.885 & 86.033 & 84.818 & 91.114 & 81.581 & 75.414 & 73.449 & 77.768 & 1582.587 & 1571.82 & 1568.49 & 1572.815 & 1567.047 & 1566.207 & 1563.842 & 1566.127 \\
\hline $8 / 14 / 162: 00$ & 75.87 & 85.998 & 84.788 & 91.088 & 81.532 & 75.403 & 73.44 & 77.729 & 1582.602 & 1571.855 & 1568.52 & 1572.841 & 1567.096 & 1566.218 & 1563.851 & 1566.166 \\
\hline $8 / 14 / 163: 00$ & 75.859 & 85.967 & 84.758 & 91.062 & 81.483 & 75.398 & 73.437 & 77.701 & 1582.613 & 1571.886 & 1568.55 & 1572.867 & 1567.145 & 1566.223 & 1563.854 & 1566.194 \\
\hline $8 / 14 / 164: 00$ & 75.847 & 85.93 & 84.725 & 91.044 & 81.434 & 75.384 & 73.43 & 77.673 & 1582.625 & 1571.923 & 1568.583 & 1572.885 & 1567.194 & 1566.237 & 1563.861 & 1566.222 \\
\hline $8 / 14 / 165: 00$ & 75.849 & 85.916 & 84.728 & 91.032 & 81.401 & 75.382 & 73.442 & 77.652 & 1582.623 & 1571.937 & 1568.58 & 1572.897 & 1567.227 & 1566.239 & 1563.849 & 1566.243 \\
\hline $8 / 14 / 166: 00$ & 75.854 & 85.902 & 84.718 & 91.009 & 81.375 & 75.393 & 73.454 & 77.645 & 1582.618 & 1571.951 & 1568.59 & 1572.92 & 1567.253 & 1566.228 & 1563.837 & 1566.25 \\
\hline $8 / 14 / 167: 00$ & 75.844 & 85.87 & 84.69 & 90.983 & 81.331 & 75.379 & 73.447 & 77.629 & 1582.628 & 1571.983 & 1568.618 & 1572.946 & 1567.297 & 1566.242 & 1563.844 & 1566.266 \\
\hline 8/14/16 8:00 & 75.835 & 85.844 & 84.669 & 90.967 & 81.287 & 75.37 & 73.442 & 77.601 & 1582.637 & 1572.009 & 1568.639 & 1572.962 & 1567.341 & 1566.251 & 1563.849 & 1566.294 \\
\hline 8/14/16 9:00 & 75.833 & 85.823 & 84.646 & 90.953 & 81.247 & 75.368 & 73.445 & 77.578 & 1582.639 & 1572.03 & 1568.662 & 1572.976 & 1567.381 & 1566.253 & 1563.846 & 1566.317 \\
\hline $8 / 14 / 1610: 00$ & 75.833 & 85.807 & 84.627 & 90.946 & 81.217 & 75.368 & 73.452 & 77.553 & 1582.639 & 1572.046 & 1568.681 & 1572.983 & 1567.411 & 1566.253 & 1563.839 & 1566.342 \\
\hline 8/14/16 11:00 & 75.833 & 85.786 & 84.613 & 90.936 & 81.184 & 75.368 & 73.461 & 77.543 & 1582.639 & 1572.067 & 1568.695 & 1572.993 & 1567.444 & 1566.253 & 1563.83 & 1566.352 \\
\hline
\end{tabular}


TABLE S1.1 Automatically recorded groundwater levels in the unconfined aquifer.

\begin{tabular}{|c|c|c|c|c|c|c|c|c|c|c|c|c|c|c|c|c|}
\hline \multirow[b]{2}{*}{ Date and Time } & \multicolumn{8}{|c|}{ Measured Depth to Water (ft TOC ${ }^{\mathrm{a}}$ ) } & \multicolumn{8}{|c|}{ Groundwater Elevation ( $\mathrm{ft} \mathrm{AMSL}^{\mathrm{b}}$ ) } \\
\hline & DL1-C & DL2-C1 & DL3-C1 & DL4-C1 & DL5-C1 & DL6-C & DL7-C & DL8-C1 & DL1-C & DL2-C1 & DL3-C1 & DL4-C1 & DL5-C1 & DL6-C & DL7-C & DL8-C1 \\
\hline $8 / 14 / 1612: 00$ & 75.819 & 85.756 & 84.595 & 90.918 & 81.138 & 75.356 & 73.447 & 77.527 & 1582.653 & 1572.097 & 1568.713 & 1573.011 & 1567.49 & 1566.265 & 1563.844 & 1566.368 \\
\hline 8/14/16 13:00 & 75.797 & 85.716 & 84.55 & 90.892 & 81.082 & 75.335 & 73.428 & 77.485 & 1582.675 & 1572.137 & 1568.758 & 1573.037 & 1567.546 & 1566.286 & 1563.863 & 1566.41 \\
\hline $8 / 14 / 1614: 00$ & 75.778 & 85.677 & 84.511 & 90.871 & 81.028 & 75.319 & 73.414 & 77.45 & 1582.694 & 1572.176 & 1568.797 & 1573.058 & 1567.6 & 1566.302 & 1563.877 & 1566.445 \\
\hline $8 / 14 / 1615: 00$ & 75.764 & 85.642 & 84.462 & 90.855 & 80.979 & 75.307 & 73.406 & 77.418 & 1582.708 & 1572.211 & 1568.846 & 1573.074 & 1567.649 & 1566.314 & 1563.885 & 1566.477 \\
\hline $8 / 14 / 1616: 00$ & 75.748 & 85.605 & 84.425 & 90.831 & 80.925 & 75.293 & 73.397 & 77.392 & 1582.724 & 1572.248 & 1568.883 & 1573.098 & 1567.703 & 1566.328 & 1563.894 & 1566.503 \\
\hline $8 / 14 / 1617: 00$ & 75.738 & 85.565 & 84.387 & 90.808 & 80.874 & 75.284 & 73.387 & 77.362 & 1582.734 & 1572.288 & 1568.921 & 1573.121 & 1567.754 & 1566.337 & 1563.904 & 1566.533 \\
\hline $8 / 14 / 1618: 00$ & 75.731 & 85.53 & 84.357 & 90.792 & 80.823 & 75.272 & 73.385 & 77.327 & 1582.741 & 1572.323 & 1568.951 & 1573.137 & 1567.805 & 1566.349 & 1563.906 & 1566.568 \\
\hline 8/14/16 19:00 & 75.722 & 85.502 & 84.329 & 90.773 & 80.781 & 75.267 & 73.382 & 77.309 & 1582.75 & 1572.351 & 1568.979 & 1573.156 & 1567.847 & 1566.354 & 1563.909 & 1566.586 \\
\hline $8 / 14 / 1620: 00$ & 75.722 & 85.474 & 84.306 & 90.759 & 80.741 & 75.263 & 73.385 & 77.29 & 1582.75 & 1572.379 & 1569.002 & 1573.17 & 1567.887 & 1566.358 & 1563.906 & 1566.605 \\
\hline $8 / 14 / 1621: 00$ & 75.722 & 85.453 & 84.285 & 90.754 & 80.702 & 75.263 & 73.394 & 77.267 & 1582.75 & 1572.4 & 1569.023 & 1573.175 & 1567.926 & 1566.358 & 1563.897 & 1566.628 \\
\hline $8 / 14 / 1622: 00$ & 75.727 & 85.439 & 84.271 & 90.745 & 80.674 & 75.265 & 73.406 & 77.26 & 1582.745 & 1572.414 & 1569.037 & 1573.184 & 1567.954 & 1566.356 & 1563.885 & 1566.635 \\
\hline $8 / 14 / 1623: 00$ & 75.731 & 85.423 & 84.257 & 90.736 & 80.643 & 75.263 & 73.414 & 77.246 & 1582.741 & 1572.43 & 1569.051 & 1573.193 & 1567.985 & 1566.358 & 1563.877 & 1566.649 \\
\hline $8 / 15 / 160: 00$ & 75.724 & 85.402 & 84.236 & 90.719 & 80.608 & 75.256 & 73.414 & 77.225 & 1582.748 & 1572.451 & 1569.072 & 1573.21 & 1568.02 & 1566.365 & 1563.877 & 1566.67 \\
\hline $8 / 15 / 161: 00$ & 75.717 & 85.374 & 84.208 & 90.703 & 80.564 & 75.249 & 73.406 & 77.2 & 1582.755 & 1572.479 & 1569.1 & 1573.226 & 1568.064 & 1566.372 & 1563.885 & 1566.695 \\
\hline $8 / 15 / 162: 00$ & 75.705 & 85.342 & 84.254 & 90.689 & 80.522 & 75.237 & 73.397 & 77.183 & 1582.767 & 1572.511 & 1569.054 & 1573.24 & 1568.106 & 1566.384 & 1563.894 & 1566.712 \\
\hline 8/15/16 3:00 & 75.698 & 85.321 & 84.233 & 90.677 & 80.487 & 75.235 & 73.402 & 77.172 & 1582.774 & 1572.532 & 1569.075 & 1573.252 & 1568.141 & 1566.386 & 1563.889 & 1566.723 \\
\hline $8 / 15 / 164: 00$ & 75.694 & 85.295 & 84.217 & 90.663 & 80.45 & 75.226 & 73.394 & 77.135 & 1582.778 & 1572.558 & 1569.091 & 1573.266 & 1568.178 & 1566.395 & 1563.897 & 1566.76 \\
\hline $8 / 15 / 165: 00$ & 75.689 & 85.272 & 84.224 & 90.656 & 80.41 & 75.221 & 73.394 & 77.132 & 1582.783 & 1572.581 & 1569.084 & 1573.273 & 1568.218 & 1566.4 & 1563.897 & 1566.763 \\
\hline $8 / 15 / 166: 00$ & 75.687 & 85.251 & 84.163 & 90.644 & 80.373 & 75.216 & 73.397 & 77.104 & 1582.785 & 1572.602 & 1569.145 & 1573.285 & 1568.255 & 1566.405 & 1563.894 & 1566.791 \\
\hline $8 / 15 / 167: 00$ & 75.684 & 85.228 & 84.11 & 90.64 & 80.338 & 75.221 & 73.394 & 77.111 & 1582.788 & 1572.625 & 1569.198 & 1573.289 & 1568.29 & 1566.4 & 1563.897 & 1566.784 \\
\hline $8 / 15 / 168: 00$ & 75.679 & 85.207 & 84.091 & 90.626 & 80.303 & 75.219 & 73.39 & 77.1 & 1582.793 & 1572.646 & 1569.217 & 1573.303 & 1568.325 & 1566.402 & 1563.901 & 1566.795 \\
\hline $8 / 15 / 169: 00$ & 75.677 & 85.191 & 84.08 & 90.626 & 80.268 & 75.216 & 73.397 & 77.084 & 1582.795 & 1572.662 & 1569.228 & 1573.303 & 1568.36 & 1566.405 & 1563.894 & 1566.811 \\
\hline $8 / 15 / 1610: 00$ & 75.663 & 85.153 & 84.052 & 90.614 & 80.223 & 75.205 & 73.375 & 77.088 & 1582.809 & 1572.7 & 1569.256 & 1573.315 & 1568.405 & 1566.416 & 1563.916 & 1566.807 \\
\hline $8 / 15 / 1611: 00$ & 75.675 & 85.149 & 84.045 & 90.612 & 80.2 & 75.205 & 73.392 & 77.077 & 1582.797 & 1572.704 & 1569.263 & 1573.317 & 1568.428 & 1566.416 & 1563.899 & 1566.818 \\
\hline $8 / 15 / 1612: 00$ & 75.67 & 85.125 & 84.028 & 90.605 & 80.163 & 75.191 & 73.385 & 77.032 & 1582.802 & 1572.728 & 1569.28 & 1573.324 & 1568.465 & 1566.43 & 1563.906 & 1566.863 \\
\hline 8/15/16 13:00 & 75.661 & 85.098 & 84 & 90.584 & 80.126 & 75.184 & 73.378 & 77.123 & 1582.811 & 1572.755 & 1569.308 & 1573.345 & 1568.502 & 1566.437 & 1563.913 & 1566.772 \\
\hline $8 / 15 / 1614: 00$ & 75.658 & 85.077 & 83.977 & 90.577 & 80.088 & 75.174 & 73.373 & 76.993 & 1582.814 & 1572.776 & 1569.331 & 1573.352 & 1568.54 & 1566.447 & 1563.918 & 1566.902 \\
\hline $8 / 15 / 1615: 00$ & 75.651 & 85.051 & 83.933 & 90.563 & 80.053 & 75.163 & 73.371 & 76.96 & 1582.821 & 1572.802 & 1569.375 & 1573.366 & 1568.575 & 1566.458 & 1563.92 & 1566.935 \\
\hline $8 / 15 / 1616: 00$ & 75.654 & 85.032 & 83.919 & 90.551 & 80.028 & 75.158 & 73.373 & 76.963 & 1582.818 & 1572.821 & 1569.389 & 1573.378 & 1568.6 & 1566.463 & 1563.918 & 1566.932 \\
\hline $8 / 15 / 1617: 00$ & 75.649 & 85.004 & 83.898 & 90.535 & 79.993 & 75.156 & 73.363 & 76.923 & 1582.823 & 1572.849 & 1569.41 & 1573.394 & 1568.635 & 1566.465 & 1563.928 & 1566.972 \\
\hline $8 / 15 / 1618: 00$ & 75.646 & 84.979 & 83.87 & 90.544 & 79.955 & 75.14 & 73.351 & 76.916 & 1582.826 & 1572.874 & 1569.438 & 1573.385 & 1568.673 & 1566.481 & 1563.94 & 1566.979 \\
\hline $8 / 15 / 16$ 19:00 & 75.644 & 84.958 & 83.842 & 90.544 & 79.918 & 75.135 & 73.349 & 76.886 & 1582.828 & 1572.895 & 1569.466 & 1573.385 & 1568.71 & 1566.486 & 1563.942 & 1567.009 \\
\hline $8 / 15 / 1620: 00$ & 75.649 & 84.932 & 83.823 & 90.542 & 79.888 & 75.13 & 73.351 & 76.865 & 1582.823 & 1572.921 & 1569.485 & 1573.387 & 1568.74 & 1566.491 & 1563.94 & 1567.03 \\
\hline $8 / 15 / 1621: 00$ & 75.658 & 84.928 & 83.812 & 90.514 & 79.869 & 75.133 & 73.363 & 76.847 & 1582.814 & 1572.925 & 1569.496 & 1573.415 & 1568.759 & 1566.488 & 1563.928 & 1567.048 \\
\hline $8 / 15 / 1622: 00$ & 75.672 & 84.918 & 83.798 & 90.5 & 79.846 & 75.135 & 73.373 & 76.844 & 1582.8 & 1572.935 & 1569.51 & 1573.429 & 1568.782 & 1566.486 & 1563.918 & 1567.051 \\
\hline $8 / 15 / 1623: 00$ & 75.682 & 84.907 & 83.788 & 90.523 & 79.825 & 75.133 & 73.375 & 76.835 & 1582.79 & 1572.946 & 1569.52 & 1573.406 & 1568.803 & 1566.488 & 1563.916 & 1567.06 \\
\hline $8 / 16 / 160: 00$ & 75.694 & 84.902 & 83.779 & 90.532 & 79.808 & 75.133 & 73.382 & 76.83 & 1582.778 & 1572.951 & 1569.529 & 1573.397 & 1568.82 & 1566.488 & 1563.909 & 1567.065 \\
\hline $8 / 16 / 161: 00$ & 75.691 & 84.877 & 83.758 & 90.525 & 79.775 & 75.123 & 73.371 & 76.803 & 1582.781 & 1572.976 & 1569.55 & 1573.404 & 1568.853 & 1566.498 & 1563.92 & 1567.092 \\
\hline $8 / 16 / 162: 00$ & 75.694 & 84.867 & 83.735 & 90.514 & 79.748 & 75.116 & 73.366 & 76.793 & 1582.778 & 1572.986 & 1569.573 & 1573.415 & 1568.88 & 1566.505 & 1563.925 & 1567.102 \\
\hline 8/16/16 3:00 & 75.703 & 84.856 & 83.723 & 90.502 & 79.729 & 75.116 & 73.371 & 76.777 & 1582.769 & 1572.997 & 1569.585 & 1573.427 & 1568.899 & 1566.505 & 1563.92 & 1567.118 \\
\hline $8 / 16 / 164: 00$ & 75.724 & 84.867 & 83.723 & 90.511 & 79.722 & 75.126 & 73.392 & 76.779 & 1582.748 & 1572.986 & 1569.585 & 1573.418 & 1568.906 & 1566.495 & 1563.899 & 1567.116 \\
\hline $8 / 16 / 165: 00$ & 75.738 & 84.863 & 83.751 & 90.504 & 79.724 & 75.133 & 73.399 & 76.791 & 1582.734 & 1572.99 & 1569.557 & 1573.425 & 1568.904 & 1566.488 & 1563.892 & 1567.104 \\
\hline $8 / 16 / 166: 00$ & 75.745 & 84.853 & 83.735 & 90.483 & 79.706 & 75.121 & 73.397 & 76.731 & 1582.727 & 1573 & 1569.573 & 1573.446 & 1568.922 & 1566.5 & 1563.894 & 1567.164 \\
\hline $8 / 16 / 167: 00$ & 75.738 & 84.823 & 83.711 & 90.46 & 79.673 & 75.105 & 73.375 & 76.745 & 1582.734 & 1573.03 & 1569.597 & 1573.469 & 1568.955 & 1566.516 & 1563.916 & 1567.15 \\
\hline $8 / 16 / 168: 00$ & 75.727 & 84.804 & 83.688 & 90.448 & 79.641 & 75.093 & 73.359 & 76.721 & 1582.745 & 1573.049 & 1569.62 & 1573.481 & 1568.987 & 1566.528 & 1563.932 & 1567.174 \\
\hline 8/16/16 9:00 & 75.764 & 84.828 & 83.704 & 90.474 & 79.657 & 75.121 & 73.406 & 76.742 & 1582.708 & 1573.025 & 1569.604 & 1573.455 & 1568.971 & 1566.5 & 1563.885 & 1567.153 \\
\hline 8/16/16 10:00 & 75.762 & 84.811 & 83.697 & 90.455 & 79.636 & 75.119 & 73.39 & 76.789 & 1582.71 & 1573.042 & 1569.611 & 1573.474 & 1568.992 & 1566.502 & 1563.901 & 1567.106 \\
\hline $8 / 16 / 1611: 00$ & 75.743 & 84.779 & 83.667 & 90.432 & 79.596 & 75.095 & 73.359 & 76.796 & 1582.729 & 1573.074 & 1569.641 & 1573.497 & 1569.032 & 1566.526 & 1563.932 & 1567.099 \\
\hline $8 / 16 / 1612: 00$ & 75.745 & 84.765 & 83.669 & 90.43 & 79.575 & 75.095 & 73.361 & 76.738 & 1582.727 & 1573.088 & 1569.639 & 1573.499 & 1569.053 & 1566.526 & 1563.93 & 1567.157 \\
\hline
\end{tabular}


TABLE S1.1 Automatically recorded groundwater levels in the unconfined aquifer.

\begin{tabular}{|c|c|c|c|c|c|c|c|c|c|c|c|c|c|c|c|c|}
\hline \multirow[b]{2}{*}{ Date and Time } & \multicolumn{8}{|c|}{ Measured Depth to Water ( $\mathrm{ft}$ TOC ${ }^{\mathrm{a}}$ ) } & \multicolumn{8}{|c|}{ Groundwater Elevation (ft AMSL ${ }^{\mathrm{b}}$ ) } \\
\hline & DL1-C & DL2-C1 & DL3-C1 & DL4-C1 & DL5-C1 & DL6-C & DL7-C & DL8-C1 & DL1-C & DL2-C1 & DL3-C1 & DL4-C1 & DL5-C1 & DL6-C & DL7-C & DL8-C1 \\
\hline 8/16/16 13:00 & 75.736 & 84.744 & 83.634 & 90.416 & 79.545 & 75.074 & 73.349 & 76.689 & 1582.736 & 1573.109 & 1569.674 & 1573.513 & 1569.083 & 1566.547 & 1563.942 & 1567.206 \\
\hline 8/16/16 14:00 & 75.755 & 84.749 & 83.639 & 90.425 & 79.545 & 75.086 & 73.366 & 76.761 & 1582.717 & 1573.104 & 1569.669 & 1573.504 & 1569.083 & 1566.535 & 1563.925 & 1567.134 \\
\hline 8/16/16 15:00 & 75.75 & 84.73 & 83.599 & 90.413 & 79.522 & 75.074 & 73.354 & 76.68 & 1582.722 & 1573.123 & 1569.709 & 1573.516 & 1569.106 & 1566.547 & 1563.937 & 1567.215 \\
\hline 8/16/16 16:00 & 75.741 & 84.707 & 83.571 & 90.399 & 79.491 & 75.06 & 73.342 & 76.647 & 1582.731 & 1573.146 & 1569.737 & 1573.53 & 1569.137 & 1566.561 & 1563.949 & 1567.248 \\
\hline 8/16/16 17:00 & 75.736 & 84.69 & 83.553 & 90.39 & 79.468 & 75.056 & 73.337 & 76.703 & 1582.736 & 1573.163 & 1569.755 & 1573.539 & 1569.16 & 1566.565 & 1563.954 & 1567.192 \\
\hline 8/16/16 18:00 & 75.738 & 84.67 & 83.539 & 90.385 & 79.447 & 75.056 & 73.335 & 76.638 & 1582.734 & 1573.183 & 1569.769 & 1573.544 & 1569.181 & 1566.565 & 1563.956 & 1567.257 \\
\hline 8/16/16 19:00 & 75.724 & 84.649 & 83.509 & 90.367 & 79.419 & 75.032 & 73.318 & 76.587 & 1582.748 & 1573.204 & 1569.799 & 1573.562 & 1569.209 & 1566.589 & 1563.973 & 1567.308 \\
\hline $8 / 16 / 1620: 00$ & 75.736 & 84.649 & 83.499 & 90.374 & 79.412 & 75.044 & 73.337 & 76.577 & 1582.736 & 1573.204 & 1569.809 & 1573.555 & 1569.216 & 1566.577 & 1563.954 & 1567.318 \\
\hline 8/16/16 21:00 & 75.764 & 84.665 & 83.513 & 90.385 & 79.421 & 75.063 & 73.368 & 76.591 & 1582.708 & 1573.188 & 1569.795 & 1573.544 & 1569.207 & 1566.558 & 1563.923 & 1567.304 \\
\hline 8/16/16 22:00 & 75.781 & 84.674 & 83.52 & 90.392 & 79.428 & 75.077 & 73.387 & 76.598 & 1582.691 & 1573.179 & 1569.788 & 1573.537 & 1569.2 & 1566.544 & 1563.904 & 1567.297 \\
\hline 8/16/16 23:00 & 75.774 & 84.665 & 83.506 & 90.378 & 79.414 & 75.06 & 73.373 & 76.587 & 1582.698 & 1573.188 & 1569.802 & 1573.551 & 1569.214 & 1566.561 & 1563.918 & 1567.308 \\
\hline 8/17/16 0:00 & 75.774 & 84.66 & 83.499 & 90.378 & 79.403 & 75.063 & 73.375 & 76.577 & 1582.698 & 1573.193 & 1569.809 & 1573.551 & 1569.225 & 1566.558 & 1563.916 & 1567.318 \\
\hline 8/17/16 1:00 & 75.767 & 84.651 & 83.485 & 90.369 & 79.388 & 75.056 & 73.371 & 76.573 & 1582.705 & 1573.202 & 1569.823 & 1573.56 & 1569.24 & 1566.565 & 1563.92 & 1567.322 \\
\hline $8 / 17 / 162: 00$ & 75.762 & 84.642 & 83.548 & 90.362 & 79.375 & 75.053 & 73.366 & 76.559 & 1582.71 & 1573.211 & 1569.76 & 1573.567 & 1569.253 & 1566.568 & 1563.925 & 1567.336 \\
\hline $8 / 17 / 163: 00$ & 75.755 & 84.63 & 83.534 & 90.355 & 79.356 & 75.046 & 73.359 & 76.556 & 1582.717 & 1573.223 & 1569.774 & 1573.574 & 1569.272 & 1566.575 & 1563.932 & 1567.339 \\
\hline 8/17/16 4:00 & 75.743 & 84.609 & 83.516 & 90.341 & 79.335 & 75.03 & 73.347 & 76.543 & 1582.729 & 1573.244 & 1569.792 & 1573.588 & 1569.293 & 1566.591 & 1563.944 & 1567.352 \\
\hline $8 / 17 / 165: 00$ & 75.741 & 84.597 & 83.539 & 90.339 & 79.321 & 75.028 & 73.344 & 76.533 & 1582.731 & 1573.256 & 1569.769 & 1573.59 & 1569.307 & 1566.593 & 1563.947 & 1567.362 \\
\hline $8 / 17 / 166: 00$ & 75.748 & 84.593 & 83.488 & 90.339 & 79.316 & 75.032 & 73.349 & 76.491 & 1582.724 & 1573.26 & 1569.82 & 1573.59 & 1569.312 & 1566.589 & 1563.942 & 1567.404 \\
\hline $8 / 17 / 167: 00$ & 75.762 & 84.6 & 83.45 & 90.343 & 79.314 & 75.035 & 73.363 & 76.508 & 1582.71 & 1573.253 & 1569.858 & 1573.586 & 1569.314 & 1566.586 & 1563.928 & 1567.387 \\
\hline 8/17/16 8:00 & 75.774 & 84.607 & 83.455 & 90.348 & 79.314 & 75.044 & 73.373 & 76.501 & 1582.698 & 1573.246 & 1569.853 & 1573.581 & 1569.314 & 1566.577 & 1563.918 & 1567.394 \\
\hline 8/17/16 9:00 & 75.786 & 84.611 & 83.471 & 90.353 & 79.314 & 75.056 & 73.385 & 76.601 & 1582.686 & 1573.242 & 1569.837 & 1573.576 & 1569.314 & 1566.565 & 1563.906 & 1567.294 \\
\hline 8/17/16 10:00 & 75.793 & 84.618 & 83.464 & 90.35 & 79.312 & 75.063 & 73.39 & 76.58 & 1582.679 & 1573.235 & 1569.844 & 1573.579 & 1569.316 & 1566.558 & 1563.901 & 1567.315 \\
\hline 8/17/16 11:00 & 75.79 & 84.609 & 83.471 & 90.346 & 79.475 & 75.046 & 73.378 & 76.54 & 1582.682 & 1573.244 & 1569.837 & 1573.583 & 1569.153 & 1566.575 & 1563.913 & 1567.355 \\
\hline 8/17/16 12:00 & 75.776 & 84.593 & 83.46 & 90.376 & 79.288 & 75.032 & 73.359 & 76.556 & 1582.696 & 1573.26 & 1569.848 & 1573.553 & 1569.34 & 1566.589 & 1563.932 & 1567.339 \\
\hline 8/17/16 13:00 & 75.762 & 84.574 & 83.425 & 90.346 & 79.258 & 75.018 & 73.34 & 76.494 & 1582.71 & 1573.279 & 1569.883 & 1573.583 & 1569.37 & 1566.603 & 1563.951 & 1567.401 \\
\hline 8/17/16 14:00 & 75.75 & 84.551 & 83.397 & 90.327 & 79.232 & 75.007 & 73.328 & 76.496 & 1582.722 & 1573.302 & 1569.911 & 1573.602 & 1569.396 & 1566.614 & 1563.963 & 1567.399 \\
\hline $8 / 17 / 16$ 15:00 & 75.755 & 84.532 & 83.362 & 90.313 & 79.216 & 74.993 & 73.316 & 76.433 & 1582.717 & 1573.321 & 1569.946 & 1573.616 & 1569.412 & 1566.628 & 1563.975 & 1567.462 \\
\hline 8/17/16 16:00 & 75.767 & 84.514 & 83.338 & 90.299 & 79.202 & 74.988 & 73.306 & 76.452 & 1582.705 & 1573.339 & 1569.97 & 1573.63 & 1569.426 & 1566.633 & 1563.985 & 1567.443 \\
\hline 8/17/16 17:00 & 75.786 & 84.502 & 83.317 & 90.29 & 79.197 & 74.988 & 73.308 & 76.447 & 1582.686 & 1573.351 & 1569.991 & 1573.639 & 1569.431 & 1566.633 & 1563.983 & 1567.448 \\
\hline $8 / 17 / 16$ 18:00 & 75.804 & 84.486 & 83.294 & 90.306 & 79.188 & 74.974 & 73.301 & 76.387 & 1582.668 & 1573.367 & 1570.014 & 1573.623 & 1569.44 & 1566.647 & 1563.99 & 1567.508 \\
\hline 8/17/16 19:00 & 75.823 & 84.474 & 83.278 & 90.311 & 79.191 & 74.967 & 73.299 & 76.364 & 1582.649 & 1573.379 & 1570.03 & 1573.618 & 1569.437 & 1566.654 & 1563.992 & 1567.531 \\
\hline $8 / 17 / 1620: 00$ & 75.842 & 84.47 & 83.264 & 90.311 & 79.181 & 74.965 & 73.301 & 76.354 & 1582.63 & 1573.383 & 1570.044 & 1573.618 & 1569.447 & 1566.656 & 1563.99 & 1567.541 \\
\hline 8/17/16 21:00 & 75.868 & 84.472 & 83.259 & 90.294 & 79.193 & 74.972 & 73.313 & 76.35 & 1582.604 & 1573.381 & 1570.049 & 1573.635 & 1569.435 & 1566.649 & 1563.978 & 1567.545 \\
\hline $8 / 17 / 1622: 00$ & 75.896 & 84.486 & 83.266 & 90.306 & 79.211 & 74.984 & 73.332 & 76.357 & 1582.576 & 1573.367 & 1570.042 & 1573.623 & 1569.417 & 1566.637 & 1563.959 & 1567.538 \\
\hline 8/17/16 23:00 & 75.91 & 84.486 & 83.259 & 90.313 & 79.218 & 74.979 & 73.33 & 76.357 & 1582.562 & 1573.367 & 1570.049 & 1573.616 & 1569.41 & 1566.642 & 1563.961 & 1567.538 \\
\hline 8/18/16 0:00 & 75.922 & 84.486 & 83.252 & 90.306 & 79.225 & 74.977 & 73.332 & 76.345 & 1582.55 & 1573.367 & 1570.056 & 1573.623 & 1569.403 & 1566.644 & 1563.959 & 1567.55 \\
\hline $8 / 18 / 161: 00$ & 75.925 & 84.477 & 83.24 & 90.301 & 79.221 & 74.969 & 73.328 & 76.331 & 1582.547 & 1573.376 & 1570.068 & 1573.628 & 1569.407 & 1566.652 & 1563.963 & 1567.564 \\
\hline 8/18/16 2:00 & 75.922 & 84.46 & 83.219 & 90.285 & 79.214 & 74.953 & 73.308 & 76.315 & 1582.55 & 1573.393 & 1570.089 & 1573.644 & 1569.414 & 1566.668 & 1563.983 & 1567.58 \\
\hline 8/18/16 3:00 & 75.917 & 84.449 & 83.208 & 90.278 & 79.197 & 74.948 & 73.301 & 76.306 & 1582.555 & 1573.404 & 1570.1 & 1573.651 & 1569.431 & 1566.673 & 1563.99 & 1567.589 \\
\hline 8/18/16 4:00 & 75.903 & 84.439 & 83.196 & 90.271 & 79.184 & 74.944 & 73.297 & 76.292 & 1582.569 & 1573.414 & 1570.112 & 1573.658 & 1569.444 & 1566.677 & 1563.994 & 1567.603 \\
\hline $8 / 18 / 165: 00$ & 75.885 & 84.416 & 83.182 & 90.238 & 79.167 & 74.928 & 73.28 & 76.275 & 1582.587 & 1573.437 & 1570.126 & 1573.691 & 1569.461 & 1566.693 & 1564.011 & 1567.62 \\
\hline $8 / 18 / 166: 00$ & 75.877 & 84.407 & 83.166 & 90.229 & 79.151 & 74.927 & 73.282 & 76.257 & 1582.595 & 1573.446 & 1570.142 & 1573.7 & 1569.477 & 1566.694 & 1564.009 & 1567.638 \\
\hline 8/18/16 7:00 & 75.885 & 84.421 & 83.166 & 90.236 & 79.156 & 74.939 & 73.308 & 76.255 & 1582.587 & 1573.432 & 1570.142 & 1573.693 & 1569.472 & 1566.682 & 1563.983 & 1567.64 \\
\hline 8/18/16 8:00 & 75.894 & 84.432 & 83.175 & 90.238 & 79.158 & 74.953 & 73.323 & 76.264 & 1582.578 & 1573.421 & 1570.133 & 1573.691 & 1569.47 & 1566.668 & 1563.968 & 1567.631 \\
\hline 8/18/16 9:00 & 75.894 & 84.43 & 83.178 & 90.236 & 79.151 & 74.956 & 73.323 & 76.347 & 1582.578 & 1573.423 & 1570.13 & 1573.693 & 1569.477 & 1566.665 & 1563.968 & 1567.548 \\
\hline 8/18/16 10:00 & 75.887 & 84.418 & 83.168 & 90.224 & 79.137 & 74.951 & 73.308 & 76.348 & 1582.585 & 1573.435 & 1570.14 & 1573.705 & 1569.491 & 1566.67 & 1563.983 & 1567.547 \\
\hline 8/18/16 11:00 & 75.877 & 84.402 & 83.152 & 90.215 & 79.116 & 74.935 & 73.299 & 76.266 & 1582.595 & 1573.451 & 1570.156 & 1573.714 & 1569.512 & 1566.686 & 1563.992 & 1567.629 \\
\hline 8/18/16 12:00 & 75.866 & 84.388 & 83.145 & 90.208 & 79.1 & 74.932 & 73.289 & 76.269 & 1582.606 & 1573.465 & 1570.163 & 1573.721 & 1569.528 & 1566.689 & 1564.002 & 1567.626 \\
\hline 8/18/16 13:00 & 75.87 & 84.374 & 83.122 & 90.192 & 79.086 & 74.925 & 73.28 & 76.278 & 1582.602 & 1573.479 & 1570.186 & 1573.737 & 1569.542 & 1566.696 & 1564.011 & 1567.617 \\
\hline
\end{tabular}


TABLE S1.1 Automatically recorded groundwater levels in the unconfined aquifer.

\begin{tabular}{|c|c|c|c|c|c|c|c|c|c|c|c|c|c|c|c|c|}
\hline \multirow[b]{2}{*}{ Date and Time } & \multicolumn{8}{|c|}{ Measured Depth to Water (ft TOC ${ }^{\mathrm{a}}$ ) } & \multicolumn{8}{|c|}{ Groundwater Elevation (ft AMSL') } \\
\hline & DL1-C & DL2-C1 & DL3-C1 & DL4-C1 & DL5-C1 & DL6-C & DL7-C & DL8-C1 & DL1-C & DL2-C1 & DL3-C1 & DL4-C1 & DL5-C1 & DL6-C & DL7-C & DL8-C1 \\
\hline 8/18/16 14:00 & 75.887 & 84.36 & 83.112 & 90.185 & 79.079 & 74.909 & 73.273 & 76.215 & 1582.585 & 1573.493 & 1570.196 & 1573.744 & 1569.549 & 1566.712 & 1564.018 & 1567.68 \\
\hline $8 / 18 / 1615: 00$ & 75.899 & 84.351 & 83.089 & 90.175 & 79.079 & 74.907 & 73.27 & 76.215 & 1582.573 & 1573.502 & 1570.219 & 1573.754 & 1569.549 & 1566.714 & 1564.021 & 1567.68 \\
\hline $8 / 18 / 1616: 00$ & 75.903 & 84.332 & 83.066 & 90.164 & 79.067 & 74.893 & 73.256 & 76.176 & 1582.569 & 1573.521 & 1570.242 & 1573.765 & 1569.561 & 1566.728 & 1564.035 & 1567.719 \\
\hline 8/18/16 17:00 & 75.91 & 84.321 & 83.047 & 90.154 & 79.062 & 74.883 & 73.251 & 76.166 & 1582.562 & 1573.532 & 1570.261 & 1573.775 & 1569.566 & 1566.738 & 1564.04 & 1567.729 \\
\hline 8/18/16 18:00 & 75.917 & 84.304 & 83.033 & 90.14 & 79.055 & 74.881 & 73.246 & 76.15 & 1582.555 & 1573.549 & 1570.275 & 1573.789 & 1569.573 & 1566.74 & 1564.045 & 1567.745 \\
\hline 8/18/16 19:00 & 75.92 & 84.29 & 83.017 & 90.145 & 79.046 & 74.872 & 73.239 & 76.122 & 1582.552 & 1573.563 & 1570.291 & 1573.784 & 1569.582 & 1566.749 & 1564.052 & 1567.773 \\
\hline $8 / 18 / 1620: 00$ & 75.932 & 84.29 & 83.01 & 90.157 & 79.048 & 74.872 & 73.249 & 76.111 & 1582.54 & 1573.563 & 1570.298 & 1573.772 & 1569.58 & 1566.749 & 1564.042 & 1567.784 \\
\hline 8/18/16 21:00 & 75.946 & 84.295 & 83.007 & 90.157 & 79.058 & 74.879 & 73.261 & 76.108 & 1582.526 & 1573.558 & 1570.301 & 1573.772 & 1569.57 & 1566.742 & 1564.03 & 1567.787 \\
\hline $8 / 18 / 1622: 00$ & 75.965 & 84.3 & 83.01 & 90.166 & 79.067 & 74.886 & 73.27 & 76.108 & 1582.507 & 1573.553 & 1570.298 & 1573.763 & 1569.561 & 1566.735 & 1564.021 & 1567.787 \\
\hline 8/18/16 23:00 & 75.991 & 84.316 & 83.019 & 90.182 & 79.086 & 74.897 & 73.292 & 76.125 & 1582.481 & 1573.537 & 1570.289 & 1573.747 & 1569.542 & 1566.724 & 1563.999 & 1567.77 \\
\hline 8/19/16 0:00 & 75.993 & 84.314 & 83.014 & 90.175 & 79.086 & 74.89 & 73.289 & 76.064 & 1582.479 & 1573.539 & 1570.294 & 1573.754 & 1569.542 & 1566.731 & 1564.002 & 1567.831 \\
\hline $8 / 19 / 161: 00$ & 75.995 & 84.318 & 83.01 & 90.157 & 79.09 & 74.893 & 73.289 & 76.115 & 1582.477 & 1573.535 & 1570.298 & 1573.772 & 1569.538 & 1566.728 & 1564.002 & 1567.78 \\
\hline $8 / 19 / 162: 00$ & 75.972 & 84.307 & 83.07 & 90.131 & 79.074 & 74.883 & 73.277 & 76.099 & 1582.5 & 1573.546 & 1570.238 & 1573.798 & 1569.554 & 1566.738 & 1564.014 & 1567.796 \\
\hline 8/19/16 3:00 & 75.965 & 84.314 & 83.077 & 90.133 & 79.062 & 74.886 & 73.285 & 76.104 & 1582.507 & 1573.539 & 1570.231 & 1573.796 & 1569.566 & 1566.735 & 1564.006 & 1567.791 \\
\hline 8/19/16 4:00 & 75.962 & 84.323 & 83.077 & 90.136 & 79.069 & 74.897 & 73.301 & 76.132 & 1582.51 & 1573.53 & 1570.231 & 1573.793 & 1569.559 & 1566.724 & 1563.99 & 1567.763 \\
\hline 8/19/16 5:00 & 75.925 & 84.29 & 83.07 & 90.112 & 79.034 & 74.874 & 73.27 & 76.106 & 1582.547 & 1573.563 & 1570.238 & 1573.817 & 1569.594 & 1566.747 & 1564.021 & 1567.789 \\
\hline 8/19/16 6:00 & 75.91 & 84.279 & 83.01 & 90.105 & 79.013 & 74.867 & 73.265 & 76.078 & 1582.562 & 1573.574 & 1570.298 & 1573.824 & 1569.615 & 1566.754 & 1564.026 & 1567.817 \\
\hline $8 / 19 / 167: 00$ & 75.906 & 84.279 & 82.968 & 90.103 & 79.002 & 74.869 & 73.265 & 76.069 & 1582.566 & 1573.574 & 1570.34 & 1573.826 & 1569.626 & 1566.752 & 1564.026 & 1567.826 \\
\hline 8/19/16 8:00 & 75.887 & 84.263 & 82.947 & 90.093 & 78.971 & 74.858 & 73.254 & 76.046 & 1582.585 & 1573.59 & 1570.361 & 1573.836 & 1569.657 & 1566.763 & 1564.037 & 1567.849 \\
\hline 8/19/16 9:00 & 75.882 & 84.26 & 82.94 & 90.093 & 78.96 & 74.865 & 73.256 & 76.076 & 1582.59 & 1573.593 & 1570.368 & 1573.836 & 1569.668 & 1566.756 & 1564.035 & 1567.819 \\
\hline $8 / 19 / 1610: 00$ & 75.877 & 84.251 & 82.93 & 90.086 & 78.946 & 74.855 & 73.254 & 76.039 & 1582.595 & 1573.602 & 1570.378 & 1573.843 & 1569.682 & 1566.766 & 1564.037 & 1567.856 \\
\hline 8/19/16 11:00 & 75.88 & 84.253 & 82.933 & 90.089 & 78.946 & 74.865 & 73.27 & 76.032 & 1582.592 & 1573.6 & 1570.375 & 1573.84 & 1569.682 & 1566.756 & 1564.021 & 1567.863 \\
\hline 8/19/16 12:00 & 75.88 & 84.256 & 82.926 & 90.091 & 78.934 & 74.872 & 73.273 & 76.108 & 1582.592 & 1573.597 & 1570.382 & 1573.838 & 1569.694 & 1566.749 & 1564.018 & 1567.787 \\
\hline 8/19/16 13:00 & 75.866 & 84.239 & 82.914 & 90.077 & 78.913 & 74.858 & 73.261 & 76.036 & 1582.606 & 1573.614 & 1570.394 & 1573.852 & 1569.715 & 1566.763 & 1564.03 & 1567.859 \\
\hline 8/19/16 14:00 & 75.87 & 84.246 & 82.916 & 90.086 & 78.911 & 74.867 & 73.277 & 76.022 & 1582.602 & 1573.607 & 1570.392 & 1573.843 & 1569.717 & 1566.754 & 1564.014 & 1567.873 \\
\hline 8/19/16 15:00 & 75.847 & 84.214 & 82.891 & 90.061 & 78.876 & 74.839 & 73.246 & 75.997 & 1582.625 & 1573.639 & 1570.417 & 1573.868 & 1569.752 & 1566.782 & 1564.045 & 1567.898 \\
\hline $8 / 19 / 1616: 00$ & 75.852 & 84.228 & 82.889 & 90.072 & 78.878 & 74.858 & 73.273 & 75.995 & 1582.62 & 1573.625 & 1570.419 & 1573.857 & 1569.75 & 1566.763 & 1564.018 & 1567.9 \\
\hline 8/19/16 17:00 & 75.814 & 84.179 & 82.849 & 90.028 & 78.827 & 74.82 & 73.225 & 75.96 & 1582.658 & 1573.674 & 1570.459 & 1573.901 & 1569.801 & 1566.801 & 1564.066 & 1567.935 \\
\hline 8/19/16 18:00 & 75.807 & 84.172 & 82.83 & 90.058 & 78.803 & 74.827 & 73.234 & 75.939 & 1582.665 & 1573.681 & 1570.478 & 1573.871 & 1569.825 & 1566.794 & 1564.057 & 1567.956 \\
\hline 8/19/16 19:00 & 75.797 & 84.16 & 82.821 & 90.065 & 78.785 & 74.818 & 73.23 & 75.93 & 1582.675 & 1573.693 & 1570.487 & 1573.864 & 1569.843 & 1566.803 & 1564.061 & 1567.965 \\
\hline 8/19/16 20:00 & 75.811 & 84.172 & 82.83 & 90.079 & 78.789 & 74.834 & 73.256 & 75.944 & 1582.661 & 1573.681 & 1570.478 & 1573.85 & 1569.839 & 1566.787 & 1564.035 & 1567.951 \\
\hline 8/19/16 21:00 & 75.828 & 84.188 & 82.844 & 90.075 & 78.794 & 74.851 & 73.28 & 75.957 & 1582.644 & 1573.665 & 1570.464 & 1573.854 & 1569.834 & 1566.77 & 1564.011 & 1567.938 \\
\hline 8/19/16 22:00 & 75.837 & 84.204 & 82.858 & 90.079 & 78.801 & 74.862 & 73.299 & 75.969 & 1582.635 & 1573.649 & 1570.45 & 1573.85 & 1569.827 & 1566.759 & 1563.992 & 1567.926 \\
\hline 8/19/16 23:00 & 75.835 & 84.202 & 82.856 & 90.089 & 78.799 & 74.862 & 73.301 & 75.969 & 1582.637 & 1573.651 & 1570.452 & 1573.84 & 1569.829 & 1566.759 & 1563.99 & 1567.926 \\
\hline $8 / 20 / 160: 00$ & 75.835 & 84.214 & 82.861 & 90.086 & 78.796 & 74.865 & 73.304 & 75.971 & 1582.637 & 1573.639 & 1570.447 & 1573.843 & 1569.832 & 1566.756 & 1563.987 & 1567.924 \\
\hline $8 / 20 / 161: 00$ & 75.835 & 84.216 & 82.861 & 90.089 & 78.794 & 74.872 & 73.308 & 75.976 & 1582.637 & 1573.637 & 1570.447 & 1573.84 & 1569.834 & 1566.749 & 1563.983 & 1567.919 \\
\hline $8 / 20 / 162: 00$ & 75.826 & 84.209 & 82.854 & 90.082 & 78.778 & 74.865 & 73.301 & 75.962 & 1582.646 & 1573.644 & 1570.454 & 1573.847 & 1569.85 & 1566.756 & 1563.99 & 1567.933 \\
\hline $8 / 20 / 163: 00$ & 75.819 & 84.211 & 82.856 & 90.077 & 78.773 & 74.865 & 73.304 & 75.969 & 1582.653 & 1573.642 & 1570.452 & 1573.852 & 1569.855 & 1566.756 & 1563.987 & 1567.926 \\
\hline $8 / 20 / 164: 00$ & 75.819 & 84.214 & 82.858 & 90.082 & 78.768 & 74.876 & 73.308 & 75.981 & 1582.653 & 1573.639 & 1570.45 & 1573.847 & 1569.86 & 1566.745 & 1563.983 & 1567.914 \\
\hline $8 / 20 / 165: 00$ & 75.807 & 84.207 & 82.868 & 90.056 & 78.752 & 74.862 & 73.304 & 75.962 & 1582.665 & 1573.646 & 1570.44 & 1573.873 & 1569.876 & 1566.759 & 1563.987 & 1567.933 \\
\hline $8 / 20 / 166: 00$ & 75.8 & 84.197 & 82.851 & 90.04 & 78.736 & 74.858 & 73.294 & 75.955 & 1582.672 & 1573.656 & 1570.457 & 1573.889 & 1569.892 & 1566.763 & 1563.997 & 1567.94 \\
\hline $8 / 20 / 167: 00$ & 75.809 & 84.209 & 82.861 & 90.049 & 78.738 & 74.872 & 73.316 & 75.957 & 1582.663 & 1573.644 & 1570.447 & 1573.88 & 1569.89 & 1566.749 & 1563.975 & 1567.938 \\
\hline 8/20/16 8:00 & 75.802 & 84.209 & 82.861 & 90.044 & 78.733 & 74.872 & 73.313 & 75.957 & 1582.67 & 1573.644 & 1570.447 & 1573.885 & 1569.895 & 1566.749 & 1563.978 & 1567.938 \\
\hline 8/20/16 9:00 & 75.8 & 84.204 & 82.858 & 90.042 & 78.719 & 74.869 & 73.316 & 75.955 & 1582.672 & 1573.649 & 1570.45 & 1573.887 & 1569.909 & 1566.752 & 1563.975 & 1567.94 \\
\hline $8 / 20 / 1610: 00$ & 75.786 & 84.186 & 82.844 & 90.028 & 78.698 & 74.855 & 73.297 & 75.946 & 1582.686 & 1573.667 & 1570.464 & 1573.901 & 1569.93 & 1566.766 & 1563.994 & 1567.949 \\
\hline $8 / 20 / 1611: 00$ & 75.776 & 84.181 & 82.84 & 90.026 & 78.685 & 74.858 & 73.297 & 75.937 & 1582.696 & 1573.672 & 1570.468 & 1573.903 & 1569.943 & 1566.763 & 1563.994 & 1567.958 \\
\hline 8/20/16 12:00 & 75.771 & 84.179 & 82.842 & 90.021 & 78.673 & 74.855 & 73.294 & 75.925 & 1582.701 & 1573.674 & 1570.466 & 1573.908 & 1569.955 & 1566.766 & 1563.997 & 1567.97 \\
\hline $8 / 20 / 1613: 00$ & 75.755 & 84.16 & 82.816 & 90.009 & 78.65 & 74.841 & 73.28 & 75.911 & 1582.717 & 1573.693 & 1570.492 & 1573.92 & 1569.978 & 1566.78 & 1564.011 & 1567.984 \\
\hline $8 / 20 / 1614: 00$ & 75.743 & 84.144 & 82.798 & 90.002 & 78.626 & 74.83 & 73.268 & 75.888 & 1582.729 & 1573.709 & 1570.51 & 1573.927 & 1570.002 & 1566.791 & 1564.023 & 1568.007 \\
\hline
\end{tabular}


TABLE S1.1 Automatically recorded groundwater levels in the unconfined aquifer.

\begin{tabular}{|c|c|c|c|c|c|c|c|c|c|c|c|c|c|c|c|c|}
\hline \multirow[b]{2}{*}{ Date and Time } & \multicolumn{8}{|c|}{ Measured Depth to Water (ft TOC ${ }^{\mathrm{a}}$ ) } & \multicolumn{8}{|c|}{ Groundwater Elevation (ft AMSL') } \\
\hline & DL1-C & DL2-C1 & DL3-C1 & DL4-C1 & DL5-C1 & DL6-C & DL7-C & DL8-C1 & DL1-C & DL2-C1 & DL3-C1 & DL4-C1 & DL5-C1 & DL6-C & DL7-C & DL8-C1 \\
\hline $8 / 20 / 1615: 00$ & 75.734 & 84.13 & 82.767 & 89.993 & 78.608 & 74.827 & 73.268 & 75.876 & 1582.738 & 1573.723 & 1570.541 & 1573.936 & 1570.02 & 1566.794 & 1564.023 & 1568.019 \\
\hline $8 / 20 / 1616: 00$ & 75.727 & 84.116 & 82.749 & 89.988 & 78.587 & 74.823 & 73.263 & 75.858 & 1582.745 & 1573.737 & 1570.559 & 1573.941 & 1570.041 & 1566.798 & 1564.028 & 1568.037 \\
\hline $8 / 20 / 1617: 00$ & 75.724 & 84.107 & 82.737 & 89.981 & 78.568 & 74.82 & 73.261 & 75.851 & 1582.748 & 1573.746 & 1570.571 & 1573.948 & 1570.06 & 1566.801 & 1564.03 & 1568.044 \\
\hline $8 / 20 / 1618: 00$ & 75.724 & 84.1 & 82.725 & 89.977 & 78.552 & 74.818 & 73.263 & 75.841 & 1582.748 & 1573.753 & 1570.583 & 1573.952 & 1570.076 & 1566.803 & 1564.028 & 1568.054 \\
\hline 8/20/16 19:00 & 75.724 & 84.088 & 82.718 & 89.981 & 78.535 & 74.818 & 73.263 & 75.834 & 1582.748 & 1573.765 & 1570.59 & 1573.948 & 1570.093 & 1566.803 & 1564.028 & 1568.061 \\
\hline $8 / 20 / 1620: 00$ & 75.724 & 84.09 & 82.709 & 89.991 & 78.526 & 74.813 & 73.268 & 75.827 & 1582.748 & 1573.763 & 1570.599 & 1573.938 & 1570.102 & 1566.808 & 1564.023 & 1568.068 \\
\hline $8 / 20 / 1621: 00$ & 75.731 & 84.088 & 82.709 & 89.993 & 78.521 & 74.82 & 73.28 & 75.825 & 1582.741 & 1573.765 & 1570.599 & 1573.936 & 1570.107 & 1566.801 & 1564.011 & 1568.07 \\
\hline $8 / 20 / 1622: 00$ & 75.741 & 84.093 & 82.714 & 90.002 & 78.519 & 74.83 & 73.292 & 75.83 & 1582.731 & 1573.76 & 1570.594 & 1573.927 & 1570.109 & 1566.791 & 1563.999 & 1568.065 \\
\hline $8 / 20 / 1623: 00$ & 75.741 & 84.093 & 82.709 & 90.009 & 78.512 & 74.823 & 73.289 & 75.776 & 1582.731 & 1573.76 & 1570.599 & 1573.92 & 1570.116 & 1566.798 & 1564.002 & 1568.119 \\
\hline $8 / 21 / 160: 00$ & 75.738 & 84.088 & 82.704 & 90.002 & 78.5 & 74.825 & 73.287 & 75.827 & 1582.734 & 1573.765 & 1570.604 & 1573.927 & 1570.128 & 1566.796 & 1564.004 & 1568.068 \\
\hline $8 / 21 / 161: 00$ & 75.738 & 84.09 & 82.707 & 89.991 & 78.496 & 74.83 & 73.297 & 75.823 & 1582.734 & 1573.763 & 1570.601 & 1573.938 & 1570.132 & 1566.791 & 1563.994 & 1568.072 \\
\hline $8 / 21 / 162: 00$ & 75.738 & 84.086 & 82.7 & 89.977 & 78.484 & 74.825 & 73.297 & 75.82 & 1582.734 & 1573.767 & 1570.608 & 1573.952 & 1570.144 & 1566.796 & 1563.994 & 1568.075 \\
\hline $8 / 21 / 163: 00$ & 75.736 & 84.086 & 82.697 & 89.972 & 78.477 & 74.827 & 73.297 & 75.827 & 1582.736 & 1573.767 & 1570.611 & 1573.957 & 1570.151 & 1566.794 & 1563.994 & 1568.068 \\
\hline $8 / 21 / 164: 00$ & 75.731 & 84.081 & 82.695 & 89.963 & 78.465 & 74.823 & 73.294 & 75.825 & 1582.741 & 1573.772 & 1570.613 & 1573.966 & 1570.163 & 1566.798 & 1563.997 & 1568.07 \\
\hline $8 / 21 / 165: 00$ & 75.727 & 84.072 & 82.709 & 89.958 & 78.456 & 74.825 & 73.294 & 75.818 & 1582.745 & 1573.781 & 1570.599 & 1573.971 & 1570.172 & 1566.796 & 1563.997 & 1568.077 \\
\hline $8 / 21 / 166: 00$ & 75.727 & 84.072 & 82.697 & 89.956 & 78.444 & 74.823 & 73.297 & 75.804 & 1582.745 & 1573.781 & 1570.611 & 1573.973 & 1570.184 & 1566.798 & 1563.994 & 1568.091 \\
\hline $8 / 21 / 167: 00$ & 75.729 & 84.072 & 82.7 & 89.96 & 78.447 & 74.827 & 73.304 & 75.804 & 1582.743 & 1573.781 & 1570.608 & 1573.969 & 1570.181 & 1566.794 & 1563.987 & 1568.091 \\
\hline $8 / 21 / 168: 00$ & 75.729 & 84.079 & 82.704 & 89.963 & 78.437 & 74.827 & 73.308 & 75.807 & 1582.743 & 1573.774 & 1570.604 & 1573.966 & 1570.191 & 1566.794 & 1563.983 & 1568.088 \\
\hline 8/21/16 9:00 & 75.731 & 84.081 & 82.704 & 89.963 & 78.433 & 74.834 & 73.313 & 75.809 & 1582.741 & 1573.772 & 1570.604 & 1573.966 & 1570.195 & 1566.787 & 1563.978 & 1568.086 \\
\hline $8 / 21 / 1610: 00$ & 75.722 & 84.074 & 82.697 & 89.956 & 78.421 & 74.827 & 73.301 & 75.827 & 1582.75 & 1573.779 & 1570.611 & 1573.973 & 1570.207 & 1566.794 & 1563.99 & 1568.068 \\
\hline $8 / 21 / 1611: 00$ & 75.717 & 84.065 & 82.69 & 89.949 & 78.405 & 74.82 & 73.297 & 75.793 & 1582.755 & 1573.788 & 1570.618 & 1573.98 & 1570.223 & 1566.801 & 1563.994 & 1568.102 \\
\hline $8 / 21 / 1612: 00$ & 75.712 & 84.049 & 82.69 & 89.942 & 78.386 & 74.813 & 73.289 & 75.781 & 1582.76 & 1573.804 & 1570.618 & 1573.987 & 1570.242 & 1566.808 & 1564.002 & 1568.114 \\
\hline $8 / 21 / 1613: 00$ & 75.698 & 84.035 & 82.665 & 89.935 & 78.365 & 74.804 & 73.275 & 75.762 & 1582.774 & 1573.818 & 1570.643 & 1573.994 & 1570.263 & 1566.817 & 1564.016 & 1568.133 \\
\hline $8 / 21 / 1614: 00$ & 75.691 & 84.021 & 82.648 & 89.923 & 78.339 & 74.793 & 73.263 & 75.737 & 1582.781 & 1573.832 & 1570.66 & 1574.006 & 1570.289 & 1566.828 & 1564.028 & 1568.158 \\
\hline $8 / 21 / 1615: 00$ & 75.675 & 83.993 & 82.609 & 89.909 & 78.311 & 74.781 & 73.242 & 75.858 & 1582.797 & 1573.86 & 1570.699 & 1574.02 & 1570.317 & 1566.84 & 1564.049 & 1568.037 \\
\hline $8 / 21 / 1616: 00$ & 75.663 & 83.962 & 82.583 & 89.895 & 78.283 & 74.765 & 73.225 & 75.723 & 1582.809 & 1573.891 & 1570.725 & 1574.034 & 1570.345 & 1566.856 & 1564.066 & 1568.172 \\
\hline $8 / 21 / 1617: 00$ & 75.658 & 83.939 & 82.56 & 89.881 & 78.26 & 74.75 & 73.218 & 75.697 & 1582.814 & 1573.914 & 1570.748 & 1574.048 & 1570.368 & 1566.871 & 1564.073 & 1568.198 \\
\hline $8 / 21 / 1618: 00$ & 75.661 & 83.93 & 82.546 & 89.904 & 78.241 & 74.753 & 73.22 & 75.69 & 1582.811 & 1573.923 & 1570.762 & 1574.025 & 1570.387 & 1566.868 & 1564.071 & 1568.205 \\
\hline $8 / 21 / 1619: 00$ & 75.661 & 83.916 & 82.534 & 89.909 & 78.223 & 74.748 & 73.22 & 75.677 & 1582.811 & 1573.937 & 1570.774 & 1574.02 & 1570.405 & 1566.873 & 1564.071 & 1568.218 \\
\hline $8 / 21 / 1620: 00$ & 75.661 & 83.904 & 82.52 & 89.909 & 78.211 & 74.741 & 73.218 & 75.649 & 1582.811 & 1573.949 & 1570.788 & 1574.02 & 1570.417 & 1566.88 & 1564.073 & 1568.246 \\
\hline $8 / 21 / 1621: 00$ & 75.668 & 83.9 & 82.511 & 89.888 & 78.197 & 74.746 & 73.223 & 75.642 & 1582.804 & 1573.953 & 1570.797 & 1574.041 & 1570.431 & 1566.875 & 1564.068 & 1568.253 \\
\hline $8 / 21 / 1622: 00$ & 75.682 & 83.909 & 82.513 & 89.897 & 78.199 & 74.753 & 73.242 & 75.639 & 1582.79 & 1573.944 & 1570.795 & 1574.032 & 1570.429 & 1566.868 & 1564.049 & 1568.256 \\
\hline $8 / 21 / 1623: 00$ & 75.687 & 83.9 & 82.506 & 89.904 & 78.183 & 74.748 & 73.239 & 75.632 & 1582.785 & 1573.953 & 1570.802 & 1574.025 & 1570.445 & 1566.873 & 1564.052 & 1568.263 \\
\hline $8 / 22 / 160: 00$ & 75.691 & 83.895 & 82.504 & 89.9 & 78.172 & 74.744 & 73.242 & 75.611 & 1582.781 & 1573.958 & 1570.804 & 1574.029 & 1570.456 & 1566.877 & 1564.049 & 1568.284 \\
\hline $8 / 22 / 161: 00$ & 75.691 & 83.89 & 82.492 & 89.897 & 78.16 & 74.746 & 73.239 & 75.625 & 1582.781 & 1573.963 & 1570.816 & 1574.032 & 1570.468 & 1566.875 & 1564.052 & 1568.27 \\
\hline $8 / 22 / 162: 00$ & 75.684 & 83.876 & 82.553 & 89.888 & 78.141 & 74.737 & 73.23 & 75.609 & 1582.788 & 1573.977 & 1570.755 & 1574.041 & 1570.487 & 1566.884 & 1564.061 & 1568.286 \\
\hline $8 / 22 / 163: 00$ & 75.687 & 83.867 & 82.553 & 89.881 & 78.132 & 74.732 & 73.227 & 75.614 & 1582.785 & 1573.986 & 1570.755 & 1574.048 & 1570.496 & 1566.889 & 1564.064 & 1568.281 \\
\hline $8 / 22 / 164: 00$ & 75.684 & 83.862 & 82.534 & 89.883 & 78.118 & 74.727 & 73.225 & 75.612 & 1582.788 & 1573.991 & 1570.774 & 1574.046 & 1570.51 & 1566.894 & 1564.066 & 1568.283 \\
\hline $8 / 22 / 165: 00$ & 75.682 & 83.853 & 82.565 & 89.858 & 78.102 & 74.725 & 73.223 & 75.602 & 1582.79 & 1574 & 1570.743 & 1574.071 & 1570.526 & 1566.896 & 1564.068 & 1568.293 \\
\hline $8 / 22 / 166: 00$ & 75.687 & 83.853 & 82.516 & 89.848 & 78.09 & 74.723 & 73.223 & 75.549 & 1582.785 & 1574 & 1570.792 & 1574.081 & 1570.538 & 1566.898 & 1564.068 & 1568.346 \\
\hline $8 / 22 / 167: 00$ & 75.689 & 83.844 & 82.481 & 89.848 & 78.085 & 74.744 & 73.23 & 75.67 & 1582.783 & 1574.009 & 1570.827 & 1574.081 & 1570.543 & 1566.877 & 1564.061 & 1568.225 \\
\hline $8 / 22 / 168: 00$ & 75.696 & 83.846 & 82.506 & 89.848 & 78.081 & 74.762 & 73.237 & 75.762 & 1582.776 & 1574.007 & 1570.802 & 1574.081 & 1570.547 & 1566.859 & 1564.054 & 1568.133 \\
\hline 8/22/16 9:00 & 75.701 & 83.846 & 82.509 & 89.844 & 78.074 & 74.799 & 73.239 & 75.978 & 1582.771 & 1574.007 & 1570.799 & 1574.085 & 1570.554 & 1566.822 & 1564.052 & 1567.917 \\
\hline $8 / 22 / 1610: 00$ & 75.698 & 83.837 & 82.509 & 89.839 & 78.067 & 74.795 & 73.23 & 75.953 & 1582.774 & 1574.016 & 1570.799 & 1574.09 & 1570.561 & 1566.826 & 1564.061 & 1567.942 \\
\hline $8 / 22 / 1611: 00$ & 75.696 & 83.837 & 82.518 & 89.837 & 78.071 & 74.807 & 73.23 & 75.904 & 1582.776 & 1574.016 & 1570.79 & 1574.092 & 1570.557 & 1566.814 & 1564.061 & 1567.991 \\
\hline $8 / 22 / 1612: 00$ & 75.694 & 83.823 & 82.523 & 89.83 & 78.078 & 74.804 & 73.225 & 75.885 & 1582.778 & 1574.03 & 1570.785 & 1574.099 & 1570.55 & 1566.817 & 1564.066 & 1568.01 \\
\hline $8 / 22 / 1613: 00$ & 75.684 & 83.814 & 82.516 & 89.825 & 78.092 & 74.816 & 73.215 & 76.004 & 1582.788 & 1574.039 & 1570.792 & 1574.104 & 1570.536 & 1566.805 & 1564.076 & 1567.891 \\
\hline $8 / 22 / 1614: 00$ & 75.684 & 83.8 & 82.511 & 89.818 & 78.099 & 74.816 & 73.211 & 75.978 & 1582.788 & 1574.053 & 1570.797 & 1574.111 & 1570.529 & 1566.805 & 1564.08 & 1567.917 \\
\hline $8 / 22 / 1615: 00$ & 75.677 & 83.786 & 82.499 & 89.813 & 78.109 & 74.832 & 73.199 & 76.041 & 1582.795 & 1574.067 & 1570.809 & 1574.116 & 1570.519 & 1566.789 & 1564.092 & 1567.854 \\
\hline
\end{tabular}


TABLE S1.1 Automatically recorded groundwater levels in the unconfined aquifer.

\begin{tabular}{|c|c|c|c|c|c|c|c|c|c|c|c|c|c|c|c|c|}
\hline \multirow[b]{2}{*}{ Date and Time } & \multicolumn{8}{|c|}{ Measured Depth to Water (ft TOC ${ }^{\mathrm{a}}$ ) } & \multicolumn{8}{|c|}{ Groundwater Elevation ( $\mathrm{ft} \mathrm{AMSL}^{\mathrm{b}}$ ) } \\
\hline & DL1-C & DL2-C1 & DL3-C1 & DL4-C1 & DL5-C1 & DL6-C & DL7-C & DL8-C1 & DL1-C & DL2-C1 & DL3-C1 & DL4-C1 & DL5-C1 & DL6-C & DL7-C & DL8-C1 \\
\hline $8 / 22 / 1616: 00$ & 75.668 & 83.769 & 82.488 & 89.797 & 78.116 & 74.825 & 73.189 & 76.009 & 1582.804 & 1574.084 & 1570.82 & 1574.132 & 1570.512 & 1566.796 & 1564.102 & 1567.886 \\
\hline $8 / 22 / 1617: 00$ & 75.675 & 83.76 & 82.483 & 89.804 & 78.13 & 74.83 & 73.191 & 76.101 & 1582.797 & 1574.093 & 1570.825 & 1574.125 & 1570.498 & 1566.791 & 1564.1 & 1567.794 \\
\hline $8 / 22 / 1618: 00$ & 75.68 & 83.762 & 82.492 & 89.802 & 78.153 & 74.846 & 73.201 & 76.046 & 1582.792 & 1574.091 & 1570.816 & 1574.127 & 1570.475 & 1566.775 & 1564.09 & 1567.849 \\
\hline $8 / 22 / 1619: 00$ & 75.682 & 83.751 & 82.485 & 89.806 & 78.169 & 74.844 & 73.199 & 76.094 & 1582.79 & 1574.102 & 1570.823 & 1574.123 & 1570.459 & 1566.777 & 1564.092 & 1567.801 \\
\hline $8 / 22 / 1620: 00$ & 75.691 & 83.753 & 82.492 & 89.818 & 78.202 & 74.851 & 73.203 & 76.016 & 1582.781 & 1574.1 & 1570.816 & 1574.111 & 1570.426 & 1566.77 & 1564.088 & 1567.879 \\
\hline $8 / 22 / 1621: 00$ & 75.705 & 83.767 & 82.492 & 89.825 & 78.237 & 74.834 & 73.22 & 75.885 & 1582.767 & 1574.086 & 1570.816 & 1574.104 & 1570.391 & 1566.787 & 1564.071 & 1568.01 \\
\hline $8 / 22 / 1622: 00$ & 75.731 & 83.783 & 82.504 & 89.844 & 78.286 & 74.841 & 73.244 & 75.86 & 1582.741 & 1574.07 & 1570.804 & 1574.085 & 1570.342 & 1566.78 & 1564.047 & 1568.035 \\
\hline $8 / 22 / 1623: 00$ & 75.734 & 83.788 & 82.499 & 89.846 & 78.316 & 74.83 & 73.234 & 75.837 & 1582.738 & 1574.065 & 1570.809 & 1574.083 & 1570.312 & 1566.791 & 1564.057 & 1568.058 \\
\hline $8 / 23 / 160: 00$ & 75.734 & 83.783 & 82.49 & 89.844 & 78.337 & 74.818 & 73.23 & 75.772 & 1582.738 & 1574.07 & 1570.818 & 1574.085 & 1570.291 & 1566.803 & 1564.061 & 1568.123 \\
\hline $8 / 23 / 161: 00$ & 75.731 & 83.779 & 82.476 & 89.823 & 78.353 & 74.811 & 73.22 & 75.793 & 1582.741 & 1574.074 & 1570.832 & 1574.106 & 1570.275 & 1566.81 & 1564.071 & 1568.102 \\
\hline $8 / 23 / 162: 00$ & 75.727 & 83.769 & 82.464 & 89.802 & 78.374 & 74.797 & 73.211 & 75.769 & 1582.745 & 1574.084 & 1570.844 & 1574.127 & 1570.254 & 1566.824 & 1564.08 & 1568.126 \\
\hline $8 / 23 / 163: 00$ & 75.722 & 83.758 & 82.453 & 89.788 & 78.384 & 74.783 & 73.199 & 75.758 & 1582.75 & 1574.095 & 1570.855 & 1574.141 & 1570.244 & 1566.838 & 1564.092 & 1568.137 \\
\hline $8 / 23 / 164: 00$ & 75.727 & 83.758 & 82.448 & 89.788 & 78.402 & 74.781 & 73.199 & 75.737 & 1582.745 & 1574.095 & 1570.86 & 1574.141 & 1570.226 & 1566.84 & 1564.092 & 1568.158 \\
\hline $8 / 23 / 165: 00$ & 75.727 & 83.751 & 82.462 & 89.783 & 78.419 & 74.774 & 73.194 & 75.73 & 1582.745 & 1574.102 & 1570.846 & 1574.146 & 1570.209 & 1566.847 & 1564.097 & 1568.165 \\
\hline $8 / 23 / 166: 00$ & 75.731 & 83.748 & 82.453 & 89.783 & 78.437 & 74.769 & 73.196 & 75.709 & 1582.741 & 1574.105 & 1570.855 & 1574.146 & 1570.191 & 1566.852 & 1564.095 & 1568.186 \\
\hline $8 / 23 / 167: 00$ & 75.736 & 83.744 & 82.446 & 89.778 & 78.451 & 74.76 & 73.191 & 75.688 & 1582.736 & 1574.109 & 1570.862 & 1574.151 & 1570.177 & 1566.861 & 1564.1 & 1568.207 \\
\hline $8 / 23 / 168: 00$ & 75.75 & 83.76 & 82.868 & 89.788 & 78.5 & 74.772 & 73.201 & 75.888 & 1582.722 & 1574.093 & 1570.44 & 1574.141 & 1570.128 & 1566.849 & 1564.09 & 1568.007 \\
\hline 8/23/16 9:00 & 75.748 & 83.762 & 83.182 & 89.783 & 78.559 & 74.771 & 73.194 & 76.087 & 1582.724 & 1574.091 & 1570.126 & 1574.146 & 1570.069 & 1566.85 & 1564.097 & 1567.808 \\
\hline $8 / 23 / 1610: 00$ & 75.743 & 83.767 & 83.299 & 89.788 & 78.622 & 74.771 & 73.187 & 76.201 & 1582.729 & 1574.086 & 1570.009 & 1574.141 & 1570.006 & 1566.85 & 1564.104 & 1567.694 \\
\hline $8 / 23 / 1611: 00$ & 75.75 & 83.779 & 83.394 & 89.792 & 78.687 & 74.804 & 73.187 & 76.413 & 1582.722 & 1574.074 & 1569.914 & 1574.137 & 1569.941 & 1566.817 & 1564.104 & 1567.482 \\
\hline $8 / 23 / 1612: 00$ & 75.748 & 83.786 & 83.483 & 89.792 & 78.743 & 74.825 & 73.177 & 76.589 & 1582.724 & 1574.067 & 1569.825 & 1574.137 & 1569.885 & 1566.796 & 1564.114 & 1567.306 \\
\hline $8 / 23 / 1613: 00$ & 75.743 & 83.786 & 83.529 & 89.79 & 78.799 & 74.832 & 73.165 & 76.642 & 1582.729 & 1574.067 & 1569.779 & 1574.139 & 1569.829 & 1566.789 & 1564.126 & 1567.253 \\
\hline $8 / 23 / 1614: 00$ & 75.743 & 83.793 & 83.583 & 89.792 & 78.855 & 74.841 & 73.163 & 76.71 & 1582.729 & 1574.06 & 1569.725 & 1574.137 & 1569.773 & 1566.78 & 1564.128 & 1567.185 \\
\hline $8 / 23 / 1615: 00$ & 75.748 & 83.802 & 83.625 & 89.799 & 78.915 & 74.855 & 73.165 & 76.803 & 1582.724 & 1574.051 & 1569.683 & 1574.13 & 1569.713 & 1566.766 & 1564.126 & 1567.092 \\
\hline $8 / 23 / 1616: 00$ & 75.75 & 83.818 & 83.66 & 89.804 & 78.974 & 74.869 & 73.168 & 76.863 & 1582.722 & 1574.035 & 1569.648 & 1574.125 & 1569.654 & 1566.752 & 1564.123 & 1567.032 \\
\hline $8 / 23 / 1617: 00$ & 75.755 & 83.825 & 83.711 & 89.809 & 79.041 & 74.89 & 73.17 & 76.956 & 1582.717 & 1574.028 & 1569.597 & 1574.12 & 1569.587 & 1566.731 & 1564.121 & 1566.939 \\
\hline $8 / 23 / 1618: 00$ & 75.757 & 83.832 & 83.742 & 89.832 & 79.09 & 74.895 & 73.163 & 76.984 & 1582.715 & 1574.021 & 1569.566 & 1574.097 & 1569.538 & 1566.726 & 1564.128 & 1566.911 \\
\hline $8 / 23 / 1619: 00$ & 75.755 & 83.839 & 83.777 & 89.846 & 79.142 & 74.902 & 73.151 & 77.032 & 1582.717 & 1574.014 & 1569.531 & 1574.083 & 1569.486 & 1566.719 & 1564.14 & 1566.863 \\
\hline $8 / 23 / 1620: 00$ & 75.776 & 83.883 & 83.839 & 89.886 & 79.228 & 74.941 & 73.196 & 77.1 & 1582.696 & 1573.97 & 1569.469 & 1574.043 & 1569.4 & 1566.68 & 1564.095 & 1566.795 \\
\hline $8 / 23 / 1621: 00$ & 75.783 & 83.902 & 83.884 & 89.862 & 79.288 & 74.956 & 73.194 & 77.16 & 1582.689 & 1573.951 & 1569.424 & 1574.067 & 1569.34 & 1566.665 & 1564.097 & 1566.735 \\
\hline $8 / 23 / 1622: 00$ & 75.804 & 83.942 & 83.944 & 89.89 & 79.365 & 74.984 & 73.223 & 77.232 & 1582.668 & 1573.911 & 1569.364 & 1574.039 & 1569.263 & 1566.637 & 1564.068 & 1566.663 \\
\hline $8 / 23 / 1623: 00$ & 75.83 & 83.988 & 84.01 & 89.923 & 79.454 & 75.018 & 73.249 & 77.306 & 1582.642 & 1573.865 & 1569.298 & 1574.006 & 1569.174 & 1566.603 & 1564.042 & 1566.589 \\
\hline $8 / 24 / 160: 00$ & 75.854 & 84.044 & 84.073 & 89.949 & 79.543 & 75.056 & 73.285 & 77.378 & 1582.618 & 1573.809 & 1569.235 & 1573.98 & 1569.085 & 1566.565 & 1564.006 & 1566.517 \\
\hline $8 / 24 / 161: 00$ & 75.866 & 84.079 & 84.131 & 89.96 & 79.615 & 75.077 & 73.289 & 77.448 & 1582.606 & 1573.774 & 1569.177 & 1573.969 & 1569.013 & 1566.544 & 1564.002 & 1566.447 \\
\hline $8 / 24 / 162: 00$ & 75.852 & 84.088 & 84.247 & 89.953 & 79.662 & 75.074 & 73.265 & 77.494 & 1582.62 & 1573.765 & 1569.061 & 1573.976 & 1568.966 & 1566.547 & 1564.026 & 1566.401 \\
\hline $8 / 24 / 163: 00$ & 75.873 & 84.146 & 84.324 & 89.986 & 79.752 & 75.116 & 73.306 & 77.58 & 1582.599 & 1573.707 & 1568.984 & 1573.943 & 1568.876 & 1566.505 & 1563.985 & 1566.315 \\
\hline $8 / 24 / 164: 00$ & 75.877 & 84.174 & 84.376 & 89.998 & 79.815 & 75.133 & 73.306 & 77.631 & 1582.595 & 1573.679 & 1568.932 & 1573.931 & 1568.813 & 1566.488 & 1563.985 & 1566.264 \\
\hline $8 / 24 / 165: 00$ & 75.87 & 84.186 & 84.439 & 89.979 & 79.864 & 75.135 & 73.289 & 77.65 & 1582.602 & 1573.667 & 1568.869 & 1573.95 & 1568.764 & 1566.486 & 1564.002 & 1566.245 \\
\hline $8 / 24 / 166: 00$ & 75.877 & 84.223 & 84.427 & 89.991 & 79.927 & 75.13 & 73.304 & 77.604 & 1582.595 & 1573.63 & 1568.881 & 1573.938 & 1568.701 & 1566.491 & 1563.987 & 1566.291 \\
\hline $8 / 24 / 167: 00$ & 75.894 & 84.263 & 84.434 & 90.012 & 80 & 75.135 & 73.323 & 77.585 & 1582.578 & 1573.59 & 1568.874 & 1573.917 & 1568.628 & 1566.486 & 1563.968 & 1566.31 \\
\hline $8 / 24 / 168: 00$ & 75.906 & 84.297 & 84.473 & 90.03 & 80.063 & 75.149 & 73.335 & 77.615 & 1582.566 & 1573.556 & 1568.835 & 1573.899 & 1568.565 & 1566.472 & 1563.956 & 1566.28 \\
\hline $8 / 24 / 169: 00$ & 75.915 & 84.325 & 84.504 & 90.037 & 80.116 & 75.151 & 73.328 & 77.694 & 1582.557 & 1573.528 & 1568.804 & 1573.892 & 1568.512 & 1566.47 & 1563.963 & 1566.201 \\
\hline $8 / 24 / 1610: 00$ & 75.946 & 84.358 & 84.571 & 90.182 & 80.165 & 75.167 & 73.33 & 77.736 & 1582.526 & 1573.495 & 1568.737 & 1573.747 & 1568.463 & 1566.454 & 1563.961 & 1566.159 \\
\hline $8 / 24 / 1611: 00$ & 75.983 & 84.414 & 84.669 & 90.36 & 80.228 & 75.198 & 73.335 & 77.85 & 1582.489 & 1573.439 & 1568.639 & 1573.569 & 1568.4 & 1566.423 & 1563.956 & 1566.045 \\
\hline $8 / 24 / 1612: 00$ & 76.019 & 84.493 & 84.77 & 90.483 & 80.298 & 75.228 & 73.354 & 77.919 & 1582.453 & 1573.36 & 1568.538 & 1573.446 & 1568.33 & 1566.393 & 1563.937 & 1565.976 \\
\hline $8 / 24 / 1613: 00$ & 76.038 & 84.546 & 84.816 & 90.56 & 80.349 & 75.237 & 73.349 & 77.97 & 1582.434 & 1573.307 & 1568.492 & 1573.369 & 1568.279 & 1566.384 & 1563.942 & 1565.925 \\
\hline $8 / 24 / 1614: 00$ & 76.061 & 84.614 & 84.879 & 90.637 & 80.405 & 75.26 & 73.361 & 78.022 & 1582.411 & 1573.239 & 1568.429 & 1573.292 & 1568.223 & 1566.361 & 1563.93 & 1565.873 \\
\hline $8 / 24 / 1615: 00$ & 76.078 & 84.674 & 84.956 & 90.696 & 80.454 & 75.27 & 73.361 & 78.063 & 1582.394 & 1573.179 & 1568.352 & 1573.233 & 1568.174 & 1566.351 & 1563.93 & 1565.832 \\
\hline $8 / 24 / 1616: 00$ & 76.087 & 84.728 & 85.014 & 90.775 & 80.496 & 75.279 & 73.361 & 78.133 & 1582.385 & 1573.125 & 1568.294 & 1573.154 & 1568.132 & 1566.342 & 1563.93 & 1565.762 \\
\hline
\end{tabular}


TABLE S1.1 Automatically recorded groundwater levels in the unconfined aquifer.

\begin{tabular}{|c|c|c|c|c|c|c|c|c|c|c|c|c|c|c|c|c|}
\hline \multirow[b]{2}{*}{ Date and Time } & \multicolumn{8}{|c|}{ Measured Depth to Water (ft TOC ${ }^{\mathrm{a}}$ ) } & \multicolumn{8}{|c|}{ Groundwater Elevation ( $\mathrm{ft} \mathrm{AMSL}^{\mathrm{b}}$ ) } \\
\hline & DL1-C & DL2-C1 & DL3-C1 & DL4-C1 & DL5-C1 & DL6-C & DL7-C & DL8-C1 & DL1-C & DL2-C1 & DL3-C1 & DL4-C1 & DL5-C1 & DL6-C & DL7-C & DL8-C1 \\
\hline $8 / 24 / 1617: 00$ & 76.073 & 84.753 & 85.005 & 90.68 & 80.51 & 75.272 & 73.323 & 78.154 & 1582.399 & 1573.1 & 1568.303 & 1573.249 & 1568.118 & 1566.349 & 1563.968 & 1565.741 \\
\hline $8 / 24 / 1618: 00$ & 76.087 & 84.781 & 84.991 & 90.563 & 80.552 & 75.286 & 73.337 & 78.154 & 1582.385 & 1573.072 & 1568.317 & 1573.366 & 1568.076 & 1566.335 & 1563.954 & 1565.741 \\
\hline $8 / 24 / 1619: 00$ & 76.111 & 84.821 & 84.996 & 90.521 & 80.601 & 75.307 & 73.351 & 78.189 & 1582.361 & 1573.032 & 1568.312 & 1573.408 & 1568.027 & 1566.314 & 1563.94 & 1565.706 \\
\hline $8 / 24 / 1620: 00$ & 76.113 & 84.832 & 84.989 & 90.479 & 80.632 & 75.309 & 73.344 & 78.212 & 1582.359 & 1573.021 & 1568.319 & 1573.45 & 1567.996 & 1566.312 & 1563.947 & 1565.683 \\
\hline $8 / 24 / 1621: 00$ & 76.125 & 84.86 & 85 & 90.465 & 80.674 & 75.321 & 73.361 & 78.226 & 1582.347 & 1572.993 & 1568.308 & 1573.464 & 1567.954 & 1566.3 & 1563.93 & 1565.669 \\
\hline $8 / 24 / 1622: 00$ & 76.13 & 84.872 & 85.005 & 90.448 & 80.704 & 75.331 & 73.354 & 78.263 & 1582.342 & 1572.981 & 1568.303 & 1573.481 & 1567.924 & 1566.29 & 1563.937 & 1565.632 \\
\hline $8 / 24 / 1623: 00$ & 76.127 & 84.874 & 85.01 & 90.442 & 80.725 & 75.335 & 73.344 & 78.282 & 1582.345 & 1572.979 & 1568.298 & 1573.487 & 1567.903 & 1566.286 & 1563.947 & 1565.613 \\
\hline $8 / 25 / 160: 00$ & 76.134 & 84.891 & 85.028 & 90.432 & 80.755 & 75.347 & 73.351 & 78.312 & 1582.338 & 1572.962 & 1568.28 & 1573.497 & 1567.873 & 1566.274 & 1563.94 & 1565.583 \\
\hline $8 / 25 / 161: 00$ & 76.139 & 84.909 & 85.056 & 90.416 & 80.792 & 75.358 & 73.361 & 78.342 & 1582.333 & 1572.944 & 1568.252 & 1573.513 & 1567.836 & 1566.263 & 1563.93 & 1565.553 \\
\hline $8 / 25 / 162: 00$ & 76.153 & 84.935 & 85.084 & 90.409 & 80.832 & 75.372 & 73.371 & 78.372 & 1582.319 & 1572.918 & 1568.224 & 1573.52 & 1567.796 & 1566.249 & 1563.92 & 1565.523 \\
\hline $8 / 25 / 163: 00$ & 76.148 & 84.93 & 85.093 & 90.395 & 80.853 & 75.375 & 73.361 & 78.4 & 1582.324 & 1572.923 & 1568.215 & 1573.534 & 1567.775 & 1566.246 & 1563.93 & 1565.495 \\
\hline $8 / 25 / 164: 00$ & 76.155 & 84.944 & 85.103 & 90.397 & 80.886 & 75.384 & 73.363 & 78.423 & 1582.317 & 1572.909 & 1568.205 & 1573.532 & 1567.742 & 1566.237 & 1563.928 & 1565.472 \\
\hline $8 / 25 / 165: 00$ & 76.16 & 84.958 & 85.112 & 90.395 & 80.911 & 75.396 & 73.371 & 78.439 & 1582.312 & 1572.895 & 1568.196 & 1573.534 & 1567.717 & 1566.225 & 1563.92 & 1565.456 \\
\hline $8 / 25 / 166: 00$ & 76.17 & 84.97 & 85.133 & 90.397 & 80.944 & 75.405 & 73.378 & 78.456 & 1582.302 & 1572.883 & 1568.175 & 1573.532 & 1567.684 & 1566.216 & 1563.913 & 1565.439 \\
\hline $8 / 25 / 167: 00$ & 76.184 & 84.988 & 85.147 & 90.406 & 80.977 & 75.421 & 73.385 & 78.481 & 1582.288 & 1572.865 & 1568.161 & 1573.523 & 1567.651 & 1566.2 & 1563.906 & 1565.414 \\
\hline 8/25/16 8:00 & 76.184 & 84.995 & 85.184 & 90.404 & 81 & 75.421 & 73.385 & 78.507 & 1582.288 & 1572.858 & 1568.124 & 1573.525 & 1567.628 & 1566.2 & 1563.906 & 1565.388 \\
\hline 8/25/16 9:00 & 76.191 & 85.021 & 85.189 & 90.416 & 81.033 & 75.438 & 73.397 & 78.579 & 1582.281 & 1572.832 & 1568.119 & 1573.513 & 1567.595 & 1566.183 & 1563.894 & 1565.316 \\
\hline $8 / 25 / 1610: 00$ & 76.212 & 85.056 & 85.24 & 90.502 & 81.075 & 75.463 & 73.423 & 78.588 & 1582.26 & 1572.797 & 1568.068 & 1573.427 & 1567.553 & 1566.158 & 1563.868 & 1565.307 \\
\hline $8 / 25 / 1611: 00$ & 76.221 & 85.098 & 85.329 & 90.68 & 81.107 & 75.487 & 73.425 & 78.718 & 1582.251 & 1572.755 & 1567.979 & 1573.249 & 1567.521 & 1566.134 & 1563.866 & 1565.177 \\
\hline $8 / 25 / 1612: 00$ & 76.224 & 85.149 & 85.394 & 90.787 & 81.14 & 75.489 & 73.428 & 78.693 & 1582.248 & 1572.704 & 1567.914 & 1573.142 & 1567.488 & 1566.132 & 1563.863 & 1565.202 \\
\hline $8 / 25 / 1613: 00$ & 76.214 & 85.17 & 85.429 & 90.848 & 81.152 & 75.477 & 73.409 & 78.648 & 1582.258 & 1572.683 & 1567.879 & 1573.081 & 1567.476 & 1566.144 & 1563.882 & 1565.247 \\
\hline $8 / 25 / 1614: 00$ & 76.221 & 85.2 & 85.464 & 90.899 & 81.173 & 75.475 & 73.399 & 78.658 & 1582.251 & 1572.653 & 1567.844 & 1573.03 & 1567.455 & 1566.146 & 1563.892 & 1565.237 \\
\hline $8 / 25 / 1615: 00$ & 76.231 & 85.232 & 85.494 & 90.943 & 81.196 & 75.477 & 73.39 & 78.69 & 1582.241 & 1572.621 & 1567.814 & 1572.986 & 1567.432 & 1566.144 & 1563.901 & 1565.205 \\
\hline $8 / 25 / 1616: 00$ & 76.25 & 85.27 & 85.525 & 90.988 & 81.226 & 75.477 & 73.392 & 78.672 & 1582.222 & 1572.583 & 1567.783 & 1572.941 & 1567.402 & 1566.144 & 1563.899 & 1565.223 \\
\hline $8 / 25 / 1617: 00$ & 76.262 & 85.307 & 85.576 & 91.013 & 81.254 & 75.494 & 73.39 & 78.755 & 1582.21 & 1572.546 & 1567.732 & 1572.916 & 1567.374 & 1566.127 & 1563.901 & 1565.14 \\
\hline $8 / 25 / 1618: 00$ & 76.269 & 85.314 & 85.546 & 90.876 & 81.28 & 75.477 & 73.378 & 78.704 & 1582.203 & 1572.539 & 1567.762 & 1573.053 & 1567.348 & 1566.144 & 1563.913 & 1565.191 \\
\hline 8/25/16 19:00 & 76.283 & 85.325 & 85.527 & 90.806 & 81.303 & 75.484 & 73.38 & 78.704 & 1582.189 & 1572.528 & 1567.781 & 1573.123 & 1567.325 & 1566.137 & 1563.911 & 1565.191 \\
\hline $8 / 25 / 1620: 00$ & 76.302 & 85.344 & 85.52 & 90.771 & 81.336 & 75.493 & 73.397 & 78.72 & 1582.17 & 1572.509 & 1567.788 & 1573.158 & 1567.292 & 1566.128 & 1563.894 & 1565.175 \\
\hline $8 / 25 / 1621: 00$ & 76.337 & 85.377 & 85.534 & 90.736 & 81.385 & 75.521 & 73.428 & 78.751 & 1582.135 & 1572.476 & 1567.774 & 1573.193 & 1567.243 & 1566.1 & 1563.863 & 1565.144 \\
\hline $8 / 25 / 1622: 00$ & 76.363 & 85.395 & 85.553 & 90.722 & 81.427 & 75.531 & 73.437 & 78.783 & 1582.109 & 1572.458 & 1567.755 & 1573.207 & 1567.201 & 1566.09 & 1563.854 & 1565.112 \\
\hline $8 / 25 / 1623: 00$ & 76.37 & 85.412 & 85.555 & 90.722 & 81.459 & 75.538 & 73.442 & 78.804 & 1582.102 & 1572.441 & 1567.753 & 1573.207 & 1567.169 & 1566.083 & 1563.849 & 1565.091 \\
\hline $8 / 26 / 160: 00$ & 76.361 & 85.412 & 85.56 & 90.701 & 81.473 & 75.538 & 73.435 & 78.804 & 1582.111 & 1572.441 & 1567.748 & 1573.228 & 1567.155 & 1566.083 & 1563.856 & 1565.091 \\
\hline $8 / 26 / 161: 00$ & 76.349 & 85.416 & 85.557 & 90.694 & 81.485 & 75.54 & 73.437 & 78.82 & 1582.123 & 1572.437 & 1567.751 & 1573.235 & 1567.143 & 1566.081 & 1563.854 & 1565.075 \\
\hline $8 / 26 / 162: 00$ & 76.339 & 85.421 & 85.646 & 90.691 & 81.497 & 75.545 & 73.435 & 78.836 & 1582.133 & 1572.432 & 1567.662 & 1573.238 & 1567.131 & 1566.076 & 1563.856 & 1565.059 \\
\hline 8/26/16 3:00 & 76.313 & 85.407 & 85.641 & 90.665 & 81.49 & 75.533 & 73.414 & 78.846 & 1582.159 & 1572.446 & 1567.667 & 1573.264 & 1567.138 & 1566.088 & 1563.877 & 1565.049 \\
\hline $8 / 26 / 164: 00$ & 76.304 & 85.405 & 85.644 & 90.67 & 81.494 & 75.538 & 73.411 & 78.855 & 1582.168 & 1572.448 & 1567.664 & 1573.259 & 1567.134 & 1566.083 & 1563.88 & 1565.04 \\
\hline $8 / 26 / 165: 00$ & 76.311 & 85.419 & 85.655 & 90.656 & 81.513 & 75.549 & 73.43 & 78.874 & 1582.161 & 1572.434 & 1567.653 & 1573.273 & 1567.115 & 1566.072 & 1563.861 & 1565.021 \\
\hline $8 / 26 / 166: 00$ & 76.316 & 85.428 & 85.18 & 90.651 & 81.499 & 75.556 & 73.44 & 78.628 & 1582.156 & 1572.425 & 1568.128 & 1573.278 & 1567.129 & 1566.065 & 1563.851 & 1565.267 \\
\hline $8 / 26 / 167: 00$ & 76.328 & 85.432 & 84.909 & 90.651 & 81.464 & 75.547 & 73.452 & 78.463 & 1582.144 & 1572.421 & 1568.399 & 1573.278 & 1567.164 & 1566.074 & 1563.839 & 1565.432 \\
\hline 8/26/16 8:00 & 76.306 & 85.402 & 84.76 & 90.623 & 81.385 & 75.5 & 73.423 & 78.247 & 1582.166 & 1572.451 & 1568.548 & 1573.306 & 1567.243 & 1566.121 & 1563.868 & 1565.648 \\
\hline 8/26/16 9:00 & 76.304 & 85.386 & 84.672 & 90.621 & 81.336 & 75.493 & 73.428 & 78.27 & 1582.168 & 1572.467 & 1568.636 & 1573.308 & 1567.292 & 1566.128 & 1563.863 & 1565.625 \\
\hline $8 / 26 / 1610: 00$ & 76.299 & 85.384 & 84.606 & 90.614 & 81.291 & 75.482 & 73.425 & 78.063 & 1582.173 & 1572.469 & 1568.702 & 1573.315 & 1567.337 & 1566.139 & 1563.866 & 1565.832 \\
\hline $8 / 26 / 1611: 00$ & 76.285 & 85.356 & 84.534 & 90.593 & 81.242 & 75.463 & 73.414 & 78.049 & 1582.187 & 1572.497 & 1568.774 & 1573.336 & 1567.386 & 1566.158 & 1563.877 & 1565.846 \\
\hline $8 / 26 / 1612: 00$ & 76.28 & 85.346 & 84.476 & 90.589 & 81.205 & 75.442 & 73.406 & 77.894 & 1582.192 & 1572.507 & 1568.832 & 1573.34 & 1567.423 & 1566.179 & 1563.885 & 1566.001 \\
\hline $8 / 26 / 1613: 00$ & 76.276 & 85.321 & 84.42 & 90.574 & 81.168 & 75.426 & 73.404 & 77.81 & 1582.196 & 1572.532 & 1568.888 & 1573.355 & 1567.46 & 1566.195 & 1563.887 & 1566.085 \\
\hline $8 / 26 / 1614: 00$ & 76.266 & 85.3 & 84.364 & 90.563 & 81.121 & 75.407 & 73.394 & 77.752 & 1582.206 & 1572.553 & 1568.944 & 1573.366 & 1567.507 & 1566.214 & 1563.897 & 1566.143 \\
\hline $8 / 26 / 1615: 00$ & 76.266 & 85.288 & 84.38 & 90.56 & 81.091 & 75.403 & 73.397 & 77.813 & 1582.206 & 1572.565 & 1568.928 & 1573.369 & 1567.537 & 1566.218 & 1563.894 & 1566.082 \\
\hline $8 / 26 / 1616: 00$ & 76.266 & 85.272 & 84.303 & 90.553 & 81.054 & 75.389 & 73.397 & 77.657 & 1582.206 & 1572.581 & 1569.005 & 1573.376 & 1567.574 & 1566.232 & 1563.894 & 1566.238 \\
\hline $8 / 26 / 1617: 00$ & 76.257 & 85.249 & 84.254 & 90.537 & 81.014 & 75.372 & 73.385 & 77.587 & 1582.215 & 1572.604 & 1569.054 & 1573.392 & 1567.614 & 1566.249 & 1563.906 & 1566.308 \\
\hline
\end{tabular}


TABLE S1.1 Automatically recorded groundwater levels in the unconfined aquifer.

\begin{tabular}{|c|c|c|c|c|c|c|c|c|c|c|c|c|c|c|c|c|}
\hline \multirow[b]{2}{*}{ Date and Time } & \multicolumn{8}{|c|}{ Measured Depth to Water (ft TOC ${ }^{\mathrm{a}}$ ) } & \multicolumn{8}{|c|}{ Groundwater Elevation ( $\mathrm{ft} \mathrm{AMSL}^{\mathrm{b}}$ ) } \\
\hline & DL1-C & DL2-C1 & DL3-C1 & DL4-C1 & DL5-C1 & DL6-C & DL7-C & DL8-C1 & DL1-C & DL2-C1 & DL3-C1 & DL4-C1 & DL5-C1 & DL6-C & DL7-C & DL8-C1 \\
\hline $8 / 26 / 1618: 00$ & 76.25 & 85.226 & 84.196 & 90.523 & 80.967 & 75.351 & 73.375 & 77.525 & 1582.222 & 1572.627 & 1569.112 & 1573.406 & 1567.661 & 1566.27 & 1563.916 & 1566.37 \\
\hline 8/26/16 19:00 & 76.252 & 85.212 & 84.163 & 90.535 & 80.939 & 75.344 & 73.378 & 77.488 & 1582.22 & 1572.641 & 1569.145 & 1573.394 & 1567.689 & 1566.277 & 1563.913 & 1566.407 \\
\hline $8 / 26 / 1620: 00$ & 76.266 & 85.214 & 84.143 & 90.549 & 80.923 & 75.347 & 73.399 & 77.46 & 1582.206 & 1572.639 & 1569.165 & 1573.38 & 1567.705 & 1566.274 & 1563.892 & 1566.435 \\
\hline 8/26/16 21:00 & 76.283 & 85.221 & 84.131 & 90.556 & 80.909 & 75.351 & 73.418 & 77.425 & 1582.189 & 1572.632 & 1569.177 & 1573.373 & 1567.719 & 1566.27 & 1563.873 & 1566.47 \\
\hline 8/26/16 22:00 & 76.276 & 85.205 & 84.101 & 90.549 & 80.879 & 75.335 & 73.406 & 77.399 & 1582.196 & 1572.648 & 1569.207 & 1573.38 & 1567.749 & 1566.286 & 1563.885 & 1566.496 \\
\hline $8 / 26 / 1623: 00$ & 76.269 & 85.186 & 84.07 & 90.549 & 80.846 & 75.324 & 73.402 & 77.36 & 1582.203 & 1572.667 & 1569.238 & 1573.38 & 1567.782 & 1566.297 & 1563.889 & 1566.535 \\
\hline $8 / 27 / 160: 00$ & 76.276 & 85.186 & 84.052 & 90.549 & 80.827 & 75.324 & 73.411 & 77.339 & 1582.196 & 1572.667 & 1569.256 & 1573.38 & 1567.801 & 1566.297 & 1563.88 & 1566.556 \\
\hline $8 / 27 / 161: 00$ & 76.264 & 85.167 & 84.019 & 90.518 & 80.795 & 75.305 & 73.394 & 77.29 & 1582.208 & 1572.686 & 1569.289 & 1573.411 & 1567.833 & 1566.316 & 1563.897 & 1566.605 \\
\hline $8 / 27 / 162: 00$ & 76.245 & 85.139 & 83.979 & 90.49 & 80.753 & 75.282 & 73.378 & 77.232 & 1582.227 & 1572.714 & 1569.329 & 1573.439 & 1567.875 & 1566.339 & 1563.913 & 1566.663 \\
\hline $8 / 27 / 163: 00$ & 76.233 & 85.123 & 83.954 & 90.479 & 80.72 & 75.272 & 73.371 & 77.221 & 1582.239 & 1572.73 & 1569.354 & 1573.45 & 1567.908 & 1566.349 & 1563.92 & 1566.674 \\
\hline $8 / 27 / 164: 00$ & 76.243 & 85.116 & 83.944 & 90.479 & 80.708 & 75.279 & 73.394 & 77.207 & 1582.229 & 1572.737 & 1569.364 & 1573.45 & 1567.92 & 1566.342 & 1563.897 & 1566.688 \\
\hline $8 / 27 / 165: 00$ & 76.247 & 85.116 & 83.949 & 90.483 & 80.692 & 75.279 & 73.404 & 77.181 & 1582.225 & 1572.737 & 1569.359 & 1573.446 & 1567.936 & 1566.342 & 1563.887 & 1566.714 \\
\hline $8 / 27 / 166: 00$ & 76.254 & 85.119 & 83.935 & 90.483 & 80.685 & 75.279 & 73.414 & 77.162 & 1582.218 & 1572.734 & 1569.373 & 1573.446 & 1567.943 & 1566.342 & 1563.877 & 1566.733 \\
\hline $8 / 27 / 167: 00$ & 76.257 & 85.116 & 83.926 & 90.486 & 80.674 & 75.279 & 73.423 & 77.144 & 1582.215 & 1572.737 & 1569.382 & 1573.443 & 1567.954 & 1566.342 & 1563.868 & 1566.751 \\
\hline $8 / 27 / 168: 00$ & 76.264 & 85.118 & 83.921 & 90.486 & 80.667 & 75.284 & 73.428 & 77.132 & 1582.208 & 1572.735 & 1569.387 & 1573.443 & 1567.961 & 1566.337 & 1563.863 & 1566.763 \\
\hline 8/27/169:00 & 76.264 & 85.121 & 83.914 & 90.486 & 80.653 & 75.284 & 73.44 & 77.211 & 1582.208 & 1572.732 & 1569.394 & 1573.443 & 1567.975 & 1566.337 & 1563.851 & 1566.684 \\
\hline $8 / 27 / 1610: 00$ & 76.262 & 85.116 & 83.905 & 90.481 & 80.625 & 75.282 & 73.435 & 77.125 & 1582.21 & 1572.737 & 1569.403 & 1573.448 & 1568.003 & 1566.339 & 1563.856 & 1566.77 \\
\hline $8 / 27 / 1611: 00$ & 76.252 & 85.1 & 83.886 & 90.476 & 80.58 & 75.272 & 73.428 & 77.139 & 1582.22 & 1572.753 & 1569.422 & 1573.453 & 1568.048 & 1566.349 & 1563.863 & 1566.756 \\
\hline $8 / 27 / 1612: 00$ & 76.238 & 85.088 & 83.872 & 90.465 & 80.536 & 75.256 & 73.418 & 77.067 & 1582.234 & 1572.765 & 1569.436 & 1573.464 & 1568.092 & 1566.365 & 1563.873 & 1566.828 \\
\hline $8 / 27 / 1613: 00$ & 76.231 & 85.074 & 83.823 & 90.458 & 80.499 & 75.249 & 73.414 & 77.039 & 1582.241 & 1572.779 & 1569.485 & 1573.471 & 1568.129 & 1566.372 & 1563.877 & 1566.856 \\
\hline $8 / 27 / 1614: 00$ & 76.226 & 85.063 & 83.8 & 90.453 & 80.461 & 75.242 & 73.414 & 77.012 & 1582.246 & 1572.79 & 1569.508 & 1573.476 & 1568.167 & 1566.379 & 1563.877 & 1566.883 \\
\hline $8 / 27 / 1615: 00$ & 76.217 & 85.042 & 83.772 & 90.441 & 80.417 & 75.23 & 73.404 & 76.986 & 1582.255 & 1572.811 & 1569.536 & 1573.488 & 1568.211 & 1566.391 & 1563.887 & 1566.909 \\
\hline $8 / 27 / 1616: 00$ & 76.214 & 85.021 & 83.744 & 90.43 & 80.37 & 75.221 & 73.397 & 76.965 & 1582.258 & 1572.832 & 1569.564 & 1573.499 & 1568.258 & 1566.4 & 1563.894 & 1566.93 \\
\hline $8 / 27 / 1617: 00$ & 76.207 & 85.002 & 83.718 & 90.425 & 80.328 & 75.207 & 73.387 & 76.94 & 1582.265 & 1572.851 & 1569.59 & 1573.504 & 1568.3 & 1566.414 & 1563.904 & 1566.955 \\
\hline $8 / 27 / 1618: 00$ & 76.224 & 84.988 & 83.693 & 90.439 & 80.298 & 75.202 & 73.387 & 76.914 & 1582.248 & 1572.865 & 1569.615 & 1573.49 & 1568.33 & 1566.419 & 1563.904 & 1566.981 \\
\hline 8/27/16 19:00 & 76.247 & 84.979 & 83.676 & 90.448 & 80.282 & 75.202 & 73.39 & 76.891 & 1582.225 & 1572.874 & 1569.632 & 1573.481 & 1568.346 & 1566.419 & 1563.901 & 1567.004 \\
\hline $8 / 27 / 1620: 00$ & 76.271 & 84.977 & 83.662 & 90.453 & 80.258 & 75.198 & 73.399 & 76.87 & 1582.201 & 1572.876 & 1569.646 & 1573.476 & 1568.37 & 1566.423 & 1563.892 & 1567.025 \\
\hline $8 / 27 / 1621: 00$ & 76.302 & 84.988 & 83.66 & 90.444 & 80.258 & 75.209 & 73.418 & 76.861 & 1582.17 & 1572.865 & 1569.648 & 1573.485 & 1568.37 & 1566.412 & 1563.873 & 1567.034 \\
\hline $8 / 27 / 1622: 00$ & 76.335 & 85.009 & 83.665 & 90.451 & 80.263 & 75.221 & 73.445 & 76.858 & 1582.137 & 1572.844 & 1569.643 & 1573.478 & 1568.365 & 1566.4 & 1563.846 & 1567.037 \\
\hline $8 / 27 / 1623: 00$ & 76.361 & 85.021 & 83.667 & 90.472 & 80.263 & 75.23 & 73.456 & 76.858 & 1582.111 & 1572.832 & 1569.641 & 1573.457 & 1568.365 & 1566.391 & 1563.835 & 1567.037 \\
\hline $8 / 28 / 160: 00$ & 76.368 & 85.014 & 83.658 & 90.465 & 80.254 & 75.223 & 73.452 & 76.854 & 1582.104 & 1572.839 & 1569.65 & 1573.464 & 1568.374 & 1566.398 & 1563.839 & 1567.041 \\
\hline $8 / 28 / 161: 00$ & 76.363 & 85.002 & 83.639 & 90.453 & 80.233 & 75.212 & 73.442 & 76.826 & 1582.109 & 1572.851 & 1569.669 & 1573.476 & 1568.395 & 1566.409 & 1563.849 & 1567.069 \\
\hline $8 / 28 / 162: 00$ & 76.353 & 84.991 & 83.62 & 90.446 & 80.205 & 75.2 & 73.433 & 76.807 & 1582.119 & 1572.862 & 1569.688 & 1573.483 & 1568.423 & 1566.421 & 1563.858 & 1567.088 \\
\hline 8/28/16 3:00 & 76.337 & 84.981 & 83.604 & 90.434 & 80.172 & 75.193 & 73.425 & 76.796 & 1582.135 & 1572.872 & 1569.704 & 1573.495 & 1568.456 & 1566.428 & 1563.866 & 1567.099 \\
\hline $8 / 28 / 164: 00$ & 76.325 & 84.977 & 83.597 & 90.441 & 80.154 & 75.198 & 73.437 & 76.789 & 1582.147 & 1572.876 & 1569.711 & 1573.488 & 1568.474 & 1566.423 & 1563.854 & 1567.106 \\
\hline $8 / 28 / 165: 00$ & 76.313 & 84.972 & 83.585 & 90.416 & 80.128 & 75.193 & 73.43 & 76.772 & 1582.159 & 1572.881 & 1569.723 & 1573.513 & 1568.5 & 1566.428 & 1563.861 & 1567.123 \\
\hline $8 / 28 / 166: 00$ & 76.302 & 84.958 & 83.567 & 90.402 & 80.1 & 75.181 & 73.428 & 76.712 & 1582.17 & 1572.895 & 1569.741 & 1573.527 & 1568.528 & 1566.44 & 1563.863 & 1567.183 \\
\hline $8 / 28 / 167: 00$ & 76.299 & 84.956 & 83.562 & 90.4 & 80.079 & 75.186 & 73.435 & 76.74 & 1582.173 & 1572.897 & 1569.746 & 1573.529 & 1568.549 & 1566.435 & 1563.856 & 1567.155 \\
\hline 8/28/16 8:00 & 76.302 & 84.96 & 83.557 & 90.404 & 80.063 & 75.191 & 73.447 & 76.74 & 1582.17 & 1572.893 & 1569.751 & 1573.525 & 1568.565 & 1566.43 & 1563.844 & 1567.155 \\
\hline 8/28/16 9:00 & 76.295 & 84.953 & 83.546 & 90.397 & 80.044 & 75.186 & 73.447 & 76.721 & 1582.177 & 1572.9 & 1569.762 & 1573.532 & 1568.584 & 1566.435 & 1563.844 & 1567.174 \\
\hline $8 / 28 / 1610: 00$ & 76.29 & 84.949 & 83.534 & 90.392 & 80.014 & 75.184 & 73.445 & 76.707 & 1582.182 & 1572.904 & 1569.774 & 1573.537 & 1568.614 & 1566.437 & 1563.846 & 1567.188 \\
\hline $8 / 28 / 1611: 00$ & 76.283 & 84.932 & 83.52 & 90.383 & 79.988 & 75.177 & 73.442 & 76.7 & 1582.189 & 1572.921 & 1569.788 & 1573.546 & 1568.64 & 1566.444 & 1563.849 & 1567.195 \\
\hline $8 / 28 / 1612: 00$ & 76.271 & 84.925 & 83.506 & 90.378 & 79.962 & 75.172 & 73.44 & 76.679 & 1582.201 & 1572.928 & 1569.802 & 1573.551 & 1568.666 & 1566.449 & 1563.851 & 1567.216 \\
\hline 8/28/16 13:00 & 76.262 & 84.911 & 83.485 & 90.367 & 79.93 & 75.163 & 73.43 & 76.656 & 1582.21 & 1572.942 & 1569.823 & 1573.562 & 1568.698 & 1566.458 & 1563.861 & 1567.239 \\
\hline $8 / 28 / 1614: 00$ & 76.243 & 84.884 & 83.464 & 90.353 & 79.89 & 75.147 & 73.409 & 76.64 & 1582.229 & 1572.969 & 1569.844 & 1573.576 & 1568.738 & 1566.474 & 1563.882 & 1567.255 \\
\hline $8 / 28 / 1615: 00$ & 76.221 & 84.858 & 83.434 & 90.339 & 79.845 & 75.128 & 73.392 & 76.61 & 1582.251 & 1572.995 & 1569.874 & 1573.59 & 1568.783 & 1566.493 & 1563.899 & 1567.285 \\
\hline $8 / 28 / 1616: 00$ & 76.21 & 84.83 & 83.406 & 90.327 & 79.806 & 75.116 & 73.38 & 76.582 & 1582.262 & 1573.023 & 1569.902 & 1573.602 & 1568.822 & 1566.505 & 1563.911 & 1567.313 \\
\hline $8 / 28 / 1617: 00$ & 76.2 & 84.807 & 83.385 & 90.313 & 79.771 & 75.107 & 73.375 & 76.563 & 1582.272 & 1573.046 & 1569.923 & 1573.616 & 1568.857 & 1566.514 & 1563.916 & 1567.332 \\
\hline $8 / 28 / 1618: 00$ & 76.203 & 84.8 & 83.376 & 90.32 & 79.748 & 75.114 & 73.385 & 76.559 & 1582.269 & 1573.053 & 1569.932 & 1573.609 & 1568.88 & 1566.507 & 1563.906 & 1567.336 \\
\hline
\end{tabular}


TABLE S1.1 Automatically recorded groundwater levels in the unconfined aquifer.

\begin{tabular}{|c|c|c|c|c|c|c|c|c|c|c|c|c|c|c|c|c|}
\hline \multirow[b]{2}{*}{ Date and Time } & \multicolumn{8}{|c|}{ Measured Depth to Water (ft TOC ${ }^{\mathrm{a}}$ ) } & \multicolumn{8}{|c|}{ Groundwater Elevation ( $\mathrm{ft} \mathrm{AMSL}^{\mathrm{b}}$ ) } \\
\hline & DL1-C & DL2-C1 & DL3-C1 & DL4-C1 & DL5-C1 & DL6-C & DL7-C & DL8-C1 & DL1-C & DL2-C1 & DL3-C1 & DL4-C1 & DL5-C1 & DL6-C & DL7-C & DL8-C1 \\
\hline 8/28/16 19:00 & 76.2 & 84.791 & 83.366 & 90.325 & 79.722 & 75.111 & 73.392 & 76.543 & 1582.272 & 1573.062 & 1569.942 & 1573.604 & 1568.906 & 1566.51 & 1563.899 & 1567.352 \\
\hline $8 / 28 / 1620: 00$ & 76.221 & 84.811 & 83.378 & 90.353 & 79.722 & 75.133 & 73.428 & 76.54 & 1582.251 & 1573.042 & 1569.93 & 1573.576 & 1568.906 & 1566.488 & 1563.863 & 1567.355 \\
\hline $8 / 28 / 1621: 00$ & 76.226 & 84.809 & 83.373 & 90.35 & 79.703 & 75.135 & 73.435 & 76.54 & 1582.246 & 1573.044 & 1569.935 & 1573.579 & 1568.925 & 1566.486 & 1563.856 & 1567.355 \\
\hline $8 / 28 / 1622: 00$ & 76.245 & 84.83 & 83.387 & 90.369 & 79.706 & 75.151 & 73.461 & 76.55 & 1582.227 & 1573.023 & 1569.921 & 1573.56 & 1568.922 & 1566.47 & 1563.83 & 1567.345 \\
\hline $8 / 28 / 1623: 00$ & 76.24 & 84.823 & 83.378 & 90.369 & 79.683 & 75.146 & 73.459 & 76.54 & 1582.232 & 1573.03 & 1569.93 & 1573.56 & 1568.945 & 1566.475 & 1563.832 & 1567.355 \\
\hline $8 / 29 / 160: 00$ & 76.238 & 84.821 & 83.371 & 90.367 & 79.666 & 75.146 & 73.459 & 76.533 & 1582.234 & 1573.032 & 1569.937 & 1573.562 & 1568.962 & 1566.475 & 1563.832 & 1567.362 \\
\hline 8/29/16 1:00 & 76.224 & 84.807 & 83.355 & 90.341 & 79.631 & 75.135 & 73.445 & 76.517 & 1582.248 & 1573.046 & 1569.953 & 1573.588 & 1568.997 & 1566.486 & 1563.846 & 1567.378 \\
\hline $8 / 29 / 162: 00$ & 76.219 & 84.798 & 83.341 & 90.322 & 79.608 & 75.13 & 73.445 & 76.478 & 1582.253 & 1573.055 & 1569.967 & 1573.607 & 1569.02 & 1566.491 & 1563.846 & 1567.417 \\
\hline 8/29/16 3:00 & 76.205 & 84.774 & 83.327 & 90.306 & 79.575 & 75.118 & 73.43 & 76.491 & 1582.267 & 1573.079 & 1569.981 & 1573.623 & 1569.053 & 1566.503 & 1563.861 & 1567.404 \\
\hline 8/29/16 4:00 & 76.179 & 84.746 & 83.294 & 90.283 & 79.526 & 75.098 & 73.406 & 76.429 & 1582.293 & 1573.107 & 1570.014 & 1573.646 & 1569.102 & 1566.523 & 1563.885 & 1567.466 \\
\hline 8/29/16 5:00 & 76.186 & 84.746 & 83.31 & 90.292 & 79.505 & 75.107 & 73.423 & 76.459 & 1582.286 & 1573.107 & 1569.998 & 1573.637 & 1569.123 & 1566.514 & 1563.868 & 1567.436 \\
\hline $8 / 29 / 166: 00$ & 76.193 & 84.746 & 83.306 & 90.292 & 79.491 & 75.111 & 73.433 & 76.443 & 1582.279 & 1573.107 & 1570.002 & 1573.637 & 1569.137 & 1566.51 & 1563.858 & 1567.452 \\
\hline $8 / 29 / 167: 00$ & 76.2 & 84.744 & 83.301 & 90.297 & 79.477 & 75.111 & 73.442 & 76.44 & 1582.272 & 1573.109 & 1570.007 & 1573.632 & 1569.151 & 1566.51 & 1563.849 & 1567.455 \\
\hline $8 / 29 / 168: 00$ & 76.21 & 84.751 & 83.306 & 90.301 & 79.463 & 75.119 & 73.452 & 76.438 & 1582.262 & 1573.102 & 1570.002 & 1573.628 & 1569.165 & 1566.502 & 1563.839 & 1567.457 \\
\hline 8/29/16 9:00 & 76.214 & 84.744 & 83.303 & 90.301 & 79.442 & 75.125 & 73.452 & 76.522 & 1582.258 & 1573.109 & 1570.005 & 1573.628 & 1569.186 & 1566.496 & 1563.839 & 1567.373 \\
\hline $8 / 29 / 1610: 00$ & 76.212 & 84.737 & 83.31 & 90.297 & 79.424 & 75.137 & 73.449 & 76.619 & 1582.26 & 1573.116 & 1569.998 & 1573.632 & 1569.204 & 1566.484 & 1563.842 & 1567.276 \\
\hline $8 / 29 / 1611: 00$ & 76.207 & 84.725 & 83.331 & 90.297 & 79.4 & 75.116 & 73.447 & 76.454 & 1582.265 & 1573.128 & 1569.977 & 1573.632 & 1569.228 & 1566.505 & 1563.844 & 1567.441 \\
\hline $8 / 29 / 1612: 00$ & 76.203 & 84.721 & 83.371 & 90.297 & 79.379 & 75.119 & 73.445 & 76.556 & 1582.269 & 1573.132 & 1569.937 & 1573.632 & 1569.249 & 1566.502 & 1563.846 & 1567.339 \\
\hline $8 / 29 / 1613: 00$ & 76.186 & 84.702 & 83.334 & 90.285 & 79.347 & 75.107 & 73.433 & 76.487 & 1582.286 & 1573.151 & 1569.974 & 1573.644 & 1569.281 & 1566.514 & 1563.858 & 1567.408 \\
\hline $8 / 29 / 1614: 00$ & 76.163 & 84.674 & 83.315 & 90.269 & 79.307 & 75.09 & 73.411 & 76.443 & 1582.309 & 1573.179 & 1569.993 & 1573.66 & 1569.321 & 1566.531 & 1563.88 & 1567.452 \\
\hline $8 / 29 / 1615: 00$ & 76.137 & 84.642 & 83.31 & 90.25 & 79.26 & 75.065 & 73.385 & 76.389 & 1582.335 & 1573.211 & 1569.998 & 1573.679 & 1569.368 & 1566.556 & 1563.906 & 1567.506 \\
\hline $8 / 29 / 1616: 00$ & 76.115 & 84.607 & 83.203 & 90.236 & 79.218 & 75.049 & 73.38 & 76.343 & 1582.357 & 1573.246 & 1570.105 & 1573.693 & 1569.41 & 1566.572 & 1563.911 & 1567.552 \\
\hline 8/29/16 17:00 & 76.111 & 84.581 & 83.14 & 90.227 & 79.181 & 75.035 & 73.363 & 76.299 & 1582.361 & 1573.272 & 1570.168 & 1573.702 & 1569.447 & 1566.586 & 1563.928 & 1567.596 \\
\hline $8 / 29 / 1618: 00$ & 76.134 & 84.602 & 83.145 & 90.271 & 79.184 & 75.058 & 73.406 & 76.308 & 1582.338 & 1573.251 & 1570.163 & 1573.658 & 1569.444 & 1566.563 & 1563.885 & 1567.587 \\
\hline 8/29/16 19:00 & 76.148 & 84.602 & 83.143 & 90.285 & 79.174 & 75.065 & 73.414 & 76.303 & 1582.324 & 1573.251 & 1570.165 & 1573.644 & 1569.454 & 1566.556 & 1563.877 & 1567.592 \\
\hline 8/29/16 20:00 & 76.163 & 84.604 & 83.143 & 90.292 & 79.165 & 75.074 & 73.428 & 76.303 & 1582.309 & 1573.249 & 1570.165 & 1573.637 & 1569.463 & 1566.547 & 1563.863 & 1567.592 \\
\hline $8 / 29 / 1621: 00$ & 76.146 & 84.577 & 83.122 & 90.252 & 79.132 & 75.051 & 73.402 & 76.282 & 1582.326 & 1573.276 & 1570.186 & 1573.677 & 1569.496 & 1566.57 & 1563.889 & 1567.613 \\
\hline $8 / 29 / 1622: 00$ & 76.151 & 84.572 & 83.108 & 90.259 & 79.114 & 75.051 & 73.409 & 76.273 & 1582.321 & 1573.281 & 1570.2 & 1573.67 & 1569.514 & 1566.57 & 1563.882 & 1567.622 \\
\hline 8/29/16 23:00 & 76.165 & 84.584 & 83.112 & 90.276 & 79.114 & 75.065 & 73.428 & 76.278 & 1582.307 & 1573.269 & 1570.196 & 1573.653 & 1569.514 & 1566.556 & 1563.863 & 1567.617 \\
\hline $8 / 30 / 160: 00$ & 76.167 & 84.577 & 83.108 & 90.269 & 79.1 & 75.065 & 73.43 & 76.271 & 1582.305 & 1573.276 & 1570.2 & 1573.66 & 1569.528 & 1566.556 & 1563.861 & 1567.624 \\
\hline $8 / 30 / 161: 00$ & 76.158 & 84.56 & 83.096 & 90.252 & 79.079 & 75.056 & 73.416 & 76.262 & 1582.314 & 1573.293 & 1570.212 & 1573.677 & 1569.549 & 1566.565 & 1563.875 & 1567.633 \\
\hline $8 / 30 / 162: 00$ & 76.139 & 84.535 & 83.066 & 90.234 & 79.046 & 75.032 & 73.397 & 76.234 & 1582.333 & 1573.318 & 1570.242 & 1573.695 & 1569.582 & 1566.589 & 1563.894 & 1567.661 \\
\hline $8 / 30 / 163: 00$ & 76.123 & 84.507 & 83.04 & 90.215 & 79.009 & 75.023 & 73.378 & 76.208 & 1582.349 & 1573.346 & 1570.268 & 1573.714 & 1569.619 & 1566.598 & 1563.913 & 1567.687 \\
\hline 8/30/16 4:00 & 76.118 & 84.493 & 83.024 & 90.215 & 78.988 & 75.016 & 73.378 & 76.187 & 1582.354 & 1573.36 & 1570.284 & 1573.714 & 1569.64 & 1566.605 & 1563.913 & 1567.708 \\
\hline $8 / 30 / 165: 00$ & 76.115 & 84.477 & 83.01 & 90.189 & 78.964 & 75.016 & 73.378 & 76.18 & 1582.357 & 1573.376 & 1570.298 & 1573.74 & 1569.664 & 1566.605 & 1563.913 & 1567.715 \\
\hline $8 / 30 / 166: 00$ & 76.123 & 84.47 & 83.003 & 90.18 & 78.95 & 75.014 & 73.387 & 76.173 & 1582.349 & 1573.383 & 1570.305 & 1573.749 & 1569.678 & 1566.607 & 1563.904 & 1567.722 \\
\hline $8 / 30 / 167: 00$ & 76.139 & 84.477 & 83.007 & 90.182 & 78.953 & 75.028 & 73.406 & 76.176 & 1582.333 & 1573.376 & 1570.301 & 1573.747 & 1569.675 & 1566.593 & 1563.885 & 1567.719 \\
\hline $8 / 30 / 168: 00$ & 76.151 & 84.483 & 83.017 & 90.185 & 78.946 & 75.035 & 73.416 & 76.183 & 1582.321 & 1573.37 & 1570.291 & 1573.744 & 1569.682 & 1566.586 & 1563.875 & 1567.712 \\
\hline 8/30/16 9:00 & 76.146 & 84.467 & 83.003 & 90.173 & 78.929 & 75.028 & 73.409 & 76.173 & 1582.326 & 1573.386 & 1570.305 & 1573.756 & 1569.699 & 1566.593 & 1563.882 & 1567.722 \\
\hline $8 / 30 / 1610: 00$ & 76.144 & 84.456 & 82.996 & 90.171 & 78.915 & 75.025 & 73.404 & 76.169 & 1582.328 & 1573.397 & 1570.312 & 1573.758 & 1569.713 & 1566.596 & 1563.887 & 1567.726 \\
\hline $8 / 30 / 1611: 00$ & 76.144 & 84.451 & 82.989 & 90.175 & 78.904 & 75.025 & 73.406 & 76.173 & 1582.328 & 1573.402 & 1570.319 & 1573.754 & 1569.724 & 1566.596 & 1563.885 & 1567.722 \\
\hline $8 / 30 / 1612: 00$ & 76.146 & 84.446 & 83 & 90.164 & 78.897 & 75.025 & 73.409 & 76.12 & 1582.326 & 1573.407 & 1570.308 & 1573.765 & 1569.731 & 1566.596 & 1563.882 & 1567.775 \\
\hline 8/30/16 13:00 & 76.132 & 84.425 & 82.975 & 90.152 & 78.871 & 75.011 & 73.392 & 76.148 & 1582.34 & 1573.428 & 1570.333 & 1573.777 & 1569.757 & 1566.61 & 1563.899 & 1567.747 \\
\hline $8 / 30 / 1614: 00$ & 76.12 & 84.407 & 82.947 & 90.152 & 78.841 & 75 & 73.375 & 76.129 & 1582.352 & 1573.446 & 1570.361 & 1573.777 & 1569.787 & 1566.621 & 1563.916 & 1567.766 \\
\hline $8 / 30 / 16$ 15:00 & 76.087 & 84.353 & 82.909 & 90.107 & 78.796 & 74.965 & 73.34 & 76.097 & 1582.385 & 1573.5 & 1570.399 & 1573.822 & 1569.832 & 1566.656 & 1563.951 & 1567.798 \\
\hline $8 / 30 / 1616: 00$ & 76.087 & 84.346 & 82.889 & 90.115 & 78.78 & 74.972 & 73.347 & 76.025 & 1582.385 & 1573.507 & 1570.419 & 1573.814 & 1569.848 & 1566.649 & 1563.944 & 1567.87 \\
\hline $8 / 30 / 1617: 00$ & 76.087 & 84.335 & 82.882 & 90.107 & 78.761 & 74.97 & 73.349 & 76.062 & 1582.385 & 1573.518 & 1570.426 & 1573.822 & 1569.867 & 1566.651 & 1563.942 & 1567.833 \\
\hline $8 / 30 / 1618: 00$ & 76.09 & 84.321 & 82.87 & 90.1 & 78.745 & 74.967 & 73.349 & 76.053 & 1582.382 & 1573.532 & 1570.438 & 1573.829 & 1569.883 & 1566.654 & 1563.942 & 1567.842 \\
\hline 8/30/16 19:00 & 76.085 & 84.302 & 82.854 & 90.103 & 78.724 & 74.958 & 73.34 & 76.039 & 1582.387 & 1573.551 & 1570.454 & 1573.826 & 1569.904 & 1566.663 & 1563.951 & 1567.856 \\
\hline
\end{tabular}


TABLE S1.1 Automatically recorded groundwater levels in the unconfined aquifer.

\begin{tabular}{|c|c|c|c|c|c|c|c|c|c|c|c|c|c|c|c|c|}
\hline \multirow[b]{2}{*}{ Date and Time } & \multicolumn{8}{|c|}{ Measured Depth to Water (ft TOC ${ }^{\mathrm{a}}$ ) } & \multicolumn{8}{|c|}{ Groundwater Elevation (ft AMSL') } \\
\hline & DL1-C & DL2-C1 & DL3-C1 & DL4-C1 & DL5-C1 & DL6-C & DL7-C & DL8-C1 & DL1-C & DL2-C1 & DL3-C1 & DL4-C1 & DL5-C1 & DL6-C & DL7-C & DL8-C1 \\
\hline $8 / 30 / 1620: 00$ & 76.085 & 84.293 & 82.844 & 90.112 & 78.712 & 74.963 & 73.349 & 76.032 & 1582.387 & 1573.56 & 1570.464 & 1573.817 & 1569.916 & 1566.658 & 1563.942 & 1567.863 \\
\hline $8 / 30 / 1621: 00$ & 76.113 & 84.314 & 82.858 & 90.131 & 78.724 & 74.981 & 73.385 & 76.043 & 1582.359 & 1573.539 & 1570.45 & 1573.798 & 1569.904 & 1566.64 & 1563.906 & 1567.852 \\
\hline $8 / 30 / 1622: 00$ & 76.127 & 84.323 & 82.868 & 90.138 & 78.729 & 74.988 & 73.394 & 76.013 & 1582.345 & 1573.53 & 1570.44 & 1573.791 & 1569.899 & 1566.633 & 1563.897 & 1567.882 \\
\hline 8/30/16 23:00 & 76.118 & 84.307 & 82.854 & 90.136 & 78.71 & 74.979 & 73.38 & 76.043 & 1582.354 & 1573.546 & 1570.454 & 1573.793 & 1569.918 & 1566.642 & 1563.911 & 1567.852 \\
\hline $8 / 31 / 160: 00$ & 76.106 & 84.293 & 82.837 & 90.124 & 78.687 & 74.969 & 73.366 & 76.027 & 1582.366 & 1573.56 & 1570.471 & 1573.805 & 1569.941 & 1566.652 & 1563.925 & 1567.868 \\
\hline $8 / 31 / 161: 00$ & 76.106 & 84.286 & 82.83 & 90.11 & 78.68 & 74.97 & 73.368 & 76.022 & 1582.366 & 1573.567 & 1570.478 & 1573.819 & 1569.948 & 1566.651 & 1563.923 & 1567.873 \\
\hline $8 / 31 / 162: 00$ & 76.099 & 84.269 & 82.893 & 90.089 & 78.666 & 74.963 & 73.363 & 76.016 & 1582.373 & 1573.584 & 1570.415 & 1573.84 & 1569.962 & 1566.658 & 1563.928 & 1567.879 \\
\hline $8 / 31 / 163: 00$ & 76.09 & 84.253 & 82.872 & 90.075 & 78.645 & 74.953 & 73.351 & 76.006 & 1582.382 & 1573.6 & 1570.436 & 1573.854 & 1569.983 & 1566.668 & 1563.94 & 1567.889 \\
\hline $8 / 31 / 164: 00$ & 76.097 & 84.256 & 82.872 & 90.077 & 78.643 & 74.96 & 73.363 & 76.002 & 1582.375 & 1573.597 & 1570.436 & 1573.852 & 1569.985 & 1566.661 & 1563.928 & 1567.893 \\
\hline $8 / 31 / 165: 00$ & 76.094 & 84.249 & 82.909 & 90.072 & 78.633 & 74.963 & 73.363 & 76.006 & 1582.378 & 1573.604 & 1570.399 & 1573.857 & 1569.995 & 1566.658 & 1563.928 & 1567.889 \\
\hline $8 / 31 / 166: 00$ & 76.101 & 84.249 & 82.858 & 90.075 & 78.631 & 74.96 & 73.368 & 75.99 & 1582.371 & 1573.604 & 1570.45 & 1573.854 & 1569.997 & 1566.661 & 1563.923 & 1567.905 \\
\hline $8 / 31 / 167: 00$ & 76.094 & 84.237 & 82.814 & 90.065 & 78.617 & 74.951 & 73.363 & 75.969 & 1582.378 & 1573.616 & 1570.494 & 1573.864 & 1570.011 & 1566.67 & 1563.928 & 1567.926 \\
\hline $8 / 31 / 168: 00$ & 76.099 & 84.239 & 82.842 & 90.073 & 78.615 & 74.951 & 73.368 & 75.985 & 1582.373 & 1573.614 & 1570.466 & 1573.856 & 1570.013 & 1566.67 & 1563.923 & 1567.91 \\
\hline 8/31/16 9:00 & 76.101 & 84.237 & 82.83 & 90.07 & 78.608 & 74.97 & 73.371 & 76.097 & 1582.371 & 1573.616 & 1570.478 & 1573.859 & 1570.02 & 1566.651 & 1563.92 & 1567.798 \\
\hline $8 / 31 / 1610: 00$ & 76.108 & 84.239 & 83.063 & 90.077 & 78.629 & 74.99 & 73.38 & 76.238 & 1582.364 & 1573.614 & 1570.245 & 1573.852 & 1569.999 & 1566.631 & 1563.911 & 1567.657 \\
\hline $8 / 31 / 1611: 00$ & 76.115 & 84.253 & 83.457 & 90.082 & 78.687 & 74.993 & 73.382 & 76.385 & 1582.357 & 1573.6 & 1569.851 & 1573.847 & 1569.941 & 1566.628 & 1563.909 & 1567.51 \\
\hline $8 / 31 / 1612: 00$ & 76.115 & 84.263 & 83.609 & 90.084 & 78.752 & 74.995 & 73.38 & 76.485 & 1582.357 & 1573.59 & 1569.699 & 1573.845 & 1569.876 & 1566.626 & 1563.911 & 1567.41 \\
\hline $8 / 31 / 1613: 00$ & 76.111 & 84.258 & 83.66 & 90.079 & 78.813 & 74.993 & 73.366 & 76.559 & 1582.361 & 1573.595 & 1569.648 & 1573.85 & 1569.815 & 1566.628 & 1563.925 & 1567.336 \\
\hline $8 / 31 / 1614: 00$ & 76.099 & 84.253 & 83.711 & 90.075 & 78.866 & 74.986 & 73.351 & 76.628 & 1582.373 & 1573.6 & 1569.597 & 1573.854 & 1569.762 & 1566.635 & 1563.94 & 1567.267 \\
\hline $8 / 31 / 1615: 00$ & 76.094 & 84.246 & 83.758 & 90.073 & 78.92 & 74.981 & 73.342 & 76.675 & 1582.378 & 1573.607 & 1569.55 & 1573.856 & 1569.708 & 1566.64 & 1563.949 & 1567.22 \\
\hline $8 / 31 / 1616: 00$ & 76.09 & 84.242 & 83.795 & 90.068 & 78.971 & 74.979 & 73.332 & 76.705 & 1582.382 & 1573.611 & 1569.513 & 1573.861 & 1569.657 & 1566.642 & 1563.959 & 1567.19 \\
\hline $8 / 31 / 1617: 00$ & 76.097 & 84.244 & 83.826 & 90.068 & 79.03 & 74.981 & 73.335 & 76.742 & 1582.375 & 1573.609 & 1569.482 & 1573.861 & 1569.598 & 1566.64 & 1563.956 & 1567.153 \\
\hline $8 / 31 / 1618: 00$ & 76.104 & 84.246 & 83.863 & 90.075 & 79.088 & 74.988 & 73.332 & 76.784 & 1582.368 & 1573.607 & 1569.445 & 1573.854 & 1569.54 & 1566.633 & 1563.959 & 1567.111 \\
\hline $8 / 31 / 1619: 00$ & 76.12 & 84.258 & 83.9 & 90.077 & 79.151 & 74.995 & 73.337 & 76.824 & 1582.352 & 1573.595 & 1569.408 & 1573.852 & 1569.477 & 1566.626 & 1563.954 & 1567.071 \\
\hline $8 / 31 / 1620: 00$ & 76.134 & 84.274 & 83.94 & 90.094 & 79.214 & 75.004 & 73.349 & 76.868 & 1582.338 & 1573.579 & 1569.368 & 1573.835 & 1569.414 & 1566.617 & 1563.942 & 1567.027 \\
\hline $8 / 31 / 1621: 00$ & 76.153 & 84.297 & 83.986 & 90.103 & 79.286 & 75.021 & 73.363 & 76.907 & 1582.319 & 1573.556 & 1569.322 & 1573.826 & 1569.342 & 1566.6 & 1563.928 & 1566.988 \\
\hline $8 / 31 / 1622: 00$ & 76.177 & 84.325 & 84.04 & 90.136 & 79.363 & 75.037 & 73.375 & 76.96 & 1582.295 & 1573.528 & 1569.268 & 1573.793 & 1569.265 & 1566.584 & 1563.916 & 1566.935 \\
\hline $8 / 31 / 1623: 00$ & 76.189 & 84.342 & 84.075 & 90.154 & 79.428 & 75.044 & 73.378 & 76.995 & 1582.283 & 1573.511 & 1569.233 & 1573.775 & 1569.2 & 1566.577 & 1563.913 & 1566.9 \\
\hline 9/1/16 0:00 & 76.198 & 84.36 & 84.11 & 90.168 & 79.489 & 75.051 & 73.382 & 77.037 & 1582.274 & 1573.493 & 1569.198 & 1573.761 & 1569.139 & 1566.57 & 1563.909 & 1566.858 \\
\hline 9/1/16 1:00 & 76.203 & 84.374 & 84.145 & 90.168 & 79.547 & 75.053 & 73.378 & 77.067 & 1582.269 & 1573.479 & 1569.163 & 1573.761 & 1569.081 & 1566.568 & 1563.913 & 1566.828 \\
\hline 9/1/16 2:00 & 76.21 & 84.39 & 84.187 & 90.175 & 79.608 & 75.058 & 73.378 & 77.104 & 1582.262 & 1573.463 & 1569.121 & 1573.754 & 1569.02 & 1566.563 & 1563.913 & 1566.791 \\
\hline 9/1/16 3:00 & 76.21 & 84.404 & 84.219 & 90.18 & 79.659 & 75.058 & 73.371 & 77.142 & 1582.262 & 1573.449 & 1569.089 & 1573.749 & 1568.969 & 1566.563 & 1563.92 & 1566.753 \\
\hline $9 / 1 / 164: 00$ & 76.212 & 84.411 & 84.25 & 90.187 & 79.708 & 75.06 & 73.371 & 77.165 & 1582.26 & 1573.442 & 1569.058 & 1573.742 & 1568.92 & 1566.561 & 1563.92 & 1566.73 \\
\hline 9/1/16 5:00 & 76.224 & 84.435 & 84.28 & 90.175 & 79.769 & 75.07 & 73.373 & 77.197 & 1582.248 & 1573.418 & 1569.028 & 1573.754 & 1568.859 & 1566.551 & 1563.918 & 1566.698 \\
\hline $9 / 1 / 166: 00$ & 76.233 & 84.453 & 84.308 & 90.18 & 79.825 & 75.079 & 73.38 & 77.223 & 1582.239 & 1573.4 & 1569 & 1573.749 & 1568.803 & 1566.542 & 1563.911 & 1566.672 \\
\hline 9/1/16 7:00 & 76.247 & 84.474 & 84.343 & 90.187 & 79.881 & 75.105 & 73.385 & 77.337 & 1582.225 & 1573.379 & 1568.965 & 1573.742 & 1568.747 & 1566.516 & 1563.906 & 1566.558 \\
\hline 9/1/16 8:00 & 76.259 & 84.5 & 84.392 & 90.201 & 79.941 & 75.139 & 73.394 & 77.46 & 1582.213 & 1573.353 & 1568.916 & 1573.728 & 1568.687 & 1566.482 & 1563.897 & 1566.435 \\
\hline 9/1/16 9:00 & 76.269 & 84.521 & 84.427 & 90.208 & 80 & 75.158 & 73.397 & 77.55 & 1582.203 & 1573.332 & 1568.881 & 1573.721 & 1568.628 & 1566.463 & 1563.894 & 1566.345 \\
\hline $9 / 1 / 1610: 00$ & 76.278 & 84.535 & 84.466 & 90.21 & 80.046 & 75.177 & 73.394 & 77.608 & 1582.194 & 1573.318 & 1568.842 & 1573.719 & 1568.582 & 1566.444 & 1563.897 & 1566.287 \\
\hline 9/1/16 11:00 & 76.283 & 84.558 & 84.501 & 90.22 & 80.105 & 75.191 & 73.394 & 77.641 & 1582.189 & 1573.295 & 1568.807 & 1573.709 & 1568.523 & 1566.43 & 1563.897 & 1566.254 \\
\hline $9 / 1 / 1612: 00$ & 76.28 & 84.565 & 84.522 & 90.222 & 80.147 & 75.198 & 73.385 & 77.701 & 1582.192 & 1573.288 & 1568.786 & 1573.707 & 1568.481 & 1566.423 & 1563.906 & 1566.194 \\
\hline $9 / 1 / 1613: 00$ & 76.276 & 84.572 & 84.543 & 90.222 & 80.186 & 75.205 & 73.375 & 77.736 & 1582.196 & 1573.281 & 1568.765 & 1573.707 & 1568.442 & 1566.416 & 1563.916 & 1566.159 \\
\hline $9 / 1 / 1614: 00$ & 76.271 & 84.577 & 84.562 & 90.224 & 80.221 & 75.214 & 73.366 & 77.787 & 1582.201 & 1573.276 & 1568.746 & 1573.705 & 1568.407 & 1566.407 & 1563.925 & 1566.108 \\
\hline $9 / 1 / 1615: 00$ & 76.271 & 84.586 & 84.583 & 90.229 & 80.261 & 75.216 & 73.361 & 77.803 & 1582.201 & 1573.267 & 1568.725 & 1573.7 & 1568.367 & 1566.405 & 1563.93 & 1566.092 \\
\hline $9 / 1 / 1616: 00$ & 76.271 & 84.586 & 84.595 & 90.227 & 80.289 & 75.223 & 73.349 & 77.82 & 1582.201 & 1573.267 & 1568.713 & 1573.702 & 1568.339 & 1566.398 & 1563.942 & 1566.075 \\
\hline $9 / 1 / 1617: 00$ & 76.266 & 84.59 & 84.609 & 90.231 & 80.324 & 75.23 & 73.344 & 77.847 & 1582.206 & 1573.263 & 1568.699 & 1573.698 & 1568.304 & 1566.391 & 1563.947 & 1566.048 \\
\hline $9 / 1 / 1618: 00$ & 76.278 & 84.604 & 84.623 & 90.264 & 80.366 & 75.244 & 73.351 & 77.871 & 1582.194 & 1573.249 & 1568.685 & 1573.665 & 1568.262 & 1566.377 & 1563.94 & 1566.024 \\
\hline $9 / 1 / 1619: 00$ & 76.285 & 84.618 & 84.641 & 90.287 & 80.41 & 75.256 & 73.356 & 77.903 & 1582.187 & 1573.235 & 1568.667 & 1573.642 & 1568.218 & 1566.365 & 1563.935 & 1565.992 \\
\hline $9 / 1 / 1620: 00$ & 76.295 & 84.639 & 84.669 & 90.304 & 80.45 & 75.277 & 73.366 & 77.931 & 1582.177 & 1573.214 & 1568.639 & 1573.625 & 1568.178 & 1566.344 & 1563.925 & 1565.964 \\
\hline
\end{tabular}


TABLE S1.1 Automatically recorded groundwater levels in the unconfined aquifer.

\begin{tabular}{|c|c|c|c|c|c|c|c|c|c|c|c|c|c|c|c|c|}
\hline \multirow[b]{2}{*}{ Date and Time } & \multicolumn{8}{|c|}{ Measured Depth to Water (ft TOC ${ }^{\mathrm{a}}$ ) } & \multicolumn{8}{|c|}{ Groundwater Elevation ( $\mathrm{ft} \mathrm{AMSL}^{\mathrm{b}}$ ) } \\
\hline & DL1-C & DL2-C1 & DL3-C1 & DL4-C1 & DL5-C1 & DL6-C & DL7-C & DL8-C1 & DL1-C & DL2-C1 & DL3-C1 & DL4-C1 & DL5-C1 & DL6-C & DL7-C & DL8-C1 \\
\hline 9/1/16 21:00 & 76.313 & 84.674 & 84.707 & 90.299 & 80.503 & 75.296 & 73.387 & 77.975 & 1582.159 & 1573.179 & 1568.601 & 1573.63 & 1568.125 & 1566.325 & 1563.904 & 1565.92 \\
\hline $9 / 1 / 1622: 00$ & 76.328 & 84.697 & 84.742 & 90.318 & 80.555 & 75.314 & 73.397 & 78.019 & 1582.144 & 1573.156 & 1568.566 & 1573.611 & 1568.073 & 1566.307 & 1563.894 & 1565.876 \\
\hline 9/1/16 23:00 & 76.335 & 84.723 & 84.772 & 90.339 & 80.599 & 75.326 & 73.402 & 78.061 & 1582.137 & 1573.13 & 1568.536 & 1573.59 & 1568.029 & 1566.295 & 1563.889 & 1565.834 \\
\hline 9/2/16 0:00 & 76.339 & 84.742 & 84.811 & 90.346 & 80.643 & 75.34 & 73.404 & 78.096 & 1582.133 & 1573.111 & 1568.497 & 1573.583 & 1567.985 & 1566.281 & 1563.887 & 1565.799 \\
\hline 9/2/16 1:00 & 76.344 & 84.763 & 84.846 & 90.353 & 80.683 & 75.351 & 73.406 & 78.126 & 1582.128 & 1573.09 & 1568.462 & 1573.576 & 1567.945 & 1566.27 & 1563.885 & 1565.769 \\
\hline 9/2/16 2:00 & 76.346 & 84.786 & 84.937 & 90.364 & 80.727 & 75.365 & 73.414 & 78.172 & 1582.126 & 1573.067 & 1568.371 & 1573.565 & 1567.901 & 1566.256 & 1563.877 & 1565.723 \\
\hline 9/2/16 3:00 & 76.346 & 84.807 & 84.977 & 90.369 & 80.767 & 75.379 & 73.416 & 78.217 & 1582.126 & 1573.046 & 1568.331 & 1573.56 & 1567.861 & 1566.242 & 1563.875 & 1565.678 \\
\hline 9/2/16 4:00 & 76.349 & 84.825 & 84.998 & 90.385 & 80.806 & 75.391 & 73.421 & 78.24 & 1582.123 & 1573.028 & 1568.31 & 1573.544 & 1567.822 & 1566.23 & 1563.87 & 1565.655 \\
\hline 9/2/16 5:00 & 76.346 & 84.842 & 85.035 & 90.367 & 80.844 & 75.396 & 73.418 & 78.275 & 1582.126 & 1573.011 & 1568.273 & 1573.562 & 1567.784 & 1566.225 & 1563.873 & 1565.62 \\
\hline 9/2/16 6:00 & 76.356 & 84.865 & 85.01 & 90.374 & 80.888 & 75.407 & 73.428 & 78.284 & 1582.116 & 1572.988 & 1568.298 & 1573.555 & 1567.74 & 1566.214 & 1563.863 & 1565.611 \\
\hline 9/2/16 7:00 & 76.358 & 84.881 & 84.996 & 90.376 & 80.923 & 75.419 & 73.43 & 78.284 & 1582.114 & 1572.972 & 1568.312 & 1573.553 & 1567.705 & 1566.202 & 1563.861 & 1565.611 \\
\hline $9 / 2 / 168: 00$ & 76.361 & 84.898 & 85.014 & 90.385 & 80.956 & 75.433 & 73.433 & 78.335 & 1582.111 & 1572.955 & 1568.294 & 1573.544 & 1567.672 & 1566.188 & 1563.858 & 1565.56 \\
\hline 9/2/16 9:00 & 76.363 & 84.916 & 85.033 & 90.395 & 80.991 & 75.442 & 73.437 & 78.358 & 1582.109 & 1572.937 & 1568.275 & 1573.534 & 1567.637 & 1566.179 & 1563.854 & 1565.537 \\
\hline $9 / 2 / 1610: 00$ & 76.368 & 84.937 & 85.049 & 90.399 & 81.026 & 75.431 & 73.442 & 78.282 & 1582.104 & 1572.916 & 1568.259 & 1573.53 & 1567.602 & 1566.19 & 1563.849 & 1565.613 \\
\hline 9/2/16 11:00 & 76.368 & 84.949 & 85.054 & 90.404 & 81.058 & 75.417 & 73.44 & 78.231 & 1582.104 & 1572.904 & 1568.254 & 1573.525 & 1567.57 & 1566.204 & 1563.851 & 1565.664 \\
\hline $9 / 2 / 1612: 00$ & 76.356 & 84.956 & 85.054 & 90.399 & 81.072 & 75.403 & 73.425 & 78.154 & 1582.116 & 1572.897 & 1568.254 & 1573.53 & 1567.556 & 1566.218 & 1563.866 & 1565.741 \\
\hline $9 / 2 / 1613: 00$ & 76.349 & 84.96 & 85.054 & 90.402 & 81.089 & 75.391 & 73.418 & 78.175 & 1582.123 & 1572.893 & 1568.254 & 1573.527 & 1567.539 & 1566.23 & 1563.873 & 1565.72 \\
\hline $9 / 2 / 1614: 00$ & 76.339 & 84.958 & 85.047 & 90.402 & 81.1 & 75.382 & 73.414 & 78.154 & 1582.133 & 1572.895 & 1568.261 & 1573.527 & 1567.528 & 1566.239 & 1563.877 & 1565.741 \\
\hline $9 / 2 / 1615: 00$ & 76.33 & 84.956 & 85.047 & 90.4 & 81.105 & 75.375 & 73.399 & 78.189 & 1582.142 & 1572.897 & 1568.261 & 1573.529 & 1567.523 & 1566.246 & 1563.892 & 1565.706 \\
\hline $9 / 2 / 1616: 00$ & 76.313 & 84.949 & 85.035 & 90.395 & 81.105 & 75.358 & 73.385 & 78.124 & 1582.159 & 1572.904 & 1568.273 & 1573.534 & 1567.523 & 1566.263 & 1563.906 & 1565.771 \\
\hline $9 / 2 / 1617: 00$ & 76.309 & 84.944 & 85.04 & 90.39 & 81.11 & 75.347 & 73.375 & 78.11 & 1582.163 & 1572.909 & 1568.268 & 1573.539 & 1567.518 & 1566.274 & 1563.916 & 1565.785 \\
\hline $9 / 2 / 1618: 00$ & 76.304 & 84.946 & 85.042 & 90.393 & 81.114 & 75.34 & 73.375 & 78.11 & 1582.168 & 1572.907 & 1568.266 & 1573.536 & 1567.514 & 1566.281 & 1563.916 & 1565.785 \\
\hline $9 / 2 / 1619: 00$ & 76.302 & 84.949 & 85.045 & 90.409 & 81.128 & 75.335 & 73.373 & 78.096 & 1582.17 & 1572.904 & 1568.263 & 1573.52 & 1567.5 & 1566.286 & 1563.918 & 1565.799 \\
\hline $9 / 2 / 1620: 00$ & 76.304 & 84.953 & 85.052 & 90.421 & 81.14 & 75.333 & 73.373 & 78.091 & 1582.168 & 1572.9 & 1568.256 & 1573.508 & 1567.488 & 1566.288 & 1563.918 & 1565.804 \\
\hline 9/2/16 21:00 & 76.32 & 84.986 & 85.075 & 90.444 & 81.17 & 75.349 & 73.404 & 78.107 & 1582.152 & 1572.867 & 1568.233 & 1573.485 & 1567.458 & 1566.272 & 1563.887 & 1565.788 \\
\hline $9 / 2 / 1622: 00$ & 76.335 & 85.014 & 85.103 & 90.46 & 81.203 & 75.363 & 73.421 & 78.1 & 1582.137 & 1572.839 & 1568.205 & 1573.469 & 1567.425 & 1566.258 & 1563.87 & 1565.795 \\
\hline $9 / 2 / 1623: 00$ & 76.335 & 85.028 & 85.114 & 90.472 & 81.221 & 75.361 & 73.418 & 78.126 & 1582.137 & 1572.825 & 1568.194 & 1573.457 & 1567.407 & 1566.26 & 1563.873 & 1565.769 \\
\hline 9/3/16 0:00 & 76.342 & 85.049 & 85.135 & 90.483 & 81.245 & 75.368 & 73.433 & 78.135 & 1582.13 & 1572.804 & 1568.173 & 1573.446 & 1567.383 & 1566.253 & 1563.858 & 1565.76 \\
\hline 9/3/16 1:00 & 76.328 & 85.051 & 85.138 & 90.46 & 81.249 & 75.356 & 73.416 & 78.131 & 1582.144 & 1572.802 & 1568.17 & 1573.469 & 1567.379 & 1566.265 & 1563.875 & 1565.764 \\
\hline 9/3/16 2:00 & 76.309 & 85.042 & 85.128 & 90.444 & 81.242 & 75.342 & 73.402 & 78.117 & 1582.163 & 1572.811 & 1568.18 & 1573.485 & 1567.386 & 1566.279 & 1563.889 & 1565.778 \\
\hline 9/3/16 3:00 & 76.311 & 85.06 & 85.14 & 90.451 & 81.252 & 75.347 & 73.414 & 78.128 & 1582.161 & 1572.793 & 1568.168 & 1573.478 & 1567.376 & 1566.274 & 1563.877 & 1565.767 \\
\hline 9/3/16 4:00 & 76.318 & 85.074 & 85.163 & 90.458 & 81.273 & 75.356 & 73.428 & 78.142 & 1582.154 & 1572.779 & 1568.145 & 1573.471 & 1567.355 & 1566.265 & 1563.863 & 1565.753 \\
\hline $9 / 3 / 165: 00$ & 76.313 & 85.086 & 85.187 & 90.458 & 81.277 & 75.354 & 73.425 & 78.152 & 1582.159 & 1572.767 & 1568.121 & 1573.471 & 1567.351 & 1566.267 & 1563.866 & 1565.743 \\
\hline 9/3/16 6:00 & 76.306 & 85.093 & 85.189 & 90.46 & 81.28 & 75.351 & 73.421 & 78.14 & 1582.166 & 1572.76 & 1568.119 & 1573.469 & 1567.348 & 1566.27 & 1563.87 & 1565.755 \\
\hline 9/3/16 7:00 & 76.299 & 85.093 & 85.184 & 90.458 & 81.275 & 75.347 & 73.416 & 78.131 & 1582.173 & 1572.76 & 1568.124 & 1573.471 & 1567.353 & 1566.274 & 1563.875 & 1565.764 \\
\hline 9/3/16 8:00 & 76.299 & 85.102 & 84.681 & 90.465 & 81.252 & 75.342 & 73.423 & 77.857 & 1582.173 & 1572.751 & 1568.627 & 1573.464 & 1567.376 & 1566.279 & 1563.868 & 1566.038 \\
\hline 9/3/16 9:00 & 76.29 & 85.093 & 84.478 & 90.46 & 81.203 & 75.328 & 73.418 & 77.736 & 1582.182 & 1572.76 & 1568.83 & 1573.469 & 1567.425 & 1566.293 & 1563.873 & 1566.159 \\
\hline $9 / 3 / 1610: 00$ & 76.295 & 85.093 & 84.383 & 90.458 & 81.161 & 75.33 & 73.425 & 77.643 & 1582.177 & 1572.76 & 1568.925 & 1573.471 & 1567.467 & 1566.291 & 1563.866 & 1566.252 \\
\hline 9/3/16 11:00 & 76.29 & 85.091 & 84.31 & 90.455 & 81.105 & 75.316 & 73.421 & 77.506 & 1582.182 & 1572.762 & 1568.998 & 1573.474 & 1567.523 & 1566.305 & 1563.87 & 1566.389 \\
\hline $9 / 3 / 1612: 00$ & 76.29 & 85.086 & 84.257 & 90.453 & 81.049 & 75.314 & 73.425 & 77.495 & 1582.182 & 1572.767 & 1569.051 & 1573.476 & 1567.579 & 1566.307 & 1563.866 & 1566.4 \\
\hline $9 / 3 / 1613: 00$ & 76.297 & 85.086 & 84.215 & 90.455 & 80.998 & 75.312 & 73.432 & 77.448 & 1582.175 & 1572.767 & 1569.093 & 1573.474 & 1567.63 & 1566.309 & 1563.859 & 1566.447 \\
\hline $9 / 3 / 1614: 00$ & 76.28 & 85.065 & 84.156 & 90.437 & 80.925 & 75.291 & 73.414 & 77.383 & 1582.192 & 1572.788 & 1569.152 & 1573.492 & 1567.703 & 1566.33 & 1563.877 & 1566.512 \\
\hline $9 / 3 / 1615: 00$ & 76.269 & 85.049 & 84.096 & 90.43 & 80.855 & 75.267 & 73.402 & 77.311 & 1582.203 & 1572.804 & 1569.212 & 1573.499 & 1567.773 & 1566.354 & 1563.889 & 1566.584 \\
\hline $9 / 3 / 1616: 00$ & 76.233 & 85.007 & 84.021 & 90.395 & 80.767 & 75.247 & 73.39 & 77.225 & 1582.239 & 1572.846 & 1569.287 & 1573.534 & 1567.861 & 1566.374 & 1563.901 & 1566.67 \\
\hline $9 / 3 / 1617: 00$ & 76.217 & 84.993 & 83.984 & 90.393 & 80.704 & 75.237 & 73.38 & 77.197 & 1582.255 & 1572.86 & 1569.324 & 1573.536 & 1567.924 & 1566.384 & 1563.911 & 1566.698 \\
\hline $9 / 3 / 1618: 00$ & 76.186 & 84.974 & 83.949 & 90.402 & 80.646 & 75.23 & 73.375 & 77.158 & 1582.286 & 1572.879 & 1569.359 & 1573.527 & 1567.982 & 1566.391 & 1563.916 & 1566.737 \\
\hline $9 / 3 / 1619: 00$ & 76.144 & 84.939 & 83.9 & 90.395 & 80.573 & 75.207 & 73.359 & 77.102 & 1582.328 & 1572.914 & 1569.408 & 1573.534 & 1568.055 & 1566.414 & 1563.932 & 1566.793 \\
\hline $9 / 3 / 1620: 00$ & 76.125 & 84.93 & 83.867 & 90.397 & 80.522 & 75.207 & 73.363 & 77.056 & 1582.347 & 1572.923 & 1569.441 & 1573.532 & 1568.106 & 1566.414 & 1563.928 & 1566.839 \\
\hline 9/3/16 21:00 & 76.111 & 84.925 & 83.839 & 90.374 & 80.475 & 75.209 & 73.373 & 77.03 & 1582.361 & 1572.928 & 1569.469 & 1573.555 & 1568.153 & 1566.412 & 1563.918 & 1566.865 \\
\hline
\end{tabular}


TABLE S1.1 Automatically recorded groundwater levels in the unconfined aquifer.

\begin{tabular}{|c|c|c|c|c|c|c|c|c|c|c|c|c|c|c|c|c|}
\hline \multirow[b]{2}{*}{ Date and Time } & \multicolumn{8}{|c|}{ Measured Depth to Water ( $\mathrm{ft}$ TOC ${ }^{\mathrm{a}}$ ) } & \multicolumn{8}{|c|}{ Groundwater Elevation (ft $\mathrm{AMSL}^{\mathrm{b}}$ ) } \\
\hline & DL1-C & DL2-C1 & DL3-C1 & DL4-C1 & DL5-C1 & DL6-C & DL7-C & DL8-C1 & DL1-C & $\mathrm{DL} 2-\mathrm{C} 1$ & DL3-C1 & DL4-C1 & DL5-C1 & DL6-C & DL7-C & DL8-C1 \\
\hline 9/3/16 22:00 & 76.097 & 84.909 & 83.812 & 90.371 & 80.422 & 75.205 & 73.38 & 77.002 & 1582.375 & 1572.944 & 1569.496 & 1573.558 & 1568.206 & 1566.416 & 1563.911 & 1566.893 \\
\hline 9/3/16 23:00 & 76.097 & 84.914 & 83.798 & 90.388 & 80.387 & 75.214 & 73.399 & 76.981 & 1582.375 & 1572.939 & 1569.51 & 1573.541 & 1568.241 & 1566.407 & 1563.892 & 1566.914 \\
\hline 9/4/16 0:00 & 76.085 & 84.902 & 83.777 & 90.381 & 80.345 & 75.212 & 73.406 & 76.928 & 1582.387 & 1572.951 & 1569.531 & 1573.548 & 1568.283 & 1566.409 & 1563.885 & 1566.967 \\
\hline 9/4/16 1:00 & 76.071 & 84.891 & 83.753 & 90.371 & 80.3 & 75.207 & 73.402 & 76.93 & 1582.401 & 1572.962 & 1569.555 & 1573.558 & 1568.328 & 1566.414 & 1563.889 & 1566.965 \\
\hline 9/4/16 2:00 & 76.038 & 84.846 & 83.711 & 90.336 & 80.228 & 75.179 & 73.366 & 76.889 & 1582.434 & 1573.007 & 1569.597 & 1573.593 & 1568.4 & 1566.442 & 1563.925 & 1567.006 \\
\hline $9 / 4 / 163: 00$ & 76.052 & 84.867 & 83.704 & 90.362 & 80.207 & 75.205 & 73.416 & 76.882 & 1582.42 & 1572.986 & 1569.604 & 1573.567 & 1568.421 & 1566.416 & 1563.875 & 1567.013 \\
\hline 9/4/16 4:00 & 76.021 & 84.828 & 83.665 & 90.334 & 80.14 & 75.174 & 73.385 & 76.842 & 1582.451 & 1573.025 & 1569.643 & 1573.595 & 1568.488 & 1566.447 & 1563.906 & 1567.053 \\
\hline 9/4/16 5:00 & 75.976 & 84.772 & 83.618 & 90.283 & 80.056 & 75.144 & 73.351 & 76.793 & 1582.496 & 1573.081 & 1569.69 & 1573.646 & 1568.572 & 1566.477 & 1563.94 & 1567.102 \\
\hline 9/4/16 6:00 & 76 & 84.797 & 83.611 & 90.306 & 80.046 & 75.177 & 73.404 & 76.777 & 1582.472 & 1573.056 & 1569.697 & 1573.623 & 1568.582 & 1566.444 & 1563.887 & 1567.118 \\
\hline 9/4/16 7:00 & 76 & 84.786 & 83.592 & 90.29 & 80.002 & 75.17 & 73.402 & 76.758 & 1582.472 & 1573.067 & 1569.716 & 1573.639 & 1568.626 & 1566.451 & 1563.889 & 1567.137 \\
\hline 9/4/16 8:00 & 75.998 & 84.779 & 83.578 & 90.292 & 79.969 & 75.174 & 73.414 & 76.747 & 1582.474 & 1573.074 & 1569.73 & 1573.637 & 1568.659 & 1566.447 & 1563.877 & 1567.148 \\
\hline 9/4/16 9:00 & 75.986 & 84.76 & 83.555 & 90.273 & 79.92 & 75.165 & 73.404 & 76.719 & 1582.486 & 1573.093 & 1569.753 & 1573.656 & 1568.708 & 1566.456 & 1563.887 & 1567.176 \\
\hline 9/4/16 10:00 & 75.979 & 84.746 & 83.534 & 90.269 & 79.876 & 75.163 & 73.409 & 76.696 & 1582.493 & 1573.107 & 1569.774 & 1573.66 & 1568.752 & 1566.458 & 1563.882 & 1567.199 \\
\hline 9/4/16 11:00 & 75.969 & 84.732 & 83.516 & 90.259 & 79.834 & 75.158 & 73.409 & 76.679 & 1582.503 & 1573.121 & 1569.792 & 1573.67 & 1568.794 & 1566.463 & 1563.882 & 1567.216 \\
\hline 9/4/16 12:00 & 75.96 & 84.714 & 83.497 & 90.257 & 79.794 & 75.154 & 73.406 & 76.654 & 1582.512 & 1573.139 & 1569.811 & 1573.672 & 1568.834 & 1566.467 & 1563.885 & 1567.241 \\
\hline 9/4/16 13:00 & 75.941 & 84.684 & 83.46 & 90.234 & 79.734 & 75.133 & 73.387 & 76.619 & 1582.531 & 1573.169 & 1569.848 & 1573.695 & 1568.894 & 1566.488 & 1563.904 & 1567.276 \\
\hline 9/4/16 14:00 & 75.925 & 84.656 & 83.427 & 90.22 & 79.685 & 75.121 & 73.38 & 76.591 & 1582.547 & 1573.197 & 1569.881 & 1573.709 & 1568.943 & 1566.5 & 1563.911 & 1567.304 \\
\hline $9 / 4 / 1615: 00$ & 75.903 & 84.625 & 83.394 & 90.201 & 79.627 & 75.104 & 73.363 & 76.559 & 1582.569 & 1573.228 & 1569.914 & 1573.728 & 1569.001 & 1566.517 & 1563.928 & 1567.336 \\
\hline 9/4/16 16:00 & 75.88 & 84.584 & 83.35 & 90.182 & 79.559 & 75.081 & 73.342 & 76.508 & 1582.592 & 1573.269 & 1569.958 & 1573.747 & 1569.069 & 1566.54 & 1563.949 & 1567.387 \\
\hline 9/4/16 17:00 & 75.866 & 84.553 & 83.317 & 90.171 & 79.505 & 75.074 & 73.337 & 76.48 & 1582.606 & 1573.3 & 1569.991 & 1573.758 & 1569.123 & 1566.547 & 1563.954 & 1567.415 \\
\hline $9 / 4 / 1618: 00$ & 75.866 & 84.539 & 83.301 & 90.166 & 79.466 & 75.077 & 73.351 & 76.459 & 1582.606 & 1573.314 & 1570.007 & 1573.763 & 1569.162 & 1566.544 & 1563.94 & 1567.436 \\
\hline 9/4/16 19:00 & 75.861 & 84.518 & 83.278 & 90.175 & 79.421 & 75.07 & 73.349 & 76.436 & 1582.611 & 1573.335 & 1570.03 & 1573.754 & 1569.207 & 1566.551 & 1563.942 & 1567.459 \\
\hline $9 / 4 / 1620: 00$ & 75.859 & 84.502 & 83.259 & 90.175 & 79.384 & 75.065 & 73.351 & 76.375 & 1582.613 & 1573.351 & 1570.049 & 1573.754 & 1569.244 & 1566.556 & 1563.94 & 1567.52 \\
\hline $9 / 4 / 1621: 00$ & 75.849 & 84.479 & 83.233 & 90.164 & 79.333 & 75.058 & 73.347 & 76.392 & 1582.623 & 1573.374 & 1570.075 & 1573.765 & 1569.295 & 1566.563 & 1563.944 & 1567.503 \\
\hline 9/4/16 22:00 & 75.87 & 84.491 & 83.236 & 90.182 & 79.319 & 75.077 & 73.38 & 76.401 & 1582.602 & 1573.362 & 1570.072 & 1573.747 & 1569.309 & 1566.544 & 1563.911 & 1567.494 \\
\hline 9/4/16 23:00 & 75.882 & 84.491 & 83.233 & 90.192 & 79.295 & 75.083 & 73.394 & 76.394 & 1582.59 & 1573.362 & 1570.075 & 1573.737 & 1569.333 & 1566.538 & 1563.897 & 1567.501 \\
\hline 9/5/16 0:00 & 75.873 & 84.479 & 83.219 & 90.182 & 79.263 & 75.079 & 73.392 & 76.378 & 1582.599 & 1573.374 & 1570.089 & 1573.747 & 1569.365 & 1566.542 & 1563.899 & 1567.517 \\
\hline 9/5/16 1:00 & 75.861 & 84.46 & 83.199 & 90.157 & 79.218 & 75.07 & 73.385 & 76.359 & 1582.611 & 1573.393 & 1570.109 & 1573.772 & 1569.41 & 1566.551 & 1563.906 & 1567.536 \\
\hline 9/5/16 2:00 & 75.856 & 84.446 & 83.254 & 90.14 & 79.181 & 75.063 & 73.382 & 76.345 & 1582.616 & 1573.407 & 1570.054 & 1573.789 & 1569.447 & 1566.558 & 1563.909 & 1567.55 \\
\hline 9/5/16 3:00 & 75.835 & 84.416 & 83.233 & 90.119 & 79.135 & 75.046 & 73.366 & 76.327 & 1582.637 & 1573.437 & 1570.075 & 1573.81 & 1569.493 & 1566.575 & 1563.925 & 1567.568 \\
\hline 9/5/16 4:00 & 75.83 & 84.4 & 83.213 & 90.11 & 79.1 & 75.039 & 73.363 & 76.306 & 1582.642 & 1573.453 & 1570.095 & 1573.819 & 1569.528 & 1566.582 & 1563.928 & 1567.589 \\
\hline 9/5/16 5:00 & 75.826 & 84.39 & 83.238 & 90.108 & 79.06 & 75.042 & 73.368 & 76.303 & 1582.646 & 1573.463 & 1570.07 & 1573.821 & 1569.568 & 1566.579 & 1563.923 & 1567.592 \\
\hline 9/5/16 6:00 & 75.835 & 84.383 & 83.178 & 90.105 & 79.037 & 75.046 & 73.38 & 76.28 & 1582.637 & 1573.47 & 1570.13 & 1573.824 & 1569.591 & 1566.575 & 1563.911 & 1567.615 \\
\hline 9/5/16 7:00 & 75.837 & 84.379 & 83.133 & 90.105 & 79.011 & 75.051 & 73.392 & 76.273 & 1582.635 & 1573.474 & 1570.175 & 1573.824 & 1569.617 & 1566.57 & 1563.899 & 1567.622 \\
\hline 9/5/16 8:00 & 75.837 & 84.374 & 83.112 & 90.11 & 78.99 & 75.051 & 73.399 & 76.259 & 1582.635 & 1573.479 & 1570.196 & 1573.819 & 1569.638 & 1566.57 & 1563.892 & 1567.636 \\
\hline 9/5/16 9:00 & 75.837 & 84.37 & 83.096 & 90.101 & 78.957 & 75.049 & 73.397 & 76.257 & 1582.635 & 1573.483 & 1570.212 & 1573.828 & 1569.671 & 1566.572 & 1563.894 & 1567.638 \\
\hline 9/5/16 10:00 & 75.83 & 84.353 & 83.075 & 90.091 & 78.922 & 75.042 & 73.394 & 76.231 & 1582.642 & 1573.5 & 1570.233 & 1573.838 & 1569.706 & 1566.579 & 1563.897 & 1567.664 \\
\hline 9/5/16 11:00 & 75.826 & 84.346 & 83.066 & 90.086 & 78.899 & 75.042 & 73.399 & 76.22 & 1582.646 & 1573.507 & 1570.242 & 1573.843 & 1569.729 & 1566.579 & 1563.892 & 1567.675 \\
\hline 9/5/16 12:00 & 75.821 & 84.335 & 83.047 & 90.082 & 78.866 & 75.037 & 73.397 & 76.211 & 1582.651 & 1573.518 & 1570.261 & 1573.847 & 1569.762 & 1566.584 & 1563.894 & 1567.684 \\
\hline 9/5/16 13:00 & 75.816 & 84.318 & 83.033 & 90.075 & 78.836 & 75.032 & 73.394 & 76.197 & 1582.656 & 1573.535 & 1570.275 & 1573.854 & 1569.792 & 1566.589 & 1563.897 & 1567.698 \\
\hline 9/5/16 14:00 & 75.804 & 84.297 & 83.012 & 90.061 & 78.796 & 75.023 & 73.382 & 76.155 & 1582.668 & 1573.556 & 1570.296 & 1573.868 & 1569.832 & 1566.598 & 1563.909 & 1567.74 \\
\hline 9/5/16 15:00 & 75.795 & 84.281 & 82.989 & 90.054 & 78.766 & 75.011 & 73.375 & 76.111 & 1582.677 & 1573.572 & 1570.319 & 1573.875 & 1569.862 & 1566.61 & 1563.916 & 1567.784 \\
\hline $9 / 5 / 1616: 00$ & 75.786 & 84.251 & 82.961 & 90.04 & 78.719 & 74.997 & 73.361 & 76.12 & 1582.686 & 1573.602 & 1570.347 & 1573.889 & 1569.909 & 1566.624 & 1563.93 & 1567.775 \\
\hline 9/5/16 17:00 & 75.776 & 84.235 & 82.942 & 90.028 & 78.687 & 74.993 & 73.359 & 76.111 & 1582.696 & 1573.618 & 1570.366 & 1573.901 & 1569.941 & 1566.628 & 1563.932 & 1567.784 \\
\hline $9 / 5 / 1618: 00$ & 75.774 & 84.223 & 82.928 & 90.054 & 78.661 & 74.993 & 73.366 & 76.097 & 1582.698 & 1573.63 & 1570.38 & 1573.875 & 1569.967 & 1566.628 & 1563.925 & 1567.798 \\
\hline 9/5/16 19:00 & 75.771 & 84.216 & 82.921 & 90.063 & 78.636 & 74.997 & 73.373 & 76.081 & 1582.701 & 1573.637 & 1570.387 & 1573.866 & 1569.992 & 1566.624 & 1563.918 & 1567.814 \\
\hline 9/5/16 20:00 & 75.778 & 84.218 & 82.914 & 90.073 & 78.617 & 75.002 & 73.39 & 76.076 & 1582.694 & 1573.635 & 1570.394 & 1573.856 & 1570.011 & 1566.619 & 1563.901 & 1567.819 \\
\hline 9/5/16 21:00 & 75.781 & 84.211 & 82.912 & 90.049 & 78.603 & 75.007 & 73.394 & 76.078 & 1582.691 & 1573.642 & 1570.396 & 1573.88 & 1570.025 & 1566.614 & 1563.897 & 1567.817 \\
\hline 9/5/16 22:00 & 75.793 & 84.218 & 82.914 & 90.059 & 78.596 & 75.014 & 73.411 & 76.083 & 1582.679 & 1573.635 & 1570.394 & 1573.87 & 1570.032 & 1566.607 & 1563.88 & 1567.812 \\
\hline
\end{tabular}


TABLE S1.1 Automatically recorded groundwater levels in the unconfined aquifer.

\begin{tabular}{|c|c|c|c|c|c|c|c|c|c|c|c|c|c|c|c|c|}
\hline \multirow[b]{2}{*}{ Date and Time } & \multicolumn{8}{|c|}{ Measured Depth to Water (ft TOC ${ }^{\mathrm{a}}$ ) } & \multicolumn{8}{|c|}{ Groundwater Elevation (ft AMSL') } \\
\hline & DL1-C & DL2-C1 & DL3-C1 & DL4-C1 & DL5-C1 & DL6-C & DL7-C & DL8-C1 & DL1-C & DL2-C1 & DL3-C1 & DL4-C1 & DL5-C1 & DL6-C & DL7-C & DL8-C1 \\
\hline 9/5/16 23:00 & 75.795 & 84.221 & 82.912 & 90.07 & 78.582 & 75.016 & 73.416 & 76.078 & 1582.677 & 1573.632 & 1570.396 & 1573.859 & 1570.046 & 1566.605 & 1563.875 & 1567.817 \\
\hline 9/6/16 0:00 & 75.788 & 84.204 & 82.898 & 90.056 & 78.549 & 75.007 & 73.404 & 76.071 & 1582.684 & 1573.649 & 1570.41 & 1573.873 & 1570.079 & 1566.614 & 1563.887 & 1567.824 \\
\hline 9/6/16 1:00 & 75.767 & 84.176 & 82.87 & 90.042 & 78.514 & 74.988 & 73.39 & 76.036 & 1582.705 & 1573.677 & 1570.438 & 1573.887 & 1570.114 & 1566.633 & 1563.901 & 1567.859 \\
\hline 9/6/16 2:00 & 75.755 & 84.163 & 82.854 & 90.033 & 78.484 & 74.986 & 73.382 & 76.02 & 1582.717 & 1573.69 & 1570.454 & 1573.896 & 1570.144 & 1566.635 & 1563.909 & 1567.875 \\
\hline 9/6/16 3:00 & 75.745 & 84.142 & 82.835 & 90.021 & 78.449 & 74.976 & 73.375 & 76.011 & 1582.727 & 1573.711 & 1570.473 & 1573.908 & 1570.179 & 1566.645 & 1563.916 & 1567.884 \\
\hline 9/6/16 4:00 & 75.743 & 84.137 & 82.828 & 90.028 & 78.43 & 74.976 & 73.38 & 76.004 & 1582.729 & 1573.716 & 1570.48 & 1573.901 & 1570.198 & 1566.645 & 1563.911 & 1567.891 \\
\hline 9/6/16 5:00 & 75.738 & 84.123 & 82.814 & 89.996 & 78.4 & 74.969 & 73.375 & 75.99 & 1582.734 & 1573.73 & 1570.494 & 1573.933 & 1570.228 & 1566.652 & 1563.916 & 1567.905 \\
\hline 9/6/16 6:00 & 75.736 & 84.109 & 82.795 & 89.984 & 78.374 & 74.967 & 73.375 & 75.971 & 1582.736 & 1573.744 & 1570.513 & 1573.945 & 1570.254 & 1566.654 & 1563.916 & 1567.924 \\
\hline 9/6/16 7:00 & 75.762 & 84.128 & 82.807 & 90.003 & 78.381 & 74.995 & 73.421 & 75.981 & 1582.71 & 1573.725 & 1570.501 & 1573.926 & 1570.247 & 1566.626 & 1563.87 & 1567.914 \\
\hline 9/6/16 8:00 & 75.762 & 84.121 & 82.807 & 89.993 & 78.363 & 74.986 & 73.409 & 75.985 & 1582.71 & 1573.732 & 1570.501 & 1573.936 & 1570.265 & 1566.635 & 1563.882 & 1567.91 \\
\hline 9/6/169:00 & 75.75 & 84.104 & 82.791 & 89.979 & 78.335 & 74.988 & 73.399 & 76.076 & 1582.722 & 1573.749 & 1570.517 & 1573.95 & 1570.293 & 1566.633 & 1563.892 & 1567.819 \\
\hline $9 / 6 / 16$ 10:00 & 75.75 & 84.1 & 82.795 & 89.979 & 78.318 & 74.997 & 73.406 & 76.067 & 1582.722 & 1573.753 & 1570.513 & 1573.95 & 1570.31 & 1566.624 & 1563.885 & 1567.828 \\
\hline 9/6/16 11:00 & 75.75 & 84.095 & 82.786 & 89.975 & 78.3 & 74.986 & 73.404 & 76.004 & 1582.722 & 1573.758 & 1570.522 & 1573.954 & 1570.328 & 1566.635 & 1563.887 & 1567.891 \\
\hline $9 / 6 / 1612: 00$ & 75.748 & 84.088 & 82.772 & 89.975 & 78.279 & 74.979 & 73.406 & 75.962 & 1582.724 & 1573.765 & 1570.536 & 1573.954 & 1570.349 & 1566.642 & 1563.885 & 1567.933 \\
\hline 9/6/16 13:00 & 75.734 & 84.069 & 82.756 & 89.961 & 78.251 & 74.972 & 73.387 & 75.983 & 1582.738 & 1573.784 & 1570.552 & 1573.968 & 1570.377 & 1566.649 & 1563.904 & 1567.912 \\
\hline 9/6/16 14:00 & 75.727 & 84.062 & 82.749 & 89.958 & 78.234 & 74.967 & 73.392 & 75.946 & 1582.745 & 1573.791 & 1570.559 & 1573.971 & 1570.394 & 1566.654 & 1563.899 & 1567.949 \\
\hline $9 / 6 / 1615: 00$ & 75.715 & 84.039 & 82.728 & 89.944 & 78.204 & 74.958 & 73.378 & 75.944 & 1582.757 & 1573.814 & 1570.58 & 1573.985 & 1570.424 & 1566.663 & 1563.913 & 1567.951 \\
\hline $9 / 6 / 1616: 00$ & 75.703 & 84.023 & 82.707 & 89.94 & 78.174 & 74.949 & 73.373 & 75.934 & 1582.769 & 1573.83 & 1570.601 & 1573.989 & 1570.454 & 1566.672 & 1563.918 & 1567.961 \\
\hline 9/6/16 17:00 & 75.696 & 84.002 & 82.686 & 89.926 & 78.144 & 74.942 & 73.366 & 75.895 & 1582.776 & 1573.851 & 1570.622 & 1574.003 & 1570.484 & 1566.679 & 1563.925 & 1568 \\
\hline 9/6/16 18:00 & 75.689 & 83.983 & 82.669 & 89.919 & 78.116 & 74.932 & 73.361 & 75.862 & 1582.783 & 1573.87 & 1570.639 & 1574.01 & 1570.512 & 1566.689 & 1563.93 & 1568.033 \\
\hline 9/6/16 19:00 & 75.682 & 83.967 & 82.648 & 89.928 & 78.085 & 74.923 & 73.356 & 75.839 & 1582.79 & 1573.886 & 1570.66 & 1574.001 & 1570.543 & 1566.698 & 1563.935 & 1568.056 \\
\hline 9/6/16 20:00 & 75.694 & 83.974 & 82.651 & 89.947 & 78.081 & 74.941 & 73.38 & 75.841 & 1582.778 & 1573.879 & 1570.657 & 1573.982 & 1570.547 & 1566.68 & 1563.911 & 1568.054 \\
\hline 9/6/16 21:00 & 75.708 & 83.986 & 82.658 & 89.951 & 78.076 & 74.951 & 73.402 & 75.855 & 1582.764 & 1573.867 & 1570.65 & 1573.978 & 1570.552 & 1566.67 & 1563.889 & 1568.04 \\
\hline $9 / 6 / 1622: 00$ & 75.703 & 83.974 & 82.648 & 89.951 & 78.057 & 74.946 & 73.399 & 75.834 & 1582.769 & 1573.879 & 1570.66 & 1573.978 & 1570.571 & 1566.675 & 1563.892 & 1568.061 \\
\hline 9/6/16 23:00 & 75.689 & 83.955 & 82.627 & 89.942 & 78.027 & 74.93 & 73.382 & 75.765 & 1582.783 & 1573.898 & 1570.681 & 1573.987 & 1570.601 & 1566.691 & 1563.909 & 1568.13 \\
\hline 9/7/16 0:00 & 75.675 & 83.935 & 82.609 & 89.933 & 77.994 & 74.92 & 73.366 & 75.795 & 1582.797 & 1573.918 & 1570.699 & 1573.996 & 1570.634 & 1566.701 & 1563.925 & 1568.1 \\
\hline 9/7/16 1:00 & 75.654 & 83.907 & 82.576 & 89.897 & 77.955 & 74.9 & 73.347 & 75.762 & 1582.818 & 1573.946 & 1570.732 & 1574.032 & 1570.673 & 1566.721 & 1563.944 & 1568.133 \\
\hline 9/7/16 2:00 & 75.672 & 83.925 & 82.653 & 89.902 & 77.95 & 74.921 & 73.387 & 75.767 & 1582.8 & 1573.928 & 1570.655 & 1574.027 & 1570.678 & 1566.7 & 1563.904 & 1568.128 \\
\hline 9/7/16 3:00 & 75.694 & 83.937 & 82.679 & 89.907 & 77.962 & 74.937 & 73.404 & 75.802 & 1582.778 & 1573.916 & 1570.629 & 1574.022 & 1570.666 & 1566.684 & 1563.887 & 1568.093 \\
\hline 9/7/16 4:00 & 75.639 & 83.862 & 82.613 & 89.851 & 77.885 & 74.879 & 73.328 & 75.751 & 1582.833 & 1573.991 & 1570.695 & 1574.078 & 1570.743 & 1566.742 & 1563.963 & 1568.144 \\
\hline 9/7/16 5:00 & 75.658 & 83.886 & 82.667 & 89.881 & 77.892 & 74.907 & 73.378 & 75.751 & 1582.814 & 1573.967 & 1570.641 & 1574.048 & 1570.736 & 1566.714 & 1563.913 & 1568.144 \\
\hline 9/7/16 6:00 & 75.663 & 83.883 & 82.616 & 89.872 & 77.882 & 74.911 & 73.38 & 75.744 & 1582.809 & 1573.97 & 1570.692 & 1574.057 & 1570.746 & 1566.71 & 1563.911 & 1568.151 \\
\hline $9 / 7 / 167: 00$ & 75.672 & 83.888 & 82.585 & 89.876 & 77.875 & 74.916 & 73.39 & 75.739 & 1582.8 & 1573.965 & 1570.723 & 1574.053 & 1570.753 & 1566.705 & 1563.901 & 1568.156 \\
\hline 9/7/16 8:00 & 75.663 & 83.869 & 82.551 & 89.865 & 77.843 & 74.9 & 73.375 & 75.725 & 1582.809 & 1573.984 & 1570.757 & 1574.064 & 1570.785 & 1566.721 & 1563.916 & 1568.17 \\
\hline 9/7/16 9:00 & 75.656 & 83.858 & 82.532 & 89.853 & 77.822 & 74.895 & 73.366 & 75.658 & 1582.816 & 1573.995 & 1570.776 & 1574.076 & 1570.806 & 1566.726 & 1563.925 & 1568.237 \\
\hline $9 / 7 / 1610: 00$ & 75.642 & 83.837 & 82.506 & 89.842 & 77.789 & 74.881 & 73.354 & 75.679 & 1582.83 & 1574.016 & 1570.802 & 1574.087 & 1570.839 & 1566.74 & 1563.937 & 1568.216 \\
\hline 9/7/16 11:00 & 75.635 & 83.818 & 82.485 & 89.83 & 77.763 & 74.874 & 73.351 & 75.663 & 1582.837 & 1574.035 & 1570.823 & 1574.099 & 1570.865 & 1566.747 & 1563.94 & 1568.232 \\
\hline $9 / 7 / 1612: 00$ & 75.618 & 83.797 & 82.467 & 89.827 & 77.733 & 74.867 & 73.347 & 75.646 & 1582.854 & 1574.056 & 1570.841 & 1574.102 & 1570.895 & 1566.754 & 1563.944 & 1568.249 \\
\hline 9/7/16 13:00 & 75.595 & 83.762 & 82.427 & 89.795 & 77.682 & 74.837 & 73.311 & 75.612 & 1582.877 & 1574.091 & 1570.881 & 1574.134 & 1570.946 & 1566.784 & 1563.98 & 1568.283 \\
\hline 9/7/16 14:00 & 75.581 & 83.737 & 82.397 & 89.788 & 77.649 & 74.825 & 73.304 & 75.563 & 1582.891 & 1574.116 & 1570.911 & 1574.141 & 1570.979 & 1566.796 & 1563.987 & 1568.332 \\
\hline 9/7/16 15:00 & 75.576 & 83.714 & 82.378 & 89.781 & 77.617 & 74.82 & 73.299 & 75.567 & 1582.896 & 1574.139 & 1570.93 & 1574.148 & 1571.011 & 1566.801 & 1563.992 & 1568.328 \\
\hline $9 / 7 / 1616: 00$ & 75.566 & 83.69 & 82.355 & 89.769 & 77.582 & 74.809 & 73.292 & 75.535 & 1582.906 & 1574.163 & 1570.953 & 1574.16 & 1571.046 & 1566.812 & 1563.999 & 1568.36 \\
\hline $9 / 7 / 1617: 00$ & 75.562 & 83.667 & 82.334 & 89.76 & 77.551 & 74.797 & 73.287 & 75.523 & 1582.91 & 1574.186 & 1570.974 & 1574.169 & 1571.077 & 1566.824 & 1564.004 & 1568.372 \\
\hline $9 / 7 / 1618: 00$ & 75.559 & 83.655 & 82.317 & 89.781 & 77.523 & 74.792 & 73.289 & 75.488 & 1582.913 & 1574.198 & 1570.991 & 1574.148 & 1571.105 & 1566.829 & 1564.002 & 1568.407 \\
\hline $9 / 7 / 1619: 00$ & 75.571 & 83.653 & 82.313 & 89.795 & 77.512 & 74.799 & 73.301 & 75.491 & 1582.901 & 1574.2 & 1570.995 & 1574.134 & 1571.116 & 1566.822 & 1563.99 & 1568.404 \\
\hline $9 / 7 / 1620: 00$ & 75.576 & 83.646 & 82.303 & 89.785 & 77.491 & 74.802 & 73.306 & 75.488 & 1582.896 & 1574.207 & 1571.005 & 1574.144 & 1571.137 & 1566.819 & 1563.985 & 1568.407 \\
\hline 9/7/16 21:00 & 75.588 & 83.653 & 82.308 & 89.774 & 77.491 & 74.813 & 73.328 & 75.491 & 1582.884 & 1574.2 & 1571 & 1574.155 & 1571.137 & 1566.808 & 1563.963 & 1568.404 \\
\hline $9 / 7 / 1622: 00$ & 75.599 & 83.66 & 82.31 & 89.783 & 77.481 & 74.818 & 73.34 & 75.479 & 1582.873 & 1574.193 & 1570.998 & 1574.146 & 1571.147 & 1566.803 & 1563.951 & 1568.416 \\
\hline 9/7/16 23:00 & 75.597 & 83.651 & 82.301 & 89.792 & 77.47 & 74.813 & 73.337 & 75.484 & 1582.875 & 1574.202 & 1571.007 & 1574.137 & 1571.158 & 1566.808 & 1563.954 & 1568.411 \\
\hline
\end{tabular}


TABLE S1.1 Automatically recorded groundwater levels in the unconfined aquifer.

\begin{tabular}{|c|c|c|c|c|c|c|c|c|c|c|c|c|c|c|c|c|}
\hline \multirow[b]{2}{*}{ Date and Time } & \multicolumn{8}{|c|}{ Measured Depth to Water (ft TOC ${ }^{\mathrm{a}}$ ) } & \multicolumn{8}{|c|}{ Groundwater Elevation ( $\mathrm{ft} \mathrm{AMSL}^{\mathrm{b}}$ ) } \\
\hline & DL1-C & DL2-C1 & DL3-C1 & DL4-C1 & DL5-C1 & DL6-C & DL7-C & DL8-C1 & DL1-C & DL2-C1 & DL3-C1 & DL4-C1 & DL5-C1 & DL6-C & DL7-C & DL8-C1 \\
\hline $9 / 8 / 160: 00$ & 75.599 & 83.653 & 82.299 & 89.795 & 77.453 & 74.816 & 73.347 & 75.475 & 1582.873 & 1574.2 & 1571.009 & 1574.134 & 1571.175 & 1566.805 & 1563.944 & 1568.42 \\
\hline 9/8/16 1:00 & 75.595 & 83.641 & 82.289 & 89.783 & 77.439 & 74.809 & 73.335 & 75.47 & 1582.877 & 1574.212 & 1571.019 & 1574.146 & 1571.189 & 1566.812 & 1563.956 & 1568.425 \\
\hline 9/8/16 2:00 & 75.585 & 83.632 & 82.278 & 89.778 & 77.414 & 74.802 & 73.332 & 75.456 & 1582.887 & 1574.221 & 1571.03 & 1574.151 & 1571.214 & 1566.819 & 1563.959 & 1568.439 \\
\hline 9/8/16 3:00 & 75.585 & 83.625 & 82.276 & 89.771 & 77.404 & 74.804 & 73.335 & 75.461 & 1582.887 & 1574.228 & 1571.032 & 1574.158 & 1571.224 & 1566.817 & 1563.956 & 1568.434 \\
\hline $9 / 8 / 164: 00$ & 75.583 & 83.621 & 82.266 & 89.774 & 77.386 & 74.799 & 73.335 & 75.456 & 1582.889 & 1574.232 & 1571.042 & 1574.155 & 1571.242 & 1566.822 & 1563.956 & 1568.439 \\
\hline 9/8/16 5:00 & 75.592 & 83.63 & 82.271 & 89.762 & 77.381 & 74.811 & 73.351 & 75.454 & 1582.88 & 1574.223 & 1571.037 & 1574.167 & 1571.247 & 1566.81 & 1563.94 & 1568.441 \\
\hline 9/8/16 6:00 & 75.595 & 83.628 & 82.266 & 89.753 & 77.379 & 74.809 & 73.356 & 75.449 & 1582.877 & 1574.225 & 1571.042 & 1574.176 & 1571.249 & 1566.812 & 1563.935 & 1568.446 \\
\hline 9/8/16 7:00 & 75.597 & 83.632 & 82.266 & 89.75 & 77.367 & 74.816 & 73.366 & 75.447 & 1582.875 & 1574.221 & 1571.042 & 1574.179 & 1571.261 & 1566.805 & 1563.925 & 1568.448 \\
\hline 9/8/16 8:00 & 75.609 & 83.641 & 82.271 & 89.757 & 77.367 & 74.82 & 73.378 & 75.456 & 1582.863 & 1574.212 & 1571.037 & 1574.172 & 1571.261 & 1566.801 & 1563.913 & 1568.439 \\
\hline 9/8/16 9:00 & 75.597 & 83.63 & 82.262 & 89.743 & 77.351 & 74.816 & 73.363 & 75.449 & 1582.875 & 1574.223 & 1571.046 & 1574.186 & 1571.277 & 1566.805 & 1563.928 & 1568.446 \\
\hline $9 / 8 / 1610: 00$ & 75.599 & 83.632 & 82.259 & 89.743 & 77.344 & 74.816 & 73.371 & 75.437 & 1582.873 & 1574.221 & 1571.049 & 1574.186 & 1571.284 & 1566.805 & 1563.92 & 1568.458 \\
\hline 9/8/16 11:00 & 75.585 & 83.611 & 82.241 & 89.727 & 77.318 & 74.802 & 73.351 & 75.421 & 1582.887 & 1574.242 & 1571.067 & 1574.202 & 1571.31 & 1566.819 & 1563.94 & 1568.474 \\
\hline $9 / 8 / 1612: 00$ & 75.564 & 83.59 & 82.22 & 89.715 & 77.29 & 74.785 & 73.33 & 75.403 & 1582.908 & 1574.263 & 1571.088 & 1574.214 & 1571.338 & 1566.836 & 1563.961 & 1568.492 \\
\hline 9/8/16 13:00 & 75.54 & 83.555 & 82.192 & 89.694 & 77.25 & 74.762 & 73.304 & 75.37 & 1582.932 & 1574.298 & 1571.116 & 1574.235 & 1571.378 & 1566.859 & 1563.987 & 1568.525 \\
\hline 9/8/16 14:00 & 75.522 & 83.528 & 82.166 & 89.673 & 77.213 & 74.746 & 73.289 & 75.338 & 1582.95 & 1574.325 & 1571.142 & 1574.256 & 1571.415 & 1566.875 & 1564.002 & 1568.557 \\
\hline $9 / 8 / 1615: 00$ & 75.503 & 83.49 & 82.129 & 89.666 & 77.171 & 74.727 & 73.265 & 75.317 & 1582.969 & 1574.363 & 1571.179 & 1574.263 & 1571.457 & 1566.894 & 1564.026 & 1568.578 \\
\hline $9 / 8 / 1616: 00$ & 75.491 & 83.46 & 82.103 & 89.652 & 77.136 & 74.716 & 73.254 & 75.289 & 1582.981 & 1574.393 & 1571.205 & 1574.277 & 1571.492 & 1566.905 & 1564.037 & 1568.606 \\
\hline 9/8/16 17:00 & 75.482 & 83.432 & 82.066 & 89.634 & 77.097 & 74.699 & 73.239 & 75.252 & 1582.99 & 1574.421 & 1571.242 & 1574.295 & 1571.531 & 1566.922 & 1564.052 & 1568.643 \\
\hline $9 / 8 / 1618: 00$ & 75.484 & 83.411 & 82.047 & 89.629 & 77.071 & 74.69 & 73.239 & 75.233 & 1582.988 & 1574.442 & 1571.261 & 1574.3 & 1571.557 & 1566.931 & 1564.052 & 1568.662 \\
\hline 9/8/16 19:00 & 75.489 & 83.395 & 82.033 & 89.641 & 77.045 & 74.685 & 73.239 & 75.173 & 1582.983 & 1574.458 & 1571.275 & 1574.288 & 1571.583 & 1566.936 & 1564.052 & 1568.722 \\
\hline $9 / 8 / 1620: 00$ & 75.486 & 83.383 & 82.019 & 89.645 & 77.027 & 74.683 & 73.239 & 75.201 & 1582.986 & 1574.47 & 1571.289 & 1574.284 & 1571.601 & 1566.938 & 1564.052 & 1568.694 \\
\hline 9/8/16 21:00 & 75.496 & 83.383 & 82.014 & 89.645 & 77.013 & 74.688 & 73.254 & 75.191 & 1582.976 & 1574.47 & 1571.294 & 1574.284 & 1571.615 & 1566.933 & 1564.037 & 1568.704 \\
\hline $9 / 8 / 1622: 00$ & 75.498 & 83.372 & 82.005 & 89.648 & 76.994 & 74.678 & 73.256 & 75.131 & 1582.974 & 1574.481 & 1571.303 & 1574.281 & 1571.634 & 1566.943 & 1564.035 & 1568.764 \\
\hline $9 / 8 / 1623: 00$ & 75.5 & 83.365 & 81.996 & 89.645 & 76.978 & 74.676 & 73.256 & 75.168 & 1582.972 & 1574.488 & 1571.312 & 1574.284 & 1571.65 & 1566.945 & 1564.035 & 1568.727 \\
\hline 9/9/16 0:00 & 75.5 & 83.355 & 81.984 & 89.645 & 76.961 & 74.676 & 73.256 & 75.163 & 1582.972 & 1574.498 & 1571.324 & 1574.284 & 1571.667 & 1566.945 & 1564.035 & 1568.732 \\
\hline 9/9/16 1:00 & 75.491 & 83.334 & 81.968 & 89.617 & 76.936 & 74.664 & 73.242 & 75.145 & 1582.981 & 1574.519 & 1571.34 & 1574.312 & 1571.692 & 1566.957 & 1564.049 & 1568.75 \\
\hline 9/9/16 2:00 & 75.489 & 83.328 & 82.019 & 89.603 & 76.917 & 74.662 & 73.244 & 75.14 & 1582.983 & 1574.525 & 1571.289 & 1574.326 & 1571.711 & 1566.959 & 1564.047 & 1568.755 \\
\hline 9/9/16 3:00 & 75.491 & 83.323 & 82.033 & 89.594 & 76.903 & 74.657 & 73.246 & 75.14 & 1582.981 & 1574.53 & 1571.275 & 1574.335 & 1571.725 & 1566.964 & 1564.045 & 1568.755 \\
\hline 9/9/16 4:00 & 75.486 & 83.311 & 82.026 & 89.585 & 76.884 & 74.655 & 73.244 & 75.133 & 1582.986 & 1574.542 & 1571.282 & 1574.344 & 1571.744 & 1566.966 & 1564.047 & 1568.762 \\
\hline 9/9/16 5:00 & 75.498 & 83.316 & 82.04 & 89.592 & 76.884 & 74.662 & 73.261 & 75.147 & 1582.974 & 1574.537 & 1571.268 & 1574.337 & 1571.744 & 1566.959 & 1564.03 & 1568.748 \\
\hline 9/9/16 6:00 & 75.498 & 83.309 & 81.979 & 89.589 & 76.87 & 74.662 & 73.261 & 75.119 & 1582.974 & 1574.544 & 1571.329 & 1574.34 & 1571.758 & 1566.959 & 1564.03 & 1568.776 \\
\hline 9/9/16 7:00 & 75.507 & 83.314 & 81.945 & 89.589 & 76.863 & 74.664 & 73.268 & 75.115 & 1582.965 & 1574.539 & 1571.363 & 1574.34 & 1571.765 & 1566.957 & 1564.023 & 1568.78 \\
\hline 9/9/16 8:00 & 75.51 & 83.314 & 81.94 & 89.594 & 76.856 & 74.664 & 73.273 & 75.115 & 1582.962 & 1574.539 & 1571.368 & 1574.335 & 1571.772 & 1566.957 & 1564.018 & 1568.78 \\
\hline 9/9/16 9:00 & 75.51 & 83.309 & 81.933 & 89.589 & 76.847 & 74.664 & 73.273 & 75.105 & 1582.962 & 1574.544 & 1571.375 & 1574.34 & 1571.781 & 1566.957 & 1564.018 & 1568.79 \\
\hline $9 / 9 / 1610: 00$ & 75.515 & 83.311 & 81.933 & 89.592 & 76.845 & 74.667 & 73.282 & 75.105 & 1582.957 & 1574.542 & 1571.375 & 1574.337 & 1571.783 & 1566.954 & 1564.009 & 1568.79 \\
\hline 9/9/16 11:00 & 75.484 & 83.274 & 81.896 & 89.552 & 76.8 & 74.632 & 73.23 & 75.082 & 1582.988 & 1574.579 & 1571.412 & 1574.377 & 1571.828 & 1566.989 & 1564.061 & 1568.813 \\
\hline 9/9/16 12:00 & 75.482 & 83.272 & 81.884 & 89.564 & 76.786 & 74.634 & 73.242 & 75.066 & 1582.99 & 1574.581 & 1571.424 & 1574.365 & 1571.842 & 1566.987 & 1564.049 & 1568.829 \\
\hline 9/9/16 13:00 & 75.491 & 83.283 & 81.9 & 89.578 & 76.796 & 74.657 & 73.285 & 75.068 & 1582.981 & 1574.57 & 1571.408 & 1574.351 & 1571.832 & 1566.964 & 1564.006 & 1568.827 \\
\hline 9/9/16 14:00 & 75.486 & 83.279 & 81.889 & 89.566 & 76.779 & 74.655 & 73.287 & 75.064 & 1582.986 & 1574.574 & 1571.419 & 1574.363 & 1571.849 & 1566.966 & 1564.004 & 1568.831 \\
\hline 9/9/16 15:00 & 75.498 & 83.288 & 81.893 & 89.571 & 76.777 & 74.657 & 73.287 & 75.073 & 1582.974 & 1574.565 & 1571.415 & 1574.358 & 1571.851 & 1566.964 & 1564.004 & 1568.822 \\
\hline 9/9/16 16:00 & 75.512 & 83.302 & 81.903 & 89.58 & 76.782 & 74.669 & 73.308 & 75.077 & 1582.96 & 1574.551 & 1571.405 & 1574.349 & 1571.846 & 1566.952 & 1563.983 & 1568.818 \\
\hline $9 / 9 / 1617: 00$ & 75.545 & 83.341 & 81.938 & 89.608 & 76.814 & 74.699 & 73.344 & 75.119 & 1582.927 & 1574.512 & 1571.37 & 1574.321 & 1571.814 & 1566.922 & 1563.947 & 1568.776 \\
\hline 9/9/16 18:00 & 75.545 & 83.348 & 81.942 & 89.622 & 76.81 & 74.695 & 73.332 & 75.129 & 1582.927 & 1574.505 & 1571.366 & 1574.307 & 1571.818 & 1566.926 & 1563.959 & 1568.766 \\
\hline $9 / 9 / 1619: 00$ & 75.555 & 83.362 & 81.954 & 89.641 & 76.819 & 74.704 & 73.347 & 75.11 & 1582.917 & 1574.491 & 1571.354 & 1574.288 & 1571.809 & 1566.917 & 1563.944 & 1568.785 \\
\hline $9 / 9 / 1620: 00$ & 75.559 & 83.376 & 81.963 & 89.645 & 76.831 & 74.713 & 73.356 & 75.147 & 1582.913 & 1574.477 & 1571.345 & 1574.284 & 1571.797 & 1566.908 & 1563.935 & 1568.748 \\
\hline 9/9/16 21:00 & 75.559 & 83.381 & 81.968 & 89.624 & 76.831 & 74.713 & 73.354 & 75.156 & 1582.913 & 1574.472 & 1571.34 & 1574.305 & 1571.797 & 1566.908 & 1563.937 & 1568.739 \\
\hline 9/9/16 22:00 & 75.559 & 83.393 & 81.968 & 89.643 & 76.831 & 74.723 & 73.373 & 75.147 & 1582.913 & 1574.46 & 1571.34 & 1574.286 & 1571.797 & 1566.898 & 1563.918 & 1568.748 \\
\hline 9/9/16 23:00 & 75.583 & 83.425 & 81.998 & 89.671 & 76.859 & 74.746 & 73.392 & 75.177 & 1582.889 & 1574.428 & 1571.31 & 1574.258 & 1571.769 & 1566.875 & 1563.899 & 1568.718 \\
\hline 9/10/16 0:00 & 75.557 & 83.402 & 81.979 & 89.645 & 76.833 & 74.718 & 73.356 & 75.166 & 1582.915 & 1574.451 & 1571.329 & 1574.284 & 1571.795 & 1566.903 & 1563.935 & 1568.729 \\
\hline
\end{tabular}


TABLE S1.1 Automatically recorded groundwater levels in the unconfined aquifer.

\begin{tabular}{|c|c|c|c|c|c|c|c|c|c|c|c|c|c|c|c|c|}
\hline \multirow[b]{2}{*}{ Date and Time } & \multicolumn{8}{|c|}{ Measured Depth to Water (ft TOC ${ }^{\mathrm{a}}$ ) } & \multicolumn{8}{|c|}{ Groundwater Elevation ( $\mathrm{ft} \mathrm{AMSL}^{\mathrm{b}}$ ) } \\
\hline & DL1-C & DL2-C1 & DL3-C1 & DL4-C1 & DL5-C1 & DL6-C & DL7-C & DL8-C1 & DL1-C & DL2-C1 & DL3-C1 & DL4-C1 & DL5-C1 & DL6-C & DL7-C & DL8-C1 \\
\hline 9/10/16 1:00 & 75.54 & 83.39 & 81.966 & 89.641 & 76.817 & 74.709 & 73.342 & 75.152 & 1582.932 & 1574.463 & 1571.342 & 1574.288 & 1571.811 & 1566.912 & 1563.949 & 1568.743 \\
\hline $9 / 10 / 162: 00$ & 75.529 & 83.381 & 81.952 & 89.631 & 76.803 & 74.697 & 73.33 & 75.138 & 1582.943 & 1574.472 & 1571.356 & 1574.298 & 1571.825 & 1566.924 & 1563.961 & 1568.757 \\
\hline 9/10/16 3:00 & 75.526 & 83.379 & 81.949 & 89.629 & 76.796 & 74.699 & 73.33 & 75.14 & 1582.946 & 1574.474 & 1571.359 & 1574.3 & 1571.832 & 1566.922 & 1563.961 & 1568.755 \\
\hline 9/10/16 4:00 & 75.517 & 83.365 & 81.94 & 89.629 & 76.782 & 74.695 & 73.328 & 75.129 & 1582.955 & 1574.488 & 1571.368 & 1574.3 & 1571.846 & 1566.926 & 1563.963 & 1568.766 \\
\hline $9 / 10 / 165: 00$ & 75.517 & 83.372 & 81.952 & 89.61 & 76.777 & 74.697 & 73.33 & 75.117 & 1582.955 & 1574.481 & 1571.356 & 1574.319 & 1571.851 & 1566.924 & 1563.961 & 1568.778 \\
\hline $9 / 10 / 166: 00$ & 75.519 & 83.372 & 81.954 & 89.603 & 76.77 & 74.697 & 73.33 & 75.126 & 1582.953 & 1574.481 & 1571.354 & 1574.326 & 1571.858 & 1566.924 & 1563.961 & 1568.769 \\
\hline 9/10/16 7:00 & 75.522 & 83.372 & 81.954 & 89.603 & 76.772 & 74.699 & 73.34 & 75.136 & 1582.95 & 1574.481 & 1571.354 & 1574.326 & 1571.856 & 1566.922 & 1563.951 & 1568.759 \\
\hline 9/10/16 8:00 & 75.524 & 83.374 & 81.949 & 89.608 & 76.768 & 74.699 & 73.34 & 75.133 & 1582.948 & 1574.479 & 1571.359 & 1574.321 & 1571.86 & 1566.922 & 1563.951 & 1568.762 \\
\hline 9/10/16 9:00 & 75.524 & 83.374 & 81.94 & 89.603 & 76.761 & 74.702 & 73.342 & 75.131 & 1582.948 & 1574.479 & 1571.368 & 1574.326 & 1571.867 & 1566.919 & 1563.949 & 1568.764 \\
\hline 9/10/16 10:00 & 75.524 & 83.372 & 81.938 & 89.599 & 76.761 & 74.702 & 73.342 & 75.126 & 1582.948 & 1574.481 & 1571.37 & 1574.33 & 1571.867 & 1566.919 & 1563.949 & 1568.769 \\
\hline 9/10/16 11:00 & 75.517 & 83.362 & 81.933 & 89.589 & 76.744 & 74.692 & 73.335 & 75.119 & 1582.955 & 1574.491 & 1571.375 & 1574.34 & 1571.884 & 1566.929 & 1563.956 & 1568.776 \\
\hline 9/10/16 12:00 & 75.498 & 83.339 & 81.907 & 89.573 & 76.721 & 74.678 & 73.311 & 75.098 & 1582.974 & 1574.514 & 1571.401 & 1574.356 & 1571.907 & 1566.943 & 1563.98 & 1568.797 \\
\hline 9/10/16 13:00 & 75.477 & 83.314 & 81.882 & 89.559 & 76.693 & 74.657 & 73.289 & 75.075 & 1582.995 & 1574.539 & 1571.426 & 1574.37 & 1571.935 & 1566.964 & 1564.002 & 1568.82 \\
\hline $9 / 10 / 1614: 00$ & 75.463 & 83.295 & 81.861 & 89.549 & 76.668 & 74.646 & 73.277 & 75.052 & 1583.009 & 1574.558 & 1571.447 & 1574.38 & 1571.96 & 1566.975 & 1564.014 & 1568.843 \\
\hline 9/10/16 15:00 & 75.449 & 83.269 & 81.837 & 89.533 & 76.64 & 74.63 & 73.258 & 75.026 & 1583.023 & 1574.584 & 1571.471 & 1574.396 & 1571.988 & 1566.991 & 1564.033 & 1568.869 \\
\hline $9 / 10 / 1616: 00$ & 75.434 & 83.241 & 81.814 & 89.524 & 76.609 & 74.615 & 73.244 & 75.003 & 1583.038 & 1574.612 & 1571.494 & 1574.405 & 1572.019 & 1567.006 & 1564.047 & 1568.892 \\
\hline 9/10/16 17:00 & 75.423 & 83.214 & 81.788 & 89.507 & 76.577 & 74.599 & 73.227 & 74.978 & 1583.049 & 1574.639 & 1571.52 & 1574.422 & 1572.051 & 1567.022 & 1564.064 & 1568.917 \\
\hline 9/10/16 18:00 & 75.413 & 83.186 & 81.763 & 89.493 & 76.549 & 74.585 & 73.218 & 74.922 & 1583.059 & 1574.667 & 1571.545 & 1574.436 & 1572.079 & 1567.036 & 1564.073 & 1568.973 \\
\hline 9/10/16 19:00 & 75.411 & 83.169 & 81.744 & 89.507 & 76.528 & 74.585 & 73.218 & 74.945 & 1583.061 & 1574.684 & 1571.564 & 1574.422 & 1572.1 & 1567.036 & 1564.073 & 1568.95 \\
\hline 9/10/16 20:00 & 75.411 & 83.155 & 81.73 & 89.512 & 76.504 & 74.578 & 73.215 & 74.892 & 1583.061 & 1574.698 & 1571.578 & 1574.417 & 1572.124 & 1567.043 & 1564.076 & 1569.003 \\
\hline $9 / 10 / 1621: 00$ & 75.413 & 83.151 & 81.723 & 89.51 & 76.495 & 74.578 & 73.223 & 74.915 & 1583.059 & 1574.702 & 1571.585 & 1574.419 & 1572.133 & 1567.043 & 1564.068 & 1568.98 \\
\hline $9 / 10 / 1622: 00$ & 75.42 & 83.141 & 81.718 & 89.514 & 76.481 & 74.578 & 73.225 & 74.906 & 1583.052 & 1574.712 & 1571.59 & 1574.415 & 1572.147 & 1567.043 & 1564.066 & 1568.989 \\
\hline 9/10/16 23:00 & 75.423 & 83.141 & 81.716 & 89.521 & 76.474 & 74.578 & 73.234 & 74.903 & 1583.049 & 1574.712 & 1571.592 & 1574.408 & 1572.154 & 1567.043 & 1564.057 & 1568.992 \\
\hline $9 / 11 / 160: 00$ & 75.418 & 83.12 & 81.697 & 89.51 & 76.453 & 74.564 & 73.22 & 74.845 & 1583.054 & 1574.733 & 1571.611 & 1574.419 & 1572.175 & 1567.057 & 1564.071 & 1569.05 \\
\hline 9/11/16 1:00 & 75.413 & 83.114 & 81.688 & 89.491 & 76.439 & 74.562 & 73.218 & 74.882 & 1583.059 & 1574.739 & 1571.62 & 1574.438 & 1572.189 & 1567.059 & 1564.073 & 1569.013 \\
\hline $9 / 11 / 162: 00$ & 75.404 & 83.09 & 81.669 & 89.465 & 76.411 & 74.546 & 73.201 & 74.866 & 1583.068 & 1574.763 & 1571.639 & 1574.464 & 1572.217 & 1567.075 & 1564.09 & 1569.029 \\
\hline 9/11/16 3:00 & 75.399 & 83.079 & 81.656 & 89.454 & 76.397 & 74.546 & 73.201 & 74.813 & 1583.073 & 1574.774 & 1571.652 & 1574.475 & 1572.231 & 1567.075 & 1564.09 & 1569.082 \\
\hline 9/11/16 4:00 & 75.401 & 83.074 & 81.649 & 89.451 & 76.381 & 74.541 & 73.203 & 74.815 & 1583.071 & 1574.779 & 1571.659 & 1574.478 & 1572.247 & 1567.08 & 1564.088 & 1569.08 \\
\hline $9 / 11 / 165: 00$ & 75.387 & 83.044 & 81.625 & 89.437 & 76.353 & 74.525 & 73.184 & 74.813 & 1583.085 & 1574.809 & 1571.683 & 1574.492 & 1572.275 & 1567.096 & 1564.107 & 1569.082 \\
\hline $9 / 11 / 166: 00$ & 75.387 & 83.037 & 81.611 & 89.433 & 76.336 & 74.525 & 73.184 & 74.804 & 1583.085 & 1574.816 & 1571.697 & 1574.496 & 1572.292 & 1567.096 & 1564.107 & 1569.091 \\
\hline 9/11/16 7:00 & 75.385 & 83.025 & 81.602 & 89.426 & 76.322 & 74.52 & 73.189 & 74.794 & 1583.087 & 1574.828 & 1571.706 & 1574.503 & 1572.306 & 1567.101 & 1564.102 & 1569.101 \\
\hline 9/11/16 8:00 & 75.387 & 83.013 & 81.593 & 89.428 & 76.306 & 74.518 & 73.187 & 74.79 & 1583.085 & 1574.84 & 1571.715 & 1574.501 & 1572.322 & 1567.103 & 1564.104 & 1569.105 \\
\hline 9/11/16 9:00 & 75.383 & 83.004 & 81.579 & 89.414 & 76.285 & 74.504 & 73.177 & 74.718 & 1583.089 & 1574.849 & 1571.729 & 1574.515 & 1572.343 & 1567.117 & 1564.114 & 1569.177 \\
\hline 9/11/16 10:00 & 75.378 & 82.993 & 81.567 & 89.412 & 76.273 & 74.504 & 73.175 & 74.759 & 1583.094 & 1574.86 & 1571.741 & 1574.517 & 1572.355 & 1567.117 & 1564.116 & 1569.136 \\
\hline 9/11/16 11:00 & 75.373 & 82.976 & 81.555 & 89.402 & 76.252 & 74.494 & 73.168 & 74.743 & 1583.099 & 1574.877 & 1571.753 & 1574.527 & 1572.376 & 1567.127 & 1564.123 & 1569.152 \\
\hline 9/11/16 12:00 & 75.361 & 82.953 & 81.532 & 89.391 & 76.224 & 74.478 & 73.151 & 74.72 & 1583.111 & 1574.9 & 1571.776 & 1574.538 & 1572.404 & 1567.143 & 1564.14 & 1569.175 \\
\hline 9/11/16 13:00 & 75.34 & 82.92 & 81.502 & 89.37 & 76.187 & 74.455 & 73.129 & 74.655 & 1583.132 & 1574.933 & 1571.806 & 1574.559 & 1572.441 & 1567.166 & 1564.162 & 1569.24 \\
\hline $9 / 11 / 1614: 00$ & 75.321 & 82.888 & 81.471 & 89.353 & 76.152 & 74.436 & 73.106 & 74.666 & 1583.151 & 1574.965 & 1571.837 & 1574.576 & 1572.476 & 1567.185 & 1564.185 & 1569.229 \\
\hline 9/11/16 15:00 & 75.3 & 82.858 & 81.439 & 89.342 & 76.115 & 74.42 & 73.089 & 74.625 & 1583.172 & 1574.995 & 1571.869 & 1574.587 & 1572.513 & 1567.201 & 1564.202 & 1569.27 \\
\hline $9 / 11 / 1616: 00$ & 75.293 & 82.832 & 81.413 & 89.332 & 76.085 & 74.408 & 73.082 & 74.553 & 1583.179 & 1575.021 & 1571.895 & 1574.597 & 1572.543 & 1567.213 & 1564.209 & 1569.342 \\
\hline 9/11/16 17:00 & 75.286 & 82.816 & 81.399 & 89.325 & 76.061 & 74.401 & 73.082 & 74.592 & 1583.186 & 1575.037 & 1571.909 & 1574.604 & 1572.567 & 1567.22 & 1564.209 & 1569.303 \\
\hline $9 / 11 / 1618: 00$ & 75.288 & 82.799 & 81.383 & 89.344 & 76.038 & 74.399 & 73.079 & 74.564 & 1583.184 & 1575.054 & 1571.925 & 1574.585 & 1572.59 & 1567.222 & 1564.212 & 1569.331 \\
\hline 9/11/16 19:00 & 75.291 & 82.788 & 81.371 & 89.356 & 76.017 & 74.396 & 73.082 & 74.555 & 1583.181 & 1575.065 & 1571.937 & 1574.573 & 1572.611 & 1567.225 & 1564.209 & 1569.34 \\
\hline 9/11/16 20:00 & 75.295 & 82.786 & 81.366 & 89.349 & 76.01 & 74.399 & 73.089 & 74.548 & 1583.177 & 1575.067 & 1571.942 & 1574.58 & 1572.618 & 1567.222 & 1564.202 & 1569.347 \\
\hline $9 / 11 / 1621: 00$ & 75.298 & 82.781 & 81.357 & 89.325 & 75.996 & 74.394 & 73.094 & 74.541 & 1583.174 & 1575.072 & 1571.951 & 1574.604 & 1572.632 & 1567.227 & 1564.197 & 1569.354 \\
\hline 9/11/16 22:00 & 75.305 & 82.774 & 81.352 & 89.318 & 75.989 & 74.394 & 73.096 & 74.527 & 1583.167 & 1575.079 & 1571.956 & 1574.611 & 1572.639 & 1567.227 & 1564.195 & 1569.368 \\
\hline 9/11/16 23:00 & 75.302 & 82.765 & 81.343 & 89.307 & 75.968 & 74.389 & 73.091 & 74.525 & 1583.17 & 1575.088 & 1571.965 & 1574.622 & 1572.66 & 1567.232 & 1564.2 & 1569.37 \\
\hline $9 / 12 / 160: 00$ & 75.298 & 82.755 & 81.332 & 89.302 & 75.956 & 74.382 & 73.091 & 74.516 & 1583.174 & 1575.098 & 1571.976 & 1574.627 & 1572.672 & 1567.239 & 1564.2 & 1569.379 \\
\hline 9/12/16 1:00 & 75.295 & 82.746 & 81.32 & 89.297 & 75.942 & 74.378 & 73.084 & 74.502 & 1583.177 & 1575.107 & 1571.988 & 1574.632 & 1572.686 & 1567.243 & 1564.207 & 1569.393 \\
\hline
\end{tabular}


TABLE S1.1 Automatically recorded groundwater levels in the unconfined aquifer.

\begin{tabular}{|c|c|c|c|c|c|c|c|c|c|c|c|c|c|c|c|c|}
\hline \multirow[b]{2}{*}{ Date and Time } & \multicolumn{8}{|c|}{ Measured Depth to Water ( $\mathrm{ft}$ TOC ${ }^{\mathrm{a}}$ ) } & \multicolumn{8}{|c|}{ Groundwater Elevation (ft AMSL ${ }^{\mathrm{b}}$ ) } \\
\hline & DL1-C & DL2-C1 & DL3-C1 & DL4-C1 & DL5-C1 & DL6-C & DL7-C & DL8-C1 & DL1-C & DL2-C1 & DL3-C1 & DL4-C1 & DL5-C1 & DL6-C & DL7-C & DL8-C1 \\
\hline 9/12/16 2:00 & 75.295 & 82.741 & 81.376 & 89.295 & 75.928 & 74.373 & 73.084 & 74.495 & 1583.177 & 1575.112 & 1571.932 & 1574.634 & 1572.7 & 1567.248 & 1564.207 & 1569.4 \\
\hline 9/12/16 3:00 & 75.288 & 82.727 & 81.38 & 89.286 & 75.907 & 74.362 & 73.072 & 74.485 & 1583.184 & 1575.126 & 1571.928 & 1574.643 & 1572.721 & 1567.259 & 1564.219 & 1569.41 \\
\hline 9/12/16 4:00 & 75.277 & 82.711 & 81.35 & 89.274 & 75.886 & 74.355 & 73.063 & 74.472 & 1583.195 & 1575.142 & 1571.958 & 1574.655 & 1572.742 & 1567.266 & 1564.228 & 1569.423 \\
\hline 9/12/16 5:00 & 75.274 & 82.704 & 81.385 & 89.274 & 75.872 & 74.35 & 73.06 & 74.469 & 1583.198 & 1575.149 & 1571.923 & 1574.655 & 1572.756 & 1567.271 & 1564.231 & 1569.426 \\
\hline $9 / 12 / 166: 00$ & 75.279 & 82.695 & 81.329 & 89.272 & 75.861 & 74.348 & 73.063 & 74.458 & 1583.193 & 1575.158 & 1571.979 & 1574.657 & 1572.767 & 1567.273 & 1564.228 & 1569.437 \\
\hline 9/12/16 7:00 & 75.277 & 82.688 & 81.29 & 89.267 & 75.849 & 74.348 & 73.065 & 74.441 & 1583.195 & 1575.165 & 1572.018 & 1574.662 & 1572.779 & 1567.273 & 1564.226 & 1569.454 \\
\hline $9 / 12 / 168: 00$ & 75.281 & 82.693 & 81.285 & 89.272 & 75.842 & 74.345 & 73.065 & 74.439 & 1583.191 & 1575.16 & 1572.023 & 1574.657 & 1572.786 & 1567.276 & 1564.226 & 1569.456 \\
\hline 9/12/16 9:00 & 75.291 & 82.695 & 81.271 & 89.276 & 75.835 & 74.345 & 73.072 & 74.437 & 1583.181 & 1575.158 & 1572.037 & 1574.653 & 1572.793 & 1567.276 & 1564.219 & 1569.458 \\
\hline 9/12/16 10:00 & 75.314 & 82.725 & 81.285 & 89.297 & 75.856 & 74.373 & 73.11 & 74.453 & 1583.158 & 1575.128 & 1572.023 & 1574.632 & 1572.772 & 1567.248 & 1564.181 & 1569.442 \\
\hline 9/12/16 11:00 & 75.326 & 82.732 & 81.294 & 89.293 & 75.863 & 74.376 & 73.115 & 74.467 & 1583.146 & 1575.121 & 1572.014 & 1574.636 & 1572.765 & 1567.245 & 1564.176 & 1569.428 \\
\hline 9/12/16 12:00 & 75.321 & 82.73 & 81.287 & 89.286 & 75.854 & 74.369 & 73.106 & 74.458 & 1583.151 & 1575.123 & 1572.021 & 1574.643 & 1572.774 & 1567.252 & 1564.185 & 1569.437 \\
\hline 9/12/16 13:00 & 75.317 & 82.727 & 81.285 & 89.283 & 75.847 & 74.366 & 73.101 & 74.455 & 1583.155 & 1575.126 & 1572.023 & 1574.646 & 1572.781 & 1567.255 & 1564.19 & 1569.44 \\
\hline 9/12/16 14:00 & 75.314 & 82.73 & 81.271 & 89.281 & 75.84 & 74.362 & 73.101 & 74.451 & 1583.158 & 1575.123 & 1572.037 & 1574.648 & 1572.788 & 1567.259 & 1564.19 & 1569.444 \\
\hline 9/12/16 15:00 & 75.331 & 82.753 & 81.301 & 89.297 & 75.861 & 74.382 & 73.129 & 74.478 & 1583.141 & 1575.1 & 1572.007 & 1574.632 & 1572.767 & 1567.239 & 1564.162 & 1569.417 \\
\hline 9/12/16 16:00 & 75.338 & 82.767 & 81.308 & 89.3 & 75.863 & 74.385 & 73.134 & 74.481 & 1583.134 & 1575.086 & 1572 & 1574.629 & 1572.765 & 1567.236 & 1564.157 & 1569.414 \\
\hline 9/12/16 17:00 & 75.331 & 82.753 & 81.299 & 89.29 & 75.854 & 74.376 & 73.117 & 74.481 & 1583.141 & 1575.1 & 1572.009 & 1574.639 & 1572.774 & 1567.245 & 1564.174 & 1569.414 \\
\hline 9/12/16 18:00 & 75.324 & 82.758 & 81.287 & 89.288 & 75.847 & 74.376 & 73.117 & 74.441 & 1583.148 & 1575.095 & 1572.021 & 1574.641 & 1572.781 & 1567.245 & 1564.174 & 1569.454 \\
\hline 9/12/16 19:00 & 75.35 & 82.786 & 81.315 & 89.318 & 75.87 & 74.403 & 73.153 & 74.497 & 1583.122 & 1575.067 & 1571.993 & 1574.611 & 1572.758 & 1567.218 & 1564.138 & 1569.398 \\
\hline 9/12/16 20:00 & 75.364 & 82.809 & 81.329 & 89.342 & 75.886 & 74.41 & 73.163 & 74.516 & 1583.108 & 1575.044 & 1571.979 & 1574.587 & 1572.742 & 1567.211 & 1564.128 & 1569.379 \\
\hline 9/12/16 21:00 & 75.359 & 82.809 & 81.332 & 89.33 & 75.886 & 74.408 & 73.158 & 74.52 & 1583.113 & 1575.044 & 1571.976 & 1574.599 & 1572.742 & 1567.213 & 1564.133 & 1569.375 \\
\hline 9/12/16 22:00 & 75.328 & 82.797 & 81.301 & 89.311 & 75.87 & 74.385 & 73.187 & 74.462 & 1583.144 & 1575.056 & 1572.007 & 1574.618 & 1572.758 & 1567.236 & 1564.104 & 1569.433 \\
\hline 9/12/16 23:00 & 75.347 & 82.825 & 81.336 & 89.314 & 75.886 & 74.413 & 73.175 & 74.527 & 1583.125 & 1575.028 & 1571.972 & 1574.615 & 1572.742 & 1567.208 & 1564.116 & 1569.368 \\
\hline 9/13/16 0:00 & 75.345 & 82.825 & 81.346 & 89.311 & 75.886 & 74.415 & 73.17 & 74.539 & 1583.127 & 1575.028 & 1571.962 & 1574.618 & 1572.742 & 1567.206 & 1564.121 & 1569.356 \\
\hline 9/13/16 1:00 & 75.347 & 82.837 & 81.348 & 89.314 & 75.891 & 74.417 & 73.165 & 74.543 & 1583.125 & 1575.016 & 1571.96 & 1574.615 & 1572.737 & 1567.204 & 1564.126 & 1569.352 \\
\hline 9/13/16 2:00 & 75.333 & 82.82 & 81.336 & 89.304 & 75.875 & 74.408 & 73.151 & 74.534 & 1583.139 & 1575.033 & 1571.972 & 1574.625 & 1572.753 & 1567.213 & 1564.14 & 1569.361 \\
\hline 9/13/16 3:00 & 75.324 & 82.813 & 81.327 & 89.3 & 75.865 & 74.396 & 73.139 & 74.525 & 1583.148 & 1575.04 & 1571.981 & 1574.629 & 1572.763 & 1567.225 & 1564.152 & 1569.37 \\
\hline 9/13/16 4:00 & 75.328 & 82.823 & 81.332 & 89.307 & 75.872 & 74.408 & 73.153 & 74.527 & 1583.144 & 1575.03 & 1571.976 & 1574.622 & 1572.756 & 1567.213 & 1564.138 & 1569.368 \\
\hline 9/13/16 5:00 & 75.331 & 82.823 & 81.332 & 89.307 & 75.867 & 74.41 & 73.156 & 74.534 & 1583.141 & 1575.03 & 1571.976 & 1574.622 & 1572.761 & 1567.211 & 1564.135 & 1569.361 \\
\hline 9/13/16 6:00 & 75.338 & 82.837 & 81.338 & 89.309 & 75.875 & 74.415 & 73.163 & 74.537 & 1583.134 & 1575.016 & 1571.97 & 1574.62 & 1572.753 & 1567.206 & 1564.128 & 1569.358 \\
\hline 9/13/16 7:00 & 75.352 & 82.848 & 81.352 & 89.321 & 75.889 & 74.424 & 73.175 & 74.56 & 1583.12 & 1575.005 & 1571.956 & 1574.608 & 1572.739 & 1567.197 & 1564.116 & 1569.335 \\
\hline 9/13/16 8:00 & 75.366 & 82.869 & 81.387 & 89.335 & 75.907 & 74.443 & 73.196 & 74.576 & 1583.106 & 1574.984 & 1571.921 & 1574.594 & 1572.721 & 1567.178 & 1564.095 & 1569.319 \\
\hline 9/13/16 9:00 & 75.35 & 82.851 & 81.376 & 89.321 & 75.9 & 74.434 & 73.182 & 74.576 & 1583.122 & 1575.002 & 1571.932 & 1574.608 & 1572.728 & 1567.187 & 1564.109 & 1569.319 \\
\hline 9/13/16 10:00 & 75.347 & 82.86 & 81.352 & 89.321 & 75.882 & 74.422 & 73.168 & 74.555 & 1583.125 & 1574.993 & 1571.956 & 1574.608 & 1572.746 & 1567.199 & 1564.123 & 1569.34 \\
\hline 9/13/16 11:00 & 75.359 & 82.872 & 81.378 & 89.325 & 75.903 & 74.438 & 73.189 & 74.578 & 1583.113 & 1574.981 & 1571.93 & 1574.604 & 1572.725 & 1567.183 & 1564.102 & 1569.317 \\
\hline 9/13/16 12:00 & 75.352 & 82.869 & 81.366 & 89.323 & 75.896 & 74.427 & 73.175 & 74.52 & 1583.12 & 1574.984 & 1571.942 & 1574.606 & 1572.732 & 1567.194 & 1564.116 & 1569.375 \\
\hline 9/13/16 13:00 & 75.34 & 82.862 & 81.364 & 89.314 & 75.886 & 74.415 & 73.165 & 74.562 & 1583.132 & 1574.991 & 1571.944 & 1574.615 & 1572.742 & 1567.206 & 1564.126 & 1569.333 \\
\hline 9/13/16 14:00 & 75.328 & 82.851 & 81.352 & 89.302 & 75.877 & 74.413 & 73.156 & 74.553 & 1583.144 & 1575.002 & 1571.956 & 1574.627 & 1572.751 & 1567.208 & 1564.135 & 1569.342 \\
\hline 9/13/16 15:00 & 75.305 & 82.825 & 81.329 & 89.293 & 75.846 & 74.401 & 73.132 & 74.548 & 1583.167 & 1575.028 & 1571.979 & 1574.636 & 1572.782 & 1567.22 & 1564.159 & 1569.347 \\
\hline 9/13/16 16:00 & 75.312 & 82.83 & 81.329 & 89.3 & 75.847 & 74.399 & 73.146 & 74.532 & 1583.16 & 1575.023 & 1571.979 & 1574.629 & 1572.781 & 1567.222 & 1564.145 & 1569.363 \\
\hline 9/13/16 17:00 & 75.305 & 82.823 & 81.322 & 89.293 & 75.835 & 74.394 & 73.137 & 74.534 & 1583.167 & 1575.03 & 1571.986 & 1574.636 & 1572.793 & 1567.227 & 1564.154 & 1569.361 \\
\hline 9/13/16 18:00 & 75.307 & 82.816 & 81.322 & 89.316 & 75.83 & 74.396 & 73.139 & 74.539 & 1583.165 & 1575.037 & 1571.986 & 1574.613 & 1572.798 & 1567.225 & 1564.152 & 1569.356 \\
\hline 9/13/16 19:00 & 75.305 & 82.816 & 81.32 & 89.318 & 75.828 & 74.399 & 73.137 & 74.532 & 1583.167 & 1575.037 & 1571.988 & 1574.611 & 1572.8 & 1567.222 & 1564.154 & 1569.363 \\
\hline 9/13/16 20:00 & 75.302 & 82.804 & 81.311 & 89.295 & 75.816 & 74.387 & 73.132 & 74.525 & 1583.17 & 1575.049 & 1571.997 & 1574.634 & 1572.812 & 1567.234 & 1564.159 & 1569.37 \\
\hline 9/13/16 21:00 & 75.321 & 82.83 & 81.325 & 89.304 & 75.835 & 74.408 & 73.158 & 74.537 & 1583.151 & 1575.023 & 1571.983 & 1574.625 & 1572.793 & 1567.213 & 1564.133 & 1569.358 \\
\hline 9/13/16 22:00 & 75.326 & 82.832 & 81.336 & 89.302 & 75.84 & 74.41 & 73.16 & 74.548 & 1583.146 & 1575.021 & 1571.972 & 1574.627 & 1572.788 & 1567.211 & 1564.131 & 1569.347 \\
\hline 9/13/16 23:00 & 75.328 & 82.837 & 81.336 & 89.3 & 75.837 & 74.41 & 73.16 & 74.541 & 1583.144 & 1575.016 & 1571.972 & 1574.629 & 1572.791 & 1567.211 & 1564.131 & 1569.354 \\
\hline 9/14/16 0:00 & 75.321 & 82.825 & 81.327 & 89.291 & 75.83 & 74.406 & 73.153 & 74.543 & 1583.151 & 1575.028 & 1571.981 & 1574.638 & 1572.798 & 1567.215 & 1564.138 & 1569.352 \\
\hline 9/14/16 1:00 & 75.312 & 82.82 & 81.318 & 89.288 & 75.819 & 74.399 & 73.146 & 74.541 & 1583.16 & 1575.033 & 1571.99 & 1574.641 & 1572.809 & 1567.222 & 1564.145 & 1569.354 \\
\hline 9/14/16 2:00 & 75.298 & 82.8 & 81.366 & 89.276 & 75.802 & 74.385 & 73.127 & 74.527 & 1583.174 & 1575.053 & 1571.942 & 1574.653 & 1572.826 & 1567.236 & 1564.164 & 1569.368 \\
\hline
\end{tabular}


TABLE S1.1 Automatically recorded groundwater levels in the unconfined aquifer.

\begin{tabular}{|c|c|c|c|c|c|c|c|c|c|c|c|c|c|c|c|c|}
\hline \multirow[b]{2}{*}{ Date and Time } & \multicolumn{8}{|c|}{ Measured Depth to Water ( $\mathrm{ft}$ TOC ${ }^{\mathrm{a}}$ ) } & \multicolumn{8}{|c|}{ Groundwater Elevation (ft AMSL ${ }^{\mathrm{b}}$ ) } \\
\hline & DL1-C & DL2-C1 & DL3-C1 & DL4-C1 & DL5-C1 & DL6-C & DL7-C & DL8-C1 & DL1-C & DL2-C1 & DL3-C1 & DL4-C1 & DL5-C1 & DL6-C & DL7-C & DL8-C1 \\
\hline 9/14/16 3:00 & 75.291 & 82.797 & 81.373 & 89.274 & 75.793 & 74.383 & 73.125 & 74.523 & 1583.181 & 1575.056 & 1571.935 & 1574.655 & 1572.835 & 1567.238 & 1564.166 & 1569.372 \\
\hline 9/14/16 4:00 & 75.284 & 82.783 & 81.371 & 89.267 & 75.777 & 74.373 & 73.113 & 74.513 & 1583.188 & 1575.07 & 1571.937 & 1574.662 & 1572.851 & 1567.248 & 1564.178 & 1569.382 \\
\hline 9/14/16 5:00 & 75.279 & 82.774 & 81.39 & 89.262 & 75.765 & 74.369 & 73.108 & 74.495 & 1583.193 & 1575.079 & 1571.918 & 1574.667 & 1572.863 & 1567.252 & 1564.183 & 1569.4 \\
\hline 9/14/16 6:00 & 75.274 & 82.765 & 81.332 & 89.26 & 75.753 & 74.364 & 73.103 & 74.492 & 1583.198 & 1575.088 & 1571.976 & 1574.669 & 1572.875 & 1567.257 & 1564.188 & 1569.403 \\
\hline 9/14/16 7:00 & 75.281 & 82.765 & 81.297 & 89.265 & 75.749 & 74.366 & 73.11 & 74.49 & 1583.191 & 1575.088 & 1572.011 & 1574.664 & 1572.879 & 1567.255 & 1564.181 & 1569.405 \\
\hline 9/14/16 8:00 & 75.286 & 82.769 & 81.29 & 89.269 & 75.749 & 74.371 & 73.117 & 74.495 & 1583.186 & 1575.084 & 1572.018 & 1574.66 & 1572.879 & 1567.25 & 1564.174 & 1569.4 \\
\hline 9/14/16 9:00 & 75.284 & 82.758 & 81.276 & 89.26 & 75.737 & 74.364 & 73.11 & 74.485 & 1583.188 & 1575.095 & 1572.032 & 1574.669 & 1572.891 & 1567.257 & 1564.181 & 1569.41 \\
\hline 9/14/16 10:00 & 75.279 & 82.751 & 81.262 & 89.255 & 75.73 & 74.357 & 73.106 & 74.472 & 1583.193 & 1575.102 & 1572.046 & 1574.674 & 1572.898 & 1567.264 & 1564.185 & 1569.423 \\
\hline 9/14/16 11:00 & 75.272 & 82.741 & 81.252 & 89.248 & 75.716 & 74.352 & 73.098 & 74.472 & 1583.2 & 1575.112 & 1572.056 & 1574.681 & 1572.912 & 1567.269 & 1564.193 & 1569.423 \\
\hline 9/14/16 12:00 & 75.265 & 82.727 & 81.238 & 89.241 & 75.7 & 74.343 & 73.086 & 74.453 & 1583.207 & 1575.126 & 1572.07 & 1574.688 & 1572.928 & 1567.278 & 1564.205 & 1569.442 \\
\hline 9/14/16 13:00 & 75.253 & 82.706 & 81.217 & 89.225 & 75.679 & 74.329 & 73.07 & 74.432 & 1583.219 & 1575.147 & 1572.091 & 1574.704 & 1572.949 & 1567.292 & 1564.221 & 1569.463 \\
\hline 9/14/16 14:00 & 75.234 & 82.681 & 81.194 & 89.213 & 75.656 & 74.313 & 73.055 & 74.413 & 1583.238 & 1575.172 & 1572.114 & 1574.716 & 1572.972 & 1567.308 & 1564.236 & 1569.482 \\
\hline 9/14/16 15:00 & 75.215 & 82.648 & 81.164 & 89.195 & 75.623 & 74.294 & 73.029 & 74.383 & 1583.257 & 1575.205 & 1572.144 & 1574.734 & 1573.005 & 1567.327 & 1564.262 & 1569.512 \\
\hline 9/14/16 16:00 & 75.196 & 82.623 & 81.138 & 89.183 & 75.593 & 74.278 & 73.015 & 74.353 & 1583.276 & 1575.23 & 1572.17 & 1574.746 & 1573.035 & 1567.343 & 1564.276 & 1569.542 \\
\hline 9/14/16 17:00 & 75.187 & 82.597 & 81.119 & 89.171 & 75.562 & 74.266 & 73 & 74.332 & 1583.285 & 1575.256 & 1572.189 & 1574.758 & 1573.066 & 1567.355 & 1564.291 & 1569.563 \\
\hline 9/14/16 18:00 & 75.187 & 82.581 & 81.101 & 89.167 & 75.541 & 74.262 & 73.003 & 74.321 & 1583.285 & 1575.272 & 1572.207 & 1574.762 & 1573.087 & 1567.359 & 1564.288 & 1569.574 \\
\hline 9/14/16 19:00 & 75.194 & 82.572 & 81.089 & 89.167 & 75.53 & 74.261 & 73.005 & 74.309 & 1583.278 & 1575.281 & 1572.219 & 1574.762 & 1573.098 & 1567.36 & 1564.286 & 1569.586 \\
\hline $9 / 14 / 1620: 00$ & 75.201 & 82.565 & 81.084 & 89.167 & 75.52 & 74.264 & 73.01 & 74.272 & 1583.271 & 1575.288 & 1572.224 & 1574.762 & 1573.108 & 1567.357 & 1564.281 & 1569.623 \\
\hline 9/14/16 21:00 & 75.206 & 82.565 & 81.082 & 89.167 & 75.513 & 74.266 & 73.02 & 74.302 & 1583.266 & 1575.288 & 1572.226 & 1574.762 & 1573.115 & 1567.355 & 1564.271 & 1569.593 \\
\hline 9/14/16 22:00 & 75.215 & 82.567 & 81.08 & 89.169 & 75.511 & 74.266 & 73.029 & 74.293 & 1583.257 & 1575.286 & 1572.228 & 1574.76 & 1573.117 & 1567.355 & 1564.262 & 1569.602 \\
\hline 9/14/16 23:00 & 75.22 & 82.565 & 81.077 & 89.169 & 75.509 & 74.268 & 73.032 & 74.29 & 1583.252 & 1575.288 & 1572.231 & 1574.76 & 1573.119 & 1567.353 & 1564.259 & 1569.605 \\
\hline 9/15/16 0:00 & 75.218 & 82.562 & 81.075 & 89.164 & 75.499 & 74.261 & 73.027 & 74.293 & 1583.254 & 1575.291 & 1572.233 & 1574.765 & 1573.129 & 1567.36 & 1564.264 & 1569.602 \\
\hline 9/15/16 1:00 & 75.208 & 82.551 & 81.061 & 89.157 & 75.483 & 74.257 & 73.015 & 74.27 & 1583.264 & 1575.302 & 1572.247 & 1574.772 & 1573.145 & 1567.364 & 1564.276 & 1569.625 \\
\hline 9/15/16 2:00 & 75.196 & 82.53 & 81.047 & 89.146 & 75.464 & 74.243 & 73.003 & 74.26 & 1583.276 & 1575.323 & 1572.261 & 1574.783 & 1573.164 & 1567.378 & 1564.288 & 1569.635 \\
\hline 9/15/16 3:00 & 75.187 & 82.52 & 81.033 & 89.136 & 75.448 & 74.238 & 72.996 & 74.246 & 1583.285 & 1575.333 & 1572.275 & 1574.793 & 1573.18 & 1567.383 & 1564.295 & 1569.649 \\
\hline 9/15/16 4:00 & 75.18 & 82.499 & 81.017 & 89.127 & 75.422 & 74.224 & 72.981 & 74.225 & 1583.292 & 1575.354 & 1572.291 & 1574.802 & 1573.206 & 1567.397 & 1564.31 & 1569.67 \\
\hline 9/15/16 5:00 & 75.161 & 82.474 & 80.991 & 89.113 & 75.397 & 74.208 & 72.965 & 74.207 & 1583.311 & 1575.379 & 1572.317 & 1574.816 & 1573.231 & 1567.413 & 1564.326 & 1569.688 \\
\hline 9/15/16 6:00 & 75.154 & 82.455 & 80.97 & 89.111 & 75.371 & 74.201 & 72.955 & 74.191 & 1583.318 & 1575.398 & 1572.338 & 1574.818 & 1573.257 & 1567.42 & 1564.336 & 1569.704 \\
\hline 9/15/16 7:00 & 75.161 & 82.448 & 80.963 & 89.106 & 75.362 & 74.199 & 72.958 & 74.179 & 1583.311 & 1575.405 & 1572.345 & 1574.823 & 1573.266 & 1567.422 & 1564.333 & 1569.716 \\
\hline 9/15/16 8:00 & 75.168 & 82.446 & 80.963 & 89.111 & 75.362 & 74.203 & 72.969 & 74.167 & 1583.304 & 1575.407 & 1572.345 & 1574.818 & 1573.266 & 1567.418 & 1564.322 & 1569.728 \\
\hline 9/15/16 9:00 & 75.166 & 82.441 & 80.959 & 89.106 & 75.343 & 74.199 & 72.962 & 74.167 & 1583.306 & 1575.412 & 1572.349 & 1574.823 & 1573.285 & 1567.422 & 1564.329 & 1569.728 \\
\hline 9/15/16 10:00 & 75.166 & 82.434 & 80.947 & 89.101 & 75.334 & 74.192 & 72.96 & 74.158 & 1583.306 & 1575.419 & 1572.361 & 1574.828 & 1573.294 & 1567.429 & 1564.331 & 1569.737 \\
\hline 9/15/16 11:00 & 75.163 & 82.425 & 80.94 & 89.097 & 75.322 & 74.189 & 72.958 & 74.151 & 1583.309 & 1575.428 & 1572.368 & 1574.832 & 1573.306 & 1567.432 & 1564.333 & 1569.744 \\
\hline 9/15/16 12:00 & 75.154 & 82.402 & 80.919 & 89.083 & 75.299 & 74.173 & 72.943 & 74.133 & 1583.318 & 1575.451 & 1572.389 & 1574.846 & 1573.329 & 1567.448 & 1564.348 & 1569.762 \\
\hline 9/15/16 13:00 & 75.147 & 82.392 & 80.903 & 89.078 & 75.278 & 74.166 & 72.931 & 74.116 & 1583.325 & 1575.461 & 1572.405 & 1574.851 & 1573.35 & 1567.455 & 1564.36 & 1569.779 \\
\hline 9/15/16 14:00 & 75.135 & 82.369 & 80.882 & 89.064 & 75.257 & 74.15 & 72.915 & 74.095 & 1583.337 & 1575.484 & 1572.426 & 1574.865 & 1573.371 & 1567.471 & 1564.376 & 1569.8 \\
\hline 9/15/16 15:00 & 75.121 & 82.344 & 80.861 & 89.055 & 75.226 & 74.136 & 72.898 & 74.07 & 1583.351 & 1575.509 & 1572.447 & 1574.874 & 1573.402 & 1567.485 & 1564.393 & 1569.825 \\
\hline 9/15/16 16:00 & 75.114 & 82.325 & 80.837 & 89.045 & 75.208 & 74.126 & 72.891 & 74.049 & 1583.358 & 1575.528 & 1572.471 & 1574.884 & 1573.42 & 1567.495 & 1564.4 & 1569.846 \\
\hline 9/15/16 17:00 & 75.105 & 82.304 & 80.835 & 89.034 & 75.18 & 74.112 & 72.879 & 74.028 & 1583.367 & 1575.549 & 1572.473 & 1574.895 & 1573.448 & 1567.509 & 1564.412 & 1569.867 \\
\hline 9/15/16 18:00 & 75.105 & 82.295 & 80.807 & 89.031 & 75.164 & 74.11 & 72.879 & 74.012 & 1583.367 & 1575.558 & 1572.501 & 1574.898 & 1573.464 & 1567.511 & 1564.412 & 1569.883 \\
\hline 9/15/16 19:00 & 75.116 & 82.295 & 80.807 & 89.038 & 75.159 & 74.119 & 72.888 & 74.012 & 1583.356 & 1575.558 & 1572.501 & 1574.891 & 1573.469 & 1567.502 & 1564.403 & 1569.883 \\
\hline 9/15/16 20:00 & 75.126 & 82.299 & 80.807 & 89.045 & 75.159 & 74.124 & 72.902 & 74.016 & 1583.346 & 1575.554 & 1572.501 & 1574.884 & 1573.469 & 1567.497 & 1564.389 & 1569.879 \\
\hline 9/15/16 21:00 & 75.123 & 82.295 & 80.8 & 89.031 & 75.145 & 74.115 & 72.895 & 74.014 & 1583.349 & 1575.558 & 1572.508 & 1574.898 & 1573.483 & 1567.506 & 1564.396 & 1569.881 \\
\hline 9/15/16 22:00 & 75.123 & 82.281 & 80.788 & 89.027 & 75.136 & 74.108 & 72.891 & 73.998 & 1583.349 & 1575.572 & 1572.52 & 1574.902 & 1573.492 & 1567.513 & 1564.4 & 1569.897 \\
\hline 9/15/16 23:00 & 75.161 & 82.32 & 80.816 & 89.057 & 75.161 & 74.145 & 72.938 & 74.028 & 1583.311 & 1575.533 & 1572.492 & 1574.872 & 1573.467 & 1567.476 & 1564.353 & 1569.867 \\
\hline 9/16/16 0:00 & 75.253 & 82.43 & 80.914 & 89.132 & 75.261 & 74.226 & 73.048 & 74.114 & 1583.219 & 1575.423 & 1572.394 & 1574.797 & 1573.367 & 1567.395 & 1564.243 & 1569.781 \\
\hline 9/16/16 1:00 & 75.251 & 82.441 & 80.926 & 89.113 & 75.266 & 74.212 & 73.039 & 74.135 & 1583.221 & 1575.412 & 1572.382 & 1574.816 & 1573.362 & 1567.409 & 1564.252 & 1569.76 \\
\hline 9/16/16 2:00 & 75.18 & 82.376 & 80.928 & 89.052 & 75.196 & 74.145 & 72.943 & 74.086 & 1583.292 & 1575.477 & 1572.38 & 1574.877 & 1573.432 & 1567.476 & 1564.348 & 1569.809 \\
\hline 9/16/16 3:00 & 75.163 & 82.367 & 80.938 & 89.048 & 75.189 & 74.14 & 72.919 & 74.028 & 1583.309 & 1575.486 & 1572.37 & 1574.881 & 1573.439 & 1567.481 & 1564.372 & 1569.867 \\
\hline
\end{tabular}


TABLE S1.1 Automatically recorded groundwater levels in the unconfined aquifer.

\begin{tabular}{|c|c|c|c|c|c|c|c|c|c|c|c|c|c|c|c|c|}
\hline \multirow[b]{2}{*}{ Date and Time } & \multicolumn{8}{|c|}{ Measured Depth to Water ( $\mathrm{ft}$ TOC ${ }^{\mathrm{a}}$ ) } & \multicolumn{8}{|c|}{ Groundwater Elevation (ft AMSL ${ }^{\mathrm{b}}$ ) } \\
\hline & DL1-C & DL2-C1 & DL3-C1 & DL4-C1 & DL5-C1 & DL6-C & DL7-C & DL8-C1 & DL1-C & DL2-C1 & DL3-C1 & DL4-C1 & DL5-C1 & DL6-C & DL7-C & DL8-C1 \\
\hline 9/16/16 4:00 & 75.161 & 82.36 & 80.926 & 89.055 & 75.184 & 74.145 & 72.934 & 74.077 & 1583.311 & 1575.493 & 1572.382 & 1574.874 & 1573.444 & 1567.476 & 1564.357 & 1569.818 \\
\hline 9/16/16 5:00 & 75.168 & 82.379 & 80.954 & 89.066 & 75.196 & 74.159 & 72.955 & 74.077 & 1583.304 & 1575.474 & 1572.354 & 1574.863 & 1573.432 & 1567.462 & 1564.336 & 1569.818 \\
\hline 9/16/16 6:00 & 75.178 & 82.385 & 80.912 & 89.071 & 75.196 & 74.164 & 72.955 & 74.086 & 1583.294 & 1575.468 & 1572.396 & 1574.858 & 1573.432 & 1567.457 & 1564.336 & 1569.809 \\
\hline 9/16/16 7:00 & 75.178 & 82.383 & 80.877 & 89.066 & 75.198 & 74.161 & 72.958 & 74.086 & 1583.294 & 1575.47 & 1572.431 & 1574.863 & 1573.43 & 1567.46 & 1564.333 & 1569.809 \\
\hline 9/16/16 8:00 & 75.182 & 82.397 & 80.879 & 89.073 & 75.201 & 74.168 & 72.967 & 74.093 & 1583.29 & 1575.456 & 1572.429 & 1574.856 & 1573.427 & 1567.453 & 1564.324 & 1569.802 \\
\hline 9/16/16 9:00 & 75.18 & 82.392 & 80.879 & 89.069 & 75.201 & 74.164 & 72.955 & 74.091 & 1583.292 & 1575.461 & 1572.429 & 1574.86 & 1573.427 & 1567.457 & 1564.336 & 1569.804 \\
\hline 9/16/16 10:00 & 75.175 & 82.397 & 80.877 & 89.069 & 75.196 & 74.161 & 72.955 & 74.042 & 1583.297 & 1575.456 & 1572.431 & 1574.86 & 1573.432 & 1567.46 & 1564.336 & 1569.853 \\
\hline 9/16/16 11:00 & 75.175 & 82.395 & 80.877 & 89.069 & 75.191 & 74.161 & 72.953 & 74.105 & 1583.297 & 1575.458 & 1572.431 & 1574.86 & 1573.437 & 1567.46 & 1564.338 & 1569.79 \\
\hline 9/16/16 12:00 & 75.161 & 82.379 & 80.865 & 89.052 & 75.177 & 74.147 & 72.934 & 74.095 & 1583.311 & 1575.474 & 1572.443 & 1574.877 & 1573.451 & 1567.474 & 1564.357 & 1569.8 \\
\hline 9/16/16 13:00 & 75.154 & 82.369 & 80.854 & 89.048 & 75.166 & 74.138 & 72.922 & 74.081 & 1583.318 & 1575.484 & 1572.454 & 1574.881 & 1573.462 & 1567.483 & 1564.369 & 1569.814 \\
\hline 9/16/16 14:00 & 75.145 & 82.36 & 80.842 & 89.045 & 75.15 & 74.133 & 72.917 & 74.068 & 1583.327 & 1575.493 & 1572.466 & 1574.884 & 1573.478 & 1567.488 & 1564.374 & 1569.827 \\
\hline 9/16/16 15:00 & 75.14 & 82.351 & 80.835 & 89.043 & 75.14 & 74.129 & 72.915 & 74.054 & 1583.332 & 1575.502 & 1572.473 & 1574.886 & 1573.488 & 1567.492 & 1564.376 & 1569.841 \\
\hline 9/16/16 16:00 & 75.135 & 82.344 & 80.819 & 89.038 & 75.133 & 74.122 & 72.91 & 74.044 & 1583.337 & 1575.509 & 1572.489 & 1574.891 & 1573.495 & 1567.499 & 1564.381 & 1569.851 \\
\hline 9/16/16 17:00 & 75.133 & 82.332 & 80.809 & 89.031 & 75.119 & 74.115 & 72.9 & 74.03 & 1583.339 & 1575.521 & 1572.499 & 1574.898 & 1573.509 & 1567.506 & 1564.391 & 1569.865 \\
\hline 9/16/16 18:00 & 75.135 & 82.334 & 80.807 & 89.034 & 75.112 & 74.117 & 72.907 & 74.028 & 1583.337 & 1575.519 & 1572.501 & 1574.895 & 1573.516 & 1567.504 & 1564.384 & 1569.867 \\
\hline 9/16/16 19:00 & 75.14 & 82.334 & 80.807 & 89.038 & 75.117 & 74.124 & 72.915 & 74.026 & 1583.332 & 1575.519 & 1572.501 & 1574.891 & 1573.511 & 1567.497 & 1564.376 & 1569.869 \\
\hline 9/16/16 20:00 & 75.147 & 82.337 & 80.809 & 89.038 & 75.117 & 74.124 & 72.917 & 74.019 & 1583.325 & 1575.516 & 1572.499 & 1574.891 & 1573.511 & 1567.497 & 1564.374 & 1569.876 \\
\hline 9/16/16 21:00 & 75.156 & 82.353 & 80.821 & 89.045 & 75.126 & 74.138 & 72.934 & 74.023 & 1583.316 & 1575.5 & 1572.487 & 1574.884 & 1573.502 & 1567.483 & 1564.357 & 1569.872 \\
\hline 9/16/16 22:00 & 75.163 & 82.36 & 80.826 & 89.048 & 75.129 & 74.143 & 72.936 & 74.047 & 1583.309 & 1575.493 & 1572.482 & 1574.881 & 1573.499 & 1567.478 & 1564.355 & 1569.848 \\
\hline 9/16/16 23:00 & 75.166 & 82.36 & 80.828 & 89.045 & 75.131 & 74.138 & 72.934 & 74.051 & 1583.306 & 1575.493 & 1572.48 & 1574.884 & 1573.497 & 1567.483 & 1564.357 & 1569.844 \\
\hline 9/17/16 0:00 & 75.161 & 82.362 & 80.828 & 89.048 & 75.126 & 74.133 & 72.931 & 74.035 & 1583.311 & 1575.491 & 1572.48 & 1574.881 & 1573.502 & 1567.488 & 1564.36 & 1569.86 \\
\hline 9/17/16 1:00 & 75.161 & 82.365 & 80.826 & 89.043 & 75.126 & 74.133 & 72.929 & 74.051 & 1583.311 & 1575.488 & 1572.482 & 1574.886 & 1573.502 & 1567.488 & 1564.362 & 1569.844 \\
\hline 9/17/16 2:00 & 75.168 & 82.374 & 80.833 & 89.048 & 75.133 & 74.14 & 72.941 & 74.058 & 1583.304 & 1575.479 & 1572.475 & 1574.881 & 1573.495 & 1567.481 & 1564.35 & 1569.837 \\
\hline 9/17/16 3:00 & 75.156 & 82.362 & 80.826 & 89.041 & 75.119 & 74.133 & 72.929 & 74.051 & 1583.316 & 1575.491 & 1572.482 & 1574.888 & 1573.509 & 1567.488 & 1564.362 & 1569.844 \\
\hline 9/17/16 4:00 & 75.147 & 82.355 & 80.812 & 89.031 & 75.112 & 74.124 & 72.917 & 74.042 & 1583.325 & 1575.498 & 1572.496 & 1574.898 & 1573.516 & 1567.497 & 1564.374 & 1569.853 \\
\hline 9/17/16 5:00 & 75.142 & 82.353 & 80.812 & 89.031 & 75.105 & 74.124 & 72.915 & 74.03 & 1583.33 & 1575.5 & 1572.496 & 1574.898 & 1573.523 & 1567.497 & 1564.376 & 1569.865 \\
\hline 9/17/16 6:00 & 75.156 & 82.365 & 80.819 & 89.041 & 75.115 & 74.133 & 72.934 & 74.047 & 1583.316 & 1575.488 & 1572.489 & 1574.888 & 1573.513 & 1567.488 & 1564.357 & 1569.848 \\
\hline 9/17/16 7:00 & 75.159 & 82.372 & 80.823 & 89.043 & 75.115 & 74.138 & 72.936 & 74.049 & 1583.313 & 1575.481 & 1572.485 & 1574.886 & 1573.513 & 1567.483 & 1564.355 & 1569.846 \\
\hline 9/17/16 8:00 & 75.161 & 82.374 & 80.83 & 89.043 & 75.122 & 74.143 & 72.941 & 74.054 & 1583.311 & 1575.479 & 1572.478 & 1574.886 & 1573.506 & 1567.478 & 1564.35 & 1569.841 \\
\hline 9/17/16 9:00 & 75.168 & 82.383 & 80.835 & 89.045 & 75.124 & 74.143 & 72.943 & 74.058 & 1583.304 & 1575.47 & 1572.473 & 1574.884 & 1573.504 & 1567.478 & 1564.348 & 1569.837 \\
\hline 9/17/16 10:00 & 75.166 & 82.386 & 80.837 & 89.048 & 75.129 & 74.145 & 72.941 & 74.077 & 1583.306 & 1575.467 & 1572.471 & 1574.881 & 1573.499 & 1567.476 & 1564.35 & 1569.818 \\
\hline 9/17/16 11:00 & 75.166 & 82.381 & 80.84 & 89.043 & 75.126 & 74.14 & 72.938 & 74.061 & 1583.306 & 1575.472 & 1572.468 & 1574.886 & 1573.502 & 1567.481 & 1564.353 & 1569.834 \\
\hline 9/17/16 12:00 & 75.154 & 82.379 & 80.833 & 89.036 & 75.115 & 74.126 & 72.926 & 74.061 & 1583.318 & 1575.474 & 1572.475 & 1574.893 & 1573.513 & 1567.495 & 1564.365 & 1569.834 \\
\hline 9/17/16 13:00 & 75.137 & 82.358 & 80.814 & 89.027 & 75.098 & 74.117 & 72.905 & 74.049 & 1583.335 & 1575.495 & 1572.494 & 1574.902 & 1573.53 & 1567.504 & 1564.386 & 1569.846 \\
\hline 9/17/16 14:00 & 75.123 & 82.339 & 80.798 & 89.01 & 75.077 & 74.108 & 72.888 & 74.016 & 1583.349 & 1575.514 & 1572.51 & 1574.919 & 1573.551 & 1567.513 & 1564.403 & 1569.879 \\
\hline 9/17/16 15:00 & 75.105 & 82.318 & 80.779 & 89.001 & 75.056 & 74.094 & 72.869 & 74.023 & 1583.367 & 1575.535 & 1572.529 & 1574.928 & 1573.572 & 1567.527 & 1564.422 & 1569.872 \\
\hline 9/17/16 16:00 & 75.097 & 82.302 & 80.763 & 88.994 & 75.038 & 74.075 & 72.862 & 73.995 & 1583.375 & 1575.551 & 1572.545 & 1574.935 & 1573.59 & 1567.546 & 1564.429 & 1569.9 \\
\hline 9/17/16 17:00 & 75.086 & 82.274 & 80.739 & 88.984 & 75.014 & 74.07 & 72.841 & 73.979 & 1583.386 & 1575.579 & 1572.569 & 1574.945 & 1573.614 & 1567.551 & 1564.45 & 1569.916 \\
\hline 9/17/16 18:00 & 75.074 & 82.258 & 80.725 & 88.973 & 74.996 & 74.061 & 72.833 & 73.954 & 1583.398 & 1575.595 & 1572.583 & 1574.956 & 1573.632 & 1567.56 & 1564.458 & 1569.941 \\
\hline 9/17/16 19:00 & 75.083 & 82.258 & 80.723 & 88.977 & 74.993 & 74.068 & 72.843 & 73.921 & 1583.389 & 1575.595 & 1572.585 & 1574.952 & 1573.635 & 1567.553 & 1564.448 & 1569.974 \\
\hline 9/17/16 20:00 & 75.097 & 82.267 & 80.732 & 88.989 & 74.998 & 74.075 & 72.862 & 73.968 & 1583.375 & 1575.586 & 1572.576 & 1574.94 & 1573.63 & 1567.546 & 1564.429 & 1569.927 \\
\hline 9/17/16 21:00 & 75.107 & 82.269 & 80.732 & 88.989 & 74.998 & 74.078 & 72.869 & 73.975 & 1583.365 & 1575.584 & 1572.576 & 1574.94 & 1573.63 & 1567.543 & 1564.422 & 1569.92 \\
\hline 9/17/16 22:00 & 75.112 & 82.269 & 80.737 & 88.989 & 75 & 74.077 & 72.867 & 73.977 & 1583.36 & 1575.584 & 1572.571 & 1574.94 & 1573.628 & 1567.544 & 1564.424 & 1569.918 \\
\hline 9/17/16 23:00 & 75.119 & 82.276 & 80.746 & 88.996 & 75.01 & 74.087 & 72.881 & 73.986 & 1583.353 & 1575.577 & 1572.562 & 1574.933 & 1573.618 & 1567.534 & 1564.41 & 1569.909 \\
\hline 9/18/16 0:00 & 75.107 & 82.265 & 80.732 & 88.98 & 74.991 & 74.073 & 72.86 & 73.979 & 1583.365 & 1575.588 & 1572.576 & 1574.949 & 1573.637 & 1567.548 & 1564.431 & 1569.916 \\
\hline 9/18/16 1:00 & 75.093 & 82.244 & 80.716 & 88.975 & 74.975 & 74.056 & 72.843 & 73.963 & 1583.379 & 1575.609 & 1572.592 & 1574.954 & 1573.653 & 1567.565 & 1564.448 & 1569.932 \\
\hline $9 / 18 / 162: 00$ & 75.088 & 82.237 & 80.707 & 88.97 & 74.963 & 74.049 & 72.841 & 73.889 & 1583.384 & 1575.616 & 1572.601 & 1574.959 & 1573.665 & 1567.572 & 1564.45 & 1570.006 \\
\hline 9/18/16 3:00 & 75.083 & 82.23 & 80.695 & 88.966 & 74.951 & 74.052 & 72.836 & 73.935 & 1583.389 & 1575.623 & 1572.613 & 1574.963 & 1573.677 & 1567.569 & 1564.455 & 1569.96 \\
\hline 9/18/16 4:00 & 75.079 & 82.216 & 80.688 & 88.959 & 74.942 & 74.045 & 72.824 & 73.926 & 1583.393 & 1575.637 & 1572.62 & 1574.97 & 1573.686 & 1567.576 & 1564.467 & 1569.969 \\
\hline
\end{tabular}


TABLE S1.1 Automatically recorded groundwater levels in the unconfined aquifer.

\begin{tabular}{|c|c|c|c|c|c|c|c|c|c|c|c|c|c|c|c|c|}
\hline \multirow[b]{2}{*}{ Date and Time } & \multicolumn{8}{|c|}{ Measured Depth to Water ( $\mathrm{ft}$ TOC ${ }^{\mathrm{a}}$ ) } & \multicolumn{8}{|c|}{ Groundwater Elevation (ft $\mathrm{AMSL}^{\mathrm{b}}$ ) } \\
\hline & DL1-C & DL2-C1 & DL3-C1 & DL4-C1 & DL5-C1 & DL6-C & DL7-C & DL8-C1 & DL1-C & DL2-C1 & DL3-C1 & DL4-C1 & DL5-C1 & DL6-C & DL7-C & DL8-C1 \\
\hline 9/18/16 5:00 & 75.076 & 82.211 & 80.679 & 88.954 & 74.93 & 74.042 & 72.824 & 73.914 & 1583.396 & 1575.642 & 1572.629 & 1574.975 & 1573.698 & 1567.579 & 1564.467 & 1569.981 \\
\hline 9/18/16 6:00 & 75.074 & 82.204 & 80.67 & 88.949 & 74.923 & 74.04 & 72.821 & 73.912 & 1583.398 & 1575.649 & 1572.638 & 1574.98 & 1573.705 & 1567.581 & 1564.47 & 1569.983 \\
\hline 9/18/16 7:00 & 75.083 & 82.211 & 80.679 & 88.959 & 74.926 & 74.047 & 72.836 & 73.917 & 1583.389 & 1575.642 & 1572.629 & 1574.97 & 1573.702 & 1567.574 & 1564.455 & 1569.978 \\
\hline 9/18/16 8:00 & 75.093 & 82.216 & 80.681 & 88.961 & 74.93 & 74.045 & 72.841 & 73.907 & 1583.379 & 1575.637 & 1572.627 & 1574.968 & 1573.698 & 1567.576 & 1564.45 & 1569.988 \\
\hline 9/18/16 9:00 & 75.095 & 82.213 & 80.681 & 88.961 & 74.928 & 74.049 & 72.843 & 73.921 & 1583.377 & 1575.64 & 1572.627 & 1574.968 & 1573.7 & 1567.572 & 1564.448 & 1569.974 \\
\hline 9/18/16 10:00 & 75.102 & 82.225 & 80.695 & 88.963 & 74.933 & 74.054 & 72.848 & 73.933 & 1583.37 & 1575.628 & 1572.613 & 1574.966 & 1573.695 & 1567.567 & 1564.443 & 1569.962 \\
\hline 9/18/16 11:00 & 75.097 & 82.216 & 80.686 & 88.959 & 74.93 & 74.052 & 72.841 & 73.924 & 1583.375 & 1575.637 & 1572.622 & 1574.97 & 1573.698 & 1567.569 & 1564.45 & 1569.971 \\
\hline 9/18/16 12:00 & 75.081 & 82.199 & 80.67 & 88.942 & 74.907 & 74.031 & 72.817 & 73.914 & 1583.391 & 1575.654 & 1572.638 & 1574.987 & 1573.721 & 1567.59 & 1564.474 & 1569.981 \\
\hline 9/18/16 13:00 & 75.067 & 82.178 & 80.651 & 88.933 & 74.884 & 74.017 & 72.798 & 73.896 & 1583.405 & 1575.675 & 1572.657 & 1574.996 & 1573.744 & 1567.604 & 1564.493 & 1569.999 \\
\hline 9/18/16 14:00 & 75.055 & 82.165 & 80.632 & 88.926 & 74.867 & 74.01 & 72.786 & 73.87 & 1583.417 & 1575.688 & 1572.676 & 1575.003 & 1573.761 & 1567.611 & 1564.505 & 1570.025 \\
\hline 9/18/16 15:00 & 75.046 & 82.148 & 80.614 & 88.917 & 74.849 & 73.996 & 72.776 & 73.852 & 1583.426 & 1575.705 & 1572.694 & 1575.012 & 1573.779 & 1567.625 & 1564.515 & 1570.043 \\
\hline 9/18/16 16:00 & 75.046 & 82.141 & 80.607 & 88.919 & 74.839 & 73.998 & 72.778 & 73.854 & 1583.426 & 1575.712 & 1572.701 & 1575.01 & 1573.789 & 1567.623 & 1564.513 & 1570.041 \\
\hline 9/18/16 17:00 & 75.043 & 82.127 & 80.593 & 88.912 & 74.825 & 73.991 & 72.769 & 73.824 & 1583.429 & 1575.726 & 1572.715 & 1575.017 & 1573.803 & 1567.63 & 1564.522 & 1570.071 \\
\hline 9/18/16 18:00 & 75.041 & 82.12 & 80.581 & 88.907 & 74.814 & 73.989 & 72.771 & 73.821 & 1583.431 & 1575.733 & 1572.727 & 1575.022 & 1573.814 & 1567.632 & 1564.52 & 1570.074 \\
\hline 9/18/16 19:00 & 75.057 & 82.132 & 80.595 & 88.921 & 74.823 & 74.003 & 72.79 & 73.831 & 1583.415 & 1575.721 & 1572.713 & 1575.008 & 1573.805 & 1567.618 & 1564.501 & 1570.064 \\
\hline 9/18/16 20:00 & 75.067 & 82.141 & 80.602 & 88.924 & 74.825 & 74.003 & 72.8 & 73.8 & 1583.405 & 1575.712 & 1572.706 & 1575.005 & 1573.803 & 1567.618 & 1564.491 & 1570.095 \\
\hline 9/18/16 21:00 & 75.083 & 82.16 & 80.616 & 88.935 & 74.842 & 74.022 & 72.821 & 73.849 & 1583.389 & 1575.693 & 1572.692 & 1574.994 & 1573.786 & 1567.599 & 1564.47 & 1570.046 \\
\hline 9/18/16 22:00 & 75.093 & 82.169 & 80.625 & 88.935 & 74.849 & 74.024 & 72.826 & 73.866 & 1583.379 & 1575.684 & 1572.683 & 1574.994 & 1573.779 & 1567.597 & 1564.465 & 1570.029 \\
\hline 9/18/16 23:00 & 75.095 & 82.178 & 80.628 & 88.938 & 74.853 & 74.026 & 72.826 & 73.868 & 1583.377 & 1575.675 & 1572.68 & 1574.991 & 1573.775 & 1567.595 & 1564.465 & 1570.027 \\
\hline 9/19/16 0:00 & 75.093 & 82.178 & 80.63 & 88.938 & 74.851 & 74.022 & 72.821 & 73.868 & 1583.379 & 1575.675 & 1572.678 & 1574.991 & 1573.777 & 1567.599 & 1564.47 & 1570.027 \\
\hline 9/19/16 1:00 & 75.09 & 82.178 & 80.628 & 88.935 & 74.851 & 74.022 & 72.819 & 73.863 & 1583.382 & 1575.675 & 1572.68 & 1574.994 & 1573.777 & 1567.599 & 1564.472 & 1570.032 \\
\hline 9/19/16 2:00 & 75.083 & 82.171 & 80.7 & 88.928 & 74.842 & 74.012 & 72.809 & 73.868 & 1583.389 & 1575.682 & 1572.608 & 1575.001 & 1573.786 & 1567.609 & 1564.482 & 1570.027 \\
\hline 9/19/16 3:00 & 75.076 & 82.167 & 80.693 & 88.926 & 74.835 & 74.01 & 72.802 & 73.866 & 1583.396 & 1575.686 & 1572.615 & 1575.003 & 1573.793 & 1567.611 & 1564.489 & 1570.029 \\
\hline 9/19/16 4:00 & 75.074 & 82.169 & 80.7 & 88.924 & 74.835 & 74.01 & 72.805 & 73.865 & 1583.398 & 1575.684 & 1572.608 & 1575.005 & 1573.793 & 1567.611 & 1564.486 & 1570.03 \\
\hline 9/19/16 5:00 & 75.069 & 82.162 & 80.705 & 88.917 & 74.825 & 74.003 & 72.795 & 73.868 & 1583.403 & 1575.691 & 1572.603 & 1575.012 & 1573.803 & 1567.618 & 1564.496 & 1570.027 \\
\hline 9/19/16 6:00 & 75.069 & 82.16 & 80.646 & 88.914 & 74.821 & 74.003 & 72.793 & 73.835 & 1583.403 & 1575.693 & 1572.662 & 1575.015 & 1573.807 & 1567.618 & 1564.498 & 1570.06 \\
\hline 9/19/16 7:00 & 75.067 & 82.158 & 80.611 & 88.924 & 74.816 & 73.998 & 72.793 & 73.854 & 1583.405 & 1575.695 & 1572.697 & 1575.005 & 1573.812 & 1567.623 & 1564.498 & 1570.041 \\
\hline 9/19/16 8:00 & 75.097 & 82.199 & 80.635 & 88.947 & 74.849 & 74.031 & 72.848 & 73.868 & 1583.375 & 1575.654 & 1572.673 & 1574.982 & 1573.779 & 1567.59 & 1564.443 & 1570.027 \\
\hline 9/19/16 9:00 & 75.109 & 82.211 & 80.656 & 88.949 & 74.858 & 74.038 & 72.845 & 73.889 & 1583.363 & 1575.642 & 1572.652 & 1574.98 & 1573.77 & 1567.583 & 1564.446 & 1570.006 \\
\hline 9/19/16 10:00 & 75.126 & 82.241 & 80.672 & 88.963 & 74.879 & 74.049 & 72.864 & 73.907 & 1583.346 & 1575.612 & 1572.636 & 1574.966 & 1573.749 & 1567.572 & 1564.427 & 1569.988 \\
\hline 9/19/16 11:00 & 75.137 & 82.255 & 80.695 & 88.973 & 74.902 & 74.064 & 72.876 & 73.93 & 1583.335 & 1575.598 & 1572.613 & 1574.956 & 1573.726 & 1567.557 & 1564.415 & 1569.965 \\
\hline 9/19/16 12:00 & 75.145 & 82.274 & 80.702 & 88.98 & 74.916 & 74.07 & 72.883 & 73.944 & 1583.327 & 1575.579 & 1572.606 & 1574.949 & 1573.712 & 1567.551 & 1564.408 & 1569.951 \\
\hline 9/19/16 13:00 & 75.14 & 82.274 & 80.707 & 88.973 & 74.916 & 74.063 & 72.874 & 73.949 & 1583.332 & 1575.579 & 1572.601 & 1574.956 & 1573.712 & 1567.558 & 1564.417 & 1569.946 \\
\hline 9/19/16 14:00 & 75.13 & 82.276 & 80.709 & 88.973 & 74.919 & 74.063 & 72.867 & 73.951 & 1583.342 & 1575.577 & 1572.599 & 1574.956 & 1573.709 & 1567.558 & 1564.424 & 1569.944 \\
\hline 9/19/16 15:00 & 75.121 & 82.274 & 80.705 & 88.968 & 74.919 & 74.059 & 72.86 & 73.956 & 1583.351 & 1575.579 & 1572.603 & 1574.961 & 1573.709 & 1567.562 & 1564.431 & 1569.939 \\
\hline 9/19/16 16:00 & 75.112 & 82.272 & 80.707 & 88.961 & 74.916 & 74.049 & 72.85 & 73.949 & 1583.36 & 1575.581 & 1572.601 & 1574.968 & 1573.712 & 1567.572 & 1564.441 & 1569.946 \\
\hline 9/19/16 17:00 & 75.109 & 82.272 & 80.705 & 88.961 & 74.921 & 74.052 & 72.852 & 73.947 & 1583.363 & 1575.581 & 1572.603 & 1574.968 & 1573.707 & 1567.569 & 1564.439 & 1569.948 \\
\hline 9/19/16 18:00 & 75.109 & 82.272 & 80.702 & 88.961 & 74.919 & 74.052 & 72.85 & 73.944 & 1583.363 & 1575.581 & 1572.606 & 1574.968 & 1573.709 & 1567.569 & 1564.441 & 1569.951 \\
\hline 9/19/16 19:00 & 75.105 & 82.272 & 80.702 & 88.961 & 74.916 & 74.054 & 72.848 & 73.951 & 1583.367 & 1575.581 & 1572.606 & 1574.968 & 1573.712 & 1567.567 & 1564.443 & 1569.944 \\
\hline 9/19/16 20:00 & 75.1 & 82.269 & 80.695 & 88.956 & 74.912 & 74.047 & 72.843 & 73.944 & 1583.372 & 1575.584 & 1572.613 & 1574.973 & 1573.716 & 1567.574 & 1564.448 & 1569.951 \\
\hline 9/19/16 21:00 & 75.107 & 82.278 & 80.702 & 88.961 & 74.919 & 74.054 & 72.855 & 73.949 & 1583.365 & 1575.575 & 1572.606 & 1574.968 & 1573.709 & 1567.567 & 1564.436 & 1569.946 \\
\hline 9/19/16 22:00 & 75.114 & 82.288 & 80.709 & 88.966 & 74.928 & 74.063 & 72.862 & 73.958 & 1583.358 & 1575.565 & 1572.599 & 1574.963 & 1573.7 & 1567.558 & 1564.429 & 1569.937 \\
\hline 9/19/16 23:00 & 75.123 & 82.299 & 80.723 & 88.973 & 74.935 & 74.066 & 72.872 & 73.954 & 1583.349 & 1575.554 & 1572.585 & 1574.956 & 1573.693 & 1567.555 & 1564.419 & 1569.941 \\
\hline $9 / 20 / 160: 00$ & 75.121 & 82.297 & 80.721 & 88.968 & 74.935 & 74.066 & 72.864 & 73.975 & 1583.351 & 1575.556 & 1572.587 & 1574.961 & 1573.693 & 1567.555 & 1564.427 & 1569.92 \\
\hline $9 / 20 / 161: 00$ & 75.112 & 82.292 & 80.714 & 88.961 & 74.933 & 74.059 & 72.855 & 73.937 & 1583.36 & 1575.561 & 1572.594 & 1574.968 & 1573.695 & 1567.562 & 1564.436 & 1569.958 \\
\hline 9/20/16 2:00 & 75.102 & 82.281 & 80.707 & 88.956 & 74.921 & 74.052 & 72.845 & 73.965 & 1583.37 & 1575.572 & 1572.601 & 1574.973 & 1573.707 & 1567.569 & 1564.446 & 1569.93 \\
\hline 9/20/16 3:00 & 75.093 & 82.276 & 80.7 & 88.952 & 74.914 & 74.042 & 72.836 & 73.944 & 1583.379 & 1575.577 & 1572.608 & 1574.977 & 1573.714 & 1567.579 & 1564.455 & 1569.951 \\
\hline $9 / 20 / 164: 00$ & 75.072 & 82.251 & 80.679 & 88.935 & 74.891 & 74.029 & 72.812 & 73.937 & 1583.4 & 1575.602 & 1572.629 & 1574.994 & 1573.737 & 1567.592 & 1564.479 & 1569.958 \\
\hline 9/20/16 5:00 & 75.067 & 82.246 & 80.665 & 88.933 & 74.874 & 74.022 & 72.807 & 73.917 & 1583.405 & 1575.607 & 1572.643 & 1574.996 & 1573.754 & 1567.599 & 1564.484 & 1569.978 \\
\hline
\end{tabular}


TABLE S1.1 Automatically recorded groundwater levels in the unconfined aquifer.

\begin{tabular}{|c|c|c|c|c|c|c|c|c|c|c|c|c|c|c|c|c|}
\hline \multirow[b]{2}{*}{ Date and Time } & \multicolumn{8}{|c|}{ Measured Depth to Water (ft TOC ${ }^{\mathrm{a}}$ ) } & \multicolumn{8}{|c|}{ Groundwater Elevation ( $\mathrm{ft} \mathrm{AMSL}^{\mathrm{b}}$ ) } \\
\hline & DL1-C & DL2-C1 & DL3-C1 & DL4-C1 & DL5-C1 & DL6-C & DL7-C & DL8-C1 & DL1-C & DL2-C1 & DL3-C1 & DL4-C1 & DL5-C1 & DL6-C & DL7-C & DL8-C1 \\
\hline $9 / 20 / 166: 00$ & 75.064 & 82.239 & 80.663 & 88.931 & 74.872 & 74.022 & 72.807 & 73.917 & 1583.408 & 1575.614 & 1572.645 & 1574.998 & 1573.756 & 1567.599 & 1564.484 & 1569.978 \\
\hline $9 / 20 / 167: 00$ & 75.064 & 82.232 & 80.656 & 88.926 & 74.863 & 74.019 & 72.802 & 73.907 & 1583.408 & 1575.621 & 1572.652 & 1575.003 & 1573.765 & 1567.602 & 1564.489 & 1569.988 \\
\hline 9/20/16 8:00 & 75.062 & 82.225 & 80.653 & 88.926 & 74.856 & 74.017 & 72.802 & 73.903 & 1583.41 & 1575.628 & 1572.655 & 1575.003 & 1573.772 & 1567.604 & 1564.489 & 1569.992 \\
\hline 9/20/16 9:00 & 75.053 & 82.216 & 80.642 & 88.917 & 74.842 & 74.008 & 72.793 & 73.893 & 1583.419 & 1575.637 & 1572.666 & 1575.012 & 1573.786 & 1567.613 & 1564.498 & 1570.002 \\
\hline 9/20/16 10:00 & 75.05 & 82.209 & 80.632 & 88.914 & 74.835 & 74.005 & 72.79 & 73.889 & 1583.422 & 1575.644 & 1572.676 & 1575.015 & 1573.793 & 1567.616 & 1564.501 & 1570.006 \\
\hline 9/20/16 11:00 & 75.05 & 82.199 & 80.635 & 88.91 & 74.823 & 74.008 & 72.786 & 73.914 & 1583.422 & 1575.654 & 1572.673 & 1575.019 & 1573.805 & 1567.613 & 1564.505 & 1569.981 \\
\hline 9/20/16 12:00 & 75.036 & 82.176 & 80.614 & 88.898 & 74.8 & 73.987 & 72.762 & 73.896 & 1583.436 & 1575.677 & 1572.694 & 1575.031 & 1573.828 & 1567.634 & 1564.529 & 1569.999 \\
\hline 9/20/16 13:00 & 75.02 & 82.144 & 80.583 & 88.877 & 74.769 & 73.968 & 72.738 & 73.847 & 1583.452 & 1575.709 & 1572.725 & 1575.052 & 1573.859 & 1567.653 & 1564.553 & 1570.048 \\
\hline 9/20/16 14:00 & 75.003 & 82.12 & 80.562 & 88.87 & 74.744 & 73.954 & 72.721 & 73.817 & 1583.469 & 1575.733 & 1572.746 & 1575.059 & 1573.884 & 1567.667 & 1564.57 & 1570.078 \\
\hline 9/20/16 15:00 & 74.987 & 82.088 & 80.534 & 88.851 & 74.713 & 73.935 & 72.7 & 73.789 & 1583.485 & 1575.765 & 1572.774 & 1575.078 & 1573.915 & 1567.686 & 1564.591 & 1570.106 \\
\hline 9/20/16 16:00 & 74.989 & 82.085 & 80.534 & 88.854 & 74.702 & 73.935 & 72.704 & 73.763 & 1583.483 & 1575.768 & 1572.774 & 1575.075 & 1573.926 & 1567.686 & 1564.587 & 1570.132 \\
\hline 9/20/16 17:00 & 74.991 & 82.076 & 80.516 & 88.854 & 74.69 & 73.935 & 72.709 & 73.777 & 1583.481 & 1575.777 & 1572.792 & 1575.075 & 1573.938 & 1567.686 & 1564.582 & 1570.118 \\
\hline $9 / 20 / 1618: 00$ & 75.001 & 82.069 & 80.511 & 88.854 & 74.683 & 73.94 & 72.714 & 73.766 & 1583.471 & 1575.784 & 1572.797 & 1575.075 & 1573.945 & 1567.681 & 1564.577 & 1570.129 \\
\hline 9/20/16 19:00 & 75.01 & 82.074 & 80.511 & 88.875 & 74.683 & 73.938 & 72.724 & 73.768 & 1583.462 & 1575.779 & 1572.797 & 1575.054 & 1573.945 & 1567.683 & 1564.567 & 1570.127 \\
\hline $9 / 20 / 1620: 00$ & 75.015 & 82.081 & 80.513 & 88.872 & 74.681 & 73.942 & 72.731 & 73.763 & 1583.457 & 1575.772 & 1572.795 & 1575.057 & 1573.947 & 1567.679 & 1564.56 & 1570.132 \\
\hline 9/20/16 21:00 & 75.027 & 82.085 & 80.52 & 88.87 & 74.685 & 73.949 & 72.745 & 73.777 & 1583.445 & 1575.768 & 1572.788 & 1575.059 & 1573.943 & 1567.672 & 1564.546 & 1570.118 \\
\hline $9 / 20 / 1622: 00$ & 75.041 & 82.099 & 80.53 & 88.873 & 74.695 & 73.956 & 72.757 & 73.78 & 1583.431 & 1575.754 & 1572.778 & 1575.056 & 1573.933 & 1567.665 & 1564.534 & 1570.115 \\
\hline $9 / 20 / 1623: 00$ & 75.041 & 82.095 & 80.53 & 88.872 & 74.692 & 73.956 & 72.752 & 73.777 & 1583.431 & 1575.758 & 1572.778 & 1575.057 & 1573.936 & 1567.665 & 1564.539 & 1570.118 \\
\hline $9 / 21 / 160: 00$ & 75.036 & 82.095 & 80.527 & 88.865 & 74.688 & 73.949 & 72.745 & 73.733 & 1583.436 & 1575.758 & 1572.781 & 1575.064 & 1573.94 & 1567.672 & 1564.546 & 1570.162 \\
\hline 9/21/16 1:00 & 75.031 & 82.085 & 80.516 & 88.858 & 74.676 & 73.94 & 72.735 & 73.77 & 1583.441 & 1575.768 & 1572.792 & 1575.071 & 1573.952 & 1567.681 & 1564.556 & 1570.125 \\
\hline $9 / 21 / 162: 00$ & 75.024 & 82.079 & 80.586 & 88.854 & 74.669 & 73.938 & 72.731 & 73.768 & 1583.448 & 1575.774 & 1572.722 & 1575.075 & 1573.959 & 1567.683 & 1564.56 & 1570.127 \\
\hline $9 / 21 / 163: 00$ & 75.022 & 82.076 & 80.581 & 88.854 & 74.662 & 73.935 & 72.728 & 73.77 & 1583.45 & 1575.777 & 1572.727 & 1575.075 & 1573.966 & 1567.686 & 1564.563 & 1570.125 \\
\hline $9 / 21 / 164: 00$ & 75.017 & 82.067 & 80.583 & 88.849 & 74.653 & 73.933 & 72.724 & 73.759 & 1583.455 & 1575.786 & 1572.725 & 1575.08 & 1573.975 & 1567.688 & 1564.567 & 1570.136 \\
\hline $9 / 21 / 165: 00$ & 75.017 & 82.071 & 80.593 & 88.849 & 74.648 & 73.931 & 72.723 & 73.759 & 1583.455 & 1575.782 & 1572.715 & 1575.08 & 1573.98 & 1567.69 & 1564.568 & 1570.136 \\
\hline $9 / 21 / 166: 00$ & 75.022 & 82.069 & 80.539 & 88.847 & 74.646 & 73.928 & 72.724 & 73.749 & 1583.45 & 1575.784 & 1572.769 & 1575.082 & 1573.982 & 1567.693 & 1564.567 & 1570.146 \\
\hline $9 / 21 / 167: 00$ & 75.02 & 82.06 & 80.497 & 88.84 & 74.634 & 73.924 & 72.716 & 73.742 & 1583.452 & 1575.793 & 1572.811 & 1575.089 & 1573.994 & 1567.697 & 1564.575 & 1570.153 \\
\hline $9 / 21 / 168: 00$ & 75.02 & 82.055 & 80.49 & 88.847 & 74.632 & 73.924 & 72.716 & 73.735 & 1583.452 & 1575.798 & 1572.818 & 1575.082 & 1573.996 & 1567.697 & 1564.575 & 1570.16 \\
\hline 9/21/16 9:00 & 75.017 & 82.055 & 80.485 & 88.847 & 74.627 & 73.924 & 72.716 & 73.733 & 1583.455 & 1575.798 & 1572.823 & 1575.082 & 1574.001 & 1567.697 & 1564.575 & 1570.162 \\
\hline $9 / 21 / 1610: 00$ & 75.017 & 82.058 & 80.485 & 88.849 & 74.623 & 73.921 & 72.719 & 73.731 & 1583.455 & 1575.795 & 1572.823 & 1575.08 & 1574.005 & 1567.7 & 1564.572 & 1570.164 \\
\hline 9/21/16 11:00 & 75.017 & 82.058 & 80.483 & 88.847 & 74.62 & 73.924 & 72.716 & 73.733 & 1583.455 & 1575.795 & 1572.825 & 1575.082 & 1574.008 & 1567.697 & 1564.575 & 1570.162 \\
\hline $9 / 21 / 1612: 00$ & 75.015 & 82.051 & 80.478 & 88.842 & 74.613 & 73.919 & 72.712 & 73.733 & 1583.457 & 1575.802 & 1572.83 & 1575.087 & 1574.015 & 1567.702 & 1564.579 & 1570.162 \\
\hline $9 / 21 / 1613: 00$ & 75.006 & 82.039 & 80.464 & 88.837 & 74.595 & 73.907 & 72.695 & 73.708 & 1583.466 & 1575.814 & 1572.844 & 1575.092 & 1574.033 & 1567.714 & 1564.596 & 1570.187 \\
\hline $9 / 21 / 1614: 00$ & 74.998 & 82.027 & 80.455 & 88.833 & 74.585 & 73.898 & 72.688 & 73.717 & 1583.474 & 1575.826 & 1572.853 & 1575.096 & 1574.043 & 1567.723 & 1564.603 & 1570.178 \\
\hline 9/21/16 15:00 & 74.991 & 82.011 & 80.448 & 88.826 & 74.571 & 73.893 & 72.683 & 73.703 & 1583.481 & 1575.842 & 1572.86 & 1575.103 & 1574.057 & 1567.728 & 1564.608 & 1570.192 \\
\hline $9 / 21 / 1616: 00$ & 74.996 & 82.016 & 80.443 & 88.826 & 74.567 & 73.893 & 72.683 & 73.705 & 1583.476 & 1575.837 & 1572.865 & 1575.103 & 1574.061 & 1567.728 & 1564.608 & 1570.19 \\
\hline $9 / 21 / 1617: 00$ & 75.001 & 82.018 & 80.446 & 88.828 & 74.567 & 73.898 & 72.692 & 73.705 & 1583.471 & 1575.835 & 1572.862 & 1575.101 & 1574.061 & 1567.723 & 1564.599 & 1570.19 \\
\hline $9 / 21 / 1618: 00$ & 74.991 & 81.999 & 80.432 & 88.812 & 74.548 & 73.884 & 72.666 & 73.68 & 1583.481 & 1575.854 & 1572.876 & 1575.117 & 1574.08 & 1567.737 & 1564.625 & 1570.215 \\
\hline 9/21/16 19:00 & 75.003 & 82.013 & 80.436 & 88.823 & 74.557 & 73.894 & 72.688 & 73.638 & 1583.469 & 1575.84 & 1572.872 & 1575.106 & 1574.071 & 1567.727 & 1564.603 & 1570.257 \\
\hline $9 / 21 / 1620: 00$ & 75.006 & 82.011 & 80.436 & 88.821 & 74.56 & 73.893 & 72.69 & 73.687 & 1583.466 & 1575.842 & 1572.872 & 1575.108 & 1574.068 & 1567.728 & 1564.601 & 1570.208 \\
\hline $9 / 21 / 1621: 00$ & 75.006 & 82.013 & 80.434 & 88.819 & 74.555 & 73.896 & 72.692 & 73.68 & 1583.466 & 1575.84 & 1572.874 & 1575.11 & 1574.073 & 1567.725 & 1564.599 & 1570.215 \\
\hline $9 / 21 / 1622: 00$ & 75.006 & 82.009 & 80.434 & 88.821 & 74.55 & 73.891 & 72.688 & 73.638 & 1583.466 & 1575.844 & 1572.874 & 1575.108 & 1574.078 & 1567.73 & 1564.603 & 1570.257 \\
\hline $9 / 21 / 1623: 00$ & 74.996 & 81.997 & 80.42 & 88.809 & 74.539 & 73.879 & 72.673 & 73.666 & 1583.476 & 1575.856 & 1572.888 & 1575.12 & 1574.089 & 1567.742 & 1564.618 & 1570.229 \\
\hline $9 / 22 / 160: 00$ & 74.996 & 81.995 & 80.413 & 88.809 & 74.532 & 73.882 & 72.673 & 73.659 & 1583.476 & 1575.858 & 1572.895 & 1575.12 & 1574.096 & 1567.739 & 1564.618 & 1570.236 \\
\hline $9 / 22 / 161: 00$ & 74.994 & 81.997 & 80.415 & 88.807 & 74.534 & 73.884 & 72.676 & 73.659 & 1583.478 & 1575.856 & 1572.893 & 1575.122 & 1574.094 & 1567.737 & 1564.615 & 1570.236 \\
\hline $9 / 22 / 162: 00$ & 74.987 & 81.985 & 80.404 & 88.798 & 74.52 & 73.872 & 72.659 & 73.608 & 1583.485 & 1575.868 & 1572.904 & 1575.131 & 1574.108 & 1567.749 & 1564.632 & 1570.287 \\
\hline 9/22/16 3:00 & 74.977 & 81.969 & 80.395 & 88.791 & 74.504 & 73.858 & 72.647 & 73.65 & 1583.495 & 1575.884 & 1572.913 & 1575.138 & 1574.124 & 1567.763 & 1564.644 & 1570.245 \\
\hline $9 / 22 / 164: 00$ & 74.973 & 81.965 & 80.39 & 88.788 & 74.492 & 73.861 & 72.645 & 73.643 & 1583.499 & 1575.888 & 1572.918 & 1575.141 & 1574.136 & 1567.76 & 1564.646 & 1570.252 \\
\hline $9 / 22 / 165: 00$ & 74.982 & 81.969 & 80.39 & 88.793 & 74.497 & 73.865 & 72.654 & 73.645 & 1583.49 & 1575.884 & 1572.918 & 1575.136 & 1574.131 & 1567.756 & 1564.637 & 1570.25 \\
\hline $9 / 22 / 166: 00$ & 74.991 & 81.981 & 80.395 & 88.8 & 74.501 & 73.872 & 72.666 & 73.652 & 1583.481 & 1575.872 & 1572.913 & 1575.129 & 1574.127 & 1567.749 & 1564.625 & 1570.243 \\
\hline
\end{tabular}


TABLE S1.1 Automatically recorded groundwater levels in the unconfined aquifer.

\begin{tabular}{|c|c|c|c|c|c|c|c|c|c|c|c|c|c|c|c|c|}
\hline \multirow[b]{2}{*}{ Date and Time } & \multicolumn{8}{|c|}{ Measured Depth to Water (ft TOC ${ }^{\mathrm{a}}$ ) } & \multicolumn{8}{|c|}{ Groundwater Elevation (ft AMSL) } \\
\hline & DL1-C & DL2-C1 & DL3-C1 & DL4-C1 & DL5-C1 & DL6-C & DL7-C & DL8-C1 & DL1-C & DL2-C1 & DL3-C1 & DL4-C1 & DL5-C1 & DL6-C & DL7-C & DL8-C1 \\
\hline 9/22/16 7:00 & 74.998 & 81.981 & 80.395 & 88.798 & 74.504 & 73.875 & 72.669 & 73.643 & 1583.474 & 1575.872 & 1572.913 & 1575.131 & 1574.124 & 1567.746 & 1564.622 & 1570.252 \\
\hline $9 / 22 / 168: 00$ & 75.003 & 81.988 & 80.404 & 88.802 & 74.508 & 73.879 & 72.673 & 73.638 & 1583.469 & 1575.865 & 1572.904 & 1575.127 & 1574.12 & 1567.742 & 1564.618 & 1570.257 \\
\hline 9/22/16 9:00 & 75.006 & 81.985 & 80.402 & 88.798 & 74.504 & 73.877 & 72.671 & 73.65 & 1583.466 & 1575.868 & 1572.906 & 1575.131 & 1574.124 & 1567.744 & 1564.62 & 1570.245 \\
\hline 9/22/16 10:00 & 75.001 & 81.99 & 80.402 & 88.798 & 74.504 & 73.872 & 72.671 & 73.645 & 1583.471 & 1575.863 & 1572.906 & 1575.131 & 1574.124 & 1567.749 & 1564.62 & 1570.25 \\
\hline 9/22/16 11:00 & 75.008 & 81.997 & 80.406 & 88.802 & 74.508 & 73.882 & 72.678 & 73.652 & 1583.464 & 1575.856 & 1572.902 & 1575.127 & 1574.12 & 1567.739 & 1564.613 & 1570.243 \\
\hline 9/22/16 12:00 & 75.006 & 81.995 & 80.406 & 88.8 & 74.506 & 73.877 & 72.671 & 73.65 & 1583.466 & 1575.858 & 1572.902 & 1575.129 & 1574.122 & 1567.744 & 1564.62 & 1570.245 \\
\hline 9/22/16 13:00 & 74.999 & 81.99 & 80.402 & 88.795 & 74.501 & 73.87 & 72.664 & 73.643 & 1583.473 & 1575.863 & 1572.906 & 1575.134 & 1574.127 & 1567.751 & 1564.627 & 1570.252 \\
\hline 9/22/16 14:00 & 74.994 & 81.988 & 80.397 & 88.795 & 74.49 & 73.868 & 72.661 & 73.647 & 1583.478 & 1575.865 & 1572.911 & 1575.134 & 1574.138 & 1567.753 & 1564.63 & 1570.248 \\
\hline 9/22/16 15:00 & 74.987 & 81.976 & 80.388 & 88.784 & 74.485 & 73.863 & 72.652 & 73.65 & 1583.485 & 1575.877 & 1572.92 & 1575.145 & 1574.143 & 1567.758 & 1564.639 & 1570.245 \\
\hline $9 / 22 / 1616: 00$ & 74.982 & 81.976 & 80.383 & 88.784 & 74.476 & 73.858 & 72.65 & 73.645 & 1583.49 & 1575.877 & 1572.925 & 1575.145 & 1574.152 & 1567.763 & 1564.641 & 1570.25 \\
\hline 9/22/16 17:00 & 74.977 & 81.964 & 80.385 & 88.781 & 74.469 & 73.856 & 72.642 & 73.636 & 1583.495 & 1575.889 & 1572.923 & 1575.148 & 1574.159 & 1567.765 & 1564.649 & 1570.259 \\
\hline 9/22/16 18:00 & 74.984 & 81.967 & 80.378 & 88.784 & 74.469 & 73.858 & 72.647 & 73.619 & 1583.488 & 1575.886 & 1572.93 & 1575.145 & 1574.159 & 1567.763 & 1564.644 & 1570.276 \\
\hline 9/22/16 19:00 & 74.987 & 81.969 & 80.378 & 88.786 & 74.469 & 73.861 & 72.652 & 73.626 & 1583.485 & 1575.884 & 1572.93 & 1575.143 & 1574.159 & 1567.76 & 1564.639 & 1570.269 \\
\hline 9/22/16 20:00 & 74.987 & 81.969 & 80.374 & 88.784 & 74.464 & 73.858 & 72.65 & 73.622 & 1583.485 & 1575.884 & 1572.934 & 1575.145 & 1574.164 & 1567.763 & 1564.641 & 1570.273 \\
\hline 9/22/16 21:00 & 74.994 & 81.978 & 80.381 & 88.788 & 74.471 & 73.863 & 72.659 & 73.626 & 1583.478 & 1575.875 & 1572.927 & 1575.141 & 1574.157 & 1567.758 & 1564.632 & 1570.269 \\
\hline $9 / 22 / 1622: 00$ & 75.008 & 81.99 & 80.392 & 88.795 & 74.48 & 73.875 & 72.673 & 73.64 & 1583.464 & 1575.863 & 1572.916 & 1575.134 & 1574.148 & 1567.746 & 1564.618 & 1570.255 \\
\hline 9/22/16 23:00 & 75.006 & 81.992 & 80.395 & 88.793 & 74.48 & 73.875 & 72.671 & 73.645 & 1583.466 & 1575.861 & 1572.913 & 1575.136 & 1574.148 & 1567.746 & 1564.62 & 1570.25 \\
\hline $9 / 23 / 160: 00$ & 75.001 & 81.995 & 80.392 & 88.793 & 74.485 & 73.87 & 72.671 & 73.643 & 1583.471 & 1575.858 & 1572.916 & 1575.136 & 1574.143 & 1567.751 & 1564.62 & 1570.252 \\
\hline 9/23/16 1:00 & 74.998 & 81.992 & 80.39 & 88.788 & 74.48 & 73.868 & 72.666 & 73.631 & 1583.474 & 1575.861 & 1572.918 & 1575.141 & 1574.148 & 1567.753 & 1564.625 & 1570.264 \\
\hline $9 / 23 / 162: 00$ & 74.998 & 81.995 & 80.467 & 88.788 & 74.48 & 73.868 & 72.664 & 73.647 & 1583.474 & 1575.858 & 1572.841 & 1575.141 & 1574.148 & 1567.753 & 1564.627 & 1570.248 \\
\hline 9/23/16 3:00 & 75.001 & 81.999 & 80.474 & 88.791 & 74.485 & 73.872 & 72.669 & 73.666 & 1583.471 & 1575.854 & 1572.834 & 1575.138 & 1574.143 & 1567.749 & 1564.622 & 1570.229 \\
\hline 9/23/16 4:00 & 74.999 & 81.997 & 80.488 & 88.791 & 74.483 & 73.87 & 72.661 & 73.675 & 1583.473 & 1575.856 & 1572.82 & 1575.138 & 1574.145 & 1567.751 & 1564.63 & 1570.22 \\
\hline $9 / 23 / 165: 00$ & 74.991 & 81.992 & 80.492 & 88.786 & 74.478 & 73.868 & 72.657 & 73.67 & 1583.481 & 1575.861 & 1572.816 & 1575.143 & 1574.15 & 1567.753 & 1564.634 & 1570.225 \\
\hline $9 / 23 / 166: 00$ & 74.996 & 81.999 & 80.443 & 88.786 & 74.48 & 73.87 & 72.664 & 73.659 & 1583.476 & 1575.854 & 1572.865 & 1575.143 & 1574.148 & 1567.751 & 1564.627 & 1570.236 \\
\hline 9/23/16 7:00 & 75.001 & 82.009 & 80.415 & 88.795 & 74.487 & 73.875 & 72.671 & 73.664 & 1583.471 & 1575.844 & 1572.893 & 1575.134 & 1574.141 & 1567.746 & 1564.62 & 1570.231 \\
\hline $9 / 23 / 168: 00$ & 75.01 & 82.016 & 80.42 & 88.802 & 74.497 & 73.882 & 72.683 & 73.677 & 1583.462 & 1575.837 & 1572.888 & 1575.127 & 1574.131 & 1567.739 & 1564.608 & 1570.218 \\
\hline 9/23/16 9:00 & 75.01 & 82.02 & 80.422 & 88.798 & 74.499 & 73.879 & 72.678 & 73.659 & 1583.462 & 1575.833 & 1572.886 & 1575.131 & 1574.129 & 1567.742 & 1564.613 & 1570.236 \\
\hline 9/23/16 10:00 & 75.006 & 82.023 & 80.422 & 88.798 & 74.499 & 73.877 & 72.678 & 73.67 & 1583.466 & 1575.83 & 1572.886 & 1575.131 & 1574.129 & 1567.744 & 1564.613 & 1570.225 \\
\hline 9/23/16 11:00 & 75.008 & 82.023 & 80.42 & 88.793 & 74.497 & 73.872 & 72.671 & 73.673 & 1583.464 & 1575.83 & 1572.888 & 1575.136 & 1574.131 & 1567.749 & 1564.62 & 1570.222 \\
\hline 9/23/16 12:00 & 74.989 & 82.006 & 80.404 & 88.781 & 74.478 & 73.856 & 72.652 & 73.657 & 1583.483 & 1575.847 & 1572.904 & 1575.148 & 1574.15 & 1567.765 & 1564.639 & 1570.238 \\
\hline 9/23/16 13:00 & 74.975 & 81.985 & 80.39 & 88.772 & 74.459 & 73.849 & 72.635 & 73.647 & 1583.497 & 1575.868 & 1572.918 & 1575.157 & 1574.169 & 1567.772 & 1564.656 & 1570.248 \\
\hline $9 / 23 / 1614: 00$ & 74.958 & 81.967 & 80.369 & 88.76 & 74.443 & 73.833 & 72.611 & 73.629 & 1583.514 & 1575.886 & 1572.939 & 1575.169 & 1574.185 & 1567.788 & 1564.68 & 1570.266 \\
\hline $9 / 23 / 1615: 00$ & 74.942 & 81.946 & 80.353 & 88.749 & 74.422 & 73.824 & 72.597 & 73.615 & 1583.53 & 1575.907 & 1572.955 & 1575.18 & 1574.206 & 1567.797 & 1564.694 & 1570.28 \\
\hline $9 / 23 / 1616: 00$ & 74.937 & 81.937 & 80.336 & 88.744 & 74.413 & 73.817 & 72.59 & 73.603 & 1583.535 & 1575.916 & 1572.972 & 1575.185 & 1574.215 & 1567.804 & 1564.701 & 1570.292 \\
\hline 9/23/16 17:00 & 74.94 & 81.925 & 80.334 & 88.742 & 74.406 & 73.817 & 72.59 & 73.608 & 1583.532 & 1575.928 & 1572.974 & 1575.187 & 1574.222 & 1567.804 & 1564.701 & 1570.287 \\
\hline 9/23/16 18:00 & 74.94 & 81.92 & 80.332 & 88.744 & 74.399 & 73.819 & 72.592 & 73.598 & 1583.532 & 1575.933 & 1572.976 & 1575.185 & 1574.229 & 1567.802 & 1564.699 & 1570.297 \\
\hline 9/23/16 19:00 & 74.947 & 81.918 & 80.329 & 88.744 & 74.403 & 73.819 & 72.599 & 73.592 & 1583.525 & 1575.935 & 1572.979 & 1575.185 & 1574.225 & 1567.802 & 1564.692 & 1570.303 \\
\hline 9/23/16 20:00 & 74.956 & 81.925 & 80.332 & 88.751 & 74.408 & 73.826 & 72.609 & 73.589 & 1583.516 & 1575.928 & 1572.976 & 1575.178 & 1574.22 & 1567.795 & 1564.682 & 1570.306 \\
\hline 9/23/16 21:00 & 74.968 & 81.934 & 80.341 & 88.756 & 74.422 & 73.835 & 72.621 & 73.598 & 1583.504 & 1575.919 & 1572.967 & 1575.173 & 1574.206 & 1567.786 & 1564.67 & 1570.297 \\
\hline 9/23/16 22:00 & 74.977 & 81.946 & 80.348 & 88.76 & 74.424 & 73.84 & 72.633 & 73.603 & 1583.495 & 1575.907 & 1572.96 & 1575.169 & 1574.204 & 1567.781 & 1564.658 & 1570.292 \\
\hline 9/23/16 23:00 & 74.98 & 81.948 & 80.35 & 88.758 & 74.427 & 73.835 & 72.63 & 73.608 & 1583.492 & 1575.905 & 1572.958 & 1575.171 & 1574.201 & 1567.786 & 1564.661 & 1570.287 \\
\hline $9 / 24 / 160: 00$ & 74.977 & 81.948 & 80.353 & 88.756 & 74.427 & 73.838 & 72.626 & 73.605 & 1583.495 & 1575.905 & 1572.955 & 1575.173 & 1574.201 & 1567.783 & 1564.665 & 1570.29 \\
\hline 9/24/16 1:00 & 74.973 & 81.946 & 80.348 & 88.756 & 74.422 & 73.835 & 72.626 & 73.605 & 1583.499 & 1575.907 & 1572.96 & 1575.173 & 1574.206 & 1567.786 & 1564.665 & 1570.29 \\
\hline $9 / 24 / 162: 00$ & 74.973 & 81.948 & 80.346 & 88.756 & 74.42 & 73.831 & 72.623 & 73.605 & 1583.499 & 1575.905 & 1572.962 & 1575.173 & 1574.208 & 1567.79 & 1564.668 & 1570.29 \\
\hline 9/24/16 3:00 & 74.968 & 81.944 & 80.35 & 88.751 & 74.42 & 73.831 & 72.618 & 73.612 & 1583.504 & 1575.909 & 1572.958 & 1575.178 & 1574.208 & 1567.79 & 1564.673 & 1570.283 \\
\hline $9 / 24 / 164: 00$ & 74.958 & 81.932 & 80.339 & 88.742 & 74.403 & 73.819 & 72.604 & 73.603 & 1583.514 & 1575.921 & 1572.969 & 1575.187 & 1574.225 & 1567.802 & 1564.687 & 1570.292 \\
\hline $9 / 24 / 165: 00$ & 74.956 & 81.93 & 80.334 & 88.739 & 74.401 & 73.819 & 72.602 & 73.598 & 1583.516 & 1575.923 & 1572.974 & 1575.19 & 1574.227 & 1567.802 & 1564.689 & 1570.297 \\
\hline $9 / 24 / 166: 00$ & 74.951 & 81.923 & 80.322 & 88.737 & 74.392 & 73.814 & 72.595 & 73.571 & 1583.521 & 1575.93 & 1572.986 & 1575.192 & 1574.236 & 1567.807 & 1564.696 & 1570.324 \\
\hline $9 / 24 / 167: 00$ & 74.947 & 81.918 & 80.315 & 88.735 & 74.387 & 73.807 & 72.59 & 73.575 & 1583.525 & 1575.935 & 1572.993 & 1575.194 & 1574.241 & 1567.814 & 1564.701 & 1570.32 \\
\hline
\end{tabular}


TABLE S1.1 Automatically recorded groundwater levels in the unconfined aquifer.

\begin{tabular}{|c|c|c|c|c|c|c|c|c|c|c|c|c|c|c|c|c|}
\hline \multirow[b]{2}{*}{ Date and Time } & \multicolumn{8}{|c|}{ Measured Depth to Water (ft TOC ${ }^{\mathrm{a}}$ ) } & \multicolumn{8}{|c|}{ Groundwater Elevation (ft AMSL) } \\
\hline & DL1-C & DL2-C1 & DL3-C1 & DL4-C1 & DL5-C1 & DL6-C & DL7-C & DL8-C1 & DL1-C & DL2-C1 & DL3-C1 & DL4-C1 & DL5-C1 & DL6-C & DL7-C & DL8-C1 \\
\hline 9/24/16 8:00 & 74.961 & 81.93 & 80.32 & 88.744 & 74.396 & 73.819 & 72.611 & 73.575 & 1583.511 & 1575.923 & 1572.988 & 1575.185 & 1574.232 & 1567.802 & 1564.68 & 1570.32 \\
\hline 9/24/16 9:00 & 74.958 & 81.923 & 80.325 & 88.737 & 74.392 & 73.817 & 72.599 & 73.58 & 1583.514 & 1575.93 & 1572.983 & 1575.192 & 1574.236 & 1567.804 & 1564.692 & 1570.315 \\
\hline 9/24/16 10:00 & 74.954 & 81.923 & 80.32 & 88.737 & 74.387 & 73.814 & 72.602 & 73.573 & 1583.518 & 1575.93 & 1572.988 & 1575.192 & 1574.241 & 1567.807 & 1564.689 & 1570.322 \\
\hline 9/24/16 11:00 & 74.958 & 81.925 & 80.322 & 88.737 & 74.387 & 73.814 & 72.604 & 73.575 & 1583.514 & 1575.928 & 1572.986 & 1575.192 & 1574.241 & 1567.807 & 1564.687 & 1570.32 \\
\hline 9/24/16 12:00 & 74.977 & 81.948 & 80.339 & 88.753 & 74.408 & 73.831 & 72.63 & 73.592 & 1583.495 & 1575.905 & 1572.969 & 1575.176 & 1574.22 & 1567.79 & 1564.661 & 1570.303 \\
\hline 9/24/16 13:00 & 74.968 & 81.937 & 80.332 & 88.737 & 74.396 & 73.824 & 72.616 & 73.594 & 1583.504 & 1575.916 & 1572.976 & 1575.192 & 1574.232 & 1567.797 & 1564.675 & 1570.301 \\
\hline 9/24/16 14:00 & 74.937 & 81.899 & 80.308 & 88.714 & 74.366 & 73.793 & 72.571 & 73.578 & 1583.535 & 1575.954 & 1573 & 1575.215 & 1574.262 & 1567.828 & 1564.72 & 1570.317 \\
\hline 9/24/16 15:00 & 74.914 & 81.878 & 80.28 & 88.702 & 74.336 & 73.777 & 72.549 & 73.54 & 1583.558 & 1575.975 & 1573.028 & 1575.227 & 1574.292 & 1567.844 & 1564.742 & 1570.355 \\
\hline $9 / 24 / 1616: 00$ & 74.925 & 81.885 & 80.278 & 88.714 & 74.343 & 73.796 & 72.573 & 73.543 & 1583.547 & 1575.968 & 1573.03 & 1575.215 & 1574.285 & 1567.825 & 1564.718 & 1570.352 \\
\hline 9/24/16 17:00 & 74.944 & 81.899 & 80.29 & 88.725 & 74.35 & 73.803 & 72.587 & 73.538 & 1583.528 & 1575.954 & 1573.018 & 1575.204 & 1574.278 & 1567.818 & 1564.704 & 1570.357 \\
\hline $9 / 24 / 1618: 00$ & 74.94 & 81.897 & 80.29 & 88.716 & 74.345 & 73.796 & 72.58 & 73.547 & 1583.532 & 1575.956 & 1573.018 & 1575.213 & 1574.283 & 1567.825 & 1564.711 & 1570.348 \\
\hline 9/24/16 19:00 & 74.944 & 81.895 & 80.29 & 88.721 & 74.345 & 73.798 & 72.585 & 73.545 & 1583.528 & 1575.958 & 1573.018 & 1575.208 & 1574.283 & 1567.823 & 1564.706 & 1570.35 \\
\hline 9/24/16 20:00 & 74.951 & 81.902 & 80.292 & 88.723 & 74.35 & 73.803 & 72.592 & 73.552 & 1583.521 & 1575.951 & 1573.016 & 1575.206 & 1574.278 & 1567.818 & 1564.699 & 1570.343 \\
\hline 9/24/16 21:00 & 74.968 & 81.923 & 80.311 & 88.735 & 74.364 & 73.817 & 72.616 & 73.566 & 1583.504 & 1575.93 & 1572.997 & 1575.194 & 1574.264 & 1567.804 & 1564.675 & 1570.329 \\
\hline 9/24/16 22:00 & 74.982 & 81.941 & 80.325 & 88.746 & 74.382 & 73.828 & 72.63 & 73.582 & 1583.49 & 1575.912 & 1572.983 & 1575.183 & 1574.246 & 1567.793 & 1564.661 & 1570.313 \\
\hline 9/24/16 23:00 & 74.98 & 81.946 & 80.332 & 88.742 & 74.385 & 73.826 & 72.626 & 73.587 & 1583.492 & 1575.907 & 1572.976 & 1575.187 & 1574.243 & 1567.795 & 1564.665 & 1570.308 \\
\hline $9 / 25 / 160: 00$ & 74.98 & 81.946 & 80.334 & 88.742 & 74.387 & 73.824 & 72.626 & 73.587 & 1583.492 & 1575.907 & 1572.974 & 1575.187 & 1574.241 & 1567.797 & 1564.665 & 1570.308 \\
\hline 9/25/16 1:00 & 74.98 & 81.951 & 80.332 & 88.744 & 74.392 & 73.828 & 72.626 & 73.58 & 1583.492 & 1575.902 & 1572.976 & 1575.185 & 1574.236 & 1567.793 & 1564.665 & 1570.315 \\
\hline $9 / 25 / 162: 00$ & 74.996 & 81.988 & 80.353 & 88.763 & 74.41 & 73.847 & 72.657 & 73.612 & 1583.476 & 1575.865 & 1572.955 & 1575.166 & 1574.218 & 1567.774 & 1564.634 & 1570.283 \\
\hline 9/25/16 3:00 & 75.015 & 82.009 & 80.388 & 88.774 & 74.438 & 73.865 & 72.676 & 73.645 & 1583.457 & 1575.844 & 1572.92 & 1575.155 & 1574.19 & 1567.756 & 1564.615 & 1570.25 \\
\hline 9/25/16 4:00 & 75.013 & 82.011 & 80.392 & 88.767 & 74.443 & 73.861 & 72.666 & 73.657 & 1583.459 & 1575.842 & 1572.916 & 1575.162 & 1574.185 & 1567.76 & 1564.625 & 1570.238 \\
\hline 9/25/16 5:00 & 75.015 & 82.025 & 80.404 & 88.777 & 74.455 & 73.868 & 72.673 & 73.673 & 1583.457 & 1575.828 & 1572.904 & 1575.152 & 1574.173 & 1567.753 & 1564.618 & 1570.222 \\
\hline $9 / 25 / 166: 00$ & 75.027 & 82.048 & 80.42 & 88.791 & 74.476 & 73.884 & 72.7 & 73.682 & 1583.445 & 1575.805 & 1572.888 & 1575.138 & 1574.152 & 1567.737 & 1564.591 & 1570.213 \\
\hline $9 / 25 / 167: 00$ & 75.027 & 82.062 & 80.432 & 88.791 & 74.485 & 73.884 & 72.695 & 73.696 & 1583.445 & 1575.791 & 1572.876 & 1575.138 & 1574.143 & 1567.737 & 1564.596 & 1570.199 \\
\hline $9 / 25 / 168: 00$ & 75.031 & 82.072 & 80.441 & 88.798 & 74.497 & 73.886 & 72.702 & 73.703 & 1583.441 & 1575.781 & 1572.867 & 1575.131 & 1574.131 & 1567.735 & 1564.589 & 1570.192 \\
\hline 9/25/16 9:00 & 75.027 & 82.078 & 80.446 & 88.795 & 74.504 & 73.889 & 72.7 & 73.708 & 1583.445 & 1575.775 & 1572.862 & 1575.134 & 1574.124 & 1567.732 & 1564.591 & 1570.187 \\
\hline 9/25/16 10:00 & 75.024 & 82.081 & 80.448 & 88.795 & 74.504 & 73.886 & 72.697 & 73.682 & 1583.448 & 1575.772 & 1572.86 & 1575.134 & 1574.124 & 1567.735 & 1564.594 & 1570.213 \\
\hline 9/25/16 11:00 & 75.022 & 82.083 & 80.45 & 88.793 & 74.511 & 73.886 & 72.695 & 73.715 & 1583.45 & 1575.77 & 1572.858 & 1575.136 & 1574.117 & 1567.735 & 1564.596 & 1570.18 \\
\hline 9/25/16 12:00 & 75.01 & 82.074 & 80.443 & 88.788 & 74.503 & 73.879 & 72.683 & 73.715 & 1583.462 & 1575.779 & 1572.865 & 1575.141 & 1574.125 & 1567.742 & 1564.608 & 1570.18 \\
\hline 9/25/16 13:00 & 74.996 & 82.067 & 80.434 & 88.779 & 74.492 & 73.87 & 72.671 & 73.701 & 1583.476 & 1575.786 & 1572.874 & 1575.15 & 1574.136 & 1567.751 & 1564.62 & 1570.194 \\
\hline 9/25/16 14:00 & 74.982 & 82.053 & 80.422 & 88.767 & 74.478 & 73.858 & 72.654 & 73.689 & 1583.49 & 1575.8 & 1572.886 & 1575.162 & 1574.15 & 1567.763 & 1564.637 & 1570.206 \\
\hline $9 / 25 / 1615: 00$ & 74.963 & 82.03 & 80.404 & 88.756 & 74.459 & 73.842 & 72.63 & 73.67 & 1583.509 & 1575.823 & 1572.904 & 1575.173 & 1574.169 & 1567.779 & 1564.661 & 1570.225 \\
\hline $9 / 25 / 1616: 00$ & 74.951 & 82.013 & 80.388 & 88.749 & 74.443 & 73.833 & 72.618 & 73.654 & 1583.521 & 1575.84 & 1572.92 & 1575.18 & 1574.185 & 1567.788 & 1564.673 & 1570.241 \\
\hline 9/25/16 17:00 & 74.949 & 82.011 & 80.383 & 88.753 & 74.434 & 73.838 & 72.618 & 73.659 & 1583.523 & 1575.842 & 1572.925 & 1575.176 & 1574.194 & 1567.783 & 1564.673 & 1570.236 \\
\hline 9/25/16 18:00 & 74.958 & 82.013 & 80.385 & 88.758 & 74.443 & 73.845 & 72.635 & 73.661 & 1583.514 & 1575.84 & 1572.923 & 1575.171 & 1574.185 & 1567.776 & 1564.656 & 1570.234 \\
\hline 9/25/16 19:00 & 74.977 & 82.034 & 80.404 & 88.767 & 74.457 & 73.858 & 72.654 & 73.673 & 1583.495 & 1575.819 & 1572.904 & 1575.162 & 1574.171 & 1567.763 & 1564.637 & 1570.222 \\
\hline 9/25/16 20:00 & 74.989 & 82.046 & 80.418 & 88.777 & 74.466 & 73.863 & 72.666 & 73.684 & 1583.483 & 1575.807 & 1572.89 & 1575.152 & 1574.162 & 1567.758 & 1564.625 & 1570.211 \\
\hline 9/25/16 21:00 & 75.001 & 82.06 & 80.427 & 88.781 & 74.48 & 73.875 & 72.678 & 73.696 & 1583.471 & 1575.793 & 1572.881 & 1575.148 & 1574.148 & 1567.746 & 1564.613 & 1570.199 \\
\hline 9/25/16 22:00 & 75.001 & 82.062 & 80.436 & 88.781 & 74.487 & 73.877 & 72.678 & 73.703 & 1583.471 & 1575.791 & 1572.872 & 1575.148 & 1574.141 & 1567.744 & 1564.613 & 1570.192 \\
\hline 9/25/16 23:00 & 74.994 & 82.06 & 80.429 & 88.772 & 74.48 & 73.868 & 72.666 & 73.687 & 1583.478 & 1575.793 & 1572.879 & 1575.157 & 1574.148 & 1567.753 & 1564.625 & 1570.208 \\
\hline $9 / 26 / 160: 00$ & 74.991 & 82.058 & 80.429 & 88.774 & 74.483 & 73.868 & 72.669 & 73.698 & 1583.481 & 1575.795 & 1572.879 & 1575.155 & 1574.145 & 1567.753 & 1564.622 & 1570.197 \\
\hline 9/26/16 1:00 & 74.98 & 82.053 & 80.42 & 88.767 & 74.473 & 73.861 & 72.659 & 73.691 & 1583.492 & 1575.8 & 1572.888 & 1575.162 & 1574.155 & 1567.76 & 1564.632 & 1570.204 \\
\hline $9 / 26 / 162: 00$ & 74.975 & 82.048 & 80.483 & 88.765 & 74.469 & 73.858 & 72.652 & 73.696 & 1583.497 & 1575.805 & 1572.825 & 1575.164 & 1574.159 & 1567.763 & 1564.639 & 1570.199 \\
\hline 9/26/16 3:00 & 74.968 & 82.034 & 80.497 & 88.758 & 74.459 & 73.851 & 72.642 & 73.701 & 1583.504 & 1575.819 & 1572.811 & 1575.171 & 1574.169 & 1567.77 & 1564.649 & 1570.194 \\
\hline $9 / 26 / 164: 00$ & 74.954 & 82.018 & 80.476 & 88.746 & 74.441 & 73.838 & 72.623 & 73.689 & 1583.518 & 1575.835 & 1572.832 & 1575.183 & 1574.187 & 1567.783 & 1564.668 & 1570.206 \\
\hline $9 / 26 / 165: 00$ & 74.956 & 82.023 & 80.502 & 88.753 & 74.445 & 73.845 & 72.633 & 73.694 & 1583.516 & 1575.83 & 1572.806 & 1575.176 & 1574.183 & 1567.776 & 1564.658 & 1570.201 \\
\hline $9 / 26 / 166: 00$ & 74.958 & 82.018 & 80.448 & 88.751 & 74.441 & 73.84 & 72.628 & 73.682 & 1583.514 & 1575.835 & 1572.86 & 1575.178 & 1574.187 & 1567.781 & 1564.663 & 1570.213 \\
\hline $9 / 26 / 167: 00$ & 74.958 & 82.016 & 80.406 & 88.753 & 74.438 & 73.84 & 72.63 & 73.673 & 1583.514 & 1575.837 & 1572.902 & 1575.176 & 1574.19 & 1567.781 & 1564.661 & 1570.222 \\
\hline $9 / 26 / 168: 00$ & 74.956 & 82.016 & 80.399 & 88.751 & 74.436 & 73.838 & 72.626 & 73.666 & 1583.516 & 1575.837 & 1572.909 & 1575.178 & 1574.192 & 1567.783 & 1564.665 & 1570.229 \\
\hline
\end{tabular}


TABLE S1.1 Automatically recorded groundwater levels in the unconfined aquifer.

\begin{tabular}{|c|c|c|c|c|c|c|c|c|c|c|c|c|c|c|c|c|}
\hline \multirow[b]{2}{*}{ Date and Time } & \multicolumn{8}{|c|}{ Measured Depth to Water ( $\mathrm{ft}$ TOC ${ }^{\mathrm{a}}$ ) } & \multicolumn{8}{|c|}{ Groundwater Elevation (ft AMSL ${ }^{\mathrm{b}}$ ) } \\
\hline & DL1-C & DL2-C1 & DL3-C1 & DL4-C1 & DL5-C1 & DL6-C & DL7-C & DL8-C1 & DL1-C & DL2-C1 & DL3-C1 & DL4-C1 & DL5-C1 & DL6-C & DL7-C & DL8-C1 \\
\hline 9/26/16 9:00 & 74.951 & 82.004 & 80.392 & 88.744 & 74.42 & 73.828 & 72.614 & 73.67 & 1583.521 & 1575.849 & 1572.916 & 1575.185 & 1574.208 & 1567.793 & 1564.677 & 1570.225 \\
\hline 9/26/16 10:00 & 74.944 & 81.99 & 80.381 & 88.737 & 74.415 & 73.821 & 72.611 & 73.652 & 1583.528 & 1575.863 & 1572.927 & 1575.192 & 1574.213 & 1567.8 & 1564.68 & 1570.243 \\
\hline 9/26/16 11:00 & 74.94 & 81.981 & 80.374 & 88.732 & 74.403 & 73.821 & 72.602 & 73.647 & 1583.532 & 1575.872 & 1572.934 & 1575.197 & 1574.225 & 1567.8 & 1564.689 & 1570.248 \\
\hline 9/26/16 12:00 & 74.928 & 81.965 & 80.355 & 88.72 & 74.389 & 73.805 & 72.585 & 73.622 & 1583.544 & 1575.888 & 1572.953 & 1575.209 & 1574.239 & 1567.816 & 1564.706 & 1570.273 \\
\hline 9/26/16 13:00 & 74.907 & 81.932 & 80.325 & 88.7 & 74.354 & 73.784 & 72.554 & 73.594 & 1583.565 & 1575.921 & 1572.983 & 1575.229 & 1574.274 & 1567.837 & 1564.737 & 1570.301 \\
\hline 9/26/16 14:00 & 74.89 & 81.902 & 80.299 & 88.683 & 74.324 & 73.768 & 72.533 & 73.571 & 1583.582 & 1575.951 & 1573.009 & 1575.246 & 1574.304 & 1567.853 & 1564.758 & 1570.324 \\
\hline 9/26/16 15:00 & 74.869 & 81.865 & 80.264 & 88.665 & 74.287 & 73.747 & 72.504 & 73.543 & 1583.603 & 1575.988 & 1573.044 & 1575.264 & 1574.341 & 1567.874 & 1564.787 & 1570.352 \\
\hline 9/26/16 16:00 & 74.857 & 81.839 & 80.243 & 88.655 & 74.263 & 73.73 & 72.49 & 73.513 & 1583.615 & 1576.014 & 1573.065 & 1575.274 & 1574.365 & 1567.891 & 1564.801 & 1570.382 \\
\hline 9/26/16 17:00 & 74.85 & 81.818 & 80.222 & 88.653 & 74.238 & 73.728 & 72.482 & 73.503 & 1583.622 & 1576.035 & 1573.086 & 1575.276 & 1574.39 & 1567.893 & 1564.809 & 1570.392 \\
\hline 9/26/16 18:00 & 74.848 & 81.797 & 80.208 & 88.646 & 74.217 & 73.719 & 72.473 & 73.492 & 1583.624 & 1576.056 & 1573.1 & 1575.283 & 1574.411 & 1567.902 & 1564.818 & 1570.403 \\
\hline 9/26/16 19:00 & 74.852 & 81.792 & 80.194 & 88.644 & 74.205 & 73.716 & 72.478 & 73.468 & 1583.62 & 1576.061 & 1573.114 & 1575.285 & 1574.423 & 1567.905 & 1564.813 & 1570.427 \\
\hline 9/26/16 20:00 & 74.874 & 81.797 & 80.199 & 88.658 & 74.21 & 73.728 & 72.499 & 73.466 & 1583.598 & 1576.056 & 1573.109 & 1575.271 & 1574.418 & 1567.893 & 1564.792 & 1570.429 \\
\hline 9/26/16 21:00 & 74.89 & 81.811 & 80.21 & 88.662 & 74.219 & 73.742 & 72.518 & 73.466 & 1583.582 & 1576.042 & 1573.098 & 1575.267 & 1574.409 & 1567.879 & 1564.773 & 1570.429 \\
\hline 9/26/16 22:00 & 74.897 & 81.813 & 80.213 & 88.665 & 74.219 & 73.737 & 72.518 & 73.475 & 1583.575 & 1576.04 & 1573.095 & 1575.264 & 1574.409 & 1567.884 & 1564.773 & 1570.42 \\
\hline 9/26/16 23:00 & 74.897 & 81.806 & 80.206 & 88.658 & 74.207 & 73.73 & 72.511 & 73.468 & 1583.575 & 1576.047 & 1573.102 & 1575.271 & 1574.421 & 1567.891 & 1564.78 & 1570.427 \\
\hline 9/27/16 0:00 & 74.89 & 81.795 & 80.196 & 88.648 & 74.198 & 73.726 & 72.501 & 73.462 & 1583.582 & 1576.058 & 1573.112 & 1575.281 & 1574.43 & 1567.895 & 1564.79 & 1570.433 \\
\hline 9/27/16 1:00 & 74.881 & 81.781 & 80.182 & 88.646 & 74.184 & 73.716 & 72.492 & 73.443 & 1583.591 & 1576.072 & 1573.126 & 1575.283 & 1574.444 & 1567.905 & 1564.799 & 1570.452 \\
\hline $9 / 27 / 162: 00$ & 74.878 & 81.776 & 80.173 & 88.644 & 74.175 & 73.712 & 72.49 & 73.434 & 1583.594 & 1576.077 & 1573.135 & 1575.285 & 1574.453 & 1567.909 & 1564.801 & 1570.461 \\
\hline 9/27/16 3:00 & 74.878 & 81.774 & 80.168 & 88.639 & 74.17 & 73.712 & 72.49 & 73.434 & 1583.594 & 1576.079 & 1573.14 & 1575.29 & 1574.458 & 1567.909 & 1564.801 & 1570.461 \\
\hline 9/27/16 4:00 & 74.874 & 81.764 & 80.161 & 88.637 & 74.156 & 73.705 & 72.482 & 73.424 & 1583.598 & 1576.089 & 1573.147 & 1575.292 & 1574.472 & 1567.916 & 1564.809 & 1570.471 \\
\hline 9/27/16 5:00 & 74.871 & 81.755 & 80.154 & 88.63 & 74.147 & 73.7 & 72.475 & 73.413 & 1583.601 & 1576.098 & 1573.154 & 1575.299 & 1574.481 & 1567.921 & 1564.816 & 1570.482 \\
\hline $9 / 27 / 166: 00$ & 74.871 & 81.748 & 80.145 & 88.625 & 74.138 & 73.695 & 72.473 & 73.41 & 1583.601 & 1576.105 & 1573.163 & 1575.304 & 1574.49 & 1567.926 & 1564.818 & 1570.485 \\
\hline $9 / 27 / 167: 00$ & 74.876 & 81.748 & 80.145 & 88.627 & 74.133 & 73.7 & 72.478 & 73.406 & 1583.596 & 1576.105 & 1573.163 & 1575.302 & 1574.495 & 1567.921 & 1564.813 & 1570.489 \\
\hline 9/27/16 8:00 & 74.883 & 81.753 & 80.15 & 88.632 & 74.135 & 73.695 & 72.482 & 73.394 & 1583.589 & 1576.1 & 1573.158 & 1575.297 & 1574.493 & 1567.926 & 1564.809 & 1570.501 \\
\hline 9/27/16 9:00 & 74.888 & 81.753 & 80.145 & 88.63 & 74.133 & 73.7 & 72.482 & 73.406 & 1583.584 & 1576.1 & 1573.163 & 1575.299 & 1574.495 & 1567.921 & 1564.809 & 1570.489 \\
\hline 9/27/16 10:00 & 74.892 & 81.753 & 80.147 & 88.634 & 74.133 & 73.705 & 72.485 & 73.406 & 1583.58 & 1576.1 & 1573.161 & 1575.295 & 1574.495 & 1567.916 & 1564.806 & 1570.489 \\
\hline 9/27/16 11:00 & 74.89 & 81.751 & 80.147 & 88.63 & 74.128 & 73.695 & 72.48 & 73.401 & 1583.582 & 1576.102 & 1573.161 & 1575.299 & 1574.5 & 1567.926 & 1564.811 & 1570.494 \\
\hline 9/27/16 12:00 & 74.871 & 81.73 & 80.129 & 88.613 & 74.107 & 73.681 & 72.454 & 73.38 & 1583.601 & 1576.123 & 1573.179 & 1575.316 & 1574.521 & 1567.94 & 1564.837 & 1570.515 \\
\hline 9/27/16 13:00 & 74.855 & 81.704 & 80.11 & 88.599 & 74.086 & 73.663 & 72.43 & 73.369 & 1583.617 & 1576.149 & 1573.198 & 1575.33 & 1574.542 & 1567.958 & 1564.861 & 1570.526 \\
\hline 9/27/16 14:00 & 74.838 & 81.683 & 80.087 & 88.588 & 74.068 & 73.649 & 72.413 & 73.352 & 1583.634 & 1576.17 & 1573.221 & 1575.341 & 1574.56 & 1567.972 & 1564.878 & 1570.543 \\
\hline 9/27/16 15:00 & 74.819 & 81.653 & 80.064 & 88.574 & 74.04 & 73.633 & 72.392 & 73.338 & 1583.653 & 1576.2 & 1573.244 & 1575.355 & 1574.588 & 1567.988 & 1564.899 & 1570.557 \\
\hline 9/27/16 16:00 & 74.81 & 81.637 & 80.047 & 88.567 & 74.019 & 73.626 & 72.385 & 73.325 & 1583.662 & 1576.216 & 1573.261 & 1575.362 & 1574.609 & 1567.995 & 1564.906 & 1570.57 \\
\hline 9/27/16 17:00 & 74.808 & 81.62 & 80.038 & 88.562 & 74.002 & 73.626 & 72.377 & 73.306 & 1583.664 & 1576.233 & 1573.27 & 1575.367 & 1574.626 & 1567.995 & 1564.914 & 1570.589 \\
\hline 9/27/16 18:00 & 74.81 & 81.613 & 80.022 & 88.559 & 73.991 & 73.614 & 72.373 & 73.283 & 1583.662 & 1576.24 & 1573.286 & 1575.37 & 1574.637 & 1568.007 & 1564.918 & 1570.612 \\
\hline 9/27/16 19:00 & 74.812 & 81.602 & 80.008 & 88.555 & 73.979 & 73.612 & 72.37 & 73.269 & 1583.66 & 1576.251 & 1573.3 & 1575.374 & 1574.649 & 1568.009 & 1564.921 & 1570.626 \\
\hline 9/27/16 20:00 & 74.822 & 81.604 & 80.01 & 88.562 & 73.977 & 73.619 & 72.382 & 73.264 & 1583.65 & 1576.249 & 1573.298 & 1575.367 & 1574.651 & 1568.002 & 1564.909 & 1570.631 \\
\hline 9/27/16 21:00 & 74.843 & 81.623 & 80.024 & 88.578 & 73.988 & 73.635 & 72.408 & 73.26 & 1583.629 & 1576.23 & 1573.284 & 1575.351 & 1574.64 & 1567.986 & 1564.883 & 1570.635 \\
\hline 9/27/16 22:00 & 74.869 & 81.648 & 80.047 & 88.59 & 74.012 & 73.656 & 72.432 & 73.301 & 1583.603 & 1576.205 & 1573.261 & 1575.339 & 1574.616 & 1567.965 & 1564.859 & 1570.594 \\
\hline 9/27/16 23:00 & 74.874 & 81.66 & 80.054 & 88.59 & 74.021 & 73.654 & 72.439 & 73.313 & 1583.598 & 1576.193 & 1573.254 & 1575.339 & 1574.607 & 1567.967 & 1564.852 & 1570.582 \\
\hline 9/28/16 0:00 & 74.888 & 81.674 & 80.066 & 88.599 & 74.03 & 73.665 & 72.454 & 73.318 & 1583.584 & 1576.179 & 1573.242 & 1575.33 & 1574.598 & 1567.956 & 1564.837 & 1570.577 \\
\hline 9/28/16 1:00 & 74.89 & 81.688 & 80.073 & 88.604 & 74.04 & 73.67 & 72.456 & 73.322 & 1583.582 & 1576.165 & 1573.235 & 1575.325 & 1574.588 & 1567.951 & 1564.835 & 1570.573 \\
\hline $9 / 28 / 162: 00$ & 74.902 & 81.706 & 80.147 & 88.609 & 74.054 & 73.679 & 72.47 & 73.345 & 1583.57 & 1576.147 & 1573.161 & 1575.32 & 1574.574 & 1567.942 & 1564.821 & 1570.55 \\
\hline 9/28/16 3:00 & 74.907 & 81.718 & 80.185 & 88.613 & 74.067 & 73.686 & 72.478 & 73.371 & 1583.565 & 1576.135 & 1573.123 & 1575.316 & 1574.561 & 1567.935 & 1564.813 & 1570.524 \\
\hline 9/28/16 4:00 & 74.914 & 81.739 & 80.194 & 88.62 & 74.077 & 73.693 & 72.49 & 73.394 & 1583.558 & 1576.114 & 1573.114 & 1575.309 & 1574.551 & 1567.928 & 1564.801 & 1570.501 \\
\hline 9/28/16 5:00 & 74.93 & 81.767 & 80.241 & 88.637 & 74.103 & 73.709 & 72.513 & 73.422 & 1583.542 & 1576.086 & 1573.067 & 1575.292 & 1574.525 & 1567.912 & 1564.778 & 1570.473 \\
\hline $9 / 28 / 166: 00$ & 74.94 & 81.785 & 80.21 & 88.648 & 74.124 & 73.719 & 72.525 & 73.429 & 1583.532 & 1576.068 & 1573.098 & 1575.281 & 1574.504 & 1567.902 & 1564.766 & 1570.466 \\
\hline 9/28/16 7:00 & 74.949 & 81.809 & 80.189 & 88.653 & 74.147 & 73.73 & 72.542 & 73.434 & 1583.523 & 1576.044 & 1573.119 & 1575.276 & 1574.481 & 1567.891 & 1564.749 & 1570.461 \\
\hline 9/28/16 8:00 & 74.966 & 81.841 & 80.21 & 88.669 & 74.17 & 73.747 & 72.561 & 73.466 & 1583.506 & 1576.012 & 1573.098 & 1575.26 & 1574.458 & 1567.874 & 1564.73 & 1570.429 \\
\hline 9/28/16 9:00 & 74.975 & 81.867 & 80.229 & 88.681 & 74.193 & 73.761 & 72.578 & 73.487 & 1583.497 & 1575.986 & 1573.079 & 1575.248 & 1574.435 & 1567.86 & 1564.713 & 1570.408 \\
\hline
\end{tabular}


TABLE S1.1 Automatically recorded groundwater levels in the unconfined aquifer.

\begin{tabular}{|c|c|c|c|c|c|c|c|c|c|c|c|c|c|c|c|c|}
\hline \multirow[b]{2}{*}{ Date and Time } & \multicolumn{8}{|c|}{ Measured Depth to Water ( $\mathrm{ft}$ TOC ${ }^{\mathrm{a}}$ ) } & \multicolumn{8}{|c|}{ Groundwater Elevation (ft $\mathrm{AMSL}^{\mathrm{b}}$ ) } \\
\hline & DL1-C & DL2-C1 & DL3-C1 & DL4-C1 & DL5-C1 & DL6-C & DL7-C & DL8-C1 & DL1-C & $\mathrm{DL} 2-\mathrm{C} 1$ & DL3-C1 & DL4-C1 & DL5-C1 & DL6-C & DL7-C & DL8-C1 \\
\hline 9/28/16 10:00 & 74.989 & 81.892 & 80.255 & 88.693 & 74.221 & 73.777 & 72.595 & 73.52 & 1583.483 & 1575.961 & 1573.053 & 1575.236 & 1574.407 & 1567.844 & 1564.696 & 1570.375 \\
\hline 9/28/16 11:00 & 74.987 & 81.906 & 80.266 & 88.695 & 74.235 & 73.779 & 72.597 & 73.529 & 1583.485 & 1575.947 & 1573.042 & 1575.234 & 1574.393 & 1567.842 & 1564.694 & 1570.366 \\
\hline 9/28/16 12:00 & 74.977 & 81.911 & 80.269 & 88.69 & 74.24 & 73.775 & 72.59 & 73.529 & 1583.495 & 1575.942 & 1573.039 & 1575.239 & 1574.388 & 1567.846 & 1564.701 & 1570.366 \\
\hline 9/28/16 13:00 & 74.963 & 81.909 & 80.264 & 88.686 & 74.235 & 73.768 & 72.576 & 73.533 & 1583.509 & 1575.944 & 1573.044 & 1575.243 & 1574.393 & 1567.853 & 1564.715 & 1570.362 \\
\hline 9/28/16 14:00 & 74.944 & 81.897 & 80.269 & 88.674 & 74.226 & 73.756 & 72.556 & 73.524 & 1583.528 & 1575.956 & 1573.039 & 1575.255 & 1574.402 & 1567.865 & 1564.735 & 1570.371 \\
\hline $9 / 28 / 1615: 00$ & 74.935 & 81.888 & 80.248 & 88.672 & 74.219 & 73.749 & 72.547 & 73.513 & 1583.537 & 1575.965 & 1573.06 & 1575.257 & 1574.409 & 1567.872 & 1564.744 & 1570.382 \\
\hline 9/28/16 16:00 & 74.925 & 81.885 & 80.25 & 88.669 & 74.217 & 73.749 & 72.544 & 73.515 & 1583.547 & 1575.968 & 1573.058 & 1575.26 & 1574.411 & 1567.872 & 1564.747 & 1570.38 \\
\hline 9/28/16 17:00 & 74.928 & 81.89 & 80.259 & 88.672 & 74.217 & 73.754 & 72.549 & 73.524 & 1583.544 & 1575.963 & 1573.049 & 1575.257 & 1574.411 & 1567.867 & 1564.742 & 1570.371 \\
\hline $9 / 28 / 1618: 00$ & 74.937 & 81.904 & 80.259 & 88.681 & 74.233 & 73.763 & 72.564 & 73.54 & 1583.535 & 1575.949 & 1573.049 & 1575.248 & 1574.395 & 1567.858 & 1564.727 & 1570.355 \\
\hline 9/28/16 19:00 & 74.944 & 81.911 & 80.269 & 88.685 & 74.24 & 73.768 & 72.571 & 73.54 & 1583.528 & 1575.942 & 1573.039 & 1575.244 & 1574.388 & 1567.853 & 1564.72 & 1570.355 \\
\hline 9/28/16 20:00 & 74.947 & 81.916 & 80.273 & 88.688 & 74.249 & 73.77 & 72.576 & 73.545 & 1583.525 & 1575.937 & 1573.035 & 1575.241 & 1574.379 & 1567.851 & 1564.715 & 1570.35 \\
\hline 9/28/16 21:00 & 74.961 & 81.941 & 80.292 & 88.702 & 74.27 & 73.782 & 72.592 & 73.566 & 1583.511 & 1575.912 & 1573.016 & 1575.227 & 1574.358 & 1567.839 & 1564.699 & 1570.329 \\
\hline 9/28/16 22:00 & 74.97 & 81.958 & 80.308 & 88.716 & 74.287 & 73.793 & 72.604 & 73.58 & 1583.502 & 1575.895 & 1573 & 1575.213 & 1574.341 & 1567.828 & 1564.687 & 1570.315 \\
\hline 9/28/16 23:00 & 74.97 & 81.962 & 80.315 & 88.716 & 74.294 & 73.791 & 72.602 & 73.589 & 1583.502 & 1575.891 & 1572.993 & 1575.213 & 1574.334 & 1567.83 & 1564.689 & 1570.306 \\
\hline 9/29/16 0:00 & 74.963 & 81.964 & 80.313 & 88.709 & 74.294 & 73.789 & 72.597 & 73.591 & 1583.509 & 1575.889 & 1572.995 & 1575.22 & 1574.334 & 1567.832 & 1564.694 & 1570.304 \\
\hline $9 / 29 / 161: 00$ & 74.951 & 81.96 & 80.306 & 88.7 & 74.291 & 73.779 & 72.585 & 73.585 & 1583.521 & 1575.893 & 1573.002 & 1575.229 & 1574.337 & 1567.842 & 1564.706 & 1570.31 \\
\hline 9/29/16 2:00 & 74.944 & 81.958 & 80.304 & 88.7 & 74.289 & 73.777 & 72.58 & 73.58 & 1583.528 & 1575.895 & 1573.004 & 1575.229 & 1574.339 & 1567.844 & 1564.711 & 1570.315 \\
\hline 9/29/16 3:00 & 74.94 & 81.951 & 80.306 & 88.695 & 74.284 & 73.779 & 72.576 & 73.589 & 1583.532 & 1575.902 & 1573.002 & 1575.234 & 1574.344 & 1567.842 & 1564.715 & 1570.306 \\
\hline 9/29/16 4:00 & 74.935 & 81.951 & 80.308 & 88.695 & 74.28 & 73.775 & 72.573 & 73.557 & 1583.537 & 1575.902 & 1573 & 1575.234 & 1574.348 & 1567.846 & 1564.718 & 1570.338 \\
\hline 9/29/16 5:00 & 74.935 & 81.953 & 80.311 & 88.695 & 74.289 & 73.777 & 72.573 & 73.596 & 1583.537 & 1575.9 & 1572.997 & 1575.234 & 1574.339 & 1567.844 & 1564.718 & 1570.299 \\
\hline $9 / 29 / 166: 00$ & 74.94 & 81.953 & 80.308 & 88.7 & 74.289 & 73.779 & 72.576 & 73.589 & 1583.532 & 1575.9 & 1573 & 1575.229 & 1574.339 & 1567.842 & 1564.715 & 1570.306 \\
\hline 9/29/16 7:00 & 74.947 & 81.967 & 80.32 & 88.704 & 74.301 & 73.786 & 72.587 & 73.598 & 1583.525 & 1575.886 & 1572.988 & 1575.225 & 1574.327 & 1567.835 & 1564.704 & 1570.297 \\
\hline 9/29/16 8:00 & 74.947 & 81.964 & 80.322 & 88.702 & 74.303 & 73.786 & 72.587 & 73.598 & 1583.525 & 1575.889 & 1572.986 & 1575.227 & 1574.325 & 1567.835 & 1564.704 & 1570.297 \\
\hline 9/29/16 9:00 & 74.951 & 81.974 & 80.327 & 88.707 & 74.308 & 73.789 & 72.592 & 73.603 & 1583.521 & 1575.879 & 1572.981 & 1575.222 & 1574.32 & 1567.832 & 1564.699 & 1570.292 \\
\hline 9/29/16 10:00 & 74.954 & 81.976 & 80.332 & 88.704 & 74.315 & 73.791 & 72.592 & 73.612 & 1583.518 & 1575.877 & 1572.976 & 1575.225 & 1574.313 & 1567.83 & 1564.699 & 1570.283 \\
\hline 9/29/16 11:00 & 74.942 & 81.967 & 80.322 & 88.697 & 74.305 & 73.779 & 72.578 & 73.603 & 1583.53 & 1575.886 & 1572.986 & 1575.232 & 1574.323 & 1567.842 & 1564.713 & 1570.292 \\
\hline 9/29/16 12:00 & 74.928 & 81.951 & 80.308 & 88.683 & 74.291 & 73.768 & 72.559 & 73.587 & 1583.544 & 1575.902 & 1573 & 1575.246 & 1574.337 & 1567.853 & 1564.732 & 1570.308 \\
\hline 9/29/16 13:00 & 74.907 & 81.927 & 80.292 & 88.674 & 74.273 & 73.751 & 72.537 & 73.568 & 1583.565 & 1575.926 & 1573.016 & 1575.255 & 1574.355 & 1567.87 & 1564.754 & 1570.327 \\
\hline 9/29/16 14:00 & 74.883 & 81.892 & 80.262 & 88.653 & 74.242 & 73.73 & 72.504 & 73.533 & 1583.589 & 1575.961 & 1573.046 & 1575.276 & 1574.386 & 1567.891 & 1564.787 & 1570.362 \\
\hline 9/29/16 15:00 & 74.859 & 81.865 & 80.234 & 88.637 & 74.21 & 73.709 & 72.478 & 73.517 & 1583.613 & 1575.988 & 1573.074 & 1575.292 & 1574.418 & 1567.912 & 1564.813 & 1570.378 \\
\hline $9 / 29 / 1616: 00$ & 74.848 & 81.834 & 80.21 & 88.625 & 74.186 & 73.695 & 72.459 & 73.496 & 1583.624 & 1576.019 & 1573.098 & 1575.304 & 1574.442 & 1567.926 & 1564.832 & 1570.399 \\
\hline 9/29/16 17:00 & 74.838 & 81.816 & 80.194 & 88.618 & 74.172 & 73.686 & 72.447 & 73.487 & 1583.634 & 1576.037 & 1573.114 & 1575.311 & 1574.456 & 1567.935 & 1564.844 & 1570.408 \\
\hline $9 / 29 / 1618: 00$ & 74.838 & 81.802 & 80.18 & 88.613 & 74.161 & 73.686 & 72.444 & 73.452 & 1583.634 & 1576.051 & 1573.128 & 1575.316 & 1574.467 & 1567.935 & 1564.847 & 1570.443 \\
\hline 9/29/16 19:00 & 74.845 & 81.795 & 80.173 & 88.616 & 74.149 & 73.684 & 72.442 & 73.45 & 1583.627 & 1576.058 & 1573.135 & 1575.313 & 1574.479 & 1567.937 & 1564.849 & 1570.445 \\
\hline 9/29/16 20:00 & 74.852 & 81.795 & 80.171 & 88.618 & 74.147 & 73.686 & 72.449 & 73.45 & 1583.62 & 1576.058 & 1573.137 & 1575.311 & 1574.481 & 1567.935 & 1564.842 & 1570.445 \\
\hline 9/29/16 21:00 & 74.859 & 81.795 & 80.171 & 88.62 & 74.147 & 73.688 & 72.456 & 73.45 & 1583.613 & 1576.058 & 1573.137 & 1575.309 & 1574.481 & 1567.933 & 1564.835 & 1570.445 \\
\hline 9/29/16 22:00 & 74.876 & 81.799 & 80.175 & 88.625 & 74.152 & 73.693 & 72.466 & 73.452 & 1583.596 & 1576.054 & 1573.133 & 1575.304 & 1574.476 & 1567.928 & 1564.825 & 1570.443 \\
\hline 9/29/16 23:00 & 74.871 & 81.797 & 80.173 & 88.623 & 74.145 & 73.691 & 72.459 & 73.45 & 1583.601 & 1576.056 & 1573.135 & 1575.306 & 1574.483 & 1567.93 & 1564.832 & 1570.445 \\
\hline 9/30/16 0:00 & 74.871 & 81.79 & 80.166 & 88.616 & 74.14 & 73.688 & 72.454 & 73.445 & 1583.601 & 1576.063 & 1573.142 & 1575.313 & 1574.488 & 1567.933 & 1564.837 & 1570.45 \\
\hline 9/30/16 1:00 & 74.862 & 81.783 & 80.157 & 88.613 & 74.126 & 73.679 & 72.447 & 73.431 & 1583.61 & 1576.07 & 1573.151 & 1575.316 & 1574.502 & 1567.942 & 1564.844 & 1570.464 \\
\hline $9 / 30 / 162: 00$ & 74.857 & 81.771 & 80.208 & 88.606 & 74.114 & 73.672 & 72.437 & 73.429 & 1583.615 & 1576.082 & 1573.1 & 1575.323 & 1574.514 & 1567.949 & 1564.854 & 1570.466 \\
\hline 9/30/16 3:00 & 74.855 & 81.767 & 80.224 & 88.602 & 74.11 & 73.67 & 72.435 & 73.424 & 1583.617 & 1576.086 & 1573.084 & 1575.327 & 1574.518 & 1567.951 & 1564.856 & 1570.471 \\
\hline 9/30/16 4:00 & 74.845 & 81.748 & 80.213 & 88.595 & 74.096 & 73.656 & 72.423 & 73.422 & 1583.627 & 1576.105 & 1573.095 & 1575.334 & 1574.532 & 1567.965 & 1564.868 & 1570.473 \\
\hline 9/30/16 5:00 & 74.841 & 81.739 & 80.222 & 88.59 & 74.089 & 73.656 & 72.413 & 73.413 & 1583.631 & 1576.114 & 1573.086 & 1575.339 & 1574.539 & 1567.965 & 1564.878 & 1570.482 \\
\hline 9/30/16 6:00 & 74.845 & 81.734 & 80.168 & 88.59 & 74.084 & 73.656 & 72.415 & 73.399 & 1583.627 & 1576.119 & 1573.14 & 1575.339 & 1574.544 & 1567.965 & 1564.876 & 1570.496 \\
\hline 9/30/16 7:00 & 74.852 & 81.737 & 80.129 & 88.59 & 74.082 & 73.656 & 72.418 & 73.394 & 1583.62 & 1576.116 & 1573.179 & 1575.339 & 1574.546 & 1567.965 & 1564.873 & 1570.501 \\
\hline 9/30/16 8:00 & 74.859 & 81.741 & 80.129 & 88.594 & 74.082 & 73.658 & 72.425 & 73.394 & 1583.613 & 1576.112 & 1573.179 & 1575.335 & 1574.546 & 1567.963 & 1564.866 & 1570.501 \\
\hline 9/30/16 9:00 & 74.862 & 81.744 & 80.124 & 88.597 & 74.084 & 73.661 & 72.427 & 73.396 & 1583.61 & 1576.109 & 1573.184 & 1575.332 & 1574.544 & 1567.96 & 1564.864 & 1570.499 \\
\hline 9/30/16 10:00 & 74.859 & 81.737 & 80.119 & 88.592 & 74.077 & 73.654 & 72.418 & 73.39 & 1583.613 & 1576.116 & 1573.189 & 1575.337 & 1574.551 & 1567.967 & 1564.873 & 1570.505 \\
\hline
\end{tabular}


TABLE S1.1 Automatically recorded groundwater levels in the unconfined aquifer.

\begin{tabular}{|c|c|c|c|c|c|c|c|c|c|c|c|c|c|c|c|c|}
\hline \multirow[b]{2}{*}{ Date and Time } & \multicolumn{8}{|c|}{ Measured Depth to Water ( $\mathrm{ft}$ TOC ${ }^{\mathrm{a}}$ ) } & \multicolumn{8}{|c|}{ Groundwater Elevation (ft AMSL ${ }^{\mathrm{b}}$ ) } \\
\hline & DL1-C & DL2-C1 & DL3-C1 & DL4-C1 & DL5-C1 & DL6-C & DL7-C & DL8-C1 & DL1-C & DL2-C1 & DL3-C1 & DL4-C1 & DL5-C1 & DL6-C & DL7-C & DL8-C1 \\
\hline 9/30/16 11:00 & 74.852 & 81.725 & 80.11 & 88.585 & 74.063 & 73.647 & 72.408 & 73.373 & 1583.62 & 1576.128 & 1573.198 & 1575.344 & 1574.565 & 1567.974 & 1564.883 & 1570.522 \\
\hline 9/30/16 12:00 & 74.831 & 81.702 & 80.085 & 88.571 & 74.04 & 73.623 & 72.38 & 73.357 & 1583.641 & 1576.151 & 1573.223 & 1575.358 & 1574.588 & 1567.998 & 1564.911 & 1570.538 \\
\hline 9/30/16 13:00 & 74.819 & 81.681 & 80.064 & 88.56 & 74.016 & 73.616 & 72.37 & 73.334 & 1583.653 & 1576.172 & 1573.244 & 1575.369 & 1574.612 & 1568.005 & 1564.921 & 1570.561 \\
\hline 9/30/16 14:00 & 74.808 & 81.657 & 80.043 & 88.548 & 73.995 & 73.602 & 72.349 & 73.311 & 1583.664 & 1576.196 & 1573.265 & 1575.381 & 1574.633 & 1568.019 & 1564.942 & 1570.584 \\
\hline 9/30/16 15:00 & 74.793 & 81.637 & 80.019 & 88.536 & 73.97 & 73.586 & 72.332 & 73.29 & 1583.679 & 1576.216 & 1573.289 & 1575.393 & 1574.658 & 1568.035 & 1564.959 & 1570.605 \\
\hline 9/30/16 16:00 & 74.789 & 81.623 & 80.01 & 88.532 & 73.949 & 73.581 & 72.327 & 73.287 & 1583.683 & 1576.23 & 1573.298 & 1575.397 & 1574.679 & 1568.04 & 1564.964 & 1570.608 \\
\hline 9/30/16 17:00 & 74.793 & 81.613 & 80.001 & 88.534 & 73.942 & 73.586 & 72.332 & 73.28 & 1583.679 & 1576.24 & 1573.307 & 1575.395 & 1574.686 & 1568.035 & 1564.959 & 1570.615 \\
\hline 9/30/16 18:00 & 74.801 & 81.609 & 79.998 & 88.536 & 73.937 & 73.584 & 72.334 & 73.264 & 1583.671 & 1576.244 & 1573.31 & 1575.393 & 1574.691 & 1568.037 & 1564.957 & 1570.631 \\
\hline 9/30/16 19:00 & 74.805 & 81.609 & 79.991 & 88.532 & 73.93 & 73.584 & 72.337 & 73.25 & 1583.667 & 1576.244 & 1573.317 & 1575.397 & 1574.698 & 1568.037 & 1564.954 & 1570.645 \\
\hline 9/30/16 20:00 & 74.803 & 81.599 & 79.982 & 88.527 & 73.918 & 73.577 & 72.327 & 73.246 & 1583.669 & 1576.254 & 1573.326 & 1575.402 & 1574.71 & 1568.044 & 1564.964 & 1570.649 \\
\hline 9/30/16 21:00 & 74.812 & 81.602 & 79.982 & 88.532 & 73.918 & 73.581 & 72.339 & 73.246 & 1583.66 & 1576.251 & 1573.326 & 1575.397 & 1574.71 & 1568.04 & 1564.952 & 1570.649 \\
\hline 9/30/16 22:00 & 74.824 & 81.609 & 79.987 & 88.536 & 73.923 & 73.586 & 72.346 & 73.222 & 1583.648 & 1576.244 & 1573.321 & 1575.393 & 1574.705 & 1568.035 & 1564.945 & 1570.673 \\
\hline 9/30/16 23:00 & 74.831 & 81.616 & 79.994 & 88.541 & 73.928 & 73.593 & 72.353 & 73.26 & 1583.641 & 1576.237 & 1573.314 & 1575.388 & 1574.7 & 1568.028 & 1564.938 & 1570.635 \\
\hline 10/1/16 0:00 & 74.834 & 81.616 & 79.994 & 88.538 & 73.928 & 73.591 & 72.356 & 73.262 & 1583.638 & 1576.237 & 1573.314 & 1575.391 & 1574.7 & 1568.03 & 1564.935 & 1570.633 \\
\hline 10/1/16 1:00 & 74.841 & 81.623 & 79.996 & 88.541 & 73.932 & 73.595 & 72.36 & 73.257 & 1583.631 & 1576.23 & 1573.312 & 1575.388 & 1574.696 & 1568.026 & 1564.931 & 1570.638 \\
\hline $10 / 1 / 162: 00$ & 74.834 & 81.62 & 79.996 & 88.538 & 73.925 & 73.591 & 72.356 & 73.25 & 1583.638 & 1576.233 & 1573.312 & 1575.391 & 1574.703 & 1568.03 & 1564.935 & 1570.645 \\
\hline $10 / 1 / 163: 00$ & 74.826 & 81.616 & 79.991 & 88.534 & 73.918 & 73.584 & 72.346 & 73.257 & 1583.646 & 1576.237 & 1573.317 & 1575.395 & 1574.71 & 1568.037 & 1564.945 & 1570.638 \\
\hline $10 / 1 / 164: 00$ & 74.826 & 81.611 & 79.987 & 88.534 & 73.914 & 73.584 & 72.344 & 73.259 & 1583.646 & 1576.242 & 1573.321 & 1575.395 & 1574.714 & 1568.037 & 1564.947 & 1570.636 \\
\hline 10/1/16 5:00 & 74.824 & 81.613 & 79.987 & 88.532 & 73.909 & 73.581 & 72.344 & 73.255 & 1583.648 & 1576.24 & 1573.321 & 1575.397 & 1574.719 & 1568.04 & 1564.947 & 1570.64 \\
\hline $10 / 1 / 166: 00$ & 74.822 & 81.606 & 79.977 & 88.527 & 73.902 & 73.572 & 72.334 & 73.204 & 1583.65 & 1576.247 & 1573.331 & 1575.402 & 1574.726 & 1568.049 & 1564.957 & 1570.691 \\
\hline 10/1/16 7:00 & 74.822 & 81.604 & 79.973 & 88.527 & 73.9 & 73.577 & 72.339 & 73.211 & 1583.65 & 1576.249 & 1573.335 & 1575.402 & 1574.728 & 1568.044 & 1564.952 & 1570.684 \\
\hline $10 / 1 / 168: 00$ & 74.834 & 81.618 & 79.987 & 88.539 & 73.909 & 73.588 & 72.353 & 73.248 & 1583.638 & 1576.235 & 1573.321 & 1575.39 & 1574.719 & 1568.033 & 1564.938 & 1570.647 \\
\hline 10/1/16 9:00 & 74.834 & 81.62 & 79.984 & 88.538 & 73.909 & 73.586 & 72.351 & 73.248 & 1583.638 & 1576.233 & 1573.324 & 1575.391 & 1574.719 & 1568.035 & 1564.94 & 1570.647 \\
\hline 10/1/16 10:00 & 74.831 & 81.618 & 79.982 & 88.531 & 73.904 & 73.579 & 72.344 & 73.243 & 1583.641 & 1576.235 & 1573.326 & 1575.398 & 1574.724 & 1568.042 & 1564.947 & 1570.652 \\
\hline 10/1/16 11:00 & 74.834 & 81.618 & 79.98 & 88.532 & 73.902 & 73.581 & 72.341 & 73.25 & 1583.638 & 1576.235 & 1573.328 & 1575.397 & 1574.726 & 1568.04 & 1564.95 & 1570.645 \\
\hline 10/1/16 12:00 & 74.829 & 81.609 & 79.975 & 88.527 & 73.895 & 73.572 & 72.334 & 73.239 & 1583.643 & 1576.244 & 1573.333 & 1575.402 & 1574.733 & 1568.049 & 1564.957 & 1570.656 \\
\hline $10 / 1 / 1613: 00$ & 74.81 & 81.59 & 79.956 & 88.511 & 73.876 & 73.558 & 72.31 & 73.222 & 1583.662 & 1576.263 & 1573.352 & 1575.418 & 1574.752 & 1568.063 & 1564.981 & 1570.673 \\
\hline 10/1/16 14:00 & 74.796 & 81.571 & 79.938 & 88.504 & 73.858 & 73.547 & 72.296 & 73.201 & 1583.676 & 1576.282 & 1573.37 & 1575.425 & 1574.77 & 1568.074 & 1564.995 & 1570.694 \\
\hline 10/1/16 15:00 & 74.789 & 81.557 & 79.924 & 88.497 & 73.841 & 73.539 & 72.286 & 73.185 & 1583.683 & 1576.296 & 1573.384 & 1575.432 & 1574.787 & 1568.082 & 1565.005 & 1570.71 \\
\hline $10 / 1 / 1616: 00$ & 74.782 & 81.548 & 79.912 & 88.49 & 73.83 & 73.532 & 72.282 & 73.174 & 1583.69 & 1576.305 & 1573.396 & 1575.439 & 1574.798 & 1568.089 & 1565.009 & 1570.721 \\
\hline 10/1/16 17:00 & 74.784 & 81.539 & 79.903 & 88.487 & 73.818 & 73.53 & 72.277 & 73.169 & 1583.688 & 1576.314 & 1573.405 & 1575.442 & 1574.81 & 1568.091 & 1565.014 & 1570.726 \\
\hline $10 / 1 / 1618: 00$ & 74.784 & 81.532 & 79.896 & 88.487 & 73.811 & 73.528 & 72.275 & 73.16 & 1583.688 & 1576.321 & 1573.412 & 1575.442 & 1574.817 & 1568.093 & 1565.016 & 1570.735 \\
\hline 10/1/16 19:00 & 74.786 & 81.534 & 79.896 & 88.487 & 73.811 & 73.53 & 72.282 & 73.155 & 1583.686 & 1576.319 & 1573.412 & 1575.442 & 1574.817 & 1568.091 & 1565.009 & 1570.74 \\
\hline $10 / 1 / 1620: 00$ & 74.803 & 81.546 & 79.91 & 88.499 & 73.82 & 73.542 & 72.296 & 73.162 & 1583.669 & 1576.307 & 1573.398 & 1575.43 & 1574.808 & 1568.079 & 1564.995 & 1570.733 \\
\hline 10/1/16 21:00 & 74.817 & 81.56 & 79.921 & 88.504 & 73.832 & 73.556 & 72.313 & 73.181 & 1583.655 & 1576.293 & 1573.387 & 1575.425 & 1574.796 & 1568.065 & 1564.978 & 1570.714 \\
\hline 10/1/16 22:00 & 74.826 & 81.569 & 79.928 & 88.508 & 73.841 & 73.556 & 72.32 & 73.19 & 1583.646 & 1576.284 & 1573.38 & 1575.421 & 1574.787 & 1568.065 & 1564.971 & 1570.705 \\
\hline $10 / 1 / 1623: 00$ & 74.829 & 81.574 & 79.933 & 88.511 & 73.844 & 73.553 & 72.32 & 73.194 & 1583.643 & 1576.279 & 1573.375 & 1575.418 & 1574.784 & 1568.068 & 1564.971 & 1570.701 \\
\hline 10/2/16 0:00 & 74.834 & 81.583 & 79.94 & 88.518 & 73.848 & 73.563 & 72.329 & 73.199 & 1583.638 & 1576.27 & 1573.368 & 1575.411 & 1574.78 & 1568.058 & 1564.962 & 1570.696 \\
\hline $10 / 2 / 161: 00$ & 74.838 & 81.592 & 79.945 & 88.513 & 73.853 & 73.565 & 72.332 & 73.204 & 1583.634 & 1576.261 & 1573.363 & 1575.416 & 1574.775 & 1568.056 & 1564.959 & 1570.691 \\
\hline $10 / 2 / 162: 00$ & 74.834 & 81.59 & 79.942 & 88.518 & 73.855 & 73.563 & 72.33 & 73.204 & 1583.638 & 1576.263 & 1573.366 & 1575.411 & 1574.773 & 1568.058 & 1564.961 & 1570.691 \\
\hline $10 / 2 / 163: 00$ & 74.831 & 81.592 & 79.945 & 88.511 & 73.853 & 73.558 & 72.327 & 73.208 & 1583.641 & 1576.261 & 1573.363 & 1575.418 & 1574.775 & 1568.063 & 1564.964 & 1570.687 \\
\hline $10 / 2 / 164: 00$ & 74.822 & 81.59 & 79.938 & 88.504 & 73.851 & 73.551 & 72.318 & 73.194 & 1583.65 & 1576.263 & 1573.37 & 1575.425 & 1574.777 & 1568.07 & 1564.973 & 1570.701 \\
\hline 10/2/16 5:00 & 74.819 & 81.588 & 79.935 & 88.504 & 73.846 & 73.551 & 72.315 & 73.197 & 1583.653 & 1576.265 & 1573.373 & 1575.425 & 1574.782 & 1568.07 & 1564.976 & 1570.698 \\
\hline $10 / 2 / 166: 00$ & 74.819 & 81.592 & 79.935 & 88.508 & 73.844 & 73.553 & 72.318 & 73.19 & 1583.653 & 1576.261 & 1573.373 & 1575.421 & 1574.784 & 1568.068 & 1564.973 & 1570.705 \\
\hline $10 / 2 / 167: 00$ & 74.826 & 81.597 & 79.942 & 88.513 & 73.851 & 73.558 & 72.327 & 73.211 & 1583.646 & 1576.256 & 1573.366 & 1575.416 & 1574.777 & 1568.063 & 1564.964 & 1570.684 \\
\hline $10 / 2 / 168: 00$ & 74.829 & 81.604 & 79.945 & 88.511 & 73.855 & 73.56 & 72.327 & 73.208 & 1583.643 & 1576.249 & 1573.363 & 1575.418 & 1574.773 & 1568.061 & 1564.964 & 1570.687 \\
\hline 10/2/16 9:00 & 74.829 & 81.604 & 79.947 & 88.513 & 73.855 & 73.558 & 72.325 & 73.211 & 1583.643 & 1576.249 & 1573.361 & 1575.416 & 1574.773 & 1568.063 & 1564.966 & 1570.684 \\
\hline 10/2/16 10:00 & 74.831 & 81.609 & 79.952 & 88.515 & 73.86 & 73.56 & 72.33 & 73.215 & 1583.641 & 1576.244 & 1573.356 & 1575.414 & 1574.768 & 1568.061 & 1564.961 & 1570.68 \\
\hline 10/2/16 11:00 & 74.829 & 81.613 & 79.952 & 88.513 & 73.86 & 73.563 & 72.327 & 73.213 & 1583.643 & 1576.24 & 1573.356 & 1575.416 & 1574.768 & 1568.058 & 1564.964 & 1570.682 \\
\hline
\end{tabular}


TABLE S1.1 Automatically recorded groundwater levels in the unconfined aquifer.

\begin{tabular}{|c|c|c|c|c|c|c|c|c|c|c|c|c|c|c|c|c|}
\hline \multirow[b]{2}{*}{ Date and Time } & \multicolumn{8}{|c|}{ Measured Depth to Water ( $\mathrm{ft}$ TOC ${ }^{\mathrm{a}}$ ) } & \multicolumn{8}{|c|}{ Groundwater Elevation (ft AMSL ${ }^{\mathrm{b}}$ ) } \\
\hline & DL1-C & DL2-C1 & DL3-C1 & DL4-C1 & DL5-C1 & DL6-C & DL7-C & DL8-C1 & DL1-C & DL2-C1 & DL3-C1 & DL4-C1 & DL5-C1 & DL6-C & DL7-C & DL8-C1 \\
\hline 10/2/16 12:00 & 74.826 & 81.606 & 79.947 & 88.506 & 73.853 & 73.553 & 72.318 & 73.213 & 1583.646 & 1576.247 & 1573.361 & 1575.423 & 1574.775 & 1568.068 & 1564.973 & 1570.682 \\
\hline 10/2/16 13:00 & 74.815 & 81.59 & 79.933 & 88.501 & 73.841 & 73.542 & 72.301 & 73.201 & 1583.657 & 1576.263 & 1573.375 & 1575.428 & 1574.787 & 1568.079 & 1564.99 & 1570.694 \\
\hline 10/2/16 14:00 & 74.801 & 81.569 & 79.917 & 88.487 & 73.823 & 73.528 & 72.277 & 73.185 & 1583.671 & 1576.284 & 1573.391 & 1575.442 & 1574.805 & 1568.093 & 1565.014 & 1570.71 \\
\hline 10/2/16 15:00 & 74.782 & 81.548 & 79.898 & 88.473 & 73.797 & 73.514 & 72.258 & 73.178 & 1583.69 & 1576.305 & 1573.41 & 1575.456 & 1574.831 & 1568.107 & 1565.033 & 1570.717 \\
\hline 10/2/16 16:00 & 74.77 & 81.532 & 79.884 & 88.469 & 73.783 & 73.507 & 72.246 & 73.157 & 1583.702 & 1576.321 & 1573.424 & 1575.46 & 1574.845 & 1568.114 & 1565.045 & 1570.738 \\
\hline 10/2/16 17:00 & 74.768 & 81.52 & 79.875 & 88.466 & 73.771 & 73.502 & 72.244 & 73.148 & 1583.704 & 1576.333 & 1573.433 & 1575.463 & 1574.857 & 1568.119 & 1565.047 & 1570.747 \\
\hline $10 / 2 / 1618: 00$ & 74.775 & 81.523 & 79.872 & 88.469 & 73.771 & 73.504 & 72.248 & 73.104 & 1583.697 & 1576.33 & 1573.436 & 1575.46 & 1574.857 & 1568.117 & 1565.043 & 1570.791 \\
\hline $10 / 2 / 1619: 00$ & 74.782 & 81.53 & 79.877 & 88.471 & 73.774 & 73.514 & 72.26 & 73.141 & 1583.69 & 1576.323 & 1573.431 & 1575.458 & 1574.854 & 1568.107 & 1565.031 & 1570.754 \\
\hline $10 / 2 / 1620: 00$ & 74.789 & 81.53 & 79.879 & 88.473 & 73.774 & 73.516 & 72.268 & 73.143 & 1583.683 & 1576.323 & 1573.429 & 1575.456 & 1574.854 & 1568.105 & 1565.023 & 1570.752 \\
\hline $10 / 2 / 1621: 00$ & 74.801 & 81.541 & 79.886 & 88.48 & 73.783 & 73.521 & 72.274 & 73.148 & 1583.671 & 1576.312 & 1573.422 & 1575.449 & 1574.845 & 1568.1 & 1565.017 & 1570.747 \\
\hline $10 / 2 / 1622: 00$ & 74.803 & 81.546 & 79.891 & 88.482 & 73.783 & 73.523 & 72.279 & 73.153 & 1583.669 & 1576.307 & 1573.417 & 1575.447 & 1574.845 & 1568.098 & 1565.012 & 1570.742 \\
\hline 10/2/16 23:00 & 74.805 & 81.548 & 79.891 & 88.48 & 73.785 & 73.521 & 72.277 & 73.157 & 1583.667 & 1576.305 & 1573.417 & 1575.449 & 1574.843 & 1568.1 & 1565.014 & 1570.738 \\
\hline 10/3/16 0:00 & 74.805 & 81.548 & 79.889 & 88.478 & 73.785 & 73.521 & 72.275 & 73.153 & 1583.667 & 1576.305 & 1573.419 & 1575.451 & 1574.843 & 1568.1 & 1565.016 & 1570.742 \\
\hline 10/3/16 1:00 & 74.798 & 81.541 & 79.882 & 88.473 & 73.776 & 73.511 & 72.267 & 73.146 & 1583.674 & 1576.312 & 1573.426 & 1575.456 & 1574.852 & 1568.11 & 1565.024 & 1570.749 \\
\hline $10 / 3 / 162: 00$ & 74.791 & 81.534 & 79.954 & 88.471 & 73.771 & 73.511 & 72.263 & 73.143 & 1583.681 & 1576.319 & 1573.354 & 1575.458 & 1574.857 & 1568.11 & 1565.028 & 1570.752 \\
\hline $10 / 3 / 163: 00$ & 74.786 & 81.527 & 79.94 & 88.464 & 73.762 & 73.507 & 72.255 & 73.143 & 1583.686 & 1576.326 & 1573.368 & 1575.465 & 1574.866 & 1568.114 & 1565.036 & 1570.752 \\
\hline $10 / 3 / 164: 00$ & 74.775 & 81.511 & 79.94 & 88.457 & 73.75 & 73.493 & 72.239 & 73.127 & 1583.697 & 1576.342 & 1573.368 & 1575.472 & 1574.878 & 1568.128 & 1565.052 & 1570.768 \\
\hline $10 / 3 / 165: 00$ & 74.77 & 81.504 & 79.942 & 88.452 & 73.741 & 73.488 & 72.234 & 73.125 & 1583.702 & 1576.349 & 1573.366 & 1575.477 & 1574.887 & 1568.133 & 1565.057 & 1570.77 \\
\hline $10 / 3 / 166: 00$ & 74.768 & 81.499 & 79.891 & 88.447 & 73.729 & 73.486 & 72.232 & 73.111 & 1583.704 & 1576.354 & 1573.417 & 1575.482 & 1574.899 & 1568.135 & 1565.059 & 1570.784 \\
\hline $10 / 3 / 167: 00$ & 74.768 & 81.492 & 79.847 & 88.445 & 73.72 & 73.481 & 72.224 & 73.109 & 1583.704 & 1576.361 & 1573.461 & 1575.484 & 1574.908 & 1568.14 & 1565.067 & 1570.786 \\
\hline 10/3/16 8:00 & 74.763 & 81.481 & 79.837 & 88.443 & 73.715 & 73.479 & 72.22 & 73.097 & 1583.709 & 1576.372 & 1573.471 & 1575.486 & 1574.913 & 1568.142 & 1565.071 & 1570.798 \\
\hline $10 / 3 / 169: 00$ & 74.758 & 81.476 & 79.826 & 88.438 & 73.704 & 73.472 & 72.215 & 73.048 & 1583.714 & 1576.377 & 1573.482 & 1575.491 & 1574.924 & 1568.149 & 1565.076 & 1570.847 \\
\hline 10/3/16 10:00 & 74.756 & 81.464 & 79.814 & 88.431 & 73.694 & 73.465 & 72.203 & 73.076 & 1583.716 & 1576.389 & 1573.494 & 1575.498 & 1574.934 & 1568.156 & 1565.088 & 1570.819 \\
\hline 10/3/16 11:00 & 74.751 & 81.457 & 79.807 & 88.429 & 73.685 & 73.46 & 72.193 & 73.078 & 1583.721 & 1576.396 & 1573.501 & 1575.5 & 1574.943 & 1568.161 & 1565.098 & 1570.817 \\
\hline $10 / 3 / 1612: 00$ & 74.739 & 81.43 & 79.786 & 88.412 & 73.664 & 73.442 & 72.172 & 73.048 & 1583.733 & 1576.423 & 1573.522 & 1575.517 & 1574.964 & 1568.179 & 1565.119 & 1570.847 \\
\hline 10/3/16 13:00 & 74.72 & 81.404 & 79.756 & 88.401 & 73.632 & 73.423 & 72.148 & 73.018 & 1583.752 & 1576.449 & 1573.552 & 1575.528 & 1574.996 & 1568.198 & 1565.143 & 1570.877 \\
\hline $10 / 3 / 1614: 00$ & 74.706 & 81.374 & 79.733 & 88.384 & 73.606 & 73.404 & 72.124 & 72.99 & 1583.766 & 1576.479 & 1573.575 & 1575.545 & 1575.022 & 1568.217 & 1565.167 & 1570.905 \\
\hline 10/3/16 15:00 & 74.687 & 81.346 & 79.705 & 88.368 & 73.576 & 73.383 & 72.1 & 72.965 & 1583.785 & 1576.507 & 1573.603 & 1575.561 & 1575.052 & 1568.238 & 1565.191 & 1570.93 \\
\hline 10/3/16 16:00 & 74.68 & 81.327 & 79.684 & 88.361 & 73.552 & 73.379 & 72.091 & 72.951 & 1583.792 & 1576.526 & 1573.624 & 1575.568 & 1575.076 & 1568.242 & 1565.2 & 1570.944 \\
\hline 10/3/16 17:00 & 74.676 & 81.304 & 79.681 & 88.356 & 73.531 & 73.372 & 72.081 & 72.939 & 1583.796 & 1576.549 & 1573.627 & 1575.573 & 1575.097 & 1568.249 & 1565.21 & 1570.956 \\
\hline 10/3/16 18:00 & 74.683 & 81.299 & 79.66 & 88.356 & 73.52 & 73.369 & 72.081 & 72.918 & 1583.789 & 1576.554 & 1573.648 & 1575.573 & 1575.108 & 1568.252 & 1565.21 & 1570.977 \\
\hline $10 / 3 / 1619: 00$ & 74.687 & 81.295 & 79.651 & 88.354 & 73.51 & 73.369 & 72.084 & 72.907 & 1583.785 & 1576.558 & 1573.657 & 1575.575 & 1575.118 & 1568.252 & 1565.207 & 1570.988 \\
\hline $10 / 3 / 1620: 00$ & 74.697 & 81.297 & 79.649 & 88.354 & 73.51 & 73.374 & 72.093 & 72.897 & 1583.775 & 1576.556 & 1573.659 & 1575.575 & 1575.118 & 1568.247 & 1565.198 & 1570.998 \\
\hline $10 / 3 / 1621: 00$ & 74.713 & 81.306 & 79.656 & 88.368 & 73.517 & 73.383 & 72.108 & 72.911 & 1583.759 & 1576.547 & 1573.652 & 1575.561 & 1575.111 & 1568.238 & 1565.183 & 1570.984 \\
\hline $10 / 3 / 1622: 00$ & 74.718 & 81.304 & 79.663 & 88.368 & 73.515 & 73.381 & 72.103 & 72.916 & 1583.754 & 1576.549 & 1573.645 & 1575.561 & 1575.113 & 1568.24 & 1565.188 & 1570.979 \\
\hline 10/3/16 23:00 & 74.711 & 81.29 & 79.646 & 88.363 & 73.501 & 73.369 & 72.091 & 72.9 & 1583.761 & 1576.563 & 1573.662 & 1575.566 & 1575.127 & 1568.252 & 1565.2 & 1570.995 \\
\hline $10 / 4 / 160: 00$ & 74.711 & 81.292 & 79.642 & 88.359 & 73.499 & 73.369 & 72.093 & 72.895 & 1583.761 & 1576.561 & 1573.666 & 1575.57 & 1575.129 & 1568.252 & 1565.198 & 1571 \\
\hline 10/4/16 1:00 & 74.716 & 81.295 & 79.649 & 88.363 & 73.496 & 73.374 & 72.095 & 72.897 & 1583.756 & 1576.558 & 1573.659 & 1575.566 & 1575.132 & 1568.247 & 1565.196 & 1570.998 \\
\hline $10 / 4 / 162: 00$ & 74.709 & 81.278 & 79.632 & 88.349 & 73.482 & 73.358 & 72.079 & 72.883 & 1583.763 & 1576.575 & 1573.676 & 1575.58 & 1575.146 & 1568.263 & 1565.212 & 1571.012 \\
\hline 10/4/16 3:00 & 74.706 & 81.283 & 79.621 & 88.347 & 73.473 & 73.355 & 72.076 & 72.874 & 1583.766 & 1576.57 & 1573.687 & 1575.582 & 1575.155 & 1568.266 & 1565.215 & 1571.021 \\
\hline $10 / 4 / 164: 00$ & 74.718 & 81.299 & 79.635 & 88.354 & 73.485 & 73.372 & 72.098 & 72.886 & 1583.754 & 1576.554 & 1573.673 & 1575.575 & 1575.143 & 1568.249 & 1565.193 & 1571.009 \\
\hline $10 / 4 / 165: 00$ & 74.732 & 81.306 & 79.644 & 88.361 & 73.496 & 73.381 & 72.115 & 72.895 & 1583.74 & 1576.547 & 1573.664 & 1575.568 & 1575.132 & 1568.24 & 1565.176 & 1571 \\
\hline $10 / 4 / 166: 00$ & 74.735 & 81.313 & 79.632 & 88.508 & 73.492 & 73.379 & 72.146 & 72.879 & 1583.737 & 1576.54 & 1573.676 & 1575.421 & 1575.136 & 1568.242 & 1565.145 & 1571.016 \\
\hline $10 / 4 / 167: 00$ & 74.739 & 81.327 & 79.658 & 88.476 & 73.503 & 73.39 & 72.148 & 72.902 & 1583.733 & 1576.526 & 1573.65 & 1575.453 & 1575.125 & 1568.231 & 1565.143 & 1570.993 \\
\hline 10/4/16 8:00 & 74.737 & 81.325 & 79.66 & 88.396 & 73.501 & 73.383 & 72.131 & 72.911 & 1583.735 & 1576.528 & 1573.648 & 1575.533 & 1575.127 & 1568.238 & 1565.16 & 1570.984 \\
\hline 10/4/16 9:00 & 74.742 & 81.336 & 79.674 & 88.375 & 73.51 & 73.393 & 72.134 & 72.918 & 1583.73 & 1576.517 & 1573.634 & 1575.554 & 1575.118 & 1568.228 & 1565.157 & 1570.977 \\
\hline 10/4/16 10:00 & 74.737 & 81.33 & 79.674 & 88.37 & 73.506 & 73.386 & 72.119 & 72.925 & 1583.735 & 1576.523 & 1573.634 & 1575.559 & 1575.122 & 1568.235 & 1565.172 & 1570.97 \\
\hline 10/4/16 11:00 & 74.744 & 81.339 & 79.681 & 88.368 & 73.515 & 73.39 & 72.127 & 72.934 & 1583.728 & 1576.514 & 1573.627 & 1575.561 & 1575.113 & 1568.231 & 1565.164 & 1570.961 \\
\hline $10 / 4 / 1612: 00$ & 74.737 & 81.334 & 79.672 & 88.366 & 73.508 & 73.386 & 72.122 & 72.925 & 1583.735 & 1576.519 & 1573.636 & 1575.563 & 1575.12 & 1568.235 & 1565.169 & 1570.97 \\
\hline
\end{tabular}


TABLE S1.1 Automatically recorded groundwater levels in the unconfined aquifer.

\begin{tabular}{|c|c|c|c|c|c|c|c|c|c|c|c|c|c|c|c|c|}
\hline \multirow[b]{2}{*}{ Date and Time } & \multicolumn{8}{|c|}{ Measured Depth to Water (ft TOC ${ }^{\mathrm{a}}$ ) } & \multicolumn{8}{|c|}{ Groundwater Elevation (ft AMSL) } \\
\hline & DL1-C & DL2-C1 & DL3-C1 & DL4-C1 & DL5-C1 & DL6-C & DL7-C & DL8-C1 & DL1-C & DL2-C1 & DL3-C1 & DL4-C1 & DL5-C1 & DL6-C & DL7-C & DL8-C1 \\
\hline 10/4/16 13:00 & 74.737 & 81.337 & 79.67 & 88.363 & 73.506 & 73.383 & 72.119 & 72.918 & 1583.735 & 1576.516 & 1573.638 & 1575.566 & 1575.122 & 1568.238 & 1565.172 & 1570.977 \\
\hline $10 / 4 / 1614: 00$ & 74.732 & 81.332 & 79.67 & 88.363 & 73.499 & 73.381 & 72.112 & 72.916 & 1583.74 & 1576.521 & 1573.638 & 1575.566 & 1575.129 & 1568.24 & 1565.179 & 1570.979 \\
\hline $10 / 4 / 1615: 00$ & 74.723 & 81.325 & 79.658 & 88.361 & 73.494 & 73.372 & 72.103 & 72.911 & 1583.749 & 1576.528 & 1573.65 & 1575.568 & 1575.134 & 1568.249 & 1565.188 & 1570.984 \\
\hline $10 / 4 / 1616: 00$ & 74.723 & 81.327 & 79.656 & 88.352 & 73.494 & 73.376 & 72.1 & 72.904 & 1583.749 & 1576.526 & 1573.652 & 1575.577 & 1575.134 & 1568.245 & 1565.191 & 1570.991 \\
\hline $10 / 4 / 1617: 00$ & 74.706 & 81.302 & 79.637 & 88.347 & 73.473 & 73.351 & 72.079 & 72.886 & 1583.766 & 1576.551 & 1573.671 & 1575.582 & 1575.155 & 1568.27 & 1565.212 & 1571.009 \\
\hline $10 / 4 / 1618: 00$ & 74.711 & 81.318 & 79.628 & 88.429 & 73.464 & 73.355 & 72.069 & 72.881 & 1583.761 & 1576.535 & 1573.68 & 1575.5 & 1575.164 & 1568.266 & 1565.222 & 1571.014 \\
\hline $10 / 4 / 1619: 00$ & 74.735 & 81.341 & 79.658 & 88.443 & 73.496 & 73.388 & 72.127 & 72.907 & 1583.737 & 1576.512 & 1573.65 & 1575.486 & 1575.132 & 1568.233 & 1565.164 & 1570.988 \\
\hline $10 / 4 / 1620: 00$ & 74.77 & 81.378 & 79.693 & 88.394 & 73.531 & 73.416 & 72.167 & 72.914 & 1583.702 & 1576.475 & 1573.615 & 1575.535 & 1575.097 & 1568.205 & 1565.124 & 1570.981 \\
\hline 10/4/16 21:00 & 74.777 & 81.395 & 79.714 & 88.389 & 73.548 & 73.423 & 72.177 & 72.965 & 1583.695 & 1576.458 & 1573.594 & 1575.54 & 1575.08 & 1568.198 & 1565.114 & 1570.93 \\
\hline $10 / 4 / 1622: 00$ & 74.791 & 81.418 & 79.733 & 88.401 & 73.566 & 73.437 & 72.194 & 72.985 & 1583.681 & 1576.435 & 1573.575 & 1575.528 & 1575.062 & 1568.184 & 1565.097 & 1570.91 \\
\hline $10 / 4 / 1623: 00$ & 74.808 & 81.446 & 79.756 & 88.412 & 73.597 & 73.453 & 72.215 & 72.955 & 1583.664 & 1576.407 & 1573.552 & 1575.517 & 1575.031 & 1568.168 & 1565.076 & 1570.94 \\
\hline 10/5/16 0:00 & 74.812 & 81.457 & 79.772 & 88.417 & 73.608 & 73.458 & 72.225 & 73.025 & 1583.66 & 1576.396 & 1573.536 & 1575.512 & 1575.02 & 1568.163 & 1565.066 & 1570.87 \\
\hline 10/5/16 1:00 & 74.815 & 81.476 & 79.784 & 88.422 & 73.625 & 73.465 & 72.232 & 73.002 & 1583.657 & 1576.377 & 1573.524 & 1575.507 & 1575.003 & 1568.156 & 1565.059 & 1570.893 \\
\hline 10/5/16 2:00 & 74.81 & 81.488 & 79.87 & 88.424 & 73.634 & 73.46 & 72.232 & 73.06 & 1583.662 & 1576.365 & 1573.438 & 1575.505 & 1574.994 & 1568.161 & 1565.059 & 1570.835 \\
\hline 10/5/16 3:00 & 74.815 & 81.502 & 79.886 & 88.436 & 73.65 & 73.472 & 72.241 & 73.074 & 1583.657 & 1576.351 & 1573.422 & 1575.493 & 1574.978 & 1568.149 & 1565.05 & 1570.821 \\
\hline 10/5/16 4:00 & 74.817 & 81.516 & 79.889 & 88.436 & 73.662 & 73.479 & 72.251 & 73.085 & 1583.655 & 1576.337 & 1573.419 & 1575.493 & 1574.966 & 1568.142 & 1565.04 & 1570.81 \\
\hline $10 / 5 / 165: 00$ & 74.836 & 81.555 & 79.942 & 88.459 & 73.694 & 73.5 & 72.282 & 73.113 & 1583.636 & 1576.298 & 1573.366 & 1575.47 & 1574.934 & 1568.121 & 1565.009 & 1570.782 \\
\hline $10 / 5 / 166: 00$ & 74.845 & 81.574 & 79.919 & 88.468 & 73.715 & 73.509 & 72.294 & 73.102 & 1583.627 & 1576.279 & 1573.389 & 1575.461 & 1574.913 & 1568.112 & 1564.997 & 1570.793 \\
\hline 10/5/16 7:00 & 74.859 & 81.604 & 79.903 & 88.48 & 73.743 & 73.53 & 72.32 & 73.16 & 1583.613 & 1576.249 & 1573.405 & 1575.449 & 1574.885 & 1568.091 & 1564.971 & 1570.735 \\
\hline $10 / 5 / 168: 00$ & 74.859 & 81.618 & 79.919 & 88.487 & 73.762 & 73.532 & 72.325 & 73.178 & 1583.613 & 1576.235 & 1573.389 & 1575.442 & 1574.866 & 1568.089 & 1564.966 & 1570.717 \\
\hline 10/5/16 9:00 & 74.857 & 81.634 & 79.935 & 88.494 & 73.778 & 73.537 & 72.33 & 73.19 & 1583.615 & 1576.219 & 1573.373 & 1575.435 & 1574.85 & 1568.084 & 1564.961 & 1570.705 \\
\hline $10 / 5 / 1610: 00$ & 74.855 & 81.639 & 79.94 & 88.494 & 73.788 & 73.537 & 72.33 & 73.199 & 1583.617 & 1576.214 & 1573.368 & 1575.435 & 1574.84 & 1568.084 & 1564.961 & 1570.696 \\
\hline $10 / 5 / 1611: 00$ & 74.855 & 81.651 & 79.949 & 88.499 & 73.797 & 73.542 & 72.337 & 73.218 & 1583.617 & 1576.202 & 1573.359 & 1575.43 & 1574.831 & 1568.079 & 1564.954 & 1570.677 \\
\hline $10 / 5 / 1612: 00$ & 74.836 & 81.641 & 79.942 & 88.487 & 73.792 & 73.528 & 72.315 & 73.208 & 1583.636 & 1576.212 & 1573.366 & 1575.442 & 1574.836 & 1568.093 & 1564.976 & 1570.687 \\
\hline $10 / 5 / 1613: 00$ & 74.815 & 81.625 & 79.924 & 88.473 & 73.776 & 73.512 & 72.291 & 73.194 & 1583.657 & 1576.228 & 1573.384 & 1575.456 & 1574.852 & 1568.109 & 1565 & 1570.701 \\
\hline $10 / 5 / 1614: 00$ & 74.784 & 81.595 & 79.893 & 88.452 & 73.75 & 73.484 & 72.256 & 73.167 & 1583.688 & 1576.258 & 1573.415 & 1575.477 & 1574.878 & 1568.137 & 1565.035 & 1570.728 \\
\hline $10 / 5 / 1615: 00$ & 74.756 & 81.56 & 79.865 & 88.431 & 73.718 & 73.463 & 72.222 & 73.132 & 1583.716 & 1576.293 & 1573.443 & 1575.498 & 1574.91 & 1568.158 & 1565.069 & 1570.763 \\
\hline $10 / 5 / 1616: 00$ & 74.758 & 81.56 & 79.868 & 88.438 & 73.718 & 73.467 & 72.229 & 73.127 & 1583.714 & 1576.293 & 1573.44 & 1575.491 & 1574.91 & 1568.154 & 1565.062 & 1570.768 \\
\hline $10 / 5 / 1617: 00$ & 74.753 & 81.553 & 79.854 & 88.431 & 73.715 & 73.467 & 72.222 & 73.076 & 1583.719 & 1576.3 & 1573.454 & 1575.498 & 1574.913 & 1568.154 & 1565.069 & 1570.819 \\
\hline $10 / 5 / 1618: 00$ & 74.763 & 81.553 & 79.856 & 88.433 & 73.715 & 73.47 & 72.227 & 73.12 & 1583.709 & 1576.3 & 1573.452 & 1575.496 & 1574.913 & 1568.151 & 1565.064 & 1570.775 \\
\hline 10/5/16 19:00 & 74.782 & 81.571 & 79.872 & 88.447 & 73.734 & 73.488 & 72.253 & 73.132 & 1583.69 & 1576.282 & 1573.436 & 1575.482 & 1574.894 & 1568.133 & 1565.038 & 1570.763 \\
\hline $10 / 5 / 1620: 00$ & 74.793 & 81.581 & 79.884 & 88.454 & 73.743 & 73.495 & 72.263 & 73.153 & 1583.679 & 1576.272 & 1573.424 & 1575.475 & 1574.885 & 1568.126 & 1565.028 & 1570.742 \\
\hline 10/5/16 21:00 & 74.796 & 81.588 & 79.891 & 88.457 & 73.75 & 73.498 & 72.263 & 73.157 & 1583.676 & 1576.265 & 1573.417 & 1575.472 & 1574.878 & 1568.123 & 1565.028 & 1570.738 \\
\hline $10 / 5 / 1622: 00$ & 74.793 & 81.583 & 79.891 & 88.45 & 73.748 & 73.493 & 72.258 & 73.157 & 1583.679 & 1576.27 & 1573.417 & 1575.479 & 1574.88 & 1568.128 & 1565.033 & 1570.738 \\
\hline 10/5/16 23:00 & 74.793 & 81.588 & 79.891 & 88.454 & 73.75 & 73.495 & 72.263 & 73.16 & 1583.679 & 1576.265 & 1573.417 & 1575.475 & 1574.878 & 1568.126 & 1565.028 & 1570.735 \\
\hline 10/6/16 0:00 & 74.786 & 81.585 & 79.889 & 88.452 & 73.753 & 73.488 & 72.256 & 73.164 & 1583.686 & 1576.268 & 1573.419 & 1575.477 & 1574.875 & 1568.133 & 1565.035 & 1570.731 \\
\hline 10/6/16 1:00 & 74.775 & 81.571 & 79.879 & 88.443 & 73.739 & 73.477 & 72.241 & 73.153 & 1583.697 & 1576.282 & 1573.429 & 1575.486 & 1574.889 & 1568.144 & 1565.05 & 1570.742 \\
\hline 10/6/16 2:00 & 74.753 & 81.543 & 79.858 & 88.426 & 73.715 & 73.46 & 72.21 & 73.129 & 1583.719 & 1576.31 & 1573.45 & 1575.503 & 1574.913 & 1568.161 & 1565.081 & 1570.766 \\
\hline $10 / 6 / 163: 00$ & 74.751 & 81.539 & 79.847 & 88.424 & 73.708 & 73.456 & 72.208 & 73.12 & 1583.721 & 1576.314 & 1573.461 & 1575.505 & 1574.92 & 1568.165 & 1565.083 & 1570.775 \\
\hline 10/6/16 4:00 & 74.749 & 81.532 & 79.844 & 88.422 & 73.704 & 73.453 & 72.198 & 73.113 & 1583.723 & 1576.321 & 1573.464 & 1575.507 & 1574.924 & 1568.168 & 1565.093 & 1570.782 \\
\hline $10 / 6 / 165: 00$ & 74.746 & 81.53 & 79.84 & 88.424 & 73.699 & 73.453 & 72.203 & 73.109 & 1583.726 & 1576.323 & 1573.468 & 1575.505 & 1574.929 & 1568.168 & 1565.088 & 1570.786 \\
\hline $10 / 6 / 166: 00$ & 74.749 & 81.52 & 79.83 & 88.422 & 73.692 & 73.451 & 72.201 & 73.097 & 1583.723 & 1576.333 & 1573.478 & 1575.507 & 1574.936 & 1568.17 & 1565.09 & 1570.798 \\
\hline 10/6/16 7:00 & 74.768 & 81.537 & 79.851 & 88.434 & 73.708 & 73.467 & 72.22 & 73.116 & 1583.704 & 1576.316 & 1573.457 & 1575.495 & 1574.92 & 1568.154 & 1565.071 & 1570.779 \\
\hline $10 / 6 / 168: 00$ & 74.77 & 81.544 & 79.856 & 88.433 & 73.711 & 73.467 & 72.225 & 73.123 & 1583.702 & 1576.309 & 1573.452 & 1575.496 & 1574.917 & 1568.154 & 1565.066 & 1570.772 \\
\hline 10/6/16 9:00 & 74.765 & 81.537 & 79.849 & 88.429 & 73.704 & 73.463 & 72.215 & 73.118 & 1583.707 & 1576.316 & 1573.459 & 1575.5 & 1574.924 & 1568.158 & 1565.076 & 1570.777 \\
\hline $10 / 6 / 1610: 00$ & 74.758 & 81.532 & 79.844 & 88.424 & 73.699 & 73.458 & 72.208 & 73.116 & 1583.714 & 1576.321 & 1573.464 & 1575.505 & 1574.929 & 1568.163 & 1565.083 & 1570.779 \\
\hline 10/6/16 11:00 & 74.749 & 81.52 & 79.83 & 88.41 & 73.685 & 73.446 & 72.194 & 73.109 & 1583.723 & 1576.333 & 1573.478 & 1575.519 & 1574.943 & 1568.175 & 1565.097 & 1570.786 \\
\hline $10 / 6 / 1612: 00$ & 74.728 & 81.492 & 79.809 & 88.398 & 73.664 & 73.428 & 72.165 & 73.083 & 1583.744 & 1576.361 & 1573.499 & 1575.531 & 1574.964 & 1568.193 & 1565.126 & 1570.812 \\
\hline $10 / 6 / 1613: 00$ & 74.73 & 81.485 & 79.807 & 88.398 & 73.655 & 73.428 & 72.165 & 73.081 & 1583.742 & 1576.368 & 1573.501 & 1575.531 & 1574.973 & 1568.193 & 1565.126 & 1570.814 \\
\hline
\end{tabular}


TABLE S1.1 Automatically recorded groundwater levels in the unconfined aquifer.

\begin{tabular}{|c|c|c|c|c|c|c|c|c|c|c|c|c|c|c|c|c|}
\hline \multirow[b]{2}{*}{ Date and Time } & \multicolumn{8}{|c|}{ Measured Depth to Water (ft TOC ${ }^{\mathrm{a}}$ ) } & \multicolumn{8}{|c|}{ Groundwater Elevation ( $\mathrm{ft} \mathrm{AMSL}^{\mathrm{b}}$ ) } \\
\hline & DL1-C & DL2-C1 & DL3-C1 & DL4-C1 & DL5-C1 & DL6-C & DL7-C & DL8-C1 & DL1-C & DL2-C1 & DL3-C1 & DL4-C1 & DL5-C1 & DL6-C & DL7-C & DL8-C1 \\
\hline $10 / 6 / 1614: 00$ & 74.718 & 81.471 & 79.788 & 88.389 & 73.639 & 73.416 & 72.15 & 73.074 & 1583.754 & 1576.382 & 1573.52 & 1575.54 & 1574.989 & 1568.205 & 1565.141 & 1570.821 \\
\hline $10 / 6 / 1615: 00$ & 74.725 & 81.476 & 79.789 & 88.384 & 73.641 & 73.423 & 72.165 & 73.067 & 1583.747 & 1576.377 & 1573.519 & 1575.545 & 1574.987 & 1568.198 & 1565.126 & 1570.828 \\
\hline $10 / 6 / 1616: 00$ & 74.753 & 81.502 & 79.819 & 88.408 & 73.666 & 73.449 & 72.196 & 73.083 & 1583.719 & 1576.351 & 1573.489 & 1575.521 & 1574.962 & 1568.172 & 1565.095 & 1570.812 \\
\hline $10 / 6 / 1617: 00$ & 74.772 & 81.52 & 79.842 & 88.412 & 73.678 & 73.456 & 72.208 & 73.102 & 1583.7 & 1576.333 & 1573.466 & 1575.517 & 1574.95 & 1568.165 & 1565.083 & 1570.793 \\
\hline $10 / 6 / 1618: 00$ & 74.793 & 81.541 & 79.849 & 88.429 & 73.697 & 73.472 & 72.232 & 73.116 & 1583.679 & 1576.312 & 1573.459 & 1575.5 & 1574.931 & 1568.149 & 1565.059 & 1570.779 \\
\hline $10 / 6 / 1619: 00$ & 74.812 & 81.571 & 79.875 & 88.452 & 73.727 & 73.495 & 72.265 & 73.146 & 1583.66 & 1576.282 & 1573.433 & 1575.477 & 1574.901 & 1568.126 & 1565.026 & 1570.749 \\
\hline $10 / 6 / 1620: 00$ & 74.834 & 81.611 & 79.907 & 88.468 & 73.762 & 73.518 & 72.296 & 73.174 & 1583.638 & 1576.242 & 1573.401 & 1575.461 & 1574.866 & 1568.103 & 1564.995 & 1570.721 \\
\hline $10 / 6 / 1621: 00$ & 74.871 & 81.667 & 79.956 & 88.499 & 73.811 & 73.549 & 72.342 & 73.225 & 1583.601 & 1576.186 & 1573.352 & 1575.43 & 1574.817 & 1568.072 & 1564.949 & 1570.67 \\
\hline $10 / 6 / 1622: 00$ & 74.878 & 81.685 & 79.982 & 88.515 & 73.839 & 73.56 & 72.358 & 73.253 & 1583.594 & 1576.168 & 1573.326 & 1575.414 & 1574.789 & 1568.061 & 1564.933 & 1570.642 \\
\hline $10 / 6 / 1623: 00$ & 74.871 & 81.695 & 79.991 & 88.503 & 73.844 & 73.556 & 72.353 & 73.264 & 1583.601 & 1576.158 & 1573.317 & 1575.426 & 1574.784 & 1568.065 & 1564.938 & 1570.631 \\
\hline $10 / 7 / 160: 00$ & 74.852 & 81.692 & 79.991 & 88.497 & 73.848 & 73.546 & 72.337 & 73.229 & 1583.62 & 1576.161 & 1573.317 & 1575.432 & 1574.78 & 1568.075 & 1564.954 & 1570.666 \\
\hline 10/7/16 1:00 & 74.838 & 81.685 & 79.984 & 88.489 & 73.837 & 73.537 & 72.332 & 73.239 & 1583.634 & 1576.168 & 1573.324 & 1575.44 & 1574.791 & 1568.084 & 1564.959 & 1570.656 \\
\hline $10 / 7 / 162: 00$ & 74.841 & 81.702 & 80.001 & 88.499 & 73.862 & 73.551 & 72.344 & 73.273 & 1583.631 & 1576.151 & 1573.307 & 1575.43 & 1574.766 & 1568.07 & 1564.947 & 1570.622 \\
\hline 10/7/16 3:00 & 74.843 & 81.72 & 80.012 & 88.511 & 73.872 & 73.56 & 72.361 & 73.29 & 1583.629 & 1576.133 & 1573.296 & 1575.418 & 1574.756 & 1568.061 & 1564.93 & 1570.605 \\
\hline $10 / 7 / 164: 00$ & 74.852 & 81.737 & 80.029 & 88.52 & 73.89 & 73.572 & 72.373 & 73.304 & 1583.62 & 1576.116 & 1573.279 & 1575.409 & 1574.738 & 1568.049 & 1564.918 & 1570.591 \\
\hline $10 / 7 / 165: 00$ & 74.857 & 81.748 & 80.036 & 88.522 & 73.902 & 73.574 & 72.377 & 73.315 & 1583.615 & 1576.105 & 1573.272 & 1575.407 & 1574.726 & 1568.047 & 1564.914 & 1570.58 \\
\hline $10 / 7 / 166: 00$ & 74.848 & 81.744 & 80.036 & 88.517 & 73.897 & 73.567 & 72.363 & 73.318 & 1583.624 & 1576.109 & 1573.272 & 1575.412 & 1574.731 & 1568.054 & 1564.928 & 1570.577 \\
\hline $10 / 7 / 167: 00$ & 74.838 & 81.739 & 80.031 & 88.513 & 73.9 & 73.558 & 72.356 & 73.311 & 1583.634 & 1576.114 & 1573.277 & 1575.416 & 1574.728 & 1568.063 & 1564.935 & 1570.584 \\
\hline $10 / 7 / 168: 00$ & 74.838 & 81.751 & 80.043 & 88.52 & 73.909 & 73.574 & 72.37 & 73.322 & 1583.634 & 1576.102 & 1573.265 & 1575.409 & 1574.719 & 1568.047 & 1564.921 & 1570.573 \\
\hline 10/7/16 9:00 & 74.838 & 81.755 & 80.052 & 88.525 & 73.916 & 73.574 & 72.372 & 73.334 & 1583.634 & 1576.098 & 1573.256 & 1575.404 & 1574.712 & 1568.047 & 1564.919 & 1570.561 \\
\hline $10 / 7 / 1610: 00$ & 74.836 & 81.757 & 80.057 & 88.527 & 73.918 & 73.57 & 72.37 & 73.341 & 1583.636 & 1576.096 & 1573.251 & 1575.402 & 1574.71 & 1568.051 & 1564.921 & 1570.554 \\
\hline $10 / 7 / 1611: 00$ & 74.831 & 81.753 & 80.047 & 88.522 & 73.914 & 73.568 & 72.365 & 73.331 & 1583.641 & 1576.1 & 1573.261 & 1575.407 & 1574.714 & 1568.053 & 1564.926 & 1570.564 \\
\hline $10 / 7 / 1612: 00$ & 74.815 & 81.739 & 80.038 & 88.51 & 73.902 & 73.553 & 72.344 & 73.327 & 1583.657 & 1576.114 & 1573.27 & 1575.419 & 1574.726 & 1568.068 & 1564.947 & 1570.568 \\
\hline $10 / 7 / 1613: 00$ & 74.801 & 81.72 & 80.024 & 88.499 & 73.888 & 73.542 & 72.327 & 73.311 & 1583.671 & 1576.133 & 1573.284 & 1575.43 & 1574.74 & 1568.079 & 1564.964 & 1570.584 \\
\hline $10 / 7 / 1614: 00$ & 74.777 & 81.688 & 79.998 & 88.48 & 73.862 & 73.518 & 72.294 & 73.283 & 1583.695 & 1576.165 & 1573.31 & 1575.449 & 1574.766 & 1568.103 & 1564.997 & 1570.612 \\
\hline $10 / 7 / 1615: 00$ & 74.753 & 81.667 & 79.975 & 88.466 & 73.837 & 73.504 & 72.272 & 73.257 & 1583.719 & 1576.186 & 1573.333 & 1575.463 & 1574.791 & 1568.117 & 1565.019 & 1570.638 \\
\hline $10 / 7 / 1616: 00$ & 74.737 & 81.632 & 79.949 & 88.45 & 73.811 & 73.488 & 72.248 & 73.234 & 1583.735 & 1576.221 & 1573.359 & 1575.479 & 1574.817 & 1568.133 & 1565.043 & 1570.661 \\
\hline $10 / 7 / 1617: 00$ & 74.725 & 81.618 & 79.928 & 88.44 & 73.79 & 73.474 & 72.232 & 73.218 & 1583.747 & 1576.235 & 1573.38 & 1575.489 & 1574.838 & 1568.147 & 1565.059 & 1570.677 \\
\hline $10 / 7 / 1618: 00$ & 74.725 & 81.597 & 79.917 & 88.438 & 73.771 & 73.474 & 72.225 & 73.201 & 1583.747 & 1576.256 & 1573.391 & 1575.491 & 1574.857 & 1568.147 & 1565.066 & 1570.694 \\
\hline $10 / 7 / 1619: 00$ & 74.73 & 81.592 & 79.91 & 88.433 & 73.767 & 73.472 & 72.227 & 73.194 & 1583.742 & 1576.261 & 1573.398 & 1575.496 & 1574.861 & 1568.149 & 1565.064 & 1570.701 \\
\hline $10 / 7 / 1620: 00$ & 74.742 & 81.592 & 79.912 & 88.44 & 73.771 & 73.479 & 72.232 & 73.19 & 1583.73 & 1576.261 & 1573.396 & 1575.489 & 1574.857 & 1568.142 & 1565.059 & 1570.705 \\
\hline $10 / 7 / 1621: 00$ & 74.744 & 81.588 & 79.907 & 88.436 & 73.767 & 73.474 & 72.229 & 73.194 & 1583.728 & 1576.265 & 1573.401 & 1575.493 & 1574.861 & 1568.147 & 1565.062 & 1570.701 \\
\hline $10 / 7 / 1622: 00$ & 74.746 & 81.588 & 79.905 & 88.433 & 73.76 & 73.477 & 72.232 & 73.192 & 1583.726 & 1576.265 & 1573.403 & 1575.496 & 1574.868 & 1568.144 & 1565.059 & 1570.703 \\
\hline $10 / 7 / 1623: 00$ & 74.746 & 81.581 & 79.903 & 88.433 & 73.757 & 73.47 & 72.227 & 73.183 & 1583.726 & 1576.272 & 1573.405 & 1575.496 & 1574.871 & 1568.151 & 1565.064 & 1570.712 \\
\hline $10 / 8 / 160: 00$ & 74.744 & 81.574 & 79.896 & 88.426 & 73.748 & 73.467 & 72.22 & 73.174 & 1583.728 & 1576.279 & 1573.412 & 1575.503 & 1574.88 & 1568.154 & 1565.071 & 1570.721 \\
\hline 10/8/16 1:00 & 74.737 & 81.562 & 79.886 & 88.419 & 73.739 & 73.46 & 72.21 & 73.164 & 1583.735 & 1576.291 & 1573.422 & 1575.51 & 1574.889 & 1568.161 & 1565.081 & 1570.731 \\
\hline $10 / 8 / 162: 00$ & 74.739 & 81.56 & 79.879 & 88.424 & 73.732 & 73.463 & 72.21 & 73.167 & 1583.733 & 1576.293 & 1573.429 & 1575.505 & 1574.896 & 1568.158 & 1565.081 & 1570.728 \\
\hline 10/8/16 3:00 & 74.742 & 81.56 & 79.879 & 88.422 & 73.729 & 73.463 & 72.215 & 73.157 & 1583.73 & 1576.293 & 1573.429 & 1575.507 & 1574.899 & 1568.158 & 1565.076 & 1570.738 \\
\hline $10 / 8 / 164: 00$ & 74.732 & 81.546 & 79.868 & 88.412 & 73.715 & 73.449 & 72.201 & 73.15 & 1583.74 & 1576.307 & 1573.44 & 1575.517 & 1574.913 & 1568.172 & 1565.09 & 1570.745 \\
\hline $10 / 8 / 165: 00$ & 74.732 & 81.539 & 79.861 & 88.41 & 73.708 & 73.446 & 72.198 & 73.141 & 1583.74 & 1576.314 & 1573.447 & 1575.519 & 1574.92 & 1568.175 & 1565.093 & 1570.754 \\
\hline $10 / 8 / 166: 00$ & 74.732 & 81.53 & 79.856 & 88.408 & 73.701 & 73.446 & 72.194 & 73.134 & 1583.74 & 1576.323 & 1573.452 & 1575.521 & 1574.927 & 1568.175 & 1565.097 & 1570.761 \\
\hline $10 / 8 / 167: 00$ & 74.735 & 81.53 & 79.854 & 88.408 & 73.697 & 73.444 & 72.191 & 73.129 & 1583.737 & 1576.323 & 1573.454 & 1575.521 & 1574.931 & 1568.177 & 1565.1 & 1570.766 \\
\hline 10/8/16 8:00 & 74.739 & 81.534 & 79.854 & 88.412 & 73.699 & 73.444 & 72.201 & 73.09 & 1583.733 & 1576.319 & 1573.454 & 1575.517 & 1574.929 & 1568.177 & 1565.09 & 1570.805 \\
\hline 10/8/16 9:00 & 74.744 & 81.539 & 79.858 & 88.415 & 73.701 & 73.451 & 72.205 & 73.139 & 1583.728 & 1576.314 & 1573.45 & 1575.514 & 1574.927 & 1568.17 & 1565.086 & 1570.756 \\
\hline $10 / 8 / 1610: 00$ & 74.744 & 81.537 & 79.858 & 88.41 & 73.699 & 73.449 & 72.2 & 73.129 & 1583.728 & 1576.316 & 1573.45 & 1575.519 & 1574.929 & 1568.172 & 1565.091 & 1570.766 \\
\hline $10 / 8 / 1611: 00$ & 74.739 & 81.53 & 79.849 & 88.408 & 73.692 & 73.444 & 72.194 & 73.102 & 1583.733 & 1576.323 & 1573.459 & 1575.521 & 1574.936 & 1568.177 & 1565.097 & 1570.793 \\
\hline $10 / 8 / 1612: 00$ & 74.732 & 81.516 & 79.844 & 88.401 & 73.68 & 73.435 & 72.182 & 73.12 & 1583.74 & 1576.337 & 1573.464 & 1575.528 & 1574.948 & 1568.186 & 1565.109 & 1570.775 \\
\hline $10 / 8 / 1613: 00$ & 74.72 & 81.504 & 79.826 & 88.394 & 73.662 & 73.423 & 72.169 & 73.104 & 1583.752 & 1576.349 & 1573.482 & 1575.535 & 1574.966 & 1568.198 & 1565.122 & 1570.791 \\
\hline $10 / 8 / 1614: 00$ & 74.702 & 81.474 & 79.805 & 88.375 & 73.641 & 73.407 & 72.141 & 73.081 & 1583.77 & 1576.379 & 1573.503 & 1575.554 & 1574.987 & 1568.214 & 1565.15 & 1570.814 \\
\hline
\end{tabular}


TABLE S1.1 Automatically recorded groundwater levels in the unconfined aquifer.

\begin{tabular}{|c|c|c|c|c|c|c|c|c|c|c|c|c|c|c|c|c|}
\hline \multirow[b]{2}{*}{ Date and Time } & \multicolumn{8}{|c|}{ Measured Depth to Water ( $\mathrm{ft}$ TOC ${ }^{\mathrm{a}}$ ) } & \multicolumn{8}{|c|}{ Groundwater Elevation (ft AMSL ${ }^{\mathrm{b}}$ ) } \\
\hline & DL1-C & DL2-C1 & DL3-C1 & DL4-C1 & DL5-C1 & DL6-C & DL7-C & DL8-C1 & DL1-C & DL2-C1 & DL3-C1 & DL4-C1 & DL5-C1 & DL6-C & DL7-C & DL8-C1 \\
\hline 10/8/16 15:00 & 74.69 & 81.453 & 79.782 & 88.366 & 73.618 & 73.39 & 72.124 & 73.06 & 1583.782 & 1576.4 & 1573.526 & 1575.563 & 1575.01 & 1568.231 & 1565.167 & 1570.835 \\
\hline 10/8/16 16:00 & 74.687 & 81.443 & 79.772 & 88.363 & 73.604 & 73.393 & 72.119 & 73.053 & 1583.785 & 1576.41 & 1573.536 & 1575.566 & 1575.024 & 1568.228 & 1565.172 & 1570.842 \\
\hline 10/8/16 17:00 & 74.692 & 81.436 & 79.768 & 88.363 & 73.597 & 73.39 & 72.122 & 73.044 & 1583.78 & 1576.417 & 1573.54 & 1575.566 & 1575.031 & 1568.231 & 1565.169 & 1570.851 \\
\hline 10/8/16 18:00 & 74.699 & 81.439 & 79.77 & 88.363 & 73.594 & 73.397 & 72.124 & 73.058 & 1583.773 & 1576.414 & 1573.538 & 1575.566 & 1575.034 & 1568.224 & 1565.167 & 1570.837 \\
\hline 10/8/16 19:00 & 74.713 & 81.446 & 79.775 & 88.37 & 73.599 & 73.404 & 72.139 & 73.058 & 1583.759 & 1576.407 & 1573.533 & 1575.559 & 1575.029 & 1568.217 & 1565.152 & 1570.837 \\
\hline 10/8/16 20:00 & 74.727 & 81.46 & 79.789 & 88.382 & 73.608 & 73.416 & 72.16 & 73.044 & 1583.745 & 1576.393 & 1573.519 & 1575.547 & 1575.02 & 1568.205 & 1565.131 & 1570.851 \\
\hline 10/8/16 21:00 & 74.749 & 81.488 & 79.807 & 88.394 & 73.634 & 73.432 & 72.184 & 73.085 & 1583.723 & 1576.365 & 1573.501 & 1575.535 & 1574.994 & 1568.189 & 1565.107 & 1570.81 \\
\hline 10/8/16 22:00 & 74.768 & 81.511 & 79.828 & 88.408 & 73.65 & 73.446 & 72.205 & 73.09 & 1583.704 & 1576.342 & 1573.48 & 1575.521 & 1574.978 & 1568.175 & 1565.086 & 1570.805 \\
\hline 10/8/16 23:00 & 74.768 & 81.518 & 79.837 & 88.405 & 73.66 & 73.446 & 72.208 & 73.113 & 1583.704 & 1576.335 & 1573.471 & 1575.524 & 1574.968 & 1568.175 & 1565.083 & 1570.782 \\
\hline 10/9/16 0:00 & 74.763 & 81.523 & 79.837 & 88.408 & 73.662 & 73.446 & 72.208 & 73.116 & 1583.709 & 1576.33 & 1573.471 & 1575.521 & 1574.966 & 1568.175 & 1565.083 & 1570.779 \\
\hline 10/9/16 1:00 & 74.753 & 81.516 & 79.833 & 88.398 & 73.653 & 73.437 & 72.193 & 73.109 & 1583.719 & 1576.337 & 1573.475 & 1575.531 & 1574.975 & 1568.184 & 1565.098 & 1570.786 \\
\hline 10/9/16 2:00 & 74.751 & 81.518 & 79.835 & 88.401 & 73.657 & 73.437 & 72.196 & 73.113 & 1583.721 & 1576.335 & 1573.473 & 1575.528 & 1574.971 & 1568.184 & 1565.095 & 1570.782 \\
\hline 10/9/16 3:00 & 74.749 & 81.523 & 79.833 & 88.401 & 73.662 & 73.439 & 72.196 & 73.111 & 1583.723 & 1576.33 & 1573.475 & 1575.528 & 1574.966 & 1568.182 & 1565.095 & 1570.784 \\
\hline 10/9/16 4:00 & 74.744 & 81.516 & 79.835 & 88.398 & 73.657 & 73.437 & 72.196 & 73.102 & 1583.728 & 1576.337 & 1573.473 & 1575.531 & 1574.971 & 1568.184 & 1565.095 & 1570.793 \\
\hline $10 / 9 / 165: 00$ & 74.742 & 81.518 & 79.828 & 88.396 & 73.65 & 73.432 & 72.189 & 73.106 & 1583.73 & 1576.335 & 1573.48 & 1575.533 & 1574.978 & 1568.189 & 1565.102 & 1570.789 \\
\hline $10 / 9 / 166: 00$ & 74.737 & 81.511 & 79.828 & 88.396 & 73.65 & 73.43 & 72.186 & 73.104 & 1583.735 & 1576.342 & 1573.48 & 1575.533 & 1574.978 & 1568.191 & 1565.105 & 1570.791 \\
\hline 10/9/16 7:00 & 74.73 & 81.509 & 79.821 & 88.389 & 73.641 & 73.423 & 72.179 & 73.053 & 1583.742 & 1576.344 & 1573.487 & 1575.54 & 1574.987 & 1568.198 & 1565.112 & 1570.842 \\
\hline 10/9/16 8:00 & 74.732 & 81.509 & 79.819 & 88.389 & 73.641 & 73.423 & 72.182 & 73.099 & 1583.74 & 1576.344 & 1573.489 & 1575.54 & 1574.987 & 1568.198 & 1565.109 & 1570.796 \\
\hline 10/9/16 9:00 & 74.735 & 81.506 & 79.821 & 88.394 & 73.641 & 73.428 & 72.184 & 73.099 & 1583.737 & 1576.347 & 1573.487 & 1575.535 & 1574.987 & 1568.193 & 1565.107 & 1570.796 \\
\hline 10/9/16 10:00 & 74.739 & 81.511 & 79.823 & 88.396 & 73.643 & 73.43 & 72.184 & 73.102 & 1583.733 & 1576.342 & 1573.485 & 1575.533 & 1574.985 & 1568.191 & 1565.107 & 1570.793 \\
\hline 10/9/16 11:00 & 74.72 & 81.485 & 79.807 & 88.38 & 73.622 & 73.409 & 72.158 & 73.083 & 1583.752 & 1576.368 & 1573.501 & 1575.549 & 1575.006 & 1568.212 & 1565.133 & 1570.812 \\
\hline 10/9/16 12:00 & 74.704 & 81.462 & 79.784 & 88.368 & 73.601 & 73.393 & 72.136 & 73.067 & 1583.768 & 1576.391 & 1573.524 & 1575.561 & 1575.027 & 1568.228 & 1565.155 & 1570.828 \\
\hline 10/9/16 13:00 & 74.683 & 81.436 & 79.758 & 88.352 & 73.571 & 73.372 & 72.107 & 73.037 & 1583.789 & 1576.417 & 1573.55 & 1575.577 & 1575.057 & 1568.249 & 1565.184 & 1570.858 \\
\hline 10/9/16 14:00 & 74.669 & 81.413 & 79.737 & 88.34 & 73.548 & 73.362 & 72.086 & 72.972 & 1583.803 & 1576.44 & 1573.571 & 1575.589 & 1575.08 & 1568.259 & 1565.205 & 1570.923 \\
\hline 10/9/16 15:00 & 74.657 & 81.385 & 79.714 & 88.328 & 73.522 & 73.351 & 72.069 & 72.99 & 1583.815 & 1576.468 & 1573.594 & 1575.601 & 1575.106 & 1568.27 & 1565.222 & 1570.905 \\
\hline 10/9/16 16:00 & 74.65 & 81.371 & 79.695 & 88.321 & 73.503 & 73.344 & 72.062 & 72.937 & 1583.822 & 1576.482 & 1573.613 & 1575.608 & 1575.125 & 1568.277 & 1565.229 & 1570.958 \\
\hline 10/9/16 17:00 & 74.652 & 81.357 & 79.688 & 88.317 & 73.489 & 73.339 & 72.055 & 72.976 & 1583.82 & 1576.496 & 1573.62 & 1575.612 & 1575.139 & 1568.282 & 1565.236 & 1570.919 \\
\hline 10/9/16 18:00 & 74.65 & 81.348 & 79.679 & 88.312 & 73.478 & 73.337 & 72.055 & 72.967 & 1583.822 & 1576.505 & 1573.629 & 1575.617 & 1575.15 & 1568.284 & 1565.236 & 1570.928 \\
\hline 10/9/16 19:00 & 74.664 & 81.35 & 79.677 & 88.314 & 73.478 & 73.339 & 72.062 & 72.951 & 1583.808 & 1576.503 & 1573.631 & 1575.615 & 1575.15 & 1568.282 & 1565.229 & 1570.944 \\
\hline 10/9/16 20:00 & 74.673 & 81.353 & 79.679 & 88.319 & 73.478 & 73.346 & 72.069 & 72.951 & 1583.799 & 1576.5 & 1573.629 & 1575.61 & 1575.15 & 1568.275 & 1565.222 & 1570.944 \\
\hline 10/9/16 21:00 & 74.683 & 81.362 & 79.686 & 88.328 & 73.485 & 73.355 & 72.086 & 72.958 & 1583.789 & 1576.491 & 1573.622 & 1575.601 & 1575.143 & 1568.266 & 1565.205 & 1570.937 \\
\hline 10/9/16 22:00 & 74.69 & 81.367 & 79.691 & 88.331 & 73.487 & 73.356 & 72.091 & 72.96 & 1583.782 & 1576.486 & 1573.617 & 1575.598 & 1575.141 & 1568.265 & 1565.2 & 1570.935 \\
\hline 10/9/16 23:00 & 74.687 & 81.36 & 79.686 & 88.324 & 73.482 & 73.351 & 72.083 & 72.96 & 1583.785 & 1576.493 & 1573.622 & 1575.605 & 1575.146 & 1568.27 & 1565.208 & 1570.935 \\
\hline 10/10/16 0:00 & 74.68 & 81.348 & 79.677 & 88.314 & 73.471 & 73.337 & 72.072 & 72.951 & 1583.792 & 1576.505 & 1573.631 & 1575.615 & 1575.157 & 1568.284 & 1565.219 & 1570.944 \\
\hline 10/10/16 1:00 & 74.671 & 81.339 & 79.665 & 88.31 & 73.457 & 73.334 & 72.062 & 72.934 & 1583.801 & 1576.514 & 1573.643 & 1575.619 & 1575.171 & 1568.287 & 1565.229 & 1570.961 \\
\hline 10/10/16 2:00 & 74.666 & 81.332 & 79.656 & 88.307 & 73.445 & 73.327 & 72.055 & 72.925 & 1583.806 & 1576.521 & 1573.652 & 1575.622 & 1575.183 & 1568.294 & 1565.236 & 1570.97 \\
\hline $10 / 10 / 163: 00$ & 74.664 & 81.323 & 79.649 & 88.305 & 73.438 & 73.323 & 72.048 & 72.918 & 1583.808 & 1576.53 & 1573.659 & 1575.624 & 1575.19 & 1568.298 & 1565.243 & 1570.977 \\
\hline 10/10/16 4:00 & 74.659 & 81.313 & 79.639 & 88.296 & 73.426 & 73.316 & 72.041 & 72.907 & 1583.813 & 1576.54 & 1573.669 & 1575.633 & 1575.202 & 1568.305 & 1565.25 & 1570.988 \\
\hline 10/10/16 5:00 & 74.659 & 81.309 & 79.635 & 88.3 & 73.422 & 73.316 & 72.041 & 72.902 & 1583.813 & 1576.544 & 1573.673 & 1575.629 & 1575.206 & 1568.305 & 1565.25 & 1570.993 \\
\hline 10/10/16 6:00 & 74.659 & 81.306 & 79.628 & 88.291 & 73.415 & 73.314 & 72.038 & 72.895 & 1583.813 & 1576.547 & 1573.68 & 1575.638 & 1575.213 & 1568.307 & 1565.253 & 1571 \\
\hline 10/10/16 7:00 & 74.661 & 81.297 & 79.623 & 88.291 & 73.408 & 73.309 & 72.034 & 72.893 & 1583.811 & 1576.556 & 1573.685 & 1575.638 & 1575.22 & 1568.312 & 1565.257 & 1571.002 \\
\hline $10 / 10 / 168: 00$ & 74.659 & 81.299 & 79.623 & 88.291 & 73.403 & 73.311 & 72.038 & 72.89 & 1583.813 & 1576.554 & 1573.685 & 1575.638 & 1575.225 & 1568.31 & 1565.253 & 1571.005 \\
\hline 10/10/16 9:00 & 74.666 & 81.302 & 79.623 & 88.298 & 73.403 & 73.311 & 72.043 & 72.897 & 1583.806 & 1576.551 & 1573.685 & 1575.631 & 1575.225 & 1568.31 & 1565.248 & 1570.998 \\
\hline $10 / 10 / 1610: 00$ & 74.676 & 81.306 & 79.625 & 88.298 & 73.408 & 73.316 & 72.048 & 72.897 & 1583.796 & 1576.547 & 1573.683 & 1575.631 & 1575.22 & 1568.305 & 1565.243 & 1570.998 \\
\hline 10/10/16 11:00 & 74.678 & 81.309 & 79.628 & 88.298 & 73.408 & 73.316 & 72.05 & 72.895 & 1583.794 & 1576.544 & 1573.68 & 1575.631 & 1575.22 & 1568.305 & 1565.241 & 1571 \\
\hline $10 / 10 / 1612: 00$ & 74.666 & 81.297 & 79.621 & 88.293 & 73.398 & 73.307 & 72.038 & 72.888 & 1583.806 & 1576.556 & 1573.687 & 1575.636 & 1575.23 & 1568.314 & 1565.253 & 1571.007 \\
\hline 10/10/16 13:00 & 74.666 & 81.292 & 79.611 & 88.286 & 73.389 & 73.302 & 72.031 & 72.881 & 1583.806 & 1576.561 & 1573.697 & 1575.643 & 1575.239 & 1568.319 & 1565.26 & 1571.014 \\
\hline 10/10/16 14:00 & 74.645 & 81.267 & 79.59 & 88.268 & 73.368 & 73.283 & 72.005 & 72.862 & 1583.827 & 1576.586 & 1573.718 & 1575.661 & 1575.26 & 1568.338 & 1565.286 & 1571.033 \\
\hline $10 / 10 / 1615: 00$ & 74.629 & 81.246 & 79.572 & 88.256 & 73.345 & 73.272 & 71.988 & 72.839 & 1583.843 & 1576.607 & 1573.736 & 1575.673 & 1575.283 & 1568.349 & 1565.303 & 1571.056 \\
\hline
\end{tabular}


TABLE S1.1 Automatically recorded groundwater levels in the unconfined aquifer.

\begin{tabular}{|c|c|c|c|c|c|c|c|c|c|c|c|c|c|c|c|c|}
\hline \multirow[b]{2}{*}{ Date and Time } & \multicolumn{8}{|c|}{ Measured Depth to Water (ft TOC ${ }^{\mathrm{a}}$ ) } & \multicolumn{8}{|c|}{ Groundwater Elevation (ft AMSL) } \\
\hline & DL1-C & DL2-C1 & DL3-C1 & DL4-C1 & DL5-C1 & DL6-C & DL7-C & DL8-C1 & DL1-C & DL2-C1 & DL3-C1 & DL4-C1 & DL5-C1 & DL6-C & DL7-C & DL8-C1 \\
\hline 10/10/16 16:00 & 74.626 & 81.239 & 79.56 & 88.258 & 73.333 & 73.272 & 71.986 & 72.828 & 1583.846 & 1576.614 & 1573.748 & 1575.671 & 1575.295 & 1568.349 & 1565.305 & 1571.067 \\
\hline 10/10/16 17:00 & 74.631 & 81.236 & 79.56 & 88.256 & 73.326 & 73.269 & 71.983 & 72.823 & 1583.841 & 1576.617 & 1573.748 & 1575.673 & 1575.302 & 1568.352 & 1565.308 & 1571.072 \\
\hline $10 / 10 / 1618: 00$ & 74.636 & 81.232 & 79.551 & 88.258 & 73.321 & 73.267 & 71.986 & 72.821 & 1583.836 & 1576.621 & 1573.757 & 1575.671 & 1575.307 & 1568.354 & 1565.305 & 1571.074 \\
\hline $10 / 10 / 1619: 00$ & 74.633 & 81.223 & 79.544 & 88.251 & 73.312 & 73.262 & 71.976 & 72.807 & 1583.839 & 1576.63 & 1573.764 & 1575.678 & 1575.316 & 1568.359 & 1565.315 & 1571.088 \\
\hline $10 / 10 / 1620: 00$ & 74.643 & 81.229 & 79.548 & 88.256 & 73.312 & 73.269 & 71.986 & 72.811 & 1583.829 & 1576.624 & 1573.76 & 1575.673 & 1575.316 & 1568.352 & 1565.305 & 1571.084 \\
\hline 10/10/16 21:00 & 74.65 & 81.234 & 79.551 & 88.261 & 73.319 & 73.274 & 71.998 & 72.811 & 1583.822 & 1576.619 & 1573.757 & 1575.668 & 1575.309 & 1568.347 & 1565.293 & 1571.084 \\
\hline $10 / 10 / 1622: 00$ & 74.657 & 81.239 & 79.56 & 88.263 & 73.321 & 73.274 & 72.002 & 72.825 & 1583.815 & 1576.614 & 1573.748 & 1575.666 & 1575.307 & 1568.347 & 1565.289 & 1571.07 \\
\hline $10 / 10 / 1623: 00$ & 74.652 & 81.237 & 79.553 & 88.261 & 73.314 & 73.274 & 71.995 & 72.816 & 1583.82 & 1576.616 & 1573.755 & 1575.668 & 1575.314 & 1568.347 & 1565.296 & 1571.079 \\
\hline $10 / 11 / 160: 00$ & 74.645 & 81.232 & 79.551 & 88.254 & 73.31 & 73.267 & 71.988 & 72.809 & 1583.827 & 1576.621 & 1573.757 & 1575.675 & 1575.318 & 1568.354 & 1565.303 & 1571.086 \\
\hline $10 / 11 / 161: 00$ & 74.64 & 81.22 & 79.539 & 88.249 & 73.298 & 73.26 & 71.978 & 72.802 & 1583.832 & 1576.633 & 1573.769 & 1575.68 & 1575.33 & 1568.361 & 1565.313 & 1571.093 \\
\hline $10 / 11 / 162: 00$ & 74.633 & 81.213 & 79.532 & 88.244 & 73.289 & 73.253 & 71.971 & 72.797 & 1583.839 & 1576.64 & 1573.776 & 1575.685 & 1575.339 & 1568.368 & 1565.32 & 1571.098 \\
\hline 10/11/16 3:00 & 74.633 & 81.209 & 79.525 & 88.244 & 73.282 & 73.251 & 71.969 & 72.786 & 1583.839 & 1576.644 & 1573.783 & 1575.685 & 1575.346 & 1568.37 & 1565.322 & 1571.109 \\
\hline $10 / 11 / 164: 00$ & 74.633 & 81.204 & 79.523 & 88.242 & 73.279 & 73.251 & 71.967 & 72.781 & 1583.839 & 1576.649 & 1573.785 & 1575.687 & 1575.349 & 1568.37 & 1565.324 & 1571.114 \\
\hline $10 / 11 / 165: 00$ & 74.629 & 81.202 & 79.516 & 88.242 & 73.272 & 73.248 & 71.964 & 72.753 & 1583.843 & 1576.651 & 1573.792 & 1575.687 & 1575.356 & 1568.373 & 1565.327 & 1571.142 \\
\hline $10 / 11 / 166: 00$ & 74.636 & 81.204 & 79.518 & 88.242 & 73.272 & 73.251 & 71.969 & 72.779 & 1583.836 & 1576.649 & 1573.79 & 1575.687 & 1575.356 & 1568.37 & 1565.322 & 1571.116 \\
\hline $10 / 11 / 167: 00$ & 74.659 & 81.227 & 79.534 & 88.258 & 73.291 & 73.269 & 71.995 & 72.793 & 1583.813 & 1576.626 & 1573.774 & 1575.671 & 1575.337 & 1568.352 & 1565.296 & 1571.102 \\
\hline $10 / 11 / 168: 00$ & 74.666 & 81.241 & 79.548 & 88.258 & 73.298 & 73.272 & 71.995 & 72.804 & 1583.806 & 1576.612 & 1573.76 & 1575.671 & 1575.33 & 1568.349 & 1565.296 & 1571.091 \\
\hline 10/11/16 9:00 & 74.673 & 81.248 & 79.558 & 88.268 & 73.307 & 73.281 & 72.01 & 72.816 & 1583.799 & 1576.605 & 1573.75 & 1575.661 & 1575.321 & 1568.34 & 1565.281 & 1571.079 \\
\hline 10/11/16 10:00 & 74.683 & 81.267 & 79.574 & 88.275 & 73.326 & 73.293 & 72.029 & 72.837 & 1583.789 & 1576.586 & 1573.734 & 1575.654 & 1575.302 & 1568.328 & 1565.262 & 1571.058 \\
\hline 10/11/16 11:00 & 74.683 & 81.274 & 79.583 & 88.272 & 73.331 & 73.302 & 72.029 & 72.951 & 1583.789 & 1576.579 & 1573.725 & 1575.657 & 1575.297 & 1568.319 & 1565.262 & 1570.944 \\
\hline 10/11/16 12:00 & 74.666 & 81.26 & 79.576 & 88.261 & 73.321 & 73.281 & 72.01 & 72.858 & 1583.806 & 1576.593 & 1573.732 & 1575.668 & 1575.307 & 1568.34 & 1565.281 & 1571.037 \\
\hline $10 / 11 / 1613: 00$ & 74.671 & 81.269 & 79.574 & 88.27 & 73.321 & 73.279 & 72.012 & 72.844 & 1583.801 & 1576.584 & 1573.734 & 1575.659 & 1575.307 & 1568.342 & 1565.279 & 1571.051 \\
\hline $10 / 11 / 1614: 00$ & 74.654 & 81.253 & 79.565 & 88.254 & 73.31 & 73.265 & 71.991 & 72.835 & 1583.818 & 1576.6 & 1573.743 & 1575.675 & 1575.318 & 1568.356 & 1565.3 & 1571.06 \\
\hline 10/11/16 15:00 & 74.64 & 81.232 & 79.569 & 88.244 & 73.298 & 73.26 & 71.981 & 72.821 & 1583.832 & 1576.621 & 1573.739 & 1575.685 & 1575.33 & 1568.361 & 1565.31 & 1571.074 \\
\hline $10 / 11 / 1616: 00$ & 74.643 & 81.243 & 79.574 & 88.254 & 73.303 & 73.267 & 71.983 & 72.823 & 1583.829 & 1576.61 & 1573.734 & 1575.675 & 1575.325 & 1568.354 & 1565.308 & 1571.072 \\
\hline 10/11/16 17:00 & 74.652 & 81.25 & 79.562 & 88.261 & 73.314 & 73.272 & 71.995 & 72.823 & 1583.82 & 1576.603 & 1573.746 & 1575.668 & 1575.314 & 1568.349 & 1565.296 & 1571.072 \\
\hline $10 / 11 / 1618: 00$ & 74.666 & 81.267 & 79.572 & 88.268 & 73.326 & 73.279 & 72.007 & 72.837 & 1583.806 & 1576.586 & 1573.736 & 1575.661 & 1575.302 & 1568.342 & 1565.284 & 1571.058 \\
\hline 10/11/16 19:00 & 74.68 & 81.288 & 79.588 & 88.279 & 73.342 & 73.293 & 72.031 & 72.853 & 1583.792 & 1576.565 & 1573.72 & 1575.65 & 1575.286 & 1568.328 & 1565.26 & 1571.042 \\
\hline $10 / 11 / 1620: 00$ & 74.685 & 81.29 & 79.6 & 88.279 & 73.354 & 73.297 & 72.036 & 72.865 & 1583.787 & 1576.563 & 1573.708 & 1575.65 & 1575.274 & 1568.324 & 1565.255 & 1571.03 \\
\hline 10/11/16 21:00 & 74.699 & 81.316 & 79.618 & 88.296 & 73.373 & 73.311 & 72.055 & 72.881 & 1583.773 & 1576.537 & 1573.69 & 1575.633 & 1575.255 & 1568.31 & 1565.236 & 1571.014 \\
\hline 10/11/16 22:00 & 74.725 & 81.355 & 79.644 & 88.324 & 73.405 & 73.337 & 72.088 & 72.911 & 1583.747 & 1576.498 & 1573.664 & 1575.605 & 1575.223 & 1568.284 & 1565.203 & 1570.984 \\
\hline $10 / 11 / 1623: 00$ & 74.735 & 81.381 & 79.665 & 88.333 & 73.426 & 73.342 & 72.105 & 72.932 & 1583.737 & 1576.472 & 1573.643 & 1575.596 & 1575.202 & 1568.279 & 1565.186 & 1570.963 \\
\hline 10/12/16 0:00 & 74.753 & 81.416 & 79.693 & 88.354 & 73.457 & 73.367 & 72.146 & 72.958 & 1583.719 & 1576.437 & 1573.615 & 1575.575 & 1575.171 & 1568.254 & 1565.145 & 1570.937 \\
\hline $10 / 12 / 161: 00$ & 74.746 & 81.411 & 79.702 & 88.338 & 73.464 & 73.355 & 72.124 & 72.969 & 1583.726 & 1576.442 & 1573.606 & 1575.591 & 1575.164 & 1568.266 & 1565.167 & 1570.926 \\
\hline $10 / 12 / 162: 00$ & 74.697 & 81.369 & 79.663 & 88.298 & 73.422 & 73.311 & 72.067 & 72.932 & 1583.775 & 1576.484 & 1573.645 & 1575.631 & 1575.206 & 1568.31 & 1565.224 & 1570.963 \\
\hline 10/12/16 3:00 & 74.725 & 81.418 & 79.691 & 88.34 & 73.459 & 73.355 & 72.126 & 72.955 & 1583.747 & 1576.435 & 1573.617 & 1575.589 & 1575.169 & 1568.266 & 1565.165 & 1570.94 \\
\hline $10 / 12 / 164: 00$ & 74.72 & 81.402 & 79.702 & 88.324 & 73.461 & 73.339 & 72.096 & 72.974 & 1583.752 & 1576.451 & 1573.606 & 1575.605 & 1575.167 & 1568.282 & 1565.195 & 1570.921 \\
\hline $10 / 12 / 165: 00$ & 74.728 & 81.427 & 79.707 & 88.338 & 73.473 & 73.353 & 72.119 & 72.981 & 1583.744 & 1576.426 & 1573.601 & 1575.591 & 1575.155 & 1568.268 & 1565.172 & 1570.914 \\
\hline $10 / 12 / 166: 00$ & 74.746 & 81.457 & 79.73 & 88.359 & 73.503 & 73.381 & 72.162 & 73.002 & 1583.726 & 1576.396 & 1573.578 & 1575.57 & 1575.125 & 1568.24 & 1565.129 & 1570.893 \\
\hline $10 / 12 / 167: 00$ & 74.763 & 81.483 & 79.761 & 88.37 & 73.529 & 73.393 & 72.179 & 73.032 & 1583.709 & 1576.37 & 1573.547 & 1575.559 & 1575.099 & 1568.228 & 1565.112 & 1570.863 \\
\hline $10 / 12 / 168: 00$ & 74.76 & 81.49 & 79.772 & 88.366 & 73.538 & 73.395 & 72.177 & 73.046 & 1583.712 & 1576.363 & 1573.536 & 1575.563 & 1575.09 & 1568.226 & 1565.114 & 1570.849 \\
\hline $10 / 12 / 169: 00$ & 74.775 & 81.52 & 79.798 & 88.38 & 73.566 & 73.409 & 72.198 & 73.074 & 1583.697 & 1576.333 & 1573.51 & 1575.549 & 1575.062 & 1568.212 & 1565.093 & 1570.821 \\
\hline $10 / 12 / 1610: 00$ & 74.772 & 81.527 & 79.805 & 88.384 & 73.58 & 73.407 & 72.201 & 73.083 & 1583.7 & 1576.326 & 1573.503 & 1575.545 & 1575.048 & 1568.214 & 1565.09 & 1570.812 \\
\hline $10 / 12 / 1611: 00$ & 74.763 & 81.534 & 79.809 & 88.38 & 73.58 & 73.407 & 72.196 & 73.083 & 1583.709 & 1576.319 & 1573.499 & 1575.549 & 1575.048 & 1568.214 & 1565.095 & 1570.812 \\
\hline 10/12/16 12:00 & 74.753 & 81.53 & 79.807 & 88.377 & 73.583 & 73.404 & 72.189 & 73.085 & 1583.719 & 1576.323 & 1573.501 & 1575.552 & 1575.045 & 1568.217 & 1565.102 & 1570.81 \\
\hline $10 / 12 / 1613: 00$ & 74.742 & 81.523 & 79.807 & 88.37 & 73.578 & 73.4 & 72.179 & 73.097 & 1583.73 & 1576.33 & 1573.501 & 1575.559 & 1575.05 & 1568.221 & 1565.112 & 1570.798 \\
\hline $10 / 12 / 1614: 00$ & 74.723 & 81.502 & 79.788 & 88.352 & 73.562 & 73.379 & 72.158 & 73.074 & 1583.749 & 1576.351 & 1573.52 & 1575.577 & 1575.066 & 1568.242 & 1565.133 & 1570.821 \\
\hline $10 / 12 / 1615: 00$ & 74.694 & 81.481 & 79.768 & 88.342 & 73.538 & 73.365 & 72.131 & 73.053 & 1583.778 & 1576.372 & 1573.54 & 1575.587 & 1575.09 & 1568.256 & 1565.16 & 1570.842 \\
\hline $10 / 12 / 1616: 00$ & 74.685 & 81.467 & 79.754 & 88.338 & 73.527 & 73.358 & 72.117 & 73.037 & 1583.787 & 1576.386 & 1573.554 & 1575.591 & 1575.101 & 1568.263 & 1565.174 & 1570.858 \\
\hline
\end{tabular}


TABLE S1.1 Automatically recorded groundwater levels in the unconfined aquifer.

\begin{tabular}{|c|c|c|c|c|c|c|c|c|c|c|c|c|c|c|c|c|}
\hline \multirow[b]{2}{*}{ Date and Time } & \multicolumn{8}{|c|}{ Measured Depth to Water ( $\mathrm{ft} \mathrm{TOC}^{\mathrm{a}}$ ) } & \multicolumn{8}{|c|}{ Groundwater Elevation $\left(\mathrm{ft} A M S L^{b}\right)$} \\
\hline & DL1-C & DL2-C1 & DL3-C1 & DL4-C1 & DL5-C1 & DL6-C & DL7-C & DL8-C1 & DL1-C & DL2-C1 & DL3-C1 & DL4-C1 & DL5-C1 & DL6-C & DL7-C & DL8-C1 \\
\hline $10 / 12 / 1617: 00$ & 74.68 & 81.455 & 79.744 & 88.331 & 73.52 & 73.351 & 72.11 & 73.027 & 1583.792 & 1576.398 & 1573.564 & 1575.598 & 1575.108 & 1568.27 & 1565.181 & 1570.868 \\
\hline $10 / 12 / 1618: 00$ & 74.687 & 81.455 & 79.742 & 88.331 & 73.517 & 73.355 & 72.11 & 73.025 & 1583.785 & 1576.398 & 1573.566 & 1575.598 & 1575.111 & 1568.266 & 1565.181 & 1570.87 \\
\hline 10/12/16 19:00 & 74.69 & 81.453 & 79.742 & 88.335 & 73.52 & 73.355 & 72.112 & 73.032 & 1583.782 & 1576.4 & 1573.566 & 1575.594 & 1575.108 & 1568.266 & 1565.179 & 1570.863 \\
\hline $10 / 12 / 1620: 00$ & 74.697 & 81.46 & 79.749 & 88.338 & 73.524 & 73.362 & 72.122 & 73.034 & 1583.775 & 1576.393 & 1573.559 & 1575.591 & 1575.104 & 1568.259 & 1565.169 & 1570.861 \\
\hline 10/12/16 21:00 & 74.702 & 81.464 & 79.754 & 88.338 & 73.529 & 73.362 & 72.129 & 73.034 & 1583.77 & 1576.389 & 1573.554 & 1575.591 & 1575.099 & 1568.259 & 1565.162 & 1570.861 \\
\hline 10/12/16 22:00 & 74.704 & 81.464 & 79.758 & 88.34 & 73.531 & 73.365 & 72.127 & 73.037 & 1583.768 & 1576.389 & 1573.55 & 1575.589 & 1575.097 & 1568.256 & 1565.164 & 1570.858 \\
\hline $10 / 12 / 1623: 00$ & 74.702 & 81.467 & 79.754 & 88.342 & 73.529 & 73.36 & 72.129 & 73.032 & 1583.77 & 1576.386 & 1573.554 & 1575.587 & 1575.099 & 1568.261 & 1565.162 & 1570.863 \\
\hline $10 / 13 / 160: 00$ & 74.706 & 81.469 & 79.763 & 88.34 & 73.538 & 73.367 & 72.134 & 73.048 & 1583.766 & 1576.384 & 1573.545 & 1575.589 & 1575.09 & 1568.254 & 1565.157 & 1570.847 \\
\hline 10/13/16 1:00 & 74.685 & 81.446 & 79.749 & 88.324 & 73.517 & 73.351 & 72.105 & 73.034 & 1583.787 & 1576.407 & 1573.559 & 1575.605 & 1575.111 & 1568.27 & 1565.186 & 1570.861 \\
\hline $10 / 13 / 162: 00$ & 74.676 & 81.434 & 79.735 & 88.319 & 73.506 & 73.341 & 72.093 & 73.018 & 1583.796 & 1576.419 & 1573.573 & 1575.61 & 1575.122 & 1568.28 & 1565.198 & 1570.877 \\
\hline $10 / 13 / 163: 00$ & 74.669 & 81.425 & 79.723 & 88.317 & 73.499 & 73.339 & 72.088 & 73.006 & 1583.803 & 1576.428 & 1573.585 & 1575.612 & 1575.129 & 1568.282 & 1565.203 & 1570.889 \\
\hline $10 / 13 / 164: 00$ & 74.673 & 81.425 & 79.723 & 88.317 & 73.496 & 73.337 & 72.086 & 73.009 & 1583.799 & 1576.428 & 1573.585 & 1575.612 & 1575.132 & 1568.284 & 1565.205 & 1570.886 \\
\hline $10 / 13 / 165: 00$ & 74.664 & 81.409 & 79.716 & 88.31 & 73.487 & 73.33 & 72.076 & 73.009 & 1583.808 & 1576.444 & 1573.592 & 1575.619 & 1575.141 & 1568.291 & 1565.215 & 1570.886 \\
\hline $10 / 13 / 166: 00$ & 74.664 & 81.404 & 79.709 & 88.307 & 73.482 & 73.327 & 72.072 & 73.002 & 1583.808 & 1576.449 & 1573.599 & 1575.622 & 1575.146 & 1568.294 & 1565.219 & 1570.893 \\
\hline $10 / 13 / 167: 00$ & 74.664 & 81.402 & 79.707 & 88.307 & 73.475 & 73.325 & 72.067 & 72.99 & 1583.808 & 1576.451 & 1573.601 & 1575.622 & 1575.153 & 1568.296 & 1565.224 & 1570.905 \\
\hline $10 / 13 / 168: 00$ & 74.654 & 81.383 & 79.695 & 88.296 & 73.461 & 73.318 & 72.05 & 73.002 & 1583.818 & 1576.47 & 1573.613 & 1575.633 & 1575.167 & 1568.303 & 1565.241 & 1570.893 \\
\hline 10/13/16 9:00 & 74.659 & 81.388 & 79.695 & 88.298 & 73.461 & 73.323 & 72.055 & 73.002 & 1583.813 & 1576.465 & 1573.613 & 1575.631 & 1575.167 & 1568.298 & 1565.236 & 1570.893 \\
\hline $10 / 13 / 1610: 00$ & 74.664 & 81.383 & 79.695 & 88.303 & 73.461 & 73.323 & 72.057 & 72.986 & 1583.808 & 1576.47 & 1573.613 & 1575.626 & 1575.167 & 1568.298 & 1565.234 & 1570.909 \\
\hline 10/13/16 11:00 & 74.659 & 81.376 & 79.688 & 88.293 & 73.454 & 73.314 & 72.048 & 72.988 & 1583.813 & 1576.477 & 1573.62 & 1575.636 & 1575.174 & 1568.307 & 1565.243 & 1570.907 \\
\hline $10 / 13 / 1612: 00$ & 74.647 & 81.357 & 79.672 & 88.282 & 73.433 & 73.297 & 72.026 & 72.967 & 1583.825 & 1576.496 & 1573.636 & 1575.647 & 1575.195 & 1568.324 & 1565.265 & 1570.928 \\
\hline 10/13/16 13:00 & 74.631 & 81.332 & 79.653 & 88.268 & 73.412 & 73.286 & 72.003 & 72.962 & 1583.841 & 1576.521 & 1573.655 & 1575.661 & 1575.216 & 1568.335 & 1565.288 & 1570.933 \\
\hline $10 / 13 / 1614: 00$ & 74.61 & 81.306 & 79.623 & 88.251 & 73.387 & 73.267 & 71.979 & 72.914 & 1583.862 & 1576.547 & 1573.685 & 1575.678 & 1575.241 & 1568.354 & 1565.312 & 1570.981 \\
\hline 10/13/16 15:00 & 74.6 & 81.278 & 79.6 & 88.237 & 73.356 & 73.253 & 71.96 & 72.886 & 1583.872 & 1576.575 & 1573.708 & 1575.692 & 1575.272 & 1568.368 & 1565.331 & 1571.009 \\
\hline 10/13/16 16:00 & 74.593 & 81.262 & 79.583 & 88.24 & 73.338 & 73.244 & 71.948 & 72.867 & 1583.879 & 1576.591 & 1573.725 & 1575.689 & 1575.29 & 1568.377 & 1565.343 & 1571.028 \\
\hline $10 / 13 / 1617: 00$ & 74.591 & 81.248 & 79.569 & 88.228 & 73.326 & 73.237 & 71.938 & 72.867 & 1583.881 & 1576.605 & 1573.739 & 1575.701 & 1575.302 & 1568.384 & 1565.353 & 1571.028 \\
\hline $10 / 13 / 1618: 00$ & 74.586 & 81.225 & 79.548 & 88.221 & 73.3 & 73.225 & 71.921 & 72.846 & 1583.886 & 1576.628 & 1573.76 & 1575.708 & 1575.328 & 1568.396 & 1565.37 & 1571.049 \\
\hline 10/13/16 19:00 & 74.593 & 81.22 & 79.541 & 88.221 & 73.298 & 73.227 & 71.926 & 72.83 & 1583.879 & 1576.633 & 1573.767 & 1575.708 & 1575.33 & 1568.394 & 1565.365 & 1571.065 \\
\hline $10 / 13 / 1620: 00$ & 74.603 & 81.225 & 79.541 & 88.226 & 73.298 & 73.234 & 71.936 & 72.828 & 1583.869 & 1576.628 & 1573.767 & 1575.703 & 1575.33 & 1568.387 & 1565.355 & 1571.067 \\
\hline 10/13/16 21:00 & 74.624 & 81.237 & 79.551 & 88.237 & 73.305 & 73.246 & 71.95 & 72.832 & 1583.848 & 1576.616 & 1573.757 & 1575.692 & 1575.323 & 1568.375 & 1565.341 & 1571.063 \\
\hline $10 / 13 / 1622: 00$ & 74.629 & 81.239 & 79.558 & 88.24 & 73.307 & 73.248 & 71.957 & 72.839 & 1583.843 & 1576.614 & 1573.75 & 1575.689 & 1575.321 & 1568.373 & 1565.334 & 1571.056 \\
\hline 10/13/16 23:00 & 74.619 & 81.225 & 79.546 & 88.226 & 73.296 & 73.234 & 71.943 & 72.83 & 1583.853 & 1576.628 & 1573.762 & 1575.703 & 1575.332 & 1568.387 & 1565.348 & 1571.065 \\
\hline $10 / 14 / 160: 00$ & 74.621 & 81.225 & 79.539 & 88.228 & 73.289 & 73.232 & 71.938 & 72.823 & 1583.851 & 1576.628 & 1573.769 & 1575.701 & 1575.339 & 1568.389 & 1565.353 & 1571.072 \\
\hline 10/14/16 1:00 & 74.617 & 81.225 & 79.537 & 88.226 & 73.286 & 73.232 & 71.94 & 72.814 & 1583.855 & 1576.628 & 1573.771 & 1575.703 & 1575.342 & 1568.389 & 1565.351 & 1571.081 \\
\hline $10 / 14 / 162: 00$ & 74.612 & 81.213 & 79.527 & 88.221 & 73.27 & 73.223 & 71.933 & 72.797 & 1583.86 & 1576.64 & 1573.781 & 1575.708 & 1575.358 & 1568.398 & 1565.358 & 1571.098 \\
\hline $10 / 14 / 163: 00$ & 74.61 & 81.209 & 79.523 & 88.219 & 73.27 & 73.223 & 71.931 & 72.802 & 1583.862 & 1576.644 & 1573.785 & 1575.71 & 1575.358 & 1568.398 & 1565.36 & 1571.093 \\
\hline $10 / 14 / 164: 00$ & 74.598 & 81.19 & 79.506 & 88.202 & 73.249 & 73.209 & 71.907 & 72.781 & 1583.874 & 1576.663 & 1573.802 & 1575.727 & 1575.379 & 1568.412 & 1565.384 & 1571.114 \\
\hline $10 / 14 / 165: 00$ & 74.591 & 81.176 & 79.495 & 88.2 & 73.235 & 73.202 & 71.9 & 72.77 & 1583.881 & 1576.677 & 1573.813 & 1575.729 & 1575.393 & 1568.419 & 1565.391 & 1571.125 \\
\hline $10 / 14 / 166: 00$ & 74.581 & 81.157 & 79.476 & 88.191 & 73.216 & 73.19 & 71.883 & 72.751 & 1583.891 & 1576.696 & 1573.832 & 1575.738 & 1575.412 & 1568.431 & 1565.408 & 1571.144 \\
\hline $10 / 14 / 167: 00$ & 74.588 & 81.155 & 79.469 & 88.195 & 73.209 & 73.19 & 71.885 & 72.744 & 1583.884 & 1576.698 & 1573.839 & 1575.734 & 1575.419 & 1568.431 & 1565.406 & 1571.151 \\
\hline $10 / 14 / 168: 00$ & 74.591 & 81.15 & 79.467 & 88.191 & 73.202 & 73.19 & 71.883 & 72.779 & 1583.881 & 1576.703 & 1573.841 & 1575.738 & 1575.426 & 1568.431 & 1565.408 & 1571.116 \\
\hline 10/14/16 9:00 & 74.598 & 81.162 & 79.474 & 88.195 & 73.205 & 73.195 & 71.895 & 72.751 & 1583.874 & 1576.691 & 1573.834 & 1575.734 & 1575.423 & 1568.426 & 1565.396 & 1571.144 \\
\hline $10 / 14 / 1610: 00$ & 74.588 & 81.141 & 79.455 & 88.181 & 73.186 & 73.181 & 71.869 & 72.73 & 1583.884 & 1576.712 & 1573.853 & 1575.748 & 1575.442 & 1568.44 & 1565.422 & 1571.165 \\
\hline 10/14/16 11:00 & 74.598 & 81.143 & 79.458 & 88.188 & 73.188 & 73.181 & 71.878 & 72.709 & 1583.874 & 1576.71 & 1573.85 & 1575.741 & 1575.44 & 1568.44 & 1565.413 & 1571.186 \\
\hline $10 / 14 / 1612: 00$ & 74.591 & 81.132 & 79.446 & 88.179 & 73.177 & 73.176 & 71.864 & 72.753 & 1583.881 & 1576.721 & 1573.862 & 1575.75 & 1575.451 & 1568.445 & 1565.427 & 1571.142 \\
\hline 10/14/16 13:00 & 74.577 & 81.111 & 79.43 & 88.17 & 73.158 & 73.16 & 71.85 & 72.705 & 1583.895 & 1576.742 & 1573.878 & 1575.759 & 1575.47 & 1568.461 & 1565.441 & 1571.19 \\
\hline $10 / 14 / 1614: 00$ & 74.555 & 81.083 & 79.402 & 88.153 & 73.128 & 73.141 & 71.823 & 72.674 & 1583.917 & 1576.77 & 1573.906 & 1575.776 & 1575.5 & 1568.48 & 1565.468 & 1571.221 \\
\hline $10 / 14 / 1615: 00$ & 74.551 & 81.071 & 79.388 & 88.148 & 73.112 & 73.137 & 71.816 & 72.663 & 1583.921 & 1576.782 & 1573.92 & 1575.781 & 1575.516 & 1568.484 & 1565.475 & 1571.232 \\
\hline 10/14/16 16:00 & 74.544 & 81.053 & 79.371 & 88.137 & 73.093 & 73.125 & 71.802 & 72.64 & 1583.928 & 1576.8 & 1573.937 & 1575.792 & 1575.535 & 1568.496 & 1565.489 & 1571.255 \\
\hline $10 / 14 / 1617: 00$ & 74.539 & 81.039 & 79.355 & 88.132 & 73.077 & 73.118 & 71.79 & 72.635 & 1583.933 & 1576.814 & 1573.953 & 1575.797 & 1575.551 & 1568.503 & 1565.501 & 1571.26 \\
\hline
\end{tabular}


TABLE S1.1 Automatically recorded groundwater levels in the unconfined aquifer.

\begin{tabular}{|c|c|c|c|c|c|c|c|c|c|c|c|c|c|c|c|c|}
\hline \multirow[b]{2}{*}{ Date and Time } & \multicolumn{8}{|c|}{ Measured Depth to Water (ft TOC ${ }^{\mathrm{a}}$ ) } & \multicolumn{8}{|c|}{ Groundwater Elevation (ft AMSL) } \\
\hline & DL1-C & DL2-C1 & DL3-C1 & DL4-C1 & DL5-C1 & DL6-C & DL7-C & DL8-C1 & DL1-C & DL2-C1 & DL3-C1 & DL4-C1 & DL5-C1 & DL6-C & DL7-C & DL8-C1 \\
\hline 10/14/16 18:00 & 74.541 & 81.034 & 79.353 & 88.132 & 73.067 & 73.12 & 71.792 & 72.623 & 1583.931 & 1576.819 & 1573.955 & 1575.797 & 1575.561 & 1568.501 & 1565.499 & 1571.272 \\
\hline 10/14/16 19:00 & 74.551 & 81.034 & 79.353 & 88.134 & 73.065 & 73.12 & 71.795 & 72.612 & 1583.921 & 1576.819 & 1573.955 & 1575.795 & 1575.563 & 1568.501 & 1565.496 & 1571.283 \\
\hline $10 / 14 / 1620: 00$ & 74.563 & 81.041 & 79.353 & 88.139 & 73.07 & 73.13 & 71.804 & 72.623 & 1583.909 & 1576.812 & 1573.955 & 1575.79 & 1575.558 & 1568.491 & 1565.487 & 1571.272 \\
\hline $10 / 14 / 1621: 00$ & 74.567 & 81.039 & 79.357 & 88.139 & 73.067 & 73.13 & 71.807 & 72.619 & 1583.905 & 1576.814 & 1573.951 & 1575.79 & 1575.561 & 1568.491 & 1565.484 & 1571.276 \\
\hline $10 / 14 / 1622: 00$ & 74.572 & 81.039 & 79.355 & 88.139 & 73.065 & 73.127 & 71.809 & 72.616 & 1583.9 & 1576.814 & 1573.953 & 1575.79 & 1575.563 & 1568.494 & 1565.482 & 1571.279 \\
\hline 10/14/16 23:00 & 74.56 & 81.02 & 79.339 & 88.123 & 73.049 & 73.109 & 71.783 & 72.605 & 1583.912 & 1576.833 & 1573.969 & 1575.806 & 1575.579 & 1568.512 & 1565.508 & 1571.29 \\
\hline 10/15/16 0:00 & 74.57 & 81.032 & 79.343 & 88.137 & 73.053 & 73.12 & 71.8 & 72.602 & 1583.902 & 1576.821 & 1573.965 & 1575.792 & 1575.575 & 1568.501 & 1565.491 & 1571.293 \\
\hline 10/15/16 1:00 & 74.572 & 81.032 & 79.341 & 88.132 & 73.056 & 73.118 & 71.797 & 72.558 & 1583.9 & 1576.821 & 1573.967 & 1575.797 & 1575.572 & 1568.503 & 1565.494 & 1571.337 \\
\hline $10 / 15 / 162: 00$ & 74.567 & 81.032 & 79.339 & 88.132 & 73.049 & 73.118 & 71.8 & 72.598 & 1583.905 & 1576.821 & 1573.969 & 1575.797 & 1575.579 & 1568.503 & 1565.491 & 1571.297 \\
\hline $10 / 15 / 163: 00$ & 74.567 & 81.032 & 79.336 & 88.13 & 73.044 & 73.116 & 71.795 & 72.595 & 1583.905 & 1576.821 & 1573.972 & 1575.799 & 1575.584 & 1568.505 & 1565.496 & 1571.3 \\
\hline $10 / 15 / 164: 00$ & 74.57 & 81.03 & 79.336 & 88.127 & 73.039 & 73.113 & 71.797 & 72.593 & 1583.902 & 1576.823 & 1573.972 & 1575.802 & 1575.589 & 1568.508 & 1565.494 & 1571.302 \\
\hline $10 / 15 / 165: 00$ & 74.565 & 81.022 & 79.329 & 88.123 & 73.03 & 73.109 & 71.787 & 72.584 & 1583.907 & 1576.831 & 1573.979 & 1575.806 & 1575.598 & 1568.512 & 1565.504 & 1571.311 \\
\hline $10 / 15 / 166: 00$ & 74.567 & 81.032 & 79.325 & 88.13 & 73.032 & 73.111 & 71.797 & 72.584 & 1583.905 & 1576.821 & 1573.983 & 1575.799 & 1575.596 & 1568.51 & 1565.494 & 1571.311 \\
\hline 10/15/16 7:00 & 74.577 & 81.032 & 79.336 & 88.13 & 73.042 & 73.116 & 71.802 & 72.595 & 1583.895 & 1576.821 & 1573.972 & 1575.799 & 1575.586 & 1568.505 & 1565.489 & 1571.3 \\
\hline $10 / 15 / 168: 00$ & 74.579 & 81.039 & 79.336 & 88.134 & 73.039 & 73.118 & 71.804 & 72.586 & 1583.893 & 1576.814 & 1573.972 & 1575.795 & 1575.589 & 1568.503 & 1565.487 & 1571.309 \\
\hline 10/15/16 9:00 & 74.596 & 81.062 & 79.355 & 88.148 & 73.06 & 73.13 & 71.826 & 72.616 & 1583.876 & 1576.791 & 1573.953 & 1575.781 & 1575.568 & 1568.491 & 1565.465 & 1571.279 \\
\hline $10 / 15 / 1610: 00$ & 74.603 & 81.069 & 79.364 & 88.148 & 73.065 & 73.134 & 71.828 & 72.614 & 1583.869 & 1576.784 & 1573.944 & 1575.781 & 1575.563 & 1568.487 & 1565.463 & 1571.281 \\
\hline 10/15/16 11:00 & 74.605 & 81.081 & 79.369 & 88.155 & 73.074 & 73.139 & 71.84 & 72.628 & 1583.867 & 1576.772 & 1573.939 & 1575.774 & 1575.554 & 1568.482 & 1565.451 & 1571.267 \\
\hline $10 / 15 / 1612: 00$ & 74.617 & 81.097 & 79.383 & 88.162 & 73.091 & 73.15 & 71.854 & 72.644 & 1583.855 & 1576.756 & 1573.925 & 1575.767 & 1575.537 & 1568.471 & 1565.437 & 1571.251 \\
\hline $10 / 15 / 1613: 00$ & 74.612 & 81.097 & 79.385 & 88.153 & 73.095 & 73.144 & 71.847 & 72.644 & 1583.86 & 1576.756 & 1573.923 & 1575.776 & 1575.533 & 1568.477 & 1565.444 & 1571.251 \\
\hline $10 / 15 / 1614: 00$ & 74.596 & 81.09 & 79.378 & 88.146 & 73.088 & 73.132 & 71.835 & 72.64 & 1583.876 & 1576.763 & 1573.93 & 1575.783 & 1575.54 & 1568.489 & 1565.456 & 1571.255 \\
\hline $10 / 15 / 1615: 00$ & 74.586 & 81.078 & 79.371 & 88.141 & 73.081 & 73.127 & 71.821 & 72.642 & 1583.886 & 1576.775 & 1573.937 & 1575.788 & 1575.547 & 1568.494 & 1565.47 & 1571.253 \\
\hline $10 / 15 / 1616: 00$ & 74.581 & 81.076 & 79.364 & 88.139 & 73.074 & 73.118 & 71.816 & 72.628 & 1583.891 & 1576.777 & 1573.944 & 1575.79 & 1575.554 & 1568.503 & 1565.475 & 1571.267 \\
\hline 10/15/16 17:00 & 74.605 & 81.104 & 79.388 & 88.162 & 73.1 & 73.151 & 71.854 & 72.651 & 1583.867 & 1576.749 & 1573.92 & 1575.767 & 1575.528 & 1568.47 & 1565.437 & 1571.244 \\
\hline $10 / 15 / 1618: 00$ & 74.612 & 81.113 & 79.395 & 88.162 & 73.107 & 73.144 & 71.857 & 72.654 & 1583.86 & 1576.74 & 1573.913 & 1575.767 & 1575.521 & 1568.477 & 1565.434 & 1571.241 \\
\hline $10 / 15 / 1619: 00$ & 74.624 & 81.132 & 79.411 & 88.172 & 73.126 & 73.162 & 71.876 & 72.67 & 1583.848 & 1576.721 & 1573.897 & 1575.757 & 1575.502 & 1568.459 & 1565.415 & 1571.225 \\
\hline $10 / 15 / 1620: 00$ & 74.638 & 81.157 & 79.434 & 88.184 & 73.147 & 73.174 & 71.893 & 72.688 & 1583.834 & 1576.696 & 1573.874 & 1575.745 & 1575.481 & 1568.447 & 1565.398 & 1571.207 \\
\hline 10/15/16 21:00 & 74.645 & 81.171 & 79.446 & 88.188 & 73.16 & 73.181 & 71.904 & 72.707 & 1583.827 & 1576.682 & 1573.862 & 1575.741 & 1575.468 & 1568.44 & 1565.387 & 1571.188 \\
\hline $10 / 15 / 1622: 00$ & 74.647 & 81.188 & 79.458 & 88.195 & 73.172 & 73.186 & 71.912 & 72.695 & 1583.825 & 1576.665 & 1573.85 & 1575.734 & 1575.456 & 1568.435 & 1565.379 & 1571.2 \\
\hline $10 / 15 / 1623: 00$ & 74.657 & 81.202 & 79.472 & 88.202 & 73.188 & 73.195 & 71.928 & 72.732 & 1583.815 & 1576.651 & 1573.836 & 1575.727 & 1575.44 & 1568.426 & 1565.363 & 1571.163 \\
\hline 10/16/16 0:00 & 74.65 & 81.206 & 79.476 & 88.2 & 73.195 & 73.197 & 71.926 & 72.737 & 1583.822 & 1576.647 & 1573.832 & 1575.729 & 1575.433 & 1568.424 & 1565.365 & 1571.158 \\
\hline $10 / 16 / 161: 00$ & 74.636 & 81.197 & 79.469 & 88.186 & 73.184 & 73.181 & 71.909 & 72.732 & 1583.836 & 1576.656 & 1573.839 & 1575.743 & 1575.444 & 1568.44 & 1565.382 & 1571.163 \\
\hline $10 / 16 / 162: 00$ & 74.619 & 81.185 & 79.458 & 88.181 & 73.177 & 73.174 & 71.893 & 72.719 & 1583.853 & 1576.668 & 1573.85 & 1575.748 & 1575.451 & 1568.447 & 1565.398 & 1571.176 \\
\hline $10 / 16 / 163: 00$ & 74.607 & 81.174 & 79.448 & 88.172 & 73.165 & 73.164 & 71.883 & 72.714 & 1583.865 & 1576.679 & 1573.86 & 1575.757 & 1575.463 & 1568.457 & 1565.408 & 1571.181 \\
\hline $10 / 16 / 164: 00$ & 74.593 & 81.164 & 79.432 & 88.167 & 73.154 & 73.155 & 71.869 & 72.698 & 1583.879 & 1576.689 & 1573.876 & 1575.762 & 1575.474 & 1568.466 & 1565.422 & 1571.197 \\
\hline $10 / 16 / 165: 00$ & 74.588 & 81.157 & 79.427 & 88.162 & 73.149 & 73.15 & 71.864 & 72.693 & 1583.884 & 1576.696 & 1573.881 & 1575.767 & 1575.479 & 1568.471 & 1565.427 & 1571.202 \\
\hline 10/16/16 6:00 & 74.567 & 81.127 & 79.404 & 88.141 & 73.119 & 73.127 & 71.828 & 72.67 & 1583.905 & 1576.726 & 1573.904 & 1575.788 & 1575.509 & 1568.494 & 1565.463 & 1571.225 \\
\hline 10/16/16 7:00 & 74.57 & 81.13 & 79.404 & 88.151 & 73.121 & 73.134 & 71.838 & 72.67 & 1583.902 & 1576.723 & 1573.904 & 1575.778 & 1575.507 & 1568.487 & 1565.453 & 1571.225 \\
\hline $10 / 16 / 168: 00$ & 74.567 & 81.12 & 79.397 & 88.146 & 73.112 & 73.134 & 71.828 & 72.658 & 1583.905 & 1576.733 & 1573.911 & 1575.783 & 1575.516 & 1568.487 & 1565.463 & 1571.237 \\
\hline 10/16/16 9:00 & 74.563 & 81.111 & 79.385 & 88.139 & 73.1 & 73.125 & 71.814 & 72.656 & 1583.909 & 1576.742 & 1573.923 & 1575.79 & 1575.528 & 1568.496 & 1565.477 & 1571.239 \\
\hline $10 / 16 / 1610: 00$ & 74.551 & 81.083 & 79.369 & 88.125 & 73.079 & 73.109 & 71.792 & 72.635 & 1583.921 & 1576.77 & 1573.939 & 1575.804 & 1575.549 & 1568.512 & 1565.499 & 1571.26 \\
\hline 10/16/16 11:00 & 74.551 & 81.081 & 79.357 & 88.125 & 73.067 & 73.104 & 71.79 & 72.619 & 1583.921 & 1576.772 & 1573.951 & 1575.804 & 1575.561 & 1568.517 & 1565.501 & 1571.276 \\
\hline $10 / 16 / 1612: 00$ & 74.534 & 81.055 & 79.334 & 88.109 & 73.042 & 73.088 & 71.764 & 72.6 & 1583.938 & 1576.798 & 1573.974 & 1575.82 & 1575.586 & 1568.533 & 1565.527 & 1571.295 \\
\hline $10 / 16 / 1613: 00$ & 74.52 & 81.032 & 79.315 & 88.099 & 73.025 & 73.076 & 71.744 & 72.558 & 1583.952 & 1576.821 & 1573.993 & 1575.83 & 1575.603 & 1568.545 & 1565.547 & 1571.337 \\
\hline $10 / 16 / 1614: 00$ & 74.511 & 81.006 & 79.297 & 88.088 & 73 & 73.062 & 71.723 & 72.558 & 1583.961 & 1576.847 & 1574.011 & 1575.841 & 1575.628 & 1568.559 & 1565.568 & 1571.337 \\
\hline $10 / 16 / 1615: 00$ & 74.506 & 80.988 & 79.28 & 88.078 & 72.983 & 73.053 & 71.709 & 72.544 & 1583.966 & 1576.865 & 1574.028 & 1575.851 & 1575.645 & 1568.568 & 1565.582 & 1571.351 \\
\hline 10/16/16 16:00 & 74.508 & 80.985 & 79.271 & 88.081 & 72.974 & 73.051 & 71.716 & 72.521 & 1583.964 & 1576.868 & 1574.037 & 1575.848 & 1575.654 & 1568.57 & 1565.575 & 1571.374 \\
\hline 10/16/16 17:00 & 74.515 & 80.981 & 79.269 & 88.083 & 72.965 & 73.053 & 71.713 & 72.526 & 1583.957 & 1576.872 & 1574.039 & 1575.846 & 1575.663 & 1568.568 & 1565.578 & 1571.369 \\
\hline $10 / 16 / 1618: 00$ & 74.525 & 80.985 & 79.271 & 88.088 & 72.967 & 73.06 & 71.721 & 72.528 & 1583.947 & 1576.868 & 1574.037 & 1575.841 & 1575.661 & 1568.561 & 1565.57 & 1571.367 \\
\hline
\end{tabular}


TABLE S1.1 Automatically recorded groundwater levels in the unconfined aquifer.

\begin{tabular}{|c|c|c|c|c|c|c|c|c|c|c|c|c|c|c|c|c|}
\hline \multirow[b]{2}{*}{ Date and Time } & \multicolumn{8}{|c|}{ Measured Depth to Water (ft TOC ${ }^{\mathrm{a}}$ ) } & \multicolumn{8}{|c|}{ Groundwater Elevation (ft AMSL) } \\
\hline & DL1-C & DL2-C1 & DL3-C1 & DL4-C1 & DL5-C1 & DL6-C & DL7-C & DL8-C1 & DL1-C & DL2-C1 & DL3-C1 & DL4-C1 & DL5-C1 & DL6-C & DL7-C & DL8-C1 \\
\hline 10/16/16 19:00 & 74.541 & 80.999 & 79.283 & 88.095 & 72.986 & 73.071 & 71.74 & 72.535 & 1583.931 & 1576.854 & 1574.025 & 1575.834 & 1575.642 & 1568.55 & 1565.551 & 1571.36 \\
\hline 10/16/16 20:00 & 74.555 & 81.009 & 79.301 & 88.102 & 73 & 73.081 & 71.754 & 72.549 & 1583.917 & 1576.844 & 1574.007 & 1575.827 & 1575.628 & 1568.54 & 1565.537 & 1571.346 \\
\hline 10/16/16 21:00 & 74.577 & 81.034 & 79.32 & 88.118 & 73.023 & 73.097 & 71.783 & 72.575 & 1583.895 & 1576.819 & 1573.988 & 1575.811 & 1575.605 & 1568.524 & 1565.508 & 1571.32 \\
\hline $10 / 16 / 1622: 00$ & 74.579 & 81.039 & 79.325 & 88.116 & 73.025 & 73.097 & 71.78 & 72.577 & 1583.893 & 1576.814 & 1573.983 & 1575.813 & 1575.603 & 1568.524 & 1565.511 & 1571.318 \\
\hline $10 / 16 / 1623: 00$ & 74.586 & 81.053 & 79.329 & 88.12 & 73.032 & 73.099 & 71.792 & 72.584 & 1583.886 & 1576.8 & 1573.979 & 1575.809 & 1575.596 & 1568.522 & 1565.499 & 1571.311 \\
\hline 10/17/16 0:00 & 74.57 & 81.041 & 79.325 & 88.109 & 73.021 & 73.088 & 71.776 & 72.575 & 1583.902 & 1576.812 & 1573.983 & 1575.82 & 1575.607 & 1568.533 & 1565.515 & 1571.32 \\
\hline 10/17/16 1:00 & 74.558 & 81.027 & 79.313 & 88.102 & 73.011 & 73.078 & 71.759 & 72.568 & 1583.914 & 1576.826 & 1573.995 & 1575.827 & 1575.617 & 1568.543 & 1565.532 & 1571.327 \\
\hline $10 / 17 / 162: 00$ & 74.548 & 81.013 & 79.301 & 88.095 & 72.997 & 73.064 & 71.742 & 72.558 & 1583.924 & 1576.84 & 1574.007 & 1575.834 & 1575.631 & 1568.557 & 1565.549 & 1571.337 \\
\hline 10/17/16 3:00 & 74.522 & 80.99 & 79.271 & 88.074 & 72.967 & 73.043 & 71.711 & 72.526 & 1583.95 & 1576.863 & 1574.037 & 1575.855 & 1575.661 & 1568.578 & 1565.58 & 1571.369 \\
\hline $10 / 17 / 164: 00$ & 74.497 & 80.955 & 79.241 & 88.053 & 72.939 & 73.02 & 71.68 & 72.496 & 1583.975 & 1576.898 & 1574.067 & 1575.876 & 1575.689 & 1568.601 & 1565.611 & 1571.399 \\
\hline $10 / 17 / 165: 00$ & 74.482 & 80.929 & 79.22 & 88.046 & 72.918 & 73.011 & 71.659 & 72.468 & 1583.99 & 1576.924 & 1574.088 & 1575.883 & 1575.71 & 1568.61 & 1565.632 & 1571.427 \\
\hline $10 / 17 / 166: 00$ & 74.478 & 80.918 & 79.206 & 88.039 & 72.902 & 73.004 & 71.652 & 72.458 & 1583.994 & 1576.935 & 1574.102 & 1575.89 & 1575.726 & 1568.617 & 1565.639 & 1571.437 \\
\hline 10/17/16 7:00 & 74.48 & 80.906 & 79.196 & 88.036 & 72.89 & 72.999 & 71.644 & 72.444 & 1583.992 & 1576.947 & 1574.112 & 1575.893 & 1575.738 & 1568.622 & 1565.647 & 1571.451 \\
\hline $10 / 17 / 168: 00$ & 74.478 & 80.897 & 79.187 & 88.034 & 72.878 & 72.997 & 71.64 & 72.435 & 1583.994 & 1576.956 & 1574.121 & 1575.895 & 1575.75 & 1568.624 & 1565.651 & 1571.46 \\
\hline 10/17/16 9:00 & 74.475 & 80.881 & 79.171 & 88.025 & 72.86 & 72.987 & 71.625 & 72.419 & 1583.997 & 1576.972 & 1574.137 & 1575.904 & 1575.768 & 1568.634 & 1565.666 & 1571.476 \\
\hline $10 / 17 / 1610: 00$ & 74.485 & 80.885 & 79.175 & 88.029 & 72.86 & 72.994 & 71.635 & 72.426 & 1583.987 & 1576.968 & 1574.133 & 1575.9 & 1575.768 & 1568.627 & 1565.656 & 1571.469 \\
\hline 10/17/16 11:00 & 74.497 & 80.89 & 79.178 & 88.034 & 72.862 & 73.002 & 71.64 & 72.426 & 1583.975 & 1576.963 & 1574.13 & 1575.895 & 1575.766 & 1568.619 & 1565.651 & 1571.469 \\
\hline $10 / 17 / 1612: 00$ & 74.508 & 80.897 & 79.192 & 88.041 & 72.871 & 73.011 & 71.652 & 72.477 & 1583.964 & 1576.956 & 1574.116 & 1575.888 & 1575.757 & 1568.61 & 1565.639 & 1571.418 \\
\hline 10/17/16 13:00 & 74.522 & 80.909 & 79.201 & 88.048 & 72.881 & 73.016 & 71.668 & 72.456 & 1583.95 & 1576.944 & 1574.107 & 1575.881 & 1575.747 & 1568.605 & 1565.623 & 1571.439 \\
\hline $10 / 17 / 1614: 00$ & 74.518 & 80.906 & 79.194 & 88.039 & 72.871 & 73.004 & 71.654 & 72.449 & 1583.954 & 1576.947 & 1574.114 & 1575.89 & 1575.757 & 1568.617 & 1565.637 & 1571.446 \\
\hline 10/17/16 15:00 & 74.52 & 80.906 & 79.194 & 88.046 & 72.871 & 73.008 & 71.661 & 72.444 & 1583.952 & 1576.947 & 1574.114 & 1575.883 & 1575.757 & 1568.613 & 1565.63 & 1571.451 \\
\hline $10 / 17 / 1616: 00$ & 74.541 & 80.939 & 79.215 & 88.062 & 72.899 & 73.032 & 71.695 & 72.475 & 1583.931 & 1576.914 & 1574.093 & 1575.867 & 1575.729 & 1568.589 & 1565.596 & 1571.42 \\
\hline $10 / 17 / 1617: 00$ & 74.567 & 80.969 & 79.252 & 88.081 & 72.923 & 73.055 & 71.725 & 72.496 & 1583.905 & 1576.884 & 1574.056 & 1575.848 & 1575.705 & 1568.566 & 1565.566 & 1571.399 \\
\hline 10/17/16 18:00 & 74.581 & 80.995 & 79.266 & 88.09 & 72.941 & 73.067 & 71.747 & 72.517 & 1583.891 & 1576.858 & 1574.042 & 1575.839 & 1575.687 & 1568.554 & 1565.544 & 1571.378 \\
\hline 10/17/16 19:00 & 74.6 & 81.022 & 79.287 & 88.104 & 72.967 & 73.081 & 71.769 & 72.54 & 1583.872 & 1576.831 & 1574.021 & 1575.825 & 1575.661 & 1568.54 & 1565.522 & 1571.355 \\
\hline $10 / 17 / 1620: 00$ & 74.614 & 81.05 & 79.311 & 88.113 & 72.995 & 73.095 & 71.795 & 72.563 & 1583.858 & 1576.803 & 1573.997 & 1575.816 & 1575.633 & 1568.526 & 1565.496 & 1571.332 \\
\hline $10 / 17 / 1621: 00$ & 74.631 & 81.081 & 79.339 & 88.127 & 73.021 & 73.109 & 71.818 & 72.591 & 1583.841 & 1576.772 & 1573.969 & 1575.802 & 1575.607 & 1568.512 & 1565.473 & 1571.304 \\
\hline 10/17/16 22:00 & 74.645 & 81.113 & 79.371 & 88.146 & 73.053 & 73.13 & 71.85 & 72.623 & 1583.827 & 1576.74 & 1573.937 & 1575.783 & 1575.575 & 1568.491 & 1565.441 & 1571.272 \\
\hline $10 / 17 / 1623: 00$ & 74.664 & 81.15 & 79.402 & 88.165 & 73.091 & 73.15 & 71.878 & 72.656 & 1583.808 & 1576.703 & 1573.906 & 1575.764 & 1575.537 & 1568.471 & 1565.413 & 1571.239 \\
\hline 10/18/16 0:00 & 74.683 & 81.19 & 79.437 & 88.181 & 73.126 & 73.174 & 71.914 & 72.698 & 1583.789 & 1576.663 & 1573.871 & 1575.748 & 1575.502 & 1568.447 & 1565.377 & 1571.197 \\
\hline 10/18/16 1:00 & 74.68 & 81.209 & 79.453 & 88.184 & 73.144 & 73.171 & 71.917 & 72.709 & 1583.792 & 1576.644 & 1573.855 & 1575.745 & 1575.484 & 1568.45 & 1565.374 & 1571.186 \\
\hline $10 / 18 / 162: 00$ & 74.676 & 81.225 & 79.467 & 88.188 & 73.16 & 73.176 & 71.928 & 72.73 & 1583.796 & 1576.628 & 1573.841 & 1575.741 & 1575.468 & 1568.445 & 1565.363 & 1571.165 \\
\hline 10/18/16 3:00 & 74.68 & 81.248 & 79.483 & 88.2 & 73.181 & 73.188 & 71.948 & 72.746 & 1583.792 & 1576.605 & 1573.825 & 1575.729 & 1575.447 & 1568.433 & 1565.343 & 1571.149 \\
\hline $10 / 18 / 164: 00$ & 74.683 & 81.274 & 79.504 & 88.209 & 73.2 & 73.202 & 71.964 & 72.767 & 1583.789 & 1576.579 & 1573.804 & 1575.72 & 1575.428 & 1568.419 & 1565.327 & 1571.128 \\
\hline $10 / 18 / 165: 00$ & 74.699 & 81.302 & 79.532 & 88.223 & 73.235 & 73.223 & 71.995 & 72.786 & 1583.773 & 1576.551 & 1573.776 & 1575.706 & 1575.393 & 1568.398 & 1565.296 & 1571.109 \\
\hline $10 / 18 / 166: 00$ & 74.709 & 81.33 & 79.562 & 88.24 & 73.265 & 73.239 & 72.022 & 72.828 & 1583.763 & 1576.523 & 1573.746 & 1575.689 & 1575.363 & 1568.382 & 1565.269 & 1571.067 \\
\hline 10/18/16 7:00 & 74.718 & 81.355 & 79.586 & 88.247 & 73.291 & 73.244 & 72.031 & 72.851 & 1583.754 & 1576.498 & 1573.722 & 1575.682 & 1575.337 & 1568.377 & 1565.26 & 1571.044 \\
\hline $10 / 18 / 168: 00$ & 74.716 & 81.381 & 79.6 & 88.254 & 73.307 & 73.255 & 72.05 & 72.86 & 1583.756 & 1576.472 & 1573.708 & 1575.675 & 1575.321 & 1568.366 & 1565.241 & 1571.035 \\
\hline 10/18/16 9:00 & 74.72 & 81.392 & 79.63 & 88.263 & 73.331 & 73.269 & 72.062 & 72.932 & 1583.752 & 1576.461 & 1573.678 & 1575.666 & 1575.297 & 1568.352 & 1565.229 & 1570.963 \\
\hline 10/18/16 10:00 & 74.725 & 81.418 & 79.658 & 88.275 & 73.354 & 73.29 & 72.081 & 72.979 & 1583.747 & 1576.435 & 1573.65 & 1575.654 & 1575.274 & 1568.331 & 1565.21 & 1570.916 \\
\hline 10/18/16 11:00 & 74.716 & 81.425 & 79.66 & 88.272 & 73.366 & 73.281 & 72.079 & 72.946 & 1583.756 & 1576.428 & 1573.648 & 1575.657 & 1575.262 & 1568.34 & 1565.212 & 1570.949 \\
\hline $10 / 18 / 1612: 00$ & 74.699 & 81.413 & 79.656 & 88.265 & 73.361 & 73.267 & 72.062 & 72.937 & 1583.773 & 1576.44 & 1573.652 & 1575.664 & 1575.267 & 1568.354 & 1565.229 & 1570.958 \\
\hline $10 / 18 / 1613: 00$ & 74.683 & 81.411 & 79.649 & 88.256 & 73.356 & 73.26 & 72.05 & 72.953 & 1583.789 & 1576.442 & 1573.659 & 1575.673 & 1575.272 & 1568.361 & 1565.241 & 1570.942 \\
\hline $10 / 18 / 1614: 00$ & 74.662 & 81.39 & 79.646 & 88.244 & 73.342 & 73.251 & 72.029 & 72.944 & 1583.81 & 1576.463 & 1573.662 & 1575.685 & 1575.286 & 1568.37 & 1565.262 & 1570.951 \\
\hline 10/18/16 15:00 & 74.647 & 81.378 & 79.625 & 88.242 & 73.335 & 73.241 & 72.012 & 72.916 & 1583.825 & 1576.475 & 1573.683 & 1575.687 & 1575.293 & 1568.38 & 1565.279 & 1570.979 \\
\hline $10 / 18 / 1616: 00$ & 74.633 & 81.362 & 79.609 & 88.228 & 73.321 & 73.228 & 71.995 & 72.902 & 1583.839 & 1576.491 & 1573.699 & 1575.701 & 1575.307 & 1568.393 & 1565.296 & 1570.993 \\
\hline 10/18/16 17:00 & 74.626 & 81.355 & 79.607 & 88.226 & 73.317 & 73.227 & 71.988 & 72.909 & 1583.846 & 1576.498 & 1573.701 & 1575.703 & 1575.311 & 1568.394 & 1565.303 & 1570.986 \\
\hline $10 / 18 / 1618: 00$ & 74.626 & 81.353 & 79.604 & 88.226 & 73.314 & 73.227 & 71.988 & 72.9 & 1583.846 & 1576.5 & 1573.704 & 1575.703 & 1575.314 & 1568.394 & 1565.303 & 1570.995 \\
\hline 10/18/16 19:00 & 74.638 & 81.362 & 79.611 & 88.235 & 73.326 & 73.237 & 72.003 & 72.904 & 1583.834 & 1576.491 & 1573.697 & 1575.694 & 1575.302 & 1568.384 & 1565.288 & 1570.991 \\
\hline
\end{tabular}


TABLE S1.1 Automatically recorded groundwater levels in the unconfined aquifer.

\begin{tabular}{|c|c|c|c|c|c|c|c|c|c|c|c|c|c|c|c|c|}
\hline \multirow[b]{2}{*}{ Date and Time } & \multicolumn{8}{|c|}{ Measured Depth to Water (ft TOC ${ }^{\mathrm{a}}$ ) } & \multicolumn{8}{|c|}{ Groundwater Elevation ( $\mathrm{ft} \mathrm{AMSL}^{\mathrm{b}}$ ) } \\
\hline & DL1-C & DL2-C1 & DL3-C1 & DL4-C1 & DL5-C1 & DL6-C & DL7-C & DL8-C1 & DL1-C & DL2-C1 & DL3-C1 & DL4-C1 & DL5-C1 & DL6-C & DL7-C & DL8-C1 \\
\hline $10 / 18 / 1620: 00$ & 74.652 & 81.378 & 79.628 & 88.242 & 73.342 & 73.248 & 72.019 & 72.914 & 1583.82 & 1576.475 & 1573.68 & 1575.687 & 1575.286 & 1568.373 & 1565.272 & 1570.981 \\
\hline $10 / 18 / 1621: 00$ & 74.662 & 81.383 & 79.635 & 88.247 & 73.352 & 73.251 & 72.026 & 72.93 & 1583.81 & 1576.47 & 1573.673 & 1575.682 & 1575.276 & 1568.37 & 1565.265 & 1570.965 \\
\hline $10 / 18 / 1622: 00$ & 74.652 & 81.378 & 79.635 & 88.242 & 73.352 & 73.246 & 72.017 & 72.923 & 1583.82 & 1576.475 & 1573.673 & 1575.687 & 1575.276 & 1568.375 & 1565.274 & 1570.972 \\
\hline $10 / 18 / 1623: 00$ & 74.65 & 81.381 & 79.635 & 88.24 & 73.352 & 73.244 & 72.014 & 72.872 & 1583.822 & 1576.472 & 1573.673 & 1575.689 & 1575.276 & 1568.377 & 1565.277 & 1571.023 \\
\hline 10/19/16 0:00 & 74.65 & 81.383 & 79.635 & 88.242 & 73.352 & 73.244 & 72.014 & 72.925 & 1583.822 & 1576.47 & 1573.673 & 1575.687 & 1575.276 & 1568.377 & 1565.277 & 1570.97 \\
\hline $10 / 19 / 161: 00$ & 74.645 & 81.374 & 79.632 & 88.244 & 73.352 & 73.241 & 72.009 & 72.881 & 1583.827 & 1576.479 & 1573.676 & 1575.685 & 1575.276 & 1568.38 & 1565.282 & 1571.014 \\
\hline $10 / 19 / 162: 00$ & 74.64 & 81.374 & 79.632 & 88.237 & 73.345 & 73.237 & 72.002 & 72.916 & 1583.832 & 1576.479 & 1573.676 & 1575.692 & 1575.283 & 1568.384 & 1565.289 & 1570.979 \\
\hline $10 / 19 / 163: 00$ & 74.64 & 81.374 & 79.632 & 88.237 & 73.352 & 73.241 & 72.007 & 72.921 & 1583.832 & 1576.479 & 1573.676 & 1575.692 & 1575.276 & 1568.38 & 1565.284 & 1570.974 \\
\hline $10 / 19 / 164: 00$ & 74.636 & 81.371 & 79.628 & 88.235 & 73.347 & 73.237 & 72.003 & 72.921 & 1583.836 & 1576.482 & 1573.68 & 1575.694 & 1575.281 & 1568.384 & 1565.288 & 1570.974 \\
\hline $10 / 19 / 165: 00$ & 74.638 & 81.376 & 79.63 & 88.237 & 73.349 & 73.237 & 72.007 & 72.881 & 1583.834 & 1576.477 & 1573.678 & 1575.692 & 1575.279 & 1568.384 & 1565.284 & 1571.014 \\
\hline $10 / 19 / 166: 00$ & 74.633 & 81.367 & 79.625 & 88.233 & 73.347 & 73.232 & 71.998 & 72.918 & 1583.839 & 1576.486 & 1573.683 & 1575.696 & 1575.281 & 1568.389 & 1565.293 & 1570.977 \\
\hline $10 / 19 / 167: 00$ & 74.629 & 81.362 & 79.623 & 88.228 & 73.342 & 73.23 & 71.991 & 72.902 & 1583.843 & 1576.491 & 1573.685 & 1575.701 & 1575.286 & 1568.391 & 1565.3 & 1570.993 \\
\hline $10 / 19 / 168: 00$ & 74.64 & 81.376 & 79.635 & 88.237 & 73.352 & 73.241 & 72.01 & 72.918 & 1583.832 & 1576.477 & 1573.673 & 1575.692 & 1575.276 & 1568.38 & 1565.281 & 1570.977 \\
\hline 10/19/16 9:00 & 74.654 & 81.397 & 79.651 & 88.251 & 73.37 & 73.255 & 72.029 & 72.934 & 1583.818 & 1576.456 & 1573.657 & 1575.678 & 1575.258 & 1568.366 & 1565.262 & 1570.961 \\
\hline $10 / 19 / 1610: 00$ & 74.664 & 81.406 & 79.667 & 88.258 & 73.382 & 73.265 & 72.038 & 72.951 & 1583.808 & 1576.447 & 1573.641 & 1575.671 & 1575.246 & 1568.356 & 1565.253 & 1570.944 \\
\hline 10/19/16 11:00 & 74.657 & 81.399 & 79.66 & 88.249 & 73.38 & 73.253 & 72.026 & 72.948 & 1583.815 & 1576.454 & 1573.648 & 1575.68 & 1575.248 & 1568.368 & 1565.265 & 1570.947 \\
\hline $10 / 19 / 1612: 00$ & 74.65 & 81.397 & 79.66 & 88.247 & 73.377 & 73.248 & 72.022 & 72.946 & 1583.822 & 1576.456 & 1573.648 & 1575.682 & 1575.251 & 1568.373 & 1565.269 & 1570.949 \\
\hline $10 / 19 / 1613: 00$ & 74.638 & 81.381 & 79.653 & 88.235 & 73.366 & 73.241 & 72.007 & 72.934 & 1583.834 & 1576.472 & 1573.655 & 1575.694 & 1575.262 & 1568.38 & 1565.284 & 1570.961 \\
\hline $10 / 19 / 1614: 00$ & 74.612 & 81.353 & 79.63 & 88.216 & 73.342 & 73.218 & 71.974 & 72.921 & 1583.86 & 1576.5 & 1573.678 & 1575.713 & 1575.286 & 1568.403 & 1565.317 & 1570.974 \\
\hline $10 / 19 / 1615: 00$ & 74.605 & 81.343 & 79.616 & 88.216 & 73.333 & 73.216 & 71.969 & 72.904 & 1583.867 & 1576.51 & 1573.692 & 1575.713 & 1575.295 & 1568.405 & 1565.322 & 1570.991 \\
\hline $10 / 19 / 1616: 00$ & 74.61 & 81.344 & 79.616 & 88.223 & 73.333 & 73.22 & 71.971 & 72.907 & 1583.862 & 1576.509 & 1573.692 & 1575.706 & 1575.295 & 1568.401 & 1565.32 & 1570.988 \\
\hline $10 / 19 / 1617: 00$ & 74.614 & 81.35 & 79.618 & 88.223 & 73.331 & 73.223 & 71.976 & 72.918 & 1583.858 & 1576.503 & 1573.69 & 1575.706 & 1575.297 & 1568.398 & 1565.315 & 1570.977 \\
\hline $10 / 19 / 1618: 00$ & 74.631 & 81.362 & 79.63 & 88.235 & 73.345 & 73.237 & 71.993 & 72.923 & 1583.841 & 1576.491 & 1573.678 & 1575.694 & 1575.283 & 1568.384 & 1565.298 & 1570.972 \\
\hline $10 / 19 / 1619: 00$ & 74.645 & 81.374 & 79.642 & 88.242 & 73.361 & 73.244 & 72.007 & 72.927 & 1583.827 & 1576.479 & 1573.666 & 1575.687 & 1575.267 & 1568.377 & 1565.284 & 1570.968 \\
\hline $10 / 19 / 1620: 00$ & 74.654 & 81.39 & 79.653 & 88.251 & 73.373 & 73.248 & 72.019 & 72.937 & 1583.818 & 1576.463 & 1573.655 & 1575.678 & 1575.255 & 1568.373 & 1565.272 & 1570.958 \\
\hline $10 / 19 / 1621: 00$ & 74.664 & 81.409 & 79.674 & 88.261 & 73.391 & 73.267 & 72.045 & 72.962 & 1583.808 & 1576.444 & 1573.634 & 1575.668 & 1575.237 & 1568.354 & 1565.246 & 1570.933 \\
\hline $10 / 19 / 1622: 00$ & 74.654 & 81.399 & 79.665 & 88.249 & 73.382 & 73.253 & 72.026 & 72.96 & 1583.818 & 1576.454 & 1573.643 & 1575.68 & 1575.246 & 1568.368 & 1565.265 & 1570.935 \\
\hline $10 / 19 / 1623: 00$ & 74.664 & 81.413 & 79.679 & 88.258 & 73.394 & 73.262 & 72.043 & 72.972 & 1583.808 & 1576.44 & 1573.629 & 1575.671 & 1575.234 & 1568.359 & 1565.248 & 1570.923 \\
\hline $10 / 20 / 160: 00$ & 74.664 & 81.418 & 79.684 & 88.263 & 73.401 & 73.265 & 72.045 & 72.967 & 1583.808 & 1576.435 & 1573.624 & 1575.666 & 1575.227 & 1568.356 & 1565.246 & 1570.928 \\
\hline $10 / 20 / 161: 00$ & 74.664 & 81.423 & 79.686 & 88.263 & 73.405 & 73.265 & 72.045 & 72.965 & 1583.808 & 1576.43 & 1573.622 & 1575.666 & 1575.223 & 1568.356 & 1565.246 & 1570.93 \\
\hline $10 / 20 / 162: 00$ & 74.664 & 81.427 & 79.691 & 88.265 & 73.41 & 73.267 & 72.05 & 72.979 & 1583.808 & 1576.426 & 1573.617 & 1575.664 & 1575.218 & 1568.354 & 1565.241 & 1570.916 \\
\hline $10 / 20 / 163: 00$ & 74.662 & 81.432 & 79.693 & 88.265 & 73.417 & 73.269 & 72.052 & 72.983 & 1583.81 & 1576.421 & 1573.615 & 1575.664 & 1575.211 & 1568.352 & 1565.239 & 1570.912 \\
\hline $10 / 20 / 164: 00$ & 74.657 & 81.427 & 79.691 & 88.261 & 73.412 & 73.265 & 72.048 & 72.981 & 1583.815 & 1576.426 & 1573.617 & 1575.668 & 1575.216 & 1568.356 & 1565.243 & 1570.914 \\
\hline $10 / 20 / 165: 00$ & 74.664 & 81.439 & 79.702 & 88.27 & 73.426 & 73.274 & 72.06 & 72.988 & 1583.808 & 1576.414 & 1573.606 & 1575.659 & 1575.202 & 1568.347 & 1565.231 & 1570.907 \\
\hline $10 / 20 / 166: 00$ & 74.673 & 81.455 & 79.714 & 88.277 & 73.438 & 73.283 & 72.072 & 73.006 & 1583.799 & 1576.398 & 1573.594 & 1575.652 & 1575.19 & 1568.338 & 1565.219 & 1570.889 \\
\hline $10 / 20 / 167: 00$ & 74.671 & 81.45 & 79.719 & 88.275 & 73.44 & 73.281 & 72.072 & 73.004 & 1583.801 & 1576.403 & 1573.589 & 1575.654 & 1575.188 & 1568.34 & 1565.219 & 1570.891 \\
\hline $10 / 20 / 168: 00$ & 74.664 & 81.45 & 79.714 & 88.27 & 73.433 & 73.276 & 72.067 & 73.004 & 1583.808 & 1576.403 & 1573.594 & 1575.659 & 1575.195 & 1568.345 & 1565.224 & 1570.891 \\
\hline 10/20/16 9:00 & 74.659 & 81.45 & 79.721 & 88.27 & 73.436 & 73.276 & 72.062 & 72.995 & 1583.813 & 1576.403 & 1573.587 & 1575.659 & 1575.192 & 1568.345 & 1565.229 & 1570.9 \\
\hline $10 / 20 / 1610: 00$ & 74.654 & 81.45 & 79.712 & 88.265 & 73.431 & 73.274 & 72.06 & 72.999 & 1583.818 & 1576.403 & 1573.596 & 1575.664 & 1575.197 & 1568.347 & 1565.231 & 1570.896 \\
\hline $10 / 20 / 1611: 00$ & 74.652 & 81.444 & 79.719 & 88.265 & 73.431 & 73.272 & 72.057 & 72.997 & 1583.82 & 1576.409 & 1573.589 & 1575.664 & 1575.197 & 1568.349 & 1565.234 & 1570.898 \\
\hline $10 / 20 / 1612: 00$ & 74.64 & 81.43 & 79.7 & 88.256 & 73.424 & 73.26 & 72.038 & 72.985 & 1583.832 & 1576.423 & 1573.608 & 1575.673 & 1575.204 & 1568.361 & 1565.253 & 1570.91 \\
\hline $10 / 20 / 1613: 00$ & 74.619 & 81.404 & 79.679 & 88.24 & 73.396 & 73.241 & 72.012 & 72.967 & 1583.853 & 1576.449 & 1573.629 & 1575.689 & 1575.232 & 1568.38 & 1565.279 & 1570.928 \\
\hline $10 / 20 / 1614: 00$ & 74.598 & 81.371 & 79.651 & 88.221 & 73.366 & 73.22 & 71.976 & 72.944 & 1583.874 & 1576.482 & 1573.657 & 1575.708 & 1575.262 & 1568.401 & 1565.315 & 1570.951 \\
\hline $10 / 20 / 1615: 00$ & 74.584 & 81.353 & 79.635 & 88.212 & 73.347 & 73.211 & 71.96 & 72.916 & 1583.888 & 1576.5 & 1573.673 & 1575.717 & 1575.281 & 1568.41 & 1565.331 & 1570.979 \\
\hline $10 / 20 / 1616: 00$ & 74.574 & 81.327 & 79.611 & 88.2 & 73.324 & 73.195 & 71.938 & 72.897 & 1583.898 & 1576.526 & 1573.697 & 1575.729 & 1575.304 & 1568.426 & 1565.353 & 1570.998 \\
\hline $10 / 20 / 1617: 00$ & 74.567 & 81.309 & 79.593 & 88.193 & 73.31 & 73.188 & 71.924 & 72.879 & 1583.905 & 1576.544 & 1573.715 & 1575.736 & 1575.318 & 1568.433 & 1565.367 & 1571.016 \\
\hline $10 / 20 / 1618: 00$ & 74.57 & 81.302 & 79.583 & 88.193 & 73.3 & 73.186 & 71.921 & 72.872 & 1583.902 & 1576.551 & 1573.725 & 1575.736 & 1575.328 & 1568.435 & 1565.37 & 1571.023 \\
\hline $10 / 20 / 1619: 00$ & 74.577 & 81.297 & 79.579 & 88.191 & 73.293 & 73.186 & 71.919 & 72.862 & 1583.895 & 1576.556 & 1573.729 & 1575.738 & 1575.335 & 1568.435 & 1565.372 & 1571.033 \\
\hline $10 / 20 / 1620: 00$ & 74.574 & 81.288 & 79.569 & 88.186 & 73.284 & 73.181 & 71.912 & 72.855 & 1583.898 & 1576.565 & 1573.739 & 1575.743 & 1575.344 & 1568.44 & 1565.379 & 1571.04 \\
\hline
\end{tabular}


TABLE S1.1 Automatically recorded groundwater levels in the unconfined aquifer.

\begin{tabular}{|c|c|c|c|c|c|c|c|c|c|c|c|c|c|c|c|c|}
\hline \multirow[b]{2}{*}{ Date and Time } & \multicolumn{8}{|c|}{ Measured Depth to Water (ft TOC ${ }^{\mathrm{a}}$ ) } & \multicolumn{8}{|c|}{ Groundwater Elevation ( $\mathrm{ft} \mathrm{AMSL}^{\mathrm{b}}$ ) } \\
\hline & DL1-C & DL2-C1 & DL3-C1 & DL4-C1 & DL5-C1 & DL6-C & DL7-C & DL8-C1 & DL1-C & DL2-C1 & DL3-C1 & DL4-C1 & DL5-C1 & DL6-C & DL7-C & DL8-C1 \\
\hline $10 / 20 / 1621: 00$ & 74.574 & 81.281 & 79.567 & 88.186 & 73.279 & 73.181 & 71.912 & 72.849 & 1583.898 & 1576.572 & 1573.741 & 1575.743 & 1575.349 & 1568.44 & 1565.379 & 1571.046 \\
\hline $10 / 20 / 1622: 00$ & 74.586 & 81.281 & 79.567 & 88.186 & 73.277 & 73.181 & 71.912 & 72.851 & 1583.886 & 1576.572 & 1573.741 & 1575.743 & 1575.351 & 1568.44 & 1565.379 & 1571.044 \\
\hline $10 / 20 / 1623: 00$ & 74.577 & 81.269 & 79.555 & 88.181 & 73.265 & 73.174 & 71.9 & 72.842 & 1583.895 & 1576.584 & 1573.753 & 1575.748 & 1575.363 & 1568.447 & 1565.391 & 1571.053 \\
\hline $10 / 21 / 160: 00$ & 74.572 & 81.26 & 79.546 & 88.174 & 73.254 & 73.162 & 71.888 & 72.837 & 1583.9 & 1576.593 & 1573.762 & 1575.755 & 1575.374 & 1568.459 & 1565.403 & 1571.058 \\
\hline $10 / 21 / 161: 00$ & 74.558 & 81.239 & 79.527 & 88.162 & 73.23 & 73.148 & 71.866 & 72.804 & 1583.914 & 1576.614 & 1573.781 & 1575.767 & 1575.398 & 1568.473 & 1565.425 & 1571.091 \\
\hline $10 / 21 / 162: 00$ & 74.553 & 81.223 & 79.513 & 88.155 & 73.216 & 73.144 & 71.857 & 72.793 & 1583.919 & 1576.63 & 1573.795 & 1575.774 & 1575.412 & 1568.477 & 1565.434 & 1571.102 \\
\hline $10 / 21 / 163: 00$ & 74.548 & 81.211 & 79.502 & 88.151 & 73.202 & 73.137 & 71.847 & 72.732 & 1583.924 & 1576.642 & 1573.806 & 1575.778 & 1575.426 & 1568.484 & 1565.444 & 1571.163 \\
\hline $10 / 21 / 164: 00$ & 74.548 & 81.202 & 79.488 & 88.144 & 73.191 & 73.134 & 71.838 & 72.765 & 1583.924 & 1576.651 & 1573.82 & 1575.785 & 1575.437 & 1568.487 & 1565.453 & 1571.13 \\
\hline $10 / 21 / 165: 00$ & 74.541 & 81.183 & 79.474 & 88.137 & 73.177 & 73.118 & 71.826 & 72.751 & 1583.931 & 1576.67 & 1573.834 & 1575.792 & 1575.451 & 1568.503 & 1565.465 & 1571.144 \\
\hline $10 / 21 / 166: 00$ & 74.541 & 81.171 & 79.465 & 88.132 & 73.16 & 73.116 & 71.816 & 72.746 & 1583.931 & 1576.682 & 1573.843 & 1575.797 & 1575.468 & 1568.505 & 1565.475 & 1571.149 \\
\hline $10 / 21 / 167: 00$ & 74.546 & 81.167 & 79.455 & 88.127 & 73.156 & 73.111 & 71.812 & 72.732 & 1583.926 & 1576.686 & 1573.853 & 1575.802 & 1575.472 & 1568.51 & 1565.479 & 1571.163 \\
\hline $10 / 21 / 168: 00$ & 74.537 & 81.15 & 79.444 & 88.12 & 73.137 & 73.106 & 71.8 & 72.721 & 1583.935 & 1576.703 & 1573.864 & 1575.809 & 1575.491 & 1568.515 & 1565.491 & 1571.174 \\
\hline $10 / 21 / 169: 00$ & 74.546 & 81.153 & 79.441 & 88.125 & 73.137 & 73.111 & 71.804 & 72.719 & 1583.926 & 1576.7 & 1573.867 & 1575.804 & 1575.491 & 1568.51 & 1565.487 & 1571.176 \\
\hline $10 / 21 / 1610: 00$ & 74.544 & 81.146 & 79.437 & 88.12 & 73.133 & 73.106 & 71.802 & 72.714 & 1583.928 & 1576.707 & 1573.871 & 1575.809 & 1575.495 & 1568.515 & 1565.489 & 1571.181 \\
\hline $10 / 21 / 1611: 00$ & 74.53 & 81.125 & 79.418 & 88.104 & 73.107 & 73.085 & 71.775 & 72.688 & 1583.942 & 1576.728 & 1573.89 & 1575.825 & 1575.521 & 1568.536 & 1565.516 & 1571.207 \\
\hline $10 / 21 / 1612: 00$ & 74.515 & 81.099 & 79.397 & 88.095 & 73.081 & 73.069 & 71.752 & 72.67 & 1583.957 & 1576.754 & 1573.911 & 1575.834 & 1575.547 & 1568.552 & 1565.539 & 1571.225 \\
\hline $10 / 21 / 1613: 00$ & 74.499 & 81.069 & 79.371 & 88.078 & 73.058 & 73.055 & 71.723 & 72.644 & 1583.973 & 1576.784 & 1573.937 & 1575.851 & 1575.57 & 1568.566 & 1565.568 & 1571.251 \\
\hline $10 / 21 / 1614: 00$ & 74.473 & 81.032 & 79.332 & 88.057 & 73.016 & 73.027 & 71.687 & 72.605 & 1583.999 & 1576.821 & 1573.976 & 1575.872 & 1575.612 & 1568.594 & 1565.604 & 1571.29 \\
\hline $10 / 21 / 1615: 00$ & 74.464 & 81.006 & 79.308 & 88.041 & 72.986 & 73.018 & 71.668 & 72.563 & 1584.008 & 1576.847 & 1574 & 1575.888 & 1575.642 & 1568.603 & 1565.623 & 1571.332 \\
\hline $10 / 21 / 1616: 00$ & 74.471 & 80.999 & 79.297 & 88.048 & 72.979 & 73.018 & 71.668 & 72.581 & 1584.001 & 1576.854 & 1574.011 & 1575.881 & 1575.649 & 1568.603 & 1565.623 & 1571.314 \\
\hline $10 / 21 / 1617: 00$ & 74.482 & 80.995 & 79.294 & 88.055 & 72.972 & 73.025 & 71.675 & 72.572 & 1583.99 & 1576.858 & 1574.014 & 1575.874 & 1575.656 & 1568.596 & 1565.616 & 1571.323 \\
\hline $10 / 21 / 1618: 00$ & 74.494 & 80.999 & 79.297 & 88.053 & 72.967 & 73.029 & 71.68 & 72.57 & 1583.978 & 1576.854 & 1574.011 & 1575.876 & 1575.661 & 1568.592 & 1565.611 & 1571.325 \\
\hline $10 / 21 / 1619: 00$ & 74.511 & 81.011 & 79.304 & 88.064 & 72.976 & 73.036 & 71.697 & 72.57 & 1583.961 & 1576.842 & 1574.004 & 1575.865 & 1575.652 & 1568.585 & 1565.594 & 1571.325 \\
\hline $10 / 21 / 1620: 00$ & 74.522 & 81.018 & 79.311 & 88.071 & 72.979 & 73.043 & 71.706 & 72.577 & 1583.95 & 1576.835 & 1573.997 & 1575.858 & 1575.649 & 1568.578 & 1565.585 & 1571.318 \\
\hline $10 / 21 / 1621: 00$ & 74.525 & 81.02 & 79.311 & 88.067 & 72.983 & 73.041 & 71.709 & 72.575 & 1583.947 & 1576.833 & 1573.997 & 1575.862 & 1575.645 & 1568.58 & 1565.582 & 1571.32 \\
\hline $10 / 21 / 1622: 00$ & 74.534 & 81.027 & 79.318 & 88.076 & 72.988 & 73.046 & 71.716 & 72.579 & 1583.938 & 1576.826 & 1573.99 & 1575.853 & 1575.64 & 1568.575 & 1565.575 & 1571.316 \\
\hline $10 / 21 / 1623: 00$ & 74.534 & 81.032 & 79.318 & 88.071 & 72.986 & 73.043 & 71.716 & 72.582 & 1583.938 & 1576.821 & 1573.99 & 1575.858 & 1575.642 & 1568.578 & 1565.575 & 1571.313 \\
\hline $10 / 22 / 160: 00$ & 74.532 & 81.023 & 79.313 & 88.067 & 72.981 & 73.041 & 71.711 & 72.575 & 1583.94 & 1576.83 & 1573.995 & 1575.862 & 1575.647 & 1568.58 & 1565.58 & 1571.32 \\
\hline $10 / 22 / 161: 00$ & 74.527 & 81.025 & 79.308 & 88.069 & 72.979 & 73.041 & 71.714 & 72.572 & 1583.945 & 1576.828 & 1574 & 1575.86 & 1575.649 & 1568.58 & 1565.577 & 1571.323 \\
\hline $10 / 22 / 162: 00$ & 74.53 & 81.025 & 79.306 & 88.069 & 72.974 & 73.039 & 71.711 & 72.572 & 1583.942 & 1576.828 & 1574.002 & 1575.86 & 1575.654 & 1568.582 & 1565.58 & 1571.323 \\
\hline $10 / 22 / 163: 00$ & 74.532 & 81.029 & 79.311 & 88.071 & 72.979 & 73.039 & 71.714 & 72.577 & 1583.94 & 1576.824 & 1573.997 & 1575.858 & 1575.649 & 1568.582 & 1565.577 & 1571.318 \\
\hline $10 / 22 / 164: 00$ & 74.53 & 81.027 & 79.308 & 88.067 & 72.974 & 73.037 & 71.711 & 72.568 & 1583.942 & 1576.826 & 1574 & 1575.862 & 1575.654 & 1568.584 & 1565.58 & 1571.327 \\
\hline $10 / 22 / 165: 00$ & 74.53 & 81.023 & 79.304 & 88.062 & 72.967 & 73.032 & 71.709 & 72.565 & 1583.942 & 1576.83 & 1574.004 & 1575.867 & 1575.661 & 1568.589 & 1565.582 & 1571.33 \\
\hline $10 / 22 / 166: 00$ & 74.534 & 81.032 & 79.308 & 88.069 & 72.974 & 73.039 & 71.716 & 72.572 & 1583.938 & 1576.821 & 1574 & 1575.86 & 1575.654 & 1568.582 & 1565.575 & 1571.323 \\
\hline $10 / 22 / 167: 00$ & 74.539 & 81.032 & 79.311 & 88.069 & 72.979 & 73.041 & 71.718 & 72.565 & 1583.933 & 1576.821 & 1573.997 & 1575.86 & 1575.649 & 1568.58 & 1565.573 & 1571.33 \\
\hline $10 / 22 / 168: 00$ & 74.539 & 81.039 & 79.313 & 88.071 & 72.976 & 73.041 & 71.721 & 72.575 & 1583.933 & 1576.814 & 1573.995 & 1575.858 & 1575.652 & 1568.58 & 1565.57 & 1571.32 \\
\hline $10 / 22 / 169: 00$ & 74.544 & 81.046 & 79.318 & 88.074 & 72.981 & 73.048 & 71.726 & 72.582 & 1583.928 & 1576.807 & 1573.99 & 1575.855 & 1575.647 & 1568.573 & 1565.565 & 1571.313 \\
\hline $10 / 22 / 1610: 00$ & 74.544 & 81.046 & 79.32 & 88.074 & 72.981 & 73.048 & 71.728 & 72.584 & 1583.928 & 1576.807 & 1573.988 & 1575.855 & 1575.647 & 1568.573 & 1565.563 & 1571.311 \\
\hline 10/22/16 11:00 & 74.541 & 81.05 & 79.318 & 88.074 & 72.981 & 73.039 & 71.728 & 72.575 & 1583.931 & 1576.803 & 1573.99 & 1575.855 & 1575.647 & 1568.582 & 1565.563 & 1571.32 \\
\hline $10 / 22 / 1612: 00$ & 74.52 & 81.02 & 79.297 & 88.055 & 72.958 & 73.025 & 71.699 & 72.563 & 1583.952 & 1576.833 & 1574.011 & 1575.874 & 1575.67 & 1568.596 & 1565.592 & 1571.332 \\
\hline $10 / 22 / 1613: 00$ & 74.506 & 81.011 & 79.285 & 88.053 & 72.941 & 73.013 & 71.685 & 72.558 & 1583.966 & 1576.842 & 1574.023 & 1575.876 & 1575.687 & 1568.608 & 1565.606 & 1571.337 \\
\hline $10 / 22 / 1614: 00$ & 74.482 & 80.976 & 79.259 & 88.027 & 72.909 & 72.99 & 71.647 & 72.535 & 1583.99 & 1576.877 & 1574.049 & 1575.902 & 1575.719 & 1568.631 & 1565.644 & 1571.36 \\
\hline $10 / 22 / 1615: 00$ & 74.459 & 80.939 & 79.227 & 88.011 & 72.876 & 72.969 & 71.613 & 72.507 & 1584.013 & 1576.914 & 1574.081 & 1575.918 & 1575.752 & 1568.652 & 1565.678 & 1571.388 \\
\hline $10 / 22 / 1616: 00$ & 74.454 & 80.923 & 79.213 & 88.006 & 72.857 & 72.969 & 71.604 & 72.503 & 1584.018 & 1576.93 & 1574.095 & 1575.923 & 1575.771 & 1568.652 & 1565.687 & 1571.392 \\
\hline $10 / 22 / 1617: 00$ & 74.461 & 80.92 & 79.208 & 88.011 & 72.85 & 72.967 & 71.604 & 72.484 & 1584.011 & 1576.933 & 1574.1 & 1575.918 & 1575.778 & 1568.654 & 1565.687 & 1571.411 \\
\hline $10 / 22 / 1618: 00$ & 74.468 & 80.913 & 79.203 & 88.013 & 72.848 & 72.969 & 71.606 & 72.479 & 1584.004 & 1576.94 & 1574.105 & 1575.916 & 1575.78 & 1568.652 & 1565.685 & 1571.416 \\
\hline $10 / 22 / 1619: 00$ & 74.494 & 80.936 & 79.217 & 88.027 & 72.862 & 72.985 & 71.632 & 72.491 & 1583.978 & 1576.917 & 1574.091 & 1575.902 & 1575.766 & 1568.636 & 1565.659 & 1571.404 \\
\hline $10 / 22 / 1620: 00$ & 74.506 & 80.946 & 79.236 & 88.032 & 72.876 & 72.997 & 71.644 & 72.505 & 1583.966 & 1576.907 & 1574.072 & 1575.897 & 1575.752 & 1568.624 & 1565.647 & 1571.39 \\
\hline $10 / 22 / 1621: 00$ & 74.508 & 80.946 & 79.234 & 88.029 & 72.876 & 72.987 & 71.639 & 72.484 & 1583.964 & 1576.907 & 1574.074 & 1575.9 & 1575.752 & 1568.634 & 1565.652 & 1571.411 \\
\hline
\end{tabular}


TABLE S1.1 Automatically recorded groundwater levels in the unconfined aquifer.

\begin{tabular}{|c|c|c|c|c|c|c|c|c|c|c|c|c|c|c|c|c|}
\hline \multirow[b]{2}{*}{ Date and Time } & \multicolumn{8}{|c|}{ Measured Depth to Water ( $\mathrm{ft}$ TOC ${ }^{\mathrm{a}}$ ) } & \multicolumn{8}{|c|}{ Groundwater Elevation ( $\mathrm{ft} A M S L^{\mathrm{b}}$ ) } \\
\hline & DL1-C & DL2-C1 & DL3-C1 & DL4-C1 & DL5-C1 & DL6-C & DL7-C & DL8-C1 & DL1-C & DL2-C1 & DL3-C1 & DL4-C1 & DL5-C1 & DL6-C & DL7-C & DL8-C1 \\
\hline $10 / 22 / 1622: 00$ & 74.501 & 80.939 & 79.227 & 88.025 & 72.867 & 72.987 & 71.637 & 72.496 & 1583.971 & 1576.914 & 1574.081 & 1575.904 & 1575.761 & 1568.634 & 1565.654 & 1571.399 \\
\hline $10 / 22 / 1623: 00$ & 74.506 & 80.946 & 79.231 & 88.027 & 72.874 & 72.99 & 71.642 & 72.505 & 1583.966 & 1576.907 & 1574.077 & 1575.902 & 1575.754 & 1568.631 & 1565.649 & 1571.39 \\
\hline $10 / 23 / 160: 00$ & 74.506 & 80.948 & 79.234 & 88.027 & 72.874 & 72.983 & 71.642 & 72.493 & 1583.966 & 1576.905 & 1574.074 & 1575.902 & 1575.754 & 1568.638 & 1565.649 & 1571.402 \\
\hline 10/23/16 1:00 & 74.506 & 80.95 & 79.231 & 88.029 & 72.876 & 72.987 & 71.642 & 72.503 & 1583.966 & 1576.903 & 1574.077 & 1575.9 & 1575.752 & 1568.634 & 1565.649 & 1571.392 \\
\hline $10 / 23 / 162: 00$ & 74.52 & 80.964 & 79.241 & 88.036 & 72.885 & 72.999 & 71.656 & 72.505 & 1583.952 & 1576.889 & 1574.067 & 1575.893 & 1575.743 & 1568.622 & 1565.635 & 1571.39 \\
\hline 10/23/16 3:00 & 74.539 & 80.995 & 79.264 & 88.053 & 72.911 & 73.025 & 71.685 & 72.507 & 1583.933 & 1576.858 & 1574.044 & 1575.876 & 1575.717 & 1568.596 & 1565.606 & 1571.388 \\
\hline 10/23/16 4:00 & 74.558 & 81.015 & 79.294 & 88.067 & 72.939 & 73.029 & 71.709 & 72.558 & 1583.914 & 1576.838 & 1574.014 & 1575.862 & 1575.689 & 1568.592 & 1565.582 & 1571.337 \\
\hline 10/23/16 5:00 & 74.567 & 81.036 & 79.306 & 88.076 & 72.953 & 73.043 & 71.725 & 72.579 & 1583.905 & 1576.817 & 1574.002 & 1575.853 & 1575.675 & 1568.578 & 1565.566 & 1571.316 \\
\hline 10/23/16 6:00 & 74.584 & 81.067 & 79.329 & 88.092 & 72.983 & 73.062 & 71.757 & 72.602 & 1583.888 & 1576.786 & 1573.979 & 1575.837 & 1575.645 & 1568.559 & 1565.534 & 1571.293 \\
\hline 10/23/16 7:00 & 74.603 & 81.102 & 79.367 & 88.111 & 73.014 & 73.081 & 71.783 & 72.644 & 1583.869 & 1576.751 & 1573.941 & 1575.818 & 1575.614 & 1568.54 & 1565.508 & 1571.251 \\
\hline 10/23/16 8:00 & 74.619 & 81.134 & 79.397 & 88.123 & 73.046 & 73.099 & 71.814 & 72.677 & 1583.853 & 1576.719 & 1573.911 & 1575.806 & 1575.582 & 1568.522 & 1565.477 & 1571.218 \\
\hline 10/23/16 9:00 & 74.631 & 81.162 & 79.418 & 88.134 & 73.074 & 73.113 & 71.833 & 72.693 & 1583.841 & 1576.691 & 1573.89 & 1575.795 & 1575.554 & 1568.508 & 1565.458 & 1571.202 \\
\hline $10 / 23 / 1610: 00$ & 74.636 & 81.181 & 79.439 & 88.144 & 73.098 & 73.123 & 71.85 & 72.716 & 1583.836 & 1576.672 & 1573.869 & 1575.785 & 1575.53 & 1568.498 & 1565.441 & 1571.179 \\
\hline 10/23/16 11:00 & 74.638 & 81.202 & 79.451 & 88.151 & 73.114 & 73.127 & 71.864 & 72.725 & 1583.834 & 1576.651 & 1573.857 & 1575.778 & 1575.514 & 1568.494 & 1565.427 & 1571.17 \\
\hline $10 / 23 / 1612: 00$ & 74.629 & 81.209 & 79.455 & 88.148 & 73.123 & 73.127 & 71.864 & 72.73 & 1583.843 & 1576.644 & 1573.853 & 1575.781 & 1575.505 & 1568.494 & 1565.427 & 1571.165 \\
\hline $10 / 23 / 1613: 00$ & 74.621 & 81.216 & 79.465 & 88.148 & 73.133 & 73.125 & 71.862 & 72.749 & 1583.851 & 1576.637 & 1573.843 & 1575.781 & 1575.495 & 1568.496 & 1565.429 & 1571.146 \\
\hline $10 / 23 / 1614: 00$ & 74.603 & 81.202 & 79.455 & 88.134 & 73.123 & 73.111 & 71.845 & 72.735 & 1583.869 & 1576.651 & 1573.853 & 1575.795 & 1575.505 & 1568.51 & 1565.446 & 1571.16 \\
\hline $10 / 23 / 1615: 00$ & 74.588 & 81.195 & 79.446 & 88.13 & 73.116 & 73.104 & 71.833 & 72.721 & 1583.884 & 1576.658 & 1573.862 & 1575.799 & 1575.512 & 1568.517 & 1565.458 & 1571.174 \\
\hline $10 / 23 / 1616: 00$ & 74.586 & 81.197 & 79.446 & 88.132 & 73.119 & 73.109 & 71.835 & 72.725 & 1583.886 & 1576.656 & 1573.862 & 1575.797 & 1575.509 & 1568.512 & 1565.456 & 1571.17 \\
\hline 10/23/16 17:00 & 74.596 & 81.211 & 79.458 & 88.139 & 73.133 & 73.118 & 71.847 & 72.735 & 1583.876 & 1576.642 & 1573.85 & 1575.79 & 1575.495 & 1568.503 & 1565.444 & 1571.16 \\
\hline $10 / 23 / 1618: 00$ & 74.596 & 81.218 & 79.462 & 88.141 & 73.137 & 73.118 & 71.852 & 72.742 & 1583.876 & 1576.635 & 1573.846 & 1575.788 & 1575.491 & 1568.503 & 1565.439 & 1571.153 \\
\hline 10/23/16 19:00 & 74.598 & 81.225 & 79.472 & 88.146 & 73.147 & 73.125 & 71.857 & 72.753 & 1583.874 & 1576.628 & 1573.836 & 1575.783 & 1575.481 & 1568.496 & 1565.434 & 1571.142 \\
\hline $10 / 23 / 1620: 00$ & 74.603 & 81.234 & 79.478 & 88.151 & 73.156 & 73.13 & 71.866 & 72.758 & 1583.869 & 1576.619 & 1573.83 & 1575.778 & 1575.472 & 1568.491 & 1565.425 & 1571.137 \\
\hline 10/23/16 21:00 & 74.605 & 81.246 & 79.488 & 88.155 & 73.167 & 73.137 & 71.871 & 72.767 & 1583.867 & 1576.607 & 1573.82 & 1575.774 & 1575.461 & 1568.484 & 1565.42 & 1571.128 \\
\hline $10 / 23 / 1622: 00$ & 74.607 & 81.25 & 79.497 & 88.158 & 73.181 & 73.141 & 71.883 & 72.777 & 1583.865 & 1576.603 & 1573.811 & 1575.771 & 1575.447 & 1568.48 & 1565.408 & 1571.118 \\
\hline $10 / 23 / 1623: 00$ & 74.612 & 81.262 & 79.506 & 88.162 & 73.186 & 73.144 & 71.885 & 72.786 & 1583.86 & 1576.591 & 1573.802 & 1575.767 & 1575.442 & 1568.477 & 1565.406 & 1571.109 \\
\hline $10 / 24 / 160: 00$ & 74.612 & 81.271 & 79.513 & 88.165 & 73.193 & 73.148 & 71.895 & 72.793 & 1583.86 & 1576.582 & 1573.795 & 1575.764 & 1575.435 & 1568.473 & 1565.396 & 1571.102 \\
\hline 10/24/16 1:00 & 74.607 & 81.269 & 79.516 & 88.162 & 73.2 & 73.144 & 71.895 & 72.802 & 1583.865 & 1576.584 & 1573.792 & 1575.767 & 1575.428 & 1568.477 & 1565.396 & 1571.093 \\
\hline 10/24/16 2:00 & 74.603 & 81.276 & 79.52 & 88.162 & 73.2 & 73.146 & 71.897 & 72.804 & 1583.869 & 1576.577 & 1573.788 & 1575.767 & 1575.428 & 1568.475 & 1565.394 & 1571.091 \\
\hline 10/24/16 3:00 & 74.617 & 81.292 & 79.534 & 88.174 & 73.216 & 73.158 & 71.909 & 72.814 & 1583.855 & 1576.561 & 1573.774 & 1575.755 & 1575.412 & 1568.463 & 1565.382 & 1571.081 \\
\hline 10/24/16 4:00 & 74.607 & 81.283 & 79.527 & 88.165 & 73.214 & 73.15 & 71.897 & 72.814 & 1583.865 & 1576.57 & 1573.781 & 1575.764 & 1575.414 & 1568.471 & 1565.394 & 1571.081 \\
\hline $10 / 24 / 165: 00$ & 74.603 & 81.285 & 79.53 & 88.167 & 73.216 & 73.15 & 71.902 & 72.809 & 1583.869 & 1576.568 & 1573.778 & 1575.762 & 1575.412 & 1568.471 & 1565.389 & 1571.086 \\
\hline $10 / 24 / 166: 00$ & 74.605 & 81.288 & 79.532 & 88.17 & 73.219 & 73.15 & 71.9 & 72.816 & 1583.867 & 1576.565 & 1573.776 & 1575.759 & 1575.409 & 1568.471 & 1565.391 & 1571.079 \\
\hline 10/24/16 7:00 & 74.6 & 81.283 & 79.534 & 88.165 & 73.216 & 73.146 & 71.895 & 72.823 & 1583.872 & 1576.57 & 1573.774 & 1575.764 & 1575.412 & 1568.475 & 1565.396 & 1571.072 \\
\hline 10/24/16 8:00 & 74.598 & 81.285 & 79.539 & 88.165 & 73.221 & 73.15 & 71.897 & 72.835 & 1583.874 & 1576.568 & 1573.769 & 1575.764 & 1575.407 & 1568.471 & 1565.394 & 1571.06 \\
\hline 10/24/16 9:00 & 74.6 & 81.288 & 79.541 & 88.167 & 73.223 & 73.153 & 71.9 & 72.828 & 1583.872 & 1576.565 & 1573.767 & 1575.762 & 1575.405 & 1568.468 & 1565.391 & 1571.067 \\
\hline $10 / 24 / 1610: 00$ & 74.596 & 81.288 & 79.544 & 88.165 & 73.223 & 73.15 & 71.895 & 72.823 & 1583.876 & 1576.565 & 1573.764 & 1575.764 & 1575.405 & 1568.471 & 1565.396 & 1571.072 \\
\hline $10 / 24 / 1611: 00$ & 74.586 & 81.276 & 79.534 & 88.153 & 73.212 & 73.139 & 71.883 & 72.814 & 1583.886 & 1576.577 & 1573.774 & 1575.776 & 1575.416 & 1568.482 & 1565.408 & 1571.081 \\
\hline $10 / 24 / 1612: 00$ & 74.567 & 81.253 & 79.511 & 88.141 & 73.193 & 73.118 & 71.862 & 72.749 & 1583.905 & 1576.6 & 1573.797 & 1575.788 & 1575.435 & 1568.503 & 1565.429 & 1571.146 \\
\hline $10 / 24 / 1613: 00$ & 74.548 & 81.23 & 79.492 & 88.127 & 73.172 & 73.104 & 71.833 & 72.779 & 1583.924 & 1576.623 & 1573.816 & 1575.802 & 1575.456 & 1568.517 & 1565.458 & 1571.116 \\
\hline $10 / 24 / 1614: 00$ & 74.525 & 81.192 & 79.462 & 88.106 & 73.14 & 73.085 & 71.797 & 72.765 & 1583.947 & 1576.661 & 1573.846 & 1575.823 & 1575.488 & 1568.536 & 1565.494 & 1571.13 \\
\hline $10 / 24 / 1615: 00$ & 74.508 & 81.167 & 79.434 & 88.097 & 73.114 & 73.069 & 71.771 & 72.728 & 1583.964 & 1576.686 & 1573.874 & 1575.832 & 1575.514 & 1568.552 & 1565.52 & 1571.167 \\
\hline $10 / 24 / 1616: 00$ & 74.492 & 81.141 & 79.406 & 88.083 & 73.091 & 73.055 & 71.749 & 72.698 & 1583.98 & 1576.712 & 1573.902 & 1575.846 & 1575.537 & 1568.566 & 1565.542 & 1571.197 \\
\hline $10 / 24 / 1617: 00$ & 74.494 & 81.13 & 79.397 & 88.083 & 73.074 & 73.053 & 71.745 & 72.681 & 1583.978 & 1576.723 & 1573.911 & 1575.846 & 1575.554 & 1568.568 & 1565.546 & 1571.214 \\
\hline $10 / 24 / 1618: 00$ & 74.494 & 81.116 & 79.385 & 88.071 & 73.06 & 73.046 & 71.73 & 72.665 & 1583.978 & 1576.737 & 1573.923 & 1575.858 & 1575.568 & 1568.575 & 1565.561 & 1571.23 \\
\hline $10 / 24 / 1619: 00$ & 74.499 & 81.102 & 79.378 & 88.071 & 73.053 & 73.043 & 71.723 & 72.658 & 1583.973 & 1576.751 & 1573.93 & 1575.858 & 1575.575 & 1568.578 & 1565.568 & 1571.237 \\
\hline $10 / 24 / 1620: 00$ & 74.513 & 81.106 & 79.376 & 88.078 & 73.051 & 73.05 & 71.735 & 72.609 & 1583.959 & 1576.747 & 1573.932 & 1575.851 & 1575.577 & 1568.571 & 1565.556 & 1571.286 \\
\hline $10 / 24 / 1621: 00$ & 74.508 & 81.095 & 79.367 & 88.071 & 73.039 & 73.041 & 71.723 & 72.649 & 1583.964 & 1576.758 & 1573.941 & 1575.858 & 1575.589 & 1568.58 & 1565.568 & 1571.246 \\
\hline $10 / 24 / 1622: 00$ & 74.513 & 81.097 & 79.367 & 88.074 & 73.037 & 73.041 & 71.726 & 72.644 & 1583.959 & 1576.756 & 1573.941 & 1575.855 & 1575.591 & 1568.58 & 1565.565 & 1571.251 \\
\hline
\end{tabular}


TABLE S1.1 Automatically recorded groundwater levels in the unconfined aquifer.

\begin{tabular}{|c|c|c|c|c|c|c|c|c|c|c|c|c|c|c|c|c|}
\hline \multirow[b]{2}{*}{ Date and Time } & \multicolumn{8}{|c|}{ Measured Depth to Water (ft TOC ${ }^{\mathrm{a}}$ ) } & \multicolumn{8}{|c|}{ Groundwater Elevation (ft AMSL) } \\
\hline & DL1-C & DL2-C1 & DL3-C1 & DL4-C1 & DL5-C1 & DL6-C & DL7-C & DL8-C1 & DL1-C & DL2-C1 & DL3-C1 & DL4-C1 & DL5-C1 & DL6-C & DL7-C & DL8-C1 \\
\hline 10/24/16 23:00 & 74.515 & 81.095 & 79.362 & 88.071 & 73.032 & 73.041 & 71.723 & 72.64 & 1583.957 & 1576.758 & 1573.946 & 1575.858 & 1575.596 & 1568.58 & 1565.568 & 1571.255 \\
\hline 10/25/16 0:00 & 74.508 & 81.081 & 79.353 & 88.062 & 73.023 & 73.032 & 71.714 & 72.633 & 1583.964 & 1576.772 & 1573.955 & 1575.867 & 1575.605 & 1568.589 & 1565.577 & 1571.262 \\
\hline 10/25/16 1:00 & 74.506 & 81.069 & 79.341 & 88.055 & 73.009 & 73.025 & 71.701 & 72.619 & 1583.966 & 1576.784 & 1573.967 & 1575.874 & 1575.619 & 1568.596 & 1565.59 & 1571.276 \\
\hline $10 / 25 / 162: 00$ & 74.499 & 81.064 & 79.332 & 88.05 & 72.997 & 73.023 & 71.697 & 72.612 & 1583.973 & 1576.789 & 1573.976 & 1575.879 & 1575.631 & 1568.598 & 1565.594 & 1571.283 \\
\hline 10/25/16 3:00 & 74.487 & 81.043 & 79.315 & 88.041 & 72.981 & 73.008 & 71.682 & 72.591 & 1583.985 & 1576.81 & 1573.993 & 1575.888 & 1575.647 & 1568.613 & 1565.609 & 1571.304 \\
\hline $10 / 25 / 164: 00$ & 74.475 & 81.018 & 79.294 & 88.029 & 72.958 & 72.992 & 71.654 & 72.572 & 1583.997 & 1576.835 & 1574.014 & 1575.9 & 1575.67 & 1568.629 & 1565.637 & 1571.323 \\
\hline $10 / 25 / 165: 00$ & 74.473 & 81.011 & 79.285 & 88.027 & 72.946 & 72.992 & 71.651 & 72.556 & 1583.999 & 1576.842 & 1574.023 & 1575.902 & 1575.682 & 1568.629 & 1565.64 & 1571.339 \\
\hline $10 / 25 / 166: 00$ & 74.478 & 81.004 & 79.278 & 88.027 & 72.941 & 72.987 & 71.647 & 72.554 & 1583.994 & 1576.849 & 1574.03 & 1575.902 & 1575.687 & 1568.634 & 1565.644 & 1571.341 \\
\hline 10/25/16 7:00 & 74.478 & 80.997 & 79.276 & 88.022 & 72.927 & 72.985 & 71.639 & 72.554 & 1583.994 & 1576.856 & 1574.032 & 1575.907 & 1575.701 & 1568.636 & 1565.652 & 1571.341 \\
\hline $10 / 25 / 168: 00$ & 74.473 & 80.983 & 79.266 & 88.015 & 72.916 & 72.976 & 71.63 & 72.542 & 1583.999 & 1576.87 & 1574.042 & 1575.914 & 1575.712 & 1568.645 & 1565.661 & 1571.353 \\
\hline 10/25/16 9:00 & 74.478 & 80.976 & 79.255 & 88.018 & 72.911 & 72.98 & 71.632 & 72.53 & 1583.994 & 1576.877 & 1574.053 & 1575.911 & 1575.717 & 1568.641 & 1565.659 & 1571.365 \\
\hline $10 / 25 / 1610: 00$ & 74.485 & 80.981 & 79.259 & 88.02 & 72.909 & 72.98 & 71.635 & 72.528 & 1583.987 & 1576.872 & 1574.049 & 1575.909 & 1575.719 & 1568.641 & 1565.656 & 1571.367 \\
\hline 10/25/16 11:00 & 74.468 & 80.96 & 79.238 & 88.006 & 72.89 & 72.962 & 71.613 & 72.507 & 1584.004 & 1576.893 & 1574.07 & 1575.923 & 1575.738 & 1568.659 & 1565.678 & 1571.388 \\
\hline $10 / 25 / 1612: 00$ & 74.459 & 80.939 & 79.222 & 87.994 & 72.867 & 72.95 & 71.594 & 72.486 & 1584.013 & 1576.914 & 1574.086 & 1575.935 & 1575.761 & 1568.671 & 1565.697 & 1571.409 \\
\hline $10 / 25 / 1613: 00$ & 74.442 & 80.916 & 79.196 & 87.98 & 72.841 & 72.932 & 71.566 & 72.461 & 1584.03 & 1576.937 & 1574.112 & 1575.949 & 1575.787 & 1568.689 & 1565.725 & 1571.434 \\
\hline $10 / 25 / 1614: 00$ & 74.424 & 80.883 & 79.166 & 87.964 & 72.806 & 72.913 & 71.537 & 72.431 & 1584.048 & 1576.97 & 1574.142 & 1575.965 & 1575.822 & 1568.708 & 1565.754 & 1571.464 \\
\hline $10 / 25 / 1615: 00$ & 74.416 & 80.862 & 79.145 & 87.957 & 72.785 & 72.904 & 71.52 & 72.407 & 1584.056 & 1576.991 & 1574.163 & 1575.972 & 1575.843 & 1568.717 & 1565.771 & 1571.488 \\
\hline $10 / 25 / 1616: 00$ & 74.409 & 80.839 & 79.124 & 87.948 & 72.759 & 72.89 & 71.504 & 72.396 & 1584.063 & 1577.014 & 1574.184 & 1575.981 & 1575.869 & 1568.731 & 1565.787 & 1571.499 \\
\hline $10 / 25 / 1617: 00$ & 74.416 & 80.832 & 79.122 & 87.95 & 72.752 & 72.892 & 71.506 & 72.391 & 1584.056 & 1577.021 & 1574.186 & 1575.979 & 1575.876 & 1568.729 & 1565.785 & 1571.504 \\
\hline $10 / 25 / 1618: 00$ & 74.424 & 80.829 & 79.115 & 87.95 & 72.743 & 72.892 & 71.504 & 72.38 & 1584.048 & 1577.024 & 1574.193 & 1575.979 & 1575.885 & 1568.729 & 1565.787 & 1571.515 \\
\hline 10/25/16 19:00 & 74.442 & 80.843 & 79.122 & 87.964 & 72.755 & 72.908 & 71.527 & 72.384 & 1584.03 & 1577.01 & 1574.186 & 1575.965 & 1575.873 & 1568.713 & 1565.764 & 1571.511 \\
\hline $10 / 25 / 1620: 00$ & 74.468 & 80.867 & 79.145 & 87.98 & 72.778 & 72.925 & 71.556 & 72.405 & 1584.004 & 1576.986 & 1574.163 & 1575.949 & 1575.85 & 1568.696 & 1565.735 & 1571.49 \\
\hline $10 / 25 / 1621: 00$ & 74.482 & 80.878 & 79.155 & 87.985 & 72.783 & 72.929 & 71.565 & 72.412 & 1583.99 & 1576.975 & 1574.153 & 1575.944 & 1575.845 & 1568.692 & 1565.726 & 1571.483 \\
\hline 10/25/16 22:00 & 74.49 & 80.888 & 79.164 & 87.985 & 72.792 & 72.934 & 71.575 & 72.421 & 1583.982 & 1576.965 & 1574.144 & 1575.944 & 1575.836 & 1568.687 & 1565.716 & 1571.474 \\
\hline $10 / 25 / 1623: 00$ & 74.475 & 80.871 & 79.152 & 87.973 & 72.778 & 72.92 & 71.558 & 72.412 & 1583.997 & 1576.982 & 1574.156 & 1575.956 & 1575.85 & 1568.701 & 1565.733 & 1571.483 \\
\hline 10/26/16 0:00 & 74.494 & 80.899 & 79.166 & 87.992 & 72.797 & 72.939 & 71.582 & 72.419 & 1583.978 & 1576.954 & 1574.142 & 1575.937 & 1575.831 & 1568.682 & 1565.709 & 1571.476 \\
\hline $10 / 26 / 161: 00$ & 74.499 & 80.909 & 79.175 & 87.994 & 72.806 & 72.941 & 71.589 & 72.428 & 1583.973 & 1576.944 & 1574.133 & 1575.935 & 1575.822 & 1568.68 & 1565.702 & 1571.467 \\
\hline $10 / 26 / 162: 00$ & 74.499 & 80.918 & 79.185 & 87.997 & 72.813 & 72.943 & 71.601 & 72.433 & 1583.973 & 1576.935 & 1574.123 & 1575.932 & 1575.815 & 1568.678 & 1565.69 & 1571.462 \\
\hline 10/26/16 3:00 & 74.513 & 80.936 & 79.196 & 88.008 & 72.827 & 72.955 & 71.618 & 72.456 & 1583.959 & 1576.917 & 1574.112 & 1575.921 & 1575.801 & 1568.666 & 1565.673 & 1571.439 \\
\hline $10 / 26 / 164: 00$ & 74.53 & 80.962 & 79.217 & 88.02 & 72.848 & 72.969 & 71.642 & 72.475 & 1583.942 & 1576.891 & 1574.091 & 1575.909 & 1575.78 & 1568.652 & 1565.649 & 1571.42 \\
\hline $10 / 26 / 165: 00$ & 74.544 & 80.99 & 79.243 & 88.034 & 72.876 & 72.987 & 71.666 & 72.505 & 1583.928 & 1576.863 & 1574.065 & 1575.895 & 1575.752 & 1568.634 & 1565.625 & 1571.39 \\
\hline $10 / 26 / 166: 00$ & 74.541 & 80.995 & 79.245 & 88.029 & 72.881 & 72.985 & 71.661 & 72.452 & 1583.931 & 1576.858 & 1574.063 & 1575.9 & 1575.747 & 1568.636 & 1565.63 & 1571.443 \\
\hline 10/26/16 7:00 & 74.546 & 81.006 & 79.266 & 88.036 & 72.892 & 72.992 & 71.675 & 72.53 & 1583.926 & 1576.847 & 1574.042 & 1575.893 & 1575.736 & 1568.629 & 1565.616 & 1571.365 \\
\hline $10 / 26 / 168: 00$ & 74.551 & 81.025 & 79.283 & 88.043 & 72.909 & 72.997 & 71.687 & 72.547 & 1583.921 & 1576.828 & 1574.025 & 1575.886 & 1575.719 & 1568.624 & 1565.604 & 1571.348 \\
\hline 10/26/16 9:00 & 74.565 & 81.053 & 79.301 & 88.06 & 72.937 & 73.011 & 71.713 & 72.558 & 1583.907 & 1576.8 & 1574.007 & 1575.869 & 1575.691 & 1568.61 & 1565.578 & 1571.337 \\
\hline $10 / 26 / 1610: 00$ & 74.579 & 81.076 & 79.322 & 88.071 & 72.958 & 73.032 & 71.735 & 72.584 & 1583.893 & 1576.777 & 1573.986 & 1575.858 & 1575.67 & 1568.589 & 1565.556 & 1571.311 \\
\hline 10/26/16 11:00 & 74.584 & 81.095 & 79.343 & 88.078 & 72.979 & 73.037 & 71.752 & 72.602 & 1583.888 & 1576.758 & 1573.965 & 1575.851 & 1575.649 & 1568.584 & 1565.539 & 1571.293 \\
\hline $10 / 26 / 1612: 00$ & 74.579 & 81.109 & 79.35 & 88.081 & 72.988 & 73.034 & 71.754 & 72.614 & 1583.893 & 1576.744 & 1573.958 & 1575.848 & 1575.64 & 1568.587 & 1565.537 & 1571.281 \\
\hline $10 / 26 / 1613: 00$ & 74.567 & 81.104 & 79.35 & 88.074 & 72.99 & 73.034 & 71.749 & 72.63 & 1583.905 & 1576.749 & 1573.958 & 1575.855 & 1575.638 & 1568.587 & 1565.542 & 1571.265 \\
\hline $10 / 26 / 1614: 00$ & 74.541 & 81.085 & 79.339 & 88.055 & 72.974 & 73.015 & 71.721 & 72.612 & 1583.931 & 1576.768 & 1573.969 & 1575.874 & 1575.654 & 1568.606 & 1565.57 & 1571.283 \\
\hline $10 / 26 / 1615: 00$ & 74.53 & 81.071 & 79.327 & 88.05 & 72.965 & 73.006 & 71.709 & 72.595 & 1583.942 & 1576.782 & 1573.981 & 1575.879 & 1575.663 & 1568.615 & 1565.582 & 1571.3 \\
\hline $10 / 26 / 1616: 00$ & 74.525 & 81.078 & 79.322 & 88.053 & 72.965 & 73.011 & 71.709 & 72.598 & 1583.947 & 1576.775 & 1573.986 & 1575.876 & 1575.663 & 1568.61 & 1565.582 & 1571.297 \\
\hline $10 / 26 / 1617: 00$ & 74.53 & 81.083 & 79.332 & 88.057 & 72.972 & 73.015 & 71.713 & 72.609 & 1583.942 & 1576.77 & 1573.976 & 1575.872 & 1575.656 & 1568.606 & 1565.578 & 1571.286 \\
\hline $10 / 26 / 1618: 00$ & 74.525 & 81.078 & 79.336 & 88.055 & 72.974 & 73.016 & 71.711 & 72.609 & 1583.947 & 1576.775 & 1573.972 & 1575.874 & 1575.654 & 1568.605 & 1565.58 & 1571.286 \\
\hline 10/26/16 19:00 & 74.541 & 81.097 & 79.35 & 88.071 & 72.993 & 73.027 & 71.73 & 72.626 & 1583.931 & 1576.756 & 1573.958 & 1575.858 & 1575.635 & 1568.594 & 1565.561 & 1571.269 \\
\hline $10 / 26 / 1620: 00$ & 74.551 & 81.111 & 79.362 & 88.074 & 73.004 & 73.032 & 71.742 & 72.64 & 1583.921 & 1576.742 & 1573.946 & 1575.855 & 1575.624 & 1568.589 & 1565.549 & 1571.255 \\
\hline $10 / 26 / 1621: 00$ & 74.553 & 81.118 & 79.371 & 88.078 & 73.016 & 73.041 & 71.749 & 72.651 & 1583.919 & 1576.735 & 1573.937 & 1575.851 & 1575.612 & 1568.58 & 1565.542 & 1571.244 \\
\hline $10 / 26 / 1622: 00$ & 74.56 & 81.132 & 79.383 & 88.085 & 73.03 & 73.053 & 71.764 & 72.665 & 1583.912 & 1576.721 & 1573.925 & 1575.844 & 1575.598 & 1568.568 & 1565.527 & 1571.23 \\
\hline $10 / 26 / 1623: 00$ & 74.56 & 81.136 & 79.39 & 88.085 & 73.039 & 73.057 & 71.761 & 72.679 & 1583.912 & 1576.717 & 1573.918 & 1575.844 & 1575.589 & 1568.564 & 1565.53 & 1571.216 \\
\hline
\end{tabular}


TABLE S1.1 Automatically recorded groundwater levels in the unconfined aquifer.

\begin{tabular}{|c|c|c|c|c|c|c|c|c|c|c|c|c|c|c|c|c|}
\hline \multirow[b]{2}{*}{ Date and Time } & \multicolumn{8}{|c|}{ Measured Depth to Water (ft TOC ${ }^{\mathrm{a}}$ ) } & \multicolumn{8}{|c|}{ Groundwater Elevation (ft AMSL') } \\
\hline & DL1-C & DL2-C1 & DL3-C1 & DL4-C1 & DL5-C1 & DL6-C & DL7-C & DL8-C1 & DL1-C & DL2-C1 & DL3-C1 & DL4-C1 & DL5-C1 & DL6-C & DL7-C & DL8-C1 \\
\hline $10 / 27 / 160: 00$ & 74.558 & 81.136 & 79.395 & 88.081 & 73.044 & 73.046 & 71.761 & 72.681 & 1583.914 & 1576.717 & 1573.913 & 1575.848 & 1575.584 & 1568.575 & 1565.53 & 1571.214 \\
\hline 10/27/16 1:00 & 74.546 & 81.132 & 79.385 & 88.078 & 73.039 & 73.044 & 71.752 & 72.672 & 1583.926 & 1576.721 & 1573.923 & 1575.851 & 1575.589 & 1568.577 & 1565.539 & 1571.223 \\
\hline 10/27/16 2:00 & 74.537 & 81.125 & 79.383 & 88.071 & 73.035 & 73.039 & 71.742 & 72.667 & 1583.935 & 1576.728 & 1573.925 & 1575.858 & 1575.593 & 1568.582 & 1565.549 & 1571.228 \\
\hline 10/27/16 3:00 & 74.527 & 81.118 & 79.376 & 88.069 & 73.03 & 73.032 & 71.735 & 72.663 & 1583.945 & 1576.735 & 1573.932 & 1575.86 & 1575.598 & 1568.589 & 1565.556 & 1571.232 \\
\hline $10 / 27 / 164: 00$ & 74.513 & 81.102 & 79.362 & 88.06 & 73.018 & 73.022 & 71.718 & 72.656 & 1583.959 & 1576.751 & 1573.946 & 1575.869 & 1575.61 & 1568.599 & 1565.573 & 1571.239 \\
\hline $10 / 27 / 165: 00$ & 74.511 & 81.102 & 79.36 & 88.057 & 73.014 & 73.023 & 71.716 & 72.651 & 1583.961 & 1576.751 & 1573.948 & 1575.872 & 1575.614 & 1568.598 & 1565.575 & 1571.244 \\
\hline $10 / 27 / 166: 00$ & 74.511 & 81.099 & 79.36 & 88.06 & 73.014 & 73.022 & 71.716 & 72.647 & 1583.961 & 1576.754 & 1573.948 & 1575.869 & 1575.614 & 1568.599 & 1565.575 & 1571.248 \\
\hline $10 / 27 / 167: 00$ & 74.508 & 81.09 & 79.357 & 88.05 & 73.007 & 73.018 & 71.704 & 72.654 & 1583.964 & 1576.763 & 1573.951 & 1575.879 & 1575.621 & 1568.603 & 1565.587 & 1571.241 \\
\hline $10 / 27 / 168: 00$ & 74.506 & 81.088 & 79.36 & 88.053 & 73.007 & 73.015 & 71.704 & 72.647 & 1583.966 & 1576.765 & 1573.948 & 1575.876 & 1575.621 & 1568.606 & 1565.587 & 1571.248 \\
\hline 10/27/16 9:00 & 74.511 & 81.09 & 79.357 & 88.057 & 73.009 & 73.018 & 71.706 & 72.642 & 1583.961 & 1576.763 & 1573.951 & 1575.872 & 1575.619 & 1568.603 & 1565.585 & 1571.253 \\
\hline 10/27/16 10:00 & 74.508 & 81.085 & 79.353 & 88.05 & 73.004 & 73.018 & 71.699 & 72.644 & 1583.964 & 1576.768 & 1573.955 & 1575.879 & 1575.624 & 1568.603 & 1565.592 & 1571.251 \\
\hline 10/27/16 11:00 & 74.499 & 81.071 & 79.341 & 88.041 & 72.993 & 73.009 & 71.683 & 72.635 & 1583.973 & 1576.782 & 1573.967 & 1575.888 & 1575.635 & 1568.612 & 1565.608 & 1571.26 \\
\hline 10/27/16 12:00 & 74.489 & 81.053 & 79.327 & 88.034 & 72.979 & 72.995 & 71.666 & 72.619 & 1583.983 & 1576.8 & 1573.981 & 1575.895 & 1575.649 & 1568.626 & 1565.625 & 1571.276 \\
\hline 10/27/16 13:00 & 74.473 & 81.032 & 79.306 & 88.02 & 72.955 & 72.978 & 71.642 & 72.595 & 1583.999 & 1576.821 & 1574.002 & 1575.909 & 1575.673 & 1568.643 & 1565.649 & 1571.3 \\
\hline 10/27/16 14:00 & 74.464 & 81.011 & 79.287 & 88.008 & 72.939 & 72.967 & 71.623 & 72.575 & 1584.008 & 1576.842 & 1574.021 & 1575.921 & 1575.689 & 1568.654 & 1565.668 & 1571.32 \\
\hline 10/27/16 15:00 & 74.447 & 80.99 & 79.269 & 87.997 & 72.916 & 72.955 & 71.601 & 72.558 & 1584.025 & 1576.863 & 1574.039 & 1575.932 & 1575.712 & 1568.666 & 1565.69 & 1571.337 \\
\hline 10/27/16 16:00 & 74.442 & 80.974 & 79.25 & 87.994 & 72.902 & 72.95 & 71.594 & 72.551 & 1584.03 & 1576.879 & 1574.058 & 1575.935 & 1575.726 & 1568.671 & 1565.697 & 1571.344 \\
\hline $10 / 27 / 1617: 00$ & 74.447 & 80.969 & 79.252 & 87.99 & 72.892 & 72.953 & 71.589 & 72.551 & 1584.025 & 1576.884 & 1574.056 & 1575.939 & 1575.736 & 1568.668 & 1565.702 & 1571.344 \\
\hline 10/27/16 18:00 & 74.449 & 80.962 & 79.243 & 87.99 & 72.883 & 72.948 & 71.582 & 72.544 & 1584.023 & 1576.891 & 1574.065 & 1575.939 & 1575.745 & 1568.673 & 1565.709 & 1571.351 \\
\hline 10/27/16 19:00 & 74.461 & 80.967 & 79.25 & 87.997 & 72.888 & 72.957 & 71.596 & 72.547 & 1584.011 & 1576.886 & 1574.058 & 1575.932 & 1575.74 & 1568.664 & 1565.695 & 1571.348 \\
\hline 10/27/16 20:00 & 74.468 & 80.969 & 79.248 & 87.999 & 72.89 & 72.957 & 71.601 & 72.521 & 1584.004 & 1576.884 & 1574.06 & 1575.93 & 1575.738 & 1568.664 & 1565.69 & 1571.374 \\
\hline 10/27/16 21:00 & 74.464 & 80.96 & 79.236 & 87.99 & 72.881 & 72.948 & 71.589 & 72.523 & 1584.008 & 1576.893 & 1574.072 & 1575.939 & 1575.747 & 1568.673 & 1565.702 & 1571.372 \\
\hline $10 / 27 / 1622: 00$ & 74.461 & 80.95 & 79.224 & 87.987 & 72.867 & 72.943 & 71.58 & 72.496 & 1584.011 & 1576.903 & 1574.084 & 1575.942 & 1575.761 & 1568.678 & 1565.711 & 1571.399 \\
\hline $10 / 27 / 1623: 00$ & 74.471 & 80.953 & 79.227 & 87.987 & 72.869 & 72.941 & 71.582 & 72.516 & 1584.001 & 1576.9 & 1574.081 & 1575.942 & 1575.759 & 1568.68 & 1565.709 & 1571.379 \\
\hline $10 / 28 / 160: 00$ & 74.464 & 80.943 & 79.217 & 87.983 & 72.86 & 72.939 & 71.573 & 72.498 & 1584.008 & 1576.91 & 1574.091 & 1575.946 & 1575.768 & 1568.682 & 1565.718 & 1571.397 \\
\hline 10/28/16 1:00 & 74.454 & 80.932 & 79.206 & 87.973 & 72.846 & 72.929 & 71.563 & 72.491 & 1584.018 & 1576.921 & 1574.102 & 1575.956 & 1575.782 & 1568.692 & 1565.728 & 1571.404 \\
\hline $10 / 28 / 162: 00$ & 74.445 & 80.916 & 79.187 & 87.966 & 72.832 & 72.918 & 71.551 & 72.472 & 1584.027 & 1576.937 & 1574.121 & 1575.963 & 1575.796 & 1568.703 & 1565.74 & 1571.423 \\
\hline 10/28/16 3:00 & 74.44 & 80.909 & 79.18 & 87.962 & 72.815 & 72.915 & 71.544 & 72.458 & 1584.032 & 1576.944 & 1574.128 & 1575.967 & 1575.813 & 1568.706 & 1565.747 & 1571.437 \\
\hline $10 / 28 / 164: 00$ & 74.438 & 80.899 & 79.171 & 87.962 & 72.808 & 72.908 & 71.537 & 72.452 & 1584.034 & 1576.954 & 1574.137 & 1575.967 & 1575.82 & 1568.713 & 1565.754 & 1571.443 \\
\hline $10 / 28 / 165: 00$ & 74.433 & 80.89 & 79.164 & 87.952 & 72.792 & 72.904 & 71.527 & 72.438 & 1584.039 & 1576.963 & 1574.144 & 1575.977 & 1575.836 & 1568.717 & 1565.764 & 1571.457 \\
\hline $10 / 28 / 166: 00$ & 74.435 & 80.883 & 79.152 & 87.95 & 72.792 & 72.899 & 71.523 & 72.433 & 1584.037 & 1576.97 & 1574.156 & 1575.979 & 1575.836 & 1568.722 & 1565.768 & 1571.462 \\
\hline $10 / 28 / 167: 00$ & 74.433 & 80.874 & 79.148 & 87.945 & 72.771 & 72.894 & 71.515 & 72.435 & 1584.039 & 1576.979 & 1574.16 & 1575.984 & 1575.857 & 1568.727 & 1565.776 & 1571.46 \\
\hline $10 / 28 / 168: 00$ & 74.426 & 80.862 & 79.141 & 87.938 & 72.764 & 72.89 & 71.51 & 72.426 & 1584.046 & 1576.991 & 1574.167 & 1575.991 & 1575.864 & 1568.731 & 1565.781 & 1571.469 \\
\hline 10/28/16 9:00 & 74.44 & 80.871 & 79.148 & 87.955 & 72.771 & 72.904 & 71.525 & 72.424 & 1584.032 & 1576.982 & 1574.16 & 1575.974 & 1575.857 & 1568.717 & 1565.766 & 1571.471 \\
\hline 10/28/16 10:00 & 74.447 & 80.876 & 79.145 & 87.95 & 72.771 & 72.901 & 71.527 & 72.421 & 1584.025 & 1576.977 & 1574.163 & 1575.979 & 1575.857 & 1568.72 & 1565.764 & 1571.474 \\
\hline 10/28/16 11:00 & 74.449 & 80.871 & 79.143 & 87.948 & 72.769 & 72.899 & 71.52 & 72.414 & 1584.023 & 1576.982 & 1574.165 & 1575.981 & 1575.859 & 1568.722 & 1565.771 & 1571.481 \\
\hline $10 / 28 / 1612: 00$ & 74.435 & 80.853 & 79.124 & 87.934 & 72.745 & 72.88 & 71.501 & 72.393 & 1584.037 & 1577 & 1574.184 & 1575.995 & 1575.883 & 1568.741 & 1565.79 & 1571.502 \\
\hline 10/28/16 13:00 & 74.433 & 80.85 & 79.122 & 87.936 & 72.743 & 72.88 & 71.499 & 72.393 & 1584.039 & 1577.003 & 1574.186 & 1575.993 & 1575.885 & 1568.741 & 1565.792 & 1571.502 \\
\hline $10 / 28 / 1614: 00$ & 74.416 & 80.825 & 79.099 & 87.913 & 72.717 & 72.862 & 71.472 & 72.363 & 1584.056 & 1577.028 & 1574.209 & 1576.016 & 1575.911 & 1568.759 & 1565.819 & 1571.532 \\
\hline 10/28/16 15:00 & 74.416 & 80.822 & 79.092 & 87.92 & 72.708 & 72.862 & 71.472 & 72.377 & 1584.056 & 1577.031 & 1574.216 & 1576.009 & 1575.92 & 1568.759 & 1565.819 & 1571.518 \\
\hline 10/28/16 16:00 & 74.421 & 80.825 & 79.092 & 87.924 & 72.708 & 72.869 & 71.48 & 72.38 & 1584.051 & 1577.028 & 1574.216 & 1576.005 & 1575.92 & 1568.752 & 1565.811 & 1571.515 \\
\hline $10 / 28 / 1617: 00$ & 74.433 & 80.829 & 79.101 & 87.929 & 72.715 & 72.88 & 71.489 & 72.387 & 1584.039 & 1577.024 & 1574.207 & 1576 & 1575.913 & 1568.741 & 1565.802 & 1571.508 \\
\hline 10/28/16 18:00 & 74.438 & 80.829 & 79.101 & 87.924 & 72.713 & 72.876 & 71.487 & 72.373 & 1584.034 & 1577.024 & 1574.207 & 1576.005 & 1575.915 & 1568.745 & 1565.804 & 1571.522 \\
\hline $10 / 28 / 1619: 00$ & 74.452 & 80.846 & 79.113 & 87.941 & 72.727 & 72.887 & 71.508 & 72.412 & 1584.02 & 1577.007 & 1574.195 & 1575.988 & 1575.901 & 1568.734 & 1565.783 & 1571.483 \\
\hline 10/28/16 20:00 & 74.473 & 80.869 & 79.143 & 87.952 & 72.75 & 72.913 & 71.532 & 72.472 & 1583.999 & 1576.984 & 1574.165 & 1575.977 & 1575.878 & 1568.708 & 1565.759 & 1571.423 \\
\hline $10 / 28 / 1621: 00$ & 74.478 & 80.881 & 79.155 & 87.955 & 72.766 & 72.911 & 71.542 & 72.435 & 1583.994 & 1576.972 & 1574.153 & 1575.974 & 1575.862 & 1568.71 & 1565.749 & 1571.46 \\
\hline 10/28/16 22:00 & 74.478 & 80.885 & 79.154 & 87.955 & 72.766 & 72.906 & 71.544 & 72.428 & 1583.994 & 1576.968 & 1574.154 & 1575.974 & 1575.862 & 1568.715 & 1565.747 & 1571.467 \\
\hline 10/28/16 23:00 & 74.475 & 80.89 & 79.152 & 87.955 & 72.769 & 72.906 & 71.546 & 72.428 & 1583.997 & 1576.963 & 1574.156 & 1575.974 & 1575.859 & 1568.715 & 1565.745 & 1571.467 \\
\hline $10 / 29 / 160: 00$ & 74.473 & 80.895 & 79.154 & 87.957 & 72.773 & 72.906 & 71.544 & 72.428 & 1583.999 & 1576.958 & 1574.154 & 1575.972 & 1575.855 & 1568.715 & 1565.747 & 1571.467 \\
\hline
\end{tabular}


TABLE S1.1 Automatically recorded groundwater levels in the unconfined aquifer.

\begin{tabular}{|c|c|c|c|c|c|c|c|c|c|c|c|c|c|c|c|c|}
\hline \multirow[b]{2}{*}{ Date and Time } & \multicolumn{8}{|c|}{ Measured Depth to Water (ft TOC ${ }^{\mathrm{a}}$ ) } & \multicolumn{8}{|c|}{ Groundwater Elevation (ft AMSL) } \\
\hline & DL1-C & DL2-C1 & DL3-C1 & DL4-C1 & DL5-C1 & DL6-C & DL7-C & DL8-C1 & DL1-C & DL2-C1 & DL3-C1 & DL4-C1 & DL5-C1 & DL6-C & DL7-C & DL8-C1 \\
\hline 10/29/16 1:00 & 74.471 & 80.895 & 79.152 & 87.955 & 72.771 & 72.906 & 71.549 & 72.421 & 1584.001 & 1576.958 & 1574.156 & 1575.974 & 1575.857 & 1568.715 & 1565.742 & 1571.474 \\
\hline $10 / 29 / 162: 00$ & 74.468 & 80.902 & 79.157 & 87.955 & 72.773 & 72.911 & 71.554 & 72.417 & 1584.004 & 1576.951 & 1574.151 & 1575.974 & 1575.855 & 1568.71 & 1565.737 & 1571.478 \\
\hline 10/29/16 3:00 & 74.468 & 80.902 & 79.157 & 87.955 & 72.776 & 72.906 & 71.551 & 72.431 & 1584.004 & 1576.951 & 1574.151 & 1575.974 & 1575.852 & 1568.715 & 1565.74 & 1571.464 \\
\hline $10 / 29 / 164: 00$ & 74.459 & 80.892 & 79.15 & 87.948 & 72.766 & 72.899 & 71.539 & 72.424 & 1584.013 & 1576.961 & 1574.158 & 1575.981 & 1575.862 & 1568.722 & 1565.752 & 1571.471 \\
\hline $10 / 29 / 165: 00$ & 74.447 & 80.883 & 79.136 & 87.941 & 72.757 & 72.892 & 71.53 & 72.407 & 1584.025 & 1576.97 & 1574.172 & 1575.988 & 1575.871 & 1568.729 & 1565.761 & 1571.488 \\
\hline $10 / 29 / 166: 00$ & 74.464 & 80.906 & 79.155 & 87.955 & 72.773 & 72.911 & 71.551 & 72.428 & 1584.008 & 1576.947 & 1574.153 & 1575.974 & 1575.855 & 1568.71 & 1565.74 & 1571.467 \\
\hline 10/29/16 7:00 & 74.468 & 80.909 & 79.164 & 87.955 & 72.776 & 72.911 & 71.556 & 72.44 & 1584.004 & 1576.944 & 1574.144 & 1575.974 & 1575.852 & 1568.71 & 1565.735 & 1571.455 \\
\hline $10 / 29 / 168: 00$ & 74.466 & 80.906 & 79.166 & 87.952 & 72.773 & 72.908 & 71.553 & 72.442 & 1584.006 & 1576.947 & 1574.142 & 1575.977 & 1575.855 & 1568.713 & 1565.738 & 1571.453 \\
\hline 10/29/16 9:00 & 74.473 & 80.916 & 79.169 & 87.957 & 72.78 & 72.913 & 71.558 & 72.449 & 1583.999 & 1576.937 & 1574.139 & 1575.972 & 1575.848 & 1568.708 & 1565.733 & 1571.446 \\
\hline $10 / 29 / 1610: 00$ & 74.48 & 80.929 & 79.175 & 87.964 & 72.792 & 72.92 & 71.57 & 72.449 & 1583.992 & 1576.924 & 1574.133 & 1575.965 & 1575.836 & 1568.701 & 1565.721 & 1571.446 \\
\hline 10/29/16 11:00 & 74.489 & 80.941 & 79.192 & 87.971 & 72.804 & 72.927 & 71.582 & 72.468 & 1583.983 & 1576.912 & 1574.116 & 1575.958 & 1575.824 & 1568.694 & 1565.709 & 1571.427 \\
\hline $10 / 29 / 1612: 00$ & 74.49 & 80.946 & 79.192 & 87.973 & 72.804 & 72.925 & 71.582 & 72.465 & 1583.982 & 1576.907 & 1574.116 & 1575.956 & 1575.824 & 1568.696 & 1565.709 & 1571.43 \\
\hline 10/29/16 13:00 & 74.473 & 80.929 & 79.182 & 87.957 & 72.794 & 72.913 & 71.565 & 72.458 & 1583.999 & 1576.924 & 1574.126 & 1575.972 & 1575.834 & 1568.708 & 1565.726 & 1571.437 \\
\hline $10 / 29 / 1614: 00$ & 74.482 & 80.95 & 79.185 & 87.971 & 72.804 & 72.92 & 71.582 & 72.456 & 1583.99 & 1576.903 & 1574.123 & 1575.958 & 1575.824 & 1568.701 & 1565.709 & 1571.439 \\
\hline $10 / 29 / 1615: 00$ & 74.494 & 80.96 & 79.196 & 87.978 & 72.811 & 72.932 & 71.592 & 72.472 & 1583.978 & 1576.893 & 1574.112 & 1575.951 & 1575.817 & 1568.689 & 1565.699 & 1571.423 \\
\hline $10 / 29 / 1616: 00$ & 74.497 & 80.967 & 79.208 & 87.985 & 72.822 & 72.936 & 71.604 & 72.482 & 1583.975 & 1576.886 & 1574.1 & 1575.944 & 1575.806 & 1568.685 & 1565.687 & 1571.413 \\
\hline $10 / 29 / 1617: 00$ & 74.511 & 80.995 & 79.229 & 87.994 & 72.843 & 72.957 & 71.63 & 72.503 & 1583.961 & 1576.858 & 1574.079 & 1575.935 & 1575.785 & 1568.664 & 1565.661 & 1571.392 \\
\hline $10 / 29 / 1618: 00$ & 74.537 & 81.032 & 79.262 & 88.018 & 72.883 & 72.978 & 71.671 & 72.537 & 1583.935 & 1576.821 & 1574.046 & 1575.911 & 1575.745 & 1568.643 & 1565.62 & 1571.358 \\
\hline $10 / 29 / 1619: 00$ & 74.555 & 81.064 & 79.287 & 88.034 & 72.909 & 72.997 & 71.697 & 72.563 & 1583.917 & 1576.789 & 1574.021 & 1575.895 & 1575.719 & 1568.624 & 1565.594 & 1571.332 \\
\hline $10 / 29 / 1620: 00$ & 74.553 & 81.074 & 79.304 & 88.034 & 72.923 & 72.997 & 71.699 & 72.584 & 1583.919 & 1576.779 & 1574.004 & 1575.895 & 1575.705 & 1568.624 & 1565.592 & 1571.311 \\
\hline 10/29/16 21:00 & 74.574 & 81.118 & 79.336 & 88.057 & 72.96 & 73.027 & 71.747 & 72.612 & 1583.898 & 1576.735 & 1573.972 & 1575.872 & 1575.668 & 1568.594 & 1565.544 & 1571.283 \\
\hline $10 / 29 / 1622: 00$ & 74.581 & 81.136 & 79.362 & 88.064 & 72.983 & 73.037 & 71.759 & 72.621 & 1583.891 & 1576.717 & 1573.946 & 1575.865 & 1575.645 & 1568.584 & 1565.532 & 1571.274 \\
\hline $10 / 29 / 1623: 00$ & 74.586 & 81.155 & 79.374 & 88.069 & 73.002 & 73.037 & 71.773 & 72.651 & 1583.886 & 1576.698 & 1573.934 & 1575.86 & 1575.626 & 1568.584 & 1565.518 & 1571.244 \\
\hline 10/30/16 0:00 & 74.572 & 81.153 & 79.376 & 88.067 & 73.004 & 73.034 & 71.766 & 72.658 & 1583.9 & 1576.7 & 1573.932 & 1575.862 & 1575.624 & 1568.587 & 1565.525 & 1571.237 \\
\hline $10 / 30 / 161: 00$ & 74.565 & 81.164 & 79.385 & 88.067 & 73.011 & 73.036 & 71.773 & 72.663 & 1583.907 & 1576.689 & 1573.923 & 1575.862 & 1575.617 & 1568.585 & 1565.518 & 1571.232 \\
\hline $10 / 30 / 162: 00$ & 74.555 & 81.16 & 79.383 & 88.062 & 73.011 & 73.032 & 71.766 & 72.663 & 1583.917 & 1576.693 & 1573.925 & 1575.867 & 1575.617 & 1568.589 & 1565.525 & 1571.232 \\
\hline 10/30/16 3:00 & 74.548 & 81.16 & 79.385 & 88.062 & 73.016 & 73.029 & 71.764 & 72.665 & 1583.924 & 1576.693 & 1573.923 & 1575.867 & 1575.612 & 1568.592 & 1565.527 & 1571.23 \\
\hline $10 / 30 / 164: 00$ & 74.534 & 81.153 & 79.376 & 88.055 & 73.007 & 73.02 & 71.752 & 72.66 & 1583.938 & 1576.7 & 1573.932 & 1575.874 & 1575.621 & 1568.601 & 1565.539 & 1571.235 \\
\hline $10 / 30 / 165: 00$ & 74.544 & 81.171 & 79.392 & 88.067 & 73.023 & 73.036 & 71.775 & 72.67 & 1583.928 & 1576.682 & 1573.916 & 1575.862 & 1575.605 & 1568.585 & 1565.516 & 1571.225 \\
\hline $10 / 30 / 166: 00$ & 74.541 & 81.171 & 79.395 & 88.067 & 73.028 & 73.034 & 71.768 & 72.677 & 1583.931 & 1576.682 & 1573.913 & 1575.862 & 1575.6 & 1568.587 & 1565.523 & 1571.218 \\
\hline $10 / 30 / 167: 00$ & 74.546 & 81.178 & 79.404 & 88.071 & 73.037 & 73.039 & 71.78 & 72.693 & 1583.926 & 1576.675 & 1573.904 & 1575.858 & 1575.591 & 1568.582 & 1565.511 & 1571.202 \\
\hline $10 / 30 / 168: 00$ & 74.539 & 81.174 & 79.406 & 88.064 & 73.032 & 73.034 & 71.771 & 72.688 & 1583.933 & 1576.679 & 1573.902 & 1575.865 & 1575.596 & 1568.587 & 1565.52 & 1571.207 \\
\hline 10/30/16 9:00 & 74.544 & 81.183 & 79.413 & 88.076 & 73.046 & 73.046 & 71.785 & 72.688 & 1583.928 & 1576.67 & 1573.895 & 1575.853 & 1575.582 & 1568.575 & 1565.506 & 1571.207 \\
\hline $10 / 30 / 1610: 00$ & 74.548 & 81.195 & 79.418 & 88.074 & 73.053 & 73.048 & 71.79 & 72.702 & 1583.924 & 1576.658 & 1573.89 & 1575.855 & 1575.575 & 1568.573 & 1565.501 & 1571.193 \\
\hline 10/30/16 11:00 & 74.534 & 81.174 & 79.413 & 88.062 & 73.044 & 73.034 & 71.768 & 72.693 & 1583.938 & 1576.679 & 1573.895 & 1575.867 & 1575.584 & 1568.587 & 1565.523 & 1571.202 \\
\hline $10 / 30 / 1612: 00$ & 74.518 & 81.164 & 79.397 & 88.055 & 73.032 & 73.02 & 71.752 & 72.679 & 1583.954 & 1576.689 & 1573.911 & 1575.874 & 1575.596 & 1568.601 & 1565.539 & 1571.216 \\
\hline $10 / 30 / 1613: 00$ & 74.494 & 81.132 & 79.369 & 88.034 & 73.002 & 72.999 & 71.718 & 72.656 & 1583.978 & 1576.721 & 1573.939 & 1575.895 & 1575.626 & 1568.622 & 1565.573 & 1571.239 \\
\hline $10 / 30 / 1614: 00$ & 74.466 & 81.095 & 79.339 & 88.011 & 72.969 & 72.974 & 71.68 & 72.623 & 1584.006 & 1576.758 & 1573.969 & 1575.918 & 1575.659 & 1568.647 & 1565.611 & 1571.272 \\
\hline $10 / 30 / 1615: 00$ & 74.447 & 81.064 & 79.306 & 87.999 & 72.939 & 72.955 & 71.647 & 72.589 & 1584.025 & 1576.789 & 1574.002 & 1575.93 & 1575.689 & 1568.666 & 1565.644 & 1571.306 \\
\hline 10/30/16 16:00 & 74.431 & 81.036 & 79.283 & 87.983 & 72.913 & 72.943 & 71.625 & 72.565 & 1584.041 & 1576.817 & 1574.025 & 1575.946 & 1575.715 & 1568.678 & 1565.666 & 1571.33 \\
\hline $10 / 30 / 1617: 00$ & 74.416 & 81.004 & 79.255 & 87.969 & 72.883 & 72.922 & 71.597 & 72.537 & 1584.056 & 1576.849 & 1574.053 & 1575.96 & 1575.745 & 1568.699 & 1565.694 & 1571.358 \\
\hline $10 / 30 / 1618: 00$ & 74.419 & 80.99 & 79.241 & 87.969 & 72.869 & 72.92 & 71.589 & 72.514 & 1584.053 & 1576.863 & 1574.067 & 1575.96 & 1575.759 & 1568.701 & 1565.702 & 1571.381 \\
\hline $10 / 30 / 1619: 00$ & 74.421 & 80.985 & 79.231 & 87.966 & 72.857 & 72.92 & 71.584 & 72.512 & 1584.051 & 1576.868 & 1574.077 & 1575.963 & 1575.771 & 1568.701 & 1565.707 & 1571.383 \\
\hline $10 / 30 / 1620: 00$ & 74.426 & 80.971 & 79.227 & 87.966 & 72.848 & 72.918 & 71.58 & 72.507 & 1584.046 & 1576.882 & 1574.081 & 1575.963 & 1575.78 & 1568.703 & 1565.711 & 1571.388 \\
\hline 10/30/16 21:00 & 74.423 & 80.953 & 79.213 & 87.952 & 72.832 & 72.906 & 71.563 & 72.491 & 1584.049 & 1576.9 & 1574.095 & 1575.977 & 1575.796 & 1568.715 & 1565.728 & 1571.404 \\
\hline $10 / 30 / 1622: 00$ & 74.414 & 80.936 & 79.196 & 87.948 & 72.818 & 72.899 & 71.546 & 72.475 & 1584.058 & 1576.917 & 1574.112 & 1575.981 & 1575.81 & 1568.722 & 1565.745 & 1571.42 \\
\hline $10 / 30 / 1623: 00$ & 74.412 & 80.918 & 79.182 & 87.938 & 72.799 & 72.89 & 71.532 & 72.458 & 1584.06 & 1576.935 & 1574.126 & 1575.991 & 1575.829 & 1568.731 & 1565.759 & 1571.437 \\
\hline 10/31/16 0:00 & 74.405 & 80.902 & 79.164 & 87.931 & 72.778 & 72.876 & 71.518 & 72.442 & 1584.067 & 1576.951 & 1574.144 & 1575.998 & 1575.85 & 1568.745 & 1565.773 & 1571.453 \\
\hline $10 / 31 / 161: 00$ & 74.391 & 80.874 & 79.138 & 87.915 & 72.752 & 72.862 & 71.491 & 72.414 & 1584.081 & 1576.979 & 1574.17 & 1576.014 & 1575.876 & 1568.759 & 1565.8 & 1571.481 \\
\hline
\end{tabular}


TABLE S1.1 Automatically recorded groundwater levels in the unconfined aquifer.

\begin{tabular}{|c|c|c|c|c|c|c|c|c|c|c|c|c|c|c|c|c|}
\hline \multirow[b]{2}{*}{ Date and Time } & \multicolumn{8}{|c|}{ Measured Depth to Water ( $\mathrm{ft}$ TOC ${ }^{\mathrm{a}}$ ) } & \multicolumn{8}{|c|}{ Groundwater Elevation (ft AMSL ${ }^{\mathrm{b}}$ ) } \\
\hline & DL1-C & DL2-C1 & DL3-C1 & DL4-C1 & DL5-C1 & DL6-C & DL7-C & DL8-C1 & DL1-C & DL2-C1 & DL3-C1 & DL4-C1 & DL5-C1 & DL6-C & DL7-C & DL8-C1 \\
\hline 10/31/16 2:00 & 74.393 & 80.867 & 79.129 & 87.917 & 72.741 & 72.859 & 71.487 & 72.403 & 1584.079 & 1576.986 & 1574.179 & 1576.012 & 1575.887 & 1568.762 & 1565.804 & 1571.492 \\
\hline 10/31/16 3:00 & 74.376 & 80.836 & 79.103 & 87.898 & 72.713 & 72.841 & 71.46 & 72.375 & 1584.096 & 1577.017 & 1574.205 & 1576.031 & 1575.915 & 1568.78 & 1565.831 & 1571.52 \\
\hline 10/31/16 4:00 & 74.369 & 80.811 & 79.08 & 87.887 & 72.687 & 72.827 & 71.439 & 72.352 & 1584.103 & 1577.042 & 1574.228 & 1576.042 & 1575.941 & 1568.794 & 1565.852 & 1571.543 \\
\hline 10/31/16 5:00 & 74.355 & 80.785 & 79.057 & 87.875 & 72.659 & 72.808 & 71.408 & 72.326 & 1584.117 & 1577.068 & 1574.251 & 1576.054 & 1575.969 & 1568.813 & 1565.883 & 1571.569 \\
\hline 10/31/16 6:00 & 74.35 & 80.76 & 79.036 & 87.863 & 72.636 & 72.801 & 71.396 & 72.301 & 1584.122 & 1577.093 & 1574.272 & 1576.066 & 1575.992 & 1568.82 & 1565.895 & 1571.594 \\
\hline 10/31/16 7:00 & 74.346 & 80.746 & 79.015 & 87.859 & 72.615 & 72.79 & 71.387 & 72.282 & 1584.126 & 1577.107 & 1574.293 & 1576.07 & 1576.013 & 1568.831 & 1565.904 & 1571.613 \\
\hline 10/31/16 8:00 & 74.325 & 80.702 & 78.98 & 87.835 & 72.571 & 72.764 & 71.341 & 72.245 & 1584.147 & 1577.151 & 1574.328 & 1576.094 & 1576.057 & 1568.857 & 1565.95 & 1571.65 \\
\hline 10/31/16 9:00 & 74.346 & 80.711 & 78.987 & 87.854 & 72.582 & 72.785 & 71.37 & 72.252 & 1584.126 & 1577.142 & 1574.321 & 1576.075 & 1576.046 & 1568.836 & 1565.921 & 1571.643 \\
\hline 10/31/16 10:00 & 74.358 & 80.706 & 78.987 & 87.854 & 72.573 & 72.783 & 71.363 & 72.243 & 1584.114 & 1577.147 & 1574.321 & 1576.075 & 1576.055 & 1568.838 & 1565.928 & 1571.652 \\
\hline 10/31/16 11:00 & 74.355 & 80.699 & 78.973 & 87.852 & 72.557 & 72.771 & 71.351 & 72.233 & 1584.117 & 1577.154 & 1574.335 & 1576.077 & 1576.071 & 1568.85 & 1565.94 & 1571.662 \\
\hline 10/31/16 12:00 & 74.355 & 80.683 & 78.963 & 87.847 & 72.543 & 72.762 & 71.336 & 72.222 & 1584.117 & 1577.17 & 1574.345 & 1576.082 & 1576.085 & 1568.859 & 1565.955 & 1571.673 \\
\hline 10/31/16 13:00 & 74.348 & 80.664 & 78.942 & 87.828 & 72.517 & 72.75 & 71.317 & 72.198 & 1584.124 & 1577.189 & 1574.366 & 1576.101 & 1576.111 & 1568.871 & 1565.974 & 1571.697 \\
\hline 10/31/16 14:00 & 74.332 & 80.639 & 78.919 & 87.814 & 72.494 & 72.729 & 71.291 & 72.177 & 1584.14 & 1577.214 & 1574.389 & 1576.115 & 1576.134 & 1568.892 & 1566 & 1571.718 \\
\hline 10/31/16 15:00 & 74.32 & 80.615 & 78.896 & 87.81 & 72.468 & 72.717 & 71.277 & 72.145 & 1584.152 & 1577.238 & 1574.412 & 1576.119 & 1576.16 & 1568.904 & 1566.014 & 1571.75 \\
\hline 10/31/16 16:00 & 74.332 & 80.615 & 78.891 & 87.814 & 72.466 & 72.724 & 71.284 & 72.096 & 1584.14 & 1577.238 & 1574.417 & 1576.115 & 1576.162 & 1568.897 & 1566.007 & 1571.799 \\
\hline 10/31/16 17:00 & 74.372 & 80.66 & 78.919 & 87.849 & 72.494 & 72.764 & 71.331 & 72.131 & 1584.1 & 1577.193 & 1574.389 & 1576.08 & 1576.134 & 1568.857 & 1565.96 & 1571.764 \\
\hline 10/31/16 18:00 & 74.383 & 80.662 & 78.931 & 87.845 & 72.501 & 72.757 & 71.334 & 72.182 & 1584.089 & 1577.191 & 1574.377 & 1576.084 & 1576.127 & 1568.864 & 1565.957 & 1571.713 \\
\hline 10/31/16 19:00 & 74.409 & 80.692 & 78.954 & 87.859 & 72.526 & 72.778 & 71.365 & 72.205 & 1584.063 & 1577.161 & 1574.354 & 1576.07 & 1576.102 & 1568.843 & 1565.926 & 1571.69 \\
\hline 10/31/16 20:00 & 74.428 & 80.72 & 78.975 & 87.873 & 72.554 & 72.792 & 71.389 & 72.222 & 1584.044 & 1577.133 & 1574.333 & 1576.056 & 1576.074 & 1568.829 & 1565.902 & 1571.673 \\
\hline $10 / 31 / 1621: 00$ & 74.435 & 80.727 & 78.987 & 87.87 & 72.568 & 72.794 & 71.396 & 72.233 & 1584.037 & 1577.126 & 1574.321 & 1576.059 & 1576.06 & 1568.827 & 1565.895 & 1571.662 \\
\hline 10/31/16 22:00 & 74.44 & 80.743 & 78.998 & 87.882 & 72.578 & 72.801 & 71.41 & 72.245 & 1584.032 & 1577.11 & 1574.31 & 1576.047 & 1576.05 & 1568.82 & 1565.881 & 1571.65 \\
\hline 10/31/16 23:00 & 74.449 & 80.767 & 79.012 & 87.896 & 72.594 & 72.808 & 71.427 & 72.259 & 1584.023 & 1577.086 & 1574.296 & 1576.033 & 1576.034 & 1568.813 & 1565.864 & 1571.636 \\
\hline $11 / 1 / 160: 00$ & 74.461 & 80.79 & 79.033 & 87.901 & 72.613 & 72.82 & 71.444 & 72.277 & 1584.011 & 1577.063 & 1574.275 & 1576.028 & 1576.015 & 1568.801 & 1565.847 & 1571.618 \\
\hline $11 / 1 / 161: 00$ & 74.464 & 80.799 & 79.04 & 87.906 & 72.624 & 72.824 & 71.453 & 72.284 & 1584.008 & 1577.054 & 1574.268 & 1576.023 & 1576.004 & 1568.797 & 1565.838 & 1571.611 \\
\hline $11 / 1 / 162: 00$ & 74.473 & 80.825 & 79.059 & 87.915 & 72.643 & 72.839 & 71.477 & 72.305 & 1583.999 & 1577.028 & 1574.249 & 1576.014 & 1575.985 & 1568.782 & 1565.814 & 1571.59 \\
\hline 11/1/16 3:00 & 74.482 & 80.848 & 79.08 & 87.924 & 72.666 & 72.853 & 71.496 & 72.331 & 1583.99 & 1577.005 & 1574.228 & 1576.005 & 1575.962 & 1568.768 & 1565.795 & 1571.564 \\
\hline $11 / 1 / 164: 00$ & 74.482 & 80.86 & 79.092 & 87.924 & 72.678 & 72.855 & 71.504 & 72.34 & 1583.99 & 1576.993 & 1574.216 & 1576.005 & 1575.95 & 1568.766 & 1565.787 & 1571.555 \\
\hline $11 / 1 / 165: 00$ & 74.475 & 80.86 & 79.094 & 87.924 & 72.68 & 72.85 & 71.506 & 72.291 & 1583.997 & 1576.993 & 1574.214 & 1576.005 & 1575.948 & 1568.771 & 1565.785 & 1571.604 \\
\hline $11 / 1 / 166: 00$ & 74.48 & 80.878 & 79.106 & 87.931 & 72.696 & 72.862 & 71.518 & 72.352 & 1583.992 & 1576.975 & 1574.202 & 1575.998 & 1575.932 & 1568.759 & 1565.773 & 1571.543 \\
\hline $11 / 1 / 167: 00$ & 74.497 & 80.906 & 79.124 & 87.941 & 72.715 & 72.873 & 71.539 & 72.384 & 1583.975 & 1576.947 & 1574.184 & 1575.988 & 1575.913 & 1568.748 & 1565.752 & 1571.511 \\
\hline $11 / 1 / 168: 00$ & 74.497 & 80.92 & 79.145 & 87.955 & 72.738 & 72.883 & 71.554 & 72.4 & 1583.975 & 1576.933 & 1574.163 & 1575.974 & 1575.89 & 1568.738 & 1565.737 & 1571.495 \\
\hline $11 / 1 / 169: 00$ & 74.504 & 80.939 & 79.159 & 87.964 & 72.752 & 72.892 & 71.568 & 72.412 & 1583.968 & 1576.914 & 1574.149 & 1575.965 & 1575.876 & 1568.729 & 1565.723 & 1571.483 \\
\hline 11/1/16 10:00 & 74.506 & 80.953 & 79.173 & 87.969 & 72.766 & 72.897 & 71.58 & 72.428 & 1583.966 & 1576.9 & 1574.135 & 1575.96 & 1575.862 & 1568.724 & 1565.711 & 1571.467 \\
\hline 11/1/16 11:00 & 74.497 & 80.957 & 79.178 & 87.962 & 72.773 & 72.894 & 71.58 & 72.431 & 1583.975 & 1576.896 & 1574.13 & 1575.967 & 1575.855 & 1568.727 & 1565.711 & 1571.464 \\
\hline $11 / 1 / 1612: 00$ & 74.497 & 80.962 & 79.187 & 87.962 & 72.778 & 72.897 & 71.575 & 72.438 & 1583.975 & 1576.891 & 1574.121 & 1575.967 & 1575.85 & 1568.724 & 1565.716 & 1571.457 \\
\hline 11/1/16 13:00 & 74.475 & 80.948 & 79.171 & 87.948 & 72.762 & 72.883 & 71.556 & 72.386 & 1583.997 & 1576.905 & 1574.137 & 1575.981 & 1575.866 & 1568.738 & 1565.735 & 1571.509 \\
\hline $11 / 1 / 16$ 14:00 & 74.454 & 80.925 & 79.152 & 87.936 & 72.748 & 72.862 & 71.53 & 72.41 & 1584.018 & 1576.928 & 1574.156 & 1575.993 & 1575.88 & 1568.759 & 1565.761 & 1571.485 \\
\hline 11/1/16 15:00 & 74.442 & 80.92 & 79.141 & 87.931 & 72.738 & 72.86 & 71.522 & 72.398 & 1584.03 & 1576.933 & 1574.167 & 1575.998 & 1575.89 & 1568.761 & 1565.769 & 1571.497 \\
\hline $11 / 1 / 1616: 00$ & 74.442 & 80.918 & 79.138 & 87.931 & 72.738 & 72.862 & 71.522 & 72.396 & 1584.03 & 1576.935 & 1574.17 & 1575.998 & 1575.89 & 1568.759 & 1565.769 & 1571.499 \\
\hline 11/1/16 17:00 & 74.449 & 80.925 & 79.157 & 87.938 & 72.743 & 72.867 & 71.527 & 72.398 & 1584.023 & 1576.928 & 1574.151 & 1575.991 & 1575.885 & 1568.754 & 1565.764 & 1571.497 \\
\hline $11 / 1 / 1618: 00$ & 74.464 & 80.941 & 79.159 & 87.948 & 72.762 & 72.88 & 71.549 & 72.419 & 1584.008 & 1576.912 & 1574.149 & 1575.981 & 1575.866 & 1568.741 & 1565.742 & 1571.476 \\
\hline $11 / 1 / 16$ 19:00 & 74.475 & 80.955 & 79.171 & 87.955 & 72.776 & 72.887 & 71.563 & 72.428 & 1583.997 & 1576.898 & 1574.137 & 1575.974 & 1575.852 & 1568.734 & 1565.728 & 1571.467 \\
\hline $11 / 1 / 1620: 00$ & 74.485 & 80.969 & 79.185 & 87.964 & 72.785 & 72.894 & 71.573 & 72.442 & 1583.987 & 1576.884 & 1574.123 & 1575.965 & 1575.843 & 1568.727 & 1565.718 & 1571.453 \\
\hline 11/1/16 21:00 & 74.475 & 80.96 & 79.178 & 87.952 & 72.783 & 72.885 & 71.561 & 72.438 & 1583.997 & 1576.893 & 1574.13 & 1575.977 & 1575.845 & 1568.736 & 1565.73 & 1571.457 \\
\hline 11/1/16 22:00 & 74.478 & 80.971 & 79.185 & 87.957 & 72.79 & 72.89 & 71.568 & 72.445 & 1583.994 & 1576.882 & 1574.123 & 1575.972 & 1575.838 & 1568.731 & 1565.723 & 1571.45 \\
\hline $11 / 1 / 1623: 00$ & 74.48 & 80.976 & 79.196 & 87.962 & 72.801 & 72.897 & 71.575 & 72.456 & 1583.992 & 1576.877 & 1574.112 & 1575.967 & 1575.827 & 1568.724 & 1565.716 & 1571.439 \\
\hline 11/2/16 0:00 & 74.468 & 80.967 & 79.182 & 87.952 & 72.792 & 72.88 & 71.558 & 72.405 & 1584.004 & 1576.886 & 1574.126 & 1575.977 & 1575.836 & 1568.741 & 1565.733 & 1571.49 \\
\hline 11/2/16 1:00 & 74.464 & 80.969 & 79.185 & 87.952 & 72.79 & 72.88 & 71.558 & 72.444 & 1584.008 & 1576.884 & 1574.123 & 1575.977 & 1575.838 & 1568.741 & 1565.733 & 1571.451 \\
\hline $11 / 2 / 162: 00$ & 74.471 & 80.976 & 79.192 & 87.957 & 72.804 & 72.894 & 71.575 & 72.458 & 1584.001 & 1576.877 & 1574.116 & 1575.972 & 1575.824 & 1568.727 & 1565.716 & 1571.437 \\
\hline
\end{tabular}


TABLE S1.1 Automatically recorded groundwater levels in the unconfined aquifer.

\begin{tabular}{|c|c|c|c|c|c|c|c|c|c|c|c|c|c|c|c|c|}
\hline \multirow[b]{2}{*}{ Date and Time } & \multicolumn{8}{|c|}{ Measured Depth to Water (ft TOC ${ }^{\mathrm{a}}$ ) } & \multicolumn{8}{|c|}{ Groundwater Elevation ( $\mathrm{ft} \mathrm{AMSL}^{\mathrm{b}}$ ) } \\
\hline & DL1-C & DL2-C1 & DL3-C1 & DL4-C1 & DL5-C1 & DL6-C & DL7-C & DL8-C1 & DL1-C & DL2-C1 & DL3-C1 & DL4-C1 & DL5-C1 & DL6-C & DL7-C & DL8-C1 \\
\hline $11 / 2 / 163: 00$ & 74.473 & 80.983 & 79.196 & 87.962 & 72.808 & 72.897 & 71.577 & 72.461 & 1583.999 & 1576.87 & 1574.112 & 1575.967 & 1575.82 & 1568.724 & 1565.714 & 1571.434 \\
\hline $11 / 2 / 164: 00$ & 74.473 & 80.983 & 79.201 & 87.964 & 72.811 & 72.897 & 71.578 & 72.465 & 1583.999 & 1576.87 & 1574.107 & 1575.965 & 1575.817 & 1568.724 & 1565.713 & 1571.43 \\
\hline $11 / 2 / 165: 00$ & 74.471 & 80.985 & 79.199 & 87.959 & 72.808 & 72.892 & 71.575 & 72.461 & 1584.001 & 1576.868 & 1574.109 & 1575.97 & 1575.82 & 1568.729 & 1565.716 & 1571.434 \\
\hline $11 / 2 / 166: 00$ & 74.475 & 80.992 & 79.203 & 87.964 & 72.818 & 72.899 & 71.587 & 72.472 & 1583.997 & 1576.861 & 1574.105 & 1575.965 & 1575.81 & 1568.722 & 1565.704 & 1571.423 \\
\hline $11 / 2 / 167: 00$ & 74.485 & 81.002 & 79.222 & 87.971 & 72.834 & 72.908 & 71.601 & 72.489 & 1583.987 & 1576.851 & 1574.086 & 1575.958 & 1575.794 & 1568.713 & 1565.69 & 1571.406 \\
\hline $11 / 2 / 168: 00$ & 74.492 & 81.016 & 79.234 & 87.976 & 72.841 & 72.918 & 71.608 & 72.496 & 1583.98 & 1576.837 & 1574.074 & 1575.953 & 1575.787 & 1568.703 & 1565.683 & 1571.399 \\
\hline $11 / 2 / 169: 00$ & 74.501 & 81.036 & 79.245 & 87.992 & 72.86 & 72.929 & 71.63 & 72.572 & 1583.971 & 1576.817 & 1574.063 & 1575.937 & 1575.768 & 1568.692 & 1565.661 & 1571.323 \\
\hline $11 / 2 / 1610: 00$ & 74.527 & 81.069 & 79.287 & 88.008 & 72.892 & 72.96 & 71.666 & 72.598 & 1583.945 & 1576.784 & 1574.021 & 1575.921 & 1575.736 & 1568.661 & 1565.625 & 1571.297 \\
\hline $11 / 2 / 1611: 00$ & 74.527 & 81.078 & 79.297 & 88.011 & 72.904 & 72.957 & 71.671 & 72.577 & 1583.945 & 1576.775 & 1574.011 & 1575.918 & 1575.724 & 1568.664 & 1565.62 & 1571.318 \\
\hline $11 / 2 / 1612: 00$ & & 81.074 & 79.294 & 88.004 & 72.902 & 72.95 & 71.659 & 72.616 & & 1576.779 & 1574.014 & 1575.925 & 1575.726 & 1568.671 & 1565.632 & 1571.279 \\
\hline $11 / 2 / 1613: 00$ & & 81.071 & 79.297 & 87.999 & 72.902 & 72.946 & 71.654 & 72.577 & & 1576.782 & 1574.011 & 1575.93 & 1575.726 & 1568.675 & 1565.637 & 1571.318 \\
\hline $11 / 2 / 1614: 00$ & & 81.062 & 79.292 & & 72.892 & & & 72.563 & & 1576.791 & 1574.016 & & 1575.736 & & & 1571.332 \\
\hline $11 / 2 / 1615: 00$ & & 81.05 & 79.273 & & 72.881 & & & 72.551 & & 1576.803 & 1574.035 & & 1575.747 & & & 1571.344 \\
\hline $11 / 2 / 1616: 00$ & & 81.064 & 79.278 & & 72.89 & & & 72.558 & & 1576.789 & 1574.03 & & 1575.738 & & & 1571.337 \\
\hline $11 / 2 / 1617: 00$ & & 81.083 & 79.306 & & 72.913 & & & 72.672 & & 1576.77 & 1574.002 & & 1575.715 & & & 1571.223 \\
\hline $11 / 2 / 1618: 00$ & & 81.095 & 79.315 & & 72.923 & & & 72.612 & & 1576.758 & 1573.993 & & 1575.705 & & & 1571.283 \\
\hline $11 / 2 / 1619: 00$ & & 81.102 & 79.32 & & 72.934 & & & 72.607 & & 1576.751 & 1573.988 & & 1575.694 & & & 1571.288 \\
\hline $11 / 2 / 1620: 00$ & & 81.104 & 79.325 & & 72.939 & & & 72.614 & & 1576.749 & 1573.983 & & 1575.689 & & & 1571.281 \\
\hline $11 / 2 / 1621: 00$ & & 81.113 & 79.329 & & 72.948 & & & 72.616 & & 1576.74 & 1573.979 & & 1575.68 & & & 1571.279 \\
\hline $11 / 2 / 1622: 00$ & & 81.118 & 79.339 & & 72.955 & & & 72.621 & & 1576.735 & 1573.969 & & 1575.673 & & & 1571.274 \\
\hline $11 / 2 / 1623: 00$ & & 81.127 & 79.343 & & 72.96 & & & 72.628 & & 1576.726 & 1573.965 & & 1575.668 & & & 1571.267 \\
\hline $11 / 3 / 160: 00$ & & 81.122 & 79.341 & & 72.962 & & & 72.626 & & 1576.731 & 1573.967 & & 1575.666 & & & 1571.269 \\
\hline $11 / 3 / 161: 00$ & & 81.123 & 79.341 & & 72.962 & & & 72.621 & & 1576.73 & 1573.967 & & 1575.666 & & & 1571.274 \\
\hline $11 / 3 / 162: 00$ & & 81.118 & 79.336 & & 72.958 & & & 72.621 & & 1576.735 & 1573.972 & & 1575.67 & & & 1571.274 \\
\hline $11 / 3 / 163: 00$ & & 81.12 & 79.341 & & 72.962 & & & 72.626 & & 1576.733 & 1573.967 & & 1575.666 & & & 1571.269 \\
\hline $11 / 3 / 164: 00$ & & 81.125 & 79.346 & & 72.965 & & & 72.628 & & 1576.728 & 1573.962 & & 1575.663 & & & 1571.267 \\
\hline $11 / 3 / 165: 00$ & & 81.125 & 79.343 & & 72.965 & & & 72.623 & & 1576.728 & 1573.965 & & 1575.663 & & & 1571.272 \\
\hline $11 / 3 / 166: 00$ & & 81.12 & 79.341 & & 72.962 & & & 72.626 & & 1576.733 & 1573.967 & & 1575.666 & & & 1571.269 \\
\hline $11 / 3 / 167: 00$ & & 81.118 & 79.339 & & 72.96 & & & 72.616 & & 1576.735 & 1573.969 & & 1575.668 & & & 1571.279 \\
\hline $11 / 3 / 168: 00$ & & 81.12 & 79.341 & & 72.967 & & & 72.626 & & 1576.733 & 1573.967 & & 1575.661 & & & 1571.269 \\
\hline $11 / 3 / 169: 00$ & & 81.129 & 79.35 & & 72.972 & & & 72.63 & & 1576.724 & 1573.958 & & 1575.656 & & & 1571.265 \\
\hline $11 / 3 / 1610: 00$ & & 81.134 & 79.364 & & 72.979 & & & 72.635 & & 1576.719 & 1573.944 & & 1575.649 & & & 1571.26 \\
\hline $11 / 3 / 1611: 00$ & & 81.136 & & & & & & 72.635 & & 1576.717 & & & & & & 1571.26 \\
\hline
\end{tabular}

a TOC, top of casing.

b AMSL, above mean sea level. 
TABLE S1.2 Automatically recorded groundwater levels in the confined aquifers.

\begin{tabular}{|c|c|c|c|c|c|c|}
\hline \multirow[b]{2}{*}{ Date-Time } & \multicolumn{3}{|c|}{ Measured Depth to Water (ft TOC ${ }^{a}$ ) } & \multicolumn{3}{|c|}{ Groundwater Elevation (ft AMSL ${ }^{b}$ ) } \\
\hline & DL2-CU & DL7-CU & DL7-CL & DL2-CU & DL7-CU & DL7-CL \\
\hline 7/8/16 18:00 & 91.344 & & & 1566.547 & & \\
\hline 7/8/16 19:00 & 91.269 & & & 1566.622 & & \\
\hline 7/8/16 20:00 & 91.189 & & & 1566.702 & & \\
\hline 7/8/16 21:00 & 91.113 & & & 1566.778 & & \\
\hline $7 / 8 / 16$ 22:00 & 91.036 & & & 1566.855 & & \\
\hline $7 / 8 / 16$ 23:00 & 90.96 & & & 1566.931 & & \\
\hline 7/9/16 0:00 & 90.885 & & & 1567.006 & & \\
\hline $7 / 9 / 16$ 1:00 & 90.812 & & & 1567.079 & & \\
\hline 7/9/16 2:00 & 90.732 & & & 1567.159 & & \\
\hline 7/9/16 3:00 & 90.662 & & & 1567.229 & & \\
\hline 7/9/16 4:00 & 90.586 & & & 1567.305 & & \\
\hline 7/9/16 5:00 & 90.513 & & & 1567.378 & & \\
\hline 7/9/16 6:00 & 90.445 & & & 1567.446 & & \\
\hline 7/9/16 7:00 & 90.377 & & & 1567.514 & & \\
\hline 7/9/16 8:00 & 90.311 & & & 1567.58 & & \\
\hline 7/9/16 9:00 & 90.243 & & & 1567.648 & & \\
\hline 7/9/16 10:00 & 90.184 & & & 1567.707 & & \\
\hline $7 / 9 / 16$ 11:00 & 90.128 & & & 1567.763 & & \\
\hline 7/9/16 12:00 & 90.073 & & & 1567.818 & & \\
\hline 7/9/16 13:00 & 90.022 & & & 1567.869 & & \\
\hline 7/9/16 14:00 & 89.972 & & & 1567.919 & & \\
\hline 7/9/16 15:00 & 89.923 & & & 1567.968 & & \\
\hline 7/9/16 16:00 & 89.871 & & & 1568.02 & & \\
\hline 7/9/16 17:00 & 89.819 & & & 1568.072 & & \\
\hline 7/9/16 18:00 & 89.768 & & & 1568.123 & & \\
\hline 7/9/16 19:00 & 89.716 & & & 1568.175 & & \\
\hline 7/9/16 20:00 & 89.664 & & & 1568.227 & & \\
\hline 7/9/16 21:00 & 89.612 & & & 1568.279 & & \\
\hline 7/9/16 22:00 & 89.565 & & & 1568.326 & & \\
\hline 7/9/16 23:00 & 89.518 & & & 1568.373 & & \\
\hline 7/10/16 0:00 & 89.469 & & & 1568.422 & & \\
\hline 7/10/16 1:00 & 89.42 & & & 1568.471 & & \\
\hline 7/10/16 2:00 & 89.37 & & & 1568.521 & & \\
\hline 7/10/16 3:00 & 89.318 & & & 1568.573 & & \\
\hline 7/10/16 4:00 & 89.274 & & & 1568.617 & & \\
\hline 7/10/16 5:00 & 89.227 & & & 1568.664 & & \\
\hline 7/10/16 6:00 & 89.182 & & & 1568.709 & & \\
\hline 7/10/16 7:00 & 89.14 & & & 1568.751 & & \\
\hline 7/10/16 8:00 & 89.093 & & & 1568.798 & & \\
\hline 7/10/16 9:00 & 89.045 & & & 1568.846 & & \\
\hline 7/10/16 10:00 & 89.003 & & & 1568.888 & & \\
\hline 7/10/16 11:00 & 88.958 & & & 1568.933 & & \\
\hline 7/10/16 12:00 & 88.921 & & & 1568.97 & & \\
\hline 7/10/16 13:00 & 88.881 & & & 1569.01 & & \\
\hline 7/10/16 14:00 & 88.843 & & & 1569.048 & & \\
\hline 7/10/16 15:00 & 88.803 & & & 1569.088 & & \\
\hline 7/10/16 16:00 & 88.763 & & & 1569.128 & & \\
\hline
\end{tabular}


TABLE S1.2 Automatically recorded groundwater levels in the confined aquifers.

\begin{tabular}{|c|c|c|c|c|c|c|}
\hline \multirow[b]{2}{*}{ Date-Time } & \multicolumn{3}{|c|}{ Measured Depth to Water (ft TOC ${ }^{\mathrm{a}}$ ) } & \multicolumn{3}{|c|}{ Groundwater Elevation (ft $A M S L^{b}$ ) } \\
\hline & DL2-CU & DL7-CU & DL7-CL & DL2-CU & DL7-CU & DL7-CL \\
\hline 7/10/16 17:00 & 88.721 & & & 1569.17 & & \\
\hline 7/10/16 18:00 & 88.681 & & & 1569.21 & & \\
\hline 7/10/16 19:00 & 88.639 & & & 1569.252 & & \\
\hline 7/10/16 20:00 & 88.599 & & & 1569.292 & & \\
\hline $7 / 10 / 16$ 21:00 & 88.556 & & & 1569.335 & & \\
\hline $7 / 10 / 16$ 22:00 & 88.516 & & & 1569.375 & & \\
\hline $7 / 10 / 16$ 23:00 & 88.476 & & & 1569.415 & & \\
\hline $7 / 11 / 16$ 0:00 & 88.436 & & & 1569.455 & & \\
\hline $7 / 11 / 16$ 1:00 & 88.399 & & & 1569.492 & & \\
\hline $7 / 11 / 16$ 2:00 & 88.359 & & & 1569.532 & & \\
\hline $7 / 11 / 16$ 3:00 & 88.321 & & & 1569.57 & & \\
\hline $7 / 11 / 164: 00$ & 88.288 & & & 1569.603 & & \\
\hline $7 / 11 / 16$ 5:00 & 88.253 & & & 1569.638 & & \\
\hline $7 / 11 / 16$ 6:00 & 88.227 & & & 1569.664 & & \\
\hline $7 / 11 / 16$ 7:00 & 88.208 & & & 1569.683 & & \\
\hline 7/11/16 8:00 & 88.194 & & & 1569.697 & & \\
\hline 7/11/16 9:00 & 88.185 & & & 1569.706 & & \\
\hline 7/11/16 10:00 & 88.18 & & & 1569.711 & & \\
\hline 7/11/16 11:00 & 88.175 & & & 1569.716 & & \\
\hline $7 / 11 / 16$ 12:00 & 88.17 & & & 1569.721 & & \\
\hline 7/11/16 13:00 & 88.166 & & & 1569.725 & & \\
\hline 7/11/16 14:00 & 88.154 & & & 1569.737 & & \\
\hline 7/11/16 15:00 & 88.137 & & & 1569.754 & & \\
\hline 7/11/16 16:00 & 88.116 & & & 1569.775 & & \\
\hline 7/11/16 17:00 & 88.095 & & & 1569.796 & & \\
\hline 7/11/16 18:00 & 88.074 & & & 1569.817 & & \\
\hline 7/11/16 19:00 & 88.057 & & & 1569.834 & & \\
\hline $7 / 11 / 16$ 20:00 & 88.039 & & & 1569.852 & & \\
\hline 7/11/16 21:00 & 88.02 & & & 1569.871 & & \\
\hline $7 / 11 / 16$ 22:00 & 87.999 & & & 1569.892 & & \\
\hline 7/11/16 23:00 & 87.978 & & & 1569.913 & & \\
\hline $7 / 12 / 16$ 0:00 & 87.952 & & & 1569.939 & & \\
\hline $7 / 12 / 16$ 1:00 & 87.93 & & & 1569.961 & & \\
\hline $7 / 12 / 16$ 2:00 & 87.912 & & & 1569.979 & & \\
\hline $7 / 12 / 163: 00$ & 87.883 & & & 1570.008 & & \\
\hline $7 / 12 / 164: 00$ & 87.869 & & & 1570.022 & & \\
\hline $7 / 12 / 165: 00$ & 87.846 & & & 1570.045 & & \\
\hline $7 / 12 / 166: 00$ & 87.829 & & & 1570.062 & & \\
\hline $7 / 12 / 16$ 7:00 & 87.806 & & & 1570.085 & & \\
\hline $7 / 12 / 168: 00$ & 87.789 & & & 1570.102 & & \\
\hline 7/12/16 9:00 & 87.775 & & & 1570.116 & & \\
\hline 7/12/16 10:00 & 87.763 & & & 1570.128 & & \\
\hline 7/12/16 11:00 & 87.756 & & & 1570.135 & & \\
\hline 7/12/16 12:00 & 87.749 & & & 1570.142 & & \\
\hline 7/12/16 13:00 & 87.738 & & & 1570.153 & & \\
\hline 7/12/16 14:00 & 87.728 & & & 1570.163 & & \\
\hline $7 / 12 / 16$ 15:00 & 87.714 & & & 1570.177 & & \\
\hline
\end{tabular}


TABLE S1.2 Automatically recorded groundwater levels in the confined aquifers.

\begin{tabular}{|c|c|c|c|c|c|c|}
\hline \multirow[b]{2}{*}{ Date-Time } & \multicolumn{3}{|c|}{ Measured Depth to Water (ft TOC ${ }^{\mathrm{a}}$ ) } & \multicolumn{3}{|c|}{ Groundwater Elevation (ft AMSL ${ }^{\mathrm{b}}$ ) } \\
\hline & DL2-CU & DL7-CU & DL7-CL & DL2-CU & DL7-CU & DL7-CL \\
\hline 7/12/16 16:00 & 87.7 & & 115.432 & 1570.191 & & 1521.719 \\
\hline $7 / 12 / 16$ 17:00 & 87.686 & 79.17 & 115.38 & 1570.205 & 1558.086 & 1521.771 \\
\hline $7 / 12 / 16$ 18:00 & 87.667 & 79.175 & 115.345 & 1570.224 & 1558.081 & 1521.806 \\
\hline 7/12/16 19:00 & 87.653 & 79.18 & 115.298 & 1570.238 & 1558.076 & 1521.853 \\
\hline 7/12/16 20:00 & 87.639 & 79.182 & 115.256 & 1570.252 & 1558.074 & 1521.895 \\
\hline 7/12/16 21:00 & 87.627 & 79.2 & 115.214 & 1570.264 & 1558.056 & 1521.937 \\
\hline 7/12/16 22:00 & 87.62 & 79.222 & 115.172 & 1570.271 & 1558.034 & 1521.979 \\
\hline 7/12/16 23:00 & 87.613 & 79.247 & 115.13 & 1570.278 & 1558.009 & 1522.021 \\
\hline 7/13/16 0:00 & 87.603 & 79.275 & 115.085 & 1570.288 & 1557.981 & 1522.066 \\
\hline 7/13/16 1:00 & 87.596 & 79.294 & 115.039 & 1570.295 & 1557.962 & 1522.112 \\
\hline $7 / 13 / 16$ 2:00 & 87.589 & 79.315 & 114.994 & 1570.302 & 1557.941 & 1522.157 \\
\hline 7/13/16 3:00 & 87.587 & 79.328 & 114.947 & 1570.304 & 1557.928 & 1522.204 \\
\hline $7 / 13 / 164: 00$ & 87.589 & 79.375 & 114.907 & 1570.302 & 1557.881 & 1522.244 \\
\hline 7/13/16 5:00 & 87.599 & 79.421 & 114.868 & 1570.292 & 1557.835 & 1522.283 \\
\hline 7/13/16 6:00 & 87.608 & 79.452 & 114.826 & 1570.283 & 1557.804 & 1522.325 \\
\hline 7/13/16 7:00 & 87.627 & 79.494 & 114.791 & 1570.264 & 1557.762 & 1522.36 \\
\hline 7/13/16 8:00 & 87.646 & 79.54 & 114.753 & 1570.245 & 1557.716 & 1522.398 \\
\hline 7/13/16 9:00 & 87.672 & 79.57 & 114.72 & 1570.219 & 1557.686 & 1522.431 \\
\hline $7 / 13 / 16$ 10:00 & 87.702 & 79.61 & 114.69 & 1570.189 & 1557.646 & 1522.461 \\
\hline $7 / 13 / 16$ 11:00 & 87.738 & 79.659 & 114.669 & 1570.153 & 1557.597 & 1522.482 \\
\hline $7 / 13 / 16$ 12:00 & 87.763 & 79.705 & 114.652 & 1570.128 & 1557.551 & 1522.499 \\
\hline 7/13/16 13:00 & 87.796 & 79.754 & 114.636 & 1570.095 & 1557.502 & 1522.515 \\
\hline 7/13/16 14:00 & 87.832 & 79.805 & 114.615 & 1570.059 & 1557.451 & 1522.536 \\
\hline 7/13/16 15:00 & 88.043 & 79.858 & 114.596 & 1569.848 & 1557.398 & 1522.555 \\
\hline 7/13/16 16:00 & 88.396 & 79.919 & 114.573 & 1569.495 & 1557.337 & 1522.578 \\
\hline 7/13/16 17:00 & 88.768 & 79.984 & 114.556 & 1569.123 & 1557.272 & 1522.595 \\
\hline 7/13/16 18:00 & 89.13 & 80.084 & 114.566 & 1568.761 & 1557.172 & 1522.585 \\
\hline 7/13/16 19:00 & 89.474 & 80.291 & 114.599 & 1568.417 & 1556.965 & 1522.552 \\
\hline $7 / 13 / 16$ 20:00 & 89.805 & 80.57 & 114.634 & 1568.086 & 1556.686 & 1522.517 \\
\hline 7/13/16 21:00 & 90.121 & 80.879 & 114.673 & 1567.77 & 1556.377 & 1522.478 \\
\hline 7/13/16 22:00 & 90.426 & 81.202 & 114.713 & 1567.465 & 1556.054 & 1522.438 \\
\hline 7/13/16 23:00 & 90.716 & 81.521 & 114.744 & 1567.175 & 1555.735 & 1522.407 \\
\hline 7/14/16 0:00 & 90.996 & 81.818 & 114.767 & 1566.895 & 1555.438 & 1522.384 \\
\hline 7/14/16 1:00 & 91.259 & 82.088 & 114.798 & 1566.632 & 1555.168 & 1522.353 \\
\hline 7/14/16 2:00 & 91.511 & 82.33 & 114.821 & 1566.38 & 1554.926 & 1522.33 \\
\hline 7/14/16 3:00 & 91.758 & 82.553 & 114.851 & 1566.133 & 1554.703 & 1522.3 \\
\hline 7/14/16 4:00 & 91.988 & 82.755 & 114.886 & 1565.903 & 1554.501 & 1522.265 \\
\hline 7/14/16 5:00 & 92.202 & 82.948 & 114.917 & 1565.689 & 1554.308 & 1522.234 \\
\hline 7/14/16 6:00 & 92.407 & 83.143 & 114.971 & 1565.484 & 1554.113 & 1522.18 \\
\hline 7/14/16 7:00 & 92.581 & 83.324 & 115.011 & 1565.31 & 1553.932 & 1522.14 \\
\hline 7/14/16 8:00 & 92.856 & 83.499 & 115.06 & 1565.035 & 1553.757 & 1522.091 \\
\hline 7/14/16 9:00 & 93.143 & 83.68 & 115.116 & 1564.748 & 1553.576 & 1522.035 \\
\hline $7 / 14 / 16$ 10:00 & 93.379 & 83.861 & 115.177 & 1564.512 & 1553.395 & 1521.974 \\
\hline 7/14/16 11:00 & 93.56 & 84.057 & 115.245 & 1564.331 & 1553.199 & 1521.906 \\
\hline 7/14/16 12:00 & 93.739 & 84.245 & 115.298 & 1564.152 & 1553.011 & 1521.853 \\
\hline 7/14/16 13:00 & 93.995 & 84.477 & 115.357 & 1563.896 & 1552.779 & 1521.794 \\
\hline $7 / 14 / 16$ 14:00 & 94.322 & 84.761 & 115.406 & 1563.569 & 1552.495 & 1521.745 \\
\hline
\end{tabular}


TABLE S1.2 Automatically recorded groundwater levels in the confined aquifers.

\begin{tabular}{|c|c|c|c|c|c|c|}
\hline \multirow[b]{2}{*}{ Date-Time } & \multicolumn{3}{|c|}{ Measured Depth to Water (ft TOC ${ }^{a}$ ) } & \multicolumn{3}{|c|}{ Groundwater Elevation (ft AMSL ${ }^{\mathrm{b}}$ ) } \\
\hline & DL2-CU & DL7-CU & DL7-CL & DL2-CU & DL7-CU & DL7-CL \\
\hline $7 / 14 / 16$ 15:00 & 94.689 & 85.075 & 115.458 & 1563.202 & 1552.181 & 1521.693 \\
\hline $7 / 14 / 16$ 16:00 & 95.084 & 85.407 & 115.514 & 1562.807 & 1551.849 & 1521.637 \\
\hline 7/14/16 17:00 & 95.47 & 85.756 & 115.568 & 1562.421 & 1551.5 & 1521.583 \\
\hline 7/14/16 18:00 & 95.86 & 86.093 & 115.619 & 1562.031 & 1551.163 & 1521.532 \\
\hline 7/14/16 19:00 & 96.251 & 86.43 & 115.666 & 1561.64 & 1550.826 & 1521.485 \\
\hline 7/14/16 20:00 & 96.644 & 86.749 & 115.71 & 1561.247 & 1550.507 & 1521.441 \\
\hline 7/14/16 21:00 & 97.044 & 87.067 & 115.753 & 1560.847 & 1550.189 & 1521.398 \\
\hline 7/14/16 22:00 & 97.446 & 87.376 & 115.799 & 1560.445 & 1549.88 & 1521.352 \\
\hline 7/14/16 23:00 & 97.879 & 87.672 & 115.839 & 1560.012 & 1549.584 & 1521.312 \\
\hline 7/15/16 0:00 & 98.312 & 87.953 & 115.886 & 1559.579 & 1549.303 & 1521.265 \\
\hline 7/15/16 1:00 & 98.733 & 88.199 & 115.923 & 1559.158 & 1549.057 & 1521.228 \\
\hline $7 / 15 / 16$ 2:00 & 99.144 & 88.429 & 115.954 & 1558.747 & 1548.827 & 1521.197 \\
\hline 7/15/16 3:00 & 99.549 & 88.636 & 115.977 & 1558.342 & 1548.62 & 1521.174 \\
\hline 7/15/16 4:00 & 99.947 & 88.836 & 116.017 & 1557.944 & 1548.42 & 1521.134 \\
\hline 7/15/16 5:00 & 100.335 & 89.027 & 116.064 & 1557.556 & 1548.229 & 1521.087 \\
\hline $7 / 15 / 16$ 6:00 & 100.695 & 89.21 & 116.125 & 1557.196 & 1548.046 & 1521.026 \\
\hline 7/15/16 7:00 & 101.024 & 89.382 & 116.179 & 1556.867 & 1547.874 & 1520.972 \\
\hline 7/15/16 8:00 & 101.332 & 89.559 & 116.258 & 1556.559 & 1547.697 & 1520.893 \\
\hline 7/15/16 9:00 & 101.631 & 89.745 & 116.359 & 1556.26 & 1547.511 & 1520.792 \\
\hline $7 / 15 / 16$ 10:00 & 101.812 & 89.922 & 116.455 & 1556.079 & 1547.334 & 1520.696 \\
\hline 7/15/16 11:00 & 101.899 & 90.077 & 116.548 & 1555.992 & 1547.179 & 1520.603 \\
\hline $7 / 15 / 16$ 12:00 & 101.911 & 90.233 & 116.623 & 1555.98 & 1547.023 & 1520.528 \\
\hline 7/15/16 13:00 & 101.671 & 90.338 & 116.698 & 1556.22 & 1546.918 & 1520.453 \\
\hline 7/15/16 14:00 & 101.353 & 90.405 & 116.764 & 1556.538 & 1546.851 & 1520.387 \\
\hline $7 / 15 / 16$ 15:00 & 100.984 & 90.47 & 116.839 & 1556.907 & 1546.786 & 1520.312 \\
\hline 7/15/16 16:00 & 100.57 & 90.526 & 116.895 & 1557.321 & 1546.73 & 1520.256 \\
\hline 7/15/16 17:00 & 100.177 & 90.589 & 116.97 & 1557.714 & 1546.667 & 1520.181 \\
\hline 7/15/16 18:00 & 99.794 & 90.647 & 117.028 & 1558.097 & 1546.609 & 1520.123 \\
\hline 7/15/16 19:00 & 99.448 & 90.698 & 117.094 & 1558.443 & 1546.558 & 1520.057 \\
\hline $7 / 15 / 16$ 20:00 & 99.126 & 90.733 & 117.145 & 1558.765 & 1546.523 & 1520.006 \\
\hline $7 / 15 / 16$ 21:00 & 98.834 & 90.752 & 117.194 & 1559.057 & 1546.504 & 1519.957 \\
\hline 7/15/16 22:00 & 98.566 & 90.77 & 117.244 & 1559.325 & 1546.486 & 1519.907 \\
\hline 7/15/16 23:00 & 98.316 & 90.773 & 117.302 & 1559.575 & 1546.483 & 1519.849 \\
\hline 7/16/16 0:00 & 98.086 & 90.754 & 117.358 & 1559.805 & 1546.502 & 1519.793 \\
\hline 7/16/16 1:00 & 97.869 & 90.707 & 117.428 & 1560.022 & 1546.549 & 1519.723 \\
\hline 7/16/16 2:00 & 97.665 & 90.635 & 117.485 & 1560.226 & 1546.621 & 1519.666 \\
\hline 7/16/16 3:00 & 97.472 & 90.54 & 117.543 & 1560.419 & 1546.716 & 1519.608 \\
\hline 7/16/16 4:00 & 97.293 & 90.468 & 117.609 & 1560.598 & 1546.788 & 1519.542 \\
\hline 7/16/16 5:00 & 97.126 & 90.391 & 117.667 & 1560.765 & 1546.865 & 1519.484 \\
\hline 7/16/16 6:00 & 96.971 & 90.301 & 117.735 & 1560.92 & 1546.955 & 1519.416 \\
\hline 7/16/16 7:00 & 96.825 & 90.203 & 117.81 & 1561.066 & 1547.053 & 1519.341 \\
\hline 7/16/16 8:00 & 96.684 & 90.108 & 117.897 & 1561.207 & 1547.148 & 1519.254 \\
\hline 7/16/16 9:00 & 96.554 & 90.017 & 118.037 & 1561.337 & 1547.239 & 1519.114 \\
\hline $7 / 16 / 16$ 10:00 & 96.425 & 89.931 & 118.222 & 1561.466 & 1547.325 & 1518.929 \\
\hline 7/16/16 11:00 & 96.305 & 89.857 & 118.386 & 1561.586 & 1547.399 & 1518.765 \\
\hline 7/16/16 12:00 & 96.187 & 89.796 & 118.449 & 1561.704 & 1547.46 & 1518.702 \\
\hline $7 / 16 / 16$ 13:00 & 96.074 & 89.74 & 118.482 & 1561.817 & 1547.516 & 1518.669 \\
\hline
\end{tabular}


TABLE S1.2 Automatically recorded groundwater levels in the confined aquifers.

\begin{tabular}{|c|c|c|c|c|c|c|}
\hline \multirow[b]{2}{*}{ Date-Time } & \multicolumn{3}{|c|}{ Measured Depth to Water (ft TOC ${ }^{a}$ ) } & \multicolumn{3}{|c|}{ Groundwater Elevation (ft AMSL ${ }^{\mathrm{b}}$ ) } \\
\hline & DL2-CU & DL7-CU & DL7-CL & DL2-CU & DL7-CU & DL7-CL \\
\hline 7/16/16 14:00 & 95.966 & 89.687 & 118.5 & 1561.925 & 1547.569 & 1518.651 \\
\hline $7 / 16 / 16$ 15:00 & 95.86 & 89.645 & 118.531 & 1562.031 & 1547.611 & 1518.62 \\
\hline $7 / 16 / 16$ 16:00 & 95.755 & 89.613 & 118.545 & 1562.136 & 1547.643 & 1518.606 \\
\hline 7/16/16 17:00 & 95.649 & 89.587 & 118.564 & 1562.242 & 1547.669 & 1518.587 \\
\hline $7 / 16 / 16$ 18:00 & 95.538 & 89.561 & 118.585 & 1562.353 & 1547.695 & 1518.566 \\
\hline 7/16/16 19:00 & 95.432 & 89.545 & 118.596 & 1562.459 & 1547.711 & 1518.555 \\
\hline 7/16/16 20:00 & 95.322 & 89.524 & 118.608 & 1562.569 & 1547.732 & 1518.543 \\
\hline 7/16/16 21:00 & 95.216 & 89.499 & 118.615 & 1562.675 & 1547.757 & 1518.536 \\
\hline 7/16/16 22:00 & 95.112 & 89.475 & 118.622 & 1562.779 & 1547.781 & 1518.529 \\
\hline 7/16/16 23:00 & 95.011 & 89.448 & 118.629 & 1562.88 & 1547.808 & 1518.522 \\
\hline 7/17/16 0:00 & 94.905 & 89.403 & 118.622 & 1562.986 & 1547.853 & 1518.529 \\
\hline 7/17/16 1:00 & 94.804 & 89.336 & 118.608 & 1563.087 & 1547.92 & 1518.543 \\
\hline $7 / 17 / 16$ 2:00 & 94.701 & 89.255 & 118.632 & 1563.19 & 1548.001 & 1518.519 \\
\hline 7/17/16 3:00 & 94.602 & 89.162 & 118.732 & 1563.289 & 1548.094 & 1518.419 \\
\hline 7/17/16 4:00 & 94.505 & 89.048 & 118.821 & 1563.386 & 1548.208 & 1518.33 \\
\hline 7/17/16 5:00 & 94.409 & 88.943 & 118.891 & 1563.482 & 1548.313 & 1518.26 \\
\hline $7 / 17 / 16$ 6:00 & 94.322 & 88.838 & 118.983 & 1563.569 & 1548.418 & 1518.168 \\
\hline 7/17/16 7:00 & 94.24 & 88.743 & 119.079 & 1563.651 & 1548.513 & 1518.072 \\
\hline 7/17/16 8:00 & 94.16 & 88.662 & 119.175 & 1563.731 & 1548.594 & 1517.976 \\
\hline 7/17/16 9:00 & 94.08 & 88.583 & 119.264 & 1563.811 & 1548.673 & 1517.887 \\
\hline $7 / 17 / 16$ 10:00 & 94.007 & 88.522 & 119.287 & 1563.884 & 1548.734 & 1517.864 \\
\hline 7/17/16 11:00 & 93.934 & 88.476 & 119.289 & 1563.957 & 1548.78 & 1517.862 \\
\hline 7/17/16 12:00 & 93.859 & 88.432 & 119.285 & 1564.032 & 1548.824 & 1517.866 \\
\hline 7/17/16 13:00 & 93.79 & 88.401 & 119.264 & 1564.101 & 1548.855 & 1517.887 \\
\hline 7/17/16 14:00 & 93.72 & 88.367 & 119.242 & 1564.171 & 1548.889 & 1517.909 \\
\hline 7/17/16 15:00 & 93.649 & 88.339 & 119.224 & 1564.242 & 1548.917 & 1517.927 \\
\hline 7/17/16 16:00 & 93.581 & 88.325 & 119.203 & 1564.31 & 1548.931 & 1517.948 \\
\hline 7/17/16 17:00 & 93.515 & 88.318 & 119.184 & 1564.376 & 1548.938 & 1517.967 \\
\hline 7/17/16 18:00 & 93.454 & 88.315 & 119.172 & 1564.437 & 1548.941 & 1517.979 \\
\hline 7/17/16 19:00 & 93.393 & 88.32 & 119.168 & 1564.498 & 1548.936 & 1517.983 \\
\hline 7/17/16 20:00 & 93.336 & 88.315 & 119.17 & 1564.555 & 1548.941 & 1517.981 \\
\hline 7/17/16 21:00 & 93.28 & 88.311 & 119.165 & 1564.611 & 1548.945 & 1517.986 \\
\hline $7 / 17 / 16$ 22:00 & 93.228 & 88.304 & 119.151 & 1564.663 & 1548.952 & 1518 \\
\hline $7 / 17 / 16$ 23:00 & 93.181 & 88.301 & 119.135 & 1564.71 & 1548.955 & 1518.016 \\
\hline 7/18/16 0:00 & 93.129 & 88.283 & 119.107 & 1564.762 & 1548.973 & 1518.044 \\
\hline 7/18/16 1:00 & 93.08 & 88.248 & 119.065 & 1564.811 & 1549.008 & 1518.086 \\
\hline 7/18/16 2:00 & 93.028 & 88.188 & 119.027 & 1564.863 & 1549.068 & 1518.124 \\
\hline 7/18/16 3:00 & 92.981 & 88.113 & 118.978 & 1564.91 & 1549.143 & 1518.173 \\
\hline 7/18/16 4:00 & 92.939 & 88.06 & 118.943 & 1564.952 & 1549.196 & 1518.208 \\
\hline 7/18/16 5:00 & 92.894 & 88.018 & 118.917 & 1564.997 & 1549.238 & 1518.234 \\
\hline $7 / 18 / 16$ 6:00 & 92.856 & 87.948 & 118.905 & 1565.035 & 1549.308 & 1518.246 \\
\hline 7/18/16 7:00 & 92.828 & 87.888 & 118.912 & 1565.063 & 1549.368 & 1518.239 \\
\hline 7/18/16 8:00 & 92.807 & 87.867 & 118.922 & 1565.084 & 1549.389 & 1518.229 \\
\hline 7/18/16 9:00 & 92.802 & 87.904 & 118.933 & 1565.089 & 1549.352 & 1518.218 \\
\hline 7/18/16 10:00 & 92.8 & 87.974 & 118.938 & 1565.091 & 1549.282 & 1518.213 \\
\hline 7/18/16 11:00 & 92.809 & 88.057 & 118.941 & 1565.082 & 1549.199 & 1518.21 \\
\hline 7/18/16 12:00 & 92.819 & 88.139 & 118.936 & 1565.072 & 1549.117 & 1518.215 \\
\hline
\end{tabular}


TABLE S1.2 Automatically recorded groundwater levels in the confined aquifers.

\begin{tabular}{|c|c|c|c|c|c|c|}
\hline \multirow[b]{2}{*}{ Date-Time } & \multicolumn{3}{|c|}{ Measured Depth to Water (ft TOC ${ }^{\mathrm{a}}$ ) } & \multicolumn{3}{|c|}{ Groundwater Elevation (ft AMSL ${ }^{\mathrm{b}}$ ) } \\
\hline & DL2-CU & DL7-CU & DL7-CL & DL2-CU & DL7-CU & DL7-CL \\
\hline $7 / 18 / 16$ 13:00 & 92.828 & 88.222 & 118.926 & 1565.063 & 1549.034 & 1518.225 \\
\hline $7 / 18 / 16$ 14:00 & 92.84 & 88.301 & 118.917 & 1565.051 & 1548.955 & 1518.234 \\
\hline $7 / 18 / 16$ 15:00 & 92.828 & 88.378 & 118.91 & 1565.063 & 1548.878 & 1518.241 \\
\hline 7/18/16 16:00 & 92.805 & 88.469 & 118.903 & 1565.086 & 1548.787 & 1518.248 \\
\hline 7/18/16 17:00 & 92.783 & 88.569 & 118.905 & 1565.108 & 1548.687 & 1518.246 \\
\hline 7/18/16 18:00 & 92.772 & 88.673 & 118.898 & 1565.119 & 1548.583 & 1518.253 \\
\hline 7/18/16 19:00 & 92.805 & 88.78 & 118.894 & 1565.086 & 1548.476 & 1518.257 \\
\hline 7/18/16 20:00 & 92.892 & 88.883 & 118.889 & 1564.999 & 1548.373 & 1518.262 \\
\hline 7/18/16 21:00 & 93.023 & 88.969 & 118.884 & 1564.868 & 1548.287 & 1518.267 \\
\hline 7/18/16 22:00 & 93.179 & 89.062 & 118.887 & 1564.712 & 1548.194 & 1518.264 \\
\hline 7/18/16 23:00 & 93.35 & 89.145 & 118.894 & 1564.541 & 1548.111 & 1518.257 \\
\hline 7/19/16 0:00 & 93.529 & 89.213 & 118.894 & 1564.362 & 1548.043 & 1518.257 \\
\hline 7/19/16 1:00 & 93.703 & 89.266 & 118.894 & 1564.188 & 1547.99 & 1518.257 \\
\hline 7/19/16 2:00 & 93.877 & 89.294 & 118.88 & 1564.014 & 1547.962 & 1518.271 \\
\hline 7/19/16 3:00 & 94.051 & 89.31 & 118.868 & 1563.84 & 1547.946 & 1518.283 \\
\hline $7 / 19 / 164: 00$ & 94.221 & 89.324 & 118.856 & 1563.67 & 1547.932 & 1518.295 \\
\hline 7/19/16 5:00 & 94.381 & 89.329 & 118.852 & 1563.51 & 1547.927 & 1518.299 \\
\hline $7 / 19 / 16$ 6:00 & 94.527 & 89.341 & 118.852 & 1563.364 & 1547.915 & 1518.299 \\
\hline 7/19/16 7:00 & 94.661 & 89.357 & 118.856 & 1563.23 & 1547.899 & 1518.295 \\
\hline 7/19/16 8:00 & 94.91 & 89.38 & 118.859 & 1562.981 & 1547.876 & 1518.292 \\
\hline 7/19/16 9:00 & 95.152 & 89.429 & 118.882 & 1562.739 & 1547.827 & 1518.269 \\
\hline $7 / 19 / 16$ 10:00 & 95.378 & 89.499 & 118.917 & 1562.513 & 1547.757 & 1518.234 \\
\hline 7/19/16 11:00 & 95.609 & 89.585 & 118.959 & 1562.282 & 1547.671 & 1518.192 \\
\hline 7/19/16 12:00 & 95.818 & 89.682 & 118.999 & 1562.073 & 1547.574 & 1518.152 \\
\hline 7/19/16 13:00 & 95.87 & 89.796 & 119.032 & 1562.021 & 1547.46 & 1518.119 \\
\hline 7/19/16 14:00 & 96.009 & 89.903 & 119.062 & 1561.882 & 1547.353 & 1518.089 \\
\hline 7/19/16 15:00 & 96.143 & 89.998 & 119.058 & 1561.748 & 1547.258 & 1518.093 \\
\hline 7/19/16 16:00 & 96.43 & 90.026 & 119.018 & 1561.461 & 1547.23 & 1518.133 \\
\hline 7/19/16 17:00 & 96.747 & 90.012 & 118.985 & 1561.144 & 1547.244 & 1518.166 \\
\hline 7/19/16 18:00 & 96.935 & 89.971 & 118.952 & 1560.956 & 1547.285 & 1518.199 \\
\hline 7/19/16 19:00 & 97.013 & 89.912 & 118.922 & 1560.878 & 1547.344 & 1518.229 \\
\hline 7/19/16 20:00 & 97.119 & 89.871 & 118.957 & 1560.772 & 1547.385 & 1518.194 \\
\hline 7/19/16 21:00 & 97.229 & 89.875 & 118.994 & 1560.662 & 1547.381 & 1518.157 \\
\hline 7/19/16 22:00 & 97.335 & 89.905 & 119.029 & 1560.556 & 1547.351 & 1518.122 \\
\hline 7/19/16 23:00 & 97.434 & 89.952 & 119.062 & 1560.457 & 1547.304 & 1518.089 \\
\hline 7/20/16 0:00 & 97.528 & 89.998 & 119.086 & 1560.363 & 1547.258 & 1518.065 \\
\hline 7/20/16 1:00 & 97.625 & 90.036 & 119.104 & 1560.266 & 1547.22 & 1518.047 \\
\hline 7/20/16 2:00 & 97.719 & 90.066 & 119.118 & 1560.172 & 1547.19 & 1518.033 \\
\hline 7/20/16 3:00 & 97.818 & 90.101 & 119.132 & 1560.073 & 1547.155 & 1518.019 \\
\hline 7/20/16 4:00 & 97.916 & 90.15 & 119.156 & 1559.975 & 1547.106 & 1517.995 \\
\hline 7/20/16 5:00 & 98.02 & 90.196 & 119.184 & 1559.871 & 1547.06 & 1517.967 \\
\hline 7/20/16 6:00 & 98.13 & 90.222 & 119.231 & 1559.761 & 1547.034 & 1517.92 \\
\hline 7/20/16 7:00 & 98.241 & 90.219 & 119.278 & 1559.65 & 1547.037 & 1517.873 \\
\hline 7/20/16 8:00 & 98.356 & 90.236 & 119.327 & 1559.535 & 1547.02 & 1517.824 \\
\hline 7/20/16 9:00 & 98.479 & 90.333 & 119.402 & 1559.412 & 1546.923 & 1517.749 \\
\hline $7 / 20 / 16$ 10:00 & 98.617 & 90.475 & 119.584 & 1559.274 & 1546.781 & 1517.567 \\
\hline $7 / 20 / 16$ 11:00 & 98.761 & 90.605 & 119.809 & 1559.13 & 1546.651 & 1517.342 \\
\hline
\end{tabular}


TABLE S1.2 Automatically recorded groundwater levels in the confined aquifers.

\begin{tabular}{|c|c|c|c|c|c|c|}
\hline \multirow[b]{2}{*}{ Date-Time } & \multicolumn{3}{|c|}{ Measured Depth to Water (ft TOC ${ }^{a}$ ) } & \multicolumn{3}{|c|}{ Groundwater Elevation (ft AMSL ${ }^{\mathrm{b}}$ ) } \\
\hline & DL2-CU & DL7-CU & DL7-CL & DL2-CU & DL7-CU & DL7-CL \\
\hline $7 / 20 / 16$ 12:00 & 98.897 & 90.724 & 120.029 & 1558.994 & 1546.532 & 1517.122 \\
\hline $7 / 20 / 16$ 13:00 & 99.029 & 90.824 & 120.099 & 1558.862 & 1546.432 & 1517.052 \\
\hline $7 / 20 / 16$ 14:00 & 99.158 & 90.886 & 120.101 & 1558.733 & 1546.37 & 1517.05 \\
\hline 7/20/16 15:00 & 99.413 & 90.912 & 120.083 & 1558.478 & 1546.344 & 1517.068 \\
\hline $7 / 20 / 16$ 16:00 & 99.695 & 90.919 & 120.062 & 1558.196 & 1546.337 & 1517.089 \\
\hline 7/20/16 17:00 & 99.951 & 90.933 & 120.029 & 1557.94 & 1546.323 & 1517.122 \\
\hline 7/20/16 18:00 & 100.172 & 90.914 & 119.989 & 1557.719 & 1546.342 & 1517.162 \\
\hline 7/20/16 19:00 & 100.358 & 90.842 & 119.959 & 1557.533 & 1546.414 & 1517.192 \\
\hline 7/20/16 20:00 & 100.537 & 90.745 & 119.994 & 1557.354 & 1546.511 & 1517.157 \\
\hline $7 / 20 / 16$ 21:00 & 100.704 & 90.645 & 120.141 & 1557.187 & 1546.611 & 1517.01 \\
\hline $7 / 20 / 16$ 22:00 & 100.859 & 90.594 & 120.277 & 1557.032 & 1546.662 & 1516.874 \\
\hline 7/20/16 23:00 & 101.005 & 90.61 & 120.305 & 1556.886 & 1546.646 & 1516.846 \\
\hline $7 / 21 / 16$ 0:00 & 101.137 & 90.666 & 120.354 & 1556.754 & 1546.59 & 1516.797 \\
\hline 7/21/16 1:00 & 101.266 & 90.731 & 120.523 & 1556.625 & 1546.525 & 1516.628 \\
\hline $7 / 21 / 16$ 2:00 & 101.396 & 90.796 & 120.705 & 1556.495 & 1546.46 & 1516.446 \\
\hline $7 / 21 / 16$ 3:00 & 101.525 & 90.873 & 120.897 & 1556.366 & 1546.383 & 1516.254 \\
\hline $7 / 21 / 164: 00$ & 101.657 & 90.956 & 121.096 & 1556.234 & 1546.3 & 1516.055 \\
\hline $7 / 21 / 16$ 5:00 & 101.781 & 91.054 & 121.298 & 1556.11 & 1546.202 & 1515.853 \\
\hline $7 / 21 / 16$ 6:00 & 101.913 & 91.165 & 121.497 & 1555.978 & 1546.091 & 1515.654 \\
\hline $7 / 21 / 16$ 7:00 & 102.052 & 91.277 & 121.686 & 1555.839 & 1545.979 & 1515.465 \\
\hline 7/21/16 8:00 & 102.235 & 91.396 & 121.773 & 1555.656 & 1545.86 & 1515.378 \\
\hline 7/21/16 9:00 & 102.513 & 91.563 & 121.798 & 1555.378 & 1545.693 & 1515.353 \\
\hline $7 / 21 / 16$ 10:00 & 102.859 & 91.781 & 121.803 & 1555.032 & 1545.475 & 1515.348 \\
\hline 7/21/16 11:00 & 103.343 & 92.037 & 121.796 & 1554.548 & 1545.219 & 1515.355 \\
\hline $7 / 21 / 16$ 12:00 & 103.753 & 92.295 & 121.777 & 1554.138 & 1544.961 & 1515.374 \\
\hline 7/21/16 13:00 & 104.176 & 92.567 & 121.747 & 1553.715 & 1544.689 & 1515.404 \\
\hline 7/21/16 14:00 & 104.597 & 92.832 & 121.712 & 1553.294 & 1544.424 & 1515.439 \\
\hline 7/21/16 15:00 & 105.014 & 93.093 & 121.667 & 1552.877 & 1544.163 & 1515.484 \\
\hline 7/21/16 16:00 & 105.416 & 93.341 & 121.616 & 1552.475 & 1543.915 & 1515.535 \\
\hline 7/21/16 17:00 & 105.679 & 93.569 & 121.562 & 1552.212 & 1543.687 & 1515.589 \\
\hline 7/21/16 18:00 & 105.889 & 93.778 & 121.511 & 1552.002 & 1543.478 & 1515.64 \\
\hline 7/21/16 19:00 & 106.114 & 93.978 & 121.468 & 1551.777 & 1543.278 & 1515.683 \\
\hline $7 / 21 / 16$ 20:00 & 106.343 & 94.18 & 121.426 & 1551.548 & 1543.076 & 1515.725 \\
\hline $7 / 21 / 16$ 21:00 & 106.566 & 94.376 & 121.391 & 1551.325 & 1542.88 & 1515.76 \\
\hline $7 / 21 / 16$ 22:00 & 106.79 & 94.534 & 121.368 & 1551.101 & 1542.722 & 1515.783 \\
\hline 7/21/16 23:00 & 106.994 & 94.65 & 121.354 & 1550.897 & 1542.606 & 1515.797 \\
\hline $7 / 22 / 160: 00$ & 107.182 & 94.752 & 121.349 & 1550.709 & 1542.504 & 1515.802 \\
\hline 7/22/16 1:00 & 107.359 & 94.871 & 121.344 & 1550.532 & 1542.385 & 1515.807 \\
\hline $7 / 22 / 16$ 2:00 & 107.526 & 95.003 & 121.344 & 1550.365 & 1542.253 & 1515.807 \\
\hline 7/22/16 3:00 & 107.686 & 95.152 & 121.347 & 1550.205 & 1542.104 & 1515.804 \\
\hline $7 / 22 / 164: 00$ & 107.848 & 95.338 & 121.368 & 1550.043 & 1541.918 & 1515.783 \\
\hline $7 / 22 / 16$ 5:00 & 108.015 & 95.585 & 121.401 & 1549.876 & 1541.671 & 1515.75 \\
\hline $7 / 22 / 16$ 6:00 & 108.189 & 95.843 & 121.457 & 1549.702 & 1541.413 & 1515.694 \\
\hline $7 / 22 / 16$ 7:00 & 108.363 & 96.108 & 121.511 & 1549.528 & 1541.148 & 1515.64 \\
\hline 7/22/16 8:00 & 108.537 & 96.377 & 121.564 & 1549.354 & 1540.879 & 1515.587 \\
\hline $7 / 22 / 16$ 9:00 & 108.712 & 96.64 & 121.602 & 1549.179 & 1540.616 & 1515.549 \\
\hline $7 / 22 / 16$ 10:00 & 108.879 & 96.896 & 121.632 & 1549.012 & 1540.36 & 1515.519 \\
\hline
\end{tabular}


TABLE S1.2 Automatically recorded groundwater levels in the confined aquifers.

\begin{tabular}{|c|c|c|c|c|c|c|}
\hline \multirow[b]{2}{*}{ Date-Time } & \multicolumn{3}{|c|}{ Measured Depth to Water (ft TOC ${ }^{a}$ ) } & \multicolumn{3}{|c|}{ Groundwater Elevation (ft AMSL ${ }^{b}$ ) } \\
\hline & DL2-CU & DL7-CU & DL7-CL & DL2-CU & DL7-CU & DL7-CL \\
\hline 7/22/16 11:00 & 109.034 & 97.119 & 121.674 & 1548.857 & 1540.137 & 1515.477 \\
\hline $7 / 22 / 16$ 12:00 & 109.163 & 97.309 & 121.719 & 1548.728 & 1539.947 & 1515.432 \\
\hline $7 / 22 / 16$ 13:00 & 109.276 & 97.491 & 121.761 & 1548.615 & 1539.765 & 1515.39 \\
\hline $7 / 22 / 1614: 00$ & 109.384 & 97.677 & 121.798 & 1548.507 & 1539.579 & 1515.353 \\
\hline $7 / 22 / 16$ 15:00 & 109.497 & 97.863 & 121.834 & 1548.394 & 1539.393 & 1515.317 \\
\hline $7 / 22 / 16$ 16:00 & 109.754 & 98.044 & 121.862 & 1548.137 & 1539.212 & 1515.289 \\
\hline 7/22/16 17:00 & 110.194 & 98.214 & 121.892 & 1547.697 & 1539.042 & 1515.259 \\
\hline $7 / 22 / 16$ 18:00 & 110.763 & 98.383 & 121.923 & 1547.128 & 1538.873 & 1515.228 \\
\hline 7/22/16 19:00 & 111.318 & 98.551 & 121.958 & 1546.573 & 1538.705 & 1515.193 \\
\hline 7/22/16 20:00 & 111.836 & 98.723 & 122.004 & 1546.055 & 1538.533 & 1515.147 \\
\hline $7 / 22 / 16$ 21:00 & 112.318 & 98.909 & 122.047 & 1545.573 & 1538.347 & 1515.104 \\
\hline $7 / 22 / 16$ 22:00 & 112.798 & 99.111 & 122.103 & 1545.093 & 1538.145 & 1515.048 \\
\hline $7 / 22 / 16$ 23:00 & 113.261 & 99.325 & 122.166 & 1544.63 & 1537.931 & 1514.985 \\
\hline 7/23/16 0:00 & 113.692 & 99.539 & 122.231 & 1544.199 & 1537.717 & 1514.92 \\
\hline 7/23/16 1:00 & 114.08 & 99.771 & 122.302 & 1543.811 & 1537.485 & 1514.849 \\
\hline $7 / 23 / 16$ 2:00 & 114.433 & 100.011 & 122.381 & 1543.458 & 1537.245 & 1514.77 \\
\hline 7/23/16 3:00 & 114.743 & 100.243 & 122.452 & 1543.148 & 1537.013 & 1514.699 \\
\hline 7/23/16 4:00 & 115.032 & 100.489 & 122.54 & 1542.859 & 1536.767 & 1514.611 \\
\hline $7 / 23 / 16$ 5:00 & 115.275 & 100.738 & 122.627 & 1542.616 & 1536.518 & 1514.524 \\
\hline $7 / 23 / 16$ 6:00 & 115.477 & 100.989 & 122.718 & 1542.414 & 1536.267 & 1514.433 \\
\hline 7/23/16 7:00 & 115.646 & 101.257 & 122.819 & 1542.245 & 1535.999 & 1514.332 \\
\hline 7/23/16 8:00 & 115.802 & 101.543 & 122.917 & 1542.089 & 1535.713 & 1514.234 \\
\hline 7/23/16 9:00 & 115.943 & 101.817 & 123.013 & 1541.948 & 1535.439 & 1514.138 \\
\hline $7 / 23 / 16$ 10:00 & 116.063 & 102.059 & 123.114 & 1541.828 & 1535.197 & 1514.037 \\
\hline 7/23/16 11:00 & 116.18 & 102.272 & 123.215 & 1541.711 & 1534.984 & 1513.936 \\
\hline $7 / 23 / 16$ 12:00 & 116.272 & 102.465 & 123.318 & 1541.619 & 1534.791 & 1513.833 \\
\hline 7/23/16 13:00 & 116.352 & 102.644 & 123.423 & 1541.539 & 1534.612 & 1513.728 \\
\hline 7/23/16 14:00 & 116.449 & 102.793 & 123.512 & 1541.442 & 1534.463 & 1513.639 \\
\hline 7/23/16 15:00 & 116.543 & 102.921 & 123.596 & 1541.348 & 1534.335 & 1513.555 \\
\hline 7/23/16 16:00 & 116.594 & 103.021 & 123.662 & 1541.297 & 1534.235 & 1513.489 \\
\hline 7/23/16 17:00 & 116.585 & 103.072 & 123.72 & 1541.306 & 1534.184 & 1513.431 \\
\hline 7/23/16 18:00 & 116.531 & 103.088 & 123.779 & 1541.36 & 1534.168 & 1513.372 \\
\hline 7/23/16 19:00 & 116.472 & 103.06 & 123.832 & 1541.419 & 1534.196 & 1513.319 \\
\hline 7/23/16 20:00 & 116.418 & 103.023 & 123.886 & 1541.473 & 1534.233 & 1513.265 \\
\hline 7/23/16 21:00 & 116.364 & 102.986 & 123.938 & 1541.527 & 1534.27 & 1513.213 \\
\hline $7 / 23 / 16$ 22:00 & 116.322 & 102.951 & 124.001 & 1541.569 & 1534.305 & 1513.15 \\
\hline $7 / 23 / 16$ 23:00 & 116.284 & 102.935 & 124.053 & 1541.607 & 1534.321 & 1513.098 \\
\hline $7 / 24 / 16$ 0:00 & 116.256 & 102.928 & 124.116 & 1541.635 & 1534.328 & 1513.035 \\
\hline $7 / 24 / 16$ 1:00 & 116.251 & 102.947 & 124.184 & 1541.64 & 1534.309 & 1512.967 \\
\hline $7 / 24 / 16$ 2:00 & 116.232 & 102.984 & 124.244 & 1541.659 & 1534.272 & 1512.907 \\
\hline $7 / 24 / 16$ 3:00 & 116.202 & 103.042 & 124.305 & 1541.689 & 1534.214 & 1512.846 \\
\hline $7 / 24 / 164: 00$ & 116.159 & 103.109 & 124.371 & 1541.732 & 1534.147 & 1512.78 \\
\hline $7 / 24 / 16$ 5:00 & 116.11 & 103.186 & 124.439 & 1541.781 & 1534.07 & 1512.712 \\
\hline $7 / 24 / 16$ 6:00 & 116.063 & 103.274 & 124.521 & 1541.828 & 1533.982 & 1512.63 \\
\hline 7/24/16 7:00 & 116.023 & 103.374 & 124.605 & 1541.868 & 1533.882 & 1512.546 \\
\hline 7/24/16 8:00 & 115.971 & 103.474 & 124.689 & 1541.92 & 1533.782 & 1512.462 \\
\hline 7/24/16 9:00 & 115.649 & 103.57 & 124.778 & 1542.242 & 1533.686 & 1512.373 \\
\hline
\end{tabular}


TABLE S1.2 Automatically recorded groundwater levels in the confined aquifers.

\begin{tabular}{|c|c|c|c|c|c|c|}
\hline \multirow[b]{2}{*}{ Date-Time } & \multicolumn{3}{|c|}{ Measured Depth to Water (ft TOC ${ }^{\mathrm{a}}$ ) } & \multicolumn{3}{|c|}{ Groundwater Elevation (ft AMSL ${ }^{\mathrm{b}}$ ) } \\
\hline & DL2-CU & DL7-CU & DL7-CL & DL2-CU & DL7-CU & DL7-CL \\
\hline 7/24/16 10:00 & 115.188 & 103.66 & 124.865 & 1542.703 & 1533.596 & 1512.286 \\
\hline $7 / 24 / 16$ 11:00 & 114.743 & 103.737 & 124.951 & 1543.148 & 1533.519 & 1512.2 \\
\hline $7 / 24 / 16$ 12:00 & 114.338 & 103.807 & 125.033 & 1543.553 & 1533.449 & 1512.118 \\
\hline $7 / 24 / 16$ 13:00 & 113.983 & 103.869 & 125.113 & 1543.908 & 1533.387 & 1512.038 \\
\hline $7 / 24 / 16$ 14:00 & 113.666 & 103.918 & 125.19 & 1544.225 & 1533.338 & 1511.961 \\
\hline $7 / 24 / 16$ 15:00 & 113.379 & 103.951 & 125.256 & 1544.512 & 1533.305 & 1511.895 \\
\hline $7 / 24 / 16$ 16:00 & 113.122 & 103.962 & 125.312 & 1544.769 & 1533.294 & 1511.839 \\
\hline 7/24/16 17:00 & 112.868 & 103.962 & 125.361 & 1545.023 & 1533.294 & 1511.79 \\
\hline $7 / 24 / 16$ 18:00 & 112.614 & 103.942 & 125.412 & 1545.277 & 1533.314 & 1511.739 \\
\hline 7/24/16 19:00 & 112.377 & 103.914 & 125.452 & 1545.514 & 1533.342 & 1511.699 \\
\hline $7 / 24 / 16$ 20:00 & 112.146 & 103.851 & 125.499 & 1545.745 & 1533.405 & 1511.652 \\
\hline $7 / 24 / 16$ 21:00 & 111.908 & 103.725 & 125.541 & 1545.983 & 1533.531 & 1511.61 \\
\hline $7 / 24 / 16$ 22:00 & 111.586 & 103.567 & 125.597 & 1546.305 & 1533.689 & 1511.554 \\
\hline $7 / 24 / 16$ 23:00 & 111.222 & 103.391 & 125.647 & 1546.669 & 1533.865 & 1511.504 \\
\hline $7 / 25 / 16$ 0:00 & 110.897 & 103.216 & 125.698 & 1546.994 & 1534.04 & 1511.453 \\
\hline $7 / 25 / 16$ 1:00 & 110.603 & 103.047 & 125.752 & 1547.288 & 1534.209 & 1511.399 \\
\hline $7 / 25 / 16$ 2:00 & 110.33 & 102.888 & 125.799 & 1547.561 & 1534.368 & 1511.352 \\
\hline $7 / 25 / 163: 00$ & 110.078 & 102.744 & 125.843 & 1547.813 & 1534.512 & 1511.308 \\
\hline $7 / 25 / 164: 00$ & 109.848 & 102.64 & 125.902 & 1548.043 & 1534.616 & 1511.249 \\
\hline $7 / 25 / 16$ 5:00 & 109.636 & 102.561 & 125.981 & 1548.255 & 1534.695 & 1511.17 \\
\hline 7/25/16 6:00 & 109.431 & 102.484 & 126.068 & 1548.46 & 1534.772 & 1511.083 \\
\hline 7/25/16 7:00 & 109.257 & 102.433 & 126.168 & 1548.634 & 1534.823 & 1510.983 \\
\hline 7/25/16 8:00 & 109.111 & 102.412 & 126.271 & 1548.78 & 1534.844 & 1510.88 \\
\hline 7/25/16 9:00 & 109.003 & 102.405 & 126.374 & 1548.888 & 1534.851 & 1510.777 \\
\hline 7/25/16 10:00 & 109.159 & 102.384 & 126.466 & 1548.732 & 1534.872 & 1510.685 \\
\hline 7/25/16 11:00 & 109.535 & 102.333 & 126.576 & 1548.356 & 1534.923 & 1510.575 \\
\hline $7 / 25 / 16$ 12:00 & 109.907 & 102.27 & 126.742 & 1547.984 & 1534.986 & 1510.409 \\
\hline 7/25/16 13:00 & 110.231 & 102.231 & 126.819 & 1547.66 & 1535.025 & 1510.332 \\
\hline 7/25/16 14:00 & 110.513 & 102.193 & 126.868 & 1547.378 & 1535.063 & 1510.283 \\
\hline 7/25/16 15:00 & 110.763 & 102.14 & 126.911 & 1547.128 & 1535.116 & 1510.24 \\
\hline 7/25/16 16:00 & 110.993 & 102.096 & 126.932 & 1546.898 & 1535.16 & 1510.219 \\
\hline 7/25/16 17:00 & 111.196 & 102.028 & 126.943 & 1546.695 & 1535.228 & 1510.208 \\
\hline $7 / 25 / 16$ 18:00 & 111.262 & 101.954 & 126.953 & 1546.629 & 1535.302 & 1510.198 \\
\hline 7/25/16 19:00 & 111.367 & 101.877 & 126.96 & 1546.524 & 1535.379 & 1510.191 \\
\hline 7/25/16 20:00 & 111.513 & 101.789 & 126.967 & 1546.378 & 1535.467 & 1510.184 \\
\hline $7 / 25 / 16$ 21:00 & 111.516 & 101.696 & 126.969 & 1546.375 & 1535.56 & 1510.182 \\
\hline 7/25/16 22:00 & 111.294 & 101.617 & 126.981 & 1546.597 & 1535.639 & 1510.17 \\
\hline 7/25/16 23:00 & 111.057 & 101.549 & 126.992 & 1546.834 & 1535.707 & 1510.159 \\
\hline 7/26/16 0:00 & 110.857 & 101.491 & 126.999 & 1547.034 & 1535.765 & 1510.152 \\
\hline 7/26/16 1:00 & 110.69 & 101.452 & 127.018 & 1547.201 & 1535.804 & 1510.133 \\
\hline $7 / 26 / 16$ 2:00 & 110.537 & 101.422 & 127.077 & 1547.354 & 1535.834 & 1510.074 \\
\hline 7/26/16 3:00 & 110.394 & 101.389 & 127.248 & 1547.497 & 1535.867 & 1509.903 \\
\hline $7 / 26 / 164: 00$ & 110.255 & 101.354 & 127.442 & 1547.636 & 1535.902 & 1509.709 \\
\hline 7/26/16 5:00 & 110.123 & 101.31 & 127.645 & 1547.768 & 1535.946 & 1509.506 \\
\hline 7/26/16 6:00 & 109.994 & 101.273 & 127.847 & 1547.897 & 1535.983 & 1509.304 \\
\hline 7/26/16 7:00 & 109.869 & 101.243 & 128.046 & 1548.022 & 1536.013 & 1509.105 \\
\hline 7/26/16 8:00 & 109.749 & 101.198 & 128.231 & 1548.142 & 1536.058 & 1508.92 \\
\hline
\end{tabular}


TABLE S1.2 Automatically recorded groundwater levels in the confined aquifers.

\begin{tabular}{|c|c|c|c|c|c|c|}
\hline \multirow[b]{2}{*}{ Date-Time } & \multicolumn{3}{|c|}{ Measured Depth to Water (ft TOC ${ }^{a}$ ) } & \multicolumn{3}{|c|}{ Groundwater Elevation (ft AMSL ${ }^{b}$ ) } \\
\hline & DL2-CU & DL7-CU & DL7-CL & DL2-CU & DL7-CU & DL7-CL \\
\hline 7/26/16 9:00 & 109.645 & 101.117 & 128.38 & 1548.246 & 1536.139 & 1508.771 \\
\hline $7 / 26 / 16$ 10:00 & 109.61 & 101.006 & 128.42 & 1548.281 & 1536.25 & 1508.731 \\
\hline 7/26/16 11:00 & 109.62 & 100.878 & 128.437 & 1548.271 & 1536.378 & 1508.714 \\
\hline 7/26/16 12:00 & 109.62 & 100.757 & 128.434 & 1548.271 & 1536.499 & 1508.717 \\
\hline 7/26/16 13:00 & 109.622 & 100.648 & 128.423 & 1548.269 & 1536.608 & 1508.728 \\
\hline 7/26/16 14:00 & 109.65 & 100.541 & 128.394 & 1548.241 & 1536.715 & 1508.757 \\
\hline $7 / 26 / 16$ 15:00 & 109.709 & 100.434 & 128.366 & 1548.182 & 1536.822 & 1508.785 \\
\hline 7/26/16 16:00 & 109.787 & 100.315 & 128.324 & 1548.104 & 1536.941 & 1508.827 \\
\hline 7/26/16 17:00 & 109.874 & 100.173 & 128.277 & 1548.017 & 1537.083 & 1508.874 \\
\hline 7/26/16 18:00 & 109.951 & 100.041 & 128.235 & 1547.94 & 1537.215 & 1508.916 \\
\hline 7/26/16 19:00 & 110.036 & 99.911 & 128.188 & 1547.855 & 1537.345 & 1508.963 \\
\hline $7 / 26 / 16$ 20:00 & 110.135 & 99.794 & 128.149 & 1547.756 & 1537.462 & 1509.002 \\
\hline $7 / 26 / 16$ 21:00 & 110.234 & 99.699 & 128.107 & 1547.657 & 1537.557 & 1509.044 \\
\hline 7/26/16 22:00 & 110.321 & 99.629 & 128.078 & 1547.57 & 1537.627 & 1509.073 \\
\hline 7/26/16 23:00 & 110.394 & 99.581 & 128.057 & 1547.497 & 1537.675 & 1509.094 \\
\hline 7/27/16 0:00 & 110.452 & 99.55 & 128.041 & 1547.439 & 1537.706 & 1509.11 \\
\hline 7/27/16 1:00 & 110.506 & 99.539 & 128.022 & 1547.385 & 1537.717 & 1509.129 \\
\hline 7/27/16 2:00 & 110.563 & 99.55 & 128.011 & 1547.328 & 1537.706 & 1509.14 \\
\hline $7 / 27 / 16$ 3:00 & 110.622 & 99.585 & 128.006 & 1547.269 & 1537.671 & 1509.145 \\
\hline $7 / 27 / 164: 00$ & 110.662 & 99.636 & 127.987 & 1547.229 & 1537.62 & 1509.164 \\
\hline $7 / 27 / 16$ 5:00 & 110.695 & 99.72 & 127.987 & 1547.196 & 1537.536 & 1509.164 \\
\hline $7 / 27 / 16$ 6:00 & 110.763 & 99.843 & 128.011 & 1547.128 & 1537.413 & 1509.14 \\
\hline 7/27/16 7:00 & 110.85 & 99.992 & 128.048 & 1547.041 & 1537.264 & 1509.103 \\
\hline 7/27/16 8:00 & 110.958 & 100.157 & 128.093 & 1546.933 & 1537.099 & 1509.058 \\
\hline 7/27/16 9:00 & 111.069 & 100.317 & 128.132 & 1546.822 & 1536.939 & 1509.019 \\
\hline 7/27/16 10:00 & 111.175 & 100.462 & 128.139 & 1546.716 & 1536.794 & 1509.012 \\
\hline 7/27/16 11:00 & 111.271 & 100.566 & 128.151 & 1546.62 & 1536.69 & 1509 \\
\hline 7/27/16 12:00 & 111.363 & 100.657 & 128.156 & 1546.528 & 1536.599 & 1508.995 \\
\hline 7/27/16 13:00 & 111.466 & 100.731 & 128.156 & 1546.425 & 1536.525 & 1508.995 \\
\hline 7/27/16 14:00 & 111.586 & 100.787 & 128.153 & 1546.305 & 1536.469 & 1508.998 \\
\hline $7 / 27 / 16$ 15:00 & 111.72 & 100.834 & 128.149 & 1546.171 & 1536.422 & 1509.002 \\
\hline 7/27/16 16:00 & 111.883 & 100.873 & 128.149 & 1546.008 & 1536.383 & 1509.002 \\
\hline 7/27/16 17:00 & 112.106 & 100.929 & 128.135 & 1545.785 & 1536.327 & 1509.016 \\
\hline 7/27/16 18:00 & 112.365 & 101.029 & 128.116 & 1545.526 & 1536.227 & 1509.035 \\
\hline 7/27/16 19:00 & 112.635 & 101.15 & 128.104 & 1545.256 & 1536.106 & 1509.047 \\
\hline 7/27/16 20:00 & 112.913 & 101.291 & 128.102 & 1544.978 & 1535.965 & 1509.049 \\
\hline $7 / 27 / 16$ 21:00 & 113.179 & 101.454 & 128.095 & 1544.712 & 1535.802 & 1509.056 \\
\hline $7 / 27 / 16$ 22:00 & 113.428 & 101.642 & 128.093 & 1544.463 & 1535.614 & 1509.058 \\
\hline $7 / 27 / 16$ 23:00 & 113.661 & 101.828 & 128.102 & 1544.23 & 1535.428 & 1509.049 \\
\hline 7/28/16 0:00 & 113.87 & 102.007 & 128.114 & 1544.021 & 1535.249 & 1509.037 \\
\hline $7 / 28 / 16$ 1:00 & 114.068 & 102.21 & 128.132 & 1543.823 & 1535.046 & 1509.019 \\
\hline $7 / 28 / 16$ 2:00 & 114.251 & 102.421 & 128.156 & 1543.64 & 1534.835 & 1508.995 \\
\hline 7/28/16 3:00 & 114.435 & 102.642 & 128.186 & 1543.456 & 1534.614 & 1508.965 \\
\hline $7 / 28 / 164: 00$ & 114.6 & 102.847 & 128.21 & 1543.291 & 1534.409 & 1508.941 \\
\hline 7/28/16 5:00 & 114.769 & 103.067 & 128.256 & 1543.122 & 1534.189 & 1508.895 \\
\hline 7/28/16 6:00 & 114.927 & 103.295 & 128.315 & 1542.964 & 1533.961 & 1508.836 \\
\hline 7/28/16 7:00 & 115.089 & 103.537 & 128.378 & 1542.802 & 1533.719 & 1508.773 \\
\hline
\end{tabular}


TABLE S1.2 Automatically recorded groundwater levels in the confined aquifers.

\begin{tabular}{|c|c|c|c|c|c|c|}
\hline \multirow[b]{2}{*}{ Date-Time } & \multicolumn{3}{|c|}{ Measured Depth to Water (ft TOC ${ }^{\mathrm{a}}$ ) } & \multicolumn{3}{|c|}{ Groundwater Elevation (ft AMSL ${ }^{b}$ ) } \\
\hline & DL2-CU & DL7-CU & DL7-CL & DL2-CU & DL7-CU & DL7-CL \\
\hline 7/28/16 8:00 & 115.23 & 103.795 & 128.434 & 1542.661 & 1533.461 & 1508.717 \\
\hline 7/28/16 9:00 & 115.289 & 104.048 & 128.502 & 1542.602 & 1533.208 & 1508.649 \\
\hline $7 / 28 / 16$ 10:00 & 115.31 & 104.29 & 128.577 & 1542.581 & 1532.966 & 1508.574 \\
\hline 7/28/16 11:00 & 115.338 & 104.518 & 128.657 & 1542.553 & 1532.738 & 1508.494 \\
\hline $7 / 28 / 1612: 00$ & 115.348 & 104.734 & 128.743 & 1542.543 & 1532.522 & 1508.408 \\
\hline 7/28/16 13:00 & 115.324 & 104.936 & 128.834 & 1542.567 & 1532.32 & 1508.317 \\
\hline 7/28/16 14:00 & 115.27 & 105.134 & 128.928 & 1542.621 & 1532.122 & 1508.223 \\
\hline 7/28/16 15:00 & 115.209 & 105.325 & 129.031 & 1542.682 & 1531.931 & 1508.12 \\
\hline 7/28/16 16:00 & 115.19 & 105.487 & 129.129 & 1542.701 & 1531.769 & 1508.022 \\
\hline 7/28/16 17:00 & 115.39 & 105.608 & 129.214 & 1542.501 & 1531.648 & 1507.937 \\
\hline $7 / 28 / 16$ 18:00 & 115.658 & 105.648 & 129.303 & 1542.233 & 1531.608 & 1507.848 \\
\hline 7/28/16 19:00 & 115.948 & 105.634 & 129.387 & 1541.943 & 1531.622 & 1507.764 \\
\hline $7 / 28 / 16$ 20:00 & 116.218 & 105.583 & 129.476 & 1541.673 & 1531.673 & 1507.675 \\
\hline 7/28/16 21:00 & 116.453 & 105.543 & 129.576 & 1541.438 & 1531.713 & 1507.575 \\
\hline 7/28/16 22:00 & 116.649 & 105.508 & 129.684 & 1541.242 & 1531.748 & 1507.467 \\
\hline 7/28/16 23:00 & 116.806 & 105.494 & 129.799 & 1541.085 & 1531.762 & 1507.352 \\
\hline 7/29/16 0:00 & 116.931 & 105.485 & 129.902 & 1540.96 & 1531.771 & 1507.249 \\
\hline 7/29/16 1:00 & 117.032 & 105.506 & 130.005 & 1540.859 & 1531.75 & 1507.146 \\
\hline $7 / 29 / 16$ 2:00 & 117.086 & 105.559 & 130.11 & 1540.805 & 1531.697 & 1507.041 \\
\hline 7/29/16 3:00 & 117.056 & 105.636 & 130.218 & 1540.835 & 1531.62 & 1506.933 \\
\hline 7/29/16 4:00 & 116.952 & 105.743 & 130.337 & 1540.939 & 1531.513 & 1506.814 \\
\hline 7/29/16 5:00 & 116.83 & 105.876 & 130.457 & 1541.061 & 1531.38 & 1506.694 \\
\hline 7/29/16 6:00 & 116.743 & 106.013 & 130.592 & 1541.148 & 1531.243 & 1506.559 \\
\hline 7/29/16 7:00 & 116.698 & 106.169 & 130.737 & 1541.193 & 1531.087 & 1506.414 \\
\hline 7/29/16 8:00 & 116.674 & 106.306 & 130.887 & 1541.217 & 1530.95 & 1506.264 \\
\hline 7/29/16 9:00 & 116.663 & 106.415 & 131.044 & 1541.228 & 1530.841 & 1506.107 \\
\hline 7/29/16 10:00 & 116.78 & 106.515 & 131.238 & 1541.111 & 1530.741 & 1505.913 \\
\hline 7/29/16 11:00 & 116.858 & 106.638 & 131.505 & 1541.033 & 1530.618 & 1505.646 \\
\hline 7/29/16 12:00 & 116.914 & 106.782 & 131.737 & 1540.977 & 1530.474 & 1505.414 \\
\hline 7/29/16 13:00 & 116.957 & 106.938 & 131.856 & 1540.934 & 1530.318 & 1505.295 \\
\hline 7/29/16 14:00 & 116.997 & 107.089 & 131.945 & 1540.894 & 1530.167 & 1505.206 \\
\hline 7/29/16 15:00 & 117.041 & 107.215 & 132.018 & 1540.85 & 1530.041 & 1505.133 \\
\hline 7/29/16 16:00 & 117.088 & 107.326 & 132.072 & 1540.803 & 1529.93 & 1505.079 \\
\hline 7/29/16 17:00 & 117.145 & 107.41 & 132.111 & 1540.746 & 1529.846 & 1505.04 \\
\hline 7/29/16 18:00 & 117.246 & 107.487 & 132.151 & 1540.645 & 1529.769 & 1505 \\
\hline 7/29/16 19:00 & 117.415 & 107.556 & 132.182 & 1540.476 & 1529.7 & 1504.969 \\
\hline $7 / 29 / 16$ 20:00 & 117.639 & 107.619 & 132.212 & 1540.252 & 1529.637 & 1504.939 \\
\hline $7 / 29 / 16$ 21:00 & 117.876 & 107.689 & 132.24 & 1540.015 & 1529.567 & 1504.911 \\
\hline 7/29/16 22:00 & 118.114 & 107.754 & 132.273 & 1539.777 & 1529.502 & 1504.878 \\
\hline 7/29/16 23:00 & 118.345 & 107.812 & 132.301 & 1539.546 & 1529.444 & 1504.85 \\
\hline $7 / 30 / 160: 00$ & 118.563 & 107.863 & 132.331 & 1539.328 & 1529.393 & 1504.82 \\
\hline $7 / 30 / 16$ 1:00 & 118.777 & 107.912 & 132.36 & 1539.114 & 1529.344 & 1504.791 \\
\hline $7 / 30 / 16$ 2:00 & 118.987 & 107.949 & 132.392 & 1538.904 & 1529.307 & 1504.759 \\
\hline 7/30/16 3:00 & 119.187 & 107.989 & 132.479 & 1538.704 & 1529.267 & 1504.672 \\
\hline $7 / 30 / 164: 00$ & 119.373 & 108.007 & 132.664 & 1538.518 & 1529.249 & 1504.487 \\
\hline 7/30/16 5:00 & 119.54 & 108.024 & 132.879 & 1538.351 & 1529.232 & 1504.272 \\
\hline 7/30/16 6:00 & 119.685 & 108.045 & 133.102 & 1538.206 & 1529.211 & 1504.049 \\
\hline
\end{tabular}


TABLE S1.2 Automatically recorded groundwater levels in the confined aquifers.

\begin{tabular}{|c|c|c|c|c|c|c|}
\hline \multirow[b]{2}{*}{ Date-Time } & \multicolumn{3}{|c|}{ Measured Depth to Water (ft TOC ${ }^{\mathrm{a}}$ ) } & \multicolumn{3}{|c|}{ Groundwater Elevation (ft AMSL ${ }^{\mathrm{b}}$ ) } \\
\hline & DL2-CU & DL7-CU & DL7-CL & DL2-CU & DL7-CU & DL7-CL \\
\hline 7/30/16 7:00 & 119.796 & 108.086 & 133.336 & 1538.095 & 1529.17 & 1503.815 \\
\hline $7 / 30 / 16$ 8:00 & 119.725 & 108.135 & 133.553 & 1538.166 & 1529.121 & 1503.598 \\
\hline 7/30/16 9:00 & 119.669 & 108.189 & 133.649 & 1538.222 & 1529.067 & 1503.502 \\
\hline $7 / 30 / 16$ 10:00 & 119.594 & 108.251 & 133.705 & 1538.297 & 1529.005 & 1503.446 \\
\hline 7/30/16 11:00 & 119.469 & 108.272 & 133.731 & 1538.422 & 1528.984 & 1503.42 \\
\hline $7 / 30 / 16$ 12:00 & 119.311 & 108.286 & 133.769 & 1538.58 & 1528.97 & 1503.382 \\
\hline 7/30/16 13:00 & 119.121 & 108.286 & 133.804 & 1538.77 & 1528.97 & 1503.347 \\
\hline 7/30/16 14:00 & 118.909 & 108.258 & 133.825 & 1538.982 & 1528.998 & 1503.326 \\
\hline 7/30/16 15:00 & 118.697 & 108.205 & 133.832 & 1539.194 & 1529.051 & 1503.319 \\
\hline 7/30/16 16:00 & 118.519 & 108.121 & 133.841 & 1539.372 & 1529.135 & 1503.31 \\
\hline 7/30/16 17:00 & 118.378 & 108.005 & 133.836 & 1539.513 & 1529.251 & 1503.315 \\
\hline 7/30/16 18:00 & 118.269 & 107.865 & 133.829 & 1539.622 & 1529.391 & 1503.322 \\
\hline 7/30/16 19:00 & 118.081 & 107.717 & 133.822 & 1539.81 & 1529.539 & 1503.329 \\
\hline 7/30/16 20:00 & 117.669 & 107.563 & 133.82 & 1540.222 & 1529.693 & 1503.331 \\
\hline 7/30/16 21:00 & 117.246 & 107.426 & 133.818 & 1540.645 & 1529.83 & 1503.333 \\
\hline $7 / 30 / 16$ 22:00 & 116.87 & 107.315 & 133.82 & 1541.021 & 1529.941 & 1503.331 \\
\hline $7 / 30 / 16$ 23:00 & 116.536 & 107.21 & 133.825 & 1541.355 & 1530.046 & 1503.326 \\
\hline $7 / 31 / 16$ 0:00 & 116.244 & 107.119 & 133.829 & 1541.647 & 1530.137 & 1503.322 \\
\hline 7/31/16 1:00 & 115.985 & 107.033 & 133.837 & 1541.906 & 1530.223 & 1503.314 \\
\hline $7 / 31 / 16$ 2:00 & 115.755 & 106.95 & 133.844 & 1542.136 & 1530.306 & 1503.307 \\
\hline 7/31/16 3:00 & 115.548 & 106.861 & 133.853 & 1542.343 & 1530.395 & 1503.298 \\
\hline 7/31/16 4:00 & 115.359 & 106.768 & 133.862 & 1542.532 & 1530.488 & 1503.289 \\
\hline 7/31/16 5:00 & 115.188 & 106.673 & 133.869 & 1542.703 & 1530.583 & 1503.282 \\
\hline 7/31/16 6:00 & 115.028 & 106.599 & 133.89 & 1542.863 & 1530.657 & 1503.261 \\
\hline 7/31/16 7:00 & 114.884 & 106.547 & 133.921 & 1543.007 & 1530.709 & 1503.23 \\
\hline 7/31/16 8:00 & 114.75 & 106.522 & 133.956 & 1543.141 & 1530.734 & 1503.195 \\
\hline 7/31/16 9:00 & 114.59 & 106.501 & 134 & 1543.301 & 1530.755 & 1503.151 \\
\hline 7/31/16 10:00 & 114.367 & 106.457 & 134.038 & 1543.524 & 1530.799 & 1503.113 \\
\hline 7/31/16 11:00 & 114.139 & 106.399 & 134.096 & 1543.752 & 1530.857 & 1503.055 \\
\hline 7/31/16 12:00 & 113.906 & 106.317 & 134.143 & 1543.985 & 1530.939 & 1503.008 \\
\hline $7 / 31 / 16$ 13:00 & 113.689 & 106.208 & 134.181 & 1544.202 & 1531.048 & 1502.97 \\
\hline 7/31/16 14:00 & 113.482 & 106.062 & 134.213 & 1544.409 & 1531.194 & 1502.938 \\
\hline 7/31/16 15:00 & 113.285 & 105.845 & 134.232 & 1544.606 & 1531.411 & 1502.919 \\
\hline 7/31/16 16:00 & 113.106 & 105.574 & 134.241 & 1544.785 & 1531.682 & 1502.91 \\
\hline 7/31/16 17:00 & 112.96 & 105.283 & 134.241 & 1544.931 & 1531.973 & 1502.91 \\
\hline 7/31/16 18:00 & 112.835 & 104.974 & 134.241 & 1545.056 & 1532.282 & 1502.91 \\
\hline 7/31/16 19:00 & 112.725 & 104.667 & 134.232 & 1545.166 & 1532.589 & 1502.919 \\
\hline 7/31/16 20:00 & 112.619 & 104.365 & 134.218 & 1545.272 & 1532.891 & 1502.933 \\
\hline 7/31/16 21:00 & 112.52 & 104.074 & 134.216 & 1545.371 & 1533.182 & 1502.935 \\
\hline 7/31/16 22:00 & 112.424 & 103.8 & 134.209 & 1545.467 & 1533.456 & 1502.942 \\
\hline 7/31/16 23:00 & 112.325 & 103.539 & 134.204 & 1545.566 & 1533.717 & 1502.947 \\
\hline 8/1/16 0:00 & 112.217 & 103.302 & 134.197 & 1545.674 & 1533.954 & 1502.954 \\
\hline 8/1/16 1:00 & 112.094 & 103.074 & 134.192 & 1545.797 & 1534.182 & 1502.959 \\
\hline $8 / 1 / 16$ 2:00 & 111.951 & 102.865 & 134.185 & 1545.94 & 1534.391 & 1502.966 \\
\hline 8/1/16 3:00 & 111.803 & 102.677 & 134.176 & 1546.088 & 1534.579 & 1502.975 \\
\hline $8 / 1 / 164: 00$ & 111.645 & 102.489 & 134.162 & 1546.246 & 1534.767 & 1502.989 \\
\hline 8/1/16 5:00 & 111.485 & 102.31 & 134.15 & 1546.406 & 1534.946 & 1503.001 \\
\hline
\end{tabular}


TABLE S1.2 Automatically recorded groundwater levels in the confined aquifers.

\begin{tabular}{|c|c|c|c|c|c|c|}
\hline \multirow[b]{2}{*}{ Date-Time } & \multicolumn{3}{|c|}{ Measured Depth to Water (ft TOC ${ }^{\mathrm{a}}$ ) } & \multicolumn{3}{|c|}{ Groundwater Elevation (ft AMSL ${ }^{b}$ ) } \\
\hline & DL2-CU & DL7-CU & DL7-CL & DL2-CU & DL7-CU & DL7-CL \\
\hline 8/1/16 6:00 & 111.33 & 102.159 & 134.169 & 1546.561 & 1535.097 & 1502.982 \\
\hline $8 / 1 / 167: 00$ & 111.179 & 102.026 & 134.211 & 1546.712 & 1535.23 & 1502.94 \\
\hline 8/1/16 8:00 & 111.024 & 101.903 & 134.265 & 1546.867 & 1535.353 & 1502.886 \\
\hline 8/1/16 9:00 & 110.885 & 101.798 & 134.349 & 1547.006 & 1535.458 & 1502.802 \\
\hline 8/1/16 10:00 & 110.793 & 101.689 & 134.555 & 1547.098 & 1535.567 & 1502.596 \\
\hline $8 / 1 / 16$ 11:00 & 110.749 & 101.566 & 134.745 & 1547.142 & 1535.69 & 1502.406 \\
\hline $8 / 1 / 16$ 12:00 & 110.739 & 101.459 & 134.824 & 1547.152 & 1535.797 & 1502.327 \\
\hline 8/1/16 13:00 & 110.751 & 101.364 & 134.866 & 1547.14 & 1535.892 & 1502.285 \\
\hline 8/1/16 14:00 & 110.793 & 101.271 & 134.887 & 1547.098 & 1535.985 & 1502.264 \\
\hline 8/1/16 15:00 & 110.864 & 101.191 & 134.894 & 1547.027 & 1536.065 & 1502.257 \\
\hline $8 / 1 / 16$ 16:00 & 110.972 & 101.15 & 134.878 & 1546.919 & 1536.106 & 1502.273 \\
\hline 8/1/16 17:00 & 111.118 & 101.136 & 134.862 & 1546.773 & 1536.12 & 1502.289 \\
\hline 8/1/16 18:00 & 111.292 & 101.129 & 134.892 & 1546.599 & 1536.127 & 1502.259 \\
\hline 8/1/16 19:00 & 111.485 & 101.087 & 134.974 & 1546.406 & 1536.169 & 1502.177 \\
\hline 8/1/16 20:00 & 111.687 & 101.052 & 134.972 & 1546.204 & 1536.204 & 1502.179 \\
\hline 8/1/16 21:00 & 111.89 & 101.059 & 134.953 & 1546.001 & 1536.197 & 1502.198 \\
\hline $8 / 1 / 16$ 22:00 & 112.094 & 101.094 & 134.934 & 1545.797 & 1536.162 & 1502.217 \\
\hline 8/1/16 23:00 & 112.299 & 101.143 & 134.906 & 1545.592 & 1536.113 & 1502.245 \\
\hline $8 / 2 / 16$ 0:00 & 112.494 & 101.208 & 134.885 & 1545.397 & 1536.048 & 1502.266 \\
\hline $8 / 2 / 16$ 1:00 & 112.675 & 101.284 & 134.859 & 1545.216 & 1535.972 & 1502.292 \\
\hline $8 / 2 / 162: 00$ & 112.854 & 101.394 & 134.859 & 1545.037 & 1535.862 & 1502.292 \\
\hline 8/2/16 3:00 & 112.962 & 101.468 & 134.913 & 1544.929 & 1535.788 & 1502.238 \\
\hline $8 / 2 / 164: 00$ & 113.073 & 101.549 & 135.03 & 1544.818 & 1535.707 & 1502.121 \\
\hline 8/2/16 5:00 & 113.167 & 101.584 & 135.157 & 1544.724 & 1535.672 & 1501.994 \\
\hline $8 / 2 / 166: 00$ & 113.231 & 101.591 & 135.278 & 1544.66 & 1535.665 & 1501.873 \\
\hline 8/2/16 7:00 & 113.292 & 101.619 & 135.416 & 1544.599 & 1535.637 & 1501.735 \\
\hline 8/2/16 8:00 & 113.346 & 101.649 & 135.55 & 1544.545 & 1535.607 & 1501.601 \\
\hline 8/2/16 9:00 & 113.402 & 101.691 & 135.681 & 1544.489 & 1535.565 & 1501.47 \\
\hline $8 / 2 / 16$ 10:00 & 113.454 & 101.74 & 135.73 & 1544.437 & 1535.516 & 1501.421 \\
\hline 8/2/16 11:00 & 113.508 & 101.791 & 135.737 & 1544.383 & 1535.465 & 1501.414 \\
\hline $8 / 2 / 16$ 12:00 & 113.567 & 101.838 & 135.732 & 1544.324 & 1535.418 & 1501.419 \\
\hline 8/2/16 13:00 & 113.63 & 101.947 & 135.709 & 1544.261 & 1535.309 & 1501.442 \\
\hline $8 / 2 / 16$ 14:00 & 113.72 & 102.112 & 135.679 & 1544.171 & 1535.144 & 1501.472 \\
\hline $8 / 2 / 16$ 15:00 & 113.804 & 102.293 & 135.634 & 1544.087 & 1534.963 & 1501.517 \\
\hline 8/2/16 16:00 & 113.873 & 102.456 & 135.585 & 1544.018 & 1534.8 & 1501.566 \\
\hline 8/2/16 17:00 & 113.934 & 102.589 & 135.512 & 1543.957 & 1534.667 & 1501.639 \\
\hline $8 / 2 / 16$ 18:00 & 113.986 & 102.64 & 135.393 & 1543.905 & 1534.616 & 1501.758 \\
\hline $8 / 2 / 16$ 19:00 & 114.03 & 102.603 & 135.274 & 1543.861 & 1534.653 & 1501.877 \\
\hline $8 / 2 / 16$ 20:00 & 114.073 & 102.526 & 135.157 & 1543.818 & 1534.73 & 1501.994 \\
\hline $8 / 2 / 16$ 21:00 & 114.115 & 102.442 & 135.054 & 1543.776 & 1534.814 & 1502.097 \\
\hline $8 / 2 / 1622: 00$ & 114.15 & 102.349 & 134.948 & 1543.741 & 1534.907 & 1502.203 \\
\hline $8 / 2 / 16$ 23:00 & 114.162 & 102.254 & 134.85 & 1543.729 & 1535.002 & 1502.301 \\
\hline 8/3/16 0:00 & 114.162 & 102.159 & 134.749 & 1543.729 & 1535.097 & 1502.402 \\
\hline $8 / 3 / 16$ 1:00 & 114.15 & 102.054 & 134.66 & 1543.741 & 1535.202 & 1502.491 \\
\hline $8 / 3 / 16$ 2:00 & 114.129 & 101.959 & 134.569 & 1543.762 & 1535.297 & 1502.582 \\
\hline 8/3/16 3:00 & 114.103 & 101.859 & 134.478 & 1543.788 & 1535.397 & 1502.673 \\
\hline 8/3/16 4:00 & 114.073 & 101.766 & 134.396 & 1543.818 & 1535.49 & 1502.755 \\
\hline
\end{tabular}


TABLE S1.2 Automatically recorded groundwater levels in the confined aquifers.

\begin{tabular}{|c|c|c|c|c|c|c|}
\hline \multirow[b]{2}{*}{ Date-Time } & \multicolumn{3}{|c|}{ Measured Depth to Water (ft TOC ${ }^{\mathrm{a}}$ ) } & \multicolumn{3}{|c|}{ Groundwater Elevation (ft AMSL ${ }^{\mathrm{b}}$ ) } \\
\hline & DL2-CU & DL7-CU & DL7-CL & DL2-CU & DL7-CU & DL7-CL \\
\hline 8/3/16 5:00 & 114.035 & 101.67 & 134.316 & 1543.856 & 1535.586 & 1502.835 \\
\hline $8 / 3 / 16$ 6:00 & 114.004 & 101.587 & 134.246 & 1543.887 & 1535.669 & 1502.905 \\
\hline $8 / 3 / 16$ 7:00 & 113.981 & 101.524 & 134.176 & 1543.91 & 1535.732 & 1502.975 \\
\hline 8/3/16 8:00 & 113.972 & 101.478 & 134.113 & 1543.919 & 1535.778 & 1503.038 \\
\hline 8/3/16 9:00 & 113.981 & 101.415 & 134.066 & 1543.91 & 1535.841 & 1503.085 \\
\hline $8 / 3 / 16$ 10:00 & 114.016 & 101.347 & 134.024 & 1543.875 & 1535.909 & 1503.127 \\
\hline 8/3/16 11:00 & 114.059 & 101.273 & 133.982 & 1543.832 & 1535.983 & 1503.169 \\
\hline $8 / 3 / 16$ 12:00 & 114.099 & 101.199 & 133.951 & 1543.792 & 1536.057 & 1503.2 \\
\hline 8/3/16 13:00 & 114.136 & 101.117 & 133.918 & 1543.755 & 1536.139 & 1503.233 \\
\hline 8/3/16 14:00 & 114.176 & 101.041 & 133.881 & 1543.715 & 1536.215 & 1503.27 \\
\hline $8 / 3 / 16$ 15:00 & 114.221 & 100.968 & 133.844 & 1543.67 & 1536.288 & 1503.307 \\
\hline $8 / 3 / 16$ 16:00 & 114.263 & 100.894 & 133.801 & 1543.628 & 1536.362 & 1503.35 \\
\hline 8/3/16 17:00 & 114.31 & 100.824 & 133.762 & 1543.581 & 1536.432 & 1503.389 \\
\hline 8/3/16 18:00 & 114.36 & 100.757 & 133.724 & 1543.531 & 1536.499 & 1503.427 \\
\hline 8/3/16 19:00 & 114.418 & 100.694 & 133.677 & 1543.473 & 1536.562 & 1503.474 \\
\hline $8 / 3 / 16$ 20:00 & 114.491 & 100.638 & 133.633 & 1543.4 & 1536.618 & 1503.518 \\
\hline $8 / 3 / 16$ 21:00 & 114.567 & 100.585 & 133.591 & 1543.324 & 1536.671 & 1503.56 \\
\hline $8 / 3 / 16$ 22:00 & 114.618 & 100.534 & 133.546 & 1543.273 & 1536.722 & 1503.605 \\
\hline $8 / 3 / 16$ 23:00 & 114.649 & 100.489 & 133.504 & 1543.242 & 1536.767 & 1503.647 \\
\hline 8/4/16 0:00 & 114.67 & 100.443 & 133.457 & 1543.221 & 1536.813 & 1503.694 \\
\hline $8 / 4 / 16$ 1:00 & 114.694 & 100.403 & 133.413 & 1543.197 & 1536.853 & 1503.738 \\
\hline $8 / 4 / 16$ 2:00 & 114.71 & 100.357 & 133.371 & 1543.181 & 1536.899 & 1503.78 \\
\hline 8/4/16 3:00 & 114.713 & 100.313 & 133.326 & 1543.178 & 1536.943 & 1503.825 \\
\hline 8/4/16 4:00 & 114.694 & 100.262 & 133.289 & 1543.197 & 1536.994 & 1503.862 \\
\hline $8 / 4 / 16$ 5:00 & 114.654 & 100.208 & 133.251 & 1543.237 & 1537.048 & 1503.9 \\
\hline 8/4/16 6:00 & 114.611 & 100.146 & 133.223 & 1543.28 & 1537.11 & 1503.928 \\
\hline 8/4/16 7:00 & 114.585 & 100.085 & 133.197 & 1543.306 & 1537.171 & 1503.954 \\
\hline 8/4/16 8:00 & 114.585 & 100.006 & 133.176 & 1543.306 & 1537.25 & 1503.975 \\
\hline 8/4/16 9:00 & 114.597 & 99.871 & 133.162 & 1543.294 & 1537.385 & 1503.989 \\
\hline 8/4/16 10:00 & 114.6 & 99.727 & 133.193 & 1543.291 & 1537.529 & 1503.958 \\
\hline 8/4/16 11:00 & 114.623 & 99.634 & 133.237 & 1543.268 & 1537.622 & 1503.914 \\
\hline 8/4/16 12:00 & 114.68 & 99.581 & 133.277 & 1543.211 & 1537.675 & 1503.874 \\
\hline 8/4/16 13:00 & 114.762 & 99.553 & 133.333 & 1543.129 & 1537.703 & 1503.818 \\
\hline 8/4/16 14:00 & 114.868 & 99.557 & 133.382 & 1543.023 & 1537.699 & 1503.769 \\
\hline 8/4/16 15:00 & 114.981 & 99.578 & 133.434 & 1542.91 & 1537.678 & 1503.717 \\
\hline 8/4/16 16:00 & 115.091 & 99.613 & 133.483 & 1542.8 & 1537.643 & 1503.668 \\
\hline 8/4/16 17:00 & 115.164 & 99.653 & 133.518 & 1542.727 & 1537.603 & 1503.633 \\
\hline 8/4/16 18:00 & 115.183 & 99.697 & 133.553 & 1542.708 & 1537.559 & 1503.598 \\
\hline 8/4/16 19:00 & 115.159 & 99.729 & 133.584 & 1542.732 & 1537.527 & 1503.567 \\
\hline 8/4/16 20:00 & 115.112 & 99.753 & 133.607 & 1542.779 & 1537.503 & 1503.544 \\
\hline 8/4/16 21:00 & 115.065 & 99.769 & 133.624 & 1542.826 & 1537.487 & 1503.527 \\
\hline 8/4/16 22:00 & 115.018 & 99.778 & 133.638 & 1542.873 & 1537.478 & 1503.513 \\
\hline 8/4/16 23:00 & 114.971 & 99.78 & 133.659 & 1542.92 & 1537.476 & 1503.492 \\
\hline 8/5/16 0:00 & 114.903 & 99.769 & 133.668 & 1542.988 & 1537.487 & 1503.483 \\
\hline 8/5/16 1:00 & 114.804 & 99.736 & 133.694 & 1543.087 & 1537.52 & 1503.457 \\
\hline 8/5/16 2:00 & 114.687 & 99.685 & 133.715 & 1543.204 & 1537.571 & 1503.436 \\
\hline 8/5/16 3:00 & 114.545 & 99.62 & 133.726 & 1543.346 & 1537.636 & 1503.425 \\
\hline
\end{tabular}


TABLE S1.2 Automatically recorded groundwater levels in the confined aquifers.

\begin{tabular}{|c|c|c|c|c|c|c|}
\hline \multirow[b]{2}{*}{ Date-Time } & \multicolumn{3}{|c|}{ Measured Depth to Water (ft TOC ${ }^{\mathrm{a}}$ ) } & \multicolumn{3}{|c|}{ Groundwater Elevation (ft AMSL ${ }^{\mathrm{b}}$ ) } \\
\hline & DL2-CU & DL7-CU & DL7-CL & DL2-CU & DL7-CU & DL7-CL \\
\hline 8/5/16 4:00 & 114.39 & 99.546 & 133.738 & 1543.501 & 1537.71 & 1503.413 \\
\hline 8/5/16 5:00 & 114.226 & 99.462 & 133.764 & 1543.665 & 1537.794 & 1503.387 \\
\hline 8/5/16 6:00 & 114.051 & 99.383 & 133.804 & 1543.84 & 1537.873 & 1503.347 \\
\hline 8/5/16 7:00 & 113.896 & 99.346 & 133.858 & 1543.995 & 1537.91 & 1503.293 \\
\hline 8/5/16 8:00 & 113.776 & 99.336 & 133.925 & 1544.115 & 1537.92 & 1503.226 \\
\hline 8/5/16 9:00 & 113.687 & 99.327 & 134.026 & 1544.204 & 1537.929 & 1503.125 \\
\hline 8/5/16 10:00 & 113.605 & 99.295 & 134.199 & 1544.286 & 1537.961 & 1502.952 \\
\hline $8 / 5 / 16$ 11:00 & 113.517 & 99.248 & 134.3 & 1544.374 & 1538.008 & 1502.851 \\
\hline $8 / 5 / 16$ 12:00 & 113.419 & 99.206 & 134.358 & 1544.472 & 1538.05 & 1502.793 \\
\hline 8/5/16 13:00 & 113.32 & 99.169 & 134.396 & 1544.571 & 1538.087 & 1502.755 \\
\hline $8 / 5 / 1614: 00$ & 113.226 & 99.134 & 134.424 & 1544.665 & 1538.122 & 1502.727 \\
\hline $8 / 5 / 16$ 15:00 & 113.141 & 99.111 & 134.45 & 1544.75 & 1538.145 & 1502.701 \\
\hline $8 / 5 / 16$ 16:00 & 113.068 & 99.104 & 134.471 & 1544.823 & 1538.152 & 1502.68 \\
\hline 8/5/16 17:00 & 113.005 & 99.116 & 134.48 & 1544.886 & 1538.14 & 1502.671 \\
\hline 8/5/16 18:00 & 112.948 & 99.132 & 134.483 & 1544.943 & 1538.124 & 1502.668 \\
\hline 8/5/16 19:00 & 112.906 & 99.146 & 134.478 & 1544.985 & 1538.11 & 1502.673 \\
\hline 8/5/16 20:00 & 112.873 & 99.172 & 134.471 & 1545.018 & 1538.084 & 1502.68 \\
\hline 8/5/16 21:00 & 112.852 & 99.188 & 134.452 & 1545.039 & 1538.068 & 1502.699 \\
\hline $8 / 5 / 16$ 22:00 & 112.838 & 99.213 & 134.44 & 1545.053 & 1538.043 & 1502.711 \\
\hline 8/5/16 23:00 & 112.831 & 99.237 & 134.422 & 1545.06 & 1538.019 & 1502.729 \\
\hline 8/6/16 0:00 & 112.828 & 99.251 & 134.389 & 1545.063 & 1538.005 & 1502.762 \\
\hline $8 / 6 / 16$ 1:00 & 112.828 & 99.248 & 134.349 & 1545.063 & 1538.008 & 1502.802 \\
\hline 8/6/16 2:00 & 112.833 & 99.239 & 134.316 & 1545.058 & 1538.017 & 1502.835 \\
\hline 8/6/16 3:00 & 112.835 & 99.211 & 134.356 & 1545.056 & 1538.045 & 1502.795 \\
\hline 8/6/16 4:00 & 112.84 & 99.172 & 134.44 & 1545.051 & 1538.084 & 1502.711 \\
\hline 8/6/16 5:00 & 112.84 & 99.116 & 134.527 & 1545.051 & 1538.14 & 1502.624 \\
\hline 8/6/16 6:00 & 112.835 & 99.065 & 134.618 & 1545.056 & 1538.191 & 1502.533 \\
\hline 8/6/16 7:00 & 112.826 & 99.025 & 134.724 & 1545.065 & 1538.231 & 1502.427 \\
\hline 8/6/16 8:00 & 112.805 & 99.039 & 134.824 & 1545.086 & 1538.217 & 1502.327 \\
\hline 8/6/16 9:00 & 112.772 & 99.118 & 134.895 & 1545.119 & 1538.138 & 1502.256 \\
\hline 8/6/16 10:00 & 112.72 & 99.248 & 134.88 & 1545.171 & 1538.008 & 1502.271 \\
\hline 8/6/16 11:00 & 112.661 & 99.406 & 134.857 & 1545.23 & 1537.85 & 1502.294 \\
\hline $8 / 6 / 16$ 12:00 & 112.6 & 99.571 & 134.824 & 1545.291 & 1537.685 & 1502.327 \\
\hline 8/6/16 13:00 & 112.541 & 99.727 & 134.789 & 1545.35 & 1537.529 & 1502.362 \\
\hline $8 / 6 / 16$ 14:00 & 112.487 & 99.883 & 134.749 & 1545.404 & 1537.373 & 1502.402 \\
\hline $8 / 6 / 16$ 15:00 & 112.438 & 100.036 & 134.712 & 1545.453 & 1537.22 & 1502.439 \\
\hline $8 / 6 / 16$ 16:00 & 112.388 & 100.201 & 134.679 & 1545.503 & 1537.055 & 1502.472 \\
\hline $8 / 6 / 16$ 17:00 & 112.346 & 100.364 & 134.646 & 1545.545 & 1536.892 & 1502.505 \\
\hline $8 / 6 / 16$ 18:00 & 112.299 & 100.522 & 134.607 & 1545.592 & 1536.734 & 1502.544 \\
\hline 8/6/16 19:00 & 112.257 & 100.664 & 134.564 & 1545.634 & 1536.592 & 1502.587 \\
\hline $8 / 6 / 16$ 20:00 & 112.231 & 100.801 & 134.52 & 1545.66 & 1536.455 & 1502.631 \\
\hline $8 / 6 / 16$ 21:00 & 112.226 & 100.917 & 134.476 & 1545.665 & 1536.339 & 1502.675 \\
\hline $8 / 6 / 16$ 22:00 & 112.203 & 101.029 & 134.436 & 1545.688 & 1536.227 & 1502.715 \\
\hline 8/6/16 23:00 & 112.158 & 101.134 & 134.389 & 1545.733 & 1536.122 & 1502.762 \\
\hline 8/7/16 0:00 & 112.092 & 101.226 & 134.337 & 1545.799 & 1536.03 & 1502.814 \\
\hline 8/7/16 1:00 & 112.017 & 101.289 & 134.281 & 1545.874 & 1535.967 & 1502.87 \\
\hline 8/7/16 2:00 & 111.939 & 101.343 & 134.218 & 1545.952 & 1535.913 & 1502.933 \\
\hline
\end{tabular}


TABLE S1.2 Automatically recorded groundwater levels in the confined aquifers.

\begin{tabular}{|c|c|c|c|c|c|c|}
\hline \multirow[b]{2}{*}{ Date-Time } & \multicolumn{3}{|c|}{ Measured Depth to Water (ft TOC ${ }^{\mathrm{a}}$ ) } & \multicolumn{3}{|c|}{ Groundwater Elevation (ft AMSL ${ }^{b}$ ) } \\
\hline & DL2-CU & DL7-CU & DL7-CL & DL2-CU & DL7-CU & DL7-CL \\
\hline 8/7/16 3:00 & 111.861 & 101.371 & 134.174 & 1546.03 & 1535.885 & 1502.977 \\
\hline $8 / 7 / 164: 00$ & 111.784 & 101.382 & 134.152 & 1546.107 & 1535.874 & 1502.999 \\
\hline 8/7/16 5:00 & 111.704 & 101.382 & 134.138 & 1546.187 & 1535.874 & 1503.013 \\
\hline 8/7/16 6:00 & 111.624 & 101.329 & 134.138 & 1546.267 & 1535.927 & 1503.013 \\
\hline 8/7/16 7:00 & 111.534 & 101.205 & 134.15 & 1546.357 & 1536.051 & 1503.001 \\
\hline 8/7/16 8:00 & 111.429 & 101.043 & 134.167 & 1546.462 & 1536.213 & 1502.984 \\
\hline 8/7/16 9:00 & 111.311 & 100.889 & 134.188 & 1546.58 & 1536.367 & 1502.963 \\
\hline 8/7/16 10:00 & 111.184 & 100.757 & 134.183 & 1546.707 & 1536.499 & 1502.968 \\
\hline 8/7/16 11:00 & 111.055 & 100.662 & 134.181 & 1546.836 & 1536.594 & 1502.97 \\
\hline 8/7/16 12:00 & 110.928 & 100.606 & 134.164 & 1546.963 & 1536.65 & 1502.987 \\
\hline 8/7/16 13:00 & 110.808 & 100.587 & 134.155 & 1547.083 & 1536.669 & 1502.996 \\
\hline 8/7/16 14:00 & 110.706 & 100.601 & 134.145 & 1547.185 & 1536.655 & 1503.006 \\
\hline $8 / 7 / 16$ 15:00 & 110.622 & 100.636 & 134.143 & 1547.269 & 1536.62 & 1503.008 \\
\hline 8/7/16 16:00 & 110.551 & 100.706 & 134.148 & 1547.34 & 1536.55 & 1503.003 \\
\hline 8/7/16 17:00 & 110.49 & 100.803 & 134.148 & 1547.401 & 1536.453 & 1503.003 \\
\hline 8/7/16 18:00 & 110.436 & 100.91 & 134.143 & 1547.455 & 1536.346 & 1503.008 \\
\hline 8/7/16 19:00 & 110.384 & 101.015 & 134.138 & 1547.507 & 1536.241 & 1503.013 \\
\hline 8/7/16 20:00 & 110.342 & 101.11 & 134.138 & 1547.549 & 1536.146 & 1503.013 \\
\hline 8/7/16 21:00 & 110.299 & 101.182 & 134.134 & 1547.592 & 1536.074 & 1503.017 \\
\hline 8/7/16 22:00 & 110.264 & 101.24 & 134.138 & 1547.627 & 1536.016 & 1503.013 \\
\hline 8/7/16 23:00 & 110.234 & 101.294 & 134.143 & 1547.657 & 1535.962 & 1503.008 \\
\hline 8/8/16 0:00 & 110.203 & 101.329 & 134.143 & 1547.688 & 1535.927 & 1503.008 \\
\hline 8/8/16 1:00 & 110.177 & 101.345 & 134.134 & 1547.714 & 1535.911 & 1503.017 \\
\hline 8/8/16 2:00 & 110.158 & 101.347 & 134.124 & 1547.733 & 1535.909 & 1503.027 \\
\hline 8/8/16 3:00 & 110.139 & 101.338 & 134.108 & 1547.752 & 1535.918 & 1503.043 \\
\hline 8/8/16 4:00 & 110.116 & 101.315 & 134.092 & 1547.775 & 1535.941 & 1503.059 \\
\hline 8/8/16 5:00 & 110.097 & 101.289 & 134.075 & 1547.794 & 1535.967 & 1503.076 \\
\hline 8/8/16 6:00 & 110.116 & 101.289 & 134.061 & 1547.775 & 1535.967 & 1503.09 \\
\hline 8/8/16 7:00 & 110.177 & 101.336 & 134.068 & 1547.714 & 1535.92 & 1503.083 \\
\hline 8/8/16 8:00 & 110.269 & 101.403 & 134.059 & 1547.622 & 1535.853 & 1503.092 \\
\hline 8/8/16 9:00 & 110.365 & 101.487 & 134.075 & 1547.526 & 1535.769 & 1503.076 \\
\hline 8/8/16 10:00 & 110.443 & 101.556 & 134.099 & 1547.448 & 1535.7 & 1503.052 \\
\hline 8/8/16 11:00 & 110.709 & 101.636 & 134.148 & 1547.182 & 1535.62 & 1503.003 \\
\hline 8/8/16 12:00 & 111.085 & 101.722 & 134.199 & 1546.806 & 1535.534 & 1502.952 \\
\hline 8/8/16 13:00 & 111.462 & 101.819 & 134.241 & 1546.429 & 1535.437 & 1502.91 \\
\hline 8/8/16 14:00 & 111.821 & 101.919 & 134.284 & 1546.07 & 1535.337 & 1502.867 \\
\hline $8 / 8 / 16$ 15:00 & 112.151 & 102.019 & 134.323 & 1545.74 & 1535.237 & 1502.828 \\
\hline 8/8/16 16:00 & 112.447 & 102.159 & 134.373 & 1545.444 & 1535.097 & 1502.778 \\
\hline 8/8/16 17:00 & 112.706 & 102.372 & 134.403 & 1545.185 & 1534.884 & 1502.748 \\
\hline $8 / 8 / 16$ 18:00 & 112.908 & 102.616 & 134.426 & 1544.983 & 1534.64 & 1502.725 \\
\hline 8/8/16 19:00 & 113.007 & 102.861 & 134.443 & 1544.884 & 1534.395 & 1502.708 \\
\hline 8/8/16 20:00 & 113.035 & 103.088 & 134.459 & 1544.856 & 1534.168 & 1502.692 \\
\hline $8 / 8 / 16$ 21:00 & 113.045 & 103.3 & 134.471 & 1544.846 & 1533.956 & 1502.68 \\
\hline $8 / 8 / 16$ 22:00 & 113.054 & 103.474 & 134.478 & 1544.837 & 1533.782 & 1502.673 \\
\hline 8/8/16 23:00 & 113.063 & 103.628 & 134.478 & 1544.828 & 1533.628 & 1502.673 \\
\hline 8/9/16 0:00 & 113.08 & 103.76 & 134.471 & 1544.811 & 1533.496 & 1502.68 \\
\hline 8/9/16 1:00 & 113.111 & 103.879 & 134.473 & 1544.78 & 1533.377 & 1502.678 \\
\hline
\end{tabular}


TABLE S1.2 Automatically recorded groundwater levels in the confined aquifers.

\begin{tabular}{|c|c|c|c|c|c|c|}
\hline \multirow[b]{2}{*}{ Date-Time } & \multicolumn{3}{|c|}{ Measured Depth to Water (ft TOC ${ }^{\mathrm{a}}$ ) } & \multicolumn{3}{|c|}{ Groundwater Elevation (ft AMSL ${ }^{\mathrm{b}}$ ) } \\
\hline & DL2-CU & DL7-CU & DL7-CL & DL2-CU & DL7-CU & DL7-CL \\
\hline 8/9/16 2:00 & 113.148 & 103.993 & 134.466 & 1544.743 & 1533.263 & 1502.685 \\
\hline 8/9/16 3:00 & 113.195 & 104.1 & 134.459 & 1544.696 & 1533.156 & 1502.692 \\
\hline 8/9/16 4:00 & 113.247 & 104.204 & 134.468 & 1544.644 & 1533.052 & 1502.683 \\
\hline 8/9/16 5:00 & 113.296 & 104.327 & 134.494 & 1544.595 & 1532.929 & 1502.657 \\
\hline 8/9/16 6:00 & 113.329 & 104.458 & 134.546 & 1544.562 & 1532.798 & 1502.605 \\
\hline 8/9/16 7:00 & 113.353 & 104.595 & 134.616 & 1544.538 & 1532.661 & 1502.535 \\
\hline 8/9/16 8:00 & 113.379 & 104.727 & 134.703 & 1544.512 & 1532.529 & 1502.448 \\
\hline 8/9/16 9:00 & 113.412 & 104.862 & 134.794 & 1544.479 & 1532.394 & 1502.357 \\
\hline 8/9/16 10:00 & 113.527 & 105.013 & 134.892 & 1544.364 & 1532.243 & 1502.259 \\
\hline 8/9/16 11:00 & 113.696 & 105.155 & 134.965 & 1544.195 & 1532.101 & 1502.186 \\
\hline $8 / 9 / 16$ 12:00 & 113.861 & 105.299 & 135.061 & 1544.03 & 1531.957 & 1502.09 \\
\hline 8/9/16 13:00 & 114.099 & 105.432 & 135.239 & 1543.792 & 1531.824 & 1501.912 \\
\hline 8/9/16 14:00 & 114.428 & 105.562 & 135.424 & 1543.463 & 1531.694 & 1501.727 \\
\hline 8/9/16 15:00 & 114.807 & 105.704 & 135.487 & 1543.084 & 1531.552 & 1501.664 \\
\hline 8/9/16 16:00 & 115.207 & 105.783 & 135.489 & 1542.684 & 1531.473 & 1501.662 \\
\hline 8/9/16 17:00 & 115.613 & 105.813 & 135.468 & 1542.278 & 1531.443 & 1501.683 \\
\hline 8/9/16 18:00 & 116.032 & 105.808 & 135.449 & 1541.859 & 1531.448 & 1501.702 \\
\hline 8/9/16 19:00 & 116.465 & 105.806 & 135.431 & 1541.426 & 1531.45 & 1501.72 \\
\hline 8/9/16 20:00 & 116.893 & 105.804 & 135.414 & 1540.998 & 1531.452 & 1501.737 \\
\hline 8/9/16 21:00 & 117.314 & 105.815 & 135.466 & 1540.577 & 1531.441 & 1501.685 \\
\hline 8/9/16 22:00 & 117.733 & 105.787 & 135.559 & 1540.158 & 1531.469 & 1501.592 \\
\hline 8/9/16 23:00 & 118.142 & 105.732 & 135.648 & 1539.749 & 1531.524 & 1501.503 \\
\hline 8/10/16 0:00 & 118.542 & 105.669 & 135.737 & 1539.349 & 1531.587 & 1501.414 \\
\hline 8/10/16 1:00 & 118.93 & 105.606 & 135.826 & 1538.961 & 1531.65 & 1501.325 \\
\hline $8 / 10 / 16$ 2:00 & 119.309 & 105.546 & 135.91 & 1538.582 & 1531.71 & 1501.241 \\
\hline 8/10/16 3:00 & 119.678 & 105.453 & 136.002 & 1538.213 & 1531.803 & 1501.149 \\
\hline $8 / 10 / 164: 00$ & 120.027 & 105.304 & 136.102 & 1537.864 & 1531.952 & 1501.049 \\
\hline 8/10/16 5:00 & 120.354 & 105.136 & 136.203 & 1537.537 & 1532.12 & 1500.948 \\
\hline 8/10/16 6:00 & 120.655 & 104.983 & 136.306 & 1537.236 & 1532.273 & 1500.845 \\
\hline 8/10/16 7:00 & 120.949 & 104.89 & 136.442 & 1536.942 & 1532.366 & 1500.709 \\
\hline 8/10/16 8:00 & 121.252 & 104.895 & 136.58 & 1536.639 & 1532.361 & 1500.571 \\
\hline 8/10/16 9:00 & 121.567 & 104.932 & 136.702 & 1536.324 & 1532.324 & 1500.449 \\
\hline $8 / 10 / 16$ 10:00 & 121.878 & 104.941 & 136.718 & 1536.013 & 1532.315 & 1500.433 \\
\hline 8/10/16 11:00 & 122.174 & 104.976 & 136.697 & 1535.717 & 1532.28 & 1500.454 \\
\hline 8/10/16 12:00 & 122.454 & 105.071 & 136.699 & 1535.437 & 1532.185 & 1500.452 \\
\hline 8/10/16 13:00 & 122.666 & 105.229 & 136.713 & 1535.225 & 1532.027 & 1500.438 \\
\hline 8/10/16 14:00 & 122.593 & 105.385 & 136.692 & 1535.298 & 1531.871 & 1500.459 \\
\hline 8/10/16 15:00 & 122.471 & 105.497 & 136.666 & 1535.42 & 1531.759 & 1500.485 \\
\hline 8/10/16 16:00 & 122.391 & 105.587 & 136.659 & 1535.5 & 1531.669 & 1500.492 \\
\hline 8/10/16 17:00 & 122.348 & 105.678 & 136.634 & 1535.543 & 1531.578 & 1500.517 \\
\hline 8/10/16 18:00 & 122.323 & 105.72 & 136.613 & 1535.568 & 1531.536 & 1500.538 \\
\hline 8/10/16 19:00 & 122.33 & 105.713 & 136.617 & 1535.561 & 1531.543 & 1500.534 \\
\hline $8 / 10 / 16$ 20:00 & 122.367 & 105.68 & 136.643 & 1535.524 & 1531.576 & 1500.508 \\
\hline 8/10/16 21:00 & 122.421 & 105.638 & 136.671 & 1535.47 & 1531.618 & 1500.48 \\
\hline 8/10/16 22:00 & 122.487 & 105.592 & 136.709 & 1535.404 & 1531.664 & 1500.442 \\
\hline 8/10/16 23:00 & 122.548 & 105.543 & 136.727 & 1535.343 & 1531.713 & 1500.424 \\
\hline 8/11/16 0:00 & 122.593 & 105.497 & 136.716 & 1535.298 & 1531.759 & 1500.435 \\
\hline
\end{tabular}


TABLE S1.2 Automatically recorded groundwater levels in the confined aquifers.

\begin{tabular}{|c|c|c|c|c|c|c|}
\hline \multirow[b]{2}{*}{ Date-Time } & \multicolumn{3}{|c|}{ Measured Depth to Water (ft TOC ${ }^{\mathrm{a}}$ ) } & \multicolumn{3}{|c|}{ Groundwater Elevation (ft AMSL ${ }^{\mathrm{b}}$ ) } \\
\hline & DL2-CU & DL7-CU & DL7-CL & DL2-CU & DL7-CU & DL7-CL \\
\hline 8/11/16 1:00 & 122.61 & 105.455 & 136.692 & 1535.281 & 1531.801 & 1500.459 \\
\hline $8 / 11 / 16$ 2:00 & 122.61 & 105.397 & 136.666 & 1535.281 & 1531.859 & 1500.485 \\
\hline 8/11/16 3:00 & 122.579 & 105.283 & 136.65 & 1535.312 & 1531.973 & 1500.501 \\
\hline $8 / 11 / 164: 00$ & 122.53 & 105.125 & 136.676 & 1535.361 & 1532.131 & 1500.475 \\
\hline $8 / 11 / 16$ 5:00 & 122.461 & 104.951 & 136.711 & 1535.43 & 1532.305 & 1500.44 \\
\hline $8 / 11 / 16$ 6:00 & 122.384 & 104.776 & 136.762 & 1535.507 & 1532.48 & 1500.389 \\
\hline 8/11/16 7:00 & 122.304 & 104.611 & 136.812 & 1535.587 & 1532.645 & 1500.339 \\
\hline 8/11/16 8:00 & 122.224 & 104.476 & 136.87 & 1535.667 & 1532.78 & 1500.281 \\
\hline 8/11/16 9:00 & 122.153 & 104.402 & 136.924 & 1535.738 & 1532.854 & 1500.227 \\
\hline 8/11/16 10:00 & 122.104 & 104.381 & 136.954 & 1535.787 & 1532.875 & 1500.197 \\
\hline 8/11/16 11:00 & 122.071 & 104.383 & 136.968 & 1535.82 & 1532.873 & 1500.183 \\
\hline 8/11/16 12:00 & 122.047 & 104.446 & 136.996 & 1535.844 & 1532.81 & 1500.155 \\
\hline 8/11/16 13:00 & 122.033 & 104.581 & 137.062 & 1535.858 & 1532.675 & 1500.089 \\
\hline 8/11/16 14:00 & 122.036 & 104.799 & 137.137 & 1535.855 & 1532.457 & 1500.014 \\
\hline 8/11/16 15:00 & 122.054 & 105.048 & 137.207 & 1535.837 & 1532.208 & 1499.944 \\
\hline 8/11/16 16:00 & 122.076 & 105.304 & 137.277 & 1535.815 & 1531.952 & 1499.874 \\
\hline 8/11/16 17:00 & 121.937 & 105.571 & 137.364 & 1535.954 & 1531.685 & 1499.787 \\
\hline 8/11/16 18:00 & 121.84 & 105.822 & 137.427 & 1536.051 & 1531.434 & 1499.724 \\
\hline 8/11/16 19:00 & 121.781 & 106.006 & 137.448 & 1536.11 & 1531.25 & 1499.703 \\
\hline 8/11/16 20:00 & 121.744 & 106.113 & 137.453 & 1536.147 & 1531.143 & 1499.698 \\
\hline 8/11/16 21:00 & 121.711 & 106.208 & 137.488 & 1536.18 & 1531.048 & 1499.663 \\
\hline 8/11/16 22:00 & 121.652 & 106.306 & 137.521 & 1536.239 & 1530.95 & 1499.63 \\
\hline 8/11/16 23:00 & 121.57 & 106.42 & 137.558 & 1536.321 & 1530.836 & 1499.593 \\
\hline 8/12/16 0:00 & 121.445 & 106.541 & 137.6 & 1536.446 & 1530.715 & 1499.551 \\
\hline 8/12/16 1:00 & 121.285 & 106.647 & 137.64 & 1536.606 & 1530.609 & 1499.511 \\
\hline $8 / 12 / 16$ 2:00 & 121.104 & 106.682 & 137.687 & 1536.787 & 1530.574 & 1499.464 \\
\hline 8/12/16 3:00 & 120.932 & 106.668 & 137.731 & 1536.959 & 1530.588 & 1499.42 \\
\hline 8/12/16 4:00 & 120.664 & 106.552 & 137.708 & 1537.227 & 1530.704 & 1499.443 \\
\hline $8 / 12 / 165: 00$ & 120.436 & 106.494 & 137.741 & 1537.455 & 1530.762 & 1499.41 \\
\hline $8 / 12 / 16$ 6:00 & 120.184 & 106.445 & 137.788 & 1537.707 & 1530.811 & 1499.363 \\
\hline 8/12/16 7:00 & 119.925 & 106.385 & 137.82 & 1537.966 & 1530.871 & 1499.331 \\
\hline 8/12/16 8:00 & 119.664 & 106.338 & 137.879 & 1538.227 & 1530.918 & 1499.272 \\
\hline 8/12/16 9:00 & 119.347 & 106.294 & 137.933 & 1538.544 & 1530.962 & 1499.218 \\
\hline 8/12/16 10:00 & 118.968 & 106.248 & 137.991 & 1538.923 & 1531.008 & 1499.16 \\
\hline 8/12/16 11:00 & 118.53 & 106.196 & 138.068 & 1539.361 & 1531.06 & 1499.083 \\
\hline 8/12/16 12:00 & 118.044 & 106.143 & 138.141 & 1539.847 & 1531.113 & 1499.01 \\
\hline 8/12/16 13:00 & 117.517 & 106.076 & 138.218 & 1540.374 & 1531.18 & 1498.933 \\
\hline 8/12/16 14:00 & 116.971 & 106.001 & 138.305 & 1540.92 & 1531.255 & 1498.846 \\
\hline 8/12/16 15:00 & 116.423 & 105.852 & 138.342 & 1541.468 & 1531.404 & 1498.809 \\
\hline 8/12/16 16:00 & 115.875 & 105.604 & 138.349 & 1542.016 & 1531.652 & 1498.802 \\
\hline 8/12/16 17:00 & 115.338 & 105.297 & 138.352 & 1542.553 & 1531.959 & 1498.799 \\
\hline 8/12/16 18:00 & 114.811 & 104.957 & 138.347 & 1543.08 & 1532.299 & 1498.804 \\
\hline 8/12/16 19:00 & 114.298 & 104.604 & 138.338 & 1543.593 & 1532.652 & 1498.813 \\
\hline 8/12/16 20:00 & 113.804 & 104.251 & 138.324 & 1544.087 & 1533.005 & 1498.827 \\
\hline 8/12/16 21:00 & 113.322 & 103.902 & 138.31 & 1544.569 & 1533.354 & 1498.841 \\
\hline 8/12/16 22:00 & 112.859 & 103.553 & 138.293 & 1545.032 & 1533.703 & 1498.858 \\
\hline 8/12/16 23:00 & 112.41 & 103.212 & 138.267 & 1545.481 & 1534.044 & 1498.884 \\
\hline
\end{tabular}


TABLE S1.2 Automatically recorded groundwater levels in the confined aquifers.

\begin{tabular}{|c|c|c|c|c|c|c|}
\hline \multirow[b]{2}{*}{ Date-Time } & \multicolumn{3}{|c|}{ Measured Depth to Water (ft TOC ${ }^{a}$ ) } & \multicolumn{3}{|c|}{ Groundwater Elevation (ft AMSL ${ }^{b}$ ) } \\
\hline & DL2-CU & DL7-CU & DL7-CL & DL2-CU & DL7-CU & DL7-CL \\
\hline 8/13/16 0:00 & 111.97 & 102.872 & 138.239 & 1545.921 & 1534.384 & 1498.912 \\
\hline $8 / 13 / 161: 00$ & 111.546 & 102.542 & 138.207 & 1546.345 & 1534.714 & 1498.944 \\
\hline $8 / 13 / 162: 00$ & 111.13 & 102.219 & 138.174 & 1546.761 & 1535.037 & 1498.977 \\
\hline 8/13/16 3:00 & 110.73 & 101.903 & 138.148 & 1547.161 & 1535.353 & 1499.003 \\
\hline $8 / 13 / 164: 00$ & 110.344 & 101.589 & 138.141 & 1547.547 & 1535.667 & 1499.01 \\
\hline $8 / 13 / 165: 00$ & 109.97 & 101.287 & 138.15 & 1547.921 & 1535.969 & 1499.001 \\
\hline 8/13/16 6:00 & 109.605 & 100.992 & 138.164 & 1548.286 & 1536.264 & 1498.987 \\
\hline 8/13/16 7:00 & 109.26 & 100.701 & 138.193 & 1548.631 & 1536.555 & 1498.958 \\
\hline 8/13/16 8:00 & 108.921 & 100.413 & 138.225 & 1548.97 & 1536.843 & 1498.926 \\
\hline 8/13/16 9:00 & 108.591 & 100.134 & 138.258 & 1549.3 & 1537.122 & 1498.893 \\
\hline $8 / 13 / 16$ 10:00 & 108.272 & 99.899 & 138.31 & 1549.619 & 1537.357 & 1498.841 \\
\hline 8/13/16 11:00 & 107.959 & 99.753 & 138.368 & 1549.932 & 1537.503 & 1498.783 \\
\hline $8 / 13 / 16$ 12:00 & 107.655 & 99.674 & 138.417 & 1550.236 & 1537.582 & 1498.734 \\
\hline 8/13/16 13:00 & 107.361 & 99.623 & 138.569 & 1550.53 & 1537.633 & 1498.582 \\
\hline 8/13/16 14:00 & 107.074 & 99.569 & 138.738 & 1550.817 & 1537.687 & 1498.413 \\
\hline 8/13/16 15:00 & 106.794 & 99.518 & 138.787 & 1551.097 & 1537.738 & 1498.364 \\
\hline 8/13/16 16:00 & 106.526 & 99.441 & 138.778 & 1551.365 & 1537.815 & 1498.373 \\
\hline 8/13/16 17:00 & 106.263 & 99.271 & 138.731 & 1551.628 & 1537.985 & 1498.42 \\
\hline $8 / 13 / 16$ 18:00 & 106.011 & 99.025 & 138.677 & 1551.88 & 1538.231 & 1498.474 \\
\hline $8 / 13 / 16$ 19:00 & 105.766 & 98.76 & 138.616 & 1552.125 & 1538.496 & 1498.535 \\
\hline $8 / 13 / 16$ 20:00 & 105.531 & 98.472 & 138.558 & 1552.36 & 1538.784 & 1498.593 \\
\hline $8 / 13 / 16$ 21:00 & 105.301 & 98.181 & 138.494 & 1552.59 & 1539.075 & 1498.657 \\
\hline 8/13/16 22:00 & 105.079 & 97.898 & 138.436 & 1552.812 & 1539.358 & 1498.715 \\
\hline 8/13/16 23:00 & 104.858 & 97.616 & 138.363 & 1553.033 & 1539.64 & 1498.788 \\
\hline 8/14/16 0:00 & 104.64 & 97.337 & 138.295 & 1553.251 & 1539.919 & 1498.856 \\
\hline 8/14/16 1:00 & 104.428 & 97.068 & 138.223 & 1553.463 & 1540.188 & 1498.928 \\
\hline $8 / 14 / 16$ 2:00 & 104.216 & 96.805 & 138.157 & 1553.675 & 1540.451 & 1498.994 \\
\hline 8/14/16 3:00 & 104.009 & 96.554 & 138.218 & 1553.882 & 1540.702 & 1498.933 \\
\hline 8/14/16 4:00 & 103.809 & 96.301 & 138.328 & 1554.082 & 1540.955 & 1498.823 \\
\hline 8/14/16 5:00 & 103.614 & 96.059 & 138.455 & 1554.277 & 1541.197 & 1498.696 \\
\hline 8/14/16 6:00 & 103.426 & 95.824 & 138.581 & 1554.465 & 1541.432 & 1498.57 \\
\hline 8/14/16 7:00 & 103.238 & 95.592 & 138.7 & 1554.653 & 1541.664 & 1498.451 \\
\hline 8/14/16 8:00 & 103.052 & 95.361 & 138.714 & 1554.839 & 1541.895 & 1498.437 \\
\hline 8/14/16 9:00 & 102.875 & 95.145 & 138.663 & 1555.016 & 1542.111 & 1498.488 \\
\hline 8/14/16 10:00 & 102.699 & 94.938 & 138.602 & 1555.192 & 1542.318 & 1498.549 \\
\hline $8 / 14 / 16$ 11:00 & 102.527 & 94.738 & 138.546 & 1555.364 & 1542.518 & 1498.605 \\
\hline $8 / 14 / 16$ 12:00 & 102.353 & 94.539 & 138.611 & 1555.538 & 1542.717 & 1498.54 \\
\hline $8 / 14 / 16$ 13:00 & 102.181 & 94.329 & 138.686 & 1555.71 & 1542.927 & 1498.465 \\
\hline $8 / 14 / 16$ 14:00 & 102.01 & 94.127 & 138.644 & 1555.881 & 1543.129 & 1498.507 \\
\hline $8 / 14 / 16$ 15:00 & 101.843 & 93.934 & 138.565 & 1556.048 & 1543.322 & 1498.586 \\
\hline $8 / 14 / 16$ 16:00 & 101.676 & 93.746 & 138.483 & 1556.215 & 1543.51 & 1498.668 \\
\hline $8 / 14 / 16$ 17:00 & 101.511 & 93.562 & 138.384 & 1556.38 & 1543.694 & 1498.767 \\
\hline $8 / 14 / 16$ 18:00 & 101.349 & 93.381 & 138.296 & 1556.542 & 1543.875 & 1498.855 \\
\hline 8/14/16 19:00 & 101.191 & 93.211 & 138.303 & 1556.7 & 1544.045 & 1498.848 \\
\hline 8/14/16 20:00 & 101.038 & 93.032 & 138.387 & 1556.853 & 1544.224 & 1498.764 \\
\hline 8/14/16 21:00 & 100.89 & 92.863 & 138.49 & 1557.001 & 1544.393 & 1498.661 \\
\hline 8/14/16 22:00 & 100.742 & 92.695 & 138.586 & 1557.149 & 1544.561 & 1498.565 \\
\hline
\end{tabular}


TABLE S1.2 Automatically recorded groundwater levels in the confined aquifers.

\begin{tabular}{|c|c|c|c|c|c|c|}
\hline \multirow[b]{2}{*}{ Date-Time } & \multicolumn{3}{|c|}{ Measured Depth to Water (ft TOC ${ }^{\mathrm{a}}$ ) } & \multicolumn{3}{|c|}{ Groundwater Elevation (ft AMSL ${ }^{\mathrm{b}}$ ) } \\
\hline & DL2-CU & DL7-CU & DL7-CL & DL2-CU & DL7-CU & DL7-CL \\
\hline 8/14/16 23:00 & 100.596 & 92.523 & 138.665 & 1557.295 & 1544.733 & 1498.486 \\
\hline 8/15/16 0:00 & 100.452 & 92.36 & 138.626 & 1557.439 & 1544.896 & 1498.525 \\
\hline 8/15/16 1:00 & 100.309 & 92.195 & 138.546 & 1557.582 & 1545.061 & 1498.605 \\
\hline $8 / 15 / 16$ 2:00 & 100.168 & 92.044 & 138.462 & 1557.723 & 1545.212 & 1498.689 \\
\hline 8/15/16 3:00 & 100.029 & 91.893 & 138.501 & 1557.862 & 1545.363 & 1498.65 \\
\hline $8 / 15 / 164: 00$ & 99.895 & 91.74 & 138.581 & 1557.996 & 1545.516 & 1498.57 \\
\hline 8/15/16 5:00 & 99.768 & 91.586 & 138.672 & 1558.123 & 1545.67 & 1498.479 \\
\hline $8 / 15 / 16$ 6:00 & 99.669 & 91.44 & 138.771 & 1558.222 & 1545.816 & 1498.38 \\
\hline 8/15/16 7:00 & 99.608 & 91.293 & 138.867 & 1558.283 & 1545.963 & 1498.284 \\
\hline 8/15/16 8:00 & 99.575 & 91.159 & 138.97 & 1558.316 & 1546.097 & 1498.181 \\
\hline 8/15/16 9:00 & 99.556 & 91.077 & 139.052 & 1558.335 & 1546.179 & 1498.099 \\
\hline $8 / 15 / 16$ 10:00 & 99.544 & 91.091 & 139.037 & 1558.347 & 1546.165 & 1498.114 \\
\hline $8 / 15 / 16$ 11:00 & 99.544 & 91.142 & 138.995 & 1558.347 & 1546.114 & 1498.156 \\
\hline $8 / 15 / 16$ 12:00 & 99.544 & 91.152 & 138.906 & 1558.347 & 1546.104 & 1498.245 \\
\hline 8/15/16 13:00 & 99.554 & 91.117 & 138.796 & 1558.337 & 1546.139 & 1498.355 \\
\hline $8 / 15 / 16$ 14:00 & 99.563 & 91.061 & 138.679 & 1558.328 & 1546.195 & 1498.472 \\
\hline $8 / 15 / 16$ 15:00 & 99.547 & 90.987 & 138.555 & 1558.344 & 1546.269 & 1498.596 \\
\hline $8 / 15 / 16$ 16:00 & 99.507 & 90.912 & 138.429 & 1558.384 & 1546.344 & 1498.722 \\
\hline $8 / 15 / 16$ 17:00 & 99.46 & 90.826 & 138.298 & 1558.431 & 1546.43 & 1498.853 \\
\hline $8 / 15 / 16$ 18:00 & 99.434 & 90.733 & 138.171 & 1558.457 & 1546.523 & 1498.98 \\
\hline $8 / 15 / 16$ 19:00 & 99.427 & 90.638 & 138.047 & 1558.464 & 1546.618 & 1499.104 \\
\hline $8 / 15 / 16$ 20:00 & 99.434 & 90.545 & 137.928 & 1558.457 & 1546.711 & 1499.223 \\
\hline 8/15/16 21:00 & 99.434 & 90.456 & 137.813 & 1558.457 & 1546.8 & 1499.338 \\
\hline $8 / 15 / 16$ 22:00 & 99.41 & 90.371 & 137.699 & 1558.481 & 1546.885 & 1499.452 \\
\hline $8 / 15 / 16$ 23:00 & 99.394 & 90.292 & 137.589 & 1558.497 & 1546.964 & 1499.562 \\
\hline 8/16/16 0:00 & 99.41 & 90.212 & 137.476 & 1558.481 & 1547.044 & 1499.675 \\
\hline 8/16/16 1:00 & 99.46 & 90.133 & 137.366 & 1558.431 & 1547.123 & 1499.785 \\
\hline 8/16/16 2:00 & 99.537 & 90.059 & 137.259 & 1558.354 & 1547.197 & 1499.892 \\
\hline 8/16/16 3:00 & 99.629 & 89.989 & 137.153 & 1558.262 & 1547.267 & 1499.998 \\
\hline 8/16/16 4:00 & 99.739 & 89.927 & 137.055 & 1558.152 & 1547.329 & 1500.096 \\
\hline 8/16/16 5:00 & 99.848 & 89.873 & 136.954 & 1558.043 & 1547.383 & 1500.197 \\
\hline 8/16/16 6:00 & 99.965 & 89.82 & 136.858 & 1557.926 & 1547.436 & 1500.293 \\
\hline 8/16/16 7:00 & 100.083 & 89.773 & 136.76 & 1557.808 & 1547.483 & 1500.391 \\
\hline 8/16/16 8:00 & 100.205 & 89.729 & 136.678 & 1557.686 & 1547.527 & 1500.473 \\
\hline 8/16/16 9:00 & 100.335 & 89.696 & 136.601 & 1557.556 & 1547.56 & 1500.55 \\
\hline 8/16/16 10:00 & 100.45 & 89.669 & 136.521 & 1557.441 & 1547.587 & 1500.63 \\
\hline 8/16/16 11:00 & 100.553 & 89.641 & 136.442 & 1557.338 & 1547.615 & 1500.709 \\
\hline 8/16/16 12:00 & 100.626 & 89.613 & 136.374 & 1557.265 & 1547.643 & 1500.777 \\
\hline 8/16/16 13:00 & 100.662 & 89.589 & 136.306 & 1557.229 & 1547.667 & 1500.845 \\
\hline 8/16/16 14:00 & 100.702 & 89.569 & 136.245 & 1557.189 & 1547.687 & 1500.906 \\
\hline 8/16/16 15:00 & 100.735 & 89.538 & 136.18 & 1557.156 & 1547.718 & 1500.971 \\
\hline 8/16/16 16:00 & 100.77 & 89.508 & 136.121 & 1557.121 & 1547.748 & 1501.03 \\
\hline $8 / 16 / 16$ 17:00 & 100.798 & 89.478 & 136.06 & 1557.093 & 1547.778 & 1501.091 \\
\hline $8 / 16 / 16$ 18:00 & 100.824 & 89.448 & 136.006 & 1557.067 & 1547.808 & 1501.145 \\
\hline 8/16/16 19:00 & 100.847 & 89.417 & 135.945 & 1557.044 & 1547.839 & 1501.206 \\
\hline 8/16/16 20:00 & 100.871 & 89.392 & 135.894 & 1557.02 & 1547.864 & 1501.257 \\
\hline $8 / 16 / 16$ 21:00 & 100.899 & 89.371 & 135.85 & 1556.992 & 1547.885 & 1501.301 \\
\hline
\end{tabular}


TABLE S1.2 Automatically recorded groundwater levels in the confined aquifers.

\begin{tabular}{|c|c|c|c|c|c|c|}
\hline \multirow[b]{2}{*}{ Date-Time } & \multicolumn{3}{|c|}{ Measured Depth to Water (ft TOC ${ }^{\mathrm{a}}$ ) } & \multicolumn{3}{|c|}{ Groundwater Elevation (ft AMSL ${ }^{\mathrm{b}}$ ) } \\
\hline & DL2-CU & DL7-CU & DL7-CL & DL2-CU & DL7-CU & DL7-CL \\
\hline $8 / 16 / 16$ 22:00 & 100.92 & 89.355 & 135.8 & 1556.971 & 1547.901 & 1501.351 \\
\hline $8 / 16 / 16$ 23:00 & 100.935 & 89.329 & 135.754 & 1556.956 & 1547.927 & 1501.397 \\
\hline 8/17/16 0:00 & 100.946 & 89.306 & 135.7 & 1556.945 & 1547.95 & 1501.451 \\
\hline 8/17/16 1:00 & 100.956 & 89.283 & 135.648 & 1556.935 & 1547.973 & 1501.503 \\
\hline $8 / 17 / 162: 00$ & 100.965 & 89.257 & 135.597 & 1556.926 & 1547.999 & 1501.554 \\
\hline 8/17/16 3:00 & 100.97 & 89.234 & 135.548 & 1556.921 & 1548.022 & 1501.603 \\
\hline 8/17/16 4:00 & 100.972 & 89.213 & 135.494 & 1556.919 & 1548.043 & 1501.657 \\
\hline 8/17/16 5:00 & 100.977 & 89.189 & 135.442 & 1556.914 & 1548.067 & 1501.709 \\
\hline 8/17/16 6:00 & 100.984 & 89.173 & 135.393 & 1556.907 & 1548.083 & 1501.758 \\
\hline 8/17/16 7:00 & 100.996 & 89.162 & 135.353 & 1556.895 & 1548.094 & 1501.798 \\
\hline 8/17/16 8:00 & 101.012 & 89.148 & 135.32 & 1556.879 & 1548.108 & 1501.831 \\
\hline 8/17/16 9:00 & 101.033 & 89.143 & 135.297 & 1556.858 & 1548.113 & 1501.854 \\
\hline $8 / 17 / 16$ 10:00 & 101.066 & 89.139 & 135.285 & 1556.825 & 1548.117 & 1501.866 \\
\hline 8/17/16 11:00 & 101.097 & 89.141 & 135.29 & 1556.794 & 1548.115 & 1501.861 \\
\hline 8/17/16 12:00 & 101.156 & 89.143 & 135.302 & 1556.735 & 1548.113 & 1501.849 \\
\hline $8 / 17 / 16$ 13:00 & 101.182 & 89.139 & 135.311 & 1556.709 & 1548.117 & 1501.84 \\
\hline $8 / 17 / 16$ 14:00 & 101.207 & 89.143 & 135.306 & 1556.684 & 1548.113 & 1501.845 \\
\hline $8 / 17 / 16$ 15:00 & 101.233 & 89.15 & 135.302 & 1556.658 & 1548.106 & 1501.849 \\
\hline $8 / 17 / 16$ 16:00 & 101.266 & 89.164 & 135.285 & 1556.625 & 1548.092 & 1501.866 \\
\hline $8 / 17 / 16$ 17:00 & 101.32 & 89.178 & 135.269 & 1556.571 & 1548.078 & 1501.882 \\
\hline 8/17/16 18:00 & 101.389 & 89.192 & 135.248 & 1556.502 & 1548.064 & 1501.903 \\
\hline 8/17/16 19:00 & 101.469 & 89.213 & 135.222 & 1556.422 & 1548.043 & 1501.929 \\
\hline 8/17/16 20:00 & 101.558 & 89.241 & 135.201 & 1556.333 & 1548.015 & 1501.95 \\
\hline 8/17/16 21:00 & 101.654 & 89.266 & 135.182 & 1556.237 & 1547.99 & 1501.969 \\
\hline $8 / 17 / 16$ 22:00 & 101.751 & 89.301 & 135.18 & 1556.14 & 1547.955 & 1501.971 \\
\hline 8/17/16 23:00 & 101.84 & 89.334 & 135.196 & 1556.051 & 1547.922 & 1501.955 \\
\hline 8/18/16 0:00 & 101.918 & 89.362 & 135.206 & 1555.973 & 1547.894 & 1501.945 \\
\hline 8/18/16 1:00 & 101.988 & 89.387 & 135.213 & 1555.903 & 1547.869 & 1501.938 \\
\hline $8 / 18 / 16$ 2:00 & 102.047 & 89.415 & 135.206 & 1555.844 & 1547.841 & 1501.945 \\
\hline 8/18/16 3:00 & 102.104 & 89.441 & 135.196 & 1555.787 & 1547.815 & 1501.955 \\
\hline 8/18/16 4:00 & 102.144 & 89.469 & 135.189 & 1555.747 & 1547.787 & 1501.962 \\
\hline 8/18/16 5:00 & 102.165 & 89.499 & 135.192 & 1555.726 & 1547.757 & 1501.959 \\
\hline 8/18/16 6:00 & 102.174 & 89.524 & 135.215 & 1555.717 & 1547.732 & 1501.936 \\
\hline 8/18/16 7:00 & 102.167 & 89.545 & 135.236 & 1555.724 & 1547.711 & 1501.915 \\
\hline 8/18/16 8:00 & 102.17 & 89.564 & 135.271 & 1555.721 & 1547.692 & 1501.88 \\
\hline 8/18/16 9:00 & 102.167 & 89.587 & 135.328 & 1555.724 & 1547.669 & 1501.823 \\
\hline 8/18/16 10:00 & 102.165 & 89.594 & 135.37 & 1555.726 & 1547.662 & 1501.781 \\
\hline 8/18/16 11:00 & 102.16 & 89.603 & 135.398 & 1555.731 & 1547.653 & 1501.753 \\
\hline 8/18/16 12:00 & 102.158 & 89.608 & 135.416 & 1555.733 & 1547.648 & 1501.735 \\
\hline 8/18/16 13:00 & 102.155 & 89.61 & 135.421 & 1555.736 & 1547.646 & 1501.73 \\
\hline 8/18/16 14:00 & 102.137 & 89.61 & 135.421 & 1555.754 & 1547.646 & 1501.73 \\
\hline 8/18/16 15:00 & 102.101 & 89.61 & 135.414 & 1555.79 & 1547.646 & 1501.737 \\
\hline $8 / 18 / 16$ 16:00 & 102.057 & 89.601 & 135.4 & 1555.834 & 1547.655 & 1501.751 \\
\hline $8 / 18 / 16$ 17:00 & 102.01 & 89.594 & 135.374 & 1555.881 & 1547.662 & 1501.777 \\
\hline 8/18/16 18:00 & 101.965 & 89.58 & 135.351 & 1555.926 & 1547.676 & 1501.8 \\
\hline 8/18/16 19:00 & 101.918 & 89.569 & 135.323 & 1555.973 & 1547.687 & 1501.828 \\
\hline $8 / 18 / 16$ 20:00 & 101.88 & 89.555 & 135.292 & 1556.011 & 1547.701 & 1501.859 \\
\hline
\end{tabular}


TABLE S1.2 Automatically recorded groundwater levels in the confined aquifers.

\begin{tabular}{|c|c|c|c|c|c|c|}
\hline \multirow[b]{2}{*}{ Date-Time } & \multicolumn{3}{|c|}{ Measured Depth to Water (ft TOC ${ }^{a}$ ) } & \multicolumn{3}{|c|}{ Groundwater Elevation (ft AMSL ${ }^{\mathrm{b}}$ ) } \\
\hline & DL2-CU & DL7-CU & DL7-CL & DL2-CU & DL7-CU & DL7-CL \\
\hline 8/18/16 21:00 & 101.847 & 89.545 & 135.262 & 1556.044 & 1547.711 & 1501.889 \\
\hline $8 / 18 / 16$ 22:00 & 101.821 & 89.534 & 135.236 & 1556.07 & 1547.722 & 1501.915 \\
\hline $8 / 18 / 16$ 23:00 & 101.795 & 89.527 & 135.206 & 1556.096 & 1547.729 & 1501.945 \\
\hline 8/19/16 0:00 & 101.767 & 89.503 & 135.166 & 1556.124 & 1547.753 & 1501.985 \\
\hline 8/19/16 1:00 & 101.737 & 89.49 & 135.136 & 1556.154 & 1547.766 & 1502.015 \\
\hline $8 / 19 / 16$ 2:00 & 101.697 & 89.473 & 135.203 & 1556.194 & 1547.783 & 1501.948 \\
\hline 8/19/16 3:00 & 101.638 & 89.45 & 135.353 & 1556.253 & 1547.806 & 1501.798 \\
\hline 8/19/16 4:00 & 101.563 & 89.422 & 135.517 & 1556.328 & 1547.834 & 1501.634 \\
\hline 8/19/16 5:00 & 101.466 & 89.383 & 135.676 & 1556.425 & 1547.873 & 1501.475 \\
\hline 8/19/16 6:00 & 101.367 & 89.35 & 135.84 & 1556.524 & 1547.906 & 1501.311 \\
\hline 8/19/16 7:00 & 101.266 & 89.318 & 136.011 & 1556.625 & 1547.938 & 1501.14 \\
\hline 8/19/16 8:00 & 101.167 & 89.283 & 136.168 & 1556.724 & 1547.973 & 1500.983 \\
\hline 8/19/16 9:00 & 101.104 & 89.245 & 136.273 & 1556.787 & 1548.011 & 1500.878 \\
\hline 8/19/16 10:00 & 101.047 & 89.213 & 136.264 & 1556.844 & 1548.043 & 1500.887 \\
\hline 8/19/16 11:00 & 100.982 & 89.19 & 136.231 & 1556.909 & 1548.066 & 1500.92 \\
\hline $8 / 19 / 16$ 12:00 & 100.902 & 89.166 & 136.184 & 1556.989 & 1548.09 & 1500.967 \\
\hline $8 / 19 / 16$ 13:00 & 100.81 & 89.141 & 136.128 & 1557.081 & 1548.115 & 1501.023 \\
\hline $8 / 19 / 16$ 14:00 & 100.718 & 89.12 & 136.067 & 1557.173 & 1548.136 & 1501.084 \\
\hline $8 / 19 / 16$ 15:00 & 100.612 & 89.085 & 135.995 & 1557.279 & 1548.171 & 1501.156 \\
\hline 8/19/16 16:00 & 100.513 & 89.05 & 135.922 & 1557.378 & 1548.206 & 1501.229 \\
\hline 8/19/16 17:00 & 100.389 & 88.999 & 135.831 & 1557.502 & 1548.257 & 1501.32 \\
\hline 8/19/16 18:00 & 100.283 & 88.962 & 135.754 & 1557.608 & 1548.294 & 1501.397 \\
\hline 8/19/16 19:00 & 100.175 & 88.927 & 135.676 & 1557.716 & 1548.329 & 1501.475 \\
\hline 8/19/16 20:00 & 100.071 & 88.894 & 135.604 & 1557.82 & 1548.362 & 1501.547 \\
\hline 8/19/16 21:00 & 99.97 & 88.86 & 135.534 & 1557.921 & 1548.396 & 1501.617 \\
\hline 8/19/16 22:00 & 99.869 & 88.825 & 135.47 & 1558.022 & 1548.431 & 1501.681 \\
\hline 8/19/16 23:00 & 99.768 & 88.785 & 135.409 & 1558.123 & 1548.471 & 1501.742 \\
\hline 8/20/16 0:00 & 99.667 & 88.739 & 135.353 & 1558.224 & 1548.517 & 1501.798 \\
\hline $8 / 20 / 16$ 1:00 & 99.565 & 88.692 & 135.304 & 1558.326 & 1548.564 & 1501.847 \\
\hline $8 / 20 / 16$ 2:00 & 99.46 & 88.639 & 135.25 & 1558.431 & 1548.617 & 1501.901 \\
\hline 8/20/16 3:00 & 99.358 & 88.585 & 135.201 & 1558.533 & 1548.671 & 1501.95 \\
\hline $8 / 20 / 164: 00$ & 99.255 & 88.529 & 135.152 & 1558.636 & 1548.727 & 1501.999 \\
\hline 8/20/16 5:00 & 99.151 & 88.469 & 135.103 & 1558.74 & 1548.787 & 1502.048 \\
\hline $8 / 20 / 16$ 6:00 & 99.05 & 88.423 & 135.054 & 1558.841 & 1548.833 & 1502.097 \\
\hline $8 / 20 / 16$ 7:00 & 98.961 & 88.381 & 135.009 & 1558.93 & 1548.875 & 1502.142 \\
\hline $8 / 20 / 16$ 8:00 & 98.869 & 88.323 & 134.974 & 1559.022 & 1548.933 & 1502.177 \\
\hline 8/20/16 9:00 & 98.777 & 88.269 & 134.934 & 1559.114 & 1548.987 & 1502.217 \\
\hline $8 / 20 / 16$ 10:00 & 98.681 & 88.202 & 134.909 & 1559.21 & 1549.054 & 1502.242 \\
\hline $8 / 20 / 16$ 11:00 & 98.587 & 88.141 & 134.902 & 1559.304 & 1549.115 & 1502.249 \\
\hline $8 / 20 / 16$ 12:00 & 98.497 & 88.072 & 134.906 & 1559.394 & 1549.184 & 1502.245 \\
\hline $8 / 20 / 16$ 13:00 & 98.41 & 87.999 & 134.911 & 1559.481 & 1549.257 & 1502.24 \\
\hline $8 / 20 / 16$ 14:00 & 98.337 & 87.927 & 134.909 & 1559.554 & 1549.329 & 1502.242 \\
\hline $8 / 20 / 16$ 15:00 & 98.276 & 87.848 & 134.895 & 1559.615 & 1549.408 & 1502.256 \\
\hline $8 / 20 / 16$ 16:00 & 98.217 & 87.772 & 134.873 & 1559.674 & 1549.484 & 1502.278 \\
\hline $8 / 20 / 16$ 17:00 & 98.166 & 87.697 & 134.838 & 1559.725 & 1549.559 & 1502.313 \\
\hline 8/20/16 18:00 & 98.116 & 87.618 & 134.803 & 1559.775 & 1549.638 & 1502.348 \\
\hline 8/20/16 19:00 & 98.069 & 87.539 & 134.761 & 1559.822 & 1549.717 & 1502.39 \\
\hline
\end{tabular}


TABLE S1.2 Automatically recorded groundwater levels in the confined aquifers.

\begin{tabular}{|c|c|c|c|c|c|c|}
\hline \multirow[b]{2}{*}{ Date-Time } & \multicolumn{3}{|c|}{ Measured Depth to Water (ft TOC ${ }^{a}$ ) } & \multicolumn{3}{|c|}{ Groundwater Elevation (ft AMSL ${ }^{\mathrm{b}}$ ) } \\
\hline & DL2-CU & DL7-CU & DL7-CL & DL2-CU & DL7-CU & DL7-CL \\
\hline $8 / 20 / 16$ 20:00 & 98.022 & 87.465 & 134.712 & 1559.869 & 1549.791 & 1502.439 \\
\hline $8 / 20 / 16$ 21:00 & 97.98 & 87.393 & 134.665 & 1559.911 & 1549.863 & 1502.486 \\
\hline $8 / 20 / 1622: 00$ & 97.94 & 87.328 & 134.618 & 1559.951 & 1549.928 & 1502.533 \\
\hline $8 / 20 / 16$ 23:00 & 97.9 & 87.26 & 134.567 & 1559.991 & 1549.996 & 1502.584 \\
\hline 8/21/16 0:00 & 97.86 & 87.197 & 134.513 & 1560.031 & 1550.059 & 1502.638 \\
\hline 8/21/16 1:00 & 97.822 & 87.139 & 134.454 & 1560.069 & 1550.117 & 1502.697 \\
\hline $8 / 21 / 16$ 2:00 & 97.782 & 87.077 & 134.401 & 1560.109 & 1550.179 & 1502.75 \\
\hline 8/21/16 3:00 & 97.742 & 87.021 & 134.34 & 1560.149 & 1550.235 & 1502.811 \\
\hline $8 / 21 / 164: 00$ & 97.7 & 86.96 & 134.286 & 1560.191 & 1550.296 & 1502.865 \\
\hline 8/21/16 5:00 & 97.658 & 86.9 & 134.244 & 1560.233 & 1550.356 & 1502.907 \\
\hline $8 / 21 / 16$ 6:00 & 97.61 & 86.835 & 134.213 & 1560.281 & 1550.421 & 1502.938 \\
\hline 8/21/16 7:00 & 97.564 & 86.772 & 134.19 & 1560.327 & 1550.484 & 1502.961 \\
\hline 8/21/16 8:00 & 97.519 & 86.712 & 134.164 & 1560.372 & 1550.544 & 1502.987 \\
\hline 8/21/16 9:00 & 97.472 & 86.644 & 134.134 & 1560.419 & 1550.612 & 1503.017 \\
\hline $8 / 21 / 16$ 10:00 & 97.422 & 86.584 & 134.094 & 1560.469 & 1550.672 & 1503.057 \\
\hline $8 / 21 / 16$ 11:00 & 97.373 & 86.53 & 134.11 & 1560.518 & 1550.726 & 1503.041 \\
\hline $8 / 21 / 16$ 12:00 & 97.326 & 86.475 & 134.246 & 1560.565 & 1550.781 & 1502.905 \\
\hline $8 / 21 / 16$ 13:00 & 97.284 & 86.405 & 134.316 & 1560.607 & 1550.851 & 1502.835 \\
\hline $8 / 21 / 16$ 14:00 & 97.244 & 86.354 & 134.293 & 1560.647 & 1550.902 & 1502.858 \\
\hline $8 / 21 / 16$ 15:00 & 97.211 & 86.291 & 134.239 & 1560.68 & 1550.965 & 1502.912 \\
\hline $8 / 21 / 16$ 16:00 & 97.18 & 86.247 & 134.192 & 1560.711 & 1551.009 & 1502.959 \\
\hline $8 / 21 / 16$ 17:00 & 97.157 & 86.193 & 134.131 & 1560.734 & 1551.063 & 1503.02 \\
\hline 8/21/16 18:00 & 97.138 & 86.156 & 134.164 & 1560.753 & 1551.1 & 1502.987 \\
\hline 8/21/16 19:00 & 97.121 & 86.112 & 134.286 & 1560.77 & 1551.144 & 1502.865 \\
\hline $8 / 21 / 16$ 20:00 & 97.107 & 86.054 & 134.33 & 1560.784 & 1551.202 & 1502.821 \\
\hline 8/21/16 21:00 & 97.095 & 86.012 & 134.286 & 1560.796 & 1551.244 & 1502.865 \\
\hline $8 / 21 / 16$ 22:00 & 97.091 & 85.979 & 134.23 & 1560.8 & 1551.277 & 1502.921 \\
\hline 8/21/16 23:00 & 97.081 & 85.951 & 134.164 & 1560.81 & 1551.305 & 1502.987 \\
\hline $8 / 22 / 16$ 0:00 & 97.072 & 85.928 & 134.092 & 1560.819 & 1551.328 & 1503.059 \\
\hline $8 / 22 / 16$ 1:00 & 97.065 & 85.91 & 134.017 & 1560.826 & 1551.346 & 1503.134 \\
\hline $8 / 22 / 16$ 2:00 & 97.053 & 85.898 & 134.031 & 1560.838 & 1551.358 & 1503.12 \\
\hline $8 / 22 / 16$ 3:00 & 97.044 & 85.884 & 134.146 & 1560.847 & 1551.372 & 1503.005 \\
\hline $8 / 22 / 164: 00$ & 97.03 & 85.87 & 134.272 & 1560.861 & 1551.386 & 1502.879 \\
\hline $8 / 22 / 165: 00$ & 97.015 & 85.861 & 134.408 & 1560.876 & 1551.395 & 1502.743 \\
\hline $8 / 22 / 16$ 6:00 & 97.001 & 85.842 & 134.541 & 1560.89 & 1551.414 & 1502.61 \\
\hline $8 / 22 / 16$ 7:00 & 96.992 & 85.845 & 134.693 & 1560.899 & 1551.411 & 1502.458 \\
\hline $8 / 22 / 16$ 8:00 & 96.992 & 85.884 & 134.836 & 1560.899 & 1551.372 & 1502.315 \\
\hline 8/22/16 9:00 & 97.008 & 86.007 & 134.958 & 1560.883 & 1551.249 & 1502.193 \\
\hline $8 / 22 / 16$ 10:00 & 97.041 & 86.158 & 134.951 & 1560.85 & 1551.098 & 1502.2 \\
\hline 8/22/16 11:00 & 97.077 & 86.316 & 134.897 & 1560.814 & 1550.94 & 1502.254 \\
\hline $8 / 22 / 16$ 12:00 & 97.14 & 86.454 & 134.829 & 1560.751 & 1550.802 & 1502.322 \\
\hline $8 / 22 / 16$ 13:00 & 97.222 & 86.588 & 134.759 & 1560.669 & 1550.668 & 1502.392 \\
\hline $8 / 22 / 16$ 14:00 & 97.333 & 86.714 & 134.686 & 1560.558 & 1550.542 & 1502.465 \\
\hline $8 / 22 / 16$ 15:00 & 97.455 & 86.833 & 134.618 & 1560.436 & 1550.423 & 1502.533 \\
\hline $8 / 22 / 16$ 16:00 & 97.578 & 86.953 & 134.541 & 1560.313 & 1550.303 & 1502.61 \\
\hline $8 / 22 / 16$ 17:00 & 97.707 & 87.072 & 134.469 & 1560.184 & 1550.184 & 1502.682 \\
\hline 8/22/16 18:00 & 97.836 & 87.202 & 134.396 & 1560.055 & 1550.054 & 1502.755 \\
\hline
\end{tabular}


TABLE S1.2 Automatically recorded groundwater levels in the confined aquifers.

\begin{tabular}{|c|c|c|c|c|c|c|}
\hline \multirow[b]{2}{*}{ Date-Time } & \multicolumn{3}{|c|}{ Measured Depth to Water (ft TOC ${ }^{a}$ ) } & \multicolumn{3}{|c|}{ Groundwater Elevation (ft AMSL ${ }^{\mathrm{b}}$ ) } \\
\hline & DL2-CU & DL7-CU & DL7-CL & DL2-CU & DL7-CU & DL7-CL \\
\hline $8 / 22 / 16$ 19:00 & 97.959 & 87.33 & 134.319 & 1559.932 & 1549.926 & 1502.832 \\
\hline $8 / 22 / 16$ 20:00 & 98.083 & 87.458 & 134.251 & 1559.808 & 1549.798 & 1502.9 \\
\hline $8 / 22 / 1621: 00$ & 98.206 & 87.562 & 134.183 & 1559.685 & 1549.694 & 1502.968 \\
\hline $8 / 22 / 16$ 22:00 & 98.33 & 87.618 & 134.127 & 1559.561 & 1549.638 & 1503.024 \\
\hline $8 / 22 / 16$ 23:00 & 98.439 & 87.623 & 134.068 & 1559.452 & 1549.633 & 1503.083 \\
\hline 8/23/16 0:00 & 98.537 & 87.623 & 134.01 & 1559.354 & 1549.633 & 1503.141 \\
\hline 8/23/16 1:00 & 98.629 & 87.621 & 133.956 & 1559.262 & 1549.635 & 1503.195 \\
\hline $8 / 23 / 16$ 2:00 & 98.712 & 87.621 & 133.9 & 1559.179 & 1549.635 & 1503.251 \\
\hline 8/23/16 3:00 & 98.796 & 87.628 & 133.844 & 1559.095 & 1549.628 & 1503.307 \\
\hline $8 / 23 / 164: 00$ & 98.879 & 87.639 & 133.801 & 1559.012 & 1549.617 & 1503.35 \\
\hline $8 / 23 / 16$ 5:00 & 98.956 & 87.658 & 133.752 & 1558.935 & 1549.598 & 1503.399 \\
\hline $8 / 23 / 16$ 6:00 & 99.034 & 87.688 & 133.708 & 1558.857 & 1549.568 & 1503.443 \\
\hline $8 / 23 / 16$ 7:00 & 99.111 & 87.716 & 133.67 & 1558.78 & 1549.54 & 1503.481 \\
\hline 8/23/16 8:00 & 99.194 & 87.755 & 133.635 & 1558.697 & 1549.501 & 1503.516 \\
\hline 8/23/16 9:00 & 99.316 & 87.802 & 133.6 & 1558.575 & 1549.454 & 1503.551 \\
\hline 8/23/16 10:00 & 99.488 & 87.853 & 133.577 & 1558.403 & 1549.403 & 1503.574 \\
\hline 8/23/16 11:00 & 99.676 & 87.925 & 133.572 & 1558.215 & 1549.331 & 1503.579 \\
\hline $8 / 23 / 16$ 12:00 & 99.869 & 88.055 & 133.553 & 1558.022 & 1549.201 & 1503.598 \\
\hline $8 / 23 / 16$ 13:00 & 100.074 & 88.237 & 133.539 & 1557.817 & 1549.019 & 1503.612 \\
\hline $8 / 23 / 16$ 14:00 & 100.288 & 88.448 & 133.523 & 1557.603 & 1548.808 & 1503.628 \\
\hline $8 / 23 / 16$ 15:00 & 100.516 & 88.671 & 133.521 & 1557.375 & 1548.585 & 1503.63 \\
\hline $8 / 23 / 16$ 16:00 & 100.746 & 88.901 & 133.523 & 1557.145 & 1548.355 & 1503.628 \\
\hline $8 / 23 / 16$ 17:00 & 100.977 & 89.132 & 133.528 & 1556.914 & 1548.124 & 1503.623 \\
\hline $8 / 23 / 16$ 18:00 & 101.207 & 89.355 & 133.521 & 1556.684 & 1547.901 & 1503.63 \\
\hline $8 / 23 / 16$ 19:00 & 101.426 & 89.571 & 133.509 & 1556.465 & 1547.685 & 1503.642 \\
\hline $8 / 23 / 16$ 20:00 & 101.65 & 89.787 & 133.507 & 1556.241 & 1547.469 & 1503.644 \\
\hline $8 / 23 / 16$ 21:00 & 101.859 & 89.985 & 133.492 & 1556.032 & 1547.271 & 1503.659 \\
\hline 8/23/16 22:00 & 102.066 & 90.185 & 133.481 & 1555.825 & 1547.071 & 1503.67 \\
\hline 8/23/16 23:00 & 102.266 & 90.378 & 133.469 & 1555.625 & 1546.878 & 1503.682 \\
\hline $8 / 24 / 16$ 0:00 & 102.461 & 90.561 & 133.471 & 1555.43 & 1546.695 & 1503.68 \\
\hline $8 / 24 / 16$ 1:00 & 102.645 & 90.738 & 133.483 & 1555.246 & 1546.518 & 1503.668 \\
\hline $8 / 24 / 16$ 2:00 & 102.816 & 90.898 & 133.497 & 1555.075 & 1546.358 & 1503.654 \\
\hline 8/24/16 3:00 & 102.993 & 91.068 & 133.521 & 1554.898 & 1546.188 & 1503.63 \\
\hline $8 / 24 / 164: 00$ & 103.158 & 91.221 & 133.532 & 1554.733 & 1546.035 & 1503.619 \\
\hline $8 / 24 / 16$ 5:00 & 103.313 & 91.372 & 133.558 & 1554.578 & 1545.884 & 1503.593 \\
\hline $8 / 24 / 16$ 6:00 & 103.47 & 91.517 & 133.586 & 1554.421 & 1545.739 & 1503.565 \\
\hline $8 / 24 / 16$ 7:00 & 103.635 & 91.644 & 133.635 & 1554.256 & 1545.612 & 1503.516 \\
\hline 8/24/16 8:00 & 103.814 & 91.733 & 133.687 & 1554.077 & 1545.523 & 1503.464 \\
\hline 8/24/16 9:00 & 104.021 & 91.796 & 133.762 & 1553.87 & 1545.46 & 1503.389 \\
\hline $8 / 24 / 16$ 10:00 & 104.263 & 91.816 & 133.839 & 1553.628 & 1545.44 & 1503.312 \\
\hline $8 / 24 / 16$ 11:00 & 104.522 & 91.828 & 133.886 & 1553.369 & 1545.428 & 1503.265 \\
\hline $8 / 24 / 16$ 12:00 & 104.788 & 91.898 & 133.916 & 1553.103 & 1545.358 & 1503.235 \\
\hline $8 / 24 / 16$ 13:00 & 105.061 & 92.002 & 133.933 & 1552.83 & 1545.254 & 1503.218 \\
\hline $8 / 24 / 16$ 14:00 & 105.343 & 92.126 & 133.944 & 1552.548 & 1545.13 & 1503.207 \\
\hline $8 / 24 / 16$ 15:00 & 105.62 & 92.253 & 133.954 & 1552.271 & 1545.003 & 1503.197 \\
\hline $8 / 24 / 16$ 16:00 & 105.884 & 92.381 & 133.949 & 1552.007 & 1544.875 & 1503.202 \\
\hline 8/24/16 17:00 & 106.091 & 92.5 & 133.944 & 1551.8 & 1544.756 & 1503.207 \\
\hline
\end{tabular}


TABLE S1.2 Automatically recorded groundwater levels in the confined aquifers.

\begin{tabular}{|c|c|c|c|c|c|c|}
\hline \multirow[b]{2}{*}{ Date-Time } & \multicolumn{3}{|c|}{ Measured Depth to Water (ft TOC ${ }^{\mathrm{a}}$ ) } & \multicolumn{3}{|c|}{ Groundwater Elevation (ft AMSL ${ }^{\mathrm{b}}$ ) } \\
\hline & DL2-CU & DL7-CU & DL7-CL & DL2-CU & DL7-CU & DL7-CL \\
\hline $8 / 24 / 16$ 18:00 & 106.248 & 92.623 & 133.942 & 1551.643 & 1544.633 & 1503.209 \\
\hline $8 / 24 / 16$ 19:00 & 106.38 & 92.749 & 133.935 & 1551.511 & 1544.507 & 1503.216 \\
\hline $8 / 24 / 16$ 20:00 & 106.474 & 92.863 & 133.923 & 1551.417 & 1544.393 & 1503.228 \\
\hline 8/24/16 21:00 & 106.552 & 92.976 & 133.911 & 1551.339 & 1544.28 & 1503.24 \\
\hline $8 / 24 / 16$ 22:00 & 106.62 & 93.09 & 133.902 & 1551.271 & 1544.166 & 1503.249 \\
\hline $8 / 24 / 16$ 23:00 & 106.681 & 93.195 & 133.886 & 1551.21 & 1544.061 & 1503.265 \\
\hline $8 / 25 / 160: 00$ & 106.745 & 93.311 & 133.897 & 1551.146 & 1543.945 & 1503.254 \\
\hline $8 / 25 / 16$ 1:00 & 106.811 & 93.413 & 134.047 & 1551.08 & 1543.843 & 1503.104 \\
\hline $8 / 25 / 16$ 2:00 & 106.877 & 93.518 & 134.234 & 1551.014 & 1543.738 & 1502.917 \\
\hline $8 / 25 / 16$ 3:00 & 106.938 & 93.611 & 134.426 & 1550.953 & 1543.645 & 1502.725 \\
\hline $8 / 25 / 164: 00$ & 106.999 & 93.704 & 134.623 & 1550.892 & 1543.552 & 1502.528 \\
\hline $8 / 25 / 16$ 5:00 & 107.055 & 93.797 & 134.824 & 1550.836 & 1543.459 & 1502.327 \\
\hline $8 / 25 / 16$ 6:00 & 107.105 & 93.888 & 135.028 & 1550.786 & 1543.368 & 1502.123 \\
\hline $8 / 25 / 16$ 7:00 & 107.152 & 93.978 & 135.225 & 1550.739 & 1543.278 & 1501.926 \\
\hline $8 / 25 / 16$ 8:00 & 107.194 & 94.062 & 135.414 & 1550.697 & 1543.194 & 1501.737 \\
\hline 8/25/16 9:00 & 107.234 & 94.143 & 135.496 & 1550.657 & 1543.113 & 1501.655 \\
\hline $8 / 25 / 16$ 10:00 & 107.276 & 94.229 & 135.505 & 1550.615 & 1543.027 & 1501.646 \\
\hline $8 / 25 / 16$ 11:00 & 107.345 & 94.32 & 135.498 & 1550.546 & 1542.936 & 1501.653 \\
\hline $8 / 25 / 16$ 12:00 & 107.387 & 94.401 & 135.48 & 1550.504 & 1542.855 & 1501.671 \\
\hline $8 / 25 / 16$ 13:00 & 107.408 & 94.478 & 135.459 & 1550.483 & 1542.778 & 1501.692 \\
\hline $8 / 25 / 16$ 14:00 & 107.439 & 94.557 & 135.442 & 1550.452 & 1542.699 & 1501.709 \\
\hline $8 / 25 / 16$ 15:00 & 107.495 & 94.634 & 135.421 & 1550.396 & 1542.622 & 1501.73 \\
\hline $8 / 25 / 16$ 16:00 & 107.587 & 94.706 & 135.398 & 1550.304 & 1542.55 & 1501.753 \\
\hline $8 / 25 / 16$ 17:00 & 107.709 & 94.771 & 135.37 & 1550.182 & 1542.485 & 1501.781 \\
\hline $8 / 25 / 16$ 18:00 & 107.789 & 94.82 & 135.346 & 1550.102 & 1542.436 & 1501.805 \\
\hline $8 / 25 / 16$ 19:00 & 107.891 & 94.871 & 135.321 & 1550 & 1542.385 & 1501.83 \\
\hline $8 / 25 / 16$ 20:00 & 108.006 & 94.915 & 135.295 & 1549.885 & 1542.341 & 1501.856 \\
\hline $8 / 25 / 16$ 21:00 & 108.128 & 94.962 & 135.274 & 1549.763 & 1542.294 & 1501.877 \\
\hline $8 / 25 / 16$ 22:00 & 108.25 & 95.01 & 135.255 & 1549.641 & 1542.246 & 1501.896 \\
\hline $8 / 25 / 16$ 23:00 & 108.37 & 95.05 & 135.239 & 1549.521 & 1542.206 & 1501.912 \\
\hline 8/26/16 0:00 & 108.481 & 95.087 & 135.213 & 1549.41 & 1542.169 & 1501.938 \\
\hline $8 / 26 / 16$ 1:00 & 108.573 & 95.129 & 135.192 & 1549.318 & 1542.127 & 1501.959 \\
\hline $8 / 26 / 16$ 2:00 & 108.646 & 95.169 & 135.175 & 1549.245 & 1542.087 & 1501.976 \\
\hline 8/26/16 3:00 & 108.695 & 95.206 & 135.147 & 1549.196 & 1542.05 & 1502.004 \\
\hline $8 / 26 / 164: 00$ & 108.737 & 95.243 & 135.131 & 1549.154 & 1542.013 & 1502.02 \\
\hline $8 / 26 / 16$ 5:00 & 108.77 & 95.285 & 135.119 & 1549.121 & 1541.971 & 1502.032 \\
\hline $8 / 26 / 16$ 6:00 & 108.789 & 95.327 & 135.112 & 1549.102 & 1541.929 & 1502.039 \\
\hline 8/26/16 7:00 & 108.74 & 95.375 & 135.112 & 1549.151 & 1541.881 & 1502.039 \\
\hline 8/26/16 8:00 & 108.592 & 95.42 & 135.101 & 1549.299 & 1541.836 & 1502.05 \\
\hline 8/26/16 9:00 & 108.396 & 95.417 & 135.101 & 1549.495 & 1541.839 & 1502.05 \\
\hline $8 / 26 / 16$ 10:00 & 108.175 & 95.352 & 135.11 & 1549.716 & 1541.904 & 1502.041 \\
\hline $8 / 26 / 16$ 11:00 & 107.971 & 95.241 & 135.14 & 1549.92 & 1542.015 & 1502.011 \\
\hline $8 / 26 / 16$ 12:00 & 107.794 & 95.101 & 135.173 & 1550.097 & 1542.155 & 1501.978 \\
\hline $8 / 26 / 16$ 13:00 & 107.639 & 94.95 & 135.215 & 1550.252 & 1542.306 & 1501.936 \\
\hline $8 / 26 / 16$ 14:00 & 107.5 & 94.794 & 135.255 & 1550.391 & 1542.462 & 1501.896 \\
\hline $8 / 26 / 16$ 15:00 & 107.375 & 94.639 & 135.29 & 1550.516 & 1542.617 & 1501.861 \\
\hline $8 / 26 / 16$ 16:00 & 107.26 & 94.487 & 135.314 & 1550.631 & 1542.769 & 1501.837 \\
\hline
\end{tabular}


TABLE S1.2 Automatically recorded groundwater levels in the confined aquifers.

\begin{tabular}{|c|c|c|c|c|c|c|}
\hline \multirow[b]{2}{*}{ Date-Time } & \multicolumn{3}{|c|}{ Measured Depth to Water (ft TOC ${ }^{a}$ ) } & \multicolumn{3}{|c|}{ Groundwater Elevation (ft AMSL ${ }^{\mathrm{b}}$ ) } \\
\hline & DL2-CU & DL7-CU & DL7-CL & DL2-CU & DL7-CU & DL7-CL \\
\hline $8 / 26 / 16$ 17:00 & 107.147 & 94.334 & 135.328 & 1550.744 & 1542.922 & 1501.823 \\
\hline $8 / 26 / 16$ 18:00 & 107.044 & 94.192 & 135.337 & 1550.847 & 1543.064 & 1501.814 \\
\hline $8 / 26 / 16$ 19:00 & 106.945 & 94.057 & 135.337 & 1550.946 & 1543.199 & 1501.814 \\
\hline $8 / 26 / 16$ 20:00 & 106.853 & 93.932 & 135.337 & 1551.038 & 1543.324 & 1501.814 \\
\hline $8 / 26 / 16$ 21:00 & 106.764 & 93.813 & 135.332 & 1551.127 & 1543.443 & 1501.819 \\
\hline $8 / 26 / 16$ 22:00 & 106.672 & 93.69 & 135.323 & 1551.219 & 1543.566 & 1501.828 \\
\hline $8 / 26 / 16$ 23:00 & 106.585 & 93.574 & 135.299 & 1551.306 & 1543.682 & 1501.852 \\
\hline 8/27/16 0:00 & 106.503 & 93.469 & 135.285 & 1551.388 & 1543.787 & 1501.866 \\
\hline $8 / 27 / 16$ 1:00 & 106.418 & 93.355 & 135.26 & 1551.473 & 1543.901 & 1501.891 \\
\hline $8 / 27 / 16$ 2:00 & 106.324 & 93.253 & 135.227 & 1551.567 & 1544.003 & 1501.924 \\
\hline 8/27/16 3:00 & 106.223 & 93.155 & 135.213 & 1551.668 & 1544.101 & 1501.938 \\
\hline $8 / 27 / 164: 00$ & 106.112 & 93.062 & 135.208 & 1551.779 & 1544.194 & 1501.943 \\
\hline $8 / 27 / 16$ 5:00 & 105.999 & 92.976 & 135.213 & 1551.892 & 1544.28 & 1501.938 \\
\hline 8/27/16 6:00 & 105.884 & 92.886 & 135.232 & 1552.007 & 1544.37 & 1501.919 \\
\hline 8/27/16 7:00 & 105.771 & 92.793 & 135.25 & 1552.12 & 1544.463 & 1501.901 \\
\hline $8 / 27 / 16$ 8:00 & 105.66 & 92.707 & 135.288 & 1552.231 & 1544.549 & 1501.863 \\
\hline 8/27/16 9:00 & 105.552 & 92.611 & 135.466 & 1552.339 & 1544.645 & 1501.685 \\
\hline $8 / 27 / 16$ 10:00 & 105.435 & 92.512 & 135.655 & 1552.456 & 1544.744 & 1501.496 \\
\hline $8 / 27 / 16$ 11:00 & 105.286 & 92.402 & 135.714 & 1552.605 & 1544.854 & 1501.437 \\
\hline $8 / 27 / 16$ 12:00 & 105.115 & 92.3 & 135.725 & 1552.776 & 1544.956 & 1501.426 \\
\hline $8 / 27 / 16$ 13:00 & 104.938 & 92.202 & 135.721 & 1552.953 & 1545.054 & 1501.43 \\
\hline $8 / 27 / 16$ 14:00 & 104.774 & 92.107 & 135.732 & 1553.117 & 1545.149 & 1501.419 \\
\hline $8 / 27 / 16$ 15:00 & 104.623 & 92.009 & 135.868 & 1553.268 & 1545.247 & 1501.283 \\
\hline 8/27/16 16:00 & 104.489 & 91.905 & 136.023 & 1553.402 & 1545.351 & 1501.128 \\
\hline $8 / 27 / 16$ 17:00 & 104.369 & 91.793 & 136.105 & 1553.522 & 1545.463 & 1501.046 \\
\hline 8/27/16 18:00 & 104.273 & 91.696 & 136.093 & 1553.618 & 1545.56 & 1501.058 \\
\hline 8/27/16 19:00 & 104.195 & 91.598 & 136.067 & 1553.696 & 1545.658 & 1501.084 \\
\hline 8/27/16 20:00 & 104.136 & 91.503 & 136.025 & 1553.755 & 1545.753 & 1501.126 \\
\hline 8/27/16 21:00 & 104.084 & 91.417 & 136.013 & 1553.807 & 1545.839 & 1501.138 \\
\hline $8 / 27 / 16$ 22:00 & 104.042 & 91.326 & 136.126 & 1553.849 & 1545.93 & 1501.025 \\
\hline 8/27/16 23:00 & 104 & 91.226 & 136.21 & 1553.891 & 1546.03 & 1500.941 \\
\hline 8/28/16 0:00 & 103.96 & 91.133 & 136.18 & 1553.931 & 1546.123 & 1500.971 \\
\hline $8 / 28 / 16$ 1:00 & 103.915 & 91.035 & 136.13 & 1553.976 & 1546.221 & 1501.021 \\
\hline $8 / 28 / 16$ 2:00 & 103.873 & 90.947 & 136.072 & 1554.018 & 1546.309 & 1501.079 \\
\hline 8/28/16 3:00 & 103.816 & 90.861 & 136.018 & 1554.075 & 1546.395 & 1501.133 \\
\hline 8/28/16 4:00 & 103.748 & 90.777 & 136.112 & 1554.143 & 1546.479 & 1501.039 \\
\hline $8 / 28 / 16$ 5:00 & 103.663 & 90.687 & 136.238 & 1554.228 & 1546.569 & 1500.913 \\
\hline 8/28/16 6:00 & 103.569 & 90.596 & 136.371 & 1554.322 & 1546.66 & 1500.78 \\
\hline 8/28/16 7:00 & 103.473 & 90.517 & 136.51 & 1554.418 & 1546.739 & 1500.641 \\
\hline 8/28/16 8:00 & 103.374 & 90.433 & 136.648 & 1554.517 & 1546.823 & 1500.503 \\
\hline 8/28/16 9:00 & 103.273 & 90.34 & 136.699 & 1554.618 & 1546.916 & 1500.452 \\
\hline $8 / 28 / 16$ 10:00 & 103.171 & 90.261 & 136.662 & 1554.72 & 1546.995 & 1500.489 \\
\hline $8 / 28 / 16$ 11:00 & 103.07 & 90.185 & 136.601 & 1554.821 & 1547.071 & 1500.55 \\
\hline $8 / 28 / 16$ 12:00 & 102.969 & 90.101 & 136.535 & 1554.922 & 1547.155 & 1500.616 \\
\hline 8/28/16 13:00 & 102.866 & 90.024 & 136.467 & 1555.025 & 1547.232 & 1500.684 \\
\hline 8/28/16 14:00 & 102.751 & 89.943 & 136.393 & 1555.14 & 1547.313 & 1500.758 \\
\hline $8 / 28 / 16$ 15:00 & 102.624 & 89.857 & 136.318 & 1555.267 & 1547.399 & 1500.833 \\
\hline
\end{tabular}


TABLE S1.2 Automatically recorded groundwater levels in the confined aquifers.

\begin{tabular}{|c|c|c|c|c|c|c|}
\hline \multirow[b]{2}{*}{ Date-Time } & \multicolumn{3}{|c|}{ Measured Depth to Water (ft TOC ${ }^{\mathrm{a}}$ ) } & \multicolumn{3}{|c|}{ Groundwater Elevation (ft AMSL ${ }^{\mathrm{b}}$ ) } \\
\hline & DL2-CU & DL7-CU & DL7-CL & DL2-CU & DL7-CU & DL7-CL \\
\hline 8/28/16 16:00 & 102.482 & 89.773 & 136.24 & 1555.409 & 1547.483 & 1500.911 \\
\hline $8 / 28 / 16$ 17:00 & 102.332 & 89.692 & 136.163 & 1555.559 & 1547.564 & 1500.988 \\
\hline $8 / 28 / 16$ 18:00 & 102.176 & 89.61 & 136.093 & 1555.715 & 1547.646 & 1501.058 \\
\hline 8/28/16 19:00 & 102.017 & 89.527 & 136.03 & 1555.874 & 1547.729 & 1501.121 \\
\hline $8 / 28 / 16$ 20:00 & 101.859 & 89.452 & 135.976 & 1556.032 & 1547.804 & 1501.175 \\
\hline $8 / 28 / 16$ 21:00 & 101.699 & 89.376 & 135.917 & 1556.192 & 1547.88 & 1501.234 \\
\hline $8 / 28 / 16$ 22:00 & 101.544 & 89.304 & 135.871 & 1556.347 & 1547.952 & 1501.28 \\
\hline $8 / 28 / 16$ 23:00 & 101.384 & 89.229 & 135.817 & 1556.507 & 1548.027 & 1501.334 \\
\hline 8/29/16 0:00 & 101.229 & 89.157 & 135.758 & 1556.662 & 1548.099 & 1501.393 \\
\hline 8/29/16 1:00 & 101.071 & 89.09 & 135.7 & 1556.82 & 1548.166 & 1501.451 \\
\hline $8 / 29 / 16$ 2:00 & 100.92 & 89.02 & 135.639 & 1556.971 & 1548.236 & 1501.512 \\
\hline 8/29/16 3:00 & 100.767 & 88.955 & 135.576 & 1557.124 & 1548.301 & 1501.575 \\
\hline 8/29/16 4:00 & 100.619 & 88.887 & 135.512 & 1557.272 & 1548.369 & 1501.639 \\
\hline 8/29/16 5:00 & 100.481 & 88.832 & 135.459 & 1557.41 & 1548.424 & 1501.692 \\
\hline 8/29/16 6:00 & 100.344 & 88.771 & 135.407 & 1557.547 & 1548.485 & 1501.744 \\
\hline $8 / 29 / 16$ 7:00 & 100.215 & 88.72 & 135.367 & 1557.676 & 1548.536 & 1501.784 \\
\hline 8/29/16 8:00 & 100.095 & 88.674 & 135.328 & 1557.796 & 1548.582 & 1501.823 \\
\hline 8/29/16 9:00 & 100.008 & 88.636 & 135.302 & 1557.883 & 1548.62 & 1501.849 \\
\hline $8 / 29 / 16$ 10:00 & 99.958 & 88.597 & 135.278 & 1557.933 & 1548.659 & 1501.873 \\
\hline $8 / 29 / 16$ 11:00 & 99.928 & 88.55 & 135.274 & 1557.963 & 1548.706 & 1501.877 \\
\hline $8 / 29 / 16$ 12:00 & 99.89 & 88.509 & 135.271 & 1558.001 & 1548.747 & 1501.88 \\
\hline 8/29/16 13:00 & 99.848 & 88.469 & 135.257 & 1558.043 & 1548.787 & 1501.894 \\
\hline 8/29/16 14:00 & 99.808 & 88.425 & 135.236 & 1558.083 & 1548.831 & 1501.915 \\
\hline $8 / 29 / 16$ 15:00 & 99.763 & 88.39 & 135.215 & 1558.128 & 1548.866 & 1501.936 \\
\hline 8/29/16 16:00 & 99.716 & 88.369 & 135.192 & 1558.175 & 1548.887 & 1501.959 \\
\hline 8/29/16 17:00 & 99.66 & 88.339 & 135.147 & 1558.231 & 1548.917 & 1502.004 \\
\hline 8/29/16 18:00 & 99.617 & 88.348 & 135.126 & 1558.274 & 1548.908 & 1502.025 \\
\hline 8/29/16 19:00 & 99.572 & 88.36 & 135.101 & 1558.319 & 1548.896 & 1502.05 \\
\hline 8/29/16 20:00 & 99.532 & 88.388 & 135.079 & 1558.359 & 1548.868 & 1502.072 \\
\hline 8/29/16 21:00 & 99.488 & 88.413 & 135.065 & 1558.403 & 1548.843 & 1502.086 \\
\hline 8/29/16 22:00 & 99.453 & 88.453 & 135.061 & 1558.438 & 1548.803 & 1502.09 \\
\hline 8/29/16 23:00 & 99.42 & 88.497 & 135.054 & 1558.471 & 1548.759 & 1502.097 \\
\hline 8/30/16 0:00 & 99.377 & 88.543 & 135.037 & 1558.514 & 1548.713 & 1502.114 \\
\hline $8 / 30 / 16$ 1:00 & 99.304 & 88.59 & 135.021 & 1558.587 & 1548.666 & 1502.13 \\
\hline $8 / 30 / 16$ 2:00 & 99.21 & 88.641 & 134.995 & 1558.681 & 1548.615 & 1502.156 \\
\hline $8 / 30 / 16$ 3:00 & 99.102 & 88.697 & 134.972 & 1558.789 & 1548.559 & 1502.179 \\
\hline $8 / 30 / 164: 00$ & 98.996 & 88.755 & 134.965 & 1558.895 & 1548.501 & 1502.186 \\
\hline 8/30/16 5:00 & 98.89 & 88.811 & 134.969 & 1559.001 & 1548.445 & 1502.182 \\
\hline $8 / 30 / 16$ 6:00 & 98.796 & 88.876 & 134.995 & 1559.095 & 1548.38 & 1502.156 \\
\hline 8/30/16 7:00 & 98.718 & 88.98 & 135.023 & 1559.173 & 1548.276 & 1502.128 \\
\hline 8/30/16 8:00 & 98.664 & 89.115 & 135.061 & 1559.227 & 1548.141 & 1502.09 \\
\hline 8/30/16 9:00 & 98.632 & 89.266 & 135.14 & 1559.259 & 1547.99 & 1502.011 \\
\hline $8 / 30 / 16$ 10:00 & 98.613 & 89.404 & 135.339 & 1559.278 & 1547.852 & 1501.812 \\
\hline 8/30/16 11:00 & 98.592 & 89.499 & 135.508 & 1559.299 & 1547.757 & 1501.643 \\
\hline $8 / 30 / 16$ 12:00 & 98.566 & 89.578 & 135.559 & 1559.325 & 1547.678 & 1501.592 \\
\hline 8/30/16 13:00 & 98.533 & 89.634 & 135.578 & 1559.358 & 1547.622 & 1501.573 \\
\hline $8 / 30 / 16$ 14:00 & 98.5 & 89.694 & 135.58 & 1559.391 & 1547.562 & 1501.571 \\
\hline
\end{tabular}


TABLE S1.2 Automatically recorded groundwater levels in the confined aquifers.

\begin{tabular}{|c|c|c|c|c|c|c|}
\hline \multirow[b]{2}{*}{ Date-Time } & \multicolumn{3}{|c|}{ Measured Depth to Water (ft TOC ${ }^{a}$ ) } & \multicolumn{3}{|c|}{ Groundwater Elevation (ft AMSL ${ }^{\mathrm{b}}$ ) } \\
\hline & DL2-CU & DL7-CU & DL7-CL & DL2-CU & DL7-CU & DL7-CL \\
\hline $8 / 30 / 16$ 15:00 & 98.46 & 89.738 & 135.569 & 1559.431 & 1547.518 & 1501.582 \\
\hline $8 / 30 / 16$ 16:00 & 98.432 & 89.796 & 135.557 & 1559.459 & 1547.46 & 1501.594 \\
\hline $8 / 30 / 16$ 17:00 & 98.403 & 89.845 & 135.536 & 1559.488 & 1547.411 & 1501.615 \\
\hline 8/30/16 18:00 & 98.38 & 89.894 & 135.512 & 1559.511 & 1547.362 & 1501.639 \\
\hline 8/30/16 19:00 & 98.356 & 89.954 & 135.487 & 1559.535 & 1547.302 & 1501.664 \\
\hline $8 / 30 / 16$ 20:00 & 98.337 & 90.015 & 135.463 & 1559.554 & 1547.241 & 1501.688 \\
\hline $8 / 30 / 16$ 21:00 & 98.328 & 90.085 & 135.58 & 1559.563 & 1547.171 & 1501.571 \\
\hline $8 / 30 / 16$ 22:00 & 98.316 & 90.131 & 135.742 & 1559.575 & 1547.125 & 1501.409 \\
\hline $8 / 30 / 16$ 23:00 & 98.305 & 90.178 & 135.91 & 1559.586 & 1547.078 & 1501.241 \\
\hline 8/31/16 0:00 & 98.29 & 90.212 & 136.081 & 1559.601 & 1547.044 & 1501.07 \\
\hline $8 / 31 / 16$ 1:00 & 98.279 & 90.247 & 136.252 & 1559.612 & 1547.009 & 1500.899 \\
\hline $8 / 31 / 16$ 2:00 & 98.267 & 90.273 & 136.418 & 1559.624 & 1546.983 & 1500.733 \\
\hline 8/31/16 3:00 & 98.255 & 90.292 & 136.584 & 1559.636 & 1546.964 & 1500.567 \\
\hline $8 / 31 / 164: 00$ & 98.246 & 90.312 & 136.751 & 1559.645 & 1546.944 & 1500.4 \\
\hline $8 / 31 / 16$ 5:00 & 98.239 & 90.331 & 136.912 & 1559.652 & 1546.925 & 1500.239 \\
\hline $8 / 31 / 16$ 6:00 & 98.234 & 90.359 & 137.078 & 1559.657 & 1546.897 & 1500.073 \\
\hline 8/31/16 7:00 & 98.239 & 90.417 & 137.238 & 1559.652 & 1546.839 & 1499.913 \\
\hline 8/31/16 8:00 & 98.272 & 90.505 & 137.413 & 1559.619 & 1546.751 & 1499.738 \\
\hline 8/31/16 9:00 & 98.319 & 90.603 & 137.577 & 1559.572 & 1546.653 & 1499.574 \\
\hline $8 / 31 / 16$ 10:00 & 98.375 & 90.682 & 137.635 & 1559.516 & 1546.574 & 1499.516 \\
\hline 8/31/16 11:00 & 98.455 & 90.736 & 137.61 & 1559.436 & 1546.52 & 1499.541 \\
\hline $8 / 31 / 16$ 12:00 & 98.601 & 90.773 & 137.561 & 1559.29 & 1546.483 & 1499.59 \\
\hline 8/31/16 13:00 & 98.799 & 90.798 & 137.5 & 1559.092 & 1546.458 & 1499.651 \\
\hline 8/31/16 14:00 & 99.029 & 90.822 & 137.434 & 1558.862 & 1546.434 & 1499.717 \\
\hline $8 / 31 / 16$ 15:00 & 99.281 & 90.84 & 137.364 & 1558.61 & 1546.416 & 1499.787 \\
\hline 8/31/16 16:00 & 99.544 & 90.863 & 137.289 & 1558.347 & 1546.393 & 1499.862 \\
\hline 8/31/16 17:00 & 99.817 & 90.882 & 137.216 & 1558.074 & 1546.374 & 1499.935 \\
\hline 8/31/16 18:00 & 100.097 & 90.901 & 137.142 & 1557.794 & 1546.355 & 1500.009 \\
\hline 8/31/16 19:00 & 100.382 & 90.919 & 137.069 & 1557.509 & 1546.337 & 1500.082 \\
\hline 8/31/16 20:00 & 100.671 & 90.935 & 137.001 & 1557.22 & 1546.321 & 1500.15 \\
\hline 8/31/16 21:00 & 100.963 & 90.954 & 136.938 & 1556.928 & 1546.302 & 1500.213 \\
\hline 8/31/16 22:00 & 101.254 & 90.97 & 136.879 & 1556.637 & 1546.286 & 1500.272 \\
\hline 8/31/16 23:00 & 101.546 & 90.982 & 136.819 & 1556.345 & 1546.274 & 1500.332 \\
\hline 9/1/16 0:00 & 101.833 & 90.996 & 136.76 & 1556.058 & 1546.26 & 1500.391 \\
\hline $9 / 1 / 16$ 1:00 & 102.113 & 90.996 & 136.694 & 1555.778 & 1546.26 & 1500.457 \\
\hline $9 / 1 / 16$ 2:00 & 102.388 & 91.005 & 136.638 & 1555.503 & 1546.251 & 1500.513 \\
\hline 9/1/16 3:00 & 102.661 & 91.003 & 136.57 & 1555.23 & 1546.253 & 1500.581 \\
\hline $9 / 1 / 164: 00$ & 102.925 & 91.014 & 136.51 & 1554.966 & 1546.242 & 1500.641 \\
\hline 9/1/16 5:00 & 103.186 & 91.005 & 136.444 & 1554.705 & 1546.251 & 1500.707 \\
\hline 9/1/16 6:00 & 103.435 & 91.01 & 136.397 & 1554.456 & 1546.246 & 1500.754 \\
\hline $9 / 1 / 16$ 7:00 & 103.68 & 91.019 & 136.343 & 1554.211 & 1546.237 & 1500.808 \\
\hline $9 / 1 / 168: 00$ & 103.92 & 91.059 & 136.301 & 1553.971 & 1546.197 & 1500.85 \\
\hline 9/1/16 9:00 & 104.162 & 91.152 & 136.254 & 1553.729 & 1546.104 & 1500.897 \\
\hline 9/1/16 10:00 & 104.407 & 91.275 & 136.208 & 1553.484 & 1545.981 & 1500.943 \\
\hline 9/1/16 11:00 & 104.658 & 91.407 & 136.173 & 1553.233 & 1545.849 & 1500.978 \\
\hline 9/1/16 12:00 & 104.912 & 91.547 & 136.135 & 1552.979 & 1545.709 & 1501.016 \\
\hline 9/1/16 13:00 & 105.164 & 91.677 & 136.1 & 1552.727 & 1545.579 & 1501.051 \\
\hline
\end{tabular}


TABLE S1.2 Automatically recorded groundwater levels in the confined aquifers.

\begin{tabular}{|c|c|c|c|c|c|c|}
\hline \multirow[b]{2}{*}{ Date-Time } & \multicolumn{3}{|c|}{ Measured Depth to Water (ft TOC ${ }^{\mathrm{a}}$ ) } & \multicolumn{3}{|c|}{ Groundwater Elevation (ft AMSL ${ }^{b}$ ) } \\
\hline & DL2-CU & DL7-CU & DL7-CL & DL2-CU & DL7-CU & DL7-CL \\
\hline $9 / 1 / 16$ 14:00 & 105.406 & 91.812 & 136.06 & 1552.485 & 1545.444 & 1501.091 \\
\hline $9 / 1 / 16$ 15:00 & 105.639 & 91.937 & 136.02 & 1552.252 & 1545.319 & 1501.131 \\
\hline 9/1/16 16:00 & 105.858 & 92.056 & 135.978 & 1552.033 & 1545.2 & 1501.173 \\
\hline 9/1/16 17:00 & 106.065 & 92.163 & 135.938 & 1551.826 & 1545.093 & 1501.213 \\
\hline 9/1/16 18:00 & 106.26 & 92.265 & 135.896 & 1551.631 & 1544.991 & 1501.255 \\
\hline 9/1/16 19:00 & 106.444 & 92.36 & 135.852 & 1551.447 & 1544.896 & 1501.299 \\
\hline 9/1/16 20:00 & 106.616 & 92.442 & 135.81 & 1551.275 & 1544.814 & 1501.341 \\
\hline 9/1/16 21:00 & 106.78 & 92.528 & 135.772 & 1551.111 & 1544.728 & 1501.379 \\
\hline 9/1/16 22:00 & 106.938 & 92.607 & 135.728 & 1550.953 & 1544.649 & 1501.423 \\
\hline 9/1/16 23:00 & 107.091 & 92.672 & 135.69 & 1550.8 & 1544.584 & 1501.461 \\
\hline $9 / 2 / 16$ 0:00 & 107.237 & 92.735 & 135.651 & 1550.654 & 1544.521 & 1501.5 \\
\hline $9 / 2 / 16$ 1:00 & 107.375 & 92.795 & 135.606 & 1550.516 & 1544.461 & 1501.545 \\
\hline $9 / 2 / 162: 00$ & 107.512 & 92.851 & 135.555 & 1550.379 & 1544.405 & 1501.596 \\
\hline 9/2/16 3:00 & 107.641 & 92.9 & 135.508 & 1550.25 & 1544.356 & 1501.643 \\
\hline $9 / 2 / 164: 00$ & 107.761 & 92.944 & 135.459 & 1550.13 & 1544.312 & 1501.692 \\
\hline $9 / 2 / 165: 00$ & 107.874 & 92.988 & 135.412 & 1550.017 & 1544.268 & 1501.739 \\
\hline $9 / 2 / 16$ 6:00 & 107.982 & 93.025 & 135.367 & 1549.909 & 1544.231 & 1501.784 \\
\hline $9 / 2 / 16$ 7:00 & 108.083 & 93.062 & 135.325 & 1549.808 & 1544.194 & 1501.826 \\
\hline $9 / 2 / 168: 00$ & 108.18 & 93.1 & 135.29 & 1549.711 & 1544.156 & 1501.861 \\
\hline 9/2/16 9:00 & 108.276 & 93.132 & 135.257 & 1549.615 & 1544.124 & 1501.894 \\
\hline 9/2/16 10:00 & 108.363 & 93.158 & 135.227 & 1549.528 & 1544.098 & 1501.924 \\
\hline 9/2/16 11:00 & 108.439 & 93.135 & 135.208 & 1549.452 & 1544.121 & 1501.943 \\
\hline 9/2/16 12:00 & 108.49 & 93.032 & 135.199 & 1549.401 & 1544.224 & 1501.952 \\
\hline 9/2/16 13:00 & 108.521 & 92.9 & 135.18 & 1549.37 & 1544.356 & 1501.971 \\
\hline 9/2/16 14:00 & 108.521 & 92.758 & 135.164 & 1549.37 & 1544.498 & 1501.987 \\
\hline 9/2/16 15:00 & 108.507 & 92.614 & 135.143 & 1549.384 & 1544.642 & 1502.008 \\
\hline 9/2/16 16:00 & 108.474 & 92.479 & 135.133 & 1549.417 & 1544.777 & 1502.018 \\
\hline 9/2/16 17:00 & 108.427 & 92.34 & 135.129 & 1549.464 & 1544.916 & 1502.022 \\
\hline 9/2/16 18:00 & 108.373 & 92.216 & 135.117 & 1549.518 & 1545.04 & 1502.034 \\
\hline 9/2/16 19:00 & 108.314 & 92.098 & 135.101 & 1549.577 & 1545.158 & 1502.05 \\
\hline 9/2/16 20:00 & 108.25 & 91.982 & 135.072 & 1549.641 & 1545.274 & 1502.079 \\
\hline 9/2/16 21:00 & 108.192 & 91.886 & 135.047 & 1549.699 & 1545.37 & 1502.104 \\
\hline 9/2/16 22:00 & 108.131 & 91.8 & 135.026 & 1549.76 & 1545.456 & 1502.125 \\
\hline 9/2/16 23:00 & 108.067 & 91.714 & 135.014 & 1549.824 & 1545.542 & 1502.137 \\
\hline 9/3/16 0:00 & 107.999 & 91.633 & 135.009 & 1549.892 & 1545.623 & 1502.142 \\
\hline $9 / 3 / 16$ 1:00 & 107.923 & 91.554 & 135 & 1549.968 & 1545.702 & 1502.151 \\
\hline $9 / 3 / 16$ 2:00 & 107.832 & 91.475 & 134.976 & 1550.059 & 1545.781 & 1502.175 \\
\hline 9/3/16 3:00 & 107.726 & 91.4 & 134.948 & 1550.165 & 1545.856 & 1502.203 \\
\hline $9 / 3 / 164: 00$ & 107.606 & 91.331 & 134.916 & 1550.285 & 1545.925 & 1502.235 \\
\hline $9 / 3 / 165: 00$ & 107.469 & 91.266 & 134.885 & 1550.422 & 1545.99 & 1502.266 \\
\hline $9 / 3 / 16$ 6:00 & 107.324 & 91.196 & 134.869 & 1550.567 & 1546.06 & 1502.282 \\
\hline $9 / 3 / 16$ 7:00 & 107.168 & 91.133 & 134.855 & 1550.723 & 1546.123 & 1502.296 \\
\hline $9 / 3 / 168: 00$ & 106.997 & 91.07 & 134.845 & 1550.894 & 1546.186 & 1502.306 \\
\hline 9/3/16 9:00 & 106.764 & 90.998 & 134.843 & 1551.127 & 1546.258 & 1502.308 \\
\hline 9/3/16 10:00 & 106.493 & 90.938 & 134.843 & 1551.398 & 1546.318 & 1502.308 \\
\hline 9/3/16 11:00 & 106.202 & 90.863 & 134.848 & 1551.689 & 1546.393 & 1502.303 \\
\hline 9/3/16 12:00 & 105.891 & 90.787 & 134.852 & 1552 & 1546.469 & 1502.299 \\
\hline
\end{tabular}


TABLE S1.2 Automatically recorded groundwater levels in the confined aquifers.

\begin{tabular}{|c|c|c|c|c|c|c|}
\hline \multirow[b]{2}{*}{ Date-Time } & \multicolumn{3}{|c|}{ Measured Depth to Water (ft TOC ${ }^{\mathrm{a}}$ ) } & \multicolumn{3}{|c|}{ Groundwater Elevation (ft AMSL ${ }^{\mathrm{b}}$ ) } \\
\hline & DL2-CU & DL7-CU & DL7-CL & DL2-CU & DL7-CU & DL7-CL \\
\hline 9/3/16 13:00 & 105.573 & 90.705 & 134.857 & 1552.318 & 1546.551 & 1502.294 \\
\hline 9/3/16 14:00 & 105.254 & 90.615 & 134.857 & 1552.637 & 1546.641 & 1502.294 \\
\hline 9/3/16 15:00 & 104.948 & 90.505 & 134.843 & 1552.943 & 1546.751 & 1502.308 \\
\hline 9/3/16 16:00 & 104.616 & 90.361 & 134.789 & 1553.275 & 1546.895 & 1502.362 \\
\hline 9/3/16 17:00 & 104.338 & 90.252 & 134.763 & 1553.553 & 1547.004 & 1502.388 \\
\hline 9/3/16 18:00 & 104.073 & 90.152 & 134.731 & 1553.818 & 1547.104 & 1502.42 \\
\hline 9/3/16 19:00 & 103.788 & 90.059 & 134.696 & 1554.103 & 1547.197 & 1502.455 \\
\hline 9/3/16 20:00 & 103.489 & 89.966 & 134.646 & 1554.402 & 1547.29 & 1502.505 \\
\hline 9/3/16 21:00 & 103.186 & 89.878 & 134.597 & 1554.705 & 1547.378 & 1502.554 \\
\hline 9/3/16 22:00 & 102.88 & 89.792 & 134.541 & 1555.011 & 1547.464 & 1502.61 \\
\hline 9/3/16 23:00 & 102.581 & 89.71 & 134.485 & 1555.31 & 1547.546 & 1502.666 \\
\hline 9/4/16 0:00 & 102.285 & 89.624 & 134.422 & 1555.606 & 1547.632 & 1502.729 \\
\hline $9 / 4 / 16$ 1:00 & 101.995 & 89.538 & 134.351 & 1555.896 & 1547.718 & 1502.8 \\
\hline $9 / 4 / 16$ 2:00 & 101.704 & 89.45 & 134.279 & 1556.187 & 1547.806 & 1502.872 \\
\hline 9/4/16 3:00 & 101.435 & 89.366 & 134.202 & 1556.456 & 1547.89 & 1502.949 \\
\hline $9 / 4 / 164: 00$ & 101.165 & 89.271 & 134.117 & 1556.726 & 1547.985 & 1503.034 \\
\hline 9/4/16 5:00 & 100.897 & 89.178 & 134.028 & 1556.994 & 1548.078 & 1503.123 \\
\hline $9 / 4 / 16$ 6:00 & 100.652 & 89.085 & 133.944 & 1557.239 & 1548.171 & 1503.207 \\
\hline 9/4/16 7:00 & 100.408 & 88.985 & 133.858 & 1557.483 & 1548.271 & 1503.293 \\
\hline 9/4/16 8:00 & 100.172 & 88.887 & 133.776 & 1557.719 & 1548.369 & 1503.375 \\
\hline 9/4/16 9:00 & 99.946 & 88.79 & 133.684 & 1557.945 & 1548.466 & 1503.467 \\
\hline 9/4/16 10:00 & 99.728 & 88.688 & 133.6 & 1558.163 & 1548.568 & 1503.551 \\
\hline 9/4/16 11:00 & 99.521 & 88.588 & 133.514 & 1558.37 & 1548.668 & 1503.637 \\
\hline 9/4/16 12:00 & 99.314 & 88.485 & 133.425 & 1558.577 & 1548.771 & 1503.726 \\
\hline 9/4/16 13:00 & 99.116 & 88.383 & 133.336 & 1558.775 & 1548.873 & 1503.815 \\
\hline 9/4/16 14:00 & 98.923 & 88.278 & 133.247 & 1558.968 & 1548.978 & 1503.904 \\
\hline 9/4/16 15:00 & 98.735 & 88.176 & 133.153 & 1559.156 & 1549.08 & 1503.998 \\
\hline 9/4/16 16:00 & 98.549 & 88.067 & 133.059 & 1559.342 & 1549.189 & 1504.092 \\
\hline 9/4/16 17:00 & 98.37 & 87.965 & 132.966 & 1559.521 & 1549.291 & 1504.185 \\
\hline 9/4/16 18:00 & 98.194 & 87.862 & 132.872 & 1559.697 & 1549.394 & 1504.279 \\
\hline 9/4/16 19:00 & 98.027 & 87.755 & 132.779 & 1559.864 & 1549.501 & 1504.372 \\
\hline 9/4/16 20:00 & 97.862 & 87.655 & 132.685 & 1560.029 & 1549.601 & 1504.466 \\
\hline 9/4/16 21:00 & 97.702 & 87.553 & 132.594 & 1560.189 & 1549.703 & 1504.557 \\
\hline 9/4/16 22:00 & 97.554 & 87.46 & 132.507 & 1560.337 & 1549.796 & 1504.644 \\
\hline 9/4/16 23:00 & 97.411 & 87.365 & 132.42 & 1560.48 & 1549.891 & 1504.731 \\
\hline 9/5/16 0:00 & 97.265 & 87.27 & 132.334 & 1560.626 & 1549.986 & 1504.817 \\
\hline 9/5/16 1:00 & 97.126 & 87.179 & 132.247 & 1560.765 & 1550.077 & 1504.904 \\
\hline $9 / 5 / 16$ 2:00 & 96.99 & 87.088 & 132.156 & 1560.901 & 1550.168 & 1504.995 \\
\hline $9 / 5 / 163: 00$ & 96.855 & 86.991 & 132.06 & 1561.036 & 1550.265 & 1505.091 \\
\hline 9/5/16 4:00 & 96.724 & 86.895 & 131.976 & 1561.167 & 1550.361 & 1505.175 \\
\hline 9/5/16 5:00 & 96.592 & 86.802 & 131.882 & 1561.299 & 1550.454 & 1505.269 \\
\hline 9/5/16 6:00 & 96.465 & 86.721 & 131.8 & 1561.426 & 1550.535 & 1505.351 \\
\hline 9/5/16 7:00 & 96.345 & 86.633 & 131.877 & 1561.546 & 1550.623 & 1505.274 \\
\hline $9 / 5 / 168: 00$ & 96.225 & 86.544 & 132.011 & 1561.666 & 1550.712 & 1505.14 \\
\hline 9/5/16 9:00 & 96.11 & 86.444 & 132.072 & 1561.781 & 1550.812 & 1505.079 \\
\hline 9/5/16 10:00 & 95.994 & 86.358 & 132.013 & 1561.897 & 1550.898 & 1505.138 \\
\hline 9/5/16 11:00 & 95.889 & 86.277 & 131.943 & 1562.002 & 1550.979 & 1505.208 \\
\hline
\end{tabular}


TABLE S1.2 Automatically recorded groundwater levels in the confined aquifers.

\begin{tabular}{|c|c|c|c|c|c|c|}
\hline \multirow[b]{2}{*}{ Date-Time } & \multicolumn{3}{|c|}{ Measured Depth to Water (ft TOC ${ }^{\mathrm{a}}$ ) } & \multicolumn{3}{|c|}{ Groundwater Elevation (ft AMSL ${ }^{\mathrm{b}}$ ) } \\
\hline & DL2-CU & DL7-CU & DL7-CL & DL2-CU & DL7-CU & DL7-CL \\
\hline 9/5/16 12:00 & 95.78 & 86.198 & 131.859 & 1562.111 & 1551.058 & 1505.292 \\
\hline 9/5/16 13:00 & 95.679 & 86.135 & 131.828 & 1562.212 & 1551.121 & 1505.323 \\
\hline 9/5/16 14:00 & 95.578 & 86.058 & 131.934 & 1562.313 & 1551.198 & 1505.217 \\
\hline 9/5/16 15:00 & 95.479 & 85.979 & 131.917 & 1562.412 & 1551.277 & 1505.234 \\
\hline 9/5/16 16:00 & 95.381 & 85.914 & 131.838 & 1562.51 & 1551.342 & 1505.313 \\
\hline 9/5/16 17:00 & 95.291 & 85.854 & 131.751 & 1562.6 & 1551.402 & 1505.4 \\
\hline 9/5/16 18:00 & 95.199 & 85.796 & 131.66 & 1562.692 & 1551.46 & 1505.491 \\
\hline 9/5/16 19:00 & 95.112 & 85.742 & 131.566 & 1562.779 & 1551.514 & 1505.585 \\
\hline 9/5/16 20:00 & 95.028 & 85.689 & 131.465 & 1562.863 & 1551.567 & 1505.686 \\
\hline 9/5/16 21:00 & 94.948 & 85.645 & 131.369 & 1562.943 & 1551.611 & 1505.782 \\
\hline 9/5/16 22:00 & 94.872 & 85.6 & 131.276 & 1563.019 & 1551.656 & 1505.875 \\
\hline 9/5/16 23:00 & 94.799 & 85.561 & 131.178 & 1563.092 & 1551.695 & 1505.973 \\
\hline 9/6/16 0:00 & 94.724 & 85.51 & 131.079 & 1563.167 & 1551.746 & 1506.072 \\
\hline 9/6/16 1:00 & 94.651 & 85.463 & 130.981 & 1563.24 & 1551.793 & 1506.17 \\
\hline $9 / 6 / 16$ 2:00 & 94.581 & 85.426 & 130.922 & 1563.31 & 1551.83 & 1506.229 \\
\hline 9/6/16 3:00 & 94.505 & 85.38 & 131.025 & 1563.386 & 1551.876 & 1506.126 \\
\hline $9 / 6 / 164: 00$ & 94.437 & 85.317 & 131.138 & 1563.454 & 1551.939 & 1506.013 \\
\hline 9/6/16 5:00 & 94.362 & 85.249 & 131.105 & 1563.529 & 1552.007 & 1506.046 \\
\hline 9/6/16 6:00 & 94.289 & 85.196 & 131.025 & 1563.602 & 1552.06 & 1506.126 \\
\hline 9/6/16 7:00 & 94.221 & 85.147 & 130.967 & 1563.67 & 1552.109 & 1506.184 \\
\hline 9/6/16 8:00 & 94.145 & 85.089 & 131.063 & 1563.746 & 1552.167 & 1506.088 \\
\hline 9/6/16 9:00 & 94.073 & 85.017 & 131.138 & 1563.818 & 1552.239 & 1506.013 \\
\hline 9/6/16 10:00 & 94.004 & 84.954 & 131.077 & 1563.887 & 1552.302 & 1506.074 \\
\hline 9/6/16 11:00 & 93.936 & 84.894 & 130.993 & 1563.955 & 1552.362 & 1506.158 \\
\hline 9/6/16 12:00 & 93.87 & 84.84 & 130.899 & 1564.021 & 1552.416 & 1506.252 \\
\hline 9/6/16 13:00 & 93.802 & 84.773 & 130.798 & 1564.089 & 1552.483 & 1506.353 \\
\hline 9/6/16 14:00 & 93.736 & 84.719 & 130.702 & 1564.155 & 1552.537 & 1506.449 \\
\hline 9/6/16 15:00 & 93.666 & 84.659 & 130.595 & 1564.225 & 1552.597 & 1506.556 \\
\hline 9/6/16 16:00 & 93.586 & 84.596 & 130.492 & 1564.305 & 1552.66 & 1506.659 \\
\hline 9/6/16 17:00 & 93.501 & 84.533 & 130.384 & 1564.39 & 1552.723 & 1506.767 \\
\hline 9/6/16 18:00 & 93.407 & 84.464 & 130.274 & 1564.484 & 1552.792 & 1506.877 \\
\hline 9/6/16 19:00 & 93.31 & 84.387 & 130.166 & 1564.581 & 1552.869 & 1506.985 \\
\hline 9/6/16 20:00 & 93.216 & 84.324 & 130.066 & 1564.675 & 1552.932 & 1507.085 \\
\hline 9/6/16 21:00 & 93.12 & 84.255 & 129.967 & 1564.771 & 1553.001 & 1507.184 \\
\hline 9/6/16 22:00 & 93.023 & 84.182 & 129.871 & 1564.868 & 1553.074 & 1507.28 \\
\hline 9/6/16 23:00 & 92.922 & 84.108 & 129.771 & 1564.969 & 1553.148 & 1507.38 \\
\hline 9/7/16 0:00 & 92.823 & 84.031 & 129.677 & 1565.068 & 1553.225 & 1507.474 \\
\hline 9/7/16 1:00 & 92.722 & 83.957 & 129.577 & 1565.169 & 1553.299 & 1507.574 \\
\hline $9 / 7 / 16$ 2:00 & 92.638 & 83.89 & 129.492 & 1565.253 & 1553.366 & 1507.659 \\
\hline 9/7/16 3:00 & 92.544 & 83.822 & 129.401 & 1565.347 & 1553.434 & 1507.75 \\
\hline 9/7/16 4:00 & 92.435 & 83.736 & 129.296 & 1565.456 & 1553.52 & 1507.855 \\
\hline 9/7/16 5:00 & 92.353 & 83.673 & 129.211 & 1565.538 & 1553.583 & 1507.94 \\
\hline $9 / 7 / 16$ 6:00 & 92.261 & 83.604 & 129.122 & 1565.63 & 1553.652 & 1508.029 \\
\hline 9/7/16 7:00 & 92.177 & 83.539 & 129.041 & 1565.714 & 1553.717 & 1508.11 \\
\hline 9/7/16 8:00 & 92.087 & 83.464 & 128.961 & 1565.804 & 1553.792 & 1508.19 \\
\hline 9/7/16 9:00 & 92 & 83.394 & 128.888 & 1565.891 & 1553.862 & 1508.263 \\
\hline 9/7/16 10:00 & 91.915 & 83.325 & 128.818 & 1565.976 & 1553.931 & 1508.333 \\
\hline
\end{tabular}


TABLE S1.2 Automatically recorded groundwater levels in the confined aquifers.

\begin{tabular}{|c|c|c|c|c|c|c|}
\hline \multirow[b]{2}{*}{ Date-Time } & \multicolumn{3}{|c|}{ Measured Depth to Water (ft TOC ${ }^{a}$ ) } & \multicolumn{3}{|c|}{ Groundwater Elevation (ft AMSL ${ }^{b}$ ) } \\
\hline & DL2-CU & DL7-CU & DL7-CL & DL2-CU & DL7-CU & DL7-CL \\
\hline 9/7/16 11:00 & 91.835 & 83.26 & 128.753 & 1566.056 & 1553.996 & 1508.398 \\
\hline 9/7/16 12:00 & 91.755 & 83.19 & 128.689 & 1566.136 & 1554.066 & 1508.462 \\
\hline 9/7/16 13:00 & 91.673 & 83.122 & 128.626 & 1566.218 & 1554.134 & 1508.525 \\
\hline 9/7/16 14:00 & 91.598 & 83.057 & 128.563 & 1566.293 & 1554.199 & 1508.588 \\
\hline 9/7/16 15:00 & 91.523 & 82.992 & 128.497 & 1566.368 & 1554.264 & 1508.654 \\
\hline 9/7/16 16:00 & 91.45 & 82.932 & 128.434 & 1566.441 & 1554.324 & 1508.717 \\
\hline 9/7/16 17:00 & 91.377 & 82.867 & 128.364 & 1566.514 & 1554.389 & 1508.787 \\
\hline 9/7/16 18:00 & 91.306 & 82.802 & 128.299 & 1566.585 & 1554.454 & 1508.852 \\
\hline 9/7/16 19:00 & 91.238 & 82.744 & 128.226 & 1566.653 & 1554.512 & 1508.925 \\
\hline 9/7/16 20:00 & 91.172 & 82.683 & 128.16 & 1566.719 & 1554.573 & 1508.991 \\
\hline 9/7/16 21:00 & 91.109 & 82.634 & 128.09 & 1566.782 & 1554.622 & 1509.061 \\
\hline 9/7/16 22:00 & 91.045 & 82.583 & 128.041 & 1566.846 & 1554.673 & 1509.11 \\
\hline 9/7/16 23:00 & 90.984 & 82.53 & 127.994 & 1566.907 & 1554.726 & 1509.157 \\
\hline 9/8/16 0:00 & 90.925 & 82.476 & 127.961 & 1566.966 & 1554.78 & 1509.19 \\
\hline 9/8/16 1:00 & 90.864 & 82.42 & 127.919 & 1567.027 & 1554.836 & 1509.232 \\
\hline $9 / 8 / 162: 00$ & 90.807 & 82.372 & 127.868 & 1567.084 & 1554.884 & 1509.283 \\
\hline 9/8/16 3:00 & 90.751 & 82.318 & 127.823 & 1567.14 & 1554.938 & 1509.328 \\
\hline $9 / 8 / 164: 00$ & 90.694 & 82.265 & 127.765 & 1567.197 & 1554.991 & 1509.386 \\
\hline 9/8/16 5:00 & 90.645 & 82.22 & 127.716 & 1567.246 & 1555.036 & 1509.435 \\
\hline $9 / 8 / 16$ 6:00 & 90.589 & 82.179 & 127.674 & 1567.302 & 1555.077 & 1509.477 \\
\hline 9/8/16 7:00 & 90.542 & 82.13 & 127.645 & 1567.349 & 1555.126 & 1509.506 \\
\hline 9/8/16 8:00 & 90.488 & 82.086 & 127.62 & 1567.403 & 1555.17 & 1509.531 \\
\hline 9/8/16 9:00 & 90.433 & 82.035 & 127.589 & 1567.458 & 1555.221 & 1509.562 \\
\hline 9/8/16 10:00 & 90.386 & 81.993 & 127.55 & 1567.505 & 1555.263 & 1509.601 \\
\hline 9/8/16 11:00 & 90.337 & 81.937 & 127.505 & 1567.554 & 1555.319 & 1509.646 \\
\hline 9/8/16 12:00 & 90.288 & 81.89 & 127.449 & 1567.603 & 1555.366 & 1509.702 \\
\hline 9/8/16 13:00 & 90.236 & 81.844 & 127.393 & 1567.655 & 1555.412 & 1509.758 \\
\hline 9/8/16 14:00 & 90.189 & 81.804 & 127.355 & 1567.702 & 1555.452 & 1509.796 \\
\hline 9/8/16 15:00 & 90.137 & 81.751 & 127.458 & 1567.754 & 1555.505 & 1509.693 \\
\hline 9/8/16 16:00 & 90.09 & 81.697 & 127.447 & 1567.801 & 1555.559 & 1509.704 \\
\hline 9/8/16 17:00 & 90.041 & 81.653 & 127.397 & 1567.85 & 1555.603 & 1509.754 \\
\hline 9/8/16 18:00 & 89.996 & 81.611 & 127.334 & 1567.895 & 1555.645 & 1509.817 \\
\hline 9/8/16 19:00 & 89.949 & 81.567 & 127.266 & 1567.942 & 1555.689 & 1509.885 \\
\hline 9/8/16 20:00 & 89.906 & 81.523 & 127.198 & 1567.985 & 1555.733 & 1509.953 \\
\hline 9/8/16 21:00 & 89.864 & 81.484 & 127.131 & 1568.027 & 1555.772 & 1510.02 \\
\hline 9/8/16 22:00 & 89.819 & 81.444 & 127.056 & 1568.072 & 1555.812 & 1510.095 \\
\hline 9/8/16 23:00 & 89.779 & 81.402 & 126.983 & 1568.112 & 1555.854 & 1510.168 \\
\hline 9/9/16 0:00 & 89.737 & 81.363 & 126.906 & 1568.154 & 1555.893 & 1510.245 \\
\hline 9/9/16 1:00 & 89.697 & 81.326 & 126.833 & 1568.194 & 1555.93 & 1510.318 \\
\hline 9/9/16 2:00 & 89.659 & 81.288 & 126.758 & 1568.232 & 1555.968 & 1510.393 \\
\hline 9/9/16 3:00 & 89.622 & 81.258 & 126.737 & 1568.269 & 1555.998 & 1510.414 \\
\hline $9 / 9 / 164: 00$ & 89.584 & 81.221 & 126.864 & 1568.307 & 1556.035 & 1510.287 \\
\hline 9/9/16 5:00 & 89.551 & 81.184 & 127.037 & 1568.34 & 1556.072 & 1510.114 \\
\hline 9/9/16 6:00 & 89.514 & 81.144 & 127.205 & 1568.377 & 1556.112 & 1509.946 \\
\hline 9/9/16 7:00 & 89.48 & 81.105 & 127.383 & 1568.411 & 1556.151 & 1509.768 \\
\hline 9/9/16 8:00 & 89.445 & 81.056 & 127.543 & 1568.446 & 1556.2 & 1509.608 \\
\hline 9/9/16 9:00 & 89.41 & 81.021 & 127.54 & 1568.481 & 1556.235 & 1509.611 \\
\hline
\end{tabular}


TABLE S1.2 Automatically recorded groundwater levels in the confined aquifers.

\begin{tabular}{|c|c|c|c|c|c|c|}
\hline \multirow[b]{2}{*}{ Date-Time } & \multicolumn{3}{|c|}{ Measured Depth to Water (ft TOC ${ }^{\mathrm{a}}$ ) } & \multicolumn{3}{|c|}{ Groundwater Elevation (ft AMSL ${ }^{\mathrm{b}}$ ) } \\
\hline & DL2-CU & DL7-CU & DL7-CL & DL2-CU & DL7-CU & DL7-CL \\
\hline 9/9/16 10:00 & 89.377 & 80.986 & 127.489 & 1568.514 & 1556.27 & 1509.662 \\
\hline 9/9/16 11:00 & 89.337 & 80.949 & 127.409 & 1568.554 & 1556.307 & 1509.742 \\
\hline 9/9/16 12:00 & 89.307 & 80.916 & 127.327 & 1568.584 & 1556.34 & 1509.824 \\
\hline 9/9/16 13:00 & 89.25 & 80.879 & 127.241 & 1568.641 & 1556.377 & 1509.91 \\
\hline 9/9/16 14:00 & 89.189 & 80.823 & 127.124 & 1568.702 & 1556.433 & 1510.027 \\
\hline 9/9/16 15:00 & 89.154 & 80.784 & 127.023 & 1568.737 & 1556.472 & 1510.128 \\
\hline 9/9/16 16:00 & 89.118 & 80.744 & 126.922 & 1568.773 & 1556.512 & 1510.229 \\
\hline 9/9/16 17:00 & 89.097 & 80.723 & 126.836 & 1568.794 & 1556.533 & 1510.315 \\
\hline 9/9/16 18:00 & 89.071 & 80.691 & 126.744 & 1568.82 & 1556.565 & 1510.407 \\
\hline 9/9/16 19:00 & 89.05 & 80.668 & 126.66 & 1568.841 & 1556.588 & 1510.491 \\
\hline 9/9/16 20:00 & 89.027 & 80.647 & 126.585 & 1568.864 & 1556.609 & 1510.566 \\
\hline 9/9/16 21:00 & 89.001 & 80.621 & 126.503 & 1568.89 & 1556.635 & 1510.648 \\
\hline 9/9/16 22:00 & 88.982 & 80.596 & 126.426 & 1568.909 & 1556.66 & 1510.725 \\
\hline 9/9/16 23:00 & 88.961 & 80.572 & 126.363 & 1568.93 & 1556.684 & 1510.788 \\
\hline 9/10/16 0:00 & 88.935 & 80.542 & 126.29 & 1568.956 & 1556.714 & 1510.861 \\
\hline 9/10/16 1:00 & 88.909 & 80.514 & 126.213 & 1568.982 & 1556.742 & 1510.938 \\
\hline 9/10/16 2:00 & 88.883 & 80.486 & 126.145 & 1569.008 & 1556.77 & 1511.006 \\
\hline 9/10/16 3:00 & 88.86 & 80.463 & 126.082 & 1569.031 & 1556.793 & 1511.069 \\
\hline 9/10/16 4:00 & 88.836 & 80.437 & 126.023 & 1569.055 & 1556.819 & 1511.128 \\
\hline 9/10/16 5:00 & 88.813 & 80.412 & 125.963 & 1569.078 & 1556.844 & 1511.188 \\
\hline 9/10/16 6:00 & 88.791 & 80.382 & 125.906 & 1569.1 & 1556.874 & 1511.245 \\
\hline 9/10/16 7:00 & 88.77 & 80.361 & 125.853 & 1569.121 & 1556.895 & 1511.298 \\
\hline 9/10/16 8:00 & 88.747 & 80.338 & 125.796 & 1569.144 & 1556.918 & 1511.355 \\
\hline 9/10/16 9:00 & 88.723 & 80.314 & 125.752 & 1569.168 & 1556.942 & 1511.399 \\
\hline 9/10/16 10:00 & 88.7 & 80.289 & 125.719 & 1569.191 & 1556.967 & 1511.432 \\
\hline 9/10/16 11:00 & 88.676 & 80.265 & 125.707 & 1569.215 & 1556.991 & 1511.444 \\
\hline 9/10/16 12:00 & 88.648 & 80.238 & 125.693 & 1569.243 & 1557.018 & 1511.458 \\
\hline 9/10/16 13:00 & 88.62 & 80.203 & 125.668 & 1569.271 & 1557.053 & 1511.483 \\
\hline 9/10/16 14:00 & 88.596 & 80.17 & 125.635 & 1569.295 & 1557.086 & 1511.516 \\
\hline 9/10/16 15:00 & 88.568 & 80.145 & 125.607 & 1569.323 & 1557.111 & 1511.544 \\
\hline 9/10/16 16:00 & 88.537 & 80.119 & 125.588 & 1569.354 & 1557.137 & 1511.563 \\
\hline 9/10/16 17:00 & 88.512 & 80.089 & 125.581 & 1569.379 & 1557.167 & 1511.57 \\
\hline 9/10/16 18:00 & 88.483 & 80.061 & 125.572 & 1569.408 & 1557.195 & 1511.579 \\
\hline 9/10/16 19:00 & 88.457 & 80.033 & 125.555 & 1569.434 & 1557.223 & 1511.596 \\
\hline 9/10/16 20:00 & 88.429 & 80 & 125.527 & 1569.462 & 1557.256 & 1511.624 \\
\hline 9/10/16 21:00 & 88.403 & 79.975 & 125.494 & 1569.488 & 1557.281 & 1511.657 \\
\hline 9/10/16 22:00 & 88.38 & 79.952 & 125.469 & 1569.511 & 1557.304 & 1511.682 \\
\hline 9/10/16 23:00 & 88.354 & 79.928 & 125.457 & 1569.537 & 1557.328 & 1511.694 \\
\hline 9/11/16 0:00 & 88.328 & 79.898 & 125.441 & 1569.563 & 1557.358 & 1511.71 \\
\hline 9/11/16 1:00 & 88.302 & 79.873 & 125.42 & 1569.589 & 1557.383 & 1511.731 \\
\hline $9 / 11 / 16$ 2:00 & 88.274 & 79.84 & 125.387 & 1569.617 & 1557.416 & 1511.764 \\
\hline 9/11/16 3:00 & 88.25 & 79.815 & 125.352 & 1569.641 & 1557.441 & 1511.799 \\
\hline 9/11/16 4:00 & 88.225 & 79.798 & 125.312 & 1569.666 & 1557.458 & 1511.839 \\
\hline 9/11/16 5:00 & 88.199 & 79.773 & 125.286 & 1569.692 & 1557.483 & 1511.865 \\
\hline 9/11/16 6:00 & 88.175 & 79.749 & 125.274 & 1569.716 & 1557.507 & 1511.877 \\
\hline 9/11/16 7:00 & 88.152 & 79.726 & 125.263 & 1569.739 & 1557.53 & 1511.888 \\
\hline 9/11/16 8:00 & 88.128 & 79.703 & 125.263 & 1569.763 & 1557.553 & 1511.888 \\
\hline
\end{tabular}


TABLE S1.2 Automatically recorded groundwater levels in the confined aquifers.

\begin{tabular}{|c|c|c|c|c|c|c|}
\hline \multirow[b]{2}{*}{ Date-Time } & \multicolumn{3}{|c|}{ Measured Depth to Water (ft TOC ${ }^{\mathrm{a}}$ ) } & \multicolumn{3}{|c|}{ Groundwater Elevation (ft AMSL ${ }^{\mathrm{b}}$ ) } \\
\hline & DL2-CU & DL7-CU & DL7-CL & DL2-CU & DL7-CU & DL7-CL \\
\hline 9/11/16 9:00 & 88.102 & 79.68 & 125.263 & 1569.789 & 1557.576 & 1511.888 \\
\hline 9/11/16 10:00 & 88.076 & 79.652 & 125.249 & 1569.815 & 1557.604 & 1511.902 \\
\hline 9/11/16 11:00 & 88.053 & 79.624 & 125.228 & 1569.838 & 1557.632 & 1511.923 \\
\hline 9/11/16 12:00 & 88.025 & 79.598 & 125.202 & 1569.866 & 1557.658 & 1511.949 \\
\hline 9/11/16 13:00 & 87.999 & 79.57 & 125.181 & 1569.892 & 1557.686 & 1511.97 \\
\hline 9/11/16 14:00 & 87.966 & 79.545 & 125.162 & 1569.925 & 1557.711 & 1511.989 \\
\hline 9/11/16 15:00 & 87.94 & 79.515 & 125.155 & 1569.951 & 1557.741 & 1511.996 \\
\hline 9/11/16 16:00 & 87.914 & 79.489 & 125.146 & 1569.977 & 1557.767 & 1512.005 \\
\hline 9/11/16 17:00 & 87.886 & 79.461 & 125.141 & 1570.005 & 1557.795 & 1512.01 \\
\hline 9/11/16 18:00 & 87.862 & 79.436 & 125.115 & 1570.029 & 1557.82 & 1512.036 \\
\hline 9/11/16 19:00 & 87.839 & 79.408 & 125.085 & 1570.052 & 1557.848 & 1512.066 \\
\hline 9/11/16 20:00 & 87.815 & 79.389 & 125.057 & 1570.076 & 1557.867 & 1512.094 \\
\hline 9/11/16 21:00 & 87.794 & 79.366 & 125.043 & 1570.097 & 1557.89 & 1512.108 \\
\hline 9/11/16 22:00 & 87.77 & 79.347 & 125.033 & 1570.121 & 1557.909 & 1512.118 \\
\hline 9/11/16 23:00 & 87.747 & 79.326 & 125.022 & 1570.144 & 1557.93 & 1512.129 \\
\hline $9 / 12 / 16$ 0:00 & 87.723 & 79.296 & 125.005 & 1570.168 & 1557.96 & 1512.146 \\
\hline 9/12/16 1:00 & 87.698 & 79.273 & 124.972 & 1570.193 & 1557.983 & 1512.179 \\
\hline $9 / 12 / 16$ 2:00 & 87.674 & 79.243 & 124.933 & 1570.217 & 1558.013 & 1512.218 \\
\hline 9/12/16 3:00 & 87.651 & 79.219 & 124.886 & 1570.24 & 1558.037 & 1512.265 \\
\hline $9 / 12 / 164: 00$ & 87.625 & 79.201 & 124.839 & 1570.266 & 1558.055 & 1512.312 \\
\hline 9/12/16 5:00 & 87.603 & 79.18 & 124.804 & 1570.288 & 1558.076 & 1512.347 \\
\hline $9 / 12 / 16$ 6:00 & 87.582 & 79.161 & 124.781 & 1570.309 & 1558.095 & 1512.37 \\
\hline 9/12/16 7:00 & 87.561 & 79.14 & 124.764 & 1570.33 & 1558.116 & 1512.387 \\
\hline 9/12/16 8:00 & 87.542 & 79.124 & 124.755 & 1570.349 & 1558.132 & 1512.396 \\
\hline 9/12/16 9:00 & 87.523 & 79.112 & 124.783 & 1570.368 & 1558.144 & 1512.368 \\
\hline 9/12/16 10:00 & 87.512 & 79.087 & 124.867 & 1570.379 & 1558.169 & 1512.284 \\
\hline 9/12/16 11:00 & 87.493 & 79.073 & 124.958 & 1570.398 & 1558.183 & 1512.193 \\
\hline 9/12/16 12:00 & 87.472 & 79.043 & 125.017 & 1570.419 & 1558.213 & 1512.134 \\
\hline 9/12/16 13:00 & 87.453 & 79.019 & 125.054 & 1570.438 & 1558.237 & 1512.097 \\
\hline 9/12/16 14:00 & 87.432 & 79.003 & 125.078 & 1570.459 & 1558.253 & 1512.073 \\
\hline 9/12/16 15:00 & 87.415 & 78.98 & 125.143 & 1570.476 & 1558.276 & 1512.008 \\
\hline 9/12/16 16:00 & 87.399 & 78.959 & 125.101 & 1570.492 & 1558.297 & 1512.05 \\
\hline 9/12/16 17:00 & 87.375 & 78.95 & 125.146 & 1570.516 & 1558.306 & 1512.005 \\
\hline 9/12/16 18:00 & 87.359 & 78.927 & 125.295 & 1570.532 & 1558.329 & 1511.856 \\
\hline 9/12/16 19:00 & 87.349 & 78.913 & 125.455 & 1570.542 & 1558.343 & 1511.696 \\
\hline 9/12/16 20:00 & 87.335 & 78.894 & 125.462 & 1570.556 & 1558.362 & 1511.689 \\
\hline 9/12/16 21:00 & 87.321 & 78.88 & 125.401 & 1570.57 & 1558.376 & 1511.75 \\
\hline 9/12/16 22:00 & 87.244 & 78.845 & 125.321 & 1570.647 & 1558.411 & 1511.83 \\
\hline 9/12/16 23:00 & 87.22 & 78.787 & 125.375 & 1570.671 & 1558.469 & 1511.776 \\
\hline 9/13/16 0:00 & 87.206 & 78.766 & 125.473 & 1570.685 & 1558.49 & 1511.678 \\
\hline 9/13/16 1:00 & 87.192 & 78.754 & 125.424 & 1570.699 & 1558.502 & 1511.727 \\
\hline 9/13/16 2:00 & 87.173 & 78.741 & 125.335 & 1570.718 & 1558.515 & 1511.816 \\
\hline 9/13/16 3:00 & 87.156 & 78.731 & 125.232 & 1570.735 & 1558.525 & 1511.919 \\
\hline 9/13/16 4:00 & 87.145 & 78.727 & 125.197 & 1570.746 & 1558.529 & 1511.954 \\
\hline 9/13/16 5:00 & 87.128 & 78.715 & 125.3 & 1570.763 & 1558.541 & 1511.851 \\
\hline 9/13/16 6:00 & 87.119 & 78.701 & 125.424 & 1570.772 & 1558.555 & 1511.727 \\
\hline 9/13/16 7:00 & 87.109 & 78.689 & 125.555 & 1570.782 & 1558.567 & 1511.596 \\
\hline
\end{tabular}


TABLE S1.2 Automatically recorded groundwater levels in the confined aquifers.

\begin{tabular}{|c|c|c|c|c|c|c|}
\hline \multirow[b]{2}{*}{ Date-Time } & \multicolumn{3}{|c|}{ Measured Depth to Water (ft TOC ${ }^{\mathrm{a}}$ ) } & \multicolumn{3}{|c|}{ Groundwater Elevation (ft AMSL ${ }^{\mathrm{b}}$ ) } \\
\hline & DL2-CU & DL7-CU & DL7-CL & DL2-CU & DL7-CU & DL7-CL \\
\hline 9/13/16 8:00 & 87.1 & 78.673 & 125.644 & 1570.791 & 1558.583 & 1511.507 \\
\hline 9/13/16 9:00 & 87.086 & 78.668 & 125.588 & 1570.805 & 1558.588 & 1511.563 \\
\hline 9/13/16 10:00 & 87.077 & 78.65 & 125.473 & 1570.814 & 1558.606 & 1511.678 \\
\hline 9/13/16 11:00 & 87.067 & 78.645 & 125.368 & 1570.824 & 1558.611 & 1511.783 \\
\hline 9/13/16 12:00 & 87.055 & 78.631 & 125.242 & 1570.836 & 1558.625 & 1511.909 \\
\hline 9/13/16 13:00 & 87.041 & 78.631 & 125.118 & 1570.85 & 1558.625 & 1512.033 \\
\hline 9/13/16 14:00 & 87.034 & 78.643 & 124.991 & 1570.857 & 1558.613 & 1512.16 \\
\hline 9/13/16 15:00 & 87.034 & 78.657 & 124.858 & 1570.857 & 1558.599 & 1512.293 \\
\hline 9/13/16 16:00 & 87.039 & 78.668 & 124.734 & 1570.852 & 1558.588 & 1512.417 \\
\hline $9 / 13 / 16$ 17:00 & 87.043 & 78.692 & 124.603 & 1570.848 & 1558.564 & 1512.548 \\
\hline 9/13/16 18:00 & 87.055 & 78.708 & 124.486 & 1570.836 & 1558.548 & 1512.665 \\
\hline 9/13/16 19:00 & 87.06 & 78.72 & 124.366 & 1570.831 & 1558.536 & 1512.785 \\
\hline 9/13/16 20:00 & 87.06 & 78.713 & 124.247 & 1570.831 & 1558.543 & 1512.904 \\
\hline 9/13/16 21:00 & 87.06 & 78.708 & 124.137 & 1570.831 & 1558.548 & 1513.014 \\
\hline 9/13/16 22:00 & 87.053 & 78.699 & 124.029 & 1570.838 & 1558.557 & 1513.122 \\
\hline 9/13/16 23:00 & 87.044 & 78.687 & 123.926 & 1570.847 & 1558.569 & 1513.225 \\
\hline 9/14/16 0:00 & 87.029 & 78.671 & 123.819 & 1570.862 & 1558.585 & 1513.332 \\
\hline 9/14/16 1:00 & 87.013 & 78.65 & 123.718 & 1570.878 & 1558.606 & 1513.433 \\
\hline $9 / 14 / 16$ 2:00 & 86.994 & 78.631 & 123.617 & 1570.897 & 1558.625 & 1513.534 \\
\hline 9/14/16 3:00 & 86.975 & 78.608 & 123.512 & 1570.916 & 1558.648 & 1513.639 \\
\hline 9/14/16 4:00 & 86.957 & 78.587 & 123.411 & 1570.934 & 1558.669 & 1513.74 \\
\hline 9/14/16 5:00 & 86.938 & 78.573 & 123.318 & 1570.953 & 1558.683 & 1513.833 \\
\hline 9/14/16 6:00 & 86.921 & 78.552 & 123.224 & 1570.97 & 1558.704 & 1513.927 \\
\hline 9/14/16 7:00 & 86.907 & 78.536 & 123.14 & 1570.984 & 1558.72 & 1514.011 \\
\hline 9/14/16 8:00 & 86.893 & 78.522 & 123.062 & 1570.998 & 1558.734 & 1514.089 \\
\hline 9/14/16 9:00 & 86.879 & 78.515 & 122.992 & 1571.012 & 1558.741 & 1514.159 \\
\hline 9/14/16 10:00 & 86.865 & 78.489 & 122.924 & 1571.026 & 1558.767 & 1514.227 \\
\hline 9/14/16 11:00 & 86.848 & 78.476 & 122.856 & 1571.043 & 1558.78 & 1514.295 \\
\hline 9/14/16 12:00 & 86.834 & 78.455 & 122.786 & 1571.057 & 1558.801 & 1514.365 \\
\hline 9/14/16 13:00 & 86.815 & 78.434 & 122.716 & 1571.076 & 1558.822 & 1514.435 \\
\hline 9/14/16 14:00 & 86.797 & 78.415 & 122.643 & 1571.094 & 1558.841 & 1514.508 \\
\hline 9/14/16 15:00 & 86.775 & 78.385 & 122.571 & 1571.116 & 1558.871 & 1514.58 \\
\hline 9/14/16 16:00 & 86.757 & 78.364 & 122.498 & 1571.134 & 1558.892 & 1514.653 \\
\hline 9/14/16 17:00 & 86.738 & 78.338 & 122.426 & 1571.153 & 1558.918 & 1514.725 \\
\hline 9/14/16 18:00 & 86.726 & 78.318 & 122.36 & 1571.165 & 1558.938 & 1514.791 \\
\hline 9/14/16 19:00 & 86.714 & 78.297 & 122.297 & 1571.177 & 1558.959 & 1514.854 \\
\hline 9/14/16 20:00 & 86.705 & 78.278 & 122.236 & 1571.186 & 1558.978 & 1514.915 \\
\hline 9/14/16 21:00 & 86.7 & 78.266 & 122.178 & 1571.191 & 1558.99 & 1514.973 \\
\hline 9/14/16 22:00 & 86.695 & 78.248 & 122.115 & 1571.196 & 1559.008 & 1515.036 \\
\hline 9/14/16 23:00 & 86.688 & 78.234 & 122.056 & 1571.203 & 1559.022 & 1515.095 \\
\hline 9/15/16 0:00 & 86.681 & 78.217 & 121.995 & 1571.21 & 1559.039 & 1515.156 \\
\hline 9/15/16 1:00 & 86.674 & 78.192 & 121.925 & 1571.217 & 1559.064 & 1515.226 \\
\hline $9 / 15 / 16$ 2:00 & 86.663 & 78.173 & 121.855 & 1571.228 & 1559.083 & 1515.296 \\
\hline 9/15/16 3:00 & 86.651 & 78.155 & 121.782 & 1571.24 & 1559.101 & 1515.369 \\
\hline 9/15/16 4:00 & 86.637 & 78.136 & 121.707 & 1571.254 & 1559.12 & 1515.444 \\
\hline 9/15/16 5:00 & 86.623 & 78.108 & 121.632 & 1571.268 & 1559.148 & 1515.519 \\
\hline 9/15/16 6:00 & 86.611 & 78.09 & 121.553 & 1571.28 & 1559.166 & 1515.598 \\
\hline
\end{tabular}


TABLE S1.2 Automatically recorded groundwater levels in the confined aquifers.

\begin{tabular}{|c|c|c|c|c|c|c|}
\hline \multirow[b]{2}{*}{ Date-Time } & \multicolumn{3}{|c|}{ Measured Depth to Water (ft TOC ${ }^{\mathrm{a}}$ ) } & \multicolumn{3}{|c|}{ Groundwater Elevation (ft AMSL ${ }^{\mathrm{b}}$ ) } \\
\hline & DL2-CU & DL7-CU & DL7-CL & DL2-CU & DL7-CU & DL7-CL \\
\hline 9/15/16 7:00 & 86.604 & 78.071 & 121.487 & 1571.287 & 1559.185 & 1515.664 \\
\hline 9/15/16 8:00 & 86.597 & 78.055 & 121.424 & 1571.294 & 1559.201 & 1515.727 \\
\hline 9/15/16 9:00 & 86.59 & 78.039 & 121.361 & 1571.301 & 1559.217 & 1515.79 \\
\hline 9/15/16 10:00 & 86.582 & 78.022 & 121.298 & 1571.309 & 1559.234 & 1515.853 \\
\hline 9/15/16 11:00 & 86.575 & 78.011 & 121.237 & 1571.316 & 1559.245 & 1515.914 \\
\hline 9/15/16 12:00 & 86.566 & 77.997 & 121.178 & 1571.325 & 1559.259 & 1515.973 \\
\hline 9/15/16 13:00 & 86.557 & 77.976 & 121.129 & 1571.334 & 1559.28 & 1516.022 \\
\hline 9/15/16 14:00 & 86.543 & 77.952 & 121.087 & 1571.348 & 1559.304 & 1516.064 \\
\hline 9/15/16 15:00 & 86.526 & 77.929 & 121.042 & 1571.365 & 1559.327 & 1516.109 \\
\hline 9/15/16 16:00 & 86.512 & 77.908 & 121 & 1571.379 & 1559.348 & 1516.151 \\
\hline 9/15/16 17:00 & 86.495 & 77.887 & 120.958 & 1571.396 & 1559.369 & 1516.193 \\
\hline 9/15/16 18:00 & 86.481 & 77.862 & 120.909 & 1571.41 & 1559.394 & 1516.242 \\
\hline 9/15/16 19:00 & 86.47 & 77.846 & 120.874 & 1571.421 & 1559.41 & 1516.277 \\
\hline 9/15/16 20:00 & 86.463 & 77.825 & 120.829 & 1571.428 & 1559.431 & 1516.322 \\
\hline 9/15/16 21:00 & 86.448 & 77.813 & 120.792 & 1571.443 & 1559.443 & 1516.359 \\
\hline $9 / 15 / 16$ 22:00 & 86.441 & 77.799 & 120.755 & 1571.45 & 1559.457 & 1516.396 \\
\hline 9/15/16 23:00 & 86.439 & 77.792 & 120.734 & 1571.452 & 1559.464 & 1516.417 \\
\hline 9/16/16 0:00 & 86.446 & 77.785 & 120.712 & 1571.445 & 1559.471 & 1516.439 \\
\hline 9/16/16 1:00 & 86.427 & 77.762 & 120.656 & 1571.464 & 1559.494 & 1516.495 \\
\hline $9 / 16 / 16$ 2:00 & 86.404 & 77.727 & 120.584 & 1571.487 & 1559.529 & 1516.567 \\
\hline 9/16/16 3:00 & 86.387 & 77.701 & 120.521 & 1571.504 & 1559.555 & 1516.63 \\
\hline $9 / 16 / 164: 00$ & 86.373 & 77.699 & 120.492 & 1571.518 & 1559.557 & 1516.659 \\
\hline 9/16/16 5:00 & 86.366 & 77.681 & 120.453 & 1571.525 & 1559.575 & 1516.698 \\
\hline 9/16/16 6:00 & 86.354 & 77.664 & 120.403 & 1571.537 & 1559.592 & 1516.748 \\
\hline 9/16/16 7:00 & 86.343 & 77.655 & 120.357 & 1571.548 & 1559.601 & 1516.794 \\
\hline 9/16/16 8:00 & 86.338 & 77.641 & 120.319 & 1571.553 & 1559.615 & 1516.832 \\
\hline 9/16/16 9:00 & 86.328 & 77.634 & 120.296 & 1571.563 & 1559.622 & 1516.855 \\
\hline 9/16/16 10:00 & 86.324 & 77.625 & 120.282 & 1571.567 & 1559.631 & 1516.869 \\
\hline 9/16/16 11:00 & 86.319 & 77.615 & 120.373 & 1571.572 & 1559.641 & 1516.778 \\
\hline 9/16/16 12:00 & 86.31 & 77.595 & 120.429 & 1571.581 & 1559.661 & 1516.722 \\
\hline 9/16/16 13:00 & 86.3 & 77.588 & 120.45 & 1571.591 & 1559.668 & 1516.701 \\
\hline 9/16/16 14:00 & 86.293 & 77.56 & 120.521 & 1571.598 & 1559.696 & 1516.63 \\
\hline 9/16/16 15:00 & 86.279 & 77.553 & 120.495 & 1571.612 & 1559.703 & 1516.656 \\
\hline 9/16/16 16:00 & 86.267 & 77.536 & 120.619 & 1571.624 & 1559.72 & 1516.532 \\
\hline 9/16/16 17:00 & 86.253 & 77.508 & 120.619 & 1571.638 & 1559.748 & 1516.532 \\
\hline 9/16/16 18:00 & 86.239 & 77.502 & 120.577 & 1571.652 & 1559.754 & 1516.574 \\
\hline 9/16/16 19:00 & 86.23 & 77.49 & 120.691 & 1571.661 & 1559.766 & 1516.46 \\
\hline 9/16/16 20:00 & 86.22 & 77.467 & 120.684 & 1571.671 & 1559.789 & 1516.467 \\
\hline 9/16/16 21:00 & 86.213 & 77.46 & 120.614 & 1571.678 & 1559.796 & 1516.537 \\
\hline 9/16/16 22:00 & 86.206 & 77.464 & 120.64 & 1571.685 & 1559.792 & 1516.511 \\
\hline 9/16/16 23:00 & 86.199 & 77.441 & 120.698 & 1571.692 & 1559.815 & 1516.453 \\
\hline 9/17/16 0:00 & 86.192 & 77.429 & 120.631 & 1571.699 & 1559.827 & 1516.52 \\
\hline 9/17/16 1:00 & 86.185 & 77.42 & 120.546 & 1571.706 & 1559.836 & 1516.605 \\
\hline 9/17/16 2:00 & 86.178 & 77.416 & 120.453 & 1571.713 & 1559.84 & 1516.698 \\
\hline 9/17/16 3:00 & 86.166 & 77.402 & 120.347 & 1571.725 & 1559.854 & 1516.804 \\
\hline 9/17/16 4:00 & 86.15 & 77.399 & 120.31 & 1571.741 & 1559.857 & 1516.841 \\
\hline 9/17/16 5:00 & 86.138 & 77.371 & 120.333 & 1571.753 & 1559.885 & 1516.818 \\
\hline
\end{tabular}


TABLE S1.2 Automatically recorded groundwater levels in the confined aquifers.

\begin{tabular}{|c|c|c|c|c|c|c|}
\hline \multirow[b]{2}{*}{ Date-Time } & \multicolumn{3}{|c|}{ Measured Depth to Water (ft TOC ${ }^{\mathrm{a}}$ ) } & \multicolumn{3}{|c|}{ Groundwater Elevation (ft AMSL ${ }^{\mathrm{b}}$ ) } \\
\hline & DL2-CU & DL7-CU & DL7-CL & DL2-CU & DL7-CU & DL7-CL \\
\hline 9/17/16 6:00 & 86.129 & 77.36 & 120.249 & 1571.762 & 1559.896 & 1516.902 \\
\hline 9/17/16 7:00 & 86.117 & 77.346 & 120.148 & 1571.774 & 1559.91 & 1517.003 \\
\hline 9/17/16 8:00 & 86.109 & 77.348 & 120.113 & 1571.782 & 1559.908 & 1517.038 \\
\hline 9/17/16 9:00 & 86.102 & 77.327 & 120.2 & 1571.789 & 1559.929 & 1516.951 \\
\hline 9/17/16 10:00 & 86.098 & 77.323 & 120.139 & 1571.793 & 1559.933 & 1517.012 \\
\hline 9/17/16 11:00 & 86.098 & 77.329 & 120.05 & 1571.793 & 1559.927 & 1517.101 \\
\hline 9/17/16 12:00 & 86.112 & 77.383 & 119.959 & 1571.779 & 1559.873 & 1517.192 \\
\hline 9/17/16 13:00 & 86.143 & 77.45 & 119.851 & 1571.748 & 1559.806 & 1517.3 \\
\hline 9/17/16 14:00 & 86.18 & 77.506 & 119.746 & 1571.711 & 1559.75 & 1517.405 \\
\hline 9/17/16 15:00 & 86.206 & 77.536 & 119.64 & 1571.685 & 1559.72 & 1517.511 \\
\hline 9/17/16 16:00 & 86.22 & 77.553 & 119.537 & 1571.671 & 1559.703 & 1517.614 \\
\hline 9/17/16 17:00 & 86.234 & 77.585 & 119.425 & 1571.657 & 1559.671 & 1517.726 \\
\hline 9/17/16 18:00 & 86.253 & 77.611 & 119.32 & 1571.638 & 1559.645 & 1517.831 \\
\hline 9/17/16 19:00 & 86.265 & 77.62 & 119.224 & 1571.626 & 1559.636 & 1517.927 \\
\hline 9/17/16 20:00 & 86.272 & 77.632 & 119.13 & 1571.619 & 1559.624 & 1518.021 \\
\hline 9/17/16 21:00 & 86.286 & 77.664 & 119.03 & 1571.605 & 1559.592 & 1518.121 \\
\hline 9/17/16 22:00 & 86.31 & 77.701 & 118.938 & 1571.581 & 1559.555 & 1518.213 \\
\hline 9/17/16 23:00 & 86.328 & 77.715 & 118.847 & 1571.563 & 1559.541 & 1518.304 \\
\hline 9/18/16 0:00 & 86.328 & 77.708 & 118.756 & 1571.563 & 1559.548 & 1518.395 \\
\hline 9/18/16 1:00 & 86.321 & 77.69 & 118.669 & 1571.57 & 1559.566 & 1518.482 \\
\hline 9/18/16 2:00 & 86.312 & 77.674 & 118.578 & 1571.579 & 1559.582 & 1518.573 \\
\hline 9/18/16 3:00 & 86.305 & 77.678 & 118.484 & 1571.586 & 1559.578 & 1518.667 \\
\hline 9/18/16 4:00 & 86.305 & 77.69 & 118.388 & 1571.586 & 1559.566 & 1518.763 \\
\hline 9/18/16 5:00 & 86.3 & 77.676 & 118.297 & 1571.591 & 1559.58 & 1518.854 \\
\hline $9 / 18 / 16$ 6:00 & 86.288 & 77.655 & 118.201 & 1571.603 & 1559.601 & 1518.95 \\
\hline 9/18/16 7:00 & 86.274 & 77.632 & 118.114 & 1571.617 & 1559.624 & 1519.037 \\
\hline 9/18/16 8:00 & 86.26 & 77.622 & 118.03 & 1571.631 & 1559.634 & 1519.121 \\
\hline 9/18/16 9:00 & 86.26 & 77.641 & 117.941 & 1571.631 & 1559.615 & 1519.21 \\
\hline 9/18/16 10:00 & 86.27 & 77.655 & 117.859 & 1571.621 & 1559.601 & 1519.292 \\
\hline 9/18/16 11:00 & 86.272 & 77.653 & 117.782 & 1571.619 & 1559.603 & 1519.369 \\
\hline 9/18/16 12:00 & 86.267 & 77.636 & 117.695 & 1571.624 & 1559.62 & 1519.456 \\
\hline 9/18/16 13:00 & 86.258 & 77.608 & 117.611 & 1571.633 & 1559.648 & 1519.54 \\
\hline 9/18/16 14:00 & 86.253 & 77.583 & 117.534 & 1571.638 & 1559.673 & 1519.617 \\
\hline 9/18/16 15:00 & 86.246 & 77.553 & 117.45 & 1571.645 & 1559.703 & 1519.701 \\
\hline 9/18/16 16:00 & 86.239 & 77.525 & 117.37 & 1571.652 & 1559.731 & 1519.781 \\
\hline 9/18/16 17:00 & 86.227 & 77.499 & 117.29 & 1571.664 & 1559.757 & 1519.861 \\
\hline 9/18/16 18:00 & 86.213 & 77.469 & 117.208 & 1571.678 & 1559.787 & 1519.943 \\
\hline 9/18/16 19:00 & 86.206 & 77.45 & 117.131 & 1571.685 & 1559.806 & 1520.02 \\
\hline 9/18/16 20:00 & 86.204 & 77.427 & 117.054 & 1571.687 & 1559.829 & 1520.097 \\
\hline 9/18/16 21:00 & 86.201 & 77.413 & 116.981 & 1571.69 & 1559.843 & 1520.17 \\
\hline 9/18/16 22:00 & 86.199 & 77.399 & 116.914 & 1571.692 & 1559.857 & 1520.237 \\
\hline 9/18/16 23:00 & 86.197 & 77.383 & 116.843 & 1571.694 & 1559.873 & 1520.308 \\
\hline 9/19/16 0:00 & 86.194 & 77.369 & 116.775 & 1571.697 & 1559.887 & 1520.376 \\
\hline 9/19/16 1:00 & 86.194 & 77.36 & 116.705 & 1571.697 & 1559.896 & 1520.446 \\
\hline 9/19/16 2:00 & 86.187 & 77.343 & 116.637 & 1571.704 & 1559.913 & 1520.514 \\
\hline 9/19/16 3:00 & 86.173 & 77.332 & 116.567 & 1571.718 & 1559.924 & 1520.584 \\
\hline 9/19/16 4:00 & 86.159 & 77.32 & 116.495 & 1571.732 & 1559.936 & 1520.656 \\
\hline
\end{tabular}


TABLE S1.2 Automatically recorded groundwater levels in the confined aquifers.

\begin{tabular}{|c|c|c|c|c|c|c|}
\hline \multirow[b]{2}{*}{ Date-Time } & \multicolumn{3}{|c|}{ Measured Depth to Water (ft TOC ${ }^{a}$ ) } & \multicolumn{3}{|c|}{ Groundwater Elevation (ft AMSL ${ }^{\mathrm{b}}$ ) } \\
\hline & DL2-CU & DL7-CU & DL7-CL & DL2-CU & DL7-CU & DL7-CL \\
\hline 9/19/16 5:00 & 86.143 & 77.292 & 116.42 & 1571.748 & 1559.964 & 1520.731 \\
\hline 9/19/16 6:00 & 86.126 & 77.276 & 116.347 & 1571.765 & 1559.98 & 1520.804 \\
\hline 9/19/16 7:00 & 86.119 & 77.26 & 116.275 & 1571.772 & 1559.996 & 1520.876 \\
\hline 9/19/16 8:00 & 86.124 & 77.255 & 116.216 & 1571.767 & 1560.001 & 1520.935 \\
\hline 9/19/16 9:00 & 86.129 & 77.241 & 116.146 & 1571.762 & 1560.015 & 1521.005 \\
\hline 9/19/16 10:00 & 86.14 & 77.241 & 116.087 & 1571.751 & 1560.015 & 1521.064 \\
\hline 9/19/16 11:00 & 86.152 & 77.243 & 116.033 & 1571.739 & 1560.013 & 1521.118 \\
\hline 9/19/16 12:00 & 86.161 & 77.243 & 115.996 & 1571.73 & 1560.013 & 1521.155 \\
\hline 9/19/16 13:00 & 86.157 & 77.241 & 115.966 & 1571.734 & 1560.015 & 1521.185 \\
\hline 9/19/16 14:00 & 86.152 & 77.237 & 115.937 & 1571.739 & 1560.019 & 1521.214 \\
\hline 9/19/16 15:00 & 86.14 & 77.23 & 115.909 & 1571.751 & 1560.026 & 1521.242 \\
\hline 9/19/16 16:00 & 86.126 & 77.218 & 115.898 & 1571.765 & 1560.038 & 1521.253 \\
\hline 9/19/16 17:00 & 86.107 & 77.206 & 115.893 & 1571.784 & 1560.05 & 1521.258 \\
\hline 9/19/16 18:00 & 86.089 & 77.19 & 115.884 & 1571.802 & 1560.066 & 1521.267 \\
\hline 9/19/16 19:00 & 86.065 & 77.176 & 115.867 & 1571.826 & 1560.08 & 1521.284 \\
\hline 9/19/16 20:00 & 86.044 & 77.157 & 115.856 & 1571.847 & 1560.099 & 1521.295 \\
\hline 9/19/16 21:00 & 86.025 & 77.144 & 115.865 & 1571.866 & 1560.112 & 1521.286 \\
\hline 9/19/16 22:00 & 86.006 & 77.132 & 115.874 & 1571.885 & 1560.124 & 1521.277 \\
\hline 9/19/16 23:00 & 85.99 & 77.12 & 115.891 & 1571.901 & 1560.136 & 1521.26 \\
\hline 9/20/16 0:00 & 85.971 & 77.102 & 115.895 & 1571.92 & 1560.154 & 1521.256 \\
\hline 9/20/16 1:00 & 85.954 & 77.085 & 115.888 & 1571.937 & 1560.171 & 1521.263 \\
\hline $9 / 20 / 16$ 2:00 & 85.938 & 77.064 & 115.874 & 1571.953 & 1560.192 & 1521.277 \\
\hline 9/20/16 3:00 & 85.919 & 77.053 & 115.853 & 1571.972 & 1560.203 & 1521.298 \\
\hline 9/20/16 4:00 & 85.895 & 77.034 & 115.827 & 1571.996 & 1560.222 & 1521.324 \\
\hline 9/20/16 5:00 & 85.877 & 77.02 & 115.818 & 1572.014 & 1560.236 & 1521.333 \\
\hline 9/20/16 6:00 & 85.856 & 76.999 & 115.813 & 1572.035 & 1560.257 & 1521.338 \\
\hline 9/20/16 7:00 & 85.832 & 76.978 & 115.809 & 1572.059 & 1560.278 & 1521.342 \\
\hline 9/20/16 8:00 & 85.813 & 76.96 & 115.792 & 1572.078 & 1560.296 & 1521.359 \\
\hline 9/20/16 9:00 & 85.792 & 76.951 & 115.835 & 1572.099 & 1560.305 & 1521.316 \\
\hline 9/20/16 10:00 & 85.776 & 76.934 & 116.012 & 1572.115 & 1560.322 & 1521.139 \\
\hline 9/20/16 11:00 & 85.757 & 76.909 & 116.207 & 1572.134 & 1560.347 & 1520.944 \\
\hline 9/20/16 12:00 & 85.74 & 76.885 & 116.312 & 1572.151 & 1560.371 & 1520.839 \\
\hline 9/20/16 13:00 & 85.722 & 76.872 & 116.441 & 1572.169 & 1560.384 & 1520.71 \\
\hline 9/20/16 14:00 & 85.707 & 76.853 & 116.616 & 1572.184 & 1560.403 & 1520.535 \\
\hline 9/20/16 15:00 & 85.689 & 76.83 & 116.789 & 1572.202 & 1560.426 & 1520.362 \\
\hline 9/20/16 16:00 & 85.672 & 76.811 & 116.839 & 1572.219 & 1560.445 & 1520.312 \\
\hline 9/20/16 17:00 & 85.656 & 76.804 & 116.829 & 1572.235 & 1560.452 & 1520.322 \\
\hline 9/20/16 18:00 & 85.639 & 76.793 & 116.939 & 1572.252 & 1560.463 & 1520.212 \\
\hline 9/20/16 19:00 & 85.625 & 76.774 & 117.084 & 1572.266 & 1560.482 & 1520.067 \\
\hline 9/20/16 20:00 & 85.609 & 76.76 & 117.234 & 1572.282 & 1560.496 & 1519.917 \\
\hline 9/20/16 21:00 & 85.597 & 76.748 & 117.389 & 1572.294 & 1560.508 & 1519.762 \\
\hline 9/20/16 22:00 & 85.585 & 76.727 & 117.501 & 1572.306 & 1560.529 & 1519.65 \\
\hline 9/20/16 23:00 & 85.573 & 76.716 & 117.482 & 1572.318 & 1560.54 & 1519.669 \\
\hline $9 / 21 / 160: 00$ & 85.562 & 76.707 & 117.431 & 1572.329 & 1560.549 & 1519.72 \\
\hline 9/21/16 1:00 & 85.55 & 76.695 & 117.368 & 1572.341 & 1560.561 & 1519.783 \\
\hline $9 / 21 / 16$ 2:00 & 85.538 & 76.697 & 117.386 & 1572.353 & 1560.559 & 1519.765 \\
\hline 9/21/16 3:00 & 85.526 & 76.69 & 117.496 & 1572.365 & 1560.566 & 1519.655 \\
\hline
\end{tabular}


TABLE S1.2 Automatically recorded groundwater levels in the confined aquifers.

\begin{tabular}{|c|c|c|c|c|c|c|}
\hline \multirow[b]{2}{*}{ Date-Time } & \multicolumn{3}{|c|}{ Measured Depth to Water (ft TOC ${ }^{\mathrm{a}}$ ) } & \multicolumn{3}{|c|}{ Groundwater Elevation (ft AMSL ${ }^{\mathrm{b}}$ ) } \\
\hline & DL2-CU & DL7-CU & DL7-CL & DL2-CU & DL7-CU & DL7-CL \\
\hline $9 / 21 / 164: 00$ & 85.514 & 76.676 & 117.618 & 1572.377 & 1560.58 & 1519.533 \\
\hline $9 / 21 / 16$ 5:00 & 85.503 & 76.662 & 117.744 & 1572.388 & 1560.594 & 1519.407 \\
\hline $9 / 21 / 16$ 6:00 & 85.491 & 76.648 & 117.869 & 1572.4 & 1560.608 & 1519.282 \\
\hline 9/21/16 7:00 & 85.475 & 76.632 & 117.99 & 1572.416 & 1560.624 & 1519.161 \\
\hline 9/21/16 8:00 & 85.463 & 76.618 & 118.119 & 1572.428 & 1560.638 & 1519.032 \\
\hline 9/21/16 9:00 & 85.453 & 76.597 & 118.185 & 1572.438 & 1560.659 & 1518.966 \\
\hline 9/21/16 10:00 & 85.458 & 76.586 & 118.133 & 1572.433 & 1560.67 & 1519.018 \\
\hline 9/21/16 11:00 & 85.491 & 76.576 & 118.058 & 1572.4 & 1560.68 & 1519.093 \\
\hline 9/21/16 12:00 & 85.538 & 76.569 & 117.967 & 1572.353 & 1560.687 & 1519.184 \\
\hline 9/21/16 13:00 & 85.578 & 76.562 & 117.869 & 1572.313 & 1560.694 & 1519.282 \\
\hline 9/21/16 14:00 & 85.623 & 76.558 & 117.766 & 1572.268 & 1560.698 & 1519.385 \\
\hline 9/21/16 15:00 & 85.672 & 76.548 & 117.658 & 1572.219 & 1560.708 & 1519.493 \\
\hline 9/21/16 16:00 & 85.705 & 76.544 & 117.55 & 1572.186 & 1560.712 & 1519.601 \\
\hline 9/21/16 17:00 & 85.714 & 76.539 & 117.443 & 1572.177 & 1560.717 & 1519.708 \\
\hline 9/21/16 18:00 & 85.703 & 76.53 & 117.328 & 1572.188 & 1560.726 & 1519.823 \\
\hline 9/21/16 19:00 & 85.684 & 76.528 & 117.23 & 1572.207 & 1560.728 & 1519.921 \\
\hline 9/21/16 20:00 & 85.658 & 76.525 & 117.124 & 1572.233 & 1560.731 & 1520.027 \\
\hline 9/21/16 21:00 & 85.632 & 76.525 & 117.031 & 1572.259 & 1560.731 & 1520.12 \\
\hline $9 / 21 / 16$ 22:00 & 85.606 & 76.518 & 116.939 & 1572.285 & 1560.738 & 1520.212 \\
\hline 9/21/16 23:00 & 85.578 & 76.514 & 116.855 & 1572.313 & 1560.742 & 1520.296 \\
\hline 9/22/16 0:00 & 85.555 & 76.504 & 116.778 & 1572.336 & 1560.752 & 1520.373 \\
\hline $9 / 22 / 16$ 1:00 & 85.531 & 76.497 & 116.701 & 1572.36 & 1560.759 & 1520.45 \\
\hline $9 / 22 / 162: 00$ & 85.507 & 76.49 & 116.623 & 1572.384 & 1560.766 & 1520.528 \\
\hline 9/22/16 3:00 & 85.486 & 76.486 & 116.546 & 1572.405 & 1560.77 & 1520.605 \\
\hline $9 / 22 / 164: 00$ & 85.463 & 76.476 & 116.481 & 1572.428 & 1560.78 & 1520.67 \\
\hline 9/22/16 5:00 & 85.446 & 76.469 & 116.413 & 1572.445 & 1560.787 & 1520.738 \\
\hline $9 / 22 / 16$ 6:00 & 85.43 & 76.462 & 116.349 & 1572.461 & 1560.794 & 1520.802 \\
\hline 9/22/16 7:00 & 85.409 & 76.458 & 116.282 & 1572.482 & 1560.798 & 1520.869 \\
\hline $9 / 22 / 168: 00$ & 85.392 & 76.453 & 116.216 & 1572.499 & 1560.803 & 1520.935 \\
\hline 9/22/16 9:00 & 85.373 & 76.439 & 116.162 & 1572.518 & 1560.817 & 1520.989 \\
\hline 9/22/16 10:00 & 85.357 & 76.43 & 116.106 & 1572.534 & 1560.826 & 1521.045 \\
\hline 9/22/16 11:00 & 85.343 & 76.425 & 116.059 & 1572.548 & 1560.831 & 1521.092 \\
\hline 9/22/16 12:00 & 85.331 & 76.411 & 116.019 & 1572.56 & 1560.845 & 1521.132 \\
\hline 9/22/16 13:00 & 85.319 & 76.404 & 115.989 & 1572.572 & 1560.852 & 1521.162 \\
\hline 9/22/16 14:00 & 85.305 & 76.395 & 115.961 & 1572.586 & 1560.861 & 1521.19 \\
\hline $9 / 22 / 16$ 15:00 & 85.293 & 76.386 & 115.935 & 1572.598 & 1560.87 & 1521.216 \\
\hline $9 / 22 / 16$ 16:00 & 85.282 & 76.374 & 115.905 & 1572.609 & 1560.882 & 1521.246 \\
\hline $9 / 22 / 16$ 17:00 & 85.27 & 76.362 & 115.874 & 1572.621 & 1560.894 & 1521.277 \\
\hline $9 / 22 / 16$ 18:00 & 85.263 & 76.355 & 115.839 & 1572.628 & 1560.901 & 1521.312 \\
\hline 9/22/16 19:00 & 85.253 & 76.349 & 115.809 & 1572.638 & 1560.907 & 1521.342 \\
\hline $9 / 22 / 16$ 20:00 & 85.242 & 76.339 & 115.785 & 1572.649 & 1560.917 & 1521.366 \\
\hline $9 / 22 / 16$ 21:00 & 85.232 & 76.337 & 115.762 & 1572.659 & 1560.919 & 1521.389 \\
\hline $9 / 22 / 1622: 00$ & 85.225 & 76.328 & 115.736 & 1572.666 & 1560.928 & 1521.415 \\
\hline 9/22/16 23:00 & 85.213 & 76.318 & 115.708 & 1572.678 & 1560.938 & 1521.443 \\
\hline 9/23/16 0:00 & 85.206 & 76.311 & 115.675 & 1572.685 & 1560.945 & 1521.476 \\
\hline 9/23/16 1:00 & 85.197 & 76.304 & 115.638 & 1572.694 & 1560.952 & 1521.513 \\
\hline $9 / 23 / 16$ 2:00 & 85.19 & 76.297 & 115.605 & 1572.701 & 1560.959 & 1521.546 \\
\hline
\end{tabular}


TABLE S1.2 Automatically recorded groundwater levels in the confined aquifers.

\begin{tabular}{|c|c|c|c|c|c|c|}
\hline \multirow[b]{2}{*}{ Date-Time } & \multicolumn{3}{|c|}{ Measured Depth to Water (ft TOC ${ }^{\mathrm{a}}$ ) } & \multicolumn{3}{|c|}{ Groundwater Elevation (ft AMSL ${ }^{\mathrm{b}}$ ) } \\
\hline & DL2-CU & DL7-CU & DL7-CL & DL2-CU & DL7-CU & DL7-CL \\
\hline 9/23/16 3:00 & 85.183 & 76.295 & 115.572 & 1572.708 & 1560.961 & 1521.579 \\
\hline $9 / 23 / 164: 00$ & 85.176 & 76.288 & 115.537 & 1572.715 & 1560.968 & 1521.614 \\
\hline 9/23/16 5:00 & 85.169 & 76.283 & 115.497 & 1572.722 & 1560.973 & 1521.654 \\
\hline 9/23/16 6:00 & 85.164 & 76.279 & 115.467 & 1572.727 & 1560.977 & 1521.684 \\
\hline 9/23/16 7:00 & 85.159 & 76.276 & 115.437 & 1572.732 & 1560.98 & 1521.714 \\
\hline 9/23/16 8:00 & 85.155 & 76.269 & 115.413 & 1572.736 & 1560.987 & 1521.738 \\
\hline 9/23/16 9:00 & 85.145 & 76.269 & 115.394 & 1572.746 & 1560.987 & 1521.757 \\
\hline 9/23/16 10:00 & 85.145 & 76.286 & 115.401 & 1572.746 & 1560.97 & 1521.75 \\
\hline 9/23/16 11:00 & 85.162 & 76.332 & 115.425 & 1572.729 & 1560.924 & 1521.726 \\
\hline 9/23/16 12:00 & 85.187 & 76.39 & 115.441 & 1572.704 & 1560.866 & 1521.71 \\
\hline 9/23/16 13:00 & 85.218 & 76.437 & 115.46 & 1572.673 & 1560.819 & 1521.691 \\
\hline 9/23/16 14:00 & 85.242 & 76.467 & 115.497 & 1572.649 & 1560.789 & 1521.654 \\
\hline 9/23/16 15:00 & 85.253 & 76.483 & 115.547 & 1572.638 & 1560.773 & 1521.604 \\
\hline 9/23/16 16:00 & 85.256 & 76.479 & 115.607 & 1572.635 & 1560.777 & 1521.544 \\
\hline 9/23/16 17:00 & 85.251 & 76.469 & 115.659 & 1572.64 & 1560.787 & 1521.492 \\
\hline $9 / 23 / 16$ 18:00 & 85.239 & 76.458 & 115.692 & 1572.652 & 1560.798 & 1521.459 \\
\hline 9/23/16 19:00 & 85.223 & 76.444 & 115.734 & 1572.668 & 1560.812 & 1521.417 \\
\hline $9 / 23 / 16$ 20:00 & 85.209 & 76.432 & 115.797 & 1572.682 & 1560.824 & 1521.354 \\
\hline 9/23/16 21:00 & 85.188 & 76.418 & 115.856 & 1572.703 & 1560.838 & 1521.295 \\
\hline $9 / 23 / 1622: 00$ & 85.169 & 76.402 & 115.919 & 1572.722 & 1560.854 & 1521.232 \\
\hline 9/23/16 23:00 & 85.148 & 76.388 & 115.98 & 1572.743 & 1560.868 & 1521.171 \\
\hline 9/24/16 0:00 & 85.124 & 76.367 & 116.038 & 1572.767 & 1560.889 & 1521.113 \\
\hline 9/24/16 1:00 & 85.103 & 76.346 & 116.064 & 1572.788 & 1560.91 & 1521.087 \\
\hline $9 / 24 / 16$ 2:00 & 85.079 & 76.335 & 116.08 & 1572.812 & 1560.921 & 1521.071 \\
\hline 9/24/16 3:00 & 85.06 & 76.323 & 116.113 & 1572.831 & 1560.933 & 1521.038 \\
\hline 9/24/16 4:00 & 85.037 & 76.304 & 116.153 & 1572.854 & 1560.952 & 1520.998 \\
\hline 9/24/16 5:00 & 85.02 & 76.297 & 116.193 & 1572.871 & 1560.959 & 1520.958 \\
\hline 9/24/16 6:00 & 84.999 & 76.286 & 116.239 & 1572.892 & 1560.97 & 1520.912 \\
\hline 9/24/16 7:00 & 84.98 & 76.265 & 116.286 & 1572.911 & 1560.991 & 1520.865 \\
\hline 9/24/16 8:00 & 84.959 & 76.235 & 116.3 & 1572.932 & 1561.021 & 1520.851 \\
\hline 9/24/16 9:00 & 84.938 & 76.218 & 116.293 & 1572.953 & 1561.038 & 1520.858 \\
\hline 9/24/16 10:00 & 84.919 & 76.204 & 116.268 & 1572.972 & 1561.052 & 1520.883 \\
\hline 9/24/16 11:00 & 84.905 & 76.195 & 116.23 & 1572.986 & 1561.061 & 1520.921 \\
\hline 9/24/16 12:00 & 84.891 & 76.186 & 116.186 & 1573 & 1561.07 & 1520.965 \\
\hline 9/24/16 13:00 & 84.858 & 76.165 & 116.125 & 1573.033 & 1561.091 & 1521.026 \\
\hline 9/24/16 14:00 & 84.835 & 76.146 & 116.059 & 1573.056 & 1561.11 & 1521.092 \\
\hline 9/24/16 15:00 & 84.818 & 76.132 & 115.989 & 1573.073 & 1561.124 & 1521.162 \\
\hline 9/24/16 16:00 & 84.806 & 76.125 & 115.923 & 1573.085 & 1561.131 & 1521.228 \\
\hline 9/24/16 17:00 & 84.795 & 76.118 & 115.86 & 1573.096 & 1561.138 & 1521.291 \\
\hline 9/24/16 18:00 & 84.781 & 76.107 & 115.79 & 1573.11 & 1561.149 & 1521.361 \\
\hline 9/24/16 19:00 & 84.766 & 76.102 & 115.724 & 1573.125 & 1561.154 & 1521.427 \\
\hline 9/24/16 20:00 & 84.755 & 76.097 & 115.652 & 1573.136 & 1561.159 & 1521.499 \\
\hline 9/24/16 21:00 & 84.75 & 76.093 & 115.584 & 1573.141 & 1561.163 & 1521.567 \\
\hline 9/24/16 22:00 & 84.741 & 76.086 & 115.519 & 1573.15 & 1561.17 & 1521.632 \\
\hline 9/24/16 23:00 & 84.726 & 76.084 & 115.448 & 1573.165 & 1561.172 & 1521.703 \\
\hline 9/25/16 0:00 & 84.715 & 76.074 & 115.38 & 1573.176 & 1561.182 & 1521.771 \\
\hline 9/25/16 1:00 & 84.705 & 76.067 & 115.317 & 1573.186 & 1561.189 & 1521.834 \\
\hline
\end{tabular}


TABLE S1.2 Automatically recorded groundwater levels in the confined aquifers.

\begin{tabular}{|c|c|c|c|c|c|c|}
\hline \multirow[b]{2}{*}{ Date-Time } & \multicolumn{3}{|c|}{ Measured Depth to Water (ft TOC ${ }^{a}$ ) } & \multicolumn{3}{|c|}{ Groundwater Elevation (ft AMSL ${ }^{\mathrm{b}}$ ) } \\
\hline & DL2-CU & DL7-CU & DL7-CL & DL2-CU & DL7-CU & DL7-CL \\
\hline $9 / 25 / 16$ 2:00 & 84.701 & 76.063 & 115.254 & 1573.19 & 1561.193 & 1521.897 \\
\hline 9/25/16 3:00 & 84.696 & 76.067 & 115.2 & 1573.195 & 1561.189 & 1521.951 \\
\hline $9 / 25 / 164: 00$ & 84.689 & 76.056 & 115.135 & 1573.202 & 1561.2 & 1522.016 \\
\hline 9/25/16 5:00 & 84.682 & 76.056 & 115.071 & 1573.209 & 1561.2 & 1522.08 \\
\hline 9/25/16 6:00 & 84.682 & 76.058 & 115.008 & 1573.209 & 1561.198 & 1522.143 \\
\hline 9/25/16 7:00 & 84.675 & 76.051 & 114.94 & 1573.216 & 1561.205 & 1522.211 \\
\hline 9/25/16 8:00 & 84.672 & 76.053 & 114.872 & 1573.219 & 1561.203 & 1522.279 \\
\hline 9/25/16 9:00 & 84.668 & 76.058 & 114.802 & 1573.223 & 1561.198 & 1522.349 \\
\hline 9/25/16 10:00 & 84.663 & 76.058 & 114.741 & 1573.228 & 1561.198 & 1522.41 \\
\hline 9/25/16 11:00 & 84.654 & 76.056 & 114.709 & 1573.237 & 1561.2 & 1522.442 \\
\hline $9 / 25 / 16$ 12:00 & 84.647 & 76.046 & 114.671 & 1573.244 & 1561.21 & 1522.48 \\
\hline 9/25/16 13:00 & 84.637 & 76.039 & 114.627 & 1573.254 & 1561.217 & 1522.524 \\
\hline 9/25/16 14:00 & 84.628 & 76.035 & 114.578 & 1573.263 & 1561.221 & 1522.573 \\
\hline 9/25/16 15:00 & 84.618 & 76.025 & 114.542 & 1573.273 & 1561.231 & 1522.609 \\
\hline 9/25/16 16:00 & 84.609 & 76.018 & 114.517 & 1573.282 & 1561.238 & 1522.634 \\
\hline 9/25/16 17:00 & 84.599 & 76.007 & 114.489 & 1573.292 & 1561.249 & 1522.662 \\
\hline 9/25/16 18:00 & 84.594 & 76.007 & 114.458 & 1573.297 & 1561.249 & 1522.693 \\
\hline 9/25/16 19:00 & 84.592 & 76.007 & 114.435 & 1573.299 & 1561.249 & 1522.716 \\
\hline $9 / 25 / 16$ 20:00 & 84.588 & 76.009 & 114.423 & 1573.303 & 1561.247 & 1522.728 \\
\hline 9/25/16 21:00 & 84.585 & 76.007 & 114.416 & 1573.306 & 1561.249 & 1522.735 \\
\hline $9 / 25 / 16$ 22:00 & 84.583 & 76.004 & 114.402 & 1573.308 & 1561.252 & 1522.749 \\
\hline 9/25/16 23:00 & 84.576 & 75.995 & 114.372 & 1573.315 & 1561.261 & 1522.779 \\
\hline 9/26/16 0:00 & 84.569 & 75.995 & 114.341 & 1573.322 & 1561.261 & 1522.81 \\
\hline 9/26/16 1:00 & 84.562 & 75.988 & 114.313 & 1573.329 & 1561.268 & 1522.838 \\
\hline $9 / 26 / 16$ 2:00 & 84.552 & 75.984 & 114.285 & 1573.339 & 1561.272 & 1522.866 \\
\hline 9/26/16 3:00 & 84.545 & 75.977 & 114.243 & 1573.346 & 1561.279 & 1522.908 \\
\hline 9/26/16 4:00 & 84.536 & 75.97 & 114.196 & 1573.355 & 1561.286 & 1522.955 \\
\hline 9/26/16 5:00 & 84.531 & 75.97 & 114.159 & 1573.36 & 1561.286 & 1522.992 \\
\hline 9/26/16 6:00 & 84.527 & 75.967 & 114.131 & 1573.364 & 1561.289 & 1523.02 \\
\hline 9/26/16 7:00 & 84.522 & 75.967 & 114.123 & 1573.369 & 1561.289 & 1523.028 \\
\hline 9/26/16 8:00 & 84.519 & 75.965 & 114.107 & 1573.372 & 1561.291 & 1523.044 \\
\hline 9/26/16 9:00 & 84.512 & 75.958 & 114.105 & 1573.379 & 1561.298 & 1523.046 \\
\hline 9/26/16 10:00 & 84.51 & 75.965 & 114.17 & 1573.381 & 1561.291 & 1522.981 \\
\hline 9/26/16 11:00 & 84.505 & 75.956 & 114.341 & 1573.386 & 1561.3 & 1522.81 \\
\hline $9 / 26 / 16$ 12:00 & 84.496 & 75.939 & 114.503 & 1573.395 & 1561.317 & 1522.648 \\
\hline 9/26/16 13:00 & 84.484 & 75.928 & 114.669 & 1573.407 & 1561.328 & 1522.482 \\
\hline 9/26/16 14:00 & 84.472 & 75.907 & 114.786 & 1573.419 & 1561.349 & 1522.365 \\
\hline $9 / 26 / 16$ 15:00 & 84.458 & 75.898 & 114.784 & 1573.433 & 1561.358 & 1522.367 \\
\hline $9 / 26 / 16$ 16:00 & 84.444 & 75.891 & 114.898 & 1573.447 & 1561.365 & 1522.253 \\
\hline 9/26/16 17:00 & 84.435 & 75.881 & 115.032 & 1573.456 & 1561.375 & 1522.119 \\
\hline 9/26/16 18:00 & 84.423 & 75.872 & 115.165 & 1573.468 & 1561.384 & 1521.986 \\
\hline 9/26/16 19:00 & 84.414 & 75.856 & 115.221 & 1573.477 & 1561.4 & 1521.93 \\
\hline $9 / 26 / 16$ 20:00 & 84.411 & 75.858 & 115.184 & 1573.48 & 1561.398 & 1521.967 \\
\hline 9/26/16 21:00 & 84.406 & 75.865 & 115.259 & 1573.485 & 1561.391 & 1521.892 \\
\hline 9/26/16 22:00 & 84.402 & 75.86 & 115.378 & 1573.489 & 1561.396 & 1521.773 \\
\hline 9/26/16 23:00 & 84.395 & 75.853 & 115.481 & 1573.496 & 1561.403 & 1521.67 \\
\hline 9/27/16 0:00 & 84.385 & 75.847 & 115.561 & 1573.506 & 1561.409 & 1521.59 \\
\hline
\end{tabular}


TABLE S1.2 Automatically recorded groundwater levels in the confined aquifers.

\begin{tabular}{|c|c|c|c|c|c|c|}
\hline \multirow[b]{2}{*}{ Date-Time } & \multicolumn{3}{|c|}{ Measured Depth to Water (ft TOC ${ }^{\mathrm{a}}$ ) } & \multicolumn{3}{|c|}{ Groundwater Elevation (ft AMSL ${ }^{\mathrm{b}}$ ) } \\
\hline & DL2-CU & DL7-CU & DL7-CL & DL2-CU & DL7-CU & DL7-CL \\
\hline 9/27/16 1:00 & 84.376 & 75.839 & 115.661 & 1573.515 & 1561.417 & 1521.49 \\
\hline $9 / 27 / 16$ 2:00 & 84.369 & 75.832 & 115.764 & 1573.522 & 1561.424 & 1521.387 \\
\hline 9/27/16 3:00 & 84.362 & 75.821 & 115.851 & 1573.529 & 1561.435 & 1521.3 \\
\hline 9/27/16 4:00 & 84.355 & 75.809 & 115.809 & 1573.536 & 1561.447 & 1521.342 \\
\hline 9/27/16 5:00 & 84.35 & 75.812 & 115.736 & 1573.541 & 1561.444 & 1521.415 \\
\hline 9/27/16 6:00 & 84.345 & 75.809 & 115.792 & 1573.546 & 1561.447 & 1521.359 \\
\hline 9/27/16 7:00 & 84.345 & 75.809 & 115.877 & 1573.546 & 1561.447 & 1521.274 \\
\hline 9/27/16 8:00 & 84.345 & 75.814 & 115.968 & 1573.546 & 1561.442 & 1521.183 \\
\hline 9/27/16 9:00 & 84.357 & 75.844 & 115.937 & 1573.534 & 1561.412 & 1521.214 \\
\hline 9/27/16 10:00 & 84.381 & 75.893 & 115.839 & 1573.51 & 1561.363 & 1521.312 \\
\hline 9/27/16 11:00 & 84.407 & 75.935 & 115.727 & 1573.484 & 1561.321 & 1521.424 \\
\hline 9/27/16 12:00 & 84.425 & 75.965 & 115.598 & 1573.466 & 1561.291 & 1521.553 \\
\hline 9/27/16 13:00 & 84.442 & 75.991 & 115.472 & 1573.449 & 1561.265 & 1521.679 \\
\hline 9/27/16 14:00 & 84.449 & 75.997 & 115.345 & 1573.442 & 1561.259 & 1521.806 \\
\hline 9/27/16 15:00 & 84.444 & 75.988 & 115.219 & 1573.447 & 1561.268 & 1521.932 \\
\hline 9/27/16 16:00 & 84.442 & 75.984 & 115.095 & 1573.449 & 1561.272 & 1522.056 \\
\hline 9/27/16 17:00 & 84.43 & 75.972 & 114.983 & 1573.461 & 1561.284 & 1522.168 \\
\hline 9/27/16 18:00 & 84.418 & 75.96 & 114.877 & 1573.473 & 1561.296 & 1522.274 \\
\hline 9/27/16 19:00 & 84.409 & 75.946 & 114.774 & 1573.482 & 1561.31 & 1522.377 \\
\hline $9 / 27 / 16$ 20:00 & 84.397 & 75.935 & 114.681 & 1573.494 & 1561.321 & 1522.47 \\
\hline 9/27/16 21:00 & 84.392 & 75.93 & 114.594 & 1573.499 & 1561.326 & 1522.557 \\
\hline $9 / 27 / 1622: 00$ & 84.39 & 75.923 & 114.51 & 1573.501 & 1561.333 & 1522.641 \\
\hline 9/27/16 23:00 & 84.381 & 75.914 & 114.423 & 1573.51 & 1561.342 & 1522.728 \\
\hline 9/28/16 0:00 & 84.373 & 75.907 & 114.344 & 1573.518 & 1561.349 & 1522.807 \\
\hline $9 / 28 / 16$ 1:00 & 84.364 & 75.9 & 114.262 & 1573.527 & 1561.356 & 1522.889 \\
\hline $9 / 28 / 16$ 2:00 & 84.357 & 75.884 & 114.187 & 1573.534 & 1561.372 & 1522.964 \\
\hline 9/28/16 3:00 & 84.35 & 75.877 & 114.116 & 1573.541 & 1561.379 & 1523.035 \\
\hline $9 / 28 / 164: 00$ & 84.348 & 75.87 & 114.046 & 1573.543 & 1561.386 & 1523.105 \\
\hline 9/28/16 5:00 & 84.348 & 75.865 & 113.976 & 1573.543 & 1561.391 & 1523.175 \\
\hline 9/28/16 6:00 & 84.345 & 75.86 & 113.913 & 1573.546 & 1561.396 & 1523.238 \\
\hline 9/28/16 7:00 & 84.348 & 75.856 & 113.847 & 1573.543 & 1561.4 & 1523.304 \\
\hline 9/28/16 8:00 & 84.355 & 75.86 & 113.786 & 1573.536 & 1561.396 & 1523.365 \\
\hline 9/28/16 9:00 & 84.362 & 75.863 & 113.73 & 1573.529 & 1561.393 & 1523.421 \\
\hline 9/28/16 10:00 & 84.369 & 75.87 & 113.683 & 1573.522 & 1561.386 & 1523.468 \\
\hline 9/28/16 11:00 & 84.374 & 75.874 & 113.66 & 1573.517 & 1561.382 & 1523.491 \\
\hline 9/28/16 12:00 & 84.376 & 75.872 & 113.646 & 1573.515 & 1561.384 & 1523.505 \\
\hline 9/28/16 13:00 & 84.376 & 75.867 & 113.637 & 1573.515 & 1561.389 & 1523.514 \\
\hline 9/28/16 14:00 & 84.376 & 75.858 & 113.62 & 1573.515 & 1561.398 & 1523.531 \\
\hline 9/28/16 15:00 & 84.369 & 75.853 & 113.611 & 1573.522 & 1561.403 & 1523.54 \\
\hline 9/28/16 16:00 & 84.369 & 75.851 & 113.606 & 1573.522 & 1561.405 & 1523.545 \\
\hline 9/28/16 17:00 & 84.367 & 75.849 & 113.609 & 1573.524 & 1561.407 & 1523.542 \\
\hline 9/28/16 18:00 & 84.367 & 75.844 & 113.62 & 1573.524 & 1561.412 & 1523.531 \\
\hline 9/28/16 19:00 & 84.367 & 75.846 & 113.634 & 1573.524 & 1561.41 & 1523.517 \\
\hline 9/28/16 20:00 & 84.367 & 75.844 & 113.653 & 1573.524 & 1561.412 & 1523.498 \\
\hline 9/28/16 21:00 & 84.371 & 75.846 & 113.674 & 1573.52 & 1561.41 & 1523.477 \\
\hline 9/28/16 22:00 & 84.385 & 75.849 & 113.695 & 1573.506 & 1561.407 & 1523.456 \\
\hline $9 / 28 / 16$ 23:00 & 84.421 & 75.851 & 113.709 & 1573.47 & 1561.405 & 1523.442 \\
\hline
\end{tabular}


TABLE S1.2 Automatically recorded groundwater levels in the confined aquifers.

\begin{tabular}{|c|c|c|c|c|c|c|}
\hline \multirow[b]{2}{*}{ Date-Time } & \multicolumn{3}{|c|}{ Measured Depth to Water (ft TOC ${ }^{\mathrm{a}}$ ) } & \multicolumn{3}{|c|}{ Groundwater Elevation (ft AMSL ${ }^{\mathrm{b}}$ ) } \\
\hline & DL2-CU & DL7-CU & DL7-CL & DL2-CU & DL7-CU & DL7-CL \\
\hline 9/29/16 0:00 & 84.475 & 75.846 & 113.726 & 1573.416 & 1561.41 & 1523.425 \\
\hline 9/29/16 1:00 & 84.517 & 75.844 & 113.737 & 1573.374 & 1561.412 & 1523.414 \\
\hline 9/29/16 2:00 & 84.538 & 75.839 & 113.735 & 1573.353 & 1561.417 & 1523.416 \\
\hline 9/29/16 3:00 & 84.543 & 75.837 & 113.719 & 1573.348 & 1561.419 & 1523.432 \\
\hline 9/29/16 4:00 & 84.541 & 75.835 & 113.714 & 1573.35 & 1561.421 & 1523.437 \\
\hline 9/29/16 5:00 & 84.534 & 75.835 & 113.714 & 1573.357 & 1561.421 & 1523.437 \\
\hline 9/29/16 6:00 & 84.524 & 75.837 & 113.719 & 1573.367 & 1561.419 & 1523.432 \\
\hline 9/29/16 7:00 & 84.519 & 75.842 & 113.73 & 1573.372 & 1561.414 & 1523.421 \\
\hline 9/29/16 8:00 & 84.51 & 75.844 & 113.74 & 1573.381 & 1561.412 & 1523.411 \\
\hline 9/29/16 9:00 & 84.505 & 75.851 & 113.756 & 1573.386 & 1561.405 & 1523.395 \\
\hline 9/29/16 10:00 & 84.5 & 75.851 & 113.768 & 1573.391 & 1561.405 & 1523.383 \\
\hline 9/29/16 11:00 & 84.494 & 75.853 & 113.768 & 1573.397 & 1561.403 & 1523.383 \\
\hline 9/29/16 12:00 & 84.487 & 75.853 & 113.854 & 1573.404 & 1561.403 & 1523.297 \\
\hline 9/29/16 13:00 & 84.479 & 75.846 & 114.016 & 1573.412 & 1561.41 & 1523.135 \\
\hline 9/29/16 14:00 & 84.468 & 75.832 & 114.131 & 1573.423 & 1561.424 & 1523.02 \\
\hline 9/29/16 15:00 & 84.454 & 75.816 & 114.114 & 1573.437 & 1561.44 & 1523.037 \\
\hline 9/29/16 16:00 & 84.444 & 75.816 & 114.142 & 1573.447 & 1561.44 & 1523.009 \\
\hline 9/29/16 17:00 & 84.435 & 75.809 & 114.269 & 1573.456 & 1561.447 & 1522.882 \\
\hline 9/29/16 18:00 & 84.428 & 75.802 & 114.409 & 1573.463 & 1561.454 & 1522.742 \\
\hline 9/29/16 19:00 & 84.423 & 75.795 & 114.554 & 1573.468 & 1561.461 & 1522.597 \\
\hline 9/29/16 20:00 & 84.423 & 75.795 & 114.695 & 1573.468 & 1561.461 & 1522.456 \\
\hline 9/29/16 21:00 & 84.423 & 75.784 & 114.767 & 1573.468 & 1561.472 & 1522.384 \\
\hline 9/29/16 22:00 & 84.425 & 75.788 & 114.856 & 1573.466 & 1561.468 & 1522.295 \\
\hline 9/29/16 23:00 & 84.428 & 75.786 & 114.976 & 1573.463 & 1561.47 & 1522.175 \\
\hline 9/30/16 0:00 & 84.425 & 75.772 & 115.029 & 1573.466 & 1561.484 & 1522.122 \\
\hline 9/30/16 1:00 & 84.425 & 75.774 & 115.006 & 1573.466 & 1561.482 & 1522.145 \\
\hline $9 / 30 / 16$ 2:00 & 84.423 & 75.767 & 115.085 & 1573.468 & 1561.489 & 1522.066 \\
\hline 9/30/16 3:00 & 84.418 & 75.765 & 115.181 & 1573.473 & 1561.491 & 1521.97 \\
\hline $9 / 30 / 164: 00$ & 84.414 & 75.753 & 115.277 & 1573.477 & 1561.503 & 1521.874 \\
\hline 9/30/16 5:00 & 84.409 & 75.744 & 115.376 & 1573.482 & 1561.512 & 1521.775 \\
\hline $9 / 30 / 16$ 6:00 & 84.404 & 75.73 & 115.472 & 1573.487 & 1561.526 & 1521.679 \\
\hline 9/30/16 7:00 & 84.404 & 75.73 & 115.568 & 1573.487 & 1561.526 & 1521.583 \\
\hline 9/30/16 8:00 & 84.404 & 75.728 & 115.666 & 1573.487 & 1561.528 & 1521.485 \\
\hline 9/30/16 9:00 & 84.406 & 75.719 & 115.741 & 1573.485 & 1561.537 & 1521.41 \\
\hline 9/30/16 10:00 & 84.409 & 75.716 & 115.678 & 1573.482 & 1561.54 & 1521.473 \\
\hline 9/30/16 11:00 & 84.409 & 75.714 & 115.568 & 1573.482 & 1561.542 & 1521.583 \\
\hline 9/30/16 12:00 & 84.409 & 75.714 & 115.446 & 1573.482 & 1561.542 & 1521.705 \\
\hline 9/30/16 13:00 & 84.407 & 75.714 & 115.32 & 1573.484 & 1561.542 & 1521.831 \\
\hline 9/30/16 14:00 & 84.404 & 75.705 & 115.186 & 1573.487 & 1561.551 & 1521.965 \\
\hline 9/30/16 15:00 & 84.397 & 75.7 & 115.046 & 1573.494 & 1561.556 & 1522.105 \\
\hline 9/30/16 16:00 & 84.392 & 75.693 & 114.915 & 1573.499 & 1561.563 & 1522.236 \\
\hline 9/30/16 17:00 & 84.39 & 75.688 & 114.781 & 1573.501 & 1561.568 & 1522.37 \\
\hline 9/30/16 18:00 & 84.385 & 75.681 & 114.645 & 1573.506 & 1561.575 & 1522.506 \\
\hline 9/30/16 19:00 & 84.385 & 75.677 & 114.519 & 1573.506 & 1561.579 & 1522.632 \\
\hline 9/30/16 20:00 & 84.381 & 75.674 & 114.402 & 1573.51 & 1561.582 & 1522.749 \\
\hline 9/30/16 21:00 & 84.383 & 75.672 & 114.285 & 1573.508 & 1561.584 & 1522.866 \\
\hline 9/30/16 22:00 & 84.385 & 75.67 & 114.168 & 1573.506 & 1561.586 & 1522.983 \\
\hline
\end{tabular}


TABLE S1.2 Automatically recorded groundwater levels in the confined aquifers.

\begin{tabular}{|c|c|c|c|c|c|c|}
\hline \multirow[b]{2}{*}{ Date-Time } & \multicolumn{3}{|c|}{ Measured Depth to Water (ft TOC ${ }^{a}$ ) } & \multicolumn{3}{|c|}{ Groundwater Elevation (ft AMSL ${ }^{\mathrm{b}}$ ) } \\
\hline & DL2-CU & DL7-CU & DL7-CL & DL2-CU & DL7-CU & DL7-CL \\
\hline 9/30/16 23:00 & 84.388 & 75.67 & 114.063 & 1573.503 & 1561.586 & 1523.088 \\
\hline $10 / 1 / 16$ 0:00 & 84.388 & 75.67 & 113.96 & 1573.503 & 1561.586 & 1523.191 \\
\hline 10/1/16 1:00 & 84.387 & 75.66 & 113.854 & 1573.504 & 1561.596 & 1523.297 \\
\hline $10 / 1 / 162: 00$ & 84.383 & 75.66 & 113.744 & 1573.508 & 1561.596 & 1523.407 \\
\hline $10 / 1 / 163: 00$ & 84.378 & 75.651 & 113.644 & 1573.513 & 1561.605 & 1523.507 \\
\hline $10 / 1 / 164: 00$ & 84.374 & 75.646 & 113.538 & 1573.517 & 1561.61 & 1523.613 \\
\hline $10 / 1 / 16$ 5:00 & 84.369 & 75.644 & 113.435 & 1573.522 & 1561.612 & 1523.716 \\
\hline $10 / 1 / 16$ 6:00 & 84.36 & 75.633 & 113.332 & 1573.531 & 1561.623 & 1523.819 \\
\hline $10 / 1 / 16$ 7:00 & 84.36 & 75.628 & 113.241 & 1573.531 & 1561.628 & 1523.91 \\
\hline $10 / 1 / 168: 00$ & 84.357 & 75.626 & 113.15 & 1573.534 & 1561.63 & 1524.001 \\
\hline 10/1/16 9:00 & 84.357 & 75.621 & 113.066 & 1573.534 & 1561.635 & 1524.085 \\
\hline 10/1/16 10:00 & 84.355 & 75.621 & 112.991 & 1573.536 & 1561.635 & 1524.16 \\
\hline 10/1/16 11:00 & 84.355 & 75.621 & 112.942 & 1573.536 & 1561.635 & 1524.209 \\
\hline 10/1/16 12:00 & 84.352 & 75.621 & 112.902 & 1573.539 & 1561.635 & 1524.249 \\
\hline 10/1/16 13:00 & 84.347 & 75.612 & 112.874 & 1573.544 & 1561.644 & 1524.277 \\
\hline 10/1/16 14:00 & 84.343 & 75.607 & 112.845 & 1573.548 & 1561.649 & 1524.306 \\
\hline 10/1/16 15:00 & 84.338 & 75.593 & 112.827 & 1573.553 & 1561.663 & 1524.324 \\
\hline 10/1/16 16:00 & 84.334 & 75.581 & 112.792 & 1573.557 & 1561.675 & 1524.359 \\
\hline 10/1/16 17:00 & 84.326 & 75.574 & 112.757 & 1573.565 & 1561.682 & 1524.394 \\
\hline 10/1/16 18:00 & 84.317 & 75.56 & 112.724 & 1573.574 & 1561.696 & 1524.427 \\
\hline 10/1/16 19:00 & 84.31 & 75.56 & 112.717 & 1573.581 & 1561.696 & 1524.434 \\
\hline 10/1/16 20:00 & 84.31 & 75.551 & 112.7 & 1573.581 & 1561.705 & 1524.451 \\
\hline 10/1/16 21:00 & 84.308 & 75.547 & 112.686 & 1573.583 & 1561.709 & 1524.465 \\
\hline 10/1/16 22:00 & 84.308 & 75.549 & 112.656 & 1573.583 & 1561.707 & 1524.495 \\
\hline 10/1/16 23:00 & 84.305 & 75.547 & 112.635 & 1573.586 & 1561.709 & 1524.516 \\
\hline $10 / 2 / 16$ 0:00 & 84.305 & 75.54 & 112.623 & 1573.586 & 1561.716 & 1524.528 \\
\hline $10 / 2 / 16$ 1:00 & 84.301 & 75.535 & 112.595 & 1573.59 & 1561.721 & 1524.556 \\
\hline $10 / 2 / 162: 00$ & 84.296 & 75.528 & 112.558 & 1573.595 & 1561.728 & 1524.593 \\
\hline $10 / 2 / 163: 00$ & 84.291 & 75.521 & 112.511 & 1573.6 & 1561.735 & 1524.64 \\
\hline $10 / 2 / 164: 00$ & 84.284 & 75.509 & 112.466 & 1573.607 & 1561.747 & 1524.685 \\
\hline $10 / 2 / 16$ 5:00 & 84.275 & 75.512 & 112.438 & 1573.616 & 1561.744 & 1524.713 \\
\hline $10 / 2 / 166: 00$ & 84.268 & 75.502 & 112.419 & 1573.623 & 1561.754 & 1524.732 \\
\hline $10 / 2 / 167: 00$ & 84.263 & 75.498 & 112.403 & 1573.628 & 1561.758 & 1524.748 \\
\hline $10 / 2 / 168: 00$ & 84.258 & 75.486 & 112.377 & 1573.633 & 1561.77 & 1524.774 \\
\hline 10/2/16 9:00 & 84.256 & 75.491 & 112.436 & 1573.635 & 1561.765 & 1524.715 \\
\hline 10/2/16 10:00 & 84.254 & 75.484 & 112.595 & 1573.637 & 1561.772 & 1524.556 \\
\hline 10/2/16 11:00 & 84.251 & 75.474 & 112.764 & 1573.64 & 1561.782 & 1524.387 \\
\hline 10/2/16 12:00 & 84.249 & 75.467 & 112.923 & 1573.642 & 1561.789 & 1524.228 \\
\hline 10/2/16 13:00 & 84.244 & 75.449 & 112.956 & 1573.647 & 1561.807 & 1524.195 \\
\hline 10/2/16 14:00 & 84.24 & 75.451 & 112.965 & 1573.651 & 1561.805 & 1524.186 \\
\hline 10/2/16 15:00 & 84.228 & 75.442 & 113.082 & 1573.663 & 1561.814 & 1524.069 \\
\hline 10/2/16 16:00 & 84.221 & 75.43 & 113.218 & 1573.67 & 1561.826 & 1523.933 \\
\hline 10/2/16 17:00 & 84.214 & 75.421 & 113.351 & 1573.677 & 1561.835 & 1523.8 \\
\hline 10/2/16 18:00 & 84.207 & 75.414 & 113.492 & 1573.684 & 1561.842 & 1523.659 \\
\hline 10/2/16 19:00 & 84.199 & 75.405 & 113.625 & 1573.692 & 1561.851 & 1523.526 \\
\hline $10 / 2 / 1620: 00$ & 84.195 & 75.395 & 113.756 & 1573.696 & 1561.861 & 1523.395 \\
\hline 10/2/16 21:00 & 84.192 & 75.395 & 113.885 & 1573.699 & 1561.861 & 1523.266 \\
\hline
\end{tabular}


TABLE S1.2 Automatically recorded groundwater levels in the confined aquifers.

\begin{tabular}{|c|c|c|c|c|c|c|}
\hline \multirow[b]{2}{*}{ Date-Time } & \multicolumn{3}{|c|}{ Measured Depth to Water (ft TOC ${ }^{a}$ ) } & \multicolumn{3}{|c|}{ Groundwater Elevation (ft AMSL ${ }^{\mathrm{b}}$ ) } \\
\hline & DL2-CU & DL7-CU & DL7-CL & DL2-CU & DL7-CU & DL7-CL \\
\hline $10 / 2 / 1622: 00$ & 84.19 & 75.386 & 113.999 & 1573.701 & 1561.87 & 1523.152 \\
\hline $10 / 2 / 1623: 00$ & 84.188 & 75.381 & 113.983 & 1573.703 & 1561.875 & 1523.168 \\
\hline 10/3/16 0:00 & 84.183 & 75.379 & 113.899 & 1573.708 & 1561.877 & 1523.252 \\
\hline $10 / 3 / 16$ 1:00 & 84.178 & 75.374 & 113.798 & 1573.713 & 1561.882 & 1523.353 \\
\hline $10 / 3 / 162: 00$ & 84.173 & 75.379 & 113.69 & 1573.718 & 1561.877 & 1523.461 \\
\hline $10 / 3 / 163: 00$ & 84.166 & 75.374 & 113.707 & 1573.725 & 1561.882 & 1523.444 \\
\hline $10 / 3 / 164: 00$ & 84.157 & 75.365 & 113.775 & 1573.734 & 1561.891 & 1523.376 \\
\hline $10 / 3 / 165: 00$ & 84.148 & 75.356 & 113.861 & 1573.743 & 1561.9 & 1523.29 \\
\hline $10 / 3 / 16$ 6:00 & 84.138 & 75.347 & 113.953 & 1573.753 & 1561.909 & 1523.198 \\
\hline $10 / 3 / 16$ 7:00 & 84.129 & 75.335 & 114.042 & 1573.762 & 1561.921 & 1523.109 \\
\hline $10 / 3 / 168: 00$ & 84.122 & 75.328 & 114.138 & 1573.769 & 1561.928 & 1523.013 \\
\hline 10/3/16 9:00 & 84.115 & 75.309 & 114.208 & 1573.776 & 1561.947 & 1522.943 \\
\hline 10/3/16 10:00 & 84.11 & 75.302 & 114.138 & 1573.781 & 1561.954 & 1523.013 \\
\hline 10/3/16 11:00 & 84.103 & 75.298 & 114.032 & 1573.788 & 1561.958 & 1523.119 \\
\hline 10/3/16 12:00 & 84.096 & 75.295 & 113.908 & 1573.795 & 1561.961 & 1523.243 \\
\hline 10/3/16 13:00 & 84.089 & 75.291 & 113.777 & 1573.802 & 1561.965 & 1523.374 \\
\hline 10/3/16 14:00 & 84.08 & 75.284 & 113.651 & 1573.811 & 1561.972 & 1523.5 \\
\hline 10/3/16 15:00 & 84.07 & 75.277 & 113.517 & 1573.821 & 1561.979 & 1523.634 \\
\hline $10 / 3 / 16$ 16:00 & 84.058 & 75.268 & 113.391 & 1573.833 & 1561.988 & 1523.76 \\
\hline $10 / 3 / 16$ 17:00 & 84.051 & 75.256 & 113.269 & 1573.84 & 1562 & 1523.882 \\
\hline $10 / 3 / 16$ 18:00 & 84.042 & 75.249 & 113.145 & 1573.849 & 1562.007 & 1524.006 \\
\hline 10/3/16 19:00 & 84.035 & 75.24 & 113.03 & 1573.856 & 1562.016 & 1524.121 \\
\hline $10 / 3 / 1620: 00$ & 84.028 & 75.233 & 112.916 & 1573.863 & 1562.023 & 1524.235 \\
\hline 10/3/16 21:00 & 84.025 & 75.226 & 112.81 & 1573.866 & 1562.03 & 1524.341 \\
\hline $10 / 3 / 16$ 22:00 & 84.03 & 75.223 & 112.707 & 1573.861 & 1562.033 & 1524.444 \\
\hline 10/3/16 23:00 & 84.061 & 75.214 & 112.604 & 1573.83 & 1562.042 & 1524.547 \\
\hline $10 / 4 / 16$ 0:00 & 84.113 & 75.209 & 112.506 & 1573.778 & 1562.047 & 1524.645 \\
\hline 10/4/16 1:00 & 84.167 & 75.207 & 112.42 & 1573.724 & 1562.049 & 1524.731 \\
\hline $10 / 4 / 16$ 2:00 & 84.19 & 75.198 & 112.324 & 1573.701 & 1562.058 & 1524.827 \\
\hline 10/4/16 3:00 & 84.2 & 75.191 & 112.235 & 1573.691 & 1562.065 & 1524.916 \\
\hline 10/4/16 4:00 & 84.19 & 75.186 & 112.148 & 1573.701 & 1562.07 & 1525.003 \\
\hline 10/4/16 5:00 & 84.167 & 75.177 & 112.05 & 1573.724 & 1562.079 & 1525.101 \\
\hline 10/4/16 6:00 & 84.115 & 75.13 & 111.93 & 1573.776 & 1562.126 & 1525.221 \\
\hline $10 / 4 / 16$ 7:00 & 84.077 & 75.107 & 111.823 & 1573.814 & 1562.149 & 1525.328 \\
\hline 10/4/16 8:00 & 84.058 & 75.091 & 111.729 & 1573.833 & 1562.165 & 1525.422 \\
\hline 10/4/16 9:00 & 84.049 & 75.086 & 111.649 & 1573.842 & 1562.17 & 1525.502 \\
\hline 10/4/16 10:00 & 84.035 & 75.089 & 111.579 & 1573.856 & 1562.167 & 1525.572 \\
\hline 10/4/16 11:00 & 84.025 & 75.086 & 111.509 & 1573.866 & 1562.17 & 1525.642 \\
\hline 10/4/16 12:00 & 84.014 & 75.089 & 111.446 & 1573.877 & 1562.167 & 1525.705 \\
\hline 10/4/16 13:00 & 84.007 & 75.089 & 111.399 & 1573.884 & 1562.167 & 1525.752 \\
\hline 10/4/16 14:00 & 83.997 & 75.086 & 111.343 & 1573.894 & 1562.17 & 1525.808 \\
\hline 10/4/16 15:00 & 83.985 & 75.079 & 111.284 & 1573.906 & 1562.177 & 1525.867 \\
\hline 10/4/16 16:00 & 83.978 & 75.075 & 111.235 & 1573.913 & 1562.181 & 1525.916 \\
\hline 10/4/16 17:00 & 83.957 & 75.065 & 111.184 & 1573.934 & 1562.191 & 1525.967 \\
\hline 10/4/16 18:00 & 83.941 & 75.026 & 111.102 & 1573.95 & 1562.23 & 1526.049 \\
\hline 10/4/16 19:00 & 83.913 & 75.017 & 111.041 & 1573.978 & 1562.239 & 1526.11 \\
\hline 10/4/16 20:00 & 83.91 & 75.014 & 110.985 & 1573.981 & 1562.242 & 1526.166 \\
\hline
\end{tabular}


TABLE S1.2 Automatically recorded groundwater levels in the confined aquifers.

\begin{tabular}{|c|c|c|c|c|c|c|}
\hline \multirow[b]{2}{*}{ Date-Time } & \multicolumn{3}{|c|}{ Measured Depth to Water (ft TOC ${ }^{a}$ ) } & \multicolumn{3}{|c|}{ Groundwater Elevation (ft AMSL ${ }^{\mathrm{b}}$ ) } \\
\hline & DL2-CU & DL7-CU & DL7-CL & DL2-CU & DL7-CU & DL7-CL \\
\hline 10/4/16 21:00 & 83.908 & 75.017 & 110.94 & 1573.983 & 1562.239 & 1526.211 \\
\hline $10 / 4 / 16$ 22:00 & 83.917 & 75.021 & 110.903 & 1573.974 & 1562.235 & 1526.248 \\
\hline 10/4/16 23:00 & 83.95 & 75.028 & 110.875 & 1573.941 & 1562.228 & 1526.276 \\
\hline 10/5/16 0:00 & 83.981 & 75.028 & 110.842 & 1573.91 & 1562.228 & 1526.309 \\
\hline 10/5/16 1:00 & 83.999 & 75.023 & 110.804 & 1573.892 & 1562.233 & 1526.347 \\
\hline $10 / 5 / 162: 00$ & 84.004 & 75.03 & 110.751 & 1573.887 & 1562.226 & 1526.4 \\
\hline 10/5/16 3:00 & 84.007 & 75.035 & 110.72 & 1573.884 & 1562.221 & 1526.431 \\
\hline $10 / 5 / 164: 00$ & 84 & 75.033 & 110.687 & 1573.891 & 1562.223 & 1526.464 \\
\hline 10/5/16 5:00 & 84 & 75.037 & 110.655 & 1573.891 & 1562.219 & 1526.496 \\
\hline $10 / 5 / 16$ 6:00 & 83.995 & 75.035 & 110.608 & 1573.896 & 1562.221 & 1526.543 \\
\hline 10/5/16 7:00 & 83.988 & 75.04 & 110.563 & 1573.903 & 1562.216 & 1526.588 \\
\hline 10/5/16 8:00 & 83.983 & 75.04 & 110.524 & 1573.908 & 1562.216 & 1526.627 \\
\hline 10/5/16 9:00 & 83.978 & 75.047 & 110.502 & 1573.913 & 1562.209 & 1526.649 \\
\hline 10/5/16 10:00 & 83.976 & 75.044 & 110.481 & 1573.915 & 1562.212 & 1526.67 \\
\hline 10/5/16 11:00 & 83.971 & 75.049 & 110.481 & 1573.92 & 1562.207 & 1526.67 \\
\hline 10/5/16 12:00 & 83.967 & 75.047 & 110.603 & 1573.924 & 1562.209 & 1526.548 \\
\hline 10/5/16 13:00 & 83.96 & 75.04 & 110.615 & 1573.931 & 1562.216 & 1526.536 \\
\hline 10/5/16 14:00 & 83.953 & 75.033 & 110.58 & 1573.938 & 1562.223 & 1526.571 \\
\hline $10 / 5 / 16$ 15:00 & 83.943 & 75.028 & 110.556 & 1573.948 & 1562.228 & 1526.595 \\
\hline 10/5/16 16:00 & 83.941 & 75.028 & 110.68 & 1573.95 & 1562.228 & 1526.471 \\
\hline 10/5/16 17:00 & 83.934 & 75.014 & 110.793 & 1573.957 & 1562.242 & 1526.358 \\
\hline 10/5/16 18:00 & 83.929 & 75.01 & 110.767 & 1573.962 & 1562.246 & 1526.384 \\
\hline 10/5/16 19:00 & 83.929 & 75.017 & 110.746 & 1573.962 & 1562.239 & 1526.405 \\
\hline 10/5/16 20:00 & 83.929 & 75.021 & 110.861 & 1573.962 & 1562.235 & 1526.29 \\
\hline 10/5/16 21:00 & 83.924 & 75.01 & 110.914 & 1573.967 & 1562.246 & 1526.237 \\
\hline 10/5/16 22:00 & 83.922 & 75.014 & 110.903 & 1573.969 & 1562.242 & 1526.248 \\
\hline 10/5/16 23:00 & 83.92 & 75.017 & 110.98 & 1573.971 & 1562.239 & 1526.171 \\
\hline 10/6/16 0:00 & 83.915 & 75.005 & 111.034 & 1573.976 & 1562.251 & 1526.117 \\
\hline 10/6/16 1:00 & 83.913 & 75.003 & 110.978 & 1573.978 & 1562.253 & 1526.173 \\
\hline $10 / 6 / 16$ 2:00 & 83.905 & 74.993 & 110.893 & 1573.986 & 1562.263 & 1526.258 \\
\hline 10/6/16 3:00 & 83.901 & 74.996 & 110.807 & 1573.99 & 1562.26 & 1526.344 \\
\hline 10/6/16 4:00 & 83.896 & 74.993 & 110.713 & 1573.995 & 1562.263 & 1526.438 \\
\hline 10/6/16 5:00 & 83.889 & 74.993 & 110.613 & 1574.002 & 1562.263 & 1526.538 \\
\hline 10/6/16 6:00 & 83.882 & 74.989 & 110.512 & 1574.009 & 1562.267 & 1526.639 \\
\hline 10/6/16 7:00 & 83.879 & 74.991 & 110.418 & 1574.012 & 1562.265 & 1526.733 \\
\hline 10/6/16 8:00 & 83.872 & 74.993 & 110.428 & 1574.019 & 1562.263 & 1526.723 \\
\hline 10/6/16 9:00 & 83.866 & 74.982 & 110.467 & 1574.025 & 1562.274 & 1526.684 \\
\hline 10/6/16 10:00 & 83.861 & 74.972 & 110.411 & 1574.03 & 1562.284 & 1526.74 \\
\hline 10/6/16 11:00 & 83.856 & 74.97 & 110.32 & 1574.035 & 1562.286 & 1526.831 \\
\hline 10/6/16 12:00 & 83.847 & 74.961 & 110.219 & 1574.044 & 1562.295 & 1526.932 \\
\hline 10/6/16 13:00 & 83.842 & 74.956 & 110.13 & 1574.049 & 1562.3 & 1527.021 \\
\hline 10/6/16 14:00 & 83.835 & 74.954 & 110.034 & 1574.056 & 1562.302 & 1527.117 \\
\hline 10/6/16 15:00 & 83.832 & 74.944 & 109.943 & 1574.059 & 1562.312 & 1527.208 \\
\hline 10/6/16 16:00 & 83.83 & 74.947 & 109.859 & 1574.061 & 1562.309 & 1527.292 \\
\hline 10/6/16 17:00 & 83.825 & 74.942 & 109.77 & 1574.066 & 1562.314 & 1527.381 \\
\hline 10/6/16 18:00 & 83.826 & 74.94 & 109.688 & 1574.065 & 1562.316 & 1527.463 \\
\hline 10/6/16 19:00 & 83.828 & 74.944 & 109.606 & 1574.063 & 1562.312 & 1527.545 \\
\hline
\end{tabular}


TABLE S1.2 Automatically recorded groundwater levels in the confined aquifers.

\begin{tabular}{|c|c|c|c|c|c|c|}
\hline \multirow[b]{2}{*}{ Date-Time } & \multicolumn{3}{|c|}{ Measured Depth to Water (ft TOC ${ }^{a}$ ) } & \multicolumn{3}{|c|}{ Groundwater Elevation (ft AMSL ${ }^{\mathrm{b}}$ ) } \\
\hline & DL2-CU & DL7-CU & DL7-CL & DL2-CU & DL7-CU & DL7-CL \\
\hline 10/6/16 20:00 & 83.832 & 74.949 & 109.531 & 1574.059 & 1562.307 & 1527.62 \\
\hline 10/6/16 21:00 & 83.842 & 74.954 & 109.463 & 1574.049 & 1562.302 & 1527.688 \\
\hline 10/6/16 22:00 & 83.842 & 74.958 & 109.386 & 1574.049 & 1562.298 & 1527.765 \\
\hline 10/6/16 23:00 & 83.844 & 74.956 & 109.313 & 1574.047 & 1562.3 & 1527.838 \\
\hline 10/7/16 0:00 & 83.842 & 74.956 & 109.246 & 1574.049 & 1562.3 & 1527.905 \\
\hline 10/7/16 1:00 & 83.842 & 74.956 & 109.168 & 1574.049 & 1562.3 & 1527.983 \\
\hline $10 / 7 / 16$ 2:00 & 83.844 & 74.958 & 109.1 & 1574.047 & 1562.298 & 1528.051 \\
\hline 10/7/16 3:00 & 83.849 & 74.961 & 109.04 & 1574.042 & 1562.295 & 1528.111 \\
\hline $10 / 7 / 164: 00$ & 83.851 & 74.965 & 108.965 & 1574.04 & 1562.291 & 1528.186 \\
\hline 10/7/16 5:00 & 83.854 & 74.961 & 108.899 & 1574.037 & 1562.295 & 1528.252 \\
\hline $10 / 7 / 16$ 6:00 & 83.849 & 74.958 & 108.824 & 1574.042 & 1562.298 & 1528.327 \\
\hline 10/7/16 7:00 & 83.847 & 74.956 & 108.752 & 1574.044 & 1562.3 & 1528.399 \\
\hline 10/7/16 8:00 & 83.847 & 74.958 & 108.684 & 1574.044 & 1562.298 & 1528.467 \\
\hline 10/7/16 9:00 & 83.844 & 74.954 & 108.614 & 1574.047 & 1562.302 & 1528.537 \\
\hline 10/7/16 10:00 & 83.844 & 74.958 & 108.55 & 1574.047 & 1562.298 & 1528.601 \\
\hline 10/7/16 11:00 & 83.842 & 74.956 & 108.499 & 1574.049 & 1562.3 & 1528.652 \\
\hline 10/7/16 12:00 & 83.84 & 74.947 & 108.445 & 1574.051 & 1562.309 & 1528.706 \\
\hline 10/7/16 13:00 & 83.835 & 74.942 & 108.396 & 1574.056 & 1562.314 & 1528.755 \\
\hline 10/7/16 14:00 & 83.828 & 74.942 & 108.347 & 1574.063 & 1562.314 & 1528.804 \\
\hline $10 / 7 / 16$ 15:00 & 83.821 & 74.937 & 108.314 & 1574.07 & 1562.319 & 1528.837 \\
\hline 10/7/16 16:00 & 83.811 & 74.93 & 108.288 & 1574.08 & 1562.326 & 1528.863 \\
\hline 10/7/16 17:00 & 83.8 & 74.921 & 108.269 & 1574.091 & 1562.335 & 1528.882 \\
\hline 10/7/16 18:00 & 83.79 & 74.914 & 108.244 & 1574.101 & 1562.342 & 1528.907 \\
\hline 10/7/16 19:00 & 83.781 & 74.91 & 108.211 & 1574.11 & 1562.346 & 1528.94 \\
\hline 10/7/16 20:00 & 83.769 & 74.903 & 108.178 & 1574.122 & 1562.353 & 1528.973 \\
\hline 10/7/16 21:00 & 83.757 & 74.903 & 108.145 & 1574.134 & 1562.353 & 1529.006 \\
\hline 10/7/16 22:00 & 83.745 & 74.898 & 108.124 & 1574.146 & 1562.358 & 1529.027 \\
\hline 10/7/16 23:00 & 83.734 & 74.898 & 108.108 & 1574.157 & 1562.358 & 1529.043 \\
\hline 10/8/16 0:00 & 83.722 & 74.896 & 108.082 & 1574.169 & 1562.36 & 1529.069 \\
\hline 10/8/16 1:00 & 83.71 & 74.882 & 108.047 & 1574.181 & 1562.374 & 1529.104 \\
\hline 10/8/16 2:00 & 83.698 & 74.889 & 108.012 & 1574.193 & 1562.367 & 1529.139 \\
\hline 10/8/16 3:00 & 83.687 & 74.884 & 107.972 & 1574.204 & 1562.372 & 1529.179 \\
\hline 10/8/16 4:00 & 83.675 & 74.886 & 107.923 & 1574.216 & 1562.37 & 1529.228 \\
\hline 10/8/16 5:00 & 83.663 & 74.879 & 107.874 & 1574.228 & 1562.377 & 1529.277 \\
\hline 10/8/16 6:00 & 83.651 & 74.877 & 107.818 & 1574.24 & 1562.379 & 1529.333 \\
\hline 10/8/16 7:00 & 83.64 & 74.872 & 107.762 & 1574.251 & 1562.384 & 1529.389 \\
\hline 10/8/16 8:00 & 83.63 & 74.873 & 107.71 & 1574.261 & 1562.383 & 1529.441 \\
\hline 10/8/16 9:00 & 83.618 & 74.877 & 107.738 & 1574.273 & 1562.379 & 1529.413 \\
\hline 10/8/16 10:00 & 83.609 & 74.875 & 107.839 & 1574.282 & 1562.381 & 1529.312 \\
\hline 10/8/16 11:00 & 83.597 & 74.858 & 107.909 & 1574.294 & 1562.398 & 1529.242 \\
\hline 10/8/16 12:00 & 83.588 & 74.854 & 107.883 & 1574.303 & 1562.402 & 1529.268 \\
\hline 10/8/16 13:00 & 83.576 & 74.847 & 107.836 & 1574.315 & 1562.409 & 1529.315 \\
\hline 10/8/16 14:00 & 83.564 & 74.851 & 107.82 & 1574.327 & 1562.405 & 1529.331 \\
\hline 10/8/16 15:00 & 83.553 & 74.844 & 107.902 & 1574.338 & 1562.412 & 1529.249 \\
\hline 10/8/16 16:00 & 83.543 & 74.838 & 108.005 & 1574.348 & 1562.418 & 1529.146 \\
\hline 10/8/16 17:00 & 83.534 & 74.835 & 108.096 & 1574.357 & 1562.421 & 1529.055 \\
\hline 10/8/16 18:00 & 83.527 & 74.826 & 108.127 & 1574.364 & 1562.43 & 1529.024 \\
\hline
\end{tabular}


TABLE S1.2 Automatically recorded groundwater levels in the confined aquifers.

\begin{tabular}{|c|c|c|c|c|c|c|}
\hline \multirow[b]{2}{*}{ Date-Time } & \multicolumn{3}{|c|}{ Measured Depth to Water (ft TOC ${ }^{a}$ ) } & \multicolumn{3}{|c|}{ Groundwater Elevation (ft AMSL ${ }^{\mathrm{b}}$ ) } \\
\hline & DL2-CU & DL7-CU & DL7-CL & DL2-CU & DL7-CU & DL7-CL \\
\hline 10/8/16 19:00 & 83.52 & 74.824 & 108.078 & 1574.371 & 1562.432 & 1529.073 \\
\hline 10/8/16 20:00 & 83.515 & 74.826 & 108.021 & 1574.376 & 1562.43 & 1529.13 \\
\hline 10/8/16 21:00 & 83.513 & 74.833 & 107.951 & 1574.378 & 1562.423 & 1529.2 \\
\hline 10/8/16 22:00 & 83.51 & 74.84 & 107.904 & 1574.381 & 1562.416 & 1529.247 \\
\hline $10 / 8 / 16$ 23:00 & 83.506 & 74.842 & 107.968 & 1574.385 & 1562.414 & 1529.183 \\
\hline $10 / 9 / 160: 00$ & 83.501 & 74.838 & 108.047 & 1574.39 & 1562.418 & 1529.104 \\
\hline 10/9/16 1:00 & 83.494 & 74.828 & 108.038 & 1574.397 & 1562.428 & 1529.113 \\
\hline $10 / 9 / 162: 00$ & 83.489 & 74.831 & 107.975 & 1574.402 & 1562.425 & 1529.176 \\
\hline 10/9/16 3:00 & 83.487 & 74.831 & 107.9 & 1574.404 & 1562.425 & 1529.251 \\
\hline 10/9/16 4:00 & 83.482 & 74.831 & 107.822 & 1574.409 & 1562.425 & 1529.329 \\
\hline 10/9/16 5:00 & 83.477 & 74.833 & 107.733 & 1574.414 & 1562.423 & 1529.418 \\
\hline 10/9/16 6:00 & 83.473 & 74.835 & 107.654 & 1574.418 & 1562.421 & 1529.497 \\
\hline 10/9/16 7:00 & 83.468 & 74.833 & 107.565 & 1574.423 & 1562.423 & 1529.586 \\
\hline 10/9/16 8:00 & 83.463 & 74.831 & 107.478 & 1574.428 & 1562.425 & 1529.673 \\
\hline 10/9/16 9:00 & 83.459 & 74.833 & 107.394 & 1574.432 & 1562.423 & 1529.757 \\
\hline 10/9/16 10:00 & 83.454 & 74.831 & 107.31 & 1574.437 & 1562.425 & 1529.841 \\
\hline 10/9/16 11:00 & 83.444 & 74.833 & 107.221 & 1574.447 & 1562.423 & 1529.93 \\
\hline 10/9/16 12:00 & 83.435 & 74.819 & 107.137 & 1574.456 & 1562.437 & 1530.014 \\
\hline 10/9/16 13:00 & 83.425 & 74.814 & 107.05 & 1574.466 & 1562.442 & 1530.101 \\
\hline 10/9/16 14:00 & 83.416 & 74.81 & 106.977 & 1574.475 & 1562.446 & 1530.174 \\
\hline 10/9/16 15:00 & 83.407 & 74.803 & 106.905 & 1574.484 & 1562.453 & 1530.246 \\
\hline 10/9/16 16:00 & 83.402 & 74.798 & 106.835 & 1574.489 & 1562.458 & 1530.316 \\
\hline 10/9/16 17:00 & 83.393 & 74.791 & 106.764 & 1574.498 & 1562.465 & 1530.387 \\
\hline 10/9/16 18:00 & 83.388 & 74.791 & 106.708 & 1574.503 & 1562.465 & 1530.443 \\
\hline 10/9/16 19:00 & 83.383 & 74.791 & 106.652 & 1574.508 & 1562.465 & 1530.499 \\
\hline 10/9/16 20:00 & 83.378 & 74.782 & 106.596 & 1574.513 & 1562.474 & 1530.555 \\
\hline 10/9/16 21:00 & 83.376 & 74.784 & 106.549 & 1574.515 & 1562.472 & 1530.602 \\
\hline 10/9/16 22:00 & 83.371 & 74.782 & 106.498 & 1574.52 & 1562.474 & 1530.653 \\
\hline 10/9/16 23:00 & 83.367 & 74.777 & 106.448 & 1574.524 & 1562.479 & 1530.703 \\
\hline $10 / 10 / 160: 00$ & 83.36 & 74.772 & 106.399 & 1574.531 & 1562.484 & 1530.752 \\
\hline 10/10/16 1:00 & 83.353 & 74.77 & 106.348 & 1574.538 & 1562.486 & 1530.803 \\
\hline 10/10/16 2:00 & 83.346 & 74.765 & 106.303 & 1574.545 & 1562.491 & 1530.848 \\
\hline 10/10/16 3:00 & 83.341 & 74.758 & 106.257 & 1574.55 & 1562.498 & 1530.894 \\
\hline 10/10/16 4:00 & 83.336 & 74.754 & 106.205 & 1574.555 & 1562.502 & 1530.946 \\
\hline 10/10/16 5:00 & 83.332 & 74.756 & 106.156 & 1574.559 & 1562.5 & 1530.995 \\
\hline 10/10/16 6:00 & 83.327 & 74.747 & 106.111 & 1574.564 & 1562.509 & 1531.04 \\
\hline 10/10/16 7:00 & 83.322 & 74.747 & 106.067 & 1574.569 & 1562.509 & 1531.084 \\
\hline 10/10/16 8:00 & 83.32 & 74.749 & 106.025 & 1574.571 & 1562.507 & 1531.126 \\
\hline 10/10/16 9:00 & 83.315 & 74.747 & 105.99 & 1574.576 & 1562.509 & 1531.161 \\
\hline 10/10/16 10:00 & 83.31 & 74.747 & 105.952 & 1574.581 & 1562.509 & 1531.199 \\
\hline $10 / 10 / 1611: 00$ & 83.31 & 74.742 & 105.931 & 1574.581 & 1562.514 & 1531.22 \\
\hline $10 / 10 / 16$ 12:00 & 83.299 & 74.735 & 105.924 & 1574.592 & 1562.521 & 1531.227 \\
\hline 10/10/16 13:00 & 83.294 & 74.726 & 105.905 & 1574.597 & 1562.53 & 1531.246 \\
\hline $10 / 10 / 16$ 14:00 & 83.284 & 74.724 & 105.88 & 1574.607 & 1562.532 & 1531.271 \\
\hline $10 / 10 / 16$ 15:00 & 83.275 & 74.712 & 105.861 & 1574.616 & 1562.544 & 1531.29 \\
\hline $10 / 10 / 16$ 16:00 & 83.27 & 74.707 & 105.854 & 1574.621 & 1562.549 & 1531.297 \\
\hline $10 / 10 / 16$ 17:00 & 83.265 & 74.705 & 105.859 & 1574.626 & 1562.551 & 1531.292 \\
\hline
\end{tabular}


TABLE S1.2 Automatically recorded groundwater levels in the confined aquifers.

\begin{tabular}{|c|c|c|c|c|c|c|}
\hline \multirow[b]{2}{*}{ Date-Time } & \multicolumn{3}{|c|}{ Measured Depth to Water (ft TOC ${ }^{\mathrm{a}}$ ) } & \multicolumn{3}{|c|}{ Groundwater Elevation (ft $\mathrm{AMSL}^{\mathrm{b}}$ ) } \\
\hline & DL2-CU & DL7-CU & DL7-CL & DL2-CU & DL7-CU & DL7-CL \\
\hline 10/10/16 18:00 & 83.261 & 74.7 & 105.859 & 1574.63 & 1562.556 & 1531.292 \\
\hline 10/10/16 19:00 & 83.256 & 74.696 & 105.852 & 1574.635 & 1562.56 & 1531.299 \\
\hline $10 / 10 / 1620: 00$ & 83.254 & 74.691 & 105.838 & 1574.637 & 1562.565 & 1531.313 \\
\hline $10 / 10 / 1621: 00$ & 83.252 & 74.686 & 105.821 & 1574.639 & 1562.57 & 1531.33 \\
\hline $10 / 10 / 1622: 00$ & 83.252 & 74.686 & 105.814 & 1574.639 & 1562.57 & 1531.337 \\
\hline $10 / 10 / 1623: 00$ & 83.244 & 74.686 & 105.8 & 1574.647 & 1562.57 & 1531.351 \\
\hline $10 / 11 / 160: 00$ & 83.24 & 74.679 & 105.795 & 1574.651 & 1562.577 & 1531.356 \\
\hline 10/11/16 1:00 & 83.235 & 74.675 & 105.781 & 1574.656 & 1562.581 & 1531.37 \\
\hline 10/11/16 2:00 & 83.23 & 74.672 & 105.774 & 1574.661 & 1562.584 & 1531.377 \\
\hline 10/11/16 3:00 & 83.226 & 74.663 & 105.756 & 1574.665 & 1562.593 & 1531.395 \\
\hline 10/11/16 4:00 & 83.226 & 74.661 & 105.744 & 1574.665 & 1562.595 & 1531.407 \\
\hline 10/11/16 5:00 & 83.223 & 74.663 & 105.739 & 1574.668 & 1562.593 & 1531.412 \\
\hline 10/11/16 6:00 & 83.226 & 74.661 & 105.746 & 1574.665 & 1562.595 & 1531.405 \\
\hline 10/11/16 7:00 & 83.232 & 74.661 & 105.751 & 1574.659 & 1562.595 & 1531.4 \\
\hline 10/11/16 8:00 & 83.233 & 74.665 & 105.828 & 1574.658 & 1562.591 & 1531.323 \\
\hline 10/11/16 9:00 & 83.233 & 74.663 & 105.976 & 1574.658 & 1562.593 & 1531.175 \\
\hline 10/11/16 10:00 & 83.235 & 74.663 & 106.128 & 1574.656 & 1562.593 & 1531.023 \\
\hline 10/11/16 11:00 & 83.235 & 74.659 & 106.268 & 1574.656 & 1562.597 & 1530.883 \\
\hline $10 / 11 / 16$ 12:00 & 83.232 & 74.652 & 106.402 & 1574.659 & 1562.604 & 1530.749 \\
\hline 10/11/16 13:00 & 83.233 & 74.649 & 106.53 & 1574.658 & 1562.607 & 1530.621 \\
\hline $10 / 11 / 16$ 14:00 & 83.223 & 74.642 & 106.65 & 1574.668 & 1562.614 & 1530.501 \\
\hline $10 / 11 / 16$ 15:00 & 83.216 & 74.633 & 106.718 & 1574.675 & 1562.623 & 1530.433 \\
\hline 10/11/16 16:00 & 83.214 & 74.626 & 106.685 & 1574.677 & 1562.63 & 1530.466 \\
\hline 10/11/16 17:00 & 83.214 & 74.638 & 106.69 & 1574.677 & 1562.618 & 1530.461 \\
\hline 10/11/16 18:00 & 83.214 & 74.642 & 106.776 & 1574.677 & 1562.614 & 1530.375 \\
\hline 10/11/16 19:00 & 83.218 & 74.647 & 106.881 & 1574.673 & 1562.609 & 1530.27 \\
\hline 10/11/16 20:00 & 83.219 & 74.647 & 106.977 & 1574.672 & 1562.609 & 1530.174 \\
\hline 10/11/16 21:00 & 83.221 & 74.649 & 107.029 & 1574.67 & 1562.607 & 1530.122 \\
\hline $10 / 11 / 16$ 22:00 & 83.235 & 74.659 & 107.08 & 1574.656 & 1562.597 & 1530.071 \\
\hline 10/11/16 23:00 & 83.268 & 74.663 & 107.162 & 1574.623 & 1562.593 & 1529.989 \\
\hline 10/12/16 0:00 & 83.329 & 74.668 & 107.235 & 1574.562 & 1562.588 & 1529.916 \\
\hline 10/12/16 1:00 & 83.383 & 74.661 & 107.23 & 1574.508 & 1562.595 & 1529.921 \\
\hline 10/12/16 2:00 & 83.421 & 74.654 & 107.153 & 1574.47 & 1562.602 & 1529.998 \\
\hline 10/12/16 3:00 & 83.447 & 74.665 & 107.076 & 1574.444 & 1562.591 & 1530.075 \\
\hline 10/12/16 4:00 & 83.444 & 74.672 & 107.073 & 1574.447 & 1562.584 & 1530.078 \\
\hline 10/12/16 5:00 & 83.447 & 74.677 & 107.139 & 1574.444 & 1562.579 & 1530.012 \\
\hline 10/12/16 6:00 & 83.444 & 74.693 & 107.221 & 1574.447 & 1562.563 & 1529.93 \\
\hline 10/12/16 7:00 & 83.437 & 74.693 & 107.296 & 1574.454 & 1562.563 & 1529.855 \\
\hline 10/12/16 8:00 & 83.423 & 74.696 & 107.366 & 1574.468 & 1562.56 & 1529.785 \\
\hline 10/12/16 9:00 & 83.421 & 74.7 & 107.436 & 1574.47 & 1562.556 & 1529.715 \\
\hline $10 / 12 / 16$ 10:00 & 83.411 & 74.71 & 107.403 & 1574.48 & 1562.546 & 1529.748 \\
\hline 10/12/16 11:00 & 83.407 & 74.71 & 107.319 & 1574.484 & 1562.546 & 1529.832 \\
\hline $10 / 12 / 16$ 12:00 & 83.395 & 74.719 & 107.226 & 1574.496 & 1562.537 & 1529.925 \\
\hline 10/12/16 13:00 & 83.383 & 74.719 & 107.125 & 1574.508 & 1562.537 & 1530.026 \\
\hline 10/12/16 14:00 & 83.374 & 74.721 & 107.022 & 1574.517 & 1562.535 & 1530.129 \\
\hline $10 / 12 / 16$ 15:00 & 83.36 & 74.719 & 106.914 & 1574.531 & 1562.537 & 1530.237 \\
\hline 10/12/16 16:00 & 83.353 & 74.717 & 106.818 & 1574.538 & 1562.539 & 1530.333 \\
\hline
\end{tabular}


TABLE S1.2 Automatically recorded groundwater levels in the confined aquifers.

\begin{tabular}{|c|c|c|c|c|c|c|}
\hline \multirow[b]{2}{*}{ Date-Time } & \multicolumn{3}{|c|}{ Measured Depth to Water (ft TOC ${ }^{\mathrm{a}}$ ) } & \multicolumn{3}{|c|}{ Groundwater Elevation (ft $\mathrm{AMSL}^{\mathrm{b}}$ ) } \\
\hline & DL2-CU & DL7-CU & DL7-CL & DL2-CU & DL7-CU & DL7-CL \\
\hline 10/12/16 17:00 & 83.348 & 74.714 & 106.72 & 1574.543 & 1562.542 & 1530.431 \\
\hline $10 / 12 / 16$ 18:00 & 83.346 & 74.717 & 106.622 & 1574.545 & 1562.539 & 1530.529 \\
\hline 10/12/16 19:00 & 83.345 & 74.717 & 106.54 & 1574.546 & 1562.539 & 1530.611 \\
\hline 10/12/16 20:00 & 83.35 & 74.717 & 106.455 & 1574.541 & 1562.539 & 1530.696 \\
\hline 10/12/16 21:00 & 83.355 & 74.719 & 106.374 & 1574.536 & 1562.537 & 1530.777 \\
\hline $10 / 12 / 16$ 22:00 & 83.362 & 74.742 & 106.296 & 1574.529 & 1562.514 & 1530.855 \\
\hline $10 / 12 / 16$ 23:00 & 83.386 & 74.793 & 106.217 & 1574.505 & 1562.463 & 1530.934 \\
\hline 10/13/16 0:00 & 83.423 & 74.856 & 106.144 & 1574.468 & 1562.4 & 1531.007 \\
\hline 10/13/16 1:00 & 83.459 & 74.9 & 106.065 & 1574.432 & 1562.356 & 1531.086 \\
\hline $10 / 13 / 162: 00$ & 83.484 & 74.917 & 105.99 & 1574.407 & 1562.339 & 1531.161 \\
\hline 10/13/16 3:00 & 83.503 & 74.919 & 105.922 & 1574.388 & 1562.337 & 1531.229 \\
\hline 10/13/16 4:00 & 83.51 & 74.917 & 105.849 & 1574.381 & 1562.339 & 1531.302 \\
\hline 10/13/16 5:00 & 83.513 & 74.907 & 105.777 & 1574.378 & 1562.349 & 1531.374 \\
\hline 10/13/16 6:00 & 83.515 & 74.896 & 105.711 & 1574.376 & 1562.36 & 1531.44 \\
\hline 10/13/16 7:00 & 83.513 & 74.884 & 105.643 & 1574.378 & 1562.372 & 1531.508 \\
\hline 10/13/16 8:00 & 83.51 & 74.875 & 105.578 & 1574.381 & 1562.381 & 1531.573 \\
\hline 10/13/16 9:00 & 83.513 & 74.868 & 105.519 & 1574.378 & 1562.388 & 1531.632 \\
\hline 10/13/16 10:00 & 83.51 & 74.854 & 105.463 & 1574.381 & 1562.402 & 1531.688 \\
\hline 10/13/16 11:00 & 83.506 & 74.84 & 105.409 & 1574.385 & 1562.416 & 1531.742 \\
\hline $10 / 13 / 16$ 12:00 & 83.499 & 74.828 & 105.36 & 1574.392 & 1562.428 & 1531.791 \\
\hline 10/13/16 13:00 & 83.489 & 74.814 & 105.325 & 1574.402 & 1562.442 & 1531.826 \\
\hline $10 / 13 / 1614: 00$ & 83.477 & 74.794 & 105.29 & 1574.414 & 1562.462 & 1531.861 \\
\hline 10/13/16 15:00 & 83.468 & 74.775 & 105.255 & 1574.423 & 1562.481 & 1531.896 \\
\hline 10/13/16 16:00 & 83.456 & 74.756 & 105.22 & 1574.435 & 1562.5 & 1531.931 \\
\hline 10/13/16 17:00 & 83.447 & 74.742 & 105.189 & 1574.444 & 1562.514 & 1531.962 \\
\hline 10/13/16 18:00 & 83.44 & 74.726 & 105.166 & 1574.451 & 1562.53 & 1531.985 \\
\hline 10/13/16 19:00 & 83.432 & 74.712 & 105.145 & 1574.459 & 1562.544 & 1532.006 \\
\hline $10 / 13 / 16$ 20:00 & 83.43 & 74.703 & 105.119 & 1574.461 & 1562.553 & 1532.032 \\
\hline $10 / 13 / 16$ 21:00 & 83.428 & 74.698 & 105.093 & 1574.463 & 1562.558 & 1532.058 \\
\hline $10 / 13 / 16$ 22:00 & 83.428 & 74.689 & 105.074 & 1574.463 & 1562.567 & 1532.077 \\
\hline $10 / 13 / 16$ 23:00 & 83.423 & 74.684 & 105.063 & 1574.468 & 1562.572 & 1532.088 \\
\hline 10/14/16 0:00 & 83.419 & 74.672 & 105.051 & 1574.472 & 1562.584 & 1532.1 \\
\hline 10/14/16 1:00 & 83.411 & 74.656 & 105.03 & 1574.48 & 1562.6 & 1532.121 \\
\hline 10/14/16 2:00 & 83.402 & 74.645 & 104.997 & 1574.489 & 1562.611 & 1532.154 \\
\hline 10/14/16 3:00 & 83.397 & 74.629 & 104.96 & 1574.494 & 1562.627 & 1532.191 \\
\hline 10/14/16 4:00 & 83.383 & 74.615 & 104.918 & 1574.508 & 1562.641 & 1532.233 \\
\hline 10/14/16 5:00 & 83.376 & 74.603 & 104.871 & 1574.515 & 1562.653 & 1532.28 \\
\hline 10/14/16 6:00 & 83.367 & 74.591 & 104.819 & 1574.524 & 1562.665 & 1532.332 \\
\hline 10/14/16 7:00 & 83.36 & 74.58 & 104.773 & 1574.531 & 1562.676 & 1532.378 \\
\hline 10/14/16 8:00 & 83.357 & 74.575 & 104.73 & 1574.534 & 1562.681 & 1532.421 \\
\hline 10/14/16 9:00 & 83.355 & 74.582 & 104.754 & 1574.536 & 1562.674 & 1532.397 \\
\hline 10/14/16 10:00 & 83.353 & 74.573 & 104.876 & 1574.538 & 1562.683 & 1532.275 \\
\hline 10/14/16 11:00 & 83.348 & 74.556 & 105.007 & 1574.543 & 1562.7 & 1532.144 \\
\hline $10 / 14 / 16$ 12:00 & 83.343 & 74.545 & 105.037 & 1574.548 & 1562.711 & 1532.114 \\
\hline 10/14/16 13:00 & 83.336 & 74.54 & 105.126 & 1574.555 & 1562.716 & 1532.025 \\
\hline 10/14/16 14:00 & 83.324 & 74.517 & 105.236 & 1574.567 & 1562.739 & 1531.915 \\
\hline $10 / 14 / 16$ 15:00 & 83.315 & 74.508 & 105.353 & 1574.576 & 1562.748 & 1531.798 \\
\hline
\end{tabular}


TABLE S1.2 Automatically recorded groundwater levels in the confined aquifers.

\begin{tabular}{|c|c|c|c|c|c|c|}
\hline \multirow[b]{2}{*}{ Date-Time } & \multicolumn{3}{|c|}{ Measured Depth to Water (ft TOC ${ }^{\mathrm{a}}$ ) } & \multicolumn{3}{|c|}{ Groundwater Elevation (ft $\mathrm{AMSL}^{\mathrm{b}}$ ) } \\
\hline & DL2-CU & DL7-CU & DL7-CL & DL2-CU & DL7-CU & DL7-CL \\
\hline 10/14/16 16:00 & 83.305 & 74.491 & 105.465 & 1574.586 & 1562.765 & 1531.686 \\
\hline 10/14/16 17:00 & 83.296 & 74.475 & 105.564 & 1574.595 & 1562.781 & 1531.587 \\
\hline $10 / 14 / 16$ 18:00 & 83.289 & 74.459 & 105.557 & 1574.602 & 1562.797 & 1531.594 \\
\hline 10/14/16 19:00 & 83.284 & 74.459 & 105.515 & 1574.607 & 1562.797 & 1531.636 \\
\hline $10 / 14 / 16$ 20:00 & 83.282 & 74.456 & 105.578 & 1574.609 & 1562.8 & 1531.573 \\
\hline $10 / 14 / 16$ 21:00 & 83.28 & 74.456 & 105.671 & 1574.611 & 1562.8 & 1531.48 \\
\hline $10 / 14 / 16$ 22:00 & 83.275 & 74.449 & 105.77 & 1574.616 & 1562.807 & 1531.381 \\
\hline $10 / 14 / 16$ 23:00 & 83.268 & 74.433 & 105.852 & 1574.623 & 1562.823 & 1531.299 \\
\hline 10/15/16 0:00 & 83.268 & 74.428 & 105.826 & 1574.623 & 1562.828 & 1531.325 \\
\hline 10/15/16 1:00 & 83.263 & 74.429 & 105.756 & 1574.628 & 1562.827 & 1531.395 \\
\hline $10 / 15 / 162: 00$ & 83.256 & 74.417 & 105.678 & 1574.635 & 1562.839 & 1531.473 \\
\hline 10/15/16 3:00 & 83.247 & 74.412 & 105.582 & 1574.644 & 1562.844 & 1531.569 \\
\hline 10/15/16 4:00 & 83.237 & 74.4 & 105.489 & 1574.654 & 1562.856 & 1531.662 \\
\hline $10 / 15 / 16$ 5:00 & 83.228 & 74.391 & 105.393 & 1574.663 & 1562.865 & 1531.758 \\
\hline 10/15/16 6:00 & 83.221 & 74.387 & 105.299 & 1574.67 & 1562.869 & 1531.852 \\
\hline 10/15/16 7:00 & 83.216 & 74.387 & 105.208 & 1574.675 & 1562.869 & 1531.943 \\
\hline 10/15/16 8:00 & 83.216 & 74.384 & 105.231 & 1574.675 & 1562.872 & 1531.92 \\
\hline 10/15/16 9:00 & 83.214 & 74.382 & 105.309 & 1574.677 & 1562.874 & 1531.842 \\
\hline 10/15/16 10:00 & 83.216 & 74.38 & 105.397 & 1574.675 & 1562.876 & 1531.754 \\
\hline 10/15/16 11:00 & 83.216 & 74.375 & 105.486 & 1574.675 & 1562.881 & 1531.665 \\
\hline $10 / 15 / 16$ 12:00 & 83.219 & 74.373 & 105.578 & 1574.672 & 1562.883 & 1531.573 \\
\hline 10/15/16 13:00 & 83.216 & 74.366 & 105.66 & 1574.675 & 1562.89 & 1531.491 \\
\hline $10 / 15 / 16$ 14:00 & 83.212 & 74.357 & 105.709 & 1574.679 & 1562.899 & 1531.442 \\
\hline $10 / 15 / 16$ 15:00 & 83.207 & 74.342 & 105.646 & 1574.684 & 1562.914 & 1531.505 \\
\hline $10 / 15 / 16$ 16:00 & 83.2 & 74.34 & 105.571 & 1574.691 & 1562.916 & 1531.58 \\
\hline $10 / 15 / 16$ 17:00 & 83.2 & 74.347 & 105.592 & 1574.691 & 1562.909 & 1531.559 \\
\hline 10/15/16 18:00 & 83.195 & 74.338 & 105.657 & 1574.696 & 1562.918 & 1531.494 \\
\hline 10/15/16 19:00 & 83.195 & 74.328 & 105.65 & 1574.696 & 1562.928 & 1531.501 \\
\hline $10 / 15 / 16$ 20:00 & 83.197 & 74.331 & 105.575 & 1574.694 & 1562.925 & 1531.576 \\
\hline $10 / 15 / 16$ 21:00 & 83.2 & 74.335 & 105.5 & 1574.691 & 1562.921 & 1531.651 \\
\hline $10 / 15 / 16$ 22:00 & 83.207 & 74.342 & 105.54 & 1574.684 & 1562.914 & 1531.611 \\
\hline $10 / 15 / 16$ 23:00 & 83.209 & 74.349 & 105.62 & 1574.682 & 1562.907 & 1531.531 \\
\hline 10/16/16 0:00 & 83.212 & 74.345 & 105.695 & 1574.679 & 1562.911 & 1531.456 \\
\hline 10/16/16 1:00 & 83.209 & 74.328 & 105.732 & 1574.682 & 1562.928 & 1531.419 \\
\hline 10/16/16 2:00 & 83.204 & 74.324 & 105.662 & 1574.687 & 1562.932 & 1531.489 \\
\hline 10/16/16 3:00 & 83.197 & 74.317 & 105.568 & 1574.694 & 1562.939 & 1531.583 \\
\hline 10/16/16 4:00 & 83.19 & 74.312 & 105.465 & 1574.701 & 1562.944 & 1531.686 \\
\hline 10/16/16 5:00 & 83.181 & 74.305 & 105.36 & 1574.71 & 1562.951 & 1531.791 \\
\hline 10/16/16 6:00 & 83.172 & 74.298 & 105.252 & 1574.719 & 1562.958 & 1531.899 \\
\hline 10/16/16 7:00 & 83.167 & 74.296 & 105.145 & 1574.724 & 1562.96 & 1532.006 \\
\hline 10/16/16 8:00 & 83.162 & 74.294 & 105.126 & 1574.729 & 1562.962 & 1532.025 \\
\hline 10/16/16 9:00 & 83.157 & 74.282 & 105.096 & 1574.734 & 1562.974 & 1532.055 \\
\hline $10 / 16 / 16$ 10:00 & 83.153 & 74.275 & 105.014 & 1574.738 & 1562.981 & 1532.137 \\
\hline 10/16/16 11:00 & 83.153 & 74.275 & 104.92 & 1574.738 & 1562.981 & 1532.231 \\
\hline $10 / 16 / 16$ 12:00 & 83.146 & 74.271 & 104.826 & 1574.745 & 1562.985 & 1532.325 \\
\hline 10/16/16 13:00 & 83.141 & 74.261 & 104.733 & 1574.75 & 1562.995 & 1532.418 \\
\hline 10/16/16 14:00 & 83.132 & 74.257 & 104.639 & 1574.759 & 1562.999 & 1532.512 \\
\hline
\end{tabular}


TABLE S1.2 Automatically recorded groundwater levels in the confined aquifers.

\begin{tabular}{|c|c|c|c|c|c|c|}
\hline \multirow[b]{2}{*}{ Date-Time } & \multicolumn{3}{|c|}{ Measured Depth to Water (ft TOC ${ }^{a}$ ) } & \multicolumn{3}{|c|}{ Groundwater Elevation (ft AMSL ${ }^{\mathrm{b}}$ ) } \\
\hline & DL2-CU & DL7-CU & DL7-CL & DL2-CU & DL7-CU & DL7-CL \\
\hline 10/16/16 15:00 & 83.122 & 74.249 & 104.55 & 1574.769 & 1563.007 & 1532.601 \\
\hline $10 / 16 / 16$ 16:00 & 83.117 & 74.24 & 104.464 & 1574.774 & 1563.016 & 1532.687 \\
\hline $10 / 16 / 16$ 17:00 & 83.11 & 74.233 & 104.384 & 1574.781 & 1563.023 & 1532.767 \\
\hline 10/16/16 18:00 & 83.103 & 74.224 & 104.307 & 1574.788 & 1563.032 & 1532.844 \\
\hline 10/16/16 19:00 & 83.101 & 74.219 & 104.23 & 1574.79 & 1563.037 & 1532.921 \\
\hline $10 / 16 / 16$ 20:00 & 83.099 & 74.217 & 104.162 & 1574.792 & 1563.039 & 1532.989 \\
\hline $10 / 16 / 16$ 21:00 & 83.101 & 74.221 & 104.103 & 1574.79 & 1563.035 & 1533.048 \\
\hline $10 / 16 / 16$ 22:00 & 83.101 & 74.219 & 104.045 & 1574.79 & 1563.037 & 1533.106 \\
\hline 10/16/16 23:00 & 83.103 & 74.217 & 103.988 & 1574.788 & 1563.039 & 1533.163 \\
\hline 10/17/16 0:00 & 83.101 & 74.217 & 103.925 & 1574.79 & 1563.039 & 1533.226 \\
\hline 10/17/16 1:00 & 83.099 & 74.21 & 103.874 & 1574.792 & 1563.046 & 1533.277 \\
\hline 10/17/16 2:00 & 83.091 & 74.203 & 103.815 & 1574.8 & 1563.053 & 1533.336 \\
\hline 10/17/16 3:00 & 83.082 & 74.191 & 103.759 & 1574.809 & 1563.065 & 1533.392 \\
\hline 10/17/16 4:00 & 83.07 & 74.177 & 103.693 & 1574.821 & 1563.079 & 1533.458 \\
\hline 10/17/16 5:00 & 83.061 & 74.161 & 103.635 & 1574.83 & 1563.095 & 1533.516 \\
\hline 10/17/16 6:00 & 83.049 & 74.152 & 103.569 & 1574.842 & 1563.104 & 1533.582 \\
\hline 10/17/16 7:00 & 83.037 & 74.142 & 103.511 & 1574.854 & 1563.114 & 1533.64 \\
\hline 10/17/16 8:00 & 83.03 & 74.138 & 103.45 & 1574.861 & 1563.118 & 1533.701 \\
\hline 10/17/16 9:00 & 83.021 & 74.129 & 103.396 & 1574.87 & 1563.127 & 1533.755 \\
\hline 10/17/16 10:00 & 83.019 & 74.129 & 103.349 & 1574.872 & 1563.127 & 1533.802 \\
\hline 10/17/16 11:00 & 83.018 & 74.124 & 103.319 & 1574.873 & 1563.132 & 1533.832 \\
\hline $10 / 17 / 16$ 12:00 & 83.021 & 74.124 & 103.303 & 1574.87 & 1563.132 & 1533.848 \\
\hline 10/17/16 13:00 & 83.021 & 74.126 & 103.296 & 1574.87 & 1563.13 & 1533.855 \\
\hline 10/17/16 14:00 & 83.016 & 74.115 & 103.282 & 1574.875 & 1563.141 & 1533.869 \\
\hline $10 / 17 / 16$ 15:00 & 83.014 & 74.11 & 103.265 & 1574.877 & 1563.146 & 1533.886 \\
\hline 10/17/16 16:00 & 83.016 & 74.11 & 103.251 & 1574.875 & 1563.146 & 1533.9 \\
\hline 10/17/16 17:00 & 83.016 & 74.11 & 103.256 & 1574.875 & 1563.146 & 1533.895 \\
\hline 10/17/16 18:00 & 83.014 & 74.112 & 103.258 & 1574.877 & 1563.144 & 1533.893 \\
\hline 10/17/16 19:00 & 83.014 & 74.108 & 103.268 & 1574.877 & 1563.148 & 1533.883 \\
\hline 10/17/16 20:00 & 83.016 & 74.103 & 103.27 & 1574.875 & 1563.153 & 1533.881 \\
\hline 10/17/16 21:00 & 83.021 & 74.103 & 103.263 & 1574.87 & 1563.153 & 1533.888 \\
\hline $10 / 17 / 16$ 22:00 & 83.026 & 74.11 & 103.26 & 1574.865 & 1563.146 & 1533.891 \\
\hline 10/17/16 23:00 & 83.035 & 74.117 & 103.265 & 1574.856 & 1563.139 & 1533.886 \\
\hline 10/18/16 0:00 & 83.044 & 74.126 & 103.277 & 1574.847 & 1563.13 & 1533.874 \\
\hline 10/18/16 1:00 & 83.052 & 74.129 & 103.289 & 1574.839 & 1563.127 & 1533.862 \\
\hline 10/18/16 2:00 & 83.054 & 74.129 & 103.282 & 1574.837 & 1563.127 & 1533.869 \\
\hline 10/18/16 3:00 & 83.059 & 74.133 & 103.268 & 1574.832 & 1563.123 & 1533.883 \\
\hline 10/18/16 4:00 & 83.063 & 74.129 & 103.246 & 1574.828 & 1563.127 & 1533.905 \\
\hline 10/18/16 5:00 & 83.066 & 74.131 & 103.221 & 1574.825 & 1563.125 & 1533.93 \\
\hline 10/18/16 6:00 & 83.068 & 74.138 & 103.197 & 1574.823 & 1563.118 & 1533.954 \\
\hline 10/18/16 7:00 & 83.07 & 74.14 & 103.183 & 1574.821 & 1563.116 & 1533.968 \\
\hline 10/18/16 8:00 & 83.07 & 74.145 & 103.183 & 1574.821 & 1563.111 & 1533.968 \\
\hline 10/18/16 9:00 & 83.075 & 74.154 & 103.221 & 1574.816 & 1563.102 & 1533.93 \\
\hline $10 / 18 / 16$ 10:00 & 83.082 & 74.156 & 103.373 & 1574.809 & 1563.1 & 1533.778 \\
\hline 10/18/16 11:00 & 83.089 & 74.154 & 103.541 & 1574.802 & 1563.102 & 1533.61 \\
\hline $10 / 18 / 16$ 12:00 & 83.094 & 74.152 & 103.693 & 1574.797 & 1563.104 & 1533.458 \\
\hline 10/18/16 13:00 & 83.101 & 74.152 & 103.846 & 1574.79 & 1563.104 & 1533.305 \\
\hline
\end{tabular}


TABLE S1.2 Automatically recorded groundwater levels in the confined aquifers.

\begin{tabular}{|c|c|c|c|c|c|c|}
\hline \multirow[b]{2}{*}{ Date-Time } & \multicolumn{3}{|c|}{ Measured Depth to Water (ft TOC ${ }^{a}$ ) } & \multicolumn{3}{|c|}{ Groundwater Elevation (ft AMSL ${ }^{\mathrm{b}}$ ) } \\
\hline & DL2-CU & DL7-CU & DL7-CL & DL2-CU & DL7-CU & DL7-CL \\
\hline 10/18/16 14:00 & 83.101 & 74.154 & 103.993 & 1574.79 & 1563.102 & 1533.158 \\
\hline $10 / 18 / 16$ 15:00 & 83.101 & 74.145 & 104.119 & 1574.79 & 1563.111 & 1533.032 \\
\hline $10 / 18 / 16$ 16:00 & 83.099 & 74.135 & 104.159 & 1574.792 & 1563.121 & 1532.992 \\
\hline 10/18/16 17:00 & 83.096 & 74.133 & 104.127 & 1574.795 & 1563.123 & 1533.024 \\
\hline 10/18/16 18:00 & 83.096 & 74.145 & 104.094 & 1574.795 & 1563.111 & 1533.057 \\
\hline 10/18/16 19:00 & 83.096 & 74.147 & 104.173 & 1574.795 & 1563.109 & 1532.978 \\
\hline $10 / 18 / 16$ 20:00 & 83.098 & 74.152 & 104.283 & 1574.793 & 1563.104 & 1532.868 \\
\hline $10 / 18 / 16$ 21:00 & 83.101 & 74.145 & 104.396 & 1574.79 & 1563.111 & 1532.755 \\
\hline $10 / 18 / 16$ 22:00 & 83.101 & 74.145 & 104.424 & 1574.79 & 1563.111 & 1532.727 \\
\hline $10 / 18 / 16$ 23:00 & 83.103 & 74.147 & 104.375 & 1574.788 & 1563.109 & 1532.776 \\
\hline 10/19/16 0:00 & 83.106 & 74.149 & 104.314 & 1574.785 & 1563.107 & 1532.837 \\
\hline 10/19/16 1:00 & 83.11 & 74.156 & 104.248 & 1574.781 & 1563.1 & 1532.903 \\
\hline 10/19/16 2:00 & 83.108 & 74.159 & 104.171 & 1574.783 & 1563.097 & 1532.98 \\
\hline 10/19/16 3:00 & 83.11 & 74.168 & 104.166 & 1574.781 & 1563.088 & 1532.985 \\
\hline 10/19/16 4:00 & 83.108 & 74.166 & 104.244 & 1574.783 & 1563.09 & 1532.907 \\
\hline 10/19/16 5:00 & 83.106 & 74.156 & 104.283 & 1574.785 & 1563.1 & 1532.868 \\
\hline 10/19/16 6:00 & 83.103 & 74.152 & 104.22 & 1574.788 & 1563.104 & 1532.931 \\
\hline 10/19/16 7:00 & 83.099 & 74.147 & 104.141 & 1574.792 & 1563.109 & 1533.01 \\
\hline 10/19/16 8:00 & 83.096 & 74.156 & 104.141 & 1574.795 & 1563.1 & 1533.01 \\
\hline 10/19/16 9:00 & 83.096 & 74.149 & 104.199 & 1574.795 & 1563.107 & 1532.952 \\
\hline 10/19/16 10:00 & 83.096 & 74.147 & 104.15 & 1574.795 & 1563.109 & 1533.001 \\
\hline 10/19/16 11:00 & 83.096 & 74.149 & 104.077 & 1574.795 & 1563.107 & 1533.074 \\
\hline $10 / 19 / 1612: 00$ & 83.096 & 74.152 & 104 & 1574.795 & 1563.104 & 1533.151 \\
\hline 10/19/16 13:00 & 83.099 & 74.154 & 103.923 & 1574.792 & 1563.102 & 1533.228 \\
\hline 10/19/16 14:00 & 83.092 & 74.154 & 103.843 & 1574.799 & 1563.102 & 1533.308 \\
\hline $10 / 19 / 16$ 15:00 & 83.094 & 74.152 & 103.766 & 1574.797 & 1563.104 & 1533.385 \\
\hline 10/19/16 16:00 & 83.094 & 74.152 & 103.698 & 1574.797 & 1563.104 & 1533.453 \\
\hline 10/19/16 17:00 & 83.092 & 74.152 & 103.628 & 1574.799 & 1563.104 & 1533.523 \\
\hline 10/19/16 18:00 & 83.094 & 74.149 & 103.562 & 1574.797 & 1563.107 & 1533.589 \\
\hline 10/19/16 19:00 & 83.096 & 74.152 & 103.502 & 1574.795 & 1563.104 & 1533.649 \\
\hline 10/19/16 20:00 & 83.099 & 74.147 & 103.438 & 1574.792 & 1563.109 & 1533.713 \\
\hline 10/19/16 21:00 & 83.096 & 74.152 & 103.38 & 1574.795 & 1563.104 & 1533.771 \\
\hline $10 / 19 / 16$ 22:00 & 83.092 & 74.145 & 103.321 & 1574.799 & 1563.111 & 1533.83 \\
\hline 10/19/16 23:00 & 83.096 & 74.149 & 103.268 & 1574.795 & 1563.107 & 1533.883 \\
\hline 10/20/16 0:00 & 83.096 & 74.154 & 103.218 & 1574.795 & 1563.102 & 1533.933 \\
\hline 10/20/16 1:00 & 83.099 & 74.156 & 103.169 & 1574.792 & 1563.1 & 1533.982 \\
\hline $10 / 20 / 162: 00$ & 83.103 & 74.154 & 103.12 & 1574.788 & 1563.102 & 1534.031 \\
\hline 10/20/16 3:00 & 83.103 & 74.159 & 103.076 & 1574.788 & 1563.097 & 1534.075 \\
\hline 10/20/16 4:00 & 83.103 & 74.154 & 103.026 & 1574.788 & 1563.102 & 1534.125 \\
\hline 10/20/16 5:00 & 83.103 & 74.156 & 102.98 & 1574.788 & 1563.1 & 1534.171 \\
\hline 10/20/16 6:00 & 83.103 & 74.159 & 102.93 & 1574.788 & 1563.097 & 1534.221 \\
\hline 10/20/16 7:00 & 83.101 & 74.154 & 102.881 & 1574.79 & 1563.102 & 1534.27 \\
\hline 10/20/16 8:00 & 83.096 & 74.152 & 102.832 & 1574.795 & 1563.104 & 1534.319 \\
\hline $10 / 20 / 169: 00$ & 83.096 & 74.154 & 102.797 & 1574.795 & 1563.102 & 1534.354 \\
\hline $10 / 20 / 16$ 10:00 & 83.092 & 74.154 & 102.895 & 1574.799 & 1563.102 & 1534.256 \\
\hline $10 / 20 / 1611: 00$ & 83.092 & 74.149 & 103.017 & 1574.799 & 1563.107 & 1534.134 \\
\hline $10 / 20 / 1612: 00$ & 83.089 & 74.135 & 103.038 & 1574.802 & 1563.121 & 1534.113 \\
\hline
\end{tabular}


TABLE S1.2 Automatically recorded groundwater levels in the confined aquifers.

\begin{tabular}{|c|c|c|c|c|c|c|}
\hline \multirow[b]{2}{*}{ Date-Time } & \multicolumn{3}{|c|}{ Measured Depth to Water (ft TOC ${ }^{a}$ ) } & \multicolumn{3}{|c|}{ Groundwater Elevation (ft AMSL ${ }^{\mathrm{b}}$ ) } \\
\hline & DL2-CU & DL7-CU & DL7-CL & DL2-CU & DL7-CU & DL7-CL \\
\hline 10/20/16 13:00 & 83.085 & 74.138 & 103.022 & 1574.806 & 1563.118 & 1534.129 \\
\hline $10 / 20 / 1614: 00$ & 83.08 & 74.135 & 103.111 & 1574.811 & 1563.121 & 1534.04 \\
\hline $10 / 20 / 16$ 15:00 & 83.077 & 74.129 & 103.235 & 1574.814 & 1563.127 & 1533.916 \\
\hline $10 / 20 / 16$ 16:00 & 83.075 & 74.117 & 103.265 & 1574.816 & 1563.139 & 1533.886 \\
\hline $10 / 20 / 16$ 17:00 & 83.068 & 74.11 & 103.232 & 1574.823 & 1563.146 & 1533.919 \\
\hline $10 / 20 / 16$ 18:00 & 83.066 & 74.115 & 103.277 & 1574.825 & 1563.141 & 1533.874 \\
\hline 10/20/16 19:00 & 83.061 & 74.112 & 103.389 & 1574.83 & 1563.144 & 1533.762 \\
\hline $10 / 20 / 16$ 20:00 & 83.056 & 74.103 & 103.511 & 1574.835 & 1563.153 & 1533.64 \\
\hline $10 / 20 / 1621: 00$ & 83.054 & 74.089 & 103.548 & 1574.837 & 1563.167 & 1533.603 \\
\hline $10 / 20 / 16$ 22:00 & 83.051 & 74.087 & 103.513 & 1574.84 & 1563.169 & 1533.638 \\
\hline $10 / 20 / 16$ 23:00 & 83.044 & 74.089 & 103.455 & 1574.847 & 1563.167 & 1533.696 \\
\hline 10/21/16 0:00 & 83.042 & 74.087 & 103.392 & 1574.849 & 1563.169 & 1533.759 \\
\hline 10/21/16 1:00 & 83.037 & 74.084 & 103.321 & 1574.854 & 1563.172 & 1533.83 \\
\hline 10/21/16 2:00 & 83.033 & 74.082 & 103.249 & 1574.858 & 1563.174 & 1533.902 \\
\hline 10/21/16 3:00 & 83.03 & 74.077 & 103.169 & 1574.861 & 1563.179 & 1533.982 \\
\hline 10/21/16 4:00 & 83.026 & 74.075 & 103.09 & 1574.865 & 1563.181 & 1534.061 \\
\hline 10/21/16 5:00 & 83.019 & 74.075 & 103.019 & 1574.872 & 1563.181 & 1534.132 \\
\hline 10/21/16 6:00 & 83.011 & 74.075 & 103.054 & 1574.88 & 1563.181 & 1534.097 \\
\hline 10/21/16 7:00 & 83.007 & 74.073 & 103.146 & 1574.884 & 1563.183 & 1534.005 \\
\hline 10/21/16 8:00 & 83 & 74.056 & 103.244 & 1574.891 & 1563.2 & 1533.907 \\
\hline 10/21/16 9:00 & 82.993 & 74.047 & 103.249 & 1574.898 & 1563.209 & 1533.902 \\
\hline $10 / 21 / 1610: 00$ & 82.988 & 74.045 & 103.193 & 1574.903 & 1563.211 & 1533.958 \\
\hline $10 / 21 / 1611: 00$ & 82.979 & 74.036 & 103.129 & 1574.912 & 1563.22 & 1534.022 \\
\hline $10 / 21 / 1612: 00$ & 82.969 & 74.029 & 103.083 & 1574.922 & 1563.227 & 1534.068 \\
\hline $10 / 21 / 16$ 13:00 & 82.962 & 74.022 & 103.029 & 1574.929 & 1563.234 & 1534.122 \\
\hline $10 / 21 / 1614: 00$ & 82.953 & 74.015 & 102.973 & 1574.938 & 1563.241 & 1534.178 \\
\hline $10 / 21 / 16$ 15:00 & 82.943 & 74.003 & 102.919 & 1574.948 & 1563.253 & 1534.232 \\
\hline 10/21/16 16:00 & 82.941 & 74.003 & 102.874 & 1574.95 & 1563.253 & 1534.277 \\
\hline 10/21/16 17:00 & 82.936 & 73.999 & 102.844 & 1574.955 & 1563.257 & 1534.307 \\
\hline $10 / 21 / 1618: 00$ & 82.932 & 73.994 & 102.813 & 1574.959 & 1563.262 & 1534.338 \\
\hline 10/21/16 19:00 & 82.929 & 73.987 & 102.778 & 1574.962 & 1563.269 & 1534.373 \\
\hline $10 / 21 / 16$ 20:00 & 82.927 & 73.984 & 102.734 & 1574.964 & 1563.272 & 1534.417 \\
\hline $10 / 21 / 16$ 21:00 & 82.922 & 73.98 & 102.696 & 1574.969 & 1563.276 & 1534.455 \\
\hline $10 / 21 / 16$ 22:00 & 82.92 & 73.977 & 102.661 & 1574.971 & 1563.279 & 1534.49 \\
\hline $10 / 21 / 16$ 23:00 & 82.915 & 73.975 & 102.638 & 1574.976 & 1563.281 & 1534.513 \\
\hline 10/22/16 0:00 & 82.91 & 73.975 & 102.626 & 1574.981 & 1563.281 & 1534.525 \\
\hline 10/22/16 1:00 & 82.908 & 73.97 & 102.617 & 1574.983 & 1563.286 & 1534.534 \\
\hline 10/22/16 2:00 & 82.906 & 73.966 & 102.61 & 1574.985 & 1563.29 & 1534.541 \\
\hline 10/22/16 3:00 & 82.903 & 73.961 & 102.61 & 1574.988 & 1563.295 & 1534.541 \\
\hline 10/22/16 4:00 & 82.901 & 73.956 & 102.589 & 1574.99 & 1563.3 & 1534.562 \\
\hline 10/22/16 5:00 & 82.899 & 73.95 & 102.561 & 1574.992 & 1563.306 & 1534.59 \\
\hline 10/22/16 6:00 & 82.898 & 73.947 & 102.53 & 1574.993 & 1563.309 & 1534.621 \\
\hline 10/22/16 7:00 & 82.894 & 73.943 & 102.49 & 1574.997 & 1563.313 & 1534.661 \\
\hline $10 / 22 / 168: 00$ & 82.892 & 73.938 & 102.446 & 1574.999 & 1563.318 & 1534.705 \\
\hline 10/22/16 9:00 & 82.887 & 73.936 & 102.404 & 1575.004 & 1563.32 & 1534.747 \\
\hline $10 / 22 / 16$ 10:00 & 82.885 & 73.938 & 102.369 & 1575.006 & 1563.318 & 1534.782 \\
\hline 10/22/16 11:00 & 82.882 & 73.94 & 102.348 & 1575.009 & 1563.316 & 1534.803 \\
\hline
\end{tabular}


TABLE S1.2 Automatically recorded groundwater levels in the confined aquifers.

\begin{tabular}{|c|c|c|c|c|c|c|}
\hline \multirow[b]{2}{*}{ Date-Time } & \multicolumn{3}{|c|}{ Measured Depth to Water (ft TOC ${ }^{\mathrm{a}}$ ) } & \multicolumn{3}{|c|}{ Groundwater Elevation (ft $\mathrm{AMSL}^{\mathrm{b}}$ ) } \\
\hline & DL2-CU & DL7-CU & DL7-CL & DL2-CU & DL7-CU & DL7-CL \\
\hline 10/22/16 12:00 & 82.882 & 73.954 & 102.312 & 1575.009 & 1563.302 & 1534.839 \\
\hline $10 / 22 / 16$ 13:00 & 82.891 & 73.97 & 102.277 & 1575 & 1563.286 & 1534.874 \\
\hline $10 / 22 / 1614: 00$ & 82.903 & 74.001 & 102.228 & 1574.988 & 1563.255 & 1534.923 \\
\hline $10 / 22 / 1615: 00$ & 82.925 & 74.047 & 102.174 & 1574.966 & 1563.209 & 1534.977 \\
\hline $10 / 22 / 16$ 16:00 & 82.95 & 74.089 & 102.125 & 1574.941 & 1563.167 & 1535.026 \\
\hline $10 / 22 / 16$ 17:00 & 82.974 & 74.11 & 102.081 & 1574.917 & 1563.146 & 1535.07 \\
\hline $10 / 22 / 16$ 18:00 & 82.997 & 74.154 & 102.025 & 1574.894 & 1563.102 & 1535.126 \\
\hline 10/22/16 19:00 & 83.033 & 74.203 & 101.975 & 1574.858 & 1563.053 & 1535.176 \\
\hline $10 / 22 / 16$ 20:00 & 83.061 & 74.233 & 101.924 & 1574.83 & 1563.023 & 1535.227 \\
\hline $10 / 22 / 16$ 21:00 & 83.077 & 74.263 & 101.868 & 1574.814 & 1562.993 & 1535.283 \\
\hline $10 / 22 / 16$ 22:00 & 83.099 & 74.298 & 101.809 & 1574.792 & 1562.958 & 1535.342 \\
\hline $10 / 22 / 16$ 23:00 & 83.125 & 74.326 & 101.758 & 1574.766 & 1562.93 & 1535.393 \\
\hline $10 / 23 / 160: 00$ & 83.138 & 74.345 & 101.704 & 1574.753 & 1562.911 & 1535.447 \\
\hline 10/23/16 1:00 & 83.157 & 74.384 & 101.645 & 1574.734 & 1562.872 & 1535.506 \\
\hline $10 / 23 / 162: 00$ & 83.186 & 74.414 & 101.592 & 1574.705 & 1562.842 & 1535.559 \\
\hline 10/23/16 3:00 & 83.207 & 74.428 & 101.538 & 1574.684 & 1562.828 & 1535.613 \\
\hline $10 / 23 / 164: 00$ & 83.216 & 74.433 & 101.496 & 1574.675 & 1562.823 & 1535.655 \\
\hline $10 / 23 / 165: 00$ & 83.226 & 74.449 & 101.442 & 1574.665 & 1562.807 & 1535.709 \\
\hline 10/23/16 6:00 & 83.24 & 74.468 & 101.395 & 1574.651 & 1562.788 & 1535.756 \\
\hline 10/23/16 7:00 & 83.254 & 74.477 & 101.344 & 1574.637 & 1562.779 & 1535.807 \\
\hline $10 / 23 / 168: 00$ & 83.258 & 74.466 & 101.299 & 1574.633 & 1562.79 & 1535.852 \\
\hline 10/23/16 9:00 & 83.256 & 74.454 & 101.252 & 1574.635 & 1562.802 & 1535.899 \\
\hline 10/23/16 10:00 & 83.249 & 74.442 & 101.205 & 1574.642 & 1562.814 & 1535.946 \\
\hline 10/23/16 11:00 & 83.237 & 74.419 & 101.156 & 1574.654 & 1562.837 & 1535.995 \\
\hline $10 / 23 / 1612: 00$ & 83.225 & 74.396 & 101.109 & 1574.666 & 1562.86 & 1536.042 \\
\hline 10/23/16 13:00 & 83.212 & 74.377 & 101.063 & 1574.679 & 1562.879 & 1536.088 \\
\hline $10 / 23 / 16$ 14:00 & 83.195 & 74.352 & 101.02 & 1574.696 & 1562.904 & 1536.131 \\
\hline $10 / 23 / 16$ 15:00 & 83.179 & 74.331 & 100.981 & 1574.712 & 1562.925 & 1536.17 \\
\hline $10 / 23 / 16$ 16:00 & 83.167 & 74.305 & 100.95 & 1574.724 & 1562.951 & 1536.201 \\
\hline $10 / 23 / 16$ 17:00 & 83.155 & 74.294 & 100.92 & 1574.736 & 1562.962 & 1536.231 \\
\hline $10 / 23 / 16$ 18:00 & 83.143 & 74.275 & 100.889 & 1574.748 & 1562.981 & 1536.262 \\
\hline 10/23/16 19:00 & 83.134 & 74.261 & 100.864 & 1574.757 & 1562.995 & 1536.287 \\
\hline $10 / 23 / 16$ 20:00 & 83.125 & 74.247 & 100.84 & 1574.766 & 1563.009 & 1536.311 \\
\hline $10 / 23 / 1621: 00$ & 83.117 & 74.231 & 100.817 & 1574.774 & 1563.025 & 1536.334 \\
\hline $10 / 23 / 1622: 00$ & 83.108 & 74.221 & 100.796 & 1574.783 & 1563.035 & 1536.355 \\
\hline $10 / 23 / 16$ 23:00 & 83.101 & 74.208 & 100.775 & 1574.79 & 1563.048 & 1536.376 \\
\hline $10 / 24 / 160: 00$ & 83.092 & 74.198 & 100.758 & 1574.799 & 1563.058 & 1536.393 \\
\hline 10/24/16 1:00 & 83.082 & 74.18 & 100.735 & 1574.809 & 1563.076 & 1536.416 \\
\hline 10/24/16 2:00 & 83.073 & 74.171 & 100.716 & 1574.818 & 1563.085 & 1536.435 \\
\hline 10/24/16 3:00 & 83.068 & 74.161 & 100.697 & 1574.823 & 1563.095 & 1536.454 \\
\hline 10/24/16 4:00 & 83.059 & 74.152 & 100.669 & 1574.832 & 1563.104 & 1536.482 \\
\hline 10/24/16 5:00 & 83.054 & 74.145 & 100.66 & 1574.837 & 1563.111 & 1536.491 \\
\hline 10/24/16 6:00 & 83.049 & 74.133 & 100.637 & 1574.842 & 1563.123 & 1536.514 \\
\hline 10/24/16 7:00 & 83.042 & 74.126 & 100.618 & 1574.849 & 1563.13 & 1536.533 \\
\hline 10/24/16 8:00 & 83.037 & 74.117 & 100.592 & 1574.854 & 1563.139 & 1536.559 \\
\hline 10/24/16 9:00 & 83.035 & 74.112 & 100.576 & 1574.856 & 1563.144 & 1536.575 \\
\hline $10 / 24 / 16$ 10:00 & 83.028 & 74.108 & 100.559 & 1574.863 & 1563.148 & 1536.592 \\
\hline
\end{tabular}


TABLE S1.2 Automatically recorded groundwater levels in the confined aquifers.

\begin{tabular}{|c|c|c|c|c|c|c|}
\hline \multirow[b]{2}{*}{ Date-Time } & \multicolumn{3}{|c|}{ Measured Depth to Water (ft TOC ${ }^{\mathrm{a}}$ ) } & \multicolumn{3}{|c|}{ Groundwater Elevation (ft AMSL ${ }^{\mathrm{b}}$ ) } \\
\hline & DL2-CU & DL7-CU & DL7-CL & DL2-CU & DL7-CU & DL7-CL \\
\hline 10/24/16 11:00 & 83.023 & 74.096 & 100.55 & 1574.868 & 1563.16 & 1536.601 \\
\hline $10 / 24 / 1612: 00$ & 83.014 & 74.087 & 100.55 & 1574.877 & 1563.169 & 1536.601 \\
\hline $10 / 24 / 1613: 00$ & 83.004 & 74.073 & 100.559 & 1574.887 & 1563.183 & 1536.592 \\
\hline $10 / 24 / 1614: 00$ & 82.99 & 74.059 & 100.562 & 1574.901 & 1563.197 & 1536.589 \\
\hline $10 / 24 / 16$ 15:00 & 82.981 & 74.047 & 100.564 & 1574.91 & 1563.209 & 1536.587 \\
\hline $10 / 24 / 16$ 16:00 & 82.969 & 74.036 & 100.576 & 1574.922 & 1563.22 & 1536.575 \\
\hline 10/24/16 17:00 & 82.962 & 74.026 & 100.602 & 1574.929 & 1563.23 & 1536.549 \\
\hline $10 / 24 / 16$ 18:00 & 82.955 & 74.015 & 100.625 & 1574.936 & 1563.241 & 1536.526 \\
\hline 10/24/16 19:00 & 82.948 & 74.01 & 100.653 & 1574.943 & 1563.246 & 1536.498 \\
\hline $10 / 24 / 16$ 20:00 & 82.946 & 74.001 & 100.672 & 1574.945 & 1563.255 & 1536.479 \\
\hline $10 / 24 / 1621: 00$ & 82.938 & 73.989 & 100.683 & 1574.953 & 1563.267 & 1536.468 \\
\hline $10 / 24 / 1622: 00$ & 82.934 & 73.987 & 100.688 & 1574.957 & 1563.269 & 1536.463 \\
\hline $10 / 24 / 16$ 23:00 & 82.927 & 73.98 & 100.7 & 1574.964 & 1563.276 & 1536.451 \\
\hline 10/25/16 0:00 & 82.917 & 73.97 & 100.723 & 1574.974 & 1563.286 & 1536.428 \\
\hline 10/25/16 1:00 & 82.908 & 73.961 & 100.747 & 1574.983 & 1563.295 & 1536.404 \\
\hline 10/25/16 2:00 & 82.901 & 73.952 & 100.756 & 1574.99 & 1563.304 & 1536.395 \\
\hline 10/25/16 3:00 & 82.892 & 73.94 & 100.758 & 1574.999 & 1563.316 & 1536.393 \\
\hline $10 / 25 / 164: 00$ & 82.88 & 73.929 & 100.747 & 1575.011 & 1563.327 & 1536.404 \\
\hline $10 / 25 / 16$ 5:00 & 82.875 & 73.922 & 100.737 & 1575.016 & 1563.334 & 1536.414 \\
\hline 10/25/16 6:00 & 82.868 & 73.913 & 100.721 & 1575.023 & 1563.343 & 1536.43 \\
\hline 10/25/16 7:00 & 82.861 & 73.905 & 100.702 & 1575.03 & 1563.351 & 1536.449 \\
\hline $10 / 25 / 168: 00$ & 82.856 & 73.905 & 100.697 & 1575.035 & 1563.351 & 1536.454 \\
\hline 10/25/16 9:00 & 82.852 & 73.903 & 100.707 & 1575.039 & 1563.353 & 1536.444 \\
\hline $10 / 25 / 16$ 10:00 & 82.847 & 73.898 & 100.726 & 1575.044 & 1563.358 & 1536.425 \\
\hline $10 / 25 / 1611: 00$ & 82.84 & 73.889 & 100.737 & 1575.051 & 1563.367 & 1536.414 \\
\hline $10 / 25 / 1612: 00$ & 82.835 & 73.877 & 100.74 & 1575.056 & 1563.379 & 1536.411 \\
\hline $10 / 25 / 16$ 13:00 & 82.833 & 73.868 & 100.749 & 1575.058 & 1563.388 & 1536.402 \\
\hline $10 / 25 / 1614: 00$ & 82.833 & 73.857 & 100.747 & 1575.058 & 1563.399 & 1536.404 \\
\hline $10 / 25 / 16$ 15:00 & 82.835 & 73.852 & 100.749 & 1575.056 & 1563.404 & 1536.402 \\
\hline 10/25/16 16:00 & 82.84 & 73.843 & 100.756 & 1575.051 & 1563.413 & 1536.395 \\
\hline $10 / 25 / 16$ 17:00 & 82.845 & 73.843 & 100.749 & 1575.046 & 1563.413 & 1536.402 \\
\hline 10/25/16 18:00 & 82.852 & 73.843 & 100.735 & 1575.039 & 1563.413 & 1536.416 \\
\hline 10/25/16 19:00 & 82.861 & 73.857 & 100.742 & 1575.03 & 1563.399 & 1536.409 \\
\hline $10 / 25 / 16$ 20:00 & 82.87 & 73.866 & 100.756 & 1575.021 & 1563.39 & 1536.395 \\
\hline $10 / 25 / 1621: 00$ & 82.882 & 73.873 & 100.77 & 1575.009 & 1563.383 & 1536.381 \\
\hline $10 / 25 / 16$ 22:00 & 82.894 & 73.882 & 100.761 & 1574.997 & 1563.374 & 1536.39 \\
\hline $10 / 25 / 16$ 23:00 & 82.896 & 73.889 & 100.754 & 1574.995 & 1563.367 & 1536.397 \\
\hline 10/26/16 0:00 & 82.91 & 73.903 & 100.768 & 1574.981 & 1563.353 & 1536.383 \\
\hline 10/26/16 1:00 & 82.92 & 73.912 & 100.775 & 1574.971 & 1563.344 & 1536.376 \\
\hline 10/26/16 2:00 & 82.929 & 73.922 & 100.772 & 1574.962 & 1563.334 & 1536.379 \\
\hline 10/26/16 3:00 & 82.941 & 73.926 & 100.756 & 1574.95 & 1563.33 & 1536.395 \\
\hline 10/26/16 4:00 & 82.948 & 73.943 & 100.733 & 1574.943 & 1563.313 & 1536.418 \\
\hline 10/26/16 5:00 & 82.955 & 73.961 & 100.709 & 1574.936 & 1563.295 & 1536.442 \\
\hline 10/26/16 6:00 & 82.955 & 73.973 & 100.7 & 1574.936 & 1563.283 & 1536.451 \\
\hline 10/26/16 7:00 & 82.957 & 73.982 & 100.693 & 1574.934 & 1563.274 & 1536.458 \\
\hline 10/26/16 8:00 & 82.967 & 73.998 & 100.676 & 1574.924 & 1563.258 & 1536.475 \\
\hline 10/26/16 9:00 & 82.978 & 74.017 & 100.702 & 1574.913 & 1563.239 & 1536.449 \\
\hline
\end{tabular}


TABLE S1.2 Automatically recorded groundwater levels in the confined aquifers.

\begin{tabular}{|c|c|c|c|c|c|c|}
\hline \multirow[b]{2}{*}{ Date-Time } & \multicolumn{3}{|c|}{ Measured Depth to Water $\left(\mathrm{ft} \mathrm{TOC}^{\mathrm{a}}\right.$ ) } & \multicolumn{3}{|c|}{ Groundwater Elevation (ft $A M S L^{b}$ ) } \\
\hline & DL2-CU & DL7-CU & DL7-CL & DL2-CU & DL7-CU & DL7-CL \\
\hline 10/26/16 10:00 & 82.988 & 74.038 & 100.726 & 1574.903 & 1563.218 & 1536.425 \\
\hline 10/26/16 11:00 & 83.007 & 74.077 & 100.775 & 1574.884 & 1563.179 & 1536.376 \\
\hline $10 / 26 / 16$ 12:00 & 83.026 & 74.119 & 100.88 & 1574.865 & 1563.137 & 1536.271 \\
\hline 10/26/16 13:00 & 83.051 & 74.187 & 100.871 & 1574.84 & 1563.069 & 1536.28 \\
\hline $10 / 26 / 16$ 14:00 & 83.077 & 74.247 & 100.84 & 1574.814 & 1563.009 & 1536.311 \\
\hline $10 / 26 / 16$ 15:00 & 83.096 & 74.296 & 100.922 & 1574.795 & 1562.96 & 1536.229 \\
\hline 10/26/16 16:00 & 83.122 & 74.352 & 100.946 & 1574.769 & 1562.904 & 1536.205 \\
\hline $10 / 26 / 16$ 17:00 & 83.15 & 74.414 & 100.882 & 1574.741 & 1562.842 & 1536.269 \\
\hline 10/26/16 18:00 & 83.174 & 74.461 & 100.868 & 1574.717 & 1562.795 & 1536.283 \\
\hline 10/26/16 19:00 & 83.192 & 74.5 & 100.948 & 1574.699 & 1562.756 & 1536.203 \\
\hline 10/26/16 20:00 & 83.216 & 74.556 & 101.03 & 1574.675 & 1562.7 & 1536.121 \\
\hline 10/26/16 21:00 & 83.249 & 74.628 & 101.011 & 1574.642 & 1562.628 & 1536.14 \\
\hline $10 / 26 / 16$ 22:00 & 83.282 & 74.682 & 100.936 & 1574.609 & 1562.574 & 1536.215 \\
\hline 10/26/16 23:00 & 83.301 & 74.705 & 100.854 & 1574.59 & 1562.551 & 1536.297 \\
\hline 10/27/16 0:00 & 83.303 & 74.719 & 100.871 & 1574.588 & 1562.537 & 1536.28 \\
\hline $10 / 27 / 16$ 1:00 & 83.299 & 74.726 & 100.861 & 1574.592 & 1562.53 & 1536.29 \\
\hline $10 / 27 / 16$ 2:00 & 83.299 & 74.742 & 100.784 & 1574.592 & 1562.514 & 1536.367 \\
\hline 10/27/16 3:00 & 83.296 & 74.742 & 100.69 & 1574.595 & 1562.514 & 1536.461 \\
\hline $10 / 27 / 164: 00$ & 83.28 & 74.728 & 100.604 & 1574.611 & 1562.528 & 1536.547 \\
\hline $10 / 27 / 165: 00$ & 83.263 & 74.707 & 100.522 & 1574.628 & 1562.549 & 1536.629 \\
\hline $10 / 27 / 16$ 6:00 & 83.24 & 74.679 & 100.44 & 1574.651 & 1562.577 & 1536.711 \\
\hline $10 / 27 / 16$ 7:00 & 83.212 & 74.659 & 100.398 & 1574.679 & 1562.597 & 1536.753 \\
\hline 10/27/16 8:00 & 83.193 & 74.649 & 100.47 & 1574.698 & 1562.607 & 1536.681 \\
\hline 10/27/16 9:00 & 83.188 & 74.668 & 100.527 & 1574.703 & 1562.588 & 1536.624 \\
\hline 10/27/16 10:00 & 83.2 & 74.71 & 100.466 & 1574.691 & 1562.546 & 1536.685 \\
\hline 10/27/16 11:00 & 83.212 & 74.735 & 100.386 & 1574.679 & 1562.521 & 1536.765 \\
\hline $10 / 27 / 1612: 00$ & 83.214 & 74.731 & 100.304 & 1574.677 & 1562.525 & 1536.847 \\
\hline 10/27/16 13:00 & 83.2 & 74.717 & 100.22 & 1574.691 & 1562.539 & 1536.931 \\
\hline 10/27/16 14:00 & 83.183 & 74.691 & 100.136 & 1574.708 & 1562.565 & 1537.015 \\
\hline 10/27/16 15:00 & 83.157 & 74.661 & 100.061 & 1574.734 & 1562.595 & 1537.09 \\
\hline 10/27/16 16:00 & 83.132 & 74.629 & 99.986 & 1574.759 & 1562.627 & 1537.165 \\
\hline 10/27/16 17:00 & 83.106 & 74.6 & 99.916 & 1574.785 & 1562.656 & 1537.235 \\
\hline 10/27/16 18:00 & 83.077 & 74.57 & 99.85 & 1574.814 & 1562.686 & 1537.301 \\
\hline 10/27/16 19:00 & 83.056 & 74.545 & 99.794 & 1574.835 & 1562.711 & 1537.357 \\
\hline $10 / 27 / 16$ 20:00 & 83.035 & 74.519 & 99.738 & 1574.856 & 1562.737 & 1537.413 \\
\hline $10 / 27 / 16$ 21:00 & 83.012 & 74.496 & 99.684 & 1574.879 & 1562.76 & 1537.467 \\
\hline $10 / 27 / 16$ 22:00 & 82.99 & 74.468 & 99.635 & 1574.901 & 1562.788 & 1537.516 \\
\hline 10/27/16 23:00 & 82.969 & 74.447 & 99.59 & 1574.922 & 1562.809 & 1537.561 \\
\hline $10 / 28 / 160: 00$ & 82.945 & 74.426 & 99.546 & 1574.946 & 1562.83 & 1537.605 \\
\hline $10 / 28 / 16$ 1:00 & 82.925 & 74.398 & 99.499 & 1574.966 & 1562.858 & 1537.652 \\
\hline $10 / 28 / 162: 00$ & 82.901 & 74.38 & 99.45 & 1574.99 & 1562.876 & 1537.701 \\
\hline 10/28/16 3:00 & 82.878 & 74.356 & 99.41 & 1575.013 & 1562.9 & 1537.741 \\
\hline $10 / 28 / 164: 00$ & 82.856 & 74.331 & 99.366 & 1575.035 & 1562.925 & 1537.785 \\
\hline $10 / 28 / 165: 00$ & 82.835 & 74.308 & 99.328 & 1575.056 & 1562.948 & 1537.823 \\
\hline $10 / 28 / 16$ 6:00 & 82.818 & 74.294 & 99.291 & 1575.073 & 1562.962 & 1537.86 \\
\hline 10/28/16 7:00 & 82.8 & 74.273 & 99.256 & 1575.091 & 1562.983 & 1537.895 \\
\hline 10/28/16 8:00 & 82.783 & 74.256 & 99.225 & 1575.108 & 1563 & 1537.926 \\
\hline
\end{tabular}


TABLE S1.2 Automatically recorded groundwater levels in the confined aquifers.

\begin{tabular}{|c|c|c|c|c|c|c|}
\hline \multirow[b]{2}{*}{ Date-Time } & \multicolumn{3}{|c|}{ Measured Depth to Water (ft TOC ${ }^{\mathrm{a}}$ ) } & \multicolumn{3}{|c|}{ Groundwater Elevation (ft AMSL ${ }^{\mathrm{b}}$ ) } \\
\hline & DL2-CU & DL7-CU & DL7-CL & DL2-CU & DL7-CU & DL7-CL \\
\hline 10/28/16 9:00 & 82.771 & 74.245 & 99.202 & 1575.12 & 1563.011 & 1537.949 \\
\hline $10 / 28 / 1610: 00$ & 82.762 & 74.236 & 99.176 & 1575.129 & 1563.02 & 1537.975 \\
\hline $10 / 28 / 1611: 00$ & 82.75 & 74.224 & 99.15 & 1575.141 & 1563.032 & 1538.001 \\
\hline $10 / 28 / 16$ 12:00 & 82.736 & 74.208 & 99.129 & 1575.155 & 1563.048 & 1538.022 \\
\hline 10/28/16 13:00 & 82.727 & 74.194 & 99.113 & 1575.164 & 1563.062 & 1538.038 \\
\hline $10 / 28 / 16$ 14:00 & 82.708 & 74.178 & 99.089 & 1575.183 & 1563.078 & 1538.062 \\
\hline $10 / 28 / 16$ 15:00 & 82.696 & 74.164 & 99.075 & 1575.195 & 1563.092 & 1538.076 \\
\hline $10 / 28 / 16$ 16:00 & 82.685 & 74.149 & 99.066 & 1575.206 & 1563.107 & 1538.085 \\
\hline $10 / 28 / 16$ 17:00 & 82.675 & 74.142 & 99.061 & 1575.216 & 1563.114 & 1538.09 \\
\hline 10/28/16 18:00 & 82.663 & 74.126 & 99.059 & 1575.228 & 1563.13 & 1538.092 \\
\hline 10/28/16 19:00 & 82.659 & 74.119 & 99.061 & 1575.232 & 1563.137 & 1538.09 \\
\hline $10 / 28 / 16$ 20:00 & 82.654 & 74.112 & 99.066 & 1575.237 & 1563.144 & 1538.085 \\
\hline $10 / 28 / 1621: 00$ & 82.651 & 74.105 & 99.073 & 1575.24 & 1563.151 & 1538.078 \\
\hline $10 / 28 / 1622: 00$ & 82.647 & 74.096 & 99.08 & 1575.244 & 1563.16 & 1538.071 \\
\hline $10 / 28 / 16$ 23:00 & 82.64 & 74.089 & 99.078 & 1575.251 & 1563.167 & 1538.073 \\
\hline 10/29/16 0:00 & 82.633 & 74.08 & 99.08 & 1575.258 & 1563.176 & 1538.071 \\
\hline 10/29/16 1:00 & 82.628 & 74.07 & 99.078 & 1575.263 & 1563.186 & 1538.073 \\
\hline $10 / 29 / 162: 00$ & 82.618 & 74.056 & 99.071 & 1575.273 & 1563.2 & 1538.08 \\
\hline 10/29/16 3:00 & 82.609 & 74.045 & 99.066 & 1575.282 & 1563.211 & 1538.085 \\
\hline 10/29/16 4:00 & 82.597 & 74.029 & 99.059 & 1575.294 & 1563.227 & 1538.092 \\
\hline 10/29/16 5:00 & 82.586 & 74.012 & 99.045 & 1575.305 & 1563.244 & 1538.106 \\
\hline 10/29/16 6:00 & 82.581 & 74.005 & 99.04 & 1575.31 & 1563.251 & 1538.111 \\
\hline 10/29/16 7:00 & 82.574 & 73.996 & 99.029 & 1575.317 & 1563.26 & 1538.122 \\
\hline 10/29/16 8:00 & 82.569 & 73.982 & 99.019 & 1575.322 & 1563.274 & 1538.132 \\
\hline 10/29/16 9:00 & 82.564 & 73.985 & 99.019 & 1575.327 & 1563.271 & 1538.132 \\
\hline 10/29/16 10:00 & 82.565 & 73.982 & 99.125 & 1575.326 & 1563.274 & 1538.026 \\
\hline 10/29/16 11:00 & 82.562 & 73.977 & 99.307 & 1575.329 & 1563.279 & 1537.844 \\
\hline 10/29/16 12:00 & 82.56 & 73.966 & 99.49 & 1575.331 & 1563.29 & 1537.661 \\
\hline 10/29/16 13:00 & 82.553 & 73.952 & 99.679 & 1575.338 & 1563.304 & 1537.472 \\
\hline 10/29/16 14:00 & 82.551 & 73.943 & 99.867 & 1575.34 & 1563.313 & 1537.284 \\
\hline 10/29/16 15:00 & 82.546 & 73.931 & 100.049 & 1575.345 & 1563.325 & 1537.102 \\
\hline 10/29/16 16:00 & 82.541 & 73.92 & 100.229 & 1575.35 & 1563.336 & 1536.922 \\
\hline 10/29/16 17:00 & 82.538 & 73.917 & 100.41 & 1575.353 & 1563.339 & 1536.741 \\
\hline $10 / 29 / 16$ 18:00 & 82.541 & 73.912 & 100.585 & 1575.35 & 1563.344 & 1536.566 \\
\hline 10/29/16 19:00 & 82.541 & 73.912 & 100.751 & 1575.35 & 1563.344 & 1536.4 \\
\hline $10 / 29 / 16$ 20:00 & 82.541 & 73.91 & 100.915 & 1575.35 & 1563.346 & 1536.236 \\
\hline $10 / 29 / 16$ 21:00 & 82.546 & 73.915 & 101.077 & 1575.345 & 1563.341 & 1536.074 \\
\hline $10 / 29 / 16$ 22:00 & 82.551 & 73.912 & 101.224 & 1575.34 & 1563.344 & 1535.927 \\
\hline 10/29/16 23:00 & 82.553 & 73.908 & 101.348 & 1575.338 & 1563.348 & 1535.803 \\
\hline 10/30/16 0:00 & 82.551 & 73.903 & 101.344 & 1575.34 & 1563.353 & 1535.807 \\
\hline 10/30/16 1:00 & 82.553 & 73.908 & 101.304 & 1575.338 & 1563.348 & 1535.847 \\
\hline $10 / 30 / 162: 00$ & 82.548 & 73.903 & 101.248 & 1575.343 & 1563.353 & 1535.903 \\
\hline 10/30/16 3:00 & 82.543 & 73.903 & 101.257 & 1575.348 & 1563.353 & 1535.894 \\
\hline 10/30/16 4:00 & 82.536 & 73.899 & 101.346 & 1575.355 & 1563.357 & 1535.805 \\
\hline 10/30/16 5:00 & 82.534 & 73.892 & 101.449 & 1575.357 & 1563.364 & 1535.702 \\
\hline 10/30/16 6:00 & 82.529 & 73.882 & 101.442 & 1575.362 & 1563.374 & 1535.709 \\
\hline 10/30/16 7:00 & 82.527 & 73.882 & 101.383 & 1575.364 & 1563.374 & 1535.768 \\
\hline
\end{tabular}


TABLE S1.2 Automatically recorded groundwater levels in the confined aquifers.

\begin{tabular}{|c|c|c|c|c|c|c|}
\hline \multirow[b]{2}{*}{ Date-Time } & \multicolumn{3}{|c|}{ Measured Depth to Water (ft TOC ${ }^{\mathrm{a}}$ ) } & \multicolumn{3}{|c|}{ Groundwater Elevation (ft $\mathrm{AMSL}^{\mathrm{b}}$ ) } \\
\hline & DL2-CU & DL7-CU & DL7-CL & DL2-CU & DL7-CU & DL7-CL \\
\hline 10/30/16 8:00 & 82.525 & 73.875 & 101.313 & 1575.366 & 1563.381 & 1535.838 \\
\hline 10/30/16 9:00 & 82.525 & 73.882 & 101.24 & 1575.366 & 1563.374 & 1535.911 \\
\hline 10/30/16 10:00 & 82.522 & 73.884 & 101.173 & 1575.369 & 1563.372 & 1535.978 \\
\hline $10 / 30 / 1611: 00$ & 82.52 & 73.884 & 101.114 & 1575.371 & 1563.372 & 1536.037 \\
\hline $10 / 30 / 16$ 12:00 & 82.518 & 73.877 & 101.067 & 1575.373 & 1563.379 & 1536.084 \\
\hline 10/30/16 13:00 & 82.513 & 73.871 & 101.011 & 1575.378 & 1563.385 & 1536.14 \\
\hline $10 / 30 / 1614: 00$ & 82.506 & 73.859 & 100.941 & 1575.385 & 1563.397 & 1536.21 \\
\hline $10 / 30 / 16$ 15:00 & 82.499 & 73.85 & 100.875 & 1575.392 & 1563.406 & 1536.276 \\
\hline 10/30/16 16:00 & 82.494 & 73.84 & 100.822 & 1575.397 & 1563.416 & 1536.329 \\
\hline $10 / 30 / 16$ 17:00 & 82.487 & 73.831 & 100.775 & 1575.404 & 1563.425 & 1536.376 \\
\hline $10 / 30 / 16$ 18:00 & 82.485 & 73.824 & 100.735 & 1575.406 & 1563.432 & 1536.416 \\
\hline 10/30/16 19:00 & 82.487 & 73.808 & 100.69 & 1575.404 & 1563.448 & 1536.461 \\
\hline $10 / 30 / 16$ 20:00 & 82.487 & 73.805 & 100.639 & 1575.404 & 1563.451 & 1536.512 \\
\hline $10 / 30 / 1621: 00$ & 82.489 & 73.806 & 100.595 & 1575.402 & 1563.45 & 1536.556 \\
\hline $10 / 30 / 1622: 00$ & 82.489 & 73.799 & 100.566 & 1575.402 & 1563.457 & 1536.585 \\
\hline $10 / 30 / 16$ 23:00 & 82.492 & 73.791 & 100.541 & 1575.399 & 1563.465 & 1536.61 \\
\hline 10/31/16 0:00 & 82.494 & 73.785 & 100.508 & 1575.397 & 1563.471 & 1536.643 \\
\hline 10/31/16 1:00 & 82.494 & 73.771 & 100.463 & 1575.397 & 1563.485 & 1536.688 \\
\hline 10/31/16 2:00 & 82.494 & 73.764 & 100.419 & 1575.397 & 1563.492 & 1536.732 \\
\hline 10/31/16 3:00 & 82.489 & 73.75 & 100.367 & 1575.402 & 1563.506 & 1536.784 \\
\hline 10/31/16 4:00 & 82.484 & 73.738 & 100.302 & 1575.407 & 1563.518 & 1536.849 \\
\hline 10/31/16 5:00 & 82.478 & 73.729 & 100.241 & 1575.413 & 1563.527 & 1536.91 \\
\hline 10/31/16 6:00 & 82.473 & 73.715 & 100.176 & 1575.418 & 1563.541 & 1536.975 \\
\hline 10/31/16 7:00 & 82.468 & 73.703 & 100.11 & 1575.423 & 1563.553 & 1537.041 \\
\hline 10/31/16 8:00 & 82.461 & 73.694 & 100.049 & 1575.43 & 1563.562 & 1537.102 \\
\hline 10/31/16 9:00 & 82.463 & 73.691 & 100.016 & 1575.428 & 1563.565 & 1537.135 \\
\hline $10 / 31 / 16$ 10:00 & 82.466 & 73.687 & 99.981 & 1575.425 & 1563.569 & 1537.17 \\
\hline 10/31/16 11:00 & 82.475 & 73.678 & 99.944 & 1575.416 & 1563.578 & 1537.207 \\
\hline $10 / 31 / 16$ 12:00 & 82.501 & 73.671 & 99.899 & 1575.39 & 1563.585 & 1537.252 \\
\hline 10/31/16 13:00 & 82.543 & 73.661 & 99.85 & 1575.348 & 1563.595 & 1537.301 \\
\hline $10 / 31 / 1614: 00$ & 82.572 & 73.654 & 99.794 & 1575.319 & 1563.602 & 1537.357 \\
\hline $10 / 31 / 16$ 15:00 & 82.586 & 73.643 & 99.747 & 1575.305 & 1563.613 & 1537.404 \\
\hline $10 / 31 / 1616: 00$ & 82.605 & 73.636 & 99.698 & 1575.286 & 1563.62 & 1537.453 \\
\hline $10 / 31 / 16$ 17:00 & 82.628 & 73.64 & 99.644 & 1575.263 & 1563.616 & 1537.507 \\
\hline $10 / 31 / 16$ 18:00 & 82.652 & 73.64 & 99.588 & 1575.239 & 1563.616 & 1537.563 \\
\hline 10/31/16 19:00 & 82.694 & 73.65 & 99.534 & 1575.197 & 1563.606 & 1537.617 \\
\hline $10 / 31 / 16$ 20:00 & 82.725 & 73.645 & 99.478 & 1575.166 & 1563.611 & 1537.673 \\
\hline 10/31/16 21:00 & 82.734 & 73.645 & 99.422 & 1575.157 & 1563.611 & 1537.729 \\
\hline 10/31/16 22:00 & 82.736 & 73.652 & 99.37 & 1575.155 & 1563.604 & 1537.781 \\
\hline 10/31/16 23:00 & 82.746 & 73.654 & 99.314 & 1575.145 & 1563.602 & 1537.837 \\
\hline 11/1/16 0:00 & 82.774 & 73.661 & 99.263 & 1575.117 & 1563.595 & 1537.888 \\
\hline $11 / 1 / 16$ 1:00 & 82.795 & 73.659 & 99.202 & 1575.096 & 1563.597 & 1537.949 \\
\hline 11/1/16 2:00 & 82.804 & 73.666 & 99.15 & 1575.087 & 1563.59 & 1538.001 \\
\hline 11/1/16 3:00 & 82.8 & 73.673 & 99.089 & 1575.091 & 1563.583 & 1538.062 \\
\hline 11/1/16 4:00 & 82.79 & 73.673 & 99.033 & 1575.101 & 1563.583 & 1538.118 \\
\hline 11/1/16 5:00 & 82.776 & 73.671 & 98.972 & 1575.115 & 1563.585 & 1538.179 \\
\hline $11 / 1 / 16$ 6:00 & 82.762 & 73.671 & 98.914 & 1575.129 & 1563.585 & 1538.237 \\
\hline
\end{tabular}


TABLE S1.2 Automatically recorded groundwater levels in the confined aquifers.

\begin{tabular}{|c|c|c|c|c|c|c|}
\hline \multirow[b]{2}{*}{ Date-Time } & \multicolumn{3}{|c|}{ Measured Depth to Water (ft TOC ${ }^{\mathrm{a}}$ ) } & \multicolumn{3}{|c|}{ Groundwater Elevation (ft $\mathrm{AMSL}^{\mathrm{b}}$ ) } \\
\hline & DL2-CU & DL7-CU & DL7-CL & DL2-CU & DL7-CU & DL7-CL \\
\hline 11/1/16 7:00 & 82.75 & 73.668 & 98.851 & 1575.141 & 1563.588 & 1538.3 \\
\hline $11 / 1 / 168: 00$ & 82.743 & 73.668 & 98.801 & 1575.148 & 1563.588 & 1538.35 \\
\hline 11/1/16 9:00 & 82.76 & 73.675 & 98.745 & 1575.131 & 1563.581 & 1538.406 \\
\hline 11/1/16 10:00 & 82.798 & 73.678 & 98.699 & 1575.093 & 1563.578 & 1538.452 \\
\hline 11/1/16 11:00 & 82.825 & 73.678 & 98.642 & 1575.066 & 1563.578 & 1538.509 \\
\hline $11 / 1 / 1612: 00$ & 82.84 & 73.682 & 98.588 & 1575.051 & 1563.574 & 1538.563 \\
\hline 11/1/16 13:00 & 82.835 & 73.682 & 98.539 & 1575.056 & 1563.574 & 1538.612 \\
\hline $11 / 1 / 1614: 00$ & 82.823 & 73.678 & 98.483 & 1575.068 & 1563.578 & 1538.668 \\
\hline 11/1/16 15:00 & 82.812 & 73.678 & 98.436 & 1575.079 & 1563.578 & 1538.715 \\
\hline 11/1/16 16:00 & 82.797 & 73.678 & 98.39 & 1575.094 & 1563.578 & 1538.761 \\
\hline 11/1/16 17:00 & 82.783 & 73.678 & 98.35 & 1575.108 & 1563.578 & 1538.801 \\
\hline $11 / 1 / 1618: 00$ & 82.772 & 73.678 & 98.315 & 1575.119 & 1563.578 & 1538.836 \\
\hline 11/1/16 19:00 & 82.76 & 73.68 & 98.28 & 1575.131 & 1563.576 & 1538.871 \\
\hline 11/1/16 20:00 & 82.75 & 73.675 & 98.249 & 1575.141 & 1563.581 & 1538.902 \\
\hline 11/1/16 21:00 & 82.739 & 73.675 & 98.219 & 1575.152 & 1563.581 & 1538.932 \\
\hline $11 / 1 / 1622: 00$ & 82.732 & 73.68 & 98.2 & 1575.159 & 1563.576 & 1538.951 \\
\hline $11 / 1 / 1623: 00$ & 82.727 & 73.682 & 98.184 & 1575.164 & 1563.574 & 1538.967 \\
\hline $11 / 2 / 16$ 0:00 & 82.718 & 73.68 & 98.165 & 1575.173 & 1563.576 & 1538.986 \\
\hline $11 / 2 / 161: 00$ & 82.71 & 73.68 & 98.153 & 1575.181 & 1563.576 & 1538.998 \\
\hline $11 / 2 / 162: 00$ & 82.706 & 73.682 & 98.141 & 1575.185 & 1563.574 & 1539.01 \\
\hline $11 / 2 / 163: 00$ & 82.699 & 73.675 & 98.127 & 1575.192 & 1563.581 & 1539.024 \\
\hline $11 / 2 / 164: 00$ & 82.689 & 73.673 & 98.111 & 1575.202 & 1563.583 & 1539.04 \\
\hline $11 / 2 / 165: 00$ & 82.68 & 73.666 & 98.088 & 1575.211 & 1563.59 & 1539.063 \\
\hline $11 / 2 / 166: 00$ & 82.673 & 73.666 & 98.071 & 1575.218 & 1563.59 & 1539.08 \\
\hline $11 / 2 / 167: 00$ & 82.666 & 73.659 & 98.057 & 1575.225 & 1563.597 & 1539.094 \\
\hline $11 / 2 / 168: 00$ & 82.658 & 73.657 & 98.043 & 1575.233 & 1563.599 & 1539.108 \\
\hline 11/2/16 9:00 & 82.656 & 73.657 & 98.034 & 1575.235 & 1563.599 & 1539.117 \\
\hline 11/2/16 10:00 & 82.659 & 73.661 & 98.029 & 1575.232 & 1563.595 & 1539.122 \\
\hline 11/2/16 11:00 & 82.659 & 73.664 & 98.034 & 1575.232 & 1563.592 & 1539.117 \\
\hline $11 / 2 / 1612: 00$ & 82.656 & 73.664 & 98.031 & 1575.235 & 1563.592 & 1539.12 \\
\hline 11/2/16 13:00 & 82.654 & 73.659 & 98.034 & 1575.237 & 1563.597 & 1539.117 \\
\hline 11/2/16 14:00 & 82.652 & & & 1575.239 & & \\
\hline 11/2/16 15:00 & 82.645 & & & 1575.246 & & \\
\hline 11/2/16 16:00 & 82.645 & & & 1575.246 & & \\
\hline 11/2/16 17:00 & 82.647 & & & 1575.244 & & \\
\hline 11/2/16 18:00 & 82.647 & & & 1575.244 & & \\
\hline 11/2/16 19:00 & 82.645 & & & 1575.246 & & \\
\hline 11/2/16 20:00 & 82.642 & & & 1575.249 & & \\
\hline 11/2/16 21:00 & 82.642 & & & 1575.249 & & \\
\hline $11 / 2 / 1622: 00$ & 82.642 & & & 1575.249 & & \\
\hline 11/2/16 23:00 & 82.644 & & & 1575.247 & & \\
\hline $11 / 3 / 16$ 0:00 & 82.644 & & & 1575.247 & & \\
\hline $11 / 3 / 16$ 1:00 & 82.645 & & & 1575.246 & & \\
\hline $11 / 3 / 162: 00$ & 82.645 & & & 1575.246 & & \\
\hline $11 / 3 / 163: 00$ & 82.642 & & & 1575.249 & & \\
\hline $11 / 3 / 164: 00$ & 82.64 & & & 1575.251 & & \\
\hline $11 / 3 / 16$ 5:00 & 82.638 & & & 1575.253 & & \\
\hline
\end{tabular}


TABLE S1.2 Automatically recorded groundwater levels in the confined aquifers.

\begin{tabular}{|c|c|c|c|c|c|c|}
\hline \multirow[b]{2}{*}{ Date-Time } & \multicolumn{3}{|c|}{ Measured Depth to Water (ft TOC ${ }^{\mathrm{a}}$ ) } & \multicolumn{3}{|c|}{ Groundwater Elevation (ft $\mathrm{AMSL}^{\mathrm{b}}$ ) } \\
\hline & DL2-CU & DL7-CU & DL7-CL & DL2-CU & DL7-CU & DL7-CL \\
\hline $11 / 3 / 16$ 6:00 & 82.633 & & & 1575.258 & & \\
\hline $11 / 3 / 16$ 7:00 & 82.626 & & & 1575.265 & & \\
\hline $11 / 3 / 168: 00$ & 82.626 & & & 1575.265 & & \\
\hline $11 / 3 / 169: 00$ & 82.623 & & & 1575.268 & & \\
\hline $11 / 3 / 16$ 10:00 & 82.623 & & & 1575.268 & & \\
\hline 11/3/16 11:00 & 82.621 & & & 1575.27 & & \\
\hline
\end{tabular}

a TOC, top of casing.

b AMSL, above mean sea level. 
TABLE S1.3 Reported precipitation events for the weather station at York Nebraska. ${ }^{a}$

\begin{tabular}{cccc}
\hline & $\begin{array}{c}\text { Total } \\
\text { Precipitation } \\
\text { (in.) }\end{array}$ & Date & $\begin{array}{c}\text { Total } \\
\text { Precipitation } \\
\text { (in.) }\end{array}$ \\
\hline $07 / 02 / 16$ & 0.68 & $09 / 02 / 16$ & 0.03 \\
$07 / 04 / 16$ & 0.01 & $09 / 03 / 16$ & 0.69 \\
$07 / 07 / 16$ & 2.26 & $09 / 04 / 16$ & 0.01 \\
$07 / 12 / 16$ & 0.00 & $09 / 07 / 16$ & 0.06 \\
$07 / 13 / 16$ & 0.03 & $09 / 09 / 16$ & 0.95 \\
$07 / 14 / 16$ & 0.02 & $09 / 12 / 16$ & 1.10 \\
$07 / 15 / 16$ & 0.36 & $09 / 13 / 16$ & 0.04 \\
$07 / 30 / 16$ & 0.09 & $09 / 15 / 16$ & 0.09 \\
$07 / 31 / 16$ & 0.01 & $09 / 16 / 16$ & 0.24 \\
$08 / 02 / 16$ & 0.22 & $09 / 24 / 16$ & 0.31 \\
$08 / 07 / 16$ & 0.03 & $10 / 04 / 16$ & 1.28 \\
$08 / 12 / 16$ & 0.45 & $10 / 05 / 16$ & 0.01 \\
$08 / 18 / 16$ & 0.24 & $10 / 06 / 16$ & 0.07 \\
$08 / 19 / 16$ & 0.32 & $10 / 12 / 16$ & 0.05 \\
$08 / 23 / 16$ & 0.07 & $10 / 19 / 16$ & 0.04 \\
$08 / 24 / 16$ & 0.01 & $11 / 07 / 16$ & 0.17 \\
$08 / 27 / 16$ & 0.02 & $11 / 09 / 16$ & 0.03 \\
$08 / 30 / 16$ & 0.05 & & \\
\hline
\end{tabular}

a Data source: Weather Underground (www.wunderground.com/us/ne/york/zmw:68467.1.99999). 


\section{Supplement 2:}

Quality Control for Sample Collection, Handling, and Analysis 


\section{Supplement 2:}

\section{Quality Control for Sample Collection, Handling, and Analysis}

This supplement documents the quality of the analytical data generated during the 20152016 investigation at York. The QA/QC procedures for sample collection, handling, and analysis during the investigation were described in detail in the Master Work Plan (Argonne 2002). The analytical results for quality control samples (replicates, duplicates, and verification samples) confirm that the data generated during the investigation reliably and accurately characterize the environmental conditions at the site. Field replicate samples verify the reproducibility of the sampling methodology, while laboratory duplicate samples verify the reproducibility of the analytical results obtained at the AGEM Laboratory. Verification samples verify the accuracy of the analytical data.

The AGEM QA/QC program (described in the Master Work Plan; Argonne 2002) is based on EPA (2008) guidelines. The QA/QC samples are listed in Table S2.1.

\section{S2.1 Sampling to Monitor Sampling Collection and Handling Activities}

Sample collection and handling activities were monitored by the documentation of samples as they were collected and the use of chain-of-custody (COC) forms and custody seals to ensure sample integrity during handling and shipment. Equipment blanks, equipment rinsates, and trip blanks were also collected to monitor sample collection and handling activities. Analytical results for carbon tetrachloride and chloroform in QA/QC samples collected to monitor sample collection and handling activities are in Table S2.2.

\section{S2.1.1 Equipment Blanks}

To verify that contamination detected did not derive from water used for equipment decontamination and the drilling and installation of monitoring wells (Sections 2.2.1, 3.3, and 3.4.4), two equipment blanks were collected of waters used during these activities (Table S2.1). Neither carbon tetrachloride nor chloroform was detected in the equipment blanks (Table S2.2). 


\section{S2.1.2 Equipment Rinsates}

To verify that equipment decontamination procedures were adequate during the field efforts, eight equipment rinsates were collected (Table S2.1). Neither carbon tetrachloride nor chloroform was detected in the rinsate samples (Table S2.2).

\section{S2.1.3 Trip Blanks}

As an indicator of potential cross-contamination of samples during shipment, 61 trip blanks were prepared and included in shipments of soil or water samples to laboratories for organic analysis. Included in this total were 37 water trip blanks and seven soil trip blanks sent to the AGEM Laboratory; two water trip blanks sent to Pace Analytical Services; and 14 water trip blanks and one soil (methanol) trip blank sent to TestAmerica. The June 2016 water trip blanks to Pace were inadvertently assigned the same sample ID as trip blanks shipped to AGEM Laboratory; the ID of the two trip blanks to Pace were therefore appended with "PACE" to distinguish these trip blanks (Table S2.1). Neither carbon tetrachloride nor chloroform was detected in the soil or water trip blanks sent to the AGEM Laboratory or TestAmerica, with the exception of a trace detection of chloroform in trip blank YKQCTB-W-37869 sent with water samples to TestAmerica (Table S2.2). Trace concentrations of methylene chloride were also detected in three of the trip blanks shipped with water samples to TestAmerica for verification analysis; however, all of these detections are marked with a B qualifier, indicating that methylene chloride was also detected in associated laboratory blanks analyzed with these samples. Overall, the analytical results indicate that sample handling procedures were followed during the investigation and that cross-contamination of samples did not occur during shipment.

\section{S2.1.4 Replicate Samples and Duplicate Analyses}

As an indicator of the consistency of the sampling methodology followed and to provide a measure of analytical precision, replicate soil, groundwater, indoor air, and sub-slab soil gas samples were collected for organic analysis. Soil and groundwater samples were also selected by the AGEM Laboratory for duplicate organic analysis. In addition, selected soil and groundwater samples were submitted for verification organic analysis at a secondary laboratory. Replicate samples, samples selected for duplicate analysis, and samples selected for verification organic analysis are listed in Table S2.1. Results for replicate samples and duplicate analyses are discussed in Section S2.2, and results for verification samples are discussed in Section S2.3. 


\section{S2.1.5 Background Air Sampling}

In conjunction with collecting indoor air samples, ambient (outdoor) air samples were collected to compare the concentrations of chemicals detected inside the structure with concentrations of chemicals that might be present in ambient air. Neither carbon tetrachloride nor chloroform was detected in any of the ambient air samples collected during indoor air sampling (Table H.1).

\section{S2.2 Quality Control for Organic Analysis of Soil and Water Samples at the AGEM Laboratory}

Soil samples collected during the investigation were quick-frozen on dry ice as they were collected and were shipped to the AGEM Laboratory for VOCs analysis by two analytical methods: (1) the headspace method to provide a rapid-turnaround qualitative measure of contaminant distribution for evaluation in the field, and (2) the purge-and-trap method to provide a quantitative measure of contaminant distribution.

The soil samples were first analyzed at the AGEM Laboratory (on a rapid-turnaround basis) by using a modification of the protocol in EPA Method 5021 (headspace analysis on a GC with electron capture detection). An 11-point calibration of the GC system was established on the basis of the mass of known quantities of carbon tetrachloride and chloroform ranging from 0.125 to 4.0 nanograms. Typical detection limits achieved were $0.1 \mu \mathrm{g} / \mathrm{kg}$ for carbon tetrachloride and $0.75 \mu \mathrm{g} / \mathrm{kg}$ for chloroform. The analytical data obtained with this method are acceptable for qualitative determination of contaminant distribution.

Aliquots of soil samples were subsequently analyzed for carbon tetrachloride, chloroform, and methylene chloride (contaminants of concern in the investigation) by a modification of EPA Method 8260B (purge-and-trap method), as referenced in the EPA's SW-846 (EPA 1998), to achieve a method detection limit of $10 \mu \mathrm{g} / \mathrm{kg}$. At the laboratory, the VOCs in each soil sample were extracted with methanol from the sample matrix. For the purgeand-trap soil analyses, an aliquot of the methanol extract was purged, and the volatile species were transferred to a sorbent tube. After purging, the sorbent tube was heated and backflushed with an inert gas to desorb the components into the GC-MS system. 
Groundwater samples (and associated QC samples) were sent to the AGEM Laboratory for VOCs analysis by modified EPA Method 524.2 (EPA 1995) to achieve a method detection limit of $1 \mu \mathrm{g} / \mathrm{L}$. For the purge-and-trap analyses on a GC-MS system, the VOCs present in each groundwater sample were extracted (purged) from the sample matrix by bubbling an inert gas through the sample. The purged components were trapped in a sorbent tube, and the sorbent tube was heated and backflushed with an inert gas to desorb the components into the GC-MS system.

In total, 82 soil samples were analyzed for VOCs, including 4 replicate soil samples. A total of 144 groundwater samples were collected for VOCs analysis, including 35 replicate samples.

For both the soil and water analyses, the compounds eluting from the GC column were identified by retention time and by comparison with reference library spectra. The concentration of each component was calculated by comparison of the MS response for the quantitation ion to corresponding calibration curves, the response for internal standards, or both. The internal standard recovery limits were $80-120 \%$. Calibration checks with each sample delivery group (SDG) were required to be within $\pm 20 \%$ of the standard.

Every analytical laboratory statistically defines analyte-specific instrument detection limits and method detection limits. The instrument detection limit is less than the method detection limit. An instrument detection limit is the analyte concentration required to produce a signal three times the signal-to-noise ratio. Noise - a measure of the fluctuation in background signal that occurs even in the absence of an analyte - is dependent on instrument and laboratory conditions. The noise value is calculated from the standard deviation of a certain number of measurements of the background signal. The instrument detection limit is specific to each instrument and can vary by a small degree with each sample and analysis performed on that instrument.

A method detection limit is defined as "the minimum concentration of a substance that can be measured and reported with $99 \%$ confidence that the analyte concentration is greater than zero" (EPA 1997). The method detection limit is defined after the analyte in the sample has been extracted, isolated, and analyzed, thus taking into account matrix effects due to analysis of a lessthan-pure analyte. Given matrix effects and unavoidable analyte loss through all steps of the analytical procedure, the method detection limit can be 2 to 100 times higher than the instrument 
detection limit. For the AGEM Laboratory, the method detection limit is typically approximately 10 times the instrument detection limit.

A concentration in a sample below the method detection limit that does not produce a signal distinguishable from background noise is reported as non-detectable, with notation ND. For carbon tetrachloride and chloroform analyses at the AGEM Laboratory, such concentrations are $<1 \mu \mathrm{g} / \mathrm{L}$ in groundwater or $<10 \mu \mathrm{g} / \mathrm{kg}$ in soil.

A concentration in a sample above the instrument detection limit and below the method detection limit that produces a signal distinguishable from background noise is marked with a $\mathbf{J}$ qualifier. These results are associated with a relatively high degree of uncertainty, because they are below the $99 \%$ confidence level specified by the method detection limit. Since results with a $\mathrm{J}$ qualifier are below the method detection limit, they can also be reported with the notation ND.

Samples submitted to the AGEM Laboratory for organic analysis by the purge-and-trap method were analyzed in 48 SDGs, as shown in Table S2.3. The QA/QC procedures followed included analysis of instrument calibration check standards, analysis of laboratory blanks, monitoring of surrogate spike recovery, and duplicate laboratory analyses. Significant results include the following:

- All samples were received with custody seals intact and at the appropriate preservation conditions, with the exception of the samples shipped with COC YK29161700, which were received frozen. One vial of each sample in this shipment remained unbroken and was analyzed. Samples were analyzed within required holding times.

- Carbon tetrachloride and chloroform were not detected in laboratory method blanks analyzed with the samples.

- For each SDG, analytical instrument calibration was monitored by the analysis of calibration check standards. Table $\mathrm{S} 2.3$ shows the relative percent difference (RPD) between the known and calculated concentrations of the standards. The concentrations of calibration check standards measured in all SDGs were within the acceptable range of $\pm 20 \%$. 
- Surrogate standard determinations were performed on samples and blanks by using surrogate spike compounds fluorobenzene, dichlorobenzene-d4, and bromofluorobenzene. Table S2.3 shows the percent recovery of these systemmonitoring compounds for each of the analyses. The surrogate recoveries were within the specified range of $80-120 \%$ for all but one sample in either the initial analysis or a successful reanalysis; trip blank YKQCTB-W-38095 was not reanalyzed after failure to meet surrogate recovery limits. In addition, the samples in SDG 16-05-26 were not reanalyzed after failure to meet surrogate recovery limits for the laboratory $20 \mathrm{ppb}$ standard.

- Secondary QC analyses of soil and groundwater samples at the AGEM Laboratory and indoor air and sub-slab soil gas samples at TestAmerica were conducted throughout the investigation as a measure of the consistency in the sampling and analytical methodologies. This was accomplished through the analysis of replicate samples submitted to the laboratory or (for soil and groundwater samples at the AGEM Laboratory) duplicate analysis of samples selected by the laboratory. Table S2.4 summarizes the analytical results for carbon tetrachloride and chloroform in the primary samples and their associated replicate or duplicate analyses. Consistency in both the sampling and analytical methodologies is indicated by the average RPD values of $13 \%$ for carbon tetrachloride and $18 \%$ for chloroform in dual analyses with the contaminants present above the respective method detection limits.

The analytical data from the AGEM Laboratory are acceptable for quantitative determination of contaminant distribution.

\section{S2.3 Quality Control for Verification Organic Analysis of Soil and Groundwater Samples by TestAmerica}

In accordance with the QA/QC procedures defined in the Master Work Plan (Argonne 2002), selected soil and groundwater samples analyzed at the AGEM Laboratory were subjected to verification analysis at a second laboratory with EPA Contract Laboratory Program methodology. Eight of the 78 vertical-profile soil samples (10\% of the soil samples) and 30 of the 109 groundwater samples (28\% of the groundwater samples) analyzed at the AGEM Laboratory were also analyzed at TestAmerica. Table S2.5 compares the results from the two 
laboratories for the soil and groundwater samples submitted for verification organic analysis. These analytical results are in 15 data packages from TestAmerica that are provided as Supplement 4 (on CD).

The QA/QC procedures followed at TestAmerica included initial and continuing calibration of instruments, analysis of laboratory blanks, monitoring of surrogate spike recovery, and analyses of laboratory control samples. Significant results included the following:

- Soil and groundwater samples shipped to TestAmerica were received with custody seals intact and at the appropriate preservation conditions, with the exception of the samples shipped with COC 4916, which were received at a temperature of $8.6^{\circ} \mathrm{C}$. Samples were analyzed within required holding times, with the exception of sample YKTI14-136-W-37954 and trip blank YKQCTB-W-37960 shipped with COC YK828151310; the three samples and one trip blank shipped with COC YK915151755; and samples YKDL1C-108W-38091 and YKDL1B-88-W-38093 shipped with COC YK531161735, which were received outside of required holding times.

- Analytical instruments were properly tuned; initial and continuing calibration checks remained within the allowable range. Surrogate standard determinations were within allowable limits.

- None of the contaminants of interest was detected in the blanks analyzed with the soil or groundwater samples, with the exception of trace concentrations of methylene chloride detected in the laboratory blanks analyzed with three trip blanks and with sample YKDL1C-108-W-38091. The results for this one sample and three trip blanks are marked with the B qualifier (Table S2.5).

Agreement between the two laboratories is good over the range of contaminant concentrations detected, with average RPD values of $16 \%$ for carbon tetrachloride and $12 \%$ for chloroform in verification analyses with the contaminants present above the respective method detection limits. The concentrations detected in analyses of soil and groundwater samples at the AGEM Laboratory are supported by the verification analyses at TestAmerica. 
TABLE S2.1 Quality control samples collected during the 2015-2016 investigation.

\begin{tabular}{|c|c|c|c|c|c|c|c|c|}
\hline $\begin{array}{l}\text { Sample Date } \\
\text { and Time }\end{array}$ & Location & Sample & $\begin{array}{c}\text { Sample } \\
\text { Type }^{a}\end{array}$ & $\begin{array}{c}\text { Depth (ft } \\
\text { BGL) }\end{array}$ & Matrix $^{b}$ & Chain of Custody & $\begin{array}{l}\text { Shipping } \\
\text { Date }\end{array}$ & Sample Description \\
\hline \multicolumn{9}{|c|}{ Replicate soil, groundwater, indoor air, and sub-slab soil gas samples } \\
\hline 7/7/15 13:08 & TI01 & YKTI01-S-37752 & DUP-F & 32 & SOIL & 7234 & $7 / 7 / 15$ & Field replicate. \\
\hline 7/7/15 15:28 & TI02 & YKTI02-S-37776 & DUP-F & 20 & SOIL & 7235 & $7 / 7 / 15$ & Field replicate. \\
\hline $7 / 8 / 15$ 13:30 & TI04 & YKTI04-S-37799 & DUP-F & 40 & SOIL & 7238 & $7 / 8 / 15$ & Field replicate. \\
\hline $7 / 9 / 15$ 10:44 & TI03 & YKTI03-S-37824 & DUP-F & 48 & SOIL & 7239 & $7 / 9 / 15$ & Field replicate. \\
\hline $7 / 9 / 15$ 15:54 & TIO4 & YKTI04-W-37834 & DUP-F & $74.6-79.6$ & WG & 7241 & $7 / 9 / 15$ & Field replicate. \\
\hline 7/9/15 16:30 & TI03 & YKTI03-W-37837 & DUP-F & $76.8-81.8$ & WG & 7241 & $7 / 9 / 15$ & Field replicate. \\
\hline 7/10/15 13:10 & TI01 & YKTI01-W-37845 & DUP-F & $77.4-82.4$ & WG & 7243 & $7 / 10 / 15$ & Field replicate. \\
\hline $7 / 10 / 15$ 14:44 & TIO2 & YKTI02-W-37848 & DUP-F & 77-82 & WG & 7243 & $7 / 10 / 15$ & Field replicate. \\
\hline $7 / 13 / 15$ 20:00 & TI02 & YKTI02100-W-37850 & DUP-F & $100-103.5$ & WG & 7244 & $7 / 15 / 15$ & Field replicate. \\
\hline 7/16/15 9:15 & TI01 & YKTI01100-W-37858 & DUP-F & 88-98 & WG & 7246 & $7 / 16 / 15$ & Field replicate. \\
\hline 7/16/15 16:00 & TI04 & YKTI04100-W-37862 & DUP-F & $89-99$ & WG & 7246 & $7 / 16 / 15$ & Field replicate. \\
\hline 7/17/15 15:35 & TI05 & YKTI05100-W-37867 & DUP-F & 88-98 & WG & 7247 & $7 / 17 / 15$ & Field replicate. \\
\hline 7/19/15 16:40 & TI06 & YKTI06100-W-37874 & DUP-F & $90-100$ & WG & 7249 & $7 / 20 / 15$ & Field replicate. \\
\hline $7 / 20 / 15$ 14:50 & TI07 & YKTI07120-W-37878 & DUP-F & $110-120$ & WG & 7249 & $7 / 20 / 15$ & Field replicate. \\
\hline $7 / 21 / 15$ 16:15 & TI08 & YKTI08107-W37884 & DUP-F & $97-107$ & WG & 7250 & $7 / 22 / 15$ & Field replicate. \\
\hline 8/5/15 11:10 & FITWORX & YKFITWORX-G-37901 & DUP-F & - & AIR & TA-06Aug2015-1 & 8/6/15 & $\begin{array}{l}\text { Field replicate. Sample collected over } \\
24 \mathrm{hr} \text {. Begin pressure }-30 \mathrm{in} . \mathrm{Hg} \text {. End } \\
\text { pressure }-6 \text { in. } \mathrm{Hg} \text {. }\end{array}$ \\
\hline 8/5/15 13:54 & DW39 & YKNORDLUNDDUP-W-37919 & DUP-F & - & WG & YK810151705 & 8/10/15 & Field replicate. \\
\hline 8/7/15 12:00 & SB77 & YKSB77DUP-W-37922 & DUP-F & $75.8-79$ & WG & YK810151705 & $8 / 10 / 15$ & Field replicate. \\
\hline 8/13/15 11:30 & TI09 & YKTI09-80DUP-W-37927 & DUP-F & $80-85$ & WG & YK814151700 & $8 / 14 / 15$ & Field replicate. \\
\hline 8/14/15 13:30 & TI10 & YKTI10-95DUP-W-37932 & DUP-F & $95-105$ & WG & YK814151700 & $8 / 14 / 15$ & Field replicate. \\
\hline $8 / 20 / 15$ 11:45 & TI12 & YKTI12-DUP92-W-37941 & DUP-F & $92-102$ & WG & YK820151750 & $8 / 20 / 15$ & Field replicate. \\
\hline 8/22/15 10:30 & TI13 & YKTI13-111DUP-W-37949 & DUP-F & $111-121$ & WG & YK8222151150 & $8 / 22 / 15$ & Field replicate. \\
\hline 8/23/15 12:30 & TI14 & YKTI14-113DUP-W-37953 & DUP-F & $113-123$ & WG & YK824151700 & $8 / 24 / 15$ & Field replicate. \\
\hline $8 / 24 / 1514: 15$ & TI15 & YKTI15-134DUP-W-37959 & DUP-F & $134-144$ & WG & YK824151700 & $8 / 24 / 15$ & Field replicate. \\
\hline 8/25/15 10:00 & TI16 & YKTI16-86DUP-W-37962 & DUP-F & 86-96 & WG & YK825151700 & $8 / 25 / 15$ & Field replicate. \\
\hline 8/26/15 16:35 & TI17 & YKTI17-133DUP-W-37968 & DUP-F & $133-143$ & WG & YK826151700 & $8 / 26 / 15$ & Field replicate. \\
\hline 8/27/15 18:00 & TI18 & YKTI18-95DUP-W-37972 & DUP-F & $95-105$ & WG & YK828151300 & $8 / 28 / 15$ & Field replicate. \\
\hline 9/9/15 14:00 & TI19 & YKTI19-129DUP-W-37978 & DUP-F & $129-139$ & WG & YK99151700 & 9/9/15 & Field replicate. \\
\hline 9/10/15 15:00 & TI20 & YKTI20-131DUP-W-37983 & DUP-F & $131-141$ & WG & YK910151700 & $9 / 10 / 15$ & Field replicate. \\
\hline 9/11/15 17:45 & $\mathrm{TI} 21$ & YKTI21-115DUP-W-37988 & DUP-F & $115-120$ & WG & YK911151800 & $9 / 11 / 15$ & Field replicate. \\
\hline 9/14/15 16:30 & TI22 & YKTI22-98DUP-W-37993 & DUP-F & 98-108 & WG & YK915151800 & $9 / 15 / 15$ & Field replicate. \\
\hline
\end{tabular}




\begin{tabular}{|c|c|c|c|c|c|c|c|c|}
\hline 9/15/15 15:15 & TI23 & YKTI23-99DUP-W-37997 & DUP-F & 99-109 & WG & YK915151800 & 9/15/15 & Field replicate. \\
\hline 1/20/16 15:40 & TI24 & YKTI24-80DUP-W-38020 & DUP-F & $80-90$ & WG & YK120161700 & $1 / 20 / 16$ & Field replicate. \\
\hline 1/21/16 15:27 & TI25 & YKTI25-98DUP-W-38025 & DUP-F & 98-108 & WG & YK121161800 & $1 / 21 / 16$ & Field replicate. \\
\hline 1/25/16 15:15 & TI26 & YKTI26-96DUP-W-38031 & DUP-F & $96-106$ & WG & YK126161700 & $1 / 26 / 16$ & Field replicate. \\
\hline 1/25/16 17:55 & TI26 & YKTI26-113DUP-W-38034 & DUP-F & $113-123$ & WG & YK126161700 & $1 / 26 / 16$ & Field replicate. \\
\hline $1 / 28 / 168: 24$ & GARRETSON & YKGARRETSON-G-38038 & DUP-F & - & AIR & YK128161000 & $1 / 28 / 16$ & $\begin{array}{l}\text { Field replicate. Sample collected over } \\
24 \mathrm{hr} \text {. Begin pressure }-26 \text { in. } \mathrm{Hg} \text {. End } \\
\text { pressure }-3 \text { in. } \mathrm{Hg} \text {. }\end{array}$ \\
\hline 1/28/16 9:32 & FITWORX & YKFITWORX-G-38041 & DUP-F & - & AIR & YK128161000 & $1 / 28 / 16$ & $\begin{array}{l}\text { Field replicate. Sample collected over } \\
24 \mathrm{hr} \text {. Begin pressure }-27 \text { in. } \mathrm{Hg} \text {. End } \\
\text { pressure }-4 \text { in. } \mathrm{Hg} \text {. }\end{array}$ \\
\hline 2/9/16 14:45 & TI27 & YKTI27-77DUP-W-38051 & DUP-F & $77-87$ & WG & YK29161700 & $2 / 9 / 16$ & $\begin{array}{l}\text { Field replicate. Sample frozen; one vial } \\
\text { was unbroken and analyzed. }\end{array}$ \\
\hline 2/9/16 16:30 & TI27 & YKTI27-100DUP-W-38054 & DUP-F & $100-110$ & WG & YK29161700 & $2 / 9 / 16$ & $\begin{array}{l}\text { Field replicate. Sample frozen; one vial } \\
\text { was unbroken and analyzed. }\end{array}$ \\
\hline 3/2/16 18:30 & $\mathrm{TI} 28$ & YKTI28-90-DUP-W-38077 & DUP-F & $90-100$ & WG & YK33161400 & $3 / 3 / 16$ & Field replicate. \\
\hline $4 / 28 / 1613: 57$ & FITWORX & YKFITWORX-G-38082 & DUP-F & - & AIR & YK428161700 & $4 / 28 / 16$ & $\begin{array}{l}\text { Field replicate. Sample collected over } \\
24 \mathrm{hr} \text {. Begin pressure }-31 \mathrm{in} . \mathrm{Hg} \text {. End } \\
\text { pressure }-5 \text { in. } \mathrm{Hg} \text {. }\end{array}$ \\
\hline 4/28/16 14:38 & FITWORX & YKFITWORX-G-38088 & DUP-F & - & SOIL GAS & YK428161700 & $4 / 28 / 16$ & $\begin{array}{l}\text { Field replicate. Begin pressure }-28 \text { in. } \\
\mathrm{Hg} \text {. End pressure } 1 \mathrm{in.} \mathrm{Hg} \text {. }\end{array}$ \\
\hline 5/31/16 17:00 & DL2CU & YKDL2CU-215-DUP-W-38098 & DUP-F & $215-225$ & WG & YK531161800 & $5 / 31 / 16$ & Field replicate. \\
\hline 8/10/16 10:43 & GARRETSON & YKGARRETSON-G-38139 & DUP-F & - & AIR & YK810161710 & $8 / 10 / 16$ & $\begin{array}{l}\text { Field replicate from Garretson } \\
\text { basement. Sample collected over } 24 \\
\text { hr. Begin pressure }-30 \text { in. Hg. End } \\
\text { pressure }-4 \text { in. Hg. Results not } \\
\text { reported by TestAmerica because } \\
\text { sample was contaminated with } \\
\text { laboratory standard due to instrument } \\
\text { malfunction. }\end{array}$ \\
\hline 8/10/16 11:15 & FITWORX & YKFITWORX-G-38149 & DUP-F & - & SOIL GAS & YK810161710 & $8 / 10 / 16$ & $\begin{array}{l}\text { Field replicate from FitnessWorx } \\
\text { stairwell. Begin pressure }-30 \text { in. } \mathrm{Hg} \text {. } \\
\text { End pressure } 0 \text { in. } \mathrm{Hg} \text {. }\end{array}$ \\
\hline 8/10/16 11:31 & FITWORX & YKFITWORX-G-38143 & DUP-F & - & AIR & YK810161710 & $8 / 10 / 16$ & $\begin{array}{l}\text { Field replicate from FitnessWorx } \\
\text { basement. Sample collected over } 24 \\
\text { hr. Begin pressure }-29 \text { in. } \mathrm{Hg} \text {. End } \\
\text { pressure }-5 \text { in. } \mathrm{Hg} \text {. }\end{array}$ \\
\hline
\end{tabular}




\section{2/14/16 10:18 GARRETSON YKGARRETSON-G-39534}

12/14/16 11:08 FITWORX

12/14/16 11:34 FITWORX

$5 / 17 / 16$ 15:30 QC

Equipment rinsates

7/9/15 16:41 QC

7/15/15 16:20 QC

7/16/15 14:00 QC

1/20/16 11:30 QC

1/21/16 16:45 OC

1/25/16 16:30 QC
YKFITWORX-G-39544

DUP-F

DUP-F $-$ SOIL GAS

1214161710

12/14/16 Field replicate of soil gas sample. Begin pressure -26 in. $\mathrm{Hg}$. End pressure 0 in. $\mathrm{Hg}$.
EB

EB

RI

$\mathrm{RI}$

RI

$\mathrm{RI}$

RI
YKCITY-W-38089

YKTRUCK-W-38090

YKQCBR-W-37839

YKQCIR-W-38032

WG

WG

YK523161710

YK523161710

$-$

$-$

$-$

WQC

7241

YKQCIR-W-38022

YKQCIR-W-38026
7244

7246

$-$

WQC

WQC

WQC

YK120161700

WQC

YK121161800

WQC
5/23/16 Sample collected from the city fire hydrant east of the county shop.

5/23/16 Sample collected from the Cascade Drilling water truck.

7/9/15 Rinsate of decontaminated bailer after samples YKTI03-W-37836 and YKTI03W-37837.

7/15/15 Rinsate of decontaminated bailer after sample YKTI01120-W-37855.

7/16/15 Rinsate of decontaminated bailer after sample YKTI04120-W-37860.

1/20/16 Rinsate of decontaminated bailer after sample YKTI24-90-W-38018.

1/21/16 Rinsate of decontaminated bailer after samples YKTI25-98-W-38024 and YKTI25-98DUP-W-38025.

1/26/16 Rinsate of decontaminated bailer after samples YKTI26-96-W-38030 and YKTI26-96DUP-W-38031. 
Trip blanks

\begin{tabular}{|c|c|c|}
\hline $7 / 7 / 1514: 38$ & QC & YKQCTB-S-37763 \\
\hline 7/7/15 15:13 & QC & YKQCTB-S-37777 \\
\hline 7/7/15 17:12 & QC & YKQCTB-S-37784 \\
\hline 7/8/15 13:27 & QC & YKQCTB-S-37804 \\
\hline 7/9/15 9:49 & QC & YKQCTB-S-37825 \\
\hline 7/9/15 13:00 & QC & YKQCTB-W-37835 \\
\hline $7 / 9 / 1514: 37$ & QC & YKQCTB-S-37832 \\
\hline $7 / 10 / 15$ 9:22 & QC & YKQCTB-S-37840 \\
\hline 7/10/15 13:30 & QC & YKQCTB-W-37846 \\
\hline 7/13/15 9:18 & QC & YKQCTB-W-37851 \\
\hline $7 / 14 / 1515: 30$ & QC & YKQCTB-W-37853 \\
\hline $7 / 16 / 159: 42$ & QC & YKQCTB-W-37859 \\
\hline $7 / 16 / 159: 50$ & QC & YK-S-MEOHBLANK16JUL2015 \\
\hline 7/17/15 12:43 & QC & YKQCTB-W-37866 \\
\hline $7 / 17 / 1512: 52$ & $\mathrm{QC}$ & YKQCTB-W-37869 \\
\hline
\end{tabular}

\begin{tabular}{|c|c|c|c|}
\hline TB & - & SOIL & 7234 \\
\hline TB & - & SOIL & 7235 \\
\hline TB & - & SOIL & 7237 \\
\hline TB & - & SOIL & 7238 \\
\hline TB & - & SOIL & 7239 \\
\hline TB & - & WQC & 7241 \\
\hline TB & - & SOIL & 7240 \\
\hline TB & - & SOIL & 7242 \\
\hline TB & - & WQC & 7243 \\
\hline TB & - & WQC & 7244 \\
\hline TB & - & WQC & 7245 \\
\hline TB & - & WQC & 7246 \\
\hline TB & - & $\mathrm{MEOH}$ & 4916 \\
\hline TB & - & WQC & 7247 \\
\hline TB & - & WQC & 72 \\
\hline
\end{tabular}

2/9/16 Rinsate of decontaminated bailer after samples YKTI27-77-W-38050 and YKTI27-77DUP-W-38051. Sample frozen; one vial was unbroken and analyzed.

3/3/16 Rinsate of decontaminated bailer after sample YKTI28-78-W-38075.

7/7/15 Trip blank with soil samples shipped to AGEM Laboratory.

7/7/15 Trip blank with soil samples shipped to AGEM Laboratory.

7/8/15 Trip blank with soil samples shipped to AGEM Laboratory.

7/8/15 Trip blank with soil samples shipped to AGEM Laboratory.

7/9/15 Trip blank with soil samples shipped to AGEM Laboratory.

7/9/15 Trip blank with water samples shipped to AGEM Laboratory.

7/9/15 Trip blank with soil samples shipped to AGEM Laboratory.

7/10/15 Trip blank with soil samples shipped to AGEM Laboratory.

7/10/15 Trip blank with water samples shipped to AGEM Laboratory.

7/15/15 Trip blank with water samples shipped to AGEM Laboratory.

7/15/15 Trip blank with water samples shipped to TestAmerica Laboratory.

7/16/15 Trip blank with water samples shipped to AGEM Laboratory.

7/16/15 Trip blank with soil samples shipped to TestAmerica Laboratory.

7/17/15 Trip blank with water samples shipped to AGEM Laboratory.

7/20/15 Trip blank with water samples shipped to TestAmerica Laboratory. 


\begin{tabular}{|c|c|c|}
\hline 7/18/15 10:31 & QC & YKQCTB-W-37871 \\
\hline $7 / 21 / 15$ 12:12 & QC & YKQCTB-W-37883 \\
\hline 8/5/15 12:00 & QC & YKQCTB-W-37925 \\
\hline 8/13/15 8:00 & $\mathrm{QC}$ & YKQCTB-W-37934 \\
\hline 8/17/15 17:30 & QC & YKQCTB-W-37938 \\
\hline 8/20/15 9:00 & QC & YKQCTB-W-37944 \\
\hline 8/22/15 8:00 & QC & YKQCTB-W-37950 \\
\hline 8/23/15 9:00 & $\mathrm{QC}$ & YKQCTB-W-37960 \\
\hline 8/25/15 9:00 & $\mathrm{QC}$ & YKQCTB-W-37965 \\
\hline 8/26/15 9:00 & $\mathrm{QC}$ & YKQCTB-W-37969 \\
\hline 8/27/15 9:00 & QC & YKQCTB-W-37974 \\
\hline 9/9/15 8:00 & QC & YKQCTB-W-37979 \\
\hline 9/10/15 8:00 & $\mathrm{QC}$ & YKQCTB-W-37984 \\
\hline 9/11/15 8:00 & $\mathrm{QC}$ & YKQCTB-W-37989 \\
\hline 9/14/15 8:00 & QC & YKQCTB-W-37994 \\
\hline 9/16/15 9:00 & QC & YKQCTB-W-38000 \\
\hline 1/20/16 9:00 & QC & YKQCTB-W-38021 \\
\hline $1 / 21 / 169: 00$ & QC & YKQCTB-W-38028 \\
\hline $1 / 25 / 168: 00$ & QC & YKQCTB-W-38035 \\
\hline
\end{tabular}

\begin{tabular}{|c|c|c|c|}
\hline TB & - & WQC & 7249 \\
\hline TB & - & WQC & 7250 \\
\hline TB & - & WQC & YK810151705 \\
\hline TB & - & WQC & YK814151700 \\
\hline TB & - & WQC & YK819151710 \\
\hline TB & - & WQC & YK820151750 \\
\hline TB & - & WQC & YK8222151150 \\
\hline TB & - & WQC & YK824151700 \\
\hline TB & - & WQC & YK825151700 \\
\hline TB & - & WQC & YK826151700 \\
\hline TB & - & WQC & YK828151300 \\
\hline TB & - & WQC & YK99151700 \\
\hline TB & - & WQC & YK910151700 \\
\hline TB & - & WQC & YK911151800 \\
\hline TB & - & WQC & YK915151800 \\
\hline TB & - & WQC & YK916151700 \\
\hline TB & - & WQC & YK120161700 \\
\hline TB & - & WQC & YK121161800 \\
\hline TB & - & WQC & YK126161700 \\
\hline
\end{tabular}

7/20/15 Trip blank with water samples shipped to AGEM Laboratory.

7/22/15 Trip blank with water samples shipped to AGEM Laboratory.

8/10/15 Trip blank with water samples shipped to AGEM Laboratory.

8/14/15 Trip blank with water samples shipped to AGEM Laboratory.

8/19/15 Trip blank with water samples shipped to AGEM Laboratory.

8/20/15 Trip blank with water samples shipped to AGEM Laboratory.

8/22/15 Trip blank with water samples shipped to AGEM Laboratory.

8/24/15 Trip blank with water samples shipped to AGEM Laboratory.

8/25/15 Trip blank with water samples shipped to AGEM Laboratory.

8/26/15 Trip blank with water samples shipped to AGEM Laboratory.

8/28/15 Trip blank with water samples shipped to AGEM Laboratory.

9/9/15 Trip blank with water samples shipped to AGEM Laboratory.

9/10/15 Trip blank with water samples shipped to AGEM Laboratory.

9/11/15 Trip blank with water samples shipped to AGEM Laboratory.

9/15/15 Trip blank with water samples shipped to AGEM Laboratory.

9/16/15 Trip blank with water samples shipped to AGEM Laboratory.

1/20/16 Trip blank with water samples shipped to AGEM Laboratory.

1/21/16 Trip blank with water samples shipped to AGEM Laboratory.

1/26/16 Trip blank with water samples shipped to AGEM Laboratory. 


$\begin{array}{rllllll}\text { 2/9/16 13:00 QC } & \text { YKQCTB-W-38055 } & \text { TB } & - & \text { WQC } & \text { YK29161700 } \\ \text { 2/10/16 10:00 QC } & \text { YKQCTB-W-38058 } & \text { TB } & - & \text { WQC } & \text { YK210161200 } \\ \text { 3/2/16 13:00 } & \text { QC } & \text { YKQCTB-W-38080 } & \text { TB } & - & \text { WQC } & \text { YK33161400 } \\ \text { 5/17/16 13:00 QC } & \text { YKQCTB-W-38092 } & \text { TB } & - & \text { WQC } & \text { YK523161710 } \\ \text { 5/25/16 15:30 } & \text { QC } & \text { YKQCTB-W-38095 } & \text { TB } & - & \text { WQC } & \text { YK525161800 } \\ \text { 5/31/16 16:00 } & \text { QC } & \text { YKQCTB-W-38099 } & \text { TB } & - & \text { WQC } & \text { YK531161800 } \\ \text { 6/2/16 19:00 } & \text { QC } & \text { YKQCTB-W-38101 } & \text { TB } & - & \text { WQC } & \text { YK62161910 } \\ \text { 6/6/16 10:20 } & \text { QC } & \text { YKQCTB-W-38105 } & \text { TB } & - & \text { WQC } & \text { YK66161800 } \\ \text { 6/7/16 8:05 } & \text { QC } & \text { YKQCTB-W-38107 } & \text { TB } & - & \text { WQC } & \text { YK67161530 } \\ 6 / 9 / 1617: 30 & \text { QC } & \text { YKQCTB-W-38110 } & \text { TB } & - & \text { WQC } & \text { YK69161730 } \\ 6 / 9 / 16 ~ 17: 30 & \text { QC } & \text { YKQCTB-W-38110PACE } & \text { TB } & - & \text { WQC } & \text { Pace-9Jun2016 } \\ \text { 6/13/16 14:30 } & \text { QC } & \text { YKQCTB-W-38114 } & \text { TB } & - & \text { WQC } & \text { YK613161500 } \\ 6 / 16 / 16 ~ 14: 50 & \text { QC } & \text { YKQCTB-W-38117 } & \text { TB } & - & \text { WQC } & \text { YK616161500 } \\ 6 / 21 / 169: 00 & \text { QC } & \text { YKQCTB-W-38120 } & \text { TB } & - & \text { WQC } & \text { YK621161730 } \\ 6 / 21 / 169: 00 & \text { QC } & \text { YKQCTB-W-38120PACE } & \text { TB } & - & \text { WQC } & \text { Pace-21Jun2016 }\end{array}$

Soil and groundwater samples selected for duplicate organic analysis by the AGEM Laboratory

$\begin{array}{lllllll}\text { 7/7/15 12:58 } & \text { TI01 } & \text { YKTI01-S-37749DUP } & \text { DUP-L } & 24 & \text { SOIL } & 7234 \\ \text { 7/7/15 15:53 TI02 } & \text { YKTIO2-S-37772DUP } & \text { DUP-L } & 36 & \text { SOIL } & 7235 \\ 7 / 7 / 15 ~ 17: 05 & \text { TI02 } & \text { YKTI02-S-37781DUP } & \text { DUP-L } & 64 & \text { SOIL } & 7237\end{array}$

2/9/16 Trip blank with water samples shipped to AGEM Laboratory. Sample frozen; one vial was unbroken and analyzed.

2/10/16 Trip blank with water samples shipped to AGEM Laboratory.

3/3/16 Trip blank with water samples shipped to AGEM Laboratory.

5/23/16 Trip blank with water samples shipped to AGEM Laboratory.

5/25/16 Trip blank with water samples shipped to AGEM Laboratory.

5/31/16 Trip blank with water samples shipped to AGEM Laboratory.

6/2/16 Trip blank with water samples shipped to AGEM Laboratory.

6/6/16 Trip blank with water samples shipped to AGEM Laboratory.

6/7/16 Trip blank with water samples shipped to AGEM Laboratory.

6/9/16 Trip blank with water samples shipped to AGEM Laboratory.

6/9/16 Trip blank with water samples shipped to Pace Analytical Services, Inc.

6/13/16 Trip blank with water samples shipped to AGEM Laboratory.

6/16/16 Trip blank with water samples shipped to AGEM Laboratory.

6/21/16 Trip blank with water samples shipped to AGEM Laboratory.

6/21/16 Trip blank with water samples shipped to Pace Analytical Services, Inc.

7/7/15 Duplicate laboratory analysis.

7/7/15 Duplicate laboratory analysis.

7/8/15 Duplicate laboratory analysis. 


\begin{tabular}{|c|c|}
\hline 7/9/15 12:02 & TIO3 \\
\hline 7/10/15 9:14 & TI01 \\
\hline 7/10/15 10:05 & TI01 \\
\hline 7/10/15 14:44 & TIO2 \\
\hline 7/15/15 16:36 & TI01 \\
\hline 7/16/15 16:00 & TI04 \\
\hline 7/17/15 15:35 & TI05 \\
\hline $7 / 18 / 15$ & TI05 \\
\hline 7/19/15 & TI06 \\
\hline 7/20/15 14:50 & TIO7 \\
\hline 7/21/15 14:45 & TI08 \\
\hline 8/7/15 12:00 & SB77 \\
\hline 8/7/15 12:00 & SB77 \\
\hline $8 / 13 / 151$ & TI09 \\
\hline $8 / 14 / 151$ & TI10 \\
\hline 8/18/15 1 & TI11 \\
\hline $8 / 18 / 15$ & TI11 \\
\hline 8/20/15 9:30 & TI12 \\
\hline $8 / 22 / 158: 30$ & TI13 \\
\hline 8/24/15 11:30 & TI15 \\
\hline 8/25/15 11:45 & TI16 \\
\hline 8/26/15 & TI17 \\
\hline 8/28/15 11:00 & TI18 \\
\hline 9/9/15 11:30 & TI19 \\
\hline 9/10/15 12:00 & TI20 \\
\hline 9/11/15 15:30 & TI21 \\
\hline 9/15/15 13:40 & TI23 \\
\hline 9/16/15 11:00 & TI23 \\
\hline 1/20/16 10:55 & TI24 \\
\hline 1/21/16 17:45 & TI25 \\
\hline 1/25/16 13:20 & TI26 \\
\hline 2/9/16 16:30 & TI27 \\
\hline 2/9/16 16:30 & TI27 \\
\hline $3 / 2 / 1617: 00$ & TI28 \\
\hline 5/23/16 17:00 & DL1C \\
\hline 5/31/16 16:45 & DL2C2 \\
\hline 6/2/16 9:15 & DL2C1 \\
\hline 6/6/16 8:05 & DL3C1 \\
\hline $6 / 7 / 16$ 14:25 & DL8C1 \\
\hline 6/9/16 17:10 & DL7CL \\
\hline
\end{tabular}

YKTI03-S-37826DUP YKTI01-S-37757DUP YKTI01-S-37761DUP YKTI02-W-37848DUP YKTI01120-W-37855DUP YKTI04100-W-37862DUP YKTI05100-W-37867DUP YKTI0580-W-37868DUP YKTI06100-W-37873DUP YKTI07120-W-37878DUP YKTI0885-W-37879DUP YKSB77DUP-W-37922DUP YKSB77-W-37921DUP

YKTI09-135-W-37929DUP YKTI10-117-W-37933DUP YKTI11-92-W-37936DUP YKTI11-112-W-37937DUP YKTI12-80-W-37939DUP YKTI13-93-W-37947DUP YKTI15-113-W-37957DUP YKTI16-105-W-37963DUP YKTI17-133-W-37968DUP YKTI18-114-W-37973DUP YKTI19-107-W-37976DUP YKTI20-105-W-37981DUP YKTI21-99-W-37986DUP YKTI23-80-W-37995DUP YKTI23-145-W-37999DUP YKTI24-90-W-38018DUP YKTI25-108-W-38027DUP YKTI26-78-W-38029DUP YKTI27-100-W-38053DUP YKTI27-100-W-38057DUP YKTI28-78-W-38075DUP YKDL1C-108-W-38091DUP YKDL2C2-136-W-38096DUP YKDL2-C1-112-W-38100DUP YKDL3-C1-113-W-38103DUP YKDL8C1-104-W-38106DUP YKDL7-CL-265-W-38108DUP

$\begin{array}{lccc}\text { DUP-L } & 63.5 & \text { SOIL } & 7240 \\ \text { DUP-L } & 52 & \text { SOIL } & 7242 \\ \text { DUP-L } & 68 & \text { SOIL } & 7242 \\ \text { DUP-L } & 77-82 & \text { WG } & 7243 \\ \text { DUP-L } & 108-118 & \text { WG } & 7244 \\ \text { DUP-L } & 89-99 & \text { WG } & 7246 \\ \text { DUP-L } & 88-98 & \text { WG } & 7247 \\ \text { DUP-L } & 76-81 & \text { WG } & 7249 \\ \text { DUP-L } & 90-100 & \text { WG } & 7249 \\ \text { DUP-L } & 110-120 & \text { WG } & 7249 \\ \text { DUP-L } & 80-85 & \text { WG } & 7250 \\ \text { DUP-L } & 75.8-79 & \text { WG } & \text { YK810151705 } \\ \text { DUP-L } & 75.8-79 & \text { WG } & \text { YK810151705 } \\ \text { DUP-L } & 135-145 & \text { WG } & \text { YK814151700 } \\ \text { DUP-L } & 117-127 & \text { WG } & \text { YK814151700 } \\ \text { DUP-L } & 92-102 & \text { WG } & \text { YK819151710 } \\ \text { DUP-L } & 112-122 & \text { WG } & \text { YK819151710 } \\ \text { DUP-L } & 80-85 & \text { WG } & \text { YK820151750 } \\ \text { DUP-L } & 93-103 & \text { WG } & \text { YK8222151150 } \\ \text { DUP-L } & 113-123 & \text { WG } & \text { YK824151700 } \\ \text { DUP-L } & 105-115 & \text { WG } & \text { YK825151700 } \\ \text { DUP-L } & 133-143 & \text { WG } & \text { YK826151700 } \\ \text { DUP-L } & 114-124 & \text { WG } & \text { YK828151300 } \\ \text { DUP-L } & 107-117 & \text { WG } & \text { YK99151700 } \\ \text { DUP-L } & 105-115 & \text { WG } & \text { YK910151700 } \\ \text { DUP-L } & 99-109 & \text { WG } & \text { YK911151800 } \\ \text { DUP-L } & 80-90 & \text { WG } & \text { YK915151800 } \\ \text { DUP-L } & 145-155 & \text { WG } & \text { YK916151700 } \\ \text { DUP-L } & 90-100 & \text { WG } & \text { YK120161700 } \\ \text { DUP-L } & 113-118 & \text { WG } & \text { YK121161800 } \\ \text { DUP-L } & 78-88 & \text { WG } & \text { YK126161700 } \\ \text { DUP-L } & 100-110 & \text { WG } & \text { YK29161700 } \\ \text { DUP-L } & 100-110 & \text { WG } & \text { YK210161200 } \\ \text { DUP-L } & 78-88 & \text { WG } & \text { YK33161400 } \\ \text { DUP-L } & 108-118 & \text { WG } & \text { YK523161710 } \\ \text { DUP-L } & 136-146 & \text { WG } & \text { YK531161800 } \\ \text { DUP-L } & 112-122 & \text { WG } & \text { YK62161910 } \\ \text { DUP-L } & 113-123 & \text { WG } & \text { YK66161800 } \\ \text { DUP-L } & 104-114 & \text { WG } & \text { YK67161530 } \\ \text { DUP-L } & 265-275 & \text { WG } & \text { YK69161730 }\end{array}$

7/9/15 Duplicate laboratory analysis 7/10/15 Duplicate laboratory analysis. 7/10/15 Duplicate laboratory analysis. 7/10/15 Duplicate laboratory analysis. 7/15/15 Duplicate laboratory analysis. 7/16/15 Duplicate laboratory analysis. 7/17/15 Duplicate laboratory analysis. 7/20/15 Duplicate laboratory analysis. 7/20/15 Duplicate laboratory analysis. $7 / 20 / 15$ Duplicate laboratory analysis. 7/22/15 Duplicate laboratory analysis. 8/10/15 Duplicate laboratory analysis. 8/10/15 Duplicate laboratory analysis. 8/14/15 Duplicate laboratory analysis. 8/14/15 Duplicate laboratory analysis. 8/19/15 Duplicate laboratory analysis. 8/19/15 Duplicate laboratory analysis. 8/20/15 Duplicate laboratory analysis. 8/22/15 Duplicate laboratory analysis. 8/24/15 Duplicate laboratory analysis. 8/25/15 Duplicate laboratory analysis. 8/26/15 Duplicate laboratory analysis. 8/28/15 Duplicate laboratory analysis. 9/9/15 Duplicate laboratory analysis. 9/10/15 Duplicate laboratory analysis. 9/11/15 Duplicate laboratory analysis. 9/15/15 Duplicate laboratory analysis. 9/16/15 Duplicate laboratory analysis. 1/20/16 Duplicate laboratory analysis. 1/21/16 Duplicate laboratory analysis. 1/26/16 Duplicate laboratory analysis. 2/9/16 Duplicate laboratory analysis. 2/10/16 Duplicate laboratory analysis. 3/3/16 Duplicate laboratory analysis. 5/23/16 Duplicate laboratory analysis. 5/31/16 Duplicate laboratory analysis. 6/2/16 Duplicate laboratory analysis. 6/6/16 Duplicate laboratory analysis. 6/7/16 Duplicate laboratory analysis. 6/9/16 Duplicate laboratory analysis. 
6/12/16 11:50 DL7C

YKDL7-C-100-W-38111DUP

YKDL5-C1-106-W-38118DUP

DUP-L

$100-110$

6/21/16 15:30 DL5C1

DUP-L

106-116

WG

WG

YK613161500

YK621161730

Soil and groundwater samples submitted for verification organic analysis at TestAmerica

\begin{tabular}{|c|c|c|c|c|c|c|}
\hline 7/7/15 15:02 & TI02 & YKTI02-S-37764VER & VER & 4 & SOIL & 4916 \\
\hline 7/7/15 15:24 & TIO2 & YKTI02-S-37767VER & VER & 16 & SOIL & 4916 \\
\hline 7/7/15 15:28 & TI02 & YKTI02-S-37768VER & VER & 20 & SOIL & 4916 \\
\hline 7/8/15 11:33 & TI04 & YKTI04-S-37791VER & VER & 20 & SOIL & 4916 \\
\hline 7/8/15 15:30 & TI04 & YKTI04-S-37805VER & VER & 68 & SOIL & 4916 \\
\hline 7/9/15 10:06 & TI03 & YKTI03-S-37814VER & VER & 28 & SOIL & 4916 \\
\hline 7/9/15 12:02 & TI03 & YKTI03-S-37826VER & VER & 63.5 & SOIL & 4916 \\
\hline 7/10/15 9:24 & TI01 & YKTI01-S-37758VER & VER & 56 & SOIL & 4916 \\
\hline 7/14/15 15:00 & TIO2 & YKTI02120-W-37852VER & VER & 108.5118 .5 & WG & 7245 \\
\hline 7/15/15 16:36 & TI01 & YKTI01120-W-37855VER & VER & 108-118 & WG & 7245 \\
\hline 7/18/15 9:40 & TI05 & YKTI0580-W-37868VER & VER & $76-81$ & WG & 7248 \\
\hline 7/19/15 15:50 & TI06 & YKTI0680-W-37872VER & VER & 77-82 & WG & 7248 \\
\hline 7/20/15 12:25 & TI07 & YKTI07100-W-37876VER & VER & $90-100$ & WG & 7248 \\
\hline 7/21/15 12:12 & QC & YKQCTB-W-37883VER & VER & - & WQC & 7252 \\
\hline 7/21/15 14:45 & TI08 & YKTI0885-W-37879VER & VER & $80-85$ & WG & 7252 \\
\hline 8/5/15 12:00 & QC & YKQCTB-W-37925VER & VER & - & WQC & YK810151710 \\
\hline 8/5/15 13:35 & DW38 & YKYORKCOUNTYSHOP-W-379: & VER & - & WG & YK810151710 \\
\hline
\end{tabular}

6/13/16 Duplicate laboratory analysis.

6/21/16 Duplicate laboratory analysis.
7/16/15 Verification sample to Test America, Inc., South Burlington, Vermont.

7/16/15 Verification sample to Test America, Inc., South Burlington, Vermont.

7/16/15 Verification sample to Test America Inc., South Burlington, Vermont.

7/16/15 Verification sample to Test America, Inc., South Burlington, Vermont.

7/16/15 Verification sample to Test America, Inc., South Burlington, Vermont.

7/16/15 Verification sample to Test America Inc., South Burlington, Vermont.

7/16/15 Verification sample to Test America Inc., South Burlington, Vermont.

7/16/15 Verification sample to Test America Inc., South Burlington, Vermont.

7/15/15 Verification sample to Test America Inc., South Burlington, Vermont.

7/15/15 Verification sample to Test America Inc., South Burlington, Vermont.

7/20/15 Verification sample to Test America, Inc., South Burlington, Vermont.

7/20/15 Verification sample to Test America, Inc., South Burlington, Vermont.

7/20/15 Verification sample to Test America Inc., South Burlington, Vermont.

7/22/15 Verification sample to Test America, Inc., South Burlington, Vermont.

7/22/15 Verification sample to Test America, Inc., South Burlington, Vermont.

8/10/15 Verification sample to Test America Inc., South Burlington, Vermont.

8/10/15 Verification sample to Test America Inc., South Burlington, Vermont. 


\begin{tabular}{|c|c|c|c|c|c|c|c|c|}
\hline 8/7/15 14:02 & SB62 & YKSB62-W-37924VER & VER & - & WG & YK810151710 & 8/10/15 & $\begin{array}{l}\text { Verification sample to Test America, } \\
\text { Inc., South Burlington, Vermont. }\end{array}$ \\
\hline 8/13/15 8:00 & $\mathrm{QC}$ & YKQCTB-W-37934VER & VER & - & WQC & YK814151710 & 8/14/15 & $\begin{array}{l}\text { Verification sample to Test America, } \\
\text { Inc., South Burlington, Vermont. }\end{array}$ \\
\hline 8/13/15 16:00 & TI09 & YKTI09-135-W-37929VER & VER & $135-145$ & WG & YK814151710 & 8/14/15 & $\begin{array}{l}\text { Verification sample to Test America, } \\
\text { Inc., South Burlington, Vermont. }\end{array}$ \\
\hline 8/14/15 15:30 & TI10 & YKTI10-117-W-37933VER & VER & $117-127$ & WG & YK814151710 & 8/14/15 & $\begin{array}{l}\text { Verification sample to Test America, } \\
\text { Inc., South Burlington, Vermont. }\end{array}$ \\
\hline 8/17/15 17:30 & $\mathrm{QC}$ & YKQCTB-W-37938VER & VER & - & WQC & YK820151755 & 8/20/15 & $\begin{array}{l}\text { Verification sample to Test America, } \\
\text { Inc., South Burlington, Vermont. }\end{array}$ \\
\hline 8/18/15 16:30 & TI11 & YKTI11-92-W-37936VER & VER & $92-102$ & WG & YK820151755 & 8/20/15 & $\begin{array}{l}\text { Verification sample to Test America, } \\
\text { Inc., South Burlington, Vermont. }\end{array}$ \\
\hline 8/20/15 17:15 & TI12 & YKTI12-145-W-37943VER & VER & 135-145 & WG & YK820151755 & 8/20/15 & $\begin{array}{l}\text { Verification sample to Test America, } \\
\text { Inc., South Burlington, Vermont. }\end{array}$ \\
\hline 8/23/15 9:00 & $\mathrm{QC}$ & YKQCTB-W-37960VER & VER & - & WQC & YK828151310 & 8/28/15 & $\begin{array}{l}\text { Verification sample to Test America, } \\
\text { Inc., South Burlington, Vermont. }\end{array}$ \\
\hline 8/23/15 14:45 & TI14 & YKTI14-136-W-37954VER & VER & 136-146 & WG & YK828151310 & 8/28/15 & $\begin{array}{l}\text { Verification sample to Test America, } \\
\text { Inc., South Burlington, Vermont. }\end{array}$ \\
\hline 8/25/15 14:50 & TI16 & YKTI16-136-W-37964VER & VER & $136-146$ & WG & YK828151310 & 8/28/15 & $\begin{array}{l}\text { Verification sample to Test America, } \\
\text { Inc., South Burlington, Vermont. }\end{array}$ \\
\hline 8/28/15 11:00 - - - & TI18 & YKTI18-114-W-37973VER & VER & $114-124$ & WG & YK828151310 & 8/28/15 & $\begin{array}{l}\text { Verification sample to Test America, } \\
\text { Inc., South Burlington, Vermont. }\end{array}$ \\
\hline 9/10/15 8:00 & $\mathrm{QC}$ & YKQCTB-W-37984VER & VER & - & WQC & YK915151755 & 9/15/15 & $\begin{array}{l}\text { Verification sample to Test America, } \\
\text { Inc., South Burlington, Vermont. }\end{array}$ \\
\hline 9/10/15 12:00 & TI20 & YKTI20-105-W-37981VER & VER & 105-115 & WG & YK915151755 & 9/15/15 & $\begin{array}{l}\text { Verification sample to Test America, } \\
\text { Inc., South Burlington, Vermont. }\end{array}$ \\
\hline 9/11/15 15:30 & TI21 & YKTI21-99-W-37986VER & VER & 99-109 & WG & YK915151755 & 9/15/15 & $\begin{array}{l}\text { Verification sample to Test America, } \\
\text { Inc., South Burlington, Vermont. }\end{array}$ \\
\hline 9/15/15 16:45 & TI23 & YKTI23-120-W-37998VER & VER & $120-130$ & WG & YK915151755 & 9/15/15 & $\begin{array}{l}\text { Verification sample to Test America, } \\
\text { Inc., South Burlington, Vermont. }\end{array}$ \\
\hline 1/20/16 9:00 & $\mathrm{QC}$ & YKQCTB-W-38021VER & VER & - & WQC & YK121161810 & 1/21/16 & $\begin{array}{l}\text { Verification sample to Test America, } \\
\text { Inc., South Burlington, Vermont. }\end{array}$ \\
\hline 1/20/16 13:20 & TI24 & YKTI24-100-W-38019VER & VER & $100-110$ & WG & YK121161810 & 1/21/16 & $\begin{array}{l}\text { Verification sample to Test America, } \\
\text { Inc., South Burlington, Vermont. }\end{array}$ \\
\hline 1/21/16 17:45 & TI25 & YKTI25-108-W-38027VER & VER & $113-118$ & WG & YK121161810 & $1 / 21 / 16$ & $\begin{array}{l}\text { Verification sample to Test America, } \\
\text { Inc., South Burlington, Vermont. }\end{array}$ \\
\hline 3/2/16 13:00 & $\mathrm{QC}$ & YKQCTB-W-38080VER & VER & - & WQC & YK33161410 & $3 / 3 / 16$ & $\begin{array}{l}\text { Verification sample to Test America, } \\
\text { Inc., South Burlington, Vermont. }\end{array}$ \\
\hline 3/3/16 10:50 & TI28 & YKTI28-104-W-38078VER & VER & 104-109 & WG & YK33161410 & $3 / 3 / 16$ & $\begin{array}{l}\text { Verification sample to Test America, } \\
\text { Inc., South Burlington, Vermont. }\end{array}$ \\
\hline
\end{tabular}




\begin{tabular}{|c|c|c|c|c|c|c|c|c|}
\hline 5/23/16 17:00 & DL1C & YKDL1C-108-W-38091VER & VER & 108-118 & WG & YK531161735 & $5 / 31 / 16$ & $\begin{array}{l}\text { Verification sample to Test America, } \\
\text { Inc., South Burlington, Vermont. }\end{array}$ \\
\hline $5 / 24 / 1618: 20$ & DL1B & YKDL1B-88-W-38093VER & VER & $88-98$ & WG & YK531161735 & $5 / 31 / 16$ & $\begin{array}{l}\text { Verification sample to Test America, } \\
\text { Inc., South Burlington, Vermont. }\end{array}$ \\
\hline 5/31/16 16:00 & $\mathrm{QC}$ & YKQCTB-W-38099VER & VER & - & WQC & YK531161735 & $5 / 31 / 16$ & $\begin{array}{l}\text { Verification sample to Test America, } \\
\text { Inc., South Burlington, Vermont. }\end{array}$ \\
\hline 5/31/16 16:45 & DL2C2 & YKDL2C2-136-W-38096VER & VER & $136-146$ & WG & YK531161735 & $5 / 31 / 16$ & $\begin{array}{l}\text { Verification sample to Test America, } \\
\text { Inc., South Burlington, Vermont. }\end{array}$ \\
\hline 5/31/16 17:00 & DL2CU & YKDL2CU-215-W-38097VER & VER & $215-225$ & WG & YK531161735 & 5/31/16 & $\begin{array}{l}\text { Verification sample to Test America, } \\
\text { Inc., South Burlington, Vermont. }\end{array}$ \\
\hline 6/9/16 17:10 & DL7CL & YKDL7-CL-265-W-38108VER & VER & 275 & WG & YK69161740 & $6 / 9 / 16$ & $\begin{array}{l}\text { Verification sample to Test America, } \\
\text { Inc., South Burlington, Vermont. }\end{array}$ \\
\hline 6/9/16 17:30 & $\mathrm{QC}$ & YKQCTB-W-38110VER & VER & - & WQC & YK69161740 & 6/9/16 & $\begin{array}{l}\text { Verification sample to Test America, } \\
\text { Inc., South Burlington, Vermont. }\end{array}$ \\
\hline 6/12/16 12:30 & DL7CU & YKDL7-CU-192-W-38112VER & VER & 192-202 & WG & YK613161510 & 6/13/16 & $\begin{array}{l}\text { Verification sample to Test America, } \\
\text { Inc., South Burlington, Vermont. }\end{array}$ \\
\hline 6/13/16 11:00 & DL6C & YKDL6-C-110-W-38113VER & VER & $110-120$ & WG & YK613161510 & $6 / 13 / 16$ & $\begin{array}{l}\text { Verification sample to Test America, } \\
\text { Inc., South Burlington, Vermont. }\end{array}$ \\
\hline 6/13/16 14:30 & $\mathrm{QC}$ & YKQCTB-W-38114VER & VER & - & WQC & YK613161510 & 6/13/16 & $\begin{array}{l}\text { Verification sample to Test America, } \\
\text { Inc., South Burlington, Vermont. }\end{array}$ \\
\hline 6/15/16 13:10 & DL4C2 & YKDL4-C2-145-W-38115VER & VER & 145-155 & WG & YK616161510 & 6/16/16 & $\begin{array}{l}\text { Verification sample to Test America, } \\
\text { Inc., South Burlington, Vermont. }\end{array}$ \\
\hline 6/16/16 14:30 & DL4C1 & YKDL4-C1-120-W-38116VER & VER & $120-130$ & WG & YK616161510 & $6 / 16 / 16$ & $\begin{array}{l}\text { Verification sample to Test America, } \\
\text { Inc., South Burlington, Vermont. }\end{array}$ \\
\hline 6/16/16 14:50 & $\mathrm{QC}$ & YKQCTB-W-38117VER & VER & - & WQC & YK616161510 & 6/16/16 & $\begin{array}{l}\text { Verification sample to Test America, } \\
\text { Inc., South Burlington, Vermont. }\end{array}$ \\
\hline \multicolumn{9}{|c|}{ Investigation-derived waste samples } \\
\hline 6/9/16 17:20 & $\mathrm{QC}$ & YKIDW-W-38109 & WASTE & - & WG & Pace-9Jun2016 & 6/9/16 & $\begin{array}{l}\text { Composite sample of the purge water } \\
\text { containerized on-site during monitoring } \\
\text { well development. }\end{array}$ \\
\hline 6/21/16 16:00 & QC & YKIDW-W-38119 & WASTE & - & WG & Pace-21Jun2016 & 6/21/16 & $\begin{array}{l}\text { Composite purge water sample from } \\
\text { poly tanks containing the development } \\
\text { water stored on-site during monitoring } \\
\text { well development. }\end{array}$ \\
\hline $6 / 21 / 1616: 55$ & $\mathrm{QC}$ & YKROLLFRONT-S-38121 & WASTE & - & SOIL & Pace-21Jun2016 & 6/21/16 & $\begin{array}{l}\text { Composite soil sample of the drill } \\
\text { cuttings stored on-site in a closed top } \\
\text { roll off dumpster. }\end{array}$ \\
\hline
\end{tabular}


Pace-21Jun2016

Background air samples

8/5/15 16:11 HEARTH

YKBKGND-G-37907

TA-06Aug2015-5

8/5/15 11:25 FITWORX

YKBKGND-G-37908

8/5/15 17:02 TROES

YKBKGND-G-37914

8/5/15 17:50 MILLER

YKBKGND-G-37915

1/28/16 8:40 GARRETSON YKBKGND-G-38039

1/28/16 9:39 FITWORX YKBKGND-G-38045

4/28/16 14:15 FITWORX

YKBKGND-G-38086

8/10/16 10:44 GARRETSON YKBKGND-G-38141
TA-06Aug2015-5

TA-06Aug2015-5
AIR

YK128161000

$-$

AIR

YK128161000

YK428161700

AIR

AIR
6/21/16 Composite soil sample of the drill cuttings stored on-site in a closed top roll off dumpster.

8/6/15 In alcove on north side of main entrance door. Can placed on empty coleman cooler in corner against wall. Background sample collected over

24hr. Begin pressure -26 in. Hg. End pressure 0 in. $\mathrm{Hg}$.

8/6/15 In corner against outside walls, to left of main entrance. On top of $5 \mathrm{gal}$ empty bucket. Background sample collected over $24 \mathrm{hr}$. Begin pressure $-30 \mathrm{in}$. $\mathrm{Hg}$. End pressure $-6 \mathrm{in}$. $\mathrm{Hg}$.

8/6/15 Under south edge of small open deck/porch at front door of house.

Background sample collected over 24 $\mathrm{hr}$. Begin pressure $-30 \mathrm{in}$. Hg. End pressure -5 in. $\mathrm{Hg}$.

8/6/15 Canister at north end of gravel drive, directly between two northernmost quonsets still on site. Background sample collected over $24 \mathrm{hr}$. Begin pressure -29 in. $\mathrm{Hg}$. End pressure -5 in. $\mathrm{Hg}$.

1/28/16 Garretson ambient air. Sample collected over $24 \mathrm{hr}$. Begin pressure -29 in. $\mathrm{Hg}$. End pressure -5 in. $\mathrm{Hg}$.

1/28/16 FitnessWorx ambient air. Sample collected over $24 \mathrm{hr}$. Begin pressure -30 in. $\mathrm{Hg}$. End pressure $-5 \mathrm{in}$. $\mathrm{Hg}$.

4/28/16 FitnessWorx ambient air. Sample collected over $24 \mathrm{hr}$. Begin pressure -29 in. $\mathrm{Hg}$. End pressure $-3 \mathrm{in.} \mathrm{Hg}$.

8/10/16 Garretson ambient air. Sample collected over $24 \mathrm{hr}$. Begin pressure $-24 \mathrm{in.} \mathrm{Hg}$. End pressure $0 \mathrm{in.} \mathrm{Hg}$. 
8/10/16 11:37 FITWORX YKBKGND-G-38147

12/14/16 10:20 GARRETSON YKBKGND-G-39536

12/14/16 11:16 FITWORX

YKBKGND-G-39542
$-$

$-$

$-$
AIR

AIR
YK810161710

YK1214161700

YK1214161700

YK1214161710
8/10/16 FitnessWorx ambient air. Sample collected over $24 \mathrm{hr}$. Begin pressure -29 in. $\mathrm{Hg}$. End pressure -6 in. $\mathrm{Hg}$

Garretson ambient air. Sample collected over $24 \mathrm{hr}$. Begin pressure -26 in. $\mathrm{Hg}$. End pressure $-1 \mathrm{in}$. $\mathrm{Hg}$. FitnessWorx ambient air. Sample collected over $24 \mathrm{hr}$. Begin pressure -29 in. $\mathrm{Hg}$. End pressure -4 in. $\mathrm{Hg}$.

\footnotetext{
${ }^{a}$ Sample types: DUP-F, field replicate; DUP-L, laboratory duplicate; EB, equipment blank; RI, rinsate; TB, trip blank; VER, verification sample; WASTE, waste sample.

${ }^{\text {b } M a t r i x ~ t y p e s: ~ A I R, ~ i n d o o r ~ a i r ; ~ M E O H, ~ m e t h a n o l ; ~ S O I L, ~ s o i l ; ~ S O I L ~ G A S, ~ s o i l ~ g a s ; ~ W G, ~ g r o u n d w a t e r ; ~ W Q C, ~ q u a l i t y ~ c o n t r o l ~ w a t e r ~ s a m p l e, ~ e . g ., ~ t r i p ~ b l a n k . ~}$
} 
TABLE S2.2 Analytical results for quality control samples collected to monitor sample collection and handling.

\begin{tabular}{|c|c|c|c|c|c|c|c|c|c|}
\hline \multirow[b]{2}{*}{ Sample } & \multirow[b]{2}{*}{$\begin{array}{c}\text { Sample } \\
\text { Date }\end{array}$} & \multirow[b]{2}{*}{$\begin{array}{c}\text { Sample } \\
\text { Type }^{\text {a }}\end{array}$} & \multirow[b]{2}{*}{ Matrix $^{\mathrm{b}}$} & \multirow[b]{2}{*}{$\begin{array}{l}\text { Analytical } \\
\text { Laboratory }\end{array}$} & \multicolumn{4}{|c|}{ Concentration } & \multirow[b]{2}{*}{ Units } \\
\hline & & & & & $\begin{array}{c}\text { Carbon } \\
\text { Tetrachloride } \\
\end{array}$ & Chloroform & $\begin{array}{c}\text { Methylene } \\
\text { Chloride }\end{array}$ & $\mathrm{MDL}$ & \\
\hline \multicolumn{10}{|l|}{ Equipment blanks } \\
\hline YKCITY-W-38089 & $5 / 17 / 16$ & EB & WG & AGEM & $N D^{c}$ & ND & ND & 1 & $\mu \mathrm{g} / \mathrm{L}$ \\
\hline YKTRUCK-W-38090 & $5 / 17 / 16$ & EB & WG & AGEM & ND & ND & ND & 1 & $\mu \mathrm{g} / \mathrm{L}$ \\
\hline \multicolumn{10}{|l|}{ Equipment rinsates } \\
\hline YKQCBR-W-37839 & $7 / 9 / 15$ & $\mathrm{RI}$ & WQC & AGEM & ND & ND & ND & 1 & $\mu \mathrm{g} / \mathrm{L}$ \\
\hline YKQCIR-W-37857 & $7 / 15 / 15$ & RI & WQC & AGEM & ND & ND & ND & 1 & $\mu \mathrm{g} / \mathrm{L}$ \\
\hline YKQCIR-W-37863 & $7 / 16 / 15$ & RI & WQC & AGEM & ND & ND & ND & 1 & $\mu \mathrm{g} / \mathrm{L}$ \\
\hline YKQCIR-W-38022 & $1 / 20 / 16$ & $\mathrm{RI}$ & WQC & AGEM & ND & ND & ND & 1 & $\mu g / L$ \\
\hline YKQCIR-W-38026 & $1 / 21 / 16$ & RI & WQC & AGEM & ND & ND & ND & 1 & $\mu \mathrm{g} / \mathrm{L}$ \\
\hline YKQCIR-W-38032 & $1 / 25 / 16$ & $\mathrm{RI}$ & WQC & AGEM & ND & ND & ND & 1 & $\mu \mathrm{g} / \mathrm{L}$ \\
\hline YKQCIR-W-38052 & $2 / 9 / 16$ & $\mathrm{RI}$ & WQC & AGEM & ND & ND & ND & 1 & $\mu \mathrm{g} / \mathrm{L}$ \\
\hline YKQCIR-W-38079 & $3 / 2 / 16$ & $\mathrm{RI}$ & WQC & AGEM & ND & ND & ND & 1 & $\mu \mathrm{g} / \mathrm{L}$ \\
\hline \multicolumn{10}{|l|}{ Trip blanks } \\
\hline YKQCTB-S-37763 & $7 / 7 / 15$ & TB & SOIL & AGEM & ND & ND & ND & 10 & $\mu \mathrm{g} / \mathrm{kg}$ \\
\hline YKQCTB-S-37777 & $7 / 7 / 15$ & TB & SOIL & AGEM & ND & ND & ND & 10 & $\mu \mathrm{g} / \mathrm{kg}$ \\
\hline YKQCTB-S-37784 & $7 / 7 / 15$ & TB & SOIL & AGEM & ND & ND & ND & 10 & $\mu \mathrm{g} / \mathrm{kg}$ \\
\hline YKQCTB-S-37804 & $7 / 8 / 15$ & TB & SOIL & AGEM & ND & ND & ND & 10 & $\mu \mathrm{g} / \mathrm{kg}$ \\
\hline YKQCTB-S-37825 & $7 / 9 / 15$ & TB & SOIL & AGEM & ND & ND & ND & 10 & $\mu \mathrm{g} / \mathrm{kg}$ \\
\hline YKQCTB-W-37835 & $7 / 9 / 15$ & TB & WQC & AGEM & ND & ND & ND & 1 & $\mu \mathrm{g} / \mathrm{L}$ \\
\hline YKQCTB-S-37832 & 7/9/15 & TB & SOIL & AGEM & ND & ND & ND & 10 & $\mu \mathrm{g} / \mathrm{kg}$ \\
\hline YKQCTB-S-37840 & $7 / 10 / 15$ & TB & SOIL & AGEM & ND & ND & ND & 10 & $\mu \mathrm{g} / \mathrm{kg}$ \\
\hline YKQCTB-W-37846 & $7 / 10 / 15$ & TB & WQC & AGEM & ND & ND & ND & 1 & $\mu \mathrm{g} / \mathrm{L}$ \\
\hline YKQCTB-W-37851 & $7 / 13 / 15$ & TB & WQC & AGEM & ND & ND & ND & 1 & $\mu \mathrm{g} / \mathrm{L}$ \\
\hline
\end{tabular}




\begin{tabular}{|c|c|c|c|c|c|c|c|c|c|}
\hline YKQCTB-W-37853 & $7 / 14 / 15$ & TB & WQC & TestAmerica & ND & ND & ND & 1 & $\mu \mathrm{g} / \mathrm{L}$ \\
\hline YKQCTB-W-37859 & $7 / 16 / 15$ & TB & WQC & AGEM & ND & ND & ND & 1 & $\mu \mathrm{g} / \mathrm{L}$ \\
\hline YK-S-MEOHBLANK16JUL2015 & $7 / 16 / 15$ & TB & $\mathrm{MEOH}$ & TestAmerica & ND & ND & ND & 10 & $\mu \mathrm{g} / \mathrm{kg}$ \\
\hline YKQCTB-W-37866 & $7 / 17 / 15$ & TB & WQC & AGEM & ND & ND & ND & 1 & $\mu g / L$ \\
\hline YKQCTB-W-37869 & $7 / 17 / 15$ & TB & WQC & TestAmerica & ND & $0.06 \mathrm{~J}^{\mathrm{d}}$ & ND & 1 & $\mu \mathrm{g} / \mathrm{L}$ \\
\hline YKQCTB-W-37871 & $7 / 18 / 15$ & TB & WQC & AGEM & ND & ND & ND & 1 & $\mu \mathrm{g} / \mathrm{L}$ \\
\hline YKQCTB-W-37883 & $7 / 21 / 15$ & TB & WQC & AGEM & ND & ND & ND & 1 & $\mu \mathrm{g} / \mathrm{L}$ \\
\hline YKQCTB-W-37883VER & $7 / 21 / 15$ & VER & WQC & TestAmerica & ND & ND & ND & 1 & $\mu \mathrm{g} / \mathrm{L}$ \\
\hline YKQCTB-W-37925 & $8 / 5 / 15$ & TB & WQC & AGEM & ND & ND & ND & 1 & $\mu \mathrm{g} / \mathrm{L}$ \\
\hline YKQCTB-W-37925VER & $8 / 5 / 15$ & VER & WQC & TestAmerica & ND & ND & ND & 1 & $\mu \mathrm{g} / \mathrm{L}$ \\
\hline YKQCTB-W-37934 & $8 / 13 / 15$ & TB & WQC & AGEM & ND & ND & ND & 1 & $\mu \mathrm{g} / \mathrm{L}$ \\
\hline YKQCTB-W-37934VER & $8 / 13 / 15$ & VER & WQC & TestAmerica & ND & ND & ND & 1 & $\mu \mathrm{g} / \mathrm{L}$ \\
\hline YKQCTB-W-37938 & $8 / 17 / 15$ & TB & WQC & AGEM & ND & ND & ND & 1 & $\mu \mathrm{g} / \mathrm{L}$ \\
\hline YKQCTB-W-37938VER & $8 / 17 / 15$ & VER & WQC & TestAmerica & ND & ND & ND & 1 & $\mu \mathrm{g} / \mathrm{L}$ \\
\hline YKQCTB-W-37944 & $8 / 20 / 15$ & TB & WQC & AGEM & ND & ND & ND & 1 & $\mu \mathrm{g} / \mathrm{L}$ \\
\hline YKQCTB-W-37950 & $8 / 22 / 15$ & TB & WQC & AGEM & ND & ND & ND & 1 & $\mu \mathrm{g} / \mathrm{L}$ \\
\hline YKQCTB-W-37960 & $8 / 23 / 15$ & TB & WQC & AGEM & ND & ND & ND & 1 & $\mu \mathrm{g} / \mathrm{L}$ \\
\hline YKQCTB-W-37960VER & $8 / 23 / 15$ & VER & WQC & TestAmerica & ND & ND & ND & 1 & $\mu \mathrm{g} / \mathrm{L}$ \\
\hline YKQCTB-W-37965 & $8 / 25 / 15$ & TB & WQC & AGEM & ND & ND & ND & 1 & $\mu \mathrm{g} / \mathrm{L}$ \\
\hline YKQCTB-W-37969 & $8 / 26 / 15$ & TB & WQC & AGEM & ND & ND & ND & 1 & $\mu \mathrm{g} / \mathrm{L}$ \\
\hline YKQCTB-W-37974 & $8 / 27 / 15$ & TB & WQC & AGEM & ND & ND & ND & 1 & $\mu \mathrm{g} / \mathrm{L}$ \\
\hline YKQCTB-W-37979 & 9/9/15 & TB & WQC & AGEM & ND & ND & ND & 1 & $\mu \mathrm{g} / \mathrm{L}$ \\
\hline YKQCTB-W-37984 & $9 / 10 / 15$ & TB & WQC & AGEM & ND & ND & ND & 1 & $\mu \mathrm{g} / \mathrm{L}$ \\
\hline YKQCTB-W-37984VER & 9/10/15 & VER & WQC & TestAmerica & ND & ND & ND & 1 & $\mu \mathrm{g} / \mathrm{L}$ \\
\hline YKQCTB-W-37989 & $9 / 11 / 15$ & TB & WQC & AGEM & ND & ND & ND & 1 & $\mu \mathrm{g} / \mathrm{L}$ \\
\hline YKQCTB-W-37994 & 9/14/15 & TB & WQC & AGEM & ND & ND & ND & 1 & $\mu \mathrm{g} / \mathrm{L}$ \\
\hline YKQCTB-W-38000 & 9/16/15 & TB & WQC & AGEM & ND & ND & ND & 1 & $\mu \mathrm{g} / \mathrm{L}$ \\
\hline YKQCTB-W-38021 & $1 / 20 / 16$ & TB & WQC & AGEM & ND & ND & ND & 1 & $\mu \mathrm{g} / \mathrm{L}$ \\
\hline YKQCTB-W-38021VER & $1 / 20 / 16$ & VER & WQC & TestAmerica & ND & ND & ND & 1 & $\mu \mathrm{g} / \mathrm{L}$ \\
\hline YKQCTB-W-38028 & $1 / 21 / 16$ & TB & WQC & AGEM & ND & ND & ND & 1 & $\mu \mathrm{g} / \mathrm{L}$ \\
\hline YKQCTB-W-38035 & $1 / 25 / 16$ & TB & WQC & AGEM & ND & ND & ND & 1 & $\mu \mathrm{g} / \mathrm{L}$ \\
\hline YKQCTB-W-38055 & 2/9/16 & TB & WQC & AGEM & ND & ND & ND & 1 & $\mu \mathrm{g} / \mathrm{L}$ \\
\hline YKQCTB-W-38058 & $2 / 10 / 16$ & TB & WQC & AGEM & ND & ND & ND & 1 & $\mu \mathrm{g} / \mathrm{L}$ \\
\hline YKQCTB-W-38080 & $3 / 2 / 16$ & TB & WQC & AGEM & ND & ND & ND & 1 & $\mu \mathrm{g} / \mathrm{L}$ \\
\hline
\end{tabular}




\begin{tabular}{|c|c|c|c|c|c|c|c|c|c|}
\hline YKQCTB-W-38080VER & $3 / 2 / 16$ & VER & WQC & TestAmerica & ND & ND & ND & 1 & $\mu \mathrm{g} / \mathrm{L}$ \\
\hline YKQCTB-W-38092 & $5 / 17 / 16$ & TB & WQC & AGEM & ND & ND & ND & 1 & $\mu \mathrm{g} / \mathrm{L}$ \\
\hline YKQCTB-W-38095 & $5 / 25 / 16$ & TB & WQC & AGEM & ND & ND & ND & 1 & $\mu \mathrm{g} / \mathrm{L}$ \\
\hline YKQCTB-W-38099 & $5 / 31 / 16$ & TB & WQC & AGEM & ND & ND & ND & 1 & $\mu \mathrm{g} / \mathrm{L}$ \\
\hline YKQCTB-W-38099VER & $5 / 31 / 16$ & VER & WQC & TestAmerica & ND & ND & $0.02 \mathrm{JB}^{\mathrm{e}}$ & 1 & $\mu \mathrm{g} / \mathrm{L}$ \\
\hline YKQCTB-W-38101 & $6 / 2 / 16$ & TB & WQC & AGEM & ND & ND & ND & 1 & $\mu \mathrm{g} / \mathrm{L}$ \\
\hline YKQCTB-W-38105 & $6 / 6 / 16$ & TB & WQC & AGEM & ND & ND & ND & 1 & $\mu \mathrm{g} / \mathrm{L}$ \\
\hline YKQCTB-W-38107 & $6 / 7 / 16$ & TB & WQC & AGEM & ND & ND & ND & 1 & $\mu \mathrm{g} / \mathrm{L}$ \\
\hline YKQCTB-W-38110 & $6 / 9 / 16$ & TB & WQC & AGEM & ND & ND & ND & 1 & $\mu \mathrm{g} / \mathrm{L}$ \\
\hline YKQCTB-W-38110PACE & $6 / 9 / 16$ & TB & WQC & Pace & ND & ND & ND & 1 & $\mu \mathrm{g} / \mathrm{L}$ \\
\hline YKQCTB-W-38110VER & $6 / 9 / 16$ & VER & WQC & TestAmerica & ND & ND & $0.02 \mathrm{JB}$ & 1 & $\mu \mathrm{g} / \mathrm{L}$ \\
\hline YKQCTB-W-38114 & $6 / 13 / 16$ & TB & WQC & AGEM & ND & ND & ND & 1 & $\mu \mathrm{g} / \mathrm{L}$ \\
\hline YKQCTB-W-38114VER & $6 / 13 / 16$ & VER & WQC & TestAmerica & ND & ND & ND & 1 & $\mu \mathrm{g} / \mathrm{L}$ \\
\hline YKQCTB-W-38117 & $6 / 16 / 16$ & TB & WQC & AGEM & ND & ND & ND & 1 & $\mu \mathrm{g} / \mathrm{L}$ \\
\hline YKQCTB-W-38117VER & $6 / 16 / 16$ & VER & WQC & TestAmerica & ND & ND & $0.02 \mathrm{JB}$ & 1 & $\mu \mathrm{g} / \mathrm{L}$ \\
\hline YKQCTB-W-38120 & $6 / 21 / 16$ & TB & WQC & AGEM & ND & ND & ND & 1 & $\mu \mathrm{g} / \mathrm{L}$ \\
\hline YKQCTB-W-38120PACE & $6 / 21 / 16$ & TB & WQC & Pace & ND & ND & ND & 1 & $\mu \mathrm{g} / \mathrm{L}$ \\
\hline
\end{tabular}

a Sample types: EB, equipment blank; RI, rinsate; TB, trip blank; VER, verification sample.

b Matrix types: MEOH, soil-methanol; SOIL, soil; WG, groundwater; WQC, water quality control sample.

c ND, compound analyzed for but not detected at a level greater than or equal to the indicated method detection limit.

d $\mathrm{J}$, compound identified with an estimated concentration between the instrument detection limit and the method detection limit.

e $\mathrm{B}$, compound detected in associated laboratory blank. 
TABLE S2.3 Calibration and surrogate recovery during organic analyses of soil and water samples at AGEM Laboratory.

Fecovery of Surrogate Compounds ${ }^{\mathrm{a}}$

Measured Concentration of Calibration Check

\begin{tabular}{cccc}
\multicolumn{4}{c}{$\begin{array}{c}\text { Measured Concentration of Calibration Check } \\
\text { Standards }\end{array}$} \\
\hline $\begin{array}{c}\text { Carbon } \\
\begin{array}{c}\text { Tetrachloride } \\
(\mathrm{ppb})\end{array}\end{array}$ & RPD & Chloroform \\
& & $(\mathrm{ppb})$ & RPD \\
\hline
\end{tabular}

SDG 15-07-09a, Analysis Date July 9, 2015

\begin{tabular}{|c|c|c|c|}
\hline 20 ppb & $100 \%$ & $100 \%$ & $100 \%$ \\
\hline Meoh Blank & $110 \%$ & $115 \%$ & $112 \%$ \\
\hline YKTI02-S-37780 & $113 \%$ & $111 \%$ & $110 \%$ \\
\hline YKTI01-S-37746 & $109 \%$ & $115 \%$ & $116 \%$ \\
\hline YKTI02-S-37775 & $115 \%$ & $118 \%$ & $124 \%$ \\
\hline YKTI01-S-37747 & $106 \%$ & $113 \%$ & $117 \%$ \\
\hline YKTI02-S-37768 & $104 \%$ & $112 \%$ & $112 \%$ \\
\hline YKTI02-S-37772 & $100 \%$ & $112 \%$ & $113 \%$ \\
\hline YKTI02-S-37772DUP & $99 \%$ & $104 \%$ & $105 \%$ \\
\hline YKTI01-S-37751 & $97 \%$ & $101 \%$ & $100 \%$ \\
\hline YKTI02-S-37775 & $94 \%$ & $93 \%$ & $93 \%$ \\
\hline YKTI02-S-37779 & $94 \%$ & $95 \%$ & $97 \%$ \\
\hline YKTI02-S-37776 & $95 \%$ & $91 \%$ & $97 \%$ \\
\hline YKTI02-S-37774 & $90 \%$ & $90 \%$ & $93 \%$ \\
\hline YKTI02-S-37766 & $91 \%$ & $91 \%$ & $95 \%$ \\
\hline YKTI02-S-37773 & $89 \%$ & $94 \%$ & $94 \%$ \\
\hline YKTI01-S-37756 & $90 \%$ & $85 \%$ & $88 \%$ \\
\hline \multicolumn{4}{|c|}{ SDG 15-07-09b, Analysis Date July 9, 2015} \\
\hline $20 \mathrm{ppb}$ & $100 \%$ & $100 \%$ & $100 \%$ \\
\hline Meoh Blank & $100 \%$ & $100 \%$ & $100 \%$ \\
\hline YKTI01-S-37752 & $82 \%$ & $81 \%$ & $88 \%$ \\
\hline YKTI01-S-37748 & $88 \%$ & $82 \%$ & $89 \%$ \\
\hline YKTI01-S-37744 & $100 \%$ & $94 \%$ & $99 \%$ \\
\hline YKTI01-S-37755 & $113 \%$ & $104 \%$ & $114 \%$ \\
\hline YKTI01-S-37750 & $98 \%$ & $99 \%$ & $109 \%$ \\
\hline YKTI01-S-37745 & $90 \%$ & $90 \%$ & $101 \%$ \\
\hline YKTI01-S-37749 & $96 \%$ & $94 \%$ & $105 \%$ \\
\hline YKTI01-S-37749DUP & $95 \%$ & $92 \%$ & $101 \%$ \\
\hline YKTI02-S-37769 & $107 \%$ & $94 \%$ & $103 \%$ \\
\hline YKTI01-S-37754 & $95 \%$ & $86 \%$ & $97 \%$ \\
\hline
\end{tabular}


TABLE S2.3 Calibration and surrogate recovery during organic analyses of soil and water samples at AGEM Laboratory.

\begin{tabular}{lccc}
\hline & \multicolumn{3}{c}{ Recovery of Surrogate Compounds } \\
\cline { 2 - 4 } Sample ID & Fluorobenzene & $\begin{array}{c}\text { Bromo- } \\
\text { fluorobenzene }\end{array}$ & $\begin{array}{c}\text { Dichloro- } \\
\text { benzene-d4 }\end{array}$ \\
\hline bKTI02-S-37764 & $92 \%$ & $86 \%$ & $102 \%$ \\
YKTI02-S-37770 & $93 \%$ & $87 \%$ & $96 \%$ \\
YKTI02-S-37774 & $91 \%$ & $87 \%$ & $98 \%$ \\
YKTI01-S-37753 & $111 \%$ & $97 \%$ & $113 \%$ \\
YKTI02-S-37765 & $98 \%$ & $94 \%$ & $106 \%$
\end{tabular}

SDG 15-07-10a, Analysis Date July 10, 2015

$\begin{array}{lccc}20 \text { ppb } & 96 \% & 96 \% & 92 \% \\ \text { Lab Blank } & 100 \% & 100 \% & 100 \% \\ \text { YKTIO4-W-37833 } & 98 \% & 99 \% & 105 \% \\ \text { YKTIO4-W-37834 } & 105 \% & 109 \% & 115 \% \\ \text { YKTIO3-W-37836 } & 102 \% & 99 \% & 105 \% \\ \text { YKTIO3-W-37837 } & 102 \% & 102 \% & 105 \% \\ \text { YKTIO3-W-37837DUP } & 99 \% & 97 \% & 106 \% \\ \text { YKQCTB-W-37835 } & 101 \% & 98 \% & 101 \% \\ \text { YKQCBR-W-37839 } & 99 \% & 95 \% & 96 \% \\ \text { Meoh Blank } & 100 \% & 100 \% & 100 \% \\ \text { YKTIO2-S-37767 } & 99 \% & 101 \% & 108 \% \\ \text { YKQCTB-S-37777 } & 104 \% & 108 \% & 107 \% \\ \text { YKQCTB-S-37763 } & 99 \% & 106 \% & 99 \% \\ \text { YKTI04-S-37803 } & 0 \% & 0 \% & 0 \% \\ \text { YKTIO4-S-37823 } & 102 \% & 111 \% & 115 \% \\ \text { YKTI04-S-37802 } & 100 \% & 101 \% & 103 \% \\ \text { YKTIO4-S-37798 } & 97 \% & 97 \% & 107 \% \\ \text { Lab Blank } 2 & 87 \% & 86 \% & 87 \% \\ \text { YKTI04-W-37833 } & 96 \% & 97 \% & 100 \% \\ \text { YKTI04-W-37834 } & 100 \% & 97 \% & 100 \% \\ \text { YKTI03-W-37836 } & 103 \% & 101 \% & 105 \% \\ \text { YKTI03-W-37837 } & 99 \% & 98 \% & 103 \%\end{array}$

\begin{tabular}{cccc}
\multicolumn{3}{c}{$\begin{array}{c}\text { Measured Concentration of Calibration Check } \\
\text { Standards }\end{array}$} \\
\hline $\begin{array}{c}\text { Carbon } \\
\begin{array}{c}\text { Tetrachloride } \\
(\mathrm{ppb})\end{array}\end{array}$ & RPD & Chloroform & \\
& & $(\mathrm{ppb})$ & RPD \\
\hline
\end{tabular}

YKTI03-W-37837

$118 \%$

$109 \%$

21.50

$7.5 \%$

$18.69-6.5 \%$

Outside calibration for CT and CF. Analyzed at dilution in same SDG.

Outside calibration for CT and CF. Analyzed at dilution in same SDG.

Outside calibration for CT and CF. Analyzed at dilution in same SDG.

Outside calibration for CT and CF. Analyzed at dilution in same SDG.

Outside calibration for $\mathrm{CT}$ and $\mathrm{CF}$.

Re-analyzed in SDG 15-07-13a with recovery limit met.

Analysis at DF 10

Analysis at DF 10.

Analysis at DF 10.

Analysis at DF 10.

20 ppb

$96 \%$

$118 \%$

21.50

YK.TableS2.3_edit.xlsx April 19, 2007 
TABLE S2.3 Calibration and surrogate recovery during organic analyses of soil and water samples at AGEM Laboratory.

\begin{tabular}{|c|c|c|c|c|c|c|c|}
\hline \multirow[b]{2}{*}{ Sample ID } & \multicolumn{3}{|c|}{ Recovery of Surrogate Compounds ${ }^{a}$} & \multicolumn{4}{|c|}{$\begin{array}{c}\text { Measured Concentration of Calibration Check } \\
\text { Standards }{ }^{\text {b }}\end{array}$} \\
\hline & Fluorobenzene & $\begin{array}{l}\text { Bromo- } \\
\text { fluorobenzene }\end{array}$ & $\begin{array}{l}\text { Dichloro- } \\
\text { benzene-d4 }\end{array}$ & $\begin{array}{c}\text { Carbon } \\
\text { Tetrachloride } \\
\text { (ppb) }\end{array}$ & RPD & $\begin{array}{l}\text { Chloroforn } \\
\qquad(\mathrm{ppb})\end{array}$ & RPD \\
\hline Meoh Blank & $100 \%$ & $100 \%$ & $100 \%$ & & & & \\
\hline YKTI04-S-37796 & $97 \%$ & $94 \%$ & $103 \%$ & & & & \\
\hline YKTI04-S-37787 & $95 \%$ & $95 \%$ & $101 \%$ & & & & \\
\hline YKTI04-S-37799 & $97 \%$ & $95 \%$ & $102 \%$ & & & & \\
\hline YKTI02-S-37781 & $98 \%$ & $92 \%$ & $98 \%$ & & & & \\
\hline YKTI02-S-37781DUP & $98 \%$ & $94 \%$ & $94 \%$ & & & & \\
\hline YKTI04-S-37794 & $97 \%$ & $92 \%$ & $99 \%$ & & & & \\
\hline YKTI04-S-37805 & $102 \%$ & $97 \%$ & $100 \%$ & & & & \\
\hline YKTI04-S-37797 & $103 \%$ & $96 \%$ & $107 \%$ & & & & \\
\hline YKTI04-S-37796 & $98 \%$ & $94 \%$ & $103 \%$ & & & & \\
\hline YKTI02-S-37785 & $94 \%$ & $89 \%$ & $95 \%$ & & & & \\
\hline YKTI04-S-37806 & $97 \%$ & $94 \%$ & $100 \%$ & & & & \\
\hline YKTI04-S-37801 & $99 \%$ & $93 \%$ & $96 \%$ & & & & \\
\hline YKTI02-S-37782 & $96 \%$ & $93 \%$ & $97 \%$ & & & & \\
\hline YKTI04-S-37792 & $96 \%$ & $85 \%$ & $92 \%$ & & & & \\
\hline YKTI04-S-37800 & $99 \%$ & $89 \%$ & $96 \%$ & & & & \\
\hline Meoh Blank 2 & $104 \%$ & $102 \%$ & $107 \%$ & & & & \\
\hline YKTI04-S-37791 & $99 \%$ & $102 \%$ & $111 \%$ & & & & \\
\hline YKTI04-S-37789 & $103 \%$ & $104 \%$ & $117 \%$ & & & & \\
\hline YKTI04-S-37788 & $94 \%$ & $97 \%$ & $107 \%$ & & & & \\
\hline YKTI04-S-37807 & $98 \%$ & $100 \%$ & $114 \%$ & & & & \\
\hline YKTI04-S-37790 & $99 \%$ & $100 \%$ & $109 \%$ & & & & \\
\hline YKTI02-S-37783 & $94 \%$ & $98 \%$ & $110 \%$ & & & & \\
\hline YKTI04-S-37793 & $96 \%$ & $92 \%$ & $106 \%$ & & & & \\
\hline \multicolumn{8}{|c|}{ SDG 15-07-12b, Analysis Date July 12, 2015} \\
\hline $20 \mathrm{ppb}$ & $122 \%$ & $106 \%$ & $101 \%$ & 20.94 & $4.7 \%$ & 16.96 & $-15.2 \%$ \\
\hline Lab Blank & $100 \%$ & $100 \%$ & $100 \%$ & & & & \\
\hline YKTI01-W-37844 & $106 \%$ & $115 \%$ & $120 \%$ & \multirow{5}{*}{\multicolumn{4}{|c|}{$\begin{array}{l}\text { Outside calibration for CT and CF. Analyzed at dilution in SDG 15-07-13b. } \\
\text { Outside calibration for CT and CF. } \\
\text { Outside calibration for CT and CF. Analyzed at dilution in SDG 15-07-13b. } \\
\text { Outside calibration for CT and CF. Analyzed at dilution in SDG 15-07-13b. } \\
\text { Outside calibration for CT and CF. Analyzed at dilution in SDG 15-07-13b. }\end{array}$}} \\
\hline YKTI01-W-37844DUP & $104 \%$ & $114 \%$ & $123 \%$ & & & & \\
\hline YKTI01-W-37845 & $99 \%$ & $107 \%$ & $111 \%$ & & & & \\
\hline YKTI02-W-37847 & $97 \%$ & $109 \%$ & $119 \%$ & & & & \\
\hline YKTI02-W-37848 & $95 \%$ & $103 \%$ & $109 \%$ & & & & \\
\hline
\end{tabular}


TABLE S2.3 Calibration and surrogate recovery during organic analyses of soil and water samples at AGEM Laboratory.

\begin{tabular}{|c|c|c|c|c|c|c|c|}
\hline \multirow[b]{2}{*}{ Sample ID } & \multicolumn{3}{|c|}{ Recovery of Surrogate Compounds ${ }^{a}$} & \multicolumn{4}{|c|}{$\begin{array}{c}\text { Measured Concentration of Calibration Check } \\
\text { Standards }{ }^{\mathrm{b}}\end{array}$} \\
\hline & Fluorobenzene & $\begin{array}{l}\text { Bromo- } \\
\text { fluorobenzene }\end{array}$ & $\begin{array}{c}\text { Dichloro- } \\
\text { benzene-d4 }\end{array}$ & $\begin{array}{c}\text { Carbon } \\
\text { Tetrachloride } \\
(p p b)\end{array}$ & RPD & $\begin{array}{l}\text { Chlorofor } \\
\qquad(\mathrm{ppb})\end{array}$ & RPD \\
\hline YKQCTB-W-37846 & $84 \%$ & $94 \%$ & $89 \%$ & & & & \\
\hline \multicolumn{8}{|c|}{ SDG 15-07-13a, Analysis Date July 13, 2015} \\
\hline 20 ppb & $100 \%$ & $100 \%$ & $100 \%$ & 21.39 & $7.0 \%$ & 20.38 & $1.9 \%$ \\
\hline Meoh Blank & $100 \%$ & $100 \%$ & $100 \%$ & & & & \\
\hline YKTI04-S-37803 & $105 \%$ & $112 \%$ & $106 \%$ & & & & \\
\hline YKTI03-S-37818 & $98 \%$ & $99 \%$ & $104 \%$ & & & & \\
\hline YKTI03-S-37812 & $90 \%$ & $96 \%$ & $103 \%$ & & & & \\
\hline YKTI03-S-37824 & $95 \%$ & $94 \%$ & $98 \%$ & & & & \\
\hline YKTI03-S-37826 & $89 \%$ & $96 \%$ & $95 \%$ & & & & \\
\hline YKTI03-S-37826DUP & $91 \%$ & $96 \%$ & $94 \%$ & & & & \\
\hline YKTI03-S-37829 & $90 \%$ & $90 \%$ & $97 \%$ & & & & \\
\hline YKTI03-S-37827 & $97 \%$ & $97 \%$ & $104 \%$ & & & & \\
\hline YKTI03-S-37820 & $102 \%$ & $98 \%$ & $101 \%$ & & & & \\
\hline YKTI03-S-37813 & $97 \%$ & $101 \%$ & $109 \%$ & & & & \\
\hline YKTI03-S-37809 & $97 \%$ & $100 \%$ & $105 \%$ & & & & \\
\hline YKTI03-S-37808 & $90 \%$ & $94 \%$ & $101 \%$ & & & & \\
\hline YKTI03-S-37821 & $91 \%$ & $90 \%$ & $95 \%$ & & & & \\
\hline YKTI03-S-37817 & $90 \%$ & $93 \%$ & $98 \%$ & & & & \\
\hline YKTI03-S-37816 & $93 \%$ & $91 \%$ & $100 \%$ & & & & \\
\hline \multicolumn{8}{|c|}{ SDG 15-07-13b, Analysis Date July 13, 2015} \\
\hline $20 \mathrm{ppb}$ & $116 \%$ & $116 \%$ & $106 \%$ & 18.10 & $-9.5 \%$ & 16.77 & $-16.2 \%$ \\
\hline Lab Blank & $96 \%$ & $84 \%$ & $94 \%$ & & & & \\
\hline YKTI01-W-37844 & $97 \%$ & $93 \%$ & $112 \%$ & \multicolumn{4}{|c|}{ Analysis at DF 10.} \\
\hline YKTI01-W-37845 & $104 \%$ & $102 \%$ & $115 \%$ & \multicolumn{4}{|c|}{ Analysis at DF 10.} \\
\hline YKTI02-W-37847 & $107 \%$ & $100 \%$ & $113 \%$ & \multicolumn{4}{|c|}{ Analysis at DF 10.} \\
\hline YKTI02-W-37848 & $97 \%$ & $93 \%$ & $112 \%$ & \multicolumn{4}{|c|}{ Analysis at DF 10.} \\
\hline YKTI02-W-37848 & $90 \%$ & $86 \%$ & $100 \%$ & \multicolumn{4}{|c|}{ Analysis at DF 10.} \\
\hline YKQCTB-W-37846 & $84 \%$ & $81 \%$ & $86 \%$ & & & & \\
\hline Meoh Blank & $118 \%$ & $112 \%$ & $102 \%$ & & & & \\
\hline YKQCTB-S-37784 & $181 \%$ & $324 \%$ & $213 \%$ & \multicolumn{4}{|c|}{ Re-analyzed in SDG $15-07-14 b$ with recovery limit me } \\
\hline
\end{tabular}


TABLE S2.3 Calibration and surrogate recovery during organic analyses of soil and water samples at AGEM Laboratory.

\begin{tabular}{|c|c|c|c|c|c|c|c|}
\hline \multirow[b]{2}{*}{ Sample ID } & \multicolumn{3}{|c|}{ Recovery of Surrogate Compounds ${ }^{a}$} & \multicolumn{4}{|c|}{$\begin{array}{c}\text { Measured Concentration of Calibration Check } \\
\text { Standards }{ }^{b}\end{array}$} \\
\hline & Fluorobenzene & $\begin{array}{l}\text { Bromo- } \\
\text { fluorobenzene }\end{array}$ & $\begin{array}{c}\text { Dichloro- } \\
\text { benzene-d4 }\end{array}$ & $\begin{array}{c}\text { Carbon } \\
\text { Tetrachloride } \\
(p p b)\end{array}$ & RPD & $\begin{array}{l}\text { Chlorofor } \\
\qquad(\mathrm{ppb})\end{array}$ & RPD \\
\hline YKQCTB-S-37804 & $201 \%$ & $382 \%$ & $264 \%$ & Re-analyzed in & DG $15-C$ & tb with recc & $y$ limit m \\
\hline YKTI03-S-37811 & $171 \%$ & $250 \%$ & $203 \%$ & Re-analyzed in & DG 15-C & tb with reco & $y$ limit m \\
\hline YKTI03-S-37819 & $171 \%$ & $176 \%$ & $162 \%$ & Re-analyzed in & DG 15-C & Ab with recc & $y$ limit m \\
\hline YKTI03-S-37810 & $149 \%$ & $153 \%$ & $140 \%$ & Re-analyzed in & DG 15- & tb with recc & $y$ limit $m$ \\
\hline YKTI03-S-37828 & $145 \%$ & $130 \%$ & $126 \%$ & Re-analyzed in & DG 15-C & tb with recc & $y$ limit m \\
\hline YKTI03-S-37822 & $133 \%$ & $118 \%$ & $109 \%$ & Re-analyzed in & DG 15-C & Hb with reco & $y$ limit m \\
\hline YKTI03-S-37815 & $132 \%$ & $128 \%$ & $133 \%$ & Re-analyzed in & DG 15-C & tb with recc & $y$ limit m \\
\hline \multicolumn{8}{|c|}{ SDG 15-07-14b, Analysis Date July 14, 2015} \\
\hline $20 \mathrm{ppb}$ & $100 \%$ & $100 \%$ & $100 \%$ & 19.76 & $-1.2 \%$ & 20.06 & $0.3 \%$ \\
\hline Meoh Blank & $100 \%$ & $100 \%$ & $100 \%$ & & & & \\
\hline YKTI03-S-37814 & $106 \%$ & $107 \%$ & $116 \%$ & & & & \\
\hline YKTI01-S-37759 & $93 \%$ & $95 \%$ & $98 \%$ & & & & \\
\hline YKTI01-S-37841 & $98 \%$ & $100 \%$ & $112 \%$ & & & & \\
\hline YKTI01-S-37842 & $94 \%$ & $93 \%$ & $101 \%$ & & & & \\
\hline YKTI01-S-37761 & $90 \%$ & $90 \%$ & $94 \%$ & & & & \\
\hline YKTI01-S-37761DUP & $93 \%$ & $88 \%$ & $94 \%$ & & & & \\
\hline YKTI01-S-37758 & $83 \%$ & $88 \%$ & $97 \%$ & & & & \\
\hline YKTI01-S-37762 & $99 \%$ & $94 \%$ & $102 \%$ & & & & \\
\hline YKTI01-S-37760 & $91 \%$ & $80 \%$ & $87 \%$ & & & & \\
\hline YKTI01-S-37757 & $86 \%$ & $86 \%$ & $99 \%$ & & & & \\
\hline YKTI01-S-37757 & $91 \%$ & $89 \%$ & $101 \%$ & & & & \\
\hline YKQCTB-S-37832 & $94 \%$ & $95 \%$ & $109 \%$ & Analysis at DF & & & \\
\hline YKQCTB-S-37825 & $101 \%$ & $99 \%$ & $113 \%$ & Analysis at DF & 00. & & \\
\hline Meoh Blank 2 & $108 \%$ & $111 \%$ & $130 \%$ & & & & \\
\hline YKTI03-S-37819 & $91 \%$ & $95 \%$ & $102 \%$ & & & & \\
\hline YKTI03-S-37811 & $98 \%$ & $90 \%$ & $99 \%$ & & & & \\
\hline YKTI03-S-37810 & $93 \%$ & $90 \%$ & $102 \%$ & & & & \\
\hline YKTI03-S-37828 & $90 \%$ & $92 \%$ & $99 \%$ & & & & \\
\hline YKTI03-S-37822 & $92 \%$ & $88 \%$ & $96 \%$ & & & & \\
\hline YKTI03-S-37815 & $88 \%$ & $90 \%$ & $99 \%$ & & & & \\
\hline YKQCTB-S-37784 & $102 \%$ & $100 \%$ & $113 \%$ & Analysis at DF & 00. & & \\
\hline YKQCTB-S-37804 & $116 \%$ & $107 \%$ & $116 \%$ & Analysis at DF & 00. & & \\
\hline
\end{tabular}


TABLE S2.3 Calibration and surrogate recovery during organic analyses of soil and water samples at AGEM Laboratory.

\begin{tabular}{|c|c|c|c|c|c|c|c|}
\hline \multirow[b]{2}{*}{ Sample ID } & \multicolumn{3}{|c|}{ Recovery of Surrogate Compounds ${ }^{a}$} & \multicolumn{4}{|c|}{$\begin{array}{c}\text { Measured Concentration of Calibration Check } \\
\text { Standards }{ }^{b}\end{array}$} \\
\hline & Fluorobenzene & $\begin{array}{l}\text { Bromo- } \\
\text { fluorobenzene }\end{array}$ & $\begin{array}{c}\text { Dichloro- } \\
\text { benzene-d4 }\end{array}$ & $\begin{array}{c}\text { Carbon } \\
\text { Tetrachloride } \\
(p p b)\end{array}$ & RPD & $\begin{array}{l}\text { Chlorofor } \\
\text { (ppb) }\end{array}$ & RPD \\
\hline YKQCTB-S-37840 & $104 \%$ & $100 \%$ & $109 \%$ & Analysis at DF 1 & 00 & & \\
\hline \multicolumn{8}{|c|}{ SDG 15-07-16b, Analysis Date July 16, 2015} \\
\hline 20 ppb & $99 \%$ & $112 \%$ & $82 \%$ & 19.57 & $-2.2 \%$ & 16.33 & $-18.4 \%$ \\
\hline Lab Blank & $98 \%$ & $90 \%$ & $86 \%$ & & & & \\
\hline YKTI02100-W-37849 & $103 \%$ & $114 \%$ & $116 \%$ & & & & \\
\hline YKTI02100-W-37850 & $103 \%$ & $130 \%$ & $120 \%$ & \multicolumn{4}{|c|}{ Re-analyzed in same SDG with recovery limit met. } \\
\hline YKQCTB-W-37851 & $98 \%$ & $103 \%$ & $94 \%$ & & & & \\
\hline YKTI02120-W-37852 & $88 \%$ & $82 \%$ & $80 \%$ & & & & \\
\hline YKQCIR-W-37857 & $92 \%$ & $86 \%$ & $88 \%$ & & & & \\
\hline YKTI01120-W-37855 & $87 \%$ & $98 \%$ & $106 \%$ & & & & \\
\hline YKTI01120-W-37855DUP & $89 \%$ & $109 \%$ & $109 \%$ & & & & \\
\hline YKTI02100-W-37850 & $88 \%$ & $102 \%$ & $96 \%$ & & & & \\
\hline YKTI02100-W-37850DUP & $107 \%$ & $140 \%$ & $132 \%$ & & & & \\
\hline \multicolumn{8}{|c|}{ SDG 15-07-17b, Analysis Date July 17, 2015} \\
\hline 20 ppb & $96 \%$ & $100 \%$ & $101 \%$ & 23.59 & $18.0 \%$ & 22.78 & $13.9 \%$ \\
\hline Lab Blank & $100 \%$ & $100 \%$ & $100 \%$ & & & & \\
\hline YKTI01100-W-37854 & $94 \%$ & $94 \%$ & $95 \%$ & & & & \\
\hline YKTI01100-W-37858 & $92 \%$ & $93 \%$ & $97 \%$ & & & & \\
\hline YKTI04120-W-37860 & $91 \%$ & $93 \%$ & $94 \%$ & & & & \\
\hline YKTI04100-W-37861 & $90 \%$ & $89 \%$ & $90 \%$ & & & & \\
\hline YKTI04100-W-37862 & $87 \%$ & $89 \%$ & $92 \%$ & & & & \\
\hline YKTI04100-W-37862DUP & $86 \%$ & $85 \%$ & $88 \%$ & & & & \\
\hline YKQCIR-W-37863 & $87 \%$ & $82 \%$ & $86 \%$ & & & & \\
\hline YKQCTB-W-37859 & $85 \%$ & $83 \%$ & $82 \%$ & & & & \\
\hline \multicolumn{8}{|c|}{ SDG 15-07-18a, Analysis Date July 18, 2015} \\
\hline 20 ppb & $101 \%$ & $101 \%$ & $95 \%$ & 21.36 & $6.8 \%$ & 20.90 & $4.5 \%$ \\
\hline Lab Blank & $100 \%$ & $100 \%$ & $100 \%$ & & & & \\
\hline YKTI05120-W-37864 & $109 \%$ & $108 \%$ & $106 \%$ & & & & \\
\hline
\end{tabular}


TABLE S2.3 Calibration and surrogate recovery during organic analyses of soil and water samples at AGEM Laboratory.

\begin{tabular}{|c|c|c|c|c|c|c|c|}
\hline \multirow[b]{2}{*}{ Sample ID } & \multicolumn{3}{|c|}{ Recovery of Surrogate Compounds ${ }^{a}$} & \multicolumn{4}{|c|}{$\begin{array}{c}\text { Measured Concentration of Calibration Check } \\
\text { Standards }{ }^{\text {b }} \\
\end{array}$} \\
\hline & Fluorobenzene & $\begin{array}{l}\text { Bromo- } \\
\text { fluorobenzene }\end{array}$ & $\begin{array}{l}\text { Dichloro- } \\
\text { benzene-d4 }\end{array}$ & $\begin{array}{c}\text { Carbon } \\
\text { Tetrachloride } \\
\quad(p p b)\end{array}$ & RPD & $\begin{array}{l}\text { Chlorofor } \\
\qquad(\mathrm{ppb})\end{array}$ & RPD \\
\hline YKTI05100-W-37865 & $93 \%$ & $93 \%$ & $95 \%$ & & & & \\
\hline YKTI05100-W-37867 & $111 \%$ & $113 \%$ & $114 \%$ & & & & \\
\hline YKTI05100-W-37867DUP & $103 \%$ & $101 \%$ & $102 \%$ & & & & \\
\hline YKQCTB-W-37866 & $104 \%$ & $99 \%$ & $97 \%$ & & & & \\
\hline \multicolumn{8}{|c|}{ SDG 15-07-21a, Analysis Date July 21, 2015} \\
\hline 20 ppb & $105 \%$ & $108 \%$ & $111 \%$ & 20.10 & $0.5 \%$ & 18.65 & $-6.8 \%$ \\
\hline Lab Blank & $107 \%$ & $104 \%$ & $104 \%$ & & & & \\
\hline YKTI0580-W-37868 & $121 \%$ & $121 \%$ & $129 \%$ & \multirow{2}{*}{\multicolumn{4}{|c|}{$\begin{array}{l}\text { Outside calibration for CT and CF. Re-analyzed at dilution in same SDG with recover: } \\
\text { Re-analyzed in same SDG with recovery limit met. }\end{array}$}} \\
\hline YKTI06120-W-37870 & $43 \%$ & $118 \%$ & $117 \%$ & & & & \\
\hline YKTI0680-W-37872 & $111 \%$ & $117 \%$ & $120 \%$ & & & & \\
\hline YKTI06100-W-37873 & $85 \%$ & $82 \%$ & $86 \%$ & & & & \\
\hline YKTI06100-W-37874 & $99 \%$ & $98 \%$ & $101 \%$ & & & & \\
\hline YKTI0780-W-37875 & $94 \%$ & $89 \%$ & $93 \%$ & & & & \\
\hline YKTI07100-W-37876 & $95 \%$ & $90 \%$ & $91 \%$ & & & & \\
\hline YKTI07120-W-37877 & $96 \%$ & $93 \%$ & $94 \%$ & & & & \\
\hline YKTI07120-W-37878 & $91 \%$ & $88 \%$ & $89 \%$ & & & & \\
\hline YKTI07120-W-37878DUP & $94 \%$ & $92 \%$ & $93 \%$ & & & & \\
\hline YKQCTB-W-37871 & $89 \%$ & $81 \%$ & $87 \%$ & & & & \\
\hline YKTI0580-W-37868 & $84 \%$ & $81 \%$ & $82 \%$ & \multicolumn{2}{|l|}{ Analysis at DF 5.} & & \\
\hline YKTI0580-W-37868DUP & $94 \%$ & $90 \%$ & $89 \%$ & \multicolumn{2}{|l|}{ Analysis at DF 5.} & & \\
\hline YKTI06120-W-37870 & $91 \%$ & $89 \%$ & $87 \%$ & \multicolumn{2}{|l|}{ Analysis at DF 5.} & & \\
\hline YKTI06100-W-37873 & $94 \%$ & $93 \%$ & $94 \%$ & & & & \\
\hline Lab Blank 2 & $94 \%$ & $89 \%$ & $87 \%$ & & & & \\
\hline \multicolumn{8}{|c|}{ SDG 15-07-23a, Analysis Date July 23, 2015} \\
\hline $20 \mathrm{ppb}$ & $95 \%$ & $92 \%$ & $91 \%$ & 21.70 & $8.5 \%$ & 21.26 & $6.3 \%$ \\
\hline Lab Blank & $100 \%$ & $100 \%$ & $100 \%$ & & & & \\
\hline YKTI0885-W-37879 & $101 \%$ & $95 \%$ & $99 \%$ & & & & \\
\hline YKTI0885-W-37879DUP & $92 \%$ & $94 \%$ & $99 \%$ & & & & \\
\hline YKTI08107-W-37881 & $96 \%$ & $94 \%$ & $101 \%$ & & & & \\
\hline YKTI08125-W-37882 & $88 \%$ & $88 \%$ & $91 \%$ & & & & \\
\hline
\end{tabular}


TABLE S2.3 Calibration and surrogate recovery during organic analyses of soil and water samples at AGEM Laboratory.

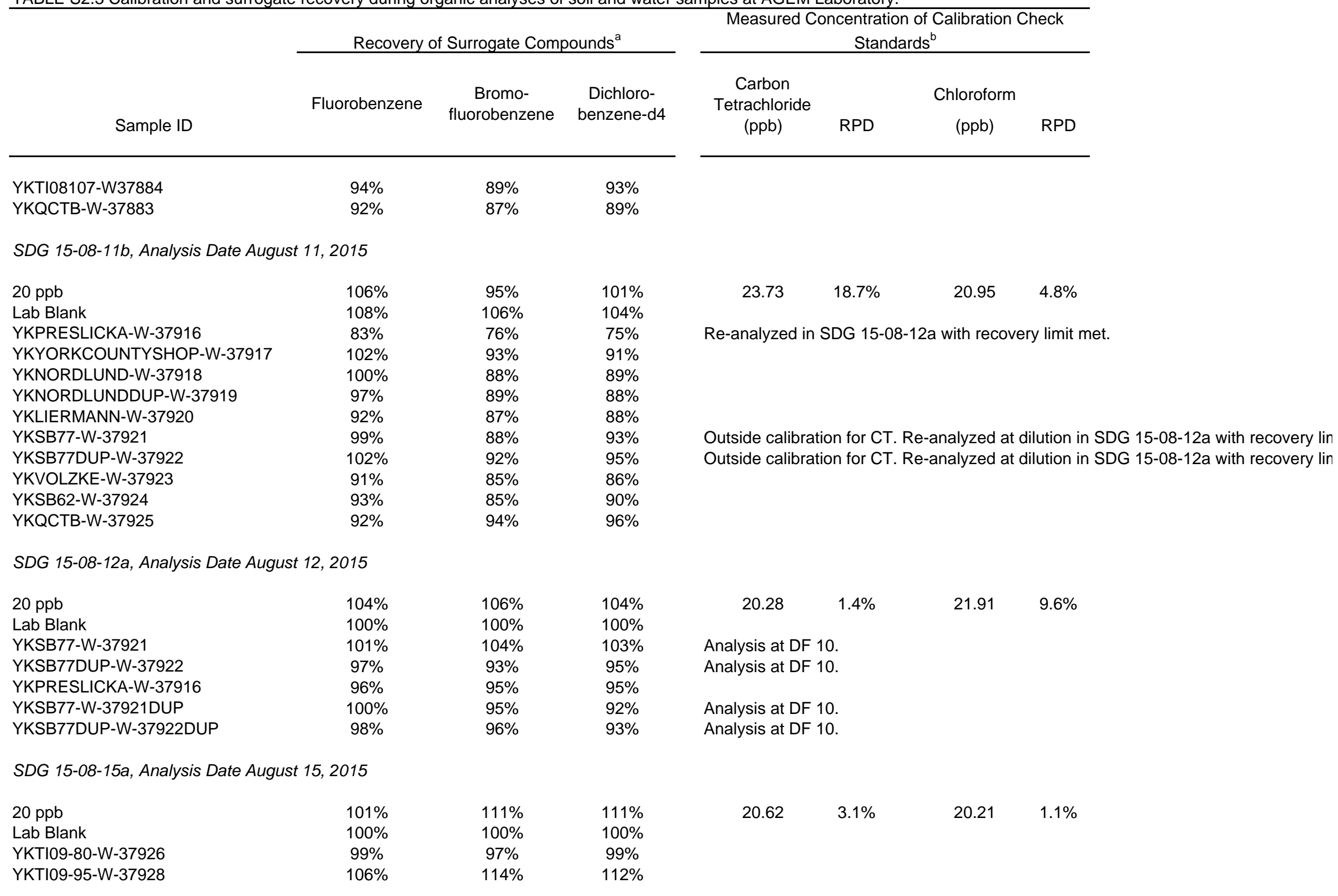


TABLE S2.3 Calibration and surrogate recovery during organic analyses of soil and water samples at AGEM Laboratory.

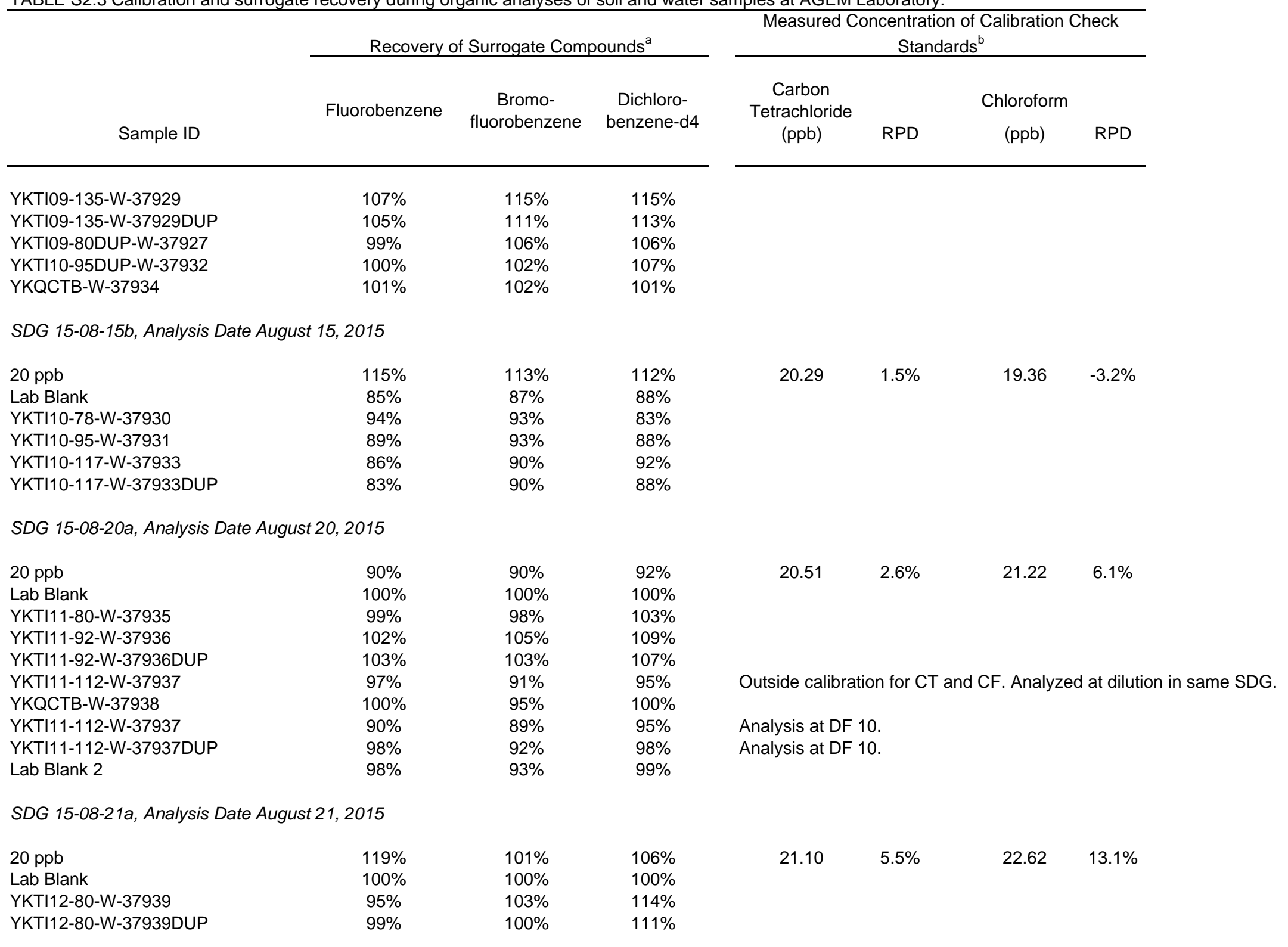


TABLE S2.3 Calibration and surrogate recovery during organic analyses of soil and water samples at AGEM Laboratory.

\begin{tabular}{|c|c|c|c|c|c|c|c|}
\hline \multirow[b]{2}{*}{ Sample ID } & \multicolumn{3}{|c|}{ Recovery of Surrogate Compounds ${ }^{a}$} & \multicolumn{4}{|c|}{$\begin{array}{c}\text { Measured Concentration of Calibration Check } \\
\text { Standards }{ }^{\text {b }} \\
\end{array}$} \\
\hline & Fluorobenzene & $\begin{array}{l}\text { Bromo- } \\
\text { fluorobenzene }\end{array}$ & $\begin{array}{c}\text { Dichloro- } \\
\text { benzene-d4 }\end{array}$ & $\begin{array}{c}\text { Carbon } \\
\text { Tetrachloride } \\
(p p b)\end{array}$ & RPD & $\begin{array}{l}\text { Chlorofor } \\
\qquad(\mathrm{ppb})\end{array}$ & RPD \\
\hline YKTI12-92-W-37940 & $99 \%$ & $98 \%$ & $97 \%$ & & & & \\
\hline YKTI12-DUP92-W-37941 & $95 \%$ & $95 \%$ & $92 \%$ & & & & \\
\hline YKTI12-120-W-37942 & $97 \%$ & $89 \%$ & $90 \%$ & & & & \\
\hline YKTI12-145-W-37943 & $93 \%$ & $85 \%$ & $87 \%$ & & & & \\
\hline YKQCTB-W-37944 & $88 \%$ & $85 \%$ & $84 \%$ & & & & \\
\hline \multicolumn{8}{|c|}{ SDG 15-08-24b, Analysis Date August 24, 2015} \\
\hline $20 \mathrm{ppb}$ & $97 \%$ & $88 \%$ & $100 \%$ & 18.94 & $-5.3 \%$ & 20.09 & $0.4 \%$ \\
\hline Lab Blank & $91 \%$ & $97 \%$ & $95 \%$ & & & & \\
\hline YKTI13-93-W-37947 & $91 \%$ & $100 \%$ & $111 \%$ & & & & \\
\hline YKTI13-93-W-37947DUP & $109 \%$ & $103 \%$ & $116 \%$ & & & & \\
\hline YKTI13-111-W-37948 & $82 \%$ & $88 \%$ & $93 \%$ & & & & \\
\hline YKTI13-111DUP-W-37949 & $80 \%$ & $90 \%$ & $87 \%$ & & & & \\
\hline YKQCTB-W-37950 & $91 \%$ & $90 \%$ & $90 \%$ & & & & \\
\hline Lab Blank 2 & $93 \%$ & $82 \%$ & $82 \%$ & & & & \\
\hline \multicolumn{8}{|c|}{ SDG 15-08-25b, Analysis Date August 25, 2015} \\
\hline $20 \mathrm{ppb}$ & $91 \%$ & $112 \%$ & $99 \%$ & 19.25 & $-3.8 \%$ & 19.94 & $-0.3 \%$ \\
\hline Lab Blank & $91 \%$ & $88 \%$ & $85 \%$ & & & & \\
\hline YKTI14-86-W-37951 & $83 \%$ & $85 \%$ & $92 \%$ & & & & \\
\hline YKTI14-113-W-37952 & $89 \%$ & $83 \%$ & $90 \%$ & & & & \\
\hline YKTI14-113DUP-W-37953 & $99 \%$ & $93 \%$ & $87 \%$ & & & & \\
\hline YKTI14-136-W-37954 & $89 \%$ & $90 \%$ & $92 \%$ & & & & \\
\hline YKTI13-82-W-37955 & $89 \%$ & $88 \%$ & $87 \%$ & & & & \\
\hline YKTI15-90-W-37956 & $109 \%$ & $112 \%$ & $115 \%$ & & & & \\
\hline YKTI15-113-W-37957 & $97 \%$ & $104 \%$ & $100 \%$ & & & & \\
\hline YKTI15-113-W-37957DUP & $94 \%$ & $97 \%$ & $92 \%$ & & & & \\
\hline YKTI15-134-W-37958 & $106 \%$ & $118 \%$ & $112 \%$ & & & & \\
\hline YKTI15-134DUP-W-37959 & $100 \%$ & $115 \%$ & $112 \%$ & & & & \\
\hline YKTI13-81-W-37945 & $111 \%$ & $101 \%$ & $98 \%$ & & & & \\
\hline YKQCTB-W-37946 & $80 \%$ & $77 \%$ & $77 \%$ & Re-analyzed in & SDG 15- & with rec & $y$ limit \\
\hline YKQCTB-W-37960 & $82 \%$ & $78 \%$ & $77 \%$ & Re-analyzed in & SDG 15- & a with reco & $y$ limit \\
\hline
\end{tabular}


TABLE S2.3 Calibration and surrogate recovery during organic analyses of soil and water samples at AGEM Laboratory.

\begin{tabular}{|c|c|c|c|c|c|c|c|}
\hline \multirow[b]{2}{*}{ Sample ID } & \multicolumn{3}{|c|}{ Recovery of Surrogate Compounds ${ }^{a}$} & \multicolumn{4}{|c|}{$\begin{array}{c}\text { Measured Concentration of Calibration Check } \\
\text { Standards }{ }^{\text {b }} \\
\end{array}$} \\
\hline & Fluorobenzene & $\begin{array}{l}\text { Bromo- } \\
\text { fluorobenzene }\end{array}$ & $\begin{array}{l}\text { Dichloro- } \\
\text { benzene-d4 }\end{array}$ & $\begin{array}{c}\text { Carbon } \\
\text { Tetrachloride } \\
(p p b)\end{array}$ & RPD & $\begin{array}{l}\text { Chloroforn } \\
\qquad(\mathrm{ppb})\end{array}$ & RPD \\
\hline Lab Blank 2 & $78 \%$ & $78 \%$ & $79 \%$ & & & & \\
\hline \multicolumn{8}{|c|}{ SDG 15-08-26a, Analysis Date August 26, 2015} \\
\hline 20 ppb & $99 \%$ & $111 \%$ & $104 \%$ & 20.33 & $1.6 \%$ & 21.22 & $6.1 \%$ \\
\hline Lab Blank & $100 \%$ & $100 \%$ & $100 \%$ & & & & \\
\hline YKTI16-86-W-37961 & $93 \%$ & $96 \%$ & $93 \%$ & & & & \\
\hline YKTI16-86DUP-W-37962 & $97 \%$ & $100 \%$ & $101 \%$ & & & & \\
\hline YKTI16-105-W-37963 & $103 \%$ & $108 \%$ & $113 \%$ & & & & \\
\hline YKTI16-105-W-37963DUP & $89 \%$ & $93 \%$ & $92 \%$ & & & & \\
\hline YKTI16-136-W-37964 & $99 \%$ & $97 \%$ & $102 \%$ & & & & \\
\hline YKQCTB-W-37965 & $93 \%$ & $92 \%$ & $92 \%$ & & & & \\
\hline YKQCTB-W-37946 & $102 \%$ & $103 \%$ & $102 \%$ & & & & \\
\hline YKQCTB-W-37960 & $101 \%$ & $99 \%$ & $96 \%$ & & & & \\
\hline \multicolumn{8}{|c|}{ SDG 15-08-27a, Analysis Date August 27, 2015} \\
\hline 20 ppb & $106 \%$ & $104 \%$ & $107 \%$ & 21.98 & $9.9 \%$ & 21.72 & $8.6 \%$ \\
\hline Lab Blank & $100 \%$ & $100 \%$ & $100 \%$ & & & & \\
\hline YKTI17-84-W-37966 & $112 \%$ & $114 \%$ & $111 \%$ & & & & \\
\hline YKTI17-104-W-37967 & $110 \%$ & $112 \%$ & $115 \%$ & & & & \\
\hline YKTI17-133-W-37968 & $107 \%$ & $111 \%$ & $115 \%$ & & & & \\
\hline YKTI17-133-W-37968DUP & $107 \%$ & $105 \%$ & $109 \%$ & & & & \\
\hline YKTI17-133DUP-W-37968 & $107 \%$ & $104 \%$ & $108 \%$ & & & & \\
\hline YKQCTB-W-37969 & $105 \%$ & $101 \%$ & $99 \%$ & & & & \\
\hline \multicolumn{8}{|c|}{ SDG 15-08-31a, Analysis Date August 31, 2015} \\
\hline $20 \mathrm{ppb}$ & $103 \%$ & $99 \%$ & $107 \%$ & 22.84 & $14.2 \%$ & 22.90 & $14.5 \%$ \\
\hline Lab Blank & $100 \%$ & $100 \%$ & $100 \%$ & & & & \\
\hline YKTI18-81-W-37970 & $99 \%$ & $101 \%$ & $101 \%$ & & & & \\
\hline YKTI18-95-W-37971 & $96 \%$ & $99 \%$ & $103 \%$ & & & & \\
\hline YKTI18-95DUP-W-37972 & $95 \%$ & $96 \%$ & $101 \%$ & & & & \\
\hline YKTI18-114-W-37973 & $92 \%$ & $90 \%$ & $96 \%$ & & & & \\
\hline
\end{tabular}


TABLE S2.3 Calibration and surrogate recovery during organic analyses of soil and water samples at AGEM Laboratory.

\begin{tabular}{|c|c|c|c|c|c|c|c|}
\hline \multirow[b]{2}{*}{ Sample ID } & \multicolumn{3}{|c|}{ Recovery of Surrogate Compounds ${ }^{a}$} & \multicolumn{4}{|c|}{$\begin{array}{c}\text { Measured Concentration of Calibration Check } \\
\text { Standards }{ }^{b}\end{array}$} \\
\hline & Fluorobenzene & $\begin{array}{l}\text { Bromo- } \\
\text { fluorobenzene }\end{array}$ & $\begin{array}{c}\text { Dichloro- } \\
\text { benzene-d4 }\end{array}$ & $\begin{array}{c}\text { Carbon } \\
\text { Tetrachloride } \\
(p p b)\end{array}$ & RPD & $\begin{array}{l}\text { Chloroform } \\
\text { (ppb) }\end{array}$ & RPD \\
\hline YKTI18-114-W-37973DUP & $93 \%$ & $89 \%$ & $98 \%$ & & & & \\
\hline YKQCTB-W-37974 & $91 \%$ & $87 \%$ & $93 \%$ & & & & \\
\hline \multicolumn{8}{|c|}{ SDG 15-09-10a, Analysis Date September 10, 2015} \\
\hline $20 \mathrm{ppb}$ & $120 \%$ & $107 \%$ & $120 \%$ & 20.53 & $2.7 \%$ & 20.92 & $4.6 \%$ \\
\hline Lab Blank & $100 \%$ & $100 \%$ & $100 \%$ & & & & \\
\hline YKTI19-84-W-37975 & $87 \%$ & $87 \%$ & $89 \%$ & & & & \\
\hline YKTI19-107-W-37976 & $95 \%$ & $86 \%$ & $90 \%$ & & & & \\
\hline YKTI19-107-W-37976DUP & $90 \%$ & $85 \%$ & $89 \%$ & & & & \\
\hline YKTI19-129-W-37977 & $90 \%$ & $92 \%$ & $92 \%$ & & & & \\
\hline YKTI19-129DUP-W-37978 & $107 \%$ & $115 \%$ & $114 \%$ & & & & \\
\hline YKQCTB-W-37979 & $99 \%$ & $103 \%$ & $106 \%$ & & & & \\
\hline Lab Blank 2 & $100 \%$ & $98 \%$ & $103 \%$ & & & & \\
\hline \multicolumn{8}{|c|}{ SDG 15-09-11a, Analysis Date September 11, 2015} \\
\hline $20 \mathrm{ppb}$ & $99 \%$ & $105 \%$ & $104 \%$ & 23.65 & $18.3 \%$ & 23.51 & $17.6 \%$ \\
\hline Lab Blank & $100 \%$ & $100 \%$ & $100 \%$ & & & & \\
\hline YKTI20-81-W-37980 & $94 \%$ & $96 \%$ & $95 \%$ & & & & \\
\hline YKTI20-105-W-37981 & $98 \%$ & $101 \%$ & $106 \%$ & & & & \\
\hline YKTI20-105-W-37981DUP & $96 \%$ & $97 \%$ & $100 \%$ & & & & \\
\hline YKTI20-131-W-37982 & $95 \%$ & $97 \%$ & $99 \%$ & & & & \\
\hline YKTI20-131DUP-W-37983 & $92 \%$ & $90 \%$ & $92 \%$ & & & & \\
\hline YKQCTB-W-37984 & $93 \%$ & $97 \%$ & $97 \%$ & & & & \\
\hline \multicolumn{8}{|c|}{ SDG 15-09-14a, Analysis Date September 14, 2015} \\
\hline $20 \mathrm{ppb}$ & $102 \%$ & $102 \%$ & $105 \%$ & 23.55 & $17.8 \%$ & 23.14 & $15.7 \%$ \\
\hline Lab Blank & $100 \%$ & $100 \%$ & $100 \%$ & & & & \\
\hline YKTI21-77-W-37985 & $99 \%$ & $97 \%$ & $100 \%$ & & & & \\
\hline YKTI21-99-W-37986 & $101 \%$ & $104 \%$ & $105 \%$ & & & & \\
\hline YKTI21-115-W-37987 & $100 \%$ & $102 \%$ & $106 \%$ & & & & \\
\hline YKTI21-99-W-37986DUP & $100 \%$ & $97 \%$ & $101 \%$ & & & & \\
\hline
\end{tabular}


TABLE S2.3 Calibration and surrogate recovery during organic analyses of soil and water samples at AGEM Laboratory.

\begin{tabular}{|c|c|c|c|c|c|c|c|}
\hline \multirow[b]{2}{*}{ Sample ID } & \multicolumn{3}{|c|}{ Recovery of Surrogate Compounds ${ }^{a}$} & \multicolumn{4}{|c|}{$\begin{array}{c}\text { Measured Concentration of Calibration Check } \\
\text { Standards }{ }^{\text {b }}\end{array}$} \\
\hline & Fluorobenzene & $\begin{array}{l}\text { Bromo- } \\
\text { fluorobenzene }\end{array}$ & $\begin{array}{l}\text { Dichloro- } \\
\text { benzene-d4 }\end{array}$ & $\begin{array}{c}\text { Carbon } \\
\text { Tetrachloride } \\
(p p b)\end{array}$ & RPD & $\begin{array}{l}\text { Chloroform } \\
\qquad(\mathrm{ppb})\end{array}$ & RPD \\
\hline YKTI21-115DUP-W-37988 & $96 \%$ & $92 \%$ & $95 \%$ & & & & \\
\hline YKQCTB-W-37989 & $100 \%$ & $96 \%$ & $96 \%$ & & & & \\
\hline \multicolumn{8}{|c|}{ SDG 15-09-16a, Analysis Date September 16, 2015} \\
\hline 20 ppb & $93 \%$ & $100 \%$ & $101 \%$ & 22.18 & $10.9 \%$ & 22.18 & $10.9 \%$ \\
\hline Lab Blank & $100 \%$ & $100 \%$ & $100 \%$ & & & & \\
\hline YKTI22-79-W-37990 & $95 \%$ & $100 \%$ & $102 \%$ & & & & \\
\hline YKTI22-98-W-37991 & $106 \%$ & $108 \%$ & $117 \%$ & & & & \\
\hline YKTI22-113-W-37992 & $101 \%$ & $101 \%$ & $107 \%$ & & & & \\
\hline YKTI22-98DUP-W-37993 & $99 \%$ & $97 \%$ & $103 \%$ & & & & \\
\hline YKQCTB-W-37994 & $94 \%$ & $94 \%$ & $94 \%$ & & & & \\
\hline YKTI23-80-W-37995 & $93 \%$ & $95 \%$ & $101 \%$ & & & & \\
\hline YKTI23-80-W-37995DUP & $93 \%$ & $97 \%$ & $102 \%$ & & & & \\
\hline YKTI23-99-W-37996 & $92 \%$ & $92 \%$ & $94 \%$ & & & & \\
\hline YKTI23-99DUP-W-37997 & $93 \%$ & $92 \%$ & $95 \%$ & & & & \\
\hline YKTI23-120-W-37998 & $90 \%$ & $86 \%$ & $89 \%$ & & & & \\
\hline \multicolumn{8}{|c|}{ SDG 15-09-17a, Analysis Date September 17, 2015} \\
\hline 20 ppb & $102 \%$ & $98 \%$ & $99 \%$ & 22.58 & $12.9 \%$ & 22.15 & $10.8 \%$ \\
\hline Lab Blank & $100 \%$ & $100 \%$ & $100 \%$ & & & & \\
\hline YKTI23-145-W-37999 & $100 \%$ & $104 \%$ & $106 \%$ & & & & \\
\hline YKTI23-145-W-37999DUP & $102 \%$ & $107 \%$ & $114 \%$ & & & & \\
\hline YKQCTB-W-38000 & $92 \%$ & $94 \%$ & $95 \%$ & & & & \\
\hline \multicolumn{8}{|c|}{ SDG 16-01-21b, Analysis Date January 21, 2016} \\
\hline 20 ppb & $100 \%$ & $100 \%$ & $100 \%$ & 17.77 & $-11.2 \%$ & 18.21 & $-9.0 \%$ \\
\hline Lab Blank & $100 \%$ & $100 \%$ & $100 \%$ & & & & \\
\hline YKTI24-80-W-38017 & $96 \%$ & $110 \%$ & $110 \%$ & & & & \\
\hline YKTI24-90-W-38018 & $96 \%$ & $95 \%$ & $100 \%$ & & & & \\
\hline YKTI24-90-W-38018DUP & $85 \%$ & $92 \%$ & $94 \%$ & & & & \\
\hline YKTI24-100-W-38019 & $90 \%$ & $94 \%$ & $94 \%$ & & & & \\
\hline
\end{tabular}


TABLE S2.3 Calibration and surrogate recovery during organic analyses of soil and water samples at AGEM Laboratory.

\begin{tabular}{|c|c|c|c|c|c|c|c|}
\hline \multirow[b]{2}{*}{ Sample ID } & \multicolumn{3}{|c|}{ Recovery of Surrogate Compounds ${ }^{a}$} & \multicolumn{4}{|c|}{$\begin{array}{c}\text { Measured Concentration of Calibration Check } \\
\text { Standards }{ }^{\text {b }} \\
\end{array}$} \\
\hline & Fluorobenzene & $\begin{array}{l}\text { Bromo- } \\
\text { fluorobenzene }\end{array}$ & $\begin{array}{c}\text { Dichloro- } \\
\text { benzene-d4 }\end{array}$ & $\begin{array}{c}\text { Carbon } \\
\text { Tetrachloride } \\
\text { (ppb) }\end{array}$ & RPD & $\begin{array}{l}\text { Chloroform } \\
\qquad(\mathrm{ppb})\end{array}$ & RPD \\
\hline YKTI24-80DUP-W-38020 & $90 \%$ & $102 \%$ & $103 \%$ & & & & \\
\hline YKQCTB-W-38021 & $93 \%$ & $98 \%$ & $97 \%$ & & & & \\
\hline YKQCIR-W-38022 & $89 \%$ & $88 \%$ & $91 \%$ & & & & \\
\hline \multicolumn{8}{|c|}{ SDG 16-01-22b, Analysis Date January 22, 2016} \\
\hline 20 ppb & $98 \%$ & $105 \%$ & $108 \%$ & 16.55 & $-17.3 \%$ & 17.83 & $-10.9 \%$ \\
\hline Lab Blank & $100 \%$ & $100 \%$ & $100 \%$ & & & & \\
\hline YKTI25-77-W-38023 & $92 \%$ & $98 \%$ & $100 \%$ & & & & \\
\hline YKTI25-98-W-38024 & $93 \%$ & $103 \%$ & $100 \%$ & & & & \\
\hline YKTI25-108-W-38027 & $87 \%$ & $97 \%$ & $93 \%$ & & & & \\
\hline YKTI25-108-W-38027DUP & $89 \%$ & $93 \%$ & $95 \%$ & & & & \\
\hline YKTI25-98DUP-W-38025 & $88 \%$ & $99 \%$ & $95 \%$ & & & & \\
\hline YKQCIR-W-38026 & $91 \%$ & $98 \%$ & $98 \%$ & & & & \\
\hline YKQCTB-W-38028 & $91 \%$ & $98 \%$ & $97 \%$ & & & & \\
\hline \multicolumn{8}{|c|}{ SDG 16-01-27a, Analysis Date January 27, 2016} \\
\hline 20 ppb & $98 \%$ & $114 \%$ & $96 \%$ & 23.76 & $18.8 \%$ & 23.33 & $16.7 \%$ \\
\hline Lab Blank & $100 \%$ & $100 \%$ & $100 \%$ & & & & \\
\hline YKTI26-78-W-38029 & $96 \%$ & $104 \%$ & $93 \%$ & & & & \\
\hline YKTI26-78-W-38029DUP & $93 \%$ & $106 \%$ & $99 \%$ & & & & \\
\hline YKTI26-96-W-38030 & $92 \%$ & $110 \%$ & $104 \%$ & & & & \\
\hline YKTI26-113-W-38033 & $85 \%$ & $98 \%$ & $86 \%$ & & & & \\
\hline YKTI26-113DUP-W-38034 & $86 \%$ & $98 \%$ & $90 \%$ & & & & \\
\hline YKTI26-96DUP-W-38031 & $91 \%$ & $104 \%$ & $95 \%$ & & & & \\
\hline YKQCIR-W-38032 & $85 \%$ & $93 \%$ & $83 \%$ & & & & \\
\hline YKQCTB-W-38035 & $85 \%$ & $90 \%$ & $80 \%$ & & & & \\
\hline \multicolumn{8}{|c|}{ SDG 16-02-10a, Analysis Date February 10, 2016} \\
\hline 20 ppb & $105 \%$ & $109 \%$ & $105 \%$ & 22.24 & $11.2 \%$ & 21.83 & $9.1 \%$ \\
\hline Lab Blank & $100 \%$ & $100 \%$ & $100 \%$ & & & & \\
\hline YKTI27-77-W-38050 & $96 \%$ & $100 \%$ & $100 \%$ & & & & \\
\hline
\end{tabular}


TABLE S2.3 Calibration and surrogate recovery during organic analyses of soil and water samples at AGEM Laboratory.

\begin{tabular}{|c|c|c|c|c|c|c|c|}
\hline \multirow[b]{2}{*}{ Sample ID } & \multicolumn{3}{|c|}{ Recovery of Surrogate Compounds ${ }^{a}$} & \multicolumn{4}{|c|}{$\begin{array}{c}\text { Measured Concentration of Calibration Check } \\
\text { Standards }{ }^{\text {b }} \\
\end{array}$} \\
\hline & Fluorobenzene & $\begin{array}{l}\text { Bromo- } \\
\text { fluorobenzene }\end{array}$ & $\begin{array}{c}\text { Dichloro- } \\
\text { benzene-d4 }\end{array}$ & $\begin{array}{c}\text { Carbon } \\
\text { Tetrachloride } \\
(p p b)\end{array}$ & RPD & $\begin{array}{l}\text { Chloroforn } \\
\qquad(\mathrm{ppb})\end{array}$ & RPD \\
\hline YKTI27-100-W-38053 & $92 \%$ & $98 \%$ & $99 \%$ & & & & \\
\hline YKTI27-100-W-38053DUP & $90 \%$ & $101 \%$ & $105 \%$ & & & & \\
\hline YKTI27-77DUP-W-38051 & $88 \%$ & $94 \%$ & $97 \%$ & & & & \\
\hline YKTI27-100DUP-W-38054 & $87 \%$ & $94 \%$ & $96 \%$ & & & & \\
\hline YKQCTB-W-38055 & $89 \%$ & $92 \%$ & $94 \%$ & & & & \\
\hline YKQCIR-W-38052 & $87 \%$ & $89 \%$ & $87 \%$ & & & & \\
\hline \multicolumn{8}{|c|}{ SDG 16-02-11a, Analysis Date February 11, 2016} \\
\hline 20 ppb & $98 \%$ & $96 \%$ & $92 \%$ & 23.66 & $18.3 \%$ & 23.65 & $18.3 \%$ \\
\hline Lab Blank & $100 \%$ & $100 \%$ & $100 \%$ & & & & \\
\hline YKTI27-77-W-38056 & $106 \%$ & $100 \%$ & $103 \%$ & & & & \\
\hline YKTI27-100-W-38057 & $108 \%$ & $109 \%$ & $119 \%$ & & & & \\
\hline YKTI27-100-W-38057DUP & $102 \%$ & $101 \%$ & $107 \%$ & & & & \\
\hline YKQCTB-W-38058 & $100 \%$ & $99 \%$ & $104 \%$ & & & & \\
\hline \multicolumn{8}{|c|}{ SDG 16-03-04b, Analysis Date March 4, 2016} \\
\hline 20 ppb & $109 \%$ & $103 \%$ & $101 \%$ & 16.66 & $-16.7 \%$ & 19.22 & $-3.9 \%$ \\
\hline Lab Blank & $100 \%$ & $100 \%$ & $100 \%$ & & & & \\
\hline YKTI28-78-W-38075 & $109 \%$ & $101 \%$ & $98 \%$ & & & & \\
\hline YKTI28-78-W-38075DUP & $103 \%$ & $98 \%$ & $99 \%$ & & & & \\
\hline YKTI28-90-W-38076 & $93 \%$ & $80 \%$ & $85 \%$ & & & & \\
\hline YKTI28-90-DUP-W-38077 & $99 \%$ & $87 \%$ & $93 \%$ & & & & \\
\hline YKTI28-104-W-38078 & $96 \%$ & $89 \%$ & $96 \%$ & & & & \\
\hline YKQCIR-W-38079 & $96 \%$ & $89 \%$ & $95 \%$ & & & & \\
\hline YKQCTB-W-38080 & $105 \%$ & $96 \%$ & $105 \%$ & & & & \\
\hline \multicolumn{8}{|c|}{ SDG 16-03-04b, Analysis Date March 4, 2016} \\
\hline $20 \mathrm{ppb}$ & $96 \%$ & $117 \%$ & $98 \%$ & 19.79 & $-1.1 \%$ & 19.43 & $-2.9 \%$ \\
\hline Lab Blank & $107 \%$ & $112 \%$ & $107 \%$ & & & & \\
\hline YKDL2C2-136-W-38096 & $95 \%$ & $98 \%$ & $97 \%$ & & & & \\
\hline YKDL2C2-136-W-38096DUP & $99 \%$ & $99 \%$ & $99 \%$ & & & & \\
\hline
\end{tabular}


TABLE S2.3 Calibration and surrogate recovery during organic analyses of soil and water samples at AGEM Laboratory.

\begin{tabular}{|c|c|c|c|c|c|c|c|}
\hline \multirow[b]{2}{*}{ Sample ID } & \multicolumn{3}{|c|}{ Recovery of Surrogate Compounds ${ }^{a}$} & \multicolumn{4}{|c|}{$\begin{array}{c}\text { Measured Concentration of Calibration Check } \\
\text { Standards }{ }^{\text {b }} \\
\end{array}$} \\
\hline & Fluorobenzene & $\begin{array}{l}\text { Bromo- } \\
\text { fluorobenzene }\end{array}$ & $\begin{array}{l}\text { Dichloro- } \\
\text { benzene-d4 }\end{array}$ & $\begin{array}{c}\text { Carbon } \\
\text { Tetrachloride } \\
(p p b)\end{array}$ & RPD & $\begin{array}{l}\text { Chlorofor } \\
\text { (ppb) }\end{array}$ & RPD \\
\hline YKDL2CU-215-W-38097 & $91 \%$ & $92 \%$ & $95 \%$ & & & & \\
\hline YKDL2CU-215-DUP-W-38098 & $93 \%$ & $88 \%$ & $93 \%$ & & & & \\
\hline YKQCTB-W-38099 & $0 \%$ & $0 \%$ & $0 \%$ & & & & \\
\hline \multicolumn{8}{|c|}{ SDG 16-05-24, Analysis Date May 24, 2016} \\
\hline 20 ppb & $94 \%$ & $113 \%$ & $98 \%$ & 22.22 & $11.1 \%$ & 22.26 & $11.3 \%$ \\
\hline Lab Blank & $100 \%$ & $100 \%$ & $100 \%$ & & & & \\
\hline YKDL1C-108-W-38091 & $94 \%$ & $96 \%$ & $100 \%$ & & & & \\
\hline YKDL1C-108-W-38091DUP & $98 \%$ & $101 \%$ & $106 \%$ & & & & \\
\hline YKCITY-W-38089 & $89 \%$ & $85 \%$ & $87 \%$ & & & & \\
\hline YKTRUCK-W-38090 & $85 \%$ & $76 \%$ & $82 \%$ & \multirow{3}{*}{\multicolumn{4}{|c|}{ Re-analyzed in same SDG with recovery limit met. }} \\
\hline YKQCTB-W-38092 & $90 \%$ & $92 \%$ & $94 \%$ & & & & \\
\hline YKTRUCK-W-38090 & $93 \%$ & $90 \%$ & $93 \%$ & & & & \\
\hline \multicolumn{8}{|c|}{ SDG 16-05-26, Analysis Date May 26, 2016} \\
\hline $20 \mathrm{ppb}^{\mathrm{c}}$ & $114 \%$ & $132 \%$ & $116 \%$ & 19.51 & $-2.4 \%$ & 18.97 & $-5.2 \%$ \\
\hline Lab Blank & $100 \%$ & $100 \%$ & $100 \%$ & & & & \\
\hline YKDL1A-75-W-38094 & $117 \%$ & $114 \%$ & $116 \%$ & \multirow{2}{*}{\multicolumn{4}{|c|}{ Outside calibration for CT and CF. Analyzed at dilution in same SDG. }} \\
\hline YKDL1B-88-W-38093 & $109 \%$ & $99 \%$ & $117 \%$ & & & & \\
\hline YKDL1A-75-W-38094 & $117 \%$ & $110 \%$ & $120 \%$ & \multicolumn{4}{|c|}{ Analysis at DF 20.} \\
\hline Lab Blank 2 & $100 \%$ & $100 \%$ & $100 \%$ & & & & \\
\hline YKQCTB-W-38095 & $112 \%$ & $107 \%$ & $123 \%$ & \multicolumn{4}{|c|}{ Sample not reanalyzed. } \\
\hline \multicolumn{8}{|c|}{ SDG 16-06-01b, Analysis Date June 1, 2016} \\
\hline $20 \mathrm{ppb}$ & $96 \%$ & $117 \%$ & $98 \%$ & 19.79 & $-1.1 \%$ & 19.43 & $-2.9 \%$ \\
\hline Lab Blank & $107 \%$ & $112 \%$ & $107 \%$ & & & & \\
\hline YKDL2C2-136-W-38096 & $95 \%$ & $98 \%$ & $97 \%$ & & & & \\
\hline YKDL2C2-136-W-38096DUP & $99 \%$ & $99 \%$ & $99 \%$ & & & & \\
\hline YKDL2CU-215-W-38097 & $91 \%$ & $92 \%$ & $95 \%$ & & & & \\
\hline YKDL2CU-215-DUP-W-38098 & $93 \%$ & $88 \%$ & $93 \%$ & & & & \\
\hline YKQCTB-W-38099 & $0 \%$ & $0 \%$ & $0 \%$ & \multicolumn{4}{|c|}{ Re-analyzed in SDG $16-06-02$ b with recovery limit met. } \\
\hline
\end{tabular}


TABLE S2.3 Calibration and surrogate recovery during organic analyses of soil and water samples at AGEM Laboratory.

\begin{tabular}{|c|c|c|c|}
\hline \multirow[b]{2}{*}{ Sample ID } & \multicolumn{3}{|c|}{ Recovery of Surrogate Compounds ${ }^{a}$} \\
\hline & Fluorobenzene & $\begin{array}{l}\text { Bromo- } \\
\text { fluorobenzene }\end{array}$ & $\begin{array}{l}\text { Dichloro- } \\
\text { benzene-d4 }\end{array}$ \\
\hline
\end{tabular}

\begin{tabular}{cccc}
\multicolumn{3}{c}{ Measured Concentration of Calibration Check } \\
Standards
\end{tabular}

SDG 16-06-02b, Analysis Date June 2, 2016

$\begin{array}{lc}20 \text { ppb } & 98 \% \\ \text { Lab Blank } & 100 \% \\ \text { YKQCTB-W-38099 } & 92 \%\end{array}$

$\begin{array}{cc}116 \% & 101 \% \\ 100 \% & 100 \% \\ 97 \% & 96 \%\end{array}$

SDG 16-06-03b, Analysis Date June 3, 2016

$20 \mathrm{ppb}$

$88 \%$

Lab Blank

$100 \%$

YKDL2-C1-112-W-38100

YKDL2-C1-112-W-38100DUP

$93 \%$

YKQCTB-W-38101

$89 \%$

$\begin{array}{cc}108 \% & 99 \% \\ 100 \% & 100 \% \\ 92 \% & 97 \% \\ 92 \% & 97 \% \\ 100 \% & 100 \%\end{array}$

SDG 16-06-07b, Analysis Date June 7, 2016

20 ppb

Lab Blank

YKDL3-C2-134-W-38102

YKDL3-C1-113-W-38103

YKDL3-C1-113-W-38103DUP

YKDL8-C2-125-W-38104

\section{$8 \%$}

$92 \%$

$100 \%$
$96 \%$

YKQCTB-W-38105

$96 \%$

$100 \%$

$93 \%$

$95 \%$

$89 \%$

$88 \%$

$89 \%$

\section{$115 \%$}

$100 \%$

$93 \%$

$89 \%$

$87 \%$

$83 \%$

$110 \%$

$100 \%$

SDG 16-06-08b, Analysis Date June 8, 2016

$20 \mathrm{ppb}$

$91 \%$

Lab Blank

$100 \%$

$92 \%$

$96 \%$

YKDL8C1-104-W-38106DUP

$86 \%$

$100 \%$

$100 \%$

$99 \%$

$103 \%$

$106 \%$

$94 \%$

$100 \%$

$90 \%$

$92 \%$

$86 \%$

$85 \%$

$106 \%$

SDG 16-06-10b, Analysis Date June 10, 2016 
TABLE S2.3 Calibration and surrogate recovery during organic analyses of soil and water samples at AGEM Laboratory.

\begin{tabular}{|c|c|c|c|c|c|c|c|}
\hline \multirow[b]{2}{*}{ Sample ID } & \multicolumn{3}{|c|}{ Recovery of Surrogate Compounds ${ }^{a}$} & \multicolumn{4}{|c|}{$\begin{array}{c}\text { Measured Concentration of Calibration Check } \\
\text { Standards }{ }^{\text {b }} \\
\end{array}$} \\
\hline & Fluorobenzene & $\begin{array}{l}\text { Bromo- } \\
\text { fluorobenzene }\end{array}$ & $\begin{array}{c}\text { Dichloro- } \\
\text { benzene-d4 }\end{array}$ & $\begin{array}{c}\text { Carbon } \\
\text { Tetrachloride } \\
(p p b)\end{array}$ & RPD & $\begin{array}{l}\text { Chloroform } \\
\qquad(\mathrm{ppb})\end{array}$ & RPD \\
\hline $20 \mathrm{ppb}$ & $101 \%$ & $103 \%$ & $103 \%$ & 20.89 & $4.5 \%$ & 20.76 & $3.8 \%$ \\
\hline Lab Blank & $100 \%$ & $100 \%$ & $100 \%$ & & & & \\
\hline YKDL7-CL-265-W-38108 & $93 \%$ & $94 \%$ & $97 \%$ & & & & \\
\hline YKDL7-CL-265-W-38108DUP & $97 \%$ & $94 \%$ & $102 \%$ & & & & \\
\hline YKQCTB-W-38110 & $88 \%$ & $95 \%$ & $99 \%$ & & & & \\
\hline \multicolumn{8}{|c|}{ SDG 16-06-14b, Analysis Date June 14, 2016} \\
\hline $20 \mathrm{ppb}$ & $98 \%$ & $119 \%$ & $94 \%$ & 20.92 & $4.6 \%$ & 20.05 & $0.3 \%$ \\
\hline Lab Blank & $100 \%$ & $100 \%$ & $100 \%$ & & & & \\
\hline YKDL7-C-100-W-38111 & $97 \%$ & $91 \%$ & $92 \%$ & & & & \\
\hline YKDL7-C-100-W-38111DUP & $93 \%$ & $86 \%$ & $93 \%$ & & & & \\
\hline YKDL7-CU-192-W-38112 & $88 \%$ & $81 \%$ & $84 \%$ & & & & \\
\hline YKDL6-C-110-W-38113 & $91 \%$ & $80 \%$ & $84 \%$ & & & & \\
\hline YKQCTB-W-38114 & $91 \%$ & $88 \%$ & $90 \%$ & & & & \\
\hline \multicolumn{8}{|c|}{ SDG 16-06-17b, Analysis Date June 17, 2016} \\
\hline $20 \mathrm{ppb}$ & $91 \%$ & $115 \%$ & $93 \%$ & 19.14 & $-4.3 \%$ & 18.76 & $-6.2 \%$ \\
\hline Lab Blank & $105 \%$ & $105 \%$ & $104 \%$ & & & & \\
\hline YKDL4-C2-145-W-38115 & $99 \%$ & $100 \%$ & $107 \%$ & & & & \\
\hline YKDL4-C1-120-W-38116 & $103 \%$ & $95 \%$ & $105 \%$ & & & & \\
\hline YKDL4-C1-120-W-38116DUP & $84 \%$ & $78 \%$ & $85 \%$ & & & & \\
\hline YKQCTB-W-38117 & $95 \%$ & $95 \%$ & $99 \%$ & & & & \\
\hline \multicolumn{8}{|c|}{ SDG 16-06-22a, Analysis Date June 22, 2016} \\
\hline $20 \mathrm{ppb}$ & $89 \%$ & $98 \%$ & $99 \%$ & 22.97 & $14.9 \%$ & 23.97 & $19.9 \%$ \\
\hline Lab Blank & $100 \%$ & $100 \%$ & $100 \%$ & & & & \\
\hline YKQCTB-W-38120 & $87 \%$ & $90 \%$ & $95 \%$ & & & & \\
\hline
\end{tabular}

SDG 16-06-22b, Analysis Date June 22, 2016 
TABLE S2.3 Calibration and surrogate recovery during organic analyses of soil and water samples at AGEM Laboratory.

\begin{tabular}{|c|c|c|c|c|c|c|c|}
\hline \multirow[b]{2}{*}{ Sample ID } & \multicolumn{3}{|c|}{ Recovery of Surrogate Compounds ${ }^{a}$} & \multicolumn{4}{|c|}{$\begin{array}{c}\text { Measured Concentration of Calibration Check } \\
\text { Standards }{ }^{\mathrm{b}}\end{array}$} \\
\hline & Fluorobenzene & $\begin{array}{l}\text { Bromo- } \\
\text { fluorobenzene }\end{array}$ & $\begin{array}{c}\text { Dichloro- } \\
\text { benzene-d4 }\end{array}$ & $\begin{array}{c}\text { Carbon } \\
\text { Tetrachloride } \\
(p p b)\end{array}$ & RPD & $\begin{array}{l}\text { Chloroforr } \\
\qquad(p p b)\end{array}$ & RPD \\
\hline 20 ppb & $113 \%$ & $117 \%$ & $109 \%$ & 19.45 & $-2.8 \%$ & 19.63 & $-1.9 \%$ \\
\hline Lab Blank & $111 \%$ & $117 \%$ & $106 \%$ & & & & \\
\hline YKDL5-C1-106-W-38118 & $115 \%$ & $116 \%$ & $118 \%$ & & & & \\
\hline YKDL5-C1-106-W-38118DUP & $100 \%$ & $105 \%$ & $101 \%$ & & & & \\
\hline
\end{tabular}

${ }^{\text {a }}$ QC Limit for $\%$ Recovery of Surrogate Compounds $=80 \%$ to $120 \%$.

${ }^{b}$ QC Limit for Calibration Check Standard Relative Percent Difference (RPD) $= \pm 20 \%$

${ }^{\mathrm{c}}$ Surrogate recovery exceeded QC limit; sample group not reanalyzed. 
TABLE S2.4 Analytical results from AGEM Laboratory and TestAmerica for duplicate quality control analyses.

\begin{tabular}{|c|c|c|c|c|c|c|c|c|c|}
\hline \multirow[b]{2}{*}{ Location } & \multirow[b]{2}{*}{ Sample } & \multirow[b]{2}{*}{$\begin{array}{c}\text { Sample } \\
\text { Date }\end{array}$} & \multirow[b]{2}{*}{ Sample Type $^{a}$} & \multirow[b]{2}{*}{$\begin{array}{c}\text { Depth (ft } \\
\text { BGL) }\end{array}$} & \multirow[b]{2}{*}{ Matrix $^{\mathrm{b}}$} & \multicolumn{3}{|c|}{ Concentration } & \multirow[b]{2}{*}{ Units } \\
\hline & & & & & & $\begin{array}{c}\text { Carbon } \\
\text { Tetrachloride } \\
\end{array}$ & Chloroform & $\begin{array}{c}\text { Methylene } \\
\text { Chloride }\end{array}$ & \\
\hline TI01 & YKTI01-S-37749 & 7/7/15 & $\mathrm{N}$ & 24 & SOIL & 139 & $7.9 \mathrm{~J}$ & ND (10) & $\mu \mathrm{g} / \mathrm{kg}$ \\
\hline TI01 & YKTI01-S-37749DUP & $7 / 7 / 15$ & DUP-L & 24 & SOIL & 150 & $9.1 \mathrm{~J}$ & ND (10) & $\mu \mathrm{g} / \mathrm{kg}$ \\
\hline TI01 & YKTI01-S-37751 & 7/7/15 & $\mathrm{N}$ & 32 & SOIL & 121 & 12 & ND (10) & $\mu \mathrm{g} / \mathrm{kg}$ \\
\hline TI01 & YKTI01-S-37752 & $7 / 7 / 15$ & DUP-F & 32 & SOIL & 188 & 10 & ND (10) & $\mu \mathrm{g} / \mathrm{kg}$ \\
\hline TI02 & YKTI02-S-37768 & 7/7/15 & $\mathrm{N}$ & 20 & SOIL & $\mathrm{ND}^{\mathrm{c}}(10)$ & ND (10) & ND (10) & $\mu \mathrm{g} / \mathrm{kg}$ \\
\hline TIO2 & YKTI02-S-37776 & $7 / 7 / 15$ & DUP-F & 20 & SOIL & ND (10) & ND (10) & ND (10) & $\mu \mathrm{g} / \mathrm{kg}$ \\
\hline TI02 & YKTI02-S-37772 & 7/7/15 & $\mathrm{N}$ & 36 & SOIL & $5.9 \mathrm{~J}$ & ND (10) & ND (10) & $\mu \mathrm{g} / \mathrm{kg}$ \\
\hline TIO2 & YKTI02-S-37772DUP & $7 / 7 / 15$ & DUP-L & 36 & SOIL & $7.5 \mathrm{~J}$ & ND (10) & ND (10) & $\mu \mathrm{g} / \mathrm{kg}$ \\
\hline TI02 & YKTI02-S-37781 & $7 / 7 / 15$ & $\mathrm{~N}$ & 64 & SOIL & ND (10) & ND (10) & ND (10) & $\mu \mathrm{g} / \mathrm{kg}$ \\
\hline TI02 & YKTI02-S-37781DUP & $7 / 7 / 15$ & DUP-L & 64 & SOIL & ND (10) & ND (10) & ND (10) & $\mu \mathrm{g} / \mathrm{kg}$ \\
\hline TI04 & YKTI04-S-37796 & 7/8/15 & $\mathrm{N}$ & 40 & SOIL & 59 & ND (10) & ND (10) & $\mu \mathrm{g} / \mathrm{kg}$ \\
\hline TI04 & YKTI04-S-37799 & $7 / 8 / 15$ & DUP-F & 40 & SOIL & 43 & ND (10) & ND (10) & $\mu \mathrm{g} / \mathrm{kg}$ \\
\hline TI03 & YKTI03-S-37819 & 7/9/15 & $\mathrm{N}$ & 48 & SOIL & ND (10) & ND (10) & ND (10) & $\mu \mathrm{g} / \mathrm{kg}$ \\
\hline TI03 & YKTI03-S-37824 & $7 / 9 / 15$ & DUP-F & 48 & SOIL & ND (10) & ND (10) & ND (10) & $\mu \mathrm{g} / \mathrm{kg}$ \\
\hline TI03 & YKTI03-S-37826 & 7/9/15 & $\mathrm{N}$ & 63.5 & SOIL & ND (10) & ND (10) & ND (10) & $\mu \mathrm{g} / \mathrm{kg}$ \\
\hline TI03 & YKTI03-S-37826DUP & $7 / 9 / 15$ & DUP-L & 63.5 & SOIL & ND (10) & ND (10) & ND (10) & $\mu \mathrm{g} / \mathrm{kg}$ \\
\hline TI04 & YKTI04-W-37833 & 7/9/15 & $\mathrm{N}$ & $74.6-79.6$ & WG & $159 \mathrm{D}$ & 164 D & ND (1) & $\mu \mathrm{g} / \mathrm{L}$ \\
\hline TIO4 & YKTI04-W-37834 & $7 / 9 / 15$ & DUP-F & $74.6-79.6$ & WG & 206 D & $140 \mathrm{D}$ & ND (1) & $\mu \mathrm{g} / \mathrm{L}$ \\
\hline TI03 & YKTI03-W-37836 & $7 / 9 / 15$ & $\mathrm{~N}$ & $76.8-81.8$ & WG & $150 \mathrm{D}$ & 11 & ND (1) & $\mu g / L$ \\
\hline
\end{tabular}




\begin{tabular}{|c|c|c|c|c|c|c|c|c|c|}
\hline TIO3 & YKTI03-W-37837 & $7 / 9 / 15$ & DUP-F & 76.881 .8 & WG & $141 \mathrm{D}$ & 8.3 & ND (1) & $\mu g / L$ \\
\hline TI01 & YKTI01-S-37757 & $7 / 10 / 15$ & $\mathrm{~N}$ & 52 & SOIL & 53 & 17 & ND (10) & $\mu \mathrm{g} / \mathrm{kg}$ \\
\hline TI01 & YKTI01-S-37757DUP & $7 / 10 / 15$ & DUP-L & 52 & SOIL & 44 & 16 & ND (10) & $\mu \mathrm{g} / \mathrm{kg}$ \\
\hline TI01 & YKTI01-S-37761 & $7 / 10 / 15$ & $\mathrm{~N}$ & 68 & SOIL & ND (10) & ND (10) & ND (10) & $\mu \mathrm{g} / \mathrm{kg}$ \\
\hline TI01 & YKTI01-S-37761DUP & $7 / 10 / 15$ & DUP-L & 68 & SOIL & ND (10) & ND (10) & ND (10) & $\mu \mathrm{g} / \mathrm{kg}$ \\
\hline TI01 & YKTI01-W-37844 & $7 / 10 / 15$ & $\mathrm{~N}$ & $77.4-82.4$ & WG & $373 D^{d}$ & $108 \mathrm{D}$ & ND (1) & $\mu g / L$ \\
\hline TI01 & YKTI01-W-37845 & $7 / 10 / 15$ & DUP-F & $77.4-82.4$ & WG & $360 \mathrm{D}$ & $113 \mathrm{D}$ & ND (1) & $\mu \mathrm{g} / \mathrm{L}$ \\
\hline TI02 & YKTI02-W-37847 & $7 / 10 / 15$ & $\mathrm{~N}$ & $77-82$ & WG & $200 \mathrm{D}$ & 54 & ND (1) & $\mu \mathrm{g} / \mathrm{L}$ \\
\hline TIO2 & YKTIO2-W-37848 & $7 / 10 / 15$ & DUP-F & $77-82$ & WG & $201 \mathrm{D}$ & 68 & ND (1) & $\mu g / L$ \\
\hline TI02 & YKTI02-W-37848DUP & $7 / 10 / 15$ & DUP-L & $77-82$ & WG & $205 \mathrm{D}$ & $69 \mathrm{D}$ & ND (1) & $\mu \mathrm{g} / \mathrm{L}$ \\
\hline TIO2 & YKTI02100-W-37849 & $7 / 13 / 15$ & $\mathrm{~N}$ & $100-103.5$ & WG & 1.4 & 7.2 & ND (1) & $\mu g / L$ \\
\hline $\mathrm{TIO}$ & YKTI02100-W-37850 & $7 / 13 / 15$ & DUP-F & $100-103.5$ & WG & 7.8 & 17 & ND (1) & $\mu \mathrm{g} / \mathrm{L}$ \\
\hline TI01 & YKTI01120-W-37855 & $7 / 15 / 15$ & $\mathrm{~N}$ & $108-118$ & WG & $0.9 \mathrm{~J}^{\mathrm{e}}$ & 2.0 & ND (1) & $\mu g / L$ \\
\hline TIO1 & YKTI01120-W-37855DUP & $7 / 15 / 15$ & DUP-L & $108-118$ & WG & ND (1) & 1.8 & ND (1) & $\mu \mathrm{g} / \mathrm{L}$ \\
\hline TIO1 & YKTI01100-W-37854 & $7 / 16 / 15$ & $\mathrm{~N}$ & $88-98$ & WG & ND (1) & $0.6 \mathrm{~J}$ & ND (1) & $\mu g / L$ \\
\hline TIO1 & YKTI01100-W-37858 & $7 / 16 / 15$ & DUP-F & $88-98$ & WG & ND (1) & 1.1 & ND (1) & $\mu \mathrm{g} / \mathrm{L}$ \\
\hline TI04 & YKTI04100-W-37861 & $7 / 16 / 15$ & $\mathrm{~N}$ & $89-99$ & WG & ND (1) & $0.8 \mathrm{~J}$ & ND (1) & $\mu g / L$ \\
\hline TIO4 & YKTI04100-W-37862 & $7 / 16 / 15$ & DUP-F & $89-99$ & WG & ND (1) & $0.6 \mathrm{~J}$ & ND (1) & $\mu \mathrm{g} / \mathrm{L}$ \\
\hline TIO4 & YKTI04100-W-37862DUP & $7 / 16 / 15$ & DUP-L & $89-99$ & WG & ND (1) & $0.5 \mathrm{~J}$ & ND (1) & $\mu \mathrm{g} / \mathrm{L}$ \\
\hline TI05 & YKTI05100-W-37865 & $7 / 17 / 15$ & $\mathrm{~N}$ & $88-98$ & WG & 82 & 6.7 & ND (1) & $\mu g / L$ \\
\hline TI05 & YKTI05100-W-37867 & $7 / 17 / 15$ & DUP-F & $88-98$ & WG & 52 & 3.2 & ND (1) & $\mu \mathrm{g} / \mathrm{L}$ \\
\hline TI05 & YKTI05100-W-37867DUP & $7 / 17 / 15$ & DUP-L & $88-98$ & WG & 54 & 3.2 & ND (1) & $\mu \mathrm{g} / \mathrm{L}$ \\
\hline TI05 & YKTI0580-W-37868 & $7 / 18 / 15$ & $\mathrm{~N}$ & $76-81$ & WG & $254 \mathrm{D}$ & $69 \mathrm{D}$ & ND (1) D & $\mu \mathrm{g} / \mathrm{L}$ \\
\hline TI05 & YKTI0580-W-37868DUP & $7 / 18 / 15$ & DUP-L & $76-81$ & WG & $228 \mathrm{D}$ & $62 \mathrm{D}$ & ND (1) D & $\mu g / L$ \\
\hline
\end{tabular}




\begin{tabular}{|c|c|c|c|c|c|c|c|c|c|}
\hline TI06 & YKTI06100-W-37873 & 7/19/15 & $\mathrm{N}$ & $90-100$ & WG & $0.8 \mathrm{~J}$ & 2.7 & ND (1) & $\mu g / L$ \\
\hline TI06 & YKTI06100-W-37874 & 7/19/15 & DUP-F & $90-100$ & WG & $0.6 \mathrm{~J}$ & 2.3 & ND (1) & $\mu \mathrm{g} / \mathrm{L}$ \\
\hline TI06 & YKTI06100-W-37873DUP & 7/19/15 & DUP-L & $90-100$ & WG & $0.6 \mathrm{~J}$ & 2.4 & ND (1) & $\mu \mathrm{g} / \mathrm{L}$ \\
\hline TI07 & YKTI07120-W-37877 & 7/20/15 & $\mathrm{N}$ & $110-120$ & WG & ND (1) & ND (1) & ND (1) & $\mu \mathrm{g} / \mathrm{L}$ \\
\hline TI07 & YKTI07120-W-37878 & 7/20/15 & DUP-F & $110-120$ & WG & ND (1) & ND (1) & ND (1) & $\mu \mathrm{g} / \mathrm{L}$ \\
\hline TI07 & YKTI07120-W-37878DUP & 7/20/15 & DUP-L & $110-120$ & WG & ND (1) & ND (1) & ND (1) & $\mu \mathrm{g} / \mathrm{L}$ \\
\hline TI08 & YKTI0885-W-37879 & 7/21/15 & $\mathrm{N}$ & $80-85$ & WG & ND (1) & ND (1) & ND (1) & $\mu \mathrm{g} / \mathrm{L}$ \\
\hline TI08 & YKTI0885-W-37879DUP & 7/21/15 & DUP-L & $80-85$ & WG & ND (1) & ND (1) & ND (1) & $\mu \mathrm{g} / \mathrm{L}$ \\
\hline TI08 & YKTI08107-W-37881 & 7/21/15 & $\mathrm{N}$ & $97-107$ & WG & 27 & $0.9 \mathrm{~J}$ & ND (1) & $\mu \mathrm{g} / \mathrm{L}$ \\
\hline TI08 & YKTI08107-W37884 & 7/21/15 & DUP-F & $97-107$ & WG & 29 & $0.8 \mathrm{~J}$ & ND (1) & $\mu g / L$ \\
\hline FITWORX & YKFITWORX-G-37897 & $8 / 5 / 15$ & $\mathrm{~N}$ & - & AIR & 6.3 & ND (0.98) & ND (1.7) & $\mu \mathrm{g} / \mathrm{m}^{3}$ \\
\hline FITWORX & YKFITWORX-G-37901 & $8 / 5 / 15$ & DUP-F & - & AIR & 5.9 & ND (0.98) & ND (1.7) & $\mu \mathrm{g} / \mathrm{m}^{3}$ \\
\hline DW39 & YKNORDLUND-W-37918 & $8 / 5 / 15$ & $\mathrm{~N}$ & - & WG & ND (1) & ND (1) & ND (1) & $\mu \mathrm{g} / \mathrm{L}$ \\
\hline DW39 & YKNORDLUNDDUP-W-37919 & $8 / 5 / 15$ & DUP-F & - & WG & ND (1) & ND (1) & ND (1) & $\mu \mathrm{g} / \mathrm{L}$ \\
\hline SB77 & YKSB77-W-37921 & $8 / 7 / 15$ & $\mathrm{~N}$ & 75.8-79 & WG & $220 \mathrm{D}$ & 9.8 & ND (1) & $\mu \mathrm{g} / \mathrm{L}$ \\
\hline SB77 & YKSB77DUP-W-37922 & $8 / 7 / 15$ & DUP-F & 75.8-79 & WG & $229 \mathrm{D}$ & 9.1 & ND (1) & $\mu \mathrm{g} / \mathrm{L}$ \\
\hline SB77 & YKSB77-W-37921DUP & 8/7/15 & DUP-L & 75.8-79 & WG & $229 \mathrm{D}$ & $10 \mathrm{D}$ & ND (1) D & $\mu \mathrm{g} / \mathrm{L}$ \\
\hline SB77 & YKSB77DUP-W-37922DUP & 8/7/15 & DUP-L & 75.8-79 & WG & $216 \mathrm{D}$ & $9.8 \mathrm{D}$ & ND (1) D & $\mu \mathrm{g} / \mathrm{L}$ \\
\hline TI09 & YKTI09-80-W-37926 & 8/13/15 & $\mathrm{N}$ & $80-85$ & WG & $0.7 \mathrm{~J}$ & ND (1) & ND (1) & $\mu \mathrm{g} / \mathrm{L}$ \\
\hline TI09 & YKTI09-80DUP-W-37927 & 8/13/15 & DUP-F & $80-85$ & WG & $0.6 \mathrm{~J}$ & ND (1) & ND (1) & $\mu g / L$ \\
\hline TI09 & YKTI09-135-W-37929 & 8/13/15 & $\mathrm{N}$ & 135-145 & WG & 1.8 & ND (1) & ND (1) & $\mu \mathrm{g} / \mathrm{L}$ \\
\hline TI09 & YKTI09-135-W-37929DUP & 8/13/15 & DUP-L & $135-145$ & WG & 1.8 & ND (1) & ND (1) & $\mu g / L$ \\
\hline TI10 & YKTI10-95-W-37931 & 8/14/15 & $\mathrm{N}$ & 95-105 & WG & 18 & 1.2 & ND (1) & $\mu \mathrm{g} / \mathrm{L}$ \\
\hline TI10 & YKTI10-95DUP-W-37932 & 8/14/15 & DUP-F & $95-105$ & WG & 15 & $0.9 \mathrm{~J}$ & ND (1) & $\mu \mathrm{g} / \mathrm{L}$ \\
\hline
\end{tabular}




\begin{tabular}{|c|c|c|c|c|c|c|c|c|c|}
\hline TI10 & YKTI10-117-W-37933 & 8/14/15 & $\mathrm{N}$ & $117-127$ & WG & 29 & 1.1 & ND (1) & $\mu \mathrm{g} / \mathrm{L}$ \\
\hline TI10 & YKTI10-117-W-37933DUP & 8/14/15 & DUP-L & $117-127$ & WG & 28 & 1.1 & ND (1) & $\mu \mathrm{g} / \mathrm{L}$ \\
\hline TI11 & YKTI11-92-W-37936 & 8/18/15 & $\mathrm{N}$ & $92-102$ & WG & ND (1) & ND (1) & ND (1) & $\mu \mathrm{g} / \mathrm{L}$ \\
\hline TI11 & YKTI11-92-W-37936DUP & 8/18/15 & DUP-L & $92-102$ & WG & ND (1) & ND (1) & ND (1) & $\mu \mathrm{g} / \mathrm{L}$ \\
\hline TI11 & YKTI11-112-W-37937 & 8/18/15 & $\mathrm{N}$ & $92-122$ & WG & 202 D & 5.1 & ND (1) & $\mu \mathrm{g} / \mathrm{L}$ \\
\hline TI11 & YKTI11-112-W-37937DUP & 8/18/15 & DUP-L & $92-122$ & WG & $171 \mathrm{D}$ & 4.4 D & ND (1) D & $\mu \mathrm{g} / \mathrm{L}$ \\
\hline TI12 & YKTI12-80-W-37939 & 8/20/15 & $\mathrm{N}$ & $80-85$ & WG & 6.2 & 1.2 & ND (1) & $\mu \mathrm{g} / \mathrm{L}$ \\
\hline TI12 & YKTI12-80-W-37939DUP & 8/20/15 & DUP-L & $80-85$ & WG & 5.6 & 1.1 & ND (1) & $\mu \mathrm{g} / \mathrm{L}$ \\
\hline TI12 & YKTI12-92-W-37940 & 8/20/15 & $\mathrm{N}$ & $92-102$ & WG & 45 & 1.5 & ND (1) & $\mu \mathrm{g} / \mathrm{L}$ \\
\hline TI12 & YKTI12-DUP92-W-37941 & 8/20/15 & DUP-F & $92-102$ & WG & 39 & 1.4 & ND (1) & $\mu \mathrm{g} / \mathrm{L}$ \\
\hline TI13 & YKTI13-93-W-37947 & 8/22/15 & $\mathrm{N}$ & 93-103 & WG & ND (1) & ND (1) & ND (1) & $\mu \mathrm{g} / \mathrm{L}$ \\
\hline TI13 & YKTI13-93-W-37947DUP & 8/22/15 & DUP-L & 93-103 & WG & ND (1) & ND (1) & ND (1) & $\mu \mathrm{g} / \mathrm{L}$ \\
\hline TI13 & YKTI13-111-W-37948 & 8/22/15 & $\mathrm{N}$ & 111-121 & WG & ND (1) & ND (1) & ND (1) & $\mu \mathrm{g} / \mathrm{L}$ \\
\hline TI13 & YKTI13-111DUP-W-37949 & $8 / 22 / 15$ & DUP-F & $111-121$ & WG & ND (1) & ND (1) & ND (1) & $\mu \mathrm{g} / \mathrm{L}$ \\
\hline TI14 & YKTI14-113-W-37952 & 8/23/15 & $\mathrm{N}$ & $113-123$ & WG & 36 & 1.4 & ND (1) & $\mu \mathrm{g} / \mathrm{L}$ \\
\hline TI14 & YKTI14-113DUP-W-37953 & 8/23/15 & DUP-F & $113-123$ & WG & 32 & 1.3 & ND (1) & $\mu \mathrm{g} / \mathrm{L}$ \\
\hline TI15 & YKTI15-113-W-37957 & 8/24/15 & $\mathrm{N}$ & $113-123$ & WG & 22 & $0.7 \mathrm{~J}$ & ND (1) & $\mu \mathrm{g} / \mathrm{L}$ \\
\hline TI15 & YKTI15-113-W-37957DUP & 8/24/15 & DUP-L & $113-123$ & WG & 21 & $0.7 \mathrm{~J}$ & ND (1) & $\mu \mathrm{g} / \mathrm{L}$ \\
\hline TI15 & YKTI15-134-W-37958 & 8/24/15 & $\mathrm{N}$ & 134-144 & WG & 68 & 1.7 & ND (1) & $\mu \mathrm{g} / \mathrm{L}$ \\
\hline TI15 & YKTI15-134DUP-W-37959 & 8/24/15 & DUP-F & 134-144 & WG & 69 & 1.8 & ND (1) & $\mu \mathrm{g} / \mathrm{L}$ \\
\hline TI16 & YKTI16-86-W-37961 & 8/25/15 & $\mathrm{N}$ & $86-96$ & WG & ND (1) & ND (1) & ND (1) & $\mu \mathrm{g} / \mathrm{L}$ \\
\hline TI16 & YKTI16-86DUP-W-37962 & 8/25/15 & DUP-F & $86-96$ & WG & ND (1) & ND (1) & ND (1) & $\mu \mathrm{g} / \mathrm{L}$ \\
\hline
\end{tabular}




\begin{tabular}{|c|c|c|c|c|c|c|c|c|c|}
\hline TI16 & YKTI16-105-W-37963 & $8 / 25 / 15$ & $\mathrm{~N}$ & $105-115$ & WG & 2.9 & ND (1) & ND (1) & $\mu \mathrm{g} / \mathrm{L}$ \\
\hline TI16 & YKTI16-105-W-37963DUP & $8 / 25 / 15$ & DUP-L & $105-115$ & WG & 3.2 & ND (1) & ND (1) & $\mu \mathrm{g} / \mathrm{L}$ \\
\hline TI17 & YKTI17-133-W-37968 & 8/26/15 & $\mathrm{N}$ & $133-143$ & WG & ND (1) & $0.4 \mathrm{~J}$ & ND (1) & $\mu \mathrm{g} / \mathrm{L}$ \\
\hline TI17 & YKTI17-133DUP-W-37968 & 8/26/15 & DUP-F & 133-143 & WG & ND (1) & $0.4 \mathrm{~J}$ & ND (1) & $\mu \mathrm{g} / \mathrm{L}$ \\
\hline TI17 & YKTI17-133-W-37968DUP & 8/26/15 & DUP-L & $133-143$ & WG & ND (1) & $0.4 \mathrm{~J}$ & ND (1) & $\mu \mathrm{g} / \mathrm{L}$ \\
\hline TI18 & YKTI18-95-W-37971 & $8 / 27 / 15$ & $\mathrm{~N}$ & $95-105$ & WG & 11 & $0.9 \mathrm{~J}$ & ND (1) & $\mu \mathrm{g} / \mathrm{L}$ \\
\hline TI18 & YKTI18-95DUP-W-37972 & $8 / 27 / 15$ & DUP-F & $95-105$ & WG & 10 & $0.8 \mathrm{~J}$ & ND (1) & $\mu \mathrm{g} / \mathrm{L}$ \\
\hline TI18 & YKTI18-114-W-37973 & 8/28/15 & $\mathrm{N}$ & $114-124$ & WG & 2.9 & ND (1) & ND (1) & $\mu \mathrm{g} / \mathrm{L}$ \\
\hline TI18 & YKTI18-114-W-37973DUP & 8/28/15 & DUP-L & $114-124$ & WG & 2.8 & ND (1) & ND (1) & $\mu \mathrm{g} / \mathrm{L}$ \\
\hline TI19 & YKTI19-107-W-37976 & 9/9/15 & $\mathrm{N}$ & $107-117$ & WG & 4.8 & ND (1) & ND (1) & $\mu \mathrm{g} / \mathrm{L}$ \\
\hline TI19 & YKTI19-107-W-37976DUP & 9/9/15 & DUP-L & $107-117$ & WG & 4.8 & ND (1) & ND (1) & $\mu \mathrm{g} / \mathrm{L}$ \\
\hline TI19 & YKTI19-129-W-37977 & 9/9/15 & $\mathrm{N}$ & $129-139$ & WG & 22 & $0.8 \mathrm{~J}$ & ND (1) & $\mu \mathrm{g} / \mathrm{L}$ \\
\hline TI19 & YKTI19-129DUP-W-37978 & 9/9/15 & DUP-F & $129-139$ & WG & 20 & $0.9 \mathrm{~J}$ & ND (1) & $\mu \mathrm{g} / \mathrm{L}$ \\
\hline TI20 & YKTI20-105-W-37981 & 9/10/15 & $\mathrm{N}$ & $105-115$ & WG & 8.2 & ND (1) & ND (1) & $\mu \mathrm{g} / \mathrm{L}$ \\
\hline $\mathrm{Tl} 20$ & YKTI20-105-W-37981DUP & 9/10/15 & DUP-L & $105-115$ & WG & 8.3 & ND (1) & ND (1) & $\mu \mathrm{g} / \mathrm{L}$ \\
\hline TI20 & YKTI20-131-W-37982 & 9/10/15 & $\mathrm{N}$ & $131-141$ & WG & 1.8 & ND (1) & ND (1) & $\mu \mathrm{g} / \mathrm{L}$ \\
\hline TI20 & YKTI20-131DUP-W-37983 & 9/10/15 & DUP-F & $131-141$ & WG & 1.8 & ND (1) & ND (1) & $\mu \mathrm{g} / \mathrm{L}$ \\
\hline TI21 & YKTI21-99-W-37986 & 9/11/15 & $\mathrm{N}$ & $99-109$ & WG & $0.8 \mathrm{~J}$ & ND (1) & ND (1) & $\mu \mathrm{g} / \mathrm{L}$ \\
\hline TI21 & YKTI21-99-W-37986DUP & 9/11/15 & DUP-L & 99-109 & WG & $0.8 \mathrm{~J}$ & ND (1) & ND (1) & $\mu \mathrm{g} / \mathrm{L}$ \\
\hline TI21 & YKTI21-115-W-37987 & 9/11/15 & $\mathrm{N}$ & $115-120$ & WG & ND (1) & ND (1) & ND (1) & $\mu \mathrm{g} / \mathrm{L}$ \\
\hline TI21 & YKTI21-115DUP-W-37988 & 9/11/15 & DUP-F & $115-120$ & WG & ND (1) & ND (1) & ND (1) & $\mu \mathrm{g} / \mathrm{L}$ \\
\hline TI22 & YKTI22-98-W-37991 & 9/14/15 & $\mathrm{N}$ & 98-108 & WG & 3.3 & ND (1) & ND (1) & $\mu \mathrm{g} / \mathrm{L}$ \\
\hline TI22 & YKTI22-98DUP-W-37993 & 9/14/15 & DUP-F & $98-108$ & WG & 3.4 & ND (1) & ND (1) & $\mu \mathrm{g} / \mathrm{L}$ \\
\hline
\end{tabular}




\begin{tabular}{|c|c|c|c|c|c|c|c|c|c|}
\hline TI23 & YKTI23-80-W-37995 & 9/15/15 & $\mathrm{N}$ & $80-90$ & WG & $0.5 \mathrm{~J}$ & $0.6 \mathrm{~J}$ & ND (1) & $\mu \mathrm{g} / \mathrm{L}$ \\
\hline $\mathrm{TI} 23$ & YKTI23-80-W-37995DUP & 9/15/15 & DUP-L & $80-90$ & WG & $0.4 \mathrm{~J}$ & $0.5 \mathrm{~J}$ & ND (1) & $\mu g / L$ \\
\hline TI23 & YKTI23-99-W-37996 & 9/15/15 & $\mathrm{N}$ & 99-109 & WG & ND (1) & ND (1) & ND (1) & $\mu \mathrm{g} / \mathrm{L}$ \\
\hline TI23 & YKTI23-99DUP-W-37997 & 9/15/15 & DUP-F & $99-109$ & WG & ND (1) & ND (1) & ND (1) & $\mu \mathrm{g} / \mathrm{L}$ \\
\hline TI23 & YKTI23-145-W-37999 & 9/16/15 & $\mathrm{N}$ & $145-155$ & WG & ND (1) & ND (1) & ND (1) & $\mu g / L$ \\
\hline TI23 & YKTI23-145-W-37999DUP & 9/16/15 & DUP-L & $145-155$ & WG & ND (1) & ND (1) & ND (1) & $\mu \mathrm{g} / \mathrm{L}$ \\
\hline TI24 & YKTI24-90-W-38018 & $1 / 20 / 16$ & $\mathrm{~N}$ & $90-100$ & WG & ND (1) & ND (1) & ND (1) & $\mu g / L$ \\
\hline TI24 & YKTI24-90-W-38018DUP & $1 / 20 / 16$ & DUP-L & $90-100$ & WG & ND (1) & ND (1) & ND (1) & $\mu g / L$ \\
\hline TI24 & YKTI24-80-W-38017 & $1 / 20 / 16$ & $\mathrm{~N}$ & $80-90$ & WG & ND (1) & ND (1) & ND (1) & $\mu \mathrm{g} / \mathrm{L}$ \\
\hline TI24 & YKTI24-80DUP-W-38020 & $1 / 20 / 16$ & DUP-F & $80-90$ & WG & ND (1) & ND (1) & ND (1) & $\mu \mathrm{g} / \mathrm{L}$ \\
\hline TI25 & YKTI25-98-W-38024 & $1 / 21 / 16$ & $\mathrm{~N}$ & $98-108$ & WG & 1.3 & ND (1) & ND (1) & $\mu \mathrm{g} / \mathrm{L}$ \\
\hline TI25 & YKTI25-98DUP-W-38025 & $1 / 21 / 16$ & DUP-F & $98-108$ & WG & 1.2 & ND (1) & ND (1) & $\mu g / L$ \\
\hline TI25 & YKTI25-108-W-38027 & $1 / 21 / 16$ & $\mathrm{~N}$ & $113-118$ & WG & ND (1) & ND (1) & ND (1) & $\mu \mathrm{g} / \mathrm{L}$ \\
\hline TI25 & YKTI25-108-W-38027DUP & $1 / 21 / 16$ & DUP-L & $113-118$ & WG & ND (1) & ND (1) & ND (1) & $\mu \mathrm{g} / \mathrm{L}$ \\
\hline TI26 & YKTI26-78-W-38029 & $1 / 25 / 16$ & $\mathrm{~N}$ & $78-88$ & WG & ND (1) & ND (1) & ND (1) & $\mu \mathrm{g} / \mathrm{L}$ \\
\hline TI26 & YKTI26-78-W-38029DUP & $1 / 25 / 16$ & DUP-L & $78-88$ & WG & ND (1) & ND (1) & ND (1) & $\mu \mathrm{g} / \mathrm{L}$ \\
\hline TI26 & YKTI26-96-W-38030 & $1 / 25 / 16$ & $\mathrm{~N}$ & $96-106$ & WG & 2.0 & ND (1) & ND (1) & $\mu g / L$ \\
\hline TI26 & YKTI26-96DUP-W-38031 & $1 / 25 / 16$ & DUP-F & $96-106$ & WG & 1.7 & ND (1) & ND (1) & $\mu g / L$ \\
\hline TI26 & YKTI26-113-W-38033 & $1 / 25 / 16$ & $\mathrm{~N}$ & $113-123$ & WG & $0.4 \mathrm{~J}$ & ND (1) & ND (1) & $\mu \mathrm{g} / \mathrm{L}$ \\
\hline TI26 & YKTI26-113DUP-W-38034 & $1 / 25 / 16$ & DUP-F & $113-123$ & WG & $0.4 \mathrm{~J}$ & ND (1) & ND (1) & $\mu g / L$ \\
\hline GARRETSON & YKGARRETSON-G-38037 & $1 / 28 / 16$ & $\mathrm{~N}$ & - & AIR & 28 & ND (0.98) & ND (1.7) & $\mu \mathrm{g} / \mathrm{m}^{3}$ \\
\hline GARRETSON & YKGARRETSON-G-38038 & $1 / 28 / 16$ & DUP-F & - & AIR & 28 & ND (0.98) & ND (1.7) & $\mu \mathrm{g} / \mathrm{m}^{3}$ \\
\hline FITWORX & YKFITWORX-G-38040 & $1 / 28 / 16$ & $\mathrm{~N}$ & - & AIR & 4.7 & 1.0 & ND (1.7) & $\mu \mathrm{g} / \mathrm{m}^{3}$ \\
\hline
\end{tabular}




\begin{tabular}{|c|c|c|c|c|c|c|c|c|c|}
\hline FITWORX & YKFITWORX-G-38041 & $1 / 28 / 16$ & DUP-F & - & AIR & 5.3 & 1.1 & ND (1.7) & $\mu \mathrm{g} / \mathrm{m}^{3}$ \\
\hline TI27 & YKTI27-77-W-38050 & 2/9/16 & $\mathrm{N}$ & $77-87$ & WG & ND (1) & ND (1) & ND (1) & $\mu \mathrm{g} / \mathrm{L}$ \\
\hline TI27 & YKTI27-77DUP-W-38051 & 2/9/16 & DUP-F & $77-87$ & WG & ND (1) & ND (1) & ND (1) & $\mu \mathrm{g} / \mathrm{L}$ \\
\hline TI27 & YKTI27-100-W-38053 & 2/9/16 & $\mathrm{N}$ & $100-110$ & WG & $0.4 \mathrm{~J}$ & ND (1) & ND (1) & $\mu \mathrm{g} / \mathrm{L}$ \\
\hline TI27 & YKTI27-100DUP-W-38054 & 2/9/16 & DUP-F & $100-110$ & WG & ND (1) & ND (1) & ND (1) & $\mu \mathrm{g} / \mathrm{L}$ \\
\hline TI27 & YKTI27-100-W-38053DUP & 2/9/16 & DUP-L & $100-110$ & WG & $0.4 \mathrm{~J}$ & ND (1) & ND (1) & $\mu \mathrm{g} / \mathrm{L}$ \\
\hline TI27 & YKTI27-100-W-38057 & 2/9/16 & $\mathrm{N}$ & $100-110$ & WG & ND (1) & ND (1) & ND (1) & $\mu \mathrm{g} / \mathrm{L}$ \\
\hline TI27 & YKTI27-100-W-38057DUP & 2/9/16 & DUP-L & $100-110$ & WG & ND (1) & ND (1) & ND (1) & $\mu \mathrm{g} / \mathrm{L}$ \\
\hline TI28 & YKTI28-78-W-38075 & $3 / 2 / 16$ & $\mathrm{~N}$ & $78-88$ & WG & ND (1) & ND (1) & ND (1) & $\mu \mathrm{g} / \mathrm{L}$ \\
\hline TI28 & YKTI28-78-W-38075DUP & $3 / 2 / 16$ & DUP-L & $78-88$ & WG & ND (1) & ND (1) & ND (1) & $\mu \mathrm{g} / \mathrm{L}$ \\
\hline TI28 & YKTI28-90-W-38076 & $3 / 2 / 16$ & $\mathrm{~N}$ & $90-100$ & WG & ND (1) & ND (1) & ND (1) & $\mu \mathrm{g} / \mathrm{L}$ \\
\hline TI28 & YKTI28-90-DUP-W-38077 & $3 / 2 / 16$ & DUP-F & $90-100$ & WG & ND (1) & ND (1) & ND (1) & $\mu \mathrm{g} / \mathrm{L}$ \\
\hline FITWORX & YKFITWORX-G-38081 & $4 / 28 / 16$ & $\mathrm{~N}$ & - & AIR & 2.9 & 6.3 & $\mathrm{ND}(1.7)$ & $\mu \mathrm{g} / \mathrm{m}^{3}$ \\
\hline FITWORX & YKFITWORX-G-38082 & $4 / 28 / 16$ & DUP-F & - & AIR & 3.3 & 6.9 & ND (1.7) & $\mu \mathrm{g} / \mathrm{m}^{3}$ \\
\hline FITWORX & YKFITWORX-G-38087 & 4/28/16 & $\mathrm{N}$ & - & SOIL GAS & 51 & ND (9.8) & ND (17) & $\mu \mathrm{g} / \mathrm{m}^{3}$ \\
\hline FITWORX & YKFITWORX-G-38088 & $4 / 28 / 16$ & DUP-F & - & SOIL GAS & 46 & ND (9.8) & ND (17) & $\mu \mathrm{g} / \mathrm{m}^{3}$ \\
\hline DL1C & YKDL1C-108-W-38091 & $5 / 23 / 16$ & $\mathrm{~N}$ & $108-118$ & WG & 1.9 & ND (1) & ND (1) & $\mu \mathrm{g} / \mathrm{L}$ \\
\hline DL1C & YKDL1C-108-W-38091DUP & $5 / 23 / 16$ & DUP-L & 108-118 & WG & 1.7 & ND (1) & ND (1) & $\mu g / L$ \\
\hline DL2C2 & YKDL2C2-136-W-38096 & $5 / 31 / 16$ & $\mathrm{~N}$ & 136-146 & WG & 42 & 1.0 & ND (1) & $\mu \mathrm{g} / \mathrm{L}$ \\
\hline DL2C2 & YKDL2C2-136-W-38096DUP & $5 / 31 / 16$ & DUP-L & $136-146$ & WG & 39 & $0.9 \mathrm{~J}$ & ND (1) & $\mu g / L$ \\
\hline DL2CU & YKDL2CU-215-W-38097 & $5 / 31 / 16$ & $\mathrm{~N}$ & $215-225$ & WG & ND (1) & ND (1) & ND (1) & $\mu \mathrm{g} / \mathrm{L}$ \\
\hline DL2CU & YKDL2CU-215-DUP-W-38098 & $5 / 31 / 16$ & DUP-F & $215-225$ & WG & ND (1) & ND (1) & ND (1) & $\mu \mathrm{g} / \mathrm{L}$ \\
\hline DL2C1 & YKDL2-C1-112-W-38100 & $6 / 2 / 16$ & $\mathrm{~N}$ & $112-122$ & WG & 41 & $0.7 \mathrm{~J}$ & ND (1) & $\mu \mathrm{g} / \mathrm{L}$ \\
\hline
\end{tabular}




\begin{tabular}{|c|c|c|c|c|c|c|c|c|c|}
\hline DL2C1 & YKDL2-C1-112-W-38100DUP & $6 / 2 / 16$ & DUP-L & $112-122$ & WG & 39 & $0.7 \mathrm{~J}$ & ND (1) & $\mu \mathrm{g} / \mathrm{L}$ \\
\hline DL3C1 & YKDL3-C1-113-W-38103 & $6 / 6 / 16$ & $\mathrm{~N}$ & $113-123$ & WG & 39 & $0.7 \mathrm{~J}$ & ND (1) & $\mu \mathrm{g} / \mathrm{L}$ \\
\hline DL3C1 & YKDL3-C1-113-W-38103DUP & $6 / 6 / 16$ & DUP-L & $113-123$ & WG & 38 & $0.7 \mathrm{~J}$ & ND (1) & $\mu g / L$ \\
\hline DL8C1 & YKDL8C1-104-W-38106 & $6 / 7 / 16$ & $\mathrm{~N}$ & 104-114 & WG & 16 & ND (1) & ND (1) & $\mu g / L$ \\
\hline DL8C1 & YKDL8C1-104-W-38106DUP & $6 / 7 / 16$ & DUP-L & 104-114 & WG & 14 & ND (1) & ND (1) & $\mu \mathrm{g} / \mathrm{L}$ \\
\hline DL7CL & YKDL7-CL-265-W-38108 & $6 / 9 / 16$ & $\mathrm{~N}$ & $265-275$ & WG & ND (1) & ND (1) & ND (1) & $\mu \mathrm{g} / \mathrm{L}$ \\
\hline DL7CL & YKDL7-CL-265-W-38108DUP & $6 / 9 / 16$ & DUP-L & $265-275$ & WG & ND (1) & ND (1) & ND (1) & $\mu \mathrm{g} / \mathrm{L}$ \\
\hline DL7C & YKDL7-C-100-W-38111 & $6 / 12 / 16$ & $\mathrm{~N}$ & $100-110$ & WG & ND (1) & ND (1) & ND (1) & $\mu \mathrm{g} / \mathrm{L}$ \\
\hline DL7C & YKDL7-C-100-W-38111DUP & $6 / 12 / 16$ & DUP-L & $100-110$ & WG & ND (1) & ND (1) & ND (1) & $\mu \mathrm{g} / \mathrm{L}$ \\
\hline DL5C1 & YKDL5-C1-106-W-38118 & $6 / 21 / 16$ & $N$ & $106-116$ & WG & ND (1) & ND (1) & ND (1) & $\mu \mathrm{g} / \mathrm{L}$ \\
\hline DL5C1 & YKDL5-C1-106-W-38118DUP & $6 / 21 / 16$ & DUP-L & 106-116 & WG & ND (1) & ND (1) & ND (1) & $\mu \mathrm{g} / \mathrm{L}$ \\
\hline FITWORX & YKFITWORX-G-38148 & $8 / 10 / 16$ & $\mathrm{~N}$ & - & SOIL GAS & 20 & ND (9.8) & ND (17) & $\mu \mathrm{g} / \mathrm{m}^{3}$ \\
\hline FITWORX & YKFITWORX-G-38149 & $8 / 10 / 16$ & DUP-F & - & SOIL GAS & 24 & ND (9.8) & ND (17) & $\mu \mathrm{g} / \mathrm{m}^{3}$ \\
\hline FITWORX & YKFITWORX-G-38142 & $8 / 10 / 16$ & $\mathrm{~N}$ & - & AIR & 7.2 & 9.2 & ND (1.7) & $\mu \mathrm{g} / \mathrm{m}^{3}$ \\
\hline FITWORX & YKFITWORX-G-38143 & $8 / 10 / 16$ & DUP-F & - & AIR & 6.5 & 9.5 & ND (1.7) & $\mu \mathrm{g} / \mathrm{m}^{3}$ \\
\hline GARRETSON & YKGARRETSON-G-39533 & $12 / 14 / 16$ & $\mathrm{~N}$ & - & AIR & ND (1.3) & ND (0.98) & 4.3 & $\mu \mathrm{g} / \mathrm{m}^{3}$ \\
\hline GARRETSON & YKGARRETSON-G-39534 & $12 / 14 / 16$ & DUP-F & - & AIR & ND (1.3) & ND (0.98) & 2.1 & $\mu \mathrm{g} / \mathrm{m}^{3}$ \\
\hline FITWORX & YKFITWORX-G-39537 & $12 / 14 / 16$ & $\mathrm{~N}$ & - & AIR & 3.1 & 4.5 & ND (1.7) & $\mu \mathrm{g} / \mathrm{m}^{3}$ \\
\hline FITWORX & YKFITWORX-G-39538 & $12 / 14 / 16$ & DUP-F & - & AIR & 3.2 & 4.6 & $\mathrm{ND}(1.7)$ & $\mu \mathrm{g} / \mathrm{m}^{3}$ \\
\hline FITWORX & YKFITWORX-G-39543 & $12 / 14 / 16$ & $\mathrm{~N}$ & - & SOIL GAS & ND (13) & ND (9.8) & ND (17) & $\mu \mathrm{g} / \mathrm{m}^{3}$ \\
\hline FITWORX & YKFITWORX-G-39544 & $12 / 14 / 16$ & DUP-F & - & SOIL GAS & ND (13) & ND (9.8) & ND (17) & $\mu \mathrm{g} / \mathrm{m}^{3}$ \\
\hline
\end{tabular}


a Sample types: DUP-F, field replicate; DUP-L, duplicate lab analysis; N, primary sample.

b Matrix types: AIR, indoor air; SOIL, soil; SOIL GAS, soil gas; WG, groundwater; WQC, water quality control sample.

c ND, compound analyzed for but not detected at a level greater than or equal to the indicated method detection limit.

d $D$, result from analysis at secondary dilution.

e $\mathrm{J}$, compound identified with an estimated concentration between the instrument detection limit and the method detection limit. 
TABLE S2.5 Analytical results from the AGEM Laboratory and TestAmerica for samples submitted for verification organic analysis.

\begin{tabular}{|c|c|c|c|c|c|c|c|c|c|c|}
\hline \multirow[b]{2}{*}{ Location } & \multirow[b]{2}{*}{ Sample } & \multirow[b]{2}{*}{$\begin{array}{c}\text { Sample } \\
\text { Date }\end{array}$} & \multirow[b]{2}{*}{$\begin{array}{c}\text { Sample } \\
\text { Type }^{a}\end{array}$} & \multirow[b]{2}{*}{$\begin{array}{l}\text { Depth (ft } \\
\text { BGL) }\end{array}$} & \multirow[b]{2}{*}{ Matrix $^{b}$} & \multirow[b]{2}{*}{$\begin{array}{l}\text { Analytical } \\
\text { Laboratory }\end{array}$} & \multicolumn{3}{|c|}{ Concentration } & \multirow[b]{2}{*}{ Units } \\
\hline & & & & & & & $\begin{array}{c}\text { Carbon } \\
\text { Tetrachloride }\end{array}$ & Chloroform & $\begin{array}{c}\text { Methylene } \\
\text { Chloride }\end{array}$ & \\
\hline TIO2 & YKTI02-S-37764 & $7 / 7 / 15$ & $\mathrm{~N}$ & 4 & SOIL & AGEM & $N D^{c}(10)$ & ND (10) & ND (10) & $\mu g / k g$ \\
\hline TI02 & YKTI02-S-37764VER & $7 / 7 / 15$ & VER & 4 & SOIL & TestAmerica & $2.4 \mathrm{~J}^{\mathrm{d}}$ & ND (10) & $8.6 \mathrm{~J}$ & $\mu \mathrm{g} / \mathrm{kg}$ \\
\hline TIO2 & YKTI02-S-37767 & $7 / 7 / 15$ & $\mathrm{~N}$ & 16 & SOIL & AGEM & ND (10) & ND (10) & ND (10) & $\mu g / k g$ \\
\hline TIO2 & YKTI02-S-37767VER & $7 / 7 / 15$ & VER & 16 & SOIL & TestAmerica & 9.1 & $1.8 \mathrm{~J}$ & ND (8.9) & $\mu \mathrm{g} / \mathrm{kg}$ \\
\hline TIO2 & YKTI02-S-37768 & $7 / 7 / 15$ & $\mathrm{~N}$ & 20 & SOIL & AGEM & ND (10) & ND (10) & ND (10) & $\mu g / k g$ \\
\hline TIO2 & YKTI02-S-37768VER & $7 / 7 / 15$ & VER & 20 & SOIL & TestAmerica & $6.5 \mathrm{~J}$ & ND (10) & $5.6 \mathrm{~J}$ & $\mu \mathrm{g} / \mathrm{kg}$ \\
\hline TIO4 & YKTI04-S-37791 & $7 / 8 / 15$ & $\mathrm{~N}$ & 20 & SOIL & AGEM & 27 & $5.1 \mathrm{~J}$ & ND (10) & $\mu g / k g$ \\
\hline TIO4 & YKTI04-S-37791VER & $7 / 8 / 15$ & VER & 20 & SOIL & TestAmerica & 44 & $6.7 \mathrm{~J}$ & ND (9.5) & $\mu \mathrm{g} / \mathrm{kg}$ \\
\hline TIO4 & YKTI04-S-37805 & $7 / 8 / 15$ & $\mathrm{~N}$ & 68 & SOIL & AGEM & ND (10) & ND (10) & ND (10) & $\mu \mathrm{g} / \mathrm{kg}$ \\
\hline TIO4 & YKTI04-S-37805VER & $7 / 8 / 15$ & VER & 68 & SOIL & TestAmerica & ND (9.5) & ND (9.5) & ND (9.5) & $\mu \mathrm{g} / \mathrm{kg}$ \\
\hline TI03 & YKTI03-S-37814 & $7 / 9 / 15$ & $\mathrm{~N}$ & 28 & SOIL & AGEM & ND (10) & ND (10) & ND (10) & $\mu g / k g$ \\
\hline TI03 & YKTI03-S-37814VER & $7 / 9 / 15$ & VER & 28 & SOIL & TestAmerica & $3.9 \mathrm{~J}$ & ND (11) & $7.3 \mathrm{~J}$ & $\mu \mathrm{g} / \mathrm{kg}$ \\
\hline TI03 & YKTI03-S-37826 & $7 / 9 / 15$ & $\mathrm{~N}$ & 63.5 & SOIL & AGEM & ND (10) & ND (10) & ND (10) & $\mu \mathrm{g} / \mathrm{kg}$ \\
\hline TI03 & YKTI03-S-37826VER & $7 / 9 / 15$ & VER & 63.5 & SOIL & TestAmerica & ND (9.1) & ND (9.1) & ND (9.1) & $\mu \mathrm{g} / \mathrm{kg}$ \\
\hline TI01 & YKTI01-S-37758 & $7 / 10 / 15$ & $\mathrm{~N}$ & 56 & SOIL & AGEM & ND (10) & ND (10) & ND (10) & $\mu g / k g$ \\
\hline TI01 & YKTI01-S-37758VER & $7 / 10 / 15$ & VER & 56 & SOIL & TestAmerica & ND (9.2) & ND (9.2) & ND (9.2) & $\mu \mathrm{g} / \mathrm{kg}$ \\
\hline TIO2 & YKTI02120-W-37852 & $7 / 14 / 15$ & $\mathrm{~N}$ & $108.5-118.5$ & WG & AGEM & ND (1) & ND (1) & ND (1) & $\mu \mathrm{g} / \mathrm{L}$ \\
\hline TIO2 & YKTI02120-W-37852VER & $7 / 14 / 15$ & VER & 108.5-118.5 & WG & TestAmerica & $0.03 \mathrm{~J}$ & ND (1) & ND (1) & $\mu \mathrm{g} / \mathrm{L}$ \\
\hline TI01 & YKTI01120-W-37855 & $7 / 15 / 15$ & $\mathrm{~N}$ & $108-118$ & WG & AGEM & $0.9 \mathrm{~J}$ & 2.0 & ND (1) & $\mu \mathrm{g} / \mathrm{L}$ \\
\hline
\end{tabular}




\begin{tabular}{|c|c|c|c|c|c|c|c|c|c|c|}
\hline TI01 & YKTI01120-W-37855VER & $7 / 15 / 15$ & VER & $108-118$ & WG & TestAmerica & $0.4 \mathrm{~J}$ & 1.9 & ND (1) & $\mu \mathrm{g} / \mathrm{L}$ \\
\hline TI05 & YKTI0580-W-37868 & 7/18/15 & $\mathrm{N}$ & $76-81$ & WG & AGEM & $254 D^{e}$ & $69 \mathrm{D}$ & ND (1) D & $\mu \mathrm{g} / \mathrm{L}$ \\
\hline TI05 & YKTI0580-W-37868VER & $7 / 18 / 15$ & VER & $76-81$ & WG & TestAmerica & $180 \mathrm{D}$ & $62 \mathrm{D}$ & ND (2.8) & $\mu \mathrm{g} / \mathrm{L}$ \\
\hline TI06 & YKTI0680-W-37872 & 7/19/15 & $\mathrm{N}$ & $77-82$ & WG & AGEM & 7.0 & $0.4 \mathrm{~J}$ & ND (1) & $\mu \mathrm{g} / \mathrm{L}$ \\
\hline TI06 & YKTI0680-W-37872VER & 7/19/15 & VER & $77-82$ & WG & TestAmerica & 7.0 & $0.6 \mathrm{~J}$ & $1.0 \mathrm{~J}$ & $\mu \mathrm{g} / \mathrm{L}$ \\
\hline TI07 & YKTI07100-W-37876 & 7/20/15 & $\mathrm{N}$ & $90-100$ & WG & AGEM & ND (1) & ND (1) & ND (1) & $\mu \mathrm{g} / \mathrm{L}$ \\
\hline TI07 & YKTI07100-W-37876VER & $7 / 20 / 15$ & VER & $90-100$ & WG & TestAmerica & $0.4 \mathrm{~J}$ & $0.06 \mathrm{~J}$ & ND (1) & $\mu \mathrm{g} / \mathrm{L}$ \\
\hline TI08 & YKTI0885-W-37879 & $7 / 21 / 15$ & $\mathrm{~N}$ & $80-85$ & WG & AGEM & ND (1) & ND (1) & ND (1) & $\mu \mathrm{g} / \mathrm{L}$ \\
\hline TI08 & YKTI0885-W-37879VER & $7 / 21 / 15$ & VER & $80-85$ & WG & TestAmerica & $0.3 \mathrm{~J}$ & ND (1) & ND (1) & $\mu \mathrm{g} / \mathrm{L}$ \\
\hline DW38 & YKYORKCOUNTYSHOP-W-37917 & $8 / 5 / 15$ & $\mathrm{~N}$ & - & WG & AGEM & 53 & $0.8 \mathrm{~J}$ & ND (1) & $\mu \mathrm{g} / \mathrm{L}$ \\
\hline DW38 & YKYORKCOUNTYSHOP-W-37917VER & $8 / 5 / 15$ & VER & - & WG & TestAmerica & $52 \mathrm{D}$ & 1.1 & ND (1) & $\mu \mathrm{g} / \mathrm{L}$ \\
\hline SB62 & YKSB62-W-37924 & $8 / 7 / 15$ & $\mathrm{~N}$ & $118-123$ & WG & AGEM & 23 & 2.2 & ND (1) & $\mu \mathrm{g} / \mathrm{L}$ \\
\hline SB62 & YKSB62-W-37924VER & $8 / 7 / 15$ & VER & $118-123$ & WG & TestAmerica & $23 \mathrm{D}$ & 2.5 & ND (1) & $\mu \mathrm{g} / \mathrm{L}$ \\
\hline TI09 & YKTI09-135-W-37929 & 8/13/15 & $\mathrm{N}$ & 135-145 & WG & AGEM & 1.8 & ND (1) & ND (1) & $\mu \mathrm{g} / \mathrm{L}$ \\
\hline TI09 & YKTI09-135-W-37929VER & $8 / 13 / 15$ & VER & $135-145$ & WG & TestAmerica & 2.1 & ND (1) & ND (1) & $\mu \mathrm{g} / \mathrm{L}$ \\
\hline TI10 & YKTI10-117-W-37933 & $8 / 14 / 15$ & $\mathrm{~N}$ & $117-127$ & WG & AGEM & 29 & 1.1 & ND (1) & $\mu \mathrm{g} / \mathrm{L}$ \\
\hline TI10 & YKTI10-117-W-37933VER & $8 / 14 / 15$ & VER & $117-127$ & WG & TestAmerica & 22 & $0.9 \mathrm{~J}$ & ND (1) & $\mu \mathrm{g} / \mathrm{L}$ \\
\hline TI11 & YKTI11-92-W-37936 & 8/18/15 & $\mathrm{N}$ & $92-102$ & WG & AGEM & ND (1) & ND (1) & ND (1) & $\mu \mathrm{g} / \mathrm{L}$ \\
\hline TI11 & YKTI11-92-W-37936VER & $8 / 18 / 15$ & VER & $92-102$ & WG & TestAmerica & $0.06 \mathrm{~J}$ & ND (1) & ND (1) & $\mu \mathrm{g} / \mathrm{L}$ \\
\hline TI12 & YKTI12-145-W-37943 & 8/20/15 & $\mathrm{N}$ & $135-145$ & WG & AGEM & 73 & 1.5 & ND (1) & $\mu \mathrm{g} / \mathrm{L}$ \\
\hline TI12 & YKTI12-145-W-37943VER & $8 / 20 / 15$ & VER & $135-145$ & WG & TestAmerica & $55 \mathrm{D}$ & 1.2 & ND (1) & $\mu \mathrm{g} / \mathrm{L}$ \\
\hline TI14 & YKTI14-136-W-37954 & 8/23/15 & $\mathrm{N}$ & 136-146 & WG & AGEM & 59 & $0.8 \mathrm{~J}$ & ND (1) & $\mu \mathrm{g} / \mathrm{L}$ \\
\hline TI14 & YKTI14-136-W-37954VER & $8 / 23 / 15$ & VER & $136-146$ & WG & TestAmerica & $55 \mathrm{D}$ & $0.8 \mathrm{~J}$ & ND (1.1) & $\mu \mathrm{g} / \mathrm{L}$ \\
\hline
\end{tabular}




\begin{tabular}{|c|c|c|c|c|c|c|c|c|c|c|}
\hline TI16 & YKTI16-136-W-37964 & $8 / 25 / 15$ & $\mathrm{~N}$ & $136-146$ & WG & AGEM & 35 & $0.7 \mathrm{~J}$ & ND (1) & $\mu \mathrm{g} / \mathrm{L}$ \\
\hline TI16 & YKTI16-136-W-37964VER & $8 / 25 / 15$ & VER & $136-146$ & WG & TestAmerica & $33 \mathrm{D}$ & $0.6 \mathrm{~J}$ & ND (1) & $\mu \mathrm{g} / \mathrm{L}$ \\
\hline TI18 & YKTI18-114-W-37973 & $8 / 28 / 15$ & $\mathrm{~N}$ & $114-124$ & WG & AGEM & 2.9 & ND (1) & ND (1) & $\mu \mathrm{g} / \mathrm{L}$ \\
\hline TI18 & YKTI18-114-W-37973VER & $8 / 28 / 15$ & VER & $114-124$ & WG & TestAmerica & 2.2 & ND (1) & ND (1) & $\mu \mathrm{g} / \mathrm{L}$ \\
\hline TI20 & YKTI20-105-W-37981 & 9/10/15 & $\mathrm{N}$ & $105-115$ & WG & AGEM & 8.2 & ND (1) & ND (1) & $\mu \mathrm{g} / \mathrm{L}$ \\
\hline TI20 & YKTI20-105-W-37981VER & 9/10/15 & VER & 105-115 & WG & TestAmerica & 8.6 & ND (1) & ND (1) & $\mu \mathrm{g} / \mathrm{L}$ \\
\hline TI21 & YKTI21-99-W-37986 & 9/11/15 & $\mathrm{N}$ & $99-109$ & WG & AGEM & $0.8 \mathrm{~J}$ & ND (1) & ND (1) & $\mu \mathrm{g} / \mathrm{L}$ \\
\hline TI21 & YKTI21-99-W-37986VER & 9/11/15 & VER & $99-109$ & WG & TestAmerica & $0.6 \mathrm{~J}$ & ND (1.2) & ND (1.2) & $\mu \mathrm{g} / \mathrm{L}$ \\
\hline TI23 & YKTI23-120-W-37998 & 9/15/15 & $\mathrm{N}$ & $120-130$ & WG & AGEM & ND (1) & ND (1) & ND (1) & $\mu \mathrm{g} / \mathrm{L}$ \\
\hline $\mathrm{TI} 23$ & YKTI23-120-W-37998VER & $9 / 15 / 15$ & VER & $120-130$ & WG & TestAmerica & $0.08 \mathrm{~J}$ & ND (1) & ND (1) & $\mu \mathrm{g} / \mathrm{L}$ \\
\hline TI24 & YKTI24-100-W-38019 & $1 / 20 / 16$ & $\mathrm{~N}$ & $100-110$ & WG & AGEM & ND (1) & ND (1) & ND (1) & $\mu \mathrm{g} / \mathrm{L}$ \\
\hline TI24 & YKTI24-100-W-38019VER & $1 / 20 / 16$ & VER & $100-110$ & WG & TestAmerica & $0.5 \mathrm{~J}$ & ND (1) & ND (1) & $\mu \mathrm{g} / \mathrm{L}$ \\
\hline TI25 & YKTI25-108-W-38027 & $1 / 21 / 16$ & $\mathrm{~N}$ & $113-118$ & WG & AGEM & ND (1) & ND (1) & ND (1) & $\mu \mathrm{g} / \mathrm{L}$ \\
\hline TI25 & YKTI25-108-W-38027VER & $1 / 21 / 16$ & VER & $113-118$ & WG & TestAmerica & ND (1) & ND (1) & ND (1) & $\mu \mathrm{g} / \mathrm{L}$ \\
\hline TI28 & YKTI28-104-W-38078 & $3 / 3 / 16$ & $\mathrm{~N}$ & 104-109 & WG & AGEM & ND (1) & ND (1) & ND (1) & $\mu \mathrm{g} / \mathrm{L}$ \\
\hline TI28 & YKTI28-104-W-38078VER & $3 / 3 / 16$ & VER & 104-109 & WG & TestAmerica & $0.03 \mathrm{~J}$ & ND (1) & ND (1) & $\mu \mathrm{g} / \mathrm{L}$ \\
\hline DL1C & YKDL1C-108-W-38091 & $5 / 23 / 16$ & $\mathrm{~N}$ & 108-118 & WG & AGEM & 1.9 & ND (1) & ND (1) & $\mu \mathrm{g} / \mathrm{L}$ \\
\hline DL1C & YKDL1C-108-W-38091VER & $5 / 23 / 16$ & VER & $108-118$ & WG & TestAmerica & 2.0 & $0.4 \mathrm{~J}$ & $0.02 \mathrm{JB}^{\dagger}$ & $\mu \mathrm{g} / \mathrm{L}$ \\
\hline DL1B & YKDL1B-88-W-38093 & $5 / 24 / 16$ & $\mathrm{~N}$ & $88-98$ & WG & AGEM & 1.6 & ND (1) & ND (1) & $\mu \mathrm{g} / \mathrm{L}$ \\
\hline DL1B & YKDL1B-88-W-38093VER & $5 / 24 / 16$ & VER & $88-98$ & WG & TestAmerica & 2.0 & $0.3 \mathrm{~J}$ & ND (1) & $\mu g / L$ \\
\hline DL2C2 & YKDL2C2-136-W-38096 & $5 / 31 / 16$ & $\mathrm{~N}$ & $136-146$ & WG & AGEM & 42 & 1.0 & ND (1) & $\mu \mathrm{g} / \mathrm{L}$ \\
\hline DL2C2 & YKDL2C2-136-W-38096VER & $5 / 31 / 16$ & VER & $136-146$ & WG & TestAmerica & $47 \mathrm{D}$ & 1.1 & ND (1) & $\mu \mathrm{g} / \mathrm{L}$ \\
\hline
\end{tabular}




\begin{tabular}{|c|c|c|c|c|c|c|c|c|c|c|}
\hline DL2CU & YKDL2CU-215-W-38097 & $5 / 31 / 16$ & $\mathrm{~N}$ & $215-225$ & WG & AGEM & ND (1) & ND (1) & ND (1) & $\mu \mathrm{g} / \mathrm{L}$ \\
\hline DL2CU & YKDL2CU-215-W-38097VER & $5 / 31 / 16$ & VER & $215-225$ & WG & TestAmerica & $0.005 \mathrm{~J}$ & ND (1) & ND (1) & $\mu \mathrm{g} / \mathrm{L}$ \\
\hline DL7CL & YKDL7-CL-265-W-38108 & $6 / 9 / 16$ & $\mathrm{~N}$ & $265-275$ & WG & AGEM & ND (1) & ND (1) & ND (1) & $\mu \mathrm{g} / \mathrm{L}$ \\
\hline DL7CL & YKDL7-CL-265-W-38108VER & $6 / 9 / 16$ & VER & $265-275$ & WG & TestAmerica & ND (1) & ND (1) & ND (1) & $\mu g / L$ \\
\hline DL7CU & YKDL7-CU-192-W-38112 & $6 / 12 / 16$ & $\mathrm{~N}$ & $192-202$ & WG & AGEM & ND (1) & ND (1) & ND (1) & $\mu \mathrm{g} / \mathrm{L}$ \\
\hline DL7CU & YKDL7-CU-192-W-38112VER & $6 / 12 / 16$ & VER & $192-202$ & WG & TestAmerica & ND (1) & ND (1) & ND (1) & $\mu \mathrm{g} / \mathrm{L}$ \\
\hline DL6C & YKDL6-C-110-W-38113 & $6 / 13 / 16$ & $\mathrm{~N}$ & $110-120$ & WG & AGEM & ND (1) & ND (1) & ND (1) & $\mu \mathrm{g} / \mathrm{L}$ \\
\hline DL6C & YKDL6-C-110-W-38113VER & $6 / 13 / 16$ & VER & $110-120$ & WG & TestAmerica & $0.8 \mathrm{~J}$ & ND (1) & ND (1) & $\mu \mathrm{g} / \mathrm{L}$ \\
\hline DL4C2 & YKDL4-C2-145-W-38115 & $6 / 15 / 16$ & $\mathrm{~N}$ & $145-155$ & WG & AGEM & ND (1) & ND (1) & ND (1) & $\mu \mathrm{g} / \mathrm{L}$ \\
\hline DL4C2 & YKDL4-C2-145-W-38115VER & $6 / 15 / 16$ & VER & $145-155$ & WG & TestAmerica & $0.2 \mathrm{~J}$ & ND (1) & ND (1) & $\mu g / L$ \\
\hline DL4C1 & YKDL4-C1-120-W-38116 & $6 / 16 / 16$ & $\mathrm{~N}$ & $120-130$ & WG & AGEM & ND (1) & ND (1) & ND (1) & $\mu \mathrm{g} / \mathrm{L}$ \\
\hline DL4C1 & YKDL4-C1-120-W-38116VER & $6 / 16 / 16$ & VER & $120-130$ & WG & TestAmerica & $0.2 \mathrm{~J}$ & ND (1) & ND (1) & $\mu \mathrm{g} / \mathrm{L}$ \\
\hline
\end{tabular}

a Sample types: N, primary sample; TB, trip blank; VER, verification sample.

b Matrix types: SOIL, soil; WG, groundwater; WQC, quality control water sample.

c ND, compound analyzed for but not detected at a level greater than or equal to the indicated method detection limit (MDL).

d $\mathrm{J}$, compound identified with an estimated concentration between the instrument detection limit and the method detection limit.

e $D$, result from analysis at secondary dilution.

$f B$, compound detected in associated laboratory blank. 


\section{Supplement 3:}

\section{Waste Characterization Data}


June 21, 2016

Mr. Travis Kamler

TCW Construction Inc

$141 \mathrm{M}$ Street

Lincoln, NE 68508

RE: Project: Investigation Derived Waste

Pace Project No.: 60221061

Dear Mr. Kamler:

Enclosed are the analytical results for sample(s) received by the laboratory on June 10, 2016. The results relate only to the samples included in this report. Results reported herein conform to the most current TNI standards and the laboratory's Quality Assurance Manual, where applicable, unless otherwise noted in the body of the report.

If you have any questions concerning this report, please feel free to contact me.

Sincerely,

$$
\text { sudey stipaon }
$$

Trudy Gipson

trudy.gipson@pacelabs.com

Project Manager

\section{Enclosures}

cc: Ms. Esther Bowen, Argonne National Lab Mr. David Surgnier, Delta Environmental

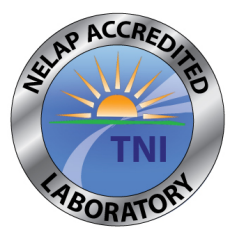




\section{CERTIFICATIONS}

Project: Investigation Derived Waste

Pace Project No.: 60221061

Kansas Certification IDs

9608 Loiret Boulevard, Lenexa, KS 66219

WY STR Certification \#: 2456.01

Arkansas Certification \#: 15-016-0

Illinois Certification \#: 003097

lowa Certification \#: 118

Kansas/NELAP Certification \#: E-10116
Louisiana Certification \#: 03055

Nevada Certification \#: KS000212008A

Oklahoma Certification \#: 9205/9935

Texas Certification \#: T104704407

Utah Certification \#: KS00021

Kansas Field Laboratory Accreditation: \# E-92587

\section{REPORT OF LABORATORY ANALYSIS}


SAMPLE SUMMARY

Project: Investigation Derived Waste

Pace Project No.: 60221061

\begin{tabular}{|c|c|c|c|c|}
\hline Lab ID & Sample ID & Matrix & Date Collected & Date Received \\
\hline 60221061001 & YKIDW-W-38109 & Water & 06/09/16 17:20 & 06/10/16 08:20 \\
\hline 60221061002 & YKQCTB-W-38110 & Water & 06/09/16 17:30 & 06/10/16 08:20 \\
\hline
\end{tabular}




\section{SAMPLE ANALYTE COUNT}

Project: Investigation Derived Waste

Pace Project No.: 60221061

\begin{tabular}{|c|c|c|c|c|}
\hline Lab ID & Sample ID & Method & Analysts & $\begin{array}{l}\text { Analytes } \\
\text { Reported }\end{array}$ \\
\hline \multirow[t]{3}{*}{60221061001} & YKIDW-W-38109 & EPA 504.1 & ACW & 1 \\
\hline & & EPA 5030B/8260 & PGH & 69 \\
\hline & & EPA 353.2 & CRS & 1 \\
\hline 60221061002 & YKQCTB-W-38110 & EPA 5030B/8260 & PGH & 69 \\
\hline
\end{tabular}

\section{REPORT OF LABORATORY ANALYSIS}




\section{ANALYTICAL RESULTS}

Project: Investigation Derived Waste

Pace Project No.: 60221061

\begin{tabular}{|c|c|c|c|c|c|c|c|c|}
\hline Sample: YKIDW-W-38109 & Lab ID: & 60221061001 & Collected: $06 / 09$ & $17: 20$ & Received: & 06/10/16 08:20 & Matrix: Water & \\
\hline Parameters & Results & Units & Report Limit & DF & Prepared & Analyzed & CAS No. & Qual \\
\hline
\end{tabular}

504 GCS EDB and DBCP

1,2-Dibromoethane (EDB)

\section{MSV}

Acetone

Benzene

Bromobenzene

Bromochloromethane

Bromodichloromethane

Bromoform

Bromomethane

2-Butanone (MEK)

n-Butylbenzene

sec-Butylbenzene

tert-Butylbenzene

Carbon disulfide

Carbon tetrachloride

Chlorobenzene

Chloroethane

Chloroform

Chloromethane

2-Chlorotoluene

4-Chlorotoluene

1,2-Dibromo-3-chloropropane

Dibromochloromethane

1,2-Dibromoethane (EDB)

Dibromomethane

1,2-Dichlorobenzene

1,3-Dichlorobenzene

1,4-Dichlorobenzene

Dichlorodifluoromethane

1,1-Dichloroethane

1,2-Dichloroethane

1,2-Dichloroethene (Total)

1,1-Dichloroethene

cis-1,2-Dichloroethene

trans-1,2-Dichloroethene

1,2-Dichloropropane

1,3-Dichloropropane

2,2-Dichloropropane

1,1-Dichloropropene

cis-1,3-Dichloropropene

trans-1,3-Dichloropropene

Ethylbenzene

Hexachloro-1,3-butadiene

2-Hexanone

Isopropylbenzene (Cumene)

p-Isopropyltoluene
Analytical Method: EPA 504.1 Preparation Method: EPA 504.1

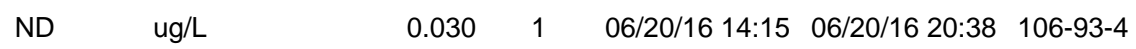

Analytical Method: EPA 5030B/8260

\begin{tabular}{|c|c|c|c|c|c|}
\hline ND & ug/L & 10.0 & 1 & 06/14/16 13:33 & $67-64-1$ \\
\hline ND & ug/L & 1.0 & 1 & 06/14/16 13:33 & $71-43-2$ \\
\hline ND & ug/L & 1.0 & 1 & 06/14/16 13:33 & $108-86-1$ \\
\hline ND & ug/L & 1.0 & 1 & 06/14/16 13:33 & $74-97-5$ \\
\hline ND & ug/L & 1.0 & 1 & 06/14/16 13:33 & $75-27-4$ \\
\hline ND & $u g / L$ & 1.0 & 1 & 06/14/16 13:33 & $75-25-2$ \\
\hline ND & ug/L & 5.0 & 1 & 06/14/16 13:33 & $74-83-9$ \\
\hline ND & ug/L & 10.0 & 1 & 06/14/16 13:33 & $78-93-3$ \\
\hline ND & $\mathrm{ug} / \mathrm{L}$ & 1.0 & 1 & 06/14/16 13:33 & $104-51-8$ \\
\hline ND & ug/L & 1.0 & 1 & 06/14/16 13:33 & $135-98-8$ \\
\hline ND & ug/L & 1.0 & 1 & 06/14/16 13:33 & $98-06-6$ \\
\hline ND & $u g / L$ & 5.0 & 1 & 06/14/16 13:33 & $75-15-0$ \\
\hline 2.7 & ug/L & 1.0 & 1 & 06/14/16 13:33 & $56-23-5$ \\
\hline ND & ug/L & 1.0 & 1 & 06/14/16 13:33 & $108-90-7$ \\
\hline ND & $\mathrm{ug} / \mathrm{L}$ & 1.0 & 1 & 06/14/16 13:33 & $75-00-3$ \\
\hline ND & ug/L & 1.0 & 1 & 06/14/16 13:33 & $67-66-3$ \\
\hline ND & ug/L & 1.0 & 1 & 06/14/16 13:33 & $74-87-3$ \\
\hline ND & ug/L & 1.0 & 1 & $06 / 14 / 16$ 13:33 & $95-49-8$ \\
\hline ND & ug/L & 1.0 & 1 & 06/14/16 13:33 & $106-43-4$ \\
\hline ND & $\mathrm{ug} / \mathrm{L}$ & 2.5 & 1 & 06/14/16 13:33 & $96-12-8$ \\
\hline ND & $\mathrm{ug} / \mathrm{L}$ & 1.0 & 1 & $06 / 14 / 1613: 33$ & $124-48-1$ \\
\hline ND & $u g / L$ & 1.0 & 1 & 06/14/16 13:33 & $106-93-4$ \\
\hline ND & ug/L & 1.0 & 1 & 06/14/16 13:33 & $74-95-3$ \\
\hline ND & ug/L & 1.0 & 1 & $06 / 14 / 1613: 33$ & $95-50-1$ \\
\hline ND & $\mathrm{ug} / \mathrm{L}$ & 1.0 & 1 & 06/14/16 13:33 & 541-73-1 \\
\hline ND & ug/L & 1.0 & 1 & 06/14/16 13:33 & $106-46-7$ \\
\hline ND & ug/L & 1.0 & 1 & 06/14/16 13:33 & $75-71-8$ \\
\hline ND & $\mathrm{ug} / \mathrm{L}$ & 1.0 & 1 & $06 / 14 / 16$ 13:33 & $75-34-3$ \\
\hline ND & $\mathrm{ug} / \mathrm{L}$ & 1.0 & 1 & 06/14/16 13:33 & $107-06-2$ \\
\hline ND & ug/L & 1.0 & 1 & $06 / 14 / 1613: 33$ & $540-59-0$ \\
\hline ND & $\mathrm{ug} / \mathrm{L}$ & 1.0 & 1 & 06/14/16 13:33 & $75-35-4$ \\
\hline ND & ug/L & 1.0 & 1 & 06/14/16 13:33 & $156-59-2$ \\
\hline ND & ug/L & 1.0 & 1 & 06/14/16 13:33 & $156-60-5$ \\
\hline ND & ug/L & 1.0 & 1 & $06 / 14 / 16$ 13:33 & $78-87-5$ \\
\hline ND & ug/L & 1.0 & 1 & $06 / 14 / 1613: 33$ & $142-28-9$ \\
\hline ND & ug/L & 1.0 & 1 & $06 / 14 / 1613: 33$ & $594-20-7$ \\
\hline ND & $\mathrm{ug} / \mathrm{L}$ & 1.0 & 1 & $06 / 14 / 1613: 33$ & $563-58-6$ \\
\hline ND & $u g / L$ & 1.0 & 1 & $06 / 14 / 1613: 33$ & $10061-01-5$ \\
\hline ND & $\mathrm{ug} / \mathrm{L}$ & 1.0 & 1 & $06 / 14 / 1613: 33$ & $10061-02-6$ \\
\hline ND & $\mathrm{ug} / \mathrm{L}$ & 1.0 & 1 & $06 / 14 / 1613: 33$ & $100-41-4$ \\
\hline ND & $\mathrm{ug} / \mathrm{L}$ & 1.0 & 1 & 06/14/16 13:33 & $87-68-3$ \\
\hline ND & ug/L & 10.0 & 1 & $06 / 14 / 16$ 13:33 & $591-78-6$ \\
\hline ND & ug/L & 1.0 & 1 & $06 / 14 / 1613: 33$ & $98-82-8$ \\
\hline ND & ug/L & 1.0 & 1 & $06 / 14 / 1613: 33$ & $99-87-6$ \\
\hline
\end{tabular}

\section{REPORT OF LABORATORY ANALYSIS}

This report shall not be reproduced, except in full,

without the written consent of Pace Analytical Services, Inc.. 


\section{ANALYTICAL RESULTS}

Project: Investigation Derived Waste

Pace Project No.: 60221061

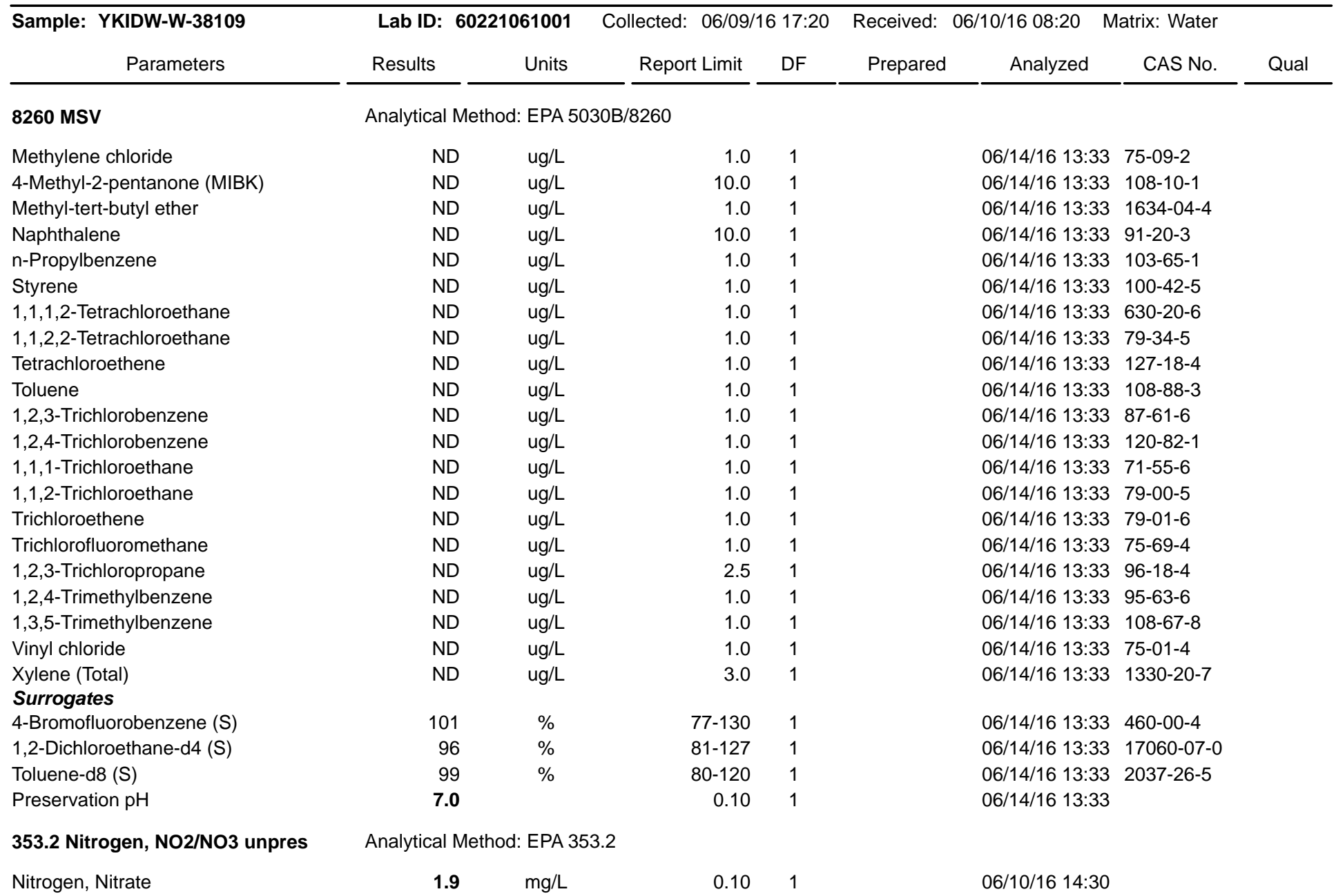

\section{REPORT OF LABORATORY ANALYSIS}




\section{ANALYTICAL RESULTS}

Project: Investigation Derived Waste

Pace Project No.: 60221061

\begin{tabular}{|c|c|c|c|c|c|c|c|c|}
\hline \multirow{2}{*}{$\begin{array}{c}\text { Sample: YKQCTB-W-38110 } \\
\text { Parameters }\end{array}$} & \multicolumn{2}{|c|}{ Lab ID: 60221061002} & \multicolumn{2}{|c|}{ Collected: $\quad$ 06/09/16 17:30 } & \multirow{2}{*}{$\begin{array}{l}\text { Received: } \\
\text { Prepared }\end{array}$} & \multirow{2}{*}{$\begin{array}{r}\text { 06/10/16 08:20 } \\
\text { Analyzed }\end{array}$} & \multirow{2}{*}{$\begin{array}{l}\text { Matrix: Water } \\
\text { CAS No. }\end{array}$} & \multirow[b]{2}{*}{ Qual } \\
\hline & Results & Units & Report Limit & DF & & & & \\
\hline 8260 MSV & Analytical M & : EPA 50 & $30 \mathrm{~B} / 8260$ & & & & & \\
\hline Acetone & ND & $u g / L$ & 10.0 & 1 & & $06 / 14 / 1612: 50$ & 67-64-1 & \\
\hline Benzene & ND & ug/L & 1.0 & 1 & & $06 / 14 / 1612: 50$ & $71-43-2$ & \\
\hline Bromobenzene & ND & $u g / L$ & 1.0 & 1 & & $06 / 14 / 1612: 50$ & 108-86-1 & \\
\hline Bromochloromethane & ND & $u g / L$ & 1.0 & 1 & & $06 / 14 / 1612: 50$ & $74-97-5$ & \\
\hline Bromodichloromethane & ND & $u g / L$ & 1.0 & 1 & & $06 / 14 / 1612: 50$ & 75-27-4 & \\
\hline Bromoform & ND & ug/L & 1.0 & 1 & & $06 / 14 / 1612: 50$ & $75-25-2$ & \\
\hline Bromomethane & ND & $\mathrm{ug} / \mathrm{L}$ & 5.0 & 1 & & $06 / 14 / 1612: 50$ & 74-83-9 & \\
\hline 2-Butanone (MEK) & ND & ug/L & 10.0 & 1 & & $06 / 14 / 1612: 50$ & 78-93-3 & \\
\hline n-Butylbenzene & ND & ug/L & 1.0 & 1 & & $06 / 14 / 1612: 50$ & $104-51-8$ & \\
\hline sec-Butylbenzene & ND & $\mathrm{ug} / \mathrm{L}$ & 1.0 & 1 & & $06 / 14 / 1612: 50$ & $135-98-8$ & \\
\hline tert-Butylbenzene & ND & $u g / L$ & 1.0 & 1 & & $06 / 14 / 1612: 50$ & $98-06-6$ & \\
\hline Carbon disulfide & ND & ug/L & 5.0 & 1 & & $06 / 14 / 1612: 50$ & $75-15-0$ & \\
\hline Carbon tetrachloride & ND & $\mathrm{ug} / \mathrm{L}$ & 1.0 & 1 & & $06 / 14 / 1612: 50$ & $56-23-5$ & \\
\hline Chlorobenzene & ND & ug/L & 1.0 & 1 & & $06 / 14 / 1612: 50$ & $108-90-7$ & \\
\hline Chloroethane & ND & $u g / L$ & 1.0 & 1 & & $06 / 14 / 1612: 50$ & $75-00-3$ & \\
\hline Chloroform & ND & $\mathrm{ug} / \mathrm{L}$ & 1.0 & 1 & & $06 / 14 / 1612: 50$ & $67-66-3$ & \\
\hline Chloromethane & ND & ug/L & 1.0 & 1 & & $06 / 14 / 1612: 50$ & $74-87-3$ & \\
\hline 2-Chlorotoluene & ND & ug/L & 1.0 & 1 & & $06 / 14 / 1612: 50$ & $95-49-8$ & \\
\hline 4-Chlorotoluene & ND & $u g / L$ & 1.0 & 1 & & $06 / 14 / 1612: 50$ & $106-43-4$ & \\
\hline 1,2-Dibromo-3-chloropropane & ND & $u g / L$ & 2.5 & 1 & & $06 / 14 / 1612: 50$ & $96-12-8$ & \\
\hline Dibromochloromethane & ND & $u g / L$ & 1.0 & 1 & & $06 / 14 / 1612: 50$ & $124-48-1$ & \\
\hline 1,2-Dibromoethane (EDB) & ND & ug/L & 1.0 & 1 & & $06 / 14 / 1612: 50$ & $106-93-4$ & \\
\hline Dibromomethane & ND & $\mathrm{ug} / \mathrm{L}$ & 1.0 & 1 & & $06 / 14 / 1612: 50$ & $74-95-3$ & \\
\hline 1,2-Dichlorobenzene & ND & ug/L & 1.0 & 1 & & $06 / 14 / 1612: 50$ & $95-50-1$ & \\
\hline 1,3-Dichlorobenzene & ND & ug/L & 1.0 & 1 & & $06 / 14 / 1612: 50$ & $541-73-1$ & \\
\hline 1,4-Dichlorobenzene & ND & $\mathrm{ug} / \mathrm{L}$ & 1.0 & 1 & & $06 / 14 / 1612: 50$ & $106-46-7$ & \\
\hline Dichlorodifluoromethane & ND & $u g / L$ & 1.0 & 1 & & $06 / 14 / 1612: 50$ & $75-71-8$ & \\
\hline 1,1-Dichloroethane & ND & ug/L & 1.0 & 1 & & $06 / 14 / 1612: 50$ & $75-34-3$ & \\
\hline 1,2-Dichloroethane & ND & $\mathrm{ug} / \mathrm{L}$ & 1.0 & 1 & & $06 / 14 / 1612: 50$ & $107-06-2$ & \\
\hline 1,2-Dichloroethene (Total) & ND & ug/L & 1.0 & 1 & & $06 / 14 / 1612: 50$ & $540-59-0$ & \\
\hline 1,1-Dichloroethene & ND & $u g / L$ & 1.0 & 1 & & $06 / 14 / 1612: 50$ & $75-35-4$ & \\
\hline cis-1,2-Dichloroethene & ND & $\mathrm{ug} / \mathrm{L}$ & 1.0 & 1 & & $06 / 14 / 1612: 50$ & $156-59-2$ & \\
\hline trans-1,2-Dichloroethene & ND & ug/L & 1.0 & 1 & & $06 / 14 / 1612: 50$ & $156-60-5$ & \\
\hline 1,2-Dichloropropane & ND & ug/L & 1.0 & 1 & & $06 / 14 / 1612: 50$ & $78-87-5$ & \\
\hline 1,3-Dichloropropane & ND & $\mathrm{ug} / \mathrm{L}$ & 1.0 & 1 & & $06 / 14 / 1612: 50$ & $142-28-9$ & \\
\hline 2,2-Dichloropropane & ND & ug/L & 1.0 & 1 & & $06 / 14 / 1612: 50$ & $594-20-7$ & \\
\hline 1,1-Dichloropropene & ND & $u g / L$ & 1.0 & 1 & & $06 / 14 / 1612: 50$ & $563-58-6$ & \\
\hline cis-1,3-Dichloropropene & ND & ug/L & 1.0 & 1 & & $06 / 14 / 1612: 50$ & $10061-01-5$ & \\
\hline trans-1,3-Dichloropropene & ND & $u g / L$ & 1.0 & 1 & & $06 / 14 / 1612: 50$ & $10061-02-6$ & \\
\hline Ethylbenzene & ND & ug/L & 1.0 & 1 & & $06 / 14 / 1612: 50$ & $100-41-4$ & \\
\hline Hexachloro-1,3-butadiene & ND & ug/L & 1.0 & 1 & & $06 / 14 / 1612: 50$ & $87-68-3$ & \\
\hline 2-Hexanone & ND & $\mathrm{ug} / \mathrm{L}$ & 10.0 & 1 & & $06 / 14 / 1612: 50$ & $591-78-6$ & \\
\hline Isopropylbenzene (Cumene) & ND & $u g / L$ & 1.0 & 1 & & $06 / 14 / 1612: 50$ & $98-82-8$ & \\
\hline p-Isopropyltoluene & ND & ug/L & 1.0 & 1 & & $06 / 14 / 1612: 50$ & $99-87-6$ & \\
\hline Methylene chloride & ND & $\mathrm{ug} / \mathrm{L}$ & 1.0 & 1 & & $06 / 14 / 1612: 50$ & $75-09-2$ & \\
\hline 4-Methyl-2-pentanone (MIBK) & ND & ug/L & 10.0 & 1 & & $06 / 14 / 1612: 50$ & $108-10-1$ & \\
\hline Methyl-tert-butyl ether & ND & $\mathrm{ug} / \mathrm{L}$ & 1.0 & 1 & & 06/14/16 12:50 & $1634-04-4$ & \\
\hline
\end{tabular}

\section{REPORT OF LABORATORY ANALYSIS}




\section{ANALYTICAL RESULTS}

Project: Investigation Derived Waste

Pace Project No.: 60221061

\begin{tabular}{|c|c|c|c|c|c|c|c|c|}
\hline \multirow{2}{*}{$\begin{array}{c}\text { Sample: YKQCTB-W-38110 } \\
\text { Parameters }\end{array}$} & \multicolumn{2}{|c|}{ Lab ID: 60221061002} & \multicolumn{2}{|c|}{ Collected: $06 / 09 / 16$ 17:30 } & \multirow{2}{*}{$\begin{array}{l}\text { Received: } \\
\text { Prepared }\end{array}$} & $06 / 10 / 1608: 20$ & \multicolumn{2}{|l|}{ Matrix: Water } \\
\hline & Results & Units & Report Limit & DF & & Analyzed & CAS No. & Qual \\
\hline 8260 MSV & \multicolumn{7}{|c|}{ Analytical Method: EPA 5030B/8260 } & \\
\hline Naphthalene & ND & $\mathrm{ug} / \mathrm{L}$ & 10.0 & 1 & & $06 / 14 / 1612: 50$ & $91-20-3$ & \\
\hline n-Propylbenzene & ND & $\mathrm{ug} / \mathrm{L}$ & 1.0 & 1 & & $06 / 14 / 1612: 50$ & $103-65-1$ & \\
\hline Styrene & ND & $\mathrm{ug} / \mathrm{L}$ & 1.0 & 1 & & $06 / 14 / 1612: 50$ & $100-42-5$ & \\
\hline 1,1,1,2-Tetrachloroethane & ND & $u g / L$ & 1.0 & 1 & & $06 / 14 / 1612: 50$ & $630-20-6$ & \\
\hline 1,1,2,2-Tetrachloroethane & ND & $\mathrm{ug} / \mathrm{L}$ & 1.0 & 1 & & $06 / 14 / 1612: 50$ & $79-34-5$ & \\
\hline Tetrachloroethene & ND & $\mathrm{ug} / \mathrm{L}$ & 1.0 & 1 & & $06 / 14 / 1612: 50$ & $127-18-4$ & \\
\hline Toluene & ND & $u g / L$ & 1.0 & 1 & & $06 / 14 / 1612: 50$ & $108-88-3$ & \\
\hline 1,2,3-Trichlorobenzene & ND & $\mathrm{ug} / \mathrm{L}$ & 1.0 & 1 & & $06 / 14 / 16 \quad 12: 50$ & $87-61-6$ & \\
\hline 1,2,4-Trichlorobenzene & ND & $\mathrm{ug} / \mathrm{L}$ & 1.0 & 1 & & $06 / 14 / 1612: 50$ & $120-82-1$ & \\
\hline 1,1,1-Trichloroethane & ND & $\mathrm{ug} / \mathrm{L}$ & 1.0 & 1 & & $06 / 14 / 1612: 50$ & $71-55-6$ & \\
\hline 1,1,2-Trichloroethane & ND & $\mathrm{ug} / \mathrm{L}$ & 1.0 & 1 & & $06 / 14 / 1612: 50$ & $79-00-5$ & \\
\hline Trichloroethene & ND & $\mathrm{ug} / \mathrm{L}$ & 1.0 & 1 & & $06 / 14 / 1612: 50$ & $79-01-6$ & \\
\hline Trichlorofluoromethane & ND & $\mathrm{ug} / \mathrm{L}$ & 1.0 & 1 & & $06 / 14 / 1612: 50$ & $75-69-4$ & \\
\hline 1,2,3-Trichloropropane & ND & ug/L & 2.5 & 1 & & $06 / 14 / 1612: 50$ & $96-18-4$ & \\
\hline $1,2,4$-Trimethylbenzene & ND & $\mathrm{ug} / \mathrm{L}$ & 1.0 & 1 & & $06 / 14 / 16 \quad 12: 50$ & $95-63-6$ & \\
\hline 1,3,5-Trimethylbenzene & ND & $\mathrm{ug} / \mathrm{L}$ & 1.0 & 1 & & $06 / 14 / 1612: 50$ & $108-67-8$ & \\
\hline Vinyl chloride & ND & ug/L & 1.0 & 1 & & $06 / 14 / 1612: 50$ & $75-01-4$ & \\
\hline Xylene (Total) & ND & $\mathrm{ug} / \mathrm{L}$ & 3.0 & 1 & & $06 / 14 / 1612: 50$ & $1330-20-7$ & \\
\hline \multicolumn{9}{|l|}{ Surrogates } \\
\hline 4-Bromofluorobenzene (S) & 99 & $\%$ & $77-130$ & 1 & & $06 / 14 / 1612: 50$ & $460-00-4$ & \\
\hline 1,2-Dichloroethane-d4 (S) & 96 & $\%$ & $81-127$ & 1 & & $06 / 14 / 1612: 50$ & $17060-07-0$ & \\
\hline Toluene-d8 (S) & 97 & $\%$ & $80-120$ & 1 & & $06 / 14 / 1612: 50$ & $2037-26-5$ & \\
\hline Preservation $\mathrm{pH}$ & 7.0 & & 0.10 & 1 & & $06 / 14 / 1612: 50$ & & \\
\hline
\end{tabular}

\section{REPORT OF LABORATORY ANALYSIS}




\section{QUALITY CONTROL DATA}

Project: Investigation Derived Waste

Pace Project No.: 60221061

\begin{tabular}{llll}
\hline QC Batch: & MSV/76405 & Analysis Method: & EPA 5030B/8260 \\
QC Batch Method: & EPA 5030B/8260 & Analysis Description: & 8260 MSV Water 7 day
\end{tabular}

Associated Lab Samples: $\quad$ 60221061001, 60221061002

METHOD BLANK: 1776035

Associated Lab Samples: $\quad$ 60221061001, 60221061002

$$
\text { Parameter }
$$

1,1,1,2-Tetrachloroethane

1,1,1-Trichloroethane

1,1,2,2-Tetrachloroethane

1,1,2-Trichloroethane

1,1-Dichloroethane

1,1-Dichloroethene

1,1-Dichloropropene

1,2,3-Trichlorobenzene

1,2,3-Trichloropropane

1,2,4-Trichlorobenzene

1,2,4-Trimethylbenzene

1,2-Dibromo-3-chloropropane

1,2-Dibromoethane (EDB)

1,2-Dichlorobenzene

1,2-Dichloroethane

1,2-Dichloroethene (Total)

1,2-Dichloropropane

1,3,5-Trimethylbenzene

1,3-Dichlorobenzene

1,3-Dichloropropane

1,4-Dichlorobenzene

2,2-Dichloropropane

2-Butanone (MEK)

2-Chlorotoluene

2-Hexanone

4-Chlorotoluene

4-Methyl-2-pentanone (MIBK)

Acetone

Benzene

Bromobenzene

Bromochloromethane

Bromodichloromethane

Bromoform

Bromomethane

Carbon disulfide

Carbon tetrachloride

Chlorobenzene

Chloroethane

Chloroform

Chloromethane

cis-1,2-Dichloroethene
Matrix: Water

Units $\quad$ Result

ug/L

ug/L

$\mathrm{ug} / \mathrm{L}$

ug/L

ug/L

ug/L

ug/L

$u g / L$

ug/L

$u g / L$

$u g / L$

ug/L

ug/L

$\mathrm{ug} / \mathrm{L}$

$u g / L$

ug/L

ug/L

ug/L

ug/L

ug/L

ug/L

ug/L

ug/L

ug/L

ug/L

ug/L

ug/L

ug/L

ug/L

ug/L

ug/L

ug/L

ug/L

ug/L

ug/L

ug/L

ug/L

ug/L

ug/L

ug/L

ug/L
Reporting

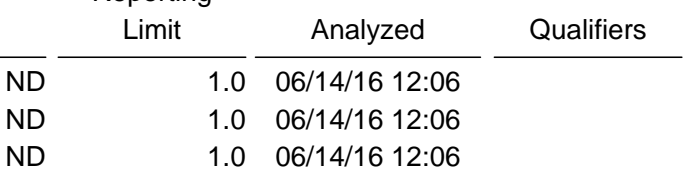

$1.0 \quad 06 / 14 / 1612 \cdot 06$

$1.0 \quad 06 / 14 / 1612: 06$

$1.0 \quad 06 / 14 / 16 \quad 12: 06$

$1.0 \quad 06 / 14 / 16 \quad 12: 06$

$1.0 \quad 06 / 14 / 16 \quad 12: 06$

$2.506 / 14 / 16$ 12:06

$1.0 \quad 06 / 14 / 16 \quad 12: 06$

$1.0 \quad 06 / 14 / 1612: 06$

$2.506 / 14 / 1612: 06$

$1.0 \quad 06 / 14 / 16 \quad 12: 06$

$1.0 \quad 06 / 14 / 16$ 12:06

$1.0 \quad 06 / 14 / 16 \quad 12: 06$

$1.0 \quad 06 / 14 / 1612: 06$

$1.0 \quad 06 / 14 / 1612: 06$

$1.0 \quad 06 / 14 / 1612: 06$

$1.0 \quad 06 / 14 / 16$ 12:06

$1.0 \quad 06 / 14 / 1612: 06$

$1.0 \quad 06 / 14 / 16 \quad 12: 06$

$1.0 \quad 06 / 14 / 1612: 06$

$10.0 \quad 06 / 14 / 1612: 06$

$1.0 \quad 06 / 14 / 1612: 06$

$10.0 \quad 06 / 14 / 1612: 06$

$1.0 \quad 06 / 14 / 16 \quad 12: 06$

$10.0 \quad 06 / 14 / 16$ 12:06

$10.0 \quad 06 / 14 / 1612: 06$

$1.0 \quad 06 / 14 / 1612: 06$

$1.0 \quad 06 / 14 / 16$ 12:06

$1.0 \quad 06 / 14 / 1612: 06$

$1.0 \quad 06 / 14 / 16 \quad 12: 06$

$1.0 \quad 06 / 14 / 1612: 06$

$5.0 \quad 06 / 14 / 1612: 06$

$5.0 \quad 06 / 14 / 1612: 06$

$1.0 \quad 06 / 14 / 1612: 06$

$1.0 \quad 06 / 14 / 1612: 06$

$1.0 \quad 06 / 14 / 16$ 12:06

$1.0 \quad 06 / 14 / 1612: 06$

$1.0 \quad 06 / 14 / 16$ 12:06

$1.0 \quad 06 / 14 / 16 \quad 12: 06$

Results presented on this page are in the units indicated by the "Units" column except where an alternate unit is presented to the right of the result.

\section{REPORT OF LABORATORY ANALYSIS}




\section{QUALITY CONTROL DATA}

Project: Investigation Derived Waste

Pace Project No.: 60221061

\begin{tabular}{|c|c|c|c|c|c|}
\hline \multicolumn{2}{|l|}{ METHOD BLANK: 1776035} & \multicolumn{2}{|c|}{ Matrix: Water } & \multirow[b]{3}{*}{ Analyzed } & \multirow[b]{3}{*}{ Qualifiers } \\
\hline Associated Lab Samples: & 60221061001,60221061002 & & & & \\
\hline Parameter & Units & $\begin{array}{l}\text { Blank } \\
\text { Result }\end{array}$ & $\begin{array}{l}\text { Reporting } \\
\text { Limit }\end{array}$ & & \\
\hline cis-1,3-Dichloropropene & $\mathrm{ug} / \mathrm{L}$ & ND & 1.0 & $06 / 14 / 1612: 06$ & \\
\hline Dibromochloromethane & $\mathrm{ug} / \mathrm{L}$ & ND & 1.0 & 06/14/16 12:06 & \\
\hline Dibromomethane & $u g / L$ & ND & 1.0 & $06 / 14 / 1612: 06$ & \\
\hline Dichlorodifluoromethane & $\mathrm{ug} / \mathrm{L}$ & ND & 1.0 & $06 / 14 / 1612: 06$ & \\
\hline Ethylbenzene & $\mathrm{ug} / \mathrm{L}$ & ND & 1.0 & 06/14/16 12:06 & \\
\hline Hexachloro-1,3-butadiene & $u g / L$ & ND & 1.0 & $06 / 14 / 1612: 06$ & \\
\hline Isopropylbenzene (Cumene) & $\mathrm{ug} / \mathrm{L}$ & ND & 1.0 & $06 / 14 / 1612: 06$ & \\
\hline Methyl-tert-butyl ether & $\mathrm{ug} / \mathrm{L}$ & ND & 1.0 & 06/14/16 12:06 & \\
\hline Methylene chloride & $u g / L$ & ND & 1.0 & 06/14/16 12:06 & \\
\hline n-Butylbenzene & $\mathrm{ug} / \mathrm{L}$ & ND & 1.0 & $06 / 14 / 1612: 06$ & \\
\hline n-Propylbenzene & $\mathrm{ug} / \mathrm{L}$ & ND & 1.0 & $06 / 14 / 1612: 06$ & \\
\hline Naphthalene & $u g / L$ & ND & 10.0 & 06/14/16 12:06 & \\
\hline p-Isopropyltoluene & $u g / L$ & ND & 1.0 & $06 / 14 / 1612: 06$ & \\
\hline sec-Butylbenzene & $\mathrm{ug} / \mathrm{L}$ & ND & 1.0 & $06 / 14 / 1612: 06$ & \\
\hline Styrene & $u g / L$ & ND & 1.0 & $06 / 14 / 1612: 06$ & \\
\hline tert-Butylbenzene & $u g / L$ & ND & 1.0 & $06 / 14 / 1612: 06$ & \\
\hline Tetrachloroethene & $\mathrm{ug} / \mathrm{L}$ & ND & 1.0 & $06 / 14 / 1612: 06$ & \\
\hline Toluene & $\mathrm{ug} / \mathrm{L}$ & ND & 1.0 & $06 / 14 / 1612: 06$ & \\
\hline trans-1,2-Dichloroethene & $u g / L$ & ND & 1.0 & $06 / 14 / 1612: 06$ & \\
\hline trans-1,3-Dichloropropene & $\mathrm{ug} / \mathrm{L}$ & ND & 1.0 & $06 / 14 / 1612: 06$ & \\
\hline Trichloroethene & $\mathrm{ug} / \mathrm{L}$ & ND & 1.0 & $06 / 14 / 1612: 06$ & \\
\hline Trichlorofluoromethane & $u g / L$ & ND & 1.0 & $06 / 14 / 1612: 06$ & \\
\hline Vinyl chloride & $\mathrm{ug} / \mathrm{L}$ & ND & 1.0 & $06 / 14 / 1612: 06$ & \\
\hline Xylene (Total) & $\mathrm{ug} / \mathrm{L}$ & ND & 3.0 & $06 / 14 / 1612: 06$ & \\
\hline 1,2-Dichloroethane-d4 (S) & $\%$ & 105 & $81-127$ & 06/14/16 12:06 & \\
\hline 4-Bromofluorobenzene (S) & $\%$ & 100 & $77-130$ & $06 / 14 / 1612: 06$ & \\
\hline Toluene-d8 (S) & $\%$ & 99 & $80-120$ & $06 / 14 / 1612: 06$ & \\
\hline
\end{tabular}

\begin{tabular}{|c|c|c|c|c|c|c|}
\hline \multirow{2}{*}{$\begin{array}{c}\text { LABORATORY CONTROL SAMPLE: } \\
\text { Parameter }\end{array}$} & \multicolumn{6}{|l|}{1776036} \\
\hline & Units & $\begin{array}{l}\text { Spike } \\
\text { Conc. }\end{array}$ & $\begin{array}{l}\text { LCS } \\
\text { Result }\end{array}$ & $\begin{array}{c}\text { LCS } \\
\% \operatorname{Rec}\end{array}$ & $\begin{array}{l}\% \text { Rec } \\
\text { Limits }\end{array}$ & Qualifiers \\
\hline 1,1,1,2-Tetrachloroethane & $u g / L$ & 20 & 20.6 & 103 & $80-112$ & \\
\hline 1,1,1-Trichloroethane & $\mathrm{ug} / \mathrm{L}$ & 20 & 20.0 & 100 & $81-116$ & \\
\hline $1,1,2,2$-Tetrachloroethane & ug/L & 20 & 20.6 & 103 & $72-117$ & \\
\hline 1,1,2-Trichloroethane & ug/L & 20 & 19.6 & 98 & $79-108$ & \\
\hline 1,1-Dichloroethane & $\mathrm{ug} / \mathrm{L}$ & 20 & 19.7 & 98 & 83-117 & \\
\hline 1,1-Dichloroethene & ug/L & 20 & 20.3 & 102 & 74-114 & \\
\hline 1,1-Dichloropropene & $\mathrm{ug} / \mathrm{L}$ & 20 & 19.8 & 99 & $80-120$ & \\
\hline 1,2,3-Trichlorobenzene & $u g / L$ & 20 & 20.3 & 102 & 74-124 & \\
\hline 1,2,3-Trichloropropane & $\mathrm{ug} / \mathrm{L}$ & 20 & 20.3 & 102 & $74-120$ & \\
\hline 1,2,4-Trichlorobenzene & $\mathrm{ug} / \mathrm{L}$ & 20 & 21.0 & 105 & $78-120$ & \\
\hline 1,2,4-Trimethylbenzene & ug/L & 20 & 20.6 & 103 & 82-114 & \\
\hline 1,2-Dibromo-3-chloropropane & $\mathrm{ug} / \mathrm{L}$ & 20 & 21.0 & 105 & 73-124 & \\
\hline 1,2-Dibromoethane (EDB) & ug/L & 20 & 20.5 & 103 & $81-120$ & \\
\hline
\end{tabular}

\section{REPORT OF LABORATORY ANALYSIS}




\section{QUALITY CONTROL DATA}

Project: Investigation Derived Waste

Pace Project No.: 60221061

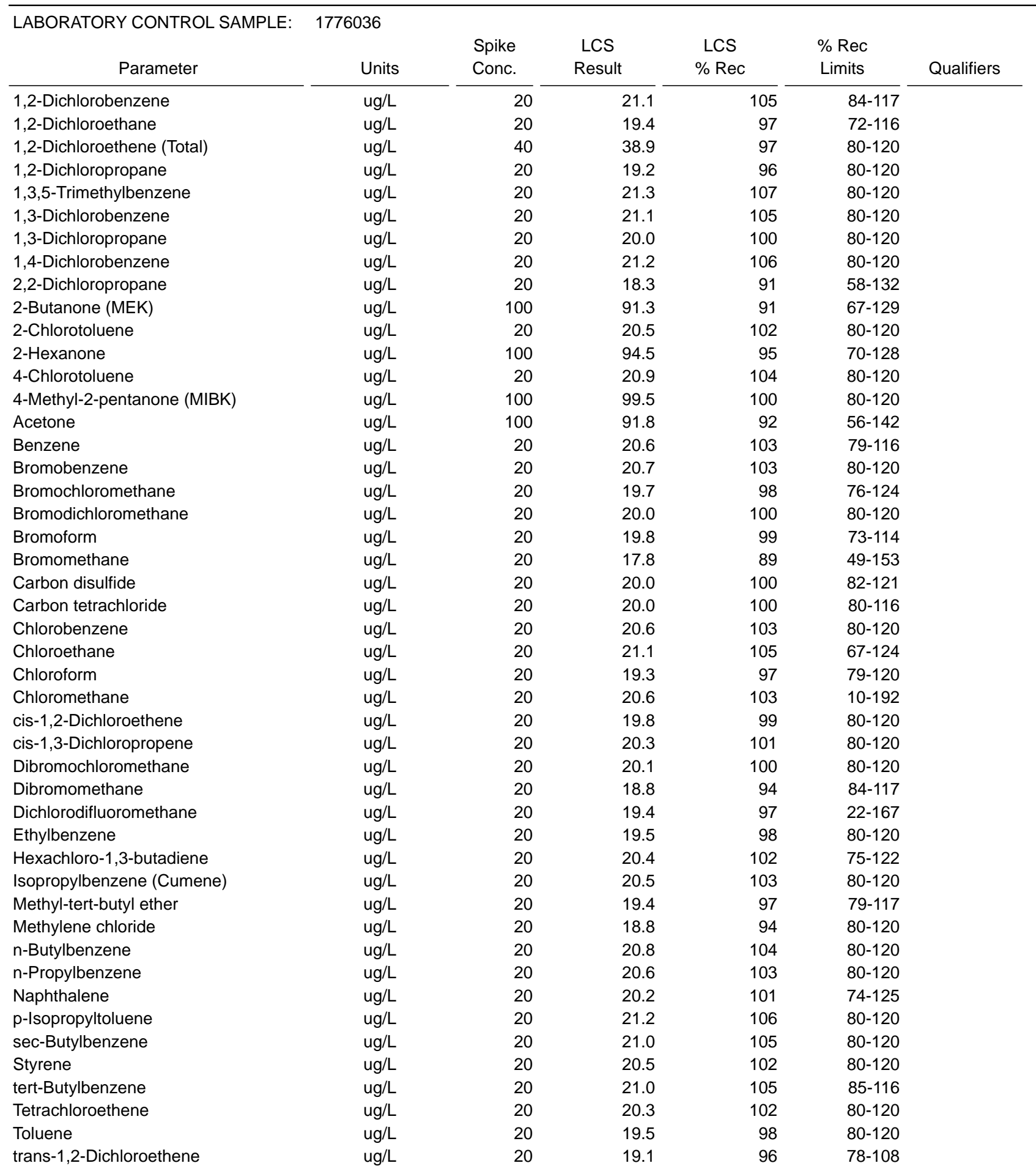




\section{QUALITY CONTROL DATA}

Project: Investigation Derived Waste

Pace Project No.: 60221061

\begin{tabular}{|c|c|c|c|c|c|c|}
\hline $\begin{array}{c}\text { LABORATORY CONTROL SAMPLE: } \\
\text { Parameter }\end{array}$ & $\begin{array}{r}1776036 \\
\text { Units }\end{array}$ & $\begin{array}{l}\text { Spike } \\
\text { Conc. }\end{array}$ & $\begin{array}{l}\text { LCS } \\
\text { Result }\end{array}$ & $\begin{array}{l}\text { LCS } \\
\% \operatorname{Rec}\end{array}$ & $\begin{array}{l}\% \text { Rec } \\
\text { Limits }\end{array}$ & Qualifiers \\
\hline trans-1,3-Dichloropropene & $\mathrm{ug} / \mathrm{L}$ & 20 & 20.2 & 101 & $85-120$ & \\
\hline Trichloroethene & $\mathrm{ug} / \mathrm{L}$ & 20 & 19.6 & 98 & $76-114$ & \\
\hline Trichlorofluoromethane & ug/L & 20 & 21.4 & 107 & $78-132$ & \\
\hline Vinyl chloride & ug/L & 20 & 21.7 & 108 & $69-129$ & \\
\hline Xylene (Total) & $u g / L$ & 60 & 60.7 & 101 & $80-120$ & \\
\hline 1,2-Dichloroethane-d4 (S) & $\%$ & & & 96 & $81-127$ & \\
\hline 4-Bromofluorobenzene (S) & $\%$ & & & 99 & $77-130$ & \\
\hline Toluene-d8 (S) & $\%$ & & & 98 & $80-120$ & \\
\hline
\end{tabular}




\section{QUALITY CONTROL DATA}

Project: Investigation Derived Waste

Pace Project No.: 60221061

\begin{tabular}{llll}
\hline QC Batch: & OEXT/54766 & Analysis Method: & EPA 504.1 \\
QC Batch Method: & EPA 504.1 & Analysis Description: & GCS 504 EDB DBCP
\end{tabular}

Associated Lab Samples: $\quad 60221061001$

METHOD BLANK: 1778591

Matrix: Water

Associated Lab Samples: $\quad 60221061001$

$\frac{\text { Parameter }}{1,2 \text {-Dibromoethane (EDB) }} \frac{\text { Units }}{\mathrm{ug} / \mathrm{L}} \frac{\begin{array}{l}\text { Blank } \\ \text { Result }\end{array}}{\mathrm{ND}} \frac{\begin{array}{c}\text { Reporting } \\ \text { Limit }\end{array}}{0.030} \frac{\text { Analyzed }}{06 / 20 / 1619: 40} \frac{\text { Qualifiers }}{-}$

\begin{tabular}{|c|c|c|c|c|c|c|c|c|c|c|}
\hline \multirow{2}{*}{ LABORATORY CONTROL SAMPLE \& LCSD: } & \multirow[t]{2}{*}{1778592} & \multicolumn{5}{|c|}{1778593} & \multirow{3}{*}{$\begin{array}{l}\% \operatorname{Rec} \\
\text { Limits }\end{array}$} & \multirow{2}{*}{\multicolumn{2}{|c|}{ Max }} & \multirow[b]{3}{*}{ Qualifiers } \\
\hline & & Spike & LCS & LCSD & LCS & LCSD & & & & \\
\hline Parameter & Units & Conc. & Result & Result & $\% \operatorname{Rec}$ & $\% \operatorname{Rec}$ & & RPD & RPD & \\
\hline 1,2-Dibromoethane (EDB) & $u g / L$ & .25 & 0.26 & 0.26 & 103 & 105 & $70-130$ & 2 & 20 & \\
\hline
\end{tabular}

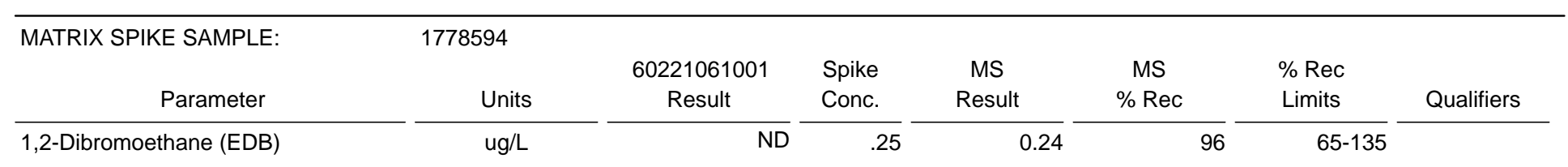

\section{REPORT OF LABORATORY ANALYSIS}




\section{QUALITY CONTROL DATA}

Project: Investigation Derived Waste

Pace Project No.: 60221061

\begin{tabular}{llll}
\hline QC Batch: & WETA/39994 & Analysis Method: & EPA 353.2 \\
QC Batch Method: & EPA 353.2 & Analysis Description: & 353.2 Nitrate + Nitrite, Unpres.
\end{tabular}

Associated Lab Samples: $\quad 60221061001$

METHOD BLANK: 1774525

Associated Lab Samples: $\quad 60221061001$

$\frac{\text { Parameter }}{\text { Nitrogen, Nitrate }} \frac{\text { Units }}{\mathrm{mg} / \mathrm{L}} \frac{\text { Result }}{\mathrm{ND}} \frac{\begin{array}{c}\text { Blank } \\ \text { Limit }\end{array}}{0.10} \frac{\text { Analyzed }}{06 / 10 / 1614: 16} \frac{\text { Qualifiers }}{-}$

\begin{tabular}{|c|c|c|c|c|c|c|}
\hline \multirow{2}{*}{ LABORATORY CONTROL SAMPLE: } & \multicolumn{6}{|l|}{1774526} \\
\hline & & Spike & LCS & LCS & $\% \operatorname{Rec}$ & \\
\hline Parameter & Units & Conc. & Result & $\% \operatorname{Rec}$ & Limits & Qualifiers \\
\hline Nitrogen, Nitrate & $\mathrm{mg} / \mathrm{L}$ & 1.6 & 1.7 & 106 & $85-115$ & \\
\hline
\end{tabular}

\begin{tabular}{|c|c|c|c|c|c|c|c|}
\hline MATRIX SPIKE SAMPLE: & 1774527 & & & & & & \\
\hline Parameter & Units & $\begin{array}{c}60221076012 \\
\text { Result }\end{array}$ & $\begin{array}{l}\text { Spike } \\
\text { Conc. }\end{array}$ & $\begin{array}{c}\text { MS } \\
\text { Result }\end{array}$ & $\begin{array}{c}\text { MS } \\
\% \operatorname{Rec}\end{array}$ & $\begin{array}{l}\% \operatorname{Rec} \\
\text { Limits }\end{array}$ & Qualifiers \\
\hline
\end{tabular}

\begin{tabular}{|c|c|c|c|c|c|c|c|}
\hline MATRIX SPIKE SAMPLE: & 1774529 & & & & & & \\
\hline & & 60221076002 & Spike & MS & MS & $\% \operatorname{Rec}$ & \\
\hline Parameter & Units & Result & Conc. & Result & $\% \operatorname{Rec}$ & Limits & Qualifiers \\
\hline Nitrogen, Nitrate & $\mathrm{mg} / \mathrm{L}$ & 8.3 & 8 & 16.8 & 106 & $85-115$ & \\
\hline
\end{tabular}

\section{SAMPLE DUPLICATE: 1774528}

\begin{tabular}{|c|c|c|c|c|c|c|}
\hline & & 60221046006 & Dup & & Max & \\
\hline Parameter & Units & Result & Result & RPD & RPD & Qualifiers \\
\hline Nitrogen, Nitrate & $\mathrm{mg} / \mathrm{L}$ & 11.1 & 10.9 & 2 & 20 & \\
\hline
\end{tabular}




\section{QUALIFIERS}

Project: Investigation Derived Waste

Pace Project No.: 60221061

\section{DEFINITIONS}

DF - Dilution Factor, if reported, represents the factor applied to the reported data due to dilution of the sample aliquot.

ND - Not Detected at or above adjusted reporting limit.

$\mathrm{J}$ - Estimated concentration above the adjusted method detection limit and below the adjusted reporting limit.

MDL - Adjusted Method Detection Limit.

PQL - Practical Quantitation Limit.

$\mathrm{RL}$ - Reporting Limit.

$S$ - Surrogate

1,2-Diphenylhydrazine decomposes to and cannot be separated from Azobenzene using Method 8270 . The result for each analyte is a combined concentration.

Consistent with EPA guidelines, unrounded data are displayed and have been used to calculate \% recovery and RPD values.

LCS(D) - Laboratory Control Sample (Duplicate)

MS(D) - Matrix Spike (Duplicate)

DUP - Sample Duplicate

RPD - Relative Percent Difference

NC - Not Calculable.

SG - Silica Gel - Clean-Up

$\mathrm{U}$ - Indicates the compound was analyzed for, but not detected.

N-Nitrosodiphenylamine decomposes and cannot be separated from Diphenylamine using Method 8270. The result reported for each analyte is a combined concentration.

Pace Analytical is TNI accredited. Contact your Pace PM for the current list of accredited analytes.

TNI - The NELAC Institute.

\section{BATCH QUALIFIERS}

Batch: MSV/76405

[M5] A matrix spike/matrix spike duplicate was not performed for this batch due to insufficient sample volume.

\section{REPORT OF LABORATORY ANALYSIS}




\section{QUALITY CONTROL DATA CROSS REFERENCE TABLE}

Project: Investigation Derived Waste

Pace Project No.: $\quad 60221061$

\begin{tabular}{llll}
\hline Lab ID & Sample ID & QC Batch Method & QC Batch \\
\hline $\mathbf{6 0 2 2 1 0 6 1 0 0 1}$ & YKIDW-W-38109 & EPA 504.1 & OEXT/54766 \\
$\mathbf{6 0 2 2 1 0 6 1 0 0 1}$ & YKIDW-W-38109 & EPA 504.1 $5030 \mathrm{~B} / 8260$ & $\mathrm{MSV} / 76405$ \\
$\mathbf{6 0 2 2 1 0 6 1 0 0 2}$ & YKQCTB-W-38110 & EPA 5030B/8260 & MSV/76405 \\
$\mathbf{6 0 2 2 1 0 6 1 0 0 1}$ & YKIDW-W-38109 & EPA 353.2 & WETA/39994
\end{tabular}

\section{REPORT OF LABORATORY ANALYSIS}


Client Name: $\quad T C \omega$

Tracking \#: $\quad 7764 \quad 88871133$

Pace Shipping Label Used? Yes $\square \quad$ No $\square$

Proj Due Date:

Custody Seal on Cooler/Box Present: $\quad$ Yes $\mathbf{Q}$ No $\square \quad$ Seals intact: Yes $\nVdash \quad$ No $\square$

Packing Material: $\quad$ Bubble Wrap $\square \quad$ Bubble Bags $\square \quad$ Foam $\square \quad$ None $\square \quad$ Other $\square$

Thermometer Used:

Cooler Temperature:

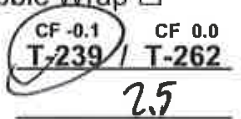

Type of Ice: Wet Blue None

$\square$ Samples received on ice, cooling process has begun.

Date and initials of person examining

Temperature should be above freezing to $6^{\circ} \mathrm{C}$

Chain of Custody present:

Chain of Custody filled out:

Chain of Custody relinquished:

Sampler name \& signature on COC:

Samples arrived within holding time:

Short Hold Time analyses $(<72 \mathrm{hr})$ :

Rush Turn Around Time requested:

Sufficient volume:

Correct containers used:

Pace containers used:

Containers intact:

Unpreserved 5035A soils frozen w/in 48hrs?

Filtered volume received for dissolved tests?

Sample labels match COC:

Includes date/time/ID/analyses

\#Yes $\square$ No $\square$ N/A 1

WYes $\square$ No $\square$ N/A 2.

Hoyes $\square$ No $\square$ N/A 3.

All containers needing preservation have been checked.

All containers needing preservation are found to be in compliance

with EPA recommendation.

Exceptions: VOA, Coliform, O\&G, WI-DRO (water)

Trip Blank present:

Pace Trip Blank lot \# (if purchased): Covered

Headspace in VOA vials $(>6 \mathrm{~mm})$ :

(

Project sampled in USDA Regulated Area:

Additional labels attached to $5035 \mathrm{~A}$ vials in the field?

Client Notification/ Resolution:

Person Contacted:

$$
\text { Copy COC to Client? Y / (D) }
$$

$\square$ Yes $\square$ No N/A 17. List State:

13.

$\square$ Yes $\square$ No $\square_{N / A}$

$\square$ Yes $\square$ No $\not N / A$

LYes $\square$ No

14.

Initial when completed

contents:

If 6110

Date/Time:

\section{Comments/ Resolution:}




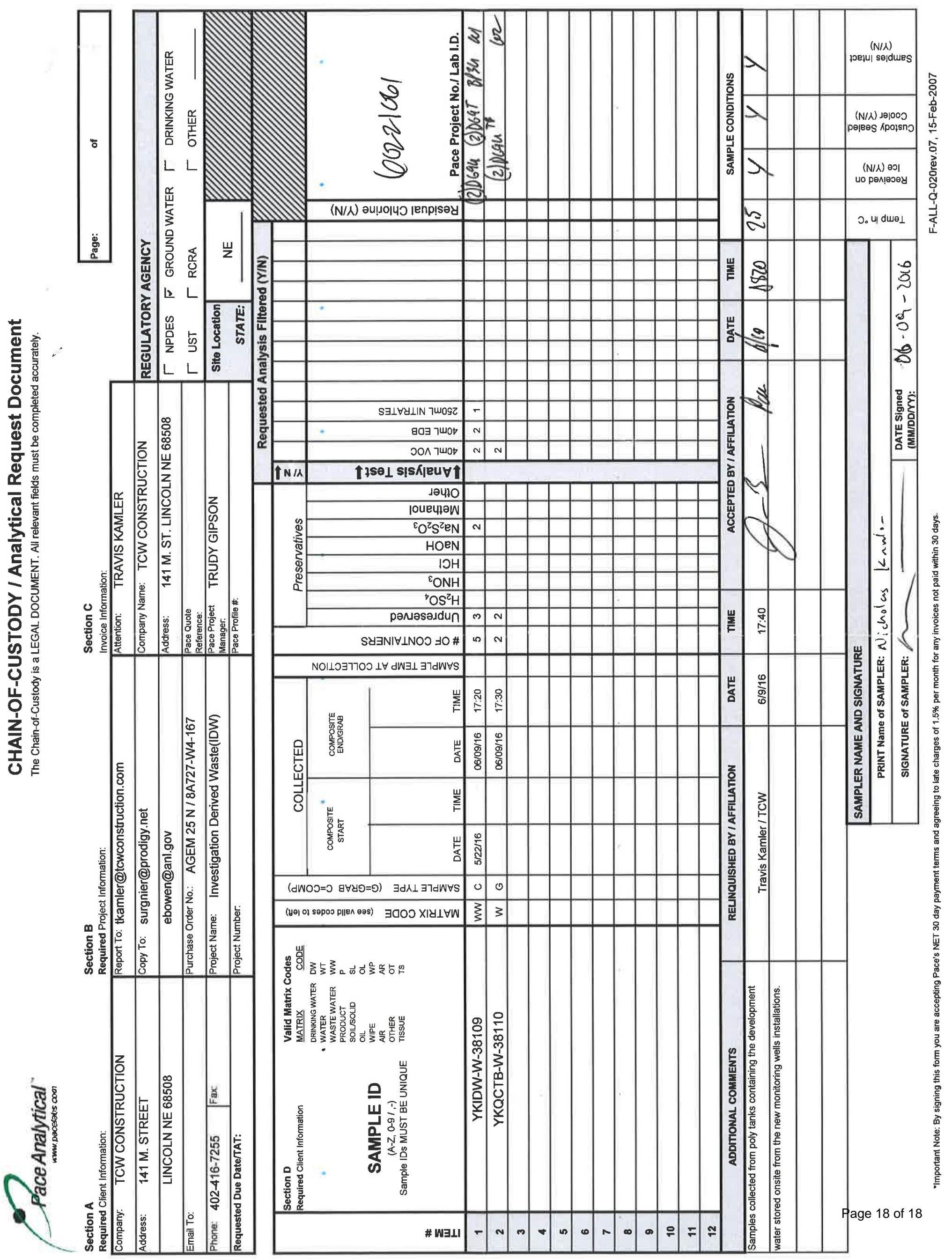


July 05, 2016

Mr. Travis Kamler

TCW Construction Inc

$141 \mathrm{M}$ Street

Lincoln, NE 68508

RE: Project: Investigation Derived Waste

Pace Project No.: 60221829

Dear Mr. Kamler:

Enclosed are the analytical results for sample(s) received by the laboratory on June 22, 2016 . The results relate only to the samples included in this report. Results reported herein conform to the most current TNI standards and the laboratory's Quality Assurance Manual, where applicable, unless otherwise noted in the body of the report.

If you have any questions concerning this report, please feel free to contact me.

Sincerely,

$$
\text { sudy stipaon }
$$

Trudy Gipson

trudy.gipson@pacelabs.com

Project Manager

\author{
Enclosures \\ cc: Ms. Esther Bowen, Argonne National Lab \\ Mr. David Surgnier, Delta Environmental
}

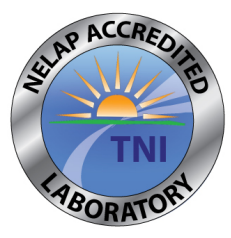




\section{CERTIFICATIONS}

Project: Investigation Derived Waste

Pace Project No.: 60221829

Kansas Certification IDs

9608 Loiret Boulevard, Lenexa, KS 66219

WY STR Certification \#: 2456.01

Arkansas Certification \#: 15-016-0

Illinois Certification \#: 003097

lowa Certification \#: 118

Kansas/NELAP Certification \#: E-10116
Louisiana Certification \#: 03055

Nevada Certification \#: KS000212008A

Oklahoma Certification \#: 9205/9935

Texas Certification \#: T104704407

Utah Certification \#: KS00021

Kansas Field Laboratory Accreditation: \# E-92587

\section{REPORT OF LABORATORY ANALYSIS}




\section{SAMPLE SUMMARY}

Project: Investigation Derived Waste

Pace Project No.: 60221829

\begin{tabular}{|c|c|c|c|c|}
\hline Lab ID & Sample ID & Matrix & Date Collected & Date Received \\
\hline 60221829001 & YKIDW-W-38119 & Water & 06/21/16 16:00 & $06 / 22 / 1608: 50$ \\
\hline 60221829002 & YKQCTB-W-38120 & Water & 06/21/16 09:00 & $06 / 22 / 1608: 50$ \\
\hline 60221829003 & YKROLLFRONT-S-38121 & Solid & 06/21/16 16:55 & $06 / 22 / 1608: 50$ \\
\hline 60221829004 & YKROLLREAR-S-38122 & Solid & 06/21/16 17:00 & $06 / 22 / 1608: 50$ \\
\hline
\end{tabular}

\section{REPORT OF LABORATORY ANALYSIS}




\section{SAMPLE ANALYTE COUNT}

Project: Investigation Derived Waste

Pace Project No.: 60221829

\begin{tabular}{|c|c|c|c|c|}
\hline Lab ID & Sample ID & Method & Analysts & $\begin{array}{l}\text { Analytes } \\
\text { Reported }\end{array}$ \\
\hline \multirow[t]{3}{*}{60221829001} & YKIDW-W-38119 & EPA 504.1 & $\mathrm{ACW}$ & 1 \\
\hline & & EPA 5030B/8260 & PGH & 69 \\
\hline & & EPA 353.2 & CRS & 1 \\
\hline 60221829002 & YKQCTB-W-38120 & EPA 5030B/8260 & PGH & 69 \\
\hline \multirow[t]{2}{*}{60221829003} & YKROLLFRONT-S-38121 & EPA 8260 & JKL & 68 \\
\hline & & ASTM D2974 & DWC & 1 \\
\hline \multirow[t]{2}{*}{60221829004} & YKROLLREAR-S-38122 & EPA 8260 & JKL & 68 \\
\hline & & ASTM D2974 & DWC & 1 \\
\hline
\end{tabular}

\section{REPORT OF LABORATORY ANALYSIS}




\section{ANALYTICAL RESULTS}

Project: Investigation Derived Waste

Pace Project No.: 60221829

\begin{tabular}{ccccccc}
\hline Sample: YKIDW-W-38119 & Lab ID: & 60221829001 & Collected: 06/21/16 16:00 & Received: & 06/22/16 08:50 & Matrix: Water \\
Parameters & Results & Units & Report Limit & DF & Prepared & Analyzed \\
\hline
\end{tabular}

\section{GCS EDB and DBCP}

1,2-Dibromoethane (EDB)

\section{MSV}

Acetone

Benzene

Bromobenzene

Bromochloromethane

Bromodichloromethane

Bromoform

Bromomethane

2-Butanone (MEK)

n-Butylbenzene

sec-Butylbenzene

tert-Butylbenzene

Carbon disulfide

Carbon tetrachloride

Chlorobenzene

Chloroethane

Chloroform

Chloromethane

2-Chlorotoluene

4-Chlorotoluene

1,2-Dibromo-3-chloropropane

Dibromochloromethane

1,2-Dibromoethane (EDB)

Dibromomethane

1,2-Dichlorobenzene

1,3-Dichlorobenzene

1,4-Dichlorobenzene

Dichlorodifluoromethane

1,1-Dichloroethane

1,2-Dichloroethane

1,2-Dichloroethene (Total)

1,1-Dichloroethene

cis-1,2-Dichloroethene

trans-1,2-Dichloroethene

1,2-Dichloropropane

1,3-Dichloropropane

2,2-Dichloropropane

1,1-Dichloropropene

cis-1,3-Dichloropropene

trans-1,3-Dichloropropene

Ethylbenzene

Hexachloro-1,3-butadiene

2-Hexanone

Isopropylbenzene (Cumene)

p-Isopropyltoluene
Analytical Method: EPA 504.1 Preparation Method: EPA 504.1

$\begin{array}{llllllll}\text { ND ug/L } & 0.030 & 1 & 06 / 30 / 16 & 15: 06 & 06 / 30 / 16 & 21: 37 & 106-93-4\end{array}$

Analytical Method: EPA 5030B/8260

\begin{tabular}{|c|c|c|c|c|c|}
\hline ND & ug/L & 10.0 & 1 & 06/24/16 13:01 & $67-64-1$ \\
\hline ND & $u g / L$ & 1.0 & 1 & 06/24/16 13:01 & $71-43-2$ \\
\hline ND & ug/L & 1.0 & 1 & 06/24/16 13:01 & $108-86-1$ \\
\hline ND & $\mathrm{ug} / \mathrm{L}$ & 1.0 & 1 & 06/24/16 13:01 & $74-97-5$ \\
\hline ND & ug/L & 1.0 & 1 & 06/24/16 13:01 & $75-27-4$ \\
\hline ND & ug/L & 1.0 & 1 & 06/24/16 13:01 & $75-25-2$ \\
\hline ND & $u g / L$ & 5.0 & 1 & 06/24/16 13:01 & $74-83-9$ \\
\hline ND & ug/L & 10.0 & 1 & 06/24/16 13:01 & $78-93-3$ \\
\hline ND & ug/L & 1.0 & 1 & 06/24/16 13:01 & $104-51-8$ \\
\hline ND & ug/L & 1.0 & 1 & 06/24/16 13:01 & $135-98-8$ \\
\hline ND & ug/L & 1.0 & 1 & 06/24/16 13:01 & $98-06-6$ \\
\hline ND & $u g / L$ & 5.0 & 1 & 06/24/16 13:01 & $75-15-0$ \\
\hline ND & ug/L & 1.0 & 1 & 06/24/16 13:01 & $56-23-5$ \\
\hline ND & ug/L & 1.0 & 1 & 06/24/16 13:01 & $108-90-7$ \\
\hline ND & $u g / L$ & 1.0 & 1 & 06/24/16 13:01 & $75-00-3$ \\
\hline ND & ug/L & 1.0 & 1 & 06/24/16 13:01 & $67-66-3$ \\
\hline ND & ug/L & 1.0 & 1 & 06/24/16 13:01 & $74-87-3$ \\
\hline ND & ug/L & 1.0 & 1 & 06/24/16 13:01 & $95-49-8$ \\
\hline ND & ug/L & 1.0 & 1 & 06/24/16 13:01 & $106-43-4$ \\
\hline ND & $\mathrm{ug} / \mathrm{L}$ & 2.5 & 1 & 06/24/16 13:01 & $96-12-8$ \\
\hline ND & ug/L & 1.0 & 1 & 06/24/16 13:01 & $124-48-1$ \\
\hline ND & ug/L & 1.0 & 1 & 06/24/16 13:01 & $106-93-4$ \\
\hline ND & $\mathrm{ug} / \mathrm{L}$ & 1.0 & 1 & 06/24/16 13:01 & $74-95-3$ \\
\hline ND & ug/L & 1.0 & 1 & 06/24/16 13:01 & $95-50-1$ \\
\hline ND & ug/L & 1.0 & 1 & 06/24/16 13:01 & 541-73-1 \\
\hline ND & $u g / L$ & 1.0 & 1 & $06 / 24 / 1613: 01$ & $106-46-7$ \\
\hline ND & ug/L & 1.0 & 1 & 06/24/16 13:01 & $75-71-8$ \\
\hline ND & $u g / L$ & 1.0 & 1 & $06 / 24 / 16$ 13:01 & $75-34-3$ \\
\hline ND & $\mathrm{ug} / \mathrm{L}$ & 1.0 & 1 & $06 / 24 / 1613: 01$ & $107-06-2$ \\
\hline ND & ug/L & 1.0 & 1 & 06/24/16 13:01 & $540-59-0$ \\
\hline ND & ug/L & 1.0 & 1 & 06/24/16 13:01 & $75-35-4$ \\
\hline ND & ug/L & 1.0 & 1 & $06 / 24 / 1613: 01$ & $156-59-2$ \\
\hline ND & ug/L & 1.0 & 1 & 06/24/16 13:01 & $156-60-5$ \\
\hline ND & ug/L & 1.0 & 1 & 06/24/16 13:01 & $78-87-5$ \\
\hline ND & ug/L & 1.0 & 1 & $06 / 24 / 1613: 01$ & $142-28-9$ \\
\hline ND & ug/L & 1.0 & 1 & 06/24/16 13:01 & $594-20-7$ \\
\hline ND & ug/L & 1.0 & 1 & 06/24/16 13:01 & $563-58-6$ \\
\hline ND & ug/L & 1.0 & 1 & $06 / 24 / 1613: 01$ & 10061-01-5 \\
\hline ND & $\mathrm{ug} / \mathrm{L}$ & 1.0 & 1 & 06/24/16 13:01 & $10061-02-6$ \\
\hline ND & ug/L & 1.0 & 1 & $06 / 24 / 16$ 13:01 & $100-41-4$ \\
\hline ND & ug/L & 1.0 & 1 & $06 / 24 / 1613: 01$ & $87-68-3$ \\
\hline ND & ug/L & 10.0 & 1 & $06 / 24 / 16$ 13:01 & $591-78-6$ \\
\hline ND & $\mathrm{ug} / \mathrm{L}$ & 1.0 & 1 & 06/24/16 13:01 & $98-82-8$ \\
\hline ND & $u g / L$ & 1.0 & 1 & 06/24/16 13:01 & $99-87-6$ \\
\hline
\end{tabular}

\section{REPORT OF LABORATORY ANALYSIS}

This report shall not be reproduced, except in full,

without the written consent of Pace Analytical Services, Inc.. 


\section{ANALYTICAL RESULTS}

Project: Investigation Derived Waste

Pace Project No.: 60221829

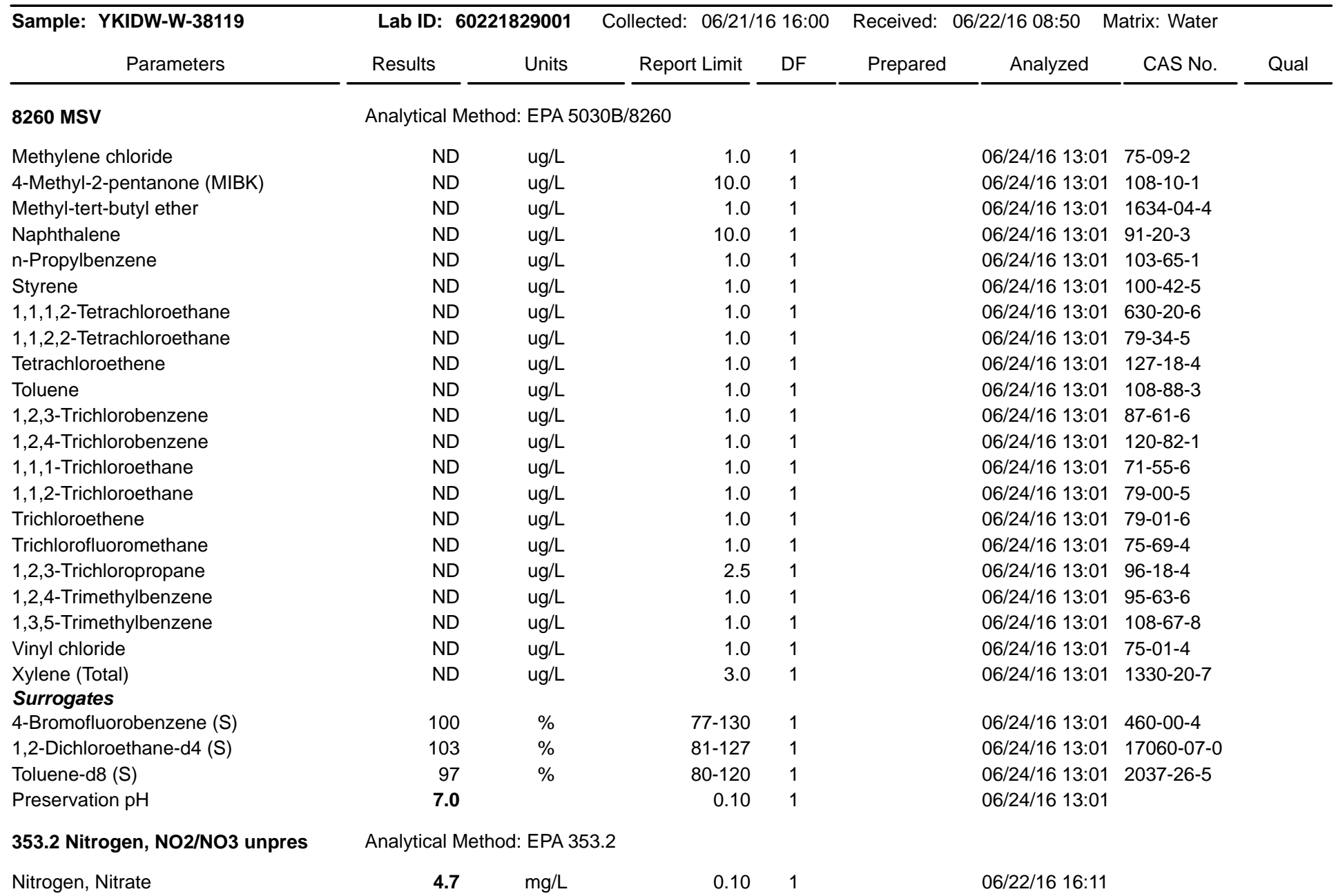

\section{REPORT OF LABORATORY ANALYSIS}




\section{ANALYTICAL RESULTS}

Project: Investigation Derived Waste

Pace Project No.: 60221829

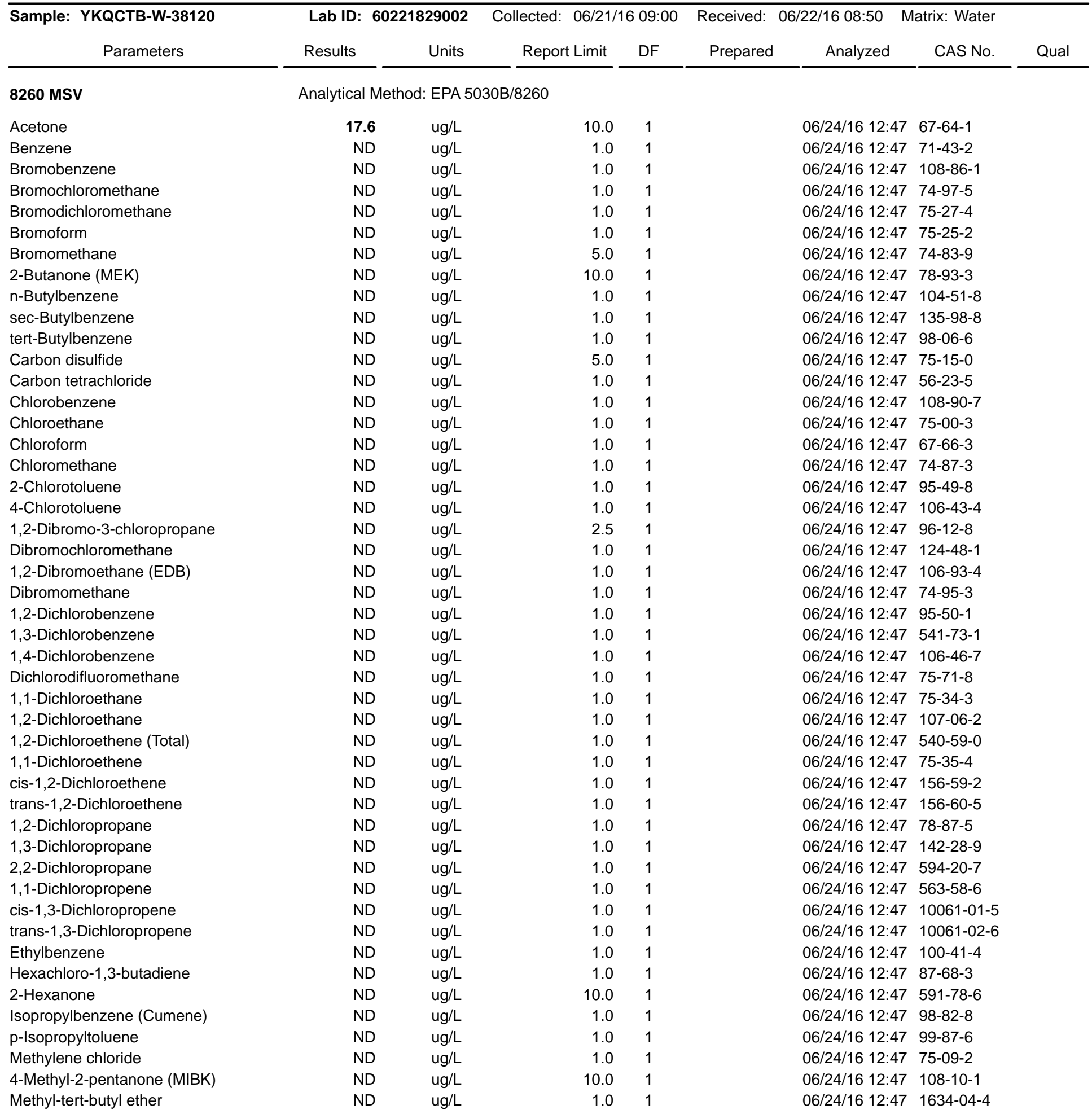

\section{REPORT OF LABORATORY ANALYSIS}




\section{ANALYTICAL RESULTS}

Project: Investigation Derived Waste

Pace Project No.: 60221829

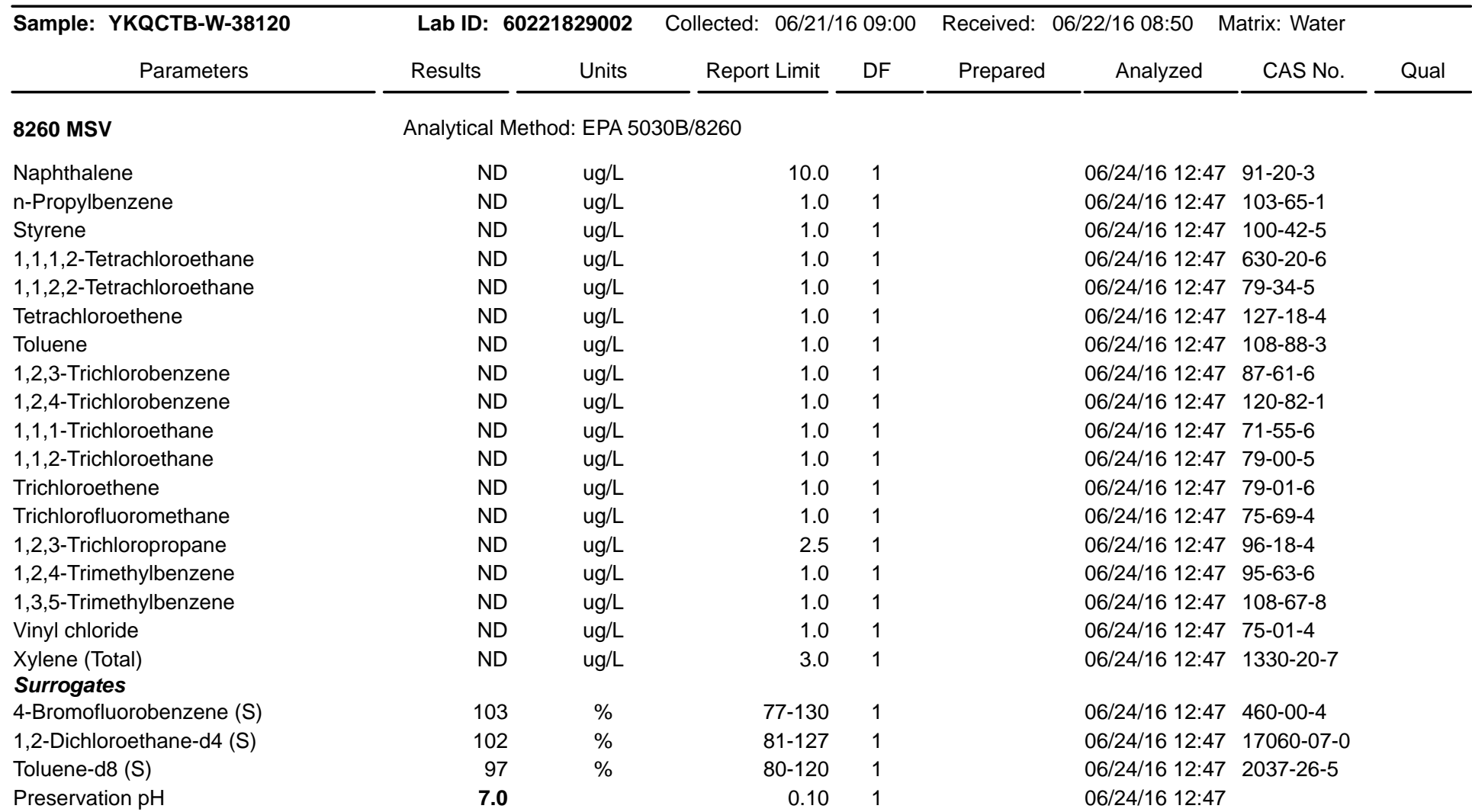

\section{REPORT OF LABORATORY ANALYSIS}




\section{ANALYTICAL RESULTS}

Project: Investigation Derived Waste

Pace Project No.: 60221829

Sample: YKROLLFRONT-S-38121 Lab ID: $60221829003 \quad$ Collected: 06/21/16 16:55 Received: 06/22/16 08:50 Matrix: Solid

Results reported on a "dry weight" basis and are adjusted for percent moisture, sample size and any dilutions.

\begin{tabular}{|c|c|c|c|c|c|c|c|c|}
\hline Parameters & Results & Units & Report Limit & DF & Prepared & Analyzed & CAS No. & Qual \\
\hline 8260 MSV 5035A VOA & Analytical M & : EPA 8260 & & & & & & \\
\hline Acetone & ND & $\mathrm{ug} / \mathrm{kg}$ & 20.2 & 1 & & 07/01/16 15:10 & $67-64-1$ & \\
\hline Benzene & ND & $\mathrm{ug} / \mathrm{kg}$ & 5.1 & 1 & & 07/01/16 15:10 & $71-43-2$ & \\
\hline Bromobenzene & ND & $\mathrm{ug} / \mathrm{kg}$ & 5.1 & 1 & & 07/01/16 15:10 & $108-86-1$ & \\
\hline Bromochloromethane & ND & $\mathrm{ug} / \mathrm{kg}$ & 5.1 & 1 & & 07/01/16 15:10 & $74-97-5$ & \\
\hline Bromodichloromethane & ND & $\mathrm{ug} / \mathrm{kg}$ & 5.1 & 1 & & 07/01/16 15:10 & $75-27-4$ & \\
\hline Bromoform & ND & $\mathrm{ug} / \mathrm{kg}$ & 5.1 & 1 & & 07/01/16 15:10 & $75-25-2$ & \\
\hline Bromomethane & ND & $\mathrm{ug} / \mathrm{kg}$ & 5.1 & 1 & & 07/01/16 15:10 & $74-83-9$ & \\
\hline 2-Butanone (MEK) & ND & $\mathrm{ug} / \mathrm{kg}$ & 10.1 & 1 & & 07/01/16 15:10 & $78-93-3$ & \\
\hline n-Butylbenzene & ND & $\mathrm{ug} / \mathrm{kg}$ & 5.1 & 1 & & 07/01/16 15:10 & $104-51-8$ & \\
\hline sec-Butylbenzene & ND & $\mathrm{ug} / \mathrm{kg}$ & 5.1 & 1 & & 07/01/16 15:10 & $135-98-8$ & \\
\hline tert-Butylbenzene & ND & $\mathrm{ug} / \mathrm{kg}$ & 5.1 & 1 & & 07/01/16 15:10 & $98-06-6$ & \\
\hline Carbon disulfide & ND & $\mathrm{ug} / \mathrm{kg}$ & 5.1 & 1 & & 07/01/16 15:10 & $75-15-0$ & \\
\hline Carbon tetrachloride & ND & $\mathrm{ug} / \mathrm{kg}$ & 5.1 & 1 & & 07/01/16 15:10 & $56-23-5$ & \\
\hline Chlorobenzene & ND & $\mathrm{ug} / \mathrm{kg}$ & 5.1 & 1 & & 07/01/16 15:10 & $108-90-7$ & \\
\hline Chloroethane & ND & $\mathrm{ug} / \mathrm{kg}$ & 5.1 & 1 & & 07/01/16 15:10 & $75-00-3$ & \\
\hline Chloroform & ND & $\mathrm{ug} / \mathrm{kg}$ & 5.1 & 1 & & 07/01/16 15:10 & $67-66-3$ & \\
\hline Chloromethane & ND & $\mathrm{ug} / \mathrm{kg}$ & 5.1 & 1 & & 07/01/16 15:10 & $74-87-3$ & \\
\hline 2-Chlorotoluene & ND & $\mathrm{ug} / \mathrm{kg}$ & 5.1 & 1 & & 07/01/16 15:10 & $95-49-8$ & \\
\hline 4-Chlorotoluene & ND & $\mathrm{ug} / \mathrm{kg}$ & 5.1 & 1 & & 07/01/16 15:10 & $106-43-4$ & \\
\hline 1,2-Dibromo-3-chloropropane & ND & $\mathrm{ug} / \mathrm{kg}$ & 10.1 & 1 & & 07/01/16 15:10 & $96-12-8$ & \\
\hline Dibromochloromethane & ND & $\mathrm{ug} / \mathrm{kg}$ & 5.1 & 1 & & 07/01/16 15:10 & $124-48-1$ & \\
\hline 1,2-Dibromoethane (EDB) & ND & $\mathrm{ug} / \mathrm{kg}$ & 5.1 & 1 & & 07/01/16 15:10 & $106-93-4$ & \\
\hline Dibromomethane & ND & $\mathrm{ug} / \mathrm{kg}$ & 5.1 & 1 & & 07/01/16 15:10 & $74-95-3$ & \\
\hline 1,2-Dichlorobenzene & ND & $\mathrm{ug} / \mathrm{kg}$ & 5.1 & 1 & & 07/01/16 15:10 & $95-50-1$ & \\
\hline 1,3-Dichlorobenzene & ND & $\mathrm{ug} / \mathrm{kg}$ & 5.1 & 1 & & 07/01/16 15:10 & $541-73-1$ & \\
\hline 1,4-Dichlorobenzene & ND & $\mathrm{ug} / \mathrm{kg}$ & 5.1 & 1 & & 07/01/16 15:10 & $106-46-7$ & \\
\hline Dichlorodifluoromethane & ND & $\mathrm{ug} / \mathrm{kg}$ & 5.1 & 1 & & 07/01/16 15:10 & $75-71-8$ & \\
\hline 1,1-Dichloroethane & ND & $\mathrm{ug} / \mathrm{kg}$ & 5.1 & 1 & & 07/01/16 15:10 & $75-34-3$ & \\
\hline 1,2-Dichloroethane & ND & $\mathrm{ug} / \mathrm{kg}$ & 5.1 & 1 & & 07/01/16 15:10 & $107-06-2$ & \\
\hline 1,2-Dichloroethene (Total) & ND & $\mathrm{ug} / \mathrm{kg}$ & 5.1 & 1 & & 07/01/16 15:10 & $540-59-0$ & \\
\hline 1,1-Dichloroethene & ND & $\mathrm{ug} / \mathrm{kg}$ & 5.1 & 1 & & 07/01/16 15:10 & $75-35-4$ & \\
\hline cis-1,2-Dichloroethene & ND & $\mathrm{ug} / \mathrm{kg}$ & 5.1 & 1 & & 07/01/16 15:10 & $156-59-2$ & \\
\hline trans-1,2-Dichloroethene & ND & $\mathrm{ug} / \mathrm{kg}$ & 5.1 & 1 & & 07/01/16 15:10 & $156-60-5$ & \\
\hline 1,2-Dichloropropane & ND & $\mathrm{ug} / \mathrm{kg}$ & 5.1 & 1 & & 07/01/16 15:10 & $78-87-5$ & \\
\hline 1,3-Dichloropropane & ND & $\mathrm{ug} / \mathrm{kg}$ & 5.1 & 1 & & 07/01/16 15:10 & $142-28-9$ & \\
\hline 2,2-Dichloropropane & ND & $\mathrm{ug} / \mathrm{kg}$ & 5.1 & 1 & & 07/01/16 15:10 & $594-20-7$ & \\
\hline 1,1-Dichloropropene & ND & $\mathrm{ug} / \mathrm{kg}$ & 5.1 & 1 & & 07/01/16 15:10 & $563-58-6$ & L2 \\
\hline cis-1,3-Dichloropropene & ND & $\mathrm{ug} / \mathrm{kg}$ & 5.1 & 1 & & 07/01/16 15:10 & $10061-01-5$ & \\
\hline trans-1,3-Dichloropropene & ND & $\mathrm{ug} / \mathrm{kg}$ & 5.1 & 1 & & 07/01/16 15:10 & $10061-02-6$ & \\
\hline Ethylbenzene & ND & $\mathrm{ug} / \mathrm{kg}$ & 5.1 & 1 & & 07/01/16 15:10 & $100-41-4$ & \\
\hline Hexachloro-1,3-butadiene & ND & $\mathrm{ug} / \mathrm{kg}$ & 5.1 & 1 & & 07/01/16 15:10 & $87-68-3$ & \\
\hline 2-Hexanone & ND & $\mathrm{ug} / \mathrm{kg}$ & 20.2 & 1 & & 07/01/16 15:10 & $591-78-6$ & \\
\hline Isopropylbenzene (Cumene) & ND & $\mathrm{ug} / \mathrm{kg}$ & 5.1 & 1 & & 07/01/16 15:10 & $98-82-8$ & \\
\hline p-Isopropyltoluene & ND & $\mathrm{ug} / \mathrm{kg}$ & 5.1 & 1 & & 07/01/16 15:10 & $99-87-6$ & \\
\hline Methylene chloride & ND & $\mathrm{ug} / \mathrm{kg}$ & 5.1 & 1 & & 07/01/16 15:10 & $75-09-2$ & \\
\hline 4-Methyl-2-pentanone (MIBK) & ND & $\mathrm{ug} / \mathrm{kg}$ & 10.1 & 1 & & 07/01/16 15:10 & $108-10-1$ & \\
\hline
\end{tabular}

\section{REPORT OF LABORATORY ANALYSIS}




\section{ANALYTICAL RESULTS}

Project: Investigation Derived Waste

Pace Project No.: 60221829

Sample: YKROLLFRONT-S-38121 Lab ID: $60221829003 \quad$ Collected: 06/21/16 16:55 Received: 06/22/16 08:50 Matrix: Solid

Results reported on a "dry weight" basis and are adjusted for percent moisture, sample size and any dilutions.

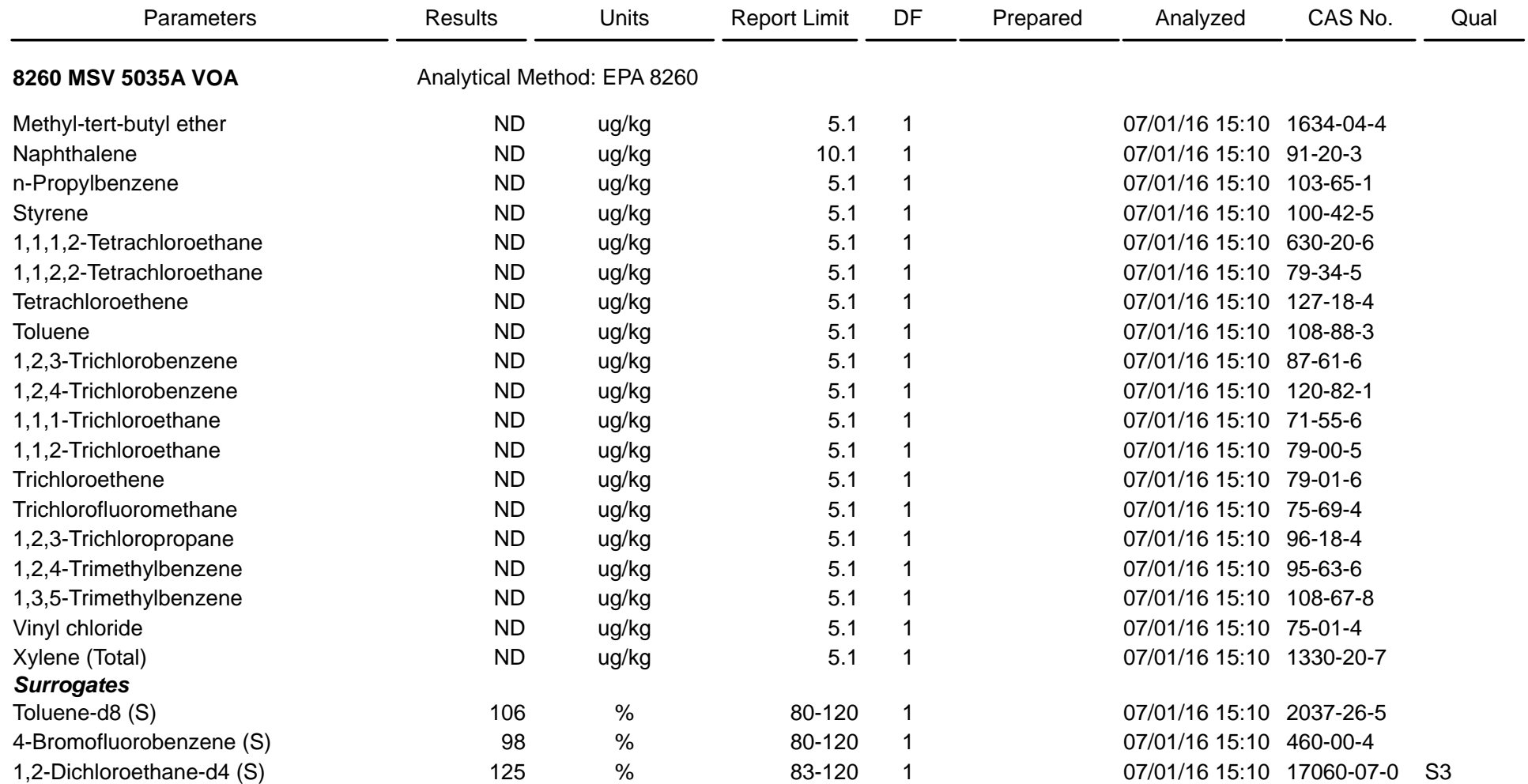

\section{Percent Moisture}

Percent Moisture
Analytical Method: ASTM D2974
1.5
$\%$

0.50

06/29/16 00:00

\section{REPORT OF LABORATORY ANALYSIS}




\section{ANALYTICAL RESULTS}

Project: Investigation Derived Waste

Pace Project No.: 60221829

Sample: YKROLLREAR-S-38122 Lab ID: 60221829004 Collected: 06/21/16 17:00 Received: 06/22/16 08:50 Matrix: Solid

Results reported on a "dry weight" basis and are adjusted for percent moisture, sample size and any dilutions.

\begin{tabular}{|c|c|c|c|c|c|c|c|c|}
\hline Parameters & Results & Units & Report Limit & DF & Prepared & Analyzed & CAS No. & Qual \\
\hline 8260 MSV 5035A VOA & \multicolumn{8}{|c|}{ Analytical Method: EPA 8260} \\
\hline Acetone & ND & $\mathrm{ug} / \mathrm{kg}$ & 26.2 & 1 & & 07/01/16 15:25 & $67-64-1$ & \\
\hline Benzene & ND & $\mathrm{ug} / \mathrm{kg}$ & 6.5 & 1 & & 07/01/16 15:25 & $71-43-2$ & \\
\hline Bromobenzene & ND & $\mathrm{ug} / \mathrm{kg}$ & 6.5 & 1 & & $07 / 01 / 1615: 25$ & $108-86-1$ & \\
\hline Bromochloromethane & ND & $\mathrm{ug} / \mathrm{kg}$ & 6.5 & 1 & & 07/01/16 15:25 & $74-97-5$ & \\
\hline Bromodichloromethane & ND & $\mathrm{ug} / \mathrm{kg}$ & 6.5 & 1 & & 07/01/16 15:25 & $75-27-4$ & \\
\hline Bromoform & ND & $\mathrm{ug} / \mathrm{kg}$ & 6.5 & 1 & & 07/01/16 15:25 & $75-25-2$ & \\
\hline Bromomethane & ND & $\mathrm{ug} / \mathrm{kg}$ & 6.5 & 1 & & 07/01/16 15:25 & $74-83-9$ & \\
\hline 2-Butanone (MEK) & ND & $\mathrm{ug} / \mathrm{kg}$ & 13.1 & 1 & & 07/01/16 15:25 & $78-93-3$ & \\
\hline n-Butylbenzene & ND & $\mathrm{ug} / \mathrm{kg}$ & 6.5 & 1 & & 07/01/16 15:25 & $104-51-8$ & \\
\hline sec-Butylbenzene & ND & $\mathrm{ug} / \mathrm{kg}$ & 6.5 & 1 & & 07/01/16 15:25 & $135-98-8$ & \\
\hline tert-Butylbenzene & ND & $\mathrm{ug} / \mathrm{kg}$ & 6.5 & 1 & & 07/01/16 15:25 & $98-06-6$ & \\
\hline Carbon disulfide & ND & $\mathrm{ug} / \mathrm{kg}$ & 6.5 & 1 & & 07/01/16 15:25 & $75-15-0$ & \\
\hline Carbon tetrachloride & ND & $\mathrm{ug} / \mathrm{kg}$ & 6.5 & 1 & & 07/01/16 15:25 & $56-23-5$ & \\
\hline Chlorobenzene & ND & $\mathrm{ug} / \mathrm{kg}$ & 6.5 & 1 & & 07/01/16 15:25 & $108-90-7$ & \\
\hline Chloroethane & ND & $\mathrm{ug} / \mathrm{kg}$ & 6.5 & 1 & & 07/01/16 15:25 & $75-00-3$ & \\
\hline Chloroform & ND & $\mathrm{ug} / \mathrm{kg}$ & 6.5 & 1 & & 07/01/16 15:25 & $67-66-3$ & \\
\hline Chloromethane & ND & $\mathrm{ug} / \mathrm{kg}$ & 6.5 & 1 & & 07/01/16 15:25 & $74-87-3$ & \\
\hline 2-Chlorotoluene & ND & $\mathrm{ug} / \mathrm{kg}$ & 6.5 & 1 & & 07/01/16 15:25 & $95-49-8$ & \\
\hline 4-Chlorotoluene & ND & $\mathrm{ug} / \mathrm{kg}$ & 6.5 & 1 & & $07 / 01 / 1615: 25$ & $106-43-4$ & \\
\hline 1,2-Dibromo-3-chloropropane & ND & $\mathrm{ug} / \mathrm{kg}$ & 13.1 & 1 & & 07/01/16 15:25 & $96-12-8$ & \\
\hline Dibromochloromethane & ND & $\mathrm{ug} / \mathrm{kg}$ & 6.5 & 1 & & 07/01/16 15:25 & $124-48-1$ & \\
\hline 1,2-Dibromoethane (EDB) & ND & $\mathrm{ug} / \mathrm{kg}$ & 6.5 & 1 & & 07/01/16 15:25 & $106-93-4$ & \\
\hline Dibromomethane & ND & $\mathrm{ug} / \mathrm{kg}$ & 6.5 & 1 & & 07/01/16 15:25 & $74-95-3$ & \\
\hline 1,2-Dichlorobenzene & ND & $\mathrm{ug} / \mathrm{kg}$ & 6.5 & 1 & & 07/01/16 15:25 & $95-50-1$ & \\
\hline 1,3-Dichlorobenzene & ND & $\mathrm{ug} / \mathrm{kg}$ & 6.5 & 1 & & 07/01/16 15:25 & $541-73-1$ & \\
\hline 1,4-Dichlorobenzene & ND & $\mathrm{ug} / \mathrm{kg}$ & 6.5 & 1 & & 07/01/16 15:25 & $106-46-7$ & \\
\hline Dichlorodifluoromethane & ND & $\mathrm{ug} / \mathrm{kg}$ & 6.5 & 1 & & 07/01/16 15:25 & $75-71-8$ & \\
\hline 1,1-Dichloroethane & ND & $\mathrm{ug} / \mathrm{kg}$ & 6.5 & 1 & & 07/01/16 15:25 & $75-34-3$ & \\
\hline 1,2-Dichloroethane & ND & $\mathrm{ug} / \mathrm{kg}$ & 6.5 & 1 & & 07/01/16 15:25 & $107-06-2$ & \\
\hline 1,2-Dichloroethene (Total) & ND & $\mathrm{ug} / \mathrm{kg}$ & 6.5 & 1 & & 07/01/16 15:25 & $540-59-0$ & \\
\hline 1,1-Dichloroethene & ND & $\mathrm{ug} / \mathrm{kg}$ & 6.5 & 1 & & 07/01/16 15:25 & $75-35-4$ & \\
\hline cis-1,2-Dichloroethene & ND & $\mathrm{ug} / \mathrm{kg}$ & 6.5 & 1 & & 07/01/16 15:25 & $156-59-2$ & \\
\hline trans-1,2-Dichloroethene & ND & $\mathrm{ug} / \mathrm{kg}$ & 6.5 & 1 & & 07/01/16 15:25 & $156-60-5$ & \\
\hline 1,2-Dichloropropane & ND & $\mathrm{ug} / \mathrm{kg}$ & 6.5 & 1 & & 07/01/16 15:25 & $78-87-5$ & \\
\hline 1,3-Dichloropropane & ND & $\mathrm{ug} / \mathrm{kg}$ & 6.5 & 1 & & 07/01/16 15:25 & $142-28-9$ & \\
\hline 2,2-Dichloropropane & ND & $\mathrm{ug} / \mathrm{kg}$ & 6.5 & 1 & & 07/01/16 15:25 & $594-20-7$ & \\
\hline 1,1-Dichloropropene & ND & $\mathrm{ug} / \mathrm{kg}$ & 6.5 & 1 & & 07/01/16 15:25 & $563-58-6$ & L2 \\
\hline cis-1,3-Dichloropropene & ND & $\mathrm{ug} / \mathrm{kg}$ & 6.5 & 1 & & 07/01/16 15:25 & $10061-01-5$ & \\
\hline trans-1,3-Dichloropropene & ND & $\mathrm{ug} / \mathrm{kg}$ & 6.5 & 1 & & 07/01/16 15:25 & $10061-02-6$ & \\
\hline Ethylbenzene & ND & $\mathrm{ug} / \mathrm{kg}$ & 6.5 & 1 & & 07/01/16 15:25 & $100-41-4$ & \\
\hline Hexachloro-1,3-butadiene & ND & $\mathrm{ug} / \mathrm{kg}$ & 6.5 & 1 & & 07/01/16 15:25 & $87-68-3$ & \\
\hline 2-Hexanone & ND & $\mathrm{ug} / \mathrm{kg}$ & 26.2 & 1 & & 07/01/16 15:25 & $591-78-6$ & \\
\hline Isopropylbenzene (Cumene) & ND & $\mathrm{ug} / \mathrm{kg}$ & 6.5 & 1 & & 07/01/16 15:25 & $98-82-8$ & \\
\hline p-Isopropyltoluene & ND & $\mathrm{ug} / \mathrm{kg}$ & 6.5 & 1 & & 07/01/16 15:25 & $99-87-6$ & \\
\hline Methylene chloride & ND & $\mathrm{ug} / \mathrm{kg}$ & 6.5 & 1 & & 07/01/16 15:25 & $75-09-2$ & \\
\hline 4-Methyl-2-pentanone (MIBK) & ND & $\mathrm{ug} / \mathrm{kg}$ & 13.1 & 1 & & 07/01/16 15:25 & $108-10-1$ & \\
\hline
\end{tabular}

\section{REPORT OF LABORATORY ANALYSIS}




\section{ANALYTICAL RESULTS}

Project: Investigation Derived Waste

Pace Project No.: 60221829

Sample: YKROLLREAR-S-38122 Lab ID: 60221829004 Collected: 06/21/16 17:00 Received: 06/22/16 08:50 Matrix: Solid

Results reported on a "dry weight" basis and are adjusted for percent moisture, sample size and any dilutions.

\begin{tabular}{|c|c|c|c|c|c|c|c|}
\hline Parameters & Results & Units & Report Limit & DF & Prepared & Analyzed & CAS No. \\
\hline 8260 MSV 5035A VOA & \multicolumn{7}{|c|}{ Analytical Method: EPA 8260} \\
\hline Methyl-tert-butyl ether & ND & ug/kg & 6.5 & 1 & & 07/01/16 15:25 & $1634-04-4$ \\
\hline Naphthalene & ND & $\mathrm{ug} / \mathrm{kg}$ & 13.1 & 1 & & 07/01/16 15:25 & $91-20-3$ \\
\hline n-Propylbenzene & ND & $\mathrm{ug} / \mathrm{kg}$ & 6.5 & 1 & & 07/01/16 15:25 & $103-65-1$ \\
\hline Styrene & ND & $\mathrm{ug} / \mathrm{kg}$ & 6.5 & 1 & & 07/01/16 15:25 & $100-42-5$ \\
\hline $1,1,1,2$-Tetrachloroethane & ND & $\mathrm{ug} / \mathrm{kg}$ & 6.5 & 1 & & 07/01/16 15:25 & $630-20-6$ \\
\hline 1,1,2,2-Tetrachloroethane & ND & $\mathrm{ug} / \mathrm{kg}$ & 6.5 & 1 & & 07/01/16 15:25 & $79-34-5$ \\
\hline Tetrachloroethene & ND & $\mathrm{ug} / \mathrm{kg}$ & 6.5 & 1 & & 07/01/16 15:25 & $127-18-4$ \\
\hline Toluene & ND & $\mathrm{ug} / \mathrm{kg}$ & 6.5 & 1 & & 07/01/16 15:25 & $108-88-3$ \\
\hline 1,2,3-Trichlorobenzene & ND & $\mathrm{ug} / \mathrm{kg}$ & 6.5 & 1 & & 07/01/16 15:25 & $87-61-6$ \\
\hline 1,2,4-Trichlorobenzene & ND & $\mathrm{ug} / \mathrm{kg}$ & 6.5 & 1 & & 07/01/16 15:25 & $120-82-1$ \\
\hline 1,1,1-Trichloroethane & ND & $\mathrm{ug} / \mathrm{kg}$ & 6.5 & 1 & & 07/01/16 15:25 & $71-55-6$ \\
\hline 1,1,2-Trichloroethane & ND & $\mathrm{ug} / \mathrm{kg}$ & 6.5 & 1 & & 07/01/16 15:25 & $79-00-5$ \\
\hline Trichloroethene & ND & $\mathrm{ug} / \mathrm{kg}$ & 6.5 & 1 & & 07/01/16 15:25 & $79-01-6$ \\
\hline Trichlorofluoromethane & ND & $\mathrm{ug} / \mathrm{kg}$ & 6.5 & 1 & & 07/01/16 15:25 & $75-69-4$ \\
\hline 1,2,3-Trichloropropane & ND & $\mathrm{ug} / \mathrm{kg}$ & 6.5 & 1 & & 07/01/16 15:25 & $96-18-4$ \\
\hline 1,2,4-Trimethylbenzene & ND & $\mathrm{ug} / \mathrm{kg}$ & 6.5 & 1 & & 07/01/16 15:25 & $95-63-6$ \\
\hline 1,3,5-Trimethylbenzene & ND & $\mathrm{ug} / \mathrm{kg}$ & 6.5 & 1 & & 07/01/16 15:25 & $108-67-8$ \\
\hline Vinyl chloride & ND & $\mathrm{ug} / \mathrm{kg}$ & 6.5 & 1 & & 07/01/16 15:25 & $75-01-4$ \\
\hline Xylene (Total) & ND & $\mathrm{ug} / \mathrm{kg}$ & 6.5 & 1 & & 07/01/16 15:25 & $1330-20-7$ \\
\hline \multicolumn{8}{|l|}{ Surrogates } \\
\hline Toluene-d8 (S) & 107 & $\%$ & $80-120$ & 1 & & 07/01/16 15:25 & $2037-26-5$ \\
\hline 4-Bromofluorobenzene (S) & 99 & $\%$ & $80-120$ & 1 & & 07/01/16 15:25 & $460-00-4$ \\
\hline 1,2-Dichloroethane-d4 (S) & 98 & $\%$ & $83-120$ & 1 & & 07/01/16 15:25 & $17060-07-0$ \\
\hline
\end{tabular}

\section{Percent Moisture}

Percent Moisture
Analytical Method: ASTM D2974

$24.1 \quad \%$

$0.50 \quad 1$

06/29/16 00:00

\section{REPORT OF LABORATORY ANALYSIS}

This report shall not be reproduced, except in full,

without the written consent of Pace Analytical Services, Inc.. 


\section{QUALITY CONTROL DATA}

Project: Investigation Derived Waste

Pace Project No.: 60221829

\begin{tabular}{llll}
\hline QC Batch: & MSV/76626 & Analysis Method: & EPA 5030B/8260 \\
QC Batch Method: & EPA 5030B/8260 & Analysis Description: & 8260 MSV Water 7 day
\end{tabular}

Associated Lab Samples: $\quad$ 60221829001, 60221829002

METHOD BLANK: 1782303

Associated Lab Samples: $\quad$ 60221829001, 60221829002

$$
\text { Parameter }
$$

1,1,1,2-Tetrachloroethane

1,1,1-Trichloroethane

1,1,2,2-Tetrachloroethane

1,1,2-Trichloroethane

1,1-Dichloroethane

1,1-Dichloroethene

1,1-Dichloropropene

1,2,3-Trichlorobenzene

1,2,3-Trichloropropane

1,2,4-Trichlorobenzene

1,2,4-Trimethylbenzene

1,2-Dibromo-3-chloropropane

1,2-Dibromoethane (EDB)

1,2-Dichlorobenzene

1,2-Dichloroethane

1,2-Dichloroethene (Total)

1,2-Dichloropropane

1,3,5-Trimethylbenzene

1,3-Dichlorobenzene

1,3-Dichloropropane

1,4-Dichlorobenzene

2,2-Dichloropropane

2-Butanone (MEK)

2-Chlorotoluene

2-Hexanone

4-Chlorotoluene

4-Methyl-2-pentanone (MIBK)

Acetone

Benzene

Bromobenzene

Bromochloromethane

Bromodichloromethane

Bromoform

Bromomethane

Carbon disulfide

Carbon tetrachloride

Chlorobenzene

Chloroethane

Chloroform

Chloromethane

cis-1,2-Dichloroethene
Matrix: Water

Units $\quad$ Result

ug/L

ug/L

$\mathrm{ug} / \mathrm{L}$

ug/L

$\mathrm{ug} / \mathrm{L}$

ug/L

ug/L

ug/L

$u g / L$

ug/L

ug/L

$\mathrm{ug} / \mathrm{L}$

ug/L

$u g / L$

ug/L

ug/L

ug/L

ug/L

ug/L

ug/L

ug/L

ug/L

ug/L

ug/L

ug/L

ug/L

ug/L

ug/L

ug/L

ug/L

ug/L

ug/L

ug/L

ug/L

ug/L

ug/L

ug/L

ug/L

ug/L

ug/L

ug/L
Reporting

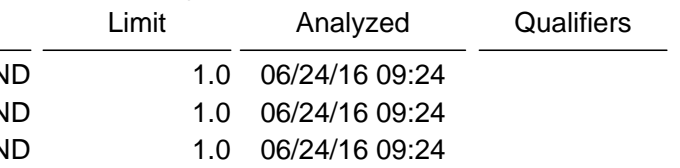

$1.0 \quad 06 / 24 / 1609 \cdot 24$

$1.0 \quad 06 / 24 / 16 \quad 09: 24$

$1.0 \quad 06 / 24 / 1609: 24$

$1.0 \quad 06 / 24 / 16 \quad 09: 24$

$1.0 \quad 06 / 24 / 1609: 24$

$2.5 \quad 06 / 24 / 1609: 24$

$1.0 \quad 06 / 24 / 1609: 24$

$1.0 \quad 06 / 24 / 16 \quad 09: 24$

$2.5 \quad 06 / 24 / 1609: 24$

$1.0 \quad 06 / 24 / 1609: 24$

$1.0 \quad 06 / 24 / 16 \quad 09: 24$

$1.0 \quad 06 / 24 / 16 \quad 09: 24$

$1.0 \quad 06 / 24 / 16 \quad 09: 24$

$1.0 \quad 06 / 24 / 1609: 24$

$1.0 \quad 06 / 24 / 16 \quad 09: 24$

$1.0 \quad 06 / 24 / 16 \quad 09: 24$

$1.0 \quad 06 / 24 / 1609: 24$

$1.0 \quad 06 / 24 / 16 \quad 09: 24$

$1.0 \quad 06 / 24 / 16 \quad 09: 24$

$10.0 \quad 06 / 24 / 1609: 24$

$1.0 \quad 06 / 24 / 16 \quad 09: 24$

$10.0 \quad 06 / 24 / 1609: 24$

$1.0 \quad 06 / 24 / 16 \quad 09: 24$

$10.0 \quad 06 / 24 / 16 \quad 09: 24$

$10.0 \quad 06 / 24 / 1609: 24$

$1.0 \quad 06 / 24 / 1609: 24$

$1.0 \quad 06 / 24 / 16 \quad 09: 24$

$1.0 \quad 06 / 24 / 16 \quad 09: 24$

$1.0 \quad 06 / 24 / 16 \quad 09: 24$

$1.0 \quad 06 / 24 / 16 \quad 09: 24$

$5.0 \quad 06 / 24 / 16 \quad 09: 24$

$5.0 \quad 06 / 24 / 16 \quad 09: 24$

$1.0 \quad 06 / 24 / 1609: 24$

$1.0 \quad 06 / 24 / 16 \quad 09: 24$

$1.0 \quad 06 / 24 / 1609: 24$

$1.0 \quad 06 / 24 / 16 \quad 09: 24$

$1.0 \quad 06 / 24 / 16 \quad 09: 24$

$1.0 \quad 06 / 24 / 16 \quad 09: 24$

Results presented on this page are in the units indicated by the "Units" column except where an alternate unit is presented to the right of the result.

\section{REPORT OF LABORATORY ANALYSIS}




\section{QUALITY CONTROL DATA}

Project: Investigation Derived Waste

Pace Project No.: 60221829

\begin{tabular}{|c|c|c|c|c|c|}
\hline \multicolumn{2}{|l|}{ METHOD BLANK: 1782303} & \multicolumn{2}{|c|}{ Matrix: Water } & \multirow[b]{3}{*}{ Analyzed } & \multirow[b]{3}{*}{ Qualifiers } \\
\hline Associated Lab Samples: & 60221829001,60221829002 & & & & \\
\hline Parameter & Units & $\begin{array}{l}\text { Blank } \\
\text { Result }\end{array}$ & $\begin{array}{l}\text { Reporting } \\
\text { Limit }\end{array}$ & & \\
\hline cis-1,3-Dichloropropene & ug/L & ND & 1.0 & 06/24/16 09:24 & \\
\hline Dibromochloromethane & $\mathrm{ug} / \mathrm{L}$ & ND & 1.0 & 06/24/16 09:24 & \\
\hline Dibromomethane & $u g / L$ & ND & 1.0 & 06/24/16 09:24 & \\
\hline Dichlorodifluoromethane & $\mathrm{ug} / \mathrm{L}$ & ND & 1.0 & $06 / 24 / 1609: 24$ & \\
\hline Ethylbenzene & $\mathrm{ug} / \mathrm{L}$ & ND & 1.0 & 06/24/16 09:24 & \\
\hline Hexachloro-1,3-butadiene & $u g / L$ & ND & 1.0 & 06/24/16 09:24 & \\
\hline Isopropylbenzene (Cumene) & $u g / L$ & ND & 1.0 & 06/24/16 09:24 & \\
\hline Methyl-tert-butyl ether & $\mathrm{ug} / \mathrm{L}$ & ND & 1.0 & 06/24/16 09:24 & \\
\hline Methylene chloride & $u g / L$ & ND & 1.0 & 06/24/16 09:24 & \\
\hline n-Butylbenzene & $\mathrm{ug} / \mathrm{L}$ & ND & 1.0 & 06/24/16 09:24 & \\
\hline n-Propylbenzene & $\mathrm{ug} / \mathrm{L}$ & ND & 1.0 & 06/24/16 09:24 & \\
\hline Naphthalene & $u g / L$ & ND & 10.0 & 06/24/16 09:24 & \\
\hline p-Isopropyltoluene & $u g / L$ & ND & 1.0 & 06/24/16 09:24 & \\
\hline sec-Butylbenzene & $\mathrm{ug} / \mathrm{L}$ & ND & 1.0 & 06/24/16 09:24 & \\
\hline Styrene & $u g / L$ & ND & 1.0 & 06/24/16 09:24 & \\
\hline tert-Butylbenzene & $u g / L$ & ND & 1.0 & 06/24/16 09:24 & \\
\hline Tetrachloroethene & $\mathrm{ug} / \mathrm{L}$ & ND & 1.0 & 06/24/16 09:24 & \\
\hline Toluene & $\mathrm{ug} / \mathrm{L}$ & ND & 1.0 & 06/24/16 09:24 & \\
\hline trans-1,2-Dichloroethene & $u g / L$ & ND & 1.0 & 06/24/16 09:24 & \\
\hline trans-1,3-Dichloropropene & $\mathrm{ug} / \mathrm{L}$ & ND & 1.0 & $06 / 24 / 1609: 24$ & \\
\hline Trichloroethene & $\mathrm{ug} / \mathrm{L}$ & ND & 1.0 & 06/24/16 09:24 & \\
\hline Trichlorofluoromethane & $u g / L$ & ND & 1.0 & 06/24/16 09:24 & \\
\hline Vinyl chloride & $u g / L$ & ND & 1.0 & 06/24/16 09:24 & \\
\hline Xylene (Total) & $\mathrm{ug} / \mathrm{L}$ & ND & 3.0 & 06/24/16 09:24 & \\
\hline 1,2-Dichloroethane-d4 (S) & $\%$ & 102 & $81-127$ & 06/24/16 09:24 & \\
\hline 4-Bromofluorobenzene (S) & $\%$ & 101 & $77-130$ & 06/24/16 09:24 & \\
\hline Toluene-d8 (S) & $\%$ & 98 & $80-120$ & 06/24/16 09:24 & \\
\hline
\end{tabular}

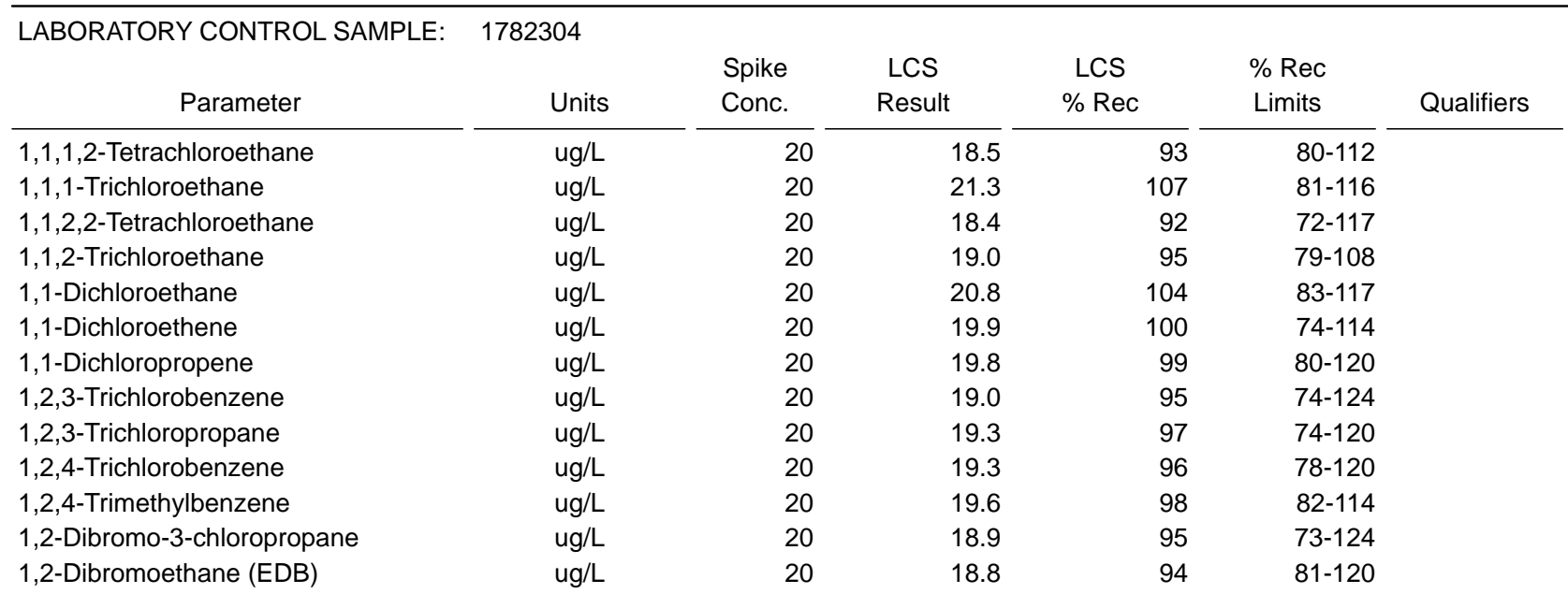

\section{REPORT OF LABORATORY ANALYSIS}




\section{QUALITY CONTROL DATA}

Project: Investigation Derived Waste

Pace Project No.: 60221829

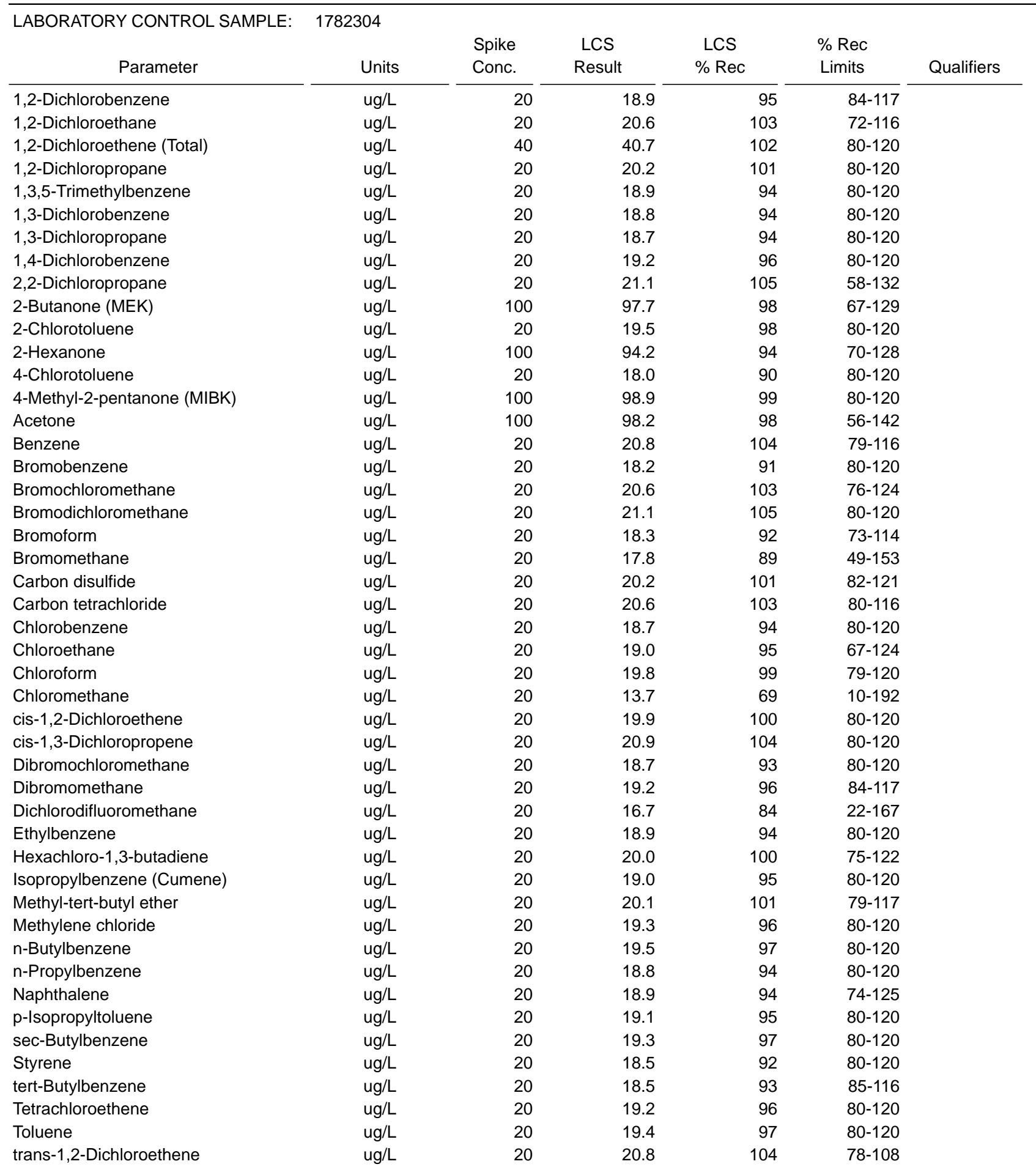




\section{QUALITY CONTROL DATA}

Project: Investigation Derived Waste

Pace Project No.: 60221829

\begin{tabular}{|c|c|c|c|c|c|c|}
\hline $\begin{array}{c}\text { LABORATORY CONTROL SAMPLE: } \\
\text { Parameter }\end{array}$ & $\begin{array}{r}1782304 \\
\text { Units }\end{array}$ & $\begin{array}{l}\text { Spike } \\
\text { Conc. }\end{array}$ & $\begin{array}{l}\text { LCS } \\
\text { Result }\end{array}$ & $\begin{array}{l}\text { LCS } \\
\% \operatorname{Rec}\end{array}$ & $\begin{array}{l}\% \text { Rec } \\
\text { Limits }\end{array}$ & Qualifiers \\
\hline trans-1,3-Dichloropropene & $\mathrm{ug} / \mathrm{L}$ & 20 & 19.7 & 99 & $85-120$ & \\
\hline Trichloroethene & $\mathrm{ug} / \mathrm{L}$ & 20 & 18.0 & 90 & $76-114$ & \\
\hline Trichlorofluoromethane & ug/L & 20 & 21.5 & 108 & $78-132$ & \\
\hline Vinyl chloride & ug/L & 20 & 20.3 & 101 & $69-129$ & \\
\hline Xylene (Total) & $u g / L$ & 60 & 56.0 & 93 & $80-120$ & \\
\hline 1,2-Dichloroethane-d4 (S) & $\%$ & & & 103 & $81-127$ & \\
\hline 4-Bromofluorobenzene (S) & $\%$ & & & 98 & $77-130$ & \\
\hline Toluene-d8 (S) & $\%$ & & & 99 & $80-120$ & \\
\hline
\end{tabular}




\section{QUALITY CONTROL DATA}

Project: Investigation Derived Waste

Pace Project No.: 60221829

\begin{tabular}{llll}
\hline QC Batch: & MSV/76784 & Analysis Method: & EPA 8260 \\
QC Batch Method: & EPA 8260 & Analysis Description: & 8260 MSV 5035A Volatile Organics
\end{tabular}

Associated Lab Samples: $\quad$ 60221829003, 60221829004

METHOD BLANK: 1786864

Associated Lab Samples: $\quad$ 60221829003, 60221829004

\section{Parameter}

1,1,1,2-Tetrachloroethane

1,1,1-Trichloroethane

1,1,2,2-Tetrachloroethane

1,1,2-Trichloroethane

1,1-Dichloroethane

1,1-Dichloroethene

1,1-Dichloropropene

1,2,3-Trichlorobenzene

1,2,3-Trichloropropane

1,2,4-Trichlorobenzene

1,2,4-Trimethylbenzene

1,2-Dibromo-3-chloropropane

1,2-Dibromoethane (EDB)

1,2-Dichlorobenzene

1,2-Dichloroethane

1,2-Dichloroethene (Total)

1,2-Dichloropropane

1,3,5-Trimethylbenzene

1,3-Dichlorobenzene

1,3-Dichloropropane

1,4-Dichlorobenzene

2,2-Dichloropropane

2-Butanone (MEK)

2-Chlorotoluene

2-Hexanone

4-Chlorotoluene

4-Methyl-2-pentanone (MIBK)

Acetone

Benzene

Bromobenzene

Bromochloromethane

Bromodichloromethane

Bromoform

Bromomethane

Carbon disulfide

Carbon tetrachloride

Chlorobenzene

Chloroethane

Chloroform

Chloromethane

cis-1,2-Dichloroethene
Matrix: Solid

$\begin{array}{ll} & \text { Blank } \\ \text { Units } & \text { Result }\end{array}$

$\mathrm{ug} / \mathrm{kg}$

$\mathrm{ug} / \mathrm{kg}$

\section{$\mathrm{ug} / \mathrm{kg}$}

$\mathrm{ug} / \mathrm{kg}$

$\mathrm{ug} / \mathrm{kg}$

$\mathrm{ug} / \mathrm{kg}$

$\mathrm{ug} / \mathrm{kg}$

$\mathrm{ug} / \mathrm{kg}$

ug/kg

$\mathrm{ug} / \mathrm{kg}$

$\mathrm{ug} / \mathrm{kg}$

ug/kg

$\mathrm{ug} / \mathrm{kg}$

$\mathrm{ug} / \mathrm{kg}$

$\mathrm{ug} / \mathrm{kg}$

ug/kg

$\mathrm{ug} / \mathrm{kg}$

$\mathrm{ug} / \mathrm{kg}$

$\mathrm{ug} / \mathrm{kg}$

$\mathrm{ug} / \mathrm{kg}$

$\mathrm{ug} / \mathrm{kg}$

$\mathrm{ug} / \mathrm{kg}$

$\mathrm{ug} / \mathrm{kg}$

ug/kg

$\mathrm{ug} / \mathrm{kg}$

$\mathrm{ug} / \mathrm{kg}$

$\mathrm{ug} / \mathrm{kg}$

$\mathrm{ug} / \mathrm{kg}$

ug/kg

$\mathrm{ug} / \mathrm{kg}$

ug/kg

$\mathrm{ug} / \mathrm{kg}$

$\mathrm{ug} / \mathrm{kg}$

$\mathrm{ug} / \mathrm{kg}$

$\mathrm{ug} / \mathrm{kg}$

ug/kg

ug/kg

$\mathrm{ug} / \mathrm{kg}$

$\mathrm{ug} / \mathrm{kg}$

$\mathrm{ug} / \mathrm{kg}$

$\mathrm{ug} / \mathrm{kg}$
Reporting

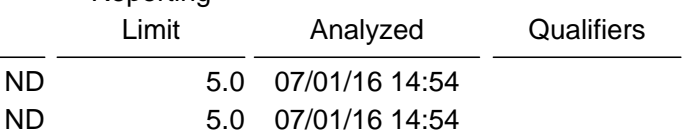

ND $\quad \begin{array}{lll}5.0 & 07 / 01 / 1614: 54\end{array}$

ND $\quad 5.0 \quad 07 / 01 / 1614: 54$

ND $\quad \begin{array}{lll}5.0 & 07 / 01 / 1614: 54\end{array}$

ND $\quad 5.0 \quad 07 / 01 / 1614: 54$

ND $\quad 5.0 \quad 07 / 01 / 1614: 54$

ND $\quad 5.0 \quad 07 / 01 / 1614: 54$

ND $\quad 5.0 \quad 07 / 01 / 1614: 54$

ND $\quad 5.0 \quad 07 / 01 / 1614: 54$

ND $\quad 5.0 \quad 07 / 01 / 1614: 54$

ND $\quad 10.0 \quad 07 / 01 / 1614: 54$

ND $\quad 5.0 \quad 07 / 01 / 1614: 54$

ND $\quad 5.0 \quad 07 / 01 / 1614: 54$

ND $\quad 5.0 \quad 07 / 01 / 1614: 54$

ND $\quad 5.0 \quad 07 / 01 / 1614: 54$

ND $\quad 5.0 \quad 07 / 01 / 1614: 54$

ND $\quad 5.0 \quad 07 / 01 / 1614: 54$

ND $\quad 5.0 \quad 07 / 01 / 1614: 54$

ND $\quad 5.0 \quad 07 / 01 / 1614: 54$

ND $\quad 5.0 \quad 07 / 01 / 1614: 54$

ND $\quad 5.0 \quad 07 / 01 / 1614: 54$

ND $\quad 10.0 \quad 07 / 01 / 1614: 54$

ND $\quad 5.0 \quad 07 / 01 / 1614: 54$

ND $\quad 20.0 \quad 07 / 01 / 1614: 54$

ND $\quad 5.0 \quad 07 / 01 / 1614: 54$

ND $\quad 10.0 \quad 07 / 01 / 1614: 54$

ND $\quad 20.0 \quad 07 / 01 / 1614: 54$

ND $\quad 5.0 \quad 07 / 01 / 1614: 54$

ND $\quad 5.0 \quad 07 / 01 / 1614: 54$

ND $\quad 5.0 \quad 07 / 01 / 1614: 54$

ND $\quad 5.0 \quad 07 / 01 / 1614: 54$

ND $\quad 5.0 \quad 07 / 01 / 1614: 54$

ND $\quad 5.0 \quad 07 / 01 / 1614: 54$

ND $\quad 5.0 \quad 07 / 01 / 1614: 54$

ND $\quad 5.0 \quad 07 / 01 / 1614: 54$

ND $\quad 5.0 \quad 07 / 01 / 1614: 54$

ND $\quad 5.0 \quad 07 / 01 / 1614: 54$

ND $\quad \begin{array}{lll}5.0 & 07 / 01 / 1614: 54\end{array}$

ND $\quad 5.0 \quad 07 / 01 / 1614: 54$

ND $\quad 5.0 \quad 07 / 01 / 1614: 54$

Results presented on this page are in the units indicated by the "Units" column except where an alternate unit is presented to the right of the result.

\section{REPORT OF LABORATORY ANALYSIS}




\section{QUALITY CONTROL DATA}

Project: $\quad$ Investigation Derived Waste

Pace Project No.: 60221829

METHOD BLANK: 1786864

Associated Lab Samples: $\quad 60221829003,60221829004$

Parameter

cis-1,3-Dichloropropene

Dibromochloromethane

Dibromomethane

Dichlorodifluoromethane

Ethylbenzene

Hexachloro-1,3-butadiene

Isopropylbenzene (Cumene)

Methyl-tert-butyl ether

Methylene chloride

n-Butylbenzene

n-Propylbenzene

Naphthalene

p-Isopropyltoluene

sec-Butylbenzene

Styrene

tert-Butylbenzene

Tetrachloroethene

Toluene

trans-1,2-Dichloroethene

trans-1,3-Dichloropropene

Trichloroethene

Trichlorofluoromethane

Vinyl chloride

Xylene (Total)

1,2-Dichloroethane-d4 (S)

4-Bromofluorobenzene (S)

Toluene-d8 (S)
Matrix: Solid

\begin{tabular}{|c|c|c|c|c|}
\hline Units & $\begin{array}{l}\text { Blank } \\
\text { Result }\end{array}$ & $\begin{array}{l}\text { Reporting } \\
\text { Limit }\end{array}$ & Analyzed & Qualifiers \\
\hline $\mathrm{ug} / \mathrm{kg}$ & ND & 5.0 & 07/01/16 14:54 & \\
\hline $\mathrm{ug} / \mathrm{kg}$ & ND & 5.0 & 07/01/16 14:54 & \\
\hline $\mathrm{ug} / \mathrm{kg}$ & ND & 5.0 & $07 / 01 / 1614: 54$ & \\
\hline $\mathrm{ug} / \mathrm{kg}$ & ND & 5.0 & 07/01/16 14:54 & \\
\hline $\mathrm{ug} / \mathrm{kg}$ & ND & 5.0 & 07/01/16 14:54 & \\
\hline $\mathrm{ug} / \mathrm{kg}$ & ND & 5.0 & $07 / 01 / 1614: 54$ & \\
\hline $\mathrm{ug} / \mathrm{kg}$ & ND & 5.0 & 07/01/16 14:54 & \\
\hline $\mathrm{ug} / \mathrm{kg}$ & 7.2 & 5.0 & 07/01/16 14:54 & \\
\hline $\mathrm{ug} / \mathrm{kg}$ & ND & 5.0 & $07 / 01 / 1614: 54$ & \\
\hline $\mathrm{ug} / \mathrm{kg}$ & ND & 5.0 & $07 / 01 / 1614: 54$ & \\
\hline $\mathrm{ug} / \mathrm{kg}$ & ND & 5.0 & 07/01/16 14:54 & \\
\hline $\mathrm{ug} / \mathrm{kg}$ & ND & 10.0 & $07 / 01 / 1614: 54$ & \\
\hline $\mathrm{ug} / \mathrm{kg}$ & ND & 5.0 & 07/01/16 14:54 & \\
\hline $\mathrm{ug} / \mathrm{kg}$ & ND & 5.0 & 07/01/16 14:54 & \\
\hline $\mathrm{ug} / \mathrm{kg}$ & ND & 5.0 & 07/01/16 14:54 & \\
\hline $\mathrm{ug} / \mathrm{kg}$ & ND & 5.0 & $07 / 01 / 1614: 54$ & \\
\hline $\mathrm{ug} / \mathrm{kg}$ & ND & 5.0 & 07/01/16 14:54 & \\
\hline $\mathrm{ug} / \mathrm{kg}$ & ND & 5.0 & $07 / 01 / 1614: 54$ & \\
\hline $\mathrm{ug} / \mathrm{kg}$ & ND & 5.0 & $07 / 01 / 1614: 54$ & \\
\hline $\mathrm{ug} / \mathrm{kg}$ & ND & 5.0 & 07/01/16 14:54 & \\
\hline $\mathrm{ug} / \mathrm{kg}$ & ND & 5.0 & 07/01/16 14:54 & \\
\hline $\mathrm{ug} / \mathrm{kg}$ & ND & 5.0 & $07 / 01 / 1614: 54$ & \\
\hline $\mathrm{ug} / \mathrm{kg}$ & ND & 5.0 & $07 / 01 / 1614: 54$ & \\
\hline $\mathrm{ug} / \mathrm{kg}$ & ND & 5.0 & 07/01/16 14:54 & \\
\hline$\%$ & 96 & $83-120$ & $07 / 01 / 1614: 54$ & \\
\hline$\%$ & 102 & $80-120$ & 07/01/16 14:54 & \\
\hline$\%$ & 103 & $80-120$ & 07/01/16 14:54 & \\
\hline
\end{tabular}

\section{LABORATORY CONTROL SAMPLE: 1786865}

\begin{tabular}{|c|c|c|c|c|c|c|}
\hline Parameter & Units & $\begin{array}{l}\text { Spike } \\
\text { Conc. }\end{array}$ & $\begin{array}{l}\text { LCS } \\
\text { Result }\end{array}$ & $\begin{array}{l}\text { LCS } \\
\% \operatorname{Rec}\end{array}$ & $\begin{array}{l}\% \operatorname{Rec} \\
\text { Limits }\end{array}$ & Qualifiers \\
\hline 1,1,1,2-Tetrachloroethane & $\mathrm{ug} / \mathrm{kg}$ & 100 & 102 & 102 & $74-117$ & \\
\hline 1,1,1-Trichloroethane & $\mathrm{ug} / \mathrm{kg}$ & 100 & 74.5 & 75 & $69-126$ & \\
\hline $1,1,2,2$-Tetrachloroethane & $\mathrm{ug} / \mathrm{kg}$ & 100 & 105 & 105 & $65-114$ & \\
\hline 1,1,2-Trichloroethane & $\mathrm{ug} / \mathrm{kg}$ & 100 & 94.4 & 94 & $69-113$ & \\
\hline 1,1-Dichloroethane & $\mathrm{ug} / \mathrm{kg}$ & 100 & 94.2 & 94 & $72-121$ & \\
\hline 1,1-Dichloroethene & $\mathrm{ug} / \mathrm{kg}$ & 100 & 89.4 & 89 & $59-131$ & \\
\hline 1,1-Dichloropropene & $\mathrm{ug} / \mathrm{kg}$ & 100 & 77.1 & 77 & $79-132$ & \\
\hline 1,2,3-Trichlorobenzene & $\mathrm{ug} / \mathrm{kg}$ & 100 & 111 & 111 & $76-128$ & \\
\hline 1,2,3-Trichloropropane & $\mathrm{ug} / \mathrm{kg}$ & 100 & 94.8 & 95 & $70-113$ & \\
\hline 1,2,4-Trichlorobenzene & $\mathrm{ug} / \mathrm{kg}$ & 100 & 107 & 107 & $76-132$ & \\
\hline 1,2,4-Trimethylbenzene & $\mathrm{ug} / \mathrm{kg}$ & 100 & 98.1 & 98 & $72-114$ & \\
\hline 1,2-Dibromo-3-chloropropane & $\mathrm{ug} / \mathrm{kg}$ & 100 & 92.8 & 93 & $67-123$ & \\
\hline 1,2-Dibromoethane (EDB) & $\mathrm{ug} / \mathrm{kg}$ & 100 & 97.4 & 97 & $79-118$ & \\
\hline
\end{tabular}

\section{REPORT OF LABORATORY ANALYSIS}




\section{QUALITY CONTROL DATA}

Project: Investigation Derived Waste

Pace Project No.: 60221829

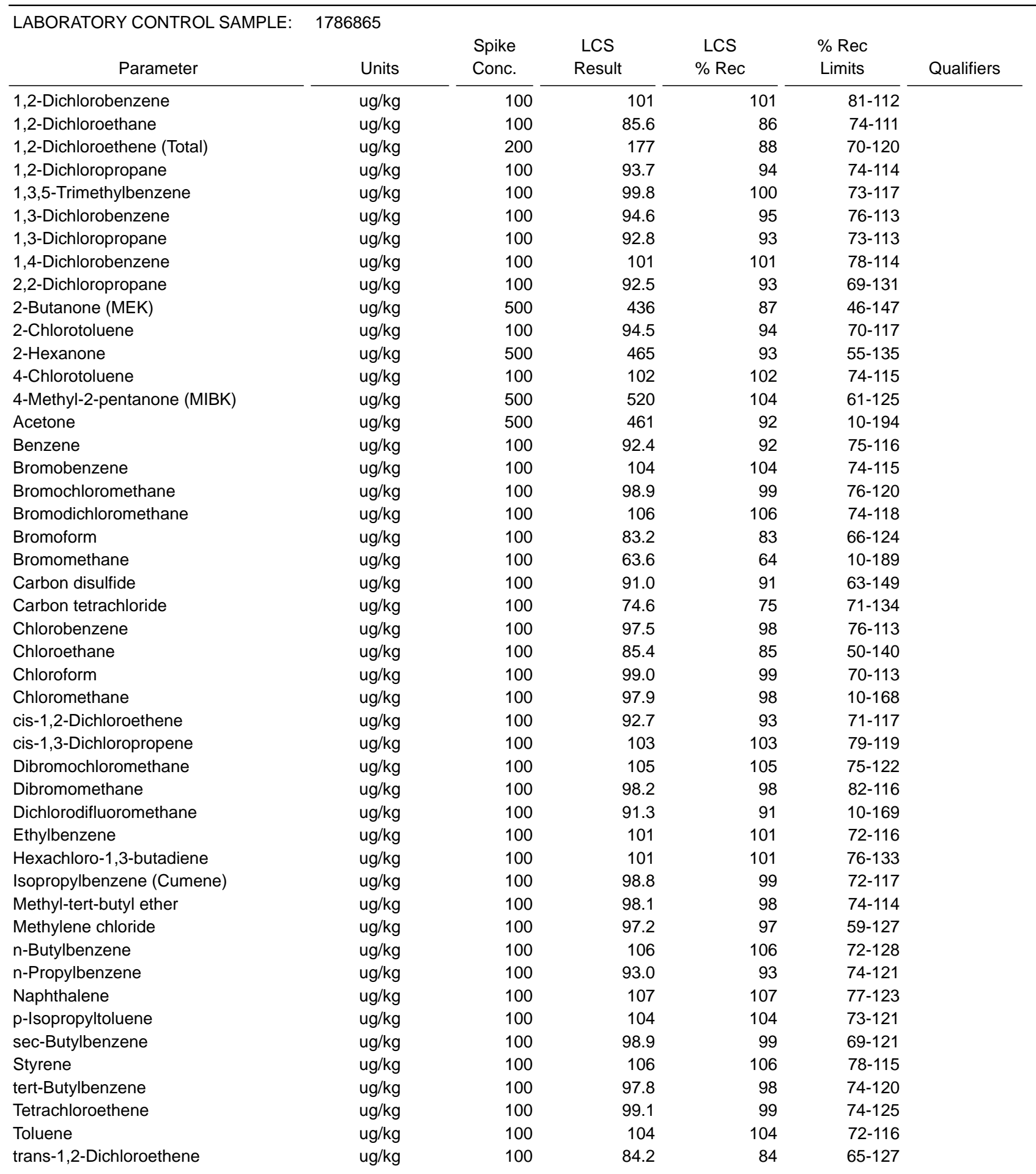




\section{QUALITY CONTROL DATA}

Project: Investigation Derived Waste

Pace Project No.: $\quad 60221829$

\begin{tabular}{|c|c|c|c|c|c|c|}
\hline LABORATORY CONTROL SAMPLE: & 1786865 & & & & & \\
\hline Parameter & Units & $\begin{array}{l}\text { Spike } \\
\text { Conc. }\end{array}$ & $\begin{array}{l}\text { LCS } \\
\text { Result }\end{array}$ & $\begin{array}{c}\text { LCS } \\
\% \operatorname{Rec}\end{array}$ & $\begin{array}{l}\% \text { Rec } \\
\text { Limits }\end{array}$ & Qualifiers \\
\hline trans-1,3-Dichloropropene & $\mathrm{ug} / \mathrm{kg}$ & 100 & 97.3 & 97 & $77-123$ & \\
\hline Trichloroethene & $\mathrm{ug} / \mathrm{kg}$ & 100 & 97.1 & 97 & $69-118$ & \\
\hline Trichlorofluoromethane & $\mathrm{ug} / \mathrm{kg}$ & 100 & 96.5 & 97 & $60-148$ & \\
\hline Vinyl chloride & $\mathrm{ug} / \mathrm{kg}$ & 100 & 91.7 & 92 & $43-159$ & \\
\hline Xylene (Total) & $\mathrm{ug} / \mathrm{kg}$ & 300 & 310 & 103 & $69-116$ & \\
\hline 1,2-Dichloroethane-d4 (S) & $\%$ & & & 93 & $83-120$ & \\
\hline 4-Bromofluorobenzene (S) & $\%$ & & & 103 & $80-120$ & \\
\hline Toluene-d8 (S) & $\%$ & & & 104 & $80-120$ & \\
\hline
\end{tabular}

MATRIX SPIKE \& MATRIX SPIKE DUPLICATE: 1786866

Parameter

1,1,1,2-Tetrachloroethane

1,1,1-Trichloroethane

1,1,2,2-Tetrachloroethane

1,1,2-Trichloroethane

1,1-Dichloroethane

1,1-Dichloroethene

1,1-Dichloropropene

1,2,3-Trichlorobenzene

1,2,3-Trichloropropane

1,2,4-Trichlorobenzene

1,2,4-Trimethylbenzene

1,2-Dibromo-3-

chloropropane

1,2-Dibromoethane (EDB)

1,2-Dichlorobenzene

1,2-Dichloroethane

1,2-Dichloroethene (Total)

1,2-Dichloropropane

1,3,5-Trimethylbenzene

1,3-Dichlorobenzene

1,3-Dichloropropane

1,4-Dichlorobenzene

2,2-Dichloropropane

2-Butanone (MEK)

2-Chlorotoluene

2-Hexanone

4-Chlorotoluene

4-Methyl-2-pentanone

(MIBK)

Acetone

Benzene

Bromobenzene

Bromochloromethane

$\mathrm{ug} / \mathrm{kg}$
60222430001 Spike Spike

Units $\frac{\text { Result }}{\text { ND }}$ Conc.

ug/kg

\section{$\mathrm{ug} / \mathrm{kg}$}

$\mathrm{ug} / \mathrm{kg}$

$\mathrm{ug} / \mathrm{kg}$

$\mathrm{ug} / \mathrm{kg}$

$\mathrm{ug} / \mathrm{kg}$

$\mathrm{ug} / \mathrm{kg}$

$\mathrm{ug} / \mathrm{kg}$

\section{$\mathrm{ug} / \mathrm{kg}$}

$\mathrm{ug} / \mathrm{kg}$

$\mathrm{ug} / \mathrm{kg}$

$\mathrm{ug} / \mathrm{kg}$

$\mathrm{ug} / \mathrm{kg}$

$\mathrm{ug} / \mathrm{kg}$

$\mathrm{ug} / \mathrm{kg}$

\section{$\mathrm{ug} / \mathrm{kg}$}

$\mathrm{ug} / \mathrm{kg}$

$\mathrm{ug} / \mathrm{kg}$

$\mathrm{ug} / \mathrm{kg}$

$\mathrm{ug} / \mathrm{kg}$

$\mathrm{ug} / \mathrm{kg}$

$\mathrm{ug} / \mathrm{kg}$

$\mathrm{ug} / \mathrm{kg}$

$\mathrm{ug} / \mathrm{kg}$

$\mathrm{ug} / \mathrm{kg}$

$\mathrm{ug} / \mathrm{kg}$

$\mathrm{ug} / \mathrm{kg}$

$\mathrm{ug} / \mathrm{kg}$

$\mathrm{ug} / \mathrm{kg}$

$\mathrm{ug} / \mathrm{kg}$

ND $\quad 4910 \quad 4910$

ND $4910 \quad 4910$

ND $\quad 4910 \quad 4910$

ND $\quad 4910 \quad 4910$

ND $\quad 4910 \quad 4910$

ND $\quad 4910 \quad 4910$

ND $\quad 4910 \quad 4910$

ND $\quad 4910 \quad 4910$

ND $\quad 4910 \quad 4910$

ND $4910 \quad 4910$

ND $\quad 4910 \quad 4910$

ND $\quad 4910$

4910

ND 4910

4910

$\begin{array}{lll}\text { ND } & 4910 & 4910 \\ \text { ND } & 9820 & 9820\end{array}$

ND $\quad 4910 \quad 4910$

ND $\quad 4910 \quad 4910$

ND $\quad 4910 \quad 4910$

ND $\quad 4910 \quad 4910$

ND $\quad 4910 \quad 4910$

ND $4910 \quad 4910$

362J $24600 \quad 24600$

ND $\quad 4910 \quad 4910$

ND $24600 \quad 24600$

ND $\quad 4910 \quad 4910$

ND $24600 \quad 24600$

ND $24600 \quad 24600$

ND $\quad 4910 \quad 4910$

ND $\quad 4910 \quad 4910$
1786867

MS MSD MS MSD \% Rec Max

Result Result \% Rec \% Rec Limits RPD RPD Qual

4740

4010

5240

4300

$$
4150
$$

4210

4410

5660

$$
4500
$$

5610

$$
4630
$$

$$
5340
$$

4680

$$
4890
$$

5360

7640

4330

4800

4600

4590

5020

3860

20500

4530

23900

4850

26000

4950

4450

5520

4790

4710

4630

3690

5540

5330

5670

4930

5490

97
82
107
88
84
86
90
115
92
114
94
109

5130

5090

4540

8630

5040

5190

4870

4940

5160

4230

22600

4780

25900

5050

28100

24200

5360

5450

4640

25000

4700

5690

5090

95
100
109
78
88
98
94
93
102
79
82
92
97
99
106
97
109
111
94

10
91
11
98
9
94
75
113
109
115
10
11
104
104
92
88
103
106
99
101
105
86
91
97
105
103
114
100
96
116
104

$\begin{array}{rrrrr}101 & 10-131 & 4 & 60 \\ 91 & 28-130 & 10 & 44 \\ 112 & 10-132 & 5 & 60 \\ 98 & 10-130 & 11 & 53\end{array}$

Results presented on this page are in the units indicated by the "Units" column except where an alternate unit is presented to the right of the result.

\section{REPORT OF LABORATORY ANALYSIS}




\section{QUALITY CONTROL DATA}

Project: Investigation Derived Waste

Pace Project No.: 60221829

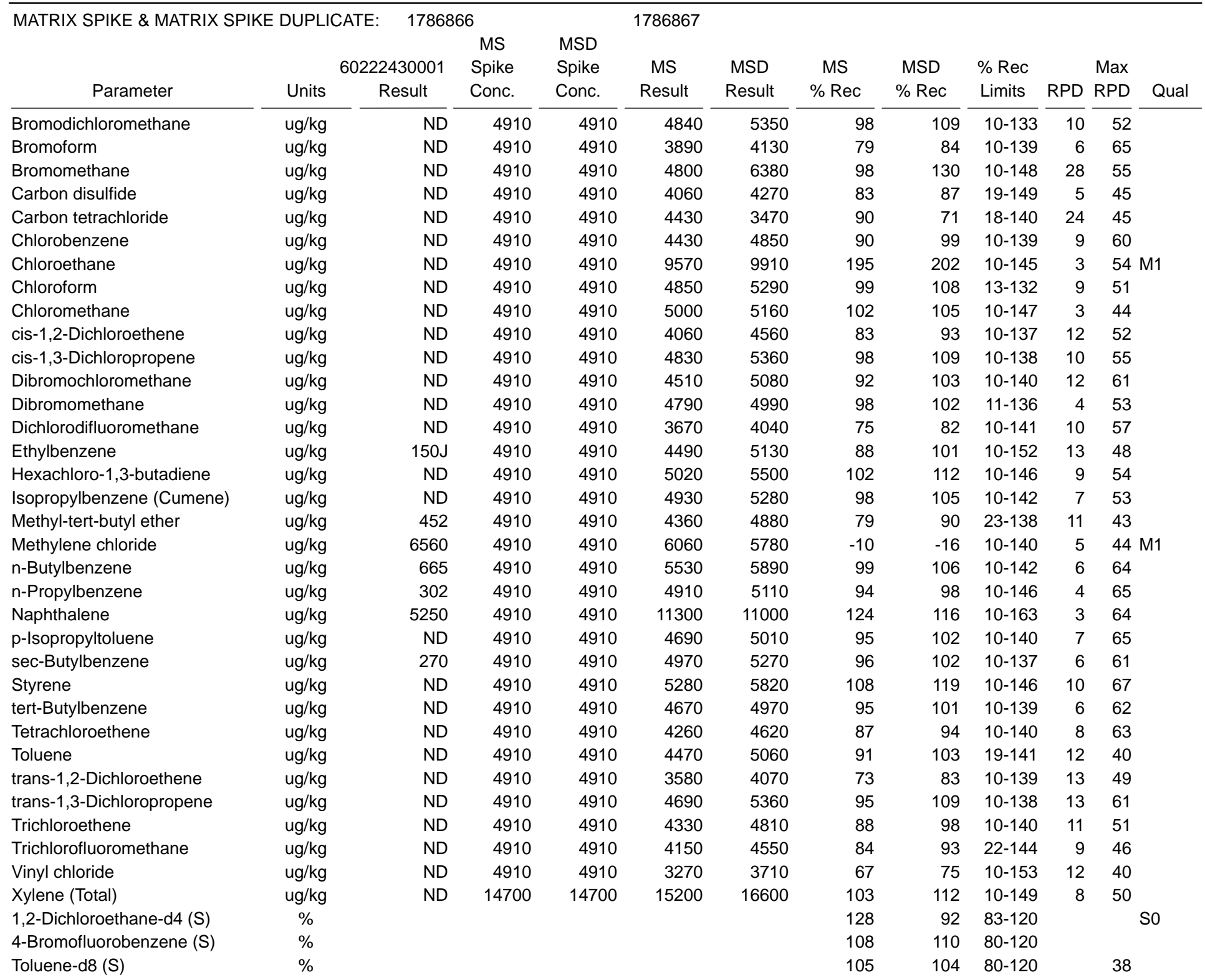




\section{QUALITY CONTROL DATA}

Project: Investigation Derived Waste

Pace Project No.: 60221829

\begin{tabular}{llll}
\hline QC Batch: & OEXT/54923 & Analysis Method: & EPA 504.1 \\
QC Batch Method: & EPA 504.1 & Analysis Description: & GCS 504 EDB DBCP
\end{tabular}

Associated Lab Samples: $\quad 60221829001$

METHOD BLANK: 1785951

Matrix: Water

Associated Lab Samples: $\quad 60221829001$

$\frac{\text { Parameter }}{1,2 \text {-Dibromoethane (EDB) }} \frac{\text { Units }}{\mathrm{ug} / \mathrm{L}} \frac{\begin{array}{l}\text { Blank } \\ \text { Result }\end{array}}{\mathrm{ND}} \frac{\begin{array}{c}\text { Reporting } \\ \text { Limit }\end{array}}{0.030} \frac{\text { Analyzed }}{06 / 30 / 1620: 50} \frac{\text { Qualifiers }}{-}$

\begin{tabular}{|c|c|c|c|c|c|c|c|c|c|c|}
\hline \multirow{2}{*}{ LABORATORY CONTROL SAMPLE \& LCSD: } & \multirow[t]{2}{*}{1785952} & \multicolumn{5}{|c|}{1785953} & \multirow{3}{*}{$\begin{array}{l}\% \text { Rec } \\
\text { Limits }\end{array}$} & \multirow{2}{*}{\multicolumn{2}{|c|}{ Max }} & \multirow[b]{3}{*}{ Qualifiers } \\
\hline & & Spike & LCS & LCSD & LCS & LCSD & & & & \\
\hline Parameter & Units & Conc. & Result & Result & $\% \operatorname{Rec}$ & $\% \operatorname{Rec}$ & & RPD & RPD & \\
\hline 1,2-Dibromoethane (EDB) & $\mathrm{ug} / \mathrm{L}$ & .25 & 0.25 & 0.27 & 98 & 107 & $70-130$ & 9 & 2 & \\
\hline
\end{tabular}

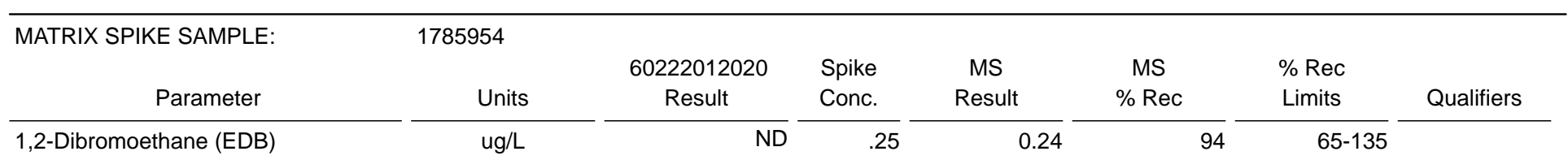

\section{REPORT OF LABORATORY ANALYSIS}




\section{QUALITY CONTROL DATA}

Project: Investigation Derived Waste

Pace Project No.: 60221829

\begin{tabular}{llll}
\hline QC Batch: & PMST/11892 & Analysis Method: & ASTM D2974 \\
QC Batch Method: & ASTM D2974 & Analysis Description: & Dry Weight/Percent Moisture
\end{tabular}

Associated Lab Samples: $\quad$ 60221829003, 60221829004

METHOD BLANK: 1784997

Associated Lab Samples: $\quad$ 60221829003, 60221829004

$\frac{\text { Parameter }}{\text { Percent Moisture }} \frac{\text { Units }}{\%}$

SAMPLE DUPLICATE: 1784998

Parameter

Percent Moisture
Matrix: Solid

Blank Reporting

Result Limit Analyzed Qualifiers
Qualifiers 


\section{QUALITY CONTROL DATA}

Project: Investigation Derived Waste

Pace Project No.: 60221829

\begin{tabular}{llll}
\hline QC Batch: & WETA/40207 & Analysis Method: & EPA 353.2 \\
QC Batch Method: & EPA 353.2 & Analysis Description: & 353.2 Nitrate + Nitrite, Unpres.
\end{tabular}

Associated Lab Samples: $\quad 60221829001$

METHOD BLANK: 1780668

Associated Lab Samples: $\quad 60221829001$

$\frac{\text { Parameter }}{\text { Nitrogen, Nitrate }} \frac{\text { Units }}{\mathrm{mg} / \mathrm{L}} \frac{\text { Result }}{\mathrm{ND}} \frac{\begin{array}{c}\text { Blank } \\ \text { Limit }\end{array}}{0.10} \frac{\text { Analyzed }}{06 / 22 / 1615: 42} \frac{\text { Qualifiers }}{-}$

\begin{tabular}{|c|c|c|c|c|c|c|}
\hline LABORATORY CONTROL SAMPLE: & 1780669 & & & & & \\
\hline & & Spike & LCS & LCS & $\% \operatorname{Rec}$ & \\
\hline Parameter & Units & Conc. & Result & $\% \operatorname{Rec}$ & Limits & Qualifiers \\
\hline Nitrogen, Nitrate & $\mathrm{mg} / \mathrm{L}$ & 1.6 & 1.6 & 101 & $85-115$ & \\
\hline
\end{tabular}

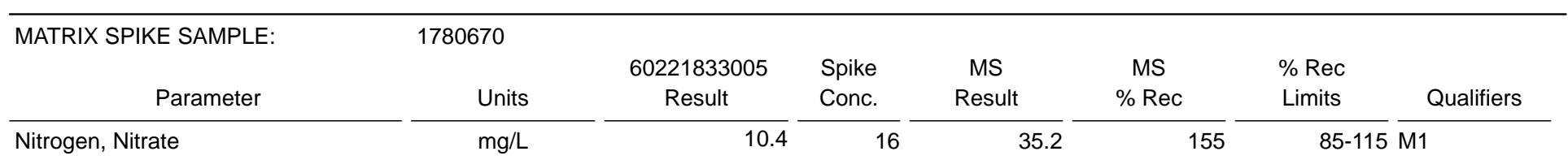

\begin{tabular}{|c|c|c|c|c|c|c|c|}
\hline MATRIX SPIKE SAMPLE: & 1780672 & & & & & & \\
\hline & & 60221779006 & Spike & MS & MS & $\% \operatorname{Rec}$ & \\
\hline Parameter & Units & Result & Conc. & Result & $\% \operatorname{Rec}$ & Limits & Qualifiers \\
\hline Nitrogen, Nitrate & $\mathrm{mg} / \mathrm{L}$ & 1.6 & 1.6 & 3.2 & 98 & $85-115$ & \\
\hline
\end{tabular}

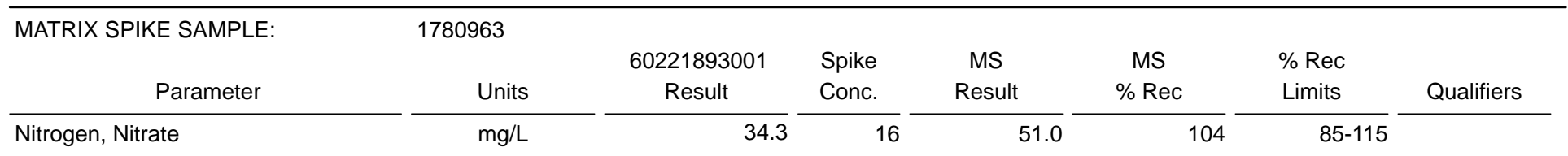

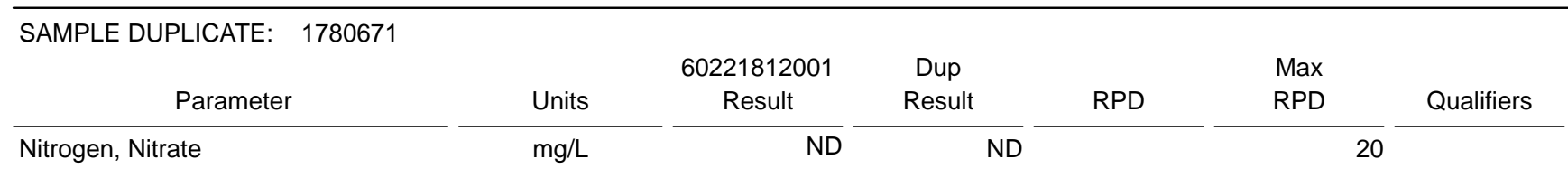

\section{REPORT OF LABORATORY ANALYSIS}




\section{QUALIFIERS}

Project: $\quad$ Investigation Derived Waste

Pace Project No.: 60221829

\section{DEFINITIONS}

DF - Dilution Factor, if reported, represents the factor applied to the reported data due to dilution of the sample aliquot.

ND - Not Detected at or above adjusted reporting limit.

$\mathrm{J}$ - Estimated concentration above the adjusted method detection limit and below the adjusted reporting limit.

MDL - Adjusted Method Detection Limit.

PQL - Practical Quantitation Limit.

$\mathrm{RL}$ - Reporting Limit.

$S$ - Surrogate

1,2-Diphenylhydrazine decomposes to and cannot be separated from Azobenzene using Method 8270 . The result for each analyte is a combined concentration.

Consistent with EPA guidelines, unrounded data are displayed and have been used to calculate \% recovery and RPD values.

LCS(D) - Laboratory Control Sample (Duplicate)

MS(D) - Matrix Spike (Duplicate)

DUP - Sample Duplicate

RPD - Relative Percent Difference

NC - Not Calculable.

SG - Silica Gel - Clean-Up

$U$ - Indicates the compound was analyzed for, but not detected.

N-Nitrosodiphenylamine decomposes and cannot be separated from Diphenylamine using Method 8270. The result reported for each analyte is a combined concentration.

Pace Analytical is TNI accredited. Contact your Pace PM for the current list of accredited analytes.

TNI - The NELAC Institute.

\section{BATCH QUALIFIERS}

Batch: MSV/76626

[M5] A matrix spike/matrix spike duplicate was not performed for this batch due to insufficient sample volume.

\section{ANALYTE QUALIFIERS}

L0 Analyte recovery in the laboratory control sample (LCS) was outside QC limits.

L2 Analyte recovery in the laboratory control sample (LCS) was below QC limits. Results for this analyte in associated samples may be biased low.

M1 Matrix spike recovery exceeded QC limits. Batch accepted based on laboratory control sample (LCS) recovery.

So Surrogate recovery outside laboratory control limits.

S3 Surrogate recovery exceeded laboratory control limits. Analyte presence below reporting limits in associated samples. Results unaffected by high bias.

\section{REPORT OF LABORATORY ANALYSIS}




\section{QUALITY CONTROL DATA CROSS REFERENCE TABLE}

Project: Investigation Derived Waste

Pace Project No.: 60221829

\begin{tabular}{|c|c|c|c|c|c|}
\hline Lab ID & Sample ID & QC Batch Method & QC Batch & Analytical Method & $\begin{array}{l}\text { Analytical } \\
\text { Batch }\end{array}$ \\
\hline 60221829001 & YKIDW-W-38119 & EPA 504.1 & OEXT/54923 & EPA 504.1 & GCSV/21368 \\
\hline 60221829001 & YKIDW-W-38119 & EPA $5030 B / 8260$ & MSV/76626 & & \\
\hline 60221829002 & YKQCTB-W-38120 & EPA 5030B/8260 & MSV/76626 & & \\
\hline 60221829003 & YKROLLFRONT-S-38121 & EPA 8260 & MSV/76784 & & \\
\hline 60221829004 & YKROLLREAR-S-38122 & EPA 8260 & MSV/76784 & & \\
\hline 60221829003 & YKROLLFRONT-S-38121 & ASTM D2974 & PMST/11892 & & \\
\hline 60221829004 & YKROLLREAR-S-38122 & ASTM D2974 & PMST/11892 & & \\
\hline 60221829001 & YKIDW-W-38119 & EPA 353.2 & WETA/40207 & & \\
\hline
\end{tabular}

\section{REPORT OF LABORATORY ANALYSIS}


Client Name:

TCW const.

Courier: $\quad$ FedEX $\otimes$ UPS $\square \quad$ VIA $\square \quad$ Clay $\square \quad$ PEX $\square \quad$ ECI $\square \quad$ Pace $\square$ Other $\square$ Client $\square$

Tracking \#: $\quad 776574714461$

Pace Shipping Label Used? Yes $\square \quad$ No $\square$

Optional

Custody Seal on Cooler/Box Present: $\quad$ Yes $\square$ No $\square$ Seals intact: Yes $\square$ No $\square$

Packing Material: $\quad$ Bubble Wrap $\square \quad$ Bubble Bags $\square \quad$ Foam $\square \quad$ None $\square \quad$ Other $\square$

Thermometer Used: $\quad$ Ty-239, T-262

Cooler Temperature: 1.1 (circle one)

Temperature should be above freezing to $6^{\circ} \mathrm{C}$

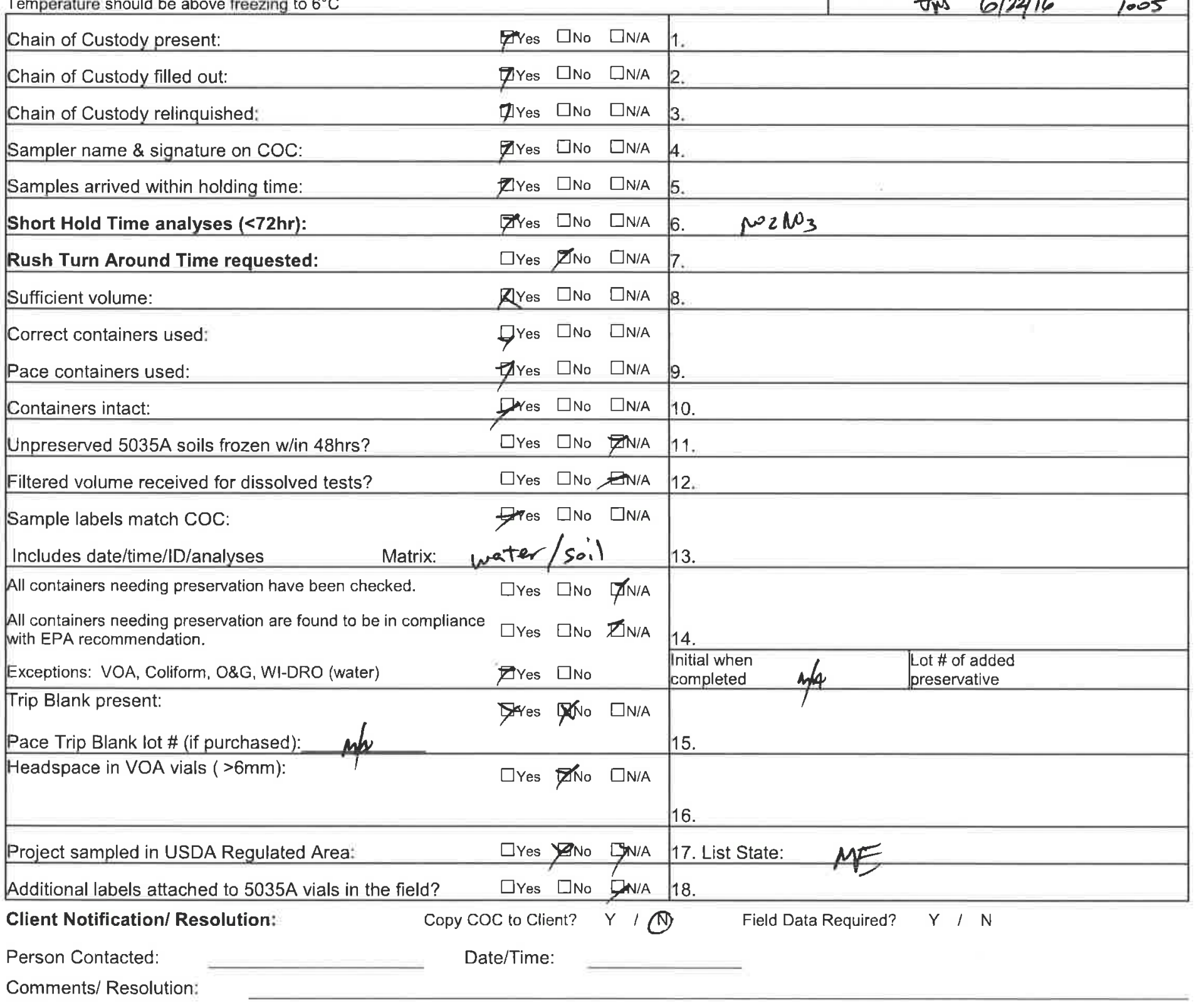

Project Manager Review:

Date:

6.22 .16

Date and initials of person examining contents:

Proj Due Date:

Proj Name: 


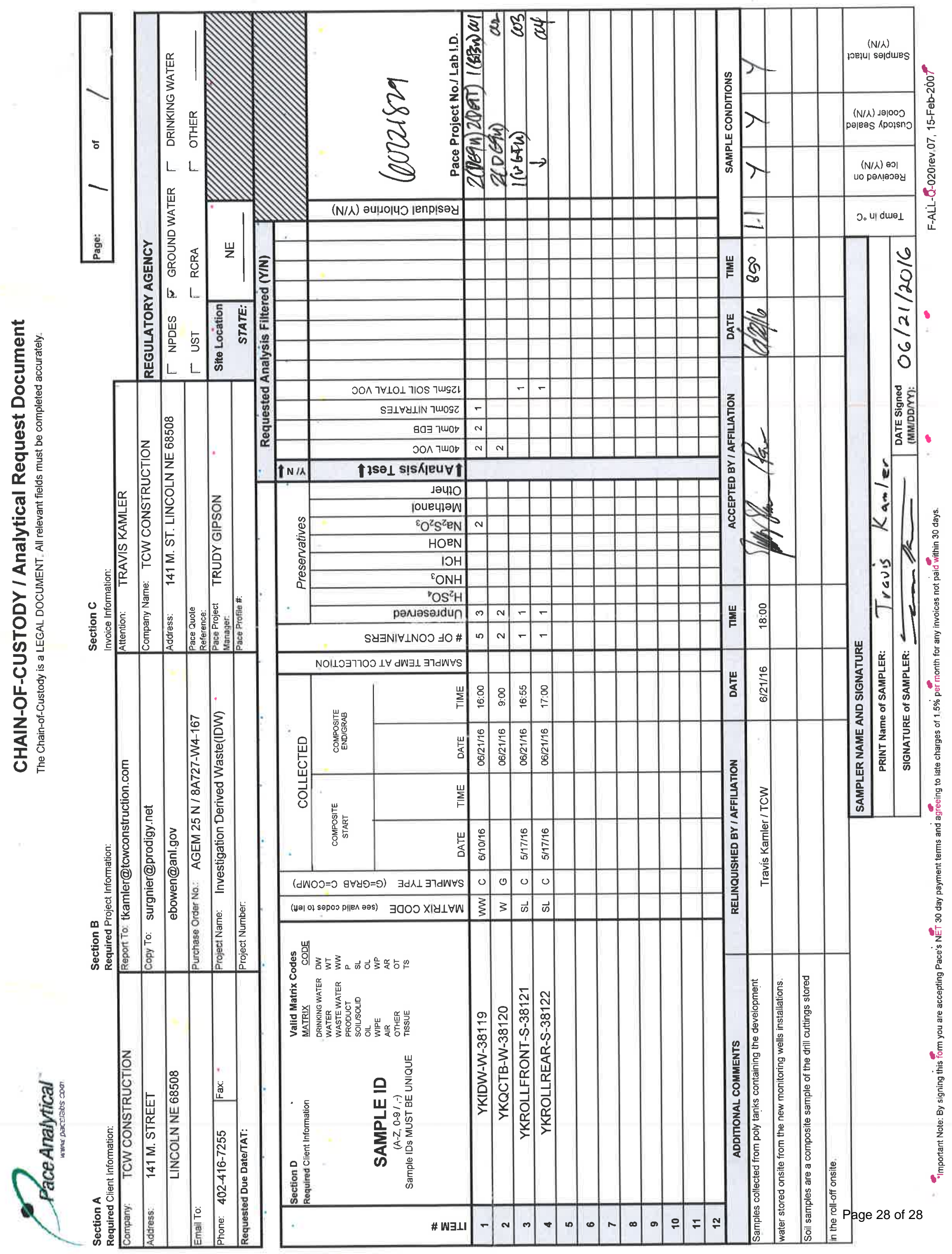




\section{Supplement 4:}

\section{TestAmerica Data}




\section{Contents}

SDG-200-28907-1..

Page 3 of 975

SDG-200-28914-1.

Page 68 of 975

SDG-200-28963-1.

Page 189 of 975

SDG-200-28988-1. Page 274 of 975

SDG-200-29233-1. Page 318 of 975

SDG-200-29312-1. Page 401 of 975

SDG-200-29407-1. Page 459 of 975

SDG-200-29506-1 Page 517 of 975

SDG-200-29764-1. Page 584 of 975

SDG-200-31673-1. Page 636 of 975

SDG-200-32333-1. Page 703 of 975

SDG-200-33767-1 Page 749 of 975

SDG-200-33933-1. Page 827 of 975

SDG-200-33957-1. Page 874 of 975

SDG-200-34024-1 Page 924 of 975 


\section{TestAmerica}

THE LEADER IN ENVIRONMENTAL TESTING

\section{ANALYTICAL REPORT}

Job Number: 200-28907-1

SDG Number: 28907

Job Description: York (200-28907)

Contract Number: 1E-30401

For:

Argonne National Laboratory

9700 South Cass Avenue

Building 203

Office B-141

Argonne, IL 60439

Attention: Ms. Esther Bowen

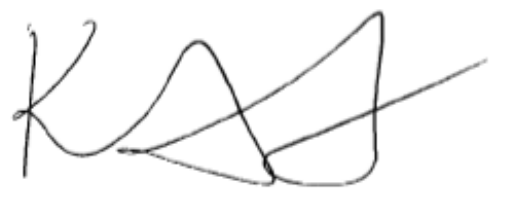

Approved for release. Kathryn A Kelly

\section{Kathryn A Kelly, Project Manager I \\ 30 Community Drive, South Burlington, VT, 05403 \\ kathryn.kelly@testamericainc.com \\ 07/31/2015}

The test results in this report relate only to sample(s) as received by the laboratory. These test results were derived under a quality system that adheres to the requirements of NELAC. Pursuant to NELAC, this report may not be produced in full without written approval from the laboratory 


\section{Table of Contents}

Cover Title Page . . . . . . . . . . . . . . . . 1

Report Narrative . . . . . . . . . . . . . . . . . 4

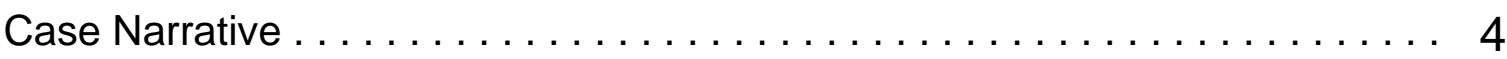

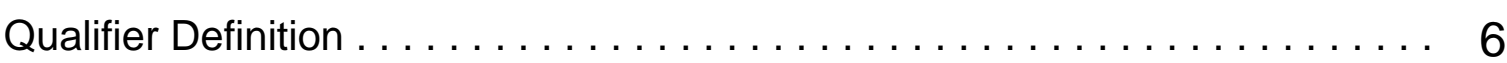

External Chain of Custody . . . . . . . . . . . . . . 7

Shipping Documentation . . . . . . . . . . . . . . 9

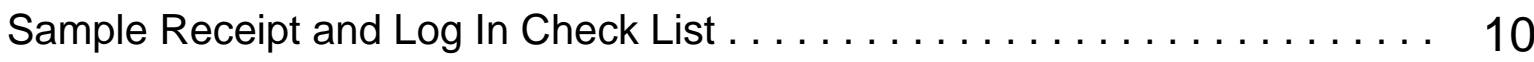

Methodology Review . . . . . . . . . . . . . . . . 11

Report Narrative . . . . . . . . . . . . . . . . . . 12

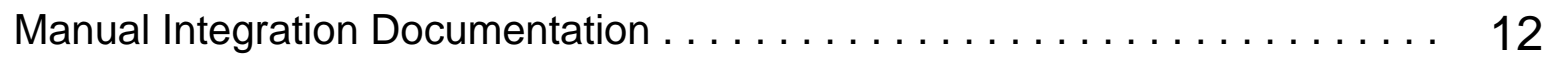

QC Summary - SOM01.2 Volatiles-Trace . . . . . . . . . . . . . 14

QC Summary - SOM01.2 Volatiles-Trace . . . . . . . . . . . . . . 14

Deuterated Monitoring Compound Summary . . . . . . . . . . . . . . . . 14

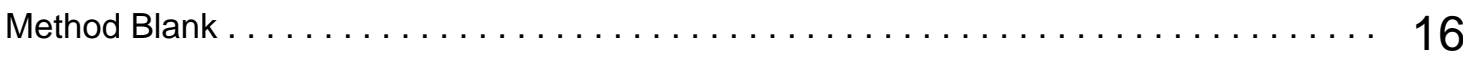

GC/MS Instrument Performance Check $\ldots \ldots \ldots \ldots \ldots \ldots \ldots \ldots \ldots \ldots \ldots \ldots \ldots \ldots$

Internal Standard Area and RT Summary $\ldots \ldots \ldots \ldots \ldots \ldots \ldots \ldots \ldots \ldots \ldots \ldots \ldots \ldots$

Sample Data - SOM01.2 Volatiles-Trace . . . . . . . . . . . 23

Sample Data - SOM01.2 Volatiles-Trace . . . . . . . . . . . . . . 23

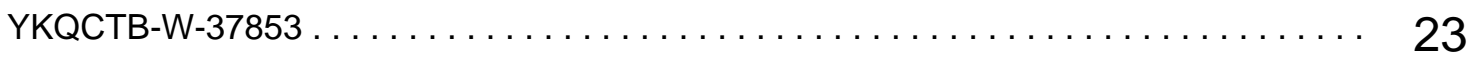

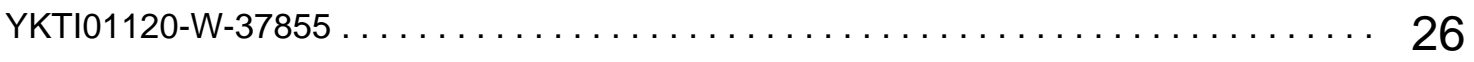

YKTI02120-W-37852 .................................... 29

Standards - SOM01.2 Volatiles-Trace . . . . . . . . . . . . . 32

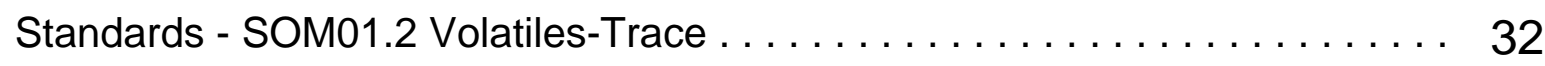

Initial Calibration Data ................................... 32

CCV Data, including closing CCV $\ldots \ldots \ldots \ldots \ldots \ldots \ldots \ldots \ldots \ldots \ldots \ldots \ldots \ldots \ldots \ldots$

Raw Qc Data - SOM01.2 Volatiles-Trace . . . . . . . . . . . . . . 47 


\section{Table of Contents}

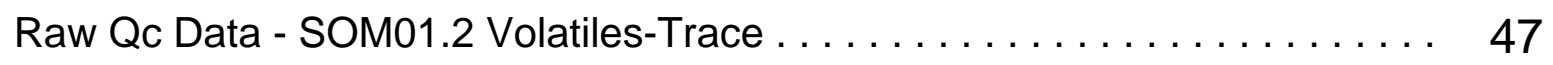

Raw Qc Data - SOM01.2 Volatiles-Trace . . . . . . . . . . . . . . . . . . . 47

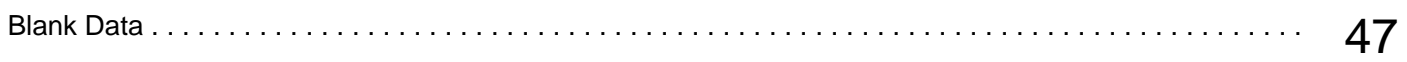

Standards Traceability . . . . . . . . . . . . . . 58 


\section{CASE NARRATIVE}

\section{Client: Argonne National Laboratory \\ Project: York (200-28907)}

\section{Report Number: 200- 28907-1}

Enclosed is the data set for the referenced project work. With the exceptions noted as flags or footnotes, standard analytical protocols were followed in performing the analytical work and the applied control limits were met.

Calculations were performed before rounding to avoid round-off errors in calculated results.

All holding times were met and proper preservation noted for the methods performed on these samples, unless otherwise detailed in the individual sections below.

\section{Receipt}

The samples were received on $07 / 14 / 2015$. Documentation of the condition of the samples at the time of their receipt and any exception to the laboratory's Sample Acceptance Policy is documented in the Shipping Documentation section of this submittal. The samples, as received, were not acid preserved. On that basis, the laboratory did provide for the analysis of the samples within seven days of sample collection.

\section{SOM01.2 Volatile Organics (Trace Level Water)}

A storage blank was prepared for volatile organics analysis, and stored in association with the storage of the samples. That storage blank, identified as VHBLK01, was carried through the holding period with the samples, and analyzed.

Each sample was analyzed without a dilution. Each of the analyses associated with the sample set exhibited an acceptable internal standard performance. There was an acceptable recovery of each deuterated monitoring compound (DMC) in the analysis of the method blank associated with the analytical work, and in the analysis of the storage blank associated with the sample set. Matrix spike and matrix spike duplicate analyses were not performed on samples in this sample set. Trace concentrations of Vinyl Chloride, 1,2,4-Trichlorobenzene and 1,2,3-Trichlorobenzene were detected in the method blanks associated with this sample set. Present in the method blank and storage blank analyses was a non-target constituent that represents a compound that is related to the DMC formulation. The fact that the presence of this compound is not within the laboratory's control is at issue.

The responses for each target analyte met the relative standard deviation criterion in the initial calibration. The response for each target analyte met the percent difference criterion in the opening/continuing calibration check acquisition. The response for each target analyte met the 50.0 percent difference criterion in the closing calibration check acquisition.

The primary quantitation mass for methylcyclohexane that is specified in the Statement of Work is mass 83 . The laboratory did identify a contribution to mass 83 from 1,2-dichloropropane- $\mathrm{d}_{6}$, one of the deuterated monitoring compounds (DMCs). The laboratory did change the primary quantitation mass assignment to mass 55 for the quantification of methylcyclohexane. 
Manual integration was employed in deriving certain of the analytical results. The values that have been derived from manual integration are qualified on the quantitation reports. Extracted ion current profiles for each manual integration are included in the data package, and further documented at the end of this submittal. 


\section{DATA REPORTING QUALIFIERS}

Client: Argonne National Laboratory

Job Number: 200-28907-1

Sdg Number: 28907

Lab Section

Qualifier

Description

GC/MS VOA

U

$\mathrm{J}$

$J$

$X$

B

$\mathrm{N}$
Analyzed for but not detected.

Indicates an Estimated Value for TICs

Indicates an estimated value.

See case narrative notes for explanation of the ' $X$ ' flag

The analyte was found in an associated blank, as well as in the sample.

This flag indicates the presumptive evidence of a compound. 


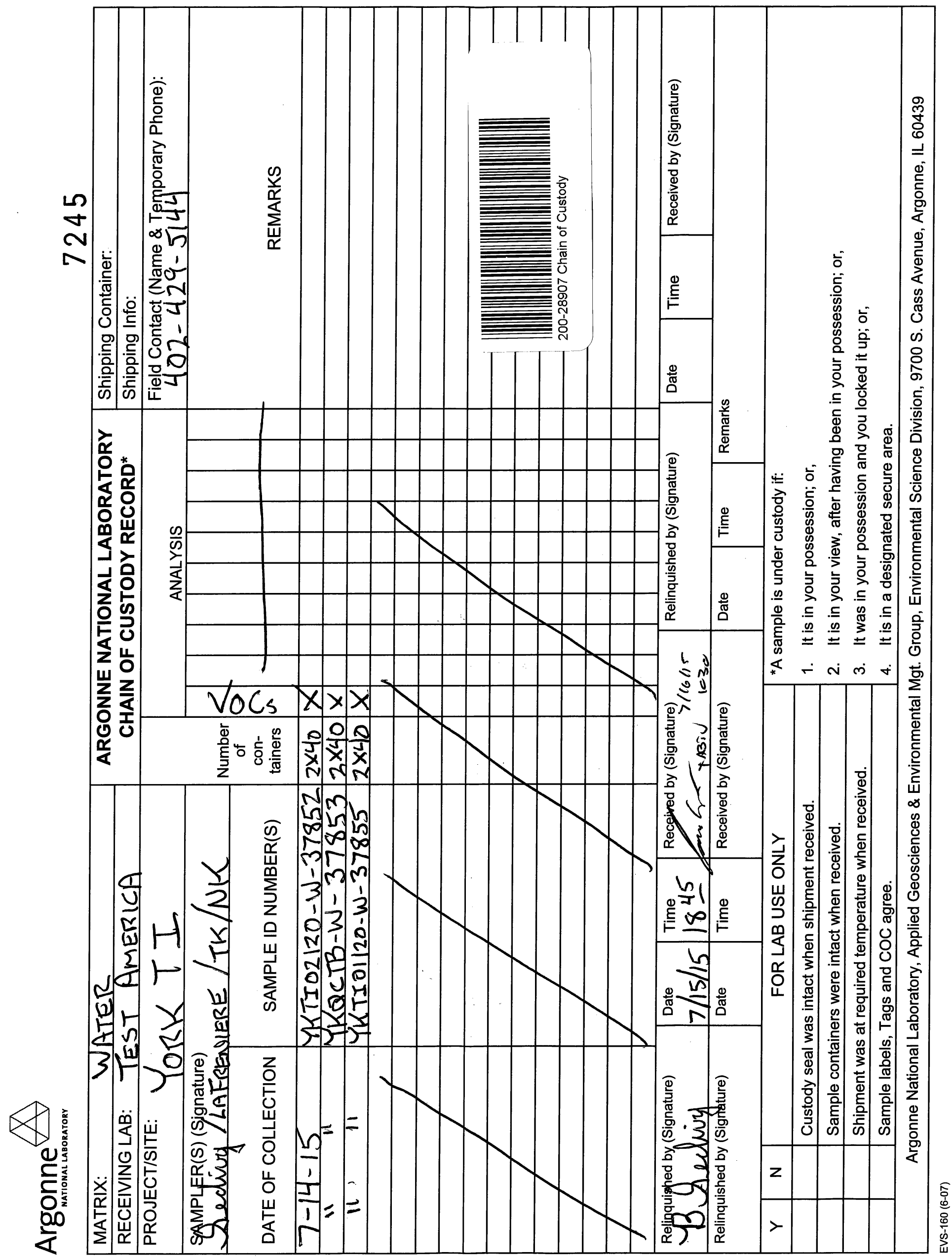




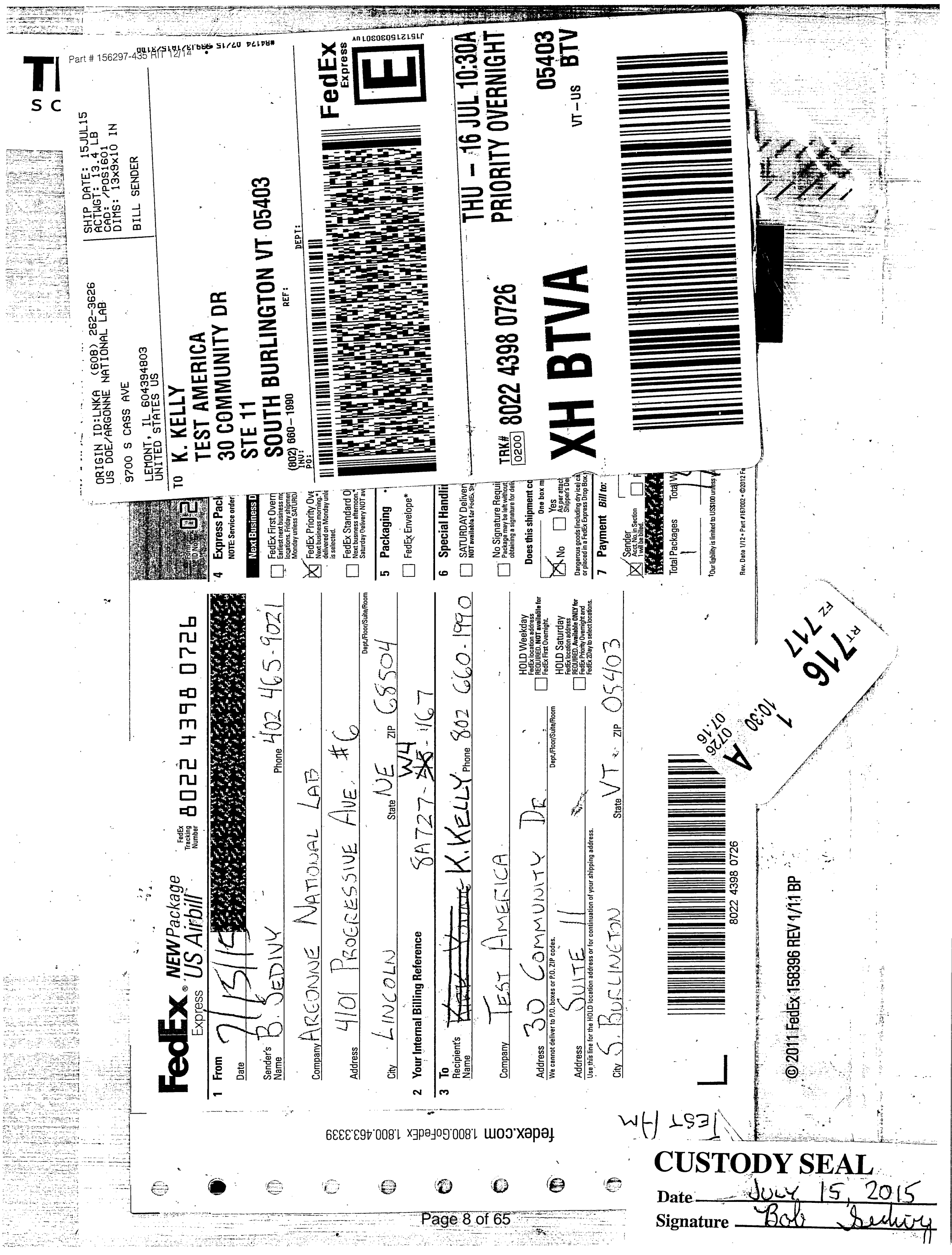




\section{Shipping and Receiving Documents}




\section{Login Sample Receipt Checklist}

Client: Argonne National Laboratory

Job Number: 200-28907-1

SDG Number: 28907

Login Number: 28907

List Source: TestAmerica Burlington

List Number: 1

Creator: Goodrich, Kenneth L

Question

Radioactivity wasn't checked or is $</=$ background as measured by a survey True meter.

The cooler's custody seal, if present, is intact.

Sample custody seals, if present, are intact.

The cooler or samples do not appear to have been compromised or tampered with.

Samples were received on ice.

Cooler Temperature is acceptable.

Cooler Temperature is recorded.

COC is present.

COC is filled out in ink and legible.

$\mathrm{COC}$ is filled out with all pertinent information.

Is the Field Sampler's name present on COC?

There are no discrepancies between the containers received and the COC.

Samples are received within Holding Time.

Sample containers have legible labels.

Containers are not broken or leaking.

Sample collection date/times are provided.

Appropriate sample containers are used.

Sample bottles are completely filled.

Sample Preservation Verified.

There is sufficient vol. for all requested analyses, incl. any requested MS/MSDs

Containers requiring zero headspace have no headspace or bubble is $<6 \mathrm{~mm}$ (1/4").

Multiphasic samples are not present.

Samples do not require splitting or compositing.

Residual Chlorine Checked.
True

True

True

True

True

True

True

True

True

True

False

True

True

True

True

True

N/A

True

True

True

True

True

N/A

Answer Comment

Lab does not accept radioactive samples.

NO NUMBERS

$3.4^{\circ} \mathrm{C}$

Refer to Job Narrative for details. 


\section{METHODOLOGY SUMMARY}

Laboratory: TestAmerica Laboratories

Location: South Burlington, Vermont
Project No:

SDG No: $\quad 28907$

\section{VOA}

Volatile Organics Trace - USEPA CLP SOM01.2 
Lab Name: TestAmerica Burlington

SDG No.: 28907

Instrument ID: CHD.i

Lab Sample ID: IC 200-91077/2

Date Analyzed: 07/14/15 11:08

\begin{tabular}{|c|r|}
\hline COMPOUND NAME & RETEN \\
TI
\end{tabular}

Lab Sample ID: IC 200-91077/3

Date Analyzed: 07/14/15 11:33 Client Sample ID: Lab File ID: 14685_003.D

Analysis Batch Number: 91077

Client Sample ID:

Lab File ID: 14685 002.D

GC Column: DB-624

ID : $0.2(\mathrm{~mm})$

\begin{tabular}{|c|c|c|c|c|}
\hline \multicolumn{1}{|c|}{ COMPOUND NAME } & RETENTION & \multicolumn{3}{|c}{ MANUAL INTEGRATION } \\
\cline { 3 - 5 } & TIME & REASON & ANALYST & DATE \\
\hline 1,2 -Dibromo-3-Chloropropane & 13.44 & Baseline & archern & $07 / 14 / 1514: 11$ \\
\hline
\end{tabular}


Lab Name: TestAmerica Burlington SDG No.: 28907

Instrument ID: CHD.i

Lab Sample ID: CCVIS 200-91426/4

Date Analyzed: 07/20/15 09:27

\begin{tabular}{|l|c|c|c|c|}
\hline \multicolumn{1}{|c|}{ COMPOUND NAME } & RETENTION & \multicolumn{3}{|c}{ MANUAL INTEGRATION } \\
\cline { 3 - 5 } & TIME & REASON & ANALYST & DATE \\
\hline Dichlorodifluoromethane & 1.48 & Baseline & archern & $07 / 20 / 15$ 09:48 \\
\hline
\end{tabular}

Job No.: 200-28907-1

Analysis Batch Number: 91426

Client Sample ID:

Lab File ID: 14808004

GC Column: DB-624

ID : $0.2(\mathrm{~mm})$ 
Lab Name: TESTAMERICA BURLINGTON

Contract: $8 \mathrm{E}-00302$

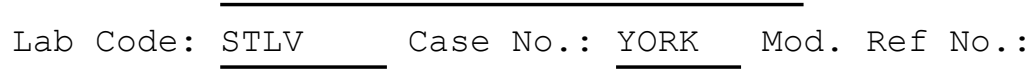
SDG No.: 28907

Level: (TRACE or LOW) TRACE

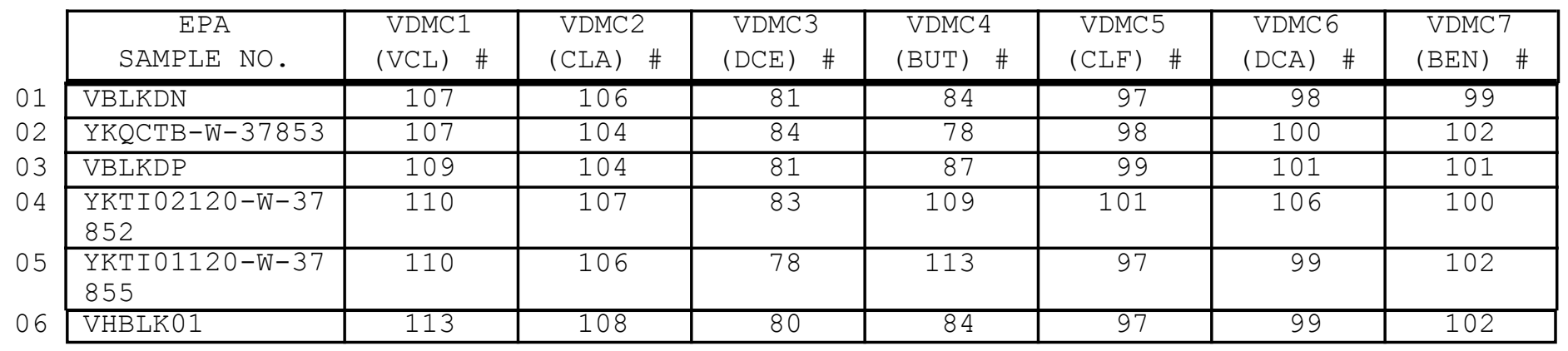

VDMC1 $(\mathrm{VCL})=$ Vinyl Chloride-d3

VDMC2 $($ CLA $)=$ Chloroethane-d5

VDMC3 $(\mathrm{DCE})=1,1-\mathrm{Dich}$ loroethene-d2

VDMC4 $\quad(B U T)=2-$ Butanone-d5

VDMC5 $(\mathrm{CLF})=$ Chloroform-d

VDMC $6 \quad(D C A)=1,2-$ Dichloroethane-d4

$\operatorname{VDMC} 7(\mathrm{BEN})=$ Benzene-d6 $\frac{\text { QC LIMITS }}{(65-131)}$

$(71-131)$

(55-104)

(49-155)

$(78-121)$

$(78-129)$

$(77-124)$

\# Column to be used to flag recovery values

* Values outside of contract required QC limits

Page 1 of 1 
Lab Name: TESTAMERICA BURLINGTON

Lab Code: STLV Case No.: YORK Mod. Ref No.:
Contract: $8 \mathrm{E}-00302$

SDG No.: 28907

Level: (TRACE or LOW) TRACE

\begin{tabular}{|c|c|c|c|c|c|c|c|c|}
\hline $\begin{array}{c}\text { EPA } \\
\text { SAMPLE NO. }\end{array}$ & $\begin{array}{l}\text { VDMC 8 } \\
(\mathrm{DPA}) \#\end{array}$ & $\begin{array}{l}\text { VDMC9 } \\
(\mathrm{TOL}) \quad \#\end{array}$ & $\begin{array}{l}\text { VDMC10 } \\
(\mathrm{TDP}) \#\end{array}$ & $\begin{array}{l}\text { VDMC11 } \\
(\operatorname{HEX}) \#\end{array}$ & $\begin{array}{l}\text { VDMC12 } \\
(\mathrm{TCA}) \quad \#\end{array}$ & $\begin{array}{l}\text { VDMC13 } \\
(\mathrm{DCZ}) \quad \#\end{array}$ & OTHER & $\begin{array}{l}\text { TOT } \\
\text { OUT }\end{array}$ \\
\hline VBLKDN & 97 & 103 & 91 & 91 & 90 & 105 & & 0 \\
\hline YKQCTB-W-37853 & 95 & 102 & 90 & 89 & 82 & 105 & & 0 \\
\hline VBLKDP & 97 & 100 & 97 & 92 & 90 & 109 & & 0 \\
\hline $\begin{array}{l}\text { YKTI02120-W-37 } \\
852\end{array}$ & 96 & 101 & 94 & 120 & 89 & 98 & & 0 \\
\hline $\begin{array}{l}\text { YKTI01120-W-37 } \\
855\end{array}$ & 95 & 102 & 91 & 119 & 92 & 97 & & 0 \\
\hline VHBLK01 & 98 & 104 & 94 & 96 & 90 & 108 & & 0 \\
\hline
\end{tabular}

\footnotetext{
$\operatorname{VDMC} 8(\mathrm{DPA})=1,2-\mathrm{Dichloropropane}-\mathrm{d} 6$

VDMC $9 \quad($ TOL $)=$ Toluene-d8

VDMC10 $(\mathrm{TDP})=$ trans $-1,3-\mathrm{Dichloropropene-d4}$

$\operatorname{VDMC1}(\mathrm{HEX})=2-$ Hexanone-d5

VDMC12 $(\mathrm{TCA})=1,1,2,2$-Tetrachloroethane-d2

VDMC13 $(\mathrm{DCZ})=1,2-\mathrm{Dichlorobenzene-d4}$
}

$$
\begin{gathered}
\frac{Q C \text { LIMITS }}{(79-124)} \\
(77-121) \\
(73-121) \\
(28-135) \\
(73-125) \\
(80-131)
\end{gathered}
$$

\# Column to be used to flag recovery values

* Values outside of contract required QC limits

Report 1,4-Dioxane-d8 for Low-Medium VOA analysis only

Page 1 of 1 
Lab Name: TESTAMERICA BURLINGTON

Contract: 8E-00302

Lab Code: STLV Case No.: YORK Mod. Ref No.: SDG No.: 28907

Lab File ID: 14793_005.D

Lab Sample ID: MB 200-91395/5

Instrument ID: CHD.i

Matrix: (SOIL/SED/WATER) Water

Date Analyzed: 07/18/2015

Level: (TRACE or LOW/MED) TRACE

Time Analyzed: 1147

GC Column: DB-624

ID $0.20 \quad(\mathrm{~mm})$

Heated Purge: ( $\mathrm{Y} / \mathrm{N}) \mathrm{N}$

\begin{tabular}{|c|c|c|c|c|}
\hline \multicolumn{1}{c|}{ EPA } & LAB & LAB & TIME \\
SAMPLE NO. & SAMPLE ID & FILE ID & ANALYZED \\
\cline { 2 - 5 } & YKQCTB-W-378 & $200-28907-2$ & $\begin{array}{l}14793 \_009 . \\
\text { D }\end{array}$ & 1326 \\
\hline
\end{tabular}

COMMENTS :

Page 1 of 1 
$4 A$ - FORM IV VOA

VOLATILE METHOD BLANK SUMMARY
EPA SAMPLE NO.

VBLKDP

Lab Name: TESTAMERICA BURLINGTON

Contract: $8 \mathrm{E}-00302$

Lab Code: STLV Case No.: YORK Mod. Ref No.: SDG No.: 28907

Lab File ID: 14808_006.D

Lab Sample ID: MB 200-91426/6

Instrument ID: CHD.i

Matrix: (SOIL/SED/WATER) Water

Date Analyzed: 07/20/2015

Level: (TRACE or LOW/MED) TRACE

Time Analyzed: 1017

GC Column: DB-624

ID $: 0.20 \quad(\mathrm{~mm})$

Heated Purge: (Y/N) N

\begin{tabular}{|c|c|c|c|}
\hline $\begin{array}{c}\text { EPA } \\
\text { SAMPLE NO. }\end{array}$ & $\begin{array}{r}\text { LAB } \\
\text { SAMPLE ID }\end{array}$ & $\begin{array}{c}\text { LAB } \\
\text { FILE ID }\end{array}$ & $\begin{array}{c}\text { TIME } \\
\text { ANALYZED }\end{array}$ \\
\hline $\begin{array}{l}\text { YKTI } 02120-W- \\
37852\end{array}$ & $200-28907-1$ & $\begin{array}{l}14808 \_009 . \\
D\end{array}$ & 1228 \\
\hline $\begin{array}{l}\text { YKTI } 01120-W- \\
37855\end{array}$ & $200-28907-3$ & $\begin{array}{l}14808 \_010 . \\
D\end{array}$ & 1253 \\
\hline VHBLK01 & $200-28907-4$ & $\begin{array}{l}14808 \_011 . \\
D\end{array}$ & 1318 \\
\hline
\end{tabular}

COMMENTS :

Page 1 of 1 
$5 A$ - FORM V VOA

VOLATILE ORGANICS INSTRUMENT

PERFORMANCE CHECK

BROMOFLUOROBENZENE (BFB)
EPA SAMPLE NO.

BFBDG
Lab Name: TESTAMERICA BURLINGTON

Lab Code: STLV

Case No.: YORK

Lab File Id: 14685_001.D

Instrument Id: CHD.i

GC Column: DB-624

ID $: 0.20$
Contract: 8E-00302 Mod. Ref No.:

SDG No.: 28907

BEB Injection Date: 07/14/2015

BFB Injection Time: 1045 $(\mathrm{mm})$

\begin{tabular}{|c|c|c|}
\hline $\mathrm{m} / \mathrm{e}$ & ION ABUNDANCE CRITERIA & $\begin{array}{l}\text { \% RELATIVE } \\
\text { ABUNDANCE }\end{array}$ \\
\hline 50 & $15.0-40.0 \%$ of mass 95 & 22.2 \\
\hline 75 & $30.0-80.0 \%$ of mass 95 & 60.1 \\
\hline 95 & Base peak, $100 \%$ relative abundance & 100 \\
\hline 96 & $5.0-9.0 \%$ of mass 95 & 6.7 \\
\hline 173 & Less than $2.0 \%$ of mass 174 & $0.7) 1$ \\
\hline 174 & $50.0-120 \%$ of mass 95 & 113 \\
\hline 175 & $5.0-9.0 \%$ of mass 174 & $8.5(7.6) 1$ \\
\hline 176 & $95.0-101 \%$ of mass 174 & $109(96.8) 1$ \\
\hline 177 & $5.0-9.0 \%$ of mass 176 & $(6.0) 2$ \\
\hline
\end{tabular}

1 - Value is omass 1742 - Value is omass 176

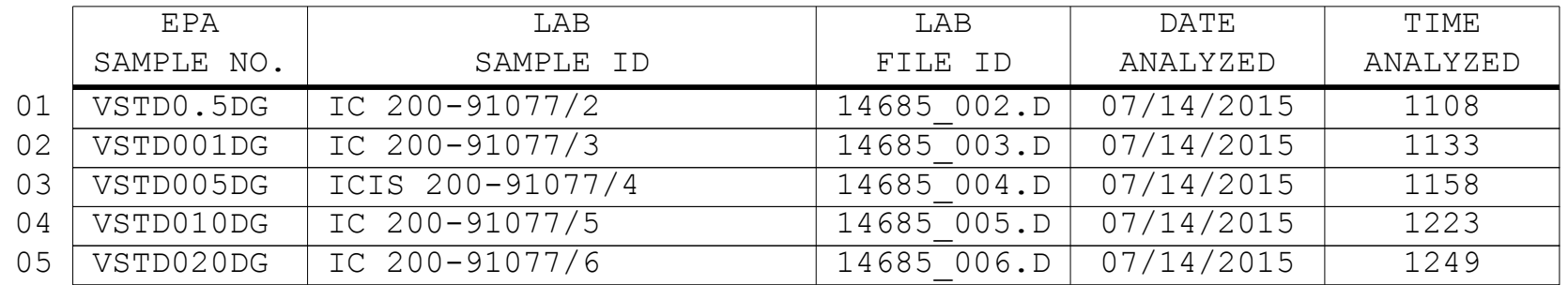


$5 A$ - FORM V VOA

VOLATILE ORGANICS INSTRUMENT

PERFORMANCE CHECK

BROMOFLUOROBENZENE (BFB)
EPA SAMPLE NO.

BFBDN
Lab Name: TESTAMERICA BURLINGTON

Lab Code: STLV Case No.: YORK

Lab File Id: 14793_001.D

Instrument Id: CHD.i

GC Column: DB-624

ID $: 0.20$
Contract: 8E-00302 Mod. Ref No.: SDG No.: 28907

BEB Injection Date: 07/18/2015

BFB Injection Time: 1012 $(\mathrm{mm})$

\begin{tabular}{|c|c|c|}
\hline $\mathrm{m} / \mathrm{e}$ & ION ABUNDANCE CRITERIA & $\begin{array}{l}\text { \% RELATIVE } \\
\text { ABUNDANCE }\end{array}$ \\
\hline 50 & $15.0-40.0 \%$ of mass 95 & 24.7 \\
\hline 75 & $30.0-80.0 \%$ of mass 95 & 64.4 \\
\hline 95 & Base peak, $100 \%$ relative abundance & 100 \\
\hline 96 & $5.0-9.0 \%$ of mass 95 & 7.2 \\
\hline 173 & Less than $2.0 \%$ of mass 174 & $1.0) 1$ \\
\hline 174 & $50.0-120 \%$ of mass 95 & 114 \\
\hline 175 & $5.0-9.0 \%$ of mass 174 & 8.11 \\
\hline 176 & $95.0-101 \%$ of mass 174 & $(98.6) 1$ \\
\hline 177 & $5.0-9.0 \%$ of mass 176 & $6.8(6.0) 2$ \\
\hline
\end{tabular}

1 - Value is omass 1742 - Value is omass 176

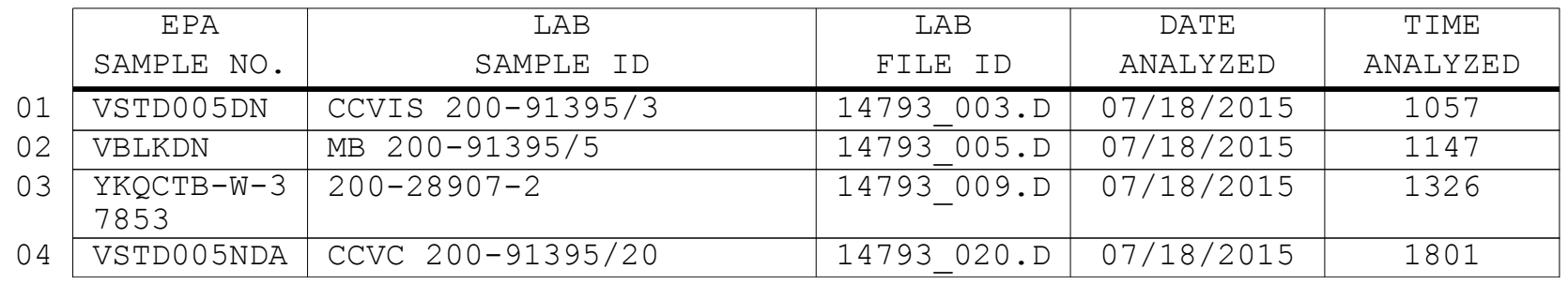


$5 A$ - FORM V VOA

VOLATILE ORGANICS INSTRUMENT

PERFORMANCE CHECK

BROMOFLUOROBENZENE (BFB)
EPA SAMPLE NO.

BFBDP
Lab Name: TESTAMERICA BURLINGTON

Lab Code: STLV Case No.: YORK

Lab File Id: 14808_002.D

Instrument Id: CHD.i

GC Column: DB-624

ID $: 0.20$
Contract: $8 \mathrm{E}-00302$ Mod. Ref No.: SDG No.: 28907

BFB Injection Date: 07/20/2015

BFB Injection Time: 0841 $(\mathrm{mm})$

\begin{tabular}{|c|c|c|}
\hline $\mathrm{m} / \mathrm{e}$ & ION ABUNDANCE CRITERIA & $\begin{array}{l}\text { \% RELATIVE } \\
\text { ABUNDANCE }\end{array}$ \\
\hline 50 & $15.0-40.0 \%$ of mass 95 & 21.5 \\
\hline 75 & $30.0-80.0 \%$ of mass 95 & 58.2 \\
\hline 95 & Base peak, $100 \%$ relative abundance & 100 \\
\hline 96 & $5.0-9.0 \%$ of mass 95 & 6.2 \\
\hline 173 & Less than $2.0 \%$ of mass 174 & $0.5) 1$ \\
\hline 174 & $50.0-120 \%$ of mass 95 & 115 \\
\hline 175 & $5.0-9.0 \%$ of mass 174 & $8.3(7.3) 1$ \\
\hline 176 & $95.0-101 \%$ of mass 174 & $110(95.8) 1$ \\
\hline 177 & $5.0-9.0 \%$ of mass 176 & $(6.7) 2$ \\
\hline
\end{tabular}

1 - Value is omass 1742 - Value is omass 176

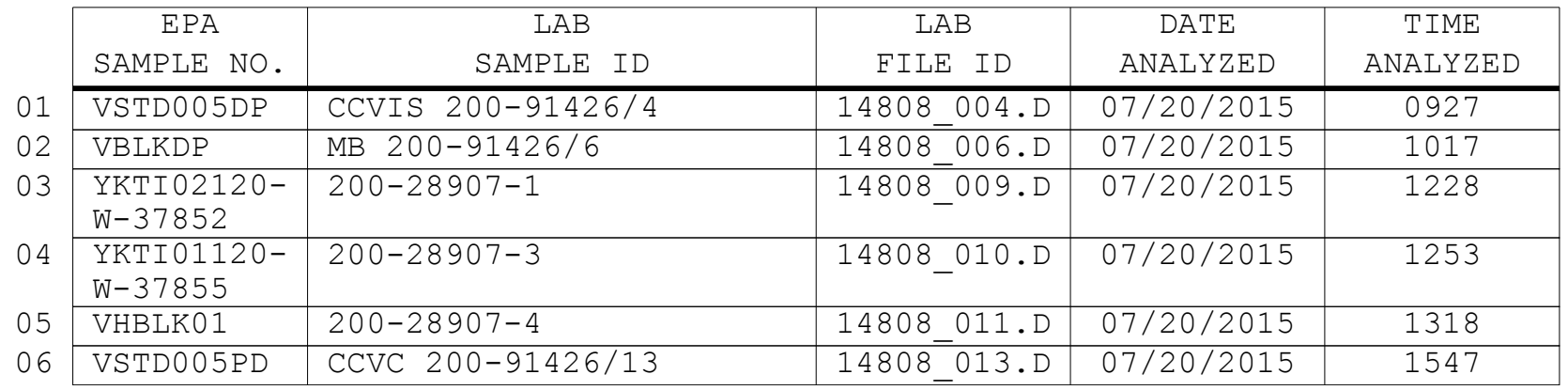


Lab Name: TESTAMERICA BURLINGTON

Contract: $8 \mathrm{E}-00302$

Lab Code: STLV Case No.: YORK Mod. Ref No.: SDG No.: 28907

GC Column: DB-624

ID: 0.20 (mm) Init. Calib. Date (s) : 07/14/2015 07/14/2015

EPA Sample No. (VSTD\#\#\#\#\#) : VSTD005DN

Lab File ID (Standard): 14793_003.D

Date Analyzed: $07 / 18 / 2015$

Instrument ID: CHD.i

Time Analyzed: 1057

Heated Purge: (Y/N) N

\begin{tabular}{|c|c|c|c|c|c|c|}
\hline & $\begin{array}{c}\text { IS1 (CBZ) } \\
\text { AREA }\end{array}$ & $\mathrm{RT}$ & $\begin{array}{c}\text { IS2 (DFB) } \\
\text { AREA }\end{array}$ & $\mathrm{RT}$ & $\begin{array}{c}\text { IS3 (DCB) } \\
\text { AREA }\end{array}$ & $\mathrm{RT}$ \\
\hline 12 HOUR STD & 219037 & 9.34 & 254077 & 5.97 & 145902 & 12.15 \\
\hline UPPER LIMIT & 306652 & 9.67 & 355708 & 6.30 & 204263 & 12.48 \\
\hline LOWER LIMIT & 131422 & 9.01 & 152446 & 5.64 & 87541 & 11.82 \\
\hline EPA SAMPLE NO. & & & & & & \\
\hline VBLKDN & 216497 & 9.34 & 246802 & 5.97 & 124992 & 12.15 \\
\hline YKQCTB-W-37853 & 211616 & 9.34 & 243540 & 5.97 & 125436 & 12.15 \\
\hline
\end{tabular}

IS1 $(\mathrm{CBZ})=$ Chlorobenzene-d5

IS2 $(\mathrm{DFB})=1,4-\mathrm{Difluorobenzene}$

IS3 $(\mathrm{DCB})=1,4-\mathrm{Dichlorobenzene-d4}$

AREA UPPER LIMIT $=140 \%$ (Trace Volatiles) of internal standard area AREA LOWER LIMIT $=60 \%$ (Trace Volatiles) of internal standard area RT UPPER LIMIT $=+0.33$ (Trace Volatiles) minutes of internal standard RT RT LOWER LIMIT $=-0.33$ (Trace Volatiles) minutes of internal standard RT

\# Column used to flag values outside contract required QC limits with an asterisk. Page 1 of 1 


\section{$8 A$ - FORM VIII VOA}

VOLATILE INTERNAL STANDARD AREA AND RETENTION TIME SUMMARY

Lab Name: TESTAMERICA BURLINGTON

Contract: $8 \mathrm{E}-00302$

Lab Code: STLV Case No.: YORK Mod. Ref No.:

SDG No.: 28907

GC Column: DB-624

ID: 0.20 (mm) Init. Calib. Date (s): 07/14/2015 07/14/2015

EPA Sample No.(VSTD\#\#\#\#\#) : VSTD005DP

Lab File ID (Standard): 14808_004.D

Date Analyzed: $07 / 20 / 2015$

Instrument ID: CHD.i

Time Analyzed: 0927

Heated Purge: ( $\mathrm{Y} / \mathrm{N}) \mathrm{N}$

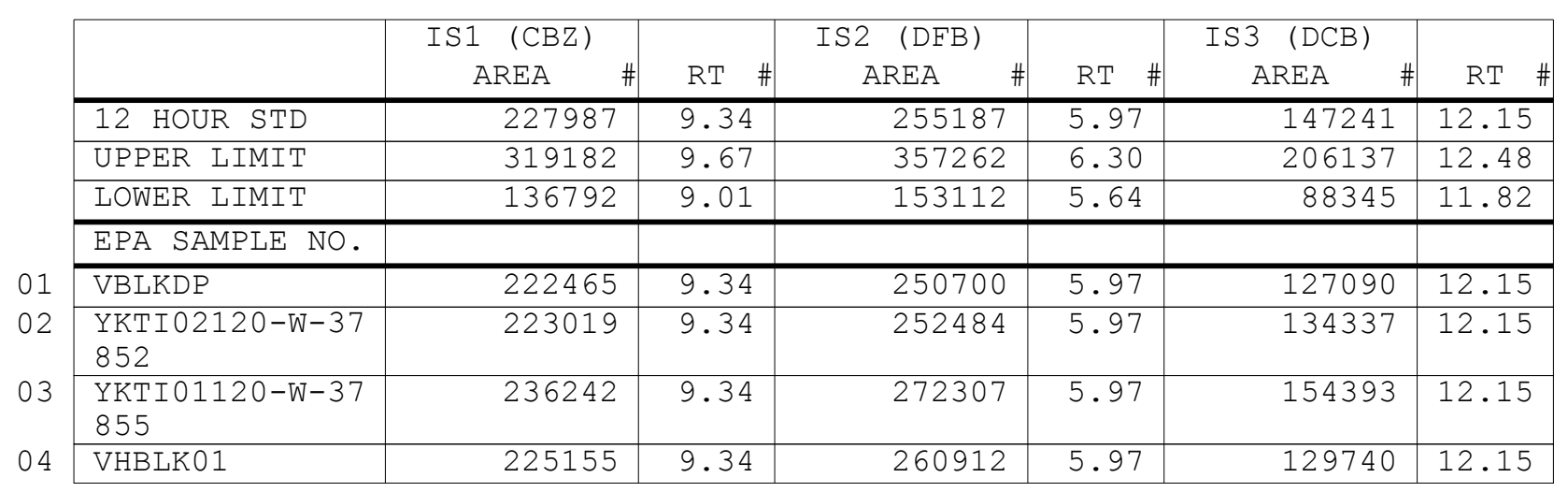

IS1 $(\mathrm{CBZ})=$ Chlorobenzene-d5

IS2 $(\mathrm{DFB})=1,4-\mathrm{Difluorobenzene}$

IS3 $(\mathrm{DCB})=1,4-\mathrm{Dichlorobenzene-d4}$

AREA UPPER LIMIT $=140 \%$ (Trace Volatiles) of internal standard area AREA LOWER LIMIT $=60 \%$ (Trace Volatiles) of internal standard area RT UPPER LIMIT $=+0.33$ (Trace Volatiles) minutes of internal standard RT RT LOWER LIMIT $=-0.33$ (Trace Volatiles) minutes of internal standard RT

\# Column used to flag values outside contract required QC limits with an asterisk. 
Lab Name: TESTAMERICA BURLINGTON

Contract: $8 \mathrm{E}-00302$

Lab Code: STLV

Case No.: YORK Mod. Ref No.:

SDG No.: 28907

Matrix: (SOIL/SED/WATER) Water

Sample wt/vol: 25.0 $(\mathrm{g} / \mathrm{mL}) \mathrm{mL}$

Level: (TRACE/LOW/MED) TRACE

\% Moisture: not dec.

GC Column: DB-624 ID: $0.20 \quad(\mathrm{~mm})$

Soil Extract Volume: (uL) $(\mathrm{mL})$

Purge Volume: 25.0
Lab Sample ID: 200-28907-2

Lab File ID: 14793_009.D

Date Received: 07/16/2015

Date Analyzed: 07/18/2015

Dilution Factor: 1.0

Soil Aliquot Volume: (uL)

\begin{tabular}{|c|c|c|c|}
\hline CAS NO. & COMPOUND & $\begin{array}{l}\text { CONCENTRATION UNITS: } \\
(\mathrm{ug} / \mathrm{L} \text { or } \mathrm{ug} / \mathrm{kg}) \mathrm{ug} / \mathrm{L}\end{array}$ & q \\
\hline $75-71-8$ & Dichlorodifluoromethane & 1.0 & $\mathrm{U}$ \\
\hline $74-87-3$ & Chloromethane & 1.0 & $\mathrm{U}$ \\
\hline $75-01-4$ & Vinyl chloride & 1.0 & $\mathrm{U}$ \\
\hline $74-83-9$ & Bromomethane & 1.0 & $\mathrm{U}$ \\
\hline $75-00-3$ & Chloroethane & 1.0 & $\mathrm{U}$ \\
\hline $75-69-4$ & Trichlorofluoromethane & 1.0 & $\mathrm{U}$ \\
\hline $75-35-4$ & 1,1-Dichloroethene & 1.0 & $\mathrm{U}$ \\
\hline $76-13-1$ & $1,1,2$-Trichloro-1,2,2-trifluoroethane & 1.0 & $\mathrm{U}$ \\
\hline $67-64-1$ & Acetone & 13 & \\
\hline $75-15-0$ & Carbon disulfide & 1.0 & $\mathrm{U}$ \\
\hline $79-20-9$ & Methyl acetate & 1.0 & $\mathrm{U}$ \\
\hline $75-09-2$ & Methylene Chloride & 1.0 & $\mathrm{U}$ \\
\hline $156-60-5$ & trans-1,2-Dichloroethene & 1.0 & $\mathrm{U}$ \\
\hline $1634-04-4$ & Methyl tert-butyl ether & 1.0 & $\mathrm{U}$ \\
\hline $75-34-3$ & 1,1-Dichloroethane & 1.0 & $\mathrm{U}$ \\
\hline $156-59-2$ & cis-1,2-Dichloroethene & 1.0 & $\mathrm{U}$ \\
\hline $78-93-3$ & 2-Butanone & 5.0 & $\mathrm{U}$ \\
\hline $74-97-5$ & Bromochloromethane & 1.0 & $\mathrm{U}$ \\
\hline $67-66-3$ & Chloroform & 1.0 & $\mathrm{U}$ \\
\hline $71-55-6$ & $1,1,1$-Trichloroethane & 1.0 & $\mathrm{U}$ \\
\hline $110-82-7$ & Cyclohexane & 1.0 & $\mathrm{U}$ \\
\hline $56-23-5$ & Carbon tetrachloride & 1.0 & $\mathrm{U}$ \\
\hline $71-43-2$ & Benzene & 0.18 & $\mathrm{~J}$ \\
\hline $107-06-2$ & 1,2-Dichloroethane & 1.0 & $\mathrm{U}$ \\
\hline
\end{tabular}

Report 1,4-Dioxane for Low-Medium VOA analysis only 
Lab Name: TESTAMERICA BURLINGTON

Contract: $8 \mathrm{E}-00302$

Lab Code: STLV

Case No.: YORK

Mod. Ref No.:

SDG No.: 28907

Matrix: (SOIL/SED/WATER) Water

Sample wt/vol: 25.0 $(\mathrm{g} / \mathrm{mL}) \mathrm{mL}$

Level: (TRACE/LOW/MED) TRACE

\% Moisture: not dec.

GC Column: DB-624 ID: $0.20 \quad(\mathrm{~mm})$

Soil Extract Volume: (uL) $(\mathrm{mL})$

Purge Volume: 25.0 (mI)
Lab Sample ID: 200-28907-2

Lab File ID: 14793_009.D

Date Received: 07/16/2015

Date Analyzed: 07/18/2015

Dilution Factor: 1.0

Soil Aliquot Volume: (uL)

\begin{tabular}{|c|c|c|c|}
\hline CAS NO. & COMPOUND & $\begin{array}{l}\text { CONCENTRATION UNITS: } \\
(\mathrm{ug} / \mathrm{L} \text { or } \mathrm{ug} / \mathrm{kg}) \mathrm{ug} / \mathrm{L}\end{array}$ & $q$ \\
\hline $79-01-6$ & Trichloroethene & 1.0 & $\mathrm{U}$ \\
\hline $108-87-2$ & Methylcyclohexane & 1.0 & $\mathrm{U}$ \\
\hline $78-87-5$ & 1,2-Dichloropropane & 1.0 & $\mathrm{U}$ \\
\hline $75-27-4$ & Bromodichloromethane & 1.0 & $\mathrm{U}$ \\
\hline $10061-01-5$ & cis-1,3-Dichloropropene & 1.0 & U \\
\hline $108-10-1$ & 4-Methyl-2-pentanone & 1.0 & $\mathrm{U}$ \\
\hline $108-88-3$ & Toluene & 0.92 & $\mathrm{~J}$ \\
\hline $10061-02-6$ & trans-1,3-Dichloropropene & 1.0 & $\mathrm{U}$ \\
\hline $79-00-5$ & $1,1,2-$ Trichloroethane & 1.0 & $\mathrm{U}$ \\
\hline $127-18-4$ & Tetrachloroethene & 1.0 & $\mathrm{U}$ \\
\hline $591-78-6$ & 2-Hexanone & 1.0 & $\mathrm{U}$ \\
\hline $124-48-1$ & Dibromochloromethane & 1.0 & $\mathrm{U}$ \\
\hline $106-93-4$ & 1,2-Dibromoethane & 1.0 & $\mathrm{U}$ \\
\hline $108-90-7$ & Chlorobenzene & 1.0 & $\mathrm{U}$ \\
\hline $100-41-4$ & Ethylbenzene & 0.063 & $\mathrm{~J}$ \\
\hline $95-47-6$ & o-Xylene & 0.092 & $\mathrm{~J}$ \\
\hline $179601-23-1$ & m,p-xylene & 0.23 & $\mathrm{~J}$ \\
\hline $100-42-5$ & Styrene & 0.018 & $\mathrm{~J}$ \\
\hline $75-25-2$ & Bromoform & 1.0 & $\mathrm{U}$ \\
\hline $98-82-8$ & Isopropylbenzene & 1.0 & $\mathrm{U}$ \\
\hline $79-34-5$ & $1,1,2,2$-Tetrachloroethane & 1.0 & $\mathrm{U}$ \\
\hline $541-73-1$ & 1,3 -Dichlorobenzene & 1.0 & $\mathrm{U}$ \\
\hline $106-46-7$ & 1,4-Dichlorobenzene & 1.0 & $\mathrm{U}$ \\
\hline $95-50-1$ & 1,2-Dichlorobenzene & 1.0 & $\mathrm{U}$ \\
\hline $96-12-8$ & 1,2-Dibromo-3-Chloropropane & 1.0 & $\mathrm{U}$ \\
\hline $120-82-1$ & $1,2,4$-Trichlorobenzene & 1.0 & $\mathrm{U}$ \\
\hline $87-61-6$ & $1,2,3$-Trichlorobenzene & 1.0 & $\mathrm{U}$ \\
\hline
\end{tabular}


Lab Name: TESTAMERICA BURLINGTON

Contract: $8 \mathrm{E}-00302$

Lab Code: STLV Case No.: YORK Mod. Ref No.: SDG No.: 28907

Matrix: (SOIL/SED/WATER) Water

Sample wt/vol: 25.0 $(\mathrm{g} / \mathrm{mL}) \mathrm{mL}$

Level: (TRACE or LOW/MED) TRACE

Moisture: not dec.

GC Column: DB-624 ID $: 0.20 \quad(\mathrm{~mm})$

Soil Extract Volume: (uL)

CONCENTRATION UNITS: (ug/L or ug/kg) ug/L
Lab Sample ID: 200-28907-2

Lab File ID: 14793_009.D

Date Received: 07/16/2015

Date Analyzed: 07/18/2015

Dilution Factor: 1.0

Soil Aliquot Volume: (uL) Purge Volume: 25.0 $(\mathrm{mL})$

01

02

\begin{tabular}{|l|l|c|c|c|}
\hline CAS NUMBER & \multicolumn{1}{|c|}{ COMPOUND NAME } & RT & EST. CONC. & Q \\
\hline & Unknown & 7.29 & 2.8 & $\mathrm{~J}$ B X \\
\hline E9667961 & Total Alkanes & N/A & & \\
\hline
\end{tabular}

1EPA-designated Registry Number. 
Lab Name: TESTAMERICA BURLINGTON

Contract: $8 \mathrm{E}-00302$

Lab Code: STLV

Case No.: YORK Mod. Ref No.:

SDG No.: 28907

Matrix: (SOIL/SED/WATER) Water

Sample wt/vol: 25.0 $(\mathrm{g} / \mathrm{mL}) \mathrm{mL}$

Level: (TRACE/LOW/MED) TRACE

\% Moisture: not dec.

GC Column: DB-624 ID: $0.20 \quad(\mathrm{~mm})$

Soil Extract Volume: (uL) $(\mathrm{mL})$

Purge Volume: 25.0
Lab Sample ID: 200-28907-3

Lab File ID: 14808_010.D

Date Received: 07/16/2015

Date Analyzed: 07/20/2015

Dilution Factor: 1.0

Soil Aliquot Volume: (uL)

\begin{tabular}{|c|c|c|c|}
\hline CAS NO. & COMPOUND & $\begin{array}{l}\text { CONCENTRATION UNITS: } \\
(\mathrm{ug} / \mathrm{L} \text { or } \mathrm{ug} / \mathrm{kg}) \mathrm{ug} / \mathrm{L}\end{array}$ & q \\
\hline $75-71-8$ & Dichlorodifluoromethane & 1.0 & $\mathrm{U}$ \\
\hline $74-87-3$ & Chloromethane & 1.0 & $\mathrm{U}$ \\
\hline $75-01-4$ & Vinyl chloride & 1.0 & $\mathrm{U}$ \\
\hline $74-83-9$ & Bromomethane & 1.0 & $\mathrm{U}$ \\
\hline $75-00-3$ & Chloroethane & 1.0 & $\mathrm{U}$ \\
\hline $75-69-4$ & Trichlorofluoromethane & 0.032 & $\mathrm{~J}$ \\
\hline $75-35-4$ & 1,1-Dichloroethene & 1.0 & $\mathrm{U}$ \\
\hline $76-13-1$ & $1,1,2$-Trichloro-1,2,2-trifluoroethane & 1.0 & $\mathrm{U}$ \\
\hline $67-64-1$ & Acetone & 4.0 & $\mathrm{~J}$ \\
\hline $75-15-0$ & Carbon disulfide & 1.0 & $\mathrm{U}$ \\
\hline $79-20-9$ & Methyl acetate & 1.0 & $\mathrm{U}$ \\
\hline $75-09-2$ & Methylene Chloride & 1.0 & $\mathrm{U}$ \\
\hline $156-60-5$ & trans-1,2-Dichloroethene & 1.0 & $\mathrm{U}$ \\
\hline $1634-04-4$ & Methyl tert-butyl ether & 1.0 & $\mathrm{U}$ \\
\hline $75-34-3$ & 1,1-Dichloroethane & 1.0 & $\mathrm{U}$ \\
\hline $156-59-2$ & cis-1,2-Dichloroethene & 1.0 & $\mathrm{U}$ \\
\hline $78-93-3$ & 2-Butanone & 2.6 & $\mathrm{~J}$ \\
\hline $74-97-5$ & Bromochloromethane & 1.0 & $\mathrm{U}$ \\
\hline $67-66-3$ & Chloroform & 1.9 & \\
\hline $71-55-6$ & $1,1,1$-Trichloroethane & 1.0 & $\mathrm{U}$ \\
\hline $110-82-7$ & Cyclohexane & 1.0 & $\mathrm{U}$ \\
\hline $56-23-5$ & Carbon tetrachloride & 0.39 & $\mathrm{~J}$ \\
\hline $71-43-2$ & Benzene & 0.053 & $\mathrm{~J}$ \\
\hline $107-06-2$ & 1,2-Dichloroethane & 1.0 & $\mathrm{U}$ \\
\hline
\end{tabular}

Report 1,4-Dioxane for Low-Medium VOA analysis only 
Lab Name: TESTAMERICA BURLINGTON

Contract: $8 \mathrm{E}-00302$

Lab Code: STLV

Case No.: YORK

Mod. Ref No.:

SDG No.: 28907

Matrix: (SOIL/SED/WATER) Water

Sample wt/vol: 25.0 $(\mathrm{g} / \mathrm{mL}) \mathrm{mL}$

Level: (TRACE/LOW/MED) TRACE

\% Moisture: not dec.

GC Column: DB-624 ID: $0.20 \quad(\mathrm{~mm})$

Soil Extract Volume: (uL) $(\mathrm{mL})$

Purge Volume: 25.0 (mI)
Lab Sample ID: 200-28907-3

Lab File ID: 14808_010.D

Date Received: 07/16/2015

Date Analyzed: 07/20/2015

Dilution Factor: 1.0

Soil Aliquot Volume: (uL)

\begin{tabular}{|c|c|c|c|}
\hline CAS NO. & COMPOUND & $\begin{array}{l}\text { CONCENTRATION UNITS: } \\
(\mathrm{ug} / \mathrm{L} \text { or } \mathrm{ug} / \mathrm{kg}) \mathrm{ug} / \mathrm{L}\end{array}$ & $q$ \\
\hline $79-01-6$ & Trichloroethene & 1.0 & $\mathrm{U}$ \\
\hline $108-87-2$ & Methylcyclohexane & 1.0 & $\mathrm{U}$ \\
\hline $78-87-5$ & 1,2-Dichloropropane & 1.0 & $\mathrm{U}$ \\
\hline $75-27-4$ & Bromodichloromethane & 1.0 & $\mathrm{U}$ \\
\hline $10061-01-5$ & cis-1,3-Dichloropropene & 1.0 & U \\
\hline $108-10-1$ & 4-Methyl-2-pentanone & 1.0 & $\mathrm{U}$ \\
\hline $108-88-3$ & Toluene & 0.69 & $\mathrm{~J}$ \\
\hline $10061-02-6$ & trans-1,3-Dichloropropene & 1.0 & $\mathrm{U}$ \\
\hline $79-00-5$ & $1,1,2-$ Trichloroethane & 1.0 & $\mathrm{U}$ \\
\hline $127-18-4$ & Tetrachloroethene & 0.028 & $\mathrm{~J}$ \\
\hline $591-78-6$ & 2-Hexanone & 1.0 & $\mathrm{U}$ \\
\hline $124-48-1$ & Dibromochloromethane & 1.0 & $\mathrm{U}$ \\
\hline $106-93-4$ & 1,2-Dibromoethane & 1.0 & $\mathrm{U}$ \\
\hline $108-90-7$ & Chlorobenzene & 1.0 & $\mathrm{U}$ \\
\hline $100-41-4$ & Ethylbenzene & 1.0 & $\mathrm{U}$ \\
\hline $95-47-6$ & o-Xylene & 0.010 & $\mathrm{~J}$ \\
\hline $179601-23-1$ & m,p-xylene & 0.045 & $\mathrm{~J}$ \\
\hline $100-42-5$ & Styrene & 1.0 & $\mathrm{U}$ \\
\hline $75-25-2$ & Bromoform & 1.0 & $\mathrm{U}$ \\
\hline $98-82-8$ & Isopropylbenzene & 1.0 & $\mathrm{U}$ \\
\hline $79-34-5$ & $1,1,2,2$-Tetrachloroethane & 1.0 & $\mathrm{U}$ \\
\hline $541-73-1$ & 1,3 -Dichlorobenzene & 1.0 & $\mathrm{U}$ \\
\hline $106-46-7$ & 1,4-Dichlorobenzene & 1.0 & $\mathrm{U}$ \\
\hline $95-50-1$ & 1,2-Dichlorobenzene & 1.0 & $\mathrm{U}$ \\
\hline $96-12-8$ & 1,2-Dibromo-3-Chloropropane & 1.0 & $\mathrm{U}$ \\
\hline $120-82-1$ & $1,2,4$-Trichlorobenzene & 1.0 & $\mathrm{U}$ \\
\hline $87-61-6$ & $1,2,3$-Trichlorobenzene & 1.0 & $\mathrm{U}$ \\
\hline
\end{tabular}


Lab Name: TESTAMERICA BURLINGTON

Contract: $8 \mathrm{E}-00302$

Lab Code: STLV Case No.: YORK Mod. Ref No.: SDG No.: 28907

Matrix: (SOIL/SED/WATER) Water

Sample wt/vol: 25.0 $(\mathrm{g} / \mathrm{mL}) \mathrm{mL}$

Level: (TRACE or LOW/MED) TRACE

\% Moisture: not dec.

GC Column: DB-624 ID $: 0.20 \quad(\mathrm{~mm})$

Soil Extract Volume: (uL)

CONCENTRATION UNITS: (ug/L or ug/kg) ug/L
Lab Sample ID: 200-28907-3

Lab File ID: 14808_010.D

Date Received: 07/16/2015

Date Analyzed: 07/20/2015

Dilution Factor: 1.0

Soil Aliquot Volume: (uL) Purge Volume: 25.0 $(\mathrm{mL})$

\begin{tabular}{|c|c|c|c|c|}
\hline CAS NUMBER & COMPOUND NAME & $\mathrm{RT}$ & EST. CONC. & Q \\
\hline 01 & Unknown & 7.29 & 2.8 & $\mathrm{~J} B \mathrm{X}$ \\
\hline $541-05-9$ & Cyclotrisiloxane, hexamethyl- & 8.22 & 1.7 & $\mathrm{~J} \quad \mathrm{~N} B$ \\
\hline 03 & Unknown & 12.02 & 0.82 & $\mathrm{~J} B$ \\
\hline $124-19-6$ & Nonanal & 13.32 & 3.5 & $\mathrm{~J} \mathrm{~N}$ \\
\hline E9667961 & Total Alkanes & $\mathrm{N} / \mathrm{A}$ & & \\
\hline
\end{tabular}

1 EPA-designated Registry Number. 
Lab Name: TESTAMERICA BURLINGTON

Contract: $8 \mathrm{E}-00302$

Lab Code: STLV

Case No.: YORK Mod. Ref No.:

SDG No.: 28907

Matrix: (SOIL/SED/WATER) Water

Sample wt/vol: 25.0 $(\mathrm{g} / \mathrm{mL}) \mathrm{mL}$

Level: (TRACE/LOW/MED) TRACE

\% Moisture: not dec.

GC Column: DB-624 ID: $0.20 \quad(\mathrm{~mm})$

Soil Extract Volume: (uL) $(\mathrm{mL})$

Purge Volume: 25.0
Lab Sample ID: 200-28907-1

Lab File ID: 14808_009.D

Date Received: 07/16/2015

Date Analyzed: 07/20/2015

Dilution Factor: 1.0

Soil Aliquot Volume: (uL)

\begin{tabular}{|c|c|c|c|}
\hline CAS NO. & COMPOUND & $\begin{array}{l}\text { CONCENTRATION UNITS: } \\
(\mathrm{ug} / \mathrm{L} \text { or } \mathrm{ug} / \mathrm{kg}) \mathrm{ug} / \mathrm{L}\end{array}$ & q \\
\hline $75-71-8$ & Dichlorodifluoromethane & 1.0 & $\mathrm{U}$ \\
\hline $74-87-3$ & Chloromethane & 1.0 & $\mathrm{U}$ \\
\hline $75-01-4$ & Vinyl chloride & 1.0 & $\mathrm{U}$ \\
\hline $74-83-9$ & Bromomethane & 1.0 & $\mathrm{U}$ \\
\hline $75-00-3$ & Chloroethane & 1.0 & $\mathrm{U}$ \\
\hline $75-69-4$ & Trichlorofluoromethane & 0.043 & $\mathrm{~J}$ \\
\hline $75-35-4$ & 1,1-Dichloroethene & 1.0 & $\mathrm{U}$ \\
\hline $76-13-1$ & $1,1,2$-Trichloro-1,2,2-trifluoroethane & 1.0 & $\mathrm{U}$ \\
\hline $67-64-1$ & Acetone & 1.6 & $\mathrm{~J}$ \\
\hline $75-15-0$ & Carbon disulfide & 1.0 & $\mathrm{U}$ \\
\hline $79-20-9$ & Methyl acetate & 1.0 & $\mathrm{U}$ \\
\hline $75-09-2$ & Methylene Chloride & 1.0 & $\mathrm{U}$ \\
\hline $156-60-5$ & trans-1,2-Dichloroethene & 1.0 & $\mathrm{U}$ \\
\hline $1634-04-4$ & Methyl tert-butyl ether & 1.0 & $\mathrm{U}$ \\
\hline $75-34-3$ & 1,1-Dichloroethane & 1.0 & $\mathrm{U}$ \\
\hline $156-59-2$ & cis-1,2-Dichloroethene & 1.0 & $\mathrm{U}$ \\
\hline $78-93-3$ & 2-Butanone & 5.0 & $\mathrm{U}$ \\
\hline $74-97-5$ & Bromochloromethane & 1.0 & $\mathrm{U}$ \\
\hline $67-66-3$ & Chloroform & 1.0 & $\mathrm{U}$ \\
\hline $71-55-6$ & $1,1,1$-Trichloroethane & 1.0 & $\mathrm{U}$ \\
\hline $110-82-7$ & Cyclohexane & 1.0 & $\mathrm{U}$ \\
\hline $56-23-5$ & Carbon tetrachloride & 0.034 & $\mathrm{~J}$ \\
\hline $71-43-2$ & Benzene & 1.0 & $\mathrm{U}$ \\
\hline $107-06-2$ & 1,2-Dichloroethane & 1.0 & $\mathrm{U}$ \\
\hline
\end{tabular}

Report 1,4-Dioxane for Low-Medium VOA analysis only 
Lab Name: TESTAMERICA BURLINGTON

Contract: $8 \mathrm{E}-00302$

Lab Code: STLV

Case No.: YORK

Mod. Ref No.:

SDG No.: 28907

Matrix: (SOIL/SED/WATER) Water

Sample wt/vol: 25.0 $(\mathrm{g} / \mathrm{mL}) \mathrm{mL}$

Level: (TRACE/LOW/MED) TRACE

\% Moisture: not dec.

GC Column: DB-624 ID: $0.20 \quad(\mathrm{~mm})$

Soil Extract Volume: (uL) $(\mathrm{mL})$

Purge Volume: 25.0 (mI)
Lab Sample ID: 200-28907-1

Lab File ID: 14808_009.D

Date Received: 07/16/2015

Date Analyzed: 07/20/2015

Dilution Factor: 1.0

Soil Aliquot Volume: (uL)

\begin{tabular}{|c|c|c|c|}
\hline CAS NO. & COMPOUND & $\begin{array}{l}\text { CONCENTRATION UNITS: } \\
(\mathrm{ug} / \mathrm{L} \text { or } \mathrm{ug} / \mathrm{kg}) \mathrm{ug} / \mathrm{L}\end{array}$ & $q$ \\
\hline $79-01-6$ & Trichloroethene & 1.0 & $\mathrm{U}$ \\
\hline $108-87-2$ & Methylcyclohexane & 1.0 & $\mathrm{U}$ \\
\hline $78-87-5$ & 1,2-Dichloropropane & 1.0 & $\mathrm{U}$ \\
\hline $75-27-4$ & Bromodichloromethane & 1.0 & $\mathrm{U}$ \\
\hline $10061-01-5$ & cis-1,3-Dichloropropene & 1.0 & U \\
\hline $108-10-1$ & 4-Methyl-2-pentanone & 1.0 & $\mathrm{U}$ \\
\hline $108-88-3$ & Toluene & 0.085 & $\mathrm{~J}$ \\
\hline $10061-02-6$ & trans-1,3-Dichloropropene & 1.0 & $\mathrm{U}$ \\
\hline $79-00-5$ & $1,1,2-$ Trichloroethane & 1.0 & $\mathrm{U}$ \\
\hline $127-18-4$ & Tetrachloroethene & 1.0 & $\mathrm{U}$ \\
\hline $591-78-6$ & 2-Hexanone & 1.0 & $\mathrm{U}$ \\
\hline $124-48-1$ & Dibromochloromethane & 1.0 & $\mathrm{U}$ \\
\hline $106-93-4$ & 1,2-Dibromoethane & 1.0 & $\mathrm{U}$ \\
\hline $108-90-7$ & Chlorobenzene & 1.0 & $\mathrm{U}$ \\
\hline $100-41-4$ & Ethylbenzene & 1.0 & $\mathrm{U}$ \\
\hline $95-47-6$ & o-Xylene & 1.0 & $\mathrm{U}$ \\
\hline $179601-23-1$ & m,p-xylene & 0.020 & $\mathrm{~J}$ \\
\hline $100-42-5$ & Styrene & 1.0 & $\mathrm{U}$ \\
\hline $75-25-2$ & Bromoform & 1.0 & $\mathrm{U}$ \\
\hline $98-82-8$ & Isopropylbenzene & 1.0 & $\mathrm{U}$ \\
\hline $79-34-5$ & $1,1,2,2$-Tetrachloroethane & 1.0 & $\mathrm{U}$ \\
\hline $541-73-1$ & 1,3 -Dichlorobenzene & 1.0 & $\mathrm{U}$ \\
\hline $106-46-7$ & 1,4-Dichlorobenzene & 1.0 & $\mathrm{U}$ \\
\hline $95-50-1$ & 1,2-Dichlorobenzene & 1.0 & $\mathrm{U}$ \\
\hline $96-12-8$ & 1,2-Dibromo-3-Chloropropane & 1.0 & $\mathrm{U}$ \\
\hline $120-82-1$ & $1,2,4$-Trichlorobenzene & 1.0 & $\mathrm{U}$ \\
\hline $87-61-6$ & $1,2,3$-Trichlorobenzene & 1.0 & $\mathrm{U}$ \\
\hline
\end{tabular}


Lab Name: TESTAMERICA BURLINGTON

Contract: $8 \mathrm{E}-00302$

Lab Code: STLV Case No.: YORK Mod. Ref No.: SDG No.: 28907

Matrix: (SOIL/SED/WATER) Water

Sample wt/vol: 25.0 $(\mathrm{g} / \mathrm{mL}) \mathrm{mL}$

Level: (TRACE or LOW/MED) TRACE

․ Moisture: not dec.

GC Column: DB-624 ID $: 0.20 \quad(\mathrm{~mm})$

Soil Extract Volume: (uL)
Lab Sample ID: 200-28907-1

Lab File ID: 14808_009.D

Date Received: 07/16/2015

Date Analyzed: 07/20/2015

Dilution Factor: 1.0

Soil Aliquot Volume: (uL)

Purge Volume: 25.0

$(\mathrm{mL})$

CONCENTRATION UNITS: (ug/L or ug/kg) ug/L

\begin{tabular}{|c|c|c|c|c|}
\hline CAS NUMBER & COMPOUND NAME & RT & EST. CONC. & $Q$ \\
\hline 01 & Unknown & 7.29 & 2.9 & $\mathrm{~J} B \mathrm{X}$ \\
\hline $541-05-9$ & Cyclotrisiloxane, hexamethyl- & 8.22 & 1.2 & $\mathrm{~J} \quad \mathrm{~N} B$ \\
\hline $556-67-2$ & Cyclotetrasiloxane, octamethyl- & 11.04 & 1.1 & $\mathrm{~J} N \mathrm{~N}$ \\
\hline E9667961 & Total Alkanes & $\mathrm{N} / \mathrm{A}$ & & \\
\hline
\end{tabular}

1 EPA-designated Registry Number. 
Lab Name: TESTAMERICA BURLINGTON

Contract: 8E-00302

Lab Code: STLV Case No.: YORK Mod. Ref No.:

SDG No.: 28907

Instrument ID: CHD.i

Calibration Date(s) : 07/14/2015 07/14/2015

Heated Purge: (Y/N) N

Purge Volume: 25.0

Calibration Time(s) : 1108

1249

GC Column: DB-624

ID: 0.20 (mL)

LAB FILE ID:

RRF5.0 = $14685004 . \mathrm{D}$

\begin{tabular}{|c|c|c|c|}
\hline RRF0. 5 & $=14685 \_002 . D$ & RRF1 . 0 & $=14685003 . \mathrm{D}$ \\
\hline REF10 & $=14685005 . D$ & RRF20 & 14685 \\
\hline
\end{tabular}

COMPOUND

-

\begin{tabular}{|c|c|c|c|c|c|c|c|}
\hline Dichlorodifluoromethane & 0.538 & 0.473 & 0.557 & 0.579 & 0.568 & 0.543 & 7.7 \\
\hline Chloromethane & 0.327 & 0.283 & 0.283 & 0.311 & 0.291 & 0.299 & 6.5 \\
\hline Vinyl chloride & 0.366 & 0.308 & 0.343 & 0.368 & 0.360 & 0.349 & 7.1 \\
\hline Bromomethane & 0.234 & 0.163 & 0.186 & 0.240 & 0.257 & 0.216 & 18.3 \\
\hline Chloroethane & 0.247 & 0.205 & 0.225 & 0.246 & 0.245 & 0.233 & 7.9 \\
\hline Trichlorofluoromethane & 0.936 & 0.791 & 0.940 & 0.963 & 0.945 & 0.915 & 7.7 \\
\hline 1,1-Dichloroethene & 0.302 & 0.288 & 0.291 & 0.303 & 0.353 & 0.307 & 8.6 \\
\hline $\begin{array}{l}1,1,2 \text {-Trichloro- } \\
1,2,2 \text {-trifluoroethane }\end{array}$ & 0.388 & 0.370 & 0.376 & 0.390 & 0.431 & 0.391 & 6.1 \\
\hline Acetone & 0.029 & 0.027 & 0.023 & 0.024 & 0.026 & 0.026 & 8.6 \\
\hline Carbon disulfide & 0.745 & 0.590 & 0.672 & 0.698 & 0.719 & 0.685 & 8.7 \\
\hline Methyl acetate & 0.041 & 0.045 & 0.036 & 0.039 & 0.040 & 0.040 & 8.3 \\
\hline Methylene Chloride & 0.237 & 0.197 & 0.215 & 0.228 & 0.222 & 0.220 & 6.8 \\
\hline trans-1,2-Dichloroethene & 0.270 & 0.249 & 0.292 & 0.298 & 0.309 & 0.284 & 8.6 \\
\hline Methyl tert-butyl ether & 0.337 & 0.335 & 0.424 & 0.447 & 0.468 & 0.402 & 15.6 \\
\hline 1,1-Dichloroethane & 0.499 & 0.433 & 0.487 & 0.514 & 0.517 & 0.490 & 6.9 \\
\hline cis-1,2-Dichloroethene & 0.254 & 0.226 & 0.275 & 0.297 & 0.297 & 0.270 & 11.2 \\
\hline 2-Butanone & 0.026 & 0.021 & 0.027 & 0.029 & 0.030 & 0.027 & 12.3 \\
\hline Bromochloromethane & 0.094 & 0.085 & 0.107 & 0.113 & 0.116 & 0.103 & 12.9 \\
\hline Chloroform & 0.598 & 0.545 & 0.642 & 0.667 & 0.655 & 0.621 & 8.0 \\
\hline 1,1,1-Trichloroethane & 0.844 & 0.717 & 0.882 & 0.907 & 0.907 & 0.851 & 9.3 \\
\hline Cyclohexane & 0.422 & 0.359 & 0.468 & 0.491 & 0.499 & 0.448 & 12.9 \\
\hline Carbon tetrachloride & 0.817 & 0.705 & 0.881 & 0.905 & 0.906 & 0.843 & 10.1 \\
\hline Benzene & 1.192 & 1.071 & 1.214 & 1.242 & 1.249 & 1.194 & 6.0 \\
\hline 1,2-Dichloroethane & 0.359 & 0.341 & 0.383 & 0.399 & 0.383 & 0.373 & 6.2 \\
\hline Trichloroethene & 0.407 & 0.333 & 0.400 & 0.409 & 0.419 & 0.394 & 8.8 \\
\hline Methylcyclohexane & 0.428 & 0.366 & 0.482 & 0.480 & 0.489 & 0.449 & 11.7 \\
\hline
\end{tabular}

Report 1,4-Dioxane for Low-Medium VOA analysis only 
Lab Name: TESTAMERICA BURLINGTON

Contract: $8 \mathrm{E}-00302$

Lab Code: STLV Case No.: YORK Mod. Ref No.:

SDG No.: 28907

Instrument ID: CHD.i

Calibration Date(s) : 07/14/2015 07/14/2015

Heated Purge: ( $\mathrm{Y} / \mathrm{N}) \mathrm{N}$

Purge Volume: 25.0

Calibration Time(s) : 1108

1249

GC Column: DB-624

ID: 0.20 $(\mathrm{mL})$

LAB FILE ID:

RRF5.0 = $14685004 . \mathrm{D}$

\begin{tabular}{|c|c|c|c|}
\hline RRF0. 5 & $=14685 \_002 . D$ & RRF1. 0 & $=14685 \ldots 03 . \mathrm{D}$ \\
\hline RRF10 & $=14685005 . \mathrm{D}$ & RRF20 & $=14685$ \\
\hline
\end{tabular}

COMPOUND

\begin{tabular}{l|l|l} 
RRF0.5 & RRE1.0 \\
\hline
\end{tabular}

RRF5. 0

\begin{tabular}{|l|l}
\hline 1,2-Dichloropropane & \\
\hline Bromodichloromethane & \\
\hline cis-1,3-Dichloropropene & \\
\hline 4-Methyl-2-pentanone & \\
\hline Toluene & \\
\hline trans-1,3-Dichloropropene & \\
\hline $1,1,2-$ Trichloroethane & \\
\hline
\end{tabular}

\begin{tabular}{|l|l|l|l|l|l|l|}
0.215 & 0.188 & 0.206 & 0.213 & 0.219 & 0.208 & 5.8
\end{tabular}

0.34450 .346

0.3110 .287

0.415

0.053

0.056

1.363

1.257

0.403

0.234

0.239

0.077

0.172

0.128

Tetrachloroethene

2-Hexanone

Dibromochloromethane

0.474

0.424

1.516

0.041

0.340

0.184

1,2-Dibromoethane

0.127

0.171

0.145

Chlorobenzene

Ethylbenzene

o-xylene

$\mathrm{m}, \mathrm{p}$-Xylene

Styrene

Bromoform

Isopropylbenzene

$1,1,2,2$-Tetrachloroethane

1,3-Dichlorobenzene

1,4-Dichlorobenzene

1,2-Dichlorobenzene

0.989

0.114

1.735

0.887

0.567

1.604

0.641

0.513

0.812

0.610

0.158

0.811

1.804

0.135

0.498

0.119

1.689

0.108

0.055

1.470

1.245

0.250

0.141

1.031

2.033

0.706

0.786

1.114

0.186

\begin{tabular}{l|l}
1.781 & 1.445
\end{tabular}

1,2-Dibromo-3-Chloropropane

$1,2,4$-Trichlorobenzene

1.178

1.166

0.043

0.031

0.821

0.701

0.593

0.485

2.356

0.135

1.596

1.627

1.344

0.040

0.905

0.646

0.21350.

0.427

0.078

1.560

$0.362 \quad 0.378$

0.447

0.443

0.443

0.082

0.151

0.378

\begin{tabular}{l|l|l|}
0.362 & 0.378 & 0 \\
\hline 0.151 & 0.150 & 0 \\
\hline 0.512 & 0.512 & 0 \\
\hline 0.059 & 0.061 & 0 \\
\hline
\end{tabular}

0.059

0.512

0.257

0.061

0.150

0.274

1.068

0.157

0.157

$\begin{array}{ll}2.150 & 2.189\end{array}$

\begin{tabular}{l|l}
2.150 & 2.189 \\
0.761 & 0.783
\end{tabular}

\begin{tabular}{l|l}
0.761 & 0.783 \\
\hline 0.819 & 0.842
\end{tabular}

0.819
1.179

\begin{tabular}{|l|l|}
\hline 1.179 & 1.197 \\
\hline 0.192 & 0.211 \\
\hline 2.515 & 2.566 \\
\hline
\end{tabular}

.842
1.197

\begin{tabular}{ll}
0.515 & 2.566 \\
\hline
\end{tabular}

0.138

.566
.144

1.652

1.707

1.649

1.684

\begin{tabular}{l|}
1.6455 \\
0.043 \\
0.988
\end{tabular}

1.684

\begin{tabular}{l|l}
1.386 & 1.2 \\
\hline 0.046 & 0.6
\end{tabular}

0.988

1.041

0.700

0.731

\begin{tabular}{|r|r|}
\hline 0.666 & 18.0 \\
\hline 0.740 & 14.4 \\
\hline 1.023 & 19.1 \\
\hline 0.176 & 16.9 \\
\hline 2.186 & 18.8 \\
\hline 0.129 & 11.5 \\
\hline 1.534 & 12.0 \\
\hline 1.637 & 7.5 \\
\hline 1.286 & 8.2 \\
\hline 0.041 & 14.3 \\
\hline 0.891 & 15.2 \\
\hline 0.631 & 15.4 \\
\hline
\end{tabular}

SOM01.2 (4/2007) 
Lab Name: TESTAMERICA BURLINGTON

Contract: $8 \mathrm{E}-00302$

Lab Code: STLV Case No.: YORK Mod. Ref No.: SDG No.: 28907

Instrument ID: CHD.i

Heated Purge: (Y/N) N

Purge Volume: 25.0

GC Column: DB-624
ID: 0.20
Calibration Date(s): 07/14/2015

Calibration Time (s) : 1108 $(\mathrm{mL})$

(mm) Length: 25
$07 / 14 / 2015$ 1249

\begin{tabular}{|c|c|c|c|c|c|c|c|}
\hline LAB FILE ID: & \multirow{2}{*}{\multicolumn{3}{|c|}{$\begin{array}{l}\operatorname{RRF}_{\underline{0.5}}=\frac{14685 \_002 . \mathrm{D}}{\operatorname{RRF10}}=14685 \_005 . \mathrm{D}\end{array}$}} & \multirow{2}{*}{\multicolumn{4}{|c|}{$\begin{array}{l}\operatorname{RRF} 1.0_{\underline{1.0}}=\frac{14685 \_003 . \mathrm{D}}{\mathrm{RRF} 20}=14685 \_006 . \mathrm{D}\end{array}$}} \\
\hline RRF5.0 $=14685 \_004 . D$ & & & & & & & \\
\hline COMPOUND & RRF0. 5 & RRF1. 0 & RRE 5.0 & RRE10 & RRE20 & $\overline{\mathrm{RRF}}$ & $\circ \mathrm{RSD}$ \\
\hline Vinyl Chloride-d3 & 0.327 & 0.271 & 0.297 & 0.312 & 0.306 & 0.303 & 6.9 \\
\hline Chloroethane-d5 & 0.284 & 0.237 & 0.267 & 0.290 & 0.295 & 0.275 & 8.5 \\
\hline 1,1-Dichloroethene-d2 & 0.762 & 0.661 & 0.721 & 0.761 & 0.808 & 0.742 & 7.4 \\
\hline 2-Butanone-d5 & 0.030 & 0.021 & 0.025 & 0.028 & 0.029 & 0.027 & 12.7 \\
\hline Chloroform-d & 0.651 & 0.577 & 0.697 & 0.721 & 0.713 & 0.672 & 8.9 \\
\hline 1,2-Dichloroethane-d4 & 0.315 & 0.272 & 0.311 & 0.318 & 0.321 & 0.307 & 6.6 \\
\hline Benzene-d6 & 1.171 & 1.017 & 1.206 & 1.226 & 1.250 & 1.174 & 7.9 \\
\hline 1,2-Dichloropropane-d6 & 0.244 & 0.209 & 0.236 & 0.245 & 0.251 & 0.237 & 6.9 \\
\hline Toluene-d8 & 1.283 & 1.087 & 1.360 & 1.403 & 1.430 & 1.312 & 10.5 \\
\hline trans-1,3-Dichloropropene-d4 & 0.244 & 0.215 & 0.294 & 0.320 & 0.343 & 0.283 & 18.6 \\
\hline 2-Hexanone-d5 & 0.020 & 0.016 & 0.025 & 0.026 & 0.028 & 0.023 & 21.2 \\
\hline $1,1,2,2$-Tetrachloroethane-d2 & 0.117 & 0.109 & 0.138 & 0.143 & 0.145 & 0.130 & 12.5 \\
\hline 1,2-Dichlorobenzene-d4 & 0.947 & 0.805 & 0.862 & 0.885 & 0.902 & 0.880 & 5.9 \\
\hline
\end{tabular}

Report 1,4-Dioxane-d8 for Low-Medium VOA analysis only 
Lab Name: TESTAMERICA BURLINGTON

Contract: $8 \mathrm{E}-00302$

Lab Code: STLV Case No.: YORK Mod. Ref No.:

SDG No.: 28907

Instrument ID: CHD.i

Calibration Date: 07/18/2015 Time: 1057

Lab File Id: 14793_003.D

Init. Calib. Date(s):07/14/2015 07/14/2015

EPA Sample No.(VSTD\#\#\#\#): VSTD005DN

Init. Calib. Time(s) : 1108 1249

Heated Purge: (Y/N) N GC Column: DB-624

ID: $0.20(\mathrm{~mm})$ Length: 25

(m)

Purge Volume: 25.0 $(\mathrm{mL})$

\begin{tabular}{|c|c|c|c|c|c|}
\hline COMPOUND & $\overline{\mathrm{RRF}}$ & RRF5.0 & $\begin{array}{l}\text { MIN } \\
\text { RRF }\end{array}$ & $\because \mathrm{D}$ & $\mathrm{MAX} \div \mathrm{D}$ \\
\hline Dichlorodifluoromethane & 0.543 & 0.673 & 0.010 & 23.9 & 40.0 \\
\hline Chloromethane & 0.299 & 0.258 & 0.010 & -13.8 & 40.0 \\
\hline Vinyl chloride & 0.349 & 0.332 & 0.100 & -4.7 & 30.0 \\
\hline Bromomethane & 0.216 & 0.141 & 0.100 & -34.7 & 30.0 \\
\hline Chloroethane & 0.233 & 0.221 & 0.010 & -5.2 & 40.0 \\
\hline Trichlorofluoromethane & 0.915 & 1.003 & 0.010 & 9.6 & 40.0 \\
\hline 1,1-Dichloroethene & 0.307 & 0.332 & 0.100 & 8.1 & 30.0 \\
\hline 1,1,2-Trichloro-1,2,2-trifluoroethane & 0.391 & 0.407 & 0.010 & 4.1 & 40.0 \\
\hline Acetone & 0.026 & 0.022 & 0.010 & -15.4 & 40.0 \\
\hline Carbon disulfide & 0.685 & 0.603 & 0.010 & -11.9 & 40.0 \\
\hline Methyl acetate & 0.040 & 0.032 & 0.010 & -20.2 & 40.0 \\
\hline Methylene Chloride & 0.220 & 0.192 & 0.010 & -12.8 & 40.0 \\
\hline trans-1,2-Dichloroethene & 0.284 & 0.266 & 0.010 & -6.0 & 40.0 \\
\hline Methyl tert-butyl ether & 0.402 & 0.335 & 0.010 & -16.8 & 40.0 \\
\hline 1,1-Dichloroethane & 0.490 & 0.460 & 0.200 & -6.1 & 30.0 \\
\hline cis-1,2-Dichloroethene & 0.270 & 0.260 & 0.010 & -3.7 & 40.0 \\
\hline 2-Butanone & 0.027 & 0.023 & 0.010 & -13.2 & 40.0 \\
\hline Bromochloromethane & 0.103 & 0.097 & 0.050 & -5.7 & 30.0 \\
\hline Chloroform & 0.621 & 0.598 & 0.200 & -3.8 & 30.0 \\
\hline 1,1,1-Trichloroethane & 0.851 & 0.894 & 0.100 & 5.0 & 30.0 \\
\hline Cyclohexane & 0.448 & 0.446 & 0.010 & -0.4 & 40.0 \\
\hline Carbon tetrachloride & 0.843 & 0.905 & 0.100 & 7.4 & 30.0 \\
\hline Benzene & 1.194 & 1.168 & 0.400 & -2.1 & 30.0 \\
\hline 1,2-Dichloroethane & 0.373 & 0.354 & 0.100 & -5.2 & 30.0 \\
\hline Trichloroethene & 0.394 & 0.386 & 0.300 & -2.0 & 30.0 \\
\hline Methylcyclohexane & 0.449 & 0.453 & 0.010 & 0.9 & 40.0 \\
\hline
\end{tabular}

Report 1,4-Dioxane for Low/Medium VOA analysis only 
Lab Name: TESTAMERICA BURLINGTON

Contract: $8 \mathrm{E}-00302$

Lab Code: STLV Case No.: YORK Mod. Ref No.:

SDG No.: 28907

Instrument ID: CHD.i

Calibration Date: 07/18/2015 Time: 1057

Lab File Id: 14793_003.D

EPA Sample No. (VSTD\#\#\#\#) : VSTD005DN Init. Calib. Date(s):07/14/2015 07/14/2015

Heated Purge: (Y/N) N GC Column: DB-624

Init. Calib. Time(s) : 1108 1249

Purge Volume: 25.0 ID: $0.20(\mathrm{~mm})$ Length: 25 (m) $(\mathrm{mL})$

\begin{tabular}{|c|c|c|c|c|c|}
\hline COMPOUND & $\overline{\mathrm{RRF}}$ & RRF5.0 & $\begin{array}{l}\text { MIN } \\
\text { RRF }\end{array}$ & $\because \mathrm{D}$ & $\operatorname{MAX} \cong \mathrm{D}$ \\
\hline 1,2-Dichloropropane & 0.208 & 0.191 & 0.010 & -8.4 & 40.0 \\
\hline Bromodichloromethane & 0.397 & 0.391 & 0.200 & -1.5 & 30.0 \\
\hline cis-1,3-Dichloropropene & 0.374 & 0.364 & 0.200 & -2.8 & 30.0 \\
\hline 4-Methyl-2-pentanone & 0.069 & 0.065 & 0.010 & -5.8 & 40.0 \\
\hline Toluene & 1.459 & 1.484 & 0.400 & 1.7 & 30.0 \\
\hline trans-1,3-Dichloropropene & 0.311 & 0.294 & 0.100 & -5.5 & 30.0 \\
\hline $1,1,2$-Trichloroethane & 0.149 & 0.130 & 0.100 & -12.6 & 30.0 \\
\hline Tetrachloroethene & 0.484 & 0.490 & 0.100 & 1.1 & 30.0 \\
\hline 2-Hexanone & 0.052 & 0.048 & 0.010 & -8.0 & 40.0 \\
\hline Dibromochloromethane & 0.227 & 0.222 & 0.100 & -2.4 & 30.0 \\
\hline 1,2-Dibromoethane & 0.138 & 0.126 & 0.010 & -8.8 & 40.0 \\
\hline Chlorobenzene & 1.009 & 0.987 & 0.500 & -2.2 & 30.0 \\
\hline Ethylbenzene & 1.942 & 2.020 & 0.100 & 4.0 & 30.0 \\
\hline o-Xylene & 0.666 & 0.676 & 0.300 & 1.5 & 30.0 \\
\hline $\mathrm{m}, \mathrm{p}$-Xylene & 0.740 & 0.762 & 0.300 & 3.0 & 30.0 \\
\hline Styrene & 1.023 & 1.043 & 0.300 & 2.0 & 30.0 \\
\hline Bromoform & 0.176 & 0.169 & 0.050 & -4.1 & 30.0 \\
\hline Isopropylbenzene & 2.186 & 2.362 & 0.010 & 8.0 & 40.0 \\
\hline $1,1,2,2$-Tetrachloroethane & 0.129 & 0.115 & 0.100 & -10.6 & 30.0 \\
\hline 1,3-Dichlorobenzene & 1.534 & 1.496 & 0.400 & -2.5 & 30.0 \\
\hline 1,4-Dichlorobenzene & 1.637 & 1.546 & 0.400 & -5.6 & 30.0 \\
\hline 1,2-Dichlorobenzene & 1.286 & 1.243 & 0.400 & -3.3 & 30.0 \\
\hline 1,2-Dibromo-3-Chloropropane & 0.041 & 0.034 & 0.010 & -16.6 & 40.0 \\
\hline 1,2,4-Trichlorobenzene & 0.891 & 0.721 & 0.200 & -19.0 & 30.0 \\
\hline $1,2,3$-Trichlorobenzene & 0.631 & 0.512 & 0.200 & $-18 \cdot 9$ & 30.0 \\
\hline
\end{tabular}


Lab Name: TESTAMERICA BURLINGTON

Contract: $8 \mathrm{E}-00302$

Lab Code: STLV Case No.: YORK Mod. Ref No.

SDG No.: 28907

Instrument ID: CHD.i

Calibration Date: 07/18/2015 Time: 1057

Lab File Id: 14793_003.D Init. Calib. Date(s):07/14/2015 07/14/2015

EPA Sample No. (VSTD\#\#\#\#) : VSTD005DN

Heated Purge: (Y/N) N GC Column: DB-624

Init. Calib. Time(s) : 1108 1249

Purge Volume: 25.0 ID: $0.20(\mathrm{~mm})$ Length: 25

(m)

\begin{tabular}{|c|c|c|c|c|c|}
\hline COMPOUND & $\overline{\mathrm{RRF}}$ & RRF5. 0 & $\begin{array}{l}\text { MIN } \\
\text { RRF }\end{array}$ & $\circ \mathrm{D}$ & $\mathrm{MAX} \div \mathrm{D}$ \\
\hline Vinyl Chloride-d3 & 0.303 & 0.317 & 0.010 & 4.7 & 30.0 \\
\hline Chloroethane-d5 & 0.275 & 0.273 & 0.010 & -0.4 & 40.0 \\
\hline 1,1-Dichloroethene-d2 & 0.742 & 0.761 & 0.010 & 2.5 & 30.0 \\
\hline 2-Butanone-d5 & 0.027 & 0.021 & 0.010 & $-21 \cdot 9$ & 40.0 \\
\hline Chloroform-d & 0.672 & 0.652 & 0.010 & -2.9 & 30.0 \\
\hline 1,2-Dichloroethane-d4 & 0.307 & 0.291 & 0.010 & -5.4 & 30.0 \\
\hline Benzene-d6 & 1.174 & 1.157 & 0.010 & $-1 \cdot 4$ & 30.0 \\
\hline 1,2-Dichloropropane-d6 & 0.237 & 0.226 & 0.010 & -4.8 & 40.0 \\
\hline Toluene-d8 & 1.312 & 1.340 & 0.010 & 2.1 & 30.0 \\
\hline trans-1,3-Dichloropropene-d4 & 0.283 & 0.263 & 0.010 & -7.2 & 30.0 \\
\hline 2-Hexanone-d5 & 0.023 & 0.021 & 0.010 & $-10 \cdot 4$ & 40.0 \\
\hline $1,1,2,2$-Tetrachloroethane-d2 & 0.130 & 0.118 & 0.010 & -9.5 & 30.0 \\
\hline 1,2-Dichlorobenzene-d4 & 0.880 & 0.775 & 0.010 & -11.9 & 30.0 \\
\hline
\end{tabular}

Report 1,4-Dioxane-d8 for Low/Medium VOA analysis only 
Lab Name: TESTAMERICA BURLINGTON

Contract: $8 \mathrm{E}-00302$

Lab Code: STLV Case No.: YORK Mod. Ref No.:

SDG No.: 28907

Instrument ID: CHD.i

Calibration Date: 07/18/2015 Time: 1801

Lab File Id: 14793_020.D

Init. Calib. Date(s):07/14/2015 07/14/2015

EPA Sample No. (VSTD\#\#\#\#) : VSTD005NDA

Heated Purge: ( $\mathrm{Y} / \mathrm{N}) \mathrm{N}$ GC Column: DB-624

Init. Calib. Time(s) : 1108 1249

Purge Volume: 25.0

ID: $0.20(\mathrm{~mm})$ Length: 25

(m)

\begin{tabular}{|c|c|c|c|c|c|}
\hline COMPOUND & $\overline{\mathrm{RRF}}$ & RRF005 & $\begin{array}{l}\text { MIN } \\
\text { RRF }\end{array}$ & $\frac{\circ}{D}$ & $\mathrm{MAX} \div \mathrm{D}$ \\
\hline Dichlorodifluoromethane & 0.543 & 0.683 & 0.010 & 25.7 & 50.0 \\
\hline Chloromethane & 0.299 & 0.249 & 0.010 & -16.8 & 50.0 \\
\hline Vinyl chloride & 0.349 & 0.341 & 0.010 & -2.3 & 50.0 \\
\hline Bromomethane & 0.216 & 0.114 & 0.010 & -47.2 & 50.0 \\
\hline Chloroethane & 0.233 & 0.223 & 0.010 & -4.6 & 50.0 \\
\hline Trichlorofluoromethane & 0.915 & 1.008 & 0.010 & 10.2 & 50.0 \\
\hline 1,1-Dichloroethene & 0.307 & 0.332 & 0.010 & 8.0 & 50.0 \\
\hline 1,1,2-Trichloro-1,2,2-trifluoroethane & 0.391 & 0.396 & 0.010 & 1.2 & 50.0 \\
\hline Acetone & 0.026 & 0.027 & 0.010 & 3.2 & 50.0 \\
\hline Carbon disulfide & 0.685 & 0.604 & 0.010 & -11.8 & 50.0 \\
\hline Methyl acetate & 0.040 & 0.039 & 0.010 & -3.3 & 50.0 \\
\hline Methylene Chloride & 0.220 & 0.206 & 0.010 & -6.1 & 50.0 \\
\hline trans-1,2-Dichloroethene & 0.284 & 0.276 & 0.010 & -2.7 & 50.0 \\
\hline Methyl tert-butyl ether & 0.402 & 0.397 & 0.010 & -1.2 & 50.0 \\
\hline 1,1-Dichloroethane & 0.490 & 0.468 & 0.010 & -4.4 & 50.0 \\
\hline cis-1,2-Dichloroethene & 0.270 & 0.265 & 0.010 & -1.5 & 50.0 \\
\hline 2-Butanone & 0.027 & 0.028 & 0.010 & 4.1 & 50.0 \\
\hline Bromochloromethane & 0.103 & 0.108 & 0.010 & 5.1 & 50.0 \\
\hline Chloroform & 0.621 & 0.621 & 0.010 & 0.0 & 50.0 \\
\hline 1,1,1-Trichloroethane & 0.851 & 0.883 & 0.010 & 3.7 & 50.0 \\
\hline Cyclohexane & 0.448 & 0.443 & 0.010 & -1.0 & 50.0 \\
\hline Carbon tetrachloride & 0.843 & 0.900 & 0.010 & 6.7 & 50.0 \\
\hline Benzene & 1.194 & 1.180 & 0.010 & -1.2 & 50.0 \\
\hline 1,2-Dichloroethane & 0.373 & 0.388 & 0.010 & 4.2 & 50.0 \\
\hline Trichloroethene & 0.394 & 0.396 & 0.010 & 0.7 & 50.0 \\
\hline Methylcyclohexane & 0.449 & 0.437 & 0.010 & $-2 \cdot 6$ & 50.0 \\
\hline
\end{tabular}

Report 1,4-Dioxane for Low/Medium VOA analysis only 
Lab Name: TESTAMERICA BURLINGTON

Contract: 8E-00302

Lab Code: STLV Case No.: YORK Mod. Ref No.:

SDG No.: 28907

Instrument ID: CHD.i

Calibration Date: 07/18/2015 Time: 1801

Lab File Id: 14793_020.D

Init. Calib. Date(s):07/14/2015 07/14/2015

EPA Sample No. (VSTD\#\#\#\#) : VSTD005NDA

Heated Purge: (Y/N) N GC Column: DB-624

Init. Calib. Time(s) : 1108 1249

Purge Volume: 25.0

ID: $0.20(\mathrm{~mm})$ Length: 25

(m)

\begin{tabular}{|c|c|c|c|c|c|}
\hline COMPOUND & $\overline{\mathrm{RRF}}$ & RRF005 & $\begin{array}{l}\text { MIN } \\
\text { RRF }\end{array}$ & $\because \mathrm{D}$ & $\operatorname{MAX} \cong \mathrm{D}$ \\
\hline 1,2-Dichloropropane & 0.208 & 0.199 & 0.010 & -4.3 & 50.0 \\
\hline Bromodichloromethane & 0.397 & 0.410 & 0.010 & 3.4 & 50.0 \\
\hline cis-1,3-Dichloropropene & 0.374 & 0.375 & 0.010 & 0.3 & 50.0 \\
\hline 4-Methyl-2-pentanone & 0.069 & 0.076 & 0.010 & 9.4 & 50.0 \\
\hline Toluene & 1.459 & 1.511 & 0.010 & 3.5 & 50.0 \\
\hline trans-1,3-Dichloropropene & 0.311 & 0.317 & 0.010 & 1.9 & 50.0 \\
\hline $1,1,2$-Trichloroethane & 0.149 & 0.142 & 0.010 & -4.5 & 50.0 \\
\hline Tetrachloroethene & 0.484 & 0.490 & 0.010 & 1.3 & 50.0 \\
\hline 2-Hexanone & 0.052 & 0.057 & 0.010 & 9.7 & 50.0 \\
\hline Dibromochloromethane & 0.227 & 0.235 & 0.010 & 3.6 & 50.0 \\
\hline 1,2-Dibromoethane & 0.138 & 0.138 & 0.010 & -0.1 & 50.0 \\
\hline Chlorobenzene & 1.009 & 1.022 & 0.010 & 1.3 & 50.0 \\
\hline Ethylbenzene & 1.942 & 2.045 & 0.010 & 5.3 & 50.0 \\
\hline o-Xylene & 0.666 & 0.695 & 0.010 & 4.4 & 50.0 \\
\hline $\mathrm{m}, \mathrm{p}$-Xylene & 0.740 & 0.773 & 0.010 & 4.5 & 50.0 \\
\hline Styrene & 1.023 & 1.092 & 0.010 & 6.8 & 50.0 \\
\hline Bromoform & 0.176 & 0.174 & 0.010 & -1.1 & 50.0 \\
\hline Isopropylbenzene & 2.186 & 2.343 & 0.010 & 7.2 & 50.0 \\
\hline $1,1,2,2$-Tetrachloroethane & 0.129 & 0.130 & 0.010 & 1.2 & 50.0 \\
\hline 1,3-Dichlorobenzene & 1.534 & 1.537 & 0.010 & 0.2 & 50.0 \\
\hline 1,4-Dichlorobenzene & 1.637 & 1.596 & 0.010 & -2.5 & 50.0 \\
\hline 1,2-Dichlorobenzene & 1.286 & 1.308 & 0.010 & 1.7 & 50.0 \\
\hline 1,2-Dibromo-3-Chloropropane & 0.041 & 0.039 & 0.010 & -3.3 & 50.0 \\
\hline 1,2,4-Trichlorobenzene & 0.891 & 0.841 & 0.010 & -5.6 & 50.0 \\
\hline $1,2,3$-Trichlorobenzene & 0.631 & 0.628 & 0.010 & -0.5 & 50.0 \\
\hline
\end{tabular}


Lab Name: TESTAMERICA BURLINGTON

Contract: $8 \mathrm{E}-00302$

Lab Code: STLV Case No.: YORK Mod. Ref No.

SDG No.: 28907

Instrument ID: CHD.i

Calibration Date: 07/18/2015 Time: 1801

Lab File Id: 14793_020.D Init. Calib. Date(s):07/14/2015 07/14/2015

EPA Sample No.(VSTD\#\#\#\#): VSTD005NDA

Heated Purge: (Y/N) N GC Column: DB-624

Init. Calib. Time(s) : 1108 1249

Purge Volume: 25.0 ID: $0.20(\mathrm{~mm})$ Length: 25

(m)

\begin{tabular}{|c|c|c|c|c|c|}
\hline COMPOUND & $\overline{\mathrm{RRF}}$ & RRF005 & $\begin{array}{l}\text { MIN } \\
\text { RRF }\end{array}$ & $\circ \mathrm{D}$ & MAX $\cong D$ \\
\hline Vinyl Chloride-d3 & 0.303 & 0.337 & 0.010 & 11.3 & 50.0 \\
\hline Chloroethane-d5 & 0.275 & 0.294 & 0.010 & 7.1 & 50.0 \\
\hline 1,1-Dichloroethene-d2 & 0.742 & 0.780 & 0.010 & 5.1 & 50.0 \\
\hline 2-Butanone-d5 & 0.027 & 0.026 & 0.010 & -2.5 & 50.0 \\
\hline Chloroform-d & 0.672 & 0.679 & 0.010 & 1.1 & 50.0 \\
\hline 1,2-Dichloroethane-d4 & 0.307 & 0.312 & 0.010 & 1.6 & 50.0 \\
\hline Benzene-d6 & 1.174 & 1.178 & 0.010 & 0.3 & 50.0 \\
\hline 1,2-Dichloropropane-d6 & 0.237 & 0.232 & 0.010 & -2.2 & 50.0 \\
\hline Toluene-d8 & 1.312 & 1.374 & 0.010 & 4.7 & 50.0 \\
\hline trans-1,3-Dichloropropene-d4 & 0.283 & 0.276 & 0.010 & -2.5 & 50.0 \\
\hline 2-Hexanone-d5 & 0.023 & 0.024 & 0.010 & 4.8 & 50.0 \\
\hline $1,1,2,2-$ Tetrachloroethane-d2 & 0.130 & 0.133 & 0.010 & 2.3 & 50.0 \\
\hline 1,2-Dichlorobenzene-d4 & 0.880 & 0.848 & 0.010 & -3.7 & 50.0 \\
\hline
\end{tabular}

Report 1,4-Dioxane-d8 for Low/Medium VOA analysis only 
Lab Name: TESTAMERICA BURLINGTON

Contract: $8 \mathrm{E}-00302$

Lab Code: STLV Case No.: YORK Mod. Ref No.:

SDG No.: 28907

Instrument ID: CHD.i

Calibration Date: 07/20/2015 Time: 0927

Lab File Id: 14808_004.D

EPA Sample No. (VSTD\#\#\#\#) : VSTD005DP

Heated Purge: ( $\mathrm{Y} / \mathrm{N}) \mathrm{N}$ GC Column: DB-624

Init. Calib. Time (s) : 1108 $07 / 14 / 2015$

Purge Volume: 25.0 $(\mathrm{mL})$

\begin{tabular}{|c|c|c|c|c|c|}
\hline COMPOUND & $\overline{\mathrm{RRF}}$ & RRF5.0 & $\begin{array}{l}\text { MIN } \\
\text { RRF }\end{array}$ & $\frac{\circ}{D}$ & $\mathrm{MAX} \div \mathrm{D}$ \\
\hline Dichlorodifluoromethane & 0.543 & 0.702 & 0.010 & 29.4 & 40.0 \\
\hline Chloromethane & 0.299 & 0.267 & 0.010 & -10.8 & 40.0 \\
\hline Vinyl chloride & 0.349 & 0.353 & 0.100 & 1.1 & 30.0 \\
\hline Bromomethane & 0.216 & 0.174 & 0.100 & -19.6 & 30.0 \\
\hline Chloroethane & 0.233 & 0.214 & 0.010 & -8.1 & 40.0 \\
\hline Trichlorofluoromethane & 0.915 & 1.013 & 0.010 & 10.7 & 40.0 \\
\hline 1,1-Dichloroethene & 0.307 & 0.322 & 0.100 & 4.6 & 30.0 \\
\hline 1,1,2-Trichloro-1,2,2-trifluoroethane & 0.391 & 0.421 & 0.010 & 7.6 & 40.0 \\
\hline Acetone & 0.026 & 0.024 & 0.010 & -7.1 & 40.0 \\
\hline Carbon disulfide & 0.685 & 0.611 & 0.010 & -10.7 & 40.0 \\
\hline Methyl acetate & 0.040 & 0.033 & 0.010 & -17.7 & 40.0 \\
\hline Methylene Chloride & 0.220 & 0.194 & 0.010 & -11.9 & 40.0 \\
\hline trans-1,2-Dichloroethene & 0.284 & 0.271 & 0.010 & -4.3 & 40.0 \\
\hline Methyl tert-butyl ether & 0.402 & 0.366 & 0.010 & -8.9 & 40.0 \\
\hline 1,1-Dichloroethane & 0.490 & 0.456 & 0.200 & -7.0 & 30.0 \\
\hline cis-1,2-Dichloroethene & 0.270 & 0.260 & 0.010 & -3.7 & 40.0 \\
\hline 2-Butanone & 0.027 & 0.023 & 0.010 & -11.9 & 40.0 \\
\hline Bromochloromethane & 0.103 & 0.102 & 0.050 & -0.8 & 30.0 \\
\hline Chloroform & 0.621 & 0.612 & 0.200 & -1.5 & 30.0 \\
\hline 1,1,1-Trichloroethane & 0.851 & 0.869 & 0.100 & 2.0 & 30.0 \\
\hline Cyclohexane & 0.448 & 0.447 & 0.010 & -0.2 & 40.0 \\
\hline Carbon tetrachloride & 0.843 & 0.876 & 0.100 & 3.9 & 30.0 \\
\hline Benzene & 1.194 & 1.133 & 0.400 & -5.1 & 30.0 \\
\hline 1,2-Dichloroethane & 0.373 & 0.360 & 0.100 & -3.5 & 30.0 \\
\hline Trichloroethene & 0.394 & 0.379 & 0.300 & -3.8 & 30.0 \\
\hline Methylcyclohexane & 0.449 & 0.438 & 0.010 & -2.5 & 40.0 \\
\hline
\end{tabular}

Report 1,4-Dioxane for Low/Medium VOA analysis only 
Lab Name: TESTAMERICA BURLINGTON

Contract: 8E-00302

Lab Code: STLV

Mod. Ref No.:

SDG No.: 28907

Instrument ID: CHD.i

Calibration Date: 07/20/2015 Time: 0927

Lab File Id: 14808_004.D

Init. Calib. Date(s):07/14/2015 07/14/2015

EPA Sample No. (VSTD\#\#\#\#) : VSTD005DP

Heated Purge: (Y/N) N GC Column: DB-624

Init. Calib. Time(s) : 1108 1249

Purge Volume: 25.0

ID: $0.20(\mathrm{~mm})$ Length: 25

(m)

\begin{tabular}{|c|c|c|c|c|c|}
\hline COMPOUND & $\overline{\mathrm{RRF}}$ & RRF5.0 & $\begin{array}{l}\text { MIN } \\
\text { RRF }\end{array}$ & $\because \mathrm{D}$ & $\operatorname{MAX} \cong \mathrm{D}$ \\
\hline 1,2-Dichloropropane & 0.208 & 0.190 & 0.010 & -9.1 & 40.0 \\
\hline Bromodichloromethane & 0.397 & 0.385 & 0.200 & -3.0 & 30.0 \\
\hline cis-1,3-Dichloropropene & 0.374 & 0.348 & 0.200 & -7.1 & 30.0 \\
\hline 4-Methyl-2-pentanone & 0.069 & 0.067 & 0.010 & -3.1 & 40.0 \\
\hline Toluene & 1.459 & 1.454 & 0.400 & -0.4 & 30.0 \\
\hline trans-1,3-Dichloropropene & 0.311 & 0.293 & 0.100 & -5.8 & 30.0 \\
\hline $1,1,2$-Trichloroethane & 0.149 & 0.134 & 0.100 & -10.1 & 30.0 \\
\hline Tetrachloroethene & 0.484 & 0.470 & 0.100 & -2.8 & 30.0 \\
\hline 2-Hexanone & 0.052 & 0.049 & 0.010 & -5.2 & 40.0 \\
\hline Dibromochloromethane & 0.227 & 0.218 & 0.100 & -4.1 & 30.0 \\
\hline 1,2-Dibromoethane & 0.138 & 0.128 & 0.010 & -6.9 & 40.0 \\
\hline Chlorobenzene & 1.009 & 0.968 & 0.500 & -4.1 & 30.0 \\
\hline Ethylbenzene & 1.942 & 1.977 & 0.100 & 1.8 & 30.0 \\
\hline o-Xylene & 0.666 & 0.665 & 0.300 & -0.1 & 30.0 \\
\hline $\mathrm{m}, \mathrm{p}$-Xylene & 0.740 & 0.732 & 0.300 & -1.0 & 30.0 \\
\hline Styrene & 1.023 & 1.001 & 0.300 & -2.1 & 30.0 \\
\hline Bromoform & 0.176 & 0.157 & 0.050 & -11.1 & 30.0 \\
\hline Isopropylbenzene & 2.186 & 2.256 & 0.010 & 3.2 & 40.0 \\
\hline $1,1,2,2$-Tetrachloroethane & 0.129 & 0.116 & 0.100 & -10.2 & 30.0 \\
\hline 1,3-Dichlorobenzene & 1.534 & 1.469 & 0.400 & -4.2 & 30.0 \\
\hline 1,4-Dichlorobenzene & 1.637 & 1.564 & 0.400 & -4.5 & 30.0 \\
\hline 1,2-Dichlorobenzene & 1.286 & 1.265 & 0.400 & -1.6 & 30.0 \\
\hline 1,2-Dibromo-3-Chloropropane & 0.041 & 0.038 & 0.010 & -7.4 & 40.0 \\
\hline 1,2,4-Trichlorobenzene & 0.891 & 0.771 & 0.200 & -13.4 & 30.0 \\
\hline $1,2,3$-Trichlorobenzene & 0.631 & 0.557 & 0.200 & -11.8 & 30.0 \\
\hline
\end{tabular}


Lab Name: TESTAMERICA BURLINGTON

Contract: $8 \mathrm{E}-00302$

Lab Code: STLV Case No.: YORK Mod. Ref No.

SDG No.: 28907

Instrument ID: CHD.i

Calibration Date: 07/20/2015 Time: 0927

Lab File Id: 14808_004.D

Init. Calib. Date(s):07/14/2015 07/14/2015

EPA Sample No. (VSTD\#\#\#\#) : VSTD005DP

Heated Purge: (Y/N) N GC Column: DB-624

Init. Calib. Time(s) : 1108 1249

Purge Volume: 25.0

ID: $0.20(\mathrm{~mm})$ Length: 25

(m)

\begin{tabular}{|c|c|c|c|c|c|}
\hline COMPOUND & $\overline{\mathrm{RRF}}$ & RRE5. 0 & $\begin{array}{l}\text { MIN } \\
\text { RRE }\end{array}$ & $\because \mathrm{D}$ & $M A X \div D$ \\
\hline Vinyl Chloride-d3 & 0.303 & 0.335 & 0.010 & 10.8 & 30.0 \\
\hline Chloroethane-d5 & 0.275 & 0.287 & 0.010 & 4.6 & 40.0 \\
\hline $1,1-$ Dichloroethene-d2 & 0.742 & 0.754 & 0.010 & 1.6 & 30.0 \\
\hline 2-Butanone-d5 & 0.027 & 0.023 & 0.010 & -12.2 & 40.0 \\
\hline Chloroform-d & 0.672 & 0.671 & 0.010 & -0.1 & 30.0 \\
\hline 1,2-Dichloroethane-d4 & 0.307 & 0.289 & 0.010 & -5.9 & 30.0 \\
\hline Benzene-d6 & 1.174 & 1.125 & 0.010 & -4.2 & 30.0 \\
\hline 1,2-Dichloropropane-d6 & 0.237 & 0.217 & 0.010 & -8.2 & 40.0 \\
\hline Toluene-d8 & 1.312 & 1.316 & 0.010 & 0.3 & 30.0 \\
\hline trans-1,3-Dichloropropene-d4 & 0.283 & 0.268 & 0.010 & -5.5 & 30.0 \\
\hline 2-Hexanone-d5 & 0.023 & 0.021 & 0.010 & -7.5 & 40.0 \\
\hline $1,1,2,2$-Tetrachloroethane-d2 & 0.130 & 0.115 & 0.010 & $-11 \cdot 7$ & 30.0 \\
\hline 1,2-Dichlorobenzene-d4 & 0.880 & 0.787 & 0.010 & -10.6 & 30.0 \\
\hline
\end{tabular}

Report 1,4-Dioxane-d8 for Low/Medium VOA analysis only 
Lab Name: TESTAMERICA BURLINGTON

Contract: $8 \mathrm{E}-00302$

Lab Code: STLV Case No.: YORK Mod. Ref No.:

SDG No.: 28907

Instrument ID: CHD.i

Calibration Date: 07/20/2015 Time: 1547

Lab File Id: 14808_013.D

EPA Sample No. (VSTD\#\#\#\#) : VSTD005PD Init. Calib. Date(s):07/14/2015 07/14/2015

Heated Purge: (Y/N) N GC Column: DB-624

Init. Calib. Time(s) : 1108 1249

Purge Volume: 25.0 ID: $0.20(\mathrm{~mm})$ Length: 25 (m) $(\mathrm{mL})$

\begin{tabular}{|c|c|c|c|c|c|}
\hline COMPOUND & $\overline{\mathrm{RRF}}$ & RRF5.0 & $\begin{array}{l}\text { MIN } \\
\text { RRF }\end{array}$ & $\frac{\circ}{D}$ & $\mathrm{MAX} \div \mathrm{D}$ \\
\hline Dichlorodifluoromethane & 0.543 & 0.697 & 0.010 & 28.4 & 50.0 \\
\hline Chloromethane & 0.299 & 0.278 & 0.010 & -7.0 & 50.0 \\
\hline Vinyl chloride & 0.349 & 0.369 & 0.010 & 5.8 & 50.0 \\
\hline Bromomethane & 0.216 & 0.227 & 0.010 & 5.0 & 50.0 \\
\hline Chloroethane & 0.233 & 0.241 & 0.010 & 3.1 & 50.0 \\
\hline Trichlorofluoromethane & 0.915 & 1.054 & 0.010 & 15.3 & 50.0 \\
\hline 1,1-Dichloroethene & 0.307 & 0.331 & 0.010 & 7.8 & 50.0 \\
\hline 1,1,2-Trichloro-1,2,2-trifluoroethane & 0.391 & 0.431 & 0.010 & 10.1 & 50.0 \\
\hline Acetone & 0.026 & 0.023 & 0.010 & $-11 \cdot 4$ & 50.0 \\
\hline Carbon disulfide & 0.685 & 0.618 & 0.010 & -9.8 & 50.0 \\
\hline Methyl acetate & 0.040 & 0.037 & 0.010 & -8.3 & 50.0 \\
\hline Methylene Chloride & 0.220 & 0.203 & 0.010 & -7.5 & 50.0 \\
\hline trans-1,2-Dichloroethene & 0.284 & 0.281 & 0.010 & -0.8 & 50.0 \\
\hline Methyl tert-butyl ether & 0.402 & 0.343 & 0.010 & -14.7 & 50.0 \\
\hline 1,1-Dichloroethane & 0.490 & 0.476 & 0.010 & -2.8 & 50.0 \\
\hline cis-1,2-Dichloroethene & 0.270 & 0.269 & 0.010 & -0.1 & 50.0 \\
\hline 2-Butanone & 0.027 & 0.023 & 0.010 & -11.7 & 50.0 \\
\hline Bromochloromethane & 0.103 & 0.100 & 0.010 & -3.0 & 50.0 \\
\hline Chloroform & 0.621 & 0.619 & 0.010 & -0.5 & 50.0 \\
\hline 1,1,1-Trichloroethane & 0.851 & 0.934 & 0.010 & 9.7 & 50.0 \\
\hline Cyclohexane & 0.448 & 0.488 & 0.010 & 9.0 & 50.0 \\
\hline Carbon tetrachloride & 0.843 & 0.936 & 0.010 & 11.0 & 50.0 \\
\hline Benzene & 1.194 & 1.204 & 0.010 & 0.8 & 50.0 \\
\hline 1,2-Dichloroethane & 0.373 & 0.367 & 0.010 & -1.6 & 50.0 \\
\hline Trichloroethene & 0.394 & 0.405 & 0.010 & 2.9 & 50.0 \\
\hline Methylcyclohexane & 0.449 & 0.483 & 0.010 & 7.6 & 50.0 \\
\hline
\end{tabular}

Report 1,4-Dioxane for Low/Medium VOA analysis only 
Lab Name: TESTAMERICA BURLINGTON

Contract: 8E-00302

Lab Code: STLV Case No.: YORK Mod. Ref No.:

SDG No.: 28907

Instrument ID: CHD.i

Calibration Date: 07/20/2015 Time: 1547

Lab File Id: 14808_013.D

EPA Sample No. (VSTD\#\#\#\#) : VSTD005PD

Init. Calib. Date(s):07/14/2015 07/14/2015

Heated Purge: (Y/N) N

GC Column: DB-624

Init. Calib. Time(s) : 1108

1249

Purge Volume: 25.0

ID: $0.20(\mathrm{~mm})$ Length: 25

(m)

$(\mathrm{mL})$

\begin{tabular}{|c|c|c|c|c|c|}
\hline COMPOUND & $\overline{\mathrm{RRF}}$ & RRF5.0 & $\begin{array}{l}\text { MIN } \\
\text { RRF }\end{array}$ & $\because \mathrm{D}$ & $\operatorname{MAX} \cong \mathrm{D}$ \\
\hline 1,2-Dichloropropane & 0.208 & 0.200 & 0.010 & -3.9 & 50.0 \\
\hline Bromodichloromethane & 0.397 & 0.399 & 0.010 & 0.6 & 50.0 \\
\hline cis-1,3-Dichloropropene & 0.374 & 0.372 & 0.010 & -0.6 & 50.0 \\
\hline 4-Methyl-2-pentanone & 0.069 & 0.065 & 0.010 & -6.6 & 50.0 \\
\hline Toluene & 1.459 & 1.546 & 0.010 & 5.9 & 50.0 \\
\hline trans-1,3-Dichloropropene & 0.311 & 0.306 & 0.010 & -1.6 & 50.0 \\
\hline $1,1,2$-Trichloroethane & 0.149 & 0.128 & 0.010 & -14.5 & 50.0 \\
\hline Tetrachloroethene & 0.484 & 0.499 & 0.010 & 3.1 & 50.0 \\
\hline 2-Hexanone & 0.052 & 0.048 & 0.010 & -8.2 & 50.0 \\
\hline Dibromochloromethane & 0.227 & 0.219 & 0.010 & -3.6 & 50.0 \\
\hline 1,2-Dibromoethane & 0.138 & 0.126 & 0.010 & -8.5 & 50.0 \\
\hline Chlorobenzene & 1.009 & 1.001 & 0.010 & -0.8 & 50.0 \\
\hline Ethylbenzene & 1.942 & 2.081 & 0.010 & 7.1 & 50.0 \\
\hline o-Xylene & 0.666 & 0.714 & 0.010 & 7.2 & 50.0 \\
\hline $\mathrm{m}, \mathrm{p}$-Xylene & 0.740 & 0.791 & 0.010 & 6.9 & 50.0 \\
\hline Styrene & 1.023 & 1.077 & 0.010 & 5.3 & 50.0 \\
\hline Bromoform & 0.176 & 0.163 & 0.010 & -7.6 & 50.0 \\
\hline Isopropylbenzene & 2.186 & 2.417 & 0.010 & 10.6 & 50.0 \\
\hline $1,1,2,2$-Tetrachloroethane & 0.129 & 0.116 & 0.010 & -10.2 & 50.0 \\
\hline 1,3-Dichlorobenzene & 1.534 & 1.547 & 0.010 & 0.8 & 50.0 \\
\hline 1,4-Dichlorobenzene & 1.637 & 1.592 & 0.010 & -2.8 & 50.0 \\
\hline 1,2-Dichlorobenzene & 1.286 & 1.264 & 0.010 & -1.7 & 50.0 \\
\hline 1,2-Dibromo-3-Chloropropane & 0.041 & 0.034 & 0.010 & -16.8 & 50.0 \\
\hline 1,2,4-Trichlorobenzene & 0.891 & 0.763 & 0.010 & -14.3 & 50.0 \\
\hline $1,2,3$-Trichlorobenzene & 0.631 & 0.538 & 0.010 & $-14 \cdot 7$ & 50.0 \\
\hline
\end{tabular}


Lab Name: TESTAMERICA BURLINGTON

Contract: $8 \mathrm{E}-00302$

Lab Code: STLV Case No.: YORK Mod. Ref No.

SDG No.: 28907

Instrument ID: CHD.i

Calibration Date: 07/20/2015 Time: 1547

Lab File Id: 14808_013.D

Init. Calib. Date(s):07/14/2015 07/14/2015

EPA Sample No. (VSTD\#\#\#\#) : VSTD005PD

Heated Purge: (Y/N) N GC Column: DB-624

Init. Calib. Time(s) : 1108 1249

Purge Volume: 25.0

ID: $0.20(\mathrm{~mm})$ Length: 25

(m)

\begin{tabular}{|c|c|c|c|c|c|}
\hline COMPOUND & $\overline{\mathrm{RRF}}$ & RRE5. 0 & $\begin{array}{l}\text { MIN } \\
\text { RRE }\end{array}$ & $\because \mathrm{D}$ & $M A X \div D$ \\
\hline Vinyl Chloride-d3 & 0.303 & 0.357 & 0.010 & 17.9 & 50.0 \\
\hline Chloroethane-d5 & 0.275 & 0.301 & 0.010 & 9.6 & 50.0 \\
\hline $1,1-$ Dichloroethene-d2 & 0.742 & 0.806 & 0.010 & 8.5 & 50.0 \\
\hline 2-Butanone-d5 & 0.027 & 0.023 & 0.010 & $-14 \cdot 3$ & 50.0 \\
\hline Chloroform-d & 0.672 & 0.689 & 0.010 & 2.6 & 50.0 \\
\hline 1,2-Dichloroethane-d4 & 0.307 & 0.295 & 0.010 & -3.9 & 50.0 \\
\hline Benzene-d6 & 1.174 & 1.211 & 0.010 & 3.1 & 50.0 \\
\hline 1,2-Dichloropropane-d6 & 0.237 & 0.240 & 0.010 & 1.2 & 50.0 \\
\hline Toluene-d8 & 1.312 & 1.387 & 0.010 & 5.7 & 50.0 \\
\hline trans-1,3-Dichloropropene-d4 & 0.283 & 0.273 & 0.010 & -3.5 & 50.0 \\
\hline 2-Hexanone-d5 & 0.023 & 0.021 & 0.010 & -9.4 & 50.0 \\
\hline $1,1,2,2$-Tetrachloroethane-d2 & 0.130 & 0.113 & 0.010 & $-13 \cdot 2$ & 50.0 \\
\hline 1,2-Dichlorobenzene-d4 & 0.880 & 0.824 & 0.010 & -6.3 & 50.0 \\
\hline
\end{tabular}

Report 1,4-Dioxane-d8 for Low/Medium VOA analysis only 
Lab Name: TESTAMERICA BURLINGTON

Contract: $8 \mathrm{E}-00302$

Lab Code: STLV

Case No.: YORK Mod. Ref No.:

SDG No.: 28907

Matrix: (SOIL/SED/WATER) Water

Sample wt/vol: 25.0 $(\mathrm{g} / \mathrm{mL}) \mathrm{mL}$

Level: (TRACE/LOW/MED) TRACE

\% Moisture: not dec.

GC Column: DB-624 ID: $0.20 \quad(\mathrm{~mm})$

Soil Extract Volume: $(u L)$ $(\mathrm{mL})$

Purge Volume: 25.0
Lab Sample ID: MB 200-91395/5

Lab File ID: 14793_005.D

Date Received:

Date Analyzed: 07/18/2015

Dilution Factor: 1.0

Soil Aliquot Volume: (uL)

\begin{tabular}{|c|c|c|c|}
\hline CAS NO. & COMPOUND & $\begin{array}{l}\text { CONCENTRATION UNITS: } \\
(\mathrm{ug} / \mathrm{L} \text { or } \mathrm{ug} / \mathrm{kg}) \mathrm{ug} / \mathrm{L}\end{array}$ & q \\
\hline $75-71-8$ & Dichlorodifluoromethane & 1.0 & $\mathrm{U}$ \\
\hline $74-87-3$ & Chloromethane & 1.0 & $\mathrm{U}$ \\
\hline $75-01-4$ & Vinyl chloride & 1.0 & $\mathrm{U}$ \\
\hline $74-83-9$ & Bromomethane & 0.12 & $\mathrm{~J}$ \\
\hline $75-00-3$ & Chloroethane & 1.0 & $\mathrm{U}$ \\
\hline $75-69-4$ & Trichlorofluoromethane & 1.0 & $\mathrm{U}$ \\
\hline $75-35-4$ & 1,1-Dichloroethene & 1.0 & $\mathrm{U}$ \\
\hline $76-13-1$ & $1,1,2$-Trichloro-1,2,2-trifluoroethane & 1.0 & $\mathrm{U}$ \\
\hline $67-64-1$ & Acetone & 5.0 & $\mathrm{U}$ \\
\hline $75-15-0$ & Carbon disulfide & 1.0 & $\mathrm{U}$ \\
\hline $79-20-9$ & Methyl acetate & 1.0 & $\mathrm{U}$ \\
\hline $75-09-2$ & Methylene Chloride & 1.0 & $\mathrm{U}$ \\
\hline $156-60-5$ & trans-1,2-Dichloroethene & 1.0 & $\mathrm{U}$ \\
\hline $1634-04-4$ & Methyl tert-butyl ether & 1.0 & $\mathrm{U}$ \\
\hline $75-34-3$ & 1,1-Dichloroethane & 1.0 & $\mathrm{U}$ \\
\hline $156-59-2$ & cis-1,2-Dichloroethene & 1.0 & $\mathrm{U}$ \\
\hline $78-93-3$ & 2-Butanone & 5.0 & $\mathrm{U}$ \\
\hline $74-97-5$ & Bromochloromethane & 1.0 & $\mathrm{U}$ \\
\hline $67-66-3$ & Chloroform & 1.0 & $\mathrm{U}$ \\
\hline $71-55-6$ & $1,1,1$-Trichloroethane & 1.0 & $\mathrm{U}$ \\
\hline $110-82-7$ & Cyclohexane & 1.0 & $\mathrm{U}$ \\
\hline $56-23-5$ & Carbon tetrachloride & 1.0 & $\mathrm{U}$ \\
\hline $71-43-2$ & Benzene & 1.0 & $\mathrm{U}$ \\
\hline $107-06-2$ & 1,2-Dichloroethane & 1.0 & $\mathrm{U}$ \\
\hline
\end{tabular}

Report 1,4-Dioxane for Low-Medium VOA analysis only 
Lab Name: TESTAMERICA BURLINGTON

Lab Code: STLV

Case No.: YORK

Mod. Ref No.:

Contract: 8E-00302

Matrix: (SOIL/SED/WATER) Water

Sample wt/vol: 25.0 $(\mathrm{g} / \mathrm{mL}) \mathrm{mL}$

Level: (TRACE/LOW/MED) TRACE

\% Moisture: not dec.

GC Column: DB-624 ID $: \underline{0.20 \quad(\mathrm{~mm})}$

Soil Extract Volume: (uL) $(\mathrm{mL})$

Purge Volume: 25.0 SDG No.: 28907

Lab Sample ID: MB 200-91395/5

Lab File ID: 14793_005.D

Date Received:

Date Analyzed: 07/18/2015

Dilution Factor: 1.0

Soil Aliquot Volume: (uL)

\begin{tabular}{|c|c|c|c|}
\hline CAS NO. & COMPOUND & $\begin{array}{l}\text { CONCENTRATION UNITS: } \\
(\mathrm{ug} / \mathrm{L} \text { or } \mathrm{ug} / \mathrm{kg}) \mathrm{ug} / \mathrm{L}\end{array}$ & $q$ \\
\hline $79-01-6$ & Trichloroethene & 1.0 & $\mathrm{U}$ \\
\hline $108-87-2$ & Methylcyclohexane & 1.0 & $\mathrm{U}$ \\
\hline $78-87-5$ & 1,2-Dichloropropane & 1.0 & $\mathrm{U}$ \\
\hline $75-27-4$ & Bromodichloromethane & 1.0 & $\mathrm{U}$ \\
\hline $10061-01-5$ & cis-1,3-Dichloropropene & 1.0 & U \\
\hline $108-10-1$ & 4-Methyl-2-pentanone & 1.0 & $\mathrm{U}$ \\
\hline $108-88-3$ & Toluene & 1.0 & $\mathrm{U}$ \\
\hline $10061-02-6$ & trans-1,3-Dichloropropene & 1.0 & $\mathrm{U}$ \\
\hline $79-00-5$ & $1,1,2-$ Trichloroethane & 1.0 & $\mathrm{U}$ \\
\hline $127-18-4$ & Tetrachloroethene & 1.0 & $\mathrm{U}$ \\
\hline $591-78-6$ & 2-Hexanone & 1.0 & $\mathrm{U}$ \\
\hline $124-48-1$ & Dibromochloromethane & 1.0 & $\mathrm{U}$ \\
\hline $106-93-4$ & 1,2-Dibromoethane & 1.0 & $\mathrm{U}$ \\
\hline $108-90-7$ & Chlorobenzene & 1.0 & $\mathrm{U}$ \\
\hline $100-41-4$ & Ethylbenzene & 1.0 & $\mathrm{U}$ \\
\hline $95-47-6$ & o-Xylene & 1.0 & $\mathrm{U}$ \\
\hline $179601-23-1$ & m,p-xylene & 1.0 & $\mathrm{U}$ \\
\hline $100-42-5$ & Styrene & 1.0 & $\mathrm{U}$ \\
\hline $75-25-2$ & Bromoform & 1.0 & $\mathrm{U}$ \\
\hline $98-82-8$ & Isopropylbenzene & 1.0 & $\mathrm{U}$ \\
\hline $79-34-5$ & $1,1,2,2$-Tetrachloroethane & 1.0 & $\mathrm{U}$ \\
\hline $541-73-1$ & 1,3 -Dichlorobenzene & 1.0 & $\mathrm{U}$ \\
\hline $106-46-7$ & 1,4-Dichlorobenzene & 1.0 & $\mathrm{U}$ \\
\hline $95-50-1$ & 1,2-Dichlorobenzene & 1.0 & $\mathrm{U}$ \\
\hline $96-12-8$ & 1,2-Dibromo-3-Chloropropane & 1.0 & $\mathrm{U}$ \\
\hline $120-82-1$ & $1,2,4$-Trichlorobenzene & 1.0 & $\mathrm{U}$ \\
\hline $87-61-6$ & $1,2,3$-Trichlorobenzene & 1.0 & $\mathrm{U}$ \\
\hline
\end{tabular}


Lab Name: TESTAMERICA BURLINGTON

Contract: $8 \mathrm{E}-00302$

Lab Code: STLV Case No.: YORK Mod. Ref No.: SDG No.: 28907

Matrix: (SOIL/SED/WATER) Water

Sample wt/vol: 25.0 $(\mathrm{g} / \mathrm{mL}) \mathrm{mL}$

Level: (TRACE or LOW/MED) TRACE

․ Moisture: not dec.

GC Column: DB-624 ID $: 0.20 \quad(\mathrm{~mm})$

Soil Extract Volume: (uL)
Lab Sample ID: MB 200-91395/5

Lab File ID: 14793_005.D

Date Received:

Date Analyzed: 07/18/2015

Dilution Factor: 1.0

Soil Aliquot Volume: (uL)

Purge Volume: 25.0 $(\mathrm{mL})$ CONCENTRATION UNITS: (ug/L or ug/kg) ug/L

\begin{tabular}{l|l|l|r|r|r|}
\cline { 2 - 6 } & CAS NUMBER & \multicolumn{1}{|c|}{ COMPOUND NAME } & RT & EST. CONC. & Q \\
\cline { 2 - 6 } 01 & & Unknown & 7.29 & 2.8 & $\mathrm{~J} X$ \\
\cline { 2 - 6 } 02 & $541-05-9$ & Cyclotrisiloxane, hexamethyl- & 8.22 & 0.96 & $\mathrm{~J} \mathrm{~N}$ \\
\cline { 2 - 6 } 03 & $556-67-2$ & Cyclotetrasiloxane, octamethyl- & 11.04 & 0.96 & $\mathrm{~J} \mathrm{~N}$ \\
\cline { 2 - 6 } 04 & E9667961 & Total Alkanes & $\mathrm{N} / \mathrm{A}$ & & \\
\cline { 2 - 6 }
\end{tabular}

1 EPA-designated Registry Number. 
Lab Name: TESTAMERICA BURLINGTON

Contract: $8 \mathrm{E}-00302$

Lab Code: STLV

Case No.: YORK Mod. Ref No.:

SDG No.: 28907

Matrix: (SOIL/SED/WATER) Water

Sample wt/vol: 25.0 $(\mathrm{g} / \mathrm{mL}) \mathrm{mL}$

Level: (TRACE/LOW/MED) TRACE

\% Moisture: not dec.

GC Column: DB-624 ID: $0.20 \quad(\mathrm{~mm})$

Soil Extract Volume: (uL) $(\mathrm{mL})$

Purge Volume: 25.0
Lab Sample ID: MB 200-91426/6

Lab File ID: 14808_006.D

Date Received:

Date Analyzed: 07/20/2015

Dilution Factor: 1.0

Soil Aliquot Volume: (uL)

\begin{tabular}{|c|c|c|c|}
\hline CAS NO. & COMPOUND & $\begin{array}{l}\text { CONCENTRATION UNITS: } \\
(\mathrm{ug} / \mathrm{L} \text { or } \mathrm{ug} / \mathrm{kg}) \mathrm{ug} / \mathrm{L}\end{array}$ & q \\
\hline $75-71-8$ & Dichlorodifluoromethane & 1.0 & $\mathrm{U}$ \\
\hline $74-87-3$ & Chloromethane & 1.0 & $\mathrm{U}$ \\
\hline $75-01-4$ & Vinyl chloride & 1.0 & $\mathrm{U}$ \\
\hline $74-83-9$ & Bromomethane & 1.0 & $\mathrm{U}$ \\
\hline $75-00-3$ & Chloroethane & 1.0 & $\mathrm{U}$ \\
\hline $75-69-4$ & Trichlorofluoromethane & 1.0 & $\mathrm{U}$ \\
\hline $75-35-4$ & 1,1-Dichloroethene & 1.0 & $\mathrm{U}$ \\
\hline $76-13-1$ & $1,1,2$-Trichloro-1,2,2-trifluoroethane & 1.0 & $\mathrm{U}$ \\
\hline $67-64-1$ & Acetone & 5.0 & $\mathrm{U}$ \\
\hline $75-15-0$ & Carbon disulfide & 1.0 & $\mathrm{U}$ \\
\hline $79-20-9$ & Methyl acetate & 1.0 & $\mathrm{U}$ \\
\hline $75-09-2$ & Methylene Chloride & 1.0 & $\mathrm{U}$ \\
\hline $156-60-5$ & trans-1,2-Dichloroethene & 1.0 & $\mathrm{U}$ \\
\hline $1634-04-4$ & Methyl tert-butyl ether & 1.0 & $\mathrm{U}$ \\
\hline $75-34-3$ & 1,1-Dichloroethane & 1.0 & $\mathrm{U}$ \\
\hline $156-59-2$ & cis-1,2-Dichloroethene & 1.0 & $\mathrm{U}$ \\
\hline $78-93-3$ & 2-Butanone & 5.0 & $\mathrm{U}$ \\
\hline $74-97-5$ & Bromochloromethane & 1.0 & $\mathrm{U}$ \\
\hline $67-66-3$ & Chloroform & 1.0 & $\mathrm{U}$ \\
\hline $71-55-6$ & $1,1,1$-Trichloroethane & 1.0 & $\mathrm{U}$ \\
\hline $110-82-7$ & Cyclohexane & 1.0 & $\mathrm{U}$ \\
\hline $56-23-5$ & Carbon tetrachloride & 1.0 & $\mathrm{U}$ \\
\hline $71-43-2$ & Benzene & 1.0 & $\mathrm{U}$ \\
\hline $107-06-2$ & 1,2-Dichloroethane & 1.0 & $\mathrm{U}$ \\
\hline
\end{tabular}

Report 1,4-Dioxane for Low-Medium VOA analysis only 
Lab Name: TESTAMERICA BURLINGTON

Contract: 8E-00302

Lab Code: STLV

Case No.: YORK

Mod. Ref No.:

SDG No.: 28907

Matrix: (SOIL/SED/WATER) Water

Sample wt/vol: 25.0 $(\mathrm{g} / \mathrm{mL}) \mathrm{mL}$

Level: (TRACE/LOW/MED) TRACE

\% Moisture: not dec.

GC Column: DB-624 ID: $0.20 \quad(\mathrm{~mm})$

Soil Extract Volume: (uL) $(\mathrm{mL})$

Purge Volume: 25.0
Lab Sample ID: MB 200-91426/6

Lab File ID: 14808_006.D

Date Received:

Date Analyzed: 07/20/2015

Dilution Factor: 1.0

Soil Aliquot Volume: (uL) (mI)

\begin{tabular}{|c|c|c|c|}
\hline CAS NO. & COMPOUND & $\begin{array}{l}\text { CONCENTRATION UNITS: } \\
(\mathrm{ug} / \mathrm{L} \text { or } \mathrm{ug} / \mathrm{kg}) \mathrm{ug} / \mathrm{L}\end{array}$ & $q$ \\
\hline $79-01-6$ & Trichloroethene & 1.0 & $\mathrm{U}$ \\
\hline $108-87-2$ & Methylcyclohexane & 1.0 & $\mathrm{U}$ \\
\hline $78-87-5$ & 1,2-Dichloropropane & 1.0 & $\mathrm{U}$ \\
\hline $75-27-4$ & Bromodichloromethane & 1.0 & $\mathrm{U}$ \\
\hline $10061-01-5$ & cis-1,3-Dichloropropene & 1.0 & U \\
\hline $108-10-1$ & 4-Methyl-2-pentanone & 1.0 & $\mathrm{U}$ \\
\hline $108-88-3$ & Toluene & 1.0 & $\mathrm{U}$ \\
\hline $10061-02-6$ & trans-1,3-Dichloropropene & 1.0 & $\mathrm{U}$ \\
\hline $79-00-5$ & $1,1,2-$ Trichloroethane & 1.0 & $\mathrm{U}$ \\
\hline $127-18-4$ & Tetrachloroethene & 1.0 & $\mathrm{U}$ \\
\hline $591-78-6$ & 2-Hexanone & 1.0 & $\mathrm{U}$ \\
\hline $124-48-1$ & Dibromochloromethane & 1.0 & $\mathrm{U}$ \\
\hline $106-93-4$ & 1,2-Dibromoethane & 1.0 & $\mathrm{U}$ \\
\hline $108-90-7$ & Chlorobenzene & 1.0 & $\mathrm{U}$ \\
\hline $100-41-4$ & Ethylbenzene & 1.0 & $\mathrm{U}$ \\
\hline $95-47-6$ & o-Xylene & 1.0 & $\mathrm{U}$ \\
\hline $179601-23-1$ & m,p-xylene & 1.0 & $\mathrm{U}$ \\
\hline $100-42-5$ & Styrene & 1.0 & $\mathrm{U}$ \\
\hline $75-25-2$ & Bromoform & 1.0 & $\mathrm{U}$ \\
\hline $98-82-8$ & Isopropylbenzene & 1.0 & $\mathrm{U}$ \\
\hline $79-34-5$ & $1,1,2,2$-Tetrachloroethane & 1.0 & $\mathrm{U}$ \\
\hline $541-73-1$ & 1,3 -Dichlorobenzene & 1.0 & $\mathrm{U}$ \\
\hline $106-46-7$ & 1,4-Dichlorobenzene & 1.0 & $\mathrm{U}$ \\
\hline $95-50-1$ & 1,2-Dichlorobenzene & 1.0 & $\mathrm{U}$ \\
\hline $96-12-8$ & 1,2-Dibromo-3-Chloropropane & 1.0 & $\mathrm{U}$ \\
\hline $120-82-1$ & $1,2,4$-Trichlorobenzene & 0.021 & $\mathrm{~J}$ \\
\hline $87-61-6$ & $1,2,3$-Trichlorobenzene & 0.046 & $\mathrm{~J}$ \\
\hline
\end{tabular}


Lab Name: TESTAMERICA BURLINGTON

Contract: $8 \mathrm{E}-00302$

Lab Code: STLV Case No.: YORK Mod. Ref No.: SDG No.: 28907

Matrix: (SOIL/SED/WATER) Water

Sample wt/vol: 25.0 $(\mathrm{g} / \mathrm{mL}) \mathrm{mL}$

Level: (TRACE or LOW/MED) TRACE

․ Moisture: not dec.

GC Column: DB-624 ID $: 0.20 \quad(\mathrm{~mm})$

Soil Extract Volume: (uL)
Lab Sample ID: MB 200-91426/6

Lab File ID: 14808_006.D

Date Received:

Date Analyzed: 07/20/2015

Dilution Factor: 1.0

Soil Aliquot Volume: (uL)

Purge Volume: 25.0 $(\mathrm{mL})$ CONCENTRATION UNITS: (ug/L or ug/kg) ug/L

\begin{tabular}{l|l|l|c|c|c|}
\cline { 2 - 6 } & CAS NUMBER & \multicolumn{1}{|c|}{ COMPOUND NAME } & RT & EST. CONC. & Q \\
\cline { 2 - 6 } 01 & & Unknown & 7.30 & 2.9 & $\mathrm{~J} X$ \\
\cline { 2 - 6 } 02 & $541-05-9$ & Cyclotrisiloxane, hexamethyl- & 8.22 & 1.2 & $\mathrm{~J} \mathrm{~N}$ \\
\cline { 2 - 6 } 03 & $556-67-2$ & Cyclotetrasiloxane, octamethyl- & 11.04 & $\mathrm{~J} \mathrm{~N}$ \\
\cline { 2 - 6 } 04 & E9667961 & Total Alkanes & $\mathrm{N} / \mathrm{A}$ & & \\
\cline { 2 - 6 }
\end{tabular}

1 EPA-designated Registry Number. 
Lab Name: TESTAMERICA BURLINGTON

Contract: $8 \mathrm{E}-00302$

Lab Code: STLV

Case No.: YORK Mod. Ref No.:

SDG No.: 28907

Matrix: (SOIL/SED/WATER) Water

Sample wt/vol: 25.0 $(\mathrm{g} / \mathrm{mL}) \mathrm{mL}$

Level: (TRACE/LOW/MED) TRACE

\% Moisture: not dec.

GC Column: DB-624 ID: $0.20 \quad(\mathrm{~mm})$

Soil Extract Volume: (uL) $(\mathrm{mL})$

Purge Volume: 25.0
Lab Sample ID: 200-28907-4

Lab File ID: 14808_011.D

Date Received:

Date Analyzed: 07/20/2015

Dilution Factor: 1.0

Soil Aliquot Volume: (uL)

\begin{tabular}{|c|c|c|c|}
\hline CAS NO. & COMPOUND & $\begin{array}{l}\text { CONCENTRATION UNITS: } \\
(\mathrm{ug} / \mathrm{L} \text { or } \mathrm{ug} / \mathrm{kg}) \mathrm{ug} / \mathrm{L}\end{array}$ & q \\
\hline $75-71-8$ & Dichlorodifluoromethane & 1.0 & $\mathrm{U}$ \\
\hline $74-87-3$ & Chloromethane & 1.0 & $\mathrm{U}$ \\
\hline $75-01-4$ & Vinyl chloride & 1.0 & $\mathrm{U}$ \\
\hline $74-83-9$ & Bromomethane & 1.0 & $\mathrm{U}$ \\
\hline $75-00-3$ & Chloroethane & 1.0 & $\mathrm{U}$ \\
\hline $75-69-4$ & Trichlorofluoromethane & 1.0 & $\mathrm{U}$ \\
\hline $75-35-4$ & 1,1-Dichloroethene & 1.0 & $\mathrm{U}$ \\
\hline $76-13-1$ & $1,1,2$-Trichloro-1,2,2-trifluoroethane & 1.0 & $\mathrm{U}$ \\
\hline $67-64-1$ & Acetone & 5.0 & $\mathrm{U}$ \\
\hline $75-15-0$ & Carbon disulfide & 1.0 & $\mathrm{U}$ \\
\hline $79-20-9$ & Methyl acetate & 1.0 & $\mathrm{U}$ \\
\hline $75-09-2$ & Methylene Chloride & 1.0 & $\mathrm{U}$ \\
\hline $156-60-5$ & trans-1,2-Dichloroethene & 1.0 & $\mathrm{U}$ \\
\hline $1634-04-4$ & Methyl tert-butyl ether & 1.0 & $\mathrm{U}$ \\
\hline $75-34-3$ & 1,1-Dichloroethane & 1.0 & $\mathrm{U}$ \\
\hline $156-59-2$ & cis-1,2-Dichloroethene & 1.0 & $\mathrm{U}$ \\
\hline $78-93-3$ & 2-Butanone & 5.0 & $\mathrm{U}$ \\
\hline $74-97-5$ & Bromochloromethane & 1.0 & $\mathrm{U}$ \\
\hline $67-66-3$ & Chloroform & 1.0 & $\mathrm{U}$ \\
\hline $71-55-6$ & $1,1,1$-Trichloroethane & 1.0 & $\mathrm{U}$ \\
\hline $110-82-7$ & Cyclohexane & 1.0 & $\mathrm{U}$ \\
\hline $56-23-5$ & Carbon tetrachloride & 1.0 & $\mathrm{U}$ \\
\hline $71-43-2$ & Benzene & 1.0 & $\mathrm{U}$ \\
\hline $107-06-2$ & 1,2-Dichloroethane & 1.0 & $\mathrm{U}$ \\
\hline
\end{tabular}

Report 1,4-Dioxane for Low-Medium VOA analysis only 
Lab Name: TESTAMERICA BURLINGTON

Lab Code: STLV

Case No.: YORK

Mod. Ref No.:

Contract: 8E-00302

Matrix: (SOIL/SED/WATER) Water

Sample wt/vol: 25.0 $(\mathrm{g} / \mathrm{mL}) \mathrm{mL}$

Level: (TRACE/LOW/MED) TRACE

\% Moisture: not dec.

GC Column: DB-624 ID $: \underline{0.20 \quad(\mathrm{~mm})}$

Soil Extract Volume: (uL) $(\mathrm{mL})$

Purge Volume: 25.0
SDG No.: 28907

Lab Sample ID: 200-28907-4

Lab File ID: 14808_011.D

Date Received:

Date Analyzed: 07/20/2015

Dilution Factor: 1.0

Soil Aliquot Volume: (uL)

\begin{tabular}{|c|c|c|c|}
\hline CAS NO. & COMPOUND & $\begin{array}{l}\text { CONCENTRATION UNITS: } \\
(\mathrm{ug} / \mathrm{L} \text { or } \mathrm{ug} / \mathrm{kg}) \mathrm{ug} / \mathrm{L}\end{array}$ & $q$ \\
\hline $79-01-6$ & Trichloroethene & 1.0 & $\mathrm{U}$ \\
\hline $108-87-2$ & Methylcyclohexane & 1.0 & $\mathrm{U}$ \\
\hline $78-87-5$ & 1,2-Dichloropropane & 1.0 & $\mathrm{U}$ \\
\hline $75-27-4$ & Bromodichloromethane & 1.0 & $\mathrm{U}$ \\
\hline $10061-01-5$ & cis-1,3-Dichloropropene & 1.0 & U \\
\hline $108-10-1$ & 4-Methyl-2-pentanone & 1.0 & $\mathrm{U}$ \\
\hline $108-88-3$ & Toluene & 0.010 & $\mathrm{~J}$ \\
\hline $10061-02-6$ & trans-1,3-Dichloropropene & 1.0 & $\mathrm{U}$ \\
\hline $79-00-5$ & $1,1,2-$ Trichloroethane & 1.0 & $\mathrm{U}$ \\
\hline $127-18-4$ & Tetrachloroethene & 1.0 & $\mathrm{U}$ \\
\hline $591-78-6$ & 2-Hexanone & 1.0 & $\mathrm{U}$ \\
\hline $124-48-1$ & Dibromochloromethane & 1.0 & $\mathrm{U}$ \\
\hline $106-93-4$ & 1,2-Dibromoethane & 1.0 & $\mathrm{U}$ \\
\hline $108-90-7$ & Chlorobenzene & 1.0 & $\mathrm{U}$ \\
\hline $100-41-4$ & Ethylbenzene & 1.0 & $\mathrm{U}$ \\
\hline $95-47-6$ & o-Xylene & 1.0 & $\mathrm{U}$ \\
\hline $179601-23-1$ & m,p-xylene & 1.0 & $\mathrm{U}$ \\
\hline $100-42-5$ & Styrene & 1.0 & $\mathrm{U}$ \\
\hline $75-25-2$ & Bromoform & 1.0 & $\mathrm{U}$ \\
\hline $98-82-8$ & Isopropylbenzene & 1.0 & $\mathrm{U}$ \\
\hline $79-34-5$ & $1,1,2,2$-Tetrachloroethane & 1.0 & $\mathrm{U}$ \\
\hline $541-73-1$ & 1,3 -Dichlorobenzene & 1.0 & $\mathrm{U}$ \\
\hline $106-46-7$ & 1,4-Dichlorobenzene & 1.0 & $\mathrm{U}$ \\
\hline $95-50-1$ & 1,2-Dichlorobenzene & 1.0 & $\mathrm{U}$ \\
\hline $96-12-8$ & 1,2-Dibromo-3-Chloropropane & 1.0 & $\mathrm{U}$ \\
\hline $120-82-1$ & $1,2,4$-Trichlorobenzene & 1.0 & $\mathrm{U}$ \\
\hline $87-61-6$ & $1,2,3$-Trichlorobenzene & 1.0 & $\mathrm{U}$ \\
\hline
\end{tabular}


Lab Name: TESTAMERICA BURLINGTON

Contract: $8 \mathrm{E}-00302$

Lab Code: STLV Case No.: YORK Mod. Ref No.: SDG No.: 28907

Matrix: (SOIL/SED/WATER) Water

Sample wt/vol: 25.0 $(\mathrm{g} / \mathrm{mL}) \mathrm{mL}$

Level: (TRACE or LOW/MED) TRACE

Moisture: not dec.

GC Column: DB-624 ID: $0.20 \quad(\mathrm{~mm})$

Soil Extract Volume: (uL)

CONCENTRATION UNITS: (ug/L or ug/kg) ug/L
Lab Sample ID: 200-28907-4

Lab File ID: 14808 _011.D

Date Received:

Date Analyzed: 07/20/2015

Dilution Factor: 1.0

Soil Aliquot Volume: (uL) Purge Volume: 25.0

\begin{tabular}{|c|c|c|c|c|}
\hline CAS NUMBER & COMPOUND NAME & RT & EST. CONC. & $Q$ \\
\hline 01 & Unknown & 7.29 & 2.9 & $\mathrm{~J} B \mathrm{X}$ \\
\hline $541-05-9$ & Cyclotrisiloxane, hexamethyl- & 8.22 & 0.85 & $\mathrm{~J} \quad \mathrm{~N} B$ \\
\hline $556-67-2$ & Cyclotetrasiloxane, octamethyl- & 11.04 & 0.51 & $\mathrm{~J} N \mathrm{~N}$ \\
\hline E9667961 & Total Alkanes & $\mathrm{N} / \mathrm{A}$ & & \\
\hline
\end{tabular}

1 EPA-designated Registry Number. 
Lab Name: TestAmerica Burlington

SDG No.: 28907

Instrument ID: CHD.i

Lab Sample ID: IC 200-91077/2

Date Analyzed: 07/14/15 11:08

\begin{tabular}{|c|r|}
\hline COMPOUND NAME & RETEN \\
TI
\end{tabular}

Lab Sample ID: IC 200-91077/3

Date Analyzed: 07/14/15 11:33 Client Sample ID: Lab File ID: 14685_003.D

Analysis Batch Number: 91077

Client Sample ID:

Lab File ID: 14685 002.D

GC Column: DB-624

ID : $0.2(\mathrm{~mm})$

\begin{tabular}{|c|c|c|c|c|}
\hline \multicolumn{1}{|c|}{ COMPOUND NAME } & RETENTION & \multicolumn{3}{|c}{ MANUAL INTEGRATION } \\
\cline { 3 - 5 } & TIME & REASON & ANALYST & DATE \\
\hline 1,2 -Dibromo-3-Chloropropane & 13.44 & Baseline & archern & $07 / 14 / 1514: 11$ \\
\hline
\end{tabular}


Lab Name: TestAmerica Burlington SDG No.: 28907

Instrument ID: CHD.i

Lab Sample ID: CCVIS 200-91426/4

Date Analyzed: 07/20/15 09:27

\begin{tabular}{|l|c|c|c|c|}
\hline \multicolumn{1}{|c|}{ COMPOUND NAME } & RETENTION & \multicolumn{3}{|c}{ MANUAL INTEGRATION } \\
\cline { 3 - 5 } & TIME & REASON & ANALYST & DATE \\
\hline Dichlorodifluoromethane & 1.48 & Baseline & archern & $07 / 20 / 15$ 09:48 \\
\hline
\end{tabular}

Job No.: 200-28907-1

Analysis Batch Number: 91426

Client Sample ID:

Lab File ID: $14808004 . \mathrm{D}$

Column: $\mathrm{DB}-624$

ID : $0.2(\mathrm{~mm})$ 
Lab Name: TestAmerica Burlington Job No.: 200-28907-1

SDG No.: 28907

\begin{tabular}{|c|c|c|c|c|}
\hline $\begin{array}{c}\text { Reagent } \\
\text { Container }\end{array}$ & $\begin{array}{l}\text { Reagent } \\
\text { ID }\end{array}$ & $\begin{array}{c}\text { Reagent } \\
\text { Description }\end{array}$ & $\begin{array}{c}\text { Preparation } \\
\text { Date }\end{array}$ & $\begin{array}{c}\text { Expiration } \\
\text { Date }\end{array}$ \\
\hline 804536 & VMSOMTRCALw_00091 & SOM TR CAL 20 PPM & $06 / 15 / 2015$ & $07 / 15 / 2015$ \\
\hline 807558 & VMBFBW_00018 & BFB TUNE 25 PPM & $06 / 23 / 2015$ & $12 / 22 / 2015$ \\
\hline 809829 & VMSOMTRISw_00101 & SOM TR ISTD 20 PPM & $06 / 27 / 2015$ & $07 / 27 / 2015$ \\
\hline 816537 & VMSOMTRCALW_00092 & SOM TR CAL 20 PPM & $07 / 16 / 2015$ & $08 / 16 / 2015$ \\
\hline 816538 & VMSOMTRSUw $\overline{00097}$ & SOM $\quad$ TR DMC 20 PPM & $07 / 16 / 2015$ & $08 / 16 / 2015$ \\
\hline 816658 & VMSOMTRCALW_00092 & SOM TR CAL 20 PPM & $07 / 16 / 2015$ & $08 / 16 / 2015$ \\
\hline
\end{tabular}


SDG No.: 28907

\begin{tabular}{|c|c|c|c|c|c|c|c|c|}
\hline \multirow[b]{2}{*}{ Reagent ID } & \multirow[b]{2}{*}{$\begin{array}{l}\text { Exp } \\
\text { Date }\end{array}$} & \multirow[b]{2}{*}{$\begin{array}{l}\text { Prep } \\
\text { Date }\end{array}$} & \multirow[b]{2}{*}{$\begin{array}{l}\text { Dilutant } \\
\text { Used }\end{array}$} & \multirow{2}{*}{$\begin{array}{l}\text { Reagent } \\
\text { Final } \\
\text { Volume }\end{array}$} & \multicolumn{2}{|c|}{ Parent Reagent } & \multirow[b]{2}{*}{ Analyte } & \multirow[b]{2}{*}{ Concentration } \\
\hline & & & & & Reagent ID & $\begin{array}{l}\text { Volume } \\
\text { Added }\end{array}$ & & \\
\hline VMBFBW_00018 & $12 / 22 / 15$ & $06 / 23 / 15$ & METHANOL, LOt 147358 & $25 \mathrm{~mL}$ & VMBFBs_00014 & $125 \mathrm{uL}$ & $\mathrm{BFB}$ & $25 \mathrm{ug} / \mathrm{mL}$ \\
\hline .VMBFBS_00014 & $12 / 22 / 15$ & & RESTEK, Lot A0106759 & & \multicolumn{2}{|c|}{ (Purchased Reagent) } & BFB & $5000 \mathrm{ug} / \mathrm{mL}$ \\
\hline \multirow[t]{33}{*}{ VMSOMTRCALW_00091 } & \multirow[t]{33}{*}{$07 / 15 / 15$} & \multirow[t]{33}{*}{$06 / 15 / 15$} & \multirow[t]{33}{*}{ METHANOL, LOt 147358} & \multirow[t]{33}{*}{4000 uL } & VM8260CAL_bs_00180 & $40 \mathrm{UL}$ & \begin{tabular}{|l|} 
Bromomethane \\
Chloroethane \\
Chloromethane \\
Dichlorodifluoromethane \\
Trichlorofluoromethane \\
Vinyl chloride
\end{tabular} & $\begin{array}{l}20 \mathrm{ug} / \mathrm{mL} \\
20 \mathrm{ug} / \mathrm{mL} \\
20 \mathrm{ug} / \mathrm{mL} \\
20 \mathrm{ug} / \mathrm{mL} \\
20 \mathrm{ug} / \mathrm{mL} \\
20 \mathrm{ug} / \mathrm{mL}\end{array}$ \\
\hline & & & & & \multirow[t]{32}{*}{ VMSOMCALas_00022 } & \multirow[t]{32}{*}{40 uL } & $\begin{array}{l}1,1,1 \text {-Trichloroethane } \\
1,1,2,2 \text {-Tetrachloroethane } \\
1,1,2 \text {-Trichloro-1,2,2-trifluor } \\
\text { oethane }\end{array}$ & $\begin{array}{l}20 \mathrm{ug} / \mathrm{mL} \\
20 \mathrm{ug} / \mathrm{mL} \\
20 \mathrm{ug} / \mathrm{mL} \\
\end{array}$ \\
\hline & & & & & & & $1,1,2$-Trichloroethane & $20 \mathrm{ug} / \mathrm{mL}$ \\
\hline & & & & & & & \begin{tabular}{|l|} 
1,1-Dichloroethane \\
1,1-Dichloroethene
\end{tabular} & $\frac{20 \mathrm{ug} / \mathrm{mL}}{20 \mathrm{ug} / \mathrm{mL}}$ \\
\hline & & & & & & & $1,2,3$-Trichlorobenzene & $20 \mathrm{ug} / \mathrm{mL}$ \\
\hline & & & & & & & 1,2,4-Trichlorobenzene & $20 \mathrm{ug} / \mathrm{mL}$ \\
\hline & & & & & & & 1,2-Dibromo-3-Chloropropane & $20 \mathrm{ug} / \mathrm{mL}$ \\
\hline & & & & & & & $\begin{array}{l}\text { 1,2-Dibromoethane } \\
1,2 \text {-Dichlorobenzene }\end{array}$ & $\frac{20 \mathrm{ug} / \mathrm{mL}}{20 \mathrm{ug} / \mathrm{mL}}$ \\
\hline & & & & & & & 1,2-Dichloroethane & $20 \mathrm{ug} / \mathrm{mL}$ \\
\hline & & & & & & & 1,2-Dichloropropane & $20 \mathrm{ug} / \mathrm{mL}$ \\
\hline & & & & & & & $\begin{array}{l}\text { 1,3-Dichlorobenzene } \\
\text { 1,4-Dichlorobenzene }\end{array}$ & $\frac{20 \mathrm{ug} / \mathrm{mL}}{20 \mathrm{ug} / \mathrm{mL}}$ \\
\hline & & & & & & & $\begin{array}{l}\text { 1,4-Dichlorobenzene } \\
\text { Benzene }\end{array}$ & $20 \mathrm{ug} / \mathrm{mL}$ \\
\hline & & & & & & & Bromochloromethane & $20 \mathrm{ug} / \mathrm{mL}$ \\
\hline & & & & & & & Bromodichloromethane & $20 \mathrm{ug} / \mathrm{mL}$ \\
\hline & & & & & & & Bromoform & $20 \mathrm{ug} / \mathrm{mL}$ \\
\hline & & & & & & & Carbon disulfide & $20 \mathrm{ug} / \mathrm{mL}$ \\
\hline & & & & & & & Carbon tetrachloride & $20 \mathrm{ug} / \mathrm{mL}$ \\
\hline & & & & & & & Chlorobenzene & $20 \mathrm{ug} / \mathrm{mL}$ \\
\hline & & & & & & & Chloroform & $20 \mathrm{ug} / \mathrm{mL}$ \\
\hline & & & & & & & cis-1,2-Dichloroethene & $20 \mathrm{ug} / \mathrm{mL}$ \\
\hline & & & & & & & $\begin{array}{l}\text { Cis-1,3-Dichloropropene } \\
\text { Cyclohexane }\end{array}$ & $20 \mathrm{ug} / \mathrm{mL}$ \\
\hline & & & & & & & Dibromochloromethane & $20 \mathrm{ug} / \mathrm{mL}$ \\
\hline & & & & & & & Ethylbenzene & $20 \mathrm{ug} / \mathrm{mL}$ \\
\hline & & & & & & & Isopropylbenzene & $20 \mathrm{ug} / \mathrm{mL}$ \\
\hline & & & & & & & $\mathrm{m}, \mathrm{p}$-Xylene & $20 \mathrm{ug} / \mathrm{mL}$ \\
\hline & & & & & & & Methyl acetate & $20 \mathrm{ug} / \mathrm{mL}$ \\
\hline & & & & & & & Methyl tert-butyl ether & $20 \mathrm{ug} / \mathrm{mL}$ \\
\hline & & & & & & & Methylcyclohexane & $20 \mathrm{ug} / \mathrm{mL}$ \\
\hline & & & & & & & Methylene Chloride & $20 \mathrm{ug} / \mathrm{mL}$ \\
\hline & & & & & & & o-Xylene & $20 \mathrm{ug} / \mathrm{mL}$ \\
\hline & & & & & & & Styrene & $20 \mathrm{ug} / \mathrm{mL}$ \\
\hline & & & & & & & Tetrachloroethene & $20 \mathrm{ug} / \mathrm{mL}$ \\
\hline & & & & & & & Toluene & $20 \mathrm{ug} / \mathrm{mL}$ \\
\hline
\end{tabular}


SDG No.: 28907

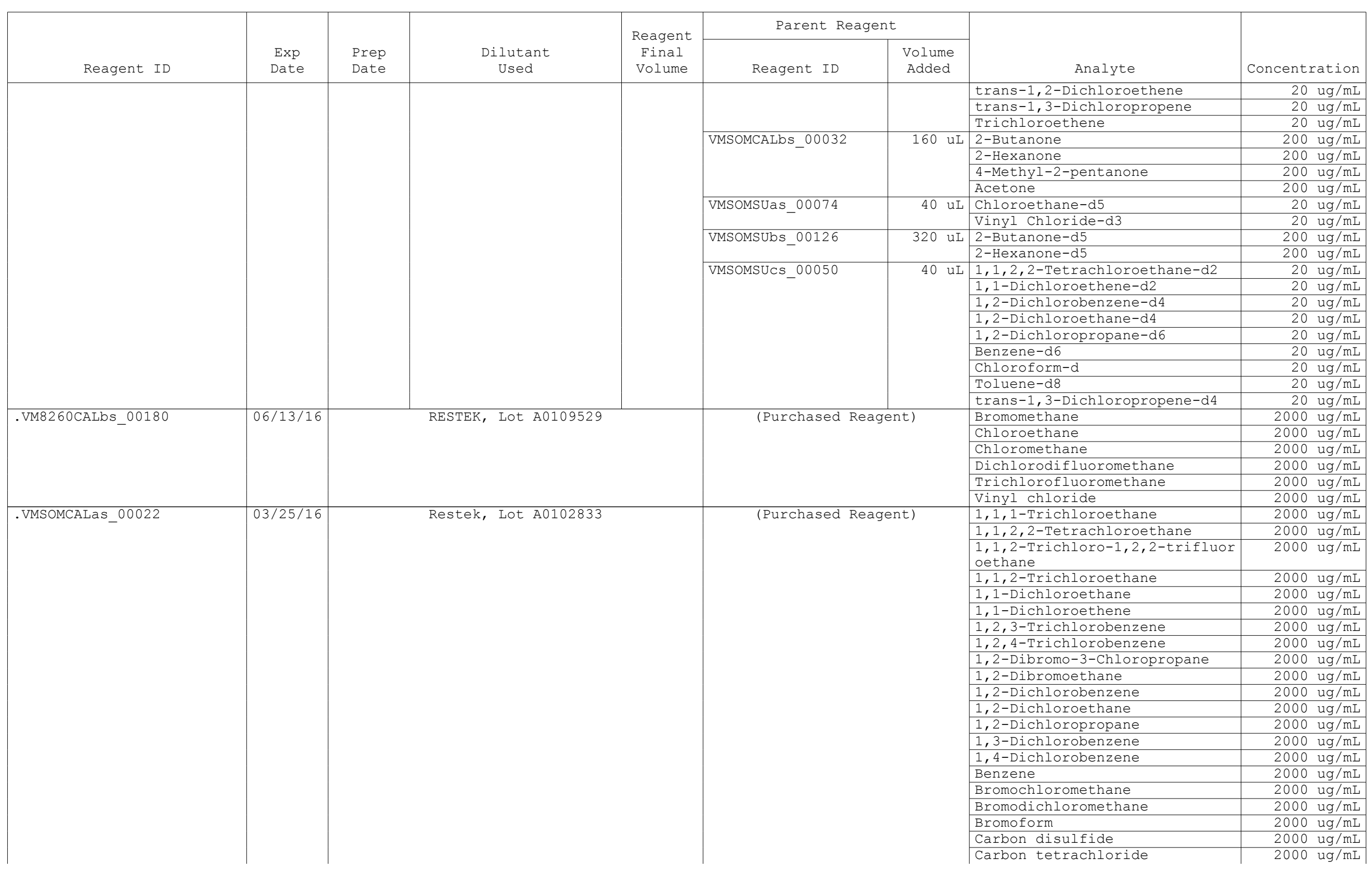


SDG No.: 28907

\begin{tabular}{|c|c|c|c|c|c|c|c|c|}
\hline \multirow[b]{2}{*}{ Reagent ID } & \multirow[b]{2}{*}{$\begin{array}{l}\text { Exp } \\
\text { Date }\end{array}$} & \multirow[b]{2}{*}{$\begin{array}{l}\text { Prep } \\
\text { Date }\end{array}$} & \multirow[b]{2}{*}{$\begin{array}{l}\text { Dilutant } \\
\text { Used }\end{array}$} & \multirow{2}{*}{$\begin{array}{l}\text { Reagent } \\
\text { Final } \\
\text { Volume }\end{array}$} & \multicolumn{2}{|c|}{ Parent Reagent } & \multirow[b]{2}{*}{ Analyte } & \multirow[b]{2}{*}{ Concentration } \\
\hline & & & & & Reagent ID & $\begin{array}{l}\text { Volume } \\
\text { Added }\end{array}$ & & \\
\hline & & & & & & & 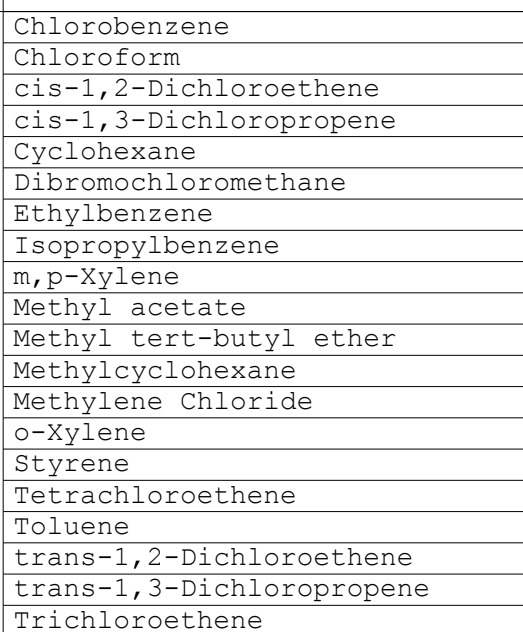 & \begin{tabular}{|l|}
$2000 \mathrm{ug} / \mathrm{mL}$ \\
$2000 \mathrm{ug} / \mathrm{mL}$ \\
$2000 \mathrm{ug} / \mathrm{mL}$ \\
$2000 \mathrm{ug} / \mathrm{mL}$ \\
$2000 \mathrm{ug} / \mathrm{mL}$ \\
$2000 \mathrm{ug} / \mathrm{mL}$ \\
$2000 \mathrm{ug} / \mathrm{mL}$ \\
$2000 \mathrm{ug} / \mathrm{mL}$ \\
$2000 \mathrm{ug} / \mathrm{mL}$ \\
$2000 \mathrm{ug} / \mathrm{mL}$ \\
$2000 \mathrm{ug} / \mathrm{mL}$ \\
$2000 \mathrm{ug} / \mathrm{mL}$ \\
$2000 \mathrm{ug} / \mathrm{mL}$ \\
$2000 \mathrm{ug} / \mathrm{mL}$ \\
$2000 \mathrm{ug} / \mathrm{mL}$ \\
$2000 \mathrm{ug} / \mathrm{mL}$ \\
$2000 \mathrm{ug} / \mathrm{mL}$ \\
$2000 \mathrm{ug} / \mathrm{mL}$ \\
$2000 \mathrm{ug} / \mathrm{mL}$ \\
$2000 \mathrm{ug} / \mathrm{mL}$
\end{tabular} \\
\hline VMSOMCALbs_00032 & $04 / 27 / 16$ & & Restek, Lot A0101160 & & (Purchased Rea & & \begin{tabular}{|l|} 
2-Butanone \\
2-Hexanone \\
4-Methyl-2-pentanone \\
\end{tabular} & \begin{tabular}{|l|}
$5000 \mathrm{ug} / \mathrm{mL}$ \\
$5000 \mathrm{ug} / \mathrm{mL}$ \\
$5000 \mathrm{ug} / \mathrm{mL}$ \\
$5000 \mathrm{lg} / \mathrm{mI}$
\end{tabular} \\
\hline .VMSOMSUas_00074 & $04 / 29 / 16$ & & Absolute, Lot 072214 & & (Purchased Rea & & $\begin{array}{l}\text { Chloroethane-d5 } \\
\text { Vinyl Chloride-d3 }\end{array}$ & $\frac{2000 \mathrm{ug} / \mathrm{mL}}{2000 \mathrm{ug} / \mathrm{mL}}$ \\
\hline .VMSOMSUbs_00126 & $06 / 13 / 16$ & & Absolute, Lot 080414 & & (Purchased Rea & & \begin{tabular}{|l|} 
2-Butanone-d5 \\
2-Hexanone-d5
\end{tabular} & $\begin{array}{l}2500 \mathrm{ug} / \mathrm{mL} \\
2500 \mathrm{ug} / \mathrm{mL}\end{array}$ \\
\hline .VMSOMSUCS_00050 & $04 / 27 / 16$ & & Absolute, Lot 011614 & & (Purchased Rea & & $\begin{array}{l}\text { 1,1,2,2-Tetrachloroethane-d2 } \\
\text { 1,1-Dichloroethene-d2 } \\
\text { 1,2-Dichlorobenzene-d4 } \\
\text { 1,2-Dichloroethane-d4 } \\
\text { 1,2-Dichloropropane-d6 } \\
\text { Benzene-d6 } \\
\text { Chloroform-d } \\
\text { Toluene-d8 } \\
\text { trans-1,3-Dichloropropene-d4 } \\
\end{array}$ & $\begin{array}{l}2000 \mathrm{ug} / \mathrm{mL} \\
2000 \mathrm{ug} / \mathrm{mL} \\
2000 \mathrm{ug} / \mathrm{mL} \\
2000 \mathrm{ug} / \mathrm{mL} \\
2000 \mathrm{ug} / \mathrm{mL} \\
2000 \mathrm{ug} / \mathrm{mL} \\
2000 \mathrm{ug} / \mathrm{mL} \\
2000 \mathrm{ug} / \mathrm{mL} \\
2000 \mathrm{ug} / \mathrm{mL} \\
\end{array}$ \\
\hline VMSOMTRCALw_00092 & $08 / 16 / 15$ & $07 / 16 / 15$ & METHANOL， Lot 147358 & 4000 uL & \begin{tabular}{|l} 
VMSOMCALaS_00022 \\
\end{tabular} & $40 \mathrm{uL}$ & \begin{tabular}{|l|} 
Bromomethane \\
Chloroethane \\
Chloromethane \\
Dichlorodifluoromethane \\
Trichlorofluoromethane \\
Vinyl chloride \\
$1,1,1-$ Trichloroethane \\
$1,1,2,2$-Tetrachloroethane \\
$1,1,2$-Trichloro-1,2,2-trifluor \\
oethane \\
$1,1,2$-Trichloroethane
\end{tabular} & \begin{tabular}{|l|}
$20 \mathrm{ug} / \mathrm{mL}$ \\
$20 \mathrm{ug} / \mathrm{mL}$ \\
$20 \mathrm{ug} / \mathrm{mL}$ \\
$20 \mathrm{ug} / \mathrm{mL}$ \\
$20 \mathrm{ug} / \mathrm{mL}$ \\
$20 \mathrm{ug} / \mathrm{mL}$ \\
$20 \mathrm{ug} / \mathrm{mL}$ \\
$20 \mathrm{ug} / \mathrm{mL}$ \\
$20 \mathrm{ug} / \mathrm{mL}$ \\
$20 \mathrm{ug} / \mathrm{mL}$ \\
\end{tabular} \\
\hline
\end{tabular}


SDG No.: 28907

\begin{tabular}{|c|c|c|c|c|c|c|c|c|}
\hline \multirow[b]{2}{*}{ Reagent ID } & \multirow[b]{2}{*}{$\begin{array}{l}\text { Exp } \\
\text { Date }\end{array}$} & \multirow[b]{2}{*}{$\begin{array}{l}\text { Prep } \\
\text { Date }\end{array}$} & \multirow[b]{2}{*}{$\begin{array}{l}\text { Dilutant } \\
\text { Used }\end{array}$} & \multirow{2}{*}{$\begin{array}{c}\text { Reagent } \\
\text { Final } \\
\text { Volume }\end{array}$} & \multicolumn{2}{|c|}{ Parent Reagent } & \multirow[b]{2}{*}{ Analyte } & \multirow[b]{2}{*}{ Concentration } \\
\hline & & & & & Reagent ID & $\begin{array}{l}\text { Volume } \\
\text { Added }\end{array}$ & & \\
\hline & & & & & & & 1,1-Dichloroethane & $20 \mathrm{ug} / \mathrm{mL}$ \\
\hline & & & & & & & 1,1-Dichloroethene & $20 \mathrm{ug} / \mathrm{mL}$ \\
\hline & & & & & & & 1,2,3-Trichlorobenzene & $20 \mathrm{ug} / \mathrm{mL}$ \\
\hline & & & & & & & 1,2,4-Trichlorobenzene & $20 \mathrm{ug} / \mathrm{mL}$ \\
\hline & & & & & & & 1,2-Dibromoethane & $20 \mathrm{ug} / \mathrm{mL}$ \\
\hline & & & & & & & 1,2-Dichlorobenzene & $20 \mathrm{ug} / \mathrm{mL}$ \\
\hline & & & & & & & 1,2-Dichloroethane & $20 \mathrm{ug} / \mathrm{mL}$ \\
\hline & & & & & & & 1,2-Dichloropropane & $20 \mathrm{ug} / \mathrm{mL}$ \\
\hline & & & & & & & 1,3-Dichlorobenzene & $20 \mathrm{ug} / \mathrm{mL}$ \\
\hline & & & & & & & 1,4-Dichlorobenzene & $20 \mathrm{ug} / \mathrm{mL}$ \\
\hline & & & & & & & Benzene & $20 \mathrm{ug} / \mathrm{mL}$ \\
\hline & & & & & & & Bromochloromethane & $20 \mathrm{ug} / \mathrm{mL}$ \\
\hline & & & & & & & Bromodichloromethane & $20 \mathrm{ug} / \mathrm{mL}$ \\
\hline & & & & & & & $\begin{array}{l}\text { Bromororm } \\
\text { Carbon disulfide }\end{array}$ & $\frac{20 \mathrm{ug} / \mathrm{mL}}{20 \mathrm{ug} / \mathrm{mL}}$ \\
\hline & & & & & & & Carbon tetrachloride & $20 \mathrm{ug} / \mathrm{mL}$ \\
\hline & & & & & & & Chlorobenzene & $20 \mathrm{ug} / \mathrm{mL}$ \\
\hline & & & & & & & Chloroform & $20 \mathrm{ug} / \mathrm{mL}$ \\
\hline & & & & & & & Cis-1,2-Dichloroethene & $20 \mathrm{ug} / \mathrm{mL}$ \\
\hline & & & & & & & Cis-1,3-Dichloropropene & $20 \mathrm{ug} / \mathrm{mL}$ \\
\hline & & & & & & & Dibromochloromethane & $\frac{20 \mathrm{ug} / \mathrm{mL}}{20 \mathrm{ug} / \mathrm{mL}}$ \\
\hline & & & & & & & Ethylbenzene & $20 \mathrm{ug} / \mathrm{mL}$ \\
\hline & & & & & & & Isopropylbenzene & $20 \mathrm{ug} / \mathrm{mL}$ \\
\hline & & & & & & & $m, p$-Xylene & $20 \mathrm{ug} / \mathrm{mL}$ \\
\hline & & & & & & & Methyl acetate & $20 \mathrm{ug} / \mathrm{mL}$ \\
\hline & & & & & & & Methyl tert-butyl ether & $20 \mathrm{ug} / \mathrm{mL}$ \\
\hline & & & & & & & Methylcyclohexane & $20 \mathrm{ug} / \mathrm{mL}$ \\
\hline & & & & & & & Methylene Chloride & $20 \mathrm{ug} / \mathrm{mL}$ \\
\hline & & & & & & & o-Xylene & $20 \mathrm{ug} / \mathrm{mL}$ \\
\hline & & & & & & & $\begin{array}{l}\text { Styrene } \\
\text { Tetrachlernethene }\end{array}$ & $\frac{20 \mathrm{ug} / \mathrm{mL}}{20 \mathrm{ug} / \mathrm{mL}}$ \\
\hline & & & & & & & $\begin{array}{l}\text { Tetracnioroetnene } \\
\text { Toluene }\end{array}$ & $20 \mathrm{ug} / \mathrm{mL}$ \\
\hline & & & & & & & trans-1,2-Dichloroethene & $20 \mathrm{ug} / \mathrm{mL}$ \\
\hline & & & & & & & trans-1,3-Dichloropropene & $20 \mathrm{ug} / \mathrm{mL}$ \\
\hline & & & & & & & Trichloroethene & $20 \mathrm{ug} / \mathrm{mL}$ \\
\hline & & & & & VMSOMCALbs_00034 & 160 uL & 2-Butanone & $200 \mathrm{ug} / \mathrm{mL}$ \\
\hline & & & & & & & 2-Hexanone & $200 \mathrm{ug} / \mathrm{mL}$ \\
\hline & & & & & & & 4-Methyl-2-pentanone & $200 \mathrm{ug} / \mathrm{mL}$ \\
\hline & & & & & & & Acetone & $200 \mathrm{ug} / \mathrm{mL}$ \\
\hline & & & & & VMSOMSUas_00074 & $40 \mathrm{uL}$ & Chloroethane-d5 & $20 \mathrm{ug} / \mathrm{mL}$ \\
\hline & & & & & & & Vinyl Chloride-d3 & $20 \mathrm{ug} / \mathrm{mL}$ \\
\hline & & & & & VMSOMSUbs_00129 & $320 \mathrm{uL}$ & 2-Butanone-d5 & $200 \mathrm{ug} / \mathrm{mL}$ \\
\hline & & & & & & & 2-Hexanone-d5 & $200 \mathrm{ug} / \mathrm{mL}$ \\
\hline & & & & & VMSOMSUCS_00050 & $40 \mathrm{uL}$ & $1,1,2,2$-Tetrachloroethane-d2 & $20 \mathrm{ug} / \mathrm{mL}$ \\
\hline & & & & & & & 1,1-Dichloroethene-d2 & $20 \mathrm{ug} / \mathrm{mL}$ \\
\hline & & & & & & & 1,2 -Dichlorobenzene-d4 & $20 \mathrm{ug} / \mathrm{mL}$ \\
\hline
\end{tabular}


SDG No.: 28907

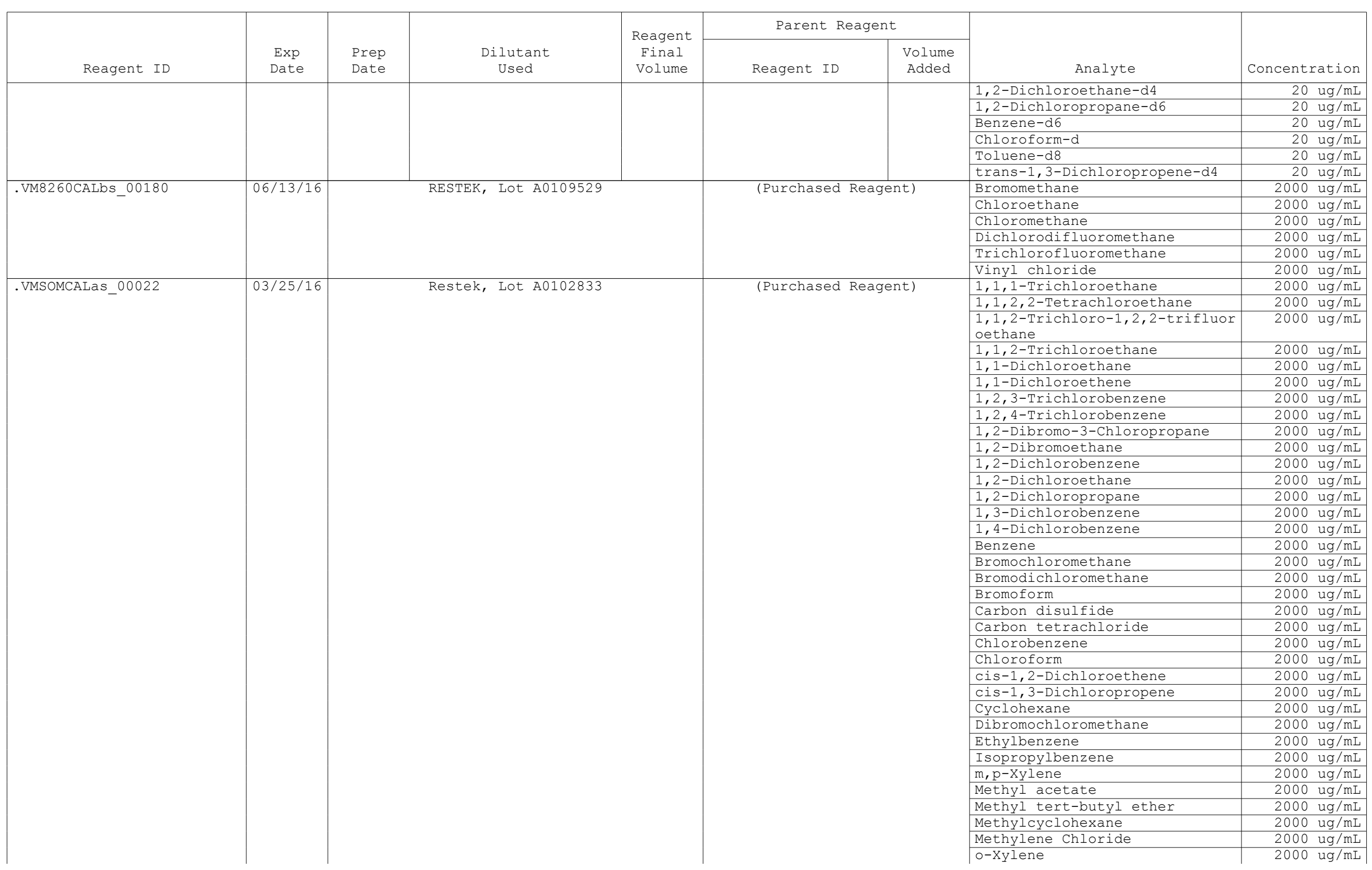


SDG No.: 28907

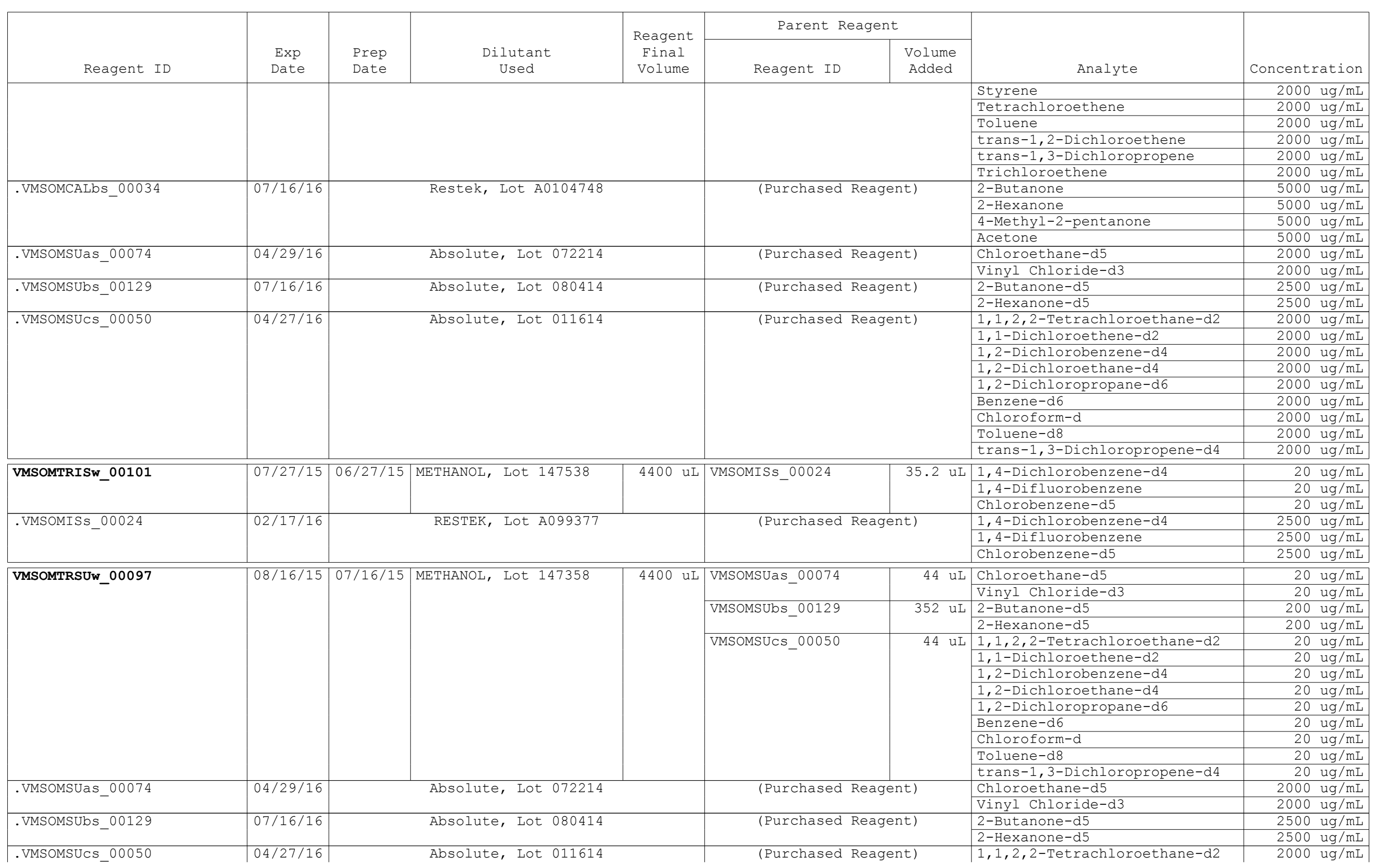


SDG No.: 28907

\begin{tabular}{|c|c|c|c|c|c|c|c|c|}
\hline \multirow[b]{2}{*}{ Reagent ID } & \multirow[b]{2}{*}{$\begin{array}{l}\text { Exp } \\
\text { Date }\end{array}$} & \multirow[b]{2}{*}{$\begin{array}{l}\text { Prep } \\
\text { Date }\end{array}$} & \multirow[b]{2}{*}{$\begin{array}{l}\text { Dilutant } \\
\text { Used }\end{array}$} & \multirow{2}{*}{$\begin{array}{c}\text { Reagent } \\
\text { Final } \\
\text { Volume }\end{array}$} & \multicolumn{2}{|c|}{ Parent Reagent } & \multirow[b]{2}{*}{ Analyte } & \multirow[b]{2}{*}{ Concentration } \\
\hline & & & & & Reagent ID & $\begin{array}{c}\text { Volume } \\
\text { Added }\end{array}$ & & \\
\hline & & & & & & & 1,1-Dichloroethene-d2 & $\begin{array}{l}2000 \mathrm{ug} / \mathrm{mL} \\
2000 \mathrm{ug} / \mathrm{mL}\end{array}$ \\
\hline & & & & & & & 1,2-Dichlorobenzene-d4 & $2000 \mathrm{ug} / \mathrm{mL}$ \\
\hline & & & & & & & 1,2-Dichloroethane-d4 & $2000 \mathrm{ug} / \mathrm{mL}$ \\
\hline & & & & & & & 1,2-Dichloropropane-d6 & $2000 \mathrm{ug} / \mathrm{mL}$ \\
\hline & & & & & & & Benzene-d6 & $2000 \mathrm{ug} / \mathrm{mL}$ \\
\hline & & & & & & & Chloroform-d & $2000 \mathrm{ug} / \mathrm{mL}$ \\
\hline & & & & & & & Toluene-d8 & $2000 \mathrm{ug} / \mathrm{mL}$ \\
\hline & & & & & & & trans-1,3-Dichloropropene-d4 & $2000 \mathrm{ug} / \mathrm{mL}$ \\
\hline
\end{tabular}




\section{TestAmerica}

THE LEADER IN ENVIRONMENTAL TESTING

\section{ANALYTICAL REPORT}

Job Number: 200-28914-1

SDG Number: 200-28914

Job Description: York (200-28914)

Contract Number: 1E-30401

For:

Argonne National Laboratory

9700 South Cass Avenue

Building 203

Office B-141

Argonne, IL 60439

Attention: Ms. Esther Bowen

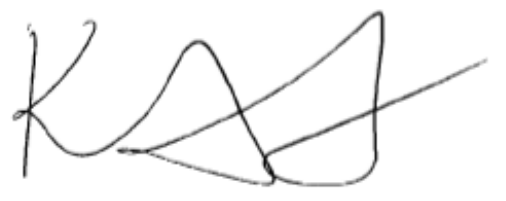

\section{Kathryn A Kelly, Project Manager I \\ 30 Community Drive, South Burlington, VT, 05403 \\ kathryn.kelly@testamericainc.com \\ $08 / 06 / 2015$}

The test results in this report relate only to sample(s) as received by the laboratory. These test results were derived under a quality system that adheres to the requirements of NELAC. Pursuant to NELAC, this report may not be produced in full without written approval from the laboratory 


\section{Table of Contents}

Cover Title Page . . . . . . . . . . . . . . . . . 1

Data Summaries . . . . . . . . . . . . . . . . . . . . . . . . 4

Report Narrative $\ldots \ldots \ldots \ldots \ldots \ldots \ldots \ldots \ldots \ldots \ldots \ldots \ldots \ldots$

Manual Integration Summary $\ldots \ldots \ldots \ldots \ldots \ldots \ldots \ldots \ldots \ldots \ldots \ldots \ldots$

Sample Summary $\ldots \ldots \ldots \ldots \ldots \ldots \ldots \ldots \ldots \ldots \ldots \ldots \ldots \ldots \ldots \ldots \ldots \ldots \ldots$

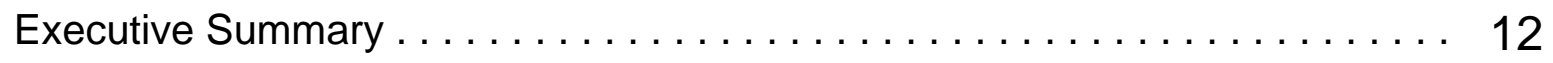

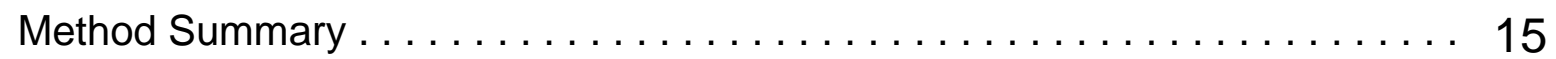

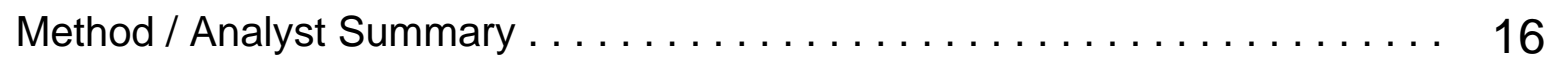

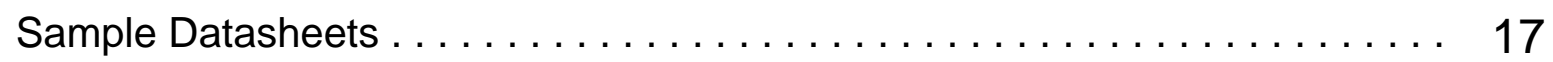

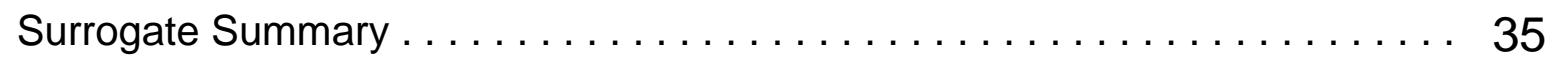

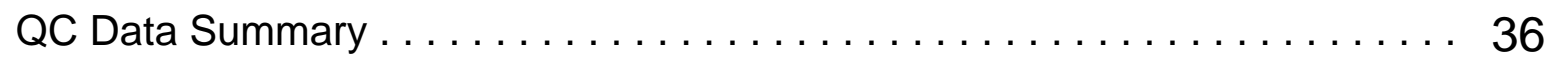

Data Qualifiers . . . . . . . . . . . . . . . . . . . 40

QC Association Summary . . . . . . . . . . . . . . . . . 41

Lab Chronicle . . . . . . . . . . . . . . . . . . . . 42

Reagent Traceability ...................... 44

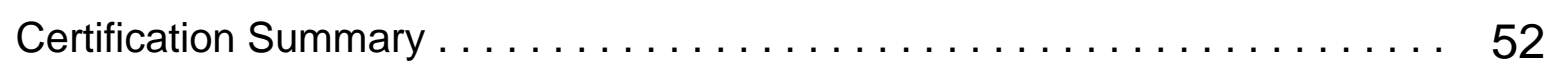

Organic Sample Data . . . . . . . . . . . . . . . . . . 53

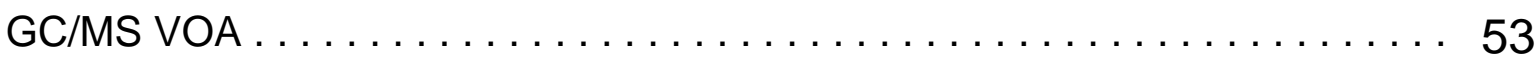

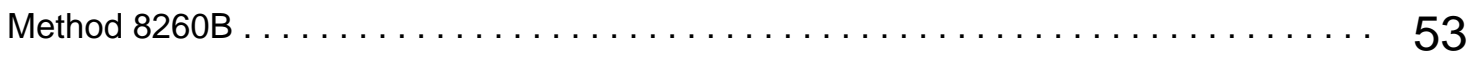

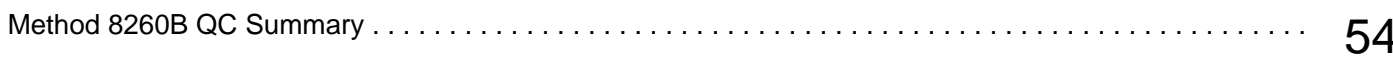

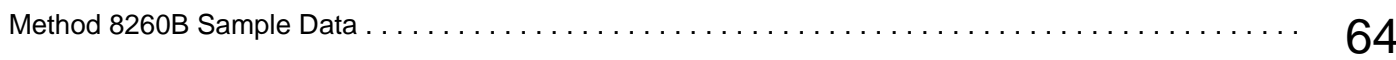

Standards Data $\ldots \ldots \ldots \ldots \ldots \ldots \ldots \ldots \ldots \ldots \ldots \ldots \ldots \ldots \ldots \ldots \ldots \ldots, \quad 91$

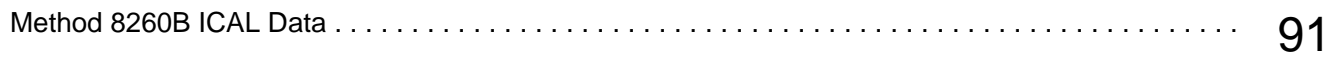

Method 8260B CCAL Data $\ldots \ldots \ldots \ldots \ldots \ldots \ldots \ldots \ldots \ldots \ldots \ldots \ldots \ldots \ldots \ldots \ldots \ldots \ldots, 103$

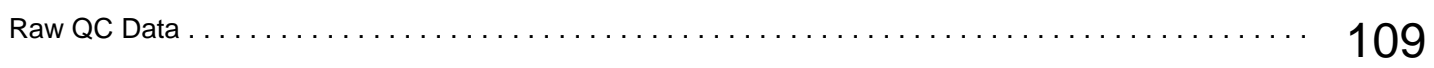

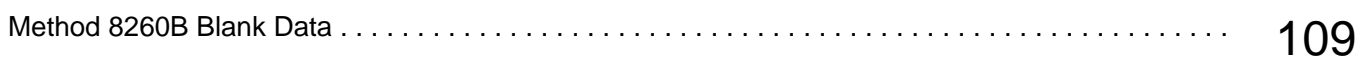




\section{Table of Contents}

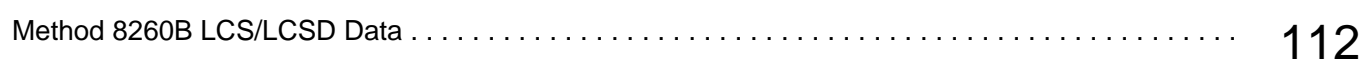

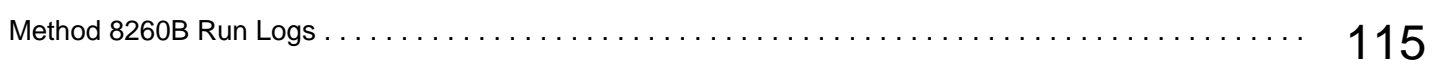

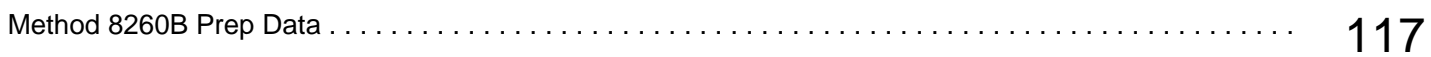

Shipping and Receiving Documents . . . . . . . . . . . . 118

Client Chain of Custody ................................. 119

Sample Receipt Checklist ............................ 121 


\title{
CASE NARRATIVE
}

\author{
Client: Argonne National Laboratory \\ Project: York (200-28914) \\ Report Number: 200- 28914-1
}

\begin{abstract}
Enclosed is the data set for the referenced project work. With the exceptions noted as flags or footnotes, standard analytical protocols were followed in performing the analytical work and the applied control limits were met.
\end{abstract}

Calculations were performed before rounding to avoid round-off errors in calculated results.

All holding times were met and proper preservation noted for the methods of analysis, unless otherwise detailed in the individual sections below.

Manual integration was employed in deriving certain of the analytical results. The values that have been derived from manual integration are qualified on the quantitation reports, and further documented with chromatographic profiles. An itemized listing of the manual integrations that were performed follows this narrative discussion, referencing the specific acquisition file names and the compounds for which manual integration was applied.

Included in this submittal is an itemized listing of the standards that were used in performing the analytical work.

\section{Receipt}

The samples were received on 07/17/2015. Documentation of the condition of the samples at the time of their receipt and any exception to the laboratory's Sample Acceptance Policy is documented in the Shipping and Receiving section of this submittal.

The samples in the set were received at the laboratory outside of temperature criteria at $8.6{ }^{\circ} \mathrm{C}$.

\section{SW846 Method(s) 5035/8260B Volatile Organics (Medium Level Soil)}

The samples were analyzed by the referenced method(s), using a low-level calibration. In performing the analytical work, 500 microliters of the methanol extract were added to the 5 milliliter purge volume. The results are reported on an as received basis, without an adjustment for moisture content.

The extract of each sample in the sample set was analyzed without a dilution. Internal responses were outside of acceptance criteria for each of the samples in this set. In the referenced analysis the response for tertiary-butyl alcohol- $d_{9}$ was outside of acceptance criteria. There was an acceptable recovery of the surrogate controls in each analysis. Several compounds were outside of QC limits in the LCS analyzed in batch 91708 . Matrix spike and matrix spike duplicate analyses were not performed on samples in this sample set. Chloromethane, Bromomethane, Methyl iodide, Ethylbenzene, Styrene, Bromobenzene, n-propylbenzene 4-Chlorotoluene, tertButylbenzene, 1,2,4-Trimethylbenzene, 1,3-Dichlorobenzene, 1,2-Dichlorobenzene, $\mathrm{n}$ Butylbenzene, 1,2,4-Trichlorobenzene, Hexachlorobutadiene and Napthalene were identified in the analysis of the methanol instrument blank associated with the analytical work. 
The initial calibration was established using five concentration levels. With the exception of those for methyl iodide, the responses for each target analyte met the 15.0 percent criterion for the application of an average response factor or met the criteria for the application of a linear calibration model. The relative standard deviation of the responses for methyl iodide was 16.0 percent. The initial calibration was verified with an analytical standard from a source different than was used for calibration. With the exception of those for Dichlorodifluoromethane, Acrolein, and Acetone, there was an acceptable performance of each target analyte in the initial calibration verification as measured against $\mathrm{a} \pm 25.0$ percent tolerance. With the exception of those for acetone and vinyl acetate there was an acceptable performance of each target analyte in the continuing calibration check acquisition as measured against $\mathrm{a} \pm 20.0$ percent tolerance. 
Lab Name: TestAmerica Burlington

Job No.: 200-28914-1

SDG No.: 200-28914

Instrument ID: CHL.i

Analysis Batch Number: 92007

Lab Sample ID: IC 200-92007/4

Client Sample ID:

Date Analyzed: 07/29/15 13:06

Lab File ID: 15008_04.D

GC Column: DB-624

ID $: 0.53(\mathrm{~mm})$

\begin{tabular}{|c|c|c|c|c|}
\hline \multirow[t]{2}{*}{ COMPOUND NAME } & \multirow{2}{*}{$\begin{array}{c}\text { RETENTION } \\
\text { TIME }\end{array}$} & \multicolumn{3}{|c|}{ MANUAL INTEGRATION } \\
\hline & & REASON & ANALYST & DATE \\
\hline 2,2-Dichloropropane & 7.75 & Assign Peak & archern & $07 / 29 / 1514: 06$ \\
\hline Tetrahydrofuran & 8.28 & Baseline & archern & $07 / 29 / 1514: 06$ \\
\hline
\end{tabular}

Lab Sample ID: IC 200-92007/5

Client Sample ID:

Date Analyzed: 07/29/15 13:38

Lab File ID: 15008 05.D

GC Column: DB-624

ID $: 0.53(\mathrm{~mm})$

\begin{tabular}{|l|r|r|c|c|}
\hline \multicolumn{1}{|c|}{ COMPOUND NAME } & RETENTION & \multicolumn{2}{|c|}{ MANUAL INTEGRATION } \\
\cline { 4 - 5 } & TIME & REASON & ANALYST & DATE \\
\hline tert-Butyl alcohol & 5.83 & Baseline & archern & $07 / 29 / 1515: 39$ \\
\hline Tetrahydrofuran & 8.28 & Baseline & archern & $07 / 29 / 1515: 39$ \\
\hline
\end{tabular}

Lab Sample ID: IC 200-92007/6

Client Sample ID:

Date Analyzed: 07/29/15 14:11

Lab File ID: 15008_06.D

GC Column: DB-624

ID $: 0.53(\mathrm{~mm})$

\begin{tabular}{|c|c|c|c|c|}
\hline \multirow[t]{2}{*}{ COMPOUND NAME } & \multirow{2}{*}{$\begin{array}{c}\text { RETENTION } \\
\text { TIME }\end{array}$} & \multicolumn{3}{|c|}{ MANUAL INTEGRATION } \\
\hline & & REASON & ANALYST & DATE \\
\hline tert-Butyl alcohol & 585 & Baseline & archern & $07 / 29 / 15 \quad 15: 36$ \\
\hline
\end{tabular}


Lab Name: TestAmerica Burlington

Job No.: 200-28914-1

SDG No.: 200-28914

Instrument ID: CHL.i

Analysis Batch Number: 92051

Lab Sample ID: LCS 200-91708/2-A

Client Sample ID:

Date Analyzed: 07/30/15 09:31

Lab File ID: 15018 03.D

GC Column: DB-624

ID $: 0.53(\mathrm{~mm})$

\begin{tabular}{|c|c|c|c|c|}
\hline \multirow[t]{2}{*}{ COMPOUND NAME } & \multirow{2}{*}{$\begin{array}{c}\text { RETENTION } \\
\text { TIME }\end{array}$} & \multicolumn{3}{|c|}{ MANUAL INTEGRATION } \\
\hline & & REASON & ANALYST & DATE \\
\hline TBA-d9 (IS) & 6.68 & Baseline & archern & $07 / 30 / 1511: 35$ \\
\hline Tetrahydrofuran & 8.31 & Baseline & archern & $07 / 30 / 15$ \\
\hline 1,4-Dioxane & 11.35 & Baseline & archern & $07 / 30 / 15$ \\
\hline
\end{tabular}

Lab Sample ID: MB 200-91708/1-A Client Sample ID:

Date Analyzed: 07/30/15 10:36

Lab File ID: 15018_05.D

GC Column: DB-624

ID $: 0.53(\mathrm{~mm})$

\begin{tabular}{|c|c|c|c|c|}
\hline \multirow[t]{2}{*}{ COMPOUND NAME } & \multirow{2}{*}{$\begin{array}{c}\text { RETENTION } \\
\text { TIME }\end{array}$} & \multicolumn{3}{|c|}{ MANUAL INTEGRATION } \\
\hline & & REASON & ANALYST & DATE \\
\hline TBA-d9 (IS) & 6.82 & Baseline & archern & $07 / 30 / 15 \quad 12: 41$ \\
\hline Dibromomethane & 10.98 & Baseline & archern & $07 / 30 / 15 \quad 12: 39$ \\
\hline 1,4-Dioxane-d8 & 11.34 & Baseline & archern & $07 / 30 / 1511: 39$ \\
\hline
\end{tabular}

Lab Sample ID: 200-28914-1

Client Sample ID: YK-S-37764

Date Analyzed: 07/30/15 12:42

Lab File ID: 15018_06.D

GC Column: DB-624

ID : $0.53(\mathrm{~mm})$

\begin{tabular}{|c|c|c|c|c|}
\hline \multirow[t]{2}{*}{ COMPOUND NAME } & \multirow{2}{*}{$\begin{array}{c}\text { RETENTION } \\
\text { TIME }\end{array}$} & \multicolumn{3}{|c|}{ MANUAL INTEGRATION } \\
\hline & & REASON & ANALYST & DATE \\
\hline Methylene Chloride & 5.47 & Baseline & archern & $07 / 31 / 15 \quad 13: 14$ \\
\hline TBA-d9 (IS) & 6.19 & Assign Peak & archern & $07 / 30 / 1513: 31$ \\
\hline 1,4-Dioxane-d8 & 11.26 & Baseline & archern & $07 / 30 / 15 \quad 13: 31$ \\
\hline
\end{tabular}


Lab Name: TestAmerica Burlington

Job No.: 200-28914-1

SDG No.: 200-28914

Instrument ID: CHL.i

Analysis Batch Number: 92051

Lab Sample ID: 200-28914-2

Client Sample ID: YK-S-37768

Date Analyzed: 07/30/15 13:15

Lab File ID: 15018 07.D

GC Column: DB-624

ID $: 0.53(\mathrm{~mm})$

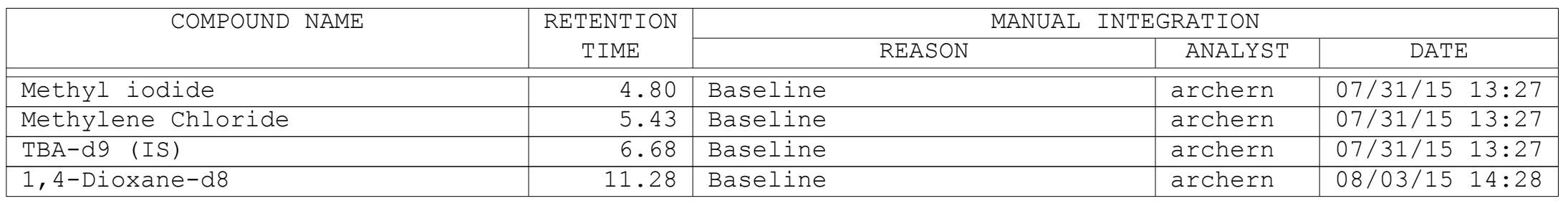

Lab Sample ID: 200-28914-3

Client Sample ID: YK-S-37767

Date Analyzed: 07/30/15 13:47

Lab File ID: 15018_08.D

GC Column: DB-624

ID $: 0.53(\mathrm{~mm})$

\begin{tabular}{|c|c|c|c|c|}
\hline \multirow[t]{2}{*}{ COMPOUND NAME } & \multirow{2}{*}{$\begin{array}{c}\text { RETENTION } \\
\text { TIME }\end{array}$} & \multicolumn{3}{|c|}{ MANUAL INTEGRATION } \\
\hline & & REASON & ANALYST & DATE \\
\hline Methyl iodide & 4.81 & Baseline & archern & $08 / 03 / 15 \quad 10: 52$ \\
\hline TBA-d9 (IS) & 6.21 & Assign Peak & archern & $08 / 03 / 15 \quad 10: 53$ \\
\hline 1,4-Dioxane-d8 & 11.27 & Baseline & archern & $08 / 03 / 1514: 29$ \\
\hline
\end{tabular}

Lab Sample ID: 200-28914-4

Client Sample ID: YK-S-37805

Date Analyzed: 07/30/15 14:20

Lab File ID: 15018_09.D

GC Column: DB-624

ID $: 0.53(\mathrm{~mm})$

\begin{tabular}{|l|r|r|c|c|}
\hline \multicolumn{1}{|c|}{ COMPOUND NAME } & RETENTION & \multicolumn{2}{|c|}{ MANUAL INTEGRATION } \\
\cline { 3 - 5 } & TIME & REASON & ANALYST & DATE \\
\hline Methyl iodide & 4.80 & Baseline & archern & $08 / 03 / 1511: 01$ \\
\hline TBA-d9 (IS) & 6.81 & Baseline & archern & $08 / 03 / 1511: 01$ \\
\hline $1,4-$ Dioxane-d8 & 11.32 & Baseline & $14: 29$ \\
\hline
\end{tabular}


Lab Name: TestAmerica Burlington

Job No.: 200-28914-1

SDG No.: 200-28914

Instrument ID: CHL.i

Analysis Batch Number: 92051

Lab Sample ID: 200-28914-5

Date Analyzed: 07/30/15 14:52

Client Sample ID: YK-S-37791

Lab File ID: 15018_10.D

GC Column: DB-624

ID $: 0.53(\mathrm{~mm})$

\begin{tabular}{|l|r|r|c|c|}
\hline \multicolumn{1}{|c|}{ COMPOUND NAME } & RETENTION & \multicolumn{2}{|c|}{ MANUAL INTEGRATION } \\
\cline { 3 - 5 } & TIME & REASON & ANALYST & DATE \\
\hline Methyl iodide & 4.82 & Baseline & archern & $08 / 03 / 1511: 03$ \\
\hline TBA-d9 (IS) & 6.21 & Baseline & archern & $08 / 03 / 1511: 03$ \\
\hline $1,4-$ Dioxane-d8 & 11.28 & Baseline & archern & $08 / 03 / 1514: 29$ \\
\hline
\end{tabular}

Lab Sample ID: 200-28914-6

Client Sample ID: YK-S-37826

Date Analyzed: 07/30/15 15:25

Lab File ID: 15018 11.D

GC Column: DB-624

ID $: 0.53(\mathrm{~mm})$

\begin{tabular}{|l|r|r|c|c|}
\hline \multicolumn{1}{|c|}{ COMPOUND NAME } & RETENTION & \multicolumn{2}{|c|}{ MANUAL INTEGRATION } \\
\cline { 3 - 5 } & TIME & & REASON & DALYST \\
\hline Methyl iodide & 4.82 & Baseline & archern & $08 / 03 / 1511: 07$ \\
\hline TBA-d9 (IS) & 6.78 & Baseline & archern & $08 / 03 / 1511: 07$ \\
\hline $1,4-$ Dioxane-d8 & 11.34 & Baseline & archern & $08 / 03 / 1514: 29$ \\
\hline
\end{tabular}

Lab Sample ID: 200-28914-7

Client Sample ID: YK-S-37814

Date Analyzed: 07/30/15 15:58

Lab File ID: 15018_12.D

GC Column: DB-624

ID $: 0.53(\mathrm{~mm})$

\begin{tabular}{|c|c|c|c|c|}
\hline \multirow[t]{2}{*}{ COMPOUND NAME } & \multirow{2}{*}{$\begin{array}{c}\text { RETENTION } \\
\text { TIME }\end{array}$} & \multicolumn{3}{|c|}{ MANUAL INTEGRATION } \\
\hline & & REASON & ANALYST & DATE \\
\hline Methyl iodide & 4.81 & Unspecified & & \\
\hline Methylene Chloride & 5.45 & Baseline & archern & $08 / 03 / 15 \quad 11: 24$ \\
\hline Carbon tetrachloride & 8.84 & Baseline & archern & $08 / 03 / 15 \quad 11: 24$ \\
\hline 1,4-Dioxane-d8 & 11.30 & Baseline & archern & $08 / 03 / 1514: 30$ \\
\hline
\end{tabular}


Lab Name: TestAmerica Burlington

SDG No.: 200-28914

Instrument ID: CHL.i

Lab Sample ID: 200-28914-8

Date Analyzed: 07/30/15 16:30

\begin{tabular}{|l|r|r|c|c|}
\hline \multicolumn{1}{|c|}{ COMPOUND NAME } & RETENTION & \multicolumn{2}{|c|}{ MANUAL INTEGRATION } \\
\cline { 4 - 5 } & TIME & REASON & ANALYST & DATE \\
\hline TBA-d9 (IS) & 6.80 & Baseline & archern & $08 / 03 / 1511: 28$ \\
\hline $1,4-D i$ mane-d8 & 11.34 & Baseline & archern & $08 / 03 / 1514: 30$ \\
\hline
\end{tabular}

Lab Sample ID: 200-28914-9 Client Sample ID: YK-S-BLANK

Date Analyzed: 07/30/15 17:03

Lab File ID: 15018_14.D

GC Column: DB-624

ID : $0.53(\mathrm{~mm})$

\begin{tabular}{|c|c|c|c|c|}
\hline \multirow[t]{2}{*}{ COMPOUND NAME } & RETENTION & \multicolumn{3}{|c|}{ MANUAL INTEGRATION } \\
\hline & TIME & REASON & ANALYST & DATE \\
\hline Methyl iodide & 4.80 & Baseline & archern & $08 / 03 / 1511: 32$ \\
\hline TBA-d9 (IS) & 6.83 & Baseline & archern & $08 / 03 / 1511: 32$ \\
\hline 1,4-Dioxane-d8 & 11.32 & Baseline & archern & $08 / 03 / 15 \quad 14: 30$ \\
\hline m\&p-Xylene & 16.11 & Baseline & archern & $08 / 03 / 1511: 32$ \\
\hline
\end{tabular}




\section{SAMPLE SUMMARY}

Client: Argonne National Laboratory

Job Number: 200-28914-1

Sdg Number: 200-28914

\begin{tabular}{|c|c|c|c|c|}
\hline Lab Sample ID & Client Sample ID & Client Matrix & $\begin{array}{c}\text { Date/Time } \\
\text { Sampled }\end{array}$ & $\begin{array}{l}\text { Date/Time } \\
\text { Received }\end{array}$ \\
\hline 200-28914-1 & YK-S-37764 & Solid & 07/16/20150000 & 07/17/2015 1010 \\
\hline 200-28914-2 & YK-S-37768 & Solid & 07/16/20150000 & $07 / 17 / 20151010$ \\
\hline 200-28914-3 & YK-S-37767 & Solid & 07/16/20150000 & $07 / 17 / 20151010$ \\
\hline 200-28914-4 & YK-S-37805 & Solid & 07/16/20150000 & 07/17/2015 1010 \\
\hline $200-28914-5$ & YK-S-37791 & Solid & 07/16/20150000 & 07/17/2015 1010 \\
\hline 200-28914-6 & YK-S-37826 & Solid & 07/16/20150000 & $07 / 17 / 20151010$ \\
\hline 200-28914-7 & YK-S-37814 & Solid & 07/16/20150000 & 07/17/2015 1010 \\
\hline 200-28914-8 & YK-S-37758 & Solid & 07/16/20150000 & 07/17/2015 1010 \\
\hline 200-28914-9TВ & YK-S-BLANK & Solid & 07/16/20150000 & 07/17/2015 1010 \\
\hline
\end{tabular}


EXECUTIVE SUMMARY - Detections

Client: Argonne National Laboratory

Job Number: $200-28914-1$

Sdg Number: 200-28914

\begin{tabular}{|c|c|c|c|c|c|}
\hline $\begin{array}{l}\text { Lab Sample ID } \\
\text { Analyte }\end{array}$ & Result & Qualifier & $\begin{array}{l}\text { Reporting } \\
\text { Limit }\end{array}$ & Units & Methoc \\
\hline 200-28914-1 & & & & & \\
\hline Chloromethane & 4.9 & $J B$ & 10 & $\mathrm{ug} / \mathrm{Kg}$ & $8260 B$ \\
\hline Bromomethane & 18 & B & 10 & $\mathrm{ug} / \mathrm{Kg}$ & $8260 B$ \\
\hline Methyl iodide & 8.9 & $J * B$ & 10 & $\mathrm{ug} / \mathrm{Kg}$ & $8260 B$ \\
\hline Carbon disulfide & 1.8 & $J^{*}$ & 10 & $\mathrm{ug} / \mathrm{Kg}$ & $8260 B$ \\
\hline Methyl acetate & 51 & * & 50 & $\mathrm{ug} / \mathrm{Kg}$ & $8260 B$ \\
\hline Methylene Chloride & 8.6 & $J$ & 10 & $\mathrm{ug} / \mathrm{Kg}$ & $8260 B$ \\
\hline trans-1,2-Dichloroethene & 1.9 & J & 10 & $\mathrm{ug} / \mathrm{Kg}$ & $8260 B$ \\
\hline cis-1,2-Dichloroethene & 2.1 & J & 10 & $\mathrm{ug} / \mathrm{Kg}$ & $8260 B$ \\
\hline Carbon tetrachloride & 2.4 & J & 10 & $\mathrm{ug} / \mathrm{Kg}$ & $8260 B$ \\
\hline Dibromomethane & 2.5 & $J$ & 10 & $\mathrm{ug} / \mathrm{Kg}$ & $8260 B$ \\
\hline cis-1,3-Dichloropropene & 2.9 & J & 10 & $\mathrm{ug} / \mathrm{Kg}$ & $8260 B$ \\
\hline trans-1,3-Dichloropropene & 4.8 & J & 10 & $\mathrm{ug} / \mathrm{Kg}$ & $8260 B$ \\
\hline Ethylbenzene & 1.2 & J B & 10 & $\mathrm{ug} / \mathrm{Kg}$ & $8260 B$ \\
\hline m\&p-Xylene & 1.4 & J & 10 & $\mathrm{ug} / \mathrm{Kg}$ & $8260 B$ \\
\hline Bromobenzene & 2.0 & J B & 10 & $\mathrm{ug} / \mathrm{Kg}$ & $8260 B$ \\
\hline 4-Chlorotoluene & 1.4 & J B & 10 & $\mathrm{ug} / \mathrm{Kg}$ & $8260 B$ \\
\hline 1,3-Dichlorobenzene & 3.3 & J B & 10 & $\mathrm{ug} / \mathrm{Kg}$ & $8260 B$ \\
\hline 1,2-Dichlorobenzene & 2.1 & J B & 10 & $\mathrm{ug} / \mathrm{Kg}$ & $8260 B$ \\
\hline n-Butylbenzene & 1.4 & J B & 10 & $\mathrm{ug} / \mathrm{Kg}$ & $8260 B$ \\
\hline 1,2,4-Trichlorobenzene & 4.5 & $\mathrm{JB}$ & 10 & $\mathrm{ug} / \mathrm{Kg}$ & $8260 B$ \\
\hline Hexachlorobutadiene & 2.3 & $\mathrm{~J} * \mathrm{~B}$ & 10 & $\mathrm{ug} / \mathrm{Kg}$ & $8260 B$ \\
\hline Naphthalene & 4.2 & $J * B$ & 10 & $\mathrm{ug} / \mathrm{Kg}$ & $8260 \mathrm{~B}$ \\
\hline 1,2,3-Trichlorobenzene & 3.6 & $\mathrm{JB}$ & 10 & $\mathrm{ug} / \mathrm{Kg}$ & $8260 B$ \\
\hline 200-28914-2 & & & & & \\
\hline Chloromethane & 4.0 & $J B$ & 10 & $\mathrm{ug} / \mathrm{Kg}$ & $8260 \mathrm{~B}$ \\
\hline Bromomethane & 17 & B & 10 & $\mathrm{ug} / \mathrm{Kg}$ & $8260 B$ \\
\hline Methyl iodide & 9.2 & $\mathrm{~J} * \mathrm{~B}$ & 10 & $\mathrm{ug} / \mathrm{Kg}$ & $8260 B$ \\
\hline Carbon disulfide & 1.1 & $\mathrm{~J}^{*}$ & 10 & $\mathrm{ug} / \mathrm{Kg}$ & $8260 B$ \\
\hline Methylene Chloride & 5.6 & $\mathrm{~J}$ & 10 & $\mathrm{ug} / \mathrm{Kg}$ & $8260 B$ \\
\hline Carbon tetrachloride & 6.5 & $J$ & 10 & $\mathrm{ug} / \mathrm{Kg}$ & $8260 B$ \\
\hline 1,2,4-Trichlorobenzene & 1.5 & $\mathrm{JB}$ & 10 & $\mathrm{ug} / \mathrm{Kg}$ & $8260 B$ \\
\hline Naphthalene & 2.2 & $\mathrm{~J} * \mathrm{~B}$ & 10 & $\mathrm{ug} / \mathrm{Kg}$ & $8260 B$ \\
\hline 1,2,3-Trichlorobenzene & 1.3 & $\mathrm{JB}$ & 10 & $\mathrm{ug} / \mathrm{Kg}$ & $8260 B$ \\
\hline
\end{tabular}




\section{EXECUTIVE SUMMARY - Detections}

Client: Argonne National Laboratory

Job Number: $200-28914-1$

Sdg Number: 200-28914

Lab Sample ID Client Sample ID
Analyte

Result Qualifier $\begin{aligned} & \text { Reporting } \\ & \text { Limit }\end{aligned}$

Units Method

200-28914-3 YK-S-37767

Bromomethane

Methyl iodide

Chloroform

Carbon tetrachloride

1,2,4-Trichlorobenzene

Naphthalene

1,2,3-Trichlorobenzene

$\begin{array}{lll}13 & \text { B } & 8.9 \\ 7.3 & \text { J * B } & 8.9 \\ 1.8 & \text { J } & 8.9 \\ 9.1 & & 8.9 \\ 1.2 & \text { J B } & 8.9 \\ 1.6 & \text { J * B } & 8.9 \\ 1.1 & \text { J B } & 8.9\end{array}$

$\begin{array}{ll}\mathrm{ug} / \mathrm{Kg} & 8260 \mathrm{~B} \\ \mathrm{ug} / \mathrm{Kg} & 8260 \mathrm{~B} \\ \mathrm{ug} / \mathrm{Kg} & 8260 \mathrm{~B} \\ \mathrm{ug} / \mathrm{Kg} & 8260 \mathrm{~B} \\ \mathrm{ug} / \mathrm{Kg} & 8260 \mathrm{~B} \\ \mathrm{ug} / \mathrm{Kg} & 8260 \mathrm{~B} \\ \mathrm{ug} / \mathrm{Kg} & 8260 \mathrm{~B}\end{array}$

200-28914-4

YK-S-37805

Chloromethane

Bromomethane

Methyl iodide

1,2,4-Trichlorobenzene

Naphthalene

$\begin{array}{lllll}5.1 & \mathrm{~J} \mathrm{~B} & 9.5 & \mathrm{ug} / \mathrm{Kg} & 8260 \mathrm{~B} \\ 20 & \mathrm{~B} & 9.5 & \mathrm{ug} / \mathrm{Kg} & 8260 \mathrm{~B} \\ 9.1 & \mathrm{~J} \text { * B } & 9.5 & \mathrm{ug} / \mathrm{Kg} & 8260 \mathrm{~B} \\ 1.2 & \mathrm{~J} \mathrm{~B} & 9.5 & \mathrm{ug} / \mathrm{Kg} & 8260 \mathrm{~B} \\ 1.5 & \mathrm{~J} \text { * B } & 9.5 & \mathrm{ug} / \mathrm{Kg} & 8260 \mathrm{~B}\end{array}$

200-28914-5

YK-S-37791

Chloromethane

3.0

15

Bromomethane

Methyl iodide

Chloroform

7.7

6.7

Carbon tetrachloride

1,2,4-Trichlorobenzene

44

Naphthalene

1,2,3-Trichlorobenzene

1.1

1.5

1.0

$\begin{array}{ll}\mathrm{J} \mathrm{B} & 9.5 \\ \mathrm{~B} & 9.5 \\ \mathrm{~J} \text { * } \mathrm{B} & 9.5 \\ \mathrm{~J} & 9.5 \\ & 9.5 \\ \mathrm{~J} \mathrm{~B} & 9.5 \\ \mathrm{~J} \text { * } \mathrm{B} & 9.5 \\ \mathrm{~J} B & 9.5\end{array}$

$\mathrm{ug} / \mathrm{Kg}$

$\mathrm{ug} / \mathrm{Kg}$

$\mathrm{ug} / \mathrm{Kg}$

$\mathrm{ug} / \mathrm{Kg}$

$\mathrm{ug} / \mathrm{Kg}$

$\mathrm{ug} / \mathrm{Kg}$

$\mathrm{ug} / \mathrm{Kg}$

$\mathrm{ug} / \mathrm{Kg}$

$8260 \mathrm{~B}$

$8260 \mathrm{~B}$

$8260 \mathrm{~B}$

$8260 \mathrm{~B}$

$8260 \mathrm{~B}$

$8260 \mathrm{~B}$

$8260 \mathrm{~B}$

$8260 \mathrm{~B}$

200-28914-6

YK-S-37826

Chloromethane

Bromomethane

Methyl iodide

1,1,1-Trichloroethane

1,2,4-Trichlorobenzene

Naphthalene

2.1

14

6.8

6.0

0.99

1.2

J B

9.1

$\mathrm{ug} / \mathrm{Kg}$

$\mathrm{ug} / \mathrm{Kg}$

$\mathrm{ug} / \mathrm{Kg}$

$\mathrm{ug} / \mathrm{Kg}$

$\mathrm{ug} / \mathrm{Kg}$

$\mathrm{ug} / \mathrm{Kg}$

$8260 \mathrm{~B}$

$8260 \mathrm{~B}$

$8260 \mathrm{~B}$

$8260 \mathrm{~B}$

$8260 \mathrm{~B}$

$J B$

9.1

$8260 \mathrm{~B}$

200-28914-7

YK-S-37814

Chloromethane

Bromomethane

Methyl iodide

Methylene Chloride

Carbon tetrachloride

Naphthalene

2.6
16
7.8
7.3
3.9
1.6

$\begin{array}{ll}J \mathrm{~B} & 11 \\ \mathrm{~B} & 11 \\ \mathrm{~J}{ }^{*} \mathrm{~B} & 11 \\ \mathrm{~J} & 11 \\ \mathrm{~J} & 11 \\ \mathrm{~J} \text { * B } & 11\end{array}$

$\mathrm{ug} / \mathrm{Kg}$

$8260 \mathrm{~B}$

$\mathrm{ug} / \mathrm{Kg}$

$8260 \mathrm{~B}$

$\mathrm{ug} / \mathrm{Kg}$

$8260 \mathrm{~B}$

$\mathrm{ug} / \mathrm{Kg}$

$8260 \mathrm{~B}$

$u g / K g$

$8260 \mathrm{~B}$

$\mathrm{ug} / \mathrm{Kg}$

$8260 \mathrm{~B}$ 


\section{EXECUTIVE SUMMARY - Detections}

Client: Argonne National Laboratory

Job Number: 200-28914-1

Sdg Number: 200-28914

\begin{tabular}{|c|c|c|c|c|c|c|}
\hline $\begin{array}{l}\text { Lab Sample ID } \\
\text { Analyte }\end{array}$ & Client Sample ID & Result & Qualifier & $\begin{array}{l}\text { Reporting } \\
\text { Limit }\end{array}$ & Units & Methoc \\
\hline 200-28914-8 & YK-S-37758 & & & & & \\
\hline Chloromethane & & 2.7 & $J B$ & 9.2 & $\mathrm{ug} / \mathrm{Kg}$ & $8260 \mathrm{~B}$ \\
\hline Bromomethane & & 14 & B & 9.2 & $\mathrm{ug} / \mathrm{Kg}$ & $8260 \mathrm{~B}$ \\
\hline Methyl iodide & & 5.7 & $J * B$ & 9.2 & $\mathrm{ug} / \mathrm{Kg}$ & $8260 \mathrm{~B}$ \\
\hline Naphthalene & & 1.1 & $\mathrm{~J} * \mathrm{~B}$ & 9.2 & $\mathrm{ug} / \mathrm{Kg}$ & $8260 \mathrm{~B}$ \\
\hline 200-28914-9TВ & YK-S-BLANK & & & & & \\
\hline Chloromethane & & 2.4 & $J B$ & 10 & $\mathrm{ug} / \mathrm{Kg}$ & $8260 \mathrm{~B}$ \\
\hline Bromomethane & & 17 & B & 10 & $\mathrm{ug} / \mathrm{Kg}$ & $8260 \mathrm{~B}$ \\
\hline Methyl iodide & & 6.7 & $J$ * $B$ & 10 & $\mathrm{ug} / \mathrm{Kg}$ & $8260 B$ \\
\hline Ethylbenzene & & 1.3 & $\mathrm{~J} B$ & 10 & $\mathrm{ug} / \mathrm{Kg}$ & $8260 B$ \\
\hline m\&p-Xylene & & 1.2 & $\mathrm{~J}$ & 10 & $\mathrm{ug} / \mathrm{Kg}$ & $8260 \mathrm{~B}$ \\
\hline
\end{tabular}


METHOD SUMMARY

Client: Argonne National Laboratory

Job Number: 200-28914-1

Sdg Number: 200-28914

Description

Lab Location Method Preparation Method

Matrix: Solid

Volatile Organic Compounds (GC/MS)

TAL BUR SW846 8260B

Closed System Purge and Trap

TAL BUR

SW846 5035

Lab References:

TAL BUR = TestAmerica Burlington

\section{Method References:}

SW846 = "Test Methods For Evaluating Solid Waste, Physical/Chemical Methods", Third Edition, November 1986 And Its Updates. 


\section{METHOD / ANALYST SUMMARY}

Client: Argonne National Laboratory

Job Number: $200-28914-1$

Sdg Number: $200-28914$

Method

SW846 8260B
Analyst

Archer, Nicholas E
Analyst ID

NEA 
Analytical Data

Client: Argonne National Laboratory

Job Number: 200-28914-1

Sdg Number: 200-28914

Client Sample ID: $\quad$ YK-S-37764

Lab Sample ID: $\quad$ 200-28914-1

Date Sampled: 07/16/2015 0000

Client Matrix:

Solid

Date Received: 07/17/2015 1010

\section{B Volatile Organic Compounds (GC/MS)}

$\begin{array}{llllll}\text { Analysis Method: } & 8260 \mathrm{~B} & \text { Analysis Batch: } & 200-92051 & \text { Instrument ID: } & \text { CHL.i } \\ \text { Prep Method: } & 5035 & \text { Prep Batch: } & 200-91708 & \text { Lab File ID: } & \text { 15018_06.D } \\ \text { Dilution: } & 1.0 & & & \text { Initial Weight/Volume: } & 10.046 \mathrm{~g} \\ \text { Analysis Date: } & 07 / 30 / 20151242 & & & \text { Final Weight/Volume: } 10 \mathrm{~mL} \\ \text { Prep Date: } & 07 / 23 / 20151500 & & & \end{array}$

Prep Date: $\quad$ 07/23/2015 1500

\begin{tabular}{|c|c|c|c|c|c|}
\hline Analyte & DryWt Corrected: N & Result (ug/Kg) & Qualifier & MDL & $\mathrm{RL}$ \\
\hline Dichlorodifluoromethane & & 10 & U & 1.7 & 10 \\
\hline Chloromethane & & 4.9 & $\mathrm{JB}$ & 2.1 & 10 \\
\hline Vinyl chloride & & 10 & $\mathrm{U}$ & 0.83 & 10 \\
\hline Bromomethane & & 18 & B & 1.0 & 10 \\
\hline Chloroethane & & 10 & $U^{*}$ & 2.5 & 10 \\
\hline Trichlorofluoromethane & & 10 & $\mathrm{U}$ & 0.82 & 10 \\
\hline 1,1-Dichloroethene & & 10 & $U^{*}$ & 1.1 & 10 \\
\hline Freon TF & & 10 & $U$ * & 0.85 & 10 \\
\hline Acetone & & 50 & U & 12 & 50 \\
\hline Methyl iodide & & 8.9 & $J * B$ & 0.75 & 10 \\
\hline Carbon disulfide & & 1.8 & $J^{*}$ & 0.87 & 10 \\
\hline Methyl acetate & & 51 & * & 9.2 & 50 \\
\hline Methylene Chloride & & 8.6 & $\mathrm{~J}$ & 2.3 & 10 \\
\hline trans-1,2-Dichloroethene & & 1.9 & $\mathrm{~J}$ & 0.99 & 10 \\
\hline Methyl t-butyl ether & & 10 & U & 1.1 & 10 \\
\hline 1,1-Dichloroethane & & 10 & U & 1.1 & 10 \\
\hline Vinyl acetate & & 50 & U & 4.5 & 50 \\
\hline 2,2-Dichloropropane & & 10 & $U$ * & 2.2 & 10 \\
\hline cis-1,2-Dichloroethene & & 2.1 & $\mathrm{~J}$ & 1.0 & 10 \\
\hline 2-Butanone & & 50 & U & 5.9 & 50 \\
\hline Bromochloromethane & & 10 & U & 1.4 & 10 \\
\hline Tetrahydrofuran & & 20 & $U$ * & 5.3 & 20 \\
\hline Chloroform & & 10 & $\mathrm{U}$ & 1.2 & 10 \\
\hline 1,1,1-Trichloroethane & & 10 & $\mathrm{U}$ & 0.85 & 10 \\
\hline Cyclohexane & & 10 & $U$ & 1.0 & 10 \\
\hline 1,1-Dichloropropene & & 10 & U & 0.97 & 10 \\
\hline Carbon tetrachloride & & 2.4 & $\mathrm{~J}$ & 2.0 & 10 \\
\hline Isobutyl alcohol & & 500 & $U$ * & 27 & 500 \\
\hline Benzene & & 10 & $U$ & 1.1 & 10 \\
\hline 1,2-Dichloroethane & & 10 & U & 1.3 & 10 \\
\hline Trichloroethene & & 10 & $\mathrm{U}$ & 0.83 & 10 \\
\hline Methylcyclohexane & & 10 & $U$ & 2.1 & 10 \\
\hline 1,2-Dichloropropane & & 10 & $\mathrm{U}$ & 1.2 & 10 \\
\hline Dibromomethane & & 2.5 & $\mathrm{~J}$ & 1.5 & 10 \\
\hline 1,4-Dioxane & & 400 & U & 65 & 400 \\
\hline Bromodichloromethane & & 10 & $U$ & 1.0 & 10 \\
\hline 2-Chloroethyl vinyl ether & & 10 & $\mathrm{U}$ & 1.9 & 10 \\
\hline cis-1,3-Dichloropropene & & 2.9 & $\mathrm{~J}$ & 1.2 & 10 \\
\hline 4-Methyl-2-pentanone & & 50 & $U^{*}$ & 4.7 & 50 \\
\hline Toluene & & 10 & U & 2.1 & 10 \\
\hline trans-1,3-Dichloropropene & & 4.8 & $\mathrm{~J}$ & 2.2 & 10 \\
\hline 1,1,2-Trichloroethane & & 10 & $U$ & 1.3 & 10 \\
\hline Tetrachloroethene & & 10 & $\mathrm{U}$ & 2.0 & 10 \\
\hline 1,3-Dichloropropane & & 10 & U & 1.1 & 10 \\
\hline 2-Hexanone & & 50 & $\mathrm{U}$ & 6.1 & 50 \\
\hline Dibromochloromethane & & 10 & $U$ & 1.1 & 10 \\
\hline
\end{tabular}


Analytical Data

Client: Argonne National Laboratory

Job Number: 200-28914-1

Sdg Number: 200-28914

Client Sample ID: $\quad$ YK-S-37764

Lab Sample ID: $\quad$ 200-28914-1

Date Sampled: 07/16/2015 0000

Client Matrix:

Solid

Date Received: 07/17/2015 1010

\section{B Volatile Organic Compounds (GC/MS)}

$\begin{array}{llllll}\text { Analysis Method: } & 8260 \mathrm{~B} & \text { Analysis Batch: } & 200-92051 & \text { Instrument ID: } & \text { CHL.i } \\ \text { Prep Method: } & 5035 & \text { Prep Batch: } & 200-91708 & \text { Lab File ID: } & \text { 15018_06.D } \\ \text { Dilution: } & 1.0 & & & \text { Initial Weight/Volume: } & 10.046 \mathrm{~g} \\ \text { Analysis Date: } & 07 / 30 / 20151242 & & & \text { Final Weight/Volume: } 10 \mathrm{~mL} \\ \text { Prep Date: } & 07 / 23 / 20151500 & & & \end{array}$

Prep Date: $\quad$ 07/23/2015 1500

\begin{tabular}{|c|c|c|c|c|}
\hline DryWt Corrected: N & Result (ug/Kg) & Qualifier & MDL & $\mathrm{RL}$ \\
\hline 1,2-Dibromoethane & 10 & U & 1.2 & 10 \\
\hline Chlorobenzene & 10 & $\mathrm{U}$ & 0.91 & 10 \\
\hline 1,1,1,2-Tetrachloroethane & 10 & $\mathrm{U}$ & 1.1 & 10 \\
\hline Ethylbenzene & 1.2 & $\mathrm{JB}$ & 0.94 & 10 \\
\hline m\&p-Xylene & 1.4 & $\mathrm{~J}$ & 1.1 & 10 \\
\hline o-Xylene & 10 & $\mathrm{U}$ & 1.0 & 10 \\
\hline Styrene & 10 & $\mathrm{U}$ & 0.98 & 10 \\
\hline Bromoform & 10 & $\mathrm{U}$ & 1.0 & 10 \\
\hline Isopropylbenzene & 10 & $U$ & 2.0 & 10 \\
\hline Bromobenzene & 2.0 & $\mathrm{JB}$ & 1.0 & 10 \\
\hline 1,1,2,2-Tetrachloroethane & 10 & $\mathrm{U}$ & 1.3 & 10 \\
\hline 1,2,3-Trichloropropane & 10 & $\mathrm{U}$ & 1.2 & 10 \\
\hline n-Propylbenzene & 10 & U & 1.1 & 10 \\
\hline 2-Chlorotoluene & 10 & $\mathrm{U}$ & 1.1 & 10 \\
\hline 4-Chlorotoluene & 1.4 & $J B$ & 0.96 & 10 \\
\hline 1,3,5-Trimethylbenzene & 10 & $\mathrm{U}$ & 2.0 & 10 \\
\hline tert-Butylbenzene & 10 & U & 1.4 & 10 \\
\hline 1,2,4-Trimethylbenzene & 10 & $\mathrm{U}$ & 1.0 & 10 \\
\hline sec-Butylbenzene & 10 & U & 2.0 & 10 \\
\hline 1,3-Dichlorobenzene & 3.3 & $\mathrm{JB}$ & 0.95 & 10 \\
\hline 4-Isopropyltoluene & 10 & $\mathrm{U}$ & 0.88 & 10 \\
\hline 1,4-Dichlorobenzene & 10 & $\mathrm{U}$ & 1.4 & 10 \\
\hline 1,2-Dichlorobenzene & 2.1 & $J B$ & 1.1 & 10 \\
\hline n-Butylbenzene & 1.4 & $J B$ & 0.90 & 10 \\
\hline 1,2-Dibromo-3-Chloropropane & 10 & $\mathrm{U}$ & 2.1 & 10 \\
\hline 1,2,4-Trichlorobenzene & 4.5 & $J B$ & 0.91 & 10 \\
\hline Hexachlorobutadiene & 2.3 & $J * B$ & 2.3 & 10 \\
\hline Naphthalene & 4.2 & $J * B$ & 1.2 & 10 \\
\hline 1,2,3-Trichlorobenzene & 3.6 & $\mathrm{JB}$ & 1.0 & 10 \\
\hline Surrogate & $\% \operatorname{Rec}$ & Qualifier & \multicolumn{2}{|c|}{ Acceptance Limits } \\
\hline 1,2-Dichloroethane-d4 & 109 & \multicolumn{3}{|c|}{$80-120$} \\
\hline Toluene-d8 & 108 & \multicolumn{3}{|c|}{$80-120$} \\
\hline Bromofluorobenzene & 111 & \multicolumn{3}{|c|}{$80-125$} \\
\hline Dibromofluoromethane (Surr) & 101 & \multicolumn{3}{|c|}{$50-150$} \\
\hline
\end{tabular}


Analytical Data

Client: Argonne National Laboratory

Job Number: 200-28914-1

Sdg Number: 200-28914

Client Sample ID: $\quad$ YK-S-37768

Lab Sample ID: 200-28914-2

Date Sampled: 07/16/2015 0000

Client Matrix:

Solid

Date Received: 07/17/2015 1010

\section{B Volatile Organic Compounds (GC/MS)}

$\begin{array}{lll}\text { Analysis Method: } & 8260 \mathrm{~B} & \\ \text { Prep Method: } & 5035 & \\ \text { Dilution: } & 1.0 & \\ \text { Analysis Date: } & 07 / 30 / 2015 & 1315 \\ \text { Prep Date: } & 07 / 23 / 2015 & 1500\end{array}$

Analysis Batch: 200-92051

Prep Batch: $\quad$ 200-91708
Instrument ID:

Lab File ID:

Initial Weight/Volume:

Final Weight/Volume:
CHL.i

15018_07.D

$9.569 \mathrm{~g}$

$10 \mathrm{~mL}$

\begin{tabular}{|c|c|c|c|c|c|}
\hline Analyte & DryWt Corrected: N & Result (ug/Kg) & Qualifier & MDL & RL \\
\hline Dichlorodifluoromethane & & 10 & U & 1.8 & 10 \\
\hline Chloromethane & & 4.0 & $\mathrm{JB}$ & 2.2 & 10 \\
\hline Vinyl chloride & & 10 & $\mathrm{U}$ & 0.87 & 10 \\
\hline Bromomethane & & 17 & B & 1.0 & 10 \\
\hline Chloroethane & & 10 & $U^{*}$ & 2.6 & 10 \\
\hline Trichlorofluoromethane & & 10 & $\mathrm{U}$ & 0.86 & 10 \\
\hline 1,1-Dichloroethene & & 10 & $U^{*}$ & 1.1 & 10 \\
\hline Freon TF & & 10 & $U^{*}$ & 0.89 & 10 \\
\hline Acetone & & 52 & $\mathrm{U}$ & 13 & 52 \\
\hline Methyl iodide & & 9.2 & $J * B$ & 0.78 & 10 \\
\hline Carbon disulfide & & 1.1 & $\mathrm{~J} *$ & 0.91 & 10 \\
\hline Methyl acetate & & 52 & $U^{*}$ & 9.6 & 52 \\
\hline Methylene Chloride & & 5.6 & $\mathrm{~J}$ & 2.4 & 10 \\
\hline trans-1,2-Dichloroethene & & 10 & $\mathrm{U}$ & 1.0 & 10 \\
\hline Methyl t-butyl ether & & 10 & $\mathrm{U}$ & 1.1 & 10 \\
\hline 1,1-Dichloroethane & & 10 & $\mathrm{U}$ & 1.1 & 10 \\
\hline Vinyl acetate & & 52 & U & 4.7 & 52 \\
\hline 2,2-Dichloropropane & & 10 & $U^{*}$ & 2.3 & 10 \\
\hline cis-1,2-Dichloroethene & & 10 & $\mathrm{U}$ & 1.0 & 10 \\
\hline 2-Butanone & & 52 & $\mathrm{U}$ & 6.2 & 52 \\
\hline Bromochloromethane & & 10 & U & 1.5 & 10 \\
\hline Tetrahydrofuran & & 21 & $U^{*}$ & 5.5 & 21 \\
\hline Chloroform & & 10 & $\mathrm{U}$ & 1.3 & 10 \\
\hline 1,1,1-Trichloroethane & & 10 & $\mathrm{U}$ & 0.89 & 10 \\
\hline Cyclohexane & & 10 & $U$ & 1.0 & 10 \\
\hline 1,1-Dichloropropene & & 10 & $\mathrm{U}$ & 1.0 & 10 \\
\hline Carbon tetrachloride & & 6.5 & $\mathrm{~J}$ & 2.1 & 10 \\
\hline Isobutyl alcohol & & 520 & $U^{*}$ & 28 & 520 \\
\hline Benzene & & 10 & $\mathrm{U}$ & 1.1 & 10 \\
\hline 1,2-Dichloroethane & & 10 & $\mathrm{U}$ & 1.4 & 10 \\
\hline Trichloroethene & & 10 & $\mathrm{U}$ & 0.87 & 10 \\
\hline Methylcyclohexane & & 10 & $\mathrm{U}$ & 2.2 & 10 \\
\hline 1,2-Dichloropropane & & 10 & $\mathrm{U}$ & 1.3 & 10 \\
\hline Dibromomethane & & 10 & U & 1.6 & 10 \\
\hline 1,4-Dioxane & & 420 & $\mathrm{U}$ & 68 & 420 \\
\hline Bromodichloromethane & & 10 & $\mathrm{U}$ & 1.0 & 10 \\
\hline 2-Chloroethyl vinyl ether & & 10 & $\mathrm{U}$ & 2.0 & 10 \\
\hline cis-1,3-Dichloropropene & & 10 & $\mathrm{U}$ & 1.3 & 10 \\
\hline 4-Methyl-2-pentanone & & 52 & $U^{*}$ & 4.9 & 52 \\
\hline Toluene & & 10 & $\mathrm{U}$ & 2.2 & 10 \\
\hline trans-1,3-Dichloropropene & & 10 & $\mathrm{U}$ & 2.3 & 10 \\
\hline 1,1,2-Trichloroethane & & 10 & $\mathrm{U}$ & 1.4 & 10 \\
\hline Tetrachloroethene & & 10 & $U$ & 2.1 & 10 \\
\hline 1,3-Dichloropropane & & 10 & U & 1.1 & 10 \\
\hline 2-Hexanone & & 52 & U & 6.4 & 52 \\
\hline Dibromochloromethane & & 10 & $\mathrm{U}$ & 1.1 & 10 \\
\hline
\end{tabular}


Analytical Data

Client: Argonne National Laboratory

Job Number: 200-28914-1

Sdg Number: 200-28914

Client Sample ID: $\quad$ YK-S-37768

Lab Sample ID: 200-28914-2

Date Sampled: 07/16/2015 0000

Client Matrix:

Solid

Date Received: 07/17/2015 1010

\section{B Volatile Organic Compounds (GC/MS)}

$\begin{array}{llllll}\text { Analysis Method: } & 8260 \mathrm{~B} & \text { Analysis Batch: } & 200-92051 & \text { Instrument ID: } & \text { CHL.i } \\ \text { Prep Method: } & 5035 & \text { Prep Batch: } & 200-91708 & \text { Lab File ID: } & 15018 \text { 07.D } \\ \text { Dilution: } & 1.0 & & & \text { Initial Weight/Volume: } & 9.569 \mathrm{~g} \\ \text { Analysis Date: } & 07 / 30 / 20151315 & & & \text { Final Weight/Volume: } 10 \mathrm{~mL} \\ \text { Prep Date: } & 07 / 23 / 20151500 & & & \end{array}$

Prep Date: $\quad$ 07/23/2015 1500

\begin{tabular}{|c|c|c|c|c|}
\hline DryWt Corrected: N & Result (ug/Kg) & Qualifier & MDL & $\mathrm{RL}$ \\
\hline 1,2-Dibromoethane & 10 & U & 1.3 & 10 \\
\hline Chlorobenzene & 10 & $\mathrm{U}$ & 0.95 & 10 \\
\hline 1,1,1,2-Tetrachloroethane & 10 & U & 1.1 & 10 \\
\hline Ethylbenzene & 10 & $\mathrm{U}$ & 0.98 & 10 \\
\hline m\&p-Xylene & 10 & U & 1.1 & 10 \\
\hline o-Xylene & 10 & $\mathrm{U}$ & 1.0 & 10 \\
\hline Styrene & 10 & $\mathrm{U}$ & 1.0 & 10 \\
\hline Bromoform & 10 & $\mathrm{U}$ & 1.0 & 10 \\
\hline Isopropylbenzene & 10 & U & 2.1 & 10 \\
\hline Bromobenzene & 10 & $\mathrm{U}$ & 1.0 & 10 \\
\hline 1,1,2,2-Tetrachloroethane & 10 & U & 1.4 & 10 \\
\hline 1,2,3-Trichloropropane & 10 & $\mathrm{U}$ & 1.3 & 10 \\
\hline n-Propylbenzene & 10 & U & 1.1 & 10 \\
\hline 2-Chlorotoluene & 10 & $\mathrm{U}$ & 1.1 & 10 \\
\hline 4-Chlorotoluene & 10 & U & 1.0 & 10 \\
\hline 1,3,5-Trimethylbenzene & 10 & $\mathrm{U}$ & 2.1 & 10 \\
\hline tert-Butylbenzene & 10 & U & 1.5 & 10 \\
\hline 1,2,4-Trimethylbenzene & 10 & U & 1.0 & 10 \\
\hline sec-Butylbenzene & 10 & U & 2.1 & 10 \\
\hline 1,3-Dichlorobenzene & 10 & U & 0.99 & 10 \\
\hline 4-Isopropyltoluene & 10 & U & 0.92 & 10 \\
\hline 1,4-Dichlorobenzene & 10 & U & 1.5 & 10 \\
\hline 1,2-Dichlorobenzene & 10 & U & 1.1 & 10 \\
\hline n-Butylbenzene & 10 & U & 0.94 & 10 \\
\hline 1,2-Dibromo-3-Chloropropane & 10 & U & 2.2 & 10 \\
\hline 1,2,4-Trichlorobenzene & 1.5 & $J B$ & 0.95 & 10 \\
\hline Hexachlorobutadiene & 10 & $U^{*}$ & 2.4 & 10 \\
\hline Naphthalene & 2.2 & $J * B$ & 1.3 & 10 \\
\hline 1,2,3-Trichlorobenzene & 1.3 & J B & 1.0 & 10 \\
\hline Surrogate & $\% \operatorname{Rec}$ & Qualifier & \multicolumn{2}{|c|}{ Acceptance Limits } \\
\hline 1,2-Dichloroethane-d4 & 106 & \multicolumn{3}{|c|}{$80-120$} \\
\hline Toluene-d8 & 111 & \multicolumn{3}{|c|}{$80-120$} \\
\hline Bromofluorobenzene & 114 & \multicolumn{3}{|c|}{$80-125$} \\
\hline Dibromofluoromethane (Surr) & 99 & \multicolumn{3}{|c|}{$50-150$} \\
\hline
\end{tabular}


Analytical Data

Client: Argonne National Laboratory

Job Number: 200-28914-1

Sdg Number: 200-28914

Client Sample ID: YK-S-37767

Lab Sample ID: $\quad$ 200-28914-3

Date Sampled: 07/16/2015 0000

Client Matrix:

Solid

Date Received: 07/17/2015 1010

\section{B Volatile Organic Compounds (GC/MS)}

$\begin{array}{lllll}\text { Analysis Method: } & 8260 \mathrm{~B} & \text { Analysis Batch: } & 200-92051 & \text { Instrument ID: } \\ \text { Prep Method: } & 5035 & \text { Prep Batch: } & \text { 200-91708 } & \text { Lab File ID: } \\ \text { Dilution: } & 1.0 & & & \text { Initial Weight/Volume: } 11.213 \mathrm{~g} \\ \text { Analysis Date: } & 07 / 30 / 20151347 & & \text { Final Weight/Volume: } 10 \mathrm{~mL} \\ \text { Prep Date: } & 07 / 23 / 20151500 & & \end{array}$

\begin{tabular}{|c|c|c|c|c|}
\hline Analyte & DryWt Corrected: N & Qualifier & MDL & $\mathrm{RL}$ \\
\hline Dichlorodifluoromethane & 8.9 & U & 1.5 & 8.9 \\
\hline Chloromethane & 8.9 & $U$ & 1.9 & 8.9 \\
\hline Vinyl chloride & 8.9 & $U$ & 0.74 & 8.9 \\
\hline Bromomethane & 13 & B & 0.89 & 8.9 \\
\hline Chloroethane & 8.9 & $U^{*}$ & 2.2 & 8.9 \\
\hline Trichlorofluoromethane & 8.9 & U & 0.73 & 8.9 \\
\hline 1,1-Dichloroethene & 8.9 & $U$ * & 0.98 & 8.9 \\
\hline Freon TF & 8.9 & $U^{*}$ & 0.76 & 8.9 \\
\hline Acetone & 45 & U & 11 & 45 \\
\hline Methyl iodide & 7.3 & $J * B$ & 0.67 & 8.9 \\
\hline Carbon disulfide & 8.9 & $U$ * & 0.78 & 8.9 \\
\hline Methyl acetate & 45 & $U^{*}$ & 8.2 & 45 \\
\hline Methylene Chloride & 8.9 & U & 2.1 & 8.9 \\
\hline trans-1,2-Dichloroethene & 8.9 & $U$ & 0.88 & 8.9 \\
\hline Methyl t-butyl ether & 8.9 & $U$ & 0.98 & 8.9 \\
\hline 1,1-Dichloroethane & 8.9 & $U$ & 0.98 & 8.9 \\
\hline Vinyl acetate & 45 & $U$ & 4.0 & 45 \\
\hline 2,2-Dichloropropane & 8.9 & $U^{*}$ & 2.0 & 8.9 \\
\hline cis-1,2-Dichloroethene & 8.9 & U & 0.89 & 8.9 \\
\hline 2-Butanone & 45 & $U$ & 5.3 & 45 \\
\hline Bromochloromethane & 8.9 & $U$ & 1.2 & 8.9 \\
\hline Tetrahydrofuran & 18 & $U^{*}$ & 4.7 & 18 \\
\hline Chloroform & 1.8 & J & 1.1 & 8.9 \\
\hline 1,1,1-Trichloroethane & 8.9 & U & 0.76 & 8.9 \\
\hline Cyclohexane & 8.9 & $U$ & 0.89 & 8.9 \\
\hline 1,1-Dichloropropene & 8.9 & $U$ & 0.87 & 8.9 \\
\hline Carbon tetrachloride & 9.1 & & 1.8 & 8.9 \\
\hline Isobutyl alcohol & 450 & $U^{*}$ & 24 & 450 \\
\hline Benzene & 8.9 & U & 0.98 & 8.9 \\
\hline 1,2-Dichloroethane & 8.9 & $U$ & 1.2 & 8.9 \\
\hline Trichloroethene & 8.9 & $U$ & 0.74 & 8.9 \\
\hline Methylcyclohexane & 8.9 & $U$ & 1.9 & 8.9 \\
\hline 1,2-Dichloropropane & 8.9 & $U$ & 1.1 & 8.9 \\
\hline Dibromomethane & 8.9 & $U$ & 1.3 & 8.9 \\
\hline 1,4-Dioxane & 360 & $U$ & 58 & 360 \\
\hline Bromodichloromethane & 8.9 & U & 0.89 & 8.9 \\
\hline 2-Chloroethyl vinyl ether & 8.9 & U & 1.7 & 8.9 \\
\hline cis-1,3-Dichloropropene & 8.9 & U & 1.1 & 8.9 \\
\hline 4-Methyl-2-pentanone & 45 & $U^{*}$ & 4.2 & 45 \\
\hline Toluene & 8.9 & $U$ & 1.9 & 8.9 \\
\hline trans-1,3-Dichloropropene & 8.9 & $U$ & 2.0 & 8.9 \\
\hline 1,1,2-Trichloroethane & 8.9 & $U$ & 1.2 & 8.9 \\
\hline Tetrachloroethene & 8.9 & U & 1.8 & 8.9 \\
\hline 1,3-Dichloropropane & 8.9 & $U$ & 0.98 & 8.9 \\
\hline 2-Hexanone & 45 & $U$ & 5.4 & 45 \\
\hline Dibromochloromethane & 8.9 & $U$ & 0.98 & 8.9 \\
\hline
\end{tabular}


Analytical Data

Client: Argonne National Laboratory

Job Number: 200-28914-1

Sdg Number: 200-28914

Client Sample ID: YK-S-37767

Lab Sample ID: $\quad$ 200-28914-3

Date Sampled: 07/16/2015 0000

Client Matrix:

Solid

Date Received: 07/17/2015 1010

\section{B Volatile Organic Compounds (GC/MS)}

$\begin{array}{llllll}\text { Analysis Method: } & 8260 \mathrm{~B} & \text { Analysis Batch: } & 200-92051 & \text { Instrument ID: } & \text { CHL.i } \\ \text { Prep Method: } & 5035 & \text { Prep Batch: } & 200-91708 & \text { Lab File ID: } \\ \text { Dilution: } & 1.0 & & & \text { Initial Weight/Volume: } 11.213 \mathrm{~g} \\ \text { Analysis Date: } & 07 / 30 / 20151347 & & & \text { Final Weight/Volume: } 10 \mathrm{~mL}\end{array}$

Prep Date: $\quad$ 07/23/2015 1500

\begin{tabular}{|c|c|c|c|c|}
\hline DryWt Corrected: N & Result (ug/Kg) & Qualifier & MDL & $\mathrm{RL}$ \\
\hline 1,2-Dibromoethane & 8.9 & U & 1.1 & 8.9 \\
\hline Chlorobenzene & 8.9 & $U$ & 0.81 & 8.9 \\
\hline 1,1,1,2-Tetrachloroethane & 8.9 & U & 0.98 & 8.9 \\
\hline Ethylbenzene & 8.9 & U & 0.84 & 8.9 \\
\hline m\&p-Xylene & 8.9 & U & 0.98 & 8.9 \\
\hline o-Xylene & 8.9 & $U$ & 0.89 & 8.9 \\
\hline Styrene & 8.9 & $U$ & 0.87 & 8.9 \\
\hline Bromoform & 8.9 & $U$ & 0.89 & 8.9 \\
\hline Isopropylbenzene & 8.9 & $U$ & 1.8 & 8.9 \\
\hline Bromobenzene & 8.9 & U & 0.89 & 8.9 \\
\hline 1,1,2,2-Tetrachloroethane & 8.9 & U & 1.2 & 8.9 \\
\hline 1,2,3-Trichloropropane & 8.9 & $\mathrm{U}$ & 1.1 & 8.9 \\
\hline n-Propylbenzene & 8.9 & $U$ & 0.98 & 8.9 \\
\hline 2-Chlorotoluene & 8.9 & $U$ & 0.98 & 8.9 \\
\hline 4-Chlorotoluene & 8.9 & $U$ & 0.86 & 8.9 \\
\hline 1,3,5-Trimethylbenzene & 8.9 & $U$ & 1.8 & 8.9 \\
\hline tert-Butylbenzene & 8.9 & U & 1.2 & 8.9 \\
\hline 1,2,4-Trimethylbenzene & 8.9 & U & 0.89 & 8.9 \\
\hline sec-Butylbenzene & 8.9 & $U$ & 1.8 & 8.9 \\
\hline 1,3-Dichlorobenzene & 8.9 & $U$ & 0.85 & 8.9 \\
\hline 4-Isopropyltoluene & 8.9 & $U$ & 0.78 & 8.9 \\
\hline 1,4-Dichlorobenzene & 8.9 & U & 1.2 & 8.9 \\
\hline 1,2-Dichlorobenzene & 8.9 & $U$ & 0.98 & 8.9 \\
\hline n-Butylbenzene & 8.9 & U & 0.80 & 8.9 \\
\hline 1,2-Dibromo-3-Chloropropane & 8.9 & $U$ & 1.9 & 8.9 \\
\hline 1,2,4-Trichlorobenzene & 1.2 & $J B$ & 0.81 & 8.9 \\
\hline Hexachlorobutadiene & 8.9 & $U$ * & 2.1 & 8.9 \\
\hline Naphthalene & 1.6 & $J * B$ & 1.1 & 8.9 \\
\hline 1,2,3-Trichlorobenzene & 1.1 & $\mathrm{JB}$ & 0.89 & 8.9 \\
\hline Surrogate & $\% \operatorname{Rec}$ & Qualifier & \multicolumn{2}{|c|}{ Acceptance Limits } \\
\hline 1,2-Dichloroethane-d4 & 107 & \multicolumn{3}{|c|}{$80-120$} \\
\hline Toluene-d8 & 110 & \multicolumn{3}{|c|}{$80-120$} \\
\hline Bromofluorobenzene & 114 & \multicolumn{3}{|c|}{$80-125$} \\
\hline Dibromofluoromethane (Surr) & 102 & \multicolumn{3}{|c|}{$50-150$} \\
\hline
\end{tabular}


Analytical Data

Client: Argonne National Laboratory

Job Number: 200-28914-1

Sdg Number: 200-28914

Client Sample ID: $\quad$ YK-S-37805

Lab Sample ID: $\quad$ 200-28914-4

Date Sampled: 07/16/2015 0000

Client Matrix:

Solid

Date Received: 07/17/2015 1010

\section{B Volatile Organic Compounds (GC/MS)}

$\begin{array}{lll}\text { Analysis Method: } & 8260 \mathrm{~B} & \\ \text { Prep Method: } & 5035 & \\ \text { Dilution: } & 1.0 & \\ \text { Analysis Date: } & 07 / 30 / 2015 & 1420 \\ \text { Prep Date: } & 07 / 23 / 2015 & 1500\end{array}$

$\begin{array}{llll}\text { Analysis Batch: } & 200-92051 & \text { Instrument ID: } & \text { CHL.i } \\ \text { Prep Batch: } & 200-91708 & \text { Lab File ID: } & 15018 \text { 09.D } \\ & & \text { Initial Weight/Volume: } & 10.554 \mathrm{~g} \\ & & \text { Final Weight/Volume: } & 10 \mathrm{~mL}\end{array}$

\begin{tabular}{|c|c|c|c|c|c|}
\hline Analyte & DryWt Corrected: N & Result (ug/Kg) & Qualifier & MDL & $\mathrm{RL}$ \\
\hline Dichlorodifluoromethane & & 9.5 & U & 1.6 & 9.5 \\
\hline Chloromethane & & 5.1 & $\mathrm{JB}$ & 2.0 & 9.5 \\
\hline Vinyl chloride & & 9.5 & $\mathrm{U}$ & 0.79 & 9.5 \\
\hline Bromomethane & & 20 & B & 0.95 & 9.5 \\
\hline Chloroethane & & 9.5 & $U^{*}$ & 2.4 & 9.5 \\
\hline Trichlorofluoromethane & & 9.5 & $\mathrm{U}$ & 0.78 & 9.5 \\
\hline 1,1-Dichloroethene & & 9.5 & $U^{*}$ & 1.0 & 9.5 \\
\hline Freon TF & & 9.5 & $U^{*}$ & 0.81 & 9.5 \\
\hline Acetone & & 47 & U & 11 & 47 \\
\hline Methyl iodide & & 9.1 & $J * B$ & 0.71 & 9.5 \\
\hline Carbon disulfide & & 9.5 & $U^{*}$ & 0.82 & 9.5 \\
\hline Methyl acetate & & 47 & $U^{*}$ & 8.7 & 47 \\
\hline Methylene Chloride & & 9.5 & $\mathrm{U}$ & 2.2 & 9.5 \\
\hline trans-1,2-Dichloroethene & & 9.5 & $\mathrm{U}$ & 0.94 & 9.5 \\
\hline Methyl t-butyl ether & & 9.5 & $U$ & 1.0 & 9.5 \\
\hline 1,1-Dichloroethane & & 9.5 & $\mathrm{U}$ & 1.0 & 9.5 \\
\hline Vinyl acetate & & 47 & $\mathrm{U}$ & 4.3 & 47 \\
\hline 2,2-Dichloropropane & & 9.5 & $U^{*}$ & 2.1 & 9.5 \\
\hline cis-1,2-Dichloroethene & & 9.5 & $\mathrm{U}$ & 0.95 & 9.5 \\
\hline 2-Butanone & & 47 & $\mathrm{U}$ & 5.6 & 47 \\
\hline Bromochloromethane & & 9.5 & $\mathrm{U}$ & 1.3 & 9.5 \\
\hline Tetrahydrofuran & & 19 & $U^{*}$ & 5.0 & 19 \\
\hline Chloroform & & 9.5 & $\mathrm{U}$ & 1.1 & 9.5 \\
\hline 1,1,1-Trichloroethane & & 9.5 & $\mathrm{U}$ & 0.81 & 9.5 \\
\hline Cyclohexane & & 9.5 & $U$ & 0.95 & 9.5 \\
\hline 1,1-Dichloropropene & & 9.5 & $\mathrm{U}$ & 0.92 & 9.5 \\
\hline Carbon tetrachloride & & 9.5 & $\mathrm{U}$ & 1.9 & 9.5 \\
\hline Isobutyl alcohol & & 470 & $U^{*}$ & 26 & 470 \\
\hline Benzene & & 9.5 & $\mathrm{U}$ & 1.0 & 9.5 \\
\hline 1,2-Dichloroethane & & 9.5 & $\mathrm{U}$ & 1.2 & 9.5 \\
\hline Trichloroethene & & 9.5 & $\mathrm{U}$ & 0.79 & 9.5 \\
\hline Methylcyclohexane & & 9.5 & $U$ & 2.0 & 9.5 \\
\hline 1,2-Dichloropropane & & 9.5 & $\mathrm{U}$ & 1.1 & 9.5 \\
\hline Dibromomethane & & 9.5 & $\mathrm{U}$ & 1.4 & 9.5 \\
\hline 1,4-Dioxane & & 380 & $\mathrm{U}$ & 62 & 380 \\
\hline Bromodichloromethane & & 9.5 & $U$ & 0.95 & 9.5 \\
\hline 2-Chloroethyl vinyl ether & & 9.5 & $\mathrm{U}$ & 1.8 & 9.5 \\
\hline cis-1,3-Dichloropropene & & 9.5 & $\mathrm{U}$ & 1.1 & 9.5 \\
\hline 4-Methyl-2-pentanone & & 47 & $U^{*}$ & 4.5 & 47 \\
\hline Toluene & & 9.5 & $\mathrm{U}$ & 2.0 & 9.5 \\
\hline trans-1,3-Dichloropropene & & 9.5 & $\mathrm{U}$ & 2.1 & 9.5 \\
\hline 1,1,2-Trichloroethane & & 9.5 & $\mathrm{U}$ & 1.2 & 9.5 \\
\hline Tetrachloroethene & & 9.5 & $U$ & 1.9 & 9.5 \\
\hline 1,3-Dichloropropane & & 9.5 & $U$ & 1.0 & 9.5 \\
\hline 2-Hexanone & & 47 & $U$ & 5.8 & 47 \\
\hline Dibromochloromethane & & 9.5 & $U$ & 1.0 & 9.5 \\
\hline
\end{tabular}


Analytical Data

Client: Argonne National Laboratory

Job Number: 200-28914-1

Sdg Number: 200-28914

Client Sample ID: $\quad$ YK-S-37805

Lab Sample ID: $\quad$ 200-28914-4

Date Sampled: 07/16/2015 0000

Client Matrix:

Solid

Date Received: 07/17/2015 1010

\section{B Volatile Organic Compounds (GC/MS)}

$\begin{array}{llllll}\text { Analysis Method: } & 8260 \mathrm{~B} & \text { Analysis Batch: } & 200-92051 & \text { Instrument ID: } & \text { CHL.i } \\ \text { Prep Method: } & 5035 & \text { Prep Batch: } & 200-91708 & \text { Lab File ID: } & \text { 15018_09.D } \\ \text { Dilution: } & 1.0 & & & \text { Initial Weight/Volume: } 10.554 \mathrm{~g} \\ \text { Analysis Date: } & 07 / 30 / 20151420 & & & \text { Final Weight/Volume: } 10 \mathrm{~mL}\end{array}$

Prep Date: $\quad$ 07/23/2015 1500

\begin{tabular}{|c|c|c|c|c|}
\hline DryWt Corrected: $\mathrm{N}$ & Result (ug/Kg) & Qualifier & MDL & $\mathrm{RL}$ \\
\hline 1,2-Dibromoethane & 9.5 & U & 1.1 & 9.5 \\
\hline Chlorobenzene & 9.5 & U & 0.86 & 9.5 \\
\hline 1,1,1,2-Tetrachloroethane & 9.5 & U & 1.0 & 9.5 \\
\hline Ethylbenzene & 9.5 & $\mathrm{U}$ & 0.89 & 9.5 \\
\hline m\&p-Xylene & 9.5 & U & 1.0 & 9.5 \\
\hline o-Xylene & 9.5 & $\mathrm{U}$ & 0.95 & 9.5 \\
\hline Styrene & 9.5 & $\mathrm{U}$ & 0.93 & 9.5 \\
\hline Bromoform & 9.5 & $\mathrm{U}$ & 0.95 & 9.5 \\
\hline Isopropylbenzene & 9.5 & $\mathrm{U}$ & 1.9 & 9.5 \\
\hline Bromobenzene & 9.5 & $\mathrm{u}$ & 0.95 & 9.5 \\
\hline 1,1,2,2-Tetrachloroethane & 9.5 & U & 1.2 & 9.5 \\
\hline 1,2,3-Trichloropropane & 9.5 & $\mathrm{U}$ & 1.1 & 9.5 \\
\hline n-Propylbenzene & 9.5 & U & 1.0 & 9.5 \\
\hline 2-Chlorotoluene & 9.5 & $\mathrm{U}$ & 1.0 & 9.5 \\
\hline 4-Chlorotoluene & 9.5 & U & 0.91 & 9.5 \\
\hline 1,3,5-Trimethylbenzene & 9.5 & $\mathrm{u}$ & 1.9 & 9.5 \\
\hline tert-Butylbenzene & 9.5 & U & 1.3 & 9.5 \\
\hline 1,2,4-Trimethylbenzene & 9.5 & U & 0.95 & 9.5 \\
\hline sec-Butylbenzene & 9.5 & U & 1.9 & 9.5 \\
\hline 1,3-Dichlorobenzene & 9.5 & U & 0.90 & 9.5 \\
\hline 4-Isopropyltoluene & 9.5 & U & 0.83 & 9.5 \\
\hline 1,4-Dichlorobenzene & 9.5 & U & 1.3 & 9.5 \\
\hline 1,2-Dichlorobenzene & 9.5 & U & 1.0 & 9.5 \\
\hline n-Butylbenzene & 9.5 & $\mathrm{U}$ & 0.85 & 9.5 \\
\hline 1,2-Dibromo-3-Chloropropane & 9.5 & U & 2.0 & 9.5 \\
\hline 1,2,4-Trichlorobenzene & 1.2 & $J B$ & 0.86 & 9.5 \\
\hline Hexachlorobutadiene & 9.5 & $U$ * & 2.2 & 9.5 \\
\hline Naphthalene & 1.5 & $J * B$ & 1.1 & 9.5 \\
\hline 1,2,3-Trichlorobenzene & 9.5 & U & 0.95 & 9.5 \\
\hline Surrogate & $\% \operatorname{Rec}$ & Qualifier & \multicolumn{2}{|c|}{ Acceptance Limits } \\
\hline 1,2-Dichloroethane-d4 & 109 & \multicolumn{3}{|c|}{$80-120$} \\
\hline Toluene-d8 & 110 & \multicolumn{3}{|c|}{$80-120$} \\
\hline Bromofluorobenzene & 113 & \multicolumn{3}{|c|}{$80-125$} \\
\hline Dibromofluoromethane (Surr) & 102 & \multicolumn{3}{|c|}{$50-150$} \\
\hline
\end{tabular}


Analytical Data

Client: Argonne National Laboratory

Job Number: 200-28914-1

Sdg Number: 200-28914

Client Sample ID: $\quad$ YK-S-37791

Lab Sample ID: $\quad$ 200-28914-5

Date Sampled: 07/16/2015 0000

Client Matrix:

Solid

Date Received: 07/17/2015 1010

\section{B Volatile Organic Compounds (GC/MS)}

$\begin{array}{llllll}\text { Analysis Method: } & 8260 \mathrm{~B} & \text { Analysis Batch: } & 200-92051 & \text { Instrument ID: } & \text { CHL.i } \\ \text { Prep Method: } & 5035 & \text { Prep Batch: } & 200-91708 & \text { Lab File ID: } & 15018 \text { 10.D } \\ \text { Dilution: } & 1.0 & & & \text { Initial Weight/Volume: } & 10.51 \mathrm{~g} \\ \text { Analysis Date: } & 07 / 30 / 20151452 & & & \text { Final Weight/Volume: } 10 \mathrm{~mL} \\ \text { Prep Date: } & 07 / 23 / 20151500 & & & \end{array}$

Prep Date: $\quad$ 07/23/2015 1500

\begin{tabular}{|c|c|c|c|c|c|}
\hline Analyte & DryWt Corrected: $\mathrm{N}$ & Result (ug/Kg) & Qualifier & MDL & RL \\
\hline Dichlorodifluoromethane & & 9.5 & U & 1.6 & 9.5 \\
\hline Chloromethane & & 3.0 & $\mathrm{JB}$ & 2.0 & 9.5 \\
\hline Vinyl chloride & & 9.5 & $\mathrm{U}$ & 0.79 & 9.5 \\
\hline Bromomethane & & 15 & B & 0.95 & 9.5 \\
\hline Chloroethane & & 9.5 & $U^{*}$ & 2.4 & 9.5 \\
\hline Trichlorofluoromethane & & 9.5 & $\mathrm{U}$ & 0.78 & 9.5 \\
\hline 1,1-Dichloroethene & & 9.5 & $U$ * & 1.0 & 9.5 \\
\hline Freon TF & & 9.5 & $U$ * & 0.81 & 9.5 \\
\hline Acetone & & 48 & $\mathrm{U}$ & 11 & 48 \\
\hline Methyl iodide & & 7.7 & $J * B$ & 0.71 & 9.5 \\
\hline Carbon disulfide & & 9.5 & $U^{*}$ & 0.83 & 9.5 \\
\hline Methyl acetate & & 48 & $U$ * & 8.8 & 48 \\
\hline Methylene Chloride & & 9.5 & $\mathrm{U}$ & 2.2 & 9.5 \\
\hline trans-1,2-Dichloroethene & & 9.5 & U & 0.94 & 9.5 \\
\hline Methyl t-butyl ether & & 9.5 & $u$ & 1.0 & 9.5 \\
\hline 1,1-Dichloroethane & & 9.5 & U & 1.0 & 9.5 \\
\hline Vinyl acetate & & 48 & $\mathrm{U}$ & 4.3 & 48 \\
\hline 2,2-Dichloropropane & & 9.5 & $U$ * & 2.1 & 9.5 \\
\hline cis-1,2-Dichloroethene & & 9.5 & $\mathrm{U}$ & 0.95 & 9.5 \\
\hline 2-Butanone & & 48 & U & 5.6 & 48 \\
\hline Bromochloromethane & & 9.5 & $\mathrm{U}$ & 1.3 & 9.5 \\
\hline Tetrahydrofuran & & 19 & $U$ * & 5.0 & 19 \\
\hline Chloroform & & 6.7 & $\mathrm{~J}$ & 1.1 & 9.5 \\
\hline 1,1,1-Trichloroethane & & 9.5 & U & 0.81 & 9.5 \\
\hline Cyclohexane & & 9.5 & U & 0.95 & 9.5 \\
\hline 1,1-Dichloropropene & & 9.5 & $\mathrm{U}$ & 0.92 & 9.5 \\
\hline Carbon tetrachloride & & 44 & & 1.9 & 9.5 \\
\hline Isobutyl alcohol & & 480 & $U$ * & 26 & 480 \\
\hline Benzene & & 9.5 & $\mathrm{U}$ & 1.0 & 9.5 \\
\hline 1,2-Dichloroethane & & 9.5 & $\mathrm{U}$ & 1.2 & 9.5 \\
\hline Trichloroethene & & 9.5 & U & 0.79 & 9.5 \\
\hline Methylcyclohexane & & 9.5 & $\mathrm{U}$ & 2.0 & 9.5 \\
\hline 1,2-Dichloropropane & & 9.5 & U & 1.1 & 9.5 \\
\hline Dibromomethane & & 9.5 & $\mathrm{U}$ & 1.4 & 9.5 \\
\hline 1,4-Dioxane & & 380 & U & 62 & 380 \\
\hline Bromodichloromethane & & 9.5 & $\mathrm{U}$ & 0.95 & 9.5 \\
\hline 2-Chloroethyl vinyl ether & & 9.5 & $\mathrm{U}$ & 1.8 & 9.5 \\
\hline cis-1,3-Dichloropropene & & 9.5 & U & 1.1 & 9.5 \\
\hline 4-Methyl-2-pentanone & & 48 & $U^{*}$ & 4.5 & 48 \\
\hline Toluene & & 9.5 & U & 2.0 & 9.5 \\
\hline trans-1,3-Dichloropropene & & 9.5 & U & 2.1 & 9.5 \\
\hline 1,1,2-Trichloroethane & & 9.5 & $\mathrm{U}$ & 1.2 & 9.5 \\
\hline Tetrachloroethene & & 9.5 & $u$ & 1.9 & 9.5 \\
\hline 1,3-Dichloropropane & & 9.5 & $\mathrm{U}$ & 1.0 & 9.5 \\
\hline 2-Hexanone & & 48 & U & 5.8 & 48 \\
\hline Dibromochloromethane & & 9.5 & U & 1.0 & 9.5 \\
\hline
\end{tabular}


Analytical Data

Client: Argonne National Laboratory

Job Number: 200-28914-1

Sdg Number: 200-28914

Client Sample ID: $\quad$ YK-S-37791

Lab Sample ID: $\quad$ 200-28914-5

Date Sampled: 07/16/2015 0000

Client Matrix:

Solid

Date Received: 07/17/2015 1010

\section{B Volatile Organic Compounds (GC/MS)}

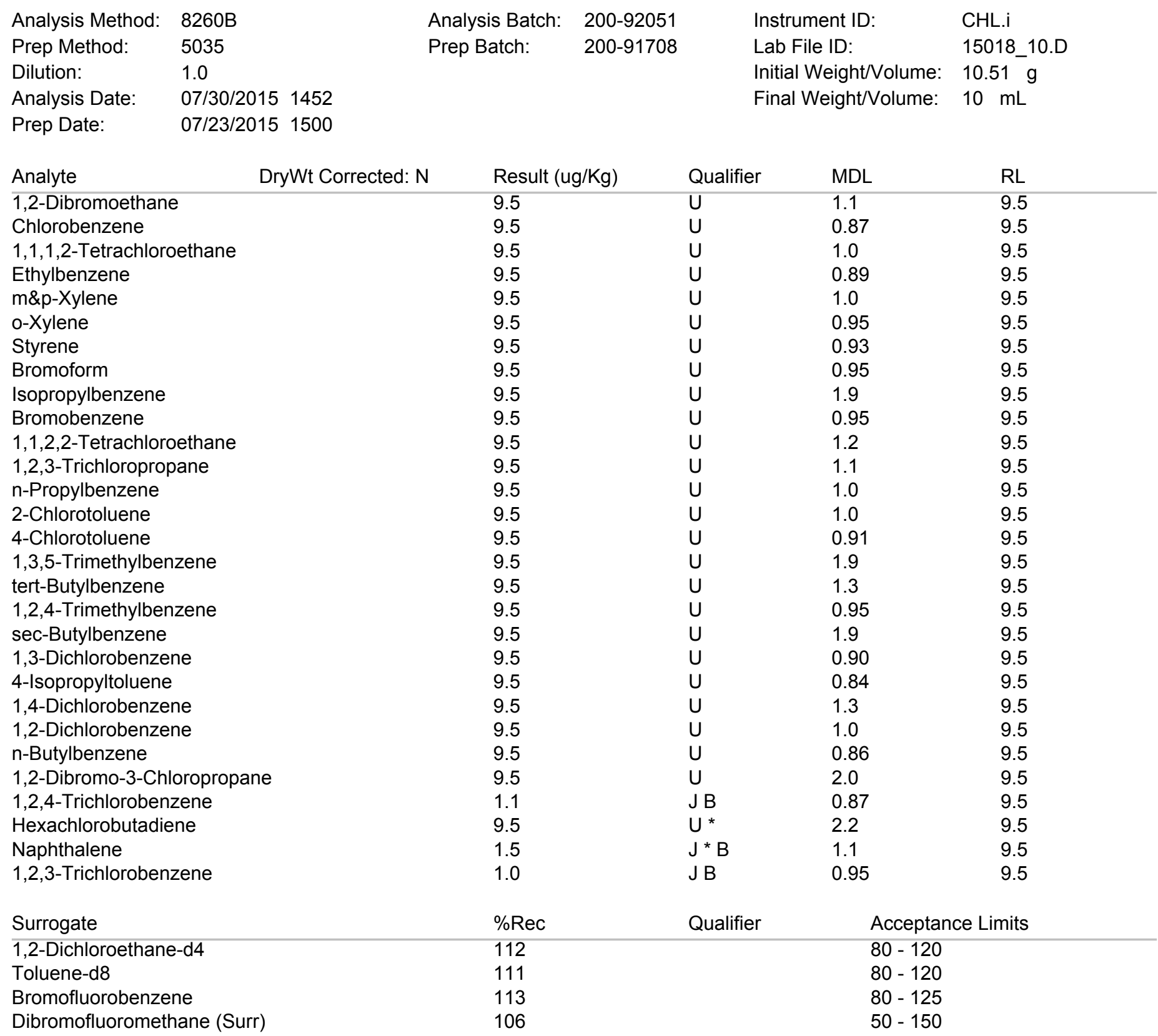


Analytical Data

Client: Argonne National Laboratory

Job Number: 200-28914-1

Sdg Number: 200-28914

Client Sample ID: YK-S-37826

Lab Sample ID: 200-28914-6

Date Sampled: 07/16/2015 0000

Client Matrix:

Solid

Date Received: 07/17/2015 1010

\section{B Volatile Organic Compounds (GC/MS)}

$\begin{array}{lllll}\text { Analysis Method: } & 8260 \mathrm{~B} & \text { Analysis Batch: } & 200-92051 & \text { Instrument ID: } \\ \text { Prep Method: } & 5035 & \text { Prep Batch: } & 200-91708 & \text { Lab File ID: } \\ \text { Dilution: } & 1.0 & & & \text { Initial Weight/Volume: 10.961 g } \\ \text { Analysis Date: } & 07 / 30 / 20151525 & & & \text { Final Weight/Volume: } 10 \mathrm{~mL}\end{array}$

Prep Date: $\quad$ 07/23/2015 1500

\begin{tabular}{|c|c|c|c|c|}
\hline Analyte & DryWt Corrected: N & Qualifier & MDL & $\mathrm{RL}$ \\
\hline Dichlorodifluoromethane & 9.1 & U & 1.6 & 9.1 \\
\hline Chloromethane & 2.1 & $J B$ & 1.9 & 9.1 \\
\hline Vinyl chloride & 9.1 & $U$ & 0.76 & 9.1 \\
\hline Bromomethane & 14 & $\mathrm{~B}$ & 0.91 & 9.1 \\
\hline Chloroethane & 9.1 & $U^{*}$ & 2.3 & 9.1 \\
\hline Trichlorofluoromethane & 9.1 & $U$ & 0.75 & 9.1 \\
\hline 1,1-Dichloroethene & 9.1 & $U$ * & 1.0 & 9.1 \\
\hline Freon TF & 9.1 & $U^{*}$ & 0.78 & 9.1 \\
\hline Acetone & 46 & U & 11 & 46 \\
\hline Methyl iodide & 6.8 & $J * B$ & 0.68 & 9.1 \\
\hline Carbon disulfide & 9.1 & $U^{*}$ & 0.79 & 9.1 \\
\hline Methyl acetate & 46 & $U^{*}$ & 8.4 & 46 \\
\hline Methylene Chloride & 9.1 & $U$ & 2.1 & 9.1 \\
\hline trans-1,2-Dichloroethene & 9.1 & $U$ & 0.90 & 9.1 \\
\hline Methyl t-butyl ether & 9.1 & U & 1.0 & 9.1 \\
\hline 1,1-Dichloroethane & 9.1 & U & 1.0 & 9.1 \\
\hline Vinyl acetate & 46 & $U$ & 4.1 & 46 \\
\hline 2,2-Dichloropropane & 9.1 & $U^{*}$ & 2.0 & 9.1 \\
\hline cis-1,2-Dichloroethene & 9.1 & $U$ & 0.91 & 9.1 \\
\hline 2-Butanone & 46 & $U$ & 5.4 & 46 \\
\hline Bromochloromethane & 9.1 & $U$ & 1.3 & 9.1 \\
\hline Tetrahydrofuran & 18 & $U^{*}$ & 4.8 & 18 \\
\hline Chloroform & 9.1 & $U$ & 1.1 & 9.1 \\
\hline 1,1,1-Trichloroethane & 6.0 & $\mathrm{~J}$ & 0.78 & 9.1 \\
\hline Cyclohexane & 9.1 & $\mathrm{U}$ & 0.91 & 9.1 \\
\hline 1,1-Dichloropropene & 9.1 & $\mathrm{U}$ & 0.88 & 9.1 \\
\hline Carbon tetrachloride & 9.1 & $U$ & 1.8 & 9.1 \\
\hline Isobutyl alcohol & 460 & $U^{*}$ & 25 & 460 \\
\hline Benzene & 9.1 & $U$ & 1.0 & 9.1 \\
\hline 1,2-Dichloroethane & 9.1 & U & 1.2 & 9.1 \\
\hline Trichloroethene & 9.1 & U & 0.76 & 9.1 \\
\hline Methylcyclohexane & 9.1 & U & 1.9 & 9.1 \\
\hline 1,2-Dichloropropane & 9.1 & U & 1.1 & 9.1 \\
\hline Dibromomethane & 9.1 & U & 1.4 & 9.1 \\
\hline 1,4-Dioxane & 360 & U & 59 & 360 \\
\hline Bromodichloromethane & 9.1 & U & 0.91 & 9.1 \\
\hline 2-Chloroethyl vinyl ether & 9.1 & U & 1.7 & 9.1 \\
\hline cis-1,3-Dichloropropene & 9.1 & $\cup$ & 1.1 & 9.1 \\
\hline 4-Methyl-2-pentanone & 46 & $U^{*}$ & 4.3 & 46 \\
\hline Toluene & 9.1 & $U$ & 1.9 & 9.1 \\
\hline trans-1,3-Dichloropropene & 9.1 & U & 2.0 & 9.1 \\
\hline $1,1,2$-Trichloroethane & 9.1 & U & 1.2 & 9.1 \\
\hline Tetrachloroethene & 9.1 & U & 1.8 & 9.1 \\
\hline 1,3-Dichloropropane & 9.1 & U & 1.0 & 9.1 \\
\hline 2-Hexanone & 46 & U & 5.6 & 46 \\
\hline Dibromochloromethane & 9.1 & U & 1.0 & 9.1 \\
\hline
\end{tabular}


Analytical Data

Client: Argonne National Laboratory

Job Number: 200-28914-1

Sdg Number: 200-28914

Client Sample ID: $\quad$ YK-S-37826

Lab Sample ID: 200-28914-6

Date Sampled: 07/16/2015 0000

Client Matrix:

Solid

Date Received: 07/17/2015 1010

\section{B Volatile Organic Compounds (GC/MS)}

$\begin{array}{llllll}\text { Analysis Method: } & 8260 \mathrm{~B} & \text { Analysis Batch: } & 200-92051 & \text { Instrument ID: } & \text { CHL.i } \\ \text { Prep Method: } & 5035 & \text { Prep Batch: } & 200-91708 & \text { Lab File ID: } & 15018 \text { 11.D } \\ \text { Dilution: } & 1.0 & & & \text { Initial Weight/Volume: } 10.961 \mathrm{~g} \\ \text { Analysis Date: } & 07 / 30 / 20151525 & & & \text { Final Weight/Volume: } 10 \mathrm{~mL}\end{array}$

Prep Date: $\quad$ 07/23/2015 1500

\begin{tabular}{|c|c|c|c|c|}
\hline DryWt Corrected: $\mathrm{N}$ & Result (ug/Kg) & Qualifier & MDL & $\mathrm{RL}$ \\
\hline 1,2-Dibromoethane & 9.1 & U & 1.1 & 9.1 \\
\hline Chlorobenzene & 9.1 & U & 0.83 & 9.1 \\
\hline 1,1,1,2-Tetrachloroethane & 9.1 & U & 1.0 & 9.1 \\
\hline Ethylbenzene & 9.1 & $\mathrm{U}$ & 0.86 & 9.1 \\
\hline m\&p-Xylene & 9.1 & $\mathrm{U}$ & 1.0 & 9.1 \\
\hline o-Xylene & 9.1 & $\mathrm{U}$ & 0.91 & 9.1 \\
\hline Styrene & 9.1 & $\mathrm{U}$ & 0.89 & 9.1 \\
\hline Bromoform & 9.1 & $\mathrm{U}$ & 0.91 & 9.1 \\
\hline Isopropylbenzene & 9.1 & $\mathrm{U}$ & 1.8 & 9.1 \\
\hline Bromobenzene & 9.1 & $\mathrm{u}$ & 0.91 & 9.1 \\
\hline 1,1,2,2-Tetrachloroethane & 9.1 & U & 1.2 & 9.1 \\
\hline 1,2,3-Trichloropropane & 9.1 & $\mathrm{U}$ & 1.1 & 9.1 \\
\hline n-Propylbenzene & 9.1 & U & 1.0 & 9.1 \\
\hline 2-Chlorotoluene & 9.1 & $\mathrm{U}$ & 1.0 & 9.1 \\
\hline 4-Chlorotoluene & 9.1 & U & 0.88 & 9.1 \\
\hline 1,3,5-Trimethylbenzene & 9.1 & $\mathrm{u}$ & 1.8 & 9.1 \\
\hline tert-Butylbenzene & 9.1 & U & 1.3 & 9.1 \\
\hline 1,2,4-Trimethylbenzene & 9.1 & $\mathrm{U}$ & 0.91 & 9.1 \\
\hline sec-Butylbenzene & 9.1 & U & 1.8 & 9.1 \\
\hline 1,3-Dichlorobenzene & 9.1 & U & 0.87 & 9.1 \\
\hline 4-Isopropyltoluene & 9.1 & U & 0.80 & 9.1 \\
\hline 1,4-Dichlorobenzene & 9.1 & U & 1.3 & 9.1 \\
\hline 1,2-Dichlorobenzene & 9.1 & U & 1.0 & 9.1 \\
\hline n-Butylbenzene & 9.1 & $\mathrm{U}$ & 0.82 & 9.1 \\
\hline 1,2-Dibromo-3-Chloropropane & 9.1 & U & 1.9 & 9.1 \\
\hline 1,2,4-Trichlorobenzene & 0.99 & $J B$ & 0.83 & 9.1 \\
\hline Hexachlorobutadiene & 9.1 & $U$ * & 2.1 & 9.1 \\
\hline Naphthalene & 1.2 & $J * B$ & 1.1 & 9.1 \\
\hline 1,2,3-Trichlorobenzene & 9.1 & U & 0.91 & 9.1 \\
\hline Surrogate & $\% \operatorname{Rec}$ & Qualifier & \multicolumn{2}{|c|}{ Acceptance Limits } \\
\hline 1,2-Dichloroethane-d4 & 103 & \multicolumn{3}{|c|}{$80-120$} \\
\hline Toluene-d8 & 109 & \multicolumn{3}{|c|}{$80-120$} \\
\hline Bromofluorobenzene & 111 & \multicolumn{3}{|c|}{$80-125$} \\
\hline Dibromofluoromethane (Surr) & 97 & \multicolumn{3}{|c|}{$50-150$} \\
\hline
\end{tabular}


Analytical Data

Client: Argonne National Laboratory

Job Number: 200-28914-1

Sdg Number: 200-28914

Client Sample ID: $\quad$ YK-S-37814

Lab Sample ID: $\quad$ 200-28914-7

Date Sampled: 07/16/2015 0000

Client Matrix:

Solid

Date Received: 07/17/2015 1010

\section{B Volatile Organic Compounds (GC/MS)}

$\begin{array}{llllll}\text { Analysis Method: } & 8260 \mathrm{~B} & \text { Analysis Batch: } & 200-92051 & \text { Instrument ID: } & \text { CHL.i } \\ \text { Prep Method: } & 5035 & \text { Prep Batch: } & 200-91708 & \text { Lab File ID: } & \text { 15018_12.D } \\ \text { Dilution: } & 1.0 & & & \text { Initial Weight/Volume: } & 9.45 \mathrm{~g} \\ \text { Analysis Date: } & 07 / 30 / 20151558 & & & \text { Final Weight/Volume: } 10 \mathrm{~mL}\end{array}$

Prep Date: $\quad$ 07/23/2015 1500

\begin{tabular}{|c|c|c|c|c|c|}
\hline Analyte & DryWt Corrected: N & Result (ug/Kg) & Qualifier & MDL & RL \\
\hline Dichlorodifluoromethane & & 11 & U & 1.8 & 11 \\
\hline Chloromethane & & 2.6 & $\mathrm{JB}$ & 2.2 & 11 \\
\hline Vinyl chloride & & 11 & $\mathrm{U}$ & 0.88 & 11 \\
\hline Bromomethane & & 16 & B & 1.1 & 11 \\
\hline Chloroethane & & 11 & $U^{*}$ & 2.6 & 11 \\
\hline Trichlorofluoromethane & & 11 & $\mathrm{U}$ & 0.87 & 11 \\
\hline 1,1-Dichloroethene & & 11 & $U^{*}$ & 1.2 & 11 \\
\hline Freon TF & & 11 & $U^{*}$ & 0.90 & 11 \\
\hline Acetone & & 53 & U & 13 & 53 \\
\hline Methyl iodide & & 7.8 & $J * B$ & 0.79 & 11 \\
\hline Carbon disulfide & & 11 & $U^{*}$ & 0.92 & 11 \\
\hline Methyl acetate & & 53 & $U^{*}$ & 9.7 & 53 \\
\hline Methylene Chloride & & 7.3 & $\mathrm{~J}$ & 2.4 & 11 \\
\hline trans-1,2-Dichloroethene & & 11 & $\mathrm{U}$ & 1.0 & 11 \\
\hline Methyl t-butyl ether & & 11 & $U$ & 1.2 & 11 \\
\hline 1,1-Dichloroethane & & 11 & $\mathrm{U}$ & 1.2 & 11 \\
\hline Vinyl acetate & & 53 & $\mathrm{U}$ & 4.8 & 53 \\
\hline 2,2-Dichloropropane & & 11 & $U^{*}$ & 2.3 & 11 \\
\hline cis-1,2-Dichloroethene & & 11 & $\mathrm{U}$ & 1.1 & 11 \\
\hline 2-Butanone & & 53 & $\mathrm{U}$ & 6.2 & 53 \\
\hline Bromochloromethane & & 11 & $\mathrm{U}$ & 1.5 & 11 \\
\hline Tetrahydrofuran & & 21 & $U^{*}$ & 5.6 & 21 \\
\hline Chloroform & & 11 & $\mathrm{U}$ & 1.3 & 11 \\
\hline 1,1,1-Trichloroethane & & 11 & $\mathrm{U}$ & 0.90 & 11 \\
\hline Cyclohexane & & 11 & $U$ & 1.1 & 11 \\
\hline 1,1-Dichloropropene & & 11 & U & 1.0 & 11 \\
\hline Carbon tetrachloride & & 3.9 & $\mathrm{~J}$ & 2.1 & 11 \\
\hline Isobutyl alcohol & & 530 & $U^{*}$ & 29 & 530 \\
\hline Benzene & & 11 & $\mathrm{U}$ & 1.2 & 11 \\
\hline 1,2-Dichloroethane & & 11 & $\mathrm{U}$ & 1.4 & 11 \\
\hline Trichloroethene & & 11 & $\mathrm{U}$ & 0.88 & 11 \\
\hline Methylcyclohexane & & 11 & $U$ & 2.2 & 11 \\
\hline 1,2-Dichloropropane & & 11 & $\mathrm{U}$ & 1.3 & 11 \\
\hline Dibromomethane & & 11 & $U$ & 1.6 & 11 \\
\hline 1,4-Dioxane & & 420 & $\mathrm{U}$ & 69 & 420 \\
\hline Bromodichloromethane & & 11 & U & 1.1 & 11 \\
\hline 2-Chloroethyl vinyl ether & & 11 & $\mathrm{U}$ & 2.0 & 11 \\
\hline cis-1,3-Dichloropropene & & 11 & U & 1.3 & 11 \\
\hline 4-Methyl-2-pentanone & & 53 & $U^{*}$ & 5.0 & 53 \\
\hline Toluene & & 11 & U & 2.2 & 11 \\
\hline trans-1,3-Dichloropropene & & 11 & $\mathrm{U}$ & 2.3 & 11 \\
\hline 1,1,2-Trichloroethane & & 11 & U & 1.4 & 11 \\
\hline Tetrachloroethene & & 11 & $U$ & 2.1 & 11 \\
\hline 1,3-Dichloropropane & & 11 & $U$ & 1.2 & 11 \\
\hline 2-Hexanone & & 53 & $\mathrm{U}$ & 6.5 & 53 \\
\hline Dibromochloromethane & & 11 & $U$ & 1.2 & 11 \\
\hline
\end{tabular}


Analytical Data

Client: Argonne National Laboratory

Job Number: 200-28914-1

Sdg Number: 200-28914

Client Sample ID: $\quad$ YK-S-37814

Lab Sample ID: $\quad$ 200-28914-7

Date Sampled: 07/16/2015 0000

Client Matrix:

Solid

Date Received: 07/17/2015 1010

\section{B Volatile Organic Compounds (GC/MS)}

$\begin{array}{llllll}\text { Analysis Method: } & 8260 \mathrm{~B} & \text { Analysis Batch: } & 200-92051 & \text { Instrument ID: } & \text { CHL.i } \\ \text { Prep Method: } & 5035 & \text { Prep Batch: } & 200-91708 & \text { Lab File ID: } & \text { 15018_12.D } \\ \text { Dilution: } & 1.0 & & & \text { Initial Weight/Volume: } & 9.45 \mathrm{~g} \\ \text { Analysis Date: } & 07 / 30 / 20151558 & & & \text { Final Weight/Volume: } 10 \mathrm{~mL}\end{array}$

Prep Date: $\quad$ 07/23/2015 1500

\begin{tabular}{|c|c|c|c|c|}
\hline DryWt Corrected: $\mathrm{N}$ & Result (ug/Kg) & Qualifier & MDL & RL \\
\hline 1,2-Dibromoethane & 11 & U & 1.3 & 11 \\
\hline Chlorobenzene & 11 & U & 0.96 & 11 \\
\hline 1,1,1,2-Tetrachloroethane & 11 & U & 1.2 & 11 \\
\hline Ethylbenzene & 11 & $\mathrm{U}$ & 0.99 & 11 \\
\hline m\&p-Xylene & 11 & U & 1.2 & 11 \\
\hline o-Xylene & 11 & $\mathrm{U}$ & 1.1 & 11 \\
\hline Styrene & 11 & $\mathrm{U}$ & 1.0 & 11 \\
\hline Bromoform & 11 & $\mathrm{U}$ & 1.1 & 11 \\
\hline Isopropylbenzene & 11 & $\mathrm{U}$ & 2.1 & 11 \\
\hline Bromobenzene & 11 & $\mathrm{u}$ & 1.1 & 11 \\
\hline 1,1,2,2-Tetrachloroethane & 11 & U & 1.4 & 11 \\
\hline 1,2,3-Trichloropropane & 11 & $\mathrm{U}$ & 1.3 & 11 \\
\hline n-Propylbenzene & 11 & U & 1.2 & 11 \\
\hline 2-Chlorotoluene & 11 & $\mathrm{U}$ & 1.2 & 11 \\
\hline 4-Chlorotoluene & 11 & $U$ & 1.0 & 11 \\
\hline 1,3,5-Trimethylbenzene & 11 & $\mathrm{u}$ & 2.1 & 11 \\
\hline tert-Butylbenzene & 11 & U & 1.5 & 11 \\
\hline 1,2,4-Trimethylbenzene & 11 & $\mathrm{U}$ & 1.1 & 11 \\
\hline sec-Butylbenzene & 11 & U & 2.1 & 11 \\
\hline 1,3-Dichlorobenzene & 11 & U & 1.0 & 11 \\
\hline 4-Isopropyltoluene & 11 & U & 0.93 & 11 \\
\hline 1,4-Dichlorobenzene & 11 & $\mathrm{U}$ & 1.5 & 11 \\
\hline 1,2-Dichlorobenzene & 11 & $\mathrm{U}$ & 1.2 & 11 \\
\hline n-Butylbenzene & 11 & $\mathrm{U}$ & 0.95 & 11 \\
\hline 1,2-Dibromo-3-Chloropropane & 11 & $\mathrm{U}$ & 2.2 & 11 \\
\hline 1,2,4-Trichlorobenzene & 11 & $\mathrm{U}$ & 0.96 & 11 \\
\hline Hexachlorobutadiene & 11 & $U$ * & 2.4 & 11 \\
\hline Naphthalene & 1.6 & $J * B$ & 1.3 & 11 \\
\hline 1,2,3-Trichlorobenzene & 11 & U & 1.1 & 11 \\
\hline Surrogate & $\% \operatorname{Rec}$ & Qualifier & \multicolumn{2}{|c|}{ Acceptance Limits } \\
\hline 1,2-Dichloroethane-d4 & 107 & \multicolumn{3}{|c|}{$80-120$} \\
\hline Toluene-d8 & 109 & \multicolumn{3}{|c|}{$80-120$} \\
\hline Bromofluorobenzene & 112 & \multicolumn{3}{|c|}{$80-125$} \\
\hline Dibromofluoromethane (Surr) & 101 & \multicolumn{3}{|c|}{$50-150$} \\
\hline
\end{tabular}


Analytical Data

Client: Argonne National Laboratory

Job Number: 200-28914-1

Sdg Number: 200-28914

Client Sample ID: YK-S-37758

Lab Sample ID: 200-28914-8

Date Sampled: 07/16/2015 0000

Client Matrix:

Solid

Date Received: 07/17/2015 1010

\section{B Volatile Organic Compounds (GC/MS)}

$\begin{array}{lll}\text { Analysis Method: } & 8260 \mathrm{~B} \\ \text { Prep Method: } & 5035 \\ \text { Dilution: } & 1.0 & \\ \text { Analysis Date: } & 07 / 30 / 20151630 \\ \text { Prep Date: } & 07 / 23 / 20151500\end{array}$

$\begin{array}{llll}\text { Analysis Batch: } & 200-92051 & \text { Instrument ID: } & \text { CHL.i } \\ \text { Prep Batch: } & 200-91708 & \text { Lab File ID: } & 15018 \_13 . D \\ & & \text { Initial Weight/Volume: } & 10.864 \mathrm{~g} \\ & & \text { Final Weight/Volume: } & 10 \mathrm{~mL}\end{array}$

\begin{tabular}{|c|c|c|c|c|}
\hline Analyte & DryWt Corrected: N & Qualifier & MDL & $\mathrm{RL}$ \\
\hline Dichlorodifluoromethane & 9.2 & U & 1.6 & 9.2 \\
\hline Chloromethane & 2.7 & J B & 1.9 & 9.2 \\
\hline Vinyl chloride & 9.2 & $U$ & 0.76 & 9.2 \\
\hline Bromomethane & 14 & B & 0.92 & 9.2 \\
\hline Chloroethane & 9.2 & $U^{*}$ & 2.3 & 9.2 \\
\hline Trichlorofluoromethane & 9.2 & $U$ & 0.75 & 9.2 \\
\hline 1,1-Dichloroethene & 9.2 & $U^{*}$ & 1.0 & 9.2 \\
\hline Freon TF & 9.2 & $U^{*}$ & 0.78 & 9.2 \\
\hline Acetone & 46 & U & 11 & 46 \\
\hline Methyl iodide & 5.7 & $J * B$ & 0.69 & 9.2 \\
\hline Carbon disulfide & 9.2 & $U$ * & 0.80 & 9.2 \\
\hline Methyl acetate & 46 & $U^{*}$ & 8.5 & 46 \\
\hline Methylene Chloride & 9.2 & U & 2.1 & 9.2 \\
\hline trans-1,2-Dichloroethene & 9.2 & $U$ & 0.91 & 9.2 \\
\hline Methyl t-butyl ether & 9.2 & $U$ & 1.0 & 9.2 \\
\hline 1,1-Dichloroethane & 9.2 & $U$ & 1.0 & 9.2 \\
\hline Vinyl acetate & 46 & U & 4.1 & 46 \\
\hline 2,2-Dichloropropane & 9.2 & $U^{*}$ & 2.0 & 9.2 \\
\hline cis-1,2-Dichloroethene & 9.2 & $U$ & 0.92 & 9.2 \\
\hline 2-Butanone & 46 & $U$ & 5.4 & 46 \\
\hline Bromochloromethane & 9.2 & $U$ & 1.3 & 9.2 \\
\hline Tetrahydrofuran & 18 & $U^{*}$ & 4.9 & 18 \\
\hline Chloroform & 9.2 & $U$ & 1.1 & 9.2 \\
\hline 1,1,1-Trichloroethane & 9.2 & U & 0.78 & 9.2 \\
\hline Cyclohexane & 9.2 & $U$ & 0.92 & 9.2 \\
\hline 1,1-Dichloropropene & 9.2 & $U$ & 0.89 & 9.2 \\
\hline Carbon tetrachloride & 9.2 & $U$ & 1.8 & 9.2 \\
\hline Isobutyl alcohol & 460 & $U^{*}$ & 25 & 460 \\
\hline Benzene & 9.2 & $U$ & 1.0 & 9.2 \\
\hline 1,2-Dichloroethane & 9.2 & U & 1.2 & 9.2 \\
\hline Trichloroethene & 9.2 & U & 0.76 & 9.2 \\
\hline Methylcyclohexane & 9.2 & U & 1.9 & 9.2 \\
\hline 1,2-Dichloropropane & 9.2 & $U$ & 1.1 & 9.2 \\
\hline Dibromomethane & 9.2 & $U$ & 1.4 & 9.2 \\
\hline 1,4-Dioxane & 370 & U & 60 & 370 \\
\hline Bromodichloromethane & 9.2 & U & 0.92 & 9.2 \\
\hline 2-Chloroethyl vinyl ether & 9.2 & U & 1.7 & 9.2 \\
\hline cis-1,3-Dichloropropene & 9.2 & $U$ & 1.1 & 9.2 \\
\hline 4-Methyl-2-pentanone & 46 & $U^{*}$ & 4.3 & 46 \\
\hline Toluene & 9.2 & $U$ & 1.9 & 9.2 \\
\hline trans-1,3-Dichloropropene & 9.2 & U & 2.0 & 9.2 \\
\hline 1,1,2-Trichloroethane & 9.2 & U & 1.2 & 9.2 \\
\hline Tetrachloroethene & 9.2 & U & 1.8 & 9.2 \\
\hline 1,3-Dichloropropane & 9.2 & U & 1.0 & 9.2 \\
\hline 2-Hexanone & 46 & U & 5.6 & 46 \\
\hline Dibromochloromethane & 9.2 & U & 1.0 & 9.2 \\
\hline
\end{tabular}


Analytical Data

Client: Argonne National Laboratory

Job Number: 200-28914-1

Sdg Number: 200-28914

Client Sample ID: YK-S-37758

Lab Sample ID: $\quad$ 200-28914-8

Date Sampled: 07/16/2015 0000

Client Matrix:

Solid

Date Received: 07/17/2015 1010

\section{B Volatile Organic Compounds (GC/MS)}

$\begin{array}{lllll}\text { Analysis Method: } & 8260 \mathrm{~B} & \text { Analysis Batch: } & 200-92051 & \text { Instrument ID: } \\ \text { Prep Method: } & 5035 & \text { Prep Batch: } & 200-91708 & \text { Lab File ID: } \\ \text { Dilution: } & 1.0 & & & \text { Initial Weight/Volume: 10.864 g } \\ \text { Analysis Date: } & 07 / 30 / 20151630 & & & \text { Final Weight/Volume: } 10 \mathrm{~mL}\end{array}$

Prep Date: $\quad$ 07/23/2015 1500

\begin{tabular}{|c|c|c|c|c|}
\hline DryWt Corrected: N & Result (ug/Kg) & Qualifier & MDL & $\mathrm{RL}$ \\
\hline 1,2-Dibromoethane & 9.2 & U & 1.1 & 9.2 \\
\hline Chlorobenzene & 9.2 & $U$ & 0.84 & 9.2 \\
\hline 1,1,1,2-Tetrachloroethane & 9.2 & U & 1.0 & 9.2 \\
\hline Ethylbenzene & 9.2 & U & 0.87 & 9.2 \\
\hline m\&p-Xylene & 9.2 & U & 1.0 & 9.2 \\
\hline o-Xylene & 9.2 & $U$ & 0.92 & 9.2 \\
\hline Styrene & 9.2 & $U$ & 0.90 & 9.2 \\
\hline Bromoform & 9.2 & $U$ & 0.92 & 9.2 \\
\hline Isopropylbenzene & 9.2 & $U$ & 1.8 & 9.2 \\
\hline Bromobenzene & 9.2 & U & 0.92 & 9.2 \\
\hline 1,1,2,2-Tetrachloroethane & 9.2 & U & 1.2 & 9.2 \\
\hline 1,2,3-Trichloropropane & 9.2 & $\mathrm{U}$ & 1.1 & 9.2 \\
\hline n-Propylbenzene & 9.2 & $U$ & 1.0 & 9.2 \\
\hline 2-Chlorotoluene & 9.2 & $U$ & 1.0 & 9.2 \\
\hline 4-Chlorotoluene & 9.2 & $U$ & 0.88 & 9.2 \\
\hline 1,3,5-Trimethylbenzene & 9.2 & $U$ & 1.8 & 9.2 \\
\hline tert-Butylbenzene & 9.2 & U & 1.3 & 9.2 \\
\hline 1,2,4-Trimethylbenzene & 9.2 & U & 0.92 & 9.2 \\
\hline sec-Butylbenzene & 9.2 & $U$ & 1.8 & 9.2 \\
\hline 1,3-Dichlorobenzene & 9.2 & $U$ & 0.87 & 9.2 \\
\hline 4-Isopropyltoluene & 9.2 & $U$ & 0.81 & 9.2 \\
\hline 1,4-Dichlorobenzene & 9.2 & U & 1.3 & 9.2 \\
\hline 1,2-Dichlorobenzene & 9.2 & $U$ & 1.0 & 9.2 \\
\hline n-Butylbenzene & 9.2 & U & 0.83 & 9.2 \\
\hline 1,2-Dibromo-3-Chloropropane & 9.2 & $U$ & 1.9 & 9.2 \\
\hline 1,2,4-Trichlorobenzene & 9.2 & $U$ & 0.84 & 9.2 \\
\hline Hexachlorobutadiene & 9.2 & $U$ * & 2.1 & 9.2 \\
\hline Naphthalene & 1.1 & $J * B$ & 1.1 & 9.2 \\
\hline 1,2,3-Trichlorobenzene & 9.2 & U & 0.92 & 9.2 \\
\hline Surrogate & $\% \operatorname{Rec}$ & Qualifier & \multicolumn{2}{|c|}{ Acceptance Limits } \\
\hline 1,2-Dichloroethane-d4 & 96 & \multicolumn{3}{|c|}{$80-120$} \\
\hline Toluene-d8 & 110 & \multicolumn{3}{|c|}{$80-120$} \\
\hline Bromofluorobenzene & 111 & \multicolumn{3}{|c|}{$80-125$} \\
\hline Dibromofluoromethane (Surr) & 97 & \multicolumn{3}{|c|}{$50-150$} \\
\hline
\end{tabular}


Analytical Data

Client: Argonne National Laboratory

Job Number: 200-28914-1

Sdg Number: 200-28914

Client Sample ID: YK-S-BLANK

Lab Sample ID: $\quad$ 200-28914-9TB

Date Sampled: 07/16/2015 0000

Client Matrix:

Solid

Date Received: 07/17/2015 1010

\section{B Volatile Organic Compounds (GC/MS)}

$\begin{array}{llllll}\text { Analysis Method: } & 8260 \mathrm{~B} & \text { Analysis Batch: } & 200-92051 & \text { Instrument ID: } & \text { CHL.i } \\ \text { Prep Method: } & 5035 & \text { Prep Batch: } & 200-91708 & \text { Lab File ID: } & \text { 15018_14.D } \\ \text { Dilution: } & 1.0 & & & \text { Initial Weight/Volume: } & 10 \mathrm{~g} \\ \text { Analysis Date: } & 07 / 30 / 20151703 & & & \text { Final Weight/Volume: } & 10 \mathrm{~mL} \\ \text { Prep Date: } & 07 / 23 / 20151500 & & & \end{array}$

\begin{tabular}{|c|c|c|c|c|c|}
\hline Analyte & DryWt Corrected: $\mathrm{N}$ & Result (ug/Kg) & Qualifier & MDL & RL \\
\hline Dichlorodifluoromethane & & 10 & U & 1.7 & 10 \\
\hline Chloromethane & & 2.4 & $J B$ & 2.1 & 10 \\
\hline Vinyl chloride & & 10 & $\mathrm{U}$ & 0.83 & 10 \\
\hline Bromomethane & & 17 & B & 1.0 & 10 \\
\hline Chloroethane & & 10 & $U$ * & 2.5 & 10 \\
\hline Trichlorofluoromethane & & 10 & U & 0.82 & 10 \\
\hline 1,1-Dichloroethene & & 10 & $U$ * & 1.1 & 10 \\
\hline Freon TF & & 10 & $U$ * & 0.85 & 10 \\
\hline Acetone & & 50 & $\mathrm{U}$ & 12 & 50 \\
\hline Methyl iodide & & 6.7 & $J * B$ & 0.75 & 10 \\
\hline Carbon disulfide & & 10 & $U^{*}$ & 0.87 & 10 \\
\hline Methyl acetate & & 50 & $U$ * & 9.2 & 50 \\
\hline Methylene Chloride & & 10 & U & 2.3 & 10 \\
\hline trans-1,2-Dichloroethene & & 10 & U & 0.99 & 10 \\
\hline Methyl t-butyl ether & & 10 & U & 1.1 & 10 \\
\hline 1,1-Dichloroethane & & 10 & U & 1.1 & 10 \\
\hline Vinyl acetate & & 50 & U & 4.5 & 50 \\
\hline 2,2-Dichloropropane & & 10 & $U^{*}$ & 2.2 & 10 \\
\hline cis-1,2-Dichloroethene & & 10 & $\mathrm{U}$ & 1.0 & 10 \\
\hline 2-Butanone & & 50 & U & 5.9 & 50 \\
\hline Bromochloromethane & & 10 & $\mathrm{U}$ & 1.4 & 10 \\
\hline Tetrahydrofuran & & 20 & $U^{*}$ & 5.3 & 20 \\
\hline Chloroform & & 10 & $\mathrm{U}$ & 1.2 & 10 \\
\hline 1,1,1-Trichloroethane & & 10 & U & 0.85 & 10 \\
\hline Cyclohexane & & 10 & U & 1.0 & 10 \\
\hline 1,1-Dichloropropene & & 10 & U & 0.97 & 10 \\
\hline Carbon tetrachloride & & 10 & $\mathrm{U}$ & 2.0 & 10 \\
\hline Isobutyl alcohol & & 500 & $U^{*}$ & 27 & 500 \\
\hline Benzene & & 10 & $\mathrm{U}$ & 1.1 & 10 \\
\hline 1,2-Dichloroethane & & 10 & U & 1.3 & 10 \\
\hline Trichloroethene & & 10 & $\mathrm{U}$ & 0.83 & 10 \\
\hline Methylcyclohexane & & 10 & $\mathrm{U}$ & 2.1 & 10 \\
\hline 1,2-Dichloropropane & & 10 & U & 1.2 & 10 \\
\hline Dibromomethane & & 10 & $U$ & 1.5 & 10 \\
\hline 1,4-Dioxane & & 400 & $\mathrm{U}$ & 65 & 400 \\
\hline Bromodichloromethane & & 10 & $\mathrm{U}$ & 1.0 & 10 \\
\hline 2-Chloroethyl vinyl ether & & 10 & $\mathrm{U}$ & 1.9 & 10 \\
\hline cis-1,3-Dichloropropene & & 10 & U & 1.2 & 10 \\
\hline 4-Methyl-2-pentanone & & 50 & $U^{*}$ & 4.7 & 50 \\
\hline Toluene & & 10 & $\mathrm{U}$ & 2.1 & 10 \\
\hline trans-1,3-Dichloropropene & & 10 & U & 2.2 & 10 \\
\hline 1,1,2-Trichloroethane & & 10 & $\mathrm{U}$ & 1.3 & 10 \\
\hline Tetrachloroethene & & 10 & $U$ & 2.0 & 10 \\
\hline 1,3-Dichloropropane & & 10 & $U$ & 1.1 & 10 \\
\hline 2-Hexanone & & 50 & $U$ & 6.1 & 50 \\
\hline Dibromochloromethane & & 10 & U & 1.1 & 10 \\
\hline
\end{tabular}


Analytical Data

Client: Argonne National Laboratory

Job Number: 200-28914-1

Sdg Number: 200-28914

Client Sample ID: YK-S-BLANK

Lab Sample ID: $\quad$ 200-28914-9TB

Date Sampled: 07/16/2015 0000

Client Matrix:

Solid

Date Received: 07/17/2015 1010

\section{B Volatile Organic Compounds (GC/MS)}

$\begin{array}{llllll}\text { Analysis Method: } & 8260 \mathrm{~B} & \text { Analysis Batch: } & 200-92051 & \text { Instrument ID: } & \text { CHL.i } \\ \text { Prep Method: } & 5035 & \text { Prep Batch: } & 200-91708 & \text { Lab File ID: } & 15018 \text { 14.D } \\ \text { Dilution: } & 1.0 & & & \text { Initial Weight/Volume: } & 10 \mathrm{~g} \\ \text { Analysis Date: } & 07 / 30 / 20151703 & & & \text { Final Weight/Volume: } 10 \mathrm{~mL} \\ \text { Prep Date: } & 07 / 23 / 20151500 & & & \end{array}$

\begin{tabular}{|c|c|c|c|c|}
\hline DryWt Corrected: $\mathrm{N}$ & Result (ug/Kg) & Qualifier & MDL & RL \\
\hline 1,2-Dibromoethane & 10 & U & 1.2 & 10 \\
\hline Chlorobenzene & 10 & U & 0.91 & 10 \\
\hline 1,1,1,2-Tetrachloroethane & 10 & $\mathrm{U}$ & 1.1 & 10 \\
\hline Ethylbenzene & 1.3 & $J B$ & 0.94 & 10 \\
\hline m\&p-Xylene & 1.2 & $\mathrm{~J}$ & 1.1 & 10 \\
\hline o-Xylene & 10 & U & 1.0 & 10 \\
\hline Styrene & 10 & U & 0.98 & 10 \\
\hline Bromoform & 10 & U & 1.0 & 10 \\
\hline Isopropylbenzene & 10 & U & 2.0 & 10 \\
\hline Bromobenzene & 10 & U & 1.0 & 10 \\
\hline 1,1,2,2-Tetrachloroethane & 10 & U & 1.3 & 10 \\
\hline 1,2,3-Trichloropropane & 10 & U & 1.2 & 10 \\
\hline n-Propylbenzene & 10 & U & 1.1 & 10 \\
\hline 2-Chlorotoluene & 10 & $\mathrm{U}$ & 1.1 & 10 \\
\hline 4-Chlorotoluene & 10 & U & 0.96 & 10 \\
\hline 1,3,5-Trimethylbenzene & 10 & U & 2.0 & 10 \\
\hline tert-Butylbenzene & 10 & U & 1.4 & 10 \\
\hline 1,2,4-Trimethylbenzene & 10 & U & 1.0 & 10 \\
\hline sec-Butylbenzene & 10 & $\mathrm{U}$ & 2.0 & 10 \\
\hline 1,3-Dichlorobenzene & 10 & $\mathrm{U}$ & 0.95 & 10 \\
\hline 4-Isopropyltoluene & 10 & U & 0.88 & 10 \\
\hline 1,4-Dichlorobenzene & 10 & U & 1.4 & 10 \\
\hline 1,2-Dichlorobenzene & 10 & $\mathrm{U}$ & 1.1 & 10 \\
\hline n-Butylbenzene & 10 & $\mathrm{U}$ & 0.90 & 10 \\
\hline 1,2-Dibromo-3-Chloropropane & 10 & U & 2.1 & 10 \\
\hline 1,2,4-Trichlorobenzene & 10 & $\mathrm{U}$ & 0.91 & 10 \\
\hline Hexachlorobutadiene & 10 & $U$ * & 2.3 & 10 \\
\hline Naphthalene & 10 & $U^{*}$ & 1.2 & 10 \\
\hline 1,2,3-Trichlorobenzene & 10 & $U$ & 1.0 & 10 \\
\hline Surrogate & $\% \operatorname{Rec}$ & Qualifier & \multicolumn{2}{|c|}{ Acceptance Limits } \\
\hline 1,2-Dichloroethane-d4 & 103 & \multicolumn{3}{|c|}{$80-120$} \\
\hline Toluene-d8 & 113 & \multicolumn{3}{|c|}{$80-120$} \\
\hline Bromofluorobenzene & 113 & \multicolumn{3}{|c|}{$80-125$} \\
\hline Dibromofluoromethane (Surr) & 97 & \multicolumn{3}{|c|}{$50-150$} \\
\hline
\end{tabular}




\section{Surrogate Recovery Report}

\section{B Volatile Organic Compounds (GC/MS)}

\section{Client Matrix: Solid}

\begin{tabular}{llcccc} 
& & DBFM & DCA & TOL & $\begin{array}{r}\text { BFB } \\
\% \text { Lab Sample ID }\end{array}$ \\
\hline $200-28914-1$ & Client Sample ID & 101 & 109 & 108 & 111 \\
$200-28914-2$ & YK-S-37764 & 99 & 106 & 111 & 114 \\
$200-28914-3$ & YK-S-37768 & 102 & 107 & 110 & 114 \\
$200-28914-4$ & YK-S-37767 & 102 & 109 & 110 & 113 \\
$200-28914-5$ & YK-S-37805 & 106 & 112 & 111 & 113 \\
$200-28914-6$ & YK-S-37791 & 97 & 103 & 109 & 111 \\
$200-28914-7$ & YK-S-37826 & 101 & 107 & 109 & 112 \\
$200-28914-8$ & YK-S-37814 & 97 & 96 & 110 & 111 \\
$200-28914-9$ & YK-S-37758 & 97 & 103 & 113 & 113 \\
MB 200-91708/1-A & YK-S-BLANK & 97 & 100 & 110 & 112 \\
LCS 200-91708/2-A & & 101 & 107 & 111 & 111
\end{tabular}

Surrogate DBFM = Dibromofluoromethane (Surr)

$\mathrm{DCA}=1,2-$-Dichloroethane-d4

$\mathrm{TOL}=$ Toluene-d8

$\mathrm{BFB}=$ Bromofluorobenzene
Acceptance Limits

50-150

80-120

$80-120$

$80-125$ 
Method Blank - Batch: 200-91708

$\begin{array}{lll}\text { Lab Sample ID: } & \text { MB 200-91708/1-A } \\ \text { Client Matrix: } & \text { Solid } \\ \text { Dilution: } & 1.0 \\ \text { Analysis Date: } & 07 / 30 / 20151036 \\ \text { Prep Date: } & 07 / 23 / 20151500 \\ \text { Leach Date: } & \text { N/A }\end{array}$

Method: $8260 \mathrm{~B}$

Preparation: 5035

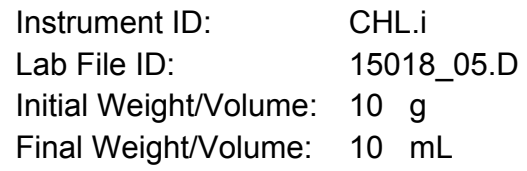

\begin{tabular}{|c|c|c|c|c|}
\hline Analyte & Result & Qual & MDL & $\mathrm{RL}$ \\
\hline Dichlorodifluoromethane & 10 & $\mathrm{U}$ & 1.7 & 10 \\
\hline Chloromethane & 11.0 & & 2.1 & 10 \\
\hline Vinyl chloride & 10 & $\mathrm{U}$ & 0.83 & 10 \\
\hline Bromomethane & 25.5 & & 1.0 & 10 \\
\hline Chloroethane & 10 & $\mathrm{U}$ & 2.5 & 10 \\
\hline Trichlorofluoromethane & 10 & $\mathrm{U}$ & 0.82 & 10 \\
\hline 1,1-Dichloroethene & 10 & $\mathrm{U}$ & 1.1 & 10 \\
\hline Freon TF & 10 & U & 0.85 & 10 \\
\hline Acetone & 50 & $\mathrm{U}$ & 12 & 50 \\
\hline Methyl iodide & 12.3 & & 0.75 & 10 \\
\hline Carbon disulfide & 10 & $\mathrm{U}$ & 0.87 & 10 \\
\hline Methyl acetate & 50 & $U$ & 9.2 & 50 \\
\hline Methylene Chloride & 10 & $\mathrm{U}$ & 2.3 & 10 \\
\hline trans-1,2-Dichloroethene & 10 & $\mathrm{U}$ & 0.99 & 10 \\
\hline Methyl t-butyl ether & 10 & $U$ & 1.1 & 10 \\
\hline 1,1-Dichloroethane & 10 & U & 1.1 & 10 \\
\hline Vinyl acetate & 50 & $\mathrm{U}$ & 4.5 & 50 \\
\hline 2,2-Dichloropropane & 10 & $u$ & 2.2 & 10 \\
\hline cis-1,2-Dichloroethene & 10 & $\mathrm{U}$ & 1.0 & 10 \\
\hline 2-Butanone & 50 & $\mathrm{U}$ & 5.9 & 50 \\
\hline Bromochloromethane & 10 & $\mathrm{U}$ & 1.4 & 10 \\
\hline Tetrahydrofuran & 20 & $\mathrm{U}$ & 5.3 & 20 \\
\hline Chloroform & 10 & $\mathrm{U}$ & 1.2 & 10 \\
\hline 1,1,1-Trichloroethane & 10 & U & 0.85 & 10 \\
\hline Cyclohexane & 10 & $\mathrm{U}$ & 1.0 & 10 \\
\hline 1,1-Dichloropropene & 10 & U & 0.97 & 10 \\
\hline Carbon tetrachloride & 10 & $\mathrm{U}$ & 2.0 & 10 \\
\hline Isobutyl alcohol & 500 & U & 27 & 500 \\
\hline Benzene & 10 & $\mathrm{U}$ & 1.1 & 10 \\
\hline 1,2-Dichloroethane & 10 & U & 1.3 & 10 \\
\hline Trichloroethene & 10 & $\mathrm{U}$ & 0.83 & 10 \\
\hline Methylcyclohexane & 10 & U & 2.1 & 10 \\
\hline 1,2-Dichloropropane & 10 & $\mathrm{U}$ & 1.2 & 10 \\
\hline Dibromomethane & 10 & U & 1.5 & 10 \\
\hline 1,4-Dioxane & 400 & U & 65 & 400 \\
\hline Bromodichloromethane & 10 & U & 1.0 & 10 \\
\hline 2-Chloroethyl vinyl ether & 10 & $\mathrm{U}$ & 1.9 & 10 \\
\hline cis-1,3-Dichloropropene & 10 & U & 1.2 & 10 \\
\hline 4-Methyl-2-pentanone & 50 & U & 4.7 & 50 \\
\hline Toluene & 10 & $\mathrm{U}$ & 2.1 & 10 \\
\hline trans-1,3-Dichloropropene & 10 & $\mathrm{U}$ & 2.2 & 10 \\
\hline 1,1,2-Trichloroethane & 10 & $\mathrm{U}$ & 1.3 & 10 \\
\hline Tetrachloroethene & 10 & $u$ & 2.0 & 10 \\
\hline 1,3-Dichloropropane & 10 & $\mathrm{U}$ & 1.1 & 10 \\
\hline 2-Hexanone & 50 & U & 6.1 & 50 \\
\hline
\end{tabular}




\section{Quality Control Results}

Client: Argonne National Laboratory

Job Number: 200-28914-1

Sdg Number: 200-28914

Method Blank - Batch: 200-91708

\section{Method: 8260B \\ Preparation: 5035}

$\begin{array}{lll}\text { Lab Sample ID: } & \text { MB 200-91708/1-A } \\ \text { Client Matrix: } & \text { Solid } & \\ \text { Dilution: } & 1.0 \\ \text { Analysis Date: } & 07 / 30 / 20151036 \\ \text { Prep Date: } & 07 / 23 / 20151500 \\ \text { Leach Date: } & \text { N/A }\end{array}$

Analysis Batch: $\quad 200-92051$

Prep Batch: $\quad$ 200-91708

Leach Batch: N/A

Units: $\begin{array}{ll}\text { Instrument ID: } & \text { CHL.i } \\ \text { Lab File ID: } & 15018 \text { 05.D } \\ \text { Initial Weight/Volume: } & 10 \mathrm{~g} \\ \text { Final Weight/Volume: } & 10 \mathrm{~mL}\end{array}$

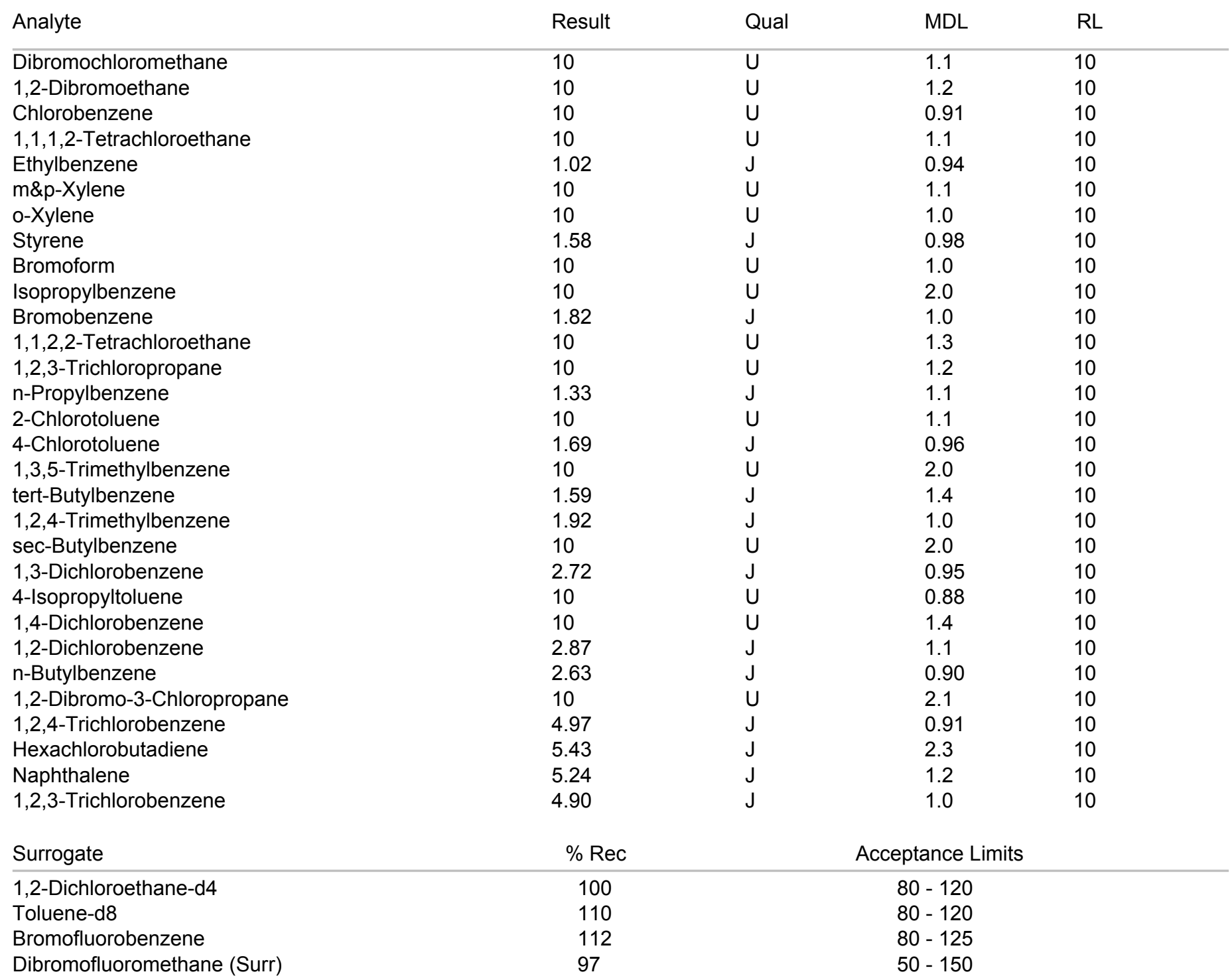




\section{Lab Control Sample - Batch: 200-91708}

$\begin{array}{ll}\text { Lab Sample ID: } & \text { LCS 200-91708/2-A } \\ \text { Client Matrix: } & \text { Solid } \\ \text { Dilution: } & 1.0 \\ \text { Analysis Date: } & 07 / 30 / 20150931 \\ \text { Prep Date: } & 07 / 23 / 20151500 \\ \text { Leach Date: } & \text { N/A }\end{array}$

\section{Method: 8260B \\ Preparation: $\mathbf{5 0 3 5}$}

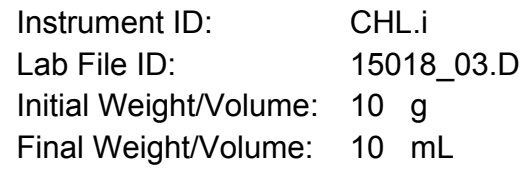

\begin{tabular}{|c|c|c|c|c|c|}
\hline Analyte & Spike Amount & Result & $\%$ Rec. & Limit & Qual \\
\hline Dichlorodifluoromethane & 248 & 281 & 113 & $35-125$ & \\
\hline Chloromethane & 248 & 284 & 114 & $65-120$ & \\
\hline Vinyl chloride & 248 & 272 & 110 & $80-130$ & \\
\hline Bromomethane & 248 & 144 & 58 & $40-120$ & \\
\hline Chloroethane & 248 & 96.9 & 39 & $40-120$ & * \\
\hline Trichlorofluoromethane & 248 & 111 & 45 & $40-120$ & \\
\hline 1,1-Dichloroethene & 248 & 97.1 & 39 & $80-120$ & * \\
\hline Freon TF & 248 & 109 & 44 & $70-120$ & * \\
\hline Acetone & 1240 & 294 & 24 & $15-200$ & \\
\hline Methyl iodide & 248 & 53.2 & 21 & $45-150$ & * \\
\hline Carbon disulfide & 248 & 90.3 & 36 & $80-120$ & * \\
\hline Methyl acetate & 1240 & 1850 & 149 & $70-120$ & * \\
\hline Methylene Chloride & 248 & 233 & 94 & $80-120$ & \\
\hline trans-1,2-Dichloroethene & 248 & 277 & 112 & $80-125$ & \\
\hline Methyl t-butyl ether & 248 & 251 & 101 & $80-120$ & \\
\hline 1,1-Dichloroethane & 248 & 203 & 82 & $80-120$ & \\
\hline Vinyl acetate & 496 & 457 & 92 & $80-200$ & \\
\hline 2,2-Dichloropropane & 248 & 402 & 162 & $80-120$ & * \\
\hline cis-1,2-Dichloroethene & 248 & 276 & 111 & $80-125$ & \\
\hline 2-Butanone & 1240 & 1650 & 133 & $60-170$ & \\
\hline Bromochloromethane & 248 & 239 & 96 & $80-130$ & \\
\hline Tetrahydrofuran & 496 & 298 & 60 & $80-120$ & * \\
\hline Chloroform & 248 & 247 & 100 & $75-120$ & \\
\hline 1,1,1-Trichloroethane & 248 & 259 & 104 & $75-120$ & \\
\hline Cyclohexane & 248 & 252 & 102 & $80-120$ & \\
\hline 1,1-Dichloropropene & 248 & 283 & 114 & $80-125$ & \\
\hline Carbon tetrachloride & 248 & 265 & 107 & $75-120$ & \\
\hline Isobutyl alcohol & 6200 & 1140 & 18 & $80-130$ & * \\
\hline Benzene & 248 & 261 & 105 & $80-125$ & \\
\hline 1,2-Dichloroethane & 248 & 250 & 101 & $70-120$ & \\
\hline Trichloroethene & 248 & 256 & 103 & $75-120$ & \\
\hline Methylcyclohexane & 248 & 259 & 104 & $80-120$ & \\
\hline 1,2-Dichloropropane & 248 & 257 & 103 & $80-125$ & \\
\hline Dibromomethane & 248 & 211 & 85 & $75-120$ & \\
\hline 1,4-Dioxane & 4960 & 5560 & 112 & $75-140$ & \\
\hline Bromodichloromethane & 248 & 254 & 102 & $80-120$ & \\
\hline 2-Chloroethyl vinyl ether & 248 & 302 & 122 & $80-125$ & \\
\hline cis-1,3-Dichloropropene & 248 & 250 & 101 & $80-125$ & \\
\hline 4-Methyl-2-pentanone & 1240 & 1780 & 144 & $80-125$ & * \\
\hline Toluene & 248 & 266 & 107 & $80-120$ & \\
\hline trans-1,3-Dichloropropene & 248 & 274 & 110 & $80-120$ & \\
\hline 1,1,2-Trichloroethane & 248 & 260 & 105 & $80-125$ & \\
\hline Tetrachloroethene & 248 & 268 & 108 & $80-120$ & \\
\hline 1,3-Dichloropropane & 248 & 258 & 104 & $80-125$ & \\
\hline 2-Hexanone & 1240 & 1650 & 133 & $75-150$ & \\
\hline Dibromochloromethane & 248 & 258 & 104 & $80-125$ & \\
\hline
\end{tabular}




\section{Lab Control Sample - Batch: 200-91708}

$\begin{array}{ll}\text { Lab Sample ID: } & \text { LCS 200-91708/2-A } \\ \text { Client Matrix: } & \text { Solid } \\ \text { Dilution: } & 1.0 \\ \text { Analysis Date: } & 07 / 30 / 20150931 \\ \text { Prep Date: } & 07 / 23 / 20151500 \\ \text { Leach Date: } & \text { N/A }\end{array}$

Method: $8260 \mathrm{~B}$

Preparation: $\mathbf{5 0 3 5}$

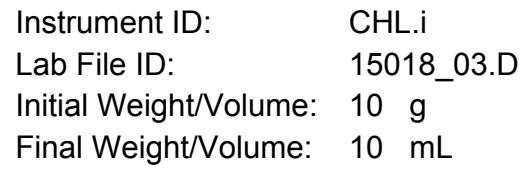

\begin{tabular}{|c|c|c|c|c|c|}
\hline Analyte & Spike Amount & Result & $\%$ Rec. & Limit & Qual \\
\hline 1,2-Dibromoethane & 248 & 264 & 106 & $80-120$ & \\
\hline Chlorobenzene & 248 & 262 & 105 & $80-120$ & \\
\hline 1,1,1,2-Tetrachloroethane & 248 & 268 & 108 & $80-120$ & \\
\hline Ethylbenzene & 248 & 262 & 106 & $80-125$ & \\
\hline m\&p-Xylene & 248 & 266 & 107 & $80-125$ & \\
\hline o-Xylene & 248 & 264 & 106 & $80-120$ & \\
\hline Styrene & 248 & 255 & 103 & $80-120$ & \\
\hline Bromoform & 248 & 224 & 90 & $80-120$ & \\
\hline Isopropylbenzene & 248 & 270 & 109 & $80-120$ & \\
\hline Bromobenzene & 248 & 270 & 109 & $80-125$ & \\
\hline 1,1,2,2-Tetrachloroethane & 248 & 238 & 96 & $80-125$ & \\
\hline 1,2,3-Trichloropropane & 248 & 247 & 100 & $80-120$ & \\
\hline n-Propylbenzene & 248 & 272 & 110 & $80-120$ & \\
\hline 2-Chlorotoluene & 248 & 263 & 106 & $80-125$ & \\
\hline 4-Chlorotoluene & 248 & 264 & 106 & $80-125$ & \\
\hline 1,3,5-Trimethylbenzene & 248 & 269 & 108 & $80-120$ & \\
\hline tert-Butylbenzene & 248 & 273 & 110 & $75-120$ & \\
\hline 1,2,4-Trimethylbenzene & 248 & 265 & 107 & $80-120$ & \\
\hline sec-Butylbenzene & 248 & 279 & 112 & $80-120$ & \\
\hline 1,3-Dichlorobenzene & 248 & 267 & 108 & $80-125$ & \\
\hline 4-Isopropyltoluene & 248 & 277 & 112 & $75-120$ & \\
\hline 1,4-Dichlorobenzene & 248 & 264 & 106 & $80-125$ & \\
\hline 1,2-Dichlorobenzene & 248 & 261 & 105 & $80-125$ & \\
\hline n-Butylbenzene & 248 & 287 & 116 & $80-120$ & \\
\hline 1,2-Dibromo-3-Chloropropane & 248 & 211 & 85 & $70-120$ & \\
\hline 1,2,4-Trichlorobenzene & 248 & 278 & 112 & $80-120$ & \\
\hline Hexachlorobutadiene & 248 & 309 & 124 & $75-120$ & * \\
\hline Naphthalene & 248 & 195 & 78 & $80-130$ & * \\
\hline 1,2,3-Trichlorobenzene & 248 & 236 & 95 & $80-120$ & \\
\hline Surrogate & \multicolumn{2}{|c|}{$\% \operatorname{Rec}$} & \multicolumn{2}{|r|}{ Acceptance Limits } & \\
\hline 1,2-Dichloroethane-d4 & \multicolumn{2}{|c|}{107} & \multicolumn{2}{|r|}{$80-120$} & \\
\hline Toluene-d8 & \multicolumn{2}{|c|}{111} & \multicolumn{2}{|r|}{$80-120$} & \\
\hline Bromofluorobenzene & \multicolumn{2}{|c|}{111} & \multicolumn{2}{|r|}{$80-125$} & \\
\hline Dibromofluoromethane (Surr) & \multicolumn{2}{|c|}{101} & \multicolumn{2}{|r|}{$50-150$} & \\
\hline
\end{tabular}




\section{DATA REPORTING QUALIFIERS}

Client: Argonne National Laboratory

Job Number: 200-28914-1

Sdg Number: $200-28914$

Description

GC/MS VOA

B

$U$

$\wedge$

*

$J$
Compound was found in the blank and sample.

Indicates the analyte was analyzed for but not detected.

Instrument related QC is outside acceptance limits.

LCS or LCSD is outside acceptance limits.

Result is less than the RL but greater than or equal to the MDL and the concentration is an approximate value. 


\section{Quality Control Results}

Client: Argonne National Laboratory

Job Number: 200-28914-1

Sdg Number: $200-28914$

QC Association Summary

\begin{tabular}{|c|c|c|c|c|c|}
\hline Lab Sample ID & Client Sample ID & $\begin{array}{l}\text { Report } \\
\text { Basis }\end{array}$ & Client Matrix & Method & Prep Batch \\
\hline \multicolumn{6}{|l|}{ GC/MS VOA } \\
\hline \multicolumn{6}{|c|}{ Prep Batch: 200-91708 } \\
\hline LCS 200-91708/2-A & Lab Control Sample & $\mathrm{T}$ & Solid & 5035 & \\
\hline MB 200-91708/1-A & Method Blank & $\mathrm{T}$ & Solid & 5035 & \\
\hline $200-28914-1$ & YK-S-37764 & $\mathrm{T}$ & Solid & 5035 & \\
\hline $200-28914-2$ & YK-S-37768 & $\mathrm{T}$ & Solid & 5035 & \\
\hline 200-28914-3 & YK-S-37767 & $\mathrm{T}$ & Solid & 5035 & \\
\hline 200-28914-4 & YK-S-37805 & $\mathrm{T}$ & Solid & 5035 & \\
\hline $200-28914-5$ & YK-S-37791 & $\mathrm{T}$ & Solid & 5035 & \\
\hline $200-28914-6$ & YK-S-37826 & $\mathrm{T}$ & Solid & 5035 & \\
\hline 200-28914-7 & YK-S-37814 & $\mathrm{T}$ & Solid & 5035 & \\
\hline 200-28914-8 & YK-S-37758 & $\mathrm{T}$ & Solid & 5035 & \\
\hline 200-28914-9TВ & YK-S-BLANK & $\mathrm{T}$ & Solid & 5035 & \\
\hline \multicolumn{6}{|c|}{ Analysis Batch:200-92051 } \\
\hline LCS 200-91708/2-A & Lab Control Sample & $\mathrm{T}$ & Solid & $8260 \mathrm{~B}$ & $200-91708$ \\
\hline MB 200-91708/1-A & Method Blank & $\mathrm{T}$ & Solid & $8260 \mathrm{~B}$ & $200-91708$ \\
\hline $200-28914-1$ & YK-S-37764 & $\mathrm{T}$ & Solid & $8260 \mathrm{~B}$ & $200-91708$ \\
\hline $200-28914-2$ & YK-S-37768 & $\mathrm{T}$ & Solid & $8260 \mathrm{~B}$ & $200-91708$ \\
\hline 200-28914-3 & YK-S-37767 & $\mathrm{T}$ & Solid & $8260 \mathrm{~B}$ & $200-91708$ \\
\hline 200-28914-4 & YK-S-37805 & $\mathrm{T}$ & Solid & $8260 \mathrm{~B}$ & $200-91708$ \\
\hline 200-28914-5 & YK-S-37791 & $\mathrm{T}$ & Solid & $8260 \mathrm{~B}$ & $200-91708$ \\
\hline $200-28914-6$ & YK-S-37826 & $\mathrm{T}$ & Solid & $8260 \mathrm{~B}$ & $200-91708$ \\
\hline 200-28914-7 & YK-S-37814 & $\mathrm{T}$ & Solid & $8260 \mathrm{~B}$ & $200-91708$ \\
\hline $200-28914-8$ & YK-S-37758 & $\mathrm{T}$ & Solid & $8260 \mathrm{~B}$ & $200-91708$ \\
\hline 200-28914-9ТВ & YK-S-BLANK & $\mathrm{T}$ & Solid & $8260 \mathrm{~B}$ & $200-91708$ \\
\hline
\end{tabular}

Report Basis

$\mathrm{T}=$ Total 


\section{Quality Control Results}

Client: Argonne National Laboratory

Job Number: 200-28914-1

SDG: $200-28914$

\section{Laboratory Chronicle}

Lab ID: $\quad$ 200-28914-1

Method

P:5035

A:8260B

Lab ID:

200-28914-2

Bottle ID

200-28914-A-1-B

200-28914-A-1-B
Client ID: YK-S-37764

Sample Date/Time: 07/16/2015 00:00 Received Date/Time: 07/17/2015 10:10

\begin{tabular}{|c|c|c|c|c|c|c|c|c|}
\hline \multirow[b]{2}{*}{ Method } & \multirow[b]{2}{*}{ Bottle ID } & \multicolumn{2}{|c|}{ Sample Date/Time: } & 07/16/2015 00:00 & \multicolumn{2}{|c|}{ Received Date/Time: } & \multicolumn{2}{|c|}{ 07/17/2015 10:10 } \\
\hline & & Run & $\begin{array}{l}\text { Analysis } \\
\text { Batch }\end{array}$ & Prep Batch & $\begin{array}{l}\text { Date Prepared / } \\
\text { Analyzed }\end{array}$ & Dil & Lab & Analyst \\
\hline$\overline{P: 5035}$ & 200-28914-A-2-B & & $200-92051$ & $200-91708$ & $07 / 23 / 2015 \quad 15: 00$ & 1 & $\overline{\text { TAL BUR }}$ & ERT \\
\hline$A: 8260 B$ & 200-28914-A-2-B & & $200-92051$ & $200-91708$ & 07/30/2015 13:15 & 1 & TAL BUR & NEA \\
\hline
\end{tabular}

Lab ID: $\quad 200-28914-3$

Client ID: YK-S-37767

Sample Date/Time: 07/16/2015 00:00 Received Date/Time: 07/17/2015 10:10

\begin{tabular}{|c|c|c|c|c|c|c|c|c|}
\hline Method & Bottle ID & Run & $\begin{array}{l}\text { Analysis } \\
\text { Batch }\end{array}$ & Prep Batch & $\begin{array}{l}\text { Date Prepared / } \\
\text { Analyzed }\end{array}$ & Dil & Lab & Analyst \\
\hline$\overline{P: 5035}$ & 200-28914-A-3-B & & $200-92051$ & $200-91708$ & $07 / 23 / 201515: 00$ & 1 & $\overline{T A L ~ B U R}$ & ERT \\
\hline$A: 8260 B$ & 200-28914-A-3-B & & $200-92051$ & $200-91708$ & 07/30/2015 13:47 & 1 & TAL BUR & NEA \\
\hline
\end{tabular}

Lab ID: $\quad 200-28914-4$

Client ID: YK-S-37805

Sample Date/Time: 07/16/2015 00:00 Received Date/Time: 07/17/2015 10:10

\begin{tabular}{|c|c|c|c|c|c|c|c|c|}
\hline Method & Bottle ID & Run & $\begin{array}{l}\text { Analysis } \\
\text { Batch }\end{array}$ & Prep Batch & $\begin{array}{l}\text { Date Prepared / } \\
\text { Analyzed }\end{array}$ & Dil & Lab & Analyst \\
\hline$P: 5035$ & 200-28914-A-4-B & & $200-92051$ & $200-91708$ & 07/23/2015 15:00 & 1 & $\overline{T A L ~ B U R}$ & ERT \\
\hline$A: 8260 B$ & 200-28914-A-4-B & & $200-92051$ & $200-91708$ & 07/30/2015 14:20 & 1 & TAL BUR & NEA \\
\hline
\end{tabular}

Lab ID: $\quad 200-28914-5$

Client ID: YK-S-37791

Sample Date/Time: 07/16/2015 00:00 Received Date/Time: 07/17/2015 10:10

\begin{tabular}{|c|c|c|c|c|c|c|c|c|}
\hline Method & Bottle ID & Run & $\begin{array}{l}\text { Analysis } \\
\text { Batch }\end{array}$ & Prep Batch & $\begin{array}{l}\text { Date Prepared / } \\
\text { Analyzed }\end{array}$ & Dil & Lab & Analyst \\
\hline$\overline{P: 5035}$ & 200-28914-A-5-B & & $200-92051$ & $200-91708$ & $07 / 23 / 201515: 00$ & 1 & $\overline{\text { TAL BUR }}$ & ERT \\
\hline$A: 8260 B$ & $200-28914-A-5-B$ & & $200-92051$ & $200-91708$ & 07/30/2015 14:52 & 1 & TAL BUR & NEA \\
\hline
\end{tabular}

Lab ID: $\quad 200-28914-6$

Client ID: YK-S-37826

Sample Date/Time: 07/16/2015 00:00 Received Date/Time: 07/17/2015 10:10

\begin{tabular}{|c|c|c|c|c|c|c|c|c|}
\hline Method & Bottle ID & Run & $\begin{array}{l}\text { Analysis } \\
\text { Batch }\end{array}$ & Prep Batch & $\begin{array}{l}\text { Date Prepared / } \\
\text { Analyzed }\end{array}$ & Dil & Lab & Analyst \\
\hline$\overline{P: 5035}$ & 200-28914-A-6-B & & $200-92051$ & $200-91708$ & $07 / 23 / 2015$ 15:00 & 1 & TAL BUR & ERT \\
\hline$A: 8260 B$ & 200-28914-A-6-B & & $200-92051$ & $200-91708$ & 07/30/2015 $15: 25$ & 1 & TAL BUR & NEA \\
\hline
\end{tabular}

TestAmerica Burlington

$A=$ Analytical Method $\quad P=$ Prep Method 


\section{Quality Control Results}

Client: Argonne National Laboratory

Job Number: 200-28914-1

SDG: $200-28914$

\section{Laboratory Chronicle}

Lab ID: $\quad 200-28914-7$

\begin{tabular}{|c|c|c|c|c|c|c|c|c|}
\hline & & Sample & Date/Time: & 7/16/2015 00:00 & Received Date & Time: & $07 / 17 / 2015$ & 10:10 \\
\hline Method & Bottle ID & Run & $\begin{array}{l}\text { Analysis } \\
\text { Batch }\end{array}$ & Prep Batch & $\begin{array}{l}\text { Date Prepared / } \\
\text { Analyzed }\end{array}$ & Dil & Lab & Analyst \\
\hline$P: 5035$ & $200-28914-A-7-B$ & & $200-92051$ & $200-91708$ & $07 / 23 / 2015$ 15:00 & 1 & $\overline{\text { TAL BUR }}$ & ERT \\
\hline$A: 8260 B$ & 200-28914-A-7-B & & $200-92051$ & $200-91708$ & 07/30/2015 $15: 58$ & 1 & TAL BUR & NEA \\
\hline
\end{tabular}

Lab ID: $\quad 200-28914-8$
Client ID: YK-S-37758

Sample Date/Time: 07/16/2015 00:00 Received Date/Time: 07/17/2015 10:10

\begin{tabular}{|c|c|c|c|c|c|c|c|c|}
\hline Method & Bottle ID & Run & $\begin{array}{l}\text { Analysis } \\
\text { Batch }\end{array}$ & Prep Batch & $\begin{array}{l}\text { Date Prepared / } \\
\text { Analyzed }\end{array}$ & Dil & Lab & Analyst \\
\hline$\overline{P: 5035}$ & 200-28914-A-8-B & & $200-92051$ & $200-91708$ & $07 / 23 / 2015$ 15:00 & 1 & TAL BUR & ERT \\
\hline$A: 8260 B$ & 200-28914-A-8-B & & $200-92051$ & $200-91708$ & 07/30/2015 16:30 & 1 & TAL BUR & NEA \\
\hline
\end{tabular}

Lab ID: $\quad 200-28914-9$

Client ID: YK-S-BLANK

Sample Date/Time: 07/16/2015 00:00 Received Date/Time: 07/17/2015 10:10

\begin{tabular}{|c|c|c|c|c|c|c|c|c|}
\hline Method & Bottle ID & Run & $\begin{array}{l}\text { Analysis } \\
\text { Batch }\end{array}$ & Prep Batch & $\begin{array}{l}\text { Date Prepared / } \\
\text { Analyzed }\end{array}$ & Dil & Lab & Analyst \\
\hline$P: 5035$ & 200-28914-A-9-B & & $200-92051$ & $200-91708$ & 07/23/2015 15:00 & 1 & $\overline{\text { TAL BUR }}$ & ERT \\
\hline$A: 8260 B$ & 200-28914-A-9-B & & $200-92051$ & $200-91708$ & 07/30/2015 17:03 & 1 & TAL BUR & NEA \\
\hline
\end{tabular}

Lab ID: MB

Client ID: N/A

Sample Date/Time: N/A

Received Date/Time: N/A

\begin{tabular}{lllllllll} 
Method & Bottle ID & Run & $\begin{array}{l}\text { Analysis } \\
\text { Batch }\end{array}$ & Prep Batch & $\begin{array}{l}\text { Date Prepared } / \\
\text { Analyzed }\end{array}$ & Dil & Lab & Analyst \\
\hline P:5035 & MB 200-91708/1-A & $200-92051$ & $200-91708$ & $07 / 23 / 2015$ & $15: 00$ & 1 & TAL BUR & ERT \\
A:8260B & MB 200-91708/1-A & $200-92051$ & $200-91708$ & $07 / 30 / 2015$ & $10: 36$ & 1 & TAL BUR & NEA \\
\hline
\end{tabular}

Lab ID: LCS

Client ID: N/A

Sample Date/Time: N/A

Received Date/Time: N/A

\begin{tabular}{|c|c|c|c|c|c|c|c|c|}
\hline Method & Bottle ID & Run & $\begin{array}{l}\text { Analysis } \\
\text { Batch }\end{array}$ & Prep Batch & $\begin{array}{l}\text { Date Prepared / } \\
\text { Analyzed }\end{array}$ & Dil & Lab & Analyst \\
\hline $\bar{P}: 5035$ & LCS 200-91708/2-A & & $200-92051$ & $200-91708$ & 07/23/2015 15:00 & 1 & $\overline{T A L ~ B U R}$ & ERT \\
\hline$A: 8260 B$ & LCS 200-91708/2-A & & $200-92051$ & $200-91708$ & 07/30/2015 09:31 & 1 & TAL BUR & NEA \\
\hline
\end{tabular}

Lab References:

TAL BUR = TestAmerica Burlington 
SDG No. : 200-28914

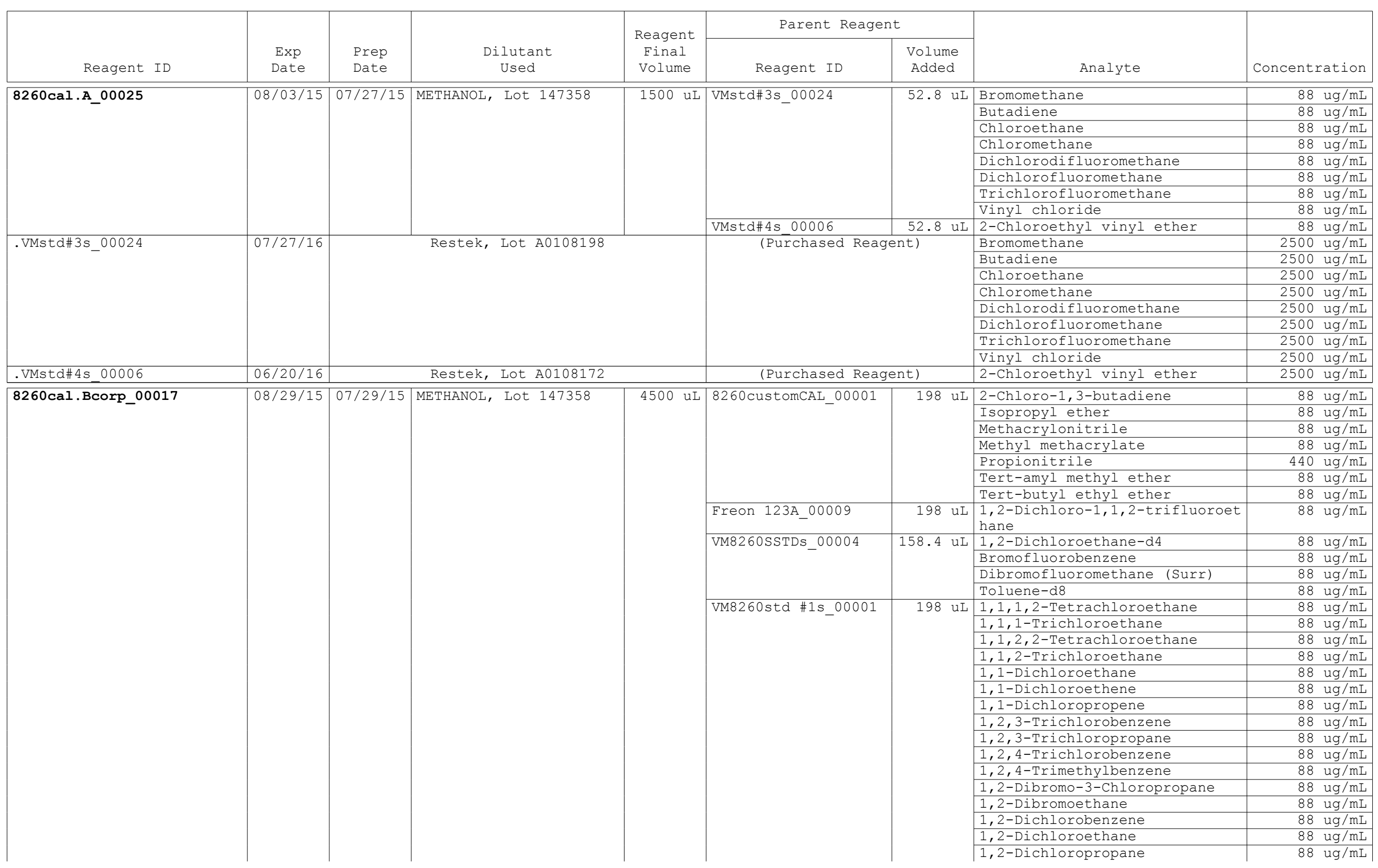


SDG No.: 200-28914

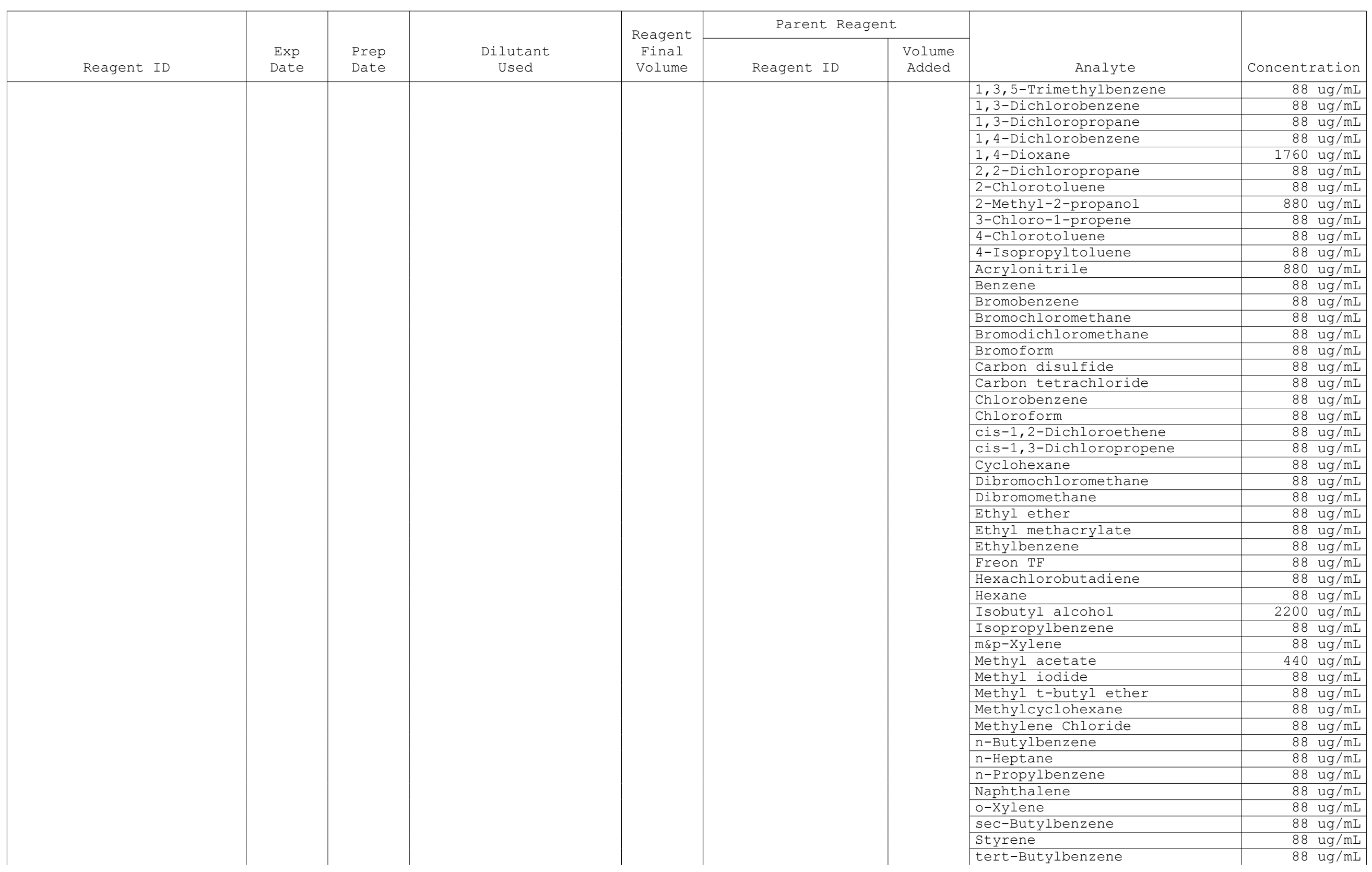


SDG No. : 200-28914

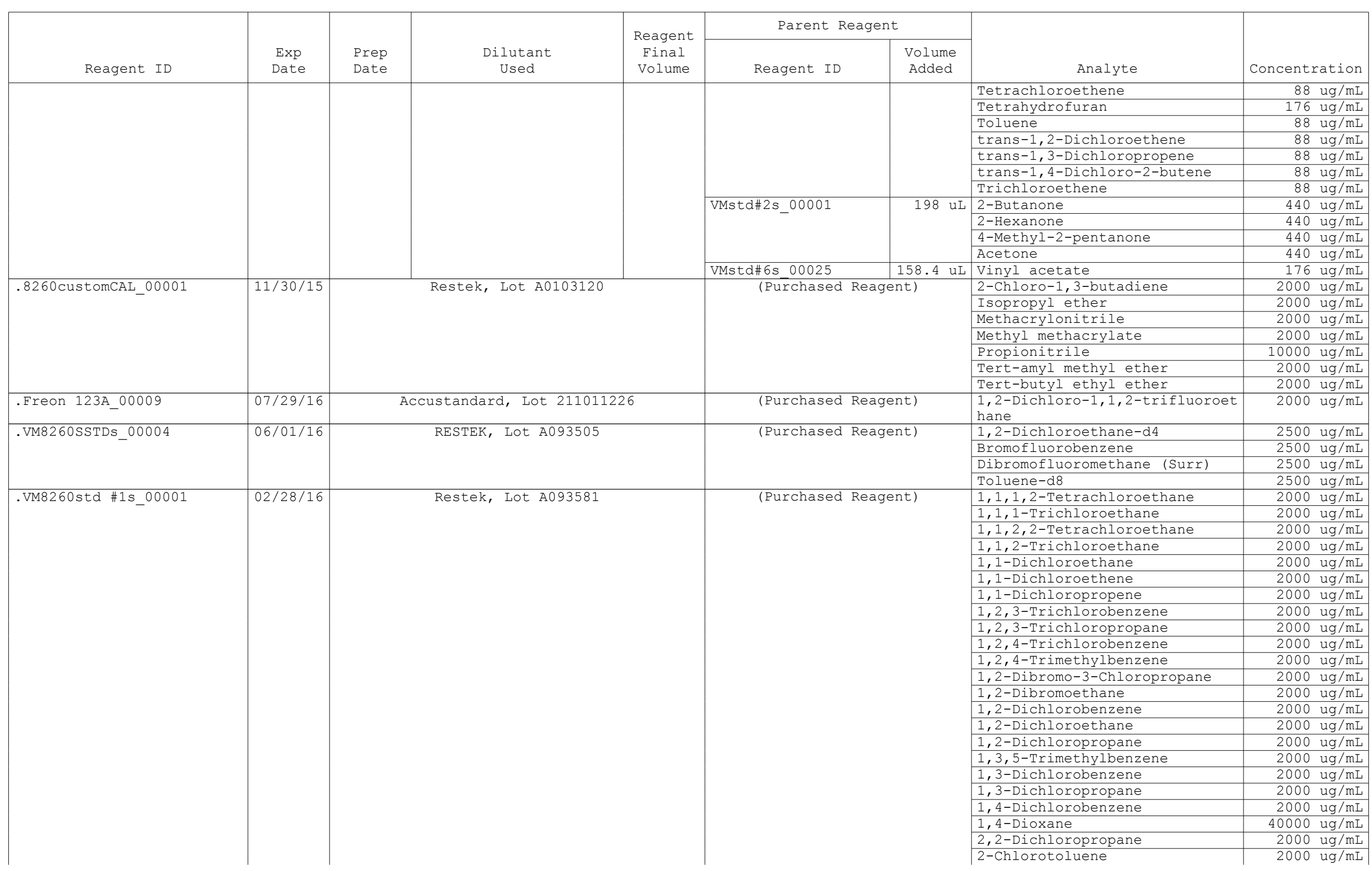


SDG No.: 200-28914

\begin{tabular}{|c|c|c|c|c|c|c|c|c|c|}
\hline \multirow[b]{2}{*}{ Reagent ID } & \multirow[b]{2}{*}{$\begin{array}{l}\text { Exp } \\
\text { Date }\end{array}$} & \multirow[b]{2}{*}{$\begin{array}{l}\text { Prep } \\
\text { Date }\end{array}$} & \multirow[b]{2}{*}{$\begin{array}{l}\text { Dilutant } \\
\text { Used }\end{array}$} & \multirow{2}{*}{$\begin{array}{c}\text { Reagent } \\
\text { Final } \\
\text { Volume }\end{array}$} & \multicolumn{2}{|c|}{ Parent Reagent } & \multirow[b]{2}{*}{ Analyte } & \multirow{2}{*}{\multicolumn{2}{|c|}{ Concentration }} \\
\hline & & & & & Reagent ID & $\begin{array}{l}\text { Volume } \\
\text { Added }\end{array}$ & & & \\
\hline & & & & & & & 2-Methyl-2-propanol & 20000 & $\mathrm{ug} / \mathrm{mL}$ \\
\hline & & & & & & & 3-Chloro-1-propene & 2000 & $\mathrm{ug} / \mathrm{mL}$ \\
\hline & & & & & & & 4-Chlorotoluene & 2000 & $\mathrm{ug} / \mathrm{mL}$ \\
\hline & & & & & & & 4-Isopropyltoluene & 2000 & $\mathrm{ug} / \mathrm{mL}$ \\
\hline & & & & & & & Acrylonitrile & 20000 & $\mathrm{ug} / \mathrm{mL}$ \\
\hline & & & & & & & Benzene & 2000 & $\mathrm{ug} / \mathrm{mL}$ \\
\hline & & & & & & & Bromobenzene & 2000 & $\mathrm{ug} / \mathrm{mL}$ \\
\hline & & & & & & & Bromochloromethane & 2000 & $\mathrm{ug} / \mathrm{mL}$ \\
\hline & & & & & & & Bromodichloromethane & 2000 & $\mathrm{ug} / \mathrm{mL}$ \\
\hline & & & & & & & Bromoform & 2000 & $\mathrm{ug} / \mathrm{mL}$ \\
\hline & & & & & & & Carbon disulfide & 2000 & $\mathrm{ug} / \mathrm{mL}$ \\
\hline & & & & & & & Carbon tetrachloride & 2000 & $\mathrm{ug} / \mathrm{mL}$ \\
\hline & & & & & & & Chlorobenzene & 2000 & $\mathrm{ug} / \mathrm{mL}$ \\
\hline & & & & & & & Chloroform & 2000 & $\mathrm{ug} / \mathrm{mL}$ \\
\hline & & & & & & & cis-1,2-Dichloroethene & 2000 & $\mathrm{ug} / \mathrm{mL}$ \\
\hline & & & & & & & cis-1,3-Dichloropropene & 2000 & $\mathrm{ug} / \mathrm{mL}$ \\
\hline & & & & & & & Cyclohexane & 2000 & $\mathrm{ug} / \mathrm{mL}$ \\
\hline & & & & & & & Dibromochloromethane & 2000 & $\mathrm{ug} / \mathrm{mL}$ \\
\hline & & & & & & & Dibromomethane & 2000 & $\mathrm{ug} / \mathrm{mL}$ \\
\hline & & & & & & & Ethyl ether & 2000 & $\mathrm{ug} / \mathrm{mL}$ \\
\hline & & & & & & & Ethyl methacrylate & 2000 & $\mathrm{ug} / \mathrm{mL}$ \\
\hline & & & & & & & Ethylbenzene & 2000 & $\mathrm{ug} / \mathrm{mL}$ \\
\hline & & & & & & & Freon TF & 2000 & $\mathrm{ug} / \mathrm{mL}$ \\
\hline & & & & & & & Hexachlorobutadiene & 2000 & $\mathrm{ug} / \mathrm{mL}$ \\
\hline & & & & & & & Hexane & 2000 & $\mathrm{ug} / \mathrm{mL}$ \\
\hline & & & & & & & Isobutyl alcohol & 50000 & $\mathrm{ug} / \mathrm{mL}$ \\
\hline & & & & & & & Isopropylbenzene & 2000 & $\mathrm{ug} / \mathrm{mL}$ \\
\hline & & & & & & & m\&p-Xylene & 2000 & $\mathrm{ug} / \mathrm{mL}$ \\
\hline & & & & & & & Methyl acetate & 10000 & $\mathrm{ug} / \mathrm{mL}$ \\
\hline & & & & & & & Methyl iodide & 2000 & $\mathrm{ug} / \mathrm{mL}$ \\
\hline & & & & & & & Methyl t-butyl ether & 2000 & $\mathrm{ug} / \mathrm{mL}$ \\
\hline & & & & & & & Methylcyclohexane & 2000 & $\mathrm{ug} / \mathrm{mL}$ \\
\hline & & & & & & & Methylene Chloride & 2000 & $\mathrm{ug} / \mathrm{mL}$ \\
\hline & & & & & & & n-Butylbenzene & 2000 & $\mathrm{ug} / \mathrm{mL}$ \\
\hline & & & & & & & n-Heptane & 2000 & $\mathrm{ug} / \mathrm{mL}$ \\
\hline & & & & & & & n-Propylbenzene & 2000 & $\mathrm{ug} / \mathrm{mL}$ \\
\hline & & & & & & & Naphthalene & 2000 & $\mathrm{ug} / \mathrm{mL}$ \\
\hline & & & & & & & o-Xylene & 2000 & $\mathrm{ug} / \mathrm{mL}$ \\
\hline & & & & & & & sec-Butylbenzene & 2000 & $\mathrm{ug} / \mathrm{mL}$ \\
\hline & & & & & & & Styrene & 2000 & $\mathrm{ug} / \mathrm{mL}$ \\
\hline & & & & & & & tert-Butylbenzene & 2000 & $\mathrm{ug} / \mathrm{mL}$ \\
\hline & & & & & & & Tetrachloroethene & 2000 & $\mathrm{ug} / \mathrm{mL}$ \\
\hline & & & & & & & Tetrahydrofuran & 4000 & $\mathrm{ug} / \mathrm{mL}$ \\
\hline & & & & & & & Toluene & 2000 & $\mathrm{ug} / \mathrm{mL}$ \\
\hline & & & & & & & trans-1,2-Dichloroethene & 2000 & $\mathrm{ug} / \mathrm{mL}$ \\
\hline & & & & & & & trans-1,3-Dichloropropene & 2000 & $\mathrm{ug} / \mathrm{mL}$ \\
\hline & & & & & & & trans-1,4-Dichloro-2-butene & 2000 & $\mathrm{ug} / \mathrm{mL}$ \\
\hline & & & & & & & Trichloroethene & 2000 & $\mathrm{ug} / \mathrm{mL}$ \\
\hline
\end{tabular}


SDG No. : 200-28914

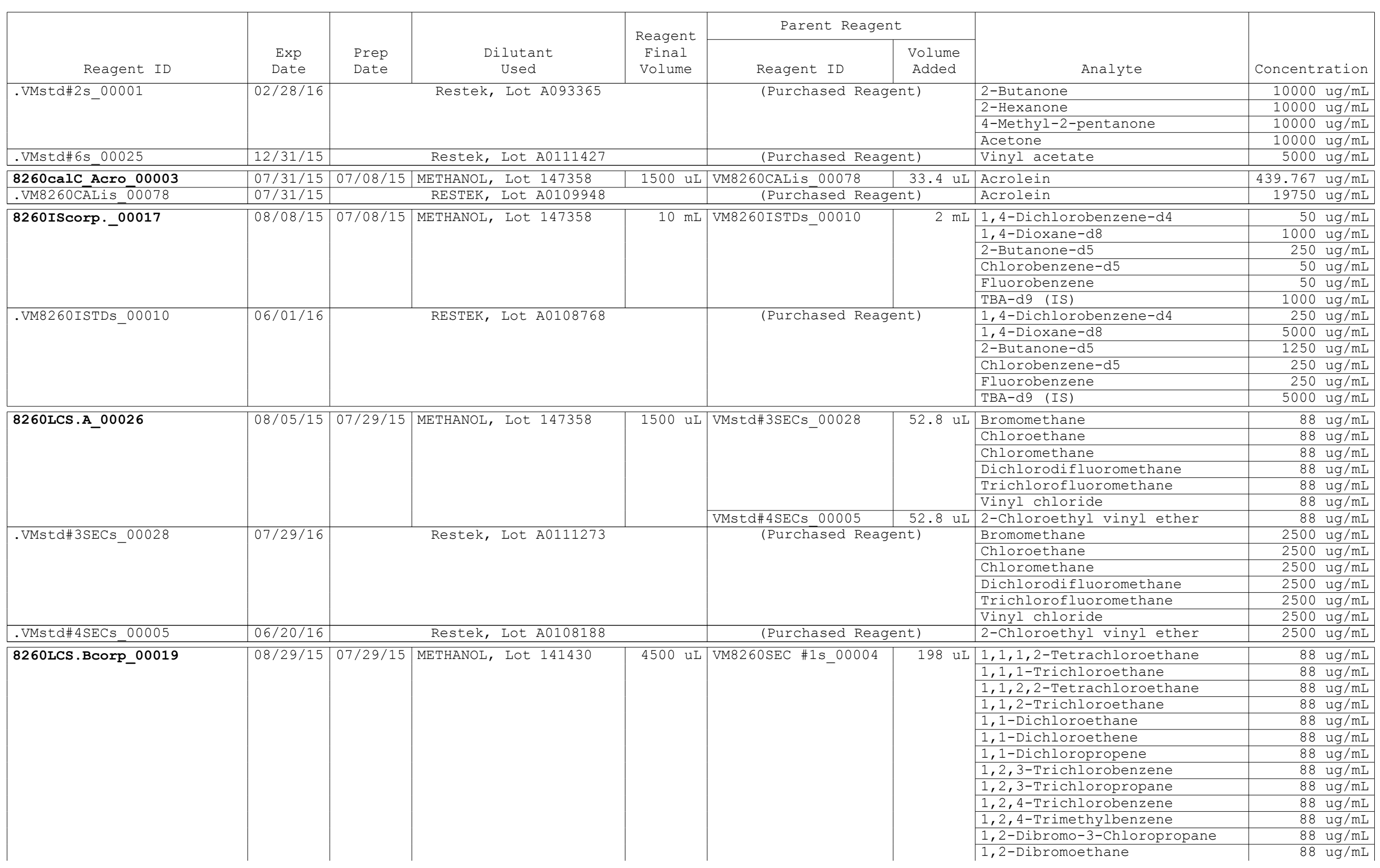


SDG No.: 200-28914

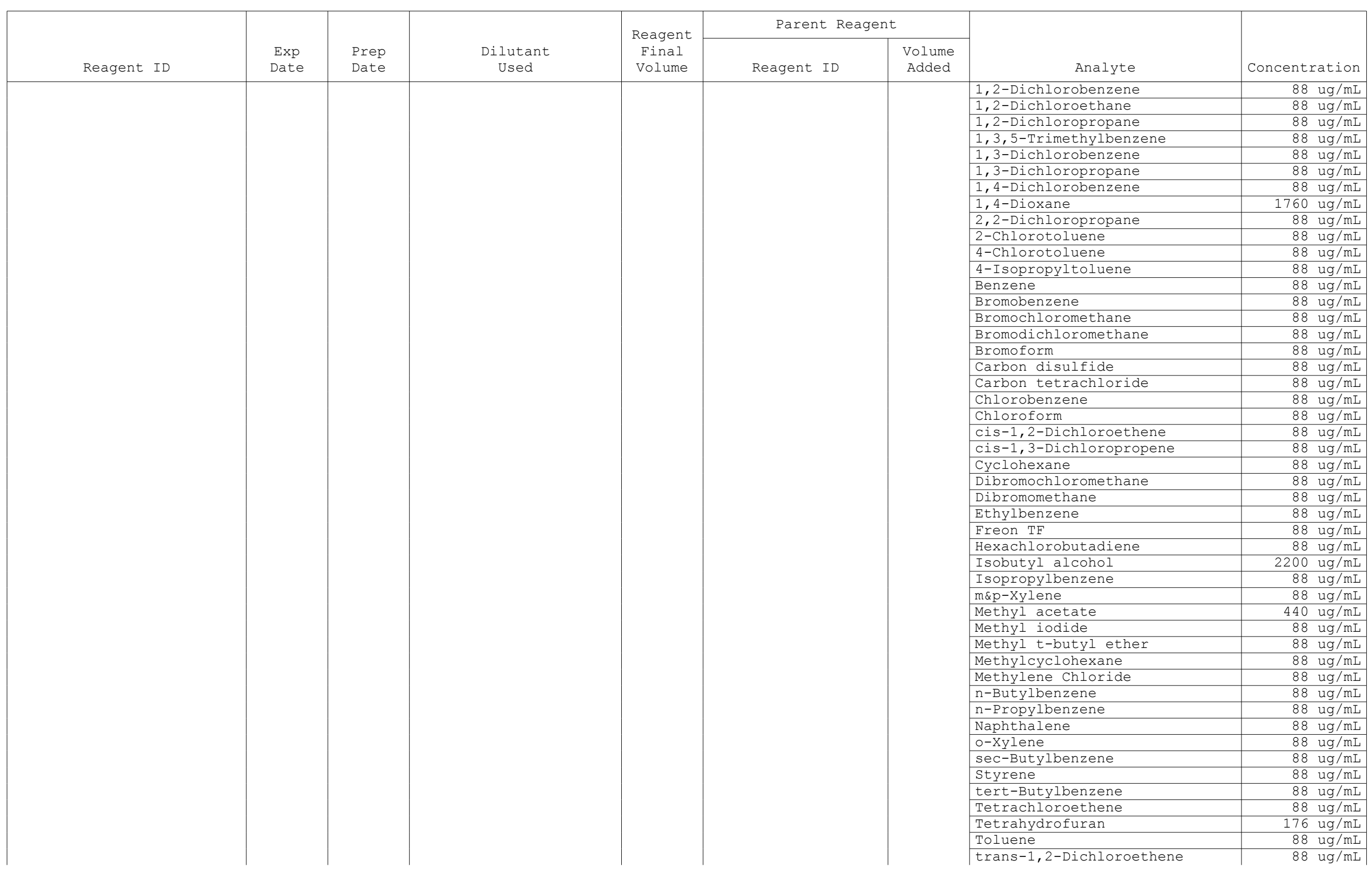


SDG No.: 200-28914

\begin{tabular}{|c|c|c|c|c|c|c|c|c|c|}
\hline \multirow[b]{2}{*}{ Reagent ID } & \multirow[b]{2}{*}{$\begin{array}{l}\text { Exp } \\
\text { Date }\end{array}$} & \multirow[b]{2}{*}{$\begin{array}{l}\text { Prep } \\
\text { Date }\end{array}$} & \multirow[b]{2}{*}{$\begin{array}{l}\text { Dilutant } \\
\text { Used }\end{array}$} & \multirow{2}{*}{$\begin{array}{c}\text { Reagent } \\
\text { Final } \\
\text { Volume }\end{array}$} & \multicolumn{2}{|c|}{ Parent Reagent } & \multirow[b]{2}{*}{ Analyte } & \multirow{2}{*}{\multicolumn{2}{|c|}{ Concentration }} \\
\hline & & & & & Reagent ID & $\begin{array}{l}\text { Volume } \\
\text { Added }\end{array}$ & & & \\
\hline & & & & & & & trans-1,3-Dichloropropene & $8 \varepsilon$ & $\mathrm{ug} / \mathrm{mL}$ \\
\hline & & & & & & & Trichloroethene & $8 \varepsilon$ & $\mathrm{ug} / \mathrm{mL}$ \\
\hline & & & & & \multirow[t]{4}{*}{ VMstd\#2SECs_00009 } & \multirow[t]{4}{*}{$198 \mathrm{uL}$} & 2-Butanone & 440 & $\mathrm{ug} / \mathrm{mL}$ \\
\hline & & & & & & & 2-Hexanone & 440 & $\mathrm{ug} / \mathrm{mL}$ \\
\hline & & & & & & & 4-Methyl-2-pentanone & 440 & $\mathrm{ug} / \mathrm{mL}$ \\
\hline & & & & & & & Acetone & 440 & $\mathrm{ug} / \mathrm{mL}$ \\
\hline & & & & & VMstd\#6SECs_00025 & $158.4 \mathrm{uL}$ & Vinyl acetate & 176 & $\mathrm{ug} / \mathrm{mL}$ \\
\hline \multirow[t]{41}{*}{.VM8260SEC \#1s_00004 } & \multirow[t]{41}{*}{$02 / 28 / 16$} & \multirow{41}{*}{\multicolumn{2}{|c|}{ Restek, Lot A093733 }} & & \multirow{41}{*}{\multicolumn{2}{|c|}{ (Purchased Reagent) }} & $1,1,1,2$-Tetrachloroethane & 2000 & $\mathrm{ug} / \mathrm{mL}$ \\
\hline & & & & & & & 1,1,1-Trichloroethane & 2000 & $\mathrm{ug} / \mathrm{mL}$ \\
\hline & & & & & & & $1,1,2,2$-Tetrachloroethane & 2000 & $\mathrm{ug} / \mathrm{mL}$ \\
\hline & & & & & & & 1,1,2-Trichloroethane & 2000 & $\mathrm{ug} / \mathrm{mL}$ \\
\hline & & & & & & & 1,1-Dichloroethane & 2000 & $\mathrm{ug} / \mathrm{mL}$ \\
\hline & & & & & & & 1,1-Dichloroethene & 2000 & $\mathrm{ug} / \mathrm{mL}$ \\
\hline & & & & & & & 1,1-Dichloropropene & 2000 & $\mathrm{ug} / \mathrm{mL}$ \\
\hline & & & & & & & 1,2,3-Trichlorobenzene & 2000 & $\mathrm{ug} / \mathrm{mL}$ \\
\hline & & & & & & & 1,2,3-Trichloropropane & 2000 & $\mathrm{ug} / \mathrm{mL}$ \\
\hline & & & & & & & 1,2,4-Trichlorobenzene & 2000 & $\mathrm{ug} / \mathrm{mL}$ \\
\hline & & & & & & & 1,2,4-Trimethylbenzene & 2000 & $\mathrm{ug} / \mathrm{mL}$ \\
\hline & & & & & & & 1,2-Dibromo-3-Chloropropane & 2000 & $\mathrm{ug} / \mathrm{mL}$ \\
\hline & & & & & & & 1,2-Dibromoethane & 2000 & $\mathrm{ug} / \mathrm{mL}$ \\
\hline & & & & & & & 1,2-Dichlorobenzene & 2000 & $\mathrm{ug} / \mathrm{mL}$ \\
\hline & & & & & & & 1,2-Dichloroethane & 2000 & $\mathrm{ug} / \mathrm{mL}$ \\
\hline & & & & & & & 1,2-Dichloropropane & 2000 & $\mathrm{ug} / \mathrm{mL}$ \\
\hline & & & & & & & 1,3,5-Trimethylbenzene & 200 & $\mathrm{ug} / \mathrm{mL}$ \\
\hline & & & & & & & 1,3-Dichlorobenzene & 200 & $\mathrm{ug} / \mathrm{mL}$ \\
\hline & & & & & & & 1,3-Dichloropropane & 200 & $\mathrm{ug} / \mathrm{mL}$ \\
\hline & & & & & & & 1,4-Dichlorobenzene & 200 & $\mathrm{ug} / \mathrm{mL}$ \\
\hline & & & & & & & 1,4-Dioxane & 4000 & $\mathrm{ug} / \mathrm{mL}$ \\
\hline & & & & & & & 2,2-Dichloropropane & 200 & $\mathrm{ug} / \mathrm{mL}$ \\
\hline & & & & & & & 2-Chlorotoluene & 200 & $\mathrm{ug} / \mathrm{mL}$ \\
\hline & & & & & & & 4-Chlorotoluene & 200 & $\mathrm{ug} / \mathrm{mL}$ \\
\hline & & & & & & & 4-Isopropyltoluene & 200 & $\mathrm{ug} / \mathrm{mL}$ \\
\hline & & & & & & & Benzene & 200 & $\mathrm{ug} / \mathrm{mL}$ \\
\hline & & & & & & & Bromobenzene & 200 & $\mathrm{ug} / \mathrm{mL}$ \\
\hline & & & & & & & Bromochloromethane & 200 & $\mathrm{ug} / \mathrm{mL}$ \\
\hline & & & & & & & Bromodichloromethane & 200 & $\mathrm{ug} / \mathrm{mL}$ \\
\hline & & & & & & & Bromoform & 200 & $\mathrm{ug} / \mathrm{mL}$ \\
\hline & & & & & & & Carbon disulfide & 200 & $\mathrm{ug} / \mathrm{mL}$ \\
\hline & & & & & & & Carbon tetrachloride & 200 & $\mathrm{ug} / \mathrm{mL}$ \\
\hline & & & & & & & \begin{tabular}{|l|} 
Chlorobenzene \\
\end{tabular} & 200 & $\mathrm{ug} / \mathrm{mL}$ \\
\hline & & & & & & & Chloroform & 200 & $\mathrm{ug} / \mathrm{mL}$ \\
\hline & & & & & & & cis-1,2-Dichloroethene & 200 & $\mathrm{ug} / \mathrm{mL}$ \\
\hline & & & & & & & Cis-1,3-Dichloropropene & 200 & $\mathrm{ug} / \mathrm{mL}$ \\
\hline & & & & & & & Cyclohexane & 200 & $\mathrm{ug} / \mathrm{mL}$ \\
\hline & & & & & & & Dibromochloromethane & 200 & $\mathrm{ug} / \mathrm{mL}$ \\
\hline & & & & & & & Dibromomethane & 200 & $\mathrm{ug} / \mathrm{mL}$ \\
\hline & & & & & & & Ethylbenzene & 200 & $\mathrm{ug} / \mathrm{mL}$ \\
\hline & & & & & & & Freon TF & 200 & $\mathrm{ug} / \mathrm{mL}$ \\
\hline
\end{tabular}


SDG No.: 200-28914

\begin{tabular}{|c|c|c|c|c|c|c|c|c|}
\hline \multirow[b]{2}{*}{ Reagent ID } & \multirow[b]{2}{*}{$\begin{array}{l}\text { Exp } \\
\text { Date }\end{array}$} & \multirow[b]{2}{*}{$\begin{array}{l}\text { Prep } \\
\text { Date }\end{array}$} & \multirow[b]{2}{*}{$\begin{array}{l}\text { Dilutant } \\
\text { Used }\end{array}$} & \multirow{2}{*}{$\begin{array}{l}\text { Reagent } \\
\text { Final } \\
\text { Volume }\end{array}$} & \multicolumn{2}{|c|}{ Parent Reagent } & \multirow[b]{2}{*}{ Analyte } & \multirow[b]{2}{*}{ Concentration } \\
\hline & & & & & Reagent ID & $\begin{array}{l}\text { Volume } \\
\text { Added }\end{array}$ & & \\
\hline & & & & & & & 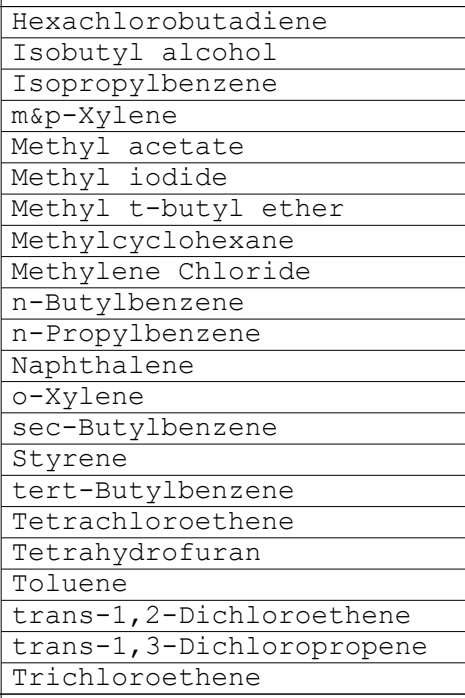 & $\begin{array}{r}2000 \mathrm{ug} / \mathrm{mL} \\
50000 \mathrm{ug} / \mathrm{mL} \\
2000 \mathrm{ug} / \mathrm{mL} \\
2000 \mathrm{ug} / \mathrm{mL} \\
10000 \mathrm{ug} / \mathrm{mL} \\
2000 \mathrm{ug} / \mathrm{mL} \\
2000 \mathrm{ug} / \mathrm{mL} \\
2000 \mathrm{ug} / \mathrm{mL} \\
2000 \mathrm{ug} / \mathrm{mL} \\
2000 \mathrm{ug} / \mathrm{mL} \\
2000 \mathrm{ug} / \mathrm{mL} \\
2000 \mathrm{ug} / \mathrm{mL} \\
2000 \mathrm{ug} / \mathrm{mL} \\
2000 \mathrm{ug} / \mathrm{mL} \\
2000 \mathrm{ug} / \mathrm{mL} \\
2000 \mathrm{ug} / \mathrm{mL} \\
2000 \mathrm{ug} / \mathrm{mL} \\
4000 \mathrm{ug} / \mathrm{mL} \\
2000 \mathrm{ug} / \mathrm{mL} \\
2000 \mathrm{ug} / \mathrm{mL} \\
2000 \mathrm{ug} / \mathrm{mL} \\
2000 \mathrm{ug} / \mathrm{mL}\end{array}$ \\
\hline .VMstd\#2SECs_00009 & $07 / 29 / 16$ & & Restek, Lot A0101295 & & (Purchased Re & & \begin{tabular}{|l|} 
2-Butanone \\
2-Hexanone \\
4-Methyl-2-pentanone \\
Acetone
\end{tabular} & $\begin{array}{l}10000 \mathrm{ug} / \mathrm{mL} \\
10000 \mathrm{ug} / \mathrm{mL} \\
10000 \mathrm{ug} / \mathrm{mL} \\
10000 \mathrm{ug} / \mathrm{mL}\end{array}$ \\
\hline .VMstd\#6SECs_00025 & $10 / 31 / 15$ & & Restek, Lot A0110474 & & (Purchased $R \epsilon$ & & Vinyl acetate & $5000 \mathrm{ug} / \mathrm{mL}$ \\
\hline 8260 SScorp_00015 & $08 / 08 / 15$ & $07 / 08 / 15$ & METHANOL, LOt 147358 & $10 \mathrm{~mL}$ & VM8260SSTDS_00004 & 200 uI & \begin{tabular}{|l} 
1,2-Dichloroethane-d4 \\
Bromofluorobenzene \\
Dibromofluoromethane (Surr) \\
Toluene-d8
\end{tabular} & $\begin{array}{l}50 \mathrm{ug} / \mathrm{mL} \\
50 \mathrm{ug} / \mathrm{mL} \\
50 \mathrm{ug} / \mathrm{mL} \\
50 \mathrm{ug} / \mathrm{mL}\end{array}$ \\
\hline .VM8260SSTDs_00004 & $06 / 01 / 16$ & & RESTEK, Lot A093505 & & (Purchased Re & & $\begin{array}{l}\text { 1,2-Dichloroethane-d4 } \\
\text { Bromofluorobenzene } \\
\text { Dibromofluoromethane (Surr) } \\
\text { Toluene-d8 }\end{array}$ & $\begin{array}{l}2500 \mathrm{ug} / \mathrm{mL} \\
2500 \mathrm{ug} / \mathrm{mL} \\
2500 \mathrm{ug} / \mathrm{mL} \\
2500 \mathrm{ug} / \mathrm{mL}\end{array}$ \\
\hline
\end{tabular}




\begin{tabular}{|c|c|c|c|c|}
\hline Laboratory & Authority & Program & EPA Region & Certification ID \\
\hline TestAmerica Burlington & Connecticut & State Program & $\overline{1}$ & $\overline{\mathrm{PH}}-0751$ \\
\hline TestAmerica Burlington & DE Haz. Subst. Cleanup Act (HSC & State Program & 3 & NA \\
\hline TestAmerica Burlington & Florida & NELAP & 4 & E87467 \\
\hline TestAmerica Burlington & L-A-B & DoD ELAP & & L2336 \\
\hline TestAmerica Burlington & Maine & State Program & 1 & VT00008 \\
\hline TestAmerica Burlington & Minnesota & NELAP & 5 & 050-999-436 \\
\hline TestAmerica Burlington & New Hampshire & NELAP & 1 & 2006 \\
\hline TestAmerica Burlington & New Jersey & NELAP & 2 & VT972 \\
\hline TestAmerica Burlington & New York & NELAP & 2 & 10391 \\
\hline TestAmerica Burlington & Pennsylvania & NELAP & 3 & $68-00489$ \\
\hline TestAmerica Burlington & Rhode Island & State Program & 1 & LAO00298 \\
\hline TestAmerica Burlington & US Fish \& Wildlife & Federal & & LE-058448-0 \\
\hline TestAmerica Burlington & USDA & Federal & & P330-11-00093 \\
\hline TestAmerica Burlington & Vermont & State Program & 1 & VT-4000 \\
\hline TestAmerica Burlington & Virginia & NELAP & 3 & 460209 \\
\hline
\end{tabular}

Accreditation may not be offered or required for all methods and analytes reported in this package. Please contact your project manager for the laboratory's current list of certified methods and analytes. 


\section{Method 8260B}

\section{Volatile Organic Compounds (GC/MS) by Method 8260B}


FORM II

GC/MS VOA SURROGATE RECOVERY

Lab Name: TestAmerica Burlington

Job No.: 200-28914-1

SDG No.: 200-28914

Matrix: Solid

Level: Medium

GC Column (1): DB-624

ID: $0.53(\mathrm{~mm})$

\begin{tabular}{|l|l|r|r|r|l|}
\hline Client Sample ID & Lab Sample ID & DBFM \# & DCA \# & TOL \# & BFB \# \\
\hline YK-S-37764 & $200-28914-1$ & 101 & 109 & 108 & 111 \\
\hline YK-S-37768 & $200-28914-2$ & 99 & 106 & 111 & 114 \\
\hline YK-S-37767 & $200-28914-3$ & 102 & 107 & 110 & 114 \\
\hline YK-S-37805 & $200-28914-4$ & 102 & 109 & 110 & 113 \\
\hline YK-S-37791 & $200-28914-5$ & 106 & 112 & 111 & 113 \\
\hline YK-S-37826 & $200-28914-6$ & 97 & 103 & 109 & 111 \\
\hline YK-S-37814 & $200-28914-7$ & 101 & 107 & 109 & 112 \\
\hline YK-S-37758 & $200-28914-8$ & 97 & 96 & 110 & 111 \\
\hline YK-S-BLANK & $200-28914-9$ & 97 & 103 & 113 & 113 \\
\hline & MB 200-91708/1-A & 97 & 100 & 110 & 112 \\
\hline & LCS & $100-91708 / 2-A$ & 107 & 111 & 111 \\
\hline
\end{tabular}

DBFM = Dibromofluoromethane (Surr)

$\mathrm{DCA}=1,2-\mathrm{Dichloroethane- \textrm {d } 4}$

TOL = Toluene-d8

$\mathrm{BFB}=$ Bromofluorobenzene
QC LIMITS

$50-150$

$80-120$

$80-120$

$80-125$

\# Column to be used to flag recovery values

FORM II $8260 B$

Page 54 of 121 
FORM III

GC/MS VOA LAB CONTROL SAMPLE RECOVERY

Lab Name: TestAmerica Burlington

Job No. : 200-28914-1

SDG No.: 200-28914

Matrix: Solid

Lab ID: LCS 200-91708/2-A
Level: Medium Lab File ID: 15018_03.D

Client ID:

\begin{tabular}{|c|c|c|c|c|c|}
\hline COMPOUND & $\begin{array}{c}\text { SPIKE } \\
\text { ADDED } \\
(\mathrm{ug} / \mathrm{Kg})\end{array}$ & $\begin{array}{c}\text { LCS } \\
\text { CONCENTRATION } \\
(\mathrm{ug} / \mathrm{Kg})\end{array}$ & $\begin{array}{l}\text { LCS } \\
\circ \\
\text { REC }\end{array}$ & $\begin{array}{c}Q C \\
\text { LIMITS } \\
\text { REC }\end{array}$ & $\#$ \\
\hline Dichlorodifluoromethane & 248 & 281 & 113 & $35-125$ & \\
\hline Chloromethane & 248 & 284 & 114 & $65-120$ & \\
\hline Vinyl chloride & 248 & 272 & 110 & $80-130$ & \\
\hline Bromomethane & 248 & 144 & 58 & $40-120$ & \\
\hline Chloroethane & 248 & 96.9 & 39 & $40-120$ & * \\
\hline Trichlorofluoromethane & 248 & 111 & 45 & $40-120$ & \\
\hline 1,1-Dichloroethene & 248 & 97.1 & 39 & $80-120$ & * \\
\hline Freon TF & 248 & 109 & 44 & $70-120$ & * \\
\hline Acetone & 1240 & 294 & 24 & $15-200$ & \\
\hline Methyl iodide & 248 & 53.2 & 21 & $45-150$ & * \\
\hline Carbon disulfide & 248 & 90.3 & 36 & $80-120$ & * \\
\hline Methyl acetate & 1240 & 1850 & 149 & $70-120$ & * \\
\hline Methylene Chloride & 248 & 233 & 94 & $80-120$ & \\
\hline trans-1,2-Dichloroethene & 248 & 277 & 112 & $80-125$ & \\
\hline Methyl t-butyl ether & 248 & 251 & 101 & $80-120$ & \\
\hline 1,1-Dichloroethane & 248 & 203 & 82 & $80-120$ & \\
\hline Vinyl acetate & 496 & 457 & 92 & $80-200$ & \\
\hline 2,2-Dichloropropane & 248 & 402 & 162 & $80-120$ & * \\
\hline cis-1,2-Dichloroethene & 248 & 276 & 111 & $80-125$ & \\
\hline 2-Butanone & 1240 & 1650 & 133 & $60-170$ & \\
\hline Bromochloromethane & 248 & 239 & 96 & $80-130$ & \\
\hline Tetrahydrofuran & 496 & 298 & 60 & $80-120$ & * \\
\hline Chloroform & 248 & 247 & 100 & $75-120$ & \\
\hline 1,1,1-Trichloroethane & 248 & 259 & 104 & $75-120$ & \\
\hline Cyclohexane & 248 & 252 & 102 & $80-120$ & \\
\hline 1,1-Dichloropropene & 248 & 283 & 114 & $80-125$ & \\
\hline Carbon tetrachloride & 248 & 265 & 107 & $75-120$ & \\
\hline Isobutyl alcohol & 6200 & 1140 & 18 & $80-130$ & * \\
\hline Benzene & 248 & 261 & 105 & $80-125$ & \\
\hline 1,2-Dichloroethane & 248 & 250 & 101 & $70-120$ & \\
\hline Trichloroethene & 248 & 256 & 103 & $75-120$ & \\
\hline Methylcyclohexane & 248 & 259 & 104 & $80-120$ & \\
\hline 1,2-Dichloropropane & 248 & 257 & 103 & $80-125$ & \\
\hline Dibromomethane & 248 & 211 & 85 & $75-120$ & \\
\hline 1,4-Dioxane & 4960 & 5560 & 112 & $75-140$ & \\
\hline Bromodichloromethane & 248 & 254 & 102 & $80-120$ & \\
\hline 2-Chloroethyl vinyl ether & 248 & 302 & 122 & $80-125$ & \\
\hline cis-1,3-Dichloropropene & 248 & 250 & 101 & $80-125$ & \\
\hline 4-Methyl-2-pentanone & 1240 & 1780 & 144 & $80-125$ & * \\
\hline Toluene & 248 & 266 & 107 & $80-120$ & \\
\hline trans-1,3-Dichloropropene & 248 & 274 & 110 & $80-120$ & \\
\hline 1,1,2-Trichloroethane & 248 & 260 & 105 & $80-125$ & \\
\hline
\end{tabular}

\# Column to be used to flag recovery and RPD values

FORM III $8260 B$

Page 55 of 121 
FORM III

GC/MS VOA LAB CONTROL SAMPLE RECOVERY

Lab Name: TestAmerica Burlington

Job No. : 200-28914-1

SDG No.: 200-28914

Matrix: Solid

Level: Medium

Lab File ID: 15018_03.D

Lab ID: LCS 200-91708/2-A

Client ID:

\begin{tabular}{|c|c|c|c|c|c|}
\hline COMPOUND & $\begin{array}{c}\text { SPIKE } \\
\text { ADDED } \\
(\mathrm{ug} / \mathrm{Kg})\end{array}$ & $\begin{array}{c}\text { LCS } \\
\text { CONCENTRATION } \\
(\mathrm{ug} / \mathrm{Kg})\end{array}$ & $\begin{array}{l}\text { LCS } \\
\stackrel{\circ}{\circ} \\
\operatorname{REC}\end{array}$ & $\begin{array}{c}\text { QC } \\
\text { LIMITS } \\
\text { REC }\end{array}$ & $\#$ \\
\hline Tetrachloroethene & 248 & 268 & 108 & $80-120$ & \\
\hline 1,3-Dichloropropane & 248 & 258 & 104 & $80-125$ & \\
\hline 2-Hexanone & 1240 & 1650 & 133 & $75-150$ & \\
\hline Dibromochloromethane & 248 & 258 & 104 & $80-125$ & \\
\hline 1,2-Dibromoethane & 248 & 264 & 106 & $80-120$ & \\
\hline Chlorobenzene & 248 & 262 & 105 & $80-120$ & \\
\hline $1,1,1,2$-Tetrachloroethane & 248 & 268 & 108 & $80-120$ & \\
\hline Ethylbenzene & 248 & 262 & 106 & $80-125$ & \\
\hline$m \& p-X y l e n e$ & 248 & 266 & 107 & $80-125$ & \\
\hline o-Xylene & 248 & 264 & 106 & $80-120$ & \\
\hline Styrene & 248 & 255 & 103 & $80-120$ & \\
\hline Bromoform & 248 & 224 & 90 & $80-120$ & \\
\hline Isopropylbenzene & 248 & 270 & 109 & $80-120$ & \\
\hline Bromobenzene & 248 & 270 & 109 & $80-125$ & \\
\hline $1,1,2,2$-Tetrachloroethane & 248 & 238 & 96 & $80-125$ & \\
\hline 1,2,3-Trichloropropane & 248 & 247 & 100 & $80-120$ & \\
\hline n-Propylbenzene & 248 & 272 & 110 & $80-120$ & \\
\hline 2-Chlorotoluene & 248 & 263 & 106 & $80-125$ & \\
\hline 4-Chlorotoluene & 248 & 264 & 106 & $80-125$ & \\
\hline 1,3,5-Trimethylbenzene & 248 & 269 & 108 & $80-120$ & \\
\hline tert-Butylbenzene & 248 & 273 & 110 & $75-120$ & \\
\hline $1,2,4$-Trimethylbenzene & 248 & 265 & 107 & $80-120$ & \\
\hline sec-Butylbenzene & 248 & 279 & 112 & $80-120$ & \\
\hline 1,3-Dichlorobenzene & 248 & 267 & 108 & $80-125$ & \\
\hline 4-Isopropyltoluene & 248 & 277 & 112 & $75-120$ & \\
\hline 1,4-Dichlorobenzene & 248 & 264 & 106 & $80-125$ & \\
\hline 1,2-Dichlorobenzene & 248 & 261 & 105 & $80-125$ & \\
\hline n-Butylbenzene & 248 & 287 & 116 & $80-120$ & \\
\hline 1,2-Dibromo-3-Chloropropane & 248 & 211 & 85 & $70-120$ & \\
\hline $1,2,4$-Trichlorobenzene & 248 & 278 & 112 & $80-120$ & \\
\hline Hexachlorobutadiene & 248 & 309 & 124 & $75-120$ & * \\
\hline Naphthalene & 248 & 195 & 78 & $80-130$ & * \\
\hline $1,2,3$-Trichlorobenzene & 248 & 236 & 95 & $80-120$ & \\
\hline
\end{tabular}

\# Column to be used to flag recovery and RPD values

FORM III $8260 B$

Page 56 of 121 
FORM IV

GC/MS VOA METHOD BLANK SUMMARY

Lab Name: TestAmerica Burlington

Job No : 200-28914-1

SDG No.: 200-28914

Lab File ID: 15018_05.D

Matrix: Solid

Instrument ID: CHL.i

Lab Sample ID: MB 200-91708/1-A

GC Column: DB-624

ID: $0.53(\mathrm{~mm})$

THIS METHOD BLANK APPLIES TO THE FOLLOWING SAMPLES:

\begin{tabular}{|c|c|c|c|c|}
\hline CLIENT SAMPLE ID & LAB SAMPLE ID & $\begin{array}{l}\text { LAB } \\
\text { FILE ID }\end{array}$ & \multicolumn{2}{|c|}{ DATE ANALYZED } \\
\hline & LCS 200-91708/2-A & 15018_03.D & $07 / 30 / 2015$ & $09: 31$ \\
\hline YK-S-37764 & $200-28914-1$ & 15018_06.D & $07 / 30 / 2015$ & $12: 42$ \\
\hline$Y K-S-37768$ & $200-28914-2$ & $15018 \quad 07 . \mathrm{D}$ & $07 / 30 / 2015$ & $13: 15$ \\
\hline YK-S-37767 & $200-28914-3$ & $1501808 . \mathrm{D}$ & $07 / 30 / 2015$ & $13: 47$ \\
\hline YK-S-37805 & $200-28914-4$ & $1501809 . \mathrm{D}$ & $07 / 30 / 2015$ & $14: 20$ \\
\hline YK-S-37791 & $200-28914-5$ & $15018 \_10 . \mathrm{D}$ & $07 / 30 / 2015$ & $14: 52$ \\
\hline YK-S-37826 & $200-28914-6$ & 15018_11.D & $07 / 30 / 2015$ & $15: 25$ \\
\hline YK-S-37814 & $200-28914-7$ & $15018 \_12 . \mathrm{D}$ & $07 / 30 / 2015$ & $15: 58$ \\
\hline$Y K-S-37758$ & $200-28914-8$ & 15018 13.D & $07 / 30 / 2015$ & $16: 30$ \\
\hline YK-S-BLANK & $200-28914-9$ & $15018 \_14 . D$ & $07 / 30 / 2015$ & $17: 03$ \\
\hline
\end{tabular}


FORM V

GC/MS VOA INSTRUMENT PERFORMANCE CHECK

BROMOFLUOROBENZENE (BFB)

Lab Name: TestAmerica Burlington

Job No : 200-28914-1

SDG No.: 200-28914

Lab File ID: 15008_01.D

Instrument ID: CHL.i

BFB Injection Date: 07/29/2015

BEB Injection Time: 11:39

Analysis Batch No.: 92007

\begin{tabular}{|c|c|c|}
\hline $\mathrm{M} / \mathrm{E}$ & ION ABUNDANCE CRITERIA & $\begin{array}{l}\text { RELATIVE } \\
\text { ABUNDANCE }\end{array}$ \\
\hline 50 & $15.0-40.0 \div$ of mass 95 & 21.5 \\
\hline 75 & $30.0-60.0 \div$ of mass 95 & 44.0 \\
\hline 95 & Base Peak, $100 \%$ relative abundance & 100.0 \\
\hline 96 & $5.0-9.0 \div$ of mass 95 & 6.7 \\
\hline 173 & Less than 2.0 \% of mass 174 & $(0.2) 1$ \\
\hline 174 & $50.0-120.00$ \% of mass 95 & 68.7 \\
\hline 175 & $5.0-9.0 \div$ of mass 174 & $(7.3) 1$ \\
\hline 176 & $95.0-101.0$ ㅇ of mass 174 & $(98.0) 1$ \\
\hline 177 & $5.0-9.0 \div$ of mass 176 & $(6.7) 2$ \\
\hline
\end{tabular}

$1-$ Value is $\%$ mass 174

2-Value is $\%$ mass 176

THIS CHECK APPLIES TO THE FOLLOWING SAMPLES, MS, MSD, BLANKS AND STANDARDS:

\begin{tabular}{|l|l|l|c|c|}
\hline CLIENT SAMPLE ID & LAB SAMPLE ID & $\begin{array}{c}\text { LAB } \\
\text { FILE ID }\end{array}$ & $\begin{array}{c}\text { DATE IME } \\
\text { ANALYED }\end{array}$ \\
ANALYED
\end{tabular}


FORM V

GC/MS VOA INSTRUMENT PERFORMANCE CHECK

BROMOFLUOROBENZENE (BFB)

Lab Name: TestAmerica Burlington

Job No : 200-28914-1

SDG No.: 200-28914

Lab File ID: 15018_01.D

Instrument ID: CHL.i

BFB Injection Date: 07/30/2015

BFB Injection Time: 08:36

Analysis Batch No.: 92051

\begin{tabular}{|c|c|c|}
\hline $\mathrm{M} / \mathrm{E}$ & ION ABUNDANCE CRITERIA & $\begin{array}{l}\text { RELATIVE } \\
\text { ABUNDANCE }\end{array}$ \\
\hline 50 & $15.0-40.0 \div$ of mass 95 & 21.2 \\
\hline 75 & $30.0-60.0 \div$ of mass 95 & 43.7 \\
\hline 95 & Base Peak, $100 \%$ relative abundance & 100.0 \\
\hline 96 & $5.0-9.0 \div$ of mass 95 & 6.7 \\
\hline 173 & Less than 2.0 \% of mass 174 & $(0.2) 1$ \\
\hline 174 & $50.0-120.00$ \% of mass 95 & 70.0 \\
\hline 175 & $5.0-9.0 \div$ of mass 174 & $(7.0) 1$ \\
\hline 176 & $95.0-101.0$ ㅇ of mass 174 & $(98.5) 1$ \\
\hline 177 & $5.0-9.0 \div$ of mass 176 & $(6.7) 2$ \\
\hline
\end{tabular}

$1-$ Value is $\%$ mass 174

2-Value is $\%$ mass 176

THIS CHECK APPLIES TO THE FOLLOWING SAMPLES, MS, MSD, BLANKS AND STANDARDS:

\begin{tabular}{|c|c|c|c|c|}
\hline CLIENT SAMPLE ID & LAB SAMPLE ID & $\begin{array}{l}\text { LAB } \\
\text { FILE ID }\end{array}$ & $\begin{array}{c}\text { DATE } \\
\text { ANALYZED }\end{array}$ & $\begin{array}{c}\text { TIME } \\
\text { ANALYZED }\end{array}$ \\
\hline & CCVIS 200-92051/2 & $1501802 . \mathrm{D}$ & $07 / 30 / 2015$ & $08: 59$ \\
\hline & LCS 200-91708/2-A & $15018 \_03 . \mathrm{D}$ & $07 / 30 / 2015$ & $09: 31$ \\
\hline & MB 200-91708/1-A & $1501805 . D$ & $07 / 30 / 2015$ & $10: 36$ \\
\hline $\mathrm{YK}-\mathrm{S}-37764$ & $200-28914-1$ & $15018-06 . D$ & $07 / 30 / 2015$ & $12: 42$ \\
\hline YK-S-37768 & $200-28914-2$ & $15018 \_07 . D$ & $07 / 30 / 2015$ & $13: 15$ \\
\hline YK-S-37767 & $200-28914-3$ & $1501808 . \mathrm{D}$ & $07 / 30 / 2015$ & $13: 47$ \\
\hline$Y K-S-37805$ & $200-28914-4$ & $15018-09 . D$ & $07 / 30 / 2015$ & $14: 20$ \\
\hline YK-S-37791 & $200-28914-5$ & $15018-10 . \mathrm{D}$ & $07 / 30 / 2015$ & $14: 52$ \\
\hline$Y K-S-37826$ & $200-28914-6$ & 15018 11.D & $07 / 30 / 2015$ & $15: 25$ \\
\hline YK-S-37814 & $200-28914-7$ & $1501812 . \mathrm{D}$ & $07 / 30 / 2015$ & $15: 58$ \\
\hline$Y K-S-37758$ & $200-28914-8$ & $15018 \_13 . \mathrm{D}$ & $07 / 30 / 2015$ & $16: 30$ \\
\hline YK-S-BLANK & $200-28914-9$ & 15018_14.D & $07 / 30 / 2015$ & $17: 03$ \\
\hline
\end{tabular}


FORM VIII

GC/MS VOA INTERNAL STANDARD AREA AND RETENTION TIME SUMMARY

Lab Name: TestAmerica Burlington

Job No : : 200-28914-1

SDG No.: 200-28914

Sample No.: ICIS 200-92007/7

Date Analyzed: 07/29/2015 14:44

Instrument ID: CHL.i

Lab File ID (Standard): 15008_07.D

GC Column: DB-624

ID $: 0.53(\mathrm{~mm})$

Calibration ID: 31571

Heated Purge: (Y/N) N

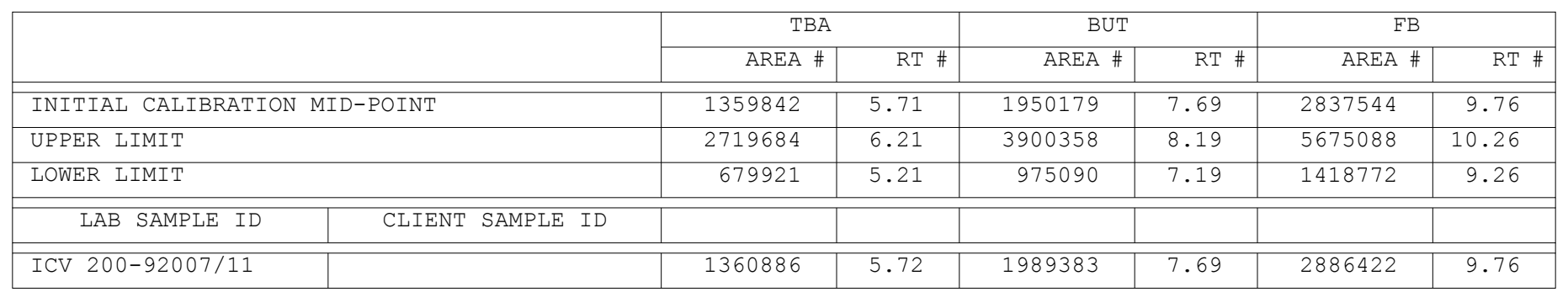

$\mathrm{TBA}=\mathrm{TBA}-\mathrm{d} 9 \quad(\mathrm{IS})$

$\mathrm{BUT}=2-$ Butanone-d5

$\mathrm{FB}=\mathrm{Fluorobenzene}$

Area Limit $=50 \%-200 \%$ of internal standard area

$\mathrm{RT}$ Limit $= \pm 0.5$ minutes of internal standard RT

\# Column used to flag values outside QC limits 
FORM VIII

GC/MS VOA INTERNAL STANDARD AREA AND RETENTION TIME SUMMARY

Lab Name: TestAmerica Burlington

Job No : : 200-28914-1

SDG No.: 200-28914

Sample No.: ICIS 200-92007/7

Date Analyzed: 07/29/2015 14:44

Instrument ID: CHL.i

Lab File ID (Standard): 15008_07.D

GC Column: DB-624

ID $: 0.53(\mathrm{~mm})$

Calibration ID: 31571

Heated Purge: (Y/N) N

\begin{tabular}{|c|c|c|c|c|c|c|c|}
\hline & & \multicolumn{2}{|c|}{ DXE } & \multicolumn{2}{|l|}{$\mathrm{CBZ}$} & \multicolumn{2}{|c|}{ DCB } \\
\hline & & AREA \# & RT \# & AREA \# & RT \# & AREA \# & RT \# \\
\hline INITIAL CALIBRATI & POINT & 136764 & 10.95 & 2278676 & 15.49 & 1249304 & 19.92 \\
\hline UPPER LIMIT & & 273528 & 11.45 & 4557352 & 15.99 & 2498608 & 20.42 \\
\hline LOWER LIMIT & & 68382 & 10.45 & 1139338 & 14.99 & 624652 & 19.42 \\
\hline LAB SAMPLE ID & CLIENT SAMPLE ID & & & & & & \\
\hline ICV 200-92007/11 & & 144094 & 10.96 & 2294117 & 15.49 & 1267188 & 19.93 \\
\hline
\end{tabular}

$\mathrm{DXE}=1,4-$ Dioxane-d8

$\mathrm{CBZ}=$ Chlorobenzene-d5

$\mathrm{DCB}=1,4-\mathrm{Dichlorobenzene-d4}$

Area Limit $=50 \%-200 \%$ of internal standard area

$\mathrm{RT}$ Limit $= \pm 0.5$ minutes of internal standard RT

\# Column used to flag values outside QC limits 
FORM VIII

GC/MS VOA INTERNAL STANDARD AREA AND RETENTION TIME SUMMARY

Lab Name: TestAmerica Burlington

SDG No.: 200-28914

Sample No.: CCVIS 200-92051/2

Instrument ID: CHL.i

Lab File ID (Standard): 15018_02.D

Calibration ID: 31571
Job No. : 200-28914-1

Date Analyzed: 07/30/2015 08:59

GC Column: DB-624

ID $: 0.53(\mathrm{~mm})$

Heated Purge: ( $\mathrm{Y} / \mathrm{N}) \mathrm{N}$

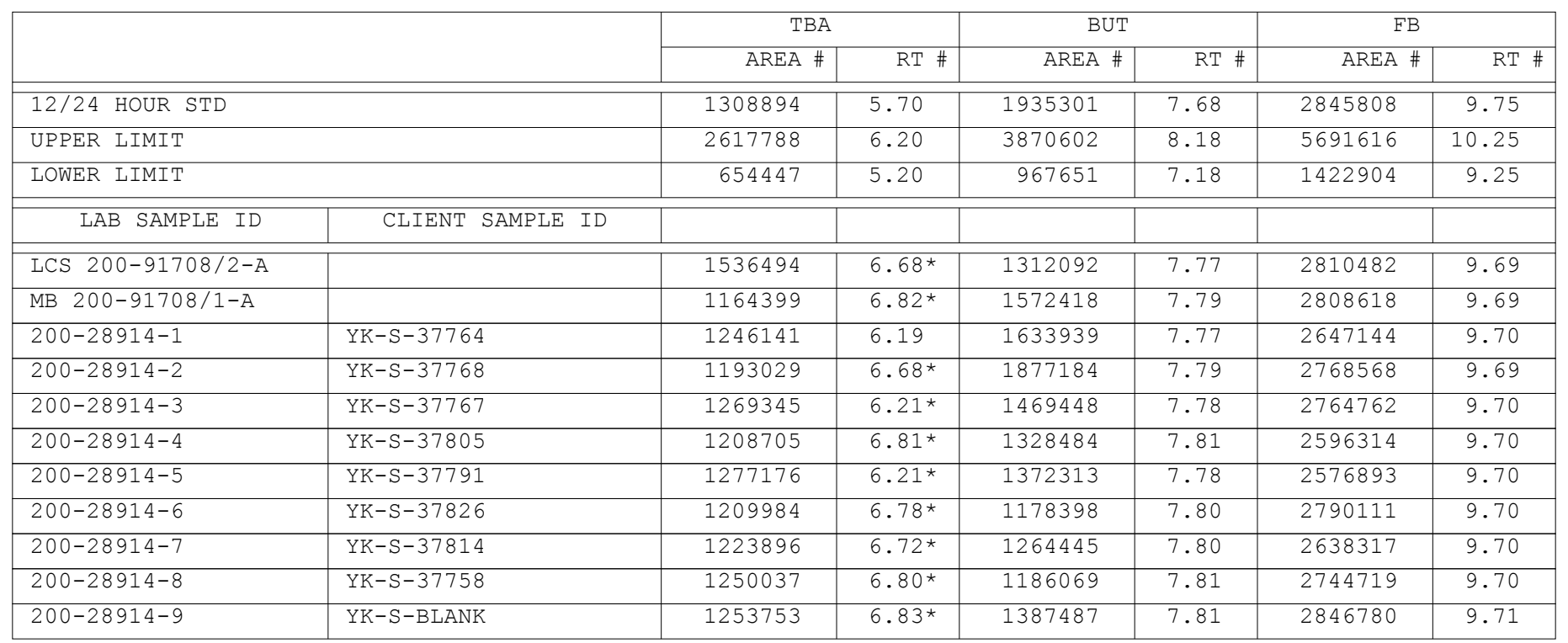

$\mathrm{TBA}=\mathrm{TBA}-\mathrm{d} 9 \quad(\mathrm{IS})$

$\mathrm{BUT}=2$-Butanone-d5

$\mathrm{FB}=\mathrm{Fluorobenzene}$

Area Limit $=50 \%-200 \%$ of internal standard area

$\mathrm{RT}$ Limit $= \pm 0.5$ minutes of internal standard RT

\# Column used to flag values outside QC limits 
FORM VIII

GC/MS VOA INTERNAL STANDARD AREA AND RETENTION TIME SUMMARY

Lab Name: TestAmerica Burlington

SDG No.: 200-28914

Sample No.: CCVIS 200-92051/2

Instrument ID: CHL.i

Lab File ID (Standard): 15018_02.D

Calibration ID: 31571
Job No. : 200-28914-1

Date Analyzed: 07/30/2015 08:59

GC Column: DB-624

ID $: 0.53(\mathrm{~mm})$

Heated Purge: (Y/N) N

\begin{tabular}{|c|c|c|c|c|c|c|c|}
\hline & & \multicolumn{2}{|l|}{ DXE } & \multicolumn{2}{|l|}{ CBZ } & \multicolumn{2}{|l|}{$\mathrm{DCB}$} \\
\hline & & AREA \# & RT \# & AREA \# & RT \# & AREA \# & RT \# \\
\hline $12 / 24$ HOUR STD & & 140789 & 10.95 & 2275683 & 15.48 & 1240430 & 19.91 \\
\hline UPPER LIMIT & & 281578 & 11.45 & 4551366 & 15.98 & 2480860 & 20.41 \\
\hline LOWER LIMIT & & 70395 & 10.45 & 1137842 & 14.98 & 620215 & 19.41 \\
\hline LAB SAMPLE ID & CLIENT SAMPLE ID & & & & & & \\
\hline LCS 200-91708/2-A & & 100592 & 11.33 & 2175294 & 15.48 & 1182011 & 19.92 \\
\hline MB 200-91708/1-A & & 131906 & 11.34 & 2184541 & 15.48 & 1155536 & 19.92 \\
\hline $200-28914-1$ & YK-S-37764 & 118293 & 11.26 & 2090366 & 15.48 & 1099650 & 19.92 \\
\hline $200-28914-2$ & YK-S-37768 & 127982 & 11.28 & 2175505 & 15.47 & 1130716 & 19.92 \\
\hline $200-28914-3$ & YK-S-37767 & 115647 & 11.27 & 2152767 & 15.48 & 1120602 & 19.92 \\
\hline $200-28914-4$ & YK-S-37805 & 103095 & 11.32 & 2016440 & 15.48 & 1072848 & 19.92 \\
\hline $200-28914-5$ & YK-S-37791 & 126843 & 11.28 & 1984258 & 15.48 & 1045048 & 19.92 \\
\hline $200-28914-6$ & YK-S-37826 & 114852 & 11.34 & 2154557 & 15.48 & 1126345 & 19.92 \\
\hline $200-28914-7$ & YK-S-37814 & 110120 & 11.30 & 2048544 & 15.48 & 1081262 & 19.92 \\
\hline $200-28914-8$ & YK-S-37758 & 126892 & 11.34 & 2119886 & 15.48 & 1120382 & 19.93 \\
\hline $200-28914-9$ & YK-S-BLANK & 132538 & 11.32 & 2215054 & 15.48 & 1164751 & 19.92 \\
\hline
\end{tabular}

DXE $=1,4-$ Dioxane-d8

$\mathrm{CBZ}=$ Chlorobenzene-d5

$\mathrm{DCB}=1,4-\mathrm{Dichlorobenzene-d4}$

Area Limit $=50 \%-200 \%$ of internal standard area

$\mathrm{RT}$ Limit $= \pm 0.5$ minutes of internal standard RT

\# Column used to flag values outside QC limits 
FORM I

GC/MS VOA ORGANICS ANALYSIS DATA SHEET

Lab Name: TestAmerica Burlington

Job No : : 200-28914-1

SDG No.: 200-28914

Client Sample ID: YK-S-37764

Matrix: Solid

Analysis Method: 8260B

Sample wt/vol: $10.046(\mathrm{~g})$

Soil Aliquot Vol: 500 (uL)

Soil Extract Vol.: $10(\mathrm{~mL})$

Moisture:

Analysis Batch No.: 92051

Lab Sample ID: 200-28914-1

Lab File ID: 15018_06.D

Date Collected: 07/16/2015 00:00

Date Analyzed: 07/30/2015 12:42

Dilution Factor: 1

GC Column: DB-624

ID $: 0.53(\mathrm{~mm})$

Level: (low/med) Medium

Units: ug/Kg

\begin{tabular}{|c|c|c|c|c|c|}
\hline CAS NO. & COMPOUND NAME & RESULT & $Q$ & $\mathrm{RL}$ & MDL \\
\hline $75-71-8$ & Dichlorodifluoromethane & 10 & $\mathrm{U}$ & 10 & 1.7 \\
\hline $74-87-3$ & Chloromethane & 4.9 & $\mathrm{~J} B$ & 10 & 2.1 \\
\hline $75-01-4$ & Vinyl chloride & 10 & $\mathrm{U}$ & 10 & 0.83 \\
\hline $74-83-9$ & Bromomethane & 18 & B & 10 & 1.0 \\
\hline $75-00-3$ & Chloroethane & 10 & $\mathrm{U} *$ & 10 & 2.5 \\
\hline $75-69-4$ & Trichlorofluoromethane & 10 & $\mathrm{U}$ & 10 & 0.82 \\
\hline $75-35-4$ & 1,1-Dichloroethene & 10 & $\mathrm{U} *$ & 10 & 1.1 \\
\hline $76-13-1$ & Freon TF & 10 & $U \star$ & 10 & 0.85 \\
\hline $67-64-1$ & Acetone & 50 & $\mathrm{U}$ & 50 & 12 \\
\hline $74-88-4$ & Methyl iodide & 8.9 & $J \star B$ & 10 & 0.75 \\
\hline $75-15-0$ & Carbon disulfide & 1.8 & $J \star$ & 10 & 0.87 \\
\hline $79-20-9$ & Methyl acetate & 51 & $\star$ & 50 & 9.2 \\
\hline $75-09-2$ & Methylene Chloride & 8.6 & $\mathrm{~J}$ & 10 & 2.3 \\
\hline $156-60-5$ & trans-1,2-Dichloroethene & 1.9 & $\mathrm{~J}$ & 10 & 0.99 \\
\hline $1634-04-4$ & Methyl t-butyl ether & 10 & $\mathrm{U}$ & 10 & 1.1 \\
\hline $75-34-3$ & 1,1-Dichloroethane & 10 & $\mathrm{U}$ & 10 & 1.1 \\
\hline $108-05-4$ & Vinyl acetate & 50 & $\mathrm{U}$ & 50 & 4.5 \\
\hline $594-20-7$ & 2,2-Dichloropropane & 10 & $\mathrm{U}$ * & 10 & 2.2 \\
\hline $156-59-2$ & cis-1,2-Dichloroethene & 2.1 & $\mathrm{~J}$ & 10 & 1.0 \\
\hline $78-93-3$ & 2-Butanone & 50 & $\mathrm{U}$ & 50 & 5.9 \\
\hline $74-97-5$ & Bromochloromethane & 10 & $\mathrm{U}$ & 10 & 1.4 \\
\hline $109-99-9$ & Tetrahydrofuran & 20 & $U \star$ & 20 & 5.3 \\
\hline $67-66-3$ & Chloroform & 10 & $\mathrm{U}$ & 10 & 1.2 \\
\hline $71-55-6$ & $1,1,1$-Trichloroethane & 10 & $\mathrm{U}$ & 10 & 0.85 \\
\hline $110-82-7$ & Cyclohexane & 10 & $\mathrm{U}$ & 10 & 1.0 \\
\hline $563-58-6$ & 1,1-Dichloropropene & 10 & $\mathrm{U}$ & 10 & 0.97 \\
\hline $56-23-5$ & Carbon tetrachloride & 2.4 & $\mathrm{~J}$ & 10 & 2.0 \\
\hline $78-83-1$ & Isobutyl alcohol & 500 & $\mathrm{U} *$ & 500 & 27 \\
\hline $71-43-2$ & Benzene & 10 & $\mathrm{U}$ & 10 & 1.1 \\
\hline $107-06-2$ & 1,2-Dichloroethane & 10 & $\mathrm{U}$ & 10 & 1.3 \\
\hline $79-01-6$ & Trichloroethene & 10 & $\mathrm{U}$ & 10 & 0.83 \\
\hline $108-87-2$ & Methylcyclohexane & 10 & $\mathrm{U}$ & 10 & 2.1 \\
\hline $78-87-5$ & 1,2-Dichloropropane & 10 & $\mathrm{U}$ & 10 & 1.2 \\
\hline $74-95-3$ & Dibromomethane & 2.5 & $\mathrm{~J}$ & 10 & 1.5 \\
\hline $123-91-1$ & 1,4-Dioxane & 400 & $\mathrm{U}$ & 400 & 65 \\
\hline $75-27-4$ & Bromodichloromethane & 10 & $\mathrm{U}$ & 10 & 1.0 \\
\hline
\end{tabular}

FORM I $8260 B$ 
FORM I

GC/MS VOA ORGANICS ANALYSIS DATA SHEET

Lab Name: TestAmerica Burlington

Job No : : 200-28914-1

SDG No.: 200-28914

Client Sample ID: YK-S-37764

Matrix: Solid

Analysis Method: 8260B

Sample wt/vol: $10.046(\mathrm{~g})$

Soil Aliquot Vol: 500 (uL)

Soil Extract Vol.: $10(\mathrm{~mL})$

Moisture:

Analysis Batch No.: 92051

Lab Sample ID: 200-28914-1

Lab File ID: 15018_06.D

Date Collected: 07/16/2015 00:00

Date Analyzed: 07/30/2015 12:42

Dilution Factor: 1

GC Column: DB-624

ID $: 0.53(\mathrm{~mm})$

Level: (low/med) Medium

Units: ug/Kg

\begin{tabular}{|c|c|c|c|c|c|}
\hline CAS NO. & COMPOUND NAME & RESULT & $Q$ & RL & MDL \\
\hline $110-75-8$ & 2-Chloroethyl vinyl ether & 10 & $\mathrm{U}$ & 10 & 1.9 \\
\hline $10061-01-5$ & cis-1,3-Dichloropropene & 2.9 & $\mathrm{~J}$ & 10 & 1.2 \\
\hline $108-10-1$ & 4-Methyl-2-pentanone & 50 & $\mathrm{U} *$ & 50 & 4.7 \\
\hline $108-88-3$ & Toluene & 10 & $\mathrm{U}$ & 10 & 2.1 \\
\hline $10061-02-6$ & trans-1,3-Dichloropropene & 4.8 & $\mathrm{~J}$ & 10 & 2.2 \\
\hline $79-00-5$ & $1,1,2$-Trichloroethane & 10 & $\mathrm{U}$ & 10 & 1.3 \\
\hline $127-18-4$ & Tetrachloroethene & 10 & $\mathrm{U}$ & 10 & 2.0 \\
\hline $142-28-9$ & 1,3-Dichloropropane & 10 & $\mathrm{U}$ & 10 & 1.1 \\
\hline $591-78-6$ & 2-Hexanone & 50 & $\mathrm{U}$ & 50 & 6.1 \\
\hline $124-48-1$ & Dibromochloromethane & 10 & $\mathrm{U}$ & 10 & 1.1 \\
\hline $106-93-4$ & 1,2-Dibromoethane & 10 & $\mathrm{U}$ & 10 & 1.2 \\
\hline $108-90-7$ & Chlorobenzene & 10 & $\mathrm{U}$ & 10 & 0.91 \\
\hline $630-20-6$ & $1,1,1,2$-Tetrachloroethane & 10 & $\mathrm{U}$ & 10 & 1.1 \\
\hline $100-41-4$ & Ethylbenzene & 1.2 & $\mathrm{~J} B$ & 10 & 0.94 \\
\hline $179601-23-1$ & m\&p-Xylene & 1.4 & $\mathrm{~J}$ & 10 & 1.1 \\
\hline $95-47-6$ & o-Xylene & 10 & $\mathrm{U}$ & 10 & 1.0 \\
\hline $100-42-5$ & Styrene & 10 & $\mathrm{U}$ & 10 & 0.98 \\
\hline $75-25-2$ & Bromoform & 10 & $\mathrm{U}$ & 10 & 1.0 \\
\hline $98-82-8$ & Isopropylbenzene & 10 & $\mathrm{U}$ & 10 & 2.0 \\
\hline $108-86-1$ & Bromobenzene & 2.0 & $\mathrm{~J} B$ & 10 & 1.0 \\
\hline $79-34-5$ & $1,1,2,2$-Tetrachloroethane & 10 & $\mathrm{U}$ & 10 & 1.3 \\
\hline $96-18-4$ & 1,2,3-Trichloropropane & 10 & $\mathrm{U}$ & 10 & 1.2 \\
\hline $103-65-1$ & n-Propylbenzene & 10 & $\mathrm{U}$ & 10 & 1.1 \\
\hline $95-49-8$ & 2-Chlorotoluene & 10 & $\mathrm{U}$ & 10 & 1.1 \\
\hline $106-43-4$ & 4-Chlorotoluene & 1.4 & $\mathrm{~J} B$ & 10 & 0.96 \\
\hline $108-67-8$ & 1,3,5-Trimethylbenzene & 10 & $\mathrm{U}$ & 10 & 2.0 \\
\hline $98-06-6$ & tert-Butylbenzene & 10 & $\mathrm{U}$ & 10 & 1.4 \\
\hline $95-63-6$ & $1,2,4$-Trimethylbenzene & 10 & $\mathrm{U}$ & 10 & 1.0 \\
\hline $135-98-8$ & sec-Butylbenzene & 10 & $\mathrm{U}$ & 10 & 2.0 \\
\hline $541-73-1$ & 1,3-Dichlorobenzene & 3.3 & $\mathrm{~J} B$ & 10 & 0.95 \\
\hline $99-87-6$ & 4-Isopropyltoluene & 10 & $\mathrm{U}$ & 10 & 0.88 \\
\hline $106-46-7$ & 1,4-Dichlorobenzene & 10 & $\mathrm{U}$ & 10 & 1.4 \\
\hline $95-50-1$ & 1,2-Dichlorobenzene & 2.1 & $\mathrm{~J} B$ & 10 & 1.1 \\
\hline $104-51-8$ & n-Butylbenzene & 1.4 & $\mathrm{~J} B$ & 10 & 0.90 \\
\hline $96-12-8$ & 1,2-Dibromo-3-Chloropropane & 10 & $\mathrm{U}$ & 10 & 2.1 \\
\hline $120-82-1$ & $1,2,4$-Trichlorobenzene & 4.5 & $\mathrm{~J} B$ & 10 & 0.91 \\
\hline
\end{tabular}

FORM I $8260 B$ 
FORM I

GC/MS VOA ORGANICS ANALYSIS DATA SHEET

Lab Name: TestAmerica Burlington

SDG No.: 200-28914

Client Sample ID: YK-S-37764

Matrix: Solid

Analysis Method: 8260B

Sample wt/vol: $10.046(\mathrm{~g})$

Soil Aliquot Vol: 500 (uL)

Soil Extract Vol.: 10 (mL)

응 Moisture:

Analysis Batch No.: 92051
Job No. : 200-28914-1

Lab Sample ID: 200-28914-1

Lab File ID: 15018_06.D

Date Collected: 07/16/2015 00:00

Date Analyzed: 07/30/2015 12:42

Dilution Factor: 1

GC Column: DB-624

ID $: 0.53(\mathrm{~mm})$

Level: (low/med) Medium

Units: ug/Kg

\begin{tabular}{|c|c|c|c|c|c|}
\hline CAS NO. & COMPOUND NAME & RESULT & Q & RL & MDL \\
\hline $87-68-3$ & Hexachlorobutadiene & 2.3 & $\mathrm{~J} \star \mathrm{B}$ & 10 & 2.3 \\
\hline $91-20-3$ & Naphthalene & 4.2 & $\mathrm{~J} \star \mathrm{B}$ & 10 & 1.2 \\
\hline $87-61-6$ & $1,2,3$-Trichlorobenzene & 3.6 & $\mathrm{~J} B$ & 10 & 1.0 \\
\hline
\end{tabular}

\begin{tabular}{|c|c|c|c|c|}
\hline CAS NO. & SURROGATE & $\because \mathrm{REC}$ & Q & LIMITS \\
\hline $17060-07-0$ & 1,2-Dichloroethane-d4 & 109 & & $80-120$ \\
\hline $2037-26-5$ & Toluene-d8 & 108 & & $80-120$ \\
\hline $460-00-4$ & Bromofluorobenzene & 111 & & $80-125$ \\
\hline $1868-53-7$ & Dibromofluoromethane (Surr) & 101 & & $50-150$ \\
\hline
\end{tabular}


FORM I

GC/MS VOA ORGANICS ANALYSIS DATA SHEET

Lab Name: TestAmerica Burlington

Job No : : 200-28914-1

SDG No.: 200-28914

Client Sample ID: YK-S-37768

Matrix: Solid

Analysis Method: 8260B

Sample wt/vol: $9.569(\mathrm{~g})$

Soil Aliquot Vol: 500 (uL)

Soil Extract Vol.: $10(\mathrm{~mL})$

응 Moisture:

Analysis Batch No.: 92051

Lab Sample ID: 200-28914-2

Lab File ID: 15018_07.D

Date Collected: 07/16/2015 00:00

Date Analyzed: 07/30/2015 13:15

Dilution Factor: 1

GC Column: DB-624

ID $: 0.53(\mathrm{~mm})$

Level: (low/med) Medium

Units: ug/Kg

\begin{tabular}{|c|c|c|c|c|c|}
\hline CAS NO. & COMPOUND NAME & RESULT & $Q$ & RL & MDL \\
\hline $75-71-8$ & Dichlorodifluoromethane & 10 & $\mathrm{U}$ & 10 & 1.8 \\
\hline $74-87-3$ & Chloromethane & 4.0 & $\mathrm{~J} B$ & 10 & 2.2 \\
\hline $75-01-4$ & Vinyl chloride & 10 & $\mathrm{U}$ & 10 & 0.87 \\
\hline $74-83-9$ & Bromomethane & 17 & B & 10 & 1.0 \\
\hline $75-00-3$ & Chloroethane & 10 & $\mathrm{U} *$ & 10 & 2.6 \\
\hline $75-69-4$ & Trichlorofluoromethane & 10 & $\mathrm{U}$ & 10 & 0.86 \\
\hline $75-35-4$ & 1,1-Dichloroethene & 10 & $\mathrm{U} *$ & 10 & 1.1 \\
\hline $76-13-1$ & Freon TF & 10 & $\mathrm{U} *$ & 10 & 0.89 \\
\hline $67-64-1$ & Acetone & 52 & $\mathrm{U}$ & 52 & 13 \\
\hline $74-88-4$ & Methyl iodide & 9.2 & $\mathrm{~J} \star \mathrm{B}$ & 10 & 0.78 \\
\hline $75-15-0$ & Carbon disulfide & 1.1 & $\mathrm{~J} \star$ & 10 & 0.91 \\
\hline $79-20-9$ & Methyl acetate & 52 & $U *$ & 52 & 9.6 \\
\hline $75-09-2$ & Methylene Chloride & 5.6 & $\mathrm{~J}$ & 10 & 2.4 \\
\hline $156-60-5$ & trans-1,2-Dichloroethene & 10 & $\mathrm{U}$ & 10 & 1.0 \\
\hline $1634-04-4$ & Methyl t-butyl ether & 10 & $\mathrm{U}$ & 10 & 1.1 \\
\hline $75-34-3$ & 1,1-Dichloroethane & 10 & $\mathrm{U}$ & 10 & 1.1 \\
\hline $108-05-4$ & Vinyl acetate & 52 & $\mathrm{U}$ & 52 & 4.7 \\
\hline $594-20-7$ & 2,2-Dichloropropane & 10 & $\mathrm{U} *$ & 10 & 2.3 \\
\hline $156-59-2$ & cis-1,2-Dichloroethene & 10 & $\mathrm{U}$ & 10 & 1.0 \\
\hline $78-93-3$ & 2-Butanone & 52 & $\mathrm{U}$ & 52 & 6.2 \\
\hline $74-97-5$ & Bromochloromethane & 10 & $\mathrm{U}$ & 10 & 1.5 \\
\hline $109-99-9$ & Tetrahydrofuran & 21 & $U \star$ & 21 & 5.5 \\
\hline $67-66-3$ & Chloroform & 10 & $\mathrm{U}$ & 10 & 1.3 \\
\hline $71-55-6$ & $1,1,1$-Trichloroethane & 10 & $\mathrm{U}$ & 10 & 0.89 \\
\hline $110-82-7$ & Cyclohexane & 10 & $\mathrm{U}$ & 10 & 1.0 \\
\hline $563-58-6$ & 1,1-Dichloropropene & 10 & $\mathrm{U}$ & 10 & 1.0 \\
\hline $56-23-5$ & Carbon tetrachloride & 6.5 & $\mathrm{~J}$ & 10 & 2.1 \\
\hline $78-83-1$ & Isobutyl alcohol & 520 & $\mathrm{U} *$ & 520 & 28 \\
\hline $71-43-2$ & Benzene & 10 & $\mathrm{U}$ & 10 & 1.1 \\
\hline $107-06-2$ & 1,2-Dichloroethane & 10 & $\mathrm{U}$ & 10 & 1.4 \\
\hline $79-01-6$ & Trichloroethene & 10 & $\mathrm{U}$ & 10 & 0.87 \\
\hline $108-87-2$ & Methylcyclohexane & 10 & $\mathrm{U}$ & 10 & 2.2 \\
\hline $78-87-5$ & 1,2-Dichloropropane & 10 & $\mathrm{U}$ & 10 & 1.3 \\
\hline $74-95-3$ & Dibromomethane & 10 & $\mathrm{U}$ & 10 & 1.6 \\
\hline $123-91-1$ & 1,4-Dioxane & 420 & $\mathrm{U}$ & 420 & 68 \\
\hline $75-27-4$ & Bromodichloromethane & 10 & $\mathrm{U}$ & 10 & 1.0 \\
\hline
\end{tabular}

FORM I $8260 B$ 
FORM I

GC/MS VOA ORGANICS ANALYSIS DATA SHEET

Lab Name: TestAmerica Burlington

Job No : : 200-28914-1

SDG No.: 200-28914

Client Sample ID: YK-S-37768

Matrix: Solid

Analysis Method: 8260B

Sample wt/vol: $9.569(\mathrm{~g})$

Soil Aliquot Vol: 500 (uL)

Soil Extract Vol.: $10(\mathrm{~mL})$

Moisture:

Analysis Batch No.: 92051

Lab Sample ID: 200-28914-2

Lab File ID: 15018_07.D

Date Collected: 07/16/2015 00:00

Date Analyzed: 07/30/2015 13:15

Dilution Factor: 1

GC Column: DB-624

ID $: 0.53(\mathrm{~mm})$

Level: (low/med) Medium

Units: ug/Kg

\begin{tabular}{|c|c|c|c|c|c|}
\hline CAS NO. & COMPOUND NAME & RESULT & $Q$ & RL & MDL \\
\hline $110-75-8$ & 2-Chloroethyl vinyl ether & 10 & $\mathrm{U}$ & 10 & 2.0 \\
\hline $10061-01-5$ & cis-1,3-Dichloropropene & 10 & $\mathrm{U}$ & 10 & 1.3 \\
\hline $108-10-1$ & 4-Methyl-2-pentanone & 52 & $\mathrm{U} *$ & 52 & 4.9 \\
\hline $108-88-3$ & Toluene & 10 & $\mathrm{U}$ & 10 & 2.2 \\
\hline $10061-02-6$ & trans-1,3-Dichloropropene & 10 & $\mathrm{U}$ & 10 & 2.3 \\
\hline $79-00-5$ & $1,1,2$-Trichloroethane & 10 & $\mathrm{U}$ & 10 & 1.4 \\
\hline $127-18-4$ & Tetrachloroethene & 10 & $\mathrm{U}$ & 10 & 2.1 \\
\hline $142-28-9$ & 1,3-Dichloropropane & 10 & $\mathrm{U}$ & 10 & 1.1 \\
\hline $591-78-6$ & 2-Hexanone & 52 & $\mathrm{U}$ & 52 & 6.4 \\
\hline $124-48-1$ & Dibromochloromethane & 10 & $\mathrm{U}$ & 10 & 1.1 \\
\hline $106-93-4$ & 1,2-Dibromoethane & 10 & $\mathrm{U}$ & 10 & 1.3 \\
\hline $108-90-7$ & Chlorobenzene & 10 & $\mathrm{U}$ & 10 & 0.95 \\
\hline $630-20-6$ & $1,1,1,2$-Tetrachloroethane & 10 & $\mathrm{U}$ & 10 & 1.1 \\
\hline $100-41-4$ & Ethylbenzene & 10 & $\mathrm{U}$ & 10 & 0.98 \\
\hline $179601-23-1$ & m\&p-Xylene & 10 & $\mathrm{U}$ & 10 & 1.1 \\
\hline $95-47-6$ & o-Xylene & 10 & $\mathrm{U}$ & 10 & 1.0 \\
\hline $100-42-5$ & Styrene & 10 & $\mathrm{U}$ & 10 & 1.0 \\
\hline $75-25-2$ & Bromoform & 10 & $\mathrm{U}$ & 10 & 1.0 \\
\hline $98-82-8$ & Isopropylbenzene & 10 & $\mathrm{U}$ & 10 & 2.1 \\
\hline $108-86-1$ & Bromobenzene & 10 & $\mathrm{U}$ & 10 & 1.0 \\
\hline $79-34-5$ & $1,1,2,2$-Tetrachloroethane & 10 & $\mathrm{U}$ & 10 & 1.4 \\
\hline $96-18-4$ & 1,2,3-Trichloropropane & 10 & $\mathrm{U}$ & 10 & 1.3 \\
\hline $103-65-1$ & n-Propylbenzene & 10 & $\mathrm{U}$ & 10 & 1.1 \\
\hline $95-49-8$ & 2-Chlorotoluene & 10 & $\mathrm{U}$ & 10 & 1.1 \\
\hline $106-43-4$ & 4-Chlorotoluene & 10 & $\mathrm{U}$ & 10 & 1.0 \\
\hline $108-67-8$ & 1,3,5-Trimethylbenzene & 10 & $\mathrm{U}$ & 10 & 2.1 \\
\hline $98-06-6$ & tert-Butylbenzene & 10 & $\mathrm{U}$ & 10 & 1.5 \\
\hline $95-63-6$ & $1,2,4$-Trimethylbenzene & 10 & $\mathrm{U}$ & 10 & 1.0 \\
\hline $135-98-8$ & sec-Butylbenzene & 10 & $\mathrm{U}$ & 10 & 2.1 \\
\hline $541-73-1$ & 1,3-Dichlorobenzene & 10 & $\mathrm{U}$ & 10 & 0.99 \\
\hline $99-87-6$ & 4-Isopropyltoluene & 10 & $\mathrm{U}$ & 10 & 0.92 \\
\hline $106-46-7$ & 1,4-Dichlorobenzene & 10 & $\mathrm{U}$ & 10 & 1.5 \\
\hline $95-50-1$ & 1,2-Dichlorobenzene & 10 & $\mathrm{U}$ & 10 & 1.1 \\
\hline $104-51-8$ & n-Butylbenzene & 10 & $\mathrm{U}$ & 10 & 0.94 \\
\hline $96-12-8$ & 1,2-Dibromo-3-Chloropropane & 10 & $\mathrm{U}$ & 10 & 2.2 \\
\hline $120-82-1$ & $1,2,4$-Trichlorobenzene & 1.5 & $\mathrm{~J} B$ & 10 & 0.95 \\
\hline
\end{tabular}

FORM I $8260 B$ 
FORM I

GC/MS VOA ORGANICS ANALYSIS DATA SHEET

Lab Name: TestAmerica Burlington

SDG No.: 200-28914

Client Sample ID: YK-S-37768

Matrix: Solid

Analysis Method: 8260B

Sample wt/vol: $9.569(\mathrm{~g})$

Soil Aliquot Vol: 500 (uL)

Soil Extract Vol.: 10 (mL)

응 Moisture:

Analysis Batch No.: 92051
Job No. : 200-28914-1

Lab Sample ID: 200-28914-2

Lab File ID: 15018_07.D

Date Collected: 07/16/2015 00:00

Date Analyzed: 07/30/2015 13:15

Dilution Factor: 1

GC Column: DB-624

ID $: 0.53(\mathrm{~mm})$

Level: (low/med) Medium

Units: ug/Kg

\begin{tabular}{|c|c|c|c|c|c|}
\hline CAS NO. & COMPOUND NAME & RESULT & Q & RL & MDL \\
\hline $87-68-3$ & Hexachlorobutadiene & 10 & $\mathrm{U} *$ & 10 & 2.4 \\
\hline $91-20-3$ & Naphthalene & 2.2 & $\mathrm{~J} \star \mathrm{B}$ & 10 & 1.3 \\
\hline $87-61-6$ & $1,2,3$-Trichlorobenzene & 1.3 & $\mathrm{~J} B$ & 10 & 1.0 \\
\hline
\end{tabular}

\begin{tabular}{|l|l|r|r|r|}
\hline \multicolumn{1}{|c|}{ CAS NO. } & \multicolumn{1}{|c|}{ SURROGATE } & \%REC & Q & LIMITS \\
\hline $17060-07-0$ & $1,2-$ Dichloroethane-d4 & 106 & \\
\hline $2037-26-5$ & Toluene-d8 & 111 & $80-120$ \\
\hline $460-00-4$ & Bromofluorobenzene & 114 & $80-120$ \\
\hline $1868-53-7$ & Dibromofluoromethane (Surr) & 99 & $80-125$ \\
\hline
\end{tabular}


FORM I

GC/MS VOA ORGANICS ANALYSIS DATA SHEET

Lab Name: TestAmerica Burlington

Job No : : 200-28914-1

SDG No.: 200-28914

Client Sample ID: YK-S-37767

Matrix: Solid

Analysis Method: 8260B

Sample wt/vol: 11.213(g)

Soil Aliquot Vol: 500 (uL)

Soil Extract Vol.: $10(\mathrm{~mL})$

Moisture:

Analysis Batch No.: 92051

Lab Sample ID: 200-28914-3

Lab File ID: 15018_08.D

Date Collected: 07/16/2015 00:00

Date Analyzed: 07/30/2015 13:47

Dilution Factor: 1

GC Column: DB-624

ID $: 0.53(\mathrm{~mm})$

Level: (low/med) Medium

Units: ug/Kg

\begin{tabular}{|c|c|c|c|c|c|}
\hline CAS NO. & COMPOUND NAME & RESULT & $Q$ & $\mathrm{RL}$ & MDL \\
\hline $75-71-8$ & Dichlorodifluoromethane & 8.9 & $\mathrm{U}$ & 8.9 & 1.5 \\
\hline $74-87-3$ & Chloromethane & 8.9 & $\mathrm{U}$ & 8.9 & 1.9 \\
\hline $75-01-4$ & Vinyl chloride & 8.9 & $\mathrm{U}$ & 8.9 & 0.74 \\
\hline $74-83-9$ & Bromomethane & 13 & B & 8.9 & 0.89 \\
\hline $75-00-3$ & Chloroethane & 8.9 & $\mathrm{U} *$ & 8.9 & 2.2 \\
\hline $75-69-4$ & Trichlorofluoromethane & 8.9 & $\mathrm{U}$ & 8.9 & 0.73 \\
\hline $75-35-4$ & 1,1-Dichloroethene & 8.9 & $U \star$ & 8.9 & 0.98 \\
\hline $76-13-1$ & Freon TF & 8.9 & $U \star$ & 8.9 & 0.76 \\
\hline $67-64-1$ & Acetone & 45 & $\mathrm{U}$ & 45 & 11 \\
\hline $74-88-4$ & Methyl iodide & 7.3 & $J \star B$ & 8.9 & 0.67 \\
\hline $75-15-0$ & Carbon disulfide & 8.9 & $\mathrm{U} \star$ & 8.9 & 0.78 \\
\hline $79-20-9$ & Methyl acetate & 45 & $U \star$ & 45 & 8.2 \\
\hline $75-09-2$ & Methylene Chloride & 8.9 & $\mathrm{U}$ & 8.9 & 2.1 \\
\hline $156-60-5$ & trans-1,2-Dichloroethene & 8.9 & $\mathrm{U}$ & 8.9 & 0.88 \\
\hline $1634-04-4$ & Methyl t-butyl ether & 8.9 & $\mathrm{U}$ & 8.9 & 0.98 \\
\hline $75-34-3$ & 1,1-Dichloroethane & 8.9 & $\mathrm{U}$ & 8.9 & 0.98 \\
\hline $108-05-4$ & Vinyl acetate & 45 & $\mathrm{U}$ & 45 & 4.0 \\
\hline $594-20-7$ & 2,2-Dichloropropane & 8.9 & $\mathrm{U}$ * & 8.9 & 2.0 \\
\hline $156-59-2$ & cis-1,2-Dichloroethene & 8.9 & $\mathrm{U}$ & 8.9 & 0.89 \\
\hline $78-93-3$ & 2-Butanone & 45 & $\mathrm{U}$ & 45 & 5.3 \\
\hline $74-97-5$ & Bromochloromethane & 8.9 & $\mathrm{U}$ & 8.9 & 1.2 \\
\hline $109-99-9$ & Tetrahydrofuran & 18 & $U \star$ & 18 & 4.7 \\
\hline $67-66-3$ & Chloroform & 1.8 & $\mathrm{~J}$ & 8.9 & 1.1 \\
\hline $71-55-6$ & $1,1,1$-Trichloroethane & 8.9 & $\mathrm{U}$ & 8.9 & 0.76 \\
\hline $110-82-7$ & Cyclohexane & 8.9 & $\mathrm{U}$ & 8.9 & 0.89 \\
\hline $563-58-6$ & 1,1-Dichloropropene & 8.9 & $\mathrm{U}$ & 8.9 & 0.87 \\
\hline $56-23-5$ & Carbon tetrachloride & 9.1 & & 8.9 & 1.8 \\
\hline $78-83-1$ & Isobutyl alcohol & 450 & $\mathrm{U} *$ & 450 & 24 \\
\hline $71-43-2$ & Benzene & 8.9 & $\mathrm{U}$ & 8.9 & 0.98 \\
\hline $107-06-2$ & 1,2-Dichloroethane & 8.9 & $\mathrm{U}$ & 8.9 & 1.2 \\
\hline $79-01-6$ & Trichloroethene & 8.9 & $\mathrm{U}$ & 8.9 & 0.74 \\
\hline $108-87-2$ & Methylcyclohexane & 8.9 & $\mathrm{U}$ & 8.9 & 1.9 \\
\hline $78-87-5$ & 1,2-Dichloropropane & 8.9 & $\mathrm{U}$ & 8.9 & 1.1 \\
\hline $74-95-3$ & Dibromomethane & 8.9 & $\mathrm{U}$ & 8.9 & 1.3 \\
\hline $123-91-1$ & 1,4-Dioxane & 360 & $\mathrm{U}$ & 360 & 58 \\
\hline $75-27-4$ & Bromodichloromethane & 8.9 & $\mathrm{U}$ & 8.9 & 0.89 \\
\hline
\end{tabular}

FORM I $8260 B$ 
FORM I

GC/MS VOA ORGANICS ANALYSIS DATA SHEET

Lab Name: TestAmerica Burlington

Job No.: 200-28914-1

SDG No.: 200-28914

Client Sample ID: YK-S-37767

Matrix: Solid

Analysis Method: 8260B

Sample wt/vol: 11.213(g)

Soil Aliquot Vol: 500 (uL)

Soil Extract Vol.: $10(\mathrm{~mL})$

o Moisture:

Analysis Batch No.: 92051

Lab Sample ID: 200-28914-3

Lab File ID: 15018_08.D

Date Collected: 07/16/2015 00:00

Date Analyzed: 07/30/2015 13:47

Dilution Factor: 1

GC Column: DB-624

ID $: 0.53(\mathrm{~mm})$

Level: (low/med) Medium

Units: ug/Kg

\begin{tabular}{|c|c|c|c|c|c|}
\hline CAS NO. & COMPOUND NAME & RESULT & $Q$ & $\mathrm{RL}$ & MDL \\
\hline $110-75-8$ & 2-Chloroethyl vinyl ether & 8.9 & $\mathrm{U}$ & 8.9 & 1.7 \\
\hline $10061-01-5$ & cis-1,3-Dichloropropene & 8.9 & $\mathrm{U}$ & 8.9 & 1.1 \\
\hline $108-10-1$ & 4-Methyl-2-pentanone & 45 & $U \star$ & 45 & 4.2 \\
\hline $108-88-3$ & Toluene & 8.9 & $\mathrm{U}$ & 8.9 & 1.9 \\
\hline $10061-02-6$ & trans-1,3-Dichloropropene & 8.9 & $\mathrm{U}$ & 8.9 & 2.0 \\
\hline $79-00-5$ & $1,1,2$-Trichloroethane & 8.9 & $\mathrm{U}$ & 8.9 & 1.2 \\
\hline $127-18-4$ & Tetrachloroethene & 8.9 & $\mathrm{U}$ & 8.9 & 1.8 \\
\hline $142-28-9$ & 1,3-Dichloropropane & 8.9 & $\mathrm{U}$ & 8.9 & 0.98 \\
\hline $591-78-6$ & 2-Hexanone & 45 & $\mathrm{U}$ & 45 & 5.4 \\
\hline $124-48-1$ & Dibromochloromethane & 8.9 & $\mathrm{U}$ & 8.9 & 0.98 \\
\hline $106-93-4$ & 1,2-Dibromoethane & 8.9 & $\mathrm{U}$ & 8.9 & 1.1 \\
\hline $108-90-7$ & Chlorobenzene & 8.9 & $\mathrm{U}$ & 8.9 & 0.81 \\
\hline $630-20-6$ & $1,1,1,2$-Tetrachloroethane & 8.9 & $\mathrm{U}$ & 8.9 & 0.98 \\
\hline $100-41-4$ & Ethylbenzene & 8.9 & $\mathrm{U}$ & 8.9 & 0.84 \\
\hline $179601-23-1$ & $\mathrm{~m} \& \mathrm{p}-\mathrm{Xy}$ lene & 8.9 & $\mathrm{U}$ & 8.9 & 0.98 \\
\hline $95-47-6$ & o-Xylene & 8.9 & $\mathrm{U}$ & 8.9 & 0.89 \\
\hline $100-42-5$ & Styrene & 8.9 & $\mathrm{U}$ & 8.9 & 0.87 \\
\hline $75-25-2$ & Bromoform & 8.9 & $\mathrm{U}$ & 8.9 & 0.89 \\
\hline $98-82-8$ & Isopropylbenzene & 8.9 & $\mathrm{U}$ & 8.9 & 1.8 \\
\hline $108-86-1$ & Bromobenzene & 8.9 & $\mathrm{U}$ & 8.9 & 0.89 \\
\hline $79-34-5$ & $1,1,2,2$-Tetrachloroethane & 8.9 & $\mathrm{U}$ & 8.9 & 1.2 \\
\hline $96-18-4$ & $1,2,3$-Trichloropropane & 8.9 & $\mathrm{U}$ & 8.9 & 1.1 \\
\hline $103-65-1$ & n-Propylbenzene & 8.9 & $\mathrm{U}$ & 8.9 & 0.98 \\
\hline $95-49-8$ & 2-Chlorotoluene & 8.9 & $\mathrm{U}$ & 8.9 & 0.98 \\
\hline $106-43-4$ & 4-Chlorotoluene & 8.9 & $\mathrm{U}$ & 8.9 & 0.86 \\
\hline $108-67-8$ & 1,3,5-Trimethylbenzene & 8.9 & $\mathrm{U}$ & 8.9 & 1.8 \\
\hline $98-06-6$ & tert-Butylbenzene & 8.9 & $\mathrm{U}$ & 8.9 & 1.2 \\
\hline $95-63-6$ & $1,2,4$-Trimethylbenzene & 8.9 & $\mathrm{U}$ & 8.9 & 0.89 \\
\hline $135-98-8$ & sec-Butylbenzene & 8.9 & $\mathrm{U}$ & 8.9 & 1.8 \\
\hline $541-73-1$ & 1,3-Dichlorobenzene & 8.9 & $\mathrm{U}$ & 8.9 & 0.85 \\
\hline $99-87-6$ & 4-Isopropyltoluene & 8.9 & $\mathrm{U}$ & 8.9 & 0.78 \\
\hline $106-46-7$ & 1,4-Dichlorobenzene & 8.9 & $\mathrm{U}$ & 8.9 & 1.2 \\
\hline $95-50-1$ & 1,2-Dichlorobenzene & 8.9 & $\mathrm{U}$ & 8.9 & 0.98 \\
\hline $104-51-8$ & n-Butylbenzene & 8.9 & $\mathrm{U}$ & 8.9 & 0.80 \\
\hline $96-12-8$ & 1,2-Dibromo-3-Chloropropane & 8.9 & $\mathrm{U}$ & 8.9 & 1.9 \\
\hline $120-82-1$ & $1,2,4$-Trichlorobenzene & 1.2 & $\mathrm{~J} B$ & 8.9 & 0.81 \\
\hline
\end{tabular}

FORM I $8260 B$ 
FORM I

GC/MS VOA ORGANICS ANALYSIS DATA SHEET

Lab Name: TestAmerica Burlington

SDG No.: 200-28914

Client Sample ID: YK-S-37767

Matrix: Solid

Analysis Method: 8260B

Sample wt/vol: 11.213(g)

Soil Aliquot Vol: 500 (uL)

Soil Extract Vol.: 10 (mL)

응 Moisture:

Analysis Batch No.: 92051
Job No. : 200-28914-1

Lab Sample ID: 200-28914-3

Lab File ID: 15018_08.D

Date Collected: 07/16/2015 00:00

Date Analyzed: 07/30/2015 13:47

Dilution Factor: 1

GC Column: DB-624

ID $: 0.53(\mathrm{~mm})$

Level: (low/med) Medium

Units: ug/Kg

\begin{tabular}{|c|c|c|c|c|c|}
\hline CAS NO. & COMPOUND NAME & RESULT & Q & RL & MDL \\
\hline $87-68-3$ & Hexachlorobutadiene & 8.9 & $\mathrm{U} *$ & 8.9 & 2.1 \\
\hline $91-20-3$ & Naphthalene & 1.6 & $\mathrm{~J} \star \mathrm{B}$ & 8.9 & 1.1 \\
\hline $87-61-6$ & $1,2,3$-Trichlorobenzene & 1.1 & $\mathrm{~J} B$ & 8.9 & 0.89 \\
\hline
\end{tabular}

\begin{tabular}{|c|c|c|c|c|}
\hline CAS NO. & SURROGATE & $\because \mathrm{REC}$ & Q & LIMITS \\
\hline $17060-07-0$ & 1,2-Dichloroethane-d4 & 107 & & $80-120$ \\
\hline $2037-26-5$ & Toluene-d8 & 110 & & $80-120$ \\
\hline $460-00-4$ & Bromofluorobenzene & 114 & & $80-125$ \\
\hline $1868-53-7$ & Dibromofluoromethane (Surr) & 102 & & $50-150$ \\
\hline
\end{tabular}


FORM I

GC/MS VOA ORGANICS ANALYSIS DATA SHEET

Lab Name: TestAmerica Burlington

Job No : : 200-28914-1

SDG No.: 200-28914

Client Sample ID: YK-S-37805

Matrix: Solid

Analysis Method: 8260B

Sample wt/vol: $10.554(\mathrm{~g})$

Soil Aliquot Vol: 500 (uL)

Soil Extract Vol.: $10(\mathrm{~mL})$

응 Moisture:

Analysis Batch No.: 92051

Lab Sample ID: 200-28914-4

Lab File ID: 15018_09.D

Date Collected: 07/16/2015 00:00

Date Analyzed: 07/30/2015 14:20

Dilution Factor: 1

GC Column: DB-624

ID $: 0.53(\mathrm{~mm})$

Level: (low/med) Medium

Units: ug/Kg

\begin{tabular}{|c|c|c|c|c|c|}
\hline CAS NO. & COMPOUND NAME & RESULT & $Q$ & $\mathrm{RL}$ & MDL \\
\hline $75-71-8$ & Dichlorodifluoromethane & 9.5 & $\mathrm{U}$ & 9.5 & 1.6 \\
\hline $74-87-3$ & Chloromethane & 5.1 & $\mathrm{~J} B$ & 9.5 & 2.0 \\
\hline $75-01-4$ & Vinyl chloride & 9.5 & $\mathrm{U}$ & 9.5 & 0.79 \\
\hline $74-83-9$ & Bromomethane & 20 & B & 9.5 & 0.95 \\
\hline $75-00-3$ & Chloroethane & 9.5 & $\mathrm{U} *$ & 9.5 & 2.4 \\
\hline $75-69-4$ & Trichlorofluoromethane & 9.5 & $\mathrm{U}$ & 9.5 & 0.78 \\
\hline $75-35-4$ & 1,1-Dichloroethene & 9.5 & $\mathrm{U} *$ & 9.5 & 1.0 \\
\hline $76-13-1$ & Freon TF & 9.5 & $U \star$ & 9.5 & 0.81 \\
\hline $67-64-1$ & Acetone & 47 & $\mathrm{U}$ & 47 & 11 \\
\hline $74-88-4$ & Methyl iodide & 9.1 & $\mathrm{~J} \star \mathrm{B}$ & 9.5 & 0.71 \\
\hline $75-15-0$ & Carbon disulfide & 9.5 & $\mathrm{U} \star$ & 9.5 & 0.82 \\
\hline $79-20-9$ & Methyl acetate & 47 & $U \star$ & 47 & 8.7 \\
\hline $75-09-2$ & Methylene Chloride & 9.5 & $\mathrm{U}$ & 9.5 & 2.2 \\
\hline $156-60-5$ & trans-1,2-Dichloroethene & 9.5 & $\mathrm{U}$ & 9.5 & 0.94 \\
\hline $1634-04-4$ & Methyl t-butyl ether & 9.5 & $\mathrm{U}$ & 9.5 & 1.0 \\
\hline $75-34-3$ & 1,1-Dichloroethane & 9.5 & $\mathrm{U}$ & 9.5 & 1.0 \\
\hline $108-05-4$ & Vinyl acetate & 47 & $\mathrm{U}$ & 47 & 4.3 \\
\hline $594-20-7$ & 2,2-Dichloropropane & 9.5 & $\mathrm{U} *$ & 9.5 & 2.1 \\
\hline $156-59-2$ & cis-1,2-Dichloroethene & 9.5 & $\mathrm{U}$ & 9.5 & 0.95 \\
\hline $78-93-3$ & 2-Butanone & 47 & $\mathrm{U}$ & 47 & 5.6 \\
\hline $74-97-5$ & Bromochloromethane & 9.5 & $\mathrm{U}$ & 9.5 & 1.3 \\
\hline $109-99-9$ & Tetrahydrofuran & 19 & $U \star$ & 19 & 5.0 \\
\hline $67-66-3$ & Chloroform & 9.5 & $\mathrm{U}$ & 9.5 & 1.1 \\
\hline $71-55-6$ & $1,1,1$-Trichloroethane & 9.5 & $\mathrm{U}$ & 9.5 & 0.81 \\
\hline $110-82-7$ & Cyclohexane & 9.5 & $\mathrm{U}$ & 9.5 & 0.95 \\
\hline $563-58-6$ & 1,1-Dichloropropene & 9.5 & $\mathrm{U}$ & 9.5 & 0.92 \\
\hline $56-23-5$ & Carbon tetrachloride & 9.5 & $\mathrm{U}$ & 9.5 & 1.9 \\
\hline $78-83-1$ & Isobutyl alcohol & 470 & $\mathrm{U} *$ & 470 & 26 \\
\hline $71-43-2$ & Benzene & 9.5 & $\mathrm{U}$ & 9.5 & 1.0 \\
\hline $107-06-2$ & 1,2-Dichloroethane & 9.5 & $\mathrm{U}$ & 9.5 & 1.2 \\
\hline $79-01-6$ & Trichloroethene & 9.5 & $\mathrm{U}$ & 9.5 & 0.79 \\
\hline $108-87-2$ & Methylcyclohexane & 9.5 & $\mathrm{U}$ & 9.5 & 2.0 \\
\hline $78-87-5$ & 1,2-Dichloropropane & 9.5 & $\mathrm{U}$ & 9.5 & 1.1 \\
\hline $74-95-3$ & Dibromomethane & 9.5 & $\mathrm{U}$ & 9.5 & 1.4 \\
\hline $123-91-1$ & 1,4-Dioxane & 380 & $\mathrm{U}$ & 380 & 62 \\
\hline $75-27-4$ & Bromodichloromethane & 9.5 & $\mathrm{U}$ & 9.5 & 0.95 \\
\hline
\end{tabular}

FORM I $8260 B$ 
FORM I

GC/MS VOA ORGANICS ANALYSIS DATA SHEET

Lab Name: TestAmerica Burlington

Job No.: 200-28914-1

SDG No.: 200-28914

Client Sample ID: YK-S-37805

Matrix: Solid

Analysis Method: 8260B

Sample wt/vol: $10.554(\mathrm{~g})$

Soil Aliquot Vol: 500 (uL)

Soil Extract Vol.: $10(\mathrm{~mL})$

o Moisture:

Analysis Batch No.: 92051

Lab Sample ID: 200-28914-4

Lab File ID: 15018_09.D

Date Collected: 07/16/2015 00:00

Date Analyzed: 07/30/2015 14:20

Dilution Factor: 1

GC Column: DB-624

ID $: 0.53(\mathrm{~mm})$

Level: (low/med) Medium

Units: ug/Kg

\begin{tabular}{|c|c|c|c|c|c|}
\hline CAS NO. & COMPOUND NAME & RESULT & $Q$ & RL & MDL \\
\hline $110-75-8$ & 2-Chloroethyl vinyl ether & 9.5 & $\mathrm{U}$ & 9.5 & 1.8 \\
\hline $10061-01-5$ & cis-1,3-Dichloropropene & 9.5 & $\mathrm{U}$ & 9.5 & 1.1 \\
\hline $108-10-1$ & 4-Methyl-2-pentanone & 47 & $\mathrm{U} *$ & 47 & 4.5 \\
\hline $108-88-3$ & Toluene & 9.5 & $\mathrm{U}$ & 9.5 & 2.0 \\
\hline $10061-02-6$ & trans-1,3-Dichloropropene & 9.5 & $\mathrm{U}$ & 9.5 & 2.1 \\
\hline $79-00-5$ & $1,1,2$-Trichloroethane & 9.5 & $\mathrm{U}$ & 9.5 & 1.2 \\
\hline $127-18-4$ & Tetrachloroethene & 9.5 & $\mathrm{U}$ & 9.5 & 1.9 \\
\hline $142-28-9$ & 1,3-Dichloropropane & 9.5 & $\mathrm{U}$ & 9.5 & 1.0 \\
\hline $591-78-6$ & 2-Hexanone & 47 & $\mathrm{U}$ & 47 & 5.8 \\
\hline $124-48-1$ & Dibromochloromethane & 9.5 & $\mathrm{U}$ & 9.5 & 1.0 \\
\hline $106-93-4$ & 1,2-Dibromoethane & 9.5 & $\mathrm{U}$ & 9.5 & 1.1 \\
\hline $108-90-7$ & Chlorobenzene & 9.5 & $\mathrm{U}$ & 9.5 & 0.86 \\
\hline $630-20-6$ & $1,1,1,2$-Tetrachloroethane & 9.5 & $\mathrm{U}$ & 9.5 & 1.0 \\
\hline $100-41-4$ & Ethylbenzene & 9.5 & $\mathrm{U}$ & 9.5 & 0.89 \\
\hline $179601-23-1$ & m\&p-Xylene & 9.5 & $\mathrm{U}$ & 9.5 & 1.0 \\
\hline $95-47-6$ & o-Xylene & 9.5 & $\mathrm{U}$ & 9.5 & 0.95 \\
\hline $100-42-5$ & Styrene & 9.5 & $\mathrm{U}$ & 9.5 & 0.93 \\
\hline $75-25-2$ & Bromoform & 9.5 & $\mathrm{U}$ & 9.5 & 0.95 \\
\hline $98-82-8$ & Isopropylbenzene & 9.5 & $\mathrm{U}$ & 9.5 & 1.9 \\
\hline $108-86-1$ & Bromobenzene & 9.5 & $\mathrm{U}$ & 9.5 & 0.95 \\
\hline $79-34-5$ & $1,1,2,2$-Tetrachloroethane & 9.5 & $\mathrm{U}$ & 9.5 & 1.2 \\
\hline $96-18-4$ & 1,2,3-Trichloropropane & 9.5 & $\mathrm{U}$ & 9.5 & 1.1 \\
\hline $103-65-1$ & n-Propylbenzene & 9.5 & $\mathrm{U}$ & 9.5 & 1.0 \\
\hline $95-49-8$ & 2-Chlorotoluene & 9.5 & $\mathrm{U}$ & 9.5 & 1.0 \\
\hline $106-43-4$ & 4-Chlorotoluene & 9.5 & $\mathrm{U}$ & 9.5 & 0.91 \\
\hline $108-67-8$ & 1,3,5-Trimethylbenzene & 9.5 & $\mathrm{U}$ & 9.5 & 1.9 \\
\hline $98-06-6$ & tert-Butylbenzene & 9.5 & $\mathrm{U}$ & 9.5 & 1.3 \\
\hline $95-63-6$ & $1,2,4$-Trimethylbenzene & 9.5 & $\mathrm{U}$ & 9.5 & 0.95 \\
\hline $135-98-8$ & sec-Butylbenzene & 9.5 & $\mathrm{U}$ & 9.5 & 1.9 \\
\hline $541-73-1$ & 1,3-Dichlorobenzene & 9.5 & $\mathrm{U}$ & 9.5 & 0.90 \\
\hline $99-87-6$ & 4-Isopropyltoluene & 9.5 & $\mathrm{U}$ & 9.5 & 0.83 \\
\hline $106-46-7$ & 1,4-Dichlorobenzene & 9.5 & $\mathrm{U}$ & 9.5 & 1.3 \\
\hline $95-50-1$ & 1,2-Dichlorobenzene & 9.5 & $\mathrm{U}$ & 9.5 & 1.0 \\
\hline $104-51-8$ & n-Butylbenzene & 9.5 & $\mathrm{U}$ & 9.5 & 0.85 \\
\hline $96-12-8$ & 1,2-Dibromo-3-Chloropropane & 9.5 & $\mathrm{U}$ & 9.5 & 2.0 \\
\hline $120-82-1$ & $1,2,4$-Trichlorobenzene & 1.2 & $\mathrm{~J} B$ & 9.5 & 0.86 \\
\hline
\end{tabular}

FORM I $8260 B$ 
FORM I

GC/MS VOA ORGANICS ANALYSIS DATA SHEET

Lab Name: TestAmerica Burlington

SDG No.: 200-28914

Client Sample ID: YK-S-37805

Matrix: Solid

Analysis Method: 8260B

Sample wt/vol: $10.554(\mathrm{~g})$

Soil Aliquot Vol: 500 (uL)

Soil Extract Vol.: 10 (mL)

응 Moisture:

Analysis Batch No.: 92051
Job No. : 200-28914-1

Lab Sample ID: 200-28914-4

Lab File ID: 15018_09.D

Date Collected: 07/16/2015 00:00

Date Analyzed: 07/30/2015 14:20

Dilution Factor: 1

GC Column: DB-624

ID $: 0.53(\mathrm{~mm})$

Level: (low/med) Medium

Units: ug/Kg

\begin{tabular}{|c|c|c|c|c|c|}
\hline CAS NO. & COMPOUND NAME & RESULT & Q & RL & MDL \\
\hline $87-68-3$ & Hexachlorobutadiene & 9.5 & $\mathrm{U} *$ & 9.5 & 2.2 \\
\hline $91-20-3$ & Naphthalene & 1.5 & $\mathrm{~J} \star \mathrm{B}$ & 9.5 & 1.1 \\
\hline $87-61-6$ & $1,2,3$-Trichlorobenzene & 9.5 & $\mathrm{U}$ & 9.5 & 0.95 \\
\hline
\end{tabular}

\begin{tabular}{|c|c|c|c|c|}
\hline CAS NO. & SURROGATE & $\because \mathrm{REC}$ & $Q$ & LIMITS \\
\hline $17060-07-0$ & 1,2-Dichloroethane-d4 & 109 & & $80-120$ \\
\hline $2037-26-5$ & Toluene-d8 & 110 & & $80-120$ \\
\hline $460-00-4$ & Bromofluorobenzene & 113 & & $80-125$ \\
\hline $1868-53-7$ & Dibromofluoromethane (Surr) & 102 & & $50-150$ \\
\hline
\end{tabular}


FORM I

GC/MS VOA ORGANICS ANALYSIS DATA SHEET

Lab Name: TestAmerica Burlington

Job No : : 200-28914-1

SDG No.: 200-28914

Client Sample ID: YK-S-37791

Matrix: Solid

Analysis Method: 8260B

Sample wt/vol: $10.51(\mathrm{~g})$

Soil Aliquot Vol: 500 (uL)

Soil Extract Vol.: $10(\mathrm{~mL})$

응 Moisture:

Analysis Batch No.: 92051

Lab Sample ID: 200-28914-5

Lab File ID: 15018_10.D

Date Collected: 07/16/2015 00:00

Date Analyzed: 07/30/2015 14:52

Dilution Factor: 1

GC Column: DB-624

ID $: 0.53(\mathrm{~mm})$

Level: (low/med) Medium

Units: ug/Kg

\begin{tabular}{|c|c|c|c|c|c|}
\hline CAS NO. & COMPOUND NAME & RESULT & $Q$ & $\mathrm{RL}$ & MDL \\
\hline $75-71-8$ & Dichlorodifluoromethane & 9.5 & $\mathrm{U}$ & 9.5 & 1.6 \\
\hline $74-87-3$ & Chloromethane & 3.0 & $\mathrm{~J} B$ & 9.5 & 2.0 \\
\hline $75-01-4$ & Vinyl chloride & 9.5 & $\mathrm{U}$ & 9.5 & 0.79 \\
\hline $74-83-9$ & Bromomethane & 15 & B & 9.5 & 0.95 \\
\hline $75-00-3$ & Chloroethane & 9.5 & $\mathrm{U} *$ & 9.5 & 2.4 \\
\hline $75-69-4$ & Trichlorofluoromethane & 9.5 & $\mathrm{U}$ & 9.5 & 0.78 \\
\hline $75-35-4$ & 1,1-Dichloroethene & 9.5 & $\mathrm{U} *$ & 9.5 & 1.0 \\
\hline $76-13-1$ & Freon TF & 9.5 & $U \star$ & 9.5 & 0.81 \\
\hline $67-64-1$ & Acetone & 48 & $\mathrm{U}$ & 48 & 11 \\
\hline $74-88-4$ & Methyl iodide & 7.7 & $\mathrm{~J} \star \mathrm{B}$ & 9.5 & 0.71 \\
\hline $75-15-0$ & Carbon disulfide & 9.5 & $\mathrm{U} \star$ & 9.5 & 0.83 \\
\hline $79-20-9$ & Methyl acetate & 48 & $U \star$ & 48 & 8.8 \\
\hline $75-09-2$ & Methylene Chloride & 9.5 & $\mathrm{U}$ & 9.5 & 2.2 \\
\hline $156-60-5$ & trans-1,2-Dichloroethene & 9.5 & $\mathrm{U}$ & 9.5 & 0.94 \\
\hline $1634-04-4$ & Methyl t-butyl ether & 9.5 & $\mathrm{U}$ & 9.5 & 1.0 \\
\hline $75-34-3$ & 1,1-Dichloroethane & 9.5 & $\mathrm{U}$ & 9.5 & 1.0 \\
\hline $108-05-4$ & Vinyl acetate & 48 & $\mathrm{U}$ & 48 & 4.3 \\
\hline $594-20-7$ & 2,2-Dichloropropane & 9.5 & $\mathrm{U}$ * & 9.5 & 2.1 \\
\hline $156-59-2$ & cis-1,2-Dichloroethene & 9.5 & $\mathrm{U}$ & 9.5 & 0.95 \\
\hline $78-93-3$ & 2-Butanone & 48 & $\mathrm{U}$ & 48 & 5.6 \\
\hline $74-97-5$ & Bromochloromethane & 9.5 & $\mathrm{U}$ & 9.5 & 1.3 \\
\hline $109-99-9$ & Tetrahydrofuran & 19 & $U \star$ & 19 & 5.0 \\
\hline $67-66-3$ & Chloroform & 6.7 & $\mathrm{~J}$ & 9.5 & 1.1 \\
\hline $71-55-6$ & $1,1,1$-Trichloroethane & 9.5 & $\mathrm{U}$ & 9.5 & 0.81 \\
\hline $110-82-7$ & Cyclohexane & 9.5 & $\mathrm{U}$ & 9.5 & 0.95 \\
\hline $563-58-6$ & 1,1-Dichloropropene & 9.5 & $\mathrm{U}$ & 9.5 & 0.92 \\
\hline $56-23-5$ & Carbon tetrachloride & 44 & & 9.5 & 1.9 \\
\hline $78-83-1$ & Isobutyl alcohol & 480 & $\mathrm{U} *$ & 480 & 26 \\
\hline $71-43-2$ & Benzene & 9.5 & $\mathrm{U}$ & 9.5 & 1.0 \\
\hline $107-06-2$ & 1,2-Dichloroethane & 9.5 & $\mathrm{U}$ & 9.5 & 1.2 \\
\hline $79-01-6$ & Trichloroethene & 9.5 & $\mathrm{U}$ & 9.5 & 0.79 \\
\hline $108-87-2$ & Methylcyclohexane & 9.5 & $\mathrm{U}$ & 9.5 & 2.0 \\
\hline $78-87-5$ & 1,2-Dichloropropane & 9.5 & $\mathrm{U}$ & 9.5 & 1.1 \\
\hline $74-95-3$ & Dibromomethane & 9.5 & $\mathrm{U}$ & 9.5 & 1.4 \\
\hline $123-91-1$ & 1,4-Dioxane & 380 & $\mathrm{U}$ & 380 & 62 \\
\hline $75-27-4$ & Bromodichloromethane & 9.5 & $\mathrm{U}$ & 9.5 & 0.95 \\
\hline
\end{tabular}

FORM I $8260 B$ 
FORM I

GC/MS VOA ORGANICS ANALYSIS DATA SHEET

Lab Name: TestAmerica Burlington

Job No.: 200-28914-1

SDG No.: 200-28914

Client Sample ID: YK-S-37791

Matrix: Solid

Analysis Method: 8260B

Sample wt/vol: $10.51(\mathrm{~g})$

Soil Aliquot Vol: 500 (uL)

Soil Extract Vol.: $10(\mathrm{~mL})$

o Moisture:

Analysis Batch No.: 92051

Lab Sample ID: 200-28914-5

Lab File ID: 15018_10.D

Date Collected: 07/16/2015 00:00

Date Analyzed: 07/30/2015 14:52

Dilution Factor: 1

GC Column: DB-624

ID $: 0.53(\mathrm{~mm})$

Level: (low/med) Medium

Units: ug/Kg

\begin{tabular}{|c|c|c|c|c|c|}
\hline CAS NO. & COMPOUND NAME & RESULT & $Q$ & RL & MDL \\
\hline $110-75-8$ & 2-Chloroethyl vinyl ether & 9.5 & $\mathrm{U}$ & 9.5 & 1.8 \\
\hline $10061-01-5$ & cis-1,3-Dichloropropene & 9.5 & $\mathrm{U}$ & 9.5 & 1.1 \\
\hline $108-10-1$ & 4-Methyl-2-pentanone & 48 & $\mathrm{U} *$ & 48 & 4.5 \\
\hline $108-88-3$ & Toluene & 9.5 & $\mathrm{U}$ & 9.5 & 2.0 \\
\hline $10061-02-6$ & trans-1,3-Dichloropropene & 9.5 & $\mathrm{U}$ & 9.5 & 2.1 \\
\hline $79-00-5$ & $1,1,2$-Trichloroethane & 9.5 & $\mathrm{U}$ & 9.5 & 1.2 \\
\hline $127-18-4$ & Tetrachloroethene & 9.5 & $\mathrm{U}$ & 9.5 & 1.9 \\
\hline $142-28-9$ & 1,3-Dichloropropane & 9.5 & $\mathrm{U}$ & 9.5 & 1.0 \\
\hline $591-78-6$ & 2-Hexanone & 48 & $\mathrm{U}$ & 48 & 5.8 \\
\hline $124-48-1$ & Dibromochloromethane & 9.5 & $\mathrm{U}$ & 9.5 & 1.0 \\
\hline $106-93-4$ & 1,2-Dibromoethane & 9.5 & $\mathrm{U}$ & 9.5 & 1.1 \\
\hline $108-90-7$ & Chlorobenzene & 9.5 & $\mathrm{U}$ & 9.5 & 0.87 \\
\hline $630-20-6$ & $1,1,1,2$-Tetrachloroethane & 9.5 & $\mathrm{U}$ & 9.5 & 1.0 \\
\hline $100-41-4$ & Ethylbenzene & 9.5 & $\mathrm{U}$ & 9.5 & 0.89 \\
\hline $179601-23-1$ & m\&p-Xylene & 9.5 & $\mathrm{U}$ & 9.5 & 1.0 \\
\hline $95-47-6$ & o-Xylene & 9.5 & $\mathrm{U}$ & 9.5 & 0.95 \\
\hline $100-42-5$ & Styrene & 9.5 & $\mathrm{U}$ & 9.5 & 0.93 \\
\hline $75-25-2$ & Bromoform & 9.5 & $\mathrm{U}$ & 9.5 & 0.95 \\
\hline $98-82-8$ & Isopropylbenzene & 9.5 & $\mathrm{U}$ & 9.5 & 1.9 \\
\hline $108-86-1$ & Bromobenzene & 9.5 & $\mathrm{U}$ & 9.5 & 0.95 \\
\hline $79-34-5$ & $1,1,2,2$-Tetrachloroethane & 9.5 & $\mathrm{U}$ & 9.5 & 1.2 \\
\hline $96-18-4$ & 1,2,3-Trichloropropane & 9.5 & $\mathrm{U}$ & 9.5 & 1.1 \\
\hline $103-65-1$ & n-Propylbenzene & 9.5 & $\mathrm{U}$ & 9.5 & 1.0 \\
\hline $95-49-8$ & 2-Chlorotoluene & 9.5 & $\mathrm{U}$ & 9.5 & 1.0 \\
\hline $106-43-4$ & 4-Chlorotoluene & 9.5 & $\mathrm{U}$ & 9.5 & 0.91 \\
\hline $108-67-8$ & 1,3,5-Trimethylbenzene & 9.5 & $\mathrm{U}$ & 9.5 & 1.9 \\
\hline $98-06-6$ & tert-Butylbenzene & 9.5 & $\mathrm{U}$ & 9.5 & 1.3 \\
\hline $95-63-6$ & $1,2,4$-Trimethylbenzene & 9.5 & $\mathrm{U}$ & 9.5 & 0.95 \\
\hline $135-98-8$ & sec-Butylbenzene & 9.5 & $\mathrm{U}$ & 9.5 & 1.9 \\
\hline $541-73-1$ & 1,3-Dichlorobenzene & 9.5 & $\mathrm{U}$ & 9.5 & 0.90 \\
\hline $99-87-6$ & 4-Isopropyltoluene & 9.5 & $\mathrm{U}$ & 9.5 & 0.84 \\
\hline $106-46-7$ & 1,4-Dichlorobenzene & 9.5 & $\mathrm{U}$ & 9.5 & 1.3 \\
\hline $95-50-1$ & 1,2-Dichlorobenzene & 9.5 & $\mathrm{U}$ & 9.5 & 1.0 \\
\hline $104-51-8$ & n-Butylbenzene & 9.5 & $\mathrm{U}$ & 9.5 & 0.86 \\
\hline $96-12-8$ & 1,2-Dibromo-3-Chloropropane & 9.5 & $\mathrm{U}$ & 9.5 & 2.0 \\
\hline $120-82-1$ & $1,2,4$-Trichlorobenzene & 1.1 & $\mathrm{~J} B$ & 9.5 & 0.87 \\
\hline
\end{tabular}

FORM I $8260 B$ 
FORM I

GC/MS VOA ORGANICS ANALYSIS DATA SHEET

Lab Name: TestAmerica Burlington

SDG No.: 200-28914

Client Sample ID: YK-S-37791

Matrix: Solid

Analysis Method: 8260B

Sample wt/vol: $10.51(\mathrm{~g})$

Soil Aliquot Vol: 500 (uL)

Soil Extract Vol.: 10 (mL)

응 Moisture:

Analysis Batch No.: 92051
Job No. : 200-28914-1

Lab Sample ID: 200-28914-5

Lab File ID: 15018_10.D

Date Collected: 07/16/2015 00:00

Date Analyzed: 07/30/2015 14:52

Dilution Factor: 1

GC Column: DB-624

ID $: 0.53(\mathrm{~mm})$

Level: (low/med) Medium

Units: ug/Kg

\begin{tabular}{|c|c|c|c|c|c|}
\hline CAS NO. & COMPOUND NAME & RESULT & Q & RL & MDL \\
\hline $87-68-3$ & Hexachlorobutadiene & 9.5 & $\mathrm{U} *$ & 9.5 & 2.2 \\
\hline $91-20-3$ & Naphthalene & 1.5 & $\mathrm{~J} \star \mathrm{B}$ & 9.5 & 1.1 \\
\hline $87-61-6$ & $1,2,3$-Trichlorobenzene & 1.0 & $\mathrm{~J} B$ & 9.5 & 0.95 \\
\hline
\end{tabular}

\begin{tabular}{|c|c|c|c|c|}
\hline CAS NO. & SURROGATE & $\because \mathrm{REC}$ & $Q$ & LIMITS \\
\hline $17060-07-0$ & 1,2-Dichloroethane-d4 & 112 & & $80-120$ \\
\hline $2037-26-5$ & Toluene-d8 & 111 & & $80-120$ \\
\hline $460-00-4$ & Bromofluorobenzene & 113 & & $80-125$ \\
\hline $1868-53-7$ & Dibromofluoromethane (Surr) & 106 & & $50-150$ \\
\hline
\end{tabular}


FORM I

GC/MS VOA ORGANICS ANALYSIS DATA SHEET

Lab Name: TestAmerica Burlington

Job No : : 200-28914-1

SDG No.: 200-28914

Client Sample ID: YK-S-37826

Matrix: Solid

Analysis Method: 8260B

Sample wt/vol: $10.961(\mathrm{~g})$

Soil Aliquot Vol: 500 (uL)

Soil Extract Vol.: $10(\mathrm{~mL})$

응 Moisture:

Analysis Batch No.: 92051

Lab Sample ID: 200-28914-6

Lab File ID: 15018_11.D

Date Collected: 07/16/2015 00:00

Date Analyzed: 07/30/2015 15:25

Dilution Factor: 1

GC Column: DB-624

ID $: 0.53(\mathrm{~mm})$

Level: (low/med) Medium

Units: ug/Kg

\begin{tabular}{|c|c|c|c|c|c|}
\hline CAS NO. & COMPOUND NAME & RESULT & $Q$ & RL & MDL \\
\hline $75-71-8$ & Dichlorodifluoromethane & 9.1 & $\mathrm{U}$ & 9.1 & 1.6 \\
\hline $74-87-3$ & Chloromethane & 2.1 & $\mathrm{~J} B$ & 9.1 & 1.9 \\
\hline $75-01-4$ & Vinyl chloride & 9.1 & $\mathrm{U}$ & 9.1 & 0.76 \\
\hline $74-83-9$ & Bromomethane & 14 & $\mathrm{~B}$ & 9.1 & 0.91 \\
\hline $75-00-3$ & Chloroethane & 9.1 & $\mathrm{U} *$ & 9.1 & 2.3 \\
\hline $75-69-4$ & Trichlorofluoromethane & 9.1 & $\mathrm{U}$ & 9.1 & 0.75 \\
\hline $75-35-4$ & 1,1-Dichloroethene & 9.1 & $\mathrm{U} *$ & 9.1 & 1.0 \\
\hline $76-13-1$ & Freon $\mathrm{TF}$ & 9.1 & $\mathrm{U} *$ & 9.1 & 0.78 \\
\hline $67-64-1$ & Acetone & 46 & $\mathrm{U}$ & 46 & 11 \\
\hline $74-88-4$ & Methyl iodide & 6.8 & $\mathrm{~J} \star \mathrm{B}$ & 9.1 & 0.68 \\
\hline $75-15-0$ & Carbon disulfide & 9.1 & $\mathrm{U} \star$ & 9.1 & 0.79 \\
\hline $79-20-9$ & Methyl acetate & 46 & $U *$ & 46 & 8.4 \\
\hline $75-09-2$ & Methylene Chloride & 9.1 & $\mathrm{U}$ & 9.1 & 2.1 \\
\hline $156-60-5$ & trans-1,2-Dichloroethene & 9.1 & $\mathrm{U}$ & 9.1 & 0.90 \\
\hline $1634-04-4$ & Methyl t-butyl ether & 9.1 & $\mathrm{U}$ & 9.1 & 1.0 \\
\hline $75-34-3$ & 1,1-Dichloroethane & 9.1 & $\mathrm{U}$ & 9.1 & 1.0 \\
\hline $108-05-4$ & Vinyl acetate & 46 & $\mathrm{U}$ & 46 & 4.1 \\
\hline $594-20-7$ & 2,2-Dichloropropane & 9.1 & $\mathrm{U} *$ & 9.1 & 2.0 \\
\hline $156-59-2$ & cis-1,2-Dichloroethene & 9.1 & $\mathrm{U}$ & 9.1 & 0.91 \\
\hline $78-93-3$ & 2-Butanone & 46 & $\mathrm{U}$ & 46 & 5.4 \\
\hline $74-97-5$ & Bromochloromethane & 9.1 & $\mathrm{U}$ & 9.1 & 1.3 \\
\hline $109-99-9$ & Tetrahydrofuran & 18 & $U \star$ & 18 & 4.8 \\
\hline $67-66-3$ & Chloroform & 9.1 & $\mathrm{U}$ & 9.1 & 1.1 \\
\hline $71-55-6$ & $1,1,1$-Trichloroethane & 6.0 & $\mathrm{~J}$ & 9.1 & 0.78 \\
\hline $110-82-7$ & Cyclohexane & 9.1 & $\mathrm{U}$ & 9.1 & 0.91 \\
\hline $563-58-6$ & 1,1-Dichloropropene & 9.1 & $\mathrm{U}$ & 9.1 & 0.88 \\
\hline $56-23-5$ & Carbon tetrachloride & 9.1 & $\mathrm{U}$ & 9.1 & 1.8 \\
\hline $78-83-1$ & Isobutyl alcohol & 460 & $\mathrm{U} *$ & 460 & 25 \\
\hline $71-43-2$ & Benzene & 9.1 & $\mathrm{U}$ & 9.1 & 1.0 \\
\hline $107-06-2$ & 1,2-Dichloroethane & 9.1 & $\mathrm{U}$ & 9.1 & 1.2 \\
\hline $79-01-6$ & Trichloroethene & 9.1 & $\mathrm{U}$ & 9.1 & 0.76 \\
\hline $108-87-2$ & Methylcyclohexane & 9.1 & $\mathrm{U}$ & 9.1 & 1.9 \\
\hline $78-87-5$ & 1,2-Dichloropropane & 9.1 & $\mathrm{U}$ & 9.1 & 1.1 \\
\hline $74-95-3$ & Dibromomethane & 9.1 & $\mathrm{U}$ & 9.1 & 1.4 \\
\hline $123-91-1$ & 1,4-Dioxane & 360 & $\mathrm{U}$ & 360 & 59 \\
\hline $75-27-4$ & Bromodichloromethane & 9.1 & $\mathrm{U}$ & 9.1 & 0.91 \\
\hline
\end{tabular}

FORM I $8260 B$ 
FORM I

GC/MS VOA ORGANICS ANALYSIS DATA SHEET

Lab Name: TestAmerica Burlington

Job No.: 200-28914-1

SDG No.: 200-28914

Client Sample ID: YK-S-37826

Matrix: Solid

Analysis Method: 8260B

Sample wt/vol: $10.961(\mathrm{~g})$

Soil Aliquot Vol: 500 (uL)

Soil Extract Vol.: $10(\mathrm{~mL})$

Moisture:

Analysis Batch No.: 92051

Lab Sample ID: 200-28914-6

Lab File ID: 15018_11.D

Date Collected: 07/16/2015 00:00

Date Analyzed: 07/30/2015 15:25

Dilution Factor: 1

GC Column: DB-624

ID $: 0.53(\mathrm{~mm})$

Level: (low/med) Medium

Units: ug/Kg

\begin{tabular}{|c|c|c|c|c|c|}
\hline CAS NO. & COMPOUND NAME & RESULT & $Q$ & $\mathrm{RL}$ & MDL \\
\hline $110-75-8$ & 2-Chloroethyl vinyl ether & 9.1 & $\mathrm{U}$ & 9.1 & 1.7 \\
\hline $10061-01-5$ & cis-1,3-Dichloropropene & 9.1 & $\mathrm{U}$ & 9.1 & 1.1 \\
\hline $108-10-1$ & 4-Methyl-2-pentanone & 46 & $U \star$ & 46 & 4.3 \\
\hline $108-88-3$ & Toluene & 9.1 & $\mathrm{U}$ & 9.1 & 1.9 \\
\hline $10061-02-6$ & trans-1,3-Dichloropropene & 9.1 & $\mathrm{U}$ & 9.1 & 2.0 \\
\hline $79-00-5$ & $1,1,2$-Trichloroethane & 9.1 & $\mathrm{U}$ & 9.1 & 1.2 \\
\hline $127-18-4$ & Tetrachloroethene & 9.1 & $\mathrm{U}$ & 9.1 & 1.8 \\
\hline $142-28-9$ & 1,3-Dichloropropane & 9.1 & $\mathrm{U}$ & 9.1 & 1.0 \\
\hline $591-78-6$ & 2-Hexanone & 46 & $\mathrm{U}$ & 46 & 5.6 \\
\hline $124-48-1$ & Dibromochloromethane & 9.1 & $\mathrm{U}$ & 9.1 & 1.0 \\
\hline $106-93-4$ & 1,2-Dibromoethane & 9.1 & $\mathrm{U}$ & 9.1 & 1.1 \\
\hline $108-90-7$ & Chlorobenzene & 9.1 & $\mathrm{U}$ & 9.1 & 0.83 \\
\hline $630-20-6$ & $1,1,1,2$-Tetrachloroethane & 9.1 & $\mathrm{U}$ & 9.1 & 1.0 \\
\hline $100-41-4$ & Ethylbenzene & 9.1 & $\mathrm{U}$ & 9.1 & 0.86 \\
\hline $179601-23-1$ & $\mathrm{~m} \& \mathrm{p}-\mathrm{Xy}$ lene & 9.1 & $\mathrm{U}$ & 9.1 & 1.0 \\
\hline $95-47-6$ & o-Xylene & 9.1 & $\mathrm{U}$ & 9.1 & 0.91 \\
\hline $100-42-5$ & Styrene & 9.1 & $\mathrm{U}$ & 9.1 & 0.89 \\
\hline $75-25-2$ & Bromoform & 9.1 & $\mathrm{U}$ & 9.1 & 0.91 \\
\hline $98-82-8$ & Isopropylbenzene & 9.1 & $\mathrm{U}$ & 9.1 & 1.8 \\
\hline $108-86-1$ & Bromobenzene & 9.1 & $\mathrm{U}$ & 9.1 & 0.91 \\
\hline $79-34-5$ & $1,1,2,2$-Tetrachloroethane & 9.1 & $\mathrm{U}$ & 9.1 & 1.2 \\
\hline $96-18-4$ & $1,2,3$-Trichloropropane & 9.1 & $\mathrm{U}$ & 9.1 & 1.1 \\
\hline $103-65-1$ & n-Propylbenzene & 9.1 & $\mathrm{U}$ & 9.1 & 1.0 \\
\hline $95-49-8$ & 2-Chlorotoluene & 9.1 & $\mathrm{U}$ & 9.1 & 1.0 \\
\hline $106-43-4$ & 4-Chlorotoluene & 9.1 & $\mathrm{U}$ & 9.1 & 0.88 \\
\hline $108-67-8$ & 1,3,5-Trimethylbenzene & 9.1 & $\mathrm{U}$ & 9.1 & 1.8 \\
\hline $98-06-6$ & tert-Butylbenzene & 9.1 & $\mathrm{U}$ & 9.1 & 1.3 \\
\hline $95-63-6$ & $1,2,4$-Trimethylbenzene & 9.1 & $\mathrm{U}$ & 9.1 & 0.91 \\
\hline $135-98-8$ & sec-Butylbenzene & 9.1 & $\mathrm{U}$ & 9.1 & 1.8 \\
\hline $541-73-1$ & 1,3-Dichlorobenzene & 9.1 & $\mathrm{U}$ & 9.1 & 0.87 \\
\hline $99-87-6$ & 4-Isopropyltoluene & 9.1 & $\mathrm{U}$ & 9.1 & 0.80 \\
\hline $106-46-7$ & 1,4-Dichlorobenzene & 9.1 & $\mathrm{U}$ & 9.1 & 1.3 \\
\hline $95-50-1$ & 1,2-Dichlorobenzene & 9.1 & $\mathrm{U}$ & 9.1 & 1.0 \\
\hline $104-51-8$ & n-Butylbenzene & 9.1 & $\mathrm{U}$ & 9.1 & 0.82 \\
\hline $96-12-8$ & 1,2-Dibromo-3-Chloropropane & 9.1 & $\mathrm{U}$ & 9.1 & 1.9 \\
\hline $120-82-1$ & $1,2,4$-Trichlorobenzene & 0.99 & $\mathrm{~J} B$ & 9.1 & 0.83 \\
\hline
\end{tabular}

FORM I $8260 B$ 
FORM I

GC/MS VOA ORGANICS ANALYSIS DATA SHEET

Lab Name: TestAmerica Burlington

SDG No.: 200-28914

Client Sample ID: YK-S-37826

Matrix: Solid

Analysis Method: 8260B

Sample wt/vol: $10.961(\mathrm{~g})$

Soil Aliquot Vol: 500 (uL)

Soil Extract Vol.: 10 (mL)

응 Moisture:

Analysis Batch No.: 92051
Job No. : 200-28914-1

Lab Sample ID: 200-28914-6

Lab File ID: 15018_11.D

Date Collected: 07/16/2015 00:00

Date Analyzed: 07/30/2015 15:25

Dilution Factor: 1

GC Column: DB-624

ID $: 0.53(\mathrm{~mm})$

Level: (low/med) Medium

Units: ug/Kg

\begin{tabular}{|c|c|c|c|c|c|}
\hline CAS NO. & COMPOUND NAME & RESULT & Q & RL & MDL \\
\hline $87-68-3$ & Hexachlorobutadiene & 9.1 & $\mathrm{U} *$ & 9.1 & 2.1 \\
\hline $91-20-3$ & Naphthalene & 1.2 & $\mathrm{~J} \star \mathrm{B}$ & 9.1 & 1.1 \\
\hline $87-61-6$ & $1,2,3$-Trichlorobenzene & 9.1 & $\mathrm{U}$ & 9.1 & 0.91 \\
\hline
\end{tabular}

\begin{tabular}{|l|l|r|r|r|}
\hline \multicolumn{1}{|c|}{ CAS NO. } & \multicolumn{1}{|c|}{ SURROGATE } & \%REC & Q & LIMITS \\
\hline $17060-07-0$ & $1,2-$ Dichloroethane-d4 & 103 & \\
\hline $2037-26-5$ & Toluene-d8 & 109 & $80-120$ \\
\hline $460-00-4$ & Bromofluorobenzene & 111 & $80-120$ \\
\hline $1868-53-7$ & Dibromofluoromethane (Surr) & 97 & $80-125$ \\
\hline
\end{tabular}


FORM I

GC/MS VOA ORGANICS ANALYSIS DATA SHEET

Lab Name: TestAmerica Burlington

Job No.: 200-28914-1

SDG No.: 200-28914

Client Sample ID: YK-S-37814

Matrix: Solid

Analysis Method: 8260B

Sample wt/vol: $9.45(\mathrm{~g})$

Soil Aliquot Vol: 500 (uL)

Soil Extract Vol.: $10(\mathrm{~mL})$

응 Moisture:

Analysis Batch No.: 92051

Lab Sample ID: 200-28914-7

Lab File ID: 15018_12.D

Date Collected: 07/16/2015 00:00

Date Analyzed: 07/30/2015 15:58

Dilution Factor: 1

GC Column: DB-624

ID $: 0.53(\mathrm{~mm})$

Level: (low/med) Medium

Units: ug/Kg

\begin{tabular}{|c|c|c|c|c|c|}
\hline CAS NO. & COMPOUND NAME & RESULT & $Q$ & $\mathrm{RL}$ & MDL \\
\hline $75-71-8$ & Dichlorodifluoromethane & 11 & $\mathrm{U}$ & 11 & 1.8 \\
\hline $74-87-3$ & Chloromethane & 2.6 & $\mathrm{~J} B$ & 11 & 2.2 \\
\hline $75-01-4$ & Vinyl chloride & 11 & $\mathrm{U}$ & 11 & 0.88 \\
\hline $74-83-9$ & Bromomethane & 16 & B & 11 & 1.1 \\
\hline $75-00-3$ & Chloroethane & 11 & $\mathrm{U} *$ & 11 & 2.6 \\
\hline $75-69-4$ & Trichlorofluoromethane & 11 & $\mathrm{U}$ & 11 & 0.87 \\
\hline $75-35-4$ & 1,1-Dichloroethene & 11 & $\mathrm{U} *$ & 11 & 1.2 \\
\hline $76-13-1$ & Freon TF & 11 & $U \star$ & 11 & 0.90 \\
\hline $67-64-1$ & Acetone & 53 & $\mathrm{U}$ & 53 & 13 \\
\hline $74-88-4$ & Methyl iodide & 7.8 & $\mathrm{~J} \star \mathrm{B}$ & 11 & 0.79 \\
\hline $75-15-0$ & Carbon disulfide & 11 & $\mathrm{U} \star$ & 11 & 0.92 \\
\hline $79-20-9$ & Methyl acetate & 53 & $U \star$ & 53 & 9.7 \\
\hline $75-09-2$ & Methylene Chloride & 7.3 & $\mathrm{~J}$ & 11 & 2.4 \\
\hline $156-60-5$ & trans-1,2-Dichloroethene & 11 & $\mathrm{U}$ & 11 & 1.0 \\
\hline $1634-04-4$ & Methyl t-butyl ether & 11 & $\mathrm{U}$ & 11 & 1.2 \\
\hline $75-34-3$ & 1,1-Dichloroethane & 11 & $\mathrm{U}$ & 11 & 1.2 \\
\hline $108-05-4$ & Vinyl acetate & 53 & $\mathrm{U}$ & 53 & 4.8 \\
\hline $594-20-7$ & 2,2-Dichloropropane & 11 & $\mathrm{U} *$ & 11 & 2.3 \\
\hline $156-59-2$ & cis-1,2-Dichloroethene & 11 & $\mathrm{U}$ & 11 & 1.1 \\
\hline $78-93-3$ & 2-Butanone & 53 & $\mathrm{U}$ & 53 & 6.2 \\
\hline $74-97-5$ & Bromochloromethane & 11 & $\mathrm{U}$ & 11 & 1.5 \\
\hline $109-99-9$ & Tetrahydrofuran & 21 & $U \star$ & 21 & 5.6 \\
\hline $67-66-3$ & Chloroform & 11 & $\mathrm{U}$ & 11 & 1.3 \\
\hline $71-55-6$ & $1,1,1$-Trichloroethane & 11 & $\mathrm{U}$ & 11 & 0.90 \\
\hline $110-82-7$ & Cyclohexane & 11 & $\mathrm{U}$ & 11 & 1.1 \\
\hline $563-58-6$ & 1,1-Dichloropropene & 11 & $\mathrm{U}$ & 11 & 1.0 \\
\hline $56-23-5$ & Carbon tetrachloride & 3.9 & $\mathrm{~J}$ & 11 & 2.1 \\
\hline $78-83-1$ & Isobutyl alcohol & 530 & $\mathrm{U} *$ & 530 & 29 \\
\hline $71-43-2$ & Benzene & 11 & $\mathrm{U}$ & 11 & 1.2 \\
\hline $107-06-2$ & 1,2-Dichloroethane & 11 & $\mathrm{U}$ & 11 & 1.4 \\
\hline $79-01-6$ & Trichloroethene & 11 & $\mathrm{U}$ & 11 & 0.88 \\
\hline $108-87-2$ & Methylcyclohexane & 11 & $\mathrm{U}$ & 11 & 2.2 \\
\hline $78-87-5$ & 1,2-Dichloropropane & 11 & $\mathrm{U}$ & 11 & 1.3 \\
\hline $74-95-3$ & Dibromomethane & 11 & $\mathrm{U}$ & 11 & 1.6 \\
\hline $123-91-1$ & 1,4-Dioxane & 420 & $\mathrm{U}$ & 420 & 69 \\
\hline $75-27-4$ & Bromodichloromethane & 11 & $\mathrm{U}$ & 11 & 1.1 \\
\hline
\end{tabular}

FORM I $8260 B$ 
FORM I

GC/MS VOA ORGANICS ANALYSIS DATA SHEET

Lab Name: TestAmerica Burlington

Job No.: 200-28914-1

SDG No.: 200-28914

Client Sample ID: YK-S-37814

Matrix: Solid

Analysis Method: 8260B

Sample wt/vol: $9.45(\mathrm{~g})$

Soil Aliquot Vol: 500 (uL)

Soil Extract Vol.: $10(\mathrm{~mL})$

Moisture:

Analysis Batch No.: 92051

Lab Sample ID: 200-28914-7

Lab File ID: 15018_12.D

Date Collected: 07/16/2015 00:00

Date Analyzed: 07/30/2015 15:58

Dilution Factor: 1

GC Column: DB-624

ID $: 0.53(\mathrm{~mm})$

Level: (low/med) Medium

Units: ug/Kg

\begin{tabular}{|c|c|c|c|c|c|}
\hline CAS NO. & COMPOUND NAME & RESULT & $Q$ & RL & MDL \\
\hline $110-75-8$ & 2-Chloroethyl vinyl ether & 11 & $\mathrm{U}$ & 11 & 2.0 \\
\hline $10061-01-5$ & cis-1,3-Dichloropropene & 11 & $\mathrm{U}$ & 11 & 1.3 \\
\hline $108-10-1$ & 4-Methyl-2-pentanone & 53 & $U \star$ & 53 & 5.0 \\
\hline $108-88-3$ & Toluene & 11 & $\mathrm{U}$ & 11 & 2.2 \\
\hline $10061-02-6$ & trans-1,3-Dichloropropene & 11 & $\mathrm{U}$ & 11 & 2.3 \\
\hline $79-00-5$ & $1,1,2$-Trichloroethane & 11 & $\mathrm{U}$ & 11 & 1.4 \\
\hline $127-18-4$ & Tetrachloroethene & 11 & $\mathrm{U}$ & 11 & 2.1 \\
\hline $142-28-9$ & 1,3-Dichloropropane & 11 & $\mathrm{U}$ & 11 & 1.2 \\
\hline $591-78-6$ & 2-Hexanone & 53 & $\mathrm{U}$ & 53 & 6.5 \\
\hline $124-48-1$ & Dibromochloromethane & 11 & $\mathrm{U}$ & 11 & 1.2 \\
\hline $106-93-4$ & 1,2-Dibromoethane & 11 & $\mathrm{U}$ & 11 & 1.3 \\
\hline $108-90-7$ & Chlorobenzene & 11 & $\mathrm{U}$ & 11 & 0.96 \\
\hline $630-20-6$ & $1,1,1,2$-Tetrachloroethane & 11 & $\mathrm{U}$ & 11 & 1.2 \\
\hline $100-41-4$ & Ethylbenzene & 11 & $\mathrm{U}$ & 11 & 0.99 \\
\hline $179601-23-1$ & $\mathrm{~m} \& \mathrm{p}-\mathrm{Xy}$ lene & 11 & $\mathrm{U}$ & 11 & 1.2 \\
\hline $95-47-6$ & o-Xylene & 11 & $\mathrm{U}$ & 11 & 1.1 \\
\hline $100-42-5$ & Styrene & 11 & $\mathrm{U}$ & 11 & 1.0 \\
\hline $75-25-2$ & Bromoform & 11 & $\mathrm{U}$ & 11 & 1.1 \\
\hline $98-82-8$ & Isopropylbenzene & 11 & $\mathrm{U}$ & 11 & 2.1 \\
\hline $108-86-1$ & Bromobenzene & 11 & $\mathrm{U}$ & 11 & 1.1 \\
\hline $79-34-5$ & $1,1,2,2$-Tetrachloroethane & 11 & $\mathrm{U}$ & 11 & 1.4 \\
\hline $96-18-4$ & $1,2,3$-Trichloropropane & 11 & $\mathrm{U}$ & 11 & 1.3 \\
\hline $103-65-1$ & n-Propylbenzene & 11 & $\mathrm{U}$ & 11 & 1.2 \\
\hline $95-49-8$ & 2-Chlorotoluene & 11 & $\mathrm{U}$ & 11 & 1.2 \\
\hline $106-43-4$ & 4-Chlorotoluene & 11 & $\mathrm{U}$ & 11 & 1.0 \\
\hline $108-67-8$ & $1,3,5$-Trimethylbenzene & 11 & $\mathrm{U}$ & 11 & 2.1 \\
\hline $98-06-6$ & tert-Butylbenzene & 11 & $\mathrm{U}$ & 11 & 1.5 \\
\hline $95-63-6$ & $1,2,4$-Trimethylbenzene & 11 & $\mathrm{U}$ & 11 & 1.1 \\
\hline $135-98-8$ & sec-Butylbenzene & 11 & $\mathrm{U}$ & 11 & 2.1 \\
\hline $541-73-1$ & 1,3-Dichlorobenzene & 11 & $\mathrm{U}$ & 11 & 1.0 \\
\hline $99-87-6$ & 4-Isopropyltoluene & 11 & $\mathrm{U}$ & 11 & 0.93 \\
\hline $106-46-7$ & 1,4-Dichlorobenzene & 11 & $\mathrm{U}$ & 11 & 1.5 \\
\hline $95-50-1$ & 1,2-Dichlorobenzene & 11 & $\mathrm{U}$ & 11 & 1.2 \\
\hline $104-51-8$ & n-Butylbenzene & 11 & $\mathrm{U}$ & 11 & 0.95 \\
\hline $96-12-8$ & 1,2-Dibromo-3-Chloropropane & 11 & $\mathrm{U}$ & 11 & 2.2 \\
\hline $120-82-1$ & $1,2,4$-Trichlorobenzene & 11 & $\mathrm{U}$ & 11 & 0.96 \\
\hline
\end{tabular}

FORM I $8260 B$ 
FORM I

GC/MS VOA ORGANICS ANALYSIS DATA SHEET

Lab Name: TestAmerica Burlington

SDG No.: 200-28914

Client Sample ID: YK-S-37814

Matrix: Solid

Analysis Method: 8260B

Sample wt/vol: $9.45(\mathrm{~g})$

Soil Aliquot Vol: 500 (uL)

Soil Extract Vol.: 10 (mL)

응 Moisture:

Analysis Batch No.: 92051
Job No. : 200-28914-1

Lab Sample ID: 200-28914-7

Lab File ID: 15018_12.D

Date Collected: 07/16/2015 00:00

Date Analyzed: 07/30/2015 15:58

Dilution Factor: 1

GC Column: DB-624

ID $: 0.53(\mathrm{~mm})$

Level: (low/med) Medium

Units: ug/Kg

\begin{tabular}{|l|l|r|r|r|r|}
\hline \multicolumn{1}{|c|}{ CAS NO. } & \multicolumn{1}{|c|}{ COMPOUND NAME } & RESULT & Q & RL & MDL \\
\hline $87-68-3$ & Hexachlorobutadiene & 11 & U * & 11 & 2.4 \\
\hline $91-20-3$ & Naphthalene & 1.6 & $\mathrm{~J} \star \mathrm{B}$ & 11 & 1.3 \\
\hline $87-61-6$ & $1,2,3-$ Trichlorobenzene & 11 & $\mathrm{U}$ & & 11 \\
\hline
\end{tabular}

\begin{tabular}{|c|c|c|c|c|}
\hline CAS NO. & SURROGATE & $\circ \mathrm{REC}$ & $Q$ & LIMITS \\
\hline $17060-07-0$ & 1,2-Dichloroethane-d4 & 107 & & $80-120$ \\
\hline $2037-26-5$ & Toluene-d8 & 109 & & $80-120$ \\
\hline $460-00-4$ & Bromofluorobenzene & 112 & & $80-125$ \\
\hline $1868-53-7$ & Dibromofluoromethane (Surr) & 101 & & $50-150$ \\
\hline
\end{tabular}


FORM I

GC/MS VOA ORGANICS ANALYSIS DATA SHEET

Lab Name: TestAmerica Burlington

Job No : : 200-28914-1

SDG No.: 200-28914

Client Sample ID: YK-S-37758

Matrix: Solid

Analysis Method: 8260B

Sample wt/vol: $10.864(\mathrm{~g})$

Soil Aliquot Vol: 500 (uL)

Soil Extract Vol.: $10(\mathrm{~mL})$

Moisture:

Analysis Batch No.: 92051

Lab Sample ID: 200-28914-8

Lab File ID: 15018_13.D

Date Collected: 07/16/2015 00:00

Date Analyzed: 07/30/2015 16:30

Dilution Factor: 1

GC Column: DB-624

ID $: 0.53(\mathrm{~mm})$

Level: (low/med) Medium

Units: ug/Kg

\begin{tabular}{|c|c|c|c|c|c|}
\hline CAS NO. & COMPOUND NAME & RESULT & $Q$ & $\mathrm{RL}$ & MDL \\
\hline $75-71-8$ & Dichlorodifluoromethane & 9.2 & $\mathrm{U}$ & 9.2 & 1.6 \\
\hline $74-87-3$ & Chloromethane & 2.7 & $\mathrm{~J} B$ & 9.2 & 1.9 \\
\hline $75-01-4$ & Vinyl chloride & 9.2 & $\mathrm{U}$ & 9.2 & 0.76 \\
\hline $74-83-9$ & Bromomethane & 14 & B & 9.2 & 0.92 \\
\hline $75-00-3$ & Chloroethane & 9.2 & $\mathrm{U} *$ & 9.2 & 2.3 \\
\hline $75-69-4$ & Trichlorofluoromethane & 9.2 & $\mathrm{U}$ & 9.2 & 0.75 \\
\hline $75-35-4$ & 1,1-Dichloroethene & 9.2 & $\mathrm{U} *$ & 9.2 & 1.0 \\
\hline $76-13-1$ & Freon TF & 9.2 & $U \star$ & 9.2 & 0.78 \\
\hline $67-64-1$ & Acetone & 46 & $\mathrm{U}$ & 46 & 11 \\
\hline $74-88-4$ & Methyl iodide & 5.7 & $\mathrm{~J} \star \mathrm{B}$ & 9.2 & 0.69 \\
\hline $75-15-0$ & Carbon disulfide & 9.2 & $\mathrm{U} \star$ & 9.2 & 0.80 \\
\hline $79-20-9$ & Methyl acetate & 46 & $U \star$ & 46 & 8.5 \\
\hline $75-09-2$ & Methylene Chloride & 9.2 & $\mathrm{U}$ & 9.2 & 2.1 \\
\hline $156-60-5$ & trans-1,2-Dichloroethene & 9.2 & $\mathrm{U}$ & 9.2 & 0.91 \\
\hline $1634-04-4$ & Methyl t-butyl ether & 9.2 & $\mathrm{U}$ & 9.2 & 1.0 \\
\hline $75-34-3$ & 1,1-Dichloroethane & 9.2 & $\mathrm{U}$ & 9.2 & 1.0 \\
\hline $108-05-4$ & Vinyl acetate & 46 & $\mathrm{U}$ & 46 & 4.1 \\
\hline $594-20-7$ & 2,2-Dichloropropane & 9.2 & $\mathrm{U} *$ & 9.2 & 2.0 \\
\hline $156-59-2$ & cis-1,2-Dichloroethene & 9.2 & $\mathrm{U}$ & 9.2 & 0.92 \\
\hline $78-93-3$ & 2-Butanone & 46 & $\mathrm{U}$ & 46 & 5.4 \\
\hline $74-97-5$ & Bromochloromethane & 9.2 & $\mathrm{U}$ & 9.2 & 1.3 \\
\hline $109-99-9$ & Tetrahydrofuran & 18 & $U \star$ & 18 & 4.9 \\
\hline $67-66-3$ & Chloroform & 9.2 & $\mathrm{U}$ & 9.2 & 1.1 \\
\hline $71-55-6$ & $1,1,1$-Trichloroethane & 9.2 & $\mathrm{U}$ & 9.2 & 0.78 \\
\hline $110-82-7$ & Cyclohexane & 9.2 & $\mathrm{U}$ & 9.2 & 0.92 \\
\hline $563-58-6$ & 1,1-Dichloropropene & 9.2 & $\mathrm{U}$ & 9.2 & 0.89 \\
\hline $56-23-5$ & Carbon tetrachloride & 9.2 & $\mathrm{U}$ & 9.2 & 1.8 \\
\hline $78-83-1$ & Isobutyl alcohol & 460 & $\mathrm{U} *$ & 460 & 25 \\
\hline $71-43-2$ & Benzene & 9.2 & $\mathrm{U}$ & 9.2 & 1.0 \\
\hline $107-06-2$ & 1,2-Dichloroethane & 9.2 & $\mathrm{U}$ & 9.2 & 1.2 \\
\hline $79-01-6$ & Trichloroethene & 9.2 & $\mathrm{U}$ & 9.2 & 0.76 \\
\hline $108-87-2$ & Methylcyclohexane & 9.2 & $\mathrm{U}$ & 9.2 & 1.9 \\
\hline $78-87-5$ & 1,2-Dichloropropane & 9.2 & $\mathrm{U}$ & 9.2 & 1.1 \\
\hline $74-95-3$ & Dibromomethane & 9.2 & $\mathrm{U}$ & 9.2 & 1.4 \\
\hline $123-91-1$ & 1,4-Dioxane & 370 & $\mathrm{U}$ & 370 & 60 \\
\hline $75-27-4$ & Bromodichloromethane & 9.2 & $\mathrm{U}$ & 9.2 & 0.92 \\
\hline
\end{tabular}

FORM I $8260 B$ 
FORM I

GC/MS VOA ORGANICS ANALYSIS DATA SHEET

Lab Name: TestAmerica Burlington

Job No.: 200-28914-1

SDG No.: 200-28914

Client Sample ID: YK-S-37758

Matrix: Solid

Analysis Method: 8260B

Sample wt/vol: $10.864(\mathrm{~g})$

Soil Aliquot Vol: 500 (uL)

Soil Extract Vol.: $10(\mathrm{~mL})$

Moisture:

Analysis Batch No.: 92051

Lab Sample ID: 200-28914-8

Lab File ID: 15018_13.D

Date Collected: 07/16/2015 00:00

Date Analyzed: 07/30/2015 16:30

Dilution Factor: 1

GC Column: DB-624

ID : $0.53(\mathrm{~mm})$

Level: (low/med) Medium

Units: ug/Kg

\begin{tabular}{|c|c|c|c|c|c|}
\hline CAS NO. & COMPOUND NAME & RESULT & $Q$ & RL & MDL \\
\hline $110-75-8$ & 2-Chloroethyl vinyl ether & 9.2 & $\mathrm{U}$ & 9.2 & 1.7 \\
\hline $10061-01-5$ & cis-1,3-Dichloropropene & 9.2 & $\mathrm{U}$ & 9.2 & 1.1 \\
\hline $108-10-1$ & 4-Methyl-2-pentanone & 46 & $\mathrm{U} *$ & 46 & 4.3 \\
\hline $108-88-3$ & Toluene & 9.2 & $\mathrm{U}$ & 9.2 & 1.9 \\
\hline $10061-02-6$ & trans-1,3-Dichloropropene & 9.2 & $\mathrm{U}$ & 9.2 & 2.0 \\
\hline $79-00-5$ & $1,1,2$-Trichloroethane & 9.2 & $\mathrm{U}$ & 9.2 & 1.2 \\
\hline $127-18-4$ & Tetrachloroethene & 9.2 & $\mathrm{U}$ & 9.2 & 1.8 \\
\hline $142-28-9$ & 1,3-Dichloropropane & 9.2 & $\mathrm{U}$ & 9.2 & 1.0 \\
\hline $591-78-6$ & 2-Hexanone & 46 & $\mathrm{U}$ & 46 & 5.6 \\
\hline $124-48-1$ & Dibromochloromethane & 9.2 & $\mathrm{U}$ & 9.2 & 1.0 \\
\hline $106-93-4$ & 1,2-Dibromoethane & 9.2 & $\mathrm{U}$ & 9.2 & 1.1 \\
\hline $108-90-7$ & Chlorobenzene & 9.2 & $\mathrm{U}$ & 9.2 & 0.84 \\
\hline $630-20-6$ & $1,1,1,2$-Tetrachloroethane & 9.2 & $\mathrm{U}$ & 9.2 & 1.0 \\
\hline $100-41-4$ & Ethylbenzene & 9.2 & $\mathrm{U}$ & 9.2 & 0.87 \\
\hline $179601-23-1$ & m\&p-Xylene & 9.2 & $\mathrm{U}$ & 9.2 & 1.0 \\
\hline $95-47-6$ & o-Xylene & 9.2 & $\mathrm{U}$ & 9.2 & 0.92 \\
\hline $100-42-5$ & Styrene & 9.2 & $\mathrm{U}$ & 9.2 & 0.90 \\
\hline $75-25-2$ & Bromoform & 9.2 & $\mathrm{U}$ & 9.2 & 0.92 \\
\hline $98-82-8$ & Isopropylbenzene & 9.2 & $\mathrm{U}$ & 9.2 & 1.8 \\
\hline $108-86-1$ & Bromobenzene & 9.2 & $\mathrm{U}$ & 9.2 & 0.92 \\
\hline $79-34-5$ & $1,1,2,2$-Tetrachloroethane & 9.2 & $\mathrm{U}$ & 9.2 & 1.2 \\
\hline $96-18-4$ & 1,2,3-Trichloropropane & 9.2 & $\mathrm{U}$ & 9.2 & 1.1 \\
\hline $103-65-1$ & n-Propylbenzene & 9.2 & $\mathrm{U}$ & 9.2 & 1.0 \\
\hline $95-49-8$ & 2-Chlorotoluene & 9.2 & $\mathrm{U}$ & 9.2 & 1.0 \\
\hline $106-43-4$ & 4-Chlorotoluene & 9.2 & $\mathrm{U}$ & 9.2 & 0.88 \\
\hline $108-67-8$ & 1,3,5-Trimethylbenzene & 9.2 & $\mathrm{U}$ & 9.2 & 1.8 \\
\hline $98-06-6$ & tert-Butylbenzene & 9.2 & $\mathrm{U}$ & 9.2 & 1.3 \\
\hline $95-63-6$ & $1,2,4$-Trimethylbenzene & 9.2 & $\mathrm{U}$ & 9.2 & 0.92 \\
\hline $135-98-8$ & sec-Butylbenzene & 9.2 & $\mathrm{U}$ & 9.2 & 1.8 \\
\hline $541-73-1$ & 1,3-Dichlorobenzene & 9.2 & $\mathrm{U}$ & 9.2 & 0.87 \\
\hline $99-87-6$ & 4-Isopropyltoluene & 9.2 & $\mathrm{U}$ & 9.2 & 0.81 \\
\hline $106-46-7$ & 1,4-Dichlorobenzene & 9.2 & $\mathrm{U}$ & 9.2 & 1.3 \\
\hline $95-50-1$ & 1,2-Dichlorobenzene & 9.2 & $\mathrm{U}$ & 9.2 & 1.0 \\
\hline $104-51-8$ & n-Butylbenzene & 9.2 & $\mathrm{U}$ & 9.2 & 0.83 \\
\hline $96-12-8$ & 1,2-Dibromo-3-Chloropropane & 9.2 & $\mathrm{U}$ & 9.2 & 1.9 \\
\hline $120-82-1$ & $1,2,4$-Trichlorobenzene & 9.2 & $\mathrm{U}$ & 9.2 & 0.84 \\
\hline
\end{tabular}

FORM I $8260 B$ 
FORM I

GC/MS VOA ORGANICS ANALYSIS DATA SHEET

Lab Name: TestAmerica Burlington

SDG No.: 200-28914

Client Sample ID: YK-S-37758

Matrix: Solid

Analysis Method: 8260B

Sample wt/vol: $10.864(\mathrm{~g})$

Soil Aliquot Vol: 500 (uL)

Soil Extract Vol.: 10 (mL)

응 Moisture:

Analysis Batch No.: 92051
Job No. : 200-28914-1

Lab Sample ID: 200-28914-8

Lab File ID: 15018_13.D

Date Collected: 07/16/2015 00:00

Date Analyzed: 07/30/2015 16:30

Dilution Factor: 1

GC Column: DB-624

ID $: 0.53(\mathrm{~mm})$

Level: (low/med) Medium

Units: ug/Kg

\begin{tabular}{|c|c|c|c|c|c|}
\hline CAS NO. & COMPOUND NAME & RESULT & Q & RL & MDL \\
\hline $87-68-3$ & Hexachlorobutadiene & 9.2 & $\mathrm{U} *$ & 9.2 & 2.1 \\
\hline $91-20-3$ & Naphthalene & 1.1 & $\mathrm{~J} \star \mathrm{B}$ & 9.2 & 1.1 \\
\hline $87-61-6$ & $1,2,3$-Trichlorobenzene & 9.2 & $\mathrm{U}$ & 9.2 & 0.92 \\
\hline
\end{tabular}

\begin{tabular}{|l|l|r|r|r|}
\hline \multicolumn{1}{|c|}{ CAS NO. } & \multicolumn{1}{|c|}{ SURROGATE } & \%REC & L & LIMITS \\
\hline $17060-07-0$ & $1,2-$ Dichloroethane-d4 & 96 & $80-120$ \\
\hline $2037-26-5$ & Toluene-d8 & 110 & $80-120$ \\
\hline $460-00-4$ & Bromofluorobenzene & 111 & 97 \\
\hline $1868-53-7$ & Dibromofluoromethane (Surr) & $90-125$ \\
\hline
\end{tabular}


FORM I

GC/MS VOA ORGANICS ANALYSIS DATA SHEET

Lab Name: TestAmerica Burlington

Job No.: 200-28914-1

SDG No.: 200-28914

Client Sample ID: YK-S-BLANK

Matrix: Solid

Analysis Method: 8260B

Sample wt/vol: $10(\mathrm{~g})$

Soil Aliquot Vol: 500 (uL)

Soil Extract Vol.: $10(\mathrm{~mL})$

Moisture:

Analysis Batch No.: 92051

Lab Sample ID: 200-28914-9

Lab File ID: 15018_14.D

Date Collected: 07/16/2015 00:00

Date Analyzed: 07/30/2015 17:03

Dilution Factor: 1

GC Column: DB-624

ID $: 0.53(\mathrm{~mm})$

Level: (low/med) Medium

Units: ug/Kg

\begin{tabular}{|c|c|c|c|c|c|}
\hline CAS NO. & COMPOUND NAME & RESULT & $Q$ & $\mathrm{RL}$ & MDL \\
\hline $75-71-8$ & Dichlorodifluoromethane & 10 & $\mathrm{U}$ & 10 & 1.7 \\
\hline $74-87-3$ & Chloromethane & 2.4 & $\mathrm{~J} B$ & 10 & 2.1 \\
\hline $75-01-4$ & Vinyl chloride & 10 & $\mathrm{U}$ & 10 & 0.83 \\
\hline $74-83-9$ & Bromomethane & 17 & B & 10 & 1.0 \\
\hline $75-00-3$ & Chloroethane & 10 & $\mathrm{U} *$ & 10 & 2.5 \\
\hline $75-69-4$ & Trichlorofluoromethane & 10 & $\mathrm{U}$ & 10 & 0.82 \\
\hline $75-35-4$ & 1,1-Dichloroethene & 10 & $\mathrm{U} *$ & 10 & 1.1 \\
\hline $76-13-1$ & Freon TF & 10 & $U \star$ & 10 & 0.85 \\
\hline $67-64-1$ & Acetone & 50 & $\mathrm{U}$ & 50 & 12 \\
\hline $74-88-4$ & Methyl iodide & 6.7 & $J \star B$ & 10 & 0.75 \\
\hline $75-15-0$ & Carbon disulfide & 10 & $\mathrm{U} \star$ & 10 & 0.87 \\
\hline $79-20-9$ & Methyl acetate & 50 & $U \star$ & 50 & 9.2 \\
\hline $75-09-2$ & Methylene Chloride & 10 & $\mathrm{U}$ & 10 & 2.3 \\
\hline $156-60-5$ & trans-1,2-Dichloroethene & 10 & $\mathrm{U}$ & 10 & 0.99 \\
\hline $1634-04-4$ & Methyl t-butyl ether & 10 & $\mathrm{U}$ & 10 & 1.1 \\
\hline $75-34-3$ & 1,1-Dichloroethane & 10 & $\mathrm{U}$ & 10 & 1.1 \\
\hline $108-05-4$ & Vinyl acetate & 50 & $\mathrm{U}$ & 50 & 4.5 \\
\hline $594-20-7$ & 2,2-Dichloropropane & 10 & $\mathrm{U}$ * & 10 & 2.2 \\
\hline $156-59-2$ & cis-1,2-Dichloroethene & 10 & $\mathrm{U}$ & 10 & 1.0 \\
\hline $78-93-3$ & 2-Butanone & 50 & $\mathrm{U}$ & 50 & 5.9 \\
\hline $74-97-5$ & Bromochloromethane & 10 & $\mathrm{U}$ & 10 & 1.4 \\
\hline $109-99-9$ & Tetrahydrofuran & 20 & $U \star$ & 20 & 5.3 \\
\hline $67-66-3$ & Chloroform & 10 & $\mathrm{U}$ & 10 & 1.2 \\
\hline $71-55-6$ & $1,1,1$-Trichloroethane & 10 & $\mathrm{U}$ & 10 & 0.85 \\
\hline $110-82-7$ & Cyclohexane & 10 & $\mathrm{U}$ & 10 & 1.0 \\
\hline $563-58-6$ & 1,1-Dichloropropene & 10 & $\mathrm{U}$ & 10 & 0.97 \\
\hline $56-23-5$ & Carbon tetrachloride & 10 & $\mathrm{U}$ & 10 & 2.0 \\
\hline $78-83-1$ & Isobutyl alcohol & 500 & $\mathrm{U} *$ & 500 & 27 \\
\hline $71-43-2$ & Benzene & 10 & $\mathrm{U}$ & 10 & 1.1 \\
\hline $107-06-2$ & 1,2-Dichloroethane & 10 & $\mathrm{U}$ & 10 & 1.3 \\
\hline $79-01-6$ & Trichloroethene & 10 & $\mathrm{U}$ & 10 & 0.83 \\
\hline $108-87-2$ & Methylcyclohexane & 10 & $\mathrm{U}$ & 10 & 2.1 \\
\hline $78-87-5$ & 1,2-Dichloropropane & 10 & $\mathrm{U}$ & 10 & 1.2 \\
\hline $74-95-3$ & Dibromomethane & 10 & $\mathrm{U}$ & 10 & 1.5 \\
\hline $123-91-1$ & 1,4-Dioxane & 400 & $\mathrm{U}$ & 400 & 65 \\
\hline $75-27-4$ & Bromodichloromethane & 10 & $\mathrm{U}$ & 10 & 1.0 \\
\hline
\end{tabular}

FORM I $8260 B$ 
FORM I

GC/MS VOA ORGANICS ANALYSIS DATA SHEET

Lab Name: TestAmerica Burlington

Job No.: 200-28914-1

SDG No.: 200-28914

Client Sample ID: YK-S-BLANK

Matrix: Solid

Analysis Method: 8260B

Sample wt/vol: $10(\mathrm{~g})$

Soil Aliquot Vol: 500 (uL)

Soil Extract Vol.: $10(\mathrm{~mL})$

Moisture:

Analysis Batch No.: 92051

Lab Sample ID: 200-28914-9

Lab File ID: 15018_14.D

Date Collected: 07/16/2015 00:00

Date Analyzed: 07/30/2015 17:03

Dilution Factor: 1

GC Column: DB-624

ID $: 0.53(\mathrm{~mm})$

Level: (low/med) Medium

Units: ug/Kg

\begin{tabular}{|c|c|c|c|c|c|}
\hline CAS NO. & COMPOUND NAME & RESULT & $Q$ & RL & MDL \\
\hline $110-75-8$ & 2-Chloroethyl vinyl ether & 10 & $\mathrm{U}$ & 10 & 1.9 \\
\hline $10061-01-5$ & cis-1,3-Dichloropropene & 10 & $\mathrm{U}$ & 10 & 1.2 \\
\hline $108-10-1$ & 4-Methyl-2-pentanone & 50 & $\mathrm{U} *$ & 50 & 4.7 \\
\hline $108-88-3$ & Toluene & 10 & $\mathrm{U}$ & 10 & 2.1 \\
\hline $10061-02-6$ & trans-1,3-Dichloropropene & 10 & $\mathrm{U}$ & 10 & 2.2 \\
\hline $79-00-5$ & $1,1,2$-Trichloroethane & 10 & $\mathrm{U}$ & 10 & 1.3 \\
\hline $127-18-4$ & Tetrachloroethene & 10 & $\mathrm{U}$ & 10 & 2.0 \\
\hline $142-28-9$ & 1,3-Dichloropropane & 10 & $\mathrm{U}$ & 10 & 1.1 \\
\hline $591-78-6$ & 2-Hexanone & 50 & $\mathrm{U}$ & 50 & 6.1 \\
\hline $124-48-1$ & Dibromochloromethane & 10 & $\mathrm{U}$ & 10 & 1.1 \\
\hline $106-93-4$ & 1,2-Dibromoethane & 10 & $\mathrm{U}$ & 10 & 1.2 \\
\hline $108-90-7$ & Chlorobenzene & 10 & $\mathrm{U}$ & 10 & 0.91 \\
\hline $630-20-6$ & $1,1,1,2$-Tetrachloroethane & 10 & $\mathrm{U}$ & 10 & 1.1 \\
\hline $100-41-4$ & Ethylbenzene & 1.3 & $\mathrm{~J} B$ & 10 & 0.94 \\
\hline $179601-23-1$ & m\&p-Xylene & 1.2 & $\mathrm{~J}$ & 10 & 1.1 \\
\hline $95-47-6$ & o-Xylene & 10 & $\mathrm{U}$ & 10 & 1.0 \\
\hline $100-42-5$ & Styrene & 10 & $\mathrm{U}$ & 10 & 0.98 \\
\hline $75-25-2$ & Bromoform & 10 & $\mathrm{U}$ & 10 & 1.0 \\
\hline $98-82-8$ & Isopropylbenzene & 10 & $\mathrm{U}$ & 10 & 2.0 \\
\hline $108-86-1$ & Bromobenzene & 10 & $\mathrm{U}$ & 10 & 1.0 \\
\hline $79-34-5$ & $1,1,2,2$-Tetrachloroethane & 10 & $\mathrm{U}$ & 10 & 1.3 \\
\hline $96-18-4$ & 1,2,3-Trichloropropane & 10 & $\mathrm{U}$ & 10 & 1.2 \\
\hline $103-65-1$ & n-Propylbenzene & 10 & $\mathrm{U}$ & 10 & 1.1 \\
\hline $95-49-8$ & 2-Chlorotoluene & 10 & $\mathrm{U}$ & 10 & 1.1 \\
\hline $106-43-4$ & 4-Chlorotoluene & 10 & $\mathrm{U}$ & 10 & 0.96 \\
\hline $108-67-8$ & 1,3,5-Trimethylbenzene & 10 & $\mathrm{U}$ & 10 & 2.0 \\
\hline $98-06-6$ & tert-Butylbenzene & 10 & $\mathrm{U}$ & 10 & 1.4 \\
\hline $95-63-6$ & $1,2,4$-Trimethylbenzene & 10 & $\mathrm{U}$ & 10 & 1.0 \\
\hline $135-98-8$ & sec-Butylbenzene & 10 & $\mathrm{U}$ & 10 & 2.0 \\
\hline $541-73-1$ & 1,3-Dichlorobenzene & 10 & $\mathrm{U}$ & 10 & 0.95 \\
\hline $99-87-6$ & 4-Isopropyltoluene & 10 & $\mathrm{U}$ & 10 & 0.88 \\
\hline $106-46-7$ & 1,4-Dichlorobenzene & 10 & $\mathrm{U}$ & 10 & 1.4 \\
\hline $95-50-1$ & 1,2-Dichlorobenzene & 10 & $\mathrm{U}$ & 10 & 1.1 \\
\hline $104-51-8$ & n-Butylbenzene & 10 & $\mathrm{U}$ & 10 & 0.90 \\
\hline $96-12-8$ & 1,2-Dibromo-3-Chloropropane & 10 & $\mathrm{U}$ & 10 & 2.1 \\
\hline $120-82-1$ & $1,2,4$-Trichlorobenzene & 10 & $\mathrm{U}$ & 10 & 0.91 \\
\hline
\end{tabular}

FORM I $8260 B$ 
FORM I

GC/MS VOA ORGANICS ANALYSIS DATA SHEET

Lab Name: TestAmerica Burlington

SDG No.: 200-28914

Client sample ID: YK-S-BLANK

Matrix: Solid

Analysis Method: 8260B

Sample wt/vol: $10(\mathrm{~g})$

Soil Aliquot Vol: 500 (uL)

Soil Extract Vol.: 10 (mL)

응 Moisture:

Analysis Batch No.: 92051
Job No. : 200-28914-1

Lab Sample ID: 200-28914-9

Lab File ID: 15018_14.D

Date Collected: 07/16/2015 00:00

Date Analyzed: 07/30/2015 17:03

Dilution Factor: 1

GC Column: DB-624

ID $: 0.53(\mathrm{~mm})$

Level: (low/med) Medium

Units: ug/Kg

\begin{tabular}{|c|c|c|c|c|c|}
\hline CAS NO. & COMPOUND NAME & RESULT & Q & RL & MDL \\
\hline $87-68-3$ & Hexachlorobutadiene & 10 & $\mathrm{U}$ * & 10 & 2.3 \\
\hline $91-20-3$ & Naphthalene & 10 & $\mathrm{U}$ * & 10 & 1.2 \\
\hline $87-61-6$ & $1,2,3$-Trichlorobenzene & 10 & $\mathrm{U}$ & 10 & 1.0 \\
\hline
\end{tabular}

\begin{tabular}{|l|l|r|r|r|}
\hline \multicolumn{1}{|c|}{ CAS NO. } & \multicolumn{1}{|c|}{ SURROGATE } & \%REC & Q & LIMITS \\
\hline $17060-07-0$ & $1,2-$ Dichloroethane-d4 & 103 & $80-120$ \\
\hline $2037-26-5$ & Toluene-d8 & 113 & $80-120$ \\
\hline $460-00-4$ & Bromofluorobenzene & 113 & $90-125$ \\
\hline $1868-53-7$ & Dibromofluoromethane (Surr) & 97 & $50-150$ \\
\hline
\end{tabular}


Lab Name: TestAmerica Burlington

SDG No.: 200-28914

Instrument ID: CHL.i

Calibration Start Date: 07/29/2015 12:33
Analy Batch No.: 92007

Heated Purge: (Y/N) N

Column: DB-624 ID: $0.53(\mathrm{~mm})$

Calibration ID: 31571

Calibration Files:

\begin{tabular}{|lll|}
\hline LEVEL: & LAB SAMPLE ID: & LAB FILE ID: \\
Level 1 & IC 200-92007/3 & 15008_03.D \\
Level 2 & IC 200-92007/4 & 15008_04.D \\
Level 3 & IC 200-92007/5 & 15008_05.D \\
Level 4 & IC 200-92007/6 & 15008_06.D \\
Level 5 & ICIS 200-92007/7 & 15008 07.D \\
Level 6 & IC 200-92007/8 & 15008_08.D \\
Level 7 & IC 200-92007/9 & 15008_09.D \\
\hline
\end{tabular}

\begin{tabular}{|c|c|c|c|c|c|c|c|c|c|c|c|c|c|c|c|c|c|}
\hline \multirow[t]{2}{*}{ ANALYTE } & \multicolumn{5}{|c|}{$\mathrm{RRF}$} & \multirow{2}{*}{$\begin{array}{l}\text { CURVE } \\
\text { TYPE }\end{array}$} & \multicolumn{3}{|c|}{ COEFFICIENT } & \multirow[t]{2}{*}{$\#$} & \multirow[t]{2}{*}{ MIN RRF } & \multirow[t]{2}{*}{$\because R S D$} & \multirow[t]{2}{*}{$\#$} & \multirow{2}{*}{$\begin{array}{l}\text { MAX } \\
\text { RSD }\end{array}$} & \multirow{2}{*}{$\begin{array}{c}R^{\wedge} 2 \\
\text { OR } C O D\end{array}$} & \multirow[t]{2}{*}{ \# } & \multirow{2}{*}{$\begin{array}{c}\text { MIN } R^{\wedge} 2 \\
\text { OR } \quad \text { COD }\end{array}$} \\
\hline & $\begin{array}{ll}\text { LVL } & 1 \\
\text { LVL } & 6\end{array}$ & $\begin{array}{ll}\text { LVL } & 2 \\
\text { LVL } & 7 \\
\end{array}$ & LVL 3 & LVL 4 & LVL 5 & & B & M1 & M2 & & & & & & & & \\
\hline Dichlorodifluoromethane & $\begin{array}{r}+++++ \\
0.4463\end{array}$ & $\begin{array}{l}0.4288 \\
0.4366\end{array}$ & 0.4461 & 0.4419 & 0.4590 & Ave & & 0.4431 & & & & 2.3 & & 15.0 & & & \\
\hline Chloromethane & $\begin{array}{r}+++++ \\
0.3243\end{array}$ & $\begin{array}{l}0.4178 \\
0.3250\end{array}$ & 0.3657 & 0.3501 & 0.3231 & Ave & & 0.3510 & & & 0.1000 & 10.5 & & 15.0 & & & \\
\hline Vinyl chloride & $\begin{array}{l}0.4372 \\
0.3506\end{array}$ & $\begin{array}{l}0.3249 \\
0.3470\end{array}$ & 0.3468 & 0.3416 & 0.3563 & Ave & & 0.3578 & & & & 10.2 & & 15.0 & & & \\
\hline Butadiene & $\begin{array}{r}+++++ \\
0.2642\end{array}$ & $\begin{array}{l}0.2627 \\
0.2589\end{array}$ & 0.2579 & 0.2583 & 0.2737 & Ave & & 0.2626 & & & & 2.3 & & 15.0 & & & \\
\hline Bromomethane & $\begin{array}{r}+++++ \\
0.2256\end{array}$ & $\begin{array}{l}0.2569 \\
0.2357\end{array}$ & 0.2110 & 0.2095 & 0.2126 & Ave & & 0.2252 & & & & 8.2 & & 15.0 & & & \\
\hline Chloroethane & $\begin{array}{r}+++++ \\
0.2115\end{array}$ & $\begin{array}{l}0.2265 \\
0.1992\end{array}$ & 0.2229 & 0.2108 & 0.2177 & Ave & & 0.2148 & & & & 4.6 & & 15.0 & & & \\
\hline Dichlorofluoromethane & $\begin{array}{r}+++++ \\
0.7505\end{array}$ & $\begin{array}{l}0.7257 \\
0.7363\end{array}$ & 0.7430 & 0.7235 & 0.7642 & Ave & & 0.7405 & & & & 2.1 & & 15.0 & & & \\
\hline Trichlorofluoromethane & $\begin{array}{r}+++++ \\
0.5577\end{array}$ & $\begin{array}{l}0.5369 \\
0.5398\end{array}$ & 0.5375 & 0.5374 & 0.5722 & Ave & & 0.5469 & & & & 2.7 & & 15.0 & & & \\
\hline Freon $123 a$ & $\begin{array}{r}+++++ \\
0.8080\end{array}$ & $\begin{array}{l}0.7274 \\
0.8011\end{array}$ & 0.7720 & 0.7601 & 0.8312 & Ave & & 0.7833 & & & & 4.8 & & 15.0 & & & \\
\hline Ethyl ether & $\begin{array}{r}+++++ \\
0.2126\end{array}$ & $\begin{array}{l}0.1861 \\
0.2061\end{array}$ & 0.2045 & 0.1954 & 0.2142 & Ave & & 0.2032 & & & & 5.3 & & 15.0 & & & \\
\hline Acrolein & $\begin{array}{r}+++++ \\
0.0155\end{array}$ & $\begin{array}{l}0.0118 \\
0.0143\end{array}$ & 0.0176 & 0.0155 & 0.0145 & Ave & & 0.0149 & & & & 13.0 & & 15.0 & & & \\
\hline 1,1-Dichloroethene & $\begin{array}{r}+++++ \\
0.3137\end{array}$ & $\begin{array}{l}0.2665 \\
0.3136\end{array}$ & 0.2992 & 0.2918 & 0.3180 & Ave & & 0.3005 & & & & 6.5 & & 15.0 & & & \\
\hline Freon TF & $\begin{array}{r}+++++ \\
0.6314\end{array}$ & $\begin{array}{l}0.5259 \\
0.6260\end{array}$ & 0.5879 & 0.5829 & 0.6483 & Ave & & 0.6004 & & & & 7.4 & & 15.0 & & & \\
\hline Acetone & $\begin{array}{r}+++++ \\
0.7329\end{array}$ & $\begin{array}{l}0.6789 \\
0.6988\end{array}$ & 0.7278 & 0.6779 & 0.7575 & Ave & & 0.7123 & & & & 4.5 & & 15.0 & & & \\
\hline
\end{tabular}


FORM VI

GC/MS VOA INITIAL CALIBRATION DATA

INTERNAL STANDARD CURVE EVALUATION

Lab Name: TestAmerica Burlington

Job No.: 200-28914-1

Analy Batch No.: 92007

SDG No.: 200-28914

Instrument ID: CHL.i

GC Column: DB-624

ID $: 0.53(\mathrm{~mm})$

Heated Purge: (Y/N) N

Calibration Start Date: 07/29/2015 12:33

Calibration End Date: 07/29/2015 15:49

Calibration ID: 31571

\begin{tabular}{|c|c|c|c|c|c|c|c|c|c|c|c|c|c|c|c|c|c|}
\hline \multirow[t]{2}{*}{ ANALYTE } & \multicolumn{5}{|c|}{$\mathrm{RRF}$} & \multirow{2}{*}{$\begin{array}{l}\text { CURVE } \\
\text { TYPE }\end{array}$} & \multicolumn{3}{|c|}{ COEFFICIENT } & \multirow[t]{2}{*}{ \# } & \multirow[t]{2}{*}{ MIN RRF } & \multirow[t]{2}{*}{$\because \mathrm{RSD}$} & \multirow[t]{2}{*}{ \# } & \multirow{2}{*}{$\begin{array}{l}\text { MAX } \\
\circ \text { RSD }\end{array}$} & \multirow{2}{*}{$\begin{array}{c}\mathrm{R}^{\wedge} 2 \\
\mathrm{OR} \quad \mathrm{COD}\end{array}$} & \multirow[t]{2}{*}{$\#$} & \multirow{2}{*}{$\begin{array}{c}\text { MIN } R^{\wedge} 2 \\
\text { OR } C O D\end{array}$} \\
\hline & $\begin{array}{ll}\text { LVL } & 1 \\
\text { LVL } & 6\end{array}$ & $\begin{array}{ll}\text { LVL } & 2 \\
\text { LVL } & 7\end{array}$ & LVL 3 & LVL 4 & LVL 5 & & B & M1 & M2 & & & & & & & & \\
\hline Methyl iodide & $\begin{array}{r}+++++ \\
0.4081 \\
\end{array}$ & $\begin{array}{l}0.3284 \\
0.4237\end{array}$ & 0.3069 & 0.2824 & 0.3558 & Ave & & 0.3509 & & & & 16.0 & * & 15.0 & & & \\
\hline Carbon disulfide & $\begin{array}{r}+++++ \\
0.8585 \\
\end{array}$ & $\begin{array}{l}0.9811 \\
0.8594 \\
\end{array}$ & 0.9007 & 0.8240 & 0.8732 & Ave & & 0.8828 & & & & 6.1 & & 15.0 & & & \\
\hline Allyl chloride & $\begin{array}{r}+++++ \\
0.6101 \\
\end{array}$ & $\begin{array}{l}0.5724 \\
0.5890 \\
\end{array}$ & 0.5972 & 0.5760 & 0.6245 & Ave & & 0.5948 & & & & 3.4 & & 15.0 & & & \\
\hline Methyl acetate & $\begin{array}{r}+++++ \\
0.2940\end{array}$ & $\begin{array}{l}0.2252 \\
0.2988\end{array}$ & 0.2644 & 0.2611 & 0.2938 & Ave & & 0.2729 & & & & 10.4 & & 15.0 & & & \\
\hline Methylene Chloride & $\begin{array}{r}+++++ \\
0.2939 \\
\end{array}$ & $\begin{array}{l}0.4024 \\
0.2894 \\
\end{array}$ & 0.3415 & 0.2971 & 0.2950 & Ave & & 0.3199 & & & & 14.0 & & 15.0 & & & \\
\hline tert-Butyl alcohol & $\begin{array}{r}+++++ \\
0.8929 \\
\end{array}$ & $\begin{array}{r}+++++ \\
0.8891 \\
\end{array}$ & 0.8435 & 0.7779 & 0.8878 & Ave & & 0.8582 & & & & 5.7 & & 15.0 & & & \\
\hline Acrylonitrile & $\begin{array}{r}+++++ \\
0.0739\end{array}$ & $\begin{array}{l}0.0475 \\
0.0727\end{array}$ & 0.0561 & 0.0575 & 0.0720 & Lin1 & -0.295 & 0.0732 & & & & & & & 0.9990 & & 0.9900 \\
\hline trans-1,2-Dichloroethene & $\begin{array}{r}+++++ \\
0.3318\end{array}$ & $\begin{array}{l}0.2815 \\
0.3283\end{array}$ & 0.3097 & 0.3042 & 0.3339 & Ave & & 0.3149 & & & & 6.5 & & 15.0 & & & \\
\hline Methyl t-butyl ether & $\begin{array}{r}+++++ \\
0.6849 \\
\end{array}$ & $\begin{array}{l}0.5904 \\
0.6651\end{array}$ & 0.6348 & 0.6221 & 0.6876 & Ave & & 0.6475 & & & & 5.9 & & 15.0 & & & \\
\hline Hexane & $\begin{array}{r}+++++ \\
0.4652 \\
\end{array}$ & $\begin{array}{l}0.4160 \\
0.4594\end{array}$ & 0.4552 & 0.4304 & 0.4778 & Ave & & 0.4507 & & & & 5.1 & & 15.0 & & & \\
\hline 1,1-Dichloroethane & $\begin{array}{r}+++++ \\
0.6661\end{array}$ & $\begin{array}{l}0.6001 \\
0.6685\end{array}$ & 0.6281 & 0.6185 & 0.6773 & Ave & & 0.6431 & & & 0.1000 & 4.9 & & 15.0 & & & \\
\hline Vinyl acetate & $\begin{array}{r}+++++ \\
0.0476 \\
\end{array}$ & $\begin{array}{l}0.0330 \\
0.0415 \\
\end{array}$ & 0.0380 & 0.0426 & 0.0451 & Ave & & 0.0413 & & & & 12.6 & & 15.0 & & & \\
\hline Chloroprene & $\begin{array}{r}+++++ \\
0.4689 \\
\end{array}$ & $\begin{array}{l}0.4156 \\
0.4641 \\
\end{array}$ & 0.4480 & 0.4279 & 0.4785 & Ave & & 0.4505 & & & & 5.5 & & 15.0 & & & \\
\hline Isopropyl ether & $\begin{array}{r}+++++ \\
1.4384\end{array}$ & $\begin{array}{l}1.2989 \\
1.4183\end{array}$ & 1.3178 & 1.2959 & 1.4453 & Ave & & 1.3691 & & & & 5.3 & & 15.0 & & & \\
\hline Tert-butyl ethyl ether & $\begin{array}{r}+++++ \\
1.0127\end{array}$ & $\begin{array}{l}0.7779 \\
0.9791\end{array}$ & 0.8655 & 0.8780 & 1.0475 & Ave & & 0.9268 & & & & 11.1 & & 15.0 & & & \\
\hline 2,2-Dichloropropane & $\begin{array}{r}+++++ \\
0.3295\end{array}$ & $\begin{array}{l}0.4246 \\
0.3143\end{array}$ & 0.4055 & 0.3525 & 0.3508 & Ave & & 0.3629 & & & & 11.9 & & 15.0 & & & \\
\hline cis-1,2-Dichloroethene & $\begin{array}{r}+++++ \\
0.3324 \\
\end{array}$ & $\begin{array}{l}0.2933 \\
0.3302 \\
\end{array}$ & 0.3068 & 0.3070 & 0.3370 & Ave & & 0.3178 & & & & 5.6 & & 15.0 & & & \\
\hline 2-Butanone & $\begin{array}{r}+++++ \\
0.2101\end{array}$ & $\begin{array}{l}0.1991 \\
0.2062\end{array}$ & 0.2042 & 0.1982 & 0.2120 & Ave & & 0.2050 & & & & 2.8 & & 15.0 & & & \\
\hline Propionitrile & $\begin{array}{r}+++++ \\
0.0255 \\
\end{array}$ & $\begin{array}{l}0.0183 \\
0.0249 \\
\end{array}$ & 0.0225 & 0.0248 & 0.0245 & Ave & & 0.0234 & & & & 11.6 & & 15.0 & & & \\
\hline Methacrylonitrile & $\begin{array}{r}+++++ \\
0.0865 \\
\end{array}$ & $\begin{array}{l}0.0703 \\
0.0838 \\
\end{array}$ & 0.0694 & 0.0790 & 0.0853 & Ave & & 0.0790 & & & & 9.6 & & 15.0 & & & \\
\hline
\end{tabular}

Note: The ml coefficient is the same as Ave RRF for an Ave curve type. 
FORM VI

GC/MS VOA INITIAL CALIBRATION DATA

INTERNAL STANDARD CURVE EVALUATION

Lab Name: TestAmerica Burlington

Job No.: 200-28914-1

Analy Batch No.: 92007

SDG No.: 200-28914

Instrument ID: CHL.i

GC Column: DB-624

ID $: 0.53(\mathrm{~mm})$

Heated Purge: (Y/N) N

Calibration Start Date: 07/29/2015 12:33

Calibration End Date: 07/29/2015 15:49

Calibration ID: 31571

\begin{tabular}{|c|c|c|c|c|c|c|c|c|c|c|c|c|c|c|c|c|c|}
\hline \multirow[t]{2}{*}{ ANALYTE } & \multicolumn{5}{|c|}{$\mathrm{RRF}$} & \multirow{2}{*}{\begin{tabular}{|l} 
CURVE \\
TYPE
\end{tabular}} & \multicolumn{3}{|c|}{ COEFFICIENT } & \multirow[t]{2}{*}{ \# } & \multirow[t]{2}{*}{ MIN RRF } & \multirow[t]{2}{*}{$\because \mathrm{RSD}$} & \multirow[t]{2}{*}{ \# } & \multirow{2}{*}{$\begin{array}{l}\text { MAX } \\
\circ \text { RSD }\end{array}$} & \multirow{2}{*}{$\begin{array}{c}\mathrm{R}^{\wedge} 2 \\
\mathrm{OR} \quad \mathrm{COD}\end{array}$} & \multirow[t]{2}{*}{$\#$} & \multirow{2}{*}{$\begin{array}{c}\text { MIN } R^{\wedge} 2 \\
\text { OR } \quad \text { COD }\end{array}$} \\
\hline & $\begin{array}{ll}\text { LVL } & 1 \\
\text { LVL } & 6\end{array}$ & $\begin{array}{ll}\text { LVL } & 2 \\
\text { LVL } & 7\end{array}$ & LVL 3 & LVL 4 & LVL 5 & & B & M1 & M2 & & & & & & & & \\
\hline Bromochloromethane & $\begin{array}{r}+++++ \\
0.1826\end{array}$ & $\begin{array}{l}0.1683 \\
0.1669\end{array}$ & 0.1790 & 0.1740 & 0.1987 & Ave & & 0.1783 & & & & 6.6 & & 15.0 & & & \\
\hline Tetrahydrofuran & $\begin{array}{r}+++++ \\
0.0900 \\
\end{array}$ & $\begin{array}{l}0.0716 \\
0.0885 \\
\end{array}$ & 0.0740 & 0.0824 & 0.0861 & Ave & & 0.0821 & & & & 9.4 & & 15.0 & & & \\
\hline Chloroform & $\begin{array}{r}+++++ \\
0.6059\end{array}$ & $\begin{array}{l}0.5224 \\
0.5941\end{array}$ & 0.5576 & 0.5514 & 0.6166 & Ave & & 0.5747 & & & & 6.4 & & 15.0 & & & \\
\hline 1,1,1-Trichloroethane & $\begin{array}{r}+++++ \\
0.5079\end{array}$ & $\begin{array}{l}0.4389 \\
0.4966\end{array}$ & 0.4585 & 0.4704 & 0.5138 & Ave & & 0.4810 & & & & 6.2 & & 15.0 & & & \\
\hline Cyclohexane & $\begin{array}{r}+++++ \\
0.6108 \\
\end{array}$ & $\begin{array}{l}0.5367 \\
0.6094 \\
\end{array}$ & 0.5649 & 0.5621 & 0.6183 & Ave & & 0.5837 & & & & 5.7 & & 15.0 & & & \\
\hline 1,1-Dichloropropene & $\begin{array}{r}+++++ \\
0.4881 \\
\end{array}$ & $\begin{array}{l}0.4039 \\
0.4856 \\
\end{array}$ & 0.4531 & 0.4457 & 0.4969 & Ave & & 0.4622 & & & & 7.6 & & 15.0 & & & \\
\hline Carbon tetrachloride & $\begin{array}{r}+++++ \\
0.4684\end{array}$ & $\begin{array}{l}0.3538 \\
0.4622\end{array}$ & 0.4190 & 0.4228 & 0.4776 & Ave & & 0.4340 & & & & 10.6 & & 15.0 & & & \\
\hline Isobutyl alcohol & $\begin{array}{r}+++++ \\
0.0135\end{array}$ & $\begin{array}{r}+++++ \\
0.0131\end{array}$ & 0.0117 & 0.0118 & 0.0130 & Ave & & 0.0126 & & & & 6.2 & & 15.0 & & & \\
\hline Benzene & $\begin{array}{r}+++++ \\
0.9455 \\
\end{array}$ & $\begin{array}{l}0.8145 \\
0.9473\end{array}$ & 0.8646 & 0.8621 & 0.9494 & Ave & & 0.8972 & & & & 6.4 & & 15.0 & & & \\
\hline 1,2-Dichloroethane & $\begin{array}{r}+++++ \\
0.3288 \\
\end{array}$ & $\begin{array}{l}0.2731 \\
0.3128 \\
\end{array}$ & 0.2994 & 0.2998 & 0.3295 & Ave & & 0.3072 & & & & 6.9 & & 15.0 & & & \\
\hline Tert-amyl methyl ether & $\begin{array}{r}+++++ \\
0.7965\end{array}$ & $\begin{array}{l}0.6751 \\
0.7814\end{array}$ & 0.7319 & 0.7250 & 0.7993 & Ave & & 0.7515 & & & & 6.6 & & 15.0 & & & \\
\hline n-Heptane & $\begin{array}{r}+++++ \\
0.5495\end{array}$ & $\begin{array}{l}0.4999 \\
0.5411\end{array}$ & 0.5370 & 0.5077 & 0.5558 & Ave & & 0.5318 & & & & 4.3 & & 15.0 & & & \\
\hline Trichloroethene & $\begin{array}{r}+++++ \\
0.3962 \\
\end{array}$ & $\begin{array}{l}0.3435 \\
0.4007 \\
\end{array}$ & 0.3680 & 0.3612 & 0.4058 & Ave & & 0.3792 & & & & 6.7 & & 15.0 & & & \\
\hline Methylcyclohexane & $\begin{array}{r}+++++ \\
0.4901\end{array}$ & $\begin{array}{l}0.4316 \\
0.4870\end{array}$ & 0.4560 & 0.4455 & 0.4997 & Ave & & 0.4683 & & & & 5.9 & & 15.0 & & & \\
\hline 1,2-Dichloropropane & $\begin{array}{r}+++++ \\
0.4188\end{array}$ & $\begin{array}{l}0.3773 \\
0.4124\end{array}$ & 0.3856 & 0.3782 & 0.4199 & Ave & & 0.3987 & & & & 5.1 & & 15.0 & & & \\
\hline Dibromomethane & $\begin{array}{r}+++++ \\
0.3047\end{array}$ & $\begin{array}{l}0.2617 \\
0.2990\end{array}$ & 0.2745 & 0.2743 & 0.3060 & Ave & & 0.2867 & & & & 6.6 & & 15.0 & & & \\
\hline Methyl methacrylate & $\begin{array}{r}+++++ \\
0.3571\end{array}$ & $\begin{array}{l}0.3100 \\
0.3480\end{array}$ & 0.3097 & 0.3170 & 0.3443 & Ave & & 0.3310 & & & & 6.4 & & 15.0 & & & \\
\hline 1,4-Dioxane & $\begin{array}{r}+++++ \\
1.0339\end{array}$ & $\begin{array}{c}+++++ \\
+++++\end{array}$ & 0.9589 & 0.9555 & 1.1015 & Ave & & 1.0124 & & & & 6.9 & & 15.0 & & & \\
\hline Bromodichloromethane & $\begin{array}{r}+++++ \\
0.6245 \\
\end{array}$ & $\begin{array}{l}0.5193 \\
0.6111 \\
\end{array}$ & 0.5447 & 0.5536 & 0.6283 & Ave & & 0.5802 & & & & 8.0 & & 15.0 & & & \\
\hline 2-Chloroethyl vinyl ether & $\begin{array}{r}+++++ \\
0.2087 \\
\end{array}$ & $\begin{array}{l}0.1639 \\
0.2096 \\
\end{array}$ & 0.1733 & 0.1767 & 0.2032 & Ave & & 0.1892 & & & & 10.7 & & 15.0 & & & \\
\hline
\end{tabular}

Note: The $m 1$ coefficient is the same as Ave RRF for an Ave curve type. 
FORM VI

GC/MS VOA INITIAL CALIBRATION DATA

INTERNAL STANDARD CURVE EVALUATION

Lab Name: TestAmerica Burlington

Job No.: 200-28914-1

Analy Batch No.: 92007

SDG No.: 200-28914

Instrument ID: CHL.i

GC Column: DB-624

ID $: 0.53(\mathrm{~mm})$

Heated Purge: (Y/N) N

Calibration Start Date: 07/29/2015 12:33

Calibration End Date: 07/29/2015 15:49

Calibration ID: 31571

\begin{tabular}{|c|c|c|c|c|c|c|c|c|c|c|c|c|c|c|c|c|c|}
\hline \multirow[t]{2}{*}{ ANALYTE } & \multicolumn{5}{|c|}{$\mathrm{RRF}$} & \multirow{2}{*}{$\begin{array}{l}\text { CURVE } \\
\text { TYPE }\end{array}$} & \multicolumn{3}{|c|}{ COEFFICIENT } & \multirow[t]{2}{*}{ \# } & \multirow[t]{2}{*}{ MIN RRF } & \multirow[t]{2}{*}{$\because \mathrm{RSD}$} & \multirow[t]{2}{*}{ \# } & \multirow{2}{*}{$\begin{array}{l}\text { MAX } \\
\circ \text { RSD }\end{array}$} & \multirow{2}{*}{$\begin{array}{c}\mathrm{R}^{\wedge} 2 \\
\mathrm{OR} \quad \mathrm{COD}\end{array}$} & \multirow[t]{2}{*}{$\#$} & \multirow{2}{*}{$\begin{array}{cc}\text { MIN } R^{\wedge} 2 \\
\text { OR }\end{array}$} \\
\hline & $\begin{array}{ll}\text { LVL } & 1 \\
\text { LVL } & 6\end{array}$ & $\begin{array}{ll}\text { LVL } & 2 \\
\text { LVL } & 7\end{array}$ & LVL 3 & LVL 4 & LVL 5 & & B & M1 & M2 & & & & & & & & \\
\hline cis-1,3-Dichloropropene & $\begin{array}{l}0.6223 \\
0.5294\end{array}$ & $\begin{array}{l}0.4460 \\
0.5131\end{array}$ & 0.4572 & 0.4655 & 0.5282 & Ave & & 0.5088 & & & & 12.0 & & 15.0 & & & \\
\hline 4-Methyl-2-pentanone & $\begin{array}{r}+++++ \\
2.7094 \\
\end{array}$ & $\begin{array}{l}2.2091 \\
2.6517 \\
\end{array}$ & 2.5956 & 2.5136 & 2.7653 & Ave & & 2.5741 & & & & 7.7 & & 15.0 & & & \\
\hline Toluene & $\begin{array}{r}+++++ \\
0.7685 \\
\end{array}$ & $\begin{array}{l}0.6397 \\
0.7659 \\
\end{array}$ & 0.6924 & 0.6971 & 0.7722 & Ave & & 0.7227 & & & & 7.5 & & 15.0 & & & \\
\hline trans-1,3-Dichloropropene & $\begin{array}{l}0.6494 \\
0.5248\end{array}$ & $\begin{array}{l}0.4344 \\
0.5120\end{array}$ & 0.4754 & 0.4775 & 0.5154 & Ave & & 0.5127 & & & & 13.2 & & 15.0 & & & \\
\hline Ethyl methacrylate & $\begin{array}{r}+++++ \\
0.3210 \\
\end{array}$ & $\begin{array}{l}0.2898 \\
0.3105 \\
\end{array}$ & 0.2855 & 0.2896 & 0.3167 & Ave & & 0.3022 & & & & 5.2 & & 15.0 & & & \\
\hline 1,1,2-Trichloroethane & $\begin{array}{r}+++++ \\
0.3607 \\
\end{array}$ & $\begin{array}{l}0.3092 \\
0.3530 \\
\end{array}$ & 0.3180 & 0.3235 & 0.3600 & Ave & & 0.3374 & & & & 6.8 & & 15.0 & & & \\
\hline Tetrachloroethene & $\begin{array}{r}+++++ \\
0.5768\end{array}$ & $\begin{array}{l}0.4577 \\
0.5757\end{array}$ & 0.5200 & 0.5164 & 0.5827 & Ave & & 0.5382 & & & & 9.2 & & 15.0 & & & \\
\hline 1,3-Dichloropropane & $\begin{array}{r}+++++ \\
0.6860\end{array}$ & $\begin{array}{l}0.5802 \\
0.6770\end{array}$ & 0.6102 & 0.6182 & 0.6913 & Ave & & 0.6438 & & & & 7.3 & & 15.0 & & & \\
\hline 2-Hexanone & $\begin{array}{r}+++++ \\
2.1641 \\
\end{array}$ & $\begin{array}{l}1.7689 \\
2.0936 \\
\end{array}$ & 2.0831 & 1.9920 & 2.2253 & Ave & & 2.0545 & & & & 7.8 & & 15.0 & & & \\
\hline Dibromochloromethane & $\begin{array}{l}0.6866 \\
0.6610\end{array}$ & $\begin{array}{l}0.4852 \\
0.6536\end{array}$ & 0.5286 & 0.5603 & 0.6578 & Ave & & 0.6047 & & & & 13.0 & & 15.0 & & & \\
\hline 1,2-Dibromoethane & $\begin{array}{r}+++++ \\
0.6020\end{array}$ & $\begin{array}{l}0.4661 \\
0.5857\end{array}$ & 0.5215 & 0.5159 & 0.5958 & Ave & & 0.5478 & & & & 10.0 & & 15.0 & & & \\
\hline Chlorobenzene & $\begin{array}{r}+++++ \\
0.9501 \\
\end{array}$ & $\begin{array}{l}0.8048 \\
0.9460\end{array}$ & 0.8703 & 0.8720 & 0.9595 & Ave & & 0.9005 & & & 0.3000 & 6.8 & & 15.0 & & & \\
\hline $1,1,1,2$-Tetrachloroethane & $\begin{array}{r}+++++ \\
0.4780\end{array}$ & $\begin{array}{l}0.3778 \\
0.4743\end{array}$ & 0.4213 & 0.4208 & 0.4803 & Ave & & 0.4421 & & & & 9.5 & & 15.0 & & & \\
\hline Ethylbenzene & $\begin{array}{r}+++++ \\
1.5489\end{array}$ & $\begin{array}{l}1.2920 \\
1.5595\end{array}$ & 1.3759 & 1.3903 & 1.5520 & Ave & & 1.4531 & & & & 7.9 & & 15.0 & & & \\
\hline$m \& p-X y l e n e$ & $\begin{array}{r}+++++ \\
0.5697 \\
\end{array}$ & $\begin{array}{l}0.4678 \\
0.5673 \\
\end{array}$ & 0.4967 & 0.4915 & 0.5735 & Ave & & 0.5277 & & & & 9.0 & & 15.0 & & & \\
\hline o-Xylene & $\begin{array}{r}+++++ \\
0.5382\end{array}$ & $\begin{array}{l}0.4324 \\
0.5363\end{array}$ & 0.4962 & 0.4912 & 0.5458 & Ave & & 0.5067 & & & & 8.5 & & 15.0 & & & \\
\hline Styrene & $\begin{array}{r}+++++ \\
0.8111\end{array}$ & $\begin{array}{l}0.6427 \\
0.8137\end{array}$ & 0.6897 & 0.7098 & 0.8058 & Ave & & 0.7455 & & & & 10.0 & & 15.0 & & & \\
\hline Bromoform & $\begin{array}{r}+++++ \\
0.4826\end{array}$ & $\begin{array}{l}0.3145 \\
0.4803\end{array}$ & 0.3714 & 0.3858 & 0.4709 & Lin1 & -0.190 & 0.4815 & & & 0.1000 & & & & 0.9990 & & 0.9900 \\
\hline Isopropylbenzene & $\begin{array}{r}+++++ \\
2.7868\end{array}$ & $\begin{array}{l}2.4441 \\
2.7811\end{array}$ & 2.6804 & 2.6394 & 2.9027 & Ave & & 2.7057 & & & & 5.8 & & 15.0 & & & \\
\hline Bromobenzene & $\begin{array}{r}+++++ \\
0.8602 \\
\end{array}$ & $\begin{array}{l}0.6976 \\
0.8451 \\
\end{array}$ & 0.8077 & 0.7919 & 0.8821 & Ave & & 0.8141 & & & & 8.1 & & 15.0 & & & \\
\hline
\end{tabular}

Note: The ml coefficient is the same as Ave RRF for an Ave curve type. 
FORM VI

GC/MS VOA INITIAL CALIBRATION DATA

INTERNAL STANDARD CURVE EVALUATION

Lab Name: TestAmerica Burlington

Job No.: 200-28914-1

Analy Batch No.: 92007

SDG No.: 200-28914

Instrument ID: CHL.i

GC Column: DB-624

ID $: 0.53(\mathrm{~mm})$

Heated Purge: (Y/N) N

Calibration Start Date: 07/29/2015 12:33

Calibration End Date: 07/29/2015 15:49

Calibration ID: 31571

\begin{tabular}{|c|c|c|c|c|c|c|c|c|c|c|c|c|c|c|c|c|c|}
\hline \multirow[t]{2}{*}{ ANALYTE } & \multicolumn{5}{|c|}{$\mathrm{RRF}$} & \multirow{2}{*}{\begin{tabular}{|l} 
CURVE \\
TYPE
\end{tabular}} & \multicolumn{3}{|c|}{ COEFFICIENT } & \multirow[t]{2}{*}{ \# } & \multirow[t]{2}{*}{ MIN RRF } & \multirow[t]{2}{*}{$\because \mathrm{RSD}$} & \multirow[t]{2}{*}{ \# } & \multirow{2}{*}{$\begin{array}{l}\text { MAX } \\
\circ \text { RSD }\end{array}$} & \multirow{2}{*}{$\begin{array}{c}\mathrm{R}^{\wedge} 2 \\
\mathrm{OR} \quad \mathrm{COD}\end{array}$} & \multirow[t]{2}{*}{$\#$} & \multirow{2}{*}{$\begin{array}{lll}\text { MIN } & R^{\wedge} 2 \\
\text { OR } & C O D\end{array}$} \\
\hline & $\begin{array}{ll}\text { LVL } & 1 \\
\text { LVL } & 6\end{array}$ & $\begin{array}{ll}\text { LVL } & 2 \\
\text { LVL } & 7\end{array}$ & LVL 3 & LVL 4 & LVL 5 & & B & M1 & M2 & & & & & & & & \\
\hline $1,1,2,2$-Tetrachloroethane & $\begin{array}{l}1.5678 \\
1.2421\end{array}$ & $\begin{array}{l}1.0918 \\
1.1806\end{array}$ & 1.1949 & 1.1719 & 1.2519 & Ave & & 1.2430 & & & 0.3000 & 12.3 & & 15.0 & & & \\
\hline 1,2,3-Trichloropropane & $\begin{array}{r}+++++ \\
0.2932 \\
\end{array}$ & $\begin{array}{l}0.2588 \\
0.2814 \\
\end{array}$ & 0.2771 & 0.2813 & 0.2993 & Ave & & 0.2818 & & & & 5.0 & & 15.0 & & & \\
\hline trans-1,4-Dichloro-2-butene & $\begin{array}{r}+++++ \\
0.2390 \\
\end{array}$ & $\begin{array}{l}0.2225 \\
0.2293 \\
\end{array}$ & 0.2190 & 0.2312 & 0.2693 & Ave & & 0.2350 & & & & 7.7 & & 15.0 & & & \\
\hline n-Propylbenzene & $\begin{array}{r}+++++ \\
0.7146\end{array}$ & $\begin{array}{l}0.6199 \\
0.7173\end{array}$ & 0.6579 & 0.6640 & 0.7359 & Ave & & 0.6849 & & & & 6.5 & & 15.0 & & & \\
\hline 2-Chlorotoluene & $\begin{array}{r}+++++ \\
0.6536 \\
\end{array}$ & $\begin{array}{l}0.5599 \\
0.6525 \\
\end{array}$ & 0.6456 & 0.6234 & 0.6783 & Ave & & 0.6356 & & & & 6.5 & & 15.0 & & & \\
\hline 1,3,5-Trimethylbenzene & $\begin{array}{r}+++++ \\
2.0428 \\
\end{array}$ & $\begin{array}{l}1.7352 \\
2.0490 \\
\end{array}$ & 1.9246 & 1.9141 & 2.0969 & Ave & & 1.9604 & & & & 6.7 & & 15.0 & & & \\
\hline 4-Chlorotoluene & $\begin{array}{r}+++++ \\
0.6807\end{array}$ & $\begin{array}{l}0.5716 \\
0.6766\end{array}$ & 0.6566 & 0.6399 & 0.6849 & Ave & & 0.6517 & & & & 6.6 & & 15.0 & & & \\
\hline tert-Butylbenzene & $\begin{array}{r}+++++ \\
2.2070\end{array}$ & $\begin{array}{l}1.9109 \\
2.2135\end{array}$ & 2.0951 & 2.0918 & 2.2853 & Ave & & 2.1339 & & & & 6.2 & & 15.0 & & & \\
\hline $1,2,4$-Trimethylbenzene & $\begin{array}{r}+++++ \\
2.0242 \\
\end{array}$ & $\begin{array}{l}1.7929 \\
2.0495 \\
\end{array}$ & 1.9010 & 1.8779 & 2.0836 & Ave & & 1.9549 & & & & 5.9 & & 15.0 & & & \\
\hline sec-Butylbenzene & $\begin{array}{r}+++++ \\
3.0933\end{array}$ & $\begin{array}{l}2.6353 \\
3.1160\end{array}$ & 2.9479 & 2.8833 & 3.1834 & Ave & & 2.9765 & & & & 6.7 & & 15.0 & & & \\
\hline 1,3-Dichlorobenzene & $\begin{array}{r}+++++ \\
1.4080\end{array}$ & $\begin{array}{l}1.2426 \\
1.3976\end{array}$ & 1.3030 & 1.2821 & 1.4236 & Ave & & 1.3428 & & & & 5.7 & & 15.0 & & & \\
\hline 4-Isopropyltoluene & $\begin{array}{r}+++++ \\
2.4114 \\
\end{array}$ & $\begin{array}{l}2.0387 \\
2.4476 \\
\end{array}$ & 2.2795 & 2.2177 & 2.4685 & Ave & & 2.3105 & & & & 7.2 & & 15.0 & & & \\
\hline 1,4-Dichlorobenzene & $\begin{array}{r}+++++ \\
1.4721\end{array}$ & $\begin{array}{l}1.3851 \\
1.4761\end{array}$ & 1.4516 & 1.4039 & 1.5409 & Ave & & 1.4550 & & & & 3.8 & & 15.0 & & & \\
\hline 1,2-Dichlorobenzene & $\begin{array}{r}+++++ \\
1.3024\end{array}$ & $\begin{array}{l}1.1928 \\
1.2941\end{array}$ & 1.2426 & 1.2252 & 1.3446 & Ave & & 1.2669 & & & & 4.4 & & 15.0 & & & \\
\hline n-Butylbenzene & $\begin{array}{r}+++++ \\
2.1504 \\
\end{array}$ & $\begin{array}{l}1.8422 \\
2.2404 \\
\end{array}$ & 1.9827 & 1.9429 & 2.1910 & Ave & & 2.0583 & & & & 7.7 & & 15.0 & & & \\
\hline 1,2-Dibromo-3-Chloropropane & $\begin{array}{r}+++++ \\
0.2310\end{array}$ & $\begin{array}{l}0.1954 \\
0.2196\end{array}$ & 0.1933 & 0.2066 & 0.2310 & Ave & & 0.2128 & & & & 8.0 & & 15.0 & & & \\
\hline 1,2,4-Trichlorobenzene & $\begin{array}{r}+++++ \\
0.8622\end{array}$ & $\begin{array}{l}0.7522 \\
0.8756\end{array}$ & 0.7672 & 0.7598 & 0.8684 & Ave & & 0.8142 & & & & 7.4 & & 15.0 & & & \\
\hline Hexachlorobutadiene & $\begin{array}{r}+++++ \\
0.4749\end{array}$ & $\begin{array}{l}0.4713 \\
0.4725\end{array}$ & 0.4817 & 0.4445 & 0.4871 & Ave & & 0.4720 & & & & 3.1 & & 15.0 & & & \\
\hline Naphthalene & $\begin{array}{r}+++++ \\
1.6843\end{array}$ & $\begin{array}{l}1.5170 \\
1.6719\end{array}$ & 1.5697 & 1.5283 & 1.6920 & Ave & & 1.6105 & & & & 5.0 & & 15.0 & & & \\
\hline $1,2,3$-Trichlorobenzene & $\begin{array}{r}+++++ \\
0.7713 \\
\end{array}$ & $\begin{array}{l}0.7013 \\
0.7765\end{array}$ & 0.7151 & 0.7082 & 0.7822 & Ave & & 0.7424 & & & & 5.1 & & 15.0 & & & \\
\hline
\end{tabular}

Note: The ml coefficient is the same as Ave RRF for an Ave curve type. 
FORM VI

GC/MS VOA INITIAL CALIBRATION DATA

INTERNAL STANDARD CURVE EVALUATION

Lab Name: TestAmerica Burlington

Job No. : 200-28914-1

Analy Batch No.: 92007

SDG No.: 200-28914

Instrument ID: CHL.i

GC Column: DB-624

ID : $0.53(\mathrm{~mm})$

Heated Purge: (Y/N) N

Calibration Start Date: 07/29/2015 12:33

Calibration End Date: 07/29/2015 15:49

Calibration ID: 31571

\begin{tabular}{|c|c|c|c|c|c|c|c|c|c|c|c|c|c|c|c|c|c|}
\hline \multirow[t]{2}{*}{ ANALYTE } & \multicolumn{5}{|c|}{$\mathrm{RRF}$} & \multirow{2}{*}{$\begin{array}{l}\text { CURVE } \\
\text { TYPE }\end{array}$} & \multicolumn{3}{|c|}{ COEFFICIENT } & \multirow[t]{2}{*}{$\#$} & \multirow[t]{2}{*}{ MIN RRF } & \multirow[t]{2}{*}{$\therefore \mathrm{RSD}$} & \multirow[t]{2}{*}{ \# } & \multirow{2}{*}{$\begin{array}{l}\text { MAX } \\
\circ R S D\end{array}$} & \multirow{2}{*}{$\begin{array}{c}R^{\wedge} 2 \\
O R \quad C O D\end{array}$} & \multirow[t]{2}{*}{$\#$} & \multirow{2}{*}{$\begin{array}{cc}\text { MIN } R^{\wedge} 2 \\
\text { OR } & \text { OOD }\end{array}$} \\
\hline & $\begin{array}{ll}\text { LVL } & 1 \\
\text { LVL } & 6\end{array}$ & $\begin{array}{ll}\text { LVL } & 2 \\
\text { LVL } & 7\end{array}$ & LVL 3 & LVL 4 & LVL 5 & & B & M1 & M2 & & & & & & & & \\
\hline Dibromofluoromethane (Surr) & $\begin{array}{r}+++++ \\
0.5122\end{array}$ & $\begin{array}{l}0.4315 \\
0.4996\end{array}$ & 0.4621 & 0.4504 & 0.5123 & Ave & & 0.4780 & & & & 7.2 & & 15.0 & & & \\
\hline 1,2-Dichloroethane-d4 & $\begin{array}{r}+++++ \\
0.2628\end{array}$ & $\begin{array}{l}0.2615 \\
0.2536\end{array}$ & 0.2557 & 0.2566 & 0.2682 & Ave & & 0.2597 & & & & 2.1 & & 15.0 & & & \\
\hline Toluene-d8 & $\begin{array}{r}+++++ \\
1.1048\end{array}$ & $\begin{array}{l}0.9249 \\
1.1083\end{array}$ & 1.0033 & 0.9936 & 1.1151 & Ave & & 1.0417 & & & & 7.6 & & 15.0 & & & \\
\hline Bromofluorobenzene & $\begin{array}{r}+++++ \\
1.2195\end{array}$ & $\begin{array}{l}1.0690 \\
1.2021\end{array}$ & 1.1958 & 1.1509 & 1.2554 & Ave & & 1.1821 & & & & 5.5 & & 15.0 & & & \\
\hline
\end{tabular}


FORM VI

GC/MS VOA INITIAL CALIBRATION DATA

INTERNAL STANDARD RESPONSE AND CONCENTRATION

Lab Name: TestAmerica Burlington

Job No. : 200-28914-1

Analy Batch No.: 92007

SDG No.: 200-28914

Instrument ID: CHL.i

GC Column: DB-624

ID $: 0.53(\mathrm{~mm})$

Heated Purge: (Y/N) N

Calibration Start Date: 07/29/2015 12:33

Calibration End Date: 07/29/2015 15:49

Calibration ID: 31571

Calibration Files:

\begin{tabular}{|lll|}
\hline LEVEL: & LAB SAMPLE ID: & LAB FILE ID: \\
Level 1 & IC 200-92007/3 & 15008_03.D \\
Level 2 & IC 200-92007/4 & 15008_04.D \\
Level 3 & IC 200-92007/5 & 15008_05.D \\
Level 4 & IC 200-92007/6 & 15008_06.D \\
Level 5 & ICIS 200-92007/7 & 15008_07.D \\
Level 6 & IC 200-92007/8 & 15008_08.D \\
Level 7 & IC 200-92007/9 & 15008_09.D \\
\hline
\end{tabular}

\begin{tabular}{|c|c|c|c|c|c|c|c|c|c|c|c|c|}
\hline \multirow[t]{2}{*}{ ANALYTE } & \multirow{2}{*}{$\begin{array}{l}\text { IS } \\
\text { REF }\end{array}$} & \multirow{2}{*}{$\begin{array}{l}\text { CURVE } \\
\text { TYPE }\end{array}$} & \multicolumn{5}{|c|}{ RESPONSE } & \multicolumn{5}{|c|}{ CONCENTRATION (UG/L) } \\
\hline & & & $\begin{array}{ll}\text { LVL } & 1 \\
\text { LVL } & 6\end{array}$ & $\begin{array}{ll}\text { LVL } & 2 \\
\text { LVL } & 7\end{array}$ & LVL 3 & LVL 4 & LVL 5 & $\begin{array}{ll}\text { LVL } & 1 \\
\text { LVL } & 6\end{array}$ & $\begin{array}{ll}\text { LVL } & 2 \\
\text { LVL } & 7\end{array}$ & LVL 3 & LVL 4 & LVL 5 \\
\hline Dichlorodifluoromethane & FB & Ave & $\begin{array}{r}+++++ \\
2590716\end{array}$ & $\begin{array}{r}37435 \\
5320305\end{array}$ & 91750 & 233062 & 1292763 & $\begin{array}{r}+++++ \\
50.0\end{array}$ & $\begin{array}{r}0.827 \\
100\end{array}$ & 1.88 & 4.80 & 24.8 \\
\hline Chloromethane & FB & Ave & $\begin{array}{r}+++++ \\
1882475\end{array}$ & $\begin{array}{r}36473 \\
3960004\end{array}$ & 75208 & 184638 & 910164 & $\begin{array}{r}+++++ \\
50.0\end{array}$ & $\begin{array}{r}0.827 \\
100 \\
\end{array}$ & 1.88 & 4.80 & 24.8 \\
\hline Vinyl chloride & FB & Ave & $\begin{array}{r}19132 \\
2035270\end{array}$ & $\begin{array}{r}28364 \\
4228333\end{array}$ & 71325 & 180160 & 1003549 & $\begin{array}{r}0.405 \\
50.0\end{array}$ & $\begin{array}{r}0.827 \\
100 \\
\end{array}$ & 1.88 & 4.80 & 24.8 \\
\hline Butadiene & FB & Ave & $\begin{array}{r}+++++ \\
1533926\end{array}$ & $\begin{array}{r}22934 \\
3154864\end{array}$ & 53048 & 136240 & 771037 & $\begin{array}{r}+++++ \\
50.0\end{array}$ & $\begin{array}{r}0.827 \\
100\end{array}$ & 1.88 & 4.80 & 24.8 \\
\hline Bromomethane & FB & Ave & $\begin{array}{r}+++++ \\
1309938\end{array}$ & $\begin{array}{r}22424 \\
2872253\end{array}$ & 43396 & 110462 & 598896 & $\begin{array}{r}+++++ \\
50.0\end{array}$ & $\begin{array}{r}0.827 \\
100\end{array}$ & 1.88 & 4.80 & 24.8 \\
\hline Chloroethane & FB & Ave & $\begin{array}{r}+++++ \\
1228008 \\
\end{array}$ & $\begin{array}{r}19772 \\
2427517 \\
\end{array}$ & 45842 & 111155 & 613172 & $\begin{array}{r}+++++ \\
50.0\end{array}$ & $\begin{array}{r}0.827 \\
100\end{array}$ & 1.88 & 4.80 & 24.8 \\
\hline Dichlorofluoromethane & FB & Ave & $\begin{array}{r}+++++ \\
4356660\end{array}$ & $\begin{array}{r}63359 \\
8972273\end{array}$ & 152814 & 381550 & 2152615 & $\begin{array}{r}+++++ \\
50.0\end{array}$ & $\begin{array}{r}0.827 \\
100\end{array}$ & 1.88 & 4.80 & 24.8 \\
\hline Trichlorofluoromethane & FB & Ave & $\begin{array}{r}+++++ \\
3237555\end{array}$ & $\begin{array}{r}46876 \\
6578290\end{array}$ & 110540 & 283407 & 1611636 & $\begin{array}{r}+++++ \\
50.0\end{array}$ & $\begin{array}{r}0.827 \\
100 \\
\end{array}$ & 1.88 & 4.80 & 24.8 \\
\hline Freon $123 a$ & FB & Ave & $\begin{array}{r}+++++ \\
4690727\end{array}$ & $\begin{array}{r}63503 \\
9761876\end{array}$ & 158767 & 400855 & 2341328 & $\begin{array}{r}+++++ \\
50.0\end{array}$ & $\begin{array}{r}0.827 \\
100\end{array}$ & 1.88 & 4.80 & 24.8 \\
\hline Ethyl ether & FB & Ave & $\begin{array}{r}+++++ \\
1234387\end{array}$ & $\begin{array}{r}16251 \\
2511873\end{array}$ & 42057 & 103069 & 603376 & $\begin{array}{r}+++++ \\
50.0\end{array}$ & $\begin{array}{r}0.827 \\
100\end{array}$ & 1.88 & 4.80 & 24.8 \\
\hline Acrolein & FB & Ave & $\begin{array}{r}+++++ \\
449992\end{array}$ & $\begin{array}{r}5131 \\
869939\end{array}$ & 18131 & 40800 & 203572 & $\begin{array}{r}++++ \\
250\end{array}$ & $\begin{array}{r}4.13 \\
500 \\
\end{array}$ & 9.41 & 24.0 & 124 \\
\hline 1,1-Dichloroethene & FB & Ave & $\begin{array}{r}+++++ \\
1821391\end{array}$ & $\begin{array}{r}23268 \\
3821278\end{array}$ & 61533 & 153863 & 895709 & $\begin{array}{r}+++++ \\
50.0\end{array}$ & $\begin{array}{r}0.827 \\
100\end{array}$ & 1.88 & 4.80 & 24.8 \\
\hline Freon TF & FB & Ave & $\begin{array}{r}+++++ \\
3665742\end{array}$ & $\begin{array}{r}45908 \\
7628426\end{array}$ & 120918 & 307408 & 1826018 & $\begin{array}{r}+++++ \\
50.0\end{array}$ & $\begin{array}{r}0.827 \\
100 \\
\end{array}$ & 1.88 & 4.80 & 24.8 \\
\hline Acetone & BUT & Ave & $\begin{array}{r}+++++ \\
3032492 \\
\end{array}$ & $\begin{array}{r}39779 \\
5900555 \\
\end{array}$ & 98847 & 246306 & 1466457 & $\begin{array}{r}+++++ \\
250 \\
\end{array}$ & $\begin{array}{r}4.14 \\
501 \\
\end{array}$ & 9.42 & 24.0 & 124 \\
\hline Methyl iodide & FB & Ave & $\begin{array}{r}+++++ \\
2368926\end{array}$ & $\begin{array}{r}28671 \\
5162962\end{array}$ & 63115 & 148916 & 1002091 & $\begin{array}{r}+++++ \\
50.0\end{array}$ & $\begin{array}{r}0.827 \\
100 \\
\end{array}$ & 1.88 & 4.80 & 24.8 \\
\hline
\end{tabular}


FORM VI

GC/MS VOA INITIAL CALIBRATION DATA

INTERNAL STANDARD RESPONSE AND CONCENTRATION

Lab Name: TestAmerica Burlington

Job No.: 200-28914-1

Analy Batch No.: 92007

SDG No.: 200-28914

Instrument ID: CHL.i

GC Column: DB-624

ID : $0.53(\mathrm{~mm})$

Heated Purge: (Y/N) N

Calibration Start Date: 07/29/2015 12:33

Calibration End Date: 07/29/2015 15:49

Calibration ID: 31571

\begin{tabular}{|c|c|c|c|c|c|c|c|c|c|c|c|c|}
\hline \multirow[t]{2}{*}{ ANALYTE } & \multirow{2}{*}{$\begin{array}{l}\text { IS } \\
\text { REF }\end{array}$} & \multirow{2}{*}{$\begin{array}{c}\text { CURVE } \\
\text { TYPE }\end{array}$} & \multicolumn{5}{|c|}{ RESPONSE } & \multicolumn{5}{|c|}{ CONCENTRATION (UG/L) } \\
\hline & & & $\begin{array}{ll}\text { LVL } & 1 \\
\text { LVL } & 6\end{array}$ & $\begin{array}{ll}\text { LVL } & 2 \\
\text { LVL } & 7\end{array}$ & LVL 3 & LVL 4 & LVL 5 & $\begin{array}{ll}\text { LVL } & 1 \\
\text { LVL } & 6\end{array}$ & $\begin{array}{l}\text { LVL } 2 \\
\text { LVL } 7\end{array}$ & LVL 3 & LVL 4 & LVL 5 \\
\hline Carbon disulfide & FB & Ave & $\begin{array}{r}+++++ \\
4984066\end{array}$ & $\begin{array}{r}85656 \\
10472434\end{array}$ & 185235 & 434519 & 2459586 & $\begin{array}{r}+++++ \\
50.0\end{array}$ & $\begin{array}{r}0.827 \\
100\end{array}$ & 1.88 & 4.80 & 24.8 \\
\hline Allyl chloride & FB & Ave & $\begin{array}{r}+++++ \\
3541744\end{array}$ & $\begin{array}{r}49970 \\
7177121\end{array}$ & 122819 & 303734 & 1758872 & $\begin{array}{r}+++++ \\
50.0\end{array}$ & $\begin{array}{r}0.827 \\
100 \\
\end{array}$ & 1.88 & 4.80 & 24.8 \\
\hline Methyl acetate & BUT & Ave & $\begin{array}{r}+++++ \\
1216753 \\
\end{array}$ & $\begin{array}{r}13194 \\
2522746 \\
\end{array}$ & 35907 & 94858 & 568682 & $\begin{array}{r}+++++ \\
250\end{array}$ & $\begin{array}{r}4.14 \\
501 \\
\end{array}$ & 9.42 & 24.0 & 124 \\
\hline Methylene Chloride & FB & Ave & $\begin{array}{r}+++++ \\
1706008 \\
\end{array}$ & $\begin{array}{r}35130 \\
3527209 \\
\end{array}$ & 70236 & 156663 & 830834 & $\begin{array}{r}+++++ \\
50.0 \\
\end{array}$ & $\begin{array}{r}0.827 \\
100 \\
\end{array}$ & 1.88 & 4.80 & 24.8 \\
\hline tert-Butyl alcohol & TBA & Ave & $\begin{array}{r}+++++ \\
1286918\end{array}$ & $\begin{array}{r}+++++ \\
2583070\end{array}$ & 41655 & 102155 & 599204 & $\begin{array}{r}+++++ \\
500\end{array}$ & $\begin{array}{r}+++++ \\
1001\end{array}$ & 18.8 & 48.0 & 248 \\
\hline Acrylonitrile & FB & Lin1 & $\begin{array}{r}+++++ \\
4287451\end{array}$ & $\begin{array}{r}41492 \\
8855413\end{array}$ & 115404 & 303096 & 2028754 & $\begin{array}{r}+++++ \\
500\end{array}$ & $\begin{array}{l}8.27 \\
1001\end{array}$ & 18.8 & 48.0 & 248 \\
\hline trans-1,2-Dichloroethene & FB & Ave & $\begin{array}{r}+++++ \\
1926070 \\
\end{array}$ & $\begin{array}{r}24576 \\
4000858 \\
\end{array}$ & 63702 & 160423 & 940472 & $\begin{array}{r}+++++ \\
50.0 \\
\end{array}$ & $\begin{array}{r}0.827 \\
100 \\
\end{array}$ & 1.88 & 4.80 & 24.8 \\
\hline Methyl t-butyl ether & FB & Ave & $\begin{array}{r}+++++ \\
3975867\end{array}$ & $\begin{array}{r}51547 \\
8105107\end{array}$ & 130556 & 328071 & 1936648 & $\begin{array}{r}+++++ \\
50.0\end{array}$ & $\begin{array}{r}0.827 \\
100 \\
\end{array}$ & 1.88 & 4.80 & 24.8 \\
\hline Hexane & FB & Ave & $\begin{array}{r}+++++ \\
2700889\end{array}$ & $\begin{array}{r}36315 \\
5598548\end{array}$ & 93607 & 226965 & 1345722 & $\begin{array}{r}+++++ \\
50.0\end{array}$ & $\begin{array}{r}0.827 \\
100\end{array}$ & 1.88 & 4.80 & 24.8 \\
\hline 1,1-Dichloroethane & FB & Ave & $\begin{array}{r}+++++ \\
3867023\end{array}$ & $\begin{array}{r}52392 \\
8146561\end{array}$ & 129176 & 326193 & 1907686 & $\begin{array}{r}+++++ \\
50.0\end{array}$ & $\begin{array}{r}0.827 \\
100 \\
\end{array}$ & 1.88 & 4.80 & 24.8 \\
\hline Vinyl acetate & FB & Ave & $\begin{array}{r}+++++ \\
552851\end{array}$ & $\begin{array}{r}5767 \\
1010779 \\
\end{array}$ & 15642 & 44962 & 253848 & $\begin{array}{l}+++++ \\
100.0\end{array}$ & $\begin{array}{r}1.65 \\
200 \\
\end{array}$ & 3.77 & 9.61 & 49.6 \\
\hline Chloroprene & FB & Ave & $\begin{array}{r}+++++ \\
2721889\end{array}$ & $\begin{array}{r}36287 \\
5655435\end{array}$ & 92140 & 225648 & 1347746 & $\begin{array}{r}+++++ \\
50.0\end{array}$ & $\begin{array}{r}0.827 \\
100\end{array}$ & 1.88 & 4.80 & 24.8 \\
\hline Isopropyl ether & FB & Ave & $\begin{array}{r}+++++ \\
8350276\end{array}$ & $\begin{array}{r}113394 \\
17283255\end{array}$ & 271022 & 683405 & 4070896 & $\begin{array}{r}+++++ \\
50.0\end{array}$ & $\begin{array}{r}0.827 \\
100\end{array}$ & 1.88 & 4.80 & 24.8 \\
\hline Tert-butyl ethyl ether & FB & Ave & $\begin{array}{r}+++++ \\
5879179\end{array}$ & $\begin{array}{r}67915 \\
11931054\end{array}$ & 177998 & 463020 & 2950549 & $\begin{array}{r}+++++ \\
50.0\end{array}$ & $\begin{array}{r}0.827 \\
100\end{array}$ & 1.88 & 4.80 & 24.8 \\
\hline 2,2-Dichloropropane & FB & Ave & $\begin{array}{r}+++++ \\
1912856\end{array}$ & $\begin{array}{r}37071 \\
3830093\end{array}$ & 83392 & 185868 & 988173 & $\begin{array}{r}+++++ \\
50.0\end{array}$ & $\begin{array}{r}0.827 \\
100\end{array}$ & 1.88 & 4.80 & 24.8 \\
\hline cis-1,2-Dichloroethene & FB & Ave & $\begin{array}{r}+++++ \\
1929948 \\
\end{array}$ & $\begin{array}{r}25610 \\
4023381 \\
\end{array}$ & 63098 & 161907 & 949241 & $\begin{array}{r}+++++ \\
50.0 \\
\end{array}$ & $\begin{array}{r}0.827 \\
100 \\
\end{array}$ & 1.88 & 4.80 & 24.8 \\
\hline 2-Butanone & BUT & Ave & $\begin{array}{r}+++++ \\
869559 \\
\end{array}$ & $\begin{array}{r}11663 \\
1740825 \\
\end{array}$ & 27737 & 72033 & 410474 & $\begin{array}{r}+++++ \\
250 \\
\end{array}$ & $\begin{array}{r}4.14 \\
501 \\
\end{array}$ & 9.42 & 24.0 & 124 \\
\hline Propionitrile & FB & Ave & $\begin{array}{r}+++++ \\
741376\end{array}$ & $\begin{array}{r}7995 \\
1516090\end{array}$ & 23097 & 65330 & 345370 & $\begin{array}{r}+++++ \\
250\end{array}$ & $\begin{array}{r}4.14 \\
501\end{array}$ & 9.42 & 24.0 & 124 \\
\hline Methacrylonitrile & FB & Ave & $\begin{array}{r}+++++ \\
502198 \\
\end{array}$ & $\begin{array}{r}6138 \\
1020620 \\
\end{array}$ & 14273 & 41659 & 240285 & $\begin{array}{r}+++++ \\
50.0 \\
\end{array}$ & $\begin{array}{r}0.827 \\
100 \\
\end{array}$ & 1.88 & 4.80 & 24.8 \\
\hline Bromochloromethane & FB & Ave & $\begin{array}{r}+++++ \\
1059848\end{array}$ & $\begin{array}{r}14692 \\
2033728\end{array}$ & 36823 & 91784 & 559699 & $\begin{array}{r}+++++ \\
50.0\end{array}$ & $\begin{array}{r}0.827 \\
100\end{array}$ & 1.88 & 4.80 & 24.8 \\
\hline Tetrahydrofuran & FB & Ave & $\begin{array}{r}+++++ \\
1045051\end{array}$ & $\begin{array}{r}12504 \\
2157749\end{array}$ & 30428 & 86907 & 484833 & $\begin{array}{l}+++++ \\
100.0\end{array}$ & $\begin{array}{r}1.65 \\
200\end{array}$ & 3.77 & 9.61 & 49.6 \\
\hline
\end{tabular}


FORM VI

GC/MS VOA INITIAL CALIBRATION DATA

INTERNAL STANDARD RESPONSE AND CONCENTRATION

Lab Name: TestAmerica Burlington

Job No. : 200-28914-1

Analy Batch No.: 92007

SDG No.: 200-28914

Instrument ID: CHL.i

GC Column: DB-624

ID : $0.53(\mathrm{~mm})$

Heated Purge: (Y/N) N

Calibration Start Date: 07/29/2015 12:33

Calibration End Date: 07/29/2015 15:49

Calibration ID: 31571

\begin{tabular}{|c|c|c|c|c|c|c|c|c|c|c|c|c|}
\hline \multirow[t]{2}{*}{ ANALYTE } & \multirow{2}{*}{$\begin{array}{l}\text { IS } \\
\text { REF }\end{array}$} & \multirow{2}{*}{$\begin{array}{l}\text { CURVE } \\
\text { TYPE }\end{array}$} & \multicolumn{5}{|c|}{ RESPONSE } & \multicolumn{5}{|c|}{ CONCENTRATION (UG/L) } \\
\hline & & & $\begin{array}{ll}\text { LVL } & 1 \\
\text { LVL } & 6\end{array}$ & $\begin{array}{ll}\text { LVL } & 2 \\
\text { LVL } & 7\end{array}$ & LVL 3 & LVL 4 & LVL 5 & $\begin{array}{ll}\text { LVL } & 1 \\
\text { LVL } & 6\end{array}$ & $\begin{array}{l}\text { LVL } 2 \\
\text { LVL } 7\end{array}$ & LVL 3 & LVL 4 & LVL 5 \\
\hline Chloroform & FB & Ave & $\begin{array}{r}+++++ \\
3517276\end{array}$ & $\begin{array}{r}45611 \\
7239122\end{array}$ & 114684 & 290809 & 1736642 & $\begin{array}{r}+++++ \\
50.0\end{array}$ & $\begin{array}{r}0.827 \\
100\end{array}$ & 1.88 & 4.80 & 24.8 \\
\hline 1,1,1-Trichloroethane & FB & Ave & $\begin{array}{r}+++++ \\
2948455\end{array}$ & $\begin{array}{r}38316 \\
6051361\end{array}$ & 94288 & 248046 & 1447263 & $\begin{array}{r}+++++ \\
50.0\end{array}$ & $\begin{array}{r}0.827 \\
100 \\
\end{array}$ & 1.88 & 4.80 & 24.8 \\
\hline Cyclohexane & FB & Ave & $\begin{array}{r}+++++ \\
3545968\end{array}$ & $\begin{array}{r}46858 \\
7426533 \\
\end{array}$ & 116184 & 296422 & 1741490 & $\begin{array}{r}+++++ \\
50.0\end{array}$ & $\begin{array}{r}0.827 \\
100\end{array}$ & 1.88 & 4.80 & 24.8 \\
\hline 1,1-Dichloropropene & FB & Ave & $\begin{array}{r}+++++ \\
2833603\end{array}$ & $\begin{array}{r}35265 \\
5917111\end{array}$ & 93184 & 235066 & 1399589 & $\begin{array}{r}+++++ \\
50.0\end{array}$ & $\begin{array}{r}0.827 \\
100\end{array}$ & 1.88 & 4.80 & 24.8 \\
\hline Carbon tetrachloride & FB & Ave & $\begin{array}{r}+++++ \\
2719464\end{array}$ & $\begin{array}{r}30890 \\
5632363\end{array}$ & 86166 & 222947 & 1345293 & $\begin{array}{r}+++++ \\
50.0\end{array}$ & $\begin{array}{r}0.827 \\
100 \\
\end{array}$ & 1.88 & 4.80 & 24.8 \\
\hline Isobutyl alcohol & FB & Ave & $\begin{array}{r}+++++ \\
1955166\end{array}$ & $\begin{array}{r}+++++ \\
3979783\end{array}$ & 60246 & 155988 & 913513 & $\begin{array}{r}+++++ \\
1250\end{array}$ & $\begin{array}{r}+++++ \\
2504\end{array}$ & 47.1 & 120 & 620 \\
\hline Benzene & FB & Ave & $\begin{array}{r}+++++ \\
5489131 \\
\end{array}$ & $\begin{array}{r}71108 \\
11543920 \\
\end{array}$ & 177812 & 454632 & 2673997 & $\begin{array}{r}+++++ \\
50.0\end{array}$ & $\begin{array}{r}0.827 \\
100 \\
\end{array}$ & 1.88 & 4.80 & 24.8 \\
\hline 1,2-Dichloroethane & FB & Ave & $\begin{array}{r}+++++ \\
1908789\end{array}$ & $\begin{array}{r}23846 \\
3812219\end{array}$ & 61584 & 158079 & 927990 & $\begin{array}{r}+++++ \\
50.0\end{array}$ & $\begin{array}{r}0.827 \\
100 \\
\end{array}$ & 1.88 & 4.80 & 24.8 \\
\hline Tert-amyl methyl ether & FB & Ave & $\begin{array}{r}+++++ \\
4624123\end{array}$ & $\begin{array}{r}58935 \\
9522508\end{array}$ & 150522 & 382319 & 2251380 & $\begin{array}{r}+++++ \\
50.0\end{array}$ & $\begin{array}{r}0.827 \\
100\end{array}$ & 1.88 & 4.80 & 24.8 \\
\hline n-Heptane & FB & Ave & $\begin{array}{r}+++++ \\
3190009 \\
\end{array}$ & $\begin{array}{r}43643 \\
6593247 \\
\end{array}$ & 110440 & 267739 & 1565454 & $\begin{array}{r}+++++ \\
50.0 \\
\end{array}$ & $\begin{array}{r}0.827 \\
100 \\
\end{array}$ & 1.88 & 4.80 & 24.8 \\
\hline Trichloroethene & FB & Ave & $\begin{array}{r}+++++ \\
2300118\end{array}$ & $\begin{array}{r}29990 \\
4882541\end{array}$ & 75690 & 190490 & 1143065 & $\begin{array}{r}+++++ \\
50.0\end{array}$ & $\begin{array}{r}0.827 \\
100 \\
\end{array}$ & 1.88 & 4.80 & 24.8 \\
\hline Methylcyclohexane & FB & Ave & $\begin{array}{r}+++++ \\
2844989\end{array}$ & $\begin{array}{r}37681 \\
5934696\end{array}$ & 93778 & 234919 & 1407540 & $\begin{array}{r}+++++ \\
50.0\end{array}$ & $\begin{array}{r}0.827 \\
100\end{array}$ & 1.88 & 4.80 & 24.8 \\
\hline 1,2-Dichloropropane & FB & Ave & $\begin{array}{r}+++++ \\
2431102\end{array}$ & $\begin{array}{r}32942 \\
5025933\end{array}$ & 79304 & 199423 & 1182687 & $\begin{array}{r}+++++ \\
50.0\end{array}$ & $\begin{array}{r}0.827 \\
100\end{array}$ & 1.88 & 4.80 & 24.8 \\
\hline Dibromomethane & FB & Ave & $\begin{array}{r}+++++ \\
1769124 \\
\end{array}$ & $\begin{array}{r}22843 \\
3643900 \\
\end{array}$ & 56450 & 144643 & 861774 & $\begin{array}{r}+++++ \\
50.0\end{array}$ & $\begin{array}{r}0.827 \\
100 \\
\end{array}$ & 1.88 & 4.80 & 24.8 \\
\hline Methyl methacrylate & CBZ & Ave & $\begin{array}{r}+++++ \\
1658331\end{array}$ & $\begin{array}{r}21911 \\
3364831\end{array}$ & 51614 & 134387 & 778874 & $\begin{array}{r}+++++ \\
50.0\end{array}$ & $\begin{array}{r}0.827 \\
100\end{array}$ & 1.88 & 4.80 & 24.8 \\
\hline 1,4-Dioxane & DXE & Ave & $\begin{array}{r}+++++ \\
311307 \\
\end{array}$ & $\begin{array}{l}+++++ \\
+++++\end{array}$ & 8934 & 25136 & 149532 & $\begin{array}{r}+++++ \\
1000 \\
\end{array}$ & $\begin{array}{l}+++++ \\
+++++\end{array}$ & 37.7 & 96.1 & 496 \\
\hline Bromodichloromethane & FB & Ave & $\begin{array}{r}+++++ \\
3625358 \\
\end{array}$ & $\begin{array}{r}45335 \\
7446904 \\
\end{array}$ & 112024 & 291962 & 1769615 & $\begin{array}{r}+++++ \\
50.0\end{array}$ & $\begin{array}{r}0.827 \\
100\end{array}$ & 1.88 & 4.80 & 24.8 \\
\hline 2-Chloroethyl vinyl ether & FB & Ave & $\begin{array}{r}+++++ \\
1211355\end{array}$ & $\begin{array}{r}14311 \\
2553639\end{array}$ & 35638 & 93179 & 572467 & $\begin{array}{r}+++++ \\
50.0\end{array}$ & $\begin{array}{r}0.827 \\
100\end{array}$ & 1.88 & 4.80 & 24.8 \\
\hline cis-1,3-Dichloropropene & FB & Ave & $\begin{array}{r}27233 \\
3073079 \\
\end{array}$ & $\begin{array}{r}38935 \\
6252444 \\
\end{array}$ & 94037 & 245501 & 1487806 & $\begin{array}{r}0.405 \\
50.0 \\
\end{array}$ & $\begin{array}{r}0.827 \\
100 \\
\end{array}$ & 1.88 & 4.80 & 24.8 \\
\hline 4-Methyl-2-pentanone & BUT & Ave & $\begin{array}{r}+++++ \\
11211217\end{array}$ & $\begin{array}{r}129436 \\
22391496\end{array}$ & 352539 & 913343 & 5353218 & $\begin{array}{r}+++++ \\
250\end{array}$ & $\begin{array}{r}4.14 \\
501\end{array}$ & 9.42 & 24.0 & 124 \\
\hline Toluene & CBZ & Ave & $\begin{array}{r}+++++ \\
3569366\end{array}$ & $\begin{array}{r}45217 \\
7404834\end{array}$ & 115388 & 295568 & 1746682 & $\begin{array}{r}+++++ \\
50.0\end{array}$ & $\begin{array}{r}0.827 \\
100\end{array}$ & 1.88 & 4.80 & 24.8 \\
\hline
\end{tabular}


FORM VI

GC/MS VOA INITIAL CALIBRATION DATA

INTERNAL STANDARD RESPONSE AND CONCENTRATION

Lab Name: TestAmerica Burlington

Job No. : 200-28914-1

Analy Batch No.: 92007

SDG No.: 200-28914

Instrument ID: CHL.i

GC Column: DB-624

ID : $0.53(\mathrm{~mm})$

Heated Purge: (Y/N) N

Calibration Start Date: 07/29/2015 12:33

Calibration End Date: 07/29/2015 15:49

Calibration ID: 31571

\begin{tabular}{|c|c|c|c|c|c|c|c|c|c|c|c|c|}
\hline \multirow[t]{2}{*}{ ANALYTE } & \multirow{2}{*}{$\begin{array}{l}\text { IS } \\
\text { REF }\end{array}$} & \multirow{2}{*}{$\begin{array}{l}\text { CURVE } \\
\text { TYPE }\end{array}$} & \multicolumn{5}{|c|}{ RESPONSE } & \multicolumn{5}{|c|}{ CONCENTRATION (UG/L) } \\
\hline & & & $\begin{array}{ll}\text { LVL } & 1 \\
\text { LVL } & 6\end{array}$ & $\begin{array}{ll}\text { LVL } & 2 \\
\text { LVL } & 7\end{array}$ & LVL 3 & LVL 4 & LVL 5 & $\begin{array}{ll}\text { LVL } & 1 \\
\text { LVL } & 6\end{array}$ & $\begin{array}{l}\text { LVL } 2 \\
\text { LVL } 7\end{array}$ & LVL 3 & LVL 4 & LVL 5 \\
\hline trans-1,3-Dichloropropene & CBZ & Ave & $\begin{array}{r}23279 \\
2437447 \\
\end{array}$ & $\begin{array}{r}30702 \\
4949815 \\
\end{array}$ & 79212 & 202476 & 1165750 & $\begin{array}{r}0.405 \\
50.0 \\
\end{array}$ & $\begin{array}{r}0.827 \\
100 \\
\end{array}$ & 1.88 & 4.80 & 24.8 \\
\hline Ethyl methacrylate & FB & Ave & $\begin{array}{r}+++++ \\
1863548\end{array}$ & $\begin{array}{r}25299 \\
3783846\end{array}$ & 58711 & 152732 & 892102 & $\begin{array}{r}+++++ \\
50.0\end{array}$ & $\begin{array}{r}0.827 \\
100 \\
\end{array}$ & 1.88 & 4.80 & 24.8 \\
\hline $1,1,2$-Trichloroethane & $\mathrm{CBZ}$ & Ave & $\begin{array}{r}+++++ \\
1675168\end{array}$ & $\begin{array}{r}21851 \\
3413028 \\
\end{array}$ & 52995 & 137167 & 814292 & $\begin{array}{r}+++++ \\
50.0\end{array}$ & $\begin{array}{r}0.827 \\
100\end{array}$ & 1.88 & 4.80 & 24.8 \\
\hline Tetrachloroethene & CBZ & Ave & $\begin{array}{r}+++++ \\
2679186\end{array}$ & $\begin{array}{r}32349 \\
5566132\end{array}$ & 86654 & 218964 & 1317975 & $\begin{array}{r}+++++ \\
50.0\end{array}$ & $\begin{array}{r}0.827 \\
100\end{array}$ & 1.88 & 4.80 & 24.8 \\
\hline 1,3-Dichloropropane & CBZ & Ave & $\begin{array}{r}+++++ \\
3186106\end{array}$ & $\begin{array}{r}41006 \\
6544972\end{array}$ & 101680 & 262125 & 1563710 & $\begin{array}{r}+++++ \\
50.0\end{array}$ & $\begin{array}{r}0.827 \\
100 \\
\end{array}$ & 1.88 & 4.80 & 24.8 \\
\hline 2-Hexanone & BUT & Ave & $\begin{array}{r}+++++ \\
8954735\end{array}$ & $\begin{array}{r}103644 \\
17678882\end{array}$ & 282931 & 723795 & 4307782 & $\begin{array}{r}+++++ \\
250\end{array}$ & $\begin{array}{r}4.14 \\
501\end{array}$ & 9.42 & 24.0 & 124 \\
\hline Dibromochloromethane & CBZ & Ave & $\begin{array}{r}24614 \\
3070134 \\
\end{array}$ & $\begin{array}{r}34295 \\
6319313 \\
\end{array}$ & 88080 & 237574 & 1487949 & $\begin{array}{r}0.405 \\
50.0\end{array}$ & $\begin{array}{r}0.827 \\
100 \\
\end{array}$ & 1.88 & 4.80 & 24.8 \\
\hline 1,2-Dibromoethane & $\mathrm{CBZ}$ & Ave & $\begin{array}{r}+++++ \\
2796108\end{array}$ & $\begin{array}{r}32945 \\
5662910\end{array}$ & 86900 & 218720 & 1347680 & $\begin{array}{r}+++++ \\
50.0\end{array}$ & $\begin{array}{r}0.827 \\
100 \\
\end{array}$ & 1.88 & 4.80 & 24.8 \\
\hline Chlorobenzene & CBZ & Ave & $\begin{array}{r}+++++ \\
4412828\end{array}$ & $\begin{array}{r}56883 \\
9146280\end{array}$ & 145029 & 369726 & 2170196 & $\begin{array}{r}+++++ \\
50.0\end{array}$ & $\begin{array}{r}0.827 \\
100\end{array}$ & 1.88 & 4.80 & 24.8 \\
\hline $1,1,1,2$-Tetrachloroethane & CBZ & Ave & $\begin{array}{r}+++++ \\
2220229\end{array}$ & $\begin{array}{r}26700 \\
4585402\end{array}$ & 70199 & 178411 & 1086342 & $\begin{array}{r}+++++ \\
50.0\end{array}$ & $\begin{array}{r}0.827 \\
100 \\
\end{array}$ & 1.88 & 4.80 & 24.8 \\
\hline Ethylbenzene & $\mathrm{CBZ}$ & Ave & $\begin{array}{r}+++++ \\
7193870\end{array}$ & $\begin{array}{r}91316 \\
15076986\end{array}$ & 229278 & 589490 & 3510457 & $\begin{array}{r}+++++ \\
50.0\end{array}$ & $\begin{array}{r}0.827 \\
100 \\
\end{array}$ & 1.88 & 4.80 & 24.8 \\
\hline$m \& p-X y l e n e$ & CBZ & Ave & $\begin{array}{r}+++++ \\
2645994\end{array}$ & $\begin{array}{r}33061 \\
5484300\end{array}$ & 82772 & 208391 & 1297120 & $\begin{array}{r}+++++ \\
50.0\end{array}$ & $\begin{array}{r}0.827 \\
100\end{array}$ & 1.88 & 4.80 & 24.8 \\
\hline o-Xylene & CBZ & Ave & $\begin{array}{r}+++++ \\
2499815\end{array}$ & $\begin{array}{r}30560 \\
5185220\end{array}$ & 82680 & 208253 & 1234619 & $\begin{array}{r}+++++ \\
50.0\end{array}$ & $\begin{array}{r}0.827 \\
100\end{array}$ & 1.88 & 4.80 & 24.8 \\
\hline Styrene & CBZ & Ave & $\begin{array}{r}+++++ \\
3767329 \\
\end{array}$ & $\begin{array}{r}45425 \\
7866392 \\
\end{array}$ & 114923 & 300961 & 1822729 & $\begin{array}{r}+++++ \\
50.0\end{array}$ & $\begin{array}{r}0.827 \\
100 \\
\end{array}$ & 1.88 & 4.80 & 24.8 \\
\hline Bromoform & CBZ & Lin1 & $\begin{array}{r}+++++ \\
2241394\end{array}$ & $\begin{array}{r}22228 \\
4643147\end{array}$ & 61889 & 163570 & 1065153 & $\begin{array}{r}+++++ \\
50.0\end{array}$ & $\begin{array}{r}0.827 \\
100\end{array}$ & 1.88 & 4.80 & 24.8 \\
\hline Isopropylbenzene & $\mathrm{DCB}$ & Ave & $\begin{array}{r}+++++ \\
7306174\end{array}$ & $\begin{array}{r}90907 \\
15364855\end{array}$ & 234066 & 599487 & 3599676 & $\begin{array}{r}+++++ \\
50.0\end{array}$ & $\begin{array}{r}0.827 \\
100 \\
\end{array}$ & 1.88 & 4.80 & 24.8 \\
\hline Bromobenzene & $\mathrm{DCB}$ & Ave & $\begin{array}{r}+++++ \\
2255232 \\
\end{array}$ & $\begin{array}{r}25947 \\
4669167 \\
\end{array}$ & 70532 & 179873 & 1093877 & $\begin{array}{r}+++++ \\
50.0\end{array}$ & $\begin{array}{r}0.827 \\
100\end{array}$ & 1.88 & 4.80 & 24.8 \\
\hline $1,1,2,2$-Tetrachloroethane & DCB & Ave & $\begin{array}{r}29421 \\
3256400\end{array}$ & $\begin{array}{r}40610 \\
6522722\end{array}$ & 104345 & 266172 & 1552547 & $\begin{array}{r}0.405 \\
50.0\end{array}$ & $\begin{array}{r}0.827 \\
100\end{array}$ & 1.88 & 4.80 & 24.8 \\
\hline 1,2,3-Trichloropropane & $\mathrm{DCB}$ & Ave & $\begin{array}{r}+++++ \\
768758 \\
\end{array}$ & $\begin{array}{r}9626 \\
1554530 \\
\end{array}$ & 24199 & 63883 & 371188 & $\begin{array}{r}+++++ \\
50.0 \\
\end{array}$ & $\begin{array}{r}0.827 \\
100 \\
\end{array}$ & 1.88 & 4.80 & 24.8 \\
\hline trans-1,4-Dichloro-2-butene & $\mathrm{DCB}$ & Ave & $\begin{array}{r}+++++ \\
626694\end{array}$ & $\begin{array}{r}8275 \\
1266608\end{array}$ & 19121 & 52511 & 333972 & $\begin{array}{r}+++++ \\
50.0\end{array}$ & $\begin{array}{r}0.827 \\
100\end{array}$ & 1.88 & 4.80 & 24.8 \\
\hline n-Propylbenzene & $\mathrm{DCB}$ & Ave & $\begin{array}{r}+++++ \\
1873614\end{array}$ & $\begin{array}{r}23056 \\
3962864\end{array}$ & 57450 & 150819 & 912610 & $\begin{array}{r}+++++ \\
50.0\end{array}$ & $\begin{array}{r}0.827 \\
100\end{array}$ & 1.88 & 4.80 & 24.8 \\
\hline
\end{tabular}


FORM VI

GC/MS VOA INITIAL CALIBRATION DATA

INTERNAL STANDARD RESPONSE AND CONCENTRATION

Lab Name: TestAmerica Burlington

Job No.: 200-28914-1

Analy Batch No.: 92007

SDG No.: 200-28914

Instrument ID: CHL.i

GC Column: DB-624

ID : $0.53(\mathrm{~mm})$

Heated Purge: (Y/N) N

Calibration Start Date: 07/29/2015 12:33

Calibration End Date: 07/29/2015 15:49

Calibration ID: 31571

\begin{tabular}{|c|c|c|c|c|c|c|c|c|c|c|c|c|}
\hline \multirow[t]{2}{*}{ ANALYTE } & \multirow{2}{*}{$\begin{array}{l}\text { IS } \\
\text { REF }\end{array}$} & \multirow{2}{*}{$\begin{array}{l}\text { CURVE } \\
\text { TYPE }\end{array}$} & \multicolumn{5}{|c|}{ RESPONSE } & \multicolumn{5}{|c|}{ CONCENTRATION (UG/L) } \\
\hline & & & $\begin{array}{ll}\text { LVL } & 1 \\
\text { LVL } & 6\end{array}$ & $\begin{array}{ll}\text { LVL } & 2 \\
\text { LVL } & 7\end{array}$ & LVL 3 & LVL 4 & LVL 5 & $\begin{array}{ll}\text { LVL } & 1 \\
\text { LVL } & 6\end{array}$ & $\begin{array}{ll}\text { LVL } 2 \\
\text { LVL } 7\end{array}$ & LVL 3 & LVL 4 & LVL 5 \\
\hline 2-Chlorotoluene & $\mathrm{DCB}$ & Ave & $\begin{array}{r}+++++ \\
1713698\end{array}$ & $\begin{array}{r}20827 \\
3604800 \\
\end{array}$ & 56373 & 141583 & 841204 & $\begin{array}{r}+++++ \\
50.0\end{array}$ & $\begin{array}{r}0.827 \\
100\end{array}$ & 1.88 & 4.80 & 24.8 \\
\hline 1,3,5-Trimethylbenzene & DCB & Ave & $\begin{array}{r}+++++ \\
5355769\end{array}$ & $\begin{array}{r}64539 \\
11320276\end{array}$ & 168065 & 434758 & 2600391 & $\begin{array}{r}+++++ \\
50.0\end{array}$ & $\begin{array}{r}0.827 \\
100\end{array}$ & 1.88 & 4.80 & 24.8 \\
\hline 4-Chlorotoluene & $\mathrm{DCB}$ & Ave & $\begin{array}{r}+++++ \\
1784520\end{array}$ & $\begin{array}{r}21261 \\
3737991\end{array}$ & 57336 & 145348 & 849372 & $\begin{array}{r}+++++ \\
50.0\end{array}$ & $\begin{array}{r}0.827 \\
100 \\
\end{array}$ & 1.88 & 4.80 & 24.8 \\
\hline tert-Butylbenzene & DCB & Ave & $\begin{array}{r}+++++ \\
5786184\end{array}$ & $\begin{array}{r}71077 \\
12229231\end{array}$ & 182954 & 475120 & 2834051 & $\begin{array}{r}+++++ \\
50.0\end{array}$ & $\begin{array}{r}0.827 \\
100\end{array}$ & 1.88 & 4.80 & 24.8 \\
\hline 1,2,4-Trimethylbenzene & DCB & Ave & $\begin{array}{r}+++++ \\
5306969\end{array}$ & $\begin{array}{r}66688 \\
11323218\end{array}$ & 166004 & 426523 & 2583898 & $\begin{array}{r}+++++ \\
50.0\end{array}$ & $\begin{array}{r}0.827 \\
100\end{array}$ & 1.88 & 4.80 & 24.8 \\
\hline sec-Butylbenzene & $\mathrm{DCB}$ & Ave & $\begin{array}{r}+++++ \\
8109865\end{array}$ & $\begin{array}{r}98020 \\
17215145\end{array}$ & 257423 & 654899 & 3947814 & $\begin{array}{r}+++++ \\
50.0\end{array}$ & $\begin{array}{r}0.827 \\
100\end{array}$ & 1.88 & 4.80 & 24.8 \\
\hline 1,3-Dichlorobenzene & $\mathrm{DCB}$ & Ave & $\begin{array}{r}+++++ \\
3691353\end{array}$ & $\begin{array}{r}46218 \\
7721245\end{array}$ & 113780 & 291206 & 1765439 & $\begin{array}{r}+++++ \\
50.0\end{array}$ & $\begin{array}{r}0.827 \\
100\end{array}$ & 1.88 & 4.80 & 24.8 \\
\hline 4-Isopropyltoluene & $\mathrm{DCB}$ & Ave & $\begin{array}{r}+++++ \\
6321967\end{array}$ & $\begin{array}{r}75828 \\
13522735\end{array}$ & 199053 & 503708 & 3061151 & $\begin{array}{r}+++++ \\
50.0\end{array}$ & $\begin{array}{r}0.827 \\
100\end{array}$ & 1.88 & 4.80 & 24.8 \\
\hline 1,4-Dichlorobenzene & $\mathrm{DCB}$ & Ave & $\begin{array}{r}+++++ \\
3859548\end{array}$ & $\begin{array}{r}51520 \\
8155061\end{array}$ & 126757 & 318876 & 1910940 & $\begin{array}{r}+++++ \\
50.0\end{array}$ & $\begin{array}{r}0.827 \\
100\end{array}$ & 1.88 & 4.80 & 24.8 \\
\hline 1,2-Dichlorobenzene & $\mathrm{DCB}$ & Ave & $\begin{array}{r}+++++ \\
3414569\end{array}$ & $\begin{array}{r}44367 \\
7149474\end{array}$ & 108509 & 278276 & 1667393 & $\begin{array}{r}+++++ \\
50.0\end{array}$ & $\begin{array}{r}0.827 \\
100\end{array}$ & 1.88 & 4.80 & 24.8 \\
\hline n-Butylbenzene & $\mathrm{DCB}$ & Ave & $\begin{array}{r}+++++ \\
5637709\end{array}$ & $\begin{array}{r}68519 \\
12378055\end{array}$ & 173136 & 441294 & 2717051 & $\begin{array}{r}+++++ \\
50.0\end{array}$ & $\begin{array}{r}0.827 \\
100 \\
\end{array}$ & 1.88 & 4.80 & 24.8 \\
\hline 1,2-Dibromo-3-Chloropropane & DCB & Ave & $\begin{array}{r}+++++ \\
605713\end{array}$ & $\begin{array}{r}7266 \\
1213220\end{array}$ & 16884 & 46920 & 286487 & $\begin{array}{r}+++++ \\
50.0\end{array}$ & $\begin{array}{r}0.827 \\
100\end{array}$ & 1.88 & 4.80 & 24.8 \\
\hline $1,2,4$-Trichlorobenzene & $\mathrm{DCB}$ & Ave & $\begin{array}{r}+++++ \\
2260578\end{array}$ & $\begin{array}{r}27979 \\
4837357\end{array}$ & 66997 & 172565 & 1076859 & $\begin{array}{r}+++++ \\
50.0\end{array}$ & $\begin{array}{r}0.827 \\
100\end{array}$ & 1.88 & 4.80 & 24.8 \\
\hline Hexachlorobutadiene & $\mathrm{DCB}$ & Ave & $\begin{array}{r}+++++ \\
1244980\end{array}$ & $\begin{array}{r}17529 \\
2610480\end{array}$ & 42064 & 100962 & 604053 & $\begin{array}{r}+++++ \\
50.0\end{array}$ & $\begin{array}{r}0.827 \\
100\end{array}$ & 1.88 & 4.80 & 24.8 \\
\hline Naphthalene & $\mathrm{DCB}$ & Ave & $\begin{array}{r}+++++ \\
4415885\end{array}$ & $\begin{array}{r}56425 \\
9237167\end{array}$ & 137070 & 347122 & 2098220 & $\begin{array}{r}+++++ \\
50.0\end{array}$ & $\begin{array}{r}0.827 \\
100\end{array}$ & 1.88 & 4.80 & 24.8 \\
\hline 1,2,3-Trichlorobenzene & DCB & Ave & $\begin{array}{r}+++++ \\
2022138\end{array}$ & $\begin{array}{r}26084 \\
4289793\end{array}$ & 62443 & 160844 & 969982 & $\begin{array}{r}+++++ \\
50.0\end{array}$ & $\begin{array}{r}0.827 \\
100\end{array}$ & 1.88 & 4.80 & 24.8 \\
\hline Dibromofluoromethane (Surr) & FB & Ave & $\begin{array}{r}+++++ \\
2973426 \\
\end{array}$ & $\begin{array}{r}37672 \\
6087543 \\
\end{array}$ & 95032 & 237540 & 1443000 & $\begin{array}{r}+++++ \\
50.0\end{array}$ & $\begin{array}{r}0.827 \\
100 \\
\end{array}$ & 1.88 & 4.80 & 24.8 \\
\hline 1,2-Dichloroethane-d4 & FB & Ave & $\begin{array}{r}+++++ \\
1525819\end{array}$ & $\begin{array}{r}22832 \\
3090098\end{array}$ & 52581 & 135312 & 755416 & $\begin{array}{r}+++++ \\
50.0\end{array}$ & $\begin{array}{r}0.827 \\
100\end{array}$ & 1.88 & 4.80 & 24.8 \\
\hline Toluene-d8 & CBZ & Ave & $\begin{array}{r}+++++ \\
5131358\end{array}$ & $\begin{array}{r}65372 \\
10715051\end{array}$ & 167195 & 421299 & 2522179 & $\begin{array}{r}+++++ \\
50.0\end{array}$ & $\begin{array}{r}0.827 \\
100 \\
\end{array}$ & 1.88 & 4.80 & 24.8 \\
\hline Bromofluorobenzene & DCB & Ave & $\begin{array}{r}+++++ \\
3197335\end{array}$ & $\begin{array}{r}39761 \\
6641390\end{array}$ & 104420 & 261416 & 1556837 & $\begin{array}{r}+++++ \\
50.0\end{array}$ & $\begin{array}{r}0.827 \\
100 \\
\end{array}$ & 1.88 & 4.80 & 24.8 \\
\hline
\end{tabular}


FORM VI

GC/MS VOA INITIAL CALIBRATION DATA

INTERNAL STANDARD RESPONSE AND CONCENTRATION

Lab Name: TestAmerica Burlington

SDG No.: 200-28914

Instrument ID: CHL.i

Calibration Start Date: 07/29/2015 12:33

Curve Type Legend:

Ave = Average ISTD

Lin1 $=$ Linear $1 /$ conc ISTD
Job No.: 200-28914-1

Analy Batch No.: 92007
GC Column: DB-624

ID : $0.53(\mathrm{~mm})$

Calibration End Date: 07/29/2015 15:49
Heated Purge: (Y/N) N

Calibration ID: 31571 
FORM VII

GC/MS VOA CONTINUING CALIBRATION DATA

Lab Name: TestAmerica Burlington

SDG No.: 200-28914

Lab Sample ID: ICV 200-92007/11

Instrument ID: CHL.i

GC Column: DB-624

Lab File ID: 15008_11.D
Job No.: 200-28914-1

Calibration Date: 07/29/2015 16:54

Calib Start Date: 07/29/2015 12:33

Calib End Date: 07/29/2015 15:49

Conc. Units: ug/L Heated Purge: (Y/N) N

\begin{tabular}{|c|c|c|c|c|c|c|c|c|}
\hline ANALYTE & $\begin{array}{l}\text { CURVE } \\
\text { TYPE }\end{array}$ & AVE RRE & $\mathrm{RRF}$ & MIN RRF & $\begin{array}{c}\text { CALC } \\
\text { AMOUNT }\end{array}$ & $\begin{array}{c}\text { SPIKE } \\
\text { AMOUNT }\end{array}$ & $\div D$ & $\begin{array}{l}\text { MAX } \\
\div D\end{array}$ \\
\hline Dichlorodifluoromethane & Ave & 0.4431 & 0.5571 & & 31.2 & 24.8 & $25.7 *$ & 25.0 \\
\hline Chloromethane & Ave & 0.3510 & 0.3544 & 0.1000 & 25.1 & 24.8 & 1.0 & 25.0 \\
\hline Vinyl chloride & Ave & 0.3578 & 0.3813 & & 26.5 & 24.8 & 6.6 & 25.0 \\
\hline Butadiene & Ave & 0.2626 & 0.2778 & & 26.2 & 24.8 & 5.8 & 25.0 \\
\hline Bromomethane & Ave & 0.2252 & 0.2487 & & 27.4 & 24.8 & 10.4 & 25.0 \\
\hline Chloroethane & Ave & 0.2148 & 0.2287 & & 26.4 & 24.8 & 6.5 & 25.0 \\
\hline Dichlorofluoromethane & Ave & 0.7405 & 0.7889 & & 26.4 & 24.8 & 6.5 & 25.0 \\
\hline Trichlorofluoromethane & Ave & 0.5469 & 0.6039 & & 27.4 & 24.8 & 10.4 & 25.0 \\
\hline Ethyl ether & Ave & 0.2032 & 0.2130 & & 26.0 & 24.8 & 4.8 & 25.0 \\
\hline Freon $123 a$ & Ave & 0.7833 & 0.8181 & & 25.9 & 24.8 & 4.4 & 25.0 \\
\hline Acrolein & Ave & 0.0149 & 0.0199 & & 166 & 124 & $33.9 *$ & 25.0 \\
\hline 1,1-Dichloroethene & Ave & 0.3005 & 0.3027 & & 25.0 & 24.8 & 0.7 & 25.0 \\
\hline Freon TF & Ave & 0.6004 & 0.6189 & & 25.6 & 24.8 & 3.1 & 25.0 \\
\hline Acetone & Ave & 0.7123 & 0.8911 & & 155 & 124 & $25.1 *$ & 25.0 \\
\hline Methyl iodide & Ave & 0.3509 & 0.3582 & & 25.3 & 24.8 & 2.1 & 25.0 \\
\hline Carbon disulfide & Ave & 0.8828 & 0.8431 & & 23.7 & 24.8 & -4.5 & 25.0 \\
\hline Allyl chloride & Ave & 0.5948 & 0.5876 & & 24.5 & 24.8 & -1.2 & 25.0 \\
\hline Methyl acetate & Ave & 0.2729 & 0.3271 & & 149 & 124 & 19.9 & 25.0 \\
\hline Methylene Chloride & Ave & 0.3199 & 0.2933 & & 22.8 & 24.8 & -8.3 & 25.0 \\
\hline tert-Butyl alcohol & Ave & 0.8582 & 0.8994 & & 260 & 248 & 4.8 & 25.0 \\
\hline Acrylonitrile & Lin1 & & 0.0693 & & 239 & 248 & -3.7 & 25.0 \\
\hline trans-1,2-Dichloroethene & Ave & 0.3149 & 0.3354 & & 26.4 & 24.8 & 6.5 & 25.0 \\
\hline Methyl t-butyl ether & Ave & 0.6475 & 0.6659 & & 25.5 & 24.8 & 2.8 & 25.0 \\
\hline Hexane & Ave & 0.4507 & 0.4976 & & 27.4 & 24.8 & 10.4 & 25.0 \\
\hline 1,1-Dichloroethane & Ave & 0.6431 & 0.6769 & 0.1000 & 26.1 & 24.8 & 5.3 & 25.0 \\
\hline Vinyl acetate & Ave & 0.0413 & 0.0417 & & 50.1 & 49.6 & 0.9 & 25.0 \\
\hline Chloroprene & Ave & 0.4505 & 0.5133 & & 28.3 & 24.8 & 13.9 & 25.0 \\
\hline Isopropyl ether & Ave & 1.369 & 1.472 & & 26.7 & 24.8 & 7.5 & 25.0 \\
\hline Tert-butyl ethyl ether & Ave & 0.9268 & 0.997 & & 26.7 & 24.8 & 7.6 & 25.0 \\
\hline 2,2-Dichloropropane & Ave & 0.3629 & 0.3613 & & 24.7 & 24.8 & -0.4 & 25.0 \\
\hline cis-1,2-Dichloroethene & Ave & 0.3178 & 0.3320 & & 25.9 & 24.8 & 4.5 & 25.0 \\
\hline 2-Butanone & Ave & 0.2050 & 0.2418 & & 146 & 124 & 18.0 & 25.0 \\
\hline Propionitrile & Ave & 0.0234 & 0.0237 & & 126 & 124 & 1.4 & 25.0 \\
\hline Methacrylonitrile & Ave & 0.0790 & 0.0830 & & 26.1 & 24.8 & 5.0 & 25.0 \\
\hline Bromochloromethane & Ave & 0.1783 & 0.1985 & & 27.6 & 24.8 & 11.4 & 25.0 \\
\hline Tetrahydrofuran & Ave & 0.0821 & 0.0827 & & 50.0 & 49.6 & 0.7 & 25.0 \\
\hline Chloroform & Ave & 0.5747 & 0.6097 & & 26.3 & 24.8 & 6.1 & 25.0 \\
\hline 1,1,1-Trichloroethane & Ave & 0.4810 & 0.5068 & & 26.1 & 24.8 & 5.4 & 25.0 \\
\hline Cyclohexane & Ave & 0.5837 & 0.6086 & & 25.9 & 24.8 & 4.3 & 25.0 \\
\hline 1,1-Dichloropropene & Ave & 0.4622 & 0.5255 & & 28.2 & 24.8 & 13.7 & 25.0 \\
\hline Carbon tetrachloride & Ave & 0.4340 & 0.4769 & & 27.3 & 24.8 & 9.9 & 25.0 \\
\hline
\end{tabular}


FORM VII

GC/MS VOA CONTINUING CALIBRATION DATA

Lab Name: TestAmerica Burlington

SDG No.: 200-28914

Lab Sample ID: ICV 200-92007/11

Instrument ID: CHL.i

GC Column: DB-624

Lab File ID: 15008_11.D
Job No.: 200-28914-1

Calibration Date: 07/29/2015 16:54

Calib Start Date: 07/29/2015 12:33

Calib End Date: 07/29/2015 15:49

Conc. Units: ug/L Heated Purge: (Y/N) N

\begin{tabular}{|c|c|c|c|c|c|c|c|c|}
\hline ANALYTE & $\begin{array}{l}\text { CURVE } \\
\text { TYPE }\end{array}$ & AVE RRE & $\mathrm{RRF}$ & MIN RRF & $\begin{array}{c}\text { CALC } \\
\text { AMOUNT }\end{array}$ & $\begin{array}{c}\text { SPIKE } \\
\text { AMOUNT }\end{array}$ & $\because \mathrm{D}$ & $\begin{array}{l}\text { MAX } \\
\div D\end{array}$ \\
\hline Isobutyl alcohol & Ave & 0.0126 & 0.0127 & & 623 & 620 & 0.4 & 25.0 \\
\hline Benzene & Ave & 0.8972 & 0.9421 & & 26.1 & 24.8 & 5.0 & 25.0 \\
\hline 1,2-Dichloroethane & Ave & 0.3072 & 0.3243 & & 26.2 & 24.8 & 5.6 & 25.0 \\
\hline Tert-amyl methyl ether & Ave & 0.7515 & 0.8137 & & 26.9 & 24.8 & 8.3 & 25.0 \\
\hline $\mathrm{n}$-Heptane & Ave & 0.5318 & 0.5819 & & 27.2 & 24.8 & 9.4 & 25.0 \\
\hline Trichloroethene & Ave & 0.3792 & 0.3892 & & 25.5 & 24.8 & 2.6 & 25.0 \\
\hline Methylcyclohexane & Ave & 0.4683 & 0.4934 & & 26.1 & 24.8 & 5.4 & 25.0 \\
\hline 1,2-Dichloropropane & Ave & 0.3987 & 0.4155 & & 25.9 & 24.8 & 4.2 & 25.0 \\
\hline Dibromomethane & Ave & 0.2867 & 0.3032 & & 26.2 & 24.8 & 5.8 & 25.0 \\
\hline Methyl methacrylate & Ave & 0.3310 & 0.3384 & & 25.4 & 24.8 & 2.2 & 25.0 \\
\hline 1,4-Dioxane & Ave & 1.012 & 0.9948 & & 488 & 496 & -1.7 & 25.0 \\
\hline Bromodichloromethane & Ave & 0.5802 & 0.6078 & & 26.0 & 24.8 & 4.7 & 25.0 \\
\hline 2-Chloroethyl vinyl ether & Ave & 0.1892 & 0.2122 & & 27.8 & 24.8 & 12.1 & 25.0 \\
\hline cis-1,3-Dichloropropene & Ave & 0.5088 & 0.5395 & & 26.3 & 24.8 & 6.0 & 25.0 \\
\hline 4-Methyl-2-pentanone & Ave & 2.574 & 2.934 & & 141 & 124 & 14.0 & 25.0 \\
\hline Toluene & Ave & 0.7227 & 0.7702 & & 26.5 & 24.8 & 6.6 & 25.0 \\
\hline trans-1,3-Dichloropropene & Ave & 0.5127 & 0.5685 & & 27.5 & 24.8 & 10.9 & 25.0 \\
\hline Ethyl methacrylate & Ave & 0.3022 & 0.3466 & & 28.5 & 24.8 & 14.7 & 25.0 \\
\hline 1,1,2-Trichloroethane & Ave & 0.3374 & 0.3635 & & 26.7 & 24.8 & 7.7 & 25.0 \\
\hline Tetrachloroethene & Ave & 0.5382 & 0.5806 & & 26.8 & 24.8 & 7.9 & 25.0 \\
\hline 1,3-Dichloropropane & Ave & 0.6438 & 0.6773 & & 26.1 & 24.8 & 5.2 & 25.0 \\
\hline 2-Hexanone & Ave & 2.054 & 2.487 & & 150 & 124 & 21.1 & 25.0 \\
\hline Dibromochloromethane & Ave & 0.6047 & 0.6536 & & 26.8 & 24.8 & 8.1 & 25.0 \\
\hline 1,2-Dibromoethane & Ave & 0.5478 & 0.5949 & & 26.9 & 24.8 & 8.6 & 25.0 \\
\hline Chlorobenzene & Ave & 0.9005 & 0.9523 & 0.3000 & 26.2 & 24.8 & 5.8 & 25.0 \\
\hline $1,1,1,2$-Tetrachloroethane & Ave & 0.4421 & 0.4773 & & 26.8 & 24.8 & 8.0 & 25.0 \\
\hline Ethylbenzene & Ave & 1.453 & 1.536 & & 26.2 & 24.8 & 5.7 & 25.0 \\
\hline $\mathrm{m} \& \mathrm{p}-\mathrm{Xylene}$ & Ave & 0.5277 & 0.5709 & & 26.8 & 24.8 & 8.2 & 25.0 \\
\hline o-Xylene & Ave & 0.5067 & 0.5422 & & 26.6 & 24.8 & 7.0 & 25.0 \\
\hline Styrene & Ave & 0.7455 & 0.7674 & & 25.5 & 24.8 & 2.9 & 25.0 \\
\hline Bromoform & Lin1 & & 0.4578 & 0.1000 & 24.0 & 24.8 & $-3 \cdot 3$ & 25.0 \\
\hline Isopropylbenzene & Ave & 2.706 & 2.868 & & 26.3 & 24.8 & 6.0 & 25.0 \\
\hline Bromobenzene & Ave & 0.8141 & 0.8706 & & 26.5 & 24.8 & 6.9 & 25.0 \\
\hline $1,1,2,2$-Tetrachloroethane & Ave & 1.243 & 1.300 & 0.3000 & 26.0 & 24.8 & 4.6 & 25.0 \\
\hline 1,2,3-Trichloropropane & Ave & 0.2818 & 0.2942 & & 25.9 & 24.8 & 4.4 & 25.0 \\
\hline trans-1,4-Dichloro-2-butene & Ave & 0.2350 & 0.2569 & & 27.1 & 24.8 & 9.3 & 25.0 \\
\hline n-Propylbenzene & Ave & 0.6849 & 0.7364 & & 26.7 & 24.8 & 7.5 & 25.0 \\
\hline 2-Chlorotoluene & Ave & 0.6356 & 0.6704 & & 26.2 & 24.8 & 5.5 & 25.0 \\
\hline 1,3,5-Trimethylbenzene & Ave & 1.960 & 2.069 & & 26.2 & 24.8 & 5.5 & 25.0 \\
\hline 4-Chlorotoluene & Ave & 0.6517 & 0.6819 & & 26.0 & 24.8 & 4.6 & 25.0 \\
\hline tert-Butylbenzene & Ave & 2.134 & 2.260 & & 26.3 & 24.8 & 5.9 & 25.0 \\
\hline
\end{tabular}


FORM VII

GC/MS VOA CONTINUING CALIBRATION DATA

Lab Name: TestAmerica Burlington

SDG No.: 200-28914

Lab Sample ID: ICV 200-92007/11

Instrument ID: CHL.i

GC Column: DB-624

ID $: 0.53(\mathrm{~mm})$

Lab File ID: 15008 11.D
Job No.: 200-28914-1

Calibration Date: 07/29/2015 16:54

Calib Start Date: 07/29/2015 12:33

Calib End Date: 07/29/2015 15:49

Conc. Units: ug/L Heated Purge: (Y/N) N

\begin{tabular}{|c|c|c|c|c|c|c|c|c|}
\hline ANALYTE & $\begin{array}{l}\text { CURVE } \\
\text { TYPE }\end{array}$ & AVE RRF & RRF & MIN RRF & $\begin{array}{c}\text { CALC } \\
\text { AMOUNT }\end{array}$ & $\begin{array}{c}\text { SPIKE } \\
\text { AMOUNT }\end{array}$ & $\div \mathrm{D}$ & $\begin{array}{l}\mathrm{MAX} \\
\div \mathrm{D}\end{array}$ \\
\hline $1,2,4$-Trimethylbenzene & Ave & 1.955 & 2.026 & & 25.7 & 24.8 & 3.6 & 25.0 \\
\hline sec-Butylbenzene & Ave & 2.977 & 3.145 & & 26.2 & 24.8 & 5.7 & 25.0 \\
\hline 1,3-Dichlorobenzene & Ave & 1.343 & 1.414 & & 26.1 & 24.8 & 5.3 & 25.0 \\
\hline 4-Isopropyltoluene & Ave & 2.311 & 2.420 & & 26.0 & 24.8 & 4.7 & 25.0 \\
\hline 1,4-Dichlorobenzene & Ave & 1.455 & 1.522 & & 26.0 & 24.8 & 4.6 & 25.0 \\
\hline 1,2-Dichlorobenzene & Ave & 1.267 & 1.332 & & 26.1 & 24.8 & 5.1 & 25.0 \\
\hline n-Butylbenzene & Ave & 2.058 & 2.146 & & 25.9 & 24.8 & 4.3 & 25.0 \\
\hline 1,2-Dibromo-3-Chloropropane & Ave & 0.2128 & 0.2339 & & 27.3 & 24.8 & 9.9 & 25.0 \\
\hline 1,2,4-Trichlorobenzene & Ave & 0.8142 & 0.8706 & & 26.5 & 24.8 & 6.9 & 25.0 \\
\hline Hexachlorobutadiene & Ave & 0.4720 & 0.4910 & & 25.8 & 24.8 & 4.0 & 25.0 \\
\hline Naphthalene & Ave & 1.611 & 1.697 & & 26.1 & 24.8 & 5.4 & 25.0 \\
\hline 1,2,3-Trichlorobenzene & Ave & 0.7424 & 0.7601 & & 25.4 & 24.8 & 2.4 & 25.0 \\
\hline Dibromofluoromethane (Surr) & Ave & 0.4780 & 0.5257 & & 27.5 & 25.0 & 10.0 & 25.0 \\
\hline 1,2-Dichloroethane-d4 & Ave & 0.2597 & 0.2731 & & 26.3 & 25.0 & 5.1 & 25.0 \\
\hline Toluene-d8 & Ave & 1.042 & 1.147 & & 27.5 & 25.0 & 10.1 & 25.0 \\
\hline Bromofluorobenzene & Ave & 1.182 & 1.276 & & 27.0 & 25.0 & 8.0 & 25.0 \\
\hline
\end{tabular}


FORM VII

GC/MS VOA CONTINUING CALIBRATION DATA

Lab Name: TestAmerica Burlington

SDG No.: 200-28914

Lab Sample ID: CCVIS 200-92051/2

Instrument ID: CHL.i

GC Column: DB-624

ID $: 0.53(\mathrm{~mm})$

Lab File ID: 15018_02.D

\begin{tabular}{|c|c|c|c|c|c|c|c|c|}
\hline ANALYTE & $\begin{array}{l}\text { CURVE } \\
\text { TYPE }\end{array}$ & AVE RRF & $\mathrm{RRF}$ & MIN RRF & $\begin{array}{c}\text { CALC } \\
\text { AMOUNT }\end{array}$ & $\begin{array}{c}\text { SPIKE } \\
\text { AMOUNT }\end{array}$ & $\div D$ & $\begin{array}{c}\mathrm{MAX} \\
\div \mathrm{D}\end{array}$ \\
\hline Dichlorodifluoromethane & Ave & 0.4431 & 0.4649 & & 26.0 & 24.8 & 4.9 & 20.0 \\
\hline Chloromethane & Ave & 0.3510 & 0.3382 & 0.1000 & 23.9 & 24.8 & -3.6 & 20.0 \\
\hline Vinyl chloride & Ave & 0.3578 & 0.3702 & & 25.7 & 24.8 & 3.5 & 20.0 \\
\hline Butadiene & Ave & 0.2626 & 0.2814 & & 26.6 & 24.8 & 7.1 & 20.0 \\
\hline Bromomethane & Ave & 0.2252 & 0.2297 & & 25.3 & 24.8 & 2.0 & 20.0 \\
\hline Chloroethane & Ave & 0.2148 & 0.2250 & & 26.0 & 24.8 & 4.8 & 20.0 \\
\hline Dichlorofluoromethane & Ave & 0.7405 & 0.7833 & & 26.3 & 24.8 & 5.8 & 20.0 \\
\hline Trichlorofluoromethane & Ave & 0.5469 & 0.5696 & & 25.8 & 24.8 & 4.2 & 20.0 \\
\hline Ethyl ether & Ave & 0.2032 & 0.2125 & & 26.0 & 24.8 & 4.6 & 20.0 \\
\hline Freon $123 a$ & Ave & 0.7833 & 0.8326 & & 26.4 & 24.8 & 6.3 & 20.0 \\
\hline Acrolein & Ave & 0.0149 & 0.0147 & & 123 & 124 & -0.7 & 20.0 \\
\hline 1,1-Dichloroethene & Ave & 0.3005 & 0.3209 & & 26.5 & 24.8 & 6.8 & 20.0 \\
\hline Freon TF & Ave & 0.6004 & 0.6474 & & 26.8 & 24.8 & 7.8 & 20.0 \\
\hline Acetone & Ave & 0.7123 & 0.4332 & & 75.5 & 124 & $-39.2 *$ & 20.0 \\
\hline Methyl iodide & Ave & 0.3509 & 0.3710 & & 22.9 & 24.8 & 5.7 & 20.0 \\
\hline Carbon disulfide & Ave & 0.8828 & 0.8989 & & 25.3 & 24.8 & 1.8 & 20.0 \\
\hline Allyl chloride & Ave & 0.5948 & 0.6638 & & 27.7 & 24.8 & 11.6 & 20.0 \\
\hline Methyl acetate & Ave & 0.2729 & 0.2962 & & 135 & 124 & 8.5 & 20.0 \\
\hline Methylene Chloride & Ave & 0.3199 & 0.3028 & & 23.5 & 24.8 & -5.4 & 20.0 \\
\hline tert-Butyl alcohol & Ave & 0.8582 & 0.9151 & & 265 & 248 & 6.6 & 20.0 \\
\hline Acrylonitrile & Lin1 & & 0.0722 & & 249 & 248 & 0.2 & 20.0 \\
\hline trans-1,2-Dichloroethene & Ave & 0.3149 & 0.3402 & & 26.8 & 24.8 & 8.0 & 20.0 \\
\hline Methyl t-butyl ether & Ave & 0.6475 & 0.6769 & & 25.9 & 24.8 & 4.5 & 20.0 \\
\hline Hexane & Ave & 0.4507 & 0.5072 & & 27.9 & 24.8 & 12.5 & 20.0 \\
\hline 1,1-Dichloroethane & Ave & 0.6431 & 0.6785 & 0.1000 & 26.2 & 24.8 & 5.5 & 20.0 \\
\hline Vinyl acetate & Ave & 0.0413 & 0.0631 & & 75.9 & 49.6 & $52.9 *$ & 20.0 \\
\hline Chloroprene & Ave & 0.4505 & 0.4818 & & 26.5 & 24.8 & 6.9 & 20.0 \\
\hline Isopropyl ether & Ave & 1.369 & 1.465 & & 26.6 & 24.8 & 7.0 & 20.0 \\
\hline Tert-butyl ethyl ether & Ave & 0.9268 & 0.9824 & & 26.3 & 24.8 & 6.0 & 20.0 \\
\hline 2,2-Dichloropropane & Ave & 0.3629 & 0.3663 & & 25.0 & 24.8 & 0.9 & 20.0 \\
\hline cis-1,2-Dichloroethene & Ave & 0.3178 & 0.3367 & & 26.3 & 24.8 & 6.0 & 20.0 \\
\hline 2-Butanone & Ave & 0.2050 & 0.1741 & & 105 & 124 & -15.1 & 20.0 \\
\hline Propionitrile & Ave & 0.0234 & 0.0245 & & 130 & 124 & 4.7 & 20.0 \\
\hline Methacrylonitrile & Ave & 0.0790 & 0.0822 & & 25.8 & 24.8 & 3.9 & 20.0 \\
\hline Bromochloromethane & Ave & 0.1783 & 0.1987 & & 27.7 & 24.8 & 11.5 & 20.0 \\
\hline Tetrahydrofuran & Ave & 0.0821 & 0.0872 & & 52.7 & 49.6 & 6.2 & 20.0 \\
\hline Chloroform & Ave & 0.5747 & 0.6140 & & 26.5 & 24.8 & 6.8 & 20.0 \\
\hline $1,1,1$-Trichloroethane & Ave & 0.4810 & 0.5155 & & 26.6 & 24.8 & 7.2 & 20.0 \\
\hline Cyclohexane & Ave & 0.5837 & 0.6324 & & 26.9 & 24.8 & 8.3 & 20.0 \\
\hline 1,1-Dichloropropene & Ave & 0.4622 & 0.5001 & & 26.8 & 24.8 & 8.2 & 20.0 \\
\hline Carbon tetrachloride & Ave & 0.4340 & 0.4720 & & 27.0 & 24.8 & 8.8 & 20.0 \\
\hline
\end{tabular}

Job No.: 200-28914-1

Calibration Date: 07/30/2015 08:59

Calib Start Date: 07/29/2015 12:33

Calib End Date: 07/29/2015 15:49

Conc. Units: ug/L Heated Purge: (Y/N) N 
FORM VII

GC/MS VOA CONTINUING CALIBRATION DATA

Lab Name: TestAmerica Burlington

SDG No.: 200-28914

Lab Sample ID: CCVIS 200-92051/2

Instrument ID: CHL.i

GC Column: DB-624

Lab File ID: 15018_02.D
Job No.: 200-28914-1

Calibration Date: 07/30/2015 08:59

Calib Start Date: 07/29/2015 12:33

Calib End Date: 07/29/2015 15:49

Conc. Units: ug/L Heated Purge: (Y/N) N

\begin{tabular}{|c|c|c|c|c|c|c|c|c|}
\hline ANALYTE & $\begin{array}{l}\text { CURVE } \\
\text { TYPE }\end{array}$ & AVE RRE & $\mathrm{RRF}$ & MIN RRF & $\begin{array}{c}\text { CALC } \\
\text { AMOUNT }\end{array}$ & $\begin{array}{c}\text { SPIKE } \\
\text { AMOUNT }\end{array}$ & $\because \mathrm{D}$ & $\begin{array}{l}\text { MAX } \\
\div D\end{array}$ \\
\hline Isobutyl alcohol & Ave & 0.0126 & 0.0127 & & 623 & 620 & 0.5 & 20.0 \\
\hline Benzene & Ave & 0.8972 & 0.9563 & & 26.4 & 24.8 & 6.6 & 20.0 \\
\hline 1,2-Dichloroethane & Ave & 0.3072 & 0.3278 & & 26.5 & 24.8 & 6.7 & 20.0 \\
\hline Tert-amyl methyl ether & Ave & 0.7515 & 0.7952 & & 26.3 & 24.8 & 5.8 & 20.0 \\
\hline $\mathrm{n}$-Heptane & Ave & 0.5318 & 0.6034 & & 28.2 & 24.8 & 13.4 & 20.0 \\
\hline Trichloroethene & Ave & 0.3792 & 0.3870 & & 25.3 & 24.8 & 2.0 & 20.0 \\
\hline Methylcyclohexane & Ave & 0.4683 & 0.5068 & & 26.9 & 24.8 & 8.2 & 20.0 \\
\hline 1,2-Dichloropropane & Ave & 0.3987 & 0.4175 & & 26.0 & 24.8 & 4.7 & 20.0 \\
\hline Dibromomethane & Ave & 0.2867 & 0.3019 & & 26.1 & 24.8 & 5.3 & 20.0 \\
\hline Methyl methacrylate & Ave & 0.3310 & 0.3448 & & 25.8 & 24.8 & 4.2 & 20.0 \\
\hline 1,4-Dioxane & Ave & 1.012 & 1.050 & & 515 & 496 & 3.7 & 20.0 \\
\hline Bromodichloromethane & Ave & 0.5802 & 0.6217 & & 26.6 & 24.8 & 7.1 & 20.0 \\
\hline 2-Chloroethyl vinyl ether & Ave & 0.1892 & 0.2012 & & 26.4 & 24.8 & 6.3 & 20.0 \\
\hline cis-1,3-Dichloropropene & Ave & 0.5088 & 0.5353 & & 26.1 & 24.8 & 5.2 & 20.0 \\
\hline 4-Methyl-2-pentanone & Ave & 2.574 & 2.676 & & 129 & 124 & 3.9 & 20.0 \\
\hline Toluene & Ave & 0.7227 & 0.7713 & & 26.5 & 24.8 & 6.7 & 20.0 \\
\hline trans-1,3-Dichloropropene & Ave & 0.5127 & 0.5281 & & 25.6 & 24.8 & 3.0 & 20.0 \\
\hline Ethyl methacrylate & Ave & 0.3022 & 0.3169 & & 26.0 & 24.8 & 4.9 & 20.0 \\
\hline 1,1,2-Trichloroethane & Ave & 0.3374 & 0.3605 & & 26.5 & 24.8 & 6.8 & 20.0 \\
\hline Tetrachloroethene & Ave & 0.5382 & 0.5783 & & 26.7 & 24.8 & 7.4 & 20.0 \\
\hline 1,3-Dichloropropane & Ave & 0.6438 & 0.6771 & & 26.1 & 24.8 & 5.2 & 20.0 \\
\hline 2-Hexanone & Ave & 2.054 & 1.796 & & 108 & 124 & -12.6 & 20.0 \\
\hline Dibromochloromethane & Ave & 0.6047 & 0.6400 & & 26.3 & 24.8 & 5.8 & 20.0 \\
\hline 1,2-Dibromoethane & Ave & 0.5478 & 0.5908 & & 26.8 & 24.8 & 7.8 & 20.0 \\
\hline Chlorobenzene & Ave & 0.9005 & 0.9570 & 0.3000 & 26.4 & 24.8 & 6.3 & 20.0 \\
\hline $1,1,1,2$-Tetrachloroethane & Ave & 0.4421 & 0.4712 & & 26.5 & 24.8 & 6.6 & 20.0 \\
\hline Ethylbenzene & Ave & 1.453 & 1.554 & & 26.5 & 24.8 & 7.0 & 20.0 \\
\hline $\mathrm{m} \& \mathrm{p}-\mathrm{Xylene}$ & Ave & 0.5277 & 0.5655 & & 26.6 & 24.8 & 7.2 & 20.0 \\
\hline o-Xylene & Ave & 0.5067 & 0.5430 & & 26.6 & 24.8 & 7.2 & 20.0 \\
\hline Styrene & Ave & 0.7455 & 0.8056 & & 26.8 & 24.8 & 8.1 & 20.0 \\
\hline Bromoform & Lin1 & & 0.4511 & 0.1000 & 23.6 & 24.8 & -4.7 & 20.0 \\
\hline Isopropylbenzene & Ave & 2.706 & 2.922 & & 26.8 & 24.8 & 8.0 & 20.0 \\
\hline Bromobenzene & Ave & 0.8141 & 0.8695 & & 26.5 & 24.8 & 6.8 & 20.0 \\
\hline $1,1,2,2$-Tetrachloroethane & Ave & 1.243 & 1.304 & 0.3000 & 26.0 & 24.8 & 4.9 & 20.0 \\
\hline 1,2,3-Trichloropropane & Ave & 0.2818 & 0.2968 & & 26.1 & 24.8 & 5.3 & 20.0 \\
\hline trans-1,4-Dichloro-2-butene & Ave & 0.2350 & 0.2541 & & 26.8 & 24.8 & 8.1 & 20.0 \\
\hline n-Propylbenzene & Ave & 0.6849 & 0.7475 & & 27.1 & 24.8 & 9.1 & 20.0 \\
\hline 2-Chlorotoluene & Ave & 0.6356 & 0.6824 & & 26.6 & 24.8 & 7.4 & 20.0 \\
\hline 1,3,5-Trimethylbenzene & Ave & 1.960 & 2.100 & & 26.6 & 24.8 & 7.1 & 20.0 \\
\hline 4-Chlorotoluene & Ave & 0.6517 & 0.6958 & & 26.5 & 24.8 & 6.8 & 20.0 \\
\hline tert-Butylbenzene & Ave & 2.134 & 2.308 & & 26.8 & 24.8 & 8.1 & 20.0 \\
\hline
\end{tabular}


FORM VII

GC/MS VOA CONTINUING CALIBRATION DATA

Lab Name: TestAmerica Burlington

SDG No.: 200-28914

Lab Sample ID: CCVIS 200-92051/2

Instrument ID: CHL.i

GC Column: DB-624

ID $: 0.53(\mathrm{~mm})$

Lab File ID: 15018 02.D
Job No.: 200-28914-1

Calibration Date: 07/30/2015 08:59

Calib Start Date: 07/29/2015 12:33

Calib End Date: 07/29/2015 15:49

Conc. Units: ug/L Heated Purge: (Y/N) N

\begin{tabular}{|c|c|c|c|c|c|c|c|c|}
\hline ANALYTE & $\begin{array}{l}\text { CURVE } \\
\text { TYPE }\end{array}$ & AVE RRF & $\mathrm{RRF}$ & MIN RRF & $\begin{array}{c}\text { CALC } \\
\text { AMOUNT }\end{array}$ & $\begin{array}{c}\text { SPIKE } \\
\text { AMOUNT }\end{array}$ & $\div D$ & $\begin{array}{l}\mathrm{MAX} \\
\div \mathrm{D}\end{array}$ \\
\hline $1,2,4$-Trimethylbenzene & Ave & 1.955 & 2.100 & & 26.7 & 24.8 & 7.4 & 20.0 \\
\hline sec-Butylbenzene & Ave & 2.977 & 3.235 & & 27.0 & 24.8 & 8.7 & 20.0 \\
\hline 1,3-Dichlorobenzene & Ave & 1.343 & 1.407 & & 26.0 & 24.8 & 4.8 & 20.0 \\
\hline 4-Isopropyltoluene & Ave & 2.311 & 2.510 & & 27.0 & 24.8 & 8.6 & 20.0 \\
\hline 1,4-Dichlorobenzene & Ave & 1.455 & 1.538 & & 26.2 & 24.8 & 5.7 & 20.0 \\
\hline 1,2-Dichlorobenzene & Ave & 1.267 & 1.314 & & 25.7 & 24.8 & 3.7 & 20.0 \\
\hline n-Butylbenzene & Ave & 2.058 & 2.254 & & 27.2 & 24.8 & 9.5 & 20.0 \\
\hline 1,2-Dibromo-3-Chloropropane & Ave & 0.2128 & 0.2239 & & 26.1 & 24.8 & 5.2 & 20.0 \\
\hline $1,2,4$-Trichlorobenzene & Ave & 0.8142 & 0.8767 & & 26.7 & 24.8 & 7.7 & 20.0 \\
\hline Hexachlorobutadiene & Ave & 0.4720 & 0.4974 & & 26.2 & 24.8 & 5.4 & 20.0 \\
\hline Naphthalene & Ave & 1.611 & 1.664 & & 25.6 & 24.8 & 3.3 & 20.0 \\
\hline $1,2,3$-Trichlorobenzene & Ave & 0.7424 & 0.7542 & & 25.2 & 24.8 & 1.6 & 20.0 \\
\hline Dibromofluoromethane (Surr) & Ave & 0.4780 & 0.5150 & & 26.7 & 24.8 & 7.7 & 20.0 \\
\hline 1,2-Dichloroethane-d4 & Ave & 0.2597 & 0.2667 & & 25.5 & 24.8 & 2.7 & 20.0 \\
\hline Toluene-d8 & Ave & 1.042 & 1.115 & & 26.6 & 24.8 & 7.0 & 20.0 \\
\hline Bromofluorobenzene & Ave & 1.182 & 1.272 & & 26.7 & 24.8 & 7.6 & 20.0 \\
\hline
\end{tabular}


FORM I

GC/MS VOA ORGANICS ANALYSIS DATA SHEET

Lab Name: TestAmerica Burlington

Job No : : 200-28914-1

SDG No.: 200-28914

Client Sample ID:

Matrix: Solid

Analysis Method: 8260B

Sample wt/vol: $10(\mathrm{~g})$

Soil Aliquot Vol: 500 (uL)

Soil Extract Vol.: $10(\mathrm{~mL})$

Moisture:

Analysis Batch No.: 92051

Lab Sample ID: MB 200-91708/1-A

Lab File ID: 15018_05.D

Date Collected:

Date Analyzed: 07/30/2015 10:36

Dilution Factor: 1

GC Column: DB-624

ID : $0.53(\mathrm{~mm})$

Level: (low/med) Medium

Units: ug/Kg

\begin{tabular}{|c|c|c|c|c|c|}
\hline CAS NO. & COMPOUND NAME & RESULT & $Q$ & RL & MDL \\
\hline $75-71-8$ & Dichlorodifluoromethane & 10 & $\mathrm{U}$ & 10 & 1.7 \\
\hline $74-87-3$ & Chloromethane & 11.0 & & 10 & 2.1 \\
\hline $75-01-4$ & Vinyl chloride & 10 & $\mathrm{U}$ & 10 & 0.83 \\
\hline $74-83-9$ & Bromomethane & 25.5 & & 10 & 1.0 \\
\hline $75-00-3$ & Chloroethane & 10 & $\mathrm{U}$ & 10 & 2.5 \\
\hline $75-69-4$ & Trichlorofluoromethane & 10 & $\mathrm{U}$ & 10 & 0.82 \\
\hline $75-35-4$ & 1,1-Dichloroethene & 10 & $\mathrm{U}$ & 10 & 1.1 \\
\hline $76-13-1$ & Freon TF & 10 & $\mathrm{U}$ & 10 & 0.85 \\
\hline $67-64-1$ & Acetone & 50 & $\mathrm{U}$ & 50 & 12 \\
\hline $74-88-4$ & Methyl iodide & 12.3 & & 10 & 0.75 \\
\hline $75-15-0$ & Carbon disulfide & 10 & $\mathrm{U}$ & 10 & 0.87 \\
\hline $79-20-9$ & Methyl acetate & 50 & $\mathrm{U}$ & 50 & 9.2 \\
\hline $75-09-2$ & Methylene Chloride & 10 & $\mathrm{U}$ & 10 & 2.3 \\
\hline $156-60-5$ & trans-1,2-Dichloroethene & 10 & $\mathrm{U}$ & 10 & 0.99 \\
\hline $1634-04-4$ & Methyl t-butyl ether & 10 & $\mathrm{U}$ & 10 & 1.1 \\
\hline $75-34-3$ & 1,1-Dichloroethane & 10 & $\mathrm{U}$ & 10 & 1.1 \\
\hline $108-05-4$ & Vinyl acetate & 50 & $\mathrm{U}$ & 50 & 4.5 \\
\hline $594-20-7$ & 2,2-Dichloropropane & 10 & $\mathrm{U}$ & 10 & 2.2 \\
\hline $156-59-2$ & cis-1,2-Dichloroethene & 10 & $\mathrm{U}$ & 10 & 1.0 \\
\hline $78-93-3$ & 2-Butanone & 50 & $\mathrm{U}$ & 50 & 5.9 \\
\hline $74-97-5$ & Bromochloromethane & 10 & $\mathrm{U}$ & 10 & 1.4 \\
\hline $109-99-9$ & Tetrahydrofuran & 20 & $\mathrm{U}$ & 20 & 5.3 \\
\hline $67-66-3$ & Chloroform & 10 & $\mathrm{U}$ & 10 & 1.2 \\
\hline $71-55-6$ & $1,1,1$-Trichloroethane & 10 & $\mathrm{U}$ & 10 & 0.85 \\
\hline $110-82-7$ & Cyclohexane & 10 & $\mathrm{U}$ & 10 & 1.0 \\
\hline $563-58-6$ & 1,1-Dichloropropene & 10 & $\mathrm{U}$ & 10 & 0.97 \\
\hline $56-23-5$ & Carbon tetrachloride & 10 & $\mathrm{U}$ & 10 & 2.0 \\
\hline $78-83-1$ & Isobutyl alcohol & 500 & $\mathrm{U}$ & 500 & 27 \\
\hline $71-43-2$ & Benzene & 10 & $\mathrm{U}$ & 10 & 1.1 \\
\hline $107-06-2$ & 1,2-Dichloroethane & 10 & $\mathrm{U}$ & 10 & 1.3 \\
\hline $79-01-6$ & Trichloroethene & 10 & $\mathrm{U}$ & 10 & 0.83 \\
\hline $108-87-2$ & Methylcyclohexane & 10 & $\mathrm{U}$ & 10 & 2.1 \\
\hline $78-87-5$ & 1,2-Dichloropropane & 10 & $\mathrm{U}$ & 10 & 1.2 \\
\hline $74-95-3$ & Dibromomethane & 10 & $\mathrm{U}$ & 10 & 1.5 \\
\hline $123-91-1$ & 1,4-Dioxane & 400 & $\mathrm{U}$ & 400 & 65 \\
\hline $75-27-4$ & Bromodichloromethane & 10 & $\mathrm{U}$ & 10 & 1.0 \\
\hline
\end{tabular}

FORM I $8260 B$ 
FORM I

GC/MS VOA ORGANICS ANALYSIS DATA SHEET

Lab Name: TestAmerica Burlington

SDG No.: 200-28914

Client Sample ID:

Matrix: Solid

Analysis Method: 8260B

Sample wt/vol: $10(\mathrm{~g})$

Soil Aliquot Vol: 500 (uL)

Soil Extract Vol.: $10(\mathrm{~mL})$

응 Moisture:

Analysis Batch No.: 92051
Job No. : 200-28914-1

Lab Sample ID: MB 200-91708/1-A

Lab File ID: 15018_05.D

Date Collected:

Date Analyzed: 07/30/2015 10:36

Dilution Factor: 1

GC Column: DB-624

ID $: 0.53(\mathrm{~mm})$

Level: (low/med) Medium

Units: ug/Kg

\begin{tabular}{|c|c|c|c|c|c|}
\hline CAS NO. & COMPOUND NAME & RESULT & $Q$ & RL & MDL \\
\hline $110-75-8$ & 2-Chloroethyl vinyl ether & 10 & $\mathrm{U}$ & 10 & 1.9 \\
\hline $10061-01-5$ & cis-1,3-Dichloropropene & 10 & $\mathrm{U}$ & 10 & 1.2 \\
\hline $108-10-1$ & 4-Methyl-2-pentanone & 50 & $\mathrm{U}$ & 50 & 4.7 \\
\hline $108-88-3$ & Toluene & 10 & $\mathrm{U}$ & 10 & 2.1 \\
\hline $10061-02-6$ & trans-1,3-Dichloropropene & 10 & $\mathrm{U}$ & 10 & 2.2 \\
\hline $79-00-5$ & 1,1,2-Trichloroethane & 10 & $\mathrm{U}$ & 10 & 1.3 \\
\hline $127-18-4$ & Tetrachloroethene & 10 & $\mathrm{U}$ & 10 & 2.0 \\
\hline $142-28-9$ & 1,3-Dichloropropane & 10 & $\mathrm{U}$ & 10 & 1.1 \\
\hline $591-78-6$ & 2-Hexanone & 50 & $\mathrm{U}$ & 50 & 6.1 \\
\hline $124-48-1$ & Dibromochloromethane & 10 & $\mathrm{U}$ & 10 & 1.1 \\
\hline $106-93-4$ & 1,2-Dibromoethane & 10 & $\mathrm{U}$ & 10 & 1.2 \\
\hline $108-90-7$ & Chlorobenzene & 10 & $\mathrm{U}$ & 10 & 0.91 \\
\hline $630-20-6$ & $1,1,1,2$-Tetrachloroethane & 10 & $\mathrm{U}$ & 10 & 1.1 \\
\hline $100-41-4$ & Ethylbenzene & 1.02 & $\mathrm{~J}$ & 10 & 0.94 \\
\hline $179601-23-1$ & $m \& p-X y l e n e$ & 10 & $\mathrm{U}$ & 10 & 1.1 \\
\hline $95-47-6$ & o-Xylene & 10 & $\mathrm{U}$ & 10 & 1.0 \\
\hline $100-42-5$ & Styrene & 1.58 & $\mathrm{~J}$ & 10 & 0.98 \\
\hline $75-25-2$ & Bromoform & 10 & $\mathrm{U}$ & 10 & 1.0 \\
\hline $98-82-8$ & Isopropylbenzene & 10 & $\mathrm{U}$ & 10 & 2.0 \\
\hline $108-86-1$ & Bromobenzene & 1.82 & $\mathrm{~J}$ & 10 & 1.0 \\
\hline $79-34-5$ & $1,1,2,2$-Tetrachloroethane & 10 & $\mathrm{U}$ & 10 & 1.3 \\
\hline $96-18-4$ & 1,2,3-Trichloropropane & 10 & $\mathrm{U}$ & 10 & 1.2 \\
\hline $103-65-1$ & n-Propylbenzene & 1.33 & $\mathrm{~J}$ & 10 & 1.1 \\
\hline $95-49-8$ & 2-Chlorotoluene & 10 & $\mathrm{U}$ & 10 & 1.1 \\
\hline $106-43-4$ & 4-Chlorotoluene & 1.69 & $\mathrm{~J}$ & 10 & 0.96 \\
\hline $108-67-8$ & 1,3,5-Trimethylbenzene & 10 & $\mathrm{U}$ & 10 & 2.0 \\
\hline $98-06-6$ & tert-Butylbenzene & 1.59 & $\mathrm{~J}$ & 10 & 1.4 \\
\hline $95-63-6$ & $1,2,4$-Trimethylbenzene & 1.92 & $\mathrm{~J}$ & 10 & 1.0 \\
\hline $135-98-8$ & sec-Butylbenzene & 10 & $\mathrm{U}$ & 10 & 2.0 \\
\hline $541-73-1$ & 1,3-Dichlorobenzene & 2.72 & $\mathrm{~J}$ & 10 & 0.95 \\
\hline $99-87-6$ & 4-Isopropyltoluene & 10 & $\mathrm{U}$ & 10 & 0.88 \\
\hline $106-46-7$ & 1,4-Dichlorobenzene & 10 & $\mathrm{U}$ & 10 & 1.4 \\
\hline $95-50-1$ & 1,2-Dichlorobenzene & 2.87 & $\mathrm{~J}$ & 10 & 1.1 \\
\hline $104-51-8$ & n-Butylbenzene & 2.63 & J & 10 & 0.90 \\
\hline $96-12-8$ & 1,2-Dibromo-3-Chloropropane & 10 & $\mathrm{U}$ & 10 & 2.1 \\
\hline $120-82-1$ & 1,2,4-Trichlorobenzene & 4.97 & $\mathrm{~J}$ & 10 & 0.91 \\
\hline
\end{tabular}

FORM I $8260 B$ 
FORM I

GC/MS VOA ORGANICS ANALYSIS DATA SHEET

Lab Name: TestAmerica Burlington

SDG No.: 200-28914

Client Sample ID:

Matrix: Solid

Analysis Method: 8260B

Sample wt/vol: $10(\mathrm{~g})$

Soil Aliquot Vol: 500 (uL)

Soil Extract Vol.: $10(\mathrm{~mL})$

응 Moisture:

Analysis Batch No.: 92051
Job No. : 200-28914-1

Lab Sample ID: MB 200-91708/1-A

Lab File ID: 15018_05.D

Date Collected:

Date Analyzed: 07/30/2015 10:36

Dilution Factor: 1

GC Column: DB-624

ID $: 0.53(\mathrm{~mm})$

Level: (low/med) Medium

Units: ug/Kg

\begin{tabular}{|l|l|r|r|r|r|}
\hline \multicolumn{1}{|c|}{ CAS NO. } & \multicolumn{1}{|c|}{ COMPOUND NAME } & RESULT & Q & RL & MDL \\
\hline $87-68-3$ & Hexachlorobutadiene & 5.43 & $\mathrm{~J}$ & & 10 \\
\hline $91-20-3$ & Naphthalene & 5.24 & $\mathrm{~J}$ & 2.3 \\
\hline $87-61-6$ & $1,2,3-$ Trichlorobenzene & 4.90 & $\mathrm{~J}$ & 10 & 1.2 \\
\hline
\end{tabular}

\begin{tabular}{|l|l|r|r|r|}
\hline \multicolumn{1}{|c|}{ CAS NO. } & \multicolumn{1}{|c|}{ SURROGATE } & \%REC & Q & LIMITS \\
\hline $17060-07-0$ & $1,2-$ Dichloroethane-d4 & 100 & $80-120$ \\
\hline $2037-26-5$ & Toluene-d8 & 110 & $80-120$ \\
\hline $460-00-4$ & Bromofluorobenzene & 112 & $80-125$ \\
\hline $1868-53-7$ & Dibromofluoromethane (Surr) & 97 & $50-150$ \\
\hline
\end{tabular}


FORM I

GC/MS VOA ORGANICS ANALYSIS DATA SHEET

Lab Name: TestAmerica Burlington

Job No.: 200-28914-1

SDG No.: 200-28914

Client Sample ID:

Matrix: Solid

Analysis Method: 8260B

Sample wt/vol: $10(\mathrm{~g})$

Soil Aliquot Vol: 500 (uL)

Soil Extract Vol.: $10(\mathrm{~mL})$

Moisture:

Analysis Batch No.: 92051

Lab Sample ID: LCS 200-91708/2-A

Lab File ID: 15018_03.D

Date Collected:

Date Analyzed: 07/30/2015 09:31

Dilution Factor: 1

GC Column: DB-624

ID : $0.53(\mathrm{~mm})$

Level: (low/med) Medium

Units: ug/Kg

\begin{tabular}{|c|c|c|c|c|c|}
\hline CAS NO. & COMPOUND NAME & RESULT & $Q$ & RL & MDL \\
\hline $75-71-8$ & Dichlorodifluoromethane & 281 & & 10 & 1.7 \\
\hline $74-87-3$ & Chloromethane & 284 & & 10 & 2.1 \\
\hline $75-01-4$ & Vinyl chloride & 272 & & 10 & 0.83 \\
\hline $74-83-9$ & Bromomethane & 144 & & 10 & 1.0 \\
\hline $75-00-3$ & Chloroethane & 96.9 & & 10 & 2.5 \\
\hline $75-69-4$ & Trichlorofluoromethane & 111 & & 10 & 0.82 \\
\hline $75-35-4$ & 1,1-Dichloroethene & 97.1 & & 10 & 1.1 \\
\hline $76-13-1$ & Freon TF & 109 & & 10 & 0.85 \\
\hline $67-64-1$ & Acetone & 294 & & 50 & 12 \\
\hline $74-88-4$ & Methyl iodide & 53.2 & & 10 & 0.75 \\
\hline $75-15-0$ & Carbon disulfide & 90.3 & & 10 & 0.87 \\
\hline $79-20-9$ & Methyl acetate & 1850 & & 50 & 9.2 \\
\hline $75-09-2$ & Methylene Chloride & 233 & & 10 & 2.3 \\
\hline $156-60-5$ & trans-1,2-Dichloroethene & 277 & & 10 & 0.99 \\
\hline $1634-04-4$ & Methyl t-butyl ether & 251 & & 10 & 1.1 \\
\hline $75-34-3$ & 1,1-Dichloroethane & 203 & & 10 & 1.1 \\
\hline $108-05-4$ & Vinyl acetate & 457 & & 50 & 4.5 \\
\hline $594-20-7$ & 2,2-Dichloropropane & 402 & & 10 & 2.2 \\
\hline $156-59-2$ & cis-1,2-Dichloroethene & 276 & & 10 & 1.0 \\
\hline $78-93-3$ & 2-Butanone & 1650 & & 50 & 5.9 \\
\hline $74-97-5$ & Bromochloromethane & 239 & & 10 & 1.4 \\
\hline $109-99-9$ & Tetrahydrofuran & 298 & & 20 & 5.3 \\
\hline $67-66-3$ & Chloroform & 247 & & 10 & 1.2 \\
\hline $71-55-6$ & 1,1,1-Trichloroethane & 259 & & 10 & 0.85 \\
\hline $110-82-7$ & Cyclohexane & 252 & & 10 & 1.0 \\
\hline $563-58-6$ & 1,1-Dichloropropene & 283 & & 10 & 0.97 \\
\hline $56-23-5$ & Carbon tetrachloride & 265 & & 10 & 2.0 \\
\hline $78-83-1$ & Isobutyl alcohol & 1140 & & 500 & 27 \\
\hline $71-43-2$ & Benzene & 261 & & 10 & 1.1 \\
\hline $107-06-2$ & 1,2-Dichloroethane & 250 & & 10 & 1.3 \\
\hline $79-01-6$ & Trichloroethene & 256 & & 10 & 0.83 \\
\hline $108-87-2$ & Methylcyclohexane & 259 & & 10 & 2.1 \\
\hline $78-87-5$ & 1,2-Dichloropropane & 257 & & 10 & 1.2 \\
\hline $74-95-3$ & Dibromomethane & 211 & & 10 & 1.5 \\
\hline $123-91-1$ & 1,4-Dioxane & 5560 & & 400 & 65 \\
\hline $75-27-4$ & Bromodichloromethane & 254 & & 10 & 1.0 \\
\hline
\end{tabular}

FORM I $8260 B$ 
FORM I

GC/MS VOA ORGANICS ANALYSIS DATA SHEET

Lab Name: TestAmerica Burlington

Job No.: 200-28914-1

SDG No.: 200-28914

Client Sample ID:

Matrix: Solid

Analysis Method: 8260B

Sample wt/vol: $10(\mathrm{~g})$

Soil Aliquot Vol: 500 (uL)

Soil Extract Vol.: $10(\mathrm{~mL})$

Moisture:

Analysis Batch No.: 92051

Lab Sample ID: LCS 200-91708/2-A

Lab File ID: 15018_03.D

Date Collected:

Date Analyzed: 07/30/2015 09:31

Dilution Factor: 1

GC Column: DB-624

ID : $0.53(\mathrm{~mm})$

Level: (low/med) Medium

Units: ug/Kg

\begin{tabular}{|c|c|c|c|c|c|}
\hline CAS NO. & COMPOUND NAME & RESULT & $Q$ & RL & MDL \\
\hline $110-75-8$ & 2-Chloroethyl vinyl ether & 302 & & 10 & 1.9 \\
\hline $10061-01-5$ & cis-1,3-Dichloropropene & 250 & & 10 & 1.2 \\
\hline $108-10-1$ & 4-Methyl-2-pentanone & 1780 & & 50 & 4.7 \\
\hline $108-88-3$ & Toluene & 266 & & 10 & 2.1 \\
\hline $10061-02-6$ & trans-1,3-Dichloropropene & 274 & & 10 & 2.2 \\
\hline $79-00-5$ & $1,1,2$-Trichloroethane & 260 & & 10 & 1.3 \\
\hline $127-18-4$ & Tetrachloroethene & 268 & & 10 & 2.0 \\
\hline $142-28-9$ & 1,3-Dichloropropane & 258 & & 10 & 1.1 \\
\hline $591-78-6$ & 2-Hexanone & 1650 & & 50 & 6.1 \\
\hline $124-48-1$ & Dibromochloromethane & 258 & & 10 & 1.1 \\
\hline $106-93-4$ & 1,2-Dibromoethane & 264 & & 10 & 1.2 \\
\hline $108-90-7$ & Chlorobenzene & 262 & & 10 & 0.91 \\
\hline $630-20-6$ & $1,1,1,2$-Tetrachloroethane & 268 & & 10 & 1.1 \\
\hline $100-41-4$ & Ethylbenzene & 262 & & 10 & 0.94 \\
\hline $179601-23-1$ & $\mathrm{~m} \& \mathrm{p}-\mathrm{Xy}$ lene & 266 & & 10 & 1.1 \\
\hline $95-47-6$ & o-Xylene & 264 & & 10 & 1.0 \\
\hline $100-42-5$ & styrene & 255 & & 10 & 0.98 \\
\hline $75-25-2$ & Bromoform & 224 & & 10 & 1.0 \\
\hline $98-82-8$ & Isopropylbenzene & 270 & & 10 & 2.0 \\
\hline $108-86-1$ & Bromobenzene & 270 & & 10 & 1.0 \\
\hline $79-34-5$ & $1,1,2,2$-Tetrachloroethane & 238 & & 10 & 1.3 \\
\hline $96-18-4$ & 1,2,3-Trichloropropane & 247 & & 10 & 1.2 \\
\hline $103-65-1$ & n-Propylbenzene & 272 & & 10 & 1.1 \\
\hline $95-49-8$ & 2-Chlorotoluene & 263 & & 10 & 1.1 \\
\hline $106-43-4$ & 4-Chlorotoluene & 264 & & 10 & 0.96 \\
\hline $108-67-8$ & 1,3,5-Trimethylbenzene & 269 & & 10 & 2.0 \\
\hline $98-06-6$ & tert-Butylbenzene & 273 & & 10 & 1.4 \\
\hline $95-63-6$ & $1,2,4$-Trimethylbenzene & 265 & & 10 & 1.0 \\
\hline $135-98-8$ & sec-Butylbenzene & 279 & & 10 & 2.0 \\
\hline $541-73-1$ & 1,3-Dichlorobenzene & 267 & & 10 & 0.95 \\
\hline $99-87-6$ & 4-Isopropyltoluene & 277 & & 10 & 0.88 \\
\hline $106-46-7$ & 1,4-Dichlorobenzene & 264 & & 10 & 1.4 \\
\hline $95-50-1$ & 1,2-Dichlorobenzene & 261 & & 10 & 1.1 \\
\hline $104-51-8$ & n-Butylbenzene & 287 & & 10 & 0.90 \\
\hline $96-12-8$ & 1,2-Dibromo-3-Chloropropane & 211 & & 10 & 2.1 \\
\hline $120-82-1$ & $1,2,4$-Trichlorobenzene & 278 & & 10 & 0.91 \\
\hline
\end{tabular}

FORM I $8260 B$ 
FORM I

GC/MS VOA ORGANICS ANALYSIS DATA SHEET

Lab Name: TestAmerica Burlington

SDG No.: 200-28914

Client Sample ID:

Matrix: Solid

Analysis Method: 8260B

Sample wt/vol: $10(\mathrm{~g})$

Soil Aliquot Vol: 500 (uL)

Soil Extract Vol.: 10 (mL)

Moisture:

Analysis Batch No.: 92051
Job No. : 200-28914-1

Lab Sample ID: LCS 200-91708/2-A

Lab File ID: 15018_03.D

Date Collected:

Date Analyzed: 07/30/2015 09:31

Dilution Factor: 1

GC Column: DB-624

ID $: 0.53(\mathrm{~mm})$

Level: (low/med) Medium

Units: ug/Kg

\begin{tabular}{|l|l|r|r|r|}
\hline \multicolumn{1}{|c|}{ CAS NO. } & \multicolumn{1}{|c|}{ COMPOUND NAME } & RESULT & Q & RL \\
\hline $87-68-3$ & Hexachlorobutadiene & 309 & & 10 \\
\hline $91-20-3$ & Naphthalene & 195 & & 2.3 \\
\hline $87-61-6$ & $1,2,3-$ Trichlorobenzene & 236 & & 10 \\
\hline
\end{tabular}

\begin{tabular}{|c|c|c|c|c|}
\hline CAS NO. & SURROGATE & $\because \mathrm{REC}$ & Q & LIMITS \\
\hline $17060-07-0$ & 1,2-Dichloroethane-d4 & 107 & & $80-120$ \\
\hline $2037-26-5$ & Toluene-d8 & 111 & & $80-120$ \\
\hline $460-00-4$ & Bromofluorobenzene & 111 & & $80-125$ \\
\hline $1868-53-7$ & Dibromofluoromethane (Surr) & 101 & & $50-150$ \\
\hline
\end{tabular}


GC/MS VOA ANALYSIS RUN LOG

Lab Name: TestAmerica Burlington

SDG No.: 200-28914

Instrument ID: CHL.i

Analysis Batch Number: 92007
Job No.: 200-28914-1

Start Date: 07/29/2015 11:39

End Date: 07/29/2015 18:31

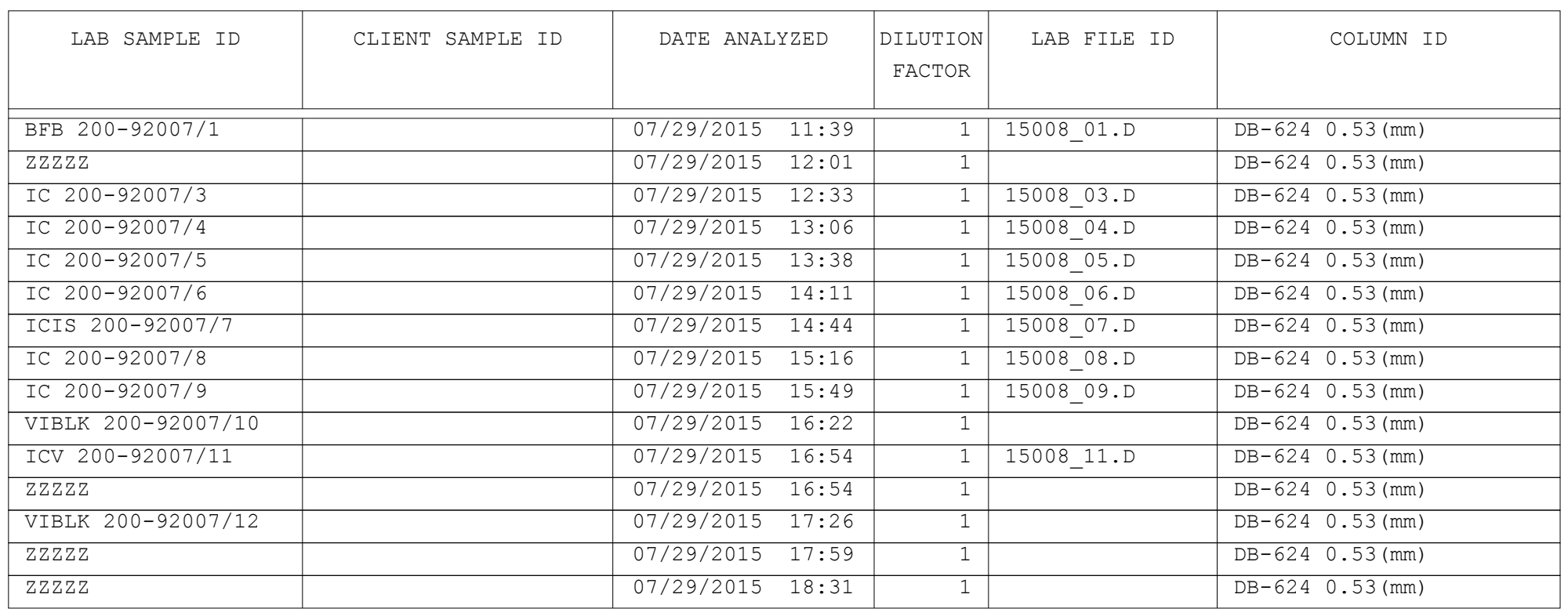


GC/MS VOA ANALYSIS RUN LOG

Lab Name: TestAmerica Burlington

SDG No.: 200-28914

Instrument ID: CHL.i

Analysis Batch Number: 92051
Job No.: 200-28914-1

Start Date: 07/30/2015 08:36

End Date: 07/30/2015 17:36

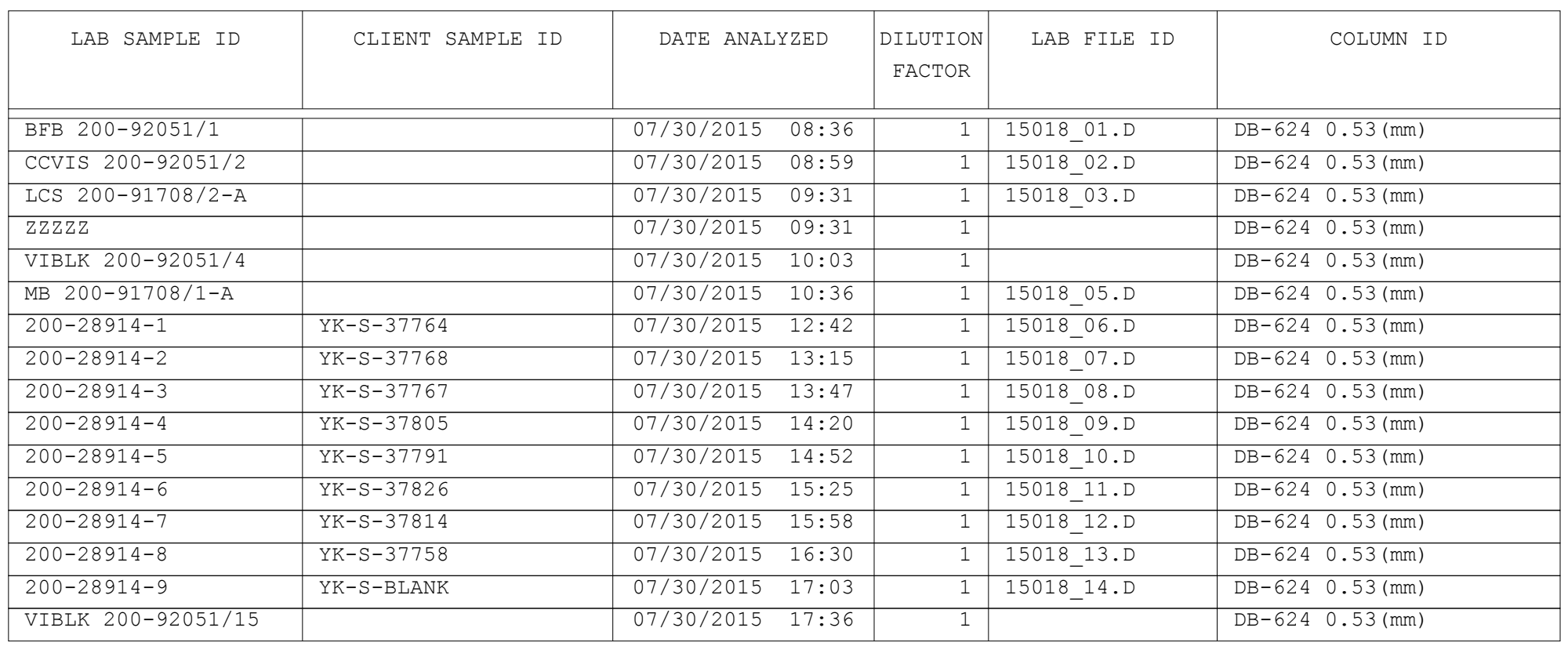


Lab Name: TestAmerica Burlington

Job No.: 200-28914-1

SDG No.: 200-28914

Batch Number: 91708

Batch Start Date: 07/23/15 11:00

Batch Analyst: Tober, Elizabeth R

Batch Method: 5035

Batch End Date: 07/23/15 11:15

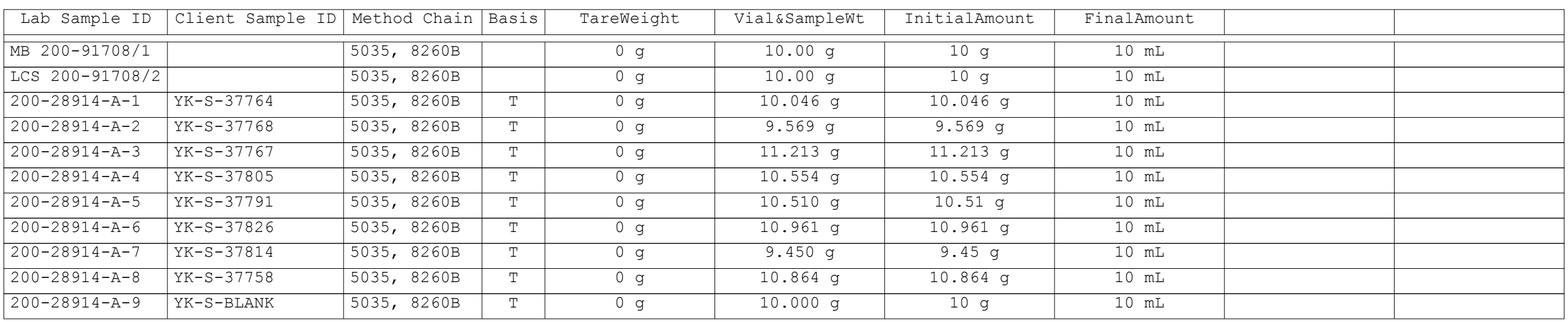

\begin{tabular}{|l|l|}
\hline Batch Notes \\
\hline \hline & \\
\hline
\end{tabular}

\begin{tabular}{|c|l|}
\hline Basis & \multicolumn{1}{|c|}{ Basis Description } \\
\hline T & Total/NA \\
\hline
\end{tabular}

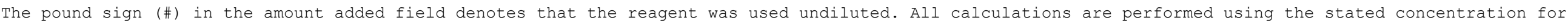
this reagent. 


\section{Shipping and Receiving Documents}




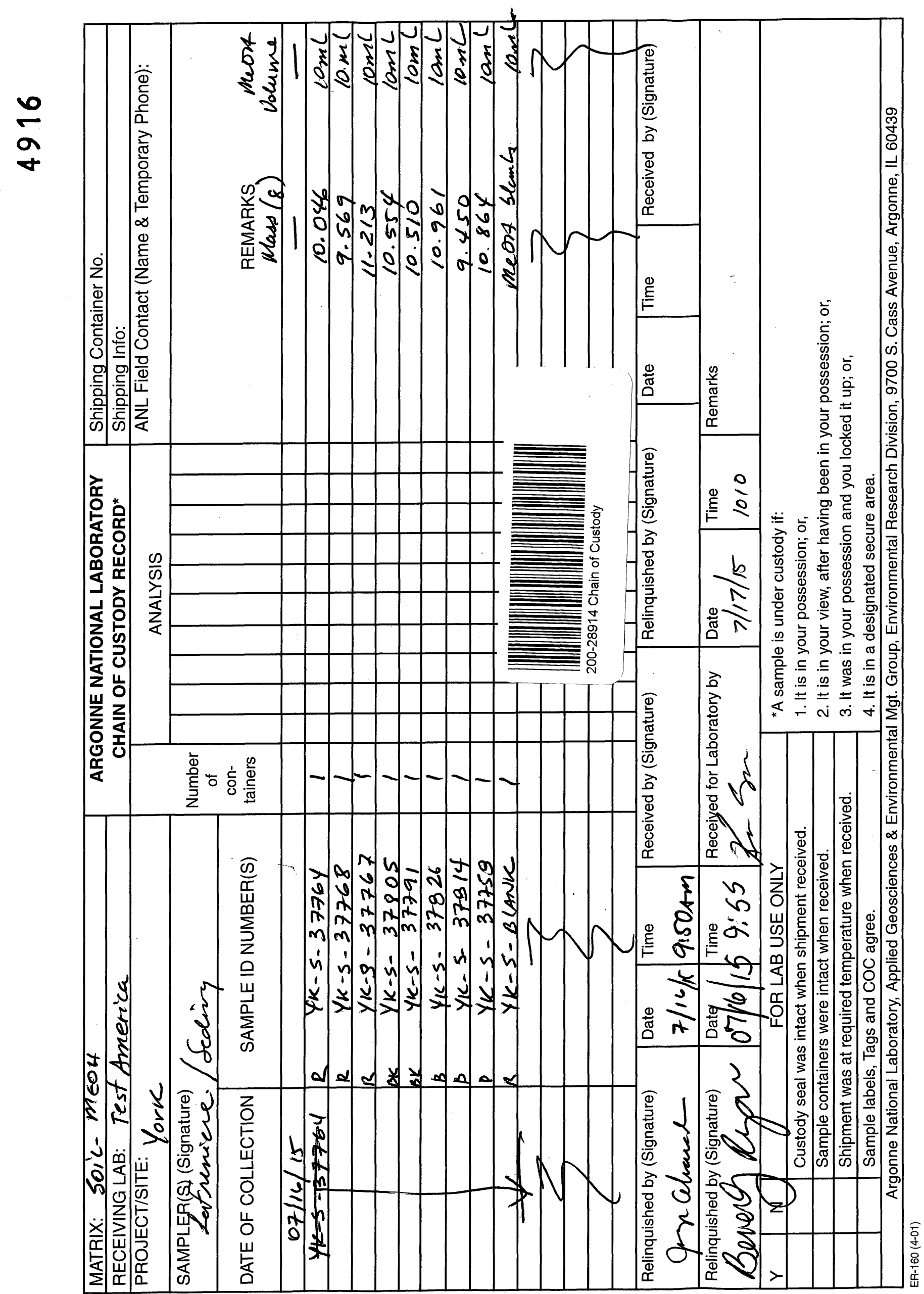




\section{Login Sample Receipt Checklist}

Client: Argonne National Laboratory

Job Number: 200-28914-1

SDG Number: 200-28914

Login Number: 28914

List Source: TestAmerica Burlington

List Number: 1

Creator: Callahan, Christopher G

Question

Radioactivity wasn't checked or is $</=$ background as measured by a survey True meter.

The cooler's custody seal, if present, is intact.

Sample custody seals, if present, are intact.

The cooler or samples do not appear to have been compromised or tampered with.

Samples were received on ice.

Cooler Temperature is acceptable.

Cooler Temperature is recorded.

$\mathrm{COC}$ is present.

$\mathrm{COC}$ is filled out in ink and legible.

$\mathrm{COC}$ is filled out with all pertinent information.

Is the Field Sampler's name present on COC?

There are no discrepancies between the containers received and the COC.

Samples are received within Holding Time.

Sample containers have legible labels.

Containers are not broken or leaking.

Sample collection date/times are provided.

Appropriate sample containers are used.

Sample bottles are completely filled.

Sample Preservation Verified.

There is sufficient vol. for all requested analyses, incl. any requested MS/MSDs

Containers requiring zero headspace have no headspace or bubble is $<6 \mathrm{~mm}$ (1/4").

Multiphasic samples are not present.

Samples do not require splitting or compositing.

Residual Chlorine Checked.
True

True

True

True

False

True

True

True

False

True

True

True

True

True

True

True

N/A

True

True

True

True

True

N/A

Answer Comment




\section{TestAmerica}

THE LEADER IN ENVIRONMENTAL TESTING

\section{ANALYTICAL REPORT}

Job Number: 200-28963-1

SDG Number: 28963

Job Description: York (200-28963)

Contract Number: 1E-30401

For:

Argonne National Laboratory

9700 South Cass Avenue

Building 203

Office B-141

Argonne, IL 60439

Attention: Ms. Esther Bowen

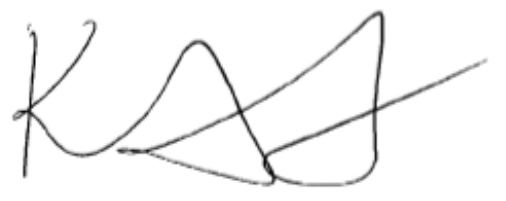

Approved for release. Kathryn A Kelly

\section{Kathryn A Kelly, Project Manager I \\ 30 Community Drive, South Burlington, VT, 05403 \\ kathryn.kelly@testamericainc.com \\ $08 / 10 / 2015$}

The test results in this report relate only to sample(s) as received by the laboratory. These test results were derived under a quality system that adheres to the requirements of NELAC. Pursuant to NELAC, this report may not be produced in full without written approval from the laboratory 


\section{Table of Contents}

Cover Title Page . . . . . . . . . . . . . . . . . 1

Report Narrative . . . . . . . . . . . . . . . 4

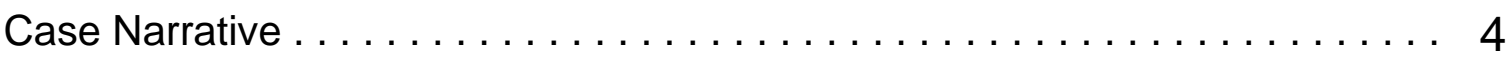

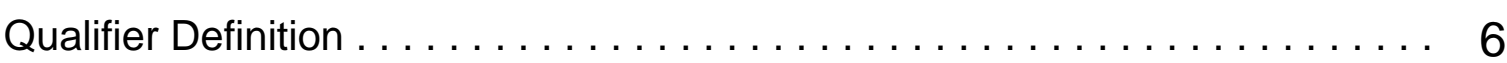

Sample List . . . . . . . . . . . . . . . . . 7

External Chain of Custody . . . . . . . . . . . . . . 8

Shipping Documentation . . . . . . . . . . . . . . . 10

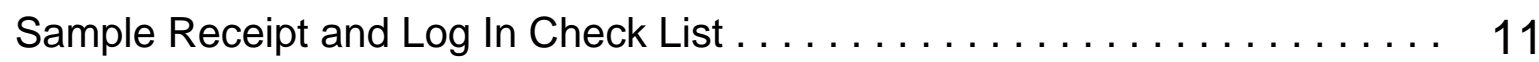

Standards Traceability . . . . . . . . . . . . . . . . 12

Methodology Review . . . . . . . . . . . . . . . . . 20

Report Narrative . . . . . . . . . . . . . . . . . 21

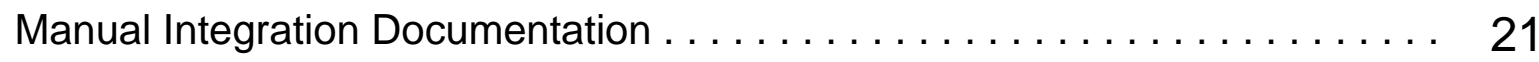

QC Summary - SOM01.2 Volatiles-Trace . . . . . . . . . . . . . 26

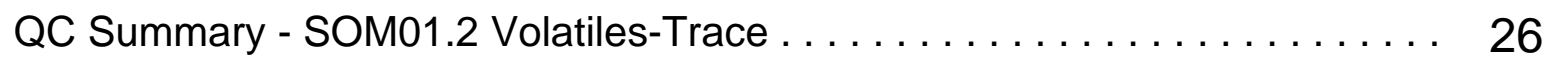

Deuterated Monitoring Compound Summary $\ldots \ldots \ldots \ldots \ldots \ldots \ldots \ldots \ldots \ldots \ldots$

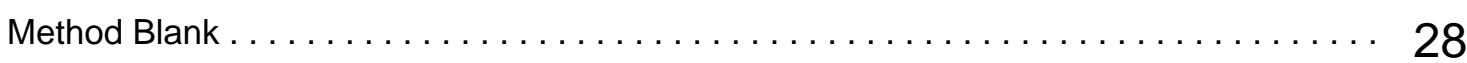

GC/MS Instrument Performance Check $\ldots \ldots \ldots \ldots \ldots \ldots \ldots \ldots \ldots \ldots \ldots \ldots \ldots \ldots \ldots \ldots$

Internal Standard Area and RT Summary . . . . . . . . . . . . . . . . . . 34

Sample Data - SOM01.2 Volatiles-Trace . . . . . . . . . . . 36

Sample Data - SOM01.2 Volatiles-Trace . . . . . . . . . . . . . 36

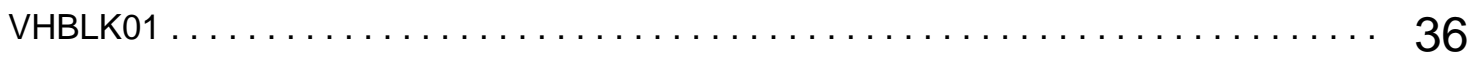

YKQCTB-W-37869 .......................................... 39

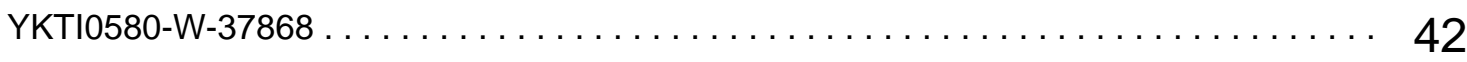

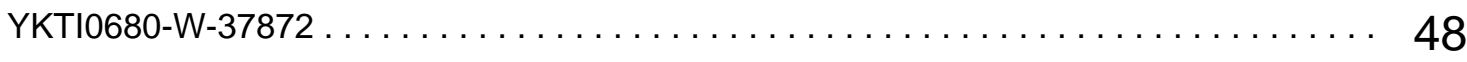

YKTI07100-W-37876 ..................................... 51

Standards - SOM01.2 Volatiles-Trace . . . . . . . . . . . . . . . . . . 54 


\section{Table of Contents}

Standards - SOM01.2 Volatiles-Trace . . . . . . . . . . . . . . . . 54

Initial Calibration Data ................................... 54

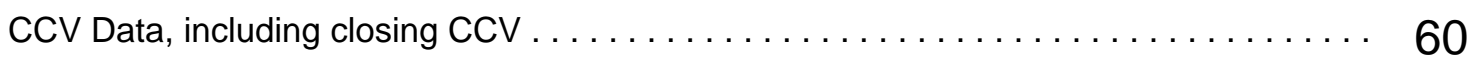

Raw Qc Data - SOM01.2 Volatiles-Trace . . . . . . . . . . . . . 72

Raw Qc Data - SOM01.2 Volatiles-Trace . . . . . . . . . . . . . 72

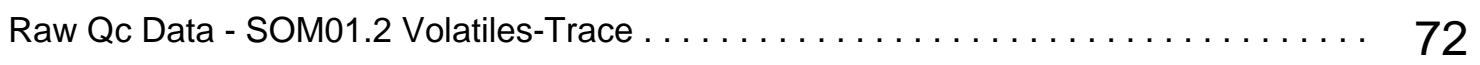

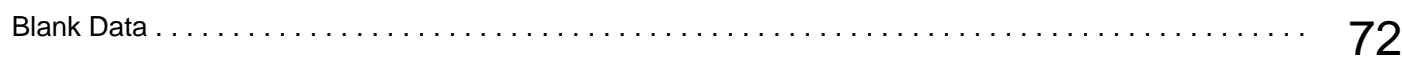




\section{CASE NARRATIVE}

\section{Client: Argonne National Laboratory \\ Project: York (200-28963)}

\section{Report Number: 200- 28963-1}

Enclosed is the data set for the referenced project work. With the exceptions noted as flags or footnotes, standard analytical protocols were followed in performing the analytical work and the applied control limits were met.

Calculations were performed before rounding to avoid round-off errors in calculated results.

All holding times were met and proper preservation noted for the methods performed on these samples, unless otherwise detailed in the individual sections below.

\section{Receipt}

The samples were received on $07 / 21 / 2015$. Documentation of the condition of the samples at the time of their receipt and any exception to the laboratory's Sample Acceptance Policy is documented in the Shipping Documentation section of this submittal. The samples, as received, were not acid preserved. On that basis, the laboratory did provide for the analysis of the samples within seven days of sample collection.

\section{SOM01.2 Volatile Organics (Trace Level Water)}

A storage blank was prepared for volatile organics analysis, and stored in association with the storage of the samples. That storage blank, identified as VHBLK01, was carried through the holding period with the samples, and analyzed.

Sample YKTI0580-W-37868 was analyzed at a 14 fold dilution due to high concentrations of target analytes. A complete more concentrated analysis could not be provided. The lab was able to provide a 5 fold more concentrated analysis. Sample YKTI0680-W-37872 was analyzed at a 1.1 fold dilution due to an excessive amount of sediment in the sample vial.

Each of the analyses associated with the sample set exhibited an acceptable internal standard performance. There was an acceptable recovery of each deuterated monitoring compound (DMC) in the analysis of the method blank associated with the analytical work, and in the analysis of the storage blank associated with the sample set. Matrix spike and matrix spike duplicate analyses were not performed on samples in this sample set. Trace concentrations of several analytes were detected in the method blanks associated with this sample set. Present in the method blank and storage blank analyses was a non-target constituent that represents a compound that is related to the DMC formulation. The fact that the presence of this compound is not within the laboratory's control is at issue.

The responses for each target analyte met the relative standard deviation criterion in the initial calibration. Bromomethane exceeded the $30.0 \%$ difference criterion in the continuing calibration verification. The response for each target analyte met the $50.0 \%$ difference criterion in the closing calibration check acquisition. 
The primary quantitation mass for methylcyclohexane that is specified in the Statement of Work is mass 83 . The laboratory did identify a contribution to mass 83 from 1,2-dichloropropane- $\mathrm{d}_{6}$, one of the deuterated monitoring compounds (DMCs). The laboratory did change the primary quantitation mass assignment to mass 55 for the quantification of methylcyclohexane.

Manual integration was employed in deriving certain of the analytical results. The values that have been derived from manual integration are qualified on the quantitation reports. Extracted ion current profiles for each manual integration are included in the data package, and further documented at the end of this submittal. 


\section{DATA REPORTING QUALIFIERS}

Client: Argonne National Laboratory

Job Number: 200-28963-1

Sdg Number: 28963

Lab Section

Qualifier

Description

GC/MS VOA

$\mathrm{U}$

$\mathrm{E}$

$J$

$J$

D

$\mathrm{X}$

$\mathrm{B}$

$\mathrm{N}$
Analyzed for but not detected.

Compound concentration exceeds the upper level of the calibration range of the instrument for that specific analysis.

Indicates an Estimated Value for TICs

Indicates an estimated value.

Sample was analyzed at a higher dilution factor.

See case narrative notes for explanation of the ' $X$ ' flag

The analyte was found in an associated blank, as well as in the sample.

This flag indicates the presumptive evidence of a compound. 


\section{Sample Login Acknowledgement}

Job 200-28963-1

\begin{tabular}{|c|c|c|c|}
\hline Client Job Description: & York (200-28963) & Report To: & Argonne National Laboratory \\
\hline Purchase Order \#: & $1 \mathrm{E}-30401$ & & Jorge Alvarado \\
\hline Work Order \#: & $1 \mathrm{E}-30401$ & & 9700 South Cass Avenue \\
\hline Project Manager: & Kathryn A Kelly & & Building 203 \\
\hline Job Due Date: & $8 / 4 / 2015$ & & Argonne, IL 60439 \\
\hline Job TAT: & 14 Days & & \\
\hline Max Deliverable Level: & IV & Bill To: & $\begin{array}{l}\text { Argonne National Laboratory } \\
\text { Accounts Payable }\end{array}$ \\
\hline Earliest Deliverable Due: & $8 / 4 / 2015$ & & $\begin{array}{l}\text { Chief Financial Offices } \\
9700 \text { S. Cass Ave. } \\
\text { Building } 201 \\
\text { Argonne, IL } 60439\end{array}$ \\
\hline
\end{tabular}

\section{Login 200-28963}

\begin{tabular}{|c|c|c|c|c|c|}
\hline $\begin{array}{l}\text { Sample Receipt: } \\
\text { Method of Delivery: }\end{array}$ & $\begin{array}{l}\text { 7/21/2015 10:15:00 AM } \\
\text { FedEx Priority Overnight }\end{array}$ & $\begin{array}{l}\text { Number of Coolers: } \\
\text { Cooler Temperature(s) }\end{array}$ & $\begin{array}{c}1 \\
\left(C^{\circ}\right): 4.0\end{array}$ & & \\
\hline $\begin{array}{l}\text { Lab Sample \# } \\
\text { Method }\end{array}$ & $\begin{array}{l}\text { Client Sample ID } \\
\text { Method Description / Work Location }\end{array}$ & Date Sampled & Matrix & Rpt Basis & Dry / Wet ** \\
\hline 200-28963-1 & YKQCTB-W-37869 & 7/17/2015 12:52:00 PM & Water & & \\
\hline SOM01.2_Vol_Tr & r SOM01.2 Trace Volatile Organics / In-Lab & & & Total & Wet \\
\hline 200-28963-2 & YKTI0580-W-37868 & $7 / 18 / 20159: 40: 00$ AM & Water & & \\
\hline SOM01.2_Vol_Tr & r SOM01.2 Trace Volatile Organics / In-Lab & & & Total & Wet \\
\hline 200-28963-3 & YKTI0680-W-37872 & 7/19/2015 3:50:00 PM & Water & & \\
\hline SOM01.2_Vol_Tr & r SOM01.2 Trace Volatile Organics / In-Lab & & & Total & Wet \\
\hline 200-28963-4 & YKTI07100-W-37876 & 7/20/2015 12:25:00 PM & Water & & \\
\hline SOM01.2_Vol_Tr & r SOM01.2 Trace Volatile Organics / In-Lab & & & Total & Wet \\
\hline 200-28963-5 & VHBLK01 & 7/21/2015 1:25:00 PM & Water & & \\
\hline SOM01.2_Vol_Tr & r SOM01.2 Trace Volatile Organics / In-Lab & & & Total & Wet \\
\hline
\end{tabular}




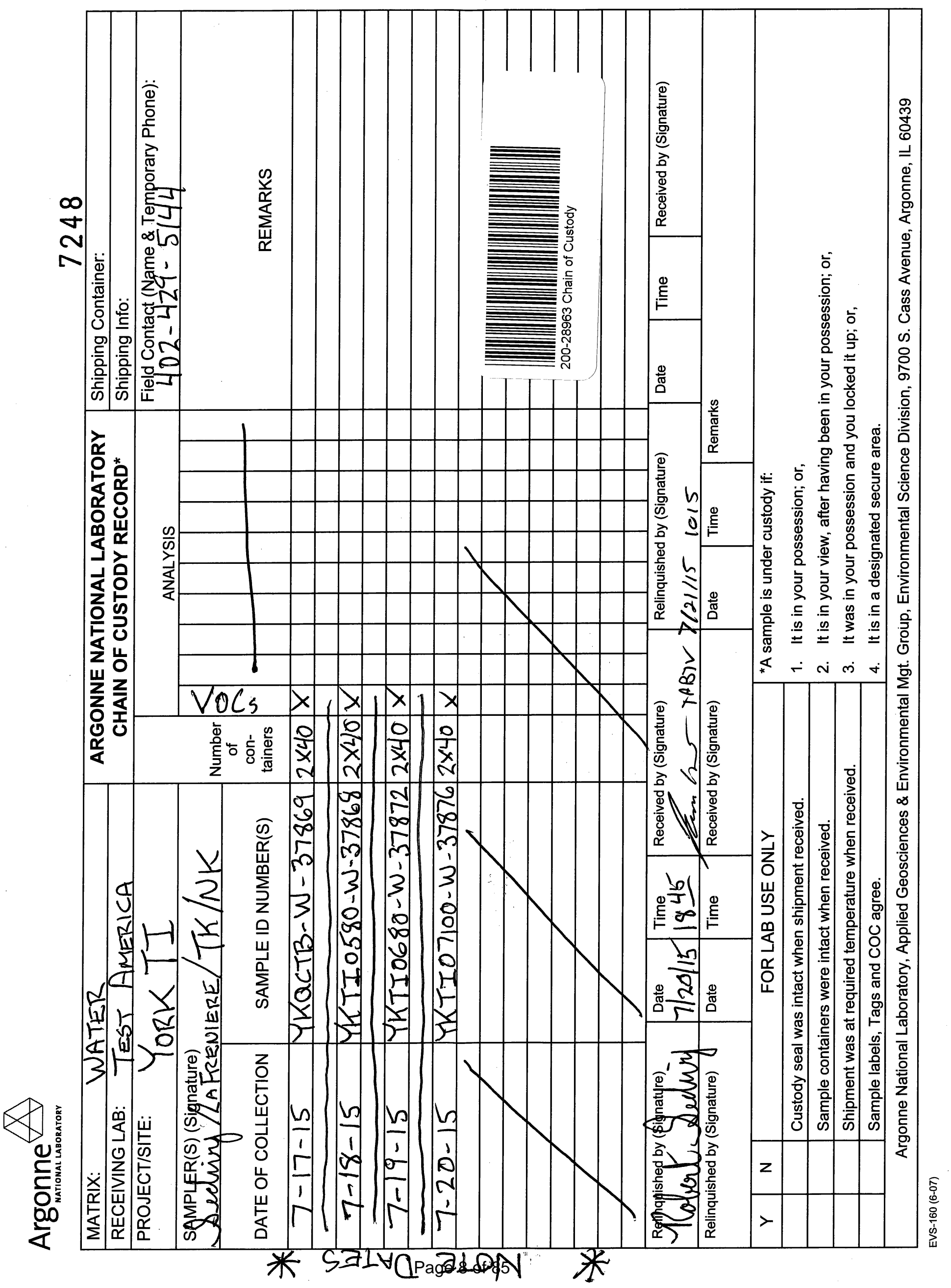



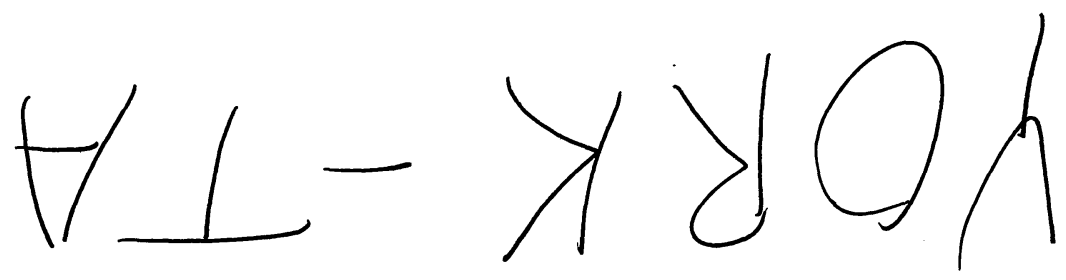

'әр!n

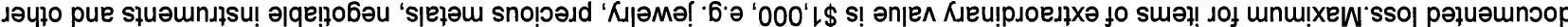

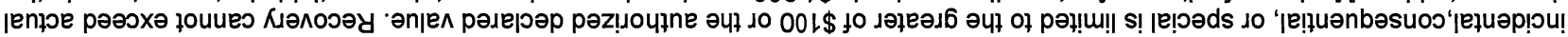

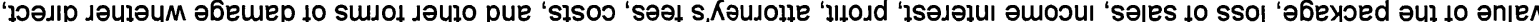

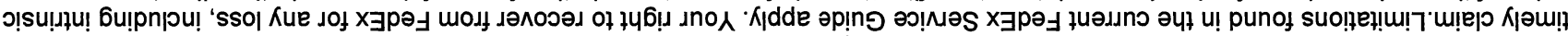

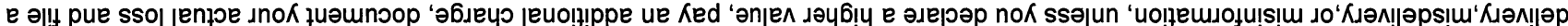

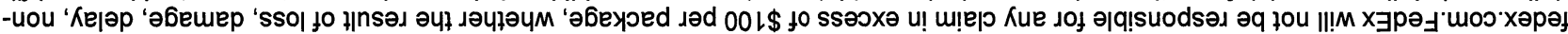
uo ә|qе|!e

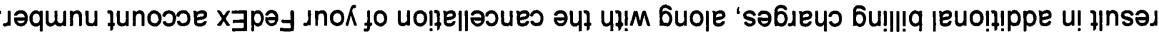

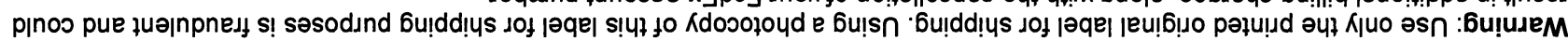

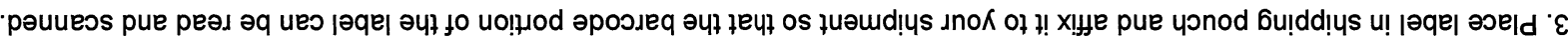

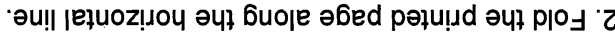

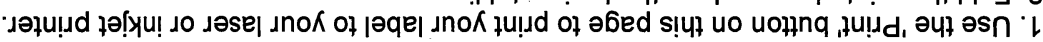

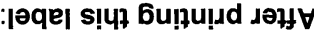

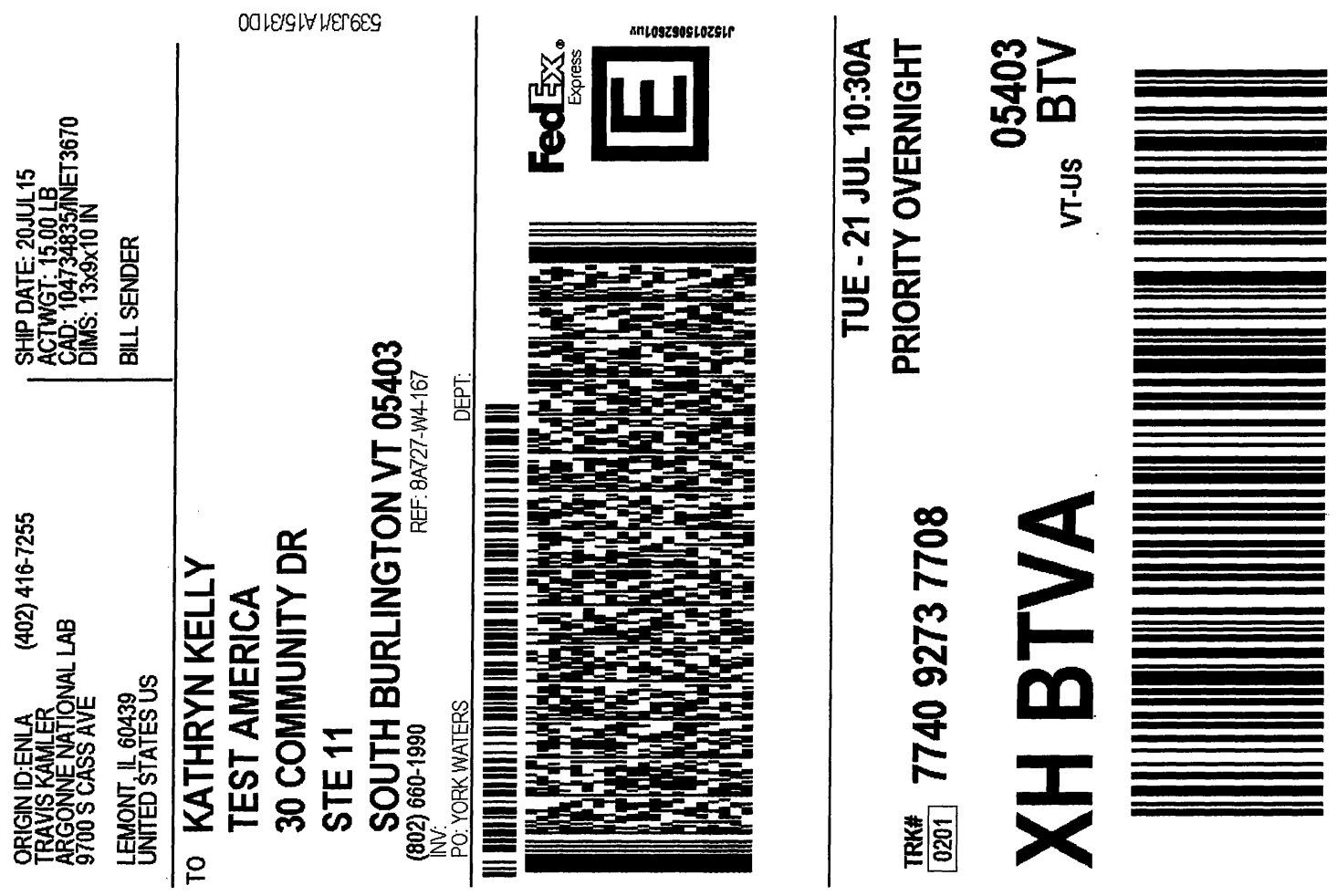




\section{Shipping and Receiving Documents}




\section{Login Sample Receipt Checklist}

Client: Argonne National Laboratory

Job Number: 200-28963-1

SDG Number: 28963

Login Number: 28963

List Source: TestAmerica Burlington

List Number: 1

Creator: Atherton, Joel E

Question

Answer

Radioactivity wasn't checked or is $</=$ background as measured by a survey True meter.

The cooler's custody seal, if present, is intact.

Sample custody seals, if present, are intact.

The cooler or samples do not appear to have been compromised or tampered with.

Samples were received on ice.

Cooler Temperature is acceptable.

Cooler Temperature is recorded.

$\mathrm{COC}$ is present.

$\mathrm{COC}$ is filled out in ink and legible.

$\mathrm{COC}$ is filled out with all pertinent information.

Is the Field Sampler's name present on COC?

There are no discrepancies between the containers received and the COC.

Samples are received within Holding Time.

Sample containers have legible labels.

Containers are not broken or leaking.

Sample collection date/times are provided.

Appropriate sample containers are used.

Sample bottles are completely filled.

Sample Preservation Verified.

There is sufficient vol. for all requested analyses, incl. any requested MS/MSDs

Containers requiring zero headspace have no headspace or bubble is $<6 \mathrm{~mm}(1 / 4 ")$.

Multiphasic samples are not present.

Samples do not require splitting or compositing.

Residual Chlorine Checked.
True

True

True

True

True

True

True

True

True

True

True

True

True

True

True

True

N/A

True

True

True

True

True

N/A 


\begin{tabular}{|c|c|c|c|c|c|c|c|c|}
\hline \multirow[b]{2}{*}{ Reagent ID } & \multirow[b]{2}{*}{$\begin{array}{l}\text { Exp } \\
\text { Date }\end{array}$} & \multirow[b]{2}{*}{$\begin{array}{l}\text { Prep } \\
\text { Date }\end{array}$} & \multirow[b]{2}{*}{$\begin{array}{l}\text { Dilutant } \\
\text { Used }\end{array}$} & \multirow{2}{*}{$\begin{array}{l}\text { Reagent } \\
\text { Final } \\
\text { Volume }\end{array}$} & \multicolumn{2}{|c|}{ Parent Reagent } & \multirow[b]{2}{*}{ Analyte } & \multirow[b]{2}{*}{ Concentration } \\
\hline & & & & & Reagent ID & $\begin{array}{l}\text { Volume } \\
\text { Added }\end{array}$ & & \\
\hline VMBFBW_00018 & $12 / 22 / 15$ & $06 / 23 / 15$ & METHANOL, LOt 147358 & $25 \mathrm{~mL}$ & VMBFBs_00014 & $125 \mathrm{uL}$ & $\mathrm{BFB}$ & $25 \mathrm{ug} / \mathrm{mL}$ \\
\hline .VMBFBS_00014 & $12 / 22 / 15$ & & RESTEK, Lot A0106759 & & \multicolumn{2}{|c|}{ (Purchased Reagent) } & BFB & $5000 \mathrm{ug} / \mathrm{mL}$ \\
\hline \multirow[t]{33}{*}{ VMSOMTRCALW_00091 } & \multirow[t]{33}{*}{$07 / 15 / 15$} & \multirow[t]{33}{*}{$06 / 15 / 15$} & \multirow[t]{33}{*}{ METHANOL, LOt 147358} & \multirow[t]{33}{*}{4000 uL } & VM8260CAL_bs_00180 & $40 \mathrm{UL}$ & \begin{tabular}{|l|} 
Bromomethane \\
Chloroethane \\
Chloromethane \\
Dichlorodifluoromethane \\
Trichlorofluoromethane \\
Vinyl chloride
\end{tabular} & $\begin{array}{l}20 \mathrm{ug} / \mathrm{mL} \\
20 \mathrm{ug} / \mathrm{mL} \\
20 \mathrm{ug} / \mathrm{mL} \\
20 \mathrm{ug} / \mathrm{mL} \\
20 \mathrm{ug} / \mathrm{mL} \\
20 \mathrm{ug} / \mathrm{mL}\end{array}$ \\
\hline & & & & & \multirow[t]{32}{*}{ VMSOMCALas_00022 } & \multirow[t]{32}{*}{40 uL } & $\begin{array}{l}1,1,1 \text {-Trichloroethane } \\
1,1,2,2 \text {-Tetrachloroethane } \\
1,1,2 \text {-Trichloro-1,2,2-trifluor } \\
\text { oethane }\end{array}$ & $\begin{array}{l}20 \mathrm{ug} / \mathrm{mL} \\
20 \mathrm{ug} / \mathrm{mL} \\
20 \mathrm{ug} / \mathrm{mL} \\
\end{array}$ \\
\hline & & & & & & & $1,1,2$-Trichloroethane & $20 \mathrm{ug} / \mathrm{mL}$ \\
\hline & & & & & & & \begin{tabular}{|l|} 
1,1-Dichloroethane \\
1,1-Dichloroethene
\end{tabular} & $\frac{20 \mathrm{ug} / \mathrm{mL}}{20 \mathrm{ug} / \mathrm{mL}}$ \\
\hline & & & & & & & $1,2,3$-Trichlorobenzene & $20 \mathrm{ug} / \mathrm{mL}$ \\
\hline & & & & & & & 1,2,4-Trichlorobenzene & $20 \mathrm{ug} / \mathrm{mL}$ \\
\hline & & & & & & & 1,2-Dibromo-3-Chloropropane & $20 \mathrm{ug} / \mathrm{mL}$ \\
\hline & & & & & & & $\begin{array}{l}\text { 1,2-Dibromoethane } \\
1,2 \text {-Dichlorobenzene }\end{array}$ & $\frac{20 \mathrm{ug} / \mathrm{mL}}{20 \mathrm{ug} / \mathrm{mL}}$ \\
\hline & & & & & & & 1,2-Dichloroethane & $20 \mathrm{ug} / \mathrm{mL}$ \\
\hline & & & & & & & 1,2-Dichloropropane & $20 \mathrm{ug} / \mathrm{mL}$ \\
\hline & & & & & & & $\begin{array}{l}\text { 1,3-Dichlorobenzene } \\
\text { 1,4-Dichlorobenzene }\end{array}$ & $\frac{20 \mathrm{ug} / \mathrm{mL}}{20 \mathrm{ug} / \mathrm{mL}}$ \\
\hline & & & & & & & $\begin{array}{l}\text { 1,4-Dichlorobenzene } \\
\text { Benzene }\end{array}$ & $20 \mathrm{ug} / \mathrm{mL}$ \\
\hline & & & & & & & Bromochloromethane & $20 \mathrm{ug} / \mathrm{mL}$ \\
\hline & & & & & & & Bromodichloromethane & $20 \mathrm{ug} / \mathrm{mL}$ \\
\hline & & & & & & & Bromoform & $20 \mathrm{ug} / \mathrm{mL}$ \\
\hline & & & & & & & Carbon disulfide & $20 \mathrm{ug} / \mathrm{mL}$ \\
\hline & & & & & & & Carbon tetrachloride & $20 \mathrm{ug} / \mathrm{mL}$ \\
\hline & & & & & & & Chlorobenzene & $20 \mathrm{ug} / \mathrm{mL}$ \\
\hline & & & & & & & Chloroform & $20 \mathrm{ug} / \mathrm{mL}$ \\
\hline & & & & & & & cis-1,2-Dichloroethene & $20 \mathrm{ug} / \mathrm{mL}$ \\
\hline & & & & & & & $\begin{array}{l}\text { Cis-1,3-Dichloropropene } \\
\text { Cyclohexane }\end{array}$ & $20 \mathrm{ug} / \mathrm{mL}$ \\
\hline & & & & & & & Dibromochloromethane & $20 \mathrm{ug} / \mathrm{mL}$ \\
\hline & & & & & & & Ethylbenzene & $20 \mathrm{ug} / \mathrm{mL}$ \\
\hline & & & & & & & Isopropylbenzene & $20 \mathrm{ug} / \mathrm{mL}$ \\
\hline & & & & & & & $\mathrm{m}, \mathrm{p}$-Xylene & $20 \mathrm{ug} / \mathrm{mL}$ \\
\hline & & & & & & & Methyl acetate & $20 \mathrm{ug} / \mathrm{mL}$ \\
\hline & & & & & & & Methyl tert-butyl ether & $20 \mathrm{ug} / \mathrm{mL}$ \\
\hline & & & & & & & Methylcyclohexane & $20 \mathrm{ug} / \mathrm{mL}$ \\
\hline & & & & & & & Methylene Chloride & $20 \mathrm{ug} / \mathrm{mL}$ \\
\hline & & & & & & & o-Xylene & $20 \mathrm{ug} / \mathrm{mL}$ \\
\hline & & & & & & & Styrene & $20 \mathrm{ug} / \mathrm{mL}$ \\
\hline & & & & & & & Tetrachloroethene & $20 \mathrm{ug} / \mathrm{mL}$ \\
\hline & & & & & & & Toluene & $20 \mathrm{ug} / \mathrm{mL}$ \\
\hline
\end{tabular}


SDG No.: 28963

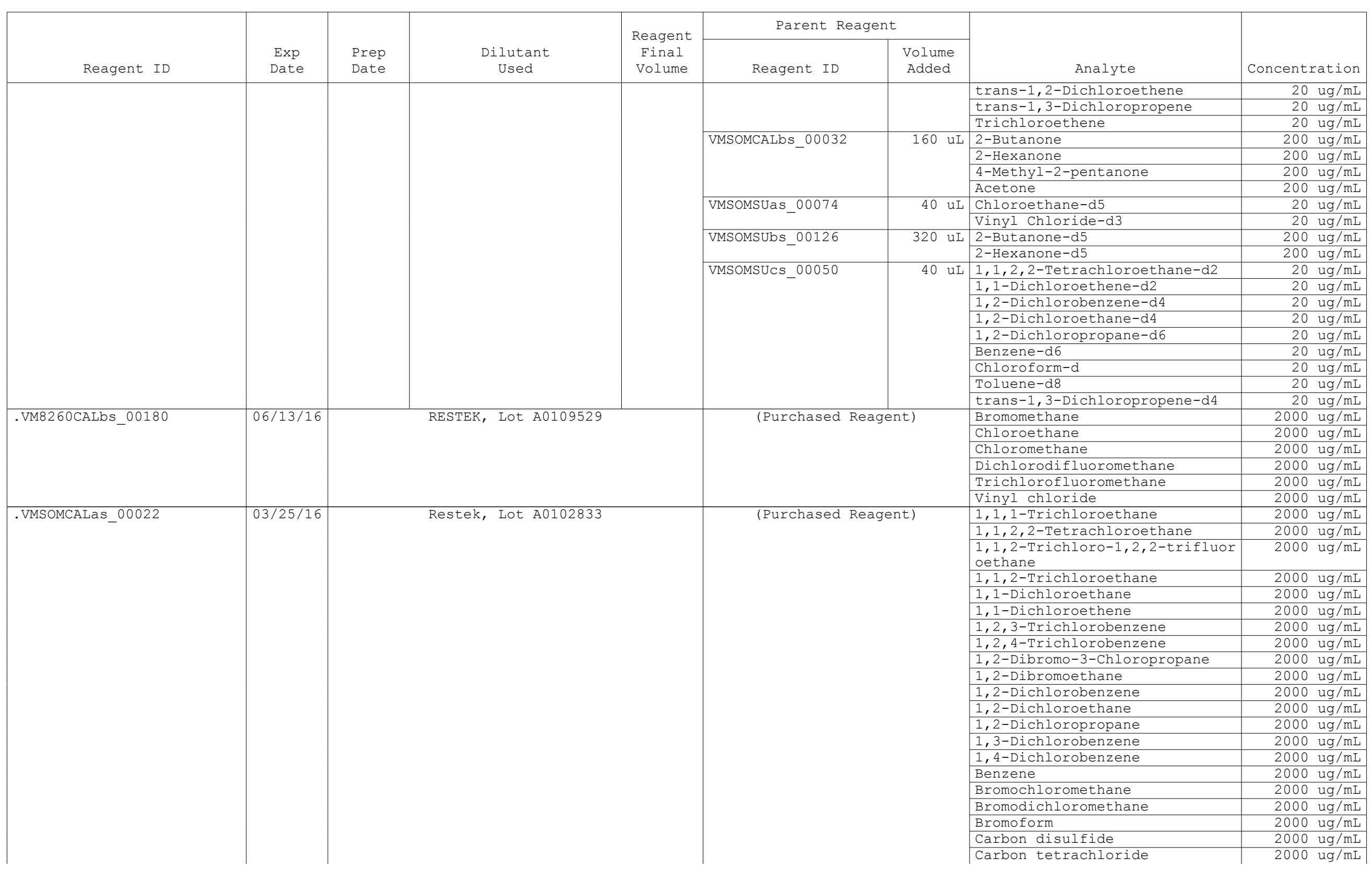


SDG No.: 28963

\begin{tabular}{|c|c|c|c|c|c|c|c|c|}
\hline \multirow[b]{2}{*}{ Reagent ID } & \multirow[b]{2}{*}{$\begin{array}{l}\text { Exp } \\
\text { Date }\end{array}$} & \multirow[b]{2}{*}{$\begin{array}{l}\text { Prep } \\
\text { Date }\end{array}$} & \multirow[b]{2}{*}{$\begin{array}{l}\text { Dilutant } \\
\text { Used }\end{array}$} & \multirow{2}{*}{$\begin{array}{l}\text { Reagent } \\
\text { Final } \\
\text { Volume }\end{array}$} & \multicolumn{2}{|c|}{ Parent Reagent } & \multirow[b]{2}{*}{ Analyte } & \multirow[b]{2}{*}{ Concentration } \\
\hline & & & & & Reagent ID & $\begin{array}{l}\text { Volume } \\
\text { Added }\end{array}$ & & \\
\hline & & & 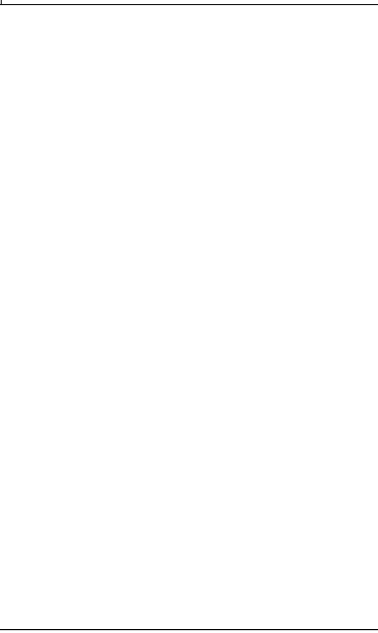 & & & & \begin{tabular}{|l} 
Chlorobenzene \\
Chloroform \\
cis-1,2-Dichloroethene \\
cis-1,3-Dichloropropene \\
Cyclohexane \\
Dibromochloromethane \\
Ethylbenzene \\
Isopropylbenzene \\
m, p-Xylene \\
Methyl acetate \\
Methyl tert-butyl ether \\
Methylyclohexane \\
Methlene Chloride \\
O-Xylene \\
Styrene \\
Tetrachloroethene \\
Toluene \\
trans-1,2-Dichloroethene \\
trans-1,3-Dichloropropene \\
Trichloroethene \\
\end{tabular} & 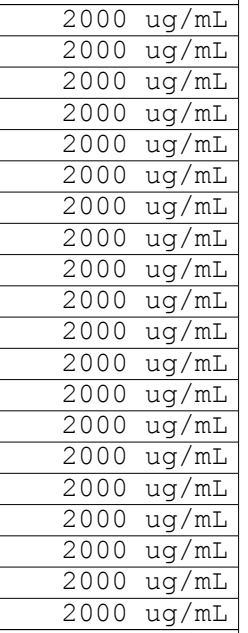 \\
\hline .VMSOMCALbs_00032 & $04 / 27 / 16$ & & Restek, Lot A0101160 & & (Purchased Rea & & $\begin{array}{l}\text { 2-Butanone } \\
\text { 2-Hexanone } \\
\text { 4-Methyl-2-pentanone } \\
\text { Acetone }\end{array}$ & $\begin{array}{l}5000 \mathrm{ug} / \mathrm{mL} \\
5000 \mathrm{ug} / \mathrm{mL} \\
5000 \mathrm{ug} / \mathrm{mL} \\
5000 \mathrm{ug} / \mathrm{mL}\end{array}$ \\
\hline VMSOMSUas_00074 & $04 / 29 / 16$ & & Absolute, Lot 072214 & & (Purchased Rea & & \begin{tabular}{|l|} 
Chloroethane-d5 \\
Vinyl Chloride-d3 \\
\end{tabular} & $\frac{2000 \mathrm{ug} / \mathrm{mL}}{2000 \mathrm{ug} / \mathrm{mL}}$ \\
\hline VMSOMSUbs_00126 & $06 / 13 / 16$ & & Absolute, Lot 080414 & & (Purchased Rea & & $\begin{array}{l}\text { 2-Butanone-d5 } \\
\text { 2-Hexanone-d5 }\end{array}$ & $\begin{array}{l}2500 \mathrm{ug} / \mathrm{mL} \\
2500 \mathrm{ug} / \mathrm{mL}\end{array}$ \\
\hline .VMSOMSUCS_00050 & $04 / 27 / 16$ & & Absolute, Lot 011614 & & (Purchased Rea & & \begin{tabular}{|l|}
$1,1,2,2$-Tetrachloroethane-d2 \\
1,1-Dichloroethene-d2 \\
1,2-Dichlorobenzene-d4 \\
1,2-Dichloroethane-d4 \\
1,2-Dichloropropane-d6 \\
Benzene-d6 \\
Chloroform-d \\
Toluene-d8 \\
trans-1,3-Dichloropropene-d4 \\
\end{tabular} & $\begin{array}{l}2000 \mathrm{ug} / \mathrm{mL} \\
2000 \mathrm{ug} / \mathrm{mL} \\
2000 \mathrm{ug} / \mathrm{mL} \\
2000 \mathrm{ug} / \mathrm{mL} \\
2000 \mathrm{ug} / \mathrm{mL} \\
2000 \mathrm{ug} / \mathrm{mL} \\
2000 \mathrm{ug} / \mathrm{mL} \\
2000 \mathrm{ug} / \mathrm{mL} \\
2000 \mathrm{ug} / \mathrm{mL} \\
\end{array}$ \\
\hline VMSOMTRCALW_00092 & $08 / 16 / 15$ & $07 / 16 / 15$ & METHANOL， Lot 147358 & 4000 uL & VM8260CALbs_00180 & 40 uL & \begin{tabular}{|l|} 
Bromomethane \\
Chloroethane \\
Chloromethane \\
Dichlorodifluoromethane \\
Trichlorofluoromethane \\
Vinyl chloride \\
$1,1,1-$ Trichloroethane \\
$1,1,2,2$-Tetrachloreethane \\
$1,1,2$-Trichloro-1,2,2-trifluor \\
oethane \\
$1,1,2$-Trichloroethane
\end{tabular} & $\begin{array}{l}20 \mathrm{ug} / \mathrm{mL} \\
20 \mathrm{ug} / \mathrm{mL} \\
20 \mathrm{ug} / \mathrm{mL} \\
20 \mathrm{ug} / \mathrm{mL} \\
20 \mathrm{ug} / \mathrm{mL} \\
20 \mathrm{ug} / \mathrm{mL} \\
20 \mathrm{ug} / \mathrm{mL} \\
20 \mathrm{ug} / \mathrm{mL} \\
20 \mathrm{ug} / \mathrm{mL} \\
\end{array}$ \\
\hline
\end{tabular}


SDG No.: 28963

\begin{tabular}{|c|c|c|c|c|c|c|c|c|}
\hline \multirow[b]{2}{*}{ Reagent ID } & \multirow[b]{2}{*}{$\begin{array}{l}\text { Exp } \\
\text { Date }\end{array}$} & \multirow[b]{2}{*}{$\begin{array}{l}\text { Prep } \\
\text { Date }\end{array}$} & \multirow[b]{2}{*}{$\begin{array}{l}\text { Dilutant } \\
\text { Used }\end{array}$} & \multirow{2}{*}{$\begin{array}{c}\text { Reagent } \\
\text { Final } \\
\text { Volume }\end{array}$} & \multicolumn{2}{|c|}{ Parent Reagent } & \multirow[b]{2}{*}{ Analyte } & \multirow[b]{2}{*}{ Concentration } \\
\hline & & & & & Reagent ID & $\begin{array}{l}\text { Volume } \\
\text { Added }\end{array}$ & & \\
\hline & & & & & & & 1,1-Dichloroethane & $20 \mathrm{ug} / \mathrm{mL}$ \\
\hline & & & & & & & 1,1-Dichloroethene & $20 \mathrm{ug} / \mathrm{mL}$ \\
\hline & & & & & & & 1,2,3-Trichlorobenzene & $20 \mathrm{ug} / \mathrm{mL}$ \\
\hline & & & & & & & 1,2,4-Trichlorobenzene & $20 \mathrm{ug} / \mathrm{mL}$ \\
\hline & & & & & & & 1,2-Dibromoethane & $20 \mathrm{ug} / \mathrm{mL}$ \\
\hline & & & & & & & 1,2-Dichlorobenzene & $20 \mathrm{ug} / \mathrm{mL}$ \\
\hline & & & & & & & 1,2-Dichloroethane & $20 \mathrm{ug} / \mathrm{mL}$ \\
\hline & & & & & & & 1,2-Dichloropropane & $20 \mathrm{ug} / \mathrm{mL}$ \\
\hline & & & & & & & 1,3-Dichlorobenzene & $20 \mathrm{ug} / \mathrm{mL}$ \\
\hline & & & & & & & 1,4-Dichlorobenzene & $20 \mathrm{ug} / \mathrm{mL}$ \\
\hline & & & & & & & Benzene & $20 \mathrm{ug} / \mathrm{mL}$ \\
\hline & & & & & & & Bromochloromethane & $20 \mathrm{ug} / \mathrm{mL}$ \\
\hline & & & & & & & Bromodichloromethane & $20 \mathrm{ug} / \mathrm{mL}$ \\
\hline & & & & & & & $\begin{array}{l}\text { Bromororm } \\
\text { Carbon disulfide }\end{array}$ & $\frac{20 \mathrm{ug} / \mathrm{mL}}{20 \mathrm{ug} / \mathrm{mL}}$ \\
\hline & & & & & & & Carbon tetrachloride & $20 \mathrm{ug} / \mathrm{mL}$ \\
\hline & & & & & & & Chlorobenzene & $20 \mathrm{ug} / \mathrm{mL}$ \\
\hline & & & & & & & Chloroform & $20 \mathrm{ug} / \mathrm{mL}$ \\
\hline & & & & & & & Cis-1,2-Dichloroethene & $20 \mathrm{ug} / \mathrm{mL}$ \\
\hline & & & & & & & Cis-1,3-Dichloropropene & $20 \mathrm{ug} / \mathrm{mL}$ \\
\hline & & & & & & & Dibromochloromethane & $\frac{20 \mathrm{ug} / \mathrm{mL}}{20 \mathrm{ug} / \mathrm{mL}}$ \\
\hline & & & & & & & Ethylbenzene & $20 \mathrm{ug} / \mathrm{mL}$ \\
\hline & & & & & & & Isopropylbenzene & $20 \mathrm{ug} / \mathrm{mL}$ \\
\hline & & & & & & & $m, p$-Xylene & $20 \mathrm{ug} / \mathrm{mL}$ \\
\hline & & & & & & & Methyl acetate & $20 \mathrm{ug} / \mathrm{mL}$ \\
\hline & & & & & & & Methyl tert-butyl ether & $20 \mathrm{ug} / \mathrm{mL}$ \\
\hline & & & & & & & Methylcyclohexane & $20 \mathrm{ug} / \mathrm{mL}$ \\
\hline & & & & & & & Methylene Chloride & $20 \mathrm{ug} / \mathrm{mL}$ \\
\hline & & & & & & & o-Xylene & $20 \mathrm{ug} / \mathrm{mL}$ \\
\hline & & & & & & & $\begin{array}{l}\text { Styrene } \\
\text { Tetrachlernethene }\end{array}$ & $\frac{20 \mathrm{ug} / \mathrm{mL}}{20 \mathrm{ug} / \mathrm{mL}}$ \\
\hline & & & & & & & $\begin{array}{l}\text { Tetracnioroetnene } \\
\text { Toluene }\end{array}$ & $20 \mathrm{ug} / \mathrm{mL}$ \\
\hline & & & & & & & trans-1,2-Dichloroethene & $20 \mathrm{ug} / \mathrm{mL}$ \\
\hline & & & & & & & trans-1,3-Dichloropropene & $20 \mathrm{ug} / \mathrm{mL}$ \\
\hline & & & & & & & Trichloroethene & $20 \mathrm{ug} / \mathrm{mL}$ \\
\hline & & & & & VMSOMCALbs_00034 & 160 uL & 2-Butanone & $200 \mathrm{ug} / \mathrm{mL}$ \\
\hline & & & & & & & 2-Hexanone & $200 \mathrm{ug} / \mathrm{mL}$ \\
\hline & & & & & & & 4-Methyl-2-pentanone & $200 \mathrm{ug} / \mathrm{mL}$ \\
\hline & & & & & & & Acetone & $200 \mathrm{ug} / \mathrm{mL}$ \\
\hline & & & & & VMSOMSUas_00074 & $40 \mathrm{uL}$ & Chloroethane-d5 & $20 \mathrm{ug} / \mathrm{mL}$ \\
\hline & & & & & & & Vinyl Chloride-d3 & $20 \mathrm{ug} / \mathrm{mL}$ \\
\hline & & & & & VMSOMSUbs_00129 & $320 \mathrm{uL}$ & 2-Butanone-d5 & $200 \mathrm{ug} / \mathrm{mL}$ \\
\hline & & & & & & & 2-Hexanone-d5 & $200 \mathrm{ug} / \mathrm{mL}$ \\
\hline & & & & & VMSOMSUCS_00050 & $40 \mathrm{uL}$ & $1,1,2,2$-Tetrachloroethane-d2 & $20 \mathrm{ug} / \mathrm{mL}$ \\
\hline & & & & & & & 1,1-Dichloroethene-d2 & $20 \mathrm{ug} / \mathrm{mL}$ \\
\hline & & & & & & & 1,2 -Dichlorobenzene-d4 & $20 \mathrm{ug} / \mathrm{mL}$ \\
\hline
\end{tabular}


SDG No.: 28963

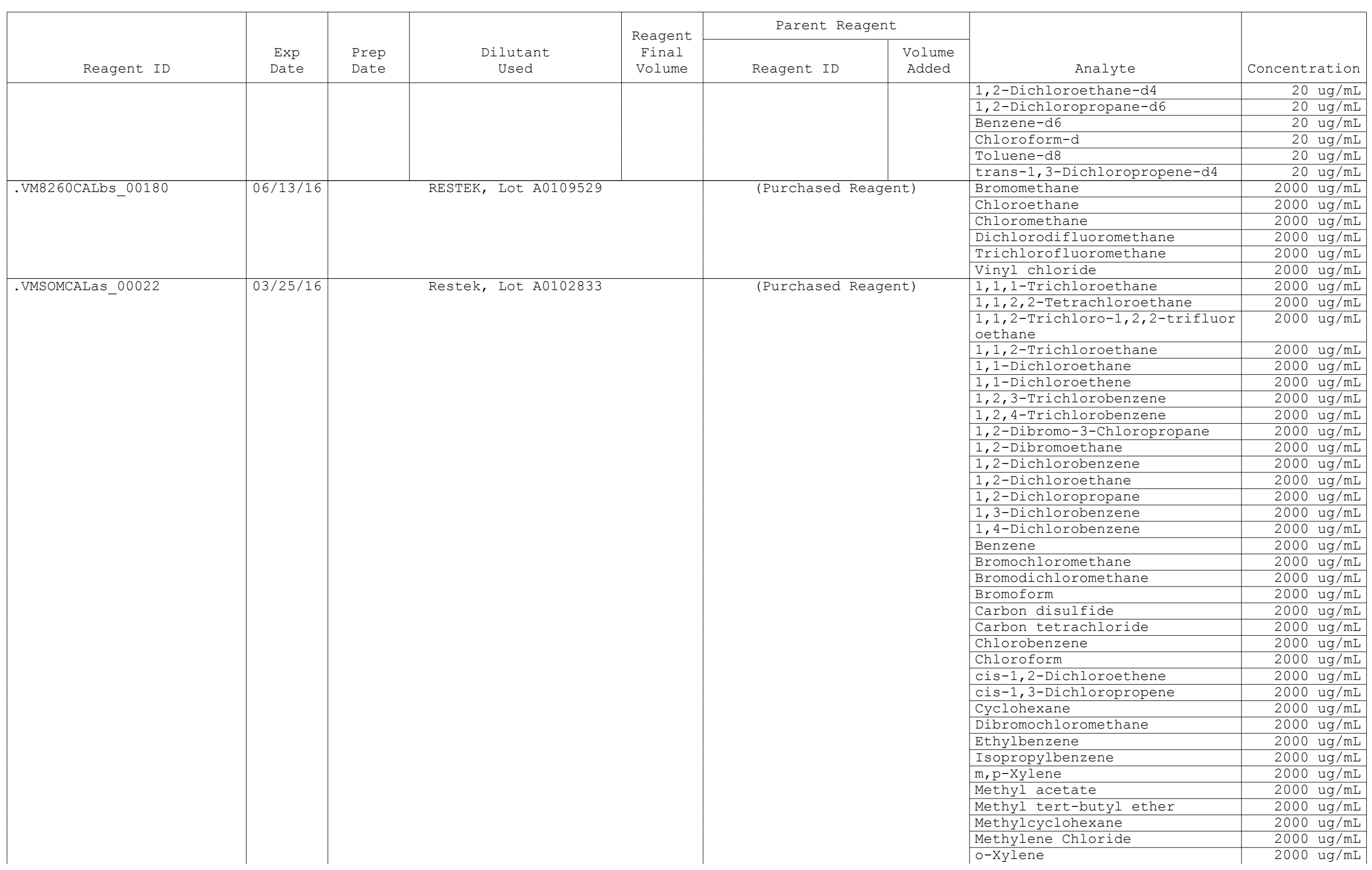


SDG No.: 28963

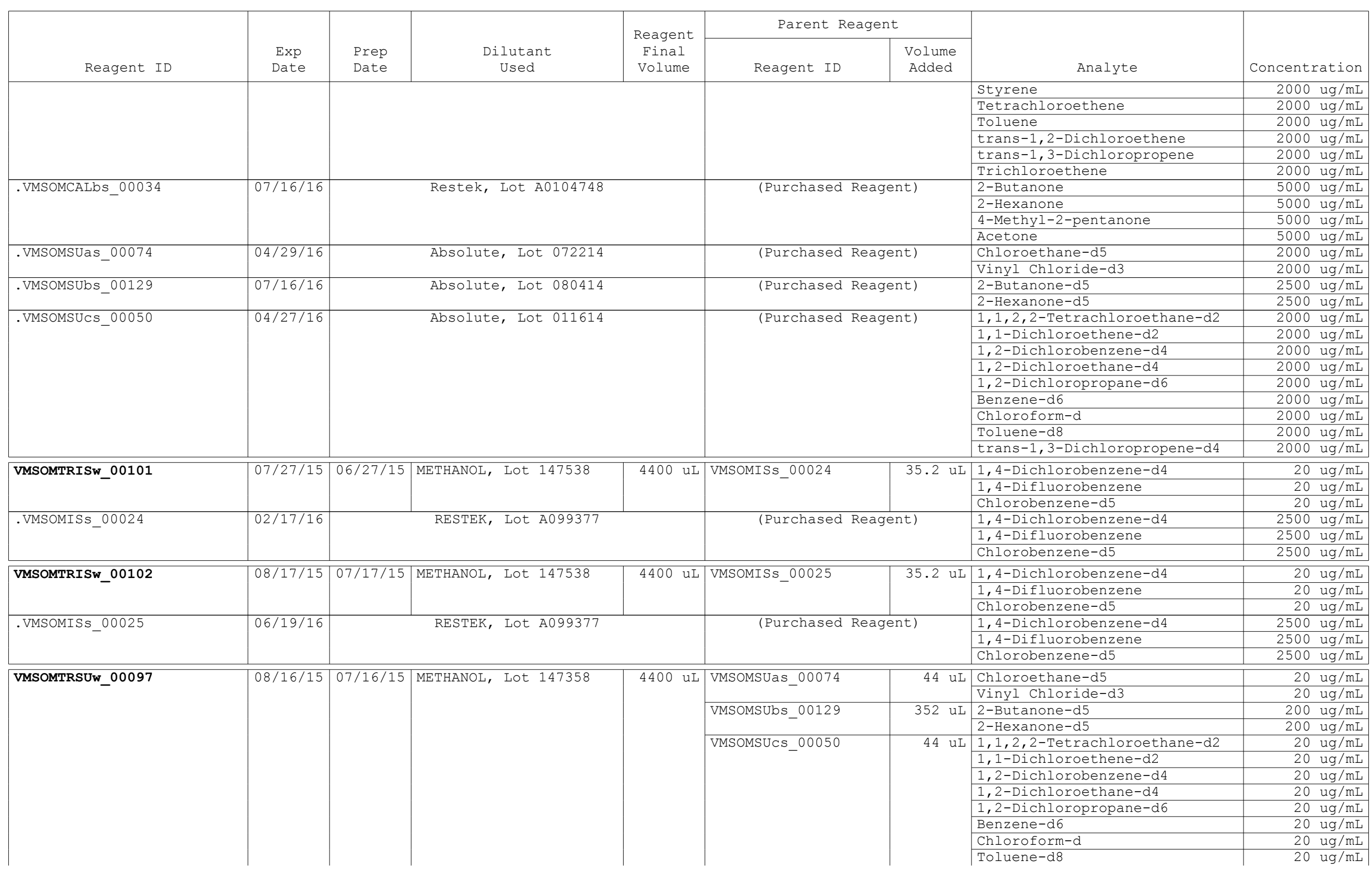


SDG No.: 28963

\begin{tabular}{|c|c|c|c|c|c|c|c|c|}
\hline \multirow[b]{2}{*}{ Reagent ID } & \multirow[b]{2}{*}{$\begin{array}{l}\text { Exp } \\
\text { Date }\end{array}$} & \multirow[b]{2}{*}{$\begin{array}{l}\text { Prep } \\
\text { Date }\end{array}$} & \multirow[b]{2}{*}{$\begin{array}{l}\text { Dilutant } \\
\text { Used }\end{array}$} & \multirow{2}{*}{$\begin{array}{l}\text { Reagent } \\
\text { Final } \\
\text { Volume }\end{array}$} & \multicolumn{2}{|c|}{ Parent Reagent } & \multirow[b]{2}{*}{ Analyte } & \multirow[b]{2}{*}{ Concentration } \\
\hline & & & & & Reagent ID & $\begin{array}{l}\text { Volume } \\
\text { Added }\end{array}$ & & \\
\hline & & & & & & & trans-1,3-Dichloropropene-d4 & $20 \mathrm{ug} / \mathrm{mI}$ \\
\hline \multirow[t]{2}{*}{.VMSOMSUas_00074 } & \multirow[t]{2}{*}{$04 / 29 / 16$} & \multirow{2}{*}{\multicolumn{3}{|c|}{ Absolute, Lot 072214}} & \multirow{2}{*}{\multicolumn{2}{|c|}{ (Purchased Reagent) }} & Chloroethane-d5 & $2000 \mathrm{ug} / \mathrm{mI}$ \\
\hline & & & & & & & Vinyl Chloride-d3 & $2000 \mathrm{ug} / \mathrm{mI}$ \\
\hline \multirow{2}{*}{.VMSOMSUbs_00129 } & \multirow{2}{*}{$07 / 16 / 16$} & \multirow{2}{*}{\multicolumn{3}{|c|}{ Absolute, Lot 080414}} & \multirow{2}{*}{\multicolumn{2}{|c|}{ (Purchased Reagent) }} & 2-Butanone-d5 & $2500 \mathrm{ug} / \mathrm{mI}$ \\
\hline & & & & & & & 2-Hexanone-d5 & $2500 \mathrm{ug} / \mathrm{mI}$ \\
\hline \multirow[t]{9}{*}{.VMSOMSUCS_00050 } & \multirow[t]{9}{*}{$04 / 27 / 16$} & \multirow{9}{*}{\multicolumn{2}{|c|}{ Absolute, Lot 011614}} & & \multirow{9}{*}{\multicolumn{2}{|c|}{ (Purchased Reagent) }} & $1,1,2,2$-Tetrachloroethane-d2 & $2000 \mathrm{ug} / \mathrm{mI}$ \\
\hline & & & & & & & 1,1-Dichloroethene-d2 & $2000 \mathrm{ug} / \mathrm{mI}$ \\
\hline & & & & & & & 1,2-Dichlorobenzene-d4 & $2000 \mathrm{ug} / \mathrm{mI}$ \\
\hline & & & & & & & 1,2-Dichloroethane-d4 & $2000 \mathrm{ug} / \mathrm{mI}$ \\
\hline & & & & & & & 1,2-Dichloropropane-d6 & $2000 \mathrm{ug} / \mathrm{mI}$ \\
\hline & & & & & & & Benzene-d6 & $2000 \mathrm{ug} / \mathrm{mI}$ \\
\hline & & & & & & & Chloroform-d & $2000 \mathrm{ug} / \mathrm{mI}$ \\
\hline & & & & & & & Toluene-d8 & $2000 \mathrm{ug} / \mathrm{mI}$ \\
\hline & & & & & & & trans-1,3-Dichloropropene-d4 & $2000 \mathrm{ug} / \mathrm{mI}$ \\
\hline
\end{tabular}


Lab Name: TestAmerica Burlington

Job No.: 200-28963-1

SDG No.: 28963

\begin{tabular}{|c|c|c|c|c|}
\hline $\begin{array}{c}\text { Reagent } \\
\text { Container }\end{array}$ & $\begin{array}{l}\text { Reagent } \\
\text { ID }\end{array}$ & $\begin{array}{c}\text { Reagent } \\
\text { Description }\end{array}$ & $\begin{array}{c}\text { Preparation } \\
\text { Date }\end{array}$ & $\begin{array}{c}\text { Expiration } \\
\text { Date }\end{array}$ \\
\hline 804536 & VMSOMTRCALw_00091 & SOM TR CAL 20 PPM & $06 / 15 / 2015$ & $07 / 15 / 2015$ \\
\hline 807558 & VMBFBW_00018 & BFB TUNE 25 PPM & $06 / 23 / 2015$ & $12 / 22 / 2015$ \\
\hline 809829 & VMSOMTRISw_00101 & SOM TR ISTD 20 PPM & $06 / 27 / 2015$ & $07 / 27 / 2015$ \\
\hline 816537 & VMSOMTRCALW_00092 & SOM TR CAL 20 PPM & $07 / 16 / 2015$ & $08 / 16 / 2015$ \\
\hline 816538 & VMSOMTRSUw $\overline{00097}$ & SOM $\quad$ TR DMC 20 PPM & $07 / 16 / 2015$ & $08 / 16 / 2015$ \\
\hline 817121 & VMSOMTRISw_00102 & SOM TR ISTD 20 PPM & $07 / 17 / 2015$ & $08 / 17 / 2015$ \\
\hline
\end{tabular}




\section{METHODOLOGY SUMMARY}

Laboratory: TestAmerica Laboratories

Location: South Burlington, Vermont
Project No:

SDG No: 28963

\section{VOA}

Volatile Organics Trace - USEPA CLP SOM01.2 
Lab Name: TestAmerica Burlington SDG No.: 28963

Instrument ID: CHD.i

Lab Sample ID: IC 200-91077/2

Date Analyzed: 07/14/15 11:08

\begin{tabular}{|c|r|}
\hline COMPOUND NAME & RETEN \\
TI
\end{tabular}

Lab Sample ID: IC 200-91077/3

Date Analyzed: 07/14/15 11:33 Client Sample ID: Lab File ID: 14685_003.D

Analysis Batch Number: 91077

Client Sample ID:

Lab File ID: 14685 002.D

GC Column: DB-624

ID: $0.2(\mathrm{~mm})$

MANUAL INTEGRATION

\begin{tabular}{|c|c|c|c|c|}
\hline \multicolumn{1}{|c|}{ COMPOUND NAME } & RETENTION & \multicolumn{3}{|c}{ MANUAL INTEGRATION } \\
\cline { 3 - 5 } & TIME & REASON & ANALYST & DATE \\
\hline 1,2 -Dibromo-3-Chloropropane & 13.44 & Baseline & archern & $07 / 14 / 1514: 11$ \\
\hline
\end{tabular}


Lab Name: TestAmerica Burlington

Job No.: 200-28963-1

SDG No.: 28963

Instrument ID: CHD.i

Analysis Batch Number: 91665

Lab Sample ID: CCVIS 200-91665/3

Client Sample ID:

Date Analyzed: 07/23/15 08:51

Lab File ID: 14885 003.D

GC Column: DB-624

ID $: 0.2(\mathrm{~mm})$

\begin{tabular}{|c|c|c|c|c|}
\hline \multirow[t]{2}{*}{ COMPOUND NAME } & RETENTION & \multicolumn{3}{|c|}{ MANUAL INTEGRATION } \\
\hline & TIME & REASON & ANALYST & DATE \\
\hline Dichlorodifluoromethane & 1.48 & Baseline & archern & $07 / 23 / 1509: 14$ \\
\hline
\end{tabular}

Lab Sample ID: CCVC 200-91665/14

Client Sample ID:

Date Analyzed: 07/23/15 14:39

Lab File ID: 14885 014.D

GC Column: DB-624

ID $: 0.2(\mathrm{~mm})$

\begin{tabular}{|l|c|c|c|c|}
\hline \multicolumn{1}{|c|}{ COMPOUND NAME } & RETENTION & \multicolumn{2}{|c|}{ MANUAL INTEGRATION } \\
\cline { 4 - 5 } & TIME & REASON & ANALYST & DATE \\
\hline Dichlorodifluoromethane & 1.48 & Baseline & archern & $07 / 23 / 1515: 02$ \\
\hline
\end{tabular}


Lab Name: TestAmerica Burlington

Job No.: 200-28963-1

SDG No.: 28963

Instrument ID: CHD.i

Analysis Batch Number: 92118

Lab Sample ID: IC 200-92118/3

Date Analyzed: 07/31/15 12:13

Client Sample ID:

Lab File ID: 15041_003.D

GC Column: DB-624

ID : $0.2(\mathrm{~mm})$

\begin{tabular}{|c|c|c|c|c|}
\hline \multirow[t]{2}{*}{ COMPOUND NAME } & \multirow{2}{*}{$\begin{array}{c}\text { RETENTION } \\
\text { TIME }\end{array}$} & \multicolumn{3}{|c|}{ MANUAL INTEGRATION } \\
\hline & & REASON & ANALYST & DATE \\
\hline Bromomethane & 2.00 & Baseline & wilburj & $08 / 05 / 15 \quad 15: 12$ \\
\hline Acetone & 2.81 & Baseline & wilburj & $08 / 05 / 15$ \\
\hline
\end{tabular}

Lab Sample ID: IC 200-92118/4

Client Sample ID:

Date Analyzed: 07/31/15 12:38

Lab File ID: $15041004 . D$

GC Column: DB-624

ID $: 0.2(\mathrm{~mm})$

\begin{tabular}{|c|c|c|c|c|}
\hline \multirow[t]{2}{*}{ COMPOUND NAME } & \multirow{2}{*}{$\begin{array}{c}\text { RETENTION } \\
\text { TIME }\end{array}$} & \multicolumn{3}{|c|}{ MANUAL INTEGRATION } \\
\hline & & REASON & ANALYST & DATE \\
\hline Dichlorodifluoromethane & 1.48 & Baseline & wilburj & $08 / 05 / 15 \quad 12: 19$ \\
\hline Bromomethane & 2.00 & Baseline & wilburj & $08 / 05 / 15 \quad 12: 19$ \\
\hline 1,2-Dibromo-3-Chloropropane & 13.43 & Baseline & wilburj & $08 / 05 / 1515: 10$ \\
\hline
\end{tabular}

Lab Sample ID: ICIS 200-92118/5

Client Sample ID:

Date Analyzed: 07/31/15 13:02

Lab File ID: 15041_005.D

GC Column: DB-624

ID $: 0.2(\mathrm{~mm})$

\begin{tabular}{|c|c|c|c|c|}
\hline \multirow[t]{2}{*}{ COMPOUND NAME } & \multirow{2}{*}{$\begin{array}{c}\text { RETENTION } \\
\text { TIME }\end{array}$} & \multicolumn{3}{|c|}{ MANUAL INTEGRATION } \\
\hline & & REASON & ANALYST & DATE \\
\hline Dichlorodifluoromethane & 1.48 & Baseline & wilburj & $08 / 05 / 15 \quad 15: 11$ \\
\hline Bromomethane & 1.99 & Baseline & wilburj & $08 / 05 / 1515: 12$ \\
\hline
\end{tabular}

Lab Sample ID: IC 200-92118/6

Client Sample ID:

Date Analyzed: 07/31/15 13:27

Lab File ID: 15041_006.D

GC Column: DB-624

ID $: 0.2(\mathrm{~mm})$

\begin{tabular}{|c|c|c|c|c|}
\hline \multirow[t]{2}{*}{ COMPOUND NAME } & \multirow{2}{*}{$\begin{array}{c}\text { RETENTION } \\
\text { TIME }\end{array}$} & \multicolumn{3}{|c|}{ MANUAL INTEGRATION } \\
\hline & & REASON & ANALYST & DATE \\
\hline Dichlorodifluoromethane & 1.48 & Baseline & wilburj & $08 / 05 / 15 \quad 15: 12$ \\
\hline Bromomethane & 1.99 & Baseline & wilburj & $08 / 05 / 15 \quad 15: 14$ \\
\hline 1,2-Dichloropropane-d6 & 6.42 & Split Peak & wilburj & $08 / 05 / 15 \quad 16: 43$ \\
\hline
\end{tabular}

SOM01.2/VOA_Tr 
Lab Name: TestAmerica Burlington

SDG No.: 28963

Instrument ID: CHD.i

Lab Sample ID: IC 200-92118/7

Date Analyzed: 07/31/15 13:52
Job No.: 200-28963-1

Analysis Batch Number: 92118

Client Sample ID:

Lab File ID: 15041 007.D
GC Column: DB-624

ID $: 0.2(\mathrm{~mm})$

\begin{tabular}{|c|c|c|c|c|}
\hline \multirow[t]{2}{*}{ COMPOUND NAME } & \multirow{2}{*}{$\begin{array}{c}\text { RETENTION } \\
\text { TIME }\end{array}$} & \multicolumn{3}{|c|}{ MANUAL INTEGRATION } \\
\hline & & REASON & ANALYST & DATE \\
\hline Dichlorodifluoromethane & 1.48 & Baseline & wilburj & $08 / 05 / 15$ \\
\hline 1,2-Dichloropropane-d6 & 6.42 & Baseline & wilburj & $08 / 05 / 15 \quad 15: 24$ \\
\hline
\end{tabular}


Lab Name: TestAmerica Burlington SDG No.: 28963

Instrument ID: CHD.i

Lab Sample ID: CCVC 200-92179/11

Date Analyzed: 08/03/15 18:38

\begin{tabular}{|c|c|c|c|c|}
\hline \multirow{2}{*}{ COMPOUND NAME } & \multirow{2}{*}{$\begin{array}{c}\text { RETENTION } \\
\text { TIME }\end{array}$} & \multicolumn{3}{|c|}{ MANUAL INTEGRATION } \\
\hline & & REASON & ANALYST & DATE \\
\hline Dichlorodifluoromethane & 1.53 & Baseline & wilburj & $08 / 05 / 15 \quad 17: 49$ \\
\hline
\end{tabular}

Job No. : 200-28963-1

Analysis Batch Number: 92179

Client Sample ID:

Lab File ID: $15058011 . \mathrm{D}$
ID : $0.2(\mathrm{~mm})$ 
Lab Name: TESTAMERICA BURLINGTON

Lab Code: STLV Case No.: YORK Mod. Ref No.: Level: (TRACE or LOW) TRACE
Contract: $8 \mathrm{E}-00302$

SDG No.: 28963

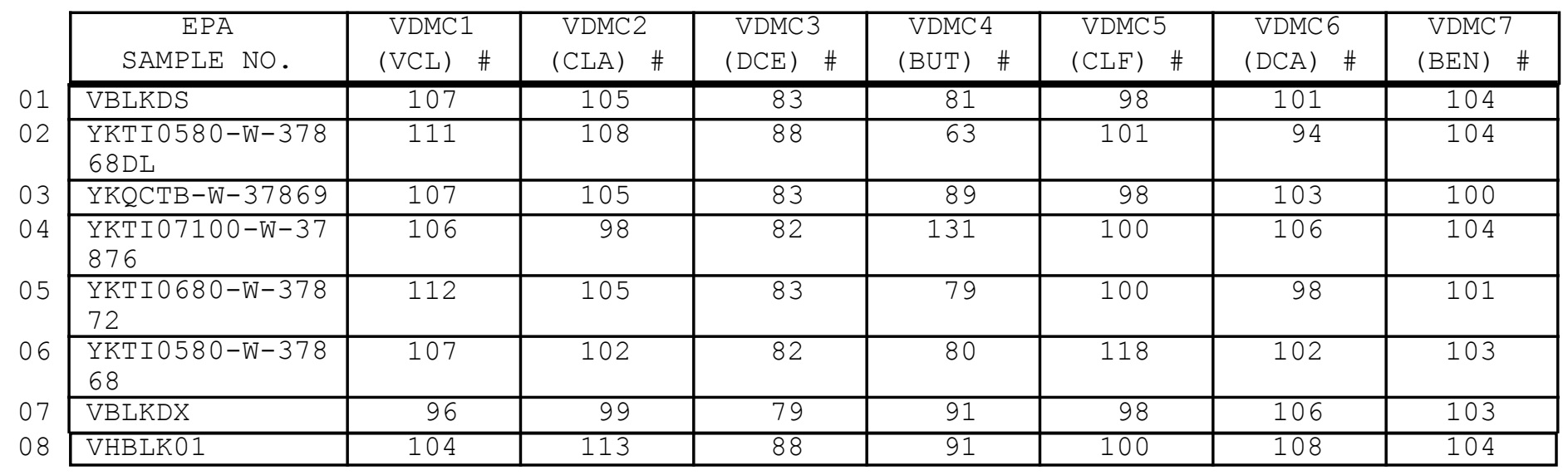

\footnotetext{
VDMC1 (VCL) = Vinyl Chloride-d3

VDMC2 $(\mathrm{CLA})=$ Chloroethane-d5

VDMC3 $(\mathrm{DCE})=1,1-\mathrm{Dichloroethene-d2}$

VDMC4 $\quad(B U T)=2-$ Butanone-d5

VDMC5 $(\mathrm{CLF})=$ Chloroform-d

VDMC $6 \quad(\mathrm{DCA})=1,2-\mathrm{Dich}$ loroethane-d4

$\operatorname{VDMC} 7(\mathrm{BEN})=$ Benzene-d6
}

$$
\begin{gathered}
\frac{\mathrm{QC} \text { LIMITS }}{(65-131)} \\
(71-131) \\
(55-104) \\
(49-155) \\
(78-121) \\
(78-129) \\
(77-124)
\end{gathered}
$$

\# Column to be used to flag recovery values

* Values outside of contract required QC limits

Page 1 of 1 
Lab Name: TESTAMERICA BURLINGTON

Lab Code: STLV Case No.: YORK Mod. Ref No.:
Contract: $8 \mathrm{E}-00302$

SDG No.: 28963

Level: (TRACE or LOW) TRACE

\begin{tabular}{|c|c|c|c|c|c|c|c|c|}
\hline $\begin{array}{c}\text { EPA } \\
\text { SAMPLE NO. }\end{array}$ & $\begin{array}{l}\text { VDMC 8 } \\
(\mathrm{DPA}) \#\end{array}$ & $\begin{array}{l}\text { VDMC9 } \\
(\text { TOL }) \#\end{array}$ & $\begin{array}{l}\text { VDMC10 } \\
(\mathrm{TDP}) \#\end{array}$ & $\begin{array}{l}\text { VDMC11 } \\
(\operatorname{HEX}) \#\end{array}$ & $\begin{array}{l}\text { VDMC12 } \\
(\mathrm{TCA}) \#\end{array}$ & $\begin{array}{l}\text { VDMC13 } \\
(\mathrm{DCZ}) \quad \#\end{array}$ & OTHER & $\begin{array}{l}\text { TOT } \\
\text { OUT }\end{array}$ \\
\hline VBLKDS & 95 & 104 & 98 & 90 & 87 & 108 & & 0 \\
\hline $\begin{array}{l}\text { YKTI0580-W-378 } \\
68 \mathrm{DL}\end{array}$ & 94 & 106 & 86 & 69 & 74 & 108 & & 0 \\
\hline YKQCTB-W-37869 & 95 & 101 & 96 & 95 & 87 & 95 & & 0 \\
\hline $\begin{array}{l}\text { YKTI07100-W-37 } \\
876\end{array}$ & 94 & 102 & 95 & 131 & 89 & 103 & & 0 \\
\hline $\begin{array}{l}\text { YKTI0680-W-378 } \\
72\end{array}$ & 95 & 102 & 92 & 80 & 83 & 102 & & 0 \\
\hline $\begin{array}{l}\text { YKTI0580-W-378 } \\
68\end{array}$ & 94 & 103 & 95 & 88 & 89 & 105 & & 0 \\
\hline VBLKDX & 102 & 103 & 95 & 94 & 95 & 102 & & 0 \\
\hline VHBLK01 & 100 & 104 & 91 & 85 & 92 & 100 & & 0 \\
\hline
\end{tabular}

\footnotetext{
$\operatorname{VDMC} 8(\mathrm{DPA})=1,2-\mathrm{Dichloropropane}-\mathrm{d} 6$

VDMC $9($ TOL $)=$ Toluene-d8

VDMC10 $(\mathrm{TDP})=$ trans $-1,3-\mathrm{Dichloropropene-d4}$

VDMC11 $(\mathrm{HEX})=2-$ Hexanone-d5

VDMC12 $(\mathrm{TCA})=1,1,2,2$-Tetrachloroethane-d2

VDMC13 $(\mathrm{DCZ})=1,2-\mathrm{Dichlorobenzene-d4}$
}

$$
\begin{gathered}
\frac{Q C \text { LIMITS }}{(79-124)} \\
(77-121) \\
(73-121) \\
(28-135) \\
(73-125) \\
(80-131)
\end{gathered}
$$

\# Column to be used to flag recovery values

* Values outside of contract required QC limits

Report 1,4-Dioxane-d8 for Low-Medium VOA analysis only

Page 1 of 1 
$4 A$ - FORM IV VOA

VOLATILE METHOD BLANK SUMMARY
EPA SAMPLE NO.

VBLKDS

Lab Name: TESTAMERICA BURLINGTON

Contract: $8 \mathrm{E}-00302$

Lab Code: STLV Case No.: YORK Mod. Ref No.: SDG No.: 28963

Lab File ID: 14885_005.D

Lab Sample ID: MB 200-91665/5

Instrument ID: CHD.i

Matrix: (SOIL/SED/WATER) Water

Date Analyzed: 07/23/2015

Level: (TRACE or LOW/MED) TRACE

Time Analyzed: 0941

GC Column: DB-624

ID $: 0.20 \quad(\mathrm{~mm})$

Heated Purge: (Y/N) N

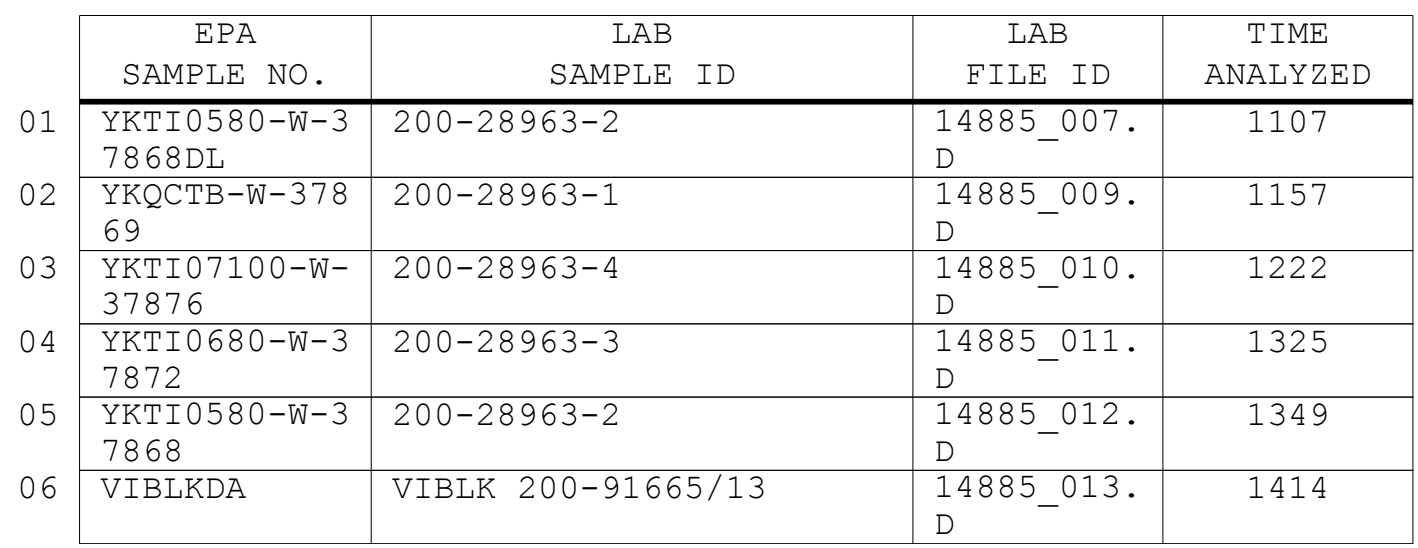

COMMENTS :

Page 1 of 1 
Lab Name: TESTAMERICA BURLINGTON

Contract: 8E-00302

Lab Code: STLV Case No.: YORK Mod. Ref No.: SDG No.: 28963

Lab File ID: 15058_005.D

Lab Sample ID: MB 200-92179/5

Instrument ID: CHD.i

Matrix: (SOIL/SED/WATER) Water

Date Analyzed: 08/03/2015

Level: (TRACE or LOW/MED) TRACE

Time Analyzed: 1127

GC Column: DB-624

ID $0.20 \quad(\mathrm{~mm})$

Heated Purge: ( $\mathrm{Y} / \mathrm{N}) \mathrm{N}$

\begin{tabular}{|c|c|c|c|}
\hline $\begin{array}{c}\text { EPA } \\
\text { SAMPLE NO. }\end{array}$ & $\begin{array}{r}\text { LAB } \\
\text { SAMPLE ID }\end{array}$ & $\begin{array}{c}\text { LAB } \\
\text { FILE ID }\end{array}$ & $\begin{array}{c}\text { TIME } \\
\text { ANALYZED }\end{array}$ \\
\hline VHBLK01 & $200-28963-5$ & $\begin{array}{l}15058 \_006 . \\
D\end{array}$ & 1634 \\
\hline
\end{tabular}

COMMENTS :

Page 1 of 1 
$5 A$ - FORM V VOA

VOLATILE ORGANICS INSTRUMENT

PERFORMANCE CHECK

BROMOFLUOROBENZENE (BFB)
EPA SAMPLE NO.

BFBDG
Lab Name: TESTAMERICA BURLINGTON

Lab Code: STLV

Case No.: YORK

Lab File Id: 14685_001.D

Instrument Id: CHD.i

GC Column: DB-624

ID $: 0.20$
Contract: 8E-00302 Mod. Ref No.:

SDG No.: 28963

BEB Injection Date: 07/14/2015

BFB Injection Time: 1045 $(\mathrm{mm})$

\begin{tabular}{|c|c|c|}
\hline $\mathrm{m} / \mathrm{e}$ & ION ABUNDANCE CRITERIA & $\begin{array}{l}\text { \% RELATIVE } \\
\text { ABUNDANCE }\end{array}$ \\
\hline 50 & $15.0-40.0 \%$ of mass 95 & 22.2 \\
\hline 75 & $30.0-80.0 \%$ of mass 95 & 60.1 \\
\hline 95 & Base peak, $100 \%$ relative abundance & 100 \\
\hline 96 & $5.0-9.0 \%$ of mass 95 & 6.7 \\
\hline 173 & Less than $2.0 \%$ of mass 174 & $0.7) 1$ \\
\hline 174 & $50.0-120 \%$ of mass 95 & 113 \\
\hline 175 & $5.0-9.0 \%$ of mass 174 & $8.5(7.6) 1$ \\
\hline 176 & $95.0-101 \%$ of mass 174 & $109(96.8) 1$ \\
\hline 177 & $5.0-9.0 \%$ of mass 176 & $(6.0) 2$ \\
\hline
\end{tabular}

1 - Value is omass 1742 - Value is omass 176

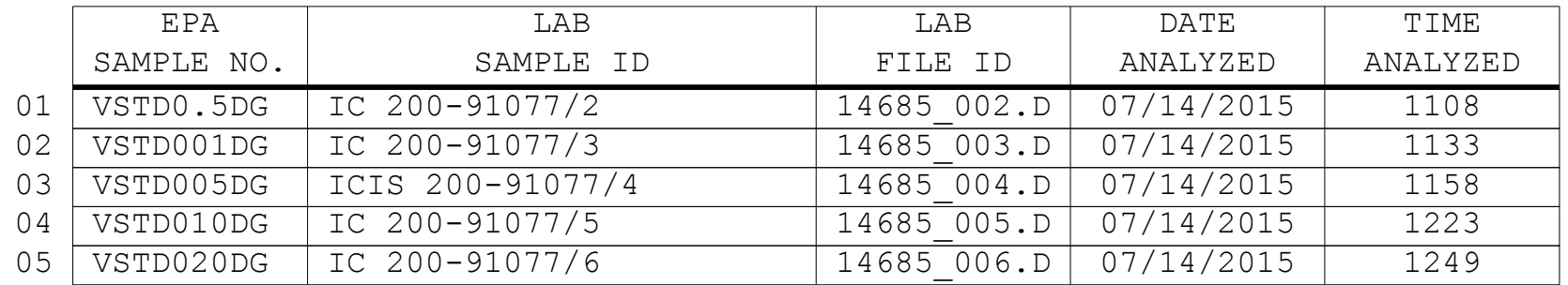


$5 A$ - FORM V VOA

VOLATILE ORGANICS INSTRUMENT

PERFORMANCE CHECK

BROMOFLUOROBENZENE (BFB)
EPA SAMPLE NO.

BFBDS
Lab Name: TESTAMERICA BURLINGTON

Lab Code: STLV

Case No.: YORK

Lab File Id: 14885_001.D

Instrument Id: CHD.i

GC Column: DB-624

ID $: 0.20$
Contract: $8 \mathrm{E}-00302$ Mod. Ref No.: SDG No.: 28963

BEB Injection Date: 07/23/2015

BFB Injection Time: 0805 (mm)

\begin{tabular}{|c|c|c|}
\hline $\mathrm{m} / \mathrm{e}$ & ION ABUNDANCE CRITERIA & $\begin{array}{l}\text { 응 RELATIVE } \\
\text { ABUNDANCE }\end{array}$ \\
\hline 50 & $15.0-40.0 \%$ of mass 95 & 24.1 \\
\hline 75 & $30.0-80.0 \%$ of mass 95 & 64.4 \\
\hline 95 & Base peak, 100\% relative abundance & 100 \\
\hline 96 & $5.0-9.0 \%$ of mass 95 & 6.0 \\
\hline 173 & Less than $2.0 \%$ of mass 174 & $1.2(1.1) 1$ \\
\hline 174 & $50.0-120 \%$ of mass 95 & 117 \\
\hline 175 & $5.0-9.0 \%$ of mass 174 & $8.7(7.4) 1$ \\
\hline 176 & $95.0-101 \%$ of mass 174 & $113(96.5) 1$ \\
\hline 177 & $5.0-9.0 \%$ of mass 176 & $7.5(6.7) 2$ \\
\hline
\end{tabular}

1 - Value is omass 174

2 - Value is omass 176

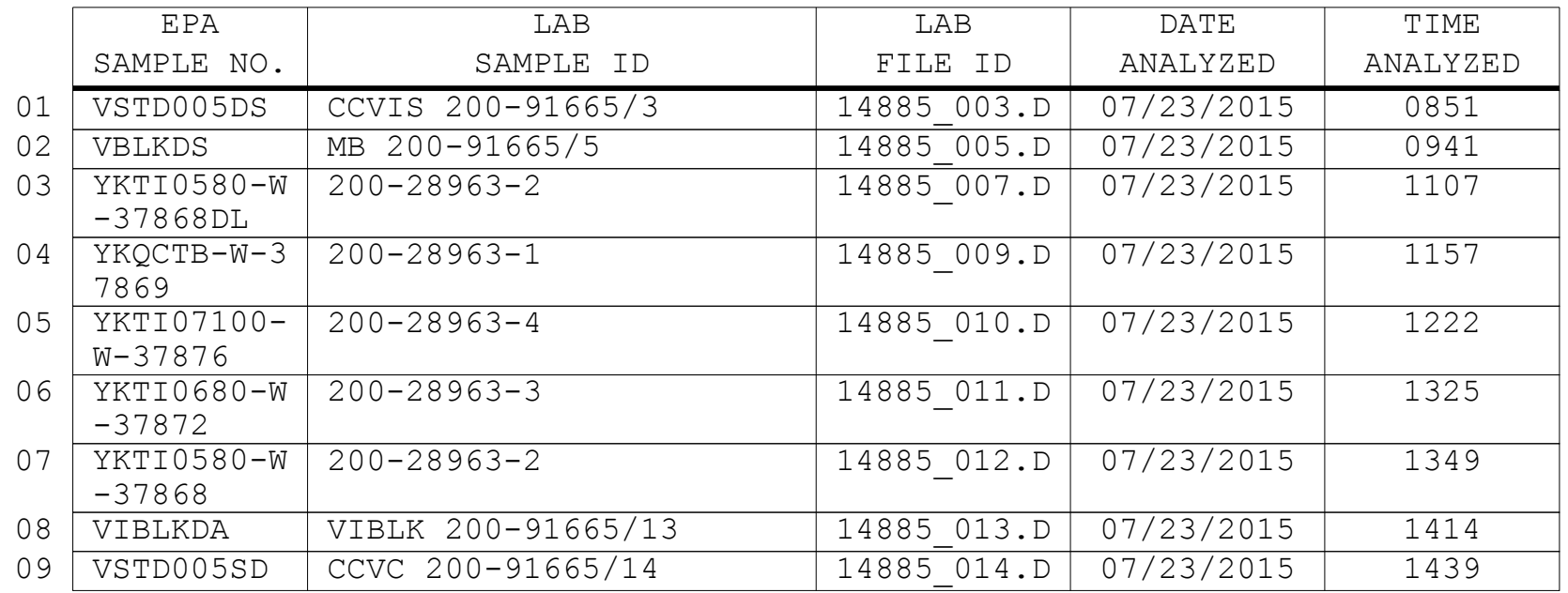


$5 A$ - FORM V VOA

VOLATILE ORGANICS INSTRUMENT

PERFORMANCE CHECK

BROMOFLUOROBENZENE (BFB)
EPA SAMPLE NO.

BFBDW
Lab Name: TESTAMERICA BURLINGTON

Lab Code: STLV Case No.: YORK

Mod.

Lab File Id: 15041_001.D

Instrument Id: CHD.i

GC Column: DB-624

ID $: 0.20$
Contract: $8 \mathrm{E}-00302$

SDG No.: 28963

BEB Injection Date: 07/31/2015

BFB Injection Time: 1119

\begin{tabular}{|c|c|c|}
\hline $\mathrm{m} / \mathrm{e}$ & ION ABUNDANCE CRITERIA & $\begin{array}{l}\text { \% RELATIVE } \\
\text { ABUNDANCE }\end{array}$ \\
\hline 50 & $15.0-40.0 \%$ of mass 95 & 18.0 \\
\hline 75 & $30.0-80.0 \%$ of mass 95 & 48.5 \\
\hline 95 & Base peak, $100 \%$ relative abundance & 100 \\
\hline 96 & $5.0-9.0 \%$ of mass 95 & 6.6 \\
\hline 173 & Less than $2.0 \%$ of mass 174 & $0.7) 1$ \\
\hline 174 & $50.0-120 \%$ of mass 95 & 87.2 \\
\hline 175 & $5.0-9.0 \%$ of mass 174 & $6.2(7.1) 1$ \\
\hline 176 & $95.0-101 \%$ of mass 174 & $84.6(97.0) 1$ \\
\hline 177 & $5.0-9.0 \%$ of mass 176 & $5.4(6.4) 2$ \\
\hline
\end{tabular}

1 - Value is omass 1742 - Value is omass 176

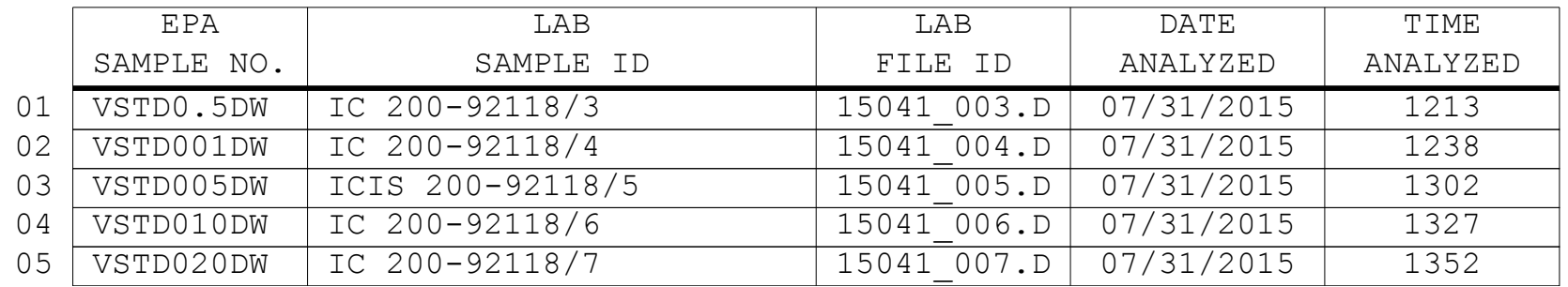

Page 1 of 1 
$5 A$ - FORM V VOA

VOLATILE ORGANICS INSTRUMENT

PERFORMANCE CHECK

BROMOFLUOROBENZENE (BFB)
EPA SAMPLE NO.

BFBDX
Lab Name: TESTAMERICA BURLINGTON

Lab Code: STLV Case No.: YORK

Lab File Id: 15058_001.D

Instrument Id: CHD.i

GC Column: DB-624

ID $: 0.20$
Contract: 8E-00302 Mod. Ref No.: SDG No.: 28963

BEB Injection Date: 08/03/2015

BFB Injection Time: 0939 $(\mathrm{mm})$

\begin{tabular}{|c|c|c|}
\hline $\mathrm{m} / \mathrm{e}$ & ION ABUNDANCE CRITERIA & $\begin{array}{l}\text { \% RELATIVE } \\
\text { ABUNDANCE }\end{array}$ \\
\hline 50 & $15.0-40.0 \%$ of mass 95 & 17.6 \\
\hline 75 & $30.0-80.0 \%$ of mass 95 & 46.9 \\
\hline 95 & Base peak, $100 \%$ relative abundance & 100 \\
\hline 96 & $5.0-9.0 \%$ of mass 95 & 6.7 \\
\hline 173 & Less than $2.0 \%$ of mass 174 & $0.7) 1$ \\
\hline 174 & $50.0-120 \%$ of mass 95 & 88.0 \\
\hline 175 & $5.0-9.0 \%$ of mass 174 & $6.0(6.8) 1$ \\
\hline 176 & $95.0-101 \%$ of mass 174 & $86.2(97.9) 1$ \\
\hline 177 & $5.0-9.0 \%$ of mass 176 & $(6.4) 2$ \\
\hline
\end{tabular}

1 - Value is omass 1742 - Value is omass 176

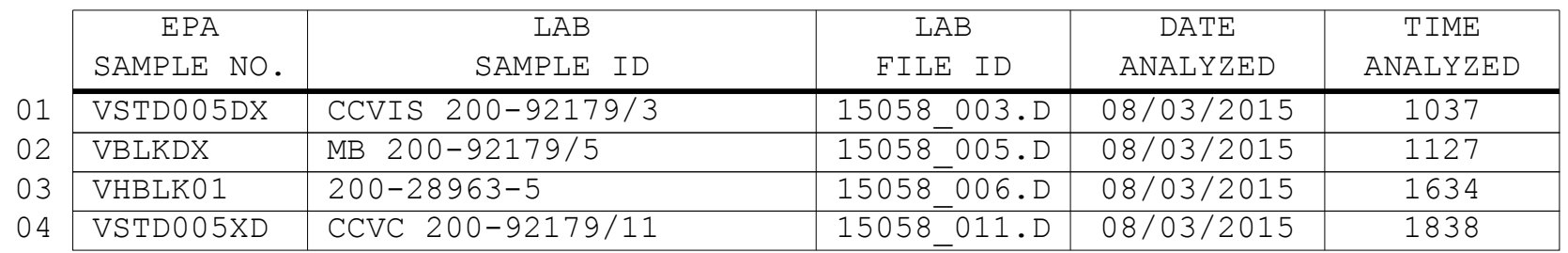




\section{$8 A$ - FORM VIII VOA}

VOLATILE INTERNAL STANDARD AREA AND RETENTION TIME SUMMARY

Lab Name: TESTAMERICA BURLINGTON

Contract: $8 \mathrm{E}-00302$

Lab Code: STLV Case No.: YORK Mod. Ref No.: SDG No.: 28963

GC Column: DB-624

ID: 0.20 (mm) Init. Calib. Date (s) : 07/14/2015 07/14/2015

EPA Sample No.(VSTD\#\#\#\#\#) : VSTD005DS

Lab File ID (Standard): 14885_003.D

Date Analyzed: $07 / 23 / 2015$

Instrument ID: CHD.i

Time Analyzed: 0851

Heated Purge: ( $\mathrm{Y} / \mathrm{N}) \mathrm{N}$

\begin{tabular}{|c|c|c|c|c|c|c|}
\hline & IS1 (CBZ) & & IS2 (DFB) & & IS3 (DCB) & \\
\hline & AREA & RT \# & AREA & RT & AREA & $\mathrm{RT}$ \\
\hline 12 HOUR STD & 227151 & 9.34 & 257597 & 5.97 & 152243 & 12.15 \\
\hline UPPER LIMIT & 318011 & 9.67 & 360636 & 6.30 & 213140 & 12.48 \\
\hline LOWER LIMIT & 136291 & 9.01 & 154558 & 5.64 & 91346 & 11.82 \\
\hline EPA SAMPLE NO. & & & & & & \\
\hline VBLKDS & 208569 & 9.34 & 239347 & 5.97 & 114642 & 12.15 \\
\hline $\begin{array}{l}\text { YKTI0580-W-378 } \\
68 \mathrm{DL}\end{array}$ & 195468 & 9.34 & 227777 & 5.97 & 108197 & 12.15 \\
\hline YKQCTB-W-37869 & 215269 & 9.34 & 241074 & 5.97 & 134188 & 12.15 \\
\hline $\begin{array}{l}\text { YKTI07100-W-37 } \\
876\end{array}$ & 206978 & 9.34 & 240306 & 5.97 & 114711 & 12.15 \\
\hline $\begin{array}{l}\text { YKTI0 } 680-W-378 \\
72\end{array}$ & 199839 & 9.33 & 231761 & 5.97 & 111507 & 12.15 \\
\hline $\begin{array}{l}\text { YKTI0580-W-378 } \\
68\end{array}$ & 204913 & 9.34 & 236107 & 5.97 & 117770 & 12.15 \\
\hline VIBLKDA & 205562 & 9.34 & 233274 & 5.97 & 116677 & 12.15 \\
\hline
\end{tabular}

IS1 $(\mathrm{CBZ})=$ Chlorobenzene-d5

IS2 $(\mathrm{DFB})=1,4-\mathrm{Difluorobenzene}$

IS3 $(\mathrm{DCB})=1,4-\mathrm{Dichlorobenzene-d4}$

AREA UPPER LIMIT $=140 \%$ (Trace Volatiles) of internal standard area AREA LOWER LIMIT $=60 \%$ (Trace Volatiles) of internal standard area RT UPPER LIMIT $=+0.33$ (Trace Volatiles) minutes of internal standard RT RT LOWER LIMIT $=-0.33$ (Trace Volatiles) minutes of internal standard RT

\# Column used to flag values outside contract required QC limits with an asterisk. Page 1 of 1 
Lab Name: TESTAMERICA BURLINGTON

Contract: $8 \mathrm{E}-00302$

Lab Code: STLV Case No.: YORK Mod. Ref No.: SDG No.: 28963

GC Column: DB-624

ID: 0.20 (mm) Init. Calib. Date (s) : 07/31/2015 07/31/2015

EPA Sample No. (VSTD\#\#\#\#\#) : VSTD005DX

Lab File ID (Standard): 15058_003.D

Instrument ID: CHD.i

Date Analyzed: $08 / 03 / 2015$

Time Analyzed: 1037

Heated Purge: (Y/N) N

\begin{tabular}{|c|c|c|c|c|c|c|}
\hline & $\begin{array}{c}\text { IS1 (CBZ) } \\
\text { AREA }\end{array}$ & RT \# & $\begin{array}{c}\text { IS2 (DFB) } \\
\text { AREA }\end{array}$ & $\mathrm{RT}$ & $\begin{array}{c}\text { IS3 ( DCB) } \\
\text { AREA }\end{array}$ & $\mathrm{RT}$ \\
\hline 12 HOUR STD & 1604922 & 9.34 & 1942414 & 5.97 & 835849 & 12.15 \\
\hline UPPER LIMIT & 2246891 & 9.67 & 2719380 & 6.30 & 1170189 & 12.48 \\
\hline LOWER LIMIT & 962953 & 9.01 & 1165448 & 5.64 & 501509 & 11.82 \\
\hline EPA SAMPLE NO. & & & & & & \\
\hline VBLKDX & 1644237 & 9.34 & 1991560 & 5.97 & 845996 & 12.15 \\
\hline VHBLK01 & 1661005 & 9.33 & 2007340 & 5.97 & 853669 & 12.15 \\
\hline
\end{tabular}

IS1 $(\mathrm{CBZ})=$ Chlorobenzene-d5

IS2 $(\mathrm{DFB})=1,4-\mathrm{Difluorobenzene}$

IS3 $(\mathrm{DCB})=1,4-\mathrm{Dichlorobenzene-d4}$

AREA UPPER LIMIT $=140 \%$ (Trace Volatiles) of internal standard area AREA LOWER LIMIT $=60 \%$ (Trace Volatiles) of internal standard area RT UPPER LIMIT $=+0.33$ (Trace Volatiles) minutes of internal standard RT RT LOWER LIMIT $=-0.33$ (Trace Volatiles) minutes of internal standard RT

\# Column used to flag values outside contract required QC limits with an asterisk. Page 1 of 1 
Lab Name: TESTAMERICA BURLINGTON

Contract: $8 \mathrm{E}-00302$

Lab Code: STLV

Case No.: YORK Mod. Ref No.: SDG No.: 28963

Matrix: (SOIL/SED/WATER) Water

Sample wt/vol: 25.0 $(\mathrm{g} / \mathrm{mL}) \mathrm{mL}$

Level: (TRACE/LOW/MED) TRACE

\% Moisture: not dec.

GC Column: DB-624 ID: $0.20 \quad(\mathrm{~mm})$

Soil Extract Volume: $(u L)$ $(\mathrm{mL})$

Purge Volume: 25.0
Lab Sample ID: 200-28963-5

Lab File ID: 15058_006.D

Date Received: 07/21/2015

Date Analyzed: 08/03/2015

Dilution Factor: 1.0

Soil Aliquot Volume: (uL)

\begin{tabular}{|c|c|c|c|}
\hline CAS NO. & COMPOUND & $\begin{array}{l}\text { CONCENTRATION UNITS: } \\
(\mathrm{ug} / \mathrm{L} \text { or } \mathrm{ug} / \mathrm{kg}) \mathrm{ug} / \mathrm{L}\end{array}$ & Q \\
\hline $75-71-8$ & Dichlorodifluoromethane & 1.0 & $\mathrm{U}$ \\
\hline $74-87-3$ & Chloromethane & 0.20 & $\mathrm{~J}$ \\
\hline $75-01-4$ & Vinyl chloride & 1.0 & $\mathrm{U}$ \\
\hline $74-83-9$ & Bromomethane & 1.0 & $\mathrm{U}$ \\
\hline $75-00-3$ & Chloroethane & 1.0 & $\mathrm{U}$ \\
\hline $75-69-4$ & Trichlorofluoromethane & 1.0 & $\mathrm{U}$ \\
\hline $75-35-4$ & 1,1-Dichloroethene & 1.0 & $\mathrm{U}$ \\
\hline $76-13-1$ & $1,1,2$-Trichloro-1,2,2-trifluoroethane & 1.0 & $\mathrm{U}$ \\
\hline $67-64-1$ & Acetone & 1.7 & $\mathrm{~J} B$ \\
\hline $75-15-0$ & Carbon disulfide & 0.083 & $\mathrm{~J} B$ \\
\hline $79-20-9$ & Methyl acetate & 1.0 & $\mathrm{U}$ \\
\hline $75-09-2$ & Methylene Chloride & 1.0 & $\mathrm{U}$ \\
\hline $156-60-5$ & trans-1,2-Dichloroethene & 1.0 & $\mathrm{U}$ \\
\hline $1634-04-4$ & Methyl tert-butyl ether & 1.0 & $\mathrm{U}$ \\
\hline $75-34-3$ & 1,1-Dichloroethane & 1.0 & $\mathrm{U}$ \\
\hline $156-59-2$ & cis-1,2-Dichloroethene & 1.0 & $\mathrm{U}$ \\
\hline $78-93-3$ & 2-Butanone & 5.0 & $\mathrm{U}$ \\
\hline $74-97-5$ & Bromochloromethane & 1.0 & $\mathrm{U}$ \\
\hline $67-66-3$ & Chloroform & 1.0 & $\mathrm{U}$ \\
\hline $71-55-6$ & $1,1,1$-Trichloroethane & 1.0 & $\mathrm{U}$ \\
\hline $110-82-7$ & Cyclohexane & 1.0 & $\mathrm{U}$ \\
\hline $56-23-5$ & Carbon tetrachloride & 1.0 & $\mathrm{U}$ \\
\hline $71-43-2$ & Benzene & 0.030 & $\mathrm{~J} B$ \\
\hline $107-06-2$ & 1,2-Dichloroethane & 1.0 & $\mathrm{U}$ \\
\hline
\end{tabular}

Report 1,4-Dioxane for Low-Medium VOA analysis only 
Lab Name: TESTAMERICA BURLINGTON

Lab Code: STLV

Case No.: YORK

Mod. Ref No.:

Contract: 8E-00302

Matrix: (SOIL/SED/WATER) Water

Sample wt/vol: 25.0 $(\mathrm{g} / \mathrm{mL}) \mathrm{mL}$

Level: (TRACE/LOW/MED) TRACE

\% Moisture: not dec.

GC Column: DB-624 ID $: \underline{0.20 \quad(\mathrm{~mm})}$

Soil Extract Volume: (uL) $(\mathrm{mL})$

Purge Volume: 25.0
SDG No.: 28963

Lab Sample ID: 200-28963-5

Lab File ID: 15058_006.D

Date Received: 07/21/2015

Date Analyzed: 08/03/2015

Dilution Factor: 1.0

Soil Aliquot Volume: (uL)

\begin{tabular}{|c|c|c|c|}
\hline CAS NO. & COMPOUND & $\begin{array}{l}\text { CONCENTRATION UNITS: } \\
(\mathrm{ug} / \mathrm{L} \text { or } \mathrm{ug} / \mathrm{kg}) \mathrm{ug} / \mathrm{L}\end{array}$ & Q \\
\hline $79-01-6$ & Trichloroethene & 0.038 & $\mathrm{~J}$ \\
\hline $108-87-2$ & Methylcyclohexane & 1.0 & $\mathrm{U}$ \\
\hline $78-87-5$ & 1,2-Dichloropropane & 1.0 & $\mathrm{U}$ \\
\hline $75-27-4$ & Bromodichloromethane & 1.0 & $\mathrm{U}$ \\
\hline $10061-01-5$ & cis-1,3-Dichloropropene & 1.0 & U \\
\hline $108-10-1$ & 4-Methyl-2-pentanone & 1.0 & $\mathrm{U}$ \\
\hline $108-88-3$ & Toluene & 0.022 & $\mathrm{~J} B$ \\
\hline $10061-02-6$ & trans-1,3-Dichloropropene & 1.0 & $\mathrm{U}$ \\
\hline $79-00-5$ & $1,1,2-$ Trichloroethane & 1.0 & $\mathrm{U}$ \\
\hline $127-18-4$ & Tetrachloroethene & 0.012 & $\mathrm{~J} B$ \\
\hline $591-78-6$ & 2-Hexanone & 1.0 & $\mathrm{U}$ \\
\hline $124-48-1$ & Dibromochloromethane & 1.0 & $\mathrm{U}$ \\
\hline $106-93-4$ & 1,2-Dibromoethane & 1.0 & $\mathrm{U}$ \\
\hline $108-90-7$ & Chlorobenzene & 1.0 & $\mathrm{U}$ \\
\hline $100-41-4$ & Ethylbenzene & 0.0085 & $\mathrm{~J} B$ \\
\hline $95-47-6$ & o-Xylene & 0.0064 & $\mathrm{~J} B$ \\
\hline $179601-23-1$ & m,p-xylene & 0.014 & $\mathrm{~J} B$ \\
\hline $100-42-5$ & Styrene & 0.014 & $\mathrm{~J}$ \\
\hline $75-25-2$ & Bromoform & 1.0 & $\mathrm{U}$ \\
\hline $98-82-8$ & Isopropylbenzene & 0.0049 & $\mathrm{~J}$ \\
\hline $79-34-5$ & $1,1,2,2$-Tetrachloroethane & 1.0 & $\mathrm{U}$ \\
\hline $541-73-1$ & 1,3 -Dichlorobenzene & 0.022 & $\mathrm{~J} B$ \\
\hline $106-46-7$ & 1,4-Dichlorobenzene & 0.026 & $\mathrm{~J} B$ \\
\hline $95-50-1$ & 1,2-Dichlorobenzene & 1.0 & $\mathrm{U}$ \\
\hline $96-12-8$ & 1,2-Dibromo-3-Chloropropane & 1.0 & $\mathrm{U}$ \\
\hline $120-82-1$ & $1,2,4$-Trichlorobenzene & 0.050 & $\mathrm{~J} B$ \\
\hline $87-61-6$ & $1,2,3$-Trichlorobenzene & 0.051 & $\mathrm{~J} B$ \\
\hline
\end{tabular}


Lab Name: TESTAMERICA BURLINGTON

Contract: $8 \mathrm{E}-00302$

Lab Code: STLV Case No.: YORK Mod. Ref No.: SDG No.: 28963

Matrix: (SOIL/SED/WATER) Water

Sample wt/vol: 25.0 $(\mathrm{g} / \mathrm{mL}) \mathrm{mL}$

Level: (TRACE or LOW/MED) TRACE

․ Moisture: not dec.

GC Column: DB-624 ID $: 0.20 \quad(\mathrm{~mm})$

Soil Extract Volume: (uL)

CONCENTRATION UNITS: (ug/L or ug/kg) ug/L
Lab Sample ID: 200-28963-5

Lab File ID: 15058_006.D

Date Received: 07/21/2015

Date Analyzed: 08/03/2015

Dilution Factor: 1.0

Soil Aliquot Volume: (uL) Purge Volume: 25.0

\begin{tabular}{|c|c|c|c|c|}
\hline CAS NUMBER & COMPOUND NAME & RT & EST. CONC. & $Q$ \\
\hline 01 & Unknown & 7.29 & 3.1 & $\mathrm{~J} B \mathrm{X}$ \\
\hline $541-05-9$ & Cyclotrisiloxane, hexamethyl- & 8.22 & 0.96 & $\mathrm{~J} \quad \mathrm{~N} B$ \\
\hline 03 & Unknown & 11.04 & 1.9 & $\mathrm{~J} \mathrm{~B}$ \\
\hline E9667961 & Total Alkanes & $\mathrm{N} / \mathrm{A}$ & & \\
\hline
\end{tabular}

1EPA-designated Registry Number. 
Lab Name: TESTAMERICA BURLINGTON

Contract: $8 \mathrm{E}-00302$

Lab Code: STLV

Case No.: YORK Mod. Ref No.: SDG No.: 28963

Matrix: (SOIL/SED/WATER) Water

Sample wt/vol: 25.0 $(\mathrm{g} / \mathrm{mL}) \mathrm{mL}$

Level: (TRACE/LOW/MED) TRACE

\% Moisture: not dec.

GC Column: DB-624 ID: $0.20 \quad(\mathrm{~mm})$

Soil Extract Volume: (uL) $(\mathrm{mL})$

Purge Volume: 25.0
Lab Sample ID: 200-28963-1

Lab File ID: 14885_009.D

Date Received: 07/21/2015

Date Analyzed: 07/23/2015

Dilution Factor: 1.0

Soil Aliquot Volume: (uL)

\begin{tabular}{|c|c|c|c|}
\hline CAS NO. & COMPOUND & $\begin{array}{l}\text { CONCENTRATION UNITS: } \\
(\mathrm{ug} / \mathrm{L} \text { or } \mathrm{ug} / \mathrm{kg}) \mathrm{ug} / \mathrm{L}\end{array}$ & q \\
\hline $75-71-8$ & Dichlorodifluoromethane & 1.0 & $\mathrm{U}$ \\
\hline $74-87-3$ & Chloromethane & 1.0 & $\mathrm{U}$ \\
\hline $75-01-4$ & Vinyl chloride & 1.0 & $\mathrm{U}$ \\
\hline $74-83-9$ & Bromomethane & 1.0 & $\mathrm{U}$ \\
\hline $75-00-3$ & Chloroethane & 1.0 & $\mathrm{U}$ \\
\hline $75-69-4$ & Trichlorofluoromethane & 1.0 & $\mathrm{U}$ \\
\hline $75-35-4$ & 1,1-Dichloroethene & 1.0 & $\mathrm{U}$ \\
\hline $76-13-1$ & $1,1,2$-Trichloro-1,2,2-trifluoroethane & 1.0 & $\mathrm{U}$ \\
\hline $67-64-1$ & Acetone & 2.7 & $\mathrm{~J}$ \\
\hline $75-15-0$ & Carbon disulfide & 0.077 & $\mathrm{~J}$ \\
\hline $79-20-9$ & Methyl acetate & 1.0 & $\mathrm{U}$ \\
\hline $75-09-2$ & Methylene Chloride & 1.0 & $\mathrm{U}$ \\
\hline $156-60-5$ & trans-1,2-Dichloroethene & 1.0 & $\mathrm{U}$ \\
\hline $1634-04-4$ & Methyl tert-butyl ether & 1.0 & $\mathrm{U}$ \\
\hline $75-34-3$ & 1,1-Dichloroethane & 1.0 & $\mathrm{U}$ \\
\hline $156-59-2$ & cis-1,2-Dichloroethene & 1.0 & $\mathrm{U}$ \\
\hline $78-93-3$ & 2-Butanone & 5.0 & $\mathrm{U}$ \\
\hline $74-97-5$ & Bromochloromethane & 1.0 & $\mathrm{U}$ \\
\hline $67-66-3$ & Chloroform & 0.064 & $\mathrm{~J}$ \\
\hline $71-55-6$ & $1,1,1$-Trichloroethane & 1.0 & $\mathrm{U}$ \\
\hline $110-82-7$ & Cyclohexane & 1.0 & $\mathrm{U}$ \\
\hline $56-23-5$ & Carbon tetrachloride & 1.0 & $\mathrm{U}$ \\
\hline $71-43-2$ & Benzene & 0.060 & $\mathrm{~J}$ \\
\hline $107-06-2$ & 1,2-Dichloroethane & 1.0 & $\mathrm{U}$ \\
\hline
\end{tabular}

Report 1,4-Dioxane for Low-Medium VOA analysis only 
Lab Name: TESTAMERICA BURLINGTON

Contract: $8 \mathrm{E}-00302$

Lab Code: STLV

Case No.: YORK

Mod. Ref No.:

SDG No.: 28963

Matrix: (SOIL/SED/WATER) Water

Sample wt/vol: 25.0 $(\mathrm{g} / \mathrm{mL}) \mathrm{mL}$

Level: (TRACE/LOW/MED) TRACE

\% Moisture: not dec.

GC Column: DB-624 ID: $0.20 \quad(\mathrm{~mm})$

Soil Extract Volume: (uL) $(\mathrm{mL})$

Purge Volume: 25.0 (mI)
Lab Sample ID: 200-28963-1

Lab File ID: 14885_009.D

Date Received: 07/21/2015

Date Analyzed: 07/23/2015

Dilution Factor: 1.0

Soil Aliquot Volume: (uL)

\begin{tabular}{|c|c|c|c|}
\hline CAS NO. & COMPOUND & $\begin{array}{l}\text { CONCENTRATION UNITS: } \\
(\mathrm{ug} / \mathrm{L} \text { or } \mathrm{ug} / \mathrm{kg}) \mathrm{ug} / \mathrm{L}\end{array}$ & $q$ \\
\hline $79-01-6$ & Trichloroethene & 1.0 & $\mathrm{U}$ \\
\hline $108-87-2$ & Methylcyclohexane & 1.0 & $\mathrm{U}$ \\
\hline $78-87-5$ & 1,2-Dichloropropane & 1.0 & $\mathrm{U}$ \\
\hline $75-27-4$ & Bromodichloromethane & 1.0 & $\mathrm{U}$ \\
\hline $10061-01-5$ & cis-1,3-Dichloropropene & 1.0 & U \\
\hline $108-10-1$ & 4-Methyl-2-pentanone & 1.0 & $\mathrm{U}$ \\
\hline $108-88-3$ & Toluene & 0.59 & $\mathrm{~J}$ \\
\hline $10061-02-6$ & trans-1,3-Dichloropropene & 1.0 & $\mathrm{U}$ \\
\hline $79-00-5$ & $1,1,2-$ Trichloroethane & 1.0 & $\mathrm{U}$ \\
\hline $127-18-4$ & Tetrachloroethene & 1.4 & \\
\hline $591-78-6$ & 2-Hexanone & 1.0 & $\mathrm{U}$ \\
\hline $124-48-1$ & Dibromochloromethane & 1.0 & $\mathrm{U}$ \\
\hline $106-93-4$ & 1,2-Dibromoethane & 1.0 & $\mathrm{U}$ \\
\hline $108-90-7$ & Chlorobenzene & 1.0 & $\mathrm{U}$ \\
\hline $100-41-4$ & Ethylbenzene & 0.052 & $\mathrm{~J}$ \\
\hline $95-47-6$ & o-Xylene & 0.16 & $\mathrm{~J}$ \\
\hline $179601-23-1$ & m,p-xylene & 0.22 & $\mathrm{~J}$ \\
\hline $100-42-5$ & Styrene & 1.0 & $\mathrm{U}$ \\
\hline $75-25-2$ & Bromoform & 1.0 & $\mathrm{U}$ \\
\hline $98-82-8$ & Isopropylbenzene & 1.0 & $\mathrm{U}$ \\
\hline $79-34-5$ & $1,1,2,2$-Tetrachloroethane & 1.0 & $\mathrm{U}$ \\
\hline $541-73-1$ & 1,3 -Dichlorobenzene & 1.0 & $\mathrm{U}$ \\
\hline $106-46-7$ & 1,4-Dichlorobenzene & 1.0 & $\mathrm{U}$ \\
\hline $95-50-1$ & 1,2-Dichlorobenzene & 1.0 & $\mathrm{U}$ \\
\hline $96-12-8$ & 1,2-Dibromo-3-Chloropropane & 1.0 & $\mathrm{U}$ \\
\hline $120-82-1$ & $1,2,4$-Trichlorobenzene & 1.0 & $\mathrm{U}$ \\
\hline $87-61-6$ & $1,2,3$-Trichlorobenzene & 1.0 & $\mathrm{U}$ \\
\hline
\end{tabular}


Lab Name: TESTAMERICA BURLINGTON

Contract: $8 \mathrm{E}-00302$

Lab Code: STLV Case No.: YORK Mod. Ref No.: SDG No.: 28963

Matrix: (SOIL/SED/WATER) Water

Sample wt/vol: 25.0 $(\mathrm{g} / \mathrm{mL}) \mathrm{mL}$

Level: (TRACE or LOW/MED) TRACE

․ Moisture: not dec.

GC Column: DB-624 ID $: 0.20 \quad(\mathrm{~mm})$

Soil Extract Volume: (uL)
Lab Sample ID: 200-28963-1

Lab File ID: 14885_009.D

Date Received: 07/21/2015

Date Analyzed: 07/23/2015

Dilution Factor: 1.0

Soil Aliquot Volume: (uL)

Purge Volume: 25.0

$(\mathrm{mL})$

CONCENTRATION UNITS: (ug/L or ug/kg) ug/L

\begin{tabular}{|c|c|c|c|c|}
\hline CAS NUMBER & COMPOUND NAME & RT & EST. CONC. & $Q$ \\
\hline 01 & Unknown & 7.29 & 2.9 & $\mathrm{~J} B \mathrm{X}$ \\
\hline $541-05-9$ & Cyclotrisiloxane, hexamethyl- & 8.22 & 2.3 & $\mathrm{~J} \quad \mathrm{~N} B$ \\
\hline $556-67-2$ & Cyclotetrasiloxane, octamethyl- & 11.04 & 1.9 & $\mathrm{~J} N \mathrm{~N}$ \\
\hline E9667961 & Total Alkanes & $\mathrm{N} / \mathrm{A}$ & & \\
\hline
\end{tabular}

1 EPA-designated Registry Number. 
Lab Name: TESTAMERICA BURLINGTON

Contract: 8E-00302

Lab Code: STLV

Case No.: YORK Mod. Ref No.: SDG No.: 28963

Matrix: (SOIL/SED/WATER) Water

Sample wt/vol: 25.0 $(\mathrm{g} / \mathrm{mL}) \mathrm{mL}$

Level: (TRACE/LOW/MED) TRACE

\% Moisture: not dec.

GC Column: DB-624 ID: $0.20 \quad(\mathrm{~mm})$

Soil Extract Volume: (uL) $(\mathrm{mL})$

Purge Volume: 25.0
Lab Sample ID: 200-28963-2

Lab File ID: 14885 _012.D

Date Received: 07/21/2015

Date Analyzed: $07 / 23 / 2015$

Dilution Factor: 2.8

Soil Aliquot Volume: (uL)

\begin{tabular}{|c|c|c|c|}
\hline CAS NO. & COMPOUND & $\begin{array}{l}\text { CONCENTRATION UNITS: } \\
(\mathrm{ug} / \mathrm{L} \text { or } \mathrm{ug} / \mathrm{kg}) \mathrm{ug} / \mathrm{L}\end{array}$ & $Q$ \\
\hline $75-71-8$ & Dichlorodifluoromethane & 2.8 & $\mathrm{U}$ \\
\hline $74-87-3$ & Chloromethane & 2.8 & $\mathrm{U}$ \\
\hline $75-01-4$ & Vinyl chloride & 2.8 & $\mathrm{U}$ \\
\hline $74-83-9$ & Bromomethane & 2.8 & $\mathrm{U}$ \\
\hline $75-00-3$ & Chloroethane & 2.8 & $\mathrm{U}$ \\
\hline $75-69-4$ & Trichlorofluoromethane & 0.16 & $\mathrm{~J}$ \\
\hline $75-35-4$ & 1,1-Dichloroethene & 2.8 & $\mathrm{U}$ \\
\hline $76-13-1$ & 1,1,2-Trichloro-1,2,2-trifluoroethane & 2.8 & $\mathrm{U}$ \\
\hline $67-64-1$ & Acetone & 17 & \\
\hline $75-15-0$ & Carbon disulfide & 2.8 & $\mathrm{U}$ \\
\hline $79-20-9$ & Methyl acetate & 2.8 & $\mathrm{U}$ \\
\hline $75-09-2$ & Methylene Chloride & 2.8 & $\mathrm{U}$ \\
\hline $156-60-5$ & trans-1,2-Dichloroethene & 2.8 & $\mathrm{U}$ \\
\hline $1634-04-4$ & Methyl tert-butyl ether & 2.8 & $\mathrm{U}$ \\
\hline $75-34-3$ & 1,1-Dichloroethane & 2.8 & $\mathrm{U}$ \\
\hline $156-59-2$ & cis-1,2-Dichloroethene & 2.8 & $\mathrm{U}$ \\
\hline $78-93-3$ & 2-Butanone & 12 & $\mathrm{~J}$ \\
\hline $74-97-5$ & Bromochloromethane & 2.8 & $\mathrm{U}$ \\
\hline $67-66-3$ & Chloroform & 58 & $\mathrm{E}$ \\
\hline $71-55-6$ & $1,1,1$-Trichloroethane & 2.8 & $\mathrm{U}$ \\
\hline $110-82-7$ & Cyclohexane & 2.8 & $\mathrm{U}$ \\
\hline $56-23-5$ & Carbon tetrachloride & 170 & $\mathrm{E}$ \\
\hline $71-43-2$ & Benzene & 2.8 & $\mathrm{U}$ \\
\hline $107-06-2$ & 1,2-Dichloroethane & 2.8 & $\mathrm{U}$ \\
\hline
\end{tabular}

Report 1,4-Dioxane for Low-Medium VOA analysis only 
Lab Name: TESTAMERICA BURLINGTON

Contract: 8E-00302

Lab Code: STLV

Case No.: YORK Mod. Ref No.:

SDG No.: 28963

Matrix: (SOIL/SED/WATER) Water

Sample wt/vol: 25.0 $(\mathrm{g} / \mathrm{mL}) \mathrm{mL}$

Level: (TRACE/LOW/MED) TRACE

\% Moisture: not dec.

GC Column: DB-624 ID: $0.20 \quad(\mathrm{~mm})$

Soil Extract Volume: (uL) $(\mathrm{mL})$

Purge Volume: 25.0
Lab Sample ID: 200-28963-2

Lab File ID: 14885_012.D

Date Received: 07/21/2015

Date Analyzed: 07/23/2015

Dilution Factor: 2.8

Soil Aliquot Volume: (uL) (mI)

\begin{tabular}{|c|c|c|c|}
\hline CAS NO. & COMPOUND & $\begin{array}{l}\text { CONCENTRATION UNITS: } \\
(\mathrm{ug} / \mathrm{L} \text { or } \mathrm{ug} / \mathrm{kg}) \mathrm{ug} / \mathrm{L}\end{array}$ & $q$ \\
\hline $79-01-6$ & Trichloroethene & 2.8 & $\mathrm{U}$ \\
\hline $108-87-2$ & Methylcyclohexane & 2.8 & $\mathrm{U}$ \\
\hline $78-87-5$ & 1,2-Dichloropropane & 2.8 & $\mathrm{U}$ \\
\hline $75-27-4$ & Bromodichloromethane & 2.8 & $\mathrm{U}$ \\
\hline $10061-01-5$ & cis-1,3-Dichloropropene & 2.8 & U \\
\hline $108-10-1$ & 4-Methyl-2-pentanone & 2.8 & $\mathrm{U}$ \\
\hline $108-88-3$ & Toluene & 4.2 & \\
\hline $10061-02-6$ & trans-1,3-Dichloropropene & 2.8 & $\mathrm{U}$ \\
\hline $79-00-5$ & $1,1,2-$ Trichloroethane & 2.8 & $\mathrm{U}$ \\
\hline $127-18-4$ & Tetrachloroethene & 0.15 & $\mathrm{~J}$ \\
\hline $591-78-6$ & 2-Hexanone & 2.8 & $\mathrm{U}$ \\
\hline $124-48-1$ & Dibromochloromethane & 2.8 & $\mathrm{U}$ \\
\hline $106-93-4$ & 1,2-Dibromoethane & 2.8 & $\mathrm{U}$ \\
\hline $108-90-7$ & Chlorobenzene & 2.8 & $\mathrm{U}$ \\
\hline $100-41-4$ & Ethylbenzene & 2.8 & $\mathrm{U}$ \\
\hline $95-47-6$ & o-Xylene & 2.8 & $\mathrm{U}$ \\
\hline $179601-23-1$ & m,p-xylene & 2.8 & $\mathrm{U}$ \\
\hline $100-42-5$ & Styrene & 2.8 & $\mathrm{U}$ \\
\hline $75-25-2$ & Bromoform & 2.8 & $\mathrm{U}$ \\
\hline $98-82-8$ & Isopropylbenzene & 2.8 & $\mathrm{U}$ \\
\hline $79-34-5$ & $1,1,2,2$-Tetrachloroethane & 2.8 & $\mathrm{U}$ \\
\hline $541-73-1$ & 1,3 -Dichlorobenzene & 2.8 & $\mathrm{U}$ \\
\hline $106-46-7$ & 1,4-Dichlorobenzene & 2.8 & $\mathrm{U}$ \\
\hline $95-50-1$ & 1,2-Dichlorobenzene & 2.8 & $\mathrm{U}$ \\
\hline $96-12-8$ & 1,2-Dibromo-3-Chloropropane & 2.8 & $\mathrm{U}$ \\
\hline $120-82-1$ & $1,2,4$-Trichlorobenzene & 2.8 & $\mathrm{U}$ \\
\hline $87-61-6$ & $1,2,3$-Trichlorobenzene & 2.8 & $\mathrm{U}$ \\
\hline
\end{tabular}


Lab Name: TESTAMERICA BURLINGTON

Contract: $8 \mathrm{E}-00302$

Lab Code: STLV Case No.: YORK Mod. Ref No.: SDG No.: 28963

Matrix: (SOIL/SED/WATER) Water

Sample wt/vol: 25.0 $(\mathrm{g} / \mathrm{mL}) \mathrm{mL}$

Level: (TRACE or LOW/MED) TRACE

Moisture: not dec.

GC Column: DB-624 ID $: 0.20 \quad(\mathrm{~mm})$

Soil Extract Volume: (uL)

CONCENTRATION UNITS: (ug/L or ug/kg) ug/L
Lab Sample ID: 200-28963-2

Lab File ID: 14885 _012.D

Date Received: 07/21/2015

Date Analyzed: 07/23/2015

Dilution Factor: 2.8

Soil Aliquot Volume: (uL)

Purge Volume: 25.0

$(\mathrm{mL})$

\begin{tabular}{l|l|l|r|r|r|}
\cline { 2 - 6 } & CAS NUMBER & \multicolumn{1}{|c|}{ COMPOUND NAME } & RT & EST. CONC. & Q \\
\cline { 2 - 6 } 01 & & Unknown & 7.29 & 8.0 & $\mathrm{~J}$ B X \\
\cline { 2 - 6 } 02 & $541-05-9$ & Cyclotrisiloxane, hexamethyl- & 8.22 & 3.8 & $\mathrm{~J} \mathrm{~N} \mathrm{~B}$ \\
\cline { 2 - 6 } 03 & $556-67-2$ & Cyclotetrasiloxane, octamethyl- & 11.04 & $\mathrm{~J} \mathrm{~N} \mathrm{~B}$ \\
\cline { 2 - 6 } 04 & E9667961 & Total Alkanes & $\mathrm{N} / \mathrm{A}$ & & \\
\cline { 2 - 6 }
\end{tabular}

1 EPA-designated Registry Number. 
Lab Name: TESTAMERICA BURLINGTON

Contract: $8 \mathrm{E}-00302$

Lab Code: STLV

Case No.: YORK

Mod. Ref No.:

SDG No.: 28963

Matrix: (SOIL/SED/WATER) Water

Sample wt/vol: 25.0 $(\mathrm{g} / \mathrm{mL}) \mathrm{mL}$

Level: (TRACE/LOW/MED) TRACE

\% Moisture: not dec.

GC Column: DB-624 ID: $0.20 \quad(\mathrm{~mm})$

Soil Extract Volume: (uL) $(\mathrm{mL})$

Purge Volume: 25.0
Lab Sample ID: 200-28963-2

Lab File ID: 14885_007.D

Date Received: 07/21/2015

Date Analyzed: 07/23/2015

Dilution Factor: 14.0

Soil Aliquot Volume: (uL)

\begin{tabular}{|c|c|c|c|}
\hline CAS NO. & COMPOUND & $\begin{array}{l}\text { CONCENTRATION UNITS: } \\
(\mathrm{ug} / \mathrm{L} \text { or } \mathrm{ug} / \mathrm{kg}) \mathrm{ug} / \mathrm{L}\end{array}$ & $Q$ \\
\hline $75-71-8$ & Dichlorodifluoromethane & 14 & $\mathrm{U}$ \\
\hline $74-87-3$ & Chloromethane & 14 & $\mathrm{U}$ \\
\hline $75-01-4$ & Vinyl chloride & 14 & $\mathrm{U}$ \\
\hline $74-83-9$ & Bromomethane & 14 & $\mathrm{U}$ \\
\hline $75-00-3$ & Chloroethane & 14 & $\mathrm{U}$ \\
\hline $75-69-4$ & Trichlorofluoromethane & 14 & $\mathrm{U}$ \\
\hline $75-35-4$ & 1,1-Dichloroethene & 14 & $\mathrm{U}$ \\
\hline $76-13-1$ & $1,1,2$-Trichloro-1,2,2-trifluoroethane & 14 & $\mathrm{U}$ \\
\hline $67-64-1$ & Acetone & 18 & $\mathrm{~J} \mathrm{D}$ \\
\hline $75-15-0$ & Carbon disulfide & 14 & $\mathrm{U}$ \\
\hline $79-20-9$ & Methyl acetate & 14 & $\mathrm{U}$ \\
\hline $75-09-2$ & Methylene Chloride & 14 & $\mathrm{U}$ \\
\hline $156-60-5$ & trans-1,2-Dichloroethene & 14 & $\mathrm{U}$ \\
\hline $1634-04-4$ & Methyl tert-butyl ether & 14 & $\mathrm{U}$ \\
\hline $75-34-3$ & 1,1-Dichloroethane & 14 & $\mathrm{U}$ \\
\hline $156-59-2$ & cis-1,2-Dichloroethene & 14 & $\mathrm{U}$ \\
\hline $78-93-3$ & 2-Butanone & 70 & $\mathrm{U}$ \\
\hline $74-97-5$ & Bromochloromethane & 14 & $\mathrm{U}$ \\
\hline $67-66-3$ & Chloroform & 62 & $\mathrm{D}$ \\
\hline $71-55-6$ & 1,1,1-Trichloroethane & 14 & $\mathrm{U}$ \\
\hline $110-82-7$ & Cyclohexane & 14 & $\mathrm{U}$ \\
\hline $56-23-5$ & Carbon tetrachloride & 180 & $\mathrm{D}$ \\
\hline $71-43-2$ & Benzene & 14 & $\mathrm{U}$ \\
\hline $107-06-2$ & 1,2-Dichloroethane & 14 & $\mathrm{U}$ \\
\hline
\end{tabular}

Report 1,4-Dioxane for Low-Medium VOA analysis only 
Lab Name: TESTAMERICA BURLINGTON

Contract: 8E-00302

Lab Code: STLV

Case No.: YORK

Mod. Ref No.:

SDG No.: 28963

Matrix: (SOIL/SED/WATER) Water

Sample wt/vol: 25.0 $(\mathrm{g} / \mathrm{mL}) \mathrm{mL}$

Level: (TRACE/LOW/MED) TRACE

\% Moisture: not dec.

GC Column: DB-624 ID: $0.20 \quad(\mathrm{~mm})$

Soil Extract Volume: (uL) $(\mathrm{mL})$

Purge Volume: 25.0
Lab Sample ID: 200-28963-2

Lab File ID: 14885_007.D

Date Received: 07/21/2015

Date Analyzed: 07/23/2015

Dilution Factor: 14.0

Soil Aliquot Volume: (uL) (mI)

\begin{tabular}{|c|c|c|c|}
\hline CAS NO. & COMPOUND & $\begin{array}{l}\text { CONCENTRATION UNITS: } \\
(\mathrm{ug} / \mathrm{L} \text { or } \mathrm{ug} / \mathrm{kg}) \mathrm{ug} / \mathrm{L}\end{array}$ & Q \\
\hline $79-01-6$ & Trichloroethene & 14 & $\mathrm{U}$ \\
\hline $108-87-2$ & Methylcyclohexane & 14 & $\mathrm{U}$ \\
\hline $78-87-5$ & 1,2-Dichloropropane & 14 & $\mathrm{U}$ \\
\hline $75-27-4$ & Bromodichloromethane & 14 & $\mathrm{U}$ \\
\hline $10061-01-5$ & cis-1,3-Dichloropropene & 14 & U \\
\hline $108-10-1$ & 4-Methyl-2-pentanone & 14 & $\mathrm{U}$ \\
\hline $108-88-3$ & Toluene & 4.8 & $\mathrm{~J} \mathrm{D}$ \\
\hline $10061-02-6$ & trans-1,3-Dichloropropene & 14 & $\mathrm{U}$ \\
\hline $79-00-5$ & $1,1,2-$ Trichloroethane & 14 & $\mathrm{U}$ \\
\hline $127-18-4$ & Tetrachloroethene & 14 & $\mathrm{U}$ \\
\hline $591-78-6$ & 2-Hexanone & 14 & $\mathrm{U}$ \\
\hline $124-48-1$ & Dibromochloromethane & 14 & $\mathrm{U}$ \\
\hline $106-93-4$ & 1,2-Dibromoethane & 14 & $\mathrm{U}$ \\
\hline $108-90-7$ & Chlorobenzene & 14 & $\mathrm{U}$ \\
\hline $100-41-4$ & Ethylbenzene & 14 & $\mathrm{U}$ \\
\hline $95-47-6$ & o-Xylene & 14 & $\mathrm{U}$ \\
\hline $179601-23-1$ & m,p-xylene & 14 & $\mathrm{U}$ \\
\hline $100-42-5$ & Styrene & 14 & $\mathrm{U}$ \\
\hline $75-25-2$ & Bromoform & 14 & $\mathrm{U}$ \\
\hline $98-82-8$ & Isopropylbenzene & 14 & $\mathrm{U}$ \\
\hline $79-34-5$ & $1,1,2,2$-Tetrachloroethane & 14 & $\mathrm{U}$ \\
\hline $541-73-1$ & 1,3 -Dichlorobenzene & 14 & $\mathrm{U}$ \\
\hline $106-46-7$ & 1,4-Dichlorobenzene & 14 & $\mathrm{U}$ \\
\hline $95-50-1$ & 1,2-Dichlorobenzene & 14 & $\mathrm{U}$ \\
\hline $96-12-8$ & 1,2-Dibromo-3-Chloropropane & 14 & $\mathrm{U}$ \\
\hline $120-82-1$ & $1,2,4$-Trichlorobenzene & 14 & $\mathrm{U}$ \\
\hline $87-61-6$ & $1,2,3$-Trichlorobenzene & 14 & $\mathrm{U}$ \\
\hline
\end{tabular}


Lab Name: TESTAMERICA BURLINGTON

Contract: $8 \mathrm{E}-00302$

Lab Code: STLV Case No.: YORK Mod. Ref No.: SDG No.: 28963

Matrix: (SOIL/SED/WATER) Water

Sample wt/vol: 25.0 $(\mathrm{g} / \mathrm{mL}) \mathrm{mL}$

Level: (TRACE or LOW/MED) TRACE

\% Moisture: not dec.

GC Column: DB-624 ID $: 0.20 \quad(\mathrm{~mm})$

Soil Extract Volume: (uL)

CONCENTRATION UNITS: (ug/L or ug/kg) ug/L
Lab Sample ID: 200-28963-2

Lab File ID: 14885_007.D

Date Received: 07/21/2015

Date Analyzed: 07/23/2015

Dilution Factor: 14.0

Soil Aliquot Volume: (uL) Purge Volume: 25.0 $(\mathrm{mL})$

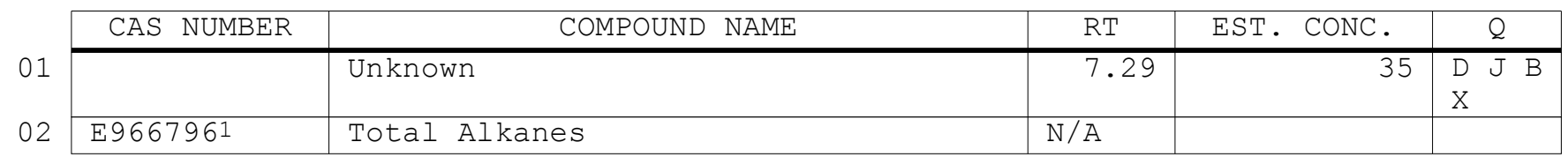

1EPA-designated Registry Number. 
Lab Name: TESTAMERICA BURLINGTON

Contract: $8 \mathrm{E}-00302$

Lab Code: STLV

Case No.: YORK Mod. Ref No.: SDG No.: 28963

Matrix: (SOIL/SED/WATER) Water

Sample wt/vol: 25.0 $(\mathrm{g} / \mathrm{mL}) \mathrm{mL}$

Level: (TRACE/LOW/MED) TRACE

\% Moisture: not dec.

GC Column: DB-624 ID: $0.20 \quad(\mathrm{~mm})$

Soil Extract Volume: $(u L)$ $(\mathrm{mL})$

Purge Volume: 25.0
Lab Sample ID: 200-28963-3

Lab File ID: 14885 _011.D

Date Received: 07/21/2015

Date Analyzed: $07 / 23 / 2015$

Dilution Factor: 1.1

Soil Aliquot Volume: (uL)

\begin{tabular}{|c|c|c|c|}
\hline CAS NO. & COMPOUND & $\begin{array}{l}\text { CONCENTRATION UNITS: } \\
(\mathrm{ug} / \mathrm{L} \text { or } \mathrm{ug} / \mathrm{kg}) \mathrm{ug} / \mathrm{L}\end{array}$ & $Q$ \\
\hline $75-71-8$ & Dichlorodifluoromethane & 1.1 & $\mathrm{U}$ \\
\hline $74-87-3$ & Chloromethane & 1.1 & $\mathrm{U}$ \\
\hline $75-01-4$ & Vinyl chloride & 1.1 & $\mathrm{U}$ \\
\hline $74-83-9$ & Bromomethane & 1.1 & $\mathrm{U}$ \\
\hline $75-00-3$ & Chloroethane & 1.1 & $\mathrm{U}$ \\
\hline $75-69-4$ & Trichlorofluoromethane & 1.1 & $\mathrm{U}$ \\
\hline $75-35-4$ & 1,1-Dichloroethene & 1.1 & $\mathrm{U}$ \\
\hline $76-13-1$ & 1,1,2-Trichloro-1,2,2-trifluoroethane & 1.1 & $\mathrm{U}$ \\
\hline $67-64-1$ & Acetone & 3.3 & $\mathrm{~J}$ \\
\hline $75-15-0$ & Carbon disulfide & 1.1 & $\mathrm{U}$ \\
\hline $79-20-9$ & Methyl acetate & 1.1 & $\mathrm{U}$ \\
\hline $75-09-2$ & Methylene Chloride & 0.97 & $\mathrm{~J}$ \\
\hline $156-60-5$ & trans-1,2-Dichloroethene & 1.1 & $\mathrm{U}$ \\
\hline $1634-04-4$ & Methyl tert-butyl ether & 1.1 & $\mathrm{U}$ \\
\hline $75-34-3$ & 1,1-Dichloroethane & 1.1 & $\mathrm{U}$ \\
\hline $156-59-2$ & cis-1,2-Dichloroethene & 1.1 & $\mathrm{U}$ \\
\hline $78-93-3$ & 2-Butanone & 5.5 & $\mathrm{U}$ \\
\hline $74-97-5$ & Bromochloromethane & 1.1 & $\mathrm{U}$ \\
\hline $67-66-3$ & Chloroform & 0.56 & $\mathrm{~J}$ \\
\hline $71-55-6$ & 1,1,1-Trichloroethane & 1.1 & $\mathrm{U}$ \\
\hline $110-82-7$ & Cyclohexane & 1.1 & $\mathrm{U}$ \\
\hline $56-23-5$ & Carbon tetrachloride & 7.0 & \\
\hline $71-43-2$ & Benzene & 0.047 & $\mathrm{~J}$ \\
\hline $107-06-2$ & 1,2-Dichloroethane & 1.1 & $\mathrm{U}$ \\
\hline
\end{tabular}

Report 1,4-Dioxane for Low-Medium VOA analysis only 
Lab Name: TESTAMERICA BURLINGTON

Contract: 8E-00302

Lab Code: STLV

Case No.: YORK Mod. Ref No.:

SDG No.: 28963

Matrix: (SOIL/SED/WATER) Water

Sample wt/vol: 25.0 $(\mathrm{g} / \mathrm{mL}) \mathrm{mL}$

Level: (TRACE/LOW/MED) TRACE

\% Moisture: not dec.

GC Column: DB-624 ID: $0.20 \quad(\mathrm{~mm})$

Soil Extract Volume: (uL) $(\mathrm{mL})$

Purge Volume: 25.0
Lab Sample ID: 200-28963-3

Lab File ID: 14885_011.D

Date Received: 07/21/2015

Date Analyzed: 07/23/2015

Dilution Factor: 1.1

Soil Aliquot Volume: (uL) (mI)

\begin{tabular}{|c|c|c|c|}
\hline CAS NO. & COMPOUND & $\begin{array}{l}\text { CONCENTRATION UNITS: } \\
(\mathrm{ug} / \mathrm{L} \text { or } \mathrm{ug} / \mathrm{kg}) \mathrm{ug} / \mathrm{L}\end{array}$ & $q$ \\
\hline $79-01-6$ & Trichloroethene & 1.1 & $\mathrm{U}$ \\
\hline $108-87-2$ & Methylcyclohexane & 1.1 & $\mathrm{U}$ \\
\hline $78-87-5$ & 1,2-Dichloropropane & 1.1 & $\mathrm{U}$ \\
\hline $75-27-4$ & Bromodichloromethane & 1.1 & $\mathrm{U}$ \\
\hline $10061-01-5$ & cis-1,3-Dichloropropene & 1.1 & U \\
\hline $108-10-1$ & 4-Methyl-2-pentanone & 1.1 & $\mathrm{U}$ \\
\hline $108-88-3$ & Toluene & 0.099 & $\mathrm{~J}$ \\
\hline $10061-02-6$ & trans-1,3-Dichloropropene & 1.1 & $\mathrm{U}$ \\
\hline $79-00-5$ & $1,1,2-$ Trichloroethane & 1.1 & $\mathrm{U}$ \\
\hline $127-18-4$ & Tetrachloroethene & 0.088 & $\mathrm{~J}$ \\
\hline $591-78-6$ & 2-Hexanone & 1.1 & $\mathrm{U}$ \\
\hline $124-48-1$ & Dibromochloromethane & 1.1 & $\mathrm{U}$ \\
\hline $106-93-4$ & 1,2-Dibromoethane & 1.1 & $\mathrm{U}$ \\
\hline $108-90-7$ & Chlorobenzene & 1.1 & $\mathrm{U}$ \\
\hline $100-41-4$ & Ethylbenzene & 1.1 & $\mathrm{U}$ \\
\hline $95-47-6$ & o-Xylene & 1.1 & $\mathrm{U}$ \\
\hline $179601-23-1$ & m,p-xylene & 1.1 & $\mathrm{U}$ \\
\hline $100-42-5$ & Styrene & 1.1 & $\mathrm{U}$ \\
\hline $75-25-2$ & Bromoform & 1.1 & $\mathrm{U}$ \\
\hline $98-82-8$ & Isopropylbenzene & 1.1 & $\mathrm{U}$ \\
\hline $79-34-5$ & $1,1,2,2$-Tetrachloroethane & 1.1 & $\mathrm{U}$ \\
\hline $541-73-1$ & 1,3 -Dichlorobenzene & 1.1 & $\mathrm{U}$ \\
\hline $106-46-7$ & 1,4-Dichlorobenzene & 1.1 & $\mathrm{U}$ \\
\hline $95-50-1$ & 1,2-Dichlorobenzene & 1.1 & $\mathrm{U}$ \\
\hline $96-12-8$ & 1,2-Dibromo-3-Chloropropane & 1.1 & $\mathrm{U}$ \\
\hline $120-82-1$ & $1,2,4$-Trichlorobenzene & 1.1 & $\mathrm{U}$ \\
\hline $87-61-6$ & $1,2,3$-Trichlorobenzene & 1.1 & $\mathrm{U}$ \\
\hline
\end{tabular}


Lab Name: TESTAMERICA BURLINGTON

Contract: $8 \mathrm{E}-00302$

Lab Code: STLV Case No.: YORK Mod. Ref No.: SDG No.: 28963

Matrix: (SOIL/SED/WATER) Water

Sample wt/vol: 25.0 $(\mathrm{g} / \mathrm{mL}) \mathrm{mL}$

Level: (TRACE or LOW/MED) TRACE

Moisture: not dec.

GC Column: DB-624 ID $: 0.20 \quad(\mathrm{~mm})$

Soil Extract Volume: (uL)

CONCENTRATION UNITS: (ug/L or ug/kg) ug/L
Lab Sample ID: 200-28963-3

Lab File ID: 14885_011.D

Date Received: 07/21/2015

Date Analyzed: 07/23/2015

Dilution Factor: 1.1

Soil Aliquot Volume: (uL) Purge Volume: 25.0 $(\mathrm{mL})$

01

02

\begin{tabular}{|l|l|c|c|c|}
\hline CAS NUMBER & \multicolumn{1}{|c|}{ COMPOUND NAME } & RT & EST. CONC. & Q \\
\hline & Unknown & 7.29 & 3.1 & $\mathrm{~J}$ B X \\
\hline E9667961 & Total Alkanes & N/A & & \\
\hline
\end{tabular}

1EPA-designated Registry Number. 
Lab Name: TESTAMERICA BURLINGTON

Contract: $8 \mathrm{E}-00302$

Lab Code: STLV

Case No.: YORK Mod. Ref No.:

SDG No.: 28963

Matrix: (SOIL/SED/WATER) Water

Sample wt/vol: 25.0 $(\mathrm{g} / \mathrm{mL}) \mathrm{mL}$

Level: (TRACE/LOW/MED) TRACE

\% Moisture: not dec.

GC Column: DB-624 ID: $0.20 \quad(\mathrm{~mm})$

Soil Extract Volume: (uL) $(\mathrm{mL})$

Purge Volume: 25.0
Lab Sample ID: 200-28963-4

Lab File ID: 14885_010.D

Date Received: 07/21/2015

Date Analyzed: 07/23/2015

Dilution Factor: 1.0

Soil Aliquot Volume: (uL)

\begin{tabular}{|c|c|c|c|}
\hline CAS NO. & COMPOUND & $\begin{array}{l}\text { CONCENTRATION UNITS: } \\
(\mathrm{ug} / \mathrm{L} \text { or } \mathrm{ug} / \mathrm{kg}) \mathrm{ug} / \mathrm{L}\end{array}$ & q \\
\hline $75-71-8$ & Dichlorodifluoromethane & 1.0 & $\mathrm{U}$ \\
\hline $74-87-3$ & Chloromethane & 1.0 & $\mathrm{U}$ \\
\hline $75-01-4$ & Vinyl chloride & 1.0 & $\mathrm{U}$ \\
\hline $74-83-9$ & Bromomethane & 1.0 & $\mathrm{U}$ \\
\hline $75-00-3$ & Chloroethane & 1.0 & $\mathrm{U}$ \\
\hline $75-69-4$ & Trichlorofluoromethane & 1.0 & $\mathrm{U}$ \\
\hline $75-35-4$ & 1,1-Dichloroethene & 1.0 & $\mathrm{U}$ \\
\hline $76-13-1$ & $1,1,2$-Trichloro-1,2,2-trifluoroethane & 1.0 & $\mathrm{U}$ \\
\hline $67-64-1$ & Acetone & 5.0 & $\mathrm{U}$ \\
\hline $75-15-0$ & Carbon disulfide & 1.0 & $\mathrm{U}$ \\
\hline $79-20-9$ & Methyl acetate & 1.0 & $\mathrm{U}$ \\
\hline $75-09-2$ & Methylene Chloride & 1.0 & $\mathrm{U}$ \\
\hline $156-60-5$ & trans-1,2-Dichloroethene & 1.0 & $\mathrm{U}$ \\
\hline $1634-04-4$ & Methyl tert-butyl ether & 1.0 & $\mathrm{U}$ \\
\hline $75-34-3$ & 1,1-Dichloroethane & 1.0 & $\mathrm{U}$ \\
\hline $156-59-2$ & cis-1,2-Dichloroethene & 1.0 & $\mathrm{U}$ \\
\hline $78-93-3$ & 2-Butanone & 5.0 & $\mathrm{U}$ \\
\hline $74-97-5$ & Bromochloromethane & 1.0 & $\mathrm{U}$ \\
\hline $67-66-3$ & Chloroform & 0.057 & $\mathrm{~J}$ \\
\hline $71-55-6$ & $1,1,1$-Trichloroethane & 1.0 & $\mathrm{U}$ \\
\hline $110-82-7$ & Cyclohexane & 1.0 & $\mathrm{U}$ \\
\hline $56-23-5$ & Carbon tetrachloride & 0.44 & $\mathrm{~J}$ \\
\hline $71-43-2$ & Benzene & 1.0 & $\mathrm{U}$ \\
\hline $107-06-2$ & 1,2-Dichloroethane & 1.0 & $\mathrm{U}$ \\
\hline
\end{tabular}

Report 1,4-Dioxane for Low-Medium VOA analysis only 
Lab Name: TESTAMERICA BURLINGTON

Contract: $8 \mathrm{E}-00302$

Lab Code: STLV

Case No.: YORK

Mod. Ref No.:

SDG No.: 28963

Matrix: (SOIL/SED/WATER) Water

Sample wt/vol: 25.0 $(\mathrm{g} / \mathrm{mL}) \mathrm{mL}$

Level: (TRACE/LOW/MED) TRACE

\% Moisture: not dec.

GC Column: DB-624 ID: $0.20 \quad(\mathrm{~mm})$

Soil Extract Volume: (uL) $(\mathrm{mL})$

Purge Volume: 25.0 (mI)
Lab Sample ID: 200-28963-4

Lab File ID: 14885_010.D

Date Received: 07/21/2015

Date Analyzed: 07/23/2015

Dilution Factor: 1.0

Soil Aliquot Volume: (uL)

\begin{tabular}{|c|c|c|c|}
\hline CAS NO. & COMPOUND & $\begin{array}{l}\text { CONCENTRATION UNITS: } \\
(\mathrm{ug} / \mathrm{L} \text { or } \mathrm{ug} / \mathrm{kg}) \mathrm{ug} / \mathrm{L}\end{array}$ & $q$ \\
\hline $79-01-6$ & Trichloroethene & 1.0 & $\mathrm{U}$ \\
\hline $108-87-2$ & Methylcyclohexane & 1.0 & $\mathrm{U}$ \\
\hline $78-87-5$ & 1,2-Dichloropropane & 1.0 & $\mathrm{U}$ \\
\hline $75-27-4$ & Bromodichloromethane & 1.0 & $\mathrm{U}$ \\
\hline $10061-01-5$ & cis-1,3-Dichloropropene & 1.0 & U \\
\hline $108-10-1$ & 4-Methyl-2-pentanone & 1.0 & $\mathrm{U}$ \\
\hline $108-88-3$ & Toluene & 0.029 & $\mathrm{~J}$ \\
\hline $10061-02-6$ & trans-1,3-Dichloropropene & 1.0 & $\mathrm{U}$ \\
\hline $79-00-5$ & $1,1,2-$ Trichloroethane & 1.0 & $\mathrm{U}$ \\
\hline $127-18-4$ & Tetrachloroethene & 0.047 & $\mathrm{~J}$ \\
\hline $591-78-6$ & 2-Hexanone & 1.0 & $\mathrm{U}$ \\
\hline $124-48-1$ & Dibromochloromethane & 1.0 & $\mathrm{U}$ \\
\hline $106-93-4$ & 1,2-Dibromoethane & 1.0 & $\mathrm{U}$ \\
\hline $108-90-7$ & Chlorobenzene & 1.0 & $\mathrm{U}$ \\
\hline $100-41-4$ & Ethylbenzene & 1.0 & $\mathrm{U}$ \\
\hline $95-47-6$ & o-Xylene & 1.0 & $\mathrm{U}$ \\
\hline $179601-23-1$ & m,p-xylene & 1.0 & $\mathrm{U}$ \\
\hline $100-42-5$ & Styrene & 1.0 & $\mathrm{U}$ \\
\hline $75-25-2$ & Bromoform & 1.0 & $\mathrm{U}$ \\
\hline $98-82-8$ & Isopropylbenzene & 1.0 & $\mathrm{U}$ \\
\hline $79-34-5$ & $1,1,2,2$-Tetrachloroethane & 1.0 & $\mathrm{U}$ \\
\hline $541-73-1$ & 1,3 -Dichlorobenzene & 1.0 & $\mathrm{U}$ \\
\hline $106-46-7$ & 1,4-Dichlorobenzene & 1.0 & $\mathrm{U}$ \\
\hline $95-50-1$ & 1,2-Dichlorobenzene & 1.0 & $\mathrm{U}$ \\
\hline $96-12-8$ & 1,2-Dibromo-3-Chloropropane & 1.0 & $\mathrm{U}$ \\
\hline $120-82-1$ & $1,2,4$-Trichlorobenzene & 1.0 & $\mathrm{U}$ \\
\hline $87-61-6$ & $1,2,3$-Trichlorobenzene & 1.0 & $\mathrm{U}$ \\
\hline
\end{tabular}


Lab Name: TESTAMERICA BURLINGTON

Contract: $8 \mathrm{E}-00302$

Lab Code: STLV Case No.: YORK Mod. Ref No.: SDG No.: 28963

Matrix: (SOIL/SED/WATER) Water

Sample wt/vol: 25.0 $(\mathrm{g} / \mathrm{mL}) \mathrm{mL}$

Level: (TRACE or LOW/MED) TRACE

․ Moisture: not dec.

GC Column: DB-624 ID $: 0.20 \quad(\mathrm{~mm})$

Soil Extract Volume: (uL)
Lab Sample ID: 200-28963-4

Lab File ID: 14885_010.D

Date Received: 07/21/2015

Date Analyzed: 07/23/2015

Dilution Factor: 1.0

Soil Aliquot Volume: (uL)

Purge Volume: 25.0 $(\mathrm{mL})$ CONCENTRATION UNITS: (ug/L or ug/kg) ug/L

\begin{tabular}{|c|c|c|c|c|}
\hline CAS NUMBER & COMPOUND NAME & RT & EST. CONC. & Q \\
\hline 01 & Unknown & 7.29 & 2.8 & $\mathrm{~J} B \mathrm{X}$ \\
\hline 02 & Unknown & 8.22 & 0.79 & $\mathrm{~J} B$ \\
\hline $556-67-2$ & Cyclotetrasiloxane, octamethyl- & 11.04 & 0.69 & $\mathrm{~J} \quad \mathrm{~N} B$ \\
\hline E9667961 & Total Alkanes & $\mathrm{N} / \mathrm{A}$ & & \\
\hline
\end{tabular}

1 EPA-designated Registry Number. 
Lab Name: TESTAMERICA BURLINGTON

Contract: 8E-00302

Lab Code: STLV Case No.: YORK Mod. Ref No.:

SDG No.: 28963

Instrument ID: CHD.i

Calibration Date(s): 07/14/2015 07/14/2015

Heated Purge: ( $\mathrm{Y} / \mathrm{N}) \mathrm{N}$

Purge Volume: 25.0

Calibration Time(s) : 1108

1249

GC Column: DB-624

ID: 0.20 (mL)

LAB FILE ID:

RRF5.0 = $14685004 . \mathrm{D}$

\begin{tabular}{|c|c|c|c|}
\hline $\operatorname{RRF} 0.5=$ & $=14685 \_002 . \mathrm{D}$ & RRF1. 0 & $=14685 \_003 . \mathrm{D}$ \\
\hline RF10 & $=14685005 . \mathrm{D}$ & RRF20 & 14685 \\
\hline
\end{tabular}

COMPOUND

\begin{tabular}{l|l|l} 
RRF0.5 & RRF1.0 & RR \\
\hline
\end{tabular}

RRE5. 0

Dichlorodifluoromethane

0.538

Chloromethane

Vinyl chloride

Bromomethane

Chloroethane

Trichlorofluoromethane

1,1-Dichloroethene

$1,1,2$-Trichloro-

$1,2,2$-trifluoroethane

Acetone

Carbon disulfide

Methyl acetate

Methylene Chloride

trans-1,2-Dichloroethene

0.327

0.473

0.283

0.366

0.308

0.247

0.163

0.936

0.205

0.302

0.791

0.388

0.288

.370

0.029

0.027

0.745

0.590

0.041

0.045

\begin{tabular}{l|l}
0.237 & 0.197
\end{tabular}

Methyl tert-butyl ether

0.270

.249

0.557

0.283

0.343

0.337

1,1-Dichloroethane

cis-1,2-Dichloroethene

\begin{tabular}{|l|l}
\hline 0.499 & 0.433 \\
\hline 0.254 & 0.226 \\
\hline 0.026 & 0.021 \\
\hline 0.094 & 0.085 \\
\hline 0.598 & 0.545 \\
\hline 0.844 & 0.717 \\
\hline 0.422 & 0.359 \\
\hline 0.817 & 0.705 \\
\hline 1.192 & 1.071 \\
\hline 0.359 & 0.341 \\
\hline 0.407 & 0.333 \\
\hline 0.428 & 0.366 \\
\hline
\end{tabular}

0.335

0.186

0.225

0.940

0.291

2-Butanone

Bromochloromethane

Chloroform

1,1,1-Trichloroethane

Cyclohexane

Carbon tetrachloride

Benzene

1,2-Dichloroethane

Trichloroethene

Methylcyclohexane

0.376

0.023

0.672

0.036

0.215

0.292

0.424

0.487

0.275

0.027

0.107

0.642

0.882

0.468

0.881

1.214

0.383

0.400

0.482

RRF10

RR

RRE20

$\overline{\mathrm{RRF}} \quad \frac{\mathrm{RSD}}{}$

\begin{tabular}{|l|l|l|l|}
\hline 0.579 & 0.568 & 0.543 & 7.7 \\
\hline 0.311 & 0.291 & 0.299 & 6.5 \\
\hline
\end{tabular}

0.311

0.368

0.291

0.240

0.360

\begin{tabular}{ll}
.368 & 0.360 \\
\hline & 0.257
\end{tabular}

\begin{tabular}{l|l|l}
0.246 & 0.245 \\
\hline 0.963 & 0.945 & 0
\end{tabular}

0.963

0.245

$\begin{array}{ll}0.303 & 0.353\end{array}$

$0.390 \quad 0.431$

0.299

$\begin{array}{ll}0.349 & 7.1\end{array}$

$0.216 \quad 18.3$

0.233

0.915

0.307

0.024

0.026

0.698

0.039

0.719

0.228

0.040

0.298

0.222

0.447

0.309

0.447
0.514
0.297

0.468

0.029

0.517

0.029

\begin{tabular}{l|l}
0.468 & 0. \\
0.517 & 0. \\
0.037 & 0. \\
0.030 & 0.
\end{tabular}

\begin{tabular}{l|l|}
0.113 & 0.116 \\
\hline 0.667 & 0.655 \\
\hline 0.907 & 0.907 \\
\hline 0.491 & 0.499
\end{tabular}

$\begin{array}{lll}0.297 & 0.03 \\ 0.116 & 0 .\end{array}$

0.907
0.491
0.905

\begin{tabular}{l|l}
0.991 \\
0.905
\end{tabular}

0.907
0.499

0.905
1.242
0.399

0.499
0.906

\begin{tabular}{l|}
1.242 \\
0.399
\end{tabular}

0.906
1.249
0.383

0.399
0.409

\begin{tabular}{l|l}
0.949 & 0.8 \\
\hline 0.383 & 0.1 \\
0.419 & 0.1 \\
\hline
\end{tabular}

0.409

\begin{tabular}{l|l}
0.383 & 0. \\
0.419 & 0.
\end{tabular}

\begin{tabular}{|c|c|}
\hline & 6.1 \\
\hline 0.026 & 8.6 \\
\hline 0.685 & 8.7 \\
\hline
\end{tabular}

\begin{tabular}{l|l|}
\hline 0.026 & 8.6 \\
\hline 0.685 & 8.7 \\
\hline 0.040 & 8.3 \\
\hline 0.220 & 6.8
\end{tabular}

\begin{tabular}{r|r}
0.040 & 8.3 \\
\hline 0.220 & 6.8 \\
\hline 0.284 & 8.6 \\
\hline 0.402 & 15.6 \\
\hline
\end{tabular}

0.284

\begin{tabular}{r|r|}
\hline 0.402 & 15.6 \\
\hline 0.490 & 6.9 \\
\hline 0.270 & 11.2 \\
\hline 0.027 & 12.3 \\
\hline
\end{tabular}

\begin{tabular}{l|r}
0.490 & 6.9 \\
\hline 0.270 & 11.2 \\
\hline 0.027 & 12.3 \\
\hline 0.103 & 12.9
\end{tabular} 
Lab Name: TESTAMERICA BURLINGTON

Contract: $8 \mathrm{E}-00302$

Lab Code: STLV Case No.: YORK Mod. Ref No.:

SDG No.: 28963

Instrument ID: CHD.i

Calibration Date(s): 07/14/2015 07/14/2015

Heated Purge: ( $\mathrm{Y} / \mathrm{N}) \mathrm{N}$

Purge Volume: 25.0

Calibration Time(s) : 1108

1249

GC Column: DB-624

ID: 0.20 $(\mathrm{mL})$

LAB FILE ID:

RRF5.0 = $14685004 . \mathrm{D}$

\begin{tabular}{|c|c|c|c|}
\hline RRF0. 5 & $=14685 \_002 . D$ & RRF1. 0 & $=14685 \ldots 03 . \mathrm{D}$ \\
\hline RRF10 & $=14685005 . \mathrm{D}$ & RRF20 & $=14685$ \\
\hline
\end{tabular}

COMPOUND

\begin{tabular}{l|l|l} 
RRF0.5 & RRE1.0 \\
\hline
\end{tabular}

RRF5. 0

\begin{tabular}{|c|c|c|c|c|c|c|c|}
\hline 1,2-Dichloropropane & 0.215 & 0.188 & 0.206 & 0.213 & 0.219 & 0.208 & 5.8 \\
\hline Bromodichloromethane & 0.344 & 0.346 & 0.415 & 0.433 & 0.447 & 0.397 & 12.3 \\
\hline cis-1,3-Dichloropropene & 0.311 & 0.287 & 0.403 & 0.427 & 0.443 & 0.374 & 18.9 \\
\hline 4-Methyl-2-pentanone & 0.053 & 0.056 & 0.077 & 0.078 & 0.082 & 0.069 & 19.6 \\
\hline Toluene & 1.363 & 1.257 & 1.516 & 1.560 & 1.599 & 1.459 & 9.9 \\
\hline trans-1,3-Dichloropropene & 0.234 & 0.239 & 0.340 & 0.362 & 0.378 & 0.311 & 22.2 \\
\hline 1,1,2-Trichloroethane & 0.172 & 0.128 & 0.145 & 0.151 & 0.150 & 0.149 & 10.6 \\
\hline Tetrachloroethene & 0.474 & 0.424 & 0.498 & 0.512 & 0.512 & 0.484 & 7.6 \\
\hline 2-Hexanone & 0.041 & 0.043 & 0.055 & 0.059 & 0.061 & 0.052 & 17.7 \\
\hline Dibromochloromethane & 0.184 & 0.171 & 0.250 & 0.257 & 0.274 & 0.227 & 20.4 \\
\hline 1,2-Dibromoethane & 0.127 & 0.114 & 0.141 & 0.150 & 0.157 & 0.138 & 12.5 \\
\hline Chlorobenzene & 0.989 & 0.887 & 1.031 & 1.068 & 1.070 & 1.009 & 7.5 \\
\hline Ethylbenzene & 1.735 & 1.604 & 2.033 & 2.150 & 2.189 & 1.942 & 13.4 \\
\hline o-Xylene & 0.567 & 0.513 & 0.706 & 0.761 & 0.783 & 0.666 & 18.0 \\
\hline $\mathrm{m}, \mathrm{p}-\mathrm{Xylene}$ & 0.641 & 0.610 & 0.786 & 0.819 & 0.842 & 0.740 & 14.4 \\
\hline Styrene & 0.812 & 0.811 & 1.114 & 1.179 & 1.197 & 1.023 & 19.1 \\
\hline Bromoform & 0.158 & 0.135 & 0.186 & 0.192 & 0.211 & 0.176 & 16.9 \\
\hline Isopropylbenzene & 1.804 & 1.689 & 2.356 & 2.515 & 2.566 & 2.186 & 18.8 \\
\hline $1,1,2,2$-Tetrachloroethane & 0.119 & 0.108 & 0.135 & 0.138 & 0.144 & 0.129 & 11.5 \\
\hline 1,3-Dichlorobenzene & 1.470 & 1.245 & 1.596 & 1.652 & 1.707 & 1.534 & 12.0 \\
\hline 1,4-Dichlorobenzene & 1.781 & 1.445 & 1.627 & 1.649 & 1.684 & 1.637 & 7.5 \\
\hline 1,2-Dichlorobenzene & 1.178 & 1.166 & 1.344 & 1.355 & 1.386 & 1.286 & 8.2 \\
\hline 1,2-Dibromo-3-Chloropropane & 0.043 & 0.031 & 0.040 & 0.043 & 0.046 & 0.041 & 14.3 \\
\hline 1,2,4-Trichlorobenzene & 0.821 & 0.701 & 0.905 & 0.988 & 1.041 & 0.891 & 15.2 \\
\hline $1,2,3$-Trichlorobenzene & 0.593 & 0.485 & 0.646 & 0.700 & 0.731 & 0.631 & 15.4 \\
\hline
\end{tabular}


Lab Name: TESTAMERICA BURLINGTON

Contract: $8 \mathrm{E}-00302$

Lab Code: STLV Case No.: YORK Mod. Ref No.: SDG No.: 28963

Instrument ID: CHD.i

Heated Purge: (Y/N) N

Purge Volume: 25.0

GC Column: DB-624
ID: 0.20
Calibration Date(s) : 07/14/2015

Calibration Time (s) : 1108 $(\mathrm{mL})$

(mm) Length: 25
$07 / 14 / 2015$ 1249

\begin{tabular}{|c|c|c|c|c|c|c|c|}
\hline LAB FILE ID: & \multirow{2}{*}{\multicolumn{3}{|c|}{$\begin{array}{l}\operatorname{RRF}_{\underline{0.5}}=\frac{14685 \_002 . \mathrm{D}}{\operatorname{RRF10}}=14685 \_005 . \mathrm{D}\end{array}$}} & \multirow{2}{*}{\multicolumn{4}{|c|}{$\begin{array}{l}\operatorname{RRF} 1.0_{\underline{1.0}}=\frac{14685 \_003 . \mathrm{D}}{\mathrm{RRF} 20}=14685 \_006 . \mathrm{D}\end{array}$}} \\
\hline RRF5.0 $=14685 \_004 . D$ & & & & & & & \\
\hline COMPOUND & RRF0. 5 & RRF1. 0 & RRE 5.0 & RRE10 & RRE20 & $\overline{\mathrm{RRF}}$ & $\circ \mathrm{RSD}$ \\
\hline Vinyl Chloride-d3 & 0.327 & 0.271 & 0.297 & 0.312 & 0.306 & 0.303 & 6.9 \\
\hline Chloroethane-d5 & 0.284 & 0.237 & 0.267 & 0.290 & 0.295 & 0.275 & 8.5 \\
\hline 1,1-Dichloroethene-d2 & 0.762 & 0.661 & 0.721 & 0.761 & 0.808 & 0.742 & 7.4 \\
\hline 2-Butanone-d5 & 0.030 & 0.021 & 0.025 & 0.028 & 0.029 & 0.027 & 12.7 \\
\hline Chloroform-d & 0.651 & 0.577 & 0.697 & 0.721 & 0.713 & 0.672 & 8.9 \\
\hline 1,2-Dichloroethane-d4 & 0.315 & 0.272 & 0.311 & 0.318 & 0.321 & 0.307 & 6.6 \\
\hline Benzene-d6 & 1.171 & 1.017 & 1.206 & 1.226 & 1.250 & 1.174 & 7.9 \\
\hline 1,2-Dichloropropane-d6 & 0.244 & 0.209 & 0.236 & 0.245 & 0.251 & 0.237 & 6.9 \\
\hline Toluene-d8 & 1.283 & 1.087 & 1.360 & 1.403 & 1.430 & 1.312 & 10.5 \\
\hline trans-1,3-Dichloropropene-d4 & 0.244 & 0.215 & 0.294 & 0.320 & 0.343 & 0.283 & 18.6 \\
\hline 2-Hexanone-d5 & 0.020 & 0.016 & 0.025 & 0.026 & 0.028 & 0.023 & 21.2 \\
\hline $1,1,2,2$-Tetrachloroethane-d2 & 0.117 & 0.109 & 0.138 & 0.143 & 0.145 & 0.130 & 12.5 \\
\hline 1,2-Dichlorobenzene-d4 & 0.947 & 0.805 & 0.862 & 0.885 & 0.902 & 0.880 & 5.9 \\
\hline
\end{tabular}

Report 1,4-Dioxane-d8 for Low-Medium VOA analysis only 
Lab Name: TESTAMERICA BURLINGTON

Contract: $8 \mathrm{E}-00302$

Lab Code: STLV Case No.: YORK Mod. Ref No.:

SDG No.: 28963

Instrument ID: CHD.i

Calibration Date(s): 07/31/2015 07/31/2015

Heated Purge: ( $\mathrm{Y} / \mathrm{N}) \mathrm{N}$

Calibration Time(s) : 1213

1352

Purge Volume: 25.0 $(\mathrm{mL})$

GC Column: DB-624

ID: $0.20 \quad$ (mm) Length: 25

$(\mathrm{m})$

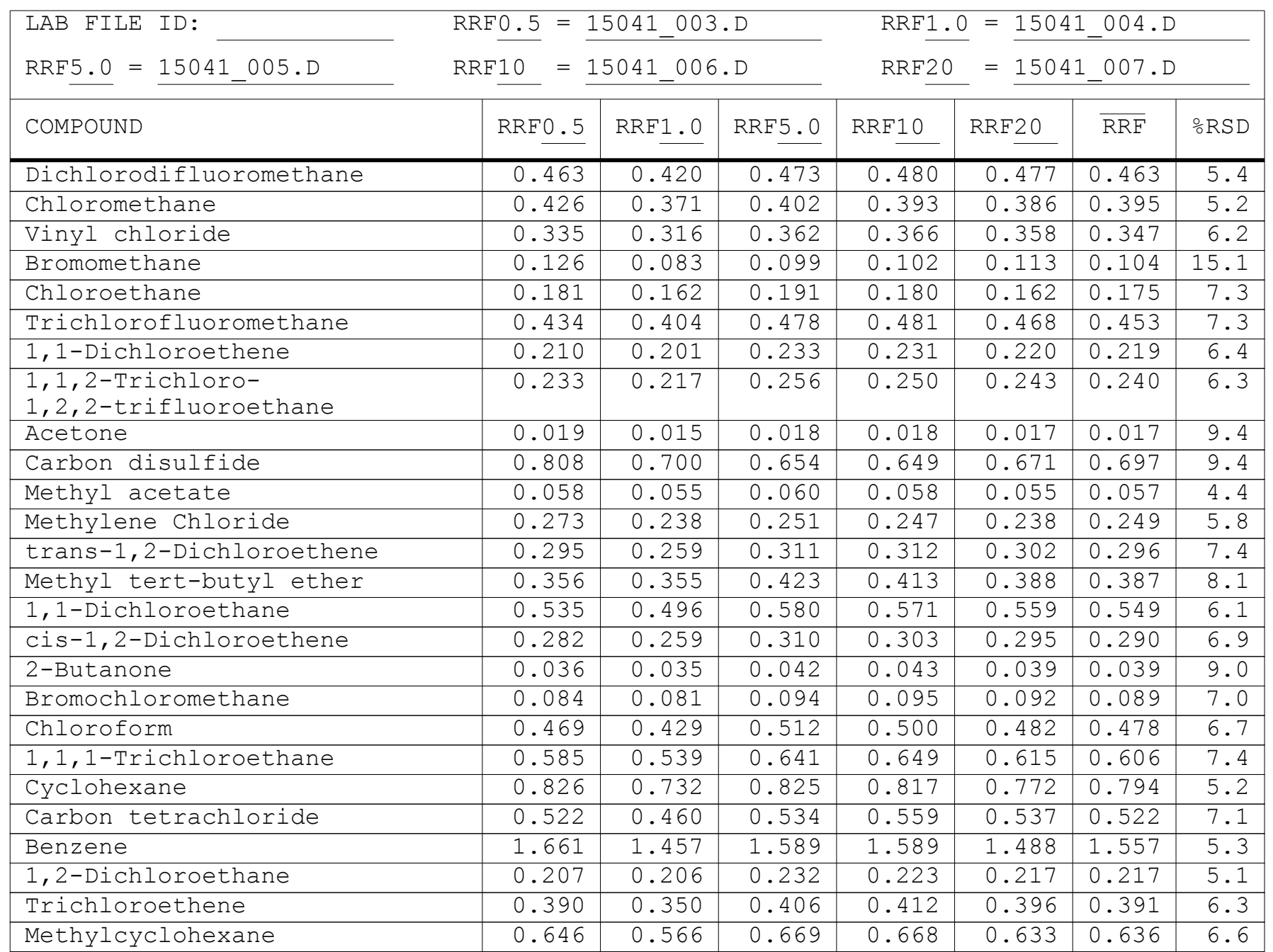

Report 1,4-Dioxane for Low-Medium VOA analysis only 
Lab Name: TESTAMERICA BURLINGTON

Contract: 8E-00302

Lab Code: STLV Case No.: YORK Mod. Ref No.:

SDG No.: 28963

Instrument ID: CHD.i

Calibration Date(s): 07/31/2015 07/31/2015

Heated Purge: ( $\mathrm{Y} / \mathrm{N}) \mathrm{N}$

Calibration Time(s) : 1213

1352

Purge Volume: 25.0 (mL)

GC Column: DB-624

ID: 0.20

$(\mathrm{mm})$

Length: 25

(m)

\begin{tabular}{|c|c|c|c|c|c|c|c|}
\hline \multirow{3}{*}{$\begin{array}{l}\text { LAB FILE ID: } \\
\text { RRF5.0 }=\text { 15041_005.D } \\
\text { COMPOUND }\end{array}$} & \multirow{2}{*}{\multicolumn{3}{|c|}{$\begin{array}{l}\operatorname{RRF0.5}=15041 \_003 . \mathrm{D} \\
\operatorname{RRF10}=15041 \_006 . \mathrm{D}\end{array}$}} & \multirow{2}{*}{\multicolumn{4}{|c|}{ 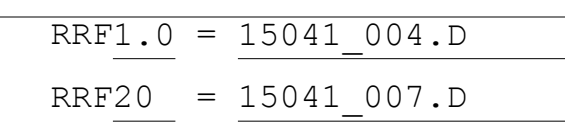 }} \\
\hline & & & & & & & \\
\hline & $\operatorname{RRF} 0.5$ & $\mathrm{RRF} 1.0$ & RRF 5.0 & RRF10 & RRF20 & $\overline{\mathrm{RRF}}$ & $\because \mathrm{RSD}$ \\
\hline 1,2-Dichloropropane & 0.316 & 0.262 & 0.335 & 0.332 & 0.311 & 0.311 & 9.4 \\
\hline Bromodichloromethane & 0.297 & 0.282 & 0.349 & 0.355 & 0.342 & 0.325 & 10.3 \\
\hline cis-1,3-Dichloropropene & 0.395 & 0.391 & 0.462 & 0.465 & 0.442 & 0.431 & 8.3 \\
\hline 4-Methyl-2-pentanone & 0.110 & 0.109 & 0.122 & 0.122 & 0.110 & 0.115 & 6.0 \\
\hline Toluene & 1.635 & 1.531 & 1.704 & 1.697 & 1.603 & 1.634 & 4.4 \\
\hline trans-1,3-Dichloropropene & 0.275 & 0.262 & 0.319 & 0.326 & 0.307 & 0.298 & 9.4 \\
\hline 1,1,2-Trichloroethane & 0.167 & 0.149 & 0.161 & 0.160 & 0.149 & 0.157 & 5.1 \\
\hline Tetrachloroethene & 0.356 & 0.320 & 0.367 & 0.378 & 0.375 & 0.359 & 6.6 \\
\hline 2-Hexanone & 0.075 & 0.073 & 0.082 & 0.082 & 0.075 & 0.077 & 5.5 \\
\hline Dibromochloromethane & 0.157 & 0.138 & 0.175 & 0.187 & 0.182 & 0.168 & 11.9 \\
\hline 1,2-Dibromoethane & 0.124 & 0.123 & 0.144 & 0.144 & 0.137 & 0.135 & 7.7 \\
\hline Chlorobenzene & 0.987 & 0.884 & 0.988 & 0.993 & 0.958 & 0.962 & 4.8 \\
\hline Ethylbenzene & 2.016 & 1.818 & 2.030 & 2.030 & 1.945 & 1.968 & 4.6 \\
\hline o-Xylene & 0.727 & 0.647 & 0.726 & 0.712 & 0.675 & 0.697 & 5.0 \\
\hline $\mathrm{m}, \mathrm{p}$-Xylene & 0.787 & 0.711 & 0.789 & 0.787 & 0.760 & 0.767 & 4.4 \\
\hline Styrene & 1.085 & 0.957 & 1.095 & 1.062 & 0.989 & 1.038 & 5.9 \\
\hline Bromoform & 0.127 & 0.111 & 0.140 & 0.154 & 0.151 & 0.137 & 12.9 \\
\hline Isopropylbenzene & 2.061 & 1.896 & 2.112 & 2.096 & 2.008 & 2.035 & 4.3 \\
\hline $1,1,2,2$-Tetrachloroethane & 0.140 & 0.130 & 0.159 & 0.159 & 0.153 & 0.148 & 8.7 \\
\hline 1,3-Dichlorobenzene & 1.581 & 1.427 & 1.640 & 1.657 & 1.629 & 1.587 & 5.9 \\
\hline 1,4-Dichlorobenzene & 1.505 & 1.388 & 1.565 & 1.576 & 1.547 & 1.516 & 5.0 \\
\hline 1,2-Dichlorobenzene & 1.216 & 1.128 & 1.282 & 1.297 & 1.251 & 1.235 & 5.5 \\
\hline 1,2-Dibromo-3-Chloropropane & 0.040 & 0.037 & 0.041 & 0.042 & 0.042 & 0.040 & 4.9 \\
\hline $1,2,4$-Trichlorobenzene & 0.784 & 0.731 & 0.836 & 0.866 & 0.858 & 0.815 & 7.0 \\
\hline 1,2,3-Trichlorobenzene & 0.528 & 0.491 & 0.584 & 0.601 & 0.588 & 0.558 & 8.4 \\
\hline
\end{tabular}


Lab Name: TESTAMERICA BURLINGTON

Contract: $8 \mathrm{E}-00302$

Lab Code: STLV Case No.: YORK Mod. Ref No.: SDG No.: 28963

Instrument ID: CHD.i

Heated Purge: (Y/N) N

Purge Volume: 25.0

GC Column: DB-624
ID: 0.20
Calibration Date(s): 07/31/2015 Calibration Time (s) : 1213 $(\mathrm{mL})$

(mm) Length: 25
$07 / 31 / 2015$ 1352

\begin{tabular}{|c|c|c|c|c|c|c|c|}
\hline LAB FILE ID: & \multirow{2}{*}{\multicolumn{3}{|c|}{ 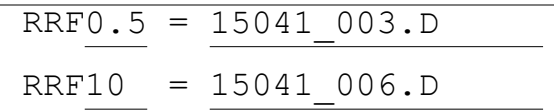 }} & \multirow{2}{*}{\multicolumn{4}{|c|}{$\begin{array}{l}\text { RRF1.0 }=\frac{15041 \_004 . D}{\text { RRF20 }}=15041 \_007 . \mathrm{D}\end{array}$}} \\
\hline RRF5.0 = 15041_005.D & & & & & & & \\
\hline COMPOUND & RRF0. 5 & RRF1 . 0 & RRF5. 0 & RRF10 & RRF20 & $\overline{\mathrm{RRF}}$ & ㅇ. RD \\
\hline Vinyl Chloride-d3 & 0.356 & 0.297 & 0.350 & 0.355 & 0.347 & 0.341 & 7.3 \\
\hline Chloroethane-d5 & 0.249 & 0.200 & 0.231 & 0.222 & 0.200 & 0.220 & 9.6 \\
\hline 1,1-Dichloroethene-d2 & 0.450 & 0.435 & 0.501 & 0.500 & 0.469 & 0.471 & 6.2 \\
\hline 2-Butanone-d5 & 0.034 & 0.033 & 0.041 & 0.040 & 0.038 & 0.037 & 9.8 \\
\hline Chloroform-d & 0.502 & 0.472 & 0.547 & 0.537 & 0.519 & 0.515 & 5.8 \\
\hline 1,2-Dichloroethane-d4 & 0.167 & 0.170 & 0.196 & 0.191 & 0.181 & 0.181 & 7.0 \\
\hline Benzene-d6 & 1.446 & 1.343 & 1.515 & 1.517 & 1.430 & 1.450 & 5.0 \\
\hline 1,2-Dichloropropane-d6 & 0.369 & 0.338 & 0.390 & 0.392 & 0.376 & 0.373 & 5.8 \\
\hline Toluene-d8 & 1.432 & 1.297 & 1.451 & 1.449 & 1.373 & 1.400 & 4.7 \\
\hline trans-1,3-Dichloropropene-d4 & 0.258 & 0.239 & 0.293 & 0.296 & 0.289 & 0.275 & 9.3 \\
\hline 2-Hexanone-d5 & 0.036 & 0.034 & 0.039 & 0.039 & 0.036 & 0.037 & 6.3 \\
\hline $1,1,2,2$-Tetrachloroethane-d2 & 0.157 & 0.139 & 0.157 & 0.158 & 0.153 & 0.153 & 5.3 \\
\hline 1,2-Dichlorobenzene-d4 & 0.864 & 0.736 & 0.819 & 0.826 & 0.800 & 0.809 & 5.8 \\
\hline
\end{tabular}

Report 1,4-Dioxane-d8 for Low-Medium VOA analysis only 
Lab Name: TESTAMERICA BURLINGTON

Contract: $8 \mathrm{E}-00302$

Lab Code: STLV Case No.: YORK Mod. Ref No.:

SDG No.: 28963

Instrument ID: CHD.i

Calibration Date: 07/23/2015 Time: 0851

Lab File Id: 14885_003.D

EPA Sample No. (VSTD\#\#\#\#) : VSTD005DS Init. Calib. Date(s):07/14/2015 07/14/2015

Heated Purge: (Y/N) N GC Column: DB-624

Init. Calib. Time(s) : 1108 1249

Purge Volume: 25.0 ID: $0.20(\mathrm{~mm})$ Length: 25 (m) $(\mathrm{mL})$

\begin{tabular}{|c|c|c|c|c|c|}
\hline COMPOUND & $\overline{\mathrm{RRF}}$ & RRF5.0 & $\begin{array}{l}\text { MIN } \\
\text { RRF }\end{array}$ & $\frac{\circ}{D}$ & $\mathrm{MAX} \div \mathrm{D}$ \\
\hline Dichlorodifluoromethane & 0.543 & 0.695 & 0.010 & 27.9 & 40.0 \\
\hline Chloromethane & 0.299 & 0.299 & 0.010 & -0.1 & 40.0 \\
\hline Vinyl chloride & 0.349 & 0.340 & 0.100 & -2.6 & 30.0 \\
\hline Bromomethane & 0.216 & 0.185 & 0.100 & -14.3 & 30.0 \\
\hline Chloroethane & 0.233 & 0.214 & 0.010 & -8.3 & 40.0 \\
\hline Trichlorofluoromethane & 0.915 & 1.012 & 0.010 & 10.7 & 40.0 \\
\hline 1,1-Dichloroethene & 0.307 & 0.288 & 0.100 & -6.4 & 30.0 \\
\hline 1,1,2-Trichloro-1,2,2-trifluoroethane & 0.391 & 0.367 & 0.010 & -6.1 & 40.0 \\
\hline Acetone & 0.026 & 0.022 & 0.010 & -13.8 & 40.0 \\
\hline Carbon disulfide & 0.685 & 0.619 & 0.010 & -9.6 & 40.0 \\
\hline Methyl acetate & 0.040 & 0.037 & 0.010 & -8.4 & 40.0 \\
\hline Methylene Chloride & 0.220 & 0.202 & 0.010 & -8.3 & 40.0 \\
\hline trans-1,2-Dichloroethene & 0.284 & 0.260 & 0.010 & -8.4 & 40.0 \\
\hline Methyl tert-butyl ether & 0.402 & 0.368 & 0.010 & -8.6 & 40.0 \\
\hline 1,1-Dichloroethane & 0.490 & 0.460 & 0.200 & -6.0 & 30.0 \\
\hline cis-1,2-Dichloroethene & 0.270 & 0.253 & 0.010 & -6.2 & 40.0 \\
\hline 2-Butanone & 0.027 & 0.024 & 0.010 & -8.9 & 40.0 \\
\hline Bromochloromethane & 0.103 & 0.103 & 0.050 & 0.1 & 30.0 \\
\hline Chloroform & 0.621 & 0.612 & 0.200 & -1.6 & 30.0 \\
\hline 1,1,1-Trichloroethane & 0.851 & 0.886 & 0.100 & 4.1 & 30.0 \\
\hline Cyclohexane & 0.448 & 0.449 & 0.010 & 0.4 & 40.0 \\
\hline Carbon tetrachloride & 0.843 & 0.902 & 0.100 & 7.0 & 30.0 \\
\hline Benzene & 1.194 & 1.141 & 0.400 & -4.4 & 30.0 \\
\hline 1,2-Dichloroethane & 0.373 & 0.362 & 0.100 & -2.8 & 30.0 \\
\hline Trichloroethene & 0.394 & 0.399 & 0.300 & 1.2 & 30.0 \\
\hline Methylcyclohexane & 0.449 & 0.452 & 0.010 & 0.8 & 40.0 \\
\hline
\end{tabular}

Report 1,4-Dioxane for Low/Medium VOA analysis only 
Lab Name: TESTAMERICA BURLINGTON

Contract: 8E-00302

Lab Code: STLV

Mod. Ref No.:

SDG No.: 28963

Instrument ID: CHD.i

Calibration Date: 07/23/2015 Time: 0851

Lab File Id: 14885_003.D

EPA Sample No. (VSTD\#\#\#\#) : VSTD005DS Init. Calib. Date(s):07/14/2015 07/14/2015

Heated Purge: ( $\mathrm{Y} / \mathrm{N}) \mathrm{N}$ GC Column: DB-624

Init. Calib. Time(s) : 1108 1249

Purge Volume: 25.0 ID: $0.20(\mathrm{~mm})$ Length: 25 (m) $(\mathrm{mL})$

\begin{tabular}{|c|c|c|c|c|c|}
\hline COMPOUND & $\overline{\mathrm{RRF}}$ & RRF5.0 & $\begin{array}{l}\text { MIN } \\
\text { RRF }\end{array}$ & $\because \mathrm{D}$ & $\operatorname{MAX} \cong \mathrm{D}$ \\
\hline 1,2-Dichloropropane & 0.208 & 0.203 & 0.010 & -2.8 & 40.0 \\
\hline Bromodichloromethane & 0.397 & 0.398 & 0.200 & 0.2 & 30.0 \\
\hline cis-1,3-Dichloropropene & 0.374 & 0.377 & 0.200 & 0.8 & 30.0 \\
\hline 4-Methyl-2-pentanone & 0.069 & 0.069 & 0.010 & -0.9 & 40.0 \\
\hline Toluene & 1.459 & 1.486 & 0.400 & 1.9 & 30.0 \\
\hline trans-1,3-Dichloropropene & 0.311 & 0.314 & 0.100 & 1.1 & 30.0 \\
\hline $1,1,2$-Trichloroethane & 0.149 & 0.135 & 0.100 & -9.6 & 30.0 \\
\hline Tetrachloroethene & 0.484 & 0.488 & 0.100 & 0.7 & 30.0 \\
\hline 2-Hexanone & 0.052 & 0.052 & 0.010 & -0.3 & 40.0 \\
\hline Dibromochloromethane & 0.227 & 0.225 & 0.100 & -0.9 & 30.0 \\
\hline 1,2-Dibromoethane & 0.138 & 0.131 & 0.010 & -5.0 & 40.0 \\
\hline Chlorobenzene & 1.009 & 0.985 & 0.500 & -2.4 & 30.0 \\
\hline Ethylbenzene & 1.942 & 1.977 & 0.100 & 1.8 & 30.0 \\
\hline o-Xylene & 0.666 & 0.671 & 0.300 & 0.8 & 30.0 \\
\hline $\mathrm{m}, \mathrm{p}$-Xylene & 0.740 & 0.755 & 0.300 & 2.1 & 30.0 \\
\hline Styrene & 1.023 & 1.033 & 0.300 & 1.1 & 30.0 \\
\hline Bromoform & 0.176 & 0.165 & 0.050 & -6.6 & 30.0 \\
\hline Isopropylbenzene & 2.186 & 2.292 & 0.010 & 4.8 & 40.0 \\
\hline $1,1,2,2$-Tetrachloroethane & 0.129 & 0.120 & 0.100 & -6.9 & 30.0 \\
\hline 1,3-Dichlorobenzene & 1.534 & 1.456 & 0.400 & -5.1 & 30.0 \\
\hline 1,4-Dichlorobenzene & 1.637 & 1.506 & 0.400 & -8.0 & 30.0 \\
\hline 1,2-Dichlorobenzene & 1.286 & 1.254 & 0.400 & -2.5 & 30.0 \\
\hline 1,2-Dibromo-3-Chloropropane & 0.041 & 0.032 & 0.010 & -22.4 & 40.0 \\
\hline 1,2,4-Trichlorobenzene & 0.891 & 0.736 & 0.200 & -17.4 & 30.0 \\
\hline $1,2,3$-Trichlorobenzene & 0.631 & 0.509 & 0.200 & -19.3 & 30.0 \\
\hline
\end{tabular}


Lab Name: TESTAMERICA BURLINGTON

Contract: $8 \mathrm{E}-00302$

Lab Code: STLV Case No.: YORK Mod. Ref No.

SDG No.: 28963

Instrument ID: CHD.i

Calibration Date: 07/23/2015 Time: 0851

Lab File Id: 14885_003.D Init. Calib. Date(s):07/14/2015 07/14/2015

EPA Sample No. (VSTD\#\#\#\#) : VSTD005DS

Heated Purge: (Y/N) N GC Column: DB-624

Init. Calib. Time(s) : 1108 1249

Purge Volume: 25.0 ID: $0.20(\mathrm{~mm})$ Length: 25

(m)

\begin{tabular}{|c|c|c|c|c|c|}
\hline COMPOUND & $\overline{\mathrm{RRF}}$ & RRE5. 0 & $\begin{array}{l}\text { MIN } \\
\text { RRE }\end{array}$ & $\div D$ & $M A X \div D$ \\
\hline Vinyl Chloride-d3 & 0.303 & 0.334 & 0.010 & 10.4 & 30.0 \\
\hline Chloroethane-d5 & 0.275 & 0.291 & 0.010 & 5.9 & 40.0 \\
\hline $1,1-$ Dichloroethene-d2 & 0.742 & 0.718 & 0.010 & -3.3 & 30.0 \\
\hline 2-Butanone-d5 & 0.027 & 0.023 & 0.010 & -15.4 & 40.0 \\
\hline Chloroform-d & 0.672 & 0.664 & 0.010 & -1.1 & 30.0 \\
\hline 1,2-Dichloroethane-d4 & 0.307 & 0.293 & 0.010 & -4.6 & 30.0 \\
\hline Benzene-d6 & 1.174 & 1.160 & 0.010 & -1.2 & 30.0 \\
\hline 1,2-Dichloropropane-d6 & 0.237 & 0.230 & 0.010 & -3.1 & 40.0 \\
\hline Toluene-d8 & 1.312 & 1.328 & 0.010 & 1.1 & 30.0 \\
\hline trans-1,3-Dichloropropene-d4 & 0.283 & 0.269 & 0.010 & -5.0 & 30.0 \\
\hline 2-Hexanone-d5 & 0.023 & 0.021 & 0.010 & -6.8 & 40.0 \\
\hline $1,1,2,2$-Tetrachloroethane-d2 & 0.130 & 0.120 & 0.010 & -7.5 & 30.0 \\
\hline 1,2-Dichlorobenzene-d4 & 0.880 & 0.793 & 0.010 & -9.9 & 30.0 \\
\hline
\end{tabular}

Report 1,4-Dioxane-d8 for Low/Medium VOA analysis only 
Lab Name: TESTAMERICA BURLINGTON

Contract: $8 \mathrm{E}-00302$

Lab Code: STLV Case No.: YORK Mod. Ref No.:

SDG No.: 28963

Instrument ID: CHD.i

Calibration Date: 07/23/2015 Time: 1439

Lab File Id: 14885_014.D

EPA Sample No. (VSTD\#\#\#\#) : VSTD005SD Init. Calib. Date(s):07/14/2015 07/14/2015

Heated Purge: (Y/N) N GC Column: DB-624

Init. Calib. Time(s) : 1108 1249

Purge Volume: 25.0 ID: $0.20(\mathrm{~mm})$ Length: 25 (m) $(\mathrm{mL})$

\begin{tabular}{|c|c|c|c|c|c|}
\hline COMPOUND & $\overline{\mathrm{RRF}}$ & RRF5.0 & $\begin{array}{l}\text { MIN } \\
\text { RRF }\end{array}$ & $\frac{\circ}{D}$ & $\mathrm{MAX} \div \mathrm{D}$ \\
\hline Dichlorodifluoromethane & 0.543 & 0.708 & 0.010 & 30.4 & 50.0 \\
\hline Chloromethane & 0.299 & 0.288 & 0.010 & -3.8 & 50.0 \\
\hline Vinyl chloride & 0.349 & 0.345 & 0.010 & -1.1 & 50.0 \\
\hline Bromomethane & 0.216 & 0.139 & 0.010 & -35.6 & 50.0 \\
\hline Chloroethane & 0.233 & 0.228 & 0.010 & -2.2 & 50.0 \\
\hline Trichlorofluoromethane & 0.915 & 1.046 & 0.010 & 14.3 & 50.0 \\
\hline 1,1-Dichloroethene & 0.307 & 0.335 & 0.010 & 8.9 & 50.0 \\
\hline 1,1,2-Trichloro-1,2,2-trifluoroethane & 0.391 & 0.429 & 0.010 & 9.6 & 50.0 \\
\hline Acetone & 0.026 & 0.022 & 0.010 & -13.6 & 50.0 \\
\hline Carbon disulfide & 0.685 & 0.629 & 0.010 & -8.1 & 50.0 \\
\hline Methyl acetate & 0.040 & 0.034 & 0.010 & -14.8 & 50.0 \\
\hline Methylene Chloride & 0.220 & 0.206 & 0.010 & -6.4 & 50.0 \\
\hline trans-1,2-Dichloroethene & 0.284 & 0.285 & 0.010 & 0.5 & 50.0 \\
\hline Methyl tert-butyl ether & 0.402 & 0.379 & 0.010 & -5.7 & 50.0 \\
\hline 1,1-Dichloroethane & 0.490 & 0.479 & 0.010 & -2.2 & 50.0 \\
\hline cis-1,2-Dichloroethene & 0.270 & 0.274 & 0.010 & 1.7 & 50.0 \\
\hline 2-Butanone & 0.027 & 0.025 & 0.010 & -6.9 & 50.0 \\
\hline Bromochloromethane & 0.103 & 0.101 & 0.010 & -1.4 & 50.0 \\
\hline Chloroform & 0.621 & 0.644 & 0.010 & 3.6 & 50.0 \\
\hline 1,1,1-Trichloroethane & 0.851 & 0.895 & 0.010 & 5.1 & 50.0 \\
\hline Cyclohexane & 0.448 & 0.455 & 0.010 & 1.6 & 50.0 \\
\hline Carbon tetrachloride & 0.843 & 0.913 & 0.010 & 8.4 & 50.0 \\
\hline Benzene & 1.194 & 1.176 & 0.010 & -1.5 & 50.0 \\
\hline 1,2-Dichloroethane & 0.373 & 0.381 & 0.010 & 2.1 & 50.0 \\
\hline Trichloroethene & 0.394 & 0.390 & 0.010 & -0.8 & 50.0 \\
\hline Methylcyclohexane & 0.449 & 0.463 & 0.010 & 3.1 & 50.0 \\
\hline
\end{tabular}

Report 1,4-Dioxane for Low/Medium VOA analysis only 
Lab Name: TESTAMERICA BURLINGTON

Contract: 8E-00302

Lab Code: STLV

Mod. Ref No.:

SDG No.: 28963

Instrument ID: CHD.i

Calibration Date: 07/23/2015 Time: 1439

Lab File Id: 14885_014.D

EPA Sample No. (VSTD\#\#\#\#) : VSTD005SD

Init. Calib. Date(s):07/14/2015 07/14/2015

Heated Purge: ( $\mathrm{Y} / \mathrm{N}) \mathrm{N}$ GC Column: DB-624

Init. Calib. Time(s) : 1108 1249

Purge Volume: 25.0

ID: $0.20(\mathrm{~mm})$ Length: 25

(m)

\begin{tabular}{|c|c|c|c|c|c|}
\hline COMPOUND & $\overline{\mathrm{RRF}}$ & RRF5.0 & $\begin{array}{l}\text { MIN } \\
\text { RRF }\end{array}$ & $\because \mathrm{D}$ & $\operatorname{MAX} \cong \mathrm{D}$ \\
\hline 1,2-Dichloropropane & 0.208 & 0.199 & 0.010 & -4.5 & 50.0 \\
\hline Bromodichloromethane & 0.397 & 0.399 & 0.010 & 0.4 & 50.0 \\
\hline cis-1,3-Dichloropropene & 0.374 & 0.375 & 0.010 & 0.2 & 50.0 \\
\hline 4-Methyl-2-pentanone & 0.069 & 0.070 & 0.010 & 0.4 & 50.0 \\
\hline Toluene & 1.459 & 1.527 & 0.010 & 4.7 & 50.0 \\
\hline trans-1,3-Dichloropropene & 0.311 & 0.309 & 0.010 & -0.7 & 50.0 \\
\hline $1,1,2$-Trichloroethane & 0.149 & 0.149 & 0.010 & 0.1 & 50.0 \\
\hline Tetrachloroethene & 0.484 & 0.498 & 0.010 & 2.9 & 50.0 \\
\hline 2-Hexanone & 0.052 & 0.053 & 0.010 & 2.6 & 50.0 \\
\hline Dibromochloromethane & 0.227 & 0.221 & 0.010 & -2.9 & 50.0 \\
\hline 1,2-Dibromoethane & 0.138 & 0.131 & 0.010 & -5.1 & 50.0 \\
\hline Chlorobenzene & 1.009 & 1.004 & 0.010 & -0.5 & 50.0 \\
\hline Ethylbenzene & 1.942 & 2.024 & 0.010 & 4.2 & 50.0 \\
\hline o-Xylene & 0.666 & 0.692 & 0.010 & 3.9 & 50.0 \\
\hline $\mathrm{m}, \mathrm{p}$-Xylene & 0.740 & 0.784 & 0.010 & 6.0 & 50.0 \\
\hline Styrene & 1.023 & 1.070 & 0.010 & 4.7 & 50.0 \\
\hline Bromoform & 0.176 & 0.160 & 0.010 & -9.5 & 50.0 \\
\hline Isopropylbenzene & 2.186 & 2.369 & 0.010 & 8.4 & 50.0 \\
\hline $1,1,2,2$-Tetrachloroethane & 0.129 & 0.123 & 0.010 & -4.7 & 50.0 \\
\hline 1,3-Dichlorobenzene & 1.534 & 1.487 & 0.010 & -3.1 & 50.0 \\
\hline 1,4-Dichlorobenzene & 1.637 & 1.581 & 0.010 & -3.5 & 50.0 \\
\hline 1,2-Dichlorobenzene & 1.286 & 1.262 & 0.010 & -1.9 & 50.0 \\
\hline 1,2-Dibromo-3-Chloropropane & 0.041 & 0.035 & 0.010 & -13.2 & 50.0 \\
\hline 1,2,4-Trichlorobenzene & 0.891 & 0.744 & 0.010 & -16.5 & 50.0 \\
\hline $1,2,3$-Trichlorobenzene & 0.631 & 0.518 & 0.010 & -17.9 & 50.0 \\
\hline
\end{tabular}


Lab Name: TESTAMERICA BURLINGTON

Contract: $8 \mathrm{E}-00302$

Lab Code: STLV Case No.: YORK Mod. Ref No.

SDG No.: 28963

Instrument ID: CHD.i

Calibration Date: 07/23/2015 Time: 1439

Lab File Id: 14885_014.D Init. Calib. Date(s):07/14/2015 07/14/2015

EPA Sample No. (VSTD\#\#\#\#) : VSTD005SD

Heated Purge: (Y/N) N GC Column: DB-624

Init. Calib. Time(s) : 1108 1249

Purge Volume: 25.0 ID: $0.20(\mathrm{~mm})$ Length: 25

(m)

\begin{tabular}{|c|c|c|c|c|c|}
\hline COMPOUND & $\overline{\mathrm{RRF}}$ & RRE5. 0 & $\begin{array}{l}\text { MIN } \\
\text { RRE }\end{array}$ & $\because \mathrm{D}$ & $M A X \div D$ \\
\hline Vinyl Chloride-d3 & 0.303 & 0.352 & 0.010 & 16.4 & 50.0 \\
\hline Chloroethane-d5 & 0.275 & 0.303 & 0.010 & 10.4 & 50.0 \\
\hline $1,1-$ Dichloroethene-d2 & 0.742 & 0.800 & 0.010 & 7.8 & 50.0 \\
\hline 2-Butanone-d5 & 0.027 & 0.024 & 0.010 & $-11 \cdot 2$ & 50.0 \\
\hline Chloroform-d & 0.672 & 0.696 & 0.010 & 3.6 & 50.0 \\
\hline 1,2-Dichloroethane-d4 & 0.307 & 0.306 & 0.010 & -0.3 & 50.0 \\
\hline Benzene-d6 & 1.174 & 1.195 & 0.010 & 1.8 & 50.0 \\
\hline 1,2-Dichloropropane-d6 & 0.237 & 0.229 & 0.010 & -3.4 & 50.0 \\
\hline Toluene-d8 & 1.312 & 1.354 & 0.010 & 3.2 & 50.0 \\
\hline trans-1,3-Dichloropropene-d4 & 0.283 & 0.285 & 0.010 & 0.7 & 50.0 \\
\hline 2-Hexanone-d5 & 0.023 & 0.022 & 0.010 & -4.8 & 50.0 \\
\hline $1,1,2,2$-Tetrachloroethane-d2 & 0.130 & 0.125 & 0.010 & -4.3 & 50.0 \\
\hline 1,2-Dichlorobenzene-d4 & 0.880 & 0.807 & 0.010 & -8.4 & 50.0 \\
\hline
\end{tabular}

Report 1,4-Dioxane-d8 for Low/Medium VOA analysis only 
Lab Name: TESTAMERICA BURLINGTON

Contract: $8 \mathrm{E}-00302$

Lab Code: STLV Case No.: YORK Mod. Ref No.:

SDG No.: 28963

Instrument ID: CHD.i

Calibration Date: 08/03/2015 Time: 1037

Lab File Id: 15058_003.D

EPA Sample No. (VSTD\#\#\#\#) : VSTD005DX Init. Calib. Date(s):07/31/2015 07/31/2015

Heated Purge: (Y/N) N GC Column: DB-624

Init. Calib. Time(s) : 1213 1352

Purge Volume: 25.0 ID: $0.20(\mathrm{~mm})$ Length: 25

(m)

\begin{tabular}{|c|c|c|c|c|c|}
\hline COMPOUND & $\overline{\mathrm{RRF}}$ & RRF5. 0 & $\begin{array}{l}\text { MIN } \\
\text { RRF }\end{array}$ & $\div \mathrm{D}$ & $\mathrm{MAX} \cong \mathrm{D}$ \\
\hline Dichlorodifluoromethane & 0.463 & 0.486 & 0.010 & 5.1 & 40.0 \\
\hline Chloromethane & 0.395 & 0.374 & 0.010 & -5.3 & 40.0 \\
\hline Vinyl chloride & 0.347 & 0.367 & 0.100 & 5.5 & 30.0 \\
\hline Bromomethane & 0.104 & 0.137 & 0.100 & 31.0 & 30.0 \\
\hline Chloroethane & 0.175 & 0.203 & 0.010 & 16.0 & 40.0 \\
\hline Trichlorofluoromethane & 0.453 & 0.483 & 0.010 & 6.6 & 40.0 \\
\hline 1,1-Dichloroethene & 0.219 & 0.240 & 0.100 & 9.8 & 30.0 \\
\hline 1,1,2-Trichloro-1,2,2-trifluoroethane & 0.240 & 0.266 & 0.010 & 11.1 & 40.0 \\
\hline Acetone & 0.017 & 0.021 & 0.010 & 23.3 & 40.0 \\
\hline Carbon disulfide & 0.697 & 0.827 & 0.010 & 18.7 & 40.0 \\
\hline Methyl acetate & 0.057 & 0.060 & 0.010 & 4.9 & 40.0 \\
\hline Methylene Chloride & 0.249 & 0.241 & 0.010 & -3.5 & 40.0 \\
\hline trans-1,2-Dichloroethene & 0.296 & 0.305 & 0.010 & 3.2 & 40.0 \\
\hline Methyl tert-butyl ether & 0.387 & 0.368 & 0.010 & -5.0 & 40.0 \\
\hline 1,1-Dichloroethane & 0.549 & 0.563 & 0.200 & 2.7 & 30.0 \\
\hline cis-1,2-Dichloroethene & 0.290 & 0.297 & 0.010 & 2.3 & 40.0 \\
\hline 2-Butanone & 0.039 & 0.038 & 0.010 & -2.9 & 40.0 \\
\hline Bromochloromethane & 0.089 & 0.089 & 0.050 & -0.1 & 30.0 \\
\hline Chloroform & 0.478 & 0.496 & 0.200 & 3.6 & 30.0 \\
\hline 1,1,1-Trichloroethane & 0.606 & 0.643 & 0.100 & 6.1 & 30.0 \\
\hline Cyclohexane & 0.794 & 0.819 & 0.010 & 3.2 & 40.0 \\
\hline Carbon tetrachloride & 0.522 & 0.544 & 0.100 & 4.1 & 30.0 \\
\hline Benzene & 1.557 & 1.578 & 0.400 & 1.4 & 30.0 \\
\hline 1,2-Dichloroethane & 0.217 & 0.223 & 0.100 & 2.4 & 30.0 \\
\hline Trichloroethene & 0.391 & 0.414 & 0.300 & 6.1 & 30.0 \\
\hline Methylcyclohexane & 0.636 & 0.667 & 0.010 & 4.8 & 40.0 \\
\hline
\end{tabular}

Report 1,4-Dioxane for Low/Medium VOA analysis only 
Lab Name: TESTAMERICA BURLINGTON

Contract: 8E-00302

Lab Code: STLV

Mod. Ref No.:

SDG No.: 28963

Instrument ID: CHD.i

Calibration Date: 08/03/2015 Time: 1037

Lab File Id: 15058_003.D

Init. Calib. Date(s):07/31/2015 07/31/2015

EPA Sample No. (VSTD\#\#\#\#) : VSTD005DX

Init. Calib. Time(s) : 1213 1352

Heated Purge: (Y/N) N GC Column: DB-624

ID: $0.20(\mathrm{~mm})$ Length: 25

(m)

Purge Volume: 25.0 $(\mathrm{mL})$

\begin{tabular}{|c|c|c|c|c|c|}
\hline COMPOUND & $\overline{\mathrm{RRF}}$ & RRF5.0 & $\begin{array}{l}\text { MIN } \\
\text { RRF }\end{array}$ & $\because \mathrm{D}$ & $\operatorname{MAX} \cong \mathrm{D}$ \\
\hline 1,2-Dichloropropane & 0.311 & 0.326 & 0.010 & 4.7 & 40.0 \\
\hline Bromodichloromethane & 0.325 & 0.338 & 0.200 & 3.9 & 30.0 \\
\hline cis-1,3-Dichloropropene & 0.431 & 0.434 & 0.200 & 0.8 & 30.0 \\
\hline 4-Methyl-2-pentanone & 0.115 & 0.112 & 0.010 & -1.9 & 40.0 \\
\hline Toluene & 1.634 & 1.729 & 0.400 & 5.9 & 30.0 \\
\hline trans-1,3-Dichloropropene & 0.298 & 0.296 & 0.100 & -0.6 & 30.0 \\
\hline $1,1,2$-Trichloroethane & 0.157 & 0.150 & 0.100 & -4.2 & 30.0 \\
\hline Tetrachloroethene & 0.359 & 0.379 & 0.100 & 5.7 & 30.0 \\
\hline 2-Hexanone & 0.077 & 0.074 & 0.010 & -4.2 & 40.0 \\
\hline Dibromochloromethane & 0.168 & 0.175 & 0.100 & 4.2 & 30.0 \\
\hline 1,2-Dibromoethane & 0.135 & 0.135 & 0.010 & 0.4 & 40.0 \\
\hline Chlorobenzene & 0.962 & 0.985 & 0.500 & 2.4 & 30.0 \\
\hline Ethylbenzene & 1.968 & 2.061 & 0.100 & 4.7 & 30.0 \\
\hline o-Xylene & 0.697 & 0.705 & 0.300 & 1.1 & 30.0 \\
\hline $\mathrm{m}, \mathrm{p}$-Xylene & 0.767 & 0.793 & 0.300 & 3.4 & 30.0 \\
\hline Styrene & 1.038 & 1.070 & 0.300 & 3.1 & 30.0 \\
\hline Bromoform & 0.137 & 0.141 & 0.050 & 3.4 & 30.0 \\
\hline Isopropylbenzene & 2.035 & 2.179 & 0.010 & 7.1 & 40.0 \\
\hline $1,1,2,2$-Tetrachloroethane & 0.148 & 0.147 & 0.100 & -0.6 & 30.0 \\
\hline 1,3-Dichlorobenzene & 1.587 & 1.625 & 0.400 & 2.4 & 30.0 \\
\hline 1,4-Dichlorobenzene & 1.516 & 1.561 & 0.400 & 2.9 & 30.0 \\
\hline 1,2-Dichlorobenzene & 1.235 & 1.269 & 0.400 & 2.8 & 30.0 \\
\hline 1,2-Dibromo-3-Chloropropane & 0.040 & 0.038 & 0.010 & -6.0 & 40.0 \\
\hline 1,2,4-Trichlorobenzene & 0.815 & 0.818 & 0.200 & 0.4 & 30.0 \\
\hline $1,2,3$-Trichlorobenzene & 0.558 & 0.571 & 0.200 & 2.3 & 30.0 \\
\hline
\end{tabular}


Lab Name: TESTAMERICA BURLINGTON

Contract: $8 \mathrm{E}-00302$

Lab Code: STLV Case No.: YORK Mod. Ref No.

SDG No.: 28963

Instrument ID: CHD.i

Calibration Date: 08/03/2015 Time: 1037

Lab File Id: 15058_003.D Init. Calib. Date(s):07/31/2015 07/31/2015

EPA Sample No. (VSTD\#\#\#\#) : VSTD005DX

Init. Calib. Time(s) : 1213 1352

Heated Purge: (Y/N) N GC Column: DB-624 ID: $0.20(\mathrm{~mm})$ Length: 25

(m)

Purge Volume: 25.0 $(\mathrm{mL})$

\begin{tabular}{|c|c|c|c|c|c|}
\hline COMPOUND & $\overline{\mathrm{RRF}}$ & RRF5.0 & $\begin{array}{l}\text { MIN } \\
\text { RRF }\end{array}$ & $\because \mathrm{D}$ & $\operatorname{MAX} \cong \mathrm{D}$ \\
\hline Vinyl Chloride-d3 & 0.341 & 0.364 & 0.010 & 6.6 & 30.0 \\
\hline Chloroethane-d5 & 0.220 & 0.236 & 0.010 & 7.0 & 40.0 \\
\hline 1,1-Dichloroethene-d2 & 0.471 & 0.517 & 0.010 & 9.8 & 30.0 \\
\hline 2-Butanone-d5 & 0.037 & 0.036 & 0.010 & -2.6 & 40.0 \\
\hline Chloroform-d & 0.515 & 0.536 & 0.010 & 4.1 & 30.0 \\
\hline 1,2-Dichloroethane-d4 & 0.181 & 0.183 & 0.010 & 1.4 & 30.0 \\
\hline Benzene-d6 & 1.450 & 1.527 & 0.010 & 5.3 & 30.0 \\
\hline 1,2-Dichloropropane-d6 & 0.373 & 0.382 & 0.010 & 2.3 & 40.0 \\
\hline Toluene-d8 & 1.400 & 1.470 & 0.010 & 5.0 & 30.0 \\
\hline trans-1,3-Dichloropropene-d4 & 0.275 & 0.275 & 0.010 & 0.0 & 30.0 \\
\hline 2-Hexanone-d5 & 0.037 & 0.035 & 0.010 & -4.0 & 40.0 \\
\hline $1,1,2,2$-Tetrachloroethane-d2 & 0.153 & 0.153 & 0.010 & 0.2 & 30.0 \\
\hline 1,2-Dichlorobenzene-d4 & 0.809 & 0.813 & 0.010 & 0.5 & 30.0 \\
\hline
\end{tabular}

Report 1,4-Dioxane-d8 for Low/Medium VOA analysis only 
Lab Name: TESTAMERICA BURLINGTON

Contract: $8 \mathrm{E}-00302$

Lab Code: STLV Case No.: YORK Mod. Ref No.:

SDG No.: 28963

Instrument ID: CHD.i

Calibration Date: 08/03/2015 Time: 1838

Lab File Id: 15058_011.D

EPA Sample No. (VSTD\#\#\#\#) : VSTD005XD Init. Calib. Date (s):07/31/2015 07/31/2015

Heated Purge: ( $\mathrm{Y} / \mathrm{N}) \mathrm{N}$ GC Column: DB-624

Init. Calib. Time(s) : 1213 1352

Purge Volume: 25.0 ID: $0.20(\mathrm{~mm})$ Length: 25

(m)

\begin{tabular}{|c|c|c|c|c|c|}
\hline COMPOUND & $\overline{\mathrm{RRF}}$ & RRF5. 0 & $\begin{array}{l}\text { MIN } \\
\text { RRF }\end{array}$ & $\div \mathrm{D}$ & $\mathrm{MAX} \cong \mathrm{D}$ \\
\hline Dichlorodifluoromethane & 0.463 & 0.492 & 0.010 & 6.4 & 50.0 \\
\hline Chloromethane & 0.395 & 0.438 & 0.010 & 10.7 & 50.0 \\
\hline Vinyl chloride & 0.347 & 0.397 & 0.010 & 14.3 & 50.0 \\
\hline Bromomethane & 0.104 & 0.136 & 0.010 & 30.4 & 50.0 \\
\hline Chloroethane & 0.175 & 0.208 & 0.010 & 18.9 & 50.0 \\
\hline Trichlorofluoromethane & 0.453 & 0.493 & 0.010 & 9.0 & 50.0 \\
\hline 1,1-Dichloroethene & 0.219 & 0.247 & 0.010 & 12.9 & 50.0 \\
\hline 1,1,2-Trichloro-1,2,2-trifluoroethane & 0.240 & 0.271 & 0.010 & 12.9 & 50.0 \\
\hline Acetone & 0.017 & 0.021 & 0.010 & 19.5 & 50.0 \\
\hline Carbon disulfide & 0.697 & 0.736 & 0.010 & 5.6 & 50.0 \\
\hline Methyl acetate & 0.057 & 0.059 & 0.010 & 2.6 & 50.0 \\
\hline Methylene Chloride & 0.249 & 0.251 & 0.010 & 0.8 & 50.0 \\
\hline trans-1,2-Dichloroethene & 0.296 & 0.304 & 0.010 & 2.8 & 50.0 \\
\hline Methyl tert-butyl ether & 0.387 & 0.384 & 0.010 & -0.7 & 50.0 \\
\hline 1,1-Dichloroethane & 0.549 & 0.567 & 0.010 & 3.4 & 50.0 \\
\hline cis-1,2-Dichloroethene & 0.290 & 0.306 & 0.010 & 5.4 & 50.0 \\
\hline 2-Butanone & 0.039 & 0.044 & 0.010 & 12.6 & 50.0 \\
\hline Bromochloromethane & 0.089 & 0.094 & 0.010 & 5.8 & 50.0 \\
\hline Chloroform & 0.478 & 0.509 & 0.010 & 6.5 & 50.0 \\
\hline 1,1,1-Trichloroethane & 0.606 & 0.594 & 0.010 & -2.0 & 50.0 \\
\hline Cyclohexane & 0.794 & 0.778 & 0.010 & -2.0 & 50.0 \\
\hline Carbon tetrachloride & 0.522 & 0.499 & 0.010 & -4.4 & 50.0 \\
\hline Benzene & 1.557 & 1.562 & 0.010 & 0.3 & 50.0 \\
\hline 1,2-Dichloroethane & 0.217 & 0.232 & 0.010 & 6.6 & 50.0 \\
\hline Trichloroethene & 0.391 & 0.395 & 0.010 & 1.1 & 50.0 \\
\hline Methylcyclohexane & 0.636 & 0.646 & 0.010 & 1.5 & 50.0 \\
\hline
\end{tabular}

Report 1,4-Dioxane for Low/Medium VOA analysis only 
Lab Name: TESTAMERICA BURLINGTON

Contract: 8E-00302

Lab Code: STLV

Mod. Ref No.:

SDG No.: 28963

Instrument ID: CHD.i

Calibration Date: 08/03/2015 Time: 1838

Lab File Id: 15058_011.D

EPA Sample No. (VSTD\#\#\#\#) : VSTD005XD

Init. Calib. Date (s):07/31/2015

$07 / 31 / 2015$

Heated Purge: (Y/N) N GC Column: DB-624

Init. Calib. Time(s) : 1213 1352

Purge Volume: 25.0

ID: $0.20(\mathrm{~mm})$ Length: 25

(m)

\begin{tabular}{|c|c|c|c|c|c|}
\hline COMPOUND & $\overline{\mathrm{RRF}}$ & RRF5.0 & $\begin{array}{l}\text { MIN } \\
\text { RRF }\end{array}$ & $\because \mathrm{D}$ & $\operatorname{MAX} \cong \mathrm{D}$ \\
\hline 1,2-Dichloropropane & 0.311 & 0.318 & 0.010 & 2.3 & 50.0 \\
\hline Bromodichloromethane & 0.325 & 0.323 & 0.010 & -0.6 & 50.0 \\
\hline cis-1,3-Dichloropropene & 0.431 & 0.431 & 0.010 & 0.0 & 50.0 \\
\hline 4-Methyl-2-pentanone & 0.115 & 0.119 & 0.010 & 4.3 & 50.0 \\
\hline Toluene & 1.634 & 1.697 & 0.010 & 3.9 & 50.0 \\
\hline trans-1,3-Dichloropropene & 0.298 & 0.295 & 0.010 & -1.0 & 50.0 \\
\hline $1,1,2$-Trichloroethane & 0.157 & 0.159 & 0.010 & 1.6 & 50.0 \\
\hline Tetrachloroethene & 0.359 & 0.371 & 0.010 & 3.2 & 50.0 \\
\hline 2-Hexanone & 0.077 & 0.082 & 0.010 & 5.9 & 50.0 \\
\hline Dibromochloromethane & 0.168 & 0.165 & 0.010 & -1.8 & 50.0 \\
\hline 1,2-Dibromoethane & 0.135 & 0.139 & 0.010 & 3.6 & 50.0 \\
\hline Chlorobenzene & 0.962 & 0.987 & 0.010 & 2.5 & 50.0 \\
\hline Ethylbenzene & 1.968 & 2.044 & 0.010 & 3.9 & 50.0 \\
\hline o-Xylene & 0.697 & 0.740 & 0.010 & 6.1 & 50.0 \\
\hline $\mathrm{m}, \mathrm{p}$-Xylene & 0.767 & 0.799 & 0.010 & 4.2 & 50.0 \\
\hline Styrene & 1.038 & 1.117 & 0.010 & 7.6 & 50.0 \\
\hline Bromoform & 0.137 & 0.123 & 0.010 & -9.6 & 50.0 \\
\hline Isopropylbenzene & 2.035 & 2.166 & 0.010 & 6.5 & 50.0 \\
\hline $1,1,2,2$-Tetrachloroethane & 0.148 & 0.160 & 0.010 & 8.1 & 50.0 \\
\hline 1,3-Dichlorobenzene & 1.587 & 1.614 & 0.010 & 1.7 & 50.0 \\
\hline 1,4-Dichlorobenzene & 1.516 & 1.554 & 0.010 & 2.5 & 50.0 \\
\hline 1,2-Dichlorobenzene & 1.235 & 1.278 & 0.010 & 3.5 & 50.0 \\
\hline 1,2-Dibromo-3-Chloropropane & 0.040 & 0.034 & 0.010 & -16.3 & 50.0 \\
\hline 1,2,4-Trichlorobenzene & 0.815 & 0.795 & 0.010 & -2.4 & 50.0 \\
\hline $1,2,3$-Trichlorobenzene & 0.558 & 0.556 & 0.010 & -0.4 & 50.0 \\
\hline
\end{tabular}


Lab Name: TESTAMERICA BURLINGTON

Contract: $8 \mathrm{E}-00302$

Lab Code: STLV Case No.: YORK Mod. Ref No.

SDG No.: 28963

Instrument ID: CHD.i

Calibration Date: 08/03/2015 Time: 1838

Lab File Id: 15058_011.D Init. Calib. Date(s):07/31/2015 07/31/2015

EPA Sample No. (VSTD\#\#\#\#) : VSTD005XD

Heated Purge: (Y/N) N GC Column: DB-624

Init. Calib. Time(s) : 1213 1352

Purge Volume: 25.0 ID: $0.20(\mathrm{~mm})$ Length: 25

(m)

\begin{tabular}{|c|c|c|c|c|c|}
\hline COMPOUND & $\overline{\mathrm{RRF}}$ & RRF 5.0 & $\begin{array}{l}\text { MIN } \\
\text { RRF }\end{array}$ & $\div D$ & $\mathrm{MAX} \div \mathrm{D}$ \\
\hline Vinyl Chloride-d3 & 0.341 & 0.385 & 0.010 & 12.8 & 50.0 \\
\hline Chloroethane-d5 & 0.220 & 0.257 & 0.010 & 16.7 & 50.0 \\
\hline 1,1-Dichloroethene-d2 & 0.471 & 0.510 & 0.010 & 8.3 & 50.0 \\
\hline 2-Butanone-d5 & 0.037 & 0.041 & 0.010 & 9.7 & 50.0 \\
\hline Chloroform-d & 0.515 & 0.545 & 0.010 & 5.8 & 50.0 \\
\hline 1,2-Dichloroethane-d4 & 0.181 & 0.196 & 0.010 & 8.4 & 50.0 \\
\hline Benzene-d6 & 1.450 & 1.483 & 0.010 & 2.3 & 50.0 \\
\hline 1,2 -Dichloropropane-d6 & 0.373 & 0.440 & 0.010 & 18.0 & 50.0 \\
\hline Toluene-d8 & 1.400 & 1.448 & 0.010 & 3.4 & 50.0 \\
\hline trans-1,3-Dichloropropene-d4 & 0.275 & 0.264 & 0.010 & -4.0 & 50.0 \\
\hline 2-Hexanone-d5 & 0.037 & 0.038 & 0.010 & 3.5 & 50.0 \\
\hline $1,1,2,2$-Tetrachloroethane-d2 & 0.153 & 0.163 & 0.010 & 6.7 & 50.0 \\
\hline 1,2-Dichlorobenzene-d4 & 0.809 & 0.838 & 0.010 & 3.6 & 50.0 \\
\hline
\end{tabular}

Report 1,4-Dioxane-d8 for Low/Medium VOA analysis only 
Lab Name: TESTAMERICA BURLINGTON

Contract: $8 \mathrm{E}-00302$

Lab Code: STLV

Case No.: YORK Mod. Ref No.:

SDG No.: 28963

Matrix: (SOIL/SED/WATER) Water

Sample wt/vol: 25.0 $(\mathrm{g} / \mathrm{mL}) \mathrm{mL}$

Level: (TRACE/LOW/MED) TRACE

\% Moisture: not dec.

GC Column: DB-624 ID: $0.20 \quad(\mathrm{~mm})$

Soil Extract Volume: (uL) $(\mathrm{mL})$

Purge Volume: 25.0
Lab Sample ID: MB 200-91665/5

Lab File ID: 14885_005.D

Date Received:

Date Analyzed: 07/23/2015

Dilution Factor: 1.0

Soil Aliquot Volume: (uL)

\begin{tabular}{|c|c|c|c|}
\hline CAS NO. & COMPOUND & $\begin{array}{l}\text { CONCENTRATION UNITS: } \\
(\mathrm{ug} / \mathrm{L} \text { or } \mathrm{ug} / \mathrm{kg}) \mathrm{ug} / \mathrm{L}\end{array}$ & q \\
\hline $75-71-8$ & Dichlorodifluoromethane & 1.0 & $\mathrm{U}$ \\
\hline $74-87-3$ & Chloromethane & 1.0 & $\mathrm{U}$ \\
\hline $75-01-4$ & Vinyl chloride & 1.0 & $\mathrm{U}$ \\
\hline $74-83-9$ & Bromomethane & 1.0 & $\mathrm{U}$ \\
\hline $75-00-3$ & Chloroethane & 1.0 & $\mathrm{U}$ \\
\hline $75-69-4$ & Trichlorofluoromethane & 1.0 & $\mathrm{U}$ \\
\hline $75-35-4$ & 1,1-Dichloroethene & 1.0 & $\mathrm{U}$ \\
\hline $76-13-1$ & $1,1,2$-Trichloro-1,2,2-trifluoroethane & 1.0 & $\mathrm{U}$ \\
\hline $67-64-1$ & Acetone & 5.0 & $\mathrm{U}$ \\
\hline $75-15-0$ & Carbon disulfide & 1.0 & $\mathrm{U}$ \\
\hline $79-20-9$ & Methyl acetate & 1.0 & $\mathrm{U}$ \\
\hline $75-09-2$ & Methylene Chloride & 1.0 & $\mathrm{U}$ \\
\hline $156-60-5$ & trans-1,2-Dichloroethene & 1.0 & $\mathrm{U}$ \\
\hline $1634-04-4$ & Methyl tert-butyl ether & 1.0 & $\mathrm{U}$ \\
\hline $75-34-3$ & 1,1-Dichloroethane & 1.0 & $\mathrm{U}$ \\
\hline $156-59-2$ & cis-1,2-Dichloroethene & 1.0 & $\mathrm{U}$ \\
\hline $78-93-3$ & 2-Butanone & 5.0 & $\mathrm{U}$ \\
\hline $74-97-5$ & Bromochloromethane & 1.0 & $\mathrm{U}$ \\
\hline $67-66-3$ & Chloroform & 1.0 & $\mathrm{U}$ \\
\hline $71-55-6$ & $1,1,1$-Trichloroethane & 1.0 & $\mathrm{U}$ \\
\hline $110-82-7$ & Cyclohexane & 1.0 & $\mathrm{U}$ \\
\hline $56-23-5$ & Carbon tetrachloride & 1.0 & $\mathrm{U}$ \\
\hline $71-43-2$ & Benzene & 1.0 & $\mathrm{U}$ \\
\hline $107-06-2$ & 1,2-Dichloroethane & 1.0 & $\mathrm{U}$ \\
\hline
\end{tabular}

Report 1,4-Dioxane for Low-Medium VOA analysis only 
Lab Name: TESTAMERICA BURLINGTON

Contract: 8E-00302

Lab Code: STLV

Case No.: YORK

Mod. Ref No.:

SDG No.: 28963

Matrix: (SOIL/SED/WATER) Water

Sample wt/vol: 25.0 $(\mathrm{g} / \mathrm{mL}) \mathrm{mL}$

Level: (TRACE/LOW/MED) TRACE

\% Moisture: not dec.

GC Column: DB-624 ID: $0.20 \quad(\mathrm{~mm})$

Soil Extract Volume: (uL) $(\mathrm{mL})$

Purge Volume: 25.0 (mI)
Lab Sample ID: MB 200-91665/5

Lab File ID: 14885_005.D

Date Received:

Date Analyzed: 07/23/2015

Dilution Factor: 1.0

Soil Aliquot Volume: (uL)

\begin{tabular}{|c|c|c|c|}
\hline CAS NO. & COMPOUND & $\begin{array}{l}\text { CONCENTRATION UNITS: } \\
(\mathrm{ug} / \mathrm{L} \text { or } \mathrm{ug} / \mathrm{kg}) \mathrm{ug} / \mathrm{L}\end{array}$ & $q$ \\
\hline $79-01-6$ & Trichloroethene & 1.0 & $\mathrm{U}$ \\
\hline $108-87-2$ & Methylcyclohexane & 1.0 & $\mathrm{U}$ \\
\hline $78-87-5$ & 1,2-Dichloropropane & 1.0 & $\mathrm{U}$ \\
\hline $75-27-4$ & Bromodichloromethane & 1.0 & $\mathrm{U}$ \\
\hline $10061-01-5$ & cis-1,3-Dichloropropene & 1.0 & U \\
\hline $108-10-1$ & 4-Methyl-2-pentanone & 1.0 & $\mathrm{U}$ \\
\hline $108-88-3$ & Toluene & 1.0 & $\mathrm{U}$ \\
\hline $10061-02-6$ & trans-1,3-Dichloropropene & 1.0 & $\mathrm{U}$ \\
\hline $79-00-5$ & $1,1,2-$ Trichloroethane & 1.0 & $\mathrm{U}$ \\
\hline $127-18-4$ & Tetrachloroethene & 1.0 & $\mathrm{U}$ \\
\hline $591-78-6$ & 2-Hexanone & 1.0 & $\mathrm{U}$ \\
\hline $124-48-1$ & Dibromochloromethane & 1.0 & $\mathrm{U}$ \\
\hline $106-93-4$ & 1,2-Dibromoethane & 1.0 & $\mathrm{U}$ \\
\hline $108-90-7$ & Chlorobenzene & 1.0 & $\mathrm{U}$ \\
\hline $100-41-4$ & Ethylbenzene & 1.0 & $\mathrm{U}$ \\
\hline $95-47-6$ & o-Xylene & 1.0 & $\mathrm{U}$ \\
\hline $179601-23-1$ & m,p-xylene & 1.0 & $\mathrm{U}$ \\
\hline $100-42-5$ & Styrene & 1.0 & $\mathrm{U}$ \\
\hline $75-25-2$ & Bromoform & 1.0 & $\mathrm{U}$ \\
\hline $98-82-8$ & Isopropylbenzene & 1.0 & $\mathrm{U}$ \\
\hline $79-34-5$ & $1,1,2,2$-Tetrachloroethane & 1.0 & $\mathrm{U}$ \\
\hline $541-73-1$ & 1,3 -Dichlorobenzene & 1.0 & $\mathrm{U}$ \\
\hline $106-46-7$ & 1,4-Dichlorobenzene & 1.0 & $\mathrm{U}$ \\
\hline $95-50-1$ & 1,2-Dichlorobenzene & 1.0 & $\mathrm{U}$ \\
\hline $96-12-8$ & 1,2-Dibromo-3-Chloropropane & 1.0 & $\mathrm{U}$ \\
\hline $120-82-1$ & $1,2,4$-Trichlorobenzene & 1.0 & $\mathrm{U}$ \\
\hline $87-61-6$ & $1,2,3$-Trichlorobenzene & 1.0 & $\mathrm{U}$ \\
\hline
\end{tabular}


Lab Name: TESTAMERICA BURLINGTON

Contract: $8 \mathrm{E}-00302$

Lab Code: STLV Case No.: YORK Mod. Ref No.: SDG No.: 28963

Matrix: (SOIL/SED/WATER) Water

Sample wt/vol: 25.0 $(\mathrm{g} / \mathrm{mL}) \mathrm{mL}$

Level: (TRACE or LOW/MED) TRACE

․ Moisture: not dec.

GC Column: DB-624 ID $: 0.20 \quad(\mathrm{~mm})$

Soil Extract Volume: (uL)
Lab Sample ID: MB 200-91665/5

Lab File ID: 14885 005.D

Date Received:

Date Analyzed: 07/23/2015

Dilution Factor: 1.0

Soil Aliquot Volume: (uL)

Purge Volume: 25.0 $(\mathrm{mL})$ CONCENTRATION UNITS: (ug/L or ug/kg) ug/L

\begin{tabular}{l|l|l|c|c|c|}
\cline { 2 - 6 } & CAS NUMBER & \multicolumn{1}{|c|}{ COMPOUND NAME } & RT & EST. CONC. & Q \\
\cline { 2 - 6 } 01 & & Unknown & 7.29 & 2.9 & $\mathrm{~J} X$ \\
\cline { 2 - 6 } 02 & $541-05-9$ & Cyclotrisiloxane, hexamethyl- & 8.22 & 1.2 & $\mathrm{~J} \mathrm{~N}$ \\
\cline { 2 - 6 } 03 & $556-67-2$ & Cyclotetrasiloxane, octamethyl- & 11.04 & $\mathrm{~J} \mathrm{~N}$ \\
\cline { 2 - 6 } 04 & E9667961 & Total Alkanes & $\mathrm{N} / \mathrm{A}$ & & \\
\cline { 2 - 6 }
\end{tabular}

1 EPA-designated Registry Number. 
Lab Name: TESTAMERICA BURLINGTON

Contract: $8 \mathrm{E}-00302$

Lab Code: STLV

Case No.: YORK Mod. Ref No.:

SDG No.: 28963

Matrix: (SOIL/SED/WATER) Water

Sample wt/vol: 25.0 $(\mathrm{g} / \mathrm{mL}) \mathrm{mL}$

Level: (TRACE/LOW/MED) TRACE

\% Moisture: not dec.

GC Column: DB-624 ID: $0.20 \quad(\mathrm{~mm})$

Soil Extract Volume: (uL) $(\mathrm{mL})$

Purge Volume: 25.0
Lab Sample ID: MB 200-92179/5

Lab File ID: 15058_005.D

Date Received:

Date Analyzed: 08/03/2015

Dilution Factor: 1.0

Soil Aliquot Volume:

(uL)

\begin{tabular}{|c|c|c|c|}
\hline CAS NO. & COMPOUND & $\begin{array}{l}\text { CONCENTRATION UNITS: } \\
(\mathrm{ug} / \mathrm{L} \text { or } \mathrm{ug} / \mathrm{kg}) \mathrm{ug} / \mathrm{L}\end{array}$ & q \\
\hline $75-71-8$ & Dichlorodifluoromethane & 1.0 & $\mathrm{U}$ \\
\hline $74-87-3$ & Chloromethane & 1.0 & $\mathrm{U}$ \\
\hline $75-01-4$ & Vinyl chloride & 1.0 & $\mathrm{U}$ \\
\hline $74-83-9$ & Bromomethane & 1.0 & $\mathrm{U}$ \\
\hline $75-00-3$ & Chloroethane & 1.0 & $\mathrm{U}$ \\
\hline $75-69-4$ & Trichlorofluoromethane & 1.0 & $\mathrm{U}$ \\
\hline $75-35-4$ & 1,1-Dichloroethene & 1.0 & $\mathrm{U}$ \\
\hline $76-13-1$ & $1,1,2$-Trichloro-1,2,2-trifluoroethane & 1.0 & $\mathrm{U}$ \\
\hline $67-64-1$ & Acetone & 1.7 & $\mathrm{~J}$ \\
\hline $75-15-0$ & Carbon disulfide & 0.040 & $\mathrm{~J}$ \\
\hline $79-20-9$ & Methyl acetate & 1.0 & $\mathrm{U}$ \\
\hline $75-09-2$ & Methylene Chloride & 1.0 & $\mathrm{U}$ \\
\hline $156-60-5$ & trans-1,2-Dichloroethene & 1.0 & $\mathrm{U}$ \\
\hline $1634-04-4$ & Methyl tert-butyl ether & 1.0 & $\mathrm{U}$ \\
\hline $75-34-3$ & 1,1-Dichloroethane & 1.0 & $\mathrm{U}$ \\
\hline $156-59-2$ & cis-1,2-Dichloroethene & 1.0 & $\mathrm{U}$ \\
\hline $78-93-3$ & 2-Butanone & 5.0 & $\mathrm{U}$ \\
\hline $74-97-5$ & Bromochloromethane & 1.0 & $\mathrm{U}$ \\
\hline $67-66-3$ & Chloroform & 1.0 & $\mathrm{U}$ \\
\hline $71-55-6$ & $1,1,1$-Trichloroethane & 1.0 & $\mathrm{U}$ \\
\hline $110-82-7$ & Cyclohexane & 1.0 & $\mathrm{U}$ \\
\hline $56-23-5$ & Carbon tetrachloride & 1.0 & $\mathrm{U}$ \\
\hline $71-43-2$ & Benzene & 0.024 & $\mathrm{~J}$ \\
\hline $107-06-2$ & 1,2-Dichloroethane & 1.0 & $\mathrm{U}$ \\
\hline
\end{tabular}

Report 1,4-Dioxane for Low-Medium VOA analysis only 
Lab Name: TESTAMERICA BURLINGTON

Lab Code: STLV

Case No.: YORK

Mod. Ref No.:

Contract: $8 \mathrm{E}-00302$

Matrix: (SOIL/SED/WATER) Water

Sample wt/vol: 25.0 $(\mathrm{g} / \mathrm{mL}) \mathrm{mL}$

Level: (TRACE/LOW/MED) TRACE

\% Moisture: not dec.

GC Column: DB-624 ID $: \underline{0.20 \quad(\mathrm{~mm})}$

Soil Extract Volume: (uL) $(\mathrm{mL})$

Purge Volume: 25.0 SDG No.: 28963

Lab Sample ID: MB 200-92179/5

Lab File ID: 15058_005.D

Date Received:

Date Analyzed: 08/03/2015

Dilution Factor: 1.0

Soil Aliquot Volume: (uL)

\begin{tabular}{|c|c|c|c|}
\hline CAS NO. & COMPOUND & $\begin{array}{l}\text { CONCENTRATION UNITS: } \\
(\mathrm{ug} / \mathrm{L} \text { or } \mathrm{ug} / \mathrm{kg}) \mathrm{ug} / \mathrm{L}\end{array}$ & 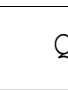 \\
\hline $79-01-6$ & Trichloroethene & $\begin{array}{r}1.0 \\
1.0\end{array}$ & $\mathrm{U}$ \\
\hline $108-87-2$ & Methylcyclohexane & 1.0 & $\mathrm{U}$ \\
\hline $78-87-5$ & 1,2-Dichloropropane & 1.0 & $\mathrm{U}$ \\
\hline $75-27-4$ & Bromodichloromethane & 1.0 & $\mathrm{U}$ \\
\hline $10061-01-5$ & cis-1,3-Dichloropropene & 1.0 & $\mathrm{U}$ \\
\hline $108-10-1$ & 4-Methyl-2-pentanone & 1.0 & $\mathrm{U}$ \\
\hline $108-88-3$ & Toluene & 0.013 & $\mathrm{~J}$ \\
\hline $10061-02-6$ & trans-1,3-Dichloropropene & 1.0 & $\mathrm{U}$ \\
\hline $79-00-5$ & $1,1,2$-Trichloroethane & 1.0 & $\mathrm{U}$ \\
\hline $127-18-4$ & Tetrachloroethene & 0.0034 & $\mathrm{~J}$ \\
\hline $591-78-6$ & 2-Hexanone & 1.0 & $\mathrm{U}$ \\
\hline $124-48-1$ & Dibromochloromethane & 1.0 & $\mathrm{U}$ \\
\hline $106-93-4$ & 1,2-Dibromoethane & 1.0 & $\mathrm{U}$ \\
\hline $108-90-7$ & Chlorobenzene & 1.0 & $\mathrm{U}$ \\
\hline $100-41-4$ & Ethylbenzene & 0.0041 & $\mathrm{~J}$ \\
\hline $95-47-6$ & o-Xylene & 0.0036 & $\mathrm{~J}$ \\
\hline $179601-23-1$ & m,p-xylene & 0.010 & $\mathrm{~J}$ \\
\hline $100-42-5$ & styrene & 1.0 & $\mathrm{U}$ \\
\hline $75-25-2$ & Bromoform & 1.0 & $\mathrm{U}$ \\
\hline $98-82-8$ & Isopropylbenzene & 1.0 & $\mathrm{U}$ \\
\hline $79-34-5$ & $1,1,2,2$-Tetrachloroethane & 1.0 & $\mathrm{U}$ \\
\hline $541-73-1$ & 1,3-Dichlorobenzene & 0.013 & $\mathrm{~J}$ \\
\hline $106-46-7$ & 1,4-Dichlorobenzene & 0.014 & $\mathrm{~J}$ \\
\hline $95-50-1$ & 1,2-Dichlorobenzene & 1.0 & $\mathrm{U}$ \\
\hline $96-12-8$ & 1,2-Dibromo-3-Chloropropane & 1.0 & $\mathrm{U}$ \\
\hline $120-82-1$ & $1,2,4$-Trichlorobenzene & 0.045 & $\mathrm{~J}$ \\
\hline $87-61-6$ & $1,2,3$-Trichlorobenzene & 0.071 & $\mathrm{~J}$ \\
\hline
\end{tabular}


Lab Name: TESTAMERICA BURLINGTON

Contract: $8 \mathrm{E}-00302$

Lab Code: STLV Case No.: YORK Mod. Ref No.: SDG No.: 28963

Matrix: (SOIL/SED/WATER) Water

Sample wt/vol: 25.0 $(\mathrm{g} / \mathrm{mL}) \mathrm{mL}$

Level: (TRACE or LOW/MED) TRACE

\% Moisture: not dec.

GC Column: DB-624 ID $: 0.20 \quad(\mathrm{~mm})$

Soil Extract Volume: (uL)

CONCENTRATION UNITS: (ug/L or ug/kg) ug/L
Lab Sample ID: MB 200-92179/5

Lab File ID: 15058_005.D

Date Received:

Date Analyzed: 08/03/2015

Dilution Factor: 1.0

Soil Aliquot Volume: (uL) Purge Volume: 25.0

$(\mathrm{mL})$

\begin{tabular}{|c|c|c|c|c|}
\hline CAS NUMBER & COMPOUND NAME & RT & EST. CONC. & Q \\
\hline $7446-09-5$ & Sulfur dioxide & 1.60 & 0.81 & $\mathrm{~J} \mathrm{~N}$ \\
\hline 02 & Unknown & 7.29 & 3.1 & $\mathrm{~J} X$ \\
\hline $541-05-9$ & Cyclotrisiloxane, hexamethyl- & 8.22 & 0.54 & $\mathrm{~J} \mathrm{~N}$ \\
\hline 04 & Unknown & 11.04 & 0.63 & $\mathrm{~J}$ \\
\hline E9667961 & Total Alkanes & $\mathrm{N} / \mathrm{A}$ & & \\
\hline
\end{tabular}

1 EPA-designated Registry Number. 
Lab Name: TESTAMERICA BURLINGTON

Contract: $8 \mathrm{E}-00302$

Lab Code: STLV

Case No.: YORK Mod. Ref No.:

SDG No.: 28963

Matrix: (SOIL/SED/WATER) Water

Sample wt/vol: 25.0 $(\mathrm{g} / \mathrm{mL}) \mathrm{mL}$

Level: (TRACE/LOW/MED) TRACE

\% Moisture: not dec.

GC Column: DB-624 ID: $0.20 \quad(\mathrm{~mm})$

Soil Extract Volume: (uL) $(\mathrm{mL})$

Purge Volume: 25.0
Lab Sample ID: VIBLK 200-91665/13

Lab File ID: 14885_013.D

Date Received:

Date Analyzed: 07/23/2015

Dilution Factor: 1.0

Soil Aliquot Volume: (uL)

\begin{tabular}{|c|c|c|c|}
\hline CAS NO. & COMPOUND & $\begin{array}{l}\text { CONCENTRATION UNITS: } \\
(\mathrm{ug} / \mathrm{L} \text { or } \mathrm{ug} / \mathrm{kg}) \mathrm{ug} / \mathrm{L}\end{array}$ & q \\
\hline $75-71-8$ & Dichlorodifluoromethane & 1.0 & $\mathrm{U}$ \\
\hline $74-87-3$ & Chloromethane & 1.0 & $\mathrm{U}$ \\
\hline $75-01-4$ & Vinyl chloride & 1.0 & $\mathrm{U}$ \\
\hline $74-83-9$ & Bromomethane & 1.0 & $\mathrm{U}$ \\
\hline $75-00-3$ & Chloroethane & 1.0 & $\mathrm{U}$ \\
\hline $75-69-4$ & Trichlorofluoromethane & 1.0 & $\mathrm{U}$ \\
\hline $75-35-4$ & 1,1-Dichloroethene & 1.0 & $\mathrm{U}$ \\
\hline $76-13-1$ & $1,1,2$-Trichloro-1,2,2-trifluoroethane & 1.0 & $\mathrm{U}$ \\
\hline $67-64-1$ & Acetone & 5.0 & $\mathrm{U}$ \\
\hline $75-15-0$ & Carbon disulfide & 1.0 & $\mathrm{U}$ \\
\hline $79-20-9$ & Methyl acetate & 1.0 & $\mathrm{U}$ \\
\hline $75-09-2$ & Methylene Chloride & 1.0 & $\mathrm{U}$ \\
\hline $156-60-5$ & trans-1,2-Dichloroethene & 1.0 & $\mathrm{U}$ \\
\hline $1634-04-4$ & Methyl tert-butyl ether & 1.0 & $\mathrm{U}$ \\
\hline $75-34-3$ & 1,1-Dichloroethane & 1.0 & $\mathrm{U}$ \\
\hline $156-59-2$ & cis-1,2-Dichloroethene & 1.0 & $\mathrm{U}$ \\
\hline $78-93-3$ & 2-Butanone & 5.0 & $\mathrm{U}$ \\
\hline $74-97-5$ & Bromochloromethane & 1.0 & $\mathrm{U}$ \\
\hline $67-66-3$ & Chloroform & 0.048 & $\mathrm{~J}$ \\
\hline $71-55-6$ & $1,1,1$-Trichloroethane & 1.0 & $\mathrm{U}$ \\
\hline $110-82-7$ & Cyclohexane & 1.0 & $\mathrm{U}$ \\
\hline $56-23-5$ & Carbon tetrachloride & 1.0 & $\mathrm{U}$ \\
\hline $71-43-2$ & Benzene & 1.0 & $\mathrm{U}$ \\
\hline $107-06-2$ & 1,2-Dichloroethane & 1.0 & $\mathrm{U}$ \\
\hline
\end{tabular}

Report 1,4-Dioxane for Low-Medium VOA analysis only 
Lab Name: TESTAMERICA BURLINGTON

Lab Code: STLV

Case No.: YORK

Mod. Ref No.:

Contract: 8E-00302

Matrix: (SOIL/SED/WATER) Water

Sample wt/vol: 25.0 $(\mathrm{g} / \mathrm{mL}) \mathrm{mL}$

Level: (TRACE/LOW/MED) TRACE

\% Moisture: not dec.

GC Column: DB-624 ID: $0.20 \quad(\mathrm{~mm})$

Soil Extract Volume: (uL) $(\mathrm{mL})$

Purge Volume: 25.0
SDG No.: 28963

Lab Sample ID: VIBLK 200-91665/13

Lab File ID: 14885_013.D

Date Received:

Date Analyzed: 07/23/2015

Dilution Factor: 1.0

Soil Aliquot Volume:

(uL)

\begin{tabular}{|c|c|c|c|}
\hline CAS NO. & COMPOUND & $\begin{array}{l}\text { CONCENTRATION UNITS: } \\
(\mathrm{ug} / \mathrm{L} \text { or } \mathrm{ug} / \mathrm{kg}) \mathrm{ug} / \mathrm{L}\end{array}$ & Q \\
\hline $79-01-6$ & Trichloroethene & 1.0 & $\mathrm{U}$ \\
\hline $108-87-2$ & Methylcyclohexane & 1.0 & $\mathrm{U}$ \\
\hline $78-87-5$ & 1,2-Dichloropropane & 1.0 & $\mathrm{U}$ \\
\hline $75-27-4$ & Bromodichloromethane & 1.0 & $\mathrm{U}$ \\
\hline $10061-01-5$ & cis-1,3-Dichloropropene & 1.0 & $\mathrm{U}$ \\
\hline $108-10-1$ & 4-Methyl-2-pentanone & 5.0 & $\mathrm{U}$ \\
\hline $108-88-3$ & Toluene & 1.0 & $\mathrm{U}$ \\
\hline $10061-02-6$ & trans-1,3-Dichloropropene & 1.0 & $\mathrm{U}$ \\
\hline $79-00-5$ & $1,1,2-T r i c h l o r o e t h a n e$ & 1.0 & $\mathrm{U}$ \\
\hline $127-18-4$ & Tetrachloroethene & 1.0 & $\mathrm{U}$ \\
\hline $591-78-6$ & 2-Hexanone & 5.0 & $\mathrm{U}$ \\
\hline $124-48-1$ & Dibromochloromethane & 1.0 & $\mathrm{U}$ \\
\hline $106-93-4$ & 1,2-Dibromoethane & 1.0 & $\mathrm{U}$ \\
\hline $108-90-7$ & Chlorobenzene & 1.0 & $\mathrm{U}$ \\
\hline $100-41-4$ & Ethylbenzene & 1.0 & $\mathrm{U}$ \\
\hline $95-47-6$ & o-Xylene & 1.0 & $\mathrm{U}$ \\
\hline $179601-23-1$ & m,p-Xylene & 1.0 & $\mathrm{U}$ \\
\hline $100-42-5$ & styrene & 1.0 & $\mathrm{U}$ \\
\hline $75-25-2$ & Bromoform & 1.0 & $\mathrm{U}$ \\
\hline $98-82-8$ & Isopropylbenzene & 1.0 & $\mathrm{U}$ \\
\hline $79-34-5$ & $1,1,2,2$-Tetrachloroethane & 1.0 & $\mathrm{U}$ \\
\hline $541-73-1$ & 1,3-Dichlorobenzene & 1.0 & $\mathrm{U}$ \\
\hline $106-46-7$ & 1,4-Dichlorobenzene & 1.0 & $\mathrm{U}$ \\
\hline $95-50-1$ & $1,2-$ Dichlorobenzene & 1.0 & $\mathrm{U}$ \\
\hline $96-12-8$ & 1,2-Dibromo-3-Chloropropane & 1.0 & $\mathrm{U}$ \\
\hline $120-82-1$ & $1,2,4$-Trichlorobenzene & 1.0 & $\mathrm{U}$ \\
\hline $87-61-6$ & $1,2,3$-Trichlorobenzene & 1.0 & $\mathrm{U}$ \\
\hline
\end{tabular}


Lab Name: TESTAMERICA BURLINGTON

Contract: $8 \mathrm{E}-00302$

Lab Code: STLV Case No.: YORK Mod. Ref No.: SDG No.: 28963

Matrix: (SOIL/SED/WATER) Water

Sample wt/vol: 25.0 $(\mathrm{g} / \mathrm{mL}) \mathrm{mL}$

Level: (TRACE or LOW/MED) TRACE

\% Moisture: not dec.

GC Column: DB-624 ID $: 0.20 \quad(\mathrm{~mm})$

Soil Extract Volume: (uL)

CONCENTRATION UNITS: (ug/L or ug/kg) ug/L
Lab Sample ID: VIBLK 200-91665/13

Lab File ID: 14885_013.D

Date Received:

Date Analyzed: 07/23/2015

Dilution Factor: 1.0

Soil Aliquot Volume: (uL) Purge Volume: 25.0 $(\mathrm{mL})$

\begin{tabular}{|c|c|c|c|c|}
\hline CAS NUMBER & COMPOUND NAME & RT & EST. CONC. & Q \\
\hline 01 & Unknown & 7.29 & 2.7 & $\mathrm{~J} B \mathrm{X}$ \\
\hline 02 & Unknown & 8.22 & 6.2 & $\mathrm{~J} B$ \\
\hline $556-67-2$ & Cyclotetrasiloxane, octamethyl- & 11.04 & 3.7 & $\mathrm{~J} \quad \mathrm{~N} B$ \\
\hline 04 & Unknown & 13.19 & 0.58 & $\mathrm{~J}$ \\
\hline E9667961 & Total Alkanes & $\mathrm{N} / \mathrm{A}$ & & \\
\hline
\end{tabular}

1 EPA-designated Registry Number. 
Lab Name: TestAmerica Burlington SDG No.: 28963

Instrument ID: CHD.i

Lab Sample ID: IC 200-91077/2

Date Analyzed: 07/14/15 11:08

\begin{tabular}{|c|r|}
\hline COMPOUND NAME & RETEN \\
TI
\end{tabular}

Lab Sample ID: IC 200-91077/3

Date Analyzed: 07/14/15 11:33 Client Sample ID: Lab File ID: 14685_003.D

Analysis Batch Number: 91077

Client Sample ID:

Lab File ID: 14685 002.D

GC Column: DB-624

ID: $0.2(\mathrm{~mm})$

MANUAL INTEGRATION

\begin{tabular}{|c|c|c|c|c|}
\hline \multicolumn{1}{|c|}{ COMPOUND NAME } & RETENTION & \multicolumn{3}{|c}{ MANUAL INTEGRATION } \\
\cline { 3 - 5 } & TIME & REASON & ANALYST & DATE \\
\hline 1,2 -Dibromo-3-Chloropropane & 13.44 & Baseline & archern & $07 / 14 / 1514: 11$ \\
\hline
\end{tabular}


Lab Name: TestAmerica Burlington

Job No.: 200-28963-1

SDG No.: 28963

Instrument ID: CHD.i

Analysis Batch Number: 91665

Lab Sample ID: CCVIS 200-91665/3

Client Sample ID:

Date Analyzed: 07/23/15 08:51

Lab File ID: 14885 003.D

GC Column: DB-624

ID $: 0.2(\mathrm{~mm})$

\begin{tabular}{|c|c|c|c|c|}
\hline \multirow[t]{2}{*}{ COMPOUND NAME } & RETENTION & \multicolumn{3}{|c|}{ MANUAL INTEGRATION } \\
\hline & TIME & REASON & ANALYST & DATE \\
\hline Dichlorodifluoromethane & 1.48 & Baseline & archern & $07 / 23 / 1509: 14$ \\
\hline
\end{tabular}

Lab Sample ID: CCVC 200-91665/14

Client Sample ID:

Date Analyzed: 07/23/15 14:39

Lab File ID: 14885 014.D

GC Column: DB-624

ID $: 0.2(\mathrm{~mm})$

\begin{tabular}{|l|c|c|c|c|}
\hline \multicolumn{1}{|c|}{ COMPOUND NAME } & RETENTION & \multicolumn{2}{|c|}{ MANUAL INTEGRATION } \\
\cline { 4 - 5 } & TIME & REASON & ANALYST & DATE \\
\hline Dichlorodifluoromethane & 1.48 & Baseline & archern & $07 / 23 / 1515: 02$ \\
\hline
\end{tabular}


Lab Name: TestAmerica Burlington

Job No.: 200-28963-1

SDG No.: 28963

Instrument ID: CHD.i

Analysis Batch Number: 92118

Lab Sample ID: IC 200-92118/3

Date Analyzed: 07/31/15 12:13

Client Sample ID:

Lab File ID: 15041_003.D

GC Column: DB-624

ID : $0.2(\mathrm{~mm})$

\begin{tabular}{|c|c|c|c|c|}
\hline \multirow[t]{2}{*}{ COMPOUND NAME } & \multirow{2}{*}{$\begin{array}{c}\text { RETENTION } \\
\text { TIME }\end{array}$} & \multicolumn{3}{|c|}{ MANUAL INTEGRATION } \\
\hline & & REASON & ANALYST & DATE \\
\hline Bromomethane & 2.00 & Baseline & wilburj & $08 / 05 / 15 \quad 15: 12$ \\
\hline Acetone & 2.81 & Baseline & wilburj & $08 / 05 / 15$ \\
\hline
\end{tabular}

Lab Sample ID: IC 200-92118/4

Client Sample ID:

Date Analyzed: 07/31/15 12:38

Lab File ID: $15041004 . D$

GC Column: DB-624

ID $: 0.2(\mathrm{~mm})$

\begin{tabular}{|c|c|c|c|c|}
\hline \multirow[t]{2}{*}{ COMPOUND NAME } & \multirow{2}{*}{$\begin{array}{c}\text { RETENTION } \\
\text { TIME }\end{array}$} & \multicolumn{3}{|c|}{ MANUAL INTEGRATION } \\
\hline & & REASON & ANALYST & DATE \\
\hline Dichlorodifluoromethane & 1.48 & Baseline & wilburj & $08 / 05 / 15 \quad 12: 19$ \\
\hline Bromomethane & 2.00 & Baseline & wilburj & $08 / 05 / 15 \quad 12: 19$ \\
\hline 1,2-Dibromo-3-Chloropropane & 13.43 & Baseline & wilburj & $08 / 05 / 1515: 10$ \\
\hline
\end{tabular}

Lab Sample ID: ICIS 200-92118/5

Client Sample ID:

Date Analyzed: 07/31/15 13:02

Lab File ID: 15041_005.D

GC Column: DB-624

ID $: 0.2(\mathrm{~mm})$

\begin{tabular}{|c|c|c|c|c|}
\hline \multirow[t]{2}{*}{ COMPOUND NAME } & \multirow{2}{*}{$\begin{array}{c}\text { RETENTION } \\
\text { TIME }\end{array}$} & \multicolumn{3}{|c|}{ MANUAL INTEGRATION } \\
\hline & & REASON & ANALYST & DATE \\
\hline Dichlorodifluoromethane & 1.48 & Baseline & wilburj & $08 / 05 / 15 \quad 15: 11$ \\
\hline Bromomethane & 1.99 & Baseline & wilburj & $08 / 05 / 1515: 12$ \\
\hline
\end{tabular}

Lab Sample ID: IC 200-92118/6

Client Sample ID:

Date Analyzed: 07/31/15 13:27

Lab File ID: 15041_006.D

GC Column: DB-624

ID $: 0.2(\mathrm{~mm})$

\begin{tabular}{|c|c|c|c|c|}
\hline \multirow[t]{2}{*}{ COMPOUND NAME } & \multirow{2}{*}{$\begin{array}{c}\text { RETENTION } \\
\text { TIME }\end{array}$} & \multicolumn{3}{|c|}{ MANUAL INTEGRATION } \\
\hline & & REASON & ANALYST & DATE \\
\hline Dichlorodifluoromethane & 1.48 & Baseline & wilburj & $08 / 05 / 15 \quad 15: 12$ \\
\hline Bromomethane & 1.99 & Baseline & wilburj & $08 / 05 / 15 \quad 15: 14$ \\
\hline 1,2-Dichloropropane-d6 & 6.42 & Split Peak & wilburj & $08 / 05 / 15 \quad 16: 43$ \\
\hline
\end{tabular}

SOM01.2/VOA_Tr 
Lab Name: TestAmerica Burlington

SDG No.: 28963

Instrument ID: CHD.i

Lab Sample ID: IC 200-92118/7

Date Analyzed: 07/31/15 13:52
Job No.: 200-28963-1

Analysis Batch Number: 92118

Client Sample ID:

Lab File ID: $15041007 . \mathrm{D}$
GC Column: DB-624

ID $: 0.2(\mathrm{~mm})$

\begin{tabular}{|c|c|c|c|c|}
\hline \multirow[t]{2}{*}{ COMPOUND NAME } & \multirow{2}{*}{$\begin{array}{c}\text { RETENTION } \\
\text { TIME }\end{array}$} & \multicolumn{3}{|c|}{ MANUAL INTEGRATION } \\
\hline & & REASON & ANALYST & DATE \\
\hline Dichlorodifluoromethane & 1.48 & Baseline & wilburj & $08 / 05 / 15$ \\
\hline 1,2-Dichloropropane-d6 & 6.42 & Baseline & wilburj & $08 / 05 / 15 \quad 15: 24$ \\
\hline
\end{tabular}


Lab Name: TestAmerica Burlington SDG No.: 28963

Instrument ID: CHD.i

Lab Sample ID: CCVC 200-92179/11

Date Analyzed: 08/03/15 18:38

\begin{tabular}{|l|c|c|c|c|}
\hline \multicolumn{1}{|c|}{ COMPOUND NAME } & RETENTION & \multicolumn{3}{|c}{ MANUAL INTEGRATION } \\
\cline { 3 - 5 } & TIME & REASON & ANALYST & DATE \\
\hline Dichlorodifluoromethane & 1.53 & Baseline & wilburj & 08/05/15 17:49 \\
\hline
\end{tabular}

Job No.: 200-28963-1

Analysis Batch Number: 92179

Client Sample ID:

Lab File ID: $15058011 . \mathrm{D}$
ID $: 0.2(\mathrm{~mm})$ 


\section{TestAmerica}

THE LEADER IN ENVIRONMENTAL TESTING

\section{ANALYTICAL REPORT}

Job Number: 200-28988-1

SDG Number: 28988

Job Description: York (200-28988)

Contract Number: 1E-30401

For:

Argonne National Laboratory

9700 South Cass Avenue

Building 203

Office B-141

Argonne, IL 60439

Attention: Ms. Esther Bowen

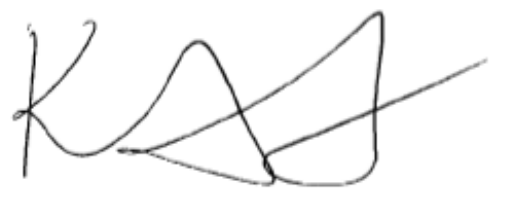

Approved for release. Kathryn A Kelly

\section{Kathryn A Kelly, Project Manager I \\ 30 Community Drive, South Burlington, VT, 05403 \\ kathryn.kelly@testamericainc.com \\ $08 / 11 / 2015$}

The test results in this report relate only to sample(s) as received by the laboratory. These test results were derived under a quality system that adheres to the requirements of NELAC. Pursuant to NELAC, this report may not be produced in full without written approval from the laboratory 


\section{Table of Contents}

Cover Title Page..$\ldots \ldots \ldots \ldots \ldots \ldots \ldots \ldots \ldots \ldots \ldots \ldots \ldots \ldots$

Report Narrative $\ldots \ldots \ldots \ldots \ldots \ldots \ldots \ldots \ldots \ldots \ldots \ldots \ldots$

Case Narrative $\ldots \ldots \ldots \ldots \ldots \ldots \ldots \ldots \ldots \ldots \ldots \ldots \ldots, 4$

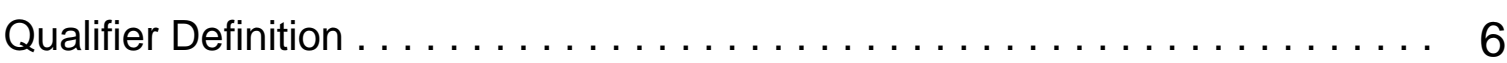

Sample List ...................... 7

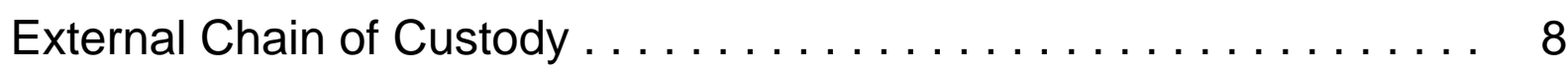

Shipping Documentation ....................... 10

Sample Receipt and Log In Check List ...................... 11

Standards Traceability . . . . . . . . . . . . . . . . . 12

Methodology Review ........................ 17

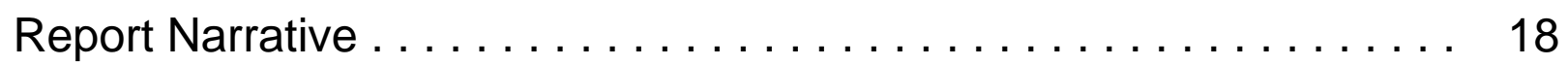

Manual Integration Documentation ........................ 18

QC Summary - SOM01.2 Volatiles-Trace . . . . . . . . . . . . 20

QC Summary - SOM01.2 Volatiles-Trace $\ldots \ldots \ldots \ldots \ldots \ldots \ldots \ldots \ldots$

Deuterated Monitoring Compound Summary $\ldots \ldots \ldots \ldots \ldots \ldots \ldots \ldots \ldots \ldots \ldots, 20$

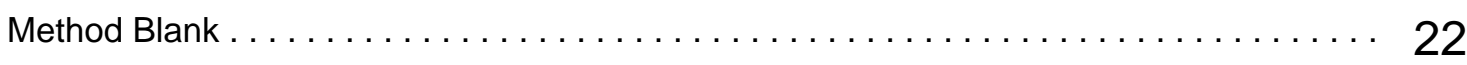

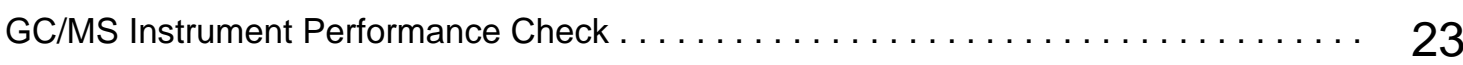

Internal Standard Area and RT Summary $\ldots \ldots \ldots \ldots \ldots \ldots \ldots \ldots \ldots \ldots \ldots \ldots \ldots \ldots \ldots$

Sample Data - SOM01.2 Volatiles-Trace ................. 25

Sample Data - SOM01.2 Volatiles-Trace ..................... 25

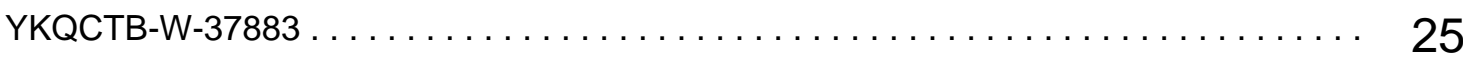

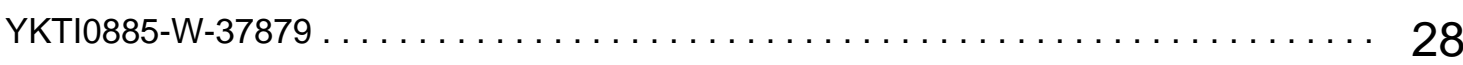

Standards - SOM01.2 Volatiles-Trace . . . . . . . . . . . . . 31

Standards - SOM01.2 Volatiles-Trace $\ldots \ldots \ldots \ldots \ldots \ldots \ldots \ldots \ldots \ldots \ldots \ldots \ldots$

Initial Calibration Data ................................... 31

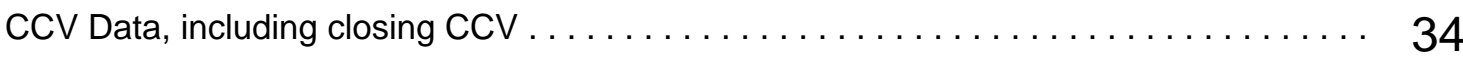




\section{Table of Contents}

Raw Qc Data - SOM01.2 Volatiles-Trace . . . . . . . . . . . . . . . 37

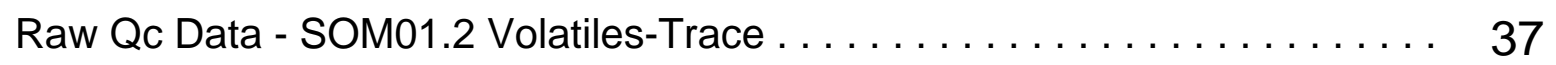

Raw Qc Data - SOM01.2 Volatiles-Trace ........................... 37

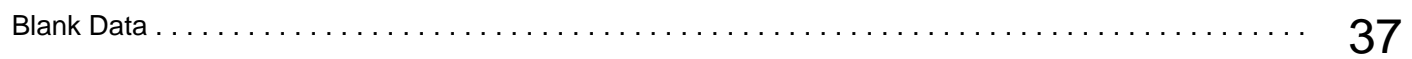




\section{CASE NARRATIVE}

\section{Client: Argonne National Laboratory \\ Project: York (200-28988)}

\section{Report Number: 200- 28988-1}

Enclosed is the data set for the referenced project work. With the exceptions noted as flags or footnotes, standard analytical protocols were followed in performing the analytical work and the applied control limits were met.

Calculations were performed before rounding to avoid round-off errors in calculated results.

All holding times were met and proper preservation noted for the methods performed on these samples, unless otherwise detailed in the individual sections below.

\section{Receipt}

The samples were received on $07 / 21 / 2015$. Documentation of the condition of the samples at the time of their receipt and any exception to the laboratory's Sample Acceptance Policy is documented in the Shipping Documentation section of this submittal. The samples, as received, were not acid preserved. On that basis, the laboratory did provide for the analysis of the samples within seven days of sample collection.

\section{SOM01.2 Volatile Organics (Trace Level Water)}

A storage blank was prepared for volatile organics analysis, and stored in association with the storage of the samples. That storage blank, identified as VHBLK01, was carried through the holding period with the samples, and analyzed.

Each of the analyses associated with the sample set exhibited an acceptable internal standard performance. There was an acceptable recovery of each deuterated monitoring compound (DMC) in the analysis of the method blank associated with the analytical work, and in the analysis of the storage blank associated with the sample set. Matrix spike and matrix spike duplicate analyses were not performed on samples in this sample set. Trace concentrations of Acetone, 1,2,4-Trichlorobenzene and 1,2,3-Trichlorobenzene were detected in the method blanks associated with this sample set. Present in the method blank and storage blank analyses was a non-target constituent that represents a compound that is related to the DMC formulation. The fact that the presence of this compound is not within the laboratory's control is at issue.

The responses for each target analyte met the relative standard deviation criterion in the initial calibration. The responses for each target analyte met the relative standard deviation criterion in the initial calibration. The response for each target analyte met the $50.0 \%$ difference criterion in the closing calibration check acquisition.

The primary quantitation mass for methylcyclohexane that is specified in the Statement of Work is mass 83 . The laboratory did identify a contribution to mass 83 from 1,2-dichloropropane- $d_{6}$, one of the deuterated monitoring compounds (DMCs). The laboratory did change the primary quantitation mass assignment to mass 55 for the quantification of methylcyclohexane. 
Manual integration was employed in deriving certain of the analytical results. The values that have been derived from manual integration are qualified on the quantitation reports. Extracted ion current profiles for each manual integration are included in the data package, and further documented at the end of this submittal. 


\section{DATA REPORTING QUALIFIERS}

Client: Argonne National Laboratory

Job Number: 200-28988-1

Sdg Number: 28988

Lab Section

Qualifier

Description

GC/MS VOA

U

$\mathrm{J}$

$\mathrm{J}$

$X$

B

$\mathrm{N}$
Analyzed for but not detected.

Indicates an Estimated Value for TICs

Indicates an estimated value.

See case narrative notes for explanation of the ' $X$ ' flag

The analyte was found in an associated blank, as well as in the sample.

This flag indicates the presumptive evidence of a compound. 


\section{Sample Login Acknowledgement}

Job 200-28988-1

\begin{tabular}{|c|c|c|c|}
\hline Client Job Description: & York (200-28988) & Report To: & Argonne National Laboratory \\
\hline Purchase Order \#: & $1 \mathrm{E}-30401$ & & Jorge Alvarado \\
\hline Work Order \#: & $1 \mathrm{E}-30401$ & & 9700 South Cass Avenue \\
\hline Project Manager: & Kathryn A Kelly & & Building 203 \\
\hline Job Due Date: & $8 / 6 / 2015$ & & $\begin{array}{l}\text { Office B-141 } \\
\text { Argonne } 60439\end{array}$ \\
\hline Job TAT: & 14 Days & & \\
\hline Max Deliverable Level: & IV & Bill To: & $\begin{array}{l}\text { Argonne National Laboratory } \\
\text { Accounts Payable }\end{array}$ \\
\hline Earliest Deliverable Due: & $8 / 6 / 2015$ & & $\begin{array}{l}\text { Chief Financial Offices } \\
9700 \text { S. Cass Ave. } \\
\text { Building } 201 \\
\text { Argonne, IL } 60439\end{array}$ \\
\hline
\end{tabular}

\section{Login 200-28988}

\section{Sample Receipt:}

Method of Delivery:

7/23/2015 10:30:00 AM
FedEx Priority Overnight

\section{Number of Coolers:}

Cooler Temperature(s) $\left(\mathbf{C}^{\circ}\right): 3.8$;

\begin{tabular}{|c|c|c|c|c|c|}
\hline $\begin{array}{l}\text { Lab Sample \# } \\
\text { Method }\end{array}$ & $\begin{array}{l}\text { Client Sample ID } \\
\text { Method Description / Work Location }\end{array}$ & Date Sampled & Matrix & Rpt Basis & Dry / Wet ** \\
\hline 200-28988-1 & YKQCTB-W-37883 & 7/21/2015 12:12:00 PM & Water & & \\
\hline 200-28988-2 & YKTI0885-W-37879 & 7/21/2015 2:45:00 PM & Water & & \\
\hline SOM01.2_Vol_Tr & ir $\quad$ SOM01.2 Trace Volatile Organics / In-Lab & & & Total & Wet \\
\hline 200-28988-3 & VHBLK01 & 7/23/2015 2:00:00 PM & Water & & \\
\hline SOM01.2_Vol_Tr & ir $\quad$ SOM01.2 Trace Volatile Organics / In-Lab & & & Total & Wet \\
\hline
\end{tabular}




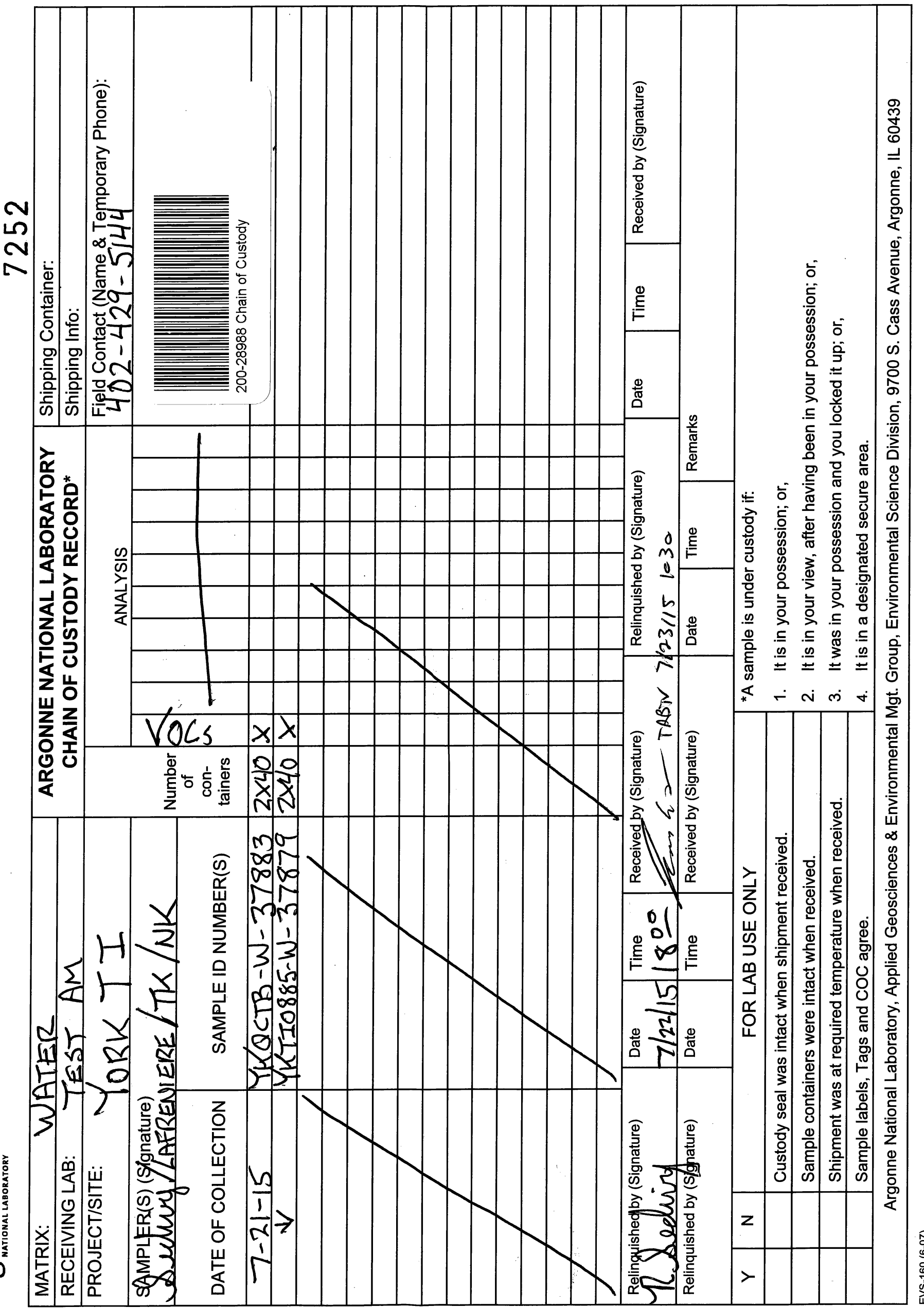



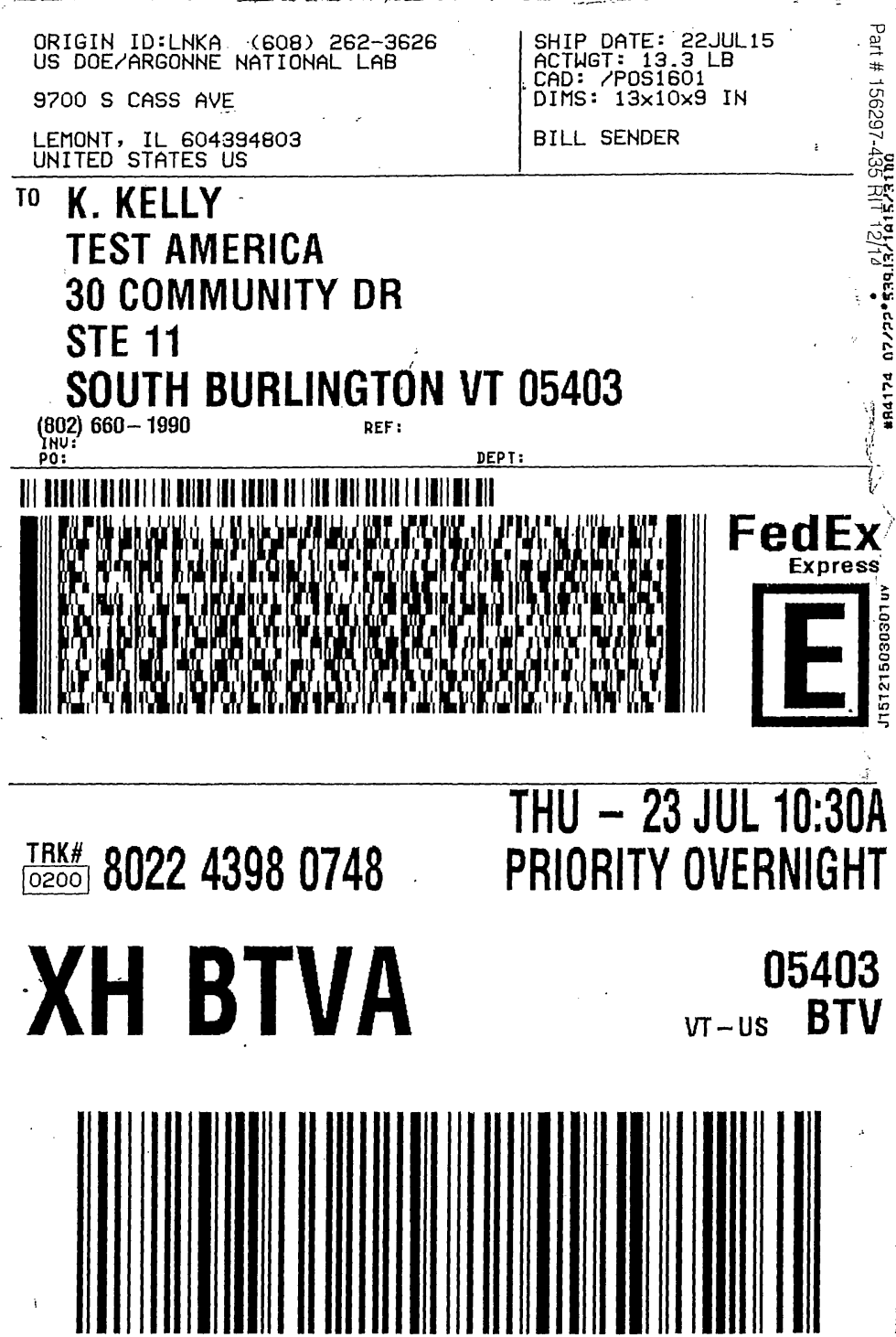


\section{Shipping and Receiving Documents}




\section{Login Sample Receipt Checklist}

Client: Argonne National Laboratory

Job Number: 200-28988-1

SDG Number: 28988

Login Number: 28988

List Source: TestAmerica Burlington

List Number: 1

Creator: Nye, Elizabeth A

Question

Radioactivity wasn't checked or is $</=$ background as measured by a survey True meter.

The cooler's custody seal, if present, is intact.

Sample custody seals, if present, are intact.

The cooler or samples do not appear to have been compromised or tampered with.

Samples were received on ice.

Cooler Temperature is acceptable.

Cooler Temperature is recorded.

$\mathrm{COC}$ is present.

$\mathrm{COC}$ is filled out in ink and legible.

$\mathrm{COC}$ is filled out with all pertinent information.

Is the Field Sampler's name present on COC?

There are no discrepancies between the containers received and the COC.

Samples are received within Holding Time.

Sample containers have legible labels.

Containers are not broken or leaking.

Sample collection date/times are provided.

Appropriate sample containers are used.

Sample bottles are completely filled.

Sample Preservation Verified.

There is sufficient vol. for all requested analyses, incl. any requested MS/MSDs

Containers requiring zero headspace have no headspace or bubble is $<6 \mathrm{~mm}(1 / 4 ")$.

Multiphasic samples are not present.

Samples do not require splitting or compositing.

Residual Chlorine Checked.

Answer Comment

True

Lab does not accept radioactive samples.

NO NUMBERS

True

True

True

True

True

$3.8^{\circ} \mathrm{C}$

True

True

True

True

True

True

True

True

True

True

N/A

True

True

True

True

True

N/A 


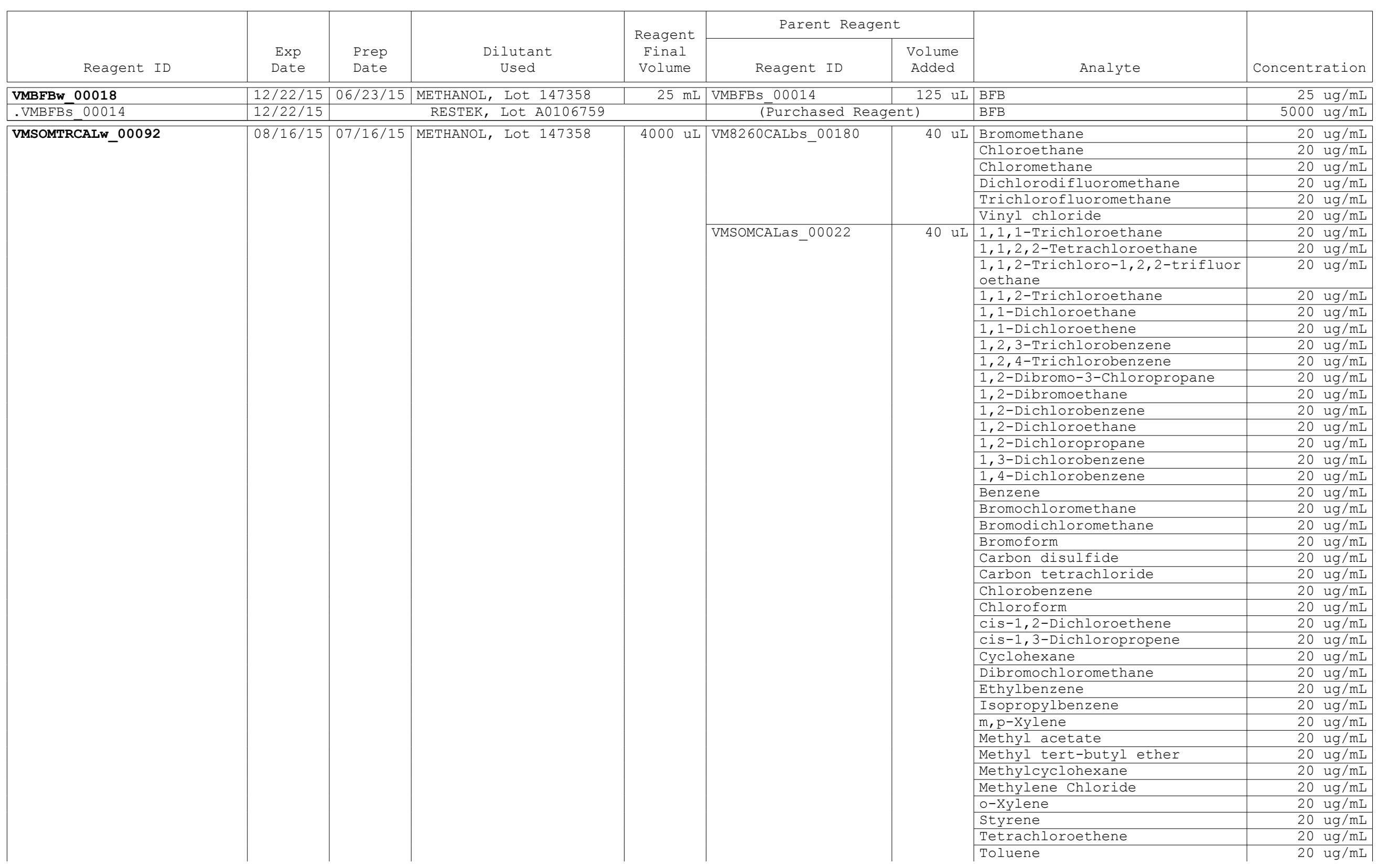


SDG No.: 28988

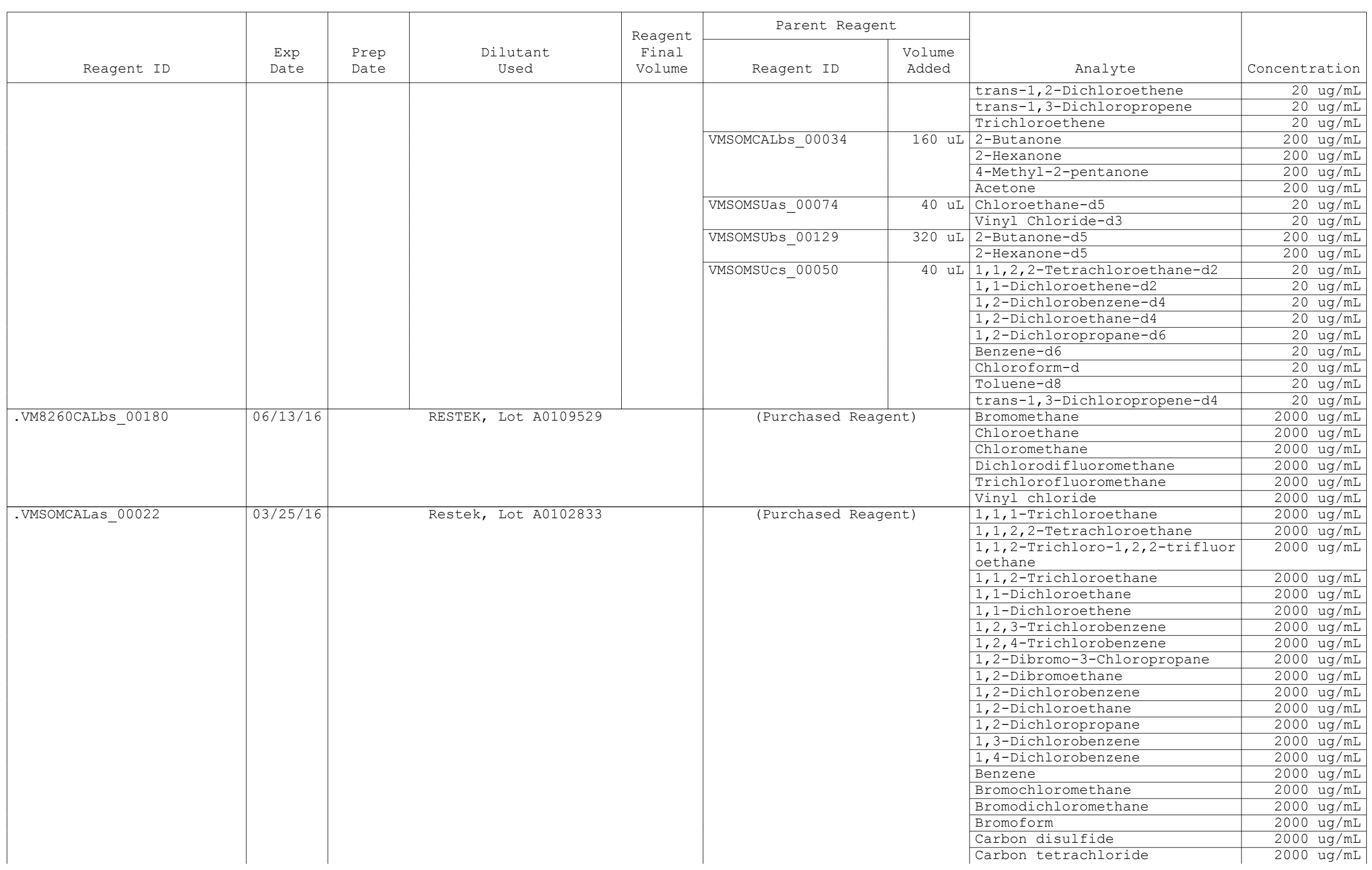


SDG No.: 28988

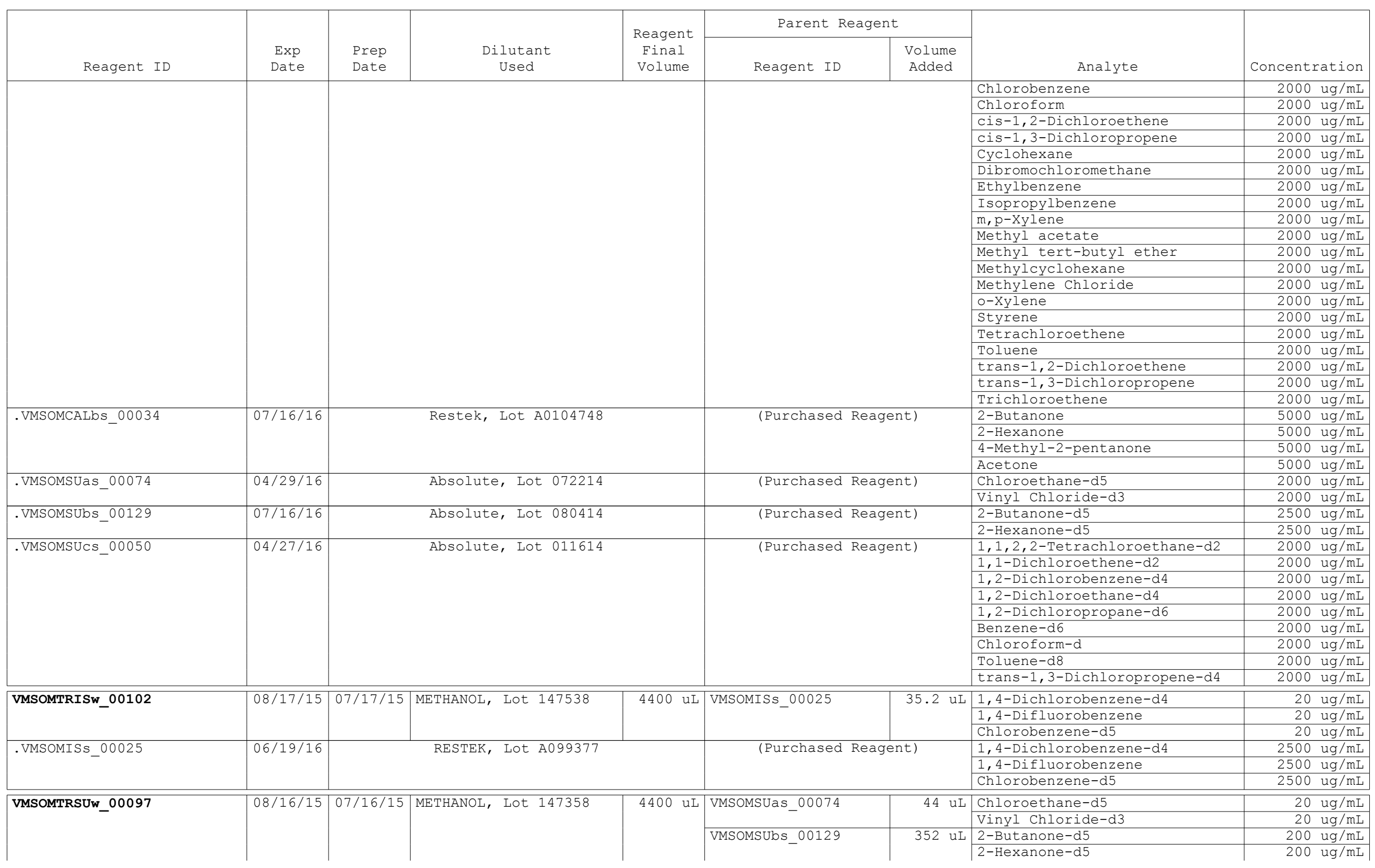


SDG No.: 28988

\begin{tabular}{|c|c|c|c|c|c|c|c|c|}
\hline \multirow[b]{2}{*}{ Reagent ID } & \multirow[b]{2}{*}{$\begin{array}{l}\text { Exp } \\
\text { Date }\end{array}$} & \multirow[b]{2}{*}{$\begin{array}{l}\text { Prep } \\
\text { Date }\end{array}$} & \multirow[b]{2}{*}{$\begin{array}{c}\text { Dilutant } \\
\text { Used }\end{array}$} & \multirow{2}{*}{$\begin{array}{l}\text { Reagent } \\
\text { Final } \\
\text { Volume }\end{array}$} & \multicolumn{2}{|c|}{ Parent Reagent } & \multirow[b]{2}{*}{ Analyte } & \multirow[b]{2}{*}{ Concentration } \\
\hline & & & & & \multirow{10}{*}{$\begin{array}{r}\text { Reagent ID } \\
\text { VMSOMSUCS_00050 }\end{array}$} & $\begin{array}{l}\text { Volume } \\
\text { Added }\end{array}$ & & \\
\hline & & & & & & $44 \mathrm{uL}$ & $1,1,2,2$-Tetrachloroethane-d2 & $20 \mathrm{ug} / \mathrm{mI}$ \\
\hline & & & & & & & 1,1-Dichloroethene-d2 & $20 \mathrm{ug} / \mathrm{mI}$ \\
\hline & & & & & & & 1,2-Dichlorobenzene-d4 & $20 \mathrm{ug} / \mathrm{mI}$ \\
\hline & & & & & & & 1,2-Dichloroethane-d4 & $20 \mathrm{ug} / \mathrm{mI}$ \\
\hline & & & & & & & 1,2-Dichloropropane-d6 & $20 \mathrm{ug} / \mathrm{mI}$ \\
\hline & & & & & & & Benzene-d6 & $20 \mathrm{ug} / \mathrm{mI}$ \\
\hline & & & & & & & Chloroform-d & $20 \mathrm{ug} / \mathrm{mI}$ \\
\hline & & & & & & & Toluene-d8 & $20 \mathrm{ug} / \mathrm{mI}$ \\
\hline & & & & & & & trans-1,3-Dichloropropene-d4 & $20 \mathrm{ug} / \mathrm{mI}$ \\
\hline \multirow[t]{2}{*}{.VMSOMSUas_00074 } & \multirow[t]{2}{*}{$04 / 29 / 16$} & \multirow{2}{*}{\multicolumn{3}{|c|}{ Absolute, Lot 072214}} & \multirow{2}{*}{\multicolumn{2}{|c|}{ (Purchased Reagent) }} & Chloroethane-d5 & $2000 \mathrm{ug} / \mathrm{mI}$ \\
\hline & & & & & & & Vinyl Chloride-d3 & $2000 \mathrm{ug} / \mathrm{mI}$ \\
\hline \multirow[t]{2}{*}{.VMSOMSUbs_00129 } & \multirow[t]{2}{*}{$07 / 16 / 16$} & \multirow{2}{*}{\multicolumn{3}{|c|}{ Absolute, Lot 080414}} & \multirow{2}{*}{\multicolumn{2}{|c|}{ (Purchased Reagent) }} & 2-Butanone-d5 & $2500 \mathrm{ug} / \mathrm{mI}$ \\
\hline & & & & & & & 2-Hexanone-d5 & $2500 \mathrm{ug} / \mathrm{mI}$ \\
\hline \multirow[t]{9}{*}{.VMSOMSUCS_00050 } & \multirow[t]{9}{*}{$04 / 27 / 16$} & \multirow{9}{*}{\multicolumn{2}{|c|}{ Absolute, Lot 011614}} & & \multirow{9}{*}{\multicolumn{2}{|c|}{ (Purchased Reagent) }} & $1,1,2,2$-Tetrachloroethane-d2 & $2000 \mathrm{ug} / \mathrm{mI}$ \\
\hline & & & & & & & 1,1-Dichloroethene-d2 & $2000 \mathrm{ug} / \mathrm{mI}$ \\
\hline & & & & & & & 1,2-Dichlorobenzene-d4 & $2000 \mathrm{ug} / \mathrm{mI}$ \\
\hline & & & & & & & 1,2-Dichloroethane-d4 & $2000 \mathrm{ug} / \mathrm{mI}$ \\
\hline & & & & & & & 1,2-Dichloropropane-d6 & $2000 \mathrm{ug} / \mathrm{mI}$ \\
\hline & & & & & & & Benzene-d6 & $2000 \mathrm{ug} / \mathrm{mI}$ \\
\hline & & & & & & & Chloroform-d & $2000 \mathrm{ug} / \mathrm{mI}$ \\
\hline & & & & & & & Toluene-d8 & $2000 \mathrm{ug} / \mathrm{mI}$ \\
\hline & & & & & & & trans-1,3-Dichloropropene-d4 & $2000 \mathrm{ug} / \mathrm{mI}$ \\
\hline
\end{tabular}


Lab Name: TestAmerica Burlington

Job No.: 200-28988-1

SDG No.: 28988

\begin{tabular}{|c|l|l|c|c|}
\hline $\begin{array}{c}\text { Reagent } \\
\text { Container }\end{array}$ & \multicolumn{1}{|c|}{$\begin{array}{c}\text { Reagent } \\
\text { ID }\end{array}$} & \multicolumn{1}{c|}{$\begin{array}{c}\text { Reagent } \\
\text { Description }\end{array}$} & $\begin{array}{c}\text { Preparation } \\
\text { Date }\end{array}$ & $\begin{array}{c}\text { Expiration } \\
\text { Date }\end{array}$ \\
\hline 807558 & VMBEBw_00018 & BFB TUNE 25 PPM & $06 / 23 / 2015$ & $12 / 22 / 2015$ \\
\hline 816537 & VMSOMTRCALw_00092 & SOM TR CAL 20 PPM & $07 / 16 / 2015$ & $08 / 16 / 2015$ \\
\hline 816538 & VMSOMTRSUw_00097 & SOM TR DMC 20 PPM & $07 / 16 / 2015$ & $08 / 16 / 2015$ \\
\hline 816658 & VMSOMTRCALw_00092 & SOM TR CAL 20 PPM & $07 / 16 / 2015$ & $08 / 16 / 2015$ \\
\hline 817121 & VMSOMTRISw_00102 & SOM TR ISTD 20 PPM & $07 / 17 / 2015$ & $08 / 17 / 2015$ \\
\hline
\end{tabular}




\section{METHODOLOGY SUMMARY}

Laboratory: TestAmerica Laboratories

Location: South Burlington, Vermont
Project No:

SDG No: 28988

VOA

Volatile Organics Trace - USEPA CLP SOM01.2 
Lab Name: TestAmerica Burlington

Job No.: 200-28988-1

SDG No.: 28988

Instrument ID: CHD.i

Analysis Batch Number: 91869

Lab Sample ID: IC 200-91869/3

Client Sample ID:

Date Analyzed: 07/27/15 13:26

Lab File ID: 14962 003.D

GC Column: DB-624

ID : $0.2(\mathrm{~mm})$

\begin{tabular}{|c|c|c|c|c|}
\hline \multirow[t]{2}{*}{ COMPOUND NAME } & \multirow{2}{*}{$\begin{array}{c}\text { RETENTION } \\
\text { TIME }\end{array}$} & \multicolumn{3}{|c|}{ MANUAL INTEGRATION } \\
\hline & & REASON & ANALYST & DATE \\
\hline Bromomethane & 2.00 & Baseline & wilburj & $08 / 06 / 15 \quad 10: 34$ \\
\hline 1,2-Dibromo-3-Chloropropane & 13.43 & Baseline & wilburj & $08 / 06 / 15 \quad 10: 34$ \\
\hline
\end{tabular}

Lab Sample ID: IC 200-91869/4 Client Sample ID:

Date Analyzed: 07/27/15 13:51

Lab File ID: 14962_004.D

GC Column: DB-624

ID $: 0.2(\mathrm{~mm})$

\begin{tabular}{|c|c|c|c|c|}
\hline \multirow[t]{2}{*}{ COMPOUND NAME } & \multirow{2}{*}{$\begin{array}{c}\text { RETENTION } \\
\text { TIME }\end{array}$} & \multicolumn{3}{|c|}{ MANUAL INTEGRATION } \\
\hline & & REASON & ANALYST & DATE \\
\hline Dichlorodifluoromethane & 1.52 & Baseline & wilburj & $08 / 06 / 15 \quad 10: 36$ \\
\hline Bromomethane & 2.00 & Baseline & wilburj & $08 / 06 / 15 \quad 10: 37$ \\
\hline 1,2-Dibromo-3-Chloropropane & 13.43 & Baseline & wilburj & $08 / 06 / 15 \quad 10: 37$ \\
\hline
\end{tabular}

Lab Sample ID: ICIS 200-91869/5

Client Sample ID:

Date Analyzed: 07/27/15 14:16

Lab File ID: 14962_005.D

GC Column: DB-624

ID $: 0.2(\mathrm{~mm})$

\begin{tabular}{|c|c|c|c|c|}
\hline \multirow[t]{2}{*}{ COMPOUND NAME } & \multirow{2}{*}{$\begin{array}{c}\text { RETENTION } \\
\text { TIME }\end{array}$} & \multicolumn{3}{|c|}{ MANUAL INTEGRATION } \\
\hline & & REASON & ANALYST & DATE \\
\hline Dichlorodifluoromethane & 1.52 & Baseline & wilburj & $08 / 06 / 15 \quad 10: 39$ \\
\hline Bromomethane & 1.99 & Baseline & wilburj & $08 / 06 / 1510: 39$ \\
\hline
\end{tabular}

Lab Sample ID: IC 200-91869/6

Client Sample ID:

Date Analyzed: 07/27/15 14:41

Lab File ID: 14962 006.D

GC Column: DB-624

ID $: 0.2(\mathrm{~mm})$

\begin{tabular}{|c|c|c|c|c|}
\hline \multirow[t]{2}{*}{ COMPOUND NAME } & \multirow{2}{*}{$\begin{array}{c}\text { RETENTION } \\
\text { TIME }\end{array}$} & \multicolumn{3}{|c|}{ MANUAL INTEGRATION } \\
\hline & & REASON & ANALYST & DATE \\
\hline Dichlorodifluoromethane & 1.52 & Baseline & wilburj & $08 / 06 / 15 \quad 10: 40$ \\
\hline Bromomethane & 1.99 & Baseline & wilburj & $08 / 06 / 15 \quad 10: 40$ \\
\hline
\end{tabular}


Lab Name: TestAmerica Burlington

SDG No.: 28988

Instrument ID: CHD.i

Lab Sample ID: IC 200-91869/7

Date Analyzed: 07/27/15 15:06

\begin{tabular}{|c|c|c|c|c|}
\hline \multirow[t]{2}{*}{ COMPOUND NAME } & \multirow{2}{*}{$\begin{array}{c}\text { RETENTION } \\
\text { TIME }\end{array}$} & \multicolumn{3}{|c|}{ MANUAL INTEGRATION } \\
\hline & & REASON & ANALYST & DATE \\
\hline Dichlorodifluoromethane & 1.53 & Baseline & wilburj & $08 / 06 / 15 \quad 10: 42$ \\
\hline Bromomethane & 1.99 & Baseline & wilburj & $08 / 06 / 15 \quad 10: 42$ \\
\hline
\end{tabular}

Lab Sample ID: CCVC 200-91869/14 Client Sample ID:

Date Analyzed: 07/27/15 18:01

Lab File ID: 14962_014.D

GC Column: DB-624

ID $: 0.2(\mathrm{~mm})$
ID $: 0.2(\mathrm{~mm})$

\begin{tabular}{|c|c|c|c|c|}
\hline \multirow[t]{2}{*}{ COMPOUND NAME } & \multirow{2}{*}{$\begin{array}{c}\text { RETENTION } \\
\text { TIME }\end{array}$} & \multicolumn{3}{|c|}{ MANUAL INTEGRATION } \\
\hline & & REASON & ANALYST & DATE \\
\hline Dichlorodifluoromethane & 1.52 & Baseline & wilburj & $08 / 06 / 15 \quad 11: 58$ \\
\hline Bromomethane & 1.99 & Baseline & wilburj & $08 / 06 / 15 \quad 11: 58$ \\
\hline
\end{tabular}


Lab Name: TESTAMERICA BURLINGTON

Lab Code: STLV Case No.: YORK Mod. Ref No.:
Contract: $8 \mathrm{E}-00302$

SDG No.: 28988

Level: (TRACE or LOW) TRACE

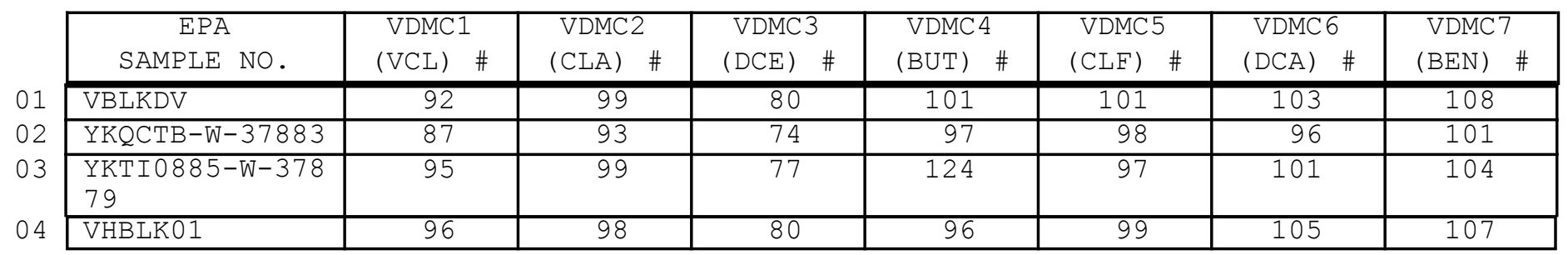

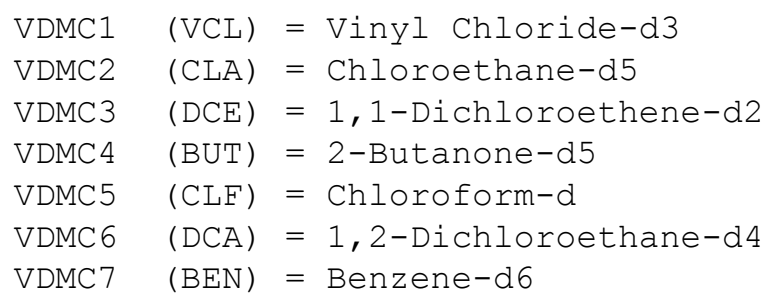

\# Column to be used to flag recovery values

* Values outside of contract required QC limits

$$
\begin{aligned}
& \frac{Q C \text { LIMITS }}{(65-131)} \\
& (71-131) \\
& (55-104) \\
& (49-155) \\
& (78-121) \\
& (78-129) \\
& (77-124)
\end{aligned}
$$

Page 1 of 1 
Lab Name: TESTAMERICA BURLINGTON

Lab Code: STLV Case No.: YORK Mod. Ref No.:
Contract: $8 \mathrm{E}-00302$

SDG No.: 28988

Level: (TRACE or LOW) TRACE

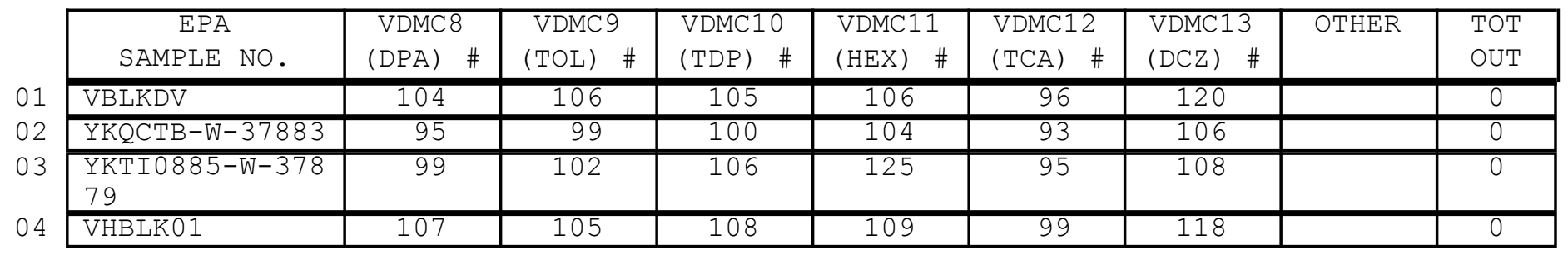

\footnotetext{
VDMC8 $(\mathrm{DPA})=1,2-\mathrm{Dich}$ loropropane-d6

VDMC $9 \quad($ TOL $)=$ Toluene-d8

VDMC10 $(\mathrm{TDP})=$ trans $-1,3-\mathrm{Dichloropropene-d4}$

$\operatorname{VDMC11}(\mathrm{HEX})=2-$ Hexanone-d5

VDMC12 $(\mathrm{TCA})=1,1,2,2$-Tetrachloroethane-d2

VDMC13 $(\mathrm{DCZ})=1,2-\mathrm{Dich})$ orobenzene-d4
}

\footnotetext{
$\frac{\text { QC LIMITS }}{(79-124)}$

$(77-121)$

$(73-121)$

$(28-135)$

$(73-125)$

$(80-131)$
}

\# Column to be used to flag recovery values

* Values outside of contract required QC limits

Report 1,4-Dioxane-d8 for Low-Medium VOA analysis only

Page 1 of 1 
$4 A$ - FORM IV VOA

VOLATILE METHOD BLANK SUMMARY
EPA SAMPLE NO.

VBLKDV

Lab Name: TESTAMERICA BURLINGTON

Contract: 8E-00302

Lab Code: STLV Case No.: YORK Mod. Ref No.: SDG No.: 28988

Lab File ID: 14962_010.D

Lab Sample ID: MB 200-91869/10

Instrument ID: CHD.i

Matrix: (SOIL/SED/WATER) Water

Date Analyzed: 07/27/2015

Level: (TRACE or LOW/MED) TRACE

Time Analyzed: 1621

GC Column: DB-624

ID $: 0.20 \quad(\mathrm{~mm})$

Heated Purge: (Y/N) N

\begin{tabular}{|c|c|c|c|}
\hline $\begin{array}{c}\text { EPA } \\
\text { SAMPLE NO. }\end{array}$ & $\begin{array}{r}\text { LAB } \\
\text { SAMPLE ID }\end{array}$ & $\begin{array}{c}\text { LAB } \\
\text { FILE ID }\end{array}$ & $\begin{array}{c}\text { TIME } \\
\text { ANALYZED }\end{array}$ \\
\hline $\begin{array}{l}\text { YKQCTB-W-378 } \\
83\end{array}$ & $200-28988-1$ & $\begin{array}{l}14962 \_011 . \\
D\end{array}$ & 1646 \\
\hline $\begin{array}{l}\text { YKTI0 885-W-3 } \\
7879\end{array}$ & $200-28988-2$ & $\begin{array}{l}14962 \_012 . \\
D\end{array}$ & 1711 \\
\hline VHBLK01 & $200-28988-3$ & $\begin{array}{l}14962 \_013 . \\
D\end{array}$ & 1736 \\
\hline
\end{tabular}

COMMENTS :

Page 1 of 1 
$5 A$ - FORM V VOA

VOLATILE ORGANICS INSTRUMENT

PERFORMANCE CHECK

BROMOFLUOROBENZENE (BFB)
EPA SAMPLE NO.

BFBDV
Lab Name: TESTAMERICA BURLINGTON

Lab Code: STLV

Case No.: YORK

Lab File Id: 14962_001.D

Instrument Id: CHD.i

GC Column: DB-624

ID $: 0.20$
Contract: $8 \mathrm{E}-00302$ Mod. Ref No.: SDG No.: 28988

BEB Injection Date: 07/27/2015

BFB Injection Time: 1238 $(\mathrm{mm})$

\begin{tabular}{|c|c|c|}
\hline $\mathrm{m} / \mathrm{e}$ & ION ABUNDANCE CRITERIA & $\begin{array}{l}\text { 응 RELATIVE } \\
\text { ABUNDANCE }\end{array}$ \\
\hline 50 & $15.0-40.0 \%$ of mass 95 & 23.5 \\
\hline 75 & $30.0-80.0 \%$ of mass 95 & 64.1 \\
\hline 95 & Base peak, 100\% relative abundance & 100 \\
\hline 96 & $5.0-9.0 \%$ of mass 95 & 5.8 \\
\hline 173 & Less than 2.0\% of mass 174 & 0) 1 \\
\hline 174 & $50.0-120 \%$ of mass 95 & 118 \\
\hline 175 & $5.0-9.0 \%$ of mass 174 & 8.2( \\
\hline 176 & $95.0-101 \%$ of mass 174 & $113(95.9) 1$ \\
\hline 177 & $5.0-9.0 \%$ of mass 176 & $7.4(6.6) 2$ \\
\hline
\end{tabular}

1 - Value is omass 174

2 - Value is omass 176

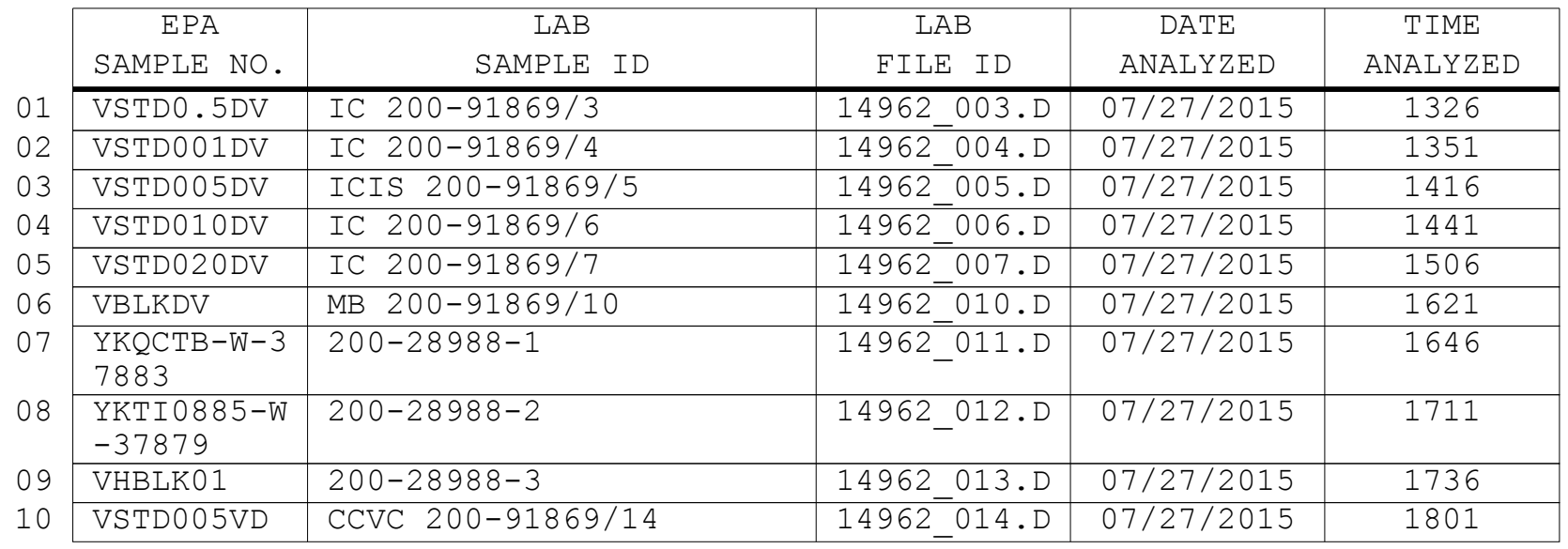




\section{$8 A$ - FORM VIII VOA}

VOLATILE INTERNAL STANDARD AREA AND RETENTION TIME SUMMARY

Lab Name: TESTAMERICA BURLINGTON

Contract: $8 \mathrm{E}-00302$

Lab Code: STLV Case No.: YORK Mod. Ref No.: SDG No.: 28988

GC Column: DB-624

ID: 0.20 (mm) Init. Calib. Date (s) : 07/27/2015 07/27/2015

EPA Sample No.(VSTD\#\#\#\#\#): VSTD005DV

Lab File ID (Standard): 14962_005.D

Instrument ID: CHD.i

Date Analyzed: 07/27/2015

Time Analyzed: 1416

Heated Purge: ( $\mathrm{Y} / \mathrm{N}) \mathrm{N}$

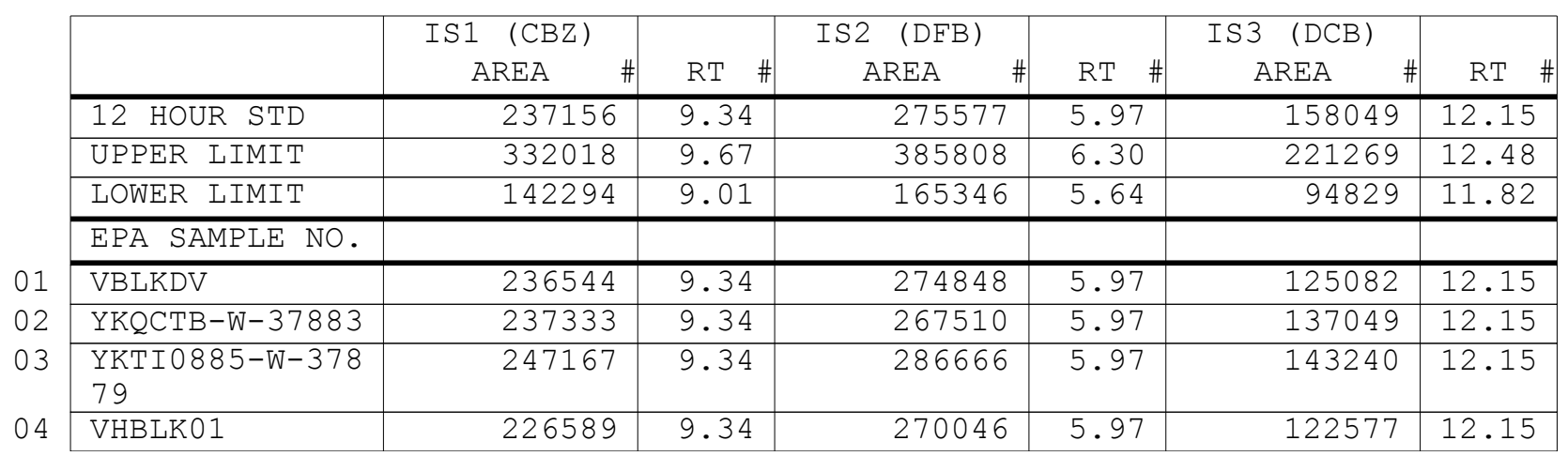

IS1 $(\mathrm{CBZ})=$ Chlorobenzene-d5

IS2 $(\mathrm{DFB})=1,4-\mathrm{Difluorobenzene}$

IS3 $(\mathrm{DCB})=1,4-\mathrm{Dichlorobenzene-d4}$

AREA UPPER LIMIT $=140 \%$ (Trace Volatiles) of internal standard area AREA LOWER LIMIT $=60 \%$ (Trace Volatiles) of internal standard area RT UPPER LIMIT $=+0.33$ (Trace Volatiles) minutes of internal standard RT RT LOWER LIMIT $=-0.33$ (Trace Volatiles) minutes of internal standard RT

\# Column used to flag values outside contract required QC limits with an asterisk. Page 1 of 1 
Lab Name: TESTAMERICA BURLINGTON

Contract: $8 \mathrm{E}-00302$

Lab Code: STLV

Case No.: YORK

Mod. Ref No.:

SDG No.: 28988

Matrix: (SOIL/SED/WATER) Water

Sample wt/vol: 25.0 $(\mathrm{g} / \mathrm{mL}) \mathrm{mL}$

Level: (TRACE/LOW/MED) TRACE

\% Moisture: not dec.

GC Column: DB-624 ID: $0.20 \quad(\mathrm{~mm})$

Soil Extract Volume: (uL) $(\mathrm{mL})$

Purge Volume: 25.0
Lab Sample ID: 200-28988-1

Lab File ID: 14962_011.D

Date Received: 07/23/2015

Date Analyzed: 07/27/2015

Dilution Factor: 1.0

Soil Aliquot Volume: (uL)

\begin{tabular}{|c|c|c|c|}
\hline CAS NO. & COMPOUND & $\begin{array}{l}\text { CONCENTRATION UNITS: } \\
(\mathrm{ug} / \mathrm{L} \text { or } \mathrm{ug} / \mathrm{kg}) \mathrm{ug} / \mathrm{L}\end{array}$ & q \\
\hline $75-71-8$ & Dichlorodifluoromethane & 1.0 & $\mathrm{U}$ \\
\hline $74-87-3$ & Chloromethane & 1.0 & $\mathrm{U}$ \\
\hline $75-01-4$ & Vinyl chloride & 1.0 & $\mathrm{U}$ \\
\hline $74-83-9$ & Bromomethane & 1.0 & $\mathrm{U}$ \\
\hline $75-00-3$ & Chloroethane & 1.0 & $\mathrm{U}$ \\
\hline $75-69-4$ & Trichlorofluoromethane & 1.0 & $\mathrm{U}$ \\
\hline $75-35-4$ & 1,1-Dichloroethene & 1.0 & $\mathrm{U}$ \\
\hline $76-13-1$ & $1,1,2$-Trichloro-1,2,2-trifluoroethane & 1.0 & $\mathrm{U}$ \\
\hline $67-64-1$ & Acetone & 47 & B \\
\hline $75-15-0$ & Carbon disulfide & 1.0 & $\mathrm{U}$ \\
\hline $79-20-9$ & Methyl acetate & 1.0 & $\mathrm{U}$ \\
\hline $75-09-2$ & Methylene Chloride & 1.0 & $\mathrm{U}$ \\
\hline $156-60-5$ & trans-1,2-Dichloroethene & 1.0 & $\mathrm{U}$ \\
\hline $1634-04-4$ & Methyl tert-butyl ether & 0.20 & $\mathrm{~J}$ \\
\hline $75-34-3$ & 1,1-Dichloroethane & 1.0 & $\mathrm{U}$ \\
\hline $156-59-2$ & cis-1,2-Dichloroethene & 1.0 & $\mathrm{U}$ \\
\hline $78-93-3$ & 2-Butanone & 5.0 & $\mathrm{U}$ \\
\hline $74-97-5$ & Bromochloromethane & 1.0 & $\mathrm{U}$ \\
\hline $67-66-3$ & Chloroform & 1.0 & $\mathrm{U}$ \\
\hline $71-55-6$ & $1,1,1$-Trichloroethane & 1.0 & $\mathrm{U}$ \\
\hline $110-82-7$ & Cyclohexane & 1.0 & $\mathrm{U}$ \\
\hline $56-23-5$ & Carbon tetrachloride & 1.0 & $\mathrm{U}$ \\
\hline $71-43-2$ & Benzene & 0.22 & $\mathrm{~J}$ \\
\hline $107-06-2$ & 1,2-Dichloroethane & 1.0 & $\mathrm{U}$ \\
\hline
\end{tabular}

Report 1,4-Dioxane for Low-Medium VOA analysis only 
Lab Name: TESTAMERICA BURLINGTON

Contract: $8 \mathrm{E}-00302$

Lab Code: STLV

Case No.: YORK

Mod. Ref No.:

SDG No.: 28988

Matrix: (SOIL/SED/WATER) Water

Sample wt/vol: 25.0 $(\mathrm{g} / \mathrm{mL}) \mathrm{mL}$

Level: (TRACE/LOW/MED) TRACE

\% Moisture: not dec.

GC Column: DB-624 ID: $0.20 \quad(\mathrm{~mm})$

Soil Extract Volume: (uL) $(\mathrm{mL})$

Purge Volume: 25.0 (mI)
Lab Sample ID: 200-28988-1

Lab File ID: 14962_011.D

Date Received: 07/23/2015

Date Analyzed: 07/27/2015

Dilution Factor: 1.0

Soil Aliquot Volume: (uL)

\begin{tabular}{|c|c|c|c|}
\hline CAS NO. & COMPOUND & $\begin{array}{l}\text { CONCENTRATION UNITS: } \\
(\mathrm{ug} / \mathrm{L} \text { or } \mathrm{ug} / \mathrm{kg}) \mathrm{ug} / \mathrm{L}\end{array}$ & $q$ \\
\hline $79-01-6$ & Trichloroethene & 1.0 & $\mathrm{U}$ \\
\hline $108-87-2$ & Methylcyclohexane & 1.0 & $\mathrm{U}$ \\
\hline $78-87-5$ & 1,2-Dichloropropane & 1.0 & $\mathrm{U}$ \\
\hline $75-27-4$ & Bromodichloromethane & 1.0 & $\mathrm{U}$ \\
\hline $10061-01-5$ & cis-1,3-Dichloropropene & 1.0 & U \\
\hline $108-10-1$ & 4-Methyl-2-pentanone & 1.0 & $\mathrm{U}$ \\
\hline $108-88-3$ & Toluene & 1.0 & $\mathrm{~J}$ \\
\hline $10061-02-6$ & trans-1,3-Dichloropropene & 1.0 & $\mathrm{U}$ \\
\hline $79-00-5$ & $1,1,2-$ Trichloroethane & 1.0 & $\mathrm{U}$ \\
\hline $127-18-4$ & Tetrachloroethene & 3.3 & \\
\hline $591-78-6$ & 2-Hexanone & 1.0 & $\mathrm{U}$ \\
\hline $124-48-1$ & Dibromochloromethane & 1.0 & $\mathrm{U}$ \\
\hline $106-93-4$ & 1,2-Dibromoethane & 1.0 & $\mathrm{U}$ \\
\hline $108-90-7$ & Chlorobenzene & 1.0 & $\mathrm{U}$ \\
\hline $100-41-4$ & Ethylbenzene & 0.089 & $\mathrm{~J}$ \\
\hline $95-47-6$ & o-Xylene & 0.11 & $\mathrm{~J}$ \\
\hline $179601-23-1$ & m,p-xylene & 0.29 & $\mathrm{~J}$ \\
\hline $100-42-5$ & Styrene & 1.0 & $\mathrm{U}$ \\
\hline $75-25-2$ & Bromoform & 1.0 & $\mathrm{U}$ \\
\hline $98-82-8$ & Isopropylbenzene & 1.0 & $\mathrm{U}$ \\
\hline $79-34-5$ & $1,1,2,2$-Tetrachloroethane & 1.0 & $\mathrm{U}$ \\
\hline $541-73-1$ & 1,3 -Dichlorobenzene & 1.0 & $\mathrm{U}$ \\
\hline $106-46-7$ & 1,4-Dichlorobenzene & 1.0 & $\mathrm{U}$ \\
\hline $95-50-1$ & 1,2-Dichlorobenzene & 1.0 & $\mathrm{U}$ \\
\hline $96-12-8$ & 1,2-Dibromo-3-Chloropropane & 1.0 & $\mathrm{U}$ \\
\hline $120-82-1$ & $1,2,4$-Trichlorobenzene & 1.0 & $\mathrm{U}$ \\
\hline $87-61-6$ & $1,2,3$-Trichlorobenzene & 1.0 & $\mathrm{U}$ \\
\hline
\end{tabular}


Lab Name: TESTAMERICA BURLINGTON

Contract: $8 \mathrm{E}-00302$

Lab Code: STLV Case No.: YORK Mod. Ref No.: SDG No.: 28988

Matrix: (SOIL/SED/WATER) Water

Sample wt/vol: 25.0 $(\mathrm{g} / \mathrm{mL}) \mathrm{mL}$

Level: (TRACE or LOW/MED) TRACE

\% Moisture: not dec.

GC Column: DB-624 ID $: 0.20 \quad(\mathrm{~mm})$

Soil Extract Volume: (uL)

CONCENTRATION UNITS: (ug/L or ug/kg) ug/L
Lab Sample ID: 200-28988-1

Lab File ID: 14962_011.D

Date Received: 07/23/2015

Date Analyzed: 07/27/2015

Dilution Factor: 1.0

Soil Aliquot Volume: (uL)

Purge Volume: 25.0

\begin{tabular}{|c|c|c|c|c|}
\hline CAS NUMBER & COMPOUND NAME & $\mathrm{RT}$ & EST. CONC. & Q \\
\hline 01 & Unknown & 1.50 & 0.53 & $\mathrm{~J}$ \\
\hline $917-92-0$ & 1-Butyne, 3,3-dimethyl- & 2.50 & 1.7 & $\mathrm{~J} \mathrm{~N}$ \\
\hline 03 & Unknown & 3.33 & 0.53 & $\mathrm{~J}$ \\
\hline 04 & Unknown & 4.37 & 0.71 & $\mathrm{~J}$ \\
\hline 05 & Unknown & 7.29 & 2.9 & $\mathrm{~J} B \mathrm{X}$ \\
\hline $541-05-9$ & Cyclotrisiloxane, hexamethyl- & 8.22 & 0.87 & $\mathrm{~J} \quad \mathrm{~N} B$ \\
\hline $556-67-2$ & Cyclotetrasiloxane, octamethyl- & 11.04 & 0.69 & $\mathrm{~J} \quad \mathrm{~N} \quad \mathrm{~B}$ \\
\hline E9667961 & Total Alkanes & $\mathrm{N} / \mathrm{A}$ & 1.6 & $\mathrm{~J}$ \\
\hline
\end{tabular}

1 EPA-designated Registry Number. 
Lab Name: TESTAMERICA BURLINGTON

Contract: $8 \mathrm{E}-00302$

Lab Code: STLV

Case No.: YORK Mod. Ref No.: SDG No.: 28988

Matrix: (SOIL/SED/WATER) Water

Sample wt/vol: 25.0 $(\mathrm{g} / \mathrm{mL}) \mathrm{mL}$

Level: (TRACE/LOW/MED) TRACE

\% Moisture: not dec.

GC Column: DB-624 ID: $0.20 \quad(\mathrm{~mm})$

Soil Extract Volume: (uL) $(\mathrm{mL})$

Purge Volume: 25.0
Lab Sample ID: 200-28988-2

Lab File ID: 14962_012.D

Date Received: 07/23/2015

Date Analyzed: 07/27/2015

Dilution Factor: 1.0

Soil Aliquot Volume: (uL)

\begin{tabular}{|c|c|c|c|}
\hline CAS NO. & COMPOUND & $\begin{array}{l}\text { CONCENTRATION UNITS: } \\
(\mathrm{ug} / \mathrm{L} \text { or } \mathrm{ug} / \mathrm{kg}) \mathrm{ug} / \mathrm{L}\end{array}$ & Q \\
\hline $75-71-8$ & Dichlorodifluoromethane & 1.0 & $\mathrm{U}$ \\
\hline $74-87-3$ & Chloromethane & 1.0 & $\mathrm{U}$ \\
\hline $75-01-4$ & Vinyl chloride & 1.0 & $\mathrm{U}$ \\
\hline $74-83-9$ & Bromomethane & 1.0 & $\mathrm{U}$ \\
\hline $75-00-3$ & Chloroethane & 1.0 & $\mathrm{U}$ \\
\hline $75-69-4$ & Trichlorofluoromethane & 0.015 & $\mathrm{~J}$ \\
\hline $75-35-4$ & 1,1-Dichloroethene & 1.0 & $\mathrm{U}$ \\
\hline $76-13-1$ & $1,1,2$-Trichloro-1,2,2-trifluoroethane & 1.0 & $\mathrm{U}$ \\
\hline $67-64-1$ & Acetone & 1.9 & $\mathrm{~J} B$ \\
\hline $75-15-0$ & Carbon disulfide & 1.0 & $\mathrm{U}$ \\
\hline $79-20-9$ & Methyl acetate & 1.0 & $\mathrm{U}$ \\
\hline $75-09-2$ & Methylene Chloride & 1.0 & $\mathrm{U}$ \\
\hline $156-60-5$ & trans-1,2-Dichloroethene & 1.0 & $\mathrm{U}$ \\
\hline $1634-04-4$ & Methyl tert-butyl ether & 1.0 & $\mathrm{U}$ \\
\hline $75-34-3$ & 1,1-Dichloroethane & 1.0 & $\mathrm{U}$ \\
\hline $156-59-2$ & cis-1,2-Dichloroethene & 1.0 & $\mathrm{U}$ \\
\hline $78-93-3$ & 2-Butanone & 5.0 & $\mathrm{U}$ \\
\hline $74-97-5$ & Bromochloromethane & 1.0 & $\mathrm{U}$ \\
\hline $67-66-3$ & Chloroform & 1.0 & $\mathrm{U}$ \\
\hline $71-55-6$ & $1,1,1$-Trichloroethane & 1.0 & $\mathrm{U}$ \\
\hline $110-82-7$ & Cyclohexane & 1.0 & $\mathrm{U}$ \\
\hline $56-23-5$ & Carbon tetrachloride & 0.26 & $\mathrm{~J}$ \\
\hline $71-43-2$ & Benzene & 0.039 & $\mathrm{~J}$ \\
\hline $107-06-2$ & 1,2-Dichloroethane & 1.0 & $\mathrm{U}$ \\
\hline
\end{tabular}

Report 1,4-Dioxane for Low-Medium VOA analysis only 
Lab Name: TESTAMERICA BURLINGTON

Lab Code: STLV

Case No.: YORK

Mod. Ref No.:

Contract: $8 \mathrm{E}-00302$

Matrix: (SOIL/SED/WATER) Water

Sample wt/vol: 25.0 $(\mathrm{g} / \mathrm{mL}) \mathrm{mL}$

Level: (TRACE/LOW/MED) TRACE

\% Moisture: not dec.

GC Column: DB-624 ID $0.20 \quad(\mathrm{~mm})$

Soil Extract Volume: (uL) $(\mathrm{mL})$

Purge Volume: 25.0 (mI)
SDG No.: 28988

Lab Sample ID: 200-28988-2

Lab File ID: 14962_012.D

Date Received: 07/23/2015

Date Analyzed: 07/27/2015

Dilution Factor: 1.0

Soil Aliquot Volume: (uL)

\begin{tabular}{|c|c|c|c|}
\hline CAS NO. & COMPOUND & $\begin{array}{l}\text { CONCENTRATION UNITS: } \\
(\mathrm{ug} / \mathrm{L} \text { or } \mathrm{ug} / \mathrm{kg}) \mathrm{ug} / \mathrm{L}\end{array}$ & $q$ \\
\hline $79-01-6$ & Trichloroethene & 1.0 & $\mathrm{U}$ \\
\hline $108-87-2$ & Methylcyclohexane & 1.0 & $\mathrm{U}$ \\
\hline $78-87-5$ & 1,2-Dichloropropane & 1.0 & $\mathrm{U}$ \\
\hline $75-27-4$ & Bromodichloromethane & 1.0 & $\mathrm{U}$ \\
\hline $10061-01-5$ & cis-1,3-Dichloropropene & 1.0 & U \\
\hline $108-10-1$ & 4-Methyl-2-pentanone & 1.0 & $\mathrm{U}$ \\
\hline $108-88-3$ & Toluene & 0.053 & $\mathrm{~J}$ \\
\hline $10061-02-6$ & trans-1,3-Dichloropropene & 1.0 & $\mathrm{U}$ \\
\hline $79-00-5$ & $1,1,2-$ Trichloroethane & 1.0 & $\mathrm{U}$ \\
\hline $127-18-4$ & Tetrachloroethene & 0.11 & $\mathrm{~J}$ \\
\hline $591-78-6$ & 2-Hexanone & 1.0 & $\mathrm{U}$ \\
\hline $124-48-1$ & Dibromochloromethane & 1.0 & $\mathrm{U}$ \\
\hline $106-93-4$ & 1,2-Dibromoethane & 1.0 & $\mathrm{U}$ \\
\hline $108-90-7$ & Chlorobenzene & 1.0 & $\mathrm{U}$ \\
\hline $100-41-4$ & Ethylbenzene & 1.0 & $\mathrm{U}$ \\
\hline $95-47-6$ & o-Xylene & 1.0 & $\mathrm{U}$ \\
\hline $179601-23-1$ & m,p-xylene & 0.021 & $\mathrm{~J}$ \\
\hline $100-42-5$ & Styrene & 1.0 & $\mathrm{U}$ \\
\hline $75-25-2$ & Bromoform & 1.0 & $\mathrm{U}$ \\
\hline $98-82-8$ & Isopropylbenzene & 1.0 & $\mathrm{U}$ \\
\hline $79-34-5$ & $1,1,2,2$-Tetrachloroethane & 1.0 & $\mathrm{U}$ \\
\hline $541-73-1$ & 1,3 -Dichlorobenzene & 1.0 & $\mathrm{U}$ \\
\hline $106-46-7$ & 1,4-Dichlorobenzene & 1.0 & $\mathrm{U}$ \\
\hline $95-50-1$ & 1,2-Dichlorobenzene & 1.0 & $\mathrm{U}$ \\
\hline $96-12-8$ & 1,2-Dibromo-3-Chloropropane & 1.0 & $\mathrm{U}$ \\
\hline $120-82-1$ & $1,2,4$-Trichlorobenzene & 1.0 & $\mathrm{U}$ \\
\hline $87-61-6$ & $1,2,3$-Trichlorobenzene & 1.0 & $\mathrm{U}$ \\
\hline
\end{tabular}


Lab Name: TESTAMERICA BURLINGTON

Contract: $8 \mathrm{E}-00302$

Lab Code: STLV Case No.: YORK Mod. Ref No.: SDG No.: 28988

Matrix: (SOIL/SED/WATER) Water

Sample wt/vol: 25.0 $(\mathrm{g} / \mathrm{mL}) \mathrm{mL}$

Level: (TRACE or LOW/MED) TRACE

\% Moisture: not dec.

GC Column: DB-624 ID $: 0.20 \quad(\mathrm{~mm})$

Soil Extract Volume: (uL)

CONCENTRATION UNITS: (ug/L or ug/kg) ug/L
Lab Sample ID: 200-28988-2

Lab File ID: 14962_012.D

Date Received: 07/23/2015

Date Analyzed: 07/27/2015

Dilution Factor: 1.0

Soil Aliquot Volume: (uL) Purge Volume: 25.0

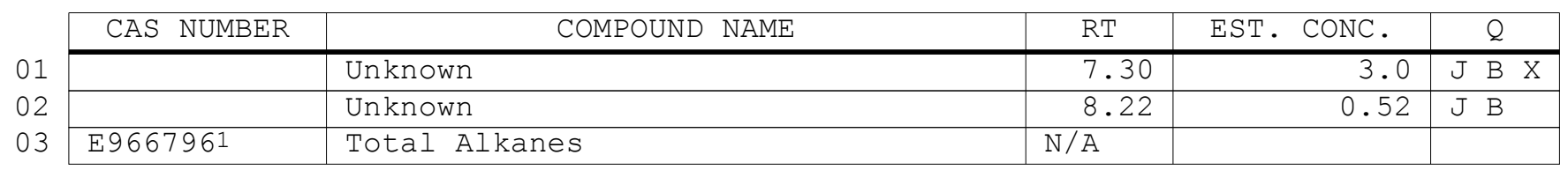

1 EPA-designated Registry Number. 
Lab Name: TESTAMERICA BURLINGTON

Contract: $8 \mathrm{E}-00302$

Lab Code: STLV Case No.: YORK Mod. Ref No.:

SDG No.: 28988

Instrument ID: CHD.i

Calibration Date(s) : 07/27/2015 07/27/2015

Heated Purge: ( $\mathrm{Y} / \mathrm{N}) \mathrm{N}$

Calibration Time(s) : 1326

1506

Purge Volume: 25.0 $(\mathrm{mL})$

GC Column: DB-624

ID: $0.20 \quad(\mathrm{~mm}) \quad$ Length: 25

$(\mathrm{m})$

\begin{tabular}{|c|c|c|c|c|c|c|c|}
\hline \multirow{2}{*}{$\begin{array}{l}\text { LAB FILE ID: } \\
\text { RRF5.0 }=14962 \_005 . D\end{array}$} & \multicolumn{3}{|c|}{$\mathrm{RRF} 0.5=14962 \_003 . \mathrm{D}$} & \multicolumn{4}{|c|}{ RRF1.0 $=14962 \_004 . D$} \\
\hline & 10 & $4962 \_006$ & & RRF20 & $=1496$ & 2007.5 & \\
\hline COMPOUND & $\mathrm{RRF} 0.5$ & $\mathrm{RRF} 1.0$ & RRF5.0 & RRF10 & RRF20 & $\overline{\mathrm{RRF}}$ & $\because \mathrm{RSD}$ \\
\hline Dichlorodifluoromethane & 0.717 & 0.701 & 0.733 & 0.752 & 0.714 & 0.723 & 2.7 \\
\hline Chloromethane & 0.468 & 0.342 & 0.356 & 0.360 & 0.343 & 0.374 & 14.3 \\
\hline Vinyl chloride & 0.332 & 0.327 & 0.373 & 0.374 & 0.377 & 0.357 & 7.0 \\
\hline Bromomethane & 0.177 & 0.113 & 0.107 & 0.119 & 0.143 & 0.132 & 21.9 \\
\hline Chloroethane & 0.245 & 0.231 & 0.240 & 0.239 & 0.245 & 0.240 & 2.6 \\
\hline Trichlorofluoromethane & 0.977 & 0.947 & 1.045 & 1.053 & 1.038 & 1.012 & 4.6 \\
\hline 1,1-Dichloroethene & 0.309 & 0.296 & 0.334 & 0.347 & 0.340 & 0.325 & 6.8 \\
\hline $\begin{array}{l}1,1,2 \text {-Trichloro- } \\
1,2,2 \text {-trifluoroethane }\end{array}$ & 0.382 & 0.400 & 0.409 & 0.422 & 0.412 & 0.405 & 3.7 \\
\hline Acetone & 0.033 & 0.025 & 0.023 & 0.024 & 0.023 & 0.026 & 17.0 \\
\hline Carbon disulfide & 0.655 & 0.566 & 0.641 & 0.668 & 0.663 & 0.639 & 6.6 \\
\hline Methyl acetate & 0.044 & 0.042 & 0.037 & 0.035 & 0.036 & 0.039 & 10.4 \\
\hline Methylene Chloride & 0.264 & 0.252 & 0.216 & 0.209 & 0.212 & 0.231 & 11.1 \\
\hline trans-1,2-Dichloroethene & 0.265 & 0.248 & 0.287 & 0.292 & 0.289 & 0.276 & 6.9 \\
\hline Methyl tert-butyl ether & 0.319 & 0.318 & 0.397 & 0.412 & 0.426 & 0.374 & 13.9 \\
\hline 1,1-Dichloroethane & 0.426 & 0.419 & 0.490 & 0.507 & 0.500 & 0.468 & 9.0 \\
\hline cis-1,2-Dichloroethene & 0.221 & 0.225 & 0.263 & 0.282 & 0.282 & 0.255 & 11.7 \\
\hline 2-Butanone & 0.021 & 0.019 & 0.025 & 0.027 & 0.027 & 0.024 & 14.0 \\
\hline Bromochloromethane & 0.092 & 0.090 & 0.101 & 0.104 & 0.106 & 0.099 & 7.3 \\
\hline Chloroform & 0.571 & 0.561 & 0.627 & 0.657 & 0.633 & 0.610 & 6.8 \\
\hline 1,1,1-Trichloroethane & 0.827 & 0.826 & 0.963 & 0.927 & 0.912 & 0.891 & 6.9 \\
\hline Cyclohexane & 0.313 & 0.359 & 0.469 & 0.474 & 0.465 & 0.416 & 18.0 \\
\hline Carbon tetrachloride & 0.821 & 0.792 & 0.970 & 0.923 & 0.918 & 0.885 & 8.5 \\
\hline Benzene & 1.139 & 1.070 & 1.232 & 1.208 & 1.214 & 1.173 & 5.7 \\
\hline 1,2-Dichloroethane & 0.334 & 0.317 & 0.381 & 0.375 & 0.374 & 0.356 & 8.1 \\
\hline Trichloroethene & 0.359 & 0.352 & 0.413 & 0.401 & 0.409 & 0.387 & 7.5 \\
\hline Methylcyclohexane & 0.354 & 0.380 & 0.467 & 0.467 & 0.472 & 0.428 & 13.3 \\
\hline
\end{tabular}

Report 1,4-Dioxane for Low-Medium VOA analysis only 
Lab Name: TESTAMERICA BURLINGTON

Contract: 8E-00302

Lab Code: STLV Case No.: YORK Mod. Ref No.:

SDG No.: 28988

Instrument ID: CHD.i

$\begin{array}{llll}\text { Calibration Date (s) : } & 07 / 27 / 2015 & \frac{07 / 27 / 2015}{1506} \\ \text { Calibration Time (s) : } & 1326 & \end{array}$

Heated Purge: ( $\mathrm{Y} / \mathrm{N}) \mathrm{N}$

Purge Volume: 25.0

GC Column: DB-624

ID: 0.20 (mL)

\begin{tabular}{|c|c|c|c|c|c|c|c|}
\hline LAB FILE ID: & \multirow{2}{*}{\multicolumn{3}{|c|}{$\begin{array}{l}\text { RRF0.5 }=\frac{14962 \_003 . \mathrm{D}}{\operatorname{RRF10}=14962 \_006 . \mathrm{D}}\end{array}$}} & \multicolumn{4}{|c|}{ RRF1.0 = 14962_004.D } \\
\hline RRF5.0 = 14962_005.D & & & & RRF2 & $=149$ & 007 . & \\
\hline COMPOUND & RRF0. 5 & RRF1. 0 & RRE5. 0 & RRF10 & RRF20 & $\overline{\mathrm{RRF}}$ & $\because \mathrm{RSD}$ \\
\hline 1,2-Dichloropropane & 0.201 & 0.181 & 0.208 & 0.198 & 0.199 & 0.198 & 5.0 \\
\hline Bromodichloromethane & 0.392 & 0.359 & 0.416 & 0.421 & 0.421 & 0.402 & 6.6 \\
\hline cis-1,3-Dichloropropene & 0.269 & 0.255 & 0.392 & 0.403 & 0.423 & 0.349 & 22.9 \\
\hline 4-Methyl-2-pentanone & 0.054 & 0.056 & 0.075 & 0.075 & 0.078 & 0.068 & 17.4 \\
\hline Toluene & 1.260 & 1.299 & 1.575 & 1.548 & 1.560 & 1.449 & 10.7 \\
\hline trans-1,3-Dichloropropene & 0.211 & 0.249 & 0.336 & 0.338 & 0.355 & 0.298 & 21.4 \\
\hline 1,1,2-Trichloroethane & 0.147 & 0.132 & 0.141 & 0.138 & 0.144 & 0.141 & 4.1 \\
\hline Tetrachloroethene & 0.434 & 0.456 & 0.502 & 0.500 & 0.506 & 0.479 & 6.8 \\
\hline 2-Hexanone & 0.040 & 0.039 & 0.056 & 0.057 & 0.058 & 0.050 & 19.6 \\
\hline Dibromochloromethane & 0.188 & 0.181 & 0.239 & 0.241 & 0.251 & 0.220 & 14.9 \\
\hline 1,2-Dibromoethane & 0.110 & 0.117 & 0.141 & 0.139 & 0.140 & 0.129 & 11.5 \\
\hline Chlorobenzene & 0.918 & 0.914 & 1.025 & 1.019 & 1.030 & 0.981 & 6.1 \\
\hline Ethylbenzene & 1.498 & 1.580 & 2.112 & 2.110 & 2.123 & 1.884 & 16.8 \\
\hline o-Xylene & 0.454 & 0.520 & 0.721 & 0.705 & 0.748 & 0.630 & 21.1 \\
\hline m,p-Xylene & 0.545 & 0.611 & 0.799 & 0.799 & 0.816 & 0.714 & 17.7 \\
\hline Styrene & 0.711 & 0.793 & 1.114 & 1.108 & 1.132 & 0.972 & 20.9 \\
\hline Bromoform & 0.146 & 0.149 & 0.165 & 0.177 & 0.188 & 0.165 & 11.0 \\
\hline Isopropylbenzene & 1.421 & 1.662 & 2.388 & 2.433 & 2.466 & 2.074 & 23.8 \\
\hline $1,1,2,2$-Tetrachloroethane & 0.106 & 0.106 & 0.125 & 0.120 & 0.128 & 0.117 & 8.9 \\
\hline 1,3-Dichlorobenzene & 1.203 & 1.289 & 1.517 & 1.605 & 1.620 & 1.447 & 13.1 \\
\hline 1,4-Dichlorobenzene & 1.607 & 1.522 & 1.570 & 1.593 & 1.589 & 1.576 & 2.1 \\
\hline 1,2-Dichlorobenzene & 1.243 & 1.184 & 1.270 & 1.290 & 1.302 & 1.258 & 3.7 \\
\hline 1,2-Dibromo-3-Chloropropane & 0.024 & 0.040 & 0.036 & 0.040 & 0.040 & 0.036 & 19.7 \\
\hline 1,2,4-Trichlorobenzene & 0.776 & 0.689 & 0.821 & 0.902 & 0.961 & 0.830 & 12.8 \\
\hline $1,2,3$-Trichlorobenzene & 0.579 & 0.502 & 0.586 & 0.641 & 0.677 & 0.597 & 11.2 \\
\hline
\end{tabular}


Lab Name: TESTAMERICA BURLINGTON

Contract: $8 \mathrm{E}-00302$

Lab Code: STLV Case No.: YORK Mod. Ref No.: SDG No.: 28988

Instrument ID: CHD.i

Heated Purge: (Y/N) N

Purge Volume: 25.0

GC Column: DB-624
ID: 0.20
Calibration Date(s): 07/27/2015

Calibration Time(s): 1326 $(\mathrm{mL})$

(mm) Length: 25
$07 / 27 / 2015$ 1506

\begin{tabular}{|c|c|c|c|c|c|c|c|}
\hline LAB FILE ID: & \multirow{2}{*}{\multicolumn{3}{|c|}{$\begin{array}{l}\operatorname{RRF}_{\underline{0.5}}=\frac{14962 \_003 . \mathrm{D}}{\operatorname{RRF10}}=14962 \_006 . \mathrm{D}\end{array}$}} & \multirow{2}{*}{\multicolumn{4}{|c|}{ 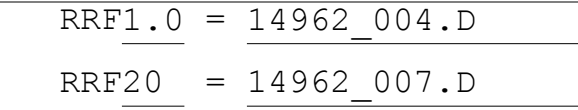 }} \\
\hline RRF5.0 = 14962_005.D & & & & & & & \\
\hline COMPOUND & RRF0. 5 & RRF1. 0 & RRE 5.0 & RRE10 & RRE20 & $\overline{\mathrm{RRF}}$ & $\circ \mathrm{RSD}$ \\
\hline Vinyl Chloride-d3 & 0.381 & 0.337 & 0.360 & 0.369 & 0.366 & 0.363 & 4.4 \\
\hline Chloroethane-d5 & 0.283 & 0.263 & 0.303 & 0.313 & 0.316 & 0.295 & 7.6 \\
\hline 1,1-Dichloroethene-d2 & 0.711 & 0.723 & 0.804 & 0.817 & 0.792 & 0.769 & 6.3 \\
\hline 2-Butanone-d5 & 0.017 & 0.018 & 0.024 & 0.025 & 0.027 & 0.022 & 19.3 \\
\hline Chloroform-d & 0.615 & 0.598 & 0.711 & 0.710 & 0.704 & 0.668 & 8.4 \\
\hline 1,2-Dichloroethane-d4 & 0.275 & 0.291 & 0.301 & 0.310 & 0.304 & 0.296 & 4.6 \\
\hline Benzene-d6 & 1.080 & 1.071 & 1.220 & 1.187 & 1.211 & 1.154 & 6.3 \\
\hline 1,2-Dichloropropane-d6 & 0.223 & 0.226 & 0.239 & 0.231 & 0.231 & 0.230 & 2.7 \\
\hline Toluene-d8 & 1.176 & 1.190 & 1.444 & 1.388 & 1.415 & 1.322 & 9.8 \\
\hline trans-1,3-Dichloropropene-d4 & 0.208 & 0.217 & 0.284 & 0.306 & 0.321 & 0.267 & 19.4 \\
\hline 2-Hexanone-d5 & 0.014 & 0.016 & 0.023 & 0.025 & 0.026 & 0.021 & 25.6 \\
\hline $1,1,2,2$-Tetrachloroethane-d2 & 0.100 & 0.104 & 0.129 & 0.125 & 0.132 & 0.118 & 12.6 \\
\hline 1,2-Dichlorobenzene-d4 & 0.857 & 0.747 & 0.823 & 0.839 & 0.835 & 0.820 & 5.2 \\
\hline
\end{tabular}

Report 1,4-Dioxane-d8 for Low-Medium VOA analysis only 
Lab Name: TESTAMERICA BURLINGTON

Contract: $8 \mathrm{E}-00302$

Lab Code: STLV Case No.: YORK Mod. Ref No.:

SDG No.: 28988

Instrument ID: CHD.i

Calibration Date: 07/27/2015 Time: 1801

Lab File Id: 14962_014.D

EPA Sample No. (VSTD\#\#\#\#) : VSTD005VD Init. Calib. Date(s):07/27/2015 07/27/2015

Heated Purge: (Y/N) N GC Column: DB-624

Init. Calib. Time(s) : 1326 1506

Purge Volume: 25.0 ID: $0.20(\mathrm{~mm})$ Length: 25

(m)

\begin{tabular}{|c|c|c|c|c|c|}
\hline COMPOUND & $\overline{\mathrm{RRF}}$ & RRF5. 0 & $\begin{array}{l}\text { MIN } \\
\text { RRF }\end{array}$ & $\because \mathrm{D}$ & $\mathrm{MAX} \cong \mathrm{D}$ \\
\hline Dichlorodifluoromethane & 0.723 & 0.722 & 0.010 & -0.2 & 50.0 \\
\hline Chloromethane & 0.374 & 0.331 & 0.010 & -11.4 & 50.0 \\
\hline Vinyl chloride & 0.357 & 0.364 & 0.010 & 2.0 & 50.0 \\
\hline Bromomethane & 0.132 & 0.127 & 0.010 & -3.3 & 50.0 \\
\hline Chloroethane & 0.240 & 0.218 & 0.010 & -9.1 & 50.0 \\
\hline Trichlorofluoromethane & 1.012 & 1.044 & 0.010 & 3.2 & 50.0 \\
\hline 1,1-Dichloroethene & 0.325 & 0.337 & 0.010 & 3.6 & 50.0 \\
\hline 1,1,2-Trichloro-1,2,2-trifluoroethane & 0.405 & 0.429 & 0.010 & 6.0 & 50.0 \\
\hline Acetone & 0.026 & 0.023 & 0.010 & -10.9 & 50.0 \\
\hline Carbon disulfide & 0.639 & 0.650 & 0.010 & 1.8 & 50.0 \\
\hline Methyl acetate & 0.039 & 0.032 & 0.010 & -16.4 & 50.0 \\
\hline Methylene Chloride & 0.231 & 0.215 & 0.010 & -6.8 & 50.0 \\
\hline trans-1,2-Dichloroethene & 0.276 & 0.277 & 0.010 & 0.2 & 50.0 \\
\hline Methyl tert-butyl ether & 0.374 & 0.382 & 0.010 & 1.9 & 50.0 \\
\hline 1,1-Dichloroethane & 0.468 & 0.473 & 0.010 & 1.1 & 50.0 \\
\hline cis-1,2-Dichloroethene & 0.255 & 0.257 & 0.010 & 0.8 & 50.0 \\
\hline 2-Butanone & 0.024 & 0.024 & 0.010 & 2.1 & 50.0 \\
\hline Bromochloromethane & 0.099 & 0.106 & 0.010 & 7.7 & 50.0 \\
\hline Chloroform & 0.610 & 0.627 & 0.010 & 2.9 & 50.0 \\
\hline 1,1,1-Trichloroethane & 0.891 & 0.883 & 0.010 & -1.0 & 50.0 \\
\hline Cyclohexane & 0.416 & 0.432 & 0.010 & 3.8 & 50.0 \\
\hline Carbon tetrachloride & 0.885 & 0.888 & 0.010 & 0.4 & 50.0 \\
\hline Benzene & 1.173 & 1.173 & 0.010 & 0.0 & 50.0 \\
\hline 1,2-Dichloroethane & 0.356 & 0.352 & 0.010 & -1.1 & 50.0 \\
\hline Trichloroethene & 0.387 & 0.384 & 0.010 & -0.6 & 50.0 \\
\hline Methylcyclohexane & 0.428 & 0.449 & 0.010 & 4.9 & 50.0 \\
\hline
\end{tabular}

Report 1,4-Dioxane for Low/Medium VOA analysis only 
Lab Name: TESTAMERICA BURLINGTON

Contract: 8E-00302

Lab Code: STLV

Mod. Ref No.:

SDG No.: 28988

Instrument ID: CHD.i

Calibration Date: 07/27/2015 Time: 1801

Lab File Id: 14962_014.D

EPA Sample No. (VSTD\#\#\#\#) : VSTD005VD Init. Calib. Date(s):07/27/2015 07/27/2015

Heated Purge: ( $\mathrm{Y} / \mathrm{N}) \mathrm{N}$ GC Column: DB-624

Init. Calib. Time(s) : 1326 1506

Purge Volume: 25.0 ID: $0.20(\mathrm{~mm})$ Length: 25

(m)

\begin{tabular}{|c|c|c|c|c|c|}
\hline COMPOUND & $\overline{\mathrm{RRF}}$ & RRF5. 0 & $\begin{array}{l}\text { MIN } \\
\text { RRF }\end{array}$ & $\because \mathrm{D}$ & $M A X \cong D$ \\
\hline 1,2-Dichloropropane & 0.198 & 0.173 & 0.010 & $-12 \cdot 3$ & 50.0 \\
\hline Bromodichloromethane & 0.402 & 0.407 & 0.010 & 1.2 & 50.0 \\
\hline cis-1,3-Dichloropropene & 0.349 & 0.376 & 0.010 & 7.8 & 50.0 \\
\hline 4-Methyl-2-pentanone & 0.068 & 0.072 & 0.010 & 6.4 & 50.0 \\
\hline Toluene & 1.449 & 1.487 & 0.010 & 2.6 & 50.0 \\
\hline trans-1,3-Dichloropropene & 0.298 & 0.313 & 0.010 & 4.9 & 50.0 \\
\hline $1,1,2$-Trichloroethane & 0.141 & 0.131 & 0.010 & -7.0 & 50.0 \\
\hline Tetrachloroethene & 0.479 & 0.470 & 0.010 & -2.0 & 50.0 \\
\hline 2-Hexanone & 0.050 & 0.051 & 0.010 & 2.4 & 50.0 \\
\hline Dibromochloromethane & 0.220 & 0.225 & 0.010 & 2.4 & 50.0 \\
\hline 1,2-Dibromoethane & 0.129 & 0.128 & 0.010 & -1.4 & 50.0 \\
\hline Chlorobenzene & 0.981 & 0.964 & 0.010 & -1.7 & 50.0 \\
\hline Ethylbenzene & 1.884 & 1.962 & 0.010 & 4.1 & 50.0 \\
\hline o-Xylene & 0.630 & 0.668 & 0.010 & 6.0 & 50.0 \\
\hline $\mathrm{m}, \mathrm{p}-\mathrm{Xylene}$ & 0.714 & 0.762 & 0.010 & 6.7 & 50.0 \\
\hline Styrene & 0.972 & 1.050 & 0.010 & 8.1 & 50.0 \\
\hline Bromoform & 0.165 & 0.166 & 0.010 & 0.3 & 50.0 \\
\hline Isopropylbenzene & 2.074 & 2.215 & 0.010 & 6.8 & 50.0 \\
\hline $1,1,2,2$-Tetrachloroethane & 0.117 & 0.114 & 0.010 & -2.3 & 50.0 \\
\hline 1,3-Dichlorobenzene & 1.447 & 1.408 & 0.010 & -2.7 & 50.0 \\
\hline 1,4-Dichlorobenzene & 1.576 & 1.499 & 0.010 & -4.9 & 50.0 \\
\hline 1,2-Dichlorobenzene & 1.258 & 1.230 & 0.010 & -2.2 & 50.0 \\
\hline 1,2-Dibromo-3-Chloropropane & 0.036 & 0.033 & 0.010 & -8.6 & 50.0 \\
\hline 1,2,4-Trichlorobenzene & 0.830 & 0.743 & 0.010 & -10.4 & 50.0 \\
\hline $1,2,3$-Trichlorobenzene & 0.597 & 0.553 & 0.010 & -7.4 & 50.0 \\
\hline
\end{tabular}


Lab Name: TESTAMERICA BURLINGTON

Contract: $8 \mathrm{E}-00302$

Lab Code: STLV Case No.: YORK Mod. Ref No.

SDG No.: 28988

Instrument ID: CHD.i

Calibration Date: 07/27/2015 Time: 1801

Lab File Id: 14962_014.D Init. Calib. Date(s):07/27/2015 07/27/2015

EPA Sample No. (VSTD\#\#\#\#) : VSTD005VD

Heated Purge: (Y/N) N GC Column: DB-624

Init. Calib. Time(s) : 1326 1506

Purge Volume: 25.0 ID: $0.20(\mathrm{~mm})$ Length: 25

(m)

\begin{tabular}{|c|c|c|c|c|c|}
\hline COMPOUND & $\overline{\mathrm{RRF}}$ & RRF 5.0 & $\begin{array}{l}\text { MIN } \\
\text { RRF }\end{array}$ & $\div D$ & $\mathrm{MAX} \div \mathrm{D}$ \\
\hline Vinyl Chloride-d3 & 0.363 & 0.363 & 0.010 & 0.0 & 50.0 \\
\hline Chloroethane-d5 & 0.295 & 0.303 & 0.010 & 2.5 & 50.0 \\
\hline 1,1-Dichloroethene-d2 & 0.769 & 0.778 & 0.010 & 1.1 & 50.0 \\
\hline 2-Butanone-d5 & 0.022 & 0.023 & 0.010 & 3.0 & 50.0 \\
\hline Chloroform-d & 0.668 & 0.704 & 0.010 & 5.4 & 50.0 \\
\hline 1,2-Dichloroethane-d4 & 0.296 & 0.300 & 0.010 & 1.1 & 50.0 \\
\hline Benzene-d6 & 1.154 & 1.161 & 0.010 & 0.6 & 50.0 \\
\hline 1,2 -Dichloropropane-d6 & 0.230 & 0.222 & 0.010 & -3.7 & 50.0 \\
\hline Toluene-d8 & 1.322 & 1.373 & 0.010 & 3.8 & 50.0 \\
\hline trans-1,3-Dichloropropene-d4 & 0.267 & 0.272 & 0.010 & 1.9 & 50.0 \\
\hline 2-Hexanone-d5 & 0.021 & 0.023 & 0.010 & 9.4 & 50.0 \\
\hline $1,1,2,2$-Tetrachloroethane-d2 & 0.118 & 0.121 & 0.010 & 2.2 & 50.0 \\
\hline 1,2-Dichlorobenzene-d4 & 0.820 & 0.811 & 0.010 & $-1 \cdot 1$ & 50.0 \\
\hline
\end{tabular}

Report 1,4-Dioxane-d8 for Low/Medium VOA analysis only 
Lab Name: TESTAMERICA BURLINGTON

Contract: $8 \mathrm{E}-00302$

Lab Code: STLV Case No.: YORK Mod. Ref No.: SDG No.: 28988

Matrix: (SOIL/SED/WATER) Water

Sample wt/vol: 25.0 $(\mathrm{g} / \mathrm{mL}) \mathrm{mL}$

Level: (TRACE/LOW/MED) TRACE

\% Moisture: not dec.

GC Column: DB-624 ID: $0.20 \quad(\mathrm{~mm})$

Soil Extract Volume: (uL) $(\mathrm{mL})$

Purge Volume: 25.0
Lab Sample ID: MB 200-91869/10

Lab File ID: 14962_010.D

Date Received:

Date Analyzed: 07/27/2015

Dilution Factor: 1.0

Soil Aliquot Volume: (uL)

\begin{tabular}{|c|c|c|c|}
\hline CAS NO. & COMPOUND & $\begin{array}{l}\text { CONCENTRATION UNITS: } \\
(\mathrm{ug} / \mathrm{L} \text { or } \mathrm{ug} / \mathrm{kg}) \mathrm{ug} / \mathrm{L}\end{array}$ & $Q$ \\
\hline $75-71-8$ & Dichlorodifluoromethane & 1.0 & $\mathrm{U}$ \\
\hline $74-87-3$ & Chloromethane & 1.0 & $\mathrm{U}$ \\
\hline $75-01-4$ & Vinyl chloride & 1.0 & $\mathrm{U}$ \\
\hline $74-83-9$ & Bromomethane & 1.0 & $\mathrm{U}$ \\
\hline $75-00-3$ & Chloroethane & 1.0 & $\mathrm{U}$ \\
\hline $75-69-4$ & Trichlorofluoromethane & 1.0 & $\mathrm{U}$ \\
\hline $75-35-4$ & 1,1-Dichloroethene & 1.0 & $\mathrm{U}$ \\
\hline $76-13-1$ & 1,1,2-Trichloro-1,2,2-trifluoroethane & 1.0 & $\mathrm{U}$ \\
\hline $67-64-1$ & Acetone & 0.87 & $\mathrm{~J}$ \\
\hline $75-15-0$ & Carbon disulfide & 1.0 & $\mathrm{U}$ \\
\hline $79-20-9$ & Methyl acetate & 1.0 & $\mathrm{U}$ \\
\hline $75-09-2$ & Methylene Chloride & 1.0 & $\mathrm{U}$ \\
\hline $156-60-5$ & trans-1,2-Dichloroethene & 1.0 & $\mathrm{U}$ \\
\hline $1634-04-4$ & Methyl tert-butyl ether & 1.0 & $\mathrm{U}$ \\
\hline $75-34-3$ & 1,1-Dichloroethane & 1.0 & $\mathrm{U}$ \\
\hline $156-59-2$ & cis-1,2-Dichloroethene & 1.0 & $\mathrm{U}$ \\
\hline $78-93-3$ & 2-Butanone & 5.0 & $\mathrm{U}$ \\
\hline $74-97-5$ & Bromochloromethane & 1.0 & $\mathrm{U}$ \\
\hline $67-66-3$ & Chloroform & 1.0 & $\mathrm{U}$ \\
\hline $71-55-6$ & 1,1,1-Trichloroethane & 1.0 & $\mathrm{U}$ \\
\hline $110-82-7$ & Cyclohexane & 1.0 & $\mathrm{U}$ \\
\hline $56-23-5$ & Carbon tetrachloride & 1.0 & $\mathrm{U}$ \\
\hline $71-43-2$ & Benzene & 1.0 & $\mathrm{U}$ \\
\hline $107-06-2$ & 1,2-Dichloroethane & 1.0 & $\mathrm{U}$ \\
\hline
\end{tabular}

Report 1,4-Dioxane for Low-Medium VOA analysis only 
Lab Name: TESTAMERICA BURLINGTON

Lab Code: STLV

Case No.: YORK

Mod. Ref No.:

Contract: 8E-00302

Matrix: (SOIL/SED/WATER) Water

Sample wt/vol: 25.0 $(\mathrm{g} / \mathrm{mL}) \mathrm{mL}$

Level: (TRACE/LOW/MED) TRACE

\% Moisture: not dec.

GC Column: DB-624 ID $: \underline{0.20 \quad(\mathrm{~mm})}$

Soil Extract Volume: (uL) $(\mathrm{mL})$

Purge Volume: 25.0
SDG No.: 28988

Lab Sample ID: MB 200-91869/10

Lab File ID: 14962_010.D

Date Received:

Date Analyzed: 07/27/2015

Dilution Factor: 1.0

Soil Aliquot Volume: (uL)

\begin{tabular}{|c|c|c|c|}
\hline CAS NO. & COMPOUND & $\begin{array}{l}\text { CONCENTRATION UNITS: } \\
(\mathrm{ug} / \mathrm{L} \text { or } \mathrm{ug} / \mathrm{kg}) \mathrm{ug} / \mathrm{L}\end{array}$ & $q$ \\
\hline $79-01-6$ & Trichloroethene & 1.0 & $\mathrm{U}$ \\
\hline $108-87-2$ & Methylcyclohexane & 1.0 & $\mathrm{U}$ \\
\hline $78-87-5$ & 1,2-Dichloropropane & 1.0 & $\mathrm{U}$ \\
\hline $75-27-4$ & Bromodichloromethane & 1.0 & $\mathrm{U}$ \\
\hline $10061-01-5$ & cis-1,3-Dichloropropene & 1.0 & U \\
\hline $108-10-1$ & 4-Methyl-2-pentanone & 1.0 & $\mathrm{U}$ \\
\hline $108-88-3$ & Toluene & 1.0 & $\mathrm{U}$ \\
\hline $10061-02-6$ & trans-1,3-Dichloropropene & 1.0 & $\mathrm{U}$ \\
\hline $79-00-5$ & $1,1,2-$ Trichloroethane & 1.0 & $\mathrm{U}$ \\
\hline $127-18-4$ & Tetrachloroethene & 1.0 & $\mathrm{U}$ \\
\hline $591-78-6$ & 2-Hexanone & 1.0 & $\mathrm{U}$ \\
\hline $124-48-1$ & Dibromochloromethane & 1.0 & $\mathrm{U}$ \\
\hline $106-93-4$ & 1,2-Dibromoethane & 1.0 & $\mathrm{U}$ \\
\hline $108-90-7$ & Chlorobenzene & 1.0 & $\mathrm{U}$ \\
\hline $100-41-4$ & Ethylbenzene & 1.0 & $\mathrm{U}$ \\
\hline $95-47-6$ & o-Xylene & 1.0 & $\mathrm{U}$ \\
\hline $179601-23-1$ & m,p-xylene & 1.0 & $\mathrm{U}$ \\
\hline $100-42-5$ & Styrene & 1.0 & $\mathrm{U}$ \\
\hline $75-25-2$ & Bromoform & 1.0 & $\mathrm{U}$ \\
\hline $98-82-8$ & Isopropylbenzene & 1.0 & $\mathrm{U}$ \\
\hline $79-34-5$ & $1,1,2,2$-Tetrachloroethane & 1.0 & $\mathrm{U}$ \\
\hline $541-73-1$ & 1,3 -Dichlorobenzene & 1.0 & $\mathrm{U}$ \\
\hline $106-46-7$ & 1,4-Dichlorobenzene & 1.0 & $\mathrm{U}$ \\
\hline $95-50-1$ & 1,2-Dichlorobenzene & 1.0 & $\mathrm{U}$ \\
\hline $96-12-8$ & 1,2-Dibromo-3-Chloropropane & 1.0 & $\mathrm{U}$ \\
\hline $120-82-1$ & $1,2,4$-Trichlorobenzene & 0.036 & $\mathrm{~J}$ \\
\hline $87-61-6$ & $1,2,3$-Trichlorobenzene & 0.063 & $\mathrm{~J}$ \\
\hline
\end{tabular}


Lab Name: TESTAMERICA BURLINGTON

Contract: $8 \mathrm{E}-00302$

Lab Code: STLV Case No.: YORK Mod. Ref No.: SDG No.: 28988

Matrix: (SOIL/SED/WATER) Water

Sample wt/vol: 25.0 $(\mathrm{g} / \mathrm{mL}) \mathrm{mL}$

Level: (TRACE or LOW/MED) TRACE

․ Moisture: not dec.

GC Column: DB-624 ID $: 0.20 \quad(\mathrm{~mm})$

Soil Extract Volume: (uL)

CONCENTRATION UNITS: (ug/L or ug/kg) ug/L
Lab Sample ID: MB 200-91869/10

Lab File ID: 14962_010.D

Date Received:

Date Analyzed: 07/27/2015

Dilution Factor: 1.0

Soil Aliquot Volume: (uL) Purge Volume: 25.0

$(\mathrm{mL})$

\begin{tabular}{|c|c|c|c|c|}
\hline CAS NUMBER & COMPOUND NAME & $\mathrm{RT}$ & EST. CONC. & Q \\
\hline 01 & Unknown & 7.29 & 2.9 & J X \\
\hline 02 & Unknown & 8.22 & 0.68 & $\mathrm{~J}$ \\
\hline 03 & Unknown & 11.04 & 0.60 & $\mathrm{~J}$ \\
\hline E9667961 & Total Alkanes & $\mathrm{N} / \mathrm{A}$ & & \\
\hline
\end{tabular}


Lab Name: TESTAMERICA BURLINGTON

Contract: $8 \mathrm{E}-00302$

Lab Code: STLV

Case No.: YORK Mod. Ref No.: SDG No.: 28988

Matrix: (SOIL/SED/WATER) Water

Sample wt/vol: 25.0 $(\mathrm{g} / \mathrm{mL}) \mathrm{mL}$

Level: (TRACE/LOW/MED) TRACE

\% Moisture: not dec.

GC Column: DB-624 ID: $0.20 \quad(\mathrm{~mm})$

Soil Extract Volume: (uL) $(\mathrm{mL})$

Purge Volume: 25.0
Lab Sample ID: 200-28988-3

Lab File ID: 14962_013.D

Date Received:

Date Analyzed: 07/27/2015

Dilution Factor: 1.0

Soil Aliquot Volume: (uL)

\begin{tabular}{|c|c|c|c|}
\hline CAS NO. & COMPOUND & $\begin{array}{l}\text { CONCENTRATION UNITS: } \\
(\mathrm{ug} / \mathrm{L} \text { or } \mathrm{ug} / \mathrm{kg}) \mathrm{ug} / \mathrm{L}\end{array}$ & q \\
\hline $75-71-8$ & Dichlorodifluoromethane & 1.0 & $\mathrm{U}$ \\
\hline $74-87-3$ & Chloromethane & 1.0 & $\mathrm{U}$ \\
\hline $75-01-4$ & Vinyl chloride & 1.0 & $\mathrm{U}$ \\
\hline $74-83-9$ & Bromomethane & 1.0 & $\mathrm{U}$ \\
\hline $75-00-3$ & Chloroethane & 1.0 & $\mathrm{U}$ \\
\hline $75-69-4$ & Trichlorofluoromethane & 1.0 & $\mathrm{U}$ \\
\hline $75-35-4$ & 1,1-Dichloroethene & 1.0 & $\mathrm{U}$ \\
\hline $76-13-1$ & $1,1,2$-Trichloro-1,2,2-trifluoroethane & 1.0 & $\mathrm{U}$ \\
\hline $67-64-1$ & Acetone & 5.0 & $\mathrm{U}$ \\
\hline $75-15-0$ & Carbon disulfide & 1.0 & $\mathrm{U}$ \\
\hline $79-20-9$ & Methyl acetate & 1.0 & $\mathrm{U}$ \\
\hline $75-09-2$ & Methylene Chloride & 1.0 & $\mathrm{U}$ \\
\hline $156-60-5$ & trans-1,2-Dichloroethene & 1.0 & $\mathrm{U}$ \\
\hline $1634-04-4$ & Methyl tert-butyl ether & 1.0 & $\mathrm{U}$ \\
\hline $75-34-3$ & 1,1-Dichloroethane & 1.0 & $\mathrm{U}$ \\
\hline $156-59-2$ & cis-1,2-Dichloroethene & 1.0 & $\mathrm{U}$ \\
\hline $78-93-3$ & 2-Butanone & 5.0 & $\mathrm{U}$ \\
\hline $74-97-5$ & Bromochloromethane & 1.0 & $\mathrm{U}$ \\
\hline $67-66-3$ & Chloroform & 1.0 & $\mathrm{U}$ \\
\hline $71-55-6$ & $1,1,1$-Trichloroethane & 1.0 & $\mathrm{U}$ \\
\hline $110-82-7$ & Cyclohexane & 1.0 & $\mathrm{U}$ \\
\hline $56-23-5$ & Carbon tetrachloride & 1.0 & $\mathrm{U}$ \\
\hline $71-43-2$ & Benzene & 0.031 & $\mathrm{~J}$ \\
\hline $107-06-2$ & 1,2-Dichloroethane & 1.0 & $\mathrm{U}$ \\
\hline
\end{tabular}

Report 1,4-Dioxane for Low-Medium VOA analysis only 
Lab Name: TESTAMERICA BURLINGTON

Lab Code: STLV

Case No.: YORK

Mod. Ref No.:

Contract: 8E-00302

Matrix: (SOIL/SED/WATER) Water

Sample wt/vol: 25.0 $(\mathrm{g} / \mathrm{mL}) \mathrm{mL}$

Level: (TRACE/LOW/MED) TRACE

\% Moisture: not dec.

GC Column: DB-624 ID $: \underline{0.20 \quad(\mathrm{~mm})}$

Soil Extract Volume: (uL) $(\mathrm{mL})$

Purge Volume: 25.0
SDG No.: 28988

Lab Sample ID: 200-28988-3

Lab File ID: 14962_013.D

Date Received:

Date Analyzed: 07/27/2015

Dilution Factor: 1.0

Soil Aliquot Volume: (uL)

\begin{tabular}{|c|c|c|c|}
\hline CAS NO. & COMPOUND & $\begin{array}{l}\text { CONCENTRATION UNITS: } \\
(\mathrm{ug} / \mathrm{L} \text { or } \mathrm{ug} / \mathrm{kg}) \mathrm{ug} / \mathrm{L}\end{array}$ & $q$ \\
\hline $79-01-6$ & Trichloroethene & 1.0 & $\mathrm{U}$ \\
\hline $108-87-2$ & Methylcyclohexane & 1.0 & $\mathrm{U}$ \\
\hline $78-87-5$ & 1,2-Dichloropropane & 1.0 & $\mathrm{U}$ \\
\hline $75-27-4$ & Bromodichloromethane & 1.0 & $\mathrm{U}$ \\
\hline $10061-01-5$ & cis-1,3-Dichloropropene & 1.0 & U \\
\hline $108-10-1$ & 4-Methyl-2-pentanone & 1.0 & $\mathrm{U}$ \\
\hline $108-88-3$ & Toluene & 0.015 & $\mathrm{~J}$ \\
\hline $10061-02-6$ & trans-1,3-Dichloropropene & 1.0 & $\mathrm{U}$ \\
\hline $79-00-5$ & $1,1,2-$ Trichloroethane & 1.0 & $\mathrm{U}$ \\
\hline $127-18-4$ & Tetrachloroethene & 1.0 & $\mathrm{U}$ \\
\hline $591-78-6$ & 2-Hexanone & 1.0 & $\mathrm{U}$ \\
\hline $124-48-1$ & Dibromochloromethane & 1.0 & $\mathrm{U}$ \\
\hline $106-93-4$ & 1,2-Dibromoethane & 1.0 & $\mathrm{U}$ \\
\hline $108-90-7$ & Chlorobenzene & 1.0 & $\mathrm{U}$ \\
\hline $100-41-4$ & Ethylbenzene & 1.0 & $\mathrm{U}$ \\
\hline $95-47-6$ & o-Xylene & 1.0 & $\mathrm{U}$ \\
\hline $179601-23-1$ & m,p-xylene & 1.0 & $\mathrm{U}$ \\
\hline $100-42-5$ & Styrene & 1.0 & $\mathrm{U}$ \\
\hline $75-25-2$ & Bromoform & 1.0 & $\mathrm{U}$ \\
\hline $98-82-8$ & Isopropylbenzene & 1.0 & $\mathrm{U}$ \\
\hline $79-34-5$ & $1,1,2,2$-Tetrachloroethane & 1.0 & $\mathrm{U}$ \\
\hline $541-73-1$ & 1,3 -Dichlorobenzene & 1.0 & $\mathrm{U}$ \\
\hline $106-46-7$ & 1,4-Dichlorobenzene & 1.0 & $\mathrm{U}$ \\
\hline $95-50-1$ & 1,2-Dichlorobenzene & 1.0 & $\mathrm{U}$ \\
\hline $96-12-8$ & 1,2-Dibromo-3-Chloropropane & 1.0 & $\mathrm{U}$ \\
\hline $120-82-1$ & $1,2,4$-Trichlorobenzene & 1.0 & $\mathrm{U}$ \\
\hline $87-61-6$ & $1,2,3$-Trichlorobenzene & 1.0 & $\mathrm{U}$ \\
\hline
\end{tabular}


Lab Name: TESTAMERICA BURLINGTON

Contract: $8 \mathrm{E}-00302$

Lab Code: STLV Case No.: YORK Mod. Ref No.: SDG No.: 28988

Matrix: (SOIL/SED/WATER) Water

Sample wt/vol: 25.0 $(\mathrm{g} / \mathrm{mL}) \mathrm{mL}$

Level: (TRACE or LOW/MED) TRACE

\% Moisture: not dec.

GC Column: DB-624 ID $: 0.20 \quad(\mathrm{~mm})$

Soil Extract Volume: (uL)

CONCENTRATION UNITS: (ug/L or ug/kg) ug/L
Lab Sample ID: 200-28988-3

Lab File ID: 14962_013.D

Date Received:

Date Analyzed: 07/27/2015

Dilution Factor: 1.0

Soil Aliquot Volume: (uL) Purge Volume: 25.0 $(\mathrm{mL})$

01

02

\begin{tabular}{|l|l|c|c|c|}
\hline CAS NUMBER & \multicolumn{1}{|c|}{ COMPOUND NAME } & RT & EST. CONC. & Q \\
\hline & Unknown & 7.29 & 2.9 & $\mathrm{~J}$ B X \\
\hline E9667961 & Total Alkanes & N/A & & \\
\hline
\end{tabular}

1EPA-designated Registry Number. 
Lab Name: TestAmerica Burlington

Job No.: 200-28988-1

SDG No.: 28988

Instrument ID: CHD.i

Analysis Batch Number: 91869

Lab Sample ID: IC 200-91869/3

Client Sample ID:

Date Analyzed: 07/27/15 13:26

Lab File ID: 14962 003.D

GC Column: DB-624

ID : $0.2(\mathrm{~mm})$

\begin{tabular}{|c|c|c|c|c|}
\hline \multirow[t]{2}{*}{ COMPOUND NAME } & \multirow{2}{*}{$\begin{array}{c}\text { RETENTION } \\
\text { TIME }\end{array}$} & \multicolumn{3}{|c|}{ MANUAL INTEGRATION } \\
\hline & & REASON & ANALYST & DATE \\
\hline Bromomethane & 2.00 & Baseline & wilburj & $08 / 06 / 15 \quad 10: 34$ \\
\hline 1,2-Dibromo-3-Chloropropane & 13.43 & Baseline & wilburj & $08 / 06 / 15 \quad 10: 34$ \\
\hline
\end{tabular}

Lab Sample ID: IC 200-91869/4 Client Sample ID:

Date Analyzed: 07/27/15 13:51

Lab File ID: 14962_004.D

GC Column: DB-624

ID $: 0.2(\mathrm{~mm})$

\begin{tabular}{|c|c|c|c|c|}
\hline \multirow[t]{2}{*}{ COMPOUND NAME } & \multirow{2}{*}{$\begin{array}{c}\text { RETENTION } \\
\text { TIME }\end{array}$} & \multicolumn{3}{|c|}{ MANUAL INTEGRATION } \\
\hline & & REASON & ANALYST & DATE \\
\hline Dichlorodifluoromethane & 1.52 & Baseline & wilburj & $08 / 06 / 15 \quad 10: 36$ \\
\hline Bromomethane & 2.00 & Baseline & wilburj & $08 / 06 / 15 \quad 10: 37$ \\
\hline 1,2-Dibromo-3-Chloropropane & 13.43 & Baseline & wilburj & $08 / 06 / 15 \quad 10: 37$ \\
\hline
\end{tabular}

Lab Sample ID: ICIS 200-91869/5

Client Sample ID:

Date Analyzed: 07/27/15 14:16

Lab File ID: 14962_005.D

GC Column: DB-624

ID $: 0.2(\mathrm{~mm})$

\begin{tabular}{|c|c|c|c|c|}
\hline \multirow[t]{2}{*}{ COMPOUND NAME } & \multirow{2}{*}{$\begin{array}{c}\text { RETENTION } \\
\text { TIME }\end{array}$} & \multicolumn{3}{|c|}{ MANUAL INTEGRATION } \\
\hline & & REASON & ANALYST & DATE \\
\hline Dichlorodifluoromethane & 1.52 & Baseline & wilburj & $08 / 06 / 15 \quad 10: 39$ \\
\hline Bromomethane & 1.99 & Baseline & wilburj & $08 / 06 / 1510: 39$ \\
\hline
\end{tabular}

Lab Sample ID: IC 200-91869/6

Client Sample ID:

Date Analyzed: 07/27/15 14:41

Lab File ID: 14962 006.D

GC Column: DB-624

ID $: 0.2(\mathrm{~mm})$

\begin{tabular}{|c|c|c|c|c|}
\hline \multirow[t]{2}{*}{ COMPOUND NAME } & \multirow{2}{*}{$\begin{array}{c}\text { RETENTION } \\
\text { TIME }\end{array}$} & \multicolumn{3}{|c|}{ MANUAL INTEGRATION } \\
\hline & & REASON & ANALYST & DATE \\
\hline Dichlorodifluoromethane & 1.52 & Baseline & wilburj & $08 / 06 / 15 \quad 10: 40$ \\
\hline Bromomethane & 1.99 & Baseline & wilburj & $08 / 06 / 15 \quad 10: 40$ \\
\hline
\end{tabular}


Lab Name: TestAmerica Burlington

Job No.: 200-28988-1

SDG No.: 28988

Instrument ID: CHD.i

Analysis Batch Number: 91869

Lab Sample ID: IC 200-91869/7

Client Sample ID:

Date Analyzed: 07/27/15 15:06

Lab File ID: 14962 007.D

GC Column: DB-624

ID $: 0.2(\mathrm{~mm})$

\begin{tabular}{|l|c|c|c|c|}
\hline \multicolumn{1}{|c|}{ COMPOUND NAME } & RETENTION & \multicolumn{2}{|c|}{ MANUAL INTEGRATION } \\
\cline { 4 - 5 } & TIME & REASON & ANALYST & DATE \\
\hline Dichlorodifluoromethane & 1.53 & Baseline & wilburj & $08 / 06 / 1510: 42$ \\
\hline Bromomethane & 1.99 & Baseline & wilburj & $08 / 06 / 1510: 42$ \\
\hline
\end{tabular}

Lab Sample ID: CCVC 200-91869/14

Client Sample ID:

Date Analyzed: 07/27/15 18:01

Lab File ID: 14962_014.D

GC Column: DB-624

ID $: 0.2(\mathrm{~mm})$

\begin{tabular}{|c|c|c|c|c|}
\hline \multirow[t]{2}{*}{ COMPOUND NAME } & \multirow{2}{*}{$\begin{array}{c}\text { RETENTION } \\
\text { TIME }\end{array}$} & \multicolumn{3}{|c|}{ MANUAL INTEGRATION } \\
\hline & & REASON & ANALYST & DATE \\
\hline Dichlorodifluoromethane & 1.52 & Baseline & wilburj & $08 / 06 / 1511: 58$ \\
\hline Bromomethane & 1.99 & Baseline & wilburj & $08 / 06 / 1511: 58$ \\
\hline
\end{tabular}




\section{TestAmerica}

THE LEADER IN ENVIRONMENTAL TESTING

\section{ANALYTICAL REPORT}

Job Number: 200-29233-1

SDG Number: 29233

Job Description: York (200-29233)

Contract Number: 1E-30401

For:

Argonne National Laboratory

9700 South Cass Avenue

Building 203

Office B-141

Argonne, IL 60439

Attention: Ms. Esther Bowen

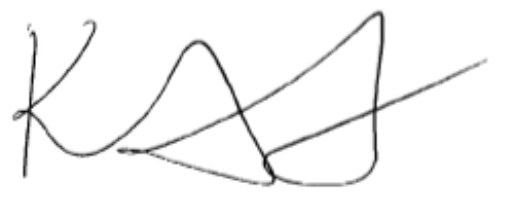

Approved for release. Kathryn A Kelly

Kathryn A Kelly, Project Manager I

30 Community Drive, South Burlington, VT, 05403

kathryn.kelly@testamericainc.com

08/28/2015

The test results in this report relate only to sample(s) as received by the laboratory. These test results were derived under a quality system that adheres to the requirements of NELAC. Pursuant to NELAC, this report may not be produced in full without written approval from the laboratory 


\section{Table of Contents}

Cover Title Page...$\ldots \ldots \ldots \ldots \ldots \ldots \ldots \ldots \ldots \ldots \ldots \ldots \ldots$

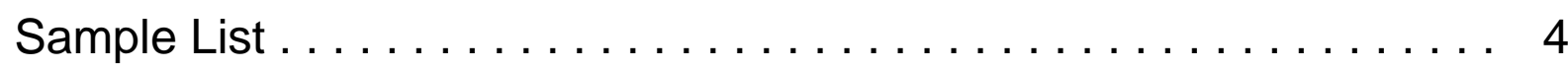

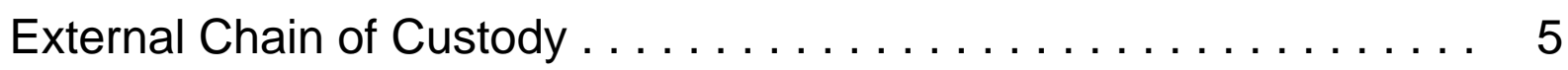

Internal Chain of Custody ..................... 7

Shipping Documentation . . . . . . . . . . . . . . . 8

Sample Receipt and Log In Check List ..................... 9

Standards Traceability . . . . . . . . . . . . . . . . . 10

Methodology Review ......................... 15

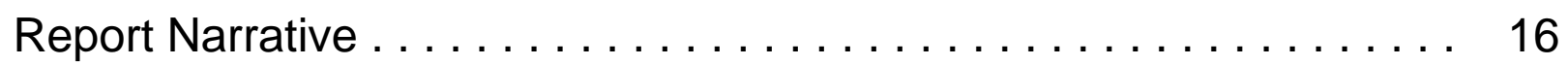

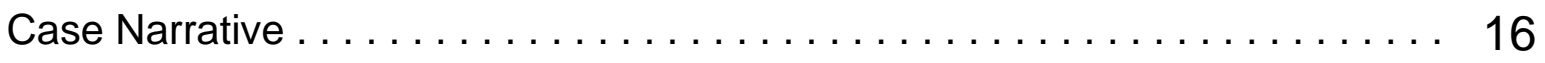

Manual Integration Documentation ....................... 17

Qualifier Definition ................................ 20

QC Summary - SOM01.2 Volatiles-Trace . . . . . . . . . . . 21

QC Summary - SOM01.2 Volatiles-Trace $\ldots \ldots \ldots \ldots \ldots \ldots \ldots \ldots \ldots .21$

Deuterated Monitoring Compound Summary $\ldots \ldots \ldots \ldots \ldots \ldots \ldots \ldots \ldots \ldots, 21$

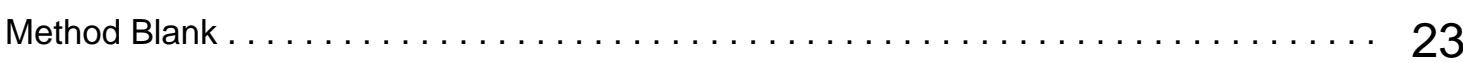

GC/MS Instrument Performance Check ........................... 25

Internal Standard Area and RT Summary $\ldots \ldots \ldots \ldots \ldots \ldots \ldots \ldots \ldots \ldots \ldots \ldots \ldots \ldots$

Sample Data - SOM01.2 Volatiles-Trace . ............. 30

Sample Data - SOM01.2 Volatiles-Trace ..................... 30

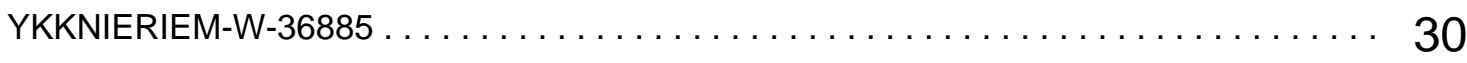

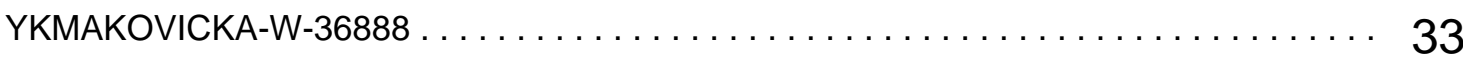

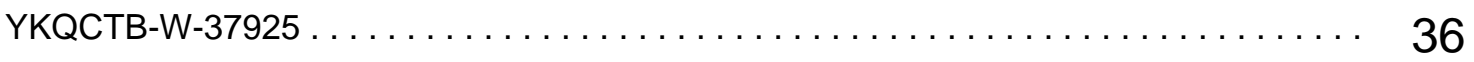

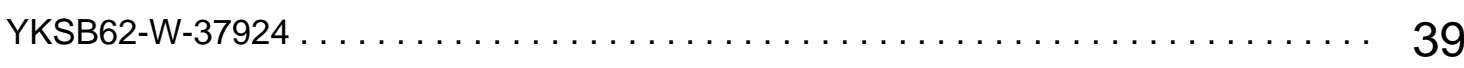

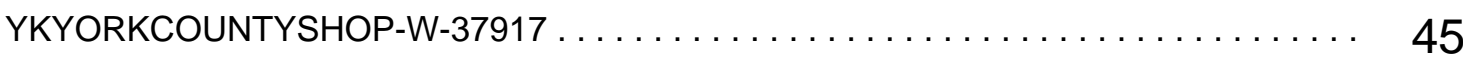

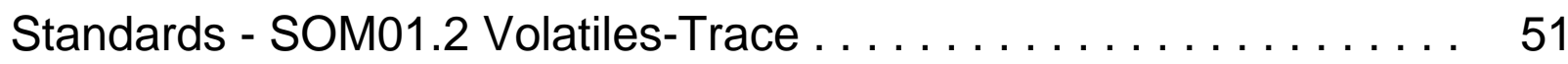




\section{Table of Contents}

Standards - SOM01.2 Volatiles-Trace $\ldots \ldots \ldots \ldots \ldots \ldots \ldots \ldots \ldots \ldots . \ldots \ldots$

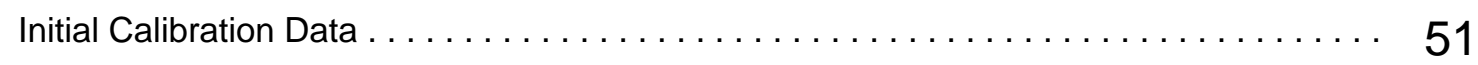

CCV Data, including closing CCV $\ldots \ldots \ldots \ldots \ldots \ldots \ldots \ldots \ldots \ldots \ldots \ldots \ldots \ldots \ldots$

Raw Qc Data - SOM01.2 Volatiles-Trace . . . . . . . . . . . . 66

Raw Qc Data - SOM01.2 Volatiles-Trace ............... 66

Raw Qc Data - SOM01.2 Volatiles-Trace $\ldots \ldots \ldots \ldots \ldots \ldots \ldots \ldots \ldots \ldots \ldots \ldots$

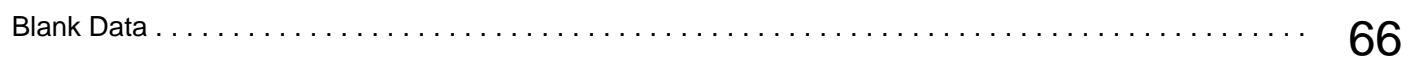




\section{Sample Login Acknowledgement}

Job 200-29233-1

\begin{tabular}{ll}
\hline Client Job Description: & York (200-29233) \\
Purchase Order \#: & 1E-30401 \\
Work Order \#: & 1E-30401 \\
Project Manager: & Kathryn A Kelly \\
Job Due Date: & $8 / 25 / 2015$ \\
Job TAT: & 14 Days \\
Max Deliverable Level: & IV
\end{tabular}

Max Deliverable Level: IV

Earliest Deliverable Due: 8/25/2015

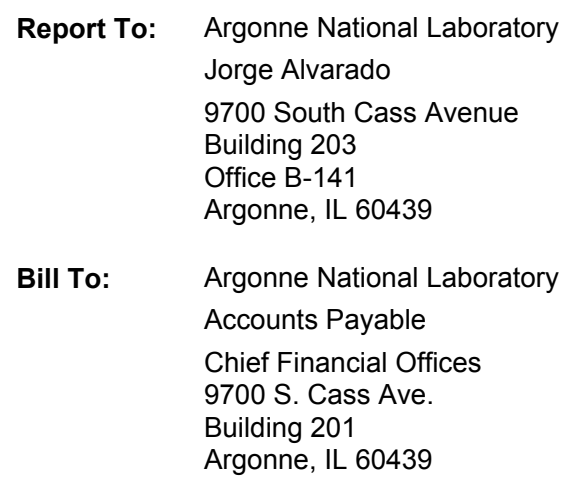

\section{Login 200-29233}

\begin{tabular}{|c|c|c|c|c|c|}
\hline Sample Receipt: & 8/11/2015 10:25:00 AM & Number of Coolers: & 1 & & \\
\hline Method of Delivery: & FedEx Priority Overnight & Cooler Temperature(s) & $\left(\mathbf{C}^{\circ}\right): 5.0$ & & \\
\hline Lab Sample \# & Client Sample ID & Date Sampled & Matrix & & \\
\hline Method & Method Description / Work Location & & & Rpt Basis & Dry / Wet ** \\
\hline 200-29233-1 & YKKNIERIEM-W-36885 & 8/6/2015 9:45:00 AM & Water & & \\
\hline SOM01.2_Vol_Tr & r SOM01.2 Trace Volatile Organics / In-Lab & & & Total & Wet \\
\hline 200-29233-2 & YKMAKOVICKA-W-36888 & 8/6/2015 10:30:00 AM & Water & & \\
\hline SOM01.2_Vol_Tr & ir SOM01.2 Trace Volatile Organics / In-Lab & & & Total & Wet \\
\hline 200-29233-3 & YKYORKCOUNTYSHOP-W-37917 & 8/5/2015 1:35:00 PM & Water & & \\
\hline SOM01.2_Vol_Tr & r SOM01.2 Trace Volatile Organics / In-Lab & & & Total & Wet \\
\hline 200-29233-4 & YKSB62-W-37924 & 8/7/2015 2:02:00 PM & Water & & \\
\hline SOM01.2_Vol_Tr & r SOM01.2 Trace Volatile Organics / In-Lab & & & Total & Wet \\
\hline 200-29233-5 & YKQCTB-W-37925 & 8/5/2015 12:00:00 PM & Water & & \\
\hline SOM01.2_Vol_Tr & ir SOM01.2 Trace Volatile Organics / In-Lab & & & Total & Wet \\
\hline 200-29233-6 & VHBLK01 & 8/11/2015 12:00:00 AM & Water & & \\
\hline SOM01.2_Vol_Tr & ir SOM01.2 Trace Volatile Organics / In-Lab & & & Total & Wet \\
\hline
\end{tabular}




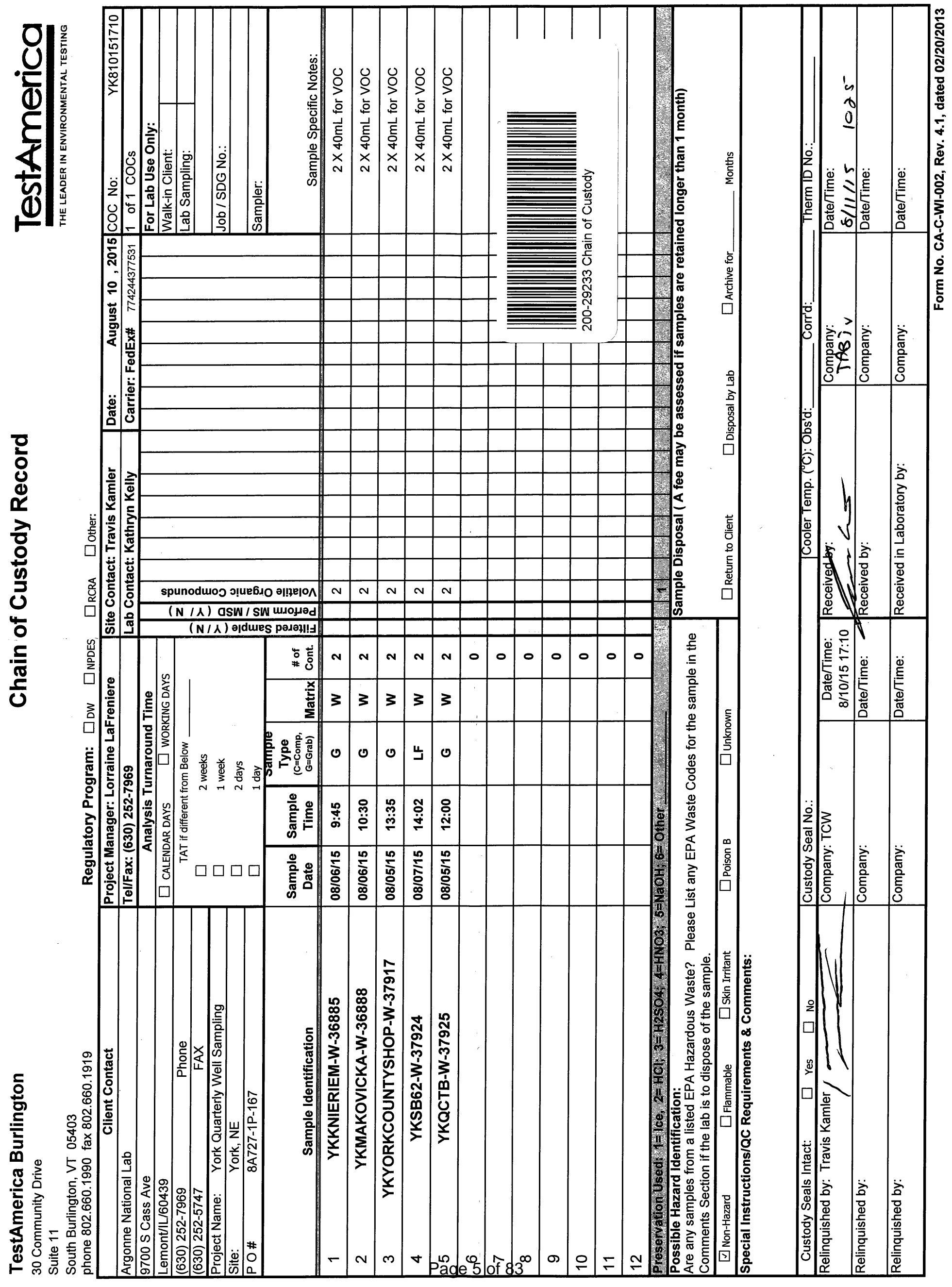


•әр!n

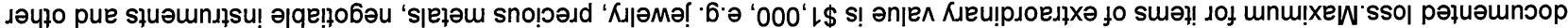

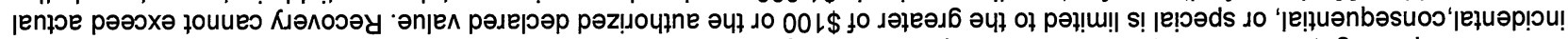

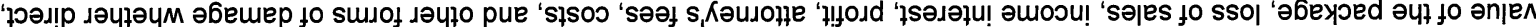

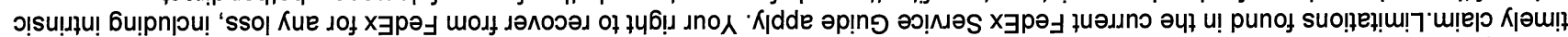

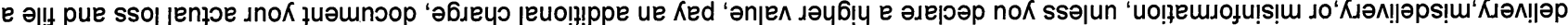

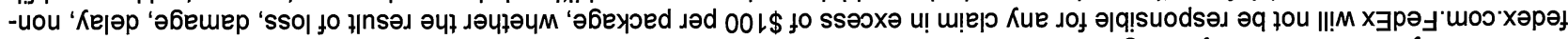

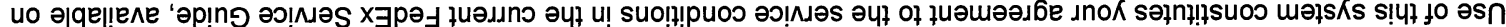

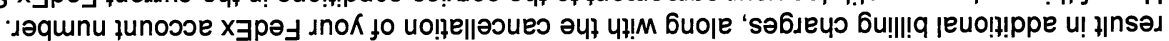
p|nos pue łuə|npneג\} s! səsodınd bu!̣d!

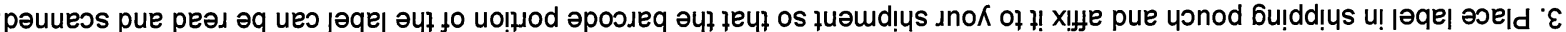

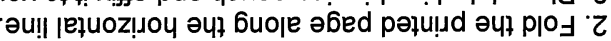

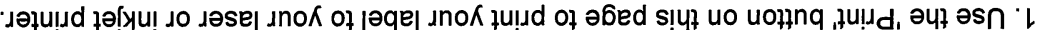

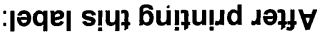

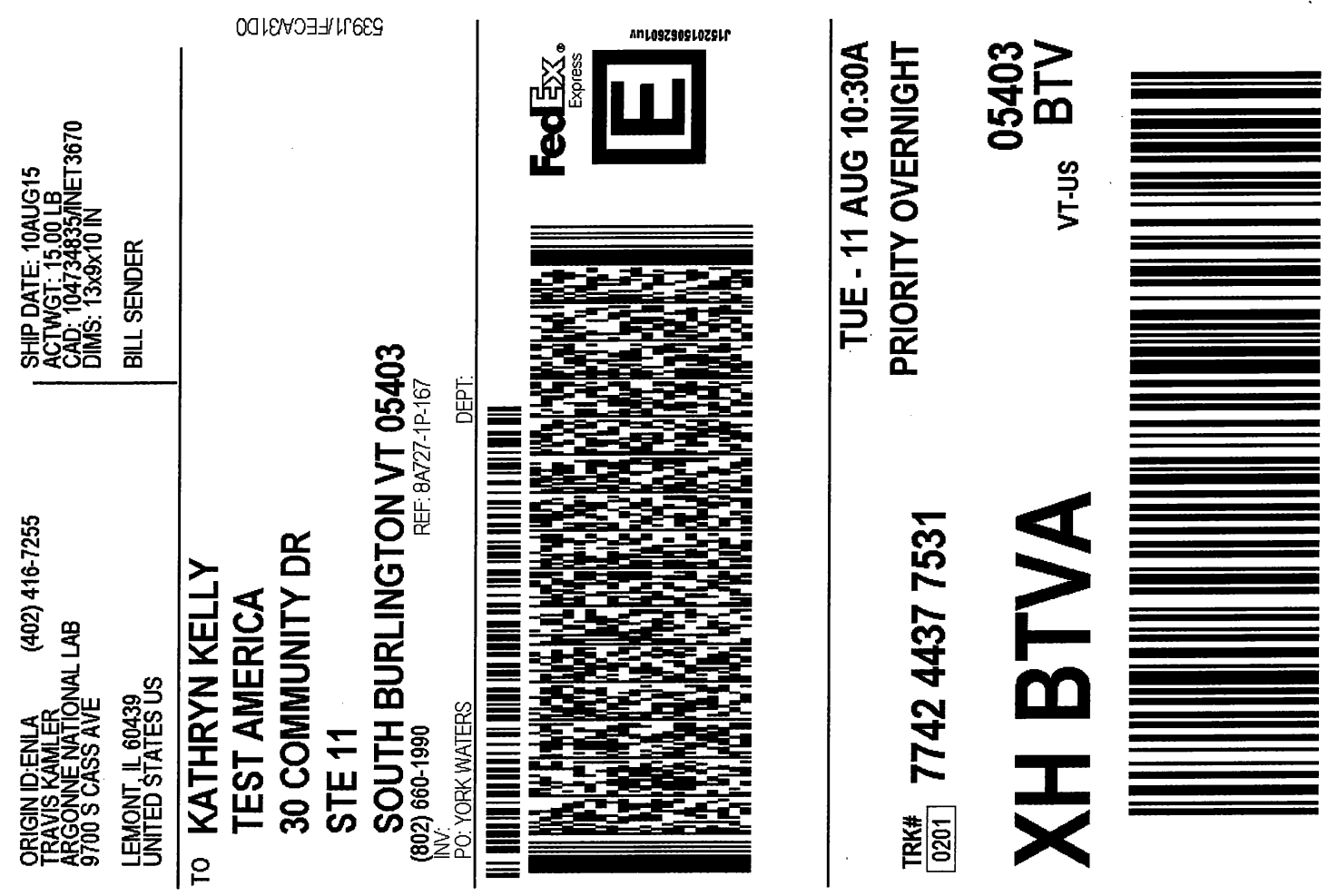




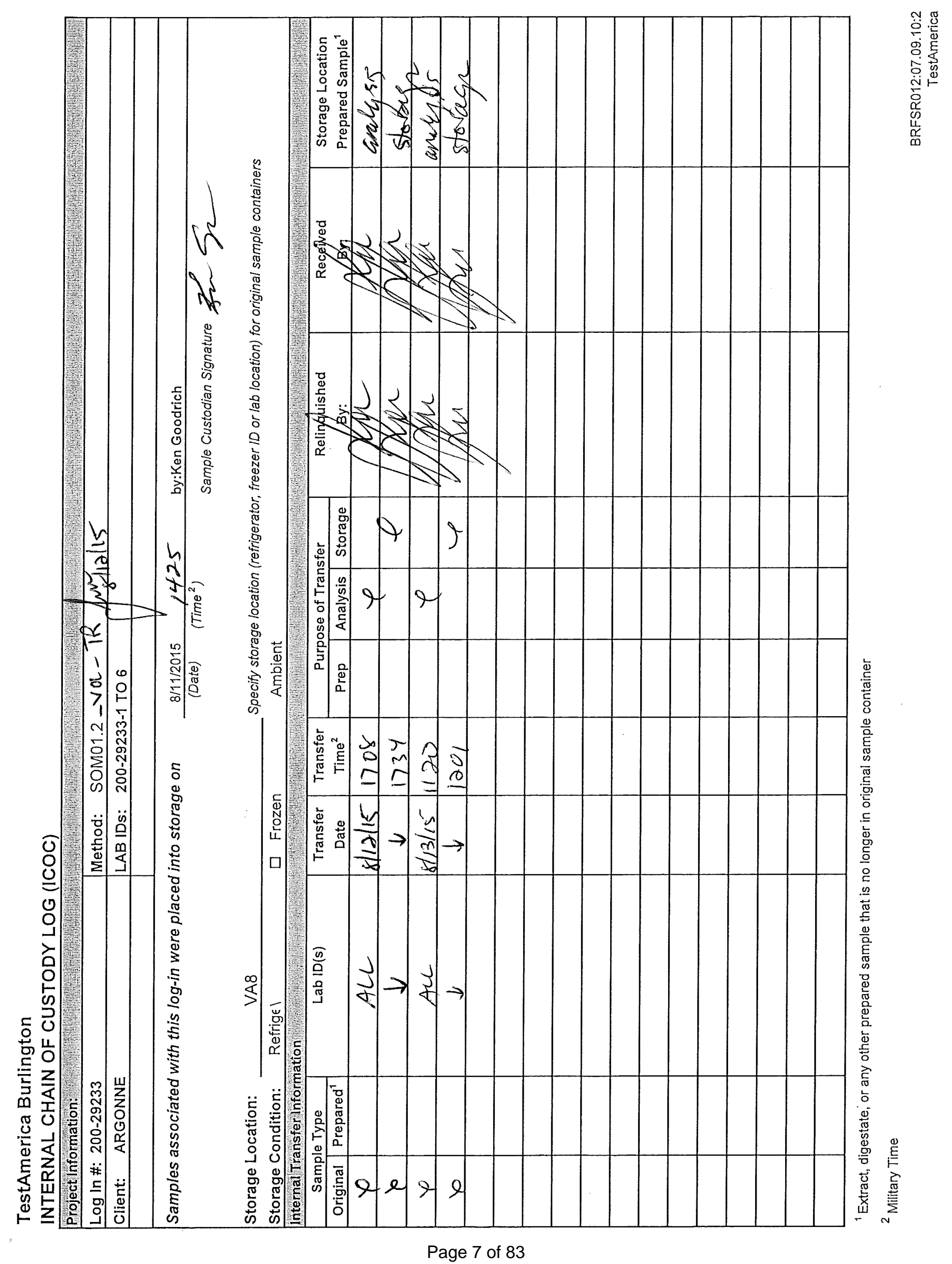




\section{Shipping and Receiving Documents}




\section{Login Sample Receipt Checklist}

Client: Argonne National Laboratory

Job Number: 200-29233-1

SDG Number: 29233

Login Number: 29233

List Source: TestAmerica Burlington

List Number: 1

Creator: Goodrich, Kenneth L

Question

Radioactivity wasn't checked or is $</=$ background as measured by a survey True meter.

The cooler's custody seal, if present, is intact.

Sample custody seals, if present, are intact.

The cooler or samples do not appear to have been compromised or tampered with.

Samples were received on ice.

Cooler Temperature is acceptable.

Cooler Temperature is recorded.

COC is present.

COC is filled out in ink and legible.

$\mathrm{COC}$ is filled out with all pertinent information.

Is the Field Sampler's name present on COC?

There are no discrepancies between the containers received and the COC.

Samples are received within Holding Time.

Sample containers have legible labels.

Containers are not broken or leaking.

Sample collection date/times are provided.

Appropriate sample containers are used.

Sample bottles are completely filled.

Sample Preservation Verified.

There is sufficient vol. for all requested analyses, incl. any requested MS/MSDs

Containers requiring zero headspace have no headspace or bubble is $<6 \mathrm{~mm}$ (1/4").

Multiphasic samples are not present.

Samples do not require splitting or compositing.

Residual Chlorine Checked.

Answer Comment

True

Lab does not accept radioactive samples.

Seal present with no number.

True

True

True

True

True

True

True

True

True

True

True

True

True

True

True

N/A

True

True

True

True

True

N/A 
SDG No.: 29233

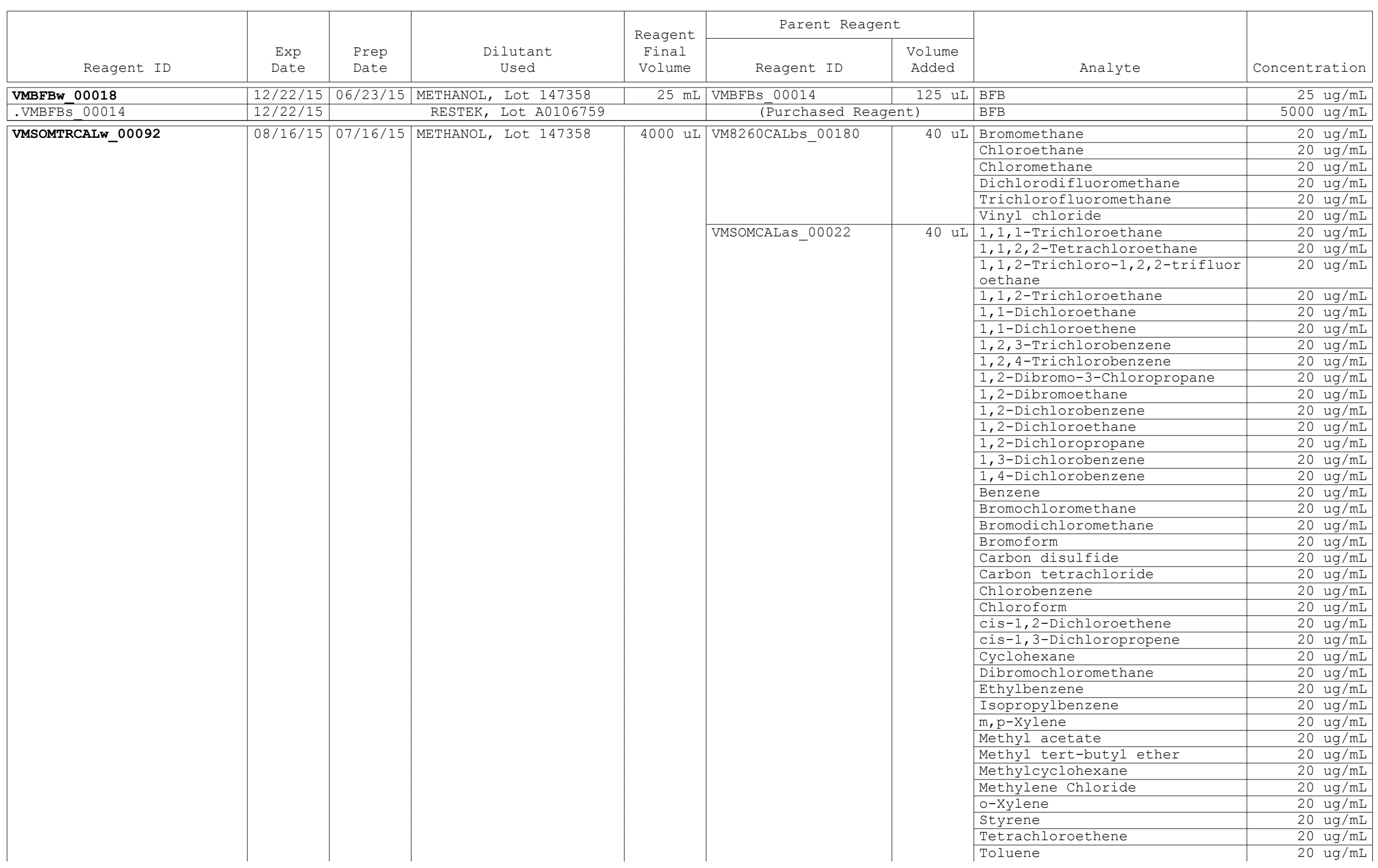


SDG No.: 29233

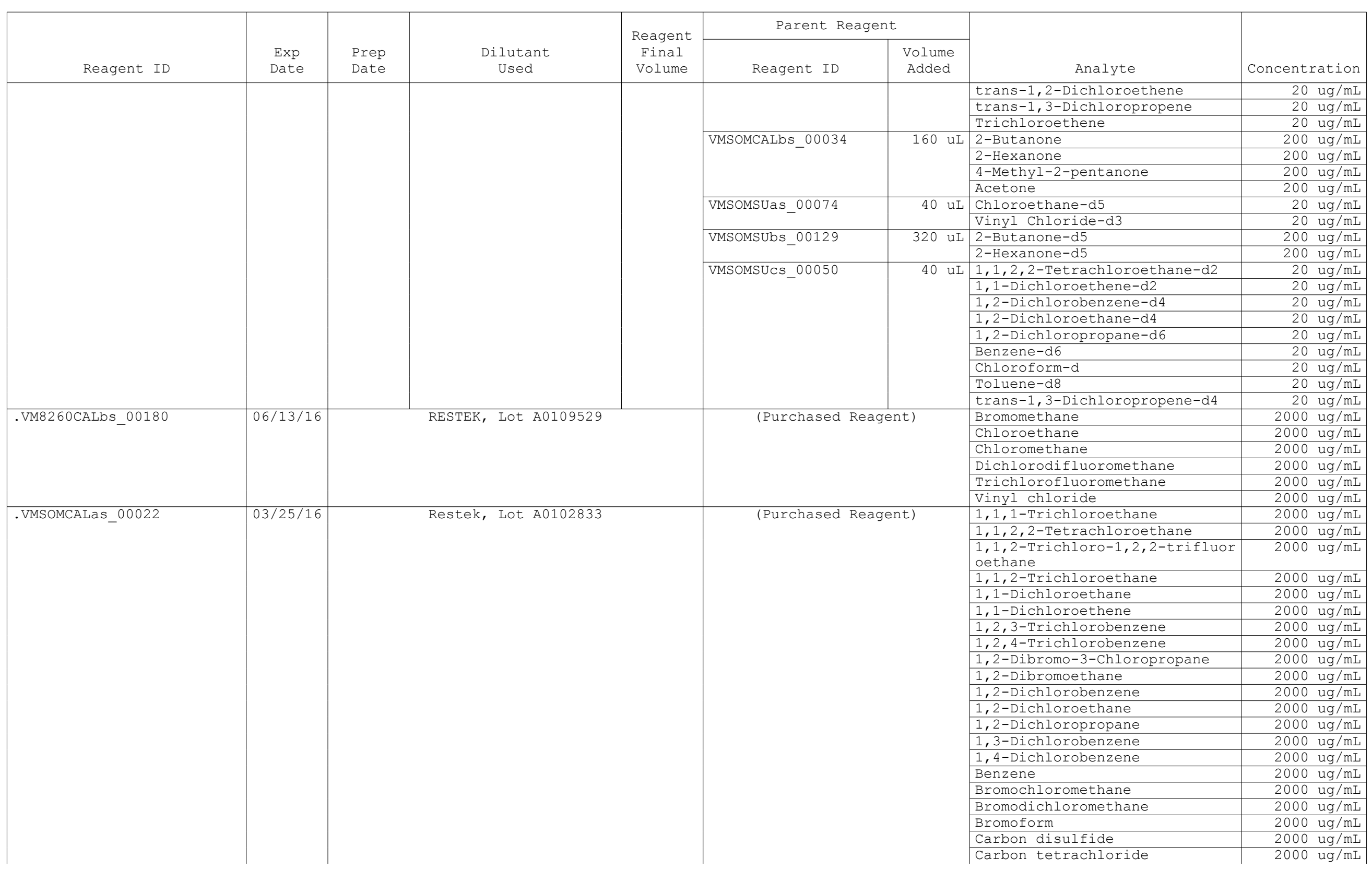


SDG No.: 29233

\begin{tabular}{|c|c|c|c|c|c|c|c|c|c|}
\hline \multirow[b]{2}{*}{ Reagent ID } & \multirow[b]{2}{*}{$\begin{array}{l}\text { Exp } \\
\text { Date }\end{array}$} & \multirow[b]{2}{*}{$\begin{array}{l}\text { Prep } \\
\text { Date }\end{array}$} & \multirow[b]{2}{*}{$\begin{array}{c}\text { Dilutant } \\
\text { Used }\end{array}$} & \multirow{2}{*}{$\begin{array}{c}\text { Reagent } \\
\text { Final } \\
\text { Volume }\end{array}$} & \multicolumn{2}{|c|}{ Parent Reagent } & \multirow[b]{2}{*}{ Analyte } & \multirow{2}{*}{\multicolumn{2}{|c|}{ Concentration }} \\
\hline & & & & & Reagent ID & $\begin{array}{l}\text { Volume } \\
\text { Added }\end{array}$ & & & \\
\hline & & & & & & & Chlorobenzene & 2000 & $\mathrm{ug} / \mathrm{mL}$ \\
\hline & & & & & & & Chloroform & 2000 & $\mathrm{ug} / \mathrm{mL}$ \\
\hline & & & & & & & cis-1,2-Dichloroethene & 2000 & $\mathrm{ug} / \mathrm{mL}$ \\
\hline & & & & & & & cis-1,3-Dichloropropene & 2000 & $\mathrm{ug} / \mathrm{mL}$ \\
\hline & & & & & & & Cyclohexane & 2000 & $\mathrm{ug} / \mathrm{mL}$ \\
\hline & & & & & & & Dibromochloromethane & 2000 & $\mathrm{ug} / \mathrm{mL}$ \\
\hline & & & & & & & Ethylbenzene & 2000 & $\mathrm{ug} / \mathrm{mL}$ \\
\hline & & & & & & & Isopropylbenzene & 2000 & $\mathrm{ug} / \mathrm{mL}$ \\
\hline & & & & & & & m,p-Xylene & 2000 & $\mathrm{ug} / \mathrm{mL}$ \\
\hline & & & & & & & Methyl acetate & 2000 & $\mathrm{ug} / \mathrm{mL}$ \\
\hline & & & & & & & Methyl tert-butyl ether & 2000 & $\mathrm{ug} / \mathrm{mL}$ \\
\hline & & & & & & & Methylcyclohexane & 2000 & $\mathrm{ug} / \mathrm{mL}$ \\
\hline & & & & & & & Methylene Chloride & 2000 & $\mathrm{ug} / \mathrm{mL}$ \\
\hline & & & & & & & o-Xylene & 2000 & $\mathrm{ug} / \mathrm{mL}$ \\
\hline & & & & & & & Styrene & 2000 & $\mathrm{ug} / \mathrm{mL}$ \\
\hline & & & & & & & Tetrachloroethene & 2000 & $\mathrm{ug} / \mathrm{mL}$ \\
\hline & & & & & & & Toluene & 2000 & $\mathrm{ug} / \mathrm{mL}$ \\
\hline & & & & & & & trans-1,2-Dichloroethene & 2000 & $\mathrm{ug} / \mathrm{mL}$ \\
\hline & & & & & & & trans-1,3-Dichloropropene & 2000 & $\mathrm{ug} / \mathrm{mL}$ \\
\hline & & & & & & & Trichloroethene & 2000 & $\mathrm{ug} / \mathrm{mL}$ \\
\hline \multirow[t]{4}{*}{.VMSOMCALbs_00034 } & \multirow[t]{4}{*}{$07 / 16 / 16$} & \multirow{4}{*}{\multicolumn{2}{|c|}{ Restek, Lot A0104748 }} & & \multirow{2}{*}{\multicolumn{2}{|c|}{ (Purchased Reagent) }} & 2-Butanone & 5000 & $\mathrm{ug} / \mathrm{mL}$ \\
\hline & & & & & & & 2-Hexanone & 5000 & $\mathrm{ug} / \mathrm{mL}$ \\
\hline & & & & & & & 4-Methyl-2-pentanone & 5000 & $\mathrm{ug} / \mathrm{mL}$ \\
\hline & & & & & & & Acetone & 5000 & $\mathrm{ug} / \mathrm{mL}$ \\
\hline \multirow[t]{2}{*}{.VMSOMSUas_00074 } & \multirow[t]{2}{*}{$04 / 29 / 16$} & \multirow{2}{*}{\multicolumn{2}{|c|}{ Absolute, Lot 072214}} & & \multirow{2}{*}{\multicolumn{2}{|c|}{ (Purchased Reagent) }} & Chloroethane-d5 & 2000 & $\mathrm{ug} / \mathrm{mL}$ \\
\hline & & & & & & & Vinyl Chloride-d3 & 2000 & $\mathrm{ug} / \mathrm{mL}$ \\
\hline \multirow[t]{2}{*}{.VMSOMSUbs_00129 } & \multirow[t]{2}{*}{$07 / 16 / 16$} & & Absolute, Lot 080414 & & (Purchased $\mathrm{F}$ & nt) & 2-Butanone-d5 & 2500 & $\mathrm{ug} / \mathrm{mL}$ \\
\hline & & & & & & & 2-Hexanone-d5 & 2500 & $\mathrm{ug} / \mathrm{mL}$ \\
\hline .VMSOMSUCS 00050 & $04 / 27 / 16$ & & Absolute, Lot 011614 & & (Purchased $\mathrm{F}$ & nt) & $1,1,2,2$-Tetrachloroethane-d2 & 2000 & $\mathrm{ug} / \mathrm{mL}$ \\
\hline & & & & & & & 1,1-Dichloroethene-d2 & 2000 & $\mathrm{ug} / \mathrm{mL}$ \\
\hline & & & & & & & 1,2-Dichlorobenzene-d4 & 2000 & $\mathrm{ug} / \mathrm{mL}$ \\
\hline & & & & & & & 1,2-Dichloroethane-d4 & 2000 & $\mathrm{ug} / \mathrm{mL}$ \\
\hline & & & & & & & 1,2-Dichloropropane-d6 & 2000 & $\mathrm{ug} / \mathrm{mL}$ \\
\hline & & & & & & & Benzene-d6 & 2000 & $\mathrm{ug} / \mathrm{mL}$ \\
\hline & & & & & & & Chloroform-d & 2000 & $\mathrm{ug} / \mathrm{mL}$ \\
\hline & & & & & & & Toluene-d8 & 2000 & $\mathrm{ug} / \mathrm{mL}$ \\
\hline & & & & & & & trans-1,3-Dichloropropene-d4 & 2000 & $\mathrm{ug} / \mathrm{mL}$ \\
\hline VMSOMTRISw_00102 & $08 / 17 / 15$ & $07 / 17 / 15$ & METHANOL, LOt 147538 & 4400 uL & VMSOMISS_00025 & $35.2 \mathrm{uL}$ & 1,4-Dichlorobenzene-d4 & 20 & $\mathrm{ug} / \mathrm{mL}$ \\
\hline & & & & & & & 1,4-Difluorobenzene & 20 & $\mathrm{ug} / \mathrm{mL}$ \\
\hline & & & & & & & Chlorobenzene-d5 & 20 & $\mathrm{ug} / \mathrm{mL}$ \\
\hline .VMSOMISs_00025 & $06 / 19 / 16$ & & RESTEK, Lot A099377 & & (Purchased $\mathrm{F}$ & nt) & 1,4-Dichlorobenzene-d4 & 2500 & $\mathrm{ug} / \mathrm{mL}$ \\
\hline & & & & & & & 1,4-Difluorobenzene & 2500 & $\mathrm{ug} / \mathrm{mL}$ \\
\hline & & & & & & & Chlorobenzene-d5 & 2500 & $\mathrm{ug} / \mathrm{mL}$ \\
\hline VMSOMTRSUw_00097 & $08 / 16 / 15$ & $07 / 16 / 15$ & METHANOL, Lot 147358 & 4400 uL & VMSOMSUas_00074 & $44 \mathrm{uL}$ & Chloroethane-d5 & 20 & $\mathrm{ug} / \mathrm{mL}$ \\
\hline & & & & & & & Vinyl Chloride-d3 & 20 & $\mathrm{ug} / \mathrm{mL}$ \\
\hline & & & & & VMSOMSUbs_00129 & $352 \mathrm{uL}$ & 2-Butanone-d5 & 200 & $\mathrm{ug} / \mathrm{mL}$ \\
\hline & & & & & & & 2-Hexanone-d5 & 200 & $\mathrm{ug} / \mathrm{mL}$ \\
\hline
\end{tabular}


SDG No.: 29233

\begin{tabular}{|c|c|c|c|c|c|c|c|c|}
\hline \multirow[b]{2}{*}{ Reagent ID } & \multirow[b]{2}{*}{$\begin{array}{l}\text { Exp } \\
\text { Date }\end{array}$} & \multirow[b]{2}{*}{$\begin{array}{l}\text { Prep } \\
\text { Date }\end{array}$} & \multirow[b]{2}{*}{$\begin{array}{c}\text { Dilutant } \\
\text { Used }\end{array}$} & \multirow{2}{*}{$\begin{array}{l}\text { Reagent } \\
\text { Final } \\
\text { Volume }\end{array}$} & \multicolumn{2}{|c|}{ Parent Reagent } & \multirow[b]{2}{*}{ Analyte } & \multirow[b]{2}{*}{ Concentration } \\
\hline & & & & & \multirow{10}{*}{$\begin{array}{r}\text { Reagent ID } \\
\text { VMSOMSUCS_00050 }\end{array}$} & $\begin{array}{l}\text { Volume } \\
\text { Added }\end{array}$ & & \\
\hline & & & & & & $44 \mathrm{uL}$ & $1,1,2,2$-Tetrachloroethane-d2 & $20 \mathrm{ug} / \mathrm{mI}$ \\
\hline & & & & & & & 1,1-Dichloroethene-d2 & $20 \mathrm{ug} / \mathrm{mI}$ \\
\hline & & & & & & & 1,2-Dichlorobenzene-d4 & $20 \mathrm{ug} / \mathrm{mI}$ \\
\hline & & & & & & & 1,2-Dichloroethane-d4 & $20 \mathrm{ug} / \mathrm{mI}$ \\
\hline & & & & & & & 1,2-Dichloropropane-d6 & $20 \mathrm{ug} / \mathrm{mI}$ \\
\hline & & & & & & & Benzene-d6 & $20 \mathrm{ug} / \mathrm{mI}$ \\
\hline & & & & & & & Chloroform-d & $20 \mathrm{ug} / \mathrm{mI}$ \\
\hline & & & & & & & Toluene-d8 & $20 \mathrm{ug} / \mathrm{mI}$ \\
\hline & & & & & & & trans-1,3-Dichloropropene-d4 & $20 \mathrm{ug} / \mathrm{mI}$ \\
\hline \multirow[t]{2}{*}{.VMSOMSUas_00074 } & \multirow[t]{2}{*}{$04 / 29 / 16$} & \multirow{2}{*}{\multicolumn{3}{|c|}{ Absolute, Lot 072214}} & \multirow{2}{*}{\multicolumn{2}{|c|}{ (Purchased Reagent) }} & Chloroethane-d5 & $2000 \mathrm{ug} / \mathrm{mI}$ \\
\hline & & & & & & & Vinyl Chloride-d3 & $2000 \mathrm{ug} / \mathrm{mI}$ \\
\hline \multirow[t]{2}{*}{.VMSOMSUbs_00129 } & \multirow[t]{2}{*}{$07 / 16 / 16$} & \multirow{2}{*}{\multicolumn{3}{|c|}{ Absolute, Lot 080414}} & \multirow{2}{*}{\multicolumn{2}{|c|}{ (Purchased Reagent) }} & 2-Butanone-d5 & $2500 \mathrm{ug} / \mathrm{mI}$ \\
\hline & & & & & & & 2-Hexanone-d5 & $2500 \mathrm{ug} / \mathrm{mI}$ \\
\hline \multirow[t]{9}{*}{.VMSOMSUCS_00050 } & \multirow[t]{9}{*}{$04 / 27 / 16$} & \multirow{9}{*}{\multicolumn{2}{|c|}{ Absolute, Lot 011614}} & & \multirow{9}{*}{\multicolumn{2}{|c|}{ (Purchased Reagent) }} & $1,1,2,2$-Tetrachloroethane-d2 & $2000 \mathrm{ug} / \mathrm{mI}$ \\
\hline & & & & & & & 1,1-Dichloroethene-d2 & $2000 \mathrm{ug} / \mathrm{mI}$ \\
\hline & & & & & & & 1,2-Dichlorobenzene-d4 & $2000 \mathrm{ug} / \mathrm{mI}$ \\
\hline & & & & & & & 1,2-Dichloroethane-d4 & $2000 \mathrm{ug} / \mathrm{mI}$ \\
\hline & & & & & & & 1,2-Dichloropropane-d6 & $2000 \mathrm{ug} / \mathrm{mI}$ \\
\hline & & & & & & & Benzene-d6 & $2000 \mathrm{ug} / \mathrm{mI}$ \\
\hline & & & & & & & Chloroform-d & $2000 \mathrm{ug} / \mathrm{mI}$ \\
\hline & & & & & & & Toluene-d8 & $2000 \mathrm{ug} / \mathrm{mI}$ \\
\hline & & & & & & & trans-1,3-Dichloropropene-d4 & $2000 \mathrm{ug} / \mathrm{mI}$ \\
\hline
\end{tabular}


Lab Name: TestAmerica Burlington

Job No.: 200-29233-1

SDG No.: 29233

\begin{tabular}{|c|l|l|c|c|}
\hline $\begin{array}{c}\text { Reagent } \\
\text { Container }\end{array}$ & \multicolumn{1}{|c|}{$\begin{array}{c}\text { Reagent } \\
\text { ID }\end{array}$} & \multicolumn{1}{c|}{$\begin{array}{c}\text { Reagent } \\
\text { Description }\end{array}$} & $\begin{array}{c}\text { Preparation } \\
\text { Date }\end{array}$ & $\begin{array}{c}\text { Expiration } \\
\text { Date }\end{array}$ \\
\hline 807558 & VMBEBw_00018 & BFB TUNE 25 PPM & $06 / 23 / 2015$ & $12 / 22 / 2015$ \\
\hline 816537 & VMSOMTRCALw_00092 & SOM TR CAL 20 PPM & $07 / 16 / 2015$ & $08 / 16 / 2015$ \\
\hline 816538 & VMSOMTRSUw_00097 & SOM TR DMC 20 PPM & $07 / 16 / 2015$ & $08 / 16 / 2015$ \\
\hline 816658 & VMSOMTRCALw_00092 & SOM TR CAL 20 PPM & $07 / 16 / 2015$ & $08 / 16 / 2015$ \\
\hline 817121 & VMSOMTRISw_00102 & SOM TR ISTD 20 PPM & $07 / 17 / 2015$ & $08 / 17 / 2015$ \\
\hline
\end{tabular}




\section{METHODOLOGY SUMMARY}

Laboratory: TestAmerica Laboratories

Location: South Burlington, Vermont
Project No:

SDG No: 29233

VOA

Volatile Organics Trace - USEPA CLP SOM01.2 


\title{
CASE NARRATIVE
}

\section{Client: Argonne National Laboratory}

\author{
Project: York (200-29233)
}

Report Number: 200-29233-1

With the exceptions noted as flags or footnotes, standard analytical protocols were followed in the analysis of the samples and no problems were encountered or anomalies observed. In addition all laboratory quality control samples were within established control limits, with any exceptions noted below. Each sample was analyzed to achieve the lowest possible reporting limit within the constraints of the method. In some cases, due to interference or analytes present at high concentrations, samples were diluted. For diluted samples, the reporting limits are adjusted relative to the dilution required.

Calculations are performed before rounding to avoid round-off errors in calculated results.

All holding times were met and proper preservation noted for the methods performed on these samples, unless otherwise detailed in the individual sections below.

\section{RECEIPT}

The samples were received on 08/11/2015; the samples arrived in good condition, properly preserved and on ice. The temperature of the cooler at receipt was $5.0^{\circ} \mathrm{C}$.

\section{VOLATILE ORGANIC COMPOUNDS - TRACE}

Samples YKKNIERIEM-W-36885, YKMAKOVICKA-W-36888, YKYORKCOUNTYSHOP-W-37917, YKSB62-W-37924, YKQCTB-W-37925 and VHBLK01 were analyzed for Volatile Organic Compounds - Trace in accordance with EPA SOW SOM01.2. The samples were analyzed on $08 / 12 / 2015$ and $08 / 13 / 2015$.

Samples YKYORKCOUNTYSHOP-W-37917 (200-29233-3) and YKQCTB-W-37925 (200-29233-5) were received with less than 2 days remaining on the holding time or less than one shift ( 8 hours) remaining on a test with a holding time of 48 hours or less. As such, the laboratory had insufficient time remaining to perform the analysis within holding time.

Reanalysis of sample YKYORKCOUNTYSHOP-W-37917 was performed outside of the analytical holding time due to the sample requiring a dilution. The more concentrated analysis was acquired within analytical holding time.

Several analytes were detected in method blank MB 200-92663/5 at levels that were above the method detection limit but below the reporting limit. The values should be considered estimates, and have been flagged. If the associated sample reported a result above the MDL and/or RL, the result has been flagged. 1,2,3-Trichlorobenzene, 1,2,4-Trichlorobenzene and Carbon disulfide were detected in method blank MB 200-92691/5 at levels that were above the method detection limit but below the reporting limit. The values should be considered estimates, and have been flagged. If the associated sample reported a result above the MDL and/or RL, the result has been flagged. Refer to the QC report for details.

13 surrogates are used for this analysis. The laboratory's SOP allows 3 of these surrogates to be outside acceptance criteria without performing re-extraction/re-analysis. The following sample contained an allowable number of surrogate compounds outside limits: YKKNIERIEM-W-36885 (200-29233-1). These results have been reported and qualified.

Samples YKYORKCOUNTYSHOP-W-37917[4.67X] and YKSB62-W-37924[1.83X] required dilution prior to analysis. The reporting limits have been adjusted accordingly.

No additional analytical or quality issues were noted, other than those described above or in the Definitions/Glossary page. 
Lab Name: TestAmerica Burlington

Job No.: 200-29233-1

SDG No.: 29233

Instrument ID: CHD.i

Analysis Batch Number: 92375

Lab Sample ID: IC 200-92375/3

Client Sample ID:

Date Analyzed: 08/06/15 10:11

Lab File ID: 15136_003.D

GC Column: DB-624

ID : $0.2(\mathrm{~mm})$

\begin{tabular}{|c|c|c|c|c|}
\hline \multirow[t]{2}{*}{ COMPOUND NAME } & \multirow{2}{*}{$\begin{array}{c}\text { RETENTION } \\
\text { TIME }\end{array}$} & \multicolumn{3}{|c|}{ MANUAL INTEGRATION } \\
\hline & & REASON & ANALYST & DATE \\
\hline 1,2-Dichloropropane-d6 & 6.42 & Split Peak & wilburj & $08 / 14 / 1515: 00$ \\
\hline 1,2-Dibromo-3-Chloropropane & 13.43 & Assign Peak & wilburj & $08 / 06 / 15 \quad 14: 13$ \\
\hline
\end{tabular}

Lab Sample ID: IC 200-92375/4 Client Sample ID:

Date Analyzed: 08/06/15 10:35

Lab File ID: 15136_004.D

GC Column: DB-624

ID $: 0.2(\mathrm{~mm})$

\begin{tabular}{|c|c|c|c|c|}
\hline \multirow[t]{2}{*}{ COMPOUND NAME } & \multirow{2}{*}{$\begin{array}{c}\text { RETENTION } \\
\text { TIME }\end{array}$} & \multicolumn{3}{|c|}{ MANUAL INTEGRATION } \\
\hline & & REASON & ANALYST & DATE \\
\hline Dichlorodifluoromethane & 1.47 & Baseline & wilburj & $08 / 06 / 15 \quad 14: 14$ \\
\hline 1,2-Dibromo-3-Chloropropane & 13.43 & Baseline & wilburj & $08 / 06 / 1514: 14$ \\
\hline
\end{tabular}

Lab Sample ID: ICIS 200-92375/5

Client Sample ID:

Date Analyzed: 08/06/15 11:00

Lab File ID: 15136 005.D

GC Column: DB-624

ID $: 0.2(\mathrm{~mm})$

\begin{tabular}{|c|c|c|c|}
\hline COMPOUND NAME & RETENTION & \multicolumn{2}{|c|}{ MANUAL INTEGRATION } \\
\cline { 3 - 4 } & TIME & REASON & ANALYST \\
\hline Dichlorodifluoromethane & 1.48 & Baseline & DATE \\
\hline
\end{tabular}

Lab Sample ID: IC 200-92375/6

Client Sample ID:

Date Analyzed: 08/06/15 11:24

Lab File ID: 15136_006.D

GC Column: DB-624

ID $: 0.2(\mathrm{~mm})$

\begin{tabular}{|c|c|c|c|c|}
\hline \multirow[t]{2}{*}{ COMPOUND NAME } & \multirow{2}{*}{$\begin{array}{c}\text { RETENTION } \\
\text { TIME }\end{array}$} & \multicolumn{3}{|c|}{ MANUAL INTEGRATION } \\
\hline & & REASON & ANALYST & DATE \\
\hline Dichlorodifluoromethane & 1.48 & Baseline & wilburj & $08 / 06 / 15 \quad 14: 15$ \\
\hline
\end{tabular}

Lab Sample ID: IC 200-92375/7

Client Sample ID:

Date Analyzed: 08/06/15 11:49

Lab File ID: 15136_007.D

GC Column: DB-624

ID $: 0.2(\mathrm{~mm})$

\begin{tabular}{|c|c|c|c|c|}
\hline \multirow[t]{2}{*}{ COMPOUND NAME } & RETENTION & \multicolumn{3}{|c|}{ MANUAL INTEGRATION } \\
\hline & TIME & REASON & ANALYST & DATE \\
\hline 1,2-Dichloropropane-d6 & 6.42 & Split Peak & wilburj & $08 / 14 / 15 \quad 15: 19$ \\
\hline
\end{tabular}

SOM01.2/VOA_Tr 
Lab Name: TestAmerica Burlington

Job No.: 200-29233-1

SDG No.: 29233

Instrument ID: CHD.i

Analysis Batch Number: 92663

Lab Sample ID: CCVIS 200-92663/3

Client Sample ID:

Date Analyzed: 08/12/15 17:53

Lab File ID: 15254 003.D

GC Column: DB-624

ID $: 0.2(\mathrm{~mm})$

\begin{tabular}{|c|c|c|c|c|}
\hline \multirow[t]{2}{*}{ COMPOUND NAME } & \multirow{2}{*}{$\begin{array}{c}\text { RETENTION } \\
\text { TIME }\end{array}$} & \multicolumn{3}{|c|}{ MANUAL INTEGRATION } \\
\hline & & REASON & ANALYST & DATE \\
\hline 1,2-Dichloropropane-d6 & 6.41 & Split Peak & wilburj & $08 / 21 / 1515: 09$ \\
\hline
\end{tabular}

Lab Sample ID: CCVC 200-92663/16

Client Sample ID:

Date Analyzed: 08/12/15 23:16

Lab File ID: 15254 016.D

GC Column: DB-624

ID $: 0.2(\mathrm{~mm})$

\begin{tabular}{|c|c|c|c|c|}
\hline \multirow[t]{2}{*}{ COMPOUND NAME } & \multirow{2}{*}{$\begin{array}{c}\text { RETENTION } \\
\text { TIME }\end{array}$} & \multicolumn{3}{|c|}{ MANUAL INTEGRATION } \\
\hline & & REASON & ANALYST & DATE \\
\hline Dichlorodifluoromethane & 1.47 & Baseline & wilburj & $08 / 21 / 15 \quad 16: 07$ \\
\hline 1,2-Dibromo-3-Chloropropane & 13.43 & Assign Peak & wilburj & $08 / 21 / 1516: 07$ \\
\hline
\end{tabular}


Lab Name: TestAmerica Burlington

SDG No.: 29233

Instrument ID: CHD.i

Lab Sample ID: CCVC 200-92691/23

Date Analyzed: 08/13/15 20:20

\begin{tabular}{|c|c|c|c|c|}
\hline \multirow[t]{2}{*}{ COMPOUND NAME } & \multirow{2}{*}{$\begin{array}{c}\text { RETENTION } \\
\text { TIME }\end{array}$} & \multicolumn{3}{|c|}{ MANUAL INTEGRATION } \\
\hline & & REASON & ANALYST & DATE \\
\hline Dichlorodifluoromethane & 1.52 & Baseline & archern & $08 / 25 / 15 \quad 11: 47$ \\
\hline 1,2-Dibromo-3-Chloropropane & 13.43 & Baseline & archern & $08 / 25 / 1511: 47$ \\
\hline
\end{tabular}

Job No.: 200-29233-1

Analysis Batch Number: 92691

Client Sample ID:

ab File ID: $15265023 . \mathrm{D}$

Column: DB-624

ID $: 0.2(\mathrm{~mm})$ 


\section{DATA REPORTING QUALIFIERS}

Client: Argonne National Laboratory

Job Number: 200-29233-1

Sdg Number: 29233

\section{Lab Section}

Qualifier

Description

\section{GC/MS VOA}

U

E

J

$\mathrm{J}$

D

$\mathrm{X}$

*

B

N
Analyzed for but not detected.

Compound concentration exceeds the upper level of the calibration range of the instrument for that specific analysis.

Indicates an Estimated Value for TICs

Indicates an estimated value.

Sample was analyzed at a higher dilution factor.

See case narrative notes for explanation of the ' $X$ ' flag

Surrogate is outside acceptance limits.

The analyte was found in an associated blank, as well as in the sample.

This flag indicates the presumptive evidence of a compound. 
Lab Name: TESTAMERICA BURLINGTON

Lab Code: STLV Case No.: YORK Mod. Ref No.: Level: (TRACE or LOW) TRACE
Contract: $8 \mathrm{E}-00302$

SDG No.: 29233

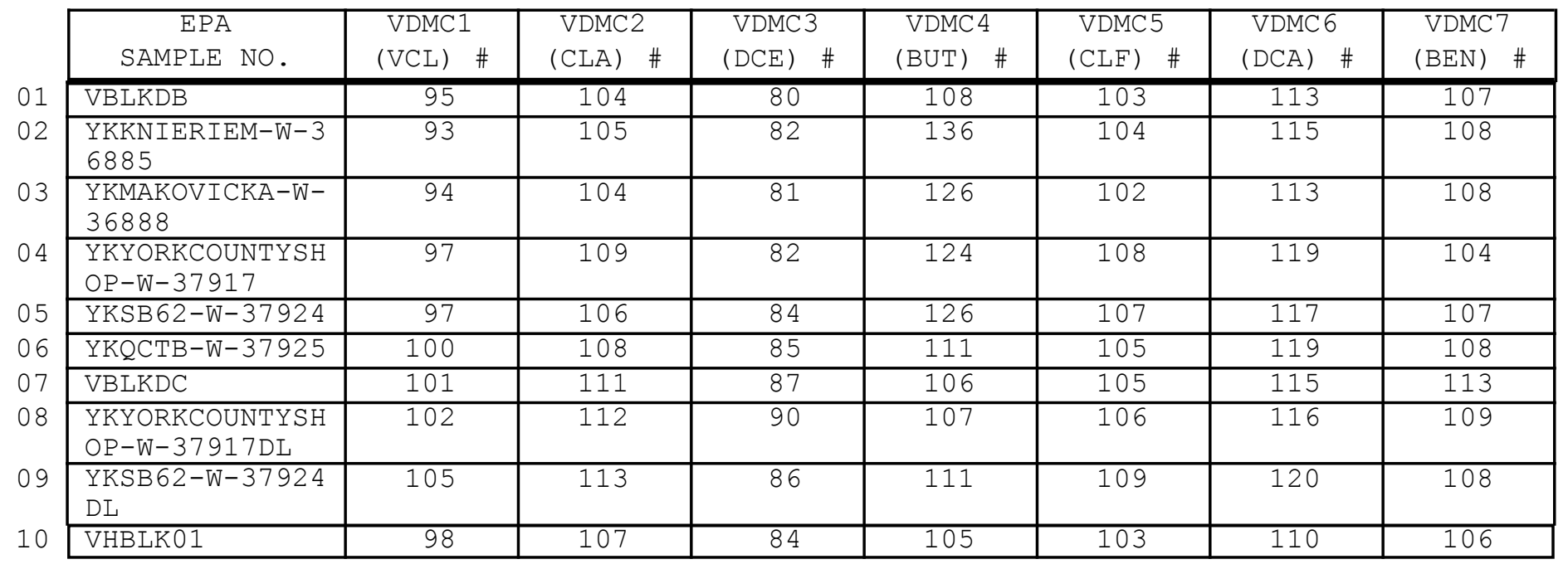

\footnotetext{
VDMC1 (VCL) = Vinyl Chloride-d3

VDMC2 $(\mathrm{CLA})=$ Chloroethane-d5

VDMC3 $(\mathrm{DCE})=1,1-\mathrm{Dichloroethene-d2}$

VDMC4 $(B U T)=2-$ Butanone-d5

VDMC5 $(\mathrm{CLF})=$ Chloroform-d

VDMC $6 \quad(\mathrm{DCA})=1,2-\mathrm{Dich}$ loroethane-d4

$\operatorname{VDMC} 7(\mathrm{BEN})=$ Benzene-d6
}

$$
\begin{gathered}
\frac{Q C \text { LIMITS }}{(65-131)} \\
(71-131) \\
(55-104) \\
(49-155) \\
(78-121) \\
(78-129) \\
(77-124)
\end{gathered}
$$

\# Column to be used to flag recovery values

* Values outside of contract required QC limits

Page 1 of 1 
Lab Name: TESTAMERICA BURLINGTON

Lab Code: STLV Case No.: YORK Mod. Ref No.:
Contract: $8 \mathrm{E}-00302$

SDG No.: 29233

Level: (TRACE or LOW) TRACE

\begin{tabular}{|c|c|c|c|c|c|c|c|c|}
\hline $\begin{array}{c}\text { EPA } \\
\text { SAMPLE NO. }\end{array}$ & $\begin{array}{l}\text { VDMC 8 } \\
(\mathrm{DPA})\end{array}$ & $\begin{array}{l}\text { VDMC9 } \\
(\mathrm{TOL})\end{array}$ & $\begin{array}{l}\text { VDMC10 } \\
(\mathrm{TDP}) \quad \#\end{array}$ & $\begin{array}{l}\text { VDMC11 } \\
(\operatorname{HEX}) \quad \#\end{array}$ & $\begin{array}{l}\text { VDMC12 } \\
(\mathrm{TCA}) \quad \#\end{array}$ & $\begin{array}{l}\text { VDMC13 } \\
\text { (DCZ) } \#\end{array}$ & OTHER & $\begin{array}{l}\text { TOT } \\
\text { OUT }\end{array}$ \\
\hline VBLKDB & 107 & 105 & 110 & 113 & 109 & 104 & & 0 \\
\hline $\begin{array}{l}\text { YKKNIERIEM-W-3 } \\
6885\end{array}$ & 110 & 107 & 114 & 140 * & 111 & 108 & & 1 \\
\hline $\begin{array}{l}\text { YKMAKOVICKA-W- } \\
36888\end{array}$ & 109 & 107 & 110 & 130 & 105 & 105 & & 0 \\
\hline $\begin{array}{l}\text { YKYORKCOUNTYSH } \\
\text { OP-W-37917 }\end{array}$ & 111 & 107 & 115 & 125 & 113 & 110 & & 0 \\
\hline YKSB 62-W-37924 & 111 & 106 & 111 & 125 & 111 & 107 & & 0 \\
\hline YKQCTB-W-37925 & 111 & 107 & 105 & 117 & 113 & 108 & & 0 \\
\hline VBLKDC & 113 & 112 & 105 & 112 & 109 & 109 & & 0 \\
\hline $\begin{array}{l}\text { YKYORKCOUNTYSH } \\
\text { OP-W-37917DL }\end{array}$ & 110 & 108 & 105 & 111 & 109 & 107 & & 0 \\
\hline $\begin{array}{l}\text { YKSB 62-W-37924 } \\
\text { DL }\end{array}$ & 113 & 106 & 108 & 114 & 114 & 109 & & 0 \\
\hline VHBLK01 & 107 & 106 & 99 & 107 & 106 & 104 & & 0 \\
\hline
\end{tabular}

VDMC8 $(\mathrm{DPA})=1,2-\mathrm{Dichloropropane}-\mathrm{d} 6$

VDMC 9 (TOL) = Toluene-d8

VDMC10 $(\mathrm{TDP})=$ trans $-1,3-\mathrm{Dichloropropene-d4}$

VDMC11 $(\mathrm{HEX})=2-$ Hexanone-d5

VDMC12 $(\mathrm{TCA})=1,1,2,2$-Tetrachloroethane-d2

VDMC13 $(\mathrm{DCZ})=1,2-\mathrm{Dichlorobenzene-d4}$
QC LIMITS

$(79-124)$

$(77-121)$

$(73-121)$

$(28-135)$

$(73-125)$

$(80-131)$

\# Column to be used to flag recovery values

* Values outside of contract required QC limits

Report 1,4-Dioxane-d8 for Low-Medium VOA analysis only

Page 1 of 1 
$4 A$ - FORM IV VOA

VOLATILE METHOD BLANK SUMMARY
EPA SAMPLE NO.

VBLKDB

Lab Name: TESTAMERICA BURLINGTON

Contract: $8 \mathrm{E}-00302$

Lab Code: STLV Case No.: YORK Mod. Ref No.: SDG No.: 29233

Lab File ID: 15254_005.D

Lab Sample ID: MB 200-92663/5

Instrument ID: CHD.i

Matrix: (SOIL/SED/WATER) Water

Level: (TRACE or LOW/MED) TRACE

Date Analyzed: 08/12/2015

GC Column: DB-624

ID $: 0.20 \quad(\mathrm{~mm})$

Time Analyzed: 1842

Heated Purge: (Y/N) N

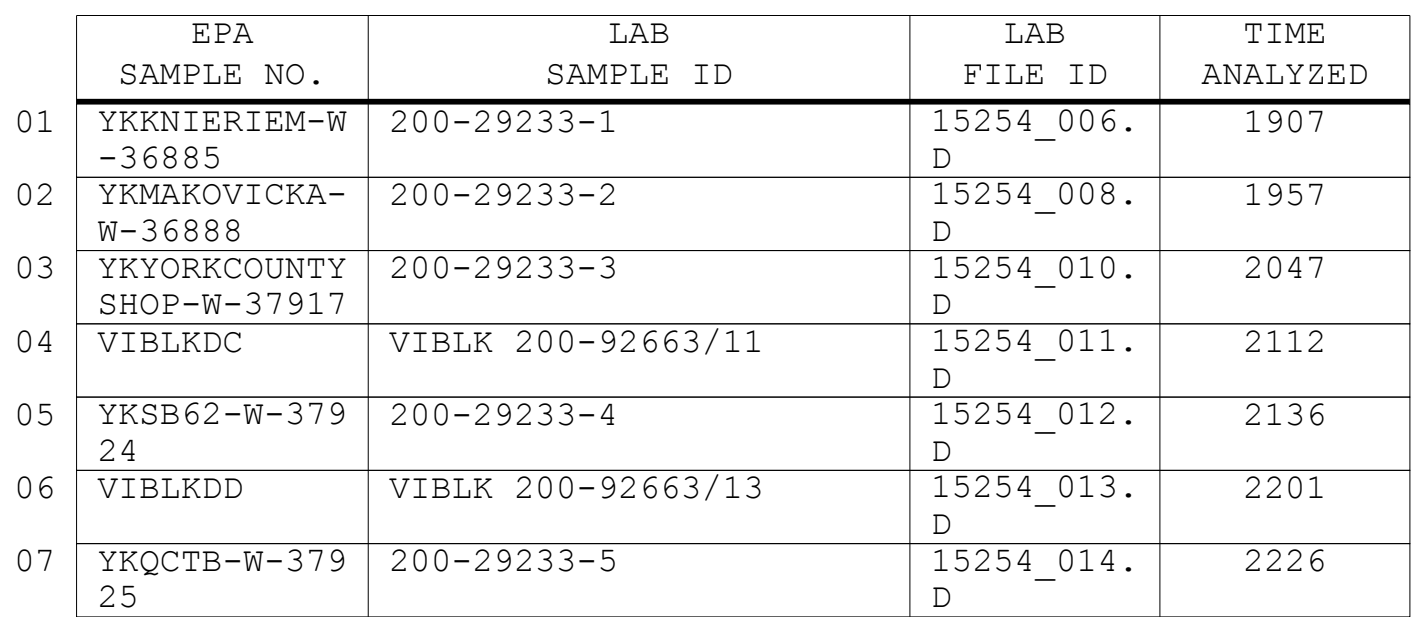

COMMENTS :

Page 1 of 1 
$4 A$ - FORM IV VOA

VOLATILE METHOD BLANK SUMMARY
EPA SAMPLE NO.

VBLKDC

Lab Name: TESTAMERICA BURLINGTON

Contract: $8 \mathrm{E}-00302$

Lab Code: STLV Case No.: YORK Mod. Ref No.: SDG No.: 29233

Lab File ID: 15265 _005.D

Lab Sample ID: MB 200-92691/5

Instrument ID: CHD.i

Matrix: (SOIL/SED/WATER) Water

Level: (TRACE or LOW/MED) TRACE

Date Analyzed: 08/13/2015

GC Column: DB-624

ID $: 0.20 \quad(\mathrm{~mm})$

Time Analyzed: 1224

Heated Purge: (Y/N) N

\begin{tabular}{|c|c|c|c|}
\hline $\begin{array}{c}\text { EPA } \\
\text { SAMPLE NO. }\end{array}$ & $\begin{array}{r}\text { LAB } \\
\text { SAMPLE ID }\end{array}$ & $\begin{array}{c}\text { LAB } \\
\text { FILE ID }\end{array}$ & $\begin{array}{c}\text { TIME } \\
\text { ANALYZED }\end{array}$ \\
\hline $\begin{array}{l}\text { YKYORKCOUNTY } \\
\text { SHOP-W-37917 } \\
\text { DL }\end{array}$ & $200-29233-3$ & $\begin{array}{l}15265 \_006 . \\
D\end{array}$ & 1248 \\
\hline $\begin{array}{l}\text { YKSB 62-W-379 } \\
24 \mathrm{DL}\end{array}$ & $200-29233-4$ & $\begin{array}{l}15265 \_007 . \\
D\end{array}$ & 1313 \\
\hline VHBLK01 & $200-29233-6$ & $\begin{array}{l}15265 \_008 . \\
D\end{array}$ & 1338 \\
\hline
\end{tabular}

COMMENTS :

Page 1 of 1 
$5 A$ - FORM V VOA

VOLATILE ORGANICS INSTRUMENT

PERFORMANCE CHECK

BROMOFLUOROBENZENE (BFB)
EPA SAMPLE NO.

BFBDA
Lab Name: TESTAMERICA BURLINGTON

Lab Code: STLV Case No.: YORK

Lab File Id: 15136_001.D

Instrument Id: CHD.i

GC Column: DB-624

ID $: 0.20$
Contract: 8E-00302 Mod. Ref No.: SDG No.: 29233

BEB Injection Date: 08/06/2015

BFB Injection Time: 0921 $(\mathrm{mm})$

\begin{tabular}{|c|c|c|}
\hline $\mathrm{m} / \mathrm{e}$ & ION ABUNDANCE CRITERIA & $\begin{array}{l}\text { 응 REATIVE } \\
\text { ABUNDANCE }\end{array}$ \\
\hline 50 & $15.0-40.0 \%$ of mass 95 & 19.3 \\
\hline 75 & $30.0-80.0 \%$ of mass 95 & 50.3 \\
\hline 95 & Base peak, $100 \%$ relative abundance & 100 \\
\hline 96 & $5.0-9.0 \%$ of mass 95 & 6.6 \\
\hline 173 & Less than $2.0 \%$ of mass 174 & $0.6) 1$ \\
\hline 174 & $50.0-120 \%$ of mass 95 & 80.8 \\
\hline 175 & $5.0-9.0 \%$ of mass 174 & 5.8( \\
\hline 176 & $95.0-101 \%$ of mass 174 & $(97.2) 1$ \\
\hline 177 & $5.0-9.0 \%$ of mass 176 & $(6.4) 2$ \\
\hline
\end{tabular}

1 - Value is omass 1742 - Value is omass 176

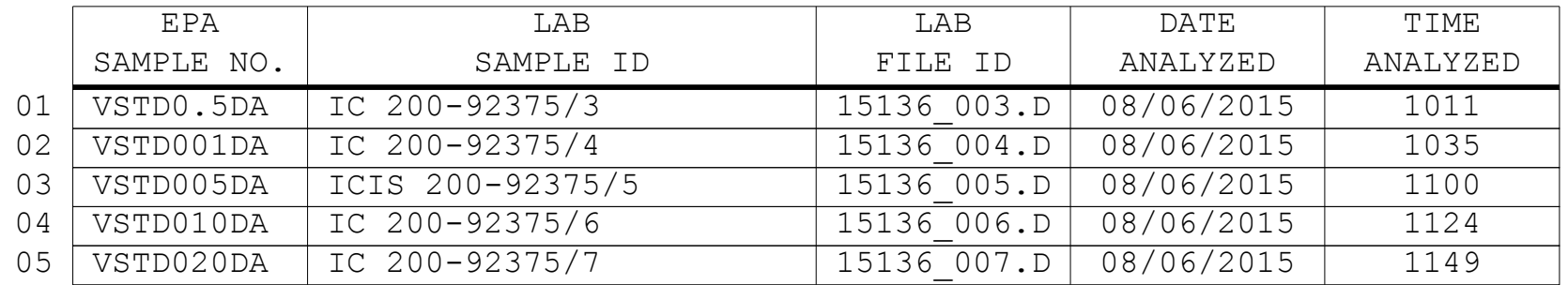


$5 A$ - FORM V VOA

VOLATILE ORGANICS INSTRUMENT

PERFORMANCE CHECK

BROMOFLUOROBENZENE (BFB)
EPA SAMPLE NO.

$\mathrm{BFBDB}$
Lab Name: TESTAMERICA BURLINGTON

Lab Code: STLV Case No.: YORK

Lab File Id: 15254_001.D

Instrument Id: CHD.i

GC Column: DB-624
ID $: 0.20$
Contract: $8 \mathrm{E}-00302$ Mod. Ref No.: SDG No.: 29233
BFB Injection Date: 08/12/2015

BFB Injection Time: 1708

\begin{tabular}{|c|c|c|}
\hline $\mathrm{m} / \mathrm{e}$ & ION ABUNDANCE CRITERIA & $\begin{array}{l}\text { 응 RELATIVE } \\
\text { ABUNDANCE }\end{array}$ \\
\hline 50 & $15.0-40.0 \%$ of mass 95 & 18.4 \\
\hline 75 & $30.0-80.0 \%$ of mass 95 & 47.9 \\
\hline 95 & Base peak, 100\% relative abundance & 100 \\
\hline 96 & $5.0-9.0 \%$ of mass 95 & 6.6 \\
\hline 173 & Less than $2.0 \%$ of mass 174 & $0.6(0.7) 1$ \\
\hline 174 & $50.0-120 \%$ of mass 95 & 85.3 \\
\hline 175 & $5.0-9.0 \%$ of mass 174 & $6.1(7.2) 1$ \\
\hline 176 & $95.0-101 \%$ of mass 174 & $83.0(97.2) 1$ \\
\hline 177 & $5.0-9.0 \%$ of mass 176 & $5.4(6.4) 2$ \\
\hline
\end{tabular}

1 - Value is omass 174

2 - Value is omass 176

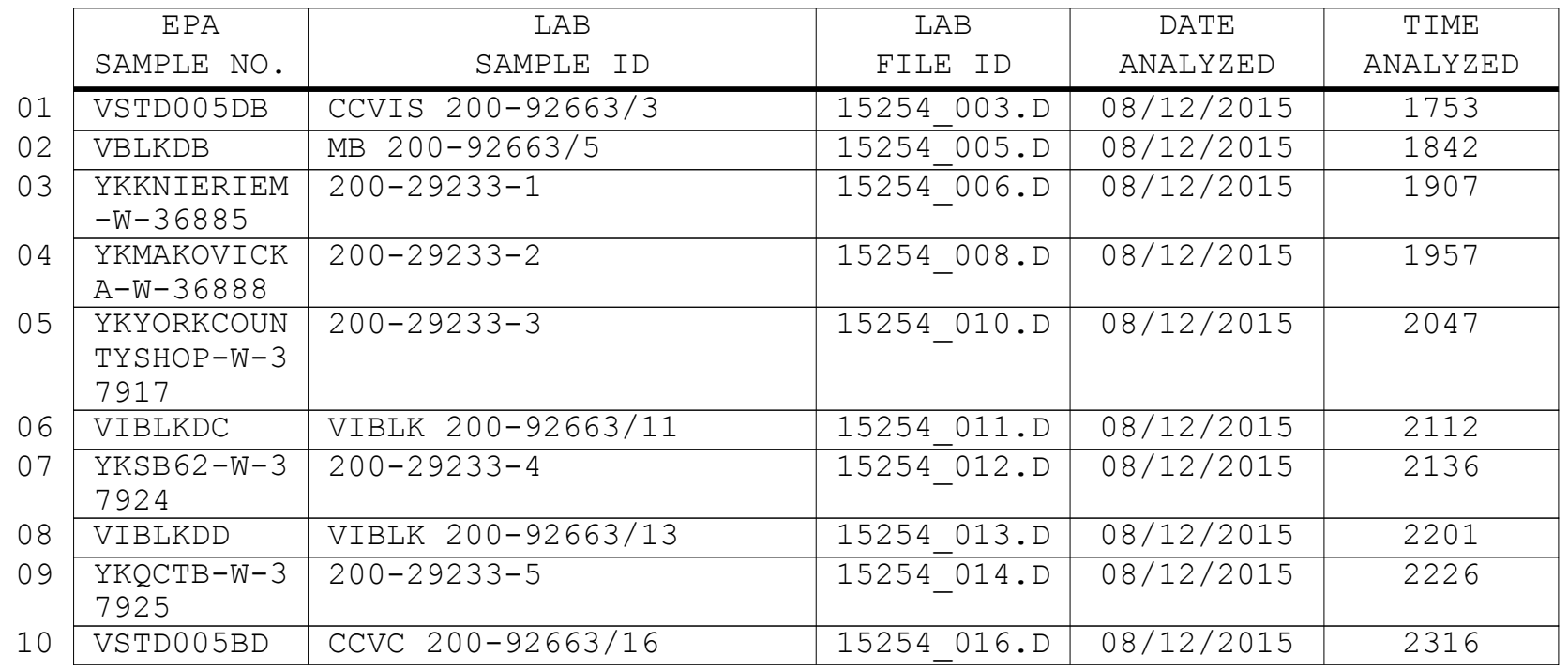

Page 1 of 1 
$5 A$ - FORM V VOA

VOLATILE ORGANICS INSTRUMENT

PERFORMANCE CHECK

BROMOFLUOROBENZENE (BFB)
EPA SAMPLE NO.

BFBDC
Lab Name: TESTAMERICA BURLINGTON

Lab Code: STLV Case No.: YORK

Lab File Id: 15265_001.D

Instrument Id: CHD.i

GC Column: DB-624

ID $: 0.20$
Contract: 8E-00302 Mod. Ref No.: SDG No.: 29233

BFB Injection Date: 08/13/2015

BFB Injection Time: 1049 $(\mathrm{mm})$

\begin{tabular}{|c|c|c|}
\hline $\mathrm{m} / \mathrm{e}$ & ION ABUNDANCE CRITERIA & $\begin{array}{l}\text { \% RELATIVE } \\
\text { ABUNDANCE }\end{array}$ \\
\hline 50 & $15.0-40.0 \%$ of mass 95 & 19.5 \\
\hline 75 & $30.0-80.0 \%$ of mass 95 & 50.1 \\
\hline 95 & Base peak, $100 \%$ relative abundance & 100 \\
\hline 96 & $5.0-9.0 \%$ of mass 95 & 6.7 \\
\hline 173 & Less than $2.0 \%$ of mass 174 & $0.7) 1$ \\
\hline 174 & $50.0-120 \%$ of mass 95 & 81.5 \\
\hline 175 & $5.0-9.0 \%$ of mass 174 & $5.8(7.1) 1$ \\
\hline 176 & $95.0-101 \%$ of mass 174 & $79.6(97.7) 1$ \\
\hline 177 & $5.0-9.0 \%$ of mass 176 & $(6.5) 2$ \\
\hline
\end{tabular}

1 - Value is omass 1742 - Value is omass 176

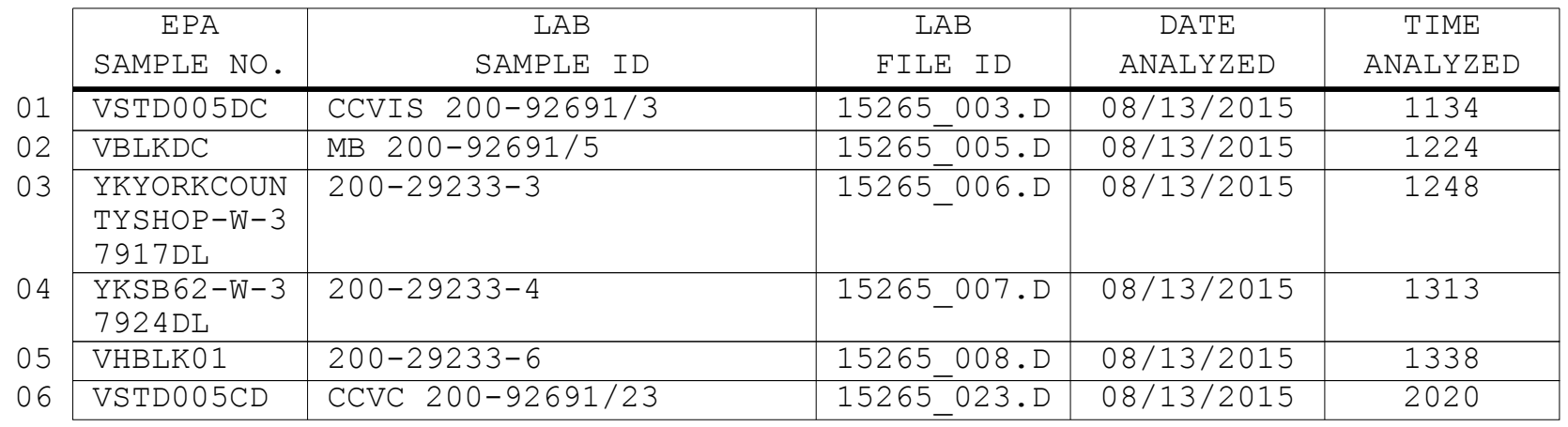




\section{$8 A$ - FORM VIII VOA}

VOLATILE INTERNAL STANDARD AREA AND RETENTION TIME SUMMARY

Lab Name: TESTAMERICA BURLINGTON

Contract: $8 \mathrm{E}-00302$

Lab Code: STLV Case No.: YORK Mod. Ref No.:

SDG No.: 29233

GC Column: DB-624

ID: 0.20 (mm) Init. Calib. Date (s) : 08/06/2015 08/06/2015

EPA Sample No.(VSTD\#\#\#\#\#): VSTD005DB

Lab File ID (Standard): 15254_003.D

Instrument ID: CHD.i

Date Analyzed: 08/12/2015

Time Analyzed: 1753

Heated Purge: ( $\mathrm{Y} / \mathrm{N}) \mathrm{N}$

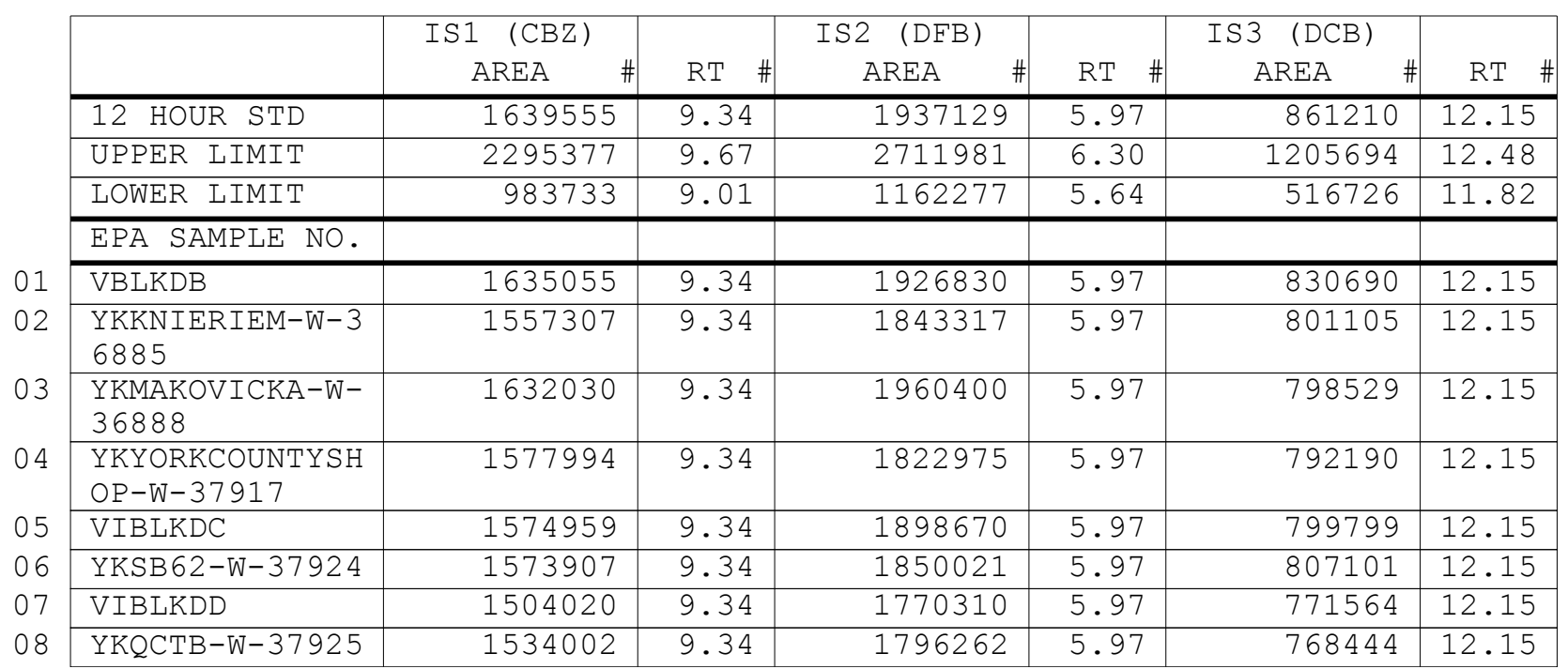

IS1 (CBZ) = Chlorobenzene-d5

IS2 $(\mathrm{DFB})=1,4-\mathrm{Difluorobenzene}$

IS3 $(\mathrm{DCB})=1,4-\mathrm{Dichlorobenzene-d4}$

AREA UPPER LIMIT $=140 \%$ (Trace Volatiles) of internal standard area AREA LOWER LIMIT $=60 \%$ (Trace Volatiles) of internal standard area RT UPPER LIMIT $=+0.33$ (Trace Volatiles) minutes of internal standard RT RT LOWER LIMIT $=-0.33$ (Trace Volatiles) minutes of internal standard RT

\# Column used to flag values outside contract required QC limits with an asterisk. Page 1 of 1 
Lab Name: TESTAMERICA BURLINGTON

Contract: $8 \mathrm{E}-00302$

Lab Code: STLV Case No.: YORK Mod. Ref No.: SDG No.: 29233

GC Column: DB-624

ID: 0.20 (mm) Init. Calib. Date $(\mathrm{s}): \underline{08 / 06 / 2015}$ 08/06/2015

EPA Sample No. (VSTD\#\#\#\#\#) : VSTD005DC

Lab File ID (Standard) : 15265_003.D

Instrument ID: CHD.i

Date Analyzed: $08 / 13 / 2015$

Time Analyzed: 1134

Heated Purge: (Y/N) N

\begin{tabular}{|c|c|c|c|c|c|c|}
\hline & $\begin{array}{c}\text { IS1 (CBZ) } \\
\text { AREA }\end{array}$ & RT \# & $\begin{array}{c}\text { IS2 (DFB) } \\
\text { AREA }\end{array}$ & $\mathrm{RT}$ & $\begin{array}{c}\text { IS3 (DCB) } \\
\text { AREA }\end{array}$ & $\mathrm{RT}$ \\
\hline 12 HOUR STD & 1488220 & 9.34 & 1729125 & 5.97 & 782013 & 12.15 \\
\hline UPPER LIMIT & 2083508 & 9.67 & 2420775 & 6.30 & 1094818 & 12.48 \\
\hline LOWER LIMIT & 892932 & 9.01 & 1037475 & 5.64 & 469208 & 11.82 \\
\hline EPA SAMPLE NO. & & & & & & \\
\hline VBLKDC & 1466940 & 9.34 & 1776877 & 5.97 & 733452 & 12.15 \\
\hline $\begin{array}{l}\text { YKYORKCOUNTYSH } \\
\text { OP-W-37917DL }\end{array}$ & 1536469 & 9.34 & 1786683 & 5.97 & 757495 & 12.15 \\
\hline $\begin{array}{l}\text { YKSB 62-W-37924 } \\
\text { DL }\end{array}$ & 1440821 & 9.34 & 1663537 & 5.97 & 740035 & 12.15 \\
\hline VHBLK01 & 1515459 & 9.34 & 1791694 & 5.97 & 774917 & 12.15 \\
\hline
\end{tabular}

IS1 $(\mathrm{CBZ})=$ Chlorobenzene-d5

IS2 $(\mathrm{DFB})=1,4-\mathrm{Difluorobenzene}$

IS3 $(\mathrm{DCB})=1,4-\mathrm{Dichlorobenzene-d4}$

AREA UPPER LIMIT $=140 \%$ (Trace Volatiles) of internal standard area AREA LOWER LIMIT $=60 \%$ (Trace Volatiles) of internal standard area RT UPPER LIMIT $=+0.33$ (Trace Volatiles) minutes of internal standard RT RT LOWER LIMIT $=-0.33$ (Trace Volatiles) minutes of internal standard RT

\# Column used to flag values outside contract required QC limits with an asterisk. 
Lab Name: TESTAMERICA BURLINGTON

Contract: $8 \mathrm{E}-00302$

Lab Code: STLV

Case No.: YORK Mod. Ref No.:

SDG No.: 29233

Matrix: (SOIL/SED/WATER) Water

Sample wt/vol: 25.0 $(\mathrm{g} / \mathrm{mL}) \mathrm{mL}$

Level: (TRACE/LOW/MED) TRACE

\% Moisture: not dec.

GC Column: DB-624 ID: $0.20 \quad(\mathrm{~mm})$

Soil Extract Volume: $(u L)$ $(\mathrm{mL})$

Purge Volume: 25.0
Lab Sample ID: 200-29233-1

Lab File ID: 15254_006.D

Date Received: 08/11/2015

Date Analyzed: 08/12/2015

Dilution Factor: 1.0

Soil Aliquot Volume: (uL)

\begin{tabular}{|c|c|c|c|}
\hline CAS NO. & COMPOUND & $\begin{array}{l}\text { CONCENTRATION UNITS: } \\
(\mathrm{ug} / \mathrm{L} \text { or } \mathrm{ug} / \mathrm{kg}) \mathrm{ug} / \mathrm{L}\end{array}$ & Q \\
\hline $75-71-8$ & Dichlorodifluoromethane & 1.0 & $\mathrm{U}$ \\
\hline $74-87-3$ & Chloromethane & 1.0 & $\mathrm{U}$ \\
\hline $75-01-4$ & Vinyl chloride & 1.0 & $\mathrm{U}$ \\
\hline $74-83-9$ & Bromomethane & 1.0 & $\mathrm{U}$ \\
\hline $75-00-3$ & Chloroethane & 1.0 & $\mathrm{U}$ \\
\hline $75-69-4$ & Trichlorofluoromethane & 1.0 & $\mathrm{U}$ \\
\hline $75-35-4$ & 1,1-Dichloroethene & 1.0 & $\mathrm{U}$ \\
\hline $76-13-1$ & $1,1,2$-Trichloro-1,2,2-trifluoroethane & 1.0 & $\mathrm{U}$ \\
\hline $67-64-1$ & Acetone & 5.0 & $\mathrm{U}$ \\
\hline $75-15-0$ & Carbon disulfide & 0.043 & $\mathrm{~J} B$ \\
\hline $79-20-9$ & Methyl acetate & 1.0 & $\mathrm{U}$ \\
\hline $75-09-2$ & Methylene Chloride & 1.0 & $\mathrm{U}$ \\
\hline $156-60-5$ & trans-1,2-Dichloroethene & 1.0 & $\mathrm{U}$ \\
\hline $1634-04-4$ & Methyl tert-butyl ether & 1.0 & $\mathrm{U}$ \\
\hline $75-34-3$ & 1,1-Dichloroethane & 1.0 & $\mathrm{U}$ \\
\hline $156-59-2$ & cis-1,2-Dichloroethene & 1.0 & $\mathrm{U}$ \\
\hline $78-93-3$ & 2-Butanone & 5.0 & $\mathrm{U}$ \\
\hline $74-97-5$ & Bromochloromethane & 1.0 & $\mathrm{U}$ \\
\hline $67-66-3$ & Chloroform & 1.0 & $\mathrm{U}$ \\
\hline $71-55-6$ & $1,1,1$-Trichloroethane & 1.0 & $\mathrm{U}$ \\
\hline $110-82-7$ & Cyclohexane & 1.0 & $\mathrm{U}$ \\
\hline $56-23-5$ & Carbon tetrachloride & 0.022 & $\mathrm{~J}$ \\
\hline $71-43-2$ & Benzene & 1.0 & $\mathrm{U}$ \\
\hline $107-06-2$ & 1,2-Dichloroethane & 1.0 & $\mathrm{U}$ \\
\hline
\end{tabular}

Report 1,4-Dioxane for Low-Medium VOA analysis only 
Lab Name: TESTAMERICA BURLINGTON

Contract: 8E-00302

Lab Code: STLV

Case No.: YORK

Mod. Ref No.:

SDG No.: 29233

Matrix: (SOIL/SED/WATER) Water

Sample wt/vol: 25.0 $(\mathrm{g} / \mathrm{mL}) \mathrm{mL}$

Level: (TRACE/LOW/MED) TRACE

\% Moisture: not dec.

GC Column: DB-624 ID: $0.20 \quad(\mathrm{~mm})$

Soil Extract Volume: (uL) $(\mathrm{mL})$

Purge Volume: 25.0 (mI)
Lab Sample ID: 200-29233-1

Lab File ID: 15254_006.D

Date Received: 08/11/2015

Date Analyzed: 08/12/2015

Dilution Factor: 1.0

Soil Aliquot Volume: (uL)

\begin{tabular}{|c|c|c|c|}
\hline CAS NO. & COMPOUND & $\begin{array}{l}\text { CONCENTRATION UNITS: } \\
(\mathrm{ug} / \mathrm{L} \text { or } \mathrm{ug} / \mathrm{kg}) \mathrm{ug} / \mathrm{L}\end{array}$ & Q \\
\hline $79-01-6$ & Trichloroethene & 1.0 & $\mathrm{U}$ \\
\hline $108-87-2$ & Methylcyclohexane & 1.0 & $\mathrm{U}$ \\
\hline $78-87-5$ & 1,2-Dichloropropane & 1.0 & $\mathrm{U}$ \\
\hline $75-27-4$ & Bromodichloromethane & 1.0 & $\mathrm{U}$ \\
\hline $10061-01-5$ & cis-1,3-Dichloropropene & 1.0 & $\mathrm{U}$ \\
\hline $108-10-1$ & 4-Methyl-2-pentanone & 1.0 & $\mathrm{U}$ \\
\hline $108-88-3$ & Toluene & 0.030 & $\mathrm{~J} B$ \\
\hline $10061-02-6$ & trans-1,3-Dichloropropene & 1.0 & $\mathrm{U}$ \\
\hline $79-00-5$ & $1,1,2-T r i c h l o r o e t h a n e$ & 1.0 & $\mathrm{U}$ \\
\hline $127-18-4$ & Tetrachloroethene & 1.0 & $\mathrm{U}$ \\
\hline $591-78-6$ & 2-Hexanone & 1.0 & $\mathrm{U}$ \\
\hline $124-48-1$ & Dibromochloromethane & 1.0 & $\mathrm{U}$ \\
\hline $106-93-4$ & 1,2-Dibromoethane & 1.0 & $\mathrm{U}$ \\
\hline $108-90-7$ & Chlorobenzene & 1.0 & $\mathrm{U}$ \\
\hline $100-41-4$ & Ethylbenzene & 0.0058 & $\mathrm{~J} B$ \\
\hline $95-47-6$ & o-Xylene & 0.0063 & $\mathrm{~J} B$ \\
\hline $179601-23-1$ & m,p-Xylene & 0.019 & $\mathrm{~J} B$ \\
\hline $100-42-5$ & Styrene & 1.0 & $\mathrm{U}$ \\
\hline $75-25-2$ & Bromoform & 1.0 & $\mathrm{U}$ \\
\hline $98-82-8$ & Isopropylbenzene & 1.0 & $\mathrm{U}$ \\
\hline $79-34-5$ & $1,1,2,2$-Tetrachloroethane & 1.0 & $\mathrm{U}$ \\
\hline $541-73-1$ & 1,3-Dichlorobenzene & 1.0 & $\mathrm{U}$ \\
\hline $106-46-7$ & 1,4-Dichlorobenzene & 1.0 & $\mathrm{U}$ \\
\hline $95-50-1$ & 1,2-Dichlorobenzene & 1.0 & $\mathrm{U}$ \\
\hline $96-12-8$ & 1,2-Dibromo-3-Chloropropane & 1.0 & $\mathrm{U}$ \\
\hline $120-82-1$ & $1,2,4$-Trichlorobenzene & 0.025 & $\mathrm{~J} B$ \\
\hline $87-61-6$ & $1,2,3$-Trichlorobenzene & 0.033 & $\mathrm{~J} B$ \\
\hline
\end{tabular}


Lab Name: TESTAMERICA BURLINGTON

Contract: $8 \mathrm{E}-00302$

Lab Code: STLV Case No.: YORK Mod. Ref No.: SDG No.: 29233

Matrix: (SOIL/SED/WATER) Water

Sample wt/vol: 25.0 $(\mathrm{g} / \mathrm{mL}) \mathrm{mL}$

Level: (TRACE or LOW/MED) TRACE

․ Moisture: not dec.

GC Column: DB-624 ID $: 0.20 \quad(\mathrm{~mm})$

Soil Extract Volume: (uL)
Lab Sample ID: 200-29233-1

Lab File ID: 15254_006.D

Date Received: 08/11/2015

Date Analyzed: 08/12/2015

Dilution Factor: 1.0

Soil Aliquot Volume: (uL) Purge Volume: 25.0 CONCENTRATION UNITS: (ug/L or ug/kg) ug/L

\begin{tabular}{|c|c|c|c|c|}
\hline CAS NUMBER & COMPOUND NAME & RT & EST. CONC. & $Q$ \\
\hline 01 & Unknown & 7.29 & 3.1 & $\mathrm{~J} B \mathrm{X}$ \\
\hline $541-05-9$ & Cyclotrisiloxane, hexamethyl- & 8.22 & 0.50 & $\mathrm{~J} \mathrm{~N} \mathrm{~B}$ \\
\hline $556-67-2$ & Cyclotetrasiloxane, octamethyl- & 11.04 & 0.53 & $\mathrm{~J} \quad \mathrm{~N} B$ \\
\hline E9667961 & Total Alkanes & $\mathrm{N} / \mathrm{A}$ & & \\
\hline
\end{tabular}


Lab Name: TESTAMERICA BURLINGTON

Contract: $8 \mathrm{E}-00302$

Lab Code: STLV

Case No.: YORK Mod. Ref No.:

SDG No.: 29233

Matrix: (SOIL/SED/WATER) Water

Sample wt/vol: 25.0 $(\mathrm{g} / \mathrm{mL}) \mathrm{mL}$

Level: (TRACE/LOW/MED) TRACE

\% Moisture: not dec.

GC Column: DB-624 ID: $0.20 \quad(\mathrm{~mm})$

Soil Extract Volume: (uL) $(\mathrm{mL})$

Purge Volume: 25.0
Lab Sample ID: 200-29233-2

Lab File ID: 15254_008.D

Date Received: 08/11/2015

Date Analyzed: 08/12/2015

Dilution Factor: 1.0

Soil Aliquot Volume: (uL)

\begin{tabular}{|c|c|c|c|}
\hline CAS NO. & COMPOUND & $\begin{array}{l}\text { CONCENTRATION UNITS: } \\
(\mathrm{ug} / \mathrm{L} \text { or } \mathrm{ug} / \mathrm{kg}) \mathrm{ug} / \mathrm{L}\end{array}$ & Q \\
\hline $75-71-8$ & Dichlorodifluoromethane & 1.0 & $\mathrm{U}$ \\
\hline $74-87-3$ & Chloromethane & 1.0 & $\mathrm{U}$ \\
\hline $75-01-4$ & Vinyl chloride & 1.0 & $\mathrm{U}$ \\
\hline $74-83-9$ & Bromomethane & 1.0 & $\mathrm{U}$ \\
\hline $75-00-3$ & Chloroethane & 1.0 & $\mathrm{U}$ \\
\hline $75-69-4$ & Trichlorofluoromethane & 1.0 & $\mathrm{U}$ \\
\hline $75-35-4$ & 1,1-Dichloroethene & 1.0 & $\mathrm{U}$ \\
\hline $76-13-1$ & $1,1,2$-Trichloro-1,2,2-trifluoroethane & 1.0 & $\mathrm{U}$ \\
\hline $67-64-1$ & Acetone & 5.0 & $\mathrm{U}$ \\
\hline $75-15-0$ & Carbon disulfide & 0.037 & $\mathrm{~J} B$ \\
\hline $79-20-9$ & Methyl acetate & 1.0 & $\mathrm{U}$ \\
\hline $75-09-2$ & Methylene Chloride & 1.0 & $\mathrm{U}$ \\
\hline $156-60-5$ & trans-1,2-Dichloroethene & 1.0 & $\mathrm{U}$ \\
\hline $1634-04-4$ & Methyl tert-butyl ether & 1.0 & $\mathrm{U}$ \\
\hline $75-34-3$ & 1,1-Dichloroethane & 1.0 & $\mathrm{U}$ \\
\hline $156-59-2$ & cis-1,2-Dichloroethene & 1.0 & $\mathrm{U}$ \\
\hline $78-93-3$ & 2-Butanone & 5.0 & $\mathrm{U}$ \\
\hline $74-97-5$ & Bromochloromethane & 1.0 & $\mathrm{U}$ \\
\hline $67-66-3$ & Chloroform & 1.0 & $\mathrm{U}$ \\
\hline $71-55-6$ & $1,1,1$-Trichloroethane & 1.0 & $\mathrm{U}$ \\
\hline $110-82-7$ & Cyclohexane & 1.0 & $\mathrm{U}$ \\
\hline $56-23-5$ & Carbon tetrachloride & 1.0 & $\mathrm{U}$ \\
\hline $71-43-2$ & Benzene & 1.0 & $\mathrm{U}$ \\
\hline $107-06-2$ & 1,2-Dichloroethane & 1.0 & $\mathrm{U}$ \\
\hline
\end{tabular}

Report 1,4-Dioxane for Low-Medium VOA analysis only 
Lab Name: TESTAMERICA BURLINGTON

Contract: $8 \mathrm{E}-00302$

Lab Code: STLV

Case No.: YORK

Mod. Ref No.:

SDG No.: 29233

Matrix: (SOIL/SED/WATER) Water

Sample wt/vol: 25.0 $(\mathrm{g} / \mathrm{mL}) \mathrm{mL}$

Level: (TRACE/LOW/MED) TRACE

\% Moisture: not dec.

GC Column: DB-624 ID: $0.20 \quad(\mathrm{~mm})$

Soil Extract Volume: (uL) $(\mathrm{mL})$

Purge Volume: 25.0 (mI)
Lab Sample ID: 200-29233-2

Lab File ID: 15254_008.D

Date Received: 08/11/2015

Date Analyzed: 08/12/2015

Dilution Factor: 1.0

Soil Aliquot Volume: (uL)

\begin{tabular}{|c|c|c|c|}
\hline CAS NO. & COMPOUND & $\begin{array}{l}\text { CONCENTRATION UNITS: } \\
(\mathrm{ug} / \mathrm{L} \text { or } \mathrm{ug} / \mathrm{kg}) \mathrm{ug} / \mathrm{L}\end{array}$ & Q \\
\hline $79-01-6$ & Trichloroethene & 1.0 & $\mathrm{U}$ \\
\hline $108-87-2$ & Methylcyclohexane & 1.0 & $\mathrm{U}$ \\
\hline $78-87-5$ & 1,2-Dichloropropane & 1.0 & $\mathrm{U}$ \\
\hline $75-27-4$ & Bromodichloromethane & 1.0 & $\mathrm{U}$ \\
\hline $10061-01-5$ & cis-1,3-Dichloropropene & 1.0 & $\mathrm{U}$ \\
\hline $108-10-1$ & 4-Methyl-2-pentanone & 1.0 & $\mathrm{U}$ \\
\hline $108-88-3$ & Toluene & 0.039 & $\mathrm{~J} B$ \\
\hline $10061-02-6$ & trans-1,3-Dichloropropene & 1.0 & $\mathrm{U}$ \\
\hline $79-00-5$ & $1,1,2-T r i c h l o r o e t h a n e$ & 1.0 & $\mathrm{U}$ \\
\hline $127-18-4$ & Tetrachloroethene & 1.0 & $\mathrm{U}$ \\
\hline $591-78-6$ & 2-Hexanone & 1.0 & $\mathrm{U}$ \\
\hline $124-48-1$ & Dibromochloromethane & 1.0 & $\mathrm{U}$ \\
\hline $106-93-4$ & 1,2-Dibromoethane & 1.0 & $\mathrm{U}$ \\
\hline $108-90-7$ & Chlorobenzene & 1.0 & $\mathrm{U}$ \\
\hline $100-41-4$ & Ethylbenzene & 0.0053 & $\mathrm{~J} B$ \\
\hline $95-47-6$ & o-Xylene & 0.0069 & $\mathrm{~J} B$ \\
\hline $179601-23-1$ & m,p-Xylene & 0.019 & $\mathrm{~J} B$ \\
\hline $100-42-5$ & Styrene & 1.0 & $\mathrm{U}$ \\
\hline $75-25-2$ & Bromoform & 1.0 & $\mathrm{U}$ \\
\hline $98-82-8$ & Isopropylbenzene & 1.0 & $\mathrm{U}$ \\
\hline $79-34-5$ & $1,1,2,2$-Tetrachloroethane & 1.0 & $\mathrm{U}$ \\
\hline $541-73-1$ & 1,3-Dichlorobenzene & 0.0057 & $\mathrm{~J} B$ \\
\hline $106-46-7$ & 1,4-Dichlorobenzene & 1.0 & $\mathrm{U}$ \\
\hline $95-50-1$ & 1,2-Dichlorobenzene & 1.0 & $\mathrm{U}$ \\
\hline $96-12-8$ & 1,2-Dibromo-3-Chloropropane & 1.0 & $\mathrm{U}$ \\
\hline $120-82-1$ & $1,2,4$-Trichlorobenzene & 0.019 & $\mathrm{~J} B$ \\
\hline $87-61-6$ & $1,2,3$-Trichlorobenzene & 0.016 & $\mathrm{~J} B$ \\
\hline
\end{tabular}


Lab Name: TESTAMERICA BURLINGTON

Contract: $8 \mathrm{E}-00302$

Lab Code: STLV Case No.: YORK Mod. Ref No.: SDG No.: 29233

Matrix: (SOIL/SED/WATER) Water

Sample wt/vol: 25.0 $(\mathrm{g} / \mathrm{mL}) \mathrm{mL}$

Level: (TRACE or LOW/MED) TRACE

Moisture: not dec.

GC Column: DB-624 ID $: 0.20 \quad(\mathrm{~mm})$

Soil Extract Volume: (uL)

CONCENTRATION UNITS: (ug/L or ug/kg) ug/L
Lab Sample ID: 200-29233-2

Lab File ID: 15254_008.D

Date Received: 08/11/2015

Date Analyzed: 08/12/2015

Dilution Factor: 1.0

Soil Aliquot Volume: (uL) Purge Volume: 25.0 $(\mathrm{mL})$

01

02

\begin{tabular}{|l|l|c|c|c|}
\hline CAS NUMBER & \multicolumn{1}{|c|}{ COMPOUND NAME } & RT & EST. CONC. & Q \\
\hline & Unknown & 7.29 & 3.0 & $\mathrm{~J}$ B X \\
\hline E9667961 & Total Alkanes & N/A & & \\
\hline
\end{tabular}

1EPA-designated Registry Number. 
Lab Name: TESTAMERICA BURLINGTON

Contract: $8 \mathrm{E}-00302$

Lab Code: STLV

Case No.: YORK Mod. Ref No.:

SDG No.: 29233

Matrix: (SOIL/SED/WATER) Water

Sample wt/vol: 25.0 $(\mathrm{g} / \mathrm{mL}) \mathrm{mL}$

Level: (TRACE/LOW/MED) TRACE

\% Moisture: not dec.

GC Column: DB-624 ID: $0.20 \quad(\mathrm{~mm})$

Soil Extract Volume: (uL) $(\mathrm{mL})$

Purge Volume: 25.0
Lab Sample ID: 200-29233-5

Lab File ID: 15254_014.D

Date Received: 08/11/2015

Date Analyzed: 08/12/2015

Dilution Factor: 1.0

Soil Aliquot Volume: (uL)

\begin{tabular}{|c|c|c|c|}
\hline CAS NO. & COMPOUND & $\begin{array}{l}\text { CONCENTRATION UNITS: } \\
(\mathrm{ug} / \mathrm{L} \text { or } \mathrm{ug} / \mathrm{kg}) \mathrm{ug} / \mathrm{L}\end{array}$ & Q \\
\hline $75-71-8$ & Dichlorodifluoromethane & 1.0 & $\mathrm{U}$ \\
\hline $74-87-3$ & Chloromethane & 1.0 & $\mathrm{U}$ \\
\hline $75-01-4$ & Vinyl chloride & 1.0 & $\mathrm{U}$ \\
\hline $74-83-9$ & Bromomethane & 1.0 & $\mathrm{U}$ \\
\hline $75-00-3$ & Chloroethane & 1.0 & $\mathrm{U}$ \\
\hline $75-69-4$ & Trichlorofluoromethane & 1.0 & $\mathrm{U}$ \\
\hline $75-35-4$ & 1,1-Dichloroethene & 1.0 & $\mathrm{U}$ \\
\hline $76-13-1$ & $1,1,2$-Trichloro-1,2,2-trifluoroethane & 1.0 & $\mathrm{U}$ \\
\hline $67-64-1$ & Acetone & 7.3 & B \\
\hline $75-15-0$ & Carbon disulfide & 0.030 & $\mathrm{~J} B$ \\
\hline $79-20-9$ & Methyl acetate & 1.0 & $\mathrm{U}$ \\
\hline $75-09-2$ & Methylene Chloride & 1.0 & $\mathrm{U}$ \\
\hline $156-60-5$ & trans-1,2-Dichloroethene & 1.0 & $\mathrm{U}$ \\
\hline $1634-04-4$ & Methyl tert-butyl ether & 1.0 & $\mathrm{U}$ \\
\hline $75-34-3$ & 1,1-Dichloroethane & 1.0 & $\mathrm{U}$ \\
\hline $156-59-2$ & cis-1,2-Dichloroethene & 1.0 & $\mathrm{U}$ \\
\hline $78-93-3$ & 2-Butanone & 5.0 & $\mathrm{U}$ \\
\hline $74-97-5$ & Bromochloromethane & 1.0 & $\mathrm{U}$ \\
\hline $67-66-3$ & Chloroform & 1.0 & $\mathrm{U}$ \\
\hline $71-55-6$ & $1,1,1$-Trichloroethane & 1.0 & $\mathrm{U}$ \\
\hline $110-82-7$ & Cyclohexane & 1.0 & $\mathrm{U}$ \\
\hline $56-23-5$ & Carbon tetrachloride & 1.0 & $\mathrm{U}$ \\
\hline $71-43-2$ & Benzene & 0.046 & $\mathrm{~J}$ \\
\hline $107-06-2$ & 1,2-Dichloroethane & 1.0 & $\mathrm{U}$ \\
\hline
\end{tabular}

Report 1,4-Dioxane for Low-Medium VOA analysis only 
Lab Name: TESTAMERICA BURLINGTON

Contract: $8 \mathrm{E}-00302$

Lab Code: STLV

Case No.: YORK

Mod. Ref No.:

SDG No.: 29233

Matrix: (SOIL/SED/WATER) Water

Sample wt/vol: 25.0 $(\mathrm{g} / \mathrm{mL}) \mathrm{mL}$

Level: (TRACE/LOW/MED) TRACE

\% Moisture: not dec.

GC Column: DB-624 ID: $0.20 \quad(\mathrm{~mm})$

Soil Extract Volume: (uL) $(\mathrm{mL})$

Purge Volume: 25.0 (mI)
Lab Sample ID: 200-29233-5

Lab File ID: 15254_014.D

Date Received: 08/11/2015

Date Analyzed: 08/12/2015

Dilution Factor: 1.0

Soil Aliquot Volume: (uL)

\begin{tabular}{|c|c|c|c|}
\hline CAS NO. & COMPOUND & $\begin{array}{l}\text { CONCENTRATION UNITS: } \\
(\mathrm{ug} / \mathrm{L} \text { or } \mathrm{ug} / \mathrm{kg}) \mathrm{ug} / \mathrm{L}\end{array}$ & Q \\
\hline $79-01-6$ & Trichloroethene & 1.0 & $\mathrm{U}$ \\
\hline $108-87-2$ & Methylcyclohexane & 1.0 & $\mathrm{U}$ \\
\hline $78-87-5$ & 1,2-Dichloropropane & 1.0 & $\mathrm{U}$ \\
\hline $75-27-4$ & Bromodichloromethane & 1.0 & $\mathrm{U}$ \\
\hline $10061-01-5$ & cis-1,3-Dichloropropene & 1.0 & U \\
\hline $108-10-1$ & 4-Methyl-2-pentanone & 1.0 & $\mathrm{U}$ \\
\hline $108-88-3$ & Toluene & 0.21 & $\mathrm{~J} B$ \\
\hline $10061-02-6$ & trans-1,3-Dichloropropene & 1.0 & $\mathrm{U}$ \\
\hline $79-00-5$ & $1,1,2-$ Trichloroethane & 1.0 & $\mathrm{U}$ \\
\hline $127-18-4$ & Tetrachloroethene & 0.0038 & $\mathrm{~J}$ \\
\hline $591-78-6$ & 2-Hexanone & 1.0 & $\mathrm{U}$ \\
\hline $124-48-1$ & Dibromochloromethane & 1.0 & $\mathrm{U}$ \\
\hline $106-93-4$ & 1,2-Dibromoethane & 1.0 & $\mathrm{U}$ \\
\hline $108-90-7$ & Chlorobenzene & 1.0 & $\mathrm{U}$ \\
\hline $100-41-4$ & Ethylbenzene & 0.011 & $\mathrm{~J} B$ \\
\hline $95-47-6$ & o-Xylene & 0.040 & $\mathrm{~J} B$ \\
\hline $179601-23-1$ & m,p-xylene & 0.041 & $\mathrm{~J} B$ \\
\hline $100-42-5$ & Styrene & 0.019 & $\mathrm{~J} B$ \\
\hline $75-25-2$ & Bromoform & 1.0 & $\mathrm{U}$ \\
\hline $98-82-8$ & Isopropylbenzene & 1.0 & $\mathrm{U}$ \\
\hline $79-34-5$ & $1,1,2,2$-Tetrachloroethane & 1.0 & $\mathrm{U}$ \\
\hline $541-73-1$ & 1,3 -Dichlorobenzene & 1.0 & $\mathrm{U}$ \\
\hline $106-46-7$ & 1,4-Dichlorobenzene & 1.0 & $\mathrm{U}$ \\
\hline $95-50-1$ & 1,2-Dichlorobenzene & 1.0 & $\mathrm{U}$ \\
\hline $96-12-8$ & 1,2-Dibromo-3-Chloropropane & 1.0 & $\mathrm{U}$ \\
\hline $120-82-1$ & $1,2,4$-Trichlorobenzene & 0.016 & $\mathrm{~J} B$ \\
\hline $87-61-6$ & $1,2,3$-Trichlorobenzene & 0.015 & $\mathrm{~J} B$ \\
\hline
\end{tabular}


Lab Name: TESTAMERICA BURLINGTON

Contract: $8 \mathrm{E}-00302$

Lab Code: STLV Case No.: YORK Mod. Ref No.: SDG No.: 29233

Matrix: (SOIL/SED/WATER) Water

Sample wt/vol: 25.0 $(\mathrm{g} / \mathrm{mL}) \mathrm{mL}$

Level: (TRACE or LOW/MED) TRACE

․ Moisture: not dec.

GC Column: DB-624 ID $: 0.20 \quad(\mathrm{~mm})$

Soil Extract Volume: (uL)
Lab Sample ID: 200-29233-5

Lab File ID: 15254_014.D

Date Received: 08/11/2015

Date Analyzed: 08/12/2015

Dilution Factor: 1.0

Soil Aliquot Volume: (uL) Purge Volume: 25.0 $(\mathrm{mL})$

01

02

\begin{tabular}{|l|l|c|c|c|}
\hline CAS NUMBER & \multicolumn{1}{|c|}{ COMPOUND NAME } & RT & EST. CONC. & Q \\
\hline & Unknown & 7.29 & 3.0 & $\mathrm{~J}$ B X \\
\hline E9667961 & Total Alkanes & N/A & & \\
\hline
\end{tabular}

1EPA-designated Registry Number. 
Lab Name: TESTAMERICA BURLINGTON

Contract: $8 \mathrm{E}-00302$

Lab Code: STLV

Case No.: YORK Mod. Ref No.:

SDG No.: 29233

Matrix: (SOIL/SED/WATER) Water

Sample wt/vol: 25.0 $(\mathrm{g} / \mathrm{mL}) \mathrm{mL}$

Level: (TRACE/LOW/MED) TRACE

\% Moisture: not dec.

GC Column: DB-624 ID: $0.20 \quad(\mathrm{~mm})$

Soil Extract Volume: (uL) $(\mathrm{mL})$

Purge Volume: 25.0
Lab Sample ID: 200-29233-4

Lab File ID: 15254_012.D

Date Received: 08/11/2015

Date Analyzed: 08/12/2015

Dilution Factor: 1.0

Soil Aliquot Volume: (uL)

\begin{tabular}{|c|c|c|c|}
\hline CAS NO. & COMPOUND & $\begin{array}{l}\text { CONCENTRATION UNITS: } \\
(\mathrm{ug} / \mathrm{L} \text { or } \mathrm{ug} / \mathrm{kg}) \mathrm{ug} / \mathrm{L}\end{array}$ & Q \\
\hline $75-71-8$ & Dichlorodifluoromethane & 1.0 & $\mathrm{U}$ \\
\hline $74-87-3$ & Chloromethane & 1.0 & $\mathrm{U}$ \\
\hline $75-01-4$ & Vinyl chloride & 1.0 & $\mathrm{U}$ \\
\hline $74-83-9$ & Bromomethane & 1.0 & $\mathrm{U}$ \\
\hline $75-00-3$ & Chloroethane & 1.0 & $\mathrm{U}$ \\
\hline $75-69-4$ & Trichlorofluoromethane & 0.082 & $\mathrm{~J}$ \\
\hline $75-35-4$ & 1,1-Dichloroethene & 1.0 & $\mathrm{U}$ \\
\hline $76-13-1$ & $1,1,2$-Trichloro-1,2,2-trifluoroethane & 1.0 & $\mathrm{U}$ \\
\hline $67-64-1$ & Acetone & 5.0 & $\mathrm{U}$ \\
\hline $75-15-0$ & Carbon disulfide & 0.031 & $\mathrm{~J} B$ \\
\hline $79-20-9$ & Methyl acetate & 1.0 & $\mathrm{U}$ \\
\hline $75-09-2$ & Methylene Chloride & 1.0 & $\mathrm{U}$ \\
\hline $156-60-5$ & trans-1,2-Dichloroethene & 1.0 & $\mathrm{U}$ \\
\hline $1634-04-4$ & Methyl tert-butyl ether & 1.0 & $\mathrm{U}$ \\
\hline $75-34-3$ & 1,1-Dichloroethane & 1.0 & $\mathrm{U}$ \\
\hline $156-59-2$ & cis-1,2-Dichloroethene & 1.0 & $\mathrm{U}$ \\
\hline $78-93-3$ & 2-Butanone & 5.0 & $\mathrm{U}$ \\
\hline $74-97-5$ & Bromochloromethane & 1.0 & $\mathrm{U}$ \\
\hline $67-66-3$ & Chloroform & 2.5 & \\
\hline $71-55-6$ & $1,1,1$-Trichloroethane & 1.0 & $\mathrm{U}$ \\
\hline $110-82-7$ & Cyclohexane & 1.0 & $\mathrm{U}$ \\
\hline $56-23-5$ & Carbon tetrachloride & 26 & $\mathrm{E}$ \\
\hline $71-43-2$ & Benzene & 1.0 & $\mathrm{U}$ \\
\hline $107-06-2$ & 1,2-Dichloroethane & 1.0 & $\mathrm{U}$ \\
\hline
\end{tabular}

Report 1,4-Dioxane for Low-Medium VOA analysis only 
Lab Name: TESTAMERICA BURLINGTON

Lab Code: STLV

Case No.: YORK

Mod. Ref No.:

Contract: $8 \mathrm{E}-00302$

Matrix: (SOIL/SED/WATER) Water

Sample wt/vol: 25.0 $(\mathrm{g} / \mathrm{mL}) \mathrm{mL}$

Level: (TRACE/LOW/MED) TRACE

\% Moisture: not dec.

GC Column: DB-624 ID: $0.20 \quad(\mathrm{~mm})$

Soil Extract Volume: (uL) $(\mathrm{mL})$

Purge Volume: 25.0
SDG No.: 29233

Lab Sample ID: 200-29233-4

Lab File ID: 15254_012.D

Date Received: 08/11/2015

Date Analyzed: 08/12/2015

Dilution Factor: 1.0

Soil Aliquot Volume: (uL) (mI)

\begin{tabular}{|c|c|c|c|}
\hline CAS NO. & COMPOUND & $\begin{array}{l}\text { CONCENTRATION UNITS: } \\
(\mathrm{ug} / \mathrm{L} \text { or } \mathrm{ug} / \mathrm{kg}) \mathrm{ug} / \mathrm{L}\end{array}$ & Q \\
\hline $79-01-6$ & Trichloroethene & 1.0 & $\mathrm{U}$ \\
\hline $108-87-2$ & Methylcyclohexane & 1.0 & $\mathrm{U}$ \\
\hline $78-87-5$ & 1,2-Dichloropropane & 1.0 & $\mathrm{U}$ \\
\hline $75-27-4$ & Bromodichloromethane & 1.0 & $\mathrm{U}$ \\
\hline $10061-01-5$ & cis-1,3-Dichloropropene & 1.0 & U \\
\hline $108-10-1$ & 4-Methyl-2-pentanone & 1.0 & $\mathrm{U}$ \\
\hline $108-88-3$ & Toluene & 0.14 & $\mathrm{~J} B$ \\
\hline $10061-02-6$ & trans-1,3-Dichloropropene & 1.0 & $\mathrm{U}$ \\
\hline $79-00-5$ & $1,1,2-$ Trichloroethane & 1.0 & $\mathrm{U}$ \\
\hline $127-18-4$ & Tetrachloroethene & 0.031 & $\mathrm{~J}$ \\
\hline $591-78-6$ & 2-Hexanone & 1.0 & $\mathrm{U}$ \\
\hline $124-48-1$ & Dibromochloromethane & 1.0 & $\mathrm{U}$ \\
\hline $106-93-4$ & 1,2-Dibromoethane & 1.0 & $\mathrm{U}$ \\
\hline $108-90-7$ & Chlorobenzene & 1.0 & $\mathrm{U}$ \\
\hline $100-41-4$ & Ethylbenzene & 0.027 & $\mathrm{~J} B$ \\
\hline $95-47-6$ & o-Xylene & 0.039 & $\mathrm{~J} B$ \\
\hline $179601-23-1$ & m,p-xylene & 0.10 & $\mathrm{~J} B$ \\
\hline $100-42-5$ & Styrene & 0.17 & $\mathrm{~J} B$ \\
\hline $75-25-2$ & Bromoform & 1.0 & $\mathrm{U}$ \\
\hline $98-82-8$ & Isopropylbenzene & 1.0 & $\mathrm{U}$ \\
\hline $79-34-5$ & $1,1,2,2$-Tetrachloroethane & 1.0 & $\mathrm{U}$ \\
\hline $541-73-1$ & 1,3 -Dichlorobenzene & 1.0 & $\mathrm{U}$ \\
\hline $106-46-7$ & 1,4-Dichlorobenzene & 1.0 & $\mathrm{U}$ \\
\hline $95-50-1$ & 1,2-Dichlorobenzene & 1.0 & $\mathrm{U}$ \\
\hline $96-12-8$ & 1,2-Dibromo-3-Chloropropane & 1.0 & $\mathrm{U}$ \\
\hline $120-82-1$ & $1,2,4$-Trichlorobenzene & 0.011 & $\mathrm{~J} B$ \\
\hline $87-61-6$ & $1,2,3$-Trichlorobenzene & 0.0095 & $\mathrm{~J} B$ \\
\hline
\end{tabular}


Lab Name: TESTAMERICA BURLINGTON

Contract: $8 \mathrm{E}-00302$

Lab Code: STLV Case No.: YORK Mod. Ref No.: SDG No.: 29233

Matrix: (SOIL/SED/WATER) Water

Sample wt/vol: 25.0 $(\mathrm{g} / \mathrm{mL}) \mathrm{mL}$

Level: (TRACE or LOW/MED) TRACE

․ Moisture: not dec.

GC Column: DB-624 ID $: 0.20 \quad(\mathrm{~mm})$

Soil Extract Volume: (uL)
Lab Sample ID: 200-29233-4

Lab File ID: 15254_012.D

Date Received: 08/11/2015

Date Analyzed: 08/12/2015

Dilution Factor: 1.0

Soil Aliquot Volume: (uL) Purge Volume: 25.0 $(\mathrm{mL})$

$\overline{(\mathrm{mL})}$
CONCENTRATION UNITS: (ug/L or ug/kg) ug/L

01

\begin{tabular}{|l|l|c|c|c|}
\hline CAS NUMBER & \multicolumn{1}{|c|}{ COMPOUND NAME } & RT & EST. CONC. & Q \\
\hline & Unknown & 7.29 & 3.1 & $\mathrm{~J}$ B X \\
\hline E9667961 & Total Alkanes & N/A & & \\
\hline
\end{tabular}

1 EPA-designated Registry Number. 
Lab Name: TESTAMERICA BURLINGTON

Contract: $8 \mathrm{E}-00302$

Lab Code: STLV

Case No.: YORK Mod. Ref No.:

SDG No.: 29233

Matrix: (SOIL/SED/WATER) Water

Sample wt/vol: 25.0 $(\mathrm{g} / \mathrm{mL}) \mathrm{mL}$

Level: (TRACE/LOW/MED) TRACE

\% Moisture: not dec.

GC Column: DB-624 ID: $0.20 \quad(\mathrm{~mm})$

Soil Extract Volume: (uL) $(\mathrm{mL})$

Purge Volume: 25.0
Lab Sample ID: 200-29233-4

Lab File ID: 15265_007.D

Date Received: 08/11/2015

Date Analyzed: 08/13/2015

Dilution Factor: 1.8

Soil Aliquot Volume: (uL)

\begin{tabular}{|c|c|c|c|}
\hline CAS NO. & COMPOUND & $\begin{array}{l}\text { CONCENTRATION UNITS: } \\
(\mathrm{ug} / \mathrm{L} \text { or } \mathrm{ug} / \mathrm{kg}) \mathrm{ug} / \mathrm{L}\end{array}$ & q \\
\hline $75-71-8$ & Dichlorodifluoromethane & 1.8 & $\mathrm{U}$ \\
\hline $74-87-3$ & Chloromethane & 1.8 & $\mathrm{U}$ \\
\hline $75-01-4$ & Vinyl chloride & 1.8 & $\mathrm{U}$ \\
\hline $74-83-9$ & Bromomethane & 1.8 & $\mathrm{U}$ \\
\hline $75-00-3$ & Chloroethane & 1.8 & $\mathrm{U}$ \\
\hline $75-69-4$ & Trichlorofluoromethane & 1.8 & $\mathrm{U}$ \\
\hline $75-35-4$ & 1,1-Dichloroethene & 1.8 & $\mathrm{U}$ \\
\hline $76-13-1$ & $1,1,2$-Trichloro-1,2,2-trifluoroethane & 1.8 & $\mathrm{U}$ \\
\hline $67-64-1$ & Acetone & 9.2 & $\mathrm{U}$ \\
\hline $75-15-0$ & Carbon disulfide & 1.8 & $\mathrm{U}$ \\
\hline $79-20-9$ & Methyl acetate & 1.8 & $\mathrm{U}$ \\
\hline $75-09-2$ & Methylene Chloride & 1.8 & $\mathrm{U}$ \\
\hline $156-60-5$ & trans-1,2-Dichloroethene & 1.8 & $\mathrm{U}$ \\
\hline $1634-04-4$ & Methyl tert-butyl ether & 1.8 & $\mathrm{U}$ \\
\hline $75-34-3$ & 1,1-Dichloroethane & 1.8 & $\mathrm{U}$ \\
\hline $156-59-2$ & cis-1,2-Dichloroethene & 1.8 & $\mathrm{U}$ \\
\hline $78-93-3$ & 2-Butanone & 9.2 & $\mathrm{U}$ \\
\hline $74-97-5$ & Bromochloromethane & 1.8 & $\mathrm{U}$ \\
\hline $67-66-3$ & Chloroform & 2.5 & $\mathrm{D}$ \\
\hline $71-55-6$ & 1,1,1-Trichloroethane & 1.8 & $\mathrm{U}$ \\
\hline $110-82-7$ & Cyclohexane & 1.8 & $\mathrm{U}$ \\
\hline $56-23-5$ & Carbon tetrachloride & 23 & $\mathrm{D}$ \\
\hline $71-43-2$ & Benzene & 1.8 & $\mathrm{U}$ \\
\hline $107-06-2$ & 1,2-Dichloroethane & 1.8 & $\mathrm{U}$ \\
\hline
\end{tabular}

Report 1,4-Dioxane for Low-Medium VOA analysis only 
Lab Name: TESTAMERICA BURLINGTON

Lab Code: STLV

Case No.: YORK

Mod. Ref No.:

Contract: $8 \mathrm{E}-00302$

Matrix: (SOIL/SED/WATER) Water

Sample wt/vol: 25.0 $(\mathrm{g} / \mathrm{mL}) \mathrm{mL}$

Level: (TRACE/LOW/MED) TRACE

\% Moisture: not dec.

GC Column: DB-624 ID $: \underline{0.20 \quad(\mathrm{~mm})}$

Soil Extract Volume: (uL) $(\mathrm{mL})$

Purge Volume: 25.0
SDG No.: 29233

Lab Sample ID: 200-29233-4

Lab File ID: 15265_007.D

Date Received: 08/11/2015

Date Analyzed: 08/13/2015

Dilution Factor: 1.8

Soil Aliquot Volume: (uL)

\begin{tabular}{|c|c|c|c|}
\hline CAS NO. & COMPOUND & $\begin{array}{l}\text { CONCENTRATION UNITS: } \\
(\mathrm{ug} / \mathrm{L} \text { or } \mathrm{ug} / \mathrm{kg}) \mathrm{ug} / \mathrm{L}\end{array}$ & Q \\
\hline $79-01-6$ & Trichloroethene & 1.8 & $\mathrm{U}$ \\
\hline $108-87-2$ & Methylcyclohexane & 1.8 & $\mathrm{U}$ \\
\hline $78-87-5$ & 1,2-Dichloropropane & 1.8 & $\mathrm{U}$ \\
\hline $75-27-4$ & Bromodichloromethane & 1.8 & $\mathrm{U}$ \\
\hline $10061-01-5$ & cis-1,3-Dichloropropene & 1.8 & U \\
\hline $108-10-1$ & 4-Methyl-2-pentanone & 1.8 & $\mathrm{U}$ \\
\hline $108-88-3$ & Toluene & 0.15 & $\mathrm{~J} \mathrm{D}$ \\
\hline $10061-02-6$ & trans-1,3-Dichloropropene & 1.8 & $\mathrm{U}$ \\
\hline $79-00-5$ & $1,1,2-$ Trichloroethane & 1.8 & $\mathrm{U}$ \\
\hline $127-18-4$ & Tetrachloroethene & 1.8 & $\mathrm{U}$ \\
\hline $591-78-6$ & 2-Hexanone & 1.8 & $\mathrm{U}$ \\
\hline $124-48-1$ & Dibromochloromethane & 1.8 & $\mathrm{U}$ \\
\hline $106-93-4$ & 1,2-Dibromoethane & 1.8 & $\mathrm{U}$ \\
\hline $108-90-7$ & Chlorobenzene & 1.8 & $\mathrm{U}$ \\
\hline $100-41-4$ & Ethylbenzene & 0.032 & $\mathrm{~J} \mathrm{D}$ \\
\hline $95-47-6$ & o-Xylene & 1.8 & $\mathrm{U}$ \\
\hline $179601-23-1$ & m,p-xylene & 1.8 & $\mathrm{U}$ \\
\hline $100-42-5$ & Styrene & 0.15 & $\mathrm{~J} D$ \\
\hline $75-25-2$ & Bromoform & 1.8 & $\mathrm{U}$ \\
\hline $98-82-8$ & Isopropylbenzene & 1.8 & $\mathrm{U}$ \\
\hline $79-34-5$ & $1,1,2,2$-Tetrachloroethane & 1.8 & $\mathrm{U}$ \\
\hline $541-73-1$ & 1,3 -Dichlorobenzene & 1.8 & $\mathrm{U}$ \\
\hline $106-46-7$ & 1,4-Dichlorobenzene & 1.8 & $\mathrm{U}$ \\
\hline $95-50-1$ & 1,2-Dichlorobenzene & 1.8 & $\mathrm{U}$ \\
\hline $96-12-8$ & 1,2-Dibromo-3-Chloropropane & 1.8 & $\mathrm{U}$ \\
\hline $120-82-1$ & $1,2,4$-Trichlorobenzene & 1.8 & $\mathrm{U}$ \\
\hline $87-61-6$ & $1,2,3$-Trichlorobenzene & 1.8 & $\mathrm{U}$ \\
\hline
\end{tabular}


Lab Name: TESTAMERICA BURLINGTON

Contract: $8 \mathrm{E}-00302$

Lab Code: STLV Case No.: YORK Mod. Ref No.: SDG No.: 29233

Matrix: (SOIL/SED/WATER) Water

Sample wt/vol: 25.0 $(\mathrm{g} / \mathrm{mL}) \mathrm{mL}$

Level: (TRACE or LOW/MED) TRACE

․ Moisture: not dec.

GC Column: DB-624 ID $: 0.20 \quad(\mathrm{~mm})$

Soil Extract Volume: (uL)
Lab Sample ID: 200-29233-4

Lab File ID: 15265_007.D

Date Received: 08/11/2015

Date Analyzed: 08/13/2015

Dilution Factor: 1.8

Soil Aliquot Volume: (uL) Purge Volume: 25.0

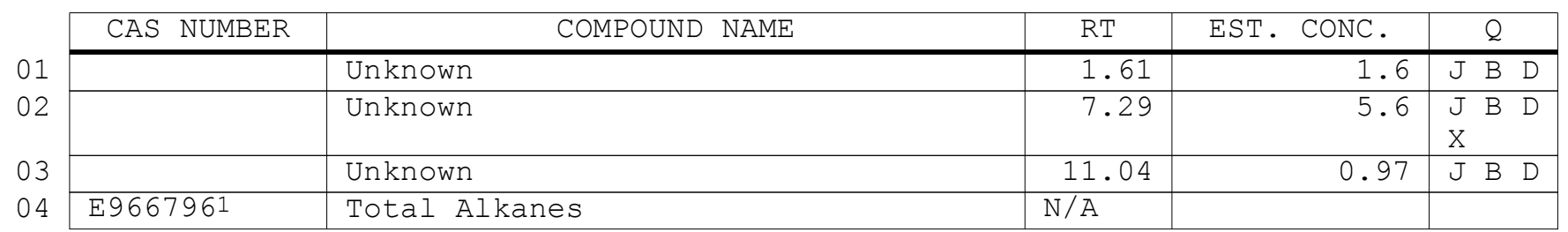

1 EPA-designated Registry Number. 
Lab Name: TESTAMERICA BURLINGTON

Contract: 8E-00302

Lab Code: STLV

Case No.: YORK Mod. Ref No.:

SDG No.: 29233

Matrix: (SOIL/SED/WATER) Water

Sample wt/vol: 25.0 $(\mathrm{g} / \mathrm{mL}) \mathrm{mL}$

Level: (TRACE/LOW/MED) TRACE

\% Moisture: not dec.

GC Column: DB-624 ID $: 0.20 \quad(\mathrm{~mm})$

Soil Extract Volume: (uL) $(\mathrm{mL})$

Purge Volume: 25.0
Lab Sample ID: 200-29233-3

Lab File ID: 15254_010.D

Date Received: 08/11/2015

Date Analyzed: 08/12/2015

Dilution Factor: 1.0

Soil Aliquot Volume: (uL)

\begin{tabular}{|c|c|c|c|}
\hline CAS NO. & COMPOUND & $\begin{array}{l}\text { CONCENTRATION UNITS: } \\
(\mathrm{ug} / \mathrm{L} \text { or } \mathrm{ug} / \mathrm{kg}) \mathrm{ug} / \mathrm{L}\end{array}$ & Q \\
\hline $75-71-8$ & Dichlorodifluoromethane & 1.0 & $\mathrm{U}$ \\
\hline $74-87-3$ & Chloromethane & 1.0 & $\mathrm{U}$ \\
\hline $75-01-4$ & Vinyl chloride & 1.0 & $\mathrm{U}$ \\
\hline $74-83-9$ & Bromomethane & 1.0 & $\mathrm{U}$ \\
\hline $75-00-3$ & Chloroethane & 1.0 & $\mathrm{U}$ \\
\hline $75-69-4$ & Trichlorofluoromethane & 0.077 & $\mathrm{~J}$ \\
\hline $75-35-4$ & 1,1-Dichloroethene & 1.0 & $\mathrm{U}$ \\
\hline $76-13-1$ & $1,1,2$-Trichloro-1,2,2-trifluoroethane & 1.0 & $\mathrm{U}$ \\
\hline $67-64-1$ & Acetone & 5.0 & $\mathrm{U}$ \\
\hline $75-15-0$ & Carbon disulfide & 0.030 & $\mathrm{~J} B$ \\
\hline $79-20-9$ & Methyl acetate & 1.0 & $\mathrm{U}$ \\
\hline $75-09-2$ & Methylene Chloride & 1.0 & $\mathrm{U}$ \\
\hline $156-60-5$ & trans-1,2-Dichloroethene & 1.0 & $\mathrm{U}$ \\
\hline $1634-04-4$ & Methyl tert-butyl ether & 1.0 & $\mathrm{U}$ \\
\hline $75-34-3$ & 1,1-Dichloroethane & 1.0 & $\mathrm{U}$ \\
\hline $156-59-2$ & cis-1,2-Dichloroethene & 1.0 & $\mathrm{U}$ \\
\hline $78-93-3$ & 2-Butanone & 5.0 & $\mathrm{U}$ \\
\hline $74-97-5$ & Bromochloromethane & 1.0 & $\mathrm{U}$ \\
\hline $67-66-3$ & Chloroform & 1.1 & \\
\hline $71-55-6$ & $1,1,1$-Trichloroethane & 1.0 & $\mathrm{U}$ \\
\hline $110-82-7$ & Cyclohexane & 1.0 & $\mathrm{U}$ \\
\hline $56-23-5$ & Carbon tetrachloride & 64 & $\mathrm{E}$ \\
\hline $71-43-2$ & Benzene & 1.0 & $\mathrm{U}$ \\
\hline $107-06-2$ & 1,2-Dichloroethane & 1.0 & $\mathrm{U}$ \\
\hline
\end{tabular}

Report 1,4-Dioxane for Low-Medium VOA analysis only 
Lab Name: TESTAMERICA BURLINGTON

Contract: $8 \mathrm{E}-00302$

Lab Code: STLV

Case No.: YORK

Mod. Ref No.:

SDG No.: 29233

Matrix: (SOIL/SED/WATER) Water

Sample wt/vol: 25.0 $(\mathrm{g} / \mathrm{mL}) \mathrm{mL}$

Level: (TRACE/LOW/MED) TRACE

\% Moisture: not dec.

GC Column: DB-624 ID $: 0.20 \quad(\mathrm{~mm})$

Soil Extract Volume: (uL) $(\mathrm{mL})$

Purge Volume: 25.0 (mI)
Lab Sample ID: 200-29233-3

Lab File ID: 15254_010.D

Date Received: 08/11/2015

Date Analyzed: 08/12/2015

Dilution Factor: 1.0

Soil Aliquot Volume: (uL)

\begin{tabular}{|c|c|c|c|}
\hline CAS NO. & COMPOUND & $\begin{array}{l}\text { CONCENTRATION UNITS: } \\
(\mathrm{ug} / \mathrm{L} \text { or } \mathrm{ug} / \mathrm{kg}) \mathrm{ug} / \mathrm{L}\end{array}$ & Q \\
\hline $79-01-6$ & Trichloroethene & 1.0 & $\mathrm{U}$ \\
\hline $108-87-2$ & Methylcyclohexane & 1.0 & $\mathrm{U}$ \\
\hline $78-87-5$ & 1,2-Dichloropropane & 1.0 & $\mathrm{U}$ \\
\hline $75-27-4$ & Bromodichloromethane & 1.0 & $\mathrm{U}$ \\
\hline $10061-01-5$ & cis-1,3-Dichloropropene & 1.0 & $\mathrm{U}$ \\
\hline $108-10-1$ & 4-Methyl-2-pentanone & 1.0 & $\mathrm{U}$ \\
\hline $108-88-3$ & Toluene & 0.065 & $\mathrm{~J} B$ \\
\hline $10061-02-6$ & trans-1,3-Dichloropropene & 1.0 & $\mathrm{U}$ \\
\hline $79-00-5$ & $1,1,2-T r i c h l o r o e t h a n e$ & 1.0 & $\mathrm{U}$ \\
\hline $127-18-4$ & Tetrachloroethene & 0.015 & $\mathrm{~J}$ \\
\hline $591-78-6$ & 2-Hexanone & 1.0 & $\mathrm{U}$ \\
\hline $124-48-1$ & Dibromochloromethane & 1.0 & $\mathrm{U}$ \\
\hline $106-93-4$ & 1,2-Dibromoethane & 1.0 & $\mathrm{U}$ \\
\hline $108-90-7$ & Chlorobenzene & 1.0 & $\mathrm{U}$ \\
\hline $100-41-4$ & Ethylbenzene & 0.0093 & $\mathrm{~J} B$ \\
\hline $95-47-6$ & o-Xylene & 0.0093 & $\mathrm{~J} B$ \\
\hline $179601-23-1$ & m,p-Xylene & 0.033 & $\mathrm{~J} B$ \\
\hline $100-42-5$ & styrene & 0.0049 & $\mathrm{~J} B$ \\
\hline $75-25-2$ & Bromoform & 1.0 & $\mathrm{U}$ \\
\hline $98-82-8$ & Isopropylbenzene & 1.0 & $\mathrm{U}$ \\
\hline $79-34-5$ & $1,1,2,2$-Tetrachloroethane & 1.0 & $\mathrm{U}$ \\
\hline $541-73-1$ & 1,3-Dichlorobenzene & 1.0 & $\mathrm{U}$ \\
\hline $106-46-7$ & 1,4-Dichlorobenzene & 1.0 & $\mathrm{U}$ \\
\hline $95-50-1$ & $1,2-$ Dichlorobenzene & 1.0 & $\mathrm{U}$ \\
\hline $96-12-8$ & 1,2-Dibromo-3-Chloropropane & 1.0 & $\mathrm{U}$ \\
\hline $120-82-1$ & $1,2,4$-Trichlorobenzene & 0.016 & $\mathrm{~J} B$ \\
\hline $87-61-6$ & $1,2,3$-Trichlorobenzene & 0.014 & $\mathrm{~J} B$ \\
\hline
\end{tabular}


Lab Name: TESTAMERICA BURLINGTON

Contract: 8E-00302

Lab Code: STLV Case No.: YORK Mod. Ref No.: SDG No.: 29233

Matrix: (SOIL/SED/WATER) Water

Sample wt/vol: 25.0 $(\mathrm{g} / \mathrm{mL}) \mathrm{mL}$

Level: (TRACE or LOW/MED) TRACE

․ Moisture: not dec.

GC Column: DB-624 ID $: 0.20 \quad(\mathrm{~mm})$

Soil Extract Volume: (uL)
Lab Sample ID: 200-29233-3

Lab File ID: 15254_010.D

Date Received: 08/11/2015

Date Analyzed: 08/12/2015

Dilution Factor: 1.0

Soil Aliquot Volume: (uL) Purge Volume: 25.0 $(\mathrm{mL})$

$\overline{(\mathrm{mL})}$
CONCENTRATION UNITS: (ug/L or ug/kg) ug/L

01

\begin{tabular}{|l|l|c|c|c|}
\hline CAS NUMBER & \multicolumn{1}{|c|}{ COMPOUND NAME } & RT & EST. CONC. & Q \\
\hline & Unknown & 7.29 & 3.2 & $\mathrm{~J}$ B X \\
\hline E9667961 & Total Alkanes & N/A & & \\
\hline
\end{tabular}

1 EPA-designated Registry Number. 
$1 A$ - FORM I VOA-1

VOLATILE ORGANICS ANALYSIS DATA SHEET
EPA SAMPLE NO.

YORKCOUNTYSHOP-W-37917

Lab Name: TESTAMERICA BURLINGTON

Contract: 8E-00302

Lab Code: STLV

Case No.: YORK

Mod. Ref No.:

SDG No.: 29233

Matrix: (SOIL/SED/WATER) Water

Sample wt/vol: 25.0 $(\mathrm{g} / \mathrm{mL}) \mathrm{mL}$

Level: (TRACE/LOW/MED) TRACE

\% Moisture: not dec.

GC Column: DB-624 ID: $0.20 \quad(\mathrm{~mm})$

Soil Extract Volume: (uL) $(\mathrm{mL})$

Purge Volume: 25.0
Lab Sample ID: 200-29233-3

Lab File ID: 15265 _006.D

Date Received: 08/11/2015

Date Analyzed: 08/13/2015

Dilution Factor: 4.7

Soil Aliquot Volume: (uL)

\begin{tabular}{|c|c|c|c|}
\hline CAS NO. & COMPOUND & $\begin{array}{l}\text { CONCENTRATION UNITS: } \\
(\mathrm{ug} / \mathrm{L} \text { or } \mathrm{ug} / \mathrm{kg}) \mathrm{ug} / \mathrm{L}\end{array}$ & q \\
\hline $75-71-8$ & Dichlorodifluoromethane & 4.7 & $\mathrm{U}$ \\
\hline $74-87-3$ & Chloromethane & 4.7 & $\mathrm{U}$ \\
\hline $75-01-4$ & Vinyl chloride & 4.7 & $\mathrm{U}$ \\
\hline $74-83-9$ & Bromomethane & 4.7 & $\mathrm{U}$ \\
\hline $75-00-3$ & Chloroethane & 4.7 & $\mathrm{U}$ \\
\hline $75-69-4$ & Trichlorofluoromethane & 4.7 & $\mathrm{U}$ \\
\hline $75-35-4$ & 1,1-Dichloroethene & 4.7 & $\mathrm{U}$ \\
\hline $76-13-1$ & $1,1,2$-Trichloro-1,2,2-trifluoroethane & 4.7 & $\mathrm{U}$ \\
\hline $67-64-1$ & Acetone & 23 & $\mathrm{U}$ \\
\hline $75-15-0$ & Carbon disulfide & 4.7 & $\mathrm{U}$ \\
\hline $79-20-9$ & Methyl acetate & 4.7 & $\mathrm{U}$ \\
\hline $75-09-2$ & Methylene Chloride & 4.7 & $\mathrm{U}$ \\
\hline $156-60-5$ & trans-1,2-Dichloroethene & 4.7 & $\mathrm{U}$ \\
\hline $1634-04-4$ & Methyl tert-butyl ether & 4.7 & $\mathrm{U}$ \\
\hline $75-34-3$ & 1,1-Dichloroethane & 4.7 & $\mathrm{U}$ \\
\hline $156-59-2$ & cis-1,2-Dichloroethene & 4.7 & $\mathrm{U}$ \\
\hline $78-93-3$ & 2-Butanone & 23 & $\mathrm{U}$ \\
\hline $74-97-5$ & Bromochloromethane & 4.7 & $\mathrm{U}$ \\
\hline $67-66-3$ & Chloroform & 4.7 & $\mathrm{U}$ \\
\hline $71-55-6$ & 1,1,1-Trichloroethane & 4.7 & $\mathrm{U}$ \\
\hline $110-82-7$ & Cyclohexane & 4.7 & $\mathrm{U}$ \\
\hline $56-23-5$ & Carbon tetrachloride & 52 & $\mathrm{D}$ \\
\hline $71-43-2$ & Benzene & 4.7 & $\mathrm{U}$ \\
\hline $107-06-2$ & 1,2-Dichloroethane & 4.7 & $\mathrm{U}$ \\
\hline
\end{tabular}

Report 1,4-Dioxane for Low-Medium VOA analysis only 
Lab Name: TESTAMERICA BURLINGTON

Contract: 8E-00302

Lab Code: STLV

Case No.: YORK

Mod. Ref No.:

SDG No.: 29233

Matrix: (SOIL/SED/WATER) Water

Sample wt/vol: 25.0 $(\mathrm{g} / \mathrm{mL}) \mathrm{mL}$

Level: (TRACE/LOW/MED) TRACE

\% Moisture: not dec.

GC Column: DB-624 ID $0.20 \quad(\mathrm{~mm})$

Soil Extract Volume: (uL) $(\mathrm{mL})$

Purge Volume: 25.0 (mI)
Lab Sample ID: 200-29233-3

Lab File ID: 15265_006.D

Date Received: 08/11/2015

Date Analyzed: 08/13/2015

Dilution Factor: 4.7

Soil Aliquot Volume: (uL)

\begin{tabular}{|c|c|c|c|}
\hline CAS NO. & COMPOUND & $\begin{array}{l}\text { CONCENTRATION UNITS: } \\
(\mathrm{ug} / \mathrm{L} \text { or } \mathrm{ug} / \mathrm{kg}) \mathrm{ug} / \mathrm{L}\end{array}$ & $q$ \\
\hline $79-01-6$ & Trichloroethene & 4.7 & $\mathrm{U}$ \\
\hline $108-87-2$ & Methylcyclohexane & 4.7 & $\mathrm{U}$ \\
\hline $78-87-5$ & 1,2-Dichloropropane & 4.7 & $\mathrm{U}$ \\
\hline $75-27-4$ & Bromodichloromethane & 4.7 & $\mathrm{U}$ \\
\hline $10061-01-5$ & cis-1,3-Dichloropropene & 4.7 & U \\
\hline $108-10-1$ & 4-Methyl-2-pentanone & 4.7 & $\mathrm{U}$ \\
\hline $108-88-3$ & Toluene & 4.7 & $\mathrm{U}$ \\
\hline $10061-02-6$ & trans-1,3-Dichloropropene & 4.7 & $\mathrm{U}$ \\
\hline $79-00-5$ & $1,1,2-$ Trichloroethane & 4.7 & $\mathrm{U}$ \\
\hline $127-18-4$ & Tetrachloroethene & 4.7 & $\mathrm{U}$ \\
\hline $591-78-6$ & 2-Hexanone & 4.7 & $\mathrm{U}$ \\
\hline $124-48-1$ & Dibromochloromethane & 4.7 & $\mathrm{U}$ \\
\hline $106-93-4$ & 1,2-Dibromoethane & 4.7 & $\mathrm{U}$ \\
\hline $108-90-7$ & Chlorobenzene & 4.7 & $\mathrm{U}$ \\
\hline $100-41-4$ & Ethylbenzene & 4.7 & $\mathrm{U}$ \\
\hline $95-47-6$ & o-Xylene & 4.7 & $\mathrm{U}$ \\
\hline $179601-23-1$ & m,p-xylene & 4.7 & $\mathrm{U}$ \\
\hline $100-42-5$ & Styrene & 4.7 & $\mathrm{U}$ \\
\hline $75-25-2$ & Bromoform & 4.7 & $\mathrm{U}$ \\
\hline $98-82-8$ & Isopropylbenzene & 4.7 & $\mathrm{U}$ \\
\hline $79-34-5$ & $1,1,2,2$-Tetrachloroethane & 4.7 & $\mathrm{U}$ \\
\hline $541-73-1$ & 1,3 -Dichlorobenzene & 4.7 & $\mathrm{U}$ \\
\hline $106-46-7$ & 1,4-Dichlorobenzene & 4.7 & $\mathrm{U}$ \\
\hline $95-50-1$ & 1,2-Dichlorobenzene & 4.7 & $\mathrm{U}$ \\
\hline $96-12-8$ & 1,2-Dibromo-3-Chloropropane & 4.7 & $\mathrm{U}$ \\
\hline $120-82-1$ & $1,2,4$-Trichlorobenzene & 4.7 & $\mathrm{U}$ \\
\hline $87-61-6$ & $1,2,3$-Trichlorobenzene & 4.7 & $\mathrm{U}$ \\
\hline
\end{tabular}


IJ - FORM I VOA-TIC

VOLATILE ORGANICS ANALYSIS DATA SHEET

TENTATIVELY IDENTIFIED COMPOUNDS

Lab Name: TESTAMERICA BURLINGTON

Contract: $8 \mathrm{E}-00302$

Lab Code: STLV Case No.: YORK Mod. Ref No.: SDG No.: 29233

Matrix: (SOIL/SED/WATER) Water

Sample wt/vol: 25.0 $(\mathrm{g} / \mathrm{mL}) \mathrm{mL}$

Level: (TRACE or LOW/MED) TRACE

․ Moisture: not dec.

GC Column: DB-624 ID $: 0.20 \quad(\mathrm{~mm})$

Soil Extract Volume: (uL)
Lab Sample ID: 200-29233-3

Lab File ID: 15265_006.D

Date Received: 08/11/2015

Date Analyzed: 08/13/2015

Dilution Factor: 4.7

Soil Aliquot Volume: (uL)

Purge Volume: 25.0 $(\mathrm{mL})$ CONCENTRATION UNITS: (ug/L or ug/kg) ug/L

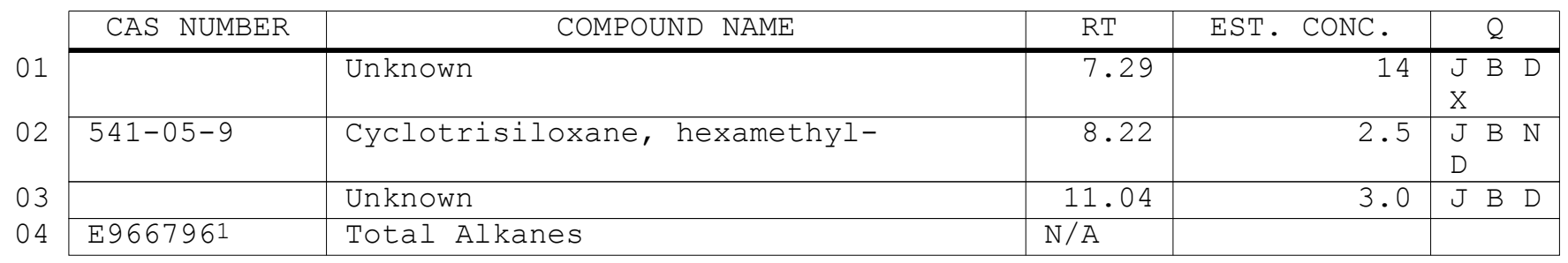

1 EPA-designated Registry Number. 
Lab Name: TESTAMERICA BURLINGTON

Contract: $8 \mathrm{E}-00302$

Lab Code: STLV Case No.: YORK Mod. Ref No.:

SDG No.: 29233

Instrument ID: CHD.i

Calibration Date(s): 08/06/2015 08/06/2015

Heated Purge: (Y/N) N

Calibration Time(s) : 1011

1149

Purge Volume: 25.0 $(\mathrm{mL})$

GC Column: DB-624

ID: $0.20 \quad$ (mm) Length: 25

$(\mathrm{m})$

\begin{tabular}{|c|c|c|c|c|c|c|c|}
\hline LAB FILE ID: & \multirow{2}{*}{\multicolumn{3}{|c|}{$\begin{array}{l}\operatorname{RRF0.5}=\frac{15136 \_003 . D}{R=15136 \_006 . D} \\
\text { RRE10 }\end{array}$}} & \multicolumn{4}{|c|}{ RRF1.0 = 15136_004.D } \\
\hline RRF5.0 = 15136_005.D & & & & RRF2C & $=151$ & 5 & \\
\hline COMPOUND & $\operatorname{RRF} 0.5$ & RRF1. 0 & RRF5. 0 & RRF10 & RRE20 & $\overline{\mathrm{RRF}}$ & $\circ \mathrm{RSD}$ \\
\hline Dichlorodifluoromethane & 0.435 & 0.440 & 0.497 & 0.485 & 0.497 & 0.471 & 6.6 \\
\hline Chloromethane & 0.439 & 0.444 & 0.448 & 0.449 & 0.406 & 0.437 & 4.1 \\
\hline Vinyl chloride & 0.352 & 0.378 & 0.424 & 0.422 & 0.409 & 0.397 & 7.9 \\
\hline Bromomethane & 0.176 & 0.139 & 0.155 & 0.164 & 0.151 & 0.157 & 9.1 \\
\hline Chloroethane & 0.178 & 0.221 & 0.220 & 0.212 & 0.163 & 0.199 & 13.4 \\
\hline Trichlorofluoromethane & 0.462 & 0.472 & 0.540 & 0.532 & 0.518 & 0.505 & 7.1 \\
\hline 1,1-Dichloroethene & 0.221 & 0.233 & 0.275 & 0.261 & 0.247 & 0.247 & 8.7 \\
\hline $\begin{array}{l}1,1,2 \text {-Trichloro- } \\
1,2,2 \text {-trifluoroethane }\end{array}$ & 0.249 & 0.265 & 0.304 & 0.294 & 0.276 & 0.278 & 7.9 \\
\hline Acetone & 0.020 & 0.018 & 0.019 & 0.020 & 0.020 & 0.019 & 3.9 \\
\hline Carbon disulfide & 0.519 & 0.556 & 0.710 & 0.673 & 0.820 & 0.656 & 18.5 \\
\hline Methyl acetate & 0.053 & 0.053 & 0.068 & 0.063 & 0.059 & 0.059 & 10.7 \\
\hline Methylene Chloride & 0.270 & 0.233 & 0.252 & 0.245 & 0.244 & 0.249 & 5.4 \\
\hline trans-1,2-Dichloroethene & 0.260 & 0.266 & 0.319 & 0.315 & 0.313 & 0.295 & 9.8 \\
\hline Methyl tert-butyl ether & 0.278 & 0.287 & 0.347 & 0.344 & 0.341 & 0.319 & 10.6 \\
\hline 1,1-Dichloroethane & 0.473 & 0.487 & 0.586 & 0.576 & 0.590 & 0.542 & 10.6 \\
\hline cis-1,2-Dichloroethene & 0.252 & 0.255 & 0.310 & 0.305 & 0.307 & 0.286 & 10.3 \\
\hline 2-Butanone & 0.029 & 0.033 & 0.041 & 0.041 & 0.041 & 0.037 & 15.3 \\
\hline Bromochloromethane & 0.069 & 0.074 & 0.091 & 0.091 & 0.090 & 0.083 & 12.6 \\
\hline Chloroform & 0.420 & 0.438 & 0.516 & 0.506 & 0.513 & 0.479 & 9.6 \\
\hline $1,1,1$-Trichloroethane & 0.446 & 0.483 & 0.593 & 0.595 & 0.617 & 0.547 & 14.1 \\
\hline Cyclohexane & 0.683 & 0.727 & 0.831 & 0.798 & 0.798 & 0.767 & 7.9 \\
\hline Carbon tetrachloride & 0.368 & 0.386 & 0.502 & 0.509 & 0.529 & 0.459 & 16.4 \\
\hline Benzene & 1.415 & 1.476 & 1.607 & 1.553 & 1.556 & 1.521 & 5.0 \\
\hline 1,2-Dichloroethane & 0.200 & 0.220 & 0.241 & 0.236 & 0.233 & 0.226 & 7.4 \\
\hline Trichloroethene & 0.331 & 0.359 & 0.412 & 0.402 & 0.411 & 0.383 & 9.4 \\
\hline Methylcyclohexane & 0.558 & 0.606 & 0.692 & 0.663 & 0.666 & 0.637 & 8.5 \\
\hline
\end{tabular}

Report 1,4-Dioxane for Low-Medium VOA analysis only 
Lab Name: TESTAMERICA BURLINGTON

Contract: $8 \mathrm{E}-00302$

Lab Code: STLV Case No.: YORK Mod. Ref No.:

SDG No.: 29233

Instrument ID: CHD.i

$\begin{array}{llll}\text { Calibration Date (s) : } & 08 / 06 / 2015 & \frac{08 / 06 / 2015}{1149} \\ \text { Calibration Time (s) : } 1011 & \frac{119}{10}\end{array}$

Heated Purge: (Y/N) N

Purge Volume: 25.0 $(\mathrm{mL})$

GC Column: DB-624

ID: 0.20

$(\mathrm{mm})$

Length: 25

(m)

\begin{tabular}{|c|c|c|c|c|c|c|c|}
\hline \multirow{2}{*}{$\begin{array}{l}\text { LAB FILE ID: } \\
\text { RRF5.0 }=\text { 15136_005.D }\end{array}$} & \multicolumn{3}{|c|}{ RRF0.5 = $15136003 . D$} & \multicolumn{4}{|c|}{ RRF1.0 = $15136004 . D$} \\
\hline & E10 & $136 \_00$ & & RRF2C & $=1513$ & - $007 . \mathrm{D}$ & \\
\hline COMPOUND & $\mathrm{RRF} 0.5$ & RRF1. 0 & RRF5.0 & RRF10 & RRF20 & $\overline{\mathrm{RRF}}$ & $\because \mathrm{RSD}$ \\
\hline 1,2-Dichloropropane & 0.261 & 0.286 & 0.328 & 0.317 & 0.314 & 0.301 & 9.2 \\
\hline Bromodichloromethane & 0.214 & 0.241 & 0.321 & 0.323 & 0.332 & 0.286 & 19.0 \\
\hline cis-1,3-Dichloropropene & 0.265 & 0.314 & 0.424 & 0.428 & 0.434 & 0.373 & 21.0 \\
\hline 4-Methyl-2-pentanone & 0.081 & 0.092 & 0.116 & 0.113 & 0.112 & 0.103 & 15.1 \\
\hline Toluene & 1.474 & 1.551 & 1.756 & 1.714 & 1.703 & 1.640 & 7.4 \\
\hline trans-1,3-Dichloropropene & 0.171 & 0.183 & 0.282 & 0.292 & 0.301 & 0.246 & 25.7 \\
\hline 1,1,2-Trichloroethane & 0.133 & 0.132 & 0.154 & 0.149 & 0.148 & 0.143 & 6.9 \\
\hline Tetrachloroethene & 0.305 & 0.323 & 0.373 & 0.371 & 0.383 & 0.351 & 9.8 \\
\hline 2-Hexanone & 0.054 & 0.060 & 0.080 & 0.077 & 0.077 & 0.070 & 17.1 \\
\hline Dibromochloromethane & 0.097 & 0.105 & 0.155 & 0.163 & 0.171 & 0.138 & 25.0 \\
\hline 1,2-Dibromoethane & 0.105 & 0.108 & 0.134 & 0.134 & 0.133 & 0.123 & 12.4 \\
\hline Chlorobenzene & 0.840 & 0.867 & 1.000 & 0.987 & 0.985 & 0.936 & 8.1 \\
\hline Ethylbenzene & 1.708 & 1.815 & 2.119 & 2.083 & 2.074 & 1.960 & 9.5 \\
\hline o-Xylene & 0.557 & 0.615 & 0.748 & 0.734 & 0.726 & 0.676 & 12.6 \\
\hline $\mathrm{m}, \mathrm{p}$-Xylene & 0.639 & 0.689 & 0.805 & 0.804 & 0.810 & 0.749 & 10.6 \\
\hline Styrene & 0.779 & 0.880 & 1.102 & 1.100 & 1.077 & 0.988 & 15.1 \\
\hline Bromoform & 0.074 & 0.075 & 0.110 & 0.122 & 0.136 & 0.104 & 26.8 \\
\hline Isopropylbenzene & 1.687 & 1.855 & 2.210 & 2.190 & 2.167 & 2.022 & 11.7 \\
\hline $1,1,2,2$-Tetrachloroethane & 0.119 & 0.124 & 0.152 & 0.151 & 0.152 & 0.140 & 11.9 \\
\hline 1,3-Dichlorobenzene & 1.359 & 1.409 & 1.640 & 1.675 & 1.699 & 1.557 & 10.3 \\
\hline 1,4-Dichlorobenzene & 1.336 & 1.374 & 1.587 & 1.592 & 1.600 & 1.498 & 8.8 \\
\hline 1,2-Dichlorobenzene & 1.093 & 1.107 & 1.287 & 1.298 & 1.292 & 1.215 & 8.7 \\
\hline 1,2-Dibromo-3-Chloropropane & 0.031 & 0.026 & 0.030 & 0.034 & 0.036 & 0.032 & 12.3 \\
\hline 1,2,4-Trichlorobenzene & 0.682 & 0.656 & 0.770 & 0.787 & 0.827 & 0.744 & 9.7 \\
\hline 1,2,3-Trichlorobenzene & 0.484 & 0.444 & 0.523 & 0.545 & 0.566 & 0.513 & 9.5 \\
\hline
\end{tabular}


Lab Name: TESTAMERICA BURLINGTON

Contract: $8 \mathrm{E}-00302$

Lab Code: STLV Case No.: YORK Mod. Ref No.: SDG No.: 29233

Instrument ID: CHD.i

Heated Purge: (Y/N) N

Purge Volume: 25.0

GC Column: DB-624
ID: 0.20
Calibration Date(s): 08/06/2015

Calibration Time(s) : 1011 $(\mathrm{mL})$

(mm) Length: 25
$08 / 06 / 2015$ 1149

\begin{tabular}{|c|c|c|c|c|c|c|c|}
\hline LAB FILE ID: & \multirow{2}{*}{\multicolumn{3}{|c|}{$\begin{array}{l}\operatorname{RRF0.5}=\frac{15136 \_003 . D}{\operatorname{RRF} 10}=15136 \_006 . \mathrm{D}\end{array}$}} & \multirow{2}{*}{\multicolumn{4}{|c|}{$\begin{array}{l}\operatorname{RRF1.0}=\underline{15136 \_004 . D} \\
\operatorname{RRF20}=15136 \_007 . \mathrm{D}\end{array}$}} \\
\hline RRF5.0 = 15136_005.D & & & & & & & \\
\hline COMPOUND & RRE0. 5 & RRE1. 0 & RRE5. 0 & RRF10 & RRE20 & $\overline{\mathrm{RRF}}$ & $\because \mathrm{RSD}$ \\
\hline Vinyl Chloride-d3 & 0.342 & 0.363 & 0.408 & 0.409 & 0.398 & 0.384 & 7.8 \\
\hline Chloroethane-d5 & 0.236 & 0.253 & 0.263 & 0.252 & 0.207 & 0.242 & 9.0 \\
\hline 1,1-Dichloroethene-d2 & 0.495 & 0.513 & 0.589 & 0.566 & 0.526 & 0.538 & 7.3 \\
\hline 2-Butanone-d5 & 0.031 & 0.031 & 0.038 & 0.038 & 0.039 & 0.035 & 12.0 \\
\hline Chloroform-d & 0.433 & 0.470 & 0.556 & 0.542 & 0.553 & 0.511 & 10.9 \\
\hline 1,2-Dichloroethane-d4 & 0.163 & 0.173 & 0.201 & 0.197 & 0.192 & 0.185 & 8.8 \\
\hline Benzene-d6 & 1.292 & 1.355 & 1.525 & 1.476 & 1.477 & 1.425 & 6.8 \\
\hline 1,2-Dichloropropane-d6 & 0.306 & 0.343 & 0.391 & 0.381 & 0.373 & 0.359 & 9.7 \\
\hline Toluene-d8 & 1.224 & 1.294 & 1.476 & 1.444 & 1.445 & 1.377 & 8.1 \\
\hline trans-1,3-Dichloropropene-d4 & 0.155 & 0.163 & 0.251 & 0.260 & 0.271 & 0.220 & 25.4 \\
\hline 2-Hexanone-d5 & 0.022 & 0.024 & 0.036 & 0.035 & 0.036 & 0.030 & 22.4 \\
\hline $1,1,2,2$-Tetrachloroethane-d2 & 0.114 & 0.114 & 0.151 & 0.152 & 0.151 & 0.136 & 14.9 \\
\hline 1,2-Dichlorobenzene-d4 & 0.745 & 0.705 & 0.816 & 0.812 & 0.819 & 0.780 & 6.7 \\
\hline
\end{tabular}

Report 1,4-Dioxane-d8 for Low-Medium VOA analysis only 
Lab Name: TESTAMERICA BURLINGTON

Contract: $8 \mathrm{E}-00302$

Lab Code: STLV Case No.: YORK Mod. Ref No.:

SDG No.: 29233

Instrument ID: CHD.i

Calibration Date: 08/12/2015 Time: 1753

Lab File Id: 15254_003.D

EPA Sample No. (VSTD\#\#\#\#) : VSTD005DB Init. Calib. Date(s):08/06/2015 08/06/2015

Heated Purge: (Y/N) N GC Column: DB-624

Init. Calib. Time(s) : 1011 1149

Purge Volume: 25.0 ID: $0.20(\mathrm{~mm})$ Length: 25 (m) $(\mathrm{mL})$

\begin{tabular}{|c|c|c|c|c|c|}
\hline COMPOUND & $\overline{\mathrm{RRF}}$ & RRF5.0 & $\begin{array}{l}\text { MIN } \\
\text { RRF }\end{array}$ & $\frac{\circ}{D}$ & $\mathrm{MAX} \div \mathrm{D}$ \\
\hline Dichlorodifluoromethane & 0.471 & 0.470 & 0.010 & -0.2 & 40.0 \\
\hline Chloromethane & 0.437 & 0.430 & 0.010 & -1.8 & 40.0 \\
\hline Vinyl chloride & 0.397 & 0.405 & 0.100 & 2.0 & 30.0 \\
\hline Bromomethane & 0.157 & 0.165 & 0.100 & 5.0 & 30.0 \\
\hline Chloroethane & 0.199 & 0.216 & 0.010 & 8.5 & 40.0 \\
\hline Trichlorofluoromethane & 0.505 & 0.519 & 0.010 & 2.8 & 40.0 \\
\hline 1,1-Dichloroethene & 0.247 & 0.254 & 0.100 & 2.6 & 30.0 \\
\hline 1,1,2-Trichloro-1,2,2-trifluoroethane & 0.278 & 0.275 & 0.010 & $-1 \cdot 1$ & 40.0 \\
\hline Acetone & 0.019 & 0.021 & 0.010 & 6.8 & 40.0 \\
\hline Carbon disulfide & 0.656 & 0.799 & 0.010 & 21.8 & 40.0 \\
\hline Methyl acetate & 0.059 & 0.063 & 0.010 & 5.5 & 40.0 \\
\hline Methylene Chloride & 0.249 & 0.246 & 0.010 & -1.0 & 40.0 \\
\hline trans-1,2-Dichloroethene & 0.295 & 0.304 & 0.010 & 3.4 & 40.0 \\
\hline Methyl tert-butyl ether & 0.319 & 0.363 & 0.010 & 13.7 & 40.0 \\
\hline 1,1-Dichloroethane & 0.542 & 0.574 & 0.200 & 5.8 & 30.0 \\
\hline cis-1,2-Dichloroethene & 0.286 & 0.293 & 0.010 & 2.6 & 40.0 \\
\hline 2-Butanone & 0.037 & 0.041 & 0.010 & 11.3 & 40.0 \\
\hline Bromochloromethane & 0.083 & 0.090 & 0.050 & 8.4 & 30.0 \\
\hline Chloroform & 0.479 & 0.504 & 0.200 & 5.3 & 30.0 \\
\hline 1,1,1-Trichloroethane & 0.547 & 0.593 & 0.100 & 8.4 & 30.0 \\
\hline Cyclohexane & 0.767 & 0.787 & 0.010 & 2.6 & 40.0 \\
\hline Carbon tetrachloride & 0.459 & 0.516 & 0.100 & 12.4 & 30.0 \\
\hline Benzene & 1.521 & 1.569 & 0.400 & 3.1 & 30.0 \\
\hline 1,2-Dichloroethane & 0.226 & 0.241 & 0.100 & 6.5 & 30.0 \\
\hline Trichloroethene & 0.383 & 0.399 & 0.300 & 4.2 & 30.0 \\
\hline Methylcyclohexane & 0.637 & 0.638 & 0.010 & 0.2 & 40.0 \\
\hline
\end{tabular}

Report 1,4-Dioxane for Low/Medium VOA analysis only 
Lab Name: TESTAMERICA BURLINGTON

Contract: 8E-00302

Lab Code: STLV

Mod. Ref No.:

SDG No.: 29233

Instrument ID: CHD.i

Calibration Date: 08/12/2015 Time: 1753

Lab File Id: 15254_003.D

Init. Calib. Date(s):08/06/2015 08/06/2015

EPA Sample No. (VSTD\#\#\#\#) : VSTD005DB

Heated Purge: (Y/N) N GC Column: DB-624

Init. Calib. Time(s) : 1011 1149

Purge Volume: 25.0 ID: $0.20(\mathrm{~mm})$ Length: 25

(m)

\begin{tabular}{|c|c|c|c|c|c|}
\hline COMPOUND & $\overline{\mathrm{RRF}}$ & RRF5. 0 & $\begin{array}{l}\text { MIN } \\
\text { RRF }\end{array}$ & $\because \mathrm{D}$ & $M A X \cong D$ \\
\hline 1,2-Dichloropropane & 0.301 & 0.318 & 0.010 & 5.5 & 40.0 \\
\hline Bromodichloromethane & 0.286 & 0.329 & 0.200 & 15.0 & 30.0 \\
\hline cis-1,3-Dichloropropene & 0.373 & 0.443 & 0.200 & 18.8 & 30.0 \\
\hline 4-Methyl-2-pentanone & 0.103 & 0.116 & 0.010 & 13.2 & 40.0 \\
\hline Toluene & 1.640 & 1.705 & 0.400 & 4.0 & 30.0 \\
\hline trans-1,3-Dichloropropene & 0.246 & 0.295 & 0.100 & 20.1 & 30.0 \\
\hline $1,1,2$-Trichloroethane & 0.143 & 0.154 & 0.100 & 7.6 & 30.0 \\
\hline Tetrachloroethene & 0.351 & 0.353 & 0.100 & 0.4 & 30.0 \\
\hline 2-Hexanone & 0.070 & 0.080 & 0.010 & 14.9 & 40.0 \\
\hline Dibromochloromethane & 0.138 & 0.171 & 0.100 & 23.5 & 30.0 \\
\hline 1,2-Dibromoethane & 0.123 & 0.135 & 0.010 & 9.8 & 40.0 \\
\hline Chlorobenzene & 0.936 & 0.963 & 0.500 & 2.9 & 30.0 \\
\hline Ethylbenzene & 1.960 & 2.024 & 0.100 & 3.3 & 30.0 \\
\hline o-Xylene & 0.676 & 0.709 & 0.300 & 4.9 & 30.0 \\
\hline $\mathrm{m}, \mathrm{p}-\mathrm{Xylene}$ & 0.749 & 0.778 & 0.300 & 3.8 & 30.0 \\
\hline Styrene & 0.988 & 1.079 & 0.300 & 9.3 & 30.0 \\
\hline Bromoform & 0.104 & 0.134 & 0.050 & 29.1 & 30.0 \\
\hline Isopropylbenzene & 2.022 & 2.093 & 0.010 & 3.5 & 40.0 \\
\hline $1,1,2,2$-Tetrachloroethane & 0.140 & 0.153 & 0.100 & 9.6 & 30.0 \\
\hline 1,3-Dichlorobenzene & 1.557 & 1.578 & 0.400 & 1.4 & 30.0 \\
\hline 1,4-Dichlorobenzene & 1.498 & 1.508 & 0.400 & 0.6 & 30.0 \\
\hline 1,2-Dichlorobenzene & 1.215 & 1.238 & 0.400 & 1.8 & 30.0 \\
\hline 1,2-Dibromo-3-Chloropropane & 0.032 & 0.036 & 0.010 & 15.0 & 40.0 \\
\hline 1,2,4-Trichlorobenzene & 0.744 & 0.745 & 0.200 & 0.1 & 30.0 \\
\hline $1,2,3$-Trichlorobenzene & 0.513 & 0.522 & 0.200 & 1.8 & 30.0 \\
\hline
\end{tabular}


Lab Name: TESTAMERICA BURLINGTON

Contract: $8 \mathrm{E}-00302$

Lab Code: STLV Case No.: YORK Mod. Ref No.

SDG No.: 29233

Instrument ID: CHD.i

Calibration Date: 08/12/2015 Time: 1753

Lab File Id: 15254_003.D Init. Calib. Date(s):08/06/2015 08/06/2015

EPA Sample No. (VSTD\#\#\#\#) : VSTD005DB

Heated Purge: (Y/N) N GC Column: DB-624

Init. Calib. Time(s) : 1011 1149

Purge Volume: 25.0 ID: $0.20(\mathrm{~mm})$ Length: 25

(m)

\begin{tabular}{|c|c|c|c|c|c|}
\hline COMPOUND & $\overline{\mathrm{RRF}}$ & RRE5. 0 & $\begin{array}{l}\text { MIN } \\
\text { RRE }\end{array}$ & $\because \mathrm{D}$ & $M A X \div D$ \\
\hline Vinyl Chloride-d3 & 0.384 & 0.388 & 0.010 & 1.0 & 30.0 \\
\hline Chloroethane-d5 & 0.242 & 0.262 & 0.010 & 8.3 & 40.0 \\
\hline $1,1-$ Dichloroethene-d2 & 0.538 & 0.562 & 0.010 & 4.4 & 30.0 \\
\hline 2-Butanone-d5 & 0.035 & 0.039 & 0.010 & 9.2 & 40.0 \\
\hline Chloroform-d & 0.511 & 0.538 & 0.010 & 5.4 & 30.0 \\
\hline 1,2-Dichloroethane-d4 & 0.185 & 0.194 & 0.010 & 4.9 & 30.0 \\
\hline Benzene-d6 & 1.425 & 1.500 & 0.010 & 5.3 & 30.0 \\
\hline 1,2-Dichloropropane-d6 & 0.359 & 0.367 & 0.010 & 2.2 & 40.0 \\
\hline Toluene-d8 & 1.377 & 1.428 & 0.010 & 3.7 & 30.0 \\
\hline trans-1,3-Dichloropropene-d4 & 0.220 & 0.266 & 0.010 & 20.6 & 30.0 \\
\hline 2-Hexanone-d5 & 0.030 & 0.036 & 0.010 & 17.5 & 40.0 \\
\hline $1,1,2,2$-Tetrachloroethane-d2 & 0.136 & 0.155 & 0.010 & 13.7 & 30.0 \\
\hline 1,2-Dichlorobenzene-d4 & 0.780 & 0.780 & 0.010 & 0.1 & 30.0 \\
\hline
\end{tabular}

Report 1,4-Dioxane-d8 for Low/Medium VOA analysis only 
Lab Name: TESTAMERICA BURLINGTON

Contract: $8 \mathrm{E}-00302$

Lab Code: STLV Case No.: YORK Mod. Ref No.:

SDG No.: 29233

Instrument ID: CHD.i

Calibration Date: 08/12/2015 Time: 2316

Lab File Id: 15254_016.D

EPA Sample No. (VSTD\#\#\#\#) : VSTD005BD Init. Calib. Date(s):08/06/2015 08/06/2015

Heated Purge: (Y/N) N GC Column: DB-624

Init. Calib. Time(s) : 1011 1149

Purge Volume: 25.0 ID: $0.20(\mathrm{~mm})$ Length: 25 (m) $(\mathrm{mL})$

\begin{tabular}{|c|c|c|c|c|c|}
\hline COMPOUND & $\overline{\mathrm{RRF}}$ & RRF5.0 & $\begin{array}{l}\text { MIN } \\
\text { RRF }\end{array}$ & $\frac{\circ}{D}$ & $\mathrm{MAX} \div \mathrm{D}$ \\
\hline Dichlorodifluoromethane & 0.471 & 0.488 & 0.010 & 3.7 & 50.0 \\
\hline Chloromethane & 0.437 & 0.439 & 0.010 & 0.3 & 50.0 \\
\hline Vinyl chloride & 0.397 & 0.418 & 0.010 & 5.2 & 50.0 \\
\hline Bromomethane & 0.157 & 0.139 & 0.010 & -11.1 & 50.0 \\
\hline Chloroethane & 0.199 & 0.223 & 0.010 & 12.1 & 50.0 \\
\hline Trichlorofluoromethane & 0.505 & 0.541 & 0.010 & 7.2 & 50.0 \\
\hline 1,1-Dichloroethene & 0.247 & 0.257 & 0.010 & 3.8 & 50.0 \\
\hline 1,1,2-Trichloro-1,2,2-trifluoroethane & 0.278 & 0.293 & 0.010 & 5.7 & 50.0 \\
\hline Acetone & 0.019 & 0.019 & 0.010 & -2.3 & 50.0 \\
\hline Carbon disulfide & 0.656 & 0.689 & 0.010 & 5.0 & 50.0 \\
\hline Methyl acetate & 0.059 & 0.057 & 0.010 & -3.6 & 50.0 \\
\hline Methylene Chloride & 0.249 & 0.248 & 0.010 & -0.2 & 50.0 \\
\hline trans-1,2-Dichloroethene & 0.295 & 0.304 & 0.010 & 3.2 & 50.0 \\
\hline Methyl tert-butyl ether & 0.319 & 0.335 & 0.010 & 4.8 & 50.0 \\
\hline 1,1-Dichloroethane & 0.542 & 0.574 & 0.010 & 5.9 & 50.0 \\
\hline cis-1,2-Dichloroethene & 0.286 & 0.302 & 0.010 & 5.6 & 50.0 \\
\hline 2-Butanone & 0.037 & 0.040 & 0.010 & 8.3 & 50.0 \\
\hline Bromochloromethane & 0.083 & 0.090 & 0.010 & 8.8 & 50.0 \\
\hline Chloroform & 0.479 & 0.510 & 0.010 & 6.5 & 50.0 \\
\hline 1,1,1-Trichloroethane & 0.547 & 0.572 & 0.010 & 4.6 & 50.0 \\
\hline Cyclohexane & 0.767 & 0.796 & 0.010 & 3.7 & 50.0 \\
\hline Carbon tetrachloride & 0.459 & 0.482 & 0.010 & 5.0 & 50.0 \\
\hline Benzene & 1.521 & 1.552 & 0.010 & 2.0 & 50.0 \\
\hline 1,2-Dichloroethane & 0.226 & 0.244 & 0.010 & 7.9 & 50.0 \\
\hline Trichloroethene & 0.383 & 0.400 & 0.010 & 4.4 & 50.0 \\
\hline Methylcyclohexane & 0.637 & 0.657 & 0.010 & 3.1 & 50.0 \\
\hline
\end{tabular}

Report 1,4-Dioxane for Low/Medium VOA analysis only 
Lab Name: TESTAMERICA BURLINGTON

Contract: 8E-00302

Lab Code: STLV

Mod. Ref No.:

SDG No.: 29233

Instrument ID: CHD.i

Calibration Date: 08/12/2015 Time: 2316

Lab File Id: 15254_016.D

EPA Sample No. (VSTD\#\#\#\#) : VSTD005BD

Init. Calib. Date (s):08/06/2015

$08 / 06 / 2015$

Heated Purge: (Y/N) N GC Column: DB-624

Init. Calib. Time(s) : 1011 1149

Purge Volume: 25.0

ID: $0.20(\mathrm{~mm})$ Length: 25

(m) $(\mathrm{mL})$

\begin{tabular}{|c|c|c|c|c|c|}
\hline COMPOUND & $\overline{\mathrm{RRF}}$ & RRF5.0 & $\begin{array}{l}\text { MIN } \\
\text { RRF }\end{array}$ & $\because \mathrm{D}$ & $\operatorname{MAX} \cong \mathrm{D}$ \\
\hline 1,2-Dichloropropane & 0.301 & 0.318 & 0.010 & 5.4 & 50.0 \\
\hline Bromodichloromethane & 0.286 & 0.299 & 0.010 & 4.4 & 50.0 \\
\hline cis-1,3-Dichloropropene & 0.373 & 0.401 & 0.010 & 7.5 & 50.0 \\
\hline 4-Methyl-2-pentanone & 0.103 & 0.116 & 0.010 & 12.7 & 50.0 \\
\hline Toluene & 1.640 & 1.727 & 0.010 & 5.3 & 50.0 \\
\hline trans-1,3-Dichloropropene & 0.246 & 0.266 & 0.010 & 8.4 & 50.0 \\
\hline $1,1,2$-Trichloroethane & 0.143 & 0.153 & 0.010 & 6.6 & 50.0 \\
\hline Tetrachloroethene & 0.351 & 0.357 & 0.010 & 1.8 & 50.0 \\
\hline 2-Hexanone & 0.070 & 0.079 & 0.010 & 13.7 & 50.0 \\
\hline Dibromochloromethane & 0.138 & 0.145 & 0.010 & 5.2 & 50.0 \\
\hline 1,2-Dibromoethane & 0.123 & 0.134 & 0.010 & 8.9 & 50.0 \\
\hline Chlorobenzene & 0.936 & 0.983 & 0.010 & 5.0 & 50.0 \\
\hline Ethylbenzene & 1.960 & 2.085 & 0.010 & 6.4 & 50.0 \\
\hline o-Xylene & 0.676 & 0.727 & 0.010 & 7.5 & 50.0 \\
\hline $\mathrm{m}, \mathrm{p}$-Xylene & 0.749 & 0.803 & 0.010 & 7.2 & 50.0 \\
\hline Styrene & 0.988 & 1.098 & 0.010 & 11.2 & 50.0 \\
\hline Bromoform & 0.104 & 0.096 & 0.010 & -7.3 & 50.0 \\
\hline Isopropylbenzene & 2.022 & 2.184 & 0.010 & 8.0 & 50.0 \\
\hline $1,1,2,2$-Tetrachloroethane & 0.140 & 0.155 & 0.010 & 11.0 & 50.0 \\
\hline 1,3-Dichlorobenzene & 1.557 & 1.601 & 0.010 & 2.9 & 50.0 \\
\hline 1,4-Dichlorobenzene & 1.498 & 1.545 & 0.010 & 3.2 & 50.0 \\
\hline 1,2-Dichlorobenzene & 1.215 & 1.278 & 0.010 & 5.1 & 50.0 \\
\hline 1,2-Dibromo-3-Chloropropane & 0.032 & 0.029 & 0.010 & -9.1 & 50.0 \\
\hline 1,2,4-Trichlorobenzene & 0.744 & 0.754 & 0.010 & 1.2 & 50.0 \\
\hline $1,2,3$-Trichlorobenzene & 0.513 & 0.511 & 0.010 & -0.2 & 50.0 \\
\hline
\end{tabular}


Lab Name: TESTAMERICA BURLINGTON

Contract: $8 \mathrm{E}-00302$

Lab Code: STLV Case No.: YORK Mod. Ref No.

SDG No.: 29233

Instrument ID: CHD.i

Calibration Date: 08/12/2015 Time: 2316

Lab File Id: 15254_016.D Init. Calib. Date(s):08/06/2015 08/06/2015

EPA Sample No. (VSTD\#\#\#\#) : VSTD005BD

Init. Calib. Time(s) : 1011 1149

Heated Purge: (Y/N) N GC Column: DB-624 ID: $0.20(\mathrm{~mm})$ Length: 25

(m)

Purge Volume: 25.0 $(\mathrm{mL})$

\begin{tabular}{|c|c|c|c|c|c|}
\hline COMPOUND & $\overline{\mathrm{RRF}}$ & RRF5.0 & $\begin{array}{l}\text { MIN } \\
\text { RRE }\end{array}$ & $\because \mathrm{D}$ & $\operatorname{MAX} \cong \mathrm{D}$ \\
\hline Vinyl Chloride-d3 & 0.384 & 0.404 & 0.010 & 5.1 & 50.0 \\
\hline Chloroethane-d5 & 0.242 & 0.268 & 0.010 & 10.8 & 50.0 \\
\hline 1,1-Dichloroethene-d2 & 0.538 & 0.579 & 0.010 & 7.5 & 50.0 \\
\hline 2-Butanone-d5 & 0.035 & 0.037 & 0.010 & 5.9 & 50.0 \\
\hline Chloroform-d & 0.511 & 0.546 & 0.010 & 7.0 & 50.0 \\
\hline 1,2-Dichloroethane-d4 & 0.185 & 0.205 & 0.010 & 10.7 & 50.0 \\
\hline Benzene-d6 & 1.425 & 1.493 & 0.010 & 4.8 & 50.0 \\
\hline 1,2-Dichloropropane-d6 & 0.359 & 0.390 & 0.010 & 8.8 & 50.0 \\
\hline Toluene-d8 & 1.377 & 1.462 & 0.010 & 6.2 & 50.0 \\
\hline trans-1,3-Dichloropropene-d4 & 0.220 & 0.235 & 0.010 & 6.7 & 50.0 \\
\hline 2-Hexanone-d5 & 0.030 & 0.035 & 0.010 & 14.5 & 50.0 \\
\hline $1,1,2,2$-Tetrachloroethane-d2 & 0.136 & 0.154 & 0.010 & 13.3 & 50.0 \\
\hline 1,2-Dichlorobenzene-d4 & 0.780 & 0.830 & 0.010 & 6.5 & 50.0 \\
\hline
\end{tabular}

Report 1,4-Dioxane-d8 for Low/Medium VOA analysis only 
Lab Name: TESTAMERICA BURLINGTON

Contract: $8 \mathrm{E}-00302$

Lab Code: STLV Case No.: YORK

Mod. Ref No.:

SDG No.: 29233

Instrument ID: CHD.i

Calibration Date: 08/13/2015 Time: 1134

Lab File Id: 15265_003.D Init. Calib. Date(s):08/06/2015 08/06/2015

EPA Sample No. (VSTD\#\#\#\#) : VSTD005DC

Heated Purge: (Y/N) N GC Column: DB-624

Init. Calib. Time(s) : 1011 1149

Purge Volume: 25.0 ID: $0.20(\mathrm{~mm})$ Length: 25 (m) $(\mathrm{mL})$

\begin{tabular}{|c|c|c|c|c|c|}
\hline COMPOUND & $\overline{\mathrm{RRF}}$ & RRF5.0 & $\begin{array}{l}\text { MIN } \\
\text { RRF }\end{array}$ & $\frac{\circ}{D}$ & $\mathrm{MAX} \div \mathrm{D}$ \\
\hline Dichlorodifluoromethane & 0.471 & 0.518 & 0.010 & 10.1 & 40.0 \\
\hline Chloromethane & 0.437 & 0.502 & 0.010 & 14.8 & 40.0 \\
\hline Vinyl chloride & 0.397 & 0.453 & 0.100 & 14.2 & 30.0 \\
\hline Bromomethane & 0.157 & 0.197 & 0.100 & 25.4 & 30.0 \\
\hline Chloroethane & 0.199 & 0.239 & 0.010 & 20.0 & 40.0 \\
\hline Trichlorofluoromethane & 0.505 & 0.578 & 0.010 & 14.4 & 40.0 \\
\hline 1,1-Dichloroethene & 0.247 & 0.268 & 0.100 & 8.2 & 30.0 \\
\hline 1,1,2-Trichloro-1,2,2-trifluoroethane & 0.278 & 0.310 & 0.010 & 11.7 & 40.0 \\
\hline Acetone & 0.019 & 0.023 & 0.010 & 18.7 & 40.0 \\
\hline Carbon disulfide & 0.656 & 0.770 & 0.010 & 17.4 & 40.0 \\
\hline Methyl acetate & 0.059 & 0.064 & 0.010 & 8.5 & 40.0 \\
\hline Methylene Chloride & 0.249 & 0.262 & 0.010 & 5.2 & 40.0 \\
\hline trans-1,2-Dichloroethene & 0.295 & 0.318 & 0.010 & 8.1 & 40.0 \\
\hline Methyl tert-butyl ether & 0.319 & 0.341 & 0.010 & 6.8 & 40.0 \\
\hline 1,1-Dichloroethane & 0.542 & 0.599 & 0.200 & 10.4 & 30.0 \\
\hline cis-1,2-Dichloroethene & 0.286 & 0.316 & 0.010 & 10.6 & 40.0 \\
\hline 2-Butanone & 0.037 & 0.041 & 0.010 & 11.3 & 40.0 \\
\hline Bromochloromethane & 0.083 & 0.092 & 0.050 & 10.5 & 30.0 \\
\hline Chloroform & 0.479 & 0.535 & 0.200 & 11.8 & 30.0 \\
\hline 1,1,1-Trichloroethane & 0.547 & 0.590 & 0.100 & 8.0 & 30.0 \\
\hline Cyclohexane & 0.767 & 0.830 & 0.010 & 8.1 & 40.0 \\
\hline Carbon tetrachloride & 0.459 & 0.506 & 0.100 & 10.4 & 30.0 \\
\hline Benzene & 1.521 & 1.623 & 0.400 & 6.7 & 30.0 \\
\hline 1,2-Dichloroethane & 0.226 & 0.256 & 0.100 & 13.0 & 30.0 \\
\hline Trichloroethene & 0.383 & 0.417 & 0.300 & 8.9 & 30.0 \\
\hline Methylcyclohexane & 0.637 & 0.687 & 0.010 & 7.8 & 40.0 \\
\hline
\end{tabular}

Report 1,4-Dioxane for Low/Medium VOA analysis only 
Lab Name: TESTAMERICA BURLINGTON

Contract: 8E-00302

Lab Code: STLV

Mod. Ref No.:

SDG No.: 29233

Instrument ID: CHD.i

Calibration Date: 08/13/2015 Time: 1134

Lab File Id: 15265_003.D

Init. Calib. Date(s):08/06/2015 08/06/2015

EPA Sample No. (VSTD\#\#\#\#) : VSTD005DC

Init. Calib. Time(s) : 1011

1149

Heated Purge: (Y/N) N GC Column: DB-624

ID: $0.20(\mathrm{~mm})$ Length: 25

(m)

Purge Volume: 25.0 $(\mathrm{mL})$

\begin{tabular}{|c|c|c|c|c|c|}
\hline COMPOUND & $\overline{\mathrm{RRF}}$ & RRF5.0 & $\begin{array}{l}\text { MIN } \\
\text { RRF }\end{array}$ & $\because \mathrm{D}$ & $\operatorname{MAX} \cong \mathrm{D}$ \\
\hline 1,2-Dichloropropane & 0.301 & 0.335 & 0.010 & 11.2 & 40.0 \\
\hline Bromodichloromethane & 0.286 & 0.317 & 0.200 & 10.9 & 30.0 \\
\hline cis-1,3-Dichloropropene & 0.373 & 0.430 & 0.200 & 15.3 & 30.0 \\
\hline 4-Methyl-2-pentanone & 0.103 & 0.117 & 0.010 & 13.9 & 40.0 \\
\hline Toluene & 1.640 & 1.777 & 0.400 & 8.3 & 30.0 \\
\hline trans-1,3-Dichloropropene & 0.246 & 0.282 & 0.100 & 14.9 & 30.0 \\
\hline $1,1,2$-Trichloroethane & 0.143 & 0.156 & 0.100 & 9.0 & 30.0 \\
\hline Tetrachloroethene & 0.351 & 0.372 & 0.100 & 6.0 & 30.0 \\
\hline 2-Hexanone & 0.070 & 0.080 & 0.010 & 14.3 & 40.0 \\
\hline Dibromochloromethane & 0.138 & 0.160 & 0.100 & 15.9 & 30.0 \\
\hline 1,2-Dibromoethane & 0.123 & 0.135 & 0.010 & 10.0 & 40.0 \\
\hline Chlorobenzene & 0.936 & 0.997 & 0.500 & 6.5 & 30.0 \\
\hline Ethylbenzene & 1.960 & 2.130 & 0.100 & 8.7 & 30.0 \\
\hline o-Xylene & 0.676 & 0.745 & 0.300 & 10.3 & 30.0 \\
\hline $\mathrm{m}, \mathrm{p}$-Xylene & 0.749 & 0.816 & 0.300 & 8.9 & 30.0 \\
\hline Styrene & 0.988 & 1.115 & 0.300 & 12.9 & 30.0 \\
\hline Bromoform & 0.104 & 0.112 & 0.050 & 7.9 & 30.0 \\
\hline Isopropylbenzene & 2.022 & 2.226 & 0.010 & 10.1 & 40.0 \\
\hline $1,1,2,2$-Tetrachloroethane & 0.140 & 0.156 & 0.100 & 12.1 & 30.0 \\
\hline 1,3-Dichlorobenzene & 1.557 & 1.621 & 0.400 & 4.1 & 30.0 \\
\hline 1,4-Dichlorobenzene & 1.498 & 1.560 & 0.400 & 4.1 & 30.0 \\
\hline 1,2-Dichlorobenzene & 1.215 & 1.286 & 0.400 & 5.8 & 30.0 \\
\hline 1,2-Dibromo-3-Chloropropane & 0.032 & 0.031 & 0.010 & -1.9 & 40.0 \\
\hline 1,2,4-Trichlorobenzene & 0.744 & 0.753 & 0.200 & 1.1 & 30.0 \\
\hline $1,2,3$-Trichlorobenzene & 0.513 & 0.509 & 0.200 & -0.7 & 30.0 \\
\hline
\end{tabular}


Lab Name: TESTAMERICA BURLINGTON

Contract: $8 \mathrm{E}-00302$

Lab Code: STLV Case No.: YORK Mod. Ref No.

SDG No.: 29233

Instrument ID: CHD.i

Calibration Date: 08/13/2015 Time: 1134

Lab File Id: 15265_003.D Init. Calib. Date(s):08/06/2015 08/06/2015

EPA Sample No. (VSTD\#\#\#\#) : VSTD005DC

Init. Calib. Time(s) : 1011 1149

Heated Purge: (Y/N) N GC Column: DB-624 ID: $0.20(\mathrm{~mm})$ Length: 25

(m)

Purge Volume: 25.0 $(\mathrm{mL})$

\begin{tabular}{|c|c|c|c|c|c|}
\hline COMPOUND & $\overline{\mathrm{RRF}}$ & RRF5.0 & $\begin{array}{l}\text { MIN } \\
\text { RRF }\end{array}$ & $\because \mathrm{D}$ & $\operatorname{MAX} \cong \mathrm{D}$ \\
\hline Vinyl Chloride-d3 & 0.384 & 0.437 & 0.010 & 13.7 & 30.0 \\
\hline Chloroethane-d5 & 0.242 & 0.285 & 0.010 & 17.8 & 40.0 \\
\hline 1,1-Dichloroethene-d2 & 0.538 & 0.625 & 0.010 & 16.2 & 30.0 \\
\hline 2-Butanone-d5 & 0.035 & 0.038 & 0.010 & 7.1 & 40.0 \\
\hline Chloroform-d & 0.511 & 0.569 & 0.010 & 11.5 & 30.0 \\
\hline 1,2-Dichloroethane-d4 & 0.185 & 0.204 & 0.010 & 9.9 & 30.0 \\
\hline Benzene-d6 & 1.425 & 1.537 & 0.010 & 7.9 & 30.0 \\
\hline 1,2-Dichloropropane-d6 & 0.359 & 0.388 & 0.010 & 8.2 & 40.0 \\
\hline Toluene-d8 & 1.377 & 1.484 & 0.010 & 7.8 & 30.0 \\
\hline trans-1,3-Dichloropropene-d4 & 0.220 & 0.248 & 0.010 & 12.6 & 30.0 \\
\hline 2-Hexanone-d5 & 0.030 & 0.034 & 0.010 & 13.1 & 40.0 \\
\hline $1,1,2,2$-Tetrachloroethane-d2 & 0.136 & 0.157 & 0.010 & 14.8 & 30.0 \\
\hline 1,2 -Dichlorobenzene-d4 & 0.780 & 0.824 & 0.010 & 5.8 & 30.0 \\
\hline
\end{tabular}

Report 1,4-Dioxane-d8 for Low/Medium VOA analysis only 
Lab Name: TESTAMERICA BURLINGTON

Contract: $8 \mathrm{E}-00302$

Lab Code: STLV Case No.: YORK Mod. Ref No.:

SDG No.: 29233

Instrument ID: CHD.i

Calibration Date: 08/13/2015 Time: 2020

Lab File Id: 15265_023.D

EPA Sample No. (VSTD\#\#\#\#) : VSTD005CD Init. Calib. Date(s):08/06/2015 08/06/2015

Heated Purge: (Y/N) N GC Column: DB-624

Init. Calib. Time(s) : 1011 1149

Purge Volume: 25.0 ID: $0.20(\mathrm{~mm})$ Length: 25 (m) $(\mathrm{mL})$

\begin{tabular}{|c|c|c|c|c|c|}
\hline COMPOUND & $\overline{\mathrm{RRF}}$ & RRF5.0 & $\begin{array}{l}\text { MIN } \\
\text { RRF }\end{array}$ & $\frac{\circ}{D}$ & $\mathrm{MAX} \div \mathrm{D}$ \\
\hline Dichlorodifluoromethane & 0.471 & 0.527 & 0.010 & 11.9 & 50.0 \\
\hline Chloromethane & 0.437 & 0.480 & 0.010 & 9.6 & 50.0 \\
\hline Vinyl chloride & 0.397 & 0.439 & 0.010 & 10.7 & 50.0 \\
\hline Bromomethane & 0.157 & 0.132 & 0.010 & -15.8 & 50.0 \\
\hline Chloroethane & 0.199 & 0.236 & 0.010 & 18.9 & 50.0 \\
\hline Trichlorofluoromethane & 0.505 & 0.574 & 0.010 & 13.7 & 50.0 \\
\hline 1,1-Dichloroethene & 0.247 & 0.266 & 0.010 & 7.5 & 50.0 \\
\hline 1,1,2-Trichloro-1,2,2-trifluoroethane & 0.278 & 0.305 & 0.010 & 10.0 & 50.0 \\
\hline Acetone & 0.019 & 0.027 & 0.010 & 40.5 & 50.0 \\
\hline Carbon disulfide & 0.656 & 0.694 & 0.010 & 5.8 & 50.0 \\
\hline Methyl acetate & 0.059 & 0.062 & 0.010 & 5.4 & 50.0 \\
\hline Methylene Chloride & 0.249 & 0.258 & 0.010 & 3.9 & 50.0 \\
\hline trans-1,2-Dichloroethene & 0.295 & 0.313 & 0.010 & 6.3 & 50.0 \\
\hline Methyl tert-butyl ether & 0.319 & 0.315 & 0.010 & -1.3 & 50.0 \\
\hline 1,1-Dichloroethane & 0.542 & 0.594 & 0.010 & 9.5 & 50.0 \\
\hline cis-1,2-Dichloroethene & 0.286 & 0.310 & 0.010 & 8.4 & 50.0 \\
\hline 2-Butanone & 0.037 & 0.043 & 0.010 & 15.5 & 50.0 \\
\hline Bromochloromethane & 0.083 & 0.092 & 0.010 & 10.5 & 50.0 \\
\hline Chloroform & 0.479 & 0.531 & 0.010 & 11.0 & 50.0 \\
\hline 1,1,1-Trichloroethane & 0.547 & 0.570 & 0.010 & 4.3 & 50.0 \\
\hline Cyclohexane & 0.767 & 0.815 & 0.010 & 6.2 & 50.0 \\
\hline Carbon tetrachloride & 0.459 & 0.476 & 0.010 & 3.7 & 50.0 \\
\hline Benzene & 1.521 & 1.596 & 0.010 & 4.9 & 50.0 \\
\hline 1,2-Dichloroethane & 0.226 & 0.247 & 0.010 & 9.1 & 50.0 \\
\hline Trichloroethene & 0.383 & 0.410 & 0.010 & 6.9 & 50.0 \\
\hline Methylcyclohexane & 0.637 & 0.674 & 0.010 & 5.8 & 50.0 \\
\hline
\end{tabular}

Report 1,4-Dioxane for Low/Medium VOA analysis only 
Lab Name: TESTAMERICA BURLINGTON

Contract: 8E-00302

Lab Code: STLV

Mod. Ref No.:

SDG No.: 29233

Instrument ID: CHD.i

Calibration Date: 08/13/2015 Time: 2020

Lab File Id: 15265_023.D

Init. Calib. Date(s):08/06/2015 08/06/2015

EPA Sample No. (VSTD\#\#\#\#) : VSTD005CD

Heated Purge: (Y/N) N GC Column: DB-624

Init. Calib. Time(s) : 1011 1149

Purge Volume: 25.0

ID: $0.20(\mathrm{~mm})$ Length: 25

(m)

\begin{tabular}{|c|c|c|c|c|c|}
\hline COMPOUND & $\overline{\mathrm{RRF}}$ & RRF5.0 & $\begin{array}{l}\text { MIN } \\
\text { RRF }\end{array}$ & $\because \mathrm{D}$ & $\operatorname{MAX} \cong \mathrm{D}$ \\
\hline 1,2-Dichloropropane & 0.301 & 0.331 & 0.010 & 9.8 & 50.0 \\
\hline Bromodichloromethane & 0.286 & 0.303 & 0.010 & 5.9 & 50.0 \\
\hline cis-1,3-Dichloropropene & 0.373 & 0.395 & 0.010 & 5.8 & 50.0 \\
\hline 4-Methyl-2-pentanone & 0.103 & 0.117 & 0.010 & 13.5 & 50.0 \\
\hline Toluene & 1.640 & 1.762 & 0.010 & 7.5 & 50.0 \\
\hline trans-1,3-Dichloropropene & 0.246 & 0.262 & 0.010 & 6.7 & 50.0 \\
\hline $1,1,2$-Trichloroethane & 0.143 & 0.154 & 0.010 & 7.8 & 50.0 \\
\hline Tetrachloroethene & 0.351 & 0.361 & 0.010 & 2.9 & 50.0 \\
\hline 2-Hexanone & 0.070 & 0.081 & 0.010 & 16.4 & 50.0 \\
\hline Dibromochloromethane & 0.138 & 0.146 & 0.010 & 5.4 & 50.0 \\
\hline 1,2-Dibromoethane & 0.123 & 0.134 & 0.010 & 9.3 & 50.0 \\
\hline Chlorobenzene & 0.936 & 0.990 & 0.010 & 5.8 & 50.0 \\
\hline Ethylbenzene & 1.960 & 2.117 & 0.010 & 8.0 & 50.0 \\
\hline o-Xylene & 0.676 & 0.745 & 0.010 & 10.3 & 50.0 \\
\hline $\mathrm{m}, \mathrm{p}$-Xylene & 0.749 & 0.805 & 0.010 & 7.4 & 50.0 \\
\hline Styrene & 0.988 & 1.125 & 0.010 & 13.9 & 50.0 \\
\hline Bromoform & 0.104 & 0.094 & 0.010 & -9.2 & 50.0 \\
\hline Isopropylbenzene & 2.022 & 2.213 & 0.010 & 9.4 & 50.0 \\
\hline $1,1,2,2$-Tetrachloroethane & 0.140 & 0.157 & 0.010 & 12.5 & 50.0 \\
\hline 1,3-Dichlorobenzene & 1.557 & 1.573 & 0.010 & 1.0 & 50.0 \\
\hline 1,4-Dichlorobenzene & 1.498 & 1.517 & 0.010 & 1.2 & 50.0 \\
\hline 1,2-Dichlorobenzene & 1.215 & 1.254 & 0.010 & 3.2 & 50.0 \\
\hline 1,2-Dibromo-3-Chloropropane & 0.032 & 0.028 & 0.010 & -12.0 & 50.0 \\
\hline 1,2,4-Trichlorobenzene & 0.744 & 0.733 & 0.010 & -1.5 & 50.0 \\
\hline $1,2,3$-Trichlorobenzene & 0.513 & 0.503 & 0.010 & -1.9 & 50.0 \\
\hline
\end{tabular}


Lab Name: TESTAMERICA BURLINGTON

Contract: $8 \mathrm{E}-00302$

Lab Code: STLV Case No.: YORK Mod. Ref No.

SDG No.: 29233

Instrument ID: CHD.i

Calibration Date: 08/13/2015 Time: 2020

Lab File Id: 15265_023.D Init. Calib. Date(s):08/06/2015 08/06/2015

EPA Sample No. (VSTD\#\#\#\#) : VSTD005CD

Init. Calib. Time(s) : 1011 1149

Heated Purge: (Y/N) N GC Column: DB-624 ID: $0.20(\mathrm{~mm})$ Length: 25

(m)

Purge Volume: 25.0 $(\mathrm{mL})$

\begin{tabular}{|c|c|c|c|c|c|}
\hline COMPOUND & $\overline{\mathrm{RRF}}$ & RRF5.0 & $\begin{array}{l}\text { MIN } \\
\text { RRE }\end{array}$ & $\because \mathrm{D}$ & $\operatorname{MAX} \cong \mathrm{D}$ \\
\hline Vinyl Chloride-d3 & 0.384 & 0.422 & 0.010 & 9.9 & 50.0 \\
\hline Chloroethane-d5 & 0.242 & 0.280 & 0.010 & 15.7 & 50.0 \\
\hline 1,1-Dichloroethene-d2 & 0.538 & 0.615 & 0.010 & 14.3 & 50.0 \\
\hline 2-Butanone-d5 & 0.035 & 0.039 & 0.010 & 10.0 & 50.0 \\
\hline Chloroform-d & 0.511 & 0.567 & 0.010 & 11.0 & 50.0 \\
\hline 1,2-Dichloroethane-d4 & 0.185 & 0.207 & 0.010 & 11.6 & 50.0 \\
\hline Benzene-d6 & 1.425 & 1.517 & 0.010 & 6.4 & 50.0 \\
\hline 1,2-Dichloropropane-d6 & 0.359 & 0.385 & 0.010 & 7.5 & 50.0 \\
\hline Toluene-d8 & 1.377 & 1.475 & 0.010 & 7.2 & 50.0 \\
\hline trans-1,3-Dichloropropene-d4 & 0.220 & 0.227 & 0.010 & 3.2 & 50.0 \\
\hline 2-Hexanone-d5 & 0.030 & 0.035 & 0.010 & 14.6 & 50.0 \\
\hline $1,1,2,2$-Tetrachloroethane-d2 & 0.136 & 0.160 & 0.010 & 17.2 & 50.0 \\
\hline 1,2-Dichlorobenzene-d4 & 0.780 & 0.807 & 0.010 & 3.5 & 50.0 \\
\hline
\end{tabular}

Report 1,4-Dioxane-d8 for Low/Medium VOA analysis only 
Lab Name: TESTAMERICA BURLINGTON

Contract: $8 \mathrm{E}-00302$

Lab Code: STLV

Case No.: YORK Mod. Ref No.:

SDG No.: 29233

Matrix: (SOIL/SED/WATER) Water

Sample wt/vol: 25.0 $(\mathrm{g} / \mathrm{mL}) \mathrm{mL}$

Level: (TRACE/LOW/MED) TRACE

\% Moisture: not dec.

GC Column: DB-624 ID: $0.20 \quad(\mathrm{~mm})$

Soil Extract Volume: (uL) $(\mathrm{mL})$

Purge Volume: 25.0
Lab Sample ID: MB 200-92663/5

Lab File ID: 15254_005.D

Date Received:

Date Analyzed: 08/12/2015

Dilution Factor: 1.0

Soil Aliquot Volume: (uL)

\begin{tabular}{|c|c|c|c|}
\hline CAS NO. & COMPOUND & $\begin{array}{l}\text { CONCENTRATION UNITS: } \\
(\mathrm{ug} / \mathrm{L} \text { or } \mathrm{ug} / \mathrm{kg}) \mathrm{ug} / \mathrm{L}\end{array}$ & q \\
\hline $75-71-8$ & Dichlorodifluoromethane & 1.0 & $\mathrm{U}$ \\
\hline $74-87-3$ & Chloromethane & 1.0 & $\mathrm{U}$ \\
\hline $75-01-4$ & Vinyl chloride & 1.0 & $\mathrm{U}$ \\
\hline $74-83-9$ & Bromomethane & 1.0 & $\mathrm{U}$ \\
\hline $75-00-3$ & Chloroethane & 1.0 & $\mathrm{U}$ \\
\hline $75-69-4$ & Trichlorofluoromethane & 1.0 & $\mathrm{U}$ \\
\hline $75-35-4$ & 1,1-Dichloroethene & 1.0 & $\mathrm{U}$ \\
\hline $76-13-1$ & $1,1,2$-Trichloro-1,2,2-trifluoroethane & 1.0 & $\mathrm{U}$ \\
\hline $67-64-1$ & Acetone & 0.98 & $\mathrm{~J}$ \\
\hline $75-15-0$ & Carbon disulfide & 0.058 & $\mathrm{~J}$ \\
\hline $79-20-9$ & Methyl acetate & 1.0 & $\mathrm{U}$ \\
\hline $75-09-2$ & Methylene Chloride & 1.0 & $\mathrm{U}$ \\
\hline $156-60-5$ & trans-1,2-Dichloroethene & 1.0 & $\mathrm{U}$ \\
\hline $1634-04-4$ & Methyl tert-butyl ether & 1.0 & $\mathrm{U}$ \\
\hline $75-34-3$ & 1,1-Dichloroethane & 1.0 & $\mathrm{U}$ \\
\hline $156-59-2$ & cis-1,2-Dichloroethene & 1.0 & $\mathrm{U}$ \\
\hline $78-93-3$ & 2-Butanone & 5.0 & $\mathrm{U}$ \\
\hline $74-97-5$ & Bromochloromethane & 1.0 & $\mathrm{U}$ \\
\hline $67-66-3$ & Chloroform & 1.0 & $\mathrm{U}$ \\
\hline $71-55-6$ & $1,1,1$-Trichloroethane & 1.0 & $\mathrm{U}$ \\
\hline $110-82-7$ & Cyclohexane & 1.0 & $\mathrm{U}$ \\
\hline $56-23-5$ & Carbon tetrachloride & 1.0 & $\mathrm{U}$ \\
\hline $71-43-2$ & Benzene & 1.0 & $\mathrm{U}$ \\
\hline $107-06-2$ & 1,2-Dichloroethane & 1.0 & $\mathrm{U}$ \\
\hline
\end{tabular}

Report 1,4-Dioxane for Low-Medium VOA analysis only 
Lab Name: TESTAMERICA BURLINGTON

Lab Code: STLV

Case No.: YORK

Mod. Ref No.:

Contract: 8E-00302

Matrix: (SOIL/SED/WATER) Water

Sample wt/vol: 25.0 $(\mathrm{g} / \mathrm{mL}) \mathrm{mL}$

Level: (TRACE/LOW/MED) TRACE

\% Moisture: not dec.

GC Column: DB-624 ID $: \underline{0.20 \quad(\mathrm{~mm})}$

Soil Extract Volume: (uL) $(\mathrm{mL})$

Purge Volume: 25.0
SDG No.: 29233

Lab Sample ID: MB 200-92663/5

Lab File ID: 15254_005.D

Date Received:

Date Analyzed: 08/12/2015

Dilution Factor: 1.0

Soil Aliquot Volume: (uL)

\begin{tabular}{|c|c|c|c|}
\hline CAS NO. & COMPOUND & $\begin{array}{l}\text { CONCENTRATION UNITS: } \\
(\mathrm{ug} / \mathrm{L} \text { or } \mathrm{ug} / \mathrm{kg}) \mathrm{ug} / \mathrm{L}\end{array}$ & Q \\
\hline $79-01-6$ & Trichloroethene & $\begin{array}{r}1.0 \\
\end{array}$ & $\mathrm{U}$ \\
\hline $108-87-2$ & Methylcyclohexane & 1.0 & $\mathrm{U}$ \\
\hline $78-87-5$ & 1,2-Dichloropropane & 1.0 & $\mathrm{U}$ \\
\hline $75-27-4$ & Bromodichloromethane & 1.0 & $\mathrm{U}$ \\
\hline $10061-01-5$ & cis-1,3-Dichloropropene & 1.0 & $\mathrm{U}$ \\
\hline $108-10-1$ & 4-Methyl-2-pentanone & 1.0 & $\mathrm{U}$ \\
\hline $108-88-3$ & Toluene & 0.014 & $\mathrm{~J}$ \\
\hline $10061-02-6$ & trans-1,3-Dichloropropene & 1.0 & $\mathrm{U}$ \\
\hline $79-00-5$ & $1,1,2-T r i c h l o r o e t h a n e$ & 1.0 & $\mathrm{U}$ \\
\hline $127-18-4$ & Tetrachloroethene & 1.0 & $\mathrm{U}$ \\
\hline $591-78-6$ & 2-Hexanone & 1.0 & $\mathrm{U}$ \\
\hline $124-48-1$ & Dibromochloromethane & 1.0 & $\mathrm{U}$ \\
\hline $106-93-4$ & 1,2-Dibromoethane & 1.0 & $\mathrm{U}$ \\
\hline $108-90-7$ & Chlorobenzene & 1.0 & $\mathrm{U}$ \\
\hline $100-41-4$ & Ethylbenzene & 0.0048 & $\mathrm{~J}$ \\
\hline $95-47-6$ & o-Xylene & 0.0037 & $\mathrm{~J}$ \\
\hline $179601-23-1$ & m,p-xylene & 0.0099 & $\mathrm{~J}$ \\
\hline $100-42-5$ & Styrene & 0.0047 & $\mathrm{~J}$ \\
\hline $75-25-2$ & Bromoform & 1.0 & $\mathrm{U}$ \\
\hline $98-82-8$ & Isopropylbenzene & 1.0 & $\mathrm{U}$ \\
\hline $79-34-5$ & $1,1,2,2$-Tetrachloroethane & 1.0 & $\mathrm{U}$ \\
\hline $541-73-1$ & 1,3 -Dichlorobenzene & 0.012 & $\mathrm{~J}$ \\
\hline $106-46-7$ & 1,4-Dichlorobenzene & 1.0 & $\mathrm{U}$ \\
\hline $95-50-1$ & 1,2-Dichlorobenzene & 1.0 & $\mathrm{U}$ \\
\hline $96-12-8$ & 1,2-Dibromo-3-Chloropropane & 1.0 & $\mathrm{U}$ \\
\hline $120-82-1$ & $1,2,4$-Trichlorobenzene & 0.048 & $\mathrm{~J}$ \\
\hline $87-61-6$ & $1,2,3$-Trichlorobenzene & 0.070 & $\mathrm{~J}$ \\
\hline
\end{tabular}


Lab Name: TESTAMERICA BURLINGTON

Contract: $8 \mathrm{E}-00302$

Lab Code: STLV Case No.: YORK Mod. Ref No.: SDG No.: 29233

Matrix: (SOIL/SED/WATER) Water

Sample wt/vol: 25.0 $(\mathrm{g} / \mathrm{mL}) \mathrm{mL}$

Level: (TRACE or LOW/MED) TRACE

․ Moisture: not dec.

GC Column: DB-624 ID $: 0.20 \quad(\mathrm{~mm})$

Soil Extract Volume: (uL)
Lab Sample ID: MB 200-92663/5

Lab File ID: 15254_005.D

Date Received:

Date Analyzed: 08/12/2015

Dilution Factor: 1.0

Soil Aliquot Volume: (uL) Purge Volume: 25.0 $(\mathrm{mL})$ CONCENTRATION UNITS: (ug/L or ug/kg) ug/L

\begin{tabular}{|c|c|c|c|c|}
\hline CAS NUMBER & COMPOUND NAME & $\mathrm{RT}$ & EST. CONC. & Q \\
\hline 01 & Unknown & 7.29 & 3.0 & $\mathrm{JX}$ \\
\hline $541-05-9$ & Cyclotrisiloxane, hexamethyl- & 8.22 & 0.57 & $\mathrm{~J} \mathrm{~N}$ \\
\hline 03 & Unknown & 11.04 & 0.71 & $\mathrm{~J}$ \\
\hline E9667961 & Total Alkanes & $\mathrm{N} / \mathrm{A}$ & & \\
\hline
\end{tabular}


Lab Name: TESTAMERICA BURLINGTON

Contract: $8 \mathrm{E}-00302$

Lab Code: STLV

Case No.: YORK Mod. Ref No.:

SDG No.: 29233

Matrix: (SOIL/SED/WATER) Water

Sample wt/vol: 25.0 $(\mathrm{g} / \mathrm{mL}) \mathrm{mL}$

Level: (TRACE/LOW/MED) TRACE

\% Moisture: not dec.

GC Column: DB-624 ID: $0.20 \quad(\mathrm{~mm})$

Soil Extract Volume: (uL) $(\mathrm{mL})$

Purge Volume: 25.0
Lab Sample ID: MB 200-92691/5

Lab File ID: 15265_005.D

Date Received:

Date Analyzed: 08/13/2015

Dilution Factor: 1.0

Soil Aliquot Volume: (uL)

\begin{tabular}{|c|c|c|c|}
\hline CAS NO. & COMPOUND & $\begin{array}{l}\text { CONCENTRATION UNITS: } \\
(\mathrm{ug} / \mathrm{L} \text { or } \mathrm{ug} / \mathrm{kg}) \mathrm{ug} / \mathrm{L}\end{array}$ & q \\
\hline $75-71-8$ & Dichlorodifluoromethane & 1.0 & $\mathrm{U}$ \\
\hline $74-87-3$ & Chloromethane & 1.0 & $\mathrm{U}$ \\
\hline $75-01-4$ & Vinyl chloride & 1.0 & $\mathrm{U}$ \\
\hline $74-83-9$ & Bromomethane & 1.0 & $\mathrm{U}$ \\
\hline $75-00-3$ & Chloroethane & 1.0 & $\mathrm{U}$ \\
\hline $75-69-4$ & Trichlorofluoromethane & 1.0 & $\mathrm{U}$ \\
\hline $75-35-4$ & 1,1-Dichloroethene & 1.0 & $\mathrm{U}$ \\
\hline $76-13-1$ & $1,1,2$-Trichloro-1,2,2-trifluoroethane & 1.0 & $\mathrm{U}$ \\
\hline $67-64-1$ & Acetone & 5.0 & $\mathrm{U}$ \\
\hline $75-15-0$ & Carbon disulfide & 0.045 & $\mathrm{~J}$ \\
\hline $79-20-9$ & Methyl acetate & 1.0 & $\mathrm{U}$ \\
\hline $75-09-2$ & Methylene Chloride & 1.0 & $\mathrm{U}$ \\
\hline $156-60-5$ & trans-1,2-Dichloroethene & 1.0 & $\mathrm{U}$ \\
\hline $1634-04-4$ & Methyl tert-butyl ether & 1.0 & $\mathrm{U}$ \\
\hline $75-34-3$ & 1,1-Dichloroethane & 1.0 & $\mathrm{U}$ \\
\hline $156-59-2$ & cis-1,2-Dichloroethene & 1.0 & $\mathrm{U}$ \\
\hline $78-93-3$ & 2-Butanone & 5.0 & $\mathrm{U}$ \\
\hline $74-97-5$ & Bromochloromethane & 1.0 & $\mathrm{U}$ \\
\hline $67-66-3$ & Chloroform & 1.0 & $\mathrm{U}$ \\
\hline $71-55-6$ & $1,1,1$-Trichloroethane & 1.0 & $\mathrm{U}$ \\
\hline $110-82-7$ & Cyclohexane & 1.0 & $\mathrm{U}$ \\
\hline $56-23-5$ & Carbon tetrachloride & 1.0 & $\mathrm{U}$ \\
\hline $71-43-2$ & Benzene & 1.0 & $\mathrm{U}$ \\
\hline $107-06-2$ & 1,2-Dichloroethane & 1.0 & $\mathrm{U}$ \\
\hline
\end{tabular}

Report 1,4-Dioxane for Low-Medium VOA analysis only 
Lab Name: TESTAMERICA BURLINGTON

Lab Code: STLV

Case No.: YORK

Mod. Ref No.:

Contract: 8E-00302

Matrix: (SOIL/SED/WATER) Water

Sample wt/vol: 25.0 $(\mathrm{g} / \mathrm{mL}) \mathrm{mL}$

Level: (TRACE/LOW/MED) TRACE

\% Moisture: not dec.

GC Column: DB-624 ID $: \underline{0.20 \quad(\mathrm{~mm})}$

Soil Extract Volume: (uL) $(\mathrm{mL})$

Purge Volume: 25.0
SDG No.: 29233

Lab Sample ID: MB 200-92691/5

Lab File ID: 15265_005.D

Date Received:

Date Analyzed: 08/13/2015

Dilution Factor: 1.0

Soil Aliquot Volume: (uL)

\begin{tabular}{|c|c|c|c|}
\hline CAS NO. & COMPOUND & $\begin{array}{l}\text { CONCENTRATION UNITS: } \\
(\mathrm{ug} / \mathrm{L} \text { or } \mathrm{ug} / \mathrm{kg}) \mathrm{ug} / \mathrm{L}\end{array}$ & Q \\
\hline $79-01-6$ & Trichloroethene & $\begin{array}{r}1.0 \\
\end{array}$ & $\mathrm{U}$ \\
\hline $108-87-2$ & Methylcyclohexane & 1.0 & $\mathrm{U}$ \\
\hline $78-87-5$ & 1,2-Dichloropropane & 1.0 & $\mathrm{U}$ \\
\hline $75-27-4$ & Bromodichloromethane & 1.0 & $\mathrm{U}$ \\
\hline $10061-01-5$ & cis-1,3-Dichloropropene & 1.0 & $\mathrm{U}$ \\
\hline $108-10-1$ & 4-Methyl-2-pentanone & 1.0 & $\mathrm{U}$ \\
\hline $108-88-3$ & Toluene & 1.0 & $\mathrm{U}$ \\
\hline $10061-02-6$ & trans-1,3-Dichloropropene & 1.0 & $\mathrm{U}$ \\
\hline $79-00-5$ & $1,1,2-T r i c h l o r o e t h a n e$ & 1.0 & $\mathrm{U}$ \\
\hline $127-18-4$ & Tetrachloroethene & 1.0 & $\mathrm{U}$ \\
\hline $591-78-6$ & 2-Hexanone & 1.0 & $\mathrm{U}$ \\
\hline $124-48-1$ & Dibromochloromethane & 1.0 & $\mathrm{U}$ \\
\hline $106-93-4$ & 1,2-Dibromoethane & 1.0 & $\mathrm{U}$ \\
\hline $108-90-7$ & Chlorobenzene & 1.0 & $\mathrm{U}$ \\
\hline $100-41-4$ & Ethylbenzene & 1.0 & $\mathrm{U}$ \\
\hline $95-47-6$ & o-Xylene & 1.0 & $\mathrm{U}$ \\
\hline $179601-23-1$ & m,p-xylene & 1.0 & $\mathrm{U}$ \\
\hline $100-42-5$ & Styrene & 1.0 & $\mathrm{U}$ \\
\hline $75-25-2$ & Bromoform & 1.0 & $\mathrm{U}$ \\
\hline $98-82-8$ & Isopropylbenzene & 1.0 & $\mathrm{U}$ \\
\hline $79-34-5$ & $1,1,2,2$-Tetrachloroethane & 1.0 & $\mathrm{U}$ \\
\hline $541-73-1$ & 1,3 -Dichlorobenzene & 1.0 & $\mathrm{U}$ \\
\hline $106-46-7$ & 1,4-Dichlorobenzene & 1.0 & $\mathrm{U}$ \\
\hline $95-50-1$ & 1,2-Dichlorobenzene & 1.0 & $\mathrm{U}$ \\
\hline $96-12-8$ & 1,2-Dibromo-3-Chloropropane & 1.0 & $\mathrm{U}$ \\
\hline $120-82-1$ & $1,2,4$-Trichlorobenzene & 0.046 & $\mathrm{~J}$ \\
\hline $87-61-6$ & $1,2,3$-Trichlorobenzene & 0.075 & $\mathrm{~J}$ \\
\hline
\end{tabular}


Lab Name: TESTAMERICA BURLINGTON

Contract: $8 \mathrm{E}-00302$

Lab Code: STLV Case No.: YORK Mod. Ref No.: SDG No.: 29233

Matrix: (SOIL/SED/WATER) Water

Sample wt/vol: 25.0 $(\mathrm{g} / \mathrm{mL}) \mathrm{mL}$

Level: (TRACE or LOW/MED) TRACE

․ Moisture: not dec.

GC Column: DB-624 ID $: 0.20 \quad(\mathrm{~mm})$

Soil Extract Volume: (uL)

CONCENTRATION UNITS: (ug/L or ug/kg) ug/L
Lab Sample ID: MB 200-92691/5

Lab File ID: 15265 005.D

Date Received:

Date Analyzed: 08/13/2015

Dilution Factor: 1.0

Soil Aliquot Volume: (uL) Purge Volume: 25.0

\begin{tabular}{|c|c|c|c|c|}
\hline CAS NUMBER & COMPOUND NAME & RT & EST. CONC. & Q \\
\hline 01 & Unknown & 7.29 & 2.9 & $\mathrm{~J} X$ \\
\hline $541-05-9$ & Cyclotrisiloxane, hexamethyl- & 8.22 & 0.54 & $\mathrm{~J} \mathrm{~N}$ \\
\hline 03 & Unknown & 11.04 & 0.68 & $\mathrm{~J}$ \\
\hline E9667961 & Total Alkanes & $\mathrm{N} / \mathrm{A}$ & & \\
\hline
\end{tabular}

1 EPA-designated Registry Number. 
Lab Name: TESTAMERICA BURLINGTON

Contract: $8 \mathrm{E}-00302$

Lab Code: STLV

Case No.: YORK Mod. Ref No.:

SDG No.: 29233

Matrix: (SOIL/SED/WATER) Water

Sample wt/vol: 25.0 $(\mathrm{g} / \mathrm{mL}) \mathrm{mL}$

Level: (TRACE/LOW/MED) TRACE

\% Moisture: not dec.

GC Column: DB-624 ID: $0.20 \quad(\mathrm{~mm})$

Soil Extract Volume: (uL) $(\mathrm{mL})$

Purge Volume: 25.0
Lab Sample ID: 200-29233-6

Lab File ID: 15265_008.D

Date Received:

Date Analyzed: 08/13/2015

Dilution Factor: 1.0

Soil Aliquot Volume: (uL)

\begin{tabular}{|c|c|c|c|}
\hline CAS NO. & COMPOUND & $\begin{array}{l}\text { CONCENTRATION UNITS: } \\
(\mathrm{ug} / \mathrm{L} \text { or } \mathrm{ug} / \mathrm{kg}) \mathrm{ug} / \mathrm{L}\end{array}$ & q \\
\hline $75-71-8$ & Dichlorodifluoromethane & 1.0 & $\mathrm{U}$ \\
\hline $74-87-3$ & Chloromethane & 1.0 & $\mathrm{U}$ \\
\hline $75-01-4$ & Vinyl chloride & 1.0 & $\mathrm{U}$ \\
\hline $74-83-9$ & Bromomethane & 1.0 & $\mathrm{U}$ \\
\hline $75-00-3$ & Chloroethane & 1.0 & $\mathrm{U}$ \\
\hline $75-69-4$ & Trichlorofluoromethane & 1.0 & $\mathrm{U}$ \\
\hline $75-35-4$ & 1,1-Dichloroethene & 1.0 & $\mathrm{U}$ \\
\hline $76-13-1$ & $1,1,2$-Trichloro-1,2,2-trifluoroethane & 1.0 & $\mathrm{U}$ \\
\hline $67-64-1$ & Acetone & 5.0 & $\mathrm{U}$ \\
\hline $75-15-0$ & Carbon disulfide & 1.0 & $\mathrm{U}$ \\
\hline $79-20-9$ & Methyl acetate & 1.0 & $\mathrm{U}$ \\
\hline $75-09-2$ & Methylene Chloride & 1.0 & $\mathrm{U}$ \\
\hline $156-60-5$ & trans-1,2-Dichloroethene & 1.0 & $\mathrm{U}$ \\
\hline $1634-04-4$ & Methyl tert-butyl ether & 1.0 & $\mathrm{U}$ \\
\hline $75-34-3$ & 1,1-Dichloroethane & 1.0 & $\mathrm{U}$ \\
\hline $156-59-2$ & cis-1,2-Dichloroethene & 1.0 & $\mathrm{U}$ \\
\hline $78-93-3$ & 2-Butanone & 5.0 & $\mathrm{U}$ \\
\hline $74-97-5$ & Bromochloromethane & 1.0 & $\mathrm{U}$ \\
\hline $67-66-3$ & Chloroform & 1.0 & $\mathrm{U}$ \\
\hline $71-55-6$ & $1,1,1$-Trichloroethane & 1.0 & $\mathrm{U}$ \\
\hline $110-82-7$ & Cyclohexane & 1.0 & $\mathrm{U}$ \\
\hline $56-23-5$ & Carbon tetrachloride & 0.029 & $\mathrm{~J}$ \\
\hline $71-43-2$ & Benzene & 1.0 & $\mathrm{U}$ \\
\hline $107-06-2$ & 1,2-Dichloroethane & 1.0 & $\mathrm{U}$ \\
\hline
\end{tabular}

Report 1,4-Dioxane for Low-Medium VOA analysis only 
Lab Name: TESTAMERICA BURLINGTON

Lab Code: STLV

Case No.: YORK

Mod. Ref No.:

Contract: 8E-00302

Matrix: (SOIL/SED/WATER) Water

Sample wt/vol: 25.0 $(\mathrm{g} / \mathrm{mL}) \mathrm{mL}$

Level: (TRACE/LOW/MED) TRACE

\% Moisture: not dec.

GC Column: DB-624 ID: $0.20 \quad(\mathrm{~mm})$

Soil Extract Volume: (uL) $(\mathrm{mL})$

Purge Volume: 25.0
SDG No.: 29233

Lab Sample ID: 200-29233-6

Lab File ID: 15265_008.D

Date Received:

Date Analyzed: 08/13/2015

Dilution Factor: 1.0

Soil Aliquot Volume:

(uL)

\begin{tabular}{|c|c|c|c|}
\hline CAS NO. & COMPOUND & $\begin{array}{l}\text { CONCENTRATION UNITS: } \\
(\mathrm{ug} / \mathrm{L} \text { or } \mathrm{ug} / \mathrm{kg}) \mathrm{ug} / \mathrm{L}\end{array}$ & $q$ \\
\hline $79-01-6$ & Trichloroethene & 1.0 & $\mathrm{U}$ \\
\hline $108-87-2$ & Methylcyclohexane & 1.0 & $\mathrm{U}$ \\
\hline $78-87-5$ & 1,2-Dichloropropane & 1.0 & $\mathrm{U}$ \\
\hline $75-27-4$ & Bromodichloromethane & 1.0 & $\mathrm{U}$ \\
\hline $10061-01-5$ & cis-1,3-Dichloropropene & 1.0 & U \\
\hline $108-10-1$ & 4-Methyl-2-pentanone & 1.0 & $\mathrm{U}$ \\
\hline $108-88-3$ & Toluene & 1.0 & $\mathrm{U}$ \\
\hline $10061-02-6$ & trans-1,3-Dichloropropene & 1.0 & $\mathrm{U}$ \\
\hline $79-00-5$ & $1,1,2-$ Trichloroethane & 1.0 & $\mathrm{U}$ \\
\hline $127-18-4$ & Tetrachloroethene & 1.0 & $\mathrm{U}$ \\
\hline $591-78-6$ & 2-Hexanone & 1.0 & $\mathrm{U}$ \\
\hline $124-48-1$ & Dibromochloromethane & 1.0 & $\mathrm{U}$ \\
\hline $106-93-4$ & 1,2-Dibromoethane & 1.0 & $\mathrm{U}$ \\
\hline $108-90-7$ & Chlorobenzene & 1.0 & $\mathrm{U}$ \\
\hline $100-41-4$ & Ethylbenzene & 1.0 & $\mathrm{U}$ \\
\hline $95-47-6$ & o-Xylene & 1.0 & $\mathrm{U}$ \\
\hline $179601-23-1$ & m,p-xylene & 1.0 & $\mathrm{U}$ \\
\hline $100-42-5$ & Styrene & 1.0 & $\mathrm{U}$ \\
\hline $75-25-2$ & Bromoform & 1.0 & $\mathrm{U}$ \\
\hline $98-82-8$ & Isopropylbenzene & 1.0 & $\mathrm{U}$ \\
\hline $79-34-5$ & $1,1,2,2$-Tetrachloroethane & 1.0 & $\mathrm{U}$ \\
\hline $541-73-1$ & 1,3 -Dichlorobenzene & 1.0 & $\mathrm{U}$ \\
\hline $106-46-7$ & 1,4-Dichlorobenzene & 1.0 & $\mathrm{U}$ \\
\hline $95-50-1$ & 1,2-Dichlorobenzene & 1.0 & $\mathrm{U}$ \\
\hline $96-12-8$ & 1,2-Dibromo-3-Chloropropane & 1.0 & $\mathrm{U}$ \\
\hline $120-82-1$ & $1,2,4$-Trichlorobenzene & 1.0 & $\mathrm{U}$ \\
\hline $87-61-6$ & $1,2,3$-Trichlorobenzene & 1.0 & $\mathrm{U}$ \\
\hline
\end{tabular}


Lab Name: TESTAMERICA BURLINGTON

Contract: $8 \mathrm{E}-00302$

Lab Code: STLV Case No.: YORK Mod. Ref No.: SDG No.: 29233

Matrix: (SOIL/SED/WATER) Water

Sample wt/vol: 25.0 $(\mathrm{g} / \mathrm{mL}) \mathrm{mL}$

Level: (TRACE or LOW/MED) TRACE

\% Moisture: not dec.

GC Column: DB-624 ID $: 0.20 \quad(\mathrm{~mm})$

Soil Extract Volume: (uL)

CONCENTRATION UNITS: (ug/L or ug/kg) ug/L
Lab Sample ID: 200-29233-6

Lab File ID: 15265 _008.D

Date Received:

Date Analyzed: 08/13/2015

Dilution Factor: 1.0

Soil Aliquot Volume: (uL) Purge Volume: 25.0 $(\mathrm{mL})$

01

02

\begin{tabular}{|l|l|c|c|c|}
\hline CAS NUMBER & \multicolumn{1}{|c|}{ COMPOUND NAME } & RT & EST. CONC. & Q \\
\hline & Unknown & 7.29 & 2.8 & $\mathrm{~J}$ B X \\
\hline E9667961 & Total Alkanes & N/A & & \\
\hline
\end{tabular}

1EPA-designated Registry Number. 
Lab Name: TESTAMERICA BURLINGTON

Contract: $8 \mathrm{E}-00302$

Lab Code: STLV

Case No.: YORK Mod. Ref No.:

SDG No.: 29233

Matrix: (SOIL/SED/WATER) Water

Sample wt/vol: 25.0 $(\mathrm{g} / \mathrm{mL}) \mathrm{mL}$

Level: (TRACE/LOW/MED) TRACE

\% Moisture: not dec.

GC Column: DB-624 ID: $0.20 \quad(\mathrm{~mm})$

Soil Extract Volume: (uL) $(\mathrm{mL})$

Purge Volume: 25.0
Lab Sample ID: VIBLK 200-92663/11

Lab File ID: 15254_011.D

Date Received:

Date Analyzed: 08/12/2015

Dilution Factor: 1.0

Soil Aliquot Volume: (uL)

\begin{tabular}{|c|c|c|c|}
\hline CAS NO. & COMPOUND & $\begin{array}{l}\text { CONCENTRATION UNITS: } \\
(\mathrm{ug} / \mathrm{L} \text { or } \mathrm{ug} / \mathrm{kg}) \mathrm{ug} / \mathrm{L}\end{array}$ & Q \\
\hline $75-71-8$ & Dichlorodifluoromethane & 1.0 & $\mathrm{U}$ \\
\hline $74-87-3$ & Chloromethane & 1.0 & $\mathrm{U}$ \\
\hline $75-01-4$ & Vinyl chloride & 1.0 & $\mathrm{U}$ \\
\hline $74-83-9$ & Bromomethane & 1.0 & $\mathrm{U}$ \\
\hline $75-00-3$ & Chloroethane & 1.0 & $\mathrm{U}$ \\
\hline $75-69-4$ & Trichlorofluoromethane & 1.0 & $\mathrm{U}$ \\
\hline $75-35-4$ & 1,1-Dichloroethene & 1.0 & $\mathrm{U}$ \\
\hline $76-13-1$ & $1,1,2$-Trichloro-1,2,2-trifluoroethane & 1.0 & $\mathrm{U}$ \\
\hline $67-64-1$ & Acetone & 5.0 & $\mathrm{U}$ \\
\hline $75-15-0$ & Carbon disulfide & 0.044 & $\mathrm{~J} B$ \\
\hline $79-20-9$ & Methyl acetate & 1.0 & $\mathrm{U}$ \\
\hline $75-09-2$ & Methylene Chloride & 1.0 & $\mathrm{U}$ \\
\hline $156-60-5$ & trans-1,2-Dichloroethene & 1.0 & $\mathrm{U}$ \\
\hline $1634-04-4$ & Methyl tert-butyl ether & 1.0 & $\mathrm{U}$ \\
\hline $75-34-3$ & 1,1-Dichloroethane & 1.0 & $\mathrm{U}$ \\
\hline $156-59-2$ & cis-1,2-Dichloroethene & 1.0 & $\mathrm{U}$ \\
\hline $78-93-3$ & 2-Butanone & 5.0 & $\mathrm{U}$ \\
\hline $74-97-5$ & Bromochloromethane & 1.0 & $\mathrm{U}$ \\
\hline $67-66-3$ & Chloroform & 1.0 & $\mathrm{U}$ \\
\hline $71-55-6$ & $1,1,1$-Trichloroethane & 1.0 & $\mathrm{U}$ \\
\hline $110-82-7$ & Cyclohexane & 1.0 & $\mathrm{U}$ \\
\hline $56-23-5$ & Carbon tetrachloride & 0.11 & $\mathrm{~J}$ \\
\hline $71-43-2$ & Benzene & 1.0 & $\mathrm{U}$ \\
\hline $107-06-2$ & 1,2-Dichloroethane & 1.0 & $\mathrm{U}$ \\
\hline
\end{tabular}

Report 1,4-Dioxane for Low-Medium VOA analysis only 
Lab Name: TESTAMERICA BURLINGTON

Lab Code: STLV

Case No.: YORK

Mod. Ref No.:

Contract: 8E-00302

Matrix: (SOIL/SED/WATER) Water

Sample wt/vol: 25.0 $(\mathrm{g} / \mathrm{mL}) \mathrm{mL}$

Level: (TRACE/LOW/MED) TRACE

\% Moisture: not dec.

GC Column: DB-624 ID: $0.20 \quad(\mathrm{~mm})$

Soil Extract Volume: (uL) $(\mathrm{mL})$

Purge Volume: 25.0
SDG No.: 29233

Lab Sample ID: VIBLK 200-92663/11

Lab File ID: 15254_011.D

Date Received:

Date Analyzed: 08/12/2015

Dilution Factor: 1.0

Soil Aliquot Volume:

(uL)

\begin{tabular}{|c|c|c|c|}
\hline CAS NO. & COMPOUND & $\begin{array}{l}\text { CONCENTRATION UNITS: } \\
(\mathrm{ug} / \mathrm{L} \text { or } \mathrm{ug} / \mathrm{kg}) \mathrm{ug} / \mathrm{L}\end{array}$ & Q \\
\hline $79-01-6$ & Trichloroethene & 1.0 & $\mathrm{U}$ \\
\hline $108-87-2$ & Methylcyclohexane & 1.0 & $\mathrm{U}$ \\
\hline $78-87-5$ & 1,2-Dichloropropane & 1.0 & $\mathrm{U}$ \\
\hline $75-27-4$ & Bromodichloromethane & 1.0 & $\mathrm{U}$ \\
\hline $10061-01-5$ & cis-1,3-Dichloropropene & 1.0 & U \\
\hline $108-10-1$ & 4-Methyl-2-pentanone & 5.0 & $\mathrm{U}$ \\
\hline $108-88-3$ & Toluene & 0.011 & $\mathrm{~J} B$ \\
\hline $10061-02-6$ & trans-1,3-Dichloropropene & 1.0 & $\mathrm{U}$ \\
\hline $79-00-5$ & $1,1,2-$ Trichloroethane & 1.0 & $\mathrm{U}$ \\
\hline $127-18-4$ & Tetrachloroethene & 1.0 & $\mathrm{U}$ \\
\hline $591-78-6$ & 2-Hexanone & 5.0 & $\mathrm{U}$ \\
\hline $124-48-1$ & Dibromochloromethane & 1.0 & $\mathrm{U}$ \\
\hline $106-93-4$ & 1,2-Dibromoethane & 1.0 & $\mathrm{U}$ \\
\hline $108-90-7$ & Chlorobenzene & 1.0 & $\mathrm{U}$ \\
\hline $100-41-4$ & Ethylbenzene & 0.0026 & $\mathrm{~J} B$ \\
\hline $95-47-6$ & o-Xylene & 1.0 & $\mathrm{U}$ \\
\hline $179601-23-1$ & m,p-xylene & 0.0075 & $\mathrm{~J} B$ \\
\hline $100-42-5$ & Styrene & 1.0 & $\mathrm{U}$ \\
\hline $75-25-2$ & Bromoform & 1.0 & $\mathrm{U}$ \\
\hline $98-82-8$ & Isopropylbenzene & 1.0 & $\mathrm{U}$ \\
\hline $79-34-5$ & $1,1,2,2$-Tetrachloroethane & 1.0 & $\mathrm{U}$ \\
\hline $541-73-1$ & 1,3 -Dichlorobenzene & 1.0 & $\mathrm{U}$ \\
\hline $106-46-7$ & 1,4-Dichlorobenzene & 1.0 & $\mathrm{U}$ \\
\hline $95-50-1$ & 1,2-Dichlorobenzene & 1.0 & $\mathrm{U}$ \\
\hline $96-12-8$ & 1,2-Dibromo-3-Chloropropane & 1.0 & $\mathrm{U}$ \\
\hline $120-82-1$ & $1,2,4$-Trichlorobenzene & 0.015 & $\mathrm{~J} B$ \\
\hline $87-61-6$ & $1,2,3$-Trichlorobenzene & 0.015 & $\mathrm{~J} B$ \\
\hline
\end{tabular}


Lab Name: TESTAMERICA BURLINGTON

Contract: $8 \mathrm{E}-00302$

Lab Code: STLV Case No.: YORK Mod. Ref No.: SDG No.: 29233

Matrix: (SOIL/SED/WATER) Water

Sample wt/vol: 25.0 $(\mathrm{g} / \mathrm{mL}) \mathrm{mL}$

Level: (TRACE or LOW/MED) TRACE

Moisture: not dec.

GC Column: DB-624 ID $: 0.20 \quad(\mathrm{~mm})$

Soil Extract Volume: (uL)

CONCENTRATION UNITS: (ug/L or ug/kg) ug/L
Lab Sample ID: VIBLK 200-92663/11

Lab File ID: 15254_011.D

Date Received:

Date Analyzed: 08/12/2015

Dilution Factor: 1.0

Soil Aliquot Volume: (uL) Purge Volume: 25.0 $(\mathrm{mL})$

01

02

\begin{tabular}{|l|l|c|c|c|}
\hline CAS NUMBER & \multicolumn{1}{|c|}{ COMPOUND NAME } & RT & EST. CONC. & Q \\
\hline & Unknown & 7.29 & 2.9 & $\mathrm{~J}$ B X \\
\hline E9667961 & Total Alkanes & N/A & & \\
\hline
\end{tabular}

1EPA-designated Registry Number. 
Lab Name: TESTAMERICA BURLINGTON

Contract: $8 \mathrm{E}-00302$

Lab Code: STLV

Case No.: YORK Mod. Ref No.:

SDG No.: 29233

Matrix: (SOIL/SED/WATER) Water

Sample wt/vol: 25.0 $(\mathrm{g} / \mathrm{mL}) \mathrm{mL}$

Level: (TRACE/LOW/MED) TRACE

\% Moisture: not dec.

GC Column: DB-624 ID: $0.20 \quad(\mathrm{~mm})$

Soil Extract Volume: $(u L)$ $(\mathrm{mL})$

Purge Volume: 25.0
Lab Sample ID: VIBLK 200-92663/13

Lab File ID: 15254_013.D

Date Received:

Date Analyzed: 08/12/2015

Dilution Factor: 1.0

Soil Aliquot Volume: (uL)

\begin{tabular}{|c|c|c|c|}
\hline CAS NO. & COMPOUND & $\begin{array}{l}\text { CONCENTRATION UNITS: } \\
(\mathrm{ug} / \mathrm{L} \text { or } \mathrm{ug} / \mathrm{kg}) \mathrm{ug} / \mathrm{L}\end{array}$ & Q \\
\hline $75-71-8$ & Dichlorodifluoromethane & 1.0 & $\mathrm{U}$ \\
\hline $74-87-3$ & Chloromethane & 1.0 & $\mathrm{U}$ \\
\hline $75-01-4$ & Vinyl chloride & 1.0 & $\mathrm{U}$ \\
\hline $74-83-9$ & Bromomethane & 1.0 & $\mathrm{U}$ \\
\hline $75-00-3$ & Chloroethane & 1.0 & $\mathrm{U}$ \\
\hline $75-69-4$ & Trichlorofluoromethane & 1.0 & $\mathrm{U}$ \\
\hline $75-35-4$ & 1,1-Dichloroethene & 1.0 & $\mathrm{U}$ \\
\hline $76-13-1$ & $1,1,2$-Trichloro-1,2,2-trifluoroethane & 1.0 & $\mathrm{U}$ \\
\hline $67-64-1$ & Acetone & 5.0 & $\mathrm{U}$ \\
\hline $75-15-0$ & Carbon disulfide & 0.039 & $\mathrm{~J} B$ \\
\hline $79-20-9$ & Methyl acetate & 1.0 & $\mathrm{U}$ \\
\hline $75-09-2$ & Methylene Chloride & 1.0 & $\mathrm{U}$ \\
\hline $156-60-5$ & trans-1,2-Dichloroethene & 1.0 & $\mathrm{U}$ \\
\hline $1634-04-4$ & Methyl tert-butyl ether & 1.0 & $\mathrm{U}$ \\
\hline $75-34-3$ & 1,1-Dichloroethane & 1.0 & $\mathrm{U}$ \\
\hline $156-59-2$ & cis-1,2-Dichloroethene & 1.0 & $\mathrm{U}$ \\
\hline $78-93-3$ & 2-Butanone & 5.0 & $\mathrm{U}$ \\
\hline $74-97-5$ & Bromochloromethane & 1.0 & $\mathrm{U}$ \\
\hline $67-66-3$ & Chloroform & 1.0 & $\mathrm{U}$ \\
\hline $71-55-6$ & $1,1,1$-Trichloroethane & 1.0 & $\mathrm{U}$ \\
\hline $110-82-7$ & Cyclohexane & 1.0 & $\mathrm{U}$ \\
\hline $56-23-5$ & Carbon tetrachloride & 0.056 & $\mathrm{~J}$ \\
\hline $71-43-2$ & Benzene & 1.0 & $\mathrm{U}$ \\
\hline $107-06-2$ & 1,2-Dichloroethane & 1.0 & $\mathrm{U}$ \\
\hline
\end{tabular}

Report 1,4-Dioxane for Low-Medium VOA analysis only 
Lab Name: TESTAMERICA BURLINGTON

Lab Code: STLV

Case No.: YORK

Mod. Ref No.:

Contract: 8E-00302

Matrix: (SOIL/SED/WATER) Water

Sample wt/vol: 25.0 $(\mathrm{g} / \mathrm{mL}) \mathrm{mL}$

Level: (TRACE/LOW/MED) TRACE

\% Moisture: not dec.

GC Column: DB-624 ID: $0.20 \quad(\mathrm{~mm})$

Soil Extract Volume: (uL) $(\mathrm{mL})$

Purge Volume: 25.0
SDG No.: 29233

Lab Sample ID: VIBLK 200-92663/13

Lab File ID: 15254_013.D

Date Received:

Date Analyzed: 08/12/2015

Dilution Factor: 1.0

Soil Aliquot Volume:

(uL)

\begin{tabular}{|c|c|c|c|}
\hline CAS NO. & COMPOUND & $\begin{array}{l}\text { CONCENTRATION UNITS: } \\
(\mathrm{ug} / \mathrm{L} \text { or } \mathrm{ug} / \mathrm{kg}) \mathrm{ug} / \mathrm{L}\end{array}$ & Q \\
\hline $79-01-6$ & Trichloroethene & 1.0 & $\mathrm{U}$ \\
\hline $108-87-2$ & Methylcyclohexane & 1.0 & $\mathrm{U}$ \\
\hline $78-87-5$ & 1,2-Dichloropropane & 1.0 & $\mathrm{U}$ \\
\hline $75-27-4$ & Bromodichloromethane & 1.0 & $\mathrm{U}$ \\
\hline $10061-01-5$ & cis-1,3-Dichloropropene & 1.0 & U \\
\hline $108-10-1$ & 4-Methyl-2-pentanone & 5.0 & $\mathrm{U}$ \\
\hline $108-88-3$ & Toluene & 0.014 & $\mathrm{~J} B$ \\
\hline $10061-02-6$ & trans-1,3-Dichloropropene & 1.0 & $\mathrm{U}$ \\
\hline $79-00-5$ & $1,1,2-$ Trichloroethane & 1.0 & $\mathrm{U}$ \\
\hline $127-18-4$ & Tetrachloroethene & 1.0 & $\mathrm{U}$ \\
\hline $591-78-6$ & 2-Hexanone & 5.0 & $\mathrm{U}$ \\
\hline $124-48-1$ & Dibromochloromethane & 1.0 & $\mathrm{U}$ \\
\hline $106-93-4$ & 1,2-Dibromoethane & 1.0 & $\mathrm{U}$ \\
\hline $108-90-7$ & Chlorobenzene & 1.0 & $\mathrm{U}$ \\
\hline $100-41-4$ & Ethylbenzene & 0.0033 & $\mathrm{~J} B$ \\
\hline $95-47-6$ & o-Xylene & 0.0034 & $\mathrm{~J} B$ \\
\hline $179601-23-1$ & m,p-xylene & 0.012 & $\mathrm{~J} B$ \\
\hline $100-42-5$ & Styrene & 1.0 & $\mathrm{U}$ \\
\hline $75-25-2$ & Bromoform & 1.0 & $\mathrm{U}$ \\
\hline $98-82-8$ & Isopropylbenzene & 1.0 & $\mathrm{U}$ \\
\hline $79-34-5$ & $1,1,2,2$-Tetrachloroethane & 1.0 & $\mathrm{U}$ \\
\hline $541-73-1$ & 1,3 -Dichlorobenzene & 1.0 & $\mathrm{U}$ \\
\hline $106-46-7$ & 1,4-Dichlorobenzene & 1.0 & $\mathrm{U}$ \\
\hline $95-50-1$ & 1,2-Dichlorobenzene & 1.0 & $\mathrm{U}$ \\
\hline $96-12-8$ & 1,2-Dibromo-3-Chloropropane & 1.0 & $\mathrm{U}$ \\
\hline $120-82-1$ & $1,2,4$-Trichlorobenzene & 0.016 & $\mathrm{~J} B$ \\
\hline $87-61-6$ & $1,2,3$-Trichlorobenzene & 0.011 & $\mathrm{~J} B$ \\
\hline
\end{tabular}


Lab Name: TESTAMERICA BURLINGTON

Contract: 8E-00302

Lab Code: STLV Case No.: YORK Mod. Ref No.: SDG No.: 29233

Matrix: (SOIL/SED/WATER) Water

Sample wt/vol: 25.0 $(\mathrm{g} / \mathrm{mL}) \mathrm{mL}$

Level: (TRACE or LOW/MED) TRACE

\% Moisture: not dec.

GC Column: DB-624 ID $: 0.20 \quad(\mathrm{~mm})$

Soil Extract Volume: (uL)

CONCENTRATION UNITS: (ug/L or ug/kg) ug/L
Lab Sample ID: VIBLK 200-92663/13

Lab File ID: 15254 013.D

Date Received:

Date Analyzed: 08/12/2015

Dilution Factor: 1.0

Soil Aliquot Volume: (uL) Purge Volume: 25.0 $(\mathrm{mL})$

01

02

\begin{tabular}{|l|l|c|c|c|}
\hline CAS NUMBER & \multicolumn{1}{|c|}{ COMPOUND NAME } & RT & EST. CONC. & Q \\
\hline & Unknown & 7.29 & 2.9 & $\mathrm{~J}$ B X \\
\hline E9667961 & Total Alkanes & N/A & & \\
\hline
\end{tabular}

1EPA-designated Registry Number. 
Lab Name: TestAmerica Burlington

Job No.: 200-29233-1

SDG No.: 29233

Instrument ID: CHD.i

Analysis Batch Number: 92375

Lab Sample ID: IC 200-92375/3

Client Sample ID:

Date Analyzed: 08/06/15 10:11

Lab File ID: 15136_003.D

GC Column: DB-624

ID : $0.2(\mathrm{~mm})$

\begin{tabular}{|c|c|c|c|c|}
\hline \multirow[t]{2}{*}{ COMPOUND NAME } & \multirow{2}{*}{$\begin{array}{c}\text { RETENTION } \\
\text { TIME }\end{array}$} & \multicolumn{3}{|c|}{ MANUAL INTEGRATION } \\
\hline & & REASON & ANALYST & DATE \\
\hline 1,2-Dichloropropane-d6 & 6.42 & Split Peak & wilburj & $08 / 14 / 1515: 00$ \\
\hline 1,2-Dibromo-3-Chloropropane & 13.43 & Assign Peak & wilburj & $08 / 06 / 15 \quad 14: 13$ \\
\hline
\end{tabular}

Lab Sample ID: IC 200-92375/4 Client Sample ID:

Date Analyzed: 08/06/15 10:35

Lab File ID: 15136_004.D

GC Column: DB-624

ID $: 0.2(\mathrm{~mm})$

\begin{tabular}{|c|c|c|c|c|}
\hline \multirow[t]{2}{*}{ COMPOUND NAME } & \multirow{2}{*}{$\begin{array}{c}\text { RETENTION } \\
\text { TIME }\end{array}$} & \multicolumn{3}{|c|}{ MANUAL INTEGRATION } \\
\hline & & REASON & ANALYST & DATE \\
\hline Dichlorodifluoromethane & 1.47 & Baseline & wilburj & $08 / 06 / 15 \quad 14: 14$ \\
\hline 1,2-Dibromo-3-Chloropropane & 13.43 & Baseline & wilburj & $08 / 06 / 1514: 14$ \\
\hline
\end{tabular}

Lab Sample ID: ICIS 200-92375/5

Client Sample ID:

Date Analyzed: 08/06/15 11:00

Lab File ID: 15136_005.D

GC Column: DB-624

ID $: 0.2(\mathrm{~mm})$

\begin{tabular}{|l|c|c|c|}
\hline \multicolumn{1}{|c|}{ COMPOUND NAME } & RETENTION & \multicolumn{2}{|c|}{ MANUAL INTEGRATION } \\
\cline { 3 - 4 } & TIME & REASON & ANALYST \\
\hline Dichlorodifluoromethane & 1.48 & Baseline & DATE \\
\hline
\end{tabular}

Lab Sample ID: IC 200-92375/6

Client Sample ID:

Date Analyzed: 08/06/15 11:24

Lab File ID: 15136_006.D

GC Column: DB-624

ID $: 0.2(\mathrm{~mm})$

\begin{tabular}{|c|c|c|c|c|}
\hline \multirow[t]{2}{*}{ COMPOUND NAME } & \multirow{2}{*}{$\begin{array}{c}\text { RETENTION } \\
\text { TIME }\end{array}$} & \multicolumn{3}{|c|}{ MANUAL INTEGRATION } \\
\hline & & REASON & ANALYST & DATE \\
\hline Dichlorodifluoromethane & 1.48 & Baseline & wilburj & $08 / 06 / 15 \quad 14: 15$ \\
\hline
\end{tabular}

Lab Sample ID: IC 200-92375/7

Client Sample ID:

Date Analyzed: 08/06/15 11:49

Lab File ID: 15136_007.D

GC Column: DB-624

ID $: 0.2(\mathrm{~mm})$

\begin{tabular}{|c|c|c|c|c|}
\hline \multirow[t]{2}{*}{ COMPOUND NAME } & RETENTION & \multicolumn{3}{|c|}{ MANUAL INTEGRATION } \\
\hline & TIME & REASON & ANALYST & DATE \\
\hline 1,2-Dichloropropane-d6 & 6.42 & Split Peak & wilburj & $08 / 14 / 15 \quad 15: 19$ \\
\hline
\end{tabular}

SOM01.2/VOA_Tr 
Lab Name: TestAmerica Burlington

Job No.: 200-29233-1

SDG No.: 29233

Instrument ID: CHD.i

Analysis Batch Number: 92663

Lab Sample ID: CCVIS 200-92663/3

Client Sample ID:

Date Analyzed: 08/12/15 17:53

Lab File ID: 15254 003.D

GC Column: DB-624

ID $: 0.2(\mathrm{~mm})$

\begin{tabular}{|c|c|c|c|c|}
\hline COMPOUND NAME & RETENTION & \multicolumn{2}{|c|}{ MANUAL INTEGRATION } \\
\cline { 3 - 4 } & TIME & REASON & ANALYST & DATE \\
\hline 1,2-Dichloropropane-d6 & 6.41 & Split Peak & wilburj & 08/21/15 15:09 \\
\hline
\end{tabular}

Lab Sample ID: CCVC 200-92663/16

Client Sample ID:

Date Analyzed: 08/12/15 23:16

Lab File ID: 15254 016.D

GC Column: DB-624

ID $: 0.2(\mathrm{~mm})$

\begin{tabular}{|c|c|c|c|c|}
\hline \multirow[t]{2}{*}{ COMPOUND NAME } & \multirow{2}{*}{$\begin{array}{c}\text { RETENTION } \\
\text { TIME }\end{array}$} & \multicolumn{3}{|c|}{ MANUAL INTEGRATION } \\
\hline & & REASON & ANALYST & DATE \\
\hline Dichlorodifluoromethane & 1.47 & Baseline & wilburj & $08 / 21 / 15 \quad 16: 07$ \\
\hline 1,2-Dibromo-3-Chloropropane & 13.43 & Assign Peak & wilburj & $08 / 21 / 1516: 07$ \\
\hline
\end{tabular}


Lab Name: TestAmerica Burlington

SDG No.: 29233

Instrument ID: CHD.i

Lab Sample ID: CCVC 200-92691/23

Date Analyzed: 08/13/15 20:20

\begin{tabular}{|c|c|c|c|c|}
\hline \multirow[t]{2}{*}{ COMPOUND NAME } & \multirow{2}{*}{$\begin{array}{c}\text { RETENTION } \\
\text { TIME }\end{array}$} & \multicolumn{3}{|c|}{ MANUAL INTEGRATION } \\
\hline & & REASON & ANALYST & DATE \\
\hline Dichlorodifluoromethane & 1.52 & Baseline & archern & $08 / 25 / 15 \quad 11: 47$ \\
\hline 1,2-Dibromo-3-Chloropropane & 13.43 & Baseline & archern & $08 / 25 / 1511: 47$ \\
\hline
\end{tabular}

Job No.: 200-29233-1

Analysis Batch Number: 92691

Client Sample ID:

tab File ID: $15265023 . \mathrm{D}$

GC Column: DB-624

ID $: 0.2(\mathrm{~mm})$ 


\section{TestAmerica}

THE LEADER IN ENVIRONMENTAL TESTING

\section{ANALYTICAL REPORT}

Job Number: 200-29312-1

SDG Number: 29312

Job Description: York (200-29312)

Contract Number: 1E-30401

For:

Argonne National Laboratory

9700 South Cass Avenue

Building 203

Office B-141

Argonne, IL 60439

Attention: Ms. Esther Bowen

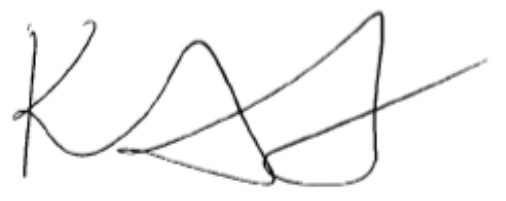

Approved for release. Kathryn A Kelly

\section{Kathryn A Kelly, Project Manager I \\ 30 Community Drive, South Burlington, VT, 05403 \\ kathryn.kelly@testamericainc.com \\ 09/02/2015}

The test results in this report relate only to sample(s) as received by the laboratory. These test results were derived under a quality system that adheres to the requirements of NELAC. Pursuant to NELAC, this report may not be produced in full without written approval from the laboratory 


\section{Table of Contents}

Cover Title Page..$\ldots \ldots \ldots \ldots \ldots \ldots \ldots \ldots \ldots \ldots \ldots \ldots \ldots \ldots$

External Chain of Custody ...................... 4

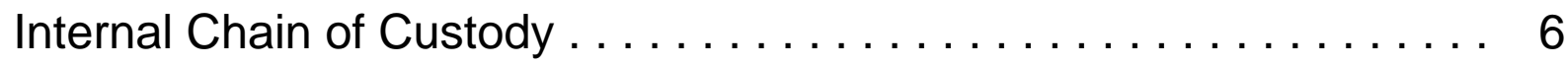

Shipping Documentation . . . . . . . . . . . . . . . 7

Sample Receipt and Log In Check List ..................... 8

Standards Traceability . . . . . . . . . . . . . . . . . 9

Methodology Review .......................... 14

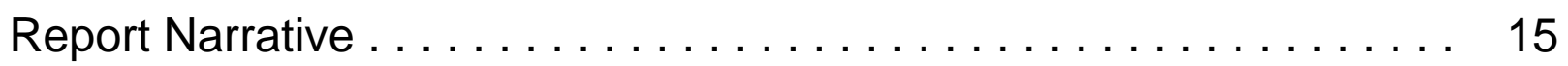

Case Narrative .................................. 15

Manual Integration Documentation ....................... 16

Qualifier Definition ............................... 19

QC Summary - SOM01.2 Volatiles-Trace . . . . . . . . . . 20

QC Summary - SOM01.2 Volatiles-Trace $\ldots \ldots \ldots \ldots \ldots \ldots \ldots \ldots .20$

Deuterated Monitoring Compound Summary $\ldots \ldots \ldots \ldots \ldots \ldots \ldots \ldots \ldots \ldots \ldots, 20$

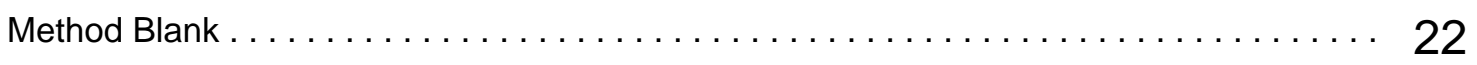

GC/MS Instrument Performance Check $\ldots \ldots \ldots \ldots \ldots \ldots \ldots \ldots \ldots \ldots \ldots \ldots \ldots \ldots \ldots$

Internal Standard Area and RT Summary $\ldots \ldots \ldots \ldots \ldots \ldots \ldots \ldots \ldots \ldots \ldots \ldots \ldots \ldots \ldots$

Sample Data - SOM01.2 Volatiles-Trace .............. 26

Sample Data - SOM01.2 Volatiles-Trace ..................... 26

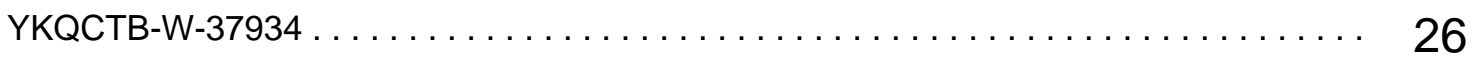

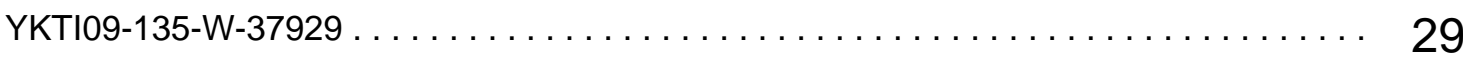

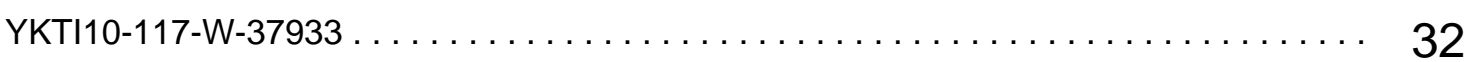

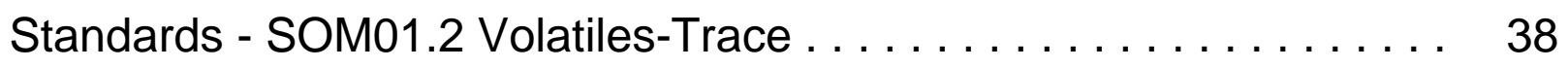

Standards - SOM01.2 Volatiles-Trace $\ldots \ldots \ldots \ldots \ldots \ldots \ldots \ldots \ldots, 38$

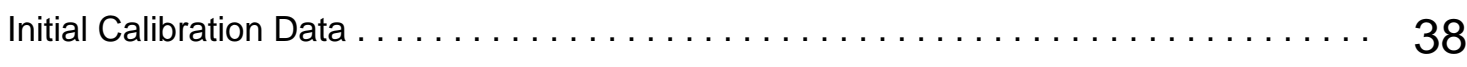

CCV Data, including closing CCV $\ldots \ldots \ldots \ldots \ldots \ldots \ldots \ldots \ldots \ldots \ldots \ldots \ldots \ldots \ldots \ldots$ 


\section{Table of Contents}

Raw Qc Data - SOM01.2 Volatiles-Trace . . . . . . . . . . . . . . 47

Raw Qc Data - SOM01.2 Volatiles-Trace . . . . . . . . . . . . . . . . 47

Raw Qc Data - SOM01.2 Volatiles-Trace . . . . . . . . . . . . . . . . . . . . 47

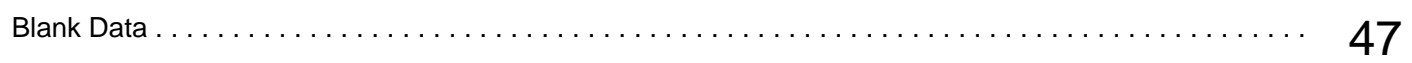




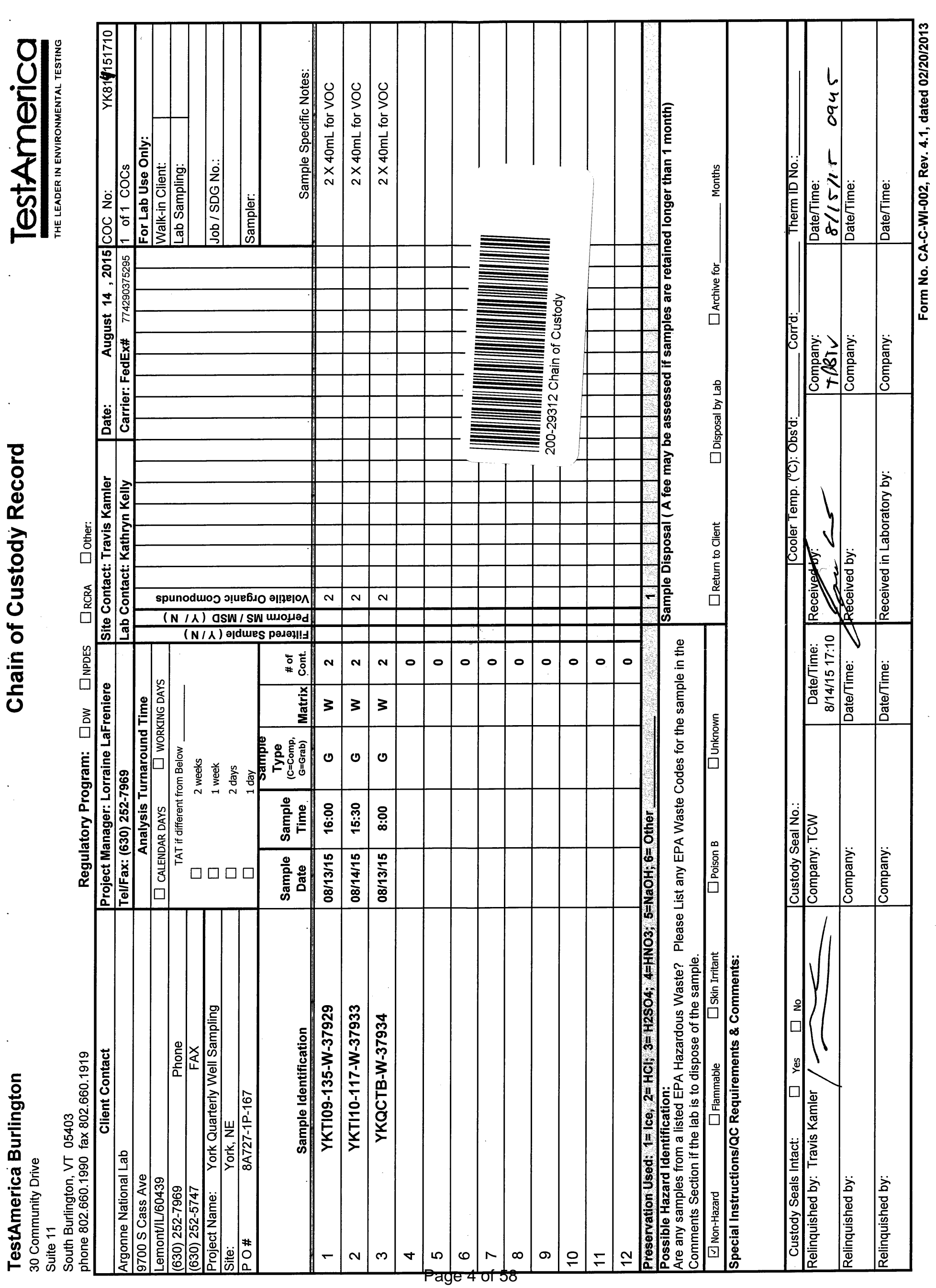




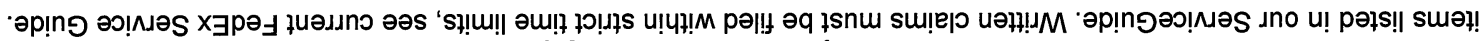

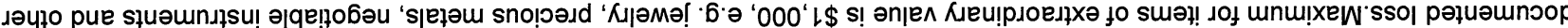

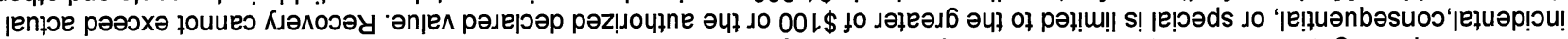

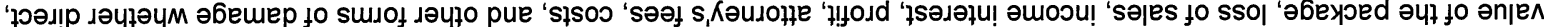

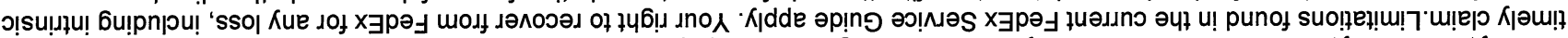

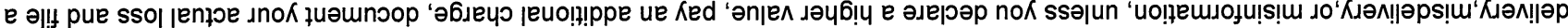

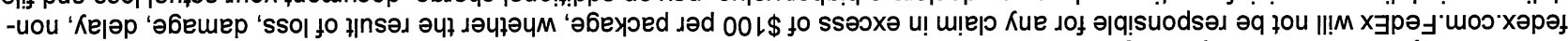
иo әाqe|le

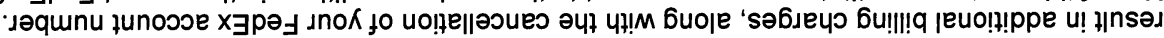

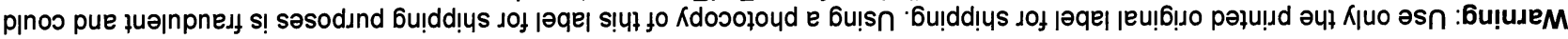

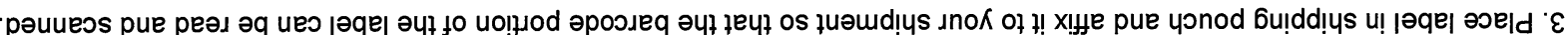

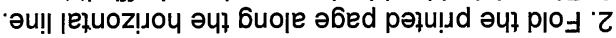

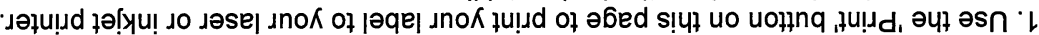

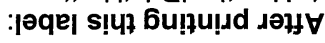

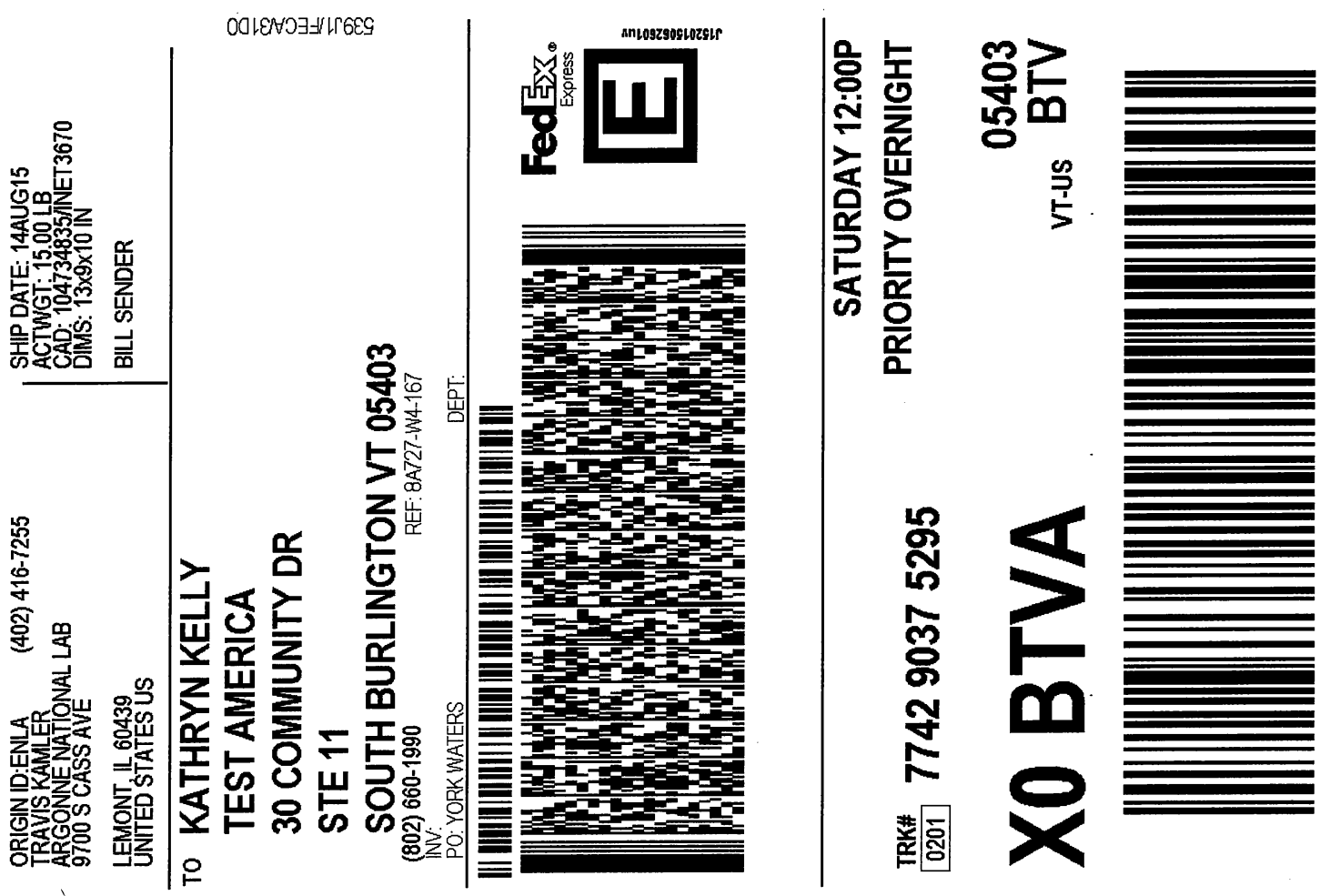




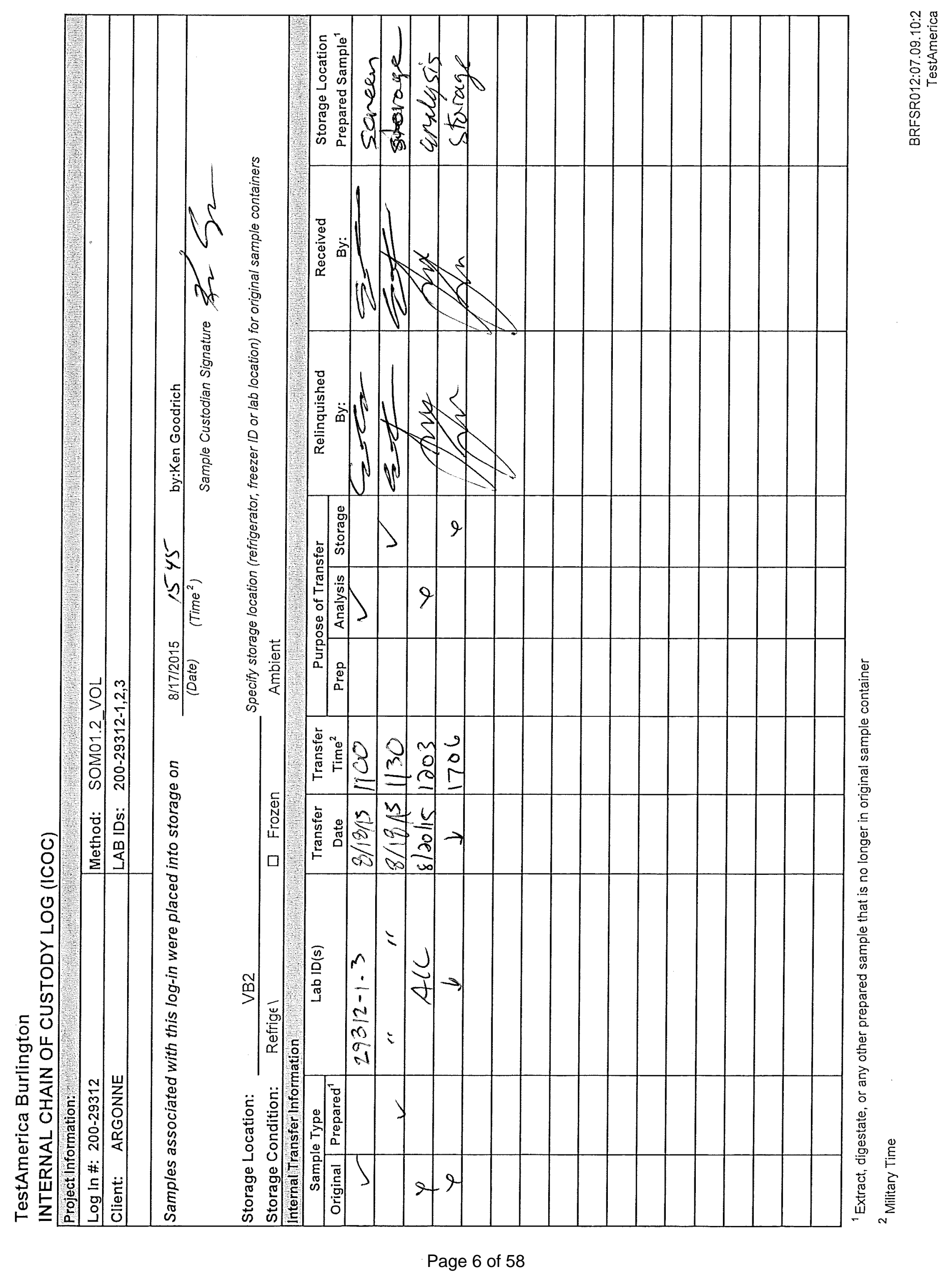




\section{Shipping and Receiving Documents}




\section{Login Sample Receipt Checklist}

Client: Argonne National Laboratory

Job Number: 200-29312-1

SDG Number: 29312

Login Number: 29312

List Source: TestAmerica Burlington

List Number: 1

Creator: Goodrich, Kenneth L

Question

Radioactivity wasn't checked or is $</=$ background as measured by a survey True meter.

The cooler's custody seal, if present, is intact.

Sample custody seals, if present, are intact.

The cooler or samples do not appear to have been compromised or tampered with.

Samples were received on ice.

Cooler Temperature is acceptable.

Cooler Temperature is recorded.

COC is present.

COC is filled out in ink and legible.

$\mathrm{COC}$ is filled out with all pertinent information.

Is the Field Sampler's name present on COC?

There are no discrepancies between the containers received and the COC.

Samples are received within Holding Time.

Sample containers have legible labels.

Containers are not broken or leaking.

Sample collection date/times are provided.

Appropriate sample containers are used.

Sample bottles are completely filled.

Sample Preservation Verified.

There is sufficient vol. for all requested analyses, incl. any requested MS/MSDs

Containers requiring zero headspace have no headspace or bubble is $<6 \mathrm{~mm}$ (1/4").

Multiphasic samples are not present.

Samples do not require splitting or compositing.

Residual Chlorine Checked.

Answer Comment

True

Lab does not accept radioactive samples.

Seal present with no number.

True

True

True

True

True

True

True

True

True

True

True

True

True

True

True

N/A

True

True

True

True

True

N/A 
SDG No.: 29312

\begin{tabular}{|c|c|c|c|c|c|c|c|c|}
\hline \multirow[b]{2}{*}{ Reagent ID } & \multirow[b]{2}{*}{$\begin{array}{l}\text { Exp } \\
\text { Date }\end{array}$} & \multirow[b]{2}{*}{$\begin{array}{l}\text { Prep } \\
\text { Date }\end{array}$} & \multirow[b]{2}{*}{$\begin{array}{l}\text { Dilutant } \\
\text { Used }\end{array}$} & \multirow{2}{*}{$\begin{array}{l}\text { Reagent } \\
\text { Final } \\
\text { Volume }\end{array}$} & \multicolumn{2}{|c|}{ Parent Reagent } & \multirow[b]{2}{*}{ Analyte } & \multirow[b]{2}{*}{ Concentration } \\
\hline & & & & & Reagent ID & $\begin{array}{l}\text { Volume } \\
\text { Added }\end{array}$ & & \\
\hline VMBFBW_00018 & $12 / 22 / 15$ & $06 / 23 / 15$ & METHANOL, LOt 147358 & $25 \mathrm{~mL}$ & VMBFBs_00014 & $125 \mathrm{uL}$ & $\mathrm{BFB}$ & $25 \mathrm{ug} / \mathrm{mL}$ \\
\hline .VMBFBS_00014 & $12 / 22 / 15$ & & RESTEK, Lot A0106759 & & \multicolumn{2}{|c|}{ (Purchased Reagent) } & BFB & $5000 \mathrm{ug} / \mathrm{mL}$ \\
\hline \multirow[t]{33}{*}{ VMSOMTRCALW_00093 } & \multirow[t]{33}{*}{$09 / 19 / 15$} & \multirow[t]{33}{*}{$08 / 19 / 15$} & \multirow[t]{33}{*}{ METHANOL, LOt 147358} & \multirow[t]{33}{*}{4000 uL } & VM8260CALbs_00175 & $40 \mathrm{uL}$ & \begin{tabular}{|l|} 
Bromomethane \\
Chloroethane \\
Chloromethane \\
Dichlorodifluoromethane \\
Trichlorofluoromethane \\
Vinyl chloride
\end{tabular} & $\begin{array}{l}20 \mathrm{ug} / \mathrm{mL} \\
20 \mathrm{ug} / \mathrm{mL} \\
20 \mathrm{ug} / \mathrm{mL} \\
20 \mathrm{ug} / \mathrm{mL} \\
20 \mathrm{ug} / \mathrm{mL} \\
20 \mathrm{ug} / \mathrm{mL}\end{array}$ \\
\hline & & & & & \multirow[t]{32}{*}{ VMSOMCALas_00023 } & \multirow[t]{32}{*}{40 uL } & $\begin{array}{l}1,1,1 \text {-Trichloroethane } \\
1,1,2,2 \text {-Tetrachloroethane } \\
1,1,2 \text {-Trichloro-1,2,2-trifluor } \\
\text { oethane }\end{array}$ & $\begin{array}{l}20 \mathrm{ug} / \mathrm{mL} \\
20 \mathrm{ug} / \mathrm{mL} \\
20 \mathrm{ug} / \mathrm{mL} \\
\end{array}$ \\
\hline & & & & & & & $1,1,2$-Trichloroethane & $20 \mathrm{ug} / \mathrm{mL}$ \\
\hline & & & & & & & \begin{tabular}{|l|} 
1,1-Dichloroethane \\
1,1-Dichloroethene
\end{tabular} & $\frac{20 \mathrm{ug} / \mathrm{mL}}{20 \mathrm{ug} / \mathrm{mL}}$ \\
\hline & & & & & & & $1,2,3$-Trichlorobenzene & $20 \mathrm{ug} / \mathrm{mL}$ \\
\hline & & & & & & & 1,2,4-Trichlorobenzene & $20 \mathrm{ug} / \mathrm{mL}$ \\
\hline & & & & & & & 1,2-Dibromo-3-Chloropropane & $\begin{array}{ll}20 \mathrm{ug} / \mathrm{mL} \\
20 \mathrm{ug} / \mathrm{mL}\end{array}$ \\
\hline & & & & & & & $\begin{array}{l}\text { 1,2-Dibromoethane } \\
1,2 \text {-Dichlorobenzene }\end{array}$ & $\begin{array}{ll}20 \mathrm{ug} / \mathrm{mL} \\
20 \mathrm{ug} / \mathrm{mL}\end{array}$ \\
\hline & & & & & & & 1,2-Dichloroethane & $20 \mathrm{ug} / \mathrm{mL}$ \\
\hline & & & & & & & 1,2-Dichloropropane & $20 \mathrm{ug} / \mathrm{mL}$ \\
\hline & & & & & & & $\begin{array}{l}\text { 1,3-Dichlorobenzene } \\
\text { 1,4-Dichlorobenzene }\end{array}$ & $\frac{20 \mathrm{ug} / \mathrm{mL}}{20 \mathrm{ug} / \mathrm{mL}}$ \\
\hline & & & & & & & $\begin{array}{l}\text { 1,4-Dichlorobenzene } \\
\text { Benzene }\end{array}$ & $20 \mathrm{ug} / \mathrm{mL}$ \\
\hline & & & & & & & Bromochloromethane & $20 \mathrm{ug} / \mathrm{mL}$ \\
\hline & & & & & & & Bromodichloromethane & $20 \mathrm{ug} / \mathrm{mL}$ \\
\hline & & & & & & & Bromoform & $20 \mathrm{ug} / \mathrm{mL}$ \\
\hline & & & & & & & Carbon disulfide & $20 \mathrm{ug} / \mathrm{mL}$ \\
\hline & & & & & & & Carbon tetrachloride & $20 \mathrm{ug} / \mathrm{mL}$ \\
\hline & & & & & & & Chlorobenzene & $20 \mathrm{ug} / \mathrm{mL}$ \\
\hline & & & & & & & Chloroform & $20 \mathrm{ug} / \mathrm{mL}$ \\
\hline & & & & & & & cis-1,2-Dichloroethene & $20 \mathrm{ug} / \mathrm{mL}$ \\
\hline & & & & & & & $\begin{array}{l}\text { Cis-1,3-Dichloropropene } \\
\text { Cyclohexane }\end{array}$ & $\frac{20 \mathrm{ug} / \mathrm{mL}}{20 \mathrm{ug} / \mathrm{mL}}$ \\
\hline & & & & & & & Dibromochloromethane & $20 \mathrm{ug} / \mathrm{mL}$ \\
\hline & & & & & & & Ethylbenzene & $20 \mathrm{ug} / \mathrm{mL}$ \\
\hline & & & & & & & Isopropylbenzene & $20 \mathrm{ug} / \mathrm{mL}$ \\
\hline & & & & & & & $\mathrm{m}, \mathrm{p}$-Xylene & $20 \mathrm{ug} / \mathrm{mL}$ \\
\hline & & & & & & & Methyl acetate & $20 \mathrm{ug} / \mathrm{mL}$ \\
\hline & & & & & & & Methyl tert-butyl ether & $20 \mathrm{ug} / \mathrm{mL}$ \\
\hline & & & & & & & Methylcyclohexane & $20 \mathrm{ug} / \mathrm{mL}$ \\
\hline & & & & & & & Methylene Chloride & $20 \mathrm{ug} / \mathrm{mL}$ \\
\hline & & & & & & & o-Xylene & $20 \mathrm{ug} / \mathrm{mL}$ \\
\hline & & & & & & & Styrene & $20 \mathrm{ug} / \mathrm{mL}$ \\
\hline & & & & & & & Tetrachloroethene & $20 \mathrm{ug} / \mathrm{mL}$ \\
\hline & & & & & & & Toluene & $20 \mathrm{ug} / \mathrm{mL}$ \\
\hline
\end{tabular}


SDG No.: 29312

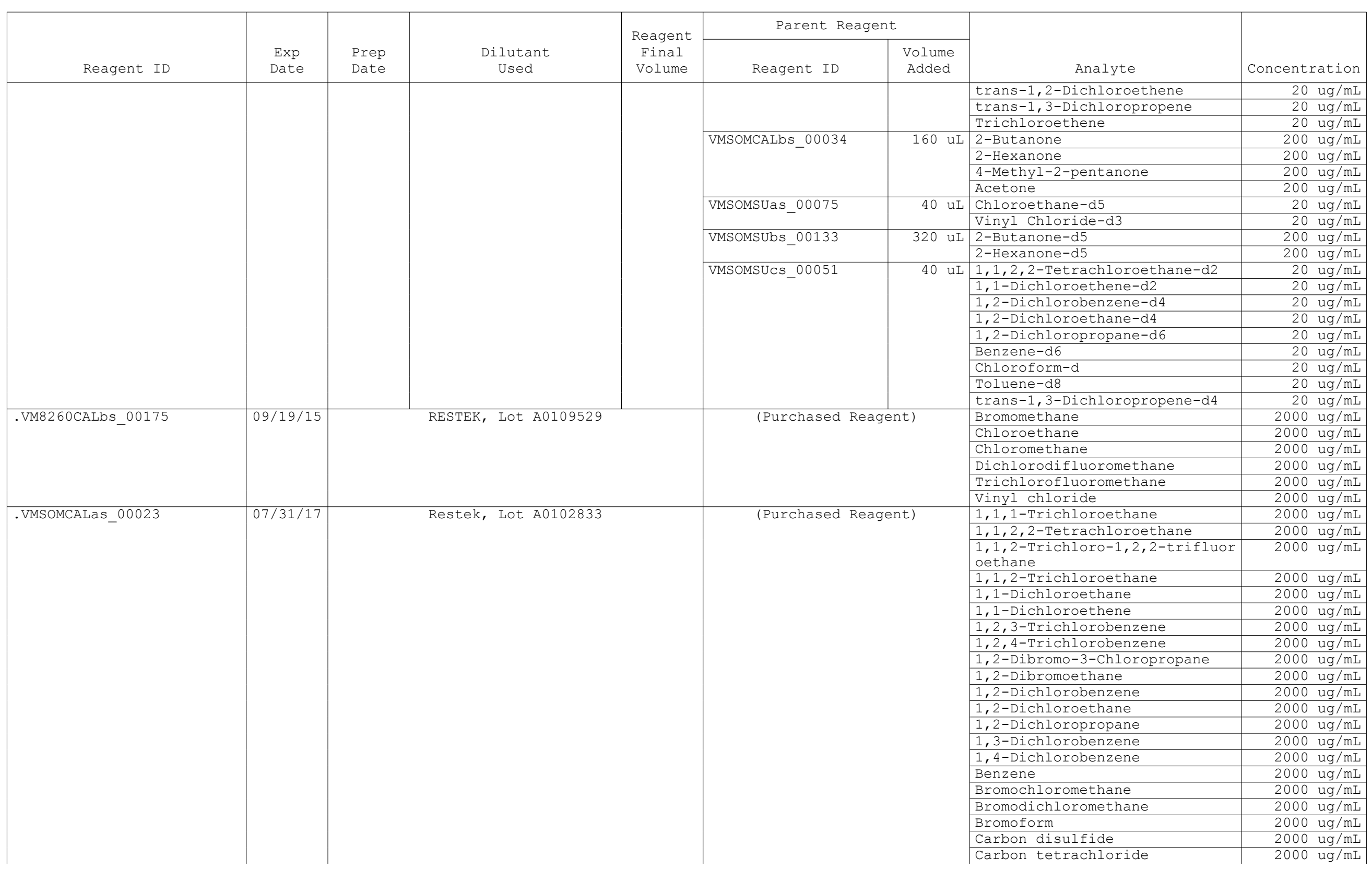


SDG No.: 29312

\begin{tabular}{|c|c|c|c|c|c|c|c|c|c|}
\hline \multirow[b]{2}{*}{ Reagent ID } & \multirow[b]{2}{*}{$\begin{array}{l}\text { Exp } \\
\text { Date }\end{array}$} & \multirow[b]{2}{*}{$\begin{array}{l}\text { Prep } \\
\text { Date }\end{array}$} & \multirow[b]{2}{*}{$\begin{array}{c}\text { Dilutant } \\
\text { Used }\end{array}$} & \multirow{2}{*}{$\begin{array}{c}\text { Reagent } \\
\text { Final } \\
\text { Volume }\end{array}$} & \multicolumn{2}{|c|}{ Parent Reagent } & \multirow[b]{2}{*}{ Analyte } & \multirow{2}{*}{\multicolumn{2}{|c|}{ Concentration }} \\
\hline & & & & & Reagent ID & $\begin{array}{l}\text { Volume } \\
\text { Added }\end{array}$ & & & \\
\hline & & & & & & & Chlorobenzene & 2000 & $\mathrm{ug} / \mathrm{mL}$ \\
\hline & & & & & & & Chloroform & 2000 & $\mathrm{ug} / \mathrm{mL}$ \\
\hline & & & & & & & cis-1,2-Dichloroethene & 2000 & $\mathrm{ug} / \mathrm{mL}$ \\
\hline & & & & & & & cis-1,3-Dichloropropene & 2000 & $\mathrm{ug} / \mathrm{mL}$ \\
\hline & & & & & & & Cyclohexane & 2000 & $\mathrm{ug} / \mathrm{mL}$ \\
\hline & & & & & & & Dibromochloromethane & 2000 & $\mathrm{ug} / \mathrm{mL}$ \\
\hline & & & & & & & Ethylbenzene & 2000 & $\mathrm{ug} / \mathrm{mL}$ \\
\hline & & & & & & & Isopropylbenzene & 2000 & $\mathrm{ug} / \mathrm{mL}$ \\
\hline & & & & & & & m,p-Xylene & 2000 & $\mathrm{ug} / \mathrm{mL}$ \\
\hline & & & & & & & Methyl acetate & 2000 & $\mathrm{ug} / \mathrm{mL}$ \\
\hline & & & & & & & Methyl tert-butyl ether & 2000 & $\mathrm{ug} / \mathrm{mL}$ \\
\hline & & & & & & & Methylcyclohexane & 2000 & $\mathrm{ug} / \mathrm{mL}$ \\
\hline & & & & & & & Methylene Chloride & 2000 & $\mathrm{ug} / \mathrm{mL}$ \\
\hline & & & & & & & o-Xylene & 2000 & $\mathrm{ug} / \mathrm{mL}$ \\
\hline & & & & & & & Styrene & 2000 & $\mathrm{ug} / \mathrm{mL}$ \\
\hline & & & & & & & Tetrachloroethene & 2000 & $\mathrm{ug} / \mathrm{mL}$ \\
\hline & & & & & & & Toluene & 2000 & $\mathrm{ug} / \mathrm{mL}$ \\
\hline & & & & & & & trans-1,2-Dichloroethene & 2000 & $\mathrm{ug} / \mathrm{mL}$ \\
\hline & & & & & & & trans-1,3-Dichloropropene & 2000 & $\mathrm{ug} / \mathrm{mL}$ \\
\hline & & & & & & & Trichloroethene & 2000 & $\mathrm{ug} / \mathrm{mL}$ \\
\hline \multirow[t]{4}{*}{.VMSOMCALbs_00034 } & \multirow[t]{4}{*}{$07 / 16 / 16$} & \multirow{4}{*}{\multicolumn{2}{|c|}{ Restek, Lot A0104748 }} & & \multirow{2}{*}{\multicolumn{2}{|c|}{ (Purchased Reagent) }} & 2-Butanone & 5000 & $\mathrm{ug} / \mathrm{mL}$ \\
\hline & & & & & & & 2-Hexanone & 5000 & $\mathrm{ug} / \mathrm{mL}$ \\
\hline & & & & & & & 4-Methyl-2-pentanone & 5000 & $\mathrm{ug} / \mathrm{mL}$ \\
\hline & & & & & & & Acetone & 5000 & $\mathrm{ug} / \mathrm{mL}$ \\
\hline \multirow[t]{2}{*}{.VMSOMSUas_00075 } & \multirow[t]{2}{*}{$07 / 22 / 17$} & \multirow{2}{*}{\multicolumn{2}{|c|}{ Absolute, Lot 072214}} & & \multicolumn{2}{|c|}{ (Purchased Reagent) } & Chloroethane-d5 & 2000 & $\mathrm{ug} / \mathrm{mL}$ \\
\hline & & & & & & & Vinyl Chloride-d3 & 2000 & $\mathrm{ug} / \mathrm{mL}$ \\
\hline \multirow[t]{2}{*}{.VMSOMSUbs_00133 } & \multirow[t]{2}{*}{$08 / 19 / 16$} & & Absolute, Lot 071515 & & (Purchased $\mathrm{F}$ & nt) & 2-Butanone-d5 & 2500 & $\mathrm{ug} / \mathrm{mL}$ \\
\hline & & & & & & & 2-Hexanone-d5 & 2500 & $\mathrm{ug} / \mathrm{mL}$ \\
\hline .VMSOMSUCS 00051 & $08 / 17 / 16$ & & Absolute, Lot 011614 & & (Purchased $\mathrm{F}$ & nt) & $1,1,2,2$-Tetrachloroethane-d2 & 2000 & $\mathrm{ug} / \mathrm{mL}$ \\
\hline & & & & & & & 1,1-Dichloroethene-d2 & 2000 & $\mathrm{ug} / \mathrm{mL}$ \\
\hline & & & & & & & 1,2-Dichlorobenzene-d4 & 2000 & $\mathrm{ug} / \mathrm{mL}$ \\
\hline & & & & & & & 1,2-Dichloroethane-d4 & 2000 & $\mathrm{ug} / \mathrm{mL}$ \\
\hline & & & & & & & 1,2-Dichloropropane-d6 & 2000 & $\mathrm{ug} / \mathrm{mL}$ \\
\hline & & & & & & & Benzene-d6 & 2000 & $\mathrm{ug} / \mathrm{mL}$ \\
\hline & & & & & & & Chloroform-d & 2000 & $\mathrm{ug} / \mathrm{mL}$ \\
\hline & & & & & & & Toluene-d8 & 2000 & $\mathrm{ug} / \mathrm{mL}$ \\
\hline & & & & & & & trans-1,3-Dichloropropene-d4 & 2000 & $\mathrm{ug} / \mathrm{mL}$ \\
\hline VMSOMTRISw_00103 & $09 / 19 / 15$ & $08 / 19 / 15$ & METHANOL, LOt 147538 & 4400 uL & VMSOMISS_00025 & $35.2 \mathrm{uL}$ & 1,4-Dichlorobenzene-d4 & 20 & $\mathrm{ug} / \mathrm{mL}$ \\
\hline & & & & & & & 1,4-Difluorobenzene & 20 & $\mathrm{ug} / \mathrm{mL}$ \\
\hline & & & & & & & Chlorobenzene-d5 & 20 & $\mathrm{ug} / \mathrm{mL}$ \\
\hline .VMSOMISs_00025 & $06 / 19 / 16$ & & RESTEK, Lot A099377 & & (Purchased $\mathrm{F}$ & nt) & 1,4-Dichlorobenzene-d4 & 2500 & $\mathrm{ug} / \mathrm{mL}$ \\
\hline & & & & & & & 1,4-Difluorobenzene & 2500 & $\mathrm{ug} / \mathrm{mL}$ \\
\hline & & & & & & & Chlorobenzene-d5 & 2500 & $\mathrm{ug} / \mathrm{mL}$ \\
\hline VMSOMTRSUw_00098 & $09 / 19 / 15$ & $08 / 19 / 15$ & METHANOL, Lot 147358 & 4400 uL & VMSOMSUas_00075 & $44 \mathrm{uL}$ & Chloroethane-d5 & 20 & $\mathrm{ug} / \mathrm{mL}$ \\
\hline & & & & & & & Vinyl Chloride-d3 & 20 & $\mathrm{ug} / \mathrm{mL}$ \\
\hline & & & & & VMSOMSUbs_00133 & $352 \mathrm{uL}$ & 2-Butanone-d5 & 200 & $\mathrm{ug} / \mathrm{mL}$ \\
\hline & & & & & & & 2-Hexanone-d5 & 200 & $\mathrm{ug} / \mathrm{mL}$ \\
\hline
\end{tabular}


SDG No.: 29312

\begin{tabular}{|c|c|c|c|c|c|c|c|c|}
\hline \multirow[b]{2}{*}{ Reagent ID } & \multirow[b]{2}{*}{$\begin{array}{l}\text { Exp } \\
\text { Date }\end{array}$} & \multirow[b]{2}{*}{$\begin{array}{l}\text { Prep } \\
\text { Date }\end{array}$} & \multirow[b]{2}{*}{$\begin{array}{c}\text { Dilutant } \\
\text { Used }\end{array}$} & \multirow{2}{*}{$\begin{array}{l}\text { Reagent } \\
\text { Final } \\
\text { Volume }\end{array}$} & \multicolumn{2}{|c|}{ Parent Reagent } & \multirow[b]{2}{*}{ Analyte } & \multirow[b]{2}{*}{ Concentration } \\
\hline & & & & & \multirow{10}{*}{$\begin{array}{r}\text { Reagent ID } \\
\text { VMSOMSUCS_00051 }\end{array}$} & $\begin{array}{l}\text { Volume } \\
\text { Added }\end{array}$ & & \\
\hline & & & & & & $44 \mathrm{uL}$ & $1,1,2,2$-Tetrachloroethane-d2 & $20 \mathrm{ug} / \mathrm{mI}$ \\
\hline & & & & & & & 1,1-Dichloroethene-d2 & $20 \mathrm{ug} / \mathrm{mI}$ \\
\hline & & & & & & & 1,2-Dichlorobenzene-d4 & $20 \mathrm{ug} / \mathrm{mI}$ \\
\hline & & & & & & & 1,2-Dichloroethane-d4 & $20 \mathrm{ug} / \mathrm{mI}$ \\
\hline & & & & & & & 1,2-Dichloropropane-d6 & $20 \mathrm{ug} / \mathrm{mI}$ \\
\hline & & & & & & & Benzene-d6 & $20 \mathrm{ug} / \mathrm{mI}$ \\
\hline & & & & & & & Chloroform-d & $20 \mathrm{ug} / \mathrm{mI}$ \\
\hline & & & & & & & Toluene-d8 & $20 \mathrm{ug} / \mathrm{mI}$ \\
\hline & & & & & & & trans-1,3-Dichloropropene-d4 & $20 \mathrm{ug} / \mathrm{mI}$ \\
\hline \multirow[t]{2}{*}{.VMSOMSUas_00075 } & \multirow[t]{2}{*}{$07 / 22 / 17$} & \multirow{2}{*}{\multicolumn{3}{|c|}{ Absolute, Lot 072214}} & \multirow{2}{*}{\multicolumn{2}{|c|}{ (Purchased Reagent) }} & Chloroethane-d5 & $2000 \mathrm{ug} / \mathrm{mI}$ \\
\hline & & & & & & & Vinyl Chloride-d3 & $2000 \mathrm{ug} / \mathrm{mI}$ \\
\hline \multirow[t]{2}{*}{.VMSOMSUbs_00133 } & \multirow[t]{2}{*}{$08 / 19 / 16$} & \multirow{2}{*}{\multicolumn{3}{|c|}{ Absolute, Lot 071515}} & \multirow{2}{*}{\multicolumn{2}{|c|}{ (Purchased Reagent) }} & 2-Butanone-d5 & $2500 \mathrm{ug} / \mathrm{mI}$ \\
\hline & & & & & & & 2-Hexanone-d5 & $2500 \mathrm{ug} / \mathrm{mI}$ \\
\hline \multirow[t]{9}{*}{.VMSOMSUCS_00051 } & \multirow[t]{9}{*}{$08 / 17 / 16$} & \multirow{9}{*}{\multicolumn{2}{|c|}{ Absolute, Lot 011614}} & & \multirow{9}{*}{\multicolumn{2}{|c|}{ (Purchased Reagent) }} & $1,1,2,2$-Tetrachloroethane-d2 & $2000 \mathrm{ug} / \mathrm{mI}$ \\
\hline & & & & & & & 1,1-Dichloroethene-d2 & $2000 \mathrm{ug} / \mathrm{mI}$ \\
\hline & & & & & & & 1,2-Dichlorobenzene-d4 & $2000 \mathrm{ug} / \mathrm{mI}$ \\
\hline & & & & & & & 1,2-Dichloroethane-d4 & $2000 \mathrm{ug} / \mathrm{mI}$ \\
\hline & & & & & & & 1,2-Dichloropropane-d6 & $2000 \mathrm{ug} / \mathrm{mI}$ \\
\hline & & & & & & & Benzene-d6 & $2000 \mathrm{ug} / \mathrm{mI}$ \\
\hline & & & & & & & Chloroform-d & $2000 \mathrm{ug} / \mathrm{mI}$ \\
\hline & & & & & & & Toluene-d8 & $2000 \mathrm{ug} / \mathrm{mI}$ \\
\hline & & & & & & & trans-1,3-Dichloropropene-d4 & $2000 \mathrm{ug} / \mathrm{mI}$ \\
\hline
\end{tabular}


Lab Name: TestAmerica Burlington

Job No.: 200-29312-1

SDG No.: 29312

\begin{tabular}{|c|l|l|c|c|}
\hline $\begin{array}{c}\text { Reagent } \\
\text { Container }\end{array}$ & \multicolumn{1}{|c|}{$\begin{array}{c}\text { Reagent } \\
\text { ID }\end{array}$} & \multicolumn{1}{c|}{$\begin{array}{c}\text { Reagent } \\
\text { Description }\end{array}$} & $\begin{array}{c}\text { Preparation } \\
\text { Date }\end{array}$ & $\begin{array}{c}\text { Expiration } \\
\text { Date }\end{array}$ \\
\hline 807558 & VMBFBw_00018 & BEB TUNE 25 PPM & $06 / 23 / 2015$ & $12 / 22 / 2015$ \\
\hline 829193 & VMSOMTRISw_00103 & SOM TR ISTD 20 PPM & $08 / 19 / 2015$ & $09 / 19 / 2015$ \\
\hline 829199 & VMSOMTRSUw_00098 & SOM TR DMC 20 PPM & $08 / 19 / 2015$ & $09 / 19 / 2015$ \\
\hline 829202 & VMSOMTRCALw_00093 & SOM TR CAL 20 - 200 PPM & $08 / 19 / 2015$ & $09 / 19 / 2015$ \\
\hline
\end{tabular}




\section{METHODOLOGY SUMMARY}

Laboratory: TestAmerica Laboratories

Location: South Burlington, Vermont
Project No:

SDG No: 29312

VOA

Volatile Organics Trace - USEPA CLP SOM01.2 


\section{CASE NARRATIVE}

\section{Client: Argonne National Laboratory}

\section{Project: York (200-29312)}

\section{Report Number: 200-29312-1}

With the exceptions noted as flags or footnotes, standard analytical protocols were followed in the analysis of the samples and no problems were encountered or anomalies observed. In addition all laboratory quality control samples were within established control limits, with any exceptions noted below. Each sample was analyzed to achieve the lowest possible reporting limit within the constraints of the method. In some cases, due to interference or analytes present at high concentrations, samples were diluted. For diluted samples, the reporting limits are adjusted relative to the dilution required.

Calculations are performed before rounding to avoid round-off errors in calculated results.

All holding times were met and proper preservation noted for the methods performed on these samples, unless otherwise detailed in the individual sections below.

\section{RECEIPT}

The samples were received on $08 / 15 / 2015$; the samples arrived in good condition, properly preserved and on ice. The temperature of the cooler at receipt was $5.6^{\circ} \mathrm{C}$.

\section{VOLATILE ORGANIC COMPOUNDS - TRACE}

Samples YKTI09-135-W-37929, YKTI10-117-W-37933, YKQCTB-W-37934 and VHBLK01 were analyzed for Volatile Organic Compounds - Trace in accordance with EPA SOW SOM01.2. The samples were analyzed on 08/20/2015.

Several analytes were detected in method blank MB 200-92982/5 at levels that were above the method detection limit but below the reporting limit. The values should be considered estimates, and have been flagged. If the associated sample reported a result above the MDL and/or RL, the result has been flagged. Refer to the $\mathrm{QC}$ report for details.

2-Hexanone-d5 failed the surrogate recovery criteria high for YKTI10-117-W-37933. Refer to the QC report for details.

Sample YKTI10-117-W-37933[1.93X] required dilution prior to analysis. The reporting limits have been adjusted accordingly.

No additional analytical or quality issues were noted, other than those described above or in the Definitions/Glossary page. 
Lab Name: TestAmerica Burlington

Job No.: 200-29312-1

SDG No.: 29312

Instrument ID: CHD.i

Analysis Batch Number: 92921

Lab Sample ID: IC 200-92921/3

Client Sample ID:

Date Analyzed: 08/19/15 15:14

Lab File ID: 15357 003.D

GC Column: DB-624

ID : $0.2(\mathrm{~mm})$

\begin{tabular}{|l|c|c|c|}
\hline \multicolumn{1}{|c|}{ COMPOUND NAME } & RETENTION & \multicolumn{2}{|c|}{ MANUAL INTEGRATION } \\
\cline { 3 - 4 } & TIME & REASON & ANALYST \\
\hline Dichlorodifluoromethane & 1.52 & Baseline & DATE \\
\hline 1,2 -Dibromo-3-Chloropropane & 13.43 & Assign Peak & 08/19/15 $16: 20$ \\
\hline
\end{tabular}

Lab Sample ID: IC 200-92921/4 Client Sample ID:

Date Analyzed: 08/19/15 15:39

Lab File ID: 15357_004.D

GC Column: DB-624

ID $: 0.2(\mathrm{~mm})$

\begin{tabular}{|c|c|c|c|c|}
\hline \multirow[t]{2}{*}{ COMPOUND NAME } & \multirow{2}{*}{$\begin{array}{c}\text { RETENTION } \\
\text { TIME }\end{array}$} & \multicolumn{3}{|c|}{ MANUAL INTEGRATION } \\
\hline & & REASON & ANALYST & DATE \\
\hline Dichlorodifluoromethane & 1.53 & Baseline & wilburj & $08 / 19 / 15 \quad 16: 21$ \\
\hline Bromomethane & 2.00 & Baseline & wilburj & $08 / 19 / 15 \quad 16: 27$ \\
\hline Chloroethane & 2.08 & Baseline & wilburj & $08 / 19 / 1516: 27$ \\
\hline 1,2-Dibromo-3-Chloropropane & 13.44 & Assign Peak & wilburj & $08 / 19 / 15 \quad 16: 27$ \\
\hline
\end{tabular}

Lab Sample ID: ICIS 200-92921/5

Client Sample ID:

Date Analyzed: 08/19/15 16:04

Lab File ID: 15357_005.D

GC Column: DB-624

ID : $0.2(\mathrm{~mm})$

\begin{tabular}{|c|c|c|c|c|}
\hline \multirow[t]{2}{*}{ COMPOUND NAME } & \multirow{2}{*}{$\begin{array}{c}\text { RETENTION } \\
\text { TIME }\end{array}$} & \multicolumn{3}{|c|}{ MANUAL INTEGRATION } \\
\hline & & REASON & ANALYST & DATE \\
\hline Dichlorodifluoromethane & 1.53 & Baseline & wilburj & $08 / 19 / 1516: 30$ \\
\hline Bromomethane & 2.00 & Baseline & wilburj & $08 / 19 / 15 \quad 17: 11$ \\
\hline
\end{tabular}

Lab Sample ID: IC 200-92921/6

Client Sample ID:

Date Analyzed: 08/19/15 16:29

Lab File ID: 15357_006.D

GC Column: DB-624

ID $: 0.2(\mathrm{~mm})$

\begin{tabular}{|c|c|c|c|c|}
\hline \multirow[t]{2}{*}{ COMPOUND NAME } & \multirow{2}{*}{$\begin{array}{c}\text { RETENTION } \\
\text { TIME }\end{array}$} & \multicolumn{3}{|c|}{ MANUAL INTEGRATION } \\
\hline & & REASON & ANALYST & DATE \\
\hline Dichlorodifluoromethane & 1.53 & Baseline & wilburj & $08 / 19 / 15 \quad 17: 12$ \\
\hline Bromomethane & 2.00 & Baseline & wilburj & $08 / 19 / 15 \quad 17: 12$ \\
\hline
\end{tabular}

SOM01.2/VOA_Tr 
Lab Name: TestAmerica Burlington

SDG No.: 29312

Instrument ID: CHD.i

Lab Sample ID: IC 200-92921/7

Date Analyzed: 08/19/15 16:54
Job No.: 200-29312-1

Analysis Batch Number: 92921

Client Sample ID:

Lab File ID: 15357 007.D
GC Column: DB-624

ID $: 0.2(\mathrm{~mm})$

\begin{tabular}{|c|c|c|c|c|}
\hline \multirow[t]{2}{*}{ COMPOUND NAME } & \multirow{2}{*}{$\begin{array}{c}\text { RETENTION } \\
\text { TIME }\end{array}$} & \multicolumn{3}{|c|}{ MANUAL INTEGRATION } \\
\hline & & REASON & ANALYST & DATE \\
\hline Dichlorodifluoromethane & 1.55 & Baseline & wilburj & $08 / 20 / 15 \quad 10: 30$ \\
\hline Vinyl Chloride-d3 & 1.71 & Baseline & wilburj & $08 / 20 / 15 \quad 10: 30$ \\
\hline Vinyl chloride & 1.72 & Baseline & wilburj & $08 / 20 / 1510: 30$ \\
\hline Bromomethane & 2.04 & Baseline & wilburj & $08 / 20 / 15 \quad 10: 30$ \\
\hline
\end{tabular}


Lab Name: TestAmerica Burlington

SDG No.: 29312

Instrument ID: CHD.i

Lab Sample ID: 200-29312-4

Date Analyzed: 08/20/15 17:44

\begin{tabular}{|c|r|}
\hline COMPOUND NAME & RETEN \\
& TIME \\
\hline Vinyl Chloride-d3 &
\end{tabular}

Lab Sample ID: CCVC 200-92982/12

Date Analyzed: 08/20/15 18:09 Lab File ID: 15379_012.D

Client Sample ID:

\begin{tabular}{|c|c|c|c|c|}
\hline \multirow[t]{2}{*}{ COMPOUND NAME } & \multirow{2}{*}{$\begin{array}{c}\text { RETENTION } \\
\text { TIME }\end{array}$} & \multicolumn{3}{|c|}{ MANUAL INTEGRATION } \\
\hline & & REASON & ANALYST & DATE \\
\hline Dichlorodifluoromethane & 1.53 & Baseline & wilburj & $08 / 21 / 1510: 10$ \\
\hline Bromomethane & 1.99 & Baseline & wilburj & $08 / 21 / 1510: 10$ \\
\hline
\end{tabular}

Analysis Batch Number: 92982

client Sample ID: VHBLKO1

Lab File ID: 15379 011.D

\begin{tabular}{l|ll|c|c|}
\multirow{2}{*}{ TION } & \multicolumn{3}{|c|}{ MANUAL INTEGRATION } \\
\cline { 2 - 5 } & REASON & ANALYST & DATE \\
\hline 1.71 & Baseline & wilburj & $08 / 21 / 1510: 11$ \\
\hline
\end{tabular}

GC Column: DB-624

ID : $0.2(\mathrm{~mm})$ 


\section{DATA REPORTING QUALIFIERS}

Client: Argonne National Laboratory

Job Number: 200-29312-1

Sdg Number: 29312

Lab Section

Qualifier

Description

GC/MS VOA

$\mathrm{U}$

E

$J$

$J$

$\mathrm{X}$

*

B

$\mathrm{N}$
Analyzed for but not detected.

Compound concentration exceeds the upper level of the calibration range of the instrument for that specific analysis.

Indicates an Estimated Value for TICs

Indicates an estimated value.

See case narrative notes for explanation of the ' $X$ ' flag

Surrogate is outside acceptance limits.

The analyte was found in an associated blank, as well as in the sample.

This flag indicates the presumptive evidence of a compound. 
Lab Name: TESTAMERICA BURLINGTON

Lab Code: STLV Case No.: YORK Mod. Ref No.: Level: (TRACE or LOW) TRACE
Contract: $8 \mathrm{E}-00302$

SDG No.: 29312

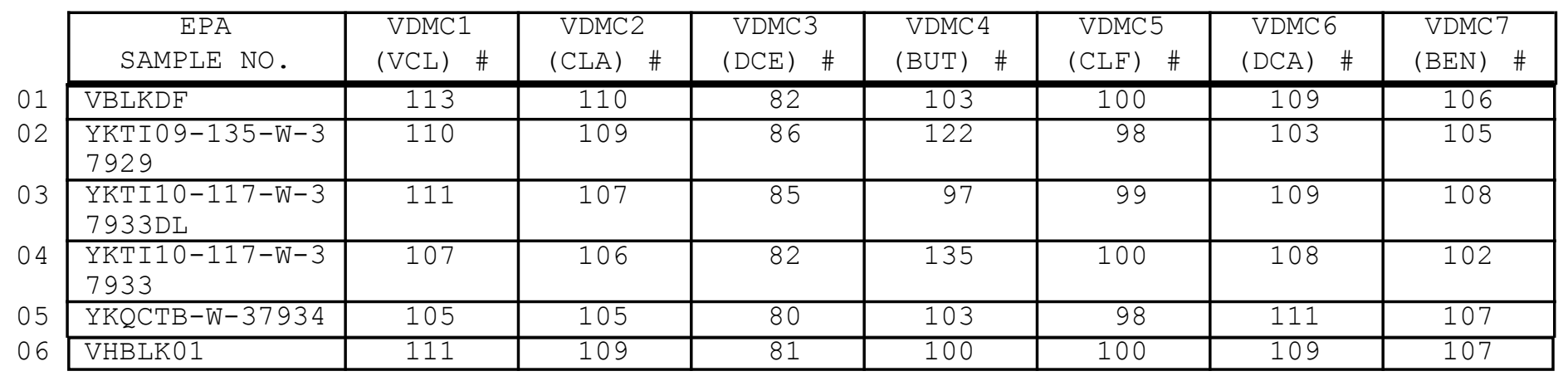

\footnotetext{
VDMC1 (VCL) = Vinyl Chloride-d3

VDMC2 $(\mathrm{CLA})=$ Chloroethane-d5

VDMC3 $(\mathrm{DCE})=1,1-\mathrm{Dichloroethene-d2}$

VDMC4 $\quad(B U T)=2-$ Butanone-d5

VDMC5 $(\mathrm{CLF})=$ Chloroform-d

VDMC $6 \quad(\mathrm{DCA})=1,2-\mathrm{Dichloroethane-d4}$

$\operatorname{VDMC} 7 \quad(\mathrm{BEN})=$ Benzene-d6
}

$$
\begin{gathered}
\frac{\mathrm{QC} \text { LIMITS }}{(65-131)} \\
(71-131) \\
(55-104) \\
(49-155) \\
(78-121) \\
(78-129) \\
(77-124)
\end{gathered}
$$

\# Column to be used to flag recovery values

* Values outside of contract required QC limits

Page 1 of 1 
Lab Name: TESTAMERICA BURLINGTON

Contract: $8 \mathrm{E}-00302$

Lab Code: STLV Case No.: YORK Mod. Ref No.: SDG No.: 29312

Level: (TRACE or LOW) TRACE

\begin{tabular}{|c|c|c|c|c|c|c|c|c|}
\hline $\begin{array}{c}\text { EPA } \\
\text { SAMPLE NO. }\end{array}$ & $\begin{array}{l}\text { VDMC8 } \\
(\mathrm{DPA}) \#\end{array}$ & $\begin{array}{l}\text { VDMC9 } \\
(\text { TOL }) \#\end{array}$ & $\begin{array}{l}\text { VDMC10 } \\
(\mathrm{TDP}) \quad \#\end{array}$ & $\begin{array}{l}\text { VDMC11 } \\
(\operatorname{HEX}) \quad \#\end{array}$ & $\begin{array}{l}\text { VDMC12 } \\
(\mathrm{TCA}) \quad \#\end{array}$ & $\begin{array}{l}\text { VDMC13 } \\
(\mathrm{DCZ})\end{array}$ & OTHER & $\begin{array}{l}\text { TOT } \\
\text { OUT }\end{array}$ \\
\hline VBLKDF & 106 & 106 & 100 & 107 & 100 & 105 & & 0 \\
\hline $\begin{array}{l}\text { YKTI09-135-W-3 } \\
7929\end{array}$ & 104 & 104 & 94 & 126 & 89 & 100 & & 0 \\
\hline $\begin{array}{l}\text { YKTI10-117-W-3 } \\
7933 \mathrm{DL}\end{array}$ & 109 & 107 & 105 & 108 & 100 & 104 & & 0 \\
\hline $\begin{array}{l}\text { YKTI10-117-W-3 } \\
7933\end{array}$ & 105 & 104 & 106 & $137 \star$ & 102 & 105 & & 1 \\
\hline YKQCTB-W-37934 & 108 & 106 & 99 & 111 & 102 & 105 & & 0 \\
\hline VHBLK01 & 107 & 105 & 94 & 106 & 101 & 106 & & 0 \\
\hline
\end{tabular}

\footnotetext{
VDMC8 $(\mathrm{DPA})=1,2-\mathrm{Dichloropropane}-\mathrm{d} 6$

VDMC $9 \quad($ TOL $)=$ Toluene-d8

VDMC10 $(\mathrm{TDP})=$ trans $-1,3-\mathrm{Dichloropropene-d4}$

VDMC11 $(\mathrm{HEX})=2-$ Hexanone-d5

VDMC12 $(\mathrm{TCA})=1,1,2,2$-Tetrachloroethane-d2

VDMC13 $(\mathrm{DCZ})=1,2-\mathrm{Dichlorobenzene-d4}$
}

$$
\begin{gathered}
\frac{Q C \text { LIMITS }}{(79-124)} \\
(77-121) \\
(73-121) \\
(28-135) \\
(73-125) \\
(80-131)
\end{gathered}
$$

\# Column to be used to flag recovery values

* Values outside of contract required QC limits

Report 1,4-Dioxane-d8 for Low-Medium VOA analysis only

Page 1 of 1 
$4 A$ - FORM IV VOA

VOLATILE METHOD BLANK SUMMARY
EPA SAMPLE NO.

VBLKDF

Lab Name: TESTAMERICA BURLINGTON

Contract: $8 \mathrm{E}-00302$

Lab Code: STLV Case No.: YORK Mod. Ref No.: SDG No.: 29312

Lab File ID: 15379_005.D

Lab Sample ID: MB 200-92982/5

Instrument ID: CHD.i

Matrix: (SOIL/SED/WATER) Water

Level: (TRACE or LOW/MED) TRACE

Date Analyzed: 08/20/2015

GC Column: DB-624

ID $: 0.20 \quad(\mathrm{~mm})$

Time Analyzed: 1403

Heated Purge: (Y/N) N

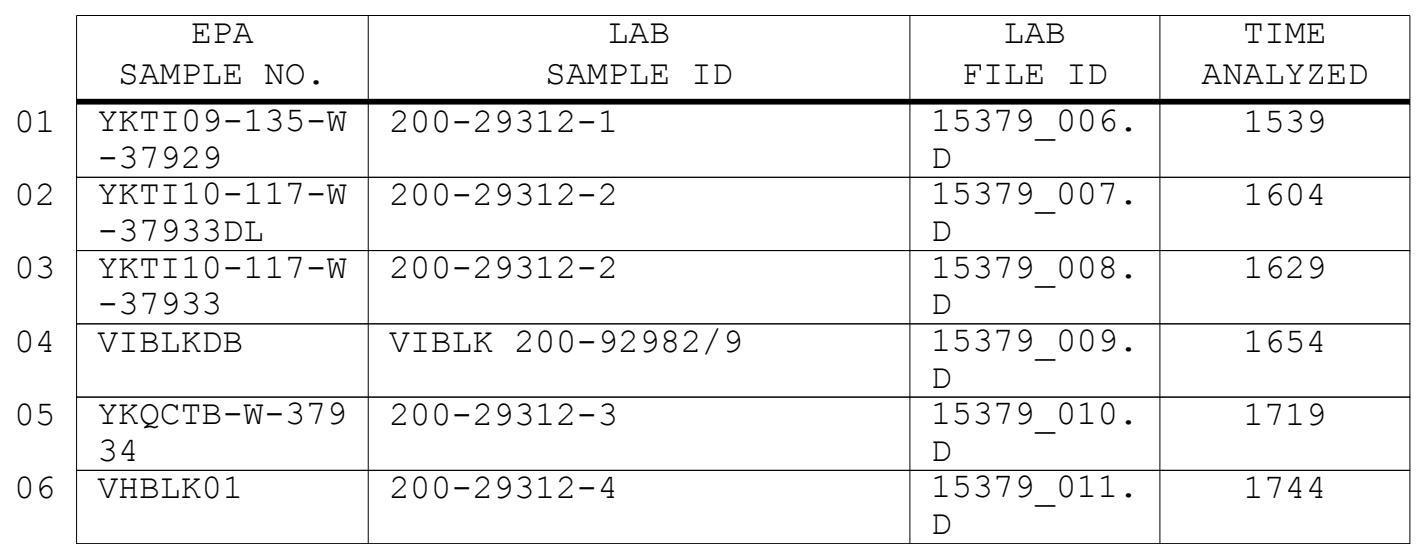

COMMENTS :

Page 1 of 1 
$5 A$ - FORM V VOA

VOLATILE ORGANICS INSTRUMENT

PERFORMANCE CHECK

BROMOFLUOROBENZENE (BFB)
EPA SAMPLE NO.

BFBDE
Lab Name: TESTAMERICA BURLINGTON

Lab Code: STLV

Case No.: YORK

Lab File Id: 15357_001.D

Instrument Id: CHD.i

GC Column: DB-624

ID $: 0.20$
Contract: 8E-00302 Mod. Ref No.:

SDG No.: 29312

BEB Injection Date: 08/19/2015

BFB Injection Time: 1435 $(\mathrm{mm})$

\begin{tabular}{|r|l|r|}
\hline m/e & ION ABUNDANCE CRITERIA & $\begin{array}{c}\text { RELATIVE } \\
\text { ABUNDANCE }\end{array}$ \\
\hline 50 & $15.0-40.0 \%$ of mass 95 & 20.1 \\
\hline 75 & $30.0-80.0 \%$ of mass 95 & 100 \\
\hline 95 & Base peak, 100\% relative abundance & 6.8 \\
\hline 96 & $5.0-9.0 \%$ of mass 95 & 0.5 \\
\hline 173 & Less than 2.0\% of mass 174 & $0.6) 1$ \\
\hline 174 & $50.0-120 \%$ of mass 95 & 79.0 \\
\hline 175 & $5.0-9.0 \%$ of mass 174 0 \\
\hline 176 & $95.0-101 \%$ of mass 174 & $(7.4) 1$ \\
\hline 177 & $5.0-9.0 \%$ of mass 176 & $(95.7) 1$ \\
\hline
\end{tabular}

1 - Value is omass 1742 - Value is mass 176

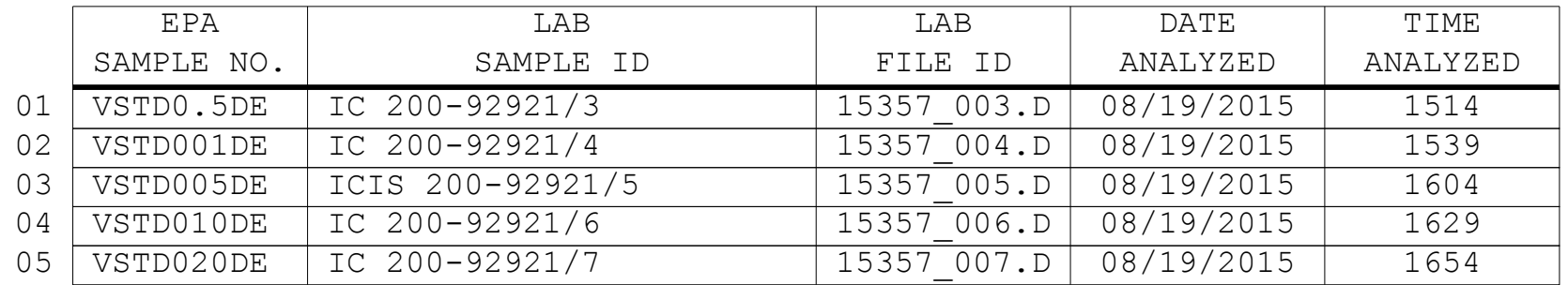


$5 A$ - FORM V VOA

VOLATILE ORGANICS INSTRUMENT

PERFORMANCE CHECK

BROMOFLUOROBENZENE (BFB)
EPA SAMPLE NO.

BFBDF
Lab Name: TESTAMERICA BURLINGTON

Lab Code: STLV

Case No.: YORK

Lab File Id: 15379_001.D

Instrument Id: CHD.i

GC Column: DB-624

ID $: 0.20$
Contract: $8 \mathrm{E}-00302$ Mod. Ref No.: SDG No.: 29312

BEB Injection Date: 08/20/2015

BFB Injection Time: 1234 $(\mathrm{mm})$

\begin{tabular}{|c|c|c|}
\hline $\mathrm{m} / \mathrm{e}$ & ION ABUNDANCE CRITERIA & $\begin{array}{l}\text { \% RELATIVE } \\
\text { ABUNDANCE }\end{array}$ \\
\hline 50 & $15.0-40.0 \%$ of mass 95 & 19.3 \\
\hline 75 & $30.0-80.0 \%$ of mass 95 & 49.9 \\
\hline 95 & Base peak, $100 \%$ relative abundance & 100 \\
\hline 96 & $5.0-9.0 \%$ of mass 95 & 6.8 \\
\hline 173 & Less than 2.0\% of mass 174 & $(0.6) 1$ \\
\hline 174 & $50.0-120 \%$ of mass 95 & 83.1 \\
\hline 175 & $5.0-9.0 \%$ of mass 174 & $7.0) 1$ \\
\hline 176 & $95.0-101 \%$ of mass 174 & $79.9(96.1) 1$ \\
\hline 177 & $5.0-9.0 \%$ of mass 176 & $5.2(6.5) 2$ \\
\hline
\end{tabular}

1 - Value is omass 174

2 - Value is omass 176

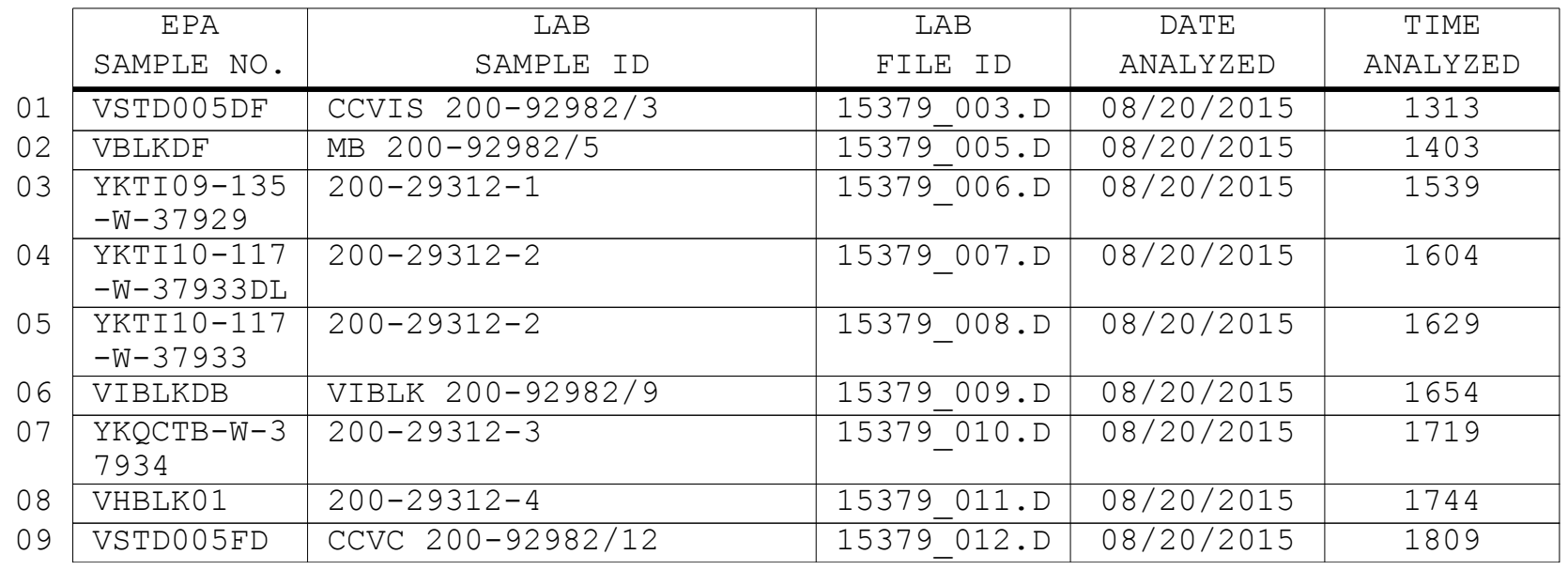




\section{$8 A$ - FORM VIII VOA}

VOLATILE INTERNAL STANDARD AREA AND RETENTION TIME SUMMARY

Lab Name: TESTAMERICA BURLINGTON

Contract: $8 \mathrm{E}-00302$

Lab Code: STLV Case No.: YORK Mod. Ref No.:

SDG No.: 29312

GC Column: DB-624

ID: 0.20 (mm) Init. Calib. Date (s) : 08/19/2015 08/19/2015

EPA Sample No. (VSTD\#\#\#\#\#) : VSTD005DF

Lab File ID (Standard): 15379_003.D

Date Analyzed: $08 / 20 / 2015$

Instrument ID: CHD.i

Time Analyzed: 1313

Heated Purge: ( $\mathrm{Y} / \mathrm{N}) \mathrm{N}$

\begin{tabular}{|c|c|c|c|c|}
\hline IS1 (CBZ) & \multicolumn{2}{|l|}{ IS2 (DFB) } & \multicolumn{2}{|l|}{ IS3 (DCB) } \\
\hline $\mathrm{RT}$ & AREA & RT \# & AREA & RT \\
\hline 9.34 & 1856685 & 5.97 & 801981 & 12.15 \\
\hline 9.67 & 2599359 & 6.30 & 1122773 & 12.48 \\
\hline 9.01 & 1114011 & 5.64 & 481189 & 11.82 \\
\hline & & & & \\
\hline 9.34 & 1777320 & 5.97 & 740336 & 12.15 \\
\hline 9.34 & 1591733 & 5.97 & 609544 & 12.15 \\
\hline 9.34 & 1850067 & 5.97 & 724222 & 12.15 \\
\hline 9.34 & 1840178 & 5.97 & 745097 & 12.15 \\
\hline 9.34 & 1962744 & 5.97 & 745476 & 12.15 \\
\hline 9.34 & 1776774 & 5.97 & 723566 & 12.15 \\
\hline 9.34 & 1685926 & 5.97 & 683345 & 12.15 \\
\hline
\end{tabular}

IS1 $(\mathrm{CBZ})=$ Chlorobenzene-d5

IS2 $(\mathrm{DFB})=1,4-\mathrm{Difluorobenzene}$

IS3 $(\mathrm{DCB})=1,4-\mathrm{Dichlorobenzene-d4}$

AREA UPPER LIMIT $=140 \%$ (Trace Volatiles) of internal standard area AREA LOWER LIMIT $=60 \%$ (Trace Volatiles) of internal standard area RT UPPER LIMIT $=+0.33$ (Trace Volatiles) minutes of internal standard RT RT LOWER LIMIT $=-0.33$ (Trace Volatiles) minutes of internal standard RT

\# Column used to flag values outside contract required QC limits with an asterisk. Page 1 of 1 
Lab Name: TESTAMERICA BURLINGTON

Contract: $8 \mathrm{E}-00302$

Lab Code: STLV

Case No.: YORK Mod. Ref No.:

SDG No.: 29312

Matrix: (SOIL/SED/WATER) Water

Sample wt/vol: 25.0 $(\mathrm{g} / \mathrm{mL}) \mathrm{mL}$

Level: (TRACE/LOW/MED) TRACE

\% Moisture: not dec.

GC Column: DB-624 ID: $0.20 \quad(\mathrm{~mm})$

Soil Extract Volume: (uL) $(\mathrm{mL})$

Purge Volume: 25.0
Lab Sample ID: 200-29312-3

Lab File ID: 15379_010.D

Date Received: 08/15/2015

Date Analyzed: 08/20/2015

Dilution Factor: 1.0

Soil Aliquot Volume: (uL)

\begin{tabular}{|c|c|c|c|}
\hline CAS NO. & COMPOUND & $\begin{array}{l}\text { CONCENTRATION UNITS: } \\
(\mathrm{ug} / \mathrm{L} \text { or } \mathrm{ug} / \mathrm{kg}) \mathrm{ug} / \mathrm{L}\end{array}$ & Q \\
\hline $75-71-8$ & Dichlorodifluoromethane & 1.0 & $\mathrm{U}$ \\
\hline $74-87-3$ & Chloromethane & 1.0 & $\mathrm{U}$ \\
\hline $75-01-4$ & Vinyl chloride & 1.0 & $\mathrm{U}$ \\
\hline $74-83-9$ & Bromomethane & 1.0 & $\mathrm{U}$ \\
\hline $75-00-3$ & Chloroethane & 1.0 & $\mathrm{U}$ \\
\hline $75-69-4$ & Trichlorofluoromethane & 1.0 & $\mathrm{U}$ \\
\hline $75-35-4$ & 1,1-Dichloroethene & 1.0 & $\mathrm{U}$ \\
\hline $76-13-1$ & $1,1,2$-Trichloro-1,2,2-trifluoroethane & 1.0 & $\mathrm{U}$ \\
\hline $67-64-1$ & Acetone & 19 & \\
\hline $75-15-0$ & Carbon disulfide & 0.018 & $\mathrm{~J} B$ \\
\hline $79-20-9$ & Methyl acetate & 1.0 & $\mathrm{U}$ \\
\hline $75-09-2$ & Methylene Chloride & 1.0 & $\mathrm{U}$ \\
\hline $156-60-5$ & trans-1,2-Dichloroethene & 1.0 & $\mathrm{U}$ \\
\hline $1634-04-4$ & Methyl tert-butyl ether & 1.0 & $\mathrm{U}$ \\
\hline $75-34-3$ & 1,1-Dichloroethane & 1.0 & $\mathrm{U}$ \\
\hline $156-59-2$ & cis-1,2-Dichloroethene & 1.0 & $\mathrm{U}$ \\
\hline $78-93-3$ & 2-Butanone & 0.78 & $\mathrm{~J}$ \\
\hline $74-97-5$ & Bromochloromethane & 1.0 & $\mathrm{U}$ \\
\hline $67-66-3$ & Chloroform & 1.0 & $\mathrm{U}$ \\
\hline $71-55-6$ & $1,1,1$-Trichloroethane & 1.0 & $\mathrm{U}$ \\
\hline $110-82-7$ & Cyclohexane & 1.0 & $\mathrm{U}$ \\
\hline $56-23-5$ & Carbon tetrachloride & 1.0 & $\mathrm{U}$ \\
\hline $71-43-2$ & Benzene & 0.032 & $\mathrm{~J}$ \\
\hline $107-06-2$ & 1,2-Dichloroethane & 1.0 & $\mathrm{U}$ \\
\hline
\end{tabular}

Report 1,4-Dioxane for Low-Medium VOA analysis only 
Lab Name: TESTAMERICA BURLINGTON

Contract: $8 \mathrm{E}-00302$

Lab Code: STLV

Case No.: YORK

Mod. Ref No.:

SDG No.: 29312

Matrix: (SOIL/SED/WATER) Water

Sample wt/vol: 25.0 $(\mathrm{g} / \mathrm{mL}) \mathrm{mL}$

Level: (TRACE/LOW/MED) TRACE

\% Moisture: not dec.

GC Column: DB-624 ID: $0.20 \quad(\mathrm{~mm})$

Soil Extract Volume: (uL) $(\mathrm{mL})$

Purge Volume: 25.0 (mI)
Lab Sample ID: 200-29312-3

Lab File ID: 15379_010.D

Date Received: 08/15/2015

Date Analyzed: 08/20/2015

Dilution Factor: 1.0

Soil Aliquot Volume: (uL)

\begin{tabular}{|c|c|c|c|}
\hline CAS NO. & COMPOUND & $\begin{array}{l}\text { CONCENTRATION UNITS: } \\
(\mathrm{ug} / \mathrm{L} \text { or } \mathrm{ug} / \mathrm{kg}) \mathrm{ug} / \mathrm{L}\end{array}$ & Q \\
\hline $79-01-6$ & Trichloroethene & 1.0 & $\mathrm{U}$ \\
\hline $108-87-2$ & Methylcyclohexane & 1.0 & $\mathrm{U}$ \\
\hline $78-87-5$ & 1,2-Dichloropropane & 1.0 & $\mathrm{U}$ \\
\hline $75-27-4$ & Bromodichloromethane & 1.0 & $\mathrm{U}$ \\
\hline $10061-01-5$ & cis-1,3-Dichloropropene & 1.0 & U \\
\hline $108-10-1$ & 4-Methyl-2-pentanone & 1.0 & $\mathrm{U}$ \\
\hline $108-88-3$ & Toluene & 0.14 & $\mathrm{~J} B$ \\
\hline $10061-02-6$ & trans-1,3-Dichloropropene & 1.0 & $\mathrm{U}$ \\
\hline $79-00-5$ & $1,1,2-$ Trichloroethane & 1.0 & $\mathrm{U}$ \\
\hline $127-18-4$ & Tetrachloroethene & 0.0033 & $\mathrm{~J}$ \\
\hline $591-78-6$ & 2-Hexanone & 1.0 & $\mathrm{U}$ \\
\hline $124-48-1$ & Dibromochloromethane & 1.0 & $\mathrm{U}$ \\
\hline $106-93-4$ & 1,2-Dibromoethane & 1.0 & $\mathrm{U}$ \\
\hline $108-90-7$ & Chlorobenzene & 1.0 & $\mathrm{U}$ \\
\hline $100-41-4$ & Ethylbenzene & 0.011 & $\mathrm{~J} B$ \\
\hline $95-47-6$ & o-Xylene & 0.029 & $\mathrm{~J} B$ \\
\hline $179601-23-1$ & m,p-xylene & 0.029 & $\mathrm{~J}$ \\
\hline $100-42-5$ & Styrene & 1.0 & $\mathrm{U}$ \\
\hline $75-25-2$ & Bromoform & 1.0 & $\mathrm{U}$ \\
\hline $98-82-8$ & Isopropylbenzene & 1.0 & $\mathrm{U}$ \\
\hline $79-34-5$ & $1,1,2,2$-Tetrachloroethane & 1.0 & $\mathrm{U}$ \\
\hline $541-73-1$ & 1,3 -Dichlorobenzene & 1.0 & $\mathrm{U}$ \\
\hline $106-46-7$ & 1,4-Dichlorobenzene & 1.0 & $\mathrm{U}$ \\
\hline $95-50-1$ & 1,2-Dichlorobenzene & 1.0 & $\mathrm{U}$ \\
\hline $96-12-8$ & 1,2-Dibromo-3-Chloropropane & 1.0 & $\mathrm{U}$ \\
\hline $120-82-1$ & $1,2,4$-Trichlorobenzene & 0.0085 & $\mathrm{~J} B$ \\
\hline $87-61-6$ & $1,2,3$-Trichlorobenzene & 0.0056 & $\mathrm{~J} B$ \\
\hline
\end{tabular}


Lab Name: TESTAMERICA BURLINGTON

Contract: $8 \mathrm{E}-00302$

Lab Code: STLV Case No.: YORK Mod. Ref No.: SDG No.: 29312

Matrix: (SOIL/SED/WATER) Water

Sample wt/vol: 25.0 $(\mathrm{g} / \mathrm{mL}) \mathrm{mL}$

Level: (TRACE or LOW/MED) TRACE

․ Moisture: not dec.

GC Column: DB-624 ID $: 0.20 \quad(\mathrm{~mm})$

Soil Extract Volume: (uL)
Lab Sample ID: 200-29312-3

Lab File ID: 15379_010.D

Date Received: 08/15/2015

Date Analyzed: 08/20/2015

Dilution Factor: 1.0

Soil Aliquot Volume: (uL) Purge Volume: 25.0 CONCENTRATION UNITS: (ug/L or ug/kg) ug/L

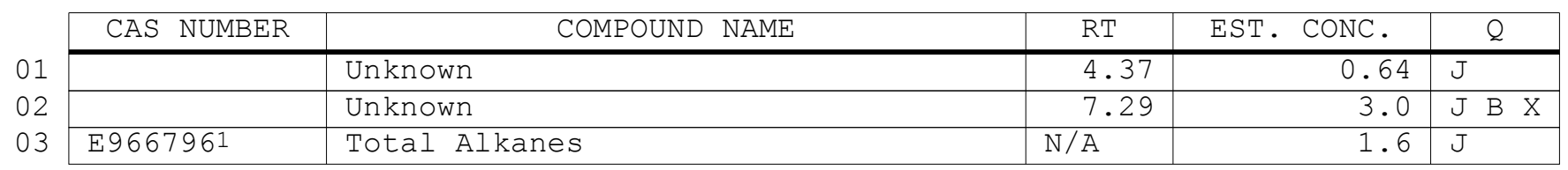


Lab Name: TESTAMERICA BURLINGTON

Contract: $8 \mathrm{E}-00302$

Lab Code: STLV

Case No.: YORK Mod. Ref No.:

SDG No.: 29312

Matrix: (SOIL/SED/WATER) Water

Sample wt/vol: 25.0 $(\mathrm{g} / \mathrm{mL}) \mathrm{mL}$

Level: (TRACE/LOW/MED) TRACE

\% Moisture: not dec.

GC Column: DB-624 ID: $0.20 \quad(\mathrm{~mm})$

Soil Extract Volume: (uL) $(\mathrm{mL})$

Purge Volume: 25.0
Lab Sample ID: 200-29312-1

Lab File ID: 15379_006.D

Date Received: 08/15/2015

Date Analyzed: 08/20/2015

Dilution Factor: 1.0

Soil Aliquot Volume: (uL)

\begin{tabular}{|c|c|c|c|}
\hline CAS NO. & COMPOUND & $\begin{array}{l}\text { CONCENTRATION UNITS: } \\
(\mathrm{ug} / \mathrm{L} \text { or } \mathrm{ug} / \mathrm{kg}) \mathrm{ug} / \mathrm{L}\end{array}$ & Q \\
\hline $75-71-8$ & Dichlorodifluoromethane & 1.0 & $\mathrm{U}$ \\
\hline $74-87-3$ & Chloromethane & 1.0 & $\mathrm{U}$ \\
\hline $75-01-4$ & Vinyl chloride & 1.0 & $\mathrm{U}$ \\
\hline $74-83-9$ & Bromomethane & 1.0 & $\mathrm{U}$ \\
\hline $75-00-3$ & Chloroethane & 1.0 & $\mathrm{U}$ \\
\hline $75-69-4$ & Trichlorofluoromethane & 0.015 & $\mathrm{~J}$ \\
\hline $75-35-4$ & 1,1-Dichloroethene & 1.0 & $\mathrm{U}$ \\
\hline $76-13-1$ & $1,1,2$-Trichloro-1,2,2-trifluoroethane & 1.0 & $\mathrm{U}$ \\
\hline $67-64-1$ & Acetone & 1.2 & $\mathrm{~J}$ \\
\hline $75-15-0$ & Carbon disulfide & 0.046 & $\mathrm{~J} B$ \\
\hline $79-20-9$ & Methyl acetate & 1.0 & $\mathrm{U}$ \\
\hline $75-09-2$ & Methylene Chloride & 1.0 & $\mathrm{U}$ \\
\hline $156-60-5$ & trans-1,2-Dichloroethene & 1.0 & $\mathrm{U}$ \\
\hline $1634-04-4$ & Methyl tert-butyl ether & 1.0 & $\mathrm{U}$ \\
\hline $75-34-3$ & 1,1-Dichloroethane & 1.0 & $\mathrm{U}$ \\
\hline $156-59-2$ & cis-1,2-Dichloroethene & 1.0 & $\mathrm{U}$ \\
\hline $78-93-3$ & 2-Butanone & 5.0 & $\mathrm{U}$ \\
\hline $74-97-5$ & Bromochloromethane & 1.0 & $\mathrm{U}$ \\
\hline $67-66-3$ & Chloroform & 1.0 & $\mathrm{U}$ \\
\hline $71-55-6$ & $1,1,1$-Trichloroethane & 0.34 & $\mathrm{~J}$ \\
\hline $110-82-7$ & Cyclohexane & 1.0 & $\mathrm{U}$ \\
\hline $56-23-5$ & Carbon tetrachloride & 2.1 & \\
\hline $71-43-2$ & Benzene & 1.0 & $\mathrm{U}$ \\
\hline $107-06-2$ & 1,2-Dichloroethane & 1.0 & $\mathrm{U}$ \\
\hline
\end{tabular}

Report 1,4-Dioxane for Low-Medium VOA analysis only 
Lab Name: TESTAMERICA BURLINGTON

Contract: 8E-00302

Lab Code: STLV

Case No.: YORK Mod. Ref No.:

SDG No.: 29312

Matrix: (SOIL/SED/WATER) Water

Sample wt/vol: 25.0 $(\mathrm{g} / \mathrm{mL}) \mathrm{mL}$

Level: (TRACE/LOW/MED) TRACE

\% Moisture: not dec.

GC Column: DB-624 ID: $0.20 \quad(\mathrm{~mm})$

Soil Extract Volume: (uL) $(\mathrm{mL})$

Purge Volume: 25.0
Lab Sample ID: 200-29312-1

Lab File ID: 15379_006.D

Date Received: 08/15/2015

Date Analyzed: 08/20/2015

Dilution Factor: 1.0

Soil Aliquot Volume: (uL)

\begin{tabular}{|c|c|c|c|}
\hline CAS NO. & COMPOUND & $\begin{array}{l}\text { CONCENTRATION UNITS: } \\
(\mathrm{ug} / \mathrm{L} \text { or } \mathrm{ug} / \mathrm{kg}) \mathrm{ug} / \mathrm{L}\end{array}$ & Q \\
\hline $79-01-6$ & Trichloroethene & 0.043 & $\mathrm{~J}$ \\
\hline $108-87-2$ & Methylcyclohexane & 1.0 & $\mathrm{U}$ \\
\hline $78-87-5$ & 1,2-Dichloropropane & 1.0 & $\mathrm{U}$ \\
\hline $75-27-4$ & Bromodichloromethane & 1.0 & $\mathrm{U}$ \\
\hline $10061-01-5$ & cis-1,3-Dichloropropene & 1.0 & $\mathrm{U}$ \\
\hline $108-10-1$ & 4-Methyl-2-pentanone & 1.0 & $\mathrm{U}$ \\
\hline $108-88-3$ & Toluene & 0.052 & $\mathrm{~J} B$ \\
\hline $10061-02-6$ & trans-1,3-Dichloropropene & 1.0 & $\mathrm{U}$ \\
\hline $79-00-5$ & $1,1,2-T r i c h l o r o e t h a n e$ & 1.0 & $\mathrm{U}$ \\
\hline $127-18-4$ & Tetrachloroethene & 0.21 & $\mathrm{~J}$ \\
\hline $591-78-6$ & 2-Hexanone & 1.0 & $\mathrm{U}$ \\
\hline $124-48-1$ & Dibromochloromethane & 1.0 & $\mathrm{U}$ \\
\hline $106-93-4$ & 1,2-Dibromoethane & 1.0 & $\mathrm{U}$ \\
\hline $108-90-7$ & Chlorobenzene & 1.0 & $\mathrm{U}$ \\
\hline $100-41-4$ & Ethylbenzene & 0.012 & $\mathrm{~J} B$ \\
\hline $95-47-6$ & o-Xylene & 0.012 & $\mathrm{~J} B$ \\
\hline $179601-23-1$ & m,p-Xylene & 0.031 & $\mathrm{~J}$ \\
\hline $100-42-5$ & styrene & 0.0097 & $\mathrm{~J}$ \\
\hline $75-25-2$ & Bromoform & 1.0 & $\mathrm{U}$ \\
\hline $98-82-8$ & Isopropylbenzene & 1.0 & $\mathrm{U}$ \\
\hline $79-34-5$ & $1,1,2,2$-Tetrachloroethane & 1.0 & $\mathrm{U}$ \\
\hline $541-73-1$ & 1,3-Dichlorobenzene & 0.020 & $\mathrm{~J} B$ \\
\hline $106-46-7$ & 1,4-Dichlorobenzene & 1.0 & $\mathrm{U}$ \\
\hline $95-50-1$ & $1,2-$ Dichlorobenzene & 1.0 & $\mathrm{U}$ \\
\hline $96-12-8$ & 1,2-Dibromo-3-Chloropropane & 1.0 & $\mathrm{U}$ \\
\hline $120-82-1$ & $1,2,4$-Trichlorobenzene & 0.036 & $\mathrm{~J} B$ \\
\hline $87-61-6$ & $1,2,3$-Trichlorobenzene & 0.035 & $\mathrm{~J} B$ \\
\hline
\end{tabular}


Lab Name: TESTAMERICA BURLINGTON

Contract: $8 \mathrm{E}-00302$

Lab Code: STLV Case No.: YORK Mod. Ref No.: SDG No.: 29312

Matrix: (SOIL/SED/WATER) Water

Sample wt/vol: 25.0 $(\mathrm{g} / \mathrm{mL}) \mathrm{mL}$

Level: (TRACE or LOW/MED) TRACE

․ Moisture: not dec.

GC Column: DB-624 ID $: 0.20 \quad(\mathrm{~mm})$

Soil Extract Volume: (uL)
Lab Sample ID: 200-29312-1

Lab File ID: 15379_006.D

Date Received: 08/15/2015

Date Analyzed: 08/20/2015

Dilution Factor: 1.0

Soil Aliquot Volume: (uL)

Purge Volume: 25.0 $(\mathrm{mL})$ CONCENTRATION UNITS: (ug/L or ug/kg) ug/L

\begin{tabular}{|c|c|c|c|c|}
\hline CAS NUMBER & COMPOUND NAME & RT & EST. CONC. & $Q$ \\
\hline 01 & Unknown & 7.29 & 2.8 & $J B X$ \\
\hline $541-05-9$ & Cyclotrisiloxane, hexamethyl- & 8.22 & 0.93 & $\mathrm{~J} B \mathrm{~N}$ \\
\hline $556-67-2$ & Cyclotetrasiloxane, octamethyl- & 11.04 & 1.7 & $\mathrm{~J} B \mathrm{~N}$ \\
\hline E9667961 & Total Alkanes & $\mathrm{N} / \mathrm{A}$ & & \\
\hline
\end{tabular}

1 EPA-designated Registry Number. 
Lab Name: TESTAMERICA BURLINGTON

Contract: $8 \mathrm{E}-00302$

Lab Code: STLV

Case No.: YORK Mod. Ref No.:

SDG No.: 29312

Matrix: (SOIL/SED/WATER) Water

Sample wt/vol: 25.0 $(\mathrm{g} / \mathrm{mL}) \mathrm{mL}$

Level: (TRACE/LOW/MED) TRACE

\% Moisture: not dec.

GC Column: DB-624 ID: $0.20 \quad(\mathrm{~mm})$

Soil Extract Volume: (uL) $(\mathrm{mL})$

Purge Volume: 25.0
Lab Sample ID: 200-29312-2

Lab File ID: 15379_008.D

Date Received: 08/15/2015

Date Analyzed: 08/20/2015

Dilution Factor: 1.0

Soil Aliquot Volume: (uL)

\begin{tabular}{|c|c|c|c|}
\hline CAS NO. & COMPOUND & $\begin{array}{l}\text { CONCENTRATION UNITS: } \\
(\mathrm{ug} / \mathrm{L} \text { or } \mathrm{ug} / \mathrm{kg}) \mathrm{ug} / \mathrm{L}\end{array}$ & Q \\
\hline $75-71-8$ & Dichlorodifluoromethane & 1.0 & $\mathrm{U}$ \\
\hline $74-87-3$ & Chloromethane & 1.0 & $\mathrm{U}$ \\
\hline $75-01-4$ & Vinyl chloride & 1.0 & $\mathrm{U}$ \\
\hline $74-83-9$ & Bromomethane & 1.0 & $\mathrm{U}$ \\
\hline $75-00-3$ & Chloroethane & 1.0 & $\mathrm{U}$ \\
\hline $75-69-4$ & Trichlorofluoromethane & 0.042 & $\mathrm{~J}$ \\
\hline $75-35-4$ & 1,1-Dichloroethene & 1.0 & $\mathrm{U}$ \\
\hline $76-13-1$ & $1,1,2$-Trichloro-1,2,2-trifluoroethane & 1.0 & $\mathrm{U}$ \\
\hline $67-64-1$ & Acetone & 0.99 & $\mathrm{~J}$ \\
\hline $75-15-0$ & Carbon disulfide & 0.021 & $\mathrm{~J} B$ \\
\hline $79-20-9$ & Methyl acetate & 1.0 & $\mathrm{U}$ \\
\hline $75-09-2$ & Methylene Chloride & 1.0 & $\mathrm{U}$ \\
\hline $156-60-5$ & trans-1,2-Dichloroethene & 1.0 & $\mathrm{U}$ \\
\hline $1634-04-4$ & Methyl tert-butyl ether & 1.0 & $\mathrm{U}$ \\
\hline $75-34-3$ & 1,1-Dichloroethane & 1.0 & $\mathrm{U}$ \\
\hline $156-59-2$ & cis-1,2-Dichloroethene & 1.0 & $\mathrm{U}$ \\
\hline $78-93-3$ & 2-Butanone & 5.0 & $\mathrm{U}$ \\
\hline $74-97-5$ & Bromochloromethane & 1.0 & $\mathrm{U}$ \\
\hline $67-66-3$ & Chloroform & 0.86 & $\mathrm{~J}$ \\
\hline $71-55-6$ & $1,1,1$-Trichloroethane & 1.0 & $\mathrm{U}$ \\
\hline $110-82-7$ & Cyclohexane & 1.0 & $\mathrm{U}$ \\
\hline $56-23-5$ & Carbon tetrachloride & 24 & $\mathrm{E}$ \\
\hline $71-43-2$ & Benzene & 1.0 & $\mathrm{U}$ \\
\hline $107-06-2$ & 1,2-Dichloroethane & 1.0 & $\mathrm{U}$ \\
\hline
\end{tabular}

Report 1,4-Dioxane for Low-Medium VOA analysis only 
Lab Name: TESTAMERICA BURLINGTON

Contract: 8E-00302

Lab Code: STLV

Case No.: YORK Mod. Ref No.:

SDG No.: 29312

Matrix: (SOIL/SED/WATER) Water

Sample wt/vol: 25.0 $(\mathrm{g} / \mathrm{mL}) \mathrm{mL}$

Level: (TRACE/LOW/MED) TRACE

\% Moisture: not dec.

GC Column: DB-624 ID: $0.20 \quad(\mathrm{~mm})$

Soil Extract Volume: (uL) $(\mathrm{mL})$

Purge Volume: 25.0
Lab Sample ID: 200-29312-2

Lab File ID: 15379_008.D

Date Received: 08/15/2015

Date Analyzed: 08/20/2015

Dilution Factor: 1.0

Soil Aliquot Volume: (uL) (mI)

\begin{tabular}{|c|c|c|c|}
\hline CAS NO. & COMPOUND & $\begin{array}{l}\text { CONCENTRATION UNITS: } \\
(\mathrm{ug} / \mathrm{L} \text { or } \mathrm{ug} / \mathrm{kg}) \mathrm{ug} / \mathrm{L}\end{array}$ & Q \\
\hline $79-01-6$ & Trichloroethene & 1.0 & $\mathrm{U}$ \\
\hline $108-87-2$ & Methylcyclohexane & 1.0 & $\mathrm{U}$ \\
\hline $78-87-5$ & 1,2-Dichloropropane & 1.0 & $\mathrm{U}$ \\
\hline $75-27-4$ & Bromodichloromethane & 1.0 & $\mathrm{U}$ \\
\hline $10061-01-5$ & cis-1,3-Dichloropropene & 1.0 & $\mathrm{U}$ \\
\hline $108-10-1$ & 4-Methyl-2-pentanone & 1.0 & $\mathrm{U}$ \\
\hline $108-88-3$ & Toluene & 0.031 & $\mathrm{~J} B$ \\
\hline $10061-02-6$ & trans-1,3-Dichloropropene & 1.0 & $\mathrm{U}$ \\
\hline $79-00-5$ & $1,1,2-T r i c h l o r o e t h a n e$ & 1.0 & $\mathrm{U}$ \\
\hline $127-18-4$ & Tetrachloroethene & 0.015 & $\mathrm{~J}$ \\
\hline $591-78-6$ & 2-Hexanone & 1.0 & $\mathrm{U}$ \\
\hline $124-48-1$ & Dibromochloromethane & 1.0 & $\mathrm{U}$ \\
\hline $106-93-4$ & 1,2-Dibromoethane & 1.0 & $\mathrm{U}$ \\
\hline $108-90-7$ & Chlorobenzene & 1.0 & $\mathrm{U}$ \\
\hline $100-41-4$ & Ethylbenzene & 0.0057 & $\mathrm{~J} B$ \\
\hline $95-47-6$ & o-Xylene & 0.0072 & $\mathrm{~J} B$ \\
\hline $179601-23-1$ & m,p-Xylene & 0.018 & $\mathrm{~J}$ \\
\hline $100-42-5$ & Styrene & 1.0 & $\mathrm{U}$ \\
\hline $75-25-2$ & Bromoform & 1.0 & $\mathrm{U}$ \\
\hline $98-82-8$ & Isopropylbenzene & 1.0 & $\mathrm{U}$ \\
\hline $79-34-5$ & $1,1,2,2$-Tetrachloroethane & 1.0 & $\mathrm{U}$ \\
\hline $541-73-1$ & 1,3-Dichlorobenzene & 1.0 & $\mathrm{U}$ \\
\hline $106-46-7$ & 1,4-Dichlorobenzene & 1.0 & $\mathrm{U}$ \\
\hline $95-50-1$ & $1,2-$ Dichlorobenzene & 1.0 & $\mathrm{U}$ \\
\hline $96-12-8$ & 1,2-Dibromo-3-Chloropropane & 1.0 & $\mathrm{U}$ \\
\hline $120-82-1$ & $1,2,4$-Trichlorobenzene & 0.0068 & $\mathrm{~J} B$ \\
\hline $87-61-6$ & $1,2,3$-Trichlorobenzene & 0.0079 & $\mathrm{~J} B$ \\
\hline
\end{tabular}


Lab Name: TESTAMERICA BURLINGTON

Contract: $8 \mathrm{E}-00302$

Lab Code: STLV Case No.: YORK Mod. Ref No.: SDG No.: 29312

Matrix: (SOIL/SED/WATER) Water

Sample wt/vol: 25.0 $(\mathrm{g} / \mathrm{mL}) \mathrm{mL}$

Level: (TRACE or LOW/MED) TRACE

Moisture: not dec.

GC Column: DB-624 ID $: 0.20 \quad(\mathrm{~mm})$

Soil Extract Volume: (uL)
Lab Sample ID: 200-29312-2

Lab File ID: 15379_008.D

Date Received: 08/15/2015

Date Analyzed: 08/20/2015

Dilution Factor: 1.0

Soil Aliquot Volume: (uL)

Purge Volume: 25.0 $(\mathrm{mL})$ CONCENTRATION UNITS: (ug/L or ug/kg) ug/L

01

02

\begin{tabular}{|l|l|c|c|c|}
\hline CAS NUMBER & \multicolumn{1}{|c|}{ COMPOUND NAME } & RT & EST. CONC. & Q \\
\hline & Unknown & 7.30 & 3.2 & $\mathrm{~J}$ B X \\
\hline E9667961 & Total Alkanes & N/A & & \\
\hline
\end{tabular}

1 EPA-designated Registry Number. 
Lab Name: TESTAMERICA BURLINGTON

Contract: $8 \mathrm{E}-00302$

Lab Code: STLV

Case No.: YORK Mod. Ref No.:

SDG No.: 29312

Matrix: (SOIL/SED/WATER) Water

Sample wt/vol: 25.0 $(\mathrm{g} / \mathrm{mL}) \mathrm{mL}$

Level: (TRACE/LOW/MED) TRACE

\% Moisture: not dec.

GC Column: DB-624 ID: $0.20 \quad(\mathrm{~mm})$

Soil Extract Volume: (uL) $(\mathrm{mL})$

Purge Volume: 25.0
Lab Sample ID: 200-29312-2

Lab File ID: 15379_007.D

Date Received: 08/15/2015

Date Analyzed: 08/20/2015

Dilution Factor: 1.9

Soil Aliquot Volume: (uL)

\begin{tabular}{|c|c|c|c|}
\hline CAS NO. & COMPOUND & $\begin{array}{l}\text { CONCENTRATION UNITS: } \\
(\mathrm{ug} / \mathrm{L} \text { or } \mathrm{ug} / \mathrm{kg}) \mathrm{ug} / \mathrm{L}\end{array}$ & Q \\
\hline $75-71-8$ & Dichlorodifluoromethane & 1.9 & $\mathrm{U}$ \\
\hline $74-87-3$ & Chloromethane & 1.9 & $\mathrm{U}$ \\
\hline $75-01-4$ & Vinyl chloride & 1.9 & $\mathrm{U}$ \\
\hline $74-83-9$ & Bromomethane & 1.9 & $\mathrm{U}$ \\
\hline $75-00-3$ & Chloroethane & 1.9 & $\mathrm{U}$ \\
\hline $75-69-4$ & Trichlorofluoromethane & 0.040 & $\mathrm{~J}$ \\
\hline $75-35-4$ & 1,1-Dichloroethene & 1.9 & $\mathrm{U}$ \\
\hline $76-13-1$ & $1,1,2$-Trichloro-1,2,2-trifluoroethane & 1.9 & $\mathrm{U}$ \\
\hline $67-64-1$ & Acetone & 2.1 & $\mathrm{~J}$ \\
\hline $75-15-0$ & Carbon disulfide & 0.041 & $\mathrm{~J} B$ \\
\hline $79-20-9$ & Methyl acetate & 1.9 & $\mathrm{U}$ \\
\hline $75-09-2$ & Methylene Chloride & 1.9 & $\mathrm{U}$ \\
\hline $156-60-5$ & trans-1,2-Dichloroethene & 1.9 & $\mathrm{U}$ \\
\hline $1634-04-4$ & Methyl tert-butyl ether & 1.9 & $\mathrm{U}$ \\
\hline $75-34-3$ & 1,1-Dichloroethane & 1.9 & $\mathrm{U}$ \\
\hline $156-59-2$ & cis-1,2-Dichloroethene & 1.9 & $\mathrm{U}$ \\
\hline $78-93-3$ & 2-Butanone & 9.6 & $\mathrm{U}$ \\
\hline $74-97-5$ & Bromochloromethane & 1.9 & $\mathrm{U}$ \\
\hline $67-66-3$ & Chloroform & 0.81 & $\mathrm{~J}$ \\
\hline $71-55-6$ & $1,1,1$-Trichloroethane & 1.9 & $\mathrm{U}$ \\
\hline $110-82-7$ & Cyclohexane & 1.9 & $\mathrm{U}$ \\
\hline $56-23-5$ & Carbon tetrachloride & 22 & \\
\hline $71-43-2$ & Benzene & 1.9 & $\mathrm{U}$ \\
\hline $107-06-2$ & 1,2-Dichloroethane & 1.9 & $\mathrm{U}$ \\
\hline
\end{tabular}

Report 1,4-Dioxane for Low-Medium VOA analysis only 
Lab Name: TESTAMERICA BURLINGTON

Lab Code: STLV

Case No.: YORK

Mod. Ref No.:

Contract: $8 \mathrm{E}-00302$

Matrix: (SOIL/SED/WATER) Water

Sample wt/vol: 25.0 $(\mathrm{g} / \mathrm{mL}) \mathrm{mL}$

Level: (TRACE/LOW/MED) TRACE

\% Moisture: not dec.

GC Column: DB-624 ID: $0.20 \quad(\mathrm{~mm})$

Soil Extract Volume: (uL) $(\mathrm{mL})$

Purge Volume: 25.0
SDG No.: 29312

Lab Sample ID: 200-29312-2

Lab File ID: 15379_007.D

Date Received: 08/15/2015

Date Analyzed: 08/20/2015

Dilution Factor: 1.9

Soil Aliquot Volume: (uL) (mI)

\begin{tabular}{|c|c|c|c|}
\hline CAS NO. & COMPOUND & $\begin{array}{l}\text { CONCENTRATION UNITS: } \\
(\mathrm{ug} / \mathrm{L} \text { or } \mathrm{ug} / \mathrm{kg}) \mathrm{ug} / \mathrm{L}\end{array}$ & Q \\
\hline $79-01-6$ & Trichloroethene & 1.9 & $\mathrm{U}$ \\
\hline $108-87-2$ & Methylcyclohexane & 1.9 & $\mathrm{U}$ \\
\hline $78-87-5$ & 1,2-Dichloropropane & 1.9 & $\mathrm{U}$ \\
\hline $75-27-4$ & Bromodichloromethane & 1.9 & $\mathrm{U}$ \\
\hline $10061-01-5$ & cis-1,3-Dichloropropene & 1.9 & U \\
\hline $108-10-1$ & 4-Methyl-2-pentanone & 1.9 & $\mathrm{U}$ \\
\hline $108-88-3$ & Toluene & 0.041 & $\mathrm{~J} B$ \\
\hline $10061-02-6$ & trans-1,3-Dichloropropene & 1.9 & $\mathrm{U}$ \\
\hline $79-00-5$ & $1,1,2-$ Trichloroethane & 1.9 & $\mathrm{U}$ \\
\hline $127-18-4$ & Tetrachloroethene & 0.014 & $\mathrm{~J}$ \\
\hline $591-78-6$ & 2-Hexanone & 1.9 & $\mathrm{U}$ \\
\hline $124-48-1$ & Dibromochloromethane & 1.9 & $\mathrm{U}$ \\
\hline $106-93-4$ & 1,2-Dibromoethane & 1.9 & $\mathrm{U}$ \\
\hline $108-90-7$ & Chlorobenzene & 1.9 & $\mathrm{U}$ \\
\hline $100-41-4$ & Ethylbenzene & 1.9 & $\mathrm{U}$ \\
\hline $95-47-6$ & o-Xylene & 0.0070 & $\mathrm{~J} B$ \\
\hline $179601-23-1$ & m,p-xylene & 0.027 & $\mathrm{~J}$ \\
\hline $100-42-5$ & Styrene & 1.9 & $\mathrm{U}$ \\
\hline $75-25-2$ & Bromoform & 1.9 & $\mathrm{U}$ \\
\hline $98-82-8$ & Isopropylbenzene & 1.9 & $\mathrm{U}$ \\
\hline $79-34-5$ & $1,1,2,2$-Tetrachloroethane & 1.9 & $\mathrm{U}$ \\
\hline $541-73-1$ & 1,3 -Dichlorobenzene & 1.9 & $\mathrm{U}$ \\
\hline $106-46-7$ & 1,4-Dichlorobenzene & 1.9 & $\mathrm{U}$ \\
\hline $95-50-1$ & 1,2-Dichlorobenzene & 1.9 & $\mathrm{U}$ \\
\hline $96-12-8$ & 1,2-Dibromo-3-Chloropropane & 1.9 & $\mathrm{U}$ \\
\hline $120-82-1$ & $1,2,4$-Trichlorobenzene & 0.018 & $\mathrm{~J} B$ \\
\hline $87-61-6$ & $1,2,3$-Trichlorobenzene & 0.020 & $\mathrm{~J} B$ \\
\hline
\end{tabular}


Lab Name: TESTAMERICA BURLINGTON

Contract: $8 \mathrm{E}-00302$

Lab Code: STLV Case No.: YORK Mod. Ref No.: SDG No.: 29312

Matrix: (SOIL/SED/WATER) Water

Sample wt/vol: 25.0 $(\mathrm{g} / \mathrm{mL}) \mathrm{mL}$

Level: (TRACE or LOW/MED) TRACE

․ Moisture: not dec.

GC Column: DB-624 ID $: 0.20 \quad(\mathrm{~mm})$

Soil Extract Volume: (uL)
Lab Sample ID: 200-29312-2

Lab File ID: 15379_007.D

Date Received: 08/15/2015

Date Analyzed: 08/20/2015

Dilution Factor: 1.9

Soil Aliquot Volume: (uL) Purge Volume: 25.0 $(\mathrm{mL})$

01

02

\begin{tabular}{|l|l|l|r|r|}
\hline CAS NUMBER & \multicolumn{1}{|c|}{ COMPOUND NAME } & RT & EST. CONC. & Q \\
\hline & Unknown & 7.29 & 5.9 & $\mathrm{~J}$ B X \\
\hline E9667961 & Total Alkanes & N/A & & \\
\hline
\end{tabular}

1EPA-designated Registry Number. 
Lab Name: TESTAMERICA BURLINGTON

Contract: 8E-00302

Lab Code: STLV Case No.: YORK Mod. Ref No.:

SDG No.: 29312

Instrument ID: CHD.i

Calibration Date(s): 08/19/2015 08/19/2015

Heated Purge: ( $\mathrm{Y} / \mathrm{N}) \mathrm{N}$

Calibration Time(s) : 1514

1654

Purge Volume: 25.0 $(\mathrm{mL})$

GC Column: DB-624

ID: 0.20

$(\mathrm{mm})$

Length: 25

$(\mathrm{m})$

\begin{tabular}{|c|c|c|c|c|c|c|c|}
\hline \multirow{3}{*}{$\begin{array}{l}\text { LAB FILE ID: } \\
\text { RRF5.0 }=\text { 15357_005.D } \\
\text { COMPOUND }\end{array}$} & \multirow{2}{*}{\multicolumn{3}{|c|}{ 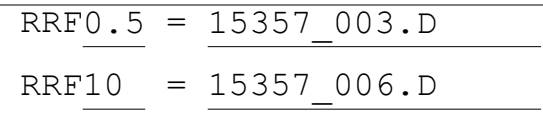 }} & \multirow{2}{*}{\multicolumn{4}{|c|}{$\begin{array}{l}\text { RRF1.0 }=\frac{15357 \_004 . \mathrm{D}}{\text { RRF20 }}=15357 \_007 . \mathrm{D}\end{array}$}} \\
\hline & & & & & & & \\
\hline & $\operatorname{RRF} 0.5$ & $\operatorname{RRF} 1.0$ & RRF5.0 & $\operatorname{RRF} 10$ & RRF20 & $\overline{\mathrm{RRF}}$ & $\because \mathrm{RSD}$ \\
\hline Dichlorodifluoromethane & 0.630 & 0.576 & 0.641 & 0.600 & 0.628 & 0.615 & 4.4 \\
\hline Chloromethane & 0.540 & 0.453 & 0.512 & 0.465 & 0.487 & 0.491 & 7.2 \\
\hline Vinyl chloride & 0.449 & 0.413 & 0.459 & 0.432 & 0.439 & 0.438 & 4.0 \\
\hline Bromomethane & 0.148 & 0.106 & 0.148 & 0.147 & 0.161 & 0.142 & 14.8 \\
\hline Chloroethane & 0.207 & 0.193 & 0.216 & 0.208 & 0.196 & 0.204 & 4.6 \\
\hline Trichlorofluoromethane & 0.558 & 0.507 & 0.584 & 0.557 & 0.568 & 0.555 & 5.2 \\
\hline 1,1-Dichloroethene & 0.290 & 0.248 & 0.281 & 0.263 & 0.265 & 0.270 & 6.2 \\
\hline $\begin{array}{l}1,1,2 \text {-Trichloro- } \\
1,2,2 \text {-trifluoroethane }\end{array}$ & 0.309 & 0.282 & 0.317 & 0.300 & 0.298 & 0.301 & 4.3 \\
\hline Acetone & 0.025 & 0.021 & 0.023 & 0.021 & 0.022 & 0.022 & 7.7 \\
\hline Carbon disulfide & 0.656 & 0.597 & 0.750 & 0.861 & 0.809 & 0.735 & 14.7 \\
\hline Methyl acetate & 0.074 & 0.060 & 0.072 & 0.065 & 0.066 & 0.067 & 8.7 \\
\hline Methylene Chloride & 0.292 & 0.261 & 0.273 & 0.266 & 0.265 & 0.271 & 4.6 \\
\hline trans-1,2-Dichloroethene & 0.296 & 0.279 & 0.328 & 0.322 & 0.320 & 0.309 & 6.7 \\
\hline Methyl tert-butyl ether & 0.349 & 0.322 & 0.364 & 0.374 & 0.388 & 0.359 & 7.0 \\
\hline 1,1-Dichloroethane & 0.554 & 0.524 & 0.605 & 0.591 & 0.606 & 0.576 & 6.3 \\
\hline cis-1,2-Dichloroethene & 0.303 & 0.269 & 0.308 & 0.317 & 0.315 & 0.302 & 6.4 \\
\hline 2-Butanone & 0.039 & 0.037 & 0.043 & 0.043 & 0.045 & 0.041 & 8.1 \\
\hline Bromochloromethane & 0.087 & 0.080 & 0.096 & 0.096 & 0.100 & 0.091 & 9.0 \\
\hline Chloroform & 0.490 & 0.490 & 0.538 & 0.531 & 0.532 & 0.516 & 4.6 \\
\hline 1,1,1-Trichloroethane & 0.524 & 0.485 & 0.591 & 0.595 & 0.613 & 0.562 & 9.7 \\
\hline Cyclohexane & 0.848 & 0.766 & 0.859 & 0.833 & 0.823 & 0.826 & 4.4 \\
\hline Carbon tetrachloride & 0.430 & 0.395 & 0.503 & 0.519 & 0.528 & 0.475 & 12.4 \\
\hline Benzene & 1.706 & 1.548 & 1.656 & 1.646 & 1.599 & 1.631 & 3.7 \\
\hline 1,2-Dichloroethane & 0.260 & 0.229 & 0.258 & 0.263 & 0.252 & 0.253 & 5.4 \\
\hline Trichloroethene & 0.401 & 0.379 & 0.416 & 0.411 & 0.406 & 0.403 & 3.5 \\
\hline Methylcyclohexane & 0.657 & 0.642 & 0.702 & 0.694 & 0.680 & 0.675 & 3.7 \\
\hline
\end{tabular}

Report 1,4-Dioxane for Low-Medium VOA analysis only 
Lab Name: TESTAMERICA BURLINGTON

Contract: $8 \mathrm{E}-00302$

Lab Code: STLV Case No.: YORK Mod. Ref No.:

SDG No.: 29312

Instrument ID: CHD.i

Calibration Date(s) : 08/19/2015 08/19/2015

Heated Purge: (Y/N) N

Calibration Time(s) : 1514

1654

Purge Volume: 25.0 (mL)

GC Column: DB-624

ID: 0.20

$(\mathrm{mm})$

Length: 25

(m)

\begin{tabular}{|c|c|c|c|c|c|c|c|}
\hline \multirow{2}{*}{$\begin{array}{l}\text { LAB FILE ID: } \\
\text { RRF5.0 }=\text { 15357_005.D }\end{array}$} & \multicolumn{3}{|c|}{ RRF0.5 = $15357003 . D$} & \multicolumn{4}{|c|}{ RRF1.0 = 15357_004.D } \\
\hline & E10 & 357_00 & & RRF20 & $=1535$ & $1007 . \mathrm{D}$ & \\
\hline COMPOUND & $\mathrm{RRF} 0.5$ & RRF1. 0 & RRF5.0 & RRF10 & RRF20 & $\overline{\mathrm{RRF}}$ & $\because \mathrm{RSD}$ \\
\hline 1,2-Dichloropropane & 0.327 & 0.313 & 0.349 & 0.344 & 0.337 & 0.334 & 4.3 \\
\hline Bromodichloromethane & 0.263 & 0.262 & 0.336 & 0.358 & 0.361 & 0.316 & 15.7 \\
\hline cis-1,3-Dichloropropene & 0.340 & 0.315 & 0.440 & 0.455 & 0.463 & 0.403 & 17.3 \\
\hline 4-Methyl-2-pentanone & 0.106 & 0.102 & 0.128 & 0.126 & 0.125 & 0.117 & 10.5 \\
\hline Toluene & 1.731 & 1.636 & 1.803 & 1.777 & 1.710 & 1.731 & 3.7 \\
\hline trans-1,3-Dichloropropene & 0.201 & 0.213 & 0.301 & 0.323 & 0.329 & 0.273 & 22.7 \\
\hline 1,1,2-Trichloroethane & 0.181 & 0.150 & 0.167 & 0.167 & 0.161 & 0.165 & 6.8 \\
\hline Tetrachloroethene & 0.357 & 0.334 & 0.367 & 0.369 & 0.366 & 0.359 & 4.0 \\
\hline 2-Hexanone & 0.073 & 0.068 & 0.087 & 0.087 & 0.086 & 0.080 & 11.1 \\
\hline Dibromochloromethane & 0.125 & 0.114 & 0.168 & 0.183 & 0.189 & 0.156 & 22.0 \\
\hline 1,2-Dibromoethane & 0.131 & 0.122 & 0.146 & 0.147 & 0.148 & 0.139 & 8.4 \\
\hline Chlorobenzene & 1.013 & 0.937 & 1.024 & 1.018 & 1.005 & 0.999 & 3.5 \\
\hline Ethylbenzene & 2.001 & 1.894 & 2.154 & 2.141 & 2.062 & 2.050 & 5.2 \\
\hline o-Xylene & 0.683 & 0.629 & 0.750 & 0.749 & 0.733 & 0.709 & 7.4 \\
\hline $\mathrm{m}, \mathrm{p}$-Xylene & 0.749 & 0.718 & 0.821 & 0.815 & 0.801 & 0.781 & 5.8 \\
\hline Styrene & 0.957 & 0.957 & 1.155 & 1.134 & 1.112 & 1.063 & 9.2 \\
\hline Bromoform & 0.094 & 0.087 & 0.118 & 0.142 & 0.153 & 0.119 & 24.2 \\
\hline Isopropylbenzene & 2.002 & 1.895 & 2.236 & 2.192 & 2.144 & 2.094 & 6.8 \\
\hline $1,1,2,2$-Tetrachloroethane & 0.159 & 0.146 & 0.172 & 0.168 & 0.172 & 0.163 & 6.8 \\
\hline 1,3-Dichlorobenzene & 1.554 & 1.483 & 1.702 & 1.712 & 1.743 & 1.639 & 6.9 \\
\hline 1,4-Dichlorobenzene & 1.614 & 1.524 & 1.654 & 1.646 & 1.666 & 1.621 & 3.5 \\
\hline 1,2-Dichlorobenzene & 1.357 & 1.238 & 1.365 & 1.353 & 1.383 & 1.339 & 4.3 \\
\hline 1,2-Dibromo-3-Chloropropane & 0.033 & 0.033 & 0.035 & 0.039 & 0.045 & 0.037 & 14.0 \\
\hline 1,2,4-Trichlorobenzene & 0.804 & 0.738 & 0.849 & 0.888 & 0.929 & 0.841 & 8.8 \\
\hline 1,2,3-Trichlorobenzene & 0.564 & 0.535 & 0.591 & 0.624 & 0.658 & 0.594 & 8.1 \\
\hline
\end{tabular}


Lab Name: TESTAMERICA BURLINGTON

Contract: $8 \mathrm{E}-00302$

Lab Code: STLV Case No.: YORK Mod. Ref No.: SDG No.: 29312

Instrument ID: CHD.i

Heated Purge: (Y/N) N

Purge Volume: 25.0

GC Column: DB-624
ID: 0.20
Calibration Date(s) : 08/19/2015

Calibration Time(s) : 1514 $(\mathrm{mL})$

(mm) Length: 25
$08 / 19 / 2015$ 1654

\begin{tabular}{|c|c|c|c|c|c|c|c|}
\hline LAB FILE ID: & \multirow{2}{*}{\multicolumn{3}{|c|}{$\begin{array}{l}\operatorname{RRF0.5}=\frac{15357 \_003 . D}{\operatorname{RRF10}=15357 \_006 . \mathrm{D}} \\
\end{array}$}} & \multirow{2}{*}{\multicolumn{4}{|c|}{$\begin{array}{l}\text { RRF1.0 }=15357 \_004 . \mathrm{D} \\
\text { RRF20 }=15357 \_007 . \mathrm{D}\end{array}$}} \\
\hline RRF5.0 = 15357_005.D & & & & & & & \\
\hline COMPOUND & RRF0. 5 & RRF1 . 0 & RRF 5.0 & RRF10 & RRF20 & $\overline{\mathrm{RRF}}$ & $\because \mathrm{RSD}$ \\
\hline Vinyl Chloride-d3 & 0.381 & 0.364 & 0.409 & 0.383 & 0.398 & 0.387 & 4.4 \\
\hline Chloroethane-d5 & 0.280 & 0.255 & 0.268 & 0.246 & 0.235 & 0.257 & 7.0 \\
\hline 1,1-Dichloroethene-d2 & 0.625 & 0.555 & 0.632 & 0.603 & 0.582 & 0.600 & 5.3 \\
\hline 2-Butanone-d5 & 0.038 & 0.036 & 0.041 & 0.041 & 0.043 & 0.040 & 6.9 \\
\hline Chloroform-d & 0.535 & 0.511 & 0.560 & 0.563 & 0.562 & 0.546 & 4.2 \\
\hline 1,2-Dichloroethane-d4 & 0.202 & 0.196 & 0.208 & 0.211 & 0.207 & 0.205 & 3.0 \\
\hline Benzene-d6 & 1.559 & 1.409 & 1.532 & 1.506 & 1.492 & 1.500 & 3.8 \\
\hline 1,2-Dichloropropane-d6 & 0.391 & 0.349 & 0.395 & 0.389 & 0.383 & 0.381 & 4.9 \\
\hline Toluene-d8 & 1.433 & 1.328 & 1.484 & 1.481 & 1.428 & 1.431 & 4.4 \\
\hline trans-1,3-Dichloropropene-d4 & 0.184 & 0.169 & 0.257 & 0.279 & 0.290 & 0.236 & 23.6 \\
\hline $2-$ Hexanone-d5 & 0.026 & 0.029 & 0.038 & 0.039 & 0.039 & 0.034 & 17.9 \\
\hline $1,1,2,2$-Tetrachloroethane-d2 & 0.149 & 0.144 & 0.170 & 0.165 & 0.171 & 0.160 & 7.8 \\
\hline 1,2-Dichlorobenzene-d4 & 0.883 & 0.800 & 0.834 & 0.838 & 0.864 & 0.844 & 3.7 \\
\hline
\end{tabular}

Report 1,4-Dioxane-d8 for Low-Medium VOA analysis only 
Lab Name: TESTAMERICA BURLINGTON

Contract: $8 \mathrm{E}-00302$

Lab Code: STLV Case No.: YORK Mod. Ref No.:

SDG No.: 29312

Instrument ID: CHD.i

Calibration Date: 08/20/2015 Time: 1313

Lab File Id: 15379_003.D

EPA Sample No. (VSTD\#\#\#\#) : VSTD005DF Init. Calib. Date(s):08/19/2015 08/19/2015

Heated Purge: (Y/N) N GC Column: DB-624

Init. Calib. Time(s) : 1514 1654

Purge Volume: 25.0 ID: $0.20(\mathrm{~mm})$ Length: 25 (m) $(\mathrm{mL})$

\begin{tabular}{|c|c|c|c|c|c|}
\hline COMPOUND & $\overline{\mathrm{RRF}}$ & RRF5.0 & $\begin{array}{l}\text { MIN } \\
\text { RRF }\end{array}$ & $\frac{\circ}{D}$ & $\mathrm{MAX} \div \mathrm{D}$ \\
\hline Dichlorodifluoromethane & 0.615 & 0.624 & 0.010 & 1.4 & 40.0 \\
\hline Chloromethane & 0.491 & 0.481 & 0.010 & -2.1 & 40.0 \\
\hline Vinyl chloride & 0.438 & 0.455 & 0.100 & 3.7 & 30.0 \\
\hline Bromomethane & 0.142 & 0.162 & 0.100 & 13.9 & 30.0 \\
\hline Chloroethane & 0.204 & 0.218 & 0.010 & 6.9 & 40.0 \\
\hline Trichlorofluoromethane & 0.555 & 0.568 & 0.010 & 2.4 & 40.0 \\
\hline 1,1-Dichloroethene & 0.270 & 0.280 & 0.100 & 3.9 & 30.0 \\
\hline 1,1,2-Trichloro-1,2,2-trifluoroethane & 0.301 & 0.319 & 0.010 & 5.8 & 40.0 \\
\hline Acetone & 0.022 & 0.023 & 0.010 & 1.7 & 40.0 \\
\hline Carbon disulfide & 0.735 & 0.771 & 0.010 & 4.9 & 40.0 \\
\hline Methyl acetate & 0.067 & 0.070 & 0.010 & 3.2 & 40.0 \\
\hline Methylene Chloride & 0.271 & 0.269 & 0.010 & -0.8 & 40.0 \\
\hline trans-1,2-Dichloroethene & 0.309 & 0.324 & 0.010 & 4.9 & 40.0 \\
\hline Methyl tert-butyl ether & 0.359 & 0.364 & 0.010 & 1.2 & 40.0 \\
\hline 1,1-Dichloroethane & 0.576 & 0.614 & 0.200 & 6.6 & 30.0 \\
\hline cis-1,2-Dichloroethene & 0.302 & 0.316 & 0.010 & 4.6 & 40.0 \\
\hline 2-Butanone & 0.041 & 0.044 & 0.010 & 6.4 & 40.0 \\
\hline Bromochloromethane & 0.091 & 0.096 & 0.050 & 4.8 & 30.0 \\
\hline Chloroform & 0.516 & 0.538 & 0.200 & 4.3 & 30.0 \\
\hline 1,1,1-Trichloroethane & 0.562 & 0.607 & 0.100 & 8.0 & 30.0 \\
\hline Cyclohexane & 0.826 & 0.876 & 0.010 & 6.1 & 40.0 \\
\hline Carbon tetrachloride & 0.475 & 0.522 & 0.100 & 10.0 & 30.0 \\
\hline Benzene & 1.631 & 1.692 & 0.400 & 3.7 & 30.0 \\
\hline 1,2-Dichloroethane & 0.253 & 0.262 & 0.100 & 3.7 & 30.0 \\
\hline Trichloroethene & 0.403 & 0.418 & 0.300 & 3.8 & 30.0 \\
\hline Methylcyclohexane & 0.675 & 0.716 & 0.010 & 6.1 & 40.0 \\
\hline
\end{tabular}

Report 1,4-Dioxane for Low/Medium VOA analysis only 
Lab Name: TESTAMERICA BURLINGTON

Contract: 8E-00302

Lab Code: STLV Case No.: YORK Mod. Ref No.:

SDG No.: 29312

Instrument ID: CHD.i

Calibration Date: 08/20/2015 Time: 1313

Lab File Id: 15379 003.D

EPA Sample No. (VSTD\#\#\#\#) : VSTD005DF

Init. Calib. Date(s):08/19/2015 08/19/2015

Heated Purge: (Y/N) N GC Column: DB-624

Init. Calib. Time(s) : 1514 1654

Purge Volume: 25.0

ID: $0.20(\mathrm{~mm})$ Length: 25

(m)

\begin{tabular}{|c|c|c|c|c|c|}
\hline COMPOUND & $\overline{\mathrm{RRF}}$ & RRF5.0 & $\begin{array}{l}\text { MIN } \\
\text { RRF }\end{array}$ & $\because \mathrm{D}$ & $\operatorname{MAX} \cong \mathrm{D}$ \\
\hline 1,2-Dichloropropane & 0.334 & 0.337 & 0.010 & 0.9 & 40.0 \\
\hline Bromodichloromethane & 0.316 & 0.348 & 0.200 & 10.2 & 30.0 \\
\hline cis-1,3-Dichloropropene & 0.403 & 0.452 & 0.200 & 12.4 & 30.0 \\
\hline 4-Methyl-2-pentanone & 0.117 & 0.125 & 0.010 & 6.7 & 40.0 \\
\hline Toluene & 1.731 & 1.821 & 0.400 & 5.2 & 30.0 \\
\hline trans-1,3-Dichloropropene & 0.273 & 0.307 & 0.100 & 12.4 & 30.0 \\
\hline $1,1,2$-Trichloroethane & 0.165 & 0.170 & 0.100 & 2.8 & 30.0 \\
\hline Tetrachloroethene & 0.359 & 0.374 & 0.100 & 4.2 & 30.0 \\
\hline 2-Hexanone & 0.080 & 0.088 & 0.010 & 9.7 & 40.0 \\
\hline Dibromochloromethane & 0.156 & 0.171 & 0.100 & 9.9 & 30.0 \\
\hline 1,2-Dibromoethane & 0.139 & 0.148 & 0.010 & 6.9 & 40.0 \\
\hline Chlorobenzene & 0.999 & 1.027 & 0.500 & 2.7 & 30.0 \\
\hline Ethylbenzene & 2.050 & 2.160 & 0.100 & 5.3 & 30.0 \\
\hline o-Xylene & 0.709 & 0.757 & 0.300 & 6.8 & 30.0 \\
\hline $\mathrm{m}, \mathrm{p}$-Xylene & 0.781 & 0.828 & 0.300 & 6.1 & 30.0 \\
\hline Styrene & 1.063 & 1.147 & 0.300 & 7.9 & 30.0 \\
\hline Bromoform & 0.119 & 0.125 & 0.050 & 5.5 & 30.0 \\
\hline Isopropylbenzene & 2.094 & 2.244 & 0.010 & 7.2 & 40.0 \\
\hline $1,1,2,2$-Tetrachloroethane & 0.163 & 0.171 & 0.100 & 4.6 & 30.0 \\
\hline 1,3-Dichlorobenzene & 1.639 & 1.695 & 0.400 & 3.4 & 30.0 \\
\hline 1,4-Dichlorobenzene & 1.621 & 1.637 & 0.400 & 1.0 & 30.0 \\
\hline 1,2-Dichlorobenzene & 1.339 & 1.353 & 0.400 & 1.1 & 30.0 \\
\hline 1,2-Dibromo-3-Chloropropane & 0.037 & 0.034 & 0.010 & -7.8 & 40.0 \\
\hline 1,2,4-Trichlorobenzene & 0.841 & 0.849 & 0.200 & 1.0 & 30.0 \\
\hline $1,2,3$-Trichlorobenzene & 0.594 & 0.591 & 0.200 & -0.6 & 30.0 \\
\hline
\end{tabular}


Lab Name: TESTAMERICA BURLINGTON

Contract: $8 \mathrm{E}-00302$

Lab Code: STLV Case No.: YORK Mod. Ref No.

SDG No.: 29312

Instrument ID: CHD.i

Calibration Date: 08/20/2015 Time: 1313

Lab File Id: 15379_003.D Init. Calib. Date(s):08/19/2015 08/19/2015

EPA Sample No. (VSTD\#\#\#\#) : VSTD005DF

Heated Purge: (Y/N) N GC Column: DB-624

Init. Calib. Time(s) : 1514 1654

Purge Volume: 25.0 ID: $0.20(\mathrm{~mm})$ Length: 25

(m)

\begin{tabular}{|c|c|c|c|c|c|}
\hline COMPOUND & $\overline{\mathrm{RRF}}$ & RRF 5.0 & $\begin{array}{l}\text { MIN } \\
\text { RRF }\end{array}$ & $\div D$ & $\mathrm{MAX} \div \mathrm{D}$ \\
\hline Vinyl Chloride-d3 & 0.387 & 0.411 & 0.010 & 6.2 & 30.0 \\
\hline Chloroethane-d5 & 0.257 & 0.267 & 0.010 & 3.9 & 40.0 \\
\hline $1,1-$ Dichloroethene-d2 & 0.600 & 0.631 & 0.010 & 5.2 & 30.0 \\
\hline 2-Butanone-d5 & 0.040 & 0.041 & 0.010 & 3.4 & 40.0 \\
\hline Chloroform-d & 0.546 & 0.569 & 0.010 & 4.2 & 30.0 \\
\hline 1,2-Dichloroethane-d4 & 0.205 & 0.214 & 0.010 & 4.3 & 30.0 \\
\hline Benzene-d6 & 1.500 & 1.553 & 0.010 & 3.5 & 30.0 \\
\hline 1,2 -Dichloropropane-d6 & 0.381 & 0.397 & 0.010 & 4.1 & 40.0 \\
\hline Toluene-d8 & 1.431 & 1.499 & 0.010 & 4.7 & 30.0 \\
\hline trans-1,3-Dichloropropene-d4 & 0.236 & 0.262 & 0.010 & 11.0 & 30.0 \\
\hline 2-Hexanone-d5 & 0.034 & 0.037 & 0.010 & 9.2 & 40.0 \\
\hline $1,1,2,2$-Tetrachloroethane-d2 & 0.160 & 0.167 & 0.010 & 4.4 & 30.0 \\
\hline 1,2-Dichlorobenzene-d4 & 0.844 & 0.842 & 0.010 & -0.2 & 30.0 \\
\hline
\end{tabular}

Report 1,4-Dioxane-d8 for Low/Medium VOA analysis only 
Lab Name: TESTAMERICA BURLINGTON

Contract: $8 \mathrm{E}-00302$

Lab Code: STLV Case No.: YORK Mod. Ref No.:

SDG No.: 29312

Instrument ID: CHD.i

Calibration Date: 08/20/2015 Time: 1809

Lab File Id: 15379 012.D

EPA Sample No.(VSTD\#\#\#\#): VSTD005FD Init. Calib. Date(s):08/19/2015 08/19/2015

Heated Purge: (Y/N) N GC Column: DB-624

Init. Calib. Time(s) : 1514 1654

Purge Volume: 25.0 ID: $0.20(\mathrm{~mm})$ Length: 25

(m)

\begin{tabular}{|c|c|c|c|c|c|}
\hline COMPOUND & $\overline{\mathrm{RRF}}$ & RRF5. 0 & $\begin{array}{l}\text { MIN } \\
\text { RRF }\end{array}$ & $\because \mathrm{D}$ & $\mathrm{MAX} \cong \mathrm{D}$ \\
\hline Dichlorodifluoromethane & 0.615 & 0.660 & 0.010 & 7.4 & 50.0 \\
\hline Chloromethane & 0.491 & 0.506 & 0.010 & 2.9 & 50.0 \\
\hline Vinyl chloride & 0.438 & 0.460 & 0.010 & 4.9 & 50.0 \\
\hline Bromomethane & 0.142 & 0.141 & 0.010 & -0.4 & 50.0 \\
\hline Chloroethane & 0.204 & 0.216 & 0.010 & 5.8 & 50.0 \\
\hline Trichlorofluoromethane & 0.555 & 0.596 & 0.010 & 7.4 & 50.0 \\
\hline 1,1-Dichloroethene & 0.270 & 0.280 & 0.010 & 3.8 & 50.0 \\
\hline 1,1,2-Trichloro-1,2,2-trifluoroethane & 0.301 & 0.310 & 0.010 & 3.1 & 50.0 \\
\hline Acetone & 0.022 & 0.021 & 0.010 & -4.5 & 50.0 \\
\hline Carbon disulfide & 0.735 & 0.779 & 0.010 & 6.0 & 50.0 \\
\hline Methyl acetate & 0.067 & 0.065 & 0.010 & -3.2 & 50.0 \\
\hline Methylene Chloride & 0.271 & 0.275 & 0.010 & 1.5 & 50.0 \\
\hline trans-1,2-Dichloroethene & 0.309 & 0.320 & 0.010 & 3.5 & 50.0 \\
\hline Methyl tert-butyl ether & 0.359 & 0.360 & 0.010 & 0.2 & 50.0 \\
\hline 1,1-Dichloroethane & 0.576 & 0.595 & 0.010 & 3.4 & 50.0 \\
\hline cis-1,2-Dichloroethene & 0.302 & 0.312 & 0.010 & 3.3 & 50.0 \\
\hline 2-Butanone & 0.041 & 0.045 & 0.010 & 8.5 & 50.0 \\
\hline Bromochloromethane & 0.091 & 0.097 & 0.010 & 5.8 & 50.0 \\
\hline Chloroform & 0.516 & 0.533 & 0.010 & 3.2 & 50.0 \\
\hline 1,1,1-Trichloroethane & 0.562 & 0.589 & 0.010 & 4.9 & 50.0 \\
\hline Cyclohexane & 0.826 & 0.873 & 0.010 & 5.7 & 50.0 \\
\hline Carbon tetrachloride & 0.475 & 0.499 & 0.010 & 5.1 & 50.0 \\
\hline Benzene & 1.631 & 1.688 & 0.010 & 3.5 & 50.0 \\
\hline 1,2-Dichloroethane & 0.253 & 0.266 & 0.010 & 5.4 & 50.0 \\
\hline Trichloroethene & 0.403 & 0.417 & 0.010 & 3.5 & 50.0 \\
\hline Methylcyclohexane & 0.675 & 0.702 & 0.010 & 4.0 & 50.0 \\
\hline
\end{tabular}

Report 1,4-Dioxane for Low/Medium VOA analysis only 
Lab Name: TESTAMERICA BURLINGTON

Contract: 8E-00302

Lab Code: STLV Case No.: YORK Mod. Ref No.:

SDG No.: 29312

Instrument ID: CHD.i

Calibration Date: 08/20/2015 Time: 1809

Lab File Id: 15379 012.D

EPA Sample No. (VSTD\#\#\#\#) : VSTD005FD Init. Calib. Date(s):08/19/2015 08/19/2015

Heated Purge: (Y/N) N GC Column: DB-624

Init. Calib. Time(s) : 1514 1654

Purge Volume: 25.0 ID: $0.20(\mathrm{~mm})$ Length: 25

(m)

\begin{tabular}{|c|c|c|c|c|c|}
\hline COMPOUND & $\overline{\mathrm{RRF}}$ & RRF 5.0 & $\begin{array}{l}\text { MIN } \\
\text { RRF }\end{array}$ & $\because \mathrm{D}$ & $\mathrm{MAX} \div \mathrm{D}$ \\
\hline 1,2-Dichloropropane & 0.334 & 0.350 & 0.010 & 4.8 & 50.0 \\
\hline Bromodichloromethane & 0.316 & 0.339 & 0.010 & 7.2 & 50.0 \\
\hline cis-1,3-Dichloropropene & 0.403 & 0.440 & 0.010 & 9.4 & 50.0 \\
\hline 4-Methyl-2-pentanone & 0.117 & 0.132 & 0.010 & 12.6 & 50.0 \\
\hline Toluene & 1.731 & 1.837 & 0.010 & 6.1 & 50.0 \\
\hline trans-1,3-Dichloropropene & 0.273 & 0.296 & 0.010 & 8.4 & 50.0 \\
\hline $1,1,2$-Trichloroethane & 0.165 & 0.169 & 0.010 & 2.5 & 50.0 \\
\hline Tetrachloroethene & 0.359 & 0.374 & 0.010 & 4.1 & 50.0 \\
\hline 2-Hexanone & 0.080 & 0.091 & 0.010 & 14.0 & 50.0 \\
\hline Dibromochloromethane & 0.156 & 0.164 & 0.010 & 5.3 & 50.0 \\
\hline 1,2-Dibromoethane & 0.139 & 0.149 & 0.010 & 7.6 & 50.0 \\
\hline Chlorobenzene & 0.999 & 1.042 & 0.010 & 4.3 & 50.0 \\
\hline Ethylbenzene & 2.050 & 2.193 & 0.010 & 7.0 & 50.0 \\
\hline o-Xylene & 0.709 & 0.764 & 0.010 & 7.8 & 50.0 \\
\hline $\mathrm{m}, \mathrm{p}-\mathrm{Xylene}$ & 0.781 & 0.840 & 0.010 & 7.6 & 50.0 \\
\hline Styrene & 1.063 & 1.173 & 0.010 & 10.4 & 50.0 \\
\hline Bromoform & 0.119 & 0.109 & 0.010 & -8.5 & 50.0 \\
\hline Isopropylbenzene & 2.094 & 2.270 & 0.010 & 8.4 & 50.0 \\
\hline $1,1,2,2$-Tetrachloroethane & 0.163 & 0.175 & 0.010 & 7.6 & 50.0 \\
\hline 1,3-Dichlorobenzene & 1.639 & 1.684 & 0.010 & 2.7 & 50.0 \\
\hline 1,4-Dichlorobenzene & 1.621 & 1.644 & 0.010 & 1.4 & 50.0 \\
\hline 1,2-Dichlorobenzene & 1.339 & 1.370 & 0.010 & 2.3 & 50.0 \\
\hline 1,2-Dibromo-3-Chloropropane & 0.037 & 0.033 & 0.010 & -10.4 & 50.0 \\
\hline 1,2,4-Trichlorobenzene & 0.841 & 0.835 & 0.010 & -0.8 & 50.0 \\
\hline 1,2,3-Trichlorobenzene & 0.594 & 0.597 & 0.010 & 0.4 & 50.0 \\
\hline
\end{tabular}


Lab Name: TESTAMERICA BURLINGTON

Contract: $8 \mathrm{E}-00302$

Lab Code: STLV Case No.: YORK Mod. Ref No.

SDG No.: 29312

Instrument ID: CHD.i

Calibration Date: 08/20/2015 Time: 1809

Lab File Id: 15379_012.D

Init. Calib. Date(s):08/19/2015 08/19/2015

EPA Sample No. (VSTD\#\#\#\#) : VSTD005ED

Init. Calib. Time(s) : $1514 \quad 1654$

Heated Purge: (Y/N) N

GC Column: DB-624

ID: $0.20(\mathrm{~mm})$ Length: 25

(m)

Purge Volume: 25.0 $(\mathrm{mL})$

\begin{tabular}{|c|c|c|c|c|c|}
\hline COMPOUND & $\overline{\mathrm{RRF}}$ & RRF5.0 & $\begin{array}{l}\text { MIN } \\
\text { RRF }\end{array}$ & $\because \mathrm{D}$ & $\operatorname{MAX} \cong \mathrm{D}$ \\
\hline Vinyl Chloride-d3 & 0.387 & 0.407 & 0.010 & 5.1 & 50.0 \\
\hline Chloroethane-d5 & 0.257 & 0.259 & 0.010 & 1.1 & 50.0 \\
\hline 1,1-Dichloroethene-d2 & 0.600 & 0.613 & 0.010 & 2.2 & 50.0 \\
\hline 2-Butanone-d5 & 0.040 & 0.042 & 0.010 & 4.8 & 50.0 \\
\hline Chloroform-d & 0.546 & 0.558 & 0.010 & 2.2 & 50.0 \\
\hline 1,2-Dichloroethane-d4 & 0.205 & 0.211 & 0.010 & 3.1 & 50.0 \\
\hline Benzene-d6 & 1.500 & 1.535 & 0.010 & 2.4 & 50.0 \\
\hline 1,2-Dichloropropane-d6 & 0.381 & 0.395 & 0.010 & 3.5 & 50.0 \\
\hline Toluene-d8 & 1.431 & 1.518 & 0.010 & 6.1 & 50.0 \\
\hline trans-1,3-Dichloropropene-d4 & 0.236 & 0.246 & 0.010 & 4.4 & 50.0 \\
\hline 2-Hexanone-d5 & 0.034 & 0.039 & 0.010 & 13.9 & 50.0 \\
\hline $1,1,2,2$-Tetrachloroethane-d2 & 0.160 & 0.173 & 0.010 & 8.3 & 50.0 \\
\hline 1,2-Dichlorobenzene-d4 & 0.844 & 0.869 & 0.010 & 2.9 & 50.0 \\
\hline
\end{tabular}

Report 1,4-Dioxane-d8 for Low/Medium VOA analysis only 
Lab Name: TESTAMERICA BURLINGTON

Contract: $8 \mathrm{E}-00302$

Lab Code: STLV

Case No.: YORK Mod. Ref No.:

SDG No.: 29312

Matrix: (SOIL/SED/WATER) Water

Sample wt/vol: 25.0 $(\mathrm{g} / \mathrm{mL}) \mathrm{mL}$

Level: (TRACE/LOW/MED) TRACE

\% Moisture: not dec.

GC Column: DB-624 ID: $0.20 \quad(\mathrm{~mm})$

Soil Extract Volume: $(u L)$ $(\mathrm{mL})$

Purge Volume: 25.0
Lab Sample ID: MB 200-92982/5

Lab File ID: 15379_005.D

Date Received:

Date Analyzed: 08/20/2015

Dilution Factor: 1.0

Soil Aliquot Volume: (uL)

\begin{tabular}{|c|c|c|c|}
\hline CAS NO. & COMPOUND & $\begin{array}{l}\text { CONCENTRATION UNITS: } \\
(\mathrm{ug} / \mathrm{L} \text { or } \mathrm{ug} / \mathrm{kg}) \mathrm{ug} / \mathrm{L}\end{array}$ & q \\
\hline $75-71-8$ & Dichlorodifluoromethane & 1.0 & $\mathrm{U}$ \\
\hline $74-87-3$ & Chloromethane & 1.0 & $\mathrm{U}$ \\
\hline $75-01-4$ & Vinyl chloride & 1.0 & $\mathrm{U}$ \\
\hline $74-83-9$ & Bromomethane & 1.0 & $\mathrm{U}$ \\
\hline $75-00-3$ & Chloroethane & 1.0 & $\mathrm{U}$ \\
\hline $75-69-4$ & Trichlorofluoromethane & 1.0 & $\mathrm{U}$ \\
\hline $75-35-4$ & 1,1-Dichloroethene & 1.0 & $\mathrm{U}$ \\
\hline $76-13-1$ & $1,1,2$-Trichloro-1,2,2-trifluoroethane & 1.0 & $\mathrm{U}$ \\
\hline $67-64-1$ & Acetone & 5.0 & $\mathrm{U}$ \\
\hline $75-15-0$ & Carbon disulfide & 0.028 & $\mathrm{~J}$ \\
\hline $79-20-9$ & Methyl acetate & 1.0 & $\mathrm{U}$ \\
\hline $75-09-2$ & Methylene Chloride & 1.0 & $\mathrm{U}$ \\
\hline $156-60-5$ & trans-1,2-Dichloroethene & 1.0 & $\mathrm{U}$ \\
\hline $1634-04-4$ & Methyl tert-butyl ether & 1.0 & $\mathrm{U}$ \\
\hline $75-34-3$ & 1,1-Dichloroethane & 1.0 & $\mathrm{U}$ \\
\hline $156-59-2$ & cis-1,2-Dichloroethene & 1.0 & $\mathrm{U}$ \\
\hline $78-93-3$ & 2-Butanone & 5.0 & $\mathrm{U}$ \\
\hline $74-97-5$ & Bromochloromethane & 1.0 & $\mathrm{U}$ \\
\hline $67-66-3$ & Chloroform & 1.0 & $\mathrm{U}$ \\
\hline $71-55-6$ & $1,1,1$-Trichloroethane & 1.0 & $\mathrm{U}$ \\
\hline $110-82-7$ & Cyclohexane & 1.0 & $\mathrm{U}$ \\
\hline $56-23-5$ & Carbon tetrachloride & 1.0 & $\mathrm{U}$ \\
\hline $71-43-2$ & Benzene & 1.0 & $\mathrm{U}$ \\
\hline $107-06-2$ & 1,2-Dichloroethane & 1.0 & $\mathrm{U}$ \\
\hline
\end{tabular}

Report 1,4-Dioxane for Low-Medium VOA analysis only 
Lab Name: TESTAMERICA BURLINGTON

Lab Code: STLV

Case No.: YORK

Mod. Ref No.:

Contract: 8E-00302

Matrix: (SOIL/SED/WATER) Water

Sample wt/vol: 25.0 $(\mathrm{g} / \mathrm{mL}) \mathrm{mL}$

Level: (TRACE/LOW/MED) TRACE

\% Moisture: not dec.

GC Column: DB-624 ID: $0.20 \quad(\mathrm{~mm})$

Soil Extract Volume: (uL) $(\mathrm{mL})$

Purge Volume: 25.0
SDG No.: 29312

Lab Sample ID: MB 200-92982/5

Lab File ID: 15379_005.D

Date Received:

Date Analyzed: 08/20/2015

Dilution Factor: 1.0

Soil Aliquot Volume: (uL)

\begin{tabular}{|c|c|c|c|}
\hline CAS NO. & COMPOUND & $\begin{array}{l}\text { CONCENTRATION UNITS: } \\
(\mathrm{ug} / \mathrm{L} \text { or } \mathrm{ug} / \mathrm{kg}) \mathrm{ug} / \mathrm{L}\end{array}$ & $q$ \\
\hline $79-01-6$ & Trichloroethene & 1.0 & $\mathrm{U}$ \\
\hline $108-87-2$ & Methylcyclohexane & 1.0 & $\mathrm{U}$ \\
\hline $78-87-5$ & 1,2-Dichloropropane & 1.0 & $\mathrm{U}$ \\
\hline $75-27-4$ & Bromodichloromethane & 1.0 & $\mathrm{U}$ \\
\hline $10061-01-5$ & cis-1,3-Dichloropropene & 1.0 & U \\
\hline $108-10-1$ & 4-Methyl-2-pentanone & 1.0 & $\mathrm{U}$ \\
\hline $108-88-3$ & Toluene & 0.010 & $\mathrm{~J}$ \\
\hline $10061-02-6$ & trans-1,3-Dichloropropene & 1.0 & $\mathrm{U}$ \\
\hline $79-00-5$ & $1,1,2-$ Trichloroethane & 1.0 & $\mathrm{U}$ \\
\hline $127-18-4$ & Tetrachloroethene & 1.0 & $\mathrm{U}$ \\
\hline $591-78-6$ & 2-Hexanone & 1.0 & $\mathrm{U}$ \\
\hline $124-48-1$ & Dibromochloromethane & 1.0 & $\mathrm{U}$ \\
\hline $106-93-4$ & 1,2-Dibromoethane & 1.0 & $\mathrm{U}$ \\
\hline $108-90-7$ & Chlorobenzene & 1.0 & $\mathrm{U}$ \\
\hline $100-41-4$ & Ethylbenzene & 0.0035 & $\mathrm{~J}$ \\
\hline $95-47-6$ & o-Xylene & 0.0028 & $\mathrm{~J}$ \\
\hline $179601-23-1$ & m,p-xylene & 1.0 & $\mathrm{U}$ \\
\hline $100-42-5$ & Styrene & 1.0 & $\mathrm{U}$ \\
\hline $75-25-2$ & Bromoform & 1.0 & $\mathrm{U}$ \\
\hline $98-82-8$ & Isopropylbenzene & 1.0 & $\mathrm{U}$ \\
\hline $79-34-5$ & $1,1,2,2$-Tetrachloroethane & 1.0 & $\mathrm{U}$ \\
\hline $541-73-1$ & 1,3 -Dichlorobenzene & 0.012 & $\mathrm{~J}$ \\
\hline $106-46-7$ & 1,4-Dichlorobenzene & 1.0 & $\mathrm{U}$ \\
\hline $95-50-1$ & 1,2-Dichlorobenzene & 1.0 & $\mathrm{U}$ \\
\hline $96-12-8$ & 1,2-Dibromo-3-Chloropropane & 1.0 & $\mathrm{U}$ \\
\hline $120-82-1$ & $1,2,4$-Trichlorobenzene & 0.039 & $\mathrm{~J}$ \\
\hline $87-61-6$ & $1,2,3$-Trichlorobenzene & 0.050 & $\mathrm{~J}$ \\
\hline
\end{tabular}


Lab Name: TESTAMERICA BURLINGTON

Contract: $8 \mathrm{E}-00302$

Lab Code: STLV Case No.: YORK Mod. Ref No.: SDG No.: 29312

Matrix: (SOIL/SED/WATER) Water

Sample wt/vol: 25.0 $(\mathrm{g} / \mathrm{mL}) \mathrm{mL}$

Level: (TRACE or LOW/MED) TRACE

․ Moisture: not dec.

GC Column: DB-624 ID $: 0.20 \quad(\mathrm{~mm})$

Soil Extract Volume: (uL)

CONCENTRATION UNITS: (ug/L or ug/kg) ug/L
Lab Sample ID: MB 200-92982/5

Lab File ID: 15379 005.D

Date Received:

Date Analyzed: 08/20/2015

Dilution Factor: 1.0

Soil Aliquot Volume: (uL) Purge Volume: 25.0

\begin{tabular}{|c|c|c|c|c|}
\hline CAS NUMBER & COMPOUND NAME & $\mathrm{RT}$ & EST. CONC. & Q \\
\hline 01 & Unknown & 7.30 & 3.0 & $\mathrm{X} \mathrm{J}$ \\
\hline $541-05-9$ & Cyclotrisiloxane, hexamethyl- & 8.22 & 0.67 & $\mathrm{~J} \mathrm{~N}$ \\
\hline 03 & Unknown & 11.04 & 0.92 & $\mathrm{~J}$ \\
\hline E9667961 & Total Alkanes & $\mathrm{N} / \mathrm{A}$ & & \\
\hline
\end{tabular}

1 EPA-designated Registry Number. 
Lab Name: TESTAMERICA BURLINGTON

Contract: $8 \mathrm{E}-00302$

Lab Code: STLV

Case No.: YORK Mod. Ref No.:

SDG No.: 29312

Matrix: (SOIL/SED/WATER) Water

Sample wt/vol: 25.0 $(\mathrm{g} / \mathrm{mL}) \mathrm{mL}$

Level: (TRACE/LOW/MED) TRACE

\% Moisture: not dec.

GC Column: DB-624 ID: $0.20 \quad(\mathrm{~mm})$

Soil Extract Volume: $(u L)$ $(\mathrm{mL})$

Purge Volume: 25.0
Lab Sample ID: 200-29312-4

Lab File ID: 15379_011.D

Date Received:

Date Analyzed: 08/20/2015

Dilution Factor: 1.0

Soil Aliquot Volume:

(uL)

\begin{tabular}{|c|c|c|c|}
\hline CAS NO. & COMPOUND & $\begin{array}{l}\text { CONCENTRATION UNITS: } \\
(\mathrm{ug} / \mathrm{L} \text { or } \mathrm{ug} / \mathrm{kg}) \mathrm{ug} / \mathrm{L}\end{array}$ & Q \\
\hline $75-71-8$ & Dichlorodifluoromethane & 1.0 & $\mathrm{U}$ \\
\hline $74-87-3$ & Chloromethane & 1.0 & $\mathrm{U}$ \\
\hline $75-01-4$ & Vinyl chloride & 1.0 & $\mathrm{U}$ \\
\hline $74-83-9$ & Bromomethane & 1.0 & $\mathrm{U}$ \\
\hline $75-00-3$ & Chloroethane & 1.0 & $\mathrm{U}$ \\
\hline $75-69-4$ & Trichlorofluoromethane & 1.0 & $\mathrm{U}$ \\
\hline $75-35-4$ & 1,1-Dichloroethene & 1.0 & $\mathrm{U}$ \\
\hline $76-13-1$ & $1,1,2$-Trichloro-1,2,2-trifluoroethane & 1.0 & $\mathrm{U}$ \\
\hline $67-64-1$ & Acetone & 1.2 & $\mathrm{~J}$ \\
\hline $75-15-0$ & Carbon disulfide & 0.017 & $\mathrm{~J} B$ \\
\hline $79-20-9$ & Methyl acetate & 1.0 & $\mathrm{U}$ \\
\hline $75-09-2$ & Methylene Chloride & 1.0 & $\mathrm{U}$ \\
\hline $156-60-5$ & trans-1,2-Dichloroethene & 1.0 & $\mathrm{U}$ \\
\hline $1634-04-4$ & Methyl tert-butyl ether & 1.0 & $\mathrm{U}$ \\
\hline $75-34-3$ & 1,1-Dichloroethane & 1.0 & $\mathrm{U}$ \\
\hline $156-59-2$ & cis-1,2-Dichloroethene & 1.0 & $\mathrm{U}$ \\
\hline $78-93-3$ & 2-Butanone & 5.0 & $\mathrm{U}$ \\
\hline $74-97-5$ & Bromochloromethane & 1.0 & $\mathrm{U}$ \\
\hline $67-66-3$ & Chloroform & 1.0 & $\mathrm{U}$ \\
\hline $71-55-6$ & $1,1,1$-Trichloroethane & 1.0 & $\mathrm{U}$ \\
\hline $110-82-7$ & Cyclohexane & 1.0 & $\mathrm{U}$ \\
\hline $56-23-5$ & Carbon tetrachloride & 1.0 & $\mathrm{U}$ \\
\hline $71-43-2$ & Benzene & 1.0 & $\mathrm{U}$ \\
\hline $107-06-2$ & 1,2-Dichloroethane & 1.0 & $\mathrm{U}$ \\
\hline
\end{tabular}

Report 1,4-Dioxane for Low-Medium VOA analysis only 
Lab Name: TESTAMERICA BURLINGTON

Lab Code: STLV

Case No.: YORK

Mod. Ref No.:

Contract: 8E-00302

Matrix: (SOIL/SED/WATER) Water

Sample wt/vol: 25.0 $(\mathrm{g} / \mathrm{mL}) \mathrm{mL}$

Level: (TRACE/LOW/MED) TRACE

\% Moisture: not dec.

GC Column: DB-624 ID: $0.20 \quad(\mathrm{~mm})$

Soil Extract Volume: (uL) $(\mathrm{mL})$

Purge Volume: 25.0 (mI)
SDG No.: 29312

Lab Sample ID: 200-29312-4

Lab File ID: 15379_011.D

Date Received:

Date Analyzed: 08/20/2015

Dilution Factor: 1.0

Soil Aliquot Volume:

(uL)

\begin{tabular}{|c|c|c|c|}
\hline CAS NO. & COMPOUND & $\begin{array}{l}\text { CONCENTRATION UNITS: } \\
(\mathrm{ug} / \mathrm{L} \text { or } \mathrm{ug} / \mathrm{kg}) \mathrm{ug} / \mathrm{L}\end{array}$ & Q \\
\hline $79-01-6$ & Trichloroethene & 1.0 & $\mathrm{U}$ \\
\hline $108-87-2$ & Methylcyclohexane & 1.0 & $\mathrm{U}$ \\
\hline $78-87-5$ & 1,2-Dichloropropane & 1.0 & $\mathrm{U}$ \\
\hline $75-27-4$ & Bromodichloromethane & 1.0 & $\mathrm{U}$ \\
\hline $10061-01-5$ & cis-1,3-Dichloropropene & 1.0 & U \\
\hline $108-10-1$ & 4-Methyl-2-pentanone & 1.0 & $\mathrm{U}$ \\
\hline $108-88-3$ & Toluene & 0.011 & $\mathrm{~J} B$ \\
\hline $10061-02-6$ & trans-1,3-Dichloropropene & 1.0 & $\mathrm{U}$ \\
\hline $79-00-5$ & $1,1,2-$ Trichloroethane & 1.0 & $\mathrm{U}$ \\
\hline $127-18-4$ & Tetrachloroethene & 1.0 & $\mathrm{U}$ \\
\hline $591-78-6$ & 2-Hexanone & 1.0 & $\mathrm{U}$ \\
\hline $124-48-1$ & Dibromochloromethane & 1.0 & $\mathrm{U}$ \\
\hline $106-93-4$ & 1,2-Dibromoethane & 1.0 & $\mathrm{U}$ \\
\hline $108-90-7$ & Chlorobenzene & 1.0 & $\mathrm{U}$ \\
\hline $100-41-4$ & Ethylbenzene & 1.0 & $\mathrm{U}$ \\
\hline $95-47-6$ & o-Xylene & 1.0 & $\mathrm{U}$ \\
\hline $179601-23-1$ & m,p-xylene & 0.0060 & $\mathrm{~J}$ \\
\hline $100-42-5$ & Styrene & 1.0 & $\mathrm{U}$ \\
\hline $75-25-2$ & Bromoform & 1.0 & $\mathrm{U}$ \\
\hline $98-82-8$ & Isopropylbenzene & 1.0 & $\mathrm{U}$ \\
\hline $79-34-5$ & $1,1,2,2$-Tetrachloroethane & 1.0 & $\mathrm{U}$ \\
\hline $541-73-1$ & 1,3 -Dichlorobenzene & 1.0 & $\mathrm{U}$ \\
\hline $106-46-7$ & 1,4-Dichlorobenzene & 1.0 & $\mathrm{U}$ \\
\hline $95-50-1$ & 1,2-Dichlorobenzene & 1.0 & $\mathrm{U}$ \\
\hline $96-12-8$ & 1,2-Dibromo-3-Chloropropane & 1.0 & $\mathrm{U}$ \\
\hline $120-82-1$ & $1,2,4$-Trichlorobenzene & 1.0 & $\mathrm{U}$ \\
\hline $87-61-6$ & $1,2,3$-Trichlorobenzene & 1.0 & $\mathrm{U}$ \\
\hline
\end{tabular}


Lab Name: TESTAMERICA BURLINGTON

Contract: $8 \mathrm{E}-00302$

Lab Code: STLV Case No.: YORK Mod. Ref No.: SDG No.: 29312

Matrix: (SOIL/SED/WATER) Water

Sample wt/vol: 25.0 $(\mathrm{g} / \mathrm{mL}) \mathrm{mL}$

Level: (TRACE or LOW/MED) TRACE

Moisture: not dec.

GC Column: DB-624 ID $: 0.20 \quad(\mathrm{~mm})$

Soil Extract Volume: (uL)
Lab Sample ID: 200-29312-4

Lab File ID: 15379_011.D

Date Received:

Date Analyzed: 08/20/2015

Dilution Factor: 1.0

Soil Aliquot Volume: (uL) Purge Volume: 25.0 $(\mathrm{mL})$

01

02

\begin{tabular}{|l|l|c|c|c|}
\hline CAS NUMBER & \multicolumn{1}{|c|}{ COMPOUND NAME } & RT & EST. CONC. & Q \\
\hline & Unknown & 7.30 & 2.8 & $\mathrm{~J}$ B X \\
\hline E9667961 & Total Alkanes & N/A & & \\
\hline
\end{tabular}

1 EPA-designated Registry Number. 
Lab Name: TESTAMERICA BURLINGTON

Contract: $8 \mathrm{E}-00302$

Lab Code: STLV

Case No.: YORK Mod. Ref No.:

SDG No.: 29312

Matrix: (SOIL/SED/WATER) Water

Sample wt/vol: 25.0 $(\mathrm{g} / \mathrm{mL}) \mathrm{mL}$

Level: (TRACE/LOW/MED) TRACE

\% Moisture: not dec.

GC Column: DB-624 ID: $0.20 \quad(\mathrm{~mm})$

Soil Extract Volume: (uL) $(\mathrm{mL})$

Purge Volume: 25.0
Lab Sample ID: VIBLK 200-92982/9

Lab File ID: 15379_009.D

Date Received:

Date Analyzed: 08/20/2015

Dilution Factor: 1.0

Soil Aliquot Volume: (uL)

\begin{tabular}{|c|c|c|c|}
\hline CAS NO. & COMPOUND & $\begin{array}{l}\text { CONCENTRATION UNITS: } \\
(\mathrm{ug} / \mathrm{L} \text { or } \mathrm{ug} / \mathrm{kg}) \mathrm{ug} / \mathrm{L}\end{array}$ & Q \\
\hline $75-71-8$ & Dichlorodifluoromethane & 1.0 & $\mathrm{U}$ \\
\hline $74-87-3$ & Chloromethane & 1.0 & $\mathrm{U}$ \\
\hline $75-01-4$ & Vinyl chloride & 1.0 & $\mathrm{U}$ \\
\hline $74-83-9$ & Bromomethane & 1.0 & $\mathrm{U}$ \\
\hline $75-00-3$ & Chloroethane & 1.0 & $\mathrm{U}$ \\
\hline $75-69-4$ & Trichlorofluoromethane & 1.0 & $\mathrm{U}$ \\
\hline $75-35-4$ & 1,1-Dichloroethene & 1.0 & $\mathrm{U}$ \\
\hline $76-13-1$ & $1,1,2$-Trichloro-1,2,2-trifluoroethane & 1.0 & $\mathrm{U}$ \\
\hline $67-64-1$ & Acetone & 0.57 & $\mathrm{~J}$ \\
\hline $75-15-0$ & Carbon disulfide & 0.020 & $\mathrm{~J} B$ \\
\hline $79-20-9$ & Methyl acetate & 1.0 & $\mathrm{U}$ \\
\hline $75-09-2$ & Methylene Chloride & 1.0 & $\mathrm{U}$ \\
\hline $156-60-5$ & trans-1,2-Dichloroethene & 1.0 & $\mathrm{U}$ \\
\hline $1634-04-4$ & Methyl tert-butyl ether & 1.0 & $\mathrm{U}$ \\
\hline $75-34-3$ & 1,1-Dichloroethane & 1.0 & $\mathrm{U}$ \\
\hline $156-59-2$ & cis-1,2-Dichloroethene & 1.0 & $\mathrm{U}$ \\
\hline $78-93-3$ & 2-Butanone & 5.0 & $\mathrm{U}$ \\
\hline $74-97-5$ & Bromochloromethane & 1.0 & $\mathrm{U}$ \\
\hline $67-66-3$ & Chloroform & 1.0 & $\mathrm{U}$ \\
\hline $71-55-6$ & $1,1,1$-Trichloroethane & 1.0 & $\mathrm{U}$ \\
\hline $110-82-7$ & Cyclohexane & 1.0 & $\mathrm{U}$ \\
\hline $56-23-5$ & Carbon tetrachloride & 0.020 & $\mathrm{~J}$ \\
\hline $71-43-2$ & Benzene & 1.0 & $\mathrm{U}$ \\
\hline $107-06-2$ & 1,2-Dichloroethane & 1.0 & $\mathrm{U}$ \\
\hline
\end{tabular}

Report 1,4-Dioxane for Low-Medium VOA analysis only 
Lab Name: TESTAMERICA BURLINGTON

Lab Code: STLV

Case No.: YORK

Mod. Ref No.:

Contract: 8E-00302

Matrix: (SOIL/SED/WATER) Water

Sample wt/vol: 25.0 $(\mathrm{g} / \mathrm{mL}) \mathrm{mL}$

Level: (TRACE/LOW/MED) TRACE

\% Moisture: not dec.

GC Column: DB-624 ID: $0.20 \quad(\mathrm{~mm})$

Soil Extract Volume: (uL) $(\mathrm{mL})$

Purge Volume: 25.0
SDG No.: 29312

Lab Sample ID: VIBLK 200-92982/9

Lab File ID: 15379_009.D

Date Received:

Date Analyzed: 08/20/2015

Dilution Factor: 1.0

Soil Aliquot Volume:

(uL)

\begin{tabular}{|c|c|c|c|}
\hline CAS NO. & COMPOUND & $\begin{array}{l}\text { CONCENTRATION UNITS: } \\
(\mathrm{ug} / \mathrm{L} \text { or } \mathrm{ug} / \mathrm{kg}) \mathrm{ug} / \mathrm{L}\end{array}$ & Q \\
\hline $79-01-6$ & Trichloroethene & 1.0 & $\mathrm{U}$ \\
\hline $108-87-2$ & Methylcyclohexane & 1.0 & $\mathrm{U}$ \\
\hline $78-87-5$ & 1,2-Dichloropropane & 1.0 & $\mathrm{U}$ \\
\hline $75-27-4$ & Bromodichloromethane & 1.0 & $\mathrm{U}$ \\
\hline $10061-01-5$ & cis-1,3-Dichloropropene & 1.0 & U \\
\hline $108-10-1$ & 4-Methyl-2-pentanone & 5.0 & $\mathrm{U}$ \\
\hline $108-88-3$ & Toluene & 0.0094 & $\mathrm{~J} B$ \\
\hline $10061-02-6$ & trans-1,3-Dichloropropene & 1.0 & $\mathrm{U}$ \\
\hline $79-00-5$ & $1,1,2-$ Trichloroethane & 1.0 & $\mathrm{U}$ \\
\hline $127-18-4$ & Tetrachloroethene & 1.0 & $\mathrm{U}$ \\
\hline $591-78-6$ & 2-Hexanone & 5.0 & $\mathrm{U}$ \\
\hline $124-48-1$ & Dibromochloromethane & 1.0 & $\mathrm{U}$ \\
\hline $106-93-4$ & 1,2-Dibromoethane & 1.0 & $\mathrm{U}$ \\
\hline $108-90-7$ & Chlorobenzene & 1.0 & $\mathrm{U}$ \\
\hline $100-41-4$ & Ethylbenzene & 0.0020 & $\mathrm{~J} B$ \\
\hline $95-47-6$ & o-Xylene & 1.0 & $\mathrm{U}$ \\
\hline $179601-23-1$ & m,p-xylene & 0.0068 & $\mathrm{~J}$ \\
\hline $100-42-5$ & Styrene & 1.0 & $\mathrm{U}$ \\
\hline $75-25-2$ & Bromoform & 1.0 & $\mathrm{U}$ \\
\hline $98-82-8$ & Isopropylbenzene & 1.0 & $\mathrm{U}$ \\
\hline $79-34-5$ & $1,1,2,2$-Tetrachloroethane & 1.0 & $\mathrm{U}$ \\
\hline $541-73-1$ & 1,3 -Dichlorobenzene & 1.0 & $\mathrm{U}$ \\
\hline $106-46-7$ & 1,4-Dichlorobenzene & 1.0 & $\mathrm{U}$ \\
\hline $95-50-1$ & 1,2-Dichlorobenzene & 1.0 & $\mathrm{U}$ \\
\hline $96-12-8$ & 1,2-Dibromo-3-Chloropropane & 1.0 & $\mathrm{U}$ \\
\hline $120-82-1$ & $1,2,4$-Trichlorobenzene & 1.0 & $\mathrm{U}$ \\
\hline $87-61-6$ & $1,2,3$-Trichlorobenzene & 1.0 & $\mathrm{U}$ \\
\hline
\end{tabular}


Lab Name: TESTAMERICA BURLINGTON

Contract: 8E-00302

Lab Code: STLV Case No.: YORK Mod. Ref No.: SDG No.: 29312

Matrix: (SOIL/SED/WATER) Water

Sample wt/vol: 25.0 $(\mathrm{g} / \mathrm{mL}) \mathrm{mL}$

Level: (TRACE or LOW/MED) TRACE

\% Moisture: not dec.

GC Column: DB-624 ID $: 0.20 \quad(\mathrm{~mm})$

Soil Extract Volume: (uL)

CONCENTRATION UNITS: (ug/L or ug/kg) ug/L
Lab Sample ID: VIBLK 200-92982/9

Lab File ID: 15379_009.D

Date Received:

Date Analyzed: 08/20/2015

Dilution Factor: 1.0

Soil Aliquot Volume: (uL) Purge Volume: 25.0 $(\mathrm{mL})$

01

02

\begin{tabular}{|l|l|c|c|c|}
\hline CAS NUMBER & \multicolumn{1}{|c|}{ COMPOUND NAME } & RT & EST. CONC. & Q \\
\hline & Unknown & 7.29 & 3.0 & $\mathrm{~J}$ B X \\
\hline E9667961 & Total Alkanes & N/A & & \\
\hline
\end{tabular}

1EPA-designated Registry Number. 
Lab Name: TestAmerica Burlington

Job No.: 200-29312-1

SDG No.: 29312

Instrument ID: CHD.i

Analysis Batch Number: 92921

Lab Sample ID: IC 200-92921/3

Client Sample ID:

Date Analyzed: 08/19/15 15:14

Lab File ID: 15357 003.D

GC Column: DB-624

ID : $0.2(\mathrm{~mm})$

\begin{tabular}{|l|c|c|c|}
\hline \multicolumn{1}{|c|}{ COMPOUND NAME } & RETENTION & \multicolumn{2}{|c|}{ MANUAL INTEGRATION } \\
\cline { 3 - 4 } & TIME & REASON & ANALYST \\
\hline Dichlorodifluoromethane & 1.52 & Baseline & DATE \\
\hline 1,2 -Dibromo-3-Chloropropane & 13.43 & Assign Peak & 08/19/15 $16: 20$ \\
\hline
\end{tabular}

Lab Sample ID: IC 200-92921/4 Client Sample ID:

Date Analyzed: 08/19/15 15:39

Lab File ID: 15357_004.D

GC Column: DB-624

ID $: 0.2(\mathrm{~mm})$

\begin{tabular}{|c|c|c|c|c|}
\hline \multirow[t]{2}{*}{ COMPOUND NAME } & \multirow{2}{*}{$\begin{array}{c}\text { RETENTION } \\
\text { TIME }\end{array}$} & \multicolumn{3}{|c|}{ MANUAL INTEGRATION } \\
\hline & & REASON & ANALYST & DATE \\
\hline Dichlorodifluoromethane & 1.53 & Baseline & wilburj & $08 / 19 / 15 \quad 16: 21$ \\
\hline Bromomethane & 2.00 & Baseline & wilburj & $08 / 19 / 15 \quad 16: 27$ \\
\hline Chloroethane & 2.08 & Baseline & wilburj & $08 / 19 / 1516: 27$ \\
\hline 1,2-Dibromo-3-Chloropropane & 13.44 & Assign Peak & wilburj & $08 / 19 / 15 \quad 16: 27$ \\
\hline
\end{tabular}

Lab Sample ID: ICIS 200-92921/5

client Sample ID:

Date Analyzed: 08/19/15 16:04

Lab File ID: 15357_005.D

GC Column: DB-624

ID : $0.2(\mathrm{~mm})$

\begin{tabular}{|c|c|c|c|c|}
\hline \multirow[t]{2}{*}{ COMPOUND NAME } & \multirow{2}{*}{$\begin{array}{c}\text { RETENTION } \\
\text { TIME }\end{array}$} & \multicolumn{3}{|c|}{ MANUAL INTEGRATION } \\
\hline & & REASON & ANALYST & DATE \\
\hline Dichlorodifluoromethane & 1.53 & Baseline & wilburj & $08 / 19 / 1516: 30$ \\
\hline Bromomethane & 2.00 & Baseline & wilburj & $08 / 19 / 15 \quad 17: 11$ \\
\hline
\end{tabular}

Lab Sample ID: IC 200-92921/6

Client Sample ID:

Date Analyzed: 08/19/15 16:29

Lab File ID: 15357_006.D

GC Column: DB-624

ID : $0.2(\mathrm{~mm})$

\begin{tabular}{|c|c|c|c|c|}
\hline \multirow[t]{2}{*}{ COMPOUND NAME } & \multirow{2}{*}{$\begin{array}{c}\text { RETENTION } \\
\text { TIME }\end{array}$} & \multicolumn{3}{|c|}{ MANUAL INTEGRATION } \\
\hline & & REASON & ANALYST & DATE \\
\hline Dichlorodifluoromethane & 1.53 & Baseline & wilburj & $08 / 19 / 15 \quad 17: 12$ \\
\hline Bromomethane & 2.00 & Baseline & wilburj & $08 / 19 / 15 \quad 17: 12$ \\
\hline
\end{tabular}

SOM01.2/VOA_Tr 
Lab Name: TestAmerica Burlington

SDG No.: 29312

Instrument ID: CHD.i

Lab Sample ID: IC 200-92921/7

Date Analyzed: 08/19/15 16:54
Job No.: 200-29312-1

Analysis Batch Number: 92921

Client Sample ID:

Lab File ID: 15357 007.D
GC Column: DB-624

ID $: 0.2(\mathrm{~mm})$

\begin{tabular}{|c|c|c|c|c|}
\hline \multirow[t]{2}{*}{ COMPOUND NAME } & \multirow{2}{*}{$\begin{array}{c}\text { RETENTION } \\
\text { TIME }\end{array}$} & \multicolumn{3}{|c|}{ MANUAL INTEGRATION } \\
\hline & & REASON & ANALYST & DATE \\
\hline Dichlorodifluoromethane & 1.55 & Baseline & wilburj & $08 / 20 / 1510: 30$ \\
\hline Vinyl Chloride-d3 & 1.71 & Baseline & wilburj & $08 / 20 / 1510: 30$ \\
\hline Vinyl chloride & 1.72 & Baseline & wilburj & $08 / 20 / 15 \quad 10: 30$ \\
\hline Bromomethane & 2.04 & Baseline & wilburj & $08 / 20 / 1510: 30$ \\
\hline
\end{tabular}


Lab Name: TestAmerica Burlington

SDG No.: 29312

Instrument ID: CHD.i

Lab Sample ID: 200-29312-4

Date Analyzed: 08/20/15 17:44

\begin{tabular}{|c|r|}
\hline COMPOUND NAME & RETEN \\
& TIME \\
\hline Vinyl Chloride-d3 &
\end{tabular}

Lab Sample ID: CCVC 200-92982/12

Date Analyzed: 08/20/15 18:09 Lab File ID: 15379_012.D

Client Sample ID:

\begin{tabular}{|c|c|c|c|c|}
\hline \multirow[t]{2}{*}{ COMPOUND NAME } & \multirow{2}{*}{$\begin{array}{c}\text { RETENTION } \\
\text { TIME }\end{array}$} & \multicolumn{3}{|c|}{ MANUAL INTEGRATION } \\
\hline & & REASON & ANALYST & DATE \\
\hline Dichlorodifluoromethane & 1.53 & Baseline & wilburj & $08 / 21 / 1510: 10$ \\
\hline Bromomethane & 1.99 & Baseline & wilburj & $08 / 21 / 1510: 10$ \\
\hline
\end{tabular}

Analysis Batch Number: 92982

client Sample ID: VHBLKO1

Lab File ID: 15379 011.D

\begin{tabular}{l|ll|c|c|}
\multirow{2}{*}{ TION } & \multicolumn{3}{|c|}{ MANUAL INTEGRATION } \\
\cline { 2 - 5 } & REASON & ANALYST & DATE \\
\hline 1.71 & Baseline & wilburj & $08 / 21 / 1510: 11$ \\
\hline
\end{tabular}

GC Column: DB-624

ID : $0.2(\mathrm{~mm})$ 


\section{TestAmerica}

THE LEADER IN ENVIRONMENTAL TESTING

\section{ANALYTICAL REPORT}

Job Number: 200-29407-1

SDG Number: 29407

Job Description: York (200-29407)

Contract Number: 1E-30401

For:

Argonne National Laboratory

9700 South Cass Avenue

Building 203

Office B-141

Argonne, IL 60439

Attention: Ms. Esther Bowen

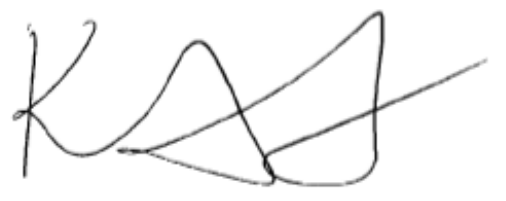

Approved for release. Kathryn A Kelly

\section{Kathryn A Kelly, Project Manager I \\ 30 Community Drive, South Burlington, VT, 05403 \\ kathryn.kelly@testamericainc.com \\ 09/02/2015}

The test results in this report relate only to sample(s) as received by the laboratory. These test results were derived under a quality system that adheres to the requirements of NELAC. Pursuant to NELAC, this report may not be produced in full without written approval from the laboratory 


\section{Table of Contents}

Cover Title Page..$\ldots \ldots \ldots \ldots \ldots \ldots \ldots \ldots \ldots \ldots \ldots \ldots \ldots \ldots$

Sample List ........................... 4

External Chain of Custody ...................... 5

Shipping Documentation . . . . . . . . . . . . . . . 7

Sample Receipt and Log In Check List ..................... 8

Standards Traceability . . . . . . . . . . . . . . . . . 9

Methodology Review .............................. 14

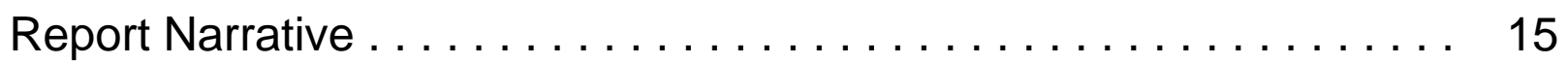

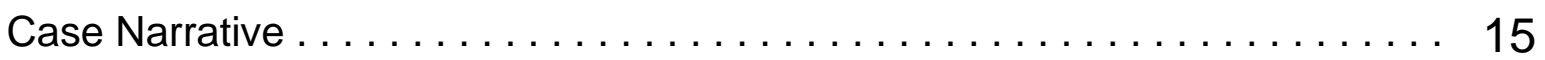

Manual Integration Documentation ....................... 16

Qualifier Definition ................................ 19

QC Summary - SOM01.2 Volatiles-Trace . . . . . . . . . . . . 20

QC Summary - SOM01.2 Volatiles-Trace .................... 20

Deuterated Monitoring Compound Summary $\ldots \ldots \ldots \ldots \ldots \ldots \ldots \ldots \ldots \ldots, 20$

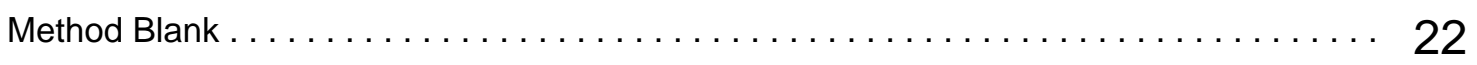

GC/MS Instrument Performance Check $\ldots \ldots \ldots \ldots \ldots \ldots \ldots \ldots \ldots \ldots \ldots \ldots \ldots \ldots$

Internal Standard Area and RT Summary $\ldots \ldots \ldots \ldots \ldots \ldots \ldots \ldots \ldots \ldots \ldots \ldots \ldots \ldots \ldots$

Sample Data - SOM01.2 Volatiles-Trace .............. 26

Sample Data - SOM01.2 Volatiles-Trace ..................... 26

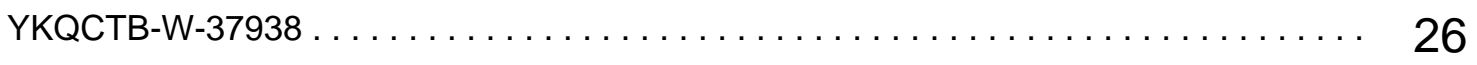

YKTI11-92-W-37936 ......................................... 29

YKTI12-145-W-37943 ................................... 32

Standards - SOM01.2 Volatiles-Trace $\ldots \ldots \ldots \ldots \ldots \ldots \ldots \ldots$

Standards - SOM01.2 Volatiles-Trace $\ldots \ldots \ldots \ldots \ldots \ldots \ldots \ldots \ldots \ldots \ldots$

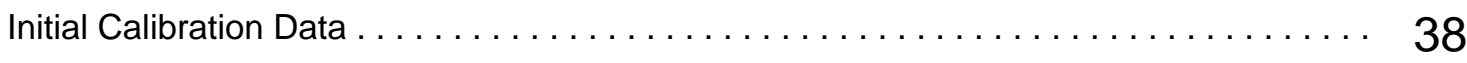

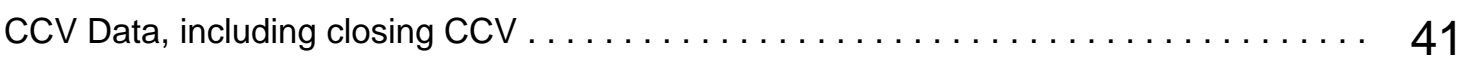




\section{Table of Contents}

Raw Qc Data - SOM01.2 Volatiles-Trace . . . . . . . . . . . . . . 47

Raw Qc Data - SOM01.2 Volatiles-Trace . . . . . . . . . . . . . . . . 47

Raw Qc Data - SOM01.2 Volatiles-Trace . . . . . . . . . . . . . . . . . . . . 47

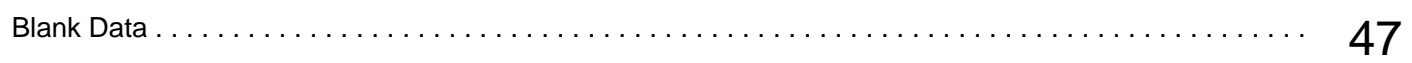




\section{ANALYTICAL DATA PACKAGE FOR THE NEW JERSEY DEPARTMENT OF ENVIRONMENTAL PROTECTION TRENTON, NEW JERSEY 08625}

\begin{tabular}{|c|c|c|c|}
\hline \multicolumn{2}{|l|}{ Agency/Division: } & \multicolumn{2}{|l|}{ Bureau/Office: } \\
\hline Project No: & & Contract No.: & \\
\hline Laboratory Name: & TestAmerica Laboratories & Laboratory Location: & South Burlington, Vermont \\
\hline SDG or Batch No.: & 29407 & NJDEP Certification No.: & VT972 \\
\hline Date of First Sample Receipt: & $08 / 21 / 2015$ & Date of Last Sample Receipt: & $08 / 21 / 2015$ \\
\hline
\end{tabular}

\begin{tabular}{|l|l|l|l|}
\hline \multicolumn{1}{|c|}{ Agency Sample Number } & \multicolumn{1}{c|}{ Laboratory Sample Number } & \multicolumn{1}{c|}{ Sample Location } & \multicolumn{1}{c|}{ Date and Time of Collection } \\
\hline VHBLK01 & $200-29407-4$ & VHBLK01 & $08 / 21 / 201500: 00$ \\
\hline YKQCTB-W-37938 & $200-29407-2$ & YKQCTB-W-37938 & $08 / 17 / 201517: 30$ \\
\hline YKTI11-92-W-37936 & $200-29407-1$ & YKTI11-92-W-37936 & $08 / 18 / 201516: 30$ \\
\hline YKTI12-145-W-37943 & $200-29407-3$ & YKTI12-145-W-37943 & $08 / 20 / 201517: 15$ \\
\hline YKTI12-145-W-37943 DL & $200-29407-3$ & YKTI12-145-W-37943 & $08 / 20 / 201517: 15$ \\
\hline $\begin{array}{l}\text { I certify that this data package is in compliance with the terms and conditions of this contract, both technically and for completeness, for other than the conditions } \\
\text { detailed above. Release of the data contained in the hardcopy data package and in the computer-readable date submitted on disk or electronically has been authorize } \\
\text { by the laboratory manager or his/her designee, as verified by the following signature. }\end{array}$ \\
\hline
\end{tabular}

\section{Laboratory Manager (Typed):}

\section{Date:}

Laboratory Manager (Signature):

Quality Assurance Manager (Typed):

Date:

Quality Assurance Manager (Signature): 


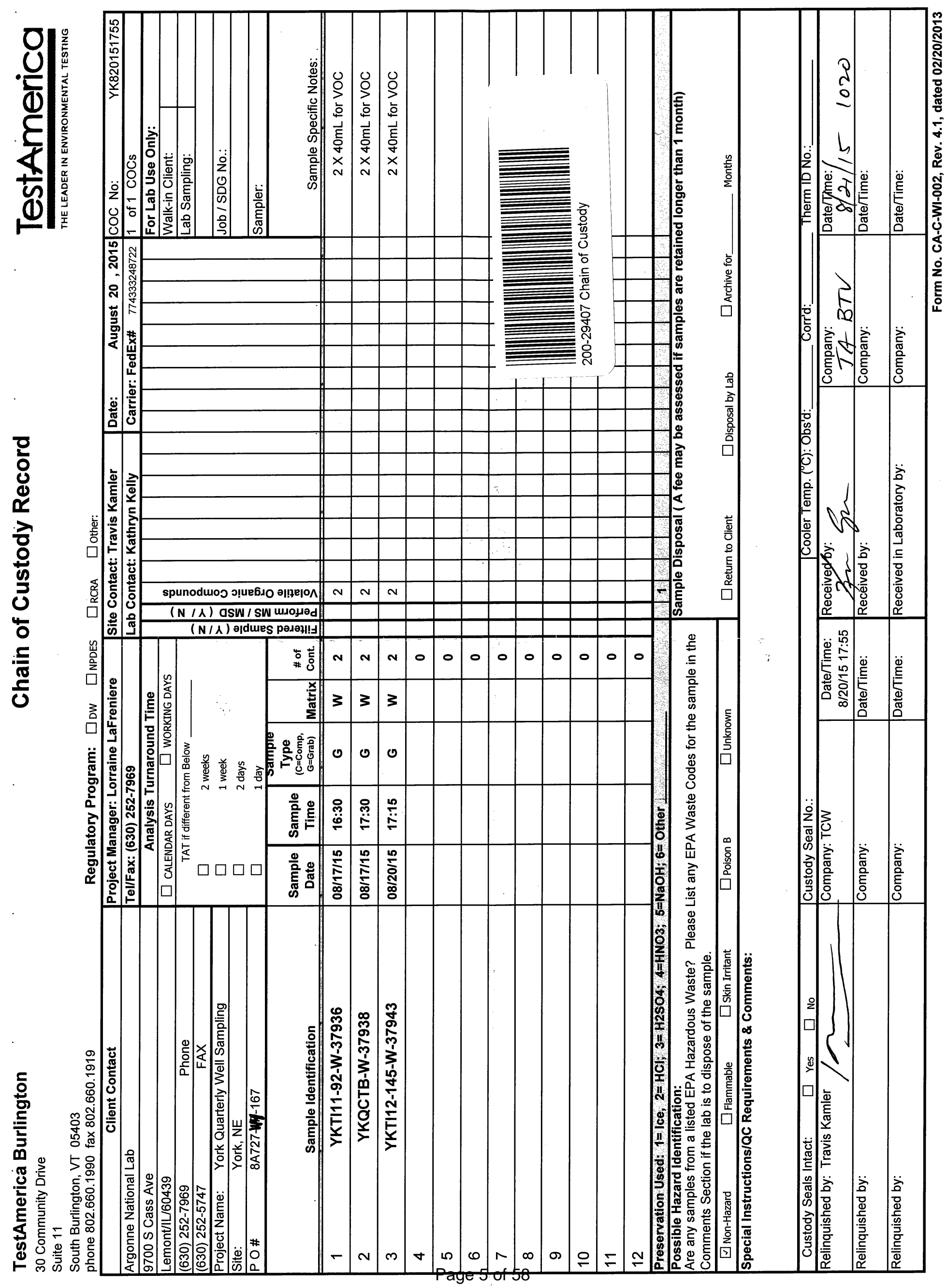




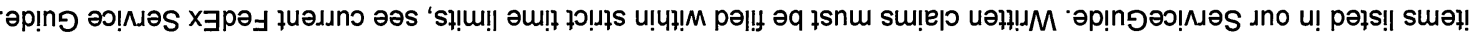

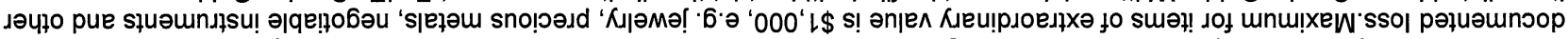

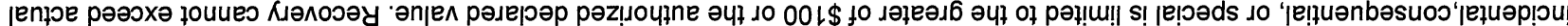

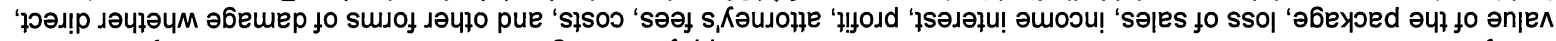

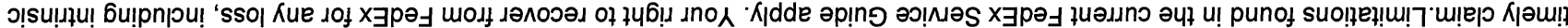

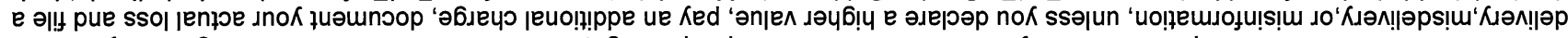

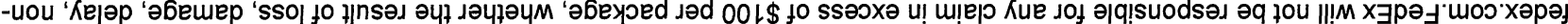
uo ә|qе|!e

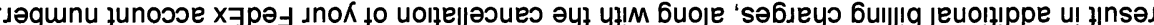

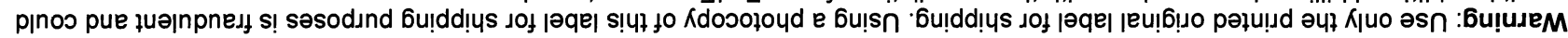

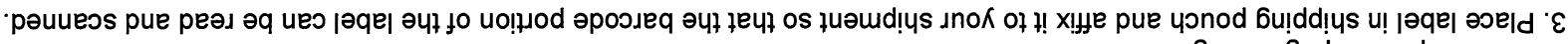

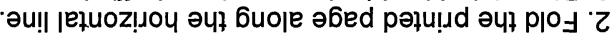

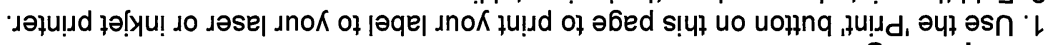

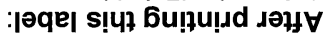

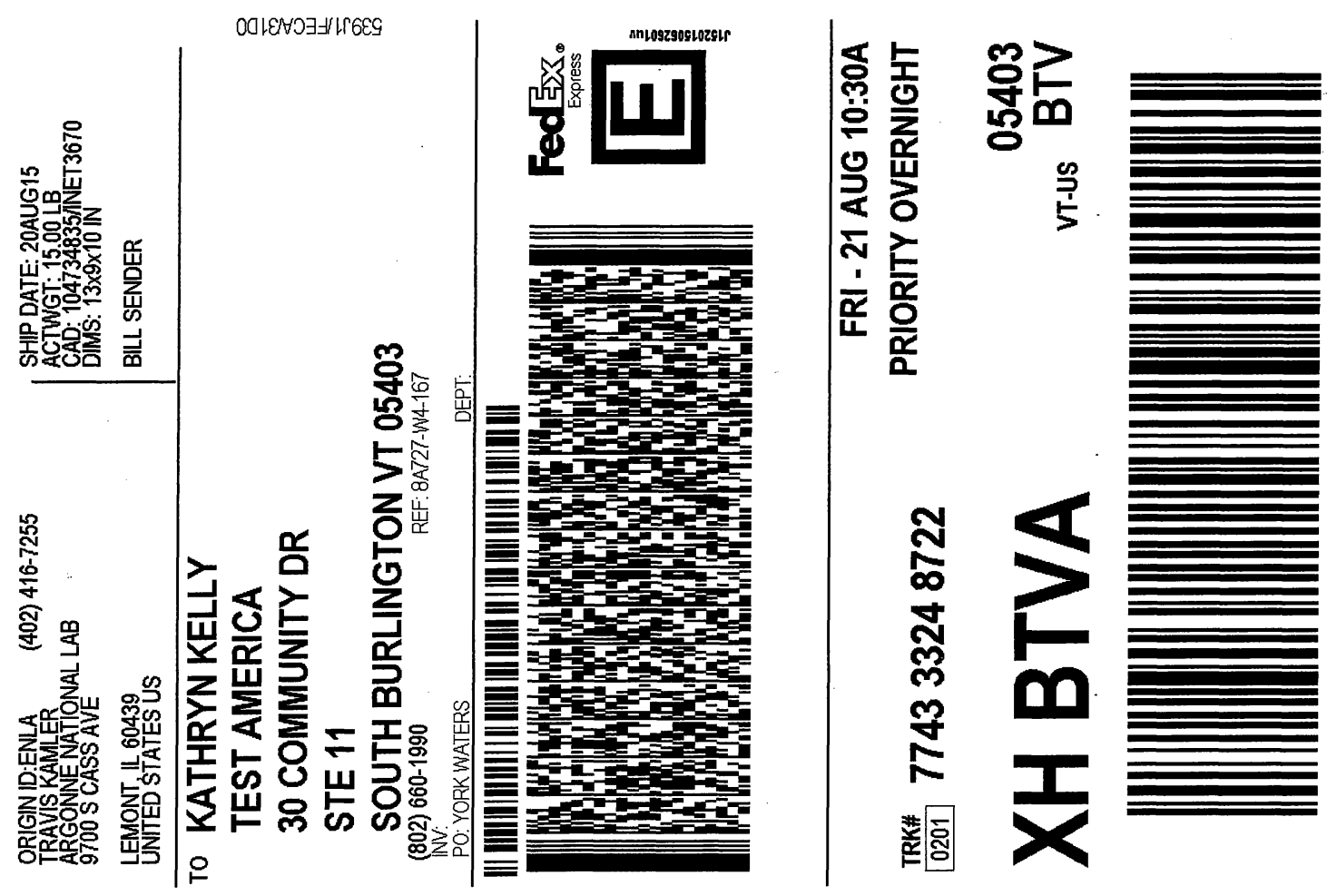




\section{Shipping and Receiving Documents}




\section{Login Sample Receipt Checklist}

Client: Argonne National Laboratory

Job Number: 200-29407-1

SDG Number: 29407

Login Number: 29407

List Source: TestAmerica Burlington

List Number: 1

Creator: Goodrich, Kenneth L

Question

Radioactivity wasn't checked or is $</=$ background as measured by a survey True meter.

The cooler's custody seal, if present, is intact.

Sample custody seals, if present, are intact.

The cooler or samples do not appear to have been compromised or tampered with.

Samples were received on ice.

Cooler Temperature is acceptable.

Cooler Temperature is recorded.

COC is present.

COC is filled out in ink and legible.

$\mathrm{COC}$ is filled out with all pertinent information.

Is the Field Sampler's name present on COC?

There are no discrepancies between the containers received and the COC.

Samples are received within Holding Time.

Sample containers have legible labels.

Containers are not broken or leaking.

Sample collection date/times are provided.

Appropriate sample containers are used.

Sample bottles are completely filled.

Sample Preservation Verified.

There is sufficient vol. for all requested analyses, incl. any requested MS/MSDs

Containers requiring zero headspace have no headspace or bubble is $<6 \mathrm{~mm}$ (1/4").

Multiphasic samples are not present.

Samples do not require splitting or compositing.

Residual Chlorine Checked.

Answer Comment

True

Lab does not accept radioactive samples.

Seal present with no number.

True

True

True

True

True

$3.2^{\circ} \mathrm{C}$

True

True

True

True

True

True

True

True

True

True

N/A

True

True

True

True

True

N/A 
SDG No.: 29407

\begin{tabular}{|c|c|c|c|c|c|c|c|c|}
\hline \multirow[b]{2}{*}{ Reagent ID } & \multirow[b]{2}{*}{$\begin{array}{l}\text { Exp } \\
\text { Date }\end{array}$} & \multirow[b]{2}{*}{$\begin{array}{l}\text { Prep } \\
\text { Date }\end{array}$} & \multirow[b]{2}{*}{$\begin{array}{l}\text { Dilutant } \\
\text { Used }\end{array}$} & \multirow{2}{*}{$\begin{array}{l}\text { Reagent } \\
\text { Final } \\
\text { Volume }\end{array}$} & \multicolumn{2}{|c|}{ Parent Reagent } & \multirow[b]{2}{*}{ Analyte } & \multirow[b]{2}{*}{ Concentration } \\
\hline & & & & & Reagent ID & $\begin{array}{l}\text { Volume } \\
\text { Added }\end{array}$ & & \\
\hline VMBFBW_00018 & $12 / 22 / 15$ & $06 / 23 / 15$ & METHANOL, LOt 147358 & $25 \mathrm{~mL}$ & VMBFBs_00014 & $125 \mathrm{uL}$ & $\mathrm{BFB}$ & $25 \mathrm{ug} / \mathrm{mL}$ \\
\hline .VMBFBS_00014 & $12 / 22 / 15$ & & RESTEK, Lot A0106759 & & \multicolumn{2}{|c|}{ (Purchased Reagent) } & BFB & $5000 \mathrm{ug} / \mathrm{mL}$ \\
\hline \multirow[t]{33}{*}{ VMSOMTRCALW_00093 } & \multirow[t]{33}{*}{$09 / 19 / 15$} & \multirow[t]{33}{*}{$08 / 19 / 15$} & \multirow[t]{33}{*}{ METHANOL, LOt 147358} & \multirow[t]{33}{*}{4000 uL } & VM8260CALbs_00175 & $40 \mathrm{uL}$ & \begin{tabular}{|l|} 
Bromomethane \\
Chloroethane \\
Chloromethane \\
Dichlorodifluoromethane \\
Trichlorofluoromethane \\
Vinyl chloride
\end{tabular} & $\begin{array}{l}20 \mathrm{ug} / \mathrm{mL} \\
20 \mathrm{ug} / \mathrm{mL} \\
20 \mathrm{ug} / \mathrm{mL} \\
20 \mathrm{ug} / \mathrm{mL} \\
20 \mathrm{ug} / \mathrm{mL} \\
20 \mathrm{ug} / \mathrm{mL}\end{array}$ \\
\hline & & & & & \multirow[t]{32}{*}{ VMSOMCALas_00023 } & \multirow[t]{32}{*}{40 uL } & $\begin{array}{l}1,1,1 \text {-Trichloroethane } \\
1,1,2,2 \text {-Tetrachloroethane } \\
1,1,2 \text {-Trichloro-1,2,2-trifluor } \\
\text { oethane }\end{array}$ & $\begin{array}{l}20 \mathrm{ug} / \mathrm{mL} \\
20 \mathrm{ug} / \mathrm{mL} \\
20 \mathrm{ug} / \mathrm{mL} \\
\end{array}$ \\
\hline & & & & & & & $1,1,2$-Trichloroethane & $20 \mathrm{ug} / \mathrm{mL}$ \\
\hline & & & & & & & \begin{tabular}{|l|} 
1,1-Dichloroethane \\
1,1-Dichloroethene
\end{tabular} & $\frac{20 \mathrm{ug} / \mathrm{mL}}{20 \mathrm{ug} / \mathrm{mL}}$ \\
\hline & & & & & & & $1,2,3$-Trichlorobenzene & $20 \mathrm{ug} / \mathrm{mL}$ \\
\hline & & & & & & & 1,2,4-Trichlorobenzene & $20 \mathrm{ug} / \mathrm{mL}$ \\
\hline & & & & & & & 1,2-Dibromo-3-Chloropropane & $\begin{array}{ll}20 \mathrm{ug} / \mathrm{mL} \\
20 \mathrm{ug} / \mathrm{mL}\end{array}$ \\
\hline & & & & & & & $\begin{array}{l}\text { 1,2-Dibromoethane } \\
1,2 \text {-Dichlorobenzene }\end{array}$ & $\begin{array}{ll}20 \mathrm{ug} / \mathrm{mL} \\
20 \mathrm{ug} / \mathrm{mL}\end{array}$ \\
\hline & & & & & & & 1,2-Dichloroethane & $20 \mathrm{ug} / \mathrm{mL}$ \\
\hline & & & & & & & 1,2-Dichloropropane & $20 \mathrm{ug} / \mathrm{mL}$ \\
\hline & & & & & & & $\begin{array}{l}\text { 1,3-Dichlorobenzene } \\
\text { 1,4-Dichlorobenzene }\end{array}$ & $\frac{20 \mathrm{ug} / \mathrm{mL}}{20 \mathrm{ug} / \mathrm{mL}}$ \\
\hline & & & & & & & $\begin{array}{l}\text { 1,4-Dichlorobenzene } \\
\text { Benzene }\end{array}$ & $20 \mathrm{ug} / \mathrm{mL}$ \\
\hline & & & & & & & Bromochloromethane & $20 \mathrm{ug} / \mathrm{mL}$ \\
\hline & & & & & & & Bromodichloromethane & $20 \mathrm{ug} / \mathrm{mL}$ \\
\hline & & & & & & & Bromoform & $20 \mathrm{ug} / \mathrm{mL}$ \\
\hline & & & & & & & Carbon disulfide & $20 \mathrm{ug} / \mathrm{mL}$ \\
\hline & & & & & & & Carbon tetrachloride & $20 \mathrm{ug} / \mathrm{mL}$ \\
\hline & & & & & & & Chlorobenzene & $20 \mathrm{ug} / \mathrm{mL}$ \\
\hline & & & & & & & Chloroform & $20 \mathrm{ug} / \mathrm{mL}$ \\
\hline & & & & & & & cis-1,2-Dichloroethene & $20 \mathrm{ug} / \mathrm{mL}$ \\
\hline & & & & & & & $\begin{array}{l}\text { Cis-1,3-Dichloropropene } \\
\text { Cyclohexane }\end{array}$ & $\frac{20 \mathrm{ug} / \mathrm{mL}}{20 \mathrm{ug} / \mathrm{mL}}$ \\
\hline & & & & & & & Dibromochloromethane & $20 \mathrm{ug} / \mathrm{mL}$ \\
\hline & & & & & & & Ethylbenzene & $20 \mathrm{ug} / \mathrm{mL}$ \\
\hline & & & & & & & Isopropylbenzene & $20 \mathrm{ug} / \mathrm{mL}$ \\
\hline & & & & & & & $\mathrm{m}, \mathrm{p}$-Xylene & $20 \mathrm{ug} / \mathrm{mL}$ \\
\hline & & & & & & & Methyl acetate & $20 \mathrm{ug} / \mathrm{mL}$ \\
\hline & & & & & & & Methyl tert-butyl ether & $20 \mathrm{ug} / \mathrm{mL}$ \\
\hline & & & & & & & Methylcyclohexane & $20 \mathrm{ug} / \mathrm{mL}$ \\
\hline & & & & & & & Methylene Chloride & $20 \mathrm{ug} / \mathrm{mL}$ \\
\hline & & & & & & & o-Xylene & $20 \mathrm{ug} / \mathrm{mL}$ \\
\hline & & & & & & & Styrene & $20 \mathrm{ug} / \mathrm{mL}$ \\
\hline & & & & & & & Tetrachloroethene & $20 \mathrm{ug} / \mathrm{mL}$ \\
\hline & & & & & & & Toluene & $20 \mathrm{ug} / \mathrm{mL}$ \\
\hline
\end{tabular}


SDG No.: 29407

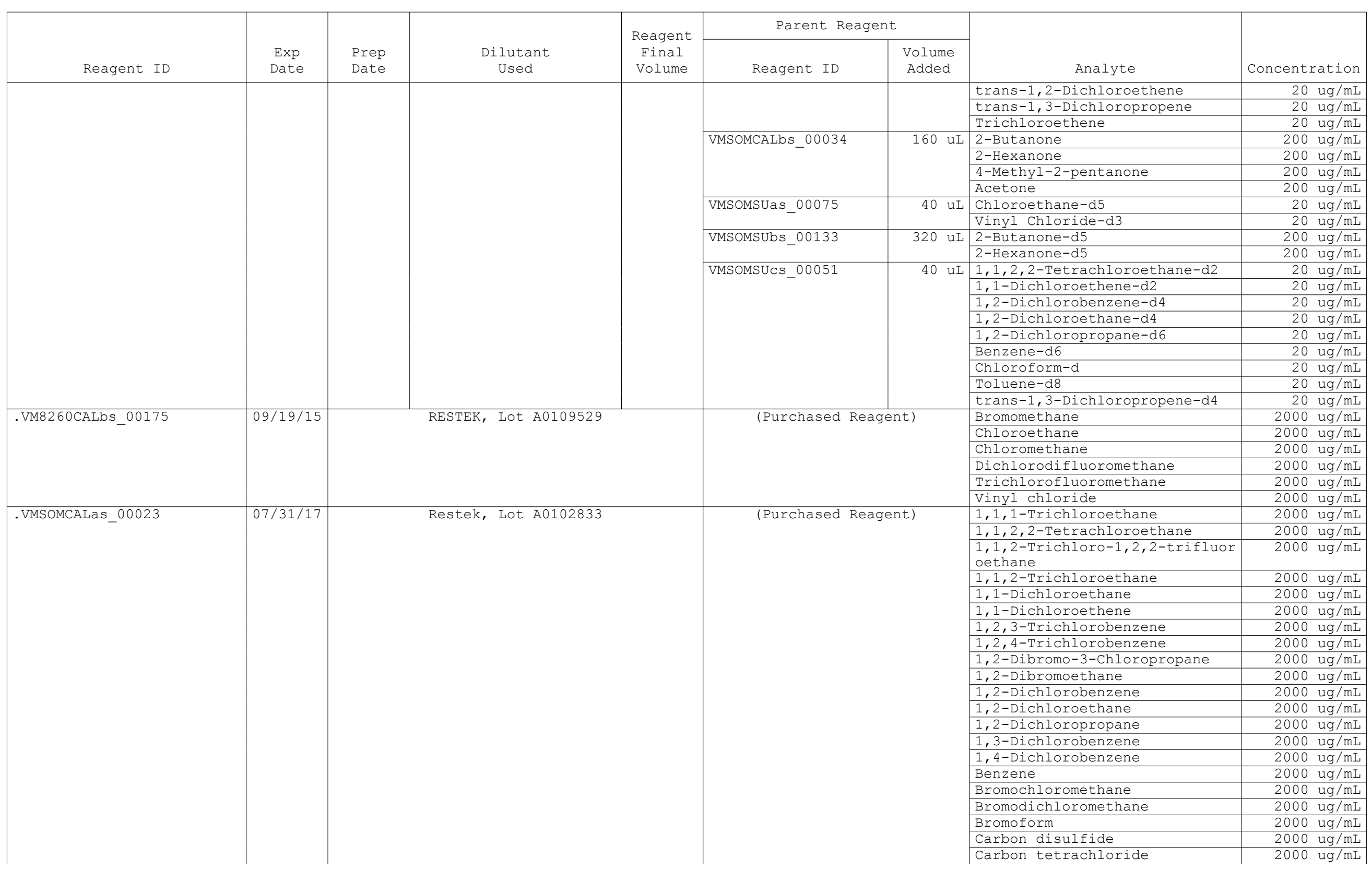


SDG No.: 29407

\begin{tabular}{|c|c|c|c|c|c|c|c|c|c|}
\hline \multirow[b]{2}{*}{ Reagent ID } & \multirow[b]{2}{*}{$\begin{array}{l}\text { Exp } \\
\text { Date }\end{array}$} & \multirow[b]{2}{*}{$\begin{array}{l}\text { Prep } \\
\text { Date }\end{array}$} & \multirow[b]{2}{*}{$\begin{array}{c}\text { Dilutant } \\
\text { Used }\end{array}$} & \multirow{2}{*}{$\begin{array}{c}\text { Reagent } \\
\text { Final } \\
\text { Volume }\end{array}$} & \multicolumn{2}{|c|}{ Parent Reagent } & \multirow[b]{2}{*}{ Analyte } & \multirow{2}{*}{\multicolumn{2}{|c|}{ Concentration }} \\
\hline & & & & & Reagent ID & $\begin{array}{l}\text { Volume } \\
\text { Added }\end{array}$ & & & \\
\hline & & & & & & & Chlorobenzene & 2000 & $\mathrm{ug} / \mathrm{mL}$ \\
\hline & & & & & & & Chloroform & 2000 & $\mathrm{ug} / \mathrm{mL}$ \\
\hline & & & & & & & cis-1,2-Dichloroethene & 2000 & $\mathrm{ug} / \mathrm{mL}$ \\
\hline & & & & & & & cis-1,3-Dichloropropene & 2000 & $\mathrm{ug} / \mathrm{mL}$ \\
\hline & & & & & & & Cyclohexane & 2000 & $\mathrm{ug} / \mathrm{mL}$ \\
\hline & & & & & & & Dibromochloromethane & 2000 & $\mathrm{ug} / \mathrm{mL}$ \\
\hline & & & & & & & Ethylbenzene & 2000 & $\mathrm{ug} / \mathrm{mL}$ \\
\hline & & & & & & & Isopropylbenzene & 2000 & $\mathrm{ug} / \mathrm{mL}$ \\
\hline & & & & & & & m,p-Xylene & 2000 & $\mathrm{ug} / \mathrm{mL}$ \\
\hline & & & & & & & Methyl acetate & 2000 & $\mathrm{ug} / \mathrm{mL}$ \\
\hline & & & & & & & Methyl tert-butyl ether & 2000 & $\mathrm{ug} / \mathrm{mL}$ \\
\hline & & & & & & & Methylcyclohexane & 2000 & $\mathrm{ug} / \mathrm{mL}$ \\
\hline & & & & & & & Methylene Chloride & 2000 & $\mathrm{ug} / \mathrm{mL}$ \\
\hline & & & & & & & o-Xylene & 2000 & $\mathrm{ug} / \mathrm{mL}$ \\
\hline & & & & & & & Styrene & 2000 & $\mathrm{ug} / \mathrm{mL}$ \\
\hline & & & & & & & Tetrachloroethene & 2000 & $\mathrm{ug} / \mathrm{mL}$ \\
\hline & & & & & & & Toluene & 2000 & $\mathrm{ug} / \mathrm{mL}$ \\
\hline & & & & & & & trans-1,2-Dichloroethene & 2000 & $\mathrm{ug} / \mathrm{mL}$ \\
\hline & & & & & & & trans-1,3-Dichloropropene & 2000 & $\mathrm{ug} / \mathrm{mL}$ \\
\hline & & & & & & & Trichloroethene & 2000 & $\mathrm{ug} / \mathrm{mL}$ \\
\hline \multirow[t]{4}{*}{.VMSOMCALbs_00034 } & \multirow[t]{4}{*}{$07 / 16 / 16$} & \multirow{4}{*}{\multicolumn{2}{|c|}{ Restek, Lot A0104748 }} & & \multirow{2}{*}{\multicolumn{2}{|c|}{ (Purchased Reagent) }} & 2-Butanone & 5000 & $\mathrm{ug} / \mathrm{mL}$ \\
\hline & & & & & & & 2-Hexanone & 5000 & $\mathrm{ug} / \mathrm{mL}$ \\
\hline & & & & & & & 4-Methyl-2-pentanone & 5000 & $\mathrm{ug} / \mathrm{mL}$ \\
\hline & & & & & & & Acetone & 5000 & $\mathrm{ug} / \mathrm{mL}$ \\
\hline \multirow[t]{2}{*}{.VMSOMSUas_00075 } & \multirow[t]{2}{*}{$07 / 22 / 17$} & \multirow{2}{*}{\multicolumn{2}{|c|}{ Absolute, Lot 072214}} & & \multicolumn{2}{|c|}{ (Purchased Reagent) } & Chloroethane-d5 & 2000 & $\mathrm{ug} / \mathrm{mL}$ \\
\hline & & & & & & & Vinyl Chloride-d3 & 2000 & $\mathrm{ug} / \mathrm{mL}$ \\
\hline \multirow[t]{2}{*}{.VMSOMSUbs_00133 } & \multirow[t]{2}{*}{$08 / 19 / 16$} & & Absolute, Lot 071515 & & (Purchased $\mathrm{F}$ & nt) & 2-Butanone-d5 & 2500 & $\mathrm{ug} / \mathrm{mL}$ \\
\hline & & & & & & & 2-Hexanone-d5 & 2500 & $\mathrm{ug} / \mathrm{mL}$ \\
\hline .VMSOMSUCS 00051 & $08 / 17 / 16$ & & Absolute, Lot 011614 & & (Purchased $\mathrm{F}$ & nt) & $1,1,2,2$-Tetrachloroethane-d2 & 2000 & $\mathrm{ug} / \mathrm{mL}$ \\
\hline & & & & & & & 1,1-Dichloroethene-d2 & 2000 & $\mathrm{ug} / \mathrm{mL}$ \\
\hline & & & & & & & 1,2-Dichlorobenzene-d4 & 2000 & $\mathrm{ug} / \mathrm{mL}$ \\
\hline & & & & & & & 1,2-Dichloroethane-d4 & 2000 & $\mathrm{ug} / \mathrm{mL}$ \\
\hline & & & & & & & 1,2-Dichloropropane-d6 & 2000 & $\mathrm{ug} / \mathrm{mL}$ \\
\hline & & & & & & & Benzene-d6 & 2000 & $\mathrm{ug} / \mathrm{mL}$ \\
\hline & & & & & & & Chloroform-d & 2000 & $\mathrm{ug} / \mathrm{mL}$ \\
\hline & & & & & & & Toluene-d8 & 2000 & $\mathrm{ug} / \mathrm{mL}$ \\
\hline & & & & & & & trans-1,3-Dichloropropene-d4 & 2000 & $\mathrm{ug} / \mathrm{mL}$ \\
\hline VMSOMTRISw_00103 & $09 / 19 / 15$ & $08 / 19 / 15$ & METHANOL, LOt 147538 & 4400 uL & VMSOMISS_00025 & $35.2 \mathrm{uL}$ & 1,4-Dichlorobenzene-d4 & 20 & $\mathrm{ug} / \mathrm{mL}$ \\
\hline & & & & & & & 1,4-Difluorobenzene & 20 & $\mathrm{ug} / \mathrm{mL}$ \\
\hline & & & & & & & Chlorobenzene-d5 & 20 & $\mathrm{ug} / \mathrm{mL}$ \\
\hline .VMSOMISs_00025 & $06 / 19 / 16$ & & RESTEK, Lot A099377 & & (Purchased $\mathrm{F}$ & nt) & 1,4-Dichlorobenzene-d4 & 2500 & $\mathrm{ug} / \mathrm{mL}$ \\
\hline & & & & & & & 1,4-Difluorobenzene & 2500 & $\mathrm{ug} / \mathrm{mL}$ \\
\hline & & & & & & & Chlorobenzene-d5 & 2500 & $\mathrm{ug} / \mathrm{mL}$ \\
\hline VMSOMTRSUw_00098 & $09 / 19 / 15$ & $08 / 19 / 15$ & METHANOL, Lot 147358 & 4400 uL & VMSOMSUas_00075 & $44 \mathrm{uL}$ & Chloroethane-d5 & 20 & $\mathrm{ug} / \mathrm{mL}$ \\
\hline & & & & & & & Vinyl Chloride-d3 & 20 & $\mathrm{ug} / \mathrm{mL}$ \\
\hline & & & & & VMSOMSUbs_00133 & $352 \mathrm{uL}$ & 2-Butanone-d5 & 200 & $\mathrm{ug} / \mathrm{mL}$ \\
\hline & & & & & & & 2-Hexanone-d5 & 200 & $\mathrm{ug} / \mathrm{mL}$ \\
\hline
\end{tabular}


SDG No.: 29407

\begin{tabular}{|c|c|c|c|c|c|c|c|c|}
\hline \multirow[b]{2}{*}{ Reagent ID } & \multirow[b]{2}{*}{$\begin{array}{l}\text { Exp } \\
\text { Date }\end{array}$} & \multirow[b]{2}{*}{$\begin{array}{l}\text { Prep } \\
\text { Date }\end{array}$} & \multirow[b]{2}{*}{$\begin{array}{c}\text { Dilutant } \\
\text { Used }\end{array}$} & \multirow{2}{*}{$\begin{array}{l}\text { Reagent } \\
\text { Final } \\
\text { Volume }\end{array}$} & \multicolumn{2}{|c|}{ Parent Reagent } & \multirow[b]{2}{*}{ Analyte } & \multirow[b]{2}{*}{ Concentration } \\
\hline & & & & & \multirow{10}{*}{$\begin{array}{r}\text { Reagent ID } \\
\text { VMSOMSUCS_00051 }\end{array}$} & $\begin{array}{l}\text { Volume } \\
\text { Added }\end{array}$ & & \\
\hline & & & & & & $44 \mathrm{uL}$ & $1,1,2,2$-Tetrachloroethane-d2 & $20 \mathrm{ug} / \mathrm{mI}$ \\
\hline & & & & & & & 1,1-Dichloroethene-d2 & $20 \mathrm{ug} / \mathrm{mI}$ \\
\hline & & & & & & & 1,2-Dichlorobenzene-d4 & $20 \mathrm{ug} / \mathrm{mI}$ \\
\hline & & & & & & & 1,2-Dichloroethane-d4 & $20 \mathrm{ug} / \mathrm{mI}$ \\
\hline & & & & & & & 1,2-Dichloropropane-d6 & $20 \mathrm{ug} / \mathrm{mI}$ \\
\hline & & & & & & & Benzene-d6 & $20 \mathrm{ug} / \mathrm{mI}$ \\
\hline & & & & & & & Chloroform-d & $20 \mathrm{ug} / \mathrm{mI}$ \\
\hline & & & & & & & Toluene-d8 & $20 \mathrm{ug} / \mathrm{mI}$ \\
\hline & & & & & & & trans-1,3-Dichloropropene-d4 & $20 \mathrm{ug} / \mathrm{mI}$ \\
\hline \multirow[t]{2}{*}{.VMSOMSUas_00075 } & \multirow[t]{2}{*}{$07 / 22 / 17$} & \multirow{2}{*}{\multicolumn{3}{|c|}{ Absolute, Lot 072214}} & \multirow{2}{*}{\multicolumn{2}{|c|}{ (Purchased Reagent) }} & Chloroethane-d5 & $2000 \mathrm{ug} / \mathrm{mI}$ \\
\hline & & & & & & & Vinyl Chloride-d3 & $2000 \mathrm{ug} / \mathrm{mI}$ \\
\hline \multirow[t]{2}{*}{.VMSOMSUbs_00133 } & \multirow[t]{2}{*}{$08 / 19 / 16$} & \multirow{2}{*}{\multicolumn{3}{|c|}{ Absolute, Lot 071515}} & \multirow{2}{*}{\multicolumn{2}{|c|}{ (Purchased Reagent) }} & 2-Butanone-d5 & $2500 \mathrm{ug} / \mathrm{mI}$ \\
\hline & & & & & & & 2-Hexanone-d5 & $2500 \mathrm{ug} / \mathrm{mI}$ \\
\hline \multirow[t]{9}{*}{.VMSOMSUCS_00051 } & \multirow[t]{9}{*}{$08 / 17 / 16$} & \multirow{9}{*}{\multicolumn{2}{|c|}{ Absolute, Lot 011614}} & & \multirow{9}{*}{\multicolumn{2}{|c|}{ (Purchased Reagent) }} & $1,1,2,2$-Tetrachloroethane-d2 & $2000 \mathrm{ug} / \mathrm{mI}$ \\
\hline & & & & & & & 1,1-Dichloroethene-d2 & $2000 \mathrm{ug} / \mathrm{mI}$ \\
\hline & & & & & & & 1,2-Dichlorobenzene-d4 & $2000 \mathrm{ug} / \mathrm{mI}$ \\
\hline & & & & & & & 1,2-Dichloroethane-d4 & $2000 \mathrm{ug} / \mathrm{mI}$ \\
\hline & & & & & & & 1,2-Dichloropropane-d6 & $2000 \mathrm{ug} / \mathrm{mI}$ \\
\hline & & & & & & & Benzene-d6 & $2000 \mathrm{ug} / \mathrm{mI}$ \\
\hline & & & & & & & Chloroform-d & $2000 \mathrm{ug} / \mathrm{mI}$ \\
\hline & & & & & & & Toluene-d8 & $2000 \mathrm{ug} / \mathrm{mI}$ \\
\hline & & & & & & & trans-1,3-Dichloropropene-d4 & $2000 \mathrm{ug} / \mathrm{mI}$ \\
\hline
\end{tabular}


Lab Name: TestAmerica Burlington

Job No.: 200-29407-1

SDG No.: 29407

\begin{tabular}{|c|l|l|c|c|}
\hline $\begin{array}{c}\text { Reagent } \\
\text { Container }\end{array}$ & \multicolumn{1}{|c|}{$\begin{array}{c}\text { Reagent } \\
\text { ID }\end{array}$} & \multicolumn{1}{c|}{$\begin{array}{c}\text { Reagent } \\
\text { Description }\end{array}$} & $\begin{array}{c}\text { Preparation } \\
\text { Date }\end{array}$ & $\begin{array}{c}\text { Expiration } \\
\text { Date }\end{array}$ \\
\hline 807558 & VMBFBw_00018 & BFB TUNE 25 PPM & $06 / 23 / 2015$ & $12 / 22 / 2015$ \\
\hline 829193 & VMSOMTRISw_00103 & SOM TR ISTD 20 PPM & $08 / 19 / 2015$ & $09 / 19 / 2015$ \\
\hline 829199 & VMSOMTRSUw_00098 & SOM TR DMC 20 PPM & $08 / 19 / 2015$ & $09 / 19 / 2015$ \\
\hline 829202 & VMSOMTRCALw_00093 & SOM TR CAL 20 - 200 PPM & $08 / 19 / 2015$ & $09 / 19 / 2015$ \\
\hline
\end{tabular}




\section{METHODOLOGY SUMMARY}

Laboratory: TestAmerica Laboratories

Location: South Burlington, Vermont
Project No:

SDG No: $\quad 29407$

VOA

Volatile Organics Trace - USEPA CLP SOM01.2 


\section{CASE NARRATIVE}

\section{Client: Argonne National Laboratory}

\section{Project: York (200-29407)}

\section{Report Number: 200-29407-1}

With the exceptions noted as flags or footnotes, standard analytical protocols were followed in the analysis of the samples and no problems were encountered or anomalies observed. In addition all laboratory quality control samples were within established control limits, with any exceptions noted below. Each sample was analyzed to achieve the lowest possible reporting limit within the constraints of the method. In some cases, due to interference or analytes present at high concentrations, samples were diluted. For diluted samples, the reporting limits are adjusted relative to the dilution required.

Calculations are performed before rounding to avoid round-off errors in calculated results.

All holding times were met and proper preservation noted for the methods performed on these samples, unless otherwise detailed in the individual sections below.

\section{RECEIPT}

The samples were received on $08 / 21 / 2015$; the samples arrived in good condition, properly preserved and on ice. The temperature of the cooler at receipt was $3.2^{\circ} \mathrm{C}$.

The client label associated with sample YKTI11-92-W-37936 has a sample collection date of 8/18/15 and the COC has 8/17/15. The information from the chain of custody was used for log-in purposes.

\section{VOLATILE ORGANIC COMPOUNDS - TRACE}

Samples YKTI11-92-W-37936, YKQCTB-W-37938, YKTI12-145-W-37943 and VHBLK01 were analyzed for Volatile Organic Compounds - Trace in accordance with EPA SOW SOM01.2. The samples were analyzed on 08/24/2015.

1,2,3-Trichlorobenzene and 1,2,4-Trichlorobenzene were detected in method blank MB 200-93092/5 at levels that were above the method detection limit but below the reporting limit. The values should be considered estimates, and have been flagged. If the associated sample reported a result above the MDL and/or RL, the result has been flagged. Refer to the QC report for details.

2-Hexanone-d5 failed the surrogate recovery criteria high for YKTI11-92-W-37936. 2-Hexanone-d5 failed the surrogate recovery criteria high for YKTI12-145-W-37943. Refer to the QC report for details.

Sample YKTI12-145-W-37943[4.15X] required dilution prior to analysis. The reporting limits have been adjusted accordingly.

No additional analytical or quality issues were noted, other than those described above or in the Definitions/Glossary page. 
Lab Name: TestAmerica Burlington

Job No.: 200-29407-1

SDG No.: 29407

Instrument ID: CHD.i

Analysis Batch Number: 92921

Lab Sample ID: IC 200-92921/3

Client Sample ID:

Date Analyzed: 08/19/15 15:14

Lab File ID: 15357 003.D

GC Column: DB-624

ID : $0.2(\mathrm{~mm})$

\begin{tabular}{|l|c|c|c|}
\hline \multicolumn{1}{|c|}{ COMPOUND NAME } & RETENTION & \multicolumn{2}{|c|}{ MANUAL INTEGRATION } \\
\cline { 3 - 4 } & TIME & REASON & ANALYST \\
\hline Dichlorodifluoromethane & 1.52 & Baseline & DATE \\
\hline 1,2 -Dibromo-3-Chloropropane & 13.43 & Assign Peak & 08/19/15 $16: 20$ \\
\hline
\end{tabular}

Lab Sample ID: IC 200-92921/4 Client Sample ID:

Date Analyzed: 08/19/15 15:39

Lab File ID: 15357_004.D

GC Column: DB-624

ID $: 0.2(\mathrm{~mm})$

\begin{tabular}{|c|c|c|c|c|}
\hline \multirow[t]{2}{*}{ COMPOUND NAME } & \multirow{2}{*}{$\begin{array}{c}\text { RETENTION } \\
\text { TIME }\end{array}$} & \multicolumn{3}{|c|}{ MANUAL INTEGRATION } \\
\hline & & REASON & ANALYST & DATE \\
\hline Dichlorodifluoromethane & 1.53 & Baseline & wilburj & $08 / 19 / 15 \quad 16: 21$ \\
\hline Bromomethane & 2.00 & Baseline & wilburj & $08 / 19 / 15 \quad 16: 27$ \\
\hline Chloroethane & 2.08 & Baseline & wilburj & $08 / 19 / 1516: 27$ \\
\hline 1,2-Dibromo-3-Chloropropane & 13.44 & Assign Peak & wilburj & $08 / 19 / 15 \quad 16: 27$ \\
\hline
\end{tabular}

Lab Sample ID: ICIS 200-92921/5

Client Sample ID:

Date Analyzed: 08/19/15 16:04

Lab File ID: 15357_005.D

GC Column: DB-624

ID : $0.2(\mathrm{~mm})$

\begin{tabular}{|c|c|c|c|c|}
\hline \multirow[t]{2}{*}{ COMPOUND NAME } & \multirow{2}{*}{$\begin{array}{c}\text { RETENTION } \\
\text { TIME }\end{array}$} & \multicolumn{3}{|c|}{ MANUAL INTEGRATION } \\
\hline & & REASON & ANALYST & DATE \\
\hline Dichlorodifluoromethane & 1.53 & Baseline & wilburj & $08 / 19 / 1516: 30$ \\
\hline Bromomethane & 2.00 & Baseline & wilburj & $08 / 19 / 15 \quad 17: 11$ \\
\hline
\end{tabular}

Lab Sample ID: IC 200-92921/6

Client Sample ID:

Date Analyzed: 08/19/15 16:29

Lab File ID: 15357_006.D

GC Column: DB-624

ID : $0.2(\mathrm{~mm})$

\begin{tabular}{|c|c|c|c|c|}
\hline \multirow[t]{2}{*}{ COMPOUND NAME } & \multirow{2}{*}{$\begin{array}{c}\text { RETENTION } \\
\text { TIME }\end{array}$} & \multicolumn{3}{|c|}{ MANUAL INTEGRATION } \\
\hline & & REASON & ANALYST & DATE \\
\hline Dichlorodifluoromethane & 1.53 & Baseline & wilburj & $08 / 19 / 15 \quad 17: 12$ \\
\hline Bromomethane & 2.00 & Baseline & wilburj & $08 / 19 / 15 \quad 17: 12$ \\
\hline
\end{tabular}

SOM01.2/VOA_Tr 
Lab Name: TestAmerica Burlington

SDG No.: 29407

Instrument ID: CHD.i

Lab Sample ID: IC 200-92921/7

Date Analyzed: 08/19/15 16:54
Job No.: 200-29407-1

Analysis Batch Number: 92921

Client Sample ID:

Lab File ID: 15357_007.D
GC Column: DB-624

ID $: 0.2(\mathrm{~mm})$

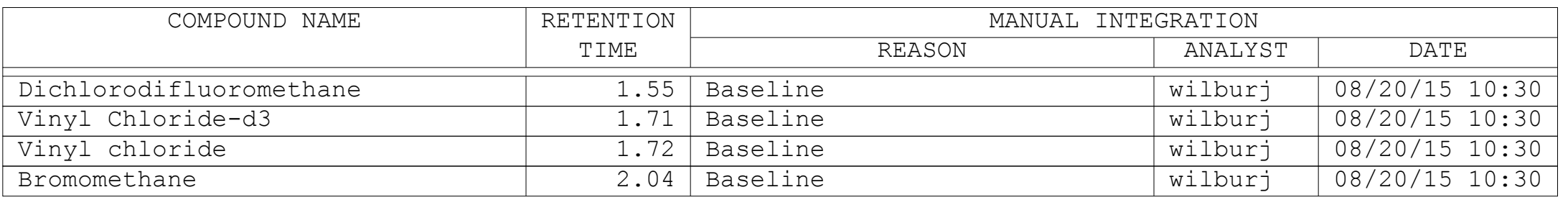


Lab Name: TestAmerica Burlington

SDG No.: 29407

Instrument ID: CHD.i

Lab Sample ID: 200-29407-3 DL

Date Analyzed: 08/24/15 15:01
Job No.: 200-29407-1

Analysis Batch Number: 93092

Client Sample ID: YKTI12-145-W-37943 DL

Lab File ID: 15421 008.D
GC Column: DB-624

ID $: 0.2(\mathrm{~mm})$

\begin{tabular}{|c|c|c|c|c|}
\hline \multirow[t]{2}{*}{ COMPOUND NAME } & RETENTION & \multicolumn{3}{|c|}{ MANUAL INTEGRATION } \\
\hline & TIME & REASON & ANALYST & DATE \\
\hline Methylene Chloride & 3.21 & Baseline & wilburj & $08 / 27 / 15 \quad 17: 17$ \\
\hline
\end{tabular}

Lab Sample ID: CCVC 200-93092/12

Client Sample ID:

Date Analyzed: 08/24/15 16:41

Lab File ID: 15421 012.D

GC Column: DB-624

ID $: 0.2(\mathrm{~mm})$

\begin{tabular}{|l|c|c|c|c|}
\hline \multicolumn{1}{|c|}{ COMPOUND NAME } & RETENTION & \multicolumn{2}{|c|}{ MANUAL INTEGRATION } \\
\cline { 3 - 4 } & TIME & REASON & ANALYST & DATE \\
\hline \hline Dichlorodifluoromethane & 1.53 & Baseline & archern & $08 / 25 / 15$ 09:43 \\
\hline
\end{tabular}




\section{DATA REPORTING QUALIFIERS}

Client: Argonne National Laboratory

Job Number: 200-29407-1

Sdg Number: 29407

\section{Lab Section}

Qualifier

Description

\section{GC/MS VOA}

U

E

J

$\mathrm{J}$

D

$\mathrm{X}$

*

B

N
Analyzed for but not detected.

Compound concentration exceeds the upper level of the calibration range of the instrument for that specific analysis.

Indicates an Estimated Value for TICs

Indicates an estimated value.

Sample was analyzed at a higher dilution factor.

See case narrative notes for explanation of the ' $X$ ' flag

Surrogate is outside acceptance limits.

The analyte was found in an associated blank, as well as in the sample.

This flag indicates the presumptive evidence of a compound. 
Lab Name: TESTAMERICA BURLINGTON

Lab Code: STLV Case No.: YORK Mod. Ref No.: Level: (TRACE or LOW) TRACE
Contract: $8 \mathrm{E}-00302$

SDG No.: 29407

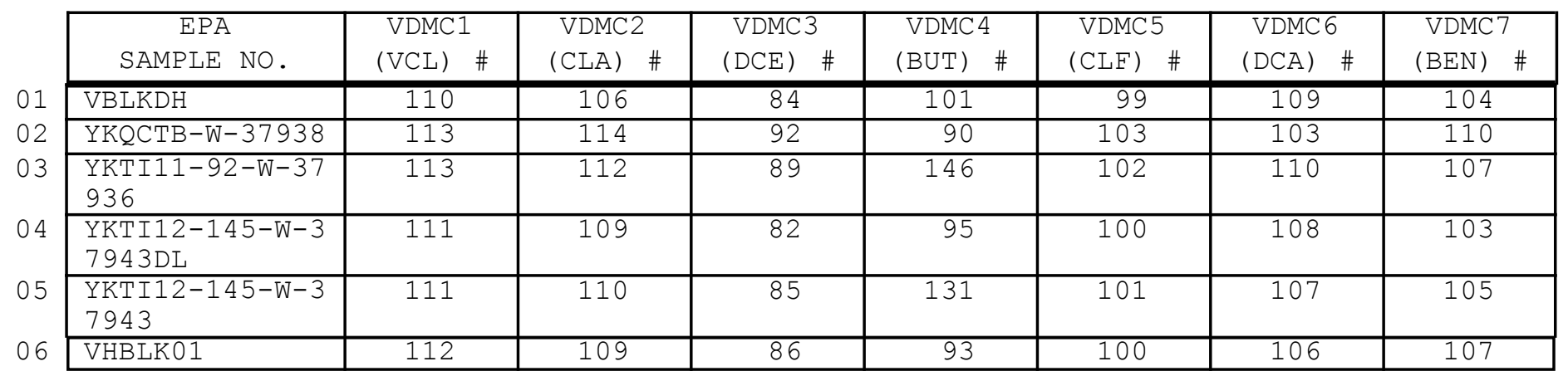

\footnotetext{
VDMC1 (VCL) = Vinyl Chloride-d3

VDMC2 $(\mathrm{CLA})=$ Chloroethane-d5

VDMC3 $(\mathrm{DCE})=1,1-\mathrm{Dichloroethene-d2}$

VDMC4 $\quad(B U T)=2-$ Butanone-d5

VDMC5 $(\mathrm{CLF})=$ Chloroform-d

VDMC $6 \quad(\mathrm{DCA})=1,2-\mathrm{Dich}$ loroethane-d4

$\operatorname{VDMC} 7 \quad(\mathrm{BEN})=$ Benzene-d6
}

$$
\begin{gathered}
\frac{\mathrm{QC} \text { LIMITS }}{(65-131)} \\
(71-131) \\
(55-104) \\
(49-155) \\
(78-121) \\
(78-129) \\
(77-124)
\end{gathered}
$$

\# Column to be used to flag recovery values

* Values outside of contract required QC limits

Page 1 of 1 
Lab Name: TESTAMERICA BURLINGTON

Lab Code: STLV Case No.: YORK Mod. Ref No.:
Contract: $8 \mathrm{E}-00302$

SDG No.: 29407

Level: (TRACE or LOW) TRACE

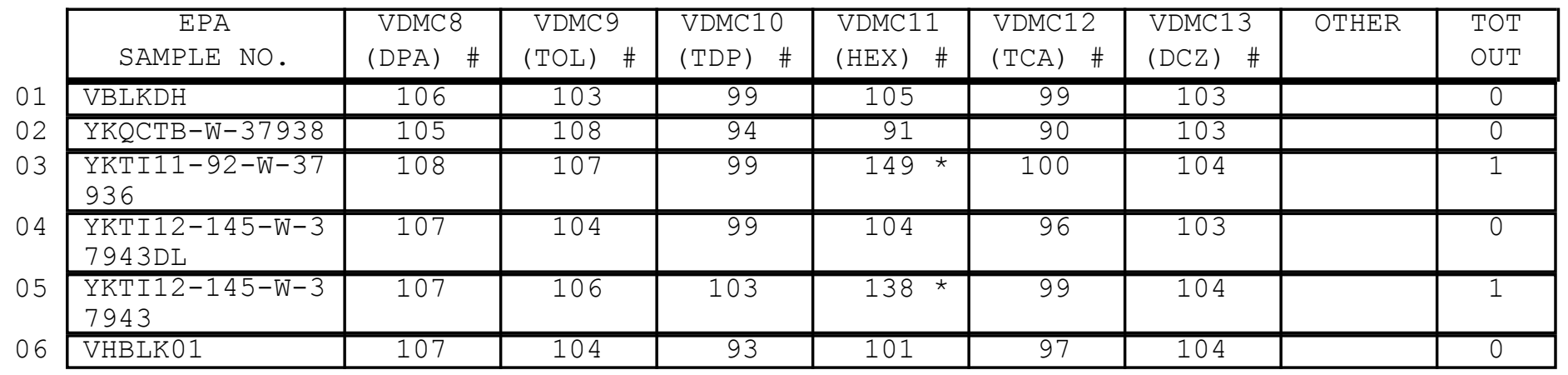

\footnotetext{
$\operatorname{VDMC} 8(\mathrm{DPA})=1,2-\mathrm{Dichloropropane}-\mathrm{d} 6$

VDMC $9 \quad($ TOL $)=$ Toluene-d8

VDMC10 $(\mathrm{TDP})=$ trans $-1,3-\mathrm{Dichloropropene-d4}$

$\operatorname{VDMC1}(\mathrm{HEX})=2-$ Hexanone-d5

VDMC12 $(\mathrm{TCA})=1,1,2,2$-Tetrachloroethane-d2

VDMC13 $(\mathrm{DCZ})=1,2-\mathrm{Dichlorobenzene-d4}$
}

$$
\begin{gathered}
\frac{Q C \text { LIMITS }}{(79-124)} \\
(77-121) \\
(73-121) \\
(28-135) \\
(73-125) \\
(80-131)
\end{gathered}
$$

\# Column to be used to flag recovery values

* Values outside of contract required QC limits

Report 1,4-Dioxane-d8 for Low-Medium VOA analysis only

Page 1 of 1 
$4 A$ - FORM IV VOA

VOLATILE METHOD BLANK SUMMARY
EPA SAMPLE NO.

VBLKDH

Lab Name: TESTAMERICA BURLINGTON

Contract: $8 \mathrm{E}-00302$

Lab Code: STLV Case No.: YORK Mod. Ref No.: SDG No.: 29407

Lab File ID: 15421_005.D

Lab Sample ID: MB 200-93092/5

Instrument ID: CHD.i

Matrix: (SOIL/SED/WATER) Water

Level: (TRACE or LOW/MED) TRACE

Date Analyzed: 08/24/2015

GC Column: DB-624

ID $: 0.20 \quad(\mathrm{~mm})$

Time Analyzed: 1245

Heated Purge: ( $\mathrm{Y} / \mathrm{N}) \mathrm{N}$

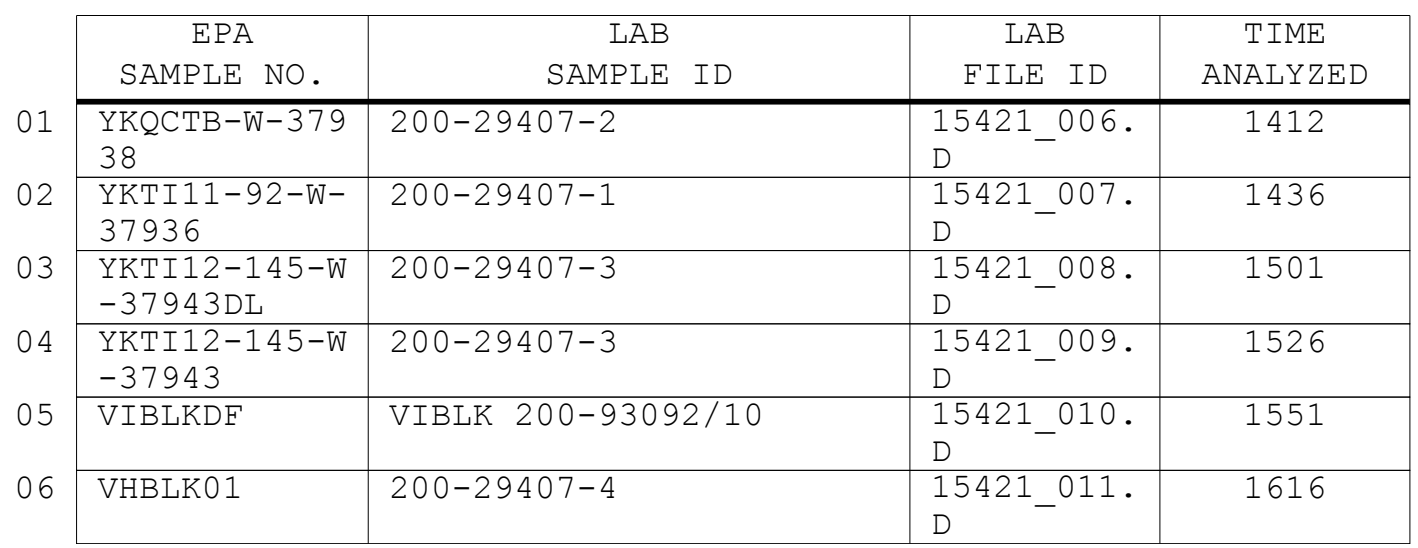

COMMENTS :

Page 1 of 1 
$5 A$ - FORM V VOA

VOLATILE ORGANICS INSTRUMENT

PERFORMANCE CHECK

BROMOFLUOROBENZENE (BFB)
EPA SAMPLE NO.

BFBDE
Lab Name: TESTAMERICA BURLINGTON

Lab Code: STLV Case No.: YORK

Lab File Id: 15357_001.D

Instrument Id: CHD.i

GC Column: DB-624

ID $: 0.20$
Contract: 8E-00302 Mod. Ref No.: SDG No.: 29407

BEB Injection Date: 08/19/2015

BFB Injection Time: 1435 $(\mathrm{mm})$

\begin{tabular}{|r|l|r|}
\hline m/e & ION ABUNDANCE CRITERIA & $\begin{array}{c}\text { RELATIVE } \\
\text { ABUNDANCE }\end{array}$ \\
\hline 50 & $15.0-40.0 \%$ of mass 95 & 20.1 \\
\hline 75 & $30.0-80.0 \%$ of mass 95 & 100 \\
\hline 95 & Base peak, 100\% relative abundance & 6.8 \\
\hline 96 & $5.0-9.0 \%$ of mass 95 & 0.5 \\
\hline 173 & Less than 2.0\% of mass 174 & $0.6) 1$ \\
\hline 174 & $50.0-120 \%$ of mass 95 & 79.0 \\
\hline 175 & $5.0-9.0 \%$ of mass 174 0 \\
\hline 176 & $95.0-101 \%$ of mass 174 & $(7.4) 1$ \\
\hline 177 & $5.0-9.0 \%$ of mass 176 & $(95.7) 1$ \\
\hline
\end{tabular}

1 - Value is omass 1742 - Value is mass 176

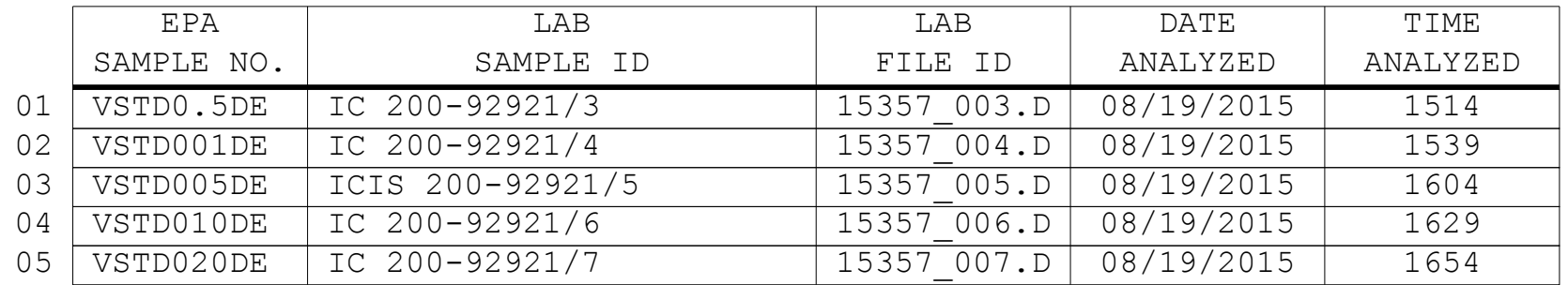


$5 A$ - FORM V VOA

VOLATILE ORGANICS INSTRUMENT

PERFORMANCE CHECK

BROMOFLUOROBENZENE (BFB)
EPA SAMPLE NO.

$\mathrm{BFBDH}$
Lab Name: TESTAMERICA BURLINGTON

Lab Code: STLV

Case No.: YORK

Lab File Id: 15421_001.D

Instrument Id: CHD.i

GC Column: DB-624

ID $: 0.20$
Contract: $8 \mathrm{E}-00302$ Mod. Ref No.:

SDG No.: 29407

BEB Injection Date: 08/24/2015

BFB Injection Time: 1109 (mm)

\begin{tabular}{|c|c|c|}
\hline $\mathrm{m} / \mathrm{e}$ & ION ABUNDANCE CRITERIA & $\begin{array}{l}\text { \% RELATIVE } \\
\text { ABUNDANCE }\end{array}$ \\
\hline 50 & $15.0-40.0 \%$ of mass 95 & 20.1 \\
\hline 75 & $30.0-80.0 \%$ of mass 95 & 51.0 \\
\hline 95 & Base peak, $100 \%$ relative abundance & 100 \\
\hline 96 & $5.0-9.0 \%$ of mass 95 & 6.3 \\
\hline 173 & Less than 2.0\% of mass 174 & $(0.8) 1$ \\
\hline 174 & $50.0-120 \%$ of mass 95 & 73.9 \\
\hline 175 & $5.0-9.0 \%$ of mass 174 & $7.0) 1$ \\
\hline 176 & $95.0-101 \%$ of mass 174 & $72.2(97.6) 1$ \\
\hline 177 & $5.0-9.0 \%$ of mass 176 & $4.6(6.4) 2$ \\
\hline
\end{tabular}

1 - Value is omass 174

2 - Value is omass 176

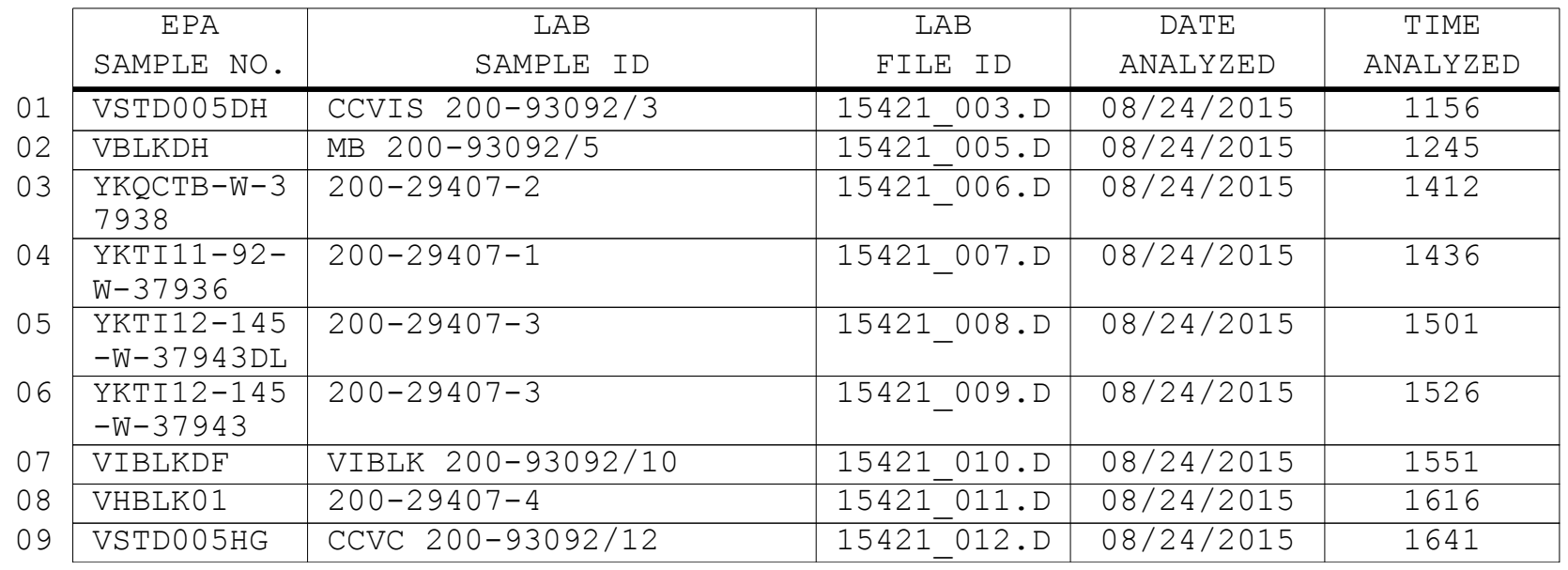

Page 1 of 1 


\section{$8 A$ - FORM VIII VOA}

VOLATILE INTERNAL STANDARD AREA AND RETENTION TIME SUMMARY

Lab Name: TESTAMERICA BURLINGTON

Contract: $8 \mathrm{E}-00302$

Lab Code: STLV Case No.: YORK Mod. Ref No.:

SDG No.: 29407

GC Column: DB-624

ID: 0.20 (mm) Init. Calib. Date (s) : 08/19/2015 08/19/2015

EPA Sample No.(VSTD\#\#\#\#\#) : VSTD005DH

Lab File ID (Standard): 15421_003.D

Date Analyzed: $08 / 24 / 2015$

Instrument ID: CHD.i

Time Analyzed: 1156

Heated Purge: ( $\mathrm{Y} / \mathrm{N}) \mathrm{N}$

\begin{tabular}{|l|l|c|c|c|c|c|c|}
\hline & $\begin{array}{c}\text { IS1 (CBZ) } \\
\text { AREA }\end{array}$ & RT \# & $\begin{array}{c}\text { IS2 } \\
\text { AREA }\end{array}$ & RT & IS3 (DCB) & AREA & RT \\
\hline
\end{tabular}

IS1 $(\mathrm{CBZ})=$ Chlorobenzene-d5

IS2 $(\mathrm{DFB})=1,4-\mathrm{Difluorobenzene}$

IS3 $(\mathrm{DCB})=1,4-\mathrm{Dichlorobenzene-d4}$

AREA UPPER LIMIT $=140 \%$ (Trace Volatiles) of internal standard area AREA LOWER LIMIT $=60 \%$ (Trace Volatiles) of internal standard area RT UPPER LIMIT $=+0.33$ (Trace Volatiles) minutes of internal standard RT RT LOWER LIMIT $=-0.33$ (Trace Volatiles) minutes of internal standard RT

\# Column used to flag values outside contract required QC limits with an asterisk. Page 1 of 1 
Lab Name: TESTAMERICA BURLINGTON

Contract: $8 \mathrm{E}-00302$

Lab Code: STLV

Case No.: YORK Mod. Ref No.:

SDG No.: 29407

Matrix: (SOIL/SED/WATER) Water

Sample wt/vol: 25.0 $(\mathrm{g} / \mathrm{mL}) \mathrm{mL}$

Level: (TRACE/LOW/MED) TRACE

\% Moisture: not dec.

GC Column: DB-624 ID: $0.20 \quad(\mathrm{~mm})$

Soil Extract Volume: (uL) $(\mathrm{mL})$

Purge Volume: 25.0
Lab Sample ID: 200-29407-2

Lab File ID: 15421_006.D

Date Received: 08/21/2015

Date Analyzed: 08/24/2015

Dilution Factor: 1.0

Soil Aliquot Volume: (uL)

\begin{tabular}{|c|c|c|c|}
\hline CAS NO. & COMPOUND & $\begin{array}{l}\text { CONCENTRATION UNITS: } \\
(\mathrm{ug} / \mathrm{L} \text { or } \mathrm{ug} / \mathrm{kg}) \mathrm{ug} / \mathrm{L}\end{array}$ & q \\
\hline $75-71-8$ & Dichlorodifluoromethane & 1.0 & $\mathrm{U}$ \\
\hline $74-87-3$ & Chloromethane & 0.087 & $\mathrm{~J}$ \\
\hline $75-01-4$ & Vinyl chloride & 1.0 & $\mathrm{U}$ \\
\hline $74-83-9$ & Bromomethane & 0.12 & $\mathrm{~J}$ \\
\hline $75-00-3$ & Chloroethane & 1.0 & $\mathrm{U}$ \\
\hline $75-69-4$ & Trichlorofluoromethane & 1.0 & $\mathrm{U}$ \\
\hline $75-35-4$ & 1,1-Dichloroethene & 1.0 & $\mathrm{U}$ \\
\hline $76-13-1$ & $1,1,2$-Trichloro-1,2,2-trifluoroethane & 1.0 & $\mathrm{U}$ \\
\hline $67-64-1$ & Acetone & 16 & \\
\hline $75-15-0$ & Carbon disulfide & 0.036 & $\mathrm{~J}$ \\
\hline $79-20-9$ & Methyl acetate & 1.0 & $\mathrm{U}$ \\
\hline $75-09-2$ & Methylene Chloride & 1.0 & $\mathrm{U}$ \\
\hline $156-60-5$ & trans-1,2-Dichloroethene & 1.0 & $\mathrm{U}$ \\
\hline $1634-04-4$ & Methyl tert-butyl ether & 1.0 & $\mathrm{U}$ \\
\hline $75-34-3$ & 1,1-Dichloroethane & 1.0 & $\mathrm{U}$ \\
\hline $156-59-2$ & cis-1,2-Dichloroethene & 1.0 & $\mathrm{U}$ \\
\hline $78-93-3$ & 2-Butanone & 1.4 & $\mathrm{~J}$ \\
\hline $74-97-5$ & Bromochloromethane & 1.0 & $\mathrm{U}$ \\
\hline $67-66-3$ & Chloroform & 1.0 & $\mathrm{U}$ \\
\hline $71-55-6$ & 1,1,1-Trichloroethane & 1.0 & $\mathrm{U}$ \\
\hline $110-82-7$ & Cyclohexane & 1.0 & $\mathrm{U}$ \\
\hline $56-23-5$ & Carbon tetrachloride & 1.0 & $\mathrm{U}$ \\
\hline $71-43-2$ & Benzene & 1.0 & $\mathrm{U}$ \\
\hline $107-06-2$ & 1,2-Dichloroethane & 1.0 & $\mathrm{U}$ \\
\hline
\end{tabular}

Report 1,4-Dioxane for Low-Medium VOA analysis only 
Lab Name: TESTAMERICA BURLINGTON

Contract: $8 \mathrm{E}-00302$

Lab Code: STLV

Case No.: YORK

Mod. Ref No.:

SDG No.: 29407

Matrix: (SOIL/SED/WATER) Water

Sample wt/vol: 25.0 $(\mathrm{g} / \mathrm{mL}) \mathrm{mL}$

Level: (TRACE/LOW/MED) TRACE

\% Moisture: not dec.

GC Column: DB-624 ID: $0.20 \quad(\mathrm{~mm})$

Soil Extract Volume: (uL) $(\mathrm{mL})$

Purge Volume: 25.0 (mI)
Lab Sample ID: 200-29407-2

Lab File ID: 15421_006.D

Date Received: 08/21/2015

Date Analyzed: 08/24/2015

Dilution Factor: 1.0

Soil Aliquot Volume: (uL)

\begin{tabular}{|c|c|c|c|}
\hline CAS NO. & COMPOUND & $\begin{array}{l}\text { CONCENTRATION UNITS: } \\
(\mathrm{ug} / \mathrm{L} \text { or } \mathrm{ug} / \mathrm{kg}) \mathrm{ug} / \mathrm{L}\end{array}$ & Q \\
\hline $79-01-6$ & Trichloroethene & 1.0 & $\mathrm{U}$ \\
\hline $108-87-2$ & Methylcyclohexane & 1.0 & $\mathrm{U}$ \\
\hline $78-87-5$ & 1,2-Dichloropropane & 1.0 & $\mathrm{U}$ \\
\hline $75-27-4$ & Bromodichloromethane & 1.0 & $\mathrm{U}$ \\
\hline $10061-01-5$ & cis-1,3-Dichloropropene & 1.0 & U \\
\hline $108-10-1$ & 4-Methyl-2-pentanone & 1.0 & $\mathrm{U}$ \\
\hline $108-88-3$ & Toluene & 0.042 & $\mathrm{~J}$ \\
\hline $10061-02-6$ & trans-1,3-Dichloropropene & 1.0 & $\mathrm{U}$ \\
\hline $79-00-5$ & $1,1,2-$ Trichloroethane & 1.0 & $\mathrm{U}$ \\
\hline $127-18-4$ & Tetrachloroethene & 0.026 & $\mathrm{~J}$ \\
\hline $591-78-6$ & 2-Hexanone & 1.0 & $\mathrm{U}$ \\
\hline $124-48-1$ & Dibromochloromethane & 1.0 & $\mathrm{U}$ \\
\hline $106-93-4$ & 1,2-Dibromoethane & 1.0 & $\mathrm{U}$ \\
\hline $108-90-7$ & Chlorobenzene & 1.0 & $\mathrm{U}$ \\
\hline $100-41-4$ & Ethylbenzene & 0.0096 & $\mathrm{~J}$ \\
\hline $95-47-6$ & o-Xylene & 0.010 & $\mathrm{~J}$ \\
\hline $179601-23-1$ & m,p-xylene & 0.021 & $\mathrm{~J}$ \\
\hline $100-42-5$ & Styrene & 0.015 & $\mathrm{~J}$ \\
\hline $75-25-2$ & Bromoform & 1.0 & $\mathrm{U}$ \\
\hline $98-82-8$ & Isopropylbenzene & 1.0 & $\mathrm{U}$ \\
\hline $79-34-5$ & $1,1,2,2$-Tetrachloroethane & 1.0 & $\mathrm{U}$ \\
\hline $541-73-1$ & 1,3 -Dichlorobenzene & 0.013 & $\mathrm{~J}$ \\
\hline $106-46-7$ & 1,4-Dichlorobenzene & 1.0 & $\mathrm{U}$ \\
\hline $95-50-1$ & 1,2-Dichlorobenzene & 1.0 & $\mathrm{U}$ \\
\hline $96-12-8$ & 1,2-Dibromo-3-Chloropropane & 1.0 & $\mathrm{U}$ \\
\hline $120-82-1$ & $1,2,4$-Trichlorobenzene & 0.028 & $\mathrm{~J} B$ \\
\hline $87-61-6$ & $1,2,3$-Trichlorobenzene & 0.032 & $\mathrm{~J} B$ \\
\hline
\end{tabular}


Lab Name: TESTAMERICA BURLINGTON

Contract: $8 \mathrm{E}-00302$

Lab Code: STLV Case No.: YORK Mod. Ref No.: SDG No.: 29407

Matrix: (SOIL/SED/WATER) Water

Sample wt/vol: 25.0 $(\mathrm{g} / \mathrm{mL}) \mathrm{mL}$

Level: (TRACE or LOW/MED) TRACE

․ Moisture: not dec.

GC Column: DB-624 ID $: 0.20 \quad(\mathrm{~mm})$

Soil Extract Volume: (uL)
Lab Sample ID: 200-29407-2

Lab File ID: 15421_006.D

Date Received: 08/21/2015

Date Analyzed: 08/24/2015

Dilution Factor: 1.0

Soil Aliquot Volume: (uL) Purge Volume: 25.0 $(\mathrm{mL})$ CONCENTRATION UNITS: (ug/L or ug/kg) ug/L

\begin{tabular}{|c|c|c|c|c|}
\hline CAS NUMBER & COMPOUND NAME & RT & EST. CONC. & $Q$ \\
\hline 01 & Unknown & 7.29 & 2.8 & $J B X$ \\
\hline $541-05-9$ & Cyclotrisiloxane, hexamethyl- & 8.22 & 0.73 & $\mathrm{~J} N$ \\
\hline 03 & Unknown & 11.04 & 1.3 & $\mathrm{~J} \mathrm{~B}$ \\
\hline E9667961 & Total Alkanes & $\mathrm{N} / \mathrm{A}$ & & \\
\hline
\end{tabular}


Lab Name: TESTAMERICA BURLINGTON

Contract: $8 \mathrm{E}-00302$

Lab Code: STLV

Case No.: YORK Mod. Ref No.:

SDG No.: 29407

Matrix: (SOIL/SED/WATER) Water

Sample wt/vol: 25.0 $(\mathrm{g} / \mathrm{mL}) \mathrm{mL}$

Level: (TRACE/LOW/MED) TRACE

\% Moisture: not dec.

GC Column: DB-624 ID: $0.20 \quad(\mathrm{~mm})$

Soil Extract Volume: (uL) $(\mathrm{mL})$

Purge Volume: 25.0
Lab Sample ID: 200-29407-1

Lab File ID: 15421_007.D

Date Received: 08/21/2015

Date Analyzed: 08/24/2015

Dilution Factor: 1.0

Soil Aliquot Volume: (uL)

\begin{tabular}{|c|c|c|c|}
\hline CAS NO. & COMPOUND & $\begin{array}{l}\text { CONCENTRATION UNITS: } \\
(\mathrm{ug} / \mathrm{L} \text { or } \mathrm{ug} / \mathrm{kg}) \mathrm{ug} / \mathrm{L}\end{array}$ & q \\
\hline $75-71-8$ & Dichlorodifluoromethane & 1.0 & $\mathrm{U}$ \\
\hline $74-87-3$ & Chloromethane & 1.0 & $\mathrm{U}$ \\
\hline $75-01-4$ & Vinyl chloride & 1.0 & $\mathrm{U}$ \\
\hline $74-83-9$ & Bromomethane & 1.0 & $\mathrm{U}$ \\
\hline $75-00-3$ & Chloroethane & 1.0 & $\mathrm{U}$ \\
\hline $75-69-4$ & Trichlorofluoromethane & 1.0 & $\mathrm{U}$ \\
\hline $75-35-4$ & 1,1-Dichloroethene & 1.0 & $\mathrm{U}$ \\
\hline $76-13-1$ & $1,1,2$-Trichloro-1,2,2-trifluoroethane & 1.0 & $\mathrm{U}$ \\
\hline $67-64-1$ & Acetone & 1.3 & $\mathrm{~J}$ \\
\hline $75-15-0$ & Carbon disulfide & 0.053 & $\mathrm{~J}$ \\
\hline $79-20-9$ & Methyl acetate & 1.0 & $\mathrm{U}$ \\
\hline $75-09-2$ & Methylene Chloride & 1.0 & $\mathrm{U}$ \\
\hline $156-60-5$ & trans-1,2-Dichloroethene & 1.0 & $\mathrm{U}$ \\
\hline $1634-04-4$ & Methyl tert-butyl ether & 1.0 & $\mathrm{U}$ \\
\hline $75-34-3$ & 1,1-Dichloroethane & 1.0 & $\mathrm{U}$ \\
\hline $156-59-2$ & cis-1,2-Dichloroethene & 1.0 & $\mathrm{U}$ \\
\hline $78-93-3$ & 2-Butanone & 5.0 & $\mathrm{U}$ \\
\hline $74-97-5$ & Bromochloromethane & 1.0 & $\mathrm{U}$ \\
\hline $67-66-3$ & Chloroform & 1.0 & $\mathrm{U}$ \\
\hline $71-55-6$ & $1,1,1$-Trichloroethane & 1.0 & $\mathrm{U}$ \\
\hline $110-82-7$ & Cyclohexane & 1.0 & $\mathrm{U}$ \\
\hline $56-23-5$ & Carbon tetrachloride & 0.060 & $\mathrm{~J}$ \\
\hline $71-43-2$ & Benzene & 1.0 & $\mathrm{U}$ \\
\hline $107-06-2$ & 1,2-Dichloroethane & 1.0 & $\mathrm{U}$ \\
\hline
\end{tabular}

Report 1,4-Dioxane for Low-Medium VOA analysis only 
Lab Name: TESTAMERICA BURLINGTON

Contract: 8E-00302

Lab Code: STLV

Case No.: YORK Mod. Ref No.:

SDG No.: 29407

Matrix: (SOIL/SED/WATER) Water

Sample wt/vol: 25.0 $(\mathrm{g} / \mathrm{mL}) \mathrm{mL}$

Level: (TRACE/LOW/MED) TRACE

\% Moisture: not dec.

GC Column: DB-624 ID: $0.20 \quad(\mathrm{~mm})$

Soil Extract Volume: (uL) $(\mathrm{mL})$

Purge Volume: 25.0
Lab Sample ID: 200-29407-1

Lab File ID: 15421_007.D

Date Received: 08/21/2015

Date Analyzed: 08/24/2015

Dilution Factor: 1.0

Soil Aliquot Volume: (uL) (mI)

\begin{tabular}{|c|c|c|c|}
\hline CAS NO. & COMPOUND & $\begin{array}{l}\text { CONCENTRATION UNITS: } \\
(\mathrm{ug} / \mathrm{L} \text { or } \mathrm{ug} / \mathrm{kg}) \mathrm{ug} / \mathrm{L}\end{array}$ & Q \\
\hline $79-01-6$ & Trichloroethene & 1.0 & $\mathrm{U}$ \\
\hline $108-87-2$ & Methylcyclohexane & 1.0 & $\mathrm{U}$ \\
\hline $78-87-5$ & 1,2-Dichloropropane & 1.0 & $\mathrm{U}$ \\
\hline $75-27-4$ & Bromodichloromethane & 1.0 & $\mathrm{U}$ \\
\hline $10061-01-5$ & cis-1,3-Dichloropropene & 1.0 & $\mathrm{U}$ \\
\hline $108-10-1$ & 4-Methyl-2-pentanone & 1.0 & $\mathrm{U}$ \\
\hline $108-88-3$ & Toluene & 0.045 & $\mathrm{~J}$ \\
\hline $10061-02-6$ & trans-1,3-Dichloropropene & 1.0 & $\mathrm{U}$ \\
\hline $79-00-5$ & $1,1,2-T r i c h l o r o e t h a n e$ & 1.0 & $\mathrm{U}$ \\
\hline $127-18-4$ & Tetrachloroethene & 0.024 & $\mathrm{~J}$ \\
\hline $591-78-6$ & 2-Hexanone & 1.0 & $\mathrm{U}$ \\
\hline $124-48-1$ & Dibromochloromethane & 1.0 & $\mathrm{U}$ \\
\hline $106-93-4$ & 1,2-Dibromoethane & 1.0 & $\mathrm{U}$ \\
\hline $108-90-7$ & Chlorobenzene & 1.0 & $\mathrm{U}$ \\
\hline $100-41-4$ & Ethylbenzene & 0.0073 & $\mathrm{~J}$ \\
\hline $95-47-6$ & o-Xylene & 0.0056 & $\mathrm{~J}$ \\
\hline $179601-23-1$ & m,p-Xylene & 0.021 & $\mathrm{~J}$ \\
\hline $100-42-5$ & styrene & 0.0049 & $\mathrm{~J}$ \\
\hline $75-25-2$ & Bromoform & 1.0 & $\mathrm{U}$ \\
\hline $98-82-8$ & Isopropylbenzene & 1.0 & $\mathrm{U}$ \\
\hline $79-34-5$ & $1,1,2,2$-Tetrachloroethane & 1.0 & $\mathrm{U}$ \\
\hline $541-73-1$ & 1,3-Dichlorobenzene & 1.0 & $\mathrm{U}$ \\
\hline $106-46-7$ & 1,4-Dichlorobenzene & 1.0 & $\mathrm{U}$ \\
\hline $95-50-1$ & $1,2-$ Dichlorobenzene & 1.0 & $\mathrm{U}$ \\
\hline $96-12-8$ & 1,2-Dibromo-3-Chloropropane & 1.0 & $\mathrm{U}$ \\
\hline $120-82-1$ & $1,2,4$-Trichlorobenzene & 0.010 & $\mathrm{~J} B$ \\
\hline $87-61-6$ & $1,2,3$-Trichlorobenzene & 0.012 & $\mathrm{~J} B$ \\
\hline
\end{tabular}


Lab Name: TESTAMERICA BURLINGTON

Contract: $8 \mathrm{E}-00302$

Lab Code: STLV Case No.: YORK Mod. Ref No.: SDG No.: 29407

Matrix: (SOIL/SED/WATER) Water

Sample wt/vol: 25.0 $(\mathrm{g} / \mathrm{mL}) \mathrm{mL}$

Level: (TRACE or LOW/MED) TRACE

\% Moisture: not dec.

GC Column: DB-624 ID $: 0.20 \quad(\mathrm{~mm})$

Soil Extract Volume: (uL)

CONCENTRATION UNITS: (ug/L or ug/kg) ug/L
Lab Sample ID: 200-29407-1

Lab File ID: 15421_007.D

Date Received: 08/21/2015

Date Analyzed: 08/24/2015

Dilution Factor: 1.0

Soil Aliquot Volume: (uL)

Purge Volume: 25.0

$(\mathrm{mL})$

01

02

\begin{tabular}{|l|l|c|c|c|}
\hline CAS NUMBER & \multicolumn{1}{|c|}{ COMPOUND NAME } & RT & EST. CONC. & Q \\
\hline & Unknown & 7.29 & 3.1 & $\mathrm{~J}$ B X \\
\hline E9667961 & Total Alkanes & N/A & & \\
\hline
\end{tabular}

1EPA-designated Registry Number. 
Lab Name: TESTAMERICA BURLINGTON

Contract: $8 \mathrm{E}-00302$

Lab Code: STLV

Case No.: YORK Mod. Ref No.:

SDG No.: 29407

Matrix: (SOIL/SED/WATER) Water

Sample wt/vol: 25.0 $(\mathrm{g} / \mathrm{mL}) \mathrm{mL}$

Level: (TRACE/LOW/MED) TRACE

\% Moisture: not dec.

GC Column: DB-624 ID: $0.20 \quad(\mathrm{~mm})$

Soil Extract Volume: (uL) $(\mathrm{mL})$

Purge Volume: 25.0
Lab Sample ID: 200-29407-3

Lab File ID: 15421_009.D

Date Received: 08/21/2015

Date Analyzed: 08/24/2015

Dilution Factor: 1.0

Soil Aliquot Volume: (uL)

\begin{tabular}{|c|c|c|c|}
\hline CAS NO. & COMPOUND & $\begin{array}{l}\text { CONCENTRATION UNITS: } \\
(\mathrm{ug} / \mathrm{L} \text { or } \mathrm{ug} / \mathrm{kg}) \mathrm{ug} / \mathrm{L}\end{array}$ & q \\
\hline $75-71-8$ & Dichlorodifluoromethane & 1.0 & $\mathrm{U}$ \\
\hline $74-87-3$ & Chloromethane & 1.0 & $\mathrm{U}$ \\
\hline $75-01-4$ & Vinyl chloride & 1.0 & $\mathrm{U}$ \\
\hline $74-83-9$ & Bromomethane & 1.0 & $\mathrm{U}$ \\
\hline $75-00-3$ & Chloroethane & 1.0 & $\mathrm{U}$ \\
\hline $75-69-4$ & Trichlorofluoromethane & 0.093 & $\mathrm{~J}$ \\
\hline $75-35-4$ & 1,1-Dichloroethene & 1.0 & $\mathrm{U}$ \\
\hline $76-13-1$ & $1,1,2$-Trichloro-1,2,2-trifluoroethane & 1.0 & $\mathrm{U}$ \\
\hline $67-64-1$ & Acetone & 0.86 & $\mathrm{~J}$ \\
\hline $75-15-0$ & Carbon disulfide & 0.018 & $\mathrm{~J}$ \\
\hline $79-20-9$ & Methyl acetate & 1.0 & $\mathrm{U}$ \\
\hline $75-09-2$ & Methylene Chloride & 1.0 & $\mathrm{U}$ \\
\hline $156-60-5$ & trans-1,2-Dichloroethene & 1.0 & $\mathrm{U}$ \\
\hline $1634-04-4$ & Methyl tert-butyl ether & 1.0 & $\mathrm{U}$ \\
\hline $75-34-3$ & 1,1-Dichloroethane & 1.0 & $\mathrm{U}$ \\
\hline $156-59-2$ & cis-1,2-Dichloroethene & 1.0 & $\mathrm{U}$ \\
\hline $78-93-3$ & 2-Butanone & 5.0 & $\mathrm{U}$ \\
\hline $74-97-5$ & Bromochloromethane & 1.0 & $\mathrm{U}$ \\
\hline $67-66-3$ & Chloroform & 1.2 & \\
\hline $71-55-6$ & $1,1,1$-Trichloroethane & 1.0 & $\mathrm{U}$ \\
\hline $110-82-7$ & Cyclohexane & 1.0 & $\mathrm{U}$ \\
\hline $56-23-5$ & Carbon tetrachloride & 65 & $\mathrm{E}$ \\
\hline $71-43-2$ & Benzene & 1.0 & $\mathrm{U}$ \\
\hline $107-06-2$ & 1,2-Dichloroethane & 1.0 & $\mathrm{U}$ \\
\hline
\end{tabular}

Report 1,4-Dioxane for Low-Medium VOA analysis only 
Lab Name: TESTAMERICA BURLINGTON

Contract: 8E-00302

Lab Code: STLV

Case No.: YORK

Mod. Ref No.:

SDG No.: 29407

Matrix: (SOIL/SED/WATER) Water

Sample wt/vol: 25.0 $(\mathrm{g} / \mathrm{mL}) \mathrm{mL}$

Level: (TRACE/LOW/MED) TRACE

\% Moisture: not dec.

GC Column: DB-624 ID: $0.20 \quad(\mathrm{~mm})$

Soil Extract Volume: (uL) $(\mathrm{mL})$

Purge Volume: 25.0 (mI)
Lab Sample ID: 200-29407-3

Lab File ID: 15421_009.D

Date Received: 08/21/2015

Date Analyzed: 08/24/2015

Dilution Factor: 1.0

Soil Aliquot Volume: (uL)

\begin{tabular}{|c|c|c|c|}
\hline CAS NO. & COMPOUND & $\begin{array}{l}\text { CONCENTRATION UNITS: } \\
(\mathrm{ug} / \mathrm{L} \text { or } \mathrm{ug} / \mathrm{kg}) \mathrm{ug} / \mathrm{L}\end{array}$ & Q \\
\hline $79-01-6$ & Trichloroethene & 1.0 & $\mathrm{U}$ \\
\hline $108-87-2$ & Methylcyclohexane & 1.0 & $\mathrm{U}$ \\
\hline $78-87-5$ & 1,2-Dichloropropane & 1.0 & $\mathrm{U}$ \\
\hline $75-27-4$ & Bromodichloromethane & 1.0 & $\mathrm{U}$ \\
\hline $10061-01-5$ & cis-1,3-Dichloropropene & 1.0 & U \\
\hline $108-10-1$ & 4-Methyl-2-pentanone & 1.0 & $\mathrm{U}$ \\
\hline $108-88-3$ & Toluene & 0.023 & $\mathrm{~J}$ \\
\hline $10061-02-6$ & trans-1,3-Dichloropropene & 1.0 & $\mathrm{U}$ \\
\hline $79-00-5$ & $1,1,2-$ Trichloroethane & 1.0 & $\mathrm{U}$ \\
\hline $127-18-4$ & Tetrachloroethene & 0.023 & $\mathrm{~J}$ \\
\hline $591-78-6$ & 2-Hexanone & 1.0 & $\mathrm{U}$ \\
\hline $124-48-1$ & Dibromochloromethane & 1.0 & $\mathrm{U}$ \\
\hline $106-93-4$ & 1,2-Dibromoethane & 1.0 & $\mathrm{U}$ \\
\hline $108-90-7$ & Chlorobenzene & 1.0 & $\mathrm{U}$ \\
\hline $100-41-4$ & Ethylbenzene & 1.0 & $\mathrm{U}$ \\
\hline $95-47-6$ & o-Xylene & 0.0040 & $\mathrm{~J}$ \\
\hline $179601-23-1$ & m,p-xylene & 0.014 & $\mathrm{~J}$ \\
\hline $100-42-5$ & Styrene & 1.0 & $\mathrm{U}$ \\
\hline $75-25-2$ & Bromoform & 1.0 & $\mathrm{U}$ \\
\hline $98-82-8$ & Isopropylbenzene & 1.0 & $\mathrm{U}$ \\
\hline $79-34-5$ & $1,1,2,2$-Tetrachloroethane & 1.0 & $\mathrm{U}$ \\
\hline $541-73-1$ & 1,3 -Dichlorobenzene & 1.0 & $\mathrm{U}$ \\
\hline $106-46-7$ & 1,4-Dichlorobenzene & 1.0 & $\mathrm{U}$ \\
\hline $95-50-1$ & 1,2-Dichlorobenzene & 1.0 & $\mathrm{U}$ \\
\hline $96-12-8$ & 1,2-Dibromo-3-Chloropropane & 1.0 & $\mathrm{U}$ \\
\hline $120-82-1$ & $1,2,4$-Trichlorobenzene & 0.0041 & $\mathrm{~J} B$ \\
\hline $87-61-6$ & $1,2,3$-Trichlorobenzene & 1.0 & $\mathrm{U}$ \\
\hline
\end{tabular}


Lab Name: TESTAMERICA BURLINGTON

Contract: $8 \mathrm{E}-00302$

Lab Code: STLV Case No.: YORK Mod. Ref No.: SDG No.: 29407

Matrix: (SOIL/SED/WATER) Water

Sample wt/vol: 25.0 $(\mathrm{g} / \mathrm{mL}) \mathrm{mL}$

Level: (TRACE or LOW/MED) TRACE

․ Moisture: not dec.

GC Column: DB-624 ID $: 0.20 \quad(\mathrm{~mm})$

Soil Extract Volume: (uL)
Lab Sample ID: 200-29407-3

Lab File ID: 15421_009.D

Date Received: 08/21/2015

Date Analyzed: 08/24/2015

Dilution Factor: 1.0

Soil Aliquot Volume: (uL)

Purge Volume: 25.0

$(\mathrm{mL})$

CONCENTRATION UNITS: (ug/L or ug/kg) ug/L

\begin{tabular}{|c|c|c|c|c|}
\hline CAS NUMBER & COMPOUND NAME & RT & EST. CONC. & $Q$ \\
\hline 01 & Unknown & 7.29 & 3.1 & $\mathrm{~J} B \mathrm{X}$ \\
\hline $541-05-9$ & Cyclotrisiloxane, hexamethyl- & 8.22 & 2.0 & $\mathrm{~J} N$ \\
\hline $556-67-2$ & Cyclotetrasiloxane, octamethyl- & 11.04 & 1.4 & $\mathrm{~J} \quad \mathrm{~N} B$ \\
\hline E9667961 & Total Alkanes & $\mathrm{N} / \mathrm{A}$ & & \\
\hline
\end{tabular}

1 EPA-designated Registry Number. 
Lab Name: TESTAMERICA BURLINGTON

Contract: $8 \mathrm{E}-00302$

Lab Code: STLV

Case No.: YORK Mod. Ref No.:

SDG No.: 29407

Matrix: (SOIL/SED/WATER) Water

Sample wt/vol: 25.0 $(\mathrm{g} / \mathrm{mL}) \mathrm{mL}$

Level: (TRACE/LOW/MED) TRACE

\% Moisture: not dec.

GC Column: DB-624 ID: $0.20 \quad(\mathrm{~mm})$

Soil Extract Volume: (uL) $(\mathrm{mL})$

Purge Volume: 25.0
Lab Sample ID: 200-29407-3

Lab File ID: 15421_008.D

Date Received: 08/21/2015

Date Analyzed: 08/24/2015

Dilution Factor: 4.2

Soil Aliquot Volume: (uL)

\begin{tabular}{|c|c|c|c|}
\hline CAS NO. & COMPOUND & $\begin{array}{l}\text { CONCENTRATION UNITS: } \\
(\mathrm{ug} / \mathrm{L} \text { or } \mathrm{ug} / \mathrm{kg}) \mathrm{ug} / \mathrm{L}\end{array}$ & Q \\
\hline $75-71-8$ & Dichlorodifluoromethane & 4.2 & $\mathrm{U}$ \\
\hline $74-87-3$ & Chloromethane & 4.2 & $\mathrm{U}$ \\
\hline $75-01-4$ & Vinyl chloride & 4.2 & $\mathrm{U}$ \\
\hline $74-83-9$ & Bromomethane & 4.2 & $\mathrm{U}$ \\
\hline $75-00-3$ & Chloroethane & 4.2 & $\mathrm{U}$ \\
\hline $75-69-4$ & Trichlorofluoromethane & 0.085 & $\mathrm{~J} \mathrm{D}$ \\
\hline $75-35-4$ & 1,1-Dichloroethene & 4.2 & $\mathrm{U}$ \\
\hline $76-13-1$ & $1,1,2$-Trichloro-1,2,2-trifluoroethane & 4.2 & $\mathrm{U}$ \\
\hline $67-64-1$ & Acetone & 20 & $\mathrm{~J} \mathrm{D}$ \\
\hline $75-15-0$ & Carbon disulfide & 0.069 & $\mathrm{~J} \mathrm{D}$ \\
\hline $79-20-9$ & Methyl acetate & 4.2 & $\mathrm{U}$ \\
\hline $75-09-2$ & Methylene Chloride & 0.45 & $\mathrm{~J} \mathrm{D}$ \\
\hline $156-60-5$ & trans-1,2-Dichloroethene & 4.2 & $\mathrm{U}$ \\
\hline $1634-04-4$ & Methyl tert-butyl ether & 4.2 & $\mathrm{U}$ \\
\hline $75-34-3$ & 1,1-Dichloroethane & 4.2 & $\mathrm{U}$ \\
\hline $156-59-2$ & cis-1,2-Dichloroethene & 4.2 & $\mathrm{U}$ \\
\hline $78-93-3$ & 2-Butanone & 21 & $\mathrm{U}$ \\
\hline $74-97-5$ & Bromochloromethane & 4.2 & $\mathrm{U}$ \\
\hline $67-66-3$ & Chloroform & 1.3 & $\mathrm{~J} \mathrm{D}$ \\
\hline $71-55-6$ & $1,1,1$-Trichloroethane & 4.2 & $\mathrm{U}$ \\
\hline $110-82-7$ & Cyclohexane & 4.2 & $\mathrm{U}$ \\
\hline $56-23-5$ & Carbon tetrachloride & 55 & $\mathrm{D}$ \\
\hline $71-43-2$ & Benzene & 4.2 & $\mathrm{U}$ \\
\hline $107-06-2$ & 1,2-Dichloroethane & 4.2 & $\mathrm{U}$ \\
\hline
\end{tabular}

Report 1,4-Dioxane for Low-Medium VOA analysis only 
Lab Name: TESTAMERICA BURLINGTON

Lab Code: STLV

Case No.: YORK

Mod. Ref No.:

Contract: $8 \mathrm{E}-00302$

Matrix: (SOIL/SED/WATER) Water

Sample wt/vol: 25.0 $(\mathrm{g} / \mathrm{mL}) \mathrm{mL}$

Level: (TRACE/LOW/MED) TRACE

\% Moisture: not dec.

GC Column: DB-624 ID: $0.20 \quad(\mathrm{~mm})$

Soil Extract Volume: (uL) $(\mathrm{mL})$

Purge Volume: 25.0
SDG No.: 29407

Lab Sample ID: 200-29407-3

Lab File ID: 15421_008.D

Date Received: 08/21/2015

Date Analyzed: 08/24/2015

Dilution Factor: 4.2

Soil Aliquot Volume: (uL) (mI)

\begin{tabular}{|c|c|c|c|}
\hline CAS NO. & COMPOUND & $\begin{array}{l}\text { CONCENTRATION UNITS: } \\
(\mathrm{ug} / \mathrm{L} \text { or } \mathrm{ug} / \mathrm{kg}) \mathrm{ug} / \mathrm{L}\end{array}$ & Q \\
\hline $79-01-6$ & Trichloroethene & 4.2 & $\mathrm{U}$ \\
\hline $108-87-2$ & Methylcyclohexane & 4.2 & $\mathrm{U}$ \\
\hline $78-87-5$ & 1,2-Dichloropropane & 4.2 & $\mathrm{U}$ \\
\hline $75-27-4$ & Bromodichloromethane & 4.2 & $\mathrm{U}$ \\
\hline $10061-01-5$ & cis-1,3-Dichloropropene & 4.2 & U \\
\hline $108-10-1$ & 4-Methyl-2-pentanone & 4.2 & $\mathrm{U}$ \\
\hline $108-88-3$ & Toluene & 0.058 & $\mathrm{~J} \quad \mathrm{D}$ \\
\hline $10061-02-6$ & trans-1,3-Dichloropropene & 4.2 & $\mathrm{U}$ \\
\hline $79-00-5$ & $1,1,2-$ Trichloroethane & 4.2 & $\mathrm{U}$ \\
\hline $127-18-4$ & Tetrachloroethene & 0.018 & $\mathrm{~J} \mathrm{D}$ \\
\hline $591-78-6$ & 2-Hexanone & 4.2 & $\mathrm{U}$ \\
\hline $124-48-1$ & Dibromochloromethane & 4.2 & $\mathrm{U}$ \\
\hline $106-93-4$ & 1,2-Dibromoethane & 4.2 & $\mathrm{U}$ \\
\hline $108-90-7$ & Chlorobenzene & 4.2 & $\mathrm{U}$ \\
\hline $100-41-4$ & Ethylbenzene & 4.2 & $\mathrm{U}$ \\
\hline $95-47-6$ & o-Xylene & 0.0092 & $\mathrm{~J} \mathrm{D}$ \\
\hline $179601-23-1$ & m,p-xylene & 0.033 & $\mathrm{~J} \mathrm{D}$ \\
\hline $100-42-5$ & Styrene & 4.2 & $\mathrm{U}$ \\
\hline $75-25-2$ & Bromoform & 4.2 & $\mathrm{U}$ \\
\hline $98-82-8$ & Isopropylbenzene & 4.2 & $\mathrm{U}$ \\
\hline $79-34-5$ & $1,1,2,2$-Tetrachloroethane & 4.2 & $\mathrm{U}$ \\
\hline $541-73-1$ & 1,3 -Dichlorobenzene & 4.2 & $\mathrm{U}$ \\
\hline $106-46-7$ & 1,4-Dichlorobenzene & 4.2 & $\mathrm{U}$ \\
\hline $95-50-1$ & 1,2-Dichlorobenzene & 4.2 & $\mathrm{U}$ \\
\hline $96-12-8$ & 1,2-Dibromo-3-Chloropropane & 4.2 & $\mathrm{U}$ \\
\hline $120-82-1$ & $1,2,4$-Trichlorobenzene & 4.2 & $\mathrm{U}$ \\
\hline $87-61-6$ & $1,2,3$-Trichlorobenzene & 0.034 & $\mathrm{~J} \quad \mathrm{D} B$ \\
\hline
\end{tabular}


Lab Name: TESTAMERICA BURLINGTON

Contract: 8E-00302

Lab Code: STLV Case No.: YORK Mod. Ref No.: SDG No.: 29407

Matrix: (SOIL/SED/WATER) Water

Sample wt/vol: 25.0 $(\mathrm{g} / \mathrm{mL}) \mathrm{mL}$

Level: (TRACE or LOW/MED) TRACE

Moisture: not dec.

GC Column: DB-624 ID $: 0.20 \quad(\mathrm{~mm})$

Soil Extract Volume: (uL)

CONCENTRATION UNITS: (ug/L or ug/kg) ug/L
Lab Sample ID: 200-29407-3

Lab File ID: 15421_008.D

Date Received: 08/21/2015

Date Analyzed: 08/24/2015

Dilution Factor: 4.2

Soil Aliquot Volume: (uL) Purge Volume: 25.0 $(\mathrm{mL})$

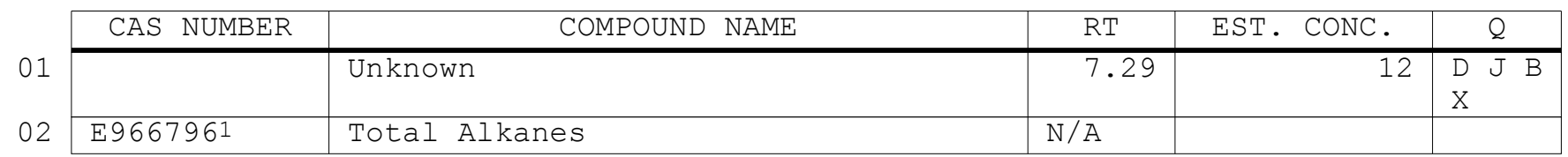

1EPA-designated Registry Number. 
Lab Name: TESTAMERICA BURLINGTON

Contract: 8E-00302

Lab Code: STLV Case No.: YORK Mod. Ref No.:

SDG No.: 29407

Instrument ID: CHD.i

Calibration Date(s): 08/19/2015 08/19/2015

Heated Purge: ( $\mathrm{Y} / \mathrm{N}) \mathrm{N}$

Calibration Time(s) : 1514

1654

Purge Volume: 25.0 $(\mathrm{mL})$

GC Column: DB-624

ID: 0.20

$(\mathrm{mm})$

Length: 25

$(\mathrm{m})$

\begin{tabular}{|c|c|c|c|c|c|c|c|}
\hline \multirow{3}{*}{$\begin{array}{l}\text { LAB FILE ID: } \\
\text { RRF5.0 }=\text { 15357_005.D } \\
\text { COMPOUND }\end{array}$} & \multirow{2}{*}{\multicolumn{3}{|c|}{ 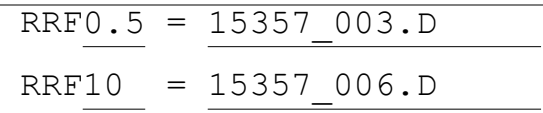 }} & \multirow{2}{*}{\multicolumn{4}{|c|}{$\begin{array}{l}\text { RRF1.0 }=\frac{15357 \_004 . \mathrm{D}}{\text { RRF20 }}=15357 \_007 . \mathrm{D}\end{array}$}} \\
\hline & & & & & & & \\
\hline & $\operatorname{RRF} 0.5$ & $\operatorname{RRF} 1.0$ & RRF5.0 & $\operatorname{RRF} 10$ & RRF20 & $\overline{\mathrm{RRF}}$ & $\because \mathrm{RSD}$ \\
\hline Dichlorodifluoromethane & 0.630 & 0.576 & 0.641 & 0.600 & 0.628 & 0.615 & 4.4 \\
\hline Chloromethane & 0.540 & 0.453 & 0.512 & 0.465 & 0.487 & 0.491 & 7.2 \\
\hline Vinyl chloride & 0.449 & 0.413 & 0.459 & 0.432 & 0.439 & 0.438 & 4.0 \\
\hline Bromomethane & 0.148 & 0.106 & 0.148 & 0.147 & 0.161 & 0.142 & 14.8 \\
\hline Chloroethane & 0.207 & 0.193 & 0.216 & 0.208 & 0.196 & 0.204 & 4.6 \\
\hline Trichlorofluoromethane & 0.558 & 0.507 & 0.584 & 0.557 & 0.568 & 0.555 & 5.2 \\
\hline 1,1-Dichloroethene & 0.290 & 0.248 & 0.281 & 0.263 & 0.265 & 0.270 & 6.2 \\
\hline $\begin{array}{l}1,1,2 \text {-Trichloro- } \\
1,2,2 \text {-trifluoroethane }\end{array}$ & 0.309 & 0.282 & 0.317 & 0.300 & 0.298 & 0.301 & 4.3 \\
\hline Acetone & 0.025 & 0.021 & 0.023 & 0.021 & 0.022 & 0.022 & 7.7 \\
\hline Carbon disulfide & 0.656 & 0.597 & 0.750 & 0.861 & 0.809 & 0.735 & 14.7 \\
\hline Methyl acetate & 0.074 & 0.060 & 0.072 & 0.065 & 0.066 & 0.067 & 8.7 \\
\hline Methylene Chloride & 0.292 & 0.261 & 0.273 & 0.266 & 0.265 & 0.271 & 4.6 \\
\hline trans-1,2-Dichloroethene & 0.296 & 0.279 & 0.328 & 0.322 & 0.320 & 0.309 & 6.7 \\
\hline Methyl tert-butyl ether & 0.349 & 0.322 & 0.364 & 0.374 & 0.388 & 0.359 & 7.0 \\
\hline 1,1-Dichloroethane & 0.554 & 0.524 & 0.605 & 0.591 & 0.606 & 0.576 & 6.3 \\
\hline cis-1,2-Dichloroethene & 0.303 & 0.269 & 0.308 & 0.317 & 0.315 & 0.302 & 6.4 \\
\hline 2-Butanone & 0.039 & 0.037 & 0.043 & 0.043 & 0.045 & 0.041 & 8.1 \\
\hline Bromochloromethane & 0.087 & 0.080 & 0.096 & 0.096 & 0.100 & 0.091 & 9.0 \\
\hline Chloroform & 0.490 & 0.490 & 0.538 & 0.531 & 0.532 & 0.516 & 4.6 \\
\hline 1,1,1-Trichloroethane & 0.524 & 0.485 & 0.591 & 0.595 & 0.613 & 0.562 & 9.7 \\
\hline Cyclohexane & 0.848 & 0.766 & 0.859 & 0.833 & 0.823 & 0.826 & 4.4 \\
\hline Carbon tetrachloride & 0.430 & 0.395 & 0.503 & 0.519 & 0.528 & 0.475 & 12.4 \\
\hline Benzene & 1.706 & 1.548 & 1.656 & 1.646 & 1.599 & 1.631 & 3.7 \\
\hline 1,2-Dichloroethane & 0.260 & 0.229 & 0.258 & 0.263 & 0.252 & 0.253 & 5.4 \\
\hline Trichloroethene & 0.401 & 0.379 & 0.416 & 0.411 & 0.406 & 0.403 & 3.5 \\
\hline Methylcyclohexane & 0.657 & 0.642 & 0.702 & 0.694 & 0.680 & 0.675 & 3.7 \\
\hline
\end{tabular}

Report 1,4-Dioxane for Low-Medium VOA analysis only 
Lab Name: TESTAMERICA BURLINGTON

Contract: $8 \mathrm{E}-00302$

Lab Code: STLV Case No.: YORK Mod. Ref No.:

SDG No.: 29407

Instrument ID: CHD.i

Calibration Date(s) : 08/19/2015 08/19/2015

Heated Purge: (Y/N) N

Calibration Time(s) : 1514

1654

Purge Volume: 25.0

GC Column: DB-624

ID: 0.20 (mL)

LAB FILE ID:

RRF5.0 = 15357_005.D

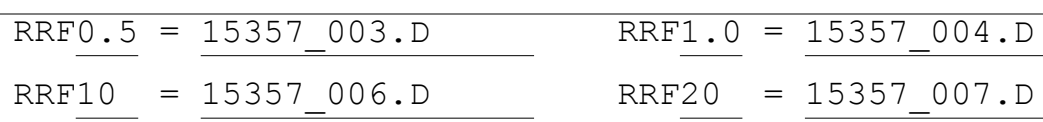

\begin{tabular}{|l|l|l|l|l|l|l|l}
\hline COMPOUND & RRF0.5 & RRF1.0 & RRF5.0 & RRF10 & RRF20 & $\overline{R R F}$ & $\frac{\circ}{0} \mathrm{RSD}$ \\
\hline
\end{tabular}

\begin{tabular}{|c|c|c|c|c|c|c|c|}
\hline 1,2-Dichloropropane & 0.327 & 0.313 & 0.349 & 0.344 & 0.337 & 0.334 & 4.3 \\
\hline Bromodichloromethane & 0.263 & 0.262 & 0.336 & 0.358 & 0.361 & 0.316 & 15.7 \\
\hline cis-1,3-Dichloropropene & 0.340 & 0.315 & 0.440 & 0.455 & 0.463 & 0.403 & 17.3 \\
\hline 4-Methyl-2-pentanone & 0.106 & 0.102 & 0.128 & 0.126 & 0.125 & 0.117 & 10.5 \\
\hline Toluene & 1.731 & 1.636 & 1.803 & 1.777 & 1.710 & 1.731 & 3.7 \\
\hline trans-1,3-Dichloropropene & 0.201 & 0.213 & 0.301 & 0.323 & 0.329 & 0.273 & 22.7 \\
\hline $1,1,2$-Trichloroethane & 0.181 & 0.150 & 0.167 & 0.167 & 0.161 & 0.165 & 6.8 \\
\hline Tetrachloroethene & 0.357 & 0.334 & 0.367 & 0.369 & 0.366 & 0.359 & 4.0 \\
\hline 2-Hexanone & 0.073 & 0.068 & 0.087 & 0.087 & 0.086 & 0.080 & 11.1 \\
\hline Dibromochloromethane & 0.125 & 0.114 & 0.168 & 0.183 & 0.189 & 0.156 & 22.0 \\
\hline 1,2-Dibromoethane & 0.131 & 0.122 & 0.146 & 0.147 & 0.148 & 0.139 & 8.4 \\
\hline Chlorobenzene & 1.013 & 0.937 & 1.024 & 1.018 & 1.005 & 0.999 & 3.5 \\
\hline Ethylbenzene & 2.001 & 1.894 & 2.154 & 2.141 & 2.062 & 2.050 & 5.2 \\
\hline o-Xylene & 0.683 & 0.629 & 0.750 & 0.749 & 0.733 & 0.709 & 7.4 \\
\hline m, p-Xylene & 0.749 & 0.718 & 0.821 & 0.815 & 0.801 & 0.781 & 5.8 \\
\hline Styrene & 0.957 & 0.957 & 1.155 & 1.134 & 1.112 & 1.063 & 9.2 \\
\hline Bromoform & 0.094 & 0.087 & 0.118 & 0.142 & 0.153 & 0.119 & 24.2 \\
\hline Isopropylbenzene & 2.002 & 1.895 & 2.236 & 2.192 & 2.144 & 2.094 & 6.8 \\
\hline $1,1,2,2$-Tetrachloroethane & 0.159 & 0.146 & 0.172 & 0.168 & 0.172 & 0.163 & 6.8 \\
\hline 1,3-Dichlorobenzene & 1.554 & 1.483 & 1.702 & 1.712 & 1.743 & 1.639 & 6.9 \\
\hline 1,4-Dichlorobenzene & 1.614 & 1.524 & 1.654 & 1.646 & 1.666 & 1.621 & 3.5 \\
\hline 1,2-Dichlorobenzene & 1.357 & 1.238 & 1.365 & 1.353 & 1.383 & 1.339 & 4.3 \\
\hline 1,2-Dibromo-3-Chloropropane & 0.033 & 0.033 & 0.035 & 0.039 & 0.045 & 0.037 & 14.0 \\
\hline $1,2,4$-Trichlorobenzene & 0.804 & 0.738 & 0.849 & 0.888 & 0.929 & 0.841 & 8.8 \\
\hline $1,2,3$-Trichlorobenzene & 0.564 & 0.535 & 0.591 & 0.624 & 0.658 & 0.594 & 8.1 \\
\hline
\end{tabular}


Lab Name: TESTAMERICA BURLINGTON

Contract: $8 \mathrm{E}-00302$

Lab Code: STLV Case No.: YORK Mod. Ref No.: SDG No.: 29407

Instrument ID: CHD.i

Heated Purge: (Y/N) N

Purge Volume: 25.0

GC Column: DB-624
ID: 0.20
Calibration Date(s) : 08/19/2015

Calibration Time(s) : 1514 $(\mathrm{mL})$

(mm) Length: 25
$08 / 19 / 2015$ 1654

\begin{tabular}{|c|c|c|c|c|c|c|c|}
\hline LAB FILE ID: & \multirow{2}{*}{\multicolumn{3}{|c|}{$\begin{array}{l}\operatorname{RRF0.5}=\frac{15357 \_003 . D}{\operatorname{RRF10}=15357 \_006 . \mathrm{D}} \\
\end{array}$}} & \multirow{2}{*}{\multicolumn{4}{|c|}{$\begin{array}{l}\text { RRF1.0 }=15357 \_004 . \mathrm{D} \\
\text { RRF20 }=15357 \_007 . \mathrm{D}\end{array}$}} \\
\hline RRF5.0 = 15357_005.D & & & & & & & \\
\hline COMPOUND & RRF0. 5 & RRF1 . 0 & RRF 5.0 & RRF10 & RRF20 & $\overline{\mathrm{RRF}}$ & $\because \mathrm{RSD}$ \\
\hline Vinyl Chloride-d3 & 0.381 & 0.364 & 0.409 & 0.383 & 0.398 & 0.387 & 4.4 \\
\hline Chloroethane-d5 & 0.280 & 0.255 & 0.268 & 0.246 & 0.235 & 0.257 & 7.0 \\
\hline 1,1-Dichloroethene-d2 & 0.625 & 0.555 & 0.632 & 0.603 & 0.582 & 0.600 & 5.3 \\
\hline 2-Butanone-d5 & 0.038 & 0.036 & 0.041 & 0.041 & 0.043 & 0.040 & 6.9 \\
\hline Chloroform-d & 0.535 & 0.511 & 0.560 & 0.563 & 0.562 & 0.546 & 4.2 \\
\hline 1,2-Dichloroethane-d4 & 0.202 & 0.196 & 0.208 & 0.211 & 0.207 & 0.205 & 3.0 \\
\hline Benzene-d6 & 1.559 & 1.409 & 1.532 & 1.506 & 1.492 & 1.500 & 3.8 \\
\hline 1,2-Dichloropropane-d6 & 0.391 & 0.349 & 0.395 & 0.389 & 0.383 & 0.381 & 4.9 \\
\hline Toluene-d8 & 1.433 & 1.328 & 1.484 & 1.481 & 1.428 & 1.431 & 4.4 \\
\hline trans-1,3-Dichloropropene-d4 & 0.184 & 0.169 & 0.257 & 0.279 & 0.290 & 0.236 & 23.6 \\
\hline $2-$ Hexanone-d5 & 0.026 & 0.029 & 0.038 & 0.039 & 0.039 & 0.034 & 17.9 \\
\hline $1,1,2,2$-Tetrachloroethane-d2 & 0.149 & 0.144 & 0.170 & 0.165 & 0.171 & 0.160 & 7.8 \\
\hline 1,2-Dichlorobenzene-d4 & 0.883 & 0.800 & 0.834 & 0.838 & 0.864 & 0.844 & 3.7 \\
\hline
\end{tabular}

Report 1,4-Dioxane-d8 for Low-Medium VOA analysis only 
Lab Name: TESTAMERICA BURLINGTON

Contract: $8 \mathrm{E}-00302$

Lab Code: STLV Case No.: YORK Mod. Ref No.:

SDG No.: 29407

Instrument ID: CHD.i

Calibration Date: 08/24/2015 Time: 1156

Lab File Id: 15421_003.D

EPA Sample No. (VSTD\#\#\#\#) : VSTD005DH Init. Calib. Date(s):08/19/2015 08/19/2015

Heated Purge: (Y/N) N GC Column: DB-624

Init. Calib. Time(s) : 1514 1654

Purge Volume: 25.0 ID: $0.20(\mathrm{~mm})$ Length: 25

(m)

\begin{tabular}{|c|c|c|c|c|c|}
\hline COMPOUND & $\overline{\mathrm{RRF}}$ & RRF5. 0 & $\begin{array}{l}\text { MIN } \\
\text { RRF }\end{array}$ & $\div \mathrm{D}$ & $\mathrm{MAX} \cong \mathrm{D}$ \\
\hline Dichlorodifluoromethane & 0.615 & 0.604 & 0.010 & -1.8 & 40.0 \\
\hline Chloromethane & 0.491 & 0.490 & 0.010 & -0.3 & 40.0 \\
\hline Vinyl chloride & 0.438 & 0.440 & 0.100 & 0.4 & 30.0 \\
\hline Bromomethane & 0.142 & 0.189 & 0.100 & 33.0 & 30.0 \\
\hline Chloroethane & 0.204 & 0.226 & 0.010 & 10.7 & 40.0 \\
\hline Trichlorofluoromethane & 0.555 & 0.591 & 0.010 & 6.5 & 40.0 \\
\hline 1,1-Dichloroethene & 0.270 & 0.279 & 0.100 & 3.4 & 30.0 \\
\hline 1,1,2-Trichloro-1,2,2-trifluoroethane & 0.301 & 0.313 & 0.010 & 4.0 & 40.0 \\
\hline Acetone & 0.022 & 0.021 & 0.010 & -6.2 & 40.0 \\
\hline Carbon disulfide & 0.735 & 0.773 & 0.010 & 5.2 & 40.0 \\
\hline Methyl acetate & 0.067 & 0.063 & 0.010 & -6.4 & 40.0 \\
\hline Methylene Chloride & 0.271 & 0.256 & 0.010 & -5.6 & 40.0 \\
\hline trans-1,2-Dichloroethene & 0.309 & 0.315 & 0.010 & 2.1 & 40.0 \\
\hline Methyl tert-butyl ether & 0.359 & 0.325 & 0.010 & -9.5 & 40.0 \\
\hline 1,1-Dichloroethane & 0.576 & 0.592 & 0.200 & 2.9 & 30.0 \\
\hline cis-1,2-Dichloroethene & 0.302 & 0.302 & 0.010 & 0.0 & 40.0 \\
\hline 2-Butanone & 0.041 & 0.038 & 0.010 & -8.0 & 40.0 \\
\hline Bromochloromethane & 0.091 & 0.086 & 0.050 & -6.2 & 30.0 \\
\hline Chloroform & 0.516 & 0.522 & 0.200 & 1.1 & 30.0 \\
\hline 1,1,1-Trichloroethane & 0.562 & 0.603 & 0.100 & 7.3 & 30.0 \\
\hline Cyclohexane & 0.826 & 0.858 & 0.010 & 3.9 & 40.0 \\
\hline Carbon tetrachloride & 0.475 & 0.521 & 0.100 & 9.7 & 30.0 \\
\hline Benzene & 1.631 & 1.657 & 0.400 & 1.6 & 30.0 \\
\hline 1,2-Dichloroethane & 0.253 & 0.243 & 0.100 & -3.6 & 30.0 \\
\hline Trichloroethene & 0.403 & 0.416 & 0.300 & 3.2 & 30.0 \\
\hline Methylcyclohexane & 0.675 & 0.716 & 0.010 & 6.1 & 40.0 \\
\hline
\end{tabular}

Report 1,4-Dioxane for Low/Medium VOA analysis only 
Lab Name: TESTAMERICA BURLINGTON

Contract: 8E-00302

Lab Code: STLV Case No.: YORK Mod. Ref No.:

SDG No.: 29407

Instrument ID: CHD.i

Calibration Date: 08/24/2015 Time: 1156

Lab File Id: 15421_003.D

EPA Sample No. (VSTD\#\#\#\#) : VSTD005DH Init. Calib. Date(s):08/19/2015 08/19/2015

Heated Purge: (Y/N) N GC Column: DB-624

Init. Calib. Time(s) : 1514 1654

Purge Volume: 25.0 ID: $0.20(\mathrm{~mm})$ Length: 25

(m)

\begin{tabular}{|c|c|c|c|c|c|}
\hline COMPOUND & $\overline{\mathrm{RRF}}$ & RRF 5.0 & $\begin{array}{l}\text { MIN } \\
\text { RRF }\end{array}$ & $\frac{\circ}{D}$ & $\mathrm{MAX} \div \mathrm{D}$ \\
\hline 1,2-Dichloropropane & 0.334 & 0.334 & 0.010 & 0.1 & 40.0 \\
\hline Bromodichloromethane & 0.316 & 0.338 & 0.200 & 7.0 & 30.0 \\
\hline cis-1,3-Dichloropropene & 0.403 & 0.434 & 0.200 & 7.9 & 30.0 \\
\hline 4-Methyl-2-pentanone & 0.117 & 0.112 & 0.010 & -4.8 & 40.0 \\
\hline Toluene & 1.731 & 1.791 & 0.400 & 3.4 & 30.0 \\
\hline trans-1,3-Dichloropropene & 0.273 & 0.292 & 0.100 & 6.9 & 30.0 \\
\hline $1,1,2$-Trichloroethane & 0.165 & 0.157 & 0.100 & -5.1 & 30.0 \\
\hline Tetrachloroethene & 0.359 & 0.357 & 0.100 & -0.5 & 30.0 \\
\hline 2-Hexanone & 0.080 & 0.078 & 0.010 & -2.0 & 40.0 \\
\hline Dibromochloromethane & 0.156 & 0.164 & 0.100 & 5.5 & 30.0 \\
\hline 1,2-Dibromoethane & 0.139 & 0.136 & 0.010 & -1.7 & 40.0 \\
\hline Chlorobenzene & 0.999 & 1.002 & 0.500 & 0.3 & 30.0 \\
\hline Ethylbenzene & 2.050 & 2.151 & 0.100 & 4.9 & 30.0 \\
\hline o-Xylene & 0.709 & 0.740 & 0.300 & 4.4 & 30.0 \\
\hline$m, p-X y l e n e$ & 0.781 & 0.804 & 0.300 & 3.0 & 30.0 \\
\hline Styrene & 1.063 & 1.125 & 0.300 & 5.9 & 30.0 \\
\hline Bromoform & 0.119 & 0.132 & 0.050 & 11.1 & 30.0 \\
\hline Isopropylbenzene & 2.094 & 2.230 & 0.010 & 6.5 & 40.0 \\
\hline $1,1,2,2$-Tetrachloroethane & 0.163 & 0.158 & 0.100 & -3.1 & 30.0 \\
\hline 1,3-Dichlorobenzene & 1.639 & 1.661 & 0.400 & 1.4 & 30.0 \\
\hline 1,4-Dichlorobenzene & 1.621 & 1.604 & 0.400 & -1.0 & 30.0 \\
\hline 1,2-Dichlorobenzene & 1.339 & 1.322 & 0.400 & -1.3 & 30.0 \\
\hline 1,2-Dibromo-3-Chloropropane & 0.037 & 0.036 & 0.010 & -2.5 & 40.0 \\
\hline $1,2,4-$ Trichlorobenzene & 0.841 & 0.783 & 0.200 & -7.0 & 30.0 \\
\hline $1,2,3$-Trichlorobenzene & 0.594 & 0.531 & 0.200 & $-10 \cdot 6$ & 30.0 \\
\hline
\end{tabular}


Lab Name: TESTAMERICA BURLINGTON

Contract: $8 \mathrm{E}-00302$

Lab Code: STLV Case No.: YORK Mod. Ref No.

SDG No.: 29407

Instrument ID: CHD.i

Calibration Date: 08/24/2015 Time: 1156

Lab File Id: 15421_003.D Init. Calib. Date(s):08/19/2015 08/19/2015

EPA Sample No. (VSTD\#\#\#\#) : VSTD005DH

Heated Purge: (Y/N) N GC Column: DB-624

Init. Calib. Time(s) : 1514 1654

Purge Volume: 25.0 ID: $0.20(\mathrm{~mm})$ Length: 25

(m)

\begin{tabular}{|c|c|c|c|c|c|}
\hline COMPOUND & $\overline{\mathrm{RRF}}$ & RRF 5.0 & $\begin{array}{l}\text { MIN } \\
\text { RRF }\end{array}$ & $\div D$ & $\mathrm{MAX} \div \mathrm{D}$ \\
\hline Vinyl Chloride-d3 & 0.387 & 0.404 & 0.010 & 4.4 & 30.0 \\
\hline Chloroethane-d5 & 0.257 & 0.263 & 0.010 & 2.5 & 40.0 \\
\hline 1,1-Dichloroethene-d2 & 0.600 & 0.637 & 0.010 & 6.3 & 30.0 \\
\hline 2-Butanone-d5 & 0.040 & 0.035 & 0.010 & $-11 \cdot 1$ & 40.0 \\
\hline Chloroform-d & 0.546 & 0.551 & 0.010 & 1.0 & 30.0 \\
\hline 1,2-Dichloroethane-d4 & 0.205 & 0.202 & 0.010 & -1.5 & 30.0 \\
\hline Benzene-d6 & 1.500 & 1.512 & 0.010 & 0.8 & 30.0 \\
\hline 1,2 -Dichloropropane-d6 & 0.381 & 0.389 & 0.010 & 2.0 & 40.0 \\
\hline Toluene-d8 & 1.431 & 1.472 & 0.010 & 2.9 & 30.0 \\
\hline trans-1,3-Dichloropropene-d4 & 0.236 & 0.252 & 0.010 & 6.6 & 30.0 \\
\hline 2-Hexanone-d5 & 0.034 & 0.033 & 0.010 & -3.4 & 40.0 \\
\hline $1,1,2,2$-Tetrachloroethane-d2 & 0.160 & 0.159 & 0.010 & -0.4 & 30.0 \\
\hline 1,2-Dichlorobenzene-d4 & 0.844 & 0.837 & 0.010 & -0.8 & 30.0 \\
\hline
\end{tabular}

Report 1,4-Dioxane-d8 for Low/Medium VOA analysis only 
Lab Name: TESTAMERICA BURLINGTON

Contract: $8 \mathrm{E}-00302$

Lab Code: STLV Case No.: YORK Mod. Ref No.:

SDG No.: 29407

Instrument ID: CHD.i

Calibration Date: 08/24/2015 Time: 1641

Lab File Id: 15421_012.D

EPA Sample No. (VSTD\#\#\#\#) : VSTD005HG Init. Calib. Date(s):08/19/2015 08/19/2015

Heated Purge: (Y/N) N GC Column: DB-624

Init. Calib. Time(s) : 1514 1654

Purge Volume: 25.0 ID: $0.20(\mathrm{~mm})$ Length: 25

(m)

\begin{tabular}{|c|c|c|c|c|c|}
\hline COMPOUND & $\overline{\mathrm{RRF}}$ & RRF5. 0 & $\begin{array}{l}\text { MIN } \\
\text { RRF }\end{array}$ & $\because \mathrm{D}$ & $\mathrm{MAX} \cong \mathrm{D}$ \\
\hline Dichlorodifluoromethane & 0.615 & 0.667 & 0.010 & 8.4 & 50.0 \\
\hline Chloromethane & 0.491 & 0.530 & 0.010 & 7.8 & 50.0 \\
\hline Vinyl chloride & 0.438 & 0.489 & 0.010 & 11.5 & 50.0 \\
\hline Bromomethane & 0.142 & 0.157 & 0.010 & 10.5 & 50.0 \\
\hline Chloroethane & 0.204 & 0.235 & 0.010 & 15.4 & 50.0 \\
\hline Trichlorofluoromethane & 0.555 & 0.631 & 0.010 & 13.7 & 50.0 \\
\hline 1,1-Dichloroethene & 0.270 & 0.296 & 0.010 & 9.9 & 50.0 \\
\hline 1,1,2-Trichloro-1,2,2-trifluoroethane & 0.301 & 0.338 & 0.010 & 12.1 & 50.0 \\
\hline Acetone & 0.022 & 0.023 & 0.010 & 3.8 & 50.0 \\
\hline Carbon disulfide & 0.735 & 0.840 & 0.010 & 14.4 & 50.0 \\
\hline Methyl acetate & 0.067 & 0.066 & 0.010 & -2.9 & 50.0 \\
\hline Methylene Chloride & 0.271 & 0.278 & 0.010 & 2.4 & 50.0 \\
\hline trans-1,2-Dichloroethene & 0.309 & 0.333 & 0.010 & 7.7 & 50.0 \\
\hline Methyl tert-butyl ether & 0.359 & 0.351 & 0.010 & -2.3 & 50.0 \\
\hline 1,1-Dichloroethane & 0.576 & 0.625 & 0.010 & 8.4 & 50.0 \\
\hline cis-1,2-Dichloroethene & 0.302 & 0.318 & 0.010 & 5.1 & 50.0 \\
\hline 2-Butanone & 0.041 & 0.045 & 0.010 & 8.4 & 50.0 \\
\hline Bromochloromethane & 0.091 & 0.094 & 0.010 & 3.3 & 50.0 \\
\hline Chloroform & 0.516 & 0.557 & 0.010 & 7.8 & 50.0 \\
\hline 1,1,1-Trichloroethane & 0.562 & 0.580 & 0.010 & 3.3 & 50.0 \\
\hline Cyclohexane & 0.826 & 0.866 & 0.010 & 4.8 & 50.0 \\
\hline Carbon tetrachloride & 0.475 & 0.503 & 0.010 & 6.0 & 50.0 \\
\hline Benzene & 1.631 & 1.648 & 0.010 & 1.1 & 50.0 \\
\hline 1,2-Dichloroethane & 0.253 & 0.269 & 0.010 & 6.6 & 50.0 \\
\hline Trichloroethene & 0.403 & 0.412 & 0.010 & 2.3 & 50.0 \\
\hline Methylcyclohexane & 0.675 & 0.693 & 0.010 & 2.7 & 50.0 \\
\hline
\end{tabular}

Report 1,4-Dioxane for Low/Medium VOA analysis only 
Lab Name: TESTAMERICA BURLINGTON

Contract: 8E-00302

Lab Code: STLV Case No.: YORK Mod. Ref No.:

SDG No.: 29407

Instrument ID: CHD.i

Calibration Date: 08/24/2015 Time: 1641

Lab File Id: 15421_012.D

EPA Sample No. (VSTD\#\#\#\#) : VSTD005HG Init. Calib. Date(s):08/19/2015 08/19/2015

Heated Purge: (Y/N) N GC Column: DB-624

Init. Calib. Time(s) : 1514 1654

Purge Volume: 25.0 ID: $0.20(\mathrm{~mm})$ Length: 25

(m)

\begin{tabular}{|c|c|c|c|c|c|}
\hline COMPOUND & $\overline{\mathrm{RRF}}$ & RRF5. 0 & $\begin{array}{l}\text { MIN } \\
\text { RRF }\end{array}$ & $\because \mathrm{D}$ & $\mathrm{MAX} \div \mathrm{D}$ \\
\hline 1,2-Dichloropropane & 0.334 & 0.329 & 0.010 & -1.4 & 50.0 \\
\hline Bromodichloromethane & 0.316 & 0.323 & 0.010 & 2.3 & 50.0 \\
\hline cis-1,3-Dichloropropene & 0.403 & 0.424 & 0.010 & 5.3 & 50.0 \\
\hline 4-Methyl-2-pentanone & 0.117 & 0.118 & 0.010 & 0.4 & 50.0 \\
\hline Toluene & 1.731 & 1.806 & 0.010 & 4.3 & 50.0 \\
\hline trans-1,3-Dichloropropene & 0.273 & 0.285 & 0.010 & 4.2 & 50.0 \\
\hline 1,1,2-Trichloroethane & 0.165 & 0.168 & 0.010 & 1.5 & 50.0 \\
\hline Tetrachloroethene & 0.359 & 0.366 & 0.010 & 2.0 & 50.0 \\
\hline 2-Hexanone & 0.080 & 0.082 & 0.010 & 2.7 & 50.0 \\
\hline Dibromochloromethane & 0.156 & 0.160 & 0.010 & 2.7 & 50.0 \\
\hline 1,2-Dibromoethane & 0.139 & 0.141 & 0.010 & 1.7 & 50.0 \\
\hline Chlorobenzene & 0.999 & 1.015 & 0.010 & 1.6 & 50.0 \\
\hline Ethylbenzene & 2.050 & 2.160 & 0.010 & 5.3 & 50.0 \\
\hline o-Xylene & 0.709 & 0.754 & 0.010 & 6.4 & 50.0 \\
\hline $\mathrm{m}, \mathrm{p}-\mathrm{Xylene}$ & 0.781 & 0.816 & 0.010 & 4.6 & 50.0 \\
\hline Styrene & 1.063 & 1.139 & 0.010 & 7.2 & 50.0 \\
\hline Bromoform & 0.119 & 0.113 & 0.010 & -4.7 & 50.0 \\
\hline Isopropylbenzene & 2.094 & 2.238 & 0.010 & 6.9 & 50.0 \\
\hline $1,1,2,2$-Tetrachloroethane & 0.163 & 0.168 & 0.010 & 2.9 & 50.0 \\
\hline 1,3-Dichlorobenzene & 1.639 & 1.652 & 0.010 & 0.8 & 50.0 \\
\hline 1,4-Dichlorobenzene & 1.621 & 1.609 & 0.010 & -0.7 & 50.0 \\
\hline 1,2-Dichlorobenzene & 1.339 & 1.338 & 0.010 & -0.1 & 50.0 \\
\hline 1,2-Dibromo-3-Chloropropane & 0.037 & 0.031 & 0.010 & -15.5 & 50.0 \\
\hline $1,2,4$-Trichlorobenzene & 0.841 & 0.782 & 0.010 & -7.1 & 50.0 \\
\hline 1,2,3-Trichlorobenzene & 0.594 & 0.550 & 0.010 & -7.4 & 50.0 \\
\hline
\end{tabular}


Lab Name: TESTAMERICA BURLINGTON

Contract: $8 \mathrm{E}-00302$

Lab Code: STLV Case No.: YORK Mod. Ref No.

SDG No.: 29407

Instrument ID: CHD.i

Calibration Date: 08/24/2015 Time: 1641

Lab File Id: 15421_012.D Init. Calib. Date(s):08/19/2015 08/19/2015

EPA Sample No. (VSTD\#\#\#\#) : VSTD005HG

Heated Purge: (Y/N) N GC Column: DB-624

Init. Calib. Time(s) : 1514 1654

Purge Volume: 25.0 ID: $0.20(\mathrm{~mm})$ Length: 25

(m)

\begin{tabular}{|c|c|c|c|c|c|}
\hline COMPOUND & $\overline{\mathrm{RRF}}$ & RRF5. 0 & $\begin{array}{l}\text { MIN } \\
\text { RRF }\end{array}$ & $\circ \mathrm{D}$ & MAX $\cong D$ \\
\hline Vinyl Chloride-d3 & 0.387 & 0.434 & 0.010 & 12.2 & 50.0 \\
\hline Chloroethane-d5 & 0.257 & 0.282 & 0.010 & 9.8 & 50.0 \\
\hline 1,1-Dichloroethene-d2 & 0.600 & 0.665 & 0.010 & 10.9 & 50.0 \\
\hline 2-Butanone-d5 & 0.040 & 0.039 & 0.010 & -2.5 & 50.0 \\
\hline Chloroform-d & 0.546 & 0.589 & 0.010 & 7.9 & 50.0 \\
\hline 1,2-Dichloroethane-d4 & 0.205 & 0.218 & 0.010 & 6.5 & 50.0 \\
\hline Benzene-d6 & 1.500 & 1.528 & 0.010 & 1.9 & 50.0 \\
\hline 1,2-Dichloropropane-d6 & 0.381 & 0.394 & 0.010 & 3.2 & 50.0 \\
\hline Toluene-d8 & 1.431 & 1.499 & 0.010 & 4.8 & 50.0 \\
\hline trans-1,3-Dichloropropene-d4 & 0.236 & 0.240 & 0.010 & 1.6 & 50.0 \\
\hline 2-Hexanone-d5 & 0.034 & 0.036 & 0.010 & 5.2 & 50.0 \\
\hline $1,1,2,2$-Tetrachloroethane-d2 & 0.160 & 0.163 & 0.010 & 2.2 & 50.0 \\
\hline 1,2-Dichlorobenzene-d4 & 0.844 & 0.836 & 0.010 & -1.0 & 50.0 \\
\hline
\end{tabular}

Report 1,4-Dioxane-d8 for Low/Medium VOA analysis only 
Lab Name: TESTAMERICA BURLINGTON

Contract: $8 \mathrm{E}-00302$

Lab Code: STLV

Case No.: YORK Mod. Ref No.:

SDG No.: 29407

Matrix: (SOIL/SED/WATER) Water

Sample wt/vol: 25.0 $(\mathrm{g} / \mathrm{mL}) \mathrm{mL}$

Level: (TRACE/LOW/MED) TRACE

\% Moisture: not dec.

GC Column: DB-624 ID: $0.20 \quad(\mathrm{~mm})$

Soil Extract Volume: (uL) $(\mathrm{mL})$

Purge Volume: 25.0
Lab Sample ID: MB 200-93092/5

Lab File ID: 15421_005.D

Date Received:

Date Analyzed: 08/24/2015

Dilution Factor: 1.0

Soil Aliquot Volume: (uL)

\begin{tabular}{|c|c|c|c|}
\hline CAS NO. & COMPOUND & $\begin{array}{l}\text { CONCENTRATION UNITS: } \\
(\mathrm{ug} / \mathrm{L} \text { or } \mathrm{ug} / \mathrm{kg}) \mathrm{ug} / \mathrm{L}\end{array}$ & q \\
\hline $75-71-8$ & Dichlorodifluoromethane & 1.0 & $\mathrm{U}$ \\
\hline $74-87-3$ & Chloromethane & 1.0 & $\mathrm{U}$ \\
\hline $75-01-4$ & Vinyl chloride & 1.0 & $\mathrm{U}$ \\
\hline $74-83-9$ & Bromomethane & 1.0 & $\mathrm{U}$ \\
\hline $75-00-3$ & Chloroethane & 1.0 & $\mathrm{U}$ \\
\hline $75-69-4$ & Trichlorofluoromethane & 1.0 & $\mathrm{U}$ \\
\hline $75-35-4$ & 1,1-Dichloroethene & 1.0 & $\mathrm{U}$ \\
\hline $76-13-1$ & $1,1,2$-Trichloro-1,2,2-trifluoroethane & 1.0 & $\mathrm{U}$ \\
\hline $67-64-1$ & Acetone & 5.0 & $\mathrm{U}$ \\
\hline $75-15-0$ & Carbon disulfide & 1.0 & $\mathrm{U}$ \\
\hline $79-20-9$ & Methyl acetate & 1.0 & $\mathrm{U}$ \\
\hline $75-09-2$ & Methylene Chloride & 1.0 & $\mathrm{U}$ \\
\hline $156-60-5$ & trans-1,2-Dichloroethene & 1.0 & $\mathrm{U}$ \\
\hline $1634-04-4$ & Methyl tert-butyl ether & 1.0 & $\mathrm{U}$ \\
\hline $75-34-3$ & 1,1-Dichloroethane & 1.0 & $\mathrm{U}$ \\
\hline $156-59-2$ & cis-1,2-Dichloroethene & 1.0 & $\mathrm{U}$ \\
\hline $78-93-3$ & 2-Butanone & 5.0 & $\mathrm{U}$ \\
\hline $74-97-5$ & Bromochloromethane & 1.0 & $\mathrm{U}$ \\
\hline $67-66-3$ & Chloroform & 1.0 & $\mathrm{U}$ \\
\hline $71-55-6$ & $1,1,1$-Trichloroethane & 1.0 & $\mathrm{U}$ \\
\hline $110-82-7$ & Cyclohexane & 1.0 & $\mathrm{U}$ \\
\hline $56-23-5$ & Carbon tetrachloride & 1.0 & $\mathrm{U}$ \\
\hline $71-43-2$ & Benzene & 1.0 & $\mathrm{U}$ \\
\hline $107-06-2$ & 1,2-Dichloroethane & 1.0 & $\mathrm{U}$ \\
\hline
\end{tabular}

Report 1,4-Dioxane for Low-Medium VOA analysis only 
Lab Name: TESTAMERICA BURLINGTON

Lab Code: STLV

Case No.: YORK

Mod. Ref No.:

Contract: 8E-00302

Matrix: (SOIL/SED/WATER) Water

Sample wt/vol: 25.0 $(\mathrm{g} / \mathrm{mL}) \mathrm{mL}$

Level: (TRACE/LOW/MED) TRACE

\% Moisture: not dec.

GC Column: DB-624 ID: $0.20 \quad(\mathrm{~mm})$

Soil Extract Volume: (uL) $(\mathrm{mL})$

Purge Volume: 25.0 SDG No.: 29407

Lab Sample ID: MB 200-93092/5

Lab File ID: 15421_005.D

Date Received:

Date Analyzed: 08/24/2015

Dilution Factor: 1.0

Soil Aliquot Volume: (uL)

\begin{tabular}{|c|c|c|c|}
\hline CAS NO. & COMPOUND & $\begin{array}{l}\text { CONCENTRATION UNITS: } \\
(\mathrm{ug} / \mathrm{L} \text { or } \mathrm{ug} / \mathrm{kg}) \mathrm{ug} / \mathrm{L}\end{array}$ & $q$ \\
\hline $79-01-6$ & Trichloroethene & 1.0 & $\mathrm{U}$ \\
\hline $108-87-2$ & Methylcyclohexane & 1.0 & $\mathrm{U}$ \\
\hline $78-87-5$ & 1,2-Dichloropropane & 1.0 & $\mathrm{U}$ \\
\hline $75-27-4$ & Bromodichloromethane & 1.0 & $\mathrm{U}$ \\
\hline $10061-01-5$ & cis-1,3-Dichloropropene & 1.0 & U \\
\hline $108-10-1$ & 4-Methyl-2-pentanone & 1.0 & $\mathrm{U}$ \\
\hline $108-88-3$ & Toluene & 1.0 & $\mathrm{U}$ \\
\hline $10061-02-6$ & trans-1,3-Dichloropropene & 1.0 & $\mathrm{U}$ \\
\hline $79-00-5$ & $1,1,2-$ Trichloroethane & 1.0 & $\mathrm{U}$ \\
\hline $127-18-4$ & Tetrachloroethene & 1.0 & $\mathrm{U}$ \\
\hline $591-78-6$ & 2-Hexanone & 1.0 & $\mathrm{U}$ \\
\hline $124-48-1$ & Dibromochloromethane & 1.0 & $\mathrm{U}$ \\
\hline $106-93-4$ & 1,2-Dibromoethane & 1.0 & $\mathrm{U}$ \\
\hline $108-90-7$ & Chlorobenzene & 1.0 & $\mathrm{U}$ \\
\hline $100-41-4$ & Ethylbenzene & 1.0 & $\mathrm{U}$ \\
\hline $95-47-6$ & o-Xylene & 1.0 & $\mathrm{U}$ \\
\hline $179601-23-1$ & m,p-xylene & 1.0 & $\mathrm{U}$ \\
\hline $100-42-5$ & Styrene & 1.0 & $\mathrm{U}$ \\
\hline $75-25-2$ & Bromoform & 1.0 & $\mathrm{U}$ \\
\hline $98-82-8$ & Isopropylbenzene & 1.0 & $\mathrm{U}$ \\
\hline $79-34-5$ & $1,1,2,2$-Tetrachloroethane & 1.0 & $\mathrm{U}$ \\
\hline $541-73-1$ & 1,3 -Dichlorobenzene & 1.0 & $\mathrm{U}$ \\
\hline $106-46-7$ & 1,4-Dichlorobenzene & 1.0 & $\mathrm{U}$ \\
\hline $95-50-1$ & 1,2-Dichlorobenzene & 1.0 & $\mathrm{U}$ \\
\hline $96-12-8$ & 1,2-Dibromo-3-Chloropropane & 1.0 & $\mathrm{U}$ \\
\hline $120-82-1$ & $1,2,4$-Trichlorobenzene & 0.037 & $\mathrm{~J}$ \\
\hline $87-61-6$ & $1,2,3$-Trichlorobenzene & 0.057 & $\mathrm{~J}$ \\
\hline
\end{tabular}


Lab Name: TESTAMERICA BURLINGTON

Contract: 8E-00302

Lab Code: STLV Case No.: YORK Mod. Ref No.: SDG No.: 29407

Matrix: (SOIL/SED/WATER) Water

Sample wt/vol: 25.0 $(\mathrm{g} / \mathrm{mL}) \mathrm{mL}$

Level: (TRACE or LOW/MED) TRACE

\% Moisture: not dec.

GC Column: DB-624 ID $: 0.20 \quad(\mathrm{~mm})$

Soil Extract Volume: (uL)

CONCENTRATION UNITS: (ug/L or ug/kg) ug/L
Lab Sample ID: MB 200-93092/5

Lab File ID: 15421_005.D

Date Received:

Date Analyzed: 08/24/2015

Dilution Factor: 1.0

Soil Aliquot Volume: (uL) Purge Volume: 25.0

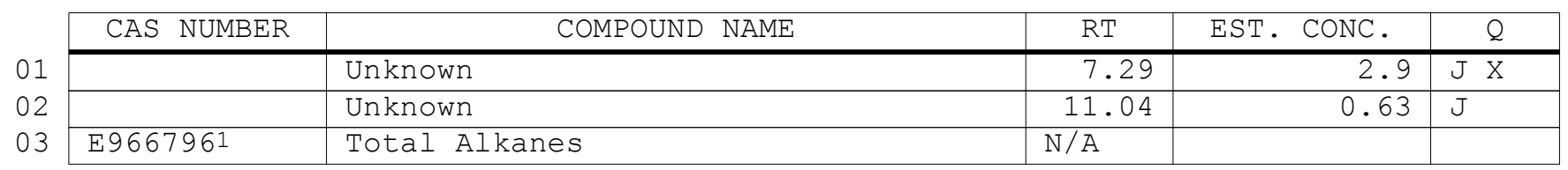

1EPA-designated Registry Number. 
Lab Name: TESTAMERICA BURLINGTON

Contract: $8 \mathrm{E}-00302$

Lab Code: STLV

Case No.: YORK Mod. Ref No.:

SDG No.: 29407

Matrix: (SOIL/SED/WATER) Water

Sample wt/vol: 25.0 $(\mathrm{g} / \mathrm{mL}) \mathrm{mL}$

Level: (TRACE/LOW/MED) TRACE

\% Moisture: not dec.

GC Column: DB-624 ID: $0.20 \quad(\mathrm{~mm})$

Soil Extract Volume: $(u L)$ $(\mathrm{mL})$

Purge Volume: 25.0
Lab Sample ID: 200-29407-4

Lab File ID: 15421_011.D

Date Received:

Date Analyzed: 08/24/2015

Dilution Factor: 1.0

Soil Aliquot Volume:

(uL)

\begin{tabular}{|c|c|c|c|}
\hline CAS NO. & COMPOUND & $\begin{array}{l}\text { CONCENTRATION UNITS: } \\
(\mathrm{ug} / \mathrm{L} \text { or } \mathrm{ug} / \mathrm{kg}) \mathrm{ug} / \mathrm{L}\end{array}$ & q \\
\hline $75-71-8$ & Dichlorodifluoromethane & 1.0 & $\mathrm{U}$ \\
\hline $74-87-3$ & Chloromethane & 0.12 & $\mathrm{~J}$ \\
\hline $75-01-4$ & Vinyl chloride & 1.0 & $\mathrm{U}$ \\
\hline $74-83-9$ & Bromomethane & 1.0 & $\mathrm{U}$ \\
\hline $75-00-3$ & Chloroethane & 1.0 & $\mathrm{U}$ \\
\hline $75-69-4$ & Trichlorofluoromethane & 1.0 & $\mathrm{U}$ \\
\hline $75-35-4$ & 1,1-Dichloroethene & 1.0 & $\mathrm{U}$ \\
\hline $76-13-1$ & $1,1,2$-Trichloro-1,2,2-trifluoroethane & 1.0 & $\mathrm{U}$ \\
\hline $67-64-1$ & Acetone & 1.1 & $\mathrm{~J}$ \\
\hline $75-15-0$ & Carbon disulfide & 0.013 & $\mathrm{~J}$ \\
\hline $79-20-9$ & Methyl acetate & 1.0 & $\mathrm{U}$ \\
\hline $75-09-2$ & Methylene Chloride & 1.0 & $\mathrm{U}$ \\
\hline $156-60-5$ & trans-1,2-Dichloroethene & 1.0 & $\mathrm{U}$ \\
\hline $1634-04-4$ & Methyl tert-butyl ether & 1.0 & $\mathrm{U}$ \\
\hline $75-34-3$ & 1,1-Dichloroethane & 1.0 & $\mathrm{U}$ \\
\hline $156-59-2$ & cis-1,2-Dichloroethene & 1.0 & $\mathrm{U}$ \\
\hline $78-93-3$ & 2-Butanone & 5.0 & $\mathrm{U}$ \\
\hline $74-97-5$ & Bromochloromethane & 1.0 & $\mathrm{U}$ \\
\hline $67-66-3$ & Chloroform & 1.0 & $\mathrm{U}$ \\
\hline $71-55-6$ & $1,1,1$-Trichloroethane & 1.0 & $\mathrm{U}$ \\
\hline $110-82-7$ & Cyclohexane & 1.0 & $\mathrm{U}$ \\
\hline $56-23-5$ & Carbon tetrachloride & 1.0 & $\mathrm{U}$ \\
\hline $71-43-2$ & Benzene & 1.0 & $\mathrm{U}$ \\
\hline $107-06-2$ & 1,2-Dichloroethane & 1.0 & $\mathrm{U}$ \\
\hline
\end{tabular}

Report 1,4-Dioxane for Low-Medium VOA analysis only 
Lab Name: TESTAMERICA BURLINGTON

Lab Code: STLV

Case No.: YORK

Mod. Ref No.:

Contract: 8E-00302

Matrix: (SOIL/SED/WATER) Water

Sample wt/vol: 25.0 $(\mathrm{g} / \mathrm{mL}) \mathrm{mL}$

Level: (TRACE/LOW/MED) TRACE

\% Moisture: not dec.

GC Column: DB-624 ID $: \underline{0.20 \quad(\mathrm{~mm})}$

Soil Extract Volume: (uL) $(\mathrm{mL})$

Purge Volume: 25.0
SDG No.: 29407

Lab Sample ID: 200-29407-4

Lab File ID: 15421_011.D

Date Received:

Date Analyzed: 08/24/2015

Dilution Factor: 1.0

Soil Aliquot Volume: (uL)

\begin{tabular}{|c|c|c|c|}
\hline CAS NO. & COMPOUND & $\begin{array}{l}\text { CONCENTRATION UNITS: } \\
(\mathrm{ug} / \mathrm{L} \text { or } \mathrm{ug} / \mathrm{kg}) \mathrm{ug} / \mathrm{L}\end{array}$ & $q$ \\
\hline $79-01-6$ & Trichloroethene & 1.0 & $\mathrm{U}$ \\
\hline $108-87-2$ & Methylcyclohexane & 1.0 & $\mathrm{U}$ \\
\hline $78-87-5$ & 1,2-Dichloropropane & 1.0 & $\mathrm{U}$ \\
\hline $75-27-4$ & Bromodichloromethane & 1.0 & $\mathrm{U}$ \\
\hline $10061-01-5$ & cis-1,3-Dichloropropene & 1.0 & U \\
\hline $108-10-1$ & 4-Methyl-2-pentanone & 1.0 & $\mathrm{U}$ \\
\hline $108-88-3$ & Toluene & 0.010 & $\mathrm{~J}$ \\
\hline $10061-02-6$ & trans-1,3-Dichloropropene & 1.0 & $\mathrm{U}$ \\
\hline $79-00-5$ & $1,1,2-$ Trichloroethane & 1.0 & $\mathrm{U}$ \\
\hline $127-18-4$ & Tetrachloroethene & 1.0 & $\mathrm{U}$ \\
\hline $591-78-6$ & 2-Hexanone & 1.0 & $\mathrm{U}$ \\
\hline $124-48-1$ & Dibromochloromethane & 1.0 & $\mathrm{U}$ \\
\hline $106-93-4$ & 1,2-Dibromoethane & 1.0 & $\mathrm{U}$ \\
\hline $108-90-7$ & Chlorobenzene & 1.0 & $\mathrm{U}$ \\
\hline $100-41-4$ & Ethylbenzene & 1.0 & $\mathrm{U}$ \\
\hline $95-47-6$ & o-Xylene & 1.0 & $\mathrm{U}$ \\
\hline $179601-23-1$ & m,p-xylene & 0.0048 & $\mathrm{~J}$ \\
\hline $100-42-5$ & Styrene & 1.0 & $\mathrm{U}$ \\
\hline $75-25-2$ & Bromoform & 1.0 & $\mathrm{U}$ \\
\hline $98-82-8$ & Isopropylbenzene & 1.0 & $\mathrm{U}$ \\
\hline $79-34-5$ & $1,1,2,2$-Tetrachloroethane & 1.0 & $\mathrm{U}$ \\
\hline $541-73-1$ & 1,3 -Dichlorobenzene & 1.0 & $\mathrm{U}$ \\
\hline $106-46-7$ & 1,4-Dichlorobenzene & 1.0 & $\mathrm{U}$ \\
\hline $95-50-1$ & 1,2-Dichlorobenzene & 1.0 & $\mathrm{U}$ \\
\hline $96-12-8$ & 1,2-Dibromo-3-Chloropropane & 1.0 & $\mathrm{U}$ \\
\hline $120-82-1$ & $1,2,4$-Trichlorobenzene & 1.0 & $\mathrm{U}$ \\
\hline $87-61-6$ & $1,2,3$-Trichlorobenzene & 1.0 & $\mathrm{U}$ \\
\hline
\end{tabular}


Lab Name: TESTAMERICA BURLINGTON

Contract: $8 \mathrm{E}-00302$

Lab Code: STLV Case No.: YORK Mod. Ref No.: SDG No.: 29407

Matrix: (SOIL/SED/WATER) Water

Sample wt/vol: 25.0 $(\mathrm{g} / \mathrm{mL}) \mathrm{mL}$

Level: (TRACE or LOW/MED) TRACE

\% Moisture: not dec.

GC Column: DB-624 ID $: 0.20 \quad(\mathrm{~mm})$

Soil Extract Volume: (uL)

CONCENTRATION UNITS: (ug/L or ug/kg) ug/L
Lab Sample ID: 200-29407-4

Lab File ID: 15421_011.D

Date Received:

Date Analyzed: 08/24/2015

Dilution Factor: 1.0

Soil Aliquot Volume: (uL) Purge Volume: 25.0

\begin{tabular}{|c|c|c|c|c|}
\hline CAS NUMBER & COMPOUND NAME & $\mathrm{RT}$ & EST. CONC. & Q \\
\hline 01 & Unknown & 7.29 & 2.8 & $\mathrm{~J} B \mathrm{X}$ \\
\hline $541-05-9$ & Cyclotrisiloxane, hexamethyl- & 8.22 & 1.4 & $\mathrm{~J} \mathrm{~N}$ \\
\hline 03 & Unknown & 11.04 & 1.1 & $\mathrm{~J} B$ \\
\hline E9667961 & Total Alkanes & $\mathrm{N} / \mathrm{A}$ & & \\
\hline
\end{tabular}

1EPA-designated Registry Number. 
Lab Name: TESTAMERICA BURLINGTON

Contract: $8 \mathrm{E}-00302$

Lab Code: STLV

Case No.: YORK Mod. Ref No.:

SDG No.: 29407

Matrix: (SOIL/SED/WATER) Water

Sample wt/vol: 25.0 $(\mathrm{g} / \mathrm{mL}) \mathrm{mL}$

Level: (TRACE/LOW/MED) TRACE

\% Moisture: not dec.

GC Column: DB-624 ID: $0.20 \quad(\mathrm{~mm})$

Soil Extract Volume: $(u L)$ $(\mathrm{mL})$

Purge Volume: 25.0
Lab Sample ID: VIBLK 200-93092/10

Lab File ID: 15421_010.D

Date Received:

Date Analyzed: 08/24/2015

Dilution Factor: 1.0

Soil Aliquot Volume:

(uL)

\begin{tabular}{|c|c|c|c|}
\hline CAS NO. & COMPOUND & $\begin{array}{l}\text { CONCENTRATION UNITS: } \\
(\mathrm{ug} / \mathrm{L} \text { or } \mathrm{ug} / \mathrm{kg}) \mathrm{ug} / \mathrm{L}\end{array}$ & q \\
\hline $75-71-8$ & Dichlorodifluoromethane & 1.0 & $\mathrm{U}$ \\
\hline $74-87-3$ & Chloromethane & 1.0 & $\mathrm{U}$ \\
\hline $75-01-4$ & Vinyl chloride & 1.0 & $\mathrm{U}$ \\
\hline $74-83-9$ & Bromomethane & 1.0 & $\mathrm{U}$ \\
\hline $75-00-3$ & Chloroethane & 1.0 & $\mathrm{U}$ \\
\hline $75-69-4$ & Trichlorofluoromethane & 1.0 & $\mathrm{U}$ \\
\hline $75-35-4$ & 1,1-Dichloroethene & 1.0 & $\mathrm{U}$ \\
\hline $76-13-1$ & $1,1,2$-Trichloro-1,2,2-trifluoroethane & 1.0 & $\mathrm{U}$ \\
\hline $67-64-1$ & Acetone & 1.1 & $\mathrm{~J}$ \\
\hline $75-15-0$ & Carbon disulfide & 0.016 & $\mathrm{~J}$ \\
\hline $79-20-9$ & Methyl acetate & 1.0 & $\mathrm{U}$ \\
\hline $75-09-2$ & Methylene Chloride & 1.0 & $\mathrm{U}$ \\
\hline $156-60-5$ & trans-1,2-Dichloroethene & 1.0 & $\mathrm{U}$ \\
\hline $1634-04-4$ & Methyl tert-butyl ether & 1.0 & $\mathrm{U}$ \\
\hline $75-34-3$ & 1,1-Dichloroethane & 1.0 & $\mathrm{U}$ \\
\hline $156-59-2$ & cis-1,2-Dichloroethene & 1.0 & $\mathrm{U}$ \\
\hline $78-93-3$ & 2-Butanone & 5.0 & $\mathrm{U}$ \\
\hline $74-97-5$ & Bromochloromethane & 1.0 & $\mathrm{U}$ \\
\hline $67-66-3$ & Chloroform & 1.0 & $\mathrm{U}$ \\
\hline $71-55-6$ & $1,1,1$-Trichloroethane & 1.0 & $\mathrm{U}$ \\
\hline $110-82-7$ & Cyclohexane & 1.0 & $\mathrm{U}$ \\
\hline $56-23-5$ & Carbon tetrachloride & 0.034 & $\mathrm{~J}$ \\
\hline $71-43-2$ & Benzene & 1.0 & $\mathrm{U}$ \\
\hline $107-06-2$ & 1,2-Dichloroethane & 1.0 & $\mathrm{U}$ \\
\hline
\end{tabular}

Report 1,4-Dioxane for Low-Medium VOA analysis only 
Lab Name: TESTAMERICA BURLINGTON

Lab Code: STLV

Case No.: YORK

Mod. Ref No.:

Contract: 8E-00302

Matrix: (SOIL/SED/WATER) Water

Sample wt/vol: 25.0 $(\mathrm{g} / \mathrm{mL}) \mathrm{mL}$

Level: (TRACE/LOW/MED) TRACE

\% Moisture: not dec.

GC Column: DB-624 ID: $0.20 \quad(\mathrm{~mm})$

Soil Extract Volume: (uL) $(\mathrm{mL})$

Purge Volume: 25.0
SDG No.: 29407

Lab Sample ID: VIBLK 200-93092/10

Lab File ID: 15421_010.D

Date Received:

Date Analyzed: 08/24/2015

Dilution Factor: 1.0

Soil Aliquot Volume:

(uL)

\begin{tabular}{|c|c|c|c|}
\hline CAS NO. & COMPOUND & $\begin{array}{l}\text { CONCENTRATION UNITS: } \\
(\mathrm{ug} / \mathrm{L} \text { or } \mathrm{ug} / \mathrm{kg}) \mathrm{ug} / \mathrm{L}\end{array}$ & Q \\
\hline $79-01-6$ & Trichloroethene & 1.0 & $\mathrm{U}$ \\
\hline $108-87-2$ & Methylcyclohexane & 1.0 & $\mathrm{U}$ \\
\hline $78-87-5$ & 1,2-Dichloropropane & 1.0 & $\mathrm{U}$ \\
\hline $75-27-4$ & Bromodichloromethane & 1.0 & $\mathrm{U}$ \\
\hline $10061-01-5$ & cis-1,3-Dichloropropene & 1.0 & $\mathrm{U}$ \\
\hline $108-10-1$ & 4-Methyl-2-pentanone & 5.0 & $\mathrm{U}$ \\
\hline $108-88-3$ & Toluene & 0.010 & $\mathrm{~J}$ \\
\hline $10061-02-6$ & trans-1,3-Dichloropropene & 1.0 & $\mathrm{U}$ \\
\hline $79-00-5$ & $1,1,2-T r i c h l o r o e t h a n e$ & 1.0 & $\mathrm{U}$ \\
\hline $127-18-4$ & Tetrachloroethene & 1.0 & $\mathrm{U}$ \\
\hline $591-78-6$ & 2-Hexanone & 5.0 & $\mathrm{U}$ \\
\hline $124-48-1$ & Dibromochloromethane & 1.0 & $\mathrm{U}$ \\
\hline $106-93-4$ & 1,2-Dibromoethane & 1.0 & $\mathrm{U}$ \\
\hline $108-90-7$ & Chlorobenzene & 1.0 & $\mathrm{U}$ \\
\hline $100-41-4$ & Ethylbenzene & 1.0 & $\mathrm{U}$ \\
\hline $95-47-6$ & o-Xylene & 1.0 & $\mathrm{U}$ \\
\hline $179601-23-1$ & m,p-Xylene & 0.0067 & $\mathrm{~J}$ \\
\hline $100-42-5$ & styrene & 1.0 & $\mathrm{U}$ \\
\hline $75-25-2$ & Bromoform & 1.0 & $\mathrm{U}$ \\
\hline $98-82-8$ & Isopropylbenzene & 1.0 & $\mathrm{U}$ \\
\hline $79-34-5$ & $1,1,2,2$-Tetrachloroethane & 1.0 & $\mathrm{U}$ \\
\hline $541-73-1$ & 1,3-Dichlorobenzene & 1.0 & $\mathrm{U}$ \\
\hline $106-46-7$ & 1,4-Dichlorobenzene & 1.0 & $\mathrm{U}$ \\
\hline $95-50-1$ & 1,2-Dichlorobenzene & 1.0 & $\mathrm{U}$ \\
\hline $96-12-8$ & 1,2-Dibromo-3-Chloropropane & 1.0 & $\mathrm{U}$ \\
\hline $120-82-1$ & $1,2,4$-Trichlorobenzene & 0.0054 & $\mathrm{~J} B$ \\
\hline $87-61-6$ & $1,2,3$-Trichlorobenzene & 1.0 & $\mathrm{U}$ \\
\hline
\end{tabular}


Lab Name: TESTAMERICA BURLINGTON

Contract: 8E-00302

Lab Code: STLV Case No.: YORK Mod. Ref No.: SDG No.: 29407

Matrix: (SOIL/SED/WATER) Water

Sample wt/vol: 25.0 $(\mathrm{g} / \mathrm{mL}) \mathrm{mL}$

Level: (TRACE or LOW/MED) TRACE

\% Moisture: not dec.

GC Column: DB-624 ID $: 0.20 \quad(\mathrm{~mm})$

Soil Extract Volume: (uL)

CONCENTRATION UNITS: (ug/L or ug/kg) ug/L
Lab Sample ID: VIBLK 200-93092/10

Lab File ID: $15421010 . \mathrm{D}$

Date Received:

Date Analyzed: 08/24/2015

Dilution Factor: 1.0

Soil Aliquot Volume: (uL) Purge Volume: 25.0 $(\mathrm{mL})$

01

02

\begin{tabular}{|l|l|c|c|c|}
\hline CAS NUMBER & \multicolumn{1}{|c|}{ COMPOUND NAME } & RT & EST. CONC. & Q \\
\hline & Unknown & 7.29 & 2.9 & $\mathrm{~J}$ B X \\
\hline E9667961 & Total Alkanes & N/A & & \\
\hline
\end{tabular}

1 EPA-designated Registry Number. 
Lab Name: TestAmerica Burlington

Job No.: 200-29407-1

SDG No.: 29407

Instrument ID: CHD.i

Analysis Batch Number: 92921

Lab Sample ID: IC 200-92921/3

Client Sample ID:

Date Analyzed: 08/19/15 15:14

Lab File ID: 15357 003.D

GC Column: DB-624

ID : $0.2(\mathrm{~mm})$

\begin{tabular}{|l|c|c|c|}
\hline \multicolumn{1}{|c|}{ COMPOUND NAME } & RETENTION & \multicolumn{2}{|c|}{ MANUAL INTEGRATION } \\
\cline { 3 - 4 } & TIME & REASON & ANALYST \\
\hline Dichlorodifluoromethane & 1.52 & Baseline & DATE \\
\hline 1,2 -Dibromo-3-Chloropropane & 13.43 & Assign Peak & 08/19/15 $16: 20$ \\
\hline
\end{tabular}

Lab Sample ID: IC 200-92921/4 Client Sample ID:

Date Analyzed: 08/19/15 15:39

Lab File ID: 15357_004.D

GC Column: DB-624

ID $: 0.2(\mathrm{~mm})$

\begin{tabular}{|c|c|c|c|c|}
\hline \multirow[t]{2}{*}{ COMPOUND NAME } & \multirow{2}{*}{$\begin{array}{c}\text { RETENTION } \\
\text { TIME }\end{array}$} & \multicolumn{3}{|c|}{ MANUAL INTEGRATION } \\
\hline & & REASON & ANALYST & DATE \\
\hline Dichlorodifluoromethane & 1.53 & Baseline & wilburj & $08 / 19 / 15 \quad 16: 21$ \\
\hline Bromomethane & 2.00 & Baseline & wilburj & $08 / 19 / 15 \quad 16: 27$ \\
\hline Chloroethane & 2.08 & Baseline & wilburj & $08 / 19 / 1516: 27$ \\
\hline 1,2-Dibromo-3-Chloropropane & 13.44 & Assign Peak & wilburj & $08 / 19 / 15 \quad 16: 27$ \\
\hline
\end{tabular}

Lab Sample ID: ICIS 200-92921/5

Client Sample ID:

Date Analyzed: 08/19/15 16:04

Lab File ID: 15357_005.D

GC Column: DB-624

ID : $0.2(\mathrm{~mm})$

\begin{tabular}{|c|c|c|c|c|}
\hline \multirow[t]{2}{*}{ COMPOUND NAME } & \multirow{2}{*}{$\begin{array}{c}\text { RETENTION } \\
\text { TIME }\end{array}$} & \multicolumn{3}{|c|}{ MANUAL INTEGRATION } \\
\hline & & REASON & ANALYST & DATE \\
\hline Dichlorodifluoromethane & 1.53 & Baseline & wilburj & $08 / 19 / 1516: 30$ \\
\hline Bromomethane & 2.00 & Baseline & wilburj & $08 / 19 / 15 \quad 17: 11$ \\
\hline
\end{tabular}

Lab Sample ID: IC 200-92921/6

Client Sample ID:

Date Analyzed: 08/19/15 16:29

Lab File ID: 15357_006.D

GC Column: DB-624

ID : $0.2(\mathrm{~mm})$

\begin{tabular}{|c|c|c|c|c|}
\hline \multirow[t]{2}{*}{ COMPOUND NAME } & \multirow{2}{*}{$\begin{array}{c}\text { RETENTION } \\
\text { TIME }\end{array}$} & \multicolumn{3}{|c|}{ MANUAL INTEGRATION } \\
\hline & & REASON & ANALYST & DATE \\
\hline Dichlorodifluoromethane & 1.53 & Baseline & wilburj & $08 / 19 / 15 \quad 17: 12$ \\
\hline Bromomethane & 2.00 & Baseline & wilburj & $08 / 19 / 15 \quad 17: 12$ \\
\hline
\end{tabular}

SOM01.2/VOA_Tr 
Lab Name: TestAmerica Burlington

SDG No.: 29407

Instrument ID: CHD.i

Lab Sample ID: IC 200-92921/7

Date Analyzed: 08/19/15 16:54
Job No.: 200-29407-1

Analysis Batch Number: 92921

Client Sample ID:

Lab File ID: 15357 007.D
GC Column: DB-624

ID $: 0.2(\mathrm{~mm})$

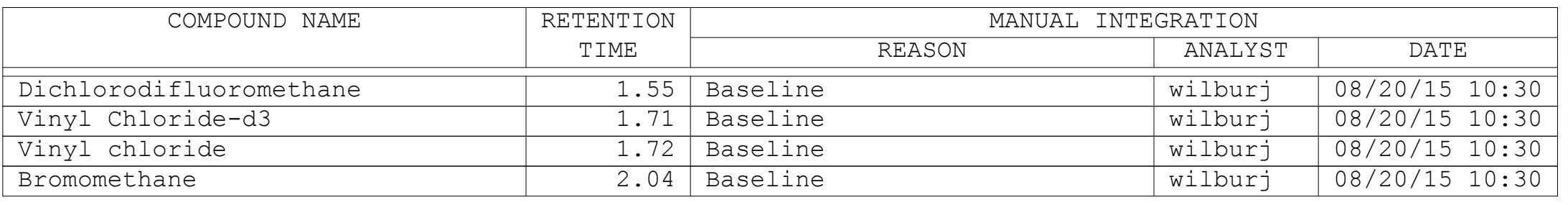


Lab Name: TestAmerica Burlington

SDG No.: 29407

Instrument ID: CHD.i

Lab Sample ID: 200-29407-3 DL

Date Analyzed: 08/24/15 15:01
Job No.: 200-29407-1

Analysis Batch Number: 93092

Client Sample ID: YKTI12-145-W-37943 DL

Lab File ID: 15421 008.D
GC Column: DB-624

ID $: 0.2(\mathrm{~mm})$

\begin{tabular}{|c|c|c|c|c|}
\hline \multirow[t]{2}{*}{ COMPOUND NAME } & RETENTION & \multicolumn{3}{|c|}{ MANUAL INTEGRATION } \\
\hline & TIME & REASON & ANALYST & DATE \\
\hline Methylene Chloride & 3.21 & Baseline & wilburj & $08 / 27 / 15 \quad 17: 17$ \\
\hline
\end{tabular}

Lab Sample ID: CCVC 200-93092/12

Client Sample ID:

Date Analyzed: 08/24/15 16:41

Lab File ID: 15421 012.D

GC Column: DB-624

ID $: 0.2(\mathrm{~mm})$

\begin{tabular}{|l|c|c|c|c|}
\hline \multicolumn{1}{|c|}{ COMPOUND NAME } & RETENTION & \multicolumn{2}{|c|}{ MANUAL INTEGRATION } \\
\cline { 3 - 4 } & TIME & REASON & ANALYST & DATE \\
\hline \hline Dichlorodifluoromethane & 1.53 & Baseline & archern & $08 / 25 / 15$ 09:43 \\
\hline
\end{tabular}




\section{TestAmerica}

THE LEADER IN ENVIRONMENTAL TESTING

\section{ANALYTICAL REPORT}

Job Number: 200-29506-1

SDG Number: York (200-29506)

Job Description: York (200-29506)

Contract Number: 1E-30401

For:

Argonne National Laboratory

9700 South Cass Avenue

Building 203

Office B-141

Argonne, IL 60439

Attention: Ms. Esther Bowen

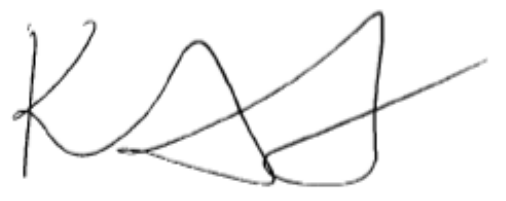

Approved for release. Kathryn A Kelly

\section{Kathryn A Kelly, Project Manager I \\ 30 Community Drive, South Burlington, VT, 05403 \\ kathryn.kelly@testamericainc.com \\ $09 / 11 / 2015$}

The test results in this report relate only to sample(s) as received by the laboratory. These test results were derived under a quality system that adheres to the requirements of NELAC. Pursuant to NELAC, this report may not be produced in full without written approval from the laboratory 


\section{Table of Contents}

Cover Title Page..$\ldots \ldots \ldots \ldots \ldots \ldots \ldots \ldots \ldots \ldots \ldots \ldots \ldots \ldots$

Sample List ........................... 4

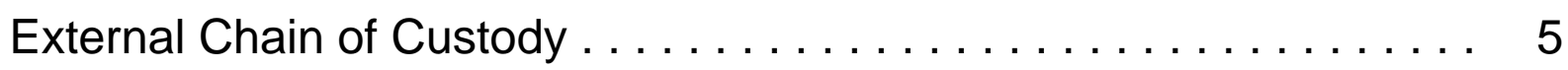

Shipping Documentation . . . . . . . . . . . . . . . 7

Sample Receipt and Log In Check List ..................... 8

Standards Traceability . . . . . . . . . . . . . . . . . 9

Methodology Review .............................. 14

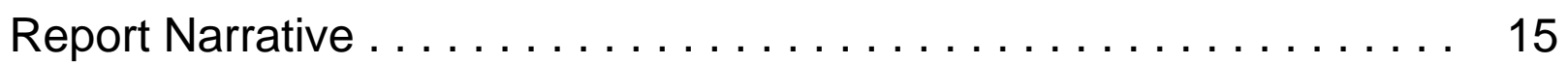

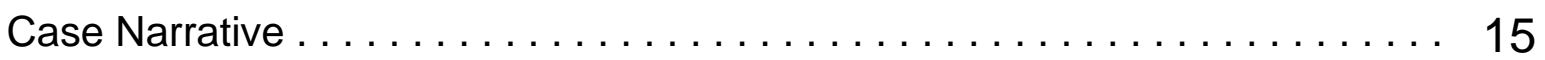

Manual Integration Documentation ....................... 16

Qualifier Definition ................................ 19

QC Summary - SOM01.2 Volatiles-Trace . . . . . . . . . . . . 20

QC Summary - SOM01.2 Volatiles-Trace .................... 20

Deuterated Monitoring Compound Summary $\ldots \ldots \ldots \ldots \ldots \ldots \ldots \ldots \ldots \ldots \ldots, 20$

Method Blank $\ldots \ldots \ldots \ldots \ldots \ldots \ldots \ldots \ldots \ldots \ldots \ldots \ldots \ldots \ldots \ldots \ldots \ldots \ldots, 22$

GC/MS Instrument Performance Check $\ldots \ldots \ldots \ldots \ldots \ldots \ldots \ldots \ldots \ldots \ldots \ldots \ldots \ldots$

Internal Standard Area and RT Summary $\ldots \ldots \ldots \ldots \ldots \ldots \ldots \ldots \ldots \ldots \ldots \ldots \ldots \ldots \ldots$

Sample Data - SOM01.2 Volatiles-Trace .............. 26

Sample Data - SOM01.2 Volatiles-Trace ..................... 26

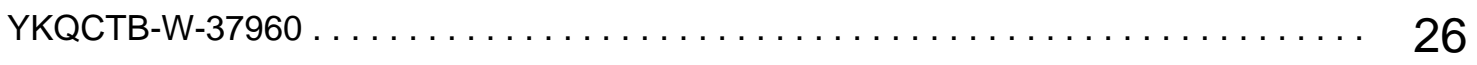

YKTI14-136-W-37954 .................................... 29

YKTI16-136-W-37964 ......................................... 35

YKTI18-114-W-37973 ..................................... 41

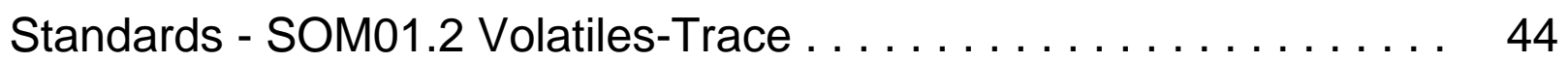

Standards - SOM01.2 Volatiles-Trace ...................... 44

Initial Calibration Data .................................. 44 


\section{Table of Contents}

CCV Data, including closing CCV $\ldots \ldots \ldots \ldots \ldots \ldots \ldots \ldots \ldots \ldots \ldots \ldots \ldots \ldots \ldots$

Raw Qc Data - SOM01.2 Volatiles-Trace . . . . . . . . . . . . 53

Raw Qc Data - SOM01.2 Volatiles-Trace ..................... 53

Raw Qc Data - SOM01.2 Volatiles-Trace $\ldots \ldots \ldots \ldots \ldots \ldots \ldots \ldots \ldots \ldots \ldots \ldots \ldots$

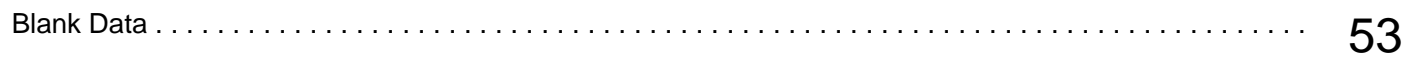




\section{Sample Login Acknowledgement}

Job 200-29506-1

\begin{tabular}{|c|c|c|c|}
\hline Client Job Description: & York (200-29506) & Report To: & Argonne National Laboratory \\
\hline Purchase Order \#: & $1 \mathrm{E}-30401$ & & Jorge Alvarado \\
\hline Work Order \#: & $1 \mathrm{E}-30401$ & & 9700 South Cass Avenue \\
\hline Project Manager: & Kathryn A Kelly & & Building 203 \\
\hline Job Due Date: & $9 / 12 / 2015$ & & Argonne, IL 60439 \\
\hline Job TAT: & 14 Days & & \\
\hline Max Deliverable Level: & IV & Bill To: & $\begin{array}{l}\text { Argonne National Laboratory } \\
\text { Accounts Payable }\end{array}$ \\
\hline Earliest Deliverable Due: & $9 / 12 / 2015$ & & $\begin{array}{l}\text { Chief Financial Offices } \\
9700 \text { S. Cass Ave. } \\
\text { Building } 201 \\
\text { Argonne, IL } 60439\end{array}$ \\
\hline
\end{tabular}

\section{Login 200-29506}

\section{Sample Receipt:}

Method of Delivery:

\begin{tabular}{|c|c|}
\hline $\begin{array}{l}\text { Lab Sample \# } \\
\text { Method }\end{array}$ & $\begin{array}{l}\text { Client Sample ID } \\
\text { Method Description / Work Location }\end{array}$ \\
\hline $200-29506-1$ & YKTI14-136-W-37954 \\
\hline SOM01.2_Vol_Tr & r SOM01.2 Trace Volatile Organics / In-Lab \\
\hline 200-29506-2 & YKQCTB-W-37960 \\
\hline SOM01.2_Vol_Tr & ir SOM01.2 Trace Volatile Organics / In-Lab \\
\hline 200-29506-3 & YKTI16-136-W-37964 \\
\hline SOM01.2_Vol_Tr & r SOM01.2 Trace Volatile Organics / In-Lab \\
\hline 200-29506-4 & YKTI18-114-W-37973 \\
\hline SOM01.2_Vol_Tr & r SOM01.2 Trace Volatile Organics / In-Lab \\
\hline 200-29506-5 & VHBLK01 \\
\hline SOM01.2_Vol_Tr & r $\quad$ SOM01.2 Trace Volatile Organics / In-Lab \\
\hline
\end{tabular}

\begin{abstract}
Number of Coolers:
1

Cooler Temperature(s) $\left(\mathbf{C}^{\circ}\right)$ : 3.0 ;
\end{abstract}

\begin{tabular}{|c|c|c|c|c|}
\hline \multirow{2}{*}{\multicolumn{2}{|c|}{ Date Sampled }} & \multirow[t]{2}{*}{ Matrix } & \multirow[b]{2}{*}{ Rpt Basis } & \multirow[b]{2}{*}{ Dry / Wet ** } \\
\hline & & & & \\
\hline \multirow{2}{*}{$8 / 23 / 2015$} & 2:45:00 PM & Water & & \\
\hline & & & Total & Wet \\
\hline \multirow[t]{2}{*}{$8 / 23 / 2015$} & 9:00:00 AM & Water & & \\
\hline & & & Total & Wet \\
\hline \multirow[t]{2}{*}{$8 / 25 / 2015$} & 2:50:00 PM & Water & & \\
\hline & & & Total & Wet \\
\hline \multirow[t]{2}{*}{$8 / 28 / 2015$} & 11:00:00 AM & Water & & \\
\hline & & & Total & Wet \\
\hline \multirow[t]{2}{*}{$8 / 31 / 2015$} & 10:25:00 AM & Water & & \\
\hline & & & Total & Wet \\
\hline
\end{tabular}




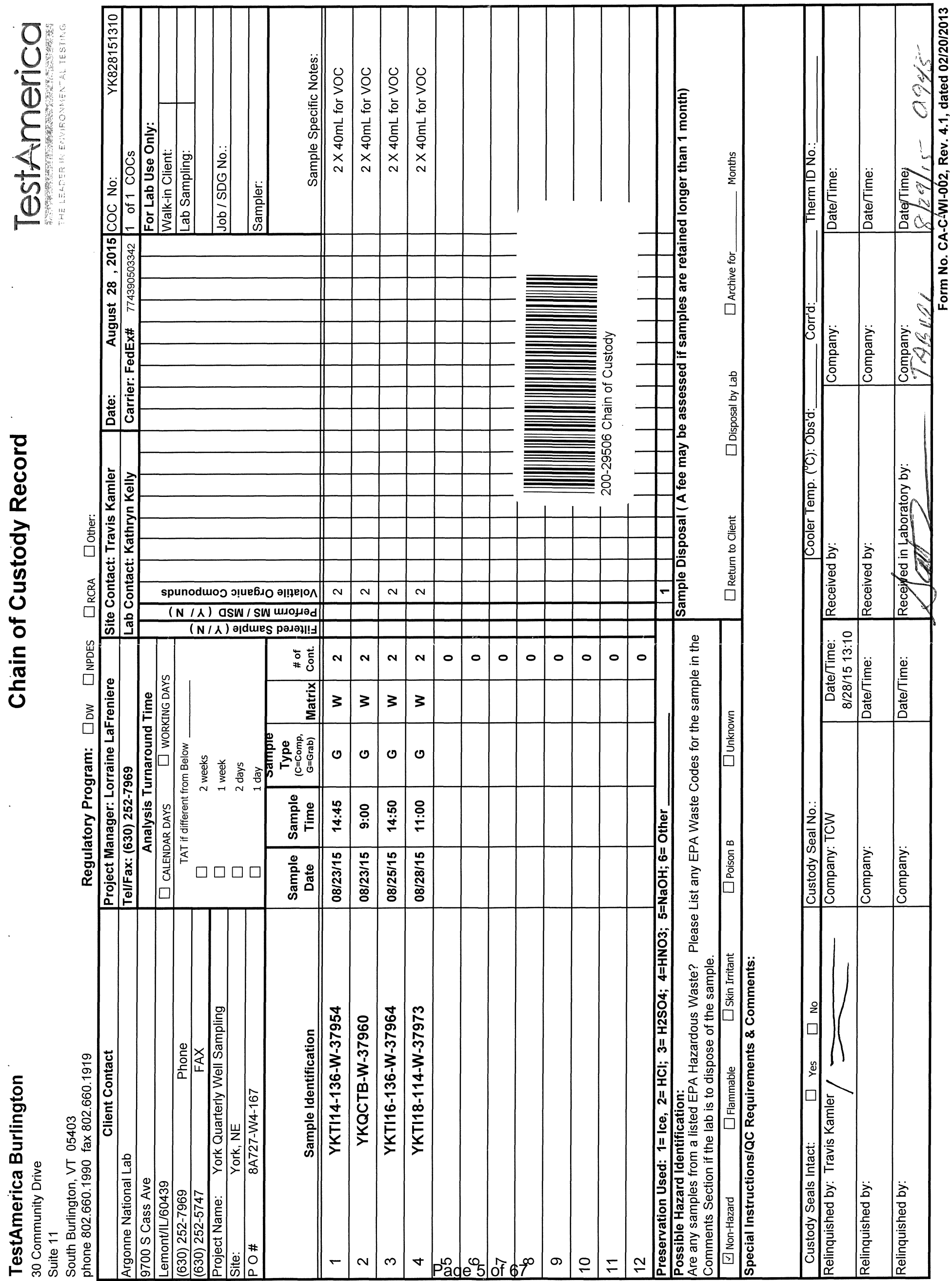




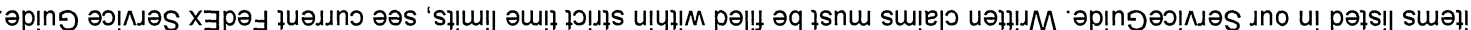

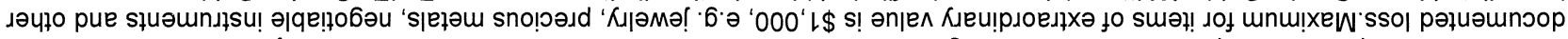

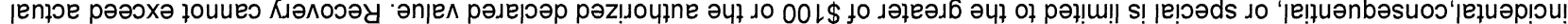

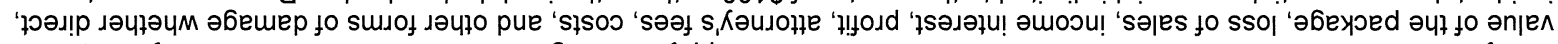

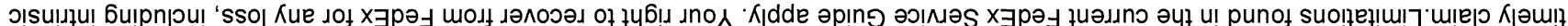

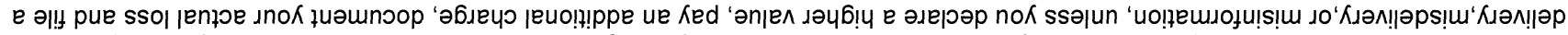

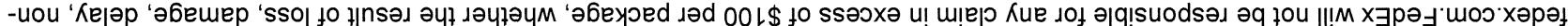

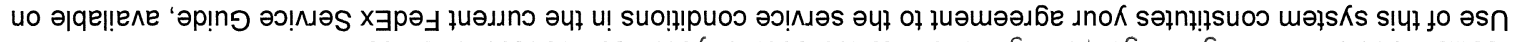

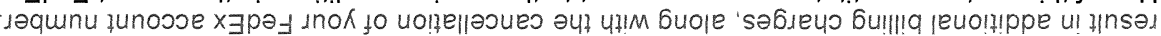

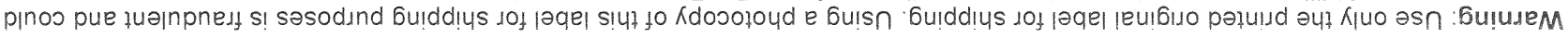

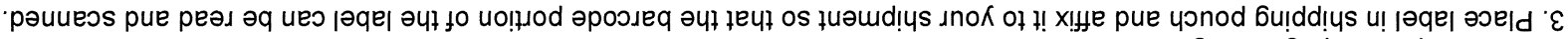

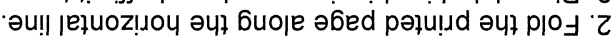

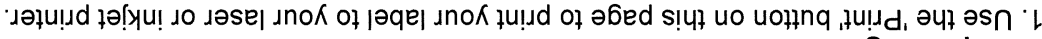

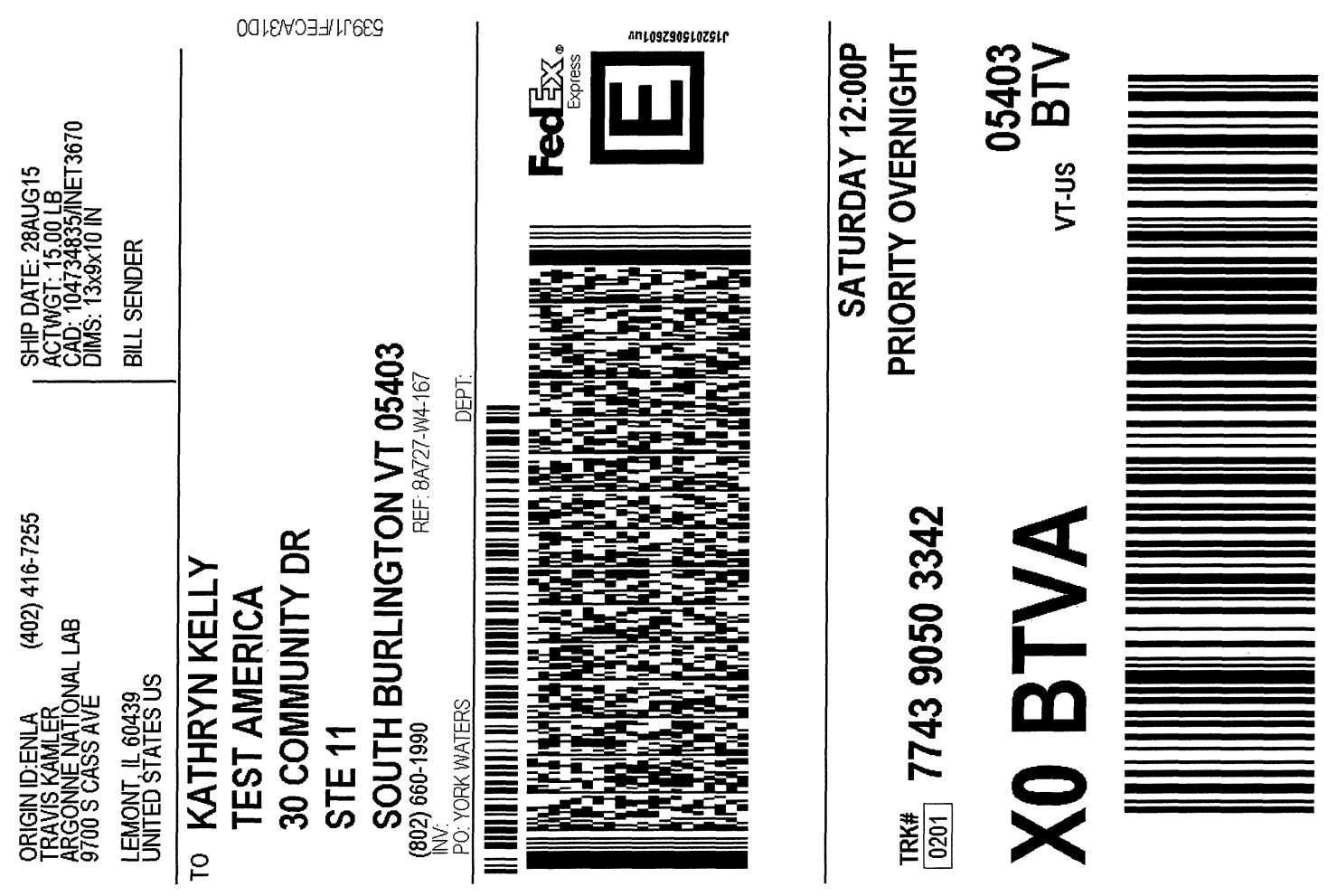




\section{Shipping and Receiving Documents}




\section{Login Sample Receipt Checklist}

Client: Argonne National Laboratory

Job Number: 200-29506-1

SDG Number: York (200-29506)

Login Number: 29506

List Source: TestAmerica Burlington

List Number: 1

Creator: Atherton, Joel E

Question

Answer

Radioactivity wasn't checked or is $</=$ background as measured by a survey True meter.

The cooler's custody seal, if present, is intact.

Sample custody seals, if present, are intact.

The cooler or samples do not appear to have been compromised or tampered with.

Samples were received on ice.

Cooler Temperature is acceptable.

Cooler Temperature is recorded.

COC is present.

COC is filled out in ink and legible.

$\mathrm{COC}$ is filled out with all pertinent information.

Is the Field Sampler's name present on COC?

There are no discrepancies between the containers received and the COC.

Samples are received within Holding Time.

Sample containers have legible labels.

Containers are not broken or leaking.

Sample collection date/times are provided.

Appropriate sample containers are used.

Sample bottles are completely filled.

Sample Preservation Verified.

There is sufficient vol. for all requested analyses, incl. any requested MS/MSDs

Containers requiring zero headspace have no headspace or bubble is $<6 \mathrm{~mm}$ (1/4").

Multiphasic samples are not present.

Samples do not require splitting or compositing.

Residual Chlorine Checked.
True

True

True

True

True

True

True

True

True

False

True

True

True

True

True

True

N/A

True

True

True

True

True

N/A 
SDG No.: York (200-29506)

\begin{tabular}{|c|c|c|c|c|c|c|c|c|}
\hline \multirow[b]{2}{*}{ Reagent ID } & \multirow[b]{2}{*}{$\begin{array}{l}\text { Exp } \\
\text { Date }\end{array}$} & \multirow[b]{2}{*}{$\begin{array}{l}\text { Prep } \\
\text { Date }\end{array}$} & \multirow[b]{2}{*}{$\begin{array}{l}\text { Dilutant } \\
\text { Used }\end{array}$} & \multirow{2}{*}{$\begin{array}{l}\text { Reagent } \\
\text { Final } \\
\text { Volume }\end{array}$} & \multicolumn{2}{|c|}{ Parent Reagent } & \multirow[b]{2}{*}{ Analyte } & \multirow[b]{2}{*}{ Concentration } \\
\hline & & & & & Reagent ID & $\begin{array}{l}\text { Volume } \\
\text { Added }\end{array}$ & & \\
\hline \multirow{2}{*}{$\begin{array}{l}\text { VMBFBW_00018 } \\
\text {.VMBFBS_00014 }\end{array}$} & $12 / 22 / 15$ & $06 / 23 / 15$ & METHANOL, Lot 147358 & $25 \mathrm{~mL}$ & VMBFBS_00014 & $125 \mathrm{uL}$ & $\mathrm{BFB}$ & $25 \mathrm{ug} / \mathrm{mL}$ \\
\hline & $12 / 22 / 15$ & & RESTEK, Lot A0106759 & & (Purchased Re & nt) & $\mathrm{BFB}$ & $5000 \mathrm{ug} / \mathrm{mL}$ \\
\hline \multirow[t]{43}{*}{ VMSOMTRCALW_00093 } & $09 / 19 / 15$ & $08 / 19 / 15$ & METHANOL, Lot 147358 & 4000 uL & VM8260CALbs_00175 & $40 \mathrm{uL}$ & Bromomethane & $20 \mathrm{ug} / \mathrm{mL}$ \\
\hline & & & & & & & Chloroethane & $20 \mathrm{ug} / \mathrm{mL}$ \\
\hline & & & & & & & Chloromethane & $20 \mathrm{ug} / \mathrm{mL}$ \\
\hline & & & & & & & Dichlorodifluoromethane & $20 \mathrm{ug} / \mathrm{mL}$ \\
\hline & & & & & & & Trichlorofluoromethane & $\frac{20 \mathrm{ug} / \mathrm{mL}}{20 \mathrm{ug} / \mathrm{mL}}$ \\
\hline & & & & & VMSOMCALas_00023 & $40 \mathrm{uL}$ & $\begin{array}{l}\text { Viny chlorlde } \\
\text { 1,1,1-Trichloroethane }\end{array}$ & $20 \mathrm{ug} / \mathrm{mL}$ \\
\hline & & & & & & & $1,1,2,2$-Tetrachloroethane & $20 \mathrm{ug} / \mathrm{mL}$ \\
\hline & & & & & & & $\begin{array}{l}\text { 1,1,2-Trichloro-1,2,2-trifluor } \\
\text { oethane }\end{array}$ & $20 \mathrm{ug} / \mathrm{mL}$ \\
\hline & & & & & & & $1,1,2$-Trichloroethane & $20 \mathrm{ug} / \mathrm{mL}$ \\
\hline & & & & & & & 1,1-Dichloroethane & $20 \mathrm{ug} / \mathrm{mL}$ \\
\hline & & & & & & & 1,1-Dichloroethene & $20 \mathrm{ug} / \mathrm{mL}$ \\
\hline & & & & & & & 1,2,3-Trichlorobenzene & $20 \mathrm{ug} / \mathrm{mL}$ \\
\hline & & & & & & & $1,2,4$-Trichlorobenzene & $20 \mathrm{ug} / \mathrm{mL}$ \\
\hline & & & & & & & 1,2-Dibromo-3-Chloropropane & $20 \mathrm{ug} / \mathrm{mL}$ \\
\hline & & & & & & & 1,2-Dibromoethane & $20 \mathrm{ug} / \mathrm{mL}$ \\
\hline & & & & & & & 1,2-Dichlorobenzene & $20 \mathrm{ug} / \mathrm{mL}$ \\
\hline & & & & & & & 1,2-Dichloroethane & $20 \mathrm{ug} / \mathrm{mL}$ \\
\hline & & & & & & & 1,2-Dichloropropane & $20 \mathrm{ug} / \mathrm{mL}$ \\
\hline & & & & & & & 1,3-Dichlorobenzene & $20 \mathrm{ug} / \mathrm{mL}$ \\
\hline & & & & & & & 1,4-Dichlorobenzene & $20 \mathrm{ug} / \mathrm{mL}$ \\
\hline & & & & & & & Benzene & $20 \mathrm{ug} / \mathrm{mL}$ \\
\hline & & & & & & & Bromochloromethane & $20 \mathrm{ug} / \mathrm{mL}$ \\
\hline & & & & & & & Bromodichloromethane & $20 \mathrm{ug} / \mathrm{mL}$ \\
\hline & & & & & & & Bromoform & $20 \mathrm{ug} / \mathrm{mL}$ \\
\hline & & & & & & & Carbon disulfide & $20 \mathrm{ug} / \mathrm{mL}$ \\
\hline & & & & & & & Carbon tetrachloride & $20 \mathrm{ug} / \mathrm{mL}$ \\
\hline & & & & & & & Chlorobenzene & $20 \mathrm{ug} / \mathrm{mL}$ \\
\hline & & & & & & & Chloroform & $20 \mathrm{ug} / \mathrm{mL}$ \\
\hline & & & & & & & cis-1,2-Dichloroethene & $20 \mathrm{ug} / \mathrm{mL}$ \\
\hline & & & & & & & cis-1,3-Dichloropropene & $20 \mathrm{ug} / \mathrm{mL}$ \\
\hline & & & & & & & Cyclohexane & $20 \mathrm{ug} / \mathrm{mL}$ \\
\hline & & & & & & & Dibromochloromethane & $20 \mathrm{ug} / \mathrm{mL}$ \\
\hline & & & & & & & Ethylbenzene & $20 \mathrm{ug} / \mathrm{mL}$ \\
\hline & & & & & & & Isopropylbenzene & $20 \mathrm{ug} / \mathrm{mL}$ \\
\hline & & & & & & & $\mathrm{m}, \mathrm{p}$-Xylene & $20 \mathrm{ug} / \mathrm{mL}$ \\
\hline & & & & & & & Methyl acetate & $20 \mathrm{ug} / \mathrm{mL}$ \\
\hline & & & & & & & Methyl tert-butyl ether & $20 \mathrm{ug} / \mathrm{mL}$ \\
\hline & & & & & & & Methylcyclohexane & $20 \mathrm{ug} / \mathrm{mL}$ \\
\hline & & & & & & & Methylene Chloride & $20 \mathrm{ug} / \mathrm{mL}$ \\
\hline & & & & & & & o-Xylene & $20 \mathrm{ug} / \mathrm{mL}$ \\
\hline & & & & & & & Styrene & $20 \mathrm{ug} / \mathrm{mL}$ \\
\hline & & & & & & & Tetrachloroethene & $20 \mathrm{ug} / \mathrm{mL}$ \\
\hline & & & & & & & Toluene & $20 \mathrm{ug}$ \\
\hline
\end{tabular}


SDG No.: York (200-29506)

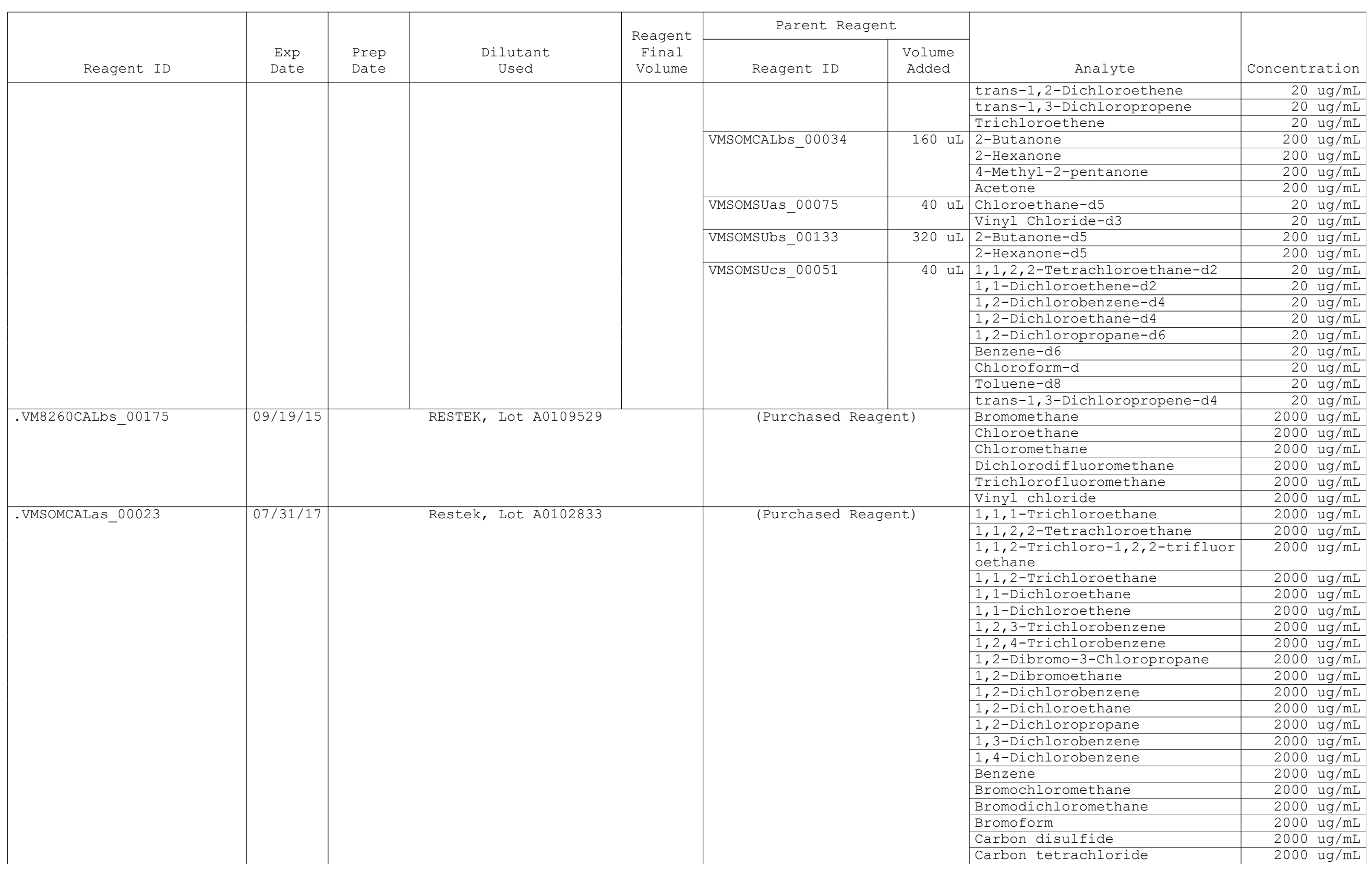


SDG No.: York (200-29506)

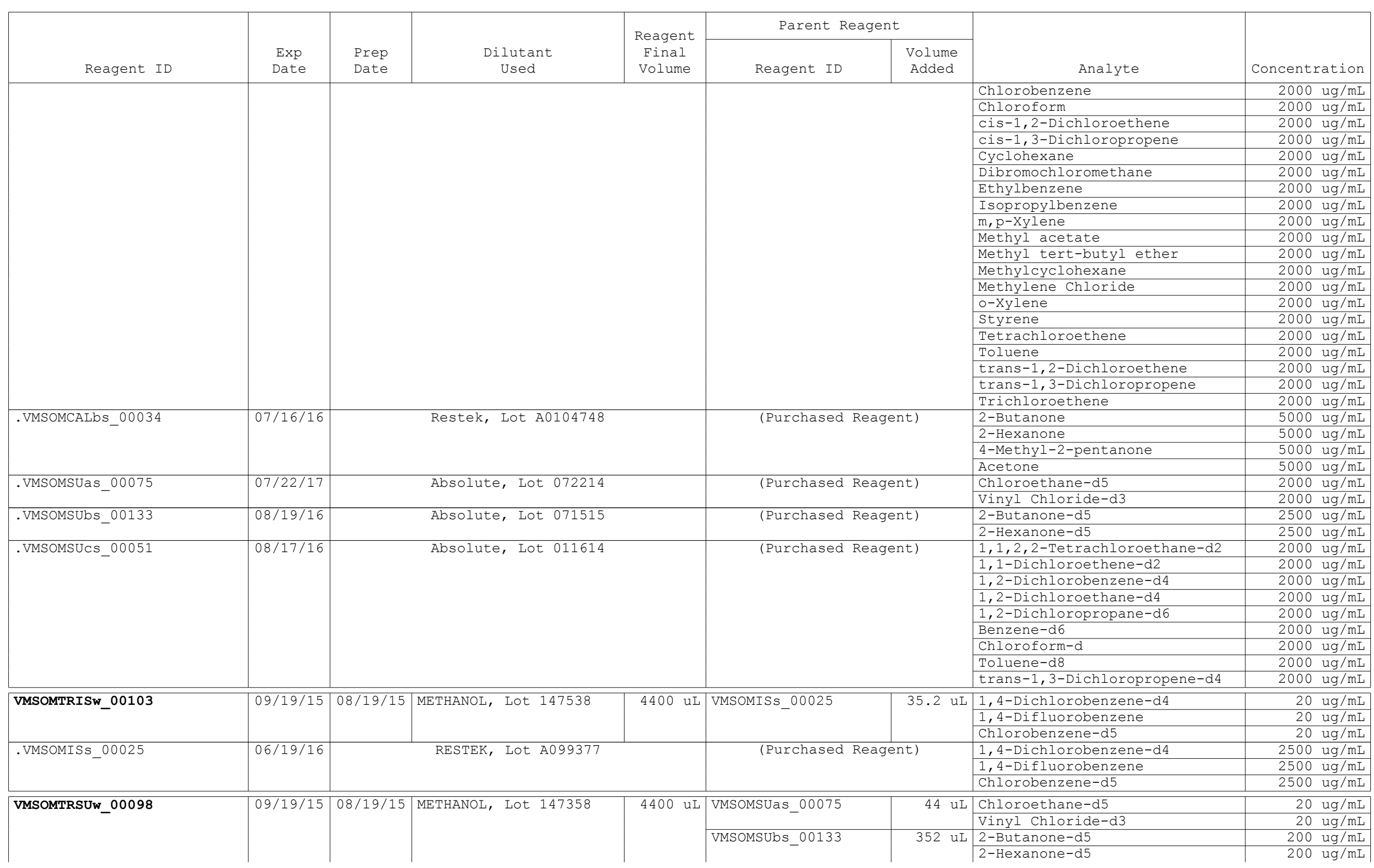


SDG No.: York (200-29506)

\begin{tabular}{|c|c|c|c|c|c|c|c|c|}
\hline \multirow[b]{2}{*}{ Reagent ID } & \multirow[b]{2}{*}{$\begin{array}{l}\text { Exp } \\
\text { Date }\end{array}$} & \multirow[b]{2}{*}{$\begin{array}{l}\text { Prep } \\
\text { Date }\end{array}$} & \multirow[b]{2}{*}{$\begin{array}{c}\text { Dilutant } \\
\text { Used }\end{array}$} & \multirow{2}{*}{$\begin{array}{l}\text { Reagent } \\
\text { Final } \\
\text { Volume }\end{array}$} & \multicolumn{2}{|c|}{ Parent Reagent } & \multirow[b]{2}{*}{ Analyte } & \multirow[b]{2}{*}{ Concentration } \\
\hline & & & & & \multirow{10}{*}{$\begin{array}{r}\text { Reagent ID } \\
\text { VMSOMSUCS_00051 }\end{array}$} & $\begin{array}{l}\text { Volume } \\
\text { Added }\end{array}$ & & \\
\hline & & & & & & $44 \mathrm{uL}$ & $1,1,2,2$-Tetrachloroethane-d2 & $20 \mathrm{ug} / \mathrm{mL}$ \\
\hline & & & & & & & 1,1-Dichloroethene-d2 & $20 \mathrm{ug} / \mathrm{mL}$ \\
\hline & & & & & & & 1,2-Dichlorobenzene-d4 & $20 \mathrm{ug} / \mathrm{mL}$ \\
\hline & & & & & & & 1,2-Dichloroethane-d4 & $20 \mathrm{ug} / \mathrm{mL}$ \\
\hline & & & & & & & 1,2-Dichloropropane-d6 & $20 \mathrm{ug} / \mathrm{mL}$ \\
\hline & & & & & & & Benzene-d6 & $20 \mathrm{ug} / \mathrm{mL}$ \\
\hline & & & & & & & Chloroform-d & $20 \mathrm{ug} / \mathrm{mL}$ \\
\hline & & & & & & & Toluene-d8 & $20 \mathrm{ug} / \mathrm{mL}$ \\
\hline & & & & & & & trans-1,3-Dichloropropene-d4 & $20 \mathrm{ug} / \mathrm{mL}$ \\
\hline \multirow[t]{2}{*}{.VMSOMSUas_00075 } & \multirow[t]{2}{*}{$07 / 22 / 17$} & \multirow{2}{*}{\multicolumn{3}{|c|}{ Absolute, Lot 072214}} & \multirow{2}{*}{\multicolumn{2}{|c|}{ (Purchased Reagent) }} & Chloroethane-d5 & $2000 \mathrm{ug} / \mathrm{mL}$ \\
\hline & & & & & & & Vinyl Chloride-d3 & $2000 \mathrm{ug} / \mathrm{mL}$ \\
\hline \multirow{2}{*}{.VMSOMSUbs_00133 } & \multirow[t]{2}{*}{$08 / 19 / 16$} & \multirow{2}{*}{\multicolumn{3}{|c|}{ Absolute, Lot 071515}} & \multirow{2}{*}{\multicolumn{2}{|c|}{ (Purchased Reagent) }} & 2-Butanone-d5 & $2500 \mathrm{ug} / \mathrm{mL}$ \\
\hline & & & & & & & 2-Hexanone-d5 & $2500 \mathrm{ug} / \mathrm{mL}$ \\
\hline \multirow[t]{9}{*}{.VMSOMSUCS_00051 } & \multirow[t]{9}{*}{$08 / 17 / 16$} & \multirow{9}{*}{\multicolumn{2}{|c|}{ Absolute, Lot 011614}} & & \multirow{9}{*}{\multicolumn{2}{|c|}{ (Purchased Reagent) }} & $1,1,2,2$-Tetrachloroethane-d2 & $2000 \mathrm{ug} / \mathrm{mL}$ \\
\hline & & & & & & & 1,1-Dichloroethene-d2 & $2000 \mathrm{ug} / \mathrm{mL}$ \\
\hline & & & & & & & 1,2-Dichlorobenzene-d4 & $2000 \mathrm{ug} / \mathrm{mL}$ \\
\hline & & & & & & & 1,2-Dichloroethane-d4 & $2000 \mathrm{ug} / \mathrm{mL}$ \\
\hline & & & & & & & 1,2-Dichloropropane-d6 & $2000 \mathrm{ug} / \mathrm{mL}$ \\
\hline & & & & & & & Benzene-d6 & $2000 \mathrm{ug} / \mathrm{mL}$ \\
\hline & & & & & & & Chloroform-d & $2000 \mathrm{ug} / \mathrm{mL}$ \\
\hline & & & & & & & Toluene-d8 & $2000 \mathrm{ug} / \mathrm{mL}$ \\
\hline & & & & & & & trans-1,3-Dichloropropene-d4 & $2000 \mathrm{ug} / \mathrm{mL}$ \\
\hline
\end{tabular}


Lab Name: TestAmerica Burlington

Job No.: 200-29506-1

SDG No.: York (200-29506)

\begin{tabular}{|c|l|l|c|c|}
\hline $\begin{array}{c}\text { Reagent } \\
\text { container }\end{array}$ & \multicolumn{1}{|c|}{$\begin{array}{c}\text { Reagent } \\
\text { ID }\end{array}$} & \multicolumn{1}{c|}{$\begin{array}{c}\text { Reagent } \\
\text { Description }\end{array}$} & $\begin{array}{c}\text { Preparation } \\
\text { Date }\end{array}$ & $\begin{array}{c}\text { Expiration } \\
\text { Date }\end{array}$ \\
\hline 807558 & VMBFB__00018 & BFB TUNE 25 PPM & $06 / 23 / 2015$ & $12 / 22 / 2015$ \\
\hline 829193 & VMSOMTRISw_00103 & SOM TR ISTD 20 PPM & $08 / 19 / 2015$ & $09 / 19 / 2015$ \\
\hline 829199 & VMSOMTRSUw_00098 & SOM TR DMC 20 PPM & $08 / 19 / 2015$ & $09 / 19 / 2015$ \\
\hline 829202 & VMSOMTRCALw_00093 & SOM TR CAL 20 - 200 PPM & $08 / 19 / 2015$ & $09 / 19 / 2015$ \\
\hline
\end{tabular}




\section{METHODOLOGY SUMMARY}

Laboratory: TestAmerica Laboratories

Location: South Burlington, Vermont
Project No:

SDG No: $\quad$ York (200-29506)

VOA

Volatile Organics Trace - USEPA CLP SOM01.2 


\section{CASE NARRATIVE}

\section{Client: Argonne National Laboratory}

\section{Project: York (200-29506)}

\section{Report Number: 200-29506-1}

With the exceptions noted as flags or footnotes, standard analytical protocols were followed in the analysis of the samples and no problems were encountered or anomalies observed. In addition all laboratory quality control samples were within established control limits, with any exceptions noted below. Each sample was analyzed to achieve the lowest possible reporting limit within the constraints of the method. In some cases, due to interference or analytes present at high concentrations, samples were diluted. For diluted samples, the reporting limits are adjusted relative to the dilution required.

Calculations are performed before rounding to avoid round-off errors in calculated results.

All holding times were met and proper preservation noted for the methods performed on these samples, unless otherwise detailed in the individual sections below.

\section{RECEIPT}

The samples were received on 08/29/2015; the samples arrived in good condition, properly preserved and on ice. The temperature of the cooler at receipt was $3.0^{\circ} \mathrm{C}$.

\section{VOLATILE ORGANIC COMPOUNDS - TRACE}

Samples YKTI14-136-W-37954, YKQCTB-W-37960, YKTI16-136-W-37964, YKTI18-114-W-37973 and VHBLK01 were analyzed for Volatile Organic Compounds - Trace in accordance with EPA SOW SOM01.2. The samples were analyzed on 08/31/2015.

The samples were received on $8 / 29 / 2015$. The lab did not initiate a holding blank for these samples until $8 / 31$ due to laboratory oversight.

The following sample(s) was received with less than one shift (8 hours) remaining on a test with a holding time of 48 hours or less. As such, the laboratory had insufficient time remaining to perform the analysis within holding time: YKTI14-136-W-37954 (200-29506-1) and YKQCTB-W-37960 (200-29506-2).

1,2,3-Trichlorobenzene, 1,2,4-Trichlorobenzene and Carbon disulfide were detected in method blank MB 200-93413/5 at levels that were above the method detection limit but below the reporting limit. The values should be considered estimates, and have been flagged. If the associated sample reported a result above the MDL and/or RL, the result has been flagged. Refer to the $\mathrm{QC}$ report for details.

Samples YKTI14-136-W-37954[1.14X], YKTI14-136-W-37954[4.52X] and YKTI16-136-W-37964[2.56X] required dilution prior to analysis. The reporting limits have been adjusted accordingly.

No additional analytical or quality issues were noted, other than those described above or in the Definitions/Glossary page. 
Lab Name: TestAmerica Burlington

Job No.: 200-29506-1

SDG No.: York (200-29506)

Instrument ID: CHD.i

Analysis Batch Number: 92921

Lab Sample ID: IC 200-92921/3

Client Sample ID:

Date Analyzed: 08/19/15 15:14

Lab File ID: 15357 003.D

GC Column: DB-624

ID $: 0.2(\mathrm{~mm})$

\begin{tabular}{|l|c|c|c|}
\hline \multicolumn{1}{|c|}{ COMPOUND NAME } & RETENTION & \multicolumn{2}{|c|}{ MANUAL INTEGRATION } \\
\cline { 3 - 4 } & TIME & REASON & ANALYST \\
\hline Dichlorodifluoromethane & 1.52 & Baseline & DATE \\
\hline 1,2 -Dibromo-3-Chloropropane & 13.43 & Assign Peak & 08/19/15 $16: 20$ \\
\hline
\end{tabular}

Lab Sample ID: IC 200-92921/4 Client Sample ID:

Date Analyzed: 08/19/15 15:39

Lab File ID: 15357_004.D

GC Column: DB-624

ID $: 0.2(\mathrm{~mm})$

\begin{tabular}{|c|c|c|c|c|}
\hline \multirow[t]{2}{*}{ COMPOUND NAME } & \multirow{2}{*}{$\begin{array}{c}\text { RETENTION } \\
\text { TIME }\end{array}$} & \multicolumn{3}{|c|}{ MANUAL INTEGRATION } \\
\hline & & REASON & ANALYST & DATE \\
\hline Dichlorodifluoromethane & 1.53 & Baseline & wilburj & $08 / 19 / 15 \quad 16: 21$ \\
\hline Bromomethane & 2.00 & Baseline & wilburj & $08 / 19 / 15 \quad 16: 27$ \\
\hline Chloroethane & 2.08 & Baseline & wilburj & $08 / 19 / 1516: 27$ \\
\hline 1,2-Dibromo-3-Chloropropane & 13.44 & Assign Peak & wilburj & $08 / 19 / 15 \quad 16: 27$ \\
\hline
\end{tabular}

Lab Sample ID: ICIS 200-92921/5

Client Sample ID:

Date Analyzed: 08/19/15 16:04

Lab File ID: 15357 005.D

GC Column: DB-624

ID $: 0.2(\mathrm{~mm})$

\begin{tabular}{|c|c|c|c|c|}
\hline \multirow[t]{2}{*}{ COMPOUND NAME } & \multirow{2}{*}{$\begin{array}{c}\text { RETENTION } \\
\text { TIME }\end{array}$} & \multicolumn{3}{|c|}{ MANUAL INTEGRATION } \\
\hline & & REASON & ANALYST & DATE \\
\hline Dichlorodifluoromethane & 1.53 & Baseline & wilburj & $08 / 19 / 1516: 30$ \\
\hline Bromomethane & 2.00 & Baseline & wilburj & $08 / 19 / 15 \quad 17: 11$ \\
\hline
\end{tabular}

Lab Sample ID: IC 200-92921/6

Client Sample ID:

Date Analyzed: 08/19/15 16:29

Lab File ID: 15357_006.D

GC Column: DB-624

ID $: 0.2(\mathrm{~mm})$

\begin{tabular}{|c|c|c|c|c|}
\hline \multirow[t]{2}{*}{ COMPOUND NAME } & \multirow{2}{*}{$\begin{array}{c}\text { RETENTION } \\
\text { TIME }\end{array}$} & \multicolumn{3}{|c|}{ MANUAL INTEGRATION } \\
\hline & & REASON & ANALYST & DATE \\
\hline Dichlorodifluoromethane & 1.53 & Baseline & wilburj & $08 / 19 / 15 \quad 17: 12$ \\
\hline Bromomethane & 2.00 & Baseline & wilburj & $08 / 19 / 15 \quad 17: 12$ \\
\hline
\end{tabular}

SOM01.2/VOA_Tr 
Lab Name: TestAmerica Burlington SDG No.: York (200-29506)

Instrument ID: CHD.i

Lab Sample ID: IC 200-92921/7

Date Analyzed: 08/19/15 16:54
Job No.: 200-29506-1

Analysis Batch Number: 92921

Client Sample ID:

Lab File ID: 15357 007.D
GC Column: DB-624

ID $: 0.2(\mathrm{~mm})$

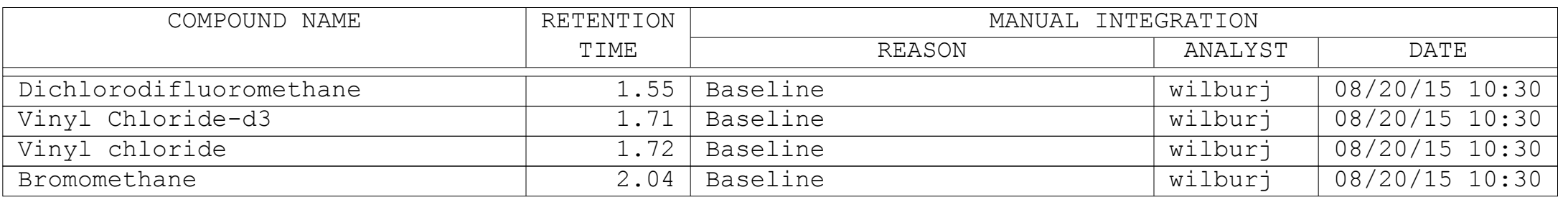


Lab Name: TestAmerica Burlington

Job No.: 200-29506-1

SDG No.: York (200-29506)

Instrument ID: CHD.i

Analysis Batch Number: 93413

Lab Sample ID: CCVIS 200-93413/3

Client Sample ID:

Date Analyzed: 08/31/15 12:54

Lab File ID: 15537 003.D

GC Column: DB-624

ID $: 0.2(\mathrm{~mm})$

\begin{tabular}{|c|c|c|c|c|}
\hline \multirow[t]{2}{*}{ COMPOUND NAME } & RETENTION & \multicolumn{3}{|c|}{ MANUAL INTEGRATION } \\
\hline & TIME & REASON & ANALYST & DATE \\
\hline Dichlorodifluoromethane & 1.48 & Assign Peak & wilburj & $08 / 31 / 1513: 41$ \\
\hline
\end{tabular}

Lab Sample ID: CCVC 200-93413/16

Client Sample ID:

Date Analyzed: 08/31/15 18:29

Lab File ID: 15537 016.D

GC Column: DB-624

ID $: 0.2(\mathrm{~mm})$

\begin{tabular}{|l|c|c|c|c|}
\hline \multicolumn{1}{|c|}{ COMPOUND NAME } & RETENTION & \multicolumn{2}{|c|}{ MANUAL INTEGRATION } \\
\cline { 4 - 5 } & TIME & REASON & ANALYST & DATE \\
\hline Dichlorodifluoromethane & 1.52 & Baseline & wilburj & $09 / 01 / 1510: 45$ \\
\hline
\end{tabular}




\section{DATA REPORTING QUALIFIERS}

Client: Argonne National Laboratory

Job Number: 200-29506-1

Sdg Number: York (200-29506)

\section{Lab Section}

Qualifier

Description

\section{GC/MS VOA}

U

E

J

$J$

D

$X$

$\mathrm{B}$

N
Analyzed for but not detected.

Compound concentration exceeds the upper level of the calibration range of the instrument for that specific analysis.

Indicates an Estimated Value for TICs

Indicates an estimated value.

Sample was analyzed at a higher dilution factor.

See case narrative notes for explanation of the ' $X$ ' flag

The analyte was found in an associated blank, as well as in the sample.

This flag indicates the presumptive evidence of a compound. 
Lab Name: TESTAMERICA BURLINGTON

Lab Code: STLV Case No.: YORK Mod. Ref No.: Level: (TRACE or LOW) TRACE
Contract: $8 \mathrm{E}-00302$

SDG NO.: YORK $(200-29$

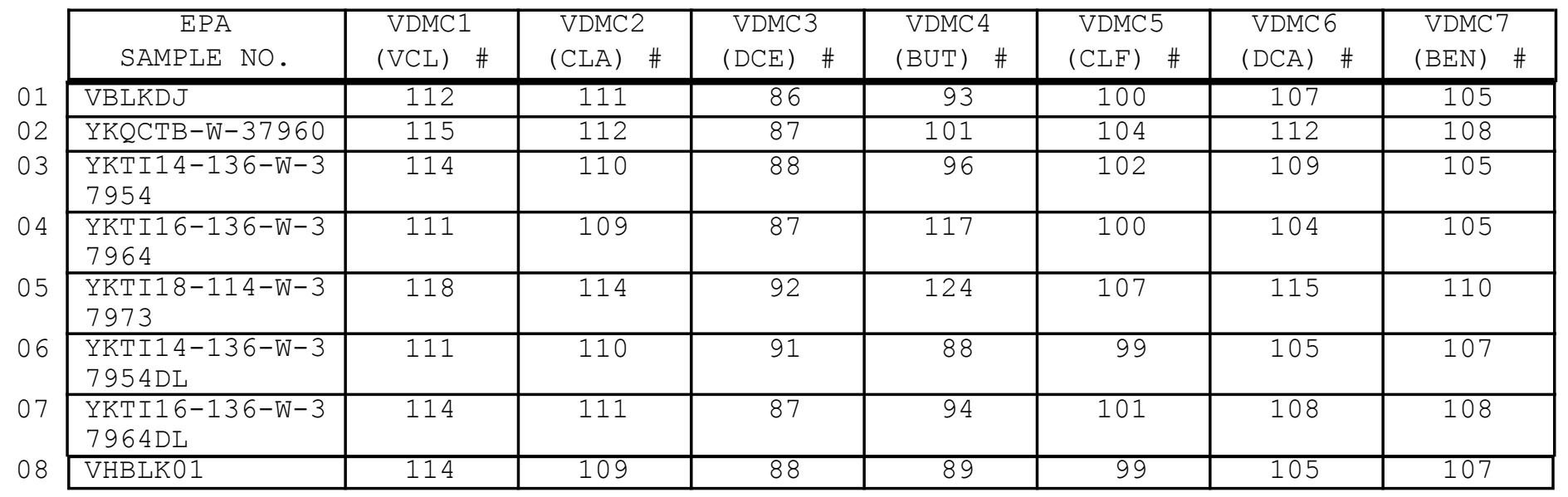

\footnotetext{
VDMC1 (VCL) = Vinyl Chloride-d3

VDMC2 $($ CLA $)=$ Chloroethane-d5

VDMC3 $(\mathrm{DCE})=1,1-\mathrm{Dichloroethene-d2}$

VDMC4 $\quad(B U T)=2-$ Butanone-d5

VDMC5 $(\mathrm{CLF})=$ Chloroform-d

VDMC $6 \quad(\mathrm{DCA})=1,2-\mathrm{Dich}$ loroethane-d4

$\operatorname{VDMC} 7 \quad(\mathrm{BEN})=$ Benzene-d6
}

$$
\begin{gathered}
\frac{\mathrm{QC} \text { LIMITS }}{(65-131)} \\
(71-131) \\
(55-104) \\
(49-155) \\
(78-121) \\
(78-129) \\
(77-124)
\end{gathered}
$$

\# Column to be used to flag recovery values

* Values outside of contract required QC limits

Page 1 of 1 
Lab Name: TESTAMERICA BURLINGTON

Lab Code: STLV Case No.: YORK Mod. Ref No.: Level: (TRACE or LOW) TRACE
Contract: $8 \mathrm{E}-00302$

SDG NO.: YORK $\quad$ (200-29

\begin{tabular}{|c|c|c|c|c|c|c|c|c|}
\hline $\begin{array}{c}\text { EPA } \\
\text { SAMPLE NO. }\end{array}$ & $\begin{array}{l}\text { VDMC8 } \\
(\mathrm{DPA}) \quad \#\end{array}$ & $\begin{array}{l}\text { VDMC9 } \\
(\mathrm{TOL}) \quad \#\end{array}$ & $\begin{array}{l}\text { VDMC10 } \\
(\mathrm{TDP})\end{array}$ & $\begin{array}{l}\text { VDMC11 } \\
(\operatorname{HEX}) \#\end{array}$ & $\begin{array}{l}\text { VDMC12 } \\
(\mathrm{TCA})\end{array}$ & $\begin{array}{l}\text { VDMC13 } \\
(\mathrm{DCZ}) \quad \#\end{array}$ & OTHER & $\begin{array}{l}\text { TOT } \\
\text { OUT }\end{array}$ \\
\hline VBLKDJ & 103 & 105 & 95 & 95 & 96 & 101 & & 0 \\
\hline YKQCTB-W-37960 & 108 & 106 & 99 & 103 & 99 & 106 & & 0 \\
\hline $\begin{array}{l}\text { YKTI14-136-W-3 } \\
7954\end{array}$ & 108 & 108 & 105 & 104 & 102 & 105 & & 0 \\
\hline $\begin{array}{l}\text { YKTI16-136-W-3 } \\
7964\end{array}$ & 105 & 106 & 99 & 123 & 97 & 100 & & 0 \\
\hline $\begin{array}{l}\text { YKTI18-114-W-3 } \\
7973\end{array}$ & 110 & 112 & 102 & 124 & 99 & 108 & & 0 \\
\hline $\begin{array}{l}\text { YKTI14-136-W-3 } \\
7954 \mathrm{DL}\end{array}$ & 103 & 107 & 93 & 91 & 91 & 104 & & 0 \\
\hline $\begin{array}{l}\text { YKTI16-136-W-3 } \\
7964 \mathrm{DL}\end{array}$ & 109 & 107 & 95 & 98 & 96 & 104 & & 0 \\
\hline \begin{tabular}{|l|} 
VHBLK01 \\
\end{tabular} & 105 & 107 & 92 & 95 & 94 & 101 & & 0 \\
\hline
\end{tabular}

\footnotetext{
$\operatorname{VDMC} 8(\mathrm{DPA})=1,2-\mathrm{Dichloropropane}-\mathrm{d} 6$

VDMC $9 \quad($ TOL $)=$ Toluene-d8

VDMC10 $(\mathrm{TDP})=$ trans $-1,3-\mathrm{Dichloropropene-d4}$

$\operatorname{VDMC1}(\mathrm{HEX})=2-$ Hexanone-d5

VDMC12 $(\mathrm{TCA})=1,1,2,2$-Tetrachloroethane-d2

VDMC13 $(\mathrm{DCZ})=1,2-\mathrm{Dichlorobenzene-d4}$
}

$$
\begin{gathered}
\frac{Q C \text { LIMITS }}{(79-124)} \\
(77-121) \\
(73-121) \\
(28-135) \\
(73-125) \\
(80-131)
\end{gathered}
$$

\# Column to be used to flag recovery values

* Values outside of contract required QC limits

Report 1,4-Dioxane-d8 for Low-Medium VOA analysis only

Page 1 of 1 
$4 \mathrm{~A}$ - FORM IV VOA

VOLATILE METHOD BLANK SUMMARY
EPA SAMPLE NO.

VBLKDJ

Lab Name: TESTAMERICA BURLINGTON

Contract: $8 \mathrm{E}-00302$

Lab Code: STLV Case No.: YORK Mod. Ref No.: SDG NO.: YORK $(200-29$

Lab File ID: 15537 005.D

Lab Sample ID: MB 200-93413/5

Instrument ID: CHD.i

Matrix: (SOIL/SED/WATER) Water

Level: (TRACE or LOW/MED) TRACE

Date Analyzed: 08/31/2015

GC Column: DB-624

ID $: 0.20 \quad(\mathrm{~mm})$

Time Analyzed: 1344

Heated Purge: (Y/N) N

\begin{tabular}{|c|c|c|c|}
\hline $\begin{array}{c}\text { EPA } \\
\text { SAMPLE NO. }\end{array}$ & $\begin{array}{r}\text { LAB } \\
\text { SAMPLE ID }\end{array}$ & $\begin{array}{c}\text { LAB } \\
\text { FILE ID }\end{array}$ & $\begin{array}{c}\text { TIME } \\
\text { ANALYZED }\end{array}$ \\
\hline $\begin{array}{l}\text { YKQCTB-W-379 } \\
60\end{array}$ & $200-29506-2$ & $\begin{array}{l}15537 \text { - } \\
D\end{array}$ & 1409 \\
\hline $\begin{array}{l}\text { YKTI14-136-W } \\
-37954\end{array}$ & $200-29506-1$ & $\begin{array}{l}15537 \_007 . \\
D\end{array}$ & 1433 \\
\hline VIBLKDI & VIBLK 200-93413/8 & $\begin{array}{l}15537 \_008 . \\
D\end{array}$ & 1458 \\
\hline $\begin{array}{l}\text { YKTI16-136-W } \\
-37964\end{array}$ & $200-29506-3$ & $\begin{array}{l}15537 \_009 . \\
D\end{array}$ & 1523 \\
\hline VIBLKDJ & VIBLK 200-93413/10 & $\begin{array}{l}15537-010 . \\
D\end{array}$ & 1548 \\
\hline $\begin{array}{l}\text { YKTI18-114-W } \\
-37973\end{array}$ & $200-29506-4$ & $\begin{array}{l}15537 \_011 . \\
\mathrm{D}\end{array}$ & 1613 \\
\hline $\begin{array}{l}\text { YKTI14-136-W } \\
-37954 \mathrm{DL}\end{array}$ & $200-29506-1$ & $\begin{array}{l}15537 \_013 . \\
D\end{array}$ & 1715 \\
\hline $\begin{array}{l}\text { YKTI16-136-W } \\
-37964 \mathrm{DL}\end{array}$ & $200-29506-3$ & $\begin{array}{l}15537 \_014 . \\
D\end{array}$ & 1740 \\
\hline VHBLK01 & $200-29506-5$ & $\begin{array}{l}15537 \_015 . \\
D\end{array}$ & 1804 \\
\hline
\end{tabular}

COMMENTS :

Page 1 of 1 
$5 A$ - FORM V VOA

VOLATILE ORGANICS INSTRUMENT

PERFORMANCE CHECK

BROMOFLUOROBENZENE (BFB)
EPA SAMPLE NO.

BFBDE
Lab Name: TESTAMERICA BURLINGTON

Lab Code: STLV Case No.: YORK

Contract: 8E-00302 Mod. Ref No.: SDG NO.: YORK $(200-29506)$

Lab File Id: 15357_001.D

BFB Injection Date: 08/19/2015

Instrument Id: CHD.i

BFB Injection Time: 1435

GC Column: DB-624

ID $: 0.20$ $(\mathrm{mm})$

\begin{tabular}{|c|c|c|}
\hline $\mathrm{m} / \mathrm{e}$ & ION ABUNDANCE CRITERIA & $\begin{array}{l}\text { \% RELATIVE } \\
\text { ABUNDANCE }\end{array}$ \\
\hline 50 & $15.0-40.0 \%$ of mass 95 & 20.1 \\
\hline 75 & $30.0-80.0 \%$ of mass 95 & 51.3 \\
\hline 95 & Base peak, 100\% relative abundance & 100 \\
\hline 96 & $5.0-9.0 \%$ of mass 95 & 6.8 \\
\hline 173 & Less than 2.0\% of mass 174 & $0.6) 1$ \\
\hline 174 & $50.0-120 \%$ of mass 95 & 79.0 \\
\hline 175 & $5.0-9.0 \%$ of mass 174 & $5.8(7.4) 1$ \\
\hline 176 & $95.0-101 \%$ of mass 174 & $75.6(95.7) 1$ \\
\hline 177 & $5.0-9.0 \%$ of mass 176 & $4.8(6.4) 2$ \\
\hline
\end{tabular}

1 - Value is omass 1742 - Value is omass 176

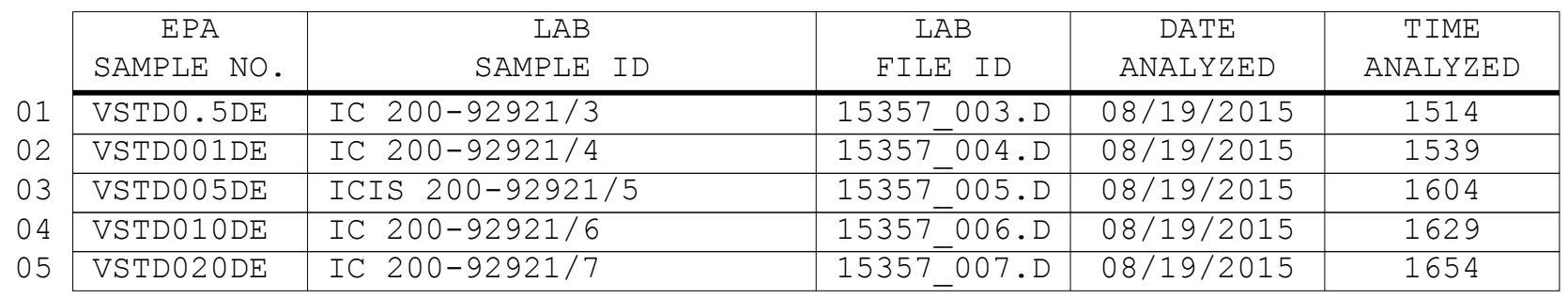

Page 1 of 1 
$5 A$ - FORM V VOA

VOLATILE ORGANICS INSTRUMENT

PERFORMANCE CHECK

BROMOFLUOROBENZENE (BFB)
EPA SAMPLE NO.

BFBDJ
Lab Name: TESTAMERICA BURLINGTON

Lab Code: STLV Case No.: YORK

Mod

Contract: 8E-00302

Lab File Id: 15537_001.D

Instrument Id: CHD.i

GC Column: DB-624

ID $: 0.20$ $(\mathrm{mm})$

\begin{tabular}{|c|c|c|}
\hline $\mathrm{m} / \mathrm{e}$ & ION ABUNDANCE CRITERIA & $\begin{array}{l}\text { \% RELATIVE } \\
\text { ABUNDANCE }\end{array}$ \\
\hline 50 & $15.0-40.0 \%$ of mass 95 & 20.0 \\
\hline 75 & $30.0-80.0 \%$ of mass 95 & 51.2 \\
\hline 95 & Base peak, 100\% relative abundance & 100 \\
\hline 96 & $5.0-9.0 \%$ of mass 95 & 6.4 \\
\hline 173 & Less than 2.0\% of mass 174 & $0.7) 1$ \\
\hline 174 & $50.0-120 \%$ of mass 95 & 78.1 \\
\hline 175 & $5.0-9.0 \%$ of mass 174 & $5.6(7.2) 1$ \\
\hline 176 & $95.0-101 \%$ of mass 174 & $76.5(98.0) 1$ \\
\hline 177 & $5.0-9.0 \%$ of mass 176 & $4.8(6.3) 2$ \\
\hline
\end{tabular}

1 - Value is omass 1742 - Value is omass 176

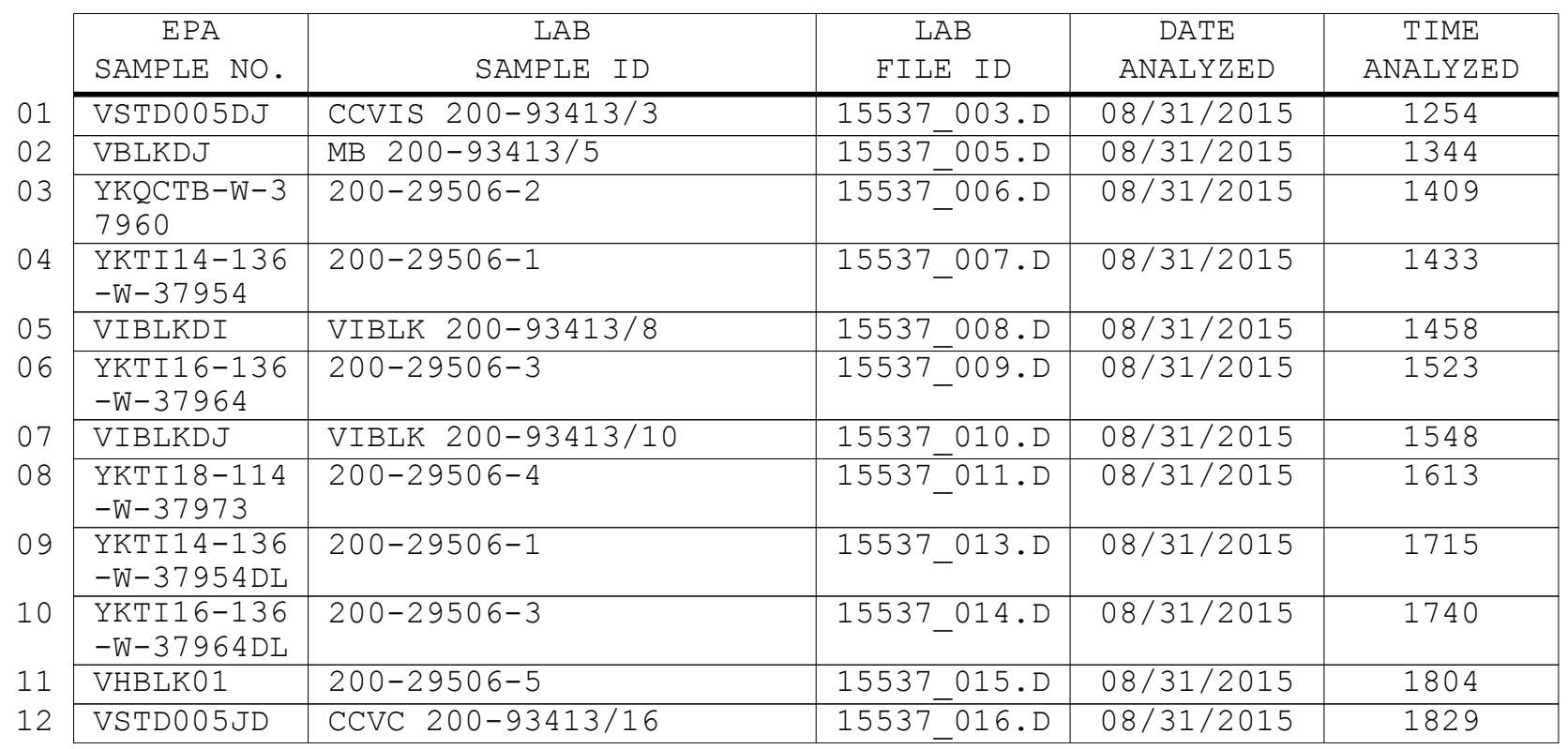

Page 1 of 1 


\section{$8 A$ - FORM VIII VOA}

VOLATILE INTERNAL STANDARD AREA AND RETENTION TIME SUMMARY

Lab Name: TESTAMERICA BURLINGTON

Contract: $8 \mathrm{E}-00302$

Lab Code: STLV Case No.: YORK Mod. Ref No.: SDG NO.: YORK (200-29

GC Column: DB-624

ID: 0.20 (mm) Init. Calib. Date (s) : 08/19/2015 08/19/2015

EPA Sample No.(VSTD\#\#\#\#\#): VSTD005DJ

Lab File ID (Standard): 15537_003.D

Instrument ID: CHD.i

Date Analyzed: 08/31/2015

Time Analyzed: 1254

Heated Purge: (Y/N) N

\begin{tabular}{|c|c|c|c|c|c|c|}
\hline & $\begin{array}{c}\text { IS1 (CBZ) } \\
\text { AREA }\end{array}$ & $\mathrm{RT} \#$ & $\begin{array}{c}\text { IS2 (DFB) } \\
\text { AREA }\end{array}$ & $\mathrm{RT}$ & $\begin{array}{c}\text { IS3 (DCB) } \\
\text { AREA }\end{array}$ & $\mathrm{RT}$ \\
\hline 12 HOUR STD & 1408019 & 9.33 & 1652054 & 5.96 & 707679 & 12.15 \\
\hline UPPER LIMIT & 1971227 & 9.66 & 2312876 & 6.29 & 990751 & 12.48 \\
\hline LOWER LIMIT & 844811 & 9.00 & 991232 & 5.63 & 424607 & 11.82 \\
\hline EPA SAMPLE NO. & & & & & & \\
\hline VBLKDJ & 1337608 & 9.33 & 1599463 & 5.97 & 639296 & 12.15 \\
\hline YKQCTB-W-37960 & 1337500 & 9.33 & 1573495 & 5.96 & 641633 & 12.15 \\
\hline $\begin{array}{l}\text { YKTI14-136-W-3 } \\
7954\end{array}$ & 1342906 & 9.33 & 1601410 & 5.97 & 647462 & 12.15 \\
\hline VIBLKDI & 1374316 & 9.33 & 1664594 & 5.97 & 639387 & 12.15 \\
\hline $\begin{array}{l}\text { YKTI16-136-W-3 } \\
7964\end{array}$ & 1416020 & 9.33 & 1706995 & 5.96 & 659112 & 12.15 \\
\hline VIBLKDJ & 1391970 & 9.33 & 1695888 & 5.96 & 635636 & 12.15 \\
\hline $\begin{array}{l}\text { YKTI18-114-W-3 } \\
7973\end{array}$ & 1393338 & 9.33 & 1651846 & 5.97 & 649668 & 12.15 \\
\hline $\begin{array}{l}\text { YKTI14-136-W-3 } \\
7954 \mathrm{DL}\end{array}$ & 1329506 & 9.33 & 1608691 & 5.96 & 624467 & 12.15 \\
\hline $\begin{array}{l}\text { YKTI16-136-W-3 } \\
7964 \mathrm{DL}\end{array}$ & 1291209 & 9.33 & 1574997 & 5.96 & 611883 & 12.15 \\
\hline VHBLK01 & 1316668 & 9.33 & 1611081 & 5.96 & 635243 & 12.15 \\
\hline
\end{tabular}

IS1 $(\mathrm{CBZ})=$ Chlorobenzene-d5

IS2 $(\mathrm{DFB})=1,4-\mathrm{Difluorobenzene}$

IS3 $(\mathrm{DCB})=1,4-\mathrm{Dichlorobenzene-d4}$

AREA UPPER LIMIT $=140 \%$ (Trace Volatiles) of internal standard area AREA LOWER LIMIT $=60 \%$ (Trace Volatiles) of internal standard area RT UPPER LIMIT $=+0.33$ (Trace Volatiles) minutes of internal standard RT RT LOWER LIMIT $=-0.33$ (Trace Volatiles) minutes of internal standard RT

\# Column used to flag values outside contract required QC limits with an asterisk. 
Lab Name: TESTAMERICA BURLINGTON

Contract: $8 \mathrm{E}-00302$

Lab Code: STLV Case No.: YORK Mod. Ref No.: SDG NO.: YORK (200-29

Matrix: (SOIL/SED/WATER) Water

Sample wt/vol: 25.0 $(\mathrm{g} / \mathrm{mL}) \mathrm{mL}$

Level: (TRACE/LOW/MED) TRACE

\% Moisture: not dec.

GC Column: DB-624 ID: $0.20 \quad(\mathrm{~mm})$

Soil Extract Volume: (uL) $(\mathrm{mL})$

Purge Volume: 25.0
Lab Sample ID: 200-29506-2

Lab File ID: 15537_006.D

Date Received: 08/29/2015

Date Analyzed: 08/31/2015

Dilution Factor: 1.0

Soil Aliquot Volume: (uL)

\begin{tabular}{|c|c|c|c|}
\hline CAS NO. & COMPOUND & $\begin{array}{l}\text { CONCENTRATION UNITS: } \\
(\mathrm{ug} / \mathrm{L} \text { or } \mathrm{ug} / \mathrm{kg}) \mathrm{ug} / \mathrm{L}\end{array}$ & q \\
\hline $75-71-8$ & Dichlorodifluoromethane & 1.0 & $\mathrm{U}$ \\
\hline $74-87-3$ & Chloromethane & 1.0 & $\mathrm{U}$ \\
\hline $75-01-4$ & Vinyl chloride & 1.0 & $\mathrm{U}$ \\
\hline $74-83-9$ & Bromomethane & 1.0 & $\mathrm{U}$ \\
\hline $75-00-3$ & Chloroethane & 1.0 & $\mathrm{U}$ \\
\hline $75-69-4$ & Trichlorofluoromethane & 1.0 & $\mathrm{U}$ \\
\hline $75-35-4$ & 1,1-Dichloroethene & 1.0 & $\mathrm{U}$ \\
\hline $76-13-1$ & $1,1,2$-Trichloro-1,2,2-trifluoroethane & 1.0 & $\mathrm{U}$ \\
\hline $67-64-1$ & Acetone & 5.0 & $\mathrm{~J}$ \\
\hline $75-15-0$ & Carbon disulfide & 1.0 & $\mathrm{U}$ \\
\hline $79-20-9$ & Methyl acetate & 1.0 & $\mathrm{U}$ \\
\hline $75-09-2$ & Methylene Chloride & 1.0 & $\mathrm{U}$ \\
\hline $156-60-5$ & trans-1,2-Dichloroethene & 1.0 & $\mathrm{U}$ \\
\hline $1634-04-4$ & Methyl tert-butyl ether & 1.0 & $\mathrm{U}$ \\
\hline $75-34-3$ & 1,1-Dichloroethane & 1.0 & $\mathrm{U}$ \\
\hline $156-59-2$ & cis-1,2-Dichloroethene & 1.0 & $\mathrm{U}$ \\
\hline $78-93-3$ & 2-Butanone & 5.0 & $\mathrm{U}$ \\
\hline $74-97-5$ & Bromochloromethane & 1.0 & $\mathrm{U}$ \\
\hline $67-66-3$ & Chloroform & 1.0 & $\mathrm{U}$ \\
\hline $71-55-6$ & $1,1,1$-Trichloroethane & 1.0 & $\mathrm{U}$ \\
\hline $110-82-7$ & Cyclohexane & 1.0 & $\mathrm{U}$ \\
\hline $56-23-5$ & Carbon tetrachloride & 1.0 & $\mathrm{U}$ \\
\hline $71-43-2$ & Benzene & 1.0 & $\mathrm{U}$ \\
\hline $107-06-2$ & 1,2-Dichloroethane & 1.0 & $\mathrm{U}$ \\
\hline
\end{tabular}

Report 1,4-Dioxane for Low-Medium VOA analysis only 
Lab Name: TESTAMERICA BURLINGTON Lab Code: STLV Case No.: YORK Mod. Ref No.:
Contract: $8 \mathrm{E}-00302$

\author{
Lab Sample ID: 200-29506-2
}

Lab File ID: 15537_006.D

Date Received: 08/29/2015

Date Analyzed: 08/31/2015

Dilution Factor: 1.0

Soil Aliquot Volume: (uL)

Soil Extract Volume: $\mathrm{ID}: \frac{0.20 \quad}{(\mathrm{~mm})}$
$(\mathrm{uL})$
$(\mathrm{mL})$ $\mathrm{ID}: \frac{0.20 \quad}{(\mathrm{~mm})}$
$(\mathrm{uL})$
$(\mathrm{mL})$

Purge Volume: 25.0

\begin{tabular}{|c|c|c|c|}
\hline CAS NO. & COMPOUND & $\begin{array}{l}\text { CONCENTRATION UNITS: } \\
(\mathrm{ug} / \mathrm{L} \text { or } \mathrm{ug} / \mathrm{kg}) \mathrm{ug} / \mathrm{L}\end{array}$ & Q \\
\hline $79-01-6$ & Trichloroethene & $\begin{array}{r}1.0 \\
1.0\end{array}$ & $\mathrm{U}$ \\
\hline $108-87-2$ & Methylcyclohexane & 1.0 & $\mathrm{U}$ \\
\hline $78-87-5$ & 1,2-Dichloropropane & 1.0 & $\mathrm{U}$ \\
\hline $75-27-4$ & Bromodichloromethane & 1.0 & $\mathrm{U}$ \\
\hline $10061-01-5$ & cis-1,3-Dichloropropene & 1.0 & $\mathrm{U}$ \\
\hline $108-10-1$ & 4-Methyl-2-pentanone & 1.0 & $\mathrm{U}$ \\
\hline $108-88-3$ & Toluene & 0.44 & $\mathrm{~J}$ \\
\hline $10061-02-6$ & trans-1,3-Dichloropropene & 1.0 & $\mathrm{U}$ \\
\hline $79-00-5$ & $1,1,2$-Trichloroethane & 1.0 & $\mathrm{U}$ \\
\hline $127-18-4$ & Tetrachloroethene & 1.0 & $\mathrm{U}$ \\
\hline $591-78-6$ & 2-Hexanone & 1.0 & $\mathrm{U}$ \\
\hline $124-48-1$ & Dibromochloromethane & 1.0 & $\mathrm{U}$ \\
\hline $106-93-4$ & 1,2-Dibromoethane & 1.0 & $\mathrm{U}$ \\
\hline $108-90-7$ & Chlorobenzene & 1.0 & $\mathrm{U}$ \\
\hline $100-41-4$ & Ethylbenzene & 0.039 & $\mathrm{~J}$ \\
\hline $95-47-6$ & o-Xylene & 0.078 & $\mathrm{~J}$ \\
\hline $179601-23-1$ & m,p-xylene & 0.13 & $\mathrm{~J}$ \\
\hline $100-42-5$ & styrene & 0.061 & $\mathrm{~J}$ \\
\hline $75-25-2$ & Bromoform & 1.0 & $\mathrm{U}$ \\
\hline $98-82-8$ & Isopropylbenzene & 1.0 & $\mathrm{U}$ \\
\hline $79-34-5$ & $1,1,2,2$-Tetrachloroethane & 1.0 & $\mathrm{U}$ \\
\hline $541-73-1$ & 1,3-Dichlorobenzene & 1.0 & $\mathrm{U}$ \\
\hline $106-46-7$ & 1,4-Dichlorobenzene & 1.0 & $\mathrm{U}$ \\
\hline $95-50-1$ & 1,2-Dichlorobenzene & 1.0 & $\mathrm{U}$ \\
\hline $96-12-8$ & 1,2-Dibromo-3-Chloropropane & 1.0 & $\mathrm{U}$ \\
\hline $120-82-1$ & $1,2,4$-Trichlorobenzene & 0.014 & $\mathrm{~J} B$ \\
\hline $87-61-6$ & $1,2,3$-Trichlorobenzene & 0.027 & $\mathrm{JB}$ \\
\hline
\end{tabular}


Lab Name: TESTAMERICA BURLINGTON

Contract: $8 \mathrm{E}-00302$

Lab Code: STLV

Case No.: YORK

Mod. Ref No.:

SDG NO.: YORK (200-29

Matrix: (SOIL/SED/WATER) Water

Lab Sample ID: 200-29506-2

Sample wt/vol: 25.0

$(\mathrm{g} / \mathrm{mL}) \mathrm{mL}$

Lab File ID: 15537_006.D

Level: (TRACE or LOW/MED) TRACE

․ Moisture: not dec.

Date Received: 08/29/2015

GC Column: DB-624 ID $: 0.20 \quad(\mathrm{~mm})$

Soil Extract Volume:

Date Analyzed: 08/31/2015

Dilution Factor: 1.0

Soil Aliquot Volume: (uL)

CONCENTRATION UNITS: (ug/L or ug/kg) ug/L

Purge Volume: 25.0

$(\mathrm{mL})$

\begin{tabular}{|c|c|c|c|c|}
\hline CAS NUMBER & COMPOUND NAME & $\mathrm{RT}$ & EST. CONC. & Q \\
\hline 01 & Unknown & 7.29 & 3.0 & $\mathrm{~B} \mathrm{X} \mathrm{J}$ \\
\hline $541-05-9$ & Cyclotrisiloxane, hexamethyl- & 8.22 & 0.52 & $\mathrm{~B} J \mathrm{~J}$ \\
\hline 03 & Unknown & 11.04 & 0.55 & $\mathrm{~B} \mathrm{~J}$ \\
\hline E9667961 & Total Alkanes & $\mathrm{N} / \mathrm{A}$ & & \\
\hline
\end{tabular}

1 EPA-designated Registry Number. 
Lab Name: TESTAMERICA BURLINGTON Lab Code: STLV Case No.: YORK Mod. Ref No.:
Contract: $8 \mathrm{E}-00302$ SDG NO.: YORK $(200-29$

Lab Sample ID: 200-29506-1

Lab File ID: 15537_007.D

Date Received: 08/29/2015

Date Analyzed: 08/31/2015

Dilution Factor: 1.1

Soil Aliquot Volume: (uL)

Soil Extract Volume: $\mathrm{ID}: \begin{aligned} & 0.20 \quad \\ & (\mathrm{~mm}) \\ & (\mathrm{uL}) \\ & (\mathrm{mL})\end{aligned}$ $\mathrm{ID}: \begin{aligned} & 0.20 \quad \\ & (\mathrm{~mm}) \\ & (\mathrm{uL}) \\ & (\mathrm{mL})\end{aligned}$

Purge Volume: 25.0

\begin{tabular}{|c|c|c|c|}
\hline CAS NO. & COMPOUND & $\begin{array}{l}\text { CONCENTRATION UNITS: } \\
(\mathrm{ug} / \mathrm{L} \text { or } \mathrm{ug} / \mathrm{kg}) \mathrm{ug} / \mathrm{L}\end{array}$ & Q \\
\hline $75-71-8$ & Dichlorodifluoromethane & 1.1 & $\bar{U}$ \\
\hline $74-87-3$ & Chloromethane & 1.1 & $\mathrm{U}$ \\
\hline $75-01-4$ & Vinyl chloride & 1.1 & $\mathrm{U}$ \\
\hline $74-83-9$ & Bromomethane & 1.1 & $\mathrm{U}$ \\
\hline $75-00-3$ & Chloroethane & 1.1 & $\mathrm{U}$ \\
\hline $75-69-4$ & Trichlorofluoromethane & 0.089 & $\mathrm{~J}$ \\
\hline $75-35-4$ & 1,1-Dichloroethene & 1.1 & $\mathrm{U}$ \\
\hline $76-13-1$ & $1,1,2$-Trichloro-1,2,2-trifluoroethane & 1.1 & $\mathrm{U}$ \\
\hline $67-64-1$ & Acetone & 3.4 & $\mathrm{~J}$ \\
\hline $75-15-0$ & Carbon disulfide & 1.1 & $\mathrm{U}$ \\
\hline $79-20-9$ & Methyl acetate & 1.1 & $\mathrm{U}$ \\
\hline $75-09-2$ & Methylene Chloride & 1.1 & $\mathrm{U}$ \\
\hline $156-60-5$ & trans-1,2-Dichloroethene & 1.1 & $\mathrm{U}$ \\
\hline $1634-04-4$ & Methyl tert-butyl ether & 1.1 & $\mathrm{U}$ \\
\hline $75-34-3$ & 1,1-Dichloroethane & 1.1 & $\mathrm{U}$ \\
\hline $156-59-2$ & cis-1,2-Dichloroethene & 1.1 & $\mathrm{U}$ \\
\hline $78-93-3$ & 2-Butanone & 5.7 & $\mathrm{U}$ \\
\hline $74-97-5$ & Bromochloromethane & 1.1 & $\mathrm{U}$ \\
\hline $67-66-3$ & Chloroform & 0.79 & $\mathrm{~J}$ \\
\hline $71-55-6$ & 1,1,1-Trichloroethane & 0.36 & $\mathrm{~J}$ \\
\hline $110-82-7$ & Cyclohexane & 1.1 & $\mathrm{U}$ \\
\hline $56-23-5$ & Carbon tetrachloride & 63 & $\mathrm{E}$ \\
\hline $71-43-2$ & Benzene & 1.1 & $\mathrm{U}$ \\
\hline $107-06-2$ & 1,2-Dichloroethane & 1.1 & $\mathrm{U}$ \\
\hline
\end{tabular}

Report 1,4-Dioxane for Low-Medium VOA analysis only 
Lab Name: TESTAMERICA BURLINGTON Lab Code: STLV Case No.: YORK Mod. Ref No.:
Contract: $8 \mathrm{E}-00302$

\author{
Lab Sample ID: 200-29506-1
}

Dilution Factor: 1.1

Soil Aliquot Volume: $(u L)$

Soil Extract Volume:

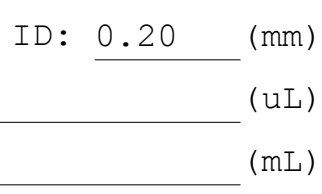

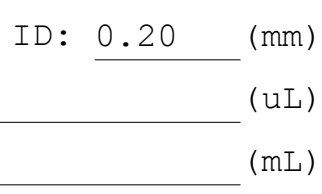

Purge Volume: 25.0

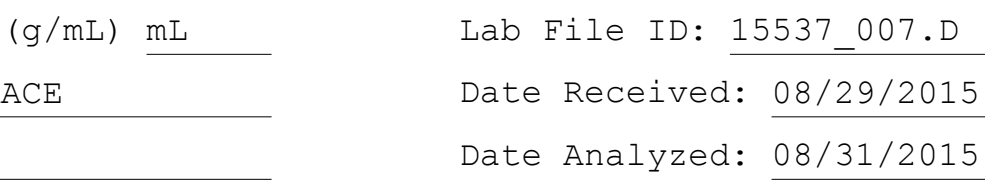

Lab File ID: 15537_007.D

Date Received: 08/29/2015

Date Analyzed: 08/31/2015 $(\mathrm{g} / \mathrm{mL}) \mathrm{mL}$

\begin{tabular}{|c|c|c|c|}
\hline CAS NO. & COMPOUND & $\begin{array}{l}\text { CONCENTRATION UNITS: } \\
(\mathrm{ug} / \mathrm{L} \text { or } \mathrm{ug} / \mathrm{kg}) \mathrm{ug} / \mathrm{L}\end{array}$ & $\S$ \\
\hline $79-01-6$ & Trichloroethene & $\begin{array}{r}1.1 \\
\end{array}$ & $\mathrm{U}$ \\
\hline $108-87-2$ & Methylcyclohexane & 1.1 & $\mathrm{U}$ \\
\hline $78-87-5$ & 1,2-Dichloropropane & 1.1 & $\mathrm{U}$ \\
\hline $75-27-4$ & Bromodichloromethane & 1.1 & $\mathrm{U}$ \\
\hline $10061-01-5$ & cis-1,3-Dichloropropene & 1.1 & $\mathrm{U}$ \\
\hline $108-10-1$ & 4-Methyl-2-pentanone & 1.1 & $\mathrm{U}$ \\
\hline $108-88-3$ & Toluene & 0.037 & $\mathrm{~J}$ \\
\hline $10061-02-6$ & trans-1,3-Dichloropropene & 1.1 & $\mathrm{U}$ \\
\hline $79-00-5$ & $1,1,2-T r i c h l o r o e t h a n e$ & 1.1 & $\mathrm{U}$ \\
\hline $127-18-4$ & Tetrachloroethene & 0.12 & $\mathrm{~J}$ \\
\hline $591-78-6$ & 2-Hexanone & 1.1 & $\mathrm{U}$ \\
\hline $124-48-1$ & Dibromochloromethane & 1.1 & $\mathrm{U}$ \\
\hline $106-93-4$ & 1,2-Dibromoethane & 1.1 & $\mathrm{U}$ \\
\hline $108-90-7$ & Chlorobenzene & 1.1 & $\mathrm{U}$ \\
\hline $100-41-4$ & Ethylbenzene & 0.0079 & $\mathrm{~J}$ \\
\hline $95-47-6$ & o-Xylene & 0.0081 & $\mathrm{~J}$ \\
\hline $179601-23-1$ & m,p-xylene & 0.022 & $\mathrm{~J}$ \\
\hline $100-42-5$ & Styrene & 1.1 & $\mathrm{U}$ \\
\hline $75-25-2$ & Bromoform & 1.1 & $\mathrm{U}$ \\
\hline $98-82-8$ & Isopropylbenzene & 1.1 & $\mathrm{U}$ \\
\hline $79-34-5$ & $1,1,2,2$-Tetrachloroethane & 1.1 & $\mathrm{U}$ \\
\hline $541-73-1$ & 1,3 -Dichlorobenzene & 1.1 & $\mathrm{U}$ \\
\hline $106-46-7$ & 1,4-Dichlorobenzene & 1.1 & $\mathrm{U}$ \\
\hline $95-50-1$ & 1,2-Dichlorobenzene & 1.1 & $\mathrm{U}$ \\
\hline $96-12-8$ & 1,2-Dibromo-3-Chloropropane & 1.1 & $\mathrm{U}$ \\
\hline $120-82-1$ & $1,2,4$-Trichlorobenzene & 1.1 & $\mathrm{U}$ \\
\hline $87-61-6$ & $1,2,3$-Trichlorobenzene & 1.1 & $\mathrm{U}$ \\
\hline
\end{tabular}


Lab Name: TESTAMERICA BURLINGTON

Contract: 8E-00302

Lab Code: STLV Case No.: YORK Mod. Ref No.: SDG NO.: YORK $(200-29$

Matrix: (SOIL/SED/WATER) Water

Sample wt/vol: 25.0 $(\mathrm{g} / \mathrm{mL}) \mathrm{mL}$

Level: (TRACE or LOW/MED) TRACE

Moisture: not dec.

GC Column: DB-624 ID $: 0.20 \quad(\mathrm{~mm})$

Soil Extract Volume: (uL)

CONCENTRATION UNITS: (ug/L or ug/kg) ug/L
Lab Sample ID: 200-29506-1

Lab File ID: 15537_007.D

Date Received: 08/29/2015

Date Analyzed: 08/31/2015

Dilution Factor: 1.1

Soil Aliquot Volume: (uL)

Purge Volume: 25.0

$(\mathrm{mL})$

01

02

\begin{tabular}{|l|l|c|c|c|}
\hline CAS NUMBER & \multicolumn{1}{|c|}{ COMPOUND NAME } & RT & EST. CONC. & Q \\
\hline & Unknown & 7.29 & 3.6 & B X J \\
\hline E9667961 & Total Alkanes & N/A & & \\
\hline
\end{tabular}

1 EPA-designated Registry Number. 
Lab Name: TESTAMERICA BURLINGTON

Lab Code: STLV

Case No.: YORK

Mod. Ref No.:

Contract: $8 \mathrm{E}-00302$

Matrix: (SOIL/SED/WATER) Water

Sample wt/vol: 25.0 $(\mathrm{g} / \mathrm{mL}) \mathrm{mL}$

Level: (TRACE/LOW/MED) TRACE

\% Moisture: not dec.

GC Column: DB-624 ID: $0.20 \quad(\mathrm{~mm})$

Soil Extract Volume: (uL) $(\mathrm{mL})$

Purge Volume: 25.0 SDG NO.: YORK (200-29

Lab Sample ID: 200-29506-1

Lab File ID: 15537_013.D

Date Received: 08/29/2015

Date Analyzed: 08/31/2015

Dilution Factor: 4.5

Soil Aliquot Volume: (uL)

\begin{tabular}{|c|c|c|c|}
\hline CAS NO. & COMPOUND & $\begin{array}{l}\text { CONCENTRATION UNITS: } \\
(\mathrm{ug} / \mathrm{L} \text { or } \mathrm{ug} / \mathrm{kg}) \mathrm{ug} / \mathrm{L}\end{array}$ & Q \\
\hline $75-71-8$ & Dichlorodifluoromethane & 4.5 & $\mathrm{U}$ \\
\hline $74-87-3$ & Chloromethane & 4.5 & $\mathrm{U}$ \\
\hline $75-01-4$ & Vinyl chloride & 4.5 & $\mathrm{U}$ \\
\hline $74-83-9$ & Bromomethane & 4.5 & $\mathrm{U}$ \\
\hline $75-00-3$ & Chloroethane & 4.5 & $\mathrm{U}$ \\
\hline $75-69-4$ & Trichlorofluoromethane & 4.5 & $\mathrm{U}$ \\
\hline $75-35-4$ & 1,1-Dichloroethene & 4.5 & $\mathrm{U}$ \\
\hline $76-13-1$ & $1,1,2$-Trichloro-1,2,2-trifluoroethane & 4.5 & $\mathrm{U}$ \\
\hline $67-64-1$ & Acetone & 23 & $\mathrm{U}$ \\
\hline $75-15-0$ & Carbon disulfide & 4.5 & $\mathrm{U}$ \\
\hline $79-20-9$ & Methyl acetate & 4.5 & $\mathrm{U}$ \\
\hline $75-09-2$ & Methylene Chloride & 4.5 & $\mathrm{U}$ \\
\hline $156-60-5$ & trans-1,2-Dichloroethene & 4.5 & $\mathrm{U}$ \\
\hline $1634-04-4$ & Methyl tert-butyl ether & 4.5 & $\mathrm{U}$ \\
\hline $75-34-3$ & 1,1-Dichloroethane & 4.5 & $\mathrm{U}$ \\
\hline $156-59-2$ & cis-1,2-Dichloroethene & 4.5 & $\mathrm{U}$ \\
\hline $78-93-3$ & 2-Butanone & 23 & $\mathrm{U}$ \\
\hline $74-97-5$ & Bromochloromethane & 4.5 & $\mathrm{U}$ \\
\hline $67-66-3$ & Chloroform & 0.78 & $\mathrm{~J} \mathrm{D}$ \\
\hline $71-55-6$ & $1,1,1$-Trichloroethane & 0.33 & $\mathrm{~J} \mathrm{D}$ \\
\hline $110-82-7$ & Cyclohexane & 4.5 & $\mathrm{U}$ \\
\hline $56-23-5$ & Carbon tetrachloride & 55 & $\mathrm{D}$ \\
\hline $71-43-2$ & Benzene & 4.5 & $\mathrm{U}$ \\
\hline $107-06-2$ & 1,2-Dichloroethane & 4.5 & $\mathrm{U}$ \\
\hline
\end{tabular}

Report 1,4-Dioxane for Low-Medium VOA analysis only 
Lab Name: TESTAMERICA BURLINGTON

Lab Code: STLV

Case No.: YORK

Mod. Ref No.:

Contract: $8 \mathrm{E}-00302$

Matrix: (SOIL/SED/WATER) Water

Sample wt/vol: 25.0 $(\mathrm{g} / \mathrm{mL}) \mathrm{mL}$

Level: (TRACE/LOW/MED) TRACE

\% Moisture: not dec.

GC Column: DB-624 ID: $0.20 \quad(\mathrm{~mm})$

Soil Extract Volume: (uL) $(\mathrm{mL})$

Purge Volume: 25.0 SDG NO.: YORK (200-29

Lab Sample ID: 200-29506-1

Lab File ID: 15537_013.D

Date Received: 08/29/2015

Date Analyzed: 08/31/2015

Dilution Factor: 4.5

Soil Aliquot Volume: (uL)

\begin{tabular}{|c|c|c|c|}
\hline CAS NO. & COMPOUND & $\begin{array}{l}\text { CONCENTRATION UNITS: } \\
(\mathrm{ug} / \mathrm{L} \text { or } \mathrm{ug} / \mathrm{kg}) \mathrm{ug} / \mathrm{L}\end{array}$ & $q$ \\
\hline $79-01-6$ & Trichloroethene & 4.5 & $\mathrm{U}$ \\
\hline $108-87-2$ & Methylcyclohexane & 4.5 & $\mathrm{U}$ \\
\hline $78-87-5$ & 1,2-Dichloropropane & 4.5 & $\mathrm{U}$ \\
\hline $75-27-4$ & Bromodichloromethane & 4.5 & $\mathrm{U}$ \\
\hline $10061-01-5$ & cis-1,3-Dichloropropene & 4.5 & U \\
\hline $108-10-1$ & 4-Methyl-2-pentanone & 4.5 & $\mathrm{U}$ \\
\hline $108-88-3$ & Toluene & 4.5 & $\mathrm{U}$ \\
\hline $10061-02-6$ & trans-1,3-Dichloropropene & 4.5 & $\mathrm{U}$ \\
\hline $79-00-5$ & $1,1,2-$ Trichloroethane & 4.5 & $\mathrm{U}$ \\
\hline $127-18-4$ & Tetrachloroethene & 4.5 & $\mathrm{U}$ \\
\hline $591-78-6$ & 2-Hexanone & 4.5 & $\mathrm{U}$ \\
\hline $124-48-1$ & Dibromochloromethane & 4.5 & $\mathrm{U}$ \\
\hline $106-93-4$ & 1,2-Dibromoethane & 4.5 & $\mathrm{U}$ \\
\hline $108-90-7$ & Chlorobenzene & 4.5 & $\mathrm{U}$ \\
\hline $100-41-4$ & Ethylbenzene & 4.5 & $\mathrm{U}$ \\
\hline $95-47-6$ & o-Xylene & 4.5 & $\mathrm{U}$ \\
\hline $179601-23-1$ & m,p-xylene & 4.5 & $\mathrm{U}$ \\
\hline $100-42-5$ & Styrene & 4.5 & $\mathrm{U}$ \\
\hline $75-25-2$ & Bromoform & 4.5 & $\mathrm{U}$ \\
\hline $98-82-8$ & Isopropylbenzene & 4.5 & $\mathrm{U}$ \\
\hline $79-34-5$ & $1,1,2,2$-Tetrachloroethane & 4.5 & $\mathrm{U}$ \\
\hline $541-73-1$ & 1,3 -Dichlorobenzene & 4.5 & $\mathrm{U}$ \\
\hline $106-46-7$ & 1,4-Dichlorobenzene & 4.5 & $\mathrm{U}$ \\
\hline $95-50-1$ & 1,2-Dichlorobenzene & 4.5 & $\mathrm{U}$ \\
\hline $96-12-8$ & 1,2-Dibromo-3-Chloropropane & 4.5 & $\mathrm{U}$ \\
\hline $120-82-1$ & $1,2,4$-Trichlorobenzene & 4.5 & $\mathrm{U}$ \\
\hline $87-61-6$ & $1,2,3$-Trichlorobenzene & 4.5 & $\mathrm{U}$ \\
\hline
\end{tabular}


Lab Name: TESTAMERICA BURLINGTON

Contract: 8E-00302

Lab Code: STLV Case No.: YORK Mod. Ref No.: SDG NO.: YORK $(200-29$

Matrix: (SOIL/SED/WATER) Water

Sample wt/vol: 25.0 $(\mathrm{g} / \mathrm{mL}) \mathrm{mL}$

Level: (TRACE or LOW/MED) TRACE

Moisture: not dec.

GC Column: DB-624 ID $: 0.20 \quad(\mathrm{~mm})$

Soil Extract Volume: (uL)

CONCENTRATION UNITS: (ug/L or ug/kg) ug/L
Lab Sample ID: 200-29506-1

Lab File ID: 15537_013.D

Date Received: 08/29/2015

Date Analyzed: 08/31/2015

Dilution Factor: 4.5

Soil Aliquot Volume: (uL) Purge Volume: 25.0 $(\mathrm{mL})$

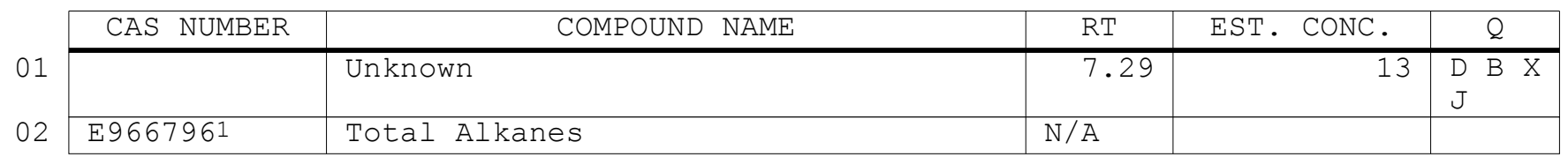

1 EPA-designated Registry Number. 
Lab Name: TESTAMERICA BURLINGTON Lab Code: STLV Case No.: YORK Mod. Ref No.:
Contract: $8 \mathrm{E}-00302$ SDG NO.: YORK $(200-29$

Lab Sample ID: 200-29506-3

Lab File ID: 15537_009.D

Date Received: 08/29/2015

Date Analyzed: 08/31/2015

Dilution Factor: 1.0

Soil Aliquot Volume: (uL)

Soil Extract Volume: $\mathrm{ID}: \begin{aligned} & 0.20 \quad \\ & (\mathrm{~mm}) \\ & (\mathrm{uL}) \\ & (\mathrm{mL})\end{aligned}$ $\mathrm{ID}: \begin{aligned} & 0.20 \quad \\ & (\mathrm{~mm}) \\ & (\mathrm{uL}) \\ & (\mathrm{mL})\end{aligned}$

Purge Volume: 25.0

\begin{tabular}{|c|c|c|c|}
\hline CAS NO. & COMPOUND & $\begin{array}{l}\text { CONCENTRATION UNITS: } \\
(\mathrm{ug} / \mathrm{L} \text { or } \mathrm{ug} / \mathrm{kg}) \mathrm{ug} / \mathrm{L}\end{array}$ & Q \\
\hline $75-71-8$ & Dichlorodifluoromethane & 1.0 & $\bar{U}$ \\
\hline $74-87-3$ & Chloromethane & 1.0 & $\mathrm{U}$ \\
\hline $75-01-4$ & Vinyl chloride & 1.0 & $\mathrm{U}$ \\
\hline $74-83-9$ & Bromomethane & 1.0 & $\mathrm{U}$ \\
\hline $75-00-3$ & Chloroethane & 1.0 & $\mathrm{U}$ \\
\hline $75-69-4$ & Trichlorofluoromethane & 0.058 & $\mathrm{~J}$ \\
\hline $75-35-4$ & 1,1-Dichloroethene & 1.0 & $\mathrm{U}$ \\
\hline $76-13-1$ & $1,1,2$-Trichloro-1,2,2-trifluoroethane & 1.0 & $\mathrm{U}$ \\
\hline $67-64-1$ & Acetone & 5.0 & $\mathrm{U}$ \\
\hline $75-15-0$ & Carbon disulfide & 1.0 & $\mathrm{U}$ \\
\hline $79-20-9$ & Methyl acetate & 1.0 & $\mathrm{U}$ \\
\hline $75-09-2$ & Methylene Chloride & 1.0 & $\mathrm{U}$ \\
\hline $156-60-5$ & trans-1,2-Dichloroethene & 1.0 & $\mathrm{U}$ \\
\hline $1634-04-4$ & Methyl tert-butyl ether & 1.0 & $\mathrm{U}$ \\
\hline $75-34-3$ & 1,1-Dichloroethane & 1.0 & $\mathrm{U}$ \\
\hline $156-59-2$ & cis-1,2-Dichloroethene & 1.0 & $\mathrm{U}$ \\
\hline $78-93-3$ & 2-Butanone & 5.0 & $\mathrm{U}$ \\
\hline $74-97-5$ & Bromochloromethane & 1.0 & $\mathrm{U}$ \\
\hline $67-66-3$ & Chloroform & 0.61 & $\mathrm{~J}$ \\
\hline $71-55-6$ & 1,1,1-Trichloroethane & 1.0 & $\mathrm{U}$ \\
\hline $110-82-7$ & Cyclohexane & 1.0 & $\mathrm{U}$ \\
\hline $56-23-5$ & Carbon tetrachloride & 36 & $\mathrm{E}$ \\
\hline $71-43-2$ & Benzene & 1.0 & $\mathrm{U}$ \\
\hline $107-06-2$ & 1,2-Dichloroethane & 1.0 & $\mathrm{U}$ \\
\hline
\end{tabular}

Report 1,4-Dioxane for Low-Medium VOA analysis only 
Lab Name: TESTAMERICA BURLINGTON Lab Code: STLV Case No.: YORK Mod. Ref No.:
Contract: $8 \mathrm{E}-00302$

\author{
Lab Sample ID: 200-29506-3
}

Dilution Factor: 1.0

Soil Aliquot Volume: (uL)

Soil Extract Volume: $\mathrm{ID}: \begin{aligned} & 0.20 \quad \\ & (\mathrm{~mm}) \\ & (\mathrm{uL}) \\ & (\mathrm{mL})\end{aligned}$ $\mathrm{ID}: \begin{aligned} & 0.20 \quad \\ & (\mathrm{~mm}) \\ & (\mathrm{uL}) \\ & (\mathrm{mL})\end{aligned}$

Purge Volume: 25.0

\section{Lab File ID: 15537_009.D \\ Date Received: 08/29/2015 \\ Date Analyzed: 08/31/2015 \\ $(\mathrm{g} / \mathrm{mL}) \mathrm{mL}$}

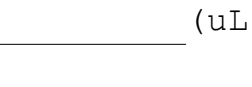

\begin{tabular}{|c|c|c|c|}
\hline CAS NO. & COMPOUND & $\begin{array}{l}\text { CONCENTRATION UNITS: } \\
(\mathrm{ug} / \mathrm{L} \text { or } \mathrm{ug} / \mathrm{kg}) \mathrm{ug} / \mathrm{L}\end{array}$ & 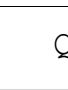 \\
\hline $79-01-6$ & Trichloroethene & $\begin{array}{r}1.0 \\
1.0\end{array}$ & $\mathrm{U}$ \\
\hline $108-87-2$ & Methylcyclohexane & 1.0 & $\mathrm{U}$ \\
\hline $78-87-5$ & 1,2-Dichloropropane & 1.0 & $\mathrm{U}$ \\
\hline $75-27-4$ & Bromodichloromethane & 1.0 & $\mathrm{U}$ \\
\hline $10061-01-5$ & cis-1,3-Dichloropropene & 1.0 & $\mathrm{U}$ \\
\hline $108-10-1$ & 4-Methyl-2-pentanone & 1.0 & $\mathrm{U}$ \\
\hline $108-88-3$ & Toluene & 1.0 & $\mathrm{U}$ \\
\hline $10061-02-6$ & trans-1,3-Dichloropropene & 1.0 & $\mathrm{U}$ \\
\hline $79-00-5$ & $1,1,2$-Trichloroethane & 1.0 & $\mathrm{U}$ \\
\hline $127-18-4$ & Tetrachloroethene & 1.0 & $\mathrm{U}$ \\
\hline $591-78-6$ & 2-Hexanone & 1.0 & $\mathrm{U}$ \\
\hline $124-48-1$ & Dibromochloromethane & 1.0 & $\mathrm{U}$ \\
\hline $106-93-4$ & 1,2-Dibromoethane & 1.0 & $\mathrm{U}$ \\
\hline $108-90-7$ & Chlorobenzene & 1.0 & $\mathrm{U}$ \\
\hline $100-41-4$ & Ethylbenzene & 1.0 & $\mathrm{U}$ \\
\hline $95-47-6$ & o-Xylene & 1.0 & $\mathrm{U}$ \\
\hline $179601-23-1$ & m,p-xylene & 1.0 & $\mathrm{U}$ \\
\hline $100-42-5$ & Styrene & 1.0 & $\mathrm{U}$ \\
\hline $75-25-2$ & Bromoform & 1.0 & $\mathrm{U}$ \\
\hline $98-82-8$ & Isopropylbenzene & 1.0 & $\mathrm{U}$ \\
\hline $79-34-5$ & $1,1,2,2$-Tetrachloroethane & 1.0 & $\mathrm{U}$ \\
\hline $541-73-1$ & 1,3-Dichlorobenzene & 1.0 & $\mathrm{U}$ \\
\hline $106-46-7$ & 1,4-Dichlorobenzene & 1.0 & $\mathrm{U}$ \\
\hline $95-50-1$ & 1,2-Dichlorobenzene & 1.0 & $\mathrm{U}$ \\
\hline $96-12-8$ & 1,2-Dibromo-3-Chloropropane & 1.0 & $\mathrm{U}$ \\
\hline $120-82-1$ & $1,2,4$-Trichlorobenzene & 1.0 & $\mathrm{U}$ \\
\hline $87-61-6$ & $1,2,3$-Trichlorobenzene & 1.0 & $\mathrm{U}$ \\
\hline
\end{tabular}


Lab Name: TESTAMERICA BURLINGTON

Contract: $8 \mathrm{E}-00302$

Lab Code: STLV

Case No.: YORK

Mod. Ref No.:

SDG NO.: YORK (200-29

Matrix: (SOIL/SED/WATER) Water

Sample wt/vol: 25.0 $(\mathrm{g} / \mathrm{mL}) \mathrm{mL}$

Level: (TRACE or LOW/MED) TRACE

\% Moisture: not dec.

GC Column: DB-624 ID $: 0.20 \quad(\mathrm{~mm})$

Soil Extract Volume: (uL)
Lab Sample ID: 200-29506-3

Lab File ID: 15537_009.D

Date Received: 08/29/2015

Date Analyzed: 08/31/2015

Dilution Factor: 1.0

Soil Aliquot Volume: (uL)

Purge Volume: 25.0

$(\mathrm{mL})$

CONCENTRATION UNITS: (ug/L or ug/kg) ug/L

\begin{tabular}{|c|c|c|c|c|}
\hline CAS NUMBER & COMPOUND NAME & RT & EST. CONC. & Q \\
\hline 01 & Unknown & 7.29 & 3.0 & $\mathrm{~B} X \mathrm{~J}$ \\
\hline $541-05-9$ & Cyclotrisiloxane, hexamethyl- & 8.22 & 0.73 & $\mathrm{~B} \quad \mathrm{~J} \mathrm{~N}$ \\
\hline $556-67-2$ & Cyclotetrasiloxane, octamethyl- & 11.04 & 0.62 & $\mathrm{~B} J \mathrm{~N}$ \\
\hline E9667961 & Total Alkanes & $\mathrm{N} / \mathrm{A}$ & & \\
\hline
\end{tabular}

1 EPA-designated Registry Number. 
Lab Name: TESTAMERICA BURLINGTON

Lab Code: STLV

Case No.: YORK

Mod. Ref No.:

Contract: $8 \mathrm{E}-00302$

Matrix: (SOIL/SED/WATER) Water

Sample wt/vol: 25.0 $(\mathrm{g} / \mathrm{mL}) \mathrm{mL}$

Level: (TRACE/LOW/MED) TRACE

\% Moisture: not dec.

GC Column: DB-624 ID: $0.20 \quad(\mathrm{~mm})$

Soil Extract Volume: (uL) $(\mathrm{mL})$

Purge Volume: 25.0 SDG NO.: YORK (200-29

Lab Sample ID: 200-29506-3

Lab File ID: 15537_014.D

Date Received: 08/29/2015

Date Analyzed: 08/31/2015

Dilution Factor: 2.6

Soil Aliquot Volume: (uL)

\begin{tabular}{|c|c|c|c|}
\hline CAS NO. & COMPOUND & $\begin{array}{l}\text { CONCENTRATION UNITS: } \\
(\mathrm{ug} / \mathrm{L} \text { or } \mathrm{ug} / \mathrm{kg}) \mathrm{ug} / \mathrm{L}\end{array}$ & $Q$ \\
\hline $75-71-8$ & Dichlorodifluoromethane & 2.6 & $\mathrm{U}$ \\
\hline $74-87-3$ & Chloromethane & 2.6 & $\mathrm{U}$ \\
\hline $75-01-4$ & Vinyl chloride & 2.6 & $\mathrm{U}$ \\
\hline $74-83-9$ & Bromomethane & 2.6 & $\mathrm{U}$ \\
\hline $75-00-3$ & Chloroethane & 2.6 & $\mathrm{U}$ \\
\hline $75-69-4$ & Trichlorofluoromethane & 2.6 & $\mathrm{U}$ \\
\hline $75-35-4$ & 1,1-Dichloroethene & 2.6 & $\mathrm{U}$ \\
\hline $76-13-1$ & $1,1,2$-Trichloro-1,2,2-trifluoroethane & 2.6 & $\mathrm{U}$ \\
\hline $67-64-1$ & Acetone & 13 & $\mathrm{U}$ \\
\hline $75-15-0$ & Carbon disulfide & 2.6 & $\mathrm{U}$ \\
\hline $79-20-9$ & Methyl acetate & 2.6 & $\mathrm{U}$ \\
\hline $75-09-2$ & Methylene Chloride & 2.6 & $\mathrm{U}$ \\
\hline $156-60-5$ & trans-1,2-Dichloroethene & 2.6 & $\mathrm{U}$ \\
\hline $1634-04-4$ & Methyl tert-butyl ether & 2.6 & $\mathrm{U}$ \\
\hline $75-34-3$ & 1,1-Dichloroethane & 2.6 & $\mathrm{U}$ \\
\hline $156-59-2$ & cis-1,2-Dichloroethene & 2.6 & $\mathrm{U}$ \\
\hline $78-93-3$ & 2-Butanone & 13 & $\mathrm{U}$ \\
\hline $74-97-5$ & Bromochloromethane & 2.6 & $\mathrm{U}$ \\
\hline $67-66-3$ & Chloroform & 0.64 & $\mathrm{~J} \mathrm{D}$ \\
\hline $71-55-6$ & $1,1,1$-Trichloroethane & 2.6 & $\mathrm{U}$ \\
\hline $110-82-7$ & Cyclohexane & 2.6 & $\mathrm{U}$ \\
\hline $56-23-5$ & Carbon tetrachloride & 33 & $\mathrm{D}$ \\
\hline $71-43-2$ & Benzene & 2.6 & $\mathrm{U}$ \\
\hline $107-06-2$ & 1,2-Dichloroethane & 2.6 & $\mathrm{U}$ \\
\hline
\end{tabular}

Report 1,4-Dioxane for Low-Medium VOA analysis only 
Lab Name: TESTAMERICA BURLINGTON

Lab Code: STLV

Case No.: YORK

Mod. Ref No.:

Contract: $8 \mathrm{E}-00302$

Matrix: (SOIL/SED/WATER) Water

Sample wt/vol: 25.0 $(\mathrm{g} / \mathrm{mL}) \mathrm{mL}$

Level: (TRACE/LOW/MED) TRACE

\% Moisture: not dec.

GC Column: DB-624 ID: $0.20 \quad(\mathrm{~mm})$

Soil Extract Volume: (uL) $(\mathrm{mL})$

Purge Volume: 25.0 SDG NO.: YORK (200-29

Lab Sample ID: 200-29506-3

Lab File ID: 15537_014.D

Date Received: 08/29/2015

Date Analyzed: 08/31/2015

Dilution Factor: 2.6

Soil Aliquot Volume: (uL)

\begin{tabular}{|c|c|c|c|}
\hline CAS NO. & COMPOUND & $\begin{array}{l}\text { CONCENTRATION UNITS: } \\
(\mathrm{ug} / \mathrm{L} \text { or } \mathrm{ug} / \mathrm{kg}) \mathrm{ug} / \mathrm{L}\end{array}$ & $q$ \\
\hline $79-01-6$ & Trichloroethene & 2.6 & $\mathrm{U}$ \\
\hline $108-87-2$ & Methylcyclohexane & 2.6 & $\mathrm{U}$ \\
\hline $78-87-5$ & 1,2-Dichloropropane & 2.6 & $\mathrm{U}$ \\
\hline $75-27-4$ & Bromodichloromethane & 2.6 & $\mathrm{U}$ \\
\hline $10061-01-5$ & cis-1,3-Dichloropropene & 2.6 & U \\
\hline $108-10-1$ & 4-Methyl-2-pentanone & 2.6 & $\mathrm{U}$ \\
\hline $108-88-3$ & Toluene & 2.6 & $\mathrm{U}$ \\
\hline $10061-02-6$ & trans-1,3-Dichloropropene & 2.6 & $\mathrm{U}$ \\
\hline $79-00-5$ & $1,1,2-$ Trichloroethane & 2.6 & $\mathrm{U}$ \\
\hline $127-18-4$ & Tetrachloroethene & 2.6 & $\mathrm{U}$ \\
\hline $591-78-6$ & 2-Hexanone & 2.6 & $\mathrm{U}$ \\
\hline $124-48-1$ & Dibromochloromethane & 2.6 & $\mathrm{U}$ \\
\hline $106-93-4$ & 1,2-Dibromoethane & 2.6 & $\mathrm{U}$ \\
\hline $108-90-7$ & Chlorobenzene & 2.6 & $\mathrm{U}$ \\
\hline $100-41-4$ & Ethylbenzene & 2.6 & $\mathrm{U}$ \\
\hline $95-47-6$ & o-Xylene & 2.6 & $\mathrm{U}$ \\
\hline $179601-23-1$ & m,p-xylene & 2.6 & $\mathrm{U}$ \\
\hline $100-42-5$ & Styrene & 2.6 & $\mathrm{U}$ \\
\hline $75-25-2$ & Bromoform & 2.6 & $\mathrm{U}$ \\
\hline $98-82-8$ & Isopropylbenzene & 2.6 & $\mathrm{U}$ \\
\hline $79-34-5$ & $1,1,2,2$-Tetrachloroethane & 2.6 & $\mathrm{U}$ \\
\hline $541-73-1$ & 1,3 -Dichlorobenzene & 2.6 & $\mathrm{U}$ \\
\hline $106-46-7$ & 1,4-Dichlorobenzene & 2.6 & $\mathrm{U}$ \\
\hline $95-50-1$ & 1,2-Dichlorobenzene & 2.6 & $\mathrm{U}$ \\
\hline $96-12-8$ & 1,2-Dibromo-3-Chloropropane & 2.6 & $\mathrm{U}$ \\
\hline $120-82-1$ & $1,2,4$-Trichlorobenzene & 2.6 & $\mathrm{U}$ \\
\hline $87-61-6$ & $1,2,3$-Trichlorobenzene & 2.6 & $\mathrm{U}$ \\
\hline
\end{tabular}


Lab Name: TESTAMERICA BURLINGTON

Contract: $8 \mathrm{E}-00302$

Lab Code: STLV Case No.: YORK Mod. Ref No.: SDG NO.: YORK $(200-29$

Matrix: (SOIL/SED/WATER) Water

Sample wt/vol: 25.0 $(\mathrm{g} / \mathrm{mL}) \mathrm{mL}$

Level: (TRACE or LOW/MED) TRACE

․ Moisture: not dec.

GC Column: DB-624 ID $: 0.20 \quad(\mathrm{~mm})$

Soil Extract Volume: (uL)
Lab Sample ID: 200-29506-3

Lab File ID: 15537_014.D

Date Received: 08/29/2015

Date Analyzed: 08/31/2015

Dilution Factor: 2.6

Soil Aliquot Volume: (uL) Purge Volume: 25.0 $(\mathrm{mL})$ CONCENTRATION UNITS: (ug/L or ug/kg) ug/L

01

\begin{tabular}{|l|l|c|c|c|}
\hline CAS NUMBER & \multicolumn{1}{|c|}{ COMPOUND NAME } & RT & EST. CONC. & Q \\
\hline & Unknown & 7.29 & 7.2 & D B X \\
& & $\mathrm{J}$ \\
\hline E9667961 & Total Alkanes & N/A & & \\
\hline
\end{tabular}

1 EPA-designated Registry Number. 
Lab Name: TESTAMERICA BURLINGTON Lab Code: STLV Case No.: YORK Mod. Ref No.:
Contract: $8 \mathrm{E}-00302$ SDG NO.: YORK $(200-29$

Lab Sample ID: 200-29506-4

Lab File ID: 15537_011.D

Date Received: 08/29/2015

Date Analyzed: 08/31/2015

Dilution Factor: 1.0

Soil Aliquot Volume: (uL)

Soil Extract Volume: $\mathrm{ID}: \begin{aligned} & 0.20 \quad \\ & (\mathrm{~mm}) \\ & (\mathrm{uL}) \\ & (\mathrm{mL})\end{aligned}$ $\mathrm{ID}: \begin{aligned} & 0.20 \quad \\ & (\mathrm{~mm}) \\ & (\mathrm{uL}) \\ & (\mathrm{mL})\end{aligned}$

Purge Volume: 25.0

\begin{tabular}{|c|c|c|c|}
\hline CAS NO. & COMPOUND & $\begin{array}{l}\text { CONCENTRATION UNITS: } \\
(\mathrm{ug} / \mathrm{L} \text { or } \mathrm{ug} / \mathrm{kg}) \mathrm{ug} / \mathrm{L}\end{array}$ & Q \\
\hline $75-71-8$ & Dichlorodifluoromethane & 1.0 & $\bar{U}$ \\
\hline $74-87-3$ & Chloromethane & 1.0 & $\mathrm{U}$ \\
\hline $75-01-4$ & Vinyl chloride & 1.0 & $\mathrm{U}$ \\
\hline $74-83-9$ & Bromomethane & 1.0 & $\mathrm{U}$ \\
\hline $75-00-3$ & Chloroethane & 1.0 & $\mathrm{U}$ \\
\hline $75-69-4$ & Trichlorofluoromethane & 1.0 & $\mathrm{U}$ \\
\hline $75-35-4$ & 1,1-Dichloroethene & 1.0 & $\mathrm{U}$ \\
\hline $76-13-1$ & $1,1,2$-Trichloro-1,2,2-trifluoroethane & 1.0 & $\mathrm{U}$ \\
\hline $67-64-1$ & Acetone & 5.0 & $\mathrm{U}$ \\
\hline $75-15-0$ & Carbon disulfide & 1.0 & $\mathrm{U}$ \\
\hline $79-20-9$ & Methyl acetate & 1.0 & $\mathrm{U}$ \\
\hline $75-09-2$ & Methylene Chloride & 1.0 & $\mathrm{U}$ \\
\hline $156-60-5$ & trans-1,2-Dichloroethene & 1.0 & $\mathrm{U}$ \\
\hline $1634-04-4$ & Methyl tert-butyl ether & 1.0 & $\mathrm{U}$ \\
\hline $75-34-3$ & 1,1-Dichloroethane & 1.0 & $\mathrm{U}$ \\
\hline $156-59-2$ & cis-1,2-Dichloroethene & 1.0 & $\mathrm{U}$ \\
\hline $78-93-3$ & 2-Butanone & 5.0 & $\mathrm{U}$ \\
\hline $74-97-5$ & Bromochloromethane & 1.0 & $\mathrm{U}$ \\
\hline $67-66-3$ & Chloroform & 1.0 & $\mathrm{U}$ \\
\hline $71-55-6$ & 1,1,1-Trichloroethane & 1.0 & $\mathrm{U}$ \\
\hline $110-82-7$ & Cyclohexane & 1.0 & $\mathrm{U}$ \\
\hline $56-23-5$ & Carbon tetrachloride & 2.2 & \\
\hline $71-43-2$ & Benzene & 1.0 & $\mathrm{U}$ \\
\hline $107-06-2$ & 1,2-Dichloroethane & 1.0 & $\mathrm{U}$ \\
\hline
\end{tabular}

Report 1,4-Dioxane for Low-Medium VOA analysis only 
Lab Name: TESTAMERICA BURLINGTON Lab Code: STLV Case No.: YORK Mod. Ref No.:
Contract: $8 \mathrm{E}-00302$

\author{
Lab Sample ID: 200-29506-4
}

Lab File ID: 15537_011.D

Date Received: 08/29/2015

Date Analyzed: 08/31/2015

Dilution Factor: 1.0

Soil Aliquot Volume: (uL)

Soil Extract Volume: $\mathrm{ID}: \begin{aligned} & 0.20 \quad \\ & (\mathrm{~mm}) \\ & (\mathrm{uL}) \\ & (\mathrm{mL})\end{aligned}$

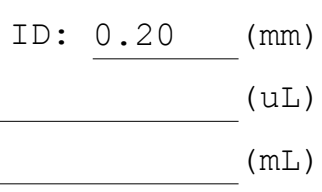

Purge Volume: 25.0

\begin{tabular}{|c|c|c|c|}
\hline CAS NO. & COMPOUND & $\begin{array}{l}\text { CONCENTRATION UNITS: } \\
(\mathrm{ug} / \mathrm{L} \text { or } \mathrm{ug} / \mathrm{kg}) \mathrm{ug} / \mathrm{L}\end{array}$ & 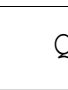 \\
\hline $79-01-6$ & Trichloroethene & $\begin{array}{r}1.0 \\
1.0\end{array}$ & $\mathrm{U}$ \\
\hline $108-87-2$ & Methylcyclohexane & 1.0 & $\mathrm{U}$ \\
\hline $78-87-5$ & 1,2-Dichloropropane & 1.0 & $\mathrm{U}$ \\
\hline $75-27-4$ & Bromodichloromethane & 1.0 & $\mathrm{U}$ \\
\hline $10061-01-5$ & cis-1,3-Dichloropropene & 1.0 & $\mathrm{U}$ \\
\hline $108-10-1$ & 4-Methyl-2-pentanone & 1.0 & $\mathrm{U}$ \\
\hline $108-88-3$ & Toluene & 1.0 & $\mathrm{U}$ \\
\hline $10061-02-6$ & trans-1,3-Dichloropropene & 1.0 & $\mathrm{U}$ \\
\hline $79-00-5$ & $1,1,2$-Trichloroethane & 1.0 & $\mathrm{U}$ \\
\hline $127-18-4$ & Tetrachloroethene & 1.0 & $\mathrm{U}$ \\
\hline $591-78-6$ & 2-Hexanone & 1.0 & $\mathrm{U}$ \\
\hline $124-48-1$ & Dibromochloromethane & 1.0 & $\mathrm{U}$ \\
\hline $106-93-4$ & 1,2-Dibromoethane & 1.0 & $\mathrm{U}$ \\
\hline $108-90-7$ & Chlorobenzene & 1.0 & $\mathrm{U}$ \\
\hline $100-41-4$ & Ethylbenzene & 1.0 & $\mathrm{U}$ \\
\hline $95-47-6$ & o-Xylene & 1.0 & $\mathrm{U}$ \\
\hline $179601-23-1$ & m,p-xylene & 1.0 & $\mathrm{U}$ \\
\hline $100-42-5$ & Styrene & 1.0 & $\mathrm{U}$ \\
\hline $75-25-2$ & Bromoform & 1.0 & $\mathrm{U}$ \\
\hline $98-82-8$ & Isopropylbenzene & 1.0 & $\mathrm{U}$ \\
\hline $79-34-5$ & $1,1,2,2$-Tetrachloroethane & 1.0 & $\mathrm{U}$ \\
\hline $541-73-1$ & 1,3-Dichlorobenzene & 1.0 & $\mathrm{U}$ \\
\hline $106-46-7$ & 1,4-Dichlorobenzene & 1.0 & $\mathrm{U}$ \\
\hline $95-50-1$ & 1,2-Dichlorobenzene & 1.0 & $\mathrm{U}$ \\
\hline $96-12-8$ & 1,2-Dibromo-3-Chloropropane & 1.0 & $\mathrm{U}$ \\
\hline $120-82-1$ & $1,2,4$-Trichlorobenzene & 1.0 & $\mathrm{U}$ \\
\hline $87-61-6$ & $1,2,3$-Trichlorobenzene & 1.0 & $\mathrm{U}$ \\
\hline
\end{tabular}


Lab Name: TESTAMERICA BURLINGTON

Contract: $8 \mathrm{E}-00302$

Lab Code: STLV Case No.: YORK Mod. Ref No.: SDG NO.: YORK $(200-29$

Matrix: (SOIL/SED/WATER) Water

Sample wt/vol: 25.0 $(\mathrm{g} / \mathrm{mL}) \mathrm{mL}$

Level: (TRACE or LOW/MED) TRACE

Moisture: not dec.

GC Column: DB-624 ID $: 0.20 \quad(\mathrm{~mm})$

Soil Extract Volume: (uL)

CONCENTRATION UNITS: (ug/L or ug/kg) ug/L
Lab Sample ID: 200-29506-4

Lab File ID: 15537_011.D

Date Received: 08/29/2015

Date Analyzed: 08/31/2015

Dilution Factor: 1.0

Soil Aliquot Volume: (uL)

Purge Volume: 25.0

01

02

\begin{tabular}{|l|l|c|c|c|}
\hline CAS NUMBER & \multicolumn{1}{|c|}{ COMPOUND NAME } & RT & EST. CONC. & Q \\
\hline & Unknown & 7.29 & 3.0 & $\mathrm{~J}$ B X \\
\hline E9667961 & Total Alkanes & N/A & & \\
\hline
\end{tabular}

1 EPA-designated Registry Number. 
Lab Name: TESTAMERICA BURLINGTON Lab Code: STLV Case No.: YORK Mod. Ref No.:

Contract: 8E-00302
SDG NO.: YORK (200-29 Calibration Date(s): 08/19/2015 08/19/2015 Calibration Time(s) : $1514 \quad 1654$ $(\mathrm{mL})$

Purge Volume: 25.0

GC Column: DB-624

ID: $0.20 \quad(\mathrm{~mm}) \quad$ Length: 25

(m)

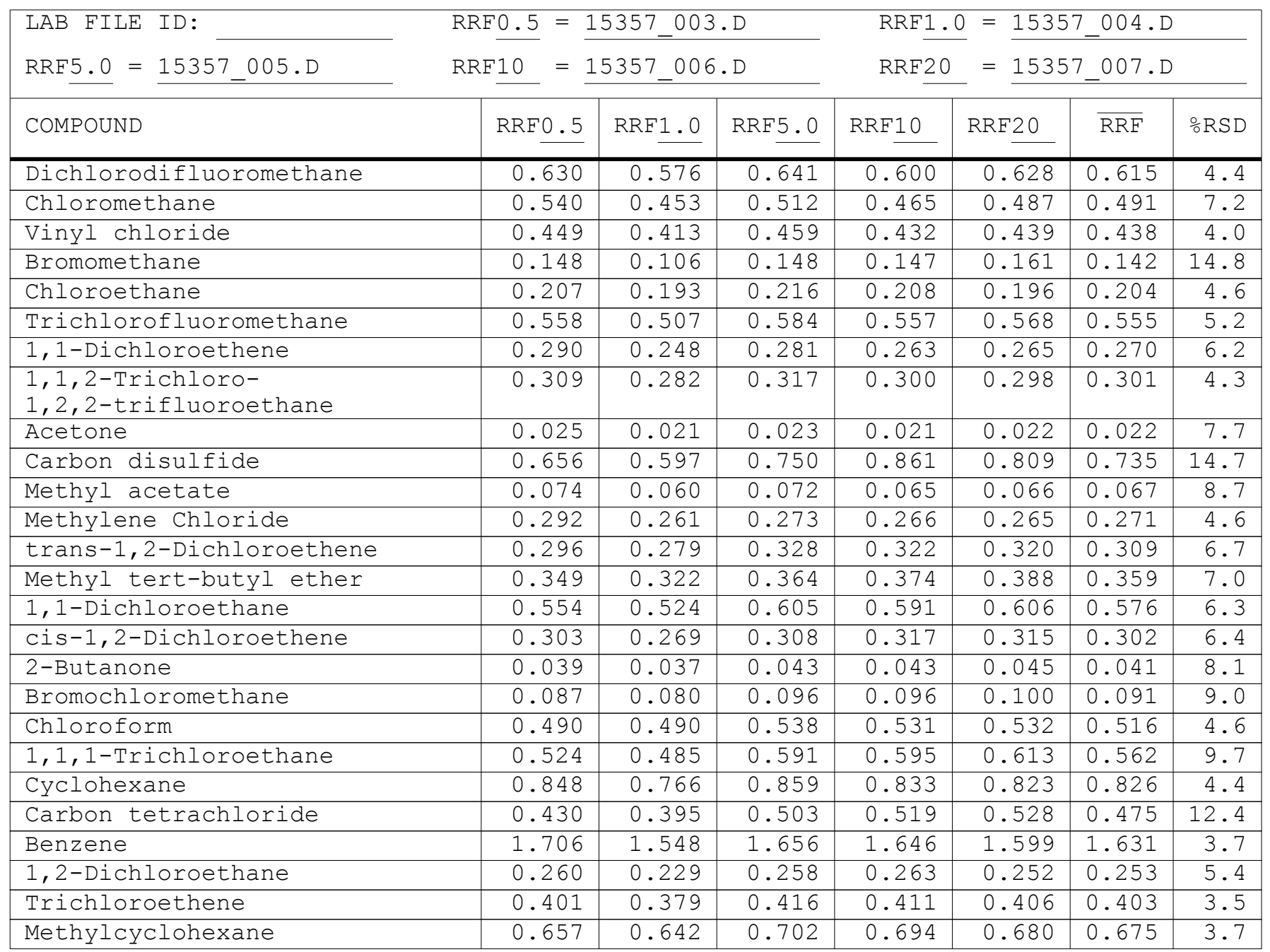

Report 1,4-Dioxane for Low-Medium VOA analysis only 
Lab Name: TESTAMERICA BURLINGTON

Contract: $8 \mathrm{E}-00302$

Lab Code: STLV Case No.: YORK Mod. Ref No.:

SDG NO.: YORK (200-29

Instrument ID: CHD.i

Calibration Date(s): 08/19/2015 08/19/2015

Heated Purge: ( $\mathrm{Y} / \mathrm{N}) \mathrm{N}$

Calibration Time(s) : 1514

1654

Purge Volume: 25.0 (mL)

GC Column: DB-624

ID: 0.20

$(\mathrm{mm})$

Length: 25

(m)

\begin{tabular}{|c|c|c|c|c|c|c|c|}
\hline \multirow{2}{*}{$\begin{array}{l}\text { LAB FILE ID: } \\
\text { RRF5.0 }=\text { 15357_005.D }\end{array}$} & \multicolumn{3}{|c|}{ RRF0.5 = $15357003 . D$} & \multicolumn{4}{|c|}{ RRF1.0 = 15357_004.D } \\
\hline & E10 & 357_00 & & RRF20 & $=1535$ & $1007 . \mathrm{D}$ & \\
\hline COMPOUND & $\mathrm{RRF} 0.5$ & RRF1. 0 & RRF5.0 & RRF10 & RRF20 & $\overline{\mathrm{RRF}}$ & $\because \mathrm{RSD}$ \\
\hline 1,2-Dichloropropane & 0.327 & 0.313 & 0.349 & 0.344 & 0.337 & 0.334 & 4.3 \\
\hline Bromodichloromethane & 0.263 & 0.262 & 0.336 & 0.358 & 0.361 & 0.316 & 15.7 \\
\hline cis-1,3-Dichloropropene & 0.340 & 0.315 & 0.440 & 0.455 & 0.463 & 0.403 & 17.3 \\
\hline 4-Methyl-2-pentanone & 0.106 & 0.102 & 0.128 & 0.126 & 0.125 & 0.117 & 10.5 \\
\hline Toluene & 1.731 & 1.636 & 1.803 & 1.777 & 1.710 & 1.731 & 3.7 \\
\hline trans-1,3-Dichloropropene & 0.201 & 0.213 & 0.301 & 0.323 & 0.329 & 0.273 & 22.7 \\
\hline 1,1,2-Trichloroethane & 0.181 & 0.150 & 0.167 & 0.167 & 0.161 & 0.165 & 6.8 \\
\hline Tetrachloroethene & 0.357 & 0.334 & 0.367 & 0.369 & 0.366 & 0.359 & 4.0 \\
\hline 2-Hexanone & 0.073 & 0.068 & 0.087 & 0.087 & 0.086 & 0.080 & 11.1 \\
\hline Dibromochloromethane & 0.125 & 0.114 & 0.168 & 0.183 & 0.189 & 0.156 & 22.0 \\
\hline 1,2-Dibromoethane & 0.131 & 0.122 & 0.146 & 0.147 & 0.148 & 0.139 & 8.4 \\
\hline Chlorobenzene & 1.013 & 0.937 & 1.024 & 1.018 & 1.005 & 0.999 & 3.5 \\
\hline Ethylbenzene & 2.001 & 1.894 & 2.154 & 2.141 & 2.062 & 2.050 & 5.2 \\
\hline o-Xylene & 0.683 & 0.629 & 0.750 & 0.749 & 0.733 & 0.709 & 7.4 \\
\hline $\mathrm{m}, \mathrm{p}$-Xylene & 0.749 & 0.718 & 0.821 & 0.815 & 0.801 & 0.781 & 5.8 \\
\hline Styrene & 0.957 & 0.957 & 1.155 & 1.134 & 1.112 & 1.063 & 9.2 \\
\hline Bromoform & 0.094 & 0.087 & 0.118 & 0.142 & 0.153 & 0.119 & 24.2 \\
\hline Isopropylbenzene & 2.002 & 1.895 & 2.236 & 2.192 & 2.144 & 2.094 & 6.8 \\
\hline $1,1,2,2$-Tetrachloroethane & 0.159 & 0.146 & 0.172 & 0.168 & 0.172 & 0.163 & 6.8 \\
\hline 1,3-Dichlorobenzene & 1.554 & 1.483 & 1.702 & 1.712 & 1.743 & 1.639 & 6.9 \\
\hline 1,4-Dichlorobenzene & 1.614 & 1.524 & 1.654 & 1.646 & 1.666 & 1.621 & 3.5 \\
\hline 1,2-Dichlorobenzene & 1.357 & 1.238 & 1.365 & 1.353 & 1.383 & 1.339 & 4.3 \\
\hline 1,2-Dibromo-3-Chloropropane & 0.033 & 0.033 & 0.035 & 0.039 & 0.045 & 0.037 & 14.0 \\
\hline 1,2,4-Trichlorobenzene & 0.804 & 0.738 & 0.849 & 0.888 & 0.929 & 0.841 & 8.8 \\
\hline 1,2,3-Trichlorobenzene & 0.564 & 0.535 & 0.591 & 0.624 & 0.658 & 0.594 & 8.1 \\
\hline
\end{tabular}


Lab Name: TESTAMERICA BURLINGTON

Contract: $8 \mathrm{E}-00302$

Lab Code: STLV Case No.: YORK Mod. Ref No.: SDG NO.: YORK (200-29

Instrument ID: CHD.i

Heated Purge: (Y/N) N

Purge Volume: 25.0

GC Column: DB-624
ID: 0.20
Calibration Date(s): 08/19/2015

Calibration Time (s) : 1514 $(\mathrm{mL})$

(mm) Length: 25
$08 / 19 / 2015$ 1654

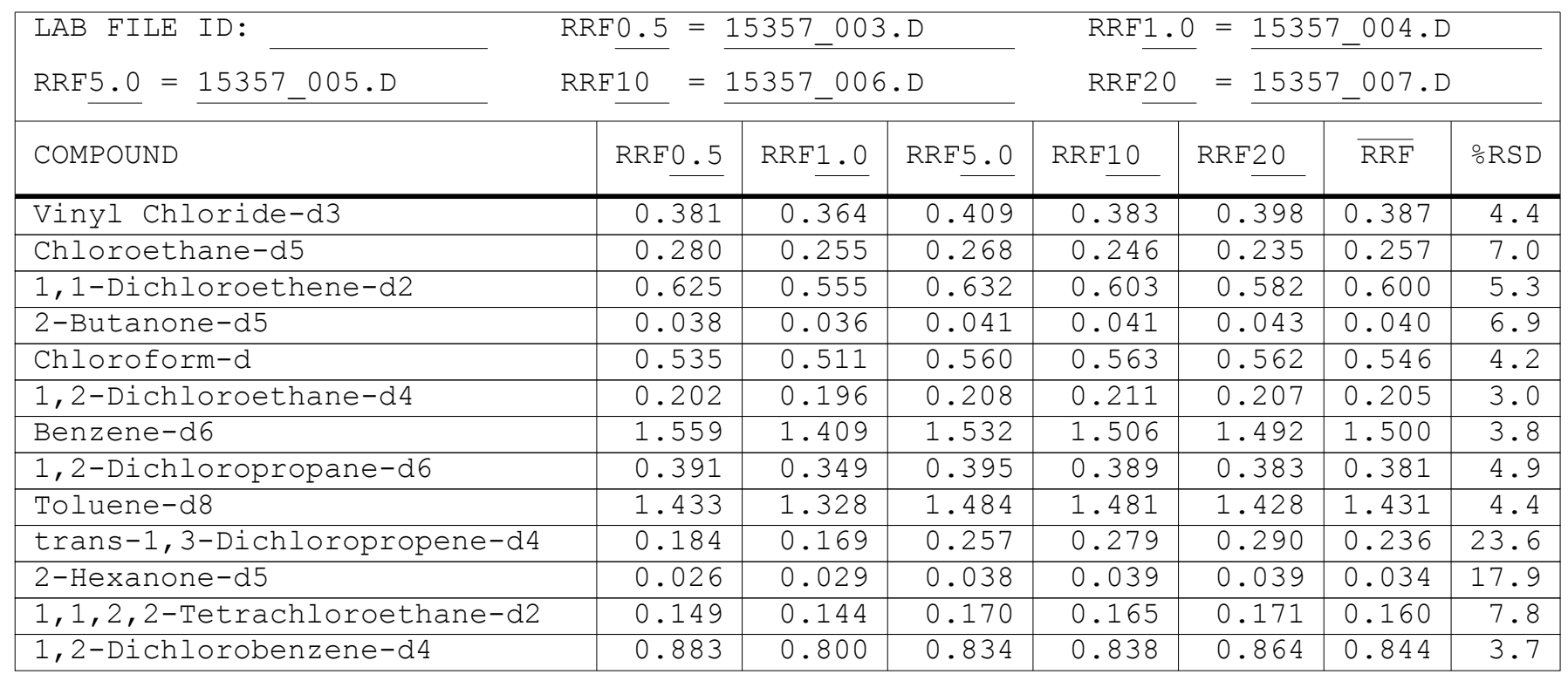

Report 1,4-Dioxane-d8 for Low-Medium VOA analysis only 
Lab Name: TESTAMERICA BURLINGTON

Contract: $8 \mathrm{E}-00302$

Lab Code: STLV Case No.: YORK Mod. Ref No.:

SDG NO.: YORK (200-29

Instrument ID: CHD.i

Calibration Date: 08/31/2015 Time: 1254

Lab File Id: 15537_003.D

EPA Sample No. (VSTD\#\#\#\#) : VSTD005DJ

Init. Calib. Date(s):08/19/2015 08/19/2015

Heated Purge: (Y/N) N GC Column: DB-624

Init. Calib. Time(s) : 1514 1654

Purge Volume: 25.0

ID: $0.20(\mathrm{~mm})$ Length: 25

(m)

\begin{tabular}{|c|c|c|c|c|c|}
\hline COMPOUND & $\overline{\mathrm{RRF}}$ & RRF5. 0 & $\begin{array}{l}\text { MIN } \\
\text { RRF }\end{array}$ & $\because \mathrm{D}$ & $\mathrm{MAX} \cong \mathrm{D}$ \\
\hline Dichlorodifluoromethane & 0.615 & 0.641 & 0.010 & 4.2 & 40.0 \\
\hline Chloromethane & 0.491 & 0.510 & 0.010 & 3.7 & 40.0 \\
\hline Vinyl chloride & 0.438 & 0.475 & 0.100 & 8.2 & 30.0 \\
\hline Bromomethane & 0.142 & 0.173 & 0.100 & 21.5 & 30.0 \\
\hline Chloroethane & 0.204 & 0.231 & 0.010 & 13.0 & 40.0 \\
\hline Trichlorofluoromethane & 0.555 & 0.614 & 0.010 & 10.6 & 40.0 \\
\hline 1,1-Dichloroethene & 0.270 & 0.292 & 0.100 & 8.4 & 30.0 \\
\hline 1,1,2-Trichloro-1,2,2-trifluoroethane & 0.301 & 0.333 & 0.010 & 10.5 & 40.0 \\
\hline Acetone & 0.022 & 0.024 & 0.010 & 5.7 & 40.0 \\
\hline Carbon disulfide & 0.735 & 0.811 & 0.010 & 10.4 & 40.0 \\
\hline Methyl acetate & 0.067 & 0.068 & 0.010 & 1.4 & 40.0 \\
\hline Methylene Chloride & 0.271 & 0.273 & 0.010 & 0.5 & 40.0 \\
\hline trans-1,2-Dichloroethene & 0.309 & 0.327 & 0.010 & 5.7 & 40.0 \\
\hline Methyl tert-butyl ether & 0.359 & 0.356 & 0.010 & -0.9 & 40.0 \\
\hline 1,1-Dichloroethane & 0.576 & 0.619 & 0.200 & 7.5 & 30.0 \\
\hline cis-1,2-Dichloroethene & 0.302 & 0.313 & 0.010 & 3.7 & 40.0 \\
\hline 2-Butanone & 0.041 & 0.042 & 0.010 & 1.8 & 40.0 \\
\hline Bromochloromethane & 0.091 & 0.096 & 0.050 & 5.1 & 30.0 \\
\hline Chloroform & 0.516 & 0.547 & 0.200 & 6.0 & 30.0 \\
\hline 1,1,1-Trichloroethane & 0.562 & 0.601 & 0.100 & 7.0 & 30.0 \\
\hline Cyclohexane & 0.826 & 0.873 & 0.010 & 5.7 & 40.0 \\
\hline Carbon tetrachloride & 0.475 & 0.525 & 0.100 & 10.6 & 30.0 \\
\hline Benzene & 1.631 & 1.677 & 0.400 & 2.8 & 30.0 \\
\hline 1,2-Dichloroethane & 0.253 & 0.259 & 0.100 & 2.7 & 30.0 \\
\hline Trichloroethene & 0.403 & 0.412 & 0.300 & 2.3 & 30.0 \\
\hline Methylcyclohexane & 0.675 & 0.707 & 0.010 & 4.7 & 40.0 \\
\hline
\end{tabular}

Report 1,4-Dioxane for Low/Medium VOA analysis only 
Lab Name: TESTAMERICA BURLINGTON

Contract: 8E-00302

Lab Code: STLV Case No.: YORK Mod. Ref No.:

SDG NO.: YORK (200-29

Instrument ID: CHD.i

Calibration Date: 08/31/2015 Time: 1254

Lab File Id: 15537_003.D

EPA Sample No. (VSTD\#\#\#\#) : VSTD005DJ

Init. Calib. Date(s):08/19/2015 08/19/2015

Heated Purge: (Y/N) N GC Column: DB-624

Init. Calib. Time(s) : 1514 1654

Purge Volume: 25.0

ID: $0.20(\mathrm{~mm})$ Length: 25

(m)

\begin{tabular}{|c|c|c|c|c|c|}
\hline COMPOUND & $\overline{\mathrm{RRF}}$ & RRF5.0 & $\begin{array}{l}\text { MIN } \\
\text { RRF }\end{array}$ & $\because \mathrm{D}$ & $\operatorname{MAX} \cong \mathrm{D}$ \\
\hline 1,2-Dichloropropane & 0.334 & 0.339 & 0.010 & 1.6 & 40.0 \\
\hline Bromodichloromethane & 0.316 & 0.348 & 0.200 & 10.3 & 30.0 \\
\hline cis-1,3-Dichloropropene & 0.403 & 0.456 & 0.200 & 13.4 & 30.0 \\
\hline 4-Methyl-2-pentanone & 0.117 & 0.118 & 0.010 & 0.2 & 40.0 \\
\hline Toluene & 1.731 & 1.832 & 0.400 & 5.8 & 30.0 \\
\hline trans-1,3-Dichloropropene & 0.273 & 0.306 & 0.100 & 12.1 & 30.0 \\
\hline $1,1,2$-Trichloroethane & 0.165 & 0.163 & 0.100 & -1.5 & 30.0 \\
\hline Tetrachloroethene & 0.359 & 0.370 & 0.100 & 3.0 & 30.0 \\
\hline 2-Hexanone & 0.080 & 0.083 & 0.010 & 3.8 & 40.0 \\
\hline Dibromochloromethane & 0.156 & 0.176 & 0.100 & 13.4 & 30.0 \\
\hline 1,2-Dibromoethane & 0.139 & 0.140 & 0.010 & 0.8 & 40.0 \\
\hline Chlorobenzene & 0.999 & 1.022 & 0.500 & 2.2 & 30.0 \\
\hline Ethylbenzene & 2.050 & 2.179 & 0.100 & 6.3 & 30.0 \\
\hline o-Xylene & 0.709 & 0.761 & 0.300 & 7.3 & 30.0 \\
\hline $\mathrm{m}, \mathrm{p}$-Xylene & 0.781 & 0.814 & 0.300 & 4.3 & 30.0 \\
\hline Styrene & 1.063 & 1.148 & 0.300 & 8.0 & 30.0 \\
\hline Bromoform & 0.119 & 0.141 & 0.050 & 18.4 & 30.0 \\
\hline Isopropylbenzene & 2.094 & 2.250 & 0.010 & 7.5 & 40.0 \\
\hline $1,1,2,2$-Tetrachloroethane & 0.163 & 0.170 & 0.100 & 4.3 & 30.0 \\
\hline 1,3-Dichlorobenzene & 1.639 & 1.712 & 0.400 & 4.5 & 30.0 \\
\hline 1,4-Dichlorobenzene & 1.621 & 1.633 & 0.400 & 0.7 & 30.0 \\
\hline 1,2-Dichlorobenzene & 1.339 & 1.350 & 0.400 & 0.8 & 30.0 \\
\hline 1,2-Dibromo-3-Chloropropane & 0.037 & 0.036 & 0.010 & -3.5 & 40.0 \\
\hline 1,2,4-Trichlorobenzene & 0.841 & 0.815 & 0.200 & -3.1 & 30.0 \\
\hline $1,2,3$-Trichlorobenzene & 0.594 & 0.559 & 0.200 & -6.0 & 30.0 \\
\hline
\end{tabular}


Lab Name: TESTAMERICA BURLINGTON

Contract: $8 \mathrm{E}-00302$

Lab Code: STLV Case No.: YORK Mod. Ref No.:

SDG NO.: YORK (200-29

Instrument ID: CHD.i

Calibration Date: 08/31/2015 Time: 1254

Lab File Id: 15537_003.D

Init. Calib. Date(s):08/19/2015 08/19/2015

EPA Sample No. (VSTD\#\#\#\#) : VSTD005DJ

Heated Purge: (Y/N) N GC Column: DB-624

Init. Calib. Time(s) : 1514 1654

Purge Volume: 25.0 ID: $0.20(\mathrm{~mm})$ Length: 25

(m)

\begin{tabular}{|c|c|c|c|c|c|}
\hline COMPOUND & $\overline{\mathrm{RRF}}$ & RRF5.0 & $\begin{array}{l}\text { MIN } \\
\text { RRF }\end{array}$ & $\because \mathrm{D}$ & $\operatorname{MAX} \cong \mathrm{D}$ \\
\hline Vinyl Chloride-d3 & 0.387 & 0.424 & 0.010 & 9.5 & 30.0 \\
\hline Chloroethane-d5 & 0.257 & 0.273 & 0.010 & 6.3 & 40.0 \\
\hline 1,1-Dichloroethene-d2 & 0.600 & 0.665 & 0.010 & 10.8 & 30.0 \\
\hline 2-Butanone-d5 & 0.040 & 0.039 & 0.010 & -1.1 & 40.0 \\
\hline Chloroform-d & 0.546 & 0.570 & 0.010 & 4.4 & 30.0 \\
\hline 1,2-Dichloroethane-d4 & 0.205 & 0.218 & 0.010 & 6.6 & 30.0 \\
\hline Benzene-d6 & 1.500 & 1.532 & 0.010 & 2.2 & 30.0 \\
\hline 1,2-Dichloropropane-d6 & 0.381 & 0.392 & 0.010 & 2.9 & 40.0 \\
\hline Toluene-d8 & 1.431 & 1.483 & 0.010 & 3.6 & 30.0 \\
\hline trans-1,3-Dichloropropene-d4 & 0.236 & 0.263 & 0.010 & 11.4 & 30.0 \\
\hline 2-Hexanone-d5 & 0.034 & 0.035 & 0.010 & 1.5 & 40.0 \\
\hline $1,1,2,2$-Tetrachloroethane-d2 & 0.160 & 0.167 & 0.010 & 4.6 & 30.0 \\
\hline 1,2-Dichlorobenzene-d4 & 0.844 & 0.833 & 0.010 & -1.3 & 30.0 \\
\hline
\end{tabular}

Report 1,4-Dioxane-d8 for Low/Medium VOA analysis only 
Lab Name: TESTAMERICA BURLINGTON

Contract: $8 \mathrm{E}-00302$

Lab Code: STLV Case No.: YORK Mod. Ref No.:

SDG NO.: YORK (200-29

Instrument ID: CHD.i

Calibration Date: 08/31/2015 Time: 1829

Lab File Id: 15537_016.D

EPA Sample No. (VSTD\#\#\#\#) : VSTD005JD

Init. Calib. Date(s):08/19/2015 08/19/2015

Heated Purge: (Y/N) N GC Column: DB-624

Init. Calib. Time(s) : 1514 1654

Purge Volume: 25.0

ID: $0.20(\mathrm{~mm})$ Length: 25

$(\mathrm{m})$

\begin{tabular}{|c|c|c|c|c|c|}
\hline COMPOUND & $\overline{\mathrm{RRF}}$ & RRF 5.0 & $\begin{array}{l}\text { MIN } \\
\text { RRF }\end{array}$ & $\because \mathrm{D}$ & $\mathrm{MAX} \div \mathrm{D}$ \\
\hline Dichlorodifluoromethane & 0.615 & 0.658 & 0.010 & 7.0 & 50.0 \\
\hline Chloromethane & 0.491 & 0.544 & 0.010 & 10.7 & 50.0 \\
\hline Vinyl chloride & 0.438 & 0.490 & 0.010 & 11.7 & 50.0 \\
\hline Bromomethane & 0.142 & 0.153 & 0.010 & 7.8 & 50.0 \\
\hline Chloroethane & 0.204 & 0.240 & 0.010 & 17.5 & 50.0 \\
\hline Trichlorofluoromethane & 0.555 & 0.627 & 0.010 & 13.0 & 50.0 \\
\hline 1,1-Dichloroethene & 0.270 & 0.295 & 0.010 & 9.3 & 50.0 \\
\hline 1,1,2-Trichloro-1,2,2-trifluoroethane & 0.301 & 0.336 & 0.010 & 11.7 & 50.0 \\
\hline Acetone & 0.022 & 0.023 & 0.010 & 3.7 & 50.0 \\
\hline Carbon disulfide & 0.735 & 0.753 & 0.010 & 2.5 & 50.0 \\
\hline Methyl acetate & 0.067 & 0.069 & 0.010 & 1.6 & 50.0 \\
\hline Methylene Chloride & 0.271 & 0.281 & 0.010 & 3.4 & 50.0 \\
\hline trans-1,2-Dichloroethene & 0.309 & 0.331 & 0.010 & 7.3 & 50.0 \\
\hline Methyl tert-butyl ether & 0.359 & 0.335 & 0.010 & -6.8 & 50.0 \\
\hline 1,1-Dichloroethane & 0.576 & 0.621 & 0.010 & 7.9 & 50.0 \\
\hline cis-1,2-Dichloroethene & 0.302 & 0.316 & 0.010 & 4.5 & 50.0 \\
\hline 2-Butanone & 0.041 & 0.042 & 0.010 & 1.0 & 50.0 \\
\hline Bromochloromethane & 0.091 & 0.096 & 0.010 & 5.3 & 50.0 \\
\hline Chloroform & 0.516 & 0.548 & 0.010 & 6.0 & 50.0 \\
\hline 1,1,1-Trichloroethane & 0.562 & 0.580 & 0.010 & 3.2 & 50.0 \\
\hline Cyclohexane & 0.826 & 0.870 & 0.010 & 5.4 & 50.0 \\
\hline Carbon tetrachloride & 0.475 & 0.500 & 0.010 & 5.2 & 50.0 \\
\hline Benzene & 1.631 & 1.687 & 0.010 & 3.5 & 50.0 \\
\hline 1,2-Dichloroethane & 0.253 & 0.256 & 0.010 & 1.3 & 50.0 \\
\hline Trichloroethene & 0.403 & 0.411 & 0.010 & 2.1 & 50.0 \\
\hline Methylcyclohexane & 0.675 & 0.712 & 0.010 & 5.4 & 50.0 \\
\hline
\end{tabular}

Report 1,4-Dioxane for Low/Medium VOA analysis only 
Lab Name: TESTAMERICA BURLINGTON

Contract: 8E-00302

Lab Code: STLV Case No.: YORK Mod. Ref No.:

SDG NO.: YORK (200-29

Instrument ID: CHD.i

Calibration Date: 08/31/2015 Time: 1829

Lab File Id: $15537016 . \mathrm{D}$

EPA Sample No. (VSTD\#\#\#\#) : VSTD005JD

Init. Calib. Date(s):08/19/2015 08/19/2015

Heated Purge: (Y/N) N

GC Column: DB-624

Init. Calib. Time(s) : 1514

1654

Purge Volume: 25.0

ID: $0.20(\mathrm{~mm})$ Length: 25

(m)

\begin{tabular}{|c|c|c|c|c|c|}
\hline COMPOUND & $\overline{\mathrm{RRF}}$ & RRF5.0 & $\begin{array}{l}\text { MIN } \\
\text { RRF }\end{array}$ & $\because \mathrm{D}$ & $\operatorname{MAX} \cong \mathrm{D}$ \\
\hline 1,2-Dichloropropane & 0.334 & 0.348 & 0.010 & 4.3 & 50.0 \\
\hline Bromodichloromethane & 0.316 & 0.327 & 0.010 & 3.5 & 50.0 \\
\hline cis-1,3-Dichloropropene & 0.403 & 0.424 & 0.010 & 5.3 & 50.0 \\
\hline 4-Methyl-2-pentanone & 0.117 & 0.117 & 0.010 & -0.5 & 50.0 \\
\hline Toluene & 1.731 & 1.831 & 0.010 & 5.8 & 50.0 \\
\hline trans-1,3-Dichloropropene & 0.273 & 0.284 & 0.010 & 4.1 & 50.0 \\
\hline $1,1,2$-Trichloroethane & 0.165 & 0.164 & 0.010 & -0.7 & 50.0 \\
\hline Tetrachloroethene & 0.359 & 0.366 & 0.010 & 2.1 & 50.0 \\
\hline 2-Hexanone & 0.080 & 0.083 & 0.010 & 3.2 & 50.0 \\
\hline Dibromochloromethane & 0.156 & 0.156 & 0.010 & 0.5 & 50.0 \\
\hline 1,2-Dibromoethane & 0.139 & 0.143 & 0.010 & 2.9 & 50.0 \\
\hline Chlorobenzene & 0.999 & 1.033 & 0.010 & 3.4 & 50.0 \\
\hline Ethylbenzene & 2.050 & 2.187 & 0.010 & 6.6 & 50.0 \\
\hline o-Xylene & 0.709 & 0.760 & 0.010 & 7.3 & 50.0 \\
\hline $\mathrm{m}, \mathrm{p}$-Xylene & 0.781 & 0.833 & 0.010 & 6.7 & 50.0 \\
\hline Styrene & 1.063 & 1.161 & 0.010 & 9.2 & 50.0 \\
\hline Bromoform & 0.119 & 0.113 & 0.010 & -4.7 & 50.0 \\
\hline Isopropylbenzene & 2.094 & 2.271 & 0.010 & 8.5 & 50.0 \\
\hline $1,1,2,2$-Tetrachloroethane & 0.163 & 0.168 & 0.010 & 3.0 & 50.0 \\
\hline 1,3-Dichlorobenzene & 1.639 & 1.661 & 0.010 & 1.3 & 50.0 \\
\hline 1,4-Dichlorobenzene & 1.621 & 1.641 & 0.010 & 1.2 & 50.0 \\
\hline 1,2-Dichlorobenzene & 1.339 & 1.356 & 0.010 & 1.2 & 50.0 \\
\hline 1,2-Dibromo-3-Chloropropane & 0.037 & 0.032 & 0.010 & $-13 \cdot 3$ & 50.0 \\
\hline 1,2,4-Trichlorobenzene & 0.841 & 0.786 & 0.010 & -6.5 & 50.0 \\
\hline $1,2,3$-Trichlorobenzene & 0.594 & 0.544 & 0.010 & -8.5 & 50.0 \\
\hline
\end{tabular}


Lab Name: TESTAMERICA BURLINGTON

Contract: $8 \mathrm{E}-00302$

Lab Code: STLV Case No.: YORK Mod. Ref No.:

SDG NO.: YORK (200-29

Instrument ID: CHD.i

Calibration Date: 08/31/2015 Time: 1829

Lab File Id: 15537_016.D

Init. Calib. Date(s):08/19/2015 08/19/2015

EPA Sample No. (VSTD\#\#\#\#) : VSTD005JD

Init. Calib. Time(s) : $1514 \quad 1654$

Heated Purge: (Y/N) N

GC Column: DB-624

ID: $0.20(\mathrm{~mm})$ Length: 25

(m)

Purge Volume: 25.0 $(\mathrm{mL})$

\begin{tabular}{|c|c|c|c|c|c|}
\hline COMPOUND & $\overline{\mathrm{RRF}}$ & RRF5.0 & $\begin{array}{l}\text { MIN } \\
\text { RRF }\end{array}$ & $\because \mathrm{D}$ & $\operatorname{MAX} \cong \mathrm{D}$ \\
\hline Vinyl Chloride-d3 & 0.387 & 0.438 & 0.010 & 13.2 & 50.0 \\
\hline Chloroethane-d5 & 0.257 & 0.283 & 0.010 & 10.1 & 50.0 \\
\hline 1,1-Dichloroethene-d2 & 0.600 & 0.685 & 0.010 & 14.3 & 50.0 \\
\hline 2-Butanone-d5 & 0.040 & 0.039 & 0.010 & -2.8 & 50.0 \\
\hline Chloroform-d & 0.546 & 0.581 & 0.010 & 6.4 & 50.0 \\
\hline 1,2-Dichloroethane-d4 & 0.205 & 0.221 & 0.010 & 7.8 & 50.0 \\
\hline Benzene-d6 & 1.500 & 1.557 & 0.010 & 3.8 & 50.0 \\
\hline 1,2-Dichloropropane-d6 & 0.381 & 0.390 & 0.010 & 2.3 & 50.0 \\
\hline Toluene-d8 & 1.431 & 1.522 & 0.010 & 6.4 & 50.0 \\
\hline trans-1,3-Dichloropropene-d4 & 0.236 & 0.235 & 0.010 & -0.2 & 50.0 \\
\hline 2-Hexanone-d5 & 0.034 & 0.035 & 0.010 & 2.7 & 50.0 \\
\hline $1,1,2,2$-Tetrachloroethane-d2 & 0.160 & 0.167 & 0.010 & 4.3 & 50.0 \\
\hline 1,2 -Dichlorobenzene-d4 & 0.844 & 0.848 & 0.010 & 0.5 & 50.0 \\
\hline
\end{tabular}

Report 1,4-Dioxane-d8 for Low/Medium VOA analysis only 
Lab Name: TESTAMERICA BURLINGTON

Contract: $8 \mathrm{E}-00302$

Lab Code: STLV Case No.: YORK Mod. Ref No.: SDG NO.: YORK $(200-29$

Matrix: (SOIL/SED/WATER) Water

Sample wt/vol: 25.0 $(\mathrm{g} / \mathrm{mL}) \mathrm{mL}$

Level: (TRACE/LOW/MED) TRACE

\% Moisture: not dec.

GC Column: DB-624 ID: $0.20 \quad(\mathrm{~mm})$

Soil Extract Volume: (uL) $(\mathrm{mL})$

Purge Volume: 25.0
Lab Sample ID: MB 200-93413/5

Lab File ID: 15537_005.D

Date Received:

Date Analyzed: 08/31/2015

Dilution Factor: 1.0

Soil Aliquot Volume: (uL)

\begin{tabular}{|c|c|c|c|}
\hline CAS NO. & COMPOUND & $\begin{array}{l}\text { CONCENTRATION UNITS: } \\
(\mathrm{ug} / \mathrm{L} \text { or } \mathrm{ug} / \mathrm{kg}) \mathrm{ug} / \mathrm{L}\end{array}$ & q \\
\hline $75-71-8$ & Dichlorodifluoromethane & 1.0 & $\mathrm{U}$ \\
\hline $74-87-3$ & Chloromethane & 1.0 & $\mathrm{U}$ \\
\hline $75-01-4$ & Vinyl chloride & 1.0 & $\mathrm{U}$ \\
\hline $74-83-9$ & Bromomethane & 1.0 & $\mathrm{U}$ \\
\hline $75-00-3$ & Chloroethane & 1.0 & $\mathrm{U}$ \\
\hline $75-69-4$ & Trichlorofluoromethane & 1.0 & $\mathrm{U}$ \\
\hline $75-35-4$ & 1,1-Dichloroethene & 1.0 & $\mathrm{U}$ \\
\hline $76-13-1$ & $1,1,2$-Trichloro-1,2,2-trifluoroethane & 1.0 & $\mathrm{U}$ \\
\hline $67-64-1$ & Acetone & 5.0 & $\mathrm{U}$ \\
\hline $75-15-0$ & Carbon disulfide & 0.027 & $\mathrm{~J}$ \\
\hline $79-20-9$ & Methyl acetate & 1.0 & $\mathrm{U}$ \\
\hline $75-09-2$ & Methylene Chloride & 1.0 & $\mathrm{U}$ \\
\hline $156-60-5$ & trans-1,2-Dichloroethene & 1.0 & $\mathrm{U}$ \\
\hline $1634-04-4$ & Methyl tert-butyl ether & 1.0 & $\mathrm{U}$ \\
\hline $75-34-3$ & 1,1-Dichloroethane & 1.0 & $\mathrm{U}$ \\
\hline $156-59-2$ & cis-1,2-Dichloroethene & 1.0 & $\mathrm{U}$ \\
\hline $78-93-3$ & 2-Butanone & 5.0 & $\mathrm{U}$ \\
\hline $74-97-5$ & Bromochloromethane & 1.0 & $\mathrm{U}$ \\
\hline $67-66-3$ & Chloroform & 1.0 & $\mathrm{U}$ \\
\hline $71-55-6$ & $1,1,1$-Trichloroethane & 1.0 & $\mathrm{U}$ \\
\hline $110-82-7$ & Cyclohexane & 1.0 & $\mathrm{U}$ \\
\hline $56-23-5$ & Carbon tetrachloride & 1.0 & $\mathrm{U}$ \\
\hline $71-43-2$ & Benzene & 1.0 & $\mathrm{U}$ \\
\hline $107-06-2$ & 1,2-Dichloroethane & 1.0 & $\mathrm{U}$ \\
\hline
\end{tabular}

Report 1,4-Dioxane for Low-Medium VOA analysis only 
Lab Name: TESTAMERICA BURLINGTON Lab Code: STLV Case No.: YORK Mod. Ref No.:
Contract: $8 \mathrm{E}-00302$

SDG NO.: YORK (200-29
Matrix: (SOIL/SED/WATER) Water

Sample wt/vol: 25.0 $(\mathrm{g} / \mathrm{mL}) \mathrm{mL}$

Level: (TRACE/LOW/MED) TRACE

\% Moisture: not dec.

GC Column: DB-624 ID $: 0.20 \quad(\mathrm{~mm})$

Soil Extract Volume: (uL) $(\mathrm{mL})$

Purge Volume: 25.0
Lab Sample ID: MB 200-93413/5

Lab File ID: 15537_005.D

Date Received:

Date Analyzed: 08/31/2015

Dilution Factor: 1.0

Soil Aliquot Volume: (uL)

\begin{tabular}{|c|c|c|c|}
\hline CAS NO. & COMPOUND & $\begin{array}{l}\text { CONCENTRATION UNITS: } \\
(\mathrm{ug} / \mathrm{L} \text { or } \mathrm{ug} / \mathrm{kg}) \mathrm{ug} / \mathrm{L}\end{array}$ & $q$ \\
\hline $79-01-6$ & Trichloroethene & 1.0 & $\mathrm{U}$ \\
\hline $108-87-2$ & Methylcyclohexane & 1.0 & $\mathrm{U}$ \\
\hline $78-87-5$ & 1,2-Dichloropropane & 1.0 & $\mathrm{U}$ \\
\hline $75-27-4$ & Bromodichloromethane & 1.0 & $\mathrm{U}$ \\
\hline $10061-01-5$ & cis-1,3-Dichloropropene & 1.0 & U \\
\hline $108-10-1$ & 4-Methyl-2-pentanone & 1.0 & $\mathrm{U}$ \\
\hline $108-88-3$ & Toluene & 1.0 & $\mathrm{U}$ \\
\hline $10061-02-6$ & trans-1,3-Dichloropropene & 1.0 & $\mathrm{U}$ \\
\hline $79-00-5$ & $1,1,2-$ Trichloroethane & 1.0 & $\mathrm{U}$ \\
\hline $127-18-4$ & Tetrachloroethene & 1.0 & $\mathrm{U}$ \\
\hline $591-78-6$ & 2-Hexanone & 1.0 & $\mathrm{U}$ \\
\hline $124-48-1$ & Dibromochloromethane & 1.0 & $\mathrm{U}$ \\
\hline $106-93-4$ & 1,2-Dibromoethane & 1.0 & $\mathrm{U}$ \\
\hline $108-90-7$ & Chlorobenzene & 1.0 & $\mathrm{U}$ \\
\hline $100-41-4$ & Ethylbenzene & 1.0 & $\mathrm{U}$ \\
\hline $95-47-6$ & o-Xylene & 1.0 & $\mathrm{U}$ \\
\hline $179601-23-1$ & m,p-xylene & 1.0 & $\mathrm{U}$ \\
\hline $100-42-5$ & Styrene & 1.0 & $\mathrm{U}$ \\
\hline $75-25-2$ & Bromoform & 1.0 & $\mathrm{U}$ \\
\hline $98-82-8$ & Isopropylbenzene & 1.0 & $\mathrm{U}$ \\
\hline $79-34-5$ & $1,1,2,2$-Tetrachloroethane & 1.0 & $\mathrm{U}$ \\
\hline $541-73-1$ & 1,3 -Dichlorobenzene & 1.0 & $\mathrm{U}$ \\
\hline $106-46-7$ & 1,4-Dichlorobenzene & 1.0 & $\mathrm{U}$ \\
\hline $95-50-1$ & 1,2-Dichlorobenzene & 1.0 & $\mathrm{U}$ \\
\hline $96-12-8$ & 1,2-Dibromo-3-Chloropropane & 1.0 & $\mathrm{U}$ \\
\hline $120-82-1$ & $1,2,4$-Trichlorobenzene & 0.046 & $\mathrm{~J}$ \\
\hline $87-61-6$ & $1,2,3$-Trichlorobenzene & 0.061 & $\mathrm{~J}$ \\
\hline
\end{tabular}


Lab Name: TESTAMERICA BURLINGTON

Contract: $8 \mathrm{E}-00302$

Lab Code: STLV

Case No.: YORK

Mod. Ref No.: SDG NO.: YORK (200-29

Matrix: (SOIL/SED/WATER) Water

Sample wt/vol: 25.0 $(\mathrm{g} / \mathrm{mL}) \mathrm{mL}$

Level: (TRACE or LOW/MED) TRACE

\% Moisture: not dec.

GC Column: DB-624 ID $: 0.20 \quad(\mathrm{~mm})$

Soil Extract Volume: (uL)
Lab Sample ID: MB 200-93413/5

Lab File ID: 15537_005.D

Date Received:

Date Analyzed: 08/31/2015

Dilution Factor: 1.0

Soil Aliquot Volume: (uL)

Purge Volume: 25.0 $(\mathrm{mL})$ CONCENTRATION UNITS: (ug/L or ug/kg) ug/L

\begin{tabular}{|c|c|c|c|c|}
\hline CAS NUMBER & COMPOUND NAME & $\mathrm{RT}$ & EST. CONC. & Q \\
\hline 01 & Unknown & 7.29 & 2.8 & $\mathrm{~J} X$ \\
\hline $541-05-9$ & Cyclotrisiloxane, hexamethyl- & 8.22 & 0.52 & $\mathrm{~J} \mathrm{~N}$ \\
\hline $556-67-2$ & Cyclotetrasiloxane, octamethyl- & 11.04 & 0.64 & $\mathrm{~J} \mathrm{~N}$ \\
\hline E9667961 & Total Alkanes & $\mathrm{N} / \mathrm{A}$ & & \\
\hline
\end{tabular}

1 EPA-designated Registry Number. 
Lab Name: TESTAMERICA BURLINGTON

Lab Code: STLV Case No.: YORK Mod. Ref No.:
Contract: $8 \mathrm{E}-00302$

SDG NO.: YORK $(200-29$

Lab Sample ID: 200-29506-5

Lab File ID: 15537 _015.D

Date Received:

Date Analyzed: 08/31/2015

Dilution Factor: 1.0

Soil Aliquot Volume: (uL)

Soil Extract Volume:

Purge Volume: 25.0 (uL)

$(\mathrm{mL})$ ID $: 0.20 \quad(\mathrm{~mm})$

\begin{tabular}{|c|c|c|c|}
\hline CAS NO. & COMPOUND & $\begin{array}{l}\text { CONCENTRATION UNITS: } \\
(\mathrm{ug} / \mathrm{L} \text { or } \mathrm{ug} / \mathrm{kg}) \mathrm{ug} / \mathrm{L}\end{array}$ & q \\
\hline $75-71-8$ & Dichlorodifluoromethane & 1.0 & $\mathrm{U}$ \\
\hline $74-87-3$ & Chloromethane & 1.0 & $\mathrm{U}$ \\
\hline $75-01-4$ & Vinyl chloride & 1.0 & $\mathrm{U}$ \\
\hline $74-83-9$ & Bromomethane & 1.0 & $\mathrm{U}$ \\
\hline $75-00-3$ & Chloroethane & 1.0 & $\mathrm{U}$ \\
\hline $75-69-4$ & Trichlorofluoromethane & 1.0 & $\mathrm{U}$ \\
\hline $75-35-4$ & 1,1-Dichloroethene & 1.0 & $\mathrm{U}$ \\
\hline $76-13-1$ & $1,1,2$-Trichloro-1,2,2-trifluoroethane & 1.0 & $\mathrm{U}$ \\
\hline $67-64-1$ & Acetone & 5.0 & $\mathrm{U}$ \\
\hline $75-15-0$ & Carbon disulfide & 1.0 & $\mathrm{U}$ \\
\hline $79-20-9$ & Methyl acetate & 1.0 & $\mathrm{U}$ \\
\hline $75-09-2$ & Methylene Chloride & 1.0 & $\mathrm{U}$ \\
\hline $156-60-5$ & trans-1,2-Dichloroethene & 1.0 & $\mathrm{U}$ \\
\hline $1634-04-4$ & Methyl tert-butyl ether & 1.0 & $\mathrm{U}$ \\
\hline $75-34-3$ & 1,1-Dichloroethane & 1.0 & $\mathrm{U}$ \\
\hline $156-59-2$ & cis-1,2-Dichloroethene & 1.0 & $\mathrm{U}$ \\
\hline $78-93-3$ & 2-Butanone & 5.0 & $\mathrm{U}$ \\
\hline $74-97-5$ & Bromochloromethane & 1.0 & $\mathrm{U}$ \\
\hline $67-66-3$ & Chloroform & 1.0 & $\mathrm{U}$ \\
\hline $71-55-6$ & $1,1,1$-Trichloroethane & 1.0 & $\mathrm{U}$ \\
\hline $110-82-7$ & Cyclohexane & 1.0 & $\mathrm{U}$ \\
\hline $56-23-5$ & Carbon tetrachloride & 1.0 & $\mathrm{U}$ \\
\hline $71-43-2$ & Benzene & 1.0 & $\mathrm{U}$ \\
\hline $107-06-2$ & 1,2-Dichloroethane & 1.0 & $\mathrm{U}$ \\
\hline
\end{tabular}

Report 1,4-Dioxane for Low-Medium VOA analysis only 
Lab Name: TESTAMERICA BURLINGTON

Lab Code: STLV

Case No.: YORK

Mod. Ref No.:

Contract: 8E-00302

Matrix: (SOIL/SED/WATER) Water

Sample wt/vol: 25.0 $(\mathrm{g} / \mathrm{mL}) \mathrm{mL}$

Level: (TRACE/LOW/MED) TRACE

\% Moisture: not dec.

GC Column: DB-624 ID: $0.20 \quad(\mathrm{~mm})$

Soil Extract Volume: (uL) $(\mathrm{mL})$

Purge Volume: 25.0 SDG NO.: YORK (200-29

Lab Sample ID: 200-29506-5

Lab File ID: 15537_015.D

Date Received:

Date Analyzed: 08/31/2015

Dilution Factor: 1.0

Soil Aliquot Volume: (uL)

\begin{tabular}{|c|c|c|c|}
\hline CAS NO. & COMPOUND & $\begin{array}{l}\text { CONCENTRATION UNITS: } \\
(\mathrm{ug} / \mathrm{L} \text { or } \mathrm{ug} / \mathrm{kg}) \mathrm{ug} / \mathrm{L}\end{array}$ & Q \\
\hline $79-01-6$ & Trichloroethene & 1.0 & $\mathrm{U}$ \\
\hline $108-87-2$ & Methylcyclohexane & 1.0 & $\mathrm{U}$ \\
\hline $78-87-5$ & 1,2-Dichloropropane & 1.0 & $\mathrm{U}$ \\
\hline $75-27-4$ & Bromodichloromethane & 1.0 & $\mathrm{U}$ \\
\hline $10061-01-5$ & cis-1,3-Dichloropropene & 1.0 & $\mathrm{U}$ \\
\hline $108-10-1$ & 4-Methyl-2-pentanone & 1.0 & $\mathrm{U}$ \\
\hline $108-88-3$ & Toluene & 1.0 & $\mathrm{U}$ \\
\hline $10061-02-6$ & trans-1,3-Dichloropropene & 1.0 & $\mathrm{U}$ \\
\hline $79-00-5$ & $1,1,2-T r i c h l o r o e t h a n e$ & 1.0 & $\mathrm{U}$ \\
\hline $127-18-4$ & Tetrachloroethene & 1.0 & $\mathrm{U}$ \\
\hline $591-78-6$ & 2-Hexanone & 1.0 & $\mathrm{U}$ \\
\hline $124-48-1$ & Dibromochloromethane & 1.0 & $\mathrm{U}$ \\
\hline $106-93-4$ & 1,2-Dibromoethane & 1.0 & $\mathrm{U}$ \\
\hline $108-90-7$ & Chlorobenzene & 1.0 & $\mathrm{U}$ \\
\hline $100-41-4$ & Ethylbenzene & 1.0 & $\mathrm{U}$ \\
\hline $95-47-6$ & o-Xylene & 1.0 & $\mathrm{U}$ \\
\hline $179601-23-1$ & m,p-Xylene & 1.0 & $\mathrm{U}$ \\
\hline $100-42-5$ & styrene & 1.0 & $\mathrm{U}$ \\
\hline $75-25-2$ & Bromoform & 1.0 & $\mathrm{U}$ \\
\hline $98-82-8$ & Isopropylbenzene & 1.0 & $\mathrm{U}$ \\
\hline $79-34-5$ & $1,1,2,2$-Tetrachloroethane & 1.0 & $\mathrm{U}$ \\
\hline $541-73-1$ & 1,3-Dichlorobenzene & 1.0 & $\mathrm{U}$ \\
\hline $106-46-7$ & 1,4-Dichlorobenzene & 1.0 & $\mathrm{U}$ \\
\hline $95-50-1$ & $1,2-$ Dichlorobenzene & 1.0 & $\mathrm{U}$ \\
\hline $96-12-8$ & 1,2-Dibromo-3-Chloropropane & 1.0 & $\mathrm{U}$ \\
\hline $120-82-1$ & $1,2,4$-Trichlorobenzene & 1.0 & $\mathrm{U}$ \\
\hline $87-61-6$ & $1,2,3$-Trichlorobenzene & 1.0 & $\mathrm{U}$ \\
\hline
\end{tabular}


Lab Name: TESTAMERICA BURLINGTON

Contract: $8 \mathrm{E}-00302$

Lab Code: STLV

Case No.: YORK

Mod. Ref No.:

SDG NO.: YORK (200-29

Matrix: (SOIL/SED/WATER) Water

Sample wt/vol: 25.0 $(\mathrm{g} / \mathrm{mL}) \mathrm{mL}$

Level: (TRACE or LOW/MED) TRACE

\% Moisture: not dec.

GC Column: DB-624 ID $: 0.20 \quad(\mathrm{~mm})$

Soil Extract Volume: (uL)
Lab Sample ID: 200-29506-5

Lab File ID: 15537_015.D

Date Received:

Date Analyzed: 08/31/2015

Dilution Factor: 1.0

Soil Aliquot Volume: (uL) Purge Volume: 25.0 CONCENTRATION UNITS: (ug/L or ug/kg) ug/L

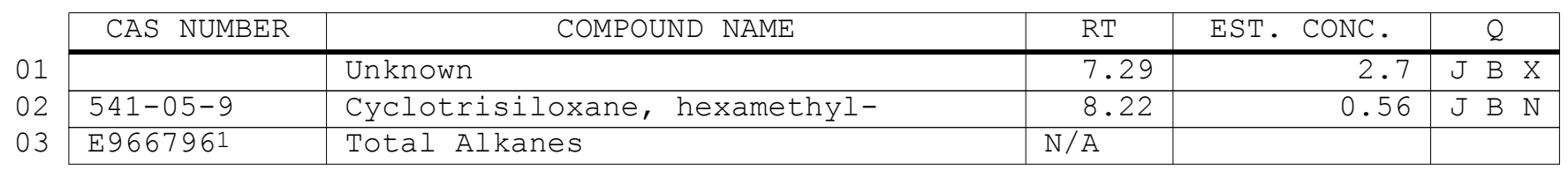


Lab Name: TESTAMERICA BURLINGTON

Lab Code: STLV Case No.: YORK Mod. Ref No.:
Contract: $8 \mathrm{E}-00302$

SDG NO.: YORK (200-29

Lab Sample ID: VIBLK 200-93413/8

Lab File ID: 15537_008.D

Date Received:

Date Analyzed: 08/31/2015

Dilution Factor: 1.0

Soil Aliquot Volume:

(uL)

Soil Extract Volume:

$(\mathrm{mL})$

Purge Volume: 25.0

$\begin{array}{ll}\mathrm{ID}: \frac{0.20}{0} & (\mathrm{~mm}) \\ (\mathrm{uL}) \\ (\mathrm{mL})\end{array}$

\begin{tabular}{|c|c|c|c|}
\hline CAS NO. & COMPOUND & $\begin{array}{l}\text { CONCENTRATION UNITS: } \\
(\mathrm{ug} / \mathrm{L} \text { or } \mathrm{ug} / \mathrm{kg}) \mathrm{ug} / \mathrm{L}\end{array}$ & 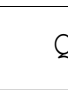 \\
\hline $75-71-8$ & Dichlorodifluoromethane & 1.0 & $\mathrm{U}$ \\
\hline $74-87-3$ & Chloromethane & 1.0 & $\mathrm{U}$ \\
\hline $75-01-4$ & Vinyl chloride & 1.0 & $\mathrm{U}$ \\
\hline $74-83-9$ & Bromomethane & 1.0 & $\mathrm{U}$ \\
\hline $75-00-3$ & Chloroethane & 1.0 & $\mathrm{U}$ \\
\hline $75-69-4$ & Trichlorofluoromethane & 1.0 & $\mathrm{U}$ \\
\hline $75-35-4$ & 1,1-Dichloroethene & 1.0 & $\mathrm{U}$ \\
\hline $76-13-1$ & $1,1,2$-Trichloro-1,2,2-trifluoroethane & 1.0 & $\mathrm{U}$ \\
\hline $67-64-1$ & Acetone & 1.0 & $\mathrm{~J}$ \\
\hline $75-15-0$ & Carbon disulfide & 1.0 & $\mathrm{U}$ \\
\hline $79-20-9$ & Methyl acetate & 1.0 & $\mathrm{U}$ \\
\hline $75-09-2$ & Methylene Chloride & 1.0 & $\mathrm{U}$ \\
\hline $156-60-5$ & trans-1,2-Dichloroethene & 1.0 & $\mathrm{U}$ \\
\hline $1634-04-4$ & Methyl tert-butyl ether & 1.0 & $\mathrm{U}$ \\
\hline $75-34-3$ & 1,1-Dichloroethane & 1.0 & $\mathrm{U}$ \\
\hline $156-59-2$ & cis-1,2-Dichloroethene & 1.0 & $\mathrm{U}$ \\
\hline $78-93-3$ & 2-Butanone & 5.0 & $\mathrm{U}$ \\
\hline $74-97-5$ & Bromochloromethane & 1.0 & $\mathrm{U}$ \\
\hline $67-66-3$ & Chloroform & 1.0 & $\mathrm{U}$ \\
\hline $71-55-6$ & $1,1,1$-Trichloroethane & 1.0 & $\mathrm{U}$ \\
\hline $110-82-7$ & Cyclohexane & 1.0 & $\mathrm{U}$ \\
\hline $56-23-5$ & Carbon tetrachloride & 0.063 & $\mathrm{~J}$ \\
\hline $71-43-2$ & Benzene & 1.0 & $\mathrm{U}$ \\
\hline $107-06-2$ & 1,2-Dichloroethane & 1.0 & $\mathrm{U}$ \\
\hline
\end{tabular}

Report 1,4-Dioxane for Low-Medium VOA analysis only 
Lab Name: TESTAMERICA BURLINGTON

Lab Code: STLV

Case No.: YORK

Mod. Ref No.:

Contract: 8E-00302

Matrix: (SOIL/SED/WATER) Water

Sample wt/vol: 25.0 $(\mathrm{g} / \mathrm{mL}) \mathrm{mL}$

Level: (TRACE/LOW/MED) TRACE

\% Moisture: not dec.

GC Column: DB-624 ID: $0.20 \quad(\mathrm{~mm})$

Soil Extract Volume: (uL) $(\mathrm{mL})$

Purge Volume: 25.0 SDG NO.: YORK (200-29

Lab Sample ID: VIBLK 200-93413/8

Lab File ID: 15537_008.D

Date Received:

Date Analyzed: 08/31/2015

Dilution Factor: 1.0

Soil Aliquot Volume: (uL)

\begin{tabular}{|c|c|c|c|}
\hline CAS NO. & COMPOUND & $\begin{array}{l}\text { CONCENTRATION UNITS: } \\
(\mathrm{ug} / \mathrm{L} \text { or } \mathrm{ug} / \mathrm{kg}) \mathrm{ug} / \mathrm{L}\end{array}$ & Q \\
\hline $79-01-6$ & Trichloroethene & 1.0 & $\mathrm{U}$ \\
\hline $108-87-2$ & Methylcyclohexane & 1.0 & $\mathrm{U}$ \\
\hline $78-87-5$ & 1,2-Dichloropropane & 1.0 & $\mathrm{U}$ \\
\hline $75-27-4$ & Bromodichloromethane & 1.0 & $\mathrm{U}$ \\
\hline $10061-01-5$ & cis-1,3-Dichloropropene & 1.0 & $\mathrm{U}$ \\
\hline $108-10-1$ & 4-Methyl-2-pentanone & 5.0 & $\mathrm{U}$ \\
\hline $108-88-3$ & Toluene & 1.0 & $\mathrm{U}$ \\
\hline $10061-02-6$ & trans-1,3-Dichloropropene & 1.0 & $\mathrm{U}$ \\
\hline $79-00-5$ & $1,1,2-T r i c h l o r o e t h a n e$ & 1.0 & $\mathrm{U}$ \\
\hline $127-18-4$ & Tetrachloroethene & 1.0 & $\mathrm{U}$ \\
\hline $591-78-6$ & 2-Hexanone & 5.0 & $\mathrm{U}$ \\
\hline $124-48-1$ & Dibromochloromethane & 1.0 & $\mathrm{U}$ \\
\hline $106-93-4$ & 1,2-Dibromoethane & 1.0 & $\mathrm{U}$ \\
\hline $108-90-7$ & Chlorobenzene & 1.0 & $\mathrm{U}$ \\
\hline $100-41-4$ & Ethylbenzene & 1.0 & $\mathrm{U}$ \\
\hline $95-47-6$ & o-Xylene & 1.0 & $\mathrm{U}$ \\
\hline $179601-23-1$ & m,p-Xylene & 1.0 & $\mathrm{U}$ \\
\hline $100-42-5$ & styrene & 1.0 & $\mathrm{U}$ \\
\hline $75-25-2$ & Bromoform & 1.0 & $\mathrm{U}$ \\
\hline $98-82-8$ & Isopropylbenzene & 1.0 & $\mathrm{U}$ \\
\hline $79-34-5$ & $1,1,2,2$-Tetrachloroethane & 1.0 & $\mathrm{U}$ \\
\hline $541-73-1$ & 1,3-Dichlorobenzene & 1.0 & $\mathrm{U}$ \\
\hline $106-46-7$ & 1,4-Dichlorobenzene & 1.0 & $\mathrm{U}$ \\
\hline $95-50-1$ & $1,2-$ Dichlorobenzene & 1.0 & $\mathrm{U}$ \\
\hline $96-12-8$ & 1,2-Dibromo-3-Chloropropane & 1.0 & $\mathrm{U}$ \\
\hline $120-82-1$ & $1,2,4$-Trichlorobenzene & 1.0 & $\mathrm{U}$ \\
\hline $87-61-6$ & $1,2,3$-Trichlorobenzene & 1.0 & $\mathrm{U}$ \\
\hline
\end{tabular}


Lab Name: TESTAMERICA BURLINGTON

Contract: $8 \mathrm{E}-00302$

Lab Code: STLV Case No.: YORK Mod. Ref No.: SDG NO.: YORK (200-29

Matrix: (SOIL/SED/WATER) Water

Sample wt/vol: 25.0 $(\mathrm{g} / \mathrm{mL}) \mathrm{mL}$

Level: (TRACE or LOW/MED) TRACE

Moisture: not dec.

GC Column: DB-624 ID: $0.20 \quad(\mathrm{~mm})$

Soil Extract Volume: (uL)

CONCENTRATION UNITS: (ug/L or ug/kg) ug/L
Lab Sample ID: VIBLK 200-93413/8

Lab File ID: $15537008 . \mathrm{D}$

Date Received:

Date Analyzed: 08/31/2015

Dilution Factor: 1.0

Soil Aliquot Volume: (uL) Purge Volume: 25.0 $(\mathrm{mL})$

01

02

\begin{tabular}{|l|l|c|c|c|}
\hline CAS NUMBER & \multicolumn{1}{|c|}{ COMPOUND NAME } & RT & EST. CONC. & Q \\
\hline & Unknown & 7.29 & 2.8 & $\mathrm{~J}$ B X \\
\hline E9667961 & Total Alkanes & N/A & & \\
\hline
\end{tabular}

1EPA-designated Registry Number. 
Lab Name: TESTAMERICA BURLINGTON

Lab Code: STLV Case No.: YORK Mod. Ref No.:
Contract: $8 \mathrm{E}-00302$

SDG NO.: YORK $(200-29$
Matrix: (SOIL/SED/WATER) Water

Sample wt/vol: 25.0 $(\mathrm{g} / \mathrm{mL}) \mathrm{mL}$

Level: (TRACE/LOW/MED) TRACE

\% Moisture: not dec.

GC Column: DB-624 ID: $0.20 \quad(\mathrm{~mm})$

Soil Extract Volume: $(u L)$ $(\mathrm{mL})$

Purge Volume: 25.0
Lab Sample ID: VIBLK 200-93413/10

Lab File ID: 15537_010.D

Date Received:

Date Analyzed: 08/31/2015

Dilution Factor: 1.0

Soil Aliquot Volume: (uL)

\begin{tabular}{|c|c|c|c|}
\hline CAS NO. & COMPOUND & $\begin{array}{l}\text { CONCENTRATION UNITS: } \\
(\mathrm{ug} / \mathrm{L} \text { or } \mathrm{ug} / \mathrm{kg}) \mathrm{ug} / \mathrm{L}\end{array}$ & q \\
\hline $75-71-8$ & Dichlorodifluoromethane & 1.0 & $\mathrm{U}$ \\
\hline $74-87-3$ & Chloromethane & 1.0 & $\mathrm{U}$ \\
\hline $75-01-4$ & Vinyl chloride & 1.0 & $\mathrm{U}$ \\
\hline $74-83-9$ & Bromomethane & 1.0 & $\mathrm{U}$ \\
\hline $75-00-3$ & Chloroethane & 1.0 & $\mathrm{U}$ \\
\hline $75-69-4$ & Trichlorofluoromethane & 1.0 & $\mathrm{U}$ \\
\hline $75-35-4$ & 1,1-Dichloroethene & 1.0 & $\mathrm{U}$ \\
\hline $76-13-1$ & $1,1,2$-Trichloro-1,2,2-trifluoroethane & 1.0 & $\mathrm{U}$ \\
\hline $67-64-1$ & Acetone & 5.0 & $\mathrm{U}$ \\
\hline $75-15-0$ & Carbon disulfide & 1.0 & $\mathrm{U}$ \\
\hline $79-20-9$ & Methyl acetate & 1.0 & $\mathrm{U}$ \\
\hline $75-09-2$ & Methylene Chloride & 1.0 & $\mathrm{U}$ \\
\hline $156-60-5$ & trans-1,2-Dichloroethene & 1.0 & $\mathrm{U}$ \\
\hline $1634-04-4$ & Methyl tert-butyl ether & 1.0 & $\mathrm{U}$ \\
\hline $75-34-3$ & 1,1-Dichloroethane & 1.0 & $\mathrm{U}$ \\
\hline $156-59-2$ & cis-1,2-Dichloroethene & 1.0 & $\mathrm{U}$ \\
\hline $78-93-3$ & 2-Butanone & 5.0 & $\mathrm{U}$ \\
\hline $74-97-5$ & Bromochloromethane & 1.0 & $\mathrm{U}$ \\
\hline $67-66-3$ & Chloroform & 1.0 & $\mathrm{U}$ \\
\hline $71-55-6$ & $1,1,1$-Trichloroethane & 1.0 & $\mathrm{U}$ \\
\hline $110-82-7$ & Cyclohexane & 1.0 & $\mathrm{U}$ \\
\hline $56-23-5$ & Carbon tetrachloride & 0.016 & $\mathrm{~J}$ \\
\hline $71-43-2$ & Benzene & 1.0 & $\mathrm{U}$ \\
\hline $107-06-2$ & 1,2-Dichloroethane & 1.0 & $\mathrm{U}$ \\
\hline
\end{tabular}

Report 1,4-Dioxane for Low-Medium VOA analysis only 
Lab Name: TESTAMERICA BURLINGTON

Lab Code: STLV

Case No.: YORK

Mod. Ref No.:

Contract: 8E-00302

Matrix: (SOIL/SED/WATER) Water

Sample wt/vol: 25.0 $(\mathrm{g} / \mathrm{mL}) \mathrm{mL}$

Level: (TRACE/LOW/MED) TRACE

\% Moisture: not dec.

GC Column: DB-624 ID: $0.20 \quad(\mathrm{~mm})$

Soil Extract Volume: (uL) $(\mathrm{mL})$

Purge Volume: 25.0 SDG NO.: YORK (200-29

Lab Sample ID: VIBLK 200-93413/10

Lab File ID: 15537_010.D

Date Received:

Date Analyzed: 08/31/2015

Dilution Factor: 1.0

Soil Aliquot Volume: (uL)

\begin{tabular}{|c|c|c|c|}
\hline CAS NO. & COMPOUND & $\begin{array}{l}\text { CONCENTRATION UNITS: } \\
(\mathrm{ug} / \mathrm{L} \text { or } \mathrm{ug} / \mathrm{kg}) \mathrm{ug} / \mathrm{L}\end{array}$ & Q \\
\hline $79-01-6$ & Trichloroethene & 1.0 & $\mathrm{U}$ \\
\hline $108-87-2$ & Methylcyclohexane & 1.0 & $\mathrm{U}$ \\
\hline $78-87-5$ & 1,2-Dichloropropane & 1.0 & $\mathrm{U}$ \\
\hline $75-27-4$ & Bromodichloromethane & 1.0 & $\mathrm{U}$ \\
\hline $10061-01-5$ & cis-1,3-Dichloropropene & 1.0 & $\mathrm{U}$ \\
\hline $108-10-1$ & 4-Methyl-2-pentanone & 5.0 & $\mathrm{U}$ \\
\hline $108-88-3$ & Toluene & 1.0 & $\mathrm{U}$ \\
\hline $10061-02-6$ & trans-1,3-Dichloropropene & 1.0 & $\mathrm{U}$ \\
\hline $79-00-5$ & $1,1,2-T r i c h l o r o e t h a n e$ & 1.0 & $\mathrm{U}$ \\
\hline $127-18-4$ & Tetrachloroethene & 1.0 & $\mathrm{U}$ \\
\hline $591-78-6$ & 2-Hexanone & 5.0 & $\mathrm{U}$ \\
\hline $124-48-1$ & Dibromochloromethane & 1.0 & $\mathrm{U}$ \\
\hline $106-93-4$ & 1,2-Dibromoethane & 1.0 & $\mathrm{U}$ \\
\hline $108-90-7$ & Chlorobenzene & 1.0 & $\mathrm{U}$ \\
\hline $100-41-4$ & Ethylbenzene & 1.0 & $\mathrm{U}$ \\
\hline $95-47-6$ & o-Xylene & 1.0 & $\mathrm{U}$ \\
\hline $179601-23-1$ & m,p-Xylene & 1.0 & $\mathrm{U}$ \\
\hline $100-42-5$ & styrene & 1.0 & $\mathrm{U}$ \\
\hline $75-25-2$ & Bromoform & 1.0 & $\mathrm{U}$ \\
\hline $98-82-8$ & Isopropylbenzene & 1.0 & $\mathrm{U}$ \\
\hline $79-34-5$ & $1,1,2,2$-Tetrachloroethane & 1.0 & $\mathrm{U}$ \\
\hline $541-73-1$ & 1,3-Dichlorobenzene & 1.0 & $\mathrm{U}$ \\
\hline $106-46-7$ & 1,4-Dichlorobenzene & 1.0 & $\mathrm{U}$ \\
\hline $95-50-1$ & $1,2-$ Dichlorobenzene & 1.0 & $\mathrm{U}$ \\
\hline $96-12-8$ & 1,2-Dibromo-3-Chloropropane & 1.0 & $\mathrm{U}$ \\
\hline $120-82-1$ & $1,2,4$-Trichlorobenzene & 1.0 & $\mathrm{U}$ \\
\hline $87-61-6$ & $1,2,3$-Trichlorobenzene & 1.0 & $\mathrm{U}$ \\
\hline
\end{tabular}


Lab Name: TESTAMERICA BURLINGTON

Contract: $8 \mathrm{E}-00302$

Lab Code: STLV Case No.: YORK Mod. Ref No.: SDG NO.: YORK (200-29

Matrix: (SOIL/SED/WATER) Water

Sample wt/vol: 25.0 $(\mathrm{g} / \mathrm{mL}) \mathrm{mL}$

Level: (TRACE or LOW/MED) TRACE

Moisture: not dec.

GC Column: DB-624 ID: $0.20 \quad(\mathrm{~mm})$

Soil Extract Volume: (uL)

CONCENTRATION UNITS: (ug/L or ug/kg) ug/L
Lab Sample ID: VIBLK 200-93413/10

Lab File ID: 15537 010.D

Date Received:

Date Analyzed: 08/31/2015

Dilution Factor: 1.0

Soil Aliquot Volume: (uL) Purge Volume: 25.0 $(\mathrm{mL})$

01

02

\begin{tabular}{|l|l|c|c|c|}
\hline CAS NUMBER & \multicolumn{1}{|c|}{ COMPOUND NAME } & RT & EST. CONC. & Q \\
\hline & Unknown & 7.29 & 2.7 & $\mathrm{~J}$ B X \\
\hline E9667961 & Total Alkanes & N/A & & \\
\hline
\end{tabular}

1 EPA-designated Registry Number. 
Lab Name: TestAmerica Burlington

Job No.: 200-29506-1

SDG No.: York (200-29506)

Instrument ID: CHD.i

Analysis Batch Number: 92921

Lab Sample ID: IC 200-92921/3

Date Analyzed: 08/19/15 15:14

Client Sample ID:

Lab File ID: 15357_003.D

GC Column: DB-624

ID $: 0.2(\mathrm{~mm})$

\begin{tabular}{|c|c|c|c|c|}
\hline \multirow[t]{2}{*}{ COMPOUND NAME } & \multirow{2}{*}{$\begin{array}{c}\text { RETENTION } \\
\text { TIME }\end{array}$} & \multicolumn{3}{|c|}{ MANUAL INTEGRATION } \\
\hline & & REASON & ANALYST & DATE \\
\hline Dichlorodifluoromethane & 1.52 & Baseline & wilburj & $08 / 19 / 15$ \\
\hline 1,2-Dibromo-3-Chloropropane & 13.43 & Assign Peak & wilburj & $08 / 19 / 1516: 20$ \\
\hline
\end{tabular}

Lab Sample ID: IC 200-92921/4 Client Sample ID:

Date Analyzed: 08/19/15 15:39

Lab File ID: 15357_004.D

GC Column: DB-624

ID $: 0.2(\mathrm{~mm})$

\begin{tabular}{|c|c|c|c|c|}
\hline \multirow[t]{2}{*}{ COMPOUND NAME } & \multirow{2}{*}{$\begin{array}{c}\text { RETENTION } \\
\text { TIME }\end{array}$} & \multicolumn{3}{|c|}{ MANUAL INTEGRATION } \\
\hline & & REASON & ANALYST & DATE \\
\hline Dichlorodifluoromethane & 1.53 & Baseline & wilburj & $08 / 19 / 15 \quad 16: 21$ \\
\hline Bromomethane & 2.00 & Baseline & wilburj & $08 / 19 / 15 \quad 16: 27$ \\
\hline Chloroethane & 2.08 & Baseline & wilburj & $08 / 19 / 1516: 27$ \\
\hline 1,2-Dibromo-3-Chloropropane & 13.44 & Assign Peak & wilburj & $08 / 19 / 15 \quad 16: 27$ \\
\hline
\end{tabular}

Lab Sample ID: ICIS 200-92921/5

Client Sample ID:

Date Analyzed: 08/19/15 16:04

Lab File ID: 15357_005.D

GC Column: DB-624

ID $: 0.2(\mathrm{~mm})$

\begin{tabular}{|c|c|c|c|c|}
\hline \multirow[t]{2}{*}{ COMPOUND NAME } & \multirow{2}{*}{$\begin{array}{c}\text { RETENTION } \\
\text { TIME }\end{array}$} & \multicolumn{3}{|c|}{ MANUAL INTEGRATION } \\
\hline & & REASON & ANALYST & DATE \\
\hline Dichlorodifluoromethane & 1.53 & Baseline & wilburj & $08 / 19 / 1516: 30$ \\
\hline Bromomethane & 2.00 & Baseline & wilburj & $08 / 19 / 15 \quad 17: 11$ \\
\hline
\end{tabular}

Lab Sample ID: IC 200-92921/6

Client Sample ID:

Date Analyzed: 08/19/15 16:29

Lab File ID: 15357_006.D

GC Column: DB-624

ID $: 0.2(\mathrm{~mm})$

\begin{tabular}{|c|c|c|c|c|}
\hline \multirow[t]{2}{*}{ COMPOUND NAME } & \multirow{2}{*}{$\begin{array}{c}\text { RETENTION } \\
\text { TIME }\end{array}$} & \multicolumn{3}{|c|}{ MANUAL INTEGRATION } \\
\hline & & REASON & ANALYST & DATE \\
\hline Dichlorodifluoromethane & 1.53 & Baseline & wilburj & $08 / 19 / 15$ \\
\hline Bromomethane & 2.00 & Baseline & wilburj & $08 / 19 / 15$ \\
\hline
\end{tabular}

SOM01.2/VOA_Tr 
Lab Name: TestAmerica Burlington SDG No.: York (200-29506)

Instrument ID: CHD.i

Lab Sample ID: IC 200-92921/7

Date Analyzed: 08/19/15 16:54
Job No.: 200-29506-1

Analysis Batch Number: 92921

client Sample ID:

Lab File ID: 15357 007.D
GC Column: DB-624

ID $: 0.2(\mathrm{~mm})$

\begin{tabular}{|c|c|c|c|c|}
\hline \multirow[t]{2}{*}{ COMPOUND NAME } & \multirow{2}{*}{$\begin{array}{c}\text { RETENTION } \\
\text { TIME }\end{array}$} & \multicolumn{3}{|c|}{ MANUAL INTEGRATION } \\
\hline & & REASON & ANALYST & DATE \\
\hline Dichlorodifluoromethane & 1.55 & Baseline & wilburj & $08 / 20 / 1510: 30$ \\
\hline Vinyl Chloride-d3 & 1.71 & Baseline & wilburj & $08 / 20 / 1510: 30$ \\
\hline Vinyl chloride & 1.72 & Baseline & wilburj & $08 / 20 / 15$ \\
\hline Bromomethane & 2.04 & Baseline & wilburj & $08 / 20 / 15 \quad 10: 30$ \\
\hline
\end{tabular}


Lab Name: TestAmerica Burlington

Job No.: 200-29506-1

SDG No.: York (200-29506)

Instrument ID: CHD.i

Analysis Batch Number: 93413

Lab Sample ID: CCVIS 200-93413/3

Client Sample ID:

Date Analyzed: 08/31/15 12:54

Lab File ID: 15537 003.D

GC Column: DB-624

ID $: 0.2(\mathrm{~mm})$

\begin{tabular}{|c|c|c|c|c|}
\hline \multirow[t]{2}{*}{ COMPOUND NAME } & RETENTION & \multicolumn{3}{|c|}{ MANUAL INTEGRATION } \\
\hline & TIME & REASON & ANALYST & DATE \\
\hline Dichlorodifluoromethane & 1.48 & Assign Peak & wilburj & $08 / 31 / 1513: 41$ \\
\hline
\end{tabular}

Lab Sample ID: CCVC 200-93413/16

Client Sample ID:

Date Analyzed: 08/31/15 18:29

Lab File ID: 15537 016.D

GC Column: DB-624

ID $: 0.2(\mathrm{~mm})$

\begin{tabular}{|l|c|c|c|c|}
\hline \multicolumn{1}{|c|}{ COMPOUND NAME } & RETENTION & \multicolumn{2}{|c|}{ MANUAL INTEGRATION } \\
\cline { 4 - 5 } & TIME & REASON & ANALYST & DATE \\
\hline Dichlorodifluoromethane & 1.52 & Baseline & wilburj & $09 / 01 / 1510: 45$ \\
\hline
\end{tabular}




\section{TestAmerica}

THE LEADER IN ENVIRONMENTAL TESTING

\section{ANALYTICAL REPORT}

Job Number: 200-29764-1

SDG Number: 29764

Job Description: York (200-29764)

Contract Number: 1E-30401

For:

Argonne National Laboratory

9700 South Cass Avenue

Building 203

Office B-141

Argonne, IL 60439

Attention: Ms. Esther Bowen

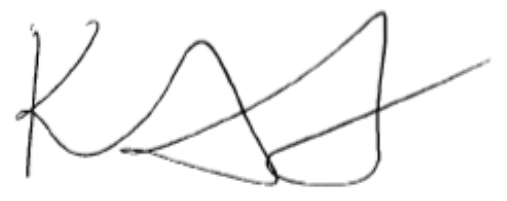

Approved for release. Kathryn A Kelly

Kathryn A Kelly, Project Manager I

30 Community Drive, South Burlington, VT, 05403

kathryn.kelly@testamericainc.com

09/30/2015

The test results in this report relate only to sample(s) as received by the laboratory. These test results were derived under a quality system that adheres to the requirements of NELAC. Pursuant to NELAC, this report may not be produced in full without written approval from the laboratory 


\section{Table of Contents}

Cover Title Page..$\ldots \ldots \ldots \ldots \ldots \ldots \ldots \ldots \ldots \ldots \ldots \ldots \ldots \ldots$

External Chain of Custody ...................... 4

Shipping Documentation . . . . . . . . . . . . . . . . 6

Sample Receipt and Log In Check List ...................... 7

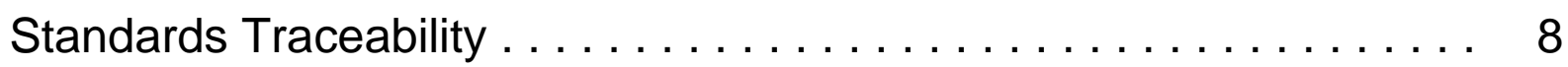

Methodology Review . . . . . . . . . . . . . . . . . . . 13

Report Narrative $\ldots \ldots \ldots \ldots \ldots \ldots \ldots \ldots \ldots \ldots \ldots \ldots \ldots \ldots \ldots$

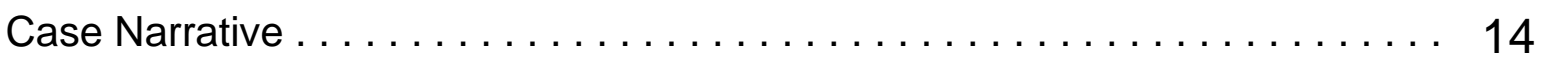

Manual Integration Documentation ........................ 15

Qualifier Definition ................................ 17

QC Summary - SOM01.2 Volatiles-Trace . . . . . . . . . . . . 18

QC Summary - SOM01.2 Volatiles-Trace .................... 18

Deuterated Monitoring Compound Summary $\ldots \ldots \ldots \ldots \ldots \ldots \ldots \ldots \ldots \ldots \ldots \ldots \ldots$

Method Blank $\ldots \ldots \ldots \ldots \ldots \ldots \ldots \ldots \ldots \ldots \ldots \ldots \ldots \ldots \ldots \ldots \ldots \ldots, 20$

GC/MS Instrument Performance Check $\ldots \ldots \ldots \ldots \ldots \ldots \ldots \ldots \ldots \ldots \ldots, 21$

Internal Standard Area and RT Summary $\ldots \ldots \ldots \ldots \ldots \ldots \ldots \ldots \ldots \ldots \ldots \ldots \ldots \ldots$

Sample Data - SOM01.2 Volatiles-Trace . . . . . . . . . . . 24

Sample Data - SOM01.2 Volatiles-Trace ...................... 24

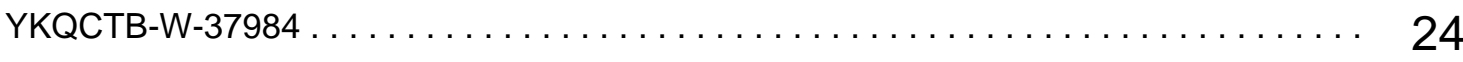

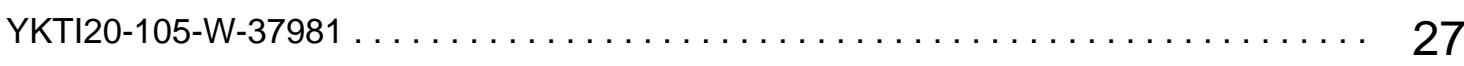

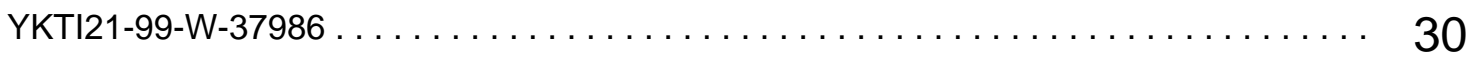

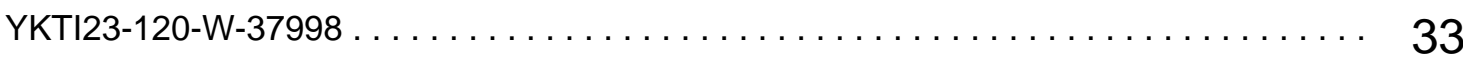

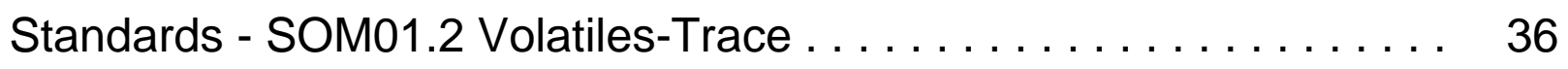

Standards - SOM01.2 Volatiles-Trace ...................... 36

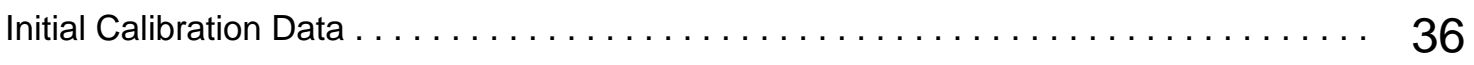

CCV Data, including closing CCV $\ldots \ldots \ldots \ldots \ldots \ldots \ldots \ldots \ldots \ldots \ldots \ldots \ldots \ldots \ldots \ldots$ 


\section{Table of Contents}

Raw Qc Data - SOM01.2 Volatiles-Trace . . . . . . . . . . . . . . . 45

Raw Qc Data - SOM01.2 Volatiles-Trace . . . . . . . . . . . . . . . . 45

Raw Qc Data - SOM01.2 Volatiles-Trace ........................... 45

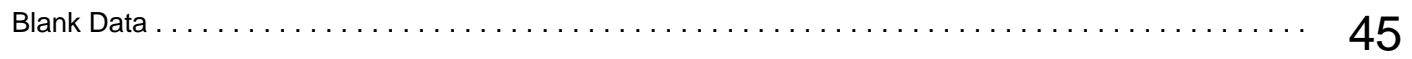




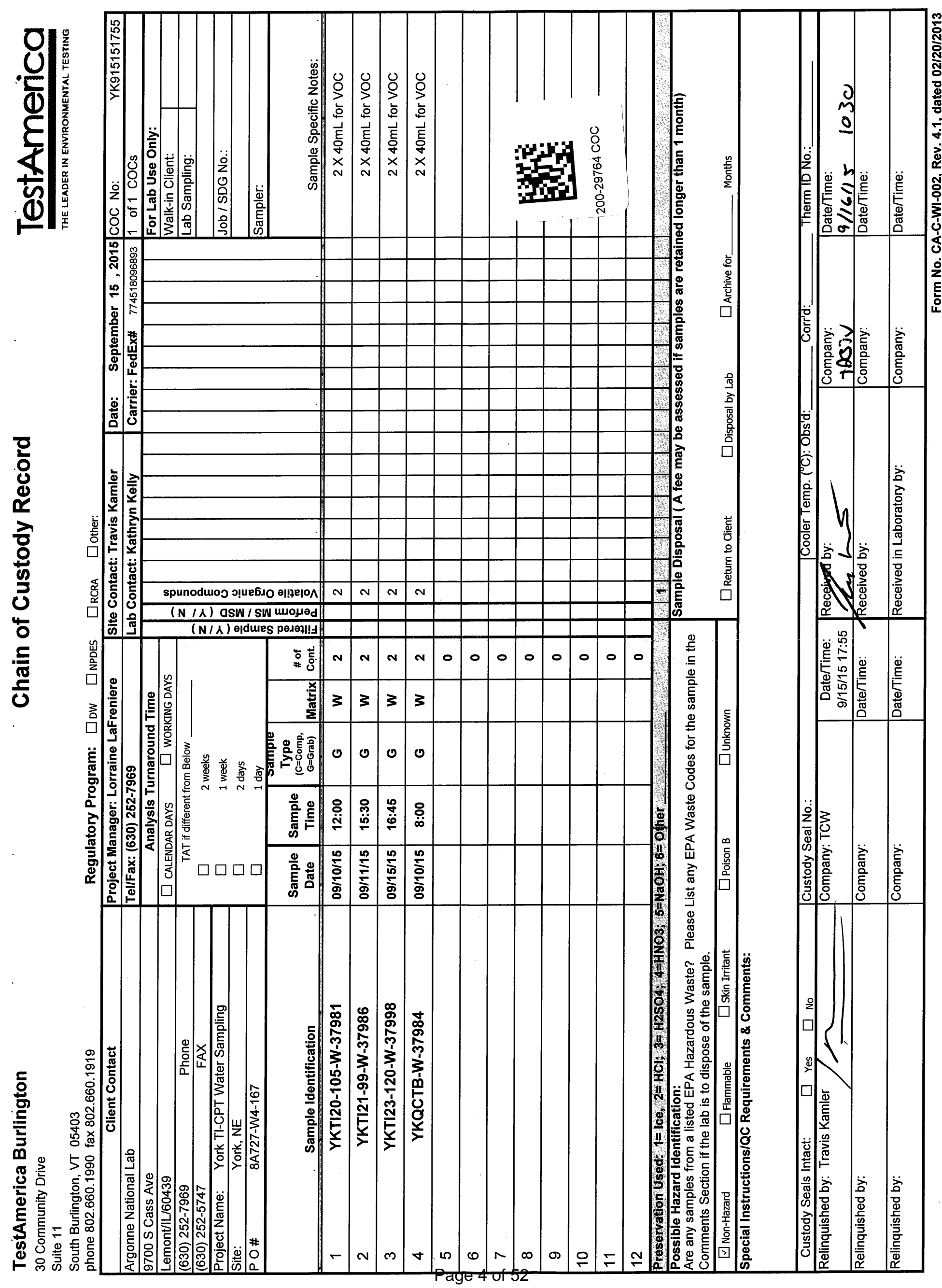


•əp!n

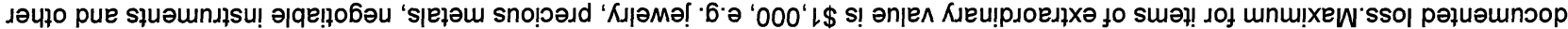

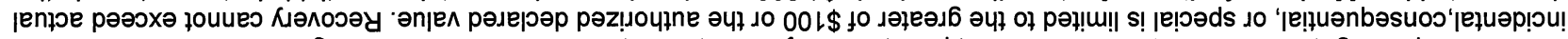

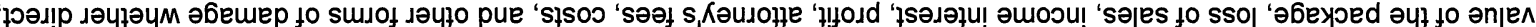

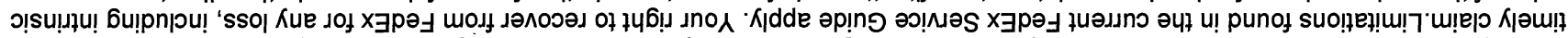

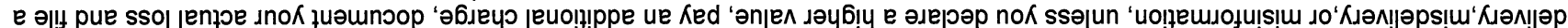

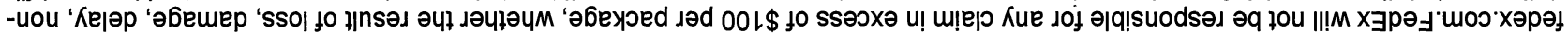

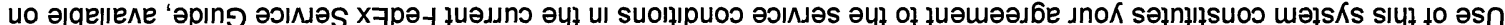

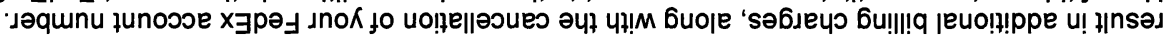

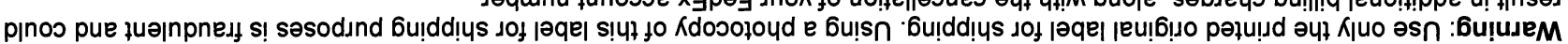

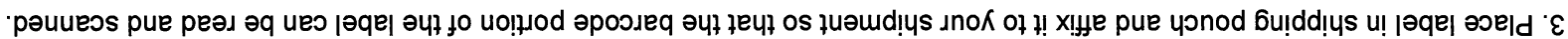

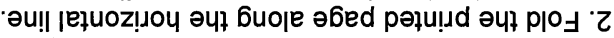

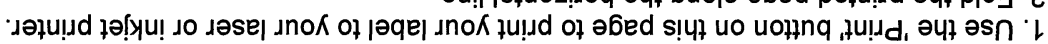

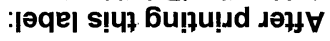

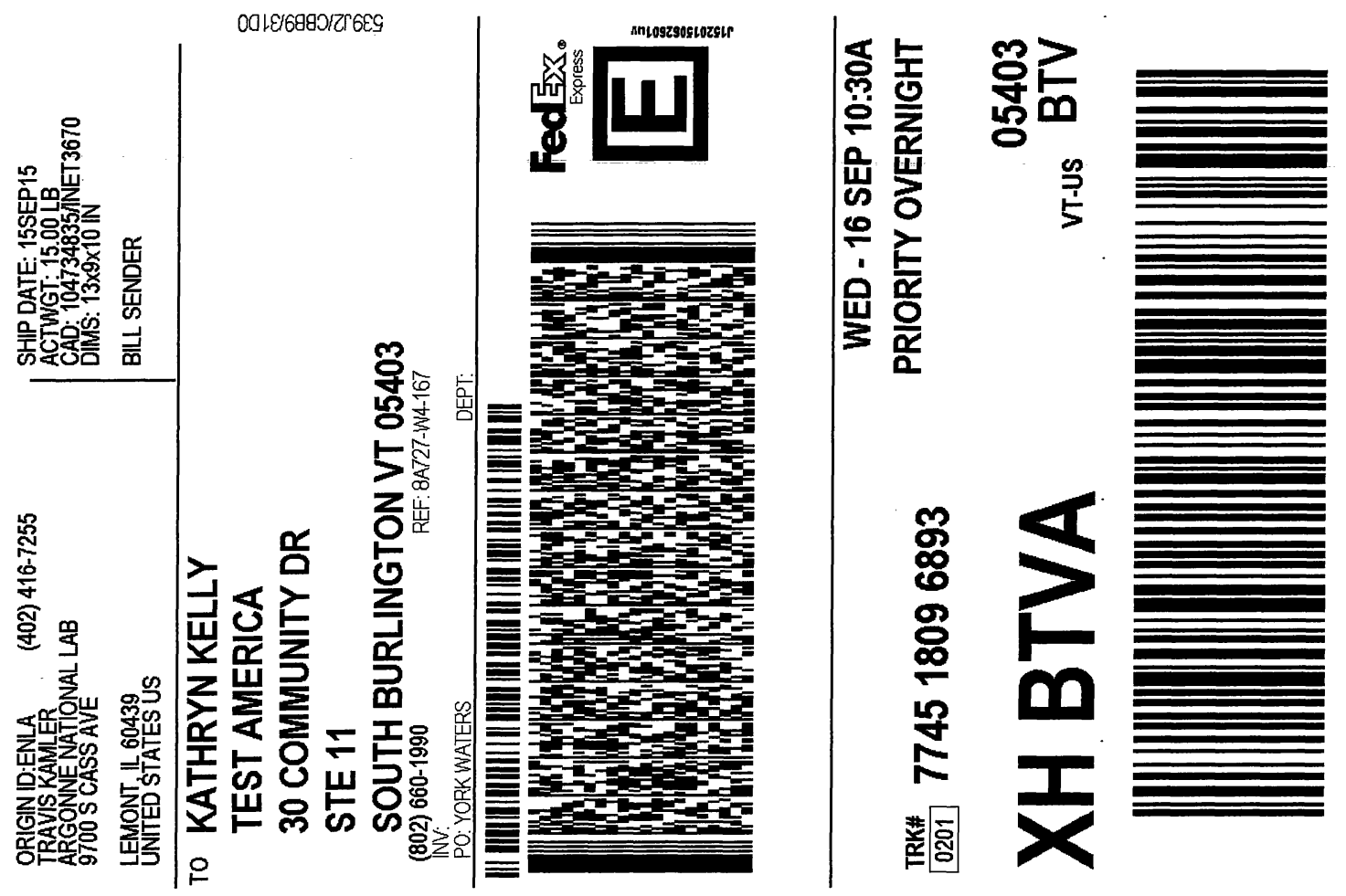




\section{Shipping and Receiving Documents}




\section{Login Sample Receipt Checklist}

Client: Argonne National Laboratory

Job Number: 200-29764-1

SDG Number: 29764

Login Number: 29764

List Source: TestAmerica Burlington

List Number: 1

Creator: Poucher, Stephanie A

Question

Radioactivity wasn't checked or is $</=$ background as measured by a survey N/A meter.

The cooler's custody seal, if present, is intact.

Sample custody seals, if present, are intact.

The cooler or samples do not appear to have been compromised or tampered with.

Samples were received on ice.

Cooler Temperature is acceptable.

Cooler Temperature is recorded.

COC is present.

COC is filled out in ink and legible.

$\mathrm{COC}$ is filled out with all pertinent information.

Is the Field Sampler's name present on COC?

There are no discrepancies between the containers received and the COC.

Samples are received within Holding Time.

Sample containers have legible labels.

Containers are not broken or leaking.

Sample collection date/times are provided.

Appropriate sample containers are used.

Sample bottles are completely filled.

Sample Preservation Verified.

There is sufficient vol. for all requested analyses, incl. any requested MS/MSDs

Containers requiring zero headspace have no headspace or bubble is $<6 \mathrm{~mm}$ (1/4").

Multiphasic samples are not present.

Samples do not require splitting or compositing.

Residual Chlorine Checked.

Answer

True

True

True

True

True

True

True

True

True

True

True

True

True

True

True

True

True

True

True

True

True

True

N/A 


\begin{tabular}{|c|c|c|c|c|c|c|c|c|}
\hline \multirow[b]{2}{*}{ Reagent ID } & \multirow[b]{2}{*}{$\begin{array}{l}\text { Exp } \\
\text { Date }\end{array}$} & \multirow[b]{2}{*}{$\begin{array}{l}\text { Prep } \\
\text { Date }\end{array}$} & \multirow[b]{2}{*}{$\begin{array}{l}\text { Dilutant } \\
\text { Used }\end{array}$} & \multirow{2}{*}{$\begin{array}{l}\text { Reagent } \\
\text { Final } \\
\text { Volume }\end{array}$} & \multicolumn{2}{|c|}{ Parent Reagent } & \multirow[b]{2}{*}{ Analyte } & \multirow[b]{2}{*}{ Concentration } \\
\hline & & & & & Reagent ID & $\begin{array}{l}\text { Volume } \\
\text { Added }\end{array}$ & & \\
\hline VMBFBW_00018 & $12 / 22 / 15$ & $06 / 23 / 15$ & METHANOL, LOt 147358 & $25 \mathrm{~mL}$ & VMBFBs_00014 & $125 \mathrm{uL}$ & $\mathrm{BFB}$ & $25 \mathrm{ug} / \mathrm{mL}$ \\
\hline .VMBFBS_00014 & $12 / 22 / 15$ & & RESTEK, Lot A0106759 & & \multicolumn{2}{|c|}{ (Purchased Reagent) } & BFB & $5000 \mathrm{ug} / \mathrm{mL}$ \\
\hline \multirow[t]{33}{*}{ VMSOMTRCALW_00093 } & \multirow[t]{33}{*}{$09 / 19 / 15$} & \multirow[t]{33}{*}{$08 / 19 / 15$} & \multirow[t]{33}{*}{ METHANOL, LOt 147358} & \multirow[t]{33}{*}{4000 uL } & VM8260CALbs_00175 & $40 \mathrm{uL}$ & \begin{tabular}{|l|} 
Bromomethane \\
Chloroethane \\
Chloromethane \\
Dichlorodifluoromethane \\
Trichlorofluoromethane \\
Vinyl chloride
\end{tabular} & $\begin{array}{l}20 \mathrm{ug} / \mathrm{mL} \\
20 \mathrm{ug} / \mathrm{mL} \\
20 \mathrm{ug} / \mathrm{mL} \\
20 \mathrm{ug} / \mathrm{mL} \\
20 \mathrm{ug} / \mathrm{mL} \\
20 \mathrm{ug} / \mathrm{mL}\end{array}$ \\
\hline & & & & & \multirow[t]{32}{*}{ VMSOMCALas_00023 } & \multirow[t]{32}{*}{40 uL } & $\begin{array}{l}1,1,1 \text {-Trichloroethane } \\
1,1,2,2 \text {-Tetrachloroethane } \\
1,1,2 \text {-Trichloro-1,2,2-trifluor } \\
\text { oethane }\end{array}$ & $\begin{array}{l}20 \mathrm{ug} / \mathrm{mL} \\
20 \mathrm{ug} / \mathrm{mL} \\
20 \mathrm{ug} / \mathrm{mL} \\
\end{array}$ \\
\hline & & & & & & & $1,1,2$-Trichloroethane & $20 \mathrm{ug} / \mathrm{mL}$ \\
\hline & & & & & & & \begin{tabular}{|l|} 
1,1-Dichloroethane \\
1,1-Dichloroethene
\end{tabular} & $\frac{20 \mathrm{ug} / \mathrm{mL}}{20 \mathrm{ug} / \mathrm{mL}}$ \\
\hline & & & & & & & $1,2,3$-Trichlorobenzene & $20 \mathrm{ug} / \mathrm{mL}$ \\
\hline & & & & & & & 1,2,4-Trichlorobenzene & $20 \mathrm{ug} / \mathrm{mL}$ \\
\hline & & & & & & & 1,2-Dibromo-3-Chloropropane & $\begin{array}{ll}20 \mathrm{ug} / \mathrm{mL} \\
20 \mathrm{ug} / \mathrm{mL}\end{array}$ \\
\hline & & & & & & & $\begin{array}{l}\text { 1,2-Dibromoethane } \\
1,2 \text {-Dichlorobenzene }\end{array}$ & $\begin{array}{ll}20 \mathrm{ug} / \mathrm{mL} \\
20 \mathrm{ug} / \mathrm{mL}\end{array}$ \\
\hline & & & & & & & 1,2-Dichloroethane & $20 \mathrm{ug} / \mathrm{mL}$ \\
\hline & & & & & & & 1,2-Dichloropropane & $20 \mathrm{ug} / \mathrm{mL}$ \\
\hline & & & & & & & $\begin{array}{l}\text { 1,3-Dichlorobenzene } \\
\text { 1,4-Dichlorobenzene }\end{array}$ & $\frac{20 \mathrm{ug} / \mathrm{mL}}{20 \mathrm{ug} / \mathrm{mL}}$ \\
\hline & & & & & & & $\begin{array}{l}\text { 1,4-Dichlorobenzene } \\
\text { Benzene }\end{array}$ & $20 \mathrm{ug} / \mathrm{mL}$ \\
\hline & & & & & & & Bromochloromethane & $20 \mathrm{ug} / \mathrm{mL}$ \\
\hline & & & & & & & Bromodichloromethane & $20 \mathrm{ug} / \mathrm{mL}$ \\
\hline & & & & & & & Bromoform & $20 \mathrm{ug} / \mathrm{mL}$ \\
\hline & & & & & & & Carbon disulfide & $20 \mathrm{ug} / \mathrm{mL}$ \\
\hline & & & & & & & Carbon tetrachloride & $20 \mathrm{ug} / \mathrm{mL}$ \\
\hline & & & & & & & Chlorobenzene & $20 \mathrm{ug} / \mathrm{mL}$ \\
\hline & & & & & & & Chloroform & $20 \mathrm{ug} / \mathrm{mL}$ \\
\hline & & & & & & & cis-1,2-Dichloroethene & $20 \mathrm{ug} / \mathrm{mL}$ \\
\hline & & & & & & & $\begin{array}{l}\text { Cis-1,3-Dichloropropene } \\
\text { Cyclohexane }\end{array}$ & $\frac{20 \mathrm{ug} / \mathrm{mL}}{20 \mathrm{ug} / \mathrm{mL}}$ \\
\hline & & & & & & & Dibromochloromethane & $20 \mathrm{ug} / \mathrm{mL}$ \\
\hline & & & & & & & Ethylbenzene & $20 \mathrm{ug} / \mathrm{mL}$ \\
\hline & & & & & & & Isopropylbenzene & $20 \mathrm{ug} / \mathrm{mL}$ \\
\hline & & & & & & & $\mathrm{m}, \mathrm{p}$-Xylene & $20 \mathrm{ug} / \mathrm{mL}$ \\
\hline & & & & & & & Methyl acetate & $20 \mathrm{ug} / \mathrm{mL}$ \\
\hline & & & & & & & Methyl tert-butyl ether & $20 \mathrm{ug} / \mathrm{mL}$ \\
\hline & & & & & & & Methylcyclohexane & $20 \mathrm{ug} / \mathrm{mL}$ \\
\hline & & & & & & & Methylene Chloride & $20 \mathrm{ug} / \mathrm{mL}$ \\
\hline & & & & & & & o-Xylene & $20 \mathrm{ug} / \mathrm{mL}$ \\
\hline & & & & & & & Styrene & $20 \mathrm{ug} / \mathrm{mL}$ \\
\hline & & & & & & & Tetrachloroethene & $20 \mathrm{ug} / \mathrm{mL}$ \\
\hline & & & & & & & Toluene & $20 \mathrm{ug} / \mathrm{mL}$ \\
\hline
\end{tabular}


SDG No.: 29764

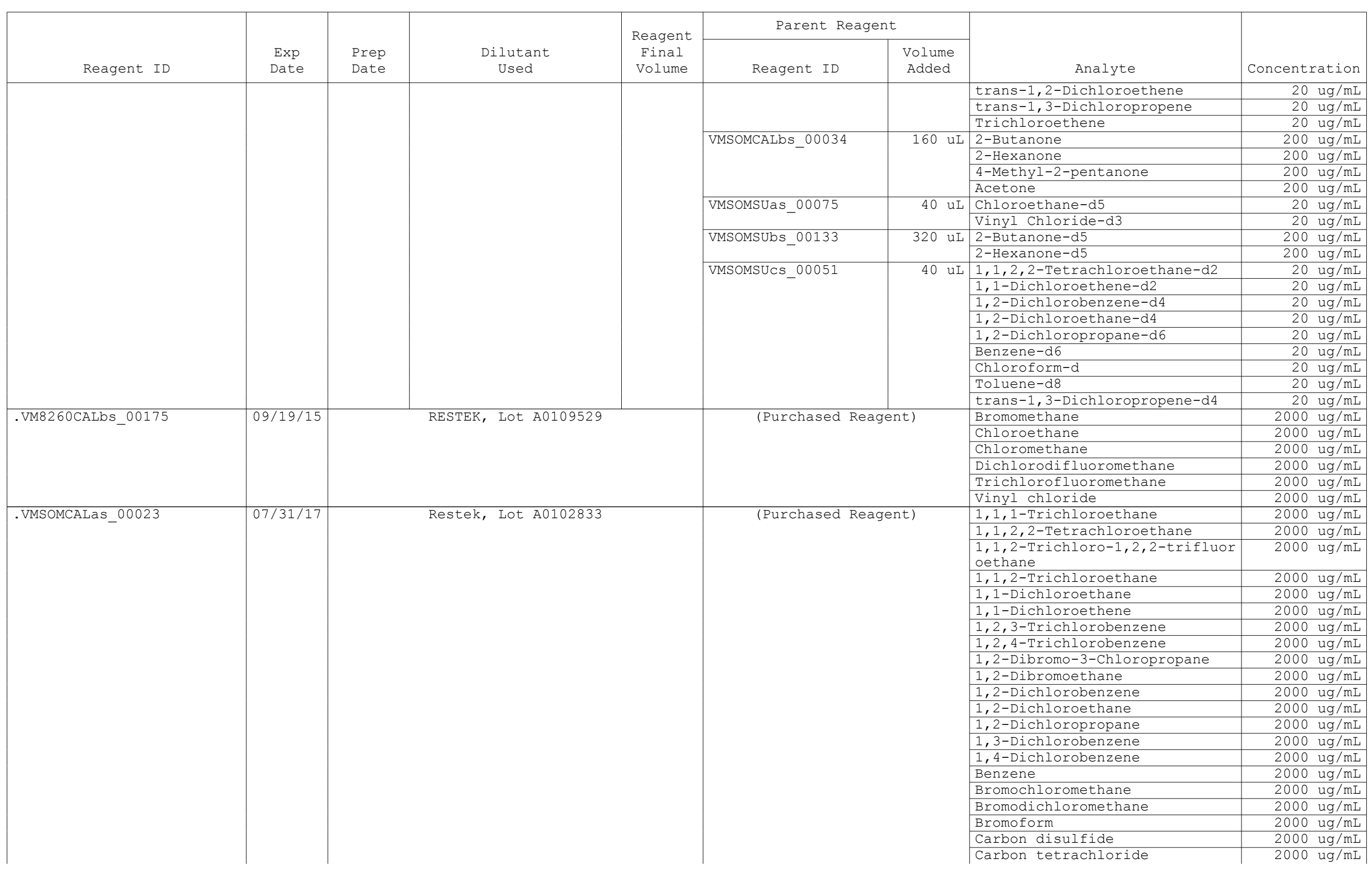


SDG No.: 29764

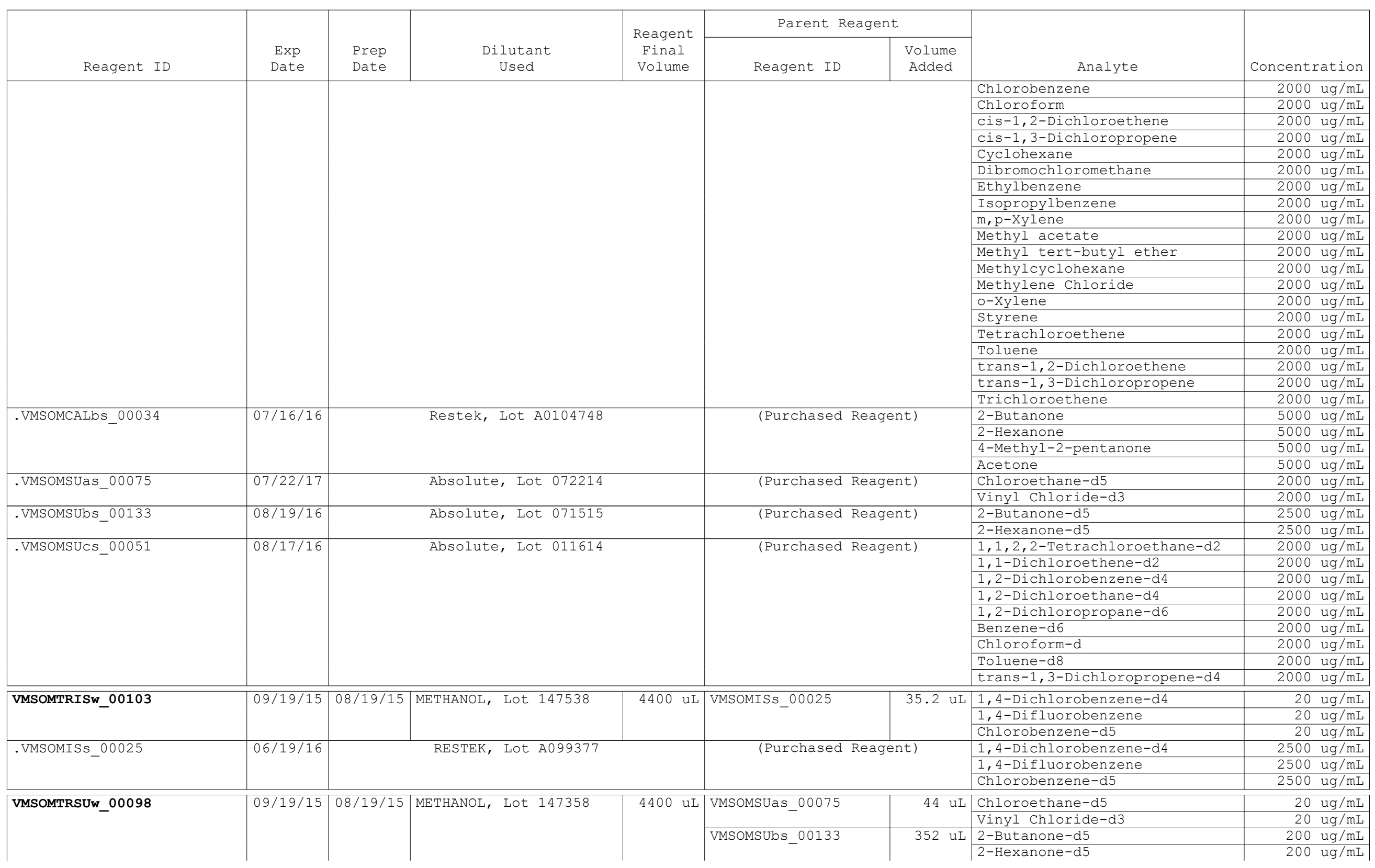


SDG No.: 29764

\begin{tabular}{|c|c|c|c|c|c|c|c|c|}
\hline \multirow[b]{2}{*}{ Reagent ID } & \multirow[b]{2}{*}{$\begin{array}{l}\text { Exp } \\
\text { Date }\end{array}$} & \multirow[b]{2}{*}{$\begin{array}{l}\text { Prep } \\
\text { Date }\end{array}$} & \multirow[b]{2}{*}{$\begin{array}{c}\text { Dilutant } \\
\text { Used }\end{array}$} & \multirow{2}{*}{$\begin{array}{l}\text { Reagent } \\
\text { Final } \\
\text { Volume }\end{array}$} & \multicolumn{2}{|c|}{ Parent Reagent } & \multirow[b]{2}{*}{ Analyte } & \multirow[b]{2}{*}{ Concentration } \\
\hline & & & & & \multirow{10}{*}{$\begin{array}{r}\text { Reagent ID } \\
\text { VMSOMSUCS_00051 }\end{array}$} & $\begin{array}{l}\text { Volume } \\
\text { Added }\end{array}$ & & \\
\hline & & & & & & $44 \mathrm{uL}$ & $1,1,2,2$-Tetrachloroethane-d2 & $20 \mathrm{ug} / \mathrm{mI}$ \\
\hline & & & & & & & 1,1-Dichloroethene-d2 & $20 \mathrm{ug} / \mathrm{mI}$ \\
\hline & & & & & & & 1,2-Dichlorobenzene-d4 & $20 \mathrm{ug} / \mathrm{mI}$ \\
\hline & & & & & & & 1,2-Dichloroethane-d4 & $20 \mathrm{ug} / \mathrm{mI}$ \\
\hline & & & & & & & 1,2-Dichloropropane-d6 & $20 \mathrm{ug} / \mathrm{mI}$ \\
\hline & & & & & & & Benzene-d6 & $20 \mathrm{ug} / \mathrm{mI}$ \\
\hline & & & & & & & Chloroform-d & $20 \mathrm{ug} / \mathrm{mI}$ \\
\hline & & & & & & & Toluene-d8 & $20 \mathrm{ug} / \mathrm{mI}$ \\
\hline & & & & & & & trans-1,3-Dichloropropene-d4 & $20 \mathrm{ug} / \mathrm{mI}$ \\
\hline \multirow[t]{2}{*}{.VMSOMSUas_00075 } & \multirow[t]{2}{*}{$07 / 22 / 17$} & \multirow{2}{*}{\multicolumn{3}{|c|}{ Absolute, Lot 072214}} & \multirow{2}{*}{\multicolumn{2}{|c|}{ (Purchased Reagent) }} & Chloroethane-d5 & $2000 \mathrm{ug} / \mathrm{mI}$ \\
\hline & & & & & & & Vinyl Chloride-d3 & $2000 \mathrm{ug} / \mathrm{mI}$ \\
\hline \multirow[t]{2}{*}{.VMSOMSUbs_00133 } & \multirow[t]{2}{*}{$08 / 19 / 16$} & \multirow{2}{*}{\multicolumn{3}{|c|}{ Absolute, Lot 071515}} & \multirow{2}{*}{\multicolumn{2}{|c|}{ (Purchased Reagent) }} & 2-Butanone-d5 & $2500 \mathrm{ug} / \mathrm{mI}$ \\
\hline & & & & & & & 2-Hexanone-d5 & $2500 \mathrm{ug} / \mathrm{mI}$ \\
\hline \multirow[t]{9}{*}{.VMSOMSUCS_00051 } & \multirow[t]{9}{*}{$08 / 17 / 16$} & \multirow{9}{*}{\multicolumn{2}{|c|}{ Absolute, Lot 011614}} & & \multirow{9}{*}{\multicolumn{2}{|c|}{ (Purchased Reagent) }} & $1,1,2,2$-Tetrachloroethane-d2 & $2000 \mathrm{ug} / \mathrm{mI}$ \\
\hline & & & & & & & 1,1-Dichloroethene-d2 & $2000 \mathrm{ug} / \mathrm{mI}$ \\
\hline & & & & & & & 1,2-Dichlorobenzene-d4 & $2000 \mathrm{ug} / \mathrm{mI}$ \\
\hline & & & & & & & 1,2-Dichloroethane-d4 & $2000 \mathrm{ug} / \mathrm{mI}$ \\
\hline & & & & & & & 1,2-Dichloropropane-d6 & $2000 \mathrm{ug} / \mathrm{mI}$ \\
\hline & & & & & & & Benzene-d6 & $2000 \mathrm{ug} / \mathrm{mI}$ \\
\hline & & & & & & & Chloroform-d & $2000 \mathrm{ug} / \mathrm{mI}$ \\
\hline & & & & & & & Toluene-d8 & $2000 \mathrm{ug} / \mathrm{mI}$ \\
\hline & & & & & & & trans-1,3-Dichloropropene-d4 & $2000 \mathrm{ug} / \mathrm{mI}$ \\
\hline
\end{tabular}


Lab Name: TestAmerica Burlington

Job No.: 200-29764-1

SDG No.: 29764

\begin{tabular}{|c|l|l|c|c|}
\hline $\begin{array}{c}\text { Reagent } \\
\text { Container }\end{array}$ & \multicolumn{1}{|c|}{$\begin{array}{c}\text { Reagent } \\
\text { ID }\end{array}$} & \multicolumn{1}{c|}{$\begin{array}{c}\text { Reagent } \\
\text { Description }\end{array}$} & $\begin{array}{c}\text { Preparation } \\
\text { Date }\end{array}$ & $\begin{array}{c}\text { Expiration } \\
\text { Date }\end{array}$ \\
\hline 807558 & VMBFBw_00018 & BFB TUNE 25 PPM & $06 / 23 / 2015$ & $12 / 22 / 2015$ \\
\hline 829193 & VMSOMTRISw_00103 & SOM TR ISTD 20 PPM & $08 / 19 / 2015$ & $09 / 19 / 2015$ \\
\hline 829199 & VMSOMTRSUw_00098 & SOM TR DMC 20 PPM & $08 / 19 / 2015$ & $09 / 19 / 2015$ \\
\hline 829202 & VMSOMTRCALw_00093 & SOM TR CAL 20 - 200 PPM & $08 / 19 / 2015$ & $09 / 19 / 2015$ \\
\hline
\end{tabular}




\section{METHODOLOGY SUMMARY}

Laboratory: TestAmerica Laboratories

Location: South Burlington, Vermont
Project No:

SDG No: 29764

VOA

Volatile Organics Trace - USEPA CLP SOM01.2 


\title{
CASE NARRATIVE
}

\section{Client: Argonne National Laboratory}

\author{
Project: York (200-29764)
}

\section{Report Number: 200-29764-1}

With the exceptions noted as flags or footnotes, standard analytical protocols were followed in the analysis of the samples and no problems were encountered or anomalies observed. In addition all laboratory quality control samples were within established control limits, with any exceptions noted below. Each sample was analyzed to achieve the lowest possible reporting limit within the constraints of the method. In some cases, due to interference or analytes present at high concentrations, samples were diluted. For diluted samples, the reporting limits are adjusted relative to the dilution required.

Calculations are performed before rounding to avoid round-off errors in calculated results.

All holding times were met and proper preservation noted for the methods performed on these samples, unless otherwise detailed in the individual sections below.

\section{RECEIPT}

The samples were received on 09/16/2015; the samples arrived in good condition, properly preserved and on ice. The temperature of the cooler at receipt was $4.4^{\circ} \mathrm{C}$.

The following sample(s) was received with less than 2 days remaining on the holding time or less than one shift (8 hours) remaining on a test with a holding time of 48 hours or less. As such, the laboratory had insufficient time remaining to perform the analysis within holding time: YKTI20-105-W-37981 (200-29764-1), YKTI21-99-W-37986 (200-29764-2), YKTI23-120-W-37998 (200-29764-3), YKQCTB-W-37984 (200-29764-4) and VHBLK01 (200-29764-5).

\section{VOLATILE ORGANIC COMPOUNDS - TRACE}

Samples YKTI20-105-W-37981, YKTI21-99-W-37986, YKTI23-120-W-37998, YKQCTB-W-37984 and VHBLK01 were analyzed for Volatile Organic Compounds - Trace in accordance with EPA SOW SOM01.2. The samples were analyzed on 09/17/2015.

Several analytes were detected in method blank MB 200-94084/5 at levels that were above the method detection limit but below the reporting limit. The values should be considered estimates, and have been flagged. If the associated sample reported a result above the MDL and/or RL, the result has been flagged. Refer to the QC report for details.

The following sample was diluted due to significant sediment deposits in the bottom of the VOA vials. YKTI21-99-W-37986 (200-29764-2). Elevated reporting limits $(\mathrm{RL})$ are provided. No more concentrated analysis is provided.

2-Hexanone-d5 failed the surrogate recovery criteria high for YKTI20-105-W-37981. 2-Butanone-d5 and 2-Hexanone-d5 failed the surrogate recovery criteria high for YKTI23-120-W-37998. 2-Hexanone-d5 failed the surrogate recovery criteria high for YKQCTB-W-37984. Refer to the QC report for details.

Sample YKTI21-99-W-37986[1.2X] required dilution prior to analysis. The reporting limits have been adjusted accordingly.

No additional analytical or quality issues were noted, other than those described above or in the Definitions/Glossary page. 
Lab Name: TestAmerica Burlington

SDG No.: 29764

Instrument ID: CHD.i

Lab Sample ID: IC 200-93966/3

Date Analyzed: 09/15/15 11:56

\begin{tabular}{|l|r|}
\hline \multicolumn{1}{|c|}{ COMPOUND NAME } & RETEN \\
& TIM \\
\hline Dichlorodifluoromethane & \\
\hline Chloroethane & \\
\hline 1,2-Dibromo-3-Chloropropane & 13 \\
\hline
\end{tabular}

Lab Sample ID: IC 200-93966/4

Date Analyzed: 09/15/15 12:20 Lab File ID: 15748 004.D

client sample ID:

Analysis Batch Number: 93966

Client Sample ID:

Lab File ID: 15748 003.D

GC Column: DB-624

ID $: 0.2(\mathrm{~mm})$

\begin{tabular}{|c|c|c|c|c|}
\hline \multirow[t]{2}{*}{ COMPOUND NAME } & \multirow{2}{*}{$\begin{array}{c}\text { RETENTION } \\
\text { TIME }\end{array}$} & \multicolumn{3}{|c|}{ MANUAL INTEGRATION } \\
\hline & & REASON & ANALYST & DATE \\
\hline 1,2-Dichloropropane-d6 & 6.40 & Split Peak & wilburj & $09 / 15 / 15 \quad 12: 53$ \\
\hline 1,2-Dibromo-3-Chloropropane & 13.43 & Assign Peak & wilburj & $09 / 15 / 1512: 53$ \\
\hline
\end{tabular}


Lab Name: TestAmerica Burlington

Job No.: 200-29764-1

SDG No.: 29764

Instrument ID: CHD.i

Analysis Batch Number: 94084

Lab Sample ID: CCVIS 200-94084/3

Client Sample ID:

Date Analyzed: 09/17/15 12:36

Lab File ID: 15795 003.D

GC Column: DB-624

ID $: 0.2(\mathrm{~mm})$

\begin{tabular}{|c|c|c|c|c|}
\hline \multirow[t]{2}{*}{ COMPOUND NAME } & \multirow{2}{*}{$\begin{array}{c}\text { RETENTION } \\
\text { TIME }\end{array}$} & \multicolumn{3}{|c|}{ MANUAL INTEGRATION } \\
\hline & & REASON & ANALYST & DATE \\
\hline Dichlorodifluoromethane & 1.51 & Baseline & wilburj & $09 / 17 / 15 \quad 12: 58$ \\
\hline 1,2-Dichloropropane-d6 & 6.41 & Split Peak & wilburj & $09 / 17 / 15 \quad 12: 58$ \\
\hline
\end{tabular}

Lab Sample ID: CCVC 200-94084/19

Client Sample ID:

Date Analyzed: 09/17/15 19:14

Lab File ID: 15795_019.D

GC Column: DB-624

ID $: 0.2(\mathrm{~mm})$

\begin{tabular}{|c|c|c|c|c|}
\hline \multirow[t]{2}{*}{ COMPOUND NAME } & \multirow{2}{*}{$\begin{array}{c}\text { RETENTION } \\
\text { TIME }\end{array}$} & \multicolumn{3}{|c|}{ MANUAL INTEGRATION } \\
\hline & & REASON & ANALYST & DATE \\
\hline Dichlorodifluoromethane & 1.47 & Incomplete Integration & wilburj & $09 / 22 / 1514: 56$ \\
\hline
\end{tabular}




\section{DATA REPORTING QUALIFIERS}

Client: Argonne National Laboratory

Job Number: 200-29764-1

Sdg Number: 29764

Lab Section

Qualifier

Description

GC/MS VOA

U

$\mathrm{J}$

$J$

$\mathrm{X}$

*

B
Analyzed for but not detected.

Indicates an Estimated Value for TICs

Indicates an estimated value.

See case narrative notes for explanation of the ' $X$ ' flag

Surrogate is outside acceptance limits.

The analyte was found in an associated blank, as well as in the sample. 
Lab Name: TESTAMERICA BURLINGTON

Lab Code: STLV Case No.: YORK Mod. Ref No.: Level: (TRACE or LOW) TRACE
Contract: $8 \mathrm{E}-00302$

SDG No.: 29764

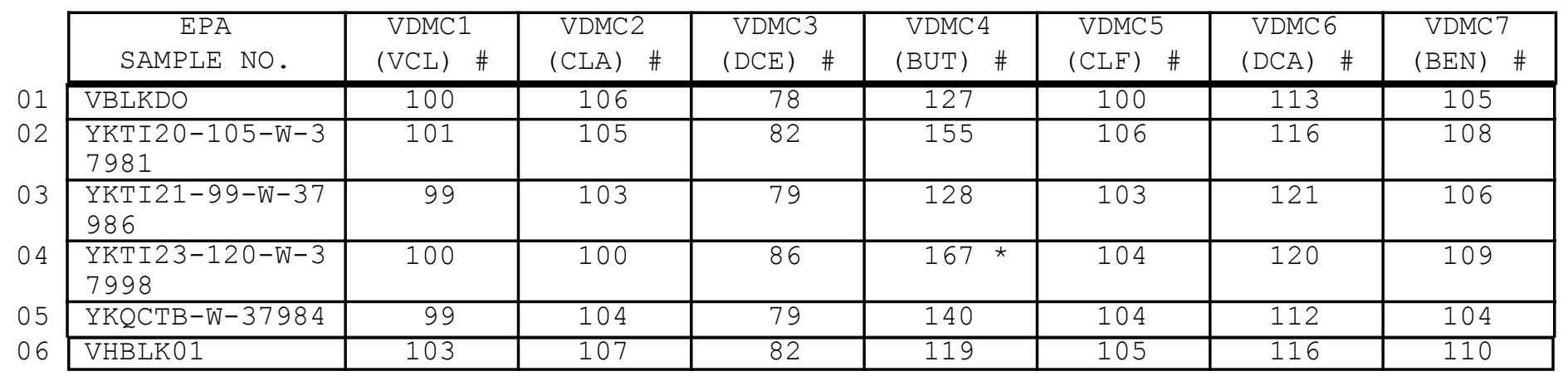

\footnotetext{
VDMC1 (VCL) = Vinyl Chloride-d3

VDMC2 $(\mathrm{CLA})=$ Chloroethane-d5

VDMC3 $(\mathrm{DCE})=1,1-\mathrm{Dichloroethene-d2}$

VDMC4 $\quad(B U T)=2-$ Butanone-d5

VDMC5 $(\mathrm{CLF})=$ Chloroform-d

VDMC $6 \quad(\mathrm{DCA})=1,2-\mathrm{Dich}$ loroethane-d4

$\operatorname{VDMC} 7 \quad(\mathrm{BEN})=$ Benzene-d6
}

$$
\begin{gathered}
\frac{\mathrm{QC} \text { LIMITS }}{(65-131)} \\
(71-131) \\
(55-104) \\
(49-155) \\
(78-121) \\
(78-129) \\
(77-124)
\end{gathered}
$$

\# Column to be used to flag recovery values

* Values outside of contract required QC limits

Page 1 of 1 
Lab Name: TESTAMERICA BURLINGTON

Contract: $8 \mathrm{E}-00302$

Lab Code: STLV Case No.: YORK Mod. Ref No.: SDG No.: 29764

Level: (TRACE or LOW) TRACE

\begin{tabular}{|c|c|c|c|c|c|c|c|c|}
\hline $\begin{array}{c}\text { EPA } \\
\text { SAMPLE NO. }\end{array}$ & $\begin{array}{l}\text { VDMC8 } \\
(\mathrm{DPA}) \#\end{array}$ & $\begin{array}{l}\text { VDMC9 } \\
(\mathrm{TOL}) \quad \#\end{array}$ & $\begin{array}{l}\text { VDMC10 } \\
(\mathrm{TDP}) \quad \#\end{array}$ & $\begin{array}{l}\text { VDMC11 } \\
(\operatorname{HEX}) \quad \#\end{array}$ & $\begin{array}{l}\text { VDMC12 } \\
(\mathrm{TCA}) \quad \#\end{array}$ & $\begin{array}{l}\text { VDMC13 } \\
(\mathrm{DCZ}) \quad \#\end{array}$ & OTHER & $\begin{array}{l}\text { TOT } \\
\text { OUT }\end{array}$ \\
\hline VBLKDO & 102 & 102 & 101 & 128 & 105 & 105 & & 0 \\
\hline $\begin{array}{l}\text { YKTI20-105-W-3 } \\
7981\end{array}$ & 104 & 107 & 105 & $166 \star$ & 108 & 106 & & 1 \\
\hline $\begin{array}{l}\text { YKTI21-99-W-37 } \\
986\end{array}$ & 105 & 104 & 107 & 132 & 113 & 107 & & 0 \\
\hline $\begin{array}{l}\text { YKTI23-120-W-3 } \\
7998\end{array}$ & 106 & 106 & 109 & $173 \star$ & 114 & 109 & & 2 \\
\hline YKQCTB-W-37984 & 103 & 106 & 101 & $143 *$ & 109 & 104 & & 1 \\
\hline VHBLK01 & 105 & 108 & 103 & 124 & 109 & 113 & & 0 \\
\hline
\end{tabular}

\footnotetext{
VDMC8 $(\mathrm{DPA})=1,2-\mathrm{Dich}$ loropropane-d6

VDMC $9 \quad($ TOL $)=$ Toluene-d8

VDMC10 $(\mathrm{TDP})=$ trans $-1,3-\mathrm{Dichloropropene-d4}$

VDMC11 $(\mathrm{HEX})=2-$ Hexanone-d5

VDMC12 $(\mathrm{TCA})=1,1,2,2$-Tetrachloroethane-d2

VDMC13 $(\mathrm{DCZ})=1,2-\mathrm{Dichlorobenzene-d4}$
}

$$
\begin{gathered}
\frac{Q C \text { LIMITS }}{(79-124)} \\
(77-121) \\
(73-121) \\
(28-135) \\
(73-125) \\
(80-131)
\end{gathered}
$$

\# Column to be used to flag recovery values

* Values outside of contract required QC limits

Report 1,4-Dioxane-d8 for Low-Medium VOA analysis only

Page 1 of 1 
$4 A$ - FORM IV VOA

VOLATILE METHOD BLANK SUMMARY
EPA SAMPLE NO.

VBLKDO

Lab Name: TESTAMERICA BURLINGTON

Contract: $8 \mathrm{E}-00302$

Lab Code: STLV Case No.: YORK Mod. Ref No.: SDG No.: 29764

Lab File ID: 15795 _005.D

Lab Sample ID: MB 200-94084/5

Instrument ID: CHD.i

Matrix: (SOIL/SED/WATER) Water

Date Analyzed: 09/17/2015

Level: (TRACE or LOW/MED) TRACE

Time Analyzed: 1325

GC Column: DB-624

ID $: 0.20 \quad(\mathrm{~mm})$

Heated Purge: (Y/N) N

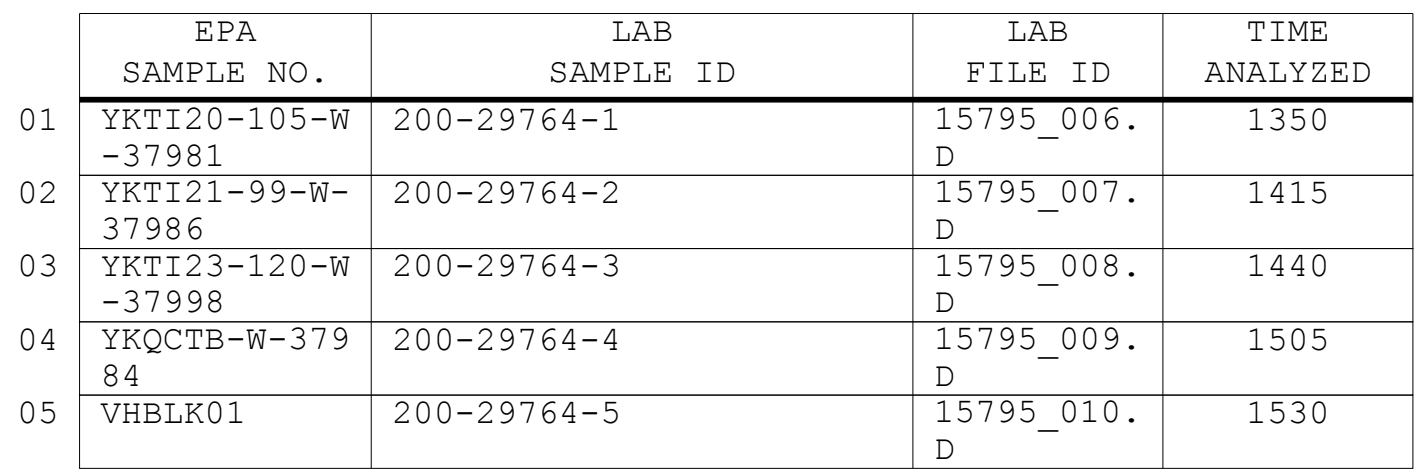

COMMENTS :

Page 1 of 1 
$5 A$ - FORM V VOA

VOLATILE ORGANICS INSTRUMENT

PERFORMANCE CHECK

BROMOFLUOROBENZENE (BFB)
EPA SAMPLE NO.

BFBDN
Lab Name: TESTAMERICA BURLINGTON

Lab Code: STLV

Case No.: YORK

Lab File Id: 15748_001.D

Instrument Id: CHD.i

GC Column: DB-624

ID $: 0.20$
Contract: 8E-00302 Mod. Ref No.:

SDG No.: 29764

BFB Injection Date: 09/15/2015

BFB Injection Time: 1108 $(\mathrm{mm})$

\begin{tabular}{|c|c|c|}
\hline $\mathrm{m} / \mathrm{e}$ & ION ABUNDANCE CRITERIA & $\begin{array}{l}\text { \% RELATIVE } \\
\text { ABUNDANCE }\end{array}$ \\
\hline 50 & $15.0-40.0 \%$ of mass 95 & 19.8 \\
\hline 75 & $30.0-80.0 \%$ of mass 95 & 50.7 \\
\hline 95 & Base peak, $100 \%$ relative abundance & 100 \\
\hline 96 & $5.0-9.0 \%$ of mass 95 & 6.5 \\
\hline 173 & Less than $2.0 \%$ of mass 174 & $0.8) 1$ \\
\hline 174 & $50.0-120 \%$ of mass 95 & 80.3 \\
\hline 175 & $5.0-9.0 \%$ of mass 174 & 5.9( \\
\hline 176 & $95.0-101 \%$ of mass 174 & $78.9(98.2) 1$ \\
\hline 177 & $5.0-9.0 \%$ of mass 176 & $(6.4) 2$ \\
\hline
\end{tabular}

1 - Value is omass 1742 - Value is omass 176

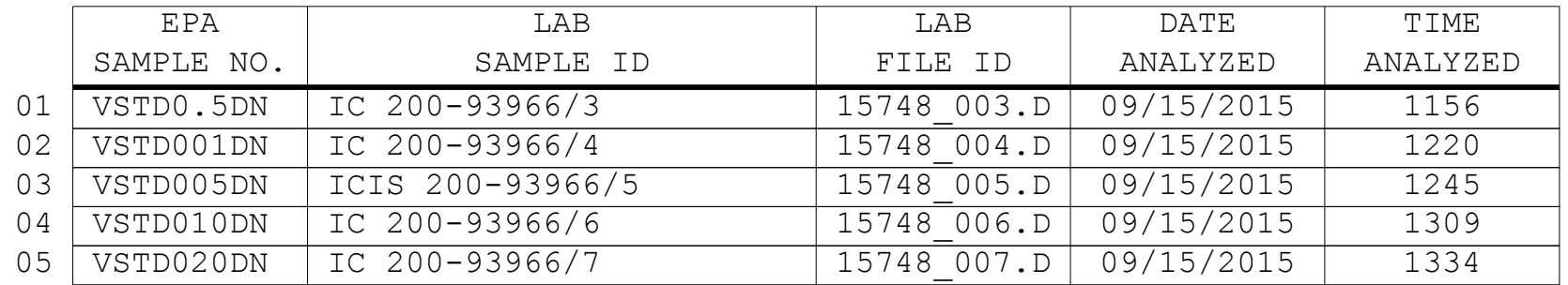


$5 A$ - FORM V VOA

VOLATILE ORGANICS INSTRUMENT

PERFORMANCE CHECK

BROMOFLUOROBENZENE (BFB)
EPA SAMPLE NO.

BFBDO
Lab Name: TESTAMERICA BURLINGTON

Lab Code: STLV

Case No.: YORK

Lab File Id: 15795_001.D

Instrument Id: CHD.i

GC Column: DB-624

ID $: 0.20$
Contract: $8 \mathrm{E}-00302$ Mod. Ref No.: SDG No.: 29764

BEB Injection Date: 09/17/2015

BFB Injection Time: 1157 $(\mathrm{mm})$

\begin{tabular}{|c|c|c|}
\hline $\mathrm{m} / \mathrm{e}$ & ION ABUNDANCE CRITERIA & $\begin{array}{l}\text { 응 RELATIVE } \\
\text { ABUNDANCE }\end{array}$ \\
\hline 50 & $15.0-40.0 \%$ of mass 95 & 20.1 \\
\hline 75 & $30.0-80.0 \%$ of mass 95 & 51.9 \\
\hline 95 & Base peak, 100\% relative abundance & 100 \\
\hline 96 & $5.0-9.0 \%$ of mass 95 & 6.9 \\
\hline 173 & Less than $2.0 \%$ of mass 174 & $0.6(0.7) 1$ \\
\hline 174 & $50.0-120 \%$ of mass 95 & 81.2 \\
\hline 175 & $5.0-9.0 \%$ of mass 174 & $5.7(7.0) 1$ \\
\hline 176 & $95.0-101 \%$ of mass 174 & $78.9(97.2) 1$ \\
\hline 177 & $5.0-9.0 \%$ of mass 176 & $5.6(7.1) 2$ \\
\hline
\end{tabular}

1 - Value is omass 174

2 - Value is omass 176

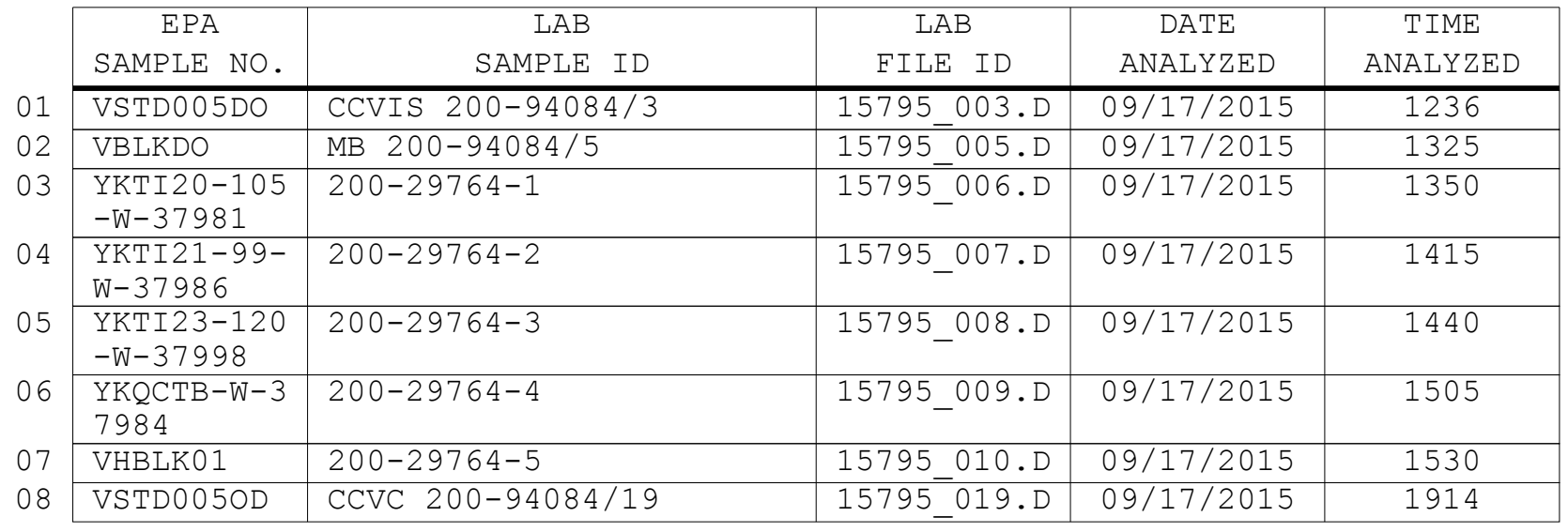




\section{$8 A$ - FORM VIII VOA}

VOLATILE INTERNAL STANDARD AREA AND RETENTION TIME SUMMARY

Lab Name: TESTAMERICA BURLINGTON

Contract: $8 \mathrm{E}-00302$

Lab Code: STLV Case No.: YORK Mod. Ref No.: SDG No.: 29764

GC Column: DB-624

ID: 0.20 (mm) Init. Calib. Date (s) : 09/15/2015 09/15/2015

EPA Sample No. (VSTD\#\#\#\#\#) : VSTD005DO

Lab File ID (Standard): 15795_003.D

Date Analyzed: 09/17/2015

Instrument ID: CHD.i

Time Analyzed: 1236

Heated Purge: (Y/N) N

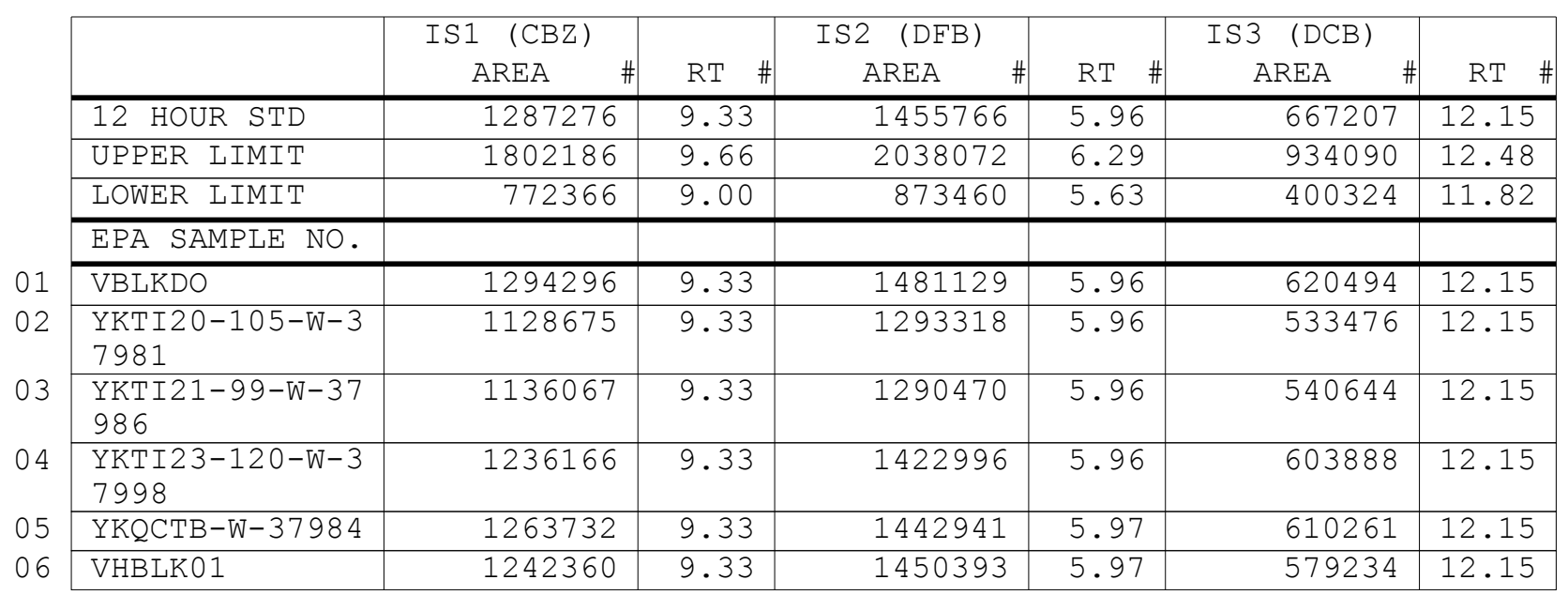

IS1 $(\mathrm{CBZ})=$ Chlorobenzene-d5

IS2 $(\mathrm{DFB})=1,4-\mathrm{Difluorobenzene}$

IS3 $(\mathrm{DCB})=1,4-\mathrm{Dichlorobenzene-d4}$

AREA UPPER LIMIT $=140 \%$ (Trace Volatiles) of internal standard area AREA LOWER LIMIT $=60 \%$ (Trace Volatiles) of internal standard area RT UPPER LIMIT $=+0.33$ (Trace Volatiles) minutes of internal standard RT RT LOWER LIMIT $=-0.33$ (Trace Volatiles) minutes of internal standard RT

\# Column used to flag values outside contract required QC limits with an asterisk. Page 1 of 1 
Lab Name: TESTAMERICA BURLINGTON

Contract: $8 \mathrm{E}-00302$

Lab Code: STLV

Case No.: YORK Mod. Ref No.:

SDG No.: 29764

Matrix: (SOIL/SED/WATER) Water

Sample wt/vol: 25.0 $(\mathrm{g} / \mathrm{mL}) \mathrm{mL}$

Level: (TRACE/LOW/MED) TRACE

\% Moisture: not dec.

GC Column: DB-624 ID: $0.20 \quad(\mathrm{~mm})$

Soil Extract Volume: (uL) $(\mathrm{mL})$

Purge Volume: 25.0
Lab Sample ID: 200-29764-4

Lab File ID: 15795_009.D

Date Received: 09/16/2015

Date Analyzed: 09/17/2015

Dilution Factor: 1.0

Soil Aliquot Volume: (uL)

\begin{tabular}{|c|c|c|c|}
\hline CAS NO. & COMPOUND & $\begin{array}{l}\text { CONCENTRATION UNITS: } \\
(\mathrm{ug} / \mathrm{L} \text { or } \mathrm{ug} / \mathrm{kg}) \mathrm{ug} / \mathrm{L}\end{array}$ & Q \\
\hline $75-71-8$ & Dichlorodifluoromethane & 1.0 & $\mathrm{U}$ \\
\hline $74-87-3$ & Chloromethane & 1.0 & $\mathrm{U}$ \\
\hline $75-01-4$ & Vinyl chloride & 1.0 & $\mathrm{U}$ \\
\hline $74-83-9$ & Bromomethane & 1.0 & $\mathrm{U}$ \\
\hline $75-00-3$ & Chloroethane & 1.0 & $\mathrm{U}$ \\
\hline $75-69-4$ & Trichlorofluoromethane & 1.0 & $\mathrm{U}$ \\
\hline $75-35-4$ & 1,1-Dichloroethene & 1.0 & $\mathrm{U}$ \\
\hline $76-13-1$ & $1,1,2$-Trichloro-1,2,2-trifluoroethane & 1.0 & $\mathrm{U}$ \\
\hline $67-64-1$ & Acetone & 13 & \\
\hline $75-15-0$ & Carbon disulfide & 0.035 & $\mathrm{~J} B$ \\
\hline $79-20-9$ & Methyl acetate & 1.0 & $\mathrm{U}$ \\
\hline $75-09-2$ & Methylene Chloride & 1.0 & $\mathrm{U}$ \\
\hline $156-60-5$ & trans-1,2-Dichloroethene & 1.0 & $\mathrm{U}$ \\
\hline $1634-04-4$ & Methyl tert-butyl ether & 1.0 & $\mathrm{U}$ \\
\hline $75-34-3$ & 1,1-Dichloroethane & 1.0 & $\mathrm{U}$ \\
\hline $156-59-2$ & cis-1,2-Dichloroethene & 1.0 & $\mathrm{U}$ \\
\hline $78-93-3$ & 2-Butanone & 5.0 & $\mathrm{U}$ \\
\hline $74-97-5$ & Bromochloromethane & 1.0 & $\mathrm{U}$ \\
\hline $67-66-3$ & Chloroform & 1.0 & $\mathrm{U}$ \\
\hline $71-55-6$ & $1,1,1$-Trichloroethane & 1.0 & $\mathrm{U}$ \\
\hline $110-82-7$ & Cyclohexane & 1.0 & $\mathrm{U}$ \\
\hline $56-23-5$ & Carbon tetrachloride & 1.0 & $\mathrm{U}$ \\
\hline $71-43-2$ & Benzene & 1.0 & $\mathrm{U}$ \\
\hline $107-06-2$ & 1,2-Dichloroethane & 1.0 & $\mathrm{U}$ \\
\hline
\end{tabular}

Report 1,4-Dioxane for Low-Medium VOA analysis only 
Lab Name: TESTAMERICA BURLINGTON

Contract: $8 \mathrm{E}-00302$

Lab Code: STLV

Case No.: YORK

Mod. Ref No.:

SDG No.: 29764

Matrix: (SOIL/SED/WATER) Water

Sample wt/vol: 25.0 $(\mathrm{g} / \mathrm{mL}) \mathrm{mL}$

Level: (TRACE/LOW/MED) TRACE

\% Moisture: not dec.

GC Column: DB-624 ID: $0.20 \quad(\mathrm{~mm})$

Soil Extract Volume: (uL) $(\mathrm{mL})$

Purge Volume: 25.0 (mI)
Lab Sample ID: 200-29764-4

Lab File ID: 15795_009.D

Date Received: 09/16/2015

Date Analyzed: 09/17/2015

Dilution Factor: 1.0

Soil Aliquot Volume: (uL)

\begin{tabular}{|c|c|c|c|}
\hline CAS NO. & COMPOUND & $\begin{array}{l}\text { CONCENTRATION UNITS: } \\
(\mathrm{ug} / \mathrm{L} \text { or } \mathrm{ug} / \mathrm{kg}) \mathrm{ug} / \mathrm{L}\end{array}$ & Q \\
\hline $79-01-6$ & Trichloroethene & 1.0 & $\mathrm{U}$ \\
\hline $108-87-2$ & Methylcyclohexane & 1.0 & $\mathrm{U}$ \\
\hline $78-87-5$ & 1,2-Dichloropropane & 1.0 & $\mathrm{U}$ \\
\hline $75-27-4$ & Bromodichloromethane & 1.0 & $\mathrm{U}$ \\
\hline $10061-01-5$ & cis-1,3-Dichloropropene & 1.0 & U \\
\hline $108-10-1$ & 4-Methyl-2-pentanone & 1.0 & $\mathrm{U}$ \\
\hline $108-88-3$ & Toluene & 0.069 & $\mathrm{~J} B$ \\
\hline $10061-02-6$ & trans-1,3-Dichloropropene & 1.0 & $\mathrm{U}$ \\
\hline $79-00-5$ & $1,1,2-$ Trichloroethane & 1.0 & $\mathrm{U}$ \\
\hline $127-18-4$ & Tetrachloroethene & 0.0090 & $\mathrm{~J}$ \\
\hline $591-78-6$ & 2-Hexanone & 1.0 & $\mathrm{U}$ \\
\hline $124-48-1$ & Dibromochloromethane & 1.0 & $\mathrm{U}$ \\
\hline $106-93-4$ & 1,2-Dibromoethane & 1.0 & $\mathrm{U}$ \\
\hline $108-90-7$ & Chlorobenzene & 1.0 & $\mathrm{U}$ \\
\hline $100-41-4$ & Ethylbenzene & 0.012 & $\mathrm{~J}$ \\
\hline $95-47-6$ & o-Xylene & 0.021 & $\mathrm{~J}$ \\
\hline $179601-23-1$ & m,p-xylene & 0.037 & $\mathrm{~J} B$ \\
\hline $100-42-5$ & Styrene & 0.013 & $\mathrm{~J}$ \\
\hline $75-25-2$ & Bromoform & 1.0 & $\mathrm{U}$ \\
\hline $98-82-8$ & Isopropylbenzene & 1.0 & $\mathrm{U}$ \\
\hline $79-34-5$ & $1,1,2,2$-Tetrachloroethane & 1.0 & $\mathrm{U}$ \\
\hline $541-73-1$ & 1,3 -Dichlorobenzene & 1.0 & $\mathrm{U}$ \\
\hline $106-46-7$ & 1,4-Dichlorobenzene & 1.0 & $\mathrm{U}$ \\
\hline $95-50-1$ & 1,2-Dichlorobenzene & 1.0 & $\mathrm{U}$ \\
\hline $96-12-8$ & 1,2-Dibromo-3-Chloropropane & 1.0 & $\mathrm{U}$ \\
\hline $120-82-1$ & $1,2,4$-Trichlorobenzene & 0.014 & $\mathrm{~J} B$ \\
\hline $87-61-6$ & $1,2,3$-Trichlorobenzene & 0.012 & $\mathrm{~J} B$ \\
\hline
\end{tabular}


Lab Name: TESTAMERICA BURLINGTON

Contract: $8 \mathrm{E}-00302$

Lab Code: STLV Case No.: YORK Mod. Ref No.: SDG No.: 29764

Matrix: (SOIL/SED/WATER) Water

Sample wt/vol: 25.0 $(\mathrm{g} / \mathrm{mL}) \mathrm{mL}$

Level: (TRACE or LOW/MED) TRACE

응 Moisture: not dec.

GC Column: DB-624 ID $: 0.20 \quad(\mathrm{~mm})$

Soil Extract Volume: (uL)

CONCENTRATION UNITS: (ug/L or ug/kg) ug/L
Lab Sample ID: 200-29764-4

Lab File ID: 15795 009.D

Date Received: 09/16/2015

Date Analyzed: 09/17/2015

Dilution Factor: 1.0

Soil Aliquot Volume: (uL) Purge Volume: 25.0

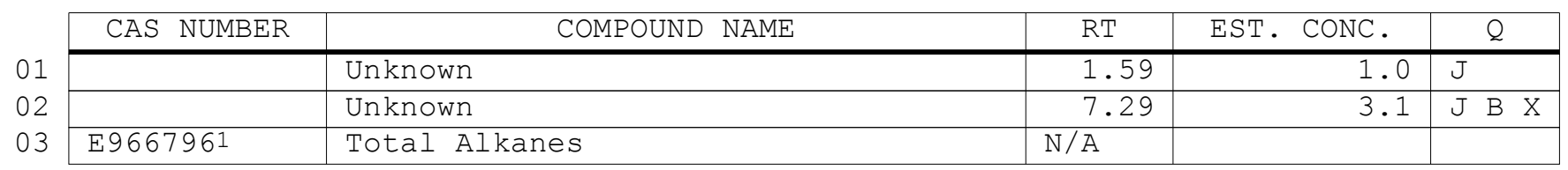

1EPA-designated Registry Number. 
Lab Name: TESTAMERICA BURLINGTON

Contract: $8 \mathrm{E}-00302$

Lab Code: STLV

Case No.: YORK Mod. Ref No.:

SDG No.: 29764

Matrix: (SOIL/SED/WATER) Water

Sample wt/vol: 25.0 $(\mathrm{g} / \mathrm{mL}) \mathrm{mL}$

Level: (TRACE/LOW/MED) TRACE

\% Moisture: not dec.

GC Column: DB-624 ID: $0.20 \quad(\mathrm{~mm})$

Soil Extract Volume: (uL) $(\mathrm{mL})$

Purge Volume: 25.0
Lab Sample ID: 200-29764-1

Lab File ID: 15795_006.D

Date Received: 09/16/2015

Date Analyzed: 09/17/2015

Dilution Factor: 1.0

Soil Aliquot Volume:

(uL)

\begin{tabular}{|c|c|c|c|}
\hline CAS NO. & COMPOUND & $\begin{array}{l}\text { CONCENTRATION UNITS: } \\
(\mathrm{ug} / \mathrm{L} \text { or } \mathrm{ug} / \mathrm{kg}) \mathrm{ug} / \mathrm{L}\end{array}$ & Q \\
\hline $75-71-8$ & Dichlorodifluoromethane & 1.0 & $\mathrm{U}$ \\
\hline $74-87-3$ & Chloromethane & 1.0 & $\mathrm{U}$ \\
\hline $75-01-4$ & Vinyl chloride & 1.0 & $\mathrm{U}$ \\
\hline $74-83-9$ & Bromomethane & 1.0 & $\mathrm{U}$ \\
\hline $75-00-3$ & Chloroethane & 1.0 & $\mathrm{U}$ \\
\hline $75-69-4$ & Trichlorofluoromethane & 1.0 & $\mathrm{U}$ \\
\hline $75-35-4$ & 1,1-Dichloroethene & 1.0 & $\mathrm{U}$ \\
\hline $76-13-1$ & $1,1,2$-Trichloro-1,2,2-trifluoroethane & 1.0 & $\mathrm{U}$ \\
\hline $67-64-1$ & Acetone & 5.0 & $\mathrm{U}$ \\
\hline $75-15-0$ & Carbon disulfide & 0.051 & $\mathrm{~J} B$ \\
\hline $79-20-9$ & Methyl acetate & 1.0 & $\mathrm{U}$ \\
\hline $75-09-2$ & Methylene Chloride & 1.0 & $\mathrm{U}$ \\
\hline $156-60-5$ & trans-1,2-Dichloroethene & 1.0 & $\mathrm{U}$ \\
\hline $1634-04-4$ & Methyl tert-butyl ether & 1.0 & $\mathrm{U}$ \\
\hline $75-34-3$ & 1,1-Dichloroethane & 1.0 & $\mathrm{U}$ \\
\hline $156-59-2$ & cis-1,2-Dichloroethene & 1.0 & $\mathrm{U}$ \\
\hline $78-93-3$ & 2-Butanone & 5.0 & $\mathrm{U}$ \\
\hline $74-97-5$ & Bromochloromethane & 1.0 & $\mathrm{U}$ \\
\hline $67-66-3$ & Chloroform & 1.0 & $\mathrm{U}$ \\
\hline $71-55-6$ & $1,1,1$-Trichloroethane & 1.0 & $\mathrm{U}$ \\
\hline $110-82-7$ & Cyclohexane & 1.0 & $\mathrm{U}$ \\
\hline $56-23-5$ & Carbon tetrachloride & 8.6 & \\
\hline $71-43-2$ & Benzene & 1.0 & $\mathrm{U}$ \\
\hline $107-06-2$ & 1,2-Dichloroethane & 1.0 & $\mathrm{U}$ \\
\hline
\end{tabular}

Report 1,4-Dioxane for Low-Medium VOA analysis only 
Lab Name: TESTAMERICA BURLINGTON

Contract: 8E-00302

Lab Code: STLV

Case No.: YORK Mod. Ref No.:

SDG No.: 29764

Matrix: (SOIL/SED/WATER) Water

Sample wt/vol: 25.0 $(\mathrm{g} / \mathrm{mL}) \mathrm{mL}$

Level: (TRACE/LOW/MED) TRACE

\% Moisture: not dec.

GC Column: DB-624 ID: $0.20 \quad(\mathrm{~mm})$

Soil Extract Volume: (uL) $(\mathrm{mL})$

Purge Volume: 25.0 (mI)
Lab Sample ID: 200-29764-1

Lab File ID: 15795_006.D

Date Received: 09/16/2015

Date Analyzed: 09/17/2015

Dilution Factor: 1.0

Soil Aliquot Volume: (uL)

\begin{tabular}{|c|c|c|c|}
\hline CAS NO. & COMPOUND & $\begin{array}{l}\text { CONCENTRATION UNITS: } \\
(\mathrm{ug} / \mathrm{L} \text { or } \mathrm{ug} / \mathrm{kg}) \mathrm{ug} / \mathrm{L}\end{array}$ & Q \\
\hline $79-01-6$ & Trichloroethene & 1.0 & $\mathrm{U}$ \\
\hline $108-87-2$ & Methylcyclohexane & 1.0 & $\mathrm{U}$ \\
\hline $78-87-5$ & 1,2-Dichloropropane & 1.0 & $\mathrm{U}$ \\
\hline $75-27-4$ & Bromodichloromethane & 1.0 & $\mathrm{U}$ \\
\hline $10061-01-5$ & cis-1,3-Dichloropropene & 1.0 & $\mathrm{U}$ \\
\hline $108-10-1$ & 4-Methyl-2-pentanone & 1.0 & $\mathrm{U}$ \\
\hline $108-88-3$ & Toluene & 0.032 & $\mathrm{~J} B$ \\
\hline $10061-02-6$ & trans-1,3-Dichloropropene & 1.0 & $\mathrm{U}$ \\
\hline $79-00-5$ & $1,1,2-T r i c h l o r o e t h a n e$ & 1.0 & $\mathrm{U}$ \\
\hline $127-18-4$ & Tetrachloroethene & 0.019 & $\mathrm{~J}$ \\
\hline $591-78-6$ & 2-Hexanone & 1.0 & $\mathrm{U}$ \\
\hline $124-48-1$ & Dibromochloromethane & 1.0 & $\mathrm{U}$ \\
\hline $106-93-4$ & 1,2-Dibromoethane & 1.0 & $\mathrm{U}$ \\
\hline $108-90-7$ & Chlorobenzene & 1.0 & $\mathrm{U}$ \\
\hline $100-41-4$ & Ethylbenzene & 1.0 & $\mathrm{U}$ \\
\hline $95-47-6$ & o-Xylene & 0.0028 & $\mathrm{~J}$ \\
\hline $179601-23-1$ & m,p-Xylene & 0.018 & $\mathrm{~J} B$ \\
\hline $100-42-5$ & Styrene & 1.0 & $\mathrm{U}$ \\
\hline $75-25-2$ & Bromoform & 1.0 & $\mathrm{U}$ \\
\hline $98-82-8$ & Isopropylbenzene & 1.0 & $\mathrm{U}$ \\
\hline $79-34-5$ & $1,1,2,2$-Tetrachloroethane & 1.0 & $\mathrm{U}$ \\
\hline $541-73-1$ & 1,3-Dichlorobenzene & 1.0 & $\mathrm{U}$ \\
\hline $106-46-7$ & 1,4-Dichlorobenzene & 1.0 & $\mathrm{U}$ \\
\hline $95-50-1$ & $1,2-$ Dichlorobenzene & 1.0 & $\mathrm{U}$ \\
\hline $96-12-8$ & 1,2-Dibromo-3-Chloropropane & 1.0 & $\mathrm{U}$ \\
\hline $120-82-1$ & $1,2,4$-Trichlorobenzene & 0.020 & $\mathrm{~J} B$ \\
\hline $87-61-6$ & $1,2,3$-Trichlorobenzene & 0.018 & $\mathrm{~J} B$ \\
\hline
\end{tabular}


Lab Name: TESTAMERICA BURLINGTON

Contract: $8 \mathrm{E}-00302$

Lab Code: STLV Case No.: YORK Mod. Ref No.: SDG No.: 29764

Matrix: (SOIL/SED/WATER) Water

Sample wt/vol: 25.0 $(\mathrm{g} / \mathrm{mL}) \mathrm{mL}$

Level: (TRACE or LOW/MED) TRACE

\% Moisture: not dec.

GC Column: DB-624 ID $: 0.20 \quad(\mathrm{~mm})$

Soil Extract Volume: (uL)

CONCENTRATION UNITS: (ug/L or ug/kg) ug/L
Lab Sample ID: 200-29764-1

Lab File ID: 15795 _006.D

Date Received: 09/16/2015

Date Analyzed: 09/17/2015

Dilution Factor: 1.0

Soil Aliquot Volume: (uL) Purge Volume: 25.0 $(\mathrm{mL})$

01

02

\begin{tabular}{|l|l|c|c|c|}
\hline CAS NUMBER & \multicolumn{1}{|c|}{ COMPOUND NAME } & RT & EST. CONC. & Q \\
\hline & Unknown & 7.29 & 3.2 & $\mathrm{~J}$ X B \\
\hline E9667961 & Total Alkanes & N/A & & \\
\hline
\end{tabular}

1EPA-designated Registry Number. 
Lab Name: TESTAMERICA BURLINGTON

Contract: $8 \mathrm{E}-00302$

Lab Code: STLV

Case No.: YORK Mod. Ref No.:

SDG No.: 29764

Matrix: (SOIL/SED/WATER) Water

Sample wt/vol: 25.0 $(\mathrm{g} / \mathrm{mL}) \mathrm{mL}$

Level: (TRACE/LOW/MED) TRACE

\% Moisture: not dec.

GC Column: DB-624 ID: $0.20 \quad(\mathrm{~mm})$

Soil Extract Volume: (uL) $(\mathrm{mL})$

Purge Volume: 25.0
Lab Sample ID: 200-29764-2

Lab File ID: 15795_007.D

Date Received: 09/16/2015

Date Analyzed: 09/17/2015

Dilution Factor: 1.2

Soil Aliquot Volume: (uL)

\begin{tabular}{|c|c|c|c|}
\hline CAS NO. & COMPOUND & $\begin{array}{l}\text { CONCENTRATION UNITS: } \\
(\mathrm{ug} / \mathrm{L} \text { or } \mathrm{ug} / \mathrm{kg}) \mathrm{ug} / \mathrm{L}\end{array}$ & Q \\
\hline $75-71-8$ & Dichlorodifluoromethane & 1.2 & $\mathrm{U}$ \\
\hline $74-87-3$ & Chloromethane & 1.2 & $\mathrm{U}$ \\
\hline $75-01-4$ & Vinyl chloride & 1.2 & $\mathrm{U}$ \\
\hline $74-83-9$ & Bromomethane & 1.2 & $\mathrm{U}$ \\
\hline $75-00-3$ & Chloroethane & 1.2 & $\mathrm{U}$ \\
\hline $75-69-4$ & Trichlorofluoromethane & 0.10 & $\mathrm{~J}$ \\
\hline $75-35-4$ & 1,1-Dichloroethene & 1.2 & $\mathrm{U}$ \\
\hline $76-13-1$ & $1,1,2$-Trichloro-1,2,2-trifluoroethane & 1.2 & $\mathrm{U}$ \\
\hline $67-64-1$ & Acetone & 2.5 & $\mathrm{~J}$ \\
\hline $75-15-0$ & Carbon disulfide & 0.076 & $\mathrm{~J} B$ \\
\hline $79-20-9$ & Methyl acetate & 1.2 & $\mathrm{U}$ \\
\hline $75-09-2$ & Methylene Chloride & 1.2 & $\mathrm{U}$ \\
\hline $156-60-5$ & trans-1,2-Dichloroethene & 1.2 & $\mathrm{U}$ \\
\hline $1634-04-4$ & Methyl tert-butyl ether & 1.2 & $\mathrm{U}$ \\
\hline $75-34-3$ & 1,1-Dichloroethane & 1.2 & $\mathrm{U}$ \\
\hline $156-59-2$ & cis-1,2-Dichloroethene & 1.2 & $\mathrm{U}$ \\
\hline $78-93-3$ & 2-Butanone & 6.0 & $\mathrm{U}$ \\
\hline $74-97-5$ & Bromochloromethane & 1.2 & $\mathrm{U}$ \\
\hline $67-66-3$ & Chloroform & 1.2 & $\mathrm{U}$ \\
\hline $71-55-6$ & $1,1,1$-Trichloroethane & 0.74 & $\mathrm{~J}$ \\
\hline $110-82-7$ & Cyclohexane & 1.2 & $\mathrm{U}$ \\
\hline $56-23-5$ & Carbon tetrachloride & 0.61 & $\mathrm{~J}$ \\
\hline $71-43-2$ & Benzene & 1.2 & $\mathrm{U}$ \\
\hline $107-06-2$ & 1,2-Dichloroethane & 1.2 & $\mathrm{U}$ \\
\hline
\end{tabular}

Report 1,4-Dioxane for Low-Medium VOA analysis only 
Lab Name: TESTAMERICA BURLINGTON

Contract: $8 \mathrm{E}-00302$

Lab Code: STLV

Case No.: YORK Mod. Ref No.:

SDG No.: 29764

Matrix: (SOIL/SED/WATER) Water

Sample wt/vol: 25.0 $(\mathrm{g} / \mathrm{mL}) \mathrm{mL}$

Level: (TRACE/LOW/MED) TRACE

\% Moisture: not dec.

GC Column: DB-624 ID: $0.20 \quad(\mathrm{~mm})$

Soil Extract Volume: (uL) $(\mathrm{mL})$

Purge Volume: 25.0
Lab Sample ID: 200-29764-2

Lab File ID: 15795_007.D

Date Received: 09/16/2015

Date Analyzed: 09/17/2015

Dilution Factor: 1.2

Soil Aliquot Volume: (uL) (mI)

\begin{tabular}{|c|c|c|c|}
\hline CAS NO. & COMPOUND & $\begin{array}{l}\text { CONCENTRATION UNITS: } \\
(\mathrm{ug} / \mathrm{L} \text { or } \mathrm{ug} / \mathrm{kg}) \mathrm{ug} / \mathrm{L}\end{array}$ & Q \\
\hline $79-01-6$ & Trichloroethene & 4.3 & \\
\hline $108-87-2$ & Methylcyclohexane & 1.2 & $\mathrm{U}$ \\
\hline $78-87-5$ & 1,2-Dichloropropane & 1.2 & $\mathrm{U}$ \\
\hline $75-27-4$ & Bromodichloromethane & 1.2 & $\mathrm{U}$ \\
\hline $10061-01-5$ & cis-1,3-Dichloropropene & 1.2 & U \\
\hline $108-10-1$ & 4-Methyl-2-pentanone & 1.2 & $\mathrm{U}$ \\
\hline $108-88-3$ & Toluene & 0.039 & $\mathrm{~J} B$ \\
\hline $10061-02-6$ & trans-1,3-Dichloropropene & 1.2 & $\mathrm{U}$ \\
\hline $79-00-5$ & $1,1,2-$ Trichloroethane & 1.2 & $\mathrm{U}$ \\
\hline $127-18-4$ & Tetrachloroethene & 0.22 & $\mathrm{~J}$ \\
\hline $591-78-6$ & 2-Hexanone & 1.2 & $\mathrm{U}$ \\
\hline $124-48-1$ & Dibromochloromethane & 1.2 & $\mathrm{U}$ \\
\hline $106-93-4$ & 1,2-Dibromoethane & 1.2 & $\mathrm{U}$ \\
\hline $108-90-7$ & Chlorobenzene & 1.2 & $\mathrm{U}$ \\
\hline $100-41-4$ & Ethylbenzene & 0.0070 & $\mathrm{~J}$ \\
\hline $95-47-6$ & o-Xylene & 0.0052 & $\mathrm{~J}$ \\
\hline $179601-23-1$ & m,p-xylene & 0.019 & $\mathrm{~J} B$ \\
\hline $100-42-5$ & Styrene & 1.2 & $\mathrm{U}$ \\
\hline $75-25-2$ & Bromoform & 1.2 & $\mathrm{U}$ \\
\hline $98-82-8$ & Isopropylbenzene & 1.2 & $\mathrm{U}$ \\
\hline $79-34-5$ & $1,1,2,2$-Tetrachloroethane & 1.2 & $\mathrm{U}$ \\
\hline $541-73-1$ & 1,3 -Dichlorobenzene & 1.2 & $\mathrm{U}$ \\
\hline $106-46-7$ & 1,4-Dichlorobenzene & 1.2 & $\mathrm{U}$ \\
\hline $95-50-1$ & 1,2-Dichlorobenzene & 1.2 & $\mathrm{U}$ \\
\hline $96-12-8$ & 1,2-Dibromo-3-Chloropropane & 1.2 & $\mathrm{U}$ \\
\hline $120-82-1$ & $1,2,4$-Trichlorobenzene & 0.021 & $\mathrm{~J} B$ \\
\hline $87-61-6$ & $1,2,3$-Trichlorobenzene & 0.020 & $\mathrm{~J} B$ \\
\hline
\end{tabular}


Lab Name: TESTAMERICA BURLINGTON

Contract: $8 \mathrm{E}-00302$

Lab Code: STLV Case No.: YORK Mod. Ref No.: SDG No.: 29764

Matrix: (SOIL/SED/WATER) Water

Sample wt/vol: 25.0 $(\mathrm{g} / \mathrm{mL}) \mathrm{mL}$

Level: (TRACE or LOW/MED) TRACE

\% Moisture: not dec.

GC Column: DB-624 ID $: 0.20 \quad(\mathrm{~mm})$

Soil Extract Volume: (uL)

CONCENTRATION UNITS: (ug/L or ug/kg) ug/L
Lab Sample ID: 200-29764-2

Lab File ID: 15795 007.D

Date Received: 09/16/2015

Date Analyzed: 09/17/2015

Dilution Factor: 1.2

Soil Aliquot Volume: (uL) Purge Volume: 25.0

$(\mathrm{mL})$

\begin{tabular}{|c|c|c|c|c|}
\hline CAS NUMBER & COMPOUND NAME & RT & EST. CONC. & $Q$ \\
\hline 01 & Unknown & 1.58 & 3.1 & $\mathrm{~J}$ \\
\hline 02 & Unknown & 1.85 & 0.99 & $\mathrm{~J}$ \\
\hline 03 & Unknown & 7.29 & 3.8 & $\mathrm{~J} B \mathrm{X}$ \\
\hline E9667961 & Total Alkanes & $\mathrm{N} / \mathrm{A}$ & & \\
\hline
\end{tabular}

1 EPA-designated Registry Number. 
Lab Name: TESTAMERICA BURLINGTON

Contract: $8 \mathrm{E}-00302$

Lab Code: STLV

Case No.: YORK Mod. Ref No.:

SDG No.: 29764

Matrix: (SOIL/SED/WATER) Water

Sample wt/vol: 25.0 $(\mathrm{g} / \mathrm{mL}) \mathrm{mL}$

Level: (TRACE/LOW/MED) TRACE

\% Moisture: not dec.

GC Column: DB-624 ID: $0.20 \quad(\mathrm{~mm})$

Soil Extract Volume: (uL) $(\mathrm{mL})$

Purge Volume: 25.0
Lab Sample ID: 200-29764-3

Lab File ID: 15795_008.D

Date Received: 09/16/2015

Date Analyzed: 09/17/2015

Dilution Factor: 1.0

Soil Aliquot Volume: (uL)

\begin{tabular}{|c|c|c|c|}
\hline CAS NO. & COMPOUND & $\begin{array}{l}\text { CONCENTRATION UNITS: } \\
(\mathrm{ug} / \mathrm{L} \text { or } \mathrm{ug} / \mathrm{kg}) \mathrm{ug} / \mathrm{L}\end{array}$ & Q \\
\hline $75-71-8$ & Dichlorodifluoromethane & 1.0 & $\mathrm{U}$ \\
\hline $74-87-3$ & Chloromethane & 1.0 & $\mathrm{U}$ \\
\hline $75-01-4$ & Vinyl chloride & 1.0 & $\mathrm{U}$ \\
\hline $74-83-9$ & Bromomethane & 1.0 & $\mathrm{U}$ \\
\hline $75-00-3$ & Chloroethane & 1.0 & $\mathrm{U}$ \\
\hline $75-69-4$ & Trichlorofluoromethane & 0.11 & $\mathrm{~J}$ \\
\hline $75-35-4$ & 1,1-Dichloroethene & 1.2 & \\
\hline $76-13-1$ & $1,1,2$-Trichloro-1,2,2-trifluoroethane & 1.0 & $\mathrm{U}$ \\
\hline $67-64-1$ & Acetone & 5.0 & $\mathrm{U}$ \\
\hline $75-15-0$ & Carbon disulfide & 0.039 & $\mathrm{~J} B$ \\
\hline $79-20-9$ & Methyl acetate & 1.0 & $\mathrm{U}$ \\
\hline $75-09-2$ & Methylene Chloride & 1.0 & $\mathrm{U}$ \\
\hline $156-60-5$ & trans-1,2-Dichloroethene & 1.0 & $\mathrm{U}$ \\
\hline $1634-04-4$ & Methyl tert-butyl ether & 1.0 & $\mathrm{U}$ \\
\hline $75-34-3$ & 1,1-Dichloroethane & 1.0 & $\mathrm{U}$ \\
\hline $156-59-2$ & cis-1,2-Dichloroethene & 1.0 & $\mathrm{U}$ \\
\hline $78-93-3$ & 2-Butanone & 5.0 & $\mathrm{U}$ \\
\hline $74-97-5$ & Bromochloromethane & 1.0 & $\mathrm{U}$ \\
\hline $67-66-3$ & Chloroform & 1.0 & $\mathrm{U}$ \\
\hline $71-55-6$ & $1,1,1$-Trichloroethane & 0.66 & $\mathrm{~J}$ \\
\hline $110-82-7$ & Cyclohexane & 1.0 & $\mathrm{U}$ \\
\hline $56-23-5$ & Carbon tetrachloride & 0.082 & $\mathrm{~J}$ \\
\hline $71-43-2$ & Benzene & 0.068 & $\mathrm{~J}$ \\
\hline $107-06-2$ & 1,2-Dichloroethane & 1.0 & $\mathrm{U}$ \\
\hline
\end{tabular}

Report 1,4-Dioxane for Low-Medium VOA analysis only 
Lab Name: TESTAMERICA BURLINGTON

Lab Code: STLV

Case No.: YORK

Mod. Ref No.:

Contract: $8 \mathrm{E}-00302$

Matrix: (SOIL/SED/WATER) Water

Sample wt/vol: 25.0 $(\mathrm{g} / \mathrm{mL}) \mathrm{mL}$

Level: (TRACE/LOW/MED) TRACE

\% Moisture: not dec.

GC Column: DB-624 ID: $0.20 \quad(\mathrm{~mm})$

Soil Extract Volume: (uL) $(\mathrm{mL})$

Purge Volume: 25.0 (mI)
SDG No.: 29764

Lab Sample ID: 200-29764-3

Lab File ID: 15795 008.D

Date Received: 09/16/2015

Date Analyzed: 09/17/2015

Dilution Factor: 1.0

Soil Aliquot Volume: (uL)

\begin{tabular}{|c|c|c|c|}
\hline CAS NO. & COMPOUND & $\begin{array}{l}\text { CONCENTRATION UNITS: } \\
(\mathrm{ug} / \mathrm{L} \text { or } \mathrm{ug} / \mathrm{kg}) \mathrm{ug} / \mathrm{L}\end{array}$ & Q \\
\hline $79-01-6$ & Trichloroethene & 1.0 & $\mathrm{U}$ \\
\hline $108-87-2$ & Methylcyclohexane & 1.0 & $\mathrm{U}$ \\
\hline $78-87-5$ & 1,2-Dichloropropane & 1.0 & $\mathrm{U}$ \\
\hline $75-27-4$ & Bromodichloromethane & 1.0 & $\mathrm{U}$ \\
\hline $10061-01-5$ & cis-1,3-Dichloropropene & 1.0 & $\mathrm{U}$ \\
\hline $108-10-1$ & 4-Methyl-2-pentanone & 1.0 & $\mathrm{U}$ \\
\hline $108-88-3$ & Toluene & 0.056 & $\mathrm{~J} B$ \\
\hline $10061-02-6$ & trans-1,3-Dichloropropene & 1.0 & $\mathrm{U}$ \\
\hline $79-00-5$ & $1,1,2-T r i c h l o r o e t h a n e$ & 1.0 & $\mathrm{U}$ \\
\hline $127-18-4$ & Tetrachloroethene & 0.32 & $\mathrm{~J}$ \\
\hline $591-78-6$ & 2-Hexanone & 1.0 & $\mathrm{U}$ \\
\hline $124-48-1$ & Dibromochloromethane & 1.0 & $\mathrm{U}$ \\
\hline $106-93-4$ & 1,2-Dibromoethane & 1.0 & $\mathrm{U}$ \\
\hline $108-90-7$ & Chlorobenzene & 1.0 & $\mathrm{U}$ \\
\hline $100-41-4$ & Ethylbenzene & 0.0077 & $\mathrm{~J}$ \\
\hline $95-47-6$ & o-Xylene & 0.0072 & $\mathrm{~J}$ \\
\hline $179601-23-1$ & m,p-Xylene & 0.026 & $\mathrm{~J} B$ \\
\hline $100-42-5$ & Styrene & 1.0 & $\mathrm{U}$ \\
\hline $75-25-2$ & Bromoform & 1.0 & $\mathrm{U}$ \\
\hline $98-82-8$ & Isopropylbenzene & 1.0 & $\mathrm{U}$ \\
\hline $79-34-5$ & $1,1,2,2$-Tetrachloroethane & 1.0 & $\mathrm{U}$ \\
\hline $541-73-1$ & 1,3-Dichlorobenzene & 1.0 & $\mathrm{U}$ \\
\hline $106-46-7$ & 1,4-Dichlorobenzene & 1.0 & $\mathrm{U}$ \\
\hline $95-50-1$ & $1,2-$ Dichlorobenzene & 1.0 & $\mathrm{U}$ \\
\hline $96-12-8$ & 1,2-Dibromo-3-Chloropropane & 1.0 & $\mathrm{U}$ \\
\hline $120-82-1$ & $1,2,4$-Trichlorobenzene & 0.014 & $\mathrm{~J} B$ \\
\hline $87-61-6$ & $1,2,3$-Trichlorobenzene & 0.011 & $\mathrm{~J} B$ \\
\hline
\end{tabular}


Lab Name: TESTAMERICA BURLINGTON

Contract: $8 \mathrm{E}-00302$

Lab Code: STLV Case No.: YORK Mod. Ref No.: SDG No.: 29764

Matrix: (SOIL/SED/WATER) Water

Sample wt/vol: 25.0 $(\mathrm{g} / \mathrm{mL}) \mathrm{mL}$

Level: (TRACE or LOW/MED) TRACE

\% Moisture: not dec.

GC Column: DB-624 ID $: 0.20 \quad(\mathrm{~mm})$

Soil Extract Volume: (uL)

CONCENTRATION UNITS: (ug/L or ug/kg) ug/L
Lab Sample ID: 200-29764-3

Lab File ID: 15795_008.D

Date Received: 09/16/2015

Date Analyzed: 09/17/2015

Dilution Factor: 1.0

Soil Aliquot Volume: (uL) Purge Volume: 25.0

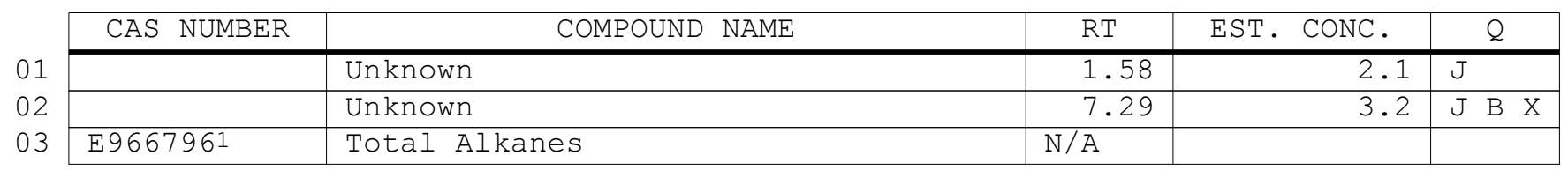

1EPA-designated Registry Number. 
Lab Name: TESTAMERICA BURLINGTON

Contract: $8 \mathrm{E}-00302$

Lab Code: STLV Case No.: YORK Mod. Ref No.:

SDG No.: 29764

Instrument ID: CHD.i

Calibration Date(s) : 09/15/2015 09/15/2015

Heated Purge: ( $\mathrm{Y} / \mathrm{N}) \mathrm{N}$

Calibration Time(s) : 1156

1334

Purge Volume: 25.0 $(\mathrm{mL})$

GC Column: DB-624

ID: $0.20 \quad$ (mm) Length: 25

$(\mathrm{m})$

\begin{tabular}{|c|c|c|c|c|c|c|c|}
\hline LAB FILE ID: & \multirow{2}{*}{\multicolumn{3}{|c|}{ 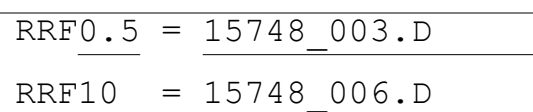 }} & \multicolumn{4}{|c|}{ RRF1.0 = 15748_004.D } \\
\hline RRF5.0 = 15748_005.D & & & & RRF2 & $=157$ & 3007.1 & \\
\hline COMPOUND & RRF0. 5 & RRE1.0 & RRF 5.0 & RRF10 & RRF20 & $\overline{\mathrm{RRF}}$ & $\because \mathrm{RSD}$ \\
\hline Dichlorodifluoromethane & 0.598 & 0.586 & 0.654 & 0.626 & 0.632 & 0.619 & 4.4 \\
\hline Chloromethane & 0.631 & 0.509 & 0.522 & 0.470 & 0.495 & 0.526 & 11.8 \\
\hline Vinyl chloride & 0.411 & 0.424 & 0.457 & 0.434 & 0.426 & 0.430 & 3.9 \\
\hline Bromomethane & 0.226 & 0.176 & 0.152 & 0.166 & 0.158 & 0.176 & 16.7 \\
\hline Chloroethane & 0.170 & 0.150 & 0.212 & 0.219 & 0.200 & 0.190 & 15.3 \\
\hline Trichlorofluoromethane & 0.543 & 0.544 & 0.615 & 0.586 & 0.591 & 0.576 & 5.5 \\
\hline 1,1-Dichloroethene & 0.267 & 0.254 & 0.288 & 0.275 & 0.267 & 0.270 & 4.5 \\
\hline $\begin{array}{l}1,1,2 \text {-Trichloro- } \\
1,2,2 \text {-trifluoroethane }\end{array}$ & 0.305 & 0.303 & 0.337 & 0.319 & 0.313 & 0.315 & 4.4 \\
\hline Acetone & 0.023 & 0.021 & 0.019 & 0.020 & 0.020 & 0.021 & 6.1 \\
\hline Carbon disulfide & 0.770 & 0.688 & 0.766 & 0.758 & 0.770 & 0.750 & 4.7 \\
\hline Methyl acetate & 0.058 & 0.064 & 0.064 & 0.065 & 0.064 & 0.063 & 4.7 \\
\hline Methylene Chloride & 0.274 & 0.256 & 0.270 & 0.269 & 0.265 & 0.267 & 2.5 \\
\hline trans-1,2-Dichloroethene & 0.317 & 0.304 & 0.328 & 0.335 & 0.337 & 0.324 & 4.2 \\
\hline Methyl tert-butyl ether & 0.284 & 0.307 & 0.337 & 0.347 & 0.358 & 0.327 & 9.3 \\
\hline 1,1-Dichloroethane & 0.556 & 0.571 & 0.620 & 0.628 & 0.626 & 0.600 & 5.7 \\
\hline cis-1,2-Dichloroethene & 0.287 & 0.302 & 0.323 & 0.326 & 0.329 & 0.313 & 5.8 \\
\hline 2-Butanone & 0.033 & 0.035 & 0.040 & 0.041 & 0.041 & 0.038 & 9.9 \\
\hline Bromochloromethane & 0.088 & 0.092 & 0.097 & 0.099 & 0.099 & 0.095 & 5.0 \\
\hline Chloroform & 0.515 & 0.517 & 0.546 & 0.558 & 0.554 & 0.538 & 3.8 \\
\hline $1,1,1$-Trichloroethane & 0.460 & 0.518 & 0.601 & 0.606 & 0.624 & 0.562 & 12.5 \\
\hline Cyclohexane & 0.724 & 0.753 & 0.870 & 0.840 & 0.852 & 0.808 & 8.1 \\
\hline Carbon tetrachloride & 0.404 & 0.429 & 0.528 & 0.519 & 0.558 & 0.488 & 13.7 \\
\hline Benzene & 1.686 & 1.677 & 1.637 & 1.593 & 1.618 & 1.642 & 2.4 \\
\hline 1,2-Dichloroethane & 0.234 & 0.253 & 0.270 & 0.268 & 0.265 & 0.258 & 5.8 \\
\hline Trichloroethene & 0.365 & 0.396 & 0.412 & 0.413 & 0.418 & 0.401 & 5.4 \\
\hline Methylcyclohexane & 0.573 & 0.590 & 0.696 & 0.656 & 0.669 & 0.637 & 8.3 \\
\hline
\end{tabular}

Report 1,4-Dioxane for Low-Medium VOA analysis only 
Lab Name: TESTAMERICA BURLINGTON

Contract: $8 \mathrm{E}-00302$

Lab Code: STLV Case No.: YORK Mod. Ref No.:

SDG No.: 29764

Instrument ID: CHD.i

Calibration Date(s) : 09/15/2015 09/15/2015

Heated Purge: ( $\mathrm{Y} / \mathrm{N}) \mathrm{N}$

Calibration Time(s) : 1156

1334

Purge Volume: 25.0 (mL)

GC Column: DB-624

ID: 0.20

$(\mathrm{mm})$

Length: 25

(m)

\begin{tabular}{|c|c|c|c|c|c|c|c|}
\hline \multirow{3}{*}{$\begin{array}{l}\text { LAB FILE ID: } \\
\text { RRF5.0 }=\underline{15748 \_005 . D} \\
\text { COMPOUND }\end{array}$} & \multirow{2}{*}{\multicolumn{3}{|c|}{$\begin{array}{l}\operatorname{RRF0.5}=15748 \_003 . \mathrm{D} \\
\operatorname{RRF10}=15748 \_006 . \mathrm{D}\end{array}$}} & \multirow{2}{*}{\multicolumn{4}{|c|}{$\begin{array}{l}\text { RRF1.0 }=\frac{15748 \_004 . D}{\underline{R R F 20}=15748 \_007 . \mathrm{D}} \\
\text { RRE }\end{array}$}} \\
\hline & & & & & & & \\
\hline & $\operatorname{RRF} 0.5$ & $\mathrm{RRF} 1.0$ & RRF 5.0 & RRF10 & RRF20 & $\overline{\mathrm{RRF}}$ & $\because \mathrm{RSD}$ \\
\hline 1,2-Dichloropropane & 0.320 & 0.321 & 0.347 & 0.340 & 0.347 & 0.335 & 4.0 \\
\hline Bromodichloromethane & 0.248 & 0.283 & 0.335 & 0.358 & 0.371 & 0.319 & 16.3 \\
\hline cis-1,3-Dichloropropene & 0.295 & 0.341 & 0.436 & 0.461 & 0.479 & 0.402 & 19.9 \\
\hline 4-Methyl-2-pentanone & 0.083 & 0.097 & 0.110 & 0.112 & 0.113 & 0.103 & 12.3 \\
\hline Toluene & 1.639 & 1.708 & 1.782 & 1.743 & 1.735 & 1.721 & 3.1 \\
\hline trans-1,3-Dichloropropene & 0.176 & 0.212 & 0.294 & 0.315 & 0.333 & 0.266 & 25.7 \\
\hline 1,1,2-Trichloroethane & 0.160 & 0.157 & 0.157 & 0.158 & 0.162 & 0.159 & 1.3 \\
\hline Tetrachloroethene & 0.327 & 0.329 & 0.354 & 0.355 & 0.373 & 0.347 & 5.6 \\
\hline 2-Hexanone & 0.053 & 0.063 & 0.076 & 0.077 & 0.079 & 0.070 & 16.3 \\
\hline Dibromochloromethane & 0.118 & 0.129 & 0.165 & 0.182 & 0.195 & 0.158 & 21.0 \\
\hline 1,2-Dibromoethane & 0.115 & 0.119 & 0.140 & 0.142 & 0.149 & 0.133 & 11.1 \\
\hline Chlorobenzene & 0.979 & 0.982 & 1.027 & 1.024 & 1.046 & 1.012 & 2.9 \\
\hline Ethylbenzene & 1.896 & 1.986 & 2.143 & 2.105 & 2.131 & 2.052 & 5.2 \\
\hline o-Xylene & 0.643 & 0.675 & 0.744 & 0.729 & 0.729 & 0.704 & 6.1 \\
\hline $\mathrm{m}, \mathrm{p}$-Xylene & 0.709 & 0.738 & 0.805 & 0.790 & 0.805 & 0.769 & 5.7 \\
\hline Styrene & 0.924 & 1.010 & 1.129 & 1.113 & 1.092 & 1.054 & 8.1 \\
\hline Bromoform & 0.095 & 0.098 & 0.129 & 0.144 & 0.160 & 0.125 & 22.7 \\
\hline Isopropylbenzene & 1.786 & 1.947 & 2.178 & 2.133 & 2.139 & 2.037 & 8.2 \\
\hline $1,1,2,2$-Tetrachloroethane & 0.145 & 0.147 & 0.162 & 0.163 & 0.166 & 0.156 & 6.3 \\
\hline 1,3-Dichlorobenzene & 1.562 & 1.601 & 1.730 & 1.713 & 1.772 & 1.675 & 5.3 \\
\hline 1,4-Dichlorobenzene & 1.652 & 1.635 & 1.709 & 1.665 & 1.692 & 1.671 & 1.8 \\
\hline 1,2-Dichlorobenzene & 1.247 & 1.318 & 1.378 & 1.365 & 1.399 & 1.341 & 4.5 \\
\hline 1,2-Dibromo-3-Chloropropane & 0.029 & 0.028 & 0.035 & 0.039 & 0.041 & 0.034 & 16.7 \\
\hline $1,2,4$-Trichlorobenzene & 0.779 & 0.767 & 0.841 & 0.872 & 0.928 & 0.837 & 7.9 \\
\hline 1,2,3-Trichlorobenzene & 0.570 & 0.539 & 0.601 & 0.620 & 0.655 & 0.597 & 7.5 \\
\hline
\end{tabular}


Lab Name: TESTAMERICA BURLINGTON

Contract: $8 \mathrm{E}-00302$

Lab Code: STLV Case No.: YORK Mod. Ref No.: SDG No.: 29764

Instrument ID: CHD.i

Heated Purge: (Y/N) N

Purge Volume: 25.0

GC Column: DB-624
ID: 0.20
Calibration Date(s) : 09/15/2015

Calibration Time(s): 1156 $(\mathrm{mL})$

(mm) Length: 25
$09 / 15 / 2015$ 1334

\begin{tabular}{|c|c|c|c|c|c|c|c|}
\hline LAB FILE ID: & \multicolumn{3}{|c|}{$\begin{array}{l}\operatorname{RRF}_{\underline{0.5}}=\frac{15748 \_003 . \mathrm{D}}{\operatorname{RRF10}}=15748 \_006 . \mathrm{D}\end{array}$} & \multicolumn{4}{|c|}{$\begin{array}{l}\text { RRF1.0 }=\frac{15748 \_004 . D}{\text { RRF20 }}=15748 \_007 . \mathrm{D}\end{array}$} \\
\hline Vinyl Chloride-d3 & 0.389 & 0.381 & 0.408 & 0.387 & 0.384 & 0.390 & 2.7 \\
\hline Chloroethane-d5 & 0.257 & 0.244 & 0.255 & 0.240 & 0.226 & 0.244 & 5.2 \\
\hline Chloroform-d & 0.526 & 0.541 & 0.572 & 0.584 & 0.583 & 0.561 & 4.7 \\
\hline 1,2-Dichloroethane-d4 & 0.200 & 0.205 & 0.214 & 0.217 & 0.215 & 0.210 & 3.4 \\
\hline Benzene-d6 & 1.411 & 1.444 & 1.525 & 1.481 & 1.514 & 1.475 & 3.2 \\
\hline 1,2-Dichloropropane-d6 & 0.366 & 0.373 & 0.396 & 0.451 & 0.411 & 0.399 & 8.5 \\
\hline Toluene-d8 & 1.293 & 1.395 & 1.466 & 1.450 & 1.455 & 1.412 & 5.1 \\
\hline 1,2-Dichlorobenzene-d4 & 0.861 & 0.829 & 0.838 & 0.843 & 0.854 & 0.845 & 1.5 \\
\hline
\end{tabular}

Report 1,4-Dioxane-d8 for Low-Medium VOA analysis only 
Lab Name: TESTAMERICA BURLINGTON

Contract: $8 \mathrm{E}-00302$

Lab Code: STLV

Mod. Ref No.:

SDG No.: 29764

Instrument ID: CHD.i

Calibration Date: 09/17/2015 Time: 1236

Lab File Id: 15795_003.D

EPA Sample No. (VSTD\#\#\#\#) : VSTD005DO

Init. Calib. Date (s):09/15/2015

$09 / 15 / 2015$

Heated Purge: (Y/N) N GC Column: DB-624

Init. Calib. Time(s) : 1156 1334

Purge Volume: 25.0

ID: $0.20(\mathrm{~mm})$ Length: 25

(m)

\begin{tabular}{|c|c|c|c|c|c|}
\hline COMPOUND & $\overline{\mathrm{RRF}}$ & RRF5.0 & $\begin{array}{l}\text { MIN } \\
\text { RRF }\end{array}$ & $\frac{\circ}{D}$ & $\mathrm{MAX} \div \mathrm{D}$ \\
\hline Dichlorodifluoromethane & 0.619 & 0.667 & 0.010 & 7.8 & 40.0 \\
\hline Chloromethane & 0.526 & 0.503 & 0.010 & -4.4 & 40.0 \\
\hline Vinyl chloride & 0.430 & 0.448 & 0.100 & 4.1 & 30.0 \\
\hline Bromomethane & 0.176 & 0.134 & 0.100 & -23.4 & 30.0 \\
\hline Chloroethane & 0.190 & 0.218 & 0.010 & 14.5 & 40.0 \\
\hline Trichlorofluoromethane & 0.576 & 0.643 & 0.010 & 11.7 & 40.0 \\
\hline 1,1-Dichloroethene & 0.270 & 0.282 & 0.100 & 4.2 & 30.0 \\
\hline 1,1,2-Trichloro-1,2,2-trifluoroethane & 0.315 & 0.336 & 0.010 & 6.5 & 40.0 \\
\hline Acetone & 0.021 & 0.022 & 0.010 & 9.4 & 40.0 \\
\hline Carbon disulfide & 0.750 & 0.740 & 0.010 & -1.4 & 40.0 \\
\hline Methyl acetate & 0.063 & 0.068 & 0.010 & 7.7 & 40.0 \\
\hline Methylene Chloride & 0.267 & 0.281 & 0.010 & 5.5 & 40.0 \\
\hline trans-1,2-Dichloroethene & 0.324 & 0.341 & 0.010 & 5.2 & 40.0 \\
\hline Methyl tert-butyl ether & 0.327 & 0.366 & 0.010 & 12.0 & 40.0 \\
\hline 1,1-Dichloroethane & 0.600 & 0.641 & 0.200 & 6.8 & 30.0 \\
\hline cis-1,2-Dichloroethene & 0.313 & 0.335 & 0.010 & 6.8 & 40.0 \\
\hline 2-Butanone & 0.038 & 0.044 & 0.010 & 15.3 & 40.0 \\
\hline Bromochloromethane & 0.095 & 0.100 & 0.050 & 5.1 & 30.0 \\
\hline Chloroform & 0.538 & 0.581 & 0.200 & 8.0 & 30.0 \\
\hline 1,1,1-Trichloroethane & 0.562 & 0.609 & 0.100 & 8.5 & 30.0 \\
\hline Cyclohexane & 0.808 & 0.866 & 0.010 & 7.2 & 40.0 \\
\hline Carbon tetrachloride & 0.488 & 0.529 & 0.100 & 8.5 & 30.0 \\
\hline Benzene & 1.642 & 1.653 & 0.400 & 0.7 & 30.0 \\
\hline 1,2-Dichloroethane & 0.258 & 0.286 & 0.100 & 10.8 & 30.0 \\
\hline Trichloroethene & 0.401 & 0.416 & 0.300 & 3.7 & 30.0 \\
\hline Methylcyclohexane & 0.637 & 0.692 & 0.010 & 8.6 & 40.0 \\
\hline
\end{tabular}

Report 1,4-Dioxane for Low/Medium VOA analysis only 
Lab Name: TESTAMERICA BURLINGTON

Contract: 8E-00302

Lab Code: STLV

Mod. Ref No.:

SDG No.: 29764

Instrument ID: CHD.i

Calibration Date: 09/17/2015 Time: 1236

Lab File Id: 15795_003.D

EPA Sample No. (VSTD\#\#\#\#): VSTD005DO

Init. Calib. Date (s):09/15/2015

$09 / 15 / 2015$

Heated Purge: (Y/N) N GC Column: DB-624

Init. Calib. Time(s) : 1156 1334

Purge Volume: 25.0

ID: $0.20(\mathrm{~mm})$ Length: 25

(m)

\begin{tabular}{|c|c|c|c|c|c|}
\hline COMPOUND & $\overline{\mathrm{RRF}}$ & RRF5.0 & $\begin{array}{l}\text { MIN } \\
\text { RRF }\end{array}$ & $\because \mathrm{D}$ & $\operatorname{MAX} \cong \mathrm{D}$ \\
\hline 1,2-Dichloropropane & 0.335 & 0.356 & 0.010 & 6.3 & 40.0 \\
\hline Bromodichloromethane & 0.319 & 0.354 & 0.200 & 11.0 & 30.0 \\
\hline cis-1,3-Dichloropropene & 0.402 & 0.453 & 0.200 & 12.5 & 30.0 \\
\hline 4-Methyl-2-pentanone & 0.103 & 0.122 & 0.010 & 18.3 & 40.0 \\
\hline Toluene & 1.721 & 1.805 & 0.400 & 4.9 & 30.0 \\
\hline trans-1,3-Dichloropropene & 0.266 & 0.305 & 0.100 & 14.7 & 30.0 \\
\hline $1,1,2$-Trichloroethane & 0.159 & 0.165 & 0.100 & 4.0 & 30.0 \\
\hline Tetrachloroethene & 0.347 & 0.357 & 0.100 & 2.7 & 30.0 \\
\hline 2-Hexanone & 0.070 & 0.085 & 0.010 & 21.6 & 40.0 \\
\hline Dibromochloromethane & 0.158 & 0.177 & 0.100 & 11.9 & 30.0 \\
\hline 1,2-Dibromoethane & 0.133 & 0.146 & 0.010 & 9.7 & 40.0 \\
\hline Chlorobenzene & 1.012 & 1.045 & 0.500 & 3.3 & 30.0 \\
\hline Ethylbenzene & 2.052 & 2.158 & 0.100 & 5.1 & 30.0 \\
\hline o-Xylene & 0.704 & 0.748 & 0.300 & 6.2 & 30.0 \\
\hline $\mathrm{m}, \mathrm{p}$-Xylene & 0.769 & 0.798 & 0.300 & 3.8 & 30.0 \\
\hline Styrene & 1.054 & 1.149 & 0.300 & 9.1 & 30.0 \\
\hline Bromoform & 0.125 & 0.127 & 0.050 & 1.6 & 30.0 \\
\hline Isopropylbenzene & 2.037 & 2.163 & 0.010 & 6.2 & 40.0 \\
\hline $1,1,2,2$-Tetrachloroethane & 0.156 & 0.169 & 0.100 & 7.9 & 30.0 \\
\hline 1,3-Dichlorobenzene & 1.675 & 1.710 & 0.400 & 2.1 & 30.0 \\
\hline 1,4-Dichlorobenzene & 1.671 & 1.671 & 0.400 & 0.0 & 30.0 \\
\hline 1,2-Dichlorobenzene & 1.341 & 1.391 & 0.400 & 3.7 & 30.0 \\
\hline 1,2-Dibromo-3-Chloropropane & 0.034 & 0.037 & 0.010 & 8.5 & 40.0 \\
\hline 1,2,4-Trichlorobenzene & 0.837 & 0.861 & 0.200 & 2.8 & 30.0 \\
\hline $1,2,3$-Trichlorobenzene & 0.597 & 0.640 & 0.200 & 7.2 & 30.0 \\
\hline
\end{tabular}


Lab Name: TESTAMERICA BURLINGTON

Contract: $8 \mathrm{E}-00302$

Lab Code: STLV Case No.: YORK Mod. Ref No.

SDG No.: 29764

Instrument ID: CHD.i

Calibration Date: 09/17/2015 Time: 1236

Lab File Id: 15795_003.D Init. Calib. Date(s):09/15/2015 09/15/2015

EPA Sample No. (VSTD\#\#\#\#) : VSTD005DO

Init. Calib. Time(s) : 1156 1334

Heated Purge: (Y/N) N GC Column: DB-624 ID: $0.20(\mathrm{~mm})$ Length: 25

(m)

Purge Volume: 25.0 $(\mathrm{mL})$

\begin{tabular}{|c|c|c|c|c|c|}
\hline COMPOUND & $\overline{\mathrm{RRF}}$ & RRF5.0 & $\begin{array}{l}\text { MIN } \\
\text { RRF }\end{array}$ & $\because \mathrm{D}$ & $\operatorname{MAX} \cong \mathrm{D}$ \\
\hline Vinyl Chloride-d3 & 0.390 & 0.396 & 0.010 & 1.7 & 30.0 \\
\hline Chloroethane-d5 & 0.244 & 0.252 & 0.010 & 2.9 & 40.0 \\
\hline 1,1-Dichloroethene-d2 & 0.613 & 0.659 & 0.010 & 7.6 & 30.0 \\
\hline 2-Butanone-d5 & 0.035 & 0.041 & 0.010 & 18.0 & 40.0 \\
\hline Chloroform-d & 0.561 & 0.607 & 0.010 & 8.1 & 30.0 \\
\hline 1,2-Dichloroethane-d4 & 0.210 & 0.230 & 0.010 & 9.6 & 30.0 \\
\hline Benzene-d6 & 1.475 & 1.513 & 0.010 & 2.6 & 30.0 \\
\hline 1,2-Dichloropropane-d6 & 0.399 & 0.416 & 0.010 & 4.2 & 40.0 \\
\hline Toluene-d8 & 1.412 & 1.485 & 0.010 & 5.2 & 30.0 \\
\hline trans-1,3-Dichloropropene-d4 & 0.231 & 0.259 & 0.010 & 12.1 & 30.0 \\
\hline 2-Hexanone-d5 & 0.029 & 0.035 & 0.010 & 20.1 & 40.0 \\
\hline $1,1,2,2$-Tetrachloroethane-d2 & 0.153 & 0.169 & 0.010 & 10.9 & 30.0 \\
\hline 1,2-Dichlorobenzene-d4 & 0.845 & 0.849 & 0.010 & 0.4 & 30.0 \\
\hline
\end{tabular}

Report 1,4-Dioxane-d8 for Low/Medium VOA analysis only 
Lab Name: TESTAMERICA BURLINGTON

Contract: $8 \mathrm{E}-00302$

Lab Code: STLV

Mod. Ref No.:

SDG No.: 29764

Instrument ID: CHD.i

Calibration Date: 09/17/2015 Time: 1914

Lab File Id: 15795_019.D

Init. Calib. Date(s):09/15/2015 09/15/2015

EPA Sample No. (VSTD\#\#\#\#) : VSTD0050D

Init. Calib. Time(s) : 1156 1334

Heated Purge: (Y/N) N GC Column: DB-624

ID: $0.20(\mathrm{~mm})$ Length: 25

(m)

Purge Volume: 25.0 $(\mathrm{mL})$

\begin{tabular}{|c|c|c|c|c|c|}
\hline COMPOUND & $\overline{\mathrm{RRF}}$ & RRF5.0 & $\begin{array}{l}\text { MIN } \\
\text { RRF }\end{array}$ & $\because \mathrm{D}$ & $\mathrm{MAX} \div \mathrm{D}$ \\
\hline Dichlorodifluoromethane & 0.619 & 0.651 & 0.010 & 5.0 & 50.0 \\
\hline Chloromethane & 0.526 & 0.519 & 0.010 & $-1 \cdot 3$ & 50.0 \\
\hline Vinyl chloride & 0.430 & 0.410 & 0.010 & -4.6 & 50.0 \\
\hline Bromomethane & 0.176 & 0.096 & 0.010 & -45.6 & 50.0 \\
\hline Chloroethane & 0.190 & 0.215 & 0.010 & 13.0 & 50.0 \\
\hline Trichlorofluoromethane & 0.576 & 0.616 & 0.010 & 7.0 & 50.0 \\
\hline 1,1-Dichloroethene & 0.270 & 0.263 & 0.010 & -2.8 & 50.0 \\
\hline 1,1,2-Trichloro-1,2,2-trifluoroethane & 0.315 & 0.313 & 0.010 & -0.7 & 50.0 \\
\hline Acetone & 0.021 & 0.021 & 0.010 & 3.5 & 50.0 \\
\hline Carbon disulfide & 0.750 & 0.657 & 0.010 & -12.5 & 50.0 \\
\hline Methyl acetate & 0.063 & 0.062 & 0.010 & -0.9 & 50.0 \\
\hline Methylene Chloride & 0.267 & 0.267 & 0.010 & 0.1 & 50.0 \\
\hline trans-1,2-Dichloroethene & 0.324 & 0.325 & 0.010 & 0.1 & 50.0 \\
\hline Methyl tert-butyl ether & 0.327 & 0.330 & 0.010 & 1.0 & 50.0 \\
\hline 1,1-Dichloroethane & 0.600 & 0.610 & 0.010 & 1.6 & 50.0 \\
\hline cis-1,2-Dichloroethene & 0.313 & 0.323 & 0.010 & 2.9 & 50.0 \\
\hline 2-Butanone & 0.038 & 0.043 & 0.010 & 11.9 & 50.0 \\
\hline Bromochloromethane & 0.095 & 0.095 & 0.010 & -0.5 & 50.0 \\
\hline Chloroform & 0.538 & 0.551 & 0.010 & 2.4 & 50.0 \\
\hline 1,1,1-Trichloroethane & 0.562 & 0.568 & 0.010 & 1.1 & 50.0 \\
\hline Cyclohexane & 0.808 & 0.850 & 0.010 & 5.3 & 50.0 \\
\hline Carbon tetrachloride & 0.488 & 0.506 & 0.010 & 3.8 & 50.0 \\
\hline Benzene & 1.642 & 1.651 & 0.010 & 0.5 & 50.0 \\
\hline 1,2-Dichloroethane & 0.258 & 0.277 & 0.010 & 7.5 & 50.0 \\
\hline Trichloroethene & 0.401 & 0.403 & 0.010 & 0.7 & 50.0 \\
\hline Methylcyclohexane & 0.637 & 0.690 & 0.010 & 8.4 & 50.0 \\
\hline
\end{tabular}

Report 1,4-Dioxane for Low/Medium VOA analysis only 
Lab Name: TESTAMERICA BURLINGTON

Contract: 8E-00302

Lab Code: STLV

Mod. Ref No.:

SDG No.: 29764

Instrument ID: CHD.i

Calibration Date: 09/17/2015 Time: 1914

Lab File Id: 15795_019.D

Init. Calib. Date(s):09/15/2015 09/15/2015

EPA Sample No. (VSTD\#\#\#\#) : VSTD0050D

Init. Calib. Time(s) : 1156 1334

Heated Purge: (Y/N) N GC Column: DB-624

ID: $0.20(\mathrm{~mm})$ Length: 25

(m)

Purge Volume: 25.0 $(\mathrm{mL})$

\begin{tabular}{|c|c|c|c|c|c|}
\hline COMPOUND & $\overline{\mathrm{RRF}}$ & RRF5.0 & $\begin{array}{l}\text { MIN } \\
\text { RRF }\end{array}$ & $\because \mathrm{D}$ & $\operatorname{MAX} \cong \mathrm{D}$ \\
\hline 1,2-Dichloropropane & 0.335 & 0.337 & 0.010 & 0.6 & 50.0 \\
\hline Bromodichloromethane & 0.319 & 0.324 & 0.010 & 1.6 & 50.0 \\
\hline cis-1,3-Dichloropropene & 0.402 & 0.423 & 0.010 & 5.0 & 50.0 \\
\hline 4-Methyl-2-pentanone & 0.103 & 0.117 & 0.010 & 13.4 & 50.0 \\
\hline Toluene & 1.721 & 1.760 & 0.010 & 2.2 & 50.0 \\
\hline trans-1,3-Dichloropropene & 0.266 & 0.285 & 0.010 & 7.0 & 50.0 \\
\hline $1,1,2$-Trichloroethane & 0.159 & 0.163 & 0.010 & 2.5 & 50.0 \\
\hline Tetrachloroethene & 0.347 & 0.351 & 0.010 & 0.9 & 50.0 \\
\hline 2-Hexanone & 0.070 & 0.083 & 0.010 & 19.2 & 50.0 \\
\hline Dibromochloromethane & 0.158 & 0.157 & 0.010 & -0.7 & 50.0 \\
\hline 1,2-Dibromoethane & 0.133 & 0.139 & 0.010 & 4.6 & 50.0 \\
\hline Chlorobenzene & 1.012 & 1.026 & 0.010 & 1.4 & 50.0 \\
\hline Ethylbenzene & 2.052 & 2.111 & 0.010 & 2.9 & 50.0 \\
\hline o-Xylene & 0.704 & 0.733 & 0.010 & 4.1 & 50.0 \\
\hline $\mathrm{m}, \mathrm{p}$-Xylene & 0.769 & 0.786 & 0.010 & 2.2 & 50.0 \\
\hline Styrene & 1.054 & 1.130 & 0.010 & 7.2 & 50.0 \\
\hline Bromoform & 0.125 & 0.109 & 0.010 & $-13 \cdot 0$ & 50.0 \\
\hline Isopropylbenzene & 2.037 & 2.143 & 0.010 & 5.2 & 50.0 \\
\hline $1,1,2,2$-Tetrachloroethane & 0.156 & 0.165 & 0.010 & 5.4 & 50.0 \\
\hline 1,3-Dichlorobenzene & 1.675 & 1.668 & 0.010 & -0.4 & 50.0 \\
\hline 1,4-Dichlorobenzene & 1.671 & 1.662 & 0.010 & -0.5 & 50.0 \\
\hline 1,2-Dichlorobenzene & 1.341 & 1.379 & 0.010 & 2.8 & 50.0 \\
\hline 1,2-Dibromo-3-Chloropropane & 0.034 & 0.034 & 0.010 & -2.5 & 50.0 \\
\hline 1,2,4-Trichlorobenzene & 0.837 & 0.840 & 0.010 & 0.4 & 50.0 \\
\hline $1,2,3$-Trichlorobenzene & 0.597 & 0.613 & 0.010 & 2.7 & 50.0 \\
\hline
\end{tabular}


Lab Name: TESTAMERICA BURLINGTON

Contract: $8 \mathrm{E}-00302$

Lab Code: STLV Case No.: YORK Mod. Ref No.

SDG No.: 29764

Instrument ID: CHD.i

Calibration Date: 09/17/2015 Time: 1914

Lab File Id: 15795_019.D Init. Calib. Date(s):09/15/2015 09/15/2015

EPA Sample No. (VSTD\#\#\#\#) : VSTD0050D

Init. Calib. Time(s) : 1156 1334

Heated Purge: (Y/N) N GC Column: DB-624 ID: $0.20(\mathrm{~mm})$ Length: 25

(m)

Purge Volume: 25.0 $(\mathrm{mL})$

\begin{tabular}{|c|c|c|c|c|c|}
\hline COMPOUND & $\overline{\mathrm{RRF}}$ & RRF5.0 & $\begin{array}{l}\text { MIN } \\
\text { RRF }\end{array}$ & $\because \mathrm{D}$ & $\operatorname{MAX} \div \mathrm{D}$ \\
\hline Vinyl Chloride-d3 & 0.390 & 0.380 & 0.010 & -2.5 & 50.0 \\
\hline Chloroethane-d5 & 0.244 & 0.233 & 0.010 & -4.7 & 50.0 \\
\hline 1,1-Dichloroethene-d2 & 0.613 & 0.618 & 0.010 & 0.8 & 50.0 \\
\hline 2-Butanone-d5 & 0.035 & 0.039 & 0.010 & 14.3 & 50.0 \\
\hline Chloroform-d & 0.561 & 0.563 & 0.010 & 0.4 & 50.0 \\
\hline 1,2-Dichloroethane-d4 & 0.210 & 0.225 & 0.010 & 6.9 & 50.0 \\
\hline Benzene-d6 & 1.475 & 1.492 & 0.010 & 1.2 & 50.0 \\
\hline 1,2-Dichloropropane-d6 & 0.399 & 0.396 & 0.010 & -0.8 & 50.0 \\
\hline Toluene-d8 & 1.412 & 1.434 & 0.010 & 1.6 & 50.0 \\
\hline trans-1,3-Dichloropropene-d4 & 0.231 & 0.232 & 0.010 & 0.4 & 50.0 \\
\hline $2-$ Hexanone-d5 & 0.029 & 0.035 & 0.010 & 21.9 & 50.0 \\
\hline $1,1,2,2$-Tetrachloroethane-d2 & 0.153 & 0.163 & 0.010 & 6.8 & 50.0 \\
\hline 1,2-Dichlorobenzene-d4 & 0.845 & 0.843 & 0.010 & -0.2 & 50.0 \\
\hline
\end{tabular}

Report 1,4-Dioxane-d8 for Low/Medium VOA analysis only 
Lab Name: TESTAMERICA BURLINGTON

Contract: $8 \mathrm{E}-00302$

Lab Code: STLV

Case No.: YORK Mod. Ref No.:

SDG No.: 29764

Matrix: (SOIL/SED/WATER) Water

Sample wt/vol: 25.0 $(\mathrm{g} / \mathrm{mL}) \mathrm{mL}$

Level: (TRACE/LOW/MED) TRACE

\% Moisture: not dec.

GC Column: DB-624 ID: $0.20 \quad(\mathrm{~mm})$

Soil Extract Volume: (uL) $(\mathrm{mL})$

Purge Volume: 25.0
Lab Sample ID: MB 200-94084/5

Lab File ID: 15795 005.D

Date Received:

Date Analyzed: 09/17/2015

Dilution Factor: 1.0

Soil Aliquot Volume: (uL)

\begin{tabular}{|c|c|c|c|}
\hline CAS NO. & COMPOUND & $\begin{array}{l}\text { CONCENTRATION UNITS: } \\
(\mathrm{ug} / \mathrm{L} \text { or } \mathrm{ug} / \mathrm{kg}) \mathrm{ug} / \mathrm{L}\end{array}$ & q \\
\hline $75-71-8$ & Dichlorodifluoromethane & 1.0 & $\mathrm{U}$ \\
\hline $74-87-3$ & Chloromethane & 1.0 & $\mathrm{U}$ \\
\hline $75-01-4$ & Vinyl chloride & 1.0 & $\mathrm{U}$ \\
\hline $74-83-9$ & Bromomethane & 0.10 & $\mathrm{~J}$ \\
\hline $75-00-3$ & Chloroethane & 1.0 & $\mathrm{U}$ \\
\hline $75-69-4$ & Trichlorofluoromethane & 1.0 & $\mathrm{U}$ \\
\hline $75-35-4$ & 1,1-Dichloroethene & 1.0 & $\mathrm{U}$ \\
\hline $76-13-1$ & $1,1,2$-Trichloro-1,2,2-trifluoroethane & 1.0 & $\mathrm{U}$ \\
\hline $67-64-1$ & Acetone & 5.0 & $\mathrm{U}$ \\
\hline $75-15-0$ & Carbon disulfide & 0.046 & $\mathrm{~J}$ \\
\hline $79-20-9$ & Methyl acetate & 1.0 & $\mathrm{U}$ \\
\hline $75-09-2$ & Methylene Chloride & 1.0 & $\mathrm{U}$ \\
\hline $156-60-5$ & trans-1,2-Dichloroethene & 1.0 & $\mathrm{U}$ \\
\hline $1634-04-4$ & Methyl tert-butyl ether & 1.0 & $\mathrm{U}$ \\
\hline $75-34-3$ & 1,1-Dichloroethane & 1.0 & $\mathrm{U}$ \\
\hline $156-59-2$ & cis-1,2-Dichloroethene & 1.0 & $\mathrm{U}$ \\
\hline $78-93-3$ & 2-Butanone & 5.0 & $\mathrm{U}$ \\
\hline $74-97-5$ & Bromochloromethane & 1.0 & $\mathrm{U}$ \\
\hline $67-66-3$ & Chloroform & 1.0 & $\mathrm{U}$ \\
\hline $71-55-6$ & $1,1,1$-Trichloroethane & 1.0 & $\mathrm{U}$ \\
\hline $110-82-7$ & Cyclohexane & 1.0 & $\mathrm{U}$ \\
\hline $56-23-5$ & Carbon tetrachloride & 1.0 & $\mathrm{U}$ \\
\hline $71-43-2$ & Benzene & 1.0 & $\mathrm{U}$ \\
\hline $107-06-2$ & 1,2-Dichloroethane & 1.0 & $\mathrm{U}$ \\
\hline
\end{tabular}

Report 1,4-Dioxane for Low-Medium VOA analysis only 
Lab Name: TESTAMERICA BURLINGTON

Lab Code: STLV

Case No.: YORK

Mod. Ref No.:

Contract: $8 \mathrm{E}-00302$

Matrix: (SOIL/SED/WATER) Water

Sample wt/vol: 25.0 $(\mathrm{g} / \mathrm{mL}) \mathrm{mL}$

Level: (TRACE/LOW/MED) TRACE

\% Moisture: not dec.

GC Column: DB-624 ID $: \underline{0.20 \quad(\mathrm{~mm})}$

Soil Extract Volume: (uL) $(\mathrm{mL})$

Purge Volume: 25.0
SDG No.: 29764

Lab Sample ID: MB 200-94084/5

Lab File ID: 15795_005.D

Date Received:

Date Analyzed: 09/17/2015

Dilution Factor: 1.0

Soil Aliquot Volume: (uL)

\begin{tabular}{|c|c|c|c|}
\hline CAS NO. & COMPOUND & $\begin{array}{l}\text { CONCENTRATION UNITS: } \\
(\mathrm{ug} / \mathrm{L} \text { or } \mathrm{ug} / \mathrm{kg}) \mathrm{ug} / \mathrm{L}\end{array}$ & Q \\
\hline $79-01-6$ & Trichloroethene & $\begin{array}{r}1.0 \\
\end{array}$ & $\mathrm{U}$ \\
\hline $108-87-2$ & Methylcyclohexane & 1.0 & $\mathrm{U}$ \\
\hline $78-87-5$ & 1,2-Dichloropropane & 1.0 & $\mathrm{U}$ \\
\hline $75-27-4$ & Bromodichloromethane & 1.0 & $\mathrm{U}$ \\
\hline $10061-01-5$ & cis-1,3-Dichloropropene & 1.0 & $\mathrm{U}$ \\
\hline $108-10-1$ & 4-Methyl-2-pentanone & 1.0 & $\mathrm{U}$ \\
\hline $108-88-3$ & Toluene & 0.012 & $\mathrm{~J}$ \\
\hline $10061-02-6$ & trans-1,3-Dichloropropene & 1.0 & $\mathrm{U}$ \\
\hline $79-00-5$ & $1,1,2-T r i c h l o r o e t h a n e$ & 1.0 & $\mathrm{U}$ \\
\hline $127-18-4$ & Tetrachloroethene & 1.0 & $\mathrm{U}$ \\
\hline $591-78-6$ & 2-Hexanone & 1.0 & $\mathrm{U}$ \\
\hline $124-48-1$ & Dibromochloromethane & 1.0 & $\mathrm{U}$ \\
\hline $106-93-4$ & 1,2-Dibromoethane & 1.0 & $\mathrm{U}$ \\
\hline $108-90-7$ & Chlorobenzene & 1.0 & $\mathrm{U}$ \\
\hline $100-41-4$ & Ethylbenzene & 1.0 & $\mathrm{U}$ \\
\hline $95-47-6$ & o-Xylene & 1.0 & $\mathrm{U}$ \\
\hline $179601-23-1$ & m,p-xylene & 0.0084 & $\mathrm{~J}$ \\
\hline $100-42-5$ & Styrene & 1.0 & $\mathrm{U}$ \\
\hline $75-25-2$ & Bromoform & 1.0 & $\mathrm{U}$ \\
\hline $98-82-8$ & Isopropylbenzene & 1.0 & $\mathrm{U}$ \\
\hline $79-34-5$ & $1,1,2,2$-Tetrachloroethane & 1.0 & $\mathrm{U}$ \\
\hline $541-73-1$ & 1,3 -Dichlorobenzene & 0.0080 & $\mathrm{~J}$ \\
\hline $106-46-7$ & 1,4-Dichlorobenzene & 1.0 & $\mathrm{U}$ \\
\hline $95-50-1$ & 1,2-Dichlorobenzene & 1.0 & $\mathrm{U}$ \\
\hline $96-12-8$ & 1,2-Dibromo-3-Chloropropane & 1.0 & $\mathrm{U}$ \\
\hline $120-82-1$ & $1,2,4$-Trichlorobenzene & 0.028 & $\mathrm{~J}$ \\
\hline $87-61-6$ & $1,2,3$-Trichlorobenzene & 0.031 & $\mathrm{~J}$ \\
\hline
\end{tabular}


Lab Name: TESTAMERICA BURLINGTON

Contract: 8E-00302

Lab Code: STLV Case No.: YORK Mod. Ref No.: SDG No.: 29764

Matrix: (SOIL/SED/WATER) Water

Sample wt/vol: 25.0 $(\mathrm{g} / \mathrm{mL}) \mathrm{mL}$

Level: (TRACE or LOW/MED) TRACE

\% Moisture: not dec.

GC Column: DB-624 ID $: 0.20 \quad(\mathrm{~mm})$

Soil Extract Volume: (uL)

CONCENTRATION UNITS: (ug/L or ug/kg) ug/L
Lab Sample ID: MB 200-94084/5

Lab File ID: 15795 005.D

Date Received:

Date Analyzed: 09/17/2015

Dilution Factor: 1.0

Soil Aliquot Volume: (uL) Purge Volume: 25.0 $(\mathrm{mL})$

01

02

\begin{tabular}{|l|l|c|c|c|}
\hline CAS NUMBER & \multicolumn{1}{|c|}{ COMPOUND NAME } & RT & EST. CONC. & Q \\
\hline & Unknown & 7.29 & 3.0 & $\mathrm{~J} X$ \\
\hline E9667961 & Total Alkanes & N/A & & \\
\hline
\end{tabular}

1EPA-designated Registry Number. 
Lab Name: TESTAMERICA BURLINGTON

Contract: $8 \mathrm{E}-00302$

Lab Code: STLV

Case No.: YORK Mod. Ref No.:

SDG No.: 29764

Matrix: (SOIL/SED/WATER) Water

Sample wt/vol: 25.0 $(\mathrm{g} / \mathrm{mL}) \mathrm{mL}$

Level: (TRACE/LOW/MED) TRACE

\% Moisture: not dec.

GC Column: DB-624 ID: $0.20 \quad(\mathrm{~mm})$

Soil Extract Volume: (uL) $(\mathrm{mL})$

Purge Volume: 25.0
Lab Sample ID: 200-29764-5

Lab File ID: 15795_010.D

Date Received:

Date Analyzed: 09/17/2015

Dilution Factor: 1.0

Soil Aliquot Volume:

(uL)

\begin{tabular}{|c|c|c|c|}
\hline CAS NO. & COMPOUND & $\begin{array}{l}\text { CONCENTRATION UNITS: } \\
(\mathrm{ug} / \mathrm{L} \text { or } \mathrm{ug} / \mathrm{kg}) \mathrm{ug} / \mathrm{L}\end{array}$ & Q \\
\hline $75-71-8$ & Dichlorodifluoromethane & 1.0 & $\mathrm{U}$ \\
\hline $74-87-3$ & Chloromethane & 1.0 & $\mathrm{U}$ \\
\hline $75-01-4$ & Vinyl chloride & 1.0 & $\mathrm{U}$ \\
\hline $74-83-9$ & Bromomethane & 1.0 & $\mathrm{U}$ \\
\hline $75-00-3$ & Chloroethane & 1.0 & $\mathrm{U}$ \\
\hline $75-69-4$ & Trichlorofluoromethane & 1.0 & $\mathrm{U}$ \\
\hline $75-35-4$ & 1,1-Dichloroethene & 1.0 & $\mathrm{U}$ \\
\hline $76-13-1$ & $1,1,2$-Trichloro-1,2,2-trifluoroethane & 1.0 & $\mathrm{U}$ \\
\hline $67-64-1$ & Acetone & 5.0 & $\mathrm{U}$ \\
\hline $75-15-0$ & Carbon disulfide & 0.031 & $\mathrm{~J} B$ \\
\hline $79-20-9$ & Methyl acetate & 1.0 & $\mathrm{U}$ \\
\hline $75-09-2$ & Methylene Chloride & 1.0 & $\mathrm{U}$ \\
\hline $156-60-5$ & trans-1,2-Dichloroethene & 1.0 & $\mathrm{U}$ \\
\hline $1634-04-4$ & Methyl tert-butyl ether & 1.0 & $\mathrm{U}$ \\
\hline $75-34-3$ & 1,1-Dichloroethane & 1.0 & $\mathrm{U}$ \\
\hline $156-59-2$ & cis-1,2-Dichloroethene & 1.0 & $\mathrm{U}$ \\
\hline $78-93-3$ & 2-Butanone & 5.0 & $\mathrm{U}$ \\
\hline $74-97-5$ & Bromochloromethane & 1.0 & $\mathrm{U}$ \\
\hline $67-66-3$ & Chloroform & 1.0 & $\mathrm{U}$ \\
\hline $71-55-6$ & $1,1,1$-Trichloroethane & 1.0 & $\mathrm{U}$ \\
\hline $110-82-7$ & Cyclohexane & 1.0 & $\mathrm{U}$ \\
\hline $56-23-5$ & Carbon tetrachloride & 1.0 & $\mathrm{U}$ \\
\hline $71-43-2$ & Benzene & 1.0 & $\mathrm{U}$ \\
\hline $107-06-2$ & 1,2-Dichloroethane & 1.0 & $\mathrm{U}$ \\
\hline
\end{tabular}

Report 1,4-Dioxane for Low-Medium VOA analysis only 
Lab Name: TESTAMERICA BURLINGTON

Lab Code: STLV

Case No.: YORK

Mod. Ref No.:

Contract: 8E-00302

Matrix: (SOIL/SED/WATER) Water

Sample wt/vol: 25.0 $(\mathrm{g} / \mathrm{mL}) \mathrm{mL}$

Level: (TRACE/LOW/MED) TRACE

\% Moisture: not dec.

GC Column: DB-624 ID: $0.20 \quad(\mathrm{~mm})$

Soil Extract Volume: (uL) $(\mathrm{mL})$

Purge Volume: 25.0
SDG No.: 29764

Lab Sample ID: 200-29764-5

Lab File ID: 15795_010.D

Date Received:

Date Analyzed: 09/17/2015

Dilution Factor: 1.0

Soil Aliquot Volume:

(uL)

\begin{tabular}{|c|c|c|c|}
\hline CAS NO. & COMPOUND & $\begin{array}{l}\text { CONCENTRATION UNITS: } \\
(\mathrm{ug} / \mathrm{L} \text { or } \mathrm{ug} / \mathrm{kg}) \mathrm{ug} / \mathrm{L}\end{array}$ & Q \\
\hline $79-01-6$ & Trichloroethene & 1.0 & $\mathrm{U}$ \\
\hline $108-87-2$ & Methylcyclohexane & 1.0 & $\mathrm{U}$ \\
\hline $78-87-5$ & 1,2-Dichloropropane & 1.0 & $\mathrm{U}$ \\
\hline $75-27-4$ & Bromodichloromethane & 1.0 & $\mathrm{U}$ \\
\hline $10061-01-5$ & cis-1,3-Dichloropropene & 1.0 & U \\
\hline $108-10-1$ & 4-Methyl-2-pentanone & 1.0 & $\mathrm{U}$ \\
\hline $108-88-3$ & Toluene & 0.012 & $\mathrm{~J} B$ \\
\hline $10061-02-6$ & trans-1,3-Dichloropropene & 1.0 & $\mathrm{U}$ \\
\hline $79-00-5$ & $1,1,2-$ Trichloroethane & 1.0 & $\mathrm{U}$ \\
\hline $127-18-4$ & Tetrachloroethene & 1.0 & $\mathrm{U}$ \\
\hline $591-78-6$ & 2-Hexanone & 1.0 & $\mathrm{U}$ \\
\hline $124-48-1$ & Dibromochloromethane & 1.0 & $\mathrm{U}$ \\
\hline $106-93-4$ & 1,2-Dibromoethane & 1.0 & $\mathrm{U}$ \\
\hline $108-90-7$ & Chlorobenzene & 1.0 & $\mathrm{U}$ \\
\hline $100-41-4$ & Ethylbenzene & 1.0 & $\mathrm{U}$ \\
\hline $95-47-6$ & o-Xylene & 1.0 & $\mathrm{U}$ \\
\hline $179601-23-1$ & m,p-xylene & 0.0078 & $\mathrm{~J} B$ \\
\hline $100-42-5$ & Styrene & 1.0 & $\mathrm{U}$ \\
\hline $75-25-2$ & Bromoform & 1.0 & $\mathrm{U}$ \\
\hline $98-82-8$ & Isopropylbenzene & 1.0 & $\mathrm{U}$ \\
\hline $79-34-5$ & $1,1,2,2$-Tetrachloroethane & 1.0 & $\mathrm{U}$ \\
\hline $541-73-1$ & 1,3 -Dichlorobenzene & 1.0 & $\mathrm{U}$ \\
\hline $106-46-7$ & 1,4-Dichlorobenzene & 1.0 & $\mathrm{U}$ \\
\hline $95-50-1$ & 1,2-Dichlorobenzene & 1.0 & $\mathrm{U}$ \\
\hline $96-12-8$ & 1,2-Dibromo-3-Chloropropane & 1.0 & $\mathrm{U}$ \\
\hline $120-82-1$ & $1,2,4$-Trichlorobenzene & 0.012 & $\mathrm{~J} B$ \\
\hline $87-61-6$ & $1,2,3$-Trichlorobenzene & 0.0093 & $\mathrm{~J} B$ \\
\hline
\end{tabular}


Lab Name: TESTAMERICA BURLINGTON

Contract: $8 \mathrm{E}-00302$

Lab Code: STLV Case No.: YORK Mod. Ref No.: SDG No.: 29764

Matrix: (SOIL/SED/WATER) Water

Sample wt/vol: 25.0 $(\mathrm{g} / \mathrm{mL}) \mathrm{mL}$

Level: (TRACE or LOW/MED) TRACE

\% Moisture: not dec.

GC Column: DB-624 ID $: 0.20 \quad(\mathrm{~mm})$

Soil Extract Volume: (uL)

CONCENTRATION UNITS: (ug/L or ug/kg) ug/L
Lab Sample ID: 200-29764-5

Lab File ID: 15795 010.D

Date Received:

Date Analyzed: 09/17/2015

Dilution Factor: 1.0

Soil Aliquot Volume: (uL) Purge Volume: 25.0 $(\mathrm{mL})$

01

02

\begin{tabular}{|l|l|c|c|c|}
\hline CAS NUMBER & \multicolumn{1}{|c|}{ COMPOUND NAME } & RT & EST. CONC. & Q \\
\hline & Unknown & 7.29 & 3.1 & $\mathrm{~J}$ B X \\
\hline E9667961 & Total Alkanes & N/A & & \\
\hline
\end{tabular}

1EPA-designated Registry Number. 
Lab Name: TestAmerica Burlington

SDG No.: 29764

Instrument ID: CHD.i

Lab Sample ID: IC 200-93966/3

Date Analyzed: 09/15/15 11:56

\begin{tabular}{|l|r|}
\hline \multicolumn{1}{|c|}{ COMPOUND NAME } & RETEN \\
& TIM \\
\hline Dichlorodifluoromethane & \\
\hline Chloroethane & \\
\hline 1,2-Dibromo-3-Chloropropane & 13 \\
\hline
\end{tabular}

Lab Sample ID: IC 200-93966/4

Date Analyzed: 09/15/15 12:20 Lab File ID: 15748 004.D

client sample ID:

Analysis Batch Number: 93966

Client Sample ID:

Lab File ID: 15748 003.D

GC Column: DB-624

ID $: 0.2(\mathrm{~mm})$

\begin{tabular}{|c|c|c|c|c|}
\hline \multirow[t]{2}{*}{ COMPOUND NAME } & \multirow{2}{*}{$\begin{array}{c}\text { RETENTION } \\
\text { TIME }\end{array}$} & \multicolumn{3}{|c|}{ MANUAL INTEGRATION } \\
\hline & & REASON & ANALYST & DATE \\
\hline 1,2-Dichloropropane-d6 & 6.40 & Split Peak & wilburj & $09 / 15 / 15 \quad 12: 53$ \\
\hline 1,2-Dibromo-3-Chloropropane & 13.43 & Assign Peak & wilburj & $09 / 15 / 1512: 53$ \\
\hline
\end{tabular}


Lab Name: TestAmerica Burlington

Job No.: 200-29764-1

SDG No.: 29764

Instrument ID: CHD.i

Analysis Batch Number: 94084

Lab Sample ID: CCVIS 200-94084/3

Client Sample ID:

Date Analyzed: 09/17/15 12:36

Lab File ID: 15795 003.D

GC Column: DB-624

ID $: 0.2(\mathrm{~mm})$

\begin{tabular}{|c|c|c|c|c|}
\hline \multirow[t]{2}{*}{ COMPOUND NAME } & \multirow{2}{*}{$\begin{array}{c}\text { RETENTION } \\
\text { TIME }\end{array}$} & \multicolumn{3}{|c|}{ MANUAL INTEGRATION } \\
\hline & & REASON & ANALYST & DATE \\
\hline Dichlorodifluoromethane & 1.51 & Baseline & wilburj & $09 / 17 / 15 \quad 12: 58$ \\
\hline 1,2-Dichloropropane-d6 & 6.41 & Split Peak & wilburj & $09 / 17 / 15 \quad 12: 58$ \\
\hline
\end{tabular}

Lab Sample ID: CCVC 200-94084/19

Client Sample ID:

Date Analyzed: 09/17/15 19:14

Lab File ID: 15795_019.D

GC Column: DB-624

ID $: 0.2(\mathrm{~mm})$

\begin{tabular}{|c|c|c|c|c|}
\hline \multirow[t]{2}{*}{ COMPOUND NAME } & \multirow{2}{*}{$\begin{array}{c}\text { RETENTION } \\
\text { TIME }\end{array}$} & \multicolumn{3}{|c|}{ MANUAL INTEGRATION } \\
\hline & & REASON & ANALYST & DATE \\
\hline Dichlorodifluoromethane & 1.47 & Incomplete Integration & wilburj & $09 / 22 / 1514: 56$ \\
\hline
\end{tabular}




\section{TestAmerica}

THE LEADER IN ENVIRONMENTAL TESTING

\section{ANALYTICAL REPORT}

Job Number: 200-31673-1

SDG Number: 31673

Job Description: York (200-31673)

Contract Number: 1E-30401

For:

Argonne National Laboratory

9700 South Cass Avenue

Building 203

Office B-141

Argonne, IL 60439

Attention: Ms. Esther Bowen

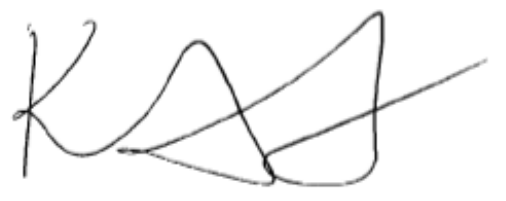

Approved for release. Kathryn A Kelly

\section{Kathryn A Kelly, Project Manager I \\ 30 Community Drive, South Burlington, VT, 05403 \\ kathryn.kelly@testamericainc.com \\ $01 / 29 / 2016$}

The test results in this report relate only to sample(s) as received by the laboratory. These test results were derived under a quality system that adheres to the requirements of NELAC. Pursuant to NELAC, this report may not be produced in full without written approval from the laboratory 


\section{Table of Contents}

Cover Title Page..$\ldots \ldots \ldots \ldots \ldots \ldots \ldots \ldots \ldots \ldots \ldots \ldots \ldots \ldots$

Sample List ........................... 4

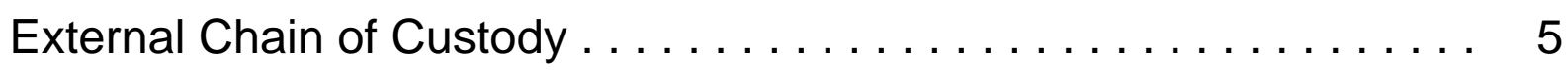

Shipping Documentation . . . . . . . . . . . . . . . 7

Sample Receipt and Log In Check List ..................... 8

Standards Traceability . . . . . . . . . . . . . . . . . . 9

Methodology Review . . . . . . . . . . . . . . . . . . . 17

Report Narrative $\ldots \ldots \ldots \ldots \ldots \ldots \ldots \ldots \ldots \ldots \ldots \ldots \ldots \ldots \ldots \ldots$

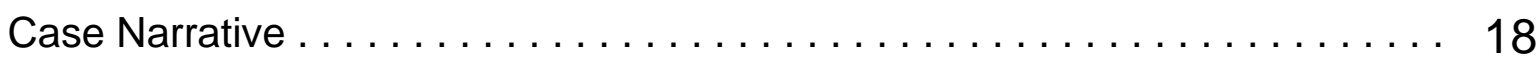

Manual Integration Documentation ....................... 19

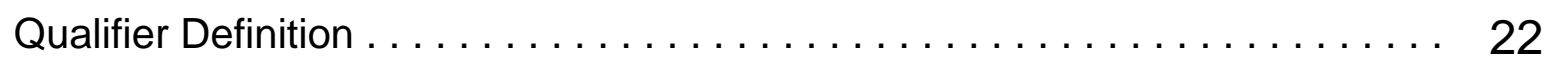

QC Summary - SOM01.2 Volatiles-Trace . . . . . . . . . . . 23

QC Summary - SOM01.2 Volatiles-Trace ................... 23

Deuterated Monitoring Compound Summary $\ldots \ldots \ldots \ldots \ldots \ldots \ldots \ldots \ldots \ldots .23$

Method Blank $\ldots \ldots \ldots \ldots \ldots \ldots \ldots \ldots \ldots \ldots \ldots \ldots \ldots \ldots \ldots \ldots, 25$

GC/MS Instrument Performance Check $\ldots \ldots \ldots \ldots \ldots \ldots \ldots \ldots \ldots \ldots \ldots \ldots \ldots \ldots \ldots \ldots$

Internal Standard Area and RT Summary $\ldots \ldots \ldots \ldots \ldots \ldots \ldots \ldots \ldots \ldots \ldots \ldots \ldots \ldots \ldots$

Sample Data - SOM01.2 Volatiles-Trace .............. 32

Sample Data - SOM01.2 Volatiles-Trace .................... 32

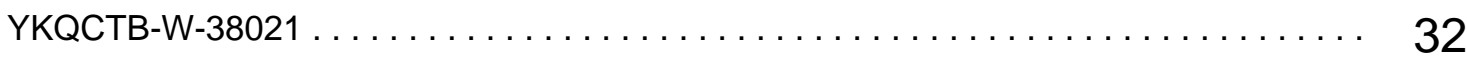

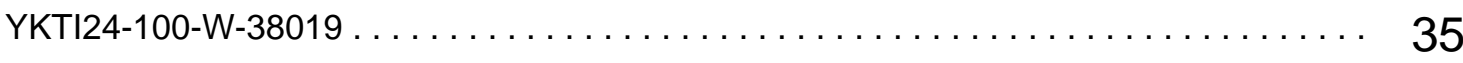

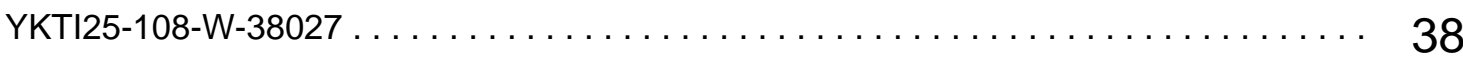

Standards - SOM01.2 Volatiles-Trace . . . . . . . . . . . . . 41

Standards - SOM01.2 Volatiles-Trace $\ldots \ldots \ldots \ldots \ldots \ldots \ldots \ldots \ldots, 41$

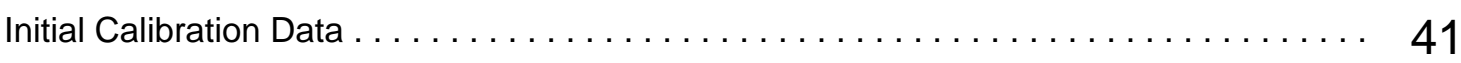

CCV Data, including closing CCV $\ldots \ldots \ldots \ldots \ldots \ldots \ldots \ldots \ldots \ldots \ldots \ldots \ldots \ldots \ldots$ 


\section{Table of Contents}

Raw Qc Data - SOM01.2 Volatiles-Trace . . . . . . . . . . . 56

Raw Qc Data - SOM01.2 Volatiles-Trace ..................... 56

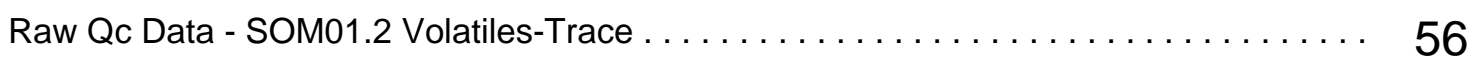

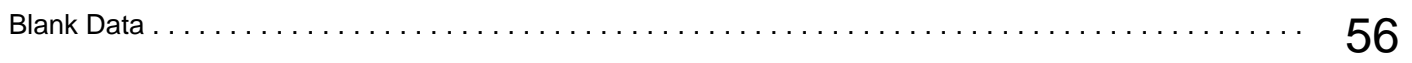




\section{Sample Login Acknowledgement}

Job 200-31673-1

\begin{tabular}{|c|c|c|c|}
\hline Client Job Description: & York (200-31673) & Report To: & Argonne National Laboratory \\
\hline Purchase Order \#: & $1 \mathrm{E}-30401$ & & Jorge Alvarado \\
\hline Work Order \#: & $1 \mathrm{E}-30401$ & & 9700 South Cass Avenue \\
\hline Project Manager: & Kathryn A Kelly & & Building 203 \\
\hline Job Due Date: & $2 / 5 / 2016$ & & $\begin{array}{l}\text { Office B-141 } \\
\text { Argonne } 60439\end{array}$ \\
\hline Job TAT: & 14 Days & & \\
\hline Max Deliverable Level: & IV & Bill To: & $\begin{array}{l}\text { Argonne National Laboratory } \\
\text { Accounts Payable }\end{array}$ \\
\hline Earliest Deliverable Due: & $2 / 5 / 2016$ & & $\begin{array}{l}\text { Chief Financial Offices } \\
9700 \text { S. Cass Ave. } \\
\text { Building } 201 \\
\text { Argonne, IL } 60439\end{array}$ \\
\hline
\end{tabular}

\section{Login 200-31673}

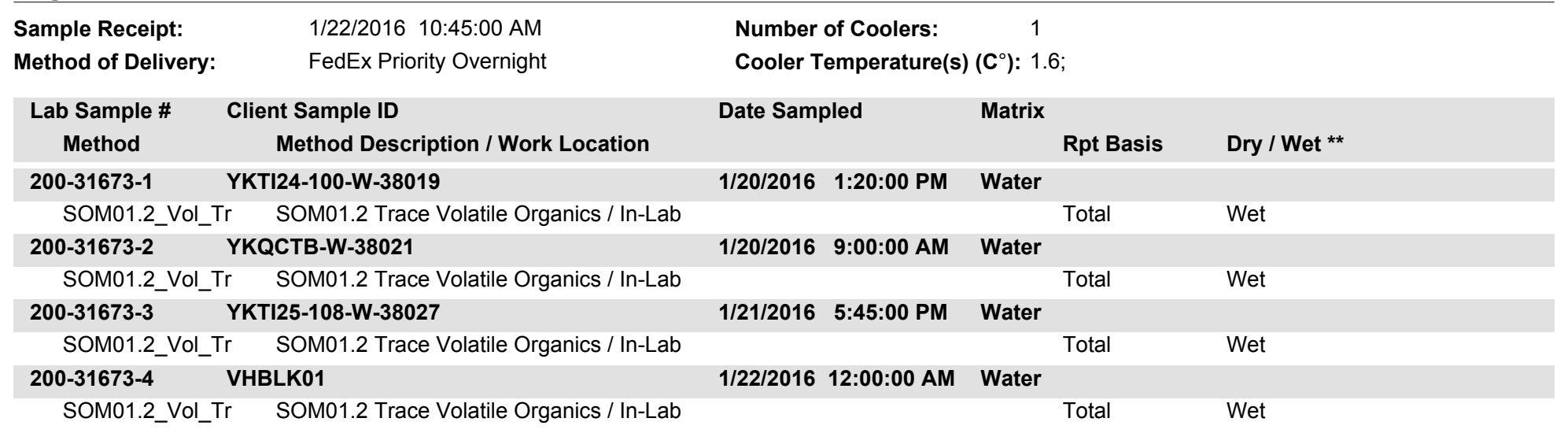




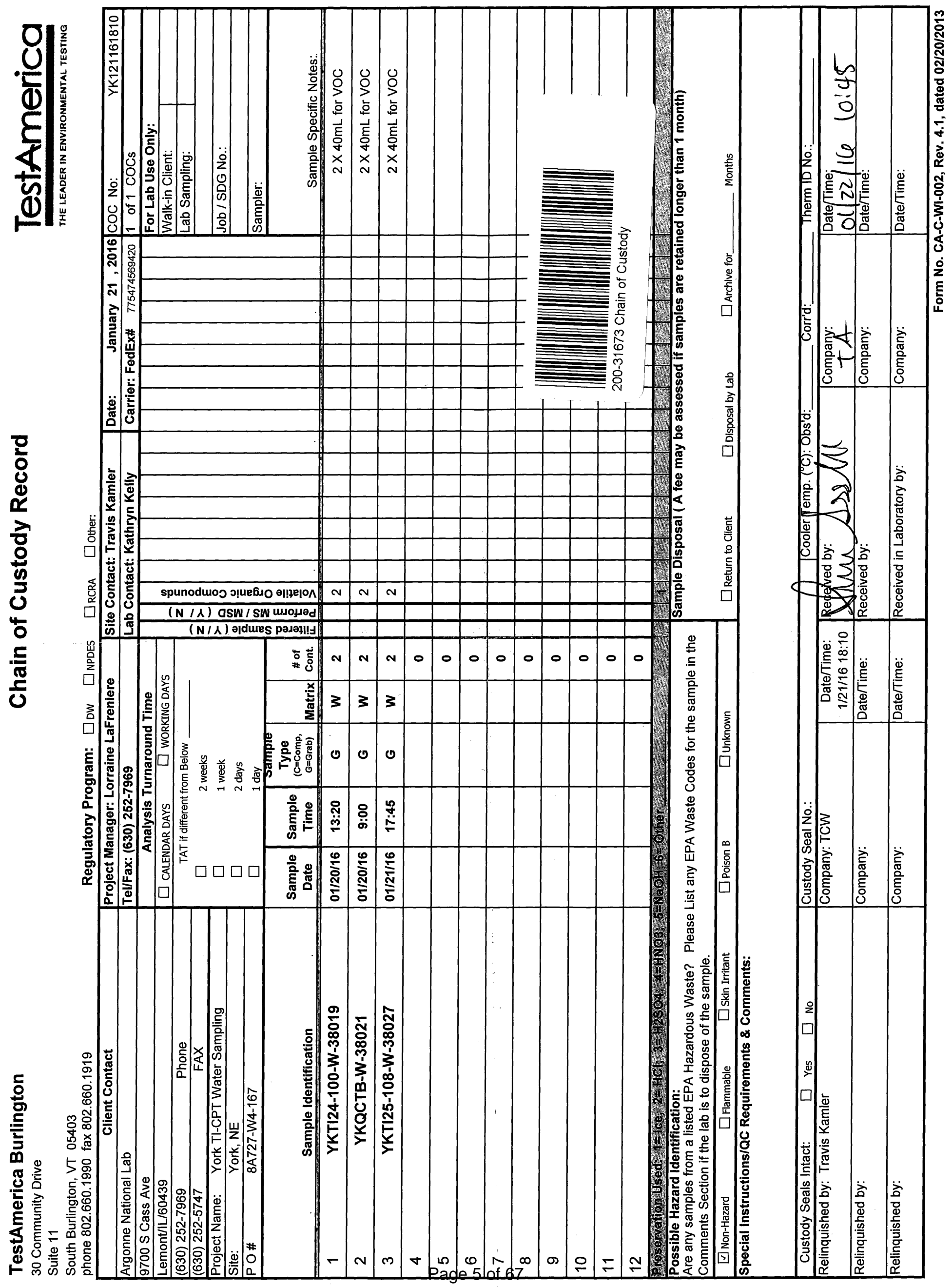


•əp!n

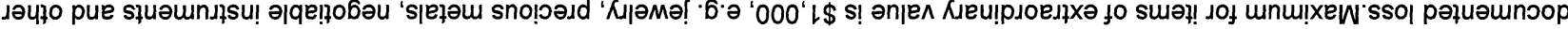

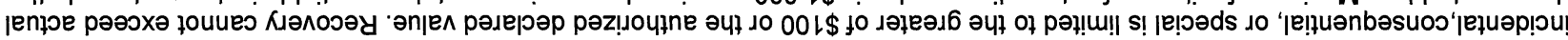

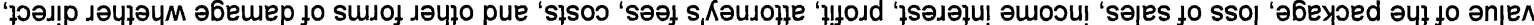

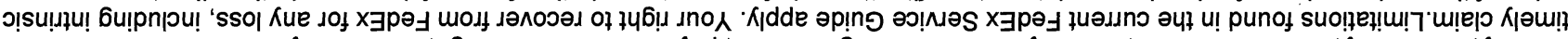

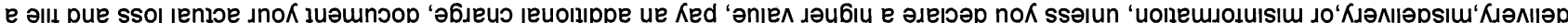

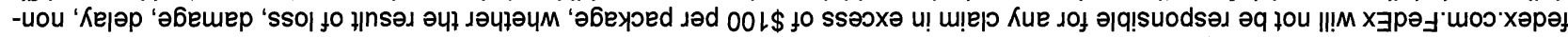
u० ә|qе|!e

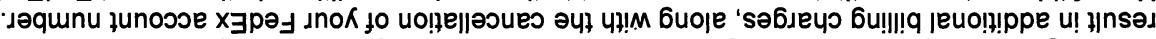

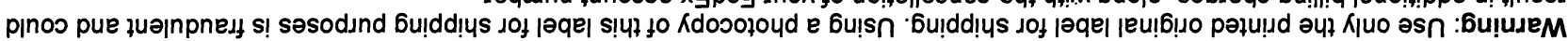

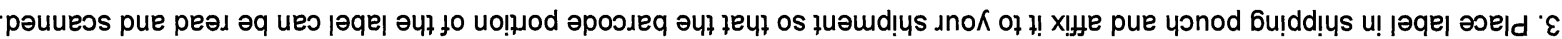

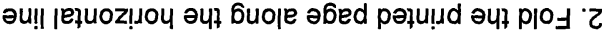

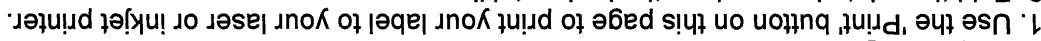

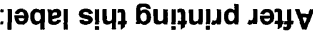

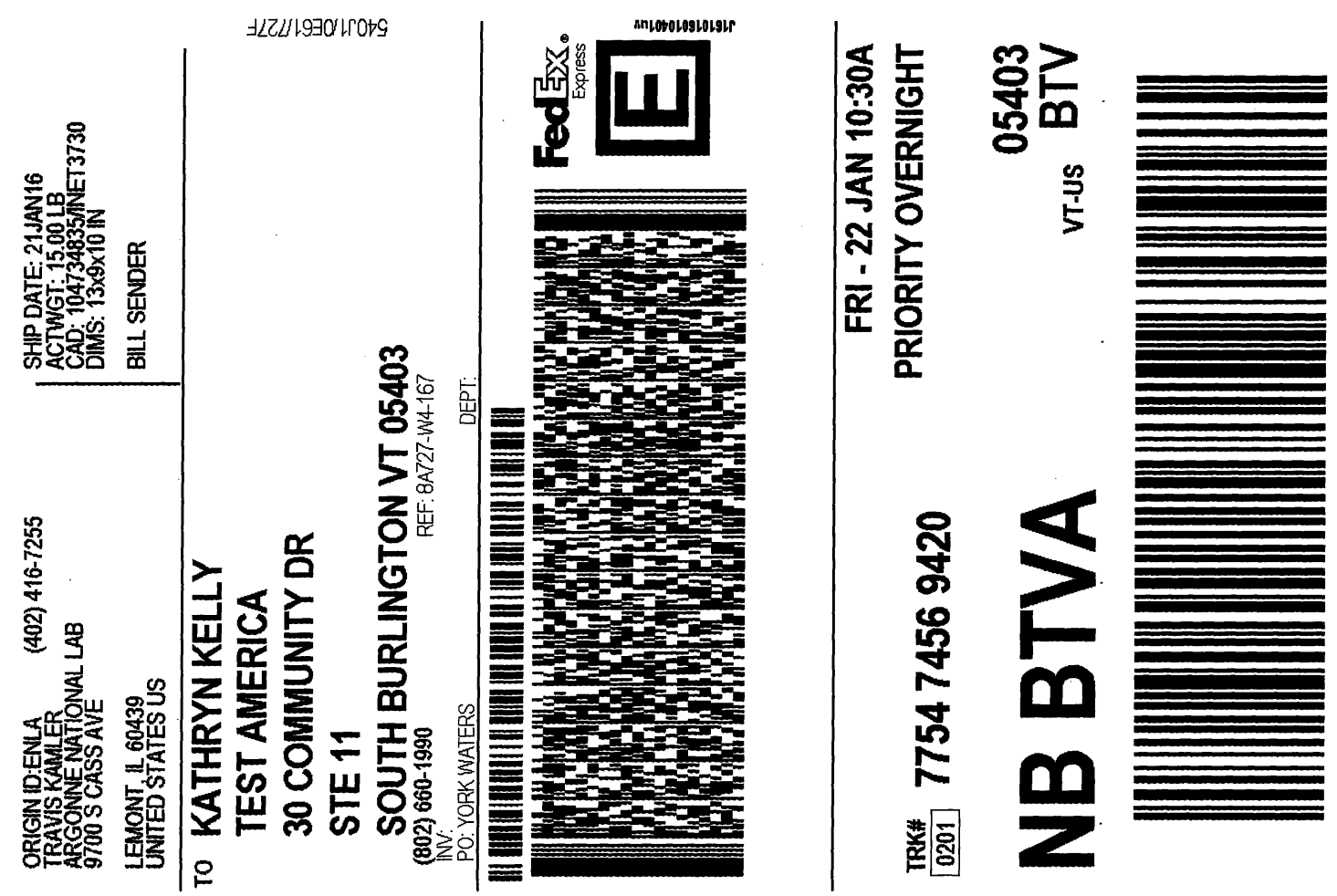




\section{Shipping and Receiving Documents}




\section{Login Sample Receipt Checklist}

Client: Argonne National Laboratory

Job Number: 200-31673-1

SDG Number: 31673

Login Number: 31673

List Source: TestAmerica Burlington

List Number: 1

Creator: Asselin, Sarah E

Question

Answer

Radioactivity wasn't checked or is $</=$ background as measured by a survey True meter.

The cooler's custody seal, if present, is intact.

Sample custody seals, if present, are intact.

The cooler or samples do not appear to have been compromised or tampered with.

Samples were received on ice.

Cooler Temperature is acceptable.

Cooler Temperature is recorded.

$\mathrm{COC}$ is present.

$\mathrm{COC}$ is filled out in ink and legible.

$\mathrm{COC}$ is filled out with all pertinent information.

Is the Field Sampler's name present on COC?

There are no discrepancies between the containers received and the COC.

Samples are received within Holding Time (excluding tests with immediate

HTs)

Sample containers have legible labels.

Containers are not broken or leaking.

Sample collection date/times are provided.

Appropriate sample containers are used.

Sample bottles are completely filled.

Sample Preservation Verified.

There is sufficient vol. for all requested analyses, incl. any requested MS/MSDs

Containers requiring zero headspace have no headspace or bubble is $<6 \mathrm{~mm}(1 / 4 ")$.

Multiphasic samples are not present.

Samples do not require splitting or compositing.

Residual Chlorine Checked.
True

True

True

True

True

True

True

True

True

True

True

True

True

True

True

True

N/A

True

True

True

True

True

N/A 


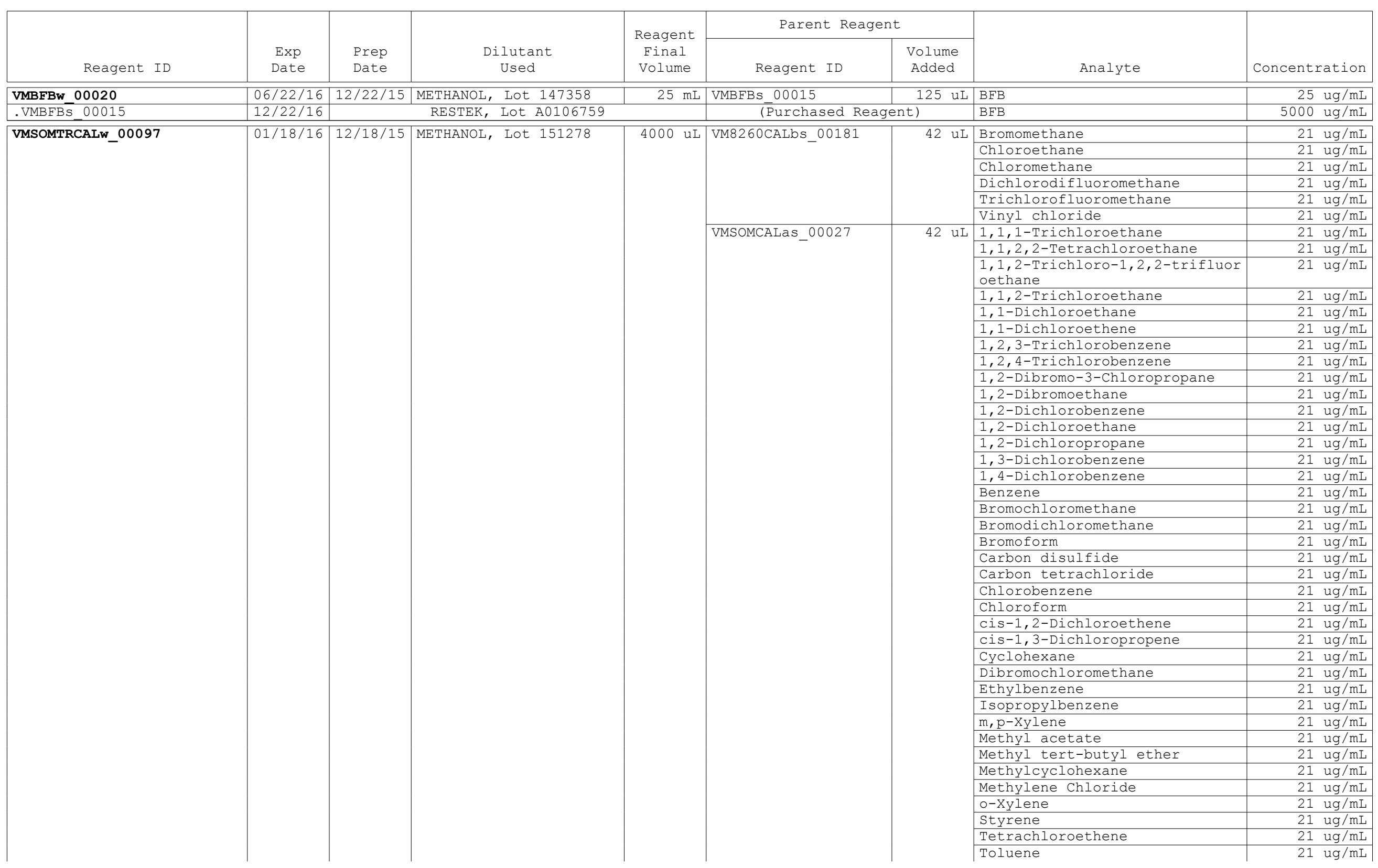


SDG No.: 31673

\begin{tabular}{|c|c|c|c|c|c|c|c|c|}
\hline \multirow[b]{2}{*}{ Reagent ID } & \multirow[b]{2}{*}{$\begin{array}{l}\text { Exp } \\
\text { Date }\end{array}$} & \multirow[b]{2}{*}{$\begin{array}{l}\text { Prep } \\
\text { Date }\end{array}$} & \multirow[b]{2}{*}{$\begin{array}{l}\text { Dilutant } \\
\text { Used }\end{array}$} & \multirow{2}{*}{$\begin{array}{c}\text { Reagent } \\
\text { Final } \\
\text { Volume }\end{array}$} & \multicolumn{2}{|l|}{ Parent Reagent } & \multirow[b]{2}{*}{ Analyte } & \multirow[b]{2}{*}{ Concentration } \\
\hline & & & & & \multirow[t]{4}{*}{ Reagent ID } & $\begin{array}{l}\text { Volume } \\
\text { Added }\end{array}$ & & \\
\hline & & & & & & & trans-1,2-Dichloroethene & $21 \mathrm{ug} / \mathrm{mL}$ \\
\hline & & & & & & & trans-1,3-Dichloropropene & $21 \mathrm{ug} / \mathrm{mL}$ \\
\hline & & & & & & \multirow{5}{*}{$168 \mathrm{uL}$} & Trichloroethene & $21 \mathrm{ug} / \mathrm{mL}$ \\
\hline & & & & & \multirow[t]{4}{*}{ VMSOMCALbs_00033 } & & 2-Butanone & $210 \mathrm{ug} / \mathrm{mL}$ \\
\hline & & & & & & & 2-Hexanone & $210 \mathrm{ug} / \mathrm{mL}$ \\
\hline & & & & & & & 4-Methyl-2-pentanone & $210 \mathrm{ug} / \mathrm{mL}$ \\
\hline & & & & & & & Acetone & $210 \mathrm{ug} / \mathrm{mL}$ \\
\hline & & & & & \multirow{2}{*}{ VMSOMSUas_00077 } & \multirow{2}{*}{42 uL } & Chloroethane-d5 & $21 \mathrm{ug} / \mathrm{mL}$ \\
\hline & & & & & & & Vinyl Chloride-d3 & $21 \mathrm{ug} / \mathrm{mL}$ \\
\hline & & & & & \multirow[t]{2}{*}{ VMSOMSUbs_00142 } & \multirow[t]{2}{*}{336 uL } & 2-Butanone-d5 & $210 \mathrm{ug} / \mathrm{mL}$ \\
\hline & & & & & & & 2-Hexanone-d5 & $210 \mathrm{ug} / \mathrm{mL}$ \\
\hline & & & & & \multirow[t]{9}{*}{ VMSOMSUCS_00055 } & \multirow[t]{9}{*}{42 uL } & $1,1,2,2$-Tetrachloroethane-d2 & $21 \mathrm{ug} / \mathrm{mL}$ \\
\hline & & & & & & & 1,1-Dichloroethene-d2 & $21 \mathrm{ug} / \mathrm{mL}$ \\
\hline & & & & & & & 1,2-Dichlorobenzene-d4 & $21 \mathrm{ug} / \mathrm{mL}$ \\
\hline & & & & & & & 1,2-Dichloroethane-d4 & $21 \mathrm{ug} / \mathrm{mL}$ \\
\hline & & & & & & & 1,2-Dichloropropane-d6 & $21 \mathrm{ug} / \mathrm{mL}$ \\
\hline & & & & & & & Benzene-d6 & $21 \mathrm{ug} / \mathrm{mL}$ \\
\hline & & & & & & & Chloroform-d & $21 \mathrm{ug} / \mathrm{mL}$ \\
\hline & & & & & & & Toluene-d8 & $21 \mathrm{ug} / \mathrm{mL}$ \\
\hline \multirow{7}{*}{.VM8260CALbs_00181 } & & & & & & & trans-1,3-Dichloropropene-d4 & $21 \mathrm{ug} / \mathrm{mL}$ \\
\hline & \multirow{6}{*}{$01 / 18 / 16$} & & RESTEK, Lot A0112834 & & (Purchased Reagen & & Bromomethane & $2000 \mathrm{ug} / \mathrm{mL}$ \\
\hline & & & & & & & Chloroethane & $2000 \mathrm{ug} / \mathrm{mL}$ \\
\hline & & & & & & & Chloromethane & $2000 \mathrm{ug} / \mathrm{mL}$ \\
\hline & & & & & & & Dichlorodifluoromethane & $2000 \mathrm{ug} / \mathrm{mL}$ \\
\hline & & & & & & & Trichlorofluoromethane & $2000 \mathrm{ug} / \mathrm{mL}$ \\
\hline & & & & & & & Vinyl chloride & $2000 \mathrm{ug} / \mathrm{mL}$ \\
\hline .VMSOMCALas_0 0027 & $10 / 22 / 16$ & & Restek, Lot A0102833 & & (Purchased Reagen & & $1,1,1$-Trichloroethane & $2000 \mathrm{ug} / \mathrm{mL}$ \\
\hline & & & & & & & 1,1,2,2-Tetrachloroethane & $2000 \mathrm{ug} / \mathrm{mL}$ \\
\hline & & & & & & & $\begin{array}{l}\text { 1,1,2-Trichloro-1,2,2-trifluor } \\
\text { oethane }\end{array}$ & $2000 \mathrm{ug} / \mathrm{mL}$ \\
\hline & & & & & & & $1,1,2$-Trichloroethane & $2000 \mathrm{ug} / \mathrm{mL}$ \\
\hline & & & & & & & 1,1-Dichloroethane & $2000 \mathrm{ug} / \mathrm{mL}$ \\
\hline & & & & & & & 1,1-Dichloroethene & $2000 \mathrm{ug} / \mathrm{mL}$ \\
\hline & & & & & & & 1,2,3-Trichlorobenzene & $2000 \mathrm{ug} / \mathrm{mL}$ \\
\hline & & & & & & & $1,2,4$-Trichlorobenzene & $2000 \mathrm{ug} / \mathrm{mL}$ \\
\hline & & & & & & & 1,2-Dibromo-3-Chloropropane & $2000 \mathrm{ug} / \mathrm{mL}$ \\
\hline & & & & & & & 1,2-Dibromoethane & $2000 \mathrm{ug} / \mathrm{mL}$ \\
\hline & & & & & & & 1,2-Dichlorobenzene & $2000 \mathrm{ug} / \mathrm{mL}$ \\
\hline & & & & & & & 1,2-Dichloroethane & $2000 \mathrm{ug} / \mathrm{mL}$ \\
\hline & & & & & & & 1,2-Dichloropropane & $2000 \mathrm{ug} / \mathrm{mL}$ \\
\hline & & & & & & & 1,3-Dichlorobenzene & $2000 \mathrm{ug} / \mathrm{mL}$ \\
\hline & & & & & & & 1,4-Dichlorobenzene & $2000 \mathrm{ug} / \mathrm{mL}$ \\
\hline & & & & & & & Benzene & $2000 \mathrm{ug} / \mathrm{mL}$ \\
\hline & & & & & & & Bromochloromethane & $2000 \mathrm{ug} / \mathrm{mL}$ \\
\hline & & & & & & & Bromodichloromethane & $2000 \mathrm{ug} / \mathrm{mL}$ \\
\hline & & & & & & & Bromoform & $2000 \mathrm{ug} / \mathrm{mL}$ \\
\hline & & & & & & & Carbon disulfide & $2000 \mathrm{ug} / \mathrm{mL}$ \\
\hline & & & & & & & Carbon tetrachloride & $2000 \mathrm{ug} / \mathrm{mL}$ \\
\hline
\end{tabular}


SDG No.: 31673

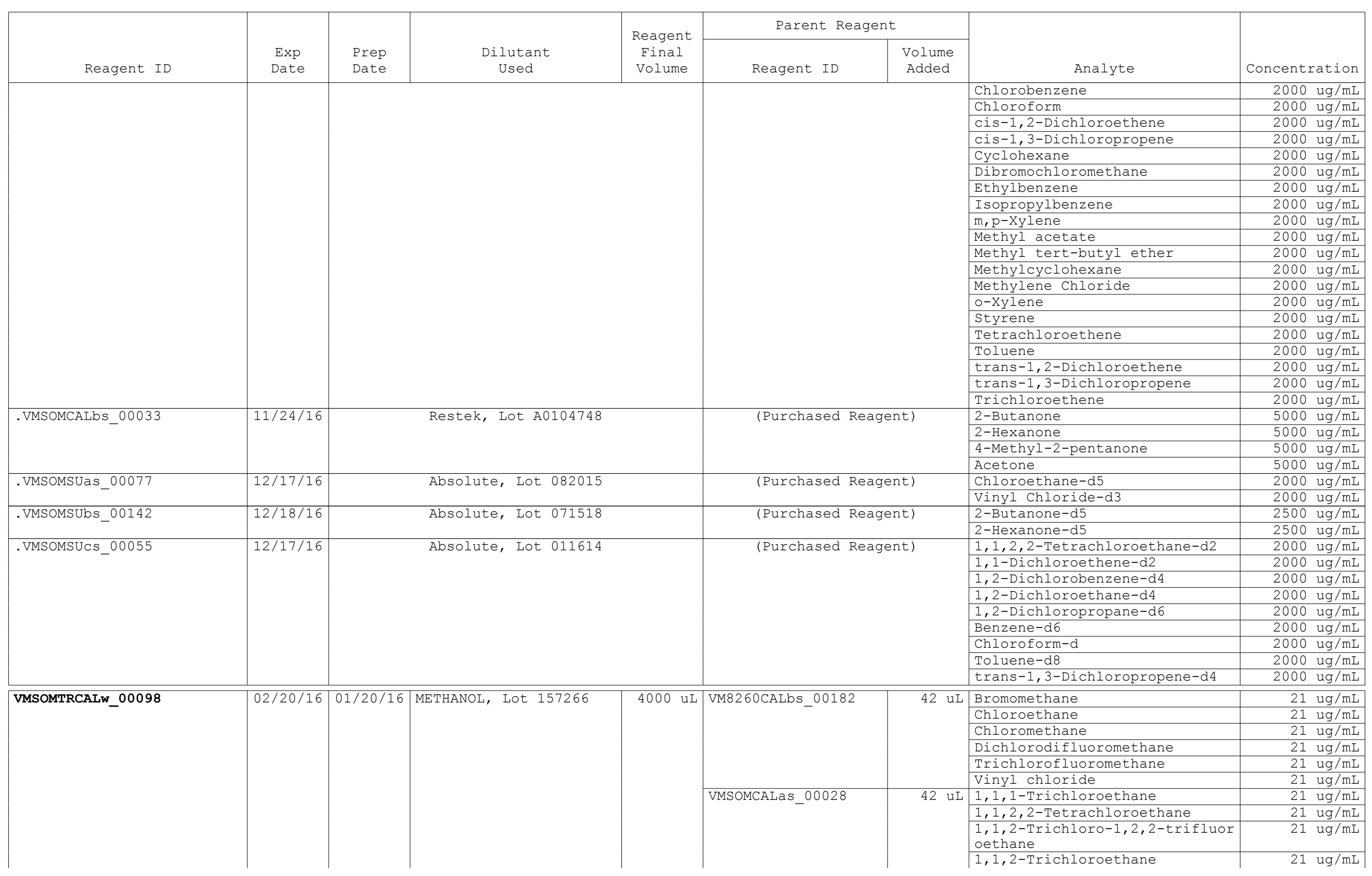


SDG No.: 31673

\begin{tabular}{|c|c|c|c|c|c|c|c|c|}
\hline \multirow[b]{2}{*}{ Reagent ID } & \multirow[b]{2}{*}{$\begin{array}{l}\text { Exp } \\
\text { Date }\end{array}$} & \multirow[b]{2}{*}{$\begin{array}{l}\text { Prep } \\
\text { Date }\end{array}$} & \multirow[b]{2}{*}{$\begin{array}{l}\text { Dilutant } \\
\text { Used }\end{array}$} & \multirow{2}{*}{$\begin{array}{c}\text { Reagent } \\
\text { Final } \\
\text { Volume }\end{array}$} & \multicolumn{2}{|c|}{ Parent Reagent } & \multirow[b]{2}{*}{ Analyte } & \multirow[b]{2}{*}{ Concentration } \\
\hline & & & & & Reagent ID & $\begin{array}{l}\text { Volume } \\
\text { Added }\end{array}$ & & \\
\hline & & & & & & & 1,1-Dichloroethane & $21 \mathrm{ug} / \mathrm{mL}$ \\
\hline & & & & & & & 1,1-Dichloroethene & $21 \mathrm{ug} / \mathrm{mL}$ \\
\hline & & & & & & & 1,2,3-Trichlorobenzene & $21 \mathrm{ug} / \mathrm{mL}$ \\
\hline & & & & & & & 1,2,4-Trichlorobenzene & $21 \mathrm{ug} / \mathrm{mL}$ \\
\hline & & & & & & & 1,2-Dibromoethane & $\begin{array}{l}21 \mathrm{ug} / \mathrm{mL} \\
21 \mathrm{ug} / \mathrm{mL}\end{array}$ \\
\hline & & & & & & & 1,2-Dichlorobenzene & $21 \mathrm{ug} / \mathrm{mL}$ \\
\hline & & & & & & & 1,2-Dichloroethane & $21 \mathrm{ug} / \mathrm{mL}$ \\
\hline & & & & & & & 1,2-Dichloropropane & $21 \mathrm{ug} / \mathrm{mL}$ \\
\hline & & & & & & & 1,3-Dichlorobenzene & $21 \mathrm{ug} / \mathrm{mL}$ \\
\hline & & & & & & & 1,4-Dichlorobenzene & $21 \mathrm{ug} / \mathrm{mL}$ \\
\hline & & & & & & & Benzene & $21 \mathrm{ug} / \mathrm{mL}$ \\
\hline & & & & & & & Bromochloromethane & $21 \mathrm{ug} / \mathrm{mL}$ \\
\hline & & & & & & & Bromodichloromethane & $21 \mathrm{ug} / \mathrm{mL}$ \\
\hline & & & & & & & Bromoform & $21 \mathrm{ug} / \mathrm{mL}$ \\
\hline & & & & & & & Carbon disulfide & $21 \mathrm{ug} / \mathrm{mL}$ \\
\hline & & & & & & & Carbon tetrachloride & $21 \mathrm{ug} / \mathrm{mL}$ \\
\hline & & & & & & & Chlorobenzene & $21 \mathrm{ug} / \mathrm{mL}$ \\
\hline & & & & & & & Chloroform & $21 \mathrm{ug} / \mathrm{mL}$ \\
\hline & & & & & & & cis-1,2-Dichloroethene & $21 \mathrm{ug} / \mathrm{mL}$ \\
\hline & & & & & & & cis-1,3-Dichloropropene & $21 \mathrm{ug} / \mathrm{mL}$ \\
\hline & & & & & & & Cyclohexane & $21 \mathrm{ug} / \mathrm{mL}$ \\
\hline & & & & & & & Dibromochloromethane & $21 \mathrm{ug} / \mathrm{mL}$ \\
\hline & & & & & & & $\begin{array}{l}\text { Ethylbenzene } \\
\text { Isopropylbenzene }\end{array}$ & $\begin{array}{l}21 \mathrm{ug} / \mathrm{mL} \\
21 \mathrm{ug} / \mathrm{mL}\end{array}$ \\
\hline & & & & & & & m, p-Xylene & $21 \mathrm{ug} / \mathrm{mL}$ \\
\hline & & & & & & & Methyl acetate & $21 \mathrm{ug} / \mathrm{mL}$ \\
\hline & & & & & & & Methyl tert-butyl ether & $21 \mathrm{ug} / \mathrm{mL}$ \\
\hline & & & & & & & Methylcyclohexane & $21 \mathrm{ug} / \mathrm{mL}$ \\
\hline & & & & & & & Methylene Chloride & $21 \mathrm{ug} / \mathrm{mL}$ \\
\hline & & & & & & & o-Xylene & $21 \mathrm{ug} / \mathrm{mL}$ \\
\hline & & & & & & & Styrene & $21 \mathrm{ug} / \mathrm{mL}$ \\
\hline & & & & & & & Tetrachloroethene & $21 \mathrm{ug} / \mathrm{mL}$ \\
\hline & & & & & & & Toluene & $21 \mathrm{ug} / \mathrm{mL}$ \\
\hline & & & & & & & trans-1,2-Dichloroethene & $21 \mathrm{ug} / \mathrm{mL}$ \\
\hline & & & & & & & trans-1,3-Dichloropropene & $21 \mathrm{ug} / \mathrm{mL}$ \\
\hline & & & & & & & Trichloroethene & $21 \mathrm{ug} / \mathrm{mL}$ \\
\hline & & & & & VMSOMCALbS_00039 & $168 \mathrm{uL}$ & 2-Butanone & $210 \mathrm{ug} / \mathrm{mL}$ \\
\hline & & & & & & & 2-Hexanone & $210 \mathrm{ug} / \mathrm{mL}$ \\
\hline & & & & & & & 4-Methyl-2-pentanone & $210 \mathrm{ug} / \mathrm{mL}$ \\
\hline & & & & & & & Acetone & $210 \mathrm{ug} / \mathrm{mL}$ \\
\hline & & & & & VMSOMSUas_00087 & 42 uL & Chloroethane-d5 & $21 \mathrm{ug} / \mathrm{mL}$ \\
\hline & & & & & & & Vinyl Chloride-d3 & $21 \mathrm{ug} / \mathrm{mL}$ \\
\hline & & & & & VMSOMSUbs_00141 & $336 \mathrm{uL}$ & 2-Butanone-d5 & $210 \mathrm{ug} / \mathrm{mL}$ \\
\hline & & & & & & & 2-Hexanone-d5 & $210 \mathrm{ug} / \mathrm{mL}$ \\
\hline & & & & & VMSOMSUCS_00062 & 42 uL & 1,1,2,2-Tetrachloroethane-d2 & $21 \mathrm{ug} / \mathrm{mL}$ \\
\hline & & & & & & & 1,1-Dichloroethene-d2 & $21 \mathrm{ug} / \mathrm{mL}$ \\
\hline & & & & & & & 1,2-Dichlorobenzene-d4 & $21 \mathrm{ug} / \mathrm{mL}$ \\
\hline
\end{tabular}


SDG No.: 31673

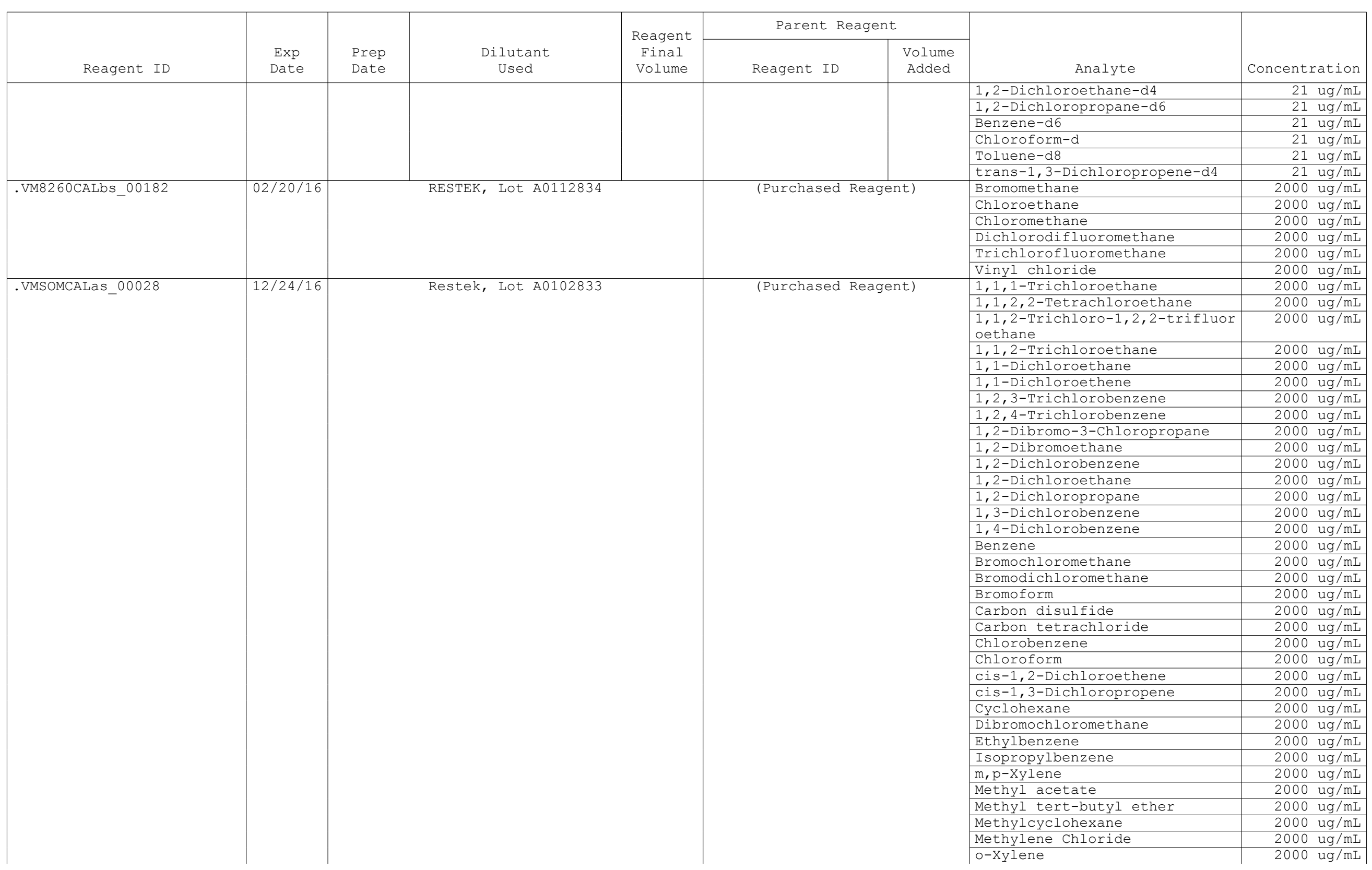


SDG No.: 31673

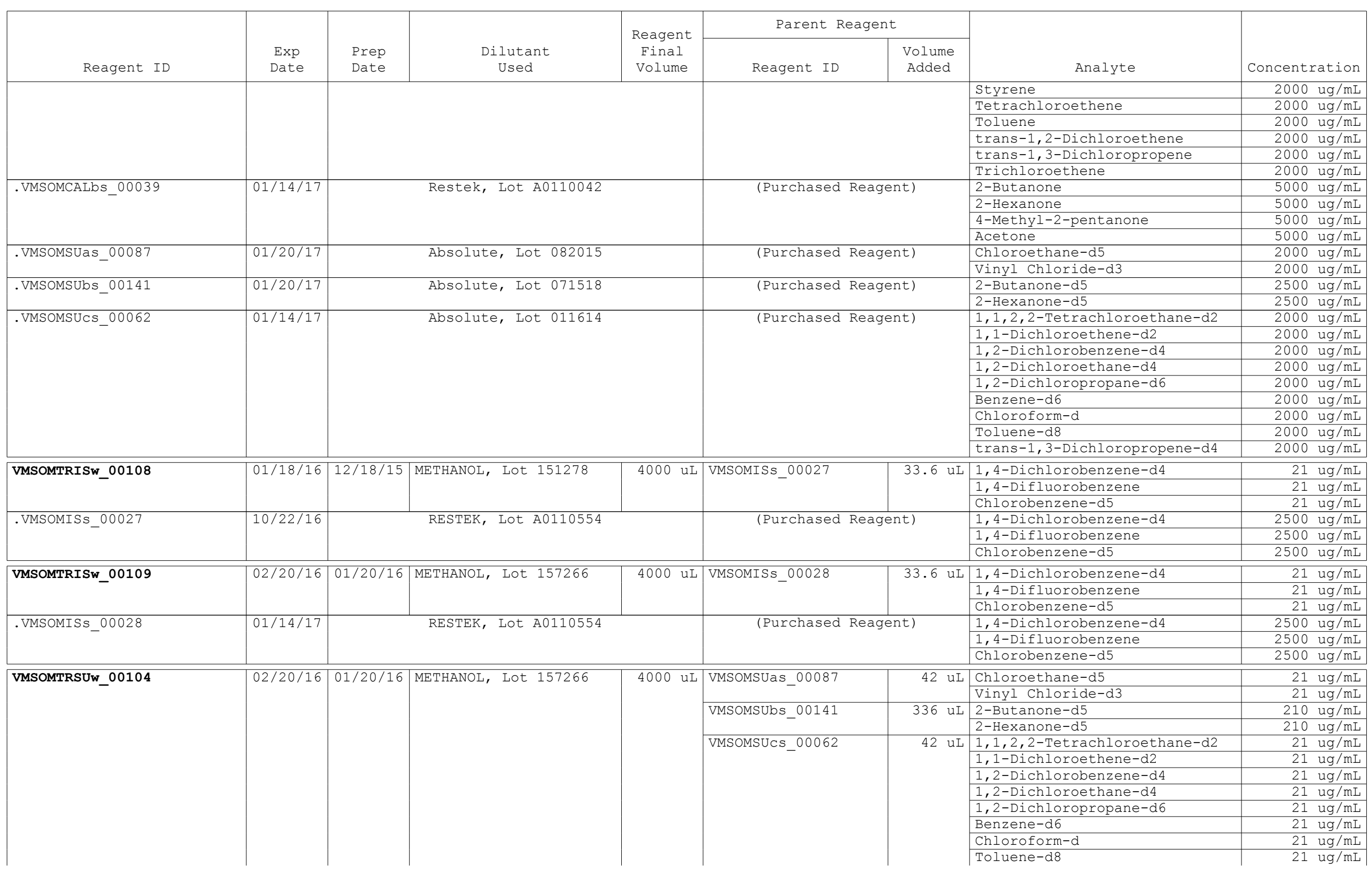


SDG No.: 31673

\begin{tabular}{|c|c|c|c|c|c|c|c|c|}
\hline \multirow[b]{2}{*}{ Reagent ID } & \multirow[b]{2}{*}{$\begin{array}{l}\text { Exp } \\
\text { Date }\end{array}$} & \multirow[b]{2}{*}{$\begin{array}{l}\text { Prep } \\
\text { Date }\end{array}$} & \multirow[b]{2}{*}{$\begin{array}{l}\text { Dilutant } \\
\text { Used }\end{array}$} & \multirow{2}{*}{$\begin{array}{c}\text { Reagent } \\
\text { Final } \\
\text { Volume }\end{array}$} & \multicolumn{2}{|c|}{ Parent Reagent } & \multirow[b]{2}{*}{ Analyte } & \multirow[b]{2}{*}{ Concentration } \\
\hline & & & & & Reagent ID & $\begin{array}{l}\text { Volume } \\
\text { Added }\end{array}$ & & \\
\hline & & & & & & & trans-1,3-Dichloropropene-d4 & $21 \mathrm{ug} / \mathrm{mL}$ \\
\hline \multirow[t]{2}{*}{.VMSOMSUas_00087 } & \multirow[t]{2}{*}{$01 / 20 / 17$} & \multirow{2}{*}{\multicolumn{3}{|c|}{ Absolute, Lot 082015}} & \multirow{2}{*}{\multicolumn{2}{|c|}{ (Purchased Reagent) }} & Chloroethane-d5 & $2000 \mathrm{ug} / \mathrm{mL}$ \\
\hline & & & & & & & Vinyl Chloride-d3 & $2000 \mathrm{ug} / \mathrm{mL}$ \\
\hline \multirow[t]{2}{*}{.VMSOMSUbs_00141 } & \multirow[t]{2}{*}{$01 / 20 / 17$} & \multirow{2}{*}{\multicolumn{3}{|c|}{ Absolute, Lot 071518}} & \multirow{2}{*}{\multicolumn{2}{|c|}{ (Purchased Reagent) }} & 2-Butanone-d5 & $2500 \mathrm{ug} / \mathrm{mL}$ \\
\hline & & & & & & & 2-Hexanone-d5 & $2500 \mathrm{ug} / \mathrm{mL}$ \\
\hline \multirow[t]{9}{*}{.VMSOMSUCS_00062 } & \multirow[t]{9}{*}{$01 / 14 / 17$} & \multirow{9}{*}{\multicolumn{2}{|c|}{ Absolute, Lot 011614}} & & \multirow{9}{*}{\multicolumn{2}{|c|}{ (Purchased Reagent) }} & $1,1,2,2$-Tetrachloroethane-d2 & $2000 \mathrm{ug} / \mathrm{mL}$ \\
\hline & & & & & & & 1,1-Dichloroethene-d2 & $2000 \mathrm{ug} / \mathrm{mL}$ \\
\hline & & & & & & & 1,2-Dichlorobenzene-d4 & $2000 \mathrm{ug} / \mathrm{mL}$ \\
\hline & & & & & & & 1,2-Dichloroethane-d4 & $2000 \mathrm{ug} / \mathrm{mL}$ \\
\hline & & & & & & & 1,2-Dichloropropane-d6 & $2000 \mathrm{ug} / \mathrm{mL}$ \\
\hline & & & & & & & Benzene-d6 & $2000 \mathrm{ug} / \mathrm{mL}$ \\
\hline & & & & & & & Chloroform-d & $2000 \mathrm{ug} / \mathrm{mL}$ \\
\hline & & & & & & & Toluene-d8 & $2000 \mathrm{ug} / \mathrm{mL}$ \\
\hline & & & & & & & trans-1,3-Dichloropropene-d4 & $2000 \mathrm{ug} / \mathrm{mL}$ \\
\hline
\end{tabular}


Lab Name: TestAmerica Burlington Job No.: 200-31673-1

SDG No.: 31673

\begin{tabular}{|c|c|c|c|c|}
\hline $\begin{array}{c}\text { Reagent } \\
\text { Container }\end{array}$ & $\begin{array}{l}\text { Reagent } \\
\text { ID }\end{array}$ & $\begin{array}{c}\text { Reagent } \\
\text { Description }\end{array}$ & $\begin{array}{c}\text { Preparation } \\
\text { Date }\end{array}$ & $\begin{array}{c}\text { Expiration } \\
\text { Date }\end{array}$ \\
\hline 885836 & VMSOMTRISW_00108 & SOM TR ISTD $21 \quad$ PPM & $12 / 18 / 2015$ & $01 / 18 / 2016$ \\
\hline 885843 & VMSOMTRCALW_00097 & SOM TR CAL $21-210$ PPM & $12 / 18 / 2015$ & $01 / 18 / 2016$ \\
\hline 886995 & VMBFBW_00020 & BEB TUNE 25 PPM & $12 / 22 / 2015$ & $06 / 22 / 2016$ \\
\hline 893452 & VMSOMTRISw_00109 & SOM TR ISTD 21 PPM & $01 / 20 / 2016$ & $02 / 20 / 2016$ \\
\hline 893453 & VMSOMTRSUW 00104 & SOM TR DMC $21 \quad$ PPM & $01 / 20 / 2016$ & $02 / 20 / 2016$ \\
\hline 893454 & VMSOMTRCALW_00098 & SOM TR CAL $21-210$ PPM & $01 / 20 / 2016$ & $02 / 20 / 2016$ \\
\hline
\end{tabular}




\section{METHODOLOGY SUMMARY}

Laboratory: TestAmerica Laboratories

Location: South Burlington, Vermont
Project No:

SDG No: $\quad 31673$

VOA

Volatile Organics Trace - USEPA CLP SOM01.2 


\section{CASE NARRATIVE}

\section{Client: Argonne National Laboratory}

\section{Project: York (200-31673)}

\section{Report Number: 200-31673-1}

With the exceptions noted as flags or footnotes, standard analytical protocols were followed in the analysis of the samples and no problems were encountered or anomalies observed. In addition all laboratory quality control samples were within established control limits, with any exceptions noted below. Each sample was analyzed to achieve the lowest possible reporting limit within the constraints of the method. In some cases, due to interference or analytes present at high concentrations, samples were diluted. For diluted samples, the reporting limits are adjusted relative to the dilution required.

Calculations are performed before rounding to avoid round-off errors in calculated results.

All holding times were met and proper preservation noted for the methods performed on these samples, unless otherwise detailed in the individual sections below.

\section{RECEIPT}

The samples were received on 01/22/2016; the samples arrived in good condition, properly preserved and on ice. The temperature of the coolers at receipt was $1.6 \mathrm{C}$.

\section{VOLATILE ORGANIC COMPOUNDS - TRACE}

Samples YKTI24-100-W-38019, YKQCTB-W-38021, YKTI25-108-W-38027 and VHBLK01 were analyzed for Volatile Organic Compounds - Trace in accordance with EPA SOW SOM01.2. The samples were analyzed on 01/26/2016 and 01/27/2016.

Toluene was detected in method blank MB 200-100004/7 at a level that was above the method detection limit but below the reporting limit. The value should be considered an estimate, and has been flagged. If the associated sample reported a result above the MDL and/or RL, the result has been flagged. m,p-Xylene, Methylene Chloride and Toluene were detected in method blank MB 200-100049/11 at levels that were above the method detection limit but below the reporting limit. The values should be considered estimates, and have been flagged. If the associated sample reported a result above the MDL and/or RL, the result has been flagged. Refer to the QC report for details.

A non-target constituent that represents a compound related to the DMC formulation is present in the samples in this set.The derived results for that compound have been qualified with an "X" qualifier to reflect the source of the contamination.

1,2-Dichloroethane-d4 failed the surrogate recovery criteria high for YKTI24-100-W-38019. 1,2-Dichloroethane-d4 failed the surrogate recovery criteria high for YKQCTB-W-38021. 1,2-Dichloroethane-d4 failed the surrogate recovery criteria high for YKTI25-108-W-38027. Refer to the QC report for details.

No additional analytical or quality issues were noted, other than those described above or in the Definitions/Glossary page. 
Lab Name: TestAmerica Burlington

SDG No.: 31673

Instrument ID: CHD.i

Lab Sample ID: IC 200-99296/7

Date Analyzed: 01/08/16 14:21

Analysis Batch Number: 99296

Client Sample ID:

Lab File ID: 17668 007.D

GC Column: DB-624

ID $: 0.2(\mathrm{~mm})$

\begin{tabular}{|l|c|c|c|c|}
\hline \multicolumn{1}{|c|}{ COMPOUND NAME } & RETENTION & \multicolumn{2}{|c|}{ MANUAL INTEGRATION } \\
\cline { 3 - 5 } & TIME & REASON & ANALYST & DATE \\
\hline Methyl acetate & 3.12 & Split Peak & wilburj & $01 / 08 / 1616: 07$ \\
\hline
\end{tabular}

Lab Sample ID: IC 200-99296/8

Client Sample ID:

Date Analyzed: 01/08/16 14:45

Lab File ID: 17668 008.D

GC Column: DB-624

ID : $0.2(\mathrm{~mm})$

\begin{tabular}{|c|c|c|c|c|}
\hline \multirow[t]{2}{*}{ COMPOUND NAME } & \multirow{2}{*}{$\begin{array}{c}\text { RETENTION } \\
\text { TIME }\end{array}$} & \multicolumn{3}{|c|}{ MANUAL INTEGRATION } \\
\hline & & REASON & ANALYST & DATE \\
\hline Methyl acetate & 3.12 & Split Peak & wilburj & $01 / 08 / 16 \quad 16: 07$ \\
\hline 1,2-Dichloropropane-d6 & 6.37 & Split Peak & wilburj & $01 / 08 / 16 \quad 16: 05$ \\
\hline
\end{tabular}

SOM01.2/VOA_Tr 
Lab Name: TestAmerica Burlington SDG No.: 31673

Instrument ID: CHD.i

Lab Sample ID: CCVC 200-100004/26

Date Analyzed: 01/26/16 21:35

\begin{tabular}{|l|r|}
\hline \multicolumn{1}{|c|}{ COMPOUND NAME } & RETEN \\
& TIM \\
\hline Dichlorodifluoromethane &
\end{tabular}

Job No.: 200-31673-1

Analysis Batch Number: 100004

Client Sample ID:

Lab File ID: 18001 026.D
GC Column: DB-624

ID $: 0.2(\mathrm{~mm})$ 
Lab Name: TestAmerica Burlington

Job No.: 200-31673-1

SDG No.: 31673

Instrument ID: CHD.i

Analysis Batch Number: 100049

Lab Sample ID: IC 200-100049/4

Client Sample ID:

Date Analyzed: 01/27/16 11:17

Lab File ID: 18020_004.D

GC Column: DB-624

ID $: 0.2(\mathrm{~mm})$

\begin{tabular}{|l|r|r|c|}
\hline \multicolumn{1}{|c|}{ COMPOUND NAME } & RETENTION & \multicolumn{2}{|c|}{ MANUAL INTEGRATION } \\
\cline { 3 - 5 } & TIME & REASON & ANALYST \\
\hline Methyl acetate & 3.12 & Unspecified & \\
\hline 1,2 -Dichloropropane & 6.47 & Unspecified & \\
\hline
\end{tabular}

Lab Sample ID: IC 200-100049/7 Client Sample ID:

Date Analyzed: 01/27/16 12:32

Lab File ID: 18020_007.D

GC Column: DB-624

ID $: 0.2(\mathrm{~mm})$

\begin{tabular}{|l|c|c|c|c|}
\hline \multicolumn{1}{|c|}{ COMPOUND NAME } & RETENTION & \multicolumn{3}{|c|}{ MANUAL INTEGRATION } \\
\cline { 3 - 5 } & TIME & REASON & ANALYST & DATE \\
\hline Dichlorodifluoromethane & 1.52 & Baseline & wilburj & $01 / 27 / 1613: 41$ \\
\hline
\end{tabular}

Lab Sample ID: IC 200-100049/8 Client Sample ID:

Date Analyzed: 01/27/16 12:57

Lab File ID: 18020_008.D

GC Column: DB-624

ID $: 0.2(\mathrm{~mm})$

\begin{tabular}{|c|c|c|c|c|}
\hline \multirow[t]{2}{*}{ COMPOUND NAME } & \multirow{2}{*}{$\begin{array}{c}\text { RETENTION } \\
\text { TIME }\end{array}$} & \multicolumn{3}{|c|}{ MANUAL INTEGRATION } \\
\hline & & REASON & ANALYST & DATE \\
\hline Dichlorodifluoromethane & 1.52 & Baseline & wilburj & $01 / 27 / 1613: 42$ \\
\hline
\end{tabular}

Lab Sample ID: MB 200-100049/11

Date Analyzed: 01/27/16 14:12

Client Sample ID:

\begin{tabular}{|l|c|c|c|c|}
\hline \multicolumn{1}{c|}{ COMPOUND NAME } & RETENTION & \multicolumn{3}{|c}{ MANUAL INTEGRATION } \\
\cline { 3 - 5 } & TIME & REASON & ANALYST & DATE \\
\hline m, p-Xylene & 9.59 & Assign Peak & wilburj & $01 / 27 / 16$ 17:23 \\
\hline
\end{tabular}

ID : $0.2(\mathrm{~mm})$

SOMO1.2/VOA_Tr 


\section{DATA REPORTING QUALIFIERS}

Client: Argonne National Laboratory

Job Number: 200-31673-1

Sdg Number: 31673

Lab Section

Qualifier

Description

GC/MS VOA

U

$\mathrm{J}$

$\mathrm{J}$

$\mathrm{X}$

*

B

$\mathrm{N}$
Analyzed for but not detected.

Indicates an Estimated Value for TICs

Indicates an estimated value.

See case narrative notes for explanation of the ' $X$ ' flag

Surrogate is outside acceptance limits.

The analyte was found in an associated blank, as well as in the sample.

This flag indicates the presumptive evidence of a compound. 
Lab Name: TESTAMERICA BURLINGTON

Lab Code: STLV Case No.: YORK Mod. Ref No.: Level: (TRACE or LOW) TRACE
Contract: $8 \mathrm{E}-00302$

SDG No.: 31673

\begin{tabular}{|c|c|c|c|c|c|c|c|}
\hline $\begin{array}{c}\text { EPA } \\
\text { SAMPLE NO. }\end{array}$ & $\begin{array}{l}\text { VDMC1 } \\
(\mathrm{VCL}) \quad \#\end{array}$ & $\begin{array}{l}\text { VDMC2 } \\
(\mathrm{CLA}) \#\end{array}$ & $\begin{array}{l}\text { VDMC3 } \\
(\mathrm{DCE}) \#\end{array}$ & $\begin{array}{l}\text { VDMC4 } \\
(\mathrm{BUT}) \#\end{array}$ & $\begin{array}{l}\text { VDMC5 } \\
(\mathrm{CLF}) \#\end{array}$ & $\begin{array}{l}\text { VDMC 6 } \\
(\mathrm{DCA})\end{array}$ & $\begin{array}{l}\text { VDMC } 7 \\
(\mathrm{BEN}) \quad \#\end{array}$ \\
\hline VBLKDG & 103 & 118 & 98 & 76 & 101 & 121 & 95 \\
\hline $\begin{array}{l}\text { YKTI24-100-W-3 } \\
8019\end{array}$ & 102 & 115 & 101 & 95 & 114 & $142 \star$ & 96 \\
\hline YKQCTB-W-38021 & 100 & 117 & 100 & 86 & 111 & $140 *$ & 92 \\
\hline $\begin{array}{l}\text { YKTI25-108-W-3 } \\
8027\end{array}$ & 99 & 117 & 103 & 97 & 115 & $145 \star$ & 94 \\
\hline VBLKDH & 101 & 106 & 79 & 108 & 100 & 110 & 109 \\
\hline VHBLK01 & 101 & 98 & 81 & 109 & 102 & 108 & 108 \\
\hline
\end{tabular}

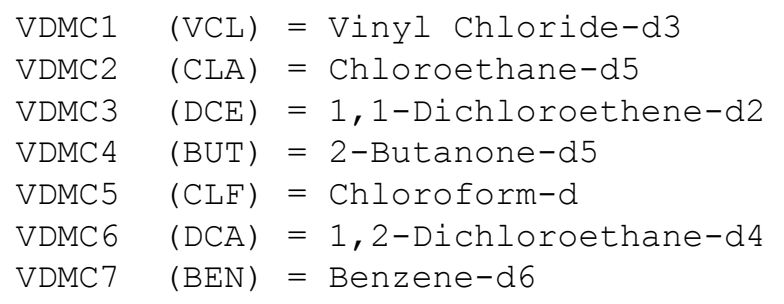

\# Column to be used to flag recovery values

* Values outside of contract required QC limits

$$
\begin{gathered}
\frac{Q C \text { LIMITS }}{(65-131)} \\
(71-131) \\
(55-104) \\
(49-155) \\
(78-121) \\
(78-129) \\
(77-124)
\end{gathered}
$$


Lab Name: TESTAMERICA BURLINGTON

Contract: $8 \mathrm{E}-00302$

Lab Code: STLV Case No.: Y YORK Mod. Ref No.: SDG No.: 31673

Level: (TRACE or LOW) TRACE

\begin{tabular}{|c|c|c|c|c|c|c|c|c|}
\hline $\begin{array}{c}\text { EPA } \\
\text { SAMPLE NO. }\end{array}$ & $\begin{array}{l}\text { VDMC8 } \\
(\mathrm{DPA}) \quad \#\end{array}$ & $\begin{array}{l}\text { VDMC9 } \\
(\text { TOL }) \#\end{array}$ & $\begin{array}{l}\text { VDMC10 } \\
(\mathrm{TDP}) \#\end{array}$ & $\begin{array}{l}\text { VDMC11 } \\
(\operatorname{HEX}) \#\end{array}$ & $\begin{array}{l}\text { VDMC12 } \\
(\mathrm{TCA}) \#\end{array}$ & $\begin{array}{l}\text { VDMC13 } \\
(\mathrm{DCZ}) \#\end{array}$ & OTHER & $\begin{array}{l}\text { TOT } \\
\text { OUT }\end{array}$ \\
\hline VBLKDG & 84 & 102 & 104 & 87 & 78 & 108 & & 0 \\
\hline $\begin{array}{l}\text { YKTI24-100-W-3 } \\
8019\end{array}$ & 87 & 103 & 109 & 104 & 89 & 113 & & 1 \\
\hline YKQCTB-W-38021 & 84 & 99 & 102 & 94 & 88 & 112 & & 1 \\
\hline $\begin{array}{l}\text { YKTI25-108-W-3 } \\
8027\end{array}$ & 87 & 103 & 108 & 104 & 90 & 120 & & 1 \\
\hline VBLKDH & 105 & 107 & 99 & 114 & 92 & 114 & & 0 \\
\hline VHBLK01 & 105 & 107 & 104 & 111 & 91 & 121 & & 0 \\
\hline
\end{tabular}

\footnotetext{
$\operatorname{VDMC} 8(\mathrm{DPA})=1,2-\mathrm{Dichloropropane}-\mathrm{d} 6$

VDMC $9 \quad($ TOL $)=$ Toluene-d8

VDMC10 $(\mathrm{TDP})=$ trans $-1,3-\mathrm{Dichloropropene-d4}$

$\operatorname{VDMC1}(\mathrm{HEX})=2-$ Hexanone-d5

VDMC12 $(\mathrm{TCA})=1,1,2,2$-Tetrachloroethane-d2

VDMC13 $(\mathrm{DCZ})=1,2-\mathrm{Dichlorobenzene-d4}$
}

$$
\begin{gathered}
\frac{Q C \text { LIMITS }}{(79-124)} \\
(77-121) \\
(73-121) \\
(28-135) \\
(73-125) \\
(80-131)
\end{gathered}
$$

\# Column to be used to flag recovery values

* Values outside of contract required QC limits

Report 1,4-Dioxane-d8 for Low-Medium VOA analysis only

Page 1 of 1 
$4 A$ - FORM IV VOA

VOLATILE METHOD BLANK SUMMARY
EPA SAMPLE NO.

VBLKDG

Lab Name: TESTAMERICA BURLINGTON

Contract: 8E-00302

Lab Code: STLV Case No.: YORK Mod. Ref No.: SDG No.: 31673

Lab File ID: 18001_007.D

Lab Sample ID: MB 200-100004/7

Instrument ID: CHD.i

Matrix: (SOIL/SED/WATER) Water

Date Analyzed: 01/26/2016

Level: (TRACE or LOW/MED) TRACE

Time Analyzed: 1327

GC Column: DB-624

ID $0.20 \quad(\mathrm{~mm})$

Heated Purge: ( $\mathrm{Y} / \mathrm{N}) \mathrm{N}$

\begin{tabular}{|c|c|c|c|}
\hline $\begin{array}{c}\text { EPA } \\
\text { SAMPLE NO. }\end{array}$ & $\begin{array}{r}\text { LAB } \\
\text { SAMPLE ID }\end{array}$ & $\begin{array}{l}\text { LAB } \\
\text { FILE ID }\end{array}$ & $\begin{array}{c}\text { TIME } \\
\text { ANALYZED }\end{array}$ \\
\hline $\begin{array}{l}\text { YKTI24-100-W } \\
-38019\end{array}$ & $200-31673-1$ & $\begin{array}{l}18001 \text { - } \\
\text { D }\end{array}$ & 1930 \\
\hline $\begin{array}{l}\text { YKQCTB-W-380 } \\
21\end{array}$ & $200-31673-2$ & $\begin{array}{l}18001+022 . \\
D\end{array}$ & 1955 \\
\hline $\begin{array}{l}\text { YKTI25-108-W } \\
-38027\end{array}$ & $200-31673-3$ & $\begin{array}{l}18001+023 . \\
D\end{array}$ & 2020 \\
\hline
\end{tabular}

COMMENTS :

Page 1 of 1 
Lab Name: TESTAMERICA BURLINGTON

Contract: 8E-00302

Lab Code: STLV Case No.: YORK Mod. Ref No.: SDG No.: 31673

Lab File ID: 18020_011.D

Lab Sample ID: MB 200-100049/11

Instrument ID: CHD.i

Matrix: (SOIL/SED/WATER) Water

Date Analyzed: 01/27/2016

Level: (TRACE or LOW/MED) TRACE

Time Analyzed: 1412

GC Column: DB-624

ID $0.20 \quad(\mathrm{~mm})$

Heated Purge: ( $\mathrm{Y} / \mathrm{N}) \mathrm{N}$

\begin{tabular}{|c|c|c|c|}
\hline $\begin{array}{c}\text { EPA } \\
\text { SAMPLE NO. }\end{array}$ & $\begin{array}{r}\text { LAB } \\
\text { SAMPLE ID }\end{array}$ & $\begin{array}{c}\text { LAB } \\
\text { FILE ID }\end{array}$ & $\begin{array}{c}\text { TIME } \\
\text { ANALYZED }\end{array}$ \\
\hline VHBLK01 & $200-31673-4$ & $\begin{array}{l}18020 \_016 . \\
D\end{array}$ & 1616 \\
\hline
\end{tabular}

COMMENTS :

Page 1 of 1 
$5 A$ - FORM V VOA

VOLATILE ORGANICS INSTRUMENT

PERFORMANCE CHECK

BROMOFLUOROBENZENE (BFB)
EPA SAMPLE NO.

BFBDZ
Lab Name: TESTAMERICA BURLINGTON

Lab Code: STLV

Case No.: YORK

Lab File Id: 17668_001.D

Instrument Id: CHD.i

GC Column: DB-624

ID $: 0.20$
Contract: 8E-00302 Mod. Ref No.: SDG No.: 31673

BEB Injection Date: 01/08/2016

BFB Injection Time: 1131 $(\mathrm{mm})$

\begin{tabular}{|c|c|c|}
\hline $\mathrm{m} / \mathrm{e}$ & ION ABUNDANCE CRITERIA & $\begin{array}{l}\text { \% RELATIVE } \\
\text { ABUNDANCE }\end{array}$ \\
\hline 50 & $15.0-40.0 \%$ of mass 95 & 20.6 \\
\hline 75 & $30.0-80.0 \%$ of mass 95 & 53.3 \\
\hline 95 & Base peak, $100 \%$ relative abundance & 100 \\
\hline 96 & $5.0-9.0 \%$ of mass 95 & 6.4 \\
\hline 173 & Less than $2.0 \%$ of mass 174 & $0.7) 1$ \\
\hline 174 & $50.0-120 \%$ of mass 95 & 79.3 \\
\hline 175 & $5.0-9.0 \%$ of mass 174 & $5.5(6.9) 1$ \\
\hline 176 & $95.0-101 \%$ of mass 174 & $76.6(96.7) 1$ \\
\hline 177 & $5.0-9.0 \%$ of mass 176 & $(6.4) 2$ \\
\hline
\end{tabular}

1 - Value is omass 1742 - Value is omass 176

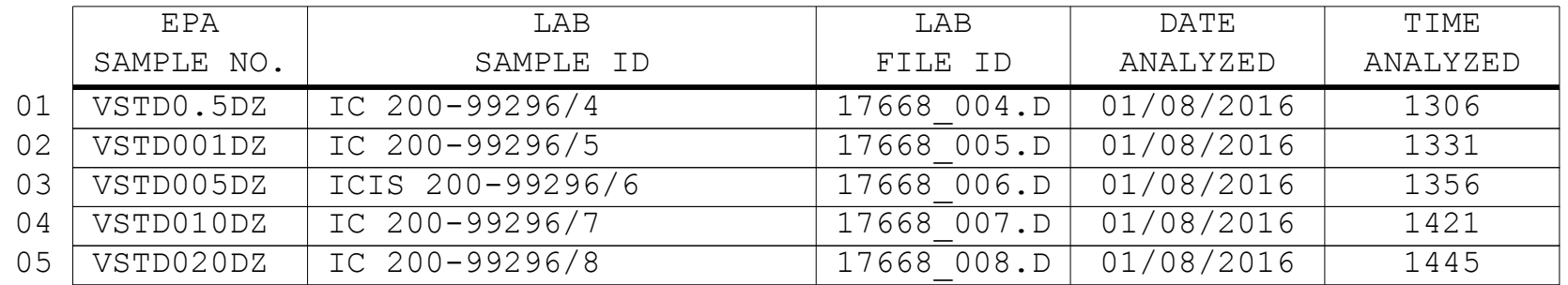


$5 A$ - FORM V VOA

VOLATILE ORGANICS INSTRUMENT

PERFORMANCE CHECK

BROMOFLUOROBENZENE (BFB)
EPA SAMPLE NO.

BFBDG
Lab Name: TESTAMERICA BURLINGTON

Lab Code: STLV Case No.: YORK

Lab File Id: 18001_001.D

Instrument Id: CHD.i

GC Column: DB-624

ID $: 0.20$
Contract: $8 \mathrm{E}-00302$ Mod. Ref No.: SDG No.: 31673

BFB Injection Date: 01/26/2016

BFB Injection Time: 1046 $(\mathrm{mm})$

\begin{tabular}{|c|c|c|}
\hline $\mathrm{m} / \mathrm{e}$ & ION ABUNDANCE CRITERIA & $\begin{array}{l}\text { \% RELATIVE } \\
\text { ABUNDANCE }\end{array}$ \\
\hline 50 & $15.0-40.0 \%$ of mass 95 & 20.7 \\
\hline 75 & $30.0-80.0 \%$ of mass 95 & 53.9 \\
\hline 95 & Base peak, $100 \%$ relative abundance & 100 \\
\hline 96 & $5.0-9.0 \%$ of mass 95 & 5.6 \\
\hline 173 & Less than $2.0 \%$ of mass 174 & $1.0) 1$ \\
\hline 174 & $50.0-120 \%$ of mass 95 & 79.7 \\
\hline 175 & $5.0-9.0 \%$ of mass 174 & $5.3(6.7) 1$ \\
\hline 176 & $95.0-101 \%$ of mass 174 & $77.8(97.7) 1$ \\
\hline 177 & $5.0-9.0 \%$ of mass 176 & $(6.1) 2$ \\
\hline
\end{tabular}

1 - Value is omass 1742 - Value is omass 176

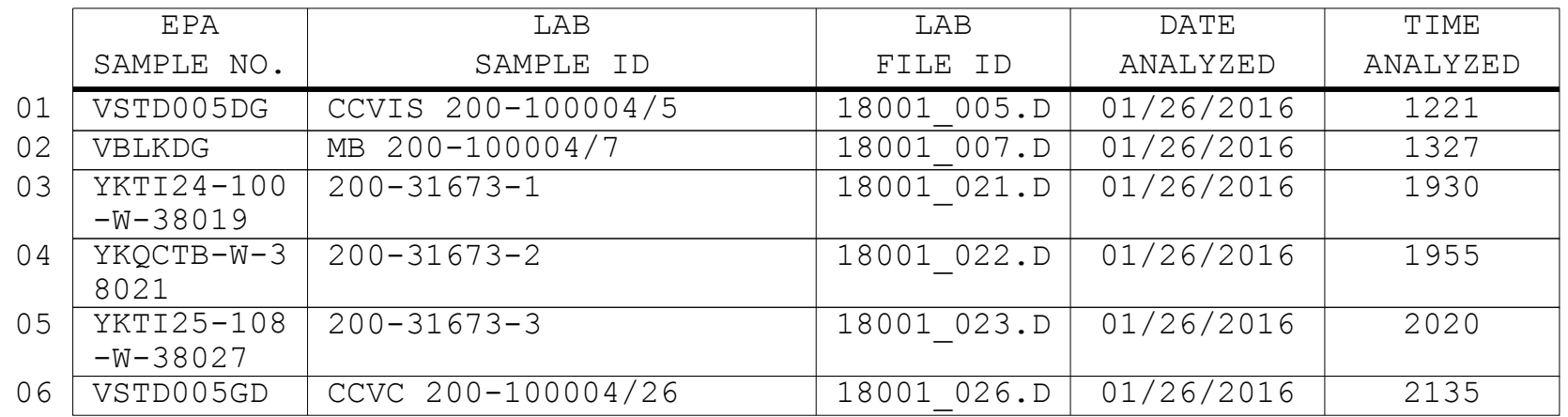


$5 A$ - FORM V VOA

VOLATILE ORGANICS INSTRUMENT

PERFORMANCE CHECK

BROMOFLUOROBENZENE (BFB)
EPA SAMPLE NO.

$\mathrm{BFBDH}$
Lab Name: TESTAMERICA BURLINGTON

Lab Code: STLV Case No.: YORK

Lab File Id: 18020_001.D

Instrument Id: CHD.i

GC Column: DB-624

ID $: 0.20$
Contract: $8 \mathrm{E}-00302$ Mod. Ref No.: SDG No.: 31673

BFB Injection Date: 01/27/2016

BFB Injection Time: 1008 (mm)

\begin{tabular}{|c|c|c|}
\hline $\mathrm{m} / \mathrm{e}$ & ION ABUNDANCE CRITERIA & $\begin{array}{l}\text { \% RELATIVE } \\
\text { ABUNDANCE }\end{array}$ \\
\hline 50 & $15.0-40.0 \%$ of mass 95 & 24.9 \\
\hline 75 & $30.0-80.0 \%$ of mass 95 & 64.2 \\
\hline 95 & Base peak, $100 \%$ relative abundance & 100 \\
\hline 96 & $5.0-9.0 \%$ of mass 95 & 6.3 \\
\hline 173 & Less than $2.0 \%$ of mass 174 & $0.6) 1$ \\
\hline 174 & $50.0-120 \%$ of mass 95 & 85.9 \\
\hline 175 & $5.0-9.0 \%$ of mass 174 & $6.2(7.2) 1$ \\
\hline 176 & $95.0-101 \%$ of mass 174 & $82.0(95.5) 1$ \\
\hline 177 & $5.0-9.0 \%$ of mass 176 & $(6.1) 2$ \\
\hline
\end{tabular}

1 - Value is omass 174

2 - Value is omass 176

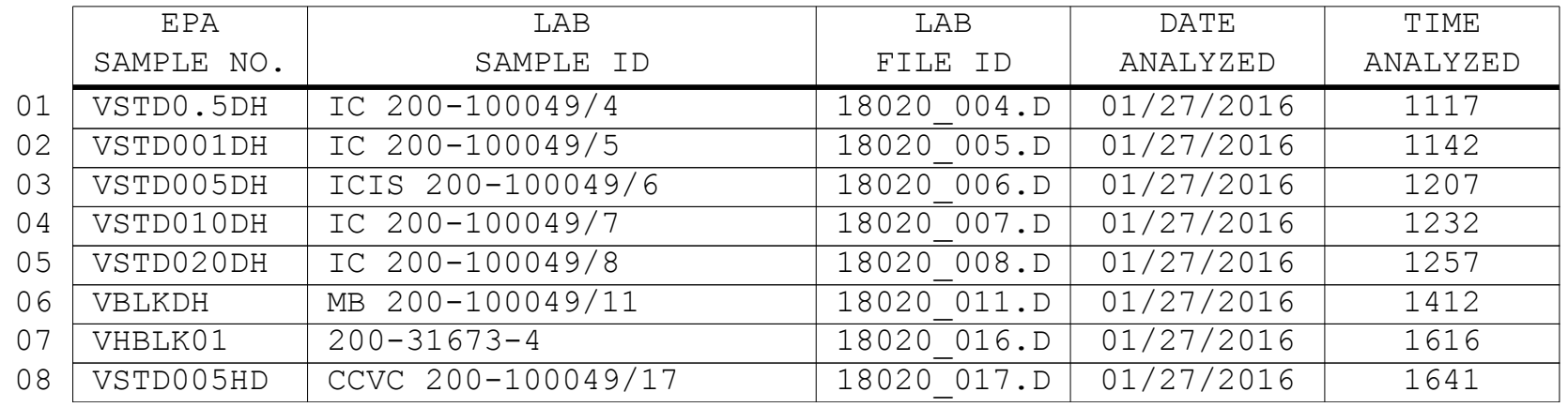


Lab Name: TESTAMERICA BURLINGTON

Contract: $8 \mathrm{E}-00302$

Lab Code: STLV Case No.: YORK Mod. Ref No.: SDG No.: 31673

GC Column: DB-624

ID: 0.20 (mm) Init. Calib. Date (s): 01/08/2016 01/08/2016

EPA Sample No. (VSTD\#\#\#\#\#) : VSTD005DG

Lab File ID (Standard): 18001_005.D

Date Analyzed: 01/26/2016

Instrument ID: CHD.i

Time Analyzed: 1221

Heated Purge: ( $\mathrm{Y} / \mathrm{N}) \mathrm{N}$

\begin{tabular}{|c|c|c|c|c|c|c|}
\hline & IS1 (CBZ) & & IS2 (DFB) & & IS3 (DCB) & \\
\hline & AREA & RT \# & AREA & $\mathrm{RT}$ & AREA & $\mathrm{RT}$ \\
\hline 12 HOUR STD & 513786 & 9.28 & 653659 & 5.93 & 250134 & 12.09 \\
\hline UPPER LIMIT & 719300 & 9.61 & 915123 & 6.26 & 350188 & 12.42 \\
\hline LOWER LIMIT & 308272 & 8.95 & 392195 & 5.60 & 150080 & 11.76 \\
\hline EPA SAMPLE NO. & & & & & & \\
\hline VBLKDG & 480349 & 9.28 & 659680 & 5.93 & 195519 & 12.09 \\
\hline $\begin{array}{l}\text { YKTI24-100-W-3 } \\
8019\end{array}$ & 440059 & 9.28 & 561747 & 5.93 & 176669 & 12.09 \\
\hline YKQCTB-W-38021 & 444990 & 9.28 & 551946 & 5.93 & 178282 & 12.09 \\
\hline $\begin{array}{l}\text { YKTI25-108-W-3 } \\
8027\end{array}$ & 464465 & 9.28 & 581672 & 5.93 & 177863 & 12.09 \\
\hline
\end{tabular}

IS1 $(\mathrm{CBZ})=$ Chlorobenzene-d5

IS2 $(\mathrm{DFB})=1,4-\mathrm{Difluorobenzene}$

IS3 $(\mathrm{DCB})=1,4-\mathrm{Dichlorobenzene-d4}$

AREA UPPER LIMIT $=140 \%$ (Trace Volatiles) of internal standard area AREA LOWER LIMIT $=60 \%$ (Trace Volatiles) of internal standard area RT UPPER LIMIT $=+0.33$ (Trace Volatiles) minutes of internal standard RT RT LOWER LIMIT $=-0.33$ (Trace Volatiles) minutes of internal standard RT

\# Column used to flag values outside contract required QC limits with an asterisk. Page 1 of 1 
Lab Name: TESTAMERICA BURLINGTON

Contract: $8 \mathrm{E}-00302$

Lab Code: STLV Case No.: YORK Mod. Ref No.: SDG No.: 31673

GC Column: DB-624

ID: 0.20 (mm) Init. Calib. Date (s) : 01/27/2016 01/27/2016

EPA Sample No. (VSTD\#\#\#\#\#) : VSTD005DH

Lab File ID (Standard) : 18020_006.D

Date Analyzed: 01/27/2016

Instrument ID: CHD.i

Time Analyzed: 1207

Heated Purge: ( $\mathrm{Y} / \mathrm{N}) \mathrm{N}$

\begin{tabular}{|c|c|c|c|c|c|c|}
\hline & $\begin{array}{c}\text { IS1 (CBZ) } \\
\text { AREA }\end{array}$ & RT \# & $\begin{array}{c}\text { IS2 (DFB) } \\
\text { AREA }\end{array}$ & $\mathrm{RT}$ & $\begin{array}{c}\text { IS3 (DCB) } \\
\text { AREA }\end{array}$ & $\mathrm{RT}$ \\
\hline 12 HOUR STD & 468047 & 9.28 & 573131 & 5.93 & 235332 & 12.09 \\
\hline UPPER LIMIT & 655266 & 9.61 & 802383 & 6.26 & 329465 & 12.42 \\
\hline LOWER LIMIT & 280828 & 8.95 & 343879 & 5.60 & 141199 & 11.76 \\
\hline EPA SAMPLE NO. & & & & & & \\
\hline VBLKDH & 441899 & 9.28 & 546095 & 5.93 & 180274 & 12.09 \\
\hline VHBLK01 & 420868 & 9.28 & 520359 & 5.93 & 168947 & 12.09 \\
\hline
\end{tabular}

IS1 $(\mathrm{CBZ})=$ Chlorobenzene-d5

IS2 $(\mathrm{DFB})=1,4-\mathrm{Difluorobenzene}$

IS3 $(\mathrm{DCB})=1,4-\mathrm{Dichlorobenzene-d4}$

AREA UPPER LIMIT $=140 \%$ (Trace Volatiles) of internal standard area AREA LOWER LIMIT $=60 \%$ (Trace Volatiles) of internal standard area RT UPPER LIMIT $=+0.33$ (Trace Volatiles) minutes of internal standard RT RT LOWER LIMIT $=-0.33$ (Trace Volatiles) minutes of internal standard RT

\# Column used to flag values outside contract required QC limits with an asterisk. Page 1 of 1 
Lab Name: TESTAMERICA BURLINGTON

Contract: $8 \mathrm{E}-00302$

Lab Code: STLV

Case No.: YORK Mod. Ref No.:

SDG No.: 31673

Matrix: (SOIL/SED/WATER) Water

Sample wt/vol: 25.0 $(\mathrm{g} / \mathrm{mL}) \mathrm{mL}$

Level: (TRACE/LOW/MED) TRACE

\% Moisture: not dec.

GC Column: DB-624 ID: $0.20 \quad(\mathrm{~mm})$

Soil Extract Volume: (uL) $(\mathrm{mL})$

Purge Volume: 25.0
Lab Sample ID: 200-31673-2

Lab File ID: 18001_022.D

Date Received: 01/22/2016

Date Analyzed: 01/26/2016

Dilution Factor: 1.0

Soil Aliquot Volume: (uL)

\begin{tabular}{|c|c|c|c|}
\hline CAS NO. & COMPOUND & $\begin{array}{l}\text { CONCENTRATION UNITS: } \\
(\mathrm{ug} / \mathrm{L} \text { or } \mathrm{ug} / \mathrm{kg}) \mathrm{ug} / \mathrm{L}\end{array}$ & q \\
\hline $75-71-8$ & Dichlorodifluoromethane & 1.0 & $\mathrm{U}$ \\
\hline $74-87-3$ & Chloromethane & 1.0 & $\mathrm{U}$ \\
\hline $75-01-4$ & Vinyl chloride & 1.0 & $\mathrm{U}$ \\
\hline $74-83-9$ & Bromomethane & 1.0 & $\mathrm{U}$ \\
\hline $75-00-3$ & Chloroethane & 1.0 & $\mathrm{U}$ \\
\hline $75-69-4$ & Trichlorofluoromethane & 1.0 & $\mathrm{U}$ \\
\hline $75-35-4$ & 1,1-Dichloroethene & 1.0 & $\mathrm{U}$ \\
\hline $76-13-1$ & $1,1,2$-Trichloro-1,2,2-trifluoroethane & 1.0 & $\mathrm{U}$ \\
\hline $67-64-1$ & Acetone & 4.6 & $\mathrm{~J}$ \\
\hline $75-15-0$ & Carbon disulfide & 1.0 & $\mathrm{U}$ \\
\hline $79-20-9$ & Methyl acetate & 1.0 & $\mathrm{U}$ \\
\hline $75-09-2$ & Methylene Chloride & 1.0 & $\mathrm{U}$ \\
\hline $156-60-5$ & trans-1,2-Dichloroethene & 1.0 & $\mathrm{U}$ \\
\hline $1634-04-4$ & Methyl tert-butyl ether & 1.0 & $\mathrm{U}$ \\
\hline $75-34-3$ & 1,1-Dichloroethane & 1.0 & $\mathrm{U}$ \\
\hline $156-59-2$ & cis-1,2-Dichloroethene & 1.0 & $\mathrm{U}$ \\
\hline $78-93-3$ & 2-Butanone & 5.0 & $\mathrm{U}$ \\
\hline $74-97-5$ & Bromochloromethane & 1.0 & $\mathrm{U}$ \\
\hline $67-66-3$ & Chloroform & 1.0 & $\mathrm{U}$ \\
\hline $71-55-6$ & $1,1,1$-Trichloroethane & 1.0 & $\mathrm{U}$ \\
\hline $110-82-7$ & Cyclohexane & 1.0 & $\mathrm{U}$ \\
\hline $56-23-5$ & Carbon tetrachloride & 1.0 & $\mathrm{U}$ \\
\hline $71-43-2$ & Benzene & 1.0 & $\mathrm{U}$ \\
\hline $107-06-2$ & 1,2-Dichloroethane & 1.0 & $\mathrm{U}$ \\
\hline
\end{tabular}

Report 1,4-Dioxane for Low-Medium VOA analysis only 
Lab Name: TESTAMERICA BURLINGTON

Contract: $8 \mathrm{E}-00302$

Lab Code: STLV

Case No.: YORK

Mod. Ref No.:

SDG No.: 31673

Matrix: (SOIL/SED/WATER) Water

Sample wt/vol: 25.0 $(\mathrm{g} / \mathrm{mL}) \mathrm{mL}$

Level: (TRACE/LOW/MED) TRACE

\% Moisture: not dec.

GC Column: DB-624 ID: $0.20 \quad(\mathrm{~mm})$

Soil Extract Volume: (uL) $(\mathrm{mL})$

Purge Volume: 25.0
Lab Sample ID: 200-31673-2

Lab File ID: 18001_022.D

Date Received: 01/22/2016

Date Analyzed: 01/26/2016

Dilution Factor: 1.0

Soil Aliquot Volume: (uL)

\begin{tabular}{|c|c|c|c|}
\hline CAS NO. & COMPOUND & $\begin{array}{l}\text { CONCENTRATION UNITS: } \\
(\mathrm{ug} / \mathrm{L} \text { or } \mathrm{ug} / \mathrm{kg}) \mathrm{ug} / \mathrm{L}\end{array}$ & Q \\
\hline $79-01-6$ & Trichloroethene & 0.093 & $\mathrm{~J}$ \\
\hline $108-87-2$ & Methylcyclohexane & 1.0 & $\mathrm{U}$ \\
\hline $78-87-5$ & 1,2-Dichloropropane & 1.0 & $\mathrm{U}$ \\
\hline $75-27-4$ & Bromodichloromethane & 1.0 & $\mathrm{U}$ \\
\hline $10061-01-5$ & cis-1,3-Dichloropropene & 1.0 & U \\
\hline $108-10-1$ & 4-Methyl-2-pentanone & 1.0 & $\mathrm{U}$ \\
\hline $108-88-3$ & Toluene & 0.15 & $\mathrm{~J} B$ \\
\hline $10061-02-6$ & trans-1,3-Dichloropropene & 1.0 & $\mathrm{U}$ \\
\hline $79-00-5$ & $1,1,2-$ Trichloroethane & 1.0 & $\mathrm{U}$ \\
\hline $127-18-4$ & Tetrachloroethene & 1.0 & $\mathrm{U}$ \\
\hline $591-78-6$ & 2-Hexanone & 1.0 & $\mathrm{U}$ \\
\hline $124-48-1$ & Dibromochloromethane & 1.0 & $\mathrm{U}$ \\
\hline $106-93-4$ & 1,2-Dibromoethane & 1.0 & $\mathrm{U}$ \\
\hline $108-90-7$ & Chlorobenzene & 1.0 & $\mathrm{U}$ \\
\hline $100-41-4$ & Ethylbenzene & 1.0 & $\mathrm{U}$ \\
\hline $95-47-6$ & o-Xylene & 0.10 & $\mathrm{~J}$ \\
\hline $179601-23-1$ & m,p-xylene & 0.048 & $\mathrm{~J}$ \\
\hline $100-42-5$ & Styrene & 1.0 & $\mathrm{U}$ \\
\hline $75-25-2$ & Bromoform & 1.0 & $\mathrm{U}$ \\
\hline $98-82-8$ & Isopropylbenzene & 1.0 & $\mathrm{U}$ \\
\hline $79-34-5$ & $1,1,2,2$-Tetrachloroethane & 1.0 & $\mathrm{U}$ \\
\hline $541-73-1$ & 1,3 -Dichlorobenzene & 1.0 & $\mathrm{U}$ \\
\hline $106-46-7$ & 1,4-Dichlorobenzene & 1.0 & $\mathrm{U}$ \\
\hline $95-50-1$ & 1,2-Dichlorobenzene & 1.0 & $\mathrm{U}$ \\
\hline $96-12-8$ & 1,2-Dibromo-3-Chloropropane & 1.0 & $\mathrm{U}$ \\
\hline $120-82-1$ & $1,2,4$-Trichlorobenzene & 1.0 & $\mathrm{U}$ \\
\hline $87-61-6$ & $1,2,3$-Trichlorobenzene & 1.0 & $\mathrm{U}$ \\
\hline
\end{tabular}


Lab Name: TESTAMERICA BURLINGTON

Contract: $8 \mathrm{E}-00302$

Lab Code: STLV Case No.: YORK Mod. Ref No.: SDG No.: 31673

Matrix: (SOIL/SED/WATER) Water

Sample wt/vol: 25.0 $(\mathrm{g} / \mathrm{mL}) \mathrm{mL}$

Level: (TRACE or LOW/MED) TRACE

응 Moisture: not dec.

GC Column: DB-624 ID $: 0.20 \quad(\mathrm{~mm})$

Soil Extract Volume: (uL)
Lab Sample ID: 200-31673-2

Lab File ID: 18001_022.D

Date Received: 01/22/2016

Date Analyzed: 01/26/2016

Dilution Factor: 1.0

Soil Aliquot Volume: (uL) Purge Volume: 25.0 CONCENTRATION UNITS: (ug/L or ug/kg) ug/L

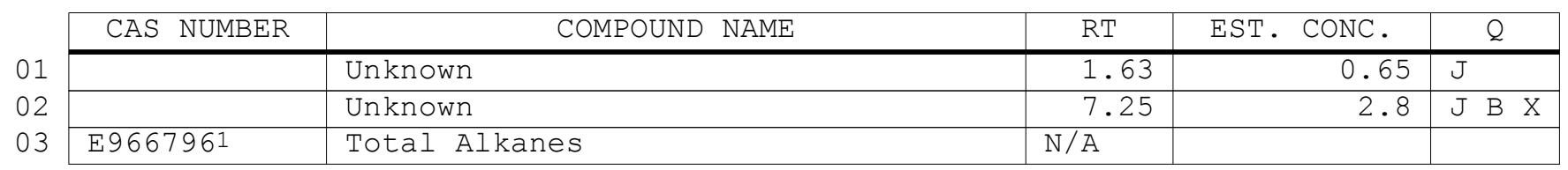


Lab Name: TESTAMERICA BURLINGTON

Contract: $8 \mathrm{E}-00302$

Lab Code: STLV

Case No.: YORK Mod. Ref No.: SDG No.: 31673

Matrix: (SOIL/SED/WATER) Water

Sample wt/vol: 25.0 $(\mathrm{g} / \mathrm{mL}) \mathrm{mL}$

Level: (TRACE/LOW/MED) TRACE

\% Moisture: not dec.

GC Column: DB-624 ID: $0.20 \quad(\mathrm{~mm})$

Soil Extract Volume: (uL) $(\mathrm{mL})$

Purge Volume: 25.0
Lab Sample ID: 200-31673-1

Lab File ID: 18001_021.D

Date Received: 01/22/2016

Date Analyzed: 01/26/2016

Dilution Factor: 1.0

Soil Aliquot Volume:

(uL)

\begin{tabular}{|c|c|c|c|}
\hline CAS NO. & COMPOUND & $\begin{array}{l}\text { CONCENTRATION UNITS: } \\
(\mathrm{ug} / \mathrm{L} \text { or } \mathrm{ug} / \mathrm{kg}) \mathrm{ug} / \mathrm{L}\end{array}$ & q \\
\hline $75-71-8$ & Dichlorodifluoromethane & 1.0 & $\mathrm{U}$ \\
\hline $74-87-3$ & Chloromethane & 1.0 & $\mathrm{U}$ \\
\hline $75-01-4$ & Vinyl chloride & 1.0 & $\mathrm{U}$ \\
\hline $74-83-9$ & Bromomethane & 1.0 & $\mathrm{U}$ \\
\hline $75-00-3$ & Chloroethane & 1.0 & $\mathrm{U}$ \\
\hline $75-69-4$ & Trichlorofluoromethane & 1.0 & $\mathrm{U}$ \\
\hline $75-35-4$ & 1,1-Dichloroethene & 1.0 & $\mathrm{U}$ \\
\hline $76-13-1$ & $1,1,2$-Trichloro-1,2,2-trifluoroethane & 1.0 & $\mathrm{U}$ \\
\hline $67-64-1$ & Acetone & 5.0 & $\mathrm{U}$ \\
\hline $75-15-0$ & Carbon disulfide & 1.0 & $\mathrm{U}$ \\
\hline $79-20-9$ & Methyl acetate & 1.0 & $\mathrm{U}$ \\
\hline $75-09-2$ & Methylene Chloride & 1.0 & $\mathrm{U}$ \\
\hline $156-60-5$ & trans-1,2-Dichloroethene & 1.0 & $\mathrm{U}$ \\
\hline $1634-04-4$ & Methyl tert-butyl ether & 1.0 & $\mathrm{U}$ \\
\hline $75-34-3$ & 1,1-Dichloroethane & 1.0 & $\mathrm{U}$ \\
\hline $156-59-2$ & cis-1,2-Dichloroethene & 1.0 & $\mathrm{U}$ \\
\hline $78-93-3$ & 2-Butanone & 5.0 & $\mathrm{U}$ \\
\hline $74-97-5$ & Bromochloromethane & 1.0 & $\mathrm{U}$ \\
\hline $67-66-3$ & Chloroform & 1.0 & $\mathrm{U}$ \\
\hline $71-55-6$ & $1,1,1$-Trichloroethane & 1.0 & $\mathrm{U}$ \\
\hline $110-82-7$ & Cyclohexane & 1.0 & $\mathrm{U}$ \\
\hline $56-23-5$ & Carbon tetrachloride & 0.51 & $\mathrm{~J}$ \\
\hline $71-43-2$ & Benzene & 1.0 & $\mathrm{U}$ \\
\hline $107-06-2$ & 1,2-Dichloroethane & 1.0 & $\mathrm{U}$ \\
\hline
\end{tabular}

Report 1,4-Dioxane for Low-Medium VOA analysis only 
Lab Name: TESTAMERICA BURLINGTON

Contract: 8E-00302

Lab Code: STLV

Case No.: YORK Mod. Ref No.:

SDG No.: 31673

Matrix: (SOIL/SED/WATER) Water

Sample wt/vol: 25.0 $(\mathrm{g} / \mathrm{mL}) \mathrm{mL}$

Level: (TRACE/LOW/MED) TRACE

\% Moisture: not dec.

GC Column: DB-624 ID: $0.20 \quad(\mathrm{~mm})$

Soil Extract Volume: (uL) $(\mathrm{mL})$

Purge Volume: 25.0
Lab Sample ID: 200-31673-1

Lab File ID: 18001_021.D

Date Received: 01/22/2016

Date Analyzed: 01/26/2016

Dilution Factor: 1.0

Soil Aliquot Volume: (uL)

\begin{tabular}{|c|c|c|c|}
\hline CAS NO. & COMPOUND & $\begin{array}{l}\text { CONCENTRATION UNITS: } \\
(\mathrm{ug} / \mathrm{L} \text { or } \mathrm{ug} / \mathrm{kg}) \mathrm{ug} / \mathrm{L}\end{array}$ & Q \\
\hline $79-01-6$ & Trichloroethene & 0.079 & $\mathrm{~J}$ \\
\hline $108-87-2$ & Methylcyclohexane & 1.0 & $\mathrm{U}$ \\
\hline $78-87-5$ & 1,2-Dichloropropane & 1.0 & $\mathrm{U}$ \\
\hline $75-27-4$ & Bromodichloromethane & 1.0 & $\mathrm{U}$ \\
\hline $10061-01-5$ & cis-1,3-Dichloropropene & 1.0 & $\mathrm{U}$ \\
\hline $108-10-1$ & 4-Methyl-2-pentanone & 1.0 & $\mathrm{U}$ \\
\hline $108-88-3$ & Toluene & 0.048 & $\mathrm{~J} B$ \\
\hline $10061-02-6$ & trans-1,3-Dichloropropene & 1.0 & $\mathrm{U}$ \\
\hline $79-00-5$ & $1,1,2-T r i c h l o r o e t h a n e$ & 1.0 & $\mathrm{U}$ \\
\hline $127-18-4$ & Tetrachloroethene & 0.035 & $\mathrm{~J}$ \\
\hline $591-78-6$ & 2-Hexanone & 1.0 & $\mathrm{U}$ \\
\hline $124-48-1$ & Dibromochloromethane & 1.0 & $\mathrm{U}$ \\
\hline $106-93-4$ & 1,2-Dibromoethane & 1.0 & $\mathrm{U}$ \\
\hline $108-90-7$ & Chlorobenzene & 1.0 & $\mathrm{U}$ \\
\hline $100-41-4$ & Ethylbenzene & 1.0 & $\mathrm{U}$ \\
\hline $95-47-6$ & o-Xylene & 1.0 & $\mathrm{U}$ \\
\hline $179601-23-1$ & m,p-Xylene & 0.012 & $\mathrm{~J}$ \\
\hline $100-42-5$ & styrene & 1.0 & $\mathrm{U}$ \\
\hline $75-25-2$ & Bromoform & 1.0 & $\mathrm{U}$ \\
\hline $98-82-8$ & Isopropylbenzene & 1.0 & $\mathrm{U}$ \\
\hline $79-34-5$ & $1,1,2,2$-Tetrachloroethane & 1.0 & $\mathrm{U}$ \\
\hline $541-73-1$ & 1,3-Dichlorobenzene & 1.0 & $\mathrm{U}$ \\
\hline $106-46-7$ & 1,4-Dichlorobenzene & 1.0 & $\mathrm{U}$ \\
\hline $95-50-1$ & $1,2-$ Dichlorobenzene & 1.0 & $\mathrm{U}$ \\
\hline $96-12-8$ & 1,2-Dibromo-3-Chloropropane & 1.0 & $\mathrm{U}$ \\
\hline $120-82-1$ & $1,2,4$-Trichlorobenzene & 1.0 & $\mathrm{U}$ \\
\hline $87-61-6$ & $1,2,3$-Trichlorobenzene & 1.0 & $\mathrm{U}$ \\
\hline
\end{tabular}


Lab Name: TESTAMERICA BURLINGTON

Contract: $8 \mathrm{E}-00302$

Lab Code: STLV Case No.: YORK Mod. Ref No.: SDG No.: 31673

Matrix: (SOIL/SED/WATER) Water

Sample wt/vol: 25.0 $(\mathrm{g} / \mathrm{mL}) \mathrm{mL}$

Level: (TRACE or LOW/MED) TRACE

․ Moisture: not dec.

GC Column: DB-624 ID $: 0.20 \quad(\mathrm{~mm})$

Soil Extract Volume: (uL)

CONCENTRATION UNITS: (ug/L or ug/kg) ug/L
Lab Sample ID: 200-31673-1

Lab File ID: 18001_021.D

Date Received: 01/22/2016

Date Analyzed: 01/26/2016

Dilution Factor: 1.0

Soil Aliquot Volume: (uL) Purge Volume: 25.0 $(\mathrm{mL})$

\begin{tabular}{|c|c|c|c|c|}
\hline CAS NUMBER & COMPOUND NAME & RT & EST. CONC. & $Q$ \\
\hline 01 & Unknown & 7.25 & 3.0 & $\mathrm{~J} B \mathrm{X}$ \\
\hline $541-05-9$ & Cyclotrisiloxane, hexamethyl- & 8.17 & 0.75 & $\mathrm{~J} \quad \mathrm{~N} B$ \\
\hline 03 & Unknown & 10.98 & 1.1 & $\mathrm{~J} \mathrm{~B}$ \\
\hline E9667961 & Total Alkanes & $\mathrm{N} / \mathrm{A}$ & & \\
\hline
\end{tabular}

1 EPA-designated Registry Number. 
Lab Name: TESTAMERICA BURLINGTON

Contract: $8 \mathrm{E}-00302$

Lab Code: STLV

Case No.: YORK Mod. Ref No.: SDG No.: 31673

Matrix: (SOIL/SED/WATER) Water

Sample wt/vol: 25.0 $(\mathrm{g} / \mathrm{mL}) \mathrm{mL}$

Level: (TRACE/LOW/MED) TRACE

\% Moisture: not dec.

GC Column: DB-624 ID: $0.20 \quad(\mathrm{~mm})$

Soil Extract Volume: (uL) $(\mathrm{mL})$

Purge Volume: 25.0
Lab Sample ID: 200-31673-3

Lab File ID: 18001_023.D

Date Received: 01/22/2016

Date Analyzed: 01/26/2016

Dilution Factor: 1.0

Soil Aliquot Volume:

(uL)

\begin{tabular}{|c|c|c|c|}
\hline CAS NO. & COMPOUND & $\begin{array}{l}\text { CONCENTRATION UNITS: } \\
(\mathrm{ug} / \mathrm{L} \text { or } \mathrm{ug} / \mathrm{kg}) \mathrm{ug} / \mathrm{L}\end{array}$ & Q \\
\hline $75-71-8$ & Dichlorodifluoromethane & 1.0 & $\mathrm{U}$ \\
\hline $74-87-3$ & Chloromethane & 1.0 & $\mathrm{U}$ \\
\hline $75-01-4$ & Vinyl chloride & 1.0 & $\mathrm{U}$ \\
\hline $74-83-9$ & Bromomethane & 1.0 & $\mathrm{U}$ \\
\hline $75-00-3$ & Chloroethane & 1.0 & $\mathrm{U}$ \\
\hline $75-69-4$ & Trichlorofluoromethane & 1.0 & $\mathrm{U}$ \\
\hline $75-35-4$ & 1,1-Dichloroethene & 1.0 & $\mathrm{U}$ \\
\hline $76-13-1$ & $1,1,2$-Trichloro-1,2,2-trifluoroethane & 1.0 & $\mathrm{U}$ \\
\hline $67-64-1$ & Acetone & 5.0 & $\mathrm{U}$ \\
\hline $75-15-0$ & Carbon disulfide & 1.0 & $\mathrm{U}$ \\
\hline $79-20-9$ & Methyl acetate & 1.0 & $\mathrm{U}$ \\
\hline $75-09-2$ & Methylene Chloride & 1.0 & $\mathrm{U}$ \\
\hline $156-60-5$ & trans-1,2-Dichloroethene & 1.0 & $\mathrm{U}$ \\
\hline $1634-04-4$ & Methyl tert-butyl ether & 1.0 & $\mathrm{U}$ \\
\hline $75-34-3$ & 1,1-Dichloroethane & 1.0 & $\mathrm{U}$ \\
\hline $156-59-2$ & cis-1,2-Dichloroethene & 1.0 & $\mathrm{U}$ \\
\hline $78-93-3$ & 2-Butanone & 5.0 & $\mathrm{U}$ \\
\hline $74-97-5$ & Bromochloromethane & 1.0 & $\mathrm{U}$ \\
\hline $67-66-3$ & Chloroform & 1.0 & $\mathrm{U}$ \\
\hline $71-55-6$ & $1,1,1$-Trichloroethane & 1.0 & $\mathrm{U}$ \\
\hline $110-82-7$ & Cyclohexane & 1.0 & $\mathrm{U}$ \\
\hline $56-23-5$ & Carbon tetrachloride & 1.0 & $\mathrm{U}$ \\
\hline $71-43-2$ & Benzene & 1.0 & $\mathrm{U}$ \\
\hline $107-06-2$ & 1,2-Dichloroethane & 1.0 & $\mathrm{U}$ \\
\hline
\end{tabular}

Report 1,4-Dioxane for Low-Medium VOA analysis only 
Lab Name: TESTAMERICA BURLINGTON

Contract: 8E-00302

Lab Code: STLV

Case No.: YORK Mod. Ref No.:

SDG No.: 31673

Matrix: (SOIL/SED/WATER) Water

Sample wt/vol: 25.0 $(\mathrm{g} / \mathrm{mL}) \mathrm{mL}$

Level: (TRACE/LOW/MED) TRACE

\% Moisture: not dec.

GC Column: DB-624 ID: $0.20 \quad(\mathrm{~mm})$

Soil Extract Volume: (uL) $(\mathrm{mL})$

Purge Volume: 25.0 (mI)
Lab Sample ID: 200-31673-3

Lab File ID: 18001_023.D

Date Received: 01/22/2016

Date Analyzed: 01/26/2016

Dilution Factor: 1.0

Soil Aliquot Volume: (uL)

\begin{tabular}{|c|c|c|c|}
\hline CAS NO. & COMPOUND & $\begin{array}{l}\text { CONCENTRATION UNITS: } \\
(\mathrm{ug} / \mathrm{L} \text { or } \mathrm{ug} / \mathrm{kg}) \mathrm{ug} / \mathrm{L}\end{array}$ & Q \\
\hline $79-01-6$ & Trichloroethene & 1.0 & $\mathrm{U}$ \\
\hline $108-87-2$ & Methylcyclohexane & 1.0 & $\mathrm{U}$ \\
\hline $78-87-5$ & 1,2-Dichloropropane & 1.0 & $\mathrm{U}$ \\
\hline $75-27-4$ & Bromodichloromethane & 1.0 & $\mathrm{U}$ \\
\hline $10061-01-5$ & cis-1,3-Dichloropropene & 1.0 & U \\
\hline $108-10-1$ & 4-Methyl-2-pentanone & 1.0 & $\mathrm{U}$ \\
\hline $108-88-3$ & Toluene & 0.024 & $\mathrm{~J} B$ \\
\hline $10061-02-6$ & trans-1,3-Dichloropropene & 1.0 & $\mathrm{U}$ \\
\hline $79-00-5$ & $1,1,2-$ Trichloroethane & 1.0 & $\mathrm{U}$ \\
\hline $127-18-4$ & Tetrachloroethene & 1.0 & $\mathrm{U}$ \\
\hline $591-78-6$ & 2-Hexanone & 1.0 & $\mathrm{U}$ \\
\hline $124-48-1$ & Dibromochloromethane & 1.0 & $\mathrm{U}$ \\
\hline $106-93-4$ & 1,2-Dibromoethane & 1.0 & $\mathrm{U}$ \\
\hline $108-90-7$ & Chlorobenzene & 1.0 & $\mathrm{U}$ \\
\hline $100-41-4$ & Ethylbenzene & 1.0 & $\mathrm{U}$ \\
\hline $95-47-6$ & o-Xylene & 1.0 & $\mathrm{U}$ \\
\hline $179601-23-1$ & m,p-xylene & 0.0076 & $\mathrm{~J}$ \\
\hline $100-42-5$ & Styrene & 1.0 & $\mathrm{U}$ \\
\hline $75-25-2$ & Bromoform & 1.0 & $\mathrm{U}$ \\
\hline $98-82-8$ & Isopropylbenzene & 1.0 & $\mathrm{U}$ \\
\hline $79-34-5$ & $1,1,2,2$-Tetrachloroethane & 1.0 & $\mathrm{U}$ \\
\hline $541-73-1$ & 1,3 -Dichlorobenzene & 1.0 & $\mathrm{U}$ \\
\hline $106-46-7$ & 1,4-Dichlorobenzene & 1.0 & $\mathrm{U}$ \\
\hline $95-50-1$ & 1,2-Dichlorobenzene & 1.0 & $\mathrm{U}$ \\
\hline $96-12-8$ & 1,2-Dibromo-3-Chloropropane & 1.0 & $\mathrm{U}$ \\
\hline $120-82-1$ & $1,2,4$-Trichlorobenzene & 1.0 & $\mathrm{U}$ \\
\hline $87-61-6$ & $1,2,3$-Trichlorobenzene & 1.0 & $\mathrm{U}$ \\
\hline
\end{tabular}


Lab Name: TESTAMERICA BURLINGTON

Contract: $8 \mathrm{E}-00302$

Lab Code: STLV Case No.: YORK Mod. Ref No.: SDG No.: 31673

Matrix: (SOIL/SED/WATER) Water

Sample wt/vol: 25.0 $(\mathrm{g} / \mathrm{mL}) \mathrm{mL}$

Level: (TRACE or LOW/MED) TRACE

․ Moisture: not dec.

GC Column: DB-624 ID $: 0.20 \quad(\mathrm{~mm})$

Soil Extract Volume: (uL)

CONCENTRATION UNITS: (ug/L or ug/kg) ug/L
Lab Sample ID: 200-31673-3

Lab File ID: 18001_023.D

Date Received: 01/22/2016

Date Analyzed: 01/26/2016

Dilution Factor: 1.0

Soil Aliquot Volume: (uL) Purge Volume: 25.0 $(\mathrm{mL})$

01

02

\begin{tabular}{|l|l|c|c|c|}
\hline CAS NUMBER & \multicolumn{1}{|c|}{ COMPOUND NAME } & RT & EST. CONC. & Q \\
\hline & Unknown & 7.25 & 3.1 & $\mathrm{~J}$ B X \\
\hline E9667961 & Total Alkanes & N/A & & \\
\hline
\end{tabular}

1EPA-designated Registry Number. 
Lab Name: TESTAMERICA BURLINGTON

Contract: $8 \mathrm{E}-00302$

Lab Code: STLV Case No.: YORK Mod. Ref No.:

SDG No.: 31673

Instrument ID: CHD.i

Calibration Date(s): 01/08/2016 01/08/2016

Heated Purge: (Y/N) N

Calibration Time(s) : 1306

1445

Purge Volume: 25.0 (mL)

GC Column: DB-624

ID: 0.20

$(\mathrm{mm})$

Length: 25

(m)

\begin{tabular}{|c|c|c|c|c|c|c|c|}
\hline \multirow{3}{*}{$\begin{array}{l}\text { LAB FILE ID: } \\
\text { RRF } \underline{5.0}=\underline{17668 \_006 . D} \\
\text { COMPOUND }\end{array}$} & \multirow{2}{*}{\multicolumn{3}{|c|}{$\begin{array}{l}\operatorname{RRF0.5}=\frac{17668 \_004 . D}{R} \\
\operatorname{RRF10}=17668 \_007 . \mathrm{D}\end{array}$}} & \multicolumn{4}{|c|}{ RRF1.0 $=17668 \_005 . \mathrm{D}$} \\
\hline & & & & RRF20 & $=176$ & 3 & \\
\hline & RRE 0.5 & RRF1.0 & RRF5.0 & RRF10 & RRF20 & $\overline{\mathrm{RRF}}$ & $\because \mathrm{RSD}$ \\
\hline Dichlorodifluoromethane & 0.687 & 0.696 & 0.701 & 0.733 & 0.724 & 0.708 & 2.7 \\
\hline Chloromethane & 0.469 & 0.434 & 0.431 & 0.438 & 0.431 & 0.441 & 3.6 \\
\hline Vinyl chloride & 0.401 & 0.387 & 0.407 & 0.429 & 0.421 & 0.409 & 4.0 \\
\hline Bromomethane & 0.151 & 0.145 & 0.142 & 0.157 & 0.167 & 0.152 & 6.6 \\
\hline Chloroethane & 0.183 & 0.258 & 0.207 & 0.226 & 0.213 & 0.217 & 12.6 \\
\hline Trichlorofluoromethane & 0.719 & 0.728 & 0.778 & 0.805 & 0.800 & 0.766 & 5.2 \\
\hline 1,1-Dichloroethene & 0.215 & 0.219 & 0.270 & 0.272 & 0.249 & 0.245 & 11.0 \\
\hline $\begin{array}{l}1,1,2 \text {-Trichloro- } \\
1,2,2 \text {-trifluoroethane }\end{array}$ & 0.284 & 0.279 & 0.329 & 0.338 & 0.317 & 0.309 & 8.6 \\
\hline Acetone & 0.022 & 0.017 & 0.024 & 0.021 & 0.022 & 0.021 & 10.8 \\
\hline Carbon disulfide & 0.506 & 0.489 & 0.538 & 0.577 & 0.596 & 0.541 & 8.4 \\
\hline Methyl acetate & 0.047 & 0.037 & 0.043 & 0.043 & 0.062 & 0.046 & 20.3 \\
\hline Methylene Chloride & 0.250 & 0.170 & 0.237 & 0.233 & 0.246 & 0.227 & 14.3 \\
\hline trans-1,2-Dichloroethene & 0.311 & 0.312 & 0.323 & 0.337 & 0.328 & 0.322 & 3.5 \\
\hline Methyl tert-butyl ether & 0.361 & 0.333 & 0.382 & 0.405 & 0.420 & 0.380 & 9.1 \\
\hline 1,1-Dichloroethane & 0.634 & 0.631 & 0.672 & 0.690 & 0.694 & 0.664 & 4.5 \\
\hline cis-1,2-Dichloroethene & 0.303 & 0.287 & 0.328 & 0.328 & 0.335 & 0.316 & 6.5 \\
\hline 2-Butanone & 0.040 & 0.033 & 0.043 & 0.042 & 0.045 & 0.040 & 11.2 \\
\hline Bromochloromethane & 0.093 & 0.084 & 0.099 & 0.099 & 0.102 & 0.095 & 7.5 \\
\hline Chloroform & 0.639 & 0.590 & 0.618 & 0.617 & 0.621 & 0.617 & 2.9 \\
\hline $1,1,1$-Trichloroethane & 0.737 & 0.776 & 0.791 & 0.834 & 0.832 & 0.794 & 5.1 \\
\hline Cyclohexane & 0.718 & 0.760 & 0.872 & 0.889 & 0.868 & 0.821 & 9.4 \\
\hline Carbon tetrachloride & 0.686 & 0.709 & 0.716 & 0.741 & 0.766 & 0.723 & 4.3 \\
\hline Benzene & 1.680 & 1.784 & 1.778 & 1.791 & 1.737 & 1.754 & 2.6 \\
\hline 1,2-Dichloroethane & 0.299 & 0.273 & 0.308 & 0.310 & 0.316 & 0.301 & 5.6 \\
\hline Trichloroethene & 0.431 & 0.452 & 0.455 & 0.474 & 0.474 & 0.457 & 3.9 \\
\hline Methylcyclohexane & 0.547 & 0.652 & 0.719 & 0.743 & 0.726 & 0.677 & 11.9 \\
\hline
\end{tabular}

Report 1,4-Dioxane for Low-Medium VOA analysis only 
Lab Name: TESTAMERICA BURLINGTON

Contract: 8E-00302

Lab Code: STLV Case No.: YORK Mod. Ref No.:

SDG No.: 31673

Instrument ID: CHD.i

Calibration Date(s): 01/08/2016 01/08/2016

Heated Purge: (Y/N) N

Calibration Time(s) : 1306

1445

Purge Volume: 25.0

GC Column: DB-624

ID: 0.20 (mL)

LAB FILE ID:

(mm) Length: 25

(m)

RRF5.0 $=17668 \_006 . \mathrm{D}$

\begin{tabular}{|c|c|c|c|}
\hline $\operatorname{RRF0.5}=$ & $=17668004 . D$ & RRF1. 0 & $=17668005 . \mathrm{D}$ \\
\hline RF10 & $=17668007 . \mathrm{D}$ & RRE20 & 17668 \\
\hline
\end{tabular}

COMPOUND

\begin{tabular}{c|c|c} 
RRF0.5 & RRE1.0 \\
\hline
\end{tabular}

RRE5. 0

\begin{tabular}{|c|c|c|c|c|c|c|c|}
\hline 1,2-Dichloropropane & 0.401 & 0.360 & 0.375 & 0.378 & 0.377 & 0.378 & 3.9 \\
\hline Bromodichloromethane & 0.391 & 0.381 & 0.410 & 0.418 & 0.446 & 0.409 & 6.2 \\
\hline cis-1,3-Dichloropropene & 0.336 & 0.380 & 0.494 & 0.511 & 0.532 & 0.451 & 19.3 \\
\hline 4-Methyl-2-pentanone & 0.094 & 0.097 & 0.123 & 0.121 & 0.123 & 0.112 & 13.2 \\
\hline Toluene & 1.570 & 1.768 & 1.878 & 1.871 & 1.812 & 1.780 & 7.1 \\
\hline trans-1,3-Dichloropropene & 0.246 & 0.270 & 0.332 & 0.357 & 0.374 & 0.316 & 17.6 \\
\hline 1,1,2-Trichloroethane & 0.199 & 0.165 & 0.173 & 0.171 & 0.171 & 0.176 & 7.6 \\
\hline Tetrachloroethene & 0.369 & 0.377 & 0.384 & 0.389 & 0.388 & 0.382 & 2.2 \\
\hline 2-Hexanone & 0.066 & 0.070 & 0.085 & 0.082 & 0.084 & 0.077 & 11.2 \\
\hline Dibromochloromethane & 0.185 & 0.164 & 0.196 & 0.203 & 0.214 & 0.192 & 9.9 \\
\hline 1,2-Dibromoethane & 0.128 & 0.122 & 0.143 & 0.147 & 0.151 & 0.138 & 9.1 \\
\hline Chlorobenzene & 1.012 & 1.000 & 1.049 & 1.047 & 1.045 & 1.031 & 2.2 \\
\hline Ethylbenzene & 1.757 & 1.910 & 2.180 & 2.197 & 2.177 & 2.044 & 9.8 \\
\hline o-Xylene & 0.499 & 0.566 & 0.704 & 0.713 & 0.706 & 0.637 & 15.5 \\
\hline $\mathrm{m}, \mathrm{p}-\mathrm{Xylene}$ & 0.591 & 0.697 & 0.789 & 0.789 & 0.785 & 0.730 & 12.0 \\
\hline Styrene & 0.764 & 0.899 & 1.084 & 1.069 & 1.056 & 0.974 & 14.3 \\
\hline Bromoform & 0.159 & 0.155 & 0.167 & 0.168 & 0.186 & 0.167 & 7.1 \\
\hline Isopropylbenzene & 1.429 & 1.719 & 2.140 & 2.183 & 2.182 & 1.931 & 17.7 \\
\hline $1,1,2,2$-Tetrachloroethane & 0.155 & 0.149 & 0.158 & 0.155 & 0.161 & 0.155 & 3.0 \\
\hline 1,3-Dichlorobenzene & 1.414 & 1.408 & 1.570 & 1.630 & 1.701 & 1.545 & 8.4 \\
\hline 1,4-Dichlorobenzene & 1.671 & 1.630 & 1.636 & 1.595 & 1.642 & 1.635 & 1.7 \\
\hline 1,2-Dichlorobenzene & 1.259 & 1.184 & 1.315 & 1.311 & 1.330 & 1.280 & 4.7 \\
\hline 1,2-Dibromo-3-Chloropropane & 0.051 & 0.043 & 0.046 & 0.047 & 0.051 & 0.048 & 7.0 \\
\hline 1,2,4-Trichlorobenzene & 0.641 & 0.554 & 0.694 & 0.766 & 0.848 & 0.701 & 16.1 \\
\hline $1,2,3$-Trichlorobenzene & 0.418 & 0.393 & 0.492 & 0.543 & 0.585 & 0.486 & 16.7 \\
\hline
\end{tabular}


Lab Name: TESTAMERICA BURLINGTON

Contract: $8 \mathrm{E}-00302$

Lab Code: STLV Case No.: YORK Mod. Ref No.: SDG No.: 31673

Instrument ID: CHD.i

Heated Purge: (Y/N) N

Purge Volume: 25.0

GC Column: DB-624
ID: 0.20
Calibration Date (s) : 01/08/2016 Calibration Time(s) : 1306 $(\mathrm{mL})$

(mm) Length: 25
$01 / 08 / 2016$ 1445

\begin{tabular}{|c|c|c|c|c|c|c|c|}
\hline LAB FILE ID: & \multicolumn{3}{|c|}{$\begin{array}{l}\operatorname{RRF}_{\underline{0.5}}=\frac{17668 \_004 . \mathrm{D}}{\operatorname{RRF10}}=17668 \_007 . \mathrm{D}\end{array}$} & \multicolumn{4}{|c|}{$\begin{array}{l}\operatorname{RRF}_{\underline{1.0}}=\frac{17668 \_005 . \mathrm{D}}{\mathrm{RRF} 20}=17668 \_008 . \mathrm{D}\end{array}$} \\
\hline Vinyl Chloride-d3 & 0.341 & 0.333 & 0.337 & 0.354 & 0.345 & 0.342 & 2.4 \\
\hline Chloroethane-d5 & 0.250 & 0.255 & 0.243 & 0.248 & 0.239 & 0.247 & 2.5 \\
\hline Chloroform-d & 0.662 & 0.622 & 0.679 & 0.673 & 0.675 & 0.662 & 3.5 \\
\hline 1,2-Dichloroethane-d4 & 0.254 & 0.224 & 0.250 & 0.250 & 0.259 & 0.247 & 5.5 \\
\hline Benzene-d6 & 1.573 & 1.623 & 1.702 & 1.660 & 1.638 & 1.639 & 2.9 \\
\hline 1,2-Dichloropropane-d6 & 0.400 & 0.415 & 0.447 & 0.440 & 0.433 & 0.427 & 4.5 \\
\hline Toluene-d8 & 1.356 & 1.449 & 1.606 & 1.624 & 1.586 & 1.524 & 7.7 \\
\hline 1,2-Dichlorobenzene-d4 & 0.781 & 0.746 & 0.806 & 0.817 & 0.835 & 0.797 & 4.4 \\
\hline
\end{tabular}

Report 1,4-Dioxane-d8 for Low-Medium VOA analysis only 
Lab Name: TESTAMERICA BURLINGTON

Contract: $8 \mathrm{E}-00302$

Lab Code: STLV Case No.: YORK Mod. Ref No.:

SDG No.: 31673

Instrument ID: CHD.i

Calibration Date(s): 01/27/2016 01/27/2016

Heated Purge: ( $\mathrm{Y} / \mathrm{N}) \mathrm{N}$

Calibration Time(s) : 1117

1257

Purge Volume: 25.0 $(\mathrm{mL})$

GC Column: DB-624

ID: $0.20 \quad$ (mm) Length: 25

$(\mathrm{m})$

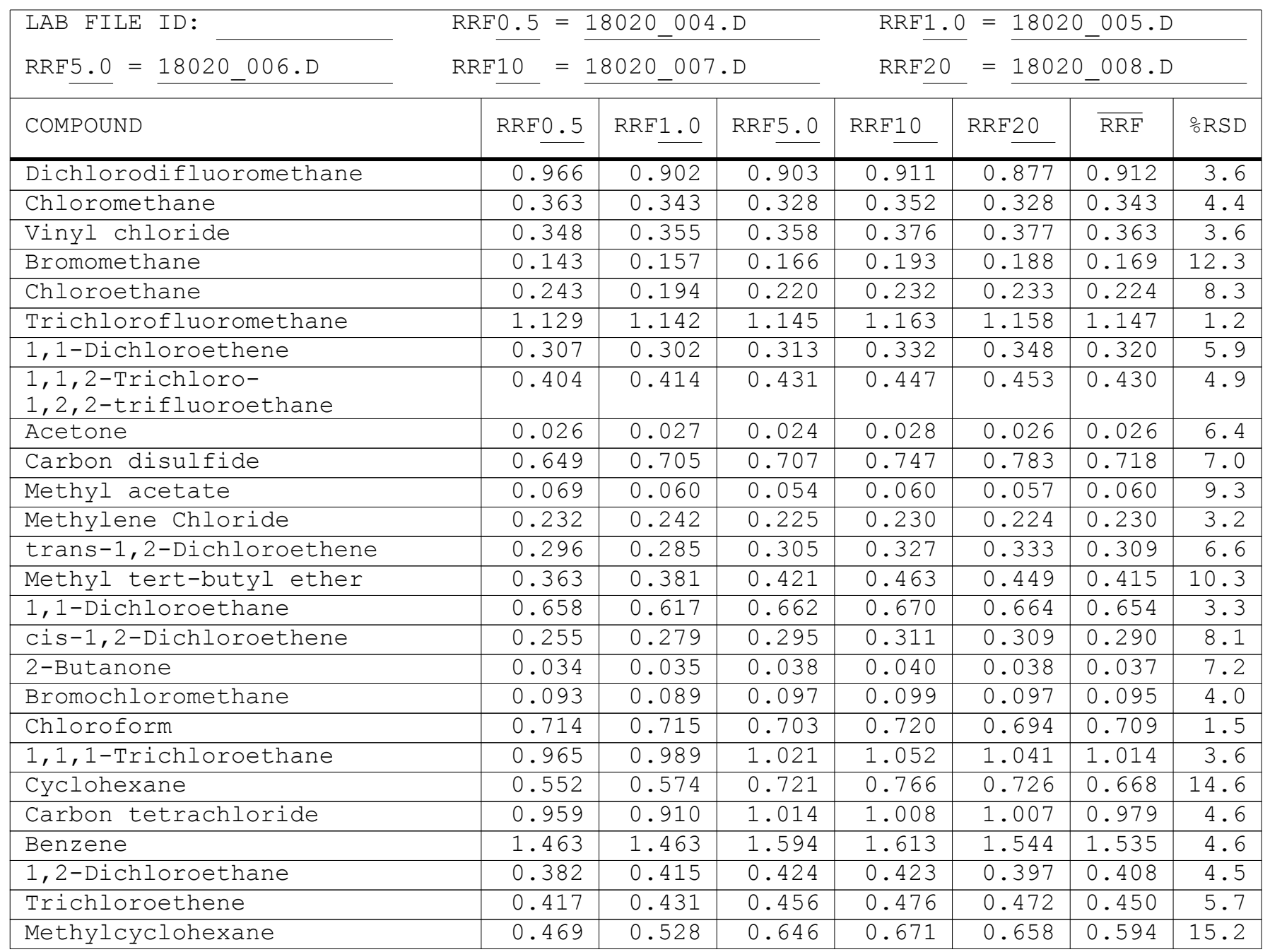

Report 1,4-Dioxane for Low-Medium VOA analysis only 
Lab Name: TESTAMERICA BURLINGTON

Contract: 8E-00302

Lab Code: STLV Case No.: YORK Mod. Ref No.:

SDG No.: 31673

Instrument ID: CHD.i

Calibration Date(s): 01/27/2016 01/27/2016

Heated Purge: ( $\mathrm{Y} / \mathrm{N}) \mathrm{N}$

Calibration Time(s) : 1117

1257

Purge Volume: 25.0 (mL)

GC Column: DB-624

ID: 0.20

$(\mathrm{mm})$

Length: 25

(m)

\begin{tabular}{|c|c|c|c|c|c|c|c|}
\hline \multirow{2}{*}{$\begin{array}{l}\text { LAB FILE ID: } \\
\text { RRF5.0 }=18020 \_006 . \mathrm{D}\end{array}$} & \multicolumn{3}{|c|}{ RRF0.5 = $18020004 . D$} & \multicolumn{4}{|c|}{ RRF1.0 = $18020005 . \mathrm{D}$} \\
\hline & E10 & $020 \_00^{\circ}$ & & RRF20 & $=1802$ & 0_008.5 & \\
\hline COMPOUND & $\mathrm{RRF} 0.5$ & RRF1. 0 & RRF5.0 & RRF10 & RRF20 & $\overline{\mathrm{RRF}}$ & $\because \mathrm{RSD}$ \\
\hline 1,2-Dichloropropane & 0.318 & 0.315 & 0.319 & 0.322 & 0.316 & 0.318 & 0.8 \\
\hline Bromodichloromethane & 0.465 & 0.471 & 0.482 & 0.505 & 0.485 & 0.482 & 3.2 \\
\hline cis-1,3-Dichloropropene & 0.342 & 0.388 & 0.497 & 0.534 & 0.518 & 0.456 & 18.8 \\
\hline 4-Methyl-2-pentanone & 0.073 & 0.088 & 0.109 & 0.115 & 0.107 & 0.099 & 17.7 \\
\hline Toluene & 1.373 & 1.564 & 1.825 & 1.863 & 1.799 & 1.685 & 12.4 \\
\hline trans-1,3-Dichloropropene & 0.263 & 0.290 & 0.363 & 0.413 & 0.403 & 0.346 & 19.4 \\
\hline 1,1,2-Trichloroethane & 0.152 & 0.168 & 0.164 & 0.166 & 0.156 & 0.161 & 4.3 \\
\hline Tetrachloroethene & 0.366 & 0.371 & 0.404 & 0.420 & 0.428 & 0.398 & 7.1 \\
\hline 2-Hexanone & 0.056 & 0.063 & 0.079 & 0.083 & 0.077 & 0.072 & 16.0 \\
\hline Dibromochloromethane & 0.206 & 0.211 & 0.227 & 0.240 & 0.235 & 0.224 & 6.7 \\
\hline 1,2-Dibromoethane & 0.141 & 0.135 & 0.142 & 0.156 & 0.149 & 0.145 & 5.5 \\
\hline Chlorobenzene & 0.949 & 1.021 & 1.060 & 1.082 & 1.064 & 1.035 & 5.1 \\
\hline Ethylbenzene & 1.601 & 1.759 & 2.247 & 2.356 & 2.350 & 2.062 & 17.3 \\
\hline o-Xylene & 0.464 & 0.520 & 0.721 & 0.769 & 0.753 & 0.645 & 22.1 \\
\hline m, p-Xylene & 0.572 & 0.648 & 0.811 & 0.838 & 0.833 & 0.740 & 16.6 \\
\hline Styrene & 0.686 & 0.932 & 1.152 & 1.190 & 1.155 & 1.023 & 21.0 \\
\hline Bromoform & 0.192 & 0.212 & 0.188 & 0.194 & 0.206 & 0.198 & 5.1 \\
\hline Isopropylbenzene & 1.394 & 1.647 & 2.272 & 2.466 & 2.500 & 2.056 & 24.5 \\
\hline $1,1,2,2$-Tetrachloroethane & 0.127 & 0.150 & 0.147 & 0.154 & 0.150 & 0.145 & 7.5 \\
\hline 1,3-Dichlorobenzene & 1.377 & 1.414 & 1.540 & 1.645 & 1.730 & 1.541 & 9.7 \\
\hline 1,4-Dichlorobenzene & 1.795 & 1.792 & 1.666 & 1.701 & 1.695 & 1.730 & 3.4 \\
\hline 1,2-Dichlorobenzene & 1.188 & 1.365 & 1.387 & 1.370 & 1.365 & 1.335 & 6.2 \\
\hline 1,2-Dibromo-3-Chloropropane & 0.052 & 0.050 & 0.052 & 0.052 & 0.057 & 0.053 & 5.2 \\
\hline 1,2,4-Trichlorobenzene & 0.632 & 0.621 & 0.668 & 0.832 & 0.877 & 0.726 & 16.5 \\
\hline 1,2,3-Trichlorobenzene & 0.491 & 0.435 & 0.480 & 0.586 & 0.612 & 0.521 & 14.4 \\
\hline
\end{tabular}


Lab Name: TESTAMERICA BURLINGTON

Lab Code: STLV Case No.: YORK

Instrument ID: CHD.i

Heated Purge: (Y/N) N

Purge Volume: 25.0

GC Column: DB-624
Mod. Ref No.:

Contract: $8 \mathrm{E}-00302$

\begin{tabular}{|c|c|c|c|c|c|c|c|}
\hline LAB FILE ID: & \multirow{2}{*}{\multicolumn{3}{|c|}{$\begin{array}{l}\operatorname{RRF}_{\underline{0.5}}=\frac{18020 \_004 . \mathrm{D}}{\operatorname{RRF10}}=18020 \_007 . \mathrm{D}\end{array}$}} & \multirow{2}{*}{\multicolumn{4}{|c|}{$\begin{array}{l}\operatorname{RRF} 1.0_{\underline{1.0}}=\frac{18020 \_005 . \mathrm{D}}{\mathrm{RRF} 20}=18020 \_008 . \mathrm{D}\end{array}$}} \\
\hline RRF5.0 = 18020_006.D & & & & & & & \\
\hline COMPOUND & RRE0. 5 & RRF1. 0 & RRE 5.0 & RRE10 & RRE20 & $\overline{\mathrm{RRF}}$ & $\circ \mathrm{RSD}$ \\
\hline Vinyl Chloride-d3 & 0.343 & 0.340 & 0.315 & 0.342 & 0.340 & 0.336 & 3.6 \\
\hline Chloroethane-d5 & 0.273 & 0.270 & 0.269 & 0.273 & 0.281 & 0.273 & 1.8 \\
\hline 1,1-Dichloroethene-d2 & 0.814 & 0.833 & 0.842 & 0.870 & 0.897 & 0.851 & 3.9 \\
\hline 2-Butanone-d5 & 0.029 & 0.029 & 0.032 & 0.035 & 0.034 & 0.032 & 8.6 \\
\hline Chloroform-d & 0.700 & 0.742 & 0.741 & 0.763 & 0.736 & 0.736 & 3.1 \\
\hline 1,2-Dichloroethane-d4 & 0.315 & 0.323 & 0.328 & 0.336 & 0.321 & 0.324 & 2.4 \\
\hline Benzene-d6 & 1.242 & 1.362 & 1.456 & 1.483 & 1.412 & 1.391 & 6.8 \\
\hline 1,2-Dichloropropane-d6 & 0.307 & 0.326 & 0.360 & 0.361 & 0.339 & 0.339 & 6.8 \\
\hline Toluene-d8 & 1.194 & 1.342 & 1.524 & 1.588 & 1.530 & 1.436 & 11.4 \\
\hline trans-1,3-Dichloropropene-d4 & 0.230 & 0.268 & 0.315 & 0.356 & 0.356 & 0.305 & 18.2 \\
\hline 2-Hexanone-d5 & 0.020 & 0.021 & 0.030 & 0.033 & 0.031 & 0.027 & 22.4 \\
\hline $1,1,2,2-$ Tetrachloroethane-d2 & 0.157 & 0.153 & 0.153 & 0.158 & 0.149 & 0.154 & 2.2 \\
\hline 1,2-Dichlorobenzene-d4 & 0.795 & 0.752 & 0.793 & 0.830 & 0.840 & 0.802 & 4.3 \\
\hline
\end{tabular}

Report 1,4-Dioxane-d8 for Low-Medium VOA analysis only 
Lab Name: TESTAMERICA BURLINGTON

Contract: $8 \mathrm{E}-00302$

Lab Code: STLV Case No.: YORK Mod. Ref No.:

SDG No.: 31673

Instrument ID: CHD.i

Calibration Date: 01/26/2016 Time: 1221

Lab File Id: 18001_005.D

EPA Sample No. (VSTD\#\#\#\#) : VSTD005DG Init. Calib. Date (s):01/08/2016 01/08/2016

Heated Purge: (Y/N) N GC Column: DB-624

Init. Calib. Time(s) : 1306 1445

Purge Volume: 25.0 ID: $0.20(\mathrm{~mm})$ Length: 25 $(\mathrm{m})$

\begin{tabular}{|c|c|c|c|c|c|}
\hline COMPOUND & $\overline{\mathrm{RRF}}$ & RRF5.0 & $\begin{array}{l}\text { MIN } \\
\text { RRF }\end{array}$ & $\frac{\circ}{D}$ & $\mathrm{MAX} \div \mathrm{D}$ \\
\hline Dichlorodifluoromethane & 0.708 & 0.843 & 0.010 & 19.0 & 40.0 \\
\hline Chloromethane & 0.441 & 0.307 & 0.010 & -30.3 & 40.0 \\
\hline Vinyl chloride & 0.409 & 0.345 & 0.100 & -15.6 & 30.0 \\
\hline Bromomethane & 0.152 & 0.143 & 0.100 & -6.0 & 30.0 \\
\hline Chloroethane & 0.217 & 0.205 & 0.010 & -5.5 & 40.0 \\
\hline Trichlorofluoromethane & 0.766 & 1.030 & 0.010 & 34.4 & 40.0 \\
\hline 1,1-Dichloroethene & 0.245 & 0.298 & 0.100 & 21.9 & 30.0 \\
\hline 1,1,2-Trichloro-1,2,2-trifluoroethane & 0.309 & 0.398 & 0.010 & 28.8 & 40.0 \\
\hline Acetone & 0.021 & 0.020 & 0.010 & -3.8 & 40.0 \\
\hline Carbon disulfide & 0.541 & 0.685 & 0.010 & 26.5 & 40.0 \\
\hline Methyl acetate & 0.046 & 0.043 & 0.010 & -7.0 & 40.0 \\
\hline Methylene Chloride & 0.227 & 0.212 & 0.010 & -6.6 & 40.0 \\
\hline trans-1,2-Dichloroethene & 0.322 & 0.305 & 0.010 & -5.3 & 40.0 \\
\hline Methyl tert-butyl ether & 0.380 & 0.397 & 0.010 & 4.4 & 40.0 \\
\hline 1,1-Dichloroethane & 0.664 & 0.632 & 0.200 & -4.8 & 30.0 \\
\hline cis-1,2-Dichloroethene & 0.316 & 0.283 & 0.010 & -10.6 & 40.0 \\
\hline 2-Butanone & 0.040 & 0.034 & 0.010 & -15.4 & 40.0 \\
\hline Bromochloromethane & 0.095 & 0.090 & 0.050 & -5.8 & 30.0 \\
\hline Chloroform & 0.617 & 0.667 & 0.200 & 8.0 & 30.0 \\
\hline 1,1,1-Trichloroethane & 0.794 & 1.014 & 0.100 & 27.7 & 30.0 \\
\hline Cyclohexane & 0.821 & 0.734 & 0.010 & $-10 \cdot 7$ & 40.0 \\
\hline Carbon tetrachloride & 0.723 & 0.974 & 0.100 & 34.6 & 30.0 \\
\hline Benzene & 1.754 & 1.604 & 0.400 & -8.5 & 30.0 \\
\hline 1,2-Dichloroethane & 0.301 & 0.391 & 0.100 & 29.9 & 30.0 \\
\hline Trichloroethene & 0.457 & 0.457 & 0.300 & -0.1 & 30.0 \\
\hline Methylcyclohexane & 0.677 & 0.668 & 0.010 & -1.4 & 40.0 \\
\hline
\end{tabular}

Report 1,4-Dioxane for Low/Medium VOA analysis only 
Lab Name: TESTAMERICA BURLINGTON

Contract: 8E-00302

Lab Code: STLV Case No.: YORK Mod. Ref No.:

SDG No.: 31673

Instrument ID: CHD.i

Calibration Date: 01/26/2016 Time: 1221

Lab File Id: 18001_005.D

EPA Sample No. (VSTD\#\#\#\#) : VSTD005DG Init. Calib. Date(s):01/08/2016 01/08/2016

Heated Purge: (Y/N) N GC Column: DB-624

Init. Calib. Time(s) : 1306 1445

Purge Volume: 25.0 ID: $0.20(\mathrm{~mm})$ Length: 25 (m)

\begin{tabular}{|c|c|c|c|c|c|}
\hline COMPOUND & $\overline{\mathrm{RRF}}$ & RRF5.0 & $\begin{array}{l}\text { MIN } \\
\text { RRF }\end{array}$ & $\because \mathrm{D}$ & $\operatorname{MAX} \cong \mathrm{D}$ \\
\hline 1,2-Dichloropropane & 0.378 & 0.340 & 0.010 & -10.1 & 40.0 \\
\hline Bromodichloromethane & 0.409 & 0.477 & 0.200 & 16.5 & 30.0 \\
\hline cis-1,3-Dichloropropene & 0.451 & 0.489 & 0.200 & 8.6 & 30.0 \\
\hline 4-Methyl-2-pentanone & 0.112 & 0.106 & 0.010 & -5.5 & 40.0 \\
\hline Toluene & 1.780 & 1.822 & 0.400 & 2.4 & 30.0 \\
\hline trans-1,3-Dichloropropene & 0.316 & 0.374 & 0.100 & 18.4 & 30.0 \\
\hline $1,1,2$-Trichloroethane & 0.176 & 0.169 & 0.100 & -3.8 & 30.0 \\
\hline Tetrachloroethene & 0.382 & 0.418 & 0.100 & 9.6 & 30.0 \\
\hline 2-Hexanone & 0.077 & 0.077 & 0.010 & -0.4 & 40.0 \\
\hline Dibromochloromethane & 0.192 & 0.220 & 0.100 & 14.5 & 30.0 \\
\hline 1,2-Dibromoethane & 0.138 & 0.145 & 0.010 & 5.3 & 40.0 \\
\hline Chlorobenzene & 1.031 & 1.034 & 0.500 & 0.4 & 30.0 \\
\hline Ethylbenzene & 2.044 & 2.203 & 0.100 & 7.8 & 30.0 \\
\hline o-Xylene & 0.637 & 0.695 & 0.300 & 9.0 & 30.0 \\
\hline $\mathrm{m}, \mathrm{p}$-Xylene & 0.730 & 0.780 & 0.300 & 6.8 & 30.0 \\
\hline Styrene & 0.974 & 1.118 & 0.300 & 14.8 & 30.0 \\
\hline Bromoform & 0.167 & 0.176 & 0.050 & 5.4 & 30.0 \\
\hline Isopropylbenzene & 1.931 & 2.274 & 0.010 & 17.8 & 40.0 \\
\hline $1,1,2,2$-Tetrachloroethane & 0.155 & 0.143 & 0.100 & -7.8 & 30.0 \\
\hline 1,3-Dichlorobenzene & 1.545 & 1.541 & 0.400 & -0.2 & 30.0 \\
\hline 1,4-Dichlorobenzene & 1.635 & 1.639 & 0.400 & 0.2 & 30.0 \\
\hline 1,2-Dichlorobenzene & 1.280 & 1.314 & 0.400 & 2.7 & 30.0 \\
\hline 1,2-Dibromo-3-Chloropropane & 0.048 & 0.052 & 0.010 & 8.1 & 40.0 \\
\hline 1,2,4-Trichlorobenzene & 0.701 & 0.734 & 0.200 & 4.7 & 30.0 \\
\hline $1,2,3$-Trichlorobenzene & 0.486 & 0.507 & 0.200 & 4.3 & 30.0 \\
\hline
\end{tabular}


Lab Name: TESTAMERICA BURLINGTON

Contract: $8 \mathrm{E}-00302$

Lab Code: STLV Case No.: YORK Mod. Ref No.

SDG No.: 31673

Instrument ID: CHD.i

Calibration Date: 01/26/2016 Time: 1221

Lab File Id: 18001_005.D Init. Calib. Date (s):01/08/2016 01/08/2016

EPA Sample No. (VSTD\#\#\#\#) : VSTD005DG

Init. Calib. Time(s) : 1306 1445

Heated Purge: (Y/N) N GC Column: DB-624 ID: $0.20(\mathrm{~mm})$ Length: 25

(m)

Purge Volume: 25.0 $(\mathrm{mL})$

\begin{tabular}{|c|c|c|c|c|c|}
\hline COMPOUND & $\overline{\mathrm{RRF}}$ & RRF5.0 & $\begin{array}{l}\text { MIN } \\
\text { RRE }\end{array}$ & $\because \mathrm{D}$ & $\operatorname{MAX} \cong \mathrm{D}$ \\
\hline Vinyl Chloride-d3 & 0.342 & 0.310 & 0.010 & -9.3 & 30.0 \\
\hline Chloroethane-d5 & 0.247 & 0.262 & 0.010 & 6.0 & 40.0 \\
\hline 1,1-Dichloroethene-d2 & 0.650 & 0.806 & 0.010 & 24.1 & 30.0 \\
\hline 2-Butanone-d5 & 0.039 & 0.031 & 0.010 & $-21 \cdot 0$ & 40.0 \\
\hline Chloroform-d & 0.662 & 0.714 & 0.010 & 7.9 & 30.0 \\
\hline 1,2-Dichloroethane-d4 & 0.247 & 0.304 & 0.010 & 22.9 & 30.0 \\
\hline Benzene-d6 & 1.639 & 1.476 & 0.010 & $-10 \cdot 0$ & 30.0 \\
\hline 1,2-Dichloropropane-d6 & 0.427 & 0.351 & 0.010 & -17.7 & 40.0 \\
\hline Toluene-d8 & 1.524 & 1.543 & 0.010 & 1.3 & 30.0 \\
\hline trans-1,3-Dichloropropene-d4 & 0.289 & 0.321 & 0.010 & 11.1 & 30.0 \\
\hline 2-Hexanone-d5 & 0.032 & 0.030 & 0.010 & -4.5 & 40.0 \\
\hline $1,1,2,2$-Tetrachloroethane-d2 & 0.161 & 0.142 & 0.010 & -11.8 & 30.0 \\
\hline 1,2-Dichlorobenzene-d4 & 0.797 & 0.801 & 0.010 & 0.4 & 30.0 \\
\hline
\end{tabular}

Report 1,4-Dioxane-d8 for Low/Medium VOA analysis only 
Lab Name: TESTAMERICA BURLINGTON

Contract: $8 \mathrm{E}-00302$

Lab Code: STLV Case No.: YORK Mod. Ref No.:

SDG No.: 31673

Instrument ID: CHD.i

Calibration Date: 01/26/2016 Time: 2135

Lab File Id: 18001_026.D

EPA Sample No. (VSTD\#\#\#\#) : VSTD005GD Init. Calib. Date(s):01/08/2016 01/08/2016

Heated Purge: ( $\mathrm{Y} / \mathrm{N}) \mathrm{N}$ GC Column: DB-624

Init. Calib. Time(s) : 1306 1445

Purge Volume: 25.0 ID: $0.20(\mathrm{~mm})$ Length: 25 $(\mathrm{m})$

\begin{tabular}{|c|c|c|c|c|c|}
\hline COMPOUND & $\overline{\mathrm{RRF}}$ & RRF5.0 & $\begin{array}{l}\text { MIN } \\
\text { RRF }\end{array}$ & $\frac{\circ}{D}$ & $\mathrm{MAX} \div \mathrm{D}$ \\
\hline Dichlorodifluoromethane & 0.708 & 0.863 & 0.010 & 21.8 & 50.0 \\
\hline Chloromethane & 0.441 & 0.310 & 0.010 & -29.6 & 50.0 \\
\hline Vinyl chloride & 0.409 & 0.355 & 0.010 & $-13 \cdot 2$ & 50.0 \\
\hline Bromomethane & 0.152 & 0.135 & 0.010 & -11.2 & 50.0 \\
\hline Chloroethane & 0.217 & 0.243 & 0.010 & 11.9 & 50.0 \\
\hline Trichlorofluoromethane & 0.766 & 1.107 & 0.010 & 44.6 & 50.0 \\
\hline 1,1-Dichloroethene & 0.245 & 0.319 & 0.010 & 30.1 & 50.0 \\
\hline 1,1,2-Trichloro-1,2,2-trifluoroethane & 0.309 & 0.407 & 0.010 & 31.7 & 50.0 \\
\hline Acetone & 0.021 & 0.026 & 0.010 & 23.3 & 50.0 \\
\hline Carbon disulfide & 0.541 & 0.725 & 0.010 & 34.0 & 50.0 \\
\hline Methyl acetate & 0.046 & 0.041 & 0.010 & -10.9 & 50.0 \\
\hline Methylene Chloride & 0.227 & 0.219 & 0.010 & -3.4 & 50.0 \\
\hline trans-1,2-Dichloroethene & 0.322 & 0.314 & 0.010 & -2.7 & 50.0 \\
\hline Methyl tert-butyl ether & 0.380 & 0.401 & 0.010 & 5.5 & 50.0 \\
\hline 1,1-Dichloroethane & 0.664 & 0.642 & 0.010 & -3.4 & 50.0 \\
\hline cis-1,2-Dichloroethene & 0.316 & 0.284 & 0.010 & -10.2 & 50.0 \\
\hline 2-Butanone & 0.040 & 0.036 & 0.010 & -11.7 & 50.0 \\
\hline Bromochloromethane & 0.095 & 0.094 & 0.010 & -1.3 & 50.0 \\
\hline Chloroform & 0.617 & 0.700 & 0.010 & 13.4 & 50.0 \\
\hline 1,1,1-Trichloroethane & 0.794 & 1.058 & 0.010 & 33.2 & 50.0 \\
\hline Cyclohexane & 0.821 & 0.724 & 0.010 & -11.8 & 50.0 \\
\hline Carbon tetrachloride & 0.723 & 1.006 & 0.010 & 39.1 & 50.0 \\
\hline Benzene & 1.754 & 1.623 & 0.010 & -7.4 & 50.0 \\
\hline 1,2-Dichloroethane & 0.301 & 0.390 & 0.010 & 29.4 & 50.0 \\
\hline Trichloroethene & 0.457 & 0.458 & 0.010 & 0.2 & 50.0 \\
\hline Methylcyclohexane & 0.677 & 0.668 & 0.010 & -1.3 & 50.0 \\
\hline
\end{tabular}

Report 1,4-Dioxane for Low/Medium VOA analysis only 
Lab Name: TESTAMERICA BURLINGTON

Contract: 8E-00302

Lab Code: STLV Case No.: YORK Mod. Ref No.:

SDG No.: 31673

Instrument ID: CHD.i

Calibration Date: 01/26/2016 Time: 2135

Lab File Id: 18001_026.D

EPA Sample No. (VSTD\#\#\#\#) : VSTD005GD Init. Calib. Date(s):01/08/2016 01/08/2016

Heated Purge: (Y/N) N GC Column: DB-624

Init. Calib. Time(s) : 1306 1445

Purge Volume: 25.0 ID: $0.20(\mathrm{~mm})$ Length: 25 (m)

\begin{tabular}{|c|c|c|c|c|c|}
\hline COMPOUND & $\overline{\mathrm{RRF}}$ & RRF5.0 & $\begin{array}{l}\text { MIN } \\
\text { RRF }\end{array}$ & $\because \mathrm{D}$ & $\operatorname{MAX} \cong \mathrm{D}$ \\
\hline 1,2-Dichloropropane & 0.378 & 0.336 & 0.010 & -11.2 & 50.0 \\
\hline Bromodichloromethane & 0.409 & 0.492 & 0.010 & 20.3 & 50.0 \\
\hline cis-1,3-Dichloropropene & 0.451 & 0.488 & 0.010 & 8.2 & 50.0 \\
\hline 4-Methyl-2-pentanone & 0.112 & 0.107 & 0.010 & -3.9 & 50.0 \\
\hline Toluene & 1.780 & 1.884 & 0.010 & 5.8 & 50.0 \\
\hline trans-1,3-Dichloropropene & 0.316 & 0.350 & 0.010 & 10.8 & 50.0 \\
\hline $1,1,2$-Trichloroethane & 0.176 & 0.166 & 0.010 & -5.6 & 50.0 \\
\hline Tetrachloroethene & 0.382 & 0.407 & 0.010 & 6.7 & 50.0 \\
\hline 2-Hexanone & 0.077 & 0.078 & 0.010 & 0.2 & 50.0 \\
\hline Dibromochloromethane & 0.192 & 0.227 & 0.010 & 17.8 & 50.0 \\
\hline 1,2-Dibromoethane & 0.138 & 0.141 & 0.010 & 2.3 & 50.0 \\
\hline Chlorobenzene & 1.031 & 1.065 & 0.010 & 3.3 & 50.0 \\
\hline Ethylbenzene & 2.044 & 2.288 & 0.010 & 11.9 & 50.0 \\
\hline o-Xylene & 0.637 & 0.719 & 0.010 & 12.8 & 50.0 \\
\hline $\mathrm{m}, \mathrm{p}$-Xylene & 0.730 & 0.810 & 0.010 & 10.9 & 50.0 \\
\hline Styrene & 0.974 & 1.144 & 0.010 & 17.4 & 50.0 \\
\hline Bromoform & 0.167 & 0.193 & 0.010 & 15.4 & 50.0 \\
\hline Isopropylbenzene & 1.931 & 2.355 & 0.010 & 22.0 & 50.0 \\
\hline $1,1,2,2$-Tetrachloroethane & 0.155 & 0.145 & 0.010 & -6.9 & 50.0 \\
\hline 1,3-Dichlorobenzene & 1.545 & 1.515 & 0.010 & -1.9 & 50.0 \\
\hline 1,4-Dichlorobenzene & 1.635 & 1.643 & 0.010 & 0.5 & 50.0 \\
\hline 1,2-Dichlorobenzene & 1.280 & 1.330 & 0.010 & 3.9 & 50.0 \\
\hline 1,2-Dibromo-3-Chloropropane & 0.048 & 0.049 & 0.010 & 3.7 & 50.0 \\
\hline 1,2,4-Trichlorobenzene & 0.701 & 0.676 & 0.010 & -3.4 & 50.0 \\
\hline $1,2,3$-Trichlorobenzene & 0.486 & 0.482 & 0.010 & -0.9 & 50.0 \\
\hline
\end{tabular}


Lab Name: TESTAMERICA BURLINGTON

Contract: $8 \mathrm{E}-00302$

Lab Code: STLV Case No.: YORK Mod. Ref No.

SDG No.: 31673

Instrument ID: CHD.i

Calibration Date: 01/26/2016 Time: 2135

Lab File Id: 18001_026.D Init. Calib. Date(s):01/08/2016 01/08/2016

EPA Sample No. (VSTD\#\#\#\#) : VSTD005GD

Heated Purge: (Y/N) N GC Column: DB-624

Init. Calib. Time(s) : 1306 1445

Purge Volume: 25.0 ID: $0.20(\mathrm{~mm})$ Length: 25

(m)

\begin{tabular}{|c|c|c|c|c|c|}
\hline COMPOUND & $\overline{\mathrm{RRF}}$ & RRE5. 0 & $\begin{array}{l}\text { MIN } \\
\text { RRE }\end{array}$ & $\because \mathrm{D}$ & $M A X \div D$ \\
\hline Vinyl Chloride-d3 & 0.342 & 0.314 & 0.010 & -8.3 & 50.0 \\
\hline Chloroethane-d5 & 0.247 & 0.270 & 0.010 & 9.3 & 50.0 \\
\hline $1,1-$ Dichloroethene-d2 & 0.650 & 0.848 & 0.010 & 30.5 & 50.0 \\
\hline 2-Butanone-d5 & 0.039 & 0.031 & 0.010 & -21.1 & 50.0 \\
\hline Chloroform-d & 0.662 & 0.732 & 0.010 & 10.6 & 50.0 \\
\hline 1,2-Dichloroethane-d4 & 0.247 & 0.322 & 0.010 & 29.9 & 50.0 \\
\hline Benzene-d6 & 1.639 & 1.495 & 0.010 & -8.8 & 50.0 \\
\hline 1,2-Dichloropropane-d6 & 0.427 & 0.352 & 0.010 & -17.5 & 50.0 \\
\hline Toluene-d8 & 1.524 & 1.585 & 0.010 & 4.0 & 50.0 \\
\hline trans-1,3-Dichloropropene-d4 & 0.289 & 0.305 & 0.010 & 5.4 & 50.0 \\
\hline 2-Hexanone-d5 & 0.032 & 0.029 & 0.010 & -8.0 & 50.0 \\
\hline $1,1,2,2$-Tetrachloroethane-d2 & 0.161 & 0.151 & 0.010 & -6.3 & 50.0 \\
\hline 1,2-Dichlorobenzene-d4 & 0.797 & 0.816 & 0.010 & 2.4 & 50.0 \\
\hline
\end{tabular}

Report 1,4-Dioxane-d8 for Low/Medium VOA analysis only 
Lab Name: TESTAMERICA BURLINGTON

Contract: $8 \mathrm{E}-00302$

Lab Code: STLV Case No.: YORK Mod. Ref No.:

SDG No.: 31673

Instrument ID: CHD.i

Calibration Date: 01/27/2016 Time: 1641

Lab File Id: 18020_017.D

EPA Sample No. (VSTD\#\#\#\#) : VSTD005HD Init. Calib. Date (s):01/27/2016 01/27/2016

Heated Purge: ( $\mathrm{Y} / \mathrm{N}) \mathrm{N}$ GC Column: DB-624

Init. Calib. Time(s) : 1117 1257

Purge Volume: 25.0 ID: $0.20(\mathrm{~mm})$ Length: 25 (m) $(\mathrm{mL})$

\begin{tabular}{|c|c|c|c|c|c|}
\hline COMPOUND & $\overline{\mathrm{RRF}}$ & RRF 5.0 & $\begin{array}{l}\text { MIN } \\
\text { RRF }\end{array}$ & $\frac{\circ}{D}$ & $\mathrm{MAX} \div \mathrm{D}$ \\
\hline Dichlorodifluoromethane & 0.912 & 0.931 & 0.010 & 2.1 & 50.0 \\
\hline Chloromethane & 0.343 & 0.332 & 0.010 & -2.9 & 50.0 \\
\hline Vinyl chloride & 0.363 & 0.363 & 0.010 & 0.1 & 50.0 \\
\hline Bromomethane & 0.169 & 0.161 & 0.010 & -5.1 & 50.0 \\
\hline Chloroethane & 0.224 & 0.211 & 0.010 & -6.2 & 50.0 \\
\hline Trichlorofluoromethane & 1.147 & 1.197 & 0.010 & 4.4 & 50.0 \\
\hline 1,1-Dichloroethene & 0.320 & 0.334 & 0.010 & 4.2 & 50.0 \\
\hline 1,1,2-Trichloro-1,2,2-trifluoroethane & 0.430 & 0.446 & 0.010 & 3.9 & 50.0 \\
\hline Acetone & 0.026 & 0.027 & 0.010 & 1.7 & 50.0 \\
\hline Carbon disulfide & 0.718 & 0.724 & 0.010 & 0.7 & 50.0 \\
\hline Methyl acetate & 0.060 & 0.051 & 0.010 & -15.1 & 50.0 \\
\hline Methylene Chloride & 0.230 & 0.223 & 0.010 & -3.0 & 50.0 \\
\hline trans-1,2-Dichloroethene & 0.309 & 0.321 & 0.010 & 3.7 & 50.0 \\
\hline Methyl tert-butyl ether & 0.415 & 0.406 & 0.010 & -2.4 & 50.0 \\
\hline 1,1-Dichloroethane & 0.654 & 0.668 & 0.010 & 2.2 & 50.0 \\
\hline cis-1,2-Dichloroethene & 0.290 & 0.296 & 0.010 & 2.2 & 50.0 \\
\hline 2-Butanone & 0.037 & 0.035 & 0.010 & -4.4 & 50.0 \\
\hline Bromochloromethane & 0.095 & 0.095 & 0.010 & -0.1 & 50.0 \\
\hline Chloroform & 0.709 & 0.716 & 0.010 & 1.0 & 50.0 \\
\hline 1,1,1-Trichloroethane & 1.014 & 1.057 & 0.010 & 4.3 & 50.0 \\
\hline Cyclohexane & 0.668 & 0.723 & 0.010 & 8.3 & 50.0 \\
\hline Carbon tetrachloride & 0.979 & 1.023 & 0.010 & 4.5 & 50.0 \\
\hline Benzene & 1.535 & 1.588 & 0.010 & 3.4 & 50.0 \\
\hline 1,2-Dichloroethane & 0.408 & 0.399 & 0.010 & -2.2 & 50.0 \\
\hline Trichloroethene & 0.450 & 0.470 & 0.010 & 4.4 & 50.0 \\
\hline Methylcyclohexane & 0.594 & 0.660 & 0.010 & 11.0 & 50.0 \\
\hline
\end{tabular}

Report 1,4-Dioxane for Low/Medium VOA analysis only 
Lab Name: TESTAMERICA BURLINGTON

Contract: 8E-00302

Lab Code: STLV Case No.: YORK Mod. Ref No.:

SDG No.: 31673

Instrument ID: CHD.i

Calibration Date: 01/27/2016 Time: 1641

Lab File Id: 18020_017.D

EPA Sample No. (VSTD\#\#\#\#) : VSTD005HD Init. Calib. Date (s):01/27/2016 01/27/2016

Heated Purge: (Y/N) N GC Column: DB-624

Init. Calib. Time(s) : 1117 1257

Purge Volume: 25.0 ID: $0.20(\mathrm{~mm})$ Length: 25 (m) $(\mathrm{mL})$

\begin{tabular}{|c|c|c|c|c|c|}
\hline COMPOUND & $\overline{\mathrm{RRF}}$ & RRF5.0 & $\begin{array}{l}\text { MIN } \\
\text { RRF }\end{array}$ & $\because \mathrm{D}$ & $\operatorname{MAX} \cong \mathrm{D}$ \\
\hline 1,2-Dichloropropane & 0.318 & 0.319 & 0.010 & 0.3 & 50.0 \\
\hline Bromodichloromethane & 0.482 & 0.483 & 0.010 & 0.4 & 50.0 \\
\hline cis-1,3-Dichloropropene & 0.456 & 0.486 & 0.010 & 6.7 & 50.0 \\
\hline 4-Methyl-2-pentanone & 0.099 & 0.107 & 0.010 & 8.7 & 50.0 \\
\hline Toluene & 1.685 & 1.852 & 0.010 & 9.9 & 50.0 \\
\hline trans-1,3-Dichloropropene & 0.346 & 0.363 & 0.010 & 4.9 & 50.0 \\
\hline $1,1,2$-Trichloroethane & 0.161 & 0.162 & 0.010 & 0.1 & 50.0 \\
\hline Tetrachloroethene & 0.398 & 0.406 & 0.010 & 2.0 & 50.0 \\
\hline 2-Hexanone & 0.072 & 0.076 & 0.010 & 5.9 & 50.0 \\
\hline Dibromochloromethane & 0.224 & 0.223 & 0.010 & -0.3 & 50.0 \\
\hline 1,2-Dibromoethane & 0.145 & 0.140 & 0.010 & -3.0 & 50.0 \\
\hline Chlorobenzene & 1.035 & 1.071 & 0.010 & 3.4 & 50.0 \\
\hline Ethylbenzene & 2.062 & 2.301 & 0.010 & 11.6 & 50.0 \\
\hline o-Xylene & 0.645 & 0.729 & 0.010 & 13.0 & 50.0 \\
\hline $\mathrm{m}, \mathrm{p}$-Xylene & 0.740 & 0.829 & 0.010 & 11.9 & 50.0 \\
\hline Styrene & 1.023 & 1.140 & 0.010 & 11.4 & 50.0 \\
\hline Bromoform & 0.198 & 0.187 & 0.010 & -5.8 & 50.0 \\
\hline Isopropylbenzene & 2.056 & 2.347 & 0.010 & 14.1 & 50.0 \\
\hline $1,1,2,2$-Tetrachloroethane & 0.145 & 0.145 & 0.010 & -0.3 & 50.0 \\
\hline 1,3-Dichlorobenzene & 1.541 & 1.574 & 0.010 & 2.1 & 50.0 \\
\hline 1,4-Dichlorobenzene & 1.730 & 1.709 & 0.010 & -1.2 & 50.0 \\
\hline 1,2-Dichlorobenzene & 1.335 & 1.383 & 0.010 & 3.6 & 50.0 \\
\hline 1,2-Dibromo-3-Chloropropane & 0.053 & 0.053 & 0.010 & 0.6 & 50.0 \\
\hline 1,2,4-Trichlorobenzene & 0.726 & 0.697 & 0.010 & -4.1 & 50.0 \\
\hline $1,2,3$-Trichlorobenzene & 0.521 & 0.495 & 0.010 & -4.9 & 50.0 \\
\hline
\end{tabular}


Lab Name: TESTAMERICA BURLINGTON

Contract: $8 \mathrm{E}-00302$

Lab Code: STLV Case No.: YORK Mod. Ref No.

SDG No.: 31673

Instrument ID: CHD.i

Calibration Date: 01/27/2016 Time: 1641

Lab File Id: 18020_017.D

Init. Calib. Date(s):01/27/2016 01/27/2016

EPA Sample No. (VSTD\#\#\#\#) : VSTD005HD

Heated Purge: (Y/N) N GC Column: DB-624

Init. Calib. Time(s) : 1117 1257

Purge Volume: 25.0

ID: $0.20(\mathrm{~mm})$ Length: 25

(m) $(\mathrm{mL})$

\begin{tabular}{|c|c|c|c|c|c|}
\hline COMPOUND & $\overline{\mathrm{RRF}}$ & RRF5.0 & $\begin{array}{l}\text { MIN } \\
\text { RRE }\end{array}$ & $\because \mathrm{D}$ & $\operatorname{MAX} \cong \mathrm{D}$ \\
\hline Vinyl Chloride-d3 & 0.336 & 0.332 & 0.010 & -1.0 & 50.0 \\
\hline Chloroethane-d5 & 0.273 & 0.270 & 0.010 & -1.3 & 50.0 \\
\hline 1,1-Dichloroethene-d2 & 0.851 & 0.883 & 0.010 & 3.7 & 50.0 \\
\hline 2-Butanone-d5 & 0.032 & 0.031 & 0.010 & -1.8 & 50.0 \\
\hline Chloroform-d & 0.736 & 0.764 & 0.010 & 3.7 & 50.0 \\
\hline 1,2-Dichloroethane-d4 & 0.324 & 0.335 & 0.010 & 3.3 & 50.0 \\
\hline Benzene-d6 & 1.391 & 1.465 & 0.010 & 5.3 & 50.0 \\
\hline 1,2-Dichloropropane-d6 & 0.339 & 0.358 & 0.010 & 5.8 & 50.0 \\
\hline Toluene-d8 & 1.436 & 1.555 & 0.010 & 8.3 & 50.0 \\
\hline trans-1,3-Dichloropropene-d4 & 0.305 & 0.308 & 0.010 & 1.1 & 50.0 \\
\hline 2-Hexanone-d5 & 0.027 & 0.029 & 0.010 & 7.0 & 50.0 \\
\hline $1,1,2,2$-Tetrachloroethane-d2 & 0.154 & 0.151 & 0.010 & -1.6 & 50.0 \\
\hline 1,2-Dichlorobenzene-d4 & 0.802 & 0.817 & 0.010 & 1.9 & 50.0 \\
\hline
\end{tabular}

Report 1,4-Dioxane-d8 for Low/Medium VOA analysis only 
Lab Name: TESTAMERICA BURLINGTON

Contract: $8 \mathrm{E}-00302$

Lab Code: STLV Case No.: YORK Mod. Ref No.: SDG No.: 31673

Matrix: (SOIL/SED/WATER) Water

Sample wt/vol: 25.0 $(\mathrm{g} / \mathrm{mL}) \mathrm{mL}$

Level: (TRACE/LOW/MED) TRACE

\% Moisture: not dec.

GC Column: DB-624 ID: $0.20 \quad(\mathrm{~mm})$

Soil Extract Volume: (uL) $(\mathrm{mL})$

Purge Volume: 25.0
Lab Sample ID: MB 200-100004/7

Lab File ID: 18001_007.D

Date Received:

Date Analyzed: 01/26/2016

Dilution Factor: 1.0

Soil Aliquot Volume: (uL)

\begin{tabular}{|c|c|c|c|}
\hline CAS NO. & COMPOUND & $\begin{array}{l}\text { CONCENTRATION UNITS: } \\
(\mathrm{ug} / \mathrm{L} \text { or } \mathrm{ug} / \mathrm{kg}) \mathrm{ug} / \mathrm{L}\end{array}$ & Q \\
\hline $75-71-8$ & Dichlorodifluoromethane & 1.0 & $\mathrm{U}$ \\
\hline $74-87-3$ & Chloromethane & 1.0 & $\mathrm{U}$ \\
\hline $75-01-4$ & Vinyl chloride & 1.0 & $\mathrm{U}$ \\
\hline $74-83-9$ & Bromomethane & 1.0 & $\mathrm{U}$ \\
\hline $75-00-3$ & Chloroethane & 1.0 & $\mathrm{U}$ \\
\hline $75-69-4$ & Trichlorofluoromethane & 1.0 & $\mathrm{U}$ \\
\hline $75-35-4$ & 1,1-Dichloroethene & 1.0 & $\mathrm{U}$ \\
\hline $76-13-1$ & $1,1,2$-Trichloro-1,2,2-trifluoroethane & 1.0 & $\mathrm{U}$ \\
\hline $67-64-1$ & Acetone & 5.0 & $\mathrm{U}$ \\
\hline $75-15-0$ & Carbon disulfide & 1.0 & $\mathrm{U}$ \\
\hline $79-20-9$ & Methyl acetate & 1.0 & $\mathrm{U}$ \\
\hline $75-09-2$ & Methylene Chloride & 1.0 & $\mathrm{U}$ \\
\hline $156-60-5$ & trans-1,2-Dichloroethene & 1.0 & $\mathrm{U}$ \\
\hline $1634-04-4$ & Methyl tert-butyl ether & 1.0 & $\mathrm{U}$ \\
\hline $75-34-3$ & 1,1-Dichloroethane & 1.0 & $\mathrm{U}$ \\
\hline $156-59-2$ & cis-1,2-Dichloroethene & 1.0 & $\mathrm{U}$ \\
\hline $78-93-3$ & 2-Butanone & 5.0 & $\mathrm{U}$ \\
\hline $74-97-5$ & Bromochloromethane & 1.0 & $\mathrm{U}$ \\
\hline $67-66-3$ & Chloroform & 1.0 & $\mathrm{U}$ \\
\hline $71-55-6$ & $1,1,1$-Trichloroethane & 1.0 & $\mathrm{U}$ \\
\hline $110-82-7$ & Cyclohexane & 1.0 & $\mathrm{U}$ \\
\hline $56-23-5$ & Carbon tetrachloride & 1.0 & $\mathrm{U}$ \\
\hline $71-43-2$ & Benzene & 1.0 & $\mathrm{U}$ \\
\hline $107-06-2$ & 1,2-Dichloroethane & 1.0 & $\mathrm{U}$ \\
\hline
\end{tabular}

Report 1,4-Dioxane for Low-Medium VOA analysis only 
Lab Name: TESTAMERICA BURLINGTON

Lab Code: STLV

Case No.: YORK

Mod. Ref No.:

Contract: 8E-00302

Matrix: (SOIL/SED/WATER) Water

Sample wt/vol: 25.0 $(\mathrm{g} / \mathrm{mL}) \mathrm{mL}$

Level: (TRACE/LOW/MED) TRACE

\% Moisture: not dec.

GC Column: DB-624 ID $: \underline{0.20 \quad(\mathrm{~mm})}$

Soil Extract Volume: (uL) $(\mathrm{mL})$

Purge Volume: 25.0
SDG No.: 31673

Lab Sample ID: MB 200-100004/7

Lab File ID: 18001_007.D

Date Received:

Date Analyzed: 01/26/2016

Dilution Factor: 1.0

Soil Aliquot Volume: (uL)

\begin{tabular}{|c|c|c|c|}
\hline CAS NO. & COMPOUND & $\begin{array}{l}\text { CONCENTRATION UNITS: } \\
(\mathrm{ug} / \mathrm{L} \text { or } \mathrm{ug} / \mathrm{kg}) \mathrm{ug} / \mathrm{L}\end{array}$ & $q$ \\
\hline $79-01-6$ & Trichloroethene & 1.0 & $\mathrm{U}$ \\
\hline $108-87-2$ & Methylcyclohexane & 1.0 & $\mathrm{U}$ \\
\hline $78-87-5$ & 1,2-Dichloropropane & 1.0 & $\mathrm{U}$ \\
\hline $75-27-4$ & Bromodichloromethane & 1.0 & $\mathrm{U}$ \\
\hline $10061-01-5$ & cis-1,3-Dichloropropene & 1.0 & U \\
\hline $108-10-1$ & 4-Methyl-2-pentanone & 1.0 & $\mathrm{U}$ \\
\hline $108-88-3$ & Toluene & 0.012 & $\mathrm{~J}$ \\
\hline $10061-02-6$ & trans-1,3-Dichloropropene & 1.0 & $\mathrm{U}$ \\
\hline $79-00-5$ & $1,1,2-$ Trichloroethane & 1.0 & $\mathrm{U}$ \\
\hline $127-18-4$ & Tetrachloroethene & 1.0 & $\mathrm{U}$ \\
\hline $591-78-6$ & 2-Hexanone & 1.0 & $\mathrm{U}$ \\
\hline $124-48-1$ & Dibromochloromethane & 1.0 & $\mathrm{U}$ \\
\hline $106-93-4$ & 1,2-Dibromoethane & 1.0 & $\mathrm{U}$ \\
\hline $108-90-7$ & Chlorobenzene & 1.0 & $\mathrm{U}$ \\
\hline $100-41-4$ & Ethylbenzene & 1.0 & $\mathrm{U}$ \\
\hline $95-47-6$ & o-Xylene & 1.0 & $\mathrm{U}$ \\
\hline $179601-23-1$ & m,p-xylene & 1.0 & $\mathrm{U}$ \\
\hline $100-42-5$ & Styrene & 1.0 & $\mathrm{U}$ \\
\hline $75-25-2$ & Bromoform & 1.0 & $\mathrm{U}$ \\
\hline $98-82-8$ & Isopropylbenzene & 1.0 & $\mathrm{U}$ \\
\hline $79-34-5$ & $1,1,2,2$-Tetrachloroethane & 1.0 & $\mathrm{U}$ \\
\hline $541-73-1$ & 1,3 -Dichlorobenzene & 1.0 & $\mathrm{U}$ \\
\hline $106-46-7$ & 1,4-Dichlorobenzene & 1.0 & $\mathrm{U}$ \\
\hline $95-50-1$ & 1,2-Dichlorobenzene & 1.0 & $\mathrm{U}$ \\
\hline $96-12-8$ & 1,2-Dibromo-3-Chloropropane & 1.0 & $\mathrm{U}$ \\
\hline $120-82-1$ & $1,2,4$-Trichlorobenzene & 1.0 & $\mathrm{U}$ \\
\hline $87-61-6$ & $1,2,3$-Trichlorobenzene & 1.0 & $\mathrm{U}$ \\
\hline
\end{tabular}


Lab Name: TESTAMERICA BURLINGTON

Contract: $8 \mathrm{E}-00302$

Lab Code: STLV Case No.: YORK Mod. Ref No.: SDG No.: 31673

Matrix: (SOIL/SED/WATER) Water

Sample wt/vol: 25.0 $(\mathrm{g} / \mathrm{mL}) \mathrm{mL}$

Level: (TRACE or LOW/MED) TRACE

Moisture: not dec.

GC Column: DB-624 ID $: 0.20 \quad(\mathrm{~mm})$

Soil Extract Volume: (uL)

CONCENTRATION UNITS: (ug/L or ug/kg) ug/L
Lab Sample ID: MB 200-100004/7

Lab File ID: 18001 007.D

Date Received:

Date Analyzed: 01/26/2016

Dilution Factor: 1.0

Soil Aliquot Volume: (uL) Purge Volume: 25.0

\begin{tabular}{l|l|l|r|r|r|}
\cline { 2 - 6 } & CAS NUMBER & \multicolumn{1}{|c|}{ COMPOUND NAME } & RT & EST. CONC. & Q \\
\cline { 2 - 6 } 01 & & Unknown & 7.25 & 2.8 & $\mathrm{~J} \mathrm{X}$ \\
\cline { 2 - 6 } 02 & $541-05-9$ & Cyclotrisiloxane, hexamethyl- & 8.17 & 0.95 & $\mathrm{~J} \mathrm{~N}$ \\
\cline { 2 - 6 } 03 & $556-67-2$ & Cyclotetrasiloxane, octamethyl- & 10.98 & $\mathrm{~J} / \mathrm{N}$ \\
\cline { 2 - 6 } 04 & E9667961 & Total Alkanes & & \\
\cline { 2 - 6 }
\end{tabular}

1EPA-designated Registry Number. 
Lab Name: TESTAMERICA BURLINGTON

Contract: $8 \mathrm{E}-00302$

Lab Code: STLV Case No.: YORK Mod. Ref No.: SDG No.: 31673

Matrix: (SOIL/SED/WATER) Water

Sample wt/vol: 25.0 $(\mathrm{g} / \mathrm{mL}) \mathrm{mL}$

Level: (TRACE/LOW/MED) TRACE

\% Moisture: not dec.

GC Column: DB-624 ID: $0.20 \quad(\mathrm{~mm})$

Soil Extract Volume: $(u L)$ $(\mathrm{mL})$

Purge Volume: 25.0
Lab Sample ID: MB 200-100049/11

Lab File ID: 18020_011.D

Date Received:

Date Analyzed: 01/27/2016

Dilution Factor: 1.0

Soil Aliquot Volume: (uL)

\begin{tabular}{|c|c|c|c|}
\hline CAS NO. & COMPOUND & $\begin{array}{l}\text { CONCENTRATION UNITS: } \\
(\mathrm{ug} / \mathrm{L} \text { or } \mathrm{ug} / \mathrm{kg}) \mathrm{ug} / \mathrm{L}\end{array}$ & q \\
\hline $75-71-8$ & Dichlorodifluoromethane & 1.0 & $\mathrm{U}$ \\
\hline $74-87-3$ & Chloromethane & 1.0 & $\mathrm{U}$ \\
\hline $75-01-4$ & Vinyl chloride & 1.0 & $\mathrm{U}$ \\
\hline $74-83-9$ & Bromomethane & 1.0 & $\mathrm{U}$ \\
\hline $75-00-3$ & Chloroethane & 1.0 & $\mathrm{U}$ \\
\hline $75-69-4$ & Trichlorofluoromethane & 1.0 & $\mathrm{U}$ \\
\hline $75-35-4$ & 1,1-Dichloroethene & 1.0 & $\mathrm{U}$ \\
\hline $76-13-1$ & $1,1,2$-Trichloro-1,2,2-trifluoroethane & 1.0 & $\mathrm{U}$ \\
\hline $67-64-1$ & Acetone & 5.0 & $\mathrm{U}$ \\
\hline $75-15-0$ & Carbon disulfide & 1.0 & $\mathrm{U}$ \\
\hline $79-20-9$ & Methyl acetate & 1.0 & $\mathrm{U}$ \\
\hline $75-09-2$ & Methylene Chloride & 0.037 & $\mathrm{~J}$ \\
\hline $156-60-5$ & trans-1,2-Dichloroethene & 1.0 & $\mathrm{U}$ \\
\hline $1634-04-4$ & Methyl tert-butyl ether & 1.0 & $\mathrm{U}$ \\
\hline $75-34-3$ & 1,1-Dichloroethane & 1.0 & $\mathrm{U}$ \\
\hline $156-59-2$ & cis-1,2-Dichloroethene & 1.0 & $\mathrm{U}$ \\
\hline $78-93-3$ & 2-Butanone & 5.0 & $\mathrm{U}$ \\
\hline $74-97-5$ & Bromochloromethane & 1.0 & $\mathrm{U}$ \\
\hline $67-66-3$ & Chloroform & 1.0 & $\mathrm{U}$ \\
\hline $71-55-6$ & $1,1,1$-Trichloroethane & 1.0 & $\mathrm{U}$ \\
\hline $110-82-7$ & Cyclohexane & 1.0 & $\mathrm{U}$ \\
\hline $56-23-5$ & Carbon tetrachloride & 1.0 & $\mathrm{U}$ \\
\hline $71-43-2$ & Benzene & 1.0 & $\mathrm{U}$ \\
\hline $107-06-2$ & 1,2-Dichloroethane & 1.0 & $\mathrm{U}$ \\
\hline
\end{tabular}

Report 1,4-Dioxane for Low-Medium VOA analysis only 
Lab Name: TESTAMERICA BURLINGTON

Lab Code: STLV

Case No.: YORK

Mod. Ref No.:
Contract: 8E-00302

SDG No.: 31673
Matrix: (SOIL/SED/WATER) Water

Sample wt/vol: 25.0 $(\mathrm{g} / \mathrm{mL}) \mathrm{mL}$

Level: (TRACE/LOW/MED) TRACE

\% Moisture: not dec.

GC Column: DB-624 ID $: \underline{0.20 \quad(\mathrm{~mm})}$

Soil Extract Volume: (uL) $(\mathrm{mL})$

Purge Volume: 25.0
Lab Sample ID: MB 200-100049/11

Lab File ID: 18020_011.D

Date Received:

Date Analyzed: 01/27/2016

Dilution Factor: 1.0

Soil Aliquot Volume: (uL)

\begin{tabular}{|c|c|c|c|}
\hline CAS NO. & COMPOUND & $\begin{array}{l}\text { CONCENTRATION UNITS: } \\
(\mathrm{ug} / \mathrm{L} \text { or } \mathrm{ug} / \mathrm{kg}) \mathrm{ug} / \mathrm{L}\end{array}$ & $q$ \\
\hline $79-01-6$ & Trichloroethene & 1.0 & $\mathrm{U}$ \\
\hline $108-87-2$ & Methylcyclohexane & 1.0 & $\mathrm{U}$ \\
\hline $78-87-5$ & 1,2-Dichloropropane & 1.0 & $\mathrm{U}$ \\
\hline $75-27-4$ & Bromodichloromethane & 1.0 & $\mathrm{U}$ \\
\hline $10061-01-5$ & cis-1,3-Dichloropropene & 1.0 & U \\
\hline $108-10-1$ & 4-Methyl-2-pentanone & 1.0 & $\mathrm{U}$ \\
\hline $108-88-3$ & Toluene & 0.010 & $\mathrm{~J}$ \\
\hline $10061-02-6$ & trans-1,3-Dichloropropene & 1.0 & $\mathrm{U}$ \\
\hline $79-00-5$ & $1,1,2-$ Trichloroethane & 1.0 & $\mathrm{U}$ \\
\hline $127-18-4$ & Tetrachloroethene & 1.0 & $\mathrm{U}$ \\
\hline $591-78-6$ & 2-Hexanone & 1.0 & $\mathrm{U}$ \\
\hline $124-48-1$ & Dibromochloromethane & 1.0 & $\mathrm{U}$ \\
\hline $106-93-4$ & 1,2-Dibromoethane & 1.0 & $\mathrm{U}$ \\
\hline $108-90-7$ & Chlorobenzene & 1.0 & $\mathrm{U}$ \\
\hline $100-41-4$ & Ethylbenzene & 1.0 & $\mathrm{U}$ \\
\hline $95-47-6$ & o-Xylene & 1.0 & $\mathrm{U}$ \\
\hline $179601-23-1$ & m,p-xylene & 0.0073 & $\mathrm{~J}$ \\
\hline $100-42-5$ & Styrene & 1.0 & $\mathrm{U}$ \\
\hline $75-25-2$ & Bromoform & 1.0 & $\mathrm{U}$ \\
\hline $98-82-8$ & Isopropylbenzene & 1.0 & $\mathrm{U}$ \\
\hline $79-34-5$ & $1,1,2,2$-Tetrachloroethane & 1.0 & $\mathrm{U}$ \\
\hline $541-73-1$ & 1,3 -Dichlorobenzene & 1.0 & $\mathrm{U}$ \\
\hline $106-46-7$ & 1,4-Dichlorobenzene & 1.0 & $\mathrm{U}$ \\
\hline $95-50-1$ & 1,2-Dichlorobenzene & 1.0 & $\mathrm{U}$ \\
\hline $96-12-8$ & 1,2-Dibromo-3-Chloropropane & 1.0 & $\mathrm{U}$ \\
\hline $120-82-1$ & $1,2,4$-Trichlorobenzene & 1.0 & $\mathrm{U}$ \\
\hline $87-61-6$ & $1,2,3$-Trichlorobenzene & 1.0 & $\mathrm{U}$ \\
\hline
\end{tabular}


Lab Name: TESTAMERICA BURLINGTON

Contract: $8 \mathrm{E}-00302$

Lab Code: STLV Case No.: YORK Mod. Ref No.: SDG No.: 31673

Matrix: (SOIL/SED/WATER) Water

Sample wt/vol: 25.0 $(\mathrm{g} / \mathrm{mL}) \mathrm{mL}$

Level: (TRACE or LOW/MED) TRACE

․ Moisture: not dec.

GC Column: DB-624 ID $: 0.20 \quad(\mathrm{~mm})$

Soil Extract Volume: (uL)
Lab Sample ID: MB 200-100049/11

Lab File ID: 18020_011.D

Date Received:

Date Analyzed: 01/27/2016

Dilution Factor: 1.0

Soil Aliquot Volume: (uL)

Purge Volume: 25.0

$(\mathrm{mL})$ CONCENTRATION UNITS: (ug/L or ug/kg) ug/L

\begin{tabular}{|c|c|c|c|c|}
\hline CAS NUMBER & COMPOUND NAME & $\mathrm{RT}$ & EST. CONC. & Q \\
\hline 01 & Unknown & 7.25 & 3.0 & $\mathrm{~J} X$ \\
\hline $541-05-9$ & Cyclotrisiloxane, hexamethyl- & 8.17 & 0.99 & $\mathrm{~J} \mathrm{~N}$ \\
\hline 03 & Unknown & 10.98 & 1.6 & $\mathrm{~J}$ \\
\hline E9667961 & Total Alkanes & $\mathrm{N} / \mathrm{A}$ & & \\
\hline
\end{tabular}

1 EPA-designated Registry Number. 
Lab Name: TESTAMERICA BURLINGTON

Contract: $8 \mathrm{E}-00302$

Lab Code: STLV

Case No.: YORK Mod. Ref No.:

SDG No.: 31673

Matrix: (SOIL/SED/WATER) Water

Sample wt/vol: 25.0 $(\mathrm{g} / \mathrm{mL}) \mathrm{mL}$

Level: (TRACE/LOW/MED) TRACE

\% Moisture: not dec.

GC Column: DB-624 ID: $0.20 \quad(\mathrm{~mm})$

Soil Extract Volume: $(u L)$ $(\mathrm{mL})$

Purge Volume: 25.0
Lab Sample ID: 200-31673-4

Lab File ID: 18020_016.D

Date Received:

Date Analyzed: 01/27/2016

Dilution Factor: 1.0

Soil Aliquot Volume:

(uL)

\begin{tabular}{|c|c|c|c|}
\hline CAS NO. & COMPOUND & $\begin{array}{l}\text { CONCENTRATION UNITS: } \\
(\mathrm{ug} / \mathrm{L} \text { or } \mathrm{ug} / \mathrm{kg}) \mathrm{ug} / \mathrm{L}\end{array}$ & q \\
\hline $75-71-8$ & Dichlorodifluoromethane & 1.0 & $\mathrm{U}$ \\
\hline $74-87-3$ & Chloromethane & 1.0 & $\mathrm{U}$ \\
\hline $75-01-4$ & Vinyl chloride & 1.0 & $\mathrm{U}$ \\
\hline $74-83-9$ & Bromomethane & 1.0 & $\mathrm{U}$ \\
\hline $75-00-3$ & Chloroethane & 1.0 & $\mathrm{U}$ \\
\hline $75-69-4$ & Trichlorofluoromethane & 1.0 & $\mathrm{U}$ \\
\hline $75-35-4$ & 1,1-Dichloroethene & 1.0 & $\mathrm{U}$ \\
\hline $76-13-1$ & $1,1,2$-Trichloro-1,2,2-trifluoroethane & 1.0 & $\mathrm{U}$ \\
\hline $67-64-1$ & Acetone & 5.0 & $\mathrm{U}$ \\
\hline $75-15-0$ & Carbon disulfide & 1.0 & $\mathrm{U}$ \\
\hline $79-20-9$ & Methyl acetate & 1.0 & $\mathrm{U}$ \\
\hline $75-09-2$ & Methylene Chloride & 1.0 & $\mathrm{U}$ \\
\hline $156-60-5$ & trans-1,2-Dichloroethene & 1.0 & $\mathrm{U}$ \\
\hline $1634-04-4$ & Methyl tert-butyl ether & 1.0 & $\mathrm{U}$ \\
\hline $75-34-3$ & 1,1-Dichloroethane & 1.0 & $\mathrm{U}$ \\
\hline $156-59-2$ & cis-1,2-Dichloroethene & 1.0 & $\mathrm{U}$ \\
\hline $78-93-3$ & 2-Butanone & 5.0 & $\mathrm{U}$ \\
\hline $74-97-5$ & Bromochloromethane & 1.0 & $\mathrm{U}$ \\
\hline $67-66-3$ & Chloroform & 1.0 & $\mathrm{U}$ \\
\hline $71-55-6$ & $1,1,1$-Trichloroethane & 1.0 & $\mathrm{U}$ \\
\hline $110-82-7$ & Cyclohexane & 1.0 & $\mathrm{U}$ \\
\hline $56-23-5$ & Carbon tetrachloride & 1.0 & $\mathrm{U}$ \\
\hline $71-43-2$ & Benzene & 1.0 & $\mathrm{U}$ \\
\hline $107-06-2$ & 1,2-Dichloroethane & 1.0 & $\mathrm{U}$ \\
\hline
\end{tabular}

Report 1,4-Dioxane for Low-Medium VOA analysis only 
Lab Name: TESTAMERICA BURLINGTON

Lab Code: STLV

Case No.: YORK

Mod. Ref No.:

Contract: 8E-00302

Matrix: (SOIL/SED/WATER) Water

Sample wt/vol: 25.0 $(\mathrm{g} / \mathrm{mL}) \mathrm{mL}$

Level: (TRACE/LOW/MED) TRACE

\% Moisture: not dec.

GC Column: DB-624 ID: $0.20 \quad(\mathrm{~mm})$

Soil Extract Volume: (uL) $(\mathrm{mL})$

Purge Volume: 25.0 (mI)
SDG No.: 31673

Lab Sample ID: 200-31673-4

Lab File ID: 18020_016.D

Date Received:

Date Analyzed: 01/27/2016

Dilution Factor: 1.0

Soil Aliquot Volume:

(uL)

\begin{tabular}{|c|c|c|c|}
\hline CAS NO. & COMPOUND & $\begin{array}{l}\text { CONCENTRATION UNITS: } \\
(\mathrm{ug} / \mathrm{L} \text { or } \mathrm{ug} / \mathrm{kg}) \mathrm{ug} / \mathrm{L}\end{array}$ & Q \\
\hline $79-01-6$ & Trichloroethene & 1.0 & $\mathrm{U}$ \\
\hline $108-87-2$ & Methylcyclohexane & 1.0 & $\mathrm{U}$ \\
\hline $78-87-5$ & 1,2-Dichloropropane & 1.0 & $\mathrm{U}$ \\
\hline $75-27-4$ & Bromodichloromethane & 1.0 & $\mathrm{U}$ \\
\hline $10061-01-5$ & cis-1,3-Dichloropropene & 1.0 & U \\
\hline $108-10-1$ & 4-Methyl-2-pentanone & 1.0 & $\mathrm{U}$ \\
\hline $108-88-3$ & Toluene & 0.0081 & $\mathrm{~J} B$ \\
\hline $10061-02-6$ & trans-1,3-Dichloropropene & 1.0 & $\mathrm{U}$ \\
\hline $79-00-5$ & $1,1,2-$ Trichloroethane & 1.0 & $\mathrm{U}$ \\
\hline $127-18-4$ & Tetrachloroethene & 1.0 & $\mathrm{U}$ \\
\hline $591-78-6$ & 2-Hexanone & 1.0 & $\mathrm{U}$ \\
\hline $124-48-1$ & Dibromochloromethane & 1.0 & $\mathrm{U}$ \\
\hline $106-93-4$ & 1,2-Dibromoethane & 1.0 & $\mathrm{U}$ \\
\hline $108-90-7$ & Chlorobenzene & 1.0 & $\mathrm{U}$ \\
\hline $100-41-4$ & Ethylbenzene & 1.0 & $\mathrm{U}$ \\
\hline $95-47-6$ & o-Xylene & 1.0 & $\mathrm{U}$ \\
\hline $179601-23-1$ & m,p-xylene & 1.0 & $\mathrm{U}$ \\
\hline $100-42-5$ & Styrene & 1.0 & $\mathrm{U}$ \\
\hline $75-25-2$ & Bromoform & 1.0 & $\mathrm{U}$ \\
\hline $98-82-8$ & Isopropylbenzene & 1.0 & $\mathrm{U}$ \\
\hline $79-34-5$ & $1,1,2,2$-Tetrachloroethane & 1.0 & $\mathrm{U}$ \\
\hline $541-73-1$ & 1,3 -Dichlorobenzene & 1.0 & $\mathrm{U}$ \\
\hline $106-46-7$ & 1,4-Dichlorobenzene & 1.0 & $\mathrm{U}$ \\
\hline $95-50-1$ & 1,2-Dichlorobenzene & 1.0 & $\mathrm{U}$ \\
\hline $96-12-8$ & 1,2-Dibromo-3-Chloropropane & 1.0 & $\mathrm{U}$ \\
\hline $120-82-1$ & $1,2,4$-Trichlorobenzene & 1.0 & $\mathrm{U}$ \\
\hline $87-61-6$ & $1,2,3$-Trichlorobenzene & 1.0 & $\mathrm{U}$ \\
\hline
\end{tabular}


Lab Name: TESTAMERICA BURLINGTON

Contract: $8 \mathrm{E}-00302$

Lab Code: STLV Case No.: YORK Mod. Ref No.: SDG No.: 31673

Matrix: (SOIL/SED/WATER) Water

Sample wt/vol: 25.0 $(\mathrm{g} / \mathrm{mL}) \mathrm{mL}$

Level: (TRACE or LOW/MED) TRACE

\% Moisture: not dec.

GC Column: DB-624 ID $: 0.20 \quad(\mathrm{~mm})$

Soil Extract Volume: (uL)
Lab Sample ID: 200-31673-4

Lab File ID: 18020_016.D

Date Received:

Date Analyzed: 01/27/2016

Dilution Factor: 1.0

Soil Aliquot Volume: (uL) Purge Volume: 25.0 CONCENTRATION UNITS: (ug/L or ug/kg) ug/L

\begin{tabular}{|c|c|c|c|c|}
\hline CAS NUMBER & COMPOUND NAME & RT & EST. CONC. & $Q$ \\
\hline 01 & Unknown & 7.25 & 3.0 & $\mathrm{~J} B \mathrm{X}$ \\
\hline $541-05-9$ & Cyclotrisiloxane, hexamethyl- & 8.17 & 0.85 & $\mathrm{~J} \quad \mathrm{~N} B$ \\
\hline $556-67-2$ & Cyclotetrasiloxane, octamethyl- & 10.98 & 1.5 & $\mathrm{~J} \quad \mathrm{~N} B$ \\
\hline E9667961 & Total Alkanes & $\mathrm{N} / \mathrm{A}$ & & \\
\hline
\end{tabular}


Lab Name: TestAmerica Burlington

SDG No.: 31673

Instrument ID: CHD.i

Lab Sample ID: IC 200-99296/7

Date Analyzed: 01/08/16 14:21

Analysis Batch Number: 99296

Client Sample ID:

Lab File ID: 17668 007.D

GC Column: DB-624

ID $: 0.2(\mathrm{~mm})$

\begin{tabular}{|l|c|c|c|c|}
\hline \multicolumn{1}{|c|}{ COMPOUND NAME } & RETENTION & \multicolumn{2}{|c|}{ MANUAL INTEGRATION } \\
\cline { 3 - 5 } & TIME & REASON & ANALYST & DATE \\
\hline Methyl acetate & 3.12 & Split Peak & wilburj & $01 / 08 / 1616: 07$ \\
\hline
\end{tabular}

Lab Sample ID: IC 200-99296/8

Client Sample ID:

Date Analyzed: 01/08/16 14:45

Lab File ID: 17668 008.D

GC Column: DB-624

ID : $0.2(\mathrm{~mm})$

\begin{tabular}{|c|c|c|c|c|}
\hline \multirow[t]{2}{*}{ COMPOUND NAME } & \multirow{2}{*}{$\begin{array}{c}\text { RETENTION } \\
\text { TIME }\end{array}$} & \multicolumn{3}{|c|}{ MANUAL INTEGRATION } \\
\hline & & REASON & ANALYST & DATE \\
\hline Methyl acetate & 3.12 & Split Peak & wilburj & $01 / 08 / 16 \quad 16: 07$ \\
\hline 1,2-Dichloropropane-d6 & 6.37 & Split Peak & wilburj & $01 / 08 / 16 \quad 16: 05$ \\
\hline
\end{tabular}


Lab Name: TestAmerica Burlington SDG No.: 31673

Instrument ID: CHD.i

Lab Sample ID: CCVC 200-100004/26

Date Analyzed: 01/26/16 21:35

\begin{tabular}{|l|c|c|c|c|}
\hline \multicolumn{1}{|c|}{ COMPOUND NAME } & RETENTION & \multicolumn{3}{|c}{ MANUAL INTEGRATION } \\
\cline { 3 - 5 } & TIME & REASON & ANALYT & DATE \\
\hline Dichlorodifluoromethane & 1.52 & Baseline & wilburj & 01/27/16 09:33 \\
\hline
\end{tabular}

Job No.: 200-31673-1

Analysis Batch Number: 100004

Client Sample ID:

Lab File ID: 18001 026.D GC Column: DB-624 ID: $0.2(\mathrm{~mm})$ 
Lab Name: TestAmerica Burlington

Job No.: 200-31673-1

SDG No.: 31673

Instrument ID: CHD.i

Analysis Batch Number: 100049

Lab Sample ID: IC 200-100049/4

Client Sample ID:

Date Analyzed: 01/27/16 11:17

Lab File ID: 18020_004.D

GC Column: DB-624

ID $: 0.2(\mathrm{~mm})$

\begin{tabular}{|l|r|r|c|}
\hline \multicolumn{1}{|c|}{ COMPOUND NAME } & RETENTION & \multicolumn{2}{|c|}{ MANUAL INTEGRATION } \\
\cline { 3 - 5 } & TIME & REASON & ANALYST \\
\hline Methyl acetate & 3.12 & Unspecified & \\
\hline 1,2 -Dichloropropane & 6.47 & Unspecified & \\
\hline
\end{tabular}

Lab Sample ID: IC 200-100049/7 Client Sample ID:

Date Analyzed: 01/27/16 12:32

Lab File ID: 18020_007.D

GC Column: DB-624

ID $: 0.2(\mathrm{~mm})$

\begin{tabular}{|l|c|c|c|c|}
\hline \multicolumn{1}{|c|}{ COMPOUND NAME } & RETENTION & \multicolumn{3}{|c|}{ MANUAL INTEGRATION } \\
\cline { 3 - 5 } & TIME & REASON & ANALYST & DATE \\
\hline Dichlorodifluoromethane & 1.52 & Baseline & wilburj & $01 / 27 / 1613: 41$ \\
\hline
\end{tabular}

Lab Sample ID: IC 200-100049/8

Client Sample ID:

Date Analyzed: 01/27/16 12:57

Lab File ID: 18020_008.D

GC Column: DB-624

ID $: 0.2(\mathrm{~mm})$

\begin{tabular}{|c|c|c|c|c|}
\hline \multirow[t]{2}{*}{ COMPOUND NAME } & \multirow{2}{*}{$\begin{array}{c}\text { RETENTION } \\
\text { TIME }\end{array}$} & \multicolumn{3}{|c|}{ MANUAL INTEGRATION } \\
\hline & & REASON & ANALYST & DATE \\
\hline Dichlorodifluoromethane & 1.52 & Baseline & wilburj & $01 / 27 / 1613: 42$ \\
\hline
\end{tabular}

Lab Sample ID: MB 200-100049/11

Client Sample ID:

Date Analyzed: 01/27/16 14:12

Lab File ID: 18020_011.D

GC Column: DB-624

ID $: 0.2(\mathrm{~mm})$

\begin{tabular}{|c|c|c|c|c|}
\hline \multicolumn{2}{|c|}{ COMPOUND NAME } & RETENTION & \multicolumn{2}{|c|}{ MANUAL INTEGRATION } \\
\cline { 3 - 5 } & TIME & REASON & ANALYST & DATE \\
\hline m,p-Xylene & 9.59 & Assign Peak & wilburj & $01 / 27 / 1617: 23$ \\
\hline
\end{tabular}




\section{TestAmerica}

THE LEADER IN ENVIRONMENTAL TESTING

\section{ANALYTICAL REPORT}

Job Number: 200-32333-1

SDG Number: 32333

Job Description: York (200-32333)

Contract Number: 1E-30401

For:

Argonne National Laboratory

9700 South Cass Avenue

Building 203

Office B-141

Argonne, IL 60439

Attention: Ms. Esther Bowen

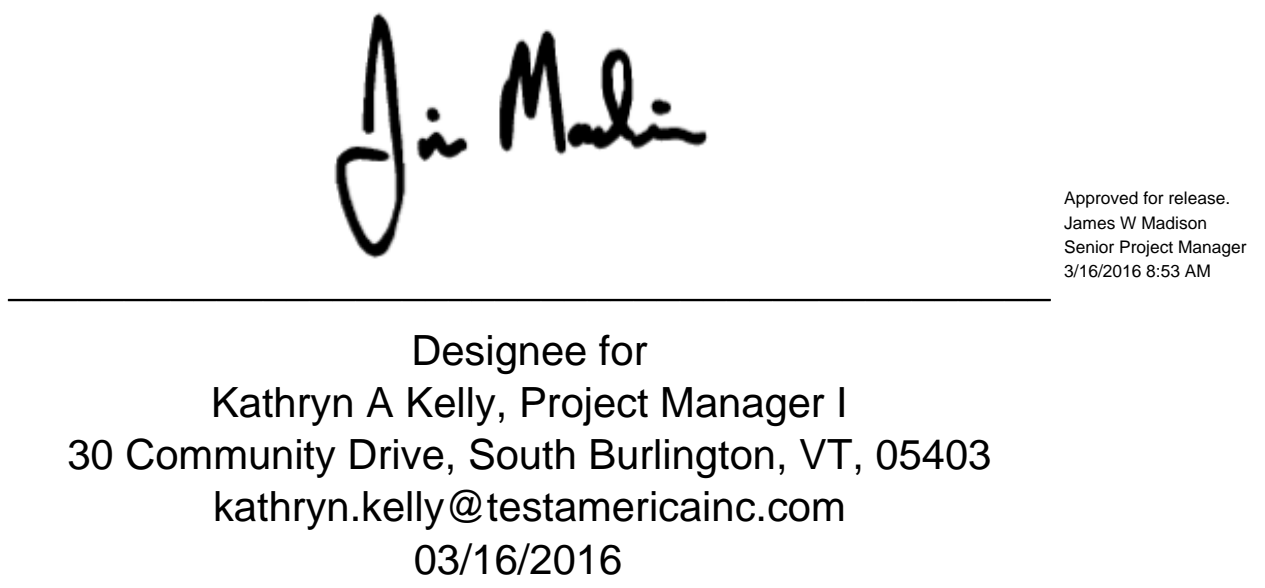

The test results in this report relate only to sample(s) as received by the laboratory. These test results were derived under a quality system that adheres to the requirements of NELAC. Pursuant to NELAC, this report may not be produced in full without written approval from the laboratory 


\section{Table of Contents}

Cover Title Page...$\ldots \ldots \ldots \ldots \ldots \ldots \ldots \ldots \ldots \ldots \ldots \ldots \ldots$

External Chain of Custody ...................... 4

Shipping Documentation . . . . . . . . . . . . . . . . 6

Sample Receipt and Log In Check List ..................... 7

Standards Traceability . . . . . . . . . . . . 8

Methodology Review . . . . . . . . . . . . . . . . . . . 13

Report Narrative $\ldots \ldots \ldots \ldots \ldots \ldots \ldots \ldots \ldots \ldots \ldots \ldots \ldots \ldots \ldots$

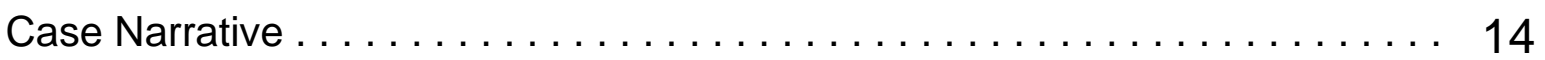

Manual Integration Documentation ........................ 15

Qualifier Definition ................................. 17

QC Summary - SOM01.2 Volatiles-Trace . . . . . . . . . . . . 18

QC Summary - SOM01.2 Volatiles-Trace .................... 18

Deuterated Monitoring Compound Summary $\ldots \ldots \ldots \ldots \ldots \ldots \ldots \ldots \ldots \ldots \ldots \ldots \ldots$

Method Blank $\ldots \ldots \ldots \ldots \ldots \ldots \ldots \ldots \ldots \ldots \ldots \ldots \ldots \ldots \ldots \ldots \ldots \ldots \ldots, 20$

GC/MS Instrument Performance Check $\ldots \ldots \ldots \ldots \ldots \ldots \ldots \ldots \ldots \ldots \ldots \ldots \ldots \ldots \ldots \ldots$

Internal Standard Area and RT Summary $\ldots \ldots \ldots \ldots \ldots \ldots \ldots \ldots \ldots \ldots \ldots \ldots \ldots \ldots$

Sample Data - SOM01.2 Volatiles-Trace ................ 24

Sample Data - SOM01.2 Volatiles-Trace ...................... 24

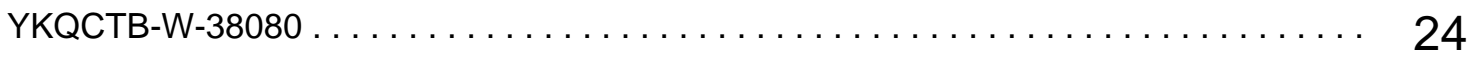

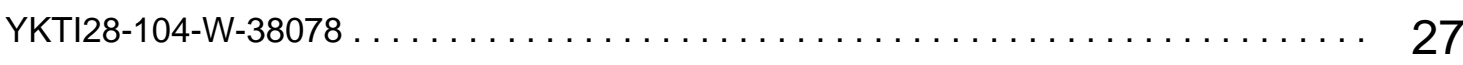

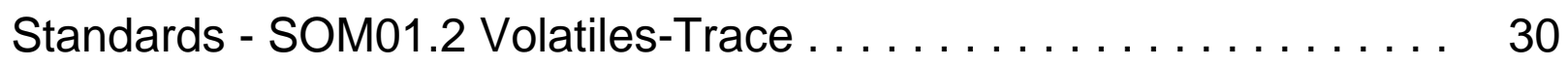

Standards - SOM01.2 Volatiles-Trace ..................... 30

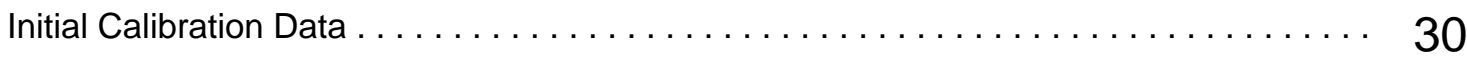

CCV Data, including closing CCV $\ldots \ldots \ldots \ldots \ldots \ldots \ldots \ldots \ldots \ldots \ldots \ldots \ldots \ldots \ldots \ldots$

Raw Qc Data - SOM01.2 Volatiles-Trace . . . . . . . . . . . . 39

Raw Qc Data - SOM01.2 Volatiles-Trace ..................... 39 


\section{Table of Contents}

Raw Qc Data - SOM01.2 Volatiles-Trace . . . . . . . . . . . . . 39

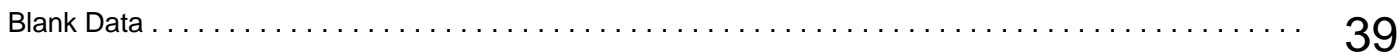




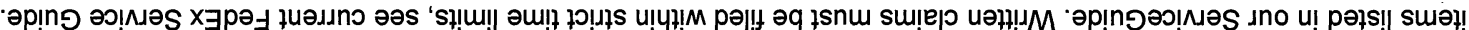

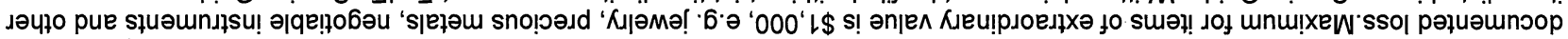

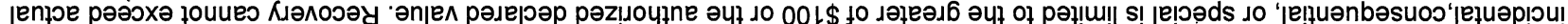

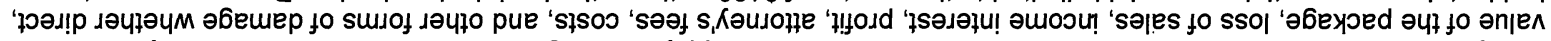

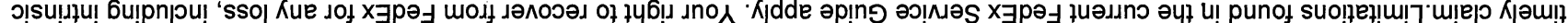

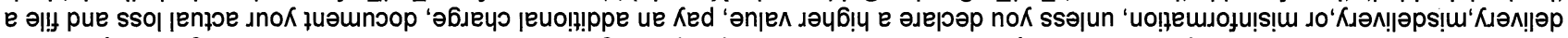

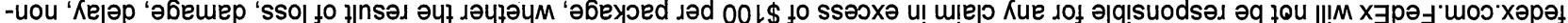

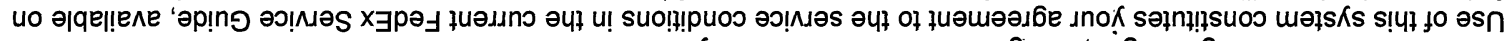

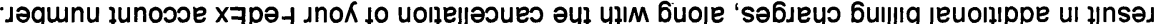

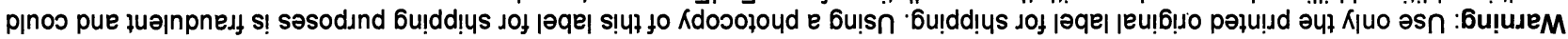

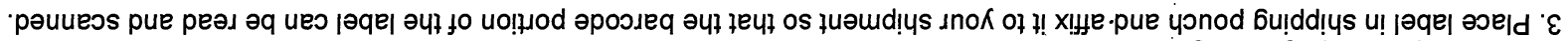

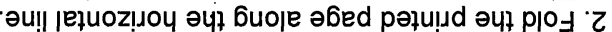

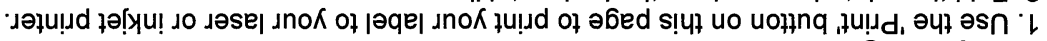

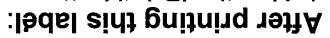

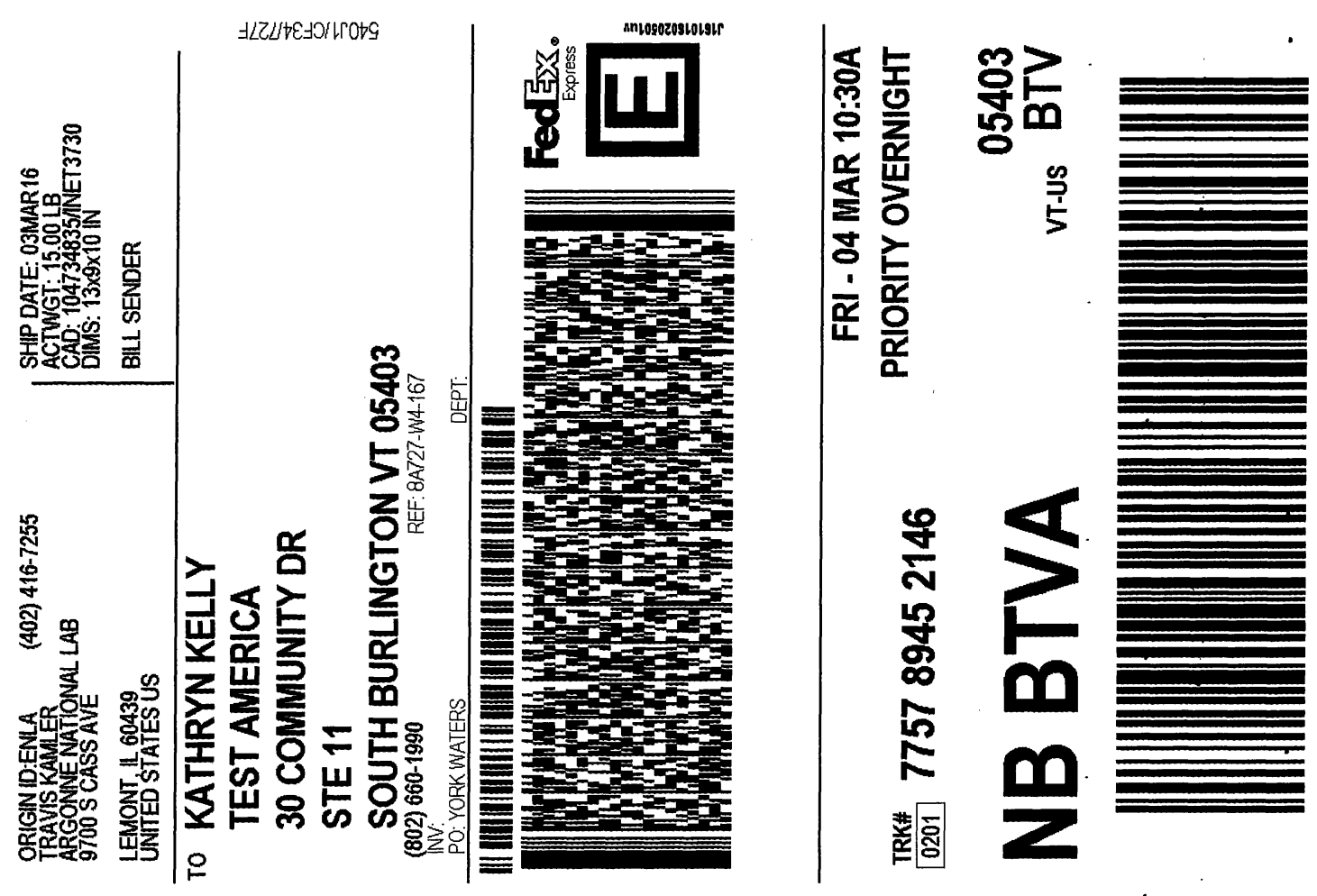




\section{Shipping and Receiving Documents}




\section{Login Sample Receipt Checklist}

Client: Argonne National Laboratory

Job Number: 200-32333-1

SDG Number: 32333

Login Number: 32333

List Source: TestAmerica Burlington

List Number: 1

Creator: Lavigne III, Scott M

Question

Radioactivity wasn't checked or is $</=$ background as measured by a survey True

Answer meter.

The cooler's custody seal, if present, is intact.

Sample custody seals, if present, are intact.

The cooler or samples do not appear to have been compromised or tampered with.

Samples were received on ice.

Cooler Temperature is acceptable.

Cooler Temperature is recorded.

COC is present.

COC is filled out in ink and legible.

$\mathrm{COC}$ is filled out with all pertinent information.

Is the Field Sampler's name present on COC?

There are no discrepancies between the containers received and the COC.

Samples are received within Holding Time (excluding tests with immediate

HTs)

Sample containers have legible labels.

Containers are not broken or leaking.

Sample collection date/times are provided.

Appropriate sample containers are used.

Sample bottles are completely filled.

Sample Preservation Verified.

There is sufficient vol. for all requested analyses, incl. any requested MS/MSDs

Containers requiring zero headspace have no headspace or bubble is $<6 \mathrm{~mm}$ (1/4").

Multiphasic samples are not present.

True

True

True

True

True

True

True

True

True

True

True

True

True

True

True

True

N/A

True

True

True

True

True

N/A

Residual Chlorine Checked.

Samples do not require splitting or compositing.
Lab does not accept radioactive samples.

Seal present with no number.

$3.0^{\circ} \mathrm{C}$ 


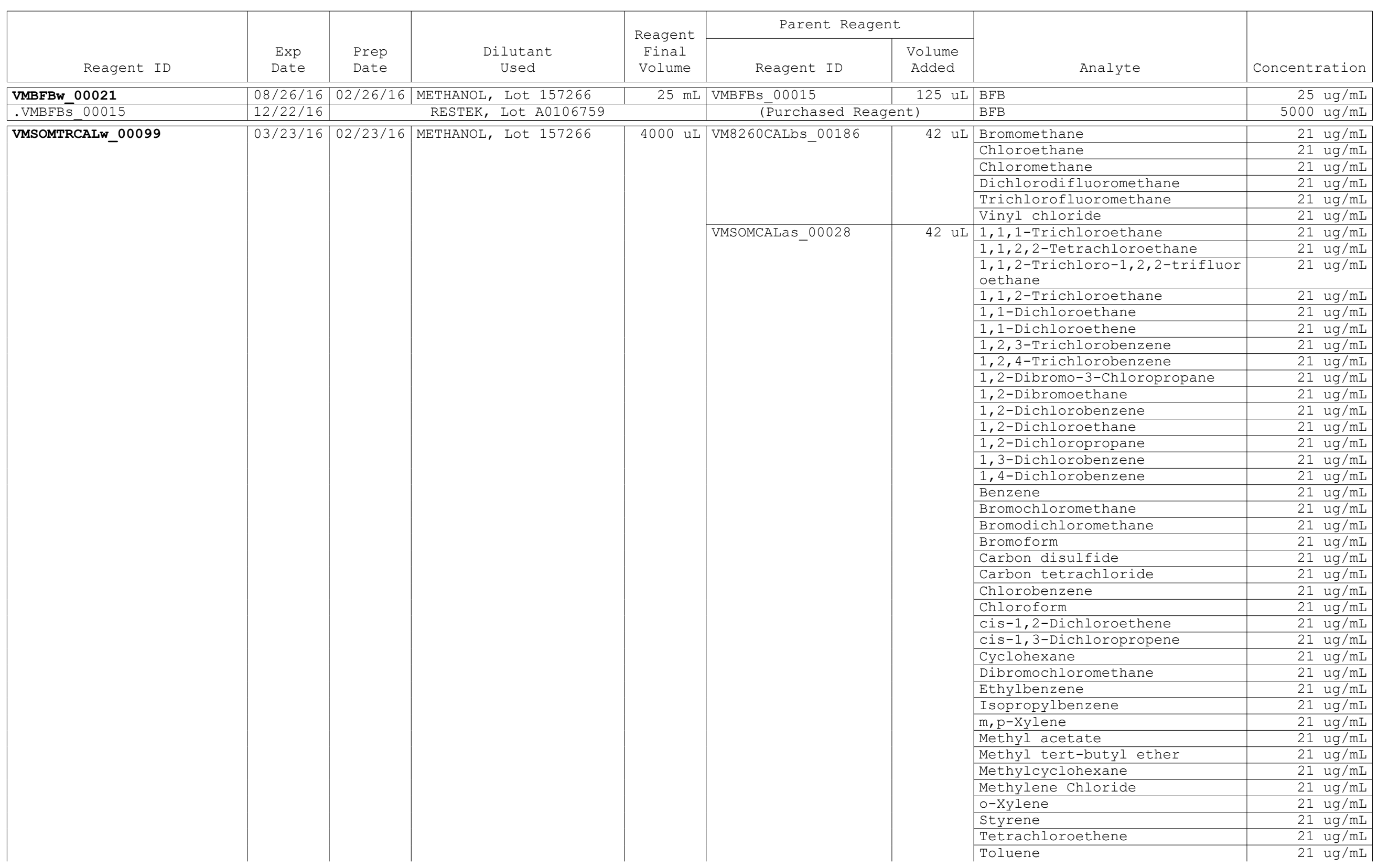


SDG No.: 32333

\begin{tabular}{|c|c|c|c|c|c|c|c|c|}
\hline \multirow[b]{2}{*}{ Reagent ID } & \multirow[b]{2}{*}{$\begin{array}{l}\text { Exp } \\
\text { Date }\end{array}$} & \multirow[b]{2}{*}{$\begin{array}{l}\text { Prep } \\
\text { Date }\end{array}$} & \multirow[b]{2}{*}{$\begin{array}{l}\text { Dilutant } \\
\text { Used }\end{array}$} & \multirow{2}{*}{$\begin{array}{c}\text { Reagent } \\
\text { Final } \\
\text { Volume }\end{array}$} & \multicolumn{2}{|l|}{ Parent Reagent } & \multirow[b]{2}{*}{ Analyte } & \multirow[b]{2}{*}{ Concentration } \\
\hline & & & & & \multirow[t]{4}{*}{ Reagent ID } & $\begin{array}{l}\text { Volume } \\
\text { Added }\end{array}$ & & \\
\hline & & & & & & & trans-1,2-Dichloroethene & $21 \mathrm{ug} / \mathrm{mL}$ \\
\hline & & & & & & & trans-1,3-Dichloropropene & $21 \mathrm{ug} / \mathrm{mL}$ \\
\hline & & & & & & \multirow{5}{*}{$168 \mathrm{uL}$} & Trichloroethene & $21 \mathrm{ug} / \mathrm{mL}$ \\
\hline & & & & & \multirow[t]{4}{*}{ VMSOMCALbs_00039 } & & 2-Butanone & $210 \mathrm{ug} / \mathrm{mL}$ \\
\hline & & & & & & & 2-Hexanone & $210 \mathrm{ug} / \mathrm{mL}$ \\
\hline & & & & & & & 4-Methyl-2-pentanone & $210 \mathrm{ug} / \mathrm{mL}$ \\
\hline & & & & & & & Acetone & $210 \mathrm{ug} / \mathrm{mL}$ \\
\hline & & & & & \multirow{2}{*}{ VMSOMSUas_00087 } & \multirow{2}{*}{42 uL } & Chloroethane-d5 & $21 \mathrm{ug} / \mathrm{mL}$ \\
\hline & & & & & & & Vinyl Chloride-d3 & $21 \mathrm{ug} / \mathrm{mL}$ \\
\hline & & & & & \multirow[t]{2}{*}{ VMSOMSUbs_00151 } & \multirow[t]{2}{*}{336 uL } & 2-Butanone-d5 & $210 \mathrm{ug} / \mathrm{mL}$ \\
\hline & & & & & & & 2-Hexanone-d5 & $210 \mathrm{ug} / \mathrm{mL}$ \\
\hline & & & & & \multirow[t]{9}{*}{ VMSOMSUCS_00062 } & \multirow[t]{9}{*}{42 uL } & $1,1,2,2$-Tetrachloroethane-d2 & $21 \mathrm{ug} / \mathrm{mL}$ \\
\hline & & & & & & & 1,1-Dichloroethene-d2 & $21 \mathrm{ug} / \mathrm{mL}$ \\
\hline & & & & & & & 1,2-Dichlorobenzene-d4 & $21 \mathrm{ug} / \mathrm{mL}$ \\
\hline & & & & & & & 1,2-Dichloroethane-d4 & $21 \mathrm{ug} / \mathrm{mL}$ \\
\hline & & & & & & & 1,2-Dichloropropane-d6 & $21 \mathrm{ug} / \mathrm{mL}$ \\
\hline & & & & & & & Benzene-d6 & $21 \mathrm{ug} / \mathrm{mL}$ \\
\hline & & & & & & & Chloroform-d & $21 \mathrm{ug} / \mathrm{mL}$ \\
\hline & & & & & & & Toluene-d8 & $21 \mathrm{ug} / \mathrm{mL}$ \\
\hline \multirow{7}{*}{.VM8260CALbs_00186 } & & & & & & & trans-1,3-Dichloropropene-d4 & $21 \mathrm{ug} / \mathrm{mL}$ \\
\hline & \multirow{6}{*}{$03 / 23 / 16$} & & RESTEK, Lot A0114018 & & (Purchased Reagen & & Bromomethane & $2000 \mathrm{ug} / \mathrm{mL}$ \\
\hline & & & & & & & Chloroethane & $2000 \mathrm{ug} / \mathrm{mL}$ \\
\hline & & & & & & & Chloromethane & $2000 \mathrm{ug} / \mathrm{mL}$ \\
\hline & & & & & & & Dichlorodifluoromethane & $2000 \mathrm{ug} / \mathrm{mL}$ \\
\hline & & & & & & & Trichlorofluoromethane & $2000 \mathrm{ug} / \mathrm{mL}$ \\
\hline & & & & & & & Vinyl chloride & $2000 \mathrm{ug} / \mathrm{mL}$ \\
\hline .VMSOMCALas_0 0028 & $12 / 24 / 16$ & & Restek, Lot A0102833 & & (Purchased Reagen & & $1,1,1$-Trichloroethane & $2000 \mathrm{ug} / \mathrm{mL}$ \\
\hline & & & & & & & 1,1,2,2-Tetrachloroethane & $2000 \mathrm{ug} / \mathrm{mL}$ \\
\hline & & & & & & & $\begin{array}{l}\text { 1,1,2-Trichloro-1,2,2-trifluor } \\
\text { oethane }\end{array}$ & $2000 \mathrm{ug} / \mathrm{mL}$ \\
\hline & & & & & & & $1,1,2$-Trichloroethane & $2000 \mathrm{ug} / \mathrm{mL}$ \\
\hline & & & & & & & 1,1-Dichloroethane & $2000 \mathrm{ug} / \mathrm{mL}$ \\
\hline & & & & & & & 1,1-Dichloroethene & $2000 \mathrm{ug} / \mathrm{mL}$ \\
\hline & & & & & & & 1,2,3-Trichlorobenzene & $2000 \mathrm{ug} / \mathrm{mL}$ \\
\hline & & & & & & & $1,2,4$-Trichlorobenzene & $2000 \mathrm{ug} / \mathrm{mL}$ \\
\hline & & & & & & & 1,2-Dibromo-3-Chloropropane & $2000 \mathrm{ug} / \mathrm{mL}$ \\
\hline & & & & & & & 1,2-Dibromoethane & $2000 \mathrm{ug} / \mathrm{mL}$ \\
\hline & & & & & & & 1,2-Dichlorobenzene & $2000 \mathrm{ug} / \mathrm{mL}$ \\
\hline & & & & & & & 1,2-Dichloroethane & $2000 \mathrm{ug} / \mathrm{mL}$ \\
\hline & & & & & & & 1,2-Dichloropropane & $2000 \mathrm{ug} / \mathrm{mL}$ \\
\hline & & & & & & & 1,3-Dichlorobenzene & $2000 \mathrm{ug} / \mathrm{mL}$ \\
\hline & & & & & & & 1,4-Dichlorobenzene & $2000 \mathrm{ug} / \mathrm{mL}$ \\
\hline & & & & & & & Benzene & $2000 \mathrm{ug} / \mathrm{mL}$ \\
\hline & & & & & & & Bromochloromethane & $2000 \mathrm{ug} / \mathrm{mL}$ \\
\hline & & & & & & & Bromodichloromethane & $2000 \mathrm{ug} / \mathrm{mL}$ \\
\hline & & & & & & & Bromoform & $2000 \mathrm{ug} / \mathrm{mL}$ \\
\hline & & & & & & & Carbon disulfide & $2000 \mathrm{ug} / \mathrm{mL}$ \\
\hline & & & & & & & Carbon tetrachloride & $2000 \mathrm{ug} / \mathrm{mL}$ \\
\hline
\end{tabular}


SDG No.: 32333

\begin{tabular}{|c|c|c|c|c|c|c|c|c|c|}
\hline \multirow[b]{2}{*}{ Reagent ID } & \multirow[b]{2}{*}{$\begin{array}{l}\text { Exp } \\
\text { Date }\end{array}$} & \multirow[b]{2}{*}{$\begin{array}{l}\text { Prep } \\
\text { Date }\end{array}$} & \multirow[b]{2}{*}{$\begin{array}{l}\text { Dilutant } \\
\text { Used }\end{array}$} & \multirow{2}{*}{$\begin{array}{l}\text { Reagent } \\
\text { Final } \\
\text { Volume }\end{array}$} & \multicolumn{2}{|c|}{ Parent Reagent } & \multirow[b]{2}{*}{ Analyte } & \multirow{2}{*}{\multicolumn{2}{|c|}{ Concentration }} \\
\hline & & & & & Reagent ID & $\begin{array}{l}\text { Volume } \\
\text { Added }\end{array}$ & & & \\
\hline & & & & & & & Chlorobenzene & 2000 & $\mathrm{ug} / \mathrm{mL}$ \\
\hline & & & & & & & Chloroform & 2000 & $\mathrm{ug} / \mathrm{mL}$ \\
\hline & & & & & & & cis-1,2-Dichloroethene & 2000 & $\mathrm{ug} / \mathrm{mL}$ \\
\hline & & & & & & & cis-1,3-Dichloropropene & 2000 & $\mathrm{ug} / \mathrm{mL}$ \\
\hline & & & & & & & Cyclohexane & 2000 & $\mathrm{ug} / \mathrm{mL}$ \\
\hline & & & & & & & Dibromochloromethane & 2000 & $\mathrm{ug} / \mathrm{mL}$ \\
\hline & & & & & & & Ethylbenzene & 2000 & $\mathrm{ug} / \mathrm{mL}$ \\
\hline & & & & & & & Isopropylbenzene & 2000 & $\mathrm{ug} / \mathrm{mL}$ \\
\hline & & & & & & & m,p-xylene & 2000 & $\mathrm{ug} / \mathrm{mL}$ \\
\hline & & & & & & & Methyl acetate & 2000 & $\mathrm{ug} / \mathrm{mL}$ \\
\hline & & & & & & & Methyl tert-butyl ether & 2000 & $\mathrm{ug} / \mathrm{mL}$ \\
\hline & & & & & & & Methylcyclohexane & 2000 & $\mathrm{ug} / \mathrm{mL}$ \\
\hline & & & & & & & Methylene Chloride & 2000 & $\mathrm{ug} / \mathrm{mL}$ \\
\hline & & & & & & & o-Xylene & 2000 & $\mathrm{ug} / \mathrm{mL}$ \\
\hline & & & & & & & Styrene & 2000 & $\mathrm{ug} / \mathrm{mL}$ \\
\hline & & & & & & & Tetrachloroethene & 2000 & $\mathrm{ug} / \mathrm{mL}$ \\
\hline & & & & & & & Toluene & 2000 & $\mathrm{ug} / \mathrm{mL}$ \\
\hline & & & & & & & trans-1,2-Dichloroethene & 2000 & $\mathrm{ug} / \mathrm{mL}$ \\
\hline & & & & & & & trans-1,3-Dichloropropene & 2000 & $\mathrm{ug} / \mathrm{mL}$ \\
\hline & & & & & & & Trichloroethene & 2000 & $\mathrm{ug} / \mathrm{mL}$ \\
\hline \multirow[t]{4}{*}{.VMSOMCALbs_00039 } & \multirow[t]{4}{*}{$01 / 14 / 17$} & \multirow{4}{*}{\multicolumn{2}{|c|}{ Restek, Lot A0110042 }} & & \multicolumn{2}{|c|}{ (Purchased Reagent) } & 2-Butanone & 5000 & $\mathrm{ug} / \mathrm{mL}$ \\
\hline & & & & & & & 2-Hexanone & 5000 & $\mathrm{ug} / \mathrm{mL}$ \\
\hline & & & & & & & 4-Methyl-2-pentanone & 5000 & $\mathrm{ug} / \mathrm{mL}$ \\
\hline & & & & & & & Acetone & 5000 & $\mathrm{ug} / \mathrm{mL}$ \\
\hline \multirow[t]{2}{*}{.VMSOMSUas_00087 } & \multirow[t]{2}{*}{$01 / 20 / 17$} & \multirow{2}{*}{\multicolumn{2}{|c|}{ Absolute, Lot 082015}} & & \multirow{2}{*}{\multicolumn{2}{|c|}{ (Purchased Reagent) }} & Chloroethane-d5 & 2000 & $\mathrm{ug} / \mathrm{mL}$ \\
\hline & & & & & & & Vinyl Chloride-d3 & 2000 & $\mathrm{ug} / \mathrm{mL}$ \\
\hline \multirow[t]{2}{*}{.VMSOMSUbs_00151 } & \multirow[t]{2}{*}{$02 / 23 / 17$} & & Absolute, Lot 071515 & & (Purchased $\mathrm{F}$ & nt) & 2-Butanone-d5 & 2500 & $\mathrm{ug} / \mathrm{mL}$ \\
\hline & & & & & & & 2-Hexanone-d5 & 2500 & $\mathrm{ug} / \mathrm{mL}$ \\
\hline .VMSOMSUCS_00062 & $01 / 14 / 17$ & & Absolute, Lot 011614 & & (Purchased $\mathrm{F}$ & nt) & $1,1,2,2$-Tetrachloroethane-d2 & 2000 & $\mathrm{ug} / \mathrm{mL}$ \\
\hline & & & & & & & 1,1-Dichloroethene-d2 & 2000 & $\mathrm{ug} / \mathrm{mL}$ \\
\hline & & & & & & & 1,2-Dichlorobenzene-d4 & 2000 & $\mathrm{ug} / \mathrm{mL}$ \\
\hline & & & & & & & 1,2-Dichloroethane-d4 & 2000 & $\mathrm{ug} / \mathrm{mL}$ \\
\hline & & & & & & & 1,2-Dichloropropane-d6 & 2000 & $\mathrm{ug} / \mathrm{mL}$ \\
\hline & & & & & & & Benzene-d6 & 2000 & $\mathrm{ug} / \mathrm{mL}$ \\
\hline & & & & & & & Chloroform-d & 2000 & $\mathrm{ug} / \mathrm{mL}$ \\
\hline & & & & & & & Toluene-d8 & 2000 & $\mathrm{ug} / \mathrm{mL}$ \\
\hline & & & & & & & trans-1,3-Dichloropropene-d4 & 2000 & $\mathrm{ug} / \mathrm{mL}$ \\
\hline VMSOMTRISw_00110 & $03 / 23 / 16$ & $02 / 23 / 16$ & METHANOL, Lot 157266 & $4000 \mathrm{uL}$ & VMSOMISS_00028 & $33.6 \mathrm{uL}$ & 1,4-Dichlorobenzene-d4 & 21 & $\mathrm{ug} / \mathrm{mL}$ \\
\hline & & & & & & & 1,4-Difluorobenzene & 21 & $\mathrm{ug} / \mathrm{mL}$ \\
\hline & & & & & & & Chlorobenzene-d5 & 21 & $\mathrm{ug} / \mathrm{mL}$ \\
\hline .VMSOMISS_00028 & $01 / 14 / 17$ & & RESTEK, Lot A0110554 & & (Purchased $\mathrm{F}$ & nt) & 1,4-Dichlorobenzene-d4 & 2500 & $\mathrm{ug} / \mathrm{mL}$ \\
\hline & & & & & & & 1,4-Difluorobenzene & 2500 & $\mathrm{ug} / \mathrm{mL}$ \\
\hline & & & & & & & Chlorobenzene-d5 & 2500 & $\mathrm{ug} / \mathrm{mL}$ \\
\hline VMSOMTRSUw_00105 & $03 / 23 / 16$ & $02 / 23 / 16$ & METHANOL, Lot 157266 & $4000 \mathrm{uL}$ & VMSOMSUas_00087 & $42 \mathrm{uL}$ & Chloroethane-d5 & 21 & $\mathrm{ug} / \mathrm{mL}$ \\
\hline & & & & & & & Vinyl Chloride-d3 & 21 & $\mathrm{ug} / \mathrm{mL}$ \\
\hline & & & & & VMSOMSUbs_00151 & 336 uL & 2-Butanone-d5 & 210 & $\mathrm{ug} / \mathrm{mL}$ \\
\hline & & & & & & & 2-Hexanone-d5 & 210 & $\mathrm{ug} / \mathrm{mL}$ \\
\hline
\end{tabular}


SDG No.: 32333

\begin{tabular}{|c|c|c|c|c|c|c|c|c|}
\hline \multirow[b]{2}{*}{ Reagent ID } & \multirow[b]{2}{*}{$\begin{array}{l}\text { Exp } \\
\text { Date }\end{array}$} & \multirow[b]{2}{*}{$\begin{array}{l}\text { Prep } \\
\text { Date }\end{array}$} & \multirow[b]{2}{*}{$\begin{array}{l}\text { Dilutant } \\
\text { Used }\end{array}$} & \multirow{2}{*}{$\begin{array}{l}\text { Reagent } \\
\text { Final } \\
\text { Volume }\end{array}$} & \multicolumn{2}{|c|}{ Parent Reagent } & \multirow[b]{2}{*}{ Analyte } & \multirow[b]{2}{*}{ Concentration } \\
\hline & & & & & \multirow{2}{*}{$\begin{array}{r}\text { Reagent ID } \\
\text { VMSOMSUCS_00062 }\end{array}$} & $\begin{array}{l}\text { Volume } \\
\text { Added }\end{array}$ & & \\
\hline & & & & & & $42 \mathrm{uL}$ & $1,1,2,2$-Tetrachloroethane-d2 & $21 \mathrm{ug} / \mathrm{mL}$ \\
\hline & & & & & & & 1,1-Dichloroethene-d2 & $21 \mathrm{ug} / \mathrm{mL}$ \\
\hline & & & & & & & 1,2-Dichlorobenzene-d4 & $21 \mathrm{ug} / \mathrm{mL}$ \\
\hline & & & & & & & 1,2-Dichloroethane-d4 & $21 \mathrm{ug} / \mathrm{mL}$ \\
\hline & & & & & & & 1,2-Dichloropropane-d6 & $21 \mathrm{ug} / \mathrm{mL}$ \\
\hline & & & & & & & Benzene-d6 & $21 \mathrm{ug} / \mathrm{mL}$ \\
\hline & & & & & & & Chloroform-d & $21 \mathrm{ug} / \mathrm{mL}$ \\
\hline & & & & & & & Toluene-d8 & $21 \mathrm{ug} / \mathrm{mL}$ \\
\hline & & & & & & & trans-1,3-Dichloropropene-d4 & $21 \mathrm{ug} / \mathrm{mL}$ \\
\hline \multirow[t]{2}{*}{.VMSOMSUas_00087 } & \multirow[t]{2}{*}{$01 / 20 / 17$} & \multirow{2}{*}{\multicolumn{3}{|c|}{ Absolute, Lot 082015}} & \multirow{2}{*}{\multicolumn{2}{|c|}{ (Purchased Reagent) }} & Chloroethane-d5 & $2000 \mathrm{ug} / \mathrm{mL}$ \\
\hline & & & & & & & Vinyl Chloride-d3 & $2000 \mathrm{ug} / \mathrm{mL}$ \\
\hline \multirow{2}{*}{.VMSOMSUbs_00151 } & \multirow[t]{2}{*}{$02 / 23 / 17$} & \multirow{2}{*}{\multicolumn{3}{|c|}{ Absolute, Lot 071515}} & \multirow{2}{*}{\multicolumn{2}{|c|}{ (Purchased Reagent) }} & 2-Butanone-d5 & $2500 \mathrm{ug} / \mathrm{mL}$ \\
\hline & & & & & & & 2-Hexanone-d5 & $2500 \mathrm{ug} / \mathrm{mL}$ \\
\hline \multirow[t]{9}{*}{.VMSOMSUCS_00062 } & \multirow[t]{9}{*}{$01 / 14 / 17$} & \multirow{9}{*}{\multicolumn{2}{|c|}{ Absolute, Lot 011614}} & & \multirow{9}{*}{\multicolumn{2}{|c|}{ (Purchased Reagent) }} & $1,1,2,2$-Tetrachloroethane-d2 & $2000 \mathrm{ug} / \mathrm{mL}$ \\
\hline & & & & & & & 1,1-Dichloroethene-d2 & $2000 \mathrm{ug} / \mathrm{mL}$ \\
\hline & & & & & & & 1,2-Dichlorobenzene-d4 & $2000 \mathrm{ug} / \mathrm{mL}$ \\
\hline & & & & & & & 1,2-Dichloroethane-d4 & $2000 \mathrm{ug} / \mathrm{mL}$ \\
\hline & & & & & & & 1,2-Dichloropropane-d6 & $2000 \mathrm{ug} / \mathrm{mL}$ \\
\hline & & & & & & & Benzene-d6 & $2000 \mathrm{ug} / \mathrm{mL}$ \\
\hline & & & & & & & Chloroform-d & $2000 \mathrm{ug} / \mathrm{mL}$ \\
\hline & & & & & & & Toluene-d8 & $2000 \mathrm{ug} / \mathrm{mL}$ \\
\hline & & & & & & & trans-1,3-Dichloropropene-d4 & $2000 \mathrm{ug} / \mathrm{mL}$ \\
\hline
\end{tabular}


Lab Name: TestAmerica Burlington

Job No.: 200-32333-1

SDG No.: 32333

\begin{tabular}{|c|c|c|c|c|}
\hline $\begin{array}{c}\text { Reagent } \\
\text { Container }\end{array}$ & $\begin{array}{c}\text { Reagent } \\
\text { ID }\end{array}$ & $\begin{array}{c}\text { Reagent } \\
\text { Description }\end{array}$ & $\begin{array}{c}\text { Preparation } \\
\text { Date }\end{array}$ & $\begin{array}{c}\text { Expiration } \\
\text { Date }\end{array}$ \\
\hline 902938 & VMSOMTRISw_00110 & SOM TR ISTD 21 PPM & $02 / 23 / 2016$ & $03 / 23 / 2016$ \\
\hline 902939 & VMSOMTRSUW_00105 & SOM $\quad$ TR $\quad$ DMC $21 \quad$ PPM & $02 / 23 / 2016$ & $03 / 23 / 2016$ \\
\hline 902940 & VMSOMTRCALW_00099 & SOM TR CAL $21-210$ PPM & $02 / 23 / 2016$ & $03 / 23 / 2016$ \\
\hline 904333 & VMBFBw_00021 & BFB TUNE 25 PPM & $02 / 26 / 2016$ & $08 / 26 / 2016$ \\
\hline
\end{tabular}




\section{METHODOLOGY SUMMARY}

Laboratory: TestAmerica Laboratories

Location: South Burlington, Vermont
Project No:

SDG No: $\quad 32333$

VOA

Volatile Organics Trace - USEPA CLP SOM01.2 


\section{CASE NARRATIVE}

\section{Client: Argonne National Laboratory}

\section{Project: York (200-32333)}

\section{Report Number: 200-32333-1}

With the exceptions noted as flags or footnotes, standard analytical protocols were followed in the analysis of the samples and no problems were encountered or anomalies observed. In addition all laboratory quality control samples were within established control limits, with any exceptions noted below. Each sample was analyzed to achieve the lowest possible reporting limit within the constraints of the method. In some cases, due to interference or analytes present at high concentrations, samples were diluted. For diluted samples, the reporting limits are adjusted relative to the dilution required.

Calculations are performed before rounding to avoid round-off errors in calculated results.

All holding times were met and proper preservation noted for the methods performed on these samples, unless otherwise detailed in the individual sections below.

\section{RECEIPT}

The samples were received on 03/05/2016; the samples arrived in good condition, properly preserved and on ice. The temperature of the cooler at receipt was $3.0^{\circ} \mathrm{C}$.

\section{VOLATILE ORGANIC COMPOUNDS - TRACE}

Samples YKTI28-104-W-38078, YKQCTB-W-38080 and VHBLK01 were analyzed for Volatile Organic Compounds - Trace in accordance with EPA SOW SOM01.2. The samples were analyzed on 03/08/2016.

Several analytes were detected in method blank MB 200-101505/5 at levels that were above the method detection limit but below the reporting limit. The values should be considered estimates, and have been flagged. If the associated sample reported a result above the MDL and/or RL, the result has been flagged. Refer to the $\mathrm{QC}$ report for details.

2-Hexanone-d5 failed the surrogate recovery criteria high for YKTI28-104-W-38078. Refer to the QC report for details.

A non-target constituent that represents a compound related to the DMC formulation is present in the samples in this set. The derived results for that compound have been qualified with an " $X$ " qualifier to reflect the source of the contamination.

No additional analytical or quality issues were noted, other than those described above or in the Definitions/Glossary page. 
Lab Name: TestAmerica Burlington

Job No.: 200-32333-1

SDG No.: 32333

Instrument ID: CHD.i

Analysis Batch Number: 101161

Lab Sample ID: IC 200-101161/7

Client Sample ID:

Date Analyzed: 02/26/16 16:46

Lab File ID: 018468_007.D

GC Column: DB-624

ID : $0.2(\mathrm{~mm})$

\begin{tabular}{|l|c|c|c|c|}
\hline \multicolumn{1}{|c|}{ COMPOUND NAME } & RETENTION & \multicolumn{2}{|c|}{ MANUAL INTEGRATION } \\
\cline { 3 - 5 } & TIME & REASON & ANALYST & DATE \\
\hline Methyl acetate & 3.12 & Baseline & wilburj & $02 / 29 / 16$ 09:54 \\
\hline
\end{tabular}

Lab Sample ID: ICIS 200-101161/8

Client Sample ID:

Date Analyzed: 02/26/16 17:11

Lab File ID: 018468 008.D

GC Column: DB-624

ID $: 0.2(\mathrm{~mm})$

\begin{tabular}{|c|c|c|c|c|}
\hline \multicolumn{1}{|c|}{ COMPOUND NAME } & RETENTION & \multicolumn{2}{|c|}{ MANUAL INTEGRATION } \\
\cline { 3 - 5 } & TIME & REASON & ANALYST & DATE \\
\hline 1,2-Dichloropropane-d6 & 6.36 & Split Peak & wilburj & 02/29/16 09:55 \\
\hline
\end{tabular}

Lab Sample ID: IC 200-101161/9 Client Sample ID:

Date Analyzed: 02/26/16 17:36 Lab File ID: 018468 009.D

GC Column: DB-624

ID : $0.2(\mathrm{~mm})$

\begin{tabular}{|c|c|c|c|c|}
\hline \multirow[t]{2}{*}{ COMPOUND NAME } & \multirow{2}{*}{$\begin{array}{c}\text { RETENTION } \\
\text { TIME }\end{array}$} & \multicolumn{3}{|c|}{ MANUAL INTEGRATION } \\
\hline & & REASON & ANALYST & DATE \\
\hline 1,2-Dichloropropane-d6 & 6.36 & Split Peak & wilburj & $02 / 29 / 1609: 56$ \\
\hline
\end{tabular}

Lab Sample ID: IC 200-101161/10

Client Sample ID:

Date Analyzed: 02/26/16 18:01

Lab File ID: 018468 010.D

GC Column: DB-624

ID $: 0.2(\mathrm{~mm})$

\begin{tabular}{|l|c|c|c|c|}
\hline \multicolumn{1}{|c|}{ COMPOUND NAME } & RETENTION & \multicolumn{2}{|c|}{ MANUAL INTEGRATION } \\
\cline { 3 - 5 } & TIME & REASON & ANALYST & DATE \\
\hline Methyl acetate & 3.11 & Baseline & wilburj & $02 / 29 / 16$ 09:56 \\
\hline
\end{tabular}


Lab Name: TestAmerica Burlington

SDG No.: 32333

Instrument ID: CHD.i

Lab Sample ID: CCVC 200-101505/11

Date Analyzed: 03/08/16 15:23

\begin{tabular}{|c|c|c|c|c|}
\hline \multirow{2}{*}{ COMPOUND NAME } & RETENTION & \multicolumn{3}{|c}{ MANUAL INTEGRATION } \\
\cline { 3 - 5 } & TIME & REASON & ANALYST & DATE \\
\hline \hline 1,2-Dichloropropane-d6 & 6.36 & Split Peak & wilburj & $03 / 08 / 1616: 02$ \\
\hline
\end{tabular}

Job No.: 200-32333-1

Analysis Batch Number: 101505

Client Sample ID:

Lab File ID: $018646011 . \mathrm{D}$

GC Column: DB-624

ID $: 0.2(\mathrm{~mm})$ 


\section{DATA REPORTING QUALIFIERS}

Client: Argonne National Laboratory

Job Number: 200-32333-1

Sdg Number: 32333

\section{Lab Section}

Qualifier

Description

\section{GC/MS VOA}

U

$\mathrm{J}$

$\mathrm{J}$

$\mathrm{X}$

B

$\mathrm{N}$
Analyzed for but not detected.

Indicates an Estimated Value for TICs

Indicates an estimated value.

See case narrative notes for explanation of the ' $X$ ' flag

Surrogate is outside acceptance limits.

The analyte was found in an associated blank, as well as in the sample.

This flag indicates the presumptive evidence of a compound. 
Lab Name: TESTAMERICA BURLINGTON

Contract: $8 \mathrm{E}-00302$

Lab Code: STLV Case No.: YORK Mod. Ref No.: SDG No.: 32333

Level: (TRACE or LOW) TRACE

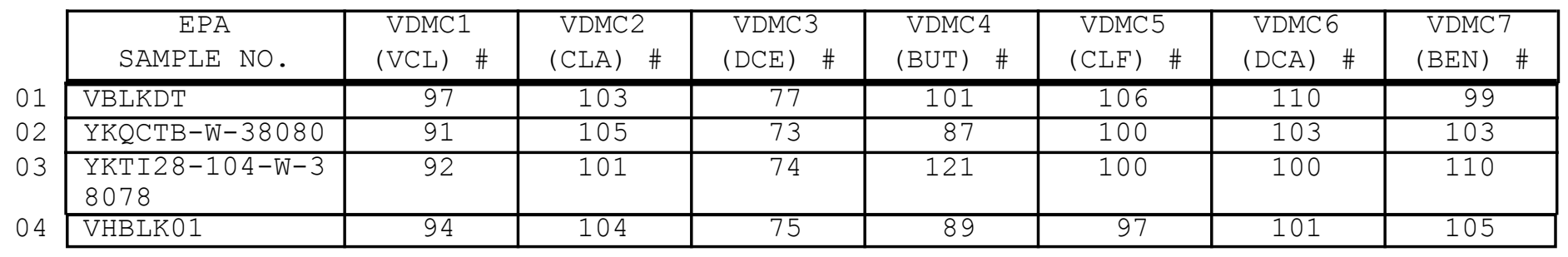

VDMC1 $(\mathrm{VCL})=$ Vinyl Chloride-d3

VDMC2 $($ CLA $)=$ Chloroethane-d5

VDMC3 $(\mathrm{DCE})=1,1-\mathrm{Dich}$ loroethene-d2

VDMC4 $\quad(B U T)=2-$ Butanone-d5

VDMC5 $(\mathrm{CLF})=$ Chloroform-d

VDMC $6(\mathrm{DCA})=1,2-\mathrm{Dich})$ loroethane-d4

$\operatorname{VDMC} 7(\mathrm{BEN})=$ Benzene-d6 $\frac{\text { QC LIMITS }}{(65-131)}$

$(71-131)$

(55-104)

(49-155)

$(78-121)$

$(78-129)$

$(77-124)$

\# Column to be used to flag recovery values

* Values outside of contract required QC limits

Page 1 of 1 
Lab Name: TESTAMERICA BURLINGTON

Contract: $8 \mathrm{E}-00302$

Lab Code: STLV Case No.: YORK Mod. Ref No.: SDG No.: 32333

Level: (TRACE or LOW) TRACE

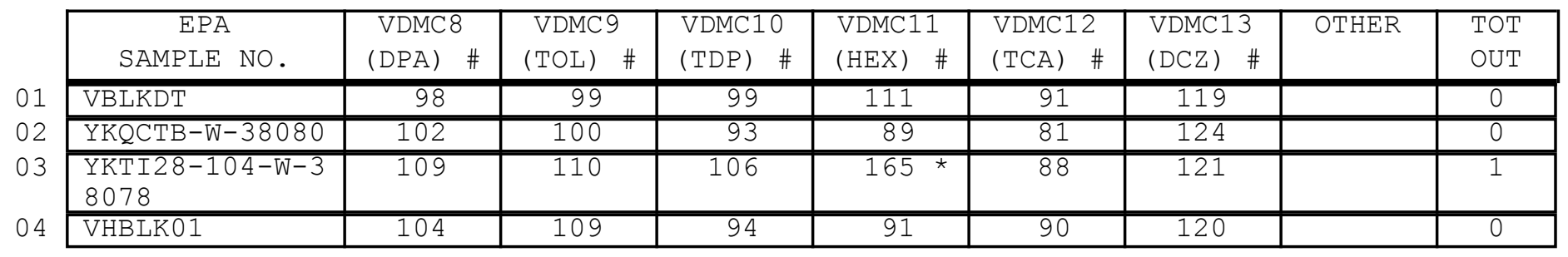

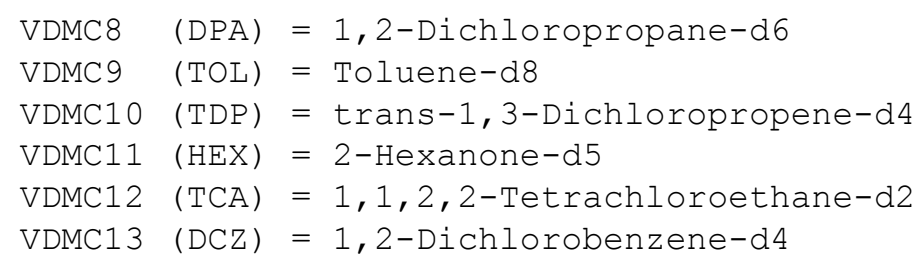

\footnotetext{
$\frac{\text { QC LIMITS }}{(79-124)}$

(77-121)

(73-121)

(28-135)

$(73-125)$

$(80-131)$
} 
$4 A$ - FORM IV VOA

VOLATILE METHOD BLANK SUMMARY
EPA SAMPLE NO.

VBLKDT

Lab Name: TESTAMERICA BURLINGTON

Contract: 8E-00302

Lab Code: STLV Case No.: YORK Mod. Ref No.: SDG No.: 32333

Lab File ID: 018646_005.D

Lab Sample ID: MB 200-101505/5

Instrument ID: CHD.i

Matrix: (SOIL/SED/WATER) Water

Date Analyzed: 03/08/2016

Level: (TRACE or LOW/MED) TRACE

Time Analyzed: 1226

GC Column: DB-624

ID $: 0.20 \quad(\mathrm{~mm})$

Heated Purge: (Y/N) N

\begin{tabular}{|c|c|c|c|}
\hline $\begin{array}{c}\text { EPA } \\
\text { SAMPLE NO. }\end{array}$ & $\begin{array}{r}\text { LAB } \\
\text { SAMPLE ID }\end{array}$ & $\begin{array}{c}\text { LAB } \\
\text { FILE ID }\end{array}$ & $\begin{array}{c}\text { TIME } \\
\text { ANALYZED }\end{array}$ \\
\hline $\begin{array}{l}\text { YKQCTB-W-380 } \\
80\end{array}$ & $200-32333-2$ & $\begin{array}{l}018646 \_008 \\
. D\end{array}$ & 1352 \\
\hline $\begin{array}{l}\text { YKTI28-104-W } \\
-38078\end{array}$ & $200-32333-1$ & $\begin{array}{l}018646 \_009 \\
. D\end{array}$ & 1417 \\
\hline VHBLK01 & $200-32333-3$ & $\begin{array}{l}018646 \_010 \\
. D\end{array}$ & 1458 \\
\hline
\end{tabular}

COMMENTS :

Page 1 of 1 
$5 A$ - FORM V VOA

VOLATILE ORGANICS INSTRUMENT

PERFORMANCE CHECK

BROMOFLUOROBENZENE (BFB)
EPA SAMPLE NO.

BFBDP
Lab Name: TESTAMERICA BURLINGTON

Lab Code: STLV Case No.: YORK

Lab File Id: 018468 003.D

Instrument Id: CHD.i

GC Column: DB-624

ID $: 0.20$
Contract: $8 \mathrm{E}-00302$ Mod. Ref No.: SDG No.: 32333

BFB Injection Date: 02/26/2016

BFB Injection Time: 1501 $(\mathrm{mm})$

\begin{tabular}{|c|c|c|}
\hline $\mathrm{m} / \mathrm{e}$ & ION ABUNDANCE CRITERIA & $\begin{array}{l}\text { \% RELATIVE } \\
\text { ABUNDANCE }\end{array}$ \\
\hline 50 & $15.0-40.0 \%$ of mass 95 & 16.1 \\
\hline 75 & $30.0-80.0 \%$ of mass 95 & 50.0 \\
\hline 95 & Base peak, $100 \%$ relative abundance & 100 \\
\hline 96 & $5.0-9.0 \%$ of mass 95 & 6.8 \\
\hline 173 & Less than $2.0 \%$ of mass 174 & $0.8) 1$ \\
\hline 174 & $50.0-120 \%$ of mass 95 & 103 \\
\hline 175 & $5.0-9.0 \%$ of mass 174 & $7.5(7.3) 1$ \\
\hline 176 & $95.0-101 \%$ of mass 174 & $100(97.7) 1$ \\
\hline 177 & $5.0-9.0 \%$ of mass 176 & $(6.6) 2$ \\
\hline
\end{tabular}

1 - Value is omass 1742 - Value is omass 176

\begin{tabular}{|c|c|c|c|c|}
\hline $\begin{array}{c}\text { EPA } \\
\text { SAMPLE NO. }\end{array}$ & $\begin{array}{r}\text { LAB } \\
\text { SAMPLE ID }\end{array}$ & $\begin{array}{l}\text { LAB } \\
\text { FILE ID }\end{array}$ & $\begin{array}{c}\text { DATE } \\
\text { ANALYZED }\end{array}$ & $\begin{array}{c}\text { TIME } \\
\text { ANALYZED }\end{array}$ \\
\hline VSTD0.5DP & IC 200-101161/6 & $\begin{array}{l}018468 \_006 . \\
D\end{array}$ & $02 / 26 / 2016$ & 1621 \\
\hline VSTD001DP & IC 200-101161/7 & $\begin{array}{l}018468 \_007 . \\
D\end{array}$ & $02 / 26 / 2016$ & 1646 \\
\hline VSTD005DP & ICIS 200-101161/8 & $\begin{array}{l}018468 \_008 . \\
D\end{array}$ & $02 / 26 / 2016$ & 1711 \\
\hline VSTD010DP & IC 200-101161/9 & $\begin{array}{l}018468 \_009 . \\
D\end{array}$ & $02 / 26 / 2016$ & 1736 \\
\hline VSTD020DP & IC 200-101161/10 & $\begin{array}{l}018468 \_010 . \\
D\end{array}$ & $02 / 26 / 2016$ & 1801 \\
\hline
\end{tabular}


$5 A$ - FORM V VOA

VOLATILE ORGANICS INSTRUMENT

PERFORMANCE CHECK

BROMOFLUOROBENZENE (BFB)
EPA SAMPLE NO.

BFBDT
Lab Name: TESTAMERICA BURLINGTON

Lab Code: STLV Case No.: YORK

Lab File Id: 018646 001.D

Instrument Id: CHD.i

GC Column: DB-624

ID $: 0.20$
Contract: $8 \mathrm{E}-00302$ Mod. Ref No.: SDG No.: 32333

BEB Injection Date: 03/08/2016

BFB Injection Time: 1046 $(\mathrm{mm})$

\begin{tabular}{|c|c|c|}
\hline $\mathrm{m} / \mathrm{e}$ & ION ABUNDANCE CRITERIA & $\begin{array}{l}\text { \% RELATIVE } \\
\text { ABUNDANCE }\end{array}$ \\
\hline 50 & $15.0-40.0 \%$ of mass 95 & 15.2 \\
\hline 75 & $30.0-80.0 \%$ of mass 95 & 49.4 \\
\hline 95 & Base peak, $100 \%$ relative abundance & 100 \\
\hline 96 & $5.0-9.0 \%$ of mass 95 & 6.7 \\
\hline 173 & Less than $2.0 \%$ of mass 174 & $0.8) 1$ \\
\hline 174 & $50.0-120 \%$ of mass 95 & 97.7 \\
\hline 175 & $5.0-9.0 \%$ of mass 174 & $6.6(6.8) 1$ \\
\hline 176 & $95.0-101 \%$ of mass 174 & $93.6(95.8) 1$ \\
\hline 177 & $5.0-9.0 \%$ of mass 176 & $(6.3) 2$ \\
\hline
\end{tabular}

1 - Value is omass 1742 - Value is omass 176

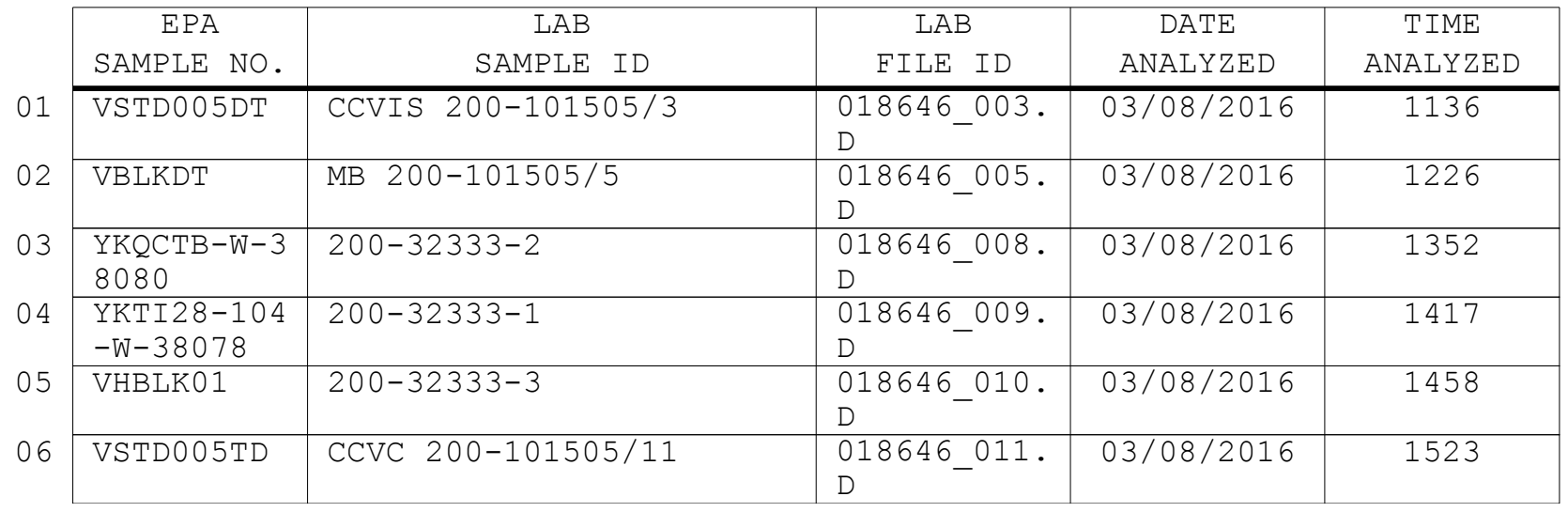




\section{$8 A$ - FORM VIII VOA}

VOLATILE INTERNAL STANDARD AREA AND RETENTION TIME SUMMARY

Lab Name: TESTAMERICA BURLINGTON

Contract: $8 \mathrm{E}-00302$

Lab Code: STLV Case No.: YORK Mod. Ref No.:

SDG No.: 32333

GC Column: DB-624

ID: 0.20 (mm) Init. Calib. Date (s): 02/26/2016 02/26/2016

EPA Sample No.(VSTD\#\#\#\#\#) : VSTD005DT

Lab File ID (Standard) : 018646_003.D

Instrument ID: CHD.i

Date Analyzed: 03/08/2016

Time Analyzed: 1136

Heated Purge: ( $\mathrm{Y} / \mathrm{N}) \mathrm{N}$

\begin{tabular}{|c|c|c|c|c|c|c|}
\hline & $\begin{array}{c}\text { IS1 (CBZ) } \\
\text { AREA }\end{array}$ & RT \# & $\begin{array}{c}\text { IS2 (DFB) } \\
\text { AREA }\end{array}$ & RT \# & $\begin{array}{c}\text { IS3 (DCB) } \\
\text { AREA }\end{array}$ & $\mathrm{RT}$ \\
\hline 12 HOUR STD & 958715 & 9.26 & 1214519 & 5.92 & 460777 & 12.08 \\
\hline UPPER LIMIT & 1342201 & 9.59 & 1700327 & 6.25 & 645088 & 12.41 \\
\hline LOWER LIMIT & 575229 & 8.93 & 728711 & 5.59 & 276466 & 11.75 \\
\hline EPA SAMPLE NO. & & & & & & \\
\hline VBLKDT & 921736 & 9.27 & 1118517 & 5.92 & 341253 & 12.08 \\
\hline YKQCTB-W-38080 & 899003 & 9.27 & 1124835 & 5.92 & 298295 & 12.08 \\
\hline $\begin{array}{l}\text { YKTI2 8-104-W-3 } \\
8078\end{array}$ & 847476 & 9.26 & 1169238 & 5.92 & 313430 & 12.08 \\
\hline VHBLK01 & 860058 & 9.26 & 1145486 & 5.92 & 304620 & 12.08 \\
\hline
\end{tabular}

IS1 (CBZ) = Chlorobenzene-d5

IS2 $(\mathrm{DFB})=1,4-\mathrm{Difluorobenzene}$

IS3 $(\mathrm{DCB})=1,4-\mathrm{Dichlorobenzene-d4}$

AREA UPPER LIMIT $=140 \%$ (Trace Volatiles) of internal standard area AREA LOWER LIMIT $=60 \%$ (Trace Volatiles) of internal standard area RT UPPER LIMIT $=+0.33$ (Trace Volatiles) minutes of internal standard RT RT LOWER LIMIT $=-0.33$ (Trace Volatiles) minutes of internal standard RT

\# Column used to flag values outside contract required QC limits with an asterisk. Page 1 of 1 
Lab Name: TESTAMERICA BURLINGTON

Contract: $8 \mathrm{E}-00302$

Lab Code: STLV

Case No.: YORK Mod. Ref No.:

SDG No.: 32333

Matrix: (SOIL/SED/WATER) Water

Sample wt/vol: 25.0 $(\mathrm{g} / \mathrm{mL}) \mathrm{mL}$

Level: (TRACE/LOW/MED) TRACE

\% Moisture: not dec.

GC Column: DB-624 ID: $0.20 \quad(\mathrm{~mm})$

Soil Extract Volume: (uL) $(\mathrm{mL})$

Purge Volume: 25.0
Lab Sample ID: 200-32333-2

Lab File ID: 018646_008.D

Date Received: 03/05/2016

Date Analyzed: 03/08/2016

Dilution Factor: 1.0

Soil Aliquot Volume:

(uL)

\begin{tabular}{|c|c|c|c|}
\hline CAS NO. & COMPOUND & $\begin{array}{l}\text { CONCENTRATION UNITS: } \\
(\mathrm{ug} / \mathrm{L} \text { or } \mathrm{ug} / \mathrm{kg}) \mathrm{ug} / \mathrm{L}\end{array}$ & Q \\
\hline $75-71-8$ & Dichlorodifluoromethane & 1.0 & $\mathrm{U}$ \\
\hline $74-87-3$ & Chloromethane & 1.0 & $\mathrm{U}$ \\
\hline $75-01-4$ & Vinyl chloride & 1.0 & $\mathrm{U}$ \\
\hline $74-83-9$ & Bromomethane & 0.067 & $\mathrm{~J} B$ \\
\hline $75-00-3$ & Chloroethane & 1.0 & $\mathrm{U}$ \\
\hline $75-69-4$ & Trichlorofluoromethane & 1.0 & $\mathrm{U}$ \\
\hline $75-35-4$ & 1,1-Dichloroethene & 1.0 & $\mathrm{U}$ \\
\hline $76-13-1$ & $1,1,2$-Trichloro-1,2,2-trifluoroethane & 1.0 & $\mathrm{U}$ \\
\hline $67-64-1$ & Acetone & 2.8 & $\mathrm{~J} B$ \\
\hline $75-15-0$ & Carbon disulfide & 0.062 & $\mathrm{~J} B$ \\
\hline $79-20-9$ & Methyl acetate & 1.0 & $\mathrm{U}$ \\
\hline $75-09-2$ & Methylene Chloride & 1.0 & $\mathrm{U}$ \\
\hline $156-60-5$ & trans-1,2-Dichloroethene & 1.0 & $\mathrm{U}$ \\
\hline $1634-04-4$ & Methyl tert-butyl ether & 1.0 & $\mathrm{U}$ \\
\hline $75-34-3$ & 1,1-Dichloroethane & 1.0 & $\mathrm{U}$ \\
\hline $156-59-2$ & cis-1,2-Dichloroethene & 1.0 & $\mathrm{U}$ \\
\hline $78-93-3$ & 2-Butanone & 5.0 & $\mathrm{U}$ \\
\hline $74-97-5$ & Bromochloromethane & 1.0 & $\mathrm{U}$ \\
\hline $67-66-3$ & Chloroform & 1.0 & $\mathrm{U}$ \\
\hline $71-55-6$ & $1,1,1$-Trichloroethane & 1.0 & $\mathrm{U}$ \\
\hline $110-82-7$ & Cyclohexane & 1.0 & $\mathrm{U}$ \\
\hline $56-23-5$ & Carbon tetrachloride & 1.0 & $\mathrm{U}$ \\
\hline $71-43-2$ & Benzene & 1.0 & $\mathrm{U}$ \\
\hline $107-06-2$ & 1,2-Dichloroethane & 1.0 & $\mathrm{U}$ \\
\hline
\end{tabular}

Report 1,4-Dioxane for Low-Medium VOA analysis only 
Lab Name: TESTAMERICA BURLINGTON

Contract: $8 \mathrm{E}-00302$

Lab Code: STLV

Case No.: YORK

Mod. Ref No.:

SDG No.: 32333

Matrix: (SOIL/SED/WATER) Water

Sample wt/vol: 25.0 $(\mathrm{g} / \mathrm{mL}) \mathrm{mL}$

Level: (TRACE/LOW/MED) TRACE

\% Moisture: not dec.

GC Column: DB-624 ID: $0.20 \quad(\mathrm{~mm})$

Soil Extract Volume: (uL) $(\mathrm{mL})$

Purge Volume: 25.0 (mI)
Lab Sample ID: 200-32333-2

Lab File ID: 018646_008.D

Date Received: 03/05/2016

Date Analyzed: 03/08/2016

Dilution Factor: 1.0

Soil Aliquot Volume: (uL)

\begin{tabular}{|c|c|c|c|}
\hline CAS NO. & COMPOUND & $\begin{array}{l}\text { CONCENTRATION UNITS: } \\
(\mathrm{ug} / \mathrm{L} \text { or } \mathrm{ug} / \mathrm{kg}) \mathrm{ug} / \mathrm{L}\end{array}$ & Q \\
\hline $79-01-6$ & Trichloroethene & 0.028 & $\mathrm{~J}$ \\
\hline $108-87-2$ & Methylcyclohexane & 1.0 & $\mathrm{U}$ \\
\hline $78-87-5$ & 1,2-Dichloropropane & 1.0 & $\mathrm{U}$ \\
\hline $75-27-4$ & Bromodichloromethane & 1.0 & $\mathrm{U}$ \\
\hline $10061-01-5$ & cis-1,3-Dichloropropene & 1.0 & U \\
\hline $108-10-1$ & 4-Methyl-2-pentanone & 1.0 & $\mathrm{U}$ \\
\hline $108-88-3$ & Toluene & 0.15 & $\mathrm{~J} B$ \\
\hline $10061-02-6$ & trans-1,3-Dichloropropene & 1.0 & $\mathrm{U}$ \\
\hline $79-00-5$ & $1,1,2-$ Trichloroethane & 1.0 & $\mathrm{U}$ \\
\hline $127-18-4$ & Tetrachloroethene & 1.0 & $\mathrm{U}$ \\
\hline $591-78-6$ & 2-Hexanone & 1.0 & $\mathrm{U}$ \\
\hline $124-48-1$ & Dibromochloromethane & 1.0 & $\mathrm{U}$ \\
\hline $106-93-4$ & 1,2-Dibromoethane & 1.0 & $\mathrm{U}$ \\
\hline $108-90-7$ & Chlorobenzene & 1.0 & $\mathrm{U}$ \\
\hline $100-41-4$ & Ethylbenzene & 0.0079 & $\mathrm{~J}$ \\
\hline $95-47-6$ & o-Xylene & 0.053 & $\mathrm{~J}$ \\
\hline $179601-23-1$ & m,p-xylene & 0.022 & $\mathrm{~J}$ \\
\hline $100-42-5$ & Styrene & 1.0 & $\mathrm{U}$ \\
\hline $75-25-2$ & Bromoform & 1.0 & $\mathrm{U}$ \\
\hline $98-82-8$ & Isopropylbenzene & 1.0 & $\mathrm{U}$ \\
\hline $79-34-5$ & $1,1,2,2$-Tetrachloroethane & 1.0 & $\mathrm{U}$ \\
\hline $541-73-1$ & 1,3 -Dichlorobenzene & 1.0 & $\mathrm{U}$ \\
\hline $106-46-7$ & 1,4-Dichlorobenzene & 0.037 & $\mathrm{~J}$ \\
\hline $95-50-1$ & 1,2-Dichlorobenzene & 1.0 & $\mathrm{U}$ \\
\hline $96-12-8$ & 1,2-Dibromo-3-Chloropropane & 1.0 & $\mathrm{U}$ \\
\hline $120-82-1$ & $1,2,4$-Trichlorobenzene & 1.0 & $\mathrm{U}$ \\
\hline $87-61-6$ & $1,2,3$-Trichlorobenzene & 1.0 & $\mathrm{U}$ \\
\hline
\end{tabular}


Lab Name: TESTAMERICA BURLINGTON

Contract: $8 \mathrm{E}-00302$

Lab Code: STLV Case No.: YORK Mod. Ref No.: SDG No.: 32333

Matrix: (SOIL/SED/WATER) Water

Sample wt/vol: 25.0 $(\mathrm{g} / \mathrm{mL}) \mathrm{mL}$

Level: (TRACE or LOW/MED) TRACE

․ Moisture: not dec.

GC Column: DB-624 ID $: 0.20 \quad(\mathrm{~mm})$

Soil Extract Volume: (uL)

CONCENTRATION UNITS: (ug/L or ug/kg) ug/L
Lab Sample ID: 200-32333-2

Lab File ID: 018646_008.D

Date Received: 03/05/2016

Date Analyzed: 03/08/2016

Dilution Factor: 1.0

Soil Aliquot Volume: (uL) Purge Volume: 25.0

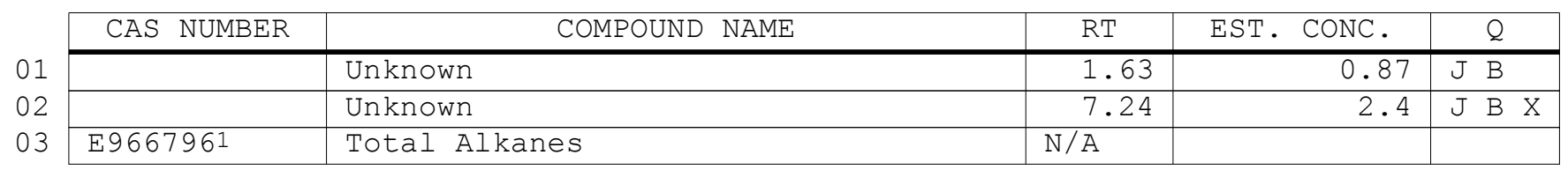

1 EPA-designated Registry Number. 
Lab Name: TESTAMERICA BURLINGTON

Contract: $8 \mathrm{E}-00302$

Lab Code: STLV

Case No.: YORK Mod. Ref No.:

SDG No.: 32333

Matrix: (SOIL/SED/WATER) Water

Sample wt/vol: 25.0 $(\mathrm{g} / \mathrm{mL}) \mathrm{mL}$

Level: (TRACE/LOW/MED) TRACE

\% Moisture: not dec.

GC Column: DB-624 ID: $0.20 \quad(\mathrm{~mm})$

Soil Extract Volume: (uL) $(\mathrm{mL})$

Purge Volume: 25.0
Lab Sample ID: 200-32333-1

Lab File ID: 018646_009.D

Date Received: 03/05/2016

Date Analyzed: 03/08/2016

Dilution Factor: 1.0

Soil Aliquot Volume:

(uL)

\begin{tabular}{|c|c|c|c|}
\hline CAS NO. & COMPOUND & $\begin{array}{l}\text { CONCENTRATION UNITS: } \\
(\mathrm{ug} / \mathrm{L} \text { or } \mathrm{ug} / \mathrm{kg}) \mathrm{ug} / \mathrm{L}\end{array}$ & Q \\
\hline $75-71-8$ & Dichlorodifluoromethane & 1.0 & $\mathrm{U}$ \\
\hline $74-87-3$ & Chloromethane & 1.0 & $\mathrm{U}$ \\
\hline $75-01-4$ & Vinyl chloride & 1.0 & $\mathrm{U}$ \\
\hline $74-83-9$ & Bromomethane & 0.051 & $\mathrm{~J} B$ \\
\hline $75-00-3$ & Chloroethane & 1.0 & $\mathrm{U}$ \\
\hline $75-69-4$ & Trichlorofluoromethane & 1.0 & $\mathrm{U}$ \\
\hline $75-35-4$ & 1,1-Dichloroethene & 1.0 & $\mathrm{U}$ \\
\hline $76-13-1$ & 1,1,2-Trichloro-1,2,2-trifluoroethane & 1.0 & $\mathrm{U}$ \\
\hline $67-64-1$ & Acetone & 1.3 & $\mathrm{~J} B$ \\
\hline $75-15-0$ & Carbon disulfide & 1.0 & $\mathrm{U}$ \\
\hline $79-20-9$ & Methyl acetate & 1.0 & $\mathrm{U}$ \\
\hline $75-09-2$ & Methylene Chloride & 1.0 & $\mathrm{U}$ \\
\hline $156-60-5$ & trans-1,2-Dichloroethene & 1.0 & $\mathrm{U}$ \\
\hline $1634-04-4$ & Methyl tert-butyl ether & 1.0 & $\mathrm{U}$ \\
\hline $75-34-3$ & 1,1-Dichloroethane & 1.0 & $\mathrm{U}$ \\
\hline $156-59-2$ & cis-1,2-Dichloroethene & 1.0 & $\mathrm{U}$ \\
\hline $78-93-3$ & 2-Butanone & 5.0 & $\mathrm{U}$ \\
\hline $74-97-5$ & Bromochloromethane & 1.0 & $\mathrm{U}$ \\
\hline $67-66-3$ & Chloroform & 1.0 & $\mathrm{U}$ \\
\hline $71-55-6$ & $1,1,1$-Trichloroethane & 1.0 & $\mathrm{U}$ \\
\hline $110-82-7$ & Cyclohexane & 1.0 & $\mathrm{U}$ \\
\hline $56-23-5$ & Carbon tetrachloride & 0.029 & $\mathrm{~J}$ \\
\hline $71-43-2$ & Benzene & 1.0 & $\mathrm{U}$ \\
\hline $107-06-2$ & 1,2-Dichloroethane & 1.0 & $\mathrm{U}$ \\
\hline
\end{tabular}

Report 1,4-Dioxane for Low-Medium VOA analysis only 
Lab Name: TESTAMERICA BURLINGTON

Contract: 8E-00302

Lab Code: STLV

Case No.: YORK

Mod. Ref No.:

SDG No.: 32333

Matrix: (SOIL/SED/WATER) Water

Sample wt/vol: 25.0 $(\mathrm{g} / \mathrm{mL}) \mathrm{mL}$

Level: (TRACE/LOW/MED) TRACE

\% Moisture: not dec.

GC Column: DB-624 ID: $0.20 \quad(\mathrm{~mm})$

Soil Extract Volume: (uL) $(\mathrm{mL})$

Purge Volume: 25.0 (mI)
Lab Sample ID: 200-32333-1

Lab File ID: 018646_009.D

Date Received: 03/05/2016

Date Analyzed: 03/08/2016

Dilution Factor: 1.0

Soil Aliquot Volume: (uL)

\begin{tabular}{|c|c|c|c|}
\hline CAS NO. & COMPOUND & $\begin{array}{l}\text { CONCENTRATION UNITS: } \\
(\mathrm{ug} / \mathrm{L} \text { or } \mathrm{ug} / \mathrm{kg}) \mathrm{ug} / \mathrm{L}\end{array}$ & Q \\
\hline $79-01-6$ & Trichloroethene & 1.0 & $\mathrm{U}$ \\
\hline $108-87-2$ & Methylcyclohexane & 1.0 & $\mathrm{U}$ \\
\hline $78-87-5$ & 1,2-Dichloropropane & 1.0 & $\mathrm{U}$ \\
\hline $75-27-4$ & Bromodichloromethane & 1.0 & $\mathrm{U}$ \\
\hline $10061-01-5$ & cis-1,3-Dichloropropene & 1.0 & U \\
\hline $108-10-1$ & 4-Methyl-2-pentanone & 1.0 & $\mathrm{U}$ \\
\hline $108-88-3$ & Toluene & 0.021 & $\mathrm{~J} B$ \\
\hline $10061-02-6$ & trans-1,3-Dichloropropene & 1.0 & $\mathrm{U}$ \\
\hline $79-00-5$ & $1,1,2-$ Trichloroethane & 1.0 & $\mathrm{U}$ \\
\hline $127-18-4$ & Tetrachloroethene & 1.0 & $\mathrm{U}$ \\
\hline $591-78-6$ & 2-Hexanone & 1.0 & $\mathrm{U}$ \\
\hline $124-48-1$ & Dibromochloromethane & 1.0 & $\mathrm{U}$ \\
\hline $106-93-4$ & 1,2-Dibromoethane & 1.0 & $\mathrm{U}$ \\
\hline $108-90-7$ & Chlorobenzene & 1.0 & $\mathrm{U}$ \\
\hline $100-41-4$ & Ethylbenzene & 1.0 & $\mathrm{U}$ \\
\hline $95-47-6$ & o-Xylene & 1.0 & $\mathrm{U}$ \\
\hline $179601-23-1$ & m,p-xylene & 0.017 & $\mathrm{~J}$ \\
\hline $100-42-5$ & Styrene & 1.0 & $\mathrm{U}$ \\
\hline $75-25-2$ & Bromoform & 1.0 & $\mathrm{U}$ \\
\hline $98-82-8$ & Isopropylbenzene & 1.0 & $\mathrm{U}$ \\
\hline $79-34-5$ & $1,1,2,2$-Tetrachloroethane & 1.0 & $\mathrm{U}$ \\
\hline $541-73-1$ & 1,3 -Dichlorobenzene & 1.0 & $\mathrm{U}$ \\
\hline $106-46-7$ & 1,4-Dichlorobenzene & 1.0 & $\mathrm{U}$ \\
\hline $95-50-1$ & 1,2-Dichlorobenzene & 1.0 & $\mathrm{U}$ \\
\hline $96-12-8$ & 1,2-Dibromo-3-Chloropropane & 1.0 & $\mathrm{U}$ \\
\hline $120-82-1$ & $1,2,4$-Trichlorobenzene & 1.0 & $\mathrm{U}$ \\
\hline $87-61-6$ & $1,2,3$-Trichlorobenzene & 1.0 & $\mathrm{U}$ \\
\hline
\end{tabular}


Lab Name: TESTAMERICA BURLINGTON

Contract: $8 \mathrm{E}-00302$

Lab Code: STLV Case No.: YORK Mod. Ref No.: SDG No.: 32333

Matrix: (SOIL/SED/WATER) Water

Sample wt/vol: 25.0 $(\mathrm{g} / \mathrm{mL}) \mathrm{mL}$

Level: (TRACE or LOW/MED) TRACE

․ Moisture: not dec.

GC Column: DB-624 ID $: 0.20 \quad(\mathrm{~mm})$

Soil Extract Volume: (uL)

CONCENTRATION UNITS: (ug/L or ug/kg) ug/L
Lab Sample ID: 200-32333-1

Lab File ID: 018646_009.D

Date Received: 03/05/2016

Date Analyzed: 03/08/2016

Dilution Factor: 1.0

Soil Aliquot Volume: (uL) Purge Volume: 25.0

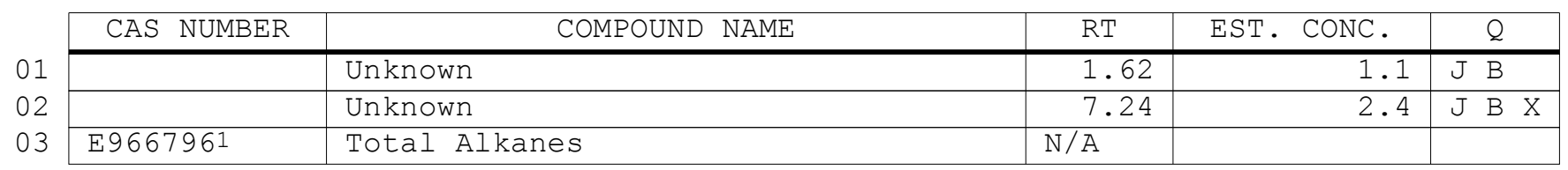

1 EPA-designated Registry Number. 
Lab Name: TESTAMERICA BURLINGTON

Contract: $8 \mathrm{E}-00302$

Lab Code: STLV Case No.: YORK Mod. Ref No.:

SDG No.: 32333

Instrument ID: CHD.i

Calibration Date(s): 02/26/2016 02/26/2016

Heated Purge: ( $\mathrm{Y} / \mathrm{N}) \mathrm{N}$

Calibration Time(s) : 1621

1801

Purge Volume: 25.0 $(\mathrm{mL})$

GC Column: DB-624

ID: $0.20 \quad(\mathrm{~mm}) \quad$ Length: 25

$(\mathrm{m})$

\begin{tabular}{|c|c|c|c|c|c|c|c|}
\hline LAB FILE ID: & \multirow{2}{*}{\multicolumn{3}{|c|}{$\begin{array}{l}\operatorname{RRF}_{\underline{0} .5}=\frac{018468 \_006 . \mathrm{D}}{\operatorname{RRF10}}=018468 \_009 . \mathrm{D}\end{array}$}} & \multicolumn{4}{|c|}{ RRF1.0 = $018468 \_007 . \mathrm{D}$} \\
\hline RRF5.0 = 018468_008.D & & & & RRF2C & $=018$ & $58 \quad 010$ & \\
\hline COMPOUND & RRF0. 5 & RRF1. 0 & RRF5. 0 & RRF10 & RRF20 & $\overline{\mathrm{RRF}}$ & $\circ \mathrm{RSD}$ \\
\hline Dichlorodifluoromethane & 0.539 & 0.581 & 0.560 & 0.514 & 0.566 & 0.552 & 4.7 \\
\hline Chloromethane & 0.317 & 0.339 & 0.278 & 0.247 & 0.256 & 0.287 & 13.8 \\
\hline Vinyl chloride & 0.301 & 0.332 & 0.317 & 0.312 & 0.333 & 0.319 & 4.2 \\
\hline Bromomethane & 0.146 & 0.148 & 0.138 & 0.143 & 0.165 & 0.148 & 6.8 \\
\hline Chloroethane & 0.203 & 0.166 & 0.200 & 0.171 & 0.179 & 0.184 & 9.1 \\
\hline Trichlorofluoromethane & 0.619 & 0.652 & 0.644 & 0.584 & 0.636 & 0.627 & 4.3 \\
\hline 1,1-Dichloroethene & 0.266 & 0.292 & 0.304 & 0.266 & 0.288 & 0.283 & 5.9 \\
\hline $\begin{array}{l}1,1,2 \text {-Trichloro- } \\
1,2,2 \text {-trifluoroethane }\end{array}$ & 0.305 & 0.324 & 0.328 & 0.307 & 0.335 & 0.320 & 4.2 \\
\hline Acetone & 0.015 & 0.015 & 0.012 & 0.010 & 0.013 & 0.013 & 13.8 \\
\hline Carbon disulfide & 0.579 & 0.666 & 0.623 & 0.624 & 0.656 & 0.630 & 5.4 \\
\hline Methyl acetate & 0.030 & 0.029 & 0.029 & 0.023 & 0.029 & 0.028 & 9.9 \\
\hline Methylene Chloride & 0.229 & 0.230 & 0.243 & 0.214 & 0.228 & 0.229 & 4.4 \\
\hline trans-1,2-Dichloroethene & 0.279 & 0.304 & 0.305 & 0.285 & 0.314 & 0.297 & 4.9 \\
\hline Methyl tert-butyl ether & 0.248 & 0.251 & 0.298 & 0.274 & 0.320 & 0.278 & 11.2 \\
\hline 1,1-Dichloroethane & 0.419 & 0.488 & 0.481 & 0.449 & 0.464 & 0.460 & 5.9 \\
\hline cis-1,2-Dichloroethene & 0.250 & 0.249 & 0.301 & 0.284 & 0.318 & 0.280 & 11.0 \\
\hline 2-Butanone & 0.020 & 0.020 & 0.022 & 0.023 & 0.026 & 0.022 & 10.1 \\
\hline Bromochloromethane & 0.095 & 0.093 & 0.105 & 0.097 & 0.101 & 0.098 & 5.0 \\
\hline Chloroform & 0.454 & 0.486 & 0.502 & 0.459 & 0.494 & 0.479 & 4.5 \\
\hline $1,1,1$-Trichloroethane & 0.636 & 0.646 & 0.691 & 0.636 & 0.645 & 0.651 & 3.5 \\
\hline Cyclohexane & 0.397 & 0.466 & 0.573 & 0.617 & 0.588 & 0.528 & 17.6 \\
\hline Carbon tetrachloride & 0.577 & 0.567 & 0.605 & 0.556 & 0.556 & 0.572 & 3.5 \\
\hline Benzene & 1.537 & 1.472 & 1.675 & 1.456 & 1.472 & 1.522 & 5.9 \\
\hline 1,2-Dichloroethane & 0.209 & 0.197 & 0.220 & 0.196 & 0.216 & 0.208 & 5.2 \\
\hline Trichloroethene & 0.374 & 0.377 & 0.410 & 0.388 & 0.408 & 0.391 & 4.3 \\
\hline Methylcyclohexane & 0.369 & 0.426 & 0.518 & 0.503 & 0.505 & 0.464 & 13.9 \\
\hline
\end{tabular}

Report 1,4-Dioxane for Low-Medium VOA analysis only 
Lab Name: TESTAMERICA BURLINGTON

Contract: $8 \mathrm{E}-00302$

Lab Code: STLV Case No.: YORK Mod. Ref No.:

SDG No.: 32333

Instrument ID: CHD.i

Calibration Date(s): 02/26/2016 02/26/2016

Heated Purge: ( $\mathrm{Y} / \mathrm{N}) \mathrm{N}$

Calibration Time(s) : 1621

1801

Purge Volume: 25.0

GC Column: DB-624

ID: 0.20 (mL)

\begin{tabular}{|c|c|c|c|c|c|c|c|}
\hline LAB FILE ID: & \multirow{2}{*}{\multicolumn{3}{|c|}{ 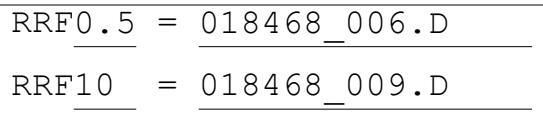 }} & \multirow{2}{*}{\multicolumn{4}{|c|}{$\begin{array}{l}\operatorname{RRF1.0}_{\underline{R}}=\frac{018468 \_007 . \mathrm{D}}{\underline{\mathrm{RRF} 20}=018468 \_010 . \mathrm{D}}\end{array}$}} \\
\hline RRE5.0 = 018468_008.D & & & & & & & \\
\hline COMPOUND & RRF0. 5 & RRF1. 0 & RRF5. 0 & RRF10 & RRF20 & $\overline{\mathrm{RRF}}$ & $\because \mathrm{RSD}$ \\
\hline 1,2-Dichloropropane & 0.262 & 0.265 & 0.301 & 0.270 & 0.278 & 0.275 & 5.8 \\
\hline Bromodichloromethane & 0.329 & 0.350 & 0.341 & 0.329 & 0.337 & 0.337 & 2.5 \\
\hline cis-1,3-Dichloropropene & 0.261 & 0.294 & 0.395 & 0.391 & 0.404 & 0.349 & 19.0 \\
\hline 4-Methyl-2-pentanone & 0.049 & 0.057 & 0.074 & 0.071 & 0.077 & 0.066 & 18.5 \\
\hline Toluene & 1.276 & 1.443 & 1.823 & 1.656 & 1.701 & 1.580 & 13.8 \\
\hline trans-1,3-Dichloropropene & 0.186 & 0.193 & 0.264 & 0.261 & 0.290 & 0.239 & 19.6 \\
\hline $1,1,2$-Trichloroethane & 0.169 & 0.158 & 0.161 & 0.157 & 0.166 & 0.162 & 3.1 \\
\hline Tetrachloroethene & 0.357 & 0.380 & 0.403 & 0.411 & 0.418 & 0.394 & 6.3 \\
\hline 2-Hexanone & 0.033 & 0.038 & 0.055 & 0.048 & 0.053 & 0.045 & 21.5 \\
\hline Dibromochloromethane & 0.170 & 0.163 & 0.187 & 0.178 & 0.195 & 0.179 & 7.1 \\
\hline 1,2-Dibromoethane & 0.126 & 0.121 & 0.144 & 0.136 & 0.142 & 0.134 & 7.6 \\
\hline Chlorobenzene & 0.961 & 1.012 & 1.128 & 1.034 & 1.103 & 1.048 & 6.5 \\
\hline Ethylbenzene & 1.306 & 1.608 & 2.000 & 1.961 & 2.087 & 1.793 & 18.3 \\
\hline o-Xylene & 0.394 & 0.465 & 0.707 & 0.693 & 0.706 & 0.593 & 25.5 \\
\hline $\mathrm{m}, \mathrm{p}-\mathrm{Xylene}$ & 0.525 & 0.599 & 0.789 & 0.807 & 0.829 & 0.710 & 19.5 \\
\hline Styrene & 0.613 & 0.772 & 1.106 & 1.094 & 1.132 & 0.943 & 25.0 \\
\hline Bromoform & 0.149 & 0.143 & 0.132 & 0.136 & 0.161 & 0.144 & 7.8 \\
\hline Isopropylbenzene & 1.079 & 1.448 & 2.107 & 2.015 & 2.134 & 1.757 & 26.8 \\
\hline $1,1,2,2$-Tetrachloroethane & 0.146 & 0.136 & 0.157 & 0.139 & 0.144 & 0.144 & 5.6 \\
\hline 1,3-Dichlorobenzene & 1.306 & 1.245 & 1.442 & 1.508 & 1.710 & 1.442 & 12.7 \\
\hline 1,4-Dichlorobenzene & 1.764 & 1.636 & 1.572 & 1.585 & 1.697 & 1.651 & 4.9 \\
\hline 1,2-Dichlorobenzene & 1.303 & 1.231 & 1.361 & 1.290 & 1.366 & 1.310 & 4.3 \\
\hline 1,2-Dibromo-3-Chloropropane & 0.043 & 0.032 & 0.035 & 0.032 & 0.035 & 0.035 & 12.5 \\
\hline $1,2,4$-Trichlorobenzene & 0.566 & 0.485 & 0.606 & 0.700 & 0.853 & 0.642 & 22.0 \\
\hline $1,2,3$-Trichlorobenzene & 0.460 & 0.363 & 0.480 & 0.480 & 0.598 & 0.476 & 17.6 \\
\hline
\end{tabular}


Lab Name: TESTAMERICA BURLINGTON

Contract: $8 \mathrm{E}-00302$

Lab Code: STLV Case No.: YORK Mod. Ref No.: SDG No.: 32333

Instrument ID: CHD.i

Heated Purge: (Y/N) N

Purge Volume: 25.0

GC Column: DB-624
ID: 0.20
Calibration Date(s): 02/26/2016

Calibration Time(s) : 1621 $(\mathrm{mL})$

(mm) Length: 25 (m)

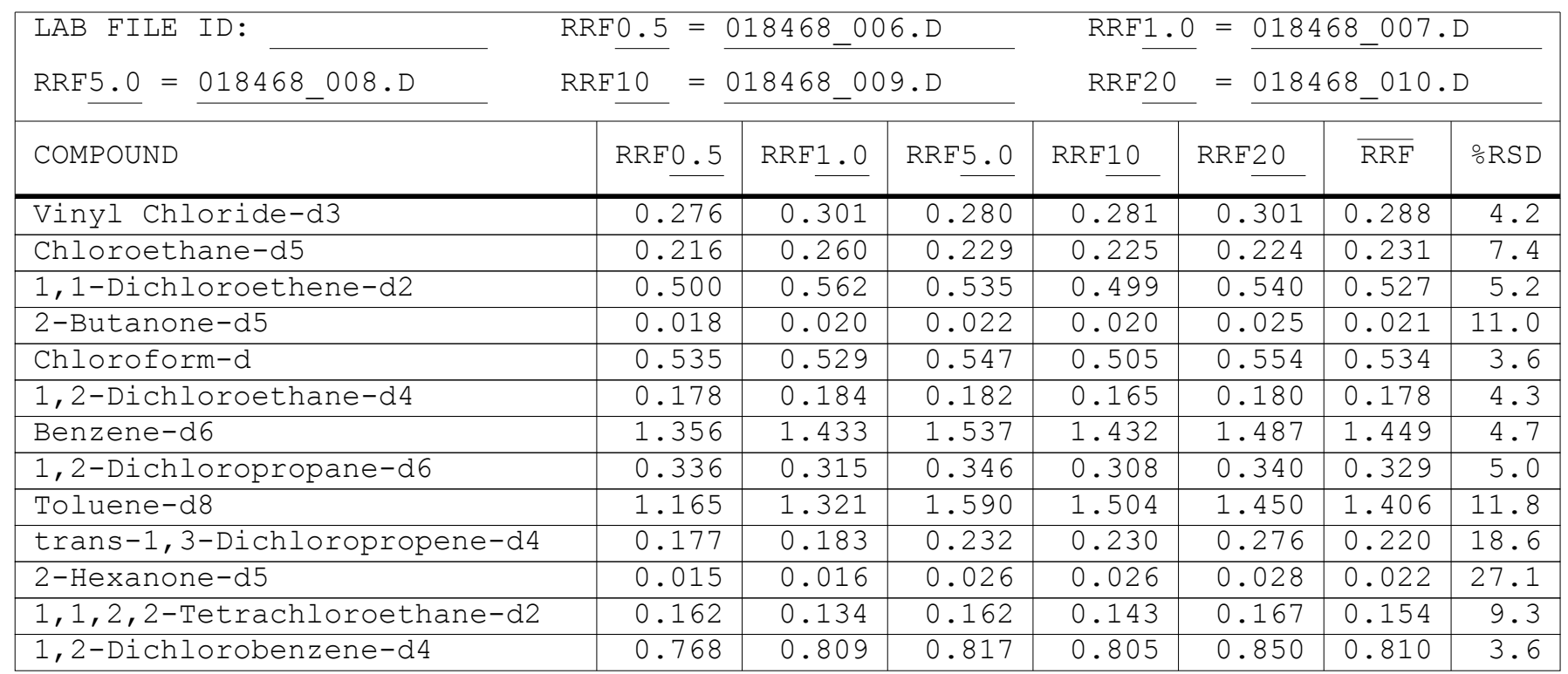

Report 1,4-Dioxane-d8 for Low-Medium VOA analysis only 
Lab Name: TESTAMERICA BURLINGTON

Contract: $8 \mathrm{E}-00302$

Lab Code: STLV

Mod. Ref No.:

SDG No.: 32333

Instrument ID: CHD.i

Calibration Date: 03/08/2016 Time: 1136

Lab File Id: 018646 003.D

Init. Calib. Date(s):02/26/2016 02/26/2016

EPA Sample No. (VSTD\#\#\#\#) : VSTD005DT

Heated Purge: ( $\mathrm{Y} / \mathrm{N}) \mathrm{N}$ GC Column: DB-624

Init. Calib. Time(s) : 1621 1801

Purge Volume: 25.0

ID: $0.20(\mathrm{~mm})$ Length: 25

(m)

\begin{tabular}{|c|c|c|c|c|c|}
\hline COMPOUND & $\overline{\mathrm{RRF}}$ & RRF5.0 & $\begin{array}{l}\text { MIN } \\
\text { RRF }\end{array}$ & $\frac{\circ}{D}$ & $\mathrm{MAX} \div \mathrm{D}$ \\
\hline Dichlorodifluoromethane & 0.552 & 0.508 & 0.010 & -8.0 & 40.0 \\
\hline Chloromethane & 0.287 & 0.228 & 0.010 & -20.7 & 40.0 \\
\hline Vinyl chloride & 0.319 & 0.296 & 0.100 & -7.1 & 30.0 \\
\hline Bromomethane & 0.148 & 0.120 & 0.100 & -19.1 & 30.0 \\
\hline Chloroethane & 0.184 & 0.173 & 0.010 & -5.8 & 40.0 \\
\hline Trichlorofluoromethane & 0.627 & 0.598 & 0.010 & -4.6 & 40.0 \\
\hline 1,1-Dichloroethene & 0.283 & 0.262 & 0.100 & -7.4 & 30.0 \\
\hline 1,1,2-Trichloro-1,2,2-trifluoroethane & 0.320 & 0.320 & 0.010 & 0.2 & 40.0 \\
\hline Acetone & 0.013 & 0.012 & 0.010 & $-11 \cdot 4$ & 40.0 \\
\hline Carbon disulfide & 0.630 & 0.598 & 0.010 & -5.1 & 40.0 \\
\hline Methyl acetate & 0.028 & 0.030 & 0.010 & 8.4 & 40.0 \\
\hline Methylene Chloride & 0.229 & 0.227 & 0.010 & -0.9 & 40.0 \\
\hline trans-1,2-Dichloroethene & 0.297 & 0.314 & 0.010 & 5.7 & 40.0 \\
\hline Methyl tert-butyl ether & 0.278 & 0.272 & 0.010 & -2.2 & 40.0 \\
\hline 1,1-Dichloroethane & 0.460 & 0.461 & 0.200 & 0.2 & 30.0 \\
\hline cis-1,2-Dichloroethene & 0.280 & 0.294 & 0.010 & 4.7 & 40.0 \\
\hline 2-Butanone & 0.022 & 0.021 & 0.010 & -3.5 & 40.0 \\
\hline Bromochloromethane & 0.098 & 0.101 & 0.050 & 2.8 & 30.0 \\
\hline Chloroform & 0.479 & 0.481 & 0.200 & 0.5 & 30.0 \\
\hline 1,1,1-Trichloroethane & 0.651 & 0.635 & 0.100 & -2.4 & 30.0 \\
\hline Cyclohexane & 0.528 & 0.576 & 0.010 & 9.0 & 40.0 \\
\hline Carbon tetrachloride & 0.572 & 0.591 & 0.100 & 3.4 & 30.0 \\
\hline Benzene & 1.522 & 1.653 & 0.400 & 8.6 & 30.0 \\
\hline 1,2-Dichloroethane & 0.208 & 0.213 & 0.100 & 2.7 & 30.0 \\
\hline Trichloroethene & 0.391 & 0.404 & 0.300 & 3.3 & 30.0 \\
\hline Methylcyclohexane & 0.464 & 0.483 & 0.010 & 4.1 & 40.0 \\
\hline
\end{tabular}

Report 1,4-Dioxane for Low/Medium VOA analysis only 
Lab Name: TESTAMERICA BURLINGTON

Contract: $8 \mathrm{E}-00302$

Lab Code: STLV Case No.: YORK Mod. Ref No.:

SDG No.: 32333

Instrument ID: CHD.i

Calibration Date: 03/08/2016 Time: 1136

Lab File Id: 018646_003.D

Init. Calib. Date(s):02/26/2016 02/26/2016

EPA Sample No.(VSTD\#\#\#\#) : VSTD005DT

Heated Purge: (Y/N) N GC Column: DB-624

Init. Calib. Time(s) : 1621 1801

Purge Volume: 25.0

ID: $0.20(\mathrm{~mm})$ Length: 25

(m)

\begin{tabular}{|c|c|c|c|c|c|}
\hline COMPOUND & $\overline{\mathrm{RRF}}$ & RRF5.0 & $\begin{array}{l}\text { MIN } \\
\text { RRF }\end{array}$ & $\because \mathrm{D}$ & $\operatorname{MAX} \cong \mathrm{D}$ \\
\hline 1,2-Dichloropropane & 0.275 & 0.277 & 0.010 & 0.5 & 40.0 \\
\hline Bromodichloromethane & 0.337 & 0.337 & 0.200 & -0.2 & 30.0 \\
\hline cis-1,3-Dichloropropene & 0.349 & 0.386 & 0.200 & 10.7 & 30.0 \\
\hline 4-Methyl-2-pentanone & 0.066 & 0.065 & 0.010 & -1.1 & 40.0 \\
\hline Toluene & 1.580 & 1.721 & 0.400 & 8.9 & 30.0 \\
\hline trans-1,3-Dichloropropene & 0.239 & 0.256 & 0.100 & 7.2 & 30.0 \\
\hline $1,1,2$-Trichloroethane & 0.162 & 0.162 & 0.100 & 0.0 & 30.0 \\
\hline Tetrachloroethene & 0.394 & 0.403 & 0.100 & 2.3 & 30.0 \\
\hline 2-Hexanone & 0.045 & 0.048 & 0.010 & 6.7 & 40.0 \\
\hline Dibromochloromethane & 0.179 & 0.192 & 0.100 & 7.3 & 30.0 \\
\hline 1,2-Dibromoethane & 0.134 & 0.133 & 0.010 & -0.6 & 40.0 \\
\hline Chlorobenzene & 1.048 & 1.049 & 0.500 & 0.1 & 30.0 \\
\hline Ethylbenzene & 1.793 & 1.966 & 0.100 & 9.7 & 30.0 \\
\hline o-Xylene & 0.593 & 0.680 & 0.300 & 14.7 & 30.0 \\
\hline $\mathrm{m}, \mathrm{p}$-Xylene & 0.710 & 0.778 & 0.300 & 9.6 & 30.0 \\
\hline Styrene & 0.943 & 1.074 & 0.300 & 13.9 & 30.0 \\
\hline Bromoform & 0.144 & 0.155 & 0.050 & 7.5 & 30.0 \\
\hline Isopropylbenzene & 1.757 & 2.009 & 0.010 & 14.4 & 40.0 \\
\hline $1,1,2,2$-Tetrachloroethane & 0.144 & 0.132 & 0.100 & -8.5 & 30.0 \\
\hline 1,3-Dichlorobenzene & 1.442 & 1.480 & 0.400 & 2.6 & 30.0 \\
\hline 1,4-Dichlorobenzene & 1.651 & 1.758 & 0.400 & 6.5 & 30.0 \\
\hline 1,2-Dichlorobenzene & 1.310 & 1.340 & 0.400 & 2.3 & 30.0 \\
\hline 1,2-Dibromo-3-Chloropropane & 0.035 & 0.032 & 0.010 & -9.4 & 40.0 \\
\hline 1,2,4-Trichlorobenzene & 0.642 & 0.653 & 0.200 & 1.7 & 30.0 \\
\hline $1,2,3$-Trichlorobenzene & 0.476 & 0.473 & 0.200 & -0.7 & 30.0 \\
\hline
\end{tabular}


Lab Name: TESTAMERICA BURLINGTON

Contract: $8 \mathrm{E}-00302$

Lab Code: STLV Case No.: YORK Mod. Ref No.

SDG No.: 32333

Instrument ID: CHD.i

Calibration Date: 03/08/2016 Time: 1136

Lab File Id: 018646 003.D Init. Calib. Date (s):02/26/2016 02/26/2016

EPA Sample No. (VSTD\#\#\#\#) : VSTD005DT

Heated Purge: (Y/N) N GC Column: DB-624

Init. Calib. Time(s) : 1621 1801

Purge Volume: 25.0 ID: $0.20(\mathrm{~mm})$ Length: 25

(m)

\begin{tabular}{|c|c|c|c|c|c|}
\hline COMPOUND & $\overline{\mathrm{RRF}}$ & RRF5. 0 & $\begin{array}{l}\text { MIN } \\
\text { RRF }\end{array}$ & $\circ \mathrm{D}$ & $\mathrm{MAX} \div \mathrm{D}$ \\
\hline Vinyl Chloride-d3 & 0.288 & 0.256 & 0.010 & -10.9 & 30.0 \\
\hline Chloroethane-d5 & 0.231 & 0.218 & 0.010 & -5.4 & 40.0 \\
\hline 1,1-Dichloroethene-d2 & 0.527 & 0.505 & 0.010 & -4.2 & 30.0 \\
\hline 2-Butanone-d5 & 0.021 & 0.019 & 0.010 & -9.4 & 40.0 \\
\hline Chloroform-d & 0.534 & 0.543 & 0.010 & 1.7 & 30.0 \\
\hline 1,2-Dichloroethane-d4 & 0.178 & 0.187 & 0.010 & 5.1 & 30.0 \\
\hline Benzene-d6 & 1.449 & 1.610 & 0.010 & 11.1 & 30.0 \\
\hline 1,2-Dichloropropane-d6 & 0.329 & 0.356 & 0.010 & 8.2 & 40.0 \\
\hline Toluene-d8 & 1.406 & 1.567 & 0.010 & 11.5 & 30.0 \\
\hline trans-1,3-Dichloropropene-d4 & 0.220 & 0.226 & 0.010 & 2.7 & 30.0 \\
\hline 2-Hexanone-d5 & 0.022 & 0.025 & 0.010 & 10.8 & 40.0 \\
\hline $1,1,2,2-$ Tetrachloroethane-d2 & 0.154 & 0.143 & 0.010 & -6.7 & 30.0 \\
\hline 1,2-Dichlorobenzene-d4 & 0.810 & 0.795 & 0.010 & -1.8 & 30.0 \\
\hline
\end{tabular}

Report 1,4-Dioxane-d8 for Low/Medium VOA analysis only 
Lab Name: TESTAMERICA BURLINGTON

Contract: $8 \mathrm{E}-00302$

Lab Code: STLV Case No.: YORK Mod. Ref No.:

SDG No.: 32333

Instrument ID: CHD.i

Calibration Date: 03/08/2016 Time: 1523

Lab File Id: 018646_011.D Init. Calib. Date(s):02/26/2016 02/26/2016

EPA Sample No. (VSTD\#\#\#\#) : VSTD005TD

Heated Purge: (Y/N) N GC Column: DB-624

Init. Calib. Time(s) : 1621 1801

Purge Volume: 25.0 ID: $0.20(\mathrm{~mm})$ Length: 25

(m)

\begin{tabular}{|c|c|c|c|c|c|}
\hline COMPOUND & $\overline{\mathrm{RRF}}$ & RRF5.0 & $\begin{array}{l}\text { MIN } \\
\text { RRF }\end{array}$ & $\frac{\circ}{D}$ & $\mathrm{MAX} \div \mathrm{D}$ \\
\hline Dichlorodifluoromethane & 0.552 & 0.532 & 0.010 & -3.6 & 50.0 \\
\hline Chloromethane & 0.287 & 0.256 & 0.010 & -10.7 & 50.0 \\
\hline Vinyl chloride & 0.319 & 0.310 & 0.010 & -2.8 & 50.0 \\
\hline Bromomethane & 0.148 & 0.091 & 0.010 & -38.6 & 50.0 \\
\hline Chloroethane & 0.184 & 0.171 & 0.010 & -7.0 & 50.0 \\
\hline Trichlorofluoromethane & 0.627 & 0.609 & 0.010 & -2.9 & 50.0 \\
\hline 1,1-Dichloroethene & 0.283 & 0.282 & 0.010 & -0.2 & 50.0 \\
\hline 1,1,2-Trichloro-1,2,2-trifluoroethane & 0.320 & 0.326 & 0.010 & 1.9 & 50.0 \\
\hline Acetone & 0.013 & 0.012 & 0.010 & -6.1 & 50.0 \\
\hline Carbon disulfide & 0.630 & 0.600 & 0.010 & -4.8 & 50.0 \\
\hline Methyl acetate & 0.028 & 0.025 & 0.010 & -9.8 & 50.0 \\
\hline Methylene Chloride & 0.229 & 0.218 & 0.010 & -4.8 & 50.0 \\
\hline trans-1,2-Dichloroethene & 0.297 & 0.313 & 0.010 & 5.2 & 50.0 \\
\hline Methyl tert-butyl ether & 0.278 & 0.275 & 0.010 & -1.4 & 50.0 \\
\hline 1,1-Dichloroethane & 0.460 & 0.464 & 0.010 & 1.0 & 50.0 \\
\hline cis-1,2-Dichloroethene & 0.280 & 0.297 & 0.010 & 5.7 & 50.0 \\
\hline 2-Butanone & 0.022 & 0.023 & 0.010 & 2.4 & 50.0 \\
\hline Bromochloromethane & 0.098 & 0.105 & 0.010 & 6.6 & 50.0 \\
\hline Chloroform & 0.479 & 0.525 & 0.010 & 9.5 & 50.0 \\
\hline 1,1,1-Trichloroethane & 0.651 & 0.671 & 0.010 & 3.1 & 50.0 \\
\hline Cyclohexane & 0.528 & 0.568 & 0.010 & 7.5 & 50.0 \\
\hline Carbon tetrachloride & 0.572 & 0.582 & 0.010 & 1.7 & 50.0 \\
\hline Benzene & 1.522 & 1.606 & 0.010 & 5.5 & 50.0 \\
\hline 1,2-Dichloroethane & 0.208 & 0.229 & 0.010 & 10.1 & 50.0 \\
\hline Trichloroethene & 0.391 & 0.390 & 0.010 & -0.5 & 50.0 \\
\hline Methylcyclohexane & 0.464 & 0.498 & 0.010 & 7.4 & 50.0 \\
\hline
\end{tabular}

Report 1,4-Dioxane for Low/Medium VOA analysis only 
Lab Name: TESTAMERICA BURLINGTON

Contract: 8E-00302

Lab Code: STLV

Mod. Ref No.:

SDG No.: 32333

Instrument ID: CHD.i

Calibration Date: 03/08/2016 Time: 1523

Lab File Id: 018646 011.D

Init. Calib. Date(s):02/26/2016 02/26/2016

EPA Sample No. (VSTD\#\#\#\#) : VSTD005TD

Heated Purge: (Y/N) N GC Column: DB-624

Init. Calib. Time(s) : 1621 1801

Purge Volume: 25.0

ID: $0.20(\mathrm{~mm})$ Length: 25

(m)

\begin{tabular}{|c|c|c|c|c|c|}
\hline COMPOUND & $\overline{\mathrm{RRF}}$ & RRF5.0 & $\begin{array}{l}\text { MIN } \\
\text { RRF }\end{array}$ & $\because \mathrm{D}$ & $\operatorname{MAX} \cong \mathrm{D}$ \\
\hline 1,2-Dichloropropane & 0.275 & 0.307 & 0.010 & 11.5 & 50.0 \\
\hline Bromodichloromethane & 0.337 & 0.362 & 0.010 & 7.5 & 50.0 \\
\hline cis-1,3-Dichloropropene & 0.349 & 0.391 & 0.010 & 12.1 & 50.0 \\
\hline 4-Methyl-2-pentanone & 0.066 & 0.067 & 0.010 & 2.4 & 50.0 \\
\hline Toluene & 1.580 & 1.763 & 0.010 & 11.6 & 50.0 \\
\hline trans-1,3-Dichloropropene & 0.239 & 0.265 & 0.010 & 11.1 & 50.0 \\
\hline $1,1,2$-Trichloroethane & 0.162 & 0.164 & 0.010 & 1.1 & 50.0 \\
\hline Tetrachloroethene & 0.394 & 0.406 & 0.010 & 3.0 & 50.0 \\
\hline 2-Hexanone & 0.045 & 0.048 & 0.010 & 5.4 & 50.0 \\
\hline Dibromochloromethane & 0.179 & 0.193 & 0.010 & 7.8 & 50.0 \\
\hline 1,2-Dibromoethane & 0.134 & 0.141 & 0.010 & 5.3 & 50.0 \\
\hline Chlorobenzene & 1.048 & 1.062 & 0.010 & 1.3 & 50.0 \\
\hline Ethylbenzene & 1.793 & 1.946 & 0.010 & 8.6 & 50.0 \\
\hline o-Xylene & 0.593 & 0.728 & 0.010 & 22.7 & 50.0 \\
\hline $\mathrm{m}, \mathrm{p}$-Xylene & 0.710 & 0.775 & 0.010 & 9.1 & 50.0 \\
\hline Styrene & 0.943 & 1.194 & 0.010 & 26.6 & 50.0 \\
\hline Bromoform & 0.144 & 0.159 & 0.010 & 9.8 & 50.0 \\
\hline Isopropylbenzene & 1.757 & 2.022 & 0.010 & 15.1 & 50.0 \\
\hline $1,1,2,2$-Tetrachloroethane & 0.144 & 0.146 & 0.010 & 1.1 & 50.0 \\
\hline 1,3-Dichlorobenzene & 1.442 & 1.326 & 0.010 & -8.0 & 50.0 \\
\hline 1,4-Dichlorobenzene & 1.651 & 1.645 & 0.010 & -0.3 & 50.0 \\
\hline 1,2-Dichlorobenzene & 1.310 & 1.370 & 0.010 & 4.6 & 50.0 \\
\hline 1,2-Dibromo-3-Chloropropane & 0.035 & 0.030 & 0.010 & -15.8 & 50.0 \\
\hline 1,2,4-Trichlorobenzene & 0.642 & 0.565 & 0.010 & $-12 \cdot 0$ & 50.0 \\
\hline $1,2,3$-Trichlorobenzene & 0.476 & 0.388 & 0.010 & -18.5 & 50.0 \\
\hline
\end{tabular}


Lab Name: TESTAMERICA BURLINGTON

Contract: $8 \mathrm{E}-00302$

Lab Code: STLV Case No.: YORK Mod. Ref No.

SDG No.: 32333

Instrument ID: CHD.i

Calibration Date: 03/08/2016 Time: 1523

Lab File Id: 018646 011.D Init. Calib. Date (s):02/26/2016 02/26/2016

EPA Sample No. (VSTD\#\#\#\#) : VSTD005TD

Heated Purge: (Y/N) N GC Column: DB-624

Init. Calib. Time(s) : 1621 1801

Purge Volume: 25.0 ID: $0.20(\mathrm{~mm})$ Length: 25

(m)

\begin{tabular}{|c|c|c|c|c|c|}
\hline COMPOUND & $\overline{\mathrm{RRF}}$ & RRF5. 0 & $\begin{array}{l}\text { MIN } \\
\text { RRF }\end{array}$ & $\circ \mathrm{D}$ & $\mathrm{MAX} \div \mathrm{D}$ \\
\hline Vinyl Chloride-d3 & 0.288 & 0.263 & 0.010 & -8.7 & 50.0 \\
\hline Chloroethane-d5 & 0.231 & 0.229 & 0.010 & -0.7 & 50.0 \\
\hline 1,1-Dichloroethene-d2 & 0.527 & 0.524 & 0.010 & -0.6 & 50.0 \\
\hline 2-Butanone-d5 & 0.021 & 0.022 & 0.010 & 2.2 & 50.0 \\
\hline Chloroform-d & 0.534 & 0.550 & 0.010 & 2.9 & 50.0 \\
\hline 1,2-Dichloroethane-d4 & 0.178 & 0.187 & 0.010 & 5.4 & 50.0 \\
\hline Benzene-d6 & 1.449 & 1.586 & 0.010 & 9.5 & 50.0 \\
\hline 1,2-Dichloropropane-d6 & 0.329 & 0.350 & 0.010 & 6.3 & 50.0 \\
\hline Toluene-d8 & 1.406 & 1.622 & 0.010 & 15.4 & 50.0 \\
\hline trans-1,3-Dichloropropene-d4 & 0.220 & 0.237 & 0.010 & 7.8 & 50.0 \\
\hline 2-Hexanone-d5 & 0.022 & 0.025 & 0.010 & 11.3 & 50.0 \\
\hline $1,1,2,2-$ Tetrachloroethane-d2 & 0.154 & 0.168 & 0.010 & 9.6 & 50.0 \\
\hline 1,2-Dichlorobenzene-d4 & 0.810 & 0.794 & 0.010 & -2.0 & 50.0 \\
\hline
\end{tabular}

Report 1,4-Dioxane-d8 for Low/Medium VOA analysis only 
Lab Name: TESTAMERICA BURLINGTON

Contract: $8 \mathrm{E}-00302$

Lab Code: STLV

Case No.: YORK Mod. Ref No.:

SDG No.: 32333

Matrix: (SOIL/SED/WATER) Water

Sample wt/vol: 25.0 $(\mathrm{g} / \mathrm{mL}) \mathrm{mL}$

Level: (TRACE/LOW/MED) TRACE

\% Moisture: not dec.

GC Column: DB-624 ID: $0.20 \quad(\mathrm{~mm})$

Soil Extract Volume: (uL) $(\mathrm{mL})$

Purge Volume: 25.0
Lab Sample ID: MB 200-101505/5

Lab File ID: $018646 \_005 . D$

Date Received:

Date Analyzed: 03/08/2016

Dilution Factor: 1.0

Soil Aliquot Volume: (uL)

\begin{tabular}{|c|c|c|c|}
\hline CAS NO. & COMPOUND & $\begin{array}{l}\text { CONCENTRATION UNITS: } \\
(\mathrm{ug} / \mathrm{L} \text { or } \mathrm{ug} / \mathrm{kg}) \mathrm{ug} / \mathrm{L}\end{array}$ & $Q$ \\
\hline $75-71-8$ & Dichlorodifluoromethane & 1.0 & $\mathrm{U}$ \\
\hline $74-87-3$ & Chloromethane & 1.0 & $\mathrm{U}$ \\
\hline $75-01-4$ & Vinyl chloride & 1.0 & $\mathrm{U}$ \\
\hline $74-83-9$ & Bromomethane & 0.12 & $\mathrm{~J}$ \\
\hline $75-00-3$ & Chloroethane & 1.0 & $\mathrm{U}$ \\
\hline $75-69-4$ & Trichlorofluoromethane & 1.0 & $\mathrm{U}$ \\
\hline $75-35-4$ & 1,1-Dichloroethene & 1.0 & $\mathrm{U}$ \\
\hline $76-13-1$ & 1,1,2-Trichloro-1,2,2-trifluoroethane & 1.0 & $\mathrm{U}$ \\
\hline $67-64-1$ & Acetone & 2.1 & $\mathrm{~J}$ \\
\hline $75-15-0$ & Carbon disulfide & 0.032 & $\mathrm{~J}$ \\
\hline $79-20-9$ & Methyl acetate & 1.0 & $\mathrm{U}$ \\
\hline $75-09-2$ & Methylene Chloride & 0.028 & $\mathrm{~J}$ \\
\hline $156-60-5$ & trans-1,2-Dichloroethene & 1.0 & $\mathrm{U}$ \\
\hline $1634-04-4$ & Methyl tert-butyl ether & 1.0 & $\mathrm{U}$ \\
\hline $75-34-3$ & 1,1-Dichloroethane & 1.0 & $\mathrm{U}$ \\
\hline $156-59-2$ & cis-1,2-Dichloroethene & 1.0 & $\mathrm{U}$ \\
\hline $78-93-3$ & 2-Butanone & 5.0 & $\mathrm{U}$ \\
\hline $74-97-5$ & Bromochloromethane & 1.0 & $\mathrm{U}$ \\
\hline $67-66-3$ & Chloroform & 1.0 & $\mathrm{U}$ \\
\hline $71-55-6$ & 1,1,1-Trichloroethane & 1.0 & $\mathrm{U}$ \\
\hline $110-82-7$ & Cyclohexane & 1.0 & $\mathrm{U}$ \\
\hline $56-23-5$ & Carbon tetrachloride & 1.0 & $\mathrm{U}$ \\
\hline $71-43-2$ & Benzene & 1.0 & $\mathrm{U}$ \\
\hline $107-06-2$ & 1,2-Dichloroethane & 1.0 & $\mathrm{U}$ \\
\hline
\end{tabular}

Report 1,4-Dioxane for Low-Medium VOA analysis only 
Lab Name: TESTAMERICA BURLINGTON

Lab Code: STLV

Case No.: YORK

Mod. Ref No.:

Contract: 8E-00302

Matrix: (SOIL/SED/WATER) Water

Sample wt/vol: 25.0 $(\mathrm{g} / \mathrm{mL}) \mathrm{mL}$

Level: (TRACE/LOW/MED) TRACE

\% Moisture: not dec.

GC Column: DB-624 ID $: \underline{0.20 \quad(\mathrm{~mm})}$

Soil Extract Volume: (uL) $(\mathrm{mL})$

Purge Volume: 25.0
SDG No.: 32333

Lab Sample ID: MB 200-101505/5

Lab File ID: 018646_005.D

Date Received:

Date Analyzed: 03/08/2016

Dilution Factor: 1.0

Soil Aliquot Volume: (uL)

\begin{tabular}{|c|c|c|c|}
\hline CAS NO. & COMPOUND & $\begin{array}{l}\text { CONCENTRATION UNITS: } \\
(\mathrm{ug} / \mathrm{L} \text { or } \mathrm{ug} / \mathrm{kg}) \mathrm{ug} / \mathrm{L}\end{array}$ & $q$ \\
\hline $79-01-6$ & Trichloroethene & 1.0 & $\mathrm{U}$ \\
\hline $108-87-2$ & Methylcyclohexane & 1.0 & $\mathrm{U}$ \\
\hline $78-87-5$ & 1,2-Dichloropropane & 1.0 & $\mathrm{U}$ \\
\hline $75-27-4$ & Bromodichloromethane & 1.0 & $\mathrm{U}$ \\
\hline $10061-01-5$ & cis-1,3-Dichloropropene & 1.0 & U \\
\hline $108-10-1$ & 4-Methyl-2-pentanone & 1.0 & $\mathrm{U}$ \\
\hline $108-88-3$ & Toluene & 0.010 & $\mathrm{~J}$ \\
\hline $10061-02-6$ & trans-1,3-Dichloropropene & 1.0 & $\mathrm{U}$ \\
\hline $79-00-5$ & $1,1,2-$ Trichloroethane & 1.0 & $\mathrm{U}$ \\
\hline $127-18-4$ & Tetrachloroethene & 1.0 & $\mathrm{U}$ \\
\hline $591-78-6$ & 2-Hexanone & 1.0 & $\mathrm{U}$ \\
\hline $124-48-1$ & Dibromochloromethane & 1.0 & $\mathrm{U}$ \\
\hline $106-93-4$ & 1,2-Dibromoethane & 1.0 & $\mathrm{U}$ \\
\hline $108-90-7$ & Chlorobenzene & 1.0 & $\mathrm{U}$ \\
\hline $100-41-4$ & Ethylbenzene & 1.0 & $\mathrm{U}$ \\
\hline $95-47-6$ & o-Xylene & 1.0 & $\mathrm{U}$ \\
\hline $179601-23-1$ & m,p-xylene & 1.0 & $\mathrm{U}$ \\
\hline $100-42-5$ & Styrene & 1.0 & $\mathrm{U}$ \\
\hline $75-25-2$ & Bromoform & 1.0 & $\mathrm{U}$ \\
\hline $98-82-8$ & Isopropylbenzene & 1.0 & $\mathrm{U}$ \\
\hline $79-34-5$ & $1,1,2,2$-Tetrachloroethane & 1.0 & $\mathrm{U}$ \\
\hline $541-73-1$ & 1,3 -Dichlorobenzene & 1.0 & $\mathrm{U}$ \\
\hline $106-46-7$ & 1,4-Dichlorobenzene & 1.0 & $\mathrm{U}$ \\
\hline $95-50-1$ & 1,2-Dichlorobenzene & 1.0 & $\mathrm{U}$ \\
\hline $96-12-8$ & 1,2-Dibromo-3-Chloropropane & 1.0 & $\mathrm{U}$ \\
\hline $120-82-1$ & $1,2,4$-Trichlorobenzene & 0.038 & $\mathrm{~J}$ \\
\hline $87-61-6$ & $1,2,3$-Trichlorobenzene & 0.057 & $\mathrm{~J}$ \\
\hline
\end{tabular}


Lab Name: TESTAMERICA BURLINGTON

Contract: $8 \mathrm{E}-00302$

Lab Code: STLV Case No.: YORK Mod. Ref No.: SDG No.: 32333

Matrix: (SOIL/SED/WATER) Water

Sample wt/vol: 25.0 $(\mathrm{g} / \mathrm{mL}) \mathrm{mL}$

Level: (TRACE or LOW/MED) TRACE

Moisture: not dec.

GC Column: DB-624 ID: $0.20 \quad(\mathrm{~mm})$

Soil Extract Volume: (uL)

CONCENTRATION UNITS: (ug/L or ug/kg) ug/L
Lab Sample ID: MB 200-101505/5

Lab File ID: 018646 005.D

Date Received:

Date Analyzed: 03/08/2016

Dilution Factor: 1.0

Soil Aliquot Volume: (uL) Purge Volume: 25.0 $(\mathrm{mL})$

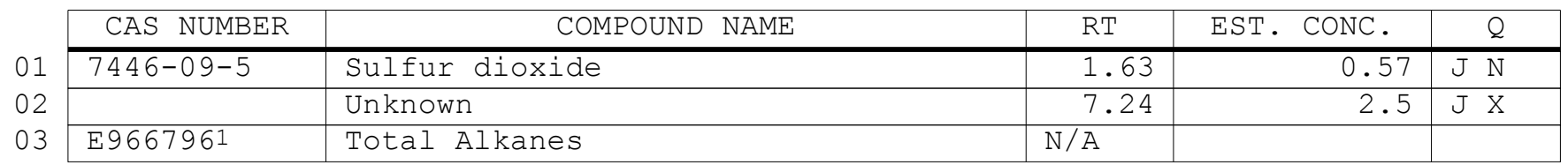

1 EPA-designated Registry Number. 
Lab Name: TESTAMERICA BURLINGTON

Contract: 8E-00302

Lab Code: STLV

Case No.: YORK Mod. Ref No.:

SDG No.: 32333

Matrix: (SOIL/SED/WATER) Water

Sample wt/vol: 25.0 $(\mathrm{g} / \mathrm{mL}) \mathrm{mL}$

Level: (TRACE/LOW/MED) TRACE

\% Moisture: not dec.

GC Column: DB-624 ID: $0.20 \quad(\mathrm{~mm})$

Soil Extract Volume: (uL) $(\mathrm{mL})$

Purge Volume: 25.0
Lab Sample ID: 200-32333-3

Lab File ID: 018646 _010.D

Date Received:

Date Analyzed: 03/08/2016

Dilution Factor: 1.0

Soil Aliquot Volume:

(uL)

\begin{tabular}{|c|c|c|c|}
\hline CAS NO. & COMPOUND & $\begin{array}{l}\text { CONCENTRATION UNITS: } \\
(\mathrm{ug} / \mathrm{L} \text { or } \mathrm{ug} / \mathrm{kg}) \mathrm{ug} / \mathrm{L}\end{array}$ & Q \\
\hline $75-71-8$ & Dichlorodifluoromethane & 1.0 & $\mathrm{U}$ \\
\hline $74-87-3$ & Chloromethane & 0.035 & $\mathrm{~J}$ \\
\hline $75-01-4$ & Vinyl chloride & 1.0 & $\mathrm{U}$ \\
\hline $74-83-9$ & Bromomethane & 0.095 & $\mathrm{~J} B$ \\
\hline $75-00-3$ & Chloroethane & 1.0 & $\mathrm{U}$ \\
\hline $75-69-4$ & Trichlorofluoromethane & 1.0 & $\mathrm{U}$ \\
\hline $75-35-4$ & 1,1-Dichloroethene & 1.0 & $\mathrm{U}$ \\
\hline $76-13-1$ & 1,1,2-Trichloro-1,2,2-trifluoroethane & 1.0 & $\mathrm{U}$ \\
\hline $67-64-1$ & Acetone & 1.3 & $\mathrm{~J} B$ \\
\hline $75-15-0$ & Carbon disulfide & 1.0 & $\mathrm{U}$ \\
\hline $79-20-9$ & Methyl acetate & 1.0 & $\mathrm{U}$ \\
\hline $75-09-2$ & Methylene Chloride & 0.033 & $\mathrm{~J} B$ \\
\hline $156-60-5$ & trans-1,2-Dichloroethene & 1.0 & $\mathrm{U}$ \\
\hline $1634-04-4$ & Methyl tert-butyl ether & 1.0 & $\mathrm{U}$ \\
\hline $75-34-3$ & 1,1-Dichloroethane & 1.0 & $\mathrm{U}$ \\
\hline $156-59-2$ & cis-1,2-Dichloroethene & 1.0 & $\mathrm{U}$ \\
\hline $78-93-3$ & 2-Butanone & 5.0 & $\mathrm{U}$ \\
\hline $74-97-5$ & Bromochloromethane & 1.0 & $\mathrm{U}$ \\
\hline $67-66-3$ & Chloroform & 1.0 & $\mathrm{U}$ \\
\hline $71-55-6$ & $1,1,1$-Trichloroethane & 1.0 & $\mathrm{U}$ \\
\hline $110-82-7$ & Cyclohexane & 1.0 & $\mathrm{U}$ \\
\hline $56-23-5$ & Carbon tetrachloride & 1.0 & $\mathrm{U}$ \\
\hline $71-43-2$ & Benzene & 1.0 & $\mathrm{U}$ \\
\hline $107-06-2$ & 1,2-Dichloroethane & 1.0 & $\mathrm{U}$ \\
\hline
\end{tabular}

Report 1,4-Dioxane for Low-Medium VOA analysis only 
Lab Name: TESTAMERICA BURLINGTON

Lab Code: STLV

Case No.: YORK

Mod. Ref No.:

Contract: $8 \mathrm{E}-00302$

Matrix: (SOIL/SED/WATER) Water

Sample wt/vol: 25.0 $(\mathrm{g} / \mathrm{mL}) \mathrm{mL}$

Level: (TRACE/LOW/MED) TRACE

\% Moisture: not dec.

GC Column: DB-624 ID: $0.20 \quad(\mathrm{~mm})$

Soil Extract Volume: (uL) $(\mathrm{mL})$

Purge Volume: 25.0 SDG No.: 32333

Lab Sample ID: 200-32333-3

Lab File ID: 018646_010.D

Date Received:

Date Analyzed: 03/08/2016

Dilution Factor: 1.0

Soil Aliquot Volume: (uL)

\begin{tabular}{|c|c|c|c|}
\hline CAS NO. & COMPOUND & $\begin{array}{l}\text { CONCENTRATION UNITS: } \\
(\mathrm{ug} / \mathrm{L} \text { or } \mathrm{ug} / \mathrm{kg}) \mathrm{ug} / \mathrm{L}\end{array}$ & Q \\
\hline $79-01-6$ & Trichloroethene & 1.0 & $\mathrm{U}$ \\
\hline $108-87-2$ & Methylcyclohexane & 1.0 & $\mathrm{U}$ \\
\hline $78-87-5$ & 1,2-Dichloropropane & 1.0 & $\mathrm{U}$ \\
\hline $75-27-4$ & Bromodichloromethane & 1.0 & $\mathrm{U}$ \\
\hline $10061-01-5$ & cis-1,3-Dichloropropene & 1.0 & $\mathrm{U}$ \\
\hline $108-10-1$ & 4-Methyl-2-pentanone & 1.0 & $\mathrm{U}$ \\
\hline $108-88-3$ & Toluene & 0.0096 & $\mathrm{~J} B$ \\
\hline $10061-02-6$ & trans-1,3-Dichloropropene & 1.0 & $\mathrm{U}$ \\
\hline $79-00-5$ & $1,1,2-T r i c h l o r o e t h a n e$ & 1.0 & $\mathrm{U}$ \\
\hline $127-18-4$ & Tetrachloroethene & 1.0 & $\mathrm{U}$ \\
\hline $591-78-6$ & 2-Hexanone & 1.0 & $\mathrm{U}$ \\
\hline $124-48-1$ & Dibromochloromethane & 1.0 & $\mathrm{U}$ \\
\hline $106-93-4$ & 1,2-Dibromoethane & 1.0 & $\mathrm{U}$ \\
\hline $108-90-7$ & Chlorobenzene & 1.0 & $\mathrm{U}$ \\
\hline $100-41-4$ & Ethylbenzene & 1.0 & $\mathrm{U}$ \\
\hline $95-47-6$ & o-Xylene & 1.0 & $\mathrm{U}$ \\
\hline $179601-23-1$ & m,p-Xylene & 0.0048 & $\mathrm{~J}$ \\
\hline $100-42-5$ & styrene & 1.0 & $\mathrm{U}$ \\
\hline $75-25-2$ & Bromoform & 1.0 & $\mathrm{U}$ \\
\hline $98-82-8$ & Isopropylbenzene & 1.0 & $\mathrm{U}$ \\
\hline $79-34-5$ & $1,1,2,2$-Tetrachloroethane & 1.0 & $\mathrm{U}$ \\
\hline $541-73-1$ & 1,3-Dichlorobenzene & 1.0 & $\mathrm{U}$ \\
\hline $106-46-7$ & 1,4-Dichlorobenzene & 1.0 & $\mathrm{U}$ \\
\hline $95-50-1$ & $1,2-$ Dichlorobenzene & 1.0 & $\mathrm{U}$ \\
\hline $96-12-8$ & 1,2-Dibromo-3-Chloropropane & 1.0 & $\mathrm{U}$ \\
\hline $120-82-1$ & $1,2,4$-Trichlorobenzene & 1.0 & $\mathrm{U}$ \\
\hline $87-61-6$ & $1,2,3$-Trichlorobenzene & 1.0 & $\mathrm{U}$ \\
\hline
\end{tabular}


Lab Name: TESTAMERICA BURLINGTON

Contract: $8 \mathrm{E}-00302$

Lab Code: STLV Case No.: YORK Mod. Ref No.: SDG No.: 32333

Matrix: (SOIL/SED/WATER) Water

Sample wt/vol: 25.0 $(\mathrm{g} / \mathrm{mL}) \mathrm{mL}$

Level: (TRACE or LOW/MED) TRACE

Moisture: not dec.

GC Column: DB-624 ID $: 0.20 \quad(\mathrm{~mm})$

Soil Extract Volume: (uL)

CONCENTRATION UNITS: (ug/L or ug/kg) ug/L
Lab Sample ID: 200-32333-3

Lab File ID: 018646_010.D

Date Received:

Date Analyzed: 03/08/2016

Dilution Factor: 1.0

Soil Aliquot Volume: (uL) Purge Volume: 25.0 $(\mathrm{mL})$

01

02

\begin{tabular}{|l|l|c|c|c|}
\hline CAS NUMBER & \multicolumn{1}{|c|}{ COMPOUND NAME } & RT & EST. CONC. & Q \\
\hline & Unknown & 7.24 & 2.3 & $\mathrm{~J}$ B X \\
\hline E9667961 & Total Alkanes & N/A & & \\
\hline
\end{tabular}

1EPA-designated Registry Number. 
Lab Name: TestAmerica Burlington

Job No.: 200-32333-1

SDG No.: 32333

Instrument ID: CHD.i

Analysis Batch Number: 101161

Lab Sample ID: IC 200-101161/7

Client Sample ID:

Date Analyzed: 02/26/16 16:46

Lab File ID: 018468_007.D

GC Column: DB-624

ID : $0.2(\mathrm{~mm})$

\begin{tabular}{|l|c|c|c|c|}
\hline \multicolumn{1}{|c|}{ COMPOUND NAME } & RETENTION & \multicolumn{2}{|c|}{ MANUAL INTEGRATION } \\
\cline { 3 - 5 } & TIME & REASON & ANALYST & DATE \\
\hline Methyl acetate & 3.12 & Baseline & wilburj & $02 / 29 / 16$ 09:54 \\
\hline
\end{tabular}

Lab Sample ID: ICIS 200-101161/8

Client Sample ID:

Date Analyzed: 02/26/16 17:11

Lab File ID: 018468 008.D

GC Column: DB-624

ID $: 0.2(\mathrm{~mm})$

\begin{tabular}{|c|c|c|c|c|}
\hline \multicolumn{1}{|c|}{ COMPOUND NAME } & RETENTION & \multicolumn{2}{|c|}{ MANUAL INTEGRATION } \\
\cline { 3 - 5 } & TIME & REASON & ANALYST & DATE \\
\hline 1,2-Dichloropropane-d6 & 6.36 & Split Peak & wilburj & 02/29/16 09:55 \\
\hline
\end{tabular}

Lab Sample ID: IC 200-101161/9 Client Sample ID:

Date Analyzed: 02/26/16 17:36

Lab File ID: $018468 \_009 . D$

GC Column: DB-624

ID $: 0.2(\mathrm{~mm})$

\begin{tabular}{|c|c|c|c|c|}
\hline \multirow[t]{2}{*}{ COMPOUND NAME } & \multirow{2}{*}{$\begin{array}{c}\text { RETENTION } \\
\text { TIME }\end{array}$} & \multicolumn{3}{|c|}{ MANUAL INTEGRATION } \\
\hline & & REASON & ANALYST & DATE \\
\hline 1,2-Dichloropropane-d6 & 6.36 & Split Peak & wilburj & $02 / 29 / 1609: 56$ \\
\hline
\end{tabular}

Lab Sample ID: IC 200-101161/10

Client Sample ID:

Date Analyzed: 02/26/16 18:01

Lab File ID: 018468 010.D

GC Column: DB-624

ID $: 0.2(\mathrm{~mm})$

\begin{tabular}{|l|c|c|c|c|}
\hline \multicolumn{1}{|c|}{ COMPOUND NAME } & RETENTION & \multicolumn{2}{|c|}{ MANUAL INTEGRATION } \\
\cline { 5 - 5 } & TIME & REASON & ANALYST & DATE \\
\hline Methyl acetate & 3.11 & Baseline & wilburj & $02 / 29 / 16$ 09:56 \\
\hline
\end{tabular}


Lab Name: TestAmerica Burlington

SDG No.: 32333

Instrument ID: CHD.i

Lab Sample ID: CCVC 200-101505/11

Date Analyzed: 03/08/16 15:23

\begin{tabular}{|c|c|c|c|c|}
\hline \multirow{2}{*}{ COMPOUND NAME } & RETENTION & \multicolumn{3}{|c}{ MANUAL INTEGRATION } \\
\cline { 3 - 5 } & TIME & REASON & ANALYST & DATE \\
\hline \hline 1,2-Dichloropropane-d6 & 6.36 & Split Peak & wilburj & $03 / 08 / 1616: 02$ \\
\hline
\end{tabular}

Job No.: 200-32333-1

Analysis Batch Number: 101505

Client Sample ID:

Lab File ID: $018646011 . \mathrm{D}$

GC Column: DB-624

ID $: 0.2(\mathrm{~mm})$ 


\section{TestAmerica}

THE LEADER IN ENVIRONMENTAL TESTING

\section{ANALYTICAL REPORT}

Job Number: 200-33767-1

SDG Number: 33767

Job Description: York (200-33767)

Contract Number: 1E-30401

For:

Argonne National Laboratory

9700 South Cass Avenue

Building 203

Office B-141

Argonne, IL 60439

Attention: Ms. Esther Bowen

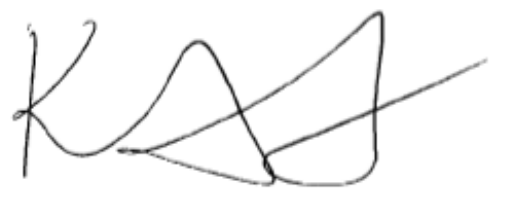

Approved for release. Kathryn A Kelly

Kathryn A Kelly, Project Manager I

30 Community Drive, South Burlington, VT, 05403

kathryn.kelly@testamericainc.com

$06 / 15 / 2016$

The test results in this report relate only to sample(s) as received by the laboratory. These test results were derived under a quality system that adheres to the requirements of NELAC. Pursuant to NELAC, this report may not be produced in full without written approval from the laboratory 


\section{Table of Contents}

Cover Title Page..$\ldots \ldots \ldots \ldots \ldots \ldots \ldots \ldots \ldots \ldots \ldots \ldots \ldots \ldots$

Sample List ........................... 4

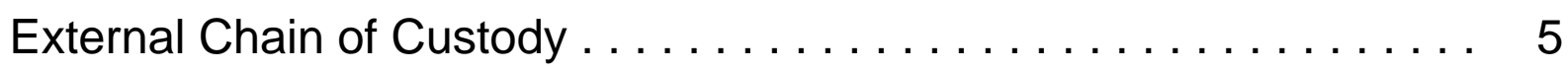

Shipping Documentation . . . . . . . . . . . . . . . 7

Sample Receipt and Log In Check List ..................... 8

Standards Traceability . . . . . . . . . . . . . . . . . 9

Methodology Review .............................. 14

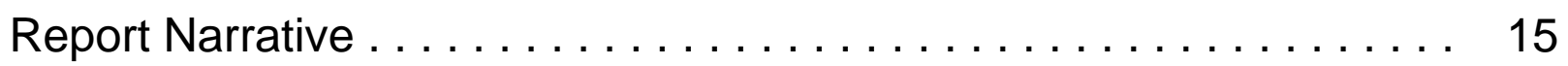

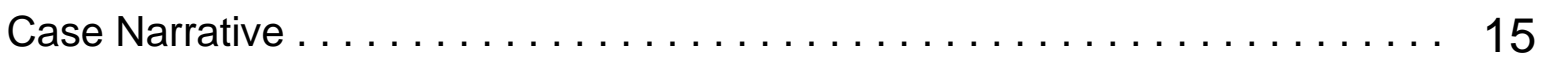

Manual Integration Documentation ....................... 16

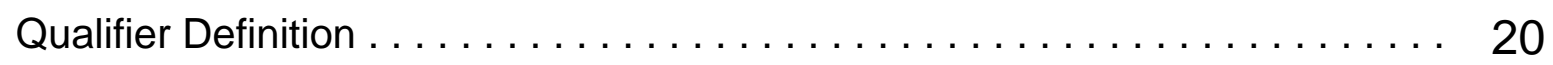

QC Summary - SOM01.2 Volatiles-Trace . . . . . . . . . . . . 21

QC Summary - SOM01.2 Volatiles-Trace $\ldots \ldots \ldots \ldots \ldots \ldots \ldots \ldots \ldots .21$

Deuterated Monitoring Compound Summary $\ldots \ldots \ldots \ldots \ldots \ldots \ldots \ldots \ldots \ldots, 21$

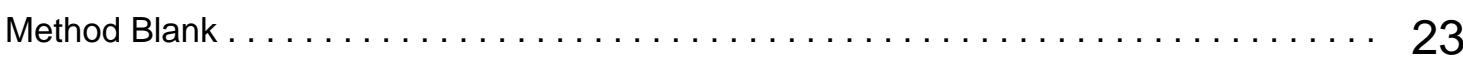

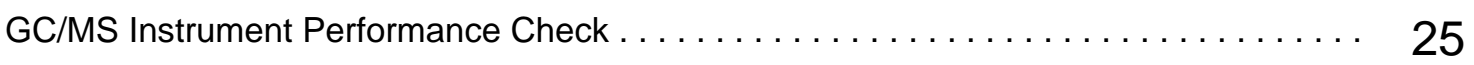

Internal Standard Area and RT Summary $\ldots \ldots \ldots \ldots \ldots \ldots \ldots \ldots \ldots \ldots \ldots \ldots \ldots \ldots \ldots$

Sample Data - SOM01.2 Volatiles-Trace .............. 30

Sample Data - SOM01.2 Volatiles-Trace ..................... 30

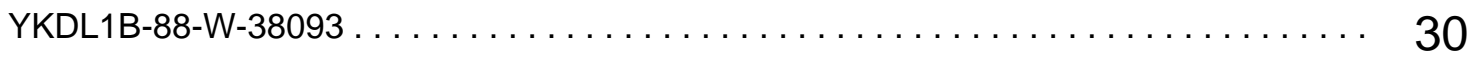

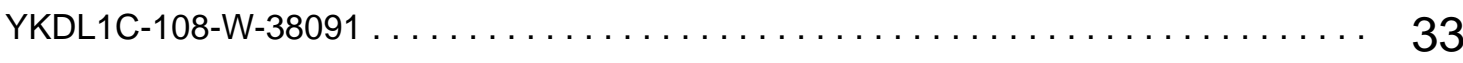

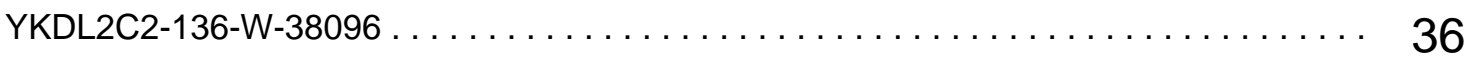

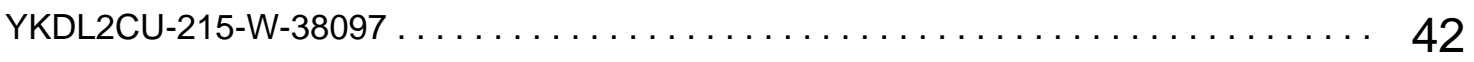

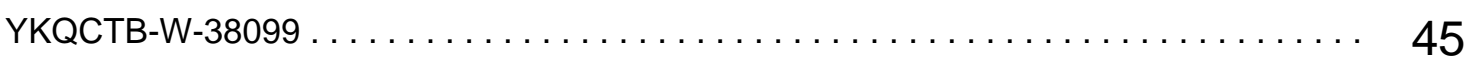

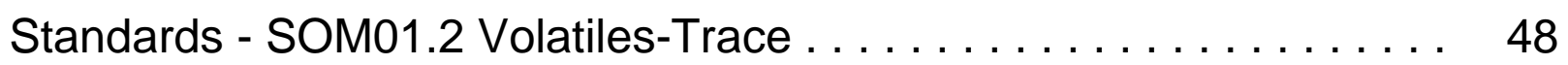

Standards - SOM01.2 Volatiles-Trace ..................... 48 


\section{Table of Contents}

Initial Calibration Data $\ldots \ldots \ldots \ldots \ldots \ldots \ldots \ldots \ldots \ldots \ldots \ldots \ldots \ldots \ldots \ldots \ldots$

CCV Data, including closing CCV $\ldots \ldots \ldots \ldots \ldots \ldots \ldots \ldots \ldots \ldots \ldots \ldots \ldots \ldots \ldots \ldots \ldots \ldots$

Raw Qc Data - SOM01.2 Volatiles-Trace . . . . . . . . . . . . 63

Raw Qc Data - SOM01.2 Volatiles-Trace ..................... 63

Raw Qc Data - SOM01.2 Volatiles-Trace $\ldots \ldots \ldots \ldots \ldots \ldots \ldots \ldots \ldots \ldots \ldots \ldots \ldots$

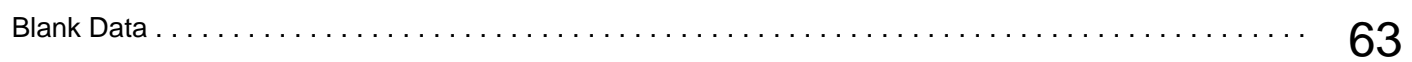




\section{Sample Login Acknowledgement}

Job 200-33767-1

\begin{tabular}{|c|c|c|c|}
\hline Client Job Description: & York (200-33767) & Report To: & Argonne National Laboratory \\
\hline Purchase Order \#: & $1 \mathrm{E}-30401$ & & Jorge Alvarado \\
\hline Work Order \#: & $1 \mathrm{E}-30401$ & & 9700 South Cass Avenue \\
\hline Project Manager: & Kathryn A Kelly & & Building 203 \\
\hline Job Due Date: & $6 / 15 / 2016$ & & Office B-141 \\
\hline . & 011012010 & & Argonne, IL 60439 \\
\hline Job TAT: & 14 Days & & \\
\hline \multirow[t]{2}{*}{ Max Deliverable Level: } & IV & Bill To: & Argonne National Laboratory \\
\hline & & & Accounts Payable \\
\hline Earliest Deliverable Due: & $6 / 15 / 2016$ & & $\begin{array}{l}\text { Chief Financial Offices } \\
9700 \text { S. Cass Ave. } \\
\text { Building } 201 \\
\text { Argonne, IL } 60439\end{array}$ \\
\hline
\end{tabular}

Login 200-33767

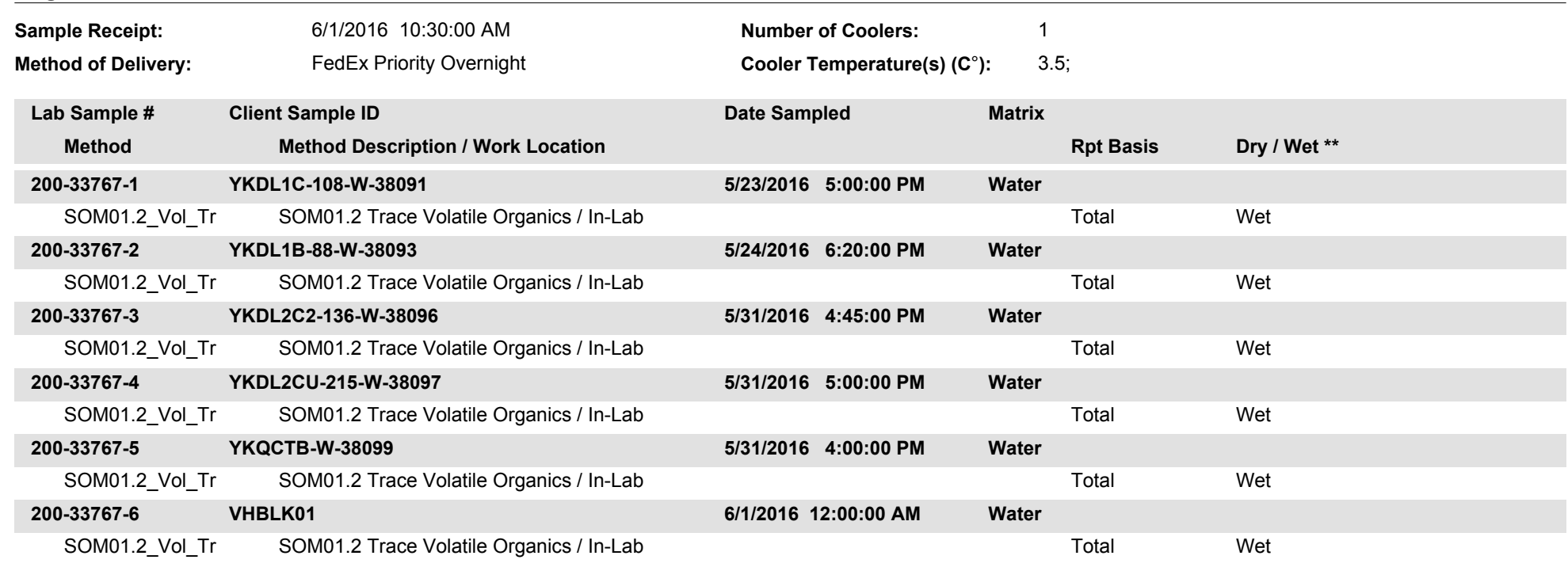




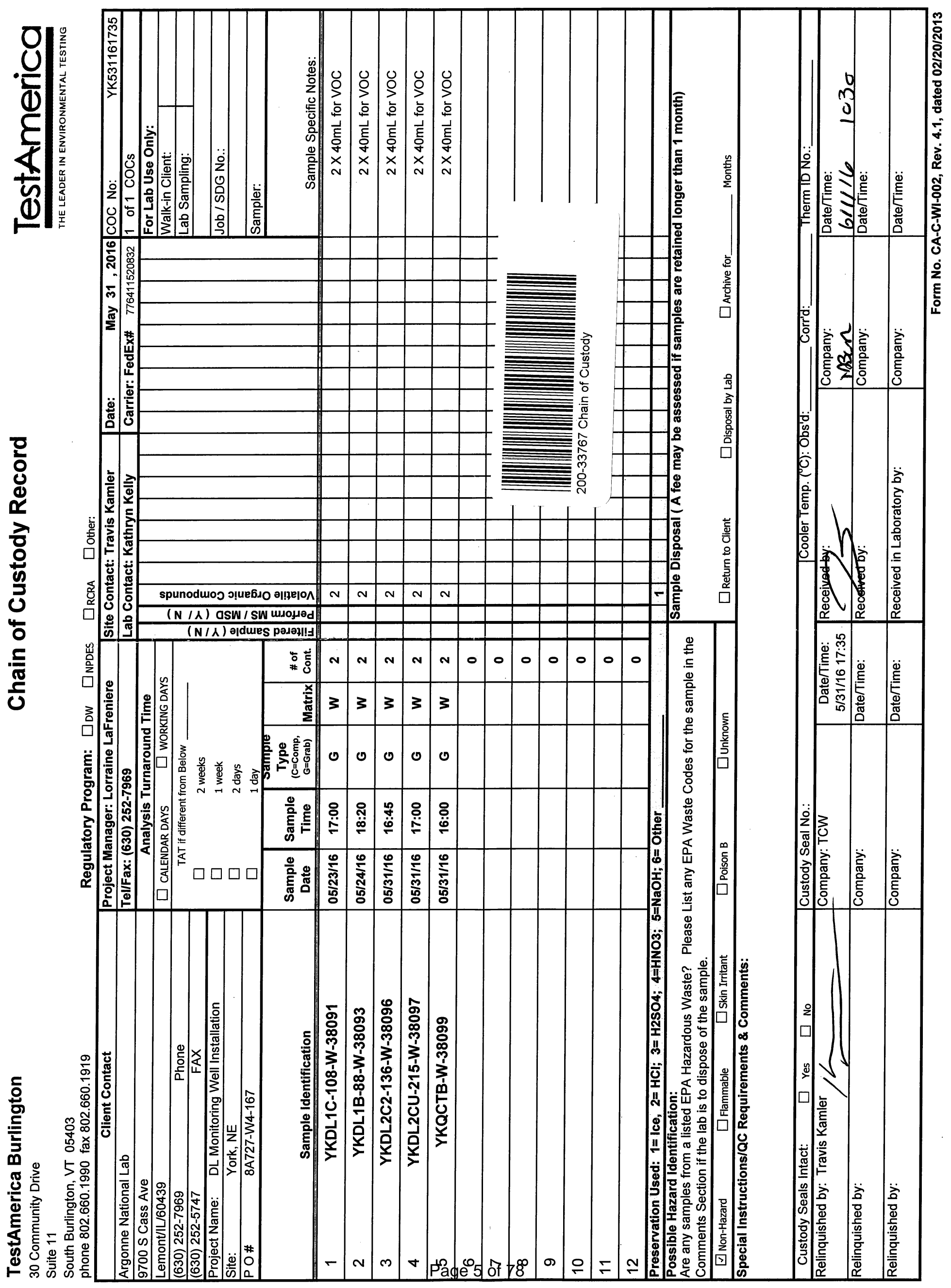




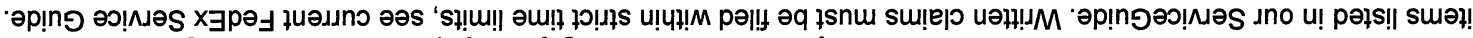

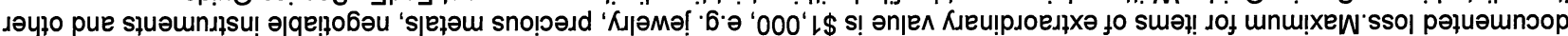

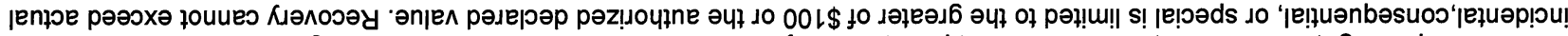

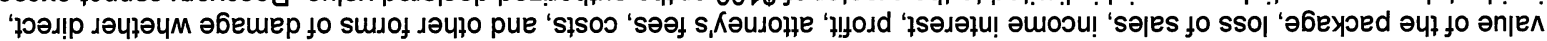

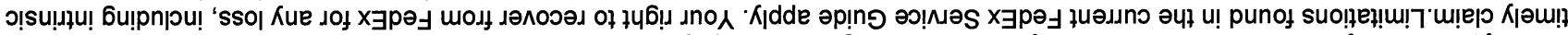

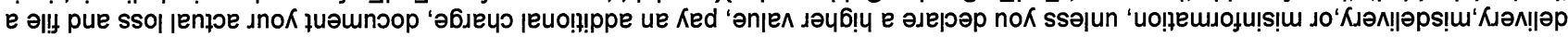

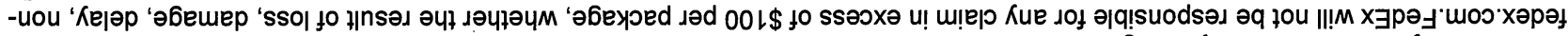
u० ә|qе|!

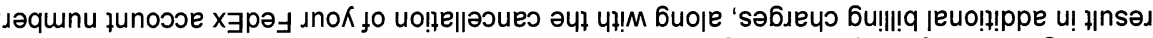

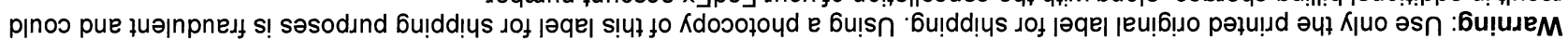

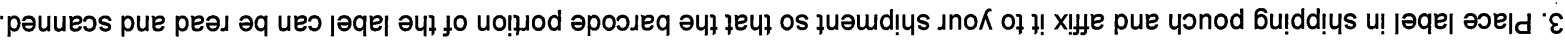

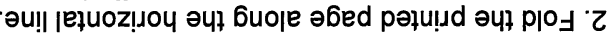

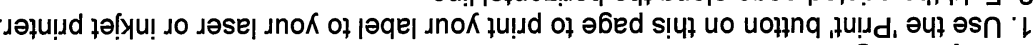

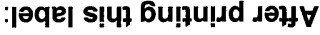

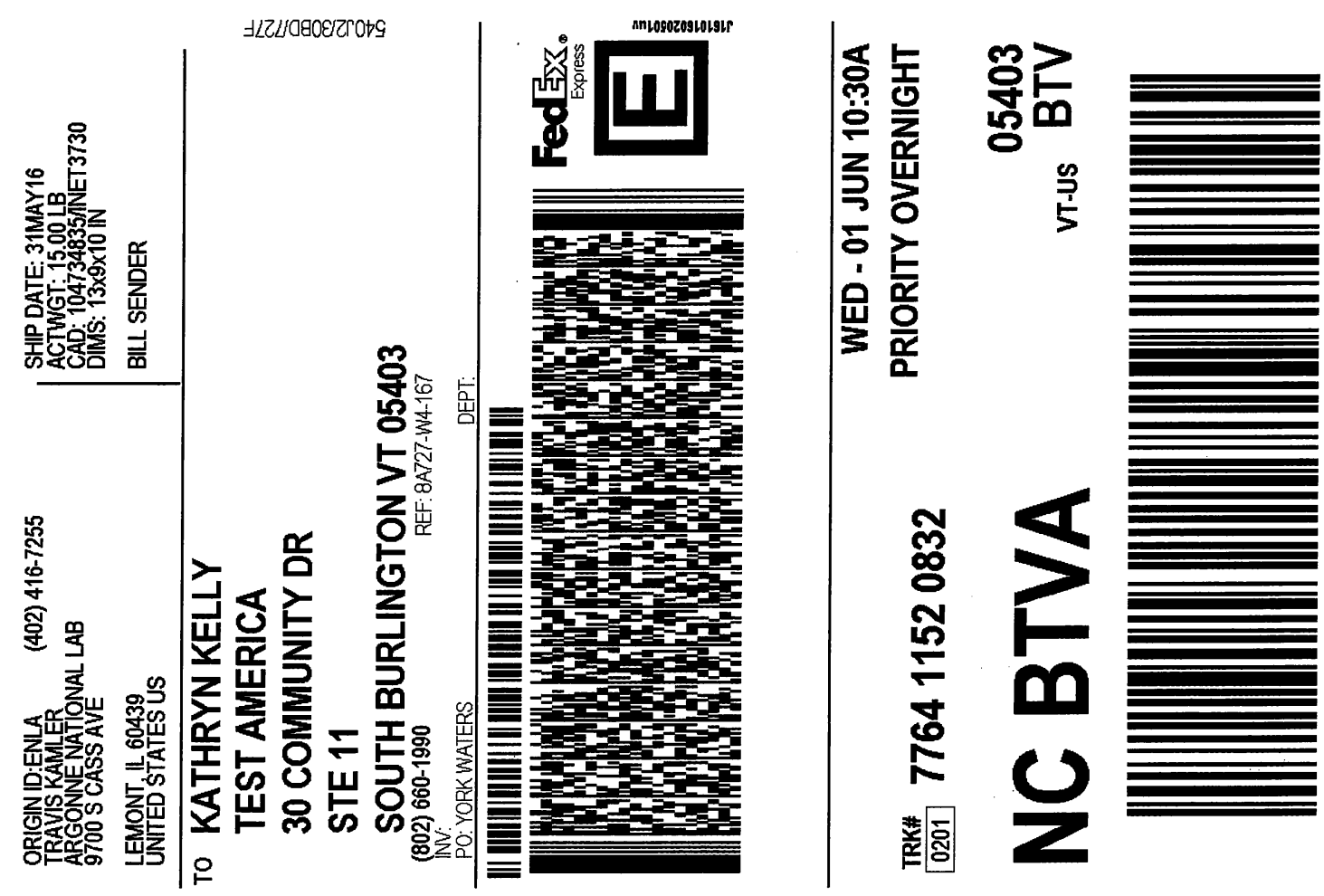




\section{Shipping and Receiving Documents}




\section{Login Sample Receipt Checklist}

Client: Argonne National Laboratory

Job Number: 200-33767-1

SDG Number: 33767

Login Number: 33767

List Source: TestAmerica Burlington

List Number: 1

Creator: Atherton, Joel E

Question

Radioactivity wasn't checked or is $</=$ background as measured by a survey meter.

The cooler's custody seal, if present, is intact.

Sample custody seals, if present, are intact.

The cooler or samples do not appear to have been compromised or tampered with.

Samples were received on ice.

Cooler Temperature is acceptable.

Cooler Temperature is recorded.

COC is present.

COC is filled out in ink and legible.

$\mathrm{COC}$ is filled out with all pertinent information.

Is the Field Sampler's name present on COC?

There are no discrepancies between the containers received and the COC.

Samples are received within Holding Time (excluding tests with immediate

HTs)

Sample containers have legible labels.

Containers are not broken or leaking.

Sample collection date/times are provided.

Appropriate sample containers are used.

Sample bottles are completely filled.

Sample Preservation Verified.

There is sufficient vol. for all requested analyses, incl. any requested MS/MSDs

Containers requiring zero headspace have no headspace or bubble is $<6 \mathrm{~mm}$ (1/4").

Multiphasic samples are not present.
Answer

True

True

True

True

True

True

True

True

True

True

True

True

False

True

True

True

True

N/A

True

True

True

True

True

N/A
Samples do not require splitting or compositing.

Residual Chlorine Checked.

\section{Comment}

Lab does not accept radioactive samples.

Seal present with no number.

$3.5^{\circ} \mathrm{C}$

Refer to Job Narrative for details. 
SDG No.: 33767

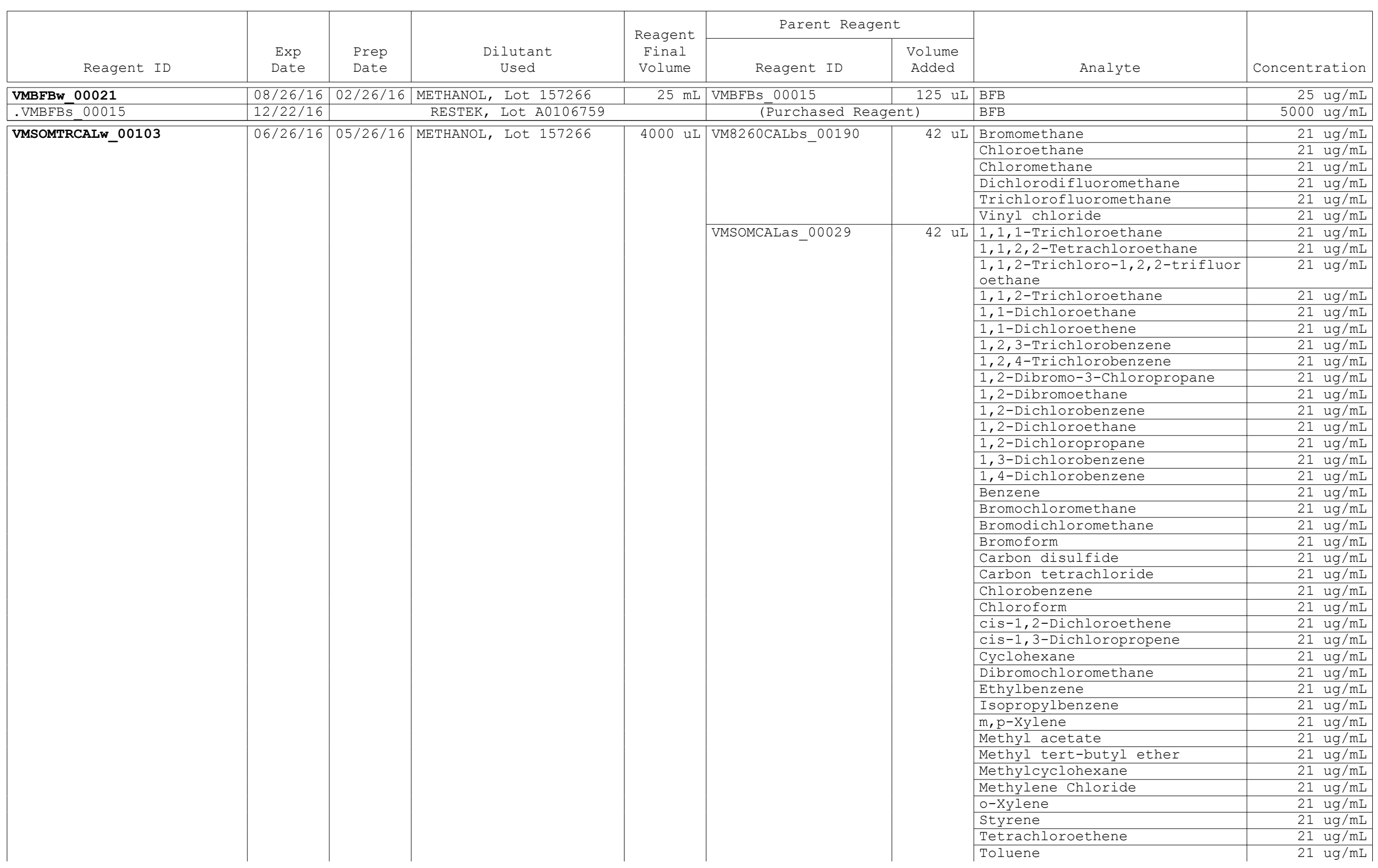


SDG No.: 33767

\begin{tabular}{|c|c|c|c|c|c|c|c|c|}
\hline \multirow[b]{2}{*}{ Reagent ID } & \multirow[b]{2}{*}{$\begin{array}{l}\text { Exp } \\
\text { Date }\end{array}$} & \multirow[b]{2}{*}{$\begin{array}{l}\text { Prep } \\
\text { Date }\end{array}$} & \multirow[b]{2}{*}{$\begin{array}{l}\text { Dilutant } \\
\text { Used }\end{array}$} & \multirow{2}{*}{$\begin{array}{c}\text { Reagent } \\
\text { Final } \\
\text { Volume }\end{array}$} & \multicolumn{2}{|l|}{ Parent Reagent } & \multirow[b]{2}{*}{ Analyte } & \multirow[b]{2}{*}{ Concentration } \\
\hline & & & & & \multirow[t]{4}{*}{ Reagent ID } & $\begin{array}{l}\text { Volume } \\
\text { Added }\end{array}$ & & \\
\hline & & & & & & & trans-1,2-Dichloroethene & $21 \mathrm{ug} / \mathrm{mL}$ \\
\hline & & & & & & & trans-1,3-Dichloropropene & $21 \mathrm{ug} / \mathrm{mL}$ \\
\hline & & & & & & & Trichloroethene & $21 \mathrm{ug} / \mathrm{mL}$ \\
\hline & & & & & VMSOMCALbS_00035 & $168 \mathrm{uL}$ & 2-Butanone & $210 \mathrm{ug} / \mathrm{mL}$ \\
\hline & & & & & & & 2-Hexanone & $210 \mathrm{ug} / \mathrm{mL}$ \\
\hline & & & & & & & 4-Methyl-2-pentanone & $210 \mathrm{ug} / \mathrm{mL}$ \\
\hline & & & & & & & Acetone & $210 \mathrm{ug} / \mathrm{mL}$ \\
\hline & & & & & VMSOMSUas_00085 & 42 uL & Chloroethane-d5 & $21 \mathrm{ug} / \mathrm{mL}$ \\
\hline & & & & & & & Vinyl Chloride-d3 & $21 \mathrm{ug} / \mathrm{mL}$ \\
\hline & & & & & VMSOMSUbs_00147 & $336 \mathrm{uL}$ & 2-Butanone-d5 & $210 \mathrm{ug} / \mathrm{mL}$ \\
\hline & & & & & & & 2-Hexanone-d5 & $210 \mathrm{ug} / \mathrm{mL}$ \\
\hline & & & & & VMSOMSUCS_00059 & 42 uL & $1,1,2,2$-Tetrachloroethane-d2 & $21 \mathrm{ug} / \mathrm{mL}$ \\
\hline & & & & & & & 1,1-Dichloroethene-d2 & $21 \mathrm{ug} / \mathrm{mL}$ \\
\hline & & & & & & & 1,2-Dichlorobenzene-d4 & $21 \mathrm{ug} / \mathrm{mL}$ \\
\hline & & & & & & & 1,2-Dichloroethane-d4 & $21 \mathrm{ug} / \mathrm{mL}$ \\
\hline & & & & & & & 1,2-Dichloropropane-d6 & $21 \mathrm{ug} / \mathrm{mL}$ \\
\hline & & & & & & & Benzene-d6 & $21 \mathrm{ug} / \mathrm{mL}$ \\
\hline & & & & & & & Chloroform-d & $21 \mathrm{ug} / \mathrm{mL}$ \\
\hline & & & & & & & Toluene-d8 & $21 \mathrm{ug} / \mathrm{mL}$ \\
\hline & & & & & & & trans-1,3-Dichloropropene-d4 & $21 \mathrm{ug} / \mathrm{mL}$ \\
\hline \multirow{6}{*}{.VM8260CALbs_00190 } & \multirow{6}{*}{$06 / 26 / 16$} & \multirow{6}{*}{\multicolumn{3}{|c|}{ RESTEK, Lot A0115510 }} & \multirow{6}{*}{\multicolumn{2}{|c|}{ (Purchased Reagent) }} & Bromomethane & $2000 \mathrm{ug} / \mathrm{mL}$ \\
\hline & & & & & & & Chloroethane & $2000 \mathrm{ug} / \mathrm{mL}$ \\
\hline & & & & & & & Chloromethane & $2000 \mathrm{ug} / \mathrm{mL}$ \\
\hline & & & & & & & Dichlorodifluoromethane & $2000 \mathrm{ug} / \mathrm{mL}$ \\
\hline & & & & & & & Trichlorofluoromethane & $2000 \mathrm{ug} / \mathrm{mL}$ \\
\hline & & & & & & & Vinyl chloride & $2000 \mathrm{ug} / \mathrm{mL}$ \\
\hline \multirow{21}{*}{.VMSOMCALas_00029 } & \multirow[t]{21}{*}{$04 / 30 / 17$} & \multirow{21}{*}{\multicolumn{2}{|c|}{ Restek, Lot A0102833 }} & & \multirow{21}{*}{\multicolumn{2}{|c|}{ (Purchased Reagent) }} & $1,1,1$-Trichloroethane & $2000 \mathrm{ug} / \mathrm{mL}$ \\
\hline & & & & & & & 1,1,2,2-Tetrachloroethane & $2000 \mathrm{ug} / \mathrm{mL}$ \\
\hline & & & & & & & $\begin{array}{l}\text { 1,1,2-Trichloro-1,2,2-trifluor } \\
\text { oethane }\end{array}$ & $2000 \mathrm{ug} / \mathrm{mL}$ \\
\hline & & & & & & & $1,1,2$-Trichloroethane & $2000 \mathrm{ug} / \mathrm{mL}$ \\
\hline & & & & & & & 1,1-Dichloroethane & $2000 \mathrm{ug} / \mathrm{mL}$ \\
\hline & & & & & & & 1,1-Dichloroethene & $2000 \mathrm{ug} / \mathrm{mL}$ \\
\hline & & & & & & & 1,2,3-Trichlorobenzene & $2000 \mathrm{ug} / \mathrm{mL}$ \\
\hline & & & & & & & 1,2,4-Trichlorobenzene & $2000 \mathrm{ug} / \mathrm{mL}$ \\
\hline & & & & & & & 1,2-Dibromo-3-Chloropropane & $2000 \mathrm{ug} / \mathrm{mL}$ \\
\hline & & & & & & & 1,2-Dibromoethane & $2000 \mathrm{ug} / \mathrm{mL}$ \\
\hline & & & & & & & 1,2-Dichlorobenzene & $2000 \mathrm{ug} / \mathrm{mL}$ \\
\hline & & & & & & & 1,2-Dichloroethane & $2000 \mathrm{ug} / \mathrm{mL}$ \\
\hline & & & & & & & 1,2-Dichloropropane & $2000 \mathrm{ug} / \mathrm{mL}$ \\
\hline & & & & & & & 1,3-Dichlorobenzene & $2000 \mathrm{ug} / \mathrm{mL}$ \\
\hline & & & & & & & 1,4-Dichlorobenzene & $2000 \mathrm{ug} / \mathrm{mL}$ \\
\hline & & & & & & & Benzene & $2000 \mathrm{ug} / \mathrm{mL}$ \\
\hline & & & & & & & Bromochloromethane & $2000 \mathrm{ug} / \mathrm{mL}$ \\
\hline & & & & & & & Bromodichloromethane & $2000 \mathrm{ug} / \mathrm{mL}$ \\
\hline & & & & & & & Bromoform & $2000 \mathrm{ug} / \mathrm{mL}$ \\
\hline & & & & & & & Carbon disulfide & $2000 \mathrm{ug} / \mathrm{mL}$ \\
\hline & & & & & & & Carbon tetrachloride & $2000 \mathrm{ug} / \mathrm{mL}$ \\
\hline
\end{tabular}


SDG No.: 33767

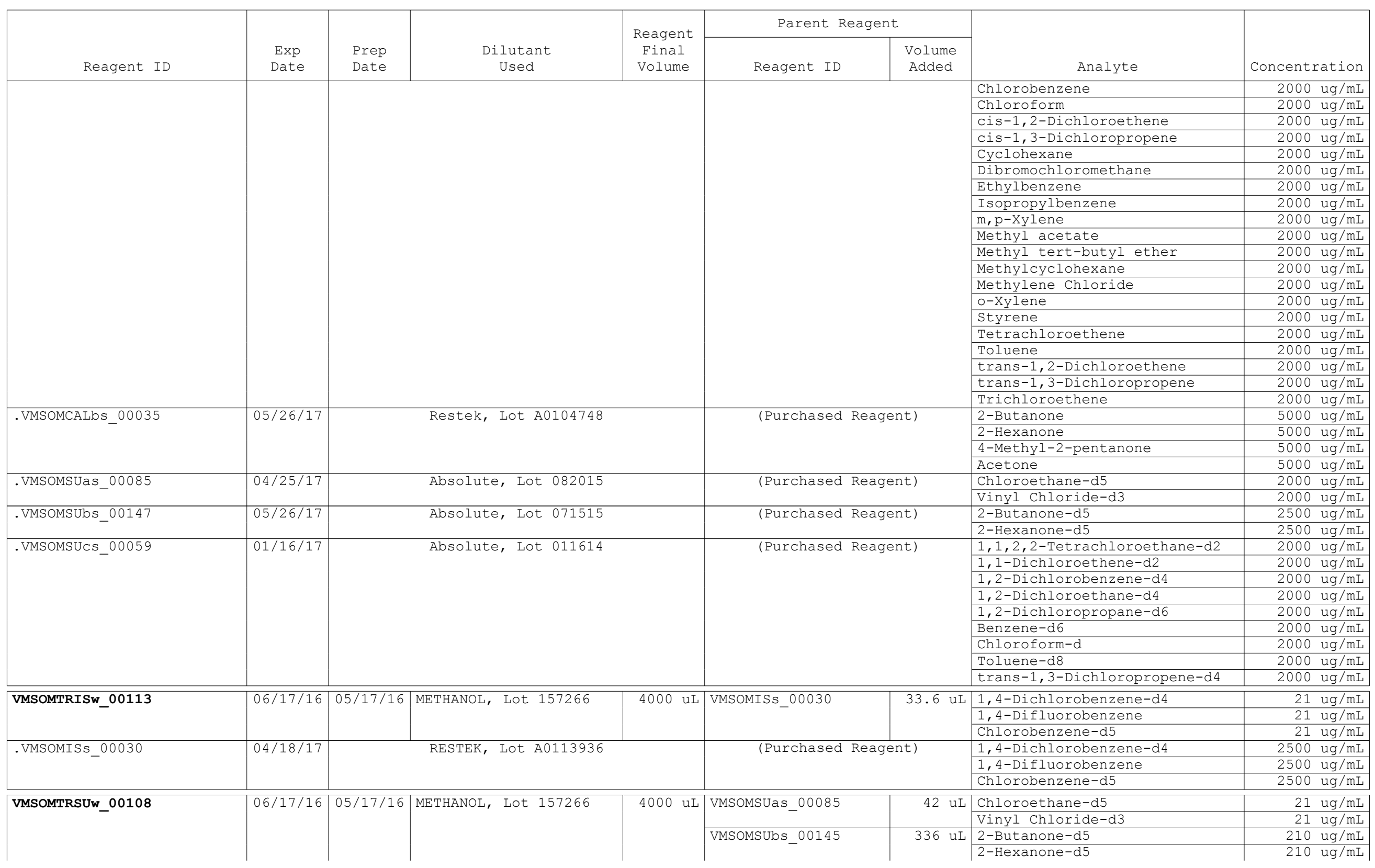


SDG No.: 33767

\begin{tabular}{|c|c|c|c|c|c|c|c|c|}
\hline \multirow[b]{2}{*}{ Reagent ID } & \multirow[b]{2}{*}{$\begin{array}{l}\text { Exp } \\
\text { Date }\end{array}$} & \multirow[b]{2}{*}{$\begin{array}{l}\text { Prep } \\
\text { Date }\end{array}$} & \multirow[b]{2}{*}{$\begin{array}{l}\text { Dilutant } \\
\text { Used }\end{array}$} & \multirow{2}{*}{$\begin{array}{c}\text { Reagent } \\
\text { Final } \\
\text { Volume }\end{array}$} & \multicolumn{2}{|c|}{ Parent Reagent } & \multirow[b]{2}{*}{ Analyte } & \multirow[b]{2}{*}{ Concentration } \\
\hline & & & & & Reagent ID & $\begin{array}{l}\text { Volume } \\
\text { Added }\end{array}$ & & \\
\hline & & & & & VMSOMSUCS_00059 & $42 \mathrm{uL}$ & $1,1,2,2$-Tetrachloroethane-d2 & $21 \mathrm{ug} / \mathrm{mL}$ \\
\hline & & & & & & & 1,1-Dichloroethene-d2 & $21 \mathrm{ug} / \mathrm{mL}$ \\
\hline & & & & & & & 1,2-Dichlorobenzene-d4 & $21 \mathrm{ug} / \mathrm{mL}$ \\
\hline & & & & & & & 1,2-Dichloroethane-d4 & $21 \mathrm{ug} / \mathrm{mL}$ \\
\hline & & & & & & & 1,2-Dichloropropane-d6 & $21 \mathrm{ug} / \mathrm{mL}$ \\
\hline & & & & & & & Benzene-d6 & $21 \mathrm{ug} / \mathrm{mL}$ \\
\hline & & & & & & & Chloroform-d & $21 \mathrm{ug} / \mathrm{mL}$ \\
\hline & & & & & & & Toluene-d8 & $21 \mathrm{ug} / \mathrm{mL}$ \\
\hline & & & & & & & trans-1,3-Dichloropropene-d4 & $21 \mathrm{ug} / \mathrm{mL}$ \\
\hline \multirow[t]{2}{*}{ VMSOMSUas_00085 } & \multirow[t]{2}{*}{$04 / 25 / 17$} & \multirow{2}{*}{\multicolumn{3}{|c|}{ Absolute, Lot 082015}} & \multirow{2}{*}{\multicolumn{2}{|c|}{ (Purchased Reagent) }} & Chloroethane-d5 & $2000 \mathrm{ug} / \mathrm{mL}$ \\
\hline & & & & & & & Vinyl Chloride-d3 & $2000 \mathrm{ug} / \mathrm{mL}$ \\
\hline \multirow[t]{2}{*}{.VMSOMSUbs_00145 } & \multirow[t]{2}{*}{$04 / 25 / 17$} & \multirow{2}{*}{\multicolumn{3}{|c|}{ Absolute, Lot 071515}} & \multirow{2}{*}{\multicolumn{2}{|c|}{ (Purchased Reagent) }} & 2-Butanone-d5 & $2500 \mathrm{ug} / \mathrm{mL}$ \\
\hline & & & & & & & 2-Hexanone-d5 & $2500 \mathrm{ug} / \mathrm{mL}$ \\
\hline \multirow[t]{9}{*}{.VMSOMSUCS_00059 } & \multirow[t]{9}{*}{$01 / 16 / 17$} & \multirow{9}{*}{\multicolumn{2}{|c|}{ Absolute, Lot 011614}} & & \multirow{9}{*}{\multicolumn{2}{|c|}{ (Purchased Reagent) }} & $1,1,2,2$-Tetrachloroethane-d2 & $2000 \mathrm{ug} / \mathrm{mL}$ \\
\hline & & & & & & & 1,1-Dichloroethene-d2 & $2000 \mathrm{ug} / \mathrm{mL}$ \\
\hline & & & & & & & 1,2-Dichlorobenzene-d4 & $2000 \mathrm{ug} / \mathrm{mL}$ \\
\hline & & & & & & & 1,2-Dichloroethane-d4 & $2000 \mathrm{ug} / \mathrm{mL}$ \\
\hline & & & & & & & 1,2-Dichloropropane-d6 & $2000 \mathrm{ug} / \mathrm{mL}$ \\
\hline & & & & & & & Benzene-d6 & $2000 \mathrm{ug} / \mathrm{mL}$ \\
\hline & & & & & & & Chloroform-d & $2000 \mathrm{ug} / \mathrm{mL}$ \\
\hline & & & & & & & Toluene-d8 & $2000 \mathrm{ug} / \mathrm{mL}$ \\
\hline & & & & & & & trans-1,3-Dichloropropene-d4 & $2000 \mathrm{ug} / \mathrm{mL}$ \\
\hline
\end{tabular}


Lab Name: TestAmerica Burlington

Job No : : 200-33767-1

SDG No.: 33767

\begin{tabular}{|c|c|c|c|c|}
\hline $\begin{array}{c}\text { Reagent } \\
\text { Container }\end{array}$ & $\begin{array}{c}\text { Reagent } \\
\text { ID }\end{array}$ & $\begin{array}{c}\text { Reagent } \\
\text { Description }\end{array}$ & $\begin{array}{c}\text { Preparation } \\
\text { Date }\end{array}$ & $\begin{array}{c}\text { Expiration } \\
\text { Date }\end{array}$ \\
\hline 904333 & VMBEBW_00021 & BFB TUNE 25 PPM & $02 / 26 / 2016$ & $08 / 26 / 2016$ \\
\hline 932091 & VMSOMTRISw_00113 & SOM TR ISTD 21 PPM & $05 / 17 / 2016$ & $06 / 17 / 2016$ \\
\hline 932092 & VMSOMTRSUw_00108 & SOM TR DMC 21 PPM & $05 / 17 / 2016$ & $06 / 17 / 2016$ \\
\hline 935531 & VMSOMTRCALW_00103 & SOM TR CAL $21-210$ PPM & $05 / 26 / 2016$ & $06 / 26 / 2016$ \\
\hline
\end{tabular}




\section{METHODOLOGY SUMMARY}

Laboratory: TestAmerica Laboratories

Location: South Burlington, Vermont
Project No:

SDG No: $\quad 33767$

\section{VOA}

Volatile Organics Trace - USEPA CLP SOM01.2 


\section{CASE NARRATIVE}

\section{Client: Argonne National Laboratory}

\section{Project: York (200-33767)}

\section{Report Number: 200-33767-1}

With the exceptions noted as flags or footnotes, standard analytical protocols were followed in the analysis of the samples and no problems were encountered or anomalies observed. In addition all laboratory quality control samples were within established control limits, with any exceptions noted below. Each sample was analyzed to achieve the lowest possible reporting limit within the constraints of the method. In some cases, due to interference or analytes present at high concentrations, samples were diluted. For diluted samples, the reporting limits are adjusted relative to the dilution required.

Calculations are performed before rounding to avoid round-off errors in calculated results.

All holding times were met and proper preservation noted for the methods performed on these samples, unless otherwise detailed in the individual sections below.

\section{RECEIPT}

The samples were received on 06/01/2016; the samples arrived in good condition, properly preserved and on ice. The temperature of the cooler at receipt was $3.5^{\circ} \mathrm{C}$.

\section{VOLATILE ORGANIC COMPOUNDS - TRACE}

Samples YKDL1C-108-W-38091, YKDL1B-88-W-38093, YKDL2C2-136-W-38096, YKDL2CU-215-W-38097, YKQCTB-W-38099 and VHBLK01 were analyzed for Volatile Organic Compounds - Trace in accordance with EPA SOW SOM01.2. The samples were analyzed on $06 / 02 / 2016$.

Several analytes were detected in method blank MB 200-105227/4 at levels that were above the method detection limit but below the reporting limit. The values should be considered estimates, and have been flagged. If the associated sample reported a result above the MDL and/or RL, the result has been flagged. Several analytes were detected in method blank MB 200-105268/3 at levels that were above the method detection limit but below the reporting limit. The values should be considered estimates, and have been flagged. If the associated sample reported a result above the MDL and/or RL, the result has been flagged. Refer to the $\mathrm{QC}$ report for details.

A non-target constituent that represents a compound related to the DMC formulation is present in the samples in this set. The derived results for that compound have been qualified with an "X" qualifier to reflect the source of the contamination.

2-Hexanone-d5 failed the surrogate recovery criteria high for YKDL2CU-215-W-38097. Refer to the QC report for details.

Sample YKDL2C2-136-W-38096[4X] required dilution prior to analysis. The reporting limits have been adjusted accordingly.

No additional analytical or quality issues were noted, other than those described above or in the Definitions/Glossary page. 
Lab Name: TestAmerica Burlington

Job No.: 200-33767-1

SDG No.: 33767

Instrument ID: CHD.i

Analysis Batch Number: 105151

Lab Sample ID: ICIS 200-105151/4

Client Sample ID:

Date Analyzed: 05/31/16 16:56

Lab File ID: 20227_04.D

GC Column: DB-624

ID $: 0.2(\mathrm{~mm})$

\begin{tabular}{|c|c|c|c|c|}
\hline \multirow[t]{2}{*}{ COMPOUND NAME } & \multirow{2}{*}{$\begin{array}{c}\text { RETENTION } \\
\text { TIME }\end{array}$} & \multicolumn{3}{|c|}{ MANUAL INTEGRATION } \\
\hline & & REASON & ANALYST & DATE \\
\hline 1,2-Dichloropropane-d6 & 6.32 & Split Peak & wilburj & $05 / 31 / 1617: 36$ \\
\hline
\end{tabular}

Lab Sample ID: IC 200-105151/5

Client Sample ID:

Date Analyzed: 05/31/16 17:21

Lab File ID: 20227 05.D

GC Column: DB-624

ID $: 0.2(\mathrm{~mm})$

\begin{tabular}{|c|c|c|c|c|}
\hline \multicolumn{1}{|c|}{ COMPOUND NAME } & RETENTION & \multicolumn{3}{|c}{ MANUAL INTEGRATION } \\
\cline { 3 - 5 } & TIME & REASON & ANALYST & DATE \\
\hline 1,2 -Dichloropropane-d6 & 6.32 & Split Peak & wilburj & 05/31/16 17:57 \\
\hline
\end{tabular}

Lab Sample ID: IC 200-105151/6

Client Sample ID:

Date Analyzed: 05/31/16 17:46

Lab File ID: 20227_06.D

GC Column: DB-624

ID $: 0.2(\mathrm{~mm})$

\begin{tabular}{|c|c|c|c|c|}
\hline \multicolumn{1}{|c|}{ COMPOUND NAME } & RETENTION & \multicolumn{3}{|c|}{ MANUAL INTEGRATION } \\
\cline { 3 - 5 } & TIME & REASON & ANALYST & DATE \\
\hline 1,2-Dichloropropane-d6 & 6.32 & Split Peak & wilburj & $05 / 31 / 16$ 18:30 \\
\hline
\end{tabular}


Lab Name: TestAmerica Burlington SDG No.: 33767

Instrument ID: CHD.i

Lab Sample ID: CCVIS 200-105227/3

Date Analyzed: 06/02/16 09:38

\begin{tabular}{|c|r|}
\hline COMPOUND NAME & RETEN \\
& TIME \\
\hline \hline 1,2-Dichloropropane-d6 & \\
\hline
\end{tabular}

Lab Sample ID: CCVC 200-105227/19

Date Analyzed: 06/02/16 16:59 Client Sample ID: Lab File ID: 20250_19.D

Analysis Batch Number: 105227

Client Sample ID:

Lab File ID: 20250 03.D

GC Column: DB-624

ID $: 0.2(\mathrm{~mm})$
DATE

ANALYST

archern

GC Column: DB-624

ID $: 0.2(\mathrm{~mm})$

\begin{tabular}{|c|c|c|c|c|}
\hline \multirow[t]{2}{*}{ COMPOUND NAME } & \multirow{2}{*}{$\begin{array}{c}\text { RETENTION } \\
\text { TIME }\end{array}$} & \multicolumn{3}{|c|}{ MANUAL INTEGRATION } \\
\hline & & REASON & ANALYST & DATE \\
\hline 1,2-Dichloropropane-d6 & 6.32 & Split Peak & wilburj & $06 / 03 / 1616: 24$ \\
\hline
\end{tabular}


Lab Name: TestAmerica Burlington

Job No.: 200-33767-1

SDG No.: 33767

Instrument ID: CHD.i

Analysis Batch Number: 105268

Lab Sample ID: CCVIS 200-105268/2

Client Sample ID:

Date Analyzed: 06/02/16 17:45

Lab File ID: 20268 02.D

GC Column: DB-624

ID $: 0.2(\mathrm{~mm})$

\begin{tabular}{|c|c|c|c|c|}
\hline \multirow[t]{2}{*}{ COMPOUND NAME } & RETENTION & \multicolumn{3}{|c|}{ MANUAL INTEGRATION } \\
\hline & TIME & REASON & ANALYST & DATE \\
\hline 1,2 -Dichloropropane-d6 & 6.32 & Assign Peak & $\begin{array}{l}\text { puangmale } \\
\text { ek }\end{array}$ & $06 / 06 / 16 \quad 07: 49$ \\
\hline
\end{tabular}

Lab Sample ID: MB 200-105268/3

Client Sample ID:

Date Analyzed: 06/02/16 18:11

Lab File ID: 20268 03.D

GC Column: DB-624

ID $: 0.2(\mathrm{~mm})$

\begin{tabular}{|c|c|c|c|c|}
\hline \multirow[t]{2}{*}{ COMPOUND NAME } & \multirow{2}{*}{$\begin{array}{c}\text { RETENTION } \\
\text { TIME }\end{array}$} & \multicolumn{3}{|c|}{ MANUAL INTEGRATION } \\
\hline & & REASON & ANALYST & DATE \\
\hline Bromomethane & 2.01 & Assign Peak & $\begin{array}{l}\text { puangmale } \\
\text { ek }\end{array}$ & $06 / 06 / 1607: 58$ \\
\hline
\end{tabular}

Lab Sample ID: 200-33767-5

Client Sample ID: YKQCTB-W-38099

Date Analyzed: 06/02/16 21:27

Lab File ID: 20268_09.D

GC Column: DB-624

ID $: 0.2(\mathrm{~mm})$

\begin{tabular}{|l|c|c|c|c|}
\hline \multicolumn{1}{|c|}{ COMPOUND NAME } & RETENTION & \multicolumn{3}{|c|}{ MANUAL INTEGRATION } \\
\cline { 3 - 5 } & TIME & REASON & ANALYST & \multicolumn{2}{|c|}{ DATE } \\
\hline Bromomethane & 2.00 & Assign Peak & $06 / 06 / 16$ 08:28 \\
\hline
\end{tabular}

Lab Sample ID: 200-33767-6

Client Sample ID: VHBLK01

Date Analyzed: 06/02/16 21:53

Lab File ID: 20268_10.D

GC Column: DB-624

ID $: 0.2(\mathrm{~mm})$

\begin{tabular}{|c|c|c|c|c|}
\hline \multirow[t]{2}{*}{ COMPOUND NAME } & \multirow{2}{*}{$\begin{array}{c}\text { RETENTION } \\
\text { TIME }\end{array}$} & \multicolumn{3}{|c|}{ MANUAL INTEGRATION } \\
\hline & & REASON & ANALYST & DATE \\
\hline Bromomethane & 2.00 & Assign Peak & $\begin{array}{l}\text { puangmale } \\
\text { ek }\end{array}$ & $06 / 06 / 1608: 37$ \\
\hline
\end{tabular}


Lab Name: TestAmerica Burlington SDG No.: 33767

Instrument ID: CHD.i

Lab Sample ID: CCVC 200-105268/24

Date Analyzed: 06/03/16 03:49

\begin{tabular}{|c|r|}
\hline COMPOUND NAME & $\begin{array}{r}\text { RETEN } \\
\text { TIME }\end{array}$ \\
\hline 1,2-Dichloropropane-d6 &
\end{tabular}

Job No.: 200-33767-1

Analysis Batch Number: 105268

Client Sample ID:

Lab File ID: 20268 24.D
ID $: 0.2(\mathrm{~mm})$ 


\section{DATA REPORTING QUALIFIERS}

Client: Argonne National Laboratory

Job Number: 200-33767-1

Sdg Number: 33767

Lab Section

Qualifier

Description

GC/MS VOA

U

E

J

$\mathrm{J}$

D

$\mathrm{X}$

*

B
Analyzed for but not detected.

Compound concentration exceeds the upper level of the calibration range of the instrument for that specific analysis.

Indicates an Estimated Value for TICs

Indicates an estimated value.

Sample was analyzed at a higher dilution factor.

See case narrative notes for explanation of the ' $X$ ' flag

Surrogate is outside acceptance limits.

The analyte was found in an associated blank, as well as in the sample. 
Lab Name: TESTAMERICA BURLINGTON

Lab Code: STLV Case No.: YORK Mod. Ref No.: Level: (TRACE or LOW) TRACE
Contract: $8 \mathrm{E}-00302$

SDG No.: 33767

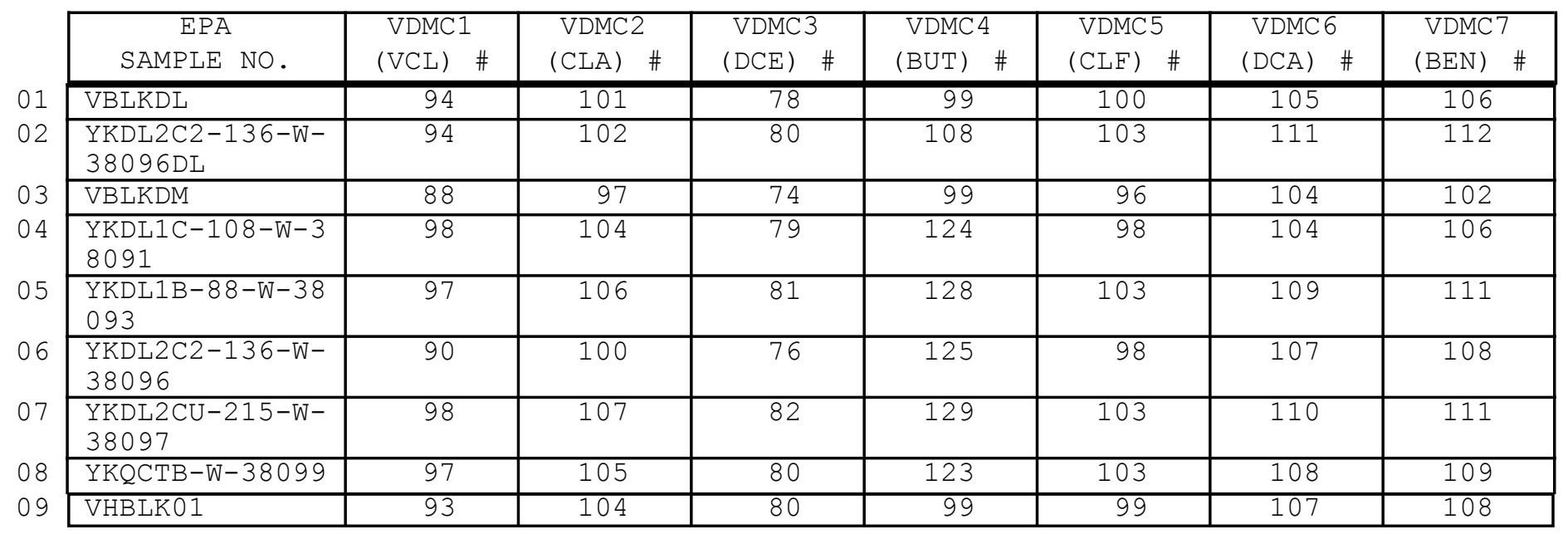

\footnotetext{
VDMC1 (VCL) = Vinyl Chloride-d3

VDMC2 $(\mathrm{CLA})=$ Chloroethane-d5

VDMC3 $(\mathrm{DCE})=1,1-\mathrm{Dichloroethene-d2}$

VDMC4 $(B U T)=2-$ Butanone-d5

VDMC5 $(\mathrm{CLF})=$ Chloroform-d

VDMC $6 \quad(\mathrm{DCA})=1,2-\mathrm{Dich}$ loroethane-d4

$\operatorname{VDMC} 7 \quad(\mathrm{BEN})=$ Benzene-d6
}

$$
\begin{gathered}
\frac{\mathrm{QC} \text { LIMITS }}{(65-131)} \\
(71-131) \\
(55-104) \\
(49-155) \\
(78-121) \\
(78-129) \\
(77-124)
\end{gathered}
$$

\# Column to be used to flag recovery values

* Values outside of contract required QC limits

Page 1 of 1 
Lab Name: TESTAMERICA BURLINGTON

Lab Code: STLV Case No.: YORK Mod. Ref No.:
Contract: $8 \mathrm{E}-00302$

SDG No.: 33767

Level: (TRACE or LOW) TRACE

\begin{tabular}{|c|c|c|c|c|c|c|c|c|}
\hline $\begin{array}{c}\text { EPA } \\
\text { SAMPLE NO. }\end{array}$ & $\begin{array}{l}\text { VDMC8 } \\
(\mathrm{DPA}) \#\end{array}$ & $\begin{array}{l}\text { VDMC9 } \\
(\text { TOL }) \#\end{array}$ & $\begin{array}{l}\text { VDMC10 } \\
(\mathrm{TDP}) \#\end{array}$ & $\begin{array}{l}\text { VDMC11 } \\
(\operatorname{HEX}) \#\end{array}$ & $\begin{array}{l}\text { VDMC12 } \\
(\mathrm{TCA}) \quad \#\end{array}$ & $\begin{array}{l}\text { VDMC13 } \\
(\mathrm{DCZ}) \quad \#\end{array}$ & OTHER & $\begin{array}{l}\text { TOT } \\
\text { OUT }\end{array}$ \\
\hline VBLKDL & 104 & 106 & 92 & 100 & 94 & 120 & & 0 \\
\hline $\begin{array}{l}\text { YKDL2C2-136-W- } \\
38096 \mathrm{DL}\end{array}$ & 110 & 110 & 94 & 115 & 101 & 124 & & 0 \\
\hline VBLKDM & 102 & 101 & 91 & 104 & 94 & 114 & & 0 \\
\hline $\begin{array}{l}\text { YKDL1C-108-W-3 } \\
8091\end{array}$ & 102 & 104 & 91 & 130 & 92 & 111 & & 0 \\
\hline $\begin{array}{l}\text { YKDL1B-88-W-38 } \\
093\end{array}$ & 108 & 109 & 95 & 135 & 98 & 119 & & 0 \\
\hline $\begin{array}{l}\text { YKDL2C2-136-W- } \\
38096\end{array}$ & 106 & 106 & 97 & 128 & 100 & 119 & & 0 \\
\hline $\begin{array}{l}\text { YKDL2CU-215-W- } \\
38097\end{array}$ & 109 & 112 & 95 & $136 *$ & 100 & 125 & & 1 \\
\hline YKQCTB-W-38099 & 106 & 108 & 93 & 127 & 97 & 112 & & 0 \\
\hline VHBLK01 & 105 & 108 & 92 & 106 & 95 & 123 & & 0 \\
\hline
\end{tabular}

\footnotetext{
VDMC8 $(\mathrm{DPA})=1,2-\mathrm{Dich}$ loropropane-d6

VDMC $9 \quad($ TOL $)=$ Toluene-d8

VDMC10 $(\mathrm{TDP})=$ trans $-1,3-\mathrm{Dichloropropene-d4}$

VDMC11 $(\mathrm{HEX})=2-$ Hexanone-d5

VDMC12 $(\mathrm{TCA})=1,1,2,2$-Tetrachloroethane-d2

VDMC13 $(\mathrm{DCZ})=1,2-\mathrm{Dichlorobenzene-d4}$
}

$$
\begin{gathered}
\frac{Q C \text { LIMITS }}{(79-124)} \\
(77-121) \\
(73-121) \\
(28-135) \\
(73-125) \\
(80-131)
\end{gathered}
$$

\# Column to be used to flag recovery values

* Values outside of contract required QC limits

Report 1,4-Dioxane-d8 for Low-Medium VOA analysis only

Page 1 of 1 
Lab Name: TESTAMERICA BURLINGTON

Contract: 8E-00302

Lab Code: STLV Case No.: YORK Mod. Ref No.: SDG No.: 33767

Lab File ID: 20250_04.D

Lab Sample ID: MB 200-105227/4

Instrument ID: CHD.i

Matrix: (SOIL/SED/WATER) Water

Date Analyzed: 06/02/2016

Level: (TRACE or LOW/MED) TRACE

Time Analyzed: 1004

GC Column: DB-624

ID $0.20 \quad(\mathrm{~mm})$

Heated Purge: ( $\mathrm{Y} / \mathrm{N}) \mathrm{N}$

\begin{tabular}{|c|c|c|c|c|}
\hline \multicolumn{1}{c|}{ EPA } & LAB & LAB & TIME \\
SAMPLE NO. & SAMPLE ID & FILE ID & ANALYZED \\
\cline { 2 - 5 } & $\begin{array}{l}\text { YKDL2C2-136- } \\
\text { W-38096DL }\end{array}$ & $200-33767-3$ & $20250 \_17 . D$ & 1607 \\
\cline { 2 - 5 }
\end{tabular}

COMMENTS :

Page 1 of 1 
$4 A$ - FORM IV VOA

VOLATILE METHOD BLANK SUMMARY
EPA SAMPLE NO.

VBLKDM

Lab Name: TESTAMERICA BURLINGTON

Contract: $8 \mathrm{E}-00302$

Lab Code: STLV Case No.: YORK Mod. Ref No.: SDG No.: 33767

Lab File ID: 20268_03.D

Lab Sample ID: MB 200-105268/3

Instrument ID: CHD.i

Matrix: (SOIL/SED/WATER) Water

Level: (TRACE or LOW/MED) TRACE

Date Analyzed: 06/02/2016

GC Column: DB-624

ID $: 0.20 \quad(\mathrm{~mm})$

Time Analyzed: 1811

Heated Purge: (Y/N) N

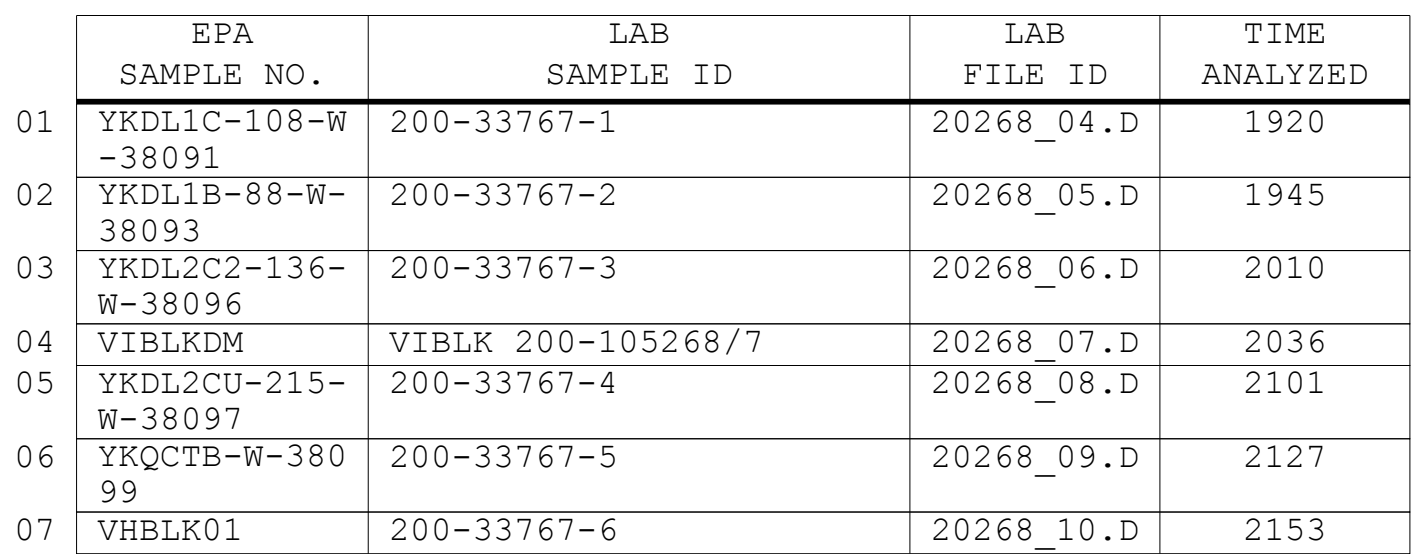

COMMENTS :

Page 1 of 1 
$5 A$ - FORM V VOA

VOLATILE ORGANICS INSTRUMENT

PERFORMANCE CHECK

BROMOFLUOROBENZENE (BFB)
EPA SAMPLE NO.

BFBDJ
Lab Name: TESTAMERICA BURLINGTON

Lab Code: STLV

Case No.: YORK

Lab File Id: $2022701 . \mathrm{D}$

Instrument Id: CHD.i

GC Column: DB-624

ID $: 0.20$
Contract: 8E-00302

Mod. Ref No.:

SDG No.: 33767

BEB Injection Date: 05/31/2016

BFB Injection Time: 1555 $(\mathrm{mm})$

\begin{tabular}{|c|c|c|}
\hline $\mathrm{m} / \mathrm{e}$ & ION ABUNDANCE CRITERIA & $\begin{array}{l}\text { \% RELATIVE } \\
\text { ABUNDANCE }\end{array}$ \\
\hline 50 & $15.0-40.0 \%$ of mass 95 & 16.9 \\
\hline 75 & $30.0-80.0 \%$ of mass 95 & 50.0 \\
\hline 95 & Base peak, $100 \%$ relative abundance & 100 \\
\hline 96 & $5.0-9.0 \%$ of mass 95 & 6.6 \\
\hline 173 & Less than $2.0 \%$ of mass 174 & $0) 1$ \\
\hline 174 & $50.0-120 \%$ of mass 95 & 90.0 \\
\hline 175 & $5.0-9.0 \%$ of mass 174 & 6.41 \\
\hline 176 & $95.0-101 \%$ of mass 174 & $87.1(96.8) 1$ \\
\hline 177 & $5.0-9.0 \%$ of mass 176 & $(6.6) 2$ \\
\hline
\end{tabular}

1 - Value is omass 1742 - Value is mass 176

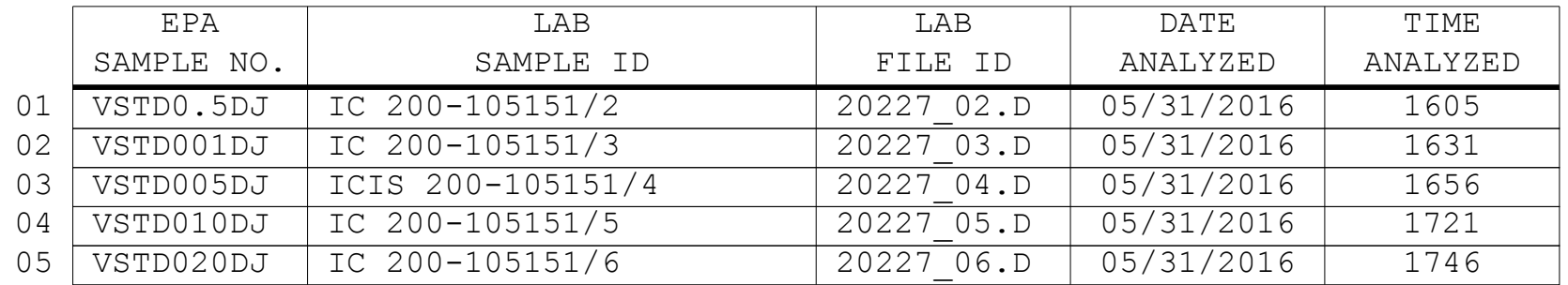


$5 A$ - FORM V VOA

VOLATILE ORGANICS INSTRUMENT

PERFORMANCE CHECK

BROMOFLUOROBENZENE (BFB)
EPA SAMPLE NO.

BFBDL
Lab Name: TESTAMERICA BURLINGTON

Lab Code: STLV Case No.: YORK

Lab File Id: 20250_01.D

Instrument Id: CHD.i

GC Column: DB-624

ID $: 0.20$
Contract: 8E-00302 Mod. Ref No.: SDG No.: 33767

BEB Injection Date: 06/02/2016

BFB Injection Time: 0849 $(\mathrm{mm})$

\begin{tabular}{|c|c|c|}
\hline $\mathrm{m} / \mathrm{e}$ & ION ABUNDANCE CRITERIA & $\begin{array}{l}\text { \% RELATIVE } \\
\text { ABUNDANCE }\end{array}$ \\
\hline 50 & $15.0-40.0 \%$ of mass 95 & 17.7 \\
\hline 75 & $30.0-80.0 \%$ of mass 95 & 51.7 \\
\hline 95 & Base peak, $100 \%$ relative abundance & 100 \\
\hline 96 & $5.0-9.0 \%$ of mass 95 & 6.5 \\
\hline 173 & Less than $2.0 \%$ of mass 174 & $1.0) 1$ \\
\hline 174 & $50.0-120 \%$ of mass 95 & 89.2 \\
\hline 175 & $5.0-9.0 \%$ of mass 174 & $6.4(7.2) 1$ \\
\hline 176 & $95.0-101 \%$ of mass 174 & $85.9(96.3) 1$ \\
\hline 177 & $5.0-9.0 \%$ of mass 176 & $(6.5) 2$ \\
\hline
\end{tabular}

1 - Value is omass 1742 - Value is omass 176

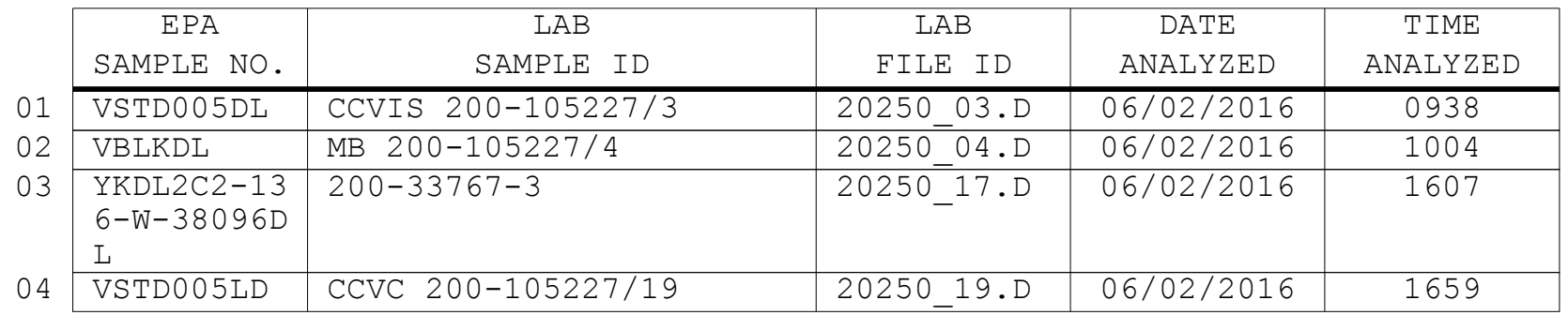


$5 A$ - FORM V VOA

VOLATILE ORGANICS INSTRUMENT

PERFORMANCE CHECK

BROMOFLUOROBENZENE (BFB)
EPA SAMPLE NO.

BFBDM
Lab Name: TESTAMERICA BURLINGTON

Lab Code: STLV

Case No.: YORK

Lab File Id: 20268_01.D

Instrument Id: CHD.i

GC Column: DB-624
ID $: 0.20$
Contract: $8 \mathrm{E}-00302$ Mod. Ref No.: SDG No.: 33767

BFB Injection Date: 06/02/2016

BFB Injection Time: 1733 (mm)

\begin{tabular}{|c|c|c|}
\hline $\mathrm{m} / \mathrm{e}$ & ION ABUNDANCE CRITERIA & $\begin{array}{l}\text { \% RELATIVE } \\
\text { ABUNDANCE }\end{array}$ \\
\hline 50 & $15.0-40.0 \%$ of mass 95 & 16.7 \\
\hline 75 & $30.0-80.0 \%$ of mass 95 & 49.9 \\
\hline 95 & Base peak, $100 \%$ relative abundance & 100 \\
\hline 96 & $5.0-9.0 \%$ of mass 95 & 6.6 \\
\hline 173 & Less than 2.0\% of mass 174 & $(1.2) 1$ \\
\hline 174 & $50.0-120 \%$ of mass 95 & 98.1 \\
\hline 175 & $5.0-9.0 \%$ of mass 174 & $(7.2) 1$ \\
\hline 176 & $95.0-101 \%$ of mass 174 & $95.2(97.1) 1$ \\
\hline 177 & $5.0-9.0 \%$ of mass 176 & $6.2(6.5) 2$ \\
\hline
\end{tabular}

1 - Value is omass 174

2 - Value is omass 176

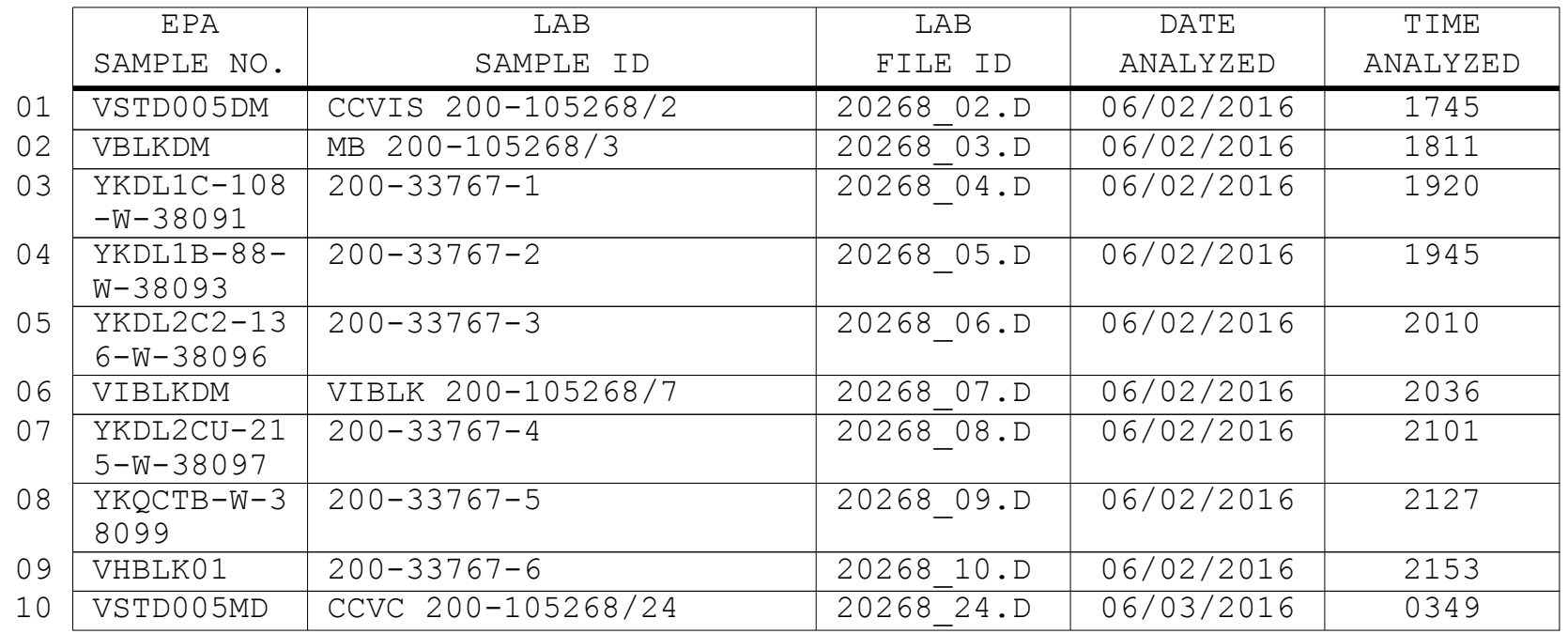


Lab Name: TESTAMERICA BURLINGTON

Contract: $8 \mathrm{E}-00302$

Lab Code: STLV Case No.: YORK Mod. Ref No.: SDG No.: 33767

GC Column: DB-624

ID: 0.20 (mm) Init. Calib. Date (s) : 05/31/2016 05/31/2016

EPA Sample No.(VSTD\#\#\#\#\#) : VSTD005DL

Lab File ID (Standard): 20250_03.D

Instrument ID: CHD.i

Date Analyzed: $06 / 02 / 2016$

Time Analyzed: 0938

Heated Purge: ( $\mathrm{Y} / \mathrm{N}) \mathrm{N}$

\begin{tabular}{|c|c|c|c|c|c|c|}
\hline & $\begin{array}{c}\text { IS1 (CBZ) } \\
\text { AREA }\end{array}$ & $\mathrm{RT} \#$ & $\begin{array}{c}\text { IS2 (DFB) } \\
\text { AREA }\end{array}$ & $\mathrm{RT}$ & $\begin{array}{c}\text { IS3 ( DCB) } \\
\text { AREA }\end{array}$ & $\mathrm{RT}$ \\
\hline 12 HOUR STD & 890929 & 9.22 & 1113752 & 5.88 & 478184 & 12.04 \\
\hline UPPER LIMIT & 1247301 & 9.55 & 1559253 & 6.21 & 669458 & 12.37 \\
\hline LOWER LIMIT & 534557 & 8.89 & 668251 & 5.55 & 286910 & 11.71 \\
\hline EPA SAMPLE NO. & & & & & & \\
\hline VBLKDL & 851662 & 9.22 & 1104589 & 5.88 & 335388 & 12.04 \\
\hline $\begin{array}{l}\text { YKDL 2C2-136-W- } \\
38096 \mathrm{DL}\end{array}$ & 835156 & 9.22 & 1113907 & 5.88 & 320630 & 12.04 \\
\hline
\end{tabular}

IS1 $(\mathrm{CBZ})=$ Chlorobenzene-d5

IS2 $(\mathrm{DFB})=1,4-\mathrm{Difluorobenzene}$

IS3 $(\mathrm{DCB})=1,4-\mathrm{Dichlorobenzene-d4}$

AREA UPPER LIMIT $=140 \%$ (Trace Volatiles) of internal standard area AREA LOWER LIMIT $=60 \%$ (Trace Volatiles) of internal standard area RT UPPER LIMIT $=+0.33$ (Trace Volatiles) minutes of internal standard RT RT LOWER LIMIT $=-0.33$ (Trace Volatiles) minutes of internal standard RT

\# Column used to flag values outside contract required QC limits with an asterisk. 


\section{$8 A$ - FORM VIII VOA}

VOLATILE INTERNAL STANDARD AREA AND RETENTION TIME SUMMARY

Lab Name: TESTAMERICA BURLINGTON

Contract: $8 \mathrm{E}-00302$

Lab Code: STLV Case No.: YORK Mod. Ref No.: SDG No.: 33767

GC Column: DB-624

ID: 0.20 (mm) Init. Calib. Date (s) : 05/31/2016 05/31/2016

EPA Sample No.(VSTD\#\#\#\#\#): VSTD005DM

Lab File ID (Standard): 20268_02.D

Date Analyzed: 06/02/2016

Instrument ID: CHD.i

Time Analyzed: 1745

Heated Purge: (Y/N) N

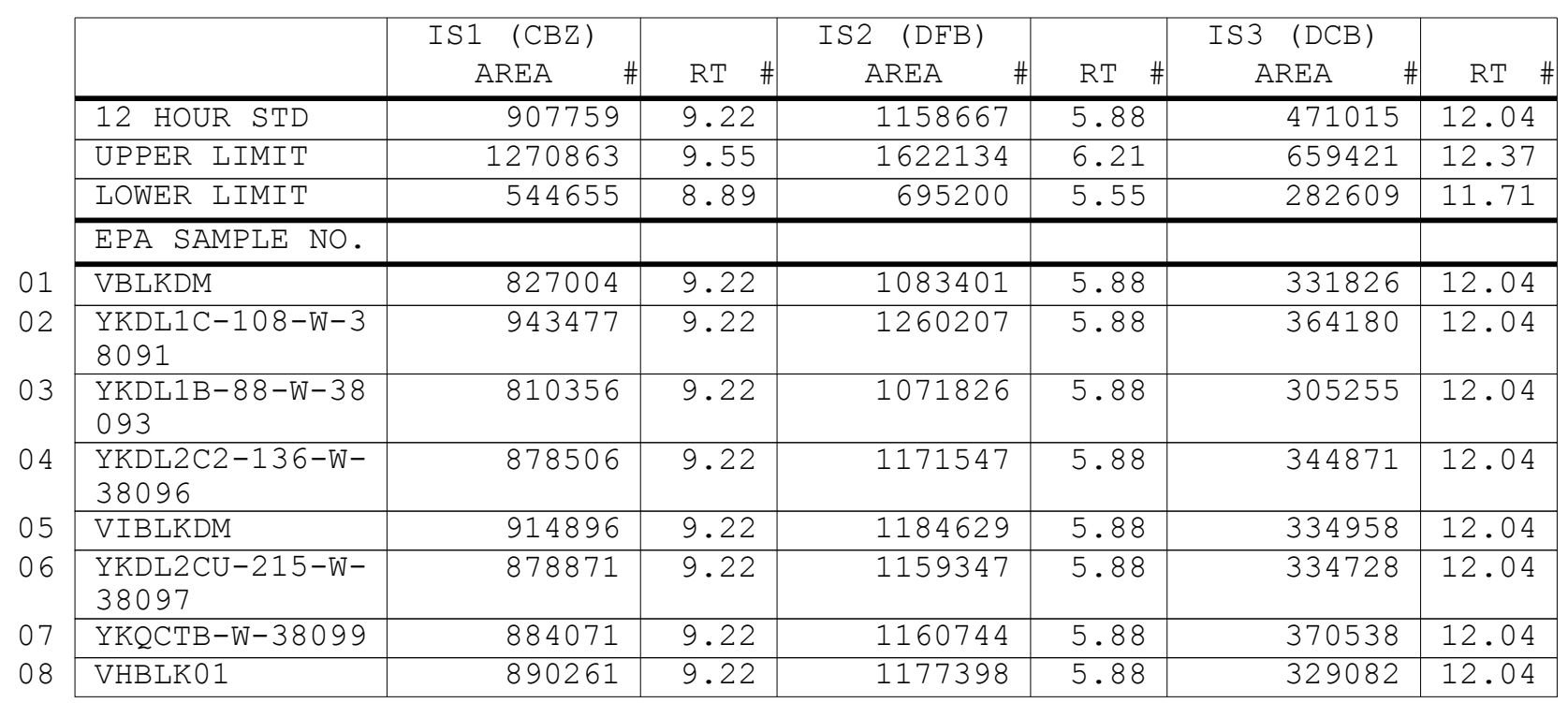

IS1 $(\mathrm{CBZ})=$ Chlorobenzene-d5

IS2 $(\mathrm{DFB})=1,4-\mathrm{Difluorobenzene}$

IS3 $(\mathrm{DCB})=1,4-\mathrm{Dichlorobenzene-d4}$

AREA UPPER LIMIT $=140 \%$ (Trace Volatiles) of internal standard area AREA LOWER LIMIT $=60 \%$ (Trace Volatiles) of internal standard area RT UPPER LIMIT $=+0.33$ (Trace Volatiles) minutes of internal standard RT RT LOWER LIMIT $=-0.33$ (Trace Volatiles) minutes of internal standard RT

\# Column used to flag values outside contract required QC limits with an asterisk. Page 1 of 1 
Lab Name: TESTAMERICA BURLINGTON

Contract: 8E-00302

Lab Code: STLV

Case No.: YORK Mod. Ref No.:

SDG No.: 33767

Matrix: (SOIL/SED/WATER) Water

Sample wt/vol: 25.0 $(\mathrm{g} / \mathrm{mL}) \mathrm{mL}$

Level: (TRACE/LOW/MED) TRACE

\% Moisture: not dec.

GC Column: DB-624 ID: $0.20 \quad(\mathrm{~mm})$

Soil Extract Volume: (uL) $(\mathrm{mL})$

Purge Volume: 25.0
Lab Sample ID: 200-33767-2

Lab File ID: 20268_05.D

Date Received: 06/01/2016

Date Analyzed: 06/02/2016

Dilution Factor: 1.0

Soil Aliquot Volume: (uL)

\begin{tabular}{|c|c|c|c|}
\hline CAS NO. & COMPOUND & $\begin{array}{l}\text { CONCENTRATION UNITS: } \\
(\mathrm{ug} / \mathrm{L} \text { or } \mathrm{ug} / \mathrm{kg}) \mathrm{ug} / \mathrm{L}\end{array}$ & Q \\
\hline $75-71-8$ & Dichlorodifluoromethane & 1.0 & $\mathrm{U}$ \\
\hline $74-87-3$ & Chloromethane & 1.0 & $\mathrm{U}$ \\
\hline $75-01-4$ & Vinyl chloride & 1.0 & $\mathrm{U}$ \\
\hline $74-83-9$ & Bromomethane & 1.0 & $\mathrm{U}$ \\
\hline $75-00-3$ & Chloroethane & 1.0 & $\mathrm{U}$ \\
\hline $75-69-4$ & Trichlorofluoromethane & 0.030 & $\mathrm{~J}$ \\
\hline $75-35-4$ & 1,1-Dichloroethene & 1.0 & $\mathrm{U}$ \\
\hline $76-13-1$ & $1,1,2$-Trichloro-1,2,2-trifluoroethane & 1.0 & $\mathrm{U}$ \\
\hline $67-64-1$ & Acetone & 5.0 & $\mathrm{U}$ \\
\hline $75-15-0$ & Carbon disulfide & 0.031 & $\mathrm{~J} B$ \\
\hline $79-20-9$ & Methyl acetate & 1.0 & $\mathrm{U}$ \\
\hline $75-09-2$ & Methylene Chloride & 1.0 & $\mathrm{U}$ \\
\hline $156-60-5$ & trans-1,2-Dichloroethene & 1.0 & $\mathrm{U}$ \\
\hline $1634-04-4$ & Methyl tert-butyl ether & 1.0 & $\mathrm{U}$ \\
\hline $75-34-3$ & 1,1-Dichloroethane & 1.0 & $\mathrm{U}$ \\
\hline $156-59-2$ & cis-1,2-Dichloroethene & 1.0 & $\mathrm{U}$ \\
\hline $78-93-3$ & 2-Butanone & 5.0 & $\mathrm{U}$ \\
\hline $74-97-5$ & Bromochloromethane & 1.0 & $\mathrm{U}$ \\
\hline $67-66-3$ & Chloroform & 0.32 & $\mathrm{~J}$ \\
\hline $71-55-6$ & $1,1,1$-Trichloroethane & 1.0 & $\mathrm{U}$ \\
\hline $110-82-7$ & Cyclohexane & 1.0 & $\mathrm{U}$ \\
\hline $56-23-5$ & Carbon tetrachloride & 2.0 & \\
\hline $71-43-2$ & Benzene & 1.0 & $\mathrm{U}$ \\
\hline $107-06-2$ & 1,2-Dichloroethane & 1.0 & $\mathrm{U}$ \\
\hline
\end{tabular}

Report 1,4-Dioxane for Low-Medium VOA analysis only 
Lab Name: TESTAMERICA BURLINGTON

Contract: 8E-00302

Lab Code: STLV

Case No.: YORK

Mod. Ref No.:

SDG No.: 33767

Matrix: (SOIL/SED/WATER) Water

Sample wt/vol: 25.0 $(\mathrm{g} / \mathrm{mL}) \mathrm{mL}$

Level: (TRACE/LOW/MED) TRACE

\% Moisture: not dec.

GC Column: DB-624 ID: $0.20 \quad(\mathrm{~mm})$

Soil Extract Volume: (uL) $(\mathrm{mL})$

Purge Volume: 25.0
Lab Sample ID: 200-33767-2

Lab File ID: 20268_05.D

Date Received: 06/01/2016

Date Analyzed: 06/02/2016

Dilution Factor: 1.0

Soil Aliquot Volume: (uL) (mI)

\begin{tabular}{|c|c|c|c|}
\hline CAS NO. & COMPOUND & $\begin{array}{l}\text { CONCENTRATION UNITS: } \\
(\mathrm{ug} / \mathrm{L} \text { or } \mathrm{ug} / \mathrm{kg}) \mathrm{ug} / \mathrm{L}\end{array}$ & Q \\
\hline $79-01-6$ & Trichloroethene & 1.0 & $\mathrm{U}$ \\
\hline $108-87-2$ & Methylcyclohexane & 1.0 & $\mathrm{U}$ \\
\hline $78-87-5$ & 1,2-Dichloropropane & 1.0 & $\mathrm{U}$ \\
\hline $75-27-4$ & Bromodichloromethane & 1.0 & $\mathrm{U}$ \\
\hline $10061-01-5$ & cis-1,3-Dichloropropene & 1.0 & U \\
\hline $108-10-1$ & 4-Methyl-2-pentanone & 1.0 & $\mathrm{U}$ \\
\hline $108-88-3$ & Toluene & 0.024 & $\mathrm{~J} B$ \\
\hline $10061-02-6$ & trans-1,3-Dichloropropene & 1.0 & $\mathrm{U}$ \\
\hline $79-00-5$ & $1,1,2-$ Trichloroethane & 1.0 & $\mathrm{U}$ \\
\hline $127-18-4$ & Tetrachloroethene & 0.012 & $\mathrm{~J} B$ \\
\hline $591-78-6$ & 2-Hexanone & 1.0 & $\mathrm{U}$ \\
\hline $124-48-1$ & Dibromochloromethane & 1.0 & $\mathrm{U}$ \\
\hline $106-93-4$ & 1,2-Dibromoethane & 1.0 & $\mathrm{U}$ \\
\hline $108-90-7$ & Chlorobenzene & 1.0 & $\mathrm{U}$ \\
\hline $100-41-4$ & Ethylbenzene & 1.0 & $\mathrm{U}$ \\
\hline $95-47-6$ & o-Xylene & 1.0 & $\mathrm{U}$ \\
\hline $179601-23-1$ & m,p-xylene & 0.0069 & $\mathrm{~J} B$ \\
\hline $100-42-5$ & Styrene & 1.0 & $\mathrm{U}$ \\
\hline $75-25-2$ & Bromoform & 1.0 & $\mathrm{U}$ \\
\hline $98-82-8$ & Isopropylbenzene & 1.0 & $\mathrm{U}$ \\
\hline $79-34-5$ & $1,1,2,2$-Tetrachloroethane & 1.0 & $\mathrm{U}$ \\
\hline $541-73-1$ & 1,3 -Dichlorobenzene & 1.0 & $\mathrm{U}$ \\
\hline $106-46-7$ & 1,4-Dichlorobenzene & 1.0 & $\mathrm{U}$ \\
\hline $95-50-1$ & 1,2-Dichlorobenzene & 1.0 & $\mathrm{U}$ \\
\hline $96-12-8$ & 1,2-Dibromo-3-Chloropropane & 1.0 & $\mathrm{U}$ \\
\hline $120-82-1$ & $1,2,4$-Trichlorobenzene & 1.0 & $\mathrm{U}$ \\
\hline $87-61-6$ & $1,2,3$-Trichlorobenzene & 1.0 & $\mathrm{U}$ \\
\hline
\end{tabular}


Lab Name: TESTAMERICA BURLINGTON

Contract: $8 \mathrm{E}-00302$

Lab Code: STLV Case No.: YORK Mod. Ref No.: SDG No.: 33767

Matrix: (SOIL/SED/WATER) Water

Sample wt/vol: 25.0 $(\mathrm{g} / \mathrm{mL}) \mathrm{mL}$

Level: (TRACE or LOW/MED) TRACE

\% Moisture: not dec.

GC Column: DB-624 ID $: 0.20 \quad(\mathrm{~mm})$

Soil Extract Volume: (uL)

CONCENTRATION UNITS: (ug/L or ug/kg) ug/L
Lab Sample ID: 200-33767-2

Lab File ID: 20268_05.D

Date Received: 06/01/2016

Date Analyzed: 06/02/2016

Dilution Factor: 1.0

Soil Aliquot Volume: (uL) Purge Volume: 25.0

$(\mathrm{mL})$

01

02

\begin{tabular}{|l|l|c|c|c|}
\hline CAS NUMBER & \multicolumn{1}{|c|}{ COMPOUND NAME } & RT & EST. CONC. & Q \\
\hline & Unknown & 7.20 & 2.6 & $\mathrm{~J}$ B X \\
\hline E9667961 & Total Alkanes & N/A & & \\
\hline
\end{tabular}

1 EPA-designated Registry Number. 
Lab Name: TESTAMERICA BURLINGTON

Contract: $8 \mathrm{E}-00302$

Lab Code: STLV

Case No.: YORK Mod. Ref No.:

SDG No.: 33767

Matrix: (SOIL/SED/WATER) Water

Sample wt/vol: 25.0 $(\mathrm{g} / \mathrm{mL}) \mathrm{mL}$

Level: (TRACE/LOW/MED) TRACE

\% Moisture: not dec.

GC Column: DB-624 ID: $0.20 \quad(\mathrm{~mm})$

Soil Extract Volume: (uL) $(\mathrm{mL})$

Purge Volume: 25.0
Lab Sample ID: 200-33767-1

Lab File ID: 20268_04.D

Date Received: 06/01/2016

Date Analyzed: 06/02/2016

Dilution Factor: 1.0

Soil Aliquot Volume: (uL)

\begin{tabular}{|c|c|c|c|}
\hline CAS NO. & COMPOUND & $\begin{array}{l}\text { CONCENTRATION UNITS: } \\
(\mathrm{ug} / \mathrm{L} \text { or } \mathrm{ug} / \mathrm{kg}) \mathrm{ug} / \mathrm{L}\end{array}$ & Q \\
\hline $75-71-8$ & Dichlorodifluoromethane & 1.0 & $\mathrm{U}$ \\
\hline $74-87-3$ & Chloromethane & 1.0 & $\mathrm{U}$ \\
\hline $75-01-4$ & Vinyl chloride & 1.0 & $\mathrm{U}$ \\
\hline $74-83-9$ & Bromomethane & 0.073 & $\mathrm{~J} B$ \\
\hline $75-00-3$ & Chloroethane & 1.0 & $\mathrm{U}$ \\
\hline $75-69-4$ & Trichlorofluoromethane & 0.035 & $\mathrm{~J}$ \\
\hline $75-35-4$ & 1,1-Dichloroethene & 1.0 & $\mathrm{U}$ \\
\hline $76-13-1$ & 1,1,2-Trichloro-1,2,2-trifluoroethane & 1.0 & $\mathrm{U}$ \\
\hline $67-64-1$ & Acetone & 5.0 & $\mathrm{U}$ \\
\hline $75-15-0$ & Carbon disulfide & 0.047 & $\mathrm{~J} B$ \\
\hline $79-20-9$ & Methyl acetate & 1.0 & $\mathrm{U}$ \\
\hline $75-09-2$ & Methylene Chloride & 0.019 & $\mathrm{~J} B$ \\
\hline $156-60-5$ & trans-1,2-Dichloroethene & 0.011 & $\mathrm{~J} B$ \\
\hline $1634-04-4$ & Methyl tert-butyl ether & 1.0 & $\mathrm{U}$ \\
\hline $75-34-3$ & 1,1-Dichloroethane & 1.0 & $\mathrm{U}$ \\
\hline $156-59-2$ & cis-1,2-Dichloroethene & 1.0 & $\mathrm{U}$ \\
\hline $78-93-3$ & 2-Butanone & 5.0 & $\mathrm{U}$ \\
\hline $74-97-5$ & Bromochloromethane & 1.0 & $\mathrm{U}$ \\
\hline $67-66-3$ & Chloroform & 0.43 & $\mathrm{~J}$ \\
\hline $71-55-6$ & 1,1,1-Trichloroethane & 1.0 & $\mathrm{U}$ \\
\hline $110-82-7$ & Cyclohexane & 1.0 & $\mathrm{U}$ \\
\hline $56-23-5$ & Carbon tetrachloride & 2.0 & \\
\hline $71-43-2$ & Benzene & 1.0 & $\mathrm{U}$ \\
\hline $107-06-2$ & 1,2-Dichloroethane & 1.0 & $\mathrm{U}$ \\
\hline
\end{tabular}

Report 1,4-Dioxane for Low-Medium VOA analysis only 
Lab Name: TESTAMERICA BURLINGTON

Contract: $8 \mathrm{E}-00302$

Lab Code: STLV

Case No.: YORK Mod. Ref No.:

SDG No.: 33767

Matrix: (SOIL/SED/WATER) Water

Sample wt/vol: 25.0 $(\mathrm{g} / \mathrm{mL}) \mathrm{mL}$

Level: (TRACE/LOW/MED) TRACE

\% Moisture: not dec.

GC Column: DB-624 ID: $0.20 \quad(\mathrm{~mm})$

Soil Extract Volume: (uL) $(\mathrm{mL})$

Purge Volume: 25.0
Lab Sample ID: 200-33767-1

Lab File ID: 20268_04.D

Date Received: 06/01/2016

Date Analyzed: 06/02/2016

Dilution Factor: 1.0

Soil Aliquot Volume: (uL)

\begin{tabular}{|c|c|c|c|}
\hline CAS NO. & COMPOUND & $\begin{array}{l}\text { CONCENTRATION UNITS: } \\
(\mathrm{ug} / \mathrm{L} \text { or } \mathrm{ug} / \mathrm{kg}) \mathrm{ug} / \mathrm{L}\end{array}$ & Q \\
\hline $79-01-6$ & Trichloroethene & 0.013 & $\mathrm{~J} B$ \\
\hline $108-87-2$ & Methylcyclohexane & 1.0 & $\mathrm{U}$ \\
\hline $78-87-5$ & 1,2-Dichloropropane & 1.0 & $\mathrm{U}$ \\
\hline $75-27-4$ & Bromodichloromethane & 1.0 & $\mathrm{U}$ \\
\hline $10061-01-5$ & cis-1,3-Dichloropropene & 1.0 & $\mathrm{U}$ \\
\hline $108-10-1$ & 4-Methyl-2-pentanone & 1.0 & $\mathrm{U}$ \\
\hline $108-88-3$ & Toluene & 0.032 & $\mathrm{~J} B$ \\
\hline $10061-02-6$ & trans-1,3-Dichloropropene & 1.0 & $\mathrm{U}$ \\
\hline $79-00-5$ & $1,1,2-T r i c h l o r o e t h a n e$ & 1.0 & $\mathrm{U}$ \\
\hline $127-18-4$ & Tetrachloroethene & 0.018 & $\mathrm{~J} B$ \\
\hline $591-78-6$ & 2-Hexanone & 1.0 & $\mathrm{U}$ \\
\hline $124-48-1$ & Dibromochloromethane & 1.0 & $\mathrm{U}$ \\
\hline $106-93-4$ & 1,2-Dibromoethane & 1.0 & $\mathrm{U}$ \\
\hline $108-90-7$ & Chlorobenzene & 1.0 & $\mathrm{U}$ \\
\hline $100-41-4$ & Ethylbenzene & 0.0067 & $\mathrm{~J} B$ \\
\hline $95-47-6$ & o-Xylene & 0.0045 & $\mathrm{~J} B$ \\
\hline $179601-23-1$ & m,p-Xylene & 0.014 & $\mathrm{~J} B$ \\
\hline $100-42-5$ & Styrene & 1.0 & $\mathrm{U}$ \\
\hline $75-25-2$ & Bromoform & 1.0 & $\mathrm{U}$ \\
\hline $98-82-8$ & Isopropylbenzene & 1.0 & $\mathrm{U}$ \\
\hline $79-34-5$ & $1,1,2,2$-Tetrachloroethane & 1.0 & $\mathrm{U}$ \\
\hline $541-73-1$ & 1,3-Dichlorobenzene & 1.0 & $\mathrm{U}$ \\
\hline $106-46-7$ & 1,4-Dichlorobenzene & 1.0 & $\mathrm{U}$ \\
\hline $95-50-1$ & 1,2-Dichlorobenzene & 1.0 & $\mathrm{U}$ \\
\hline $96-12-8$ & 1,2-Dibromo-3-Chloropropane & 1.0 & $\mathrm{U}$ \\
\hline $120-82-1$ & $1,2,4$-Trichlorobenzene & 0.040 & $\mathrm{~J} B$ \\
\hline $87-61-6$ & $1,2,3$-Trichlorobenzene & 1.0 & $\mathrm{U}$ \\
\hline
\end{tabular}


Lab Name: TESTAMERICA BURLINGTON

Contract: $8 \mathrm{E}-00302$

Lab Code: STLV Case No.: YORK Mod. Ref No.: SDG No.: 33767

Matrix: (SOIL/SED/WATER) Water

Sample wt/vol: 25.0 $(\mathrm{g} / \mathrm{mL}) \mathrm{mL}$

Level: (TRACE or LOW/MED) TRACE

\% Moisture: not dec.

GC Column: DB-624 ID $: 0.20 \quad(\mathrm{~mm})$

Soil Extract Volume: (uL)

CONCENTRATION UNITS: (ug/L or ug/kg) ug/L
Lab Sample ID: 200-33767-1

Lab File ID: 20268_04.D

Date Received: 06/01/2016

Date Analyzed: 06/02/2016

Dilution Factor: 1.0

Soil Aliquot Volume: (uL) Purge Volume: 25.0

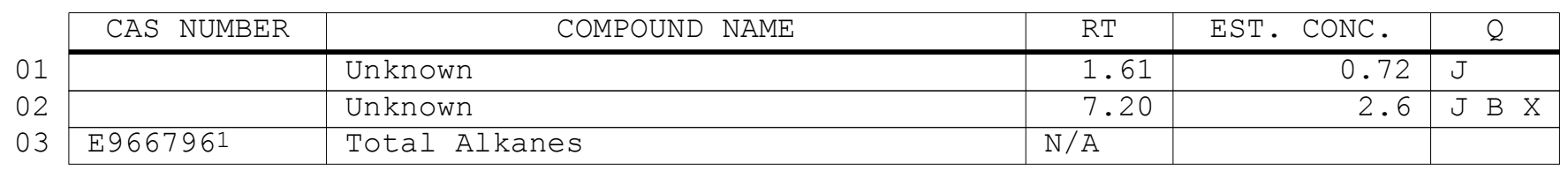

1 EPA-designated Registry Number. 
Lab Name: TESTAMERICA BURLINGTON

Contract: $8 \mathrm{E}-00302$

Lab Code: STLV

Case No.: YORK Mod. Ref No.:

SDG No.: 33767

Matrix: (SOIL/SED/WATER) Water

Sample wt/vol: 25.0 $(\mathrm{g} / \mathrm{mL}) \mathrm{mL}$

Level: (TRACE/LOW/MED) TRACE

\% Moisture: not dec.

GC Column: DB-624 ID: $0.20 \quad(\mathrm{~mm})$

Soil Extract Volume: (uL) $(\mathrm{mL})$

Purge Volume: 25.0
Lab Sample ID: 200-33767-3

Lab File ID: 20268_06.D

Date Received: 06/01/2016

Date Analyzed: 06/02/2016

Dilution Factor: 1.0

Soil Aliquot Volume:

(uL)

\begin{tabular}{|c|c|c|c|}
\hline CAS NO. & COMPOUND & $\begin{array}{l}\text { CONCENTRATION UNITS: } \\
(\mathrm{ug} / \mathrm{L} \text { or } \mathrm{ug} / \mathrm{kg}) \mathrm{ug} / \mathrm{L}\end{array}$ & Q \\
\hline $75-71-8$ & Dichlorodifluoromethane & 1.0 & $\mathrm{U}$ \\
\hline $74-87-3$ & Chloromethane & 1.0 & $\mathrm{U}$ \\
\hline $75-01-4$ & Vinyl chloride & 1.0 & $\mathrm{U}$ \\
\hline $74-83-9$ & Bromomethane & 1.0 & $\mathrm{U}$ \\
\hline $75-00-3$ & Chloroethane & 1.0 & $\mathrm{U}$ \\
\hline $75-69-4$ & Trichlorofluoromethane & 0.045 & $\mathrm{~J}$ \\
\hline $75-35-4$ & 1,1-Dichloroethene & 1.0 & $\mathrm{U}$ \\
\hline $76-13-1$ & $1,1,2$-Trichloro-1,2,2-trifluoroethane & 1.0 & $\mathrm{U}$ \\
\hline $67-64-1$ & Acetone & 5.0 & $\mathrm{U}$ \\
\hline $75-15-0$ & Carbon disulfide & 0.028 & $\mathrm{~J} B$ \\
\hline $79-20-9$ & Methyl acetate & 1.0 & $\mathrm{U}$ \\
\hline $75-09-2$ & Methylene Chloride & 1.0 & $\mathrm{U}$ \\
\hline $156-60-5$ & trans-1,2-Dichloroethene & 1.0 & $\mathrm{U}$ \\
\hline $1634-04-4$ & Methyl tert-butyl ether & 1.0 & $\mathrm{U}$ \\
\hline $75-34-3$ & 1,1-Dichloroethane & 1.0 & $\mathrm{U}$ \\
\hline $156-59-2$ & cis-1,2-Dichloroethene & 1.0 & $\mathrm{U}$ \\
\hline $78-93-3$ & 2-Butanone & 5.0 & $\mathrm{U}$ \\
\hline $74-97-5$ & Bromochloromethane & 1.0 & $\mathrm{U}$ \\
\hline $67-66-3$ & Chloroform & 1.1 & \\
\hline $71-55-6$ & $1,1,1$-Trichloroethane & 1.0 & $\mathrm{U}$ \\
\hline $110-82-7$ & Cyclohexane & 1.0 & $\mathrm{U}$ \\
\hline $56-23-5$ & Carbon tetrachloride & 50 & $\mathrm{E}$ \\
\hline $71-43-2$ & Benzene & 1.0 & $\mathrm{U}$ \\
\hline $107-06-2$ & 1,2-Dichloroethane & 1.0 & $\mathrm{U}$ \\
\hline
\end{tabular}

Report 1,4-Dioxane for Low-Medium VOA analysis only 
Lab Name: TESTAMERICA BURLINGTON

Contract: $8 \mathrm{E}-00302$

Lab Code: STLV

Case No.: YORK

Mod. Ref No.:

SDG No.: 33767

Matrix: (SOIL/SED/WATER) Water

Sample wt/vol: 25.0 $(\mathrm{g} / \mathrm{mL}) \mathrm{mL}$

Level: (TRACE/LOW/MED) TRACE

\% Moisture: not dec.

GC Column: DB-624 ID: $0.20 \quad(\mathrm{~mm})$

Soil Extract Volume: (uL) $(\mathrm{mL})$

Purge Volume: 25.0 (mI)
Lab Sample ID: 200-33767-3

Lab File ID: 20268_06.D

Date Received: 06/01/2016

Date Analyzed: 06/02/2016

Dilution Factor: 1.0

Soil Aliquot Volume: (uL)

\begin{tabular}{|c|c|c|c|}
\hline CAS NO. & COMPOUND & $\begin{array}{l}\text { CONCENTRATION UNITS: } \\
(\mathrm{ug} / \mathrm{L} \text { or } \mathrm{ug} / \mathrm{kg}) \mathrm{ug} / \mathrm{L}\end{array}$ & Q \\
\hline $79-01-6$ & Trichloroethene & 1.0 & $\mathrm{U}$ \\
\hline $108-87-2$ & Methylcyclohexane & 1.0 & $\mathrm{U}$ \\
\hline $78-87-5$ & 1,2-Dichloropropane & 1.0 & $\mathrm{U}$ \\
\hline $75-27-4$ & Bromodichloromethane & 1.0 & $\mathrm{U}$ \\
\hline $10061-01-5$ & cis-1,3-Dichloropropene & 1.0 & U \\
\hline $108-10-1$ & 4-Methyl-2-pentanone & 1.0 & $\mathrm{U}$ \\
\hline $108-88-3$ & Toluene & 0.021 & $\mathrm{~J} B$ \\
\hline $10061-02-6$ & trans-1,3-Dichloropropene & 1.0 & $\mathrm{U}$ \\
\hline $79-00-5$ & $1,1,2-$ Trichloroethane & 1.0 & $\mathrm{U}$ \\
\hline $127-18-4$ & Tetrachloroethene & 0.022 & $\mathrm{~J} B$ \\
\hline $591-78-6$ & 2-Hexanone & 1.0 & $\mathrm{U}$ \\
\hline $124-48-1$ & Dibromochloromethane & 1.0 & $\mathrm{U}$ \\
\hline $106-93-4$ & 1,2-Dibromoethane & 1.0 & $\mathrm{U}$ \\
\hline $108-90-7$ & Chlorobenzene & 1.0 & $\mathrm{U}$ \\
\hline $100-41-4$ & Ethylbenzene & 1.0 & $\mathrm{U}$ \\
\hline $95-47-6$ & o-Xylene & 1.0 & $\mathrm{U}$ \\
\hline $179601-23-1$ & m,p-xylene & 0.0096 & $\mathrm{~J} B$ \\
\hline $100-42-5$ & Styrene & 1.0 & $\mathrm{U}$ \\
\hline $75-25-2$ & Bromoform & 3.0 & \\
\hline $98-82-8$ & Isopropylbenzene & 1.0 & $\mathrm{U}$ \\
\hline $79-34-5$ & $1,1,2,2$-Tetrachloroethane & 1.0 & $\mathrm{U}$ \\
\hline $541-73-1$ & 1,3 -Dichlorobenzene & 1.0 & $\mathrm{U}$ \\
\hline $106-46-7$ & 1,4-Dichlorobenzene & 1.0 & $\mathrm{U}$ \\
\hline $95-50-1$ & 1,2-Dichlorobenzene & 1.0 & $\mathrm{U}$ \\
\hline $96-12-8$ & 1,2-Dibromo-3-Chloropropane & 1.0 & $\mathrm{U}$ \\
\hline $120-82-1$ & $1,2,4$-Trichlorobenzene & 1.0 & $\mathrm{U}$ \\
\hline $87-61-6$ & $1,2,3$-Trichlorobenzene & 1.0 & $\mathrm{U}$ \\
\hline
\end{tabular}


Lab Name: TESTAMERICA BURLINGTON

Contract: $8 \mathrm{E}-00302$

Lab Code: STLV Case No.: YORK Mod. Ref No.: SDG No.: 33767

Matrix: (SOIL/SED/WATER) Water

Sample wt/vol: 25.0 $(\mathrm{g} / \mathrm{mL}) \mathrm{mL}$

Level: (TRACE or LOW/MED) TRACE

\% Moisture: not dec.

GC Column: DB-624 ID $: 0.20 \quad(\mathrm{~mm})$

Soil Extract Volume: (uL)

CONCENTRATION UNITS: (ug/L or ug/kg) ug/L
Lab Sample ID: 200-33767-3

Lab File ID: 20268_06.D

Date Received: 06/01/2016

Date Analyzed: 06/02/2016

Dilution Factor: 1.0

Soil Aliquot Volume: (uL) Purge Volume: 25.0 $(\mathrm{mL})$

01

02

\begin{tabular}{|l|l|c|c|c|}
\hline CAS NUMBER & \multicolumn{1}{|c|}{ COMPOUND NAME } & RT & EST. CONC. & Q \\
\hline & Unknown & 7.20 & 2.7 & $\mathrm{~J}$ B X \\
\hline E9667961 & Total Alkanes & N/A & & \\
\hline
\end{tabular}

1EPA-designated Registry Number. 
Lab Name: TESTAMERICA BURLINGTON

Contract: $8 \mathrm{E}-00302$

Lab Code: STLV

Case No.: YORK Mod. Ref No.:

SDG No.: 33767

Matrix: (SOIL/SED/WATER) Water

Sample wt/vol: 25.0 $(\mathrm{g} / \mathrm{mL}) \mathrm{mL}$

Level: (TRACE/LOW/MED) TRACE

\% Moisture: not dec.

GC Column: DB-624 ID: $0.20 \quad(\mathrm{~mm})$

Soil Extract Volume: (uL) $(\mathrm{mL})$

Purge Volume: 25.0
Lab Sample ID: 200-33767-3

Lab File ID: 20250_17.D

Date Received: 06/01/2016

Date Analyzed: 06/02/2016

Dilution Factor: 4.0

Soil Aliquot Volume: (uL)

\begin{tabular}{|c|c|c|c|}
\hline CAS NO. & COMPOUND & $\begin{array}{l}\text { CONCENTRATION UNITS: } \\
(\mathrm{ug} / \mathrm{L} \text { or } \mathrm{ug} / \mathrm{kg}) \mathrm{ug} / \mathrm{L}\end{array}$ & $Q$ \\
\hline $75-71-8$ & Dichlorodifluoromethane & 4.0 & $\mathrm{U}$ \\
\hline $74-87-3$ & Chloromethane & 4.0 & $\mathrm{U}$ \\
\hline $75-01-4$ & Vinyl chloride & 4.0 & $\mathrm{U}$ \\
\hline $74-83-9$ & Bromomethane & 4.0 & $\mathrm{U}$ \\
\hline $75-00-3$ & Chloroethane & 4.0 & $\mathrm{U}$ \\
\hline $75-69-4$ & Trichlorofluoromethane & 0.045 & $\mathrm{~J} \mathrm{D}$ \\
\hline $75-35-4$ & 1,1-Dichloroethene & 4.0 & $\mathrm{U}$ \\
\hline $76-13-1$ & $1,1,2$-Trichloro-1,2,2-trifluoroethane & 4.0 & $\mathrm{U}$ \\
\hline $67-64-1$ & Acetone & 20 & $\mathrm{U}$ \\
\hline $75-15-0$ & Carbon disulfide & 0.080 & $\mathrm{~J} \quad \mathrm{D} B$ \\
\hline $79-20-9$ & Methyl acetate & 4.0 & $\mathrm{U}$ \\
\hline $75-09-2$ & Methylene Chloride & 0.066 & $\mathrm{~J} \quad \mathrm{D} \quad \mathrm{B}$ \\
\hline $156-60-5$ & trans-1,2-Dichloroethene & 4.0 & $\mathrm{U}$ \\
\hline $1634-04-4$ & Methyl tert-butyl ether & 4.0 & $\mathrm{U}$ \\
\hline $75-34-3$ & 1,1-Dichloroethane & 4.0 & $\mathrm{U}$ \\
\hline $156-59-2$ & cis-1,2-Dichloroethene & 4.0 & $\mathrm{U}$ \\
\hline $78-93-3$ & 2-Butanone & 20 & $\mathrm{U}$ \\
\hline $74-97-5$ & Bromochloromethane & 4.0 & $\mathrm{U}$ \\
\hline $67-66-3$ & Chloroform & 1.2 & $\mathrm{~J} \mathrm{D}$ \\
\hline $71-55-6$ & 1,1,1-Trichloroethane & 4.0 & $\mathrm{U}$ \\
\hline $110-82-7$ & Cyclohexane & 4.0 & $\mathrm{U}$ \\
\hline $56-23-5$ & Carbon tetrachloride & 47 & $\mathrm{D}$ \\
\hline $71-43-2$ & Benzene & 4.0 & $\mathrm{U}$ \\
\hline $107-06-2$ & 1,2-Dichloroethane & 4.0 & $\mathrm{U}$ \\
\hline
\end{tabular}

Report 1,4-Dioxane for Low-Medium VOA analysis only 
Lab Name: TESTAMERICA BURLINGTON

Contract: $8 \mathrm{E}-00302$

Lab Code: STLV

Case No.: YORK

Mod. Ref No.:

SDG No.: 33767

Matrix: (SOIL/SED/WATER) Water

Sample wt/vol: 25.0 $(\mathrm{g} / \mathrm{mL}) \mathrm{mL}$

Level: (TRACE/LOW/MED) TRACE

\% Moisture: not dec.

GC Column: DB-624 ID: $0.20 \quad(\mathrm{~mm})$

Soil Extract Volume: (uL) $(\mathrm{mL})$

Purge Volume: 25.0
Lab Sample ID: 200-33767-3

Lab File ID: 20250_17.D

Date Received: 06/01/2016

Date Analyzed: 06/02/2016

Dilution Factor: 4.0

Soil Aliquot Volume: (uL) (mI)

\begin{tabular}{|c|c|c|c|}
\hline CAS NO. & COMPOUND & $\begin{array}{l}\text { CONCENTRATION UNITS: } \\
(\mathrm{ug} / \mathrm{L} \text { or } \mathrm{ug} / \mathrm{kg}) \mathrm{ug} / \mathrm{L}\end{array}$ & Q \\
\hline $79-01-6$ & Trichloroethene & 4.0 & $\mathrm{U}$ \\
\hline $108-87-2$ & Methylcyclohexane & 4.0 & $\mathrm{U}$ \\
\hline $78-87-5$ & 1,2-Dichloropropane & 4.0 & $\mathrm{U}$ \\
\hline $75-27-4$ & Bromodichloromethane & 4.0 & $\mathrm{U}$ \\
\hline $10061-01-5$ & cis-1,3-Dichloropropene & 4.0 & U \\
\hline $108-10-1$ & 4-Methyl-2-pentanone & 4.0 & $\mathrm{U}$ \\
\hline $108-88-3$ & Toluene & 0.053 & $\mathrm{~J} \quad \mathrm{D} \quad \mathrm{B}$ \\
\hline $10061-02-6$ & trans-1,3-Dichloropropene & 4.0 & $\mathrm{U}$ \\
\hline $79-00-5$ & $1,1,2-$ Trichloroethane & 4.0 & $\mathrm{U}$ \\
\hline $127-18-4$ & Tetrachloroethene & 4.0 & $\mathrm{U}$ \\
\hline $591-78-6$ & 2-Hexanone & 4.0 & $\mathrm{U}$ \\
\hline $124-48-1$ & Dibromochloromethane & 4.0 & $\mathrm{U}$ \\
\hline $106-93-4$ & 1,2-Dibromoethane & 4.0 & $\mathrm{U}$ \\
\hline $108-90-7$ & Chlorobenzene & 4.0 & $\mathrm{U}$ \\
\hline $100-41-4$ & Ethylbenzene & 4.0 & $\mathrm{U}$ \\
\hline $95-47-6$ & o-Xylene & 4.0 & $\mathrm{U}$ \\
\hline $179601-23-1$ & m,p-xylene & 0.020 & $\mathrm{~J} \quad \mathrm{D} B$ \\
\hline $100-42-5$ & Styrene & 4.0 & $\mathrm{U}$ \\
\hline $75-25-2$ & Bromoform & 2.4 & $\mathrm{~J} \mathrm{D}$ \\
\hline $98-82-8$ & Isopropylbenzene & 4.0 & $\mathrm{U}$ \\
\hline $79-34-5$ & $1,1,2,2$-Tetrachloroethane & 4.0 & $\mathrm{U}$ \\
\hline $541-73-1$ & 1,3 -Dichlorobenzene & 4.0 & $\mathrm{U}$ \\
\hline $106-46-7$ & 1,4-Dichlorobenzene & 4.0 & $\mathrm{U}$ \\
\hline $95-50-1$ & 1,2-Dichlorobenzene & 4.0 & $\mathrm{U}$ \\
\hline $96-12-8$ & 1,2-Dibromo-3-Chloropropane & 4.0 & $\mathrm{U}$ \\
\hline $120-82-1$ & $1,2,4$-Trichlorobenzene & 4.0 & $\mathrm{U}$ \\
\hline $87-61-6$ & $1,2,3$-Trichlorobenzene & 4.0 & $\mathrm{U}$ \\
\hline
\end{tabular}


Lab Name: TESTAMERICA BURLINGTON

Contract: $8 \mathrm{E}-00302$

Lab Code: STLV Case No.: YORK Mod. Ref No.: SDG No.: 33767

Matrix: (SOIL/SED/WATER) Water

Sample wt/vol: 25.0 $(\mathrm{g} / \mathrm{mL}) \mathrm{mL}$

Level: (TRACE or LOW/MED) TRACE

\% Moisture: not dec.

GC Column: DB-624 ID $: 0.20 \quad(\mathrm{~mm})$

Soil Extract Volume: (uL)

CONCENTRATION UNITS: (ug/L or ug/kg) ug/L
Lab Sample ID: 200-33767-3

Lab File ID: 20250 17.D

Date Received: 06/01/2016

Date Analyzed: 06/02/2016

Dilution Factor: 4.0

Soil Aliquot Volume: (uL) Purge Volume: 25.0

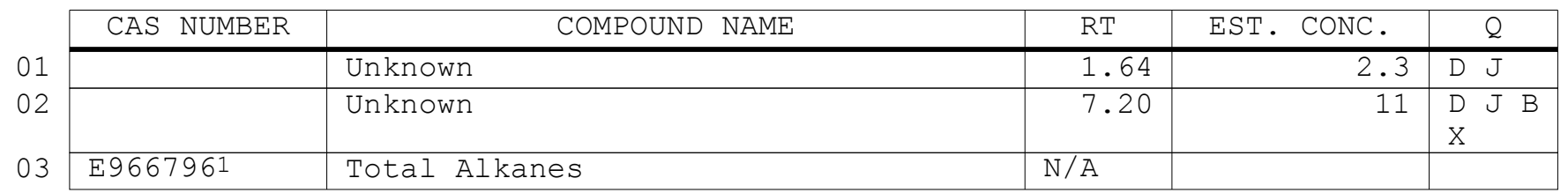

1EPA-designated Registry Number. 
Lab Name: TESTAMERICA BURLINGTON

Contract: $8 \mathrm{E}-00302$

Lab Code: STLV

Case No.: YORK Mod. Ref No.:

SDG No.: 33767

Matrix: (SOIL/SED/WATER) Water

Sample wt/vol: 25.0 $(\mathrm{g} / \mathrm{mL}) \mathrm{mL}$

Level: (TRACE/LOW/MED) TRACE

\% Moisture: not dec.

GC Column: DB-624 ID: $0.20 \quad(\mathrm{~mm})$

Soil Extract Volume: (uL) $(\mathrm{mL})$

Purge Volume: 25.0
Lab Sample ID: 200-33767-4

Lab File ID: 20268_08.D

Date Received: 06/01/2016

Date Analyzed: 06/02/2016

Dilution Factor: 1.0

Soil Aliquot Volume: (uL)

\begin{tabular}{|c|c|c|c|}
\hline CAS NO. & COMPOUND & $\begin{array}{l}\text { CONCENTRATION UNITS: } \\
(\mathrm{ug} / \mathrm{L} \text { or } \mathrm{ug} / \mathrm{kg}) \mathrm{ug} / \mathrm{L}\end{array}$ & Q \\
\hline $75-71-8$ & Dichlorodifluoromethane & 1.0 & $\mathrm{U}$ \\
\hline $74-87-3$ & Chloromethane & 1.0 & $\mathrm{U}$ \\
\hline $75-01-4$ & Vinyl chloride & 1.0 & $\mathrm{U}$ \\
\hline $74-83-9$ & Bromomethane & 1.0 & $\mathrm{U}$ \\
\hline $75-00-3$ & Chloroethane & 1.0 & $\mathrm{U}$ \\
\hline $75-69-4$ & Trichlorofluoromethane & 1.0 & $\mathrm{U}$ \\
\hline $75-35-4$ & 1,1-Dichloroethene & 1.0 & $\mathrm{U}$ \\
\hline $76-13-1$ & $1,1,2$-Trichloro-1,2,2-trifluoroethane & 1.0 & $\mathrm{U}$ \\
\hline $67-64-1$ & Acetone & 5.0 & $\mathrm{U}$ \\
\hline $75-15-0$ & Carbon disulfide & 0.023 & $\mathrm{~J} B$ \\
\hline $79-20-9$ & Methyl acetate & 1.0 & $\mathrm{U}$ \\
\hline $75-09-2$ & Methylene Chloride & 1.0 & $\mathrm{U}$ \\
\hline $156-60-5$ & trans-1,2-Dichloroethene & 1.0 & $\mathrm{U}$ \\
\hline $1634-04-4$ & Methyl tert-butyl ether & 1.0 & $\mathrm{U}$ \\
\hline $75-34-3$ & 1,1-Dichloroethane & 1.0 & $\mathrm{U}$ \\
\hline $156-59-2$ & cis-1,2-Dichloroethene & 1.0 & $\mathrm{U}$ \\
\hline $78-93-3$ & 2-Butanone & 5.0 & $\mathrm{U}$ \\
\hline $74-97-5$ & Bromochloromethane & 1.0 & $\mathrm{U}$ \\
\hline $67-66-3$ & Chloroform & 1.0 & $\mathrm{U}$ \\
\hline $71-55-6$ & $1,1,1$-Trichloroethane & 1.0 & $\mathrm{U}$ \\
\hline $110-82-7$ & Cyclohexane & 1.0 & $\mathrm{U}$ \\
\hline $56-23-5$ & Carbon tetrachloride & 0.0048 & $\mathrm{~J}$ \\
\hline $71-43-2$ & Benzene & 1.0 & $\mathrm{U}$ \\
\hline $107-06-2$ & 1,2-Dichloroethane & 0.11 & $\mathrm{~J}$ \\
\hline
\end{tabular}

Report 1,4-Dioxane for Low-Medium VOA analysis only 
Lab Name: TESTAMERICA BURLINGTON

Lab Code: STLV

Case No.: YORK

Mod. Ref No.:

Contract: $8 \mathrm{E}-00302$

Matrix: (SOIL/SED/WATER) Water

Sample wt/vol: 25.0 $(\mathrm{g} / \mathrm{mL}) \mathrm{mL}$

Level: (TRACE/LOW/MED) TRACE

\% Moisture: not dec.

GC Column: DB-624 ID $: \underline{0.20 \quad(\mathrm{~mm})}$

Soil Extract Volume: (uL) $(\mathrm{mL})$

Purge Volume: 25.0
SDG No.: 33767

Lab Sample ID: 200-33767-4

Lab File ID: 20268_08.D

Date Received: 06/01/2016

Date Analyzed: 06/02/2016

Dilution Factor: 1.0

Soil Aliquot Volume: (uL)

\begin{tabular}{|c|c|c|c|}
\hline CAS NO. & COMPOUND & $\begin{array}{l}\text { CONCENTRATION UNITS: } \\
(\mathrm{ug} / \mathrm{L} \text { or } \mathrm{ug} / \mathrm{kg}) \mathrm{ug} / \mathrm{L}\end{array}$ & Q \\
\hline $79-01-6$ & Trichloroethene & 1.0 & $\mathrm{U}$ \\
\hline $108-87-2$ & Methylcyclohexane & 1.0 & $\mathrm{U}$ \\
\hline $78-87-5$ & 1,2-Dichloropropane & 1.0 & $\mathrm{U}$ \\
\hline $75-27-4$ & Bromodichloromethane & 1.0 & $\mathrm{U}$ \\
\hline $10061-01-5$ & cis-1,3-Dichloropropene & 1.0 & U \\
\hline $108-10-1$ & 4-Methyl-2-pentanone & 1.0 & $\mathrm{U}$ \\
\hline $108-88-3$ & Toluene & 0.022 & $\mathrm{~J} B$ \\
\hline $10061-02-6$ & trans-1,3-Dichloropropene & 1.0 & $\mathrm{U}$ \\
\hline $79-00-5$ & $1,1,2-$ Trichloroethane & 1.0 & $\mathrm{U}$ \\
\hline $127-18-4$ & Tetrachloroethene & 1.0 & $\mathrm{U}$ \\
\hline $591-78-6$ & 2-Hexanone & 1.0 & $\mathrm{U}$ \\
\hline $124-48-1$ & Dibromochloromethane & 1.0 & $\mathrm{U}$ \\
\hline $106-93-4$ & 1,2-Dibromoethane & 1.0 & $\mathrm{U}$ \\
\hline $108-90-7$ & Chlorobenzene & 1.0 & $\mathrm{U}$ \\
\hline $100-41-4$ & Ethylbenzene & 1.0 & $\mathrm{U}$ \\
\hline $95-47-6$ & o-Xylene & 1.0 & $\mathrm{U}$ \\
\hline $179601-23-1$ & m,p-xylene & 0.0074 & $\mathrm{~J} B$ \\
\hline $100-42-5$ & Styrene & 1.0 & $\mathrm{U}$ \\
\hline $75-25-2$ & Bromoform & 1.0 & $\mathrm{U}$ \\
\hline $98-82-8$ & Isopropylbenzene & 1.0 & $\mathrm{U}$ \\
\hline $79-34-5$ & $1,1,2,2$-Tetrachloroethane & 1.0 & $\mathrm{U}$ \\
\hline $541-73-1$ & 1,3 -Dichlorobenzene & 1.0 & $\mathrm{U}$ \\
\hline $106-46-7$ & 1,4-Dichlorobenzene & 1.0 & $\mathrm{U}$ \\
\hline $95-50-1$ & 1,2-Dichlorobenzene & 1.0 & $\mathrm{U}$ \\
\hline $96-12-8$ & 1,2-Dibromo-3-Chloropropane & 1.0 & $\mathrm{U}$ \\
\hline $120-82-1$ & $1,2,4$-Trichlorobenzene & 1.0 & $\mathrm{U}$ \\
\hline $87-61-6$ & $1,2,3$-Trichlorobenzene & 1.0 & $\mathrm{U}$ \\
\hline
\end{tabular}


Lab Name: TESTAMERICA BURLINGTON

Contract: $8 \mathrm{E}-00302$

Lab Code: STLV Case No.: YORK Mod. Ref No.: SDG No.: 33767

Matrix: (SOIL/SED/WATER) Water

Sample wt/vol: 25.0 $(\mathrm{g} / \mathrm{mL}) \mathrm{mL}$

Level: (TRACE or LOW/MED) TRACE

\% Moisture: not dec.

GC Column: DB-624 ID $: 0.20 \quad(\mathrm{~mm})$

Soil Extract Volume: (uL)

CONCENTRATION UNITS: (ug/L or ug/kg) ug/L
Lab Sample ID: 200-33767-4

Lab File ID: 20268_08.D

Date Received: 06/01/2016

Date Analyzed: 06/02/2016

Dilution Factor: 1.0

Soil Aliquot Volume: (uL) Purge Volume: 25.0

$(\mathrm{mL})$

01

02

\begin{tabular}{|l|l|c|c|c|}
\hline CAS NUMBER & \multicolumn{1}{|c|}{ COMPOUND NAME } & RT & EST. CONC. & Q \\
\hline & Unknown & 7.20 & 2.7 & $\mathrm{~J}$ B X \\
\hline E9667961 & Total Alkanes & N/A & & \\
\hline
\end{tabular}

1 EPA-designated Registry Number. 
Lab Name: TESTAMERICA BURLINGTON

Contract: $8 \mathrm{E}-00302$

Lab Code: STLV

Case No.: YORK Mod. Ref No.:

SDG No.: 33767

Matrix: (SOIL/SED/WATER) Water

Sample wt/vol: 25.0 $(\mathrm{g} / \mathrm{mL}) \mathrm{mL}$

Level: (TRACE/LOW/MED) TRACE

\% Moisture: not dec.

GC Column: DB-624 ID: $0.20 \quad(\mathrm{~mm})$

Soil Extract Volume: (uL) $(\mathrm{mL})$

Purge Volume: 25.0
Lab Sample ID: 200-33767-5

Lab File ID: 20268_09.D

Date Received: 06/01/2016

Date Analyzed: 06/02/2016

Dilution Factor: 1.0

Soil Aliquot Volume: (uL)

\begin{tabular}{|c|c|c|c|}
\hline CAS NO. & COMPOUND & $\begin{array}{l}\text { CONCENTRATION UNITS: } \\
(\mathrm{ug} / \mathrm{L} \text { or } \mathrm{ug} / \mathrm{kg}) \mathrm{ug} / \mathrm{L}\end{array}$ & Q \\
\hline $75-71-8$ & Dichlorodifluoromethane & 1.0 & $\mathrm{U}$ \\
\hline $74-87-3$ & Chloromethane & 1.0 & $\mathrm{U}$ \\
\hline $75-01-4$ & Vinyl chloride & 1.0 & $\mathrm{U}$ \\
\hline $74-83-9$ & Bromomethane & 0.077 & $\mathrm{~J} B$ \\
\hline $75-00-3$ & Chloroethane & 1.0 & $\mathrm{U}$ \\
\hline $75-69-4$ & Trichlorofluoromethane & 1.0 & $\mathrm{U}$ \\
\hline $75-35-4$ & 1,1-Dichloroethene & 1.0 & $\mathrm{U}$ \\
\hline $76-13-1$ & $1,1,2$-Trichloro-1,2,2-trifluoroethane & 1.0 & $\mathrm{U}$ \\
\hline $67-64-1$ & Acetone & 16 & B \\
\hline $75-15-0$ & Carbon disulfide & 0.023 & $\mathrm{~J} B$ \\
\hline $79-20-9$ & Methyl acetate & 1.0 & $\mathrm{U}$ \\
\hline $75-09-2$ & Methylene Chloride & 0.015 & $\mathrm{~J} B$ \\
\hline $156-60-5$ & trans-1,2-Dichloroethene & 1.0 & $\mathrm{U}$ \\
\hline $1634-04-4$ & Methyl tert-butyl ether & 1.0 & $\mathrm{U}$ \\
\hline $75-34-3$ & 1,1-Dichloroethane & 1.0 & $\mathrm{U}$ \\
\hline $156-59-2$ & cis-1,2-Dichloroethene & 1.0 & $\mathrm{U}$ \\
\hline $78-93-3$ & 2-Butanone & 0.94 & $\mathrm{~J}$ \\
\hline $74-97-5$ & Bromochloromethane & 1.0 & $\mathrm{U}$ \\
\hline $67-66-3$ & Chloroform & 1.0 & $\mathrm{U}$ \\
\hline $71-55-6$ & 1,1,1-Trichloroethane & 1.0 & $\mathrm{U}$ \\
\hline $110-82-7$ & Cyclohexane & 1.0 & $\mathrm{U}$ \\
\hline $56-23-5$ & Carbon tetrachloride & 1.0 & $\mathrm{U}$ \\
\hline $71-43-2$ & Benzene & 1.0 & $\mathrm{U}$ \\
\hline $107-06-2$ & 1,2-Dichloroethane & 1.0 & $\mathrm{U}$ \\
\hline
\end{tabular}

Report 1,4-Dioxane for Low-Medium VOA analysis only 
Lab Name: TESTAMERICA BURLINGTON

Contract: $8 \mathrm{E}-00302$

Lab Code: STLV

Case No.: YORK

Mod. Ref No.:

SDG No.: 33767

Matrix: (SOIL/SED/WATER) Water

Sample wt/vol: 25.0 $(\mathrm{g} / \mathrm{mL}) \mathrm{mL}$

Level: (TRACE/LOW/MED) TRACE

\% Moisture: not dec.

GC Column: DB-624 ID: $0.20 \quad(\mathrm{~mm})$

Soil Extract Volume: (uL) $(\mathrm{mL})$

Purge Volume: 25.0 (mI)
Lab Sample ID: 200-33767-5

Lab File ID: 20268_09.D

Date Received: 06/01/2016

Date Analyzed: 06/02/2016

Dilution Factor: 1.0

Soil Aliquot Volume: (uL)

\begin{tabular}{|c|c|c|c|}
\hline CAS NO. & COMPOUND & $\begin{array}{l}\text { CONCENTRATION UNITS: } \\
(\mathrm{ug} / \mathrm{L} \text { or } \mathrm{ug} / \mathrm{kg}) \mathrm{ug} / \mathrm{L}\end{array}$ & Q \\
\hline $79-01-6$ & Trichloroethene & 1.0 & $\mathrm{U}$ \\
\hline $108-87-2$ & Methylcyclohexane & 1.0 & $\mathrm{U}$ \\
\hline $78-87-5$ & 1,2-Dichloropropane & 1.0 & $\mathrm{U}$ \\
\hline $75-27-4$ & Bromodichloromethane & 1.0 & $\mathrm{U}$ \\
\hline $10061-01-5$ & cis-1,3-Dichloropropene & 1.0 & U \\
\hline $108-10-1$ & 4-Methyl-2-pentanone & 1.0 & $\mathrm{U}$ \\
\hline $108-88-3$ & Toluene & 0.18 & $\mathrm{~J} B$ \\
\hline $10061-02-6$ & trans-1,3-Dichloropropene & 1.0 & $\mathrm{U}$ \\
\hline $79-00-5$ & $1,1,2-$ Trichloroethane & 1.0 & $\mathrm{U}$ \\
\hline $127-18-4$ & Tetrachloroethene & 1.0 & $\mathrm{U}$ \\
\hline $591-78-6$ & 2-Hexanone & 1.0 & $\mathrm{U}$ \\
\hline $124-48-1$ & Dibromochloromethane & 1.0 & $\mathrm{U}$ \\
\hline $106-93-4$ & 1,2-Dibromoethane & 1.0 & $\mathrm{U}$ \\
\hline $108-90-7$ & Chlorobenzene & 1.0 & $\mathrm{U}$ \\
\hline $100-41-4$ & Ethylbenzene & 0.0095 & $\mathrm{~J} B$ \\
\hline $95-47-6$ & o-Xylene & 0.071 & $\mathrm{~J} B$ \\
\hline $179601-23-1$ & m,p-xylene & 0.025 & $\mathrm{~J} B$ \\
\hline $100-42-5$ & Styrene & 0.015 & $\mathrm{~J} B$ \\
\hline $75-25-2$ & Bromoform & 1.0 & $\mathrm{U}$ \\
\hline $98-82-8$ & Isopropylbenzene & 1.0 & $\mathrm{U}$ \\
\hline $79-34-5$ & $1,1,2,2$-Tetrachloroethane & 1.0 & $\mathrm{U}$ \\
\hline $541-73-1$ & 1,3 -Dichlorobenzene & 1.0 & $\mathrm{U}$ \\
\hline $106-46-7$ & 1,4-Dichlorobenzene & 0.019 & $\mathrm{~J} B$ \\
\hline $95-50-1$ & 1,2-Dichlorobenzene & 1.0 & $\mathrm{U}$ \\
\hline $96-12-8$ & 1,2-Dibromo-3-Chloropropane & 1.0 & $\mathrm{U}$ \\
\hline $120-82-1$ & $1,2,4$-Trichlorobenzene & 1.0 & $\mathrm{U}$ \\
\hline $87-61-6$ & $1,2,3$-Trichlorobenzene & 1.0 & $\mathrm{U}$ \\
\hline
\end{tabular}


Lab Name: TESTAMERICA BURLINGTON

Contract: $8 \mathrm{E}-00302$

Lab Code: STLV Case No.: YORK Mod. Ref No.: SDG No.: 33767

Matrix: (SOIL/SED/WATER) Water

Sample wt/vol: 25.0 $(\mathrm{g} / \mathrm{mL}) \mathrm{mL}$

Level: (TRACE or LOW/MED) TRACE

․ Moisture: not dec.

GC Column: DB-624 ID $: 0.20 \quad(\mathrm{~mm})$

Soil Extract Volume: (uL)
Lab Sample ID: 200-33767-5

Lab File ID: 20268_09.D

Date Received: 06/01/2016

Date Analyzed: 06/02/2016

Dilution Factor: 1.0

Soil Aliquot Volume: (uL) Purge Volume: 25.0 CONCENTRATION UNITS: (ug/L or ug/kg) ug/L

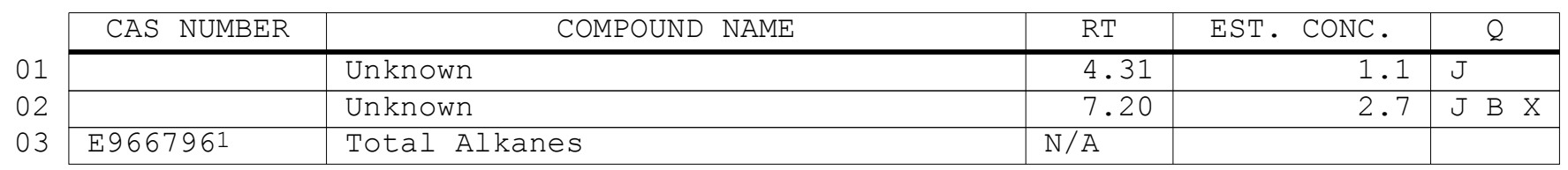


Lab Name: TESTAMERICA BURLINGTON

Contract: $8 \mathrm{E}-00302$

Lab Code: STLV Case No.: YORK Mod. Ref No.:

SDG No.: 33767

Instrument ID: CHD.i

Calibration Date(s): 05/31/2016 05/31/2016

Heated Purge: (Y/N) N

Calibration Time(s) : 1605

1746

Purge Volume: 25.0 $(\mathrm{mL})$

GC Column: DB-624

ID: $0.20 \quad$ (mm) Length: 25

$(\mathrm{m})$

\begin{tabular}{|c|c|c|c|c|c|c|c|}
\hline \multirow{3}{*}{$\begin{array}{l}\text { LAB FILE ID: } \\
\text { RRF } 5.0=20227 \_04 . D \\
\text { COMPOUND }\end{array}$} & \multirow{2}{*}{\multicolumn{3}{|c|}{ 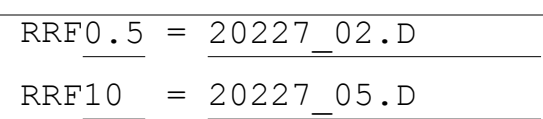 }} & \multirow{2}{*}{\multicolumn{4}{|c|}{$\mathrm{RRF} 1.0=\frac{20227 \_03 . \mathrm{D}}{}$}} \\
\hline & & & & & & & \\
\hline & RRE0.5 & RRE1.0 & RRF 5.0 & RRF10 & RRF20 & $\overline{\mathrm{RRF}}$ & $\because \mathrm{RSD}$ \\
\hline Dichlorodifluoromethane & 0.667 & 0.602 & 0.556 & 0.572 & 0.564 & 0.592 & 7.7 \\
\hline Chloromethane & 0.595 & 0.547 & 0.480 & 0.473 & 0.459 & 0.511 & 11.4 \\
\hline Vinyl chloride & 0.583 & 0.509 & 0.481 & 0.502 & 0.491 & 0.513 & 7.9 \\
\hline Bromomethane & 0.239 & 0.158 & 0.155 & 0.169 & 0.171 & 0.179 & 19.4 \\
\hline Chloroethane & 0.290 & 0.283 & 0.280 & 0.282 & 0.259 & 0.279 & 4.3 \\
\hline Trichlorofluoromethane & 0.718 & 0.675 & 0.613 & 0.618 & 0.608 & 0.646 & 7.5 \\
\hline 1,1-Dichloroethene & 0.384 & 0.348 & 0.328 & 0.331 & 0.325 & 0.343 & 7.1 \\
\hline $\begin{array}{l}1,1,2 \text {-Trichloro- } \\
1,2,2 \text {-trifluoroethane }\end{array}$ & 0.448 & 0.398 & 0.367 & 0.368 & 0.358 & 0.388 & 9.5 \\
\hline Acetone & 0.024 & 0.021 & 0.017 & 0.019 & 0.019 & 0.020 & 12.6 \\
\hline Carbon disulfide & 1.077 & 0.937 & 0.929 & 0.988 & 1.005 & 0.987 & 6.0 \\
\hline Methyl acetate & 0.059 & 0.052 & 0.050 & 0.051 & 0.050 & 0.052 & 7.1 \\
\hline Methylene Chloride & 0.324 & 0.295 & 0.272 & 0.273 & 0.270 & 0.287 & 8.1 \\
\hline trans-1,2-Dichloroethene & 0.388 & 0.352 & 0.332 & 0.335 & 0.332 & 0.348 & 6.9 \\
\hline Methyl tert-butyl ether & 0.376 & 0.353 & 0.357 & 0.373 & 0.389 & 0.370 & 4.0 \\
\hline 1,1-Dichloroethane & 0.736 & 0.665 & 0.623 & 0.637 & 0.636 & 0.660 & 6.9 \\
\hline cis-1,2-Dichloroethene & 0.352 & 0.315 & 0.332 & 0.342 & 0.331 & 0.334 & 4.2 \\
\hline 2-Butanone & 0.033 & 0.030 & 0.032 & 0.035 & 0.035 & 0.033 & 7.0 \\
\hline Bromochloromethane & 0.112 & 0.107 & 0.097 & 0.102 & 0.105 & 0.105 & 5.1 \\
\hline Chloroform & 0.634 & 0.586 & 0.556 & 0.566 & 0.576 & 0.584 & 5.2 \\
\hline 1,1,1-Trichloroethane & 0.763 & 0.693 & 0.667 & 0.679 & 0.688 & 0.698 & 5.4 \\
\hline Cyclohexane & 0.734 & 0.702 & 0.814 & 0.863 & 0.880 & 0.799 & 9.8 \\
\hline Carbon tetrachloride & 0.673 & 0.612 & 0.607 & 0.623 & 0.628 & 0.629 & 4.1 \\
\hline Benzene & 2.159 & 1.975 & 1.906 & 1.952 & 1.975 & 1.993 & 4.9 \\
\hline 1,2-Dichloroethane & 0.293 & 0.257 & 0.256 & 0.260 & 0.267 & 0.266 & 5.7 \\
\hline Trichloroethene & 0.465 & 0.422 & 0.424 & 0.440 & 0.450 & 0.440 & 4.1 \\
\hline Methylcyclohexane & 0.599 & 0.590 & 0.666 & 0.695 & 0.694 & 0.649 & 7.8 \\
\hline
\end{tabular}

Report 1,4-Dioxane for Low-Medium VOA analysis only 
Lab Name: TESTAMERICA BURLINGTON

Contract: $8 \mathrm{E}-00302$

Lab Code: STLV Case No.: YORK Mod. Ref No.:

SDG No.: 33767

Instrument ID: CHD.i

Calibration Date(s): 05/31/2016 05/31/2016

Heated Purge: (Y/N) N

Calibration Time(s) : 1605

1746

Purge Volume: 25.0

GC Column: DB-624

ID: 0.20 (mL)

LAB FILE ID:

(mm) Length: 25

(m)

$\mathrm{RRF} 5.0=20227 \_04 . \mathrm{D}$
$\mathrm{RRF} 0.5=20227 \_02 . \mathrm{D}$
RRF1.0 = 20227_03.D
$\mathrm{RRF} 10=20227$ 05.D
RRF20 = 20227_06.D

COMPOUND

\begin{tabular}{|l|l|}
\hline COMPOUND & RRF0.5 \\
\hline
\end{tabular}

\begin{tabular}{|c|c|c|c|c|c|c|c|}
\hline 1,2-Dichloropropane & 0.450 & 0.394 & 0.387 & 0.391 & 0.397 & 0.404 & 6.5 \\
\hline Bromodichloromethane & 0.396 & 0.364 & 0.368 & 0.382 & 0.395 & 0.381 & 3.9 \\
\hline cis-1,3-Dichloropropene & 0.383 & 0.368 & 0.472 & 0.517 & 0.545 & 0.457 & 17.2 \\
\hline 4-Methyl-2-pentanone & 0.072 & 0.074 & 0.094 & 0.101 & 0.105 & 0.089 & 17.2 \\
\hline Toluene & 1.692 & 1.704 & 1.891 & 1.962 & 1.990 & 1.848 & 7.7 \\
\hline trans-1,3-Dichloropropene & 0.238 & 0.235 & 0.304 & 0.343 & 0.370 & 0.298 & 20.5 \\
\hline 1,1,2-Trichloroethane & 0.209 & 0.182 & 0.169 & 0.172 & 0.171 & 0.180 & 9.2 \\
\hline Tetrachloroethene & 0.472 & 0.431 & 0.425 & 0.440 & 0.435 & 0.441 & 4.2 \\
\hline 2-Hexanone & 0.048 & 0.052 & 0.066 & 0.070 & 0.072 & 0.062 & 17.6 \\
\hline Dibromochloromethane & 0.176 & 0.164 & 0.176 & 0.190 & 0.198 & 0.181 & 7.5 \\
\hline 1,2-Dibromoethane & 0.142 & 0.128 & 0.135 & 0.138 & 0.144 & 0.137 & 4.6 \\
\hline Chlorobenzene & 1.207 & 1.138 & 1.100 & 1.124 & 1.120 & 1.138 & 3.6 \\
\hline Ethylbenzene & 1.724 & 1.752 & 2.139 & 2.283 & 2.319 & 2.043 & 14.0 \\
\hline o-Xylene & 0.488 & 0.541 & 0.751 & 0.802 & 0.804 & 0.677 & 22.3 \\
\hline $\mathrm{m}, \mathrm{p}$-Xylene & 0.627 & 0.666 & 0.841 & 0.885 & 0.893 & 0.783 & 16.2 \\
\hline Styrene & 0.803 & 0.905 & 1.184 & 1.244 & 1.250 & 1.077 & 19.4 \\
\hline Bromoform & 0.169 & 0.149 & 0.145 & 0.162 & 0.182 & 0.161 & 9.3 \\
\hline Isopropylbenzene & 1.433 & 1.568 & 2.168 & 2.351 & 2.365 & 1.977 & 22.5 \\
\hline $1,1,2,2$-Tetrachloroethane & 0.177 & 0.167 & 0.165 & 0.171 & 0.172 & 0.170 & 2.8 \\
\hline 1,3-Dichlorobenzene & 1.375 & 1.332 & 1.435 & 1.557 & 1.640 & 1.468 & 8.7 \\
\hline 1,4-Dichlorobenzene & 1.993 & 1.711 & 1.583 & 1.599 & 1.627 & 1.703 & 10.0 \\
\hline 1,2-Dichlorobenzene & 1.344 & 1.274 & 1.266 & 1.300 & 1.323 & 1.301 & 2.5 \\
\hline 1,2-Dibromo-3-Chloropropane & 0.045 & 0.040 & 0.033 & 0.036 & 0.041 & 0.039 & 11.3 \\
\hline $1,2,4$-Trichlorobenzene & 0.687 & 0.612 & 0.691 & 0.845 & 0.949 & 0.757 & 18.1 \\
\hline $1,2,3$-Trichlorobenzene & 0.470 & 0.439 & 0.518 & 0.605 & 0.671 & 0.541 & 17.8 \\
\hline
\end{tabular}


Lab Name: TESTAMERICA BURLINGTON

Contract: $8 \mathrm{E}-00302$

Lab Code: STLV Case No.: YORK Mod. Ref No.: SDG No.: 33767

Instrument ID: CHD.i

Heated Purge: (Y/N) N

Purge Volume: 25.0

GC Column: DB-624
ID: 0.20
Calibration Date(s): 05/31/2016

Calibration Time (s) : 1605 $(\mathrm{mL})$

(mm) Length: 25
$05 / 31 / 2016$ 1746

\begin{tabular}{|c|c|c|c|c|c|c|c|}
\hline LAB FILE ID: & \multirow{2}{*}{\multicolumn{3}{|c|}{$\begin{array}{l}\operatorname{RRF}_{\underline{0.5}}=\frac{20227 \_02 . \mathrm{D}}{\mathrm{RRF}_{10}}=20227 \_05 . \mathrm{D}\end{array}$}} & \multirow{2}{*}{\multicolumn{4}{|c|}{$\begin{array}{l}\text { RRF1.0 }=\frac{20227 \_03 . \mathrm{D}}{\text { RRF20 }}=20227 \text { 06.D }\end{array}$}} \\
\hline RRF5.0 = 20227_04.D & & & & & & & \\
\hline COMPOUND & RRF0. 5 & RRF1. 0 & RRE 5.0 & RRE10 & RRE20 & $\overline{\mathrm{RRF}}$ & $\circ \mathrm{RSD}$ \\
\hline Vinyl Chloride-d3 & 0.507 & 0.432 & 0.420 & 0.437 & 0.428 & 0.445 & 8.0 \\
\hline Chloroethane-d5 & 0.424 & 0.372 & 0.339 & 0.334 & 0.311 & 0.356 & 12.2 \\
\hline 1,1-Dichloroethene-d2 & 0.875 & 0.756 & 0.720 & 0.733 & 0.726 & 0.762 & 8.5 \\
\hline 2-Butanone-d5 & 0.034 & 0.028 & 0.029 & 0.032 & 0.030 & 0.030 & 7.5 \\
\hline Chloroform-d & 0.681 & 0.615 & 0.576 & 0.591 & 0.594 & 0.611 & 6.8 \\
\hline 1,2-Dichloroethane-d4 & 0.245 & 0.212 & 0.201 & 0.205 & 0.209 & 0.214 & 8.2 \\
\hline Benzene-d6 & 1.902 & 1.743 & 1.756 & 1.802 & 1.828 & 1.806 & 3.5 \\
\hline 1,2-Dichloropropane-d6 & 0.507 & 0.429 & 0.417 & 0.434 & 0.436 & 0.444 & 8.0 \\
\hline Toluene-d8 & 1.445 & 1.398 & 1.557 & 1.626 & 1.638 & 1.533 & 7.0 \\
\hline trans-1,3-Dichloropropene-d4 & 0.221 & 0.200 & 0.236 & 0.270 & 0.300 & 0.245 & 16.2 \\
\hline 2-Hexanone-d5 & 0.021 & 0.021 & 0.031 & 0.036 & 0.040 & 0.030 & 28.4 \\
\hline $1,1,2,2$-Tetrachloroethane-d2 & 0.194 & 0.163 & 0.165 & 0.166 & 0.168 & 0.171 & 7.6 \\
\hline 1,2-Dichlorobenzene-d4 & 0.917 & 0.756 & 0.750 & 0.791 & 0.823 & 0.807 & 8.5 \\
\hline
\end{tabular}

Report 1,4-Dioxane-d8 for Low-Medium VOA analysis only 
Lab Name: TESTAMERICA BURLINGTON

Contract: $8 \mathrm{E}-00302$

Lab Code: STLV

Mod. Ref No.:

SDG No.: 33767

Instrument ID: CHD.i

Calibration Date: 06/02/2016 Time: 0938

Lab File Id: 20250_03.D

Init. Calib. Date(s):05/31/2016 05/31/2016

EPA Sample No.(VSTD\#\#\#\#) : VSTD005DL

Init. Calib. Time(s) : 1605 1746

Heated Purge: (Y/N) N GC Column: DB-624

ID: $0.20(\mathrm{~mm})$ Length: 25

(m)

Purge Volume: 25.0 $(\mathrm{mL})$

\begin{tabular}{|c|c|c|c|c|c|}
\hline COMPOUND & $\overline{\mathrm{RRF}}$ & RRF5.0 & $\begin{array}{l}\text { MIN } \\
\text { RRF }\end{array}$ & $\because \mathrm{D}$ & $\mathrm{MAX} \div \mathrm{D}$ \\
\hline Dichlorodifluoromethane & 0.592 & 0.630 & 0.010 & 6.4 & 40.0 \\
\hline Chloromethane & 0.511 & 0.524 & 0.010 & 2.5 & 40.0 \\
\hline Vinyl chloride & 0.513 & 0.544 & 0.100 & 5.9 & 30.0 \\
\hline Bromomethane & 0.179 & 0.164 & 0.100 & -8.0 & 30.0 \\
\hline Chloroethane & 0.279 & 0.299 & 0.010 & 7.0 & 40.0 \\
\hline Trichlorofluoromethane & 0.646 & 0.680 & 0.010 & 5.2 & 40.0 \\
\hline 1,1-Dichloroethene & 0.343 & 0.358 & 0.100 & 4.2 & 30.0 \\
\hline 1,1,2-Trichloro-1,2,2-trifluoroethane & 0.388 & 0.405 & 0.010 & 4.4 & 40.0 \\
\hline Acetone & 0.020 & 0.019 & 0.010 & -5.5 & 40.0 \\
\hline Carbon disulfide & 0.987 & 1.041 & 0.010 & 5.4 & 40.0 \\
\hline Methyl acetate & 0.052 & 0.056 & 0.010 & 7.2 & 40.0 \\
\hline Methylene Chloride & 0.287 & 0.296 & 0.010 & 3.4 & 40.0 \\
\hline trans-1,2-Dichloroethene & 0.348 & 0.356 & 0.010 & 2.3 & 40.0 \\
\hline Methyl tert-butyl ether & 0.370 & 0.367 & 0.010 & -0.8 & 40.0 \\
\hline 1,1-Dichloroethane & 0.660 & 0.682 & 0.200 & 3.4 & 30.0 \\
\hline cis-1,2-Dichloroethene & 0.334 & 0.332 & 0.010 & -0.6 & 40.0 \\
\hline 2-Butanone & 0.033 & 0.031 & 0.010 & -5.3 & 40.0 \\
\hline Bromochloromethane & 0.105 & 0.109 & 0.050 & 4.2 & 30.0 \\
\hline Chloroform & 0.584 & 0.611 & 0.200 & 4.6 & 30.0 \\
\hline 1,1,1-Trichloroethane & 0.698 & 0.714 & 0.100 & 2.3 & 30.0 \\
\hline Cyclohexane & 0.799 & 0.875 & 0.010 & 9.5 & 40.0 \\
\hline Carbon tetrachloride & 0.629 & 0.661 & 0.100 & 5.2 & 30.0 \\
\hline Benzene & 1.993 & 2.029 & 0.400 & 1.8 & 30.0 \\
\hline 1,2-Dichloroethane & 0.266 & 0.280 & 0.100 & 5.1 & 30.0 \\
\hline Trichloroethene & 0.440 & 0.449 & 0.300 & 2.0 & 30.0 \\
\hline Methylcyclohexane & 0.649 & 0.729 & 0.010 & 12.4 & 40.0 \\
\hline
\end{tabular}

Report 1,4-Dioxane for Low/Medium VOA analysis only 
Lab Name: TESTAMERICA BURLINGTON

Contract: 8E-00302

Lab Code: STLV

Mod. Ref No.:

SDG No.: 33767

Instrument ID: CHD.i

Calibration Date: 06/02/2016 Time: 0938

Lab File Id: 20250_03.D

Init. Calib. Date(s):05/31/2016 05/31/2016

EPA Sample No. (VSTD\#\#\#\#) : VSTD005DL

Init. Calib. Time(s) : 1605 1746

Heated Purge: (Y/N) N GC Column: DB-624

ID: $0.20(\mathrm{~mm})$ Length: 25

(m)

Purge Volume: 25.0 $(\mathrm{mL})$

\begin{tabular}{|c|c|c|c|c|c|}
\hline COMPOUND & $\overline{\mathrm{RRF}}$ & RRF5.0 & $\begin{array}{l}\text { MIN } \\
\text { RRF }\end{array}$ & $\because \mathrm{D}$ & $\operatorname{MAX} \cong \mathrm{D}$ \\
\hline 1,2-Dichloropropane & 0.404 & 0.407 & 0.010 & 0.8 & 40.0 \\
\hline Bromodichloromethane & 0.381 & 0.390 & 0.200 & 2.5 & 30.0 \\
\hline cis-1,3-Dichloropropene & 0.457 & 0.497 & 0.200 & 8.7 & 30.0 \\
\hline 4-Methyl-2-pentanone & 0.089 & 0.098 & 0.010 & 9.9 & 40.0 \\
\hline Toluene & 1.848 & 2.031 & 0.400 & 9.9 & 30.0 \\
\hline trans-1,3-Dichloropropene & 0.298 & 0.317 & 0.100 & 6.4 & 30.0 \\
\hline $1,1,2$-Trichloroethane & 0.180 & 0.176 & 0.100 & -2.2 & 30.0 \\
\hline Tetrachloroethene & 0.441 & 0.454 & 0.100 & 3.0 & 30.0 \\
\hline 2-Hexanone & 0.062 & 0.069 & 0.010 & 11.7 & 40.0 \\
\hline Dibromochloromethane & 0.181 & 0.186 & 0.100 & 2.9 & 30.0 \\
\hline 1,2-Dibromoethane & 0.137 & 0.138 & 0.010 & 0.7 & 40.0 \\
\hline Chlorobenzene & 1.138 & 1.169 & 0.500 & 2.7 & 30.0 \\
\hline Ethylbenzene & 2.043 & 2.278 & 0.100 & 11.5 & 30.0 \\
\hline o-Xylene & 0.677 & 0.794 & 0.300 & 17.2 & 30.0 \\
\hline $\mathrm{m}, \mathrm{p}$-Xylene & 0.783 & 0.897 & 0.300 & 14.6 & 30.0 \\
\hline Styrene & 1.077 & 1.261 & 0.300 & 17.1 & 30.0 \\
\hline Bromoform & 0.161 & 0.164 & 0.050 & 1.6 & 30.0 \\
\hline Isopropylbenzene & 1.977 & 2.283 & 0.010 & 15.5 & 40.0 \\
\hline $1,1,2,2$-Tetrachloroethane & 0.170 & 0.175 & 0.100 & 2.8 & 30.0 \\
\hline 1,3-Dichlorobenzene & 1.468 & 1.487 & 0.400 & 1.3 & 30.0 \\
\hline 1,4-Dichlorobenzene & 1.703 & 1.673 & 0.400 & -1.8 & 30.0 \\
\hline 1,2-Dichlorobenzene & 1.301 & 1.345 & 0.400 & 3.3 & 30.0 \\
\hline 1,2-Dibromo-3-Chloropropane & 0.039 & 0.035 & 0.010 & $-10 \cdot 3$ & 40.0 \\
\hline 1,2,4-Trichlorobenzene & 0.757 & 0.701 & 0.200 & -7.4 & 30.0 \\
\hline $1,2,3$-Trichlorobenzene & 0.541 & 0.496 & 0.200 & -8.3 & 30.0 \\
\hline
\end{tabular}


Lab Name: TESTAMERICA BURLINGTON

Contract: $8 \mathrm{E}-00302$

Lab Code: STLV Case No.: YORK Mod. Ref No.

SDG No.: 33767

Instrument ID: CHD.i

Calibration Date: 06/02/2016 Time: 0938

Lab File Id: 20250_03.D

Init. Calib. Date(s):05/31/2016 05/31/2016

EPA Sample No. (VSTD\#\#\#\#) : VSTD005DL

Init. Calib. Time(s) : 1605 1746

Heated Purge: (Y/N) N GC Column: DB-624

ID: $0.20(\mathrm{~mm})$ Length: 25

(m)

Purge Volume: 25.0 $(\mathrm{mL})$

\begin{tabular}{|c|c|c|c|c|c|}
\hline COMPOUND & $\overline{\mathrm{RRF}}$ & RRF5.0 & $\begin{array}{l}\text { MIN } \\
\text { RRF }\end{array}$ & $\because \mathrm{D}$ & $\mathrm{MAX} \div \mathrm{D}$ \\
\hline Vinyl Chloride-d3 & 0.445 & 0.470 & 0.010 & 5.7 & 30.0 \\
\hline Chloroethane-d5 & 0.356 & 0.377 & 0.010 & 5.9 & 40.0 \\
\hline 1,1-Dichloroethene-d2 & 0.762 & 0.804 & 0.010 & 5.5 & 30.0 \\
\hline 2-Butanone-d5 & 0.030 & 0.030 & 0.010 & -2.5 & 40.0 \\
\hline Chloroform-d & 0.611 & 0.639 & 0.010 & 4.5 & 30.0 \\
\hline 1,2-Dichloroethane-d4 & 0.214 & 0.223 & 0.010 & 4.2 & 30.0 \\
\hline Benzene-d6 & 1.806 & 1.872 & 0.010 & 3.6 & 30.0 \\
\hline 1,2-Dichloropropane-d6 & 0.444 & 0.460 & 0.010 & 3.6 & 40.0 \\
\hline Toluene-d8 & 1.533 & 1.665 & 0.010 & 8.6 & 30.0 \\
\hline trans-1,3-Dichloropropene-d4 & 0.245 & 0.248 & 0.010 & 1.0 & 30.0 \\
\hline 2-Hexanone-d5 & 0.030 & 0.032 & 0.010 & 7.0 & 40.0 \\
\hline $1,1,2,2$-Tetrachloroethane-d2 & 0.171 & 0.175 & 0.010 & 2.0 & 30.0 \\
\hline 1,2-Dichlorobenzene-d4 & 0.807 & 0.805 & 0.010 & -0.3 & 30.0 \\
\hline
\end{tabular}

Report 1,4-Dioxane-d8 for Low/Medium VOA analysis only 
Lab Name: TESTAMERICA BURLINGTON

Contract: $8 \mathrm{E}-00302$

Lab Code: STLV Case No.: YORK Mod. Ref No.:

SDG No.: 33767

Instrument ID: CHD.i

Calibration Date: 06/02/2016 Time: 1659

Lab File Id: 20250_19.D

Init. Calib. Date(s):05/31/2016 05/31/2016

EPA Sample No.(VSTD\#\#\#\#): VSTD005LD

Init. Calib. Time(s) : 1605 1746

Heated Purge: (Y/N) N GC Column: DB-624

ID: $0.20(\mathrm{~mm})$ Length: 25

(m)

Purge Volume: 25.0 $(\mathrm{mL})$

\begin{tabular}{|c|c|c|c|c|c|}
\hline COMPOUND & $\overline{\mathrm{RRF}}$ & RRF5.0 & $\begin{array}{l}\text { MIN } \\
\text { RRF }\end{array}$ & $\because \mathrm{D}$ & $\mathrm{MAX} \div \mathrm{D}$ \\
\hline Dichlorodifluoromethane & 0.592 & 0.595 & 0.010 & 0.5 & 50.0 \\
\hline Chloromethane & 0.511 & 0.507 & 0.010 & -0.8 & 50.0 \\
\hline Vinyl chloride & 0.513 & 0.523 & 0.010 & 1.9 & 50.0 \\
\hline Bromomethane & 0.179 & 0.147 & 0.010 & -17.5 & 50.0 \\
\hline Chloroethane & 0.279 & 0.298 & 0.010 & 6.7 & 50.0 \\
\hline Trichlorofluoromethane & 0.646 & 0.661 & 0.010 & 2.2 & 50.0 \\
\hline 1,1-Dichloroethene & 0.343 & 0.348 & 0.010 & 1.4 & 50.0 \\
\hline 1,1,2-Trichloro-1,2,2-trifluoroethane & 0.388 & 0.386 & 0.010 & -0.4 & 50.0 \\
\hline Acetone & 0.020 & 0.022 & 0.010 & 9.9 & 50.0 \\
\hline Carbon disulfide & 0.987 & 0.921 & 0.010 & -6.7 & 50.0 \\
\hline Methyl acetate & 0.052 & 0.061 & 0.010 & 17.4 & 50.0 \\
\hline Methylene Chloride & 0.287 & 0.287 & 0.010 & 0.0 & 50.0 \\
\hline trans-1,2-Dichloroethene & 0.348 & 0.353 & 0.010 & 1.5 & 50.0 \\
\hline Methyl tert-butyl ether & 0.370 & 0.379 & 0.010 & 2.5 & 50.0 \\
\hline 1,1-Dichloroethane & 0.660 & 0.683 & 0.010 & 3.5 & 50.0 \\
\hline cis-1,2-Dichloroethene & 0.334 & 0.330 & 0.010 & -1.3 & 50.0 \\
\hline 2-Butanone & 0.033 & 0.033 & 0.010 & 0.8 & 50.0 \\
\hline Bromochloromethane & 0.105 & 0.107 & 0.010 & 2.0 & 50.0 \\
\hline Chloroform & 0.584 & 0.610 & 0.010 & 4.6 & 50.0 \\
\hline 1,1,1-Trichloroethane & 0.698 & 0.714 & 0.010 & 2.3 & 50.0 \\
\hline Cyclohexane & 0.799 & 0.870 & 0.010 & 8.9 & 50.0 \\
\hline Carbon tetrachloride & 0.629 & 0.657 & 0.010 & 4.5 & 50.0 \\
\hline Benzene & 1.993 & 2.048 & 0.010 & 2.7 & 50.0 \\
\hline 1,2-Dichloroethane & 0.266 & 0.279 & 0.010 & 4.9 & 50.0 \\
\hline Trichloroethene & 0.440 & 0.450 & 0.010 & 2.1 & 50.0 \\
\hline Methylcyclohexane & 0.649 & 0.690 & 0.010 & 6.4 & 50.0 \\
\hline
\end{tabular}

Report 1,4-Dioxane for Low/Medium VOA analysis only 
Lab Name: TESTAMERICA BURLINGTON

Contract: 8E-00302

Lab Code: STLV

Mod. Ref No.:

SDG No.: 33767

Instrument ID: CHD.i

Calibration Date: 06/02/2016 Time: 1659

Lab File Id: 20250_19.D

Init. Calib. Date(s):05/31/2016 05/31/2016

EPA Sample No. (VSTD\#\#\#\#) : VSTD005LD

Init. Calib. Time(s) : 1605 1746

Heated Purge: (Y/N) N GC Column: DB-624

ID: $0.20(\mathrm{~mm})$ Length: 25

(m)

Purge Volume: 25.0 $(\mathrm{mL})$

\begin{tabular}{|c|c|c|c|c|c|}
\hline COMPOUND & $\overline{\mathrm{RRF}}$ & RRF5.0 & $\begin{array}{l}\text { MIN } \\
\text { RRF }\end{array}$ & $\because \mathrm{D}$ & $\operatorname{MAX} \cong \mathrm{D}$ \\
\hline 1,2-Dichloropropane & 0.404 & 0.418 & 0.010 & 3.5 & 50.0 \\
\hline Bromodichloromethane & 0.381 & 0.392 & 0.010 & 2.9 & 50.0 \\
\hline cis-1,3-Dichloropropene & 0.457 & 0.506 & 0.010 & 10.8 & 50.0 \\
\hline 4-Methyl-2-pentanone & 0.089 & 0.105 & 0.010 & 17.8 & 50.0 \\
\hline Toluene & 1.848 & 2.043 & 0.010 & 10.6 & 50.0 \\
\hline trans-1,3-Dichloropropene & 0.298 & 0.323 & 0.010 & 8.5 & 50.0 \\
\hline $1,1,2$-Trichloroethane & 0.180 & 0.182 & 0.010 & 1.0 & 50.0 \\
\hline Tetrachloroethene & 0.441 & 0.442 & 0.010 & 0.3 & 50.0 \\
\hline 2-Hexanone & 0.062 & 0.073 & 0.010 & 17.7 & 50.0 \\
\hline Dibromochloromethane & 0.181 & 0.193 & 0.010 & 6.6 & 50.0 \\
\hline 1,2-Dibromoethane & 0.137 & 0.142 & 0.010 & 3.7 & 50.0 \\
\hline Chlorobenzene & 1.138 & 1.165 & 0.010 & 2.4 & 50.0 \\
\hline Ethylbenzene & 2.043 & 2.280 & 0.010 & 11.6 & 50.0 \\
\hline o-Xylene & 0.677 & 0.798 & 0.010 & 17.8 & 50.0 \\
\hline $\mathrm{m}, \mathrm{p}$-Xylene & 0.783 & 0.885 & 0.010 & 13.1 & 50.0 \\
\hline Styrene & 1.077 & 1.265 & 0.010 & 17.4 & 50.0 \\
\hline Bromoform & 0.161 & 0.160 & 0.010 & -0.8 & 50.0 \\
\hline Isopropylbenzene & 1.977 & 2.270 & 0.010 & 14.8 & 50.0 \\
\hline $1,1,2,2$-Tetrachloroethane & 0.170 & 0.175 & 0.010 & 2.6 & 50.0 \\
\hline 1,3-Dichlorobenzene & 1.468 & 1.472 & 0.010 & 0.3 & 50.0 \\
\hline 1,4-Dichlorobenzene & 1.703 & 1.679 & 0.010 & -1.4 & 50.0 \\
\hline 1,2-Dichlorobenzene & 1.301 & 1.366 & 0.010 & 5.0 & 50.0 \\
\hline 1,2-Dibromo-3-Chloropropane & 0.039 & 0.035 & 0.010 & -10.8 & 50.0 \\
\hline 1,2,4-Trichlorobenzene & 0.757 & 0.655 & 0.010 & -13.5 & 50.0 \\
\hline $1,2,3$-Trichlorobenzene & 0.541 & 0.469 & 0.010 & $-13 \cdot 2$ & 50.0 \\
\hline
\end{tabular}


Lab Name: TESTAMERICA BURLINGTON

Contract: $8 \mathrm{E}-00302$

Lab Code: STLV Case No.: YORK Mod. Ref No.

SDG No.: 33767

Instrument ID: CHD.i

Calibration Date: 06/02/2016 Time: 1659

Lab File Id: 20250_19.D

Init. Calib. Date (s):05/31/2016 05/31/2016

EPA Sample No. (VSTD\#\#\#\#) : VSTD005LD

Init. Calib. Time(s) : 1605 1746

Heated Purge: (Y/N) N GC Column: DB-624

ID: $0.20(\mathrm{~mm})$ Length: 25

(m)

Purge Volume: 25.0 $(\mathrm{mL})$

\begin{tabular}{|c|c|c|c|c|c|}
\hline COMPOUND & $\overline{\mathrm{RRF}}$ & RRF5.0 & $\begin{array}{l}\text { MIN } \\
\text { RRF }\end{array}$ & $\because \mathrm{D}$ & $\operatorname{MAX} \cong \mathrm{D}$ \\
\hline Vinyl Chloride-d3 & 0.445 & 0.460 & 0.010 & 3.3 & 50.0 \\
\hline Chloroethane-d5 & 0.356 & 0.370 & 0.010 & 3.8 & 50.0 \\
\hline 1,1-Dichloroethene-d2 & 0.762 & 0.797 & 0.010 & 4.6 & 50.0 \\
\hline 2-Butanone-d5 & 0.030 & 0.031 & 0.010 & 1.3 & 50.0 \\
\hline Chloroform-d & 0.611 & 0.640 & 0.010 & 4.7 & 50.0 \\
\hline 1,2-Dichloroethane-d4 & 0.214 & 0.225 & 0.010 & 4.8 & 50.0 \\
\hline Benzene-d6 & 1.806 & 1.882 & 0.010 & 4.2 & 50.0 \\
\hline 1,2-Dichloropropane-d6 & 0.444 & 0.486 & 0.010 & 9.3 & 50.0 \\
\hline Toluene-d8 & 1.533 & 1.700 & 0.010 & 10.9 & 50.0 \\
\hline trans-1,3-Dichloropropene-d4 & 0.245 & 0.244 & 0.010 & -0.5 & 50.0 \\
\hline 2-Hexanone-d5 & 0.030 & 0.034 & 0.010 & 15.1 & 50.0 \\
\hline $1,1,2,2$-Tetrachloroethane-d2 & 0.171 & 0.173 & 0.010 & 1.1 & 50.0 \\
\hline 1,2-Dichlorobenzene-d4 & 0.807 & 0.812 & 0.010 & 0.6 & 50.0 \\
\hline
\end{tabular}

Report 1,4-Dioxane-d8 for Low/Medium VOA analysis only 
Lab Name: TESTAMERICA BURLINGTON

Contract: $8 \mathrm{E}-00302$

Lab Code: STLV Case No.: YORK Mod. Ref No.:

SDG No.: 33767

Instrument ID: CHD.i

Calibration Date: 06/02/2016 Time: 1745

Lab File Id: 20268 02.D

EPA Sample No. (VSTD\#\#\#\#) : VSTD005DM Init. Calib. Date (s):05/31/2016 05/31/2016

Heated Purge: (Y/N) N GC Column: DB-624

Init. Calib. Time(s) : 1605 1746

Purge Volume: 25.0 ID: $0.20(\mathrm{~mm})$ Length: 25 (m) $(\mathrm{mL})$

\begin{tabular}{|c|c|c|c|c|c|}
\hline COMPOUND & $\overline{\mathrm{RRF}}$ & RRF5.0 & $\begin{array}{l}\text { MIN } \\
\text { RRF }\end{array}$ & $\frac{\circ}{D}$ & $\mathrm{MAX} \div \mathrm{D}$ \\
\hline Dichlorodifluoromethane & 0.592 & 0.637 & 0.010 & 7.6 & 40.0 \\
\hline Chloromethane & 0.511 & 0.533 & 0.010 & 4.3 & 40.0 \\
\hline Vinyl chloride & 0.513 & 0.552 & 0.100 & 7.5 & 30.0 \\
\hline Bromomethane & 0.179 & 0.216 & 0.100 & 20.7 & 30.0 \\
\hline Chloroethane & 0.279 & 0.306 & 0.010 & 9.6 & 40.0 \\
\hline Trichlorofluoromethane & 0.646 & 0.680 & 0.010 & 5.2 & 40.0 \\
\hline 1,1-Dichloroethene & 0.343 & 0.354 & 0.100 & 3.0 & 30.0 \\
\hline 1,1,2-Trichloro-1,2,2-trifluoroethane & 0.388 & 0.404 & 0.010 & 4.1 & 40.0 \\
\hline Acetone & 0.020 & 0.021 & 0.010 & 5.0 & 40.0 \\
\hline Carbon disulfide & 0.987 & 1.005 & 0.010 & 1.8 & 40.0 \\
\hline Methyl acetate & 0.052 & 0.059 & 0.010 & 12.2 & 40.0 \\
\hline Methylene Chloride & 0.287 & 0.302 & 0.010 & 5.3 & 40.0 \\
\hline trans-1,2-Dichloroethene & 0.348 & 0.359 & 0.010 & 3.2 & 40.0 \\
\hline Methyl tert-butyl ether & 0.370 & 0.367 & 0.010 & -0.8 & 40.0 \\
\hline 1,1-Dichloroethane & 0.660 & 0.684 & 0.200 & 3.7 & 30.0 \\
\hline cis-1,2-Dichloroethene & 0.334 & 0.359 & 0.010 & 7.2 & 40.0 \\
\hline 2-Butanone & 0.033 & 0.036 & 0.010 & 8.9 & 40.0 \\
\hline Bromochloromethane & 0.105 & 0.107 & 0.050 & 2.1 & 30.0 \\
\hline Chloroform & 0.584 & 0.618 & 0.200 & 5.8 & 30.0 \\
\hline 1,1,1-Trichloroethane & 0.698 & 0.722 & 0.100 & 3.4 & 30.0 \\
\hline Cyclohexane & 0.799 & 0.889 & 0.010 & 11.3 & 40.0 \\
\hline Carbon tetrachloride & 0.629 & 0.661 & 0.100 & 5.2 & 30.0 \\
\hline Benzene & 1.993 & 2.059 & 0.400 & 3.3 & 30.0 \\
\hline 1,2-Dichloroethane & 0.266 & 0.282 & 0.100 & 5.9 & 30.0 \\
\hline Trichloroethene & 0.440 & 0.456 & 0.300 & 3.6 & 30.0 \\
\hline Methylcyclohexane & 0.649 & 0.738 & 0.010 & 13.8 & 40.0 \\
\hline
\end{tabular}

Report 1,4-Dioxane for Low/Medium VOA analysis only 
Lab Name: TESTAMERICA BURLINGTON

Contract: 8E-00302

Lab Code: STLV

Mod. Ref No.:

SDG No.: 33767

Instrument ID: CHD.i

Calibration Date: 06/02/2016 Time: 1745

Lab File Id: 20268 02.D

EPA Sample No. (VSTD\#\#\#\#) : VSTD005DM

Init. Calib. Date (s):05/31/2016

$05 / 31 / 2016$

Heated Purge: (Y/N) N GC Column: DB-624

Init. Calib. Time(s) : 1605 1746

Purge Volume: 25.0

ID: $0.20(\mathrm{~mm})$ Length: 25

(m)

\begin{tabular}{|c|c|c|c|c|c|}
\hline COMPOUND & $\overline{\mathrm{RRF}}$ & RRF5.0 & $\begin{array}{l}\text { MIN } \\
\text { RRF }\end{array}$ & $\because \mathrm{D}$ & $\operatorname{MAX} \cong \mathrm{D}$ \\
\hline 1,2-Dichloropropane & 0.404 & 0.416 & 0.010 & 3.1 & 40.0 \\
\hline Bromodichloromethane & 0.381 & 0.392 & 0.200 & 2.8 & 30.0 \\
\hline cis-1,3-Dichloropropene & 0.457 & 0.507 & 0.200 & 10.9 & 30.0 \\
\hline 4-Methyl-2-pentanone & 0.089 & 0.101 & 0.010 & 13.5 & 40.0 \\
\hline Toluene & 1.848 & 2.064 & 0.400 & 11.7 & 30.0 \\
\hline trans-1,3-Dichloropropene & 0.298 & 0.316 & 0.100 & 6.0 & 30.0 \\
\hline $1,1,2$-Trichloroethane & 0.180 & 0.184 & 0.100 & 2.3 & 30.0 \\
\hline Tetrachloroethene & 0.441 & 0.463 & 0.100 & 5.2 & 30.0 \\
\hline 2-Hexanone & 0.062 & 0.070 & 0.010 & 13.7 & 40.0 \\
\hline Dibromochloromethane & 0.181 & 0.192 & 0.100 & 6.1 & 30.0 \\
\hline 1,2-Dibromoethane & 0.137 & 0.143 & 0.010 & 4.2 & 40.0 \\
\hline Chlorobenzene & 1.138 & 1.185 & 0.500 & 4.1 & 30.0 \\
\hline Ethylbenzene & 2.043 & 2.312 & 0.100 & 13.2 & 30.0 \\
\hline o-Xylene & 0.677 & 0.810 & 0.300 & 19.6 & 30.0 \\
\hline $\mathrm{m}, \mathrm{p}$-Xylene & 0.783 & 0.899 & 0.300 & 14.9 & 30.0 \\
\hline Styrene & 1.077 & 1.273 & 0.300 & 18.2 & 30.0 \\
\hline Bromoform & 0.161 & 0.158 & 0.050 & -1.9 & 30.0 \\
\hline Isopropylbenzene & 1.977 & 2.332 & 0.010 & 17.9 & 40.0 \\
\hline $1,1,2,2$-Tetrachloroethane & 0.170 & 0.174 & 0.100 & 2.2 & 30.0 \\
\hline 1,3-Dichlorobenzene & 1.468 & 1.542 & 0.400 & 5.1 & 30.0 \\
\hline 1,4-Dichlorobenzene & 1.703 & 1.719 & 0.400 & 1.0 & 30.0 \\
\hline 1,2-Dichlorobenzene & 1.301 & 1.372 & 0.400 & 5.4 & 30.0 \\
\hline 1,2-Dibromo-3-Chloropropane & 0.039 & 0.034 & 0.010 & $-12 \cdot 3$ & 40.0 \\
\hline 1,2,4-Trichlorobenzene & 0.757 & 0.727 & 0.200 & -4.0 & 30.0 \\
\hline $1,2,3$-Trichlorobenzene & 0.541 & 0.531 & 0.200 & -1.7 & 30.0 \\
\hline
\end{tabular}


Lab Name: TESTAMERICA BURLINGTON

Contract: $8 \mathrm{E}-00302$

Lab Code: STLV Case No.: YORK Mod. Ref No.

SDG No.: 33767

Instrument ID: CHD.i

Calibration Date: 06/02/2016 Time: 1745

Lab File Id: 20268_02.D

Init. Calib. Date(s):05/31/2016 05/31/2016

EPA Sample No. (VSTD\#\#\#\#) : VSTD005DM

Init. Calib. Time(s) : 1605 1746

Heated Purge: (Y/N) N GC Column: DB-624

ID: $0.20(\mathrm{~mm})$ Length: 25

(m)

Purge Volume: 25.0 $(\mathrm{mL})$

\begin{tabular}{|c|c|c|c|c|c|}
\hline COMPOUND & $\overline{\mathrm{RRF}}$ & RRF5.0 & $\begin{array}{l}\text { MIN } \\
\text { RRF }\end{array}$ & $\because \mathrm{D}$ & $\mathrm{MAX} \div \mathrm{D}$ \\
\hline Vinyl Chloride-d3 & 0.445 & 0.483 & 0.010 & 8.5 & 30.0 \\
\hline Chloroethane-d5 & 0.356 & 0.380 & 0.010 & 6.9 & 40.0 \\
\hline 1,1-Dichloroethene-d2 & 0.762 & 0.800 & 0.010 & 5.0 & 30.0 \\
\hline 2-Butanone-d5 & 0.030 & 0.033 & 0.010 & 8.0 & 40.0 \\
\hline Chloroform-d & 0.611 & 0.640 & 0.010 & 4.7 & 30.0 \\
\hline 1,2-Dichloroethane-d4 & 0.214 & 0.223 & 0.010 & 4.0 & 30.0 \\
\hline Benzene-d6 & 1.806 & 1.898 & 0.010 & 5.1 & 30.0 \\
\hline 1,2-Dichloropropane-d6 & 0.444 & 0.458 & 0.010 & 3.1 & 40.0 \\
\hline Toluene-d8 & 1.533 & 1.692 & 0.010 & 10.4 & 30.0 \\
\hline trans-1,3-Dichloropropene-d4 & 0.245 & 0.246 & 0.010 & 0.0 & 30.0 \\
\hline 2-Hexanone-d5 & 0.030 & 0.032 & 0.010 & 8.4 & 40.0 \\
\hline $1,1,2,2$-Tetrachloroethane-d2 & 0.171 & 0.174 & 0.010 & 1.7 & 30.0 \\
\hline 1,2-Dichlorobenzene-d4 & 0.807 & 0.812 & 0.010 & 0.5 & 30.0 \\
\hline
\end{tabular}

Report 1,4-Dioxane-d8 for Low/Medium VOA analysis only 
Lab Name: TESTAMERICA BURLINGTON

Contract: $8 \mathrm{E}-00302$

Lab Code: STLV Case No.: YORK Mod. Ref No.:

SDG No.: 33767

Instrument ID: CHD.i

Calibration Date: 06/03/2016 Time: 0349

Lab File Id: 20268_24.D

Init. Calib. Date(s) :05/31/2016 05/31/2016

EPA Sample No. (VSTD\#\#\#\#) : VSTD005MD

Init. Calib. Time(s) : 1605 1746

Heated Purge: (Y/N) N GC Column: DB-624

ID: $0.20(\mathrm{~mm})$ Length: 25

$(\mathrm{m})$

Purge Volume: 25.0 $(\mathrm{mL})$

\begin{tabular}{|c|c|c|c|c|c|}
\hline COMPOUND & $\overline{\mathrm{RRF}}$ & RRF5.0 & $\begin{array}{l}\text { MIN } \\
\text { RRF }\end{array}$ & $\because \mathrm{D}$ & $\mathrm{MAX} \div \mathrm{D}$ \\
\hline Dichlorodifluoromethane & 0.592 & 0.617 & 0.010 & 4.1 & 50.0 \\
\hline Chloromethane & 0.511 & 0.519 & 0.010 & 1.5 & 50.0 \\
\hline Vinyl chloride & 0.513 & 0.541 & 0.010 & 5.3 & 50.0 \\
\hline Bromomethane & 0.179 & 0.150 & 0.010 & -15.8 & 50.0 \\
\hline Chloroethane & 0.279 & 0.309 & 0.010 & 10.7 & 50.0 \\
\hline Trichlorofluoromethane & 0.646 & 0.687 & 0.010 & 6.2 & 50.0 \\
\hline 1,1-Dichloroethene & 0.343 & 0.357 & 0.010 & 4.1 & 50.0 \\
\hline 1,1,2-Trichloro-1,2,2-trifluoroethane & 0.388 & 0.400 & 0.010 & 3.1 & 50.0 \\
\hline Acetone & 0.020 & 0.021 & 0.010 & 7.3 & 50.0 \\
\hline Carbon disulfide & 0.987 & 0.938 & 0.010 & -4.9 & 50.0 \\
\hline Methyl acetate & 0.052 & 0.054 & 0.010 & 3.5 & 50.0 \\
\hline Methylene Chloride & 0.287 & 0.296 & 0.010 & 3.3 & 50.0 \\
\hline trans-1,2-Dichloroethene & 0.348 & 0.354 & 0.010 & 1.9 & 50.0 \\
\hline Methyl tert-butyl ether & 0.370 & 0.362 & 0.010 & -2.1 & 50.0 \\
\hline 1,1-Dichloroethane & 0.660 & 0.702 & 0.010 & 6.4 & 50.0 \\
\hline cis-1,2-Dichloroethene & 0.334 & 0.338 & 0.010 & 1.0 & 50.0 \\
\hline 2-Butanone & 0.033 & 0.033 & 0.010 & 0.0 & 50.0 \\
\hline Bromochloromethane & 0.105 & 0.108 & 0.010 & 3.8 & 50.0 \\
\hline Chloroform & 0.584 & 0.617 & 0.010 & 5.8 & 50.0 \\
\hline 1,1,1-Trichloroethane & 0.698 & 0.723 & 0.010 & 3.6 & 50.0 \\
\hline Cyclohexane & 0.799 & 0.897 & 0.010 & 12.3 & 50.0 \\
\hline Carbon tetrachloride & 0.629 & 0.674 & 0.010 & 7.2 & 50.0 \\
\hline Benzene & 1.993 & 2.096 & 0.010 & 5.1 & 50.0 \\
\hline 1,2-Dichloroethane & 0.266 & 0.287 & 0.010 & 7.6 & 50.0 \\
\hline Trichloroethene & 0.440 & 0.458 & 0.010 & 4.0 & 50.0 \\
\hline Methylcyclohexane & 0.649 & 0.726 & 0.010 & 11.9 & 50.0 \\
\hline
\end{tabular}

Report 1,4-Dioxane for Low/Medium VOA analysis only 
Lab Name: TESTAMERICA BURLINGTON

Contract: 8E-00302

Lab Code: STLV

Mod. Ref No.:

SDG No.: 33767

Instrument ID: CHD.i

Calibration Date: 06/03/2016 Time: 0349

Lab File Id: 20268_24.D

Init. Calib. Date(s):05/31/2016 05/31/2016

EPA Sample No. (VSTD\#\#\#\#) : VSTD005MD

Init. Calib. Time(s) : 1605 1746

Heated Purge: (Y/N) N GC Column: DB-624

ID: $0.20(\mathrm{~mm})$ Length: 25

(m)

Purge Volume: 25.0 $(\mathrm{mL})$

\begin{tabular}{|c|c|c|c|c|c|}
\hline COMPOUND & $\overline{\mathrm{RRF}}$ & RRF5.0 & $\begin{array}{l}\text { MIN } \\
\text { RRF }\end{array}$ & $\because \mathrm{D}$ & $\operatorname{MAX} \cong \mathrm{D}$ \\
\hline 1,2-Dichloropropane & 0.404 & 0.425 & 0.010 & 5.4 & 50.0 \\
\hline Bromodichloromethane & 0.381 & 0.397 & 0.010 & 4.1 & 50.0 \\
\hline cis-1,3-Dichloropropene & 0.457 & 0.477 & 0.010 & 4.4 & 50.0 \\
\hline 4-Methyl-2-pentanone & 0.089 & 0.100 & 0.010 & 12.0 & 50.0 \\
\hline Toluene & 1.848 & 2.096 & 0.010 & 13.4 & 50.0 \\
\hline trans-1,3-Dichloropropene & 0.298 & 0.298 & 0.010 & 0.1 & 50.0 \\
\hline $1,1,2$-Trichloroethane & 0.180 & 0.183 & 0.010 & 1.4 & 50.0 \\
\hline Tetrachloroethene & 0.441 & 0.464 & 0.010 & 5.2 & 50.0 \\
\hline 2-Hexanone & 0.062 & 0.071 & 0.010 & 15.1 & 50.0 \\
\hline Dibromochloromethane & 0.181 & 0.187 & 0.010 & 3.5 & 50.0 \\
\hline 1,2-Dibromoethane & 0.137 & 0.139 & 0.010 & 1.2 & 50.0 \\
\hline Chlorobenzene & 1.138 & 1.184 & 0.010 & 4.0 & 50.0 \\
\hline Ethylbenzene & 2.043 & 2.352 & 0.010 & 15.1 & 50.0 \\
\hline o-Xylene & 0.677 & 0.801 & 0.010 & 18.3 & 50.0 \\
\hline $\mathrm{m}, \mathrm{p}$-Xylene & 0.783 & 0.912 & 0.010 & 16.6 & 50.0 \\
\hline Styrene & 1.077 & 1.273 & 0.010 & 18.2 & 50.0 \\
\hline Bromoform & 0.161 & 0.153 & 0.010 & -5.4 & 50.0 \\
\hline Isopropylbenzene & 1.977 & 2.353 & 0.010 & 19.0 & 50.0 \\
\hline $1,1,2,2$-Tetrachloroethane & 0.170 & 0.175 & 0.010 & 2.6 & 50.0 \\
\hline 1,3-Dichlorobenzene & 1.468 & 1.525 & 0.010 & 3.9 & 50.0 \\
\hline 1,4-Dichlorobenzene & 1.703 & 1.716 & 0.010 & 0.8 & 50.0 \\
\hline 1,2-Dichlorobenzene & 1.301 & 1.357 & 0.010 & 4.2 & 50.0 \\
\hline 1,2-Dibromo-3-Chloropropane & 0.039 & 0.033 & 0.010 & -15.2 & 50.0 \\
\hline 1,2,4-Trichlorobenzene & 0.757 & 0.685 & 0.010 & -9.5 & 50.0 \\
\hline $1,2,3$-Trichlorobenzene & 0.541 & 0.497 & 0.010 & -8.0 & 50.0 \\
\hline
\end{tabular}


Lab Name: TESTAMERICA BURLINGTON

Contract: $8 \mathrm{E}-00302$

Lab Code: STLV Case No.: YORK Mod. Ref No.:

SDG No.: 33767

Instrument ID: CHD.i

Calibration Date: 06/03/2016 Time: 0349

Lab File Id: 20268_24.D

EPA Sample No. (VSTD\#\#\#\#) : VSTD005MD Init. Calib. Date(s):05/31/2016 05/31/2016

Heated Purge: (Y/N) N GC Column: DB-624

Init. Calib. Time(s) : 1605 1746

Purge Volume: 25.0 ID: $0.20(\mathrm{~mm})$ Length: 25

(m)

\begin{tabular}{|c|c|c|c|c|c|}
\hline COMPOUND & $\overline{\mathrm{RRF}}$ & RRF5. 0 & $\begin{array}{l}\text { MIN } \\
\text { RRF }\end{array}$ & $\circ \mathrm{D}$ & MAX $\cong D$ \\
\hline Vinyl Chloride-d3 & 0.445 & 0.471 & 0.010 & 5.9 & 50.0 \\
\hline Chloroethane-d5 & 0.356 & 0.382 & 0.010 & 7.4 & 50.0 \\
\hline 1,1-Dichloroethene-d2 & 0.762 & 0.816 & 0.010 & 7.1 & 50.0 \\
\hline 2-Butanone-d5 & 0.030 & 0.030 & 0.010 & $-1 \cdot 3$ & 50.0 \\
\hline Chloroform-d & 0.611 & 0.646 & 0.010 & 5.7 & 50.0 \\
\hline 1,2-Dichloroethane-d4 & 0.214 & 0.225 & 0.010 & 4.7 & 50.0 \\
\hline Benzene-d6 & 1.806 & 1.926 & 0.010 & 6.6 & 50.0 \\
\hline 1,2-Dichloropropane-d6 & 0.444 & 0.465 & 0.010 & 4.7 & 50.0 \\
\hline Toluene-d8 & 1.533 & 1.732 & 0.010 & 13.0 & 50.0 \\
\hline trans-1,3-Dichloropropene-d4 & 0.245 & 0.232 & 0.010 & -5.6 & 50.0 \\
\hline 2-Hexanone-d5 & 0.030 & 0.032 & 0.010 & 8.4 & 50.0 \\
\hline $1,1,2,2-$ Tetrachloroethane-d2 & 0.171 & 0.174 & 0.010 & 1.8 & 50.0 \\
\hline 1,2-Dichlorobenzene-d4 & 0.807 & 0.803 & 0.010 & -0.5 & 50.0 \\
\hline
\end{tabular}

Report 1,4-Dioxane-d8 for Low/Medium VOA analysis only 
Lab Name: TESTAMERICA BURLINGTON

Contract: $8 \mathrm{E}-00302$

Lab Code: STLV Case No.: YORK Mod. Ref No.:

SDG No.: 33767

Matrix: (SOIL/SED/WATER) Water

Sample wt/vol: 25.0 $(\mathrm{g} / \mathrm{mL}) \mathrm{mL}$

Level: (TRACE/LOW/MED) TRACE

\% Moisture: not dec.

GC Column: DB-624 ID: $0.20 \quad(\mathrm{~mm})$

Soil Extract Volume: (uL) $(\mathrm{mL})$

Purge Volume: 25.0
Lab Sample ID: MB 200-105227/4

Lab File ID: 20250_04.D

Date Received:

Date Analyzed: 06/02/2016

Dilution Factor: 1.0

Soil Aliquot Volume: (uL)

\begin{tabular}{|c|c|c|c|}
\hline CAS NO. & COMPOUND & $\begin{array}{l}\text { CONCENTRATION UNITS: } \\
(\mathrm{ug} / \mathrm{L} \text { or } \mathrm{ug} / \mathrm{kg}) \mathrm{ug} / \mathrm{L}\end{array}$ & q \\
\hline $75-71-8$ & Dichlorodifluoromethane & 1.0 & $\mathrm{U}$ \\
\hline $74-87-3$ & Chloromethane & 0.10 & $\mathrm{~J}$ \\
\hline $75-01-4$ & Vinyl chloride & 1.0 & $\mathrm{U}$ \\
\hline $74-83-9$ & Bromomethane & 0.14 & $\mathrm{~J}$ \\
\hline $75-00-3$ & Chloroethane & 1.0 & $\mathrm{U}$ \\
\hline $75-69-4$ & Trichlorofluoromethane & 1.0 & $\mathrm{U}$ \\
\hline $75-35-4$ & 1,1-Dichloroethene & 1.0 & $\mathrm{U}$ \\
\hline $76-13-1$ & $1,1,2$-Trichloro-1,2,2-trifluoroethane & 1.0 & $\mathrm{U}$ \\
\hline $67-64-1$ & Acetone & 1.3 & $\mathrm{~J}$ \\
\hline $75-15-0$ & Carbon disulfide & 0.048 & $\mathrm{~J}$ \\
\hline $79-20-9$ & Methyl acetate & 1.0 & $\mathrm{U}$ \\
\hline $75-09-2$ & Methylene Chloride & 0.052 & $\mathrm{~J}$ \\
\hline $156-60-5$ & trans-1,2-Dichloroethene & 0.018 & $\mathrm{~J}$ \\
\hline $1634-04-4$ & Methyl tert-butyl ether & 0.034 & $\mathrm{~J}$ \\
\hline $75-34-3$ & 1,1-Dichloroethane & 1.0 & $\mathrm{U}$ \\
\hline $156-59-2$ & cis-1,2-Dichloroethene & 1.0 & $\mathrm{U}$ \\
\hline $78-93-3$ & 2-Butanone & 5.0 & $\mathrm{U}$ \\
\hline $74-97-5$ & Bromochloromethane & 1.0 & $\mathrm{U}$ \\
\hline $67-66-3$ & Chloroform & 1.0 & $\mathrm{U}$ \\
\hline $71-55-6$ & $1,1,1$-Trichloroethane & 1.0 & $\mathrm{U}$ \\
\hline $110-82-7$ & Cyclohexane & 1.0 & $\mathrm{U}$ \\
\hline $56-23-5$ & Carbon tetrachloride & 1.0 & $\mathrm{U}$ \\
\hline $71-43-2$ & Benzene & 0.039 & $\mathrm{~J}$ \\
\hline $107-06-2$ & 1,2-Dichloroethane & 1.0 & $\mathrm{U}$ \\
\hline
\end{tabular}

Report 1,4-Dioxane for Low-Medium VOA analysis only 
Lab Name: TESTAMERICA BURLINGTON

Contract: $8 \mathrm{E}-00302$

Lab Code: STLV

Case No.: YORK

Mod. Ref No.:

SDG No.: 33767

Matrix: (SOIL/SED/WATER) Water

Sample wt/vol: 25.0 $(\mathrm{g} / \mathrm{mL}) \mathrm{mL}$

Level: (TRACE/LOW/MED) TRACE

\% Moisture: not dec.

GC Column: DB-624 ID: $0.20 \quad(\mathrm{~mm})$

Soil Extract Volume: (uL) $(\mathrm{mL})$

Purge Volume: 25.0 (mI)
Lab Sample ID: MB 200-105227/4

Lab File ID: 20250_04.D

Date Received:

Date Analyzed: 06/02/2016

Dilution Factor: 1.0

Soil Aliquot Volume: (uL)

\begin{tabular}{|c|c|c|c|}
\hline CAS NO. & COMPOUND & $\begin{array}{l}\text { CONCENTRATION UNITS: } \\
(\mathrm{ug} / \mathrm{L} \text { or } \mathrm{ug} / \mathrm{kg}) \mathrm{ug} / \mathrm{L}\end{array}$ & $q$ \\
\hline $79-01-6$ & Trichloroethene & 1.0 & $\mathrm{U}$ \\
\hline $108-87-2$ & Methylcyclohexane & 1.0 & $\mathrm{U}$ \\
\hline $78-87-5$ & 1,2-Dichloropropane & 1.0 & $\mathrm{U}$ \\
\hline $75-27-4$ & Bromodichloromethane & 1.0 & $\mathrm{U}$ \\
\hline $10061-01-5$ & cis-1,3-Dichloropropene & 1.0 & U \\
\hline $108-10-1$ & 4-Methyl-2-pentanone & 1.0 & $\mathrm{U}$ \\
\hline $108-88-3$ & Toluene & 0.029 & $\mathrm{~J}$ \\
\hline $10061-02-6$ & trans-1,3-Dichloropropene & 1.0 & $\mathrm{U}$ \\
\hline $79-00-5$ & $1,1,2-$ Trichloroethane & 1.0 & $\mathrm{U}$ \\
\hline $127-18-4$ & Tetrachloroethene & 0.014 & $\mathrm{~J}$ \\
\hline $591-78-6$ & 2-Hexanone & 1.0 & $\mathrm{U}$ \\
\hline $124-48-1$ & Dibromochloromethane & 1.0 & $\mathrm{U}$ \\
\hline $106-93-4$ & 1,2-Dibromoethane & 1.0 & $\mathrm{U}$ \\
\hline $108-90-7$ & Chlorobenzene & 1.0 & $\mathrm{U}$ \\
\hline $100-41-4$ & Ethylbenzene & 0.0099 & $\mathrm{~J}$ \\
\hline $95-47-6$ & o-Xylene & 0.010 & $\mathrm{~J}$ \\
\hline $179601-23-1$ & m,p-xylene & 0.011 & $\mathrm{~J}$ \\
\hline $100-42-5$ & Styrene & 0.015 & $\mathrm{~J}$ \\
\hline $75-25-2$ & Bromoform & 1.0 & $\mathrm{U}$ \\
\hline $98-82-8$ & Isopropylbenzene & 1.0 & $\mathrm{U}$ \\
\hline $79-34-5$ & $1,1,2,2$-Tetrachloroethane & 1.0 & $\mathrm{U}$ \\
\hline $541-73-1$ & 1,3 -Dichlorobenzene & 0.048 & $\mathrm{~J}$ \\
\hline $106-46-7$ & 1,4-Dichlorobenzene & 0.074 & $\mathrm{~J}$ \\
\hline $95-50-1$ & 1,2-Dichlorobenzene & 0.10 & $\mathrm{~J}$ \\
\hline $96-12-8$ & 1,2-Dibromo-3-Chloropropane & 1.0 & $\mathrm{U}$ \\
\hline $120-82-1$ & $1,2,4$-Trichlorobenzene & 0.20 & $\mathrm{~J}$ \\
\hline $87-61-6$ & $1,2,3$-Trichlorobenzene & 0.30 & $\mathrm{~J}$ \\
\hline
\end{tabular}


Lab Name: TESTAMERICA BURLINGTON

Contract: $8 \mathrm{E}-00302$

Lab Code: STLV Case No.: YORK Mod. Ref No.: SDG No.: 33767

Matrix: (SOIL/SED/WATER) Water

Sample wt/vol: 25.0 $(\mathrm{g} / \mathrm{mL}) \mathrm{mL}$

Level: (TRACE or LOW/MED) TRACE

Moisture: not dec.

GC Column: DB-624 ID $: 0.20 \quad(\mathrm{~mm})$

Soil Extract Volume: (uL)

CONCENTRATION UNITS: (ug/L or ug/kg) ug/L
Lab Sample ID: MB 200-105227/4

Lab File ID: 20250_04.D

Date Received:

Date Analyzed: 06/02/2016

Dilution Factor: 1.0

Soil Aliquot Volume: (uL) Purge Volume: 25.0 $(\mathrm{mL})$

01

02

\begin{tabular}{|l|l|c|c|c|}
\hline CAS NUMBER & \multicolumn{1}{|c|}{ COMPOUND NAME } & RT & EST. CONC. & Q \\
\hline & Unknown & 7.20 & 2.7 & $\mathrm{~J}$ X \\
\hline E9667961 & Total Alkanes & N/A & & \\
\hline
\end{tabular}

1 EPA-designated Registry Number. 
Lab Name: TESTAMERICA BURLINGTON

Contract: $8 \mathrm{E}-00302$

Lab Code: STLV Case No.: YORK Mod. Ref No.: SDG No.: 33767

Matrix: (SOIL/SED/WATER) Water

Sample wt/vol: 25.0 $(\mathrm{g} / \mathrm{mL}) \mathrm{mL}$

Level: (TRACE/LOW/MED) TRACE

\% Moisture: not dec.

GC Column: DB-624 ID $0.20 \quad(\mathrm{~mm})$

Soil Extract Volume: (uL) $(\mathrm{mL})$

Purge Volume: 25.0
Lab Sample ID: MB 200-105268/3

Lab File ID: 20268_03.D

Date Received:

Date Analyzed: 06/02/2016

Dilution Factor: 1.0

Soil Aliquot Volume: (uL)

\begin{tabular}{|c|c|c|c|}
\hline CAS NO. & COMPOUND & $\begin{array}{l}\text { CONCENTRATION UNITS: } \\
(\mathrm{ug} / \mathrm{L} \text { or } \mathrm{ug} / \mathrm{kg}) \mathrm{ug} / \mathrm{L}\end{array}$ & q \\
\hline $75-71-8$ & Dichlorodifluoromethane & 1.0 & $\mathrm{U}$ \\
\hline $74-87-3$ & Chloromethane & 1.0 & $\mathrm{U}$ \\
\hline $75-01-4$ & Vinyl chloride & 1.0 & $\mathrm{U}$ \\
\hline $74-83-9$ & Bromomethane & 0.16 & $\mathrm{~J}$ \\
\hline $75-00-3$ & Chloroethane & 1.0 & $\mathrm{U}$ \\
\hline $75-69-4$ & Trichlorofluoromethane & 1.0 & $\mathrm{U}$ \\
\hline $75-35-4$ & 1,1-Dichloroethene & 1.0 & $\mathrm{U}$ \\
\hline $76-13-1$ & $1,1,2$-Trichloro-1,2,2-trifluoroethane & 1.0 & $\mathrm{U}$ \\
\hline $67-64-1$ & Acetone & 2.3 & $\mathrm{~J}$ \\
\hline $75-15-0$ & Carbon disulfide & 0.049 & $\mathrm{~J}$ \\
\hline $79-20-9$ & Methyl acetate & 1.0 & $\mathrm{U}$ \\
\hline $75-09-2$ & Methylene Chloride & 0.067 & $\mathrm{~J}$ \\
\hline $156-60-5$ & trans-1,2-Dichloroethene & 0.019 & $\mathrm{~J}$ \\
\hline $1634-04-4$ & Methyl tert-butyl ether & 1.0 & $\mathrm{U}$ \\
\hline $75-34-3$ & 1,1-Dichloroethane & 1.0 & $\mathrm{U}$ \\
\hline $156-59-2$ & cis-1,2-Dichloroethene & 1.0 & $\mathrm{U}$ \\
\hline $78-93-3$ & 2-Butanone & 5.0 & $\mathrm{U}$ \\
\hline $74-97-5$ & Bromochloromethane & 0.030 & $\mathrm{~J}$ \\
\hline $67-66-3$ & Chloroform & 1.0 & $\mathrm{U}$ \\
\hline $71-55-6$ & 1,1,1-Trichloroethane & 1.0 & $\mathrm{U}$ \\
\hline $110-82-7$ & Cyclohexane & 1.0 & $\mathrm{U}$ \\
\hline $56-23-5$ & Carbon tetrachloride & 1.0 & $\mathrm{U}$ \\
\hline $71-43-2$ & Benzene & 1.0 & $\mathrm{U}$ \\
\hline $107-06-2$ & 1,2-Dichloroethane & 1.0 & $\mathrm{U}$ \\
\hline
\end{tabular}

Report 1,4-Dioxane for Low-Medium VOA analysis only 
Lab Name: TESTAMERICA BURLINGTON

Lab Code: STLV

Case No.: YORK

Mod. Ref No.:

Contract: 8E-00302

Matrix: (SOIL/SED/WATER) Water

Sample wt/vol: 25.0 $(\mathrm{g} / \mathrm{mL}) \mathrm{mL}$

Level: (TRACE/LOW/MED) TRACE

\% Moisture: not dec.

GC Column: DB-624 ID: $0.20 \quad(\mathrm{~mm})$

Soil Extract Volume: (uL) $(\mathrm{mL})$

Purge Volume: 25.0
SDG No.: 33767

Lab Sample ID: MB 200-105268/3

Lab File ID: 20268_03.D

Date Received:

Date Analyzed: 06/02/2016

Dilution Factor: 1.0

Soil Aliquot Volume:

(uL)

\begin{tabular}{|c|c|c|c|}
\hline CAS NO. & COMPOUND & $\begin{array}{l}\text { CONCENTRATION UNITS: } \\
(\mathrm{ug} / \mathrm{L} \text { or } \mathrm{ug} / \mathrm{kg}) \mathrm{ug} / \mathrm{L}\end{array}$ & Q \\
\hline $79-01-6$ & Trichloroethene & 0.014 & $\mathrm{~J}$ \\
\hline $108-87-2$ & Methylcyclohexane & 1.0 & $\mathrm{U}$ \\
\hline $78-87-5$ & 1,2-Dichloropropane & 1.0 & $\mathrm{U}$ \\
\hline $75-27-4$ & Bromodichloromethane & 1.0 & $\mathrm{U}$ \\
\hline $10061-01-5$ & cis-1,3-Dichloropropene & 1.0 & $\mathrm{U}$ \\
\hline $108-10-1$ & 4-Methyl-2-pentanone & 0.43 & $\mathrm{~J}$ \\
\hline $108-88-3$ & Toluene & 0.032 & $\mathrm{~J}$ \\
\hline $10061-02-6$ & trans-1,3-Dichloropropene & 1.0 & $\mathrm{U}$ \\
\hline $79-00-5$ & $1,1,2-T r i c h l o r o e t h a n e$ & 0.065 & $\mathrm{~J}$ \\
\hline $127-18-4$ & Tetrachloroethene & 0.016 & $\mathrm{~J}$ \\
\hline $591-78-6$ & 2-Hexanone & 1.0 & $\mathrm{U}$ \\
\hline $124-48-1$ & Dibromochloromethane & 0.024 & $\mathrm{~J}$ \\
\hline $106-93-4$ & 1,2-Dibromoethane & 1.0 & $\mathrm{U}$ \\
\hline $108-90-7$ & Chlorobenzene & 0.039 & $\mathrm{~J}$ \\
\hline $100-41-4$ & Ethylbenzene & 0.012 & $\mathrm{~J}$ \\
\hline $95-47-6$ & o-Xylene & 0.013 & $\mathrm{~J}$ \\
\hline $179601-23-1$ & m,p-Xylene & 0.015 & $\mathrm{~J}$ \\
\hline $100-42-5$ & styrene & 0.018 & $\mathrm{~J}$ \\
\hline $75-25-2$ & Bromoform & 1.0 & $\mathrm{U}$ \\
\hline $98-82-8$ & Isopropylbenzene & 1.0 & $\mathrm{U}$ \\
\hline $79-34-5$ & $1,1,2,2$-Tetrachloroethane & 1.0 & $\mathrm{U}$ \\
\hline $541-73-1$ & 1,3-Dichlorobenzene & 0.060 & $\mathrm{~J}$ \\
\hline $106-46-7$ & 1,4-Dichlorobenzene & 0.087 & $\mathrm{~J}$ \\
\hline $95-50-1$ & $1,2-$ Dichlorobenzene & 0.11 & $\mathrm{~J}$ \\
\hline $96-12-8$ & 1,2-Dibromo-3-Chloropropane & 1.0 & $\mathrm{U}$ \\
\hline $120-82-1$ & $1,2,4$-Trichlorobenzene & 0.24 & $\mathrm{~J}$ \\
\hline $87-61-6$ & $1,2,3$-Trichlorobenzene & 0.40 & $\mathrm{~J}$ \\
\hline
\end{tabular}


Lab Name: TESTAMERICA BURLINGTON

Contract: 8E-00302

Lab Code: STLV Case No.: YORK Mod. Ref No.: SDG No.: 33767

Matrix: (SOIL/SED/WATER) Water

Sample wt/vol: 25.0 $(\mathrm{g} / \mathrm{mL}) \mathrm{mL}$

Level: (TRACE or LOW/MED) TRACE

\% Moisture: not dec.

GC Column: DB-624 ID $: 0.20 \quad(\mathrm{~mm})$

Soil Extract Volume: (uL)

CONCENTRATION UNITS: (ug/L or ug/kg) ug/L
Lab Sample ID: MB 200-105268/3

Lab File ID: 20268_03.D

Date Received:

Date Analyzed: 06/02/2016

Dilution Factor: 1.0

Soil Aliquot Volume: (uL) Purge Volume: 25.0 $(\mathrm{mL})$

01

02

\begin{tabular}{|l|l|c|c|c|}
\hline CAS NUMBER & \multicolumn{1}{|c|}{ COMPOUND NAME } & RT & EST. CONC. & Q \\
\hline & Unknown & 7.20 & 2.6 & $\mathrm{~J} X$ \\
\hline E9667961 & Total Alkanes & N/A & & \\
\hline
\end{tabular}

1EPA-designated Registry Number. 
Lab Name: TESTAMERICA BURLINGTON

Contract: $8 \mathrm{E}-00302$

Lab Code: STLV

Case No.: YORK Mod. Ref No.:

SDG No.: 33767

Matrix: (SOIL/SED/WATER) Water

Sample wt/vol: 25.0 $(\mathrm{g} / \mathrm{mL}) \mathrm{mL}$

Level: (TRACE/LOW/MED) TRACE

\% Moisture: not dec.

GC Column: DB-624 ID: $0.20 \quad(\mathrm{~mm})$

Soil Extract Volume: (uL) $(\mathrm{mL})$

Purge Volume: 25.0
Lab Sample ID: 200-33767-6

Lab File ID: 20268_10.D

Date Received:

Date Analyzed: 06/02/2016

Dilution Factor: 1.0

Soil Aliquot Volume:

(uL)

\begin{tabular}{|c|c|c|c|}
\hline CAS NO. & COMPOUND & $\begin{array}{l}\text { CONCENTRATION UNITS: } \\
(\mathrm{ug} / \mathrm{L} \text { or } \mathrm{ug} / \mathrm{kg}) \mathrm{ug} / \mathrm{L}\end{array}$ & Q \\
\hline $75-71-8$ & Dichlorodifluoromethane & 1.0 & $\mathrm{U}$ \\
\hline $74-87-3$ & Chloromethane & 1.0 & $\mathrm{U}$ \\
\hline $75-01-4$ & Vinyl chloride & 1.0 & $\mathrm{U}$ \\
\hline $74-83-9$ & Bromomethane & 0.038 & $\mathrm{~J} B$ \\
\hline $75-00-3$ & Chloroethane & 1.0 & $\mathrm{U}$ \\
\hline $75-69-4$ & Trichlorofluoromethane & 1.0 & $\mathrm{U}$ \\
\hline $75-35-4$ & 1,1-Dichloroethene & 1.0 & $\mathrm{U}$ \\
\hline $76-13-1$ & 1,1,2-Trichloro-1,2,2-trifluoroethane & 1.0 & $\mathrm{U}$ \\
\hline $67-64-1$ & Acetone & 2.0 & $\mathrm{~J} B$ \\
\hline $75-15-0$ & Carbon disulfide & 0.020 & $\mathrm{~J} B$ \\
\hline $79-20-9$ & Methyl acetate & 1.0 & $\mathrm{U}$ \\
\hline $75-09-2$ & Methylene Chloride & 0.025 & $\mathrm{~J} B$ \\
\hline $156-60-5$ & trans-1,2-Dichloroethene & 1.0 & $\mathrm{U}$ \\
\hline $1634-04-4$ & Methyl tert-butyl ether & 1.0 & $\mathrm{U}$ \\
\hline $75-34-3$ & 1,1-Dichloroethane & 1.0 & $\mathrm{U}$ \\
\hline $156-59-2$ & cis-1,2-Dichloroethene & 1.0 & $\mathrm{U}$ \\
\hline $78-93-3$ & 2-Butanone & 5.0 & $\mathrm{U}$ \\
\hline $74-97-5$ & Bromochloromethane & 1.0 & $\mathrm{U}$ \\
\hline $67-66-3$ & Chloroform & 1.0 & $\mathrm{U}$ \\
\hline $71-55-6$ & $1,1,1$-Trichloroethane & 1.0 & $\mathrm{U}$ \\
\hline $110-82-7$ & Cyclohexane & 1.0 & $\mathrm{U}$ \\
\hline $56-23-5$ & Carbon tetrachloride & 1.0 & $\mathrm{U}$ \\
\hline $71-43-2$ & Benzene & 1.0 & $\mathrm{U}$ \\
\hline $107-06-2$ & 1,2-Dichloroethane & 1.0 & $\mathrm{U}$ \\
\hline
\end{tabular}

Report 1,4-Dioxane for Low-Medium VOA analysis only 
Lab Name: TESTAMERICA BURLINGTON

Lab Code: STLV

Case No.: YORK

Mod. Ref No.:

Contract: 8E-00302

Matrix: (SOIL/SED/WATER) Water

Sample wt/vol: 25.0 $(\mathrm{g} / \mathrm{mL}) \mathrm{mL}$

Level: (TRACE/LOW/MED) TRACE

\% Moisture: not dec.

GC Column: DB-624 ID: $0.20 \quad(\mathrm{~mm})$

Soil Extract Volume: (uL) $(\mathrm{mL})$

Purge Volume: 25.0 (mI)
SDG No.: 33767

Lab Sample ID: 200-33767-6

Lab File ID: 20268_10.D

Date Received:

Date Analyzed: 06/02/2016

Dilution Factor: 1.0

Soil Aliquot Volume:

(uL)

\begin{tabular}{|c|c|c|c|}
\hline CAS NO. & COMPOUND & $\begin{array}{l}\text { CONCENTRATION UNITS: } \\
(\mathrm{ug} / \mathrm{L} \text { or } \mathrm{ug} / \mathrm{kg}) \mathrm{ug} / \mathrm{L}\end{array}$ & Q \\
\hline $79-01-6$ & Trichloroethene & 1.0 & $\mathrm{U}$ \\
\hline $108-87-2$ & Methylcyclohexane & 1.0 & $\mathrm{U}$ \\
\hline $78-87-5$ & 1,2-Dichloropropane & 1.0 & $\mathrm{U}$ \\
\hline $75-27-4$ & Bromodichloromethane & 1.0 & $\mathrm{U}$ \\
\hline $10061-01-5$ & cis-1,3-Dichloropropene & 1.0 & U \\
\hline $108-10-1$ & 4-Methyl-2-pentanone & 1.0 & $\mathrm{U}$ \\
\hline $108-88-3$ & Toluene & 0.016 & $\mathrm{~J} B$ \\
\hline $10061-02-6$ & trans-1,3-Dichloropropene & 1.0 & $\mathrm{U}$ \\
\hline $79-00-5$ & $1,1,2-$ Trichloroethane & 1.0 & $\mathrm{U}$ \\
\hline $127-18-4$ & Tetrachloroethene & 1.0 & $\mathrm{U}$ \\
\hline $591-78-6$ & 2-Hexanone & 1.0 & $\mathrm{U}$ \\
\hline $124-48-1$ & Dibromochloromethane & 1.0 & $\mathrm{U}$ \\
\hline $106-93-4$ & 1,2-Dibromoethane & 1.0 & $\mathrm{U}$ \\
\hline $108-90-7$ & Chlorobenzene & 1.0 & $\mathrm{U}$ \\
\hline $100-41-4$ & Ethylbenzene & 1.0 & $\mathrm{U}$ \\
\hline $95-47-6$ & o-Xylene & 1.0 & $\mathrm{U}$ \\
\hline $179601-23-1$ & m,p-xylene & 1.0 & $\mathrm{U}$ \\
\hline $100-42-5$ & Styrene & 1.0 & $\mathrm{U}$ \\
\hline $75-25-2$ & Bromoform & 1.0 & $\mathrm{U}$ \\
\hline $98-82-8$ & Isopropylbenzene & 1.0 & $\mathrm{U}$ \\
\hline $79-34-5$ & $1,1,2,2$-Tetrachloroethane & 1.0 & $\mathrm{U}$ \\
\hline $541-73-1$ & 1,3 -Dichlorobenzene & 1.0 & $\mathrm{U}$ \\
\hline $106-46-7$ & 1,4-Dichlorobenzene & 1.0 & $\mathrm{U}$ \\
\hline $95-50-1$ & 1,2-Dichlorobenzene & 1.0 & $\mathrm{U}$ \\
\hline $96-12-8$ & 1,2-Dibromo-3-Chloropropane & 1.0 & $\mathrm{U}$ \\
\hline $120-82-1$ & $1,2,4$-Trichlorobenzene & 1.0 & $\mathrm{U}$ \\
\hline $87-61-6$ & $1,2,3$-Trichlorobenzene & 1.0 & $\mathrm{U}$ \\
\hline
\end{tabular}


Lab Name: TESTAMERICA BURLINGTON

Contract: $8 \mathrm{E}-00302$

Lab Code: STLV Case No.: YORK Mod. Ref No.: SDG No.: 33767

Matrix: (SOIL/SED/WATER) Water

Sample wt/vol: 25.0 $(\mathrm{g} / \mathrm{mL}) \mathrm{mL}$

Level: (TRACE or LOW/MED) TRACE

\% Moisture: not dec.

GC Column: DB-624 ID $: 0.20 \quad(\mathrm{~mm})$

Soil Extract Volume: (uL)

CONCENTRATION UNITS: (ug/L or ug/kg) ug/L
Lab Sample ID: 200-33767-6

Lab File ID: 20268_10.D

Date Received:

Date Analyzed: 06/02/2016

Dilution Factor: 1.0

Soil Aliquot Volume: (uL) Purge Volume: 25.0 $(\mathrm{mL})$

01

02

\begin{tabular}{|l|l|c|c|c|}
\hline CAS NUMBER & \multicolumn{1}{|c|}{ COMPOUND NAME } & RT & EST. CONC. & Q \\
\hline & Unknown & 7.20 & 2.6 & $\mathrm{~J}$ B X \\
\hline E9667961 & Total Alkanes & N/A & & \\
\hline
\end{tabular}

1EPA-designated Registry Number. 
Lab Name: TESTAMERICA BURLINGTON

Contract: 8E-00302

Lab Code: STLV

Case No.: YORK Mod. Ref No.:

SDG No.: 33767

Matrix: (SOIL/SED/WATER) Water

Sample wt/vol: 25.0 $(\mathrm{g} / \mathrm{mL}) \mathrm{mL}$

Level: (TRACE/LOW/MED) TRACE

\% Moisture: not dec.

GC Column: DB-624 ID: $0.20 \quad(\mathrm{~mm})$

Soil Extract Volume: $(u L)$ $(\mathrm{mL})$

Purge Volume: 25.0
Lab Sample ID: VIBLK 200-105268/7

Lab File ID: 20268_07.D

Date Received:

Date Analyzed: 06/02/2016

Dilution Factor: 1.0

Soil Aliquot Volume:

(uL)

\begin{tabular}{|c|c|c|c|}
\hline CAS NO. & COMPOUND & $\begin{array}{l}\text { CONCENTRATION UNITS: } \\
(\mathrm{ug} / \mathrm{L} \text { or } \mathrm{ug} / \mathrm{kg}) \mathrm{ug} / \mathrm{L}\end{array}$ & q \\
\hline $75-71-8$ & Dichlorodifluoromethane & 1.0 & $\mathrm{U}$ \\
\hline $74-87-3$ & Chloromethane & 1.0 & $\mathrm{U}$ \\
\hline $75-01-4$ & Vinyl chloride & 1.0 & $\mathrm{U}$ \\
\hline $74-83-9$ & Bromomethane & 1.0 & $\mathrm{U}$ \\
\hline $75-00-3$ & Chloroethane & 1.0 & $\mathrm{U}$ \\
\hline $75-69-4$ & Trichlorofluoromethane & 1.0 & $\mathrm{U}$ \\
\hline $75-35-4$ & 1,1-Dichloroethene & 1.0 & $\mathrm{U}$ \\
\hline $76-13-1$ & $1,1,2$-Trichloro-1,2,2-trifluoroethane & 1.0 & $\mathrm{U}$ \\
\hline $67-64-1$ & Acetone & 5.0 & $\mathrm{U}$ \\
\hline $75-15-0$ & Carbon disulfide & 1.0 & $\mathrm{U}$ \\
\hline $79-20-9$ & Methyl acetate & 1.0 & $\mathrm{U}$ \\
\hline $75-09-2$ & Methylene Chloride & 0.026 & $\mathrm{~J}$ \\
\hline $156-60-5$ & trans-1,2-Dichloroethene & 1.0 & $\mathrm{U}$ \\
\hline $1634-04-4$ & Methyl tert-butyl ether & 1.0 & $\mathrm{U}$ \\
\hline $75-34-3$ & 1,1-Dichloroethane & 1.0 & $\mathrm{U}$ \\
\hline $156-59-2$ & cis-1,2-Dichloroethene & 1.0 & $\mathrm{U}$ \\
\hline $78-93-3$ & 2-Butanone & 5.0 & $\mathrm{U}$ \\
\hline $74-97-5$ & Bromochloromethane & 1.0 & $\mathrm{U}$ \\
\hline $67-66-3$ & Chloroform & 1.0 & $\mathrm{U}$ \\
\hline $71-55-6$ & $1,1,1$-Trichloroethane & 1.0 & $\mathrm{U}$ \\
\hline $110-82-7$ & Cyclohexane & 1.0 & $\mathrm{U}$ \\
\hline $56-23-5$ & Carbon tetrachloride & 0.027 & $\mathrm{~J}$ \\
\hline $71-43-2$ & Benzene & 1.0 & $\mathrm{U}$ \\
\hline $107-06-2$ & 1,2-Dichloroethane & 1.0 & $\mathrm{U}$ \\
\hline
\end{tabular}

Report 1,4-Dioxane for Low-Medium VOA analysis only 
Lab Name: TESTAMERICA BURLINGTON

Lab Code: STLV

Case No.: YORK

Mod. Ref No.:

Contract: 8E-00302

Matrix: (SOIL/SED/WATER) Water

Sample wt/vol: 25.0 $(\mathrm{g} / \mathrm{mL}) \mathrm{mL}$

Level: (TRACE/LOW/MED) TRACE

\% Moisture: not dec.

GC Column: DB-624 ID: $0.20 \quad(\mathrm{~mm})$

Soil Extract Volume: (uL) $(\mathrm{mL})$

Purge Volume: 25.0
SDG No.: 33767

Lab Sample ID: VIBLK 200-105268/7

Lab File ID: 20268_07.D

Date Received:

Date Analyzed: 06/02/2016

Dilution Factor: 1.0

Soil Aliquot Volume:

(uL)

\begin{tabular}{|c|c|c|c|}
\hline CAS NO. & COMPOUND & $\begin{array}{l}\text { CONCENTRATION UNITS: } \\
(\mathrm{ug} / \mathrm{L} \text { or } \mathrm{ug} / \mathrm{kg}) \mathrm{ug} / \mathrm{L}\end{array}$ & Q \\
\hline $79-01-6$ & Trichloroethene & 1.0 & $\mathrm{U}$ \\
\hline $108-87-2$ & Methylcyclohexane & 1.0 & $\mathrm{U}$ \\
\hline $78-87-5$ & 1,2-Dichloropropane & 1.0 & $\mathrm{U}$ \\
\hline $75-27-4$ & Bromodichloromethane & 1.0 & $\mathrm{U}$ \\
\hline $10061-01-5$ & cis-1,3-Dichloropropene & 1.0 & $\mathrm{U}$ \\
\hline $108-10-1$ & 4-Methyl-2-pentanone & 5.0 & $\mathrm{U}$ \\
\hline $108-88-3$ & Toluene & 0.015 & $\mathrm{~J}$ \\
\hline $10061-02-6$ & trans-1,3-Dichloropropene & 1.0 & $\mathrm{U}$ \\
\hline $79-00-5$ & $1,1,2-T r i c h l o r o e t h a n e$ & 1.0 & $\mathrm{U}$ \\
\hline $127-18-4$ & Tetrachloroethene & 1.0 & $\mathrm{U}$ \\
\hline $591-78-6$ & 2-Hexanone & 5.0 & $\mathrm{U}$ \\
\hline $124-48-1$ & Dibromochloromethane & 1.0 & $\mathrm{U}$ \\
\hline $106-93-4$ & 1,2-Dibromoethane & 1.0 & $\mathrm{U}$ \\
\hline $108-90-7$ & Chlorobenzene & 1.0 & $\mathrm{U}$ \\
\hline $100-41-4$ & Ethylbenzene & 1.0 & $\mathrm{U}$ \\
\hline $95-47-6$ & o-Xylene & 1.0 & $\mathrm{U}$ \\
\hline $179601-23-1$ & m,p-Xylene & 1.0 & $\mathrm{U}$ \\
\hline $100-42-5$ & styrene & 1.0 & $\mathrm{U}$ \\
\hline $75-25-2$ & Bromoform & 1.0 & $\mathrm{U}$ \\
\hline $98-82-8$ & Isopropylbenzene & 1.0 & $\mathrm{U}$ \\
\hline $79-34-5$ & $1,1,2,2$-Tetrachloroethane & 1.0 & $\mathrm{U}$ \\
\hline $541-73-1$ & 1,3-Dichlorobenzene & 1.0 & $\mathrm{U}$ \\
\hline $106-46-7$ & 1,4-Dichlorobenzene & 1.0 & $\mathrm{U}$ \\
\hline $95-50-1$ & 1,2-Dichlorobenzene & 1.0 & $\mathrm{U}$ \\
\hline $96-12-8$ & 1,2-Dibromo-3-Chloropropane & 1.0 & $\mathrm{U}$ \\
\hline $120-82-1$ & $1,2,4$-Trichlorobenzene & 1.0 & $\mathrm{U}$ \\
\hline $87-61-6$ & $1,2,3$-Trichlorobenzene & 1.0 & $\mathrm{U}$ \\
\hline
\end{tabular}


Lab Name: TESTAMERICA BURLINGTON

Contract: 8E-00302

Lab Code: STLV Case No.: YORK Mod. Ref No.: SDG No.: 33767

Matrix: (SOIL/SED/WATER) Water

Sample wt/vol: 25.0 $(\mathrm{g} / \mathrm{mL}) \mathrm{mL}$

Level: (TRACE or LOW/MED) TRACE

\% Moisture: not dec.

GC Column: DB-624 ID $: 0.20 \quad(\mathrm{~mm})$

Soil Extract Volume: (uL)

CONCENTRATION UNITS: (ug/L or ug/kg) ug/L
Lab Sample ID: VIBLK 200-105268/7

Lab File ID: 20268_07.D

Date Received:

Date Analyzed: 06/02/2016

Dilution Factor: 1.0

Soil Aliquot Volume: (uL) Purge Volume: 25.0 $(\mathrm{mL})$

01

02

\begin{tabular}{|l|l|c|c|c|}
\hline CAS NUMBER & \multicolumn{1}{|c|}{ COMPOUND NAME } & RT & EST. CONC. & Q \\
\hline & Unknown & 7.20 & 2.7 & $\mathrm{~J}$ B X \\
\hline E9667961 & Total Alkanes & N/A & & \\
\hline
\end{tabular}

1EPA-designated Registry Number. 
Lab Name: TestAmerica Burlington

Job No.: 200-33767-1

SDG No.: 33767

Instrument ID: CHD.i

Analysis Batch Number: 105151

Lab Sample ID: ICIS 200-105151/4

Client Sample ID:

Date Analyzed: 05/31/16 16:56

Lab File ID: 20227 04.D

GC Column: DB-624

ID $: 0.2(\mathrm{~mm})$

\begin{tabular}{|c|c|c|c|c|}
\hline \multirow[t]{2}{*}{ COMPOUND NAME } & \multirow{2}{*}{$\begin{array}{c}\text { RETENTION } \\
\text { TIME }\end{array}$} & \multicolumn{3}{|c|}{ MANUAL INTEGRATION } \\
\hline & & REASON & ANALYST & DATE \\
\hline 1,2-Dichloropropane-d6 & 6.32 & Split Peak & wilburj & $05 / 31 / 1617: 36$ \\
\hline
\end{tabular}

Lab Sample ID: IC 200-105151/5

Client Sample ID:

Date Analyzed: 05/31/16 17:21

Lab File ID: 20227 05.D

GC Column: DB-624

ID $: 0.2(\mathrm{~mm})$

\begin{tabular}{|c|c|c|c|c|}
\hline \multicolumn{1}{|c|}{ COMPOUND NAME } & RETENTION & \multicolumn{3}{|c}{ MANUAL INTEGRATION } \\
\cline { 3 - 5 } & TIME & REASON & ANALYST & DATE \\
\hline 1,2 -Dichloropropane-d6 & 6.32 & Split Peak & wilburj & 05/31/16 17:57 \\
\hline
\end{tabular}

Lab Sample ID: IC 200-105151/6

Client Sample ID:

Date Analyzed: 05/31/16 17:46

Lab File ID: 20227_06.D

GC Column: DB-624

ID $: 0.2(\mathrm{~mm})$

\begin{tabular}{|c|c|c|c|c|}
\hline \multicolumn{1}{|c|}{ COMPOUND NAME } & RETENTION & \multicolumn{3}{|c|}{ MANUAL INTEGRATION } \\
\cline { 3 - 5 } & TIME & REASON & ANALYST & DATE \\
\hline 1,2-Dichloropropane-d6 & 6.32 & Split Peak & wilburj & $05 / 31 / 16$ 18:30 \\
\hline
\end{tabular}


Lab Name: TestAmerica Burlington SDG No.: 33767

Instrument ID: CHD.i

Lab Sample ID: CCVIS 200-105227/3

Date Analyzed: 06/02/16 09:38

\begin{tabular}{|c|r|}
\hline COMPOUND NAME & RETEN \\
& TIME \\
\hline \hline 1,2-Dichloropropane-d6 & \\
\hline
\end{tabular}

Lab Sample ID: CCVC 200-105227/19

Date Analyzed: 06/02/16 16:59 Client Sample ID: Lab File ID: 20250_19.D

Analysis Batch Number: 105227

Client Sample ID:

Lab File ID: 20250 03.D

GC Column: DB-624

ID $: 0.2(\mathrm{~mm})$
DATE

ANALYST

archern

GC Column: DB-624

ID $: 0.2(\mathrm{~mm})$

\begin{tabular}{|c|c|c|c|c|}
\hline \multirow[t]{2}{*}{ COMPOUND NAME } & \multirow{2}{*}{$\begin{array}{c}\text { RETENTION } \\
\text { TIME }\end{array}$} & \multicolumn{3}{|c|}{ MANUAL INTEGRATION } \\
\hline & & REASON & ANALYST & DATE \\
\hline 1,2-Dichloropropane-d6 & 6.32 & Split Peak & wilburj & $06 / 03 / 1616: 24$ \\
\hline
\end{tabular}


Lab Name: TestAmerica Burlington

Job No.: 200-33767-1

SDG No.: 33767

Instrument ID: CHD.i

Analysis Batch Number: 105268

Lab Sample ID: CCVIS 200-105268/2

Client Sample ID:

Date Analyzed: 06/02/16 17:45

Lab File ID: 20268 02.D

GC Column: DB-624

ID $: 0.2(\mathrm{~mm})$

\begin{tabular}{|c|c|c|c|c|}
\hline \multirow[t]{2}{*}{ COMPOUND NAME } & RETENTION & \multicolumn{3}{|c|}{ MANUAL INTEGRATION } \\
\hline & TIME & REASON & ANALYST & DATE \\
\hline 1,2 -Dichloropropane-d6 & 6.32 & Assign Peak & $\begin{array}{l}\text { puangmale } \\
\text { ek }\end{array}$ & $06 / 06 / 16 \quad 07: 49$ \\
\hline
\end{tabular}

Lab Sample ID: MB 200-105268/3

Client Sample ID:

Date Analyzed: 06/02/16 18:11

Lab File ID: 20268 03.D

GC Column: DB-624

ID $: 0.2(\mathrm{~mm})$

\begin{tabular}{|c|c|c|c|c|}
\hline \multirow[t]{2}{*}{ COMPOUND NAME } & \multirow{2}{*}{$\begin{array}{c}\text { RETENTION } \\
\text { TIME }\end{array}$} & \multicolumn{3}{|c|}{ MANUAL INTEGRATION } \\
\hline & & REASON & ANALYST & DATE \\
\hline Bromomethane & 2.01 & Assign Peak & $\begin{array}{l}\text { puangmale } \\
\text { ek }\end{array}$ & $06 / 06 / 1607: 58$ \\
\hline
\end{tabular}

Lab Sample ID: 200-33767-5

Client Sample ID: YKQCTB-W-38099

Date Analyzed: 06/02/16 21:27

Lab File ID: 20268_09.D

GC Column: DB-624

ID $: 0.2(\mathrm{~mm})$

\begin{tabular}{|l|c|c|c|c|}
\hline \multicolumn{1}{|c|}{ COMPOUND NAME } & RETENTION & \multicolumn{3}{|c|}{ MANUAL INTEGRATION } \\
\cline { 3 - 5 } & TIME & REASON & ANALYST & \multicolumn{2}{|c|}{ DATE } \\
\hline Bromomethane & 2.00 & Assign Peak & $06 / 06 / 16$ 08:28 \\
\hline
\end{tabular}

Lab Sample ID: 200-33767-6

Client Sample ID: VHBLK01

Date Analyzed: 06/02/16 21:53

Lab File ID: 20268_10.D

GC Column: DB-624

ID $: 0.2(\mathrm{~mm})$

\begin{tabular}{|c|c|c|c|c|}
\hline \multirow[t]{2}{*}{ COMPOUND NAME } & \multirow{2}{*}{$\begin{array}{c}\text { RETENTION } \\
\text { TIME }\end{array}$} & \multicolumn{3}{|c|}{ MANUAL INTEGRATION } \\
\hline & & REASON & ANALYST & DATE \\
\hline Bromomethane & 2.00 & Assign Peak & $\begin{array}{l}\text { puangmale } \\
\text { ek }\end{array}$ & $06 / 06 / 1608: 37$ \\
\hline
\end{tabular}


Lab Name: TestAmerica Burlington SDG No.: 33767

Instrument ID: CHD.i

Lab Sample ID: CCVC 200-105268/24

Date Analyzed: 06/03/16 03:49

\begin{tabular}{|c|c|c|c|c|}
\hline \multirow[t]{2}{*}{ COMPOUND NAME } & RETENTION & \multicolumn{3}{|c|}{ MANUAL INTEGRATION } \\
\hline & TIME & REASON & ANALYST & DATE \\
\hline 1,2 -Dichloropropane-d6 & 6.32 & Assign Peak & $\begin{array}{l}\text { puangmale } \\
\text { ek }\end{array}$ & $06 / 06 / 1609: 51$ \\
\hline
\end{tabular}

Job No.: 200-33767-1

Analysis Batch Number: 105268

Client Sample ID:

Lab File ID: $2026824 . \mathrm{D}$

GC Column: DB-624

ID : $0.2(\mathrm{~mm})$ 


\section{TestAmerica}

THE LEADER IN ENVIRONMENTAL TESTING

\section{ANALYTICAL REPORT}

Job Number: 200-33933-1

SDG Number: 33933

Job Description: York (200-33933)

Contract Number: 1E-30401

For:

Argonne National Laboratory

9700 South Cass Avenue

Building 203

Office B-141

Argonne, IL 60439

Attention: Ms. Esther Bowen

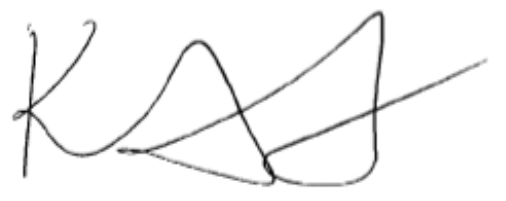

Approved for release. Kathryn A Kelly

Kathryn A Kelly, Project Manager I

30 Community Drive, South Burlington, VT, 05403

kathryn.kelly@testamericainc.com

$06 / 22 / 2016$

The test results in this report relate only to sample(s) as received by the laboratory. These test results were derived under a quality system that adheres to the requirements of NELAC. Pursuant to NELAC, this report may not be produced in full without written approval from the laboratory 


\section{Table of Contents}

Cover Title Page...$\ldots \ldots \ldots \ldots \ldots \ldots \ldots \ldots \ldots \ldots \ldots \ldots \ldots$

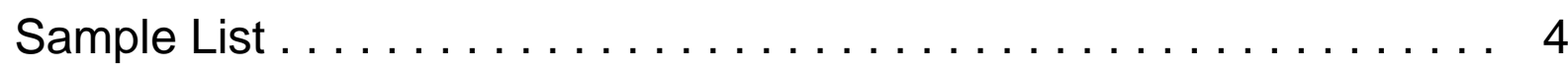

External Chain of Custody ...................... 5

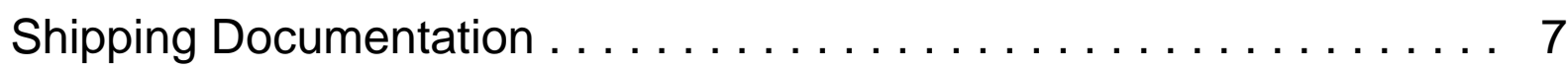

Sample Receipt and Log In Check List ..................... 8

Standards Traceability . . . . . . . . . . . . . . . . . 9

Methodology Review .............................. 14

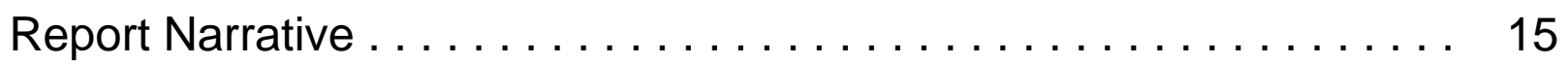

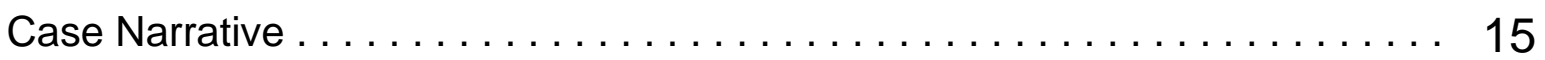

Manual Integration Documentation ....................... 16

Qualifier Definition ................................ 18

QC Summary - SOM01.2 Volatiles-Trace . . . . . . . . . . . . 19

QC Summary - SOM01.2 Volatiles-Trace ..................... 19

Deuterated Monitoring Compound Summary $\ldots \ldots \ldots \ldots \ldots \ldots \ldots \ldots \ldots \ldots \ldots$

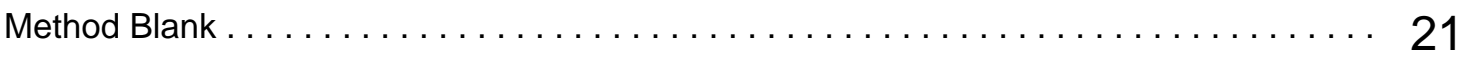

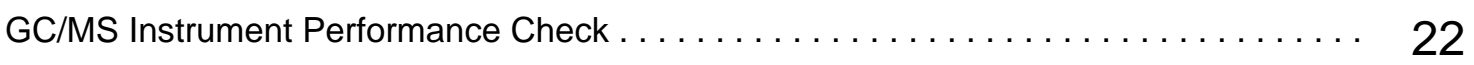

Internal Standard Area and RT Summary $\ldots \ldots \ldots \ldots \ldots \ldots \ldots \ldots \ldots \ldots \ldots \ldots \ldots \ldots \ldots$

Sample Data - SOM01.2 Volatiles-Trace ................. 25

Sample Data - SOM01.2 Volatiles-Trace ...................... 25

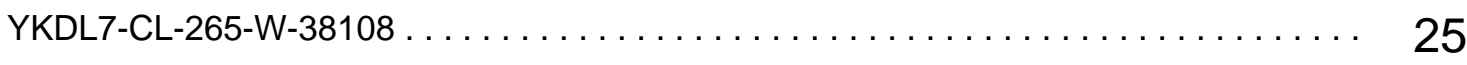

YKQCTB-W- $38110 \ldots \ldots \ldots \ldots \ldots \ldots \ldots \ldots \ldots \ldots \ldots \ldots \ldots \ldots \ldots \ldots \ldots \ldots, 28$

Standards - SOM01.2 Volatiles-Trace . . . . . . . . . . . . . 31

Standards - SOM01.2 Volatiles-Trace $\ldots \ldots \ldots \ldots \ldots \ldots \ldots \ldots \ldots \ldots \ldots \ldots$

Initial Calibration Data ................................... 31

CCV Data, including closing CCV $\ldots \ldots \ldots \ldots \ldots \ldots \ldots \ldots \ldots \ldots \ldots \ldots \ldots \ldots \ldots \ldots$

Raw Qc Data - SOM01.2 Volatiles-Trace ............... 40 


\section{Table of Contents}

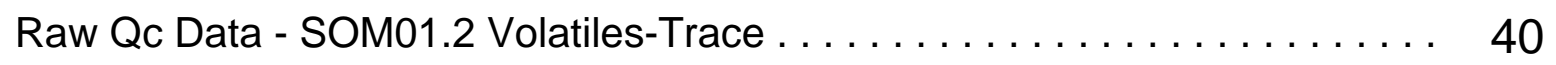

Raw Qc Data - SOM01.2 Volatiles-Trace ........................... 40

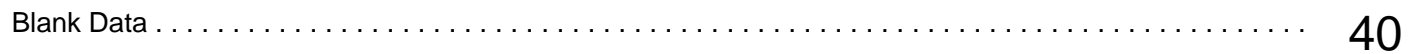




\section{Sample Login Acknowledgement}

Job 200-33933-1

\begin{tabular}{|c|c|c|c|}
\hline Client Job Description: & York (200-33933) & Report To: & Argonne National Laboratory \\
\hline Purchase Order \#: & 1E-30401 & & Jorge Alvarado \\
\hline Work Order \#: & $1 \mathrm{E}-30401$ & & 9700 South Cass Avenue \\
\hline Project Manager: & Kathryn A Kelly & & Building 203 \\
\hline Job Due Date: & $6 / 25 / 2016$ & & Office B-141 \\
\hline Job TAT: & 14 Days & & 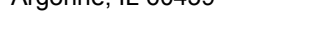 \\
\hline \multirow[t]{2}{*}{ Max Deliverable Level: } & \multirow[t]{2}{*}{ IV } & \multirow[t]{2}{*}{ Bill To: } & Argonne National Laboratory \\
\hline & & & Accounts Payable \\
\hline \multirow[t]{4}{*}{ Earliest Deliverable Due: } & \multirow[t]{4}{*}{$6 / 25 / 2016$} & & Chief Financial Offices \\
\hline & & & 9700 S. Cass Ave. \\
\hline & & & Building 201 \\
\hline & & & Argonne, IL 60439 \\
\hline
\end{tabular}

\section{Login 200-33933}

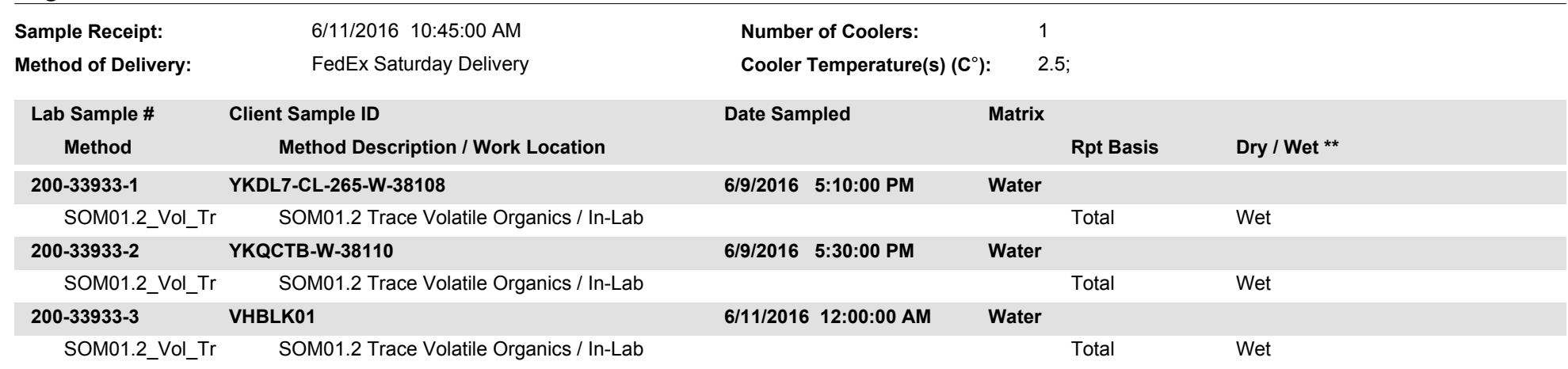




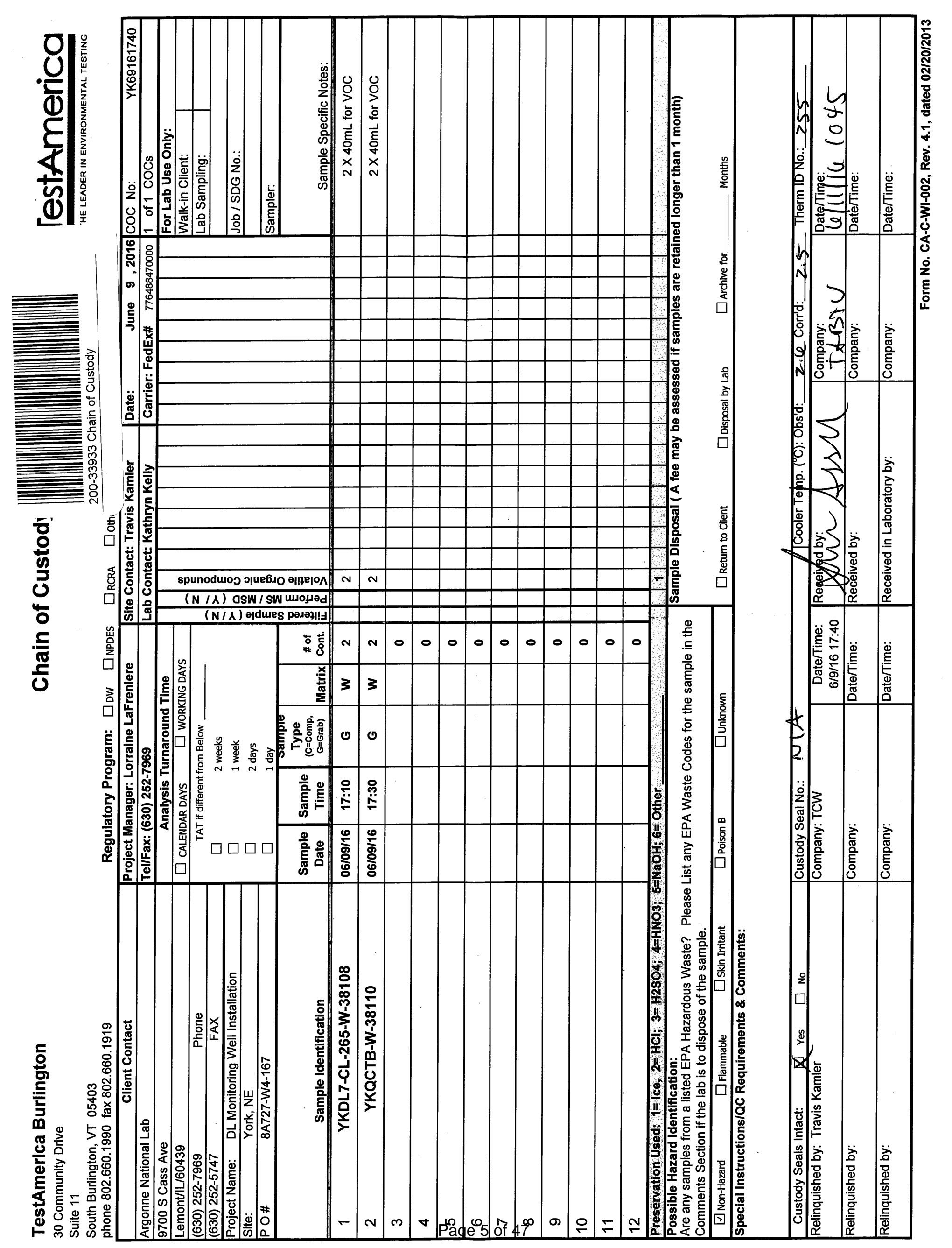




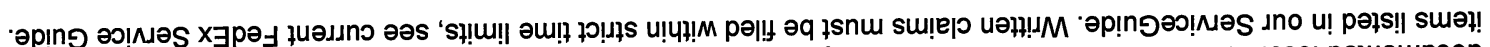

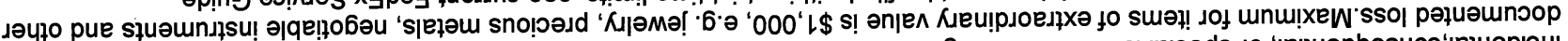

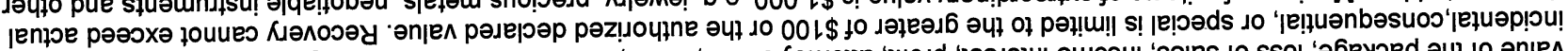

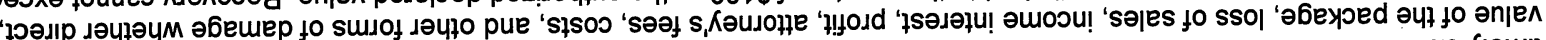

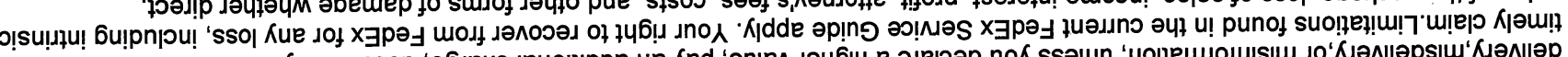

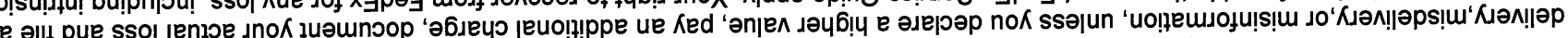

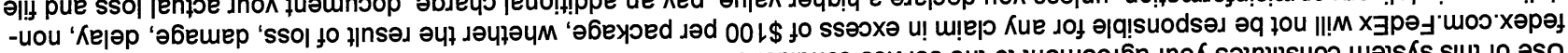

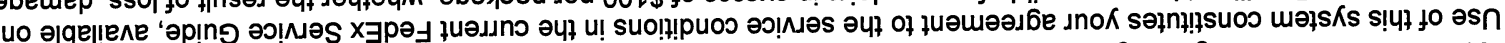

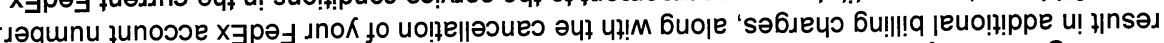

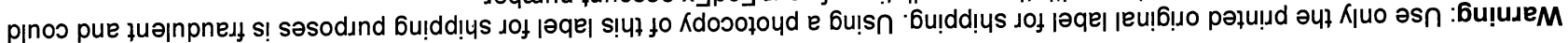

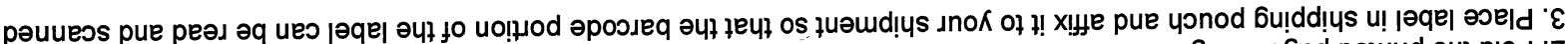

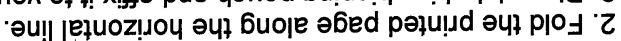

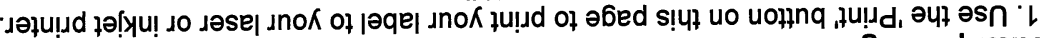

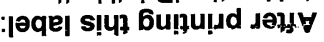

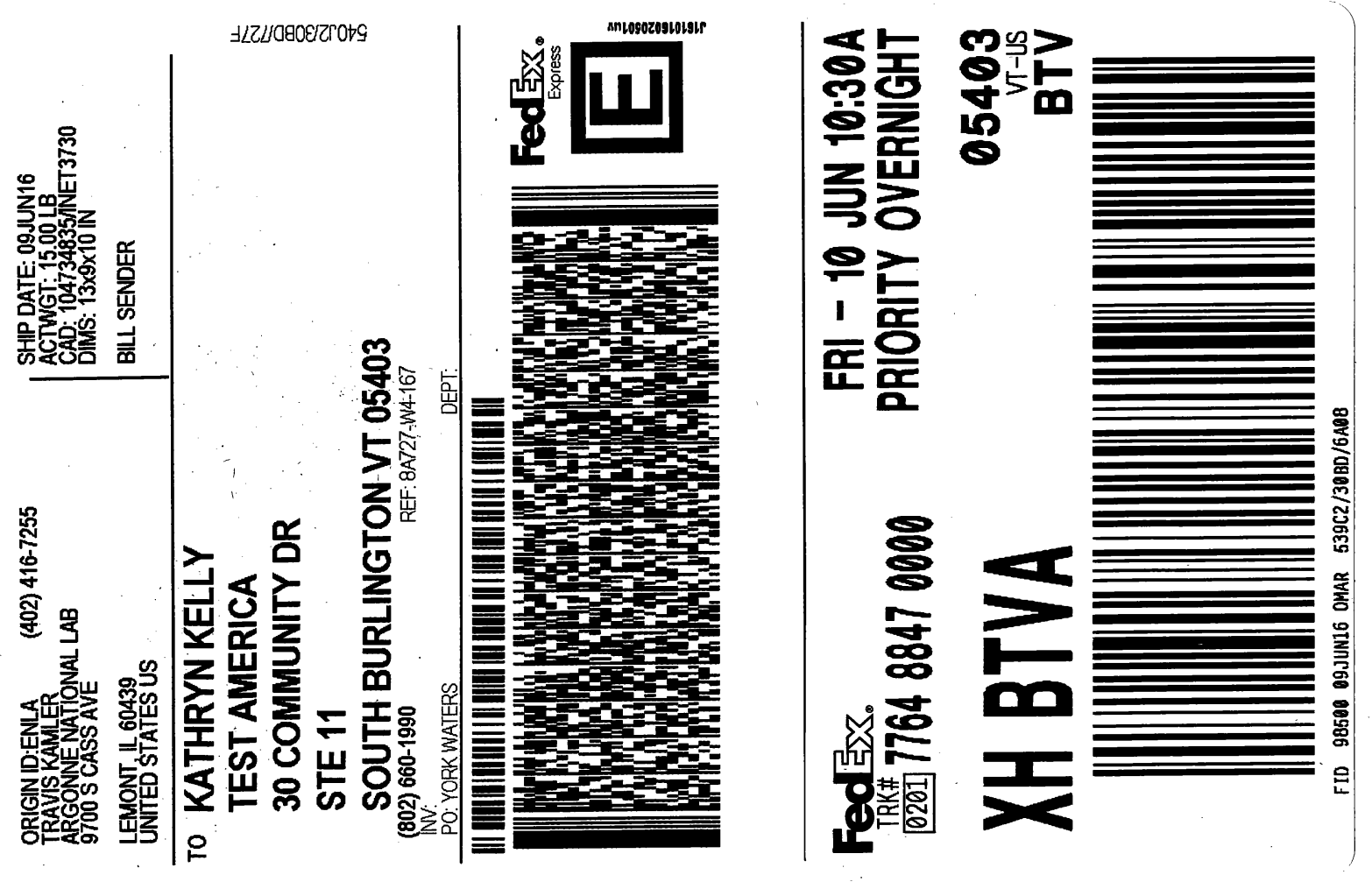




\section{Shipping and Receiving Documents}




\section{Login Sample Receipt Checklist}

Client: Argonne National Laboratory

Job Number: 200-33933-1

SDG Number: 33933

Login Number: 33933

List Source: TestAmerica Burlington

List Number: 1

Creator: Lavigne III, Scott M

Question

Radioactivity wasn't checked or is $</=$ background as measured by a survey True

Answer meter.

The cooler's custody seal, if present, is intact.

Sample custody seals, if present, are intact.

The cooler or samples do not appear to have been compromised or tampered with.

Samples were received on ice.

Cooler Temperature is acceptable.

Cooler Temperature is recorded.

$\mathrm{COC}$ is present.

$\mathrm{COC}$ is filled out in ink and legible.

$\mathrm{COC}$ is filled out with all pertinent information.

Is the Field Sampler's name present on COC?

There are no discrepancies between the containers received and the COC.

Samples are received within Holding Time (excluding tests with immediate

HTs)

Sample containers have legible labels.

Containers are not broken or leaking.

Sample collection date/times are provided.

Appropriate sample containers are used.

Sample bottles are completely filled.

Sample Preservation Verified.

There is sufficient vol. for all requested analyses, incl. any requested MS/MSDs

Containers requiring zero headspace have no headspace or bubble is $<6 \mathrm{~mm}(1 / 4 ")$.

Multiphasic samples are not present.

Samples do not require splitting or compositing.

Residual Chlorine Checked.
True

True

True

True

True

True

True

True

True

True

True

True

True

True

True

True

N/A

True

True

True

True

True

N/A 


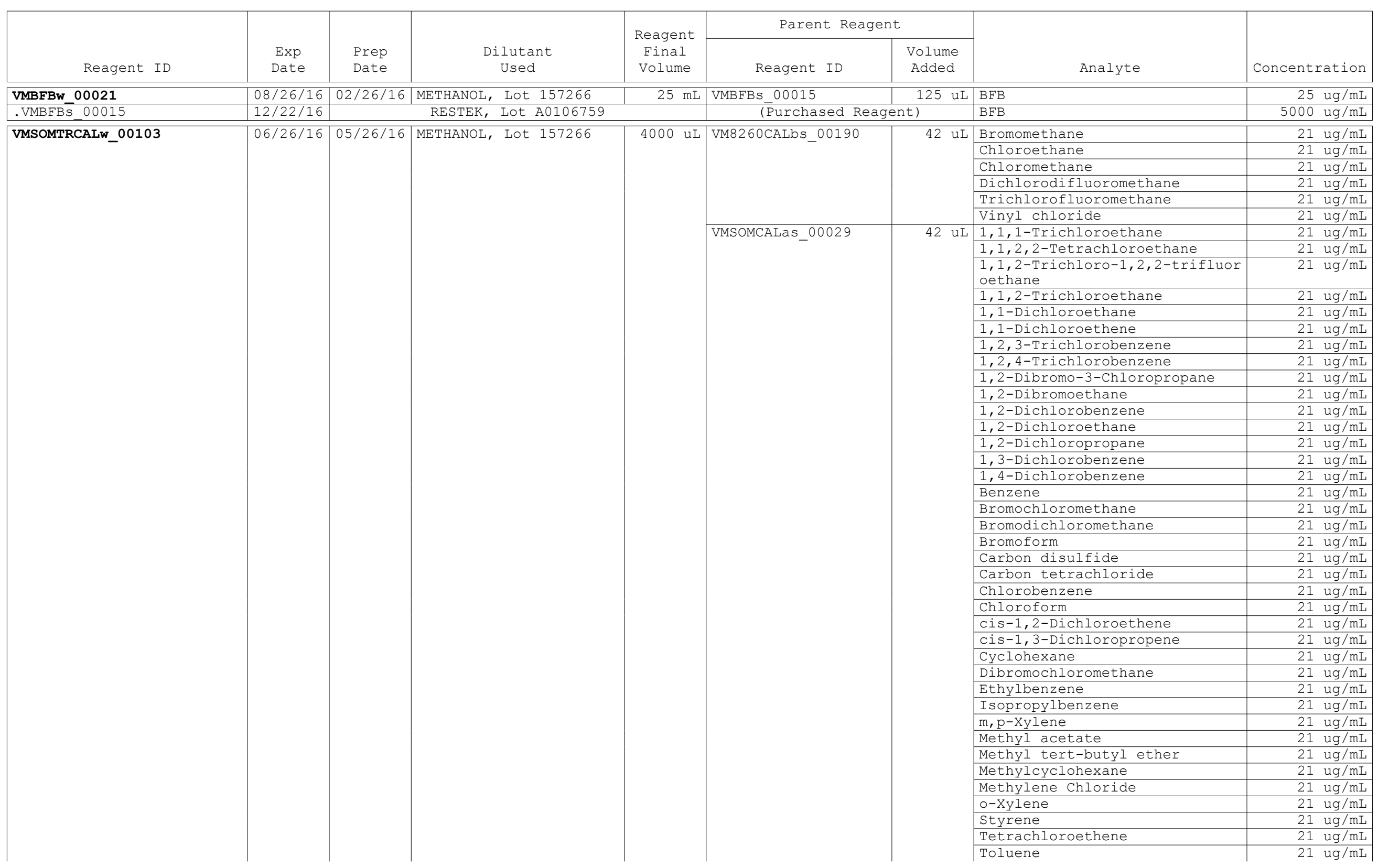


SDG No.: 33933

\begin{tabular}{|c|c|c|c|c|c|c|c|c|}
\hline \multirow[b]{2}{*}{ Reagent ID } & \multirow[b]{2}{*}{$\begin{array}{l}\text { Exp } \\
\text { Date }\end{array}$} & \multirow[b]{2}{*}{$\begin{array}{l}\text { Prep } \\
\text { Date }\end{array}$} & \multirow[b]{2}{*}{$\begin{array}{l}\text { Dilutant } \\
\text { Used }\end{array}$} & \multirow{2}{*}{$\begin{array}{c}\text { Reagent } \\
\text { Final } \\
\text { Volume }\end{array}$} & \multicolumn{2}{|l|}{ Parent Reagent } & \multirow[b]{2}{*}{ Analyte } & \multirow[b]{2}{*}{ Concentration } \\
\hline & & & & & \multirow[t]{4}{*}{ Reagent ID } & $\begin{array}{l}\text { Volume } \\
\text { Added }\end{array}$ & & \\
\hline & & & & & & & trans-1,2-Dichloroethene & $21 \mathrm{ug} / \mathrm{mL}$ \\
\hline & & & & & & & trans-1,3-Dichloropropene & $21 \mathrm{ug} / \mathrm{mL}$ \\
\hline & & & & & & & Trichloroethene & $21 \mathrm{ug} / \mathrm{mL}$ \\
\hline & & & & & VMSOMCALbS_00035 & $168 \mathrm{uL}$ & 2-Butanone & $210 \mathrm{ug} / \mathrm{mL}$ \\
\hline & & & & & & & 2-Hexanone & $210 \mathrm{ug} / \mathrm{mL}$ \\
\hline & & & & & & & 4-Methyl-2-pentanone & $210 \mathrm{ug} / \mathrm{mL}$ \\
\hline & & & & & & & Acetone & $210 \mathrm{ug} / \mathrm{mL}$ \\
\hline & & & & & VMSOMSUas_00085 & 42 uL & Chloroethane-d5 & $21 \mathrm{ug} / \mathrm{mL}$ \\
\hline & & & & & & & Vinyl Chloride-d3 & $21 \mathrm{ug} / \mathrm{mL}$ \\
\hline & & & & & VMSOMSUbs_00147 & $336 \mathrm{uL}$ & 2-Butanone-d5 & $210 \mathrm{ug} / \mathrm{mL}$ \\
\hline & & & & & & & 2-Hexanone-d5 & $210 \mathrm{ug} / \mathrm{mL}$ \\
\hline & & & & & VMSOMSUCS_00059 & 42 uL & $1,1,2,2$-Tetrachloroethane-d2 & $21 \mathrm{ug} / \mathrm{mL}$ \\
\hline & & & & & & & 1,1-Dichloroethene-d2 & $21 \mathrm{ug} / \mathrm{mL}$ \\
\hline & & & & & & & 1,2-Dichlorobenzene-d4 & $21 \mathrm{ug} / \mathrm{mL}$ \\
\hline & & & & & & & 1,2-Dichloroethane-d4 & $21 \mathrm{ug} / \mathrm{mL}$ \\
\hline & & & & & & & 1,2-Dichloropropane-d6 & $21 \mathrm{ug} / \mathrm{mL}$ \\
\hline & & & & & & & Benzene-d6 & $21 \mathrm{ug} / \mathrm{mL}$ \\
\hline & & & & & & & Chloroform-d & $21 \mathrm{ug} / \mathrm{mL}$ \\
\hline & & & & & & & Toluene-d8 & $21 \mathrm{ug} / \mathrm{mL}$ \\
\hline & & & & & & & trans-1,3-Dichloropropene-d4 & $21 \mathrm{ug} / \mathrm{mL}$ \\
\hline \multirow{6}{*}{.VM8260CALbs_00190 } & \multirow{6}{*}{$06 / 26 / 16$} & \multirow{6}{*}{\multicolumn{3}{|c|}{ RESTEK, Lot A0115510 }} & \multirow{6}{*}{\multicolumn{2}{|c|}{ (Purchased Reagent) }} & Bromomethane & $2000 \mathrm{ug} / \mathrm{mL}$ \\
\hline & & & & & & & Chloroethane & $2000 \mathrm{ug} / \mathrm{mL}$ \\
\hline & & & & & & & Chloromethane & $2000 \mathrm{ug} / \mathrm{mL}$ \\
\hline & & & & & & & Dichlorodifluoromethane & $2000 \mathrm{ug} / \mathrm{mL}$ \\
\hline & & & & & & & Trichlorofluoromethane & $2000 \mathrm{ug} / \mathrm{mL}$ \\
\hline & & & & & & & Vinyl chloride & $2000 \mathrm{ug} / \mathrm{mL}$ \\
\hline \multirow{21}{*}{.VMSOMCALas_00029 } & \multirow[t]{21}{*}{$04 / 30 / 17$} & \multirow{21}{*}{\multicolumn{2}{|c|}{ Restek, Lot A0102833 }} & & \multirow{21}{*}{\multicolumn{2}{|c|}{ (Purchased Reagent) }} & $1,1,1$-Trichloroethane & $2000 \mathrm{ug} / \mathrm{mL}$ \\
\hline & & & & & & & 1,1,2,2-Tetrachloroethane & $2000 \mathrm{ug} / \mathrm{mL}$ \\
\hline & & & & & & & $\begin{array}{l}\text { 1,1,2-Trichloro-1,2,2-trifluor } \\
\text { oethane }\end{array}$ & $2000 \mathrm{ug} / \mathrm{mL}$ \\
\hline & & & & & & & $1,1,2$-Trichloroethane & $2000 \mathrm{ug} / \mathrm{mL}$ \\
\hline & & & & & & & 1,1-Dichloroethane & $2000 \mathrm{ug} / \mathrm{mL}$ \\
\hline & & & & & & & 1,1-Dichloroethene & $2000 \mathrm{ug} / \mathrm{mL}$ \\
\hline & & & & & & & 1,2,3-Trichlorobenzene & $2000 \mathrm{ug} / \mathrm{mL}$ \\
\hline & & & & & & & 1,2,4-Trichlorobenzene & $2000 \mathrm{ug} / \mathrm{mL}$ \\
\hline & & & & & & & 1,2-Dibromo-3-Chloropropane & $2000 \mathrm{ug} / \mathrm{mL}$ \\
\hline & & & & & & & 1,2-Dibromoethane & $2000 \mathrm{ug} / \mathrm{mL}$ \\
\hline & & & & & & & 1,2-Dichlorobenzene & $2000 \mathrm{ug} / \mathrm{mL}$ \\
\hline & & & & & & & 1,2-Dichloroethane & $2000 \mathrm{ug} / \mathrm{mL}$ \\
\hline & & & & & & & 1,2-Dichloropropane & $2000 \mathrm{ug} / \mathrm{mL}$ \\
\hline & & & & & & & 1,3-Dichlorobenzene & $2000 \mathrm{ug} / \mathrm{mL}$ \\
\hline & & & & & & & 1,4-Dichlorobenzene & $2000 \mathrm{ug} / \mathrm{mL}$ \\
\hline & & & & & & & Benzene & $2000 \mathrm{ug} / \mathrm{mL}$ \\
\hline & & & & & & & Bromochloromethane & $2000 \mathrm{ug} / \mathrm{mL}$ \\
\hline & & & & & & & Bromodichloromethane & $2000 \mathrm{ug} / \mathrm{mL}$ \\
\hline & & & & & & & Bromoform & $2000 \mathrm{ug} / \mathrm{mL}$ \\
\hline & & & & & & & Carbon disulfide & $2000 \mathrm{ug} / \mathrm{mL}$ \\
\hline & & & & & & & Carbon tetrachloride & $2000 \mathrm{ug} / \mathrm{mL}$ \\
\hline
\end{tabular}


SDG No.: 33933

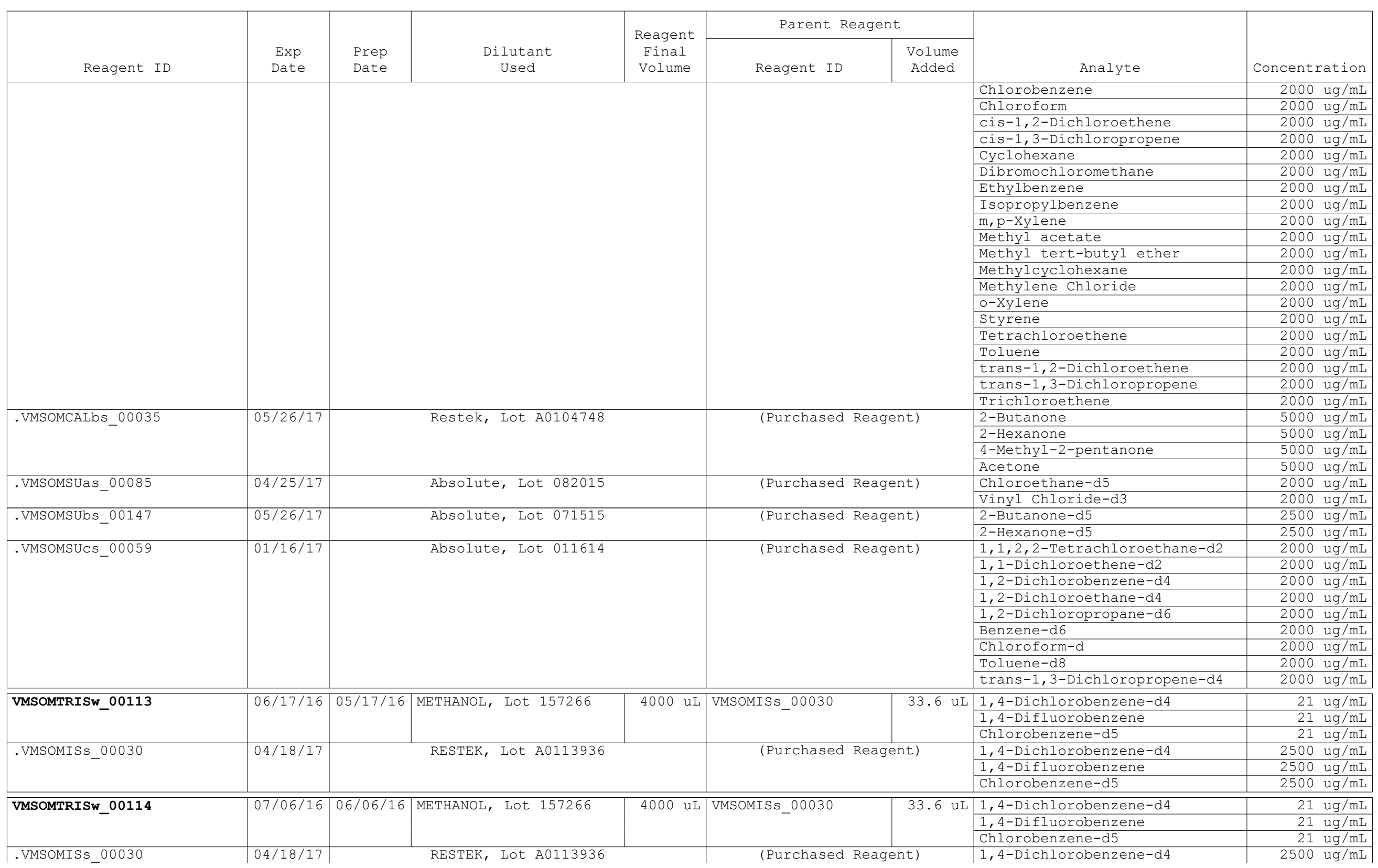


SDG No.: 33933

\begin{tabular}{|c|c|c|c|c|c|c|c|c|}
\hline \multirow[b]{2}{*}{ Reagent ID } & \multirow[b]{2}{*}{$\begin{array}{l}\text { Exp } \\
\text { Date }\end{array}$} & \multirow[b]{2}{*}{$\begin{array}{l}\text { Prep } \\
\text { Date }\end{array}$} & \multirow[b]{2}{*}{$\begin{array}{l}\text { Dilutant } \\
\text { Used }\end{array}$} & \multirow{2}{*}{$\begin{array}{l}\text { Reagent } \\
\text { Final } \\
\text { Volume }\end{array}$} & \multicolumn{2}{|c|}{ Parent Reagent } & \multirow[b]{2}{*}{ Analyte } & \multirow[b]{2}{*}{ Concentration } \\
\hline & & & & & \multicolumn{2}{|r|}{$\begin{array}{l}\text { Volume } \\
\text { Added }\end{array}$} & & \\
\hline & & & & & & & \begin{tabular}{|l} 
1,4-Difluorobenzene \\
Chlorobenzene-d5
\end{tabular} & $\begin{array}{l}2500 \mathrm{ug} / \mathrm{mL} \\
2500 \mathrm{ug} / \mathrm{mL}\end{array}$ \\
\hline \multirow[t]{10}{*}{ VMSOMTRSUw_00108 } & \multirow[t]{10}{*}{$06 / 17 / 16$} & \multirow[t]{10}{*}{$05 / 17 / 16$} & \multirow[t]{10}{*}{ METHANOL， Lot 157266} & \multirow{10}{*}{4000 UL } & VMSOMSUas_00085 & $42 \mathrm{uL}$ & Chloroethane-d5 & $21 \mathrm{ug} / \mathrm{mL}$ \\
\hline & & & & & VMSOMSUbs_00145 & $336 \mathrm{uL}$ & $\begin{array}{l}\text { Vinyl Chloride-d3 } \\
\text { 2-Butanone-d5 } \\
\text {-Hexanone-d5 }\end{array}$ & $\begin{aligned} 21 \mathrm{ug} / \mathrm{mL} \\
210 \mathrm{ug} / \mathrm{mL} \\
210 \mathrm{ug} / \mathrm{mL}\end{aligned}$ \\
\hline & & & & & \multirow[t]{8}{*}{ VMSOMSUCS_00059 } & \multirow[t]{8}{*}{42 uL } & $1,1,2,2$-Tetrachloroethane-d2 & $21 \mathrm{ug} / \mathrm{mL}$ \\
\hline & & & & & & & 1,1-Dichloroethene-d2 & $21 \mathrm{ug} / \mathrm{mL}$ \\
\hline & & & & & & & 1,2-Dichlorobenzene-d4 & $\frac{21 \mathrm{ug} / \mathrm{mL}}{21 \mathrm{ug} / \mathrm{mL}}$ \\
\hline & & & & & & & $\frac{1,2 \text {-Dichloroethane-d4 }}{1,2 \text {-Dichloropropane-d6 }}$ & $\frac{21 \mathrm{ug} / \mathrm{mL}}{21 \mathrm{ug} / \mathrm{mL}}$ \\
\hline & & & & & & & $\frac{1,2-11 C n 10}{\text { Benzene-d6 }}$ & $21 \mathrm{ug} / \mathrm{mL}$ \\
\hline & & & & & & & Chloroform-d & $21 \mathrm{ug} / \mathrm{mL}$ \\
\hline & & & & & & & Toluene-d8 & $21 \mathrm{ug} / \mathrm{mL}$ \\
\hline & & & & & & & trans-1,3-Dichloropropene-d4 & $21 \mathrm{ug} / \mathrm{mL}$ \\
\hline .VMSOMSUas_00085 & $04 / 25 / 17$ & \multicolumn{3}{|c|}{ Absolute, Lot 082015} & \multicolumn{2}{|c|}{ (Purchased Reagent) } & $\begin{array}{l}\text { Chloroethane-d5 } \\
\text { Vinvl Chloride-d3 }\end{array}$ & $2000 \mathrm{ug} / \mathrm{mL}$ \\
\hline \multirow{2}{*}{.VMSOMSUbs_00145 } & \multirow{2}{*}{$04 / 25 / 17$} & \multirow{2}{*}{\multicolumn{3}{|c|}{ Absolute, Lot 071515}} & \multirow{2}{*}{\multicolumn{2}{|c|}{ (Purchased Reagent) }} & 2-Butanone-d5 & $2500 \mathrm{ug} / \mathrm{mL}$ \\
\hline & & & & & & & 2-Hexanone-d5 & $2500 \mathrm{ug} / \mathrm{mL}$ \\
\hline \multirow[t]{7}{*}{.VMSOMSUCs_00059 } & \multirow[t]{7}{*}{$01 / 16 / 17$} & \multirow{7}{*}{\multicolumn{3}{|c|}{ Absolute, Lot 011614}} & \multirow{7}{*}{\multicolumn{2}{|c|}{ (Purchased Reagent) }} & $1,1,2,2$-Tetrachloroethane-d2 & $2000 \mathrm{ug} / \mathrm{mL}$ \\
\hline & & & & & & & 1,1-Dichloroethene-d2 & $2000 \mathrm{ug} / \mathrm{mL}$ \\
\hline & & & & & & & $\begin{array}{l}\text { 1,2-Dichlorrobenzene-d4 } \\
\text { 1,2-Dichloroethane-d4 }\end{array}$ & $2000 \mathrm{uq} / \mathrm{mL}$ \\
\hline & & & & & & & 1,2-Dichloropropane-d6 & $2000 \mathrm{ug} / \mathrm{mL}$ \\
\hline & & & & & & & Benzene-d6 & $2000 \mathrm{ug} / \mathrm{mL}$ \\
\hline & & & & & & & Chloroform-d & $2000 \mathrm{ug} / \mathrm{mL}$ \\
\hline & & & & & & & $\begin{array}{l}\frac{T}{T o l u e n e-d 8} \\
\text { trans-1,3-Dichloropropene-d4 }\end{array}$ & $\frac{2000 \mathrm{ug} / \mathrm{mL}}{2000 \mathrm{ug} / \mathrm{mL}}$ \\
\hline
\end{tabular}


Lab Name: TestAmerica Burlington

Job No.: 200-33933-1

SDG No.: 33933

\begin{tabular}{|c|c|c|c|c|}
\hline $\begin{array}{c}\text { Reagent } \\
\text { Container }\end{array}$ & $\begin{array}{c}\text { Reagent } \\
\text { ID }\end{array}$ & $\begin{array}{c}\text { Reagent } \\
\text { Description }\end{array}$ & $\begin{array}{c}\text { Preparation } \\
\text { Date }\end{array}$ & $\begin{array}{c}\text { Expiration } \\
\text { Date }\end{array}$ \\
\hline 904333 & VMBFBW 00021 & BFB TUNE 25 PPM & $02 / 26 / 2016$ & $08 / 26 / 2016$ \\
\hline 932091 & VMSOMTRISw_00113 & SOM TR ISTD 21 PPM & $05 / 17 / 2016$ & $06 / 17 / 2016$ \\
\hline 932092 & VMSOMTRSUw 00108 & SOM TR $\quad$ DMC $21 \quad$ PPM & $05 / 17 / 2016$ & $06 / 17 / 2016$ \\
\hline 935531 & VMSOMTRCALW_00103 & SOM TR CAL $21-210$ PPM & $05 / 26 / 2016$ & $06 / 26 / 2016$ \\
\hline 938927 & VMSOMTRISW $\overline{0} 0114$ & SOM TR ISTD 21 PPM & $06 / 06 / 2016$ & $07 / 06 / 2016$ \\
\hline
\end{tabular}




\section{METHODOLOGY SUMMARY}

Laboratory: TestAmerica Laboratories

Location: South Burlington, Vermont
Project No:

SDG No: 33933

VOA

Volatile Organics Trace - USEPA CLP SOM01.2 


\section{CASE NARRATIVE}

\section{Client: Argonne National Laboratory}

\section{Project: York (200-33933)}

\section{Report Number: 200-33933-1}

With the exceptions noted as flags or footnotes, standard analytical protocols were followed in the analysis of the samples and no problems were encountered or anomalies observed. In addition all laboratory quality control samples were within established control limits, with any exceptions noted below. Each sample was analyzed to achieve the lowest possible reporting limit within the constraints of the method. In some cases, due to interference or analytes present at high concentrations, samples were diluted. For diluted samples, the reporting limits are adjusted relative to the dilution required.

Calculations are performed before rounding to avoid round-off errors in calculated results.

All holding times were met and proper preservation noted for the methods performed on these samples, unless otherwise detailed in the individual sections below.

\section{RECEIPT}

The samples were received on 06/11/2016; the samples arrived in good condition, properly preserved and on ice. The temperature of the cooler at receipt was $2.5^{\circ} \mathrm{C}$.

\section{VOLATILE ORGANIC COMPOUNDS - TRACE}

Samples YKDL7-CL-265-W-38108, YKQCTB-W-38110 and VHBLK01 were analyzed for Volatile Organic Compounds - Trace in accordance with EPA SOW SOM01.2. The samples were analyzed on 06/15/2016.

Several analytes were detected in method blank MB 200-105769/4 at levels that were above the method detection limit but below the reporting limit. The values should be considered estimates, and have been flagged. If the associated sample reported a result above the MDL and/or RL, the result has been flagged. Refer to the $\mathrm{QC}$ report for details.

2-Hexanone-d5 failed the surrogate recovery criteria high for YKQCTB-W-38110. Refer to the QC report for details.

No additional analytical or quality issues were noted, other than those described above or in the Definitions/Glossary page. 
Lab Name: TestAmerica Burlington

Job No.: 200-33933-1

SDG No.: 33933

Instrument ID: CHD.i

Analysis Batch Number: 105151

Lab Sample ID: ICIS 200-105151/4

Client Sample ID:

Date Analyzed: 05/31/16 16:56

Lab File ID: 20227 04.D

GC Column: DB-624

ID $: 0.2(\mathrm{~mm})$

\begin{tabular}{|c|c|c|c|c|}
\hline \multirow[t]{2}{*}{ COMPOUND NAME } & \multirow{2}{*}{$\begin{array}{c}\text { RETENTION } \\
\text { TIME }\end{array}$} & \multicolumn{3}{|c|}{ MANUAL INTEGRATION } \\
\hline & & REASON & ANALYST & DATE \\
\hline 1,2-Dichloropropane-d6 & 6.32 & Split Peak & wilburj & $05 / 31 / 1617: 36$ \\
\hline
\end{tabular}

Lab Sample ID: IC 200-105151/5

Client Sample ID:

Date Analyzed: 05/31/16 17:21

Lab File ID: 20227 05.D

GC Column: DB-624

ID $: 0.2(\mathrm{~mm})$

\begin{tabular}{|c|c|c|c|c|}
\hline \multicolumn{1}{|c|}{ COMPOUND NAME } & RETENTION & \multicolumn{3}{|c}{ MANUAL INTEGRATION } \\
\cline { 3 - 5 } & TIME & REASON & ANALYST & DATE \\
\hline 1,2 -Dichloropropane-d6 & 6.32 & Split Peak & wilburj & 05/31/16 17:57 \\
\hline
\end{tabular}

Lab Sample ID: IC 200-105151/6

Client Sample ID:

Date Analyzed: 05/31/16 17:46

Lab File ID: 20227_06.D

GC Column: DB-624

ID $: 0.2(\mathrm{~mm})$

\begin{tabular}{|c|c|c|c|c|}
\hline \multicolumn{1}{|c|}{ COMPOUND NAME } & RETENTION & \multicolumn{3}{|c|}{ MANUAL INTEGRATION } \\
\cline { 3 - 5 } & TIME & REASON & ANALYST & DATE \\
\hline 1,2-Dichloropropane-d6 & 6.32 & Split Peak & wilburj & $05 / 31 / 16$ 18:30 \\
\hline
\end{tabular}


Lab Name: TestAmerica Burlington

Job No.: 200-33933-1

SDG No.: 33933

Instrument ID: CHD.i

Analysis Batch Number: 105769

Lab Sample ID: CCVIS 200-105769/3

Client Sample ID:

Date Analyzed: 06/15/16 13:43

Lab File ID: 20443 03.D

GC Column: DB-624

ID $: 0.2(\mathrm{~mm})$

\begin{tabular}{|c|c|c|c|c|}
\hline COMPOUND NAME & RETENTION & \multicolumn{2}{|c|}{ MANUAL INTEGRATION } \\
\cline { 3 - 5 } & TIME & REASON & ANALYST & DATE \\
\hline \hline 1,2 -Dichloropropane-d6 & 6.32 & Split Peak & wilburj & $06 / 15 / 1614: 47$ \\
\hline
\end{tabular}

Lab Sample ID: MB 200-105769/4

Client Sample ID:

Date Analyzed: 06/15/16 14:09

Lab File ID: 20443_04.D

GC Column: DB-624

ID $: 0.2(\mathrm{~mm})$

\begin{tabular}{|l|c|c|c|c|}
\hline \multirow{2}{*}{ COMPOUND NAME } & RETENTION & \multicolumn{2}{|c|}{ MANUAL INTEGRATION } \\
\cline { 3 - 5 } & TIME & \multicolumn{2}{|c|}{ REASON } & ANALYST \\
\hline Acetone & 2.79 & Assign Peak & DATE \\
\hline
\end{tabular}

Lab Sample ID: 200-33933-3

Client Sample ID: VHBLKO1

Date Analyzed: 06/15/16 16:14

Lab File ID: 20443 07.D

GC Column: DB-624

ID: $0.2(\mathrm{~mm})$

\begin{tabular}{|c|c|c|c|c|}
\hline \multirow[t]{2}{*}{ COMPOUND NAME } & \multirow{2}{*}{$\begin{array}{c}\text { RETENTION } \\
\text { TIME }\end{array}$} & \multicolumn{3}{|c|}{ MANUAL INTEGRATION } \\
\hline & & REASON & ANALYST & DATE \\
\hline Acetone & 2.79 & Assign Peak & maheseep & $06 / 16 / 1608: 56$ \\
\hline
\end{tabular}

Lab Sample ID: CCVC 200-105769/12

Client Sample ID:

Date Analyzed: 06/15/16 18:21

Lab File ID: 20443 12.D

GC Column: DB-624

ID $: 0.2(\mathrm{~mm})$

\begin{tabular}{|c|c|c|c|c|}
\hline \multirow[t]{2}{*}{ COMPOUND NAME } & \multirow{2}{*}{$\begin{array}{c}\text { RETENTION } \\
\text { TIME }\end{array}$} & \multicolumn{3}{|c|}{ MANUAL INTEGRATION } \\
\hline & & REASON & ANALYST & DATE \\
\hline Chloroethane & 2.08 & Assign Peak & maheseep & $06 / 16 / 1609: 16$ \\
\hline 1,2-Dichloropropane-d6 & 6.32 & Split Peak & wilburj & $06 / 15 / 1618: 51$ \\
\hline
\end{tabular}




\section{DATA REPORTING QUALIFIERS}

Client: Argonne National Laboratory

Job Number: 200-33933-1

Sdg Number: 33933

Description

GC/MS VOA

B

U

J

$J$

$\mathrm{X}$

*

B

N
Analyte was found in the associated method blank as well as in the sample.

Analyzed for but not detected.

Indicates an Estimated Value for TICs

Indicates an estimated value.

See case narrative notes for explanation of the ' $X$ ' flag

Surrogate is outside acceptance limits.

The analyte was found in an associated blank, as well as in the sample.

This flag indicates the presumptive evidence of a compound. 
Lab Name: TESTAMERICA BURLINGTON

Contract: $8 \mathrm{E}-00302$

Lab Code: STLV Case No.: YORK Mod. Ref No.: SDG No.: 33933

Level: (TRACE or LOW) TRACE

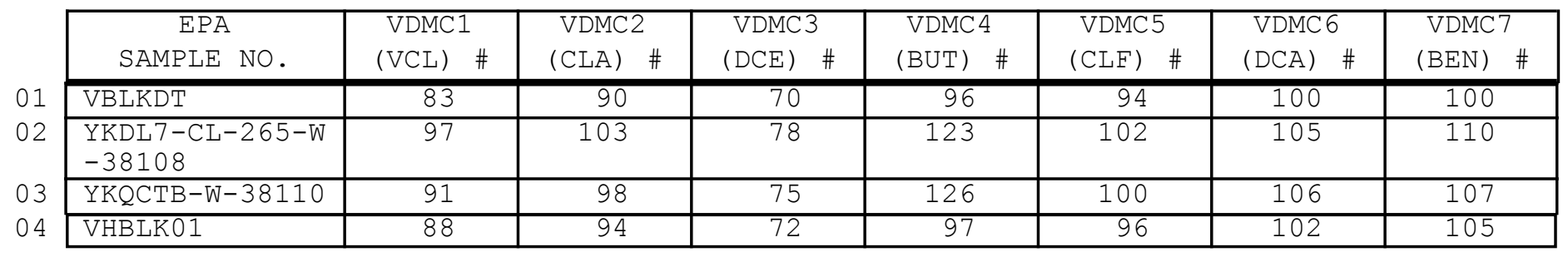

VDMC1 $(\mathrm{VCL})=$ Vinyl Chloride-d3

VDMC2 $($ CLA $)=$ Chloroethane-d5

VDMC3 $(\mathrm{DCE})=1,1-\mathrm{Dich}$ loroethene-d2

VDMC4 $\quad(B U T)=2-$ Butanone-d5

VDMC5 $(\mathrm{CLF})=$ Chloroform-d

VDMC $6 \quad(D C A)=1,2-$ Dichloroethane-d4

$\operatorname{VDMC} 7(\mathrm{BEN})=$ Benzene-d6

$$
\begin{gathered}
\frac{\mathrm{QC} \text { LIMITS }}{(65-131)} \\
(71-131) \\
(55-104) \\
(49-155) \\
(78-121) \\
(78-129) \\
(77-124)
\end{gathered}
$$

\# Column to be used to flag recovery values

* Values outside of contract required QC limits

Page 1 of 1 
Lab Name: TESTAMERICA BURLINGTON

Lab Code: STLV Case No.: YORK Mod. Ref No.:
Contract: $8 \mathrm{E}-00302$

SDG No.: 33933

Level: (TRACE or LOW) TRACE

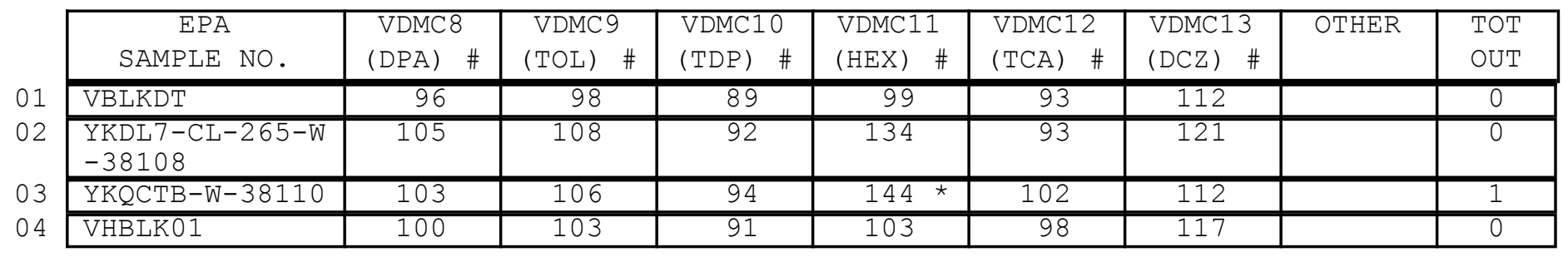

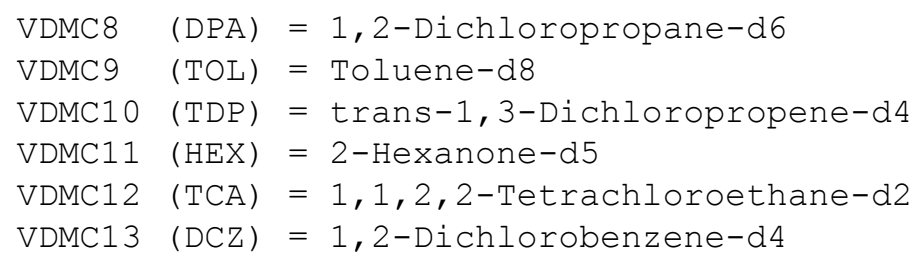

$$
\begin{gathered}
\frac{Q C \text { LIMITS }}{(79-124)} \\
(77-121) \\
(73-121) \\
(28-135) \\
(73-125) \\
(80-131)
\end{gathered}
$$


$4 A$ - FORM IV VOA

VOLATILE METHOD BLANK SUMMARY
EPA SAMPLE NO.

VBLKDT

Lab Name: TESTAMERICA BURLINGTON

Contract: 8E-00302

Lab Code: STLV Case No.: YORK Mod. Ref No.: SDG No. : 33933

Lab File ID: 20443_04.D

Lab Sample ID: MB 200-105769/4

Instrument ID: CHD.i

Matrix: (SOIL/SED/WATER) Water

Level: (TRACE or LOW/MED) TRACE

Date Analyzed: 06/15/2016

GC Column: DB-624

ID $: 0.20 \quad(\mathrm{~mm})$

Time Analyzed: 1409

Heated Purge: (Y/N) N

\begin{tabular}{|c|c|c|c|}
\hline $\begin{array}{c}\text { EPA } \\
\text { SAMPLE NO. }\end{array}$ & $\begin{array}{r}\text { LAB } \\
\text { SAMPLE ID }\end{array}$ & $\begin{array}{l}\text { LAB } \\
\text { FILE ID }\end{array}$ & $\begin{array}{c}\text { TIME } \\
\text { ANALYZED }\end{array}$ \\
\hline $\begin{array}{l}\text { YKDL 7-CL-265 } \\
-W-38108\end{array}$ & $200-33933-1$ & 20443 05.D & 1523 \\
\hline $\begin{array}{l}\text { YKQCTB-W-381 } \\
10\end{array}$ & $200-33933-2$ & 20443 _06.D & 1549 \\
\hline VHBLK01 & $200-33933-3$ & $20443-07 . D$ & 1614 \\
\hline
\end{tabular}

COMMENTS :

Page 1 of 1 
$5 A$ - FORM V VOA

VOLATILE ORGANICS INSTRUMENT

PERFORMANCE CHECK

BROMOFLUOROBENZENE (BFB)
EPA SAMPLE NO.

BFBDJ
Lab Name: TESTAMERICA BURLINGTON

Lab Code: STLV

Case No.: YORK

Lab File Id: $2022701 . \mathrm{D}$

Instrument Id: CHD.i

GC Column: DB-624

ID $: 0.20$
Contract: 8E-00302

Mod. Ref No.:

SDG No.: 33933

BEB Injection Date: 05/31/2016

BFB Injection Time: 1555 $(\mathrm{mm})$

\begin{tabular}{|c|c|c|}
\hline $\mathrm{m} / \mathrm{e}$ & ION ABUNDANCE CRITERIA & $\begin{array}{l}\text { \% RELATIVE } \\
\text { ABUNDANCE }\end{array}$ \\
\hline 50 & $15.0-40.0 \%$ of mass 95 & 16.9 \\
\hline 75 & $30.0-80.0 \%$ of mass 95 & 50.0 \\
\hline 95 & Base peak, $100 \%$ relative abundance & 100 \\
\hline 96 & $5.0-9.0 \%$ of mass 95 & 6.6 \\
\hline 173 & Less than $2.0 \%$ of mass 174 & $0) 1$ \\
\hline 174 & $50.0-120 \%$ of mass 95 & 90.0 \\
\hline 175 & $5.0-9.0 \%$ of mass 174 & $7.2) 1$ \\
\hline 176 & $95.0-101 \%$ of mass 174 & $87.1(96.8) 1$ \\
\hline 177 & $5.0-9.0 \%$ of mass 176 & $(6.6) 2$ \\
\hline
\end{tabular}

1 - Value is omass 1742 - Value is mass 176

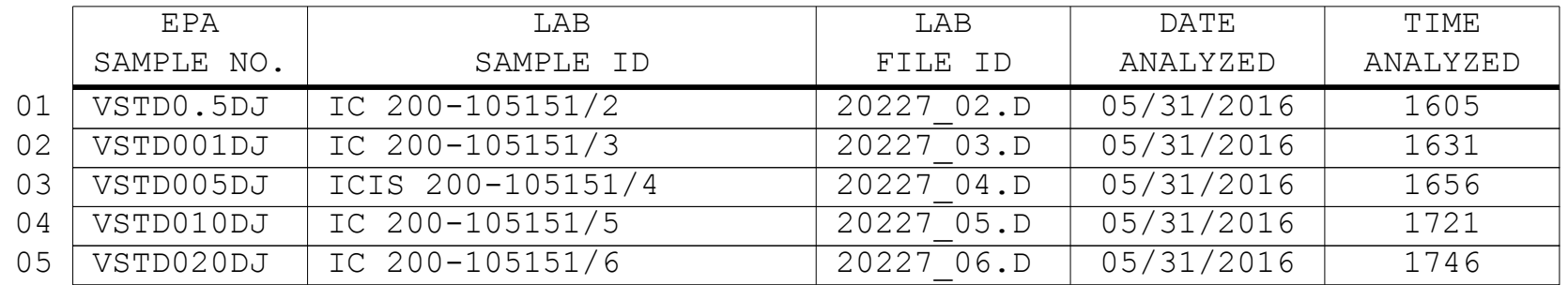


$5 A$ - FORM V VOA

VOLATILE ORGANICS INSTRUMENT

PERFORMANCE CHECK

BROMOFLUOROBENZENE (BFB)
EPA SAMPLE NO.

BFBDT
Lab Name: TESTAMERICA BURLINGTON

Lab Code: STLV Case No.: YORK

Lab File Id: 20443_01.D

Instrument Id: CHD.i

GC Column: DB-624
ID $: 0.20$
Contract: 8E-00302 Mod. Ref No.: SDG No.: 33933

BEB Injection Date: 06/15/2016

BFB Injection Time: 1305

\begin{tabular}{|c|c|c|}
\hline $\mathrm{m} / \mathrm{e}$ & ION ABUNDANCE CRITERIA & $\begin{array}{l}\text { \% RELATIVE } \\
\text { ABUNDANCE }\end{array}$ \\
\hline 50 & $15.0-40.0 \%$ of mass 95 & 17.4 \\
\hline 75 & $30.0-80.0 \%$ of mass 95 & 50.8 \\
\hline 95 & Base peak, $100 \%$ relative abundance & 100 \\
\hline 96 & $5.0-9.0 \%$ of mass 95 & 6.6 \\
\hline 173 & Less than $2.0 \%$ of mass 174 & $1.1) 1$ \\
\hline 174 & $50.0-120 \%$ of mass 95 & 93.5 \\
\hline 175 & $5.0-9.0 \%$ of mass 174 & $7.0) 1$ \\
\hline 176 & $95.0-101 \%$ of mass 174 & $(96.5) 1$ \\
\hline 177 & $5.0-9.0 \%$ of mass 176 & $5.6(6.2) 2$ \\
\hline
\end{tabular}

1 - Value is omass 1742 - Value is omass 176

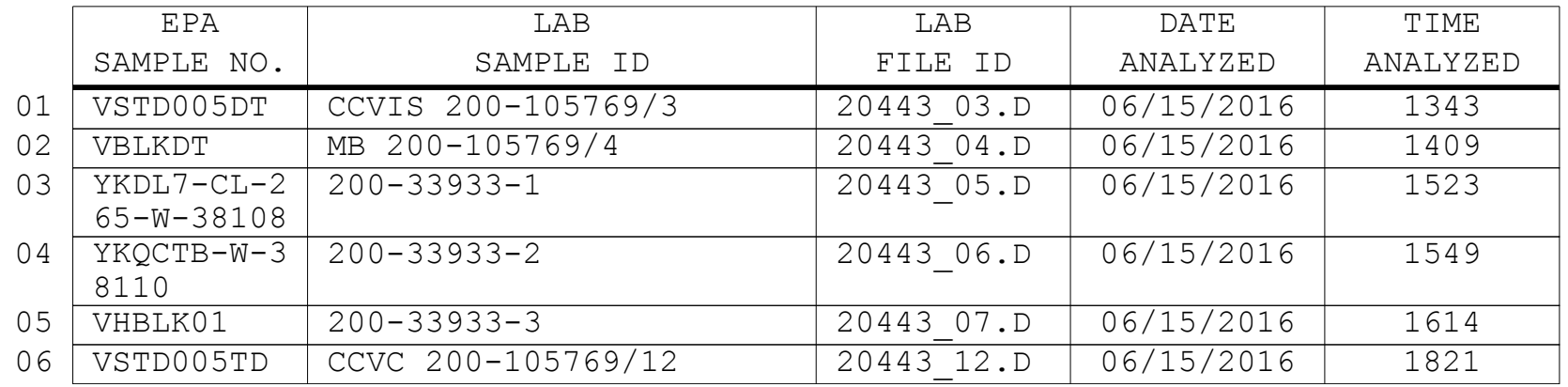


Lab Name: TESTAMERICA BURLINGTON

Contract: $8 \mathrm{E}-00302$

Lab Code: STLV Case No.: YORK Mod. Ref No.: SDG No.: 33933

GC Column: DB-624

ID: 0.20 (mm) Init. Calib. Date (s) : 05/31/2016 05/31/2016

EPA Sample No.(VSTD\#\#\#\#\#): VSTD005DT

Date Analyzed: 06/15/2016

Lab File ID (Standard): 20443_03.D

Time Analyzed: 1343

Instrument ID: CHD.i

Heated Purge: (Y/N) N

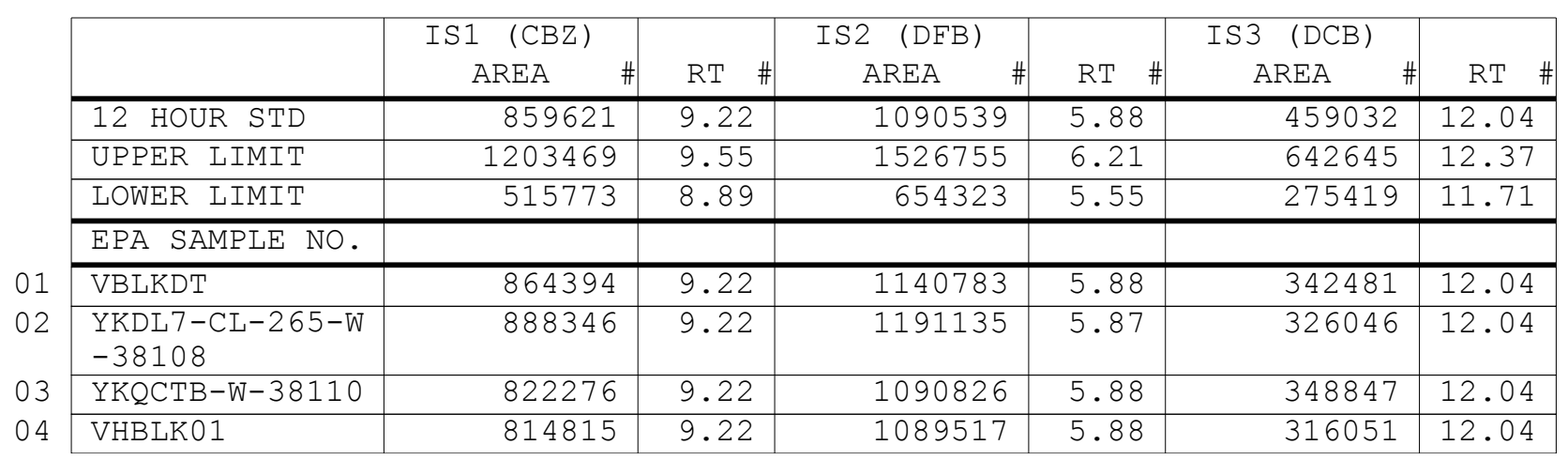

IS1 $(\mathrm{CBZ})=$ Chlorobenzene-d5

IS2 $(\mathrm{DFB})=1,4-\mathrm{Difluorobenzene}$

IS3 $(\mathrm{DCB})=1,4-\mathrm{Dichlorobenzene-d4}$

AREA UPPER LIMIT $=140 \%$ (Trace Volatiles) of internal standard area AREA LOWER LIMIT $=60 \%$ (Trace Volatiles) of internal standard area RT UPPER LIMIT $=+0.33$ (Trace Volatiles) minutes of internal standard RT RT LOWER LIMIT $=-0.33$ (Trace Volatiles) minutes of internal standard RT

\# Column used to flag values outside contract required QC limits with an asterisk. Page 1 of 1 
Lab Name: TESTAMERICA BURLINGTON

Contract: 8E-00302

Lab Code: STLV

Case No.: YORK Mod. Ref No.:

SDG No.: 33933

Matrix: (SOIL/SED/WATER) Water

Sample wt/vol: 25.0 $(\mathrm{g} / \mathrm{mL}) \mathrm{mL}$

Level: (TRACE/LOW/MED) TRACE

\% Moisture: not dec.

GC Column: DB-624 ID: $0.20 \quad(\mathrm{~mm})$

Soil Extract Volume: (uL) $(\mathrm{mL})$

Purge Volume: 25.0
Lab Sample ID: 200-33933-1

Lab File ID: 20443_05.D

Date Received: 06/11/2016

Date Analyzed: 06/15/2016

Dilution Factor: 1.0

Soil Aliquot Volume:

(uL)

\begin{tabular}{|c|c|c|c|}
\hline CAS NO. & COMPOUND & $\begin{array}{l}\text { CONCENTRATION UNITS: } \\
(\mathrm{ug} / \mathrm{L} \text { or } \mathrm{ug} / \mathrm{kg}) \mathrm{ug} / \mathrm{L}\end{array}$ & Q \\
\hline $75-71-8$ & Dichlorodifluoromethane & 1.0 & $\mathrm{U}$ \\
\hline $74-87-3$ & Chloromethane & 1.0 & $\mathrm{U}$ \\
\hline $75-01-4$ & Vinyl chloride & 1.0 & $\mathrm{U}$ \\
\hline $74-83-9$ & Bromomethane & 1.0 & $\mathrm{U}$ \\
\hline $75-00-3$ & Chloroethane & 1.0 & $\mathrm{U}$ \\
\hline $75-69-4$ & Trichlorofluoromethane & 1.0 & $\mathrm{U}$ \\
\hline $75-35-4$ & 1,1-Dichloroethene & 1.0 & $\mathrm{U}$ \\
\hline $76-13-1$ & 1,1,2-Trichloro-1,2,2-trifluoroethane & 1.0 & $\mathrm{U}$ \\
\hline $67-64-1$ & Acetone & 0.89 & $\mathrm{~J} B$ \\
\hline $75-15-0$ & Carbon disulfide & 0.036 & $\mathrm{~J} B$ \\
\hline $79-20-9$ & Methyl acetate & 1.0 & $\mathrm{U}$ \\
\hline $75-09-2$ & Methylene Chloride & 1.0 & $\mathrm{U}$ \\
\hline $156-60-5$ & trans-1,2-Dichloroethene & 0.011 & $\mathrm{~J} B$ \\
\hline $1634-04-4$ & Methyl tert-butyl ether & 1.0 & $\mathrm{U}$ \\
\hline $75-34-3$ & 1,1-Dichloroethane & 1.0 & $\mathrm{U}$ \\
\hline $156-59-2$ & cis-1,2-Dichloroethene & 1.0 & $\mathrm{U}$ \\
\hline $78-93-3$ & 2-Butanone & 5.0 & $\mathrm{U}$ \\
\hline $74-97-5$ & Bromochloromethane & 1.0 & $\mathrm{U}$ \\
\hline $67-66-3$ & Chloroform & 1.0 & $\mathrm{U}$ \\
\hline $71-55-6$ & $1,1,1$-Trichloroethane & 1.0 & $\mathrm{U}$ \\
\hline $110-82-7$ & Cyclohexane & 1.0 & $\mathrm{U}$ \\
\hline $56-23-5$ & Carbon tetrachloride & 1.0 & $\mathrm{U}$ \\
\hline $71-43-2$ & Benzene & 1.0 & $\mathrm{U}$ \\
\hline $107-06-2$ & 1,2-Dichloroethane & 1.0 & $\mathrm{U}$ \\
\hline
\end{tabular}

Report 1,4-Dioxane for Low-Medium VOA analysis only 
Lab Name: TESTAMERICA BURLINGTON

Lab Code: STLV

Case No.: YORK

Mod. Ref No.:

Contract: $8 \mathrm{E}-00302$

Matrix: (SOIL/SED/WATER) Water

Sample wt/vol: 25.0 $(\mathrm{g} / \mathrm{mL}) \mathrm{mL}$

Level: (TRACE/LOW/MED) TRACE

\% Moisture: not dec.

GC Column: DB-624 ID: $0.20 \quad(\mathrm{~mm})$

Soil Extract Volume: (uL) $(\mathrm{mL})$

Purge Volume: 25.0 SDG No.: 33933

Lab Sample ID: 200-33933-1

Lab File ID: 20443_05.D

Date Received: 06/11/2016

Date Analyzed: 06/15/2016

Dilution Factor: 1.0

Soil Aliquot Volume: (uL)

\begin{tabular}{|c|c|c|c|}
\hline CAS NO. & COMPOUND & $\begin{array}{l}\text { CONCENTRATION UNITS: } \\
(\mathrm{ug} / \mathrm{L} \text { or } \mathrm{ug} / \mathrm{kg}) \mathrm{ug} / \mathrm{L}\end{array}$ & Q \\
\hline $79-01-6$ & Trichloroethene & $\begin{array}{r}1.0 \\
\end{array}$ & $\mathrm{U}$ \\
\hline $108-87-2$ & Methylcyclohexane & 1.0 & $\mathrm{U}$ \\
\hline $78-87-5$ & 1,2-Dichloropropane & 1.0 & $\mathrm{U}$ \\
\hline $75-27-4$ & Bromodichloromethane & 1.0 & $\mathrm{U}$ \\
\hline $10061-01-5$ & cis-1,3-Dichloropropene & 1.0 & $\mathrm{U}$ \\
\hline $108-10-1$ & 4-Methyl-2-pentanone & 1.0 & $\mathrm{U}$ \\
\hline $108-88-3$ & Toluene & 0.025 & $\mathrm{~J} B$ \\
\hline $10061-02-6$ & trans-1,3-Dichloropropene & 1.0 & $\mathrm{U}$ \\
\hline $79-00-5$ & $1,1,2$-Trichloroethane & 1.0 & $\mathrm{U}$ \\
\hline $127-18-4$ & Tetrachloroethene & 1.0 & $\mathrm{U}$ \\
\hline $591-78-6$ & 2-Hexanone & 1.0 & $\mathrm{U}$ \\
\hline $124-48-1$ & Dibromochloromethane & 1.0 & $\mathrm{U}$ \\
\hline $106-93-4$ & 1,2-Dibromoethane & 1.0 & $\mathrm{U}$ \\
\hline $108-90-7$ & Chlorobenzene & 1.0 & $\mathrm{U}$ \\
\hline $100-41-4$ & Ethylbenzene & 1.0 & $\mathrm{U}$ \\
\hline $95-47-6$ & o-Xylene & 1.0 & $\mathrm{U}$ \\
\hline $179601-23-1$ & m,p-Xylene & 1.0 & $\mathrm{U}$ \\
\hline $100-42-5$ & Styrene & 1.0 & $\mathrm{U}$ \\
\hline $75-25-2$ & Bromoform & 1.0 & $\mathrm{U}$ \\
\hline $98-82-8$ & Isopropylbenzene & 1.0 & $\mathrm{U}$ \\
\hline $79-34-5$ & $1,1,2,2$-Tetrachloroethane & 1.0 & $\mathrm{U}$ \\
\hline $541-73-1$ & 1,3-Dichlorobenzene & 1.0 & $\mathrm{U}$ \\
\hline $106-46-7$ & 1,4-Dichlorobenzene & 1.0 & $\mathrm{U}$ \\
\hline $95-50-1$ & 1,2-Dichlorobenzene & 1.0 & $\mathrm{U}$ \\
\hline $96-12-8$ & 1,2-Dibromo-3-Chloropropane & 1.0 & $\mathrm{U}$ \\
\hline $120-82-1$ & $1,2,4$-Trichlorobenzene & 1.0 & $\mathrm{U}$ \\
\hline $87-61-6$ & $1,2,3$-Trichlorobenzene & 1.0 & $\mathrm{U}$ \\
\hline
\end{tabular}


Lab Name: TESTAMERICA BURLINGTON

Contract: $8 \mathrm{E}-00302$

Lab Code: STLV Case No.: YORK Mod. Ref No.: SDG No.: 33933

Matrix: (SOIL/SED/WATER) Water

Sample wt/vol: 25.0 $(\mathrm{g} / \mathrm{mL}) \mathrm{mL}$

Level: (TRACE or LOW/MED) TRACE

․ Moisture: not dec.

GC Column: DB-624 ID $: 0.20 \quad(\mathrm{~mm})$

Soil Extract Volume: (uL)
Lab Sample ID: 200-33933-1

Lab File ID: 20443_05.D

Date Received: 06/11/2016

Date Analyzed: 06/15/2016

Dilution Factor: 1.0

Soil Aliquot Volume: (uL) Purge Volume: 25.0 $(\mathrm{mL})$

01

02

\begin{tabular}{|l|l|c|c|c|}
\hline CAS NUMBER & \multicolumn{1}{|c|}{ COMPOUND NAME } & RT & EST. CONC. & Q \\
\hline & Unknown & 7.20 & 2.5 & $\mathrm{~J}$ X B \\
\hline E9667961 & Total Alkanes & N/A & & \\
\hline
\end{tabular}

1 EPA-designated Registry Number. 
Lab Name: TESTAMERICA BURLINGTON

Contract: $8 \mathrm{E}-00302$

Lab Code: STLV

Case No.: YORK Mod. Ref No.: SDG No.: 33933

Matrix: (SOIL/SED/WATER) Water

Sample wt/vol: 25.0 $(\mathrm{g} / \mathrm{mL}) \mathrm{mL}$

Level: (TRACE/LOW/MED) TRACE

\% Moisture: not dec.

GC Column: DB-624 ID: $0.20 \quad(\mathrm{~mm})$

Soil Extract Volume: (uL) $(\mathrm{mL})$

Purge Volume: 25.0
Lab Sample ID: 200-33933-2

Lab File ID: 20443_06.D

Date Received: 06/11/2016

Date Analyzed: 06/15/2016

Dilution Factor: 1.0

Soil Aliquot Volume: (uL)

\begin{tabular}{|c|c|c|c|}
\hline CAS NO. & COMPOUND & $\begin{array}{l}\text { CONCENTRATION UNITS: } \\
(\mathrm{ug} / \mathrm{L} \text { or } \mathrm{ug} / \mathrm{kg}) \mathrm{ug} / \mathrm{L}\end{array}$ & Q \\
\hline $75-71-8$ & Dichlorodifluoromethane & 1.0 & $\mathrm{U}$ \\
\hline $74-87-3$ & Chloromethane & 1.0 & $\mathrm{U}$ \\
\hline $75-01-4$ & Vinyl chloride & 1.0 & $\mathrm{U}$ \\
\hline $74-83-9$ & Bromomethane & 1.0 & $\mathrm{U}$ \\
\hline $75-00-3$ & Chloroethane & 1.0 & $\mathrm{U}$ \\
\hline $75-69-4$ & Trichlorofluoromethane & 1.0 & $\mathrm{U}$ \\
\hline $75-35-4$ & 1,1-Dichloroethene & 1.0 & $\mathrm{U}$ \\
\hline $76-13-1$ & $1,1,2$-Trichloro-1,2,2-trifluoroethane & 1.0 & $\mathrm{U}$ \\
\hline $67-64-1$ & Acetone & 4.5 & $\mathrm{~J} B$ \\
\hline $75-15-0$ & Carbon disulfide & 0.021 & $\mathrm{~J} B$ \\
\hline $79-20-9$ & Methyl acetate & 1.0 & $\mathrm{U}$ \\
\hline $75-09-2$ & Methylene Chloride & 0.021 & $\mathrm{~J} B$ \\
\hline $156-60-5$ & trans-1,2-Dichloroethene & 1.0 & $\mathrm{U}$ \\
\hline $1634-04-4$ & Methyl tert-butyl ether & 1.0 & $\mathrm{U}$ \\
\hline $75-34-3$ & 1,1-Dichloroethane & 1.0 & $\mathrm{U}$ \\
\hline $156-59-2$ & cis-1,2-Dichloroethene & 1.0 & $\mathrm{U}$ \\
\hline $78-93-3$ & 2-Butanone & 5.0 & $\mathrm{U}$ \\
\hline $74-97-5$ & Bromochloromethane & 1.0 & $\mathrm{U}$ \\
\hline $67-66-3$ & Chloroform & 1.0 & $\mathrm{U}$ \\
\hline $71-55-6$ & $1,1,1$-Trichloroethane & 1.0 & $\mathrm{U}$ \\
\hline $110-82-7$ & Cyclohexane & 1.0 & $\mathrm{U}$ \\
\hline $56-23-5$ & Carbon tetrachloride & 1.0 & $\mathrm{U}$ \\
\hline $71-43-2$ & Benzene & 1.0 & $\mathrm{U}$ \\
\hline $107-06-2$ & 1,2-Dichloroethane & 1.0 & $\mathrm{U}$ \\
\hline
\end{tabular}

Report 1,4-Dioxane for Low-Medium VOA analysis only 
Lab Name: TESTAMERICA BURLINGTON

Contract: $8 \mathrm{E}-00302$

Lab Code: STLV

Case No.: YORK

Mod. Ref No.:

SDG No.: 33933

Matrix: (SOIL/SED/WATER) Water

Sample wt/vol: 25.0 $(\mathrm{g} / \mathrm{mL}) \mathrm{mL}$

Level: (TRACE/LOW/MED) TRACE

\% Moisture: not dec.

GC Column: DB-624 ID: $0.20 \quad(\mathrm{~mm})$

Soil Extract Volume: (uL) $(\mathrm{mL})$

Purge Volume: 25.0 (mI)
Lab Sample ID: 200-33933-2

Lab File ID: 20443_06.D

Date Received: 06/11/2016

Date Analyzed: 06/15/2016

Dilution Factor: 1.0

Soil Aliquot Volume: (uL)

\begin{tabular}{|c|c|c|c|}
\hline CAS NO. & COMPOUND & $\begin{array}{l}\text { CONCENTRATION UNITS: } \\
(\mathrm{ug} / \mathrm{L} \text { or } \mathrm{ug} / \mathrm{kg}) \mathrm{ug} / \mathrm{L}\end{array}$ & Q \\
\hline $79-01-6$ & Trichloroethene & 1.0 & $\mathrm{U}$ \\
\hline $108-87-2$ & Methylcyclohexane & 1.0 & $\mathrm{U}$ \\
\hline $78-87-5$ & 1,2-Dichloropropane & 1.0 & $\mathrm{U}$ \\
\hline $75-27-4$ & Bromodichloromethane & 1.0 & $\mathrm{U}$ \\
\hline $10061-01-5$ & cis-1,3-Dichloropropene & 1.0 & U \\
\hline $108-10-1$ & 4-Methyl-2-pentanone & 1.0 & $\mathrm{U}$ \\
\hline $108-88-3$ & Toluene & 0.47 & $\mathrm{~J} B$ \\
\hline $10061-02-6$ & trans-1,3-Dichloropropene & 1.0 & $\mathrm{U}$ \\
\hline $79-00-5$ & $1,1,2-$ Trichloroethane & 1.0 & $\mathrm{U}$ \\
\hline $127-18-4$ & Tetrachloroethene & 0.037 & $\mathrm{~J} B$ \\
\hline $591-78-6$ & 2-Hexanone & 1.0 & $\mathrm{U}$ \\
\hline $124-48-1$ & Dibromochloromethane & 1.0 & $\mathrm{U}$ \\
\hline $106-93-4$ & 1,2-Dibromoethane & 1.0 & $\mathrm{U}$ \\
\hline $108-90-7$ & Chlorobenzene & 1.0 & $\mathrm{U}$ \\
\hline $100-41-4$ & Ethylbenzene & 0.043 & $\mathrm{~J} B$ \\
\hline $95-47-6$ & o-Xylene & 0.10 & $\mathrm{~J} B$ \\
\hline $179601-23-1$ & m,p-xylene & 0.12 & $\mathrm{~J} B$ \\
\hline $100-42-5$ & Styrene & 0.18 & $\mathrm{~J} B$ \\
\hline $75-25-2$ & Bromoform & 1.0 & $\mathrm{U}$ \\
\hline $98-82-8$ & Isopropylbenzene & 1.0 & $\mathrm{U}$ \\
\hline $79-34-5$ & $1,1,2,2$-Tetrachloroethane & 1.0 & $\mathrm{U}$ \\
\hline $541-73-1$ & 1,3 -Dichlorobenzene & 1.0 & $\mathrm{U}$ \\
\hline $106-46-7$ & 1,4-Dichlorobenzene & 1.0 & $\mathrm{U}$ \\
\hline $95-50-1$ & 1,2-Dichlorobenzene & 1.0 & $\mathrm{U}$ \\
\hline $96-12-8$ & 1,2-Dibromo-3-Chloropropane & 1.0 & $\mathrm{U}$ \\
\hline $120-82-1$ & $1,2,4$-Trichlorobenzene & 1.0 & $\mathrm{U}$ \\
\hline $87-61-6$ & $1,2,3$-Trichlorobenzene & 1.0 & $\mathrm{U}$ \\
\hline
\end{tabular}


Lab Name: TESTAMERICA BURLINGTON

Contract: $8 \mathrm{E}-00302$

Lab Code: STLV Case No.: YORK Mod. Ref No.: SDG No.: 33933

Matrix: (SOIL/SED/WATER) Water

Sample wt/vol: 25.0 $(\mathrm{g} / \mathrm{mL}) \mathrm{mL}$

Level: (TRACE or LOW/MED) TRACE

․ Moisture: not dec.

GC Column: DB-624 ID $: 0.20 \quad(\mathrm{~mm})$

Soil Extract Volume: (uL)

CONCENTRATION UNITS: (ug/L or ug/kg) ug/L
Lab Sample ID: 200-33933-2

Lab File ID: 20443_06.D

Date Received: 06/11/2016

Date Analyzed: 06/15/2016

Dilution Factor: 1.0

Soil Aliquot Volume: (uL) Purge Volume: 25.0

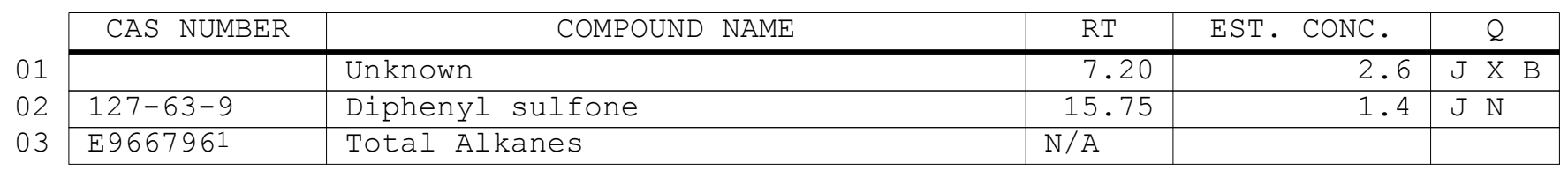

1EPA-designated Registry Number. 
Lab Name: TESTAMERICA BURLINGTON

Contract: $8 \mathrm{E}-00302$

Lab Code: STLV Case No.: YORK Mod. Ref No.:

SDG No.: 33933

Instrument ID: CHD.i

Calibration Date(s): 05/31/2016 05/31/2016

Heated Purge: (Y/N) N

Calibration Time(s) : 1605

1746

Purge Volume: 25.0 $(\mathrm{mL})$

GC Column: DB-624

ID: $0.20 \quad(\mathrm{~mm}) \quad$ Length: 25

$(\mathrm{m})$

\begin{tabular}{|c|c|c|c|c|c|c|c|}
\hline \multirow{3}{*}{$\begin{array}{l}\text { LAB FILE ID: } \\
\text { RRF } 5.0=20227 \_04 . D \\
\text { COMPOUND }\end{array}$} & \multirow{2}{*}{\multicolumn{3}{|c|}{ 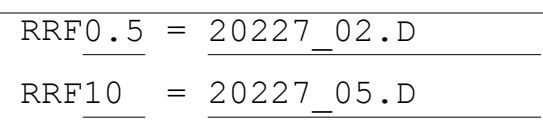 }} & \multicolumn{4}{|c|}{ RRF1.0 $=20227$ 03.D } \\
\hline & & & & RRF2 & $=202$ & DO6.D & \\
\hline & RRF0. 5 & RRF1.0 & $\operatorname{RRF} 5.0$ & RRF10 & RRF20 & $\overline{\mathrm{RRF}}$ & $\because \mathrm{RSD}$ \\
\hline Dichlorodifluoromethane & 0.667 & 0.602 & 0.556 & 0.572 & 0.564 & 0.592 & 7.7 \\
\hline Chloromethane & 0.595 & 0.547 & 0.480 & 0.473 & 0.459 & 0.511 & 11.4 \\
\hline Vinyl chloride & 0.583 & 0.509 & 0.481 & 0.502 & 0.491 & 0.513 & 7.9 \\
\hline Bromomethane & 0.239 & 0.158 & 0.155 & 0.169 & 0.171 & 0.179 & 19.4 \\
\hline Chloroethane & 0.290 & 0.283 & 0.280 & 0.282 & 0.259 & 0.279 & 4.3 \\
\hline Trichlorofluoromethane & 0.718 & 0.675 & 0.613 & 0.618 & 0.608 & 0.646 & 7.5 \\
\hline 1,1-Dichloroethene & 0.384 & 0.348 & 0.328 & 0.331 & 0.325 & 0.343 & 7.1 \\
\hline $\begin{array}{l}1,1,2 \text {-Trichloro- } \\
1,2,2 \text {-trifluoroethane }\end{array}$ & 0.448 & 0.398 & 0.367 & 0.368 & 0.358 & 0.388 & 9.5 \\
\hline Acetone & 0.024 & 0.021 & 0.017 & 0.019 & 0.019 & 0.020 & 12.6 \\
\hline Carbon disulfide & 1.077 & 0.937 & 0.929 & 0.988 & 1.005 & 0.987 & 6.0 \\
\hline Methyl acetate & 0.059 & 0.052 & 0.050 & 0.051 & 0.050 & 0.052 & 7.1 \\
\hline Methylene Chloride & 0.324 & 0.295 & 0.272 & 0.273 & 0.270 & 0.287 & 8.1 \\
\hline trans-1,2-Dichloroethene & 0.388 & 0.352 & 0.332 & 0.335 & 0.332 & 0.348 & 6.9 \\
\hline Methyl tert-butyl ether & 0.376 & 0.353 & 0.357 & 0.373 & 0.389 & 0.370 & 4.0 \\
\hline 1,1-Dichloroethane & 0.736 & 0.665 & 0.623 & 0.637 & 0.636 & 0.660 & 6.9 \\
\hline cis-1,2-Dichloroethene & 0.352 & 0.315 & 0.332 & 0.342 & 0.331 & 0.334 & 4.2 \\
\hline 2-Butanone & 0.033 & 0.030 & 0.032 & 0.035 & 0.035 & 0.033 & 7.0 \\
\hline Bromochloromethane & 0.112 & 0.107 & 0.097 & 0.102 & 0.105 & 0.105 & 5.1 \\
\hline Chloroform & 0.634 & 0.586 & 0.556 & 0.566 & 0.576 & 0.584 & 5.2 \\
\hline $1,1,1$-Trichloroethane & 0.763 & 0.693 & 0.667 & 0.679 & 0.688 & 0.698 & 5.4 \\
\hline Cyclohexane & 0.734 & 0.702 & 0.814 & 0.863 & 0.880 & 0.799 & 9.8 \\
\hline Carbon tetrachloride & 0.673 & 0.612 & 0.607 & 0.623 & 0.628 & 0.629 & 4.1 \\
\hline Benzene & 2.159 & 1.975 & 1.906 & 1.952 & 1.975 & 1.993 & 4.9 \\
\hline 1,2-Dichloroethane & 0.293 & 0.257 & 0.256 & 0.260 & 0.267 & 0.266 & 5.7 \\
\hline Trichloroethene & 0.465 & 0.422 & 0.424 & 0.440 & 0.450 & 0.440 & 4.1 \\
\hline Methylcyclohexane & 0.599 & 0.590 & 0.666 & 0.695 & 0.694 & 0.649 & 7.8 \\
\hline
\end{tabular}

Report 1,4-Dioxane for Low-Medium VOA analysis only 
Lab Name: TESTAMERICA BURLINGTON

Contract: 8E-00302

Lab Code: STLV Case No.: YORK Mod. Ref No.:

SDG No.: 33933

Instrument ID: CHD.i

Calibration Date(s): 05/31/2016 05/31/2016

Heated Purge: (Y/N) N

Purge Volume: 25.0

Calibration Time(s) : 1605

1746

GC Column: DB-624

ID: 0.20 (mL)

LAB FILE ID:

(mm) Length: 25

(m)

$\mathrm{RRF} 5.0=20227 \_04 . \mathrm{D}$
$\mathrm{RRF} 0.5=20227 \_02 . \mathrm{D}$
RRF1.0 $=20227 \_03 . \mathrm{D}$
$\mathrm{RRF} 10=20227$ 05.D
RRF20 $=2022706 . \mathrm{D}$

COMPOUND

\begin{tabular}{|l|l|}
\hline COMPOUND & RRF0.5 \\
\hline
\end{tabular}

\begin{tabular}{|c|c|c|c|c|c|c|c|}
\hline 1,2-Dichloropropane & 0.450 & 0.394 & 0.387 & 0.391 & 0.397 & 0.404 & 6.5 \\
\hline Bromodichloromethane & 0.396 & 0.364 & 0.368 & 0.382 & 0.395 & 0.381 & 3.9 \\
\hline cis-1,3-Dichloropropene & 0.383 & 0.368 & 0.472 & 0.517 & 0.545 & 0.457 & 17.2 \\
\hline 4-Methyl-2-pentanone & 0.072 & 0.074 & 0.094 & 0.101 & 0.105 & 0.089 & 17.2 \\
\hline Toluene & 1.692 & 1.704 & 1.891 & 1.962 & 1.990 & 1.848 & 7.7 \\
\hline trans-1,3-Dichloropropene & 0.238 & 0.235 & 0.304 & 0.343 & 0.370 & 0.298 & 20.5 \\
\hline 1,1,2-Trichloroethane & 0.209 & 0.182 & 0.169 & 0.172 & 0.171 & 0.180 & 9.2 \\
\hline Tetrachloroethene & 0.472 & 0.431 & 0.425 & 0.440 & 0.435 & 0.441 & 4.2 \\
\hline 2-Hexanone & 0.048 & 0.052 & 0.066 & 0.070 & 0.072 & 0.062 & 17.6 \\
\hline Dibromochloromethane & 0.176 & 0.164 & 0.176 & 0.190 & 0.198 & 0.181 & 7.5 \\
\hline 1,2-Dibromoethane & 0.142 & 0.128 & 0.135 & 0.138 & 0.144 & 0.137 & 4.6 \\
\hline Chlorobenzene & 1.207 & 1.138 & 1.100 & 1.124 & 1.120 & 1.138 & 3.6 \\
\hline Ethylbenzene & 1.724 & 1.752 & 2.139 & 2.283 & 2.319 & 2.043 & 14.0 \\
\hline o-Xylene & 0.488 & 0.541 & 0.751 & 0.802 & 0.804 & 0.677 & 22.3 \\
\hline $\mathrm{m}, \mathrm{p}$-Xylene & 0.627 & 0.666 & 0.841 & 0.885 & 0.893 & 0.783 & 16.2 \\
\hline Styrene & 0.803 & 0.905 & 1.184 & 1.244 & 1.250 & 1.077 & 19.4 \\
\hline Bromoform & 0.169 & 0.149 & 0.145 & 0.162 & 0.182 & 0.161 & 9.3 \\
\hline Isopropylbenzene & 1.433 & 1.568 & 2.168 & 2.351 & 2.365 & 1.977 & 22.5 \\
\hline $1,1,2,2$-Tetrachloroethane & 0.177 & 0.167 & 0.165 & 0.171 & 0.172 & 0.170 & 2.8 \\
\hline 1,3-Dichlorobenzene & 1.375 & 1.332 & 1.435 & 1.557 & 1.640 & 1.468 & 8.7 \\
\hline 1,4-Dichlorobenzene & 1.993 & 1.711 & 1.583 & 1.599 & 1.627 & 1.703 & 10.0 \\
\hline 1,2-Dichlorobenzene & 1.344 & 1.274 & 1.266 & 1.300 & 1.323 & 1.301 & 2.5 \\
\hline 1,2-Dibromo-3-Chloropropane & 0.045 & 0.040 & 0.033 & 0.036 & 0.041 & 0.039 & 11.3 \\
\hline $1,2,4$-Trichlorobenzene & 0.687 & 0.612 & 0.691 & 0.845 & 0.949 & 0.757 & 18.1 \\
\hline $1,2,3$-Trichlorobenzene & 0.470 & 0.439 & 0.518 & 0.605 & 0.671 & 0.541 & 17.8 \\
\hline
\end{tabular}


Lab Name: TESTAMERICA BURLINGTON

Contract: $8 \mathrm{E}-00302$

Lab Code: STLV Case No.: YORK Mod. Ref No.: SDG No.: 33933

Instrument ID: CHD.i

Heated Purge: (Y/N) N

Purge Volume: 25.0

GC Column: DB-624
ID: 0.20
Calibration Date(s): 05/31/2016

Calibration Time (s) : 1605 $(\mathrm{mL})$

(mm) Length: 25 $(\mathrm{m})$

\begin{tabular}{|c|c|c|c|c|c|c|c|}
\hline LAB FILE ID: & \multicolumn{3}{|c|}{$\begin{array}{l}\operatorname{RRF}_{\underline{0.5}}=\frac{20227 \_02 . \mathrm{D}}{\operatorname{RRF10}}=20227 \_05 . \mathrm{D}\end{array}$} & \multicolumn{4}{|c|}{$\begin{array}{l}\operatorname{RRF1.0}_{\underline{1.0}}=\frac{20227 \_03 . \mathrm{D}}{\underline{\mathrm{RRF} 20}}=20227 \_06 . \mathrm{D}\end{array}$} \\
\hline Vinyl Chloride-d3 & 0.507 & 0.432 & 0.420 & 0.437 & 0.428 & 0.445 & 8.0 \\
\hline Chloroethane-d5 & 0.424 & 0.372 & 0.339 & 0.334 & 0.311 & 0.356 & 12.2 \\
\hline Chloroform-d & 0.681 & 0.615 & 0.576 & 0.591 & 0.594 & 0.611 & 6.8 \\
\hline 1,2-Dichloroethane-d4 & 0.245 & 0.212 & 0.201 & 0.205 & 0.209 & 0.214 & 8.2 \\
\hline Benzene-d6 & 1.902 & 1.743 & 1.756 & 1.802 & 1.828 & 1.806 & 3.5 \\
\hline 1,2-Dichloropropane-d6 & 0.507 & 0.429 & 0.417 & 0.434 & 0.436 & 0.444 & 8.0 \\
\hline Toluene-d8 & 1.445 & 1.398 & 1.557 & 1.626 & 1.638 & 1.533 & 7.0 \\
\hline 1,2-Dichlorobenzene-d4 & 0.917 & 0.756 & 0.750 & 0.791 & 0.823 & 0.807 & 8.5 \\
\hline
\end{tabular}

Report 1,4-Dioxane-d8 for Low-Medium VOA analysis only 
Lab Name: TESTAMERICA BURLINGTON

Contract: $8 \mathrm{E}-00302$

Lab Code: STLV

Mod. Ref No.:

SDG No.: 33933

Instrument ID: CHD.i

Calibration Date: 06/15/2016 Time: 1343

Lab File Id: 20443_03.D

Init. Calib. Date(s):05/31/2016 05/31/2016

EPA Sample No. (VSTD\#\#\#\#) : VSTD005DT

Heated Purge: ( $\mathrm{Y} / \mathrm{N}) \mathrm{N}$ GC Column: DB-624

Init. Calib. Time(s) : 1605 1746

Purge Volume: 25.0

ID: $0.20(\mathrm{~mm})$ Length: 25

(m)

\begin{tabular}{|c|c|c|c|c|c|}
\hline COMPOUND & $\overline{\mathrm{RRF}}$ & RRF 5.0 & $\begin{array}{l}\text { MIN } \\
\text { RRE }\end{array}$ & $\because \mathrm{D}$ & $\mathrm{MAX} \div \mathrm{D}$ \\
\hline Dichlorodifluoromethane & 0.592 & 0.593 & 0.010 & 0.2 & 40.0 \\
\hline Chloromethane & 0.511 & 0.479 & 0.010 & -6.3 & 40.0 \\
\hline Vinyl chloride & 0.513 & 0.517 & 0.100 & 0.6 & 30.0 \\
\hline Bromomethane & 0.179 & 0.174 & 0.100 & -2.7 & 30.0 \\
\hline Chloroethane & 0.279 & 0.293 & 0.010 & 5.1 & 40.0 \\
\hline Trichlorofluoromethane & 0.646 & 0.664 & 0.010 & 2.7 & 40.0 \\
\hline 1,1-Dichloroethene & 0.343 & 0.351 & 0.100 & 2.3 & 30.0 \\
\hline 1,1,2-Trichloro-1,2,2-trifluoroethane & 0.388 & 0.397 & 0.010 & 2.4 & 40.0 \\
\hline Acetone & 0.020 & 0.020 & 0.010 & -0.1 & 40.0 \\
\hline Carbon disulfide & 0.987 & 1.028 & 0.010 & 4.1 & 40.0 \\
\hline Methyl acetate & 0.052 & 0.050 & 0.010 & -4.6 & 40.0 \\
\hline Methylene Chloride & 0.287 & 0.290 & 0.010 & 1.3 & 40.0 \\
\hline trans-1,2-Dichloroethene & 0.348 & 0.357 & 0.010 & 2.5 & 40.0 \\
\hline Methyl tert-butyl ether & 0.370 & 0.360 & 0.010 & -2.5 & 40.0 \\
\hline 1,1-Dichloroethane & 0.660 & 0.666 & 0.200 & 1.0 & 30.0 \\
\hline cis-1,2-Dichloroethene & 0.334 & 0.356 & 0.010 & 6.4 & 40.0 \\
\hline 2-Butanone & 0.033 & 0.035 & 0.010 & 6.5 & 40.0 \\
\hline Bromochloromethane & 0.105 & 0.108 & 0.050 & 3.0 & 30.0 \\
\hline Chloroform & 0.584 & 0.612 & 0.200 & 4.9 & 30.0 \\
\hline 1,1,1-Trichloroethane & 0.698 & 0.720 & 0.100 & 3.1 & 30.0 \\
\hline Cyclohexane & 0.799 & 0.869 & 0.010 & 8.8 & 40.0 \\
\hline Carbon tetrachloride & 0.629 & 0.676 & 0.100 & 7.5 & 30.0 \\
\hline Benzene & 1.993 & 2.068 & 0.400 & 3.7 & 30.0 \\
\hline 1,2-Dichloroethane & 0.266 & 0.281 & 0.100 & 5.5 & 30.0 \\
\hline Trichloroethene & 0.440 & 0.451 & 0.300 & 2.5 & 30.0 \\
\hline Methylcyclohexane & 0.649 & 0.717 & 0.010 & 10.5 & 40.0 \\
\hline
\end{tabular}

Report 1,4-Dioxane for Low/Medium VOA analysis only 
Lab Name: TESTAMERICA BURLINGTON

Contract: 8E-00302

Lab Code: STLV

Mod. Ref No.:

SDG No.: 33933

Instrument ID: CHD.i

Calibration Date: 06/15/2016 Time: 1343

Lab File Id: 20443_03.D

Init. Calib. Date(s):05/31/2016 05/31/2016

EPA Sample No. (VSTD\#\#\#\#) : VSTD005DT

Heated Purge: ( $\mathrm{Y} / \mathrm{N}) \mathrm{N}$ GC Column: DB-624

Init. Calib. Time(s) : 1605 1746

Purge Volume: 25.0

ID: $0.20(\mathrm{~mm})$ Length: 25

(m)

\begin{tabular}{|c|c|c|c|c|c|}
\hline COMPOUND & $\overline{\mathrm{RRF}}$ & RRF5.0 & $\begin{array}{l}\text { MIN } \\
\text { RRF }\end{array}$ & $\because \mathrm{D}$ & $\operatorname{MAX} \cong \mathrm{D}$ \\
\hline 1,2-Dichloropropane & 0.404 & 0.417 & 0.010 & 3.4 & 40.0 \\
\hline Bromodichloromethane & 0.381 & 0.402 & 0.200 & 5.4 & 30.0 \\
\hline cis-1,3-Dichloropropene & 0.457 & 0.499 & 0.200 & 9.1 & 30.0 \\
\hline 4-Methyl-2-pentanone & 0.089 & 0.099 & 0.010 & 10.9 & 40.0 \\
\hline Toluene & 1.848 & 2.065 & 0.400 & 11.8 & 30.0 \\
\hline trans-1,3-Dichloropropene & 0.298 & 0.321 & 0.100 & 7.8 & 30.0 \\
\hline $1,1,2$-Trichloroethane & 0.180 & 0.182 & 0.100 & 1.1 & 30.0 \\
\hline Tetrachloroethene & 0.441 & 0.461 & 0.100 & 4.5 & 30.0 \\
\hline 2-Hexanone & 0.062 & 0.069 & 0.010 & 12.3 & 40.0 \\
\hline Dibromochloromethane & 0.181 & 0.200 & 0.100 & 10.5 & 30.0 \\
\hline 1,2-Dibromoethane & 0.137 & 0.142 & 0.010 & 3.3 & 40.0 \\
\hline Chlorobenzene & 1.138 & 1.190 & 0.500 & 4.6 & 30.0 \\
\hline Ethylbenzene & 2.043 & 2.294 & 0.100 & 12.3 & 30.0 \\
\hline o-Xylene & 0.677 & 0.791 & 0.300 & 16.8 & 30.0 \\
\hline $\mathrm{m}, \mathrm{p}$-Xylene & 0.783 & 0.900 & 0.300 & 15.0 & 30.0 \\
\hline Styrene & 1.077 & 1.279 & 0.300 & 18.8 & 30.0 \\
\hline Bromoform & 0.161 & 0.171 & 0.050 & 5.9 & 30.0 \\
\hline Isopropylbenzene & 1.977 & 2.320 & 0.010 & 17.3 & 40.0 \\
\hline $1,1,2,2$-Tetrachloroethane & 0.170 & 0.177 & 0.100 & 3.7 & 30.0 \\
\hline 1,3-Dichlorobenzene & 1.468 & 1.490 & 0.400 & 1.5 & 30.0 \\
\hline 1,4-Dichlorobenzene & 1.703 & 1.697 & 0.400 & -0.4 & 30.0 \\
\hline 1,2-Dichlorobenzene & 1.301 & 1.387 & 0.400 & 6.6 & 30.0 \\
\hline 1,2-Dibromo-3-Chloropropane & 0.039 & 0.036 & 0.010 & -8.6 & 40.0 \\
\hline 1,2,4-Trichlorobenzene & 0.757 & 0.688 & 0.200 & -9.1 & 30.0 \\
\hline $1,2,3$-Trichlorobenzene & 0.541 & 0.504 & 0.200 & -6.7 & 30.0 \\
\hline
\end{tabular}


Lab Name: TESTAMERICA BURLINGTON

Contract: $8 \mathrm{E}-00302$

Lab Code: STLV Case No.: YORK Mod. Ref No.

SDG No.: 33933

Instrument ID: CHD.i

Calibration Date: 06/15/2016 Time: 1343

Lab File Id: 20443_03.D

Init. Calib. Date(s):05/31/2016 05/31/2016

EPA Sample No.(VSTD\#\#\#\#): VSTD005DT

Init. Calib. Time(s) : 1605 1746

Heated Purge: (Y/N) N GC Column: DB-624

ID: $0.20(\mathrm{~mm})$ Length: 25

(m)

Purge Volume: 25.0 $(\mathrm{mL})$

\begin{tabular}{|c|c|c|c|c|c|}
\hline COMPOUND & $\overline{\mathrm{RRF}}$ & RRF5. 0 & $\begin{array}{l}\text { MIN } \\
\text { RRF }\end{array}$ & $\circ \mathrm{D}$ & MAX $\cong D$ \\
\hline Vinyl Chloride-d3 & 0.445 & 0.451 & 0.010 & 1.4 & 30.0 \\
\hline Chloroethane-d5 & 0.356 & 0.363 & 0.010 & 2.0 & 40.0 \\
\hline 1,1-Dichloroethene-d2 & 0.762 & 0.768 & 0.010 & 0.7 & 30.0 \\
\hline 2-Butanone-d5 & 0.030 & 0.031 & 0.010 & 2.5 & 40.0 \\
\hline Chloroform-d & 0.611 & 0.637 & 0.010 & 4.2 & 30.0 \\
\hline 1,2-Dichloroethane-d4 & 0.214 & 0.218 & 0.010 & 1.7 & 30.0 \\
\hline Benzene-d6 & 1.806 & 1.869 & 0.010 & 3.4 & 30.0 \\
\hline 1,2-Dichloropropane-d6 & 0.444 & 0.451 & 0.010 & 1.5 & 40.0 \\
\hline Toluene-d8 & 1.533 & 1.686 & 0.010 & 10.0 & 30.0 \\
\hline trans-1,3-Dichloropropene-d4 & 0.245 & 0.243 & 0.010 & $-1 \cdot 1$ & 30.0 \\
\hline 2-Hexanone-d5 & 0.030 & 0.032 & 0.010 & 6.5 & 40.0 \\
\hline $1,1,2,2-$ Tetrachloroethane-d2 & 0.171 & 0.179 & 0.010 & 4.9 & 30.0 \\
\hline 1,2-Dichlorobenzene-d4 & 0.807 & 0.795 & 0.010 & -1.6 & 30.0 \\
\hline
\end{tabular}

Report 1,4-Dioxane-d8 for Low/Medium VOA analysis only 
Lab Name: TESTAMERICA BURLINGTON

Contract: $8 \mathrm{E}-00302$

Lab Code: STLV Case No.: YORK Mod. Ref No.:

SDG No.: 33933

Instrument ID: CHD.i

Calibration Date: 06/15/2016 Time: 1821

Lab File Id: 20443_12.D

Init. Calib. Date(s):05/31/2016 05/31/2016

EPA Sample No. (VSTD\#\#\#\#) : VSTD005TD

Init. Calib. Time(s) : 1605 1746

Heated Purge: (Y/N) N GC Column: DB-624

ID: $0.20(\mathrm{~mm})$ Length: 25

(m)

Purge Volume: 25.0 $(\mathrm{mL})$

\begin{tabular}{|c|c|c|c|c|c|}
\hline COMPOUND & $\overline{\mathrm{RRF}}$ & RRF5.0 & $\begin{array}{l}\text { MIN } \\
\text { RRF }\end{array}$ & $\frac{\circ}{D}$ & $\mathrm{MAX} \div \mathrm{D}$ \\
\hline Dichlorodifluoromethane & 0.592 & 0.585 & 0.010 & -1.2 & 50.0 \\
\hline Chloromethane & 0.511 & 0.481 & 0.010 & -5.8 & 50.0 \\
\hline Vinyl chloride & 0.513 & 0.515 & 0.010 & 0.2 & 50.0 \\
\hline Bromomethane & 0.179 & 0.159 & 0.010 & -10.8 & 50.0 \\
\hline Chloroethane & 0.279 & 0.295 & 0.010 & 5.6 & 50.0 \\
\hline Trichlorofluoromethane & 0.646 & 0.667 & 0.010 & 3.2 & 50.0 \\
\hline 1,1-Dichloroethene & 0.343 & 0.348 & 0.010 & 1.5 & 50.0 \\
\hline 1,1,2-Trichloro-1,2,2-trifluoroethane & 0.388 & 0.396 & 0.010 & 2.2 & 50.0 \\
\hline Acetone & 0.020 & 0.020 & 0.010 & -1.7 & 50.0 \\
\hline Carbon disulfide & 0.987 & 0.942 & 0.010 & -4.5 & 50.0 \\
\hline Methyl acetate & 0.052 & 0.056 & 0.010 & 7.3 & 50.0 \\
\hline Methylene Chloride & 0.287 & 0.294 & 0.010 & 2.5 & 50.0 \\
\hline trans-1,2-Dichloroethene & 0.348 & 0.351 & 0.010 & 1.0 & 50.0 \\
\hline Methyl tert-butyl ether & 0.370 & 0.368 & 0.010 & -0.5 & 50.0 \\
\hline 1,1-Dichloroethane & 0.660 & 0.668 & 0.010 & 1.2 & 50.0 \\
\hline cis-1,2-Dichloroethene & 0.334 & 0.328 & 0.010 & -2.0 & 50.0 \\
\hline 2-Butanone & 0.033 & 0.034 & 0.010 & 3.4 & 50.0 \\
\hline Bromochloromethane & 0.105 & 0.109 & 0.010 & 4.7 & 50.0 \\
\hline Chloroform & 0.584 & 0.624 & 0.010 & 7.0 & 50.0 \\
\hline 1,1,1-Trichloroethane & 0.698 & 0.718 & 0.010 & 2.8 & 50.0 \\
\hline Cyclohexane & 0.799 & 0.858 & 0.010 & 7.4 & 50.0 \\
\hline Carbon tetrachloride & 0.629 & 0.667 & 0.010 & 6.1 & 50.0 \\
\hline Benzene & 1.993 & 2.056 & 0.010 & 3.2 & 50.0 \\
\hline 1,2-Dichloroethane & 0.266 & 0.289 & 0.010 & 8.6 & 50.0 \\
\hline Trichloroethene & 0.440 & 0.448 & 0.010 & 1.7 & 50.0 \\
\hline Methylcyclohexane & 0.649 & 0.706 & 0.010 & 8.8 & 50.0 \\
\hline
\end{tabular}

Report 1,4-Dioxane for Low/Medium VOA analysis only 
Lab Name: TESTAMERICA BURLINGTON

Contract: 8E-00302

Lab Code: STLV

Mod. Ref No.:

SDG No.: 33933

Instrument ID: CHD.i

Calibration Date: 06/15/2016 Time: 1821

Lab File Id: 20443_12.D

Init. Calib. Date(s):05/31/2016 05/31/2016

EPA Sample No. (VSTD\#\#\#\#) : VSTD005TD

Init. Calib. Time(s) : 1605 1746

Heated Purge: (Y/N) N GC Column: DB-624

ID: $0.20(\mathrm{~mm})$ Length: 25

(m)

Purge Volume: 25.0 $(\mathrm{mL})$

\begin{tabular}{|c|c|c|c|c|c|}
\hline COMPOUND & $\overline{\mathrm{RRF}}$ & RRF5.0 & $\begin{array}{l}\text { MIN } \\
\text { RRF }\end{array}$ & $\because \mathrm{D}$ & $\operatorname{MAX} \cong \mathrm{D}$ \\
\hline 1,2-Dichloropropane & 0.404 & 0.414 & 0.010 & 2.5 & 50.0 \\
\hline Bromodichloromethane & 0.381 & 0.398 & 0.010 & 4.4 & 50.0 \\
\hline cis-1,3-Dichloropropene & 0.457 & 0.495 & 0.010 & 8.4 & 50.0 \\
\hline 4-Methyl-2-pentanone & 0.089 & 0.103 & 0.010 & 16.1 & 50.0 \\
\hline Toluene & 1.848 & 2.103 & 0.010 & 13.8 & 50.0 \\
\hline trans-1,3-Dichloropropene & 0.298 & 0.312 & 0.010 & 4.7 & 50.0 \\
\hline $1,1,2$-Trichloroethane & 0.180 & 0.185 & 0.010 & 2.3 & 50.0 \\
\hline Tetrachloroethene & 0.441 & 0.453 & 0.010 & 2.7 & 50.0 \\
\hline 2-Hexanone & 0.062 & 0.073 & 0.010 & 18.4 & 50.0 \\
\hline Dibromochloromethane & 0.181 & 0.193 & 0.010 & 6.9 & 50.0 \\
\hline 1,2-Dibromoethane & 0.137 & 0.145 & 0.010 & 5.8 & 50.0 \\
\hline Chlorobenzene & 1.138 & 1.189 & 0.010 & 4.5 & 50.0 \\
\hline Ethylbenzene & 2.043 & 2.304 & 0.010 & 12.8 & 50.0 \\
\hline o-Xylene & 0.677 & 0.805 & 0.010 & 18.8 & 50.0 \\
\hline $\mathrm{m}, \mathrm{p}$-Xylene & 0.783 & 0.900 & 0.010 & 15.0 & 50.0 \\
\hline Styrene & 1.077 & 1.293 & 0.010 & 20.1 & 50.0 \\
\hline Bromoform & 0.161 & 0.161 & 0.010 & 0.0 & 50.0 \\
\hline Isopropylbenzene & 1.977 & 2.307 & 0.010 & 16.7 & 50.0 \\
\hline $1,1,2,2$-Tetrachloroethane & 0.170 & 0.182 & 0.010 & 7.1 & 50.0 \\
\hline 1,3-Dichlorobenzene & 1.468 & 1.496 & 0.010 & 1.9 & 50.0 \\
\hline 1,4-Dichlorobenzene & 1.703 & 1.714 & 0.010 & 0.7 & 50.0 \\
\hline 1,2-Dichlorobenzene & 1.301 & 1.400 & 0.010 & 7.6 & 50.0 \\
\hline 1,2-Dibromo-3-Chloropropane & 0.039 & 0.036 & 0.010 & -7.5 & 50.0 \\
\hline 1,2,4-Trichlorobenzene & 0.757 & 0.673 & 0.010 & $-11 \cdot 1$ & 50.0 \\
\hline $1,2,3$-Trichlorobenzene & 0.541 & 0.507 & 0.010 & -6.2 & 50.0 \\
\hline
\end{tabular}


Lab Name: TESTAMERICA BURLINGTON

Contract: $8 \mathrm{E}-00302$

Lab Code: STLV Case No.: YORK Mod. Ref No.

SDG No.: 33933

Instrument ID: CHD.i

Calibration Date: 06/15/2016 Time: 1821

Lab File Id: 20443_12.D

Init. Calib. Date(s):05/31/2016 05/31/2016

EPA Sample No. (VSTD\#\#\#\#) : VSTD005TD

Init. Calib. Time(s) : 1605 1746

Heated Purge: (Y/N) N GC Column: DB-624

ID: $0.20(\mathrm{~mm})$ Length: 25

(m)

Purge Volume: 25.0 $(\mathrm{mL})$

\begin{tabular}{|c|c|c|c|c|c|}
\hline COMPOUND & $\overline{\mathrm{RRF}}$ & RRF5.0 & $\begin{array}{l}\text { MIN } \\
\text { RRF }\end{array}$ & $\because \mathrm{D}$ & $\operatorname{MAX} \cong \mathrm{D}$ \\
\hline Vinyl Chloride-d3 & 0.445 & 0.450 & 0.010 & 1.2 & 50.0 \\
\hline Chloroethane-d5 & 0.356 & 0.363 & 0.010 & 1.9 & 50.0 \\
\hline 1,1-Dichloroethene-d2 & 0.762 & 0.773 & 0.010 & 1.5 & 50.0 \\
\hline 2-Butanone-d5 & 0.030 & 0.030 & 0.010 & $-1 \cdot 1$ & 50.0 \\
\hline Chloroform-d & 0.611 & 0.643 & 0.010 & 5.2 & 50.0 \\
\hline 1,2-Dichloroethane-d4 & 0.214 & 0.228 & 0.010 & 6.1 & 50.0 \\
\hline Benzene-d6 & 1.806 & 1.900 & 0.010 & 5.2 & 50.0 \\
\hline 1,2-Dichloropropane-d6 & 0.444 & 0.461 & 0.010 & 3.8 & 50.0 \\
\hline Toluene-d8 & 1.533 & 1.695 & 0.010 & 10.6 & 50.0 \\
\hline trans-1,3-Dichloropropene-d4 & 0.245 & 0.239 & 0.010 & -2.5 & 50.0 \\
\hline 2-Hexanone-d5 & 0.030 & 0.033 & 0.010 & 11.4 & 50.0 \\
\hline $1,1,2,2$-Tetrachloroethane-d2 & 0.171 & 0.183 & 0.010 & 6.7 & 50.0 \\
\hline 1,2-Dichlorobenzene-d4 & 0.807 & 0.821 & 0.010 & 1.6 & 50.0 \\
\hline
\end{tabular}

Report 1,4-Dioxane-d8 for Low/Medium VOA analysis only 
Lab Name: TESTAMERICA BURLINGTON

Contract: $8 \mathrm{E}-00302$

Lab Code: STLV

Case No.: YORK Mod. Ref No.:

SDG No.: 33933

Matrix: (SOIL/SED/WATER) Water

Sample wt/vol: 25.0 $(\mathrm{g} / \mathrm{mL}) \mathrm{mL}$

Level: (TRACE/LOW/MED) TRACE

\% Moisture: not dec.

GC Column: DB-624 ID: $0.20 \quad(\mathrm{~mm})$

Soil Extract Volume: (uL) $(\mathrm{mL})$

Purge Volume: 25.0
Lab Sample ID: MB 200-105769/4

Lab File ID: 20443_04.D

Date Received:

Date Analyzed: 06/15/2016

Dilution Factor: 1.0

Soil Aliquot Volume: (uL)

\begin{tabular}{|c|c|c|c|}
\hline CAS NO. & COMPOUND & $\begin{array}{l}\text { CONCENTRATION UNITS: } \\
(\mathrm{ug} / \mathrm{L} \text { or } \mathrm{ug} / \mathrm{kg}) \mathrm{ug} / \mathrm{L}\end{array}$ & q \\
\hline $75-71-8$ & Dichlorodifluoromethane & 1.0 & $\mathrm{U}$ \\
\hline $74-87-3$ & Chloromethane & 1.0 & $\mathrm{U}$ \\
\hline $75-01-4$ & Vinyl chloride & 1.0 & $\mathrm{U}$ \\
\hline $74-83-9$ & Bromomethane & 1.0 & $\mathrm{U}$ \\
\hline $75-00-3$ & Chloroethane & 1.0 & $\mathrm{U}$ \\
\hline $75-69-4$ & Trichlorofluoromethane & 1.0 & $\mathrm{U}$ \\
\hline $75-35-4$ & 1,1-Dichloroethene & 1.0 & $\mathrm{U}$ \\
\hline $76-13-1$ & $1,1,2$-Trichloro-1,2,2-trifluoroethane & 1.0 & $\mathrm{U}$ \\
\hline $67-64-1$ & Acetone & 1.6 & $\mathrm{~J}$ \\
\hline $75-15-0$ & Carbon disulfide & 0.037 & $\mathrm{~J}$ \\
\hline $79-20-9$ & Methyl acetate & 1.0 & $\mathrm{U}$ \\
\hline $75-09-2$ & Methylene Chloride & 0.036 & $\mathrm{~J}$ \\
\hline $156-60-5$ & trans-1,2-Dichloroethene & 0.015 & $\mathrm{~J}$ \\
\hline $1634-04-4$ & Methyl tert-butyl ether & 0.034 & $\mathrm{~J}$ \\
\hline $75-34-3$ & 1,1-Dichloroethane & 1.0 & $\mathrm{U}$ \\
\hline $156-59-2$ & cis-1,2-Dichloroethene & 1.0 & $\mathrm{U}$ \\
\hline $78-93-3$ & 2-Butanone & 5.0 & $\mathrm{U}$ \\
\hline $74-97-5$ & Bromochloromethane & 1.0 & $\mathrm{U}$ \\
\hline $67-66-3$ & Chloroform & 1.0 & $\mathrm{U}$ \\
\hline $71-55-6$ & $1,1,1$-Trichloroethane & 1.0 & $\mathrm{U}$ \\
\hline $110-82-7$ & Cyclohexane & 1.0 & $\mathrm{U}$ \\
\hline $56-23-5$ & Carbon tetrachloride & 1.0 & $\mathrm{U}$ \\
\hline $71-43-2$ & Benzene & 1.0 & $\mathrm{U}$ \\
\hline $107-06-2$ & 1,2-Dichloroethane & 1.0 & $\mathrm{U}$ \\
\hline
\end{tabular}

Report 1,4-Dioxane for Low-Medium VOA analysis only 
Lab Name: TESTAMERICA BURLINGTON

Lab Code: STLV

Case No.: YORK

Mod. Ref No.:

Contract: 8E-00302

Matrix: (SOIL/SED/WATER) Water

Sample wt/vol: 25.0 $(\mathrm{g} / \mathrm{mL}) \mathrm{mL}$

Level: (TRACE/LOW/MED) TRACE

\% Moisture: not dec.

GC Column: DB-624 ID $: 0.20 \quad(\mathrm{~mm})$

Soil Extract Volume: (uL) $(\mathrm{mL})$

Purge Volume: 25.0
SDG No.: 33933

Lab Sample ID: MB 200-105769/4

Lab File ID: 20443_04.D

Date Received:

Date Analyzed: 06/15/2016

Dilution Factor: 1.0

Soil Aliquot Volume: (uL)

\begin{tabular}{|c|c|c|c|}
\hline CAS NO. & COMPOUND & $\begin{array}{l}\text { CONCENTRATION UNITS: } \\
(\mathrm{ug} / \mathrm{L} \text { or } \mathrm{ug} / \mathrm{kg}) \mathrm{ug} / \mathrm{L}\end{array}$ & $q$ \\
\hline $79-01-6$ & Trichloroethene & 0.012 & $\mathrm{~J}$ \\
\hline $108-87-2$ & Methylcyclohexane & 1.0 & $\mathrm{U}$ \\
\hline $78-87-5$ & 1,2-Dichloropropane & 1.0 & $\mathrm{U}$ \\
\hline $75-27-4$ & Bromodichloromethane & 1.0 & $\mathrm{U}$ \\
\hline $10061-01-5$ & cis-1,3-Dichloropropene & 1.0 & U \\
\hline $108-10-1$ & 4-Methyl-2-pentanone & 1.0 & $\mathrm{U}$ \\
\hline $108-88-3$ & Toluene & 0.066 & $\mathrm{~J}$ \\
\hline $10061-02-6$ & trans-1,3-Dichloropropene & 1.0 & $\mathrm{U}$ \\
\hline $79-00-5$ & $1,1,2-$ Trichloroethane & 1.0 & $\mathrm{U}$ \\
\hline $127-18-4$ & Tetrachloroethene & 0.0078 & $\mathrm{~J}$ \\
\hline $591-78-6$ & 2-Hexanone & 1.0 & $\mathrm{U}$ \\
\hline $124-48-1$ & Dibromochloromethane & 0.028 & $\mathrm{~J}$ \\
\hline $106-93-4$ & 1,2-Dibromoethane & 1.0 & $\mathrm{U}$ \\
\hline $108-90-7$ & Chlorobenzene & 1.0 & $\mathrm{U}$ \\
\hline $100-41-4$ & Ethylbenzene & 0.0096 & $\mathrm{~J}$ \\
\hline $95-47-6$ & o-Xylene & 0.011 & $\mathrm{~J}$ \\
\hline $179601-23-1$ & m,p-xylene & 0.013 & $\mathrm{~J}$ \\
\hline $100-42-5$ & Styrene & 0.013 & $\mathrm{~J}$ \\
\hline $75-25-2$ & Bromoform & 1.0 & $\mathrm{U}$ \\
\hline $98-82-8$ & Isopropylbenzene & 1.0 & $\mathrm{U}$ \\
\hline $79-34-5$ & $1,1,2,2$-Tetrachloroethane & 1.0 & $\mathrm{U}$ \\
\hline $541-73-1$ & 1,3 -Dichlorobenzene & 0.049 & $\mathrm{~J}$ \\
\hline $106-46-7$ & 1,4-Dichlorobenzene & 0.072 & $\mathrm{~J}$ \\
\hline $95-50-1$ & 1,2-Dichlorobenzene & 0.091 & $\mathrm{~J}$ \\
\hline $96-12-8$ & 1,2-Dibromo-3-Chloropropane & 1.0 & $\mathrm{U}$ \\
\hline $120-82-1$ & $1,2,4$-Trichlorobenzene & 0.18 & $\mathrm{~J}$ \\
\hline $87-61-6$ & $1,2,3$-Trichlorobenzene & 0.30 & $\mathrm{~J}$ \\
\hline
\end{tabular}


Lab Name: TESTAMERICA BURLINGTON

Contract: 8E-00302

Lab Code: STLV Case No.: YORK Mod. Ref No.: SDG No.: 33933

Matrix: (SOIL/SED/WATER) Water

Sample wt/vol: 25.0 $(\mathrm{g} / \mathrm{mL}) \mathrm{mL}$

Level: (TRACE or LOW/MED) TRACE

Moisture: not dec.

GC Column: DB-624 ID $: 0.20 \quad(\mathrm{~mm})$

Soil Extract Volume: (uL)

CONCENTRATION UNITS: (ug/L or ug/kg) ug/L
Lab Sample ID: MB 200-105769/4

Lab File ID: 20443_04.D

Date Received:

Date Analyzed: 06/15/2016

Dilution Factor: 1.0

Soil Aliquot Volume: (uL) Purge Volume: 25.0 $(\mathrm{mL})$

01

02

\begin{tabular}{|l|l|c|c|c|}
\hline CAS NUMBER & \multicolumn{1}{|c|}{ COMPOUND NAME } & RT & EST. CONC. & Q \\
\hline & Unknown & 7.20 & 2.5 & $\mathrm{~J} X$ \\
\hline E9667961 & Total Alkanes & N/A & & \\
\hline
\end{tabular}

1EPA-designated Registry Number. 
Lab Name: TESTAMERICA BURLINGTON

Contract: 8E-00302

Lab Code: STLV

Case No.: YORK Mod. Ref No.: SDG No.: 33933

Matrix: (SOIL/SED/WATER) Water

Sample wt/vol: 25.0 $(\mathrm{g} / \mathrm{mL}) \mathrm{mL}$

Level: (TRACE/LOW/MED) TRACE

\% Moisture: not dec.

GC Column: DB-624 ID: $0.20 \quad(\mathrm{~mm})$

Soil Extract Volume: $(u L)$ $(\mathrm{mL})$

Purge Volume: 25.0
Lab Sample ID: 200-33933-3

Lab File ID: 20443_07.D

Date Received:

Date Analyzed: 06/15/2016

Dilution Factor: 1.0

Soil Aliquot Volume:

(uL)

\begin{tabular}{|c|c|c|c|}
\hline CAS NO. & COMPOUND & $\begin{array}{l}\text { CONCENTRATION UNITS: } \\
(\mathrm{ug} / \mathrm{L} \text { or } \mathrm{ug} / \mathrm{kg}) \mathrm{ug} / \mathrm{L}\end{array}$ & $Q$ \\
\hline $75-71-8$ & Dichlorodifluoromethane & 1.0 & $\mathrm{U}$ \\
\hline $74-87-3$ & Chloromethane & 1.0 & $\mathrm{U}$ \\
\hline $75-01-4$ & Vinyl chloride & 1.0 & $\mathrm{U}$ \\
\hline $74-83-9$ & Bromomethane & 1.0 & $\mathrm{U}$ \\
\hline $75-00-3$ & Chloroethane & 1.0 & $\mathrm{U}$ \\
\hline $75-69-4$ & Trichlorofluoromethane & 1.0 & $\mathrm{U}$ \\
\hline $75-35-4$ & 1,1-Dichloroethene & 1.0 & $\mathrm{U}$ \\
\hline $76-13-1$ & $1,1,2$-Trichloro-1,2,2-trifluoroethane & 1.0 & $\mathrm{U}$ \\
\hline $67-64-1$ & Acetone & 1.6 & $\mathrm{~J} B$ \\
\hline $75-15-0$ & Carbon disulfide & 1.0 & $\mathrm{U}$ \\
\hline $79-20-9$ & Methyl acetate & 1.0 & $\mathrm{U}$ \\
\hline $75-09-2$ & Methylene Chloride & 0.025 & $\mathrm{~J} B$ \\
\hline $156-60-5$ & trans-1,2-Dichloroethene & 1.0 & $\mathrm{U}$ \\
\hline $1634-04-4$ & Methyl tert-butyl ether & 1.0 & $\mathrm{U}$ \\
\hline $75-34-3$ & 1,1-Dichloroethane & 1.0 & $\mathrm{U}$ \\
\hline $156-59-2$ & cis-1,2-Dichloroethene & 1.0 & $\mathrm{U}$ \\
\hline $78-93-3$ & 2-Butanone & 5.0 & $\mathrm{U}$ \\
\hline $74-97-5$ & Bromochloromethane & 1.0 & $\mathrm{U}$ \\
\hline $67-66-3$ & Chloroform & 1.0 & $\mathrm{U}$ \\
\hline $71-55-6$ & $1,1,1$-Trichloroethane & 1.0 & $\mathrm{U}$ \\
\hline $110-82-7$ & Cyclohexane & 1.0 & $\mathrm{U}$ \\
\hline $56-23-5$ & Carbon tetrachloride & 1.0 & $\mathrm{U}$ \\
\hline $71-43-2$ & Benzene & 1.0 & $\mathrm{U}$ \\
\hline $107-06-2$ & 1,2-Dichloroethane & 1.0 & $\mathrm{U}$ \\
\hline
\end{tabular}

Report 1,4-Dioxane for Low-Medium VOA analysis only 
Lab Name: TESTAMERICA BURLINGTON

Lab Code: STLV

Case No.: YORK

Mod. Ref No.:

Contract: 8E-00302

Matrix: (SOIL/SED/WATER) Water

Sample wt/vol: 25.0 $(\mathrm{g} / \mathrm{mL}) \mathrm{mL}$

Level: (TRACE/LOW/MED) TRACE

\% Moisture: not dec.

GC Column: DB-624 ID: $0.20 \quad(\mathrm{~mm})$

Soil Extract Volume: (uL) $(\mathrm{mL})$

Purge Volume: 25.0 SDG No.: 33933

Lab Sample ID: 200-33933-3

Lab File ID: 20443_07.D

Date Received:

Date Analyzed: 06/15/2016

Dilution Factor: 1.0

Soil Aliquot Volume: (uL)

\begin{tabular}{|c|c|c|c|}
\hline CAS NO. & COMPOUND & $\begin{array}{l}\text { CONCENTRATION UNITS: } \\
(\mathrm{ug} / \mathrm{L} \text { or } \mathrm{ug} / \mathrm{kg}) \mathrm{ug} / \mathrm{L}\end{array}$ & Q \\
\hline $79-01-6$ & Trichloroethene & 1.0 & $\mathrm{U}$ \\
\hline $108-87-2$ & Methylcyclohexane & 1.0 & $\mathrm{U}$ \\
\hline $78-87-5$ & 1,2-Dichloropropane & 1.0 & $\mathrm{U}$ \\
\hline $75-27-4$ & Bromodichloromethane & 1.0 & $\mathrm{U}$ \\
\hline $10061-01-5$ & cis-1,3-Dichloropropene & 1.0 & $\mathrm{U}$ \\
\hline $108-10-1$ & 4-Methyl-2-pentanone & 1.0 & $\mathrm{U}$ \\
\hline $108-88-3$ & Toluene & 0.016 & $\mathrm{~J} B$ \\
\hline $10061-02-6$ & trans-1,3-Dichloropropene & 1.0 & $\mathrm{U}$ \\
\hline $79-00-5$ & $1,1,2-T r i c h l o r o e t h a n e$ & 1.0 & $\mathrm{U}$ \\
\hline $127-18-4$ & Tetrachloroethene & 1.0 & $\mathrm{U}$ \\
\hline $591-78-6$ & 2-Hexanone & 1.0 & $\mathrm{U}$ \\
\hline $124-48-1$ & Dibromochloromethane & 1.0 & $\mathrm{U}$ \\
\hline $106-93-4$ & 1,2-Dibromoethane & 1.0 & $\mathrm{U}$ \\
\hline $108-90-7$ & Chlorobenzene & 1.0 & $\mathrm{U}$ \\
\hline $100-41-4$ & Ethylbenzene & 1.0 & $\mathrm{U}$ \\
\hline $95-47-6$ & o-Xylene & 1.0 & $\mathrm{U}$ \\
\hline $179601-23-1$ & m,p-Xylene & 1.0 & $\mathrm{U}$ \\
\hline $100-42-5$ & styrene & 1.0 & $\mathrm{U}$ \\
\hline $75-25-2$ & Bromoform & 1.0 & $\mathrm{U}$ \\
\hline $98-82-8$ & Isopropylbenzene & 1.0 & $\mathrm{U}$ \\
\hline $79-34-5$ & $1,1,2,2$-Tetrachloroethane & 1.0 & $\mathrm{U}$ \\
\hline $541-73-1$ & 1,3-Dichlorobenzene & 1.0 & $\mathrm{U}$ \\
\hline $106-46-7$ & 1,4-Dichlorobenzene & 1.0 & $\mathrm{U}$ \\
\hline $95-50-1$ & $1,2-$ Dichlorobenzene & 1.0 & $\mathrm{U}$ \\
\hline $96-12-8$ & 1,2-Dibromo-3-Chloropropane & 1.0 & $\mathrm{U}$ \\
\hline $120-82-1$ & $1,2,4$-Trichlorobenzene & 1.0 & $\mathrm{U}$ \\
\hline $87-61-6$ & $1,2,3$-Trichlorobenzene & 1.0 & $\mathrm{U}$ \\
\hline
\end{tabular}


Lab Name: TESTAMERICA BURLINGTON

Contract: $8 \mathrm{E}-00302$

Lab Code: STLV Case No.: YORK Mod. Ref No.: SDG No.: 33933

Matrix: (SOIL/SED/WATER) Water

Sample wt/vol: 25.0 $(\mathrm{g} / \mathrm{mL}) \mathrm{mL}$

Level: (TRACE or LOW/MED) TRACE

\% Moisture: not dec.

GC Column: DB-624 ID $: 0.20 \quad(\mathrm{~mm})$

Soil Extract Volume: (uL)

CONCENTRATION UNITS: (ug/L or ug/kg) ug/L
Lab Sample ID: 200-33933-3

Lab File ID: 20443_07.D

Date Received:

Date Analyzed: 06/15/2016

Dilution Factor: 1.0

Soil Aliquot Volume: (uL) Purge Volume: 25.0 $(\mathrm{mL})$

01

02

\begin{tabular}{|l|l|c|c|c|}
\hline CAS NUMBER & \multicolumn{1}{|c|}{ COMPOUND NAME } & RT & EST. CONC. & Q \\
\hline & Unknown & 7.20 & 2.5 & $\mathrm{~J}$ X B \\
\hline E9667961 & Total Alkanes & N/A & & \\
\hline
\end{tabular}

1EPA-designated Registry Number. 
Lab Name: TestAmerica Burlington

Job No.: 200-33933-1

SDG No.: 33933

Instrument ID: CHD.i

Analysis Batch Number: 105151

Lab Sample ID: ICIS 200-105151/4

Client Sample ID:

Date Analyzed: 05/31/16 16:56

Lab File ID: 20227 04.D

GC Column: DB-624

ID $: 0.2(\mathrm{~mm})$

\begin{tabular}{|c|c|c|c|c|}
\hline \multirow[t]{2}{*}{ COMPOUND NAME } & \multirow{2}{*}{$\begin{array}{c}\text { RETENTION } \\
\text { TIME }\end{array}$} & \multicolumn{3}{|c|}{ MANUAL INTEGRATION } \\
\hline & & REASON & ANALYST & DATE \\
\hline 1,2-Dichloropropane-d6 & 6.32 & Split Peak & wilburj & $05 / 31 / 1617: 36$ \\
\hline
\end{tabular}

Lab Sample ID: IC 200-105151/5

Client Sample ID:

Date Analyzed: 05/31/16 17:21

Lab File ID: 20227 05.D

GC Column: DB-624

ID $: 0.2(\mathrm{~mm})$

\begin{tabular}{|c|c|c|c|c|}
\hline \multicolumn{1}{|c|}{ COMPOUND NAME } & RETENTION & \multicolumn{3}{|c}{ MANUAL INTEGRATION } \\
\cline { 3 - 5 } & TIME & REASON & ANALYST & DATE \\
\hline 1,2 -Dichloropropane-d6 & 6.32 & Split Peak & wilburj & 05/31/16 17:57 \\
\hline
\end{tabular}

Lab Sample ID: IC 200-105151/6

Client Sample ID:

Date Analyzed: 05/31/16 17:46

Lab File ID: 20227_06.D

GC Column: DB-624

ID $: 0.2(\mathrm{~mm})$

\begin{tabular}{|c|c|c|c|c|}
\hline \multicolumn{1}{|c|}{ COMPOUND NAME } & RETENTION & \multicolumn{3}{|c|}{ MANUAL INTEGRATION } \\
\cline { 3 - 5 } & TIME & REASON & ANALYST & DATE \\
\hline 1,2-Dichloropropane-d6 & 6.32 & Split Peak & wilburj & $05 / 31 / 16$ 18:30 \\
\hline
\end{tabular}


Lab Name: TestAmerica Burlington

Job No.: 200-33933-1

SDG No.: 33933

Instrument ID: CHD.i

Analysis Batch Number: 105769

Lab Sample ID: CCVIS 200-105769/3

Client Sample ID:

Date Analyzed: 06/15/16 13:43

Lab File ID: 20443 03.D

GC Column: DB-624

ID $: 0.2(\mathrm{~mm})$

\begin{tabular}{|c|c|c|c|c|}
\hline COMPOUND NAME & RETENTION & \multicolumn{2}{|c|}{ MANUAL INTEGRATION } \\
\cline { 3 - 5 } & TIME & REASON & ANALYST & DATE \\
\hline \hline 1,2 -Dichloropropane-d6 & 6.32 & Split Peak & wilburj & $06 / 15 / 1614: 47$ \\
\hline
\end{tabular}

Lab Sample ID: MB 200-105769/4

Client Sample ID:

Date Analyzed: 06/15/16 14:09

Lab File ID: 20443_04.D

GC Column: DB-624

ID $: 0.2(\mathrm{~mm})$

\begin{tabular}{|l|c|c|c|c|}
\hline \multirow{2}{*}{ COMPOUND NAME } & RETENTION & \multicolumn{2}{|c|}{ MANUAL INTEGRATION } \\
\cline { 3 - 5 } & TIME & \multicolumn{2}{|c|}{ REASON } & ANALYST \\
\hline Acetone & 2.79 & Assign Peak & DATE \\
\hline
\end{tabular}

Lab Sample ID: 200-33933-3

Client Sample ID: VHBLKO1

Date Analyzed: 06/15/16 16:14

Lab File ID: 20443 07.D

GC Column: DB-624

ID: $0.2(\mathrm{~mm})$

\begin{tabular}{|c|c|c|c|c|}
\hline \multirow[t]{2}{*}{ COMPOUND NAME } & \multirow{2}{*}{$\begin{array}{c}\text { RETENTION } \\
\text { TIME }\end{array}$} & \multicolumn{3}{|c|}{ MANUAL INTEGRATION } \\
\hline & & REASON & ANALYST & DATE \\
\hline Acetone & 2.79 & Assign Peak & maheseep & $06 / 16 / 1608: 56$ \\
\hline
\end{tabular}

Lab Sample ID: CCVC 200-105769/12

Client Sample ID:

Date Analyzed: 06/15/16 18:21

Lab File ID: 20443 12.D

GC Column: DB-624

ID $: 0.2(\mathrm{~mm})$

\begin{tabular}{|c|c|c|c|c|}
\hline \multirow[t]{2}{*}{ COMPOUND NAME } & \multirow{2}{*}{$\begin{array}{c}\text { RETENTION } \\
\text { TIME }\end{array}$} & \multicolumn{3}{|c|}{ MANUAL INTEGRATION } \\
\hline & & REASON & ANALYST & DATE \\
\hline Chloroethane & 2.08 & Assign Peak & maheseep & $06 / 16 / 1609: 16$ \\
\hline 1,2-Dichloropropane-d6 & 6.32 & Split Peak & wilburj & $06 / 15 / 1618: 51$ \\
\hline
\end{tabular}




\section{TestAmerica}

THE LEADER IN ENVIRONMENTAL TESTING

\section{ANALYTICAL REPORT}

Job Number: 200-33957-1

SDG Number: 33957

Job Description: York (200-33957)

Contract Number: 1E-30401

For:

Argonne National Laboratory

9700 South Cass Avenue

Building 203

Office B-141

Argonne, IL 60439

Attention: Ms. Esther Bowen

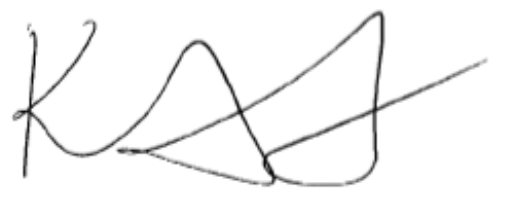

\section{Kathryn A Kelly, Project Manager I \\ 30 Community Drive, South Burlington, VT, 05403 \\ kathryn.kelly@testamericainc.com \\ $06 / 23 / 2016$}

The test results in this report relate only to sample(s) as received by the laboratory. These test results were derived under a quality system that adheres to the requirements of NELAC. Pursuant to NELAC, this report may not be produced in full without written approval from the laboratory 


\section{Table of Contents}

Cover Title Page...$\ldots \ldots \ldots \ldots \ldots \ldots \ldots \ldots \ldots \ldots \ldots \ldots \ldots$

Sample List ........................... 4

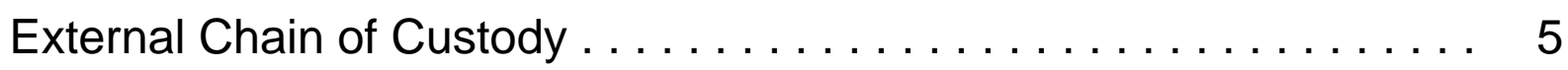

Shipping Documentation . . . . . . . . . . . . . . . 7

Sample Receipt and Log In Check List ..................... 8

Standards Traceability . . . . . . . . . . . . . . . . . 9

Methodology Review .............................. 14

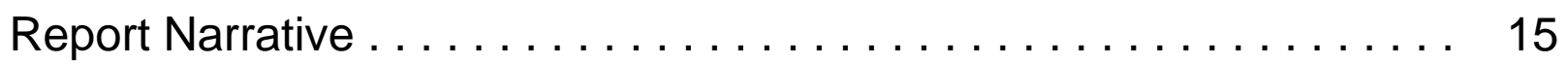

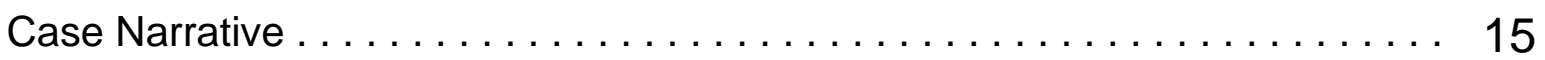

Manual Integration Documentation ....................... 16

Qualifier Definition ................................ 18

QC Summary - SOM01.2 Volatiles-Trace . . . . . . . . . . . . . 19

QC Summary - SOM01.2 Volatiles-Trace ..................... 19

Deuterated Monitoring Compound Summary $\ldots \ldots \ldots \ldots \ldots \ldots \ldots \ldots \ldots \ldots \ldots$

Method Blank $\ldots \ldots \ldots \ldots \ldots \ldots \ldots \ldots \ldots \ldots \ldots \ldots \ldots \ldots \ldots \ldots \ldots \ldots \ldots, 21$

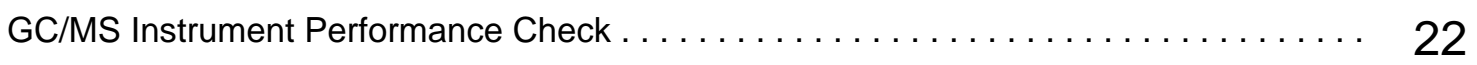

Internal Standard Area and RT Summary $\ldots \ldots \ldots \ldots \ldots \ldots \ldots \ldots \ldots \ldots \ldots \ldots \ldots \ldots \ldots$

Sample Data - SOM01.2 Volatiles-Trace ................. 25

Sample Data - SOM01.2 Volatiles-Trace ...................... 25

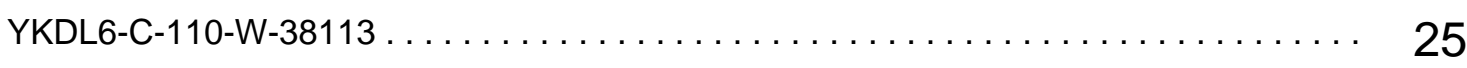

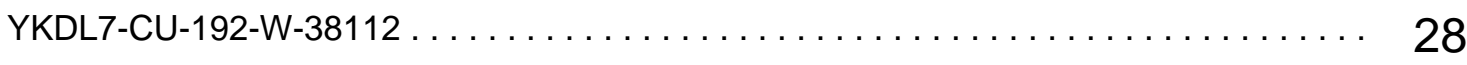

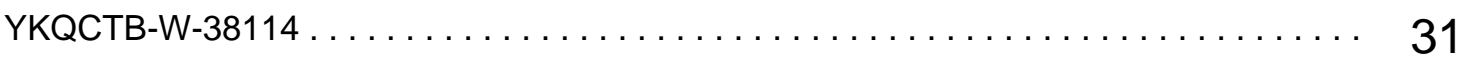

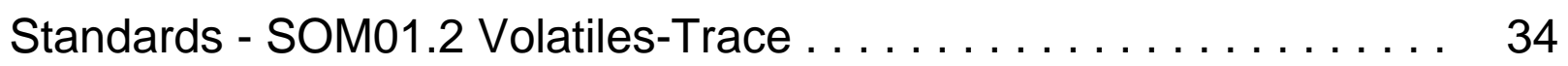

Standards - SOM01.2 Volatiles-Trace $\ldots \ldots \ldots \ldots \ldots \ldots \ldots \ldots \ldots, 34$

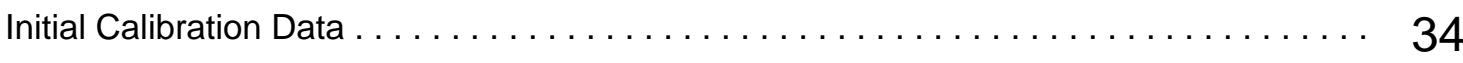

CCV Data, including closing CCV $\ldots \ldots \ldots \ldots \ldots \ldots \ldots \ldots \ldots \ldots \ldots \ldots \ldots \ldots \ldots \ldots \ldots$ 


\section{Table of Contents}

Raw Qc Data - SOM01.2 Volatiles-Trace . . . . . . . . . . . . . . 43

Raw Qc Data - SOM01.2 Volatiles-Trace . . . . . . . . . . . . . . 43

Raw Qc Data - SOM01.2 Volatiles-Trace ......................... 43

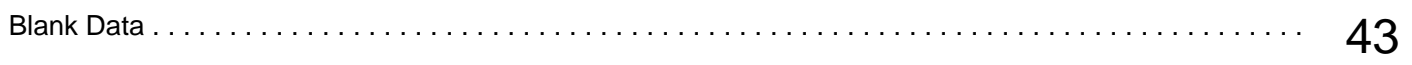




\section{Sample Login Acknowledgement}

Job 200-33957-1

\begin{tabular}{|c|c|c|c|}
\hline Client Job Description: & York (200-33957) & Report To: & Argonne National Laboratory \\
\hline Purchase Order \#: & $1 \mathrm{E}-30401$ & & Jorge Alvarado \\
\hline Work Order \#: & $1 \mathrm{E}-30401$ & & 9700 South Cass Avenue \\
\hline Project Manager: & Kathryn A Kelly & & Building 203 \\
\hline Job Due Date: & $6 / 28 / 2016$ & & Office B-141 \\
\hline Job TAT: & 14 Days & & \\
\hline \multirow[t]{2}{*}{ Max Deliverable Level: } & IV & Bill To: & Argonne National Laboratory \\
\hline & & & Accounts Payable \\
\hline Earliest Deliverable Due: & $6 / 28 / 2016$ & & Chief Financial Offices \\
\hline & & & 9700 S. Cass Ave. \\
\hline & & & Building 201 \\
\hline & & & Argonne, IL 60439 \\
\hline
\end{tabular}

\section{Login 200-33957}

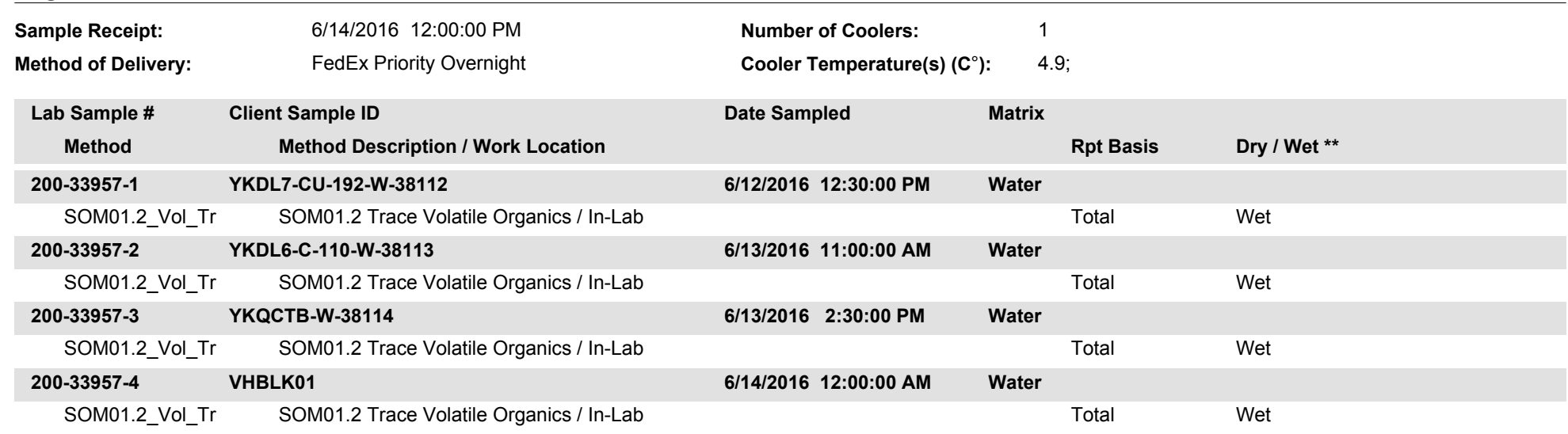




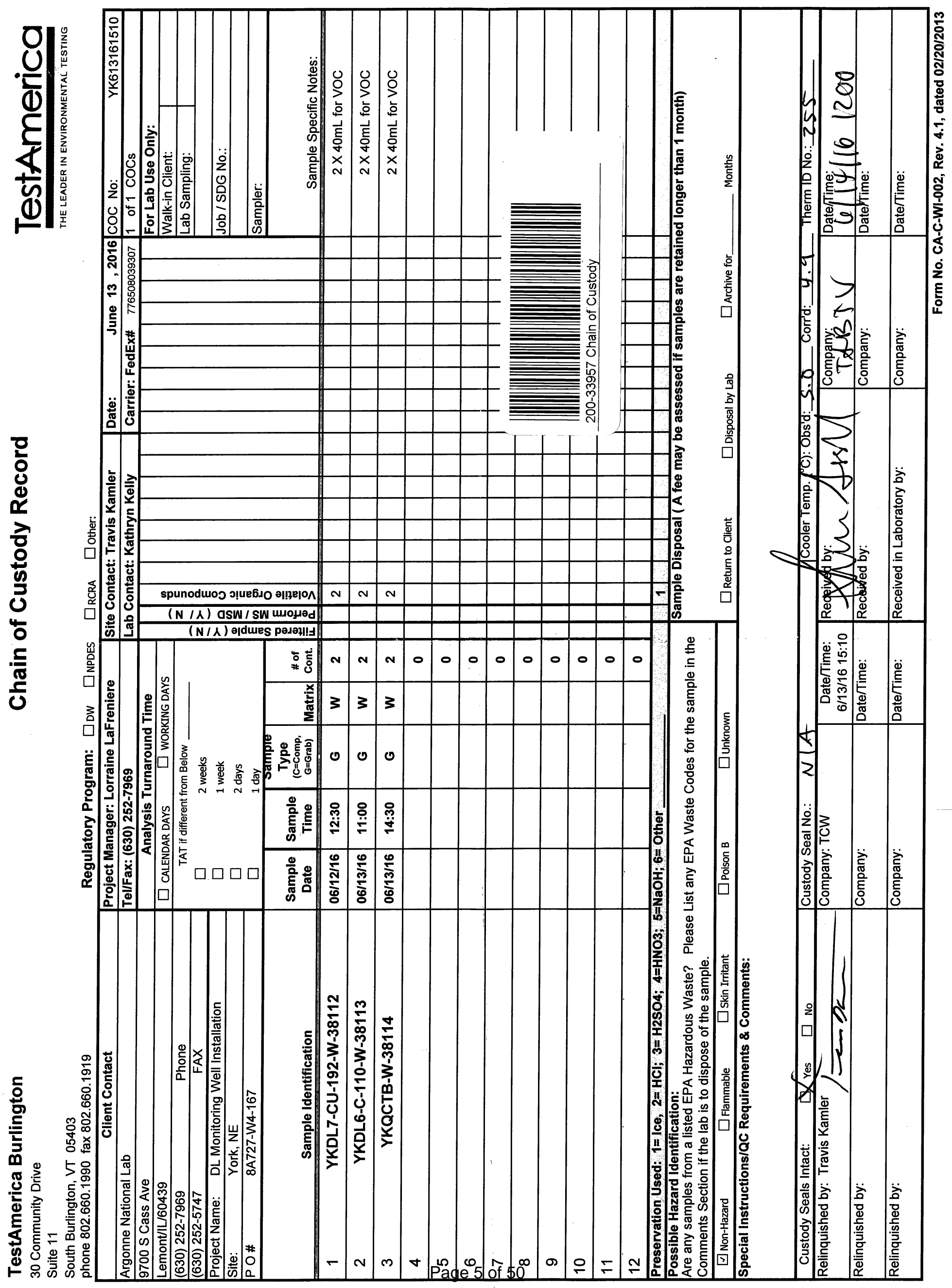




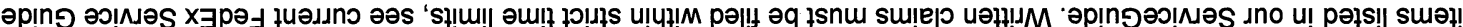

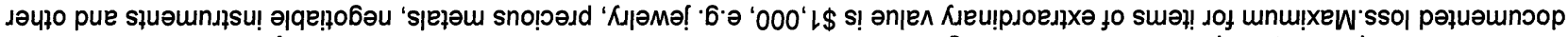

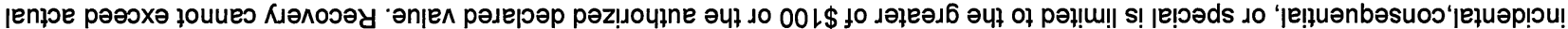

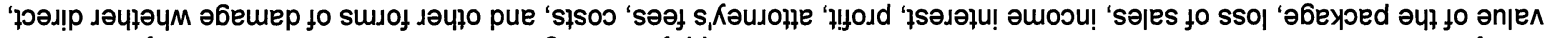

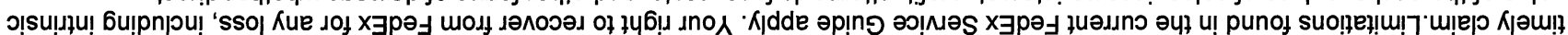

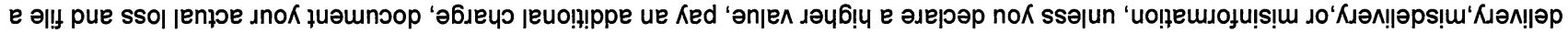

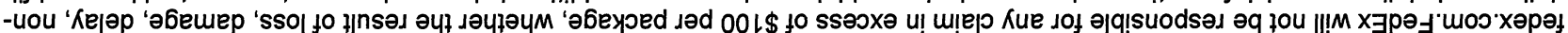
u० ә|qе|!e

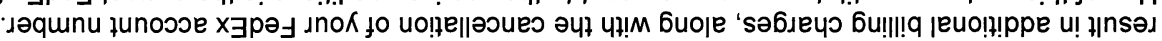

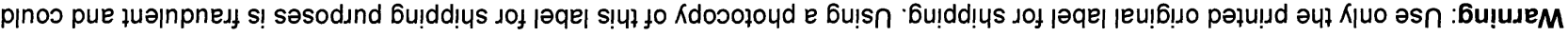

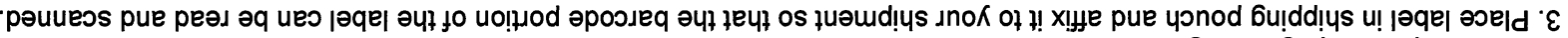

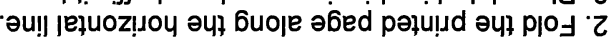

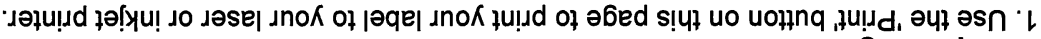

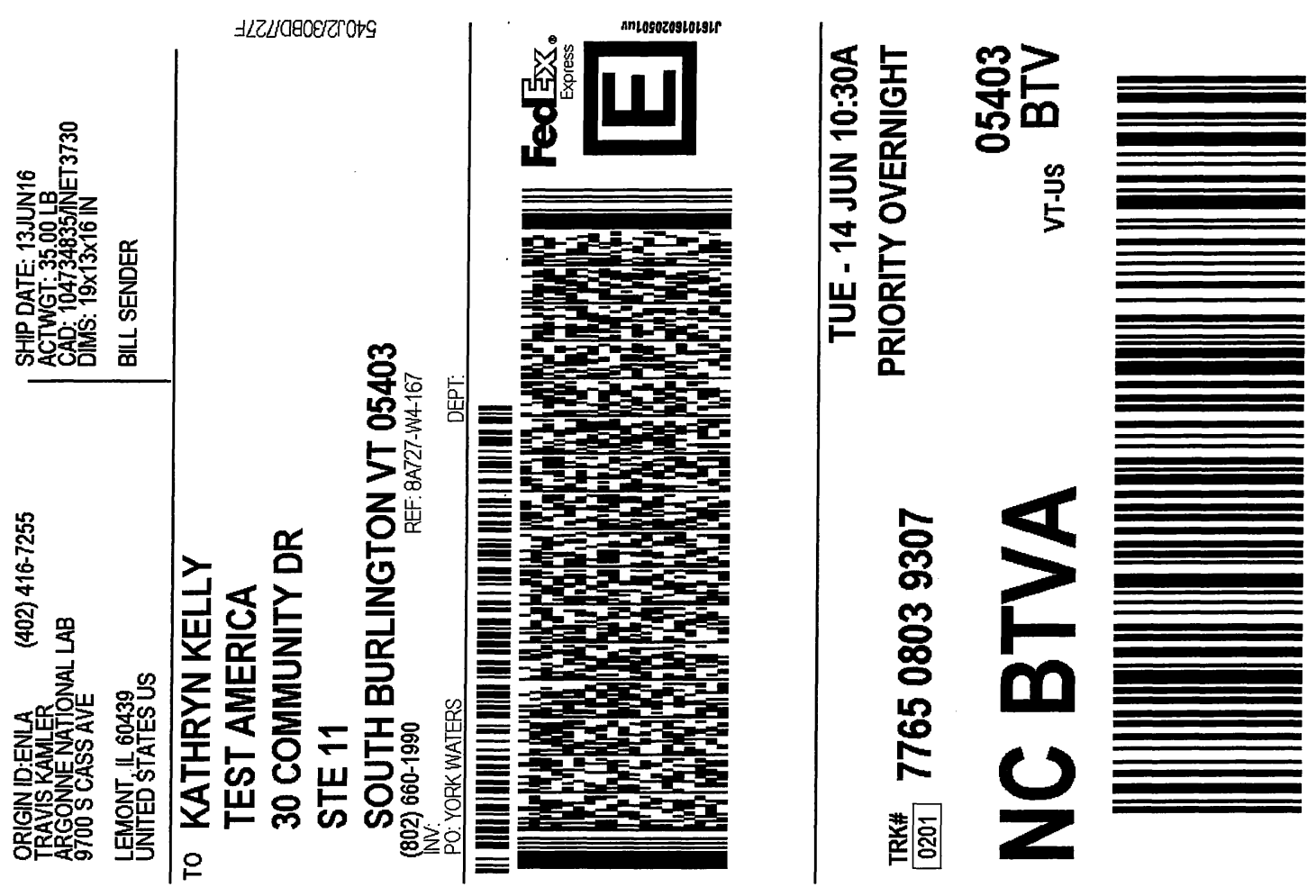




\section{Shipping and Receiving Documents}




\section{Login Sample Receipt Checklist}

Client: Argonne National Laboratory

Job Number: 200-33957-1

SDG Number: 33957

Login Number: 33957

List Source: TestAmerica Burlington

List Number: 1

Creator: Atherton, Joel E

Question

Radioactivity wasn't checked or is $</=$ background as measured by a survey meter.

The cooler's custody seal, if present, is intact.

Sample custody seals, if present, are intact.

The cooler or samples do not appear to have been compromised or tampered with.

Samples were received on ice.

Cooler Temperature is acceptable.

Cooler Temperature is recorded.

COC is present.

COC is filled out in ink and legible.

$\mathrm{COC}$ is filled out with all pertinent information.

Is the Field Sampler's name present on COC?

There are no discrepancies between the containers received and the COC.

Samples are received within Holding Time (excluding tests with immediate

HTs)

Sample containers have legible labels.

Containers are not broken or leaking.

Sample collection date/times are provided.

Appropriate sample containers are used.

Sample bottles are completely filled.

Sample Preservation Verified.

There is sufficient vol. for all requested analyses, incl. any requested MS/MSDs

Containers requiring zero headspace have no headspace or bubble is $<6 \mathrm{~mm}$ (1/4").

Multiphasic samples are not present.

Answer

True

True

True

True

True

True

True

True

True

True

True

True

True

True

True

True

True

N/A

True

True

True

True

True

N/A

Residual Chlorine Checked.

Lab does not accept radioactive samples.

Seal present with no number.

$4.9^{\circ} \mathrm{C}$

Samples do not require splitting or compositing. 


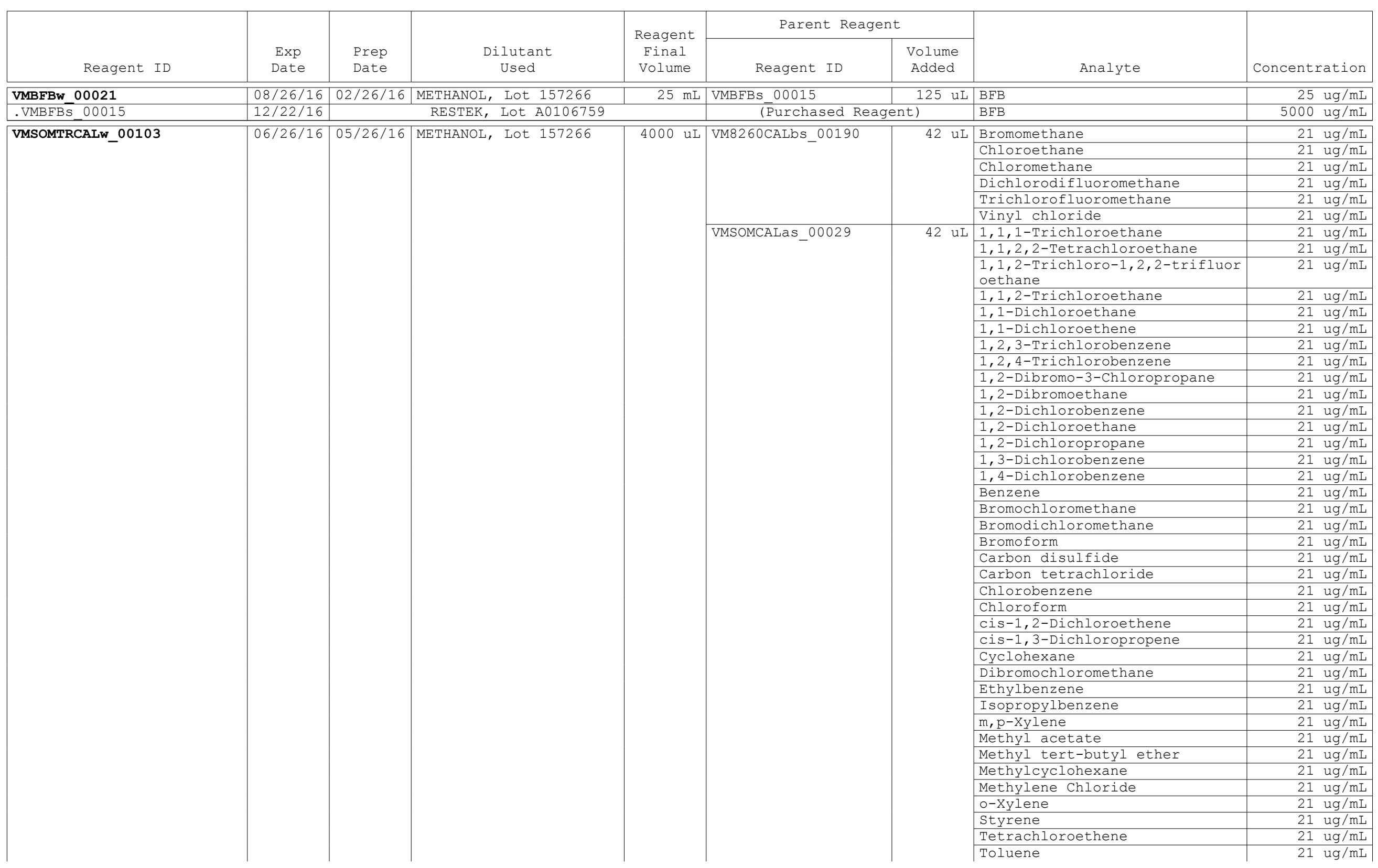


SDG No.: 33957

\begin{tabular}{|c|c|c|c|c|c|c|c|c|}
\hline \multirow[b]{2}{*}{ Reagent ID } & \multirow[b]{2}{*}{$\begin{array}{l}\text { Exp } \\
\text { Date }\end{array}$} & \multirow[b]{2}{*}{$\begin{array}{l}\text { Prep } \\
\text { Date }\end{array}$} & \multirow[b]{2}{*}{$\begin{array}{l}\text { Dilutant } \\
\text { Used }\end{array}$} & \multirow{2}{*}{$\begin{array}{c}\text { Reagent } \\
\text { Final } \\
\text { Volume }\end{array}$} & \multicolumn{2}{|l|}{ Parent Reagent } & \multirow[b]{2}{*}{ Analyte } & \multirow[b]{2}{*}{ Concentration } \\
\hline & & & & & \multirow[t]{4}{*}{ Reagent ID } & $\begin{array}{l}\text { Volume } \\
\text { Added }\end{array}$ & & \\
\hline & & & & & & & trans-1,2-Dichloroethene & $21 \mathrm{ug} / \mathrm{mL}$ \\
\hline & & & & & & & trans-1,3-Dichloropropene & $21 \mathrm{ug} / \mathrm{mL}$ \\
\hline & & & & & & & Trichloroethene & $21 \mathrm{ug} / \mathrm{mL}$ \\
\hline & & & & & VMSOMCALbS_00035 & $168 \mathrm{uL}$ & 2-Butanone & $210 \mathrm{ug} / \mathrm{mL}$ \\
\hline & & & & & & & 2-Hexanone & $210 \mathrm{ug} / \mathrm{mL}$ \\
\hline & & & & & & & 4-Methyl-2-pentanone & $210 \mathrm{ug} / \mathrm{mL}$ \\
\hline & & & & & & & Acetone & $210 \mathrm{ug} / \mathrm{mL}$ \\
\hline & & & & & VMSOMSUas_00085 & 42 uL & Chloroethane-d5 & $21 \mathrm{ug} / \mathrm{mL}$ \\
\hline & & & & & & & Vinyl Chloride-d3 & $21 \mathrm{ug} / \mathrm{mL}$ \\
\hline & & & & & VMSOMSUbs_00147 & $336 \mathrm{uL}$ & 2-Butanone-d5 & $210 \mathrm{ug} / \mathrm{mL}$ \\
\hline & & & & & & & 2-Hexanone-d5 & $210 \mathrm{ug} / \mathrm{mL}$ \\
\hline & & & & & VMSOMSUCS_00059 & 42 uL & $1,1,2,2$-Tetrachloroethane-d2 & $21 \mathrm{ug} / \mathrm{mL}$ \\
\hline & & & & & & & 1,1-Dichloroethene-d2 & $21 \mathrm{ug} / \mathrm{mL}$ \\
\hline & & & & & & & 1,2-Dichlorobenzene-d4 & $21 \mathrm{ug} / \mathrm{mL}$ \\
\hline & & & & & & & 1,2-Dichloroethane-d4 & $21 \mathrm{ug} / \mathrm{mL}$ \\
\hline & & & & & & & 1,2-Dichloropropane-d6 & $21 \mathrm{ug} / \mathrm{mL}$ \\
\hline & & & & & & & Benzene-d6 & $21 \mathrm{ug} / \mathrm{mL}$ \\
\hline & & & & & & & Chloroform-d & $21 \mathrm{ug} / \mathrm{mL}$ \\
\hline & & & & & & & Toluene-d8 & $21 \mathrm{ug} / \mathrm{mL}$ \\
\hline & & & & & & & trans-1,3-Dichloropropene-d4 & $21 \mathrm{ug} / \mathrm{mL}$ \\
\hline \multirow{6}{*}{.VM8260CALbs_00190 } & \multirow{6}{*}{$06 / 26 / 16$} & \multirow{6}{*}{\multicolumn{3}{|c|}{ RESTEK, Lot A0115510 }} & \multirow{6}{*}{\multicolumn{2}{|c|}{ (Purchased Reagent) }} & Bromomethane & $2000 \mathrm{ug} / \mathrm{mL}$ \\
\hline & & & & & & & Chloroethane & $2000 \mathrm{ug} / \mathrm{mL}$ \\
\hline & & & & & & & Chloromethane & $2000 \mathrm{ug} / \mathrm{mL}$ \\
\hline & & & & & & & Dichlorodifluoromethane & $2000 \mathrm{ug} / \mathrm{mL}$ \\
\hline & & & & & & & Trichlorofluoromethane & $2000 \mathrm{ug} / \mathrm{mL}$ \\
\hline & & & & & & & Vinyl chloride & $2000 \mathrm{ug} / \mathrm{mL}$ \\
\hline \multirow{21}{*}{.VMSOMCALas_00029 } & \multirow[t]{21}{*}{$04 / 30 / 17$} & \multirow{21}{*}{\multicolumn{2}{|c|}{ Restek, Lot A0102833 }} & & \multirow{21}{*}{\multicolumn{2}{|c|}{ (Purchased Reagent) }} & $1,1,1$-Trichloroethane & $2000 \mathrm{ug} / \mathrm{mL}$ \\
\hline & & & & & & & 1,1,2,2-Tetrachloroethane & $2000 \mathrm{ug} / \mathrm{mL}$ \\
\hline & & & & & & & $\begin{array}{l}\text { 1,1,2-Trichloro-1,2,2-trifluor } \\
\text { oethane }\end{array}$ & $2000 \mathrm{ug} / \mathrm{mL}$ \\
\hline & & & & & & & $1,1,2$-Trichloroethane & $2000 \mathrm{ug} / \mathrm{mL}$ \\
\hline & & & & & & & 1,1-Dichloroethane & $2000 \mathrm{ug} / \mathrm{mL}$ \\
\hline & & & & & & & 1,1-Dichloroethene & $2000 \mathrm{ug} / \mathrm{mL}$ \\
\hline & & & & & & & 1,2,3-Trichlorobenzene & $2000 \mathrm{ug} / \mathrm{mL}$ \\
\hline & & & & & & & 1,2,4-Trichlorobenzene & $2000 \mathrm{ug} / \mathrm{mL}$ \\
\hline & & & & & & & 1,2-Dibromo-3-Chloropropane & $2000 \mathrm{ug} / \mathrm{mL}$ \\
\hline & & & & & & & 1,2-Dibromoethane & $2000 \mathrm{ug} / \mathrm{mL}$ \\
\hline & & & & & & & 1,2-Dichlorobenzene & $2000 \mathrm{ug} / \mathrm{mL}$ \\
\hline & & & & & & & 1,2-Dichloroethane & $2000 \mathrm{ug} / \mathrm{mL}$ \\
\hline & & & & & & & 1,2-Dichloropropane & $2000 \mathrm{ug} / \mathrm{mL}$ \\
\hline & & & & & & & 1,3-Dichlorobenzene & $2000 \mathrm{ug} / \mathrm{mL}$ \\
\hline & & & & & & & 1,4-Dichlorobenzene & $2000 \mathrm{ug} / \mathrm{mL}$ \\
\hline & & & & & & & Benzene & $2000 \mathrm{ug} / \mathrm{mL}$ \\
\hline & & & & & & & Bromochloromethane & $2000 \mathrm{ug} / \mathrm{mL}$ \\
\hline & & & & & & & Bromodichloromethane & $2000 \mathrm{ug} / \mathrm{mL}$ \\
\hline & & & & & & & Bromoform & $2000 \mathrm{ug} / \mathrm{mL}$ \\
\hline & & & & & & & Carbon disulfide & $2000 \mathrm{ug} / \mathrm{mL}$ \\
\hline & & & & & & & Carbon tetrachloride & $2000 \mathrm{ug} / \mathrm{mL}$ \\
\hline
\end{tabular}


SDG No.: 33957

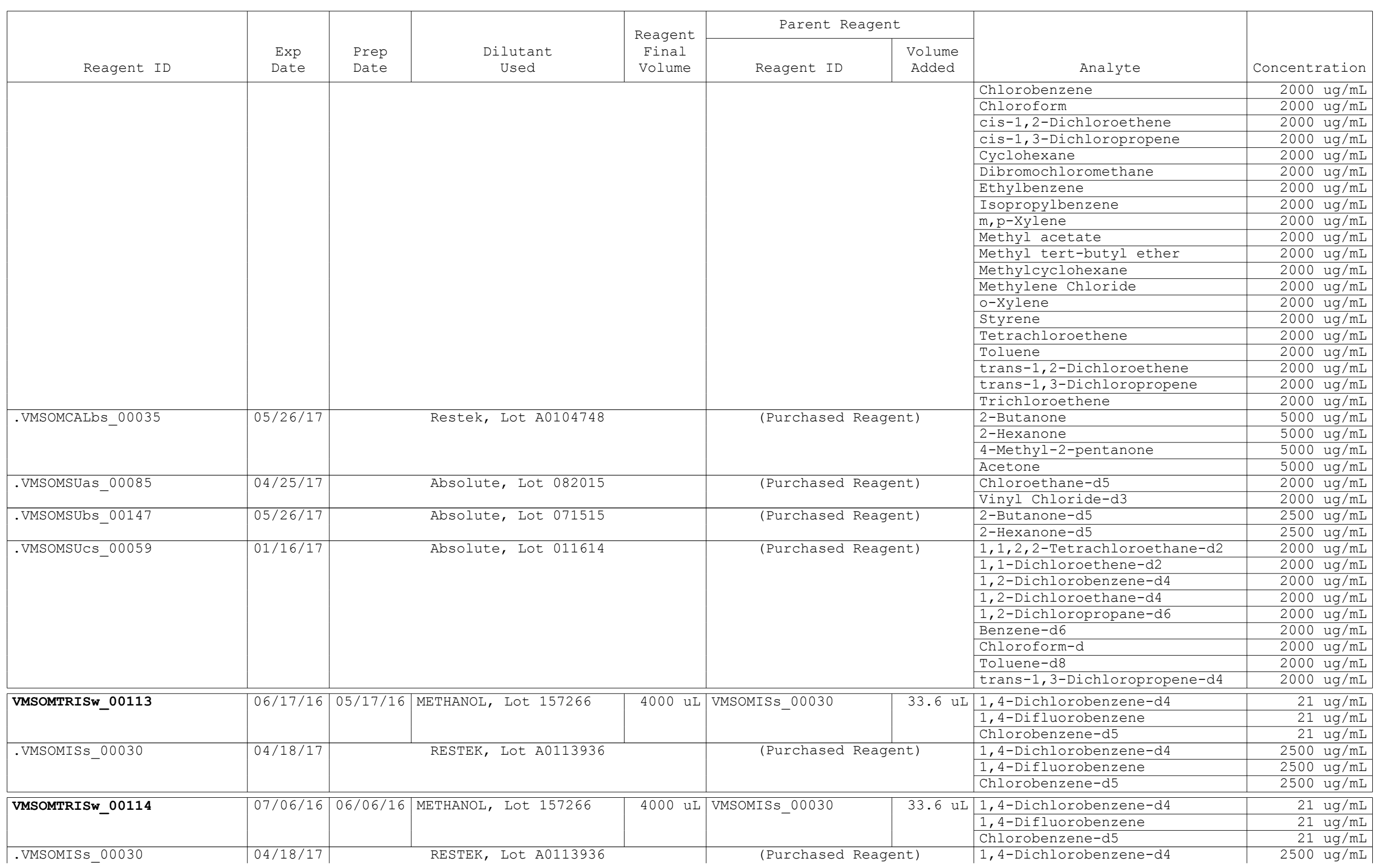


SDG No.: 33957

\begin{tabular}{|c|c|c|c|c|c|c|c|c|}
\hline \multirow[b]{2}{*}{ Reagent ID } & \multirow[b]{2}{*}{$\begin{array}{l}\text { Exp } \\
\text { Date }\end{array}$} & \multirow[b]{2}{*}{$\begin{array}{l}\text { Prep } \\
\text { Date }\end{array}$} & \multirow[b]{2}{*}{$\begin{array}{l}\text { Dilutant } \\
\text { Used }\end{array}$} & \multirow{2}{*}{$\begin{array}{l}\text { Reagent } \\
\text { Final } \\
\text { Volume }\end{array}$} & \multicolumn{2}{|c|}{ Parent Reagent } & \multirow[b]{2}{*}{ Analyte } & \multirow[b]{2}{*}{ Concentration } \\
\hline & & & & & \multicolumn{2}{|r|}{$\begin{array}{l}\text { Volume } \\
\text { Added }\end{array}$} & & \\
\hline & & & & & & & \begin{tabular}{|l} 
1,4-Difluorobenzene \\
Chlorobenzene-d5
\end{tabular} & $\begin{array}{l}2500 \mathrm{ug} / \mathrm{mL} \\
2500 \mathrm{ug} / \mathrm{mL}\end{array}$ \\
\hline \multirow[t]{10}{*}{ VMSOMTRSUw_00108 } & \multirow[t]{10}{*}{$06 / 17 / 16$} & \multirow[t]{10}{*}{$05 / 17 / 16$} & \multirow[t]{10}{*}{ METHANOL， Lot 157266} & \multirow{10}{*}{4000 UL } & VMSOMSUas_00085 & $42 \mathrm{uL}$ & Chloroethane-d5 & $21 \mathrm{ug} / \mathrm{mL}$ \\
\hline & & & & & VMSOMSUbs_00145 & $336 \mathrm{uL}$ & $\begin{array}{l}\text { Vinyl Chloride-d3 } \\
\text { 2-Butanone-d5 } \\
\text {-Hexanone-d5 }\end{array}$ & $\begin{aligned} 21 \mathrm{ug} / \mathrm{mL} \\
210 \mathrm{ug} / \mathrm{mL} \\
210 \mathrm{ug} / \mathrm{mL}\end{aligned}$ \\
\hline & & & & & \multirow[t]{8}{*}{ VMSOMSUCS_00059 } & \multirow[t]{8}{*}{42 uL } & $1,1,2,2$-Tetrachloroethane-d2 & $21 \mathrm{ug} / \mathrm{mL}$ \\
\hline & & & & & & & 1,1-Dichloroethene-d2 & $21 \mathrm{ug} / \mathrm{mL}$ \\
\hline & & & & & & & 1,2-Dichlorobenzene-d4 & $\frac{21 \mathrm{ug} / \mathrm{mL}}{21 \mathrm{ug} / \mathrm{mL}}$ \\
\hline & & & & & & & $\frac{1,2 \text {-Dichloroethane-d4 }}{1,2 \text {-Dichloropropane-d6 }}$ & $\frac{21 \mathrm{ug} / \mathrm{mL}}{21 \mathrm{ug} / \mathrm{mL}}$ \\
\hline & & & & & & & $\frac{1,2-11 C n 10}{\text { Benzene-d6 }}$ & $21 \mathrm{ug} / \mathrm{mL}$ \\
\hline & & & & & & & Chloroform-d & $21 \mathrm{ug} / \mathrm{mL}$ \\
\hline & & & & & & & Toluene-d8 & $21 \mathrm{ug} / \mathrm{mL}$ \\
\hline & & & & & & & trans-1,3-Dichloropropene-d4 & $21 \mathrm{ug} / \mathrm{mL}$ \\
\hline .VMSOMSUas_00085 & $04 / 25 / 17$ & \multicolumn{3}{|c|}{ Absolute, Lot 082015} & \multicolumn{2}{|c|}{ (Purchased Reagent) } & $\begin{array}{l}\text { Chloroethane-d5 } \\
\text { Vinvl Chloride-d3 }\end{array}$ & $2000 \mathrm{ug} / \mathrm{mL}$ \\
\hline \multirow{2}{*}{.VMSOMSUbs_00145 } & \multirow{2}{*}{$04 / 25 / 17$} & \multirow{2}{*}{\multicolumn{3}{|c|}{ Absolute, Lot 071515}} & \multirow{2}{*}{\multicolumn{2}{|c|}{ (Purchased Reagent) }} & 2-Butanone-d5 & $2500 \mathrm{ug} / \mathrm{mL}$ \\
\hline & & & & & & & 2-Hexanone-d5 & $2500 \mathrm{ug} / \mathrm{mL}$ \\
\hline \multirow[t]{7}{*}{.VMSOMSUCs_00059 } & \multirow[t]{7}{*}{$01 / 16 / 17$} & \multirow{7}{*}{\multicolumn{3}{|c|}{ Absolute, Lot 011614}} & \multirow{7}{*}{\multicolumn{2}{|c|}{ (Purchased Reagent) }} & $1,1,2,2$-Tetrachloroethane-d2 & $2000 \mathrm{ug} / \mathrm{mL}$ \\
\hline & & & & & & & 1,1-Dichloroethene-d2 & $2000 \mathrm{ug} / \mathrm{mL}$ \\
\hline & & & & & & & $\begin{array}{l}\text { 1,2-Dichlorrobenzene-d4 } \\
\text { 1,2-Dichloroethane-d4 }\end{array}$ & $2000 \mathrm{uq} / \mathrm{mL}$ \\
\hline & & & & & & & 1,2-Dichloropropane-d6 & $2000 \mathrm{ug} / \mathrm{mL}$ \\
\hline & & & & & & & Benzene-d6 & $2000 \mathrm{ug} / \mathrm{mL}$ \\
\hline & & & & & & & Chloroform-d & $2000 \mathrm{ug} / \mathrm{mL}$ \\
\hline & & & & & & & $\begin{array}{l}\frac{T}{T o l u e n e-d 8} \\
\text { trans-1,3-Dichloropropene-d4 }\end{array}$ & $\frac{2000 \mathrm{ug} / \mathrm{mL}}{2000 \mathrm{ug} / \mathrm{mL}}$ \\
\hline
\end{tabular}


Lab Name: TestAmerica Burlington

Job No.: 200-33957-1

SDG No.: 33957

\begin{tabular}{|c|c|c|c|c|}
\hline $\begin{array}{c}\text { Reagent } \\
\text { Container }\end{array}$ & $\begin{array}{c}\text { Reagent } \\
\text { ID }\end{array}$ & $\begin{array}{c}\text { Reagent } \\
\text { Description }\end{array}$ & $\begin{array}{c}\text { Preparation } \\
\text { Date }\end{array}$ & $\begin{array}{c}\text { Expiration } \\
\text { Date }\end{array}$ \\
\hline 904333 & VMBFBW 00021 & BFB TUNE 25 PPM & $02 / 26 / 2016$ & $08 / 26 / 2016$ \\
\hline 932091 & VMSOMTRISw_00113 & SOM TR ISTD 21 PPM & $05 / 17 / 2016$ & $06 / 17 / 2016$ \\
\hline 932092 & VMSOMTRSUw 00108 & SOM TR $\quad$ DMC $21 \quad$ PPM & $05 / 17 / 2016$ & $06 / 17 / 2016$ \\
\hline 935531 & VMSOMTRCALW_00103 & SOM TR CAL $21-210$ PPM & $05 / 26 / 2016$ & $06 / 26 / 2016$ \\
\hline 938927 & VMSOMTRISW $\overline{0} 0114$ & SOM TR ISTD 21 PPM & $06 / 06 / 2016$ & $07 / 06 / 2016$ \\
\hline
\end{tabular}




\section{METHODOLOGY SUMMARY}

Laboratory: TestAmerica Laboratories

Location: South Burlington, Vermont
Project No:

SDG No: $\quad 33957$

\section{VOA}

Volatile Organics Trace - USEPA CLP SOM01.2 


\section{CASE NARRATIVE}

\section{Client: Argonne National Laboratory}

\section{Project: York (200-33957)}

\section{Report Number: 200-33957-1}

With the exceptions noted as flags or footnotes, standard analytical protocols were followed in the analysis of the samples and no problems were encountered or anomalies observed. In addition all laboratory quality control samples were within established control limits, with any exceptions noted below. Each sample was analyzed to achieve the lowest possible reporting limit within the constraints of the method. In some cases, due to interference or analytes present at high concentrations, samples were diluted. For diluted samples, the reporting limits are adjusted relative to the dilution required.

Calculations are performed before rounding to avoid round-off errors in calculated results.

All holding times were met and proper preservation noted for the methods performed on these samples, unless otherwise detailed in the individual sections below.

\section{RECEIPT}

The samples were received on 06/14/2016; the samples arrived in good condition, properly preserved and on ice. The temperature of the cooler at receipt was $4.9^{\circ} \mathrm{C}$.

\section{VOLATILE ORGANIC COMPOUNDS - TRACE}

Samples YKDL7-CU-192-W-38112, YKDL6-C-110-W-38113, YKQCTB-W-38114 and VHBLK01 were analyzed for Volatile Organic Compounds - Trace in accordance with EPA SOW SOM01.2. The samples were analyzed on 06/15/2016.

Several analytes were detected in method blank MB 200-105769/4 at levels that were above the method detection limit but below the reporting limit. The values should be considered estimates, and have been flagged. If the associated sample reported a result above the MDL and/or RL, the result has been flagged. Refer to the $\mathrm{QC}$ report for details.

2-Hexanone-d5 failed the surrogate recovery criteria high for YKQCTB-W-38114. Refer to the QC report for details.

No additional analytical or quality issues were noted, other than those described above or in the Definitions/Glossary page. 
Lab Name: TestAmerica Burlington

Job No.: 200-33957-1

SDG No.: 33957

Instrument ID: CHD.i

Analysis Batch Number: 105151

Lab Sample ID: ICIS 200-105151/4

Client Sample ID:

Date Analyzed: 05/31/16 16:56

Lab File ID: 20227 04.D

GC Column: DB-624

ID $: 0.2(\mathrm{~mm})$

\begin{tabular}{|c|c|c|c|c|}
\hline \multirow[t]{2}{*}{ COMPOUND NAME } & \multirow{2}{*}{$\begin{array}{c}\text { RETENTION } \\
\text { TIME }\end{array}$} & \multicolumn{3}{|c|}{ MANUAL INTEGRATION } \\
\hline & & REASON & ANALYST & DATE \\
\hline 1,2-Dichloropropane-d6 & 6.32 & Split Peak & wilburj & $05 / 31 / 1617: 36$ \\
\hline
\end{tabular}

Lab Sample ID: IC 200-105151/5

Client Sample ID:

Date Analyzed: 05/31/16 17:21

Lab File ID: 20227 05.D

GC Column: DB-624

ID $: 0.2(\mathrm{~mm})$

\begin{tabular}{|c|c|c|c|c|}
\hline \multicolumn{1}{|c|}{ COMPOUND NAME } & RETENTION & \multicolumn{3}{|c}{ MANUAL INTEGRATION } \\
\cline { 3 - 5 } & TIME & REASON & ANALYST & DATE \\
\hline 1,2 -Dichloropropane-d6 & 6.32 & Split Peak & wilburj & 05/31/16 17:57 \\
\hline
\end{tabular}

Lab Sample ID: IC 200-105151/6

Client Sample ID:

Date Analyzed: 05/31/16 17:46

Lab File ID: 20227_06.D

GC Column: DB-624

ID $: 0.2(\mathrm{~mm})$

\begin{tabular}{|c|c|c|c|c|}
\hline \multicolumn{1}{|c|}{ COMPOUND NAME } & RETENTION & \multicolumn{3}{|c|}{ MANUAL INTEGRATION } \\
\cline { 3 - 5 } & TIME & REASON & ANALYST & DATE \\
\hline 1,2-Dichloropropane-d6 & 6.32 & Split Peak & wilburj & $05 / 31 / 16$ 18:30 \\
\hline
\end{tabular}


Lab Name: TestAmerica Burlington

Job No.: 200-33957-1

SDG No.: 33957

Instrument ID: CHD.i

Analysis Batch Number: 105769

Lab Sample ID: CCVIS 200-105769/3

Client Sample ID:

Date Analyzed: 06/15/16 13:43

Lab File ID: 20443_03.D

GC Column: DB-624

ID $: 0.2(\mathrm{~mm})$

\begin{tabular}{|c|c|c|c|c|}
\hline \multirow[t]{2}{*}{ COMPOUND NAME } & \multirow{2}{*}{$\begin{array}{c}\text { RETENTION } \\
\text { TIME }\end{array}$} & \multicolumn{3}{|c|}{ MANUAL INTEGRATION } \\
\hline & & REASON & ANALYST & DATE \\
\hline $1,2-$ Dichloropropane-d6 & 6.32 & Split Peak & wilburj & $06 / 15 / 16 \quad 14: 47$ \\
\hline
\end{tabular}

Lab Sample ID: MB 200-105769/4

Client Sample ID:

Date Analyzed: 06/15/16 14:09

Lab File ID: 20443_04.D

GC Column: DB-624

ID $: 0.2(\mathrm{~mm})$

\begin{tabular}{|l|c|c|c|c|}
\hline \multicolumn{1}{c|}{ COMPOUND NAME } & RETENTION & \multicolumn{3}{|c}{ MANUAL INTEGRATION } \\
\cline { 3 - 5 } & TIME & REASON & ANALYST & DATE \\
\hline Acetone & 2.79 & Assign Peak & maheseep & $06 / 16 / 1608: 33$ \\
\hline
\end{tabular}

Lab Sample ID: CCVC 200-105769/12

Client Sample ID:

Date Analyzed: 06/15/16 18:21

Lab File ID: 20443_12.D

GC Column: DB-624

ID : $0.2(\mathrm{~mm})$

\begin{tabular}{|c|c|c|c|c|}
\hline \multirow[t]{2}{*}{ COMPOUND NAME } & \multirow{2}{*}{$\begin{array}{c}\text { RETENTION } \\
\text { TIME }\end{array}$} & \multicolumn{3}{|c|}{ MANUAL INTEGRATION } \\
\hline & & REASON & ANALYST & DATE \\
\hline Chloroethane & 2.08 & Assign Peak & maheseep & $06 / 16 / 1609: 16$ \\
\hline 1,2-Dichloropropane-d6 & 6.32 & Split Peak & wilburj & $06 / 15 / 1618: 51$ \\
\hline
\end{tabular}




\section{DATA REPORTING QUALIFIERS}

Client: Argonne National Laboratory

Job Number: 200-33957-1

Sdg Number: 33957

Lab Section

Qualifier

Description

GC/MS VOA

B

U

$\mathrm{J}$

$J$

$\mathrm{X}$

B
Analyte was found in the associated method blank as well as in the sample.

Analyzed for but not detected.

Indicates an Estimated Value for TICs

Indicates an estimated value.

See case narrative notes for explanation of the ' $X$ ' flag

Surrogate is outside acceptance limits.

The analyte was found in an associated blank, as well as in the sample. 
Lab Name: TESTAMERICA BURLINGTON

Lab Code: STLV Case No.: YORK Mod. Ref No.: Level: (TRACE or LOW) TRACE
Contract: $8 \mathrm{E}-00302$

SDG No.: 33957

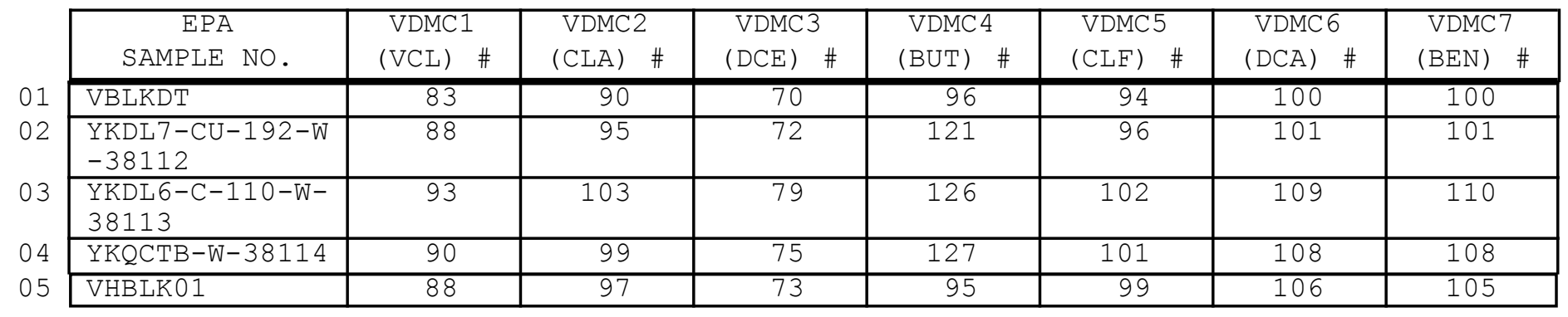

\footnotetext{
VDMC1 (VCL) = Vinyl Chloride-d3

VDMC2 $(\mathrm{CLA})=$ Chloroethane-d5

VDMC3 $(\mathrm{DCE})=1,1-\mathrm{Dichloroethene-d2}$

VDMC4 $\quad(B U T)=2-$ Butanone-d5

VDMC5 $(\mathrm{CLF})=$ Chloroform-d

VDMC $6 \quad(\mathrm{DCA})=1,2-\mathrm{Dichloroethane-d4}$

$\operatorname{VDMC} 7 \quad(\mathrm{BEN})=$ Benzene-d6
}

$$
\begin{gathered}
\frac{\mathrm{QC} \text { LIMITS }}{(65-131)} \\
(71-131) \\
(55-104) \\
(49-155) \\
(78-121) \\
(78-129) \\
(77-124)
\end{gathered}
$$

\# Column to be used to flag recovery values

* Values outside of contract required QC limits

Page 1 of 1 
Lab Name: TESTAMERICA BURLINGTON

Lab Code: STLV Case No.: YORK Mod. Ref No.:
Contract: $8 \mathrm{E}-00302$

SDG No.: 33957

Level: (TRACE or LOW) TRACE

\begin{tabular}{|c|c|c|c|c|c|c|c|c|}
\hline $\begin{array}{c}\text { EPA } \\
\text { SAMPLE NO. }\end{array}$ & $\begin{array}{l}\text { VDMC8 } \\
(\mathrm{DPA}) \#\end{array}$ & $\begin{array}{l}\text { VDMC9 } \\
(\text { TOL }) \#\end{array}$ & $\begin{array}{l}\text { VDMC10 } \\
(\mathrm{TDP}) \#\end{array}$ & $\begin{array}{l}\text { VDMC11 } \\
(\operatorname{HEX}) \quad \#\end{array}$ & $\begin{array}{l}\text { VDMC12 } \\
(\mathrm{TCA}) \quad \#\end{array}$ & $\begin{array}{l}\text { VDMC13 } \\
(\mathrm{DCZ})\end{array}$ & OTHER & $\begin{array}{l}\text { TOT } \\
\text { OUT }\end{array}$ \\
\hline VBLKDT & 96 & 98 & 89 & 99 & 93 & 112 & & 0 \\
\hline $\begin{array}{l}\text { YKDL 7-CU-192-W } \\
-38112\end{array}$ & 98 & 99 & 88 & 127 & 93 & 111 & & 0 \\
\hline $\begin{array}{l}\text { YKDL 6-C-110-W- } \\
38113\end{array}$ & 108 & 107 & 94 & 123 & 99 & 123 & & 0 \\
\hline YKQCTB-W-38114 & 105 & 107 & 96 & $140 \star$ & 101 & 124 & & 1 \\
\hline VHBLK01 & 100 & 102 & 88 & 98 & 96 & 129 & & 0 \\
\hline
\end{tabular}

\footnotetext{
$\operatorname{VDMC} 8(\mathrm{DPA})=1,2-\mathrm{Dichloropropane}-\mathrm{d} 6$

VDMC $9 \quad($ TOL $)=$ Toluene-d8

VDMC10 $(\mathrm{TDP})=$ trans $-1,3-\mathrm{Dichloropropene-d4}$

$\operatorname{VDMC1}(\mathrm{HEX})=2-$ Hexanone-d5

VDMC12 $(\mathrm{TCA})=1,1,2,2$-Tetrachloroethane-d2

VDMC13 $(\mathrm{DCZ})=1,2-\mathrm{Dichlorobenzene-d4}$
}

$$
\begin{gathered}
\frac{Q C \text { LIMITS }}{(79-124)} \\
(77-121) \\
(73-121) \\
(28-135) \\
(73-125) \\
(80-131)
\end{gathered}
$$

\# Column to be used to flag recovery values

* Values outside of contract required QC limits

Report 1,4-Dioxane-d8 for Low-Medium VOA analysis only

Page 1 of 1 
$4 \mathrm{~A}$ - FORM IV VOA

VOLATILE METHOD BLANK SUMMARY
EPA SAMPLE NO.

VBLKDT

Lab Name: TESTAMERICA BURLINGTON

Contract: $8 \mathrm{E}-00302$

Lab Code: STLV Case No.: YORK Mod. Ref No.: SDG No.: 33957

Lab File ID: 20443_04.D

Lab Sample ID: MB 200-105769/4

Instrument ID: CHD.i

Matrix: (SOIL/SED/WATER) Water

Level: (TRACE or LOW/MED) TRACE

Date Analyzed: 06/15/2016

GC Column: DB-624

ID $: 0.20 \quad(\mathrm{~mm})$

Time Analyzed: 1409

Heated Purge: (Y/N) N

\begin{tabular}{|c|c|c|c|}
\hline $\begin{array}{c}\text { EPA } \\
\text { SAMPLE NO. }\end{array}$ & $\begin{array}{r}\text { LAB } \\
\text { SAMPLE ID }\end{array}$ & $\begin{array}{l}\text { LAB } \\
\text { FILE ID }\end{array}$ & $\begin{array}{c}\text { TIME } \\
\text { ANALYZED }\end{array}$ \\
\hline $\begin{array}{l}\text { YKDL 7-CU-192 } \\
-W-38112\end{array}$ & $200-33957-1$ & 20443 _08.D & 1639 \\
\hline $\begin{array}{l}\text { YKDL 6-C-110- } \\
W-38113\end{array}$ & $200-33957-2$ & 20443_09.D & 1704 \\
\hline $\begin{array}{l}\text { YKQCTB-W-381 } \\
14\end{array}$ & $200-33957-3$ & 20443 _10.D & 1730 \\
\hline VHBLK01 & $200-33957-4$ & 20443 11.D & 1756 \\
\hline
\end{tabular}

COMMENTS :

Page 1 of 1 
$5 A$ - FORM V VOA

VOLATILE ORGANICS INSTRUMENT

PERFORMANCE CHECK

BROMOFLUOROBENZENE (BFB)
EPA SAMPLE NO.

BFBDJ
Lab Name: TESTAMERICA BURLINGTON

Lab Code: STLV

Case No.: YORK

Lab File Id: $2022701 . \mathrm{D}$

Instrument Id: CHD.i

GC Column: DB-624

ID $: 0.20$
Contract: 8E-00302

Mod. Ref No.:

SDG No.: 33957

BEB Injection Date: 05/31/2016

BFB Injection Time: 1555 $(\mathrm{mm})$

\begin{tabular}{|c|c|c|}
\hline $\mathrm{m} / \mathrm{e}$ & ION ABUNDANCE CRITERIA & $\begin{array}{l}\text { \% RELATIVE } \\
\text { ABUNDANCE }\end{array}$ \\
\hline 50 & $15.0-40.0 \%$ of mass 95 & 16.9 \\
\hline 75 & $30.0-80.0 \%$ of mass 95 & 50.0 \\
\hline 95 & Base peak, $100 \%$ relative abundance & 100 \\
\hline 96 & $5.0-9.0 \%$ of mass 95 & 6.6 \\
\hline 173 & Less than $2.0 \%$ of mass 174 & $0) 1$ \\
\hline 174 & $50.0-120 \%$ of mass 95 & 90.0 \\
\hline 175 & $5.0-9.0 \%$ of mass 174 & 6.41 \\
\hline 176 & $95.0-101 \%$ of mass 174 & $87.1(96.8) 1$ \\
\hline 177 & $5.0-9.0 \%$ of mass 176 & $(6.6) 2$ \\
\hline
\end{tabular}

1 - Value is omass 1742 - Value is mass 176

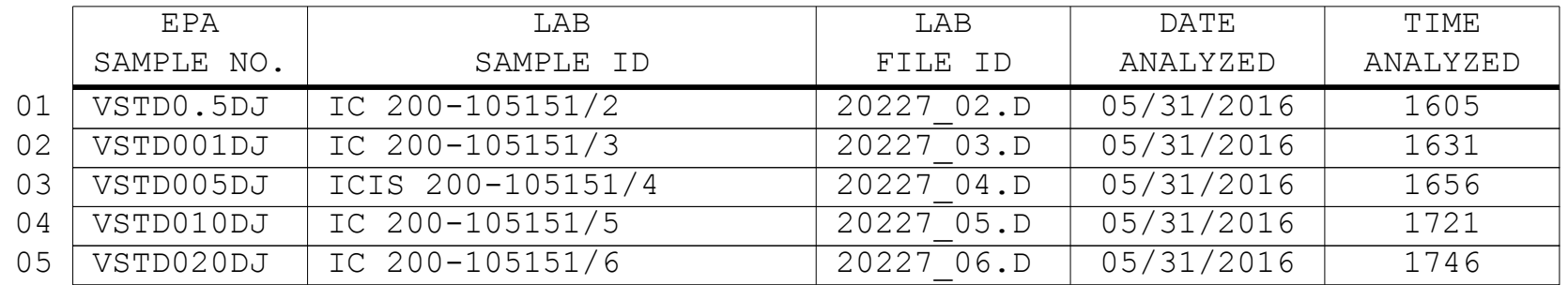


$5 A$ - FORM V VOA

VOLATILE ORGANICS INSTRUMENT

PERFORMANCE CHECK

BROMOFLUOROBENZENE (BFB)
EPA SAMPLE NO.

BFBDT
Lab Name: TESTAMERICA BURLINGTON

Lab Code: STLV Case No.: YORK

Lab File Id: 20443_01.D

Instrument Id: CHD.i

GC Column: DB-624
ID: 0.20
Contract: 8E-00302 Mod. Ref No.: SDG No.: 33957

BEB Injection Date: 06/15/2016

BFB Injection Time: 1305

\begin{tabular}{|c|c|c|}
\hline $\mathrm{m} / \mathrm{e}$ & ION ABUNDANCE CRITERIA & $\begin{array}{l}\text { \% RELATIVE } \\
\text { ABUNDANCE }\end{array}$ \\
\hline 50 & $15.0-40.0 \%$ of mass 95 & 17.4 \\
\hline 75 & $30.0-80.0 \%$ of mass 95 & 50.8 \\
\hline 95 & Base peak, $100 \%$ relative abundance & 100 \\
\hline 96 & $5.0-9.0 \%$ of mass 95 & 6.6 \\
\hline 173 & Less than $2.0 \%$ of mass 174 & $1.1) 1$ \\
\hline 174 & $50.0-120 \%$ of mass 95 & 93.5 \\
\hline 175 & $5.0-9.0 \%$ of mass 174 & $7.0) 1$ \\
\hline 176 & $95.0-101 \%$ of mass 174 & $(96.5) 1$ \\
\hline 177 & $5.0-9.0 \%$ of mass 176 & $5.6(6.2) 2$ \\
\hline
\end{tabular}

1 - Value is omass 174

2 - Value is omass 176

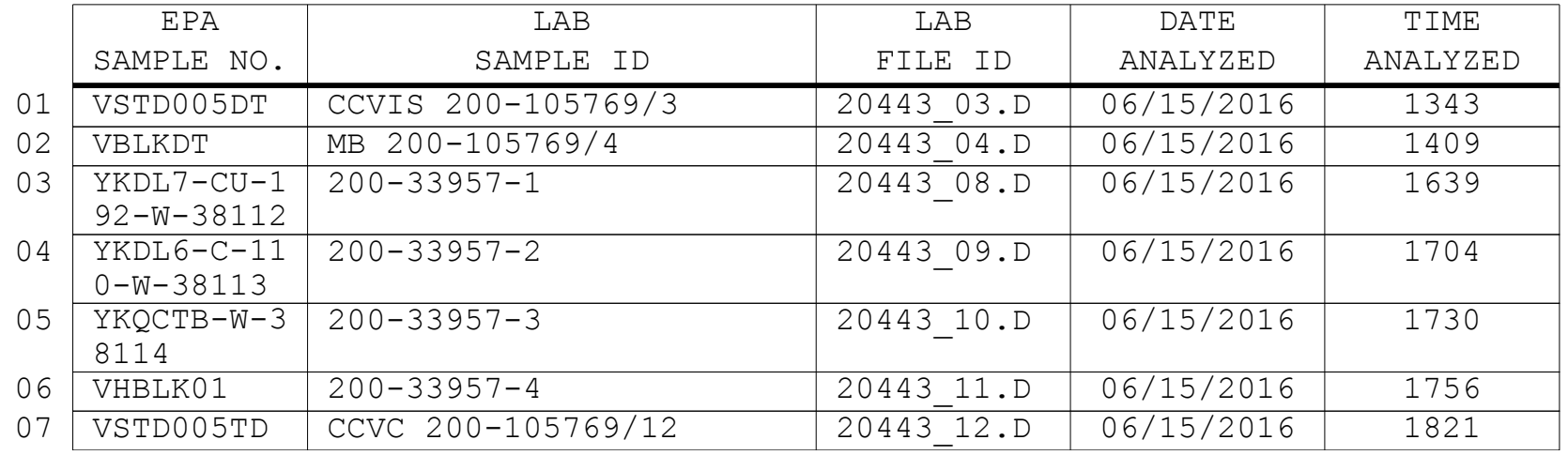




\section{$8 A$ - FORM VIII VOA}

VOLATILE INTERNAL STANDARD AREA AND RETENTION TIME SUMMARY

Lab Name: TESTAMERICA BURLINGTON

Contract: $8 \mathrm{E}-00302$

Lab Code: STLV Case No.: YORK Mod. Ref No.:

SDG No.: 33957

GC Column: DB-624

ID: 0.20 (mm) Init. Calib. Date (s): 05/31/2016 05/31/2016

EPA Sample No.(VSTD\#\#\#\#\#) : VSTD005DT

Date Analyzed: 06/15/2016

Lab File ID (Standard): 20443_03.D

Time Analyzed: 1343

Instrument ID: CHD.i

Heated Purge: ( $\mathrm{Y} / \mathrm{N}) \mathrm{N}$

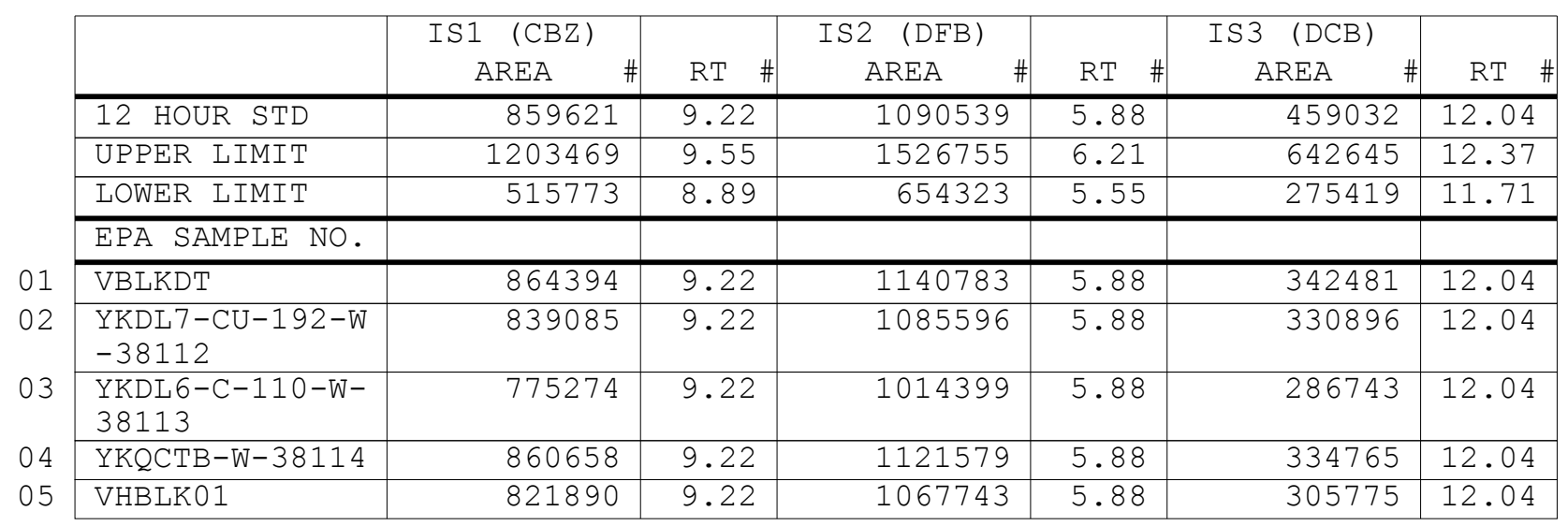

IS1 $(\mathrm{CBZ})=$ Chlorobenzene-d5

IS2 $(\mathrm{DFB})=1,4-\mathrm{Difluorobenzene}$

IS3 $(\mathrm{DCB})=1,4-\mathrm{Dichlorobenzene-d4}$

AREA UPPER LIMIT $=140 \%$ (Trace Volatiles) of internal standard area AREA LOWER LIMIT $=60 \%$ (Trace Volatiles) of internal standard area RT UPPER LIMIT $=+0.33$ (Trace Volatiles) minutes of internal standard RT RT LOWER LIMIT $=-0.33$ (Trace Volatiles) minutes of internal standard RT

\# Column used to flag values outside contract required QC limits with an asterisk. Page 1 of 1 
Lab Name: TESTAMERICA BURLINGTON

Contract: $8 \mathrm{E}-00302$

Lab Code: STLV

Case No.: YORK Mod. Ref No.:

SDG No.: 33957

Matrix: (SOIL/SED/WATER) Water

Sample wt/vol: 25.0 $(\mathrm{g} / \mathrm{mL}) \mathrm{mL}$

Level: (TRACE/LOW/MED) TRACE

\% Moisture: not dec.

GC Column: DB-624 ID: $0.20 \quad(\mathrm{~mm})$

Soil Extract Volume: (uL) $(\mathrm{mL})$

Purge Volume: 25.0
Lab Sample ID: 200-33957-2

Lab File ID: 20443_09.D

Date Received: 06/14/2016

Date Analyzed: 06/15/2016

Dilution Factor: 1.0

Soil Aliquot Volume: (uL)

\begin{tabular}{|c|c|c|c|}
\hline CAS NO. & COMPOUND & $\begin{array}{l}\text { CONCENTRATION UNITS: } \\
(\mathrm{ug} / \mathrm{L} \text { or } \mathrm{ug} / \mathrm{kg}) \mathrm{ug} / \mathrm{L}\end{array}$ & Q \\
\hline $75-71-8$ & Dichlorodifluoromethane & 1.0 & $\mathrm{U}$ \\
\hline $74-87-3$ & Chloromethane & 1.0 & $\mathrm{U}$ \\
\hline $75-01-4$ & Vinyl chloride & 1.0 & $\mathrm{U}$ \\
\hline $74-83-9$ & Bromomethane & 1.0 & $\mathrm{U}$ \\
\hline $75-00-3$ & Chloroethane & 1.0 & $\mathrm{U}$ \\
\hline $75-69-4$ & Trichlorofluoromethane & 0.084 & $\mathrm{~J}$ \\
\hline $75-35-4$ & 1,1-Dichloroethene & 0.66 & $\mathrm{~J}$ \\
\hline $76-13-1$ & $1,1,2$-Trichloro-1,2,2-trifluoroethane & 1.0 & $\mathrm{U}$ \\
\hline $67-64-1$ & Acetone & 5.0 & $\mathrm{U}$ \\
\hline $75-15-0$ & Carbon disulfide & 0.019 & $\mathrm{~J} B$ \\
\hline $79-20-9$ & Methyl acetate & 1.0 & $\mathrm{U}$ \\
\hline $75-09-2$ & Methylene Chloride & 1.0 & $\mathrm{U}$ \\
\hline $156-60-5$ & trans-1,2-Dichloroethene & 1.0 & $\mathrm{U}$ \\
\hline $1634-04-4$ & Methyl tert-butyl ether & 1.0 & $\mathrm{U}$ \\
\hline $75-34-3$ & 1,1-Dichloroethane & 1.0 & $\mathrm{U}$ \\
\hline $156-59-2$ & cis-1,2-Dichloroethene & 1.0 & $\mathrm{U}$ \\
\hline $78-93-3$ & 2-Butanone & 5.0 & $\mathrm{U}$ \\
\hline $74-97-5$ & Bromochloromethane & 1.0 & $\mathrm{U}$ \\
\hline $67-66-3$ & Chloroform & 1.0 & $\mathrm{U}$ \\
\hline $71-55-6$ & $1,1,1$-Trichloroethane & 0.64 & $\mathrm{~J}$ \\
\hline $110-82-7$ & Cyclohexane & 1.0 & $\mathrm{U}$ \\
\hline $56-23-5$ & Carbon tetrachloride & 0.84 & $\mathrm{~J}$ \\
\hline $71-43-2$ & Benzene & 1.0 & $\mathrm{U}$ \\
\hline $107-06-2$ & 1,2-Dichloroethane & 1.0 & $\mathrm{U}$ \\
\hline
\end{tabular}

Report 1,4-Dioxane for Low-Medium VOA analysis only 
Lab Name: TESTAMERICA BURLINGTON

Lab Code: STLV

Case No.: YORK

Mod. Ref No.:

Contract: $8 \mathrm{E}-00302$

Matrix: (SOIL/SED/WATER) Water

Sample wt/vol: 25.0 $(\mathrm{g} / \mathrm{mL}) \mathrm{mL}$

Level: (TRACE/LOW/MED) TRACE

\% Moisture: not dec.

GC Column: DB-624 ID $0.20 \quad(\mathrm{~mm})$

Soil Extract Volume: (uL) $(\mathrm{mL})$

Purge Volume: 25.0 (mI)
SDG No.: 33957

Lab Sample ID: 200-33957-2

Lab File ID: 20443_09.D

Date Received: 06/14/2016

Date Analyzed: 06/15/2016

Dilution Factor: 1.0

Soil Aliquot Volume: (uL)

\begin{tabular}{|c|c|c|c|}
\hline CAS NO. & COMPOUND & $\begin{array}{l}\text { CONCENTRATION UNITS: } \\
(\mathrm{ug} / \mathrm{L} \text { or } \mathrm{ug} / \mathrm{kg}) \mathrm{ug} / \mathrm{L}\end{array}$ & Q \\
\hline $79-01-6$ & Trichloroethene & 3.5 & B \\
\hline $108-87-2$ & Methylcyclohexane & 1.0 & $\mathrm{U}$ \\
\hline $78-87-5$ & 1,2-Dichloropropane & 1.0 & $\mathrm{U}$ \\
\hline $75-27-4$ & Bromodichloromethane & 1.0 & $\mathrm{U}$ \\
\hline $10061-01-5$ & cis-1,3-Dichloropropene & 1.0 & U \\
\hline $108-10-1$ & 4-Methyl-2-pentanone & 1.0 & $\mathrm{U}$ \\
\hline $108-88-3$ & Toluene & 0.021 & $\mathrm{~J} B$ \\
\hline $10061-02-6$ & trans-1,3-Dichloropropene & 1.0 & $\mathrm{U}$ \\
\hline $79-00-5$ & $1,1,2-$ Trichloroethane & 1.0 & $\mathrm{U}$ \\
\hline $127-18-4$ & Tetrachloroethene & 0.19 & $\mathrm{~J} B$ \\
\hline $591-78-6$ & 2-Hexanone & 1.0 & $\mathrm{U}$ \\
\hline $124-48-1$ & Dibromochloromethane & 1.0 & $\mathrm{U}$ \\
\hline $106-93-4$ & 1,2-Dibromoethane & 1.0 & $\mathrm{U}$ \\
\hline $108-90-7$ & Chlorobenzene & 1.0 & $\mathrm{U}$ \\
\hline $100-41-4$ & Ethylbenzene & 1.0 & $\mathrm{U}$ \\
\hline $95-47-6$ & o-Xylene & 1.0 & $\mathrm{U}$ \\
\hline $179601-23-1$ & m,p-xylene & 0.0077 & $\mathrm{~J} B$ \\
\hline $100-42-5$ & Styrene & 1.0 & $\mathrm{U}$ \\
\hline $75-25-2$ & Bromoform & 1.0 & $\mathrm{U}$ \\
\hline $98-82-8$ & Isopropylbenzene & 1.0 & $\mathrm{U}$ \\
\hline $79-34-5$ & $1,1,2,2$-Tetrachloroethane & 1.0 & $\mathrm{U}$ \\
\hline $541-73-1$ & 1,3 -Dichlorobenzene & 1.0 & $\mathrm{U}$ \\
\hline $106-46-7$ & 1,4-Dichlorobenzene & 1.0 & $\mathrm{U}$ \\
\hline $95-50-1$ & 1,2-Dichlorobenzene & 1.0 & $\mathrm{U}$ \\
\hline $96-12-8$ & 1,2-Dibromo-3-Chloropropane & 1.0 & $\mathrm{U}$ \\
\hline $120-82-1$ & $1,2,4$-Trichlorobenzene & 1.0 & $\mathrm{U}$ \\
\hline $87-61-6$ & $1,2,3$-Trichlorobenzene & 1.0 & $\mathrm{U}$ \\
\hline
\end{tabular}


Lab Name: TESTAMERICA BURLINGTON

Contract: $8 \mathrm{E}-00302$

Lab Code: STLV Case No.: YORK Mod. Ref No.: SDG No.: 33957

Matrix: (SOIL/SED/WATER) Water

Sample wt/vol: 25.0 $(\mathrm{g} / \mathrm{mL}) \mathrm{mL}$

Level: (TRACE or LOW/MED) TRACE

\% Moisture: not dec.

GC Column: DB-624 ID $: 0.20 \quad(\mathrm{~mm})$

Soil Extract Volume: (uL)

CONCENTRATION UNITS: (ug/L or ug/kg) ug/L
Lab Sample ID: 200-33957-2

Lab File ID: 20443_09.D

Date Received: 06/14/2016

Date Analyzed: 06/15/2016

Dilution Factor: 1.0

Soil Aliquot Volume: (uL) Purge Volume: 25.0 $(\mathrm{mL})$

01

02

\begin{tabular}{|l|l|c|c|c|}
\hline CAS NUMBER & \multicolumn{1}{|c|}{ COMPOUND NAME } & RT & EST. CONC. & Q \\
\hline & Unknown & 7.20 & 2.6 & $\mathrm{~J}$ X B \\
\hline E9667961 & Total Alkanes & N/A & & \\
\hline
\end{tabular}

1EPA-designated Registry Number. 
Lab Name: TESTAMERICA BURLINGTON

Contract: $8 \mathrm{E}-00302$

Lab Code: STLV

Case No.: YORK Mod. Ref No.:

SDG No.: 33957

Matrix: (SOIL/SED/WATER) Water

Sample wt/vol: 25.0 $(\mathrm{g} / \mathrm{mL}) \mathrm{mL}$

Level: (TRACE/LOW/MED) TRACE

\% Moisture: not dec.

GC Column: DB-624 ID: $0.20 \quad(\mathrm{~mm})$

Soil Extract Volume: (uL) $(\mathrm{mL})$

Purge Volume: 25.0
Lab Sample ID: 200-33957-1

Lab File ID: 20443_08.D

Date Received: 06/14/2016

Date Analyzed: 06/15/2016

Dilution Factor: 1.0

Soil Aliquot Volume: (uL)

\begin{tabular}{|c|c|c|c|}
\hline CAS NO. & COMPOUND & $\begin{array}{l}\text { CONCENTRATION UNITS: } \\
(\mathrm{ug} / \mathrm{L} \text { or } \mathrm{ug} / \mathrm{kg}) \mathrm{ug} / \mathrm{L}\end{array}$ & q \\
\hline $75-71-8$ & Dichlorodifluoromethane & 1.0 & $\mathrm{U}$ \\
\hline $74-87-3$ & Chloromethane & 1.0 & $\mathrm{U}$ \\
\hline $75-01-4$ & Vinyl chloride & 1.0 & $\mathrm{U}$ \\
\hline $74-83-9$ & Bromomethane & 1.0 & $\mathrm{U}$ \\
\hline $75-00-3$ & Chloroethane & 1.0 & $\mathrm{U}$ \\
\hline $75-69-4$ & Trichlorofluoromethane & 1.0 & $\mathrm{U}$ \\
\hline $75-35-4$ & 1,1-Dichloroethene & 1.0 & $\mathrm{U}$ \\
\hline $76-13-1$ & $1,1,2$-Trichloro-1,2,2-trifluoroethane & 1.0 & $\mathrm{U}$ \\
\hline $67-64-1$ & Acetone & 5.0 & $\mathrm{U}$ \\
\hline $75-15-0$ & Carbon disulfide & 1.0 & $\mathrm{U}$ \\
\hline $79-20-9$ & Methyl acetate & 1.0 & $\mathrm{U}$ \\
\hline $75-09-2$ & Methylene Chloride & 1.0 & $\mathrm{U}$ \\
\hline $156-60-5$ & trans-1,2-Dichloroethene & 1.0 & $\mathrm{U}$ \\
\hline $1634-04-4$ & Methyl tert-butyl ether & 1.0 & $\mathrm{U}$ \\
\hline $75-34-3$ & 1,1-Dichloroethane & 1.0 & $\mathrm{U}$ \\
\hline $156-59-2$ & cis-1,2-Dichloroethene & 1.0 & $\mathrm{U}$ \\
\hline $78-93-3$ & 2-Butanone & 5.0 & $\mathrm{U}$ \\
\hline $74-97-5$ & Bromochloromethane & 1.0 & $\mathrm{U}$ \\
\hline $67-66-3$ & Chloroform & 1.0 & $\mathrm{U}$ \\
\hline $71-55-6$ & $1,1,1$-Trichloroethane & 1.0 & $\mathrm{U}$ \\
\hline $110-82-7$ & Cyclohexane & 1.0 & $\mathrm{U}$ \\
\hline $56-23-5$ & Carbon tetrachloride & 1.0 & $\mathrm{U}$ \\
\hline $71-43-2$ & Benzene & 1.0 & $\mathrm{U}$ \\
\hline $107-06-2$ & 1,2-Dichloroethane & 1.0 & $\mathrm{U}$ \\
\hline
\end{tabular}

Report 1,4-Dioxane for Low-Medium VOA analysis only 
Lab Name: TESTAMERICA BURLINGTON

Lab Code: STLV

Case No.: YORK

Mod. Ref No.:

Contract: $8 \mathrm{E}-00302$

Matrix: (SOIL/SED/WATER) Water

Sample wt/vol: 25.0 $(\mathrm{g} / \mathrm{mL}) \mathrm{mL}$

Level: (TRACE/LOW/MED) TRACE

\% Moisture: not dec.

GC Column: DB-624 ID: $0.20 \quad(\mathrm{~mm})$

Soil Extract Volume: (uL) $(\mathrm{mL})$

Purge Volume: 25.0
SDG No.: 33957

Lab Sample ID: 200-33957-1

Lab File ID: 20443_08.D

Date Received: 06/14/2016

Date Analyzed: 06/15/2016

Dilution Factor: 1.0

Soil Aliquot Volume:

(uL) (mI)

\begin{tabular}{|c|c|c|c|}
\hline CAS NO. & COMPOUND & $\begin{array}{l}\text { CONCENTRATION UNITS: } \\
(\mathrm{ug} / \mathrm{L} \text { or } \mathrm{ug} / \mathrm{kg}) \mathrm{ug} / \mathrm{L}\end{array}$ & Q \\
\hline $79-01-6$ & Trichloroethene & 1.0 & $\mathrm{U}$ \\
\hline $108-87-2$ & Methylcyclohexane & 1.0 & $\mathrm{U}$ \\
\hline $78-87-5$ & 1,2-Dichloropropane & 1.0 & $\mathrm{U}$ \\
\hline $75-27-4$ & Bromodichloromethane & 1.0 & $\mathrm{U}$ \\
\hline $10061-01-5$ & cis-1,3-Dichloropropene & 1.0 & U \\
\hline $108-10-1$ & 4-Methyl-2-pentanone & 1.0 & $\mathrm{U}$ \\
\hline $108-88-3$ & Toluene & 0.019 & $\mathrm{~J} B$ \\
\hline $10061-02-6$ & trans-1,3-Dichloropropene & 1.0 & $\mathrm{U}$ \\
\hline $79-00-5$ & $1,1,2-$ Trichloroethane & 1.0 & $\mathrm{U}$ \\
\hline $127-18-4$ & Tetrachloroethene & 1.0 & $\mathrm{U}$ \\
\hline $591-78-6$ & 2-Hexanone & 1.0 & $\mathrm{U}$ \\
\hline $124-48-1$ & Dibromochloromethane & 1.0 & $\mathrm{U}$ \\
\hline $106-93-4$ & 1,2-Dibromoethane & 1.0 & $\mathrm{U}$ \\
\hline $108-90-7$ & Chlorobenzene & 1.0 & $\mathrm{U}$ \\
\hline $100-41-4$ & Ethylbenzene & 1.0 & $\mathrm{U}$ \\
\hline $95-47-6$ & o-Xylene & 1.0 & $\mathrm{U}$ \\
\hline $179601-23-1$ & m,p-xylene & 1.0 & $\mathrm{U}$ \\
\hline $100-42-5$ & Styrene & 1.0 & $\mathrm{U}$ \\
\hline $75-25-2$ & Bromoform & 1.0 & $\mathrm{U}$ \\
\hline $98-82-8$ & Isopropylbenzene & 1.0 & $\mathrm{U}$ \\
\hline $79-34-5$ & $1,1,2,2$-Tetrachloroethane & 1.0 & $\mathrm{U}$ \\
\hline $541-73-1$ & 1,3 -Dichlorobenzene & 1.0 & $\mathrm{U}$ \\
\hline $106-46-7$ & 1,4-Dichlorobenzene & 1.0 & $\mathrm{U}$ \\
\hline $95-50-1$ & 1,2-Dichlorobenzene & 1.0 & $\mathrm{U}$ \\
\hline $96-12-8$ & 1,2-Dibromo-3-Chloropropane & 1.0 & $\mathrm{U}$ \\
\hline $120-82-1$ & $1,2,4$-Trichlorobenzene & 1.0 & $\mathrm{U}$ \\
\hline $87-61-6$ & $1,2,3$-Trichlorobenzene & 1.0 & $\mathrm{U}$ \\
\hline
\end{tabular}


Lab Name: TESTAMERICA BURLINGTON

Contract: $8 \mathrm{E}-00302$

Lab Code: STLV Case No.: YORK Mod. Ref No.: SDG No.: 33957

Matrix: (SOIL/SED/WATER) Water

Sample wt/vol: 25.0 $(\mathrm{g} / \mathrm{mL}) \mathrm{mL}$

Level: (TRACE or LOW/MED) TRACE

․ Moisture: not dec.

GC Column: DB-624 ID $: 0.20 \quad(\mathrm{~mm})$

Soil Extract Volume: (uL)

CONCENTRATION UNITS: (ug/L or ug/kg) ug/L
Lab Sample ID: 200-33957-1

Lab File ID: 20443_08.D

Date Received: 06/14/2016

Date Analyzed: 06/15/2016

Dilution Factor: 1.0

Soil Aliquot Volume: (uL) Purge Volume: 25.0 $(\mathrm{mL})$

01

02

\begin{tabular}{|l|l|c|c|c|}
\hline CAS NUMBER & \multicolumn{1}{|c|}{ COMPOUND NAME } & RT & EST. CONC. & Q \\
\hline & Unknown & 7.20 & 2.5 & $\mathrm{~J}$ X B \\
\hline E9667961 & Total Alkanes & N/A & & \\
\hline
\end{tabular}

1EPA-designated Registry Number. 
Lab Name: TESTAMERICA BURLINGTON

Contract: $8 \mathrm{E}-00302$

Lab Code: STLV

Case No.: YORK Mod. Ref No.:

SDG No.: 33957

Matrix: (SOIL/SED/WATER) Water

Sample wt/vol: 25.0 $(\mathrm{g} / \mathrm{mL}) \mathrm{mL}$

Level: (TRACE/LOW/MED) TRACE

\% Moisture: not dec.

GC Column: DB-624 ID: $0.20 \quad(\mathrm{~mm})$

Soil Extract Volume: (uL) $(\mathrm{mL})$

Purge Volume: 25.0
Lab Sample ID: 200-33957-3

Lab File ID: 20443_10.D

Date Received: 06/14/2016

Date Analyzed: 06/15/2016

Dilution Factor: 1.0

Soil Aliquot Volume: (uL)

\begin{tabular}{|c|c|c|c|}
\hline CAS NO. & COMPOUND & $\begin{array}{l}\text { CONCENTRATION UNITS: } \\
(\mathrm{ug} / \mathrm{L} \text { or } \mathrm{ug} / \mathrm{kg}) \mathrm{ug} / \mathrm{L}\end{array}$ & q \\
\hline $75-71-8$ & Dichlorodifluoromethane & 1.0 & $\mathrm{U}$ \\
\hline $74-87-3$ & Chloromethane & 1.0 & $\mathrm{U}$ \\
\hline $75-01-4$ & Vinyl chloride & 1.0 & $\mathrm{U}$ \\
\hline $74-83-9$ & Bromomethane & 1.0 & $\mathrm{U}$ \\
\hline $75-00-3$ & Chloroethane & 1.0 & $\mathrm{U}$ \\
\hline $75-69-4$ & Trichlorofluoromethane & 1.0 & $\mathrm{U}$ \\
\hline $75-35-4$ & 1,1-Dichloroethene & 1.0 & $\mathrm{U}$ \\
\hline $76-13-1$ & $1,1,2$-Trichloro-1,2,2-trifluoroethane & 1.0 & $\mathrm{U}$ \\
\hline $67-64-1$ & Acetone & 12 & B \\
\hline $75-15-0$ & Carbon disulfide & 1.0 & $\mathrm{U}$ \\
\hline $79-20-9$ & Methyl acetate & 1.0 & $\mathrm{U}$ \\
\hline $75-09-2$ & Methylene Chloride & 1.0 & $\mathrm{U}$ \\
\hline $156-60-5$ & trans-1,2-Dichloroethene & 1.0 & $\mathrm{U}$ \\
\hline $1634-04-4$ & Methyl tert-butyl ether & 1.0 & $\mathrm{U}$ \\
\hline $75-34-3$ & 1,1-Dichloroethane & 1.0 & $\mathrm{U}$ \\
\hline $156-59-2$ & cis-1,2-Dichloroethene & 1.0 & $\mathrm{U}$ \\
\hline $78-93-3$ & 2-Butanone & 5.0 & $\mathrm{U}$ \\
\hline $74-97-5$ & Bromochloromethane & 1.0 & $\mathrm{U}$ \\
\hline $67-66-3$ & Chloroform & 1.0 & $\mathrm{U}$ \\
\hline $71-55-6$ & $1,1,1$-Trichloroethane & 1.0 & $\mathrm{U}$ \\
\hline $110-82-7$ & Cyclohexane & 1.0 & $\mathrm{U}$ \\
\hline $56-23-5$ & Carbon tetrachloride & 1.0 & $\mathrm{U}$ \\
\hline $71-43-2$ & Benzene & 1.0 & $\mathrm{U}$ \\
\hline $107-06-2$ & 1,2-Dichloroethane & 1.0 & $\mathrm{U}$ \\
\hline
\end{tabular}

Report 1,4-Dioxane for Low-Medium VOA analysis only 
Lab Name: TESTAMERICA BURLINGTON

Contract: $8 \mathrm{E}-00302$

Lab Code: STLV

Case No.: YORK

Mod. Ref No.:

SDG No.: 33957

Matrix: (SOIL/SED/WATER) Water

Sample wt/vol: 25.0 $(\mathrm{g} / \mathrm{mL}) \mathrm{mL}$

Level: (TRACE/LOW/MED) TRACE

\% Moisture: not dec.

GC Column: DB-624 ID: $0.20 \quad(\mathrm{~mm})$

Soil Extract Volume: (uL) $(\mathrm{mL})$

Purge Volume: 25.0 (mI)
Lab Sample ID: 200-33957-3

Lab File ID: 20443_10.D

Date Received: 06/14/2016

Date Analyzed: 06/15/2016

Dilution Factor: 1.0

Soil Aliquot Volume: (uL)

\begin{tabular}{|c|c|c|c|}
\hline CAS NO. & COMPOUND & $\begin{array}{l}\text { CONCENTRATION UNITS: } \\
(\mathrm{ug} / \mathrm{L} \text { or } \mathrm{ug} / \mathrm{kg}) \mathrm{ug} / \mathrm{L}\end{array}$ & Q \\
\hline $79-01-6$ & Trichloroethene & 1.0 & $\mathrm{U}$ \\
\hline $108-87-2$ & Methylcyclohexane & 1.0 & $\mathrm{U}$ \\
\hline $78-87-5$ & 1,2-Dichloropropane & 1.0 & $\mathrm{U}$ \\
\hline $75-27-4$ & Bromodichloromethane & 1.0 & $\mathrm{U}$ \\
\hline $10061-01-5$ & cis-1,3-Dichloropropene & 1.0 & U \\
\hline $108-10-1$ & 4-Methyl-2-pentanone & 1.0 & $\mathrm{U}$ \\
\hline $108-88-3$ & Toluene & 0.16 & $\mathrm{~J} B$ \\
\hline $10061-02-6$ & trans-1,3-Dichloropropene & 1.0 & $\mathrm{U}$ \\
\hline $79-00-5$ & $1,1,2-$ Trichloroethane & 1.0 & $\mathrm{U}$ \\
\hline $127-18-4$ & Tetrachloroethene & 0.023 & $\mathrm{~J} B$ \\
\hline $591-78-6$ & 2-Hexanone & 1.0 & $\mathrm{U}$ \\
\hline $124-48-1$ & Dibromochloromethane & 1.0 & $\mathrm{U}$ \\
\hline $106-93-4$ & 1,2-Dibromoethane & 1.0 & $\mathrm{U}$ \\
\hline $108-90-7$ & Chlorobenzene & 1.0 & $\mathrm{U}$ \\
\hline $100-41-4$ & Ethylbenzene & 0.021 & $\mathrm{~J} B$ \\
\hline $95-47-6$ & o-Xylene & 0.044 & $\mathrm{~J} B$ \\
\hline $179601-23-1$ & m,p-xylene & 0.066 & $\mathrm{~J} B$ \\
\hline $100-42-5$ & Styrene & 0.064 & $\mathrm{~J} B$ \\
\hline $75-25-2$ & Bromoform & 1.0 & $\mathrm{U}$ \\
\hline $98-82-8$ & Isopropylbenzene & 1.0 & $\mathrm{U}$ \\
\hline $79-34-5$ & $1,1,2,2$-Tetrachloroethane & 1.0 & $\mathrm{U}$ \\
\hline $541-73-1$ & 1,3 -Dichlorobenzene & 1.0 & $\mathrm{U}$ \\
\hline $106-46-7$ & 1,4-Dichlorobenzene & 1.0 & $\mathrm{U}$ \\
\hline $95-50-1$ & 1,2-Dichlorobenzene & 1.0 & $\mathrm{U}$ \\
\hline $96-12-8$ & 1,2-Dibromo-3-Chloropropane & 1.0 & $\mathrm{U}$ \\
\hline $120-82-1$ & $1,2,4$-Trichlorobenzene & 1.0 & $\mathrm{U}$ \\
\hline $87-61-6$ & $1,2,3$-Trichlorobenzene & 1.0 & $\mathrm{U}$ \\
\hline
\end{tabular}


Lab Name: TESTAMERICA BURLINGTON

Contract: $8 \mathrm{E}-00302$

Lab Code: STLV Case No.: YORK Mod. Ref No.: SDG No.: 33957

Matrix: (SOIL/SED/WATER) Water

Sample wt/vol: 25.0 $(\mathrm{g} / \mathrm{mL}) \mathrm{mL}$

Level: (TRACE or LOW/MED) TRACE

․ Moisture: not dec.

GC Column: DB-624 ID $: 0.20 \quad(\mathrm{~mm})$

Soil Extract Volume: (uL)
Lab Sample ID: 200-33957-3

Lab File ID: 20443_10.D

Date Received: 06/14/2016

Date Analyzed: 06/15/2016

Dilution Factor: 1.0

Soil Aliquot Volume: (uL) Purge Volume: 25.0 $(\mathrm{mL})$

01

02

\begin{tabular}{|l|l|c|c|c|}
\hline CAS NUMBER & \multicolumn{1}{|c|}{ COMPOUND NAME } & RT & EST. CONC. & Q \\
\hline & Unknown & 7.20 & 2.6 & $\mathrm{~J}$ X B \\
\hline E9667961 & Total Alkanes & N/A & & \\
\hline
\end{tabular}

1 EPA-designated Registry Number. 
Lab Name: TESTAMERICA BURLINGTON

Contract: $8 \mathrm{E}-00302$

Lab Code: STLV Case No.: YORK Mod. Ref No.:

SDG No.: 33957

Instrument ID: CHD.i

Calibration Date(s): 05/31/2016 05/31/2016

Heated Purge: ( $\mathrm{Y} / \mathrm{N}) \mathrm{N}$

Calibration Time(s) : 1605

1746

Purge Volume: 25.0 $(\mathrm{mL})$

GC Column: DB-624

ID: $0.20 \quad$ (mm) Length: 25

$(\mathrm{m})$

\begin{tabular}{|c|c|c|c|c|c|c|c|}
\hline \multirow{3}{*}{$\begin{array}{l}\text { LAB FILE ID: } \\
\text { RRF } 5.0=20227 \_04 . D \\
\text { COMPOUND }\end{array}$} & \multirow{2}{*}{\multicolumn{3}{|c|}{ 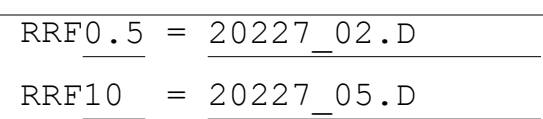 }} & \multirow{2}{*}{\multicolumn{4}{|c|}{$\mathrm{RRF} 1.0=\frac{20227 \_03 . \mathrm{D}}{}$}} \\
\hline & & & & & & & \\
\hline & RRE0.5 & RRE1.0 & RRF 5.0 & RRF10 & RRF20 & $\overline{\mathrm{RRF}}$ & $\because \mathrm{RSD}$ \\
\hline Dichlorodifluoromethane & 0.667 & 0.602 & 0.556 & 0.572 & 0.564 & 0.592 & 7.7 \\
\hline Chloromethane & 0.595 & 0.547 & 0.480 & 0.473 & 0.459 & 0.511 & 11.4 \\
\hline Vinyl chloride & 0.583 & 0.509 & 0.481 & 0.502 & 0.491 & 0.513 & 7.9 \\
\hline Bromomethane & 0.239 & 0.158 & 0.155 & 0.169 & 0.171 & 0.179 & 19.4 \\
\hline Chloroethane & 0.290 & 0.283 & 0.280 & 0.282 & 0.259 & 0.279 & 4.3 \\
\hline Trichlorofluoromethane & 0.718 & 0.675 & 0.613 & 0.618 & 0.608 & 0.646 & 7.5 \\
\hline 1,1-Dichloroethene & 0.384 & 0.348 & 0.328 & 0.331 & 0.325 & 0.343 & 7.1 \\
\hline $\begin{array}{l}1,1,2 \text {-Trichloro- } \\
1,2,2 \text {-trifluoroethane }\end{array}$ & 0.448 & 0.398 & 0.367 & 0.368 & 0.358 & 0.388 & 9.5 \\
\hline Acetone & 0.024 & 0.021 & 0.017 & 0.019 & 0.019 & 0.020 & 12.6 \\
\hline Carbon disulfide & 1.077 & 0.937 & 0.929 & 0.988 & 1.005 & 0.987 & 6.0 \\
\hline Methyl acetate & 0.059 & 0.052 & 0.050 & 0.051 & 0.050 & 0.052 & 7.1 \\
\hline Methylene Chloride & 0.324 & 0.295 & 0.272 & 0.273 & 0.270 & 0.287 & 8.1 \\
\hline trans-1,2-Dichloroethene & 0.388 & 0.352 & 0.332 & 0.335 & 0.332 & 0.348 & 6.9 \\
\hline Methyl tert-butyl ether & 0.376 & 0.353 & 0.357 & 0.373 & 0.389 & 0.370 & 4.0 \\
\hline 1,1-Dichloroethane & 0.736 & 0.665 & 0.623 & 0.637 & 0.636 & 0.660 & 6.9 \\
\hline cis-1,2-Dichloroethene & 0.352 & 0.315 & 0.332 & 0.342 & 0.331 & 0.334 & 4.2 \\
\hline 2-Butanone & 0.033 & 0.030 & 0.032 & 0.035 & 0.035 & 0.033 & 7.0 \\
\hline Bromochloromethane & 0.112 & 0.107 & 0.097 & 0.102 & 0.105 & 0.105 & 5.1 \\
\hline Chloroform & 0.634 & 0.586 & 0.556 & 0.566 & 0.576 & 0.584 & 5.2 \\
\hline 1,1,1-Trichloroethane & 0.763 & 0.693 & 0.667 & 0.679 & 0.688 & 0.698 & 5.4 \\
\hline Cyclohexane & 0.734 & 0.702 & 0.814 & 0.863 & 0.880 & 0.799 & 9.8 \\
\hline Carbon tetrachloride & 0.673 & 0.612 & 0.607 & 0.623 & 0.628 & 0.629 & 4.1 \\
\hline Benzene & 2.159 & 1.975 & 1.906 & 1.952 & 1.975 & 1.993 & 4.9 \\
\hline 1,2-Dichloroethane & 0.293 & 0.257 & 0.256 & 0.260 & 0.267 & 0.266 & 5.7 \\
\hline Trichloroethene & 0.465 & 0.422 & 0.424 & 0.440 & 0.450 & 0.440 & 4.1 \\
\hline Methylcyclohexane & 0.599 & 0.590 & 0.666 & 0.695 & 0.694 & 0.649 & 7.8 \\
\hline
\end{tabular}

Report 1,4-Dioxane for Low-Medium VOA analysis only 
Lab Name: TESTAMERICA BURLINGTON

Contract: 8E-00302

Lab Code: STLV Case No.: YORK Mod. Ref No.:

SDG No.: 33957

Instrument ID: CHD.i

Calibration Date(s): 05/31/2016 05/31/2016

Heated Purge: (Y/N) N

Purge Volume: 25.0

Calibration Time(s) : 1605

1746

GC Column: DB-624

ID: 0.20 (mL)

LAB FILE ID:

(mm) Length: 25

(m)

$\mathrm{RRF} 5.0=20227 \_04 . \mathrm{D}$
$\mathrm{RRF} 0.5=20227 \_02 . \mathrm{D}$
RRF1.0 $=20227 \_03 . \mathrm{D}$
$\mathrm{RRF} 10=20227$ 05.D
RRF20 $=2022706 . \mathrm{D}$

COMPOUND

\begin{tabular}{|l|l|}
\hline COMPOUND & RRF0.5 \\
\hline
\end{tabular}

\begin{tabular}{|c|c|c|c|c|c|c|c|}
\hline 1,2-Dichloropropane & 0.450 & 0.394 & 0.387 & 0.391 & 0.397 & 0.404 & 6.5 \\
\hline Bromodichloromethane & 0.396 & 0.364 & 0.368 & 0.382 & 0.395 & 0.381 & 3.9 \\
\hline cis-1,3-Dichloropropene & 0.383 & 0.368 & 0.472 & 0.517 & 0.545 & 0.457 & 17.2 \\
\hline 4-Methyl-2-pentanone & 0.072 & 0.074 & 0.094 & 0.101 & 0.105 & 0.089 & 17.2 \\
\hline Toluene & 1.692 & 1.704 & 1.891 & 1.962 & 1.990 & 1.848 & 7.7 \\
\hline trans-1,3-Dichloropropene & 0.238 & 0.235 & 0.304 & 0.343 & 0.370 & 0.298 & 20.5 \\
\hline 1,1,2-Trichloroethane & 0.209 & 0.182 & 0.169 & 0.172 & 0.171 & 0.180 & 9.2 \\
\hline Tetrachloroethene & 0.472 & 0.431 & 0.425 & 0.440 & 0.435 & 0.441 & 4.2 \\
\hline 2-Hexanone & 0.048 & 0.052 & 0.066 & 0.070 & 0.072 & 0.062 & 17.6 \\
\hline Dibromochloromethane & 0.176 & 0.164 & 0.176 & 0.190 & 0.198 & 0.181 & 7.5 \\
\hline 1,2-Dibromoethane & 0.142 & 0.128 & 0.135 & 0.138 & 0.144 & 0.137 & 4.6 \\
\hline Chlorobenzene & 1.207 & 1.138 & 1.100 & 1.124 & 1.120 & 1.138 & 3.6 \\
\hline Ethylbenzene & 1.724 & 1.752 & 2.139 & 2.283 & 2.319 & 2.043 & 14.0 \\
\hline o-Xylene & 0.488 & 0.541 & 0.751 & 0.802 & 0.804 & 0.677 & 22.3 \\
\hline $\mathrm{m}, \mathrm{p}$-Xylene & 0.627 & 0.666 & 0.841 & 0.885 & 0.893 & 0.783 & 16.2 \\
\hline Styrene & 0.803 & 0.905 & 1.184 & 1.244 & 1.250 & 1.077 & 19.4 \\
\hline Bromoform & 0.169 & 0.149 & 0.145 & 0.162 & 0.182 & 0.161 & 9.3 \\
\hline Isopropylbenzene & 1.433 & 1.568 & 2.168 & 2.351 & 2.365 & 1.977 & 22.5 \\
\hline $1,1,2,2$-Tetrachloroethane & 0.177 & 0.167 & 0.165 & 0.171 & 0.172 & 0.170 & 2.8 \\
\hline 1,3-Dichlorobenzene & 1.375 & 1.332 & 1.435 & 1.557 & 1.640 & 1.468 & 8.7 \\
\hline 1,4-Dichlorobenzene & 1.993 & 1.711 & 1.583 & 1.599 & 1.627 & 1.703 & 10.0 \\
\hline 1,2-Dichlorobenzene & 1.344 & 1.274 & 1.266 & 1.300 & 1.323 & 1.301 & 2.5 \\
\hline 1,2-Dibromo-3-Chloropropane & 0.045 & 0.040 & 0.033 & 0.036 & 0.041 & 0.039 & 11.3 \\
\hline $1,2,4$-Trichlorobenzene & 0.687 & 0.612 & 0.691 & 0.845 & 0.949 & 0.757 & 18.1 \\
\hline $1,2,3$-Trichlorobenzene & 0.470 & 0.439 & 0.518 & 0.605 & 0.671 & 0.541 & 17.8 \\
\hline
\end{tabular}


Lab Name: TESTAMERICA BURLINGTON

Contract: $8 \mathrm{E}-00302$

Lab Code: STLV Case No.: YORK Mod. Ref No.: SDG No.: 33957

Instrument ID: CHD.i

Heated Purge: ( $\mathrm{Y} / \mathrm{N}) \mathrm{N}$

Purge Volume: 25.0

GC Column: DB-624
ID: 0.20
Calibration Date(s): 05/31/2016

Calibration Time (s) : 1605 $(\mathrm{mL})$

(mm) Length: 25 $(\mathrm{m})$

\begin{tabular}{|c|c|c|c|c|c|c|c|}
\hline LAB FILE ID: & \multicolumn{3}{|c|}{$\begin{array}{l}\operatorname{RRF}_{\underline{0.5}}=\frac{20227 \_02 . \mathrm{D}}{\operatorname{RRF10}}=20227 \_05 . \mathrm{D}\end{array}$} & \multicolumn{4}{|c|}{$\begin{array}{l}\operatorname{RRF1.0}_{\underline{1.0}}=\frac{20227 \_03 . \mathrm{D}}{\underline{\mathrm{RRF} 20}}=20227 \_06 . \mathrm{D}\end{array}$} \\
\hline Vinyl Chloride-d3 & 0.507 & 0.432 & 0.420 & 0.437 & 0.428 & 0.445 & 8.0 \\
\hline Chloroethane-d5 & 0.424 & 0.372 & 0.339 & 0.334 & 0.311 & 0.356 & 12.2 \\
\hline Chloroform-d & 0.681 & 0.615 & 0.576 & 0.591 & 0.594 & 0.611 & 6.8 \\
\hline 1,2-Dichloroethane-d4 & 0.245 & 0.212 & 0.201 & 0.205 & 0.209 & 0.214 & 8.2 \\
\hline Benzene-d6 & 1.902 & 1.743 & 1.756 & 1.802 & 1.828 & 1.806 & 3.5 \\
\hline 1,2-Dichloropropane-d6 & 0.507 & 0.429 & 0.417 & 0.434 & 0.436 & 0.444 & 8.0 \\
\hline Toluene-d8 & 1.445 & 1.398 & 1.557 & 1.626 & 1.638 & 1.533 & 7.0 \\
\hline 1,2-Dichlorobenzene-d4 & 0.917 & 0.756 & 0.750 & 0.791 & 0.823 & 0.807 & 8.5 \\
\hline
\end{tabular}

Report 1,4-Dioxane-d8 for Low-Medium VOA analysis only 
Lab Name: TESTAMERICA BURLINGTON

Contract: $8 \mathrm{E}-00302$

Lab Code: STLV

Mod. Ref No.:

SDG No.: 33957

Instrument ID: CHD.i

Calibration Date: 06/15/2016 Time: 1343

Lab File Id: 20443_03.D

Init. Calib. Date(s):05/31/2016 05/31/2016

EPA Sample No. (VSTD\#\#\#\#) : VSTD005DT

Heated Purge: ( $\mathrm{Y} / \mathrm{N}) \mathrm{N}$ GC Column: DB-624

Init. Calib. Time(s) : 1605 1746

Purge Volume: 25.0

ID: $0.20(\mathrm{~mm})$ Length: 25

(m)

\begin{tabular}{|c|c|c|c|c|c|}
\hline COMPOUND & $\overline{\mathrm{RRF}}$ & RRF5.0 & $\begin{array}{l}\text { MIN } \\
\text { RRF }\end{array}$ & $\frac{\circ}{D}$ & $\mathrm{MAX} \div \mathrm{D}$ \\
\hline Dichlorodifluoromethane & 0.592 & 0.593 & 0.010 & 0.2 & 40.0 \\
\hline Chloromethane & 0.511 & 0.479 & 0.010 & -6.3 & 40.0 \\
\hline Vinyl chloride & 0.513 & 0.517 & 0.100 & 0.6 & 30.0 \\
\hline Bromomethane & 0.179 & 0.174 & 0.100 & -2.7 & 30.0 \\
\hline Chloroethane & 0.279 & 0.293 & 0.010 & 5.1 & 40.0 \\
\hline Trichlorofluoromethane & 0.646 & 0.664 & 0.010 & 2.7 & 40.0 \\
\hline 1,1-Dichloroethene & 0.343 & 0.351 & 0.100 & 2.3 & 30.0 \\
\hline 1,1,2-Trichloro-1,2,2-trifluoroethane & 0.388 & 0.397 & 0.010 & 2.4 & 40.0 \\
\hline Acetone & 0.020 & 0.020 & 0.010 & -0.1 & 40.0 \\
\hline Carbon disulfide & 0.987 & 1.028 & 0.010 & 4.1 & 40.0 \\
\hline Methyl acetate & 0.052 & 0.050 & 0.010 & -4.6 & 40.0 \\
\hline Methylene Chloride & 0.287 & 0.290 & 0.010 & 1.3 & 40.0 \\
\hline trans-1,2-Dichloroethene & 0.348 & 0.357 & 0.010 & 2.5 & 40.0 \\
\hline Methyl tert-butyl ether & 0.370 & 0.360 & 0.010 & -2.5 & 40.0 \\
\hline 1,1-Dichloroethane & 0.660 & 0.666 & 0.200 & 1.0 & 30.0 \\
\hline cis-1,2-Dichloroethene & 0.334 & 0.356 & 0.010 & 6.4 & 40.0 \\
\hline 2-Butanone & 0.033 & 0.035 & 0.010 & 6.5 & 40.0 \\
\hline Bromochloromethane & 0.105 & 0.108 & 0.050 & 3.0 & 30.0 \\
\hline Chloroform & 0.584 & 0.612 & 0.200 & 4.9 & 30.0 \\
\hline 1,1,1-Trichloroethane & 0.698 & 0.720 & 0.100 & 3.1 & 30.0 \\
\hline Cyclohexane & 0.799 & 0.869 & 0.010 & 8.8 & 40.0 \\
\hline Carbon tetrachloride & 0.629 & 0.676 & 0.100 & 7.5 & 30.0 \\
\hline Benzene & 1.993 & 2.068 & 0.400 & 3.7 & 30.0 \\
\hline 1,2-Dichloroethane & 0.266 & 0.281 & 0.100 & 5.5 & 30.0 \\
\hline Trichloroethene & 0.440 & 0.451 & 0.300 & 2.5 & 30.0 \\
\hline Methylcyclohexane & 0.649 & 0.717 & 0.010 & 10.5 & 40.0 \\
\hline
\end{tabular}

Report 1,4-Dioxane for Low/Medium VOA analysis only 
Lab Name: TESTAMERICA BURLINGTON

Contract: 8E-00302

Lab Code: STLV

Mod. Ref No.:

SDG No.: 33957

Instrument ID: CHD.i

Calibration Date: 06/15/2016 Time: 1343

Lab File Id: 20443_03.D

Init. Calib. Date(s):05/31/2016 05/31/2016

EPA Sample No. (VSTD\#\#\#\#) : VSTD005DT

Init. Calib. Time(s) : 1605 1746

Heated Purge: (Y/N) N GC Column: DB-624

ID: $0.20(\mathrm{~mm})$ Length: 25

(m)

Purge Volume: 25.0 $(\mathrm{mL})$

\begin{tabular}{|c|c|c|c|c|c|}
\hline COMPOUND & $\overline{\mathrm{RRF}}$ & RRF5.0 & $\begin{array}{l}\text { MIN } \\
\text { RRF }\end{array}$ & $\because \mathrm{D}$ & $\operatorname{MAX} \cong \mathrm{D}$ \\
\hline 1,2-Dichloropropane & 0.404 & 0.417 & 0.010 & 3.4 & 40.0 \\
\hline Bromodichloromethane & 0.381 & 0.402 & 0.200 & 5.4 & 30.0 \\
\hline cis-1,3-Dichloropropene & 0.457 & 0.499 & 0.200 & 9.1 & 30.0 \\
\hline 4-Methyl-2-pentanone & 0.089 & 0.099 & 0.010 & 10.9 & 40.0 \\
\hline Toluene & 1.848 & 2.065 & 0.400 & 11.8 & 30.0 \\
\hline trans-1,3-Dichloropropene & 0.298 & 0.321 & 0.100 & 7.8 & 30.0 \\
\hline $1,1,2$-Trichloroethane & 0.180 & 0.182 & 0.100 & 1.1 & 30.0 \\
\hline Tetrachloroethene & 0.441 & 0.461 & 0.100 & 4.5 & 30.0 \\
\hline 2-Hexanone & 0.062 & 0.069 & 0.010 & 12.3 & 40.0 \\
\hline Dibromochloromethane & 0.181 & 0.200 & 0.100 & 10.5 & 30.0 \\
\hline 1,2-Dibromoethane & 0.137 & 0.142 & 0.010 & 3.3 & 40.0 \\
\hline Chlorobenzene & 1.138 & 1.190 & 0.500 & 4.6 & 30.0 \\
\hline Ethylbenzene & 2.043 & 2.294 & 0.100 & 12.3 & 30.0 \\
\hline o-Xylene & 0.677 & 0.791 & 0.300 & 16.8 & 30.0 \\
\hline $\mathrm{m}, \mathrm{p}$-Xylene & 0.783 & 0.900 & 0.300 & 15.0 & 30.0 \\
\hline Styrene & 1.077 & 1.279 & 0.300 & 18.8 & 30.0 \\
\hline Bromoform & 0.161 & 0.171 & 0.050 & 5.9 & 30.0 \\
\hline Isopropylbenzene & 1.977 & 2.320 & 0.010 & 17.3 & 40.0 \\
\hline $1,1,2,2$-Tetrachloroethane & 0.170 & 0.177 & 0.100 & 3.7 & 30.0 \\
\hline 1,3-Dichlorobenzene & 1.468 & 1.490 & 0.400 & 1.5 & 30.0 \\
\hline 1,4-Dichlorobenzene & 1.703 & 1.697 & 0.400 & -0.4 & 30.0 \\
\hline 1,2-Dichlorobenzene & 1.301 & 1.387 & 0.400 & 6.6 & 30.0 \\
\hline 1,2-Dibromo-3-Chloropropane & 0.039 & 0.036 & 0.010 & -8.6 & 40.0 \\
\hline 1,2,4-Trichlorobenzene & 0.757 & 0.688 & 0.200 & -9.1 & 30.0 \\
\hline $1,2,3$-Trichlorobenzene & 0.541 & 0.504 & 0.200 & -6.7 & 30.0 \\
\hline
\end{tabular}


Lab Name: TESTAMERICA BURLINGTON

Contract: $8 \mathrm{E}-00302$

Lab Code: STLV Case No.: YORK Mod. Ref No.

SDG No.: 33957

Instrument ID: CHD.i

Calibration Date: 06/15/2016 Time: 1343

Lab File Id: 20443_03.D

Init. Calib. Date(s):05/31/2016 05/31/2016

EPA Sample No. (VSTD\#\#\#\#) : VSTD005DT

Init. Calib. Time(s) : 1605 1746

Heated Purge: (Y/N) N GC Column: DB-624

ID: $0.20(\mathrm{~mm})$ Length: 25

(m)

Purge Volume: 25.0 $(\mathrm{mL})$

\begin{tabular}{|c|c|c|c|c|c|}
\hline COMPOUND & $\overline{\mathrm{RRF}}$ & RRF5. 0 & $\begin{array}{l}\text { MIN } \\
\text { RRF }\end{array}$ & $\circ \mathrm{D}$ & MAX $\cong D$ \\
\hline Vinyl Chloride-d3 & 0.445 & 0.451 & 0.010 & 1.4 & 30.0 \\
\hline Chloroethane-d5 & 0.356 & 0.363 & 0.010 & 2.0 & 40.0 \\
\hline 1,1-Dichloroethene-d2 & 0.762 & 0.768 & 0.010 & 0.7 & 30.0 \\
\hline 2-Butanone-d5 & 0.030 & 0.031 & 0.010 & 2.5 & 40.0 \\
\hline Chloroform-d & 0.611 & 0.637 & 0.010 & 4.2 & 30.0 \\
\hline 1,2-Dichloroethane-d4 & 0.214 & 0.218 & 0.010 & 1.7 & 30.0 \\
\hline Benzene-d6 & 1.806 & 1.869 & 0.010 & 3.4 & 30.0 \\
\hline 1,2-Dichloropropane-d6 & 0.444 & 0.451 & 0.010 & 1.5 & 40.0 \\
\hline Toluene-d8 & 1.533 & 1.686 & 0.010 & 10.0 & 30.0 \\
\hline trans-1,3-Dichloropropene-d4 & 0.245 & 0.243 & 0.010 & $-1 \cdot 1$ & 30.0 \\
\hline 2-Hexanone-d5 & 0.030 & 0.032 & 0.010 & 6.5 & 40.0 \\
\hline $1,1,2,2-$ Tetrachloroethane-d2 & 0.171 & 0.179 & 0.010 & 4.9 & 30.0 \\
\hline 1,2-Dichlorobenzene-d4 & 0.807 & 0.795 & 0.010 & -1.6 & 30.0 \\
\hline
\end{tabular}

Report 1,4-Dioxane-d8 for Low/Medium VOA analysis only 
Lab Name: TESTAMERICA BURLINGTON

Contract: $8 \mathrm{E}-00302$

Lab Code: STLV Case No.: YORK Mod. Ref No.:

SDG No.: 33957

Instrument ID: CHD.i

Calibration Date: 06/15/2016 Time: 1821

Lab File Id: 20443_12.D

Init. Calib. Date(s):05/31/2016 05/31/2016

EPA Sample No. (VSTD\#\#\#\#) : VSTD005TD

Init. Calib. Time(s) : 1605 1746

Heated Purge: (Y/N) N GC Column: DB-624

ID: $0.20(\mathrm{~mm})$ Length: 25

(m)

Purge Volume: 25.0 $(\mathrm{mL})$

\begin{tabular}{|c|c|c|c|c|c|}
\hline COMPOUND & $\overline{\mathrm{RRF}}$ & RRF5.0 & $\begin{array}{l}\text { MIN } \\
\text { RRF }\end{array}$ & $\frac{\circ}{D}$ & $\mathrm{MAX} \div \mathrm{D}$ \\
\hline Dichlorodifluoromethane & 0.592 & 0.585 & 0.010 & -1.2 & 50.0 \\
\hline Chloromethane & 0.511 & 0.481 & 0.010 & -5.8 & 50.0 \\
\hline Vinyl chloride & 0.513 & 0.515 & 0.010 & 0.2 & 50.0 \\
\hline Bromomethane & 0.179 & 0.159 & 0.010 & -10.8 & 50.0 \\
\hline Chloroethane & 0.279 & 0.295 & 0.010 & 5.6 & 50.0 \\
\hline Trichlorofluoromethane & 0.646 & 0.667 & 0.010 & 3.2 & 50.0 \\
\hline 1,1-Dichloroethene & 0.343 & 0.348 & 0.010 & 1.5 & 50.0 \\
\hline 1,1,2-Trichloro-1,2,2-trifluoroethane & 0.388 & 0.396 & 0.010 & 2.2 & 50.0 \\
\hline Acetone & 0.020 & 0.020 & 0.010 & -1.7 & 50.0 \\
\hline Carbon disulfide & 0.987 & 0.942 & 0.010 & -4.5 & 50.0 \\
\hline Methyl acetate & 0.052 & 0.056 & 0.010 & 7.3 & 50.0 \\
\hline Methylene Chloride & 0.287 & 0.294 & 0.010 & 2.5 & 50.0 \\
\hline trans-1,2-Dichloroethene & 0.348 & 0.351 & 0.010 & 1.0 & 50.0 \\
\hline Methyl tert-butyl ether & 0.370 & 0.368 & 0.010 & -0.5 & 50.0 \\
\hline 1,1-Dichloroethane & 0.660 & 0.668 & 0.010 & 1.2 & 50.0 \\
\hline cis-1,2-Dichloroethene & 0.334 & 0.328 & 0.010 & -2.0 & 50.0 \\
\hline 2-Butanone & 0.033 & 0.034 & 0.010 & 3.4 & 50.0 \\
\hline Bromochloromethane & 0.105 & 0.109 & 0.010 & 4.7 & 50.0 \\
\hline Chloroform & 0.584 & 0.624 & 0.010 & 7.0 & 50.0 \\
\hline 1,1,1-Trichloroethane & 0.698 & 0.718 & 0.010 & 2.8 & 50.0 \\
\hline Cyclohexane & 0.799 & 0.858 & 0.010 & 7.4 & 50.0 \\
\hline Carbon tetrachloride & 0.629 & 0.667 & 0.010 & 6.1 & 50.0 \\
\hline Benzene & 1.993 & 2.056 & 0.010 & 3.2 & 50.0 \\
\hline 1,2-Dichloroethane & 0.266 & 0.289 & 0.010 & 8.6 & 50.0 \\
\hline Trichloroethene & 0.440 & 0.448 & 0.010 & 1.7 & 50.0 \\
\hline Methylcyclohexane & 0.649 & 0.706 & 0.010 & 8.8 & 50.0 \\
\hline
\end{tabular}

Report 1,4-Dioxane for Low/Medium VOA analysis only 
Lab Name: TESTAMERICA BURLINGTON

Contract: 8E-00302

Lab Code: STLV

Mod. Ref No.:

SDG No.: 33957

Instrument ID: CHD.i

Calibration Date: 06/15/2016 Time: 1821

Lab File Id: 20443_12.D

Init. Calib. Date(s):05/31/2016 05/31/2016

EPA Sample No. (VSTD\#\#\#\#) : VSTD005TD

Init. Calib. Time(s) : 1605 1746

Heated Purge: (Y/N) N GC Column: DB-624

ID: $0.20(\mathrm{~mm})$ Length: 25

(m)

Purge Volume: 25.0 $(\mathrm{mL})$

\begin{tabular}{|c|c|c|c|c|c|}
\hline COMPOUND & $\overline{\mathrm{RRF}}$ & RRF5.0 & $\begin{array}{l}\text { MIN } \\
\text { RRF }\end{array}$ & $\because \mathrm{D}$ & $\operatorname{MAX} \cong \mathrm{D}$ \\
\hline 1,2-Dichloropropane & 0.404 & 0.414 & 0.010 & 2.5 & 50.0 \\
\hline Bromodichloromethane & 0.381 & 0.398 & 0.010 & 4.4 & 50.0 \\
\hline cis-1,3-Dichloropropene & 0.457 & 0.495 & 0.010 & 8.4 & 50.0 \\
\hline 4-Methyl-2-pentanone & 0.089 & 0.103 & 0.010 & 16.1 & 50.0 \\
\hline Toluene & 1.848 & 2.103 & 0.010 & 13.8 & 50.0 \\
\hline trans-1,3-Dichloropropene & 0.298 & 0.312 & 0.010 & 4.7 & 50.0 \\
\hline $1,1,2$-Trichloroethane & 0.180 & 0.185 & 0.010 & 2.3 & 50.0 \\
\hline Tetrachloroethene & 0.441 & 0.453 & 0.010 & 2.7 & 50.0 \\
\hline 2-Hexanone & 0.062 & 0.073 & 0.010 & 18.4 & 50.0 \\
\hline Dibromochloromethane & 0.181 & 0.193 & 0.010 & 6.9 & 50.0 \\
\hline 1,2-Dibromoethane & 0.137 & 0.145 & 0.010 & 5.8 & 50.0 \\
\hline Chlorobenzene & 1.138 & 1.189 & 0.010 & 4.5 & 50.0 \\
\hline Ethylbenzene & 2.043 & 2.304 & 0.010 & 12.8 & 50.0 \\
\hline o-Xylene & 0.677 & 0.805 & 0.010 & 18.8 & 50.0 \\
\hline $\mathrm{m}, \mathrm{p}$-Xylene & 0.783 & 0.900 & 0.010 & 15.0 & 50.0 \\
\hline Styrene & 1.077 & 1.293 & 0.010 & 20.1 & 50.0 \\
\hline Bromoform & 0.161 & 0.161 & 0.010 & 0.0 & 50.0 \\
\hline Isopropylbenzene & 1.977 & 2.307 & 0.010 & 16.7 & 50.0 \\
\hline $1,1,2,2$-Tetrachloroethane & 0.170 & 0.182 & 0.010 & 7.1 & 50.0 \\
\hline 1,3-Dichlorobenzene & 1.468 & 1.496 & 0.010 & 1.9 & 50.0 \\
\hline 1,4-Dichlorobenzene & 1.703 & 1.714 & 0.010 & 0.7 & 50.0 \\
\hline 1,2-Dichlorobenzene & 1.301 & 1.400 & 0.010 & 7.6 & 50.0 \\
\hline 1,2-Dibromo-3-Chloropropane & 0.039 & 0.036 & 0.010 & -7.5 & 50.0 \\
\hline 1,2,4-Trichlorobenzene & 0.757 & 0.673 & 0.010 & $-11 \cdot 1$ & 50.0 \\
\hline $1,2,3$-Trichlorobenzene & 0.541 & 0.507 & 0.010 & -6.2 & 50.0 \\
\hline
\end{tabular}


Lab Name: TESTAMERICA BURLINGTON

Contract: $8 \mathrm{E}-00302$

Lab Code: STLV Case No.: YORK Mod. Ref No.

SDG No.: 33957

Instrument ID: CHD.i

Calibration Date: 06/15/2016 Time: 1821

Lab File Id: 20443_12.D

Init. Calib. Date(s):05/31/2016 05/31/2016

EPA Sample No. (VSTD\#\#\#\#) : VSTD005TD

Init. Calib. Time(s) : 1605 1746

Heated Purge: (Y/N) N GC Column: DB-624

ID: $0.20(\mathrm{~mm})$ Length: 25

(m)

Purge Volume: 25.0 $(\mathrm{mL})$

\begin{tabular}{|c|c|c|c|c|c|}
\hline COMPOUND & $\overline{\mathrm{RRF}}$ & RRF5.0 & $\begin{array}{l}\text { MIN } \\
\text { RRF }\end{array}$ & $\because \mathrm{D}$ & $\operatorname{MAX} \cong \mathrm{D}$ \\
\hline Vinyl Chloride-d3 & 0.445 & 0.450 & 0.010 & 1.2 & 50.0 \\
\hline Chloroethane-d5 & 0.356 & 0.363 & 0.010 & 1.9 & 50.0 \\
\hline 1,1-Dichloroethene-d2 & 0.762 & 0.773 & 0.010 & 1.5 & 50.0 \\
\hline 2-Butanone-d5 & 0.030 & 0.030 & 0.010 & -1.1 & 50.0 \\
\hline Chloroform-d & 0.611 & 0.643 & 0.010 & 5.2 & 50.0 \\
\hline 1,2-Dichloroethane-d4 & 0.214 & 0.228 & 0.010 & 6.1 & 50.0 \\
\hline Benzene-d6 & 1.806 & 1.900 & 0.010 & 5.2 & 50.0 \\
\hline 1,2-Dichloropropane-d6 & 0.444 & 0.461 & 0.010 & 3.8 & 50.0 \\
\hline Toluene-d8 & 1.533 & 1.695 & 0.010 & 10.6 & 50.0 \\
\hline trans-1,3-Dichloropropene-d4 & 0.245 & 0.239 & 0.010 & -2.5 & 50.0 \\
\hline 2-Hexanone-d5 & 0.030 & 0.033 & 0.010 & 11.4 & 50.0 \\
\hline $1,1,2,2$-Tetrachloroethane-d2 & 0.171 & 0.183 & 0.010 & 6.7 & 50.0 \\
\hline 1,2-Dichlorobenzene-d4 & 0.807 & 0.821 & 0.010 & 1.6 & 50.0 \\
\hline
\end{tabular}

Report 1,4-Dioxane-d8 for Low/Medium VOA analysis only 
Lab Name: TESTAMERICA BURLINGTON

Contract: $8 \mathrm{E}-00302$

Lab Code: STLV Case No.: YORK Mod. Ref No.: SDG No.: 33957

Matrix: (SOIL/SED/WATER) Water

Sample wt/vol: 25.0 $(\mathrm{g} / \mathrm{mL}) \mathrm{mL}$

Level: (TRACE/LOW/MED) TRACE

\% Moisture: not dec.

GC Column: DB-624 ID: $0.20 \quad(\mathrm{~mm})$

Soil Extract Volume: (uL) $(\mathrm{mL})$

Purge Volume: 25.0
Lab Sample ID: MB 200-105769/4

Lab File ID: 20443_04.D

Date Received:

Date Analyzed: 06/15/2016

Dilution Factor: 1.0

Soil Aliquot Volume: (uL)

\begin{tabular}{|c|c|c|c|}
\hline CAS NO. & COMPOUND & $\begin{array}{l}\text { CONCENTRATION UNITS: } \\
(\mathrm{ug} / \mathrm{L} \text { or } \mathrm{ug} / \mathrm{kg}) \mathrm{ug} / \mathrm{L}\end{array}$ & q \\
\hline $75-71-8$ & Dichlorodifluoromethane & 1.0 & $\mathrm{U}$ \\
\hline $74-87-3$ & Chloromethane & 1.0 & $\mathrm{U}$ \\
\hline $75-01-4$ & Vinyl chloride & 1.0 & $\mathrm{U}$ \\
\hline $74-83-9$ & Bromomethane & 1.0 & $\mathrm{U}$ \\
\hline $75-00-3$ & Chloroethane & 1.0 & $\mathrm{U}$ \\
\hline $75-69-4$ & Trichlorofluoromethane & 1.0 & $\mathrm{U}$ \\
\hline $75-35-4$ & 1,1-Dichloroethene & 1.0 & $\mathrm{U}$ \\
\hline $76-13-1$ & $1,1,2$-Trichloro-1,2,2-trifluoroethane & 1.0 & $\mathrm{U}$ \\
\hline $67-64-1$ & Acetone & 1.6 & $\mathrm{~J}$ \\
\hline $75-15-0$ & Carbon disulfide & 0.037 & $\mathrm{~J}$ \\
\hline $79-20-9$ & Methyl acetate & 1.0 & $\mathrm{U}$ \\
\hline $75-09-2$ & Methylene Chloride & 0.036 & $\mathrm{~J}$ \\
\hline $156-60-5$ & trans-1,2-Dichloroethene & 0.015 & $\mathrm{~J}$ \\
\hline $1634-04-4$ & Methyl tert-butyl ether & 0.034 & $\mathrm{~J}$ \\
\hline $75-34-3$ & 1,1-Dichloroethane & 1.0 & $\mathrm{U}$ \\
\hline $156-59-2$ & cis-1,2-Dichloroethene & 1.0 & $\mathrm{U}$ \\
\hline $78-93-3$ & 2-Butanone & 5.0 & $\mathrm{U}$ \\
\hline $74-97-5$ & Bromochloromethane & 1.0 & $\mathrm{U}$ \\
\hline $67-66-3$ & Chloroform & 1.0 & $\mathrm{U}$ \\
\hline $71-55-6$ & $1,1,1$-Trichloroethane & 1.0 & $\mathrm{U}$ \\
\hline $110-82-7$ & Cyclohexane & 1.0 & $\mathrm{U}$ \\
\hline $56-23-5$ & Carbon tetrachloride & 1.0 & $\mathrm{U}$ \\
\hline $71-43-2$ & Benzene & 1.0 & $\mathrm{U}$ \\
\hline $107-06-2$ & 1,2-Dichloroethane & 1.0 & $\mathrm{U}$ \\
\hline
\end{tabular}

Report 1,4-Dioxane for Low-Medium VOA analysis only 
Lab Name: TESTAMERICA BURLINGTON

Lab Code: STLV

Case No.: YORK

Mod. Ref No.:

Contract: 8E-00302

Matrix: (SOIL/SED/WATER) Water

Sample wt/vol: 25.0 $(\mathrm{g} / \mathrm{mL}) \mathrm{mL}$

Level: (TRACE/LOW/MED) TRACE

\% Moisture: not dec.

GC Column: DB-624 ID: $0.20 \quad(\mathrm{~mm})$

Soil Extract Volume: (uL) $(\mathrm{mL})$

Purge Volume: 25.0
SDG No.: 33957

Lab Sample ID: MB 200-105769/4

Lab File ID: 20443_04.D

Date Received:

Date Analyzed: 06/15/2016

Dilution Factor: 1.0

Soil Aliquot Volume: (uL)

\begin{tabular}{|c|c|c|c|}
\hline CAS NO. & COMPOUND & $\begin{array}{l}\text { CONCENTRATION UNITS: } \\
(\mathrm{ug} / \mathrm{L} \text { or } \mathrm{ug} / \mathrm{kg}) \mathrm{ug} / \mathrm{L}\end{array}$ & $q$ \\
\hline $79-01-6$ & Trichloroethene & 0.012 & $\mathrm{~J}$ \\
\hline $108-87-2$ & Methylcyclohexane & 1.0 & $\mathrm{U}$ \\
\hline $78-87-5$ & 1,2-Dichloropropane & 1.0 & $\mathrm{U}$ \\
\hline $75-27-4$ & Bromodichloromethane & 1.0 & $\mathrm{U}$ \\
\hline $10061-01-5$ & cis-1,3-Dichloropropene & 1.0 & U \\
\hline $108-10-1$ & 4-Methyl-2-pentanone & 1.0 & $\mathrm{U}$ \\
\hline $108-88-3$ & Toluene & 0.066 & $\mathrm{~J}$ \\
\hline $10061-02-6$ & trans-1,3-Dichloropropene & 1.0 & $\mathrm{U}$ \\
\hline $79-00-5$ & $1,1,2-$ Trichloroethane & 1.0 & $\mathrm{U}$ \\
\hline $127-18-4$ & Tetrachloroethene & 0.0078 & $\mathrm{~J}$ \\
\hline $591-78-6$ & 2-Hexanone & 1.0 & $\mathrm{U}$ \\
\hline $124-48-1$ & Dibromochloromethane & 0.028 & $\mathrm{~J}$ \\
\hline $106-93-4$ & 1,2-Dibromoethane & 1.0 & $\mathrm{U}$ \\
\hline $108-90-7$ & Chlorobenzene & 1.0 & $\mathrm{U}$ \\
\hline $100-41-4$ & Ethylbenzene & 0.0096 & $\mathrm{~J}$ \\
\hline $95-47-6$ & o-Xylene & 0.011 & $\mathrm{~J}$ \\
\hline $179601-23-1$ & m,p-xylene & 0.013 & $\mathrm{~J}$ \\
\hline $100-42-5$ & Styrene & 0.013 & $\mathrm{~J}$ \\
\hline $75-25-2$ & Bromoform & 1.0 & $\mathrm{U}$ \\
\hline $98-82-8$ & Isopropylbenzene & 1.0 & $\mathrm{U}$ \\
\hline $79-34-5$ & $1,1,2,2$-Tetrachloroethane & 1.0 & $\mathrm{U}$ \\
\hline $541-73-1$ & 1,3 -Dichlorobenzene & 0.049 & $\mathrm{~J}$ \\
\hline $106-46-7$ & 1,4-Dichlorobenzene & 0.072 & $\mathrm{~J}$ \\
\hline $95-50-1$ & 1,2-Dichlorobenzene & 0.091 & $\mathrm{~J}$ \\
\hline $96-12-8$ & 1,2-Dibromo-3-Chloropropane & 1.0 & $\mathrm{U}$ \\
\hline $120-82-1$ & $1,2,4$-Trichlorobenzene & 0.18 & $\mathrm{~J}$ \\
\hline $87-61-6$ & $1,2,3$-Trichlorobenzene & 0.30 & $\mathrm{~J}$ \\
\hline
\end{tabular}


Lab Name: TESTAMERICA BURLINGTON

Contract: 8E-00302

Lab Code: STLV Case No.: YORK Mod. Ref No.: SDG No.: 33957

Matrix: (SOIL/SED/WATER) Water

Sample wt/vol: 25.0 $(\mathrm{g} / \mathrm{mL}) \mathrm{mL}$

Level: (TRACE or LOW/MED) TRACE

Moisture: not dec.

GC Column: DB-624 ID $: 0.20 \quad(\mathrm{~mm})$

Soil Extract Volume: (uL)

CONCENTRATION UNITS: (ug/L or ug/kg) ug/L
Lab Sample ID: MB 200-105769/4

Lab File ID: 20443_04.D

Date Received:

Date Analyzed: 06/15/2016

Dilution Factor: 1.0

Soil Aliquot Volume: (uL) Purge Volume: 25.0 $(\mathrm{mL})$

01

02

\begin{tabular}{|l|l|c|c|c|}
\hline CAS NUMBER & \multicolumn{1}{|c|}{ COMPOUND NAME } & RT & EST. CONC. & Q \\
\hline & Unknown & 7.20 & 2.5 & $\mathrm{~J} X$ \\
\hline E9667961 & Total Alkanes & N/A & & \\
\hline
\end{tabular}

1 EPA-designated Registry Number. 
Lab Name: TESTAMERICA BURLINGTON

Contract: $8 \mathrm{E}-00302$

Lab Code: STLV

Case No.: YORK Mod. Ref No.:

SDG No.: 33957

Matrix: (SOIL/SED/WATER) Water

Sample wt/vol: 25.0 $(\mathrm{g} / \mathrm{mL}) \mathrm{mL}$

Level: (TRACE/LOW/MED) TRACE

\% Moisture: not dec.

GC Column: DB-624 ID: $0.20 \quad(\mathrm{~mm})$

Soil Extract Volume: (uL) $(\mathrm{mL})$

Purge Volume: 25.0
Lab Sample ID: 200-33957-4

Lab File ID: 20443_11.D

Date Received:

Date Analyzed: 06/15/2016

Dilution Factor: 1.0

Soil Aliquot Volume:

(uL)

\begin{tabular}{|c|c|c|c|}
\hline CAS NO. & COMPOUND & $\begin{array}{l}\text { CONCENTRATION UNITS: } \\
(\mathrm{ug} / \mathrm{L} \text { or } \mathrm{ug} / \mathrm{kg}) \mathrm{ug} / \mathrm{L}\end{array}$ & Q \\
\hline $75-71-8$ & Dichlorodifluoromethane & 1.0 & $\mathrm{U}$ \\
\hline $74-87-3$ & Chloromethane & 1.0 & $\mathrm{U}$ \\
\hline $75-01-4$ & Vinyl chloride & 1.0 & $\mathrm{U}$ \\
\hline $74-83-9$ & Bromomethane & 1.0 & $\mathrm{U}$ \\
\hline $75-00-3$ & Chloroethane & 1.0 & $\mathrm{U}$ \\
\hline $75-69-4$ & Trichlorofluoromethane & 1.0 & $\mathrm{U}$ \\
\hline $75-35-4$ & 1,1-Dichloroethene & 1.0 & $\mathrm{U}$ \\
\hline $76-13-1$ & 1,1,2-Trichloro-1,2,2-trifluoroethane & 1.0 & $\mathrm{U}$ \\
\hline $67-64-1$ & Acetone & 5.0 & $\mathrm{U}$ \\
\hline $75-15-0$ & Carbon disulfide & 1.0 & $\mathrm{U}$ \\
\hline $79-20-9$ & Methyl acetate & 1.0 & $\mathrm{U}$ \\
\hline $75-09-2$ & Methylene Chloride & 0.023 & $\mathrm{~J} B$ \\
\hline $156-60-5$ & trans-1,2-Dichloroethene & 1.0 & $\mathrm{U}$ \\
\hline $1634-04-4$ & Methyl tert-butyl ether & 1.0 & $\mathrm{U}$ \\
\hline $75-34-3$ & 1,1-Dichloroethane & 1.0 & $\mathrm{U}$ \\
\hline $156-59-2$ & cis-1,2-Dichloroethene & 1.0 & $\mathrm{U}$ \\
\hline $78-93-3$ & 2-Butanone & 5.0 & $\mathrm{U}$ \\
\hline $74-97-5$ & Bromochloromethane & 1.0 & $\mathrm{U}$ \\
\hline $67-66-3$ & Chloroform & 1.0 & $\mathrm{U}$ \\
\hline $71-55-6$ & $1,1,1$-Trichloroethane & 1.0 & $\mathrm{U}$ \\
\hline $110-82-7$ & Cyclohexane & 1.0 & $\mathrm{U}$ \\
\hline $56-23-5$ & Carbon tetrachloride & 1.0 & $\mathrm{U}$ \\
\hline $71-43-2$ & Benzene & 1.0 & $\mathrm{U}$ \\
\hline $107-06-2$ & 1,2-Dichloroethane & 1.0 & $\mathrm{U}$ \\
\hline
\end{tabular}

Report 1,4-Dioxane for Low-Medium VOA analysis only 
Lab Name: TESTAMERICA BURLINGTON

Lab Code: STLV

Case No.: YORK

Mod. Ref No.:

Contract: 8E-00302

Matrix: (SOIL/SED/WATER) Water

Sample wt/vol: 25.0 $(\mathrm{g} / \mathrm{mL}) \mathrm{mL}$

Level: (TRACE/LOW/MED) TRACE

\% Moisture: not dec.

GC Column: DB-624 ID: $0.20 \quad(\mathrm{~mm})$

Soil Extract Volume: (uL) $(\mathrm{mL})$

Purge Volume: 25.0
SDG No.: 33957

Lab Sample ID: 200-33957-4

Lab File ID: 20443_11.D

Date Received:

Date Analyzed: 06/15/2016

Dilution Factor: 1.0

Soil Aliquot Volume:

(uL)

\begin{tabular}{|c|c|c|c|}
\hline CAS NO. & COMPOUND & $\begin{array}{l}\text { CONCENTRATION UNITS: } \\
(\mathrm{ug} / \mathrm{L} \text { or } \mathrm{ug} / \mathrm{kg}) \mathrm{ug} / \mathrm{L}\end{array}$ & Q \\
\hline $79-01-6$ & Trichloroethene & 1.0 & $\mathrm{U}$ \\
\hline $108-87-2$ & Methylcyclohexane & 1.0 & $\mathrm{U}$ \\
\hline $78-87-5$ & 1,2-Dichloropropane & 1.0 & $\mathrm{U}$ \\
\hline $75-27-4$ & Bromodichloromethane & 1.0 & $\mathrm{U}$ \\
\hline $10061-01-5$ & cis-1,3-Dichloropropene & 1.0 & $\mathrm{U}$ \\
\hline $108-10-1$ & 4-Methyl-2-pentanone & 1.0 & $\mathrm{U}$ \\
\hline $108-88-3$ & Toluene & 0.012 & $\mathrm{~J} B$ \\
\hline $10061-02-6$ & trans-1,3-Dichloropropene & 1.0 & $\mathrm{U}$ \\
\hline $79-00-5$ & $1,1,2-T r i c h l o r o e t h a n e$ & 1.0 & $\mathrm{U}$ \\
\hline $127-18-4$ & Tetrachloroethene & 1.0 & $\mathrm{U}$ \\
\hline $591-78-6$ & 2-Hexanone & 1.0 & $\mathrm{U}$ \\
\hline $124-48-1$ & Dibromochloromethane & 1.0 & $\mathrm{U}$ \\
\hline $106-93-4$ & 1,2-Dibromoethane & 1.0 & $\mathrm{U}$ \\
\hline $108-90-7$ & Chlorobenzene & 1.0 & $\mathrm{U}$ \\
\hline $100-41-4$ & Ethylbenzene & 1.0 & $\mathrm{U}$ \\
\hline $95-47-6$ & o-Xylene & 1.0 & $\mathrm{U}$ \\
\hline $179601-23-1$ & m,p-Xylene & 1.0 & $\mathrm{U}$ \\
\hline $100-42-5$ & styrene & 1.0 & $\mathrm{U}$ \\
\hline $75-25-2$ & Bromoform & 1.0 & $\mathrm{U}$ \\
\hline $98-82-8$ & Isopropylbenzene & 1.0 & $\mathrm{U}$ \\
\hline $79-34-5$ & $1,1,2,2$-Tetrachloroethane & 1.0 & $\mathrm{U}$ \\
\hline $541-73-1$ & 1,3-Dichlorobenzene & 1.0 & $\mathrm{U}$ \\
\hline $106-46-7$ & 1,4-Dichlorobenzene & 1.0 & $\mathrm{U}$ \\
\hline $95-50-1$ & $1,2-$ Dichlorobenzene & 1.0 & $\mathrm{U}$ \\
\hline $96-12-8$ & 1,2-Dibromo-3-Chloropropane & 1.0 & $\mathrm{U}$ \\
\hline $120-82-1$ & $1,2,4$-Trichlorobenzene & 1.0 & $\mathrm{U}$ \\
\hline $87-61-6$ & $1,2,3$-Trichlorobenzene & 1.0 & $\mathrm{U}$ \\
\hline
\end{tabular}


Lab Name: TESTAMERICA BURLINGTON

Contract: $8 \mathrm{E}-00302$

Lab Code: STLV Case No.: YORK Mod. Ref No.: SDG No.: 33957

Matrix: (SOIL/SED/WATER) Water

Sample wt/vol: 25.0 $(\mathrm{g} / \mathrm{mL}) \mathrm{mL}$

Level: (TRACE or LOW/MED) TRACE

\% Moisture: not dec.

GC Column: DB-624 ID $: 0.20 \quad(\mathrm{~mm})$

Soil Extract Volume: (uL)

CONCENTRATION UNITS: (ug/L or ug/kg) ug/L
Lab Sample ID: 200-33957-4

Lab File ID: 20443 11.D

Date Received:

Date Analyzed: 06/15/2016

Dilution Factor: 1.0

Soil Aliquot Volume: (uL) Purge Volume: 25.0 $(\mathrm{mL})$

01

02

\begin{tabular}{|l|l|c|c|c|}
\hline CAS NUMBER & \multicolumn{1}{|c|}{ COMPOUND NAME } & RT & EST. CONC. & Q \\
\hline & Unknown & 7.20 & 2.5 & $\mathrm{~J}$ X B \\
\hline E9667961 & Total Alkanes & N/A & & \\
\hline
\end{tabular}

1EPA-designated Registry Number. 
Lab Name: TestAmerica Burlington

Job No.: 200-33957-1

SDG No.: 33957

Instrument ID: CHD.i

Analysis Batch Number: 105151

Lab Sample ID: ICIS 200-105151/4

Client Sample ID:

Date Analyzed: 05/31/16 16:56

Lab File ID: 20227 04.D

GC Column: DB-624

ID $: 0.2(\mathrm{~mm})$

\begin{tabular}{|c|c|c|c|c|}
\hline \multirow[t]{2}{*}{ COMPOUND NAME } & \multirow{2}{*}{$\begin{array}{c}\text { RETENTION } \\
\text { TIME }\end{array}$} & \multicolumn{3}{|c|}{ MANUAL INTEGRATION } \\
\hline & & REASON & ANALYST & DATE \\
\hline 1,2-Dichloropropane-d6 & 6.32 & Split Peak & wilburj & $05 / 31 / 1617: 36$ \\
\hline
\end{tabular}

Lab Sample ID: IC 200-105151/5

Client Sample ID:

Date Analyzed: 05/31/16 17:21

Lab File ID: 20227 05.D

GC Column: DB-624

ID $: 0.2(\mathrm{~mm})$

\begin{tabular}{|c|c|c|c|c|}
\hline \multicolumn{1}{|c|}{ COMPOUND NAME } & RETENTION & \multicolumn{3}{|c}{ MANUAL INTEGRATION } \\
\cline { 3 - 5 } & TIME & REASON & ANALYST & DATE \\
\hline 1,2 -Dichloropropane-d6 & 6.32 & Split Peak & wilburj & 05/31/16 17:57 \\
\hline
\end{tabular}

Lab Sample ID: IC 200-105151/6

Client Sample ID:

Date Analyzed: 05/31/16 17:46

Lab File ID: 20227_06.D

GC Column: DB-624

ID $: 0.2(\mathrm{~mm})$

\begin{tabular}{|c|c|c|c|c|}
\hline \multicolumn{1}{|c|}{ COMPOUND NAME } & RETENTION & \multicolumn{3}{|c|}{ MANUAL INTEGRATION } \\
\cline { 3 - 5 } & TIME & REASON & ANALYST & DATE \\
\hline 1,2-Dichloropropane-d6 & 6.32 & Split Peak & wilburj & $05 / 31 / 16$ 18:30 \\
\hline
\end{tabular}


Lab Name: TestAmerica Burlington

Job No.: 200-33957-1

SDG No.: 33957

Instrument ID: CHD.i

Analysis Batch Number: 105769

Lab Sample ID: CCVIS 200-105769/3

Client Sample ID:

Date Analyzed: 06/15/16 13:43

Lab File ID: 20443_03.D

GC Column: DB-624

ID $: 0.2(\mathrm{~mm})$

\begin{tabular}{|c|c|c|c|c|}
\hline \multirow[t]{2}{*}{ COMPOUND NAME } & \multirow{2}{*}{$\begin{array}{c}\text { RETENTION } \\
\text { TIME }\end{array}$} & \multicolumn{3}{|c|}{ MANUAL INTEGRATION } \\
\hline & & REASON & ANALYST & DATE \\
\hline $1,2-$ Dichloropropane-d6 & 6.32 & Split Peak & wilburj & $06 / 15 / 16 \quad 14: 47$ \\
\hline
\end{tabular}

Lab Sample ID: MB 200-105769/4

Client Sample ID:

Date Analyzed: 06/15/16 14:09

Lab File ID: 20443_04.D

GC Column: DB-624

ID $: 0.2(\mathrm{~mm})$

\begin{tabular}{|l|c|c|c|c|}
\hline \multicolumn{2}{|c|}{ COMPOUND NAME } & RETENTION & \multicolumn{2}{c|}{ MANUAL INTEGRATION } \\
\cline { 4 - 5 } & TIME & REASON & ANALYST & DATE \\
\hline Acetone & 2.79 & Assign Peak & maheseep & $06 / 16 / 1608: 33$ \\
\hline
\end{tabular}

Lab Sample ID: CCVC 200-105769/12

Client Sample ID:

Date Analyzed: 06/15/16 18:21

Lab File ID: 20443_12.D

GC Column: DB-624

ID : $0.2(\mathrm{~mm})$

\begin{tabular}{|c|c|c|c|c|}
\hline \multirow[t]{2}{*}{ COMPOUND NAME } & \multirow{2}{*}{$\begin{array}{c}\text { RETENTION } \\
\text { TIME }\end{array}$} & \multicolumn{3}{|c|}{ MANUAL INTEGRATION } \\
\hline & & REASON & ANALYST & DATE \\
\hline Chloroethane & 2.08 & Assign Peak & maheseep & $06 / 16 / 1609: 16$ \\
\hline 1,2-Dichloropropane-d6 & 6.32 & Split Peak & wilburj & $06 / 15 / 1618: 51$ \\
\hline
\end{tabular}




\section{TestAmerica}

THE LEADER IN ENVIRONMENTAL TESTING

\section{ANALYTICAL REPORT}

Job Number: 200-34024-1

SDG Number: 34024

Job Description: York (200-34024)

Contract Number: 1E-30401

For:

Argonne National Laboratory

9700 South Cass Avenue

Building 203

Office B-141

Argonne, IL 60439

Attention: Ms. Esther Bowen

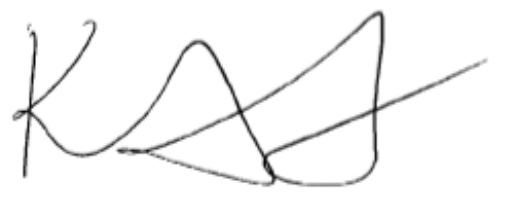

Approved for release. Kathryn A Kelly

Kathryn A Kelly, Project Manager I

30 Community Drive, South Burlington, VT, 05403

kathryn.kelly@testamericainc.com

06/28/2016

The test results in this report relate only to sample(s) as received by the laboratory. These test results were derived under a quality system that adheres to the requirements of NELAC. Pursuant to NELAC, this report may not be produced in full without written approval from the laboratory 


\section{Table of Contents}

Cover Title Page..$\ldots \ldots \ldots \ldots \ldots \ldots \ldots \ldots \ldots \ldots \ldots \ldots \ldots \ldots$

External Chain of Custody ...................... 4

Shipping Documentation . . . . . . . . . . . . . . . . . 6

Sample Receipt and Log In Check List ..................... 7

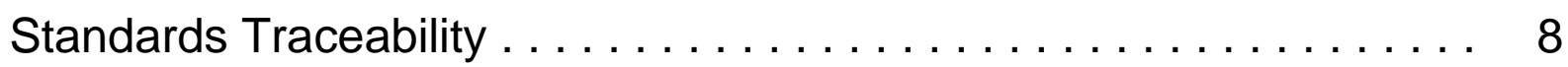

Methodology Review . . . . . . . . . . . . . . . . . . . 13

Report Narrative $\ldots \ldots \ldots \ldots \ldots \ldots \ldots \ldots \ldots \ldots \ldots \ldots \ldots \ldots \ldots$

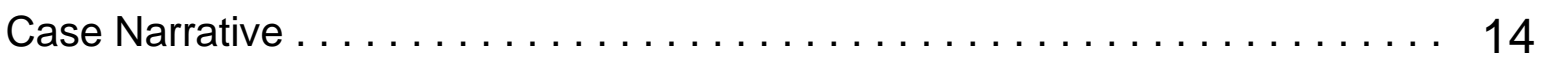

Manual Integration Documentation ........................ 15

Qualifier Definition ................................. 17

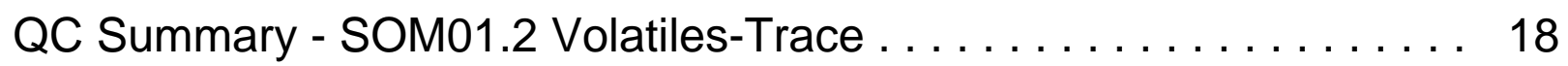

QC Summary - SOM01.2 Volatiles-Trace .................... 18

Deuterated Monitoring Compound Summary $\ldots \ldots \ldots \ldots \ldots \ldots \ldots \ldots \ldots \ldots \ldots \ldots \ldots$

Method Blank $\ldots \ldots \ldots \ldots \ldots \ldots \ldots \ldots \ldots \ldots \ldots \ldots \ldots \ldots \ldots \ldots \ldots, 20$

GC/MS Instrument Performance Check $\ldots \ldots \ldots \ldots \ldots \ldots \ldots \ldots \ldots \ldots \ldots, 21$

Internal Standard Area and RT Summary $\ldots \ldots \ldots \ldots \ldots \ldots \ldots \ldots \ldots \ldots \ldots \ldots \ldots \ldots$

Sample Data - SOM01.2 Volatiles-Trace . . . . . . . . . . . 24

Sample Data - SOM01.2 Volatiles-Trace ...................... 24

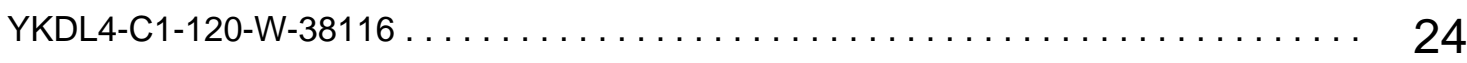

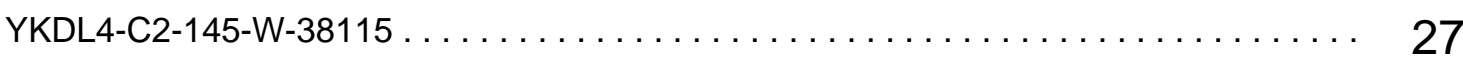

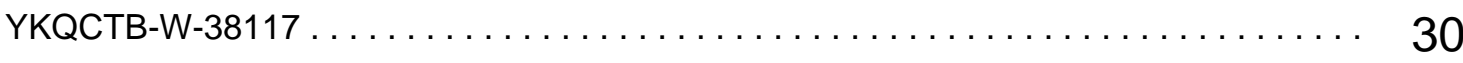

Standards - SOM01.2 Volatiles-Trace . . . . . . . . . . . . 33

Standards - SOM01.2 Volatiles-Trace $\ldots \ldots \ldots \ldots \ldots \ldots \ldots \ldots \ldots . . \ldots \ldots$

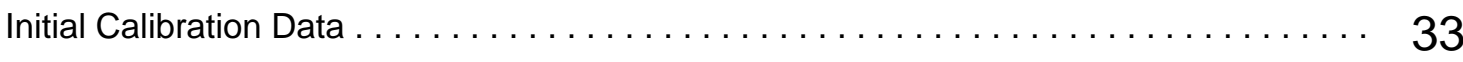

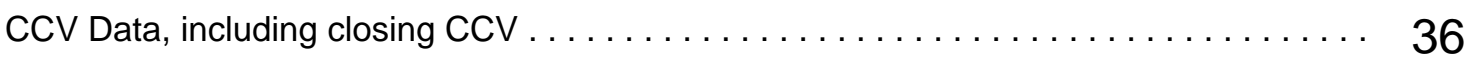

Raw Qc Data - SOM01.2 Volatiles-Trace . . . . . . . . . . . . . 45 


\section{Table of Contents}

Raw Qc Data - SOM01.2 Volatiles-Trace . . . . . . . . . . . . . 45

Raw Qc Data - SOM01.2 Volatiles-Trace .......................... 45

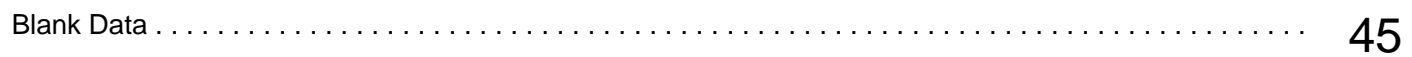




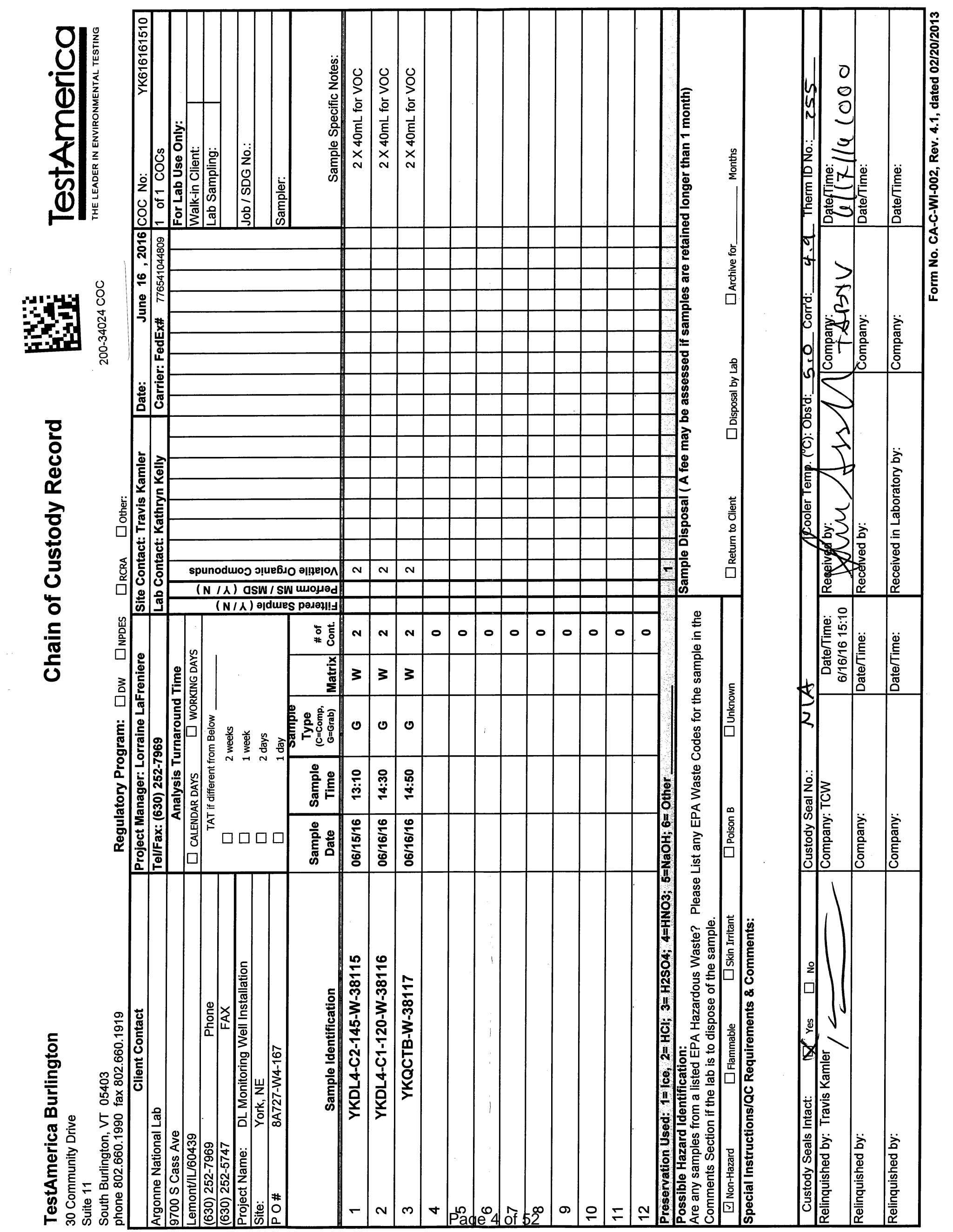




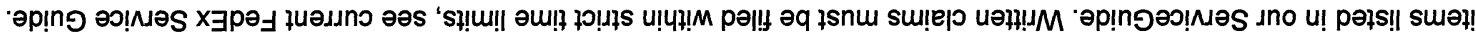
ләчұо pue słuәunд)

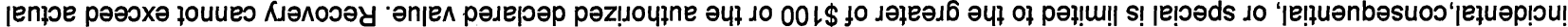

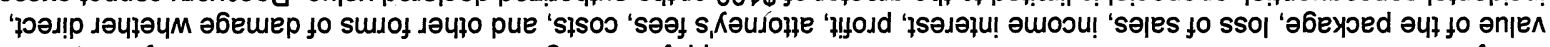

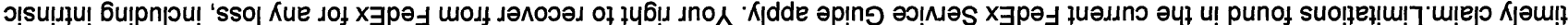

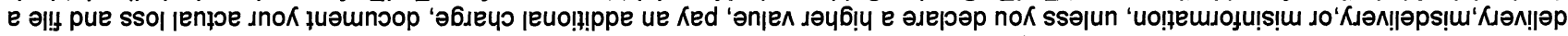

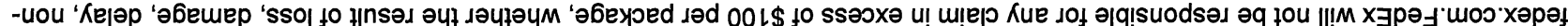

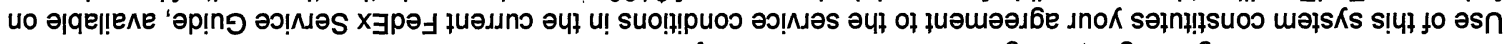

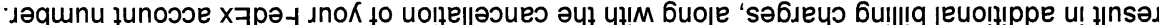

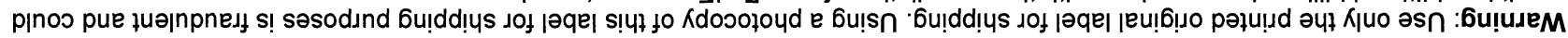

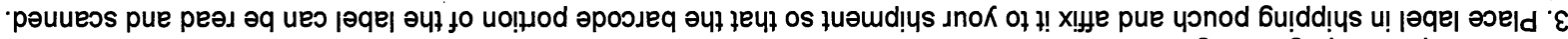

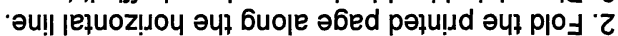

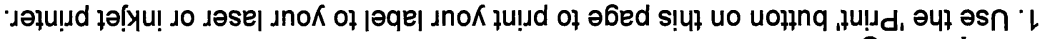

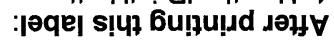

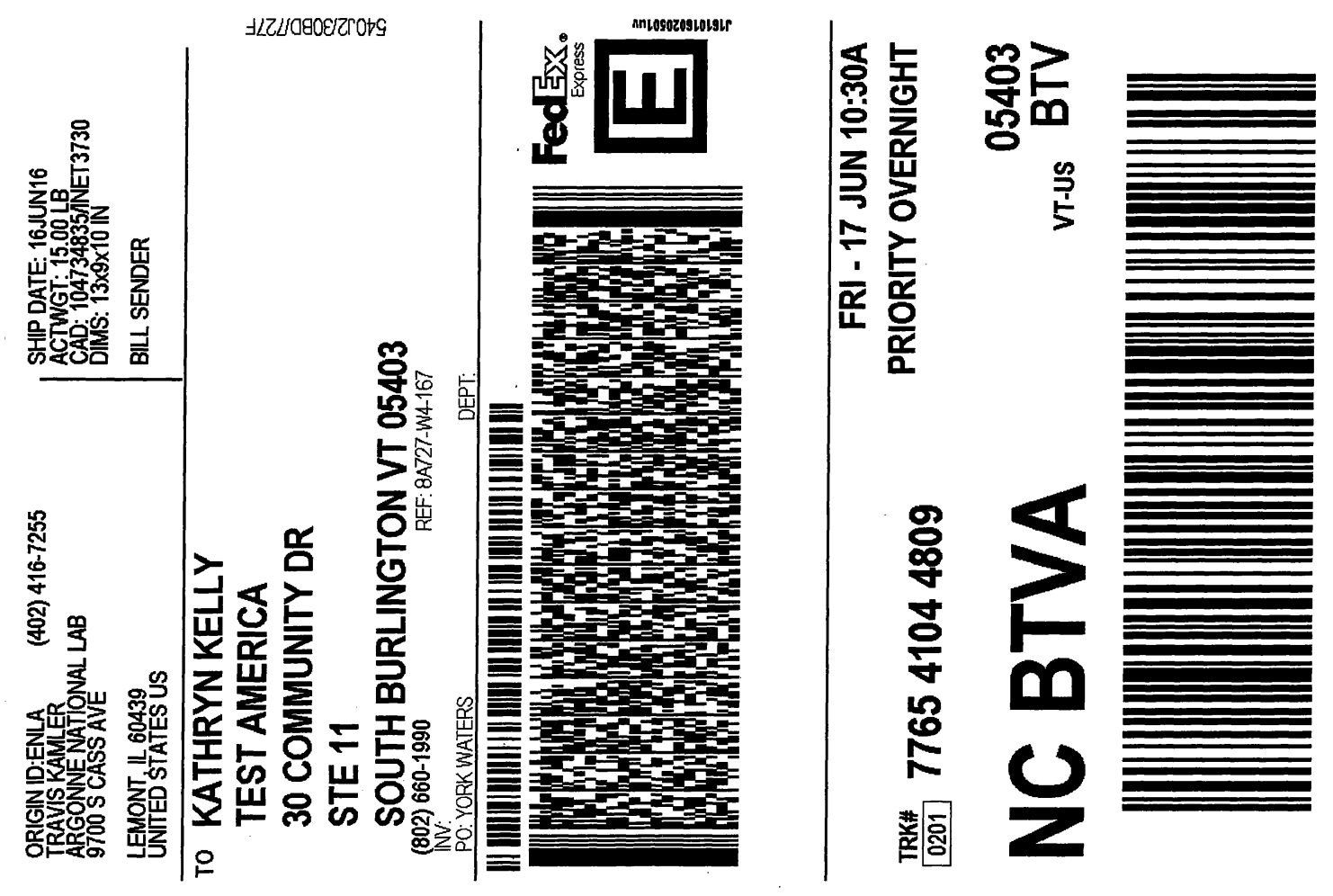




\section{Shipping and Receiving Documents}




\section{Login Sample Receipt Checklist}

Client: Argonne National Laboratory

Job Number: 200-34024-1

SDG Number: 34024

Login Number: 34024

List Source: TestAmerica Burlington

List Number: 1

Creator: Lavigne III, Scott M

Question

Radioactivity wasn't checked or is $</=$ background as measured by a survey True

Answer meter.

The cooler's custody seal, if present, is intact.

Sample custody seals, if present, are intact.

The cooler or samples do not appear to have been compromised or tampered with.

Samples were received on ice.

Cooler Temperature is acceptable.

Cooler Temperature is recorded.

$\mathrm{COC}$ is present.

$\mathrm{COC}$ is filled out in ink and legible.

$\mathrm{COC}$ is filled out with all pertinent information.

Is the Field Sampler's name present on COC?

There are no discrepancies between the containers received and the COC.

Samples are received within Holding Time (excluding tests with immediate

HTs)

Sample containers have legible labels.

Containers are not broken or leaking.

Sample collection date/times are provided.

Appropriate sample containers are used.

Sample bottles are completely filled.

Sample Preservation Verified.

There is sufficient vol. for all requested analyses, incl. any requested MS/MSDs

Containers requiring zero headspace have no headspace or bubble is $<6 \mathrm{~mm}(1 / 4 ")$.

Multiphasic samples are not present.

True

True

True

True

True

True

True

True

True

True

True

True

True

True

True

True

N/A

True

True

True

True

Samples do not require splitting or compositing.

Residual Chlorine Checked.

N/A
Lab does not accept radioactive samples.

Seal present with no number.

$4.9^{\circ} \mathrm{C}$ 


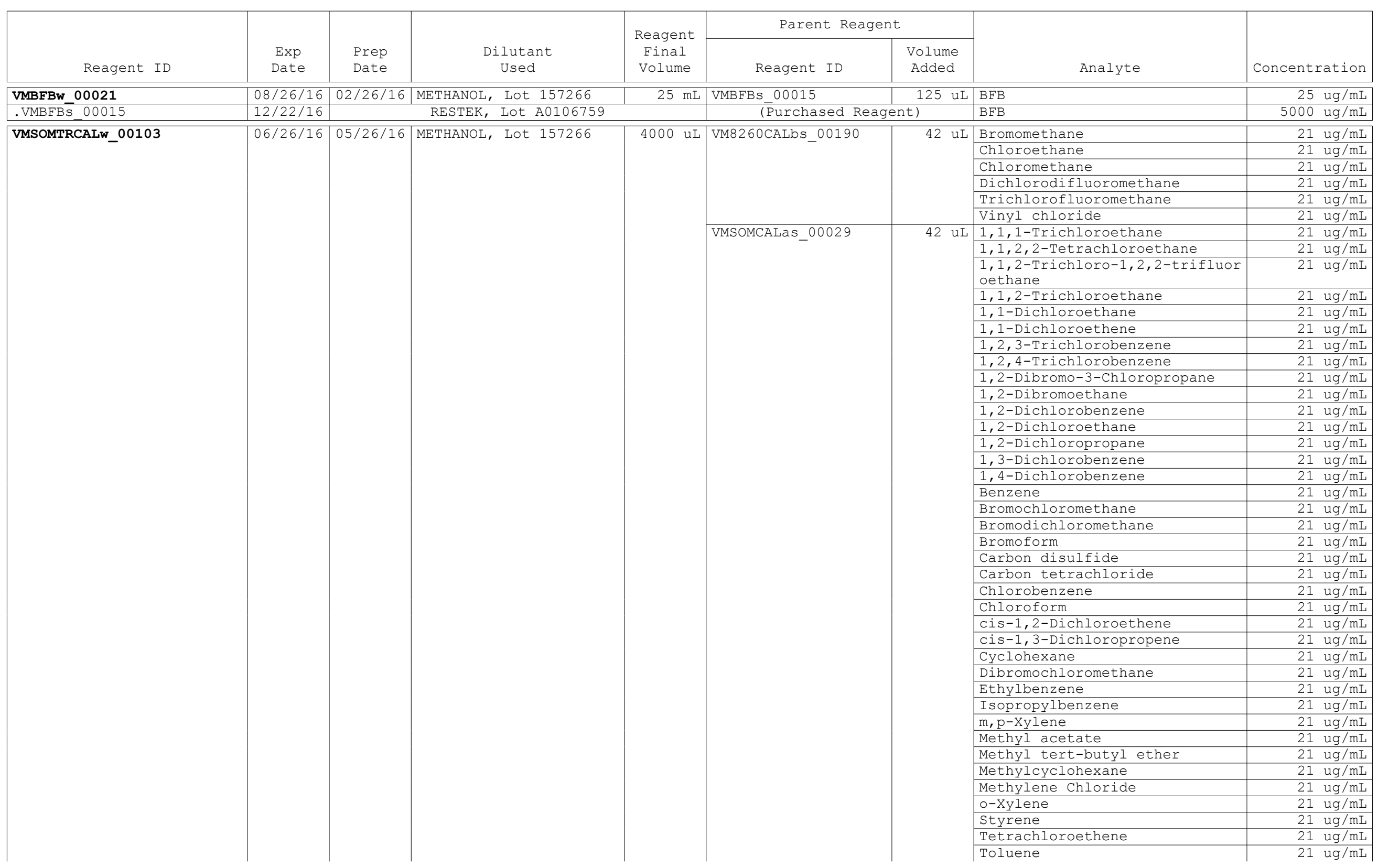


SDG No.: 34024

\begin{tabular}{|c|c|c|c|c|c|c|c|c|}
\hline \multirow[b]{2}{*}{ Reagent ID } & \multirow[b]{2}{*}{$\begin{array}{l}\text { Exp } \\
\text { Date }\end{array}$} & \multirow[b]{2}{*}{$\begin{array}{l}\text { Prep } \\
\text { Date }\end{array}$} & \multirow[b]{2}{*}{$\begin{array}{l}\text { Dilutant } \\
\text { Used }\end{array}$} & \multirow{2}{*}{$\begin{array}{c}\text { Reagent } \\
\text { Final } \\
\text { Volume }\end{array}$} & \multicolumn{2}{|l|}{ Parent Reagent } & \multirow[b]{2}{*}{ Analyte } & \multirow[b]{2}{*}{ Concentration } \\
\hline & & & & & \multirow[t]{4}{*}{ Reagent ID } & $\begin{array}{l}\text { Volume } \\
\text { Added }\end{array}$ & & \\
\hline & & & & & & & trans-1,2-Dichloroethene & $21 \mathrm{ug} / \mathrm{mL}$ \\
\hline & & & & & & & trans-1,3-Dichloropropene & $21 \mathrm{ug} / \mathrm{mL}$ \\
\hline & & & & & & & Trichloroethene & $21 \mathrm{ug} / \mathrm{mL}$ \\
\hline & & & & & VMSOMCALbS_00035 & $168 \mathrm{uL}$ & 2-Butanone & $210 \mathrm{ug} / \mathrm{mL}$ \\
\hline & & & & & & & 2-Hexanone & $210 \mathrm{ug} / \mathrm{mL}$ \\
\hline & & & & & & & 4-Methyl-2-pentanone & $210 \mathrm{ug} / \mathrm{mL}$ \\
\hline & & & & & & & Acetone & $210 \mathrm{ug} / \mathrm{mL}$ \\
\hline & & & & & VMSOMSUas_00085 & 42 uL & Chloroethane-d5 & $21 \mathrm{ug} / \mathrm{mL}$ \\
\hline & & & & & & & Vinyl Chloride-d3 & $21 \mathrm{ug} / \mathrm{mL}$ \\
\hline & & & & & VMSOMSUbs_00147 & $336 \mathrm{uL}$ & 2-Butanone-d5 & $210 \mathrm{ug} / \mathrm{mL}$ \\
\hline & & & & & & & 2-Hexanone-d5 & $210 \mathrm{ug} / \mathrm{mL}$ \\
\hline & & & & & VMSOMSUCS_00059 & 42 uL & $1,1,2,2$-Tetrachloroethane-d2 & $21 \mathrm{ug} / \mathrm{mL}$ \\
\hline & & & & & & & 1,1-Dichloroethene-d2 & $21 \mathrm{ug} / \mathrm{mL}$ \\
\hline & & & & & & & 1,2-Dichlorobenzene-d4 & $21 \mathrm{ug} / \mathrm{mL}$ \\
\hline & & & & & & & 1,2-Dichloroethane-d4 & $21 \mathrm{ug} / \mathrm{mL}$ \\
\hline & & & & & & & 1,2-Dichloropropane-d6 & $21 \mathrm{ug} / \mathrm{mL}$ \\
\hline & & & & & & & Benzene-d6 & $21 \mathrm{ug} / \mathrm{mL}$ \\
\hline & & & & & & & Chloroform-d & $21 \mathrm{ug} / \mathrm{mL}$ \\
\hline & & & & & & & Toluene-d8 & $21 \mathrm{ug} / \mathrm{mL}$ \\
\hline & & & & & & & trans-1,3-Dichloropropene-d4 & $21 \mathrm{ug} / \mathrm{mL}$ \\
\hline \multirow{6}{*}{.VM8260CALbs_00190 } & \multirow{6}{*}{$06 / 26 / 16$} & \multirow{6}{*}{\multicolumn{3}{|c|}{ RESTEK, Lot A0115510 }} & \multirow{6}{*}{\multicolumn{2}{|c|}{ (Purchased Reagent) }} & Bromomethane & $2000 \mathrm{ug} / \mathrm{mL}$ \\
\hline & & & & & & & Chloroethane & $2000 \mathrm{ug} / \mathrm{mL}$ \\
\hline & & & & & & & Chloromethane & $2000 \mathrm{ug} / \mathrm{mL}$ \\
\hline & & & & & & & Dichlorodifluoromethane & $2000 \mathrm{ug} / \mathrm{mL}$ \\
\hline & & & & & & & Trichlorofluoromethane & $2000 \mathrm{ug} / \mathrm{mL}$ \\
\hline & & & & & & & Vinyl chloride & $2000 \mathrm{ug} / \mathrm{mL}$ \\
\hline \multirow{21}{*}{.VMSOMCALas_00029 } & \multirow[t]{21}{*}{$04 / 30 / 17$} & \multirow{21}{*}{\multicolumn{2}{|c|}{ Restek, Lot A0102833 }} & & \multirow{21}{*}{\multicolumn{2}{|c|}{ (Purchased Reagent) }} & $1,1,1$-Trichloroethane & $2000 \mathrm{ug} / \mathrm{mL}$ \\
\hline & & & & & & & 1,1,2,2-Tetrachloroethane & $2000 \mathrm{ug} / \mathrm{mL}$ \\
\hline & & & & & & & $\begin{array}{l}\text { 1,1,2-Trichloro-1,2,2-trifluor } \\
\text { oethane }\end{array}$ & $2000 \mathrm{ug} / \mathrm{mL}$ \\
\hline & & & & & & & $1,1,2$-Trichloroethane & $2000 \mathrm{ug} / \mathrm{mL}$ \\
\hline & & & & & & & 1,1-Dichloroethane & $2000 \mathrm{ug} / \mathrm{mL}$ \\
\hline & & & & & & & 1,1-Dichloroethene & $2000 \mathrm{ug} / \mathrm{mL}$ \\
\hline & & & & & & & 1,2,3-Trichlorobenzene & $2000 \mathrm{ug} / \mathrm{mL}$ \\
\hline & & & & & & & 1,2,4-Trichlorobenzene & $2000 \mathrm{ug} / \mathrm{mL}$ \\
\hline & & & & & & & 1,2-Dibromo-3-Chloropropane & $2000 \mathrm{ug} / \mathrm{mL}$ \\
\hline & & & & & & & 1,2-Dibromoethane & $2000 \mathrm{ug} / \mathrm{mL}$ \\
\hline & & & & & & & 1,2-Dichlorobenzene & $2000 \mathrm{ug} / \mathrm{mL}$ \\
\hline & & & & & & & 1,2-Dichloroethane & $2000 \mathrm{ug} / \mathrm{mL}$ \\
\hline & & & & & & & 1,2-Dichloropropane & $2000 \mathrm{ug} / \mathrm{mL}$ \\
\hline & & & & & & & 1,3-Dichlorobenzene & $2000 \mathrm{ug} / \mathrm{mL}$ \\
\hline & & & & & & & 1,4-Dichlorobenzene & $2000 \mathrm{ug} / \mathrm{mL}$ \\
\hline & & & & & & & Benzene & $2000 \mathrm{ug} / \mathrm{mL}$ \\
\hline & & & & & & & Bromochloromethane & $2000 \mathrm{ug} / \mathrm{mL}$ \\
\hline & & & & & & & Bromodichloromethane & $2000 \mathrm{ug} / \mathrm{mL}$ \\
\hline & & & & & & & Bromoform & $2000 \mathrm{ug} / \mathrm{mL}$ \\
\hline & & & & & & & Carbon disulfide & $2000 \mathrm{ug} / \mathrm{mL}$ \\
\hline & & & & & & & Carbon tetrachloride & $2000 \mathrm{ug} / \mathrm{mL}$ \\
\hline
\end{tabular}


SDG No.: 34024

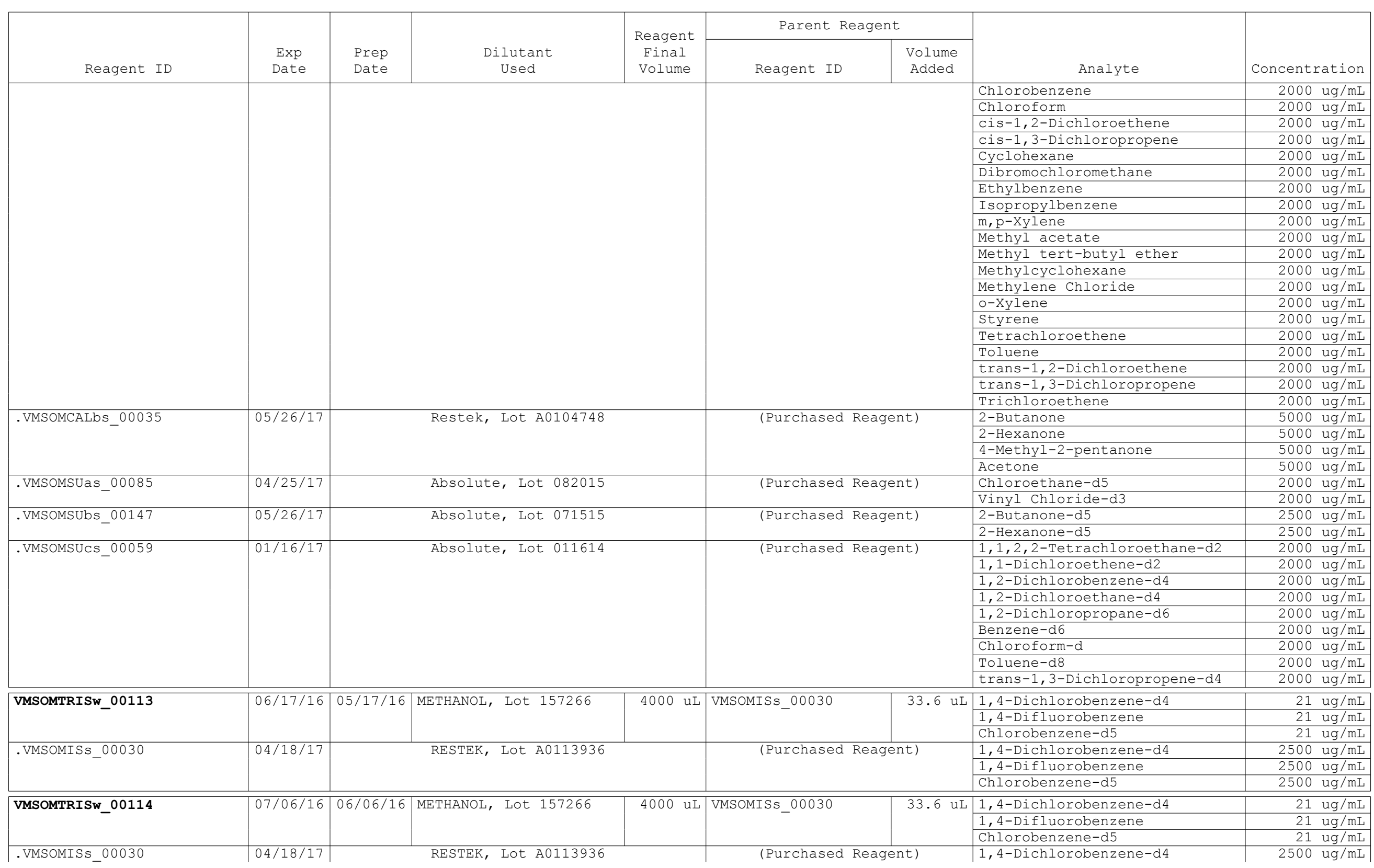


SDG No.: 34024

\begin{tabular}{|c|c|c|c|c|c|c|c|c|}
\hline \multirow[b]{2}{*}{ Reagent ID } & \multirow[b]{2}{*}{$\begin{array}{l}\text { Exp } \\
\text { Date }\end{array}$} & \multirow[b]{2}{*}{$\begin{array}{l}\text { Prep } \\
\text { Date }\end{array}$} & \multirow[b]{2}{*}{$\begin{array}{l}\text { Dilutant } \\
\text { Used }\end{array}$} & \multirow{2}{*}{$\begin{array}{l}\text { Reagent } \\
\text { Final } \\
\text { Volume }\end{array}$} & \multicolumn{2}{|c|}{ Parent Reagent } & \multirow[b]{2}{*}{ Analyte } & \multirow[b]{2}{*}{ Concentration } \\
\hline & & & & & \multicolumn{2}{|r|}{$\begin{array}{l}\text { Volume } \\
\text { Added }\end{array}$} & & \\
\hline & & & & & & & \begin{tabular}{|l} 
1,4-Difluorobenzene \\
Chlorobenzene-d5
\end{tabular} & $\begin{array}{l}2500 \mathrm{ug} / \mathrm{mL} \\
2500 \mathrm{ug} / \mathrm{mL}\end{array}$ \\
\hline \multirow[t]{10}{*}{ VMSOMTRSUw_00109 } & \multirow[t]{10}{*}{$06 / 30 / 16$} & \multirow[t]{10}{*}{$05 / 31 / 16$} & \multirow[t]{10}{*}{ METHANOL， Lot 157266} & \multirow{10}{*}{4000 UL } & VMSOMSUas_00085 & $42 \mathrm{uL}$ & Chloroethane-d5 & $21 \mathrm{ug} / \mathrm{mL}$ \\
\hline & & & & & VMSOMSUbs_00146 & $336 \mathrm{uL}$ & $\begin{array}{l}\text { Vinyl Chloride-d3 } \\
\text { 2-Butanone-d5 } \\
\text {-Hexanone-d5 }\end{array}$ & $\begin{aligned} 21 \mathrm{ug} / \mathrm{mL} \\
210 \mathrm{ug} / \mathrm{mL} \\
210 \mathrm{ug} / \mathrm{mL}\end{aligned}$ \\
\hline & & & & & \multirow[t]{8}{*}{ VMSOMSUCS_00059 } & \multirow[t]{8}{*}{42 uL } & $1,1,2,2$-Tetrachloroethane-d2 & $21 \mathrm{ug} / \mathrm{mL}$ \\
\hline & & & & & & & 1,1-Dichloroethene-d2 & $21 \mathrm{ug} / \mathrm{mL}$ \\
\hline & & & & & & & 1,2-Dichlorobenzene-d4 & $\frac{21 \mathrm{ug} / \mathrm{mL}}{21 \mathrm{ug} / \mathrm{mL}}$ \\
\hline & & & & & & & $\frac{1,2 \text {-Dichloroethane-d4 }}{1,2 \text {-Dichloropropane-d6 }}$ & $\frac{21 \mathrm{ug} / \mathrm{mL}}{21 \mathrm{ug} / \mathrm{mL}}$ \\
\hline & & & & & & & $\frac{1,2-11 C n 10}{\text { Benzene-d6 }}$ & $21 \mathrm{ug} / \mathrm{mL}$ \\
\hline & & & & & & & Chloroform-d & $21 \mathrm{ug} / \mathrm{mL}$ \\
\hline & & & & & & & Toluene-d8 & $21 \mathrm{ug} / \mathrm{mL}$ \\
\hline & & & & & & & trans-1,3-Dichloropropene-d4 & $21 \mathrm{ug} / \mathrm{mL}$ \\
\hline .VMSOMSUas_00085 & $04 / 25 / 17$ & \multicolumn{3}{|c|}{ Absolute, Lot 082015} & \multicolumn{2}{|c|}{ (Purchased Reagent) } & $\begin{array}{l}\text { Chloroethane-d5 } \\
\text { Vinvl Chloride-d3 }\end{array}$ & $2000 \mathrm{ug} / \mathrm{mL}$ \\
\hline \multirow{2}{*}{.VMSOMSUbs_00146 } & \multirow{2}{*}{$05 / 31 / 17$} & \multirow{2}{*}{\multicolumn{3}{|c|}{ Absolute, Lot 071515}} & \multirow{2}{*}{\multicolumn{2}{|c|}{ (Purchased Reagent) }} & 2-Butanone-d5 & $2500 \mathrm{ug} / \mathrm{mL}$ \\
\hline & & & & & & & 2-Hexanone-d5 & $2500 \mathrm{ug} / \mathrm{mL}$ \\
\hline \multirow[t]{7}{*}{.VMSOMSUCs_00059 } & \multirow[t]{7}{*}{$01 / 16 / 17$} & \multirow{7}{*}{\multicolumn{3}{|c|}{ Absolute, Lot 011614}} & \multirow{7}{*}{\multicolumn{2}{|c|}{ (Purchased Reagent) }} & $1,1,2,2$-Tetrachloroethane-d2 & $2000 \mathrm{ug} / \mathrm{mL}$ \\
\hline & & & & & & & 1,1-Dichloroethene-d2 & $2000 \mathrm{ug} / \mathrm{mL}$ \\
\hline & & & & & & & $\begin{array}{l}\text { 1,2-Dichlorrobenzene-d4 } \\
\text { 1,2-Dichloroethane-d4 }\end{array}$ & $2000 \mathrm{uq} / \mathrm{mL}$ \\
\hline & & & & & & & 1,2-Dichloropropane-d6 & $2000 \mathrm{ug} / \mathrm{mL}$ \\
\hline & & & & & & & Benzene-d6 & $2000 \mathrm{ug} / \mathrm{mL}$ \\
\hline & & & & & & & Chloroform-d & $2000 \mathrm{ug} / \mathrm{mL}$ \\
\hline & & & & & & & $\begin{array}{l}\frac{T}{T o l u e n e-d 8} \\
\text { trans-1,3-Dichloropropene-d4 }\end{array}$ & $\frac{2000 \mathrm{ug} / \mathrm{mL}}{2000 \mathrm{ug} / \mathrm{mL}}$ \\
\hline
\end{tabular}


Lab Name: TestAmerica Burlington

Job No.: 200-34024-1

SDG No.: 34024

\begin{tabular}{|c|c|c|c|c|}
\hline $\begin{array}{c}\text { Reagent } \\
\text { Container }\end{array}$ & $\begin{array}{c}\text { Reagent } \\
\text { ID }\end{array}$ & $\begin{array}{c}\text { Reagent } \\
\text { Description }\end{array}$ & $\begin{array}{c}\text { Preparation } \\
\text { Date }\end{array}$ & $\begin{array}{c}\text { Expiration } \\
\text { Date }\end{array}$ \\
\hline 904333 & VMBEBW 00021 & BFB TUNE 25 PPM & $02 / 26 / 2016$ & $08 / 26 / 2016$ \\
\hline 932091 & VMSOMTẼSI_00113 & SOM TR ISTD 21 PPM & $05 / 17 / 2016$ & $06 / 17 / 2016$ \\
\hline 935531 & VMSOMTRCALW 00103 & SOM TR CAL $21-210$ PPM & $05 / 26 / 2016$ & $06 / 26 / 2016$ \\
\hline 936819 & VMSOMTRSUw_ō0109 & SOM TR $\quad$ DMC 21 PPM & $05 / 31 / 2016$ & $06 / 30 / 2016$ \\
\hline 938927 & VMSOMTRISW 00114 & SOM TR ISTD 21 PPM & $06 / 06 / 2016$ & $07 / 06 / 2016$ \\
\hline
\end{tabular}




\section{METHODOLOGY SUMMARY}

Laboratory: TestAmerica Laboratories

Location: South Burlington, Vermont
Project No:

SDG No: $\quad 34024$

VOA

Volatile Organics Trace - USEPA CLP SOM01.2 


\title{
CASE NARRATIVE
}

\section{Client: Argonne National Laboratory}

\author{
Project: York (200-34024)
}

\section{Report Number: 200-34024-1}

With the exceptions noted as flags or footnotes, standard analytical protocols were followed in the analysis of the samples and no problems were encountered or anomalies observed. In addition all laboratory quality control samples were within established control limits, with any exceptions noted below. Each sample was analyzed to achieve the lowest possible reporting limit within the constraints of the method. In some cases, due to interference or analytes present at high concentrations, samples were diluted. For diluted samples, the reporting limits are adjusted relative to the dilution required.

Calculations are performed before rounding to avoid round-off errors in calculated results.

All holding times were met and proper preservation noted for the methods performed on these samples, unless otherwise detailed in the individual sections below.

\section{RECEIPT}

The samples were received on 06/17/2016; the samples arrived in good condition, properly preserved and on ice. The temperature of the cooler at receipt was $4.9^{\circ} \mathrm{C}$.

\section{VOLATILE ORGANIC COMPOUNDS - TRACE}

Samples YKDL4-C2-145-W-38115, YKDL4-C1-120-W-38116, YKQCTB-W-38117 and VHBLK01 were analyzed for Volatile Organic Compounds - Trace in accordance with EPA SOW SOM01.2. The samples were analyzed on 06/21/2016.

Several analytes were detected in method blank MB 200-105993/4 at levels that were above the method detection limit but below the reporting limit. The values should be considered estimates, and have been flagged. If the associated sample reported a result above the $\mathrm{MDL}$ and/or RL, the result has been flagged. Refer to the QC report for details.

A non-target constituent that represents a compound related to the DMC formulation is present in the samples in this set. The derived results for that compound have been qualified with an "X" qualifier to reflect the source of the contamination.

2-Hexanone-d5 failed the surrogate recovery criteria high for YKDL4-C1-120-W-38116. 2-Hexanone-d5 failed the surrogate recovery criteria high for YKQCTB-W-38117. Refer to the QC report for details.

The closing continuing calibration verification (CCV) standard associated with batch 200-105993 failed to meet acceptance limits. The associated samples were re-analyzed following a successful CCV and produced similar results, indicating that the sample matrix is adversely affecting the instrument and causing the failures. Both closing CCVs are reported.

No additional analytical or quality issues were noted, other than those described above or in the Definitions/Glossary page. 
Lab Name: TestAmerica Burlington

Job No.: 200-34024-1

SDG No.: 34024

Instrument ID: CHD.i

Analysis Batch Number: 105151

Lab Sample ID: ICIS 200-105151/4

Client Sample ID:

Date Analyzed: 05/31/16 16:56

Lab File ID: 20227 04.D

GC Column: DB-624

ID $: 0.2(\mathrm{~mm})$

\begin{tabular}{|c|c|c|c|c|}
\hline \multirow[t]{2}{*}{ COMPOUND NAME } & \multirow{2}{*}{$\begin{array}{c}\text { RETENTION } \\
\text { TIME }\end{array}$} & \multicolumn{3}{|c|}{ MANUAL INTEGRATION } \\
\hline & & REASON & ANALYST & DATE \\
\hline 1,2-Dichloropropane-d6 & 6.32 & Split Peak & wilburj & $05 / 31 / 1617: 36$ \\
\hline
\end{tabular}

Lab Sample ID: IC 200-105151/5

Client Sample ID:

Date Analyzed: 05/31/16 17:21

Lab File ID: 20227 05.D

GC Column: DB-624

ID $: 0.2(\mathrm{~mm})$

\begin{tabular}{|c|c|c|c|c|}
\hline \multicolumn{1}{|c|}{ COMPOUND NAME } & RETENTION & \multicolumn{3}{|c}{ MANUAL INTEGRATION } \\
\cline { 3 - 5 } & TIME & REASON & ANALYST & DATE \\
\hline 1,2 -Dichloropropane-d6 & 6.32 & Split Peak & wilburj & 05/31/16 17:57 \\
\hline
\end{tabular}

Lab Sample ID: IC 200-105151/6

Client Sample ID:

Date Analyzed: 05/31/16 17:46

Lab File ID: 20227_06.D

GC Column: DB-624

ID $: 0.2(\mathrm{~mm})$

\begin{tabular}{|c|c|c|c|c|}
\hline \multicolumn{1}{|c|}{ COMPOUND NAME } & RETENTION & \multicolumn{3}{|c|}{ MANUAL INTEGRATION } \\
\cline { 3 - 5 } & TIME & REASON & ANALYST & DATE \\
\hline 1,2-Dichloropropane-d6 & 6.32 & Split Peak & wilburj & $05 / 31 / 16$ 18:30 \\
\hline
\end{tabular}


Lab Name: TestAmerica Burlington

Job No.: 200-34024-1

SDG No.: 34024

Instrument ID: CHD.i

Analysis Batch Number: 105993

Lab Sample ID: CCVIS 200-105993/3

Client Sample ID:

Date Analyzed: 06/21/16 12:23

Lab File ID: 20517 03.D

GC Column: DB-624

ID $: 0.2(\mathrm{~mm})$

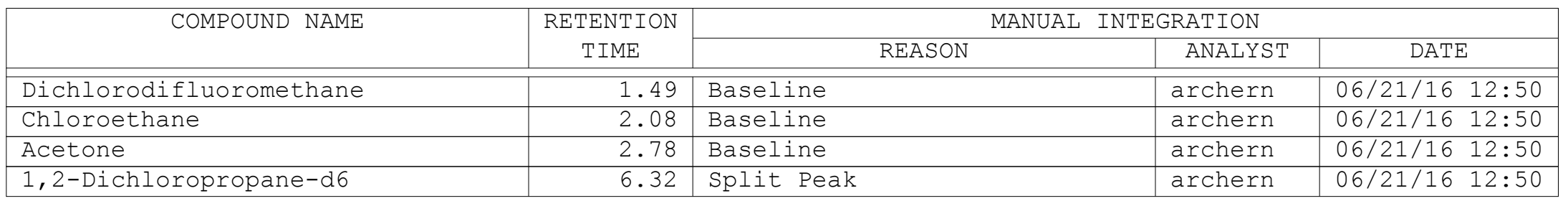

Lab Sample ID: CCVC 200-105993/20

Client Sample ID:

Date Analyzed: 06/21/16 20:11

Lab File ID: 20517_20.D

GC Column: DB-624

ID $: 0.2(\mathrm{~mm})$

\begin{tabular}{|l|c|c|c|c|}
\hline \multirow{2}{*}{ COMPOUND NAME } & RETENTION & \multicolumn{3}{|c}{ MANUAL INTEGRATION } \\
\cline { 3 - 5 } & TIME & REASON & ANALYST & DATE \\
\hline Dichlorodifluoromethane & 1.50 & Baseline & wilburj & $06 / 24 / 16$ 11:59 \\
\hline
\end{tabular}

Lab Sample ID: CCVC 200-105993/21

Client Sample ID:

Date Analyzed: 06/21/16 20:37

Lab File ID: 20517_21.D

GC Column: DB-624

ID: $0.2(\mathrm{~mm})$

\begin{tabular}{|c|c|c|c|c|}
\hline \multirow[t]{2}{*}{ COMPOUND NAME } & \multirow{2}{*}{$\begin{array}{c}\text { RETENTION } \\
\text { TIME }\end{array}$} & \multicolumn{3}{|c|}{ MANUAL INTEGRATION } \\
\hline & & REASON & ANALYST & DATE \\
\hline Dichlorodifluoromethane & 1.49 & Assign Peak & $\begin{array}{l}\text { puangmale } \\
\text { ek }\end{array}$ & $06 / 23 / 1611: 02$ \\
\hline
\end{tabular}




\section{DATA REPORTING QUALIFIERS}

Client: Argonne National Laboratory

Job Number: 200-34024-1

Sdg Number: 34024

Description

GC/MS VOA

B

U

$\mathrm{J}$

$J$

$\mathrm{X}$

*

B
Analyte was found in the associated method blank as well as in the sample.

Analyzed for but not detected.

Indicates an Estimated Value for TICs

Indicates an estimated value.

See case narrative notes for explanation of the ' $X$ ' flag

Surrogate is outside acceptance limits.

The analyte was found in an associated blank, as well as in the sample. 
Lab Name: TESTAMERICA BURLINGTON

Lab Code: STLV Case No.: YORK Mod. Ref No.: Level: (TRACE or LOW) TRACE
Contract: $8 \mathrm{E}-00302$

SDG No.: 34024

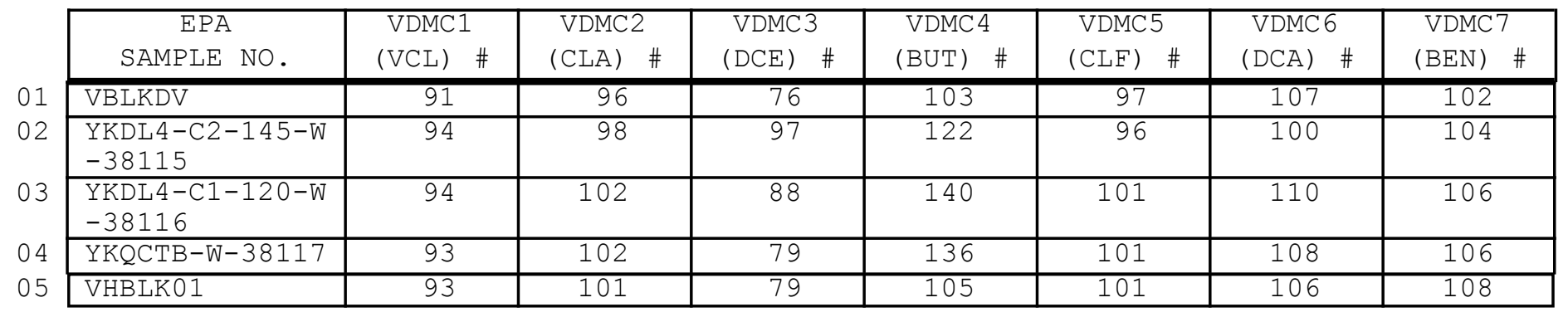

\footnotetext{
VDMC1 $(\mathrm{VCL})=$ Vinyl Chloride-d3

VDMC2 $(\mathrm{CLA})=$ Chloroethane-d5

VDMC3 $(\mathrm{DCE})=1,1-\mathrm{Dichloroethene-d2}$

VDMC4 $\quad(B U T)=2-$ Butanone-d5

VDMC5 $(\mathrm{CLF})=$ Chloroform-d

VDMC $6 \quad(\mathrm{DCA})=1,2-\mathrm{Dichloroethane-d4}$

$\operatorname{VDMC} 7 \quad(\mathrm{BEN})=$ Benzene-d6
}

$$
\begin{gathered}
\frac{\mathrm{QC} \text { LIMITS }}{(65-131)} \\
(71-131) \\
(55-104) \\
(49-155) \\
(78-121) \\
(78-129) \\
(77-124)
\end{gathered}
$$

\# Column to be used to flag recovery values

* Values outside of contract required QC limits

Page 1 of 1 
Lab Name: TESTAMERICA BURLINGTON

Lab Code: STLV Case No.: YORK Mod. Ref No.:
Contract: $8 \mathrm{E}-00302$

SDG No.: 34024

Level: (TRACE or LOW) TRACE

\begin{tabular}{|c|c|c|c|c|c|c|c|c|}
\hline $\begin{array}{c}\text { EPA } \\
\text { SAMPLE NO. }\end{array}$ & $\begin{array}{l}\text { VDMC 8 } \\
(\mathrm{DPA}) \quad \#\end{array}$ & $\begin{array}{l}\text { VDMC9 } \\
(\mathrm{TOL}) \quad \#\end{array}$ & $\begin{array}{l}\text { VDMC10 } \\
(\mathrm{TDP}) \quad \#\end{array}$ & $\begin{array}{l}\text { VDMC11 } \\
(\operatorname{HEX}) \quad \#\end{array}$ & $\begin{array}{l}\text { VDMC12 } \\
(\mathrm{TCA}) \quad \#\end{array}$ & $\begin{array}{l}\text { VDMC13 } \\
(\mathrm{DCZ}) \quad \#\end{array}$ & OTHER & $\begin{array}{l}\text { TOT } \\
\text { OUT }\end{array}$ \\
\hline VBLKDV & 101 & 102 & 93 & 106 & 96 & 123 & & 0 \\
\hline $\begin{array}{l}\text { YKDL } 4-C 2-145-W \\
-38115\end{array}$ & 100 & 102 & 89 & 124 & 90 & 113 & & 0 \\
\hline $\begin{array}{l}\text { YKDL } 4-C 1-120-W \\
-38116\end{array}$ & 103 & 103 & $\overline{96}$ & $137 \star$ & 99 & 120 & & 1 \\
\hline YKQCTB-W-38117 & 102 & 104 & 98 & $141 \star$ & 101 & 116 & & 1 \\
\hline VHBLK01 & 103 & 108 & 94 & 104 & 98 & 128 & & 0 \\
\hline
\end{tabular}

\footnotetext{
VDMC8 $(\mathrm{DPA})=1,2-\mathrm{Dichloropropane}-\mathrm{d} 6$

VDMC $9 \quad($ TOL $)=$ Toluene-d8

VDMC10 $(\mathrm{TDP})=$ trans $-1,3-\mathrm{Dichloropropene-d4}$

$\operatorname{VDMC1} 1 \mathrm{HEX})=2-$ Hexanone-d5

VDMC12 $(\mathrm{TCA})=1,1,2,2$-Tetrachloroethane-d2

VDMC13 $(\mathrm{DCZ})=1,2-\mathrm{Dichlorobenzene-d4}$
}

\footnotetext{
QC LIMITS

$(79-124)$

$(77-121)$

$(73-121)$

$(28-135)$

$(73-125)$

$(80-131)$
}

\# Column to be used to flag recovery values

* Values outside of contract required QC limits

Report 1,4-Dioxane-d8 for Low-Medium VOA analysis only

Page 1 of 1 
$4 A$ - FORM IV VOA

VOLATILE METHOD BLANK SUMMARY
EPA SAMPLE NO.

VBLKDV

Lab Name: TESTAMERICA BURLINGTON

Contract: $8 \mathrm{E}-00302$

Lab Code: STLV Case No.: YORK Mod. Ref No.: SDG No.: 34024

Lab File ID: 20517_04.D

Lab Sample ID: MB 200-105993/4

Instrument ID: CHD.i

Matrix: (SOIL/SED/WATER) Water

Date Analyzed: 06/21/2016

Level: (TRACE or LOW/MED) TRACE

Time Analyzed: 1249

GC Column: DB-624

ID $: 0.20 \quad(\mathrm{~mm})$

Heated Purge: (Y/N) N

\begin{tabular}{|c|c|c|c|}
\hline $\begin{array}{c}\text { EPA } \\
\text { SAMPLE NO. }\end{array}$ & $\begin{array}{r}\text { LAB } \\
\text { SAMPLE ID }\end{array}$ & $\begin{array}{c}\text { LAB } \\
\text { FILE ID }\end{array}$ & $\begin{array}{c}\text { TIME } \\
\text { ANALYZED }\end{array}$ \\
\hline $\begin{array}{l}\text { YKDL } 4-C 2-145 \\
-W-38115\end{array}$ & $200-34024-1$ & 20517_05.D & 1331 \\
\hline $\begin{array}{l}\text { YKDL } 4-C 1-120 \\
-W-38116\end{array}$ & $200-34024-2$ & 20517 _06.D & 1356 \\
\hline $\begin{array}{l}\text { YKQCTB-W-381 } \\
17\end{array}$ & $200-34024-3$ & $20517 \_07 . \mathrm{D}$ & 1421 \\
\hline VHBLK01 & $200-34024-4$ & 20517 _08.D & 1447 \\
\hline
\end{tabular}

COMMENTS :

Page 1 of 1 
$5 A$ - FORM V VOA

VOLATILE ORGANICS INSTRUMENT

PERFORMANCE CHECK

BROMOFLUOROBENZENE (BFB)
EPA SAMPLE NO.

BFBDJ
Lab Name: TESTAMERICA BURLINGTON

Lab Code: STLV

Case No.: YORK

Lab File Id: 20227_01.D

Instrument Id: CHD.i

GC Column: DB-624

ID $: 0.20$
Contract: 8E-00302

Mod. Ref No.:

SDG No.: 34024

BEB Injection Date: 05/31/2016

BFB Injection Time: 1555 $(\mathrm{mm})$

\begin{tabular}{|c|c|c|}
\hline $\mathrm{m} / \mathrm{e}$ & ION ABUNDANCE CRITERIA & $\begin{array}{l}\text { \% RELATIVE } \\
\text { ABUNDANCE }\end{array}$ \\
\hline 50 & $15.0-40.0 \%$ of mass 95 & 16.9 \\
\hline 75 & $30.0-80.0 \%$ of mass 95 & 50.0 \\
\hline 95 & Base peak, $100 \%$ relative abundance & 100 \\
\hline 96 & $5.0-9.0 \%$ of mass 95 & 6.6 \\
\hline 173 & Less than $2.0 \%$ of mass 174 & $0) 1$ \\
\hline 174 & $50.0-120 \%$ of mass 95 & 90.0 \\
\hline 175 & $5.0-9.0 \%$ of mass 174 & $7.2) 1$ \\
\hline 176 & $95.0-101 \%$ of mass 174 & $87.1(96.8) 1$ \\
\hline 177 & $5.0-9.0 \%$ of mass 176 & $(6.6) 2$ \\
\hline
\end{tabular}

1 - Value is omass 1742 - Value is mass 176

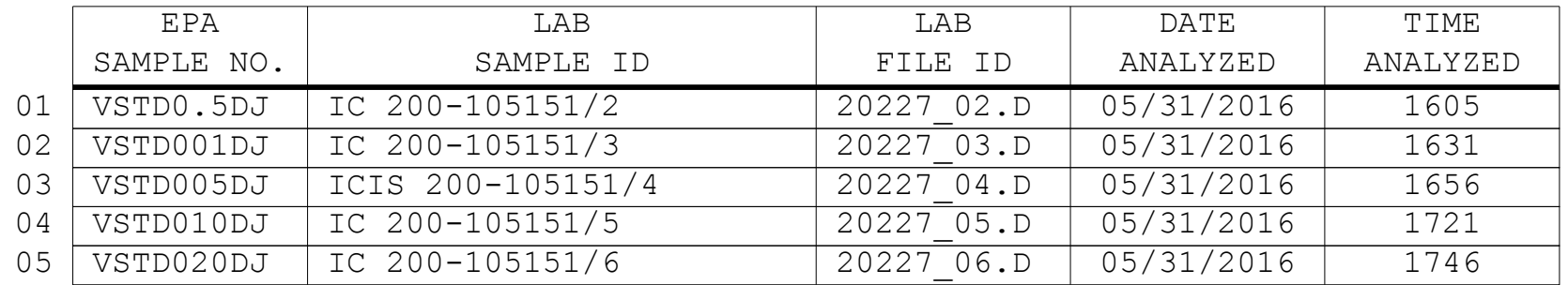


$5 A$ - FORM V VOA

VOLATILE ORGANICS INSTRUMENT

PERFORMANCE CHECK

BROMOFLUOROBENZENE (BFB)
EPA SAMPLE NO.

BFBDV
Lab Name: TESTAMERICA BURLINGTON

Lab Code: STLV Case No.: YORK

Lab File Id: 20517_01.D

Instrument Id: CHD.i

GC Column: DB-624

ID $: 0.20$
Contract: 8E-00302 Mod. Ref No.: SDG No.: 34024

BFB Injection Date: 06/21/2016

BFB Injection Time: 1136 $(\mathrm{mm})$

\begin{tabular}{|c|c|c|}
\hline $\mathrm{m} / \mathrm{e}$ & ION ABUNDANCE CRITERIA & $\begin{array}{l}\text { \% RELATIVE } \\
\text { ABUNDANCE }\end{array}$ \\
\hline 50 & $15.0-40.0 \%$ of mass 95 & 17.7 \\
\hline 75 & $30.0-80.0 \%$ of mass 95 & 51.6 \\
\hline 95 & Base peak, $100 \%$ relative abundance & 100 \\
\hline 96 & $5.0-9.0 \%$ of mass 95 & 6.7 \\
\hline 173 & Less than $2.0 \%$ of mass 174 & $1.0) 1$ \\
\hline 174 & $50.0-120 \%$ of mass 95 & 91.2 \\
\hline 175 & $5.0-9.0 \%$ of mass 174 & $6.5(7.1) 1$ \\
\hline 176 & $95.0-101 \%$ of mass 174 & $87.5(95.9) 1$ \\
\hline 177 & $5.0-9.0 \%$ of mass 176 & $(6.2) 2$ \\
\hline
\end{tabular}

1 - Value is omass 174

2 - Value is omass 176

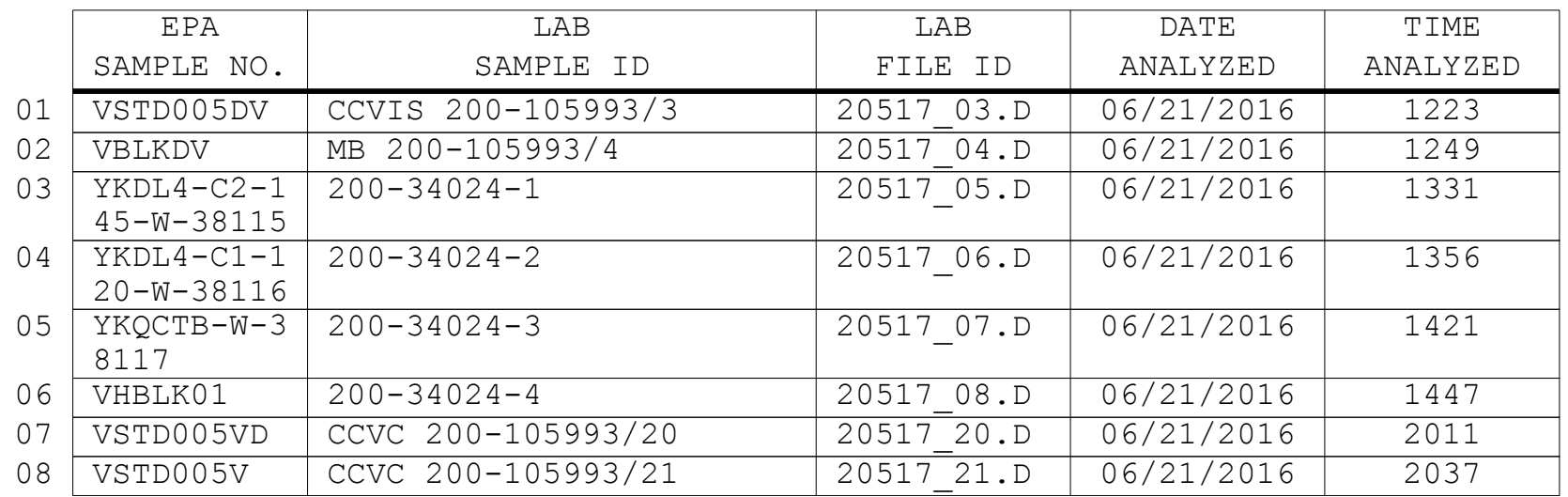




\section{$8 A$ - FORM VIII VOA}

VOLATILE INTERNAL STANDARD AREA AND RETENTION TIME SUMMARY

Lab Name: TESTAMERICA BURLINGTON

Contract: $8 \mathrm{E}-00302$

Lab Code: STLV Case No.: YORK Mod. Ref No.:

SDG No.: 34024

GC Column: DB-624

ID: 0.20 (mm) Init. Calib. Date (s): 05/31/2016 05/31/2016

EPA Sample No.(VSTD\#\#\#\#\#) : VSTD005DV

Date Analyzed: 06/21/2016

Lab File ID (Standard): 20517_03.D

Time Analyzed: 1223

Instrument ID: CHD.i

Heated Purge: ( $\mathrm{Y} / \mathrm{N}) \mathrm{N}$

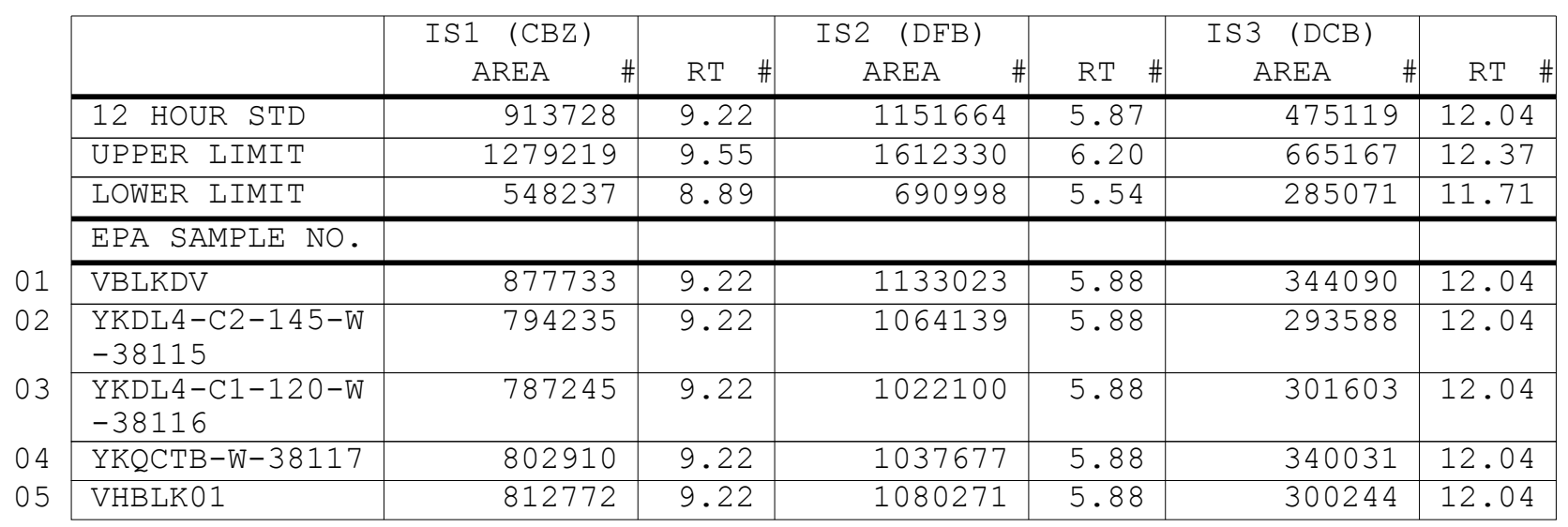

IS1 $(\mathrm{CBZ})=$ Chlorobenzene-d5

IS2 $(\mathrm{DFB})=1,4-\mathrm{Difluorobenzene}$

IS3 $(\mathrm{DCB})=1,4-\mathrm{Dichlorobenzene-d4}$

AREA UPPER LIMIT $=140 \%$ (Trace Volatiles) of internal standard area AREA LOWER LIMIT $=60 \%$ (Trace Volatiles) of internal standard area RT UPPER LIMIT $=+0.33$ (Trace Volatiles) minutes of internal standard RT RT LOWER LIMIT $=-0.33$ (Trace Volatiles) minutes of internal standard RT

\# Column used to flag values outside contract required QC limits with an asterisk. Page 1 of 1 
Lab Name: TESTAMERICA BURLINGTON

Contract: 8E-00302

Lab Code: STLV

Case No.: YORK Mod. Ref No.:

SDG No.: 34024

Matrix: (SOIL/SED/WATER) Water

Sample wt/vol: 25.0 $(\mathrm{g} / \mathrm{mL}) \mathrm{mL}$

Level: (TRACE/LOW/MED) TRACE

\% Moisture: not dec.

GC Column: DB-624 ID: $0.20 \quad(\mathrm{~mm})$

Soil Extract Volume: (uL) $(\mathrm{mL})$

Purge Volume: 25.0
Lab Sample ID: 200-34024-2

Lab File ID: 20517_06.D

Date Received: 06/17/2016

Date Analyzed: 06/21/2016

Dilution Factor: 1.0

Soil Aliquot Volume: (uL)

\begin{tabular}{|c|c|c|c|}
\hline CAS NO. & COMPOUND & $\begin{array}{l}\text { CONCENTRATION UNITS: } \\
(\mathrm{ug} / \mathrm{L} \text { or } \mathrm{ug} / \mathrm{kg}) \mathrm{ug} / \mathrm{L}\end{array}$ & Q \\
\hline $75-71-8$ & Dichlorodifluoromethane & 1.0 & $\mathrm{U}$ \\
\hline $74-87-3$ & Chloromethane & 1.0 & $\mathrm{U}$ \\
\hline $75-01-4$ & Vinyl chloride & 1.0 & $\mathrm{U}$ \\
\hline $74-83-9$ & Bromomethane & 1.0 & $\mathrm{U}$ \\
\hline $75-00-3$ & Chloroethane & 1.0 & $\mathrm{U}$ \\
\hline $75-69-4$ & Trichlorofluoromethane & 0.22 & $\mathrm{~J}$ \\
\hline $75-35-4$ & 1,1-Dichloroethene & 1.8 & \\
\hline $76-13-1$ & $1,1,2$-Trichloro-1,2,2-trifluoroethane & 1.0 & $\mathrm{U}$ \\
\hline $67-64-1$ & Acetone & 5.0 & $\mathrm{U}$ \\
\hline $75-15-0$ & Carbon disulfide & 0.71 & $\mathrm{~J} B$ \\
\hline $79-20-9$ & Methyl acetate & 1.0 & $\mathrm{U}$ \\
\hline $75-09-2$ & Methylene Chloride & 1.0 & $\mathrm{U}$ \\
\hline $156-60-5$ & trans-1,2-Dichloroethene & 1.0 & $\mathrm{U}$ \\
\hline $1634-04-4$ & Methyl tert-butyl ether & 1.0 & $\mathrm{U}$ \\
\hline $75-34-3$ & 1,1-Dichloroethane & 1.0 & $\mathrm{U}$ \\
\hline $156-59-2$ & cis-1,2-Dichloroethene & 1.0 & $\mathrm{U}$ \\
\hline $78-93-3$ & 2-Butanone & 5.0 & $\mathrm{U}$ \\
\hline $74-97-5$ & Bromochloromethane & 1.0 & $\mathrm{U}$ \\
\hline $67-66-3$ & Chloroform & 1.0 & $\mathrm{U}$ \\
\hline $71-55-6$ & $1,1,1$-Trichloroethane & 0.70 & $\mathrm{~J}$ \\
\hline $110-82-7$ & Cyclohexane & 1.0 & $\mathrm{U}$ \\
\hline $56-23-5$ & Carbon tetrachloride & 0.18 & $\mathrm{~J}$ \\
\hline $71-43-2$ & Benzene & 1.0 & $\mathrm{U}$ \\
\hline $107-06-2$ & 1,2-Dichloroethane & 1.0 & $\mathrm{U}$ \\
\hline
\end{tabular}

Report 1,4-Dioxane for Low-Medium VOA analysis only 
Lab Name: TESTAMERICA BURLINGTON

Lab Code: STLV

Case No.: YORK

Mod. Ref No.:

Contract: $8 \mathrm{E}-00302$

Matrix: (SOIL/SED/WATER) Water

Sample wt/vol: 25.0 $(\mathrm{g} / \mathrm{mL}) \mathrm{mL}$

Level: (TRACE/LOW/MED) TRACE

\% Moisture: not dec.

GC Column: DB-624 ID: $0.20 \quad(\mathrm{~mm})$

Soil Extract Volume: (uL) $(\mathrm{mL})$

Purge Volume: 25.0
SDG No.: 34024

Lab Sample ID: 200-34024-2

Lab File ID: 20517_06.D

Date Received: 06/17/2016

Date Analyzed: 06/21/2016

Dilution Factor: 1.0

Soil Aliquot Volume: (uL) (mI)

\begin{tabular}{|c|c|c|c|}
\hline CAS NO. & COMPOUND & $\begin{array}{l}\text { CONCENTRATION UNITS: } \\
(\mathrm{ug} / \mathrm{L} \text { or } \mathrm{ug} / \mathrm{kg}) \mathrm{ug} / \mathrm{L}\end{array}$ & Q \\
\hline $79-01-6$ & Trichloroethene & 0.010 & $\mathrm{~J} B$ \\
\hline $108-87-2$ & Methylcyclohexane & 1.0 & $\mathrm{U}$ \\
\hline $78-87-5$ & 1,2-Dichloropropane & 1.0 & $\mathrm{U}$ \\
\hline $75-27-4$ & Bromodichloromethane & 1.0 & $\mathrm{U}$ \\
\hline $10061-01-5$ & cis-1,3-Dichloropropene & 1.0 & U \\
\hline $108-10-1$ & 4-Methyl-2-pentanone & 1.0 & $\mathrm{U}$ \\
\hline $108-88-3$ & Toluene & 0.063 & $\mathrm{~J} B$ \\
\hline $10061-02-6$ & trans-1,3-Dichloropropene & 1.0 & $\mathrm{U}$ \\
\hline $79-00-5$ & $1,1,2-$ Trichloroethane & 1.0 & $\mathrm{U}$ \\
\hline $127-18-4$ & Tetrachloroethene & 0.29 & $\mathrm{~J}$ \\
\hline $591-78-6$ & 2-Hexanone & 1.0 & $\mathrm{U}$ \\
\hline $124-48-1$ & Dibromochloromethane & 1.0 & $\mathrm{U}$ \\
\hline $106-93-4$ & 1,2-Dibromoethane & 1.0 & $\mathrm{U}$ \\
\hline $108-90-7$ & Chlorobenzene & 1.0 & $\mathrm{U}$ \\
\hline $100-41-4$ & Ethylbenzene & 1.0 & $\mathrm{U}$ \\
\hline $95-47-6$ & o-Xylene & 1.0 & $\mathrm{U}$ \\
\hline $179601-23-1$ & m,p-xylene & 0.011 & $\mathrm{~J} B$ \\
\hline $100-42-5$ & Styrene & 1.0 & $\mathrm{U}$ \\
\hline $75-25-2$ & Bromoform & 1.0 & $\mathrm{U}$ \\
\hline $98-82-8$ & Isopropylbenzene & 1.0 & $\mathrm{U}$ \\
\hline $79-34-5$ & $1,1,2,2$-Tetrachloroethane & 1.0 & $\mathrm{U}$ \\
\hline $541-73-1$ & 1,3 -Dichlorobenzene & 1.0 & $\mathrm{U}$ \\
\hline $106-46-7$ & 1,4-Dichlorobenzene & 1.0 & $\mathrm{U}$ \\
\hline $95-50-1$ & 1,2-Dichlorobenzene & 1.0 & $\mathrm{U}$ \\
\hline $96-12-8$ & 1,2-Dibromo-3-Chloropropane & 1.0 & $\mathrm{U}$ \\
\hline $120-82-1$ & $1,2,4$-Trichlorobenzene & 1.0 & $\mathrm{U}$ \\
\hline $87-61-6$ & $1,2,3$-Trichlorobenzene & 1.0 & $\mathrm{U}$ \\
\hline
\end{tabular}


Lab Name: TESTAMERICA BURLINGTON

Contract: $8 \mathrm{E}-00302$

Lab Code: STLV Case No.: YORK Mod. Ref No.: SDG No.: 34024

Matrix: (SOIL/SED/WATER) Water

Sample wt/vol: 25.0 $(\mathrm{g} / \mathrm{mL}) \mathrm{mL}$

Level: (TRACE or LOW/MED) TRACE

\% Moisture: not dec.

GC Column: DB-624 ID $: 0.20 \quad(\mathrm{~mm})$

Soil Extract Volume: (uL)

CONCENTRATION UNITS: (ug/L or ug/kg) ug/L
Lab Sample ID: 200-34024-2

Lab File ID: 20517_06.D

Date Received: 06/17/2016

Date Analyzed: 06/21/2016

Dilution Factor: 1.0

Soil Aliquot Volume: (uL) Purge Volume: 25.0 $(\mathrm{mL})$

01

02

\begin{tabular}{|l|l|c|c|c|}
\hline CAS NUMBER & \multicolumn{1}{|c|}{ COMPOUND NAME } & RT & EST. CONC. & Q \\
\hline & Unknown & 7.20 & 2.7 & X B J \\
\hline E9667961 & Total Alkanes & N/A & & \\
\hline
\end{tabular}

1 EPA-designated Registry Number. 
Lab Name: TESTAMERICA BURLINGTON

Contract: 8E-00302

Lab Code: STLV

Case No.: YORK Mod. Ref No.:

SDG No.: 34024

Matrix: (SOIL/SED/WATER) Water

Sample wt/vol: 25.0 $(\mathrm{g} / \mathrm{mL}) \mathrm{mL}$

Level: (TRACE/LOW/MED) TRACE

\% Moisture: not dec.

GC Column: DB-624 ID: $0.20 \quad(\mathrm{~mm})$

Soil Extract Volume: $(u L)$ $(\mathrm{mL})$

Purge Volume: 25.0
Lab Sample ID: 200-34024-1

Lab File ID: 20517_05.D

Date Received: 06/17/2016

Date Analyzed: 06/21/2016

Dilution Factor: 1.0

Soil Aliquot Volume:

(uL)

\begin{tabular}{|c|c|c|c|}
\hline CAS NO. & COMPOUND & $\begin{array}{l}\text { CONCENTRATION UNITS: } \\
(\mathrm{ug} / \mathrm{L} \text { or } \mathrm{ug} / \mathrm{kg}) \mathrm{ug} / \mathrm{L}\end{array}$ & Q \\
\hline $75-71-8$ & Dichlorodifluoromethane & 1.0 & $\mathrm{U}$ \\
\hline $74-87-3$ & Chloromethane & 1.0 & $\mathrm{U}$ \\
\hline $75-01-4$ & Vinyl chloride & 1.0 & $\mathrm{U}$ \\
\hline $74-83-9$ & Bromomethane & 1.0 & $\mathrm{U}$ \\
\hline $75-00-3$ & Chloroethane & 1.0 & $\mathrm{U}$ \\
\hline $75-69-4$ & Trichlorofluoromethane & 0.16 & $\mathrm{~J}$ \\
\hline $75-35-4$ & 1,1-Dichloroethene & 3.9 & \\
\hline $76-13-1$ & $1,1,2$-Trichloro-1,2,2-trifluoroethane & 1.0 & $\mathrm{U}$ \\
\hline $67-64-1$ & Acetone & 5.0 & $\mathrm{U}$ \\
\hline $75-15-0$ & Carbon disulfide & 0.40 & $\mathrm{~J} B$ \\
\hline $79-20-9$ & Methyl acetate & 1.0 & $\mathrm{U}$ \\
\hline $75-09-2$ & Methylene Chloride & 1.0 & $\mathrm{U}$ \\
\hline $156-60-5$ & trans-1,2-Dichloroethene & 1.0 & $\mathrm{U}$ \\
\hline $1634-04-4$ & Methyl tert-butyl ether & 1.0 & $\mathrm{U}$ \\
\hline $75-34-3$ & 1,1-Dichloroethane & 1.0 & $\mathrm{U}$ \\
\hline $156-59-2$ & cis-1,2-Dichloroethene & 1.0 & $\mathrm{U}$ \\
\hline $78-93-3$ & 2-Butanone & 5.0 & $\mathrm{U}$ \\
\hline $74-97-5$ & Bromochloromethane & 1.0 & $\mathrm{U}$ \\
\hline $67-66-3$ & Chloroform & 1.0 & $\mathrm{U}$ \\
\hline $71-55-6$ & 1,1,1-Trichloroethane & 2.0 & \\
\hline $110-82-7$ & Cyclohexane & 1.0 & $\mathrm{U}$ \\
\hline $56-23-5$ & Carbon tetrachloride & 0.22 & $\mathrm{~J}$ \\
\hline $71-43-2$ & Benzene & 1.0 & $\mathrm{U}$ \\
\hline $107-06-2$ & 1,2-Dichloroethane & 1.0 & $\mathrm{U}$ \\
\hline
\end{tabular}

Report 1,4-Dioxane for Low-Medium VOA analysis only 
Lab Name: TESTAMERICA BURLINGTON

Lab Code: STLV

Case No.: YORK

Mod. Ref No.:

Contract: $8 \mathrm{E}-00302$

Matrix: (SOIL/SED/WATER) Water

Sample wt/vol: 25.0 $(\mathrm{g} / \mathrm{mL}) \mathrm{mL}$

Level: (TRACE/LOW/MED) TRACE

\% Moisture: not dec.

GC Column: DB-624 ID: $0.20 \quad(\mathrm{~mm})$

Soil Extract Volume: (uL) $(\mathrm{mL})$

Purge Volume: 25.0
SDG No.: 34024

Lab Sample ID: 200-34024-1

Lab File ID: 20517_05.D

Date Received: 06/17/2016

Date Analyzed: 06/21/2016

Dilution Factor: 1.0

Soil Aliquot Volume: (uL) (mI)

\begin{tabular}{|c|c|c|c|}
\hline CAS NO. & COMPOUND & $\begin{array}{l}\text { CONCENTRATION UNITS: } \\
(\mathrm{ug} / \mathrm{L} \text { or } \mathrm{ug} / \mathrm{kg}) \mathrm{ug} / \mathrm{L}\end{array}$ & Q \\
\hline $79-01-6$ & Trichloroethene & 0.017 & $\mathrm{~J} B$ \\
\hline $108-87-2$ & Methylcyclohexane & 1.0 & $\mathrm{U}$ \\
\hline $78-87-5$ & 1,2-Dichloropropane & 1.0 & $\mathrm{U}$ \\
\hline $75-27-4$ & Bromodichloromethane & 1.0 & $\mathrm{U}$ \\
\hline $10061-01-5$ & cis-1,3-Dichloropropene & 1.0 & U \\
\hline $108-10-1$ & 4-Methyl-2-pentanone & 1.0 & $\mathrm{U}$ \\
\hline $108-88-3$ & Toluene & 0.19 & $\mathrm{~J} B$ \\
\hline $10061-02-6$ & trans-1,3-Dichloropropene & 1.0 & $\mathrm{U}$ \\
\hline $79-00-5$ & $1,1,2-$ Trichloroethane & 1.0 & $\mathrm{U}$ \\
\hline $127-18-4$ & Tetrachloroethene & 0.61 & $\mathrm{~J}$ \\
\hline $591-78-6$ & 2-Hexanone & 1.0 & $\mathrm{U}$ \\
\hline $124-48-1$ & Dibromochloromethane & 1.0 & $\mathrm{U}$ \\
\hline $106-93-4$ & 1,2-Dibromoethane & 1.0 & $\mathrm{U}$ \\
\hline $108-90-7$ & Chlorobenzene & 1.0 & $\mathrm{U}$ \\
\hline $100-41-4$ & Ethylbenzene & 1.0 & $\mathrm{U}$ \\
\hline $95-47-6$ & o-Xylene & 1.0 & $\mathrm{U}$ \\
\hline $179601-23-1$ & m,p-xylene & 0.010 & $\mathrm{~J} B$ \\
\hline $100-42-5$ & Styrene & 1.0 & $\mathrm{U}$ \\
\hline $75-25-2$ & Bromoform & 1.0 & $\mathrm{U}$ \\
\hline $98-82-8$ & Isopropylbenzene & 1.0 & $\mathrm{U}$ \\
\hline $79-34-5$ & $1,1,2,2$-Tetrachloroethane & 1.0 & $\mathrm{U}$ \\
\hline $541-73-1$ & 1,3 -Dichlorobenzene & 1.0 & $\mathrm{U}$ \\
\hline $106-46-7$ & 1,4-Dichlorobenzene & 1.0 & $\mathrm{U}$ \\
\hline $95-50-1$ & 1,2-Dichlorobenzene & 1.0 & $\mathrm{U}$ \\
\hline $96-12-8$ & 1,2-Dibromo-3-Chloropropane & 1.0 & $\mathrm{U}$ \\
\hline $120-82-1$ & $1,2,4$-Trichlorobenzene & 1.0 & $\mathrm{U}$ \\
\hline $87-61-6$ & $1,2,3$-Trichlorobenzene & 1.0 & $\mathrm{U}$ \\
\hline
\end{tabular}


Lab Name: TESTAMERICA BURLINGTON

Contract: $8 \mathrm{E}-00302$

Lab Code: STLV Case No.: YORK Mod. Ref No.: SDG No.: 34024

Matrix: (SOIL/SED/WATER) Water

Sample wt/vol: 25.0 $(\mathrm{g} / \mathrm{mL}) \mathrm{mL}$

Level: (TRACE or LOW/MED) TRACE

․ Moisture: not dec.

GC Column: DB-624 ID $: 0.20 \quad(\mathrm{~mm})$

Soil Extract Volume: (uL)
Lab Sample ID: 200-34024-1

Lab File ID: 20517_05.D

Date Received: 06/17/2016

Date Analyzed: 06/21/2016

Dilution Factor: 1.0

Soil Aliquot Volume: (uL) Purge Volume: 25.0 $(\mathrm{mL})$

$\overline{(\mathrm{mL})}$
CONCENTRATION UNITS: (ug/L or ug/kg) ug/L

01

02

\begin{tabular}{|l|l|c|c|c|}
\hline CAS NUMBER & \multicolumn{1}{|c|}{ COMPOUND NAME } & RT & EST. CONC. & Q \\
\hline & Unknown & 7.20 & 2.4 & X B J \\
\hline E9667961 & Total Alkanes & N/A & & \\
\hline
\end{tabular}

1EPA-designated Registry Number. 
Lab Name: TESTAMERICA BURLINGTON

Contract: $8 \mathrm{E}-00302$

Lab Code: STLV

Case No.: YORK Mod. Ref No.:

SDG No.: 34024

Matrix: (SOIL/SED/WATER) Water

Sample wt/vol: 25.0 $(\mathrm{g} / \mathrm{mL}) \mathrm{mL}$

Level: (TRACE/LOW/MED) TRACE

\% Moisture: not dec.

GC Column: DB-624 ID: $0.20 \quad(\mathrm{~mm})$

Soil Extract Volume: (uL) $(\mathrm{mL})$

Purge Volume: 25.0
Lab Sample ID: 200-34024-3

Lab File ID: 20517_07.D

Date Received: 06/17/2016

Date Analyzed: 06/21/2016

Dilution Factor: 1.0

Soil Aliquot Volume: (uL)

\begin{tabular}{|c|c|c|c|}
\hline CAS NO. & COMPOUND & $\begin{array}{l}\text { CONCENTRATION UNITS: } \\
(\mathrm{ug} / \mathrm{L} \text { or } \mathrm{ug} / \mathrm{kg}) \mathrm{ug} / \mathrm{L}\end{array}$ & $Q$ \\
\hline $75-71-8$ & Dichlorodifluoromethane & 1.0 & $\mathrm{U}$ \\
\hline $74-87-3$ & Chloromethane & 0.054 & $\mathrm{~J} B$ \\
\hline $75-01-4$ & Vinyl chloride & 1.0 & $\mathrm{U}$ \\
\hline $74-83-9$ & Bromomethane & 0.060 & $\mathrm{~J}$ \\
\hline $75-00-3$ & Chloroethane & 1.0 & $\mathrm{U}$ \\
\hline $75-69-4$ & Trichlorofluoromethane & 1.0 & $\mathrm{U}$ \\
\hline $75-35-4$ & 1,1-Dichloroethene & 1.0 & $\mathrm{U}$ \\
\hline $76-13-1$ & $1,1,2$-Trichloro-1,2,2-trifluoroethane & 1.0 & $\mathrm{U}$ \\
\hline $67-64-1$ & Acetone & 4.9 & $\mathrm{~J}$ \\
\hline $75-15-0$ & Carbon disulfide & 1.0 & $\mathrm{U}$ \\
\hline $79-20-9$ & Methyl acetate & 1.0 & $\mathrm{U}$ \\
\hline $75-09-2$ & Methylene Chloride & 0.020 & $\mathrm{~J} B$ \\
\hline $156-60-5$ & trans-1,2-Dichloroethene & 1.0 & $\mathrm{U}$ \\
\hline $1634-04-4$ & Methyl tert-butyl ether & 1.0 & $\mathrm{U}$ \\
\hline $75-34-3$ & 1,1-Dichloroethane & 1.0 & $\mathrm{U}$ \\
\hline $156-59-2$ & cis-1,2-Dichloroethene & 1.0 & $\mathrm{U}$ \\
\hline $78-93-3$ & 2-Butanone & 5.0 & $\mathrm{U}$ \\
\hline $74-97-5$ & Bromochloromethane & 1.0 & $\mathrm{U}$ \\
\hline $67-66-3$ & Chloroform & 1.0 & $\mathrm{U}$ \\
\hline $71-55-6$ & $1,1,1$-Trichloroethane & 1.0 & $\mathrm{U}$ \\
\hline $110-82-7$ & Cyclohexane & 1.0 & $\mathrm{U}$ \\
\hline $56-23-5$ & Carbon tetrachloride & 1.0 & $\mathrm{U}$ \\
\hline $71-43-2$ & Benzene & 0.049 & $\mathrm{~J}$ \\
\hline $107-06-2$ & 1,2-Dichloroethane & 1.0 & $\mathrm{U}$ \\
\hline
\end{tabular}

Report 1,4-Dioxane for Low-Medium VOA analysis only 
Lab Name: TESTAMERICA BURLINGTON

Contract: $8 \mathrm{E}-00302$

Lab Code: STLV

Case No.: YORK

Mod. Ref No.:

SDG No.: 34024

Matrix: (SOIL/SED/WATER) Water

Sample wt/vol: 25.0 $(\mathrm{g} / \mathrm{mL}) \mathrm{mL}$

Level: (TRACE/LOW/MED) TRACE

\% Moisture: not dec.

GC Column: DB-624 ID: $0.20 \quad(\mathrm{~mm})$

Soil Extract Volume: (uL) $(\mathrm{mL})$

Purge Volume: 25.0 (mI)
Lab Sample ID: 200-34024-3

Lab File ID: 20517_07.D

Date Received: 06/17/2016

Date Analyzed: 06/21/2016

Dilution Factor: 1.0

Soil Aliquot Volume: (uL)

\begin{tabular}{|c|c|c|c|}
\hline CAS NO. & COMPOUND & $\begin{array}{l}\text { CONCENTRATION UNITS: } \\
(\mathrm{ug} / \mathrm{L} \text { or } \mathrm{ug} / \mathrm{kg}) \mathrm{ug} / \mathrm{L}\end{array}$ & Q \\
\hline $79-01-6$ & Trichloroethene & 1.0 & $\mathrm{U}$ \\
\hline $108-87-2$ & Methylcyclohexane & 1.0 & $\mathrm{U}$ \\
\hline $78-87-5$ & 1,2-Dichloropropane & 1.0 & $\mathrm{U}$ \\
\hline $75-27-4$ & Bromodichloromethane & 1.0 & $\mathrm{U}$ \\
\hline $10061-01-5$ & cis-1,3-Dichloropropene & 1.0 & U \\
\hline $108-10-1$ & 4-Methyl-2-pentanone & 1.0 & $\mathrm{U}$ \\
\hline $108-88-3$ & Toluene & 0.35 & $\mathrm{~J} B$ \\
\hline $10061-02-6$ & trans-1,3-Dichloropropene & 1.0 & $\mathrm{U}$ \\
\hline $79-00-5$ & $1,1,2-$ Trichloroethane & 1.0 & $\mathrm{U}$ \\
\hline $127-18-4$ & Tetrachloroethene & 0.038 & $\mathrm{~J}$ \\
\hline $591-78-6$ & 2-Hexanone & 1.0 & $\mathrm{U}$ \\
\hline $124-48-1$ & Dibromochloromethane & 1.0 & $\mathrm{U}$ \\
\hline $106-93-4$ & 1,2-Dibromoethane & 1.0 & $\mathrm{U}$ \\
\hline $108-90-7$ & Chlorobenzene & 1.0 & $\mathrm{U}$ \\
\hline $100-41-4$ & Ethylbenzene & 0.040 & $\mathrm{~J} B$ \\
\hline $95-47-6$ & o-Xylene & 0.087 & $\mathrm{~J} B$ \\
\hline $179601-23-1$ & m,p-xylene & 0.11 & $\mathrm{~J} B$ \\
\hline $100-42-5$ & Styrene & 0.18 & $\mathrm{~J} B$ \\
\hline $75-25-2$ & Bromoform & 1.0 & $\mathrm{U}$ \\
\hline $98-82-8$ & Isopropylbenzene & 1.0 & $\mathrm{U}$ \\
\hline $79-34-5$ & $1,1,2,2$-Tetrachloroethane & 1.0 & $\mathrm{U}$ \\
\hline $541-73-1$ & 1,3 -Dichlorobenzene & 1.0 & $\mathrm{U}$ \\
\hline $106-46-7$ & 1,4-Dichlorobenzene & 1.0 & $\mathrm{U}$ \\
\hline $95-50-1$ & 1,2-Dichlorobenzene & 1.0 & $\mathrm{U}$ \\
\hline $96-12-8$ & 1,2-Dibromo-3-Chloropropane & 1.0 & $\mathrm{U}$ \\
\hline $120-82-1$ & $1,2,4$-Trichlorobenzene & 1.0 & $\mathrm{U}$ \\
\hline $87-61-6$ & $1,2,3$-Trichlorobenzene & 1.0 & $\mathrm{U}$ \\
\hline
\end{tabular}


Lab Name: TESTAMERICA BURLINGTON

Contract: $8 \mathrm{E}-00302$

Lab Code: STLV Case No.: YORK Mod. Ref No.: SDG No.: 34024

Matrix: (SOIL/SED/WATER) Water

Sample wt/vol: 25.0 $(\mathrm{g} / \mathrm{mL}) \mathrm{mL}$

Level: (TRACE or LOW/MED) TRACE

․ Moisture: not dec.

GC Column: DB-624 ID $: 0.20 \quad(\mathrm{~mm})$

Soil Extract Volume: (uL)
Lab Sample ID: 200-34024-3

Lab File ID: 20517_07.D

Date Received: 06/17/2016

Date Analyzed: 06/21/2016

Dilution Factor: 1.0

Soil Aliquot Volume: (uL) Purge Volume: 25.0 $(\mathrm{mL})$

01

02

\begin{tabular}{|l|l|c|c|c|}
\hline CAS NUMBER & \multicolumn{1}{|c|}{ COMPOUND NAME } & RT & EST. CONC. & Q \\
\hline & Unknown & 7.20 & 2.6 & X B J \\
\hline E9667961 & Total Alkanes & N/A & & \\
\hline
\end{tabular}

1 EPA-designated Registry Number. 
Lab Name: TESTAMERICA BURLINGTON

Contract: $8 \mathrm{E}-00302$

Lab Code: STLV Case No.: YORK Mod. Ref No.:

SDG No.: 34024

Instrument ID: CHD.i

Calibration Date(s): 05/31/2016 05/31/2016

Heated Purge: (Y/N) N

Calibration Time(s) : 1605

1746

Purge Volume: 25.0 $(\mathrm{mL})$

GC Column: DB-624

ID: $0.20 \quad$ (mm) Length: 25

$(\mathrm{m})$

\begin{tabular}{|c|c|c|c|c|c|c|c|}
\hline \multirow{3}{*}{$\begin{array}{l}\text { LAB FILE ID: } \\
\text { RRF } 5.0=20227 \_04 . D \\
\text { COMPOUND }\end{array}$} & \multirow{2}{*}{\multicolumn{3}{|c|}{ 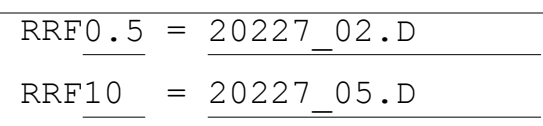 }} & \multicolumn{4}{|c|}{ RRF1.0 $=20227$ 03.D } \\
\hline & & & & RRF2 & $=202$ & DO6.D & \\
\hline & RRF0. 5 & RRF1.0 & $\operatorname{RRF} 5.0$ & RRF10 & RRF20 & $\overline{\mathrm{RRF}}$ & $\because \mathrm{RSD}$ \\
\hline Dichlorodifluoromethane & 0.667 & 0.602 & 0.556 & 0.572 & 0.564 & 0.592 & 7.7 \\
\hline Chloromethane & 0.595 & 0.547 & 0.480 & 0.473 & 0.459 & 0.511 & 11.4 \\
\hline Vinyl chloride & 0.583 & 0.509 & 0.481 & 0.502 & 0.491 & 0.513 & 7.9 \\
\hline Bromomethane & 0.239 & 0.158 & 0.155 & 0.169 & 0.171 & 0.179 & 19.4 \\
\hline Chloroethane & 0.290 & 0.283 & 0.280 & 0.282 & 0.259 & 0.279 & 4.3 \\
\hline Trichlorofluoromethane & 0.718 & 0.675 & 0.613 & 0.618 & 0.608 & 0.646 & 7.5 \\
\hline 1,1-Dichloroethene & 0.384 & 0.348 & 0.328 & 0.331 & 0.325 & 0.343 & 7.1 \\
\hline $\begin{array}{l}1,1,2 \text {-Trichloro- } \\
1,2,2 \text {-trifluoroethane }\end{array}$ & 0.448 & 0.398 & 0.367 & 0.368 & 0.358 & 0.388 & 9.5 \\
\hline Acetone & 0.024 & 0.021 & 0.017 & 0.019 & 0.019 & 0.020 & 12.6 \\
\hline Carbon disulfide & 1.077 & 0.937 & 0.929 & 0.988 & 1.005 & 0.987 & 6.0 \\
\hline Methyl acetate & 0.059 & 0.052 & 0.050 & 0.051 & 0.050 & 0.052 & 7.1 \\
\hline Methylene Chloride & 0.324 & 0.295 & 0.272 & 0.273 & 0.270 & 0.287 & 8.1 \\
\hline trans-1,2-Dichloroethene & 0.388 & 0.352 & 0.332 & 0.335 & 0.332 & 0.348 & 6.9 \\
\hline Methyl tert-butyl ether & 0.376 & 0.353 & 0.357 & 0.373 & 0.389 & 0.370 & 4.0 \\
\hline 1,1-Dichloroethane & 0.736 & 0.665 & 0.623 & 0.637 & 0.636 & 0.660 & 6.9 \\
\hline cis-1,2-Dichloroethene & 0.352 & 0.315 & 0.332 & 0.342 & 0.331 & 0.334 & 4.2 \\
\hline 2-Butanone & 0.033 & 0.030 & 0.032 & 0.035 & 0.035 & 0.033 & 7.0 \\
\hline Bromochloromethane & 0.112 & 0.107 & 0.097 & 0.102 & 0.105 & 0.105 & 5.1 \\
\hline Chloroform & 0.634 & 0.586 & 0.556 & 0.566 & 0.576 & 0.584 & 5.2 \\
\hline $1,1,1$-Trichloroethane & 0.763 & 0.693 & 0.667 & 0.679 & 0.688 & 0.698 & 5.4 \\
\hline Cyclohexane & 0.734 & 0.702 & 0.814 & 0.863 & 0.880 & 0.799 & 9.8 \\
\hline Carbon tetrachloride & 0.673 & 0.612 & 0.607 & 0.623 & 0.628 & 0.629 & 4.1 \\
\hline Benzene & 2.159 & 1.975 & 1.906 & 1.952 & 1.975 & 1.993 & 4.9 \\
\hline 1,2-Dichloroethane & 0.293 & 0.257 & 0.256 & 0.260 & 0.267 & 0.266 & 5.7 \\
\hline Trichloroethene & 0.465 & 0.422 & 0.424 & 0.440 & 0.450 & 0.440 & 4.1 \\
\hline Methylcyclohexane & 0.599 & 0.590 & 0.666 & 0.695 & 0.694 & 0.649 & 7.8 \\
\hline
\end{tabular}

Report 1,4-Dioxane for Low-Medium VOA analysis only 
Lab Name: TESTAMERICA BURLINGTON

Contract: $8 \mathrm{E}-00302$

Lab Code: STLV Case No.: YORK Mod. Ref No.:

SDG No.: 34024

Instrument ID: CHD.i

Calibration Date(s): 05/31/2016 05/31/2016

Heated Purge: (Y/N) N

Calibration Time(s) : 1605

1746

Purge Volume: 25.0 (mL)

GC Column: DB-624

ID: 0.20

$(\mathrm{mm})$

Length: 25

(m)

\begin{tabular}{|c|c|c|c|c|c|c|c|}
\hline \multirow{2}{*}{$\begin{array}{l}\text { LAB FILE ID: } \\
\text { RRF5.0 }=20227 \_04 . D\end{array}$} & \multicolumn{3}{|c|}{ RRF0.5 = 20227_02.D } & \multicolumn{4}{|c|}{ RRF1.0 = 20227_03.D } \\
\hline & F10 & $227 \_05$ & & RRF20 & $=2022$ & $7-06 . \mathrm{D}$ & \\
\hline COMPOUND & RRF0.5 & RRE1.0 & RRF5.0 & RRF10 & RRF20 & $\overline{\mathrm{RRF}}$ & $\because \mathrm{RSD}$ \\
\hline 1,2-Dichloropropane & 0.450 & 0.394 & 0.387 & 0.391 & 0.397 & 0.404 & 6.5 \\
\hline Bromodichloromethane & 0.396 & 0.364 & 0.368 & 0.382 & 0.395 & 0.381 & 3.9 \\
\hline cis-1,3-Dichloropropene & 0.383 & 0.368 & 0.472 & 0.517 & 0.545 & 0.457 & 17.2 \\
\hline 4-Methyl-2-pentanone & 0.072 & 0.074 & 0.094 & 0.101 & 0.105 & 0.089 & 17.2 \\
\hline Toluene & 1.692 & 1.704 & 1.891 & 1.962 & 1.990 & 1.848 & 7.7 \\
\hline trans-1,3-Dichloropropene & 0.238 & 0.235 & 0.304 & 0.343 & 0.370 & 0.298 & 20.5 \\
\hline 1,1,2-Trichloroethane & 0.209 & 0.182 & 0.169 & 0.172 & 0.171 & 0.180 & 9.2 \\
\hline Tetrachloroethene & 0.472 & 0.431 & 0.425 & 0.440 & 0.435 & 0.441 & 4.2 \\
\hline 2-Hexanone & 0.048 & 0.052 & 0.066 & 0.070 & 0.072 & 0.062 & 17.6 \\
\hline Dibromochloromethane & 0.176 & 0.164 & 0.176 & 0.190 & 0.198 & 0.181 & 7.5 \\
\hline 1,2-Dibromoethane & 0.142 & 0.128 & 0.135 & 0.138 & 0.144 & 0.137 & 4.6 \\
\hline Chlorobenzene & 1.207 & 1.138 & 1.100 & 1.124 & 1.120 & 1.138 & 3.6 \\
\hline Ethylbenzene & 1.724 & 1.752 & 2.139 & 2.283 & 2.319 & 2.043 & 14.0 \\
\hline o-Xylene & 0.488 & 0.541 & 0.751 & 0.802 & 0.804 & 0.677 & 22.3 \\
\hline $\mathrm{m}, \mathrm{p}$-Xylene & 0.627 & 0.666 & 0.841 & 0.885 & 0.893 & 0.783 & 16.2 \\
\hline Styrene & 0.803 & 0.905 & 1.184 & 1.244 & 1.250 & 1.077 & 19.4 \\
\hline Bromoform & 0.169 & 0.149 & 0.145 & 0.162 & 0.182 & 0.161 & 9.3 \\
\hline Isopropylbenzene & 1.433 & 1.568 & 2.168 & 2.351 & 2.365 & 1.977 & 22.5 \\
\hline $1,1,2,2$-Tetrachloroethane & 0.177 & 0.167 & 0.165 & 0.171 & 0.172 & 0.170 & 2.8 \\
\hline 1,3-Dichlorobenzene & 1.375 & 1.332 & 1.435 & 1.557 & 1.640 & 1.468 & 8.7 \\
\hline 1,4-Dichlorobenzene & 1.993 & 1.711 & 1.583 & 1.599 & 1.627 & 1.703 & 10.0 \\
\hline 1,2-Dichlorobenzene & 1.344 & 1.274 & 1.266 & 1.300 & 1.323 & 1.301 & 2.5 \\
\hline 1,2-Dibromo-3-Chloropropane & 0.045 & 0.040 & 0.033 & 0.036 & 0.041 & 0.039 & 11.3 \\
\hline 1,2,4-Trichlorobenzene & 0.687 & 0.612 & 0.691 & 0.845 & 0.949 & 0.757 & 18.1 \\
\hline 1,2,3-Trichlorobenzene & 0.470 & 0.439 & 0.518 & 0.605 & 0.671 & 0.541 & 17.8 \\
\hline
\end{tabular}


Lab Name: TESTAMERICA BURLINGTON

Contract: $8 \mathrm{E}-00302$

Lab Code: STLV Case No.: YORK Mod. Ref No.: SDG No.: 34024

Instrument ID: CHD.i

Heated Purge: (Y/N) N

Purge Volume: 25.0

GC Column: DB-624
ID: 0.20
Calibration Date(s) : 05/31/2016

Calibration Time(s) : 1605 $(\mathrm{mL})$

(mm) Length: 25 (m)

\begin{tabular}{|c|c|c|c|c|c|c|c|}
\hline LAB FILE ID: & \multicolumn{3}{|c|}{$\begin{array}{l}\operatorname{RRF}_{\underline{0.5}}=\frac{20227 \_02 . \mathrm{D}}{\operatorname{RRF10}}=20227 \_05 . \mathrm{D}\end{array}$} & \multicolumn{4}{|c|}{$\begin{array}{l}\operatorname{RRF1.0}_{\underline{1.0}}=\frac{20227 \_03 . \mathrm{D}}{\underline{\mathrm{RRF} 20}}=20227 \_06 . \mathrm{D}\end{array}$} \\
\hline Vinyl Chloride-d3 & 0.507 & 0.432 & 0.420 & 0.437 & 0.428 & 0.445 & 8.0 \\
\hline Chloroethane-d5 & 0.424 & 0.372 & 0.339 & 0.334 & 0.311 & 0.356 & 12.2 \\
\hline Chloroform-d & 0.681 & 0.615 & 0.576 & 0.591 & 0.594 & 0.611 & 6.8 \\
\hline 1,2-Dichloroethane-d4 & 0.245 & 0.212 & 0.201 & 0.205 & 0.209 & 0.214 & 8.2 \\
\hline Benzene-d6 & 1.902 & 1.743 & 1.756 & 1.802 & 1.828 & 1.806 & 3.5 \\
\hline 1,2-Dichloropropane-d6 & 0.507 & 0.429 & 0.417 & 0.434 & 0.436 & 0.444 & 8.0 \\
\hline Toluene-d8 & 1.445 & 1.398 & 1.557 & 1.626 & 1.638 & 1.533 & 7.0 \\
\hline 1,2-Dichlorobenzene-d4 & 0.917 & 0.756 & 0.750 & 0.791 & 0.823 & 0.807 & 8.5 \\
\hline
\end{tabular}

Report 1,4-Dioxane-d8 for Low-Medium VOA analysis only 
Lab Name: TESTAMERICA BURLINGTON

Contract: $8 \mathrm{E}-00302$

Lab Code: STLV Case No.: YORK Mod. Ref No.:

SDG No.: 34024

Instrument ID: CHD.i

Calibration Date: 06/21/2016 Time: 1223

Lab File Id: 20517_03.D

Init. Calib. Date(s):05/31/2016 05/31/2016

EPA Sample No. (VSTD\#\#\#\#) : VSTD005DV

Init. Calib. Time(s) : 1605 1746

Heated Purge: (Y/N) N GC Column: DB-624

ID: $0.20(\mathrm{~mm})$ Length: 25

(m)

Purge Volume: 25.0 $(\mathrm{mL})$

\begin{tabular}{|c|c|c|c|c|c|}
\hline COMPOUND & $\overline{\mathrm{RRF}}$ & RRF5.0 & $\begin{array}{l}\text { MIN } \\
\text { RRF }\end{array}$ & $\frac{\circ}{D}$ & $\mathrm{MAX} \div \mathrm{D}$ \\
\hline Dichlorodifluoromethane & 0.592 & 0.547 & 0.010 & -7.7 & 40.0 \\
\hline Chloromethane & 0.511 & 0.464 & 0.010 & -9.1 & 40.0 \\
\hline Vinyl chloride & 0.513 & 0.502 & 0.100 & -2.2 & 30.0 \\
\hline Bromomethane & 0.179 & 0.158 & 0.100 & -11.7 & 30.0 \\
\hline Chloroethane & 0.279 & 0.294 & 0.010 & 5.4 & 40.0 \\
\hline Trichlorofluoromethane & 0.646 & 0.651 & 0.010 & 0.7 & 40.0 \\
\hline 1,1-Dichloroethene & 0.343 & 0.344 & 0.100 & 0.1 & 30.0 \\
\hline 1,1,2-Trichloro-1,2,2-trifluoroethane & 0.388 & 0.386 & 0.010 & -0.6 & 40.0 \\
\hline Acetone & 0.020 & 0.020 & 0.010 & -1.9 & 40.0 \\
\hline Carbon disulfide & 0.987 & 0.980 & 0.010 & -0.8 & 40.0 \\
\hline Methyl acetate & 0.052 & 0.052 & 0.010 & -0.1 & 40.0 \\
\hline Methylene Chloride & 0.287 & 0.284 & 0.010 & -1.0 & 40.0 \\
\hline trans-1,2-Dichloroethene & 0.348 & 0.340 & 0.010 & -2.4 & 40.0 \\
\hline Methyl tert-butyl ether & 0.370 & 0.376 & 0.010 & 1.8 & 40.0 \\
\hline 1,1-Dichloroethane & 0.660 & 0.670 & 0.200 & 1.6 & 30.0 \\
\hline cis-1,2-Dichloroethene & 0.334 & 0.322 & 0.010 & -3.8 & 40.0 \\
\hline 2-Butanone & 0.033 & 0.034 & 0.010 & 2.7 & 40.0 \\
\hline Bromochloromethane & 0.105 & 0.106 & 0.050 & 1.9 & 30.0 \\
\hline Chloroform & 0.584 & 0.594 & 0.200 & 1.8 & 30.0 \\
\hline 1,1,1-Trichloroethane & 0.698 & 0.693 & 0.100 & -0.8 & 30.0 \\
\hline Cyclohexane & 0.799 & 0.838 & 0.010 & 5.0 & 40.0 \\
\hline Carbon tetrachloride & 0.629 & 0.646 & 0.100 & 2.7 & 30.0 \\
\hline Benzene & 1.993 & 1.985 & 0.400 & -0.4 & 30.0 \\
\hline 1,2-Dichloroethane & 0.266 & 0.277 & 0.100 & 4.1 & 30.0 \\
\hline Trichloroethene & 0.440 & 0.434 & 0.300 & -1.5 & 30.0 \\
\hline Methylcyclohexane & 0.649 & 0.690 & 0.010 & 6.3 & 40.0 \\
\hline
\end{tabular}

Report 1,4-Dioxane for Low/Medium VOA analysis only 
Lab Name: TESTAMERICA BURLINGTON

Contract: 8E-00302

Lab Code: STLV

Mod. Ref No.:

SDG No.: 34024

Instrument ID: CHD.i

Calibration Date: 06/21/2016 Time: 1223

Lab File Id: 20517_03.D

Init. Calib. Date(s):05/31/2016 05/31/2016

EPA Sample No. (VSTD\#\#\#\#) : VSTD005DV

Init. Calib. Time(s) : 1605 1746

Heated Purge: (Y/N) N GC Column: DB-624

ID: $0.20(\mathrm{~mm})$ Length: 25

(m)

Purge Volume: 25.0 $(\mathrm{mL})$

\begin{tabular}{|c|c|c|c|c|c|}
\hline COMPOUND & $\overline{\mathrm{RRF}}$ & RRF5.0 & $\begin{array}{l}\text { MIN } \\
\text { RRF }\end{array}$ & $\because \mathrm{D}$ & $\operatorname{MAX} \cong \mathrm{D}$ \\
\hline 1,2-Dichloropropane & 0.404 & 0.404 & 0.010 & 0.1 & 40.0 \\
\hline Bromodichloromethane & 0.381 & 0.388 & 0.200 & 1.9 & 30.0 \\
\hline cis-1,3-Dichloropropene & 0.457 & 0.490 & 0.200 & 7.3 & 30.0 \\
\hline 4-Methyl-2-pentanone & 0.089 & 0.101 & 0.010 & 13.5 & 40.0 \\
\hline Toluene & 1.848 & 1.993 & 0.400 & 7.9 & 30.0 \\
\hline trans-1,3-Dichloropropene & 0.298 & 0.316 & 0.100 & 6.0 & 30.0 \\
\hline $1,1,2$-Trichloroethane & 0.180 & 0.180 & 0.100 & -0.2 & 30.0 \\
\hline Tetrachloroethene & 0.441 & 0.441 & 0.100 & 0.1 & 30.0 \\
\hline 2-Hexanone & 0.062 & 0.070 & 0.010 & 13.6 & 40.0 \\
\hline Dibromochloromethane & 0.181 & 0.192 & 0.100 & 6.1 & 30.0 \\
\hline 1,2-Dibromoethane & 0.137 & 0.138 & 0.010 & 0.4 & 40.0 \\
\hline Chlorobenzene & 1.138 & 1.136 & 0.500 & -0.2 & 30.0 \\
\hline Ethylbenzene & 2.043 & 2.211 & 0.100 & 8.2 & 30.0 \\
\hline o-Xylene & 0.677 & 0.762 & 0.300 & 12.6 & 30.0 \\
\hline $\mathrm{m}, \mathrm{p}$-Xylene & 0.783 & 0.858 & 0.300 & 9.7 & 30.0 \\
\hline Styrene & 1.077 & 1.237 & 0.300 & 14.8 & 30.0 \\
\hline Bromoform & 0.161 & 0.172 & 0.050 & 6.6 & 30.0 \\
\hline Isopropylbenzene & 1.977 & 2.220 & 0.010 & 12.3 & 40.0 \\
\hline $1,1,2,2$-Tetrachloroethane & 0.170 & 0.173 & 0.100 & 1.6 & 30.0 \\
\hline 1,3-Dichlorobenzene & 1.468 & 1.463 & 0.400 & -0.3 & 30.0 \\
\hline 1,4-Dichlorobenzene & 1.703 & 1.681 & 0.400 & -1.3 & 30.0 \\
\hline 1,2-Dichlorobenzene & 1.301 & 1.360 & 0.400 & 4.5 & 30.0 \\
\hline 1,2-Dibromo-3-Chloropropane & 0.039 & 0.035 & 0.010 & -9.1 & 40.0 \\
\hline 1,2,4-Trichlorobenzene & 0.757 & 0.715 & 0.200 & -5.6 & 30.0 \\
\hline $1,2,3$-Trichlorobenzene & 0.541 & 0.508 & 0.200 & -5.9 & 30.0 \\
\hline
\end{tabular}


Lab Name: TESTAMERICA BURLINGTON

Contract: $8 \mathrm{E}-00302$

Lab Code: STLV Case No.: YORK Mod. Ref No.

SDG No.: 34024

Instrument ID: CHD.i

Calibration Date: 06/21/2016 Time: 1223

Lab File Id: 20517_03.D Init. Calib. Date (s):05/31/2016 05/31/2016

EPA Sample No. (VSTD\#\#\#\#) : VSTD005DV

Heated Purge: (Y/N) N GC Column: DB-624

Init. Calib. Time(s) : 1605 1746

Purge Volume: 25.0 ID: $0.20(\mathrm{~mm})$ Length: 25

(m)

\begin{tabular}{|c|c|c|c|c|c|}
\hline COMPOUND & $\overline{\mathrm{RRF}}$ & RRE5. 0 & $\begin{array}{l}\text { MIN } \\
\text { RRE }\end{array}$ & $\because \mathrm{D}$ & $M A X \div D$ \\
\hline Vinyl Chloride-d3 & 0.445 & 0.442 & 0.010 & -0.5 & 30.0 \\
\hline Chloroethane-d5 & 0.356 & 0.361 & 0.010 & 1.4 & 40.0 \\
\hline $1,1-$ Dichloroethene-d2 & 0.762 & 0.777 & 0.010 & 1.9 & 30.0 \\
\hline 2-Butanone-d5 & 0.030 & 0.030 & 0.010 & 0.0 & 40.0 \\
\hline Chloroform-d & 0.611 & 0.623 & 0.010 & 2.0 & 30.0 \\
\hline 1,2-Dichloroethane-d4 & 0.214 & 0.218 & 0.010 & 1.7 & 30.0 \\
\hline Benzene-d6 & 1.806 & 1.829 & 0.010 & 1.3 & 30.0 \\
\hline 1,2-Dichloropropane-d6 & 0.444 & 0.449 & 0.010 & 0.9 & 40.0 \\
\hline Toluene-d8 & 1.533 & 1.635 & 0.010 & 6.7 & 30.0 \\
\hline trans-1,3-Dichloropropene-d4 & 0.245 & 0.245 & 0.010 & -0.4 & 30.0 \\
\hline 2-Hexanone-d5 & 0.030 & 0.033 & 0.010 & 9.9 & 40.0 \\
\hline $1,1,2,2$-Tetrachloroethane-d2 & 0.171 & 0.175 & 0.010 & 2.1 & 30.0 \\
\hline 1,2-Dichlorobenzene-d4 & 0.807 & 0.826 & 0.010 & 2.3 & 30.0 \\
\hline
\end{tabular}

Report 1,4-Dioxane-d8 for Low/Medium VOA analysis only 
Lab Name: TESTAMERICA BURLINGTON

Contract: $8 \mathrm{E}-00302$

Lab Code: STLV

Mod. Ref No.:

SDG No.: 34024

Instrument ID: CHD.i

Calibration Date: 06/21/2016 Time: 2011

Lab File Id: 20517_20.D

EPA Sample No. (VSTD\#\#\#\#) : VSTD005VD Init. Calib. Date (s):05/31/2016 05/31/2016

Heated Purge: ( $\mathrm{Y} / \mathrm{N}) \mathrm{N}$ GC Column: DB-624

Init. Calib. Time(s) : 1605 1746

Purge Volume: 25.0 ID: $0.20(\mathrm{~mm})$ Length: 25

(m)

\begin{tabular}{|c|c|c|c|c|c|}
\hline COMPOUND & $\overline{\mathrm{RRF}}$ & RRF5.0 & $\begin{array}{l}\text { MIN } \\
\text { RRF }\end{array}$ & $\because \mathrm{D}$ & $\mathrm{MAX} \div \mathrm{D}$ \\
\hline Dichlorodifluoromethane & 0.592 & 0.549 & 0.010 & -7.4 & 50.0 \\
\hline Chloromethane & 0.511 & 0.307 & 0.010 & -39.9 & 50.0 \\
\hline Vinyl chloride & 0.513 & 0.400 & 0.010 & -22.2 & 50.0 \\
\hline Bromomethane & 0.179 & 0.011 & 0.010 & -94.1 & 50.0 \\
\hline Chloroethane & 0.279 & 0.307 & 0.010 & 9.9 & 50.0 \\
\hline Trichlorofluoromethane & 0.646 & 0.659 & 0.010 & 2.0 & 50.0 \\
\hline 1,1-Dichloroethene & 0.343 & 0.336 & 0.010 & -2.2 & 50.0 \\
\hline 1,1,2-Trichloro-1,2,2-trifluoroethane & 0.388 & 0.371 & 0.010 & -4.3 & 50.0 \\
\hline Acetone & 0.020 & 0.019 & 0.010 & -4.7 & 50.0 \\
\hline Carbon disulfide & 0.987 & 1.032 & 0.010 & 4.6 & 50.0 \\
\hline Methyl acetate & 0.052 & 0.047 & 0.010 & -10.4 & 50.0 \\
\hline Methylene Chloride & 0.287 & 0.285 & 0.010 & -0.7 & 50.0 \\
\hline trans-1,2-Dichloroethene & 0.348 & 0.335 & 0.010 & -3.8 & 50.0 \\
\hline Methyl tert-butyl ether & 0.370 & 0.361 & 0.010 & -2.3 & 50.0 \\
\hline 1,1-Dichloroethane & 0.660 & 0.671 & 0.010 & 1.7 & 50.0 \\
\hline cis-1,2-Dichloroethene & 0.334 & 0.338 & 0.010 & 1.0 & 50.0 \\
\hline 2-Butanone & 0.033 & 0.032 & 0.010 & -1.0 & 50.0 \\
\hline Bromochloromethane & 0.105 & 0.100 & 0.010 & -4.0 & 50.0 \\
\hline Chloroform & 0.584 & 0.596 & 0.010 & 2.2 & 50.0 \\
\hline 1,1,1-Trichloroethane & 0.698 & 0.729 & 0.010 & 4.4 & 50.0 \\
\hline Cyclohexane & 0.799 & 0.830 & 0.010 & 3.9 & 50.0 \\
\hline Carbon tetrachloride & 0.629 & 0.674 & 0.010 & 7.3 & 50.0 \\
\hline Benzene & 1.993 & 2.009 & 0.010 & 0.8 & 50.0 \\
\hline 1,2-Dichloroethane & 0.266 & 0.269 & 0.010 & 1.2 & 50.0 \\
\hline Trichloroethene & 0.440 & 0.426 & 0.010 & -3.2 & 50.0 \\
\hline Methylcyclohexane & 0.649 & 0.680 & 0.010 & 4.8 & 50.0 \\
\hline
\end{tabular}

Report 1,4-Dioxane for Low/Medium VOA analysis only 
Lab Name: TESTAMERICA BURLINGTON

Contract: 8E-00302

Lab Code: STLV

Mod. Ref No.:

SDG No.: 34024

Instrument ID: CHD.i

Calibration Date: 06/21/2016 Time: 2011

Lab File Id: 20517_20.D

EPA Sample No. (VSTD\#\#\#\#) : VSTD005VD Init. Calib. Date(s):05/31/2016 05/31/2016

Heated Purge: (Y/N) N GC Column: DB-624

Init. Calib. Time(s) : 1605 1746

Purge Volume: 25.0

ID: $0.20(\mathrm{~mm})$ Length: 25

(m)

\begin{tabular}{|c|c|c|c|c|c|}
\hline COMPOUND & $\overline{\mathrm{RRF}}$ & RRF5.0 & $\begin{array}{l}\text { MIN } \\
\text { RRF }\end{array}$ & $\because \mathrm{D}$ & $\operatorname{MAX} \cong \mathrm{D}$ \\
\hline 1,2-Dichloropropane & 0.404 & 0.405 & 0.010 & 0.3 & 50.0 \\
\hline Bromodichloromethane & 0.381 & 0.398 & 0.010 & 4.4 & 50.0 \\
\hline cis-1,3-Dichloropropene & 0.457 & 0.460 & 0.010 & 0.7 & 50.0 \\
\hline 4-Methyl-2-pentanone & 0.089 & 0.095 & 0.010 & 7.0 & 50.0 \\
\hline Toluene & 1.848 & 2.018 & 0.010 & 9.2 & 50.0 \\
\hline trans-1,3-Dichloropropene & 0.298 & 0.293 & 0.010 & -1.6 & 50.0 \\
\hline $1,1,2$-Trichloroethane & 0.180 & 0.182 & 0.010 & 0.9 & 50.0 \\
\hline Tetrachloroethene & 0.441 & 0.451 & 0.010 & 2.4 & 50.0 \\
\hline 2-Hexanone & 0.062 & 0.066 & 0.010 & 7.6 & 50.0 \\
\hline Dibromochloromethane & 0.181 & 0.192 & 0.010 & 6.3 & 50.0 \\
\hline 1,2-Dibromoethane & 0.137 & 0.135 & 0.010 & -1.7 & 50.0 \\
\hline Chlorobenzene & 1.138 & 1.136 & 0.010 & -0.1 & 50.0 \\
\hline Ethylbenzene & 2.043 & 2.229 & 0.010 & 9.1 & 50.0 \\
\hline o-Xylene & 0.677 & 0.766 & 0.010 & 13.2 & 50.0 \\
\hline $\mathrm{m}, \mathrm{p}$-Xylene & 0.783 & 0.870 & 0.010 & 11.2 & 50.0 \\
\hline Styrene & 1.077 & 1.226 & 0.010 & 13.8 & 50.0 \\
\hline Bromoform & 0.161 & 0.166 & 0.010 & 2.9 & 50.0 \\
\hline Isopropylbenzene & 1.977 & 2.251 & 0.010 & 13.9 & 50.0 \\
\hline $1,1,2,2$-Tetrachloroethane & 0.170 & 0.170 & 0.010 & -0.2 & 50.0 \\
\hline 1,3-Dichlorobenzene & 1.468 & 1.421 & 0.010 & -3.2 & 50.0 \\
\hline 1,4-Dichlorobenzene & 1.703 & 1.640 & 0.010 & -3.7 & 50.0 \\
\hline 1,2-Dichlorobenzene & 1.301 & 1.312 & 0.010 & 0.8 & 50.0 \\
\hline 1,2-Dibromo-3-Chloropropane & 0.039 & 0.036 & 0.010 & -7.2 & 50.0 \\
\hline 1,2,4-Trichlorobenzene & 0.757 & 0.620 & 0.010 & $-18 \cdot 0$ & 50.0 \\
\hline $1,2,3$-Trichlorobenzene & 0.541 & 0.444 & 0.010 & -17.9 & 50.0 \\
\hline
\end{tabular}


Lab Name: TESTAMERICA BURLINGTON

Contract: $8 \mathrm{E}-00302$

Lab Code: STLV Case No.: YORK Mod. Ref No.

SDG No.: 34024

Instrument ID: CHD.i

Calibration Date: 06/21/2016 Time: 2011

Lab File Id: 20517_20.D

Init. Calib. Date(s):05/31/2016 05/31/2016

EPA Sample No. (VSTD\#\#\#\#) : VSTD005VD

Init. Calib. Time(s) : 1605 1746

Heated Purge: (Y/N) N GC Column: DB-624

ID: $0.20(\mathrm{~mm})$ Length: 25

(m)

Purge Volume: 25.0 $(\mathrm{mL})$

\begin{tabular}{|c|c|c|c|c|c|}
\hline COMPOUND & $\overline{\mathrm{RRF}}$ & RRF5.0 & $\begin{array}{l}\text { MIN } \\
\text { RRF }\end{array}$ & $\because \mathrm{D}$ & $\operatorname{MAX} \cong \mathrm{D}$ \\
\hline Vinyl Chloride-d3 & 0.445 & 0.347 & 0.010 & -21.9 & 50.0 \\
\hline Chloroethane-d5 & 0.356 & 0.353 & 0.010 & -0.9 & 50.0 \\
\hline 1,1-Dichloroethene-d2 & 0.762 & 0.772 & 0.010 & 1.3 & 50.0 \\
\hline 2-Butanone-d5 & 0.030 & 0.030 & 0.010 & -1.2 & 50.0 \\
\hline Chloroform-d & 0.611 & 0.623 & 0.010 & 1.8 & 50.0 \\
\hline 1,2-Dichloroethane-d4 & 0.214 & 0.214 & 0.010 & -0.4 & 50.0 \\
\hline Benzene-d6 & 1.806 & 1.842 & 0.010 & 2.0 & 50.0 \\
\hline 1,2-Dichloropropane-d6 & 0.444 & 0.564 & 0.010 & 26.8 & 50.0 \\
\hline Toluene-d8 & 1.533 & 1.650 & 0.010 & 7.6 & 50.0 \\
\hline trans-1,3-Dichloropropene-d4 & 0.245 & 0.228 & 0.010 & -7.1 & 50.0 \\
\hline 2-Hexanone-d5 & 0.030 & 0.031 & 0.010 & 3.7 & 50.0 \\
\hline $1,1,2,2$-Tetrachloroethane-d2 & 0.171 & 0.172 & 0.010 & 0.3 & 50.0 \\
\hline 1,2-Dichlorobenzene-d4 & 0.807 & 0.761 & 0.010 & -5.7 & 50.0 \\
\hline
\end{tabular}

Report 1,4-Dioxane-d8 for Low/Medium VOA analysis only 
Lab Name: TESTAMERICA BURLINGTON

Contract: $8 \mathrm{E}-00302$

Lab Code: STLV Case No.: YORK Mod. Ref No.:

SDG No.: 34024

Instrument ID: CHD.i

Calibration Date: 06/21/2016 Time: 2037

Lab File Id: 20517_21.D

Init. Calib. Date(s):05/31/2016 05/31/2016

EPA Sample No. (VSTD\#\#\#\#) : VSTD005V

Init. Calib. Time(s) : 1605 1746

Heated Purge: (Y/N) N GC Column: DB-624

ID: $0.20(\mathrm{~mm})$ Length: 25

(m)

Purge Volume: 25.0 $(\mathrm{mL})$

\begin{tabular}{|c|c|c|c|c|c|}
\hline COMPOUND & $\overline{\mathrm{RRF}}$ & $\operatorname{RRF} 0.0$ & $\begin{array}{l}\text { MIN } \\
\text { RRF }\end{array}$ & $\because \mathrm{D}$ & $\mathrm{MAX} \div \mathrm{D}$ \\
\hline Dichlorodifluoromethane & 0.592 & 0.554 & 0.010 & -6.4 & 50.0 \\
\hline Chloromethane & 0.511 & 0.307 & 0.010 & -39.9 & 50.0 \\
\hline Vinyl chloride & 0.513 & 0.417 & 0.010 & -18.8 & 50.0 \\
\hline Bromomethane & 0.179 & 0.015 & 0.010 & -91.5 & 50.0 \\
\hline Chloroethane & 0.279 & 0.328 & 0.010 & 17.5 & 50.0 \\
\hline Trichlorofluoromethane & 0.646 & 0.674 & 0.010 & 4.3 & 50.0 \\
\hline 1,1-Dichloroethene & 0.343 & 0.348 & 0.010 & 1.3 & 50.0 \\
\hline 1,1,2-Trichloro-1,2,2-trifluoroethane & 0.388 & 0.387 & 0.010 & -0.3 & 50.0 \\
\hline Acetone & 0.020 & 0.020 & 0.010 & 1.8 & 50.0 \\
\hline Carbon disulfide & 0.987 & 1.044 & 0.010 & 5.8 & 50.0 \\
\hline Methyl acetate & 0.052 & 0.058 & 0.010 & 11.1 & 50.0 \\
\hline Methylene Chloride & 0.287 & 0.288 & 0.010 & 0.4 & 50.0 \\
\hline trans-1,2-Dichloroethene & 0.348 & 0.347 & 0.010 & -0.4 & 50.0 \\
\hline Methyl tert-butyl ether & 0.370 & 0.396 & 0.010 & 7.2 & 50.0 \\
\hline 1,1-Dichloroethane & 0.660 & 0.700 & 0.010 & 6.1 & 50.0 \\
\hline cis-1,2-Dichloroethene & 0.334 & 0.350 & 0.010 & 4.7 & 50.0 \\
\hline 2-Butanone & 0.033 & 0.037 & 0.010 & 11.9 & 50.0 \\
\hline Bromochloromethane & 0.105 & 0.104 & 0.010 & -0.4 & 50.0 \\
\hline Chloroform & 0.584 & 0.617 & 0.010 & 5.8 & 50.0 \\
\hline 1,1,1-Trichloroethane & 0.698 & 0.746 & 0.010 & 6.8 & 50.0 \\
\hline Cyclohexane & 0.799 & 0.852 & 0.010 & 6.7 & 50.0 \\
\hline Carbon tetrachloride & 0.629 & 0.684 & 0.010 & 8.8 & 50.0 \\
\hline Benzene & 1.993 & 2.059 & 0.010 & 3.3 & 50.0 \\
\hline 1,2-Dichloroethane & 0.266 & 0.290 & 0.010 & 8.7 & 50.0 \\
\hline Trichloroethene & 0.440 & 0.444 & 0.010 & 0.8 & 50.0 \\
\hline Methylcyclohexane & 0.649 & 0.701 & 0.010 & 8.1 & 50.0 \\
\hline
\end{tabular}

Report 1,4-Dioxane for Low/Medium VOA analysis only 
Lab Name: TESTAMERICA BURLINGTON

Contract: 8E-00302

Lab Code: STLV

Mod. Ref No.:

SDG No.: 34024

Instrument ID: CHD.i

Calibration Date: 06/21/2016 Time: 2037

Lab File Id: 20517_21.D

Init. Calib. Date (s):05/31/2016 05/31/2016

EPA Sample No. (VSTD\#\#\#\#) : VSTD005V

Init. Calib. Time(s) : 1605 1746

Heated Purge: (Y/N) N GC Column: DB-624

ID: $0.20(\mathrm{~mm})$ Length: 25

(m)

Purge Volume: 25.0 $(\mathrm{mL})$

\begin{tabular}{|c|c|c|c|c|c|}
\hline COMPOUND & $\overline{\mathrm{RRF}}$ & RRF0.0 & $\begin{array}{l}\text { MIN } \\
\text { RRF }\end{array}$ & $\because \mathrm{D}$ & $\operatorname{MAX} \cong \mathrm{D}$ \\
\hline 1,2-Dichloropropane & 0.404 & 0.434 & 0.010 & 7.5 & 50.0 \\
\hline Bromodichloromethane & 0.381 & 0.413 & 0.010 & 8.5 & 50.0 \\
\hline cis-1,3-Dichloropropene & 0.457 & 0.485 & 0.010 & 6.0 & 50.0 \\
\hline 4-Methyl-2-pentanone & 0.089 & 0.105 & 0.010 & 17.9 & 50.0 \\
\hline Toluene & 1.848 & 2.073 & 0.010 & 12.2 & 50.0 \\
\hline trans-1,3-Dichloropropene & 0.298 & 0.314 & 0.010 & 5.4 & 50.0 \\
\hline $1,1,2$-Trichloroethane & 0.180 & 0.196 & 0.010 & 8.4 & 50.0 \\
\hline Tetrachloroethene & 0.441 & 0.458 & 0.010 & 3.9 & 50.0 \\
\hline 2-Hexanone & 0.062 & 0.073 & 0.010 & 18.5 & 50.0 \\
\hline Dibromochloromethane & 0.181 & 0.201 & 0.010 & 11.0 & 50.0 \\
\hline 1,2-Dibromoethane & 0.137 & 0.146 & 0.010 & 6.3 & 50.0 \\
\hline Chlorobenzene & 1.138 & 1.180 & 0.010 & 3.7 & 50.0 \\
\hline Ethylbenzene & 2.043 & 2.274 & 0.010 & 11.3 & 50.0 \\
\hline o-Xylene & 0.677 & 0.795 & 0.010 & 17.3 & 50.0 \\
\hline $\mathrm{m}, \mathrm{p}$-Xylene & 0.783 & 0.898 & 0.010 & 14.7 & 50.0 \\
\hline Styrene & 1.077 & 1.279 & 0.010 & 18.8 & 50.0 \\
\hline Bromoform & 0.161 & 0.166 & 0.010 & 2.7 & 50.0 \\
\hline Isopropylbenzene & 1.977 & 2.276 & 0.010 & 15.1 & 50.0 \\
\hline $1,1,2,2$-Tetrachloroethane & 0.170 & 0.181 & 0.010 & 6.1 & 50.0 \\
\hline 1,3-Dichlorobenzene & 1.468 & 1.504 & 0.010 & 2.4 & 50.0 \\
\hline 1,4-Dichlorobenzene & 1.703 & 1.709 & 0.010 & 0.4 & 50.0 \\
\hline 1,2-Dichlorobenzene & 1.301 & 1.392 & 0.010 & 7.0 & 50.0 \\
\hline 1,2-Dibromo-3-Chloropropane & 0.039 & 0.039 & 0.010 & 1.1 & 50.0 \\
\hline 1,2,4-Trichlorobenzene & 0.757 & 0.734 & 0.010 & -3.0 & 50.0 \\
\hline $1,2,3$-Trichlorobenzene & 0.541 & 0.524 & 0.010 & -3.0 & 50.0 \\
\hline
\end{tabular}


Lab Name: TESTAMERICA BURLINGTON

Contract: $8 \mathrm{E}-00302$

Lab Code: STLV Case No.: YORK Mod. Ref No.

SDG No.: 34024

Instrument ID: CHD.i

Calibration Date: 06/21/2016 Time: 2037

Lab File Id: 20517_21.D Init. Calib. Date (s):05/31/2016 05/31/2016

EPA Sample No. (VSTD\#\#\#\#) : VSTD005V

Init. Calib. Time(s) : 1605 1746

Heated Purge: (Y/N) N GC Column: DB-624 ID: $0.20(\mathrm{~mm})$ Length: 25

(m)

Purge Volume: 25.0 $(\mathrm{mL})$

\begin{tabular}{|c|c|c|c|c|c|}
\hline COMPOUND & $\overline{\mathrm{RRF}}$ & RRF0.0 & $\begin{array}{l}\text { MIN } \\
\text { RRE }\end{array}$ & $\because \mathrm{D}$ & $\operatorname{MAX} \cong \mathrm{D}$ \\
\hline Vinyl Chloride-d3 & 0.445 & 0.370 & 0.010 & -16.7 & 50.0 \\
\hline Chloroethane-d5 & 0.356 & 0.353 & 0.010 & -0.8 & 50.0 \\
\hline 1,1-Dichloroethene-d2 & 0.762 & 0.807 & 0.010 & 5.9 & 50.0 \\
\hline 2-Butanone-d5 & 0.030 & 0.033 & 0.010 & 7.3 & 50.0 \\
\hline Chloroform-d & 0.611 & 0.641 & 0.010 & 4.9 & 50.0 \\
\hline 1,2-Dichloroethane-d4 & 0.214 & 0.226 & 0.010 & 5.4 & 50.0 \\
\hline Benzene-d6 & 1.806 & 1.890 & 0.010 & 4.6 & 50.0 \\
\hline 1,2-Dichloropropane-d6 & 0.444 & 0.583 & 0.010 & 31.2 & 50.0 \\
\hline Toluene-d8 & 1.533 & 1.681 & 0.010 & 9.7 & 50.0 \\
\hline trans-1,3-Dichloropropene-d4 & 0.245 & 0.246 & 0.010 & 0.3 & 50.0 \\
\hline 2-Hexanone-d5 & 0.030 & 0.034 & 0.010 & 15.7 & 50.0 \\
\hline $1,1,2,2$-Tetrachloroethane-d2 & 0.171 & 0.178 & 0.010 & 4.1 & 50.0 \\
\hline 1,2-Dichlorobenzene-d4 & 0.807 & 0.814 & 0.010 & 0.8 & 50.0 \\
\hline
\end{tabular}

Report 1,4-Dioxane-d8 for Low/Medium VOA analysis only 
Lab Name: TESTAMERICA BURLINGTON

Contract: $8 \mathrm{E}-00302$

Lab Code: STLV Case No.: YORK Mod. Ref No.: SDG No.: 34024

Matrix: (SOIL/SED/WATER) Water

Sample wt/vol: 25.0 $(\mathrm{g} / \mathrm{mL}) \mathrm{mL}$

Level: (TRACE/LOW/MED) TRACE

\% Moisture: not dec.

GC Column: DB-624 ID: $0.20 \quad(\mathrm{~mm})$

Soil Extract Volume: (uL) $(\mathrm{mL})$

Purge Volume: 25.0
Lab Sample ID: MB 200-105993/4

Lab File ID: 20517_04.D

Date Received:

Date Analyzed: 06/21/2016

Dilution Factor: 1.0

Soil Aliquot Volume: (uL)

\begin{tabular}{|c|c|c|c|}
\hline CAS NO. & COMPOUND & $\begin{array}{l}\text { CONCENTRATION UNITS: } \\
(\mathrm{ug} / \mathrm{L} \text { or } \mathrm{ug} / \mathrm{kg}) \mathrm{ug} / \mathrm{L}\end{array}$ & 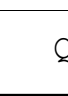 \\
\hline $75-71-8$ & Dichlorodifluoromethane & 1.0 & $\mathrm{U}$ \\
\hline $74-87-3$ & Chloromethane & 0.059 & $\mathrm{~J}$ \\
\hline $75-01-4$ & Vinyl chloride & 1.0 & $\mathrm{U}$ \\
\hline $74-83-9$ & Bromomethane & 1.0 & $\mathrm{U}$ \\
\hline $75-00-3$ & Chloroethane & 1.0 & $\mathrm{U}$ \\
\hline $75-69-4$ & Trichlorofluoromethane & 1.0 & $\mathrm{U}$ \\
\hline $75-35-4$ & 1,1-Dichloroethene & 1.0 & $\mathrm{U}$ \\
\hline $76-13-1$ & 1,1,2-Trichloro-1,2,2-trifluoroethane & 1.0 & $\mathrm{U}$ \\
\hline $67-64-1$ & Acetone & 5.0 & $\mathrm{U}$ \\
\hline $75-15-0$ & Carbon disulfide & 0.035 & $\mathrm{~J}$ \\
\hline $79-20-9$ & Methyl acetate & 1.0 & $\mathrm{U}$ \\
\hline $75-09-2$ & Methylene Chloride & 0.042 & $\mathrm{~J}$ \\
\hline $156-60-5$ & trans-1,2-Dichloroethene & 1.0 & $\mathrm{U}$ \\
\hline $1634-04-4$ & Methyl tert-butyl ether & 0.032 & $\mathrm{~J}$ \\
\hline $75-34-3$ & 1,1-Dichloroethane & 1.0 & $\mathrm{U}$ \\
\hline $156-59-2$ & cis-1,2-Dichloroethene & 1.0 & $\mathrm{U}$ \\
\hline $78-93-3$ & 2-Butanone & 5.0 & $\mathrm{U}$ \\
\hline $74-97-5$ & Bromochloromethane & 1.0 & $\mathrm{U}$ \\
\hline $67-66-3$ & Chloroform & 1.0 & $\mathrm{U}$ \\
\hline $71-55-6$ & $1,1,1$-Trichloroethane & 1.0 & $\mathrm{U}$ \\
\hline $110-82-7$ & Cyclohexane & 1.0 & $\mathrm{U}$ \\
\hline $56-23-5$ & Carbon tetrachloride & 1.0 & $\mathrm{U}$ \\
\hline $71-43-2$ & Benzene & 1.0 & $\mathrm{U}$ \\
\hline $107-06-2$ & 1,2-Dichloroethane & 1.0 & $\mathrm{U}$ \\
\hline
\end{tabular}

Report 1,4-Dioxane for Low-Medium VOA analysis only 
Lab Name: TESTAMERICA BURLINGTON

Lab Code: STLV

Case No.: YORK

Mod. Ref No.:

Contract: 8E-00302

Matrix: (SOIL/SED/WATER) Water

Sample wt/vol: 25.0 $(\mathrm{g} / \mathrm{mL}) \mathrm{mL}$

Level: (TRACE/LOW/MED) TRACE

\% Moisture: not dec.

GC Column: DB-624 ID: $0.20 \quad(\mathrm{~mm})$

Soil Extract Volume: (uL) $(\mathrm{mL})$

Purge Volume: 25.0 (mI)
SDG No.: 34024

Lab Sample ID: MB 200-105993/4

Lab File ID: 20517_04.D

Date Received:

Date Analyzed: 06/21/2016

Dilution Factor: 1.0

Soil Aliquot Volume: (uL)

\begin{tabular}{|c|c|c|c|}
\hline CAS NO. & COMPOUND & $\begin{array}{l}\text { CONCENTRATION UNITS: } \\
(\mathrm{ug} / \mathrm{L} \text { or } \mathrm{ug} / \mathrm{kg}) \mathrm{ug} / \mathrm{L}\end{array}$ & $q$ \\
\hline $79-01-6$ & Trichloroethene & 0.0080 & $\mathrm{~J}$ \\
\hline $108-87-2$ & Methylcyclohexane & 1.0 & $\mathrm{U}$ \\
\hline $78-87-5$ & 1,2-Dichloropropane & 1.0 & $\mathrm{U}$ \\
\hline $75-27-4$ & Bromodichloromethane & 1.0 & $\mathrm{U}$ \\
\hline $10061-01-5$ & cis-1,3-Dichloropropene & 1.0 & U \\
\hline $108-10-1$ & 4-Methyl-2-pentanone & 0.32 & $\mathrm{~J}$ \\
\hline $108-88-3$ & Toluene & 0.035 & $\mathrm{~J}$ \\
\hline $10061-02-6$ & trans-1,3-Dichloropropene & 0.20 & $\mathrm{~J}$ \\
\hline $79-00-5$ & $1,1,2-$ Trichloroethane & 1.0 & $\mathrm{U}$ \\
\hline $127-18-4$ & Tetrachloroethene & 1.0 & $\mathrm{U}$ \\
\hline $591-78-6$ & 2-Hexanone & 1.0 & $\mathrm{U}$ \\
\hline $124-48-1$ & Dibromochloromethane & 1.0 & $\mathrm{U}$ \\
\hline $106-93-4$ & 1,2-Dibromoethane & 1.0 & $\mathrm{U}$ \\
\hline $108-90-7$ & Chlorobenzene & 1.0 & $\mathrm{U}$ \\
\hline $100-41-4$ & Ethylbenzene & 0.0088 & $\mathrm{~J}$ \\
\hline $95-47-6$ & o-Xylene & 0.0091 & $\mathrm{~J}$ \\
\hline $179601-23-1$ & m,p-xylene & 0.0079 & $\mathrm{~J}$ \\
\hline $100-42-5$ & Styrene & 0.014 & $\mathrm{~J}$ \\
\hline $75-25-2$ & Bromoform & 1.0 & $\mathrm{U}$ \\
\hline $98-82-8$ & Isopropylbenzene & 1.0 & $\mathrm{U}$ \\
\hline $79-34-5$ & $1,1,2,2$-Tetrachloroethane & 1.0 & $\mathrm{U}$ \\
\hline $541-73-1$ & 1,3 -Dichlorobenzene & 0.041 & $\mathrm{~J}$ \\
\hline $106-46-7$ & 1,4-Dichlorobenzene & 0.068 & $\mathrm{~J}$ \\
\hline $95-50-1$ & 1,2-Dichlorobenzene & 0.098 & $\mathrm{~J}$ \\
\hline $96-12-8$ & 1,2-Dibromo-3-Chloropropane & 1.0 & $\mathrm{U}$ \\
\hline $120-82-1$ & $1,2,4$-Trichlorobenzene & 0.18 & $\mathrm{~J}$ \\
\hline $87-61-6$ & $1,2,3$-Trichlorobenzene & 0.27 & $\mathrm{~J}$ \\
\hline
\end{tabular}


Lab Name: TESTAMERICA BURLINGTON

Contract: $8 \mathrm{E}-00302$

Lab Code: STLV Case No.: YORK Mod. Ref No.: SDG No.: 34024

Matrix: (SOIL/SED/WATER) Water

Sample wt/vol: 25.0 $(\mathrm{g} / \mathrm{mL}) \mathrm{mL}$

Level: (TRACE or LOW/MED) TRACE

Moisture: not dec.

GC Column: DB-624 ID $: 0.20 \quad(\mathrm{~mm})$

Soil Extract Volume: (uL)

CONCENTRATION UNITS: (ug/L or ug/kg) ug/L
Lab Sample ID: MB 200-105993/4

Lab File ID: 20517_04.D

Date Received:

Date Analyzed: 06/21/2016

Dilution Factor: 1.0

Soil Aliquot Volume: (uL) Purge Volume: 25.0 $(\mathrm{mL})$

01

02

\begin{tabular}{|l|l|c|c|c|}
\hline CAS NUMBER & \multicolumn{1}{|c|}{ COMPOUND NAME } & RT & EST. CONC. & Q \\
\hline & Unknown & 7.20 & 2.6 & $\mathrm{~J} X$ \\
\hline E9667961 & Total Alkanes & N/A & & \\
\hline
\end{tabular}

1 EPA-designated Registry Number. 
Lab Name: TESTAMERICA BURLINGTON

Contract: $8 \mathrm{E}-00302$

Lab Code: STLV

Case No.: YORK Mod. Ref No.:

SDG No.: 34024

Matrix: (SOIL/SED/WATER) Water

Sample wt/vol: 25.0 $(\mathrm{g} / \mathrm{mL}) \mathrm{mL}$

Level: (TRACE/LOW/MED) TRACE

\% Moisture: not dec.

GC Column: DB-624 ID: $0.20 \quad(\mathrm{~mm})$

Soil Extract Volume: $(u L)$ $(\mathrm{mL})$

Purge Volume: 25.0
Lab Sample ID: 200-34024-4

Lab File ID: 20517_08.D

Date Received:

Date Analyzed: 06/21/2016

Dilution Factor: 1.0

Soil Aliquot Volume: (uL)

\begin{tabular}{|c|c|c|c|}
\hline CAS NO. & COMPOUND & $\begin{array}{l}\text { CONCENTRATION UNITS: } \\
(\mathrm{ug} / \mathrm{L} \text { or } \mathrm{ug} / \mathrm{kg}) \mathrm{ug} / \mathrm{L}\end{array}$ & Q \\
\hline $75-71-8$ & Dichlorodifluoromethane & 1.0 & $\mathrm{U}$ \\
\hline $74-87-3$ & Chloromethane & 0.066 & $\mathrm{~J} B$ \\
\hline $75-01-4$ & Vinyl chloride & 1.0 & $\mathrm{U}$ \\
\hline $74-83-9$ & Bromomethane & 0.031 & $\mathrm{~J}$ \\
\hline $75-00-3$ & Chloroethane & 1.0 & $\mathrm{U}$ \\
\hline $75-69-4$ & Trichlorofluoromethane & 1.0 & $\mathrm{U}$ \\
\hline $75-35-4$ & 1,1-Dichloroethene & 1.0 & $\mathrm{U}$ \\
\hline $76-13-1$ & $1,1,2$-Trichloro-1,2,2-trifluoroethane & 1.0 & $\mathrm{U}$ \\
\hline $67-64-1$ & Acetone & 5.0 & $\mathrm{U}$ \\
\hline $75-15-0$ & Carbon disulfide & 1.0 & $\mathrm{U}$ \\
\hline $79-20-9$ & Methyl acetate & 1.0 & $\mathrm{U}$ \\
\hline $75-09-2$ & Methylene Chloride & 0.14 & $\mathrm{~J} B$ \\
\hline $156-60-5$ & trans-1,2-Dichloroethene & 1.0 & $\mathrm{U}$ \\
\hline $1634-04-4$ & Methyl tert-butyl ether & 1.0 & $\mathrm{U}$ \\
\hline $75-34-3$ & 1,1-Dichloroethane & 1.0 & $\mathrm{U}$ \\
\hline $156-59-2$ & cis-1,2-Dichloroethene & 1.0 & $\mathrm{U}$ \\
\hline $78-93-3$ & 2-Butanone & 5.0 & $\mathrm{U}$ \\
\hline $74-97-5$ & Bromochloromethane & 1.0 & $\mathrm{U}$ \\
\hline $67-66-3$ & Chloroform & 1.0 & $\mathrm{U}$ \\
\hline $71-55-6$ & $1,1,1$-Trichloroethane & 1.0 & $\mathrm{U}$ \\
\hline $110-82-7$ & Cyclohexane & 1.0 & $\mathrm{U}$ \\
\hline $56-23-5$ & Carbon tetrachloride & 1.0 & $\mathrm{U}$ \\
\hline $71-43-2$ & Benzene & 1.0 & $\mathrm{U}$ \\
\hline $107-06-2$ & 1,2-Dichloroethane & 1.0 & $\mathrm{U}$ \\
\hline
\end{tabular}

Report 1,4-Dioxane for Low-Medium VOA analysis only 
Lab Name: TESTAMERICA BURLINGTON

Lab Code: STLV

Case No.: YORK

Mod. Ref No.:

Contract: 8E-00302

Matrix: (SOIL/SED/WATER) Water

Sample wt/vol: 25.0 $(\mathrm{g} / \mathrm{mL}) \mathrm{mL}$

Level: (TRACE/LOW/MED) TRACE

\% Moisture: not dec.

GC Column: DB-624 ID: $0.20 \quad(\mathrm{~mm})$

Soil Extract Volume: (uL) $(\mathrm{mL})$

Purge Volume: 25.0 SDG No.: 34024

Lab Sample ID: 200-34024-4

Lab File ID: 20517_08.D

Date Received:

Date Analyzed: 06/21/2016

Dilution Factor: 1.0

Soil Aliquot Volume:

(uL)

\begin{tabular}{|c|c|c|c|}
\hline CAS NO. & COMPOUND & $\begin{array}{l}\text { CONCENTRATION UNITS: } \\
(\mathrm{ug} / \mathrm{L} \text { or } \mathrm{ug} / \mathrm{kg}) \mathrm{ug} / \mathrm{L}\end{array}$ & Q \\
\hline $79-01-6$ & Trichloroethene & 1.0 & $\mathrm{U}$ \\
\hline $108-87-2$ & Methylcyclohexane & 1.0 & $\mathrm{U}$ \\
\hline $78-87-5$ & 1,2-Dichloropropane & 1.0 & $\mathrm{U}$ \\
\hline $75-27-4$ & Bromodichloromethane & 1.0 & $\mathrm{U}$ \\
\hline $10061-01-5$ & cis-1,3-Dichloropropene & 1.0 & $\mathrm{U}$ \\
\hline $108-10-1$ & 4-Methyl-2-pentanone & 1.0 & $\mathrm{U}$ \\
\hline $108-88-3$ & Toluene & 0.011 & $\mathrm{~J} B$ \\
\hline $10061-02-6$ & trans-1,3-Dichloropropene & 1.0 & $\mathrm{U}$ \\
\hline $79-00-5$ & $1,1,2-$ Trichloroethane & 1.0 & $\mathrm{U}$ \\
\hline $127-18-4$ & Tetrachloroethene & 1.0 & $\mathrm{U}$ \\
\hline $591-78-6$ & 2-Hexanone & 1.0 & $\mathrm{U}$ \\
\hline $124-48-1$ & Dibromochloromethane & 1.0 & $\mathrm{U}$ \\
\hline $106-93-4$ & 1,2-Dibromoethane & 1.0 & $\mathrm{U}$ \\
\hline $108-90-7$ & Chlorobenzene & 1.0 & $\mathrm{U}$ \\
\hline $100-41-4$ & Ethylbenzene & 1.0 & $\mathrm{U}$ \\
\hline $95-47-6$ & o-Xylene & 1.0 & $\mathrm{U}$ \\
\hline $179601-23-1$ & m,p-Xylene & 1.0 & $\mathrm{U}$ \\
\hline $100-42-5$ & Styrene & 1.0 & $\mathrm{U}$ \\
\hline $75-25-2$ & Bromoform & 1.0 & $\mathrm{U}$ \\
\hline $98-82-8$ & Isopropylbenzene & 1.0 & $\mathrm{U}$ \\
\hline $79-34-5$ & $1,1,2,2$-Tetrachloroethane & 1.0 & $\mathrm{U}$ \\
\hline $541-73-1$ & 1,3-Dichlorobenzene & 1.0 & $\mathrm{U}$ \\
\hline $106-46-7$ & 1,4-Dichlorobenzene & 1.0 & $\mathrm{U}$ \\
\hline $95-50-1$ & 1,2-Dichlorobenzene & 1.0 & $\mathrm{U}$ \\
\hline $96-12-8$ & 1,2-Dibromo-3-Chloropropane & 1.0 & $\mathrm{U}$ \\
\hline $120-82-1$ & 1,2,4-Trichlorobenzene & 1.0 & $\mathrm{U}$ \\
\hline $87-61-6$ & $1,2,3$-Trichlorobenzene & 1.0 & $\mathrm{U}$ \\
\hline
\end{tabular}


Lab Name: TESTAMERICA BURLINGTON

Contract: $8 \mathrm{E}-00302$

Lab Code: STLV Case No.: YORK Mod. Ref No.: SDG No.: 34024

Matrix: (SOIL/SED/WATER) Water

Sample wt/vol: 25.0 $(\mathrm{g} / \mathrm{mL}) \mathrm{mL}$

Level: (TRACE or LOW/MED) TRACE

Moisture: not dec.

GC Column: DB-624 ID $: 0.20 \quad(\mathrm{~mm})$

Soil Extract Volume: (uL)

CONCENTRATION UNITS: (ug/L or ug/kg) ug/L
Lab Sample ID: 200-34024-4

Lab File ID: 20517_08.D

Date Received:

Date Analyzed: 06/21/2016

Dilution Factor: 1.0

Soil Aliquot Volume: (uL) Purge Volume: 25.0 $(\mathrm{mL})$

01

02

\begin{tabular}{|l|l|c|c|c|}
\hline CAS NUMBER & \multicolumn{1}{|c|}{ COMPOUND NAME } & RT & EST. CONC. & Q \\
\hline & Unknown & 7.20 & 2.5 & X B J \\
\hline E9667961 & Total Alkanes & N/A & & \\
\hline
\end{tabular}

1EPA-designated Registry Number. 
Lab Name: TestAmerica Burlington

Job No.: 200-34024-1

SDG No.: 34024

Instrument ID: CHD.i

Analysis Batch Number: 105151

Lab Sample ID: ICIS 200-105151/4

Client Sample ID:

Date Analyzed: 05/31/16 16:56

Lab File ID: 20227 04.D

GC Column: DB-624

ID $: 0.2(\mathrm{~mm})$

\begin{tabular}{|c|c|c|c|c|}
\hline \multirow[t]{2}{*}{ COMPOUND NAME } & \multirow{2}{*}{$\begin{array}{c}\text { RETENTION } \\
\text { TIME }\end{array}$} & \multicolumn{3}{|c|}{ MANUAL INTEGRATION } \\
\hline & & REASON & ANALYST & DATE \\
\hline 1,2-Dichloropropane-d6 & 6.32 & Split Peak & wilburj & $05 / 31 / 1617: 36$ \\
\hline
\end{tabular}

Lab Sample ID: IC 200-105151/5

Client Sample ID:

Date Analyzed: 05/31/16 17:21

Lab File ID: 20227 05.D

GC Column: DB-624

ID $: 0.2(\mathrm{~mm})$

\begin{tabular}{|c|c|c|c|c|}
\hline \multicolumn{1}{|c|}{ COMPOUND NAME } & RETENTION & \multicolumn{3}{|c}{ MANUAL INTEGRATION } \\
\cline { 3 - 5 } & TIME & REASON & ANALYST & DATE \\
\hline 1,2 -Dichloropropane-d6 & 6.32 & Split Peak & wilburj & 05/31/16 17:57 \\
\hline
\end{tabular}

Lab Sample ID: IC 200-105151/6

Client Sample ID:

Date Analyzed: 05/31/16 17:46

Lab File ID: 20227_06.D

GC Column: DB-624

ID $: 0.2(\mathrm{~mm})$

\begin{tabular}{|c|c|c|c|c|}
\hline \multicolumn{1}{|c|}{ COMPOUND NAME } & RETENTION & \multicolumn{3}{|c|}{ MANUAL INTEGRATION } \\
\cline { 3 - 5 } & TIME & REASON & ANALYST & DATE \\
\hline 1,2-Dichloropropane-d6 & 6.32 & Split Peak & wilburj & $05 / 31 / 16$ 18:30 \\
\hline
\end{tabular}


Lab Name: TestAmerica Burlington

Job No.: 200-34024-1

SDG No.: 34024

Instrument ID: CHD.i

Analysis Batch Number: 105993

Lab Sample ID: CCVIS 200-105993/3

Client Sample ID:

Date Analyzed: 06/21/16 12:23

Lab File ID: 20517 03.D

GC Column: DB-624

ID $: 0.2(\mathrm{~mm})$

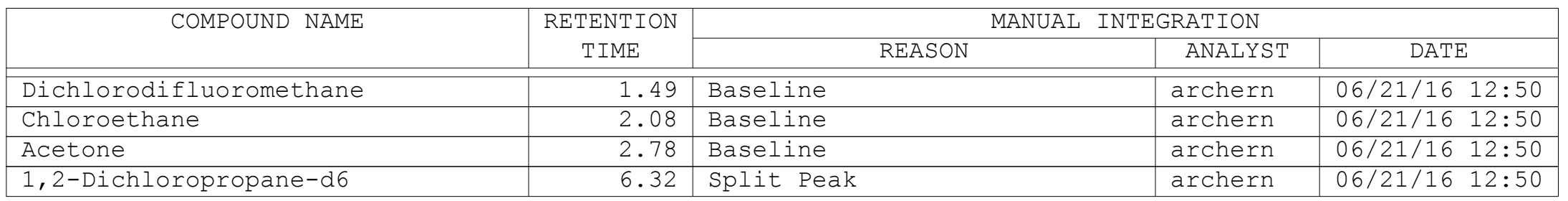

Lab Sample ID: CCVC 200-105993/20

Client Sample ID:

Date Analyzed: 06/21/16 20:11

Lab File ID: 20517_20.D

GC Column: DB-624

ID $: 0.2(\mathrm{~mm})$

\begin{tabular}{|c|c|c|c|c|}
\hline \multirow[t]{2}{*}{ COMPOUND NAME } & \multirow{2}{*}{$\begin{array}{c}\text { RETENTION } \\
\text { TIME }\end{array}$} & \multicolumn{3}{|c|}{ MANUAL INTEGRATION } \\
\hline & & REASON & ANALYST & DATE \\
\hline Dichlorodifluoromethane & 1.50 & Baseline & wilburj & $06 / 24 / 16 \quad 11: 59$ \\
\hline
\end{tabular}

Lab Sample ID: CCVC 200-105993/21

Client Sample ID:

Date Analyzed: 06/21/16 20:37

Lab File ID: 20517_21.D

GC Column: DB-624

ID $: 0.2(\mathrm{~mm})$

\begin{tabular}{|c|c|c|c|c|}
\hline \multirow[t]{2}{*}{ COMPOUND NAME } & \multirow{2}{*}{$\begin{array}{c}\text { RETENTION } \\
\text { TIME }\end{array}$} & \multicolumn{3}{|c|}{ MANUAL INTEGRATION } \\
\hline & & REASON & ANALYST & DATE \\
\hline Dichlorodifluoromethane & 1.49 & Assign Peak & $\begin{array}{l}\text { puangmale } \\
\text { ek }\end{array}$ & $06 / 23 / 1611: 02$ \\
\hline
\end{tabular}




\section{AGEM Team at Argonne}

Lorraine M. LaFreniere, Ph.D.

Principal Geological Engineer

Program Manager

Jorge S. Alvarado, Ph.D.

Chemist

Laboratory Manager and Analyst

Esther E. Bowen, M.S.

Quality Assurance/Quality Control and Records

Data Management Specialist

Manager

Laurel A. Culbert, B.A.

Graphic Artist/Designer

Report Graphics and Maps (including GIS)

James Hansen, B.A.

Program Coordinator/Manager

Site Reconnaissance and Community Relations

Louis E. Martino, M.S.

Environmental Systems Engineer/Program

Manager

Technical Lead/Remediation Specialist (Cost Engineering)

Terri Patton, M.S.

Principal Geologist

Technical Editor

Candace M. Rose, B.S.

Scientific Associate Senior

Laboratory Analyst

Robert A. Sedivy, M.S.

Hydrogeologist

Technical Lead/Hydrogeologist

Eugene Y. Yan, Ph.D.

Earth Scientist

Technical Lead/Hydrogeologist 
Argonne

Environmental Science Division

Argonne National Laboratory

9700 South Cass Avenue, BIdg. 203

Argonne, IL 60439-4843

www.anl.gov 